\title{
CLINICAL MATERNAL-FETAL MEDICINE ONLINE
}

\section{Second Edition}

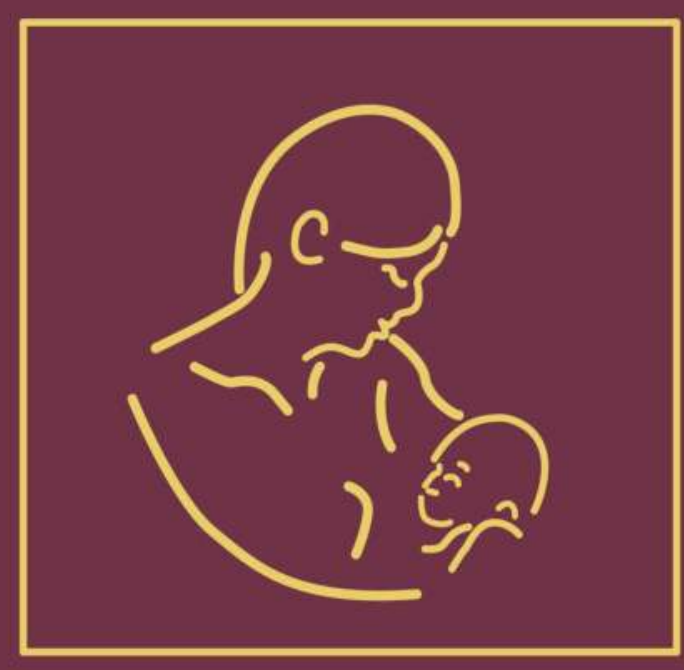

Edited by

Hung N. Winn

Frank A. Chervenak

Roberto Romero 


\section{Clinical Maternal-Fetal Medicine Online}




\section{SERIES IN MATERNAL-FETAL MEDICINE}

Published in association with the Journal of Maternal-Fetal \& Neonatal Medicine

Editors in Chief:

\section{Gian Carlo Di Renzo and Dev Maulik}

\section{Recent and Forthcoming Titles}

Vincenzo Berghella, Maternal Fetal Evidence Based Guidelines, Second Edition

ISBN 978-1-84184-826-6

Vincenzo Berghella, Obstetric Evidence Based Guidelines, Second Edition

ISBN 978-1-84184-824-2

Howard Carp, Recurrent Pregnancy Loss: Causes, Controversies and Treatment

ISBN 978-0-415-42130-0

Fabio Facchinetti, Gustaaf A Dekker, Dante Baronciani, George Saade, Stillbirth: Understanding and Management ISBN 978-0-415-47390-3

Moshe Hod, Lois Jovanovic, Gian Carlo Di Renzo, Alberto de Leiva, Oded Langer, Textbook of Diabetes

and Pregnancy, Second Edition

ISBN 978-0-415-42560-6

Michael S Marsh, Lina AM Nashef, Peter A Brex, Neurology and Pregnancy: Clinical Management ISBN 978-1-84184-652-1

Simcha Yagel, Norman H Silver, Ulrich Gembruch, Fetal Cardiology: Embryology, Genetics, Physiology, Echocardiographic Evaluation, Diagnosis and Perinatal Management of Cardiac Diseases, Second Edition

ISBN 978-0-415-43265-8 


\title{
Clinical Maternal-Fetal Medicine Online
}

\author{
Second Edition
}

\author{
Edited by \\ Hung N. Winn MD
}

Chair and David G. Hall Professor of Obstetrics and Gynecology, Department of Obstetrics, Gynecology and Women's Health, University of Missouri School of Medicine,

Columbia, Missouri, USA

\section{Frank A. Chervenak MD}

Given Foundation Professor and Chair, Department of Obstetrics and Gynecology,

New York Weill Cornell Medical Center, New York, New York, USA

\section{Roberto Romero MD}

Chief, Perinatology Research Branch and Program Research Director for Obstetrics

and Perinatology Intramural Division, Eunice Kennedy Shriver National Institute of

Child Health and Human Development, National Institutes of Health, Department of Health and Human Services, Bethesda, Maryland, USA

Professor of Molecular Obstetrics and Genetics, Center for Molecular Medicine and Genetics, Wayne State University, Detroit, Michigan, USA

Professor of Epidemiology, Michigan State University, East Lansing, Michigan, USA
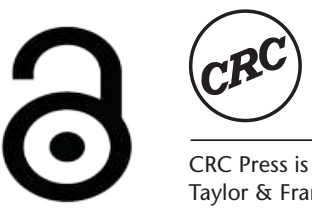
This second edition published online in 2012 by Informa Healthcare, 119 Farringdon Road, London EC1R 3DA, UK.

Simultaneously published in the USA by Informa Healthcare, 52 Vanderbilt Avenue, 7th Floor, New York, NY 10017, USA.

First edition published in 2000 by The Parthenon Publishing Group.

CRC Press

Taylor \& Francis Group

6000 Broken Sound Parkway NW, Suite 300

Boca Raton, FL 33487-2742

C 2012 by Taylor \& Francis Group, LLC

CRC Press is an imprint of Taylor \& Francis Group, an Informa business

No claim to original U.S. Government works

ISBN-13: 9781003222590 (eBook)

DOI: $10.1201 / 9781003222590$

This book contains information obtained from authentic and highly regarded sources. While all reasonable efforts have been made to publish reliable data and information, neither the author[s] nor the publisher can accept any legal respon-sibility or liability for any errors or omissions that may be made. The publishers wish to make clear that any views or opinions expressed in this book by individual editors, authors or contributors are personal to them and do not neces-sarily reflect the views/opinions of the publishers. The information or guidance contained in this book is intended for use by medical, scientific or health-care professionals and is provided strictly as a supplement to the medical or other professional's own judgement, their knowledge of the patient's medical history, relevant manufacturer's instructions and the appropriate best practice guidelines. Because of the rapid advances in medical science, any information or advice on dosages, procedures or diagnoses should be independently verified. The reader is strongly urged to consult the relevant national drug formulary and the drug companies' and device or material manufacturers' printed instructions, and their websites, before administering or utilizing any of the drugs, devices or materials mentioned in this book. This book does not indicate whether a particular treatment is appropriate or suitable for a particular individual. Ultimately it is the sole responsibility of the medical professional to make his or her own professional judgements, so as to advise and treat patients appropriately. The authors and publishers have also attempted to trace the copyright holders of all mate-rial reproduced in this publication and apologize to copyright holders if permission to publish in this form has not been obtained. If any copyright material has not been acknowledged please write and let us know so we may rectify in any future reprint.

The Open Access version of this book, available at www.taylorfrancis.com, has been made available under a Creative Commons Attribution-Non Commercial-No Derivatives 4.0 license.

Trademark Notice: Product or corporate names may be trademarks or registered trademarks, and are used only for identification and explanation without intent to infringe.

Visit the Taylor \& Francis Web site at http://www.taylorandfrancis.com

and the CRC Press Web site at http://www.crcpress.com 


\section{Contents}

Contributors

Preface

\section{OBSTETRIC DISORDERS}

1 The diagnosis and management of preterm labor with intact membranes

Roberto Romero, Tinnakorn Chaiworapongsa, Francesca Gotsch, Lami Yeo, Ichchha Madan, and Sonia S. Hassan

2 Prelabor rupture of the membranes

Roberto Romero, Lami Yeo, Francesca Gotsch, Eleazar Soto, Sonia S. Hassan, Juan Pedro Kusanovic, and Ray Bahado-Singh

3 Cervical insufficiency

Sonia S. Hassan, Roberto Romero, Francesca Gotsch, Lorraine Nikita, and Tinnakorn Chaiworapongsa

4 Recurrent pregnancy loss

Christine E. Ryan and Danny J. Schust

5 Multiple gestation Isaac Blickstein

6 Gestational hypertension and pre-eclampsia Erol Amon and Erin Dickert

7 Coagulopathy in obstetrics ${ }^{\star}$ Hung N. Winn and Roberto Romero

8 Amniotic fluid embolism ${ }^{\star}$ Hung N. Winn

9 Placenta previa and placental abruption Ann K. Lal and William J. Watson

10 Postpartum hemorrhage Wade D. Schwendemann and William J. Watson

11 Septic shock Bryan E. Freeman and Michael R. Foley

12 Invasive hemodynamic monitoring in obstetrics Luis D. Pacheco, Shannon Clark, and Gary D. V. Hankins

13 Shoulder dystocia Randall C. Floyd and James S. Smeltzer

14 Breech presentation Randall C. Floyd and Martin L. Gimovsky

15 Cesarean section and vaginal birth after cesarean section Jessica Winn and Hung N. Winn

\section{MATERNAL MEDICAL DISORDERS}

16 Diabetes in pregnancy

Donald R. Coustan
17 Chronic hypertension and acute hypertensive crisis William F. Rayburn and Lauren Plante

18 Rheumatologic diseases and antiphospholipid syndrome Thomas J. Santoro, Michiyo Tomita, and Alfonse T. Masi

19 Sickle cell disease Marc R. Parrish and John C. Morrison

20 Cardiac diseases in pregnancy Saravanan Kuppuswamy and Sudarshan Balla

21 Dermatologic diseases and pregnancy Holly Edmonds, Dana Ward, Ann G. Martin, and Susana Leal-Khouri

22 Endocrine diseases and pregnancy Stephen A. Brietzke

23 Gastrointestinal diseases and pregnancy Murtaza Arif, Anjana Sathyamurthy, Jessica Winn, and Jamal A. Ibdah

24 Hepatic disorders in pregnancy Ghassan M. Hammoud and Jamal A. Ibdah

25 Neurologic disorders in pregnancy Robert Burger, Terry Rolan, David Lardizabal, Upinder Dhand, Aarti Sarwal, and Pradeep Sahota

26 Sleep disorders and pregnancy Pradeep Sahota and Sanjay Jain

27 Pulmonary diseases in pregnancy Leah Lande, Abraham Sanders, and Dana Zappetti

28 Psychiatric disorders in pregnancy Robin Kopelman and Scott Stuart

29 Renal diseases in pregnancy Dana Negoi and Khaled Mohamed

III MATERNAL SURGICAL, MALIGNANCY, AND OTHER ISSUES

30 Acute abdomen in pregnancy Nicole Fearing and William L. Holcomb

31 Trauma in pregnancy Hung N. Winn

32 Medical evaluation and management of pregnant patients undergoing non-obstetrical surgery John G. Gianopoulos

33 Anesthesia and analgesia in pregnancy Steven T. Fogel

*This chapter is not available. 
34 Breast cancer and pregnancy

Beth Kliethermes, Dani Stramer, and Edward R. Sauter

35 Neoplasia in pregnancy

Nora T. Kizer and David G. Mutch

36 Chemotherapy in pregnancy

Mark I. Hunter

37 Maternal obesity

D. Yvette LaCoursiere and Thomas R. Moore

38 Medicolegal considerations

Erol Amon and Gilad Gross

39 Drug abuse in pregnancy: Marijuana, LSD, cocaine, amphetamines, alcohol, and opiates Jacquelyn C. Howitt and Anita Bublik-Anderson

40 Tobacco and alcohol use during pregnancy Beth A. Bailey and Robert J. Sokol

41 Exercise and pregnancy

Raul Artal

42 Ethical considerations

Frank A. Chervenak and Laurence B. McCullough

\section{GENETICS AND FETAL DISORDERS}

43 Basic genetics and patterns of inheritance Dorothy K. Grange

44 Principles of teratology of drugs and radiation Frank R. Witter

45 Fetal programming Katherine E. Pelch, Jana L. Allison, and Susan C. Nagel

46 Assessment of fetal genetic disorders Teresa Martino, J. Pratt Rossiter, and Karin J. Blakemore

47 Preimplantation diagnosis of genetic diseases ${ }^{\star}$ The-Hung Bui

48 First-trimester screening for aneuploidy Mark I. Evans and Howard S. Cuckle

49 Second-trimester screening for fetal abnormalities Jolene C. Muscat and Anthony M. Vintzileos

50 Genetic counseling Zoltán Papp, Valéria Váradi, and Júlia Hajdú

51 Fetal anomalies ${ }^{\star}$ Hung N. Winn

52 Assessment of fetal brain abnormalities Ritsuko K. Pooh

53 Erythroblastosis fetalis

Avinash Patil, Brian Brocato, Rebecca A. Uhlmann, and Giancarlo Mari

54 Fetal alloimmune thrombocytopenia Hung N. Winn
55 Nonimmune hydrops ${ }^{\star}$

Hung N. Winn

56 Fetal arrhythmias

Júlia Hajdú, Valéria Váradi, and Zoltán Papp

57 Fetal growth restriction

Daniel L. Jackson, M. Y. Divon, and Hung N. Winn

58 Fetal demise

Hung N. Winn

\section{$\mathrm{V}$ FETAL ASSESSMENT}

59 Application of 3D and 4D ultrasound in fetal medicine

Eberhard Merz

60 Prenatal diagnosis of fetal abnormality using fetal cells in maternal circulation

Gian Carlo Di Renzo, Elena Picchiassi, Michela Centra, and Giuliana Coata

61 Recent developments in fetal therapy Gihad E. Chalouhi and Yves Ville

62 Chorionic villus sampling Giovanni Monni, Maria Angelica Zoppi, and Carolina Axiana

63 Amniocentesis Aris Antsaklis and Marianna Theodora

64 Fetal echocardiography Caroline K. Lee, Erik C. Michelfelder, and Gautam K. Singh

65 Assessment of fetal behavior Asim Kurjak, Milan Stanojevic, Badreldeen Ahmed, Guillermo Azumendi, and Lara Spalldi-Barisic

66 Umbilical artery doppler sonography for fetal surveillance: Principles and practice Dev Maulik, David Mundy, and Timothy Bennett

67 Assessment of fetal well-being: Fetal heart rate monitoring and the fetal biophysical profile Yinka Oyelese, Martin Chavez, and Anthony M. Vintzileos

68 Intrapartum fetal heart rate monitoring ${ }^{\star}$ Hung N. Winn

69 Amnioinfusion James Kerns, Erol Amon, and Hung N. Winn

70 Assessment of fetal age and fetal lung maturity ${ }^{\star}$ Hung N. Winn

71 The maternal immune system during pregnancy Breton F. Barrier

72 Placental transport and metabolism Jeffrey M. Dicke

${ }^{*}$ This chapter is not available. 
73 Fertilization and normal embryonic and early fetal development

Asim Kurjak, Ritsuko K. Pooh, Aida Salihagic-Kadic, Iva Lausin, and Lara Spalldi-Barisic

\section{PERINATAL INFECTION AND NEONATAL DISEASE}

74 Group B streptococcus infection Jessica Winn and Hung N. Winn

75 Gonorrhea infection* Hung N. Winn

76 Postpartum infections Dorothea Mostello

77 Urinary tract infections in pregnancy Steven R. Allen

78 Other bacterial infection: Lyme, granuloma inguinale, gardnerella vaginale, chancroid ${ }^{*}$ Hung N. Winn

79 Bacterial vaginosis in pregnancy: Evidence-based approaches James A. McGregor and Michael W. McCullough

80 Viral hepatitis in pregnancy Dennis $\mathrm{Ng}$
81 Toxoplasmosis

Edward R. Newton

82 Herpes $^{*}$

Edward R. Newton

83 Cytomegalovirus $(\mathrm{CMV})^{\star}$

Hung N. Winn

84 Human immunodeficiency virus (HIV) Richard Basilan and William Salzer

85 Other viral infections Stanley A. Gall

86 Chlamydial infection Joyce A. Ibana and Danny J. Schust

87 Candida and parasitic infection: Helminths, trichomoniasis, lice, scabies, and malaria Jeanna M. Piper

88 Routine maternal immunizations for all pregnant women Stanley A. Gall

89 Neonatal diseases I John Pardalos

90 Neonatal diseases II Brian P. Hackett, Jeffrey Dawson, Akshaya Vach harajani, Barbara Warner, and F. Sessions Cole 


\section{Contributors}

\section{Badreldeen Ahmed}

Feto-Maternal Unit, Department of Obstetrics and Gynecology, Hamad Medical Corporation, Doha, Qatar

\section{Steven R. Allen}

Section of Maternal-Fetal Medicine, Texas A\&M Health Science Center, College of Medicine, Temple, Texas, USA

\section{Jana L. Allison}

Department of Obstetrics, Gynecology and Women's Health, University of Missouri School of Medicine, Columbia, Missouri, USA

\section{Erol Amon}

Department of Obstetrics, Gynecology and Women's Health, MaternalFetal Medicine, St. Louis University School of Medicine,

St. Louis, Missouri, USA

\section{Aris Antsaklis}

Department of Obstetrics and Gynecology, University of Athens Medical School, Athens, Greece

\section{Murtaza Arif}

Division of Gastroenterology and Hepatology, University of Missouri School of Medicine, Columbia, Missouri, USA

\section{Raul Artal}

Department of Obstetrics, Gynecology and Women's Health, St. Louis School of Medicine, St. Louis, Missouri, USA

\section{Carolina Axiana}

Department of Obstetrics and Gynecology, Prenatal and Preimplantation Genetic Diagnosis, Microcitemico Hospital, University of Cagliari, Cagliari, Italy

\section{Guillermo Azumendi}

Ultrasound Unit, Clinica Gutenberg, Malaga, Spain

\section{Ray Bahado-Singh}

Department of Obstetrics and Gynecology, Wayne State University, Detroit, Michigan, USA

\section{Beth A. Bailey}

Department of Family Medicine, James H. Quillen College of Medicine, East Tennessee State University, Johnson City, Tennessee, USA

\section{Sudarshan Balla}

Department of Internal Medicine, University of Missouri School of Medicine, Columbia, Missouri, USA

\section{Breton F. Barrier}

Department of Obstetrics, Gynecology and Women's Health, University of Missouri School of Medicine, Columbia, Missouri, USA

\section{Richard Basilan}

Department of Internal Medicine, University of Missouri School of Medicine, Columbia, Missouri, USA

\section{Timothy Bennett}

Department of Obstetrics and Gynecology, University of

Missouri-Kansas City School of Medicine, Kansas City,

Missouri, USA

\section{Karin J. Blakemore}

Department of Obstetrics and Gynecology, Johns Hopkins University Hospital, Baltimore, Maryland, USA

\section{Isaac Blickstein}

Department of Obstetrics and Gynecology, Kaplan Medical Center, Rehovot and the Hadassah-Hebrew University School of Medicine, Jerusalem, Israel

\section{Stephen A. Brietzke}

Department of Internal Medicine, University of Missouri School of Medicine, Columbia, Missouri, USA

\section{Brian Brocato}

Department of Obstetrics and Gynecology, University of Tennessee Health Science Center, Memphis, Tennessee, USA

\section{Anita Bublik-Anderson}

Private Practice, Obstetrics and Gynecology, Wausau, Wisconsin, USA

\section{Robert Burger}

Department of Neurology, University of Missouri School of Medicine, Columbia, Missouri, USA

\section{Michela Centra}

Department of Obstetrics and Gynecology, University of Perugia, Perugia, Italy

\section{Tinnakorn Chaiworapongsa}

Perinatology Research Branch, Eunice Kennedy Shriver National Institute of Child Health and Human Development, National Institutes of Health, Department of Health and Human Services, and Department of Obstetrics and Gynecology, Wayne State University, Detroit, Michigan, USA

\section{Gihad E. Chalouhi}

Department of Obstetrics and Fetal Medicine, Necker-Enfants-Malades Medical School, Paris Descartes University, Paris, France

\section{Martin Chavez}

Division of Maternal Fetal Medicine, Winthrop University Hospital, Mineola, New York, USA

\section{Frank A. Chervenak}

Department of Obstetrics and Gynecology, New York Weill Cornell Medical Center, New York, New York, USA

\section{Shannon Clark}

Department of Obstetrics and Gynecology, University of Texas Medical Branch, Galveston, Texas, USA

\section{Giuliana Coata}

Department of Obstetrics and Gynecology, University of Perugia, Perugia, Italy

\section{F. Sessions Cole}

Division of Newborn Medicine, the Edward Mallinckrodt Department of Pediatrics, Washington University School of Medicine and St. Louis Children's Hospital, St. Louis, Missouri, USA

\section{Donald R. Coustan}

Division of Maternal-Fetal Medicine, Warren Alpert Medical School of Brown University and Women \& Infants Hospital, Providence, Rhode Island, USA

\section{Howard S. Cuckle}

Department of Obstetrics and Gynecology, Columbia University, New York, New York, USA 


\section{Jeffrey Dawson}

Division of Newborn Medicine, the Edward Mallinckrodt Department of Pediatrics, Washington University School of Medicine and St. Louis Children's Hospital, St. Louis, Missouri, USA

\section{Upinder Dhand}

Department of Neurology, University of Missouri School of Medicine, Columbia, Missouri, USA

\section{Gian Carlo Di Renzo}

Department of Obstetrics and Gynecology, University of Perugia, Perugia, Italy

\section{Jeffrey M. Dicke}

Department of Obstetrics and Gynecology, Washington University School of Medicine, St. Louis, Missouri, USA

\section{Erin Dickert}

Department of Obstetrics, Gynecology and Women's Health, Maternal-Fetal Medicine, St. Louis University School of Medicine, St. Louis, Missouri, USA

\section{Y. Divon}

Lenox Hill Hospital, New York, New York, USA

\section{Holly Edmonds}

Dermatology Specialty Center, Roswell, Georgia, USA

\section{Mark I. Evans}

Comprehensive Genetics and Department of Obstetrics and Gynecology, Mt. Sinai School of Medicine, New York,

New York, USA

\section{Nicole Fearing}

Private Practice Bariatric Surgery, Malley Surgical, Mission, Kansas, USA

\section{Randall C. Floyd}

Maternal Fetal Medicine and Obstetrics, Department of Obstetrics, Gynecology and Women's Health, University of Missouri Women's and Children's Hospital, Columbia, Missouri, USA

\section{Steven T. Fogel}

Department of Anesthesiology and Perioperative Medicine, University of Missouri School of Medicine, Columbia, Missouri, USA

\section{Michael R. Foley}

Maternal-Fetal Medicine, Scottsdale Healthcare, Scottsdale, Arizona, USA

\section{Bryan E. Freeman}

Maternal-Fetal Medicine, Scottsdale Healthcare, Scottsdale, Arizona, USA

\section{Stanley A. Gall}

Obstetrics, Gynecology and Women's Health, University

of Louisville, Louisville, Kentucky, USA

\section{John G. Gianopoulos}

Obstetrics and Gynecology, Northwestern University/Fienberg School of Medicine, and Maternal/Fetal Medicine, Cook County Health and Hospital System, Chicago, Illinois, USA

\section{Martin L. Gimovsky}

Department of Obstetrics and Gynecology, Newark Beth Israel Medical Center, and Department of Obstetrics and Gynecology, Mount Sinai School of Medicine, New York, New York, USA

\section{Francesca Gotsch}

Department of Obstetrics and Gynecology, Azienda Ospedaliera Universitaria Integrata, Verona, Italy

\section{Dorothy K. Grange}

Division of Genetics and Genomic Medicine, Department of Pediatrics, Washington University School of Medicine, St. Louis, Missouri, USA

\section{Gilad Gross}

Department of Obstetrics, Gynecology, and Women's Health, Maternal-Fetal Medicine, St. Louis University School of Medicine, St. Louis, Missouri, USA

\section{Brian P. Hackett}

Division of Newborn Medicine, the Edward Mallinckrodt Department of Pediatrics, Washington University School of Medicine and St. Louis Children's Hospital, St. Louis, Missouri, USA

\section{Júlia Hajdú}

Department of Obstetrics and Gynecology, Semmelweis University Medical School, Budapest, Hungary

\section{Ghassan M. Hammoud}

Division of Gastroenterology and Hepatology, University of Missouri School of Medicine, Columbia, Missouri, USA

\section{Gary D. V. Hankins}

Department of Obstetrics and Gynecology, University of Texas Medical Branch, Galveston, Texas, USA

\section{Sonia S. Hassan}

Perinatology Research Branch, Eunice Kennedy Shriver National Institute of Child Health and Human Development, National Institutes of Health, Department of Health and Human Services, and Department of Obstetrics and Gynecology, Wayne State University, Detroit, Michigan, USA

\section{William L. Holcomb}

Private practice in Fetal Medicine, Maternal Medicine, Obstetrics and Gynecology, St. Charles, Missouri, USA

\section{Jacquelyn C. Howitt}

Department of Obstetrics and Gynecology, Rochester General Hospital and Department of Obstetrics and Gynecology, University of Rochester Medical Center, Rochester, New York, USA

\section{Mark I. Hunter}

Division of Gynecologic Oncology, Ellis Fischel Cancer Center and Department of Obstetrics, Gynecology, and Women's Health, University of Missouri School of Medicine, Columbia, Missouri, USA

Joyce A. Ibana

Department of Microbiology, Immunology and Parasitology, Louisiana State University Health Sciences Center, New Orleans, Louisiana, USA

\section{Jamal A. Ibdah}

Division of Gastroenterology and Hepatology, University of Missouri School of Medicine, Columbia, Missouri, USA

\section{Daniel L. Jackson}

Department of Obstetrics, Gynecology, and Women's Health, University of Missouri School of Medicine, Columbia, Missouri, USA

\section{Sanjay Jain}

Department of Neurology, University of Missouri School of Medicine, Columbia, Missouri, USA

\section{James Kerns}

Department of Obstetrics, Gynecology and Women's Health, University of Missouri School of Medicine, Columbia, Missouri, USA

\section{Nora T. Kizer}

Division of Gynecologic Oncology, Washington University School of Medicine, St. Louis, Missouri, USA

\section{Beth Kliethermes}

Department of Surgery, University of North Dakota School of Medicine and Health Sciences, Grand Forks, North Dakota, USA

\section{Robin Kopelman}

Department of Psychiatry, University of Iowa Carver College of Medicine, Iowa City, Iowa, USA 
Saravanan Kuppuswamy

Department of Cardiology, University of Missouri School of Medicine, Columbia, Missouri, USA

\section{Asim Kurjak}

Department of Obstetrics and Gynecology, Sveti Duh Hospital, Zagreb, Croatia

\section{Juan Pedro Kusanovic}

Perinatology Research Branch, Eunice Kennedy Shriver National Institute of Child Health and Human Development, National Institutes of Health, Department of Health and Human Services, Detroit, Michigan, USA

\section{Yvette LaCoursiere}

Division of General Obstetrics and Gynecology, Department of Reproductive Medicine, University of California at San Diego, San Diego, California, USA

\section{Ann K. Lal}

Division of Maternal Fetal Medicine, Department of Obstetrics and Gynecology, University of Illinois, Chicago, Illinois, USA

\section{David Lardizabal}

Department of Neurology, University of Missouri School of Medicine, Columbia, Missouri, USA

\section{Leah Lande}

Wynnewood Pulmonology Associates, Wynnewood, Pennsylvania, USA

Iva Lausin

Department of Obstetrics and Gynecology, Sveti Duh Hospital, Zagreb, Croatia

\section{Susana Leal-Khouri}

Division of Dermatology, University of Missouri School of Medicine, Columbia, Missouri, USA

\section{Caroline K. Lee}

Pediatric Cardiology Fellowship Program, Washington University School of Medicine, St. Louis, Missouri, USA

\section{Ichchha Madan}

Perinatology Research Branch, Eunice Kennedy Shriver National Institute of Child Health and Human Development, National Institutes of Health, Department of Health and Human Services, and Department of Obstetrics and Gynecology, Wayne State University, Detroit, Michigan, USA

\section{Giancarlo Mari}

Department of Obstetrics and Gynecology, University of Tennessee Health Science Center, Memphis, Tennessee, USA

\section{Ann G. Martin}

Division of Dermatology, University of Missouri School of Medicine, Columbia, Missouri, USA

\section{Teresa Martino}

Department of Obstetrics and Gynecology, Johns Hopkins University Hospital, Baltimore, Maryland, USA

\section{Alfonse T. Masi}

Section of Rheumatology, University of Illinois College of Medicine at Peoria, Peoria, Illinois, USA

\section{Dev Maulik}

Department of Obstetrics and Gynecology, University of MissouriKansas City School of Medicine, Kansas City, Missouri, USA

\section{Laurence B. McCullough}

Center for Medical Ethics and Health Policy, Baylor College of Medicine, Houston, Texas, USA
Michael W. McCullough

Department of Obstetrics, Gynecology and Women's Health, University of Missouri School of Medicine, Columbia, Missouri, USA

James A. McGregor

Department of Obstetrics and Gynecology, University of Colorado, Denver, Colorado, USA

\section{Eberhard Merz}

Department of Obstetrics and Gynecology, Krankenhaus Nordwest (University of Frankfurt), Frankfurt am Main, Germany

\section{Erik C. Michelfelder}

University of Cincinnati College of Medicine, and Fetal Heart Program, Cincinnati Children's Hospital Medical Center, Cincinnati, Ohio, USA

\section{Khaled Mohamed \\ Division of Nephrology, University of Missouri School of Medicine, Columbia, Missouri, USA}

\section{Giovanni Monni}

Department of Obstetrics and Gynecology, Prenatal and Preimplantation Genetic Diagnosis, Microcitemico Hospital, University of Cagliari, Cagliari, Italy

\section{Thomas R. Moore}

Division of Maternal Fetal Medicine, Department of Reproductive Medicine, University of California at San Diego, San Diego, California, USA

\section{John C. Morrison}

Division of Maternal-Fetal Medicine, Department of Obstetrics and Gynecology, University of Mississippi Medical Center, Jackson, Mississippi, USA

\section{Dorothea Mostello}

Department of Obstetrics and Gynecology and Women's Health, St. Louis University School of Medicine, St. Louis,

Missouri, USA

\section{David Mundy}

Department of Obstetrics and Gynecology, University of Missouri-Kansas City School of Medicine, Kansas City, Missouri, USA

\section{Jolene C. Muscat}

Department of Obstetrics \& Gynecology, Winthrop University Hospital, Mineola; and Department of Obstetrics, Gynecology \& Reproductive Medicine, Stony Brook University School of Medicine, Stony Brook, New York, USA

\section{David G. Mutch}

Division of Gynecologic Oncology, Washington University School of Medicine, St. Louis, Missouri, USA

\section{Susan C. Nagel}

Department of Obstetrics, Gynecology and Women's Health, University of Missouri School of Medicine, Columbia, Missouri, USA

\section{Dana Negoi}

Fletcher Allen Health Care, University of Vermont, Burlington, Vermont, USA

\section{Edward R. Newton}

Department of Obstetrics and Gynecology, Brody School of Medicine, East Carolina University, Greenville, North Carolina, USA

\section{Dennis Ng}

Division of Gastroenterology and Hepatology, Department of Obstetrics, Gynecology and Women's Health, University of Missouri School of Medicine, Columbia, Missouri, USA 


\section{Lorraine Nikita}

Perinatology Research Branch, Eunice Kennedy Shriver National Institute of Child Health and Human Development, National Institutes of Health, Department of Health and Human Services, Detroit, Michigan, USA

\section{Yinka Oyelese}

Division of Maternal Fetal Medicine, Jersey Shore University Medical Center, Neptune, New Jersey, USA

\section{Luis D. Pacheco}

Departments of Obstetrics and Gynecology and of Anesthesia,

University of Texas Medical Branch, Galveston, Texas, USA

\section{Zoltán Papp}

Department of Obstetrics and Gynecology, Semmelweis University Medical School, Budapest, Hungary

\section{John Pardalos}

Division of Neonatology, University of Missouri School of Medicine, Columbia, Missouri, USA

\section{Marc R. Parrish}

Department of Maternal Fetal Medicine, Saint Lukes Hospital-Plaza, Kansas City, Missouri, USA

\section{Avinash Patil}

Department of Obstetrics and Gynecology, University of Tennessee Health Science Center, Memphis, Tennessee, USA

\section{Katherine E. Pelch}

Department of Obstetrics, Gynecology and Women's Health, University of Missouri School of Medicine, Columbia, Missouri, USA

\section{Elena Picchiassi}

Department of Obstetrics and Gynecology, University of Perugia, Perugia, Italy

\section{Jeanna M. Piper}

Clinical Microbicide Research Branch, Division of AIDS, NIAID, NIH, Bethesda, Maryland, USA

\section{Lauren Plante}

Division of Maternal-Fetal Medicine, Drexel University College of Medicine, Philadelphia, Pennsylvania, USA

\section{Ritsuko K. Pooh}

Clinical Research Institute of Fetal Medicine PMC, Osaka, Japan

\section{William F. Rayburn}

Division of Maternal-Fetal Medicine, University of New Mexico School of Medicine, Albuquerque, New Mexico, USA

\section{Terry Rolan}

Department of Neurology, University of Missouri School of Medicine, Columbia, Missouri, USA

\section{Roberto Romero}

Perinatology Research Branch and Obstetrics and Perinatology Intramural Division, Eunice Kennedy Shriver National Institute of Child Health and Human Development, National Institutes of Health, Department of Health and Human Services, Bethesda, Maryland, USA

\section{J. Pratt Rossiter}

Private practice in Obstetrics and Gynecology, Towson, Maryland, USA

\section{Christine E. Ryan}

Department of Obstetrics, Gynecology and Women's Health, University of Missouri School of Medicine, Columbia, Missouri, USA

\section{Pradeep Sahota}

Department of Neurology, University of Missouri School of Medicine, Columbia, Missouri, USA
Aida Salihagic-Kadic

Department of Physiology, School of Medicine, University of Zagreb, Zagreb, Croatia

William Salzer

Department of Internal Medicine, University of Missouri School of Medicine, Columbia, Missouri, USA

\section{Abraham Sanders}

Weill Cornell Pulmonary Associates and New York Presbyterian Hospital, New York, New York, USA

\section{Thomas J. Santoro}

Section of Rheumatology, University of Illinois College of Medicine at Peoria, Peoria, Illinois, USA

\section{Aarti Sarwal}

Department of Neurology, University of Missouri School of Medicine, Columbia, Missouri, USA

\section{Anjana Sathyamurthy}

Department of Internal Medicine, University of Missouri School of Medicine, Columbia, Missouri, USA

\section{Edward R. Sauter}

Department of Surgery, University of North Dakota School of Medicine and Health Sciences, Grand Forks, North Dakota, USA

\section{Danny J. Schust}

Department of Obstetrics, Gynecology and Women's Health, University of Missouri School of Medicine, Columbia, Missouri, USA

\section{Wade D. Schwendemann}

Division of Perinatology, Children's Specialists of San Diego, San Diego, California, USA

\section{Gautam K. Singh}

Echocardiography Laboratory, Washington University School of Medicine, St. Louis, Missouri, USA

\section{James S. Smeltzer}

Wellstar Physicians Group, Wellstar Kennestone Hospital, Marietta, Georgia, USA

\section{Robert J. Sokol}

C.S. Mott Center for Human Growth and Development, Wayne State University School of Medicine and Department of Clinical and Translational Science, Wayne State University, Detroit, Michigan, USA

\section{Eleazar Soto}

Perinatology Research Branch, Eunice Kennedy Shriver National Institute of Child Health and Human Development, National Institutes of Health, Department of Health and Human Services, Detroit, Michigan, USA

\section{Lara Spalldi-Barisic}

Department of Obstetrics and Gynecology, Sveti Duh Hospital, Zagreb, Croatia

Milan Stanojevic

Department of Obstetrics and Gynecology, Sveti Duh Hospital, Zagreb, Croatia

\section{Dani Stramer}

Department of Surgery, University of North Dakota School of Medicine and Health Sciences, Grand Forks, North Dakota, USA

\section{Scott Stuart}

Department of Psychiatry, University of Iowa Carver College of Medicine, Iowa City, Iowa, USA

\section{Marianna Theodora}

Department of Obstetrics and Gynecology, University of Athens Medical School, Athens, Greece 


\section{Michiyo Tomita}

Section of Rheumatology, University of Illinois College of Medicine at Peoria, Peoria, Illinois, USA

\section{Rebecca A. Uhlmann}

Department of Obstetrics and Gynecology, University of Tennessee Health Science Center, Memphis, Tennessee, USA

\section{Akshaya Vachharajani}

Division of Newborn Medicine, the Edward Mallinckrodt Department of Pediatrics, Washington University School of Medicine and St. Louis Children's Hospital, St. Louis, Missouri, USA

\section{Valéria Váradi}

Department of Obstetrics and Gynecology, Semmelweis University Medical School, Budapest, Hungary

\section{Yves Ville}

Department of Obstetrics and Fetal Medicine, Necker-Enfants-Malades Medical School, Paris Descartes University, Paris, France

\section{Anthony M. Vintzileos}

Department of Obstetrics \& Gynecology, Winthrop University Hospital, Mineola; and Department of Obstetrics, Gynecology \& Reproductive Medicine, Stony Brook University School of Medicine, Stony Brook, New York, USA

\section{Dana Ward}

Division of Dermatology, University of Missouri School of Medicine, Columbia, Missouri, USA

\section{Barbara Warner}

Division of Newborn Medicine, the Edward Mallinckrodt Department of Pediatrics, Washington University School of Medicine and St. Louis Children's Hospital, St. Louis, Missouri, USA
William J. Watson

Division of Maternal Fetal Medicine, Mayo Clinic College of Medicine, Rochester, Minnesota, USA

\section{Hung N. Winn}

Department of Obstetrics, Gynecology and Women's Health, University of Missouri School of Medicine, Columbia, Missouri, USA

\section{Jessica Winn}

Department of Internal Medicine, University of Missouri School of Medicine, Columbia, Missouri, USA

\section{Frank R. Witter}

Department of Obstetrics and Gynecology, Johns Hopkins University School of Medicine, Baltimore, Maryland, USA

\section{Lami Yeo}

Perinatology Research Branch, Eunice Kennedy Shriver National Institute of Child Health and Human Development, National Institutes of Health, Department of Health and Human Services, and Department of Obstetrics and Gynecology, Wayne State University, Detroit, Michigan, USA

\section{Dana Zappetti}

Weill Cornell Pulmonary Associates and New York Presbyterian Hospital, New York, New York, USA

\section{Maria Angelica Zoppi}

Department of Obstetrics and Gynecology, Prenatal and Pre-implantation Genetic Diagnosis, Microcitemico Hospital, University of Cagliari, Cagliari, Italy 


\section{Preface}

The discipline of Maternal-Fetal Medicine has made great progress since the first edition of this textbook (Clinical Maternal-Fetal Medicine) published nearly a decade ago. Basic, clini$\mathrm{cal}$, and translation research has allowed clinicians and scientists to improve the understanding of the mechanisms of disease, and improve the care of the mother and fetus.

We are of the view that Maternal-Fetal Medicine is one of the most challenging disciplines in medicine, because we care for two patients; one of whom, the fetus, is often inaccessible, difficult to examine, and optimal medical care requires balancing the interests of mother and fetus. Yet, the importance of our discipline has become increasingly clear, as the concept that the "child is the father of the man" has gained ground through growing evidence that insults sustained during intrauterine life have a long-lasting effect and increase the risk for adult diseases (cardiovascular, metabolic, mental health, reproductive, etc.).

This book addresses the pathophysiology, diagnosis, and treatment of many important maternal medical and obstetric complications, as well as fetal complications. It is intended to provide a review of clinically relevant topics in Maternal-Fetal Medicine. In contrast to the first edition, this book is available online rather than as a traditional print book. This approach was embraced to make its use easier for the readers. You will be able to search the book across chapters and provide feedback. In addition, updates could be more frequently done as the information develops.

This book could serve as a convenient reference for clinicians, fellows, residents and students who have the privilege of caring for the mother and fetus. We hope that the constant search for new understandings of the biology of pregnancy would allow us to improve the diagnosis and treatment of complications of pregnancy, and the health of future generations.

Hung N. Winn

Frank A. Chervenak

Roberto Romero 
$\because$ Taylor \& Francis

http://taylorandfrancis.com 


\section{The diagnosis and management of preterm labor with intact membranes Roberto Romero, Tinnakorn Chaiworapongsa, Francesca Gotsch, Lami Yeo, Ichchha Madan, and Sonia S. Hassan}

\section{INTRODUCTION}

A preterm birth is one that occurs between fetal viability and 37 completed weeks of gestation (1-4). Delivery of a previable conceptus represents a spontaneous abortion rather than a preterm birth. Although the definition of "viability" varies among countries and even medical centers, the central idea is that a nonviable infant is so immature that there is no likelihood of survival in the extrauterine environment despite all medical support.

Prior to modern developments in neonatal care, a nonviable infant was defined as one weighing less than $500 \mathrm{~g}$ (see below). This definition is discouraged because preterm delivery should be defined by gestational age at birth, rather than birth weight. Although some have proposed to define a preterm birth as one that occurs between 20 and 37 weeks of gestation, we prefer to define preterm birth as one occurring between 24 and 37 weeks of gestation. Some neonates can survive if born around 24 weeks of gestation, but none at 20 weeks (5-8). This definition may need to be revised in the future if technological advances allow substantial survival of neonates born at less than 24 weeks of gestation.

A birthweight of $500 \mathrm{~g}$ has historically been used to define viability. However, this approach has limitations since viable neonates born after 24 weeks of gestation may be affected by intrauterine growth restriction (IUGR) and have birthweights below $500 \mathrm{~g}$. Conversely, some previable infants may weigh more than $500 \mathrm{~g}$. The threshold of $500 \mathrm{~g}$ is valuable when there is uncertainty about gestational age. An accurate definition of preterm birth has implications for the calculation of vital statistics and comparisons of the rates of preterm delivery among different countries and populations, an issue that is often overlooked (1).

\section{Spontaneous Vs. Indicated Preterm Birth}

Preterm births can be spontaneous or "indicated." Spontaneous preterm labor can occur with either intact or prelabor rupture of the (fetal) membranes (PROM). "Indicated" preterm births are those that result from induced preterm labor or preterm cesarean delivery for maternal or fetal indications, such as pre-eclampsia and/or IUGR $(3,9-13)$. Other causes include severe medical or surgical complications of pregnancy, some congenital anomalies, and complications of twin gestations (in particular, complications of monochorionic twins).

Of all preterm deliveries, spontaneous preterm labor with intact membranes occurs in $45 \%$ (23.2-64.1\%), spontaneous preterm labor following PROM occurs in 30\% (7.1-51.2\%), and preterm birth as a result of an indicated delivery occurs in $25 \%(18.7-35.2 \%)(14,15)$. In the United States, the rate of preterm delivery has increased by $14 \%$ since 1990 - this has been attributed to "indicated" preterm births of singleton gestations, multiple gestations, and increased numbers of older parturients (16). Preterm births have been classified by gestational age. Table 1 presents the classification of preterm birth according to gestational age at birth and the contribution of each stratum (3).

\section{THE COMMON PATHWAY OF TERM AND PRETERM LABOR}

The traditional view that has dominated the study of preterm parturition is that spontaneous labor at term and preterm labor fundamentally involves the same processes, albeit occurring at different gestational ages. Indeed, term and preterm labor share a common pathway. We have defined the "common pathway of parturition" as the anatomic, biochemical, endocrinologic, and clinical events that occur in the fetus and/or mother in both term and preterm labor (17-22). Broadly conceptualized, the common pathway of parturition can be considered to have uterine (maternal and fetal) and extrauterine components. The uterine components (which are a subject of wide attention in clinical obstetrics) include increased uterine contractility, cervical ripening, and decidual/ membrane activation $(17,18)$. The extrauterine components include endocrinologic and metabolic changes associated with labor. For example, labor is associated with increased caloric metabolic expenditures (21), and an increase in maternal and fetal cortisol (20).

A fundamental difference between term and preterm labor is that the former results from "physiologic activation" of the common pathway, while the latter results from a pathologic process (pathologic activation) that extemporaneously activates one or more components of the common pathway (22).

Activation of the uterine components of the common pathway of parturition may be synchronous or asynchronous. Synchronous activation will result in clinical spontaneous preterm labor, and asynchronous activation will result in a different phenotype. For example, predominant activation of the membranes would lead to preterm PROM, of the cervix to cervical insufficiency, or of myometrium to preterm uterine contractions (Figs. 1 and 2). The activation of each component

This work is based on several review articles and chapters published previously by the first author in other publications. This work has been modified and adapted for this textbook. The original chapters are referenced and contain a more extensive discussion of the subject. This chapter has a clinical emphasis. The work has been primarily done by Roberto Romero, who is a government employee, and therefore, is not subject to copyright. 
Table 1 Classification of Preterm Birth According to Gestational Age at Birth and the Contribution of Each Stratum

\begin{tabular}{lcc} 
Proposed classification & $\begin{array}{c}\text { Gestational age } \\
\text { at birth (in weeks) }\end{array}$ & Frequency \\
\hline Extreme preterm birth & $<28$ & $5 \%$ \\
Very early preterm birth & 28 to $30-6 / 7$ & $15 \%$ \\
Early preterm birth & 31 to $33-6 / 7$ & $20 \%$ \\
Late preterm birth & 34 to $36-6 / 7$ & $60-70 \%$
\end{tabular}

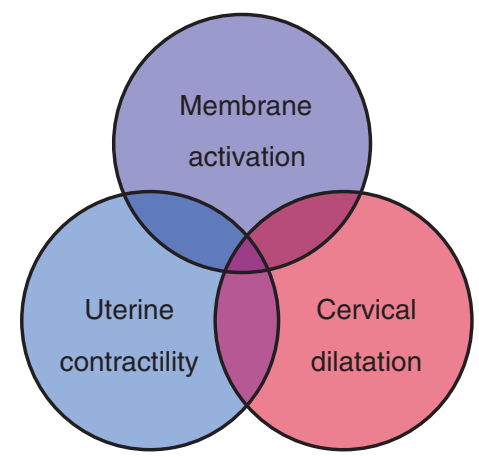

Figure 1 Uterine components of the common pathway of parturition. Source: From Ref. 22.

confers a different risk for impending preterm delivery. For example, rupture of membranes is followed by the onset of labor in most cases within a short period of time. In contrast, most patients who present with increased uterine contractility without cervical change at an early gestational age deliver at term. Acute cervical insufficiency (formerly called "cervical incompetence") may lead to late spontaneous abortion or early preterm delivery days or weeks after the diagnosis $(23,24)$.

\section{SPONTANEOUS PRETERM LABOR AS A SYNDROME}

Preterm labor with intact membranes is a "syndrome" caused by multiple etiologies. The term "syndrome" refers to a combination of symptoms or signs identified as a discrete clinical entity (25). Symptoms and signs of preterm labor include increased uterine contractility, changes in cervical status (dilatation and effacement), and/or changes in membrane status (i.e., activation, which can be expressed subclinically as a positive fetal fibronectin, or overtly, such as rupture of membranes).

The proposed etiologies of the preterm labor syndrome are presented in Figure 3. The main causes are (i) intra-amniotic infection/inflammation (26-57); (ii) vascular disease mediated through ischemia (58-64); (iii) uterine overdistension [twin gestations (65), patients with polyhydramnios $(66,67)$, and Müllerian duct abnormalities (68)]; (iv) stress (69-73); (v) cervical disease [congenital after diethylstilbestrol exposure (74-79), post-traumatic after conization (80-88), etc. (89)]; (vi) hormonal disorders that mediate a suspension of progesterone actions (90-98); (vii) allergic disease (99-107); and (viii) an abnormal allogeneic response in which there is a rejection-like mechanism by the maternal immune system of the conceptus (108-112).

We also believe that the entire spectrum of the mechanisms of the preterm labor syndrome has not been fully elucidated. In addition, some patients have preterm labor because more than

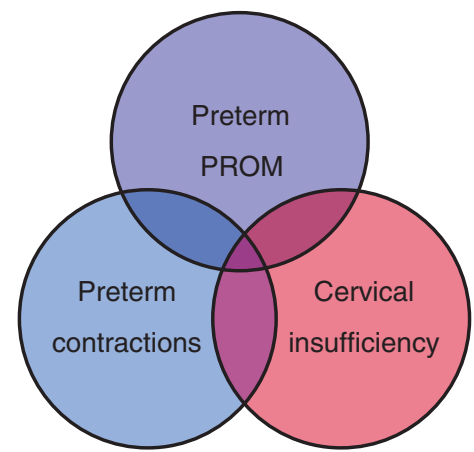

Figure 2 Clinical manifestations of preterm activation of the common pathway of parturition. Source: From Ref. 22.

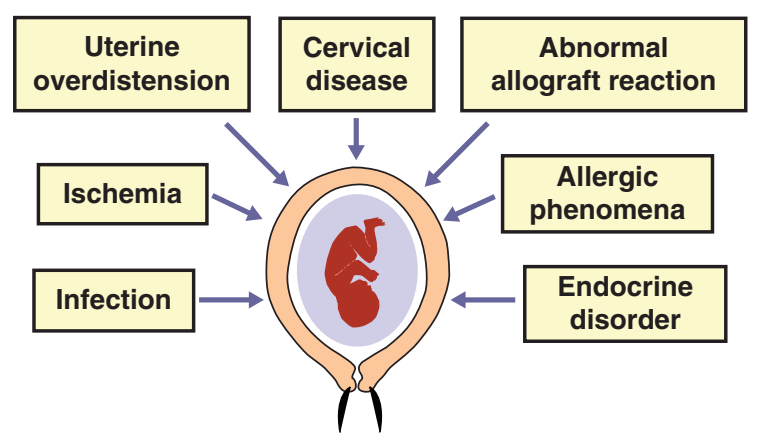

Figure 3 The preterm parturition syndrome. Source: From Ref. 25.

one mechanism of disease operates simultaneously. For example, subclinical intra-amniotic infection leads to preterm labor through an inflammatory mechanism that involves chemokines, cytokines, and prostaglandins; yet, one of the effects of IL1 $\beta$ (a cytokine involved in preterm parturition) can decrease progesterone action through activation of NFKB (113-115). This transcription factor may lead to preterm cervical ripening and also to the activation of membrane and decidua. The interested reader is referred to recent reviews of the mechanisms responsible for the preterm labor syndrome $(3,116)$.

There is an extensive body of epidemiologic literature identifying risk factors for preterm labor and delivery-for example, individuals of African-American origin (12,117$119)$, those with a low body mass index (120-124), or patients who smoke during pregnancy (125-127) are at increased risk (Table 2 lists commonly identified risk factors). Yet, the precise pathologic mechanism responsible for the increased risk has not been elucidated.

\section{THE DIAGNOSIS OF PRETERM LABOR}

\section{The Importance of Diagnosis}

The clinical diagnosis of preterm labor is important because patients at risk for preterm delivery can be offered (i) admission to the hospital, (ii) assessment for intra-amniotic infection, (iii) steroids, (iv) tocolysis, (v) antibiotic prophylaxis against group B streptococci, (vi) transport to a tertiary care center, and (vii) the option of magnesium sulfate for neuroprotection.

\section{Criteria to Increase the Index of Suspicion}

Preterm labor is suspected in patients with preterm gestations (before 36-6/7 weeks of gestation) who present with symptoms of increased uterine contractility, vaginal bleeding, pelvic 
Table 2 Risk Factors Associated with Preterm Delivery

\section{Sociobiological variables}

Age $<17$ years, $>35$ years

Low BMI or pre-pregnancy weight $<50 \mathrm{~kg}(<120 \mathrm{lbs})$

Poor nutritional status

Single marital status

Lower socioeconomic status

Lower education

Smoking

Heavy alcohol consumption

Illicit drug use (cocaine, heroin, etc.)

Social factors (e.g., poor access to care, physical abuse)

Working conditions-shift work, standing for long periods of time ( $>8$ hours)

Past obstetric/gynecologic history

Prior preterm birth

Multiple therapeutic and/or spontaneous abortions

Uterine anomalies

Cervical surgery (cone biopsy, LEEP)

Characteristics of current pregnancy

Short inter-pregnancy interval ( $<6$ months)

In vitro fertilization/assisted reproductive techniques

Multiple gestation

Fetal conditions (e.g., structural malformations, chromosomal abnormalities, IUGR, fetal demise)

Antepartum hemorrhage

Amniotic fluid abnormalities-polyhydramnios, oligohydramnios

Maternal medical conditions (e.g., hypertension, diabetes, asthma, thyroid disease)

Maternal abdominal surgery

Psychological factors (e.g., stress, depression)

Infections during pregnancy (e.g., chlamydia, gonorrhea, trichomonas, bacterial vaginosis, asymptomatic bacteriuria, urinary tract infection, pyelonephritis, severe viral infections)

Short cervical length

Positive fetal fibronectin between 22 and 34 weeks

Preterm contractions

Abbreviations: LEEP, loop electrosurgical excision procedure; IUGR, intrauterine growth retardation.

pressure and/or pain, expulsion of the mucus plug, and leakage of amniotic fluid. The latter is particularly important because if rupture of membranes is present, the management and prognosis are different from those of patients with preterm labor and intact membranes $(15,128)$. For example, tocolysis is not indicated in patients with preterm prelabor rupture of membranes (129) (see the chapter on preterm PROM in this book).

\section{"Threatened Preterm Labor," "False Preterm Labor," and "Rule out preterm labor"}

Some authors and textbooks have employed these terms because approximately $15 \%$ of pregnant women present with symptoms and signs consistent with preterm labor, yet they do not deliver preterm (130). Results of randomized clinical trials of beta-adrenergic agents have shown that $47 \%$ to $50 \%$ of patients admitted with symptoms of preterm labor randomized to placebo treatment deliver at term (131). Some investigators have interpreted such findings as indicating that these patients were in "false preterm labor" or "threatened preterm labor" because they delivered at term when treated with placebo (intravenous hydration). However, there is an implicit problem in requiring that a patient delivers preterm for the diagnosis of preterm labor to be correct: such view would imply that preterm labor inevitably leads to preterm delivery. In the pre-antibiotic era, pyelonephritis was associated with preterm labor and delivery. After the introduction of antibiotics (132), pyelonephritis has been associated with preterm labor but not necessarily preterm birth $(133,134)$. This indicates that labor has a reversible phase in which early treatment may prevent preterm delivery (135).

The pathologic process responsible for preterm labor can have a spectrum of severity. Mild insults that can be managed by the homeostatic system do not lead to preterm delivery, but more severe insults would be required for progression to preterm delivery. A pregnant woman who sustains mild abdominal trauma (such as an automobile accident or a fall) may experience increased uterine contractility which eventually subsides. However, if the trauma leads to preterm separation of the placenta (abruptio placentae), preterm labor/ delivery may occur.

\section{True or False Labor: a Diagnostic Challenge}

The diagnostic dilemma described in the previous paragraph is not exclusive to preterm labor, but it also applies to labor at term. Labor is a retrospective diagnosis that can be made with certainty only when the patient has delivered or when delivery is inevitable (e.g., a patient with preterm labor and advanced cervical dilation who has spontaneous rupture of membranes or, alternatively, a patient with preterm labor who is in the second stage of labor). Except for these rare circumstances, physicians will often encounter a patient who has some degree of activation of the three components of the terminal pathway of parturition (increased uterine contractility, cervical changes, and/or membrane decidual activation) and will need to decide whether or not to initiate therapeutic intervention.

The diagnosis of labor at term is also challenging. Nonetheless, the risk of an inaccurate diagnosis is lower because a fetus at term does not require steroids and other interventions which may be beneficial to the preterm neonate. Thus, the diagnosis of "false labor" at term carries different consequences from a misdiagnosis of preterm labor. Patients diagnosed with "false labor" at term typically present as if they were in the latent phase of labor and stop contracting after the administration of sedation, analgesia, or during a period of observation. Yet this diagnosis of "false labor" is not always correct; many labor wards have at least one story of a patient sent home who has delivered a few hours later outside the hospital or on her way back to the hospital. Since the neonates are born at term, such diagnostic errors generally do not have serious consequences; however, the possibility of shoulder dystocia, other obstetrical complications, or neonatal emergencies can transform this situation into a tragedy.

\section{Does "False Preterm Labor" have Any Consequences?}

Clinicians believe that an episode of preterm labor that resolves itself is benign. Yet these patients remain at risk for recurrent episodes of preterm labor and, more importantly, preterm delivery (136-138). An explanation for this is that a subclinical pathologic process may underline an episode of preterm labor. If the pathologic process worsens, this eventually may lead to preterm labor and delivery. 


\section{Synchronous and Asynchronous Activation of the Common Pathway}

Activation of the common pathway of labor can occur with recruitment of the three components (i.e., synchronous) or with activation of mainly one component (i.e., asynchronous) (Figs. 1 and 2). The phenotypic definitions of the preterm labor syndrome depend on the specific pathway activated.

Most physicians and patients focus on increased uterine contractility as a sign of preterm labor-such symptoms are easily detected by patients and verifiable by the health-care provider. In contrast, cervical ripening (effacement and dilatation) is largely subclinical, and its diagnosis requires a pelvic examination or transvaginal sonography. Some patients may present with a short cervix (and even visible membranes) in the absence of demonstrable contractions - this phenotype is consistent with cervical insufficiency, typically occurring in the midtrimester.

Similarly, mild activation of the decidual/membrane component of the common pathway of labor is largely subclinical and can be detected by an elevation in the concentration of molecules involved in extracellular matrix degradation, such as fetal fibronectin (139-143), IL-6 (144-148), and matrix metalloproteinases (MMPs) (149-151) in the cervicovaginal fluid. Most asymptomatic patients with a positive fetal fibronectin after 22 weeks of gestation do not deliver preterm (even though the relative risk for preterm delivery is increased) $(143,152,153)$. This indicates that decidual/membrane activation of this common pathway can occur without leading to inevitable preterm labor and delivery.

\section{The Clinical Diagnosis of Preterm Labor}

With the introduction and widespread utilization of tocolytic agents, several definitions of preterm labor were proposed. Notably, in 1975, Ingemar Ingemarsson (154) proposed that the criteria for the diagnosis should include (i) painful regular uterine contractions occurring at intervals of less than 10 minutes for at least 30 minutes by external tocography, (ii) a cervix that is effaced or almost effaced and dilated at least 1 to $2 \mathrm{~cm}$, (iii) intact membranes, and (iv) a gestational age between 28 and 36 weeks. This definition was the basis for a randomized, double-blinded clinical trial of terbutaline in women with preterm labor and intact membranes (154). The gestational age limitation reflected neonatal survival at the time. Importantly, two components of the common pathway were required for the diagnosis (increased myometrial contractility and cervical change). Gonik and Creasy wrote a clinical opinion in 1986 (155), in which they proposed a definition of preterm labor that has been used subsequently in the literature. The definition is based on uterine contractility and cervical change, and was proposed to select patients for tocolytic treatment. A change in cervical status was required because of the concern about overtreating patients with painful Braxton-Hicks contractions without other evidence of preterm labor (156-158).

\section{Uterine Contractility}

Increased uterine contractility can be present in the absence of cervical change. Two types of uterine contractions have been identified using electromyography (159): (i) "contractures," which are epochs of myometrial activity leading to a modest increase in intrauterine pressure and are of long duration, and (ii) "contractions," which are of short duration but increase intrauterine pressure. A switch from a predominant "contracture pattern" to a predominant "contraction pattern" can occur during normal labor or be induced by pathologic events such as intra-abdominal surgery, infection, or food withdrawal. The molecular basis of this switch is thought to be the development of gap junctions, which increase the transmission of cell-to-cell communication in the myometrium during labor. Efforts to differentiate contractures from contractions using clinically available external tocodynamometry have failed to distinguish between the patient at risk for preterm delivery and the one who is not at risk (160). Therefore, uterine contractility alone is not sufficient to reliably diagnose preterm labor. Recent modalities that use external electromyography may improve such a diagnosis, but these techniques are not yet clinically available (161-165).

\section{Sonographic Cervical Length}

The most important advance in the diagnosis of preterm labor has been the introduction of transvaginal sonography (79,166-168). Many investigators have shown that transvaginal sonography is more accurate than digital examination of the cervix (to assess dilatation and effacement) in predicting the risk of preterm birth $(168,169)$. The data consistently indicate that women with a long cervix (defined as $>30 \mathrm{~mm}$ ) have a likelihood of preterm delivery of less than $5 \%(169,170)$. In contrast, women with preterm uterine contractions and with a cervical length of $<15 \mathrm{~mm}$ have a probability of preterm delivery of $63 \%$ (171). Figure 4 illustrates the probability of preterm delivery in patients presenting with an episode of preterm labor - the shorter the cervix, the greater the risk of preterm delivery (168,172-174). Moreover, the shorter the cervix and the earlier the gestational age at presentation, the greater the risk of intra-amniotic infection (175).

\section{Fetal Fibronectin}

Another test frequently used to assess the risk of preterm delivery is fetal fibronectin. This glycoprotein is present in the

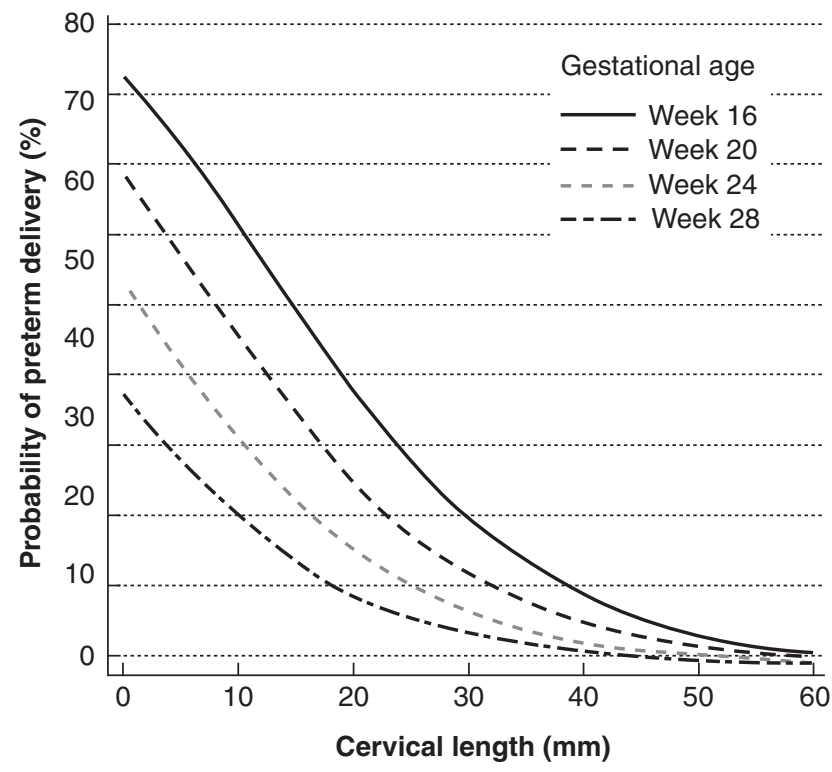

Figure 4 Predicted probability of delivery before 32 weeks of gestation, by cervical length $(\mathrm{mm})$ and time of measurement (week of pregnancy), in women with a prior preterm birth. Source: From Ref. 504. 
trophoblast-decidua interface and, like all fibronectins, acts as cellular "cement" to anchor trophoblast cells to the uterine wall (decidua). Decidual/membrane activation is a process whereby extracellular matrix is dissolved at this interface. Such a process is teleologically intended to weaken the membranes for membrane rupture during labor and also to prepare the membranes for separation from the uterine cavity after delivery. This intercellular "cement" has a unique glycosylation pattern, which is different from other fibronectins, and that is why it is called "fetal" fibronectin. Fortuitously, fetal fibronectin can be detected in vaginal and cervical fluids (142). It is interesting that fetal fibronectin can be detected between 16 and 20 weeks of gestation in normal pregnancy (176). However, normally it becomes undetectable (using a cut-off of $50 \mathrm{ng} / \mathrm{mL}$ ) after 22 weeks of gestation and becomes detectable again at term prior to the onset of labor $(142,177)$.

After 22 weeks of gestation, a positive fetal fibronectin in cervicovaginal fluid is associated with an increased risk for preterm delivery in symptomatic and asymptomatic patients $(142,178-182)$. However, the proportion of patients with a positive fetal fibronectin who will deliver preterm is small (low positive predictive value) in both symptomatic and asymptomatic patients $(143,152,153)$. In contrast, this test is considered more valuable in identifying patients at low risk for preterm delivery (high negative predictive value) (183-185). The value of this test relates to its ability to identify the patient unlikely to deliver, therefore avoiding unnecessary intervention. The results of fetal fibronectin may be affected by a history of sexual intercourse within 24 hours (186), a pelvic examination during the same period of time, or vaginal bleeding. Each of these conditions may lead to what has been called a "falsepositive" test (187). In contrast, the use of lubricant may lead to a false-negative result. Amniotic fluid contains fetal fibronectin in high concentrations (187). Therefore, a positive test can occur if there is membrane rupture or if the sample is contaminated with amniotic fluid (187).

\section{Combined Use of Cervical Sonography and Fetal Fibronectin in the Diagnosis of Preterm Labor}

Several studies have examined the value of combining these tests to predict preterm delivery. A study by Gomez et al. (171) indicated that (i) cervical length in labor is a strong predictor of preterm delivery; (ii) a short cervix (defined as a cervical length of $<15 \mathrm{~mm}$ ) identifies patients at high risk of impending preterm delivery (within 48 hours or 7 days of admission); (iii) a long cervix (defined as cervical length of $30 \mathrm{~mm}$ or more) identifies patients at low risk of preterm delivery; (iv) a positive fetal fibronectin is also associated with spontaneous preterm delivery, but the likelihood ratio of a positive test is substantially lower for fetal fibronectin than for a short cervix (i.e., 3.6 vs. 9.2 for delivering at $<35$ weeks of gestation) (see Tables 3 and 4 for a detailed comparison of cervical length and vaginal fetal fibronectin); and (v) the combined use of sonographic cervical length and fetal fibronectin improves the prediction of preterm delivery over that provided by each test alone; however, this effect was observed only when the cervical length was $<30 \mathrm{~mm}(171,188)$. Therefore, there does not seem to be a justification for performing a fetal fibronectin when the cervical length is $30 \mathrm{~mm}$ or more.

Our practice is to perform a vaginal speculum examination to exclude rupture of membranes (assess the presence of pooling and nitrazine testing, and collect samples for ferning) and obtain samples for microbiology (including group B streptococcus (GBS), and if the prevalence justifies, gonorrhea

Table 3 Risk of Spontaneous Preterm Delivery Within 48 hours, 7 days, and 14 days According to Cervical Length Results and Vaginal Fibronectin Determination

\begin{tabular}{|c|c|c|c|c|c|c|c|c|c|}
\hline & \multirow{2}{*}{$\begin{array}{c}\text { Delivery within } \\
48 \text { hours }\end{array}$} & \multicolumn{2}{|c|}{ LR } & \multirow{2}{*}{$\begin{array}{c}\text { Delivery within } \\
7 \text { days }\end{array}$} & \multicolumn{2}{|c|}{ LR } & \multirow{2}{*}{$\begin{array}{c}\text { Delivery within } \\
\text { 14days }\end{array}$} & \multicolumn{2}{|c|}{ LR } \\
\hline & & + & - & & + & - & & + & - \\
\hline Cervical length $<15 \mathrm{~mm}$ & $36.7 \%(11 / 30)$ & 6.7 & & $56.7 \%(17 / 30)$ & 8.7 & & $56.7 \%(17 / 30)$ & 6.9 & \\
\hline Cervical length $<30 \mathrm{~mm}$ & $13.9 \%(15 / 108)$ & 1.9 & & $23.1 \%(25 / 108)$ & 2.0 & & $26.9 \%(29 / 108)$ & 1.9 & \\
\hline Cervical length $\geq 30 \mathrm{~mm}$ & $1.9 \%(2 / 107)$ & & 0.2 & $2.8 \%(3 / 107)$ & & 0.2 & $4.7 \%(5 / 107)$ & & 0.3 \\
\hline Vaginal fetal fibronectin $(+)$ & $19.2 \%(10 / 52)$ & 2.8 & & $34.6 \%(18 / 52)$ & 3.5 & & $42.3 \%(22 / 52)$ & 3.9 & \\
\hline
\end{tabular}

Table 4 Risk of Spontaneous Preterm Delivery $<32$ and $<35$ weeks According to Cervical Length Results and Vaginal Fibronectin Determination

\begin{tabular}{|c|c|c|c|c|c|c|}
\hline & \multirow[b]{2}{*}{ Delivery $<32$ weeks } & \multicolumn{2}{|c|}{ LR } & \multirow[b]{2}{*}{ Delivery $<35$ weeks } & \multicolumn{2}{|c|}{ LR } \\
\hline & & + & - & & + & - \\
\hline Cervical length $<15 \mathrm{~mm}$ & $58.3 \%(7 / 12)$ & 14.3 & & $63.3 \%(19 / 30)$ & 9.2 & \\
\hline Cervical length $<30 \mathrm{~mm}$ & $18.4 \%(9 / 49)$ & 2.2 & & $27.8 \%(30 / 108)$ & 2.0 & \\
\hline Cervical length $>30 \mathrm{~mm}$ & $0 \%(0 / 52)$ & & 0.1 & $3.7 \%(4 / 107)$ & & 0.2 \\
\hline Vaginal fetal fibronectin $(+)$ & $30.4 \%(7 / 23)$ & 4.5 & & $40.4 \%(21 / 52)$ & 3.6 & \\
\hline
\end{tabular}


Table 5 Frequency of Spontaneous Preterm Delivery According to Cervical Length (Cut-off $30 \mathrm{~mm}$ ) and Vaginal Fibronectin Results

$\begin{array}{lclllll}\begin{array}{l}\text { Cervical } \\ \text { length }<\mathbf{3 0} \mathbf{m m}\end{array} & \begin{array}{c}\text { Fetal } \\ \text { fibronectin }+\end{array} & \begin{array}{c}\text { Delivery within } \\ \mathbf{4 8 h o u r s}\end{array} & \begin{array}{c}\text { Delivery within } \\ \mathbf{7} \text { days }\end{array} & \begin{array}{c}\text { Delivery within } \\ \mathbf{1 4} \text { days }\end{array} & \begin{array}{c}\text { Delivery } \\ <32 \text { weeks }\end{array} & \begin{array}{c}\text { Delivery } \\ <35 \text { weeks }\end{array} \\ \text { No } & \text { No } & 2.2 \%(2 / 93) & 2.2 \%(2 / 93) & 3.2 \%(3 / 93) & 0 \%(0 / 47) & 1.1 \%(1 / 93) \\ \text { No } & \text { Yes } & 0 \%(0 / 14) & 7.1 \%(1 / 14) & 14.3 \%(2 / 14) & 0 \%(0 / 5) & 21.4 \%(3 / 14) \\ \text { Yes } & \text { No } & 7.1 \%(5 / 70) & 11.4 \%(8 / 70) & 12.9 \%(9 / 70) & 6.5 \%(2 / 31) & 17.1 \%(12 / 70) \\ \text { Yes } & \text { Yes } & 26.3 \%(10 / 38) & 44.7 \%(17 / 38) & 52.6 \%(20 / 38) & 38.9 \%(7 / 18) & 47.4 \%(18 / 38) \\ \text { Prevalence of the outcome } & 7.9 \%(17 / 215) & 13.0 \%(28 / 215) & 15.8 \%(34 / 215) & 8.9 \%(9 / 101) & 15.8 \%(34 / 215)\end{array}$

Table 6 Frequency of Spontaneous Preterm Delivery According to Cervical Length (Cut-off $15 \mathrm{~mm}$ ) and Vaginal Fibronectin Results

\begin{tabular}{|c|c|c|c|c|c|c|}
\hline $\begin{array}{l}\text { Cervical length } \\
<15 \mathrm{~mm}\end{array}$ & $\begin{array}{c}\text { Fetal } \\
\text { fibronectin }+\end{array}$ & $\begin{array}{c}\text { Delivery within } \\
48 \text { hours }\end{array}$ & $\begin{array}{c}\text { Delivery within } \\
7 \text { days }\end{array}$ & $\begin{array}{c}\text { Delivery within } \\
\leq 14 \text { days }\end{array}$ & $\begin{array}{l}\text { Delivery } \\
\leq 32 \text { weeks }\end{array}$ & $\begin{array}{r}\text { Delivery } \\
\leq 35 \text { weeks }\end{array}$ \\
\hline No & No & $2.0 \%(3 / 149)$ & $3.4 \%(5 / 149)$ & $4.7 \%(7 / 149)$ & $1.4 \%(1 / 74)$ & $4.7 \%(7 / 149)$ \\
\hline No & Yes & $8.3 \%(3 / 36)$ & $16.7 \%(6 / 36)$ & $27.8 \%(10 / 36)$ & $6.7 \%(1 / 15)$ & $22.2 \%(8 / 36)$ \\
\hline Yes & Yes & $43.8 \%(7 / 16)$ & $75 \%(12 / 16)$ & $75 \%(12 / 16)$ & $75 \%(6 / 8)$ & $81.3 \%(13 / 16)$ \\
\hline \multicolumn{2}{|c|}{ Prevalence of the outcome } & $7.9 \%(17 / 215)$ & $13.0 \%(28 / 215)$ & $15.8 \%(34 / 215)$ & $8.9 \%(9 / 101)$ & $15.8 \%(34 / 215)$ \\
\hline
\end{tabular}

and Chlamydia trachomatis). We collect a sample using a Dacron swab, and save it to assess fetal fibronectin concentration and perform an endovaginal ultrasound examination to measure cervical length. This is followed by a digital examination if the membranes are intact.

Approximately $50 \%$ of patients presenting with preterm contractions will have a cervical length of $30 \mathrm{~mm}$ or more, and this will make testing for fetal fibronectin unnecessary (171). However, if the cervical length is $<30 \mathrm{~mm}$, a fetal fibronectin test may be performed to improve the assessment of the likelihood of preterm delivery. The magnitude of the increase is presented in Tables 5 and 6 and in Figure 5. A practical approach is to restrict fetal fibronectin testing between 20 and $30 \mathrm{~mm}$ (188), because the patients who have a cervical length below $20 \mathrm{~mm}$ are already at high risk of preterm delivery, and the management would not be affected by the improvement in risk estimation derived from the performance of the two tests. This method is consistent with the study of Hincz et al. (189), who proposed a two-step approach.

Preterm labor is a worldwide challenge, and the performance of tests for the diagnosis of preterm labor should consider the availability of resources. In most countries, assessment of preterm labor can be conducted with transvaginal sonography without the performance of a fetal fibronectin test. Cervical length can be determined with transvaginal sonography or even with transperineal sonography in centers that do not have an endovaginal probe (190). Ultrasound is widely available in virtually all obstetrical units, and the results can be obtained immediately. An immunoassay for fetal fibronectin or another analyte can be expensive and impractical. The recent development of a bedside test for fetal fibronectin has clinical appeal $(191,192)$.

\section{DIAGNOSTIC WORKUP OF PRETERM LABOR}

The clinical assessment of patients presenting with symptoms of preterm labor should include (i) a history of previous pregnancies and risk factors (e.g., prior preterm delivery, prior second-trimester abortion); (ii) current pregnancy status; (iii) presenting complaint(s); (iv) physical examination; (v) monitoring of fetal heart rate and uterine contractions; (vi) vaginal examination (speculum and/or digital); (vii) collection of cervicovaginal samples for microbiology (streptococcus group B (GBS), chlamydia, gonorrhea, Gram stain for bacterial vaginosis (BV)); (viii) urinalysis, culture, and sensitivity; (ix) amniocentesis; (x) fetal fibronectin; and (xi) ultrasound (estimated fetal weight, number of fetuses, placental location, fetal presentation and lie, amniotic fluid volume, and transvaginal cervical length).

\section{Evaluation for Subclinical Intra-amniotic Infection}

Intra-amniotic infection is a frequent and important cause of premature labor and delivery (33,193-203). Most of these infections are subclinical in nature, and only $12.5 \%$ of patients with a positive amniotic fluid culture for microorganisms will have a fever or meet the definition of clinical chorioamnionitis (193). Patients with intra-amniotic infections are more likely to deliver despite the administration of tocolytic agents (204), mothers are at an increased risk for pulmonary edema (205-211) and neonates for sepsis and other neonatal complications, such as intraventricular hemorrhage and periventricular leukomalacia.

Amniocentesis is offered to patients to assess the microbial state of the amniotic cavity and fetal lung maturity. The amniotic fluid is considered to be sterile for bacteria. Therefore, the identification of bacteria by culture $(33,193,212)$ or microbial footprints with molecular techniques (213-216) is evidence that microbial invasion of the amniotic cavity has occurred. These infections result, in the majority of cases, from microorganisms normally present in the vagina, which cross the endocervical canal and intact membranes and gain access to the amniotic cavity (196). Microorganisms can proliferate and gain access to the fetus. Aspiration of infected fluid may lead to congenital pneumonia (217), but other infections such as otitis, conjunctivitis, and omphalitis can occur by direct spread of microorganisms from infected amniotic fluid. Seeding from any of these sites to the fetal circulation may lead to bacteremia and sepsis. The frequency of intra-amniotic infection in patients with preterm labor and intact membranes is approximately $10 \%(193,218)$; however, the lower the gestational 
(A)

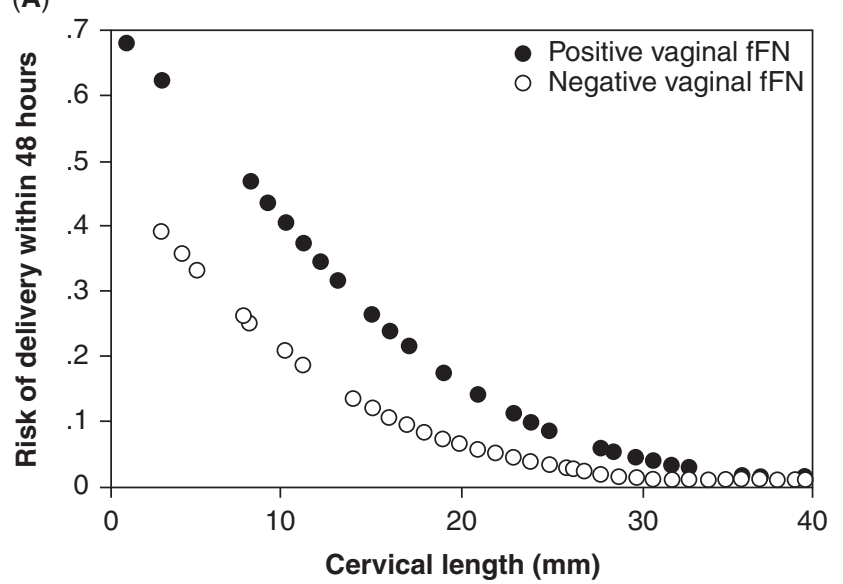

(C)

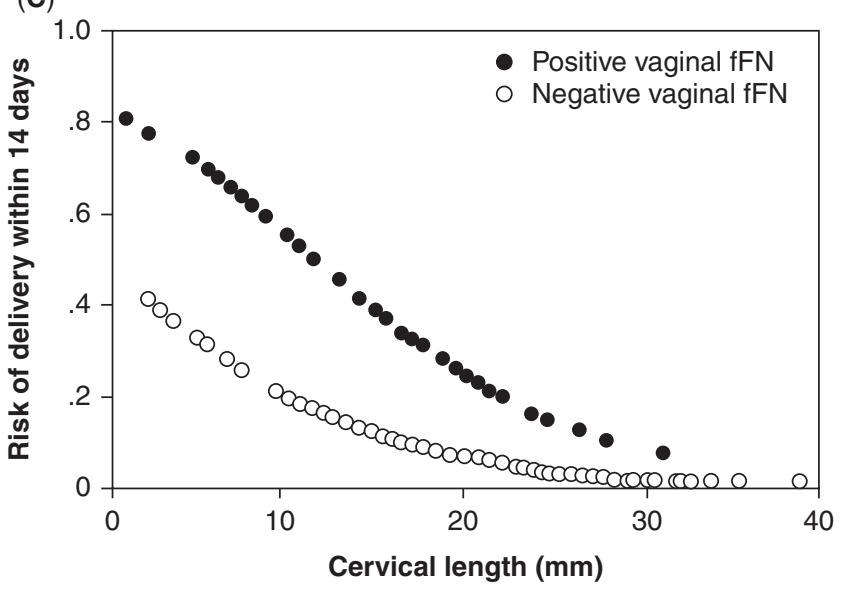

(B)

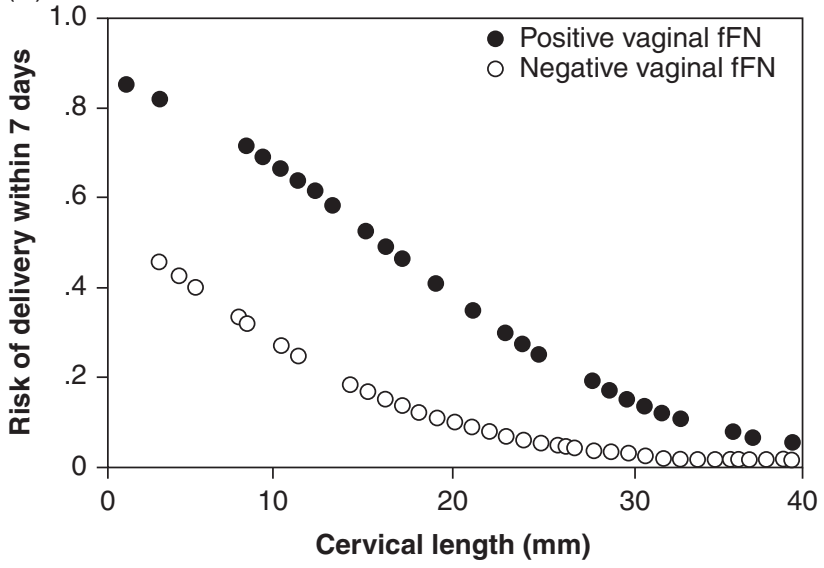

(D)

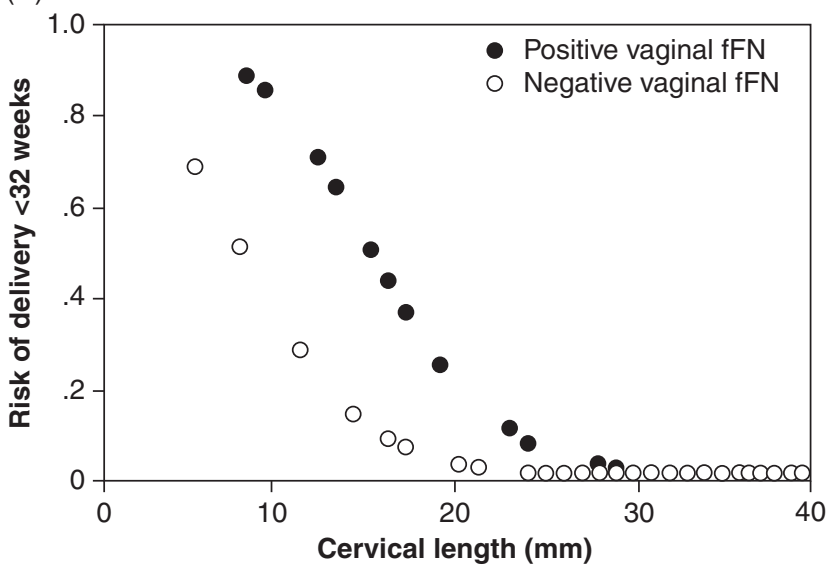

(E)

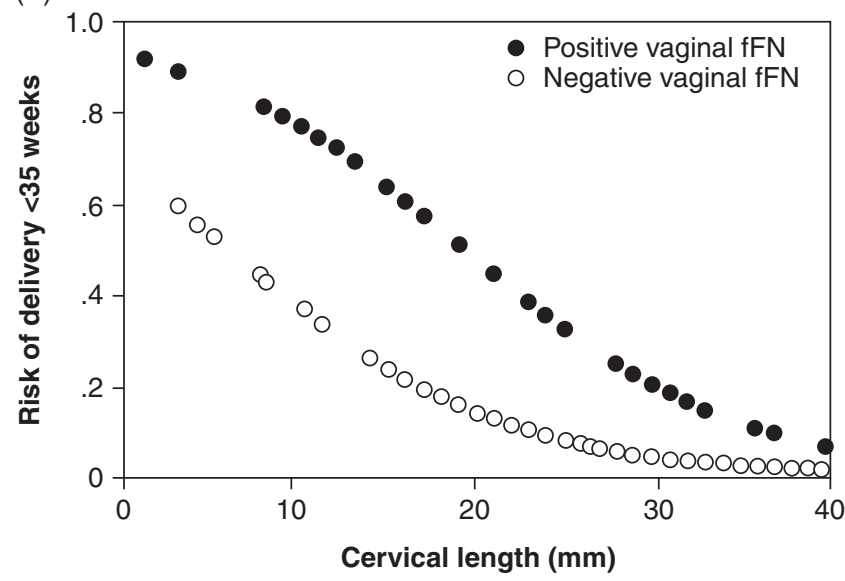

Figure 5 Risk of spontaneous preterm delivery within (A) 48 hours, (B) 7 days, (C) 14 days, (D) delivery $<32$ weeks, and (E) $<35$ weeks, according to cervical length results and vaginal fetal fibronectin (FFN) determination. Source: From Ref. 171.

age at presentation and the shorter the cervix, the higher the frequency of intra-amniotic infection and intra-amniotic inflammation (219). The organisms most frequently found in the amniotic cavity are genital mycoplasmas $(33,51,193,195,200,220)$ and aerobic and anaerobic infections. Fungi have been identified in pregnancies with a retained intrauterine contraceptive device (221-225).

Amniocentesis is easy to perform in patients with preterm labor and intact membranes. The likelihood of infection is assessed by rapid tests, such as a Gram stain of amniotic fluid (204), amniotic fluid white blood cell count (226), and a glucose determination (227). A positive Gram stain of amniotic fluid has a specificity of $99 \%$ for intra-amniotic infection (false-positive rate 1\%) (193). However, it has a limited sensitivity because genital mycoplasmas cannot be seen on a Gram stain due to their small size. Gram stain examinations must be performed by experienced laboratory staff, because artifacts and crystals of the reagents may be confused with bacteria by inexperienced individuals.

Neutrophils are not normally present in amniotic fluid. The presence of neutrophils is indicative of intra-amniotic inflammation (226). An amniotic fluid white blood cell count 
can be performed easily in a hemocytometer chamber, which is universally available in hospitals worldwide. It is the same chamber used to perform white blood cell counts in peripheral blood. In patients with intact membranes, a white blood cell count above 50 cells $/ \mathrm{mm}^{3}$ has a sensitivity of $80 \%$ and a specificity of $87.6 \%$ in the detection of a positive amniotic fluid culture (226). If patients have an elevated white blood cell count, but the Gram stain is negative, an infection with genital mycoplasmas must be suspected.

Glucose is a normal constituent of amniotic fluid, and the concentration of glucose decreases with advancing gestational age (227). In the presence of infection or inflammation, the concentrations of glucose in the amniotic fluid decreases (227). This has been attributed to a combination of the consumption of glucose by microorganisms and by activated neutrophils involved in host defense and microbial killing. A glucose concentration can be performed by standard analyzers, and it is important to alert laboratories that the amniotic fluid glucose concentration is much lower than that of plasma or serum. A glucose concentration of $<14 \mathrm{mg} / \mathrm{dL}$ has a high sensitivity and specificity for the detection of intra-amniotic infection (212,228,229).

The determination of amniotic fluid interleukin-6 (IL-6) is a sensitive method to detect intra-amniotic inflammation. A concentration above $2.6 \mathrm{ng} / \mathrm{mL}$ identified patients at risk for a positive amniotic fluid culture or impending delivery (218). Moreover, these patients are at increased risk for perinatal morbidity or mortality. Importantly, patients with an elevated amniotic fluid IL-6 and a negative amniotic fluid culture have a similar outcome as patients with a positive amniotic fluid culture (218). This suggests that there may be infections that cannot be detected with current methods (230) or, alternatively, that intra-amniotic inflammation due to non-infectious etiology is present (231-233) and this is associated with adverse outcome.

Neutrophils produce a collagenase, which is called matrix metalloproteinase 8 (MMP-8). MMP- 8 has been proven to be an excellent marker for intra-amniotic infection/inflammation $(234,235)$. Amniotic fluid MMP-8 $>30 \mathrm{ng} / \mathrm{mL}$ has a sensitivity of $82.4 \%$, specificity of $78 \%$, positive predictive value of $36 \%$, and negative predictive value of $97.7 \%$ for the prediction of intra-amniotic infection, defined as positive amniotic fluid culture (235). A rapid test (MMP-8 PTD Check test; SK Pharma Co. LTD, Korea) has been produced $(236,237)$, which has high sensitivity and specificity, as well as likelihood ratios for a positive and a negative result (238).

Amniotic fluid cultures should be performed for aerobic and anaerobic bacteria, as well as for genital mycoplasmas. Bacteria take time to grow and results may take several days. Molecular microbiologic techniques are becoming available and would allow detection of bacteria and identification of the organisms in 8 hours or less $(239,240)$.

The detection of microorganisms or intra-amniotic inflammation is a poor prognostic sign. Patients with preterm labor with intact membranes and documented intra-amniotic infection/inflammation are unlikely to respond to tocolysis $(193,218,239,241)$ and are at risk of developing pulmonary edema $(205,242,243)$ if attempts are made to delay delivery with tocolysis. Therefore, our approach is to suspend the administration of tocolysis in these patients. We continue to administer steroids because there is evidence that they cross the placenta and may downregulate the fetal inflammatory response syndrome (244). In patients with preterm PROM, the management depends upon gestational age. The higher the gestational age (e.g., >33 weeks), the better the outcome if the decision is to deliver the patient. On the other hand, at a gestational age of $<32$ weeks, treatment must be individualized. We have previously reported that it is possible to eradicate intra-amniotic infection in patients with preterm PROM $(245,246)$, as well as in patients with a sonographic short cervix with antibiotic treatment (247).

Amniotic fluid can also be tested for fetal lung maturity. This can be done with the standard tests, including the lecithin/sphingomyelin (L/S) ratio, phosphatidylglycerol, TDx-FLM surfactant albumin ratio, and lamellar body count. In the presence of lung maturity, heroic measures with aggressive tocolysis should be considered carefully.

Performance of amniocentesis in patients with preterm labor is valuable to identify patients at risk for intra-amniotic infection/inflammation and can be performed by individuals with expertise in ultrasound and invasive procedures. We explain to patients that examination of amniotic fluid is necessary to detect the presence of infection and identify the microorganism, and that infection is a frequent cause of preterm delivery. Although a randomized clinical trial of amniocentesis versus management without amniocentesis has not been performed, we believe that amniocentesis is indicated to identify intra-amniotic infection/inflammation. In any other specialty in medicine, identification of infection and the microorganism is undertaken. It is only in obstetrics that some are hesitant to identify this process. We believe that the benefits of identifying intra-amniotic infection/inflammation and establishing fetal lung maturity outweigh the risks of amniocentesis, which is today a minimally invasive procedure (248-252).

\section{TREATMENT OF PRETERM LABOR Glucocorticoids}

The idea that glucocorticoid administration could induce fetal lung maturity was first proposed by Liggins et al. more than 50 years ago (253). While studying the mechanisms of the onset of parturition in sheep, Liggins reported that administration of steroids to the fetal sheep induced preterm labor and that neonatal lambs born under these circumstances had inflated rather than collapsed lungs. Aeration of the lungs was attributed to the effects of steroids in inducing the production of pulmonary surfactant. This observation was followed by a randomized clinical trial in humans in which betamethasone was reported to decrease the rate of respiratory distress syndrome (254). Subsequently, multiple randomized clinical trials using either betamethasone or dexamethasone were reported (254-275). Systematic reviews of such trials have been performed since 1990, and they have consistently concluded that the beneficial effects of corticosteroids in neonatal outcome extend beyond inducing fetal lung maturity (276,277). Glucocorticoid receptors are expressed in many organs; and hence, the potential benefit in reducing morbidity such as intraventricular hemorrhage, necrotizing enterocolitis, etc.

The most recent systematic analysis reported by Roberts and Dalziel (276) included the review of 21 trials (3885 women and 4269 infants). Administration of corticosteroids was associated with a significant reduction in neonatal death, respiratory 


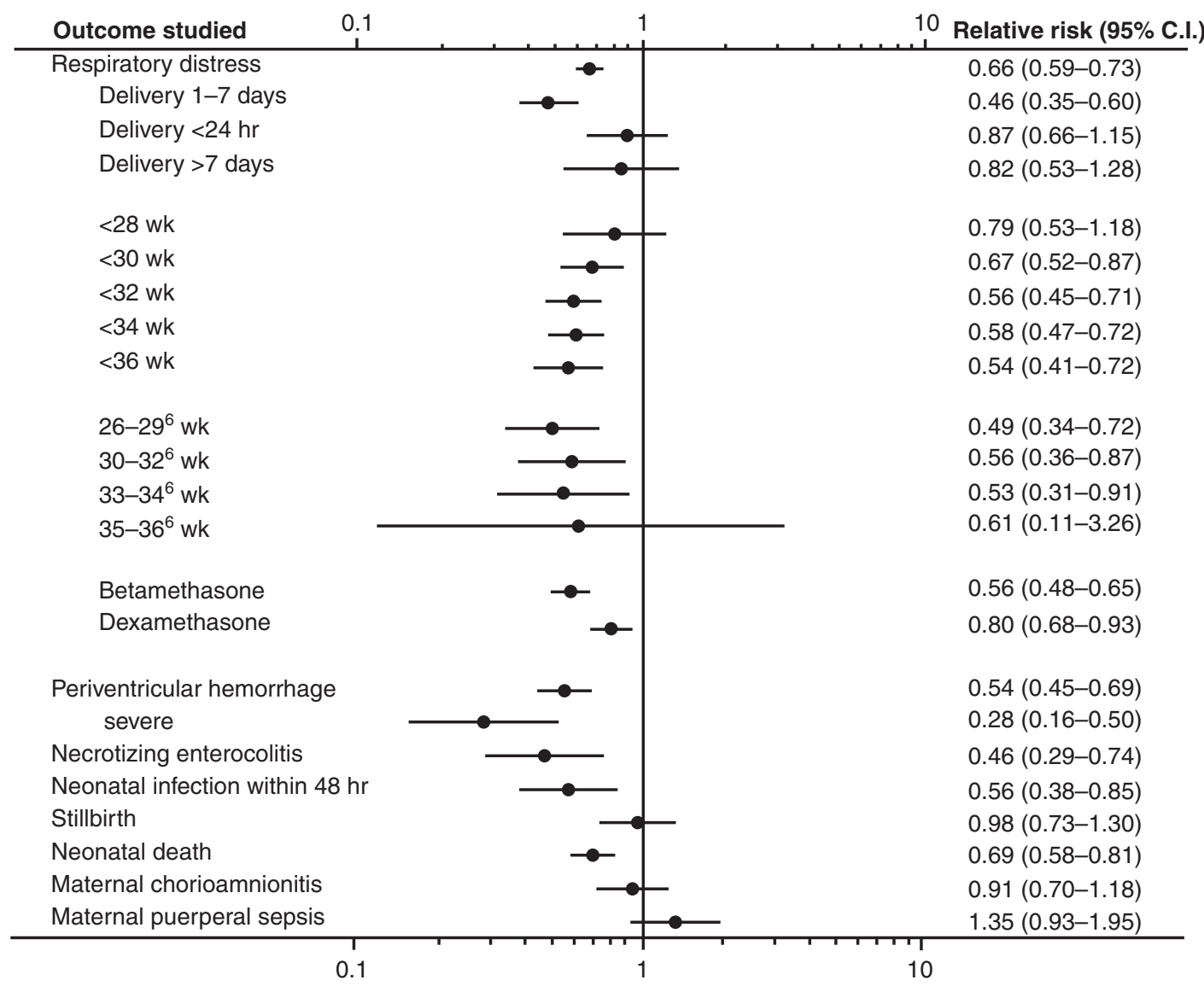

Figure 6 Impact of antenatal corticosteroids before anticipated preterm birth on perinatal outcomes. Abbreviation: C.I., confidence interval. Source: From Ref. 276.

distress syndrome, intraventricular hemorrhage, necrotizing enterocolitis, admission to neonatal intensive care unit, and neonatal infection within the first 48 hours of life (Fig. 6) (276). In addition, these benefits accrue without an increased risk of clinical chorioamnionitis, puerperal sepsis, or the risk of stillbirth.

The agents administered are either dexamethasone or betamethasone because they cross the placenta readily, are weaker immunosuppressors than other glucocorticoid steroids, and have a longer duration of action than cortisol. The standard dosage of betamethasone consists of two intramuscular 12-mg doses (24 hours apart), while four intramuscular doses of $6 \mathrm{mg}$ of dexamethasone should be administered at 6-hour intervals. Oral administration of dexamethasone is not recommended, because it has been associated with an increased risk of neonatal intraventricular hemorrhage (IVH) and sepsis compared with intramuscular administration $(278,279)$.

Betamethasone is preferred over dexamethasone because its administration results in a greater reduction in respiratory distress syndrome and puerperal infection (280) while the use of dexamethasone is associated with a lower frequency of intraventricular hemorrhage than betamethasone. However, most investigators believe that there is still equipoise between the two drugs $(255,276,280-283)$.

Glucocorticoids provide beneficial effects to patients between 24 and 33-6/7 weeks of gestation (276). Some data suggest that there are beneficial effects at 23 weeks of gestation (284), while more studies are required to establish efficacy between 34 and 36-6/7 weeks of gestation $(285,286)$.
Long-term follow-up studies have not uncovered complications in children exposed to a single course of antenatal steroids, and one study has followed these infants for 30 years (287).

Although many believe that the benefits of antenatal steroid administration occur after 24 hours of initiation of therapy, there is evidence that when delivery occurs between the first and second dose of betamethasone, there is still a reduction in the rate of neonatal death, intraventricular hemorrhage, and the need for vasopressors (288). Therefore, benefits accrue even if 48 hours have not elapsed since the administration of the first dose of steroids. On the other hand, the beneficial effects of antenatal corticosteroids are not demonstrable in babies born more than 7 days after treatment, and the birth weight is significantly reduced $(276,289)$.

Should Steroid Administration Be Repeated After 7 days? Several randomized clinical trials have been conducted to address this question. Moreover, a systematic review including five trials has been reported (290). The trial reported by Crowther et al. (291) (Australasian Collaborative Trial of Repeat Doses of Steroids) randomized 982 women (1146 neonates) at less than 32 weeks of gestation who had received a single course of antenatal corticosteroids to receive weekly betamethasone or placebo (291). Repeated corticosteroids led to a significant reduction in the rate of respiratory distress syndrome ( $33 \%$ vs. $41 \% ; p=0.01)$, severe lung disease $(12 \%$ vs. $20 \%$; $p=0.0003$ ), less frequent oxygen therapy ( $56 \%$ vs. $63 \% ; p=0.03)$, and shorter duration of mechanical ventilation ( $p=0.01)$ (291). 
In contrast, a randomized controlled trial conducted in the United States by Wapner et al. (292) randomly assigned 495 women at 23 weeks of gestation to receive either betamethasone or placebo weekly between 7 and 10 days after the initial dose of steroids. The primary outcome of the trial was neonatal morbidity as a composite score. There was no difference between those patients allocated to repeat steroids versus placebo ( $8 \%$ vs. $9.1 \% ; p=0.6$ ) (292). Neonates in the repeat steroid group had a significantly lower frequency of surfactant administration, mechanical ventilation, continuous positive airway pressure, and pneumothorax. However, the frequency of small for gestational age $(<10$ th percentile) was higher in infants exposed to repeated doses of steroids than in the control group. Importantly, the frequency of cerebral palsy was higher in neonates that received four or more courses of maternal corticosteroids, but this was not statistically significant $[2.4 \%(6 / 248)$ vs. $0.4 \%(1 / 238)](293)$. There were no differences in physical or neurocognitive outcomes in this trial. By contrast, Crowther et al. (294) noted a higher frequency of children with behavioral problems (e.g., attention deficit and emotional reactivity), but no differences in the frequency of cerebral palsy [repeat steroids, $4.2 \%(22 / 521)$ vs. $4.8 \%$ (25/528)].

There are several differences in these two trials. The dose in the study of Crowther et al. was $11.4 \mathrm{mg} /$ week of betamethasone, while the study of Wapner et al. used $24 \mathrm{mg} /$ week. Also, more women received four or more doses of steroids in the Wapner et al. trial than those in the Crowther et al. trial.

Murphy et al. (295) reported results of a randomized clinical trial of the multiple courses of antenatal corticosteroids for preterm birth. In this study, 1858 women between 25 and 32 weeks who were undelivered 14 to 21 days after the initial dose of steroids were randomized to repeat doses of steroids $(n=973)$ or placebo $(n=921)$ every 14 days until 33 weeks of gestation or delivery, whichever came first. The primary endpoint was a composite of perinatal morbidity and mortality. Infants exposed to multiple courses of steroids had similar morbidity and mortality rates to those allocated to placebo $(12.9 \%$ vs. $12.5 \%)$. In this study, exposure to steroids was associated with a lower birth weight, lower length at birth, and smaller head circumference. The authors concluded that multiple courses of steroids could not be recommended.

An individual patient meta-analysis is in progress but results are not available at the time of this writing. The American Congress of Obstetricians and Gynecologists has recommended that only one course of betamethasone (12 mg in two doses, 24 hours apart) should be given to women in preterm labor. In contrast, in Australia and New Zealand, repeat doses are given weekly to women at risk up to 32 weeks of gestation.

Another strategy for repeat steroids is what is referred to as "rescue" therapy. In this instance, repeat steroids are administered only to patients with impending delivery. In a study by Garite et al. (296), patients who were diagnosed to have an episode of preterm labor at less than 33 weeks and received a complete course steroids before 30 weeks more than two weeks ago were randomized to receive either a single "rescue" course of betamethasone (12 mg; 2 doses, 24 hours apart) or placebo. Patients with rupture of membranes, advanced cervical dilatation $(>5 \mathrm{~cm})$, and chorioamnionitis were excluded. Of the 433 patients randomized (223 to "rescue" steroids and 214 to placebo), 55\% in each group delivered at less than 34 weeks of gestation. In the rescue group there was a significant reduction in the primary outcome (composite neonatal morbidity below 34 weeks) (43.9\% vs. 63.6\%; OR 0.45; 95\% CI, 0.27-0.75; $p=0.002$ ). There was also a significant decrease in respiratory distress syndrome, ventilatory support, and surfactant use (296). This observation suggests that "rescue" therapy can be considered with an understanding that prediction of the patient who is at risk of impending delivery is difficult.

\section{TOCOLYTIC THERAPY}

The basic premise behind the use of tocolytic treatment is that the administration of pharmacologic agents can inhibit myometrial contractions, prolong pregnancy, and reduce the rate of neonatal morbidity and mortality. Intravenous alcohol was the first agent introduced to delay preterm delivery (297). Its use was accompanied by an unacceptable rate of maternal complications. Since that time, multiple pharmacologic agents have been used, including beta-adrenergic agents, magnesium sulfate, oxytocin receptor antagonists, calcium-channel blockers, nitroglycerin, and prostaglandin synthase inhibitors. Decades of research indicate that tocolysis can prolong pregnancy for 48 hours to 7 days (298-301). This prolongation of gestation is considered beneficial, because it allows the transfer of patients to a tertiary care facility and, importantly, the administration of corticosteroids. The hope that the use of tocolysis would reduce the rate of preterm birth has not been realized. It is possible that the ideal agent has not been identified. Alternatively, it is possible that tocolysis, as a strategy, would not succeed if a serious pathologic process which has led to activation of the myometrium and other components of the common pathway of parturition is not treated. For example, the administration of beta-adrenergic agents in patients with documented intrauterine infection is not effective in prolonging pregnancy and may result in an increased rate of maternal pulmonary edema $(205,242,243)$. Therefore, many studies of tocolysis are confounded by including patients who will not benefit from inhibition of uterine contractility.

\section{Beta-adrenergic Receptor Agonists}

The first and only agent approved for tocolysis in the United States by the Food and Drug Administration (FDA) was ritodrine, a selective beta-2 adrenergic agent. This drug is no longer marketed in the United States. This class of agents achieves myometrial relaxation by binding to the beta- 2 adrenergic receptor and increasing the levels of intracellular cyclic adenosine monophosphate, which, in turn, activates protein kinase, inactivates myosin light-chain kinase, and inhibits myometrial contractility (302-304). Agents in this class include terbutaline and fenoterol (4). A meta-analysis including 11 randomized clinical trials ( 1320 women) in which beta-adrenergic agents were compared with placebo indicates that these agents reduce the rate of delivery within 48 hours, but not within 7 days (300). There was no evidence of a significant decrease in perinatal or neonatal morbidity and mortality.

The use of beta-adrenergic agents is associated with maternal and fetal side effects. The most common are maternal and fetal tachycardia, tremor, headache, nausea, 
vomiting, hypokalemia (301), and hyperglycemia (300,301). Hypotension due to peripheral vasodilatation is a common problem (305). Maternal cardiac arrhythmias and myocardial ischemia have been reported during the course of tocolysis with these agents (306). The onset of chest pain should be taken seriously during the course of therapy. Because of metabolic effects (hyperglycemia) of beta-adrenergic agents, it is best to use other agents in patients with glucose intolerance. Neonatal side effects include hypoglycemia (4), hypocalcemia (4), and myocardial damage (case reports) (307).

There is no evidence that maintenance treatment with either oral or subcutaneous beta-adrenergic agents is beneficial. Some have used subcutaneous terbutaline administered via a pump; however, randomized clinical trials do not show evidence of efficacy $(308,309)$. Recently, the FDA has issued a black-label warning for terbutaline administration based upon reports of some maternal deaths associated with its use $(310,311)$. Fenoterol is used in Europe, and there is considerable experience with this agent in Germany.

A common practice in patients who present with preterm uterine contractions is to administer a single subcutaneous dose of terbutaline. Patients in whom contractions persist are at increased risk for preterm delivery (312).

\section{Magnesium Sulfate}

This tocolytic agent can cause a reduction in uterine contractility in vitro and in vivo (313). Magnesium hyperpolarizes the cellular membrane and inhibits myosin light-chain kinase activity by competing with intracellular calcium (314-316). Despite its wide utilization in the United States, the largest randomized clinical trial in which magnesium sulfate was compared with placebo concluded that magnesium sulfate was not effective in prolonging pregnancy (317). Meta-analyses of randomized trials in which magnesium was compared with placebo or other agents do not show differences in prolongation of pregnancy for 48 hours (318). Maternal and neonatal side effects of magnesium sulfate are well known since this drug is used to prevent seizures in pre-eclampsia. Common side effects include nausea and vomiting, a metallic taste in the mouth, lethargy, and hot flashes. Pulmonary edema has been reported in patients receiving this drug. Since magnesium crosses the placenta, prolonged administration can lead to hypermagnesemia in the neonate. A systematic review reported by Crowther et al. (318) in which more than 2000 women were included in 23 trials concluded that magnesium was not effective in preventing preterm delivery and was associated with an increased risk of death (fetal and pediatric) (RR 2.8; 95\% CI, 1.20-6.62). Grimes and Nanda have called for a discontinuation of magnesium sulfate as a tocolytic agent (319). There is no evidence that oral magnesium administration can prevent preterm birth or is effective for maintenance of tocolysis.

\section{Calcium-Channel Blockers}

These agents inhibit uterine contractility by blocking the calcium channels and, thereby, the influx of calcium through the cell membrane as well as the release of intracellular calcium from the sarcoplasmic reticulum $(320,321)$. Nifedipine is the most popular agent used as a tocolytic agent. Its use is based on comparative trials with beta-adrenergic agents.

A recent systematic review indicates that nifedipine is as effective as beta-adrenergic agents in delaying delivery for 48 hours (298). However, nifedipine was superior to betaadrenergic receptor agonists in delaying delivery of 7 days within initiation of therapy and also in improving clinically important neonatal outcomes such as respiratory distress syndrome, necrotizing enterocolitis, intraventricular hemorrhage, jaundice, admission to the neonatal intensive care unit (NICU), and length of stay in the NICU. Moreover, nifedipine use was associated with fewer maternal side effects and a lower rate of discontinuation of treatment than beta-adrenergic agents. Follow-up of infants exposed to either nifedipine or beta-adrenergic agents showed no difference in neurodevelopmental status at 2 years of age (322), or psychological or motor function at 9 to 12 years of age (323). There are insufficient data to assess the efficacy of nifedipine against oxytocin receptor antagonists or nitric oxide. There was no evidence that maintenance therapy with nifedipine was efficacious. The proposed dose of administration is $10 \mathrm{mg}$ orally or sublingually. If contractions persist, this dose can be repeated every 15 to 20 minutes up to a maximal total dose of $40 \mathrm{mg}$ during the first hour of treatment, then $20 \mathrm{mg}$ orally every 6 to 8 hours for 2 to 3 days. The side effects of nifedipine include headache, flushing, dizziness, hypotension, and nausea (324). Suppression of maternal heart rate, contractility, and left ventricular systolic pressure may also occur. Concurrent administration of magnesium with calcium-channel blockers may result in neuromuscular blockade and pulmonary edema $(325,326)$. However, a retrospective case-control study concluded that the use of nifedipine and magnesium sulfate together in patients with pre-eclampsia does not increase the risk of serious magnesium-related effects including neuromuscular blockade (327).

\section{Prostaglandin Synthase Inhibitors}

Prostaglandins are considered the universal mediators of labor and can induce uterine contractility and cervical ripening. The therapeutic target is the cyclooxygenase (COX) enzyme, which catalyzes the conversion of arachidonic acid to the intermediate product of prostaglandins, $\mathrm{PGH}$. There are two COX enzymes, COX-1 and COX-2. The former is the constitutive enzyme, and the second is the inducible enzyme. Indomethacin is a non-specific inhibitor of COX, while nimesulide is a specific inhibitor of COX-2. One of the randomized clinical trials in which indomethacin was compared with placebo concluded that indomethacin resulted in a reduction in the rate of preterm birth (defined as $<37$ gestational weeks) $(328,329)$. However, this trial included only 36 women, and there was no difference in neonatal outcome. Comparative trials (with either beta-adrenergic agents or magnesium sulfate) show that COX inhibitors reduced the rate of preterm birth before 37 weeks and the frequency of adverse events (329). However, there was no demonstrable improvement in neonatal outcome. Two studies comparing nonselected COX inhibitors versus COX-2 inhibitors did not demonstrate differences in maternal and neonatal outcome (329-331). It is recognized that the trials have been of small sample size.

Adverse events include nausea, vomiting, heartburn, gastrointestinal bleeding, and impairment of platelet function (prolonged bleeding time). Indomethacin should not be administered in patients with peptic ulcer, kidney, or liver 
disease. The drug crosses the placenta readily, and the most common side effects include stenosis of the fetal ductus arteriosus and oligohydramnios $(328,332)$. There is also a concern about impairment of platelet function in the neonates (333). However, a meta-analysis of 28 studies showed no significant differences in the rates of intraventricular hemorrhage, patent ductus arteriosus, necrotizing enterocolitis, and neonatal mortality between the two groups (334). The standard recommendation is not to use the agent after 32 weeks of gestation or longer than $50 \mathrm{mg}$ (4). Indomethacin is used as an acute tocolytic. The oral dose of indomethacin is a $50 \mathrm{mg}$ loading dose, followed by 25 to $50 \mathrm{mg}$ every 6 hours for 48 hours (total duration of treatment). The drug can also be administered rectally as a suppository.

\section{Nitric Oxide Donors}

Nitric oxide can induce relaxation of smooth muscle by increasing cyclic guanosine monophosphate, which inhibits myosin light-chain kinases. Nitroglycerin is a nitric oxide donor. The initial trials with nitroglycerin for tocolysis suggested that it was comparable with ritodrine (335). A randomized, double-blind, placebo-controlled trial was conducted in which patients $(\mathrm{n}=153)$ with preterm labor (between 24 and 32 weeks of gestation) were randomized to receive transdermal nitroglycerin $(0.4 \mathrm{mg} / \mathrm{h})$ or placebo patches (336). The primary endpoint of this study was a composite of neonatal morbidity and mortality. Infants born to mothers allocated to transdermal nitroglycerin had a significantly lower composite neonatal outcome compared with placebo (RR 0.29, 95\% CI 0.08-1.00; $p=0.048$ ). The number needed to treat was 10 . Preterm birth prior to 28 weeks of gestation was reduced by $50 \%$ (RR $0.50,95 \%$ CI 0.23-1.09). Patients receiving nitroglycerin had significantly more maternal side effects than those allocated to placebo. The most common side effects were headache and local irritation from the patch. Importantly, in this trial, none of the subjects received other tocolytics from randomization to delivery. Therefore, this is a unique trial in the history of tocolytic therapy. Long-term follow-up of infants enrolled in a previous trial of nitroglycerin patches versus beta-adrenergic agonists showed that there was no significant difference in psychometric testing at the age of 2 (337).

\section{Oxytocin Receptor Antagonists}

Oxytocin is a powerful stimulant of myometrial contractions. Its receptor is widely distributed in myometrium and decidua. Atosiban, an oxytocin receptor antagonist, can block spontaneous and oxytocin-induced uterine contractility. This agent is used widely in Europe, but is not available in the United States.

Comparative randomized clinical trials in which patients were allocated to beta-mimetic agents or atosiban indicated that atosiban had the same efficacy, but fewer maternal adverse events (338-341). These studies were conducted by a worldwide atosiban versus beta-adrenergic agonists study group and included comparisons of this agent with ritodrine, salbutamol, and terbutaline. Tocolytic effectiveness was similar in terms of gestational age at delivery, but maternal side effects, particularly cardiovascular adverse events, were less frequent in patients allocated to atosiban than in patients allocated to beta-adrenergic agents $(8.3 \%$ vs. $81.2 \%, p<0.001)(341)$. This was associated with a lower rate of discontinuation of treatment due to side effects $(1.1 \%$ vs. $15.4 \%, p=0.0001)$.

A large randomized clinical trial in which patients in preterm labor were allocated to either atosiban- or placebo-initiated treatment showed that atosiban-treated patients were significantly less likely to deliver within 24 hours, 48 hours, and 7 days than patients with placebo-initiated treatment (342). There was no difference in the interval from enrollment to delivery between the two groups. In this study, the rate of fetal/infant death was greater in patients allocated to atosiban than placebo. This was attributed to an excess number of patients with gestational ages less than 24 weeks allocated to the atosiban arm compared with the placebo-initiated treatment. Similarly, there was an excess of infection-associated preterm labor and delivery in patients allocated to atosiban. This concern for safety was probably the reason the FDA did not approve atosiban in the United States. Another randomized clinical trial in which patients with an episode of preterm labor were treated with atosiban and subsequently randomized to either maintenance therapy with atosiban (subcutaneous pump) or placebo showed that maintenance therapy was not associated with a reduction in the rate of preterm delivery (343). However, in this trial, prolonged exposure to atosiban did not result in an excess of fetal or infant death. This finding, coupled with the extensive experience in Europe with atosiban, makes it unlikely that there is a toxic effect of atosiban on the fetus.

\section{Antibiotics in Preterm Labor}

Several randomized clinical trials have tested the effect of antibiotics versus placebo in patients with an episode of preterm labor (344-354). A systematic review of such intervention demonstrated that antibiotic administration (ampicillin and/or erythromycin) does not reduce the rate of preterm delivery or neonatal complications. The largest trial conducted to date is the ORCALE II trial (346), in which 6295 women were randomized to placebo, amoxicillin/clavulanic acid, erythromycin, and the combination of amoxicillin/clavulanic acid and erythromycin.

The study that followed the infants enrolled in the ORACLE II trial at the age of 7 years indicated that those exposed to antibiotics have an increased rate of cerebral palsy, which was modest in magnitude, but statistically significant (355). This unexpected observation suggests that antibiotic administration to women without demonstrable infection could cause harm. However, the precise mechanism by which antibiotic administration can cause neurologic injury is unknown. One possibility is that antibiotics may cause the release of microbial products, which could initiate an inflammatory reaction, and change the nature of the host-microbial interaction in normal pregnancy and fetal life. These observations, and the lack of efficacy of adjuvant antibiotics in the prevention of preterm delivery and neonatal morbidity, suggest that antibiotics should not be used in patients with preterm labor and intact membranes. However, this does not apply to the prevention of vertical transmission of GBS. Antibiotic administration is effective in reducing the rate of neonatal GBS sepsis and should be used in patients at risk (356-361). The recommended treatment is penicillin G 5 million units intravenously, followed by 2.5 to 3 million units every 4 hours. Patients allergic 
to penicillin can receive either clindamycin (900 mg IV every 8 hours) or erythromycin (500 mg IV every 6 hours), if the isolate microorganism is susceptible to clindamycin or erythromycin, or vancomycin (1g IV every 12 hours) if the isolate microorganism is resistant to the clindamycin or erythromycin or in cases of unknown susceptibility (362).

\section{Magnesium Sulfate for Neuroprotection}

Preterm delivery is a major risk factor for cerebral palsy. Several randomized clinical trials have been conducted to test the effect of magnesium sulfate in the prevention of cerebral palsy after a report by Nelson and Grether (363) suggesting that in utero exposure to magnesium sulfate was associated with a lower rate of cerebral palsy in infants born weighing less than $1500 \mathrm{~g}$.

A systematic review of six trials (364), involving 4796 women and 5357 infants, indicated that antenatal magnesium sulfate was associated with a significant reduction in the risk of cerebral palsy (RR $0.69,95 \%$ CI $0.55-0.88$ ), moderate or severe cerebral palsy (RR $0.64,95 \%$ CI $0.44-0.92$ ), and substantial gross motor dysfunction (RR $0.60,95 \%$ CI $0.43-0.83$ ). There was no overall difference in the risk of total pediatric mortality (RR 1.01, 95\% CI 0.89-1.14). Minor side effects were more frequent among women receiving magnesium sulfate. Therefore, we recommend the use of magnesium sulfate for neuroprotection in women at high risk for preterm delivery before 34 weeks of gestation. The loading dose of magnesium sulfate is $6 \mathrm{~g}$ and the maintenance dose 1 to $2 \mathrm{~g} / \mathrm{h}$, for 24 hours. This treatment should be restricted to patients expected to deliver within 24 hours. It has been used in patients with both intact and ruptured membranes. Using this approach, it has been estimated that the incremental cost of preventing one case of cerebral palsy would be approximately \$10,291 (95\% CI \$6135-\$29,685) (364).

\section{Prediction of Spontaneous Preterm Delivery}

The risk factors associated with preterm delivery/preterm PROM (3) include a sonographic short cervix (2,166-169, 173,174,247), African-American race (365-368), and a prior history of preterm delivery $(2,369)$. There is conflicting evidence of the efficacy of antibiotic treatment for BV in women with a prior preterm birth. Some studies suggest a benefit to the use of antibiotics for these patients (370-372) and others have not shown this benefit (373-376). A systematic review of seven randomized clinical trials demonstrated no evidence of benefit for screening or treating low-risk pregnant women who are asymptomatic for BV. Furthermore, they found no advantage to screening for and treating $\mathrm{BV}$ in the general population of pregnant women who are asymptomatic for BV (373).

Fetal fibronectin has been used in the evaluation of asymptomatic women to predict their risk of preterm delivery. Numerous meta-analyses $(176,184,377-380)$ have concluded that cervicovaginal fetal fibronectin testing might be clinically useful in the prediction of preterm birth, with an emphasis given to the high negative predictive value of the test, particularly in women who are symptomatic of threatened preterm labor and for delivery within 7 to 10 days of sampling. Kurtzman et al. (381) have proposed the use of quantitative fetal fibronectin screening in asymptomatic women with a prior preterm delivery. This group demonstrated that quantitative fetal fibronectin assessment at 24 weeks of gestation could be used to predict the risk of spontaneous preterm delivery before 34 weeks of gestation, and that this risk increased as the quantity of fetal fibronectin (FFN) increased [compared with an FFN $=0$ ng/mL; RR 2.42 (FFN 1-49ng/mL; 95\% CI 0.76-5.66), 4.68 (FFN 50-199 ng/mL; 95\% CI 1.28-10.95), and 9.94 (FFN $>200 \mathrm{ng} / \mathrm{mL}$; 95\% CI 2.90-19.67)] (381).

Recently, Conde-Agudelo and Romero conducted a systematic review and meta-analysis of 15 studies investigated the accuracy of cervicovaginal fetal fibronectin in predicting preterm birth in women with multiple gestations (382). This study demonstrated that among asymptomatic women with multiple or twin pregnancies, the pooled sensitivities, specificities, and positive and negative likelihood ratios for predicting preterm birth before 32, 34, and 37 weeks of gestation ranged from $33 \%$ to $45 \%, 80 \%$ to $94 \%, 2.0$ to 5.5 , and 0.68 to 0.76 , respectively. Among women with twin pregnancies and threatened preterm labor, the test was most accurate in predicting spontaneous preterm birth within 7 days of testing (pooled sensitivity, specificity, and positive and negative likelihood ratios of $85 \%, 78 \%, 3.9$, and 0.20 , respectively).

These findings provided evidence that cervicovaginal fetal fibronectin has limited accuracy in predicting spontaneous preterm birth in both asymptomatic and symptomatic women with multiple pregnancies because the likelihood ratios for positive and negative test results generated only minimal to moderate changes in the pretest probabilities of preterm birth. The test is most accurate in predicting spontaneous preterm birth before 32 weeks of gestation in asymptomatic women with multiple or twin pregnancies, and spontaneous preterm birth within 7 days of testing in women with twin pregnancies and threatened preterm labor (382).

\section{A Short Sonographic Cervical Length: a Powerful Predictor of Preterm Delivery}

Cervical sonography is the most objective and reliable method to assess cervical length $(168,172-174,383,384)$. Furthermore, cervical length is the most powerful predictor of spontaneous preterm delivery. The shorter the sonographic cervical length in the midtrimester, the higher the risk of spontaneous preterm labor/delivery $(168,173,174,383)$. There is no agreement as to what is a sonographic short cervix. For example, Iams et al. (174) proposed that a cervix of $26 \mathrm{~mm}$ or shorter at 24 weeks of gestation increases the risk for spontaneous preterm delivery (RR 6.19, 95\% CI 3.84-9.97). The prevalence of spontaneous preterm delivery (defined as $<35$ weeks) in this study was $4.3 \%$, and the positive predictive value was $17.8 \%$ for a cervical length of $\leq 25 \mathrm{~mm}$ at 24 weeks of gestation (174). Thus, most women with a short cervix (defined as $\leq 25 \mathrm{~mm}$ ) and no history of previous preterm birth will not deliver a preterm neonate. Other investigators have proposed a cut-off of $15 \mathrm{~mm}(173,383)$, because a cervical length of $15 \mathrm{~mm}$ or less is associated with nearly a $50 \%$ risk of spontaneous preterm delivery at 32 weeks of gestation or less, when neonatal morbidity is substantial $(171,173,219,247,383,385-390)$. To et al. (385) have developed a method to assess the risk of preterm delivery for individual patients using sonographic 
cervical length and other maternal risk factors such as maternal age, ethnic group, body mass index, cigarette smoking, and previous cervical surgery. Importantly, sonographic cervical length is the single most powerful predictor for preterm birth in the index pregnancy $(172,384)$ and is far more informative than a history of previous preterm birth $(172,385,391)$. This has implications in the selection of patients for future trials and the interpretation of past trials. Specifically, all trials conducted to date (392-396), execpt one (397), have identified patients for study based upon a history of previous preterm birth. As much as preterm birth is a syndrome, it is likely that the risk conferred by a previous preterm delivery may vary within and between populations. This may explain the apparent contradictory results between trials [i.e., conflicting reports of trials of vaginal progesterone $(395,396)$ and the wide range of preterm birth among control groups (394-396)].

Sonographic cervical length is not a screening test for spontaneous preterm delivery because only some of the patients who will have a spontaneous preterm birth have a short cervix in the midtrimester. However, sonographic cervical length is a method for risk assessment for spontaneous preterm delivery. Its importance derives from the observation that, as a fraction of patients with a history of previous preterm birth who shorten their cervix during the index pregnancy may benefit from therapeutic cervical cerclage $(398,399)$, patients with a short cervix may benefit from vaginal progesterone administration to reduce the rate of spontaneous preterm birth (397).

Cervical ultrasound is a powerful tool in performing risk assessment for spontaneous preterm birth. It is simple to perform, inexpensive when performed at the time of secondtrimester screening for anomalies, informative, and can provide an estimate of risk in primigravidae. We believe that measuring cervical length should be part of standard sonographic examination in the midtrimester. Other tools may help to refine the estimation of risk. Such tools can range from vaginal fibronectin $(219,400)$, the collascope $(165,401-403)$, amniotic fluid analysis $(220,226,227,235,238,404-407)$, and the presence/absence of "amniotic fluid sludge" $(408,409)$, to genetic analysis of DNA variants of the progesterone receptor (410-413). These may help determine patients who will respond to progestogens or other treatments and those who will not and realize "personalized perinatal medicine" in the 21 st century.

\section{Amniotic Fluid "Sludge"}

Particulate matter in the amniotic fluid is present in about $4 \%$ of pregnancies during transvaginal ultrasound in the first and early second trimesters (414). Particulate matter in the first two trimesters of pregnancy has been associated with intraamniotic bleeding $(415,416)$ and the acrania-anencephaly sequence (417), and has been observed in women with high concentrations of maternal serum $\alpha$-fetoprotein (418). In contrast, in the last trimester of pregnancy, particulate matter and "echogenic amniotic fluid" have been attributed to the presence of vernix caseosa and/or meconium (419-422), and with a lecithin-sphingomyelin ratio indicative of lung maturity $(423,424)$.

Amniotic fluid "sludge" is defined as particulate matter seen in the proximity of the internal cervical os during a transvaginal sonographic examination of the cervix and occurs in $1 \%$ of uncomplicated pregnancies (408) (Fig. 7). The first description of amniotic fluid sludge was in patients in preterm labor. Espinoza et al. (408) conducted a retrospective study of 84 patients in preterm labor with intact membranes. The prevalence of amniotic fluid sludge was $22.6 \%$ (19 of 84 ) in patients with preterm labor. Patients with amniotic fluid sludge had a higher frequency of positive amniotic fluid cultures $[33.3 \%$ (6 of 18$)$ vs. $2.5 \% \quad(1 / 40), p=0.003]$ and histological chorioamnionitis $[77.8 \%$ (14 of 18 ) vs. $19 \%$ (11 of 58$), p<0.001]$, and a higher rate of spontaneous preterm delivery within 48 hours [42.9\% (6 of 14) vs. $4.4 \%$ ( 2 of 45$), p=0.001]$, within 7 days [71.4\% (10 of 14) vs. $115.6 \%$ (7 of 45 ), $p<0.001$ ], less than 32 weeks [75\% ( 9 of 12 ) vs. $25.8 \%$ ( 8 of 31$), p=0.005$ ], and less than 35 weeks [ $92.9 \%$ (13 of 14 ) vs. $37.8 \%$ (17 of 45 ), $p<0.001$ ] than those without amniotic fluid "sludge." Stepwise logistic regression analysis indicated that the presence of sludge was independently associated with the likelihood of spontaneous delivery within 48 hours and 7 days, but not less than 32 weeks or less than 35 weeks. Survival analysis demonstrated that patients with amniotic fluid "sludge" had a shorter examination-to-delivery interval compared with those without "sludge" [sludge median: 1 day

(interquartile range: 1-5 days) versus no sludge, median: 33 days (interquartile range: $18-58$ days); $p<0.001]$. These results indicate that amniotic fluid "sludge" during transvaginal examination of the cervix is a risk factor for intraamniotic infection, histological chorioamnionitis, and impending preterm delivery (408).

The presence of amniotic fluid "sludge" on transvaginal examination in asymptomatic patients is also associated with delivery within 14 days of ultrasound and preterm delivery at less than 32 and less than 34 weeks $(409,425)$. Amniotic fluid sludge has also been identified as an independent risk factor for spontaneous preterm delivery at less than 28 weeks, less than 32 weeks, and less than 35 weeks of gestation; and for preterm PROM; intra-amniotic infection; and histological chorioamnionitis (409). Furthermore, asymptomatic patients with amniotic fluid sludge had shorter ultrasound-to-delivery and ultrasoundto-preterm PROM intervals than those without sludge $(409,425)$.

Recently, amniotic fluid sludge has been demonstrated to represent a biofilm $(426,427)$. A sample of sludge was retrieved by transvaginal amniotomy under ultrasound guidance from a patient at 28 weeks with spontaneous labor and clinical chorioamnionitis. Grossly, the sludge had a "pus-like" appearance and the Gram stain showed gram-positive bacteria. The amniotic fluid culture was positive for Streptococcus mutans, Mycoplasma hominis, and Aspergillus flavus. Of interest, the results of the amniocentesis performed at the time of admission for preterm labor were negative for intraamniotic infection $(426,427)$. The aspirated sludge was then analyzed further. Scanning electron microscopy showed flocs of amniotic fluid sludge that consisted of bacterial cells and the exopolymeric matrix material that are typical of a biofilm (Fig. 8) (427). The evidence that sludge represents a biofilm in this case includes the following: (i) the presence of bacteria detected by fluorescence in situ hybridization, with the use of a probe against the conserved sequence of prokaryotes; (ii) bacterial aggregates were separated by material that resembled 


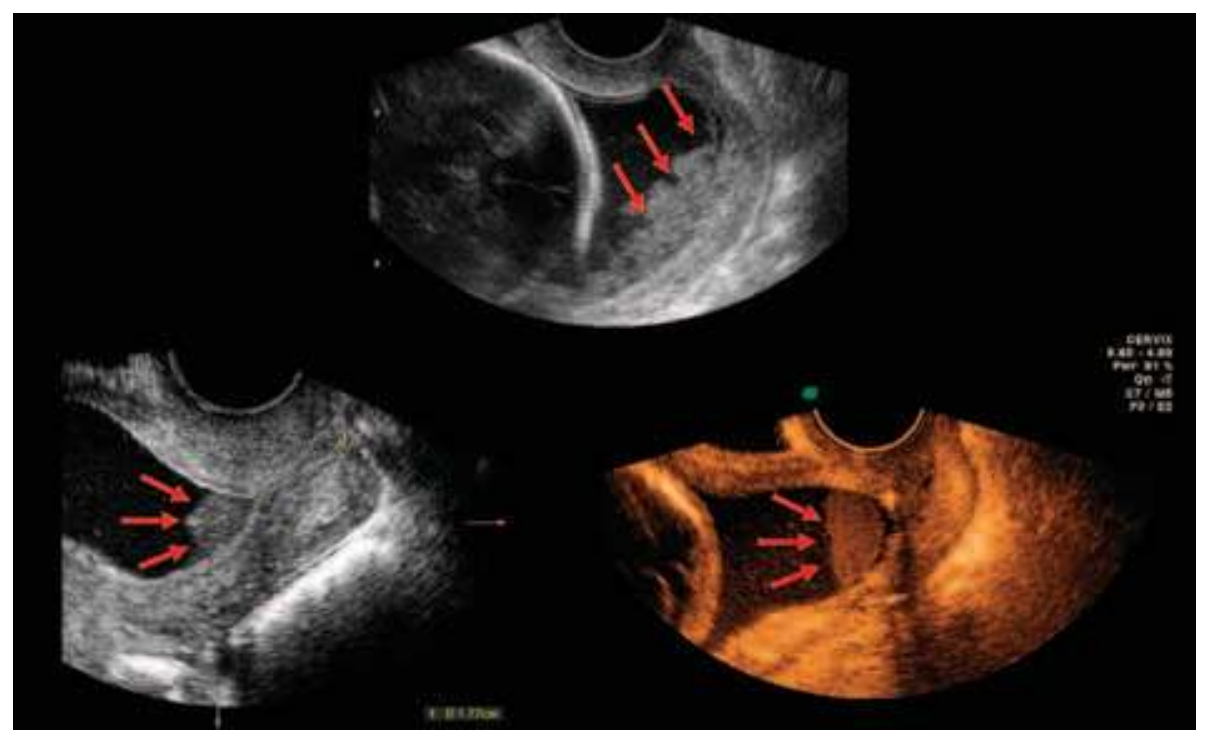

Figure 7 Amniotic fluid "sludge" seen in proximity of the internal cervical os during a transvaginal sonographic examination of the cervix. Source: From Ref. 505.

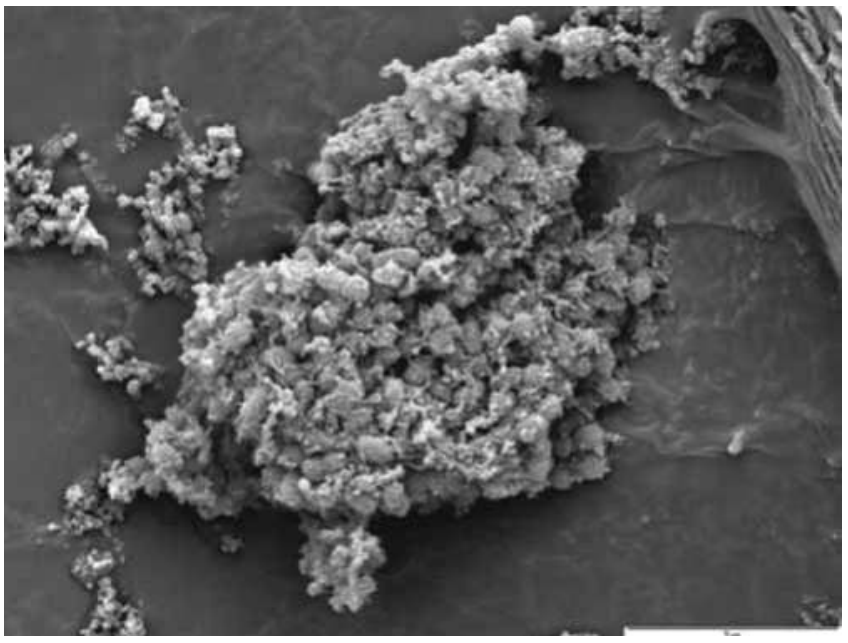

Figure 8 Examination of sludge using scanning electron microscopy. Source: From Ref. 427.

a matrix; and (iii) lectin-based identification of exopolymeric matrix that stained with wheat germ agglutinin (427).

The clinical significance of this relates to the challenges encountered with diagnosis and treatment of this condition. First, the diagnosis of microbial invasion in the presence of biofilms is extremely challenging, and current cultivation techniques are inadequate to detect such infections. The consequences are that the frequency of infection of the amniotic cavity may be underestimated and that molecular microbiologic techniques will be required to improve diagnosis. Second, the optimal treatment of biofilm-related infections represents a challenge in clinical medicine. Antimicrobial agents appear to be inactivated or fail to reach bacteria within a biofilm. Interestingly, bacteria within biofilms have increased resistance to antimicrobial compounds even though the same bacteria can be sensitive to the same agent if grown under standard conditions (428-431). Thus, the difficulties in treating intra-amniotic infection may be due to the refractoriness of biofilms to conventional antibiotic treatment. Third, biofilms in the amniotic fluid may represent a unique form of these structures, which can be dislodged by fetal movement, resulting in seeding of planktonic bacteria and the eliciting of an inflammatory response.

\section{Progestogens for the Prevention of Preterm Birth}

The importance of progesterone in the maintenance of mammalian pregnancy is well-established $(90,97,116,432-446)$, and the suspension of progesterone action is believed to be central to the initiation of parturition in most mammalian species, including primates $(90,97,410,436,438,442,443,447)$. Yet the precise mechanism for this in humans has not been elucidated (90,97,98,436,439,443,448-451).

Progestogen (a term that, like progestins, describes both "natural" progesterone and synthetic compounds with progesterone action) (452) administration to prevent spontaneous abortion (453-465) and preterm birth (392-395,397, 454,466-469) has been a subject of investigation for several decades. The use of progesterone in the first trimester of pregnancy to "support corpus luteum function" is a well-established clinical practice $(453,455,456,460,461,463,465,470)$, and formulations of progesterone for this indication have been approved by the FDA (http://www.accessdata.fda.gov/scripts/ cder/drugsatfda/index.cfm) and regulatory agencies in Europe and other countries (471).

The results of the early trials for the use of progestogens for the prevention of preterm birth or recurrent abortion were contradictory; as were the meta-analyses of such trials $(470,472,473)$. For example, the meta-analysis of Goldstein et al. (473) included trials employing several progestational agents and could not demonstrate a beneficial effect of progesterone on the prevention of preterm birth. The metaanalysis of Professor Marc Keirse (472) focused only on studies of 17 alpha-hydroxyprogesterone caproate and examined its effect on different endpoints: "early curtailment of gestation, whether by miscarriage ( $<20$ weeks or $<500 \mathrm{~g})$ or by preterm birth ( $<37$ weeks)." Despite these encouraging results, little clinical research on the subject was conducted for more than a decade. 


\section{The Use of Progestogens in Women with a Prior History of Preterm Birth}

In 2003, two trials were reported in women with a prior history of preterm birth. The first study Fonseca et al. (395) randomized 142 women with risk factors for preterm birth to receive $100 \mathrm{mg}$ vaginal progesterone or placebo between 24 and 34 weeks of gestation (395). In this trial, the rate of preterm delivery at both $<37$ and $<34$ weeks was significantly lower in women allocated to progesterone treatment than to placebo $[<37$ weeks, progesterone $13.8 \%(10 / 72)$ vs. placebo $28.5 \%$ (20/70), $p=0.03$; and $<34$ weeks, progesterone $2.8 \%$ (2/72) vs. placebo $18.6 \%(13 / 70), p=0.002]$.

Another trial (by Meis et al.) (394) evaluated the use of weekly injections of 17 alpha-hydroxyprogesterone caproate (250 mg IM between 16 and 36 weeks, $\mathrm{n}=310$; and placebo, $\mathrm{n}=153$ ) in women with a prior preterm birth. The authors reported a reduction in the rate of preterm birth before 37 (36.3\% vs. $54.9 \%$, RR $0.66,95 \%$ CI $0.54-0.81)$ and 35 (20.6\% vs. $30.7 \%$, RR $0.67,95 \%$ CI $0.48-0.93$ ) weeks of gestation.

The largest randomized clinical trial in which patients with a history of a previous spontaneous preterm delivery were allocated to receive vaginal progesterone $(90 \mathrm{mg} /$ day $)$ in a bioadhesive formulation/gel or placebo was conducted by O'Brien et al. (396) The primary endpoint for the trial was preterm delivery ( $\leq 32$ weeks). All patients had transvaginal sonography to determine the cervical length at enrollment and at 28 weeks. Randomization-to-delivery interval in patients who received tocolytic therapy for preterm labor or shortening of the cervix was considered a secondary outcome.

This multinational trial involved 53 centers and 659 women with a singleton pregnancy who were randomized, between 18 and 22 completed weeks of gestation, to receive daily treatment with vaginal progesterone gel or placebo (396). Progesterone or placebo was self-administered either until delivery, 37 weeks of gestation, or the occurrence of premature rupture of membranes. Vaginal progesterone did not reduce the rate of preterm birth at $\leq 32, \leq 35$, or $\leq 37$ weeks of gestation. Moreover, there was no difference in neonatal and maternal outcomes. The results of this study conflict with those of the initial trial reported by Fonseca et al. (395).

\section{The Use of Progestogens to Prevent Preterm Birth in Multiple Gestations}

Multiple gestation is a risk factor for preterm birth $(369,474$ 486). Uterine overdistention has been implicated as a mechanism responsible for the excess rate of preterm labor in this subset of patients $(22,487,488)$. Progesterone downregulates the expression of contraction-associated proteins (113-115,489-491); therefore, it is possible that progestogens may reduce the rate of preterm birth in multiple gestations. Consequently, investigators have tested whether progestogens can prevent preterm birth in these pregnancies $(397,466,468)$.

More than 25 years ago, Hartikainen-Sorri et al. (466) reported a double-blind, placebo-controlled clinical trial of 17 alpha-hydroxyprogesterone caproate administration in 77 women with twin pregnancies enrolled between 28 and 33 weeks of gestation. Weekly intramuscular injection of $250 \mathrm{mg}$ of 17 alpha-hydroxyprogesterone caproate or placebo were initiated at the time of enrollment and discontinued at 37 weeks of gestation or when delivery occurred before term. The administration of 17 alphahydroxyprogesterone caproate did not reduce the rate of preterm birth ( $<37$ weeks) or perinatal morbidity.

The issue has been revisited recently. Rouse et al. (468) reported a multicenter, placebo-controlled, double-blind, randomized clinical trial of 17 alpha-hydroxyprogesterone caproate for prevention of preterm birth in twin pregnancies, which included 655 women enrolled between 16 and 20 completed weeks of gestation. Patients were allocated to receive weekly injection of $250 \mathrm{mg}$ of 17 alpha-hydroxyprogesterone caproate or placebo until 34 completed weeks of gestation or delivery. The primary outcome was a composite of fetal death or delivery before 35 completed weeks of gestation, which occurred in $41.5 \%$ (135/325) of patients in the 17 alphahydroxyprogesterone caproate group and in $37.3 \%(123 / 330)$ of those in the placebo group [RR 1.1 (95\% CI 0.9-1.3)]. No benefit to the use of 17 alpha-hydroxyprogesterone caproate was demonstrated in twins.

In a sub-analysis of their randomized trial of vaginal progesterone versus placebo in 24 women with twin gestations and a short cervix $(\leq 15 \mathrm{~mm})$, Fonseca et al. (397) reported that progesterone administration was associated with a nonsignificant reduction in the rate of preterm delivery.

Norman et al. (492) reported the results of a randomized clinical trial of vaginal progesterone in 500 women with twin gestations. The use of vaginal progesterone beginning at 24 weeks of gestation was not associated with a reduction in the risk of preterm delivery before 34 weeks of gestation. Collectively, the results of these studies indicate that prophylactic progesterone administration does not reduce the rate of preterm delivery in women with twin pregnancies.

Recently, Caritis et al. (493) reported that the use of 17 alpha-hydroxyprogesterone caproate in triplet gestations did not reduce the rate of preterm delivery.

\section{Sonographic Cervical Length to Identify the Patients who may Benefit from Progesterone Treatment}

Facchinetti et al. (494) reported the results of a randomized prospective clinical trial in which women with preterm labor and intact membranes (25 to 33-6/7 weeks) were allocated to either observation or intramuscular administration of $341 \mathrm{mg}$ of 17 alpha-hydroxyprogesterone caproate twice a week until 36 weeks of gestation or delivery, and sonographic cervical length measured at discharge as well as at 7 (7-9) and 21 (18-21) days later. Patients allocated to receive 17 alphahydroxyprogesterone caproate had a longer sonographic cervical length than those in the observation group. These findings, coupled with experimental data, suggest that progesterone may have major effects in the uterine cervix.

Two clinical lines of evidence support that cervical status may identify the patient who could benefit from progestogen administration $(397,495)$. First, a recent randomized clinical trial reported by Fonseca et al. (397) indicates that vaginal progesterone reduces the rate of preterm birth by $44 \%$ (from $34.4 \%$ in the placebo group to $19.2 \%$ in the progesterone treatment group) in women with a sonographic short cervix. Second, a secondary analysis of a study by DeFranco et al. (495) suggests that patients with a short cervix may benefit from vaginal progesterone administration. 
Fonseca et al. (397), working with the Fetal Medicine Foundation Second Trimester Screening Group, conducted a randomized, double blind, placebo-controlled trial in which women with a short cervix $(\leq 15 \mathrm{~mm}$ by transvaginal ultrasound), between 20 and 25 weeks of gestation, were allocated to daily vaginal administration of $200 \mathrm{mg}$ of micronized progesterone or placebo (safflower oil) from 24 to 34 weeks. The frequency of spontaneous preterm delivery at $<34$ weeks (primary endpoint for the trial) was significantly lower in the progesterone group than that in the placebo group [19.2\% $(24 / 125)$ vs. $34.4 \%(43 / 125) ; p=0.007]$. A secondary analysis of this trial indicated that among women without a history of delivery before 34 weeks, the incidence of preterm birth was significantly lower in women receiving progesterone than in those allocated to placebo $[17.9 \%(20 / 112)$ vs. $31.2 \%(34 / 109)$; RR $0.57,95 \%$ CI $0.35-0.93 ; p=0.03)]$. The trial was not designed to test whether progesterone administration could reduce neonatal morbidity and such a reduction was not observed (397).

DeFranco et al. (495) reported a retrospective analysis of the effect of vaginal progesterone on pregnancy outcome (preterm birth and infant outcome) as a function of cervical length. The hypothesis for this study was that the effect of prophylactic vaginal progesterone may vary according to cervical length. To test this concept, all patients eligible to participate in the trial of O'Brien et al. (396) had a sonographic measurement of the cervix at the time of enrollment. Patients without a previous preterm birth and a cervical length of $\leq 25 \mathrm{~mm}$ were subjected to a separate randomization procedure (to vaginal progesterone or placebo) and excluded from the main trial. The rationale for this was that the main trial tested the effect of vaginal progesterone on patients with a history of preterm delivery regardless of cervical length. However, after the completion of the main trial, only nine patients had been enrolled in the "short cervix only" arm of the trial. Analysis of the effect of progesterone on nine patients with a short cervix was not meaningful. Therefore, the investigators modified their initial plan so that the analysis included women enrolled in the main trial (and therefore, with a history of previous preterm birth). The authors divided the patient population into quartiles according to cervical length at enrollment and tested whether outcomes were different as a function of cervical length (the lowest quartile was a cervical length of $\leq 32 \mathrm{~mm}$ ). Although there was a delay in delivery, progesterone administration did not result in a significant difference in outcome in patients in the lowest quartile.

The authors (495) then explored the effect of vaginal progesterone as a function of cervical length using two new cut-offs: $\leq 30$ and $<28 \mathrm{~mm}$. For the cut-off of $30 \mathrm{~mm}$, there was a trend for a longer randomization-to-delivery interval in women allocated to progesterone than those allocated to placebo (Wilcoxon $p=0.043$, log-rank $p=0.057$ ) (432). However, there was no difference in the frequency of preterm delivery at $\leq 32$ weeks.

With the second cut-off $(<28 \mathrm{~mm})$, patients who had received vaginal progesterone had a lower rate of spontaneous preterm delivery at $\leq 32$ weeks of gestation. Though this was not observed for preterm delivery defined as $\leq 35$ weeks or $\leq 37$ weeks. It is noteworthy that the frequency of newborn intensive care unit admission was lower in women with a cervical length of $\leq 30$ and $<28 \mathrm{~mm}$ and who had received progesterone treatment than in those allocated to the placebo group. The same was the case for the duration of newborn intensive care unit length of stay (495). This analysis provided the first hint that vaginal progesterone administration may improve infant outcome in properly selected patients. It is important to stress, however, that these conclusions were derived from a secondary analysis that was intended to be hypothesis-generating (496).

Hassan et al. (497) conducted a multicenter, randomized, placebo-controlled trial that enrolled 465 asymptomatic women with a singleton pregnancy and a sonographic short cervix (10-20 mm) at 19 to 23-6/7 weeks of gestation. Women were randomly allocated to receive vaginal progesterone gel $(\mathrm{n}=235)$ or placebo $(\mathrm{n}=223)$ daily from 20 to $23-6 / 7$ weeks to $36-6 / 7$ weeks, preterm rupture of membranes, or delivery, whichever occurred first. The primary endpoint was preterm birth before 33 weeks of gestation. Women allocated to receive vaginal progesterone had a lower risk of preterm birth before 33 weeks than those allocated to placebo $[8.9 \%(n=21)$ vs. $16.1 \%(\mathrm{n}=36)$, relative risk $(\mathrm{RR}) \quad 0.55,95 \%$ confidence interval (CI) $0.33-0.92, p=0.02]$. The effect remained significant after adjustment for co-variables (adjusted RR 0.52, $95 \%$ CI $0.31-0.91, p=0.02$ ). Vaginal progesterone was also associated with a significant reduction in the risk of preterm birth before 28 ( $5.1 \%$ vs. $10.3 \%$, RR $0.50,95 \%$ CI $0.25-0.97$, $p=0.04)$ and 35 weeks ( $14.5 \%$ vs. $23.3 \%$ RR $0.62,95 \%$ CI $0.42-0.92, p=0.02)$, respiratory distress syndrome $(3.0 \%$ vs. $7.6 \%$ RR $0.39,95 \% \mathrm{CI} 0.17-0.92, p=0.03)$, any morbidity or mortality event ( $7.7 \%$ vs. $13.5 \%$ RR $0.57,95 \%$ CI $0.33-0.99$, $p=0.04)$, and birth weight $<1500 \mathrm{~g}[6.4 \%(15 / 234)$ vs. $13.6 \%$ (30/220), RR 0.47, 95\% CI 0.26-0.85, $p=0.01]$. There were no differences in the incidence of adverse events between the groups. The conclusion of this study was that the administration of vaginal progesterone gel to women with a sonographic short cervix in the midtrimester is associated with a $45 \%$ reduction in the risk of preterm birth before 33 weeks of gestation and improved neonatal outcomes.

In conclusion, patients with a previous history of spontaneous preterm delivery can be offered 17 alpha-hydroxyprogesterone caproate for the prevention of recurrent preterm delivery (394). It is important to counsel the patients following the determinations of the FDA indicating that this agent reduces the risk of preterm birth in subsequent pregnancies (394), but there is no solid evidence of improvement in neonatal outcome (394). Patients should also be counseled about the safety signal identified by the FDA, namely, a potential increase in the risk of pregnancy loss in the midtrimester (498).

Patients with a short cervix $(10-20 \mathrm{~mm})$ should be offered vaginal progesterone for the prevention of preterm birth and improvement in infant outcome. The approach of universal screening with transvaginal cervical length in the midtrimester followed by the use of vaginal progesterone appears to be costeffective and allows the prevention of preterm delivery in nulliparous women $(497,499,500)$.

\section{Mode of Delivery}

Preterm birth per se is not an indication for cesarean delivery. In the last two decades, there has been an interest in determining whether cesarean delivery could improve the 
outcome of preterm birth. Efforts to conduct randomized clinical trials have not been effective in enrolling patients. Grant et al. (501), while conducting a systematic review of six trials of elective versus selective cesarean delivery, for infants less than 30 weeks of gestation, emphasized the difficulties in the execution of the trial and recommended that decision making should be individualized taking into account parenteral preferences.

Therefore, cesarean delivery is currently performed for obstetrical indications, such as breech presentation, and fetal distress. The performance of a cesarean section in an early preterm gestation ( $<30$ weeks) is often associated with an incision in the uterine corpus, because the lower uterine segment is not formed. This would increase the risk of uterine rupture in subsequent pregnancies, as well as complications associated with implantation of the placenta on the uterine scar (502). There is little evidence that the fetus may benefit from cesarean delivery and, therefore, the increased maternal risk for subsequent pregnancies does not appear to be justified.

In terms of the management of a vaginal delivery, some evidence suggests that delivery with intact membranes may improve umbilical artery $\mathrm{pH}$ by reducing the effect of mechanical forces on the fetus and umbilical cord. There is little evidence that the performance of a "prophylactic" outlet forceps or "elective" episiotomy improves neonatal outcome. Vacuum extraction is considered to be contraindicated in preterm neonates.

Timing of clamping of the cord deserves consideration. Randomized clinical trials of delayed versus early cord clamping indicate that delayed clamping is associated with a reduction in the need for transfusions of the infant for anemia (RR 2.01, 95\% CI 1.24-3.27), neonatal hypotension (RR 2.58, 95\% CI 1.17-5.67), and IVH (RR 1.74, 95\% CI 1.08-2.81) (503).

\section{SUMMARY}

It is becoming increasingly clear that preterm labor, preterm PROM, and cervical insufficiency are syndromes caused by multiple pathologic processes leading to increased myometrial contractility, cervical remodeling, and/or membrane activation. The clinical presentation will depend upon the nature and timing of the insults on the various components of the common pathway of parturition. This view has important implications for the understanding of the biology of preterm parturition, as well as its diagnosis, treatment, and prevention.

\section{ACKNOWLEDGEMENTS}

This work was funded in part by the Intramural Program of the Eunice Kennedy Shriver National Institute of Child Health and Human Development (NICHD) of the National Institutes of Health (NIH).

\section{REFERENCES}

1. Romero R, Lockwood CJ. Pathogenesis of spontaneous preterm labor. In: Creasy RK, Resnik R, Iams JD, Lockwood CJ, Moore TR, eds. Creasy and Resnik Maternal-Fetal Medicine: Principles and Practice, 6th edn Philadelphia: Saunders Elsevier, 2009: 521-43.

2. Mazaki-Tovi S, Romero R, Kusanovic JP, et al. Recurrent preterm birth. Semin Perinatol 2007; 31: 142-58.

3. Goldenberg RL, Culhane JF, Iams JD, Romero R. Epidemiology and causes of preterm birth. Lancet 2008; 371: 75-84
4. Iams JD, Romero R, Creasy RK. Preterm labor and birth. In: Creasy RK, Resnik R, Iams JD, Lockwood CJ, Moore TR, eds. Creasy \& Resnik's Maternal-Fetal Medicine; Principles and Practice, 6th edn. Philadelphia: Saunders Elsevier, 2009: 545-82.

5. Zayek MM, Trimm RF, Hamm CR, et al. The limit of viability: a single regional unit's experience. Arch Pediatr Adolesc Med 2011; 165: $126-33$.

6. Dani C, Poggi C, Romagnoli C, Bertini G. Survival and major disability rate in infant born at 22-25 weeks of gestation. J Perinat Med 2009; 37: 599-608.

7. Emsley HC, Wardle SP, Sims DG, Chiswick ML, D’Souza SW. Increased survival and deteriorating developmental outcome in 23 to 25 week old gestation infants, 1990-4 compared with 1984-9. Arch Dis Child Fetal Neonatal Ed 1998; 78: F99-104.

8. Hoekstra RE, Ferrara TB, Couser RJ, Payne NR, Connett JE. Survival and long-term neurodevelopmental outcome of extremely premature infants born at 23-26 weeks' gestational age at a tertiary center. Pediatrics 2004; 113(1 Pt 1): e1-6.

9. Tucker JM, Goldenberg RL, Davis RO, et al. Etiologies of preterm birth in an indigent population: is prevention a logical expectation? Obstet Gynecol 1991; 77: 343-7.

10. Ananth CV, Savitz DA, Luther ER, Bowes WA Jr. Preeclampsia and preterm birth subtypes in Nova Scotia, 1986 to 1992. Am J Perinatol 1997; 14: 17-23.

11. Mercer BM, Goldenberg RL, Moawad AH, et al. The preterm prediction study: effect of gestational age and cause of preterm birth on subsequent obstetric outcome. National Institute of Child Health and Human Development Maternal-Fetal Medicine Units Network. Am J Obstet Gynecol 1999; 181(5 Pt 1): 1216-21.

12. Berkowitz GS, Blackmore-Prince C, Lapinski RH, Savitz DA. Risk factors for preterm birth subtypes. Epidemiology 1998; 9: 279-85.

13. Savitz DA, Blackmore CA, Thorp JM. Epidemiologic characteristics of preterm delivery: etiologic heterogeneity. Am J Obstet Gynecol 1991; 164: 467-71.

14. Moutquin JM. Classification and heterogeneity of preterm birth. BJOG 2003; 110(Suppl 20): 30-3.

15. Parry S, Strauss JF III. Premature rupture of the fetal membranes. N Engl J Med 1998; 338: 663-70.

16. Martin JA, Hamilton BE, Sutton PD, et al. Births: final data for 2002. Natl Vital Stat Rep 2003; 52: 1-113.

17. Genazzani AR, Petraglia F, Facchinetti F, Galli PA, Volpe A. Lack of betaendorphin plasma level rise in oxytocin-induced labor. Gynecol Obstet Invest 1985; 19: 130-4.

18. Romero R, Mazor M, Munoz H, et al. The preterm labor syndrome. Ann NY Acad Sci 1994; 734: 414-29.

19. Petraglia F, Giardino L, Coukos G, et al. Corticotropin-releasing factor and parturition: plasma and amniotic fluid levels and placental binding sites. Obstet Gynecol 1990; 75: 784-9.

20. Ohrlander S, Gennser G, Eneroth P. Plasma cortisol levels in human fetus during parturition. Obstet Gynecol 1976; 48: 381-7.

21. Randall NJ, Bond K, Macaulay J, Steer PJ. Measuring fetal and maternal temperature differentials: a probe for clinical use during labour. J Biomed Eng 1991; 13: 481-5.

22. Romero R, Gomez R, Mazor M, Ghezzi F, Yoon BH. The preterm labor syndrome. In: Elder MG, Romero R, Lamont R, eds. Preterm Labor. New York, NY: Churchill Livingstone, 1997: 29-49.

23. Althuisius SM, Dekker GA, van Geijn HP. Cervical incompetence: a reappraisal of an obstetric controversy. Obstet Gynecol Surv 2002; 57: 377-87.

24. Iams JD, Johnson FF, Sonek J, et al. Cervical competence as a continuum: a study of ultrasonographic cervical length and obstetric performance. Am J Obstet Gynecol 1995; 172(4 Pt 1): 1097-103.

25. Romero R, Espinoza J, Mazor M, Chaiworapongsa T. The preterm parturition syndrome. In: Critchely $\mathrm{H}$, Bennett $\mathrm{P}$, Thornton $\mathrm{S}$, eds. Preterm Birth. London: RCOG Press, 2004: 28-60.

26. Elovitz MA, Mrinalini C. Animal models of preterm birth. Trends Endocrinol Metab 2004; 15: 479-87.

27. Fidel PL Jr, Romero R, Wolf N, et al. Systemic and local cytokine profiles in endotoxin-induced preterm parturition in mice. Am J Obstet Gynecol 1994; 170(5 Pt 1): 1467-75. 
28. Gravett MG, Witkin SS, Haluska GJ, et al. An experimental model for intraamniotic infection and preterm labor in rhesus monkeys. Am J Obstet Gynecol 1994; 171: 1660-7.

29. Hirsch E, Saotome I, Hirsh D. A model of intrauterine infection and preterm delivery in mice. Am J Obstet Gynecol 1995; 172: 1598-603.

30. Kullander S. Fever and parturition. An experimental study in rabbits. Acta Obstet Gynecol Scand Suppl 1977; 66: 77-85.

31. McDuffie RS Jr, Sherman MP, Gibbs RS. Amniotic fluid tumor necrosis factor-alpha and interleukin-1 in a rabbit model of bacterially induced preterm pregnancy loss. Am J Obstet Gynecol 1992; 167: 1583-8.

32. McKay DG, Wong TC. The effect of bacterial endotoxin on the placenta of the rat. Am J Pathol 1963; 42: 357-77.

33. Romero R, Mazor M, Wu YK, et al. Infection in the pathogenesis of preterm labor. Semin Perinatol 1988; 12: 262-79.

34. Gibbs RS, McDuffie RS Jr, Kunze M, et al. Experimental intrauterine infection with Prevotella bivia in New Zealand White rabbits. Am J Obstet Gynecol 2004; 190: 1082-6.

35. Gilles HM, Lawson JB, Sibelas M, Voller A, Allan N. Malaria, anaemia and pregnancy. Ann Trop Med Parasitol 1969; 63: 245-63.

36. Heard N, Jordan T. An investigation of malaria during pregnancy in Zimbabwe. Cent Afr J Med 1981; 27: 62-8.

37. Hibbard L, Thrupp L, Summeril S, Smale M, Adams R. Treatment of pyelonephritis in pregnancy. Am J Obstet Gynecol 1967; 98: 609-15.

38. Patrick MJ. Influence of maternal renal infection on the foetus and infant. Arch Dis Child 1967; 42: 208-13.

39. Wren BG. Subclinical renal infection and prematurity. Med J Aust 1969; 2: 596-600.

40. Cunningham FG, Morris GB, Mickal A. Acute pyelonephritis of pregnancy: a clinical review. Obstet Gynecol 1973; 42: 112-17.

41. Kaul AK, Khan S, Martens MG, et al. Experimental gestational pyelonephritis induces preterm births and low birth weights in $\mathrm{C} 3 \mathrm{H} /$ HeJ mice. Infect Immun 1999; 67: 5958-66.

42. Benedetti TJ, Valle R, Ledger WJ. Antepartum pneumonia in pregnancy. Am J Obstet Gynecol 1982; 144: 413-17.

43. Madinger NE, Greenspoon JS, Ellrodt AG. Pneumonia during pregnancy: has modern technology improved maternal and fetal outcome? Am J Obstet Gynecol 1989; 161: 657-62.

44. Munn MB, Groome LJ, Atterbury JL, Baker SL, Hoff C. Pneumonia as a complication of pregnancy. J Matern Fetal Med 1999; 8: 151-4.

45. Goepfert AR, Jeffcoat MK, Andrews WW, et al. Periodontal disease and upper genital tract inflammation in early spontaneous preterm birth. Obstet Gynecol 2004; 104: 777-83.

46. Jarjoura K, Devine PC, Perez-Delboy A, et al. Markers of periodontal infection and preterm birth. Am J Obstet Gynecol 2005; 192: 513-19.

47. Jeffcoat MK, Geurs NC, Reddy MS, Goldenberg RL, Hauth JC. Current evidence regarding periodontal disease as a risk factor in preterm birth. Ann Periodontol 2001; 6: 183-8.

48. Offenbacher S, Boggess KA, Murtha AP, et al. Progressive periodontal disease and risk of very preterm delivery. Obstet Gynecol 2006; 107: 29-36.

49. Xiong X, Buekens P, Fraser WD, Beck J, Offenbacher S. Periodontal disease and adverse pregnancy outcomes: a systematic review. BJOG 2006; 113: 135-43.

50. Offenbacher S. Maternal periodontal infections, prematurity, and growth restriction. Clin Obstet Gynecol 2004; 47: 808-21.

51. Gomez R, Ghezzi F, Romero R, et al. Premature labor and intraamniotic infection. Clinical aspects and role of the cytokines in diagnosis and pathophysiology. Clin Perinatol 1995; 22: 281-342.

52. Cassell GH, Davis RO, Waites KB, et al. Isolation of Mycoplasma hominis and Ureaplasma urealyticum from amniotic fluid at 16-20 weeks of gestation: potential effect on outcome of pregnancy. Sex Transm Dis 1983; 10(4 Suppl): 294-302.

53. Gray DJ, Robinson HB, Malone J, Thomson RB Jr. Adverse outcome in pregnancy following amniotic fluid isolation of Ureaplasma urealyticum. Prenat Diagn 1992; 12: 111-17.

54. Horowitz S, Mazor M, Romero R, Horowitz J, Glezerman M. Infection of the amniotic cavity with Ureaplasma urealyticum in the midtrimester of pregnancy. J Reprod Med 1995; 40: 375-9.

55. Wenstrom KD, Andrews WW, Hauth JC, et al. Elevated secondtrimester amniotic fluid interleukin-6 levels predict preterm delivery. Am J Obstet Gynecol 1998; 178: 546-50.
56. Yoon BH, Oh SY, Romero R, et al. An elevated amniotic fluid matrix metalloproteinase- 8 level at the time of mid-trimester genetic amniocentesis is a risk factor for spontaneous preterm delivery. Am J Obstet Gynecol 2001; 185: 1162-7.

57. Wang H, Hirsch E. Bacterially-induced preterm labor and regulation of prostaglandin-metabolizing enzyme expression in mice: the role of tolllike receptor 4. Biol Reprod 2003; 69: 1957-63.

58. Arias F, Rodriquez L, Rayne SC, Kraus FT. Maternal placental vasculopathy and infection: two distinct subgroups among patients with preterm labor and preterm ruptured membranes. Am J Obstet Gynecol 1993; 168: 585-91.

59. Kim YM, Chaiworapongsa T, Gomez R, et al. Failure of physiologic transformation of the spiral arteries in the placental bed in preterm premature rupture of membranes. Am J Obstet Gynecol 2002; 187: 1137-42.

60. Kim YM, Bujold E, Chaiworapongsa T, et al. Failure of physiologic transformation of the spiral arteries in patients with preterm labor and intact membranes. Am J Obstet Gynecol 2003; 189: 1063-9.

61. Salafia CM, Lopez-Zeno JA, Sherer DM, et al. Histologic evidence of old intrauterine bleeding is more frequent in prematurity. Am J Obstet Gynecol 1995; 173: 1065-70.

62. Brar HS, Medearis AL, DeVore GR, Platt LD. Maternal and fetal blood flow velocity waveforms in patients with preterm labor: prediction of successful tocolysis. Am J Obstet Gynecol 1988; 159: 947-50.

63. Strigini FA, Lencioni G, De LG, et al. Uterine artery velocimetry and spontaneous preterm delivery. Obstet Gynecol 1995; 85: 374-77.

64. Brar HS, Medearis AL, De Vore GR, Platt LD. Maternal and fetal blood flow velocity waveforms in patients with preterm labor: relationship to outcome. Am J Obstet Gynecol 1989; 161(6 Pt 1): 1519-22.

65. Besinger R, Carson N. The physiology of preterm labor. In: Keith L, Papiernik E, Keith D, Luke B, eds. Multiple Pregnancy: Epidemiology, Gestation and Perinatal Outcome. London: Parthenon Publishing, 1995: 415.

66. Hill LM, Breckle R, Thomas ML, Fries JK. Polyhydramnios: ultrasonically detected prevalence and neonatal outcome. Obstet Gynecol 1987; 69: 21-5.

67. Phelan JP, Park YW, Ahn MO, Rutherford SE. Polyhydramnios and perinatal outcome. J Perinatol 1990; 10: 347-50.

68. Ludmir J, Samuels P, Brooks S, Mennuti MT. Pregnancy outcome of patients with uncorrected uterine anomalies managed in a high-risk obstetric setting. Obstet Gynecol 1990; 75: 906-10.

69. Lockwood CJ. Stress-associated preterm delivery: the role of corticotropin-releasing hormone. Am J Obstet Gynecol 1999; 180 (1 Pt 3): S264-6.

70. Challis JR, Smith SK. Fetal endocrine signals and preterm labor. Biol Neonate 2001; 79: 163-7.

71. Hobel CJ. Stress and preterm birth. Clin Obstet Gynecol 2004; 47: 856-80.

72. Wadhwa PD, Culhane JF, Rauh V, Barve SS. Stress and preterm birth: neuroendocrine, immune/inflammatory, and vascular mechanisms. Matern Child Health J 2001; 5: 119-25.

73. Wadhwa PD, Culhane JF, Rauh V, et al. Stress, infection and preterm birth: a biobehavioural perspective. Paediatr Perinat Epidemiol 2001; 15 (Suppl 2): 17-29.

74. Barnes AB, Colton T, Gundersen J, et al. Fertility and outcome of pregnancy in women exposed in utero to diethylstilbestrol. N Engl J Med 1980; 302: 609-13.

75. Sandberg EC, Riffle NL, Higdon JV, Getman CE. Pregnancy outcome in women exposed to diethylstilbestrol in utero. Am J Obstet Gynecol 1981; 140: 194-205.

76. Jefferies JA, Robboy SJ, O'Brien PC, et al. Structural anomalies of the cervix and vagina in women enrolled in the Diethylstilbestrol Adenosis (DESAD) Project. Am J Obstet Gynecol 1984; 148: 59-66.

77. Kinch RA. Diethylstilbestrol in pregnancy: an update. Can Med Assoc J 1982; 127: 812-13.

78. Kaufman RH. Structural changes of the genital tract associated with in utero exposure to diethylstilbestrol. Obstet Gynecol Annu 1982; 11: 187-202.

79. Michaels WH, Thompson HO, Schreiber FR, et al. Ultrasound surveillance of the cervix during pregnancy in diethylstilbestrol-exposed offspring. Obstet Gynecol 1989; 73: 230-9. 
80. Armarnik S, Sheiner E, Piura B, et al. Obstetric outcome following cervical conization. Arch Gynecol Obstet 2011; 283: 765-9.

81. Nam KH, Kwon JY, Kim YH, Park YW. Pregnancy outcome after cervical conization: risk factors for preterm delivery and the efficacy of prophylactic cerclage. J Gynecol Oncol 2010; 21: 225-9.

82. Bamford PN, Hall SA. Preterm birth and previous conisation of the cervix. BJOG 2010; 117: 1158-9.

83. Jakobsson M, Gissler M, Paavonen J, Tapper AM. Loop electrosurgical excision procedure and the risk for preterm birth. Obstet Gynecol 2009; 114: 504-10.

84. Michelin MA, Merino LM, Franco CA, Murta EF. Pregnancy outcome after treatment of cervical intraepithelial neoplasia by the loop electrosurgical excision procedure and cold knife conization. Clin Exp Obstet Gynecol 2009; 36: 17-19.

85. Albrechtsen S, Rasmussen S, Thoresen S, Irgens LM, Iversen OE Pregnancy outcome in women before and after cervical conisation: population based cohort study. BMJ 2008; 337: a1343.

86. Kristensen J, Langhoff-Roos J, Wittrup M, Bock JE. Cervical conization and preterm delivery/low birth weight. A systematic review of the literature. Acta Obstet Gynecol Scand 1993; 72: 640-4.

87. Kristensen GB. The outcome of pregnancy and preterm delivery after conization of the cervix. Arch Gynecol 1985; 236: 127-30.

88. Klaritsch P, Reich O, Giuliani A, et al. Delivery outcome after cold-knife conization of the uterine cervix. Gynecol Oncol 2006; 103: 604-7.

89. Sadler L, Saftlas A. Cervical surgery and preterm birth. J Perinat Med 2007; 35: 5-9.

90. Chwalisz K. The use of progesterone antagonists for cervical ripening and as an adjunct to labour and delivery. Hum Reprod 1994; 9(Suppl 1): 131-61.

91. Puri CP, Patil RK, Elger WA, Pongubala JM. Effects of progesterone antagonist ZK 98.299 on early pregnancy and foetal outcome in bonnet monkeys. Contraception 1990; 41: 197-205.

92. Zakar T, Hertelendy F. Progesterone withdrawal: key to parturition. Am J Obstet Gynecol 2007; 196: 289-96.

93. Merlino AA, Welsh TN, Tan H, et al. Nuclear progesterone receptors in the human pregnancy myometrium: evidence that parturition involves functional progesterone withdrawal mediated by increased expression of progesterone receptor-A. J Clin Endocrinol Metab 2007; 92: 1927-33.

94. Karteris E, Zervou S, Pang Y, et al. Progesterone signaling in human myometrium through two novel membrane $G$ protein-coupled receptors: potential role in functional progesterone withdrawal at term. Mol Endocrinol 2006; 20: 1519-34.

95. Sheehan PM, Rice GE, Moses EK, Brennecke SP. 5 Beta-dihydroprogesterone and steroid 5 beta-reductase decrease in association with human parturition at term. Mol Hum Reprod 2005; 11: 495-501.

96. Dong X, Shylnova O, Challis JR, Lye SJ. Identification and characterization of the protein-associated splicing factor as a negative co-regulator of the progesterone receptor. J Biol Chem 2005; 280: 13329-40.

97. Mesiano S. Roles of estrogen and progesterone in human parturition. Front Horm Res 2001; 27: 86-104.

98. Oh SY, Kim CJ, Park I, et al. Progesterone receptor isoform (A/B) ratio of human fetal membranes increases during term parturition. Am J Obstet Gynecol 2005; 193(3 Pt 2): 1156-60.

99. Romero R, Kusanovic JP, Munoz H, et al. Allergy-induced preterm labor after the ingestion of shellfish. J Matern Fetal Neonatal Med 2010; 23: 351-9.

100. Corry DB, Kheradmand F. Induction and regulation of the IgE response. Nature 1999; 402(6760 Suppl): B18-23.

101. Jones AC, Miles EA, Warner JO, et al. Fetal peripheral blood mononuclear cell proliferative responses to mitogenic and allergenic stimuli during gestation. Pediatr Allergy Immunol 1996; 7: 109-16.

102. Rudolph MI, Reinicke K, Cruz MA, et al. Distribution of mast cells and the effect of their mediators on contractility in human myometrium. $\mathrm{Br}$ J Obstet Gynaecol 1993; 100: 1125-30.

103. Rudolph MI, Bardisa L, Cruz MA, Reinicke K. Mast cells mediators evoke contractility and potentiate each other in mouse uterine horns. Gen Pharmacol 1992; 23: 833-6.

104. Padilla L, Reinicke K, Montesino H, et al. Histamine content and mast cells distribution in mouse uterus: the effect of sexual hormones, gestation and labor. Cell Mol Biol 1990; 36: 93-100.
105. Bytautiene E, Vedernikov YP, Saade GR, Romero R, Garfield RE. Endogenous mast cell degranulation modulates cervical contractility in the guinea pig. Am J Obstet Gynecol 2002; 186: 438-45.

106. Garfield RE, Bytautiene E, Vedernikov YP, Marshall JS, Romero R. Modulation of rat uterine contractility by mast cells and their mediators. Am J Obstet Gynecol 2000; 183: 118-25.

107. Bytautiene E, Romero R, Vedernikov YP, et al. Induction of premature labor and delivery by allergic reaction and prevention by histamine $\mathrm{H} 1$ receptor antagonist. Am J Obstet Gynecol 2004; 191: 1356-61.

108. Kim MJ, Romero R, Kim CJ, et al. Villitis of unknown etiology is associated with a distinct pattern of chemokine up-regulation in the fetomaternal and placental compartments: implications for conjoint maternal allograft rejection and maternal anti-fetal graft-versus-host disease. J Immunol 2009; 182: 3919-27.

109. Ogge G, Romero R, Lee DC, et al. Chronic chorioamnionitis displays distinct alterations of the amniotic fluid proteome. J Pathol 2011; 223: 553-65.

110. Kim JS, Romero R, Kim MR, et al. Involvement of Hofbauer cells and maternal $\mathrm{T}$ cells in villitis of unknown aetiology. Histopathology 2008; 52: 457-64.

111. Kim CJ, Romero R, Kusanovic JP, et al. The frequency, clinical significance, and pathological features of chronic chorioamnionitis: a lesion associated with spontaneous preterm birth. Mod Pathol 2010; 23: $1000-11$.

112. Lee J, Romero R, Xu Y, et al. A signature of maternal anti-fetal rejection in spontaneous preterm birth: chronic chorioamnionitis, anti-human leukocyte antigen antibodies, and C4d. PLoS One 2011; 6: e16806.

113. Allport VC, Pieber D, Slater DM, et al. Human labour is associated with nuclear factor-kappaB activity which mediates cyclo-oxygenase-2 expression and is involved with the 'functional progesterone withdrawal'. Mol Hum Reprod 2001; 7: 581-6.

114. Belt AR, Baldassare JJ, Molnar M, Romero R, Hertelendy F. The nuclear transcription factor NF-kappaB mediates interleukin-1beta-induced expression of cyclooxygenase-2 in human myometrial cells. Am J Obstet Gynecol 1999; 181: 359-66.

115. Kalkhoven E, Wissink S, van der Saag PT, van der BB. Negative interaction between the $\operatorname{RelA}(\mathrm{p} 65)$ subunit of NF-kappaB and the progesterone receptor. J Biol Chem 1996; 271: 6217-24.

116. Romero R, Espinoza J, Kusanovic JP, et al. The preterm parturition syndrome. BJOG 2006; 113(Suppl 3): 17-42.

117. Blackmore CA, Savitz DA, Edwards LJ, Harlow SD, Bowes WA Jr. Racial differences in the patterns of preterm delivery in central North Carolina, USA. Paediatr Perinat Epidemiol 1995; 9: 281-95.

118. Zhang J, Savitz DA. Preterm birth subtypes among blacks and whites. Epidemiology 1992; 3: 428-33.

119. Blackmore-Prince C, Kieke B Jr, Kugaraj KA, et al. Racial differences in the patterns of singleton preterm delivery in the 1988 National Maternal and Infant Health Survey. Matern Child Health J 1999; 3: 189-97.

120. Salihu HM, Mbah AK, Alio AP, Clayton HB, Lynch O. Low prepregnancy body mass index and risk of medically indicated versus spontaneous preterm singleton birth. Eur J Obstet Gynecol Reprod Biol 2009; 144: 119-23.

121. Wise LA, Palmer JR, Heffner LJ, Rosenberg L. Prepregnancy body size, gestational weight gain, and risk of preterm birth in African-American women. Epidemiology 2010; 21: 243-52.

122. Salihu HM, Lynch O, Alio AP, et al. Extreme maternal underweight and feto-infant morbidity outcomes: a population-based study. J Matern Fetal Neonatal Med 2009; 22: 428-34.

123. Johnson TS, Rottier KJ, Luellwitz A, Kirby RS. Maternal prepregnancy body mass index and delivery of a preterm infant in missouri 1998-2000. Public Health Nurs 2009; 26: 3-13.

124. Spinillo A, Capuzzo E, Piazzi G, et al. Risk for spontaneous preterm delivery by combined body mass index and gestational weight gain patterns. Acta Obstet Gynecol Scand 1998; 77: 32-6.

125. Meyer MB, Jonas BS, Tonascia JA. Perinatal events associated with maternal smoking during pregnancy. Am J Epidemiol 1976; 103: 464-76.

126. Aliyu MH, Lynch O, Saidu R, et al. Intrauterine exposure to tobacco and risk of medically indicated and spontaneous preterm birth. Am J Perinatol 2010; 27: 405-10. 
127. McCowan LM, Dekker GA, Chan E, et al. Spontaneous preterm birth and small for gestational age infants in women who stop smoking early in pregnancy: prospective cohort study. BMJ 2009; 338: b1081.

128. Mercer BM. Preterm premature rupture of the membranes. Obstet Gynecol 2003; 101: 178-93.

129. Mercer BM. Is there a role for tocolytic therapy during conservative management of preterm premature rupture of the membranes? Clin Obstet Gynecol 2007; 50: 487-96.

130. Svigos JM, Dodd JM, Robinson JS. Threatened and actual preterm labor including mode of delivery. In: James D, Steer PJ, Weiner CP, Gonik B, Crowther CA, Robson SC, eds. High Risk Pregnancy: Management Options, 4th edn. St Louis: Elsevier Saunders, 2011: 1075-90.

131. King JF, Grant A, Keirse MJ, Chalmers I. Beta-mimetics in preterm labour: an overview of the randomized controlled trials. $\mathrm{Br}$ J Obstet Gynaecol 1988; 95: 211-22.

132. Romero R, Oyarzun E, Mazor M, et al. Meta-analysis of the relationship between asymptomatic bacteriuria and preterm delivery/low birth weight. Obstet Gynecol 1989; 73: 576-82.

133. Graham JM, Oshiro BT, Blanco JD, Magee KP. Uterine contractions after antibiotic therapy for pyelonephritis in pregnancy. Am J Obstet Gynecol 1993; 168: 577-80.

134. Millar LK, DeBuque L, Wing DA. Uterine contraction frequency during treatment of pyelonephritis in pregnancy and subsequent risk of preterm birth. J Perinat Med 2003; 31: 41-6.

135. Nagey DA. Contractions are not always labor. Am J Obstet Gynecol 1995; 173(3 Pt 1): 975-6.

136. Brustman LE, Langer O, Damus K, Anyaegbunam A, Merkatz IR. Uterine contractility patterns after an episode of preterm labor. Obstet Gynecol 1990; 75(3 Pt 1): 346-9.

137. Blondel B, Breart G, Llado J, Chartier M. Evaluation of the home-visiting system for women with threatened preterm labor: results of a randomized controlled trial. Eur J Obstet Gynecol Reprod Biol 1990; 34: 47-58.

138. Bastek JA, Sammel MD, Rebele EC, Srinivas SK, Elovitz MA. The effects of a preterm labor episode prior to 34 weeks are evident in late preterm outcomes, despite the administration of betamethasone. Am J Obstet Gynecol 2010; 203: 140-7.

139. Rizzo G, Capponi A, Arduini D, Lorido C, Romanini C. The value of fetal fibronectin in cervical and vaginal secretions and of ultrasonographic examination of the uterine cervix in predicting premature delivery for patients with preterm labor and intact membranes. Am J Obstet Gynecol 1996; 175: 1146-51.

140. Nageotte MP, Casal D, Senyei AE. Fetal fibronectin in patients at increased risk for premature birth. Am J Obstet Gynecol 1994; 170(1 Pt 1): $20-5$.

141. Goldenberg RL, Iams JD, Das A, et al. The Preterm Prediction Study: sequential cervical length and fetal fibronectin testing for the prediction of spontaneous preterm birth. National Institute of Child Health and Human Development Maternal-Fetal Medicine Units Network. Am J Obstet Gynecol 2000; 182: 636-43.

142. Lockwood CJ, Senyei AE, Dische MR, et al. Fetal fibronectin in cervical and vaginal secretions as a predictor of preterm delivery. N Engl J Med 1991; 325: 669-74.

143. Lockwood CJ, Wein R, Lapinski R, et al. The presence of cervical and vaginal fetal fibronectin predicts preterm delivery in an inner-city obstetric population. Am J Obstet Gynecol 1993; 169: 798-804.

144. Woodworth A, Moore J, G'Sell C, et al. Diagnostic accuracy of cervicovaginal interleukin-6 and interleukin-6:albumin ratio as markers of preterm delivery. Clin Chem 2007; 53: 1534-40.

145. Goffinet F, Kayem G, Maillard F, et al. Detection of interleukin 6 mRNA by RT-PCR in vaginal secretions: association with preterm delivery and neonatal infection in women with preterm labour and intact membranes. Eur J Obstet Gynecol Reprod Biol 2005; 123: 167-73.

146. Grenache DG, Hankins K, Parvin CA, Gronowski AM. Cervicovaginal interleukin-6, tumor necrosis factor-alpha, and interleukin-2 receptor as markers of preterm delivery. Clin Chem 2004; 50: 1839-42.

147. Kishida T, Yamada H, Furuta I, et al. Increased levels of interleukin-6 in cervical secretions and assessment of the uterine cervix by transvaginal ultrasonography predict preterm premature rupture of the membranes. Fetal Diagn Ther 2003; 18: 98-104.
148. Lockwood CJ, Ghidini A, Wein R, et al. Increased interleukin-6 concentrations in cervical secretions are associated with preterm delivery. Am J Obstet Gynecol 1994; 171: 1097-102.

149. az-Cueto L, Cuica-Flores A, Ziga-Cordero F, et al. Vaginal matrix metalloproteinase levels in pregnant women with bacterial vaginosis. J Soc Gynecol Investig 2006; 13: 430-4.

150. Rahkonen L, Rutanen EM, Unkila-Kallio L, et al. Factors affecting matrix metalloproteinase- 8 levels in the vaginal and cervical fluids in the first and second trimester of pregnancy. Hum Reprod 2009; 24: 2693-702.

151. Sennstrom MB, Brauner A, Bystrom B, Malmstrom A, Ekman G. Matrix metalloproteinase- 8 correlates with the cervical ripening process in humans. Acta Obstet Gynecol Scand 2003; 82: 904-11.

152. Leeson SC, Maresh MJ, Martindale EA, et al. Detection of fetal fibronectin as a predictor of preterm delivery in high risk asymptomatic pregnancies. Br J Obstet Gynaecol 1996; 103: 48-53.

153. Morrison JC, Naef RW III, Botti JJ, et al. Prediction of spontaneous preterm birth by fetal fibronectin and uterine activity. Obstet Gynecol 1996; 87(5 Pt 1): 649-55.

154. Ingemarsson I. Effect of terbutaline on premature labor. A double-blind placebo-controlled study. Am J Obstet Gynecol 1976; 125: 520-4.

155. Gonik B, Creasy RK. Preterm labor: its diagnosis and management. Am J Obstet Gynecol 1986; 154: 3-8.

156. MacKinnon K, McIntyre M. From Braxton Hicks to preterm labour: the constitution of risk in pregnancy. Can J Nurs Res 2006; 38: 56-72.

157. Day TW. Braxton Hicks vs. preterm labor. Minn Med 1990; 73: 21-2.

158. Hill WC, Lambertz EL. Let's get rid of the term "Braxton Hicks contractions." Obstet Gynecol 1990; 75: 709-10.

159. Morgan MA, Silavin SL, Wentworth RA, et al. Different patterns of myometrial activity and 24-h rhythms in myometrial contractility in the gravid baboon during the second half of pregnancy. Biol Reprod 1992; 46: 1158-64.

160. Iams JD, Johnson FF, O'Shaughnessy RW. A prospective random trial of home uterine activity monitoring in pregnancies at increased risk of preterm labor. Part II. Am J Obstet Gynecol 1988; 159: 595-603.

161. Lucovnik M, Kuon RJ, Chambliss LR, et al. Use of uterine electromyography to diagnose term and preterm labor. Acta Obstet Gynecol Scand 2011; 90: 150-7.

162. Lucovnik M, Maner WL, Chambliss LR, et al. Noninvasive uterine electromyography for prediction of preterm delivery. Am J Obstet Gynecol 2011; 204: 228-10.

163. Hassan M, Terrien J, Alexandersson A, Marque C, Karlsson B. Nonlinearity of EHG signals used to distinguish active labor from normal pregnancy contractions. Conf Proc IEEE Eng Med Biol Soc 2010; 2010: 2387-90.

164. Moslem B, Khalil M, Marque C, Diab MO. Complexity analysis of the uterine electromyography. Conf Proc IEEE Eng Med Biol Soc 2010; 2010: 2802-5.

165. Garfield RE, Maul H, Maner W, et al. Uterine electromyography and light-induced fluorescence in the management of term and preterm labor. J Soc Gynecol Investig 2002; 9: 265-75.

166. Michaels WH, Montgomery C, Karo J, et al. Ultrasound differentiation of the competent from the incompetent cervix: prevention of preterm delivery. Am J Obstet Gynecol 1986; 154: 537-46.

167. Ayers JW, DeGrood RM, Compton AA, Barclay M, Ansbacher R. Sonographic evaluation of cervical length in pregnancy: diagnosis and management of preterm cervical effacement in patients at risk for premature delivery. Obstet Gynecol 1988; 71(6 Pt 1): 939-44.

168. Andersen HF, Nugent CE, Wanty SD, Hayashi RH. Prediction of risk for preterm delivery by ultrasonographic measurement of cervical length. Am J Obstet Gynecol 1990; 163: 859-67.

169. Gomez R, Galasso M, Romero R, et al. Ultrasonographic examination of the uterine cervix is better than cervical digital examination as a predictor of the likelihood of premature delivery in patients with preterm labor and intact membranes. Am J Obstet Gynecol 1994; 171: 956-64.

170. Iams JD, Paraskos J, Landon MB, Teteris JN, Johnson FF. Cervical sonography in preterm labor. Obstet Gynecol 1994; 84: 40-6.

171. Gomez R, Romero R, Medina L, et al. Cervicovaginal fibronectin improves the prediction of preterm delivery based on sonographic cervical length in patients with preterm uterine contractions and intact membranes. Am J Obstet Gynecol 2005; 192: 350-9. 
172. Hassan SS, Romero R, Berry SM, et al. Patients with an ultrasonographic cervical length $<$ or $=15 \mathrm{~mm}$ have nearly a $50 \%$ risk of early spontaneous preterm delivery. Am J Obstet Gynecol 2000; 182: 1458-67.

173. Heath VC, Southall TR, Souka AP, Elisseou A, Nicolaides KH. Cervical length at 23 weeks of gestation: prediction of spontaneous preterm delivery. Ultrasound Obstet Gynecol 1998; 12: 312-17.

174. Iams JD, Goldenberg RL, Meis PJ, et al. The length of the cervix and the risk of spontaneous premature delivery. National Institute of Child Health and Human Development Maternal Fetal Medicine Unit Network. N Engl J Med 1996; 334: 567-72.

175. Vaisbuch E, Romero R, Erez O, et al. Clinical significance of early $(<20$ weeks) vs. late (20-24 weeks) detection of sonographic short cervix in asymptomatic women in the mid-trimester. Ultrasound Obstet Gynecol 2010; 36: 471-81.

176. Leitich H, Kaider A. Fetal fibronectin-how useful is it in the prediction of preterm birth? BJOG 2003; 110(Suppl 20): 66-70.

177. Ascarelli $\mathrm{MH}$, Morrison JC. Use of fetal fibronectin in clinical practice. Obstet Gynecol Surv 1997; 52(4 Suppl): S1-12.

178. Morrison JC, Allbert JR, McLaughlin BN, et al. Oncofetal fibronectin in patients with false labor as a predictor of preterm delivery. Am J Obstet Gynecol 1993; 168: 538-42.

179. Inglis SR, Jeremias J, Kuno K, et al. Detection of tumor necrosis factoralpha, interleukin-6, and fetal fibronectin in the lower genital tract during pregnancy: relation to outcome. Am J Obstet Gynecol 1994; 171: 5-10.

180. Iams JD, Casal D, McGregor JA, et al. Fetal fibronectin improves the accuracy of diagnosis of preterm labor. Am J Obstet Gynecol 1995; 173: 141-5.

181. Burrus DR, Ernest JM, Veille JC. Fetal fibronectin, interleukin-6, and $\mathrm{C}$-reactive protein are useful in establishing prognostic subcategories of idiopathic preterm labor. Am J Obstet Gynecol 1995; 173: 1258-62.

182. Bartnicki J, Casal D, Kreaden US, Saling E, Vetter K. Fetal fibronectin in vaginal specimens predicts preterm delivery and very-low-birth-weight infants. Am J Obstet Gynecol 1996; 174: 971-4.

183. Peaceman AM, Andrews WW, Thorp JM, et al. Fetal fibronectin as a predictor of preterm birth in patients with symptoms: a multicenter trial. Am J Obstet Gynecol 1997; 177: 13-18.

184. Honest H, Bachmann LM, Gupta JK, Kleijnen J, Khan KS. Accuracy of cervicovaginal fetal fibronectin test in predicting risk of spontaneous preterm birth: systematic review. BMJ 2002; 325: 301

185. Sanchez-Ramos L, Delke I, Zamora J, Kaunitz AM. Fetal fibronectin as a short-term predictor of preterm birth in symptomatic patients: a metaanalysis. Obstet Gynecol 2009; 114: 631-40.

186. Shimoya K, Hashimoto K, Shimizu T, Saji F, Murata Y. Effect of sexual intercourse on fetal fibronectin concentration in cervicovaginal secretions. Am J Obstet Gynecol 1998; 179: 255-6.

187. Berghella V. Preterm birth prevention and management. Hoboken: Blackwell Publishing Ltd, 2010.

188. Iams JD. Prediction and early detection of preterm labor. Obstet Gynecol 2003; 101: 402-12.

189. Hincz P, Wilczynski J, Kozarzewski M, Szaflik K. Two-step test: the combined use of fetal fibronectin and sonographic examination of the uterine cervix for prediction of preterm delivery in symptomatic patients. Acta Obstet Gynecol Scand 2002; 81: 58-63

190. Meijer-Hoogeveen M, Stoutenbeek P, Visser GH. Transperineal versus transvaginal sonographic cervical length measurement in second- and third-trimester pregnancies. Ultrasound Obstet Gynecol 2008; 32: 657-62.

191. Coleman MA, McCowan LM, Pattison NS, Mitchell M. Fetal fibronectin detection in preterm labor: evaluation of a prototype bedside dipstick technique and cervical assessment. Am J Obstet Gynecol 1998; 179(6 Pt 1): 1553-8.

192. Sullivan A, Hueppchen NA, Satin AJ. Cost effectiveness of bedside fetal fibronectin testing varies according to treatment algorithm. J Matern Fetal Med 2001; 10: 380-4.

193. Romero R, Sirtori M, Oyarzun E, et al. Infection and labor. V. Prevalence, microbiology, and clinical significance of intraamniotic infection in women with preterm labor and intact membranes. Am J Obstet Gynecol 1989; 161: 817-24.

194. Minkoff H. Prematurity: infection as an etiologic factor. Obstet Gynecol 1983; 62: 137-44.
195. Goncalves LF, Chaiworapongsa T, Romero R. Intrauterine infection and prematurity. Ment Retard Dev Disabil Res Rev 2002; 8: 3-13.

196. Hitti J, Hillier SL, Agnew KJ, et al. Vaginal indicators of amniotic fluid infection in preterm labor. Obstet Gynecol 2001; 97: 211-19.

197. Gravett MG, Adams KM, Sadowsky DW, et al. Immunomodulators plus antibiotics delay preterm delivery after experimental intraamniotic infection in a nonhuman primate model. Am J Obstet Gynecol 2007; 197: 518.

198. Novy MJ, Duffy L, Axthelm MK, et al. Ureaplasma parvum or Mycoplasma hominis as sole pathogens cause chorioamnionitis, preterm delivery, and fetal pneumonia in rhesus macaques. Reprod Sci 2009; 16: $56-70$.

199. Sperling RS, Newton E, Gibbs RS. Intraamniotic infection in low-birthweight infants. J Infect Dis 1988; 157: 113-17.

200. Romero R, Quintero R, Oyarzun E, et al. Intraamniotic infection and the onset of labor in preterm premature rupture of the membranes. Am J Obstet Gynecol 1988; 159: 661-6.

201. Gibbs RS, Cassell GH, Davis JK, St Clair PJ. Further studies on genital mycoplasmas in intra-amniotic infection: blood cultures and serologic response. Am J Obstet Gynecol 1986; 154: 717-26.

202. Gibbs RS, Romero R, Hillier SL, Eschenbach DA, Sweet RL. A review of premature birth and subclinical infection. Am J Obstet Gynecol 1992; 166: 1515-28.

203. Romero R, Espinoza J, Goncalves LF, et al. The role of inflammation and infection in preterm birth. Semin Reprod Med 2007; 25: 21-39.

204. Romero R, Emamian M, Quintero R, et al. The value and limitations of the Gram stain examination in the diagnosis of intraamniotic infection. Am J Obstet Gynecol 1988; 159: 114-19.

205. Samol JM, Lambers DS. Magnesium sulfate tocolysis and pulmonary edema: the drug or the vehicle? Am J Obstet Gynecol 2005; 192: 1430-2.

206. Vaast P, Dubreucq-Fossaert S, Houfflin-Debarge V, et al. Acute pulmonary oedema during nicardipine therapy for premature labour; Report of five cases. Eur J Obstet Gynecol Reprod Biol 2004; 113: 98-9.

207. Karaman S, Ozcan O, Akercan F, et al. Pulmonary edema after ritodrine therapy during pregnancy and subsequent cesarean section with epidural anesthesia. Clin Exp Obstet Gynecol 2004; 31: 67-9.

208. DiFederico EM, Burlingame JM, Kilpatrick SJ, Harrison M, Matthay MA. Pulmonary edema in obstetric patients is rapidly resolved except in the presence of infection or of nitroglycerin tocolysis after open fetal surgery. Am J Obstet Gynecol 1998; 179: 925-33.

209. Levy DL. Morbidity caused by terbutaline infusion pump therapy. Am J Obstet Gynecol 1994; 170: 1835

210. Lampert MB, Hibbard J, Weinert L, et al. Peripartum heart failure associated with prolonged tocolytic therapy. Am J Obstet Gynecol 1993; 168: 493-5.

211. Armson BA, Samuels P, Miller F, Verbalis J, Main EK. Evaluation of maternal fluid dynamics during tocolytic therapy with ritodrine hydrochloride and magnesium sulfate. Am J Obstet Gynecol 1992; 167: 758-65.

212. Romero R, Yoon BH, Mazor M, et al. The diagnostic and prognostic value of amniotic fluid white blood cell count, glucose, interleukin-6, and gram stain in patients with preterm labor and intact membranes. Am J Obstet Gynecol 1993; 169: 805-16.

213. DiGiulio DB, Romero R, Kusanovic JP, et al. Prevalence and diversity of microbes in the amniotic fluid, the fetal inflammatory response, and pregnancy outcome in women with preterm pre-labor rupture of membranes. Am J Reprod Immunol 2010; 64: 38-57.

214. Miralles R, Hodge R, McParland PC, et al. Relationship between antenatal inflammation and antenatal infection identified by detection of microbial genes by polymerase chain reaction. Pediatr Res 2005; 57: 570-7.

215. Gravett MG, Novy MJ, Rosenfeld RG, et al. Diagnosis of intra-amniotic infection by proteomic profiling and identification of novel biomarkers. JAMA 2004; 292: 462-9.

216. Hitti J, Riley DE, Krohn MA, et al. Broad-spectrum bacterial rDNA polymerase chain reaction assay for detecting amniotic fluid infection among women in premature labor. Clin Infect Dis 1997; 24: 1228-32.

217. Novy MJ, Duffy L, Axthelm MK, et al. Ureaplasma parvum or Mycoplasma hominis as sole pathogens cause chorioamnionitis, preterm delivery, and fetal pneumonia in rhesus macaques. Reprod Sci 2009; 16 : 56-70. 
218. Yoon BH, Romero R, Moon JB, et al. Clinical significance of intraamniotic inflammation in patients with preterm labor and intact membranes. Am J Obstet Gynecol 2001; 185: 1130-6.

219. Gomez R, Romero R, Nien JK, et al. A short cervix in women with preterm labor and intact membranes: a risk factor for microbial invasion of the amniotic cavity. Am J Obstet Gynecol 2005; 192: 678-89.

220. Yoon BH, Romero R, Lim JH, et al. The clinical significance of detecting Ureaplasma urealyticum by the polymerase chain reaction in the amniotic fluid of patients with preterm labor. Am J Obstet Gynecol 2003; 189: 919-24.

221. Kim SK, Romero R, Kusanovic JP, et al. The prognosis of pregnancy conceived despite the presence of an intrauterine device (IUD). J Perinat Med 2010; 38: 45-53.

222. Chaim W, Mazor M, Wiznitzer A. The prevalence and clinical significance of intraamniotic infection with Candida species in women with preterm labor. Arch Gynecol Obstet 1992; 251: 9-15.

223. Smith CV, Horenstein J, Platt LD. Intraamniotic infection with Candida albicans associated with a retained intrauterine contraceptive device: a case report. Am J Obstet Gynecol 1988; 159: 123-4.

224. Brandsma MA, Braaksma JT, van der Harten JJ. Immature delivery after intrauterine Candida albicans infection. Eur J Obstet Gynecol Reprod Biol 1975; 5: 331-5.

225. Marelli G, Mariani A, Frigerio L, Leone E, Ferrari A. Fetal Candida infection associated with an intrauterine contraceptive device. Eur J Obstet Gynecol Reprod Biol 1996; 68: 209-12.

226. Romero R, Quintero R, Nores J, et al. Amniotic fluid white blood cell count: a rapid and simple test to diagnose microbial invasion of the amniotic cavity and predict preterm delivery. Am J Obstet Gynecol 1991; 165(4 Pt 1): 821-30.

227. Romero R, Jimenez C, Lohda AK, et al. Amniotic fluid glucose concentration: a rapid and simple method for the detection of intraamniotic infection in preterm labor. Am J Obstet Gynecol 1990; 163: 968-74.

228. Romero R, Yoon BH, Mazor M, et al. A comparative study of the diagnostic performance of amniotic fluid glucose, white blood cell count, interleukin-6, and gram stain in the detection of microbial invasion in patients with preterm premature rupture of membranes. Am J Obstet Gynecol 1993; 169: 839-51.

229. Hussey MJ, Levy ES, Pombar X, Meyer P, Strassner HT. Evaluating rapid diagnostic tests of intra-amniotic infection: Gram stain, amniotic fluid glucose level, and amniotic fluid to serum glucose level ratio. Am J Obstet Gynecol 1998; 179(3 Pt 1): 650-6.

230. DiGiulio DB, Romero R, Amogan HP, et al. Microbial prevalence, diversity and abundance in amniotic fluid during preterm labor: a molecular and culture-based investigation. PLoS One 2008; 3: e3056.

231. Bianchi ME. DAMPs, PAMPs and alarmins: all we need to know about danger. J Leukoc Biol 2007; 81: 1-5.

232. Oppenheim JJ, Tewary P, de la RG, Yang D. Alarmins initiate host defense. Adv Exp Med Biol 2007; 601: 185-94.

233. Romero R, Chaiworapongsa T, Alpay S, et al. HMGB1, a late mediator of sepsis and death, is involved in the host response to intra-amniotic infection/inflammation in preterm gestation. Reprod Sci 2011; 18(3 Suppl): 245A.

234. Maymon E, Romero R, Pacora P, et al. Human neutrophil collagenase (matrix metalloproteinase 8) in parturition, premature rupture of the membranes, and intrauterine infection. Am J Obstet Gynecol 2000; 183 94-9.

235. Maymon E, Romero R, Chaiworapongsa T, et al. Amniotic fluid matrix metalloproteinase- 8 in preterm labor with intact membranes. Am Obstet Gynecol 2001; 185: 1149-55.

236. Kim KW, Romero R, Park HS, et al. A rapid matrix metalloproteinase- 8 bedside test for the detection of intraamniotic inflammation in women with preterm premature rupture of membranes. Am J Obstet Gynecol 2007; 197: 292-5.

237. Park CW, Lee SM, Park JS, et al. The antenatal identification of funisitis with a rapid MMP-8 bedside test. J Perinat Med 2008; 36: 497-502.

238. Nien JK, Yoon BH, Espinoza J, et al. A rapid MMP-8 bedside test for the detection of intra-amniotic inflammation identifies patients at risk for imminent preterm delivery. Am J Obstet Gynecol 2006; 195: 1025-30.
239. Foulon W, Van LD, Demanet C, et al. Markers of infection and their relationship to preterm delivery. Am J Perinatol 1995; 12: 208-11.

240. Straka M, Dela CW, Blackmon C, et al. Rapid detection of group B streptococcus and Escherichia coli in amniotic fluid using real-time fluorescent PCR. Infect Dis Obstet Gynecol 2004; 12: 109-14.

241. Hameed C, Tejani N, Verma UL, Archbald F. Silent chorioamnionitis as a cause of preterm labor refractory to tocolytic therapy. Am J Obstet Gynecol 1984; 149: 726-30.

242. Ogburn PL Jr, Hansen CA, Williams PP, et al. Magnesium sulfate and beta-mimetic dual-agent tocolysis in preterm labor after single-agent failure. J Reprod Med 1985; 30: 583-7.

243. Hatjis CG, Swain M. Systemic tocolysis for premature labor is associated with an increased incidence of pulmonary edema in the presence of maternal infection. Am J Obstet Gynecol 1988; 159: 723-8.

244. Goldenberg RL, Andrews WW, Faye-Petersen OM, et al. The Alabama preterm birth study: corticosteroids and neonatal outcomes in 23- to 32week newborns with various markers of intrauterine infection. Am J Obstet Gynecol 2006; 195: 1020-4.

245. Romero R, Scioscia AL, Edberg SC, Hobbins JC. Use of parenteral antibiotic therapy to eradicate bacterial colonization of amniotic fluid in premature rupture of membranes. Obstet Gynecol 1986; 67(3 Suppl): 15S-17S.

246. Romero R, Hagay Z, Nores J, Sepulveda W, Mazor M. Eradication of Ureaplasma urealyticum from the amniotic fluid with transplacental antibiotic treatment. Am J Obstet Gynecol 1992; 166: 618-20.

247. Hassan S, Romero R, Hendler I, et al. A sonographic short cervix as the only clinical manifestation of intra-amniotic infection. J Perinat Med 2006; 34: 13-19.

248. Picone O, Senat MV, Rosenblatt J, et al. Fear of pregnancy loss and fetal karyotyping: a place for third-trimester amniocentesis? Fetal Diagn Ther 2008; 23: 30-5.

249. Tabor A, Vestergaard CH, Lidegaard O. Fetal loss rate after chorionic villus sampling and amniocentesis: an 11-year national registry study. Ultrasound Obstet Gynecol 2009; 34: 19-24.

250. Cahill AG, Macones GA, Stamilio DM, et al. Pregnancy loss rate after mid-trimester amniocentesis in twin pregnancies. Am J Obstet Gynecol 2009; 200: 257-6.

251. Gaudry P, Grange G, Lebbar A, et al. Fetal loss after amniocentesis in a series of 5,780 procedures. Fetal Diagn Ther 2008; 23: 217-21.

252. Odibo AO, Gray DL, Dicke JM, et al. Revisiting the fetal loss rate after second-trimester genetic amniocentesis: a single center's 16-year experience. Obstet Gynecol 2008; 111: 589-95.

253. Liggins GC. Premature delivery of foetal lambs infused with glucocorticoids. J Endocrinol 1969; 45: 515-23.

254. Liggins GC, Howie RN. A controlled trial of antepartum glucocorticoid treatment for prevention of the respiratory distress syndrome in premature infants. Pediatrics 1972; 50: 515-25.

255. Elimian A, Garry D, Figueroa R, et al. Antenatal betamethasone compared with dexamethasone (betacode trial): a randomized controlled trial. Obstet Gynecol 2007; 110: 26-30.

256. Amorim MM, Santos LC, Faundes A. Corticosteroid therapy for prevention of respiratory distress syndrome in severe preeclampsia. Am J Obstet Gynecol 1999; 180: 1283-8.

257. Block MF, Kling OR, Crosby WM. Antenatal glucocorticoid therapy for the prevention of respiratory distress syndrome in the premature infant. Obstet Gynecol 1977; 50: 186-90.

258. Effect of antenatal dexamethasone administration on the prevention of respiratory distress syndrome. Am J Obstet Gynecol 1981; 141: 276-87.

259. Pattinson RC, Makin JD, Funk M, et al. The use of dexamethasone in women with preterm premature rupture of membranes-a multicentre, double-blind, placebo-controlled, randomised trial. Dexiprom Study Group. S Afr Med J 1999; 89: 865-70.

260. Doran TA, Swyer P, MacMurray B, et al. Results of a double-blind controlled study on the use of betamethasone in the prevention of respiratory distress syndrome. Am J Obstet Gynecol 1980; 136: 313-20.

261. Fekih M, Chaieb A, Sboui H, et al. Value of prenatal corticotherapy in the prevention of hyaline membrane disease in premature infants. Randomized prospective study. Tunis Med 2002; 80: 260-5.

262. Gamsu HR, Mullinger BM, Donnai P, Dash CH. Antenatal administration of betamethasone to prevent respiratory distress syndrome in 
preterm infants: report of a UK multicentre trial. Br J Obstet Gynaecol 1989; 96: 401-10.

263. Garite TJ, Rumney PJ, Briggs GG, et al. A randomized, placebocontrolled trial of betamethasone for the prevention of respiratory distress syndrome at 24 to 28 weeks' gestation. Am J Obstet Gynecol 1992; 166: 646-51.

264. Kari MA, Hallman M, Eronen M, et al. Prenatal dexamethasone treatment in conjunction with rescue therapy of human surfactant: a randomized placebo-controlled multicenter study. Pediatrics 1994; 93: 730-6.

265. Lewis DF, Brody K, Edwards MS, et al. Preterm premature ruptured membranes: a randomized trial of steroids after treatment with antibiotics. Obstet Gynecol 1996; 88: 801-5.

266. Morales WJ, Angel JL, O'Brien WF, Knuppel RA. Use of ampicillin and corticosteroids in premature rupture of membranes: a randomized study. Obstet Gynecol 1989; 73(5 Pt 1): 721-6.

267. Nelson LH, Meis PJ, Hatjis CG, et al. Premature rupture of membranes: a prospective, randomized evaluation of steroids, latent phase, and expectant management. Obstet Gynecol 1985; 66: 55-8.

268. Qublan HS, Malkawi HY, Hiasat MS, et al. The effect of antenatal corticosteroid therapy on pregnancies complicated by premature rupture of membranes. Clin Exp Obstet Gynecol 2001; 28: 183-6.

269. Schutte MF, Treffers PE, Koppe JG, Breur W. The influence of betamethasone and orciprenaline on the incidence of respiratory distress syndrome in the newborn after preterm labour. Br J Obstet Gynaecol 1980; 87: 127-31.

270. Silver RK, Vyskocil C, Solomon SL, et al. Randomized trial of antenatal dexamethasone in surfactant-treated infants delivered before 30 weeks' gestation. Obstet Gynecol 1996; 87(5 Pt 1): 683-91.

271. Taeusch HW Jr, Frigoletto F, Kitzmiller J, et al. Risk of respiratory distress syndrome after prenatal dexamethasone treatment. Pediatrics 1979; 63: 64-72.

272. Teramo K, Hallman M, Raivio KO. Maternal glucocorticoid in unplanned premature labor. Controlled study on the effects of betamethasone phosphate on the phospholipids of the gastric aspirate and on the adrenal cortical function of the newborn infant. Pediatr Res 1980; 14(4 Pt 1): 326-9.

273. Cararach V, Botet F, Sentis J, Carmona F. A multicenter, perspective randomized study in premature rupture of membranes (PROM). Maternal and perinatal complications. 267. 1991. Proceedings of the 13th World Congress of Gynaecology in Obstetrics (FIGO). Singapore, 1991.

274. Carlan SJ, Parsons M, O'Brien WF, Krammer J. Pharmacologic pulmonary maturation in preterm premature rupture of membranes. Am J Obstet Gynecol 1991; 164: 371.

275. Parsons MT, Sobel D, Cummiskey K, Constantine L, Roitman J. Steroid, antibiotic and tocolytic vs no steroid antibiotic and tocolytic management in patients with preterm PROM at 25-32 weeks. Proceedings of the 8th Annual Meeting of the Society of Perinatal Obstetricians 1988. Las Vegas: Nevada, 1988: 44.

276. Roberts D, Dalziel S. Antenatal corticosteroids for accelerating fetal lung maturation for women at risk of preterm birth. Cochrane Database Syst Rev 2006; 3: CD004454.

277. Crowley PA. Antenatal corticosteroid therapy: a meta-analysis of the randomized trials, 1972 to 1994. Am J Obstet Gynecol 1995; 173: 322-35.

278. Egerman RS, Pierce WF, Andersen RN, et al. A comparison of the bioavailability of oral and intramuscular dexamethasone in women in late pregnancy. Obstet Gynecol 1997; 89: 276-80.

279. Egerman RS, Mercer BM, Doss JL, Sibai BM. A randomized, controlled trial of oral and intramuscular dexamethasone in the prevention of neonatal respiratory distress syndrome. Am J Obstet Gynecol 1998; 179: 1120-3.

280. Brownfoot FC, Crowther CA, Middleton P. Different corticosteroids and regimens for accelerating fetal lung maturation for women at risk of preterm birth. Cochrane Database Syst Rev 2008: CD006764.

281. Baud O, Foix-L'Helias L, Kaminski M, et al. Antenatal glucocorticoid treatment and cystic periventricular leukomalacia in very premature infants. N Engl J Med 1999; 341: 1190-6.

282. Spinillo A, Viazzo F, Colleoni R, et al. Two-year infant neurodevelopmental outcome after single or multiple antenatal courses of corticosteroids to prevent complications of prematurity. Am J Obstet Gynecol 2004; 191: 217-24.
283. Lee BH, Stoll BJ, McDonald SA, Higgins RD. Adverse neonatal outcomes associated with antenatal dexamethasone versus antenatal betamethasone. Pediatrics 2006; 117: 1503-10.

284. Hayes EJ, Paul DA, Stahl GE, et al. Effect of antenatal corticosteroids on survival for neonates born at 23 weeks of gestation. Obstet Gynecol 2008; 111: 921-6.

285. Porto AM, Coutinho IC, Correia JB, Amorim MM. Effectiveness of antenatal corticosteroids in reducing respiratory disorders in late preterm infants: randomised clinical trial. BMJ 2011; 342: d1696.

286. Roberts D. Antenatal corticosteroids in late preterm infants. BMJ 2011; 342: d1614.

287. Dalziel SR, Walker NK, Parag V, et al. Cardiovascular risk factors after antenatal exposure to betamethasone: 30-year follow-up of a randomised controlled trial. Lancet 2005; 365: 1856-62.

288. Elimian A, Figueroa R, Spitzer AR, et al. Antenatal corticosteroids: are incomplete courses beneficial? Obstet Gynecol 2003; 102: 352-5.

289. McLaughlin KJ, Crowther CA, Walker N, Harding JE. Effects of a single course of corticosteroids given more than 7 days before birth: a systematic review. Aust NZ J Obstet Gynaecol 2003; 43: 101-6.

290. Crowther CA, Harding JE. Repeat doses of prenatal corticosteroids for women at risk of preterm birth for preventing neonatal respiratory disease. Cochrane Database Syst Rev 2007: CD003935.

291. Crowther CA, Haslam RR, Hiller JE, Doyle LW, Robinson JS. Neonatal respiratory distress syndrome after repeat exposure to antenatal corticosteroids: a randomised controlled trial. Lancet 2006; 367: 1913-19.

292. Wapner RJ, Sorokin Y, Thom EA, et al. Single versus weekly courses of antenatal corticosteroids: evaluation of safety and efficacy. Am J Obstet Gynecol 2006; 195: 633-42.

293. Wapner RJ, Sorokin Y, Mele L, et al. Long-term outcomes after repeat doses of antenatal corticosteroids. N Engl J Med 2007; 357: 1190-8.

294. Crowther CA, Doyle LW, Haslam RR, et al. Outcomes at 2 years of age after repeat doses of antenatal corticosteroids. N Engl J Med 2007; 357: 1179-89.

295. Murphy KE, Hannah ME, Willan AR, et al. Multiple courses of antenatal corticosteroids for preterm birth (MACS): a randomised controlled trial. Lancet 2008; 372: 2143-51.

296. Garite TJ, Kurtzman J, Maurel K, Clark R. Impact of a 'rescue course' of antenatal corticosteroids: a multicenter randomized placebo-controlled trial. Am J Obstet Gynecol 2009; 200: 248-9.

297. Fuchs F, Raiha NC, Seppala M. Ethanol administration in premature labour. Lancet 1971; 2: 312-13.

298. Conde-Agudelo A, Romero R, Kusanovic JP. Nifedipine in the management of preterm labor: a systematic review and metaanalysis. Am J Obstet Gynecol 2011; 204: 134-20.

299. Tan TC, Devendra K, Tan LK, Tan HK. Tocolytic treatment for the management of preterm labour: a systematic review. Singapore Med J 2006; 47: 361-6.

300. Anotayanonth S, Subhedar NV, Garner P, Neilson JP, Harigopal S. Betamimetics for inhibiting preterm labour. Cochrane Database Syst Rev 2004: CD004352.

301. Gyetvai K, Hannah ME, Hodnett ED, Ohlsson A. Tocolytics for preterm labor: a systematic review. Obstet Gynecol 1999; 94(5 Pt 2): 869-77.

302. Caritis SN, Chiao JP, Kridgen P. Comparison of pulsatile and continuous ritodrine administration: effects on uterine contractility and betaadrenergic receptor cascade. Am J Obstet Gynecol 1991; 164: 1005-11.

303. Caritis SN, Chiao JP, Moore JJ, Ward SM. Myometrial desensitization after ritodrine infusion. Am J Physiol 1987; 253(4 Pt 1): E410-17.

304. Caritis SN, Edelstone DI, Mueller-Heubach E. Pharmacologic inhibition of preterm labor. Am J Obstet Gynecol 1979; 133: 557-78.

305. Ferguson JE, Dyson DC, Holbrook RH Jr, Schutz T, Stevenson DK. Cardiovascular and metabolic effects associated with nifedipine and ritodrine tocolysis. Am J Obstet Gynecol 1989; 161: 788-95

306. Benedetti TJ. Maternal complications of parenteral beta-sympathomimetic therapy for premature labor. Am J Obstet Gynecol 1983; 145: 1-6.

307. Ingemarsson I, Arulkumaran S, Kottegoda SR. Complications of betamimetic therapy in preterm labour. Aust NZ J Obstet Gynaecol 1985; 25: $182-9$.

308. Wenstrom KD, Weiner CP, Merrill D, Niebyl J. A placebo-controlled randomized trial of the terbutaline pump for prevention of preterm delivery. Am J Perinatol 1997; 14: 87-91. 
309. Guinn DA, Goepfert AR, Owen J, Wenstrom KD, Hauth JC. Terbutaline pump maintenance therapy for prevention of preterm delivery: a doubleblind trial. Am J Obstet Gynecol 1998; 179: 874-8.

310. Hadi HA. Maternal death after terbutaline administration. Am J Obstet Gynecol 1994; 170: 1208-9.

311. Hudgens DR, Conradi SE. Sudden death associated with terbutaline sulfate administration. Am J Obstet Gynecol 1993; 169: 120-1.

312. Guinn DA, Goepfert AR, Owen J, Brumfield C, Hauth JC. Management options in women with preterm uterine contractions: a randomized clinical trial. Am J Obstet Gynecol 1997; 177: 814-18.

313. Kumar D, Zourlas PA, Barnes AC. In vitro and in vivo effects of magnesium sulfate on human uterine contractility. Am J Obstet Gynecol 1963; 86: 1036-40.

314. Cunze T, Rath W, Osmers R, et al. Magnesium and calcium concentration in the pregnant and non-pregnant myometrium. Int J Gynaecol Obstet 1995; 48: 9-13.

315. Lemancewicz A, Laudanska H, Laudanski T, Karpiuk A, Batra S. Permeability of fetal membranes to calcium and magnesium: possible role in preterm labour. Hum Reprod 2000; 15: 2018-22.

316. Mizuki J, Tasaka K, Masumoto N, et al. Magnesium sulfate inhibits oxytocin-induced calcium mobilization in human puerperal myometrial cells: possible involvement of intracellular free magnesium concentration. Am J Obstet Gynecol 1993; 169: 134-9.

317. Cox SM, Sherman ML, Leveno KJ. Randomized investigation of magnesium sulfate for prevention of preterm birth. Am J Obstet Gynecol 1990; 163: 767-72.

318. Crowther CA, Hiller JE, Doyle LW. Magnesium sulphate for preventing preterm birth in threatened preterm labour. Cochrane Database Syst Rev 2002: CD001060.

319. Grimes DA, Nanda K. Magnesium sulfate tocolysis: time to quit. Obstet Gynecol 2006; 108: 986-9.

320. Wray S, Jones K, Kupittayanant S, et al. Calcium signaling and uterine contractility. J Soc Gynecol Investig 2003; 10: 252-64.

321. Forman A, Andersson KE, Maigaard S. Effects of calcium channel blockers on the female genital tract. Acta Pharmacol Toxicol (Copenh) 1986; 58(Suppl 2): 183-92.

322. Van De WM, Kessel ET, De Kleine MJ, Oei SG. Tocolytic effectiveness of nifedipine versus ritodrine and follow-up of newborns: a randomised controlled trial. Acta Obstet Gynecol Scand 2008; 87: 340-5.

323. Houtzager BA, Hogendoorn SM, Papatsonis DN, et al. Long-term follow up of children exposed in utero to nifedipine or ritodrine for the management of preterm labour. BJOG 2006; 113: 324-31.

324. de Heus R, Mol BW, Erwich JJ, et al. Adverse drug reactions to tocolytic treatment for preterm labour: prospective cohort study. BMJ 2009; 338: b744.

325. Ben-Ami M, Giladi Y, Shalev E. The combination of magnesium sulphate and nifedipine: a cause of neuromuscular blockade. Br J Obstet Gynaecol 1994; 101: 262-3.

326. Snyder SW, Cardwell MS. Neuromuscular blockade with magnesium sulfate and nifedipine. Am J Obstet Gynecol 1989; 161: 35-6.

327. Magee LA, Miremadi S, Li J, et al. Therapy with both magnesium sulfate and nifedipine does not increase the risk of serious magnesium-related maternal side effects in women with preeclampsia. Am J Obstet Gynecol 2005; 193: 153-63.

328. Zuckerman H, Shalev E, Gilad G, Katzuni E. Further study of the inhibition of premature labor by indomethacin. Part II double-blind study. J Perinat Med 1984; 12: 25-9.

329. King J, Flenady V, Cole S, Thornton S. Cyclo-oxygenase (COX) inhibitors for treating preterm labour. Cochrane Database Syst Rev 2005: CD001992.

330. Sawdy RJ, Lye S, Fisk NM, Bennett PR. A double-blind randomized study of fetal side effects during and after the short-term maternal administration of indomethacin, sulindac, and nimesulide for the treatment of preterm labor. Am J Obstet Gynecol 2003; 188: 1046-51.

331. Stika CS, Gross GA, Leguizamon G, et al. A prospective randomized safety trial of celecoxib for treatment of preterm labor. Am J Obstet Gynecol 2002; 187: 653-60.

332. Simhan HN, Caritis SN. Prevention of preterm delivery. N Engl J Med 2007; 357: 477-87.
333. Corazza MS, Davis RF, Merritt TA, Bejar R, Cvetnic W. Prolonged bleeding time in preterm infants receiving indomethacin for patent ductus arteriosus. J Pediatr 1984; 105: 292-6.

334. Loe SM, Sanchez-Ramos L, Kaunitz AM. Assessing the neonatal safety of indomethacin tocolysis: a systematic review with meta-analysis. Obstet Gynecol 2005; 106: 173-9.

335. Lees CC, Lojacono A, Thompson C, et al. Glyceryl trinitrate and ritodrine in tocolysis: an international multicenter randomized study. GTN Preterm Labour Investigation Group. Obstet Gynecol 1999; 94: 403-8.

336. Smith GN, Walker MC, Ohlsson A, O'Brien K, Windrim R. Randomized double-blind placebo-controlled trial of transdermal nitroglycerin for preterm labor. Am J Obstet Gynecol 2007; 196: 37-8.

337. Gill A, Madsen G, Knox M, et al. Neonatal neurodevelopmental outcomes following tocolysis with glycerol trinitrate patches. Am J Obstet Gynecol 2006; 195: 484-7.

338. The oxytocin antagonist atosiban versus the beta-agonist terbutaline in the treatment of preterm labor. A randomized, double-blind, controlled study. Acta Obstet Gynecol Scand 2001; 80: 413-22.

339. Treatment of preterm labor with the oxytocin antagonist atosiban: a double-blind, randomized, controlled comparison with salbutamol. Eur J Obstet Gynecol Reprod Biol 2001; 98: 177-85.

340. Moutquin JM, Sherman D, Cohen H, et al. Double-blind, randomized, controlled trial of atosiban and ritodrine in the treatment of preterm labor: a multicenter effectiveness and safety study. Am J Obstet Gynecol 2000; 182: 1191-9.

341. Effectiveness and safety of the oxytocin antagonist atosiban versus betaadrenergic agonists in the treatment of preterm labour. The Worldwide Atosiban versus Beta-agonists Study Group. BJOG 2001; 108: 133-42.

342. Romero R, Sibai BM, Sanchez-Ramos L, et al. An oxytocin receptor antagonist (atosiban) in the treatment of preterm labor: a randomized, double-blind, placebo-controlled trial with tocolytic rescue. Am J Obstet Gynecol 2000; 182: 1173-83.

343. Valenzuela GJ, Sanchez-Ramos L, Romero R, et al. Maintenance treatment of preterm labor with the oxytocin antagonist atosiban. The Atosiban PTL-098 Study Group. Am J Obstet Gynecol 2000; 182: 1184-90.

344. Cox SM, Bohman VR, Sherman ML, Leveno KJ. Randomized investigation of antimicrobials for the prevention of preterm birth. Am J Obstet Gynecol 1996; 174(1 Pt 1): 206-10.

345. Gordon M, Samuels P, Shubert P, et al. A randomized, prospective study of adjunctive ceftizoxime in preterm labor. Am J Obstet Gynecol 1995; 172: 1546-52.

346. Kenyon SL, Taylor DJ, Tarnow-Mordi W. Broad-spectrum antibiotics for spontaneous preterm labour: the ORACLE II randomised trial. ORACLE Collaborative Group. Lancet 2001; 357: 989-94.

347. McGregor JA, French JI, Seo K. Adjunctive clindamycin therapy in preterm labor; results of a double-blinded randomized, placebocontrolled trial. Am J Obstet Gynecol 1991; 164(1 Pt 2): 259.

348. Newton ER, Dinsmoor MJ, Gibbs RS. A randomized, blinded, placebocontrolled trial of antibiotics in idiopathic preterm labor. Obstet Gynecol 1989; 74: 562-6.

349. Newton ER, Shields L, Ridgway LE III, Berkus MD, Elliott BD. Combination antibiotics and indomethacin in idiopathic preterm labor: a randomized double-blind clinical trial. Am J Obstet Gynecol 1991; 165 (6 Pt 1): 1753-9.

350. Norman K, Pattinson RC, de SJ, et al. Ampicillin and metronidazole treatment in preterm labour: a multicentre, randomised controlled trial. Br J Obstet Gynaecol 1994; 101: 404-8.

351. Oyarzun E, Gomez R, Rioseco A, et al. Antibiotic treatment in preterm labor and intact membranes: a randomized, double-blinded, placebocontrolled trial. J Matern Fetal Med 1998; 7: 105-10.

352. Romero R, Sibai B, Caritis S, et al. Antibiotic treatment of preterm labor with intact membranes: a multicenter, randomized, double-blinded, placebo-controlled trial. Am J Obstet Gynecol 1993; 169: 764-74.

353. Svare J, Langhoff-Roos J, Andersen LF, et al. Ampicillin-metronidazole treatment in idiopathic preterm labour: a randomised controlled multicentre trial. Br J Obstet Gynaecol 1997; 104: 892-7.

354. Watts DH, Krohn MA, Hillier SL, Eschenbach DA. Randomized trial of antibiotics in addition to tocolytic therapy to treat preterm labor. Infect Dis Obstet Gynecol 1994; 1: 220-7.

355. Kenyon S, Pike K, Jones DR, et al. Childhood outcomes after prescription of antibiotics to pregnant women with spontaneous 
preterm labour: 7-year follow-up of the ORACLE II trial. Lancet 2008; 372: 1319-27.

356. Allardice JG, Baskett TF, Seshia MM, Bowman N, Malazdrewicz R. Perinatal group B streptococcal colonization and infection. Am J Obstet Gynecol 1982; 142(6 Pt 1): 617-20.

357. Boyer KM, Gotoff SP. Prevention of early-onset neonatal group B streptococcal disease with selective intrapartum chemoprophylaxis. $\mathrm{N}$ Engl J Med 1986; 314: 1665-9.

358. Lim DV, Morales WJ, Walsh AF, Kazanis D. Reduction of morbidity and mortality rates for neonatal group B streptococcal disease through early diagnosis and chemoprophylaxis. J Clin Microbiol 1986; 23: 489-92.

359. Tuppurainen N, Hallman M. Prevention of neonatal group B streptococcal disease: intrapartum detection and chemoprophylaxis of heavily colonized parturients. Obstet Gynecol 1989; 73: 583-7.

360. Garland SM, Fliegner JR. Group B streptococcus (GBS) and neonatal infections: the case for intrapartum chemoprophylaxis. Aust NZ J Obstet Gynaecol 1991; 31: 119-22.

361. Matorras R, Garcia-Perea A, Omenaca F, et al. Intrapartum chemoprophylaxis of early-onset group B streptococcal disease. Eur J Obstet Gynecol Reprod Biol 1991; 40: 57-62.

362. Verani JR, McGee L, Schrag SJ. Prevention of perinatal group B streptococcal disease-revised guidelines from CDC, 2010. MMWR Recomm Rep 2010; 59: 1-36.

363. Nelson KB, Grether JK. Can magnesium sulfate reduce the risk of cerebral palsy in very low birthweight infants? Pediatrics 1995; 95: 263-9.

364. Conde-Agudelo A, Romero R. Antenatal magnesium sulfate for the prevention of cerebral palsy in preterm infants less than 34 weeks gestation: a systematic review and metaanalysis. Am J Obstet Gynecol 2009; 200: 595-609.

365. Alexander GR, Kogan MD, Himes JH, Mor JM, Goldenberg R. Racial differences in birthweight for gestational age and infant mortality in extremely-low-risk US populations. Paediatr Perinat Epidemiol 1999; 13 205-17.

366. Frisbie WP, Forbes D, Pullum SG. Compromised birth outcomes and infant mortality among racial and ethnic groups. Demography 1996; 33: 469-81.

367. Blackmore CA, Savitz DA, Edwards LJ, Harlow SD, Bowes WA Jr. Racial differences in the patterns of preterm delivery in central North Carolina, USA. Paediatr Perinat Epidemiol 1995; 9: 281-95.

368. Lieberman E, Ryan KJ, Monson RR, Schoenbaum SC. Risk factors accounting for racial differences in the rate of premature birth. $\mathrm{N}$ Engl J Med 1987; 317: 743-8.

369. Roberts WE, Morrison JC, Hamer C, Wiser WL. The incidence of preterm labor and specific risk factors. Obstet Gynecol 1990; 76(1 Suppl): 85S-9S.

370. Hauth JC, Goldenberg RL, Andrews WW, DuBard MB, Copper RL. Reduced incidence of preterm delivery with metronidazole and erythromycin in women with bacterial vaginosis. N Engl J Med 1995; 333: 1732-6.

371. McDonald HM, O'Loughlin JA, Vigneswaran R, et al. Impact of metronidazole therapy on preterm birth in women with bacterial vaginosis flora (Gardnerella vaginalis): a randomised, placebo controlled trial. Br J Obstet Gynaecol 1997; 104: 1391-7.

372. Kiss H, Petricevic L, Martina S, Husslein P. Reducing the rate of preterm birth through a simple antenatal screen-and-treat programme: a retrospective cohort study. Eur J Obstet Gynecol Reprod Biol 2010; 153: $38-42$.

373. Nygren P, Fu R, Freeman M, Bougatsos C, Guise JM. Screening and treatment for bacterial vaginosis in pregnancy: systematic review to update the 2001 U.S. Preventive Services Task Force Recommendation [Internet]. 2008.

374. Vermeulen GM, Bruinse HW. Prophylactic administration of clindamycin $2 \%$ vaginal cream to reduce the incidence of spontaneous preterm birth in women with an increased recurrence risk: a randomised placebo-controlled double-blind trial. Br J Obstet Gynaecol 1999; 106: 652-7.

375. Riggs MA, Klebanoff MA. Treatment of vaginal infections to prevent preterm birth: a meta-analysis. Clin Obstet Gynecol 2004; 47: 796-807.

376. Carey JC, Klebanoff MA, Hauth JC, et al. Metronidazole to prevent preterm delivery in pregnant women with asymptomatic bacterial vaginosis. National Institute of Child Health and Human Development
Network of Maternal-Fetal Medicine Units. N Engl J Med 2000; 342: 534-40.

377. Chien PF, Khan KS, Ogston S, Owen P. The diagnostic accuracy of cervico-vaginal fetal fibronectin in predicting preterm delivery: an overview. Br J Obstet Gynaecol 1997; 104: 436-44.

378. Faron G, Boulvain M, Irion O, Bernard PM, Fraser WD. Prediction of preterm delivery by fetal fibronectin: a meta-analysis. Obstet Gynecol 1998; 92: 153-8.

379. Revah A, Hannah ME, Sue AQA. Fetal fibronectin as a predictor of preterm birth: an overview. Am J Perinatol 1998; 15: 613-21.

380. Leitich H, Egarter C, Kaider A, et al. Cervicovaginal fetal fibronectin as a marker for preterm delivery: a meta-analysis. Am J Obstet Gynecol 1999; 180: 1169-76.

381. Kurtzman J, Chandiramani M, Briley A, et al. Quantitative fetal fibronectin screening in asymptomatic high-risk patients and the spectrum of risk for recurrent preterm delivery. Am J Obstet Gynecol 2009; 200: 263-6.

382. Conde-Agudelo A, Romero R. Cervicovaginal fetal fibronectin for the prediction of spontaneous preterm birth in multiple pregnancies: a systematic review and meta-analysis. J Matern Fetal Neonatal Med 2010; 23: $1365-76$.

383. Taipale P, Hiilesmaa V. Sonographic measurement of uterine cervix at 18-22 weeks' gestation and the risk of preterm delivery. Obstet Gynecol 1998; 92: 902-7.

384. Tekesin I, Eberhart LH, Schaefer V, Wallwiener D, Schmidt S. Evaluation and validation of a new risk score (CLEOPATRA score) to predict the probability of premature delivery for patients with threatened preterm labor. Ultrasound Obstet Gynecol 2005; 26: 699-706.

385. To MS, Skentou CA, Royston P, Yu CK, Nicolaides KH. Prediction of patient-specific risk of early preterm delivery using maternal history and sonographic measurement of cervical length: a population-based prospective study. Ultrasound Obstet Gynecol 2006; 27: 362-7.

386. Heath VC, Daskalakis G, Zagaliki A, Carvalho M, Nicolaides KH. Cervicovaginal fibronectin and cervical length at 23 weeks of gestation: relative risk of early preterm delivery. BJOG 2000; 107: 1276-81.

387. To MS, Palaniappan V, Skentou C, Gibb D, Nicolaides KH. Elective cerclage vs. ultrasound-indicated cerclage in high-risk pregnancies. Ultrasound Obstet Gynecol 2002; 19: 475-7.

388. To MS, Alfirevic Z, Heath VC, et al. Cervical cerclage for prevention of preterm delivery in women with short cervix: randomised controlled trial. Lancet 2004; 363: 1849-53.

389. Palma-Dias RS, Fonseca MM, Stein NR, Schmidt AP, Magalhaes JA. Relation of cervical length at 22-24 weeks of gestation to demographic characteristics and obstetric history. Braz J Med Biol Res 2004; 37: 737-44.

390. Alfirevic Z, len-Coward H, Molina F, Vinuesa CP, Nicolaides K. Targeted therapy for threatened preterm labor based on sonographic measurement of the cervical length: a randomized controlled trial. Ultrasound Obstet Gynecol 2007; 29: 47-50.

391. Owen J, Yost N, Berghella V, et al. Mid-trimester endovaginal sonography in women at high risk for spontaneous preterm birth. JAMA 2001; 286: 1340-8

392. Johnson JW, Austin KL, Jones GS, Davis GH, King TM. Efficacy of 17alpha-hydroxyprogesterone caproate in the prevention of premature labor. N Engl J Med 1975; 293: 675-80.

393. Yemini M, Borenstein R, Dreazen E, et al. Prevention of premature labor by 17 alpha-hydroxyprogesterone caproate. Am J Obstet Gynecol 1985; 151: 574-7.

394. Meis PJ, Klebanoff M, Thom E, et al. Prevention of recurrent preterm delivery by 17 alpha-hydroxyprogesterone caproate. N Engl J Med 2003; 348: 2379-85.

395. Fonseca EB, Bittar RE, Carvalho MH, Zugaib M. Prophylactic administration of progesterone by vaginal suppository to reduce the incidence of spontaneous preterm birth in women at increased risk: a randomized placebo-controlled double-blind study. Am J Obstet Gynecol 2003; 188: 419-24.

396. O'Brien JM, Adair CD, Lewis DF, et al. Progesterone vaginal gel for the reduction of recurrent preterm birth: primary results from a randomized, double-blind, placebo-controlled trial. Ultrasound Obstet Gynecol 2007; 30: 687-96. 
397. Fonseca EB, Celik E, Parra M, Singh M, Nicolaides KH. Progesterone and the risk of preterm birth among women with a short cervix. N Engl J Med 2007; 357: 462-9.

398. Althuisius S, Dekker G, Hummel P, et al. Cervical Incompetence Prevention Randomized Cerclage Trial (CIPRACT): effect of therapeutic cerclage with bed rest vs. bed rest only on cervical length. Ultrasound Obstet Gynecol 2002; 20: 163-7.

399. Owen J, Hankins G, Iams JD, et al. Multicenter randomized trial of cerclage for preterm birth prevention in high-risk women with shortened midtrimester cervical length. Am J Obstet Gynecol 2009; 201: 375-8.

400. Goldenberg RL, Mercer BM, Iams JD, et al. The preterm prediction study: patterns of cervicovaginal fetal fibronectin as predictors of spontaneous preterm delivery. National Institute of Child Health and Human Development Maternal-Fetal Medicine Units Network. Am J Obstet Gynecol 1997; 177: 8-12.

401. Garfield RE, Maul H, Shi L, et al. Methods and devices for the management of term and preterm labor. Ann NY Acad Sci 2001; 943: 203-24.

402. Fittkow CT, Maul H, Olson G, et al. Light-induced fluorescence of the human cervix decreases after prostaglandin application for induction of labor at term. Eur J Obstet Gynecol Reprod Biol 2005; 123: 62-6.

403. Maul H, Mackay L, Garfield RE. Cervical ripening: biochemical, molecular, and clinical considerations. Clin Obstet Gynecol 2006; 49: 551-63.

404. Robert JA, Romero R, Costigan K. Amniotic fluid concentrations of fibronectin and intra-amniotic infection. Am J Perinatol 1988; 5: 26-8.

405. Romero R, Avila C, Santhanam U, Sehgal PB. Amniotic fluid interleukin 6 in preterm labor. Association with infection. J Clin Invest 1990; 85: 1392-400.

406. Gomez R, Romero R, Galasso M, et al. The value of amniotic fluid interleukin-6, white blood cell count, and gram stain in the diagnosis of microbial invasion of the amniotic cavity in patients at term. Am J Reprod Immunol 1994; 32: 200-10.

407. Gomez R, Romero R, Nien JK, et al. Antibiotic administration to patients with preterm premature rupture of membranes does not eradicate intra-amniotic infection. J Matern Fetal Neonatal Med 2007; 20: $167-73$.

408. Espinoza J, Goncalves LF, Romero R, et al. The prevalence and clinical significance of amniotic fluid 'sludge' in patients with preterm labor and intact membranes. Ultrasound Obstet Gynecol 2005; 25: 346-52.

409. Kusanovic JP, Espinoza J, Romero R, et al. Clinical significance of the presence of amniotic fluid 'sludge' in asymptomatic patients at high risk for spontaneous preterm delivery. Ultrasound Obstet Gynecol 2007; 30: 706-14.

410. Mesiano S. Myometrial progesterone responsiveness and the control of human parturition. J Soc Gynecol Investig 2004; 11: 193-202.

411. Romano A, Delvoux B, Fischer DC, Groothuis P. The PROGINS polymorphism of the human progesterone receptor diminishes the response to progesterone. J Mol Endocrinol 2007; 38: 331-50.

412. Cramer DW, Hornstein MD, McShane P, et al. Human progesterone receptor polymorphisms and implantation failure during in vitro fertilization. Am J Obstet Gynecol 2003; 189: 1085-92.

413. Marshburn PB, Zhang J, Bahrani-Mostafavi Z, et al. Variant progesterone receptor $\mathrm{mRNAs}$ are co-expressed with the wild-type progesterone receptor mRNA in human endometrium during all phases of the menstrual cycle. Mol Hum Reprod 2005; 11: 809-15.

414. Zimmer EZ, Bronshtein M. Ultrasonic features of intra-amniotic 'unidentified debris' at 14-16 weeks' gestation. Ultrasound Obstet Gynecol 1996; 7: 178-81.

415. Sepulveda W, Reid R, Nicolaidis P, et al. Second-trimester echogenic bowel and intraamniotic bleeding: association between fetal bowel echogenicity and amniotic fluid spectrophotometry at $410 \mathrm{~nm}$. Am J Obstet Gynecol 1996; 174: 839-42.

416. Vengalil S, Santolaya-Forgas J, Meyer W, Myles T. Ultrasonically dense amniotic fluid in early pregnancy in asymptomatic women without vaginal bleeding. A report of two cases. J Reprod Med 1998; 43: 462-4.

417. Cafici D, Sepulveda W. First-trimester echogenic amniotic fluid in the acrania-anencephaly sequence. J Ultrasound Med 2003; 22: 1075-9.
418. Hallak M, Zador IE, Garcia EM, et al. Ultrasound-detected free-floating particles in amniotic fluid: correlation with maternal serum alphafetoprotein. Fetal Diagn Ther 1993; 8: 402-6.

419. Benacerraf BR, Gatter MA, Ginsburgh F. Ultrasound diagnosis of meconium-stained amniotic fluid. Am J Obstet Gynecol 1984; 149: 570-2.

420. DeVore GR, Platt LD. Ultrasound appearance of particulate matter in amniotic cavity: vernix or meconium? J Clin Ultrasound 1986; 14: 229-30.

421. Sepulveda WH, Quiroz VH. Sonographic detection of echogenic amniotic fluid and its clinical significance. J Perinat Med 1989; 17: 333-5.

422. Sherer DM, Abramowicz JS, Smith SA, Woods JR Jr. Sonographically homogeneous echogenic amniotic fluid in detecting meconium-stained amniotic fluid. Obstet Gynecol 1991; 78(5 Pt 1): 819-22.

423. Gross TL, Wolfson RN, Kuhnert PM, Sokol RJ. Sonographically detected free-floating particles in amniotic fluid predict a mature lecithinsphingomyelin ratio. J Clin Ultrasound 1985; 13: 405-9.

424. Mullin TJ, Gross TL, Wolfson RN. Ultrasound screening for free-floating particles and fetal lung maturity. Obstet Gynecol 1985; 66: 50-4.

425. Bujold E, Pasquier JC, Simoneau J, et al. Intra-amniotic sludge, short cervix, and risk of preterm delivery. J Obstet Gynaecol Can 2006; 28: 198-202.

426. Romero R, Kusanovic JP, Espinoza J, et al. What is amniotic fluid 'sludge'? Ultrasound Obstet Gynecol 2007; 30: 793-8.

427. Romero R, Schaudinn C, Kusanovic JP, et al. Detection of a microbial biofilm in intraamniotic infection. Am J Obstet Gynecol 2008; 198 : 135.

428. Donlan RM. Role of biofilms in antimicrobial resistance. ASAIO J 2000; 46: S47-52.

429. Stewart PS, Costerton JW. Antibiotic resistance of bacteria in biofilms. Lancet 2001; 358: 135-8.

430. Stewart PS. Mechanisms of antibiotic resistance in bacterial biofilms. Int J Med Microbiol 2002; 292: 107-13.

431. Davies D. Understanding biofilm resistance to antibacterial agents. Nat Rev Drug Discov 2003; 2: 114-22.

432. Liggins GC, Grieves SA, Kendall JZ, Knox BS. The physiological roles of progesterone, oestradiol-17 and prostaglandin F 2 in the control of ovine parturition. J Reprod Fertil Suppl 1972; 16(Suppl 10): 85-10.

433. Liggins GC, Fairclough RJ, Grieves SA, Kendall JZ, Knox BS. The mechanism of initiation of parturition in the ewe. Recent Prog Horm Res 1973; 29: 111-59.

434. Liggins GC. Initiation of parturition. Br Med Bull 1979; 35: 145-50.

435. Liggins GC, Fairclough RJ, Grieves SA, Forster CS, Knox BS. Parturition in the sheep. Ciba Found Symp 1977; 47: 5-30.

436. Stites DP, Siiteri PK. Steroids as immunosuppressants in pregnancy. Immunol Rev 1983; 75: 117-38.

437. Gorodeski IG, Geier A, Lunenfeld B, Beery R, Bahary CM. Progesterone (P) receptor dynamics in estrogen primed normal human cervix following P injection. Fertil Steril 1987; 47: 108-13.

438. Pepe GJ, Albrecht ED. Actions of placental and fetal adrenal steroid hormones in primate pregnancy. Endocr Rev 1995; 16: 608-48.

439. Stjernholm Y, Sahlin L, Akerberg S, et al. Cervical ripening in humans: potential roles of estrogen, progesterone, and insulin-like growth factorI. Am J Obstet Gynecol 1996; 174: 1065-71.

440. Lydon JP, DeMayo FJ, Conneely OM, O'Malley BW. Reproductive phenotypes of the progesterone receptor null mutant mouse. J Steroid Biochem Mol Biol 1996; 56: 67-77.

441. Reynolds LP, Redmer DA. Growth and development of the corpus luteum. J Reprod Fertil Suppl 1999; 54: 181-91.

442. Henson MC. Pregnancy maintenance and the regulation of placental progesterone biosynthesis in the baboon. Hum Reprod Update 1998; 4: 389-405.

443. Bernal AL. Overview of current research in parturition. Exp Physiol 2001; 86: 213-22.

444. Young IR. The comparative physiology of parturition in mammals. Front Horm Res 2001; 27: 10-30.

445. Rider V. Progesterone and the control of uterine cell proliferation and differentiation. Front Biosci 2002; 7: d1545-55.

446. Arck P, Hansen PJ, Mulac JB, Piccinni MP, Szekeres-Bartho J. Progesterone during pregnancy: endocrine-immune cross talk in 
mammalian species and the role of stress. Am J Reprod Immunol 2007; 58: $268-79$.

447. Fidel PI Jr, Romero R, Maymon E, Hertelendy F. Bacteria-induced or bacterial product-induced preterm parturition in mice and rabbits is preceded by a significant fall in serum progesterone concentrations. J Matern Fetal Med 1998; 7: 222-6.

448. Smith R, Mesiano S, McGrath S. Hormone trajectories leading to human birth. Regul Pept 2002; 108: 159-64.

449. Smith R. Parturition. N Engl J Med 2007; 356: 271-83.

450. Lye SJ. The initiation and inhibition of labor toward a molecular understanding. Semin Reprod Endocrinol 1994; 12: 284-97.

451. Lye SJ. The molecular basis of labor and tocolysis. Fetal Maternal Med Rev 1998; 10: 121-36.

452. Halmi NS. Definition of progestogens. N Engl J Med 1967; 277: 660.

453. Swyer GI, Daley D. Progesterone implantation in habitual abortion. $\mathrm{Br}$ Med J 1953; 1: 1073-7.

454. Brenner WE, Hendricks CH. Effect of medroxyprogesterone acetate upon the duration and characteristics of human gestation and labor. Am J Obstet Gynecol 1962; 83: 1094-8.

455. Levine L. Habitual abortion. A controlled study of progestational therapy. West J Surg Obstet Gynecol 1964; 72: 30-6.

456. Goldzieher JW. Double-blind trial of a progestin in habitual abortion. JAMA 1964; 188: 651-4.

457. Camilleri AP, Gauci NM. Progesterone depot in threatened abortion. Obstet Gynecol 1971; 38: 893-5.

458. Tognoni G, Ferrario L, Inzalaco M, Crosignani PG. Progestagens in threatened abortion. Lancet 1980; 2: 1242-3.

459. Varma TR, Morsman J. Evaluation of the use of Proluton-Depot (hydroxyprogesterone hexanoate) in early pregnancy. Int J Gynaecol Obstet 1982; 20: 13-17.

460. Balasch J, Creus M, Marquez M, Burzaco I, Vanrell JA. The significance of luteal phase deficiency on fertility: a diagnostic and therapeutic approach. Hum Reprod 1986; 1: 145-7.

461. Macdonald RR. Does treatment with progesterone prevent miscarriage? Br J Obstet Gynaecol 1989; 96: 257-60.

462. Rand SE. Recurrent spontaneous abortion: evaluation and management. Am Fam Physician 1993; 48: 1451-7.

463. Chantilis SJ, Zeitoun KM, Patel SI, et al. Use of Crinone vaginal progesterone gel for luteal support in in vitro fertilization cycles. Fertil Steril 1999; 72: 823-9.

464. Oates-Whitehead RM, Haas DM, Carrier JA. Progestogen for preventing miscarriage. Cochrane Database Syst Rev 2003: CD003511.

465. Nardo LG, Sallam HN. Progesterone supplementation to prevent recurrent miscarriage and to reduce implantation failure in assisted reproduction cycles. Reprod Biomed Online 2006; 13: 47-57.

466. Hartikainen-Sorri AL, Kauppila A, Tuimala R. Inefficacy of 17 alphahydroxyprogesterone caproate in the prevention of prematurity in twin pregnancy. Obstet Gynecol 1980; 56: 692-5.

467. Hauth JC, Gilstrap LC III, Brekken AL, Hauth JM. The effect of 17 alpha-hydroxyprogesterone caproate on pregnancy outcome in an active-duty military population. Am J Obstet Gynecol 1983; 146: 187-90.

468. Rouse DJ, Caritis SN, Peaceman AM, et al. A trial of 17 alphahydroxyprogesterone caproate to prevent prematurity in twins. N Engl J Med 2007; 357: 454-61.

469. Papiernik-Berkhauer E. Etude en double aveugle d'un medicament prevenant la survenue prematuree de l'accouchement chez les femmes a risque eleve d'accouchement premature. Edition Schering Serie IV 1970; 3: 65-8.

470. Daya S. Efficacy of progesterone support for pregnancy in women with recurrent miscarriage. A meta-analysis of controlled trials. Br J Obstet Gynaecol 1989; 96: 275-80.

471. Sweetman S. Martindale: the complete drug reference. 33rd edn. The Pharmaceutical Press, 2002.

472. Keirse MJ. Progestogen administration in pregnancy may prevent preterm delivery. Br J Obstet Gynaecol 1990; 97: 149-54

473. Goldstein P, Berrier J, Rosen S, Sacks HS, Chalmers TC. A meta-analysis of randomized control trials of progestational agents in pregnancy. $\mathrm{Br}$ J Obstet Gynaecol 1989; 96: 265-74.

474. Hoffman HJ, Bakketeig LS. Risk factors associated with the occurrence of preterm birth. Clin Obstet Gynecol 1984; 27: 539-52.
475. Heinonen KM, Jokela V. Multiple fetuses, growth deviations and mortality in a very preterm birth cohort. J Perinat Med 1994; 22: 5-11.

476. Imseis HM, Albert TA, Iams JD. Identifying twin gestations at low risk for preterm birth with a transvaginal ultrasonographic cervical measurement at 24 to 26 weeks' gestation. Am J Obstet Gynecol 1997; 177: 1149-55.

477. Wennerholm UB, Holm B, Mattsby-Baltzer I, et al. Fetal fibronectin, endotoxin, bacterial vaginosis and cervical length as predictors of preterm birth and neonatal morbidity in twin pregnancies. Br J Obstet Gynaecol 1997; 104: 1398-404.

478. Wright SP, Mitchell EA, Thompson JM, et al. Risk factors for preterm birth: a New Zealand study. NZ Med J 1998; 111: 14-16.

479. Kiely JL. What is the population-based risk of preterm birth among twins and other multiples? Clin Obstet Gynecol 1998; 41: 3-11.

480. Newman RB. Obstetric management of high-order multiple pregnancies. Baillieres Clin Obstet Gynaecol 1998; 12: 109-29.

481. Joseph KS, Allen AC, Dodds L, Vincer MJ, Armson BA. Causes and consequences of recent increases in preterm birth among twins. Obstet Gynecol 2001; 98: 57-64.

482. Ananth CV, Demissie K, Hanley ML. Birth weight discordancy and adverse perinatal outcomes among twin gestations in the United States: the effect of placental abruption. Am J Obstet Gynecol 2003; 188: 954-60.

483. Vintzileos AM, Ananth CV, Smulian JC, Scorza WE. The impact of prenatal care on preterm births among twin gestations in the United States, 1989-2000. Am J Obstet Gynecol 2003; 189: 818-23.

484. Blickstein I. Do multiple gestations raise the risk of cerebral palsy? Clin Perinatol 2004; 31: 395-408.

485. Elliott JP, Istwan NB, Collins A, Rhea D, Stanziano G. Indicated and non-indicated preterm delivery in twin gestations: impact on neonatal outcome and cost. J Perinatol 2005; 25: 4-7.

486. Ananth CV, Joseph KS, Demissie K, Vintzileos AM. Trends in twin preterm birth subtypes in the United States, 1989 through 2000: impact on perinatal mortality. Am J Obstet Gynecol 2005; 193(3 Pt 2): 1076-82.

487. Friedman EA, Sachtleben MR. The effect of uterine overdistention on labor. I. Multiple pregnancy. Obstet Gynecol 1964; 23: 164-72.

488. Dudley DJ. Immunoendocrinology of preterm labor: the link between corticotropin-releasing hormone and inflammation. Am J Obstet Gynecol 1999; 180(1 Pt 3): S251-6.

489. Pieber D, Allport VC, Hills F, Johnson M, Bennett PR. Interactions between progesterone receptor isoforms in myometrial cells in human labour. Mol Hum Reprod 2001; 7: 875-9.

490. Pieber D, Allport VC, Bennett PR. Progesterone receptor isoform A inhibits isoform B-mediated transactivation in human amnion. Eur J Pharmacol 2001; 427: 7-11.

491. Elovitz MA, Mrinalini C. The use of progestational agents for preterm birth: lessons from a mouse model. Am J Obstet Gynecol 2006; 195: 1004-10.

492. Norman JE, Mackenzie F, Owen P, et al. Progesterone for the prevention of preterm birth in twin pregnancy (STOPPIT): a randomised, doubleblind, placebo-controlled study and meta-analysis. Lancet 2009; 373: 2034-40.

493. Caritis SN, Rouse DJ, Peaceman AM, et al. Prevention of preterm birth in triplets using 17 alpha-hydroxyprogesterone caproate: a randomized controlled trial. Obstet Gynecol 2009; 113(2 Pt 1): 285-92.

494. Facchinetti F, Paganelli S, Comitini G, Dante G, Volpe A. Cervical length changes during preterm cervical ripening: effects of 17-alpha-hydroxyprogesterone caproate. Am J Obstet Gynecol 2007; 196: 453-4.

495. Defranco EA, O’Brien JM, Adair CD, et al. Vaginal progesterone is associated with a decrease in risk for early preterm birth and improved neonatal outcome in women with a short cervix: a secondary analysis from a randomized, double-blind, placebo-controlled trial. Ultrasound Obstet Gynecol 2007; 30: 697-705.

496. Klebanoff MA. Subgroup analysis in obstetrics clinical trials. Am J Obstet Gynecol 2007; 197: 119-22.

497. Hassan SS, Romero R, Vidyadhari D, et al. Vaginal progesterone for the prevention of preterm birth in women with a sonographic short cervix; a multicentre, randomised, double-blind, placebo-controlled trial. Ultrasound in Obstetrics and Gynecology 2011; 38:18-31.

498. Calda P. Safety signals of 17-OHP-C use in pregnancy and efficacy in the prevention of preterm birth. J Matern Fetal Neonatal Med 2009; 22: 540-2. 
499. Cahill AG, Odibo AO, Caughey AB, et al. Universal cervical length screening and treatment with vaginal progesterone to prevent preterm birth: a decision and economic analysis. Am J Obstet Gynecol 2010; 202: 548.

500. Werner E, Han C, Pettker C, et al. Universal cervical length screening to prevent preterm birth; a cost-effectiveness analysis. Ultrasound Obstet Gynecol 2011; In press.

501. Grant A, Glazener CM. Elective caesarean section versus expectant management for delivery of the small baby. Cochrane Database Syst Rev 2001: CD000078.
502. Silver RM. Delivery after previous cesarean: long-term maternal outcomes. Semin Perinatol 2010; 34: 258-66.

503. Rabe H, Reynolds G, az-Rossello J. Early versus delayed umbilical cord clamping in preterm infants. Cochrane Database Syst Rev 2004: CD003248.

504. Berghella V. Gestational age at cervical length measurement and incidence of preterm birth? Obstet Gynecol 2007; 110: 311-17.

505. Hassan SS, Chaiworapongsa T, Vaisbuch E, et al. Sonographic examination of the uterine cervix. In: Fleischer AC, Toy EC, Lee W, Manning FA, Romero R, eds. Sonography in Obstetrics and Gynecology: Principles and Practice, 7th edn. New York: McGraw-Hill, 2011: 816-47. 


\section{Prelabor rupture of the membranes \\ Roberto Romero, Lami Yeo, Francesca Gotsch, Eleazar Soto, Sonia S. Hassan, Juan Pedro Kusanovic, and Ray Bahado-Singh}

\begin{abstract}
INTRODUCTION
Prelabor rupture of the membranes (PROM) is rupture of the chorioamniotic membranes before the onset of labor (1). The "latency period" is the interval between PROM and the onset of labor. There is no agreement about the length of the interval between the rupture of the membranes and the onset of labor required to diagnose PROM. This period of time has varied between 1 and 12 hours in the literature (2-9). The consequences of PROM depend on gestational age; therefore, this condition has been classified as "preterm PROM" or "term PROM," depending upon whether the event occurs before or after 37 weeks of gestation (2-12). The term "previable PROM" has been applied to gestations in which this complication occurs before 23 weeks (12), while "preterm PROM remote from term" refers to the time frame between viability and about 32 weeks, and "PROM near term" is that which occurs between 32 and 36 weeks (12).
\end{abstract}

\section{FREQUENCY, TIMING, AND SITE OF MEMBRANE RUPTURE \\ Frequency}

Term PROM occurs in approximately $10 \%$ of patients, while the frequency of preterm PROM is $2 \%$ to $3.5 \%(5,13-17)$. Preterm PROM accounts for $30 \%$ to $40 \%$ of preterm deliveries and, therefore, is a leading clinically identifiable cause of preterm birth and a major contributor to perinatal morbidity and mortality $(2,5,13,14,16-19)$. It has been estimated that in the United States, approximately 150,000 women are diagnosed with preterm PROM every year (20).

Spontaneous Rupture of Membranes in Normal Pregnancy Figure 1 shows the proportion of women with spontaneous rupture of membranes as a function of cervical dilatation (21). Most patients rupture the chorioamniotic membranes at the end of the first stage of labor, hence the rationale for defining PROM as rupture of membranes before the onset of labor.

The site of rupture is generally located in the most dependent part of the uterine cavity in close proximity to the cervix (22). After invasive procedures such as fetoscopy, rupture of membranes can occur away from the cervix. The frequency with which spontaneous rupture of membranes occurs away from the most dependent part of the uterus (high leak) is unknown.

\section{PRETERM PROM AS AN OBSTETRICAL SYNDROME}

The current taxonomy of disease in obstetrics is largely based on the clinical presentation of the mother and not the mechanism responsible for disease. For example, the term "rupture of membranes" refers to a clinical condition in which amniotic fluid leaks from the amniotic cavity into the lower genital tract. However, the term ROM does not provide information about the cause (e.g., infection, a vascular insult, a weakness in the structure of the membranes, trauma caused by an invasive procedure [endoscopy], or other mechanisms of disease). We have proposed that the classification of diseases in obstetrics is at a stage in which we recognize syndromes caused by multiple mechanisms (23). The features of these obstetrical syndromes are (i) multiple etiologies, (ii) a long subclinical phase, (iii) frequent fetal involvement, (iv) adaptive clinical manifestations, and (v) predisposition due to gene-environment interaction (24).

Preterm PROM is one of the "great obstetrical syndromes" (23). Multiple pathologic processes can lead to preterm PROM. The chronic nature of the pathologic process leading to preterm PROM can be inferred from the observations that women with a short cervix $(<25 \mathrm{~mm})$ in the midtrimester $(25)$, a positive fetal fibronectin (FFN) in vaginal fluid (25), bacterial vaginosis (BV) (25), and bleeding in the first and second trimesters of pregnancy (25-27) are at risk for the subsequent development of preterm PROM. Fetal involvement has been demonstrated, as $10 \%$ of all fetuses with preterm PROM have evidence of fetal bacteremia demonstrated by cordocentesis (28). We have proposed that PROM is not the result of an accident, but rather a mechanism of host defense in the context of intrauterine infection (or other insults), and that spontaneous rupture of membranes occurs to facilitate the drainage of an infected cavity (intra-amniotic infection), as well as to initiate labor (amniotomy is often followed by the onset of labor in term or preterm gestation). Therefore, rupture of membranes may be considered adaptive in nature. Other mechanisms of disease, such as chronic chorioamnionitis, in which there is infiltration of the chorion laeve with lymphocytes but no evidence of infection, represent another potential mechanism for preterm PROM (29). The underlying mechanism of disease appears to be immune in nature: maternal rejection of the fetal allograft. Fetuses born to mothers whose placentas are affected with chronic chorioamnionitis have evidence of a fetal inflammatory response syndrome (FIRS). We propose that the

This work is based on several chapters that were published previously by the authors in other textbooks. This work has been modified and adapted for this textbook. The original chapters are referenced and contain a more extensive discussion of the subject. This chapter has a clinical emphasis. The work has been primarily done by Roberto Romero, who is a government employee, and therefore, is not subject to copyright. 


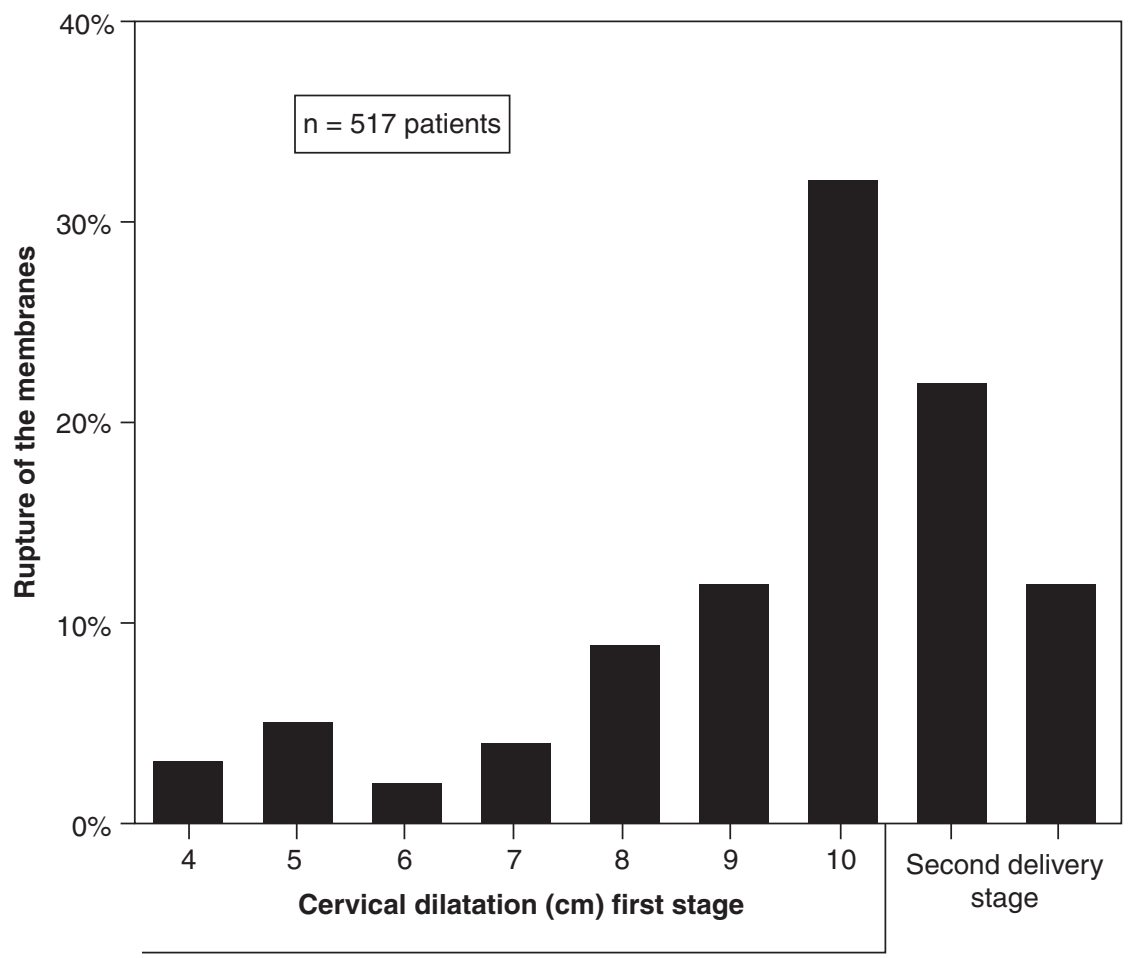

Figure 1 Rupture of membranes as a function of cervical dilatation and stage of labor. Percentage distribution for 517 normal spontaneous deliveries without medication or maneuvers; vertex presentation. Source: From Ref. 21.

initiation of labor in these cases is the result of a combination of a fetal inflammatory response and the maternal damage of chorion laeve by the lymphocytes and natural killer cells, which are capable of inducing apoptosis of the trophoblast (29). In this context, rupture of membranes and the initiation of labor may also be adaptive in nature because the fetus is in a hostile environment unrelated to infection.

Finally, there is evidence that genetic factors predispose to preterm PROM. Polymorphisms for genes coding for MMP-1 (30), MMP-9 (31), MMP-8 (32), and SERPINH1 (33) in the fetus have been associated with spontaneous rupture of membranes in case-control studies. Environmental factors, such as BV $(25,34)$ or a pro-inflammatory vaginal milieu $(35)$, have also been associated with PROM. Evidence for a gene-environment interaction for preterm birth has been demonstrated between $\mathrm{BV}$ and a polymorphism for the proinflammatory cytokine, TNF- $\alpha(36,37)$. It is also possible that gene-gene interactions are operative. The genetic predisposition for preterm PROM is likely to result from the effect of multiple alleles, which individually confer a small risk for preterm PROM. Epigenetic changes in MMP-1 have been found to be associated with preterm PROM (38). Collectively, the evidence reviewed above supports the concept that preterm PROM is not a single condition, but one of the "great obstetrical syndromes."

\section{Mechanisms of Disease Implicated in Preterm PROM}

1. Intra-amniotic infection/inflammation: Preterm PROM is associated with positive amniotic fluid cultures for bacteria at the time of admission in approximately 30\% of cases (39). With the use of molecular techniques, about $50 \%$ of cases have microbial footprints in the amniotic cavity at the time of admission (40). However, intra-amniotic infection can be a primary cause of PROM or a consequence of the rupture of membranes. There is evidence that infection precedes preterm PROM in a fraction of cases. In a study in which amniotic fluid cultures for microorganisms (41-43) and pro-inflammatory cytokines (44) were measured in the amniotic fluid of women undergoing midtrimester amniocentesis, some women with positive cultures or elevated biomarkers of inflammation subsequently developed preterm PROM (41-43).

2. Vascular pathology: In a study examining histologic lesions of the placenta, Arias et al. found that women who delivered with preterm PROM fell, in general terms, into two subgroups: those with acute histologic chorioamnionitis and those with vascular lesions of the placenta (45). Some patients have both types of lesions (46). The vascular lesions observed include "failure of physiologic transformation of the spiral arteries" $(47,48)$, atherosis, fibrinoid necrosis of the decidual vessels, and decidual vessel thrombosis consistent with decidual vasculopathy (49). Vaginal bleeding during pregnancy is a risk factor for preterm PROM (50). We have proposed that some patients who bleed in the first or second trimester of pregnancy have a disorder of decidual hemostasis (50). Vaginal bleeding may predispose to membrane rupture by causing a separation between the chorioamnion and the decidua, which weakens the fetal membranes (50). Alternatively, during the formation of a retroplacental clot, thrombin is generated (51). This enzyme can stimulate the production of MMP-1 (52) and MMP-3 (51) by decidual cells in culture media of chorioamniotic membranes (53). These MMPs can degrade fibrillar collagen (types I and III) and other components of the extracellular matrix of the chorioamniotic membranes (54). The mechanisms responsible for defective decidual hemostasis during pregnancy have not been identified. It is possible that vascular disease leading to microthrombosis in the decidua leads to local necrosis and bleeding. 
Table 1 Risk Factors Associated with Preterm PROM (at Less Than 35 weeks) Stratified by Parity (25)

\begin{tabular}{|c|c|c|c|c|}
\hline \multirow[b]{2}{*}{ Risk factor } & \multicolumn{2}{|r|}{ Nulliparous } & \multicolumn{2}{|r|}{ Multiparous } \\
\hline & Odds ratio & 95\% confidence interval & Odds ratio & 95\% confidence interval \\
\hline Cervical length $\leq 25 \mathrm{~mm}$ & 9.9 & $3.8-25.9$ & 4.2 & $2.0-8.9$ \\
\hline Previous preterm labor with intact membranes & - & - & 2.6 & $1.2-5.3$ \\
\hline Working during pregnancy & 5.3 & $1.5-18.7$ & n.s. & n.s. \\
\hline Medical complications & 4.2 & $1.1-16.0$ & n.s. & n.s. \\
\hline FFN $(+)$ and absent BV & n.s. & n.s. & 9.0 & $3.6-22.5$ \\
\hline FFN $(-)$ and present BV & n.s. & n.s. & 2.8 & $1.2-6.3$ \\
\hline
\end{tabular}

In some cases, vaginal bleeding will be the only clinical manifestation of intrauterine infection (50). This association is important because women with preterm PROM often have clinical or subclinical abruptions, and histologic examination of the membranes indicates that acute chorioamnionitis is frequent in patients with abruption (55).

3. Uterine cervical pathology: Women who had surgery in the lower genital tract, such as cervical conization $(56,57)$ or previous spontaneous abortions (58), are at greater risk for preterm PROM. Buchmayer et al. reported that a history of two or more spontaneous abortions was associated with an odds ratio of 4.1 (95\% CI: 2.2-7.8) for preterm PROM (58). We propose that some degree of cervical insufficiency (e.g., a short cervix resulting in an inadequate mucous plug) predisposes to ascending intrauterine infection $(59,60)$. This hypothesis would explain the link between a short cervix and subsequent preterm PROM (25). It is noteworthy that a cervical length $<25 \mathrm{~mm}$ confers an increased risk for preterm PROM before 35 weeks of gestation in nulliparas (OR: 9.9) as well as in multiparas (OR: 4.2) (25).

4. Acquired or congenital connective tissue disorders: There is evidence that a connective tissue disorder, which can affect the membranes (the chorioamniotic membranes are fetal tissue), may predispose to preterm PROM. For example, patients with Ehlers-Danlos syndrome are at increased risk for preterm PROM if they carry an affected fetus (61). However, the attributable risk of Mendelian disorders for preterm PROM is extremely low. It is likely that a genetic predisposition to preterm PROM can be due to the effect of multiple genes. Indeed, a relationship between polymorphisms in the promoter region of genes encoding for MMPs may also predispose to membrane rupture (MMP-1 (30), MMP-8 (32), MMP-9 (31), and SERPINH1 (33)). Polymorphisms may confer a mild to moderate risk, but this risk may be increased in patients who have a relative deficiency of vitamin $\mathrm{C}$ (environmental contribution).

\section{CLINICAL RISK FACTORS FOR MEMBRANE RUPTURE}

Harger et al. reported a comprehensive analysis of risk factors associated with preterm PROM in 341 women with preterm PROM (20-36 weeks) and 253 controls matched for maternal age, gestational age, parity, type of care (private vs. clinic), and previous vaginal or cesarean delivery (62). Three factors associated with preterm PROM were identified: (i) previous preterm delivery, (ii) vaginal bleeding during the index pregnancy, and (iii) cigarette smoking. Similar findings have been reported in a case-control study of 138 patients with preterm PROM and 267 controls (63). Vaginal bleeding, smoking, and low socioeconomic class were also found to be independent risk factors for preterm PROM.

In a large, multicenter, observational cohort study, Mercer et al. reported the risk factors for preterm PROM (less than 35 weeks), stratified according to parity (Table 1) (25). In conclusion, vaginal bleeding, a short cervix $(<25 \mathrm{~mm})$, a history of previous spontaneous preterm delivery (with intact or ruptured membranes), and smoking are risk factors for preterm PROM in the index pregnancy. A history of preterm birth with preterm PROM in a previous pregnancy confers a high risk for recurrence (approximately 20\%) (56).

\section{CLINICAL CONSEQUENCES OF PREMATURE RUPTURE OF THE MEMBRANES \\ Preterm Parturition}

Preterm PROM is followed by the onset of labor and delivery within a week in the majority of cases. The duration of the latency period is inversely related to the gestational age. The lower the gestational age, the longer the latency period (64-66). Cox et al. described the natural history of preterm PROM in 298 patients managed expectantly without the use of steroids, tocolytics or prophylactic antibiotics (64). Of the 267 patients who gave birth to infants weighing $\geq 750 \mathrm{~g}$, only $7 \%$ remained undelivered for more than 48 hours after admission.

Wilson et al. reported the outcome of 143 patients with preterm PROM managed expectantly; only $18 \%$ of patients remained undelivered 1 week after admission (65). Maternal febrile infectious morbidity (antepartum and postpartum) occurred in $10 \%$ of patients, and the neonatal death rate was $13.1 \%$.

The most comprehensive study of the natural history of preterm PROM was reported by Nelson et al., who evaluated the outcome following expectant management of 511 women with a singleton gestation and preterm PROM between 20 and 36 weeks (66). Fifty-two percent of patients delivered within 48 hours, while $12.9 \%$ remained undelivered after 1 week. The perinatal death rate was $8.4 \%$. Not surprisingly, most deaths occurred at gestational ages of less than 28 weeks $(42.7 \%)$. Maternal infection occurred in $21.7 \%$, and the 
occurrence of fetal death was strongly associated with infection. The perinatal mortality was higher in neonates born to infected mothers with preterm PROM before 28 weeks than after 28 weeks ( $46.6 \%$ vs. $1.2 \%$ ), as was infectionrelated morbidity (36\% vs. $19.8 \%$ ).

\section{Infection}

Rupture of the membranes is strongly associated with maternal or fetal infection. Maternal infection can be expressed as clinical chorioamnionitis. However, most women who have microbial invasion of the amniotic cavity (MIAC) do not have evidence of infection (67), such as fever, leukocytosis, etc. Indeed, in our experience, only $20 \%$ of patients with preterm PROM and a positive amniotic fluid culture for bacteria have clinical evidence of chorioamnionitis (68).

The prevalence of positive amniotic fluid cultures in women with preterm PROM is $32.4 \%$ (39), whereas in term PROM, the prevalence is $34.3 \%$ (69). However, this represents a minimum estimate of the frequency of infection, because the frequency is dependent upon the isolation of microorganisms with standard microbiologic techniques, which underestimate the true rate of infection. The application of molecular microbiologic techniques (cultivation independent) has yielded a higher rate of microbial footprints (50\%) (40). Patients who have a positive PCR result for microorganisms, but a negative culture, have comparable outcomes with those who have a positive amniotic fluid culture for microorganisms (70).

Genital mycoplasmas (Ureaplasma urealyticum and Mycoplasma hominis) are the most frequent isolates from the amniotic fluid, followed by Streptococcus agalactiae, Fusobacterium species, and Gardnerella vaginal is. Polymicrobial infection is found in $26.7 \%$ of cases $(28,68,71-75)$ and an inoculum size greater than $10^{5}$ colony-forming units per milliliter is found in $23 \%$ of patients (75). The most common microorganisms isolated from women with term PROM are U. urealyticum, Peptostreptococcus, Lactobacillus, Bacteroides species, and Fusobacterium (69).

Patients with intra-amniotic infection are more likely to develop chorioamnionitis, endometritis, and neonatal sepsis than patients with negative amniotic fluid cultures on admission (28,68,71-86). The frequency of respiratory distress syndrome (RDS) is twofold higher in neonates born to women with positive amniotic fluid cultures than in those born to women with negative cultures (80).

One study has examined the relationship between MIAC and the onset of preterm labor in women with PROM (68). Patients in labor on admission had a higher rate of positive amniotic fluid cultures than women admitted with preterm PROM who were not in labor ( $39 \%$ vs. $26 \%, p=0.049$ ). Moreover, $75 \%$ of patients who were not in labor on admission, but subsequently went into spontaneous labor, had a positive amniotic fluid culture around the time of the onset of labor.

Zlatnik et al. conducted a unique study in which the results of amniotic fluid culture were not used in patient management. A higher proportion of patients with positive amniotic fluid cultures delivered within 7 days, as compared with those with negative cultures (positive cultures: $89 \%$ vs. negative cultures: $45 \%, p=0.04)(75)$. These data support a relationship between MIAC and the onset of preterm labor.
Can routine antibiotic administration eradicate intraamniotic infection and prevent secondary infection? A recent study investigated the course of MIAC in 46 patients with preterm PROM (87). All underwent amniocentesis upon admission, with an $18 \%$ prevalence of intra-amniotic inflammation (defined as an amniotic fluid white blood cell (WBC) count $\geq 100 / \mathrm{mm}^{3}$ ) and a $15 \%$ prevalence of MIAC. Patients without evidence of intra-amniotic inflammation or MIAC were treated with ampicillin and erythromycin for 7 days. Those with intra-amniotic inflammation or MIAC were treated with ceftriaxone, clindamycin, and erythromycin for 10-14 days. At the time of the second amniocentesis, six of the seven patients with a prior diagnosis of MIAC were again positive for microorganisms. Of 18 patients with intraamniotic inflammation, only 3 showed no evidence of inflammation after antibiotic treatment. Of note, among patients with no evidence of intra-amniotic inflammation or MIAC at admission, 32\% developed inflammation despite therapy. Five of the nine patients in question had positive amniotic fluid cultures. These observations suggest that systemic treatment with these antibiotics may not alter the natural course of intra-amniotic infection in preterm PROM.

Evidence that fetal infection (bacteremia) is frequently present in preterm PROM was provided by Carroll et al., who performed amniocentesis and cordocentesis at the time of presentation with PROM (88). The frequency of positive fetal blood culture was $15 \%$. The authors found that for patients with positive amniotic fluid and fetal blood cultures, the median time to delivery was 2 days (range: 1-5), compared with 41 days (range: 1-161) for patients with negative cultures in both amniotic fluid and fetal blood. In the case of patients with MIAC and negative fetal blood cultures, the median interval to delivery was 9 days (range: 1-37) (88).

The microorganisms isolated from septic newborns are similar to those found in the amniotic fluid. In a study of 221 patients with preterm PROM, 6 cases with culture-proven neonatal sepsis were found (68). In five of these cases, the microorganisms were the same as those found in the amniotic fluid; in the remaining case, the amniotic fluid culture 48 hours before delivery had been negative (68). The practical implication of this observation is that an amniocentesis performed before delivery may provide microbiologic information helpful in guiding antibiotic choice for the newborn.

Fetuses with preterm PROM can mount a systemic inflammatory response (89). The term "fetal inflammatory response syndrome" refers to an elevation in the fetal plasma concentration of IL-6 $(>11 \mathrm{pg} / \mathrm{mL})$ that was associated with severe neonatal morbidity (89) and a shorter cordocentesis-todelivery interval (90).

Fetal microbial invasion or other insults result in a systemic inflammatory response that can progress toward multiple organ dysfunction, septic shock, and perhaps death in the absence of timely delivery. Evidence of multisystemic involvement in cases of FIRS includes increased concentrations of fetal plasma MMP-9 (91), an enzyme involved in the digestion of type IV collagen and in the pathophysiology of preterm PROM (92). Moreover, several fetal organs including the hematopoietic system (93-95), adrenals (96), heart (97), brain $(98,99)$, lungs $(100,101)$, and skin $(102)$ are target organs during FIRS. 
Pathologic examination of the umbilical cord is an easy approach to determine whether fetal inflammation was present before birth. Funisitis and chorionic vasculitis are the histopathologic hallmarks of FIRS (103). Funisitis is associated with endothelial activation, a key mechanism in the development of organ damage (104), and neonates with funisitis are at increased risk for neonatal sepsis (105) and long-term complications, such as bronchopulmonary dysplasia (BPD) (99) and cerebral palsy (101). Indeed, newborns with funisitis are at more than a twofold increased risk for intraventricular hemorrhage (IVH) (106) and have an 11-fold risk for the development of periventricular echolucencies (107). In the context of FIRS, the combination of inflammatory changes in the brain and fetal systemic hypotension may increase the likelihood of brain injury (108). Collectively, these observations suggest that a subset of fetuses presenting with preterm PROM have bacteremia and/or FIRS that may contribute to fetal organ damage.

The traditional view has been that MIAC is the consequence of membrane rupture. However, evidence suggests that PROM may be the result of subclinical infection and inflammation. Naeye and Peters reported in 1980 that patients with preterm PROM 1 to 4 hours before the onset of labor had a higher prevalence of histologic chorioamnionitis than patients who delivered preterm without PROM (109). Because it is unlikely that inflammation of the chorioamniotic membranes develops in 4 hours, these data suggest that, in these cases, histologic chorioamnionitis precedes rather than follows PROM. Several lines of evidence suggest that the most likely cause of histologic chorioamnionitis is subclinical infection. Bacteria have been recovered from $72 \%$ of placentas with histologic chorioamnionitis (110). Furthermore, we have demonstrated a good correlation between a positive amniotic fluid culture for microorganisms and histologic chorioamnionitis (111).

MIAC can also be the consequence of PROM. The frequency of positive amniotic fluid cultures increases with time. Indeed, $75 \%$ of patients who were quiescent on admission and subsequently went into labor had a positive amniotic fluid culture (68). Only $25 \%$ of these patients, however, had a positive culture on admission, and the remaining $50 \%$ became positive during the latency period (68). These observations are consistent with those of Naeye and Peters, who showed that the incidence of histologic chorioamnionitis increases with the duration of the latency period (109). In addition, the authors reported that histologic chorioamnionitis (defined as the presence of 4-15 neutrophils in the chorionic plate) in PROM was two- to threefold more common when rupture of membranes occurred just before the onset of labor than when it occurred after labor began (109). This suggests that inflammation (and probably infection) is, in many cases, not only a consequence of PROM, but also its cause.

\section{Abruptio Placentae}

Abruptio placentae occurs more frequently in patients with preterm PROM than in those with preterm labor and intact membranes $(2.29 \%$ vs. $0.86 \%$, respectively, RR: $3.58,95 \%$ CI: 1.74-7.39) (112). The same conclusion was reported in a systematic review (113) and a population-based epidemiologic study (114). Nelson et al. proposed that leakage of fluid after
PROM may lead to a disproportion between the placental and uterine surfaces that would favor placental separation (115). In the context of preterm PROM, the incidence of abruptio placentae increases with the severity of oligohydramnios (12.3\% for patients with a vertical pocket of $1-2 \mathrm{~cm}$ vs. $3.5 \%$ among those with a vertical pocket $>2 \mathrm{~cm}$ ) (116). However, two groups of investigators using subjective means to estimate amniotic fluid volume could not confirm this observation $(117,118)$.

An alternative hypothesis to explain the relationship between abruptio placentae and preterm PROM postulates that a disorder of decidual hemostasis leads to separation of the membranes from the decidua, with subsequent compromise of their nutritive support, weakening of the membranes, and eventual rupture. Indeed, patients with abruptio placentae after preterm PROM have a higher incidence of vaginal bleeding before rupture and during the latency period than patients without abruptio placentae.

Infection/inflammation within the decidua could also facilitate premature placental detachment. Indeed, there is an association between histologic chorioamnionitis and abruptio placentae $(55,119)$. The relative risk for abruption is 9.03 (95\% CI: $2.80-29.15)$ when chorioamnionitis is associated with preterm PROM (112).

\section{Pulmonary Hypoplasia}

The frequency of pulmonary hypoplasia is related to gestational age at the time of membrane rupture, and its presence increases the risk of neonatal death and other complications such as pneumothorax and persistent pulmonary hypertension.

Three studies examined the frequency of pulmonary hypoplasia in the context of preterm PROM. Vergani et al. conducted a prospective study of patients with PROM before 28 weeks of gestation managed conservatively and found that the frequency of pulmonary hypoplasia was 28\% (120). Gestational age at the time of PROM and presence of oligohydramnios, but not the latency period, were independent predictors of pulmonary hypoplasia (120).

Rotschild et al. studied 88 neonates born to mothers with PROM occurring before 29 weeks and a latency period of at least 1 week (121). The prevalence of pulmonary hypoplasia was $16 \%$. Gestational age at the time of PROM, but not the duration of the latency period or the severity of oligohydramnios, was associated with pulmonary hypoplasia. The risk of pulmonary hypoplasia when PROM occurs at 19 weeks was $50 \%$, whereas it was only $10 \%$ when the membranes ruptured at 25 weeks.

Winn et al. prospectively studied 163 patients with preterm PROM from 15 to 28 weeks of gestation (122). The incidence of pulmonary hypoplasia was $12.9 \%$. The authors reported that gestational age at rupture of the membranes, latency period, and either the initial or the average amniotic fluid index (AFI) had significant influence on the development of pulmonary hypoplasia.

The role of duration of rupture of membranes in the development of pulmonary hypoplasia is not clear. Univariate analysis demonstrated an association between the duration of rupture of membranes and the occurrence of pulmonary hypoplasia $(120,123-125)$. However, there is an inverse 
relationship between the duration of the latency period and the gestational age at the time of membrane rupture. Multivariate analysis has not shown a significant effect of the duration of PROM in the development of pulmonary hypoplasia (120).

Whereas Rotschild et al. (121) reported that the severity of oligohydramnios was not a factor in the development of pulmonary hypoplasia, both Vergani et al. (120) and Winn et al. (122) reported that amniotic fluid volume was an independent predictor of its development. The discrepancy between studies may be explained by the (i) determination of amniotic fluid volume, (ii) exclusion of patients, and (iii) definition of pulmonary hypoplasia.

\section{Fetal Compression Syndrome}

The fetal compression syndrome was originally described in the context of oligohydramnios and renal agenesis (126). Classically, it includes limb position deformities and craniofacial defects that are thought to result from physical compression inhibiting fetal growth and movement $(127,128)$. Nimrod et al. reported an incidence of $12 \%$ in women with preterm PROM, most occurring when the latency period was longer than 5 weeks (124). Blott and Greenough found that $46 \%$ of infants born after prolonged membrane rupture (more than 4 weeks) had limb deformities (129). The median duration of rupture in the group with deformities was 28 days, compared with 9 days in infants without deformities.

\section{Fetal Growth Restriction}

Preterm delivery resulting from preterm labor and intact membranes or preterm PROM has been associated with "fetal growth restriction." Most studies are cross-sectional and have not separated preterm PROM from preterm labor with intact membranes $(130,131)$. One interesting study determined the fetal growth rate for biometric parameters in a cohort of 69 singleton pregnancies complicated with preterm PROM (24-31 weeks) and who remained undelivered for more than 14 days. The mean growth velocity of the head and abdominal circumference was significantly lower than that of the control group ( $\mathrm{n}=345$ normal pregnancies). Neonates who had either $\mathrm{IVH}$, periventricular leukomalacia (PVL), or cerebral palsy had a lower growth velocity than those not affected with these disorders (132).

\section{Fetal Death}

The rate of fetal death in preterm PROM is approximately $1 \%$ when this complication is diagnosed after 24 weeks and 15\% if PROM occurs before (20). The etiology for fetal death remains unknown. Fetal infection, placental abruption, fetal growth restriction, umbilical cord prolapse, or accidents (resulting from compression in cases of severe oligohydramnios) have been implicated.

Fetal death, in the context of intrauterine infection, has been attributed to microbial invasion of the fetus, where the unborn child fails to deploy an inflammatory response which is sufficiently intense to stimulate labor. Indeed, the frequency of histologic chorioamnionitis (maternal inflammatory response) is nine times more frequent than funisitis (a fetal inflammatory response) in patients with stillbirth (133). In this case, in utero fetal death would represent failure of the host response mechanisms dealing with intrauterine infection.
This concept is supported by a genetic association study which demonstrated that fetal carriage of allele 2 of the gene encoding for the IL-1 receptor antagonist (IL-1 ra) is associated with fetal death (134). An excess of IL-1ra in the fetal compartment may limit the ability of the fetus to deploy a pro-inflammatory response and limit the effectiveness of the mechanisms available for host defense, including the ability to initiate labor to exit a hostile intrauterine environment.

\section{DIAGNOSIS}

\section{Presenting Symptoms}

The most common presentation of PROM is a watery vaginal discharge or a sudden gush of fluid from the vagina, as reported by the patient. Obtaining information about the timing of the initial loss of vaginal fluid, color and consistency of the discharge, and any odor may help to differentiate PROM from loss of the mucous plug in early labor, vaginal discharge associated with infection, normal leukorrhea of pregnancy, and urinary incontinence (sometimes present in pregnancy), as well as to determine the presence of blood or meconium in the amniotic fluid.

\section{Vaginal Examination}

Evaluation of the patient begins with a sterile speculum examination. Visualization of a vaginal pool or obvious leakage of fluid from the cervix into the posterior fornix is considered evidence that PROM has occurred. Increasing intra-abdominal pressure may assist in the visualization of this sign. If no fluid is present in the posterior fornix, the patient can be reexamined after resting in the supine position to allow for accumulation of fluid in the posterior fornix. Additionally, a speculum examination allows for collection of vaginal and cervical cultures and amniotic fluid to assess fetal lung maturity, as well as to rule out cord prolapse.

A sterile swab of fluid should be obtained from the posterior fornix and placed on a clean glass slide and on a piece of nitrazine paper. Amniotic fluid, when put on a slide and allowed to dry, will show arborization (ferning) under the microscope at low magnification (135). This method has an overall accuracy of 95\% (136). Rare false-positive ferning results have been described in association with fingerprints on the slide or contamination with semen and cervical mucus $(137,138)$. False negatives $(5-10 \%)$ may be caused by dry swabs or by contamination with blood $(135,139,140)$. The slide should be evaluated after at least 10 minutes of drying to decrease the false-negative rate (141).

Should a digital examination of the cervix be performed in patients with preterm PROM? The traditional view has been that "once an examination has been performed, the clock of infection starts to tick." Adoni and coworkers reported a study in which the latency period and incidence of chorioamnionitis were evaluated in patients with preterm PROM (26-34 weeks) who underwent a digital examination or a sterile speculum examination (142). The latency period was longer in patients undergoing speculum examination than in those digitally examined (9.5 days \pm 1.5 vs. 3.1 days $\pm 0.5, p<.005$ ). No significant difference in the incidence of chorioamnionitis was found. Lewis and associates prospectively collected data on 271 singleton pregnancies with preterm PROM (143). Patients 
who underwent digital examination had shorter latency periods than those who had speculum examination (digital examination: 2.1 days \pm 4.0 vs. speculum examination: 11.3 days $\pm 13.4, p<0.0001)$.

Sukcharoen et al. performed a retrospective study in which women with preterm PROM had digital examinations or speculum examinations. The authors reported no differences in latency periods or neonatal outcomes in the study groups. However, patients who underwent digital examinations had a higher frequency of chorioamnionitis (digital exam: $12 \%$ vs. speculum exam: 3.1\%, $p<0.05)(144)$.

Schutte and colleagues retrospectively examined the incidence of neonatal infection in patients with PROM according to the interval between initial digital vaginal examination and delivery (145). The incidence of neonatal infection was higher in patients examined more than 24 hours before delivery than in those whose first vaginal examination occurred less than 24 hours before delivery (33\% vs. 5\%, $p<0.0001$ ).

The only justification for performing a digital examination is to determine cervical status. In preterm gestation, this information rarely alters clinical management, but in term gestation, the cervical state may influence decisions regarding induction. There is a strong relationship between the results of sterile speculum examination and digital examination of the cervix (146-148). This was demonstrated in a study in which visual speculum and digital cervical examinations in women in labor were performed by two separate blinded examiners within 5 minutes of each other. Visual examination underestimated actual cervical dilation by only $0.6 \mathrm{~cm}$ (95\% CI: $0.58-0.62)$.

\section{Biochemical Studies to Diagnose Rupture of Membranes}

The biochemical properties of the amniotic fluid are the basis to distinguish it from other fluids that can be observed in the vagina (i.e., cervical secretions, urine, and semen). The normal $\mathrm{pH}$ of the vagina is 4.5 to 5.5 during gestation, and that of the amniotic fluid is 7.0 to 7.5. Nitrazine paper turns from yellow to blue when exposed to any alkaline fluid (i.e., $\mathrm{pH}$ of 7.0 or more) and the use of nitrazine paper has been reported to have an accuracy of $93.3 \%$ (136) to determine the presence of amniotic fluid in the vagina. False-positive results range from $1 \%$ to $17 \%$ and can result from alkaline urine, blood, semen, vaginal discharge in cases of $\mathrm{BV}$, or Trichomonas infection (149). False negatives may occur in up to $10 \%$ of cases.

Additional biochemical tests for the diagnosis of PROM include diamine oxidase (DAO) activity (150), prolactin concentration (151-153), alpha-fetoprotein (AFP; sensitivity: 94.5\% and specificity: 95.4\%) (151,154), and insulin-like growth factor-binding protein-1 (IGFBP-1) (155). AFP has been reported to be better than prolactin and more practical than DAO assay with an overall accuracy of $98 \%(156,157)$. IGFBP-1 determinations have a sensitivity of $74.4 \%$ and specificity of $92.6 \%$ (155). Overall, the sensitivity, specificity, and positive and negative predictive values of the different diagnostic tests presented today in comparison with the nitrazine test are good (158-163).

The assay for FFN is useful in the identification of patients at risk for preterm and term labor and imminent delivery. However, doubts still exist about its diagnostic value in PROM (164). Eriksen reported that for the detection of term PROM,
FFN had a sensitivity of $98.2 \%$, but a specificity of only $26.8 \%$ (165). Although the authors proposed that the false positives were the result of the detection of small amounts of amniotic fluid not detected by clinical tests (pool, nitrazine, and ferning), an alternative explanation is that a positive FFN detects degradation of the extracellular matrix in the fetalmaternal interface that precedes the clinical onset of labor rather than PROM. Support for the hypothesis derives from the observation that patients without PROM but with positive cervical FFN are more likely to deliver within 72 hours than those with negative cervical FFN (164,166-173). In conclusion, the detection of cervicovaginal FFN is not specific for PROM.

\section{Transabdominal Injection of Dye}

When the diagnosis of preterm PROM is not clear, a transabdominal injection of dye (indigo carmine, Evans blue, fluorescein) into the amniotic cavity may be used for confirmation (174-177). Methylene blue should not be used as it may cause fetal methemoglobinemia (178-180). A tampon in the vagina can document subsequent dye leakage in cases of PROM.

\section{INITIAL ASSESSMENT}

The initial evaluation of a patient with preterm PROM includes (i) accurate assessment of gestational age, (ii) estimation of fetal weight and presentation, (iii) evaluation of the risk of infection, (iv) determination of lung maturity, (v) assessment of fetal well-being, and (vi) exclusion of occult cord prolapse.

\section{Ultrasound Examination in the Evaluation of Patients with Preterm PROM}

The initial ultrasound examination aims to (i) assess fetal viability, biometry, and presentation; (ii) quantify amniotic fluid volume; (iii) rule out fetal anomalies; and (iv) confirm gestational age. The sonographic examination of fetuses with PROM may be challenging due to the reduced amniotic fluid volume. For example, sonographic estimates of fetal weight have been shown to underestimate the birth weight (181-183).

\section{Diagnosis of Intrauterine Infection in Preterm PROM}

Amniocentesis can be used for the evaluation of the microbiologic state of the amniotic cavity and of fetal lung maturity in the patient with preterm PROM (184). Results of amniotic fluid analysis provide a rational approach to the management of preterm PROM. Patients without evidence of infection/inflammation and lung immaturity could be managed expectantly while those with evidence of infection could be managed using algorithms tailored to the gestational age (see management section).

One randomized clinical trial examined the value of amniocentesis in preterm PROM (185). Forty-seven patients (26-34 weeks of gestation with an accessible amniotic fluid pocket) were randomized to amniocentesis or no amniocentesis. Indications for induction of labor included positive Gram stain of amniotic fluid or mature fetal lungs, as determined by a lecithin-to-sphingomyelin (L/S) ratio of more than 2 or positive phosphatidylglycerol (PG). Neonates born to women 
Table 2 Diagnostic Indices and Predictive Values of Different Amniotic Fluid Tests in the Detection of Positive Amniotic Fluid Culture in Patients with Premature Rupture of the Fetal Membranes

\begin{tabular}{|c|c|c|c|c|}
\hline Amniotic fluid tests & Sensitivity \% & Specificity \% & $\begin{array}{c}\text { Positive } \\
\text { predictive } \\
\text { value } \%\end{array}$ & $\begin{array}{l}\text { Negative } \\
\text { predictive } \\
\text { value } \%\end{array}$ \\
\hline Gram stain (187) & 34.8 & 96.4 & 88.9 & 63.9 \\
\hline MMP-8 (>30 ng/mL) (187) & 76.1 & 61.8 & 62.5 & 75.6 \\
\hline WBC count $(\geq 30$ cells $/ \mu \mathrm{L})(187)$ & 55.6 & 76.4 & 65.8 & 67.7 \\
\hline WBC count $(\geq 50$ cells $/ \mu \mathrm{L})(85)$ & 52.4 & 83.8 & 66.7 & 74 \\
\hline Gram stain + WBC count $(\geq 30$ cells/ $\mu \mathrm{L})(187)$ & 62.2 & 76.4 & 68.3 & 82.5 \\
\hline Gram stain + glucose $(<10 \mathrm{mg} / \mathrm{dL})(85)$ & 66.7 & 73.5 & 60.9 & 78.1 \\
\hline Gram stain + IL-6 ( $\geq 7.9$ ng/mL) (85) & 80.9 & 75 & 66.7 & 86.4 \\
\hline Gram stain+ MMP-8 (>30 ng/mL) (187) & 82.6 & 61.8 & 64.4 & 81 \\
\hline WBC count $(\geq 30$ cells/ $\mu \mathrm{L})+\mathrm{MMP}-8(>30 \mathrm{ng} / \mathrm{mL})(187)$ & 80 & 60 & 62.1 & 78.6 \\
\hline $\begin{array}{l}\text { Gram stain }+ \text { WBC count }(\geq 30 \text { cells } / \mu \mathrm{L})+ \\
\text { glucose }(<10 \mathrm{mg} / \mathrm{dL})+\mathrm{IL}-6(\geq 7.9 \mathrm{ng} / \mathrm{mL})(85)\end{array}$ & 92.9 & 47.1 & 52 & 91.4 \\
\hline
\end{tabular}

who had amniocenteses had a lower incidence of "fetal stress" during labor (diagnosed by fetal heart rate tracing) and a shorter hospital stay than those born to women who were randomized not to have amniocenteses ["fetal distress": $4 \%$ vs. $32 \%, p<0.05$; hospital stay (median): 8.5 vs. 22 days, $p<0.01]$. No differences in the rate of neonatal sepsis, maternal chorioamnionitis, or endometritis were noted between the two groups. This study had limited power to detect differences in neonatal morbidity.

The analyses of amniotic fluid used to detect the presence of MIAC or intra-amniotic inflammation include (i) Gram stain, (ii) a quantitative WBC count, (iii) glucose concentration, and (iv) microbial cultures for aerobic and anaerobic bacteria, as well as genital mycoplasmas. Patients with a negative Gram stain (read by experienced personnel) and a high WBC count (more than 30 cells $/ \mu \mathrm{L}$ ) are at a high risk of having microbial invasion with genital mycoplasmas, which are not visible on Gram stain examination. Lower concentrations of glucose in amniotic fluid $(<10 \mathrm{mg} / \mathrm{dL})$ can serve as an additional marker for MIAC. The results of amniotic fluid culture may take days to be available. Therefore, most centers rely on the determination of intra-amniotic inflammation because the outcome of preterm PROM in patients with intra-amniotic inflammation is similar to those with MIAC proven with standard microbiologic techniques (186). Table 2 summarizes the diagnostic criteria and predictive values of different amniotic fluid tests in detecting positive amniotic fluid cultures in patients with preterm PROM $(85,187)$. Amniotic fluid IL-6 performed best in detecting MIAC as well as in identifying patients at risk for impending preterm delivery and neonatal complications. We have shown that amniotic fluid IL-6 is a sensitive test for the prospective diagnosis of acute histologic chorioamnionitis (IL-6 of more than $17 \mathrm{ng} / \mathrm{mL}$ had a sensitivity of $79 \%$ and specificity of $100 \%$ ), significant neonatal morbidity (sepsis, RDS, pneumonia, IVH, BPD, and necrotizing enterocolitis (NEC)), and mortality (IL-6 of more than $17 \mathrm{ng} / \mathrm{mL}$ had a sensitivity of $69 \%$ and a specificity of 79\%) (188). Other rapid tests reported for the detection of MIAC include amniotic fluid catalase (79), alpha ${ }_{1}$-antitrypsin (84), limulus amebocyte lysate test (83), and bacterial PCR (189).

A rapid bedside test for the detection of MMP- 8 in amniotic fluid has been developed. This kit has been reported to have high accuracy in the identification of patients with MIAC and inflammation among patients with preterm labor and intact membranes (190). Future studies may determine the utility of this test in the identification of patients with intra-amniotic infection/inflammation among patients with preterm PROM.

The risk of amniocentesis, when performed by experienced individuals, appears to be extremely low. Yeast and colleagues specifically addressed this issue in 91 patients with preterm PROM in whom amniocenteses were performed (191). A retrospective review of neonatal records uncovered no evidence of fetal trauma with any procedure. This study also found that the incidence of spontaneous labor in patients who underwent amniocentesis was no different from that of patients who did not undergo amniocentesis secondary to oligohydramnios or an anterior placenta. The authors concluded that their study failed to show that amniocentesis might induce labor.

\section{Assessment of Lung Maturity}

Lung maturity can be assessed from the amniotic fluid obtained by amniocentesis or from the vaginal pool. The latter has the advantage of being less invasive and more feasible in patients with oligohydramnios. Amniotic fluid from the vaginal pool can be collected in three ways: (i) from 
the posterior vaginal fornix by sterile speculum examination, (ii) in a clean bedpan maintained under the patient, or (iii) by the use of obstetric perineal pads left in place for 12 to 24 hours to ensure saturation (192-195). The success rate in obtaining fluid within 48 hours with these noninvasive techniques ranges from $54 \%$ to $100 \%(194,195)$. Using a pad to detect PG, Estol et al. found a sensitivity of $88 \%$, specificity of $76 \%$, positive predictive value of $34 \%$, and negative predictive value of $98 \%$ (196). Lewis et al. investigated the value of a rapid antibody agglutination method (Amniostat FLM) to detect PG in vaginal pool samples (197). Of 201 patients between 26 and 36 weeks of gestation, 36 had positive PG, and none of the infants born to these mothers developed RDS. PG was detectable only after 30 weeks of gestation.

The reliability of lung maturity tests from amniotic fluid collected vaginally has been challenged $(198,199)$. This section reviews the correlation between the $\mathrm{L} / \mathrm{S}$ ratio and $\mathrm{PG}$ results in amniotic fluid obtained by amniocentesis and from the vaginal pool. Shaver and associates compared the phospholipid profile of paired amniotic fluid samples in 28 patients with preterm PROM (192). No significant difference was found in the concentrations of PG, phosphatidylinositol, phosphatidylethanolamine, and phosphatidylserine in amniotic fluid obtained by the two sampling methods. The L/S ratio was higher in fluid collected transvaginally than in fluid collected transabdominally, but this difference did not reach statistical significance. The only phospholipid clearly increased by vaginal contamination was lysolecithin.

Dombroski et al. reported a study in which amniotic fluid was obtained by amniocentesis in patients at term in labor (200). Thirty minutes after artificial rupture of membranes, a vaginal sample of amniotic fluid was collected. L/S ratios obtained from amniotic fluid in the vaginal pool samples were significantly lower than those obtained by amniocentesis. However, in 22\% of cases, L/S ratios were higher in the vaginal pool samples than amniocentesis.

Several studies have examined the value of PG determinations in amniotic fluid obtained transvaginally. Stedman et al. reported that of 25 patients with PROM between 26 and 34 weeks, $60 \%$ had positive PG and none of their neonates developed RDS (within 72 hours of the test) (193). Among the newborns of the 10 patients with negative PG, four developed RDS. Similarly, Brame and MacKenna reported no cases of neonatal RDS in 36 patients with PG found in vaginal fluid (194).

The possibility that bacterial contamination from vaginal secretions may lead to false-positive PG results has been raised by Schumacher and associates, who reported that one patient had PG detected in the fluid from the vaginal pool, but not in the fluid retrieved by transabdominal amniocentesis (199). The neonate developed respiratory insufficiency that was attributed to either RDS or pneumonia (the amniotic fluid culture was positive for bacteria). These investigators also demonstrated that bacteria might be a source of PG. Therefore, excessive bacterial contamination may alter results of PG determinations. It would seem prudent to minimize the interval between sample collection and assay in the hope of preventing bacterial growth in the sample.
Three studies have reported neonatal outcome and L/S ratio results in preterm PROM $(194,195,201)$. In two of the studies, a mature L/S ratio was an indication for delivery $(195,201)$. In the third study, the presence of PG was used as an indication for delivery (194). The data are consistent: with a mature L/S ratio, the risk of RDS is low. An $\mathrm{L} / \mathrm{S}$ ratio of more than 2 was found in 103 patients, and none of the neonates developed RDS.

The available evidence indicates that fetal lung maturity studies can be performed on amniotic fluid obtained from the vagina, and that a mature $L / S$ ratio or the presence of $P G$ is associated with a very low risk of RDS. Moreover, this noninvasive, low-risk approach allows for serial L/S and PG determinations.

A mature phospholipid test has been demonstrated in approximately $50 \%$ of patients with preterm PROM at gestational ages of less than 34 weeks $(72,74,185)$. Garite and associates reported that none of the neonates with an $\mathrm{L} / \mathrm{S}$ ratio of 1.8 or greater developed RDS (74). The incidence of this complication in neonates with an immature L/S was $33 \%$.

Two randomized clinical trials have examined the outcome of induction of labor in patients with a mature result. In the first trial, 47 patients with preterm PROM (less than 36 gestational weeks) and mature amniotic fluid indices were randomized to either prompt delivery or expectant management (202). A mature test was defined as an L/S ratio above 2 or a Foam Stability Index (FSI) of 47 or more (often from vaginal fluid). There was no difference in perinatal mortality between the two groups. There were no cases of RDS in the expectant management group, but two in the prompt delivery group. One newborn died from severe hyaline membrane disease (birth weight $900 \mathrm{~g}$, vaginal FSI $=48$ ), and the other neonate survived (birth weight $1700 \mathrm{~g}$, vaginal $\mathrm{L} / \mathrm{S}=2.0$ ). There were no differences in the rate of neonatal sepsis or other neonatal complications in the two groups. However, the only two cases of intracranial hemorrhage occurred in the prompt delivery group. Maternal chorioamnionitis was more common in the expectantly managed group than in the delivery group ( $38 \%$ vs. $8 \%, p<0.02)$.

Mercer et al. reported the results of a randomized clinical trial in which 93 women with mature amniotic fluid phospholipid studies (vaginal or transabdominal amniocentesis FSI $\geq 47$ ) were randomized to induction of labor with oxytocin or expectant management (bed rest) (203). Maternal chorioamnionitis was more frequent in the expectant group. However, this difference did not reach statistical significance. There were no significant differences in the cesarean delivery rate or in the incidence of confirmed neonatal sepsis between the groups. Suspected sepsis was higher in neonates born to women in the expectant group, as was antibiotic administration and septic workups. However, neonatologists were not blinded to treatment allocation.

\section{Assessment of Fetal Well-Being}

The goal of fetal evaluation is to identify fetal infection/ inflammation or a pathologic process, which increases the risk of antepartum or neonatal death. Methods of fetal surveillance include the non-stress test (NST) and the components of the biophysical profile (BPP). 


\section{NST}

The differential diagnosis of a nonreactive NST is (i) preterm gestation, (ii) infection, and (iii) hypoxia. The interpretation and the significance and management of fetal heart rate decelerations associated with umbilical cord compression due to oligohydramnios are also a challenge.

Fetuses with preterm PROM between 24 and 37 weeks have a significantly higher incidence of reactive tracings than gestational age-matched counterparts with intact membranes (204-206). This has been attributed to "accelerated fetal central nervous system maturation" and umbilical vein compression with resulting fetal heart rate accelerations (207). Thus, lack of reactivity should not be ascribed to preterm gestation without further investigation.

A nonreactive NST is frequently observed in fetuses with MIAC. Three studies (208-210) have found the NST to be an insensitive predictor of infection-related outcome. A major issue is the high false-positive rate (approximately 35\%) of the NST for the detection of infection. Therefore, a nonreactive NST is not sufficient to diagnose infection. Evaluation of other biophysical parameters and the results of amniocentesis are recommended before delivery can be indicated (see below).

\section{Assessment of Amniotic Fluid Volume}

Contrary to what is generally believed, rupture of membranes is not necessarily associated with oligohydramnios. Harding et al. noted that the AFI in patients with preterm PROM remains stable after the rupture of membranes, with the mean AFI on admission being $5.9 \pm 2.5 \mathrm{~cm}$ and on the day of delivery $5.4 \pm 2.0 \mathrm{~cm}$ (211). Moreover, Vintzileos et al. reported that $65.5 \%$ of patients with PROM had a vertical pocket of amniotic fluid greater than $2 \mathrm{~cm}$, while $15.5 \%$ had a vertical pocket between 1 and $2 \mathrm{~cm}$. Only 19\% had a vertical pocket less than $1 \mathrm{~cm}$ (212).

Several studies have examined the relationship between oligohydramnios and outcomes in PROM. Patients with a vertical amniotic fluid pocket $<1 \mathrm{~cm}$ have a shorter latency period and a higher incidence of chorioamnionitis and neonatal sepsis than patients with a vertical pocket greater than $2 \mathrm{~cm}$ (212). Similar findings were reported by Gonik et al. (213). Women with a vertical amniotic fluid pocket of $<1 \mathrm{~cm}$ had a higher incidence of chorioamnionitis and endometritis than those with an amniotic fluid pocket of $>1 \mathrm{~cm}$. No difference in the duration of the latency period between the two groups was found (213).

Hadi et al. reported that chorioamnionitis occurred in $26.4 \%$ of women with an amniotic fluid pocket of less than $2 \mathrm{~cm}$ (214). Similarly, Lao et al. (215) used a cutoff of $2 \mathrm{~cm}$ as the largest pocket of amniotic fluid to define oligohydramnios and found that the frequency of chorioamnionitis and funisitis was higher in patients with oligohydramnios than in those without reduced amniotic fluid volume (chorioamnionitis: $55.3 \%$ vs. $29.3 \%$; funisitis: $44.7 \%$ vs. $16.7 \%$ ). A reduction in amniotic fluid volume was also associated with MIAC.

There is an association between reduced amniotic fluid volume and maternal or neonatal infection-related morbidity and MIAC. The reason for the high rate of infection in patients with oligohydramnios is unknown. Intra-amniotic infection may alter amniotic fluid dynamics, leading to a reduction in fluid volume. Yoon et al. proposed that redistribution of blood flow away from the kidneys might take place as part of the host response to microbial products, and this may lead to oligohydramnios (216).

Patients with decelerations have a lower AFI than those without decelerations $(4.32 \mathrm{~cm} \pm 1.67$ vs. $6.47 \mathrm{~cm} \pm 3.59$, $p<0.01)$ (217). This observation suggests that cord compression due to oligohydramnios may be the mechanism behind variable decelerations observed in patients with PROM.

Preterm PROM is associated with a significant and prolonged reduction of fetal breathing movements lasting approximately 2 weeks $(218,219)$. This phenomenon seems to be related to rupture of membranes per se, rather than to infection, hypoxia, or intrauterine growth restriction, even though the precise mechanisms are unknown. Membrane rupture leads to a reduction in intra-amniotic pressure and, thus, favors loss of lung fluid. Teleologically, a reduction in fetal breathing may be a mechanism to protect against lung fluid loss and pulmonary hypoplasia.

Vintzileos was the first to document an association between infection and decreased fetal breathing activity in preterm PROM $(220,221)$. Subsequently, we confirmed these findings and documented that women with positive amniotic fluid cultures had fewer and shorter episodes of fetal breathing activity than women with negative amniotic fluid cultures (222).

The presence of fetal breathing has a very high negative predictive value (approximately 95\%) for MIAC and neonatal sepsis. However, the absence of breathing activity has a limited positive predictive value (approximately 50\%) for either of these two outcomes and, thus, it cannot be used as an indication for delivery. Therefore, the presence of breathing indicates that infection is unlikely.

Intra-amniotic infection is associated with a dramatic reduction in fetal body movements (222). Decreased fetal motion in the context of infection may be the counterpart of the reduction in motor behavior observed during the course of febrile illnesses in adults and children.

The BPP has been found to be helpful in the management of patients with PROM (204,207-210,220,222-227). Vintzileos et al., using logistic regression analysis, demonstrated that each component of the BPP contains useful information for the prediction of infection-related morbidity (defined as maternal chorioamnionitis, possible neonatal sepsis, and proven neonatal sepsis). In their first study, a modified BPP scoring system that incorporated placental grading (with a maximal score of 12) was used (220). A BPP score of 7 or less was much better than any single component of the BPP in the prediction of infection-related outcome. Placental grading was the only parameter that had no predictive value. Thus, it was excluded from subsequent studies. The diagnostic indices of a BPP score $\leq 7$ (performed 24 hours before delivery) were sensitivity $94 \%$, specificity $97 \%$, positive predictive value $95 \%$, and negative predictive value $97 \%$ in a population with a prevalence of infection-related outcome of $30 \%$. This study was observational in nature and, thus, the BPP was not used for patient management.

Subsequently, Vintzileos et al. compared the outcome of pregnancy in patients managed with serial BPPs with two historical control groups: (i) expectant management without BPP or amniocentesis and (ii) management with a single amniocentesis on admission (228). A BPP score $\leq 7$ on two 


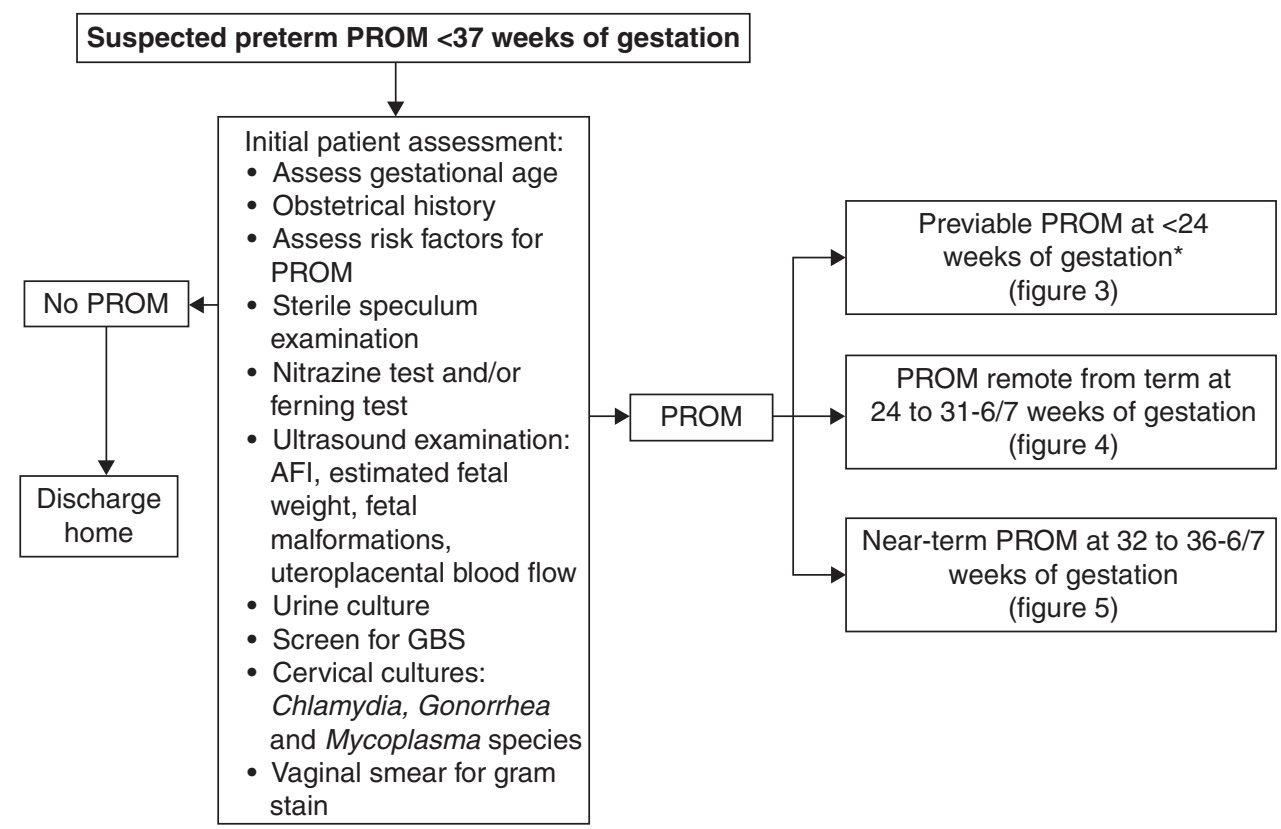

Figure 2 Initial assessment of preterm prelabor rupture of the membranes (PROM). Abbreviations: AFI, amniotic fluid index; GBS, Group B Streptococcus. Source: From Ref. 235.

${ }^{*}$ Original author used gestational age cutoff of 23 weeks.

examinations 2 hours apart was used as an indication for delivery. An abnormal score required a nonreactive NST and absence of fetal breathing. The results of this study indicated that patients managed with daily BPPs had a lower rate of overall neonatal sepsis (suspected and culture proven) than patients in either control group. This study did not provide the frequency of other indices of neonatal morbidity (e.g., RDS, IVH, duration of mechanical ventilation) in the different groups. This issue is important, since 14 patients who were delivered because of a low BPP score showed no evidence of neonatal infection and, thus, could be considered false positives. If intervention was not associated with an increased rate of other neonatal complications, management with serial BPPs would seem a reasonable approach. The investigators found that the BPP had limitations when the interval between the test and the delivery was longer than 24 hours and that maternal infection without fetal infection was not correlated with the results of the BPP scoring. Vintzileos et al. subsequently reported on 111 fetuses with preterm PROM followed with daily BPPs and found that the more biophysical activities became compromised, the higher the incidence of infectionrelated complications (207).

It is noteworthy that subsequent to this work, three studies $(223,224,227)$ reported an association between the results of the BPP and infection-related outcomes, and others could not confirm such an association $(225,226)$. Our explanation for the apparent discrepancy is that studies reporting negative results used the BPP at less frequent testing intervals (48- to 72-hour intervals) than the daily testing used in positive reports.

\section{MANAGEMENT OF PATIENTS WITH PREMATURE PROM}

The management of patients with premature PROM depends on the gestational age at the time of membrane rupture (Fig. 2).

\section{Previable PROM}

The major complications of previable PROM are maternal infection, late abortion/preterm labor, low neonatal survival, and a high risk of neurologic complications (Fig. 3) (229,230). Patients are generally offered two options: induction of labor or expectant management. The presence of intra-amniotic inflammation/infection in amniotic fluid analysis carries a poor prognosis because of the risk of spontaneous preterm labor as well as fetal morbidity. Management of these patients requires an in-depth discussion involving the parents, neonatologists, and obstetricians and careful documentation in the medical record. The value of antibiotic or corticosteroid administration in previable PROM has not been established. However, our practice is to administer antibiotics to the patient who desires expectant management. A systematic review of previable PROM indicates that the quality of the evidence to support the management is not high (231).

Leakage of amniotic fluid after second-trimester amniocentesis should be considered a separate entity from previable PROM. It occurs in $1.2 \%$ of patients and is usually transient in nature (232). The risk of delayed PROM in these cases is no different from that in the general population (233).

\section{PROM Remote from Term (24 to 31-6/7 Weeks of Gestation)}

The management goals are to (i) exclude intra-amniotic infection/inflammation and (ii) institute expectant management in patients without documented infection/inflammation (Fig. 4).

\section{Intra-amniotic Infection/Inflammation and Its Management}

The most accurate method for diagnosis of intra-amniotic infection/inflammation is amniocentesis. Once intra-amniotic infection/inflammation is identified in patients between 24 and 31 completed weeks of gestation, the optimal management is a challenge: the earlier the gestational age, the more difficult the dilemma. In patients who are close to 32 weeks 


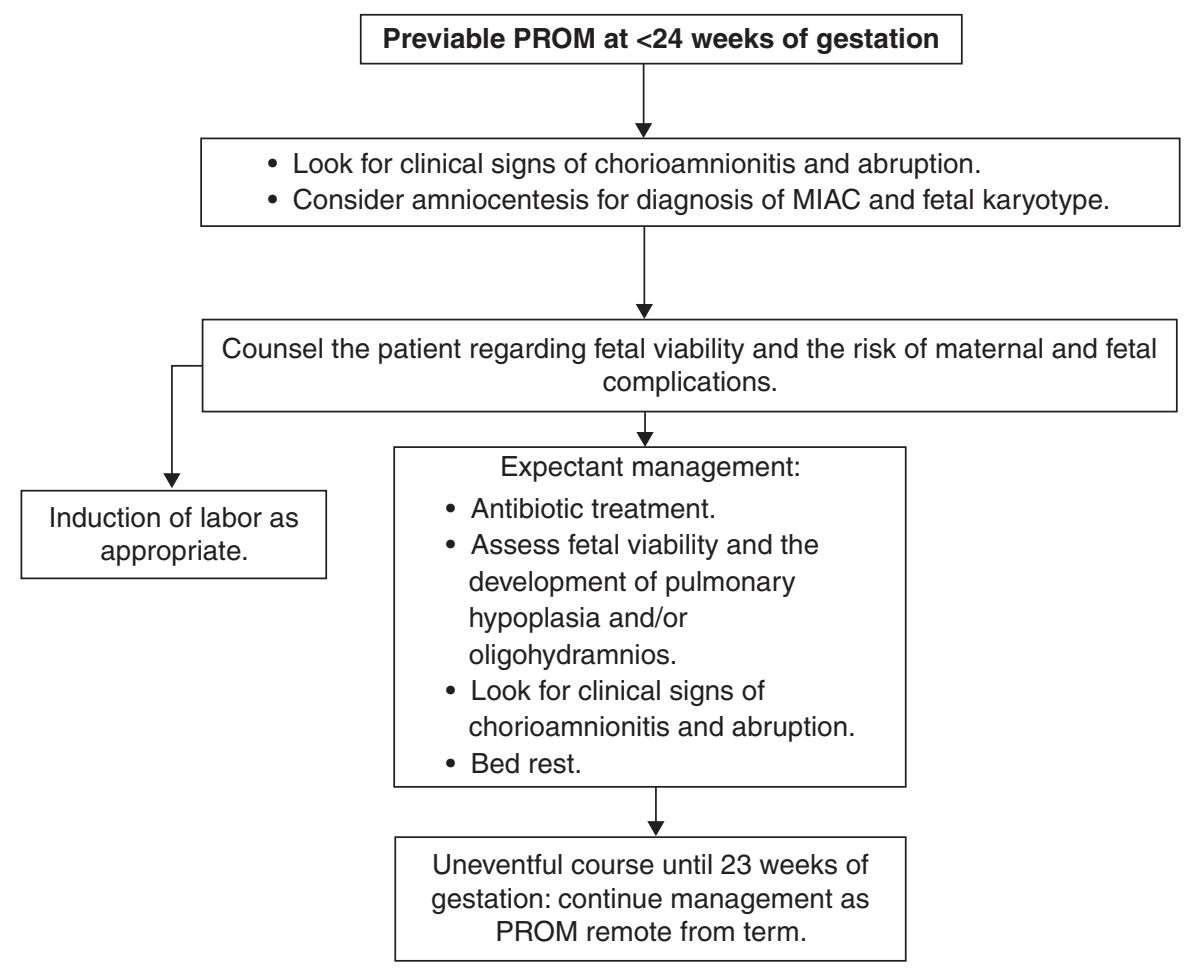

Figure 3 Management of previable prelabor rupture of the membranes (PROM) (before 24 weeks of gestation). Abbreviations: MIAC, microbial invasion of the amniotic cavity. Source: From Ref. 235.

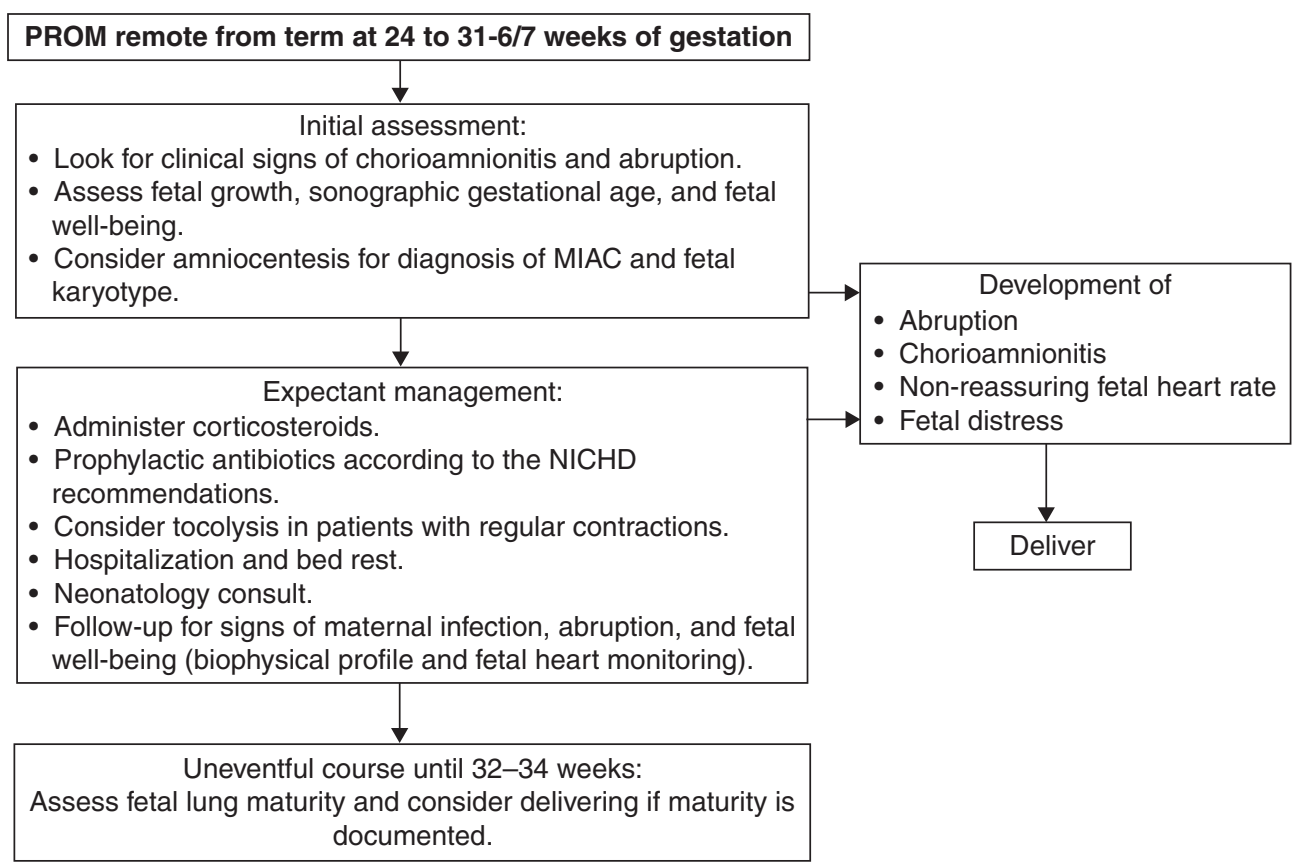

Figure 4 Management of prelabor rupture of the membranes (PROM) remote from term (between 24 and 31-6/7 weeks of gestation). Abbreviations: MIAC, microbial invasion of the amniotic cavity; NICHD, National Institute of Child Health and Human Development, NIH/DHHS. Source: From Ref. 235.

of gestation, delivery would avoid continuous exposure to microbial products and inflammatory agents and would be unlikely to increase neonatal morbidity. These patients are managed in our unit with detailed counseling, antibiotic administration, and delivery. In contrast, in patients close to 24 weeks of gestation, the option of parenteral administration of antibiotics is considered to eradicate intra-amniotic infection and inflammation. Patients are informed that this alternative may prolong pregnancy, eradicate intra-amniotic infection, and reduce the risk of extreme prematurity, but that it requires intensive surveillance and repeat evaluation of the amniotic cavity to ensure eradication of microorganisms and reduce intra-amniotic inflammation (as determined by the amniotic fluid WBC count). Despite these interventions, the risk for infection and prematurity is not eliminated. Broad coverage is recommended before the results of cultures are 
available and this approach modified once the specific microorganisms involved are identified. The choice of antibiotics is informed by the results of microbial cultures. Our choice for broad coverage antibiotics includes azithromycin, clindamycin, and ampicillin. We have also used a combination of ceftriaxone, clindamycin, and erythromycin for 10 to 14 days (87). Azithromycin is included because $U$. urealyticum is the most frequent microorganism found in the amniotic cavity (67).

Antibiotic treatment aimed at the eradication of intraamniotic infection should not be confused with the prophylactic treatment, which is now the standard of care for patients with preterm PROM, regardless of whether the inflammatory/ infection state of the amniotic fluid is known. Thus, patients in this gestational age range, without evidence of infection and inflammation, are given prophylactic treatment with antibiotics (ampicillin and erythromycin). The patient in whom an amniocentesis cannot be performed is managed taking into account the results of biophysical testing, and antibiotics and steroids are administered.

In summary, the management of PROM between 24 and 31 completed gestational weeks comprises (i) maternal and fetal inpatient surveillance in a tertiary medical center, (ii) administration of corticosteroids to accelerate fetal lung maturity (234), and (iii) antibiotic administration, which may be therapeutic or prophylactic $(235,236)$.

\section{"Prophylactic" Antibiotic Administration}

Antibiotic administration has now become standard of care in patients with preterm PROM. This practice is based upon the results of several randomized clinical trials, in which antibiotic administration is associated with prolongation of pregnancy, a reduced rate of maternal chorioamnionitis (237), and a reduced frequency of neonatal morbidity, measured as "composite neonatal outcome" (238). This approach has been often referred to as "prophylactic" antibiotic administration. However, this may be a misnomer. One-third of women with preterm PROM have a positive amniotic fluid culture on admission (39); furthermore, the frequency of MIAC increases as the patients are being observed in the antepartum ward to the point that at the time of the onset of labor, $75 \%$ of patients will have a positive amniotic fluid culture for microorganisms (68). These studies were conducted before the administration of prophylactic antibiotics and demonstrate that microorganisms are present at admission and that secondary infection of the amniotic cavity occurs during expectant management. It would be inaccurate to refer to "prophylactic" administration as therapy instituted with patients who have a proven infection (one-third of all patients). Anti-microbial therapy may prolong pregnancy by controlling microbial proliferation of an existing infection and preventing secondary infection/inflammation. However, antibiotic administration is not uniformly efficacious in eradicating microbially proven intra-amniotic infection (87).

Several investigators have conducted randomized clinical trials to assess the potential benefits of prophylactic antibiotic administration in patients with preterm PROM (238-251). Mercer et al. (251) reported a randomized clinical trial in which patients were allocated to receive intravenous ampicillin ( 2 g every 6 hours) and erythromycin ( 250 mg every 6 hours for 48 hours, followed by oral amoxicillin and erythromycin base (every 8 hours for 5 days) versus placebos. Recruitment was restricted to patients with a gestational age ranging from 24 to 32 weeks. Group B Streptococcus (GBS) carriers were identified and treated, and tocolysis and steroids were not administered after randomization. The primary outcome of the trial was a composite variable that included any of the following: fetal or infant death, RDS, severe IVH, stage II or III of NEC, or sepsis within 72 hours of birth (251). Antibiotic administration was associated with prolongation of pregnancy and a significant reduction in the rate of RDS (RR: 0.83,95\% CI: 0.69-0.99), NEC (RR: 0.4, 95\% CI: 0.17-0.95), clinical chorioamnionitis, and the composite primary outcome, which is an index of fetal/infant morbidity and mortality (RR: 0.84, 95\% CI: 0.71-0.99). These differences were not demonstrated in GBS carriers, an observation attributable to antibiotic administration to patients allocated to the placebo group for this clinical indication and thus obscuring the potential effects of antibiotic administration (251).

In the ORACLE study (238), 4826 women with preterm PROM were randomly assigned to (i) erythromycin, (ii) coamoxiclav (amoxicillin and clavulanic acid), (iii) erythromycin and co-amoxiclav, and (iv) placebo. The study included patients before 36-6/7 weeks from 161 medical centers. Tocolysis and corticosteroid administration was left to the discretion of the attending physician. The primary outcome measure was a composite variable, which included neonatal death, chronic lung disease, or major cerebral abnormality before discharge from the hospital.

Among neonates of patients with singleton gestations allocated to erythromycin only, fewer had the primary composite outcome than those in the placebo group $(11.2 \%$ vs. $14.4 \%, p=0.02)$. Erythromycin treatment alone significantly reduced the proportion of patients delivering within 48 hours in comparison with the placebo group. The combination of erythromycin with co-amoxiclav significantly reduced the proportion of patients delivering within a week of admission. Similarly, co-amoxiclav administration alone, or in combination with erythromycin, significantly reduced the proportion of patients delivering within 48 hours and within 7 days from admission, compared with the placebo group (238).

The neonatal effects of erythromycin treatment included a reduction in the need for exogenous surfactant, in neonates needing $21 \% \mathrm{O}_{2}$ administration for 48 hours after delivery, as well as a reduction of the positive neonatal blood cultures (238). Co-amoxiclav had a similar effect on the proportion of neonates needing $21 \% \mathrm{O}_{2}$ administration for 48 hours after delivery. Of note, the rate of suspected and proven NEC was significantly higher in the group of neonates whose mothers were treated with co-amoxiclav as a single or combined therapy. The authors attribute their findings to the wide and nonspecific effect of this broad-spectrum antibiotic that may change the flora of the premature neonates and induce growth of pathologic bacteria that induce NEC (238).

Lovett et al. did not demonstrate an association between prophylactic antibiotic treatment of patients with preterm PROM with co-amoxiclav and an increased incidence of NEC in comparison with placebo (252). The studies differ in the antibiotic regimen, as well as in the gestational age at inclusion and the number of patients. Therefore, comparison 
of the studies is difficult. The recommendation of the investigators in the ORACLE I trial was to use erythromycin and avoid using co-amoxiclav in patients with preterm PROM (238). Recently, a systematic review by Kenyon et al. confirmed these results (253).

According to Kenyon et al., the number of patients needed to treat to prevent one adverse outcome remains high [chorioamnionitis: 10 (95\% CI: 7-34); delivery within 48 hours: 9 (95\% CI: 6-20); delivery within 7 days: 7 (95\% CI: 5-15); neonatal infection: 17 (95\% CI: 12-50); abnormal cerebral ultrasonography before discharge: 69 (95\% CI: 35-1842)] (253). It is possible that the wide confidence intervals reflect the range of gestational ages of patients included in the systematic review.

The follow-up of children to the age of seven enrolled in the ORACLE I trial has demonstrated that any antibiotic treatment (erythromycin or co-amoxiclav) did not have a significant effect on the overall level of behavioral difficulties, on specific medical conditions on the proportion of children achieving each level in reading, writing, or doing mathematics (254). Therefore, it seems that the short-term benefits of antibiotic administration do not result in detectable differences in outcome at the age of seven.

\section{Can Antibiotic Treatment of Women with Documented} Intra-amniotic Infection Alter the Natural History of Preterm PROM?

The traditional view has been that clinical chorioamnionitis should be managed by immediate delivery, and this view has been extended to the management of subclinical intraamniotic infection (255). There is evidence that both of these conditions can be treated in utero without interruption of pregnancy. Ogita and colleagues first reported the successful treatment of established chorioamnionitis with antibiotic treatment via a transcervical catheter (256). Subsequently, we reported that giving antibiotics to a mother with preterm PROM at 29 weeks and an amniotic fluid culture positive for Bacteroides bivius, Veillonella parvula, and Peptococcus without clinical signs of chorioamnionitis resulted in eradication of MIAC (257). In a second case, we were successful at eradicating $U$. urealyticum from the amniotic cavity with antibiotic treatment (258).

The effects of antibiotics on the natural history of MIAC in patients with preterm PROM has been reported by Gomez et al. (87). Patients who underwent amniocentesis upon admission and those without evidence of intra-amniotic inflammation or MIAC were treated with ampicillin and erythromycin for 7 days. By contrast, patients with intra-amniotic inflammation or MIAC were treated with ceftriaxone, clindamycin, and erythromycin for 10 to 14 days. Patients who remained undelivered after the conclusion of the course of antibiotics underwent a second amniocentesis. Six of seven patients who had MIAC at the time of the first amniocentesis still had positive amniotic fluid cultures for microorganisms after a full course of antibiotic treatment. Of the 18 patients with intra-amniotic inflammation, most 15 still showed evidence of an elevated WBC count in amniotic fluid after antibiotic administration. Therefore, antibiotic administration did not eradicate MIAC or intra-amniotic inflammation. Moreover, among patients with no evidence of intra-amniotic inflammation, 32\% developed inflammation despite therapy, and among those without MIAC, $55 \%$ developed a positive amniotic fluid culture (87). These data raise important questions about the effect of antibiotics and the nature of the invading microorganisms in preterm PROM.

Should Corticosteroids Be Administrated to Patients with Preterm PROM Remote from Term?

A systematic review included 13 randomized clinical trials and demonstrated a reduction in the incidence of RDS, IVH, and NEC (RR: 0.56, 95\% CI: 0.46-0.70; RR: $0.47,95 \%$ CI: $0.31-0.70$; RR: $0.21,95 \%$ CI: $0.05-0.82$, respectively) in newborn to mothers who had been given corticosteroids (259). A nonsignificant trend of reduced neonatal mortality was observed; moreover, no increase in neonatal and fetal infection was observed (259). Steroid treatment was associated with a modest, yet significant, increase in the risk of puerperal endometritis (RR: 2.42, 95\% CI: 1.38-4.24), but no significant increase in neonatal sepsis. Similar findings were reported in an earlier meta-analysis, which included fewer trials, by Crowley $(260,261)$.

Clinical investigators have compared expectant management with steroid administration for 48 hours followed by delivery. However, induction of delivery immediately after steroid administration is associated with an increased risk of RDS and, therefore, is best avoided (261). The 1994 National Institutes of Health Consensus Conference recommended the use of corticosteroids in pregnancies complicated by preterm PROM with expected delivery between 24 and 30 to 32 weeks of gestation (234). This recommendation was based largely on data suggesting that the incidence of IVH was lower in neonates exposed to corticosteroids (234). The modest increased risk of puerperal infection is considered easy to manage. A meta-analysis comparing the outcome of treatment with antibiotics and steroids versus antibiotics without steroids found that steroid administration diminished the beneficial effects of antibiotics in reducing the rate of chorioamnionitis, endometritis, neonatal sepsis, and IVH (262).

\section{How Many Courses of Corticosteroids Should Be Administered?}

Repeated courses of corticosteroids have been used to enhance their effects. However, recent data have raised questions about the safety of repeated corticosteroid administration based on studies in humans and animals. Guinn et al. performed a double-blind, randomized, controlled trial, where women at risk for preterm delivery received one course of betamethasone or dexamethasone at admission and were randomly allocated for subsequent weekly courses of either betamethasone or placebo until 34 weeks of gestation or delivery (263). There were no significant differences in the frequency of composite neonatal morbidity (severe RDS, BPD, severe IVH, PVL, proven sepsis, NEC, or perinatal death) between the study groups (weekly course: $22.5 \%$ vs. single course: $28 \%, p=0.16$ ). However, when the analysis was stratified by gestational age, patients who delivered between 24 and 27 weeks who received a single course had a higher rate of composite neonatal morbidity and severe RDS than those in the weekly course group. An important limitation of this study is that the authors did not control for the use of surfactant, which was more 
frequent in the single-course group (single course: $24 \%$ vs. weekly course: $15.6 \%, p=0.01)(263)$. This subject is covered in detail in the chapter on preterm labor.

\section{Tocolysis}

Meta-analysis of the four randomized trials of tocolysis in preterm PROM (264-267) indicates that tocolysis does result in prolongation of pregnancy of more than 48 hours. However, no study has shown an improvement in maternal or neonatal outcomes. Therefore, there is no evidence to support the use of intravenous tocolysis in women with PROM.

\section{Should a Cervical Cerclage Be Removed in a Patient Who} Presents with Preterm PROM?

Cerclage removal has been advocated to reduce the risk of infection-related complications (268), while leaving the cerclage in place has been recommended to prolong pregnancy. Yeast and Garite reported the results of a casecontrol study in which the outcome of patients with cervical cerclage removed after preterm PROM was compared with that of patients with PROM of a similar gestational age (269). There was no difference in the incidence of chorioamnionitis or other infectious complications and neonatal outcome between the two groups. The interval between PROM and delivery was not significantly different between patients with and without cerclage. Blickstein and associates reported similar findings after comparing the outcome of 32 patients with cerclage and 76 without cerclage (270). In contrast, Goldman and colleagues compared the outcome of 46 women with preterm PROM in whom the cerclage was not removed with that of 46 women with preterm PROM without cerclage (271). Patients with a cerclage had a significantly shorter PROM-to-delivery interval and lower gestational age at delivery than patients without the cerclage. However, the rates of chorioamnionitis, other infection-related complications, and neonatal outcome were not different between the two groups. Ludmir et al. evaluated the role of immediate cerclage removal in preterm PROM in 30 women (272). In 20 women, the cerclage was removed immediately after the diagnosis of ruptured membranes was made, while in 10 women, the cerclage was retained after the membranes ruptured. Thirty-three patients with preterm PROM without cerclage served as controls. A greater proportion of women with a cerclage left in place delivered after 48 hours (90\% vs. 50\%, respectively). However, perinatal mortality was significantly higher in infants born to women in whom cerclage was retained in comparison with immediate removal or the control group ( $70 \%$ vs. $10 \%$ vs. $18 \%$, respectively). Seventy-one percent of neonatal morbidity was attributable to sepsis. The authors did not use broad-spectrum antibiotic treatment, which may explain this observation (272).

McElrath et al. reported a case-control study from a 12-year experience, comparing removal versus non-removal of cerclage in patients with preterm PROM (273). Eighty-one women with premature PPROM who had a cerclage were included, in which $30(37 \%)$ had their cerclage removed at presentation, and 51 (63\%) retained the cerclage until delivery. The authors concluded that retention of cervical cerclage after premature rupture of the membranes occurring before 34 completed weeks of gestation is associated with comparable clinical outcomes with respect to latency and neonatal morbidity and mortality, when compared with removal of the cerclage. The same authors compared patients with preterm PROM who had a cerclage versus those with preterm PROM without a cerclage, and reported that patients with a cerclage had a lower gestational age at membrane rupture than those without a cerclage $(29.6 \pm 3.0$ weeks vs. $31.7 \pm 2.8$ weeks $)$. However, there was no difference in the latency period between the groups. Neonatal mortality was higher among patients in the cerclage group. This difference became nonsignificant when the analysis was stratified into three gestational age groups $(<28,28-30$, and $>30$ weeks $)$. There were no significant differences in the rates of RDS, IVH and neonatal sepsis between the study groups (274).

In summary, the evidence indicates that patients with preterm PROM and a cerclage could be managed by leaving the cerclage in place and maintaining close surveillance to detect maternal and/or fetal infection. However, removal of the cerclage is a legitimate alternative.

\section{Home Care Vs. Hospital Care}

Practice Bulletin No. 80 of the American College of Obstetricians and Gynecologists (275) stated that the safety of expectant management at home has not been established, although management of selected patients at home with careful observation has been reported. The potential disadvantages of home care include the risk of delivering a preterm infant outside a tertiary center and the implicit delay in obstetric intervention if fetal distress or infection occurs. The advantages of home care include decreased costs and psychologic benefits to the patient.

Two studies have compared home care with hospital care. The first, a small, randomized trial of women with PROM at less than 37 weeks of gestation, had strict criteria for home care (276). Only patients undelivered after 72 hours $(60 \%$ delivered in less than 72 hours), in cephalic presentation, with negative amniotic fluid culture (by amniocentesis), cervical dilation of less than $4 \mathrm{~cm}$ on speculum examination, and at least one amniotic fluid pocket of more than $1 \mathrm{~cm}(22 \%$ had oligohydramnios) were eligible for participation. Follow-up included biweekly NST, weekly ultrasound examination, and corticosteroid administration. Fifty-five patients were randomized; 27 to home care and 28 to remain in-hospital. There was no difference in the latency period, gestational age at delivery, chorioamnionitis $(11.1 \%$ vs. $14.3 \%)$, neonatal morbidity (3.7\% vs. $7.1 \%)$, RDS $(3.7 \%$ vs. $7.1 \%)$, or neonatal pneumonia (18.5\% vs. $10.7 \%)$ (276).

The second study of home care was a retrospective study of patients with preterm PROM between 20 and 30 weeks of gestation, in which 19 of 21 women undelivered after 7 days were discharged home (277). All patients had "adequate or slightly diminished amniotic fluid volume.” Of 19 women, 11 delivered at term. No neonatal deaths occurred, and there was one case of maternal and neonatal sepsis in a woman managed at home (she was infected with human immunodeficiency virus). One case of neonatal sepsis occurred in an infant born to a woman who was managed in-hospital and delivered preterm.

The data available are insufficient to recommend management of preterm PROM outside tertiary-care centers. We believe that fetuses with preterm PROM require careful 


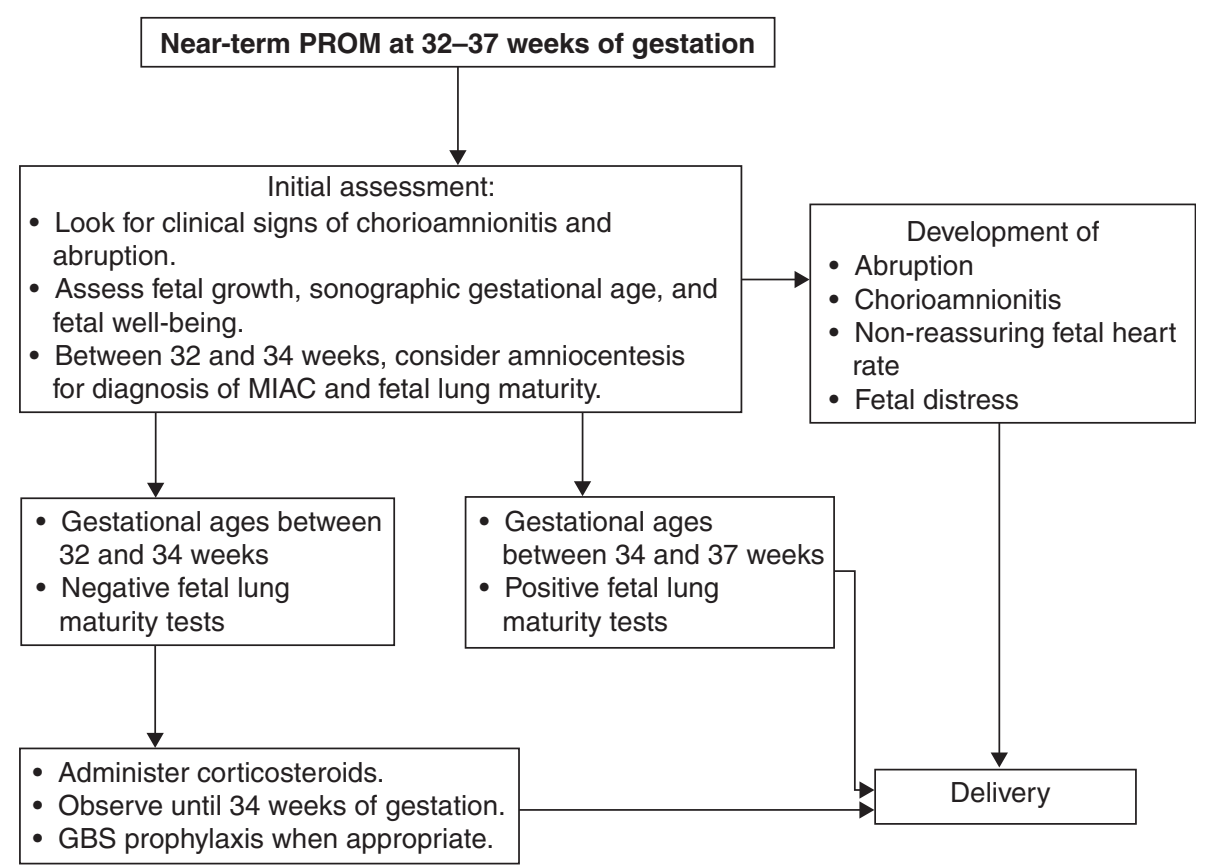

Figure 5 Management of near-term prelabor rupture of the membranes (PROM) (between 32 and 37 weeks of gestation). Abbreviations: MIAC, microbial invasion of the amniotic cavity; GBS, Group B Streptococcus. Source: From Ref. 235.

surveillance, which is rarely available outside a hospital environment.

What Is the Appropriate Time for Induction?

Today, the standard of care for patients presenting with preterm PROM remote from term is expectant management $(20,235,236)$. This management significantly reduces the neonatal complications related with prematurity $(20,235,236)$; however, the longer the latency period, the higher the risk for chorioamnionitis and abruption $(202,278-280)$.

Cox and Leveno performed a prospective study comparing induction versus expectant management of patients presenting with preterm PROM between 30 and 34 weeks. There were no significant differences in neonatal morbidity. However, the frequency of chorioamnionitis was significantly higher in the expectant management group ( $2 \%$ vs. $15 \%, p=0.009)$. Of note, $74 \%$ of the expectant management group delivered within 72 hours (281).

The timing of delivery is a determinant of the presence of major, but not minor, composite neonatal morbidity. Indeed, major composite neonatal complications (RDS, IVH, intubation, BPD, seizure, NEC, bowel perforation, retinopathy of prematurity, meningitis, pneumonia, primary pulmonary hypertension, and patent ductus arteriosus) decrease substantially after 32 weeks of gestation (282). In contrast, minor composite neonatal outcomes (hyperbilirubinemia, transient tachypnea of the newborn, hyper- or hypoglycemia and hyperor hyponatremia) decrease only after 34 weeks of gestation. Mercer et al. demonstrated that, in patients with preterm PROM, delivery after 32 weeks of gestation was not associated with a significant increase in neonatal morbidity (203). The development of chorioamnionitis, placental abruption, or non-reassuring fetal heart rate tracing often requires induction of labor and delivery, regardless of gestational age.

\section{PROM near Term (32 to 36 Weeks of Gestation)}

Mercer et al. demonstrated that there were no differences in the maternal and neonatal outcomes of expectant management and induction of labor in this gestational age window (Fig. 5) (203). However, there was a trend toward a higher incidence of chorioamnionitis in the expectant management group (203). Cox and Leveno reported a higher incidence of chorioamnionitis (281). One approach to management is to perform an amniocentesis for fetal lung maturity between 32 and 34 weeks and, if the result is mature, to proceed with induction of labor in order to reduce maternal morbidity $(235,281,283)$. In cases with negative fetal lung maturity, the management is not clear. Some physicians will choose expectant management until 34 weeks, while others will administer corticosteroids and induce labor 48 hours later (284). There are not sufficient data in the literature to support either course of action. In a survey that was conducted in the United States during 2003 among members of the Society of Maternal Fetal Medicine, $42 \%$ will induce labor in patients presenting with premature PROM and positive lung maturity at 32 weeks (284). In cases with unknown fetal lung maturity, 58\% will postpone delivery to 34 weeks of gestation (284).

In summary, patients between 32 and 34 weeks need assessment of lung maturity, and if the profile is consistent with maturity, the patients could be offered induction of labor. In cases without evidence of fetal lung maturity, there are not sufficient data as to the optimal management approach. Mercer et al. proposed that women with PROM after 34 weeks should be delivered (12).

\section{PROM AT TERM}

The management of patients with PROM at term includes (i) exclusion of cord prolapse, (ii) detection of infection, and (iii) evaluation of fetal well-being. If any evidence exists of fetal compromise or infection, induction of labor or delivery is the 
management of choice. For other patients, the relevant management issues include deciding between (i) induction or expectant management and (ii) when and how induction should be undertaken (285-308).

The natural history of term PROM is that $90 \%$ of women will be in spontaneous labor within 24 hours (308). Nulliparous women have a longer latency period than multiparous women $(291,298,303)$. Patients with an unfavorable cervix at presentation and those who are not in labor within 6 hours of rupture of membranes generally do not initiate labor spontaneously within 24 hours and represent a management dilemma (307).

The largest clinical trial in term PROM (292) included 5041 patients who were randomly allocated to four groups: (i) immediate induction with oxytocin, (ii) expectant management followed by induction with oxytocin after 4 days, (iii) induction of labor with vaginal prostaglandin $E_{2}$ (1-2 mg, followed by IV oxytocin; if not in labor in 4 hours, a second dose of prostaglandin $\mathrm{E}_{2}$ ), and (iv) expectant management followed by induction of labor with prostaglandin $E_{2}$, if labor had not begun within 4 days. The primary outcome of the trial was probable neonatal infection (clinical and laboratory signs). Secondary outcomes were the need for cesarean delivery and the patient's evaluation of their treatment. The results showed no difference in neonatal infection and cesarean delivery rate between the induction groups (oxytocin vs. prostaglandin $\mathrm{E}_{2}$ ). However, the incidence of chorioamnionitis was lower in patients allocated to induction of labor. Satisfaction with their obstetrical care was higher for patients allocated to induction of labor.

Mozurkewich and Wolf, in their meta-analysis of 23 studies including 7493 women, demonstrated that induction of labor is superior to expectant management (299). The meta-analysis compared three policies: (i) immediate induction with oxytocin, (ii) induction of labor with vaginal or endocervical prostaglandin $\mathrm{E}_{2}$ gel suppositories or tablets, and (iii) expectant management that sometimes has included delayed induction with oxytocin. The frequency of chorioamnionitis and endometritis was significantly lower in patients undergoing immediate induction of labor with oxytocin than in those managed expectantly (OR: $0.67,95 \%$ CI: $0.52-0.85$ and OR: 0.71, 95\% CI: $0.51-0.99$, respectively). The rate of chorioamnionitis was significantly higher in patients who received vaginal prostaglandins than in those induced with immediate administration of oxytocin, but lower than that of patients in the expectant management group (OR: 1.55, 95\% CI: 1.09-2.21 and OR: $0.68,95 \%$ CI: $0.51-0.91$, respectively). The rates of cesarean delivery and neonatal infection were not different among the three management schemes. Based on these data, we endorse a policy of immediate induction of labor in patients with term PROM. Antibiotic administration is justified before cesarean delivery for obstetric indications or for carriers of GBS.

Tan and Hannah (309) performed a meta-analysis of 18 trials comparing induction of labor with oxytocin versus expectant management in patients with PROM at or near term. Induction of labor with oxytocin was associated with a lower risk of chorioamnionitis (OR: $0.63,95 \%$ CI: 0.51-0.78), endometritis (OR: 0.72, 95\% CI: 0.52-0.99), and neonatal infection (OR: 0.64, 95\% CI: 0.44-0.93). Cesarean delivery rates were not statistically different. Oxytocin was associated with a more frequent use of pain medication and internal fetal heart rate monitoring. Another meta-analysis of 15 RCTs by Lin et al. included (i) six studies $(n=453)$ of misoprostol versus placebo or expectant management and (ii) nine studies $(n=1130)$ of misoprostol versus oxytocin for labor induction with term PROM (310). There were no significant differences in the frequency of chorioamnionitis, neonatal sepsis, or caesarean delivery among study groups.

Expectant management at home of patients with PROM at term is not recommended. This recommendation is based upon the report of Hanna et al. that home care was associated with an increased risk of neonatal infections (OR: 1.97, 95\% CI: 1.00-3.90) and cesarean delivery in patients not colonized with GBS (OR: 1.48, 95\% CI: 1.03-2.14) (311).

\section{ACKNOWLEDGEMENT}

This work was conducted by members of the Perinatology Research Branch of the Eunice Kennedy Shriver National Institute of Child Health and Human Development (NICHD) of the National Institutes of Health (NIH) and funded by the Intramural Program of NICHD/NIH.

\section{REFERENCES}

1. Keirse MJ, Ohlsson A, Treffers PE, Kanhani HHH. Prelabour rupture of the membranes preterm. In: Chalmers I, Enkin M, Keirse MJ, eds. Effective care in pregnancy and childbirth. Oxford: Oxford University Press, 1989: 666.

2. Johnson JW, Daikoku NH, Niebyl JR, et al. Premature rupture of the membranes and prolonged latency. Obstet Gynecol 1981; 57: 547-56.

3. Hauth JC, Cunningham FG, Whalley PJ. Early labor initiation with oral PGE2 after premature rupture of the membranes at term. Obstet Gynecol 1977; 49: 523-6.

4. Lange AP, Secher NJ, Nielsen FH, Pedersen GT. Stimulation of labor in cases of premature rupture of the membranes at or near term. A consecutive randomized study of prostaglandin E2-tablets and intravenous oxytocin. Acta Obstet Gynecol Scand 1981; 60: 207-10.

5. Lebherz TB, Hellman LP, Madding R, Anctil A, Arje SL. Double-blind study of premature rupture of the membranes. A report of 1,896 cases. Am J Obstet Gynecol 1963; 87: 218-25.

6. Magos AL, Noble MC, Wong TY, Rodeck CH. Controlled study comparing vaginal prostaglandin E2 pessaries with intravenous oxytocin for the stimulation of labour after spontaneous rupture of the membranes. Br J Obstet Gynaecol 1983; 90: 726-31.

7. Tamsen L, Lyrenas S, Cnattingius S. Premature rupture of the membranes-intervention or not. Gynecol Obstet Invest 1990; 29: 128-31.

8. van der WD, Venter PF. Management of term pregnancy with premature rupture of the membranes and unfavourable cervix. S Afr Med J 1989; 75: 54-6.

9. Westergaard JG, Lange AP, Pedersen GT, Secher NJ. Use of oral oxytocics for stimulation of labor in cases of premature rupture of the membranes at term. A randomized comparative study of prostaglandin E2 tablets and demoxytocin resoriblets. Acta Obstet Gynecol Scand 1983; 62: 111-16.

10. Knudsen FU, Steinrud J. Septicaemia of the newborn, associated with ruptured foetal membranes, discoloured amniotic fluid or maternal fever. Acta Paediatr Scand 1976; 65: 725-31.

11. Verber IG, Pearce JM, New LC, Hamilton PA, Davies EG. Prolonged rupture of the fetal membranes and neonatal outcome. J Perinat Med 1989; 17: 469-76.

12. Mercer BM. Preterm premature rupture of the membranes. Obstet Gynecol 2003; 101: 178-93.

13. Christensen KK, Christensen P, Ingemarsson I, et al. A study of complications in preterm deliveries after prolonged premature rupture of the membranes. Obstet Gynecol 1976; 48: 670-7. 
14. Daikoku NH, Kaltreider DF, Khouzami VA, Spence M, Johnson JW. Premature rupture of membranes and spontaneous preterm labor: maternal endometritis risks. Obstet Gynecol 1982; 59: 13-20.

15. Fayez JA, Hasan AA, Jonas HS, Miller GL. Management of premature rupture of the membranes. Obstet Gynecol 1978; 52: 17-21.

16. Gunn GC, Mishell DR Jr, Morton DG. Premature rupture of the fetal membranes. A review. Am J Obstet Gynecol 1970; 106: 469-83.

17. Sachs M, Baker TH. Spontaneous premature rupture of the membranes. Am J Obstet Gynecol 1967; 97: 888.

18. Gibbs RS, Blanco JD. Premature rupture of the membranes. Obstet Gynecol 1982; 60: 671-9.

19. Shubert PJ, Diss E, Iams JD. Etiology of preterm premature rupture of membranes. Obstet Gynecol Clin North Am 1992; 19: 251-63.

20. Mercer BM. Preterm premature rupture of the membranes: current approaches to evaluation and management. Obstet Gynecol Clin North Am 2005; 32: 411-28.

21. Schwarcz R, Belizan JM, Nieto F, Tenzer SM, Rios AM. Third progress report on the Latin American collaborative study on the effects of late rupture of membranes on labor and the neonate, submitted to the Director of the Pan-American Health Organization (PAHO/WHO) and the participating groups. Montevideo: Latin American Center of Perinatology and Human Development, 1974.

22. Quintero RA, Morales WJ, Kalter CS, et al. Transabdominal intraamniotic endoscopic assessment of previable premature rupture of membranes. Am J Obstet Gynecol 1998; 179: 71-6.

23. Romero R. Prenatal medicine: the child is the father of the man. Prenat Neonatal Med 1996; 1: 8-11.

24. Romero R, Espinoza J, Mazor M, Chaiworapongsa T. The preterm parturition syndrome. In: Critchley $\mathrm{H}$, Bennett $\mathrm{P}$, Thornton S, eds. Preterm birth. London, UK: RCOG Press, 2004: 28-60.

25. Mercer BM, Goldenberg RL, Meis PJ, et al. The Preterm Prediction Study: prediction of preterm premature rupture of membranes through clinical findings and ancillary testing. The National Institute of Child Health and Human Development Maternal-Fetal Medicine Units Network. Am J Obstet Gynecol 2000; 183: 738-45.

26. Weiss JL, Malone FD, Vidaver J, et al. Threatened abortion: a risk factor for poor pregnancy outcome, a population-based screening study. Am J Obstet Gynecol 2004; 190: 745-50.

27. Yang J, Hartmann KE, Savitz DA, et al. Vaginal bleeding during pregnancy and preterm birth. Am J Epidemiol 2004; 160: 118-25.

28. Carroll SG, Papaioannou S, Ntumazah IL, Philpott-Howard J, Nicolaides $\mathrm{KH}$. Lower genital tract swabs in the prediction of intrauterine infection in preterm prelabour rupture of the membranes. Br J Obstet Gynaecol 1996; 103: 54-9.

29. Kim CJ, Romero R, Kusanovic JP, et al. The frequency, clinical significance, and pathological features of chronic chorioamnionitis: a lesion associated with spontaneous preterm birth. Mod Pathol 2010; 23: 1000-11.

30. Fujimoto T, Parry S, Urbanek M, et al. A single nucleotide polymorphism in the matrix metalloproteinase-1 (MMP-1) promoter influences amnion cell MMP-1 expression and risk for preterm premature rupture of the fetal membranes. J Biol Chem 2002; 277: 6296-302.

31. Ferrand PE, Parry S, Sammel M, et al. A polymorphism in the matrix metalloproteinase- 9 promoter is associated with increased risk of preterm premature rupture of membranes in African Americans. Mol Hum Reprod 2002; 8: 494-501.

32. Wang H, Parry S, Macones G, et al. Functionally significant SNP MMP8 promoter haplotypes and preterm premature rupture of membranes (PPROM). Hum Mol Genet 2004; 13: 2659-69.

33. Wang H, Parry S, Macones G, et al. A functional SNP in the promoter of the SERPINH1 gene increases risk of preterm premature rupture of membranes in African Americans. Proc Natl Acad Sci USA 2006; 103: 13463-7.

34. Kurki T, Sivonen A, Renkonen OV, Savia E, Ylikorkala O. Bacterial vaginosis in early pregnancy and pregnancy outcome. Obstet Gynecol 1992; 80: 173-7.

35. Simhan HN, Caritis SN, Krohn MA, Hillier SL. The vaginal inflammatory milieu and the risk of early premature preterm rupture of membranes. Am J Obstet Gynecol 2005; 192: 213-18.

36. Roberts AK, Monzon-Bordonaba F, Van Deerlin PG, et al. Association of polymorphism within the promoter of the tumor necrosis factor alpha gene with increased risk of preterm premature rupture of the fetal membranes. Am J Obstet Gynecol 1999; 180: 1297-302.
37. Macones GA, Parry S, Elkousy M, et al. A polymorphism in the promoter region of TNF and bacterial vaginosis: preliminary evidence of gene-environment interaction in the etiology of spontaneous preterm birth. Am J Obstet Gynecol 2004; 190: 1504-8.

38. Wang H, Ogawa M, Wood JR, et al. Genetic and epigenetic mechanisms combine to control MMP1 expression and its association with preterm premature rupture of membranes. Hum Mol Genet 2008; 17: 1087-96.

39. Goncalves LF, Chaiworapongsa T, Romero R. Intrauterine infection and prematurity. Ment Retard Dev Disabil Res Rev 2002; 8: 3-13.

40. DiGiulio DB, Romero R, Kusanovic JP, et al. Prevalence and diversity of microbes in the amniotic fluid, the fetal inflammatory response, and pregnancy outcome in women with preterm pre-labor rupture of membranes. Am J Reprod Immunol 2010; 64: 38-57.

41. Cassell GH, Davis RO, Waites KB, et al. Isolation of Mycoplasma hominis and Ureaplasma urealyticum from amniotic fluid at 16-20 weeks of gestation: potential effect on outcome of pregnancy. Sex Transm Dis 1983; 10: 294-302.

42. Gray DJ, Robinson HB, Malone J, Thomson RB Jr. Adverse outcome in pregnancy following amniotic fluid isolation of Ureaplasma urealyticum. Prenat Diagn 1992; 12: 111-17.

43. Horowitz S, Mazor M, Romero R, Horowitz J, Glezerman M. Infection of the amniotic cavity with Ureaplasma urealyticum in the midtrimester of pregnancy. J Reprod Med 1995; 40: 375-9.

44. Yoon BH, Oh SY, Romero R, et al. An elevated amniotic fluid matrix metalloproteinase- 8 level at the time of mid-trimester genetic amniocentesis is a risk factor for spontaneous preterm delivery. Am J Obstet Gynecol 2001; 185: 1162-7.

45. Arias F, Rodriquez L, Rayne SC, Kraus FT. Maternal placental vasculopathy and infection: two distinct subgroups among patients with preterm labor and preterm ruptured membranes. Am J Obstet Gynecol 1993; 168: 585-91.

46. Arias F, Victoria A, Cho K, Kraus F. Placental histology and clinical characteristics of patients with preterm premature rupture of membranes. Obstet Gynecol 1997; 89: 265-71.

47. Romero R, Kusanovic JP, Kim CJ. Placental bed disorder in the genesis of the great obstetrical syndrome. In: Pijnenborg R, Brosens I, Romero R, eds. Placental Bed Disorder. Basic Science and its Translation to Obstetrics. Cambridge: Cambridge University Press, 2010: 271-89.

48. Brosens I, Pijnenborg R, Vercruysse L, Romero R. The "Great Obstetrical Syndromes" are associated with disorders of deep placentation. Am J Obstet Gynecol 2011; 204: 193-201.

49. Kim YM, Chaiworapongsa T, Gomez R, et al. Failure of physiologic transformation of the spiral arteries in the placental bed in preterm premature rupture of membranes. Am J Obstet Gynecol 2002; 187: $1137-42$.

50. Gomez R, Romero R, Nien JK, et al. Idiopathic vaginal bleeding during pregnancy as the only clinical manifestation of intrauterine infection. J Matern Fetal Neonatal Med 2005; 18: 31-7.

51. Mackenzie AP, Schatz F, Krikun G, et al. Mechanisms of abruptioninduced premature rupture of the fetal membranes: thrombin enhanced decidual matrix metalloproteinase-3 (stromelysin-1) expression. Am J Obstet Gynecol 2004; 191: 1996-2001.

52. Rosen T, Schatz F, Kuczynski E, et al. Thrombin-enhanced matrix metalloproteinase-1 expression: a mechanism linking placental abruption with premature rupture of the membranes. J Matern Fetal Neonatal Med 2002; 11: 11-17.

53. Stephenson CD, Lockwood CJ, Ma Y, Guller S. Thrombin-dependent regulation of matrix metalloproteinase (MMP)-9 levels in human fetal membranes. J Matern Fetal Neonatal Med 2005; 18: 17-22.

54. Curry TE Jr, Osteen KG. The matrix metalloproteinase system: changes, regulation, and impact throughout the ovarian and uterine reproductive cycle. Endocr Rev 2003; 24: 428-65.

55. Darby MJ, Caritis SN, Shen-Schwarz S. Placental abruption in the preterm gestation: an association with chorioamnionitis. Obstet Gynecol 1989; 74: 88-92.

56. Naeye RL. Factors that predispose to premature rupture of the fetal membranes. Obstet Gynecol 1982; 60: 93-8.

57. Evaldson G, Lagrelius A, Winiarski J. Premature rupture of the membranes. Acta Obstet Gynecol Scand 1980; 59: 385-93. 
58. Buchmayer SM, Sparen P, Cnattingius S. Previous pregnancy loss: risks related to severity of preterm delivery. Am J Obstet Gynecol 2004; 191: 1225-31.

59. Hassan S, Romero R, Hendler I, et al. A sonographic short cervix as the only clinical manifestation of intra-amniotic infection. J Perinat Med 2006; 34: 13-19.

60. Rizzo G, Capponi A, Vlachopoulou A, et al. Ultrasonographic assessment of the uterine cervix and interleukin- 8 concentrations in cervical secretions predict intrauterine infection in patients with preterm labor and intact membranes. Ultrasound Obstet Gynecol 1998; 12: 86-92.

61. Lind J, Wallenburg HC. Pregnancy and the Ehlers-Danlos syndrome: a retrospective study in a Dutch population. Acta Obstet Gynecol Scand 2002; 81: 293-300.

62. Harger JH, Hsing AW, Tuomala RE, et al. Risk factors for preterm premature rupture of fetal membranes: a multicenter case-control study. Am J Obstet Gynecol 1990; 163: 130-7.

63. Spinillo A, Nicola S, Piazzi G, et al. Epidemiological correlates of preterm premature rupture of membranes. Int J Gynaecol Obstet 1994; 47: 7-15.

64. Cox SM, Williams ML, Leveno KJ. The natural history of preterm ruptured membranes: what to expect of expectant management. Obstet Gynecol 1988; 71: 558-62.

65. Wilson JC, Levy DL, Wilds PL. Premature rupture of membranes prior to term: consequences of nonintervention. Obstet Gynecol 1982; 60: 601-6.

66. Nelson LH, Anderson RL, O'Shea TM, Swain M. Expectant management of preterm premature rupture of the membranes. Am J Obstet Gynecol 1994; 171: 350-6.

67. Romero R, Sirtori M, Oyarzun E, et al. Infection and labor. V. Prevalence, microbiology, and clinical significance of intraamniotic infection in women with preterm labor and intact membranes. Am J Obstet Gynecol 1989; 161: 817-24.

68. Romero R, Quintero R, Oyarzun E, et al. Intraamniotic infection and the onset of labor in preterm premature rupture of the membranes. Am J Obstet Gynecol 1988; 159: 661-6.

69. Romero R, Mazor M, Morretti R, et al. Infection and labor VII: microbial invasion of the amniotic cavity in spontaneous rupture of membranes at term. Am J Obstet Gynecol 1992; 166: 129.

70. Yoon BH, Romero R, Kim M, et al. Clinical implications of detection of Ureaplasma urealyticum in the amniotic cavity with the polymerase chain reaction. Am J Obstet Gynecol 2000; 183: 1130-7.

71. Averbuch B, Mazor M, Shoham-Vardi I, et al. Intra-uterine infection in women with preterm premature rupture of membranes: maternal and neonatal characteristics. Eur J Obstet Gynecol Reprod Biol 1995; 62: 25-9.

72. Cotton DB, Hill LM, Strassner HT, Platt LD, Ledger WJ. Use of amniocentesis in preterm gestation with ruptured membranes. Obstet Gynecol 1984; 63: 38-43.

73. Coultrip LL, Grossman JH. Evaluation of rapid diagnostic tests in the detection of microbial invasion of the amniotic cavity. Am J Obstet Gynecol 1992; 167: 1231-42.

74. Garite TJ, Freeman RK, Linzey EM, Braly P. The use of amniocentesis in patients with premature rupture of membranes. Obstet Gynecol 1979; 54: 226-30.

75. Zlatnik FJ, Cruikshank DP, Petzold CR, Galask RP. Amniocentesis in the identification of inapparent infection in preterm patients with premature rupture of the membranes. J Reprod Med 1984; 29: 656-60.

76. Broekhuizen FF, Gilman M, Hamilton PR. Amniocentesis for gram stain and culture in preterm premature rupture of the membranes. Obstet Gynecol 1985; 66: 316-21.

77. Dudley J, Malcolm G, Ellwood D. Amniocentesis in the management of preterm premature rupture of the membranes. Aust NZ J Obstet Gynaecol 1991; 31: 331-6.

78. Feinstein SJ, Vintzileos AM, Lodeiro JG, et al. Amniocentesis with premature rupture of membranes. Obstet Gynecol 1986; 68: 147-52.

79. Font GE, Gauthier DW, Meyer WJ, et al. Catalase activity as a predictor of amniotic fluid culture results in preterm labor or premature rupture of membranes. Obstet Gynecol 1995; 85: 656-8.

80. Garite TJ, Freeman RK. Chorioamnionitis in the preterm gestation Obstet Gynecol 1982; 59: 539-45.

81. Gauthier DW, Meyer WJ, Bieniarz A. Correlation of amniotic fluid glucose concentration and intraamniotic infection in patients with preterm labor or premature rupture of membranes. Am J Obstet Gynecol 1991; 165: 1105-10.
82. Gauthier DW, Meyer WJ. Comparison of gram stain, leukocyte esterase activity, and amniotic fluid glucose concentration in predicting amniotic fluid culture results in preterm premature rupture of membranes. Am J Obstet Gynecol 1992; 167: 1092-5.

83. Hazan Y, Mazor M, Horowitz S, Leiberman JR, Glezerman M. The diagnostic value of amniotic fluid Gram stain examination and limulus amebocyte lysate assay in patients with preterm birth. Acta Obstet Gynecol Scand 1995; 74: 275-80.

84. O’Brien WF, Knuppel RA, Morales WJ, Angel JL, Torres CT. Amniotic fluid alpha 1-antitrypsin concentration in premature rupture of the membranes. Am J Obstet Gynecol 1990; 162: 756-9.

85. Romero R, Yoon BH, Mazor M, et al. A comparative study of the diagnostic performance of amniotic fluid glucose, white blood cell count, interleukin-6, and gram stain in the detection of microbial invasion in patients with preterm premature rupture of membranes. Am J Obstet Gynecol 1993; 169: 839-51.

86. Vintzileos AM, Campbell WA, Nochimson DJ, et al. Qualitative amniotic fluid volume versus amniocentesis in predicting infection in preterm premature rupture of the membranes. Obstet Gynecol 1986; 67: 579-83.

87. Gomez R, Romero R, Nien JK, et al. Antibiotic administration to patients with preterm premature rupture of membranes does not eradicate intraamniotic infection. J Matern Fetal Neonatal Med 2007; 20: 167-73.

88. Carroll SG, Ville Y, Greenough A, et al. Preterm prelabour amniorrhexis: intrauterine infection and interval between membrane rupture and delivery. Arch Dis Child Fetal Neonatal Ed 1995; 72: F43-6.

89. Gomez R, Romero R, Ghezzi F, et al. The fetal inflammatory response syndrome. Am J Obstet Gynecol 1998; 179: 194-202.

90. Romero R, Gomez R, Ghezzi F, et al. A fetal systemic inflammatory response is followed by the spontaneous onset of preterm parturition. Am J Obstet Gynecol 1998; 179: 186-93.

91. Romero R, Chaiworapongsa T, Espinoza J, et al. Fetal plasma MMP-9 concentrations are elevated in preterm premature rupture of the membranes. Am J Obstet Gynecol 2002; 187: 1125-30.

92. Romero R, Athayde N, Gomez R, et al. The fetal inflammatory response syndrome is characterized by the outpouring of a potent extracellular matrix degrading enzyme into the fetal circulation. Am J Obstet Gynecol 1998; 178: S3.

93. Berry SM, Romero R, Gomez R, et al. Premature parturition is characterized by in utero activation of the fetal immune system. Am J Obstet Gynecol 1995; 173: 1315-20.

94. Berry SM, Gomez R, Athayde N, et al. The role of granulocyte colony stimulating factor in the neutrophilia observed in the fetal inflammatory response syndrome. Am J Obstet Gynecol 1998; 178: S202.

95. Gomez R, Berry S, Yoon BH, et al. The hematologic profile of the fetus with systemic inflammatory response syndrome. Am J Obstet Gynecol 1998; 178: S202.

96. Yoon BH, Romero R, Jun JK, et al. An increase in fetal plasma cortisol but not dehydroepiandrosterone sulfate is followed by the onset of preterm labor in patients with preterm premature rupture of the membranes. Am J Obstet Gynecol 1998;179: 1107-14.

97. Romero R, Espinoza J, Goncalves LF, et al. Fetal cardiac dysfunction in preterm premature rupture of membranes. J Matern Fetal Neonatal Med 2004; 16: 146-57.

98. Yoon BH, Jun JK, Romero R, et al. Amniotic fluid inflammatory cytokines (interleukin-6, interleukin-1beta, and tumor necrosis factoralpha), neonatal brain white matter lesions, and cerebral palsy. Am J Obstet Gynecol 1997; 177: 19-26.

99. Yoon BH, Romero R, Park JS, et al. Fetal exposure to an intra-amniotic inflammation and the development of cerebral palsy at the age of three years. Am J Obstet Gynecol 2000; 182: 675-81.

100. Yoon BH, Romero R, Jun JK, et al. Amniotic fluid cytokines (interleukin-6, tumor necrosis factor-alpha, interleukin-1 beta, and interleukin-8) and the risk for the development of bronchopulmonary dysplasia. Am J Obstet Gynecol 1997; 177: 825-30.

101. Yoon BH, Romero R, Kim KS, et al. A systemic fetal inflammatory response and the development of bronchopulmonary dysplasia. Am J Obstet Gynecol 1999; 181: 773-9.

102. Kim YM, Romero R, Chaiworapongsa T, et al. Dermatitis as a component of the fetal inflammatory response syndrome is associated with activation of toll-like receptors in epidermal keratinocytes. Histopathology 2006; 49: 506-14. 
103. Pacora P, Chaiworapongsa T, Maymon E, et al. Funisitis and chorionic vasculitis: the histological counterpart of the fetal inflammatory response syndrome. J Matern Fetal Neonatal Med 2002; 11: 18-25.

104. D'Alquen D, Kramer BW, Seidenspinner S, et al. Activation of umbilical cord endothelial cells and fetal inflammatory response in preterm infants with chorioamnionitis and funisitis. Pediatr Res 2005; 57: 263-9.

105. Yoon BH, Romero R, Park JS, et al. The relationship among inflammatory lesions of the umbilical cord (funisitis), umbilical cord plasma interleukin 6 concentration, amniotic fluid infection, and neonatal sepsis. Am J Obstet Gynecol 2000; 183: 1124-9.

106. Wu YW, Colford JM Jr. Chorioamnionitis as a risk factor for cerebral palsy: a meta-analysis. JAMA 2000; 284: 1417-24.

107. Leviton A, Paneth N, Reuss ML, et al. Maternal infection, fetal inflammatory response, and brain damage in very low birth weight infants. Developmental Epidemiology Network Investigators. Pediatr Res 1999; 46: 566-75.

108. Cornette L. Fetal and neonatal inflammatory response and adverse outcome. Semin Fetal Neonatal Med 2004; 9: 459-70.

109. Naeye RL, Peters EC. Causes and consequences of premature rupture of fetal membranes. Lancet 1980; 1: 192-4.

110. Pankuch GA, Appelbaum PC, Lorenz RP, et al. Placental microbiology and histology and the pathogenesis of chorioamnionitis. Obstet Gynecol 1984; 64: 802-6.

111. Romero R, Salafia CA, Athanassiadis AP, et al. The relationship between acute inflammatory lesions of the preterm placenta and amniotic fluid microbiology. Am J Obstet Gynecol 1992; 166: 1382.

112. Ananth CV, Oyelese Y, Srinivas N, Yeo L, Vintzileos AM. Preterm premature rupture of membranes, intrauterine infection, and oligohydramnios: risk factors for placental abruption. Obstet Gynecol 2004; 104: 71-7.

113. Ananth CV, Savitz DA, Williams MA. Placental abruption and its association with hypertension and prolonged rupture of membranes: a methodologic review and meta-analysis. Obstet Gynecol 1996; 88: 309-18.

114. Ananth CV, Smulian JC, Demissie K, Vintzileos AM, Knuppel RA. Placental abruption among singleton and twin births in the United States: risk factor profiles. Am J Epidemiol 2001; 153: 771-8.

115. Nelson DM, Stempel LE, Zuspan FP. Association of prolonged, preterm premature rupture of the membranes and abruptio placentae. J Reprod Med 1986; 31: 249-53.

116. Vintzileos AM, Campbell WA, Nochimson DJ, Weinbaum PJ. Preterm premature rupture of the membranes: a risk factor for the development of abruptio placentae. Am J Obstet Gynecol 1987; 156: 1235-8.

117. Gonen R, Hannah ME, Milligan JE. Does prolonged preterm premature rupture of the membranes predispose to abruptio placentae? Obstet Gynecol 1989; 74: 347-50.

118. Major CA, de Veciana M, Lewis DF, Morgan MA. Preterm premature rupture of membranes and abruptio placentae: is there an association between these pregnancy complications? Am J Obstet Gynecol 1995; 172: 672-6

119. Naeye RL. Coitus and antepartum haemorrhage. Br J Obstet Gynaecol 1981; 88: 765-70.

120. Vergani P, Ghidini A, Locatelli A, et al. Risk factors for pulmonary hypoplasia in second-trimester premature rupture of membranes. Am J Obstet Gynecol 1994; 170: 1359-64.

121. Rotschild A, Ling EW, Puterman ML, Farquharson D. Neonatal outcome after prolonged preterm rupture of the membranes. Am J Obstet Gynecol 1990; 162: 46-52.

122. Winn HN, Chen M, Amon E, et al. Neonatal pulmonary hypoplasia and perinatal mortality in patients with midtrimester rupture of amniotic membranes -a critical analysis. Am J Obstet Gynecol 2000; 182: 1638-44.

123. Johnson A, Callan NA, Bhutani VK, et al. Ultrasonic ratio of fetal thoracic to abdominal circumference: an association with fetal pulmonary hypoplasia. Am J Obstet Gynecol 1987; 157: 764-9.

124. Nimrod C, Varela-Gittings F, Machin G, Campbell D, Wesenberg R. The effect of very prolonged membrane rupture on fetal development. Am J Obstet Gynecol 1984; 148: 540-3.

125. Thibeault DW, Beatty EC Jr, Hall RT, Bowen SK, O’Neill DH. Neonatal pulmonary hypoplasia with premature rupture of fetal membranes and oligohydramnios. J Pediatr 1985; 107: 273-7.
126. Potter E. Bilateral renal agenesis. J Pediatr 1946; 29: 68.

127. Porter HJ. Pulmonary hypoplasia-size is not everything. Virch Arch 1998; 432: 3-6.

128. Wenstrom KD. Pulmonary hypoplasia and deformations related to premature rupture of membranes. Obstet Gynecol Clin North Am 1992; 19: 397-408.

129. Blott M, Greenough A, Nicolaides KH, et al. Fetal breathing movements as predictor of favourable pregnancy outcome after oligohydramnios due to membrane rupture in second trimester. Lancet 1987; 2: 129-31.

130. Tamura RK, Sabbagha RE, Depp R, et al. Diminished growth in fetuses born preterm after spontaneous labor or rupture of membranes. Am J Obstet Gynecol 1984; 148: 1105-10.

131. Bukowski R, Gahn D, Denning J, Saade G. Impairment of growth in fetuses destined to deliver preterm. Am J Obstet Gynecol 2001; 185: 463-7.

132. Spinillo A, Montanari L, Sanpaolo P, et al. Fetal growth and infant neurodevelopmental outcome after preterm premature rupture of membranes. Obstet Gynecol 2004; 103: 1286-93.

133. Blackwell S, Romero R, Chaiworapongsa T, et al. Maternal and fetal inflammatory responses in unexplained fetal death. J Matern Fetal Neonatal Med 2003; 14: 151-7.

134. Gerber S, Vardhana S, Meagher-Villemure K, et al. Association between fetal interleukin-1 receptor antagonist gene polymorphism and unexplained fetal death. Am J Obstet Gynecol 2005; 193: 1472-7.

135. Reece EA, Chervenak FA, Moya FR, Hobbins JC. Amniotic fluid arborization: effect of blood, meconium, and $\mathrm{pH}$ alterations. Obstet Gynecol 1984; 64: 248-50.

136. Friedman ML, McElin TW. Diagnosis of ruptured fetal membranes. Clinical study and review of the literature. Am J Obstet Gynecol 1969; 104: 544-50.

137. Lodeiro JG, Hsieh KA, Byers JH, Feinstein SJ. The fingerprint, a falsepositive fern test. Obstet Gynecol 1989; 73: 873-4.

138. McGregor JA, Johnson S. "Fig leaf" ferning and positive Nitrazine testing: semen as a cause of misdiagnosis of premature rupture of membranes. Am J Obstet Gynecol 1985; 151: 1142-3.

139. Brookes C, Shand K, Jones WR. A reevaluation of the ferning test to detect ruptured membranes. Aust NZ J Obstet Gynaecol 1986; 26: 260-4.

140. Rosemond RL, Lombardi SJ, Boehm FH. Ferning of amniotic fluid contaminated with blood. Obstet Gynecol 1990; 75: 338-40.

141. Bennett SL, Cullen JB, Sherer DM, Woods JR Jr. The ferning and nitrazine tests of amniotic fluid between 12 and 41 weeks gestation. Am J Perinatol 1993; 10: 101-4.

142. Adoni A, Ben Chetrit A, Zacut D, Palti Z, Hurwitz A. Prolongation of the latent period in patients with premature rupture of the membranes by avoiding digital examination. Int J Gynaecol Obstet 1990; 32: 19-21.

143. Lewis DF, Major CA, Towers CV, et al. Effects of digital vaginal examinations on latency period in preterm premature rupture of membranes. Obstet Gynecol 1992; 80: 630-4.

144. Sukcharoen N, Vasuratna A. Effects of digital cervical examinations on duration of latency period, maternal and neonatal outcome in preterm premature rupture of membranes. J Med Assoc Thai 1993; 76: 203-9.

145. Schutte MF, Treffers PE, Kloosterman GJ, Soepatmi S. Management of premature rupture of membranes: the risk of vaginal examination to the infant. Am J Obstet Gynecol 1983; 146: 395-400.

146. Munson LA, Graham A, Koos BJ, Valenzuela GJ. Is there a need for digital examination in patients with spontaneous rupture of the membranes? Am J Obstet Gynecol 1985; 153: 562-63.

147. Brown CL, Ludwiczak MH, Blanco JD, Hirsch CE. Cervical dilation: accuracy of visual and digital examinations. Obstet Gynecol 1993; 81: 215-16.

148. Morgan MA, de Veciana M, Rumney PJ, Schlinke S. Cervical assessment: visual or digital? J Perinatol 1996; 16: 103-6.

149. Smith RP. A technique for the detection of rupture of the membranes. A review and preliminary report. Obstet Gynecol 1976; 48: 172-6.

150. Gahl W, Kazina TR, Furmann D, et al. Diamine oxidase in the diagnosis of ruptured fetal membranes. Am J Obstet Gynecol 1969; 104: 544.

151. Huber JF, Bischof P, Extermann P, Beguin F, Herrmann WL. Are vaginal fluid concentrations of prolactin, alpha-fetoprotein and human placental lactogen useful for diagnosing ruptured membranes? $\mathrm{Br}$ J Obstet Gynaecol 1983; 90: 1183-5. 
152. Koninckx PR, Trappeniers H, Van Assche FA. Prolactin concentration in vaginal fluid: a new method for diagnosing ruptured membranes. $\mathrm{Br}$ J Obstet Gynaecol 1981; 88: 607-10.

153. Phocas I, Sarandakou A, Kontoravdis A, Chryssicopoulos A, Zourlas PA. Vaginal fluid prolactin: a reliable marker for the diagnosis of prematurely ruptured membranes. Comparison with vaginal fluid alpha-fetoprotein and placental lactogen. Eur J Obstet Gynecol Reprod Biol 1989; 31: 133-41.

154. Gaucherand P, Guibaud S, Rudigoz RC, Wong A. Diagnosis of premature rupture of the membranes by the identification of alphafeto-protein in vaginal secretions. Acta Obstet Gynecol Scand 1994; 73: 456-9.

155. Lockwood CJ, Wein R, Chien D, et al. Fetal membrane rupture is associated with the presence of insulin-like growth factor-binding protein-1 in vaginal secretions. Am J Obstet Gynecol 1994; 171: 146-50.

156. Kishida T, Hirao A, Matsuura T, et al. Diagnosis of premature rupture of membranes with an improved alpha-fetoprotein monoclonal antibody kit. Clin Chem 1995; 41: 1500-3.

157. Kishida T, Yamada H, Negishi H, et al. Diagnosis of preterm premature rupture of the membranes using a newly developed AFP monoclonal antibody test kit. Eur J Obstet Gynecol Reprod Biol 1995; 58: 67-72.

158. Buyukbayrak EE, Turan C, Unal O, Dansuk R, Cengizoglu B. Diagnostic power of the vaginal washing-fluid prolactin assay as an alternative method for the diagnosis of premature rupture of membranes. J Matern Fetal Neonatal Med 2004; 15: 120-5.

159. Esim E, Turan C, Unal O, Dansuk R, Cengizglu B. Diagnosis of premature rupture of membranes by identification of beta-HCG in vaginal washing fluid. Eur J Obstet Gynecol Reprod Biol 2003; 107: 37-40.

160. Erdemoglu E, Mungan T. Significance of detecting insulin-like growth factor binding protein-1 in cervicovaginal secretions: comparison with nitrazine test and amniotic fluid volume assessment. Acta Obstet Gynecol Scand 2004; 83: 622-6.

161. Anai T, Tanaka Y, Hirota Y, Miyakawa I. Vaginal fluid hCG levels for detecting premature rupture of membranes. Obstet Gynecol 1997; 89: 261-4.

162. Kishida T, Yamada H, Negishi H, et al. Diagnosis of premature rupture of the membranes in preterm patients, using an improved AFP kit: comparison with ROM-check and/or nitrazine test. Eur J Obstet Gynecol Reprod Biol 1996; 69: 77-82.

163. Atterbury JL, Groome LJ, Hoff C. Methods used to diagnose premature rupture of membranes: a national survey of 812 obstetric nurses. Obstet Gynecol 1998; 92: 384-9.

164. Lockwood CJ, Senyei AE, Dische MR, et al. Fetal fibronectin in cervical and vaginal secretions as a predictor of preterm delivery. N Engl J Med 1991; 325: 669-74.

165. Eriksen NL, Parisi VM, Daoust S, et al. Fetal fibronectin: a method for detecting the presence of amniotic fluid. Obstet Gynecol 1992; 80: 451-4.

166. Goldenberg RL, Mercer BM, Meis PJ, et al. The preterm prediction study: fetal fibronectin testing and spontaneous preterm birth. NICHD Maternal Fetal Medicine Units Network. Obstet Gynecol 1996; 87: 643-8.

167. Greenhagen JB, Van Wagoner J, Dudley D, et al. Value of fetal fibronectin as a predictor of preterm delivery for a low-risk population. Am J Obstet Gynecol 1996; 175: 1054-6.

168. Irion O, Matute S, Bischof P, Beguin F. Cervical oncofetal fibronectin in patients with false labor as a predictor of preterm delivery. Am J Obstet Gynecol 1996; 394: 384.

169. Leeson SC, Maresh MJ, Martindale EA, et al. Detection of fetal fibronectin as a predictor of preterm delivery in high risk asymptomatic pregnancies. Br J Obstet Gynaecol 1996; 103: 48-53.

170. Malak TM, Sizmur F, Bell SC, Taylor DJ. Fetal fibronectin in cervicovaginal secretions as a predictor of preterm birth. Br J Obstet Gynaecol 1996; 103: 648-53.

171. Morrison JC, Allbert JR, McLaughlin BN, et al. Oncofetal fibronectin in patients with false labor as a predictor of preterm delivery. Am J Obstet Gynecol 1993; 168: 538-42.

172. Nageotte MP, Casal D, Senyei AE. Fetal fibronectin in patients at increased risk for premature birth. Am J Obstet Gynecol 1994; 170: 20-5.

173. Rizzo G, Capponi A, Arduini D, Lorido C, Romanini C. The value of fetal fibronectin in cervical and vaginal secretions and of ultrasonographic examination of the uterine cervix in predicting premature delivery for patients with preterm labor and intact membranes. Am J Obstet Gynecol 1996; 175: 1146-51.
174. Atlay RD, Sutherst JR. Premature rupture of the fetal membranes confirmed by intra-amniotic injection of dye (Evans blue T-1824). Am J Obstet Gynecol 1970; 108: 993-4.

175. Diaz-Garzon J. Indigocarmine test of preterm rupture of membranes. Rev Columb Obstet Gynecol 1969; 20: 373.

176. Fujimoto S, Kishida T, Sagawa T, et al. Clinical usefulness of the dyeinjection method for diagnosing premature rupture of the membranes in equivocal cases. J Obstet Gynaecol 1995; 21: 215-20.

177. Meyer BA, Gonik B, Creasy RK. Evaluation of phenazopyridine hydrochloride as a tool in the diagnosis of premature rupture of the membranes. Am J Perinatol 1991; 8: 297-9.

178. Cowett RM, Hakanson DO, Kocon RW, Oh W. Untoward neonatal effect of intraamniotic administration of methylene blue. Obstet Gynecol 1976; 48: 74S-5S.

179. McEnerney J, McEnerney L. Unfavourable neonatal outcomes after intraamniotic injection of methylene blue. Obstet Gynecol 1983; 1983: 351

180. Troche BI. Methylene blue baby. N Engl J Med 1989; 320: 1756-7

181. Divon MY, Chamberlain PF, Sipos L, Platt LD. Underestimation of fetal weight in premature rupture of membranes. J Ultrasound Med 1984; 3: 529-31.

182. Ben Haroush A, Yogev Y, Bar J, et al. Accuracy of sonographically estimated fetal weight in 840 women with different pregnancy complications prior to induction of labor. Ultrasound Obstet Gynecol 2004; 23: 172-6.

183. Edwards A, Goff J, Baker L. Accuracy and modifying factors of the sonographic estimation of fetal weight in a high-risk population. Aust NZ J Obstet Gynaecol 2001; 41: 187-90.

184. Romero R, Goncalves LF, Ghezzi F, et al. Amniocenteses. In: Fleischer AC, Manning FA, Jeanty P, Romero R, eds. Sonography in obstetrics and gynecology: principles and practice. Stamford, CT: Appleton \& Lange, 1996.

185. Cotton DB, Gonik B, Bottoms SF. Conservative versus aggressive management of preterm rupture of membranes. A randomized trial of amniocentesis. Am J Perinatol 1984; 1: 322-4.

186. Shim SS, Romero R, Hong JS, et al. Clinical significance of intraamniotic inflammation in patients with preterm premature rupture of membranes. Am J Obstet Gynecol 2004; 191: 1339-45.

187. Maymon E, Romero R, Chaiworapongsa T, et al. Value of amniotic fluid neutrophil collagenase concentrations in preterm premature rupture of membranes. Am J Obstet Gynecol 2001; 185: 1143-8.

188. Yoon BH, Romero R, Kim CJ, et al. Amniotic fluid interleukin-6: a sensitive test for antenatal diagnosis of acute inflammatory lesions of preterm placenta and prediction of perinatal morbidity. Am J Obstet Gynecol 1995; 172: 960-70.

189. Jalava J, Mantymaa ML, Ekblad U, et al. Bacterial 16S rDNA polymerase chain reaction in the detection of intra-amniotic infection. Br J Obstet Gynaecol 1996; 103: 664-9.

190. Nien JK, Yoon BH, Espinoza J, et al. A rapid MMP-8 bedside test for the detection of intra-amniotic inflammation identifies patients at risk for imminent preterm delivery. Am J Obstet Gynecol 2006; 195: 1025-30.

191. Yeast JD, Garite TJ, Dorchester W. The risks of amniocentesis in the management of premature rupture of the membranes. Am J Obstet Gynecol 1984; 149: 505-8.

192. Shaver DC, Spinnato JA, Whybrew D, Williams WK, Anderson GD. Comparison of phospholipids in vaginal and amniocentesis specimens of patients with premature rupture of membranes. Am J Obstet Gynecol 1987; 156: 454-7

193. Stedman CM, Crawford S, Staten E, Cherny WB. Management of preterm premature rupture of membranes: assessing amniotic fluid in the vagina for phosphatidylglycerol. Am J Obstet Gynecol 1981; 140: 34-8.

194. Brame RG, MacKenna J. Vaginal pool phospholipids in the management of premature rupture of membranes. Am J Obstet Gynecol 1983; 145: 992-1000.

195. Golde SH. Use of obstetric perineal pads in collection of amniotic fluid in patients with rupture of the membranes. Am J Obstet Gynecol 1983; 146: 710-12.

196. Estol PC, Poseiro JJ, Schwarcz R. Phosphatidylglycerol determination in the amniotic fluid from a PAD placed over the vulva: a method for diagnosis of fetal lung maturity in cases of premature ruptured membranes. J Perinat Med 1992; 20: 65-71. 
197. Lewis DF, Towers CV, Major CA, et al. Use of Amniostat-FLM in detecting the presence of phosphatidylglycerol in vaginal pool samples in preterm premature rupture of membranes. Am J Obstet Gynecol 1993; 169: 573-6.

198. Gluck L, Kulovich MV, Borer RC Jr, Keidel WN. The interpretation and significance of the lecithin-sphingomyelin ratio in amniotic fluid. Am J Obstet Gynecol 1974; 120: 142-55.

199. Schumacher RE, Parisi VM, Steady HM, Tsao FH. Bacteria causing false positive test for phosphatidylglycerol in amniotic fluid. Am J Obstet Gynecol 1985; 151: 1067-8.

200. Dombroski RA, MacKenna J, Brame RG. Comparison of amniotic fluid lung maturity profiles in paired vaginal and amniocentesis specimens. Am J Obstet Gynecol 1981; 140: 461-4.

201. Goldstein AS, Mangurten HH, Libretti JV, Berman AM. Lecithin/ sphingomyelin ratio in amniotic fluid obtained vaginally. Am J Obstet Gynecol 1980; 138: 232-3.

202. Spinnato JA, Shaver DC, Bray EM, Lipshitz J. Preterm premature rupture of the membranes with fetal pulmonary maturity present: a prospective study. Obstet Gynecol 1987; 69: 196-201.

203. Mercer BM, Crocker LG, Boe NM, Sibai BM. Induction versus expectant management in premature rupture of the membranes with mature amniotic fluid at 32 to 36 weeks: a randomized trial. Am J Obstet Gynecol 1993; 169: 775-82.

204. Vintzileos AM, Feinstein SJ, Lodeiro JG, et al. Fetal biophysical profile and the effect of premature rupture of the membranes. Obstet Gynecol 1986; 67: 818-23.

205. Vintzileos AM, Campbell WA, Nochimson DJ, Weinbaum PJ. The use of the nonstress test in patients with premature rupture of the membranes. Am J Obstet Gynecol 1986; 155: 149-53.

206. Zeevi D, Sadovsky E, Younis J, Granat M, Ohel G. Antepartum fetal heart rate characteristics in cases of premature rupture of membranes. Am J Perinatol 1988; 5: 260-3.

207. Vintzileos AM, Knuppel RA. Fetal biophysical assessment in premature rupture of the membranes. Clin Obstet Gynecol 1995; 38: 45-58.

208. Carroll SG, Papaioannou S, Nicolaides KH. Assessment of fetal activity and amniotic fluid volume in the prediction of intrauterine infection in preterm prelabor amniorrhexis. Am J Obstet Gynecol 1995; 172: 1427-35.

209. Del Valle GO, Joffe GM, Izquierdo LA, et al. The biophysical profile and the nonstress test: poor predictors of chorioamnionitis and fetal infection in prolonged preterm premature rupture of membranes. Obstet Gynecol 1992; 80: 106-10.

210. Gauthier DW, Meyer WJ, Bieniarz A. Biophysical profile as a predictor of amniotic fluid culture results. Obstet Gynecol 1992; 80: 102-5.

211. Harding JA, Jackson DM, Lewis DF, et al. Correlation of amniotic fluid index and nonstress test in patients with preterm premature rupture of membranes. Am J Obstet Gynecol 1991; 165: 1088-94.

212. Vintzileos AM, Campbell WA, Nochimson DJ, Weinbaum PJ. Degree of oligohydramnios and pregnancy outcome in patients with premature rupture of the membranes. Obstet Gynecol 1985; 66: 162-7.

213. Gonik B, Bottoms SF, Cotton DB. Amniotic fluid volume as a risk factor in preterm premature rupture of the membranes. Obstet Gynecol 1985; 65: 456-9.

214. Hadi HA, Hodson CA, Strickland D. Premature rupture of the membranes between 20 and 25 weeks' gestation: role of amniotic fluid volume in perinatal outcome. Am J Obstet Gynecol 1994; 170: 1139-44.

215. Lao TT, Cheung VY. Expectant management of preterm prelabour rupture of membranes-the significance of oligohydramnios at presentation. Eur J Obstet Gynecol Reprod Biol 1993; 48: 87-91.

216. Yoon BH, Kim YA, Romero R, et al. Association of oligohydramnios in women with preterm premature rupture of membranes with an inflammatory response in fetal, amniotic, and maternal compartments. Am J Obstet Gynecol 1999; 181: 784-8.

217. Smith CV, Greenspoon J, Phelan JP, Platt LD. Clinical utility of the nonstress test in the conservative management of women with preterm spontaneous premature rupture of the membranes. J Reprod Med 1987; 32: $1-4$.

218. Kivikoski AI, Amon E, Vaalamo PO, Pirhonen J, Kopta MM. Effect of third-trimester premature rupture of membranes on fetal breathing movements: a prospective case-control study. Am J Obstet Gynecol 1988; 159: 1474-7.
219. Roberts AB, Goldstein I, Romero R, Hobbins JC. Fetal breathing movements after preterm premature rupture of membranes. Am J Obstet Gynecol 1991; 164: 821-5.

220. Vintzileos AM, Campbell WA, Nochimson DJ, et al. The fetal biophysical profile in patients with premature rupture of the membranes-an early predictor of fetal infection. Am J Obstet Gynecol 1985; 152: 510-16.

221. Vintzileos AM, Campbell WA, Nochimson DJ, Weinbaum PJ. Fetal breathing as a predictor of infection in premature rupture of the membranes. Obstet Gynecol 1986; 67: 813-17.

222. Goldstein I, Romero R, Merrill S, et al. Fetal body and breathing movements as predictors of intraamniotic infection in preterm premature rupture of membranes. Am J Obstet Gynecol 1988; 159: 363-8.

223. Fleming AD, Salafia CM, Vintzileos AM, et al. The relationships among umbilical artery velocimetry, fetal biophysical profile, and placental inflammation in preterm premature rupture of the membranes. Am J Obstet Gynecol 1991; 164: 38-41.

224. Mercer B, Moretti M, Deal S. Intensive antenatal testing for women with prolonged preterm premature rupture of the membranes. Abstract 420 Presented at 11th annual meeting of the Society of Perinatal Obstetricians. San Francisco, 1991.

225. Miller JM Jr, Kho MS, Brown HL, Gabert HA. Clinical chorioamnionitis is not predicted by an ultrasonic biophysical profile in patients with premature rupture of membranes. Obstet Gynecol 1990; 76: 1051-4.

226. Roberts AB, Goldstein I, Romero R, Hobbins JC. Comparison of total fetal activity measurement with the biophysical profile in predicting intra-amniotic infection in preterm premature rupture of membranes. Ultrasound Obstet Gynecol 1991; 1: 36-9.

227. Roussis P, Rosemond RL, Glass C, Boehm FH. Preterm premature rupture of membranes: detection of infection. Am J Obstet Gynecol 1991; 165: 1099-104.

228. Vintzileos AM, Bors-Koefoed R, Pelegano JF, et al. The use of fetal biophysical profile improves pregnancy outcome in premature rupture of the membranes. Am J Obstet Gynecol 1987; 157: 236-40.

229. Fanaroff AA, Hack M. Periventricular leukomalacia-prospects for prevention. N Engl J Med 1999; 341: 1229-31.

230. Hack M, Fanaroff AA. Outcomes of extremely immature infants-a perinatal dilemma. N Engl J Med 1993; 329: 1649-50.

231. Dewan H, Morris JM. A systematic review of pregnancy outcome following preterm premature rupture of membranes at a previable gestational age. Aust NZ J Obstet Gynaecol 2001; 41: 389-94.

232. Gold RB, Goyert GL, Schwartz DB, Evans MI, Seabolt LA. Conservative management of second-trimester post-amniocentesis fluid leakage. Obstet Gynecol 1989; 74: 745-7.

233. Hanson FW, Tennant FR, Zorn EM, Samuels S. Analysis of 2136 genetic amniocenteses: experience of a single physician. Am J Obstet Gynecol 1985; 152: 436-43.

234. Effect of corticosteroids for fetal maturation on perinatal outcomes. NIH Consensus Development Panel on the Effect of Corticosteroids for Fetal Maturation on Perinatal Outcomes. JAMA 1995; 273: 413-18.

235. Mercer BM. Preterm premature rupture of the membranes: diagnosis and management. Clin Perinatol 2004; 31: 765-82, vi.

236. Simhan HN, Canavan TP. Preterm premature rupture of membranes: diagnosis, evaluation and management strategies. BJOG 2005; 112(Suppl 1): $32-7$.

237. Mercer BM, Arheart KL. Antimicrobial therapy in expectant management of preterm premature rupture of the membranes. Lancet 1995; 346: 1271-9.

238. Kenyon SL, Taylor DJ, Tarnow-Mordi W. Broad-spectrum antibiotics for preterm, prelabour rupture of fetal membranes: the ORACLE I randomised trial. ORACLE Collaborative Group. Lancet 2001; 357: 979-88.

239. Amon E, Lewis SV, Sibai BM, Villar MA, Arheart KL. Ampicillin prophylaxis in preterm premature rupture of the membranes: a prospective randomized study. Am J Obstet Gynecol 1988; 159: 539-43.

240. Morales WJ, Angel JL, O’Brien WF, Knuppel RA. Use of ampicillin and corticosteroids in premature rupture of membranes: a randomized study. Obstet Gynecol 1989; 73: 721-6.

241. Johnston MM, Sanchez-Ramos L, Vaughn AJ, Todd MW, Benrubi GI. Antibiotic therapy in preterm premature rupture of membranes: a 
randomized, prospective, double-blind trial. Am J Obstet Gynecol 1990; 163: 743-7.

242. Christmas JT, Cox SM, Andrews W, et al. Expectant management of preterm ruptured membranes: effects of antimicrobial therapy. Obstet Gynecol 1992; 80: 759-62.

243. McGregor JA, French JI, Seo K. Antimicrobial therapy in preterm premature rupture of membranes: results of a prospective, double-blind, placebo-controlled trial of erythromycin. Am J Obstet Gynecol 1991; 165: 632-40.

244. Kurki T, Hallman M, Zilliacus R, Teramo K, Ylikorkala O. Premature rupture of the membranes: effect of penicillin prophylaxis and long-term outcome of the children. Am J Perinatol 1992; 9: 11-16.

245. McCaul JF, Perry KG Jr, Moore JL Jr, et al. Adjunctive antibiotic treatment of women with preterm rupture of membranes or preterm labor. Int J Gynaecol Obstet 1992; 38: 19-24.

246. Mercer BM, Moretti ML, Prevost RR, Sibai BM. Erythromycin therapy in preterm premature rupture of the membranes: a prospective, randomized trial of 220 patients. Am J Obstet Gynecol 1992; 166: 794-802.

247. Owen J, Groome LJ, Hauth JC. Randomized trial of prophylactic antibiotic therapy after preterm amnion rupture. Am J Obstet Gynecol 1993; 169: 976-81.

248. Blanco JD, Iams J, Artal R, et al. Multicentre double-blind prospective randomized trial of ceftizoxime versus placebo in women with preterm premature rupture of the membranes. Am J Obstet Gynecol 1993; 168: 378.

249. Lockwood CJ, Costigan K, Ghidini A, et al. Double-blind; placebocontrolled trial of piperacillin prophylaxis in preterm membrane rupture. Am J Obstet Gynecol 1993; 169: 970-6.

250. Ernest JM, Givner LB. A prospective, randomized, placebo-controlled trial of penicillin in preterm premature rupture of membranes. Am J Obstet Gynecol 1994; 170: 516-21.

251. Mercer BM, Miodovnik M, Thurnau GR, et al. Antibiotic therapy for reduction of infant morbidity after preterm premature rupture of the membranes. A randomized controlled trial. National Institute of Child Health and Human Development Maternal-Fetal Medicine Units Network. JAMA 1997; 278: 989-95.

252. Lovett SM, Weiss JD, Diogo MJ, Williams PT, Garite TJ. A prospective, double-blind, randomized, controlled clinical trial of ampicillinsulbactam for preterm premature rupture of membranes in women receiving antenatal corticosteroid therapy. Am J Obstet Gynecol 1997; 176: $1030-8$.

253. Kenyon S, Boulvain M, Neilson J. Antibiotics for preterm rupture of the membranes: a systematic review. Obstet Gynecol 2004; 104: 1051-7.

254. Kenyon S, Pike K, Jones DR, et al. Childhood outcomes after prescription of antibiotics to pregnant women with preterm rupture of the membranes: 7-year follow-up of the ORACLE I trial. Lancet 2008; 372: $1310-18$.

255. Brelje MC, Kaltreider DF. The use of vaginal antibiotics in premature rupture of the membranes. Am J Obstet Gynecol 1966; 94: 889.

256. Ogita S, Imanaka M, Matsumoto M, Hatanaka K. Premature rupture of the membranes managed with a new cervical catheter. Lancet 1984; 1: $1330-1$.

257. Romero R, Scioscia AL, Edberg SC, Hobbins JC. Use of parenteral antibiotic therapy to eradicate bacterial colonization of amniotic fluid in premature rupture of membranes. Obstet Gynecol 1986; 67: 15S-7S.

258. Romero R, Hagay Z, Nores J, Sepulveda W, Mazor M. Eradication of Ureaplasma urealyticum from the amniotic fluid with transplacental antibiotic treatment. Am J Obstet Gynecol 1992; 166: 618-20.

259. Harding JE, Pang J, Knight DB, Liggins GC. Do antenatal corticosteroids help in the setting of preterm rupture of membranes? Am J Obstet Gynecol 2001; 184: 131-9.

260. Crowley P. Corticosteroids after prelabour rupture of membranes [revised May 5, 1994]. In: Keirse MJ, Renfre MJ, Neilson JP, Crowther C, eds. Pregnancy and childbirth module. The Cochrane Pregnancy and Childbirth Database. The Cochrane Collaboration, Issue 2 [Available from BMJ Publishing Group, London.]. Oxford, UK, 1995.

261. Crowley P. Corticosteroids + induction of labour after PROM preterm [revised April 22, 1993]. In: Keirse MJ, Renfre MJ, Neilson JP, Crowther C, eds. Pregnancy and childbirth module. The Cochrane Pregnancy and Childbirth Database. The Cochrane Collaboration, Issue 2, [Available from BMJ Publishing Group, London.]. Oxford, UK, 1995.
262. Leitich H, Egarter C, Reisenberger K, Kaider A, Berghammer P. Concomitant use of glucocorticoids: a comparison of two metaanalyses on antibiotic treatment in preterm premature rupture of membranes. Am J Obstet Gynecol 1998; 178: 899-908.

263. Guinn DA, Atkinson MW, Sullivan L, et al. Single vs weekly courses of antenatal corticosteroids for women at risk of preterm delivery: a randomized controlled trial. JAMA 2001; 286: 1581-7.

264. Christensen KK, Ingemarsson I, Leideman T, Solum T, Svenningsen N. Effect of ritodrine on labor after premature rupture of the membranes. Obstet Gynecol 1980; 55: 187-90.

265. Garite TJ, Keegan KA, Freeman RK, Nageotte MP. A randomized trial of ritodrine tocolysis versus expectant management in patients with premature rupture of membranes at 25 to 30 weeks of gestation. Am J Obstet Gynecol 1987; 157: 388-93.

266. Matsuda Y, Ikenoue T, Hokanishi H. Premature rupture of the membranes-aggressive versus conservative approach: effect of tocolytic and antibiotic therapy. Gynecol Obstet Invest 1993; 36: 102-7.

267. Weiner CP, Renk K, Klugman M. The therapeutic efficacy and costeffectiveness of aggressive tocolysis for premature labor associated with premature rupture of the membranes. Am J Obstet Gynecol 1988; 159: 216-22.

268. Harger JH. Comparison of success and morbidity in cervical cerclage procedures. Obstet Gynecol 1980; 56: 543-8.

269. Yeast JD, Garite TR. The role of cervical cerclage in the management of preterm premature rupture of the membranes. Am J Obstet Gynecol 1988; 158: 106-10.

270. Blickstein I, Katz Z, Lancet M, Molgilner BM. The outcome of pregnancies complicated by preterm rupture of the membranes with and without cerclage. Int J Gynaecol Obstet 1989; 28: 237-42.

271. Goldman JM, Greene MF, Tuomala R, et al. Outcome of expectant management in preterm premature rupture of membranes with cervical cerclage in place. Abstract 139. Presented at the Tenth Annual Meeting of the Society of Perinatal Obstetricians. Houston, Texas, 1990.

272. Ludmir J, Bader T, Chen L, Lindenbaum C, Wong G. Poor perinatal outcome associated with retained cerclage in patients with premature rupture of membranes. Obstet Gynecol 1994; 84: 823-6.

273. McElrath TF, Norwitz ER, Lieberman ES, Heffner LJ. Management of cervical cerclage and preterm premature rupture of the membranes: should the stitch be removed? Am J Obstet Gynecol. 2000 Oct; 183(4): 840-6.

274. McElrath TF, Norwitz ER, Lieberman ES, Heffner LJ. Perinatal outcome after preterm premature rupture of membranes with in situ cervical cerclage. Am J Obstet Gynecol. 2002 Nov; 187(5): 1147-52.

275. ACOG Practice Bulletin No. 80: premature rupture of membranes. Clinical management guidelines for obstetrician-gynecologists. Obstet Gynecol 2007; 109: 1007-19.

276. Carlan SJ, O’Brien WF, Parsons MT, Lense JJ. Preterm premature rupture of membranes: a randomized study of home versus hospital management. Obstet Gynecol 1993; 81: 61-4.

277. Philipson EH, Hoffman DS, Hansen GO, Ingardia CJ. Preterm premature rupture of membranes: experience with latent periods in excess of seven days. Am J Perinatol 1994; 11: 416-19.

278. Curet LB, Rao AV, Zachman RD, et al. Association between ruptured membranes, tocolytic therapy, and respiratory distress syndrome. Am J Obstet Gynecol 1984; 148: 263-8.

279. Ustun C, Kokcu A, Cil E, Kandemir B. Relationship between endomyometritis and the duration of premature membrane rupture. J Matern Fetal Med 1998; 7: 243-6.

280. McElrath TF, Allred EN, Leviton A. Prolonged latency after preterm premature rupture of membranes: an evaluation of histologic condition and intracranial ultrasonic abnormality in the neonate born at $<28$ weeks of gestation. Am J Obstet Gynecol 2003; 189: 794-8.

281. Cox SM, Leveno KJ. Intentional delivery versus expectant management with preterm ruptured membranes at 30-34 weeks' gestation. Obstet Gynecol 1995; 86: 875-9.

282. Lieman JM, Brumfield CG, Carlo W, Ramsey PS. Preterm premature rupture of membranes: is there an optimal gestational age for delivery? Obstet Gynecol 2005; 105: 12-17.

283. ACOG practice bulletin. Premature rupture of membranes. Clinical management guidelines for obstetrician-gynecologists. Number 1, June 1998. American College of Obstetricians and Gynecologists. Int J Gynaecol Obstet 1998; 63: 75-84. 
284. Healy AJ, Veille JC, Sciscione A, McNutt LA, Dexter SC. The timing of elective delivery in preterm premature rupture of the membranes: a survey of members of the Society of Maternal-Fetal Medicine. Am J Obstet Gynecol 2004; 190: 1479-81.

285. Chua S, Arulkumaran S, Yap C, Selamat N, Ratnam SS. Premature rupture of membranes in nulliparas at term with unfavorable cervices: a double-blind randomized trial of prostaglandin and placebo. Obstet Gynecol 1995; 86: 550-4.

286. Chung T, Rogers MS, Gordon H, Chang A. Prelabour rupture of the membranes at term and unfavourable cervix; a randomized placebocontrolled trial on early intervention with intravaginal prostaglandin E2 gel. Aust NZ J Obstet Gynaecol 1992; 32: 25-7.

287. Duff P, Huff RW, Gibbs RS. Management of premature rupture of membranes and unfavorable cervix in term pregnancy. Obstet Gynecol 1984; 63: 697-702.

288. Ekman-Ordeberg G, Uldbjerg N, Ulmsten U. Comparison of intravenous oxytocin and vaginal prostaglandin E2 gel in women with unripe cervixes and premature rupture of the membranes. Obstet Gynecol 1985; 66: 307-10.

289. Gonen R, Samberg I, Degani S. Intracervical prostaglandin E2 for induction of labor in patients with premature rupture of membranes and an unripe cervix. Am J Perinatol 1994; 11: 436-8.

290. Grant JM, Serle E, Mahmood T, Sarmandal P, Conway DI. Management of prelabour rupture of the membranes in term primigravidae: report of a randomized prospective trial. Br J Obstet Gynaecol 1992; 99: 557-62.

291. Hagskog K, Nisell H, Sarman I, Westgren M. Conservative ambulatory management of prelabor rupture of the membranes at term in nulliparous women. Acta Obstet Gynecol Scand 1994; 73: 765-9.

292. Hannah ME, Ohlsson A, Farine D, et al. Induction of labor compared with expectant management for prelabor rupture of the membranes at term. TERMPROM Study Group. N Engl J Med 1996; 334: 1005-10.

293. Hjertberg R, Hammarstrom M, Moberger B, Nordlander E, Granstrom L. Premature rupture of the membranes (PROM) at term in nulliparous women with a ripe cervix. A randomized trial of 12 or 24 hours of expectant management. Acta Obstet Gynecol Scand 1996; 75: 48-53.

294. Ladfors L, Mattsson LA, Eriksson M, Fall O. A randomised trial of two expectant managements of prelabour rupture of the membranes at 34 to 42 weeks. Br J Obstet Gynaecol 1996; 103: 755-62.

295. Mahmood TA, Dick MJ, Smith NC, Templeton AA. Role of prostaglandin in the management of prelabour rupture of the membranes at term. Br J Obstet Gynaecol 1992; 99: 112-17.

296. Mahmood TA, Dick MJ. A randomized trial of management of pre-labor rupture of membranes at term in multiparous women using vaginal prostaglandin gel. Obstet Gynecol 1995; 85: 71-4.
297. Malik N, Gittens L, Gonzalez D, et al. Clinical amnionitis and endometritis in patients with premature rupture of membranes: endocervical prostaglandin E2 gel versus oxytocin for induction of labor. Obstet Gynecol 1996; 88: 540-3.

298. Morales WJ, Lazar AJ. Expectant management of rupture of membranes at term. South Med J 1986; 79: 955-8.

299. Mozurkewich E, Wolf F. Premature rupture of membranes at term: a metaanalysis of three management schemes. Obstet Gynecol 1997; 89: 1035-43.

300. Natale R, Milne JK, Campbell MK, et al. Management of premature rupture of membranes at term: randomized trial. Am J Obstet Gynecol 1994; 171: 936-9.

301. Ngai SW, To WK, Lao T, Ho PC. Cervical priming with oral misoprostol in pre-labor rupture of membranes at term. Obstet Gynecol 1996; 87: 923-6.

302. Ray DA, Garite TJ. Prostaglandin E2 for induction of labor in patients with premature rupture of membranes at term. Am J Obstet Gynecol 1992; 166: 836-43.

303. Rydhstrom H, Ingemarsson I. No benefit from conservative management in nulliparous women with premature rupture of the membranes (PROM) at term. A randomized study. Acta Obstet Gynecol Scand 1991; 70: 543-7.

304. Rymer J, Parker A. A comparison of Syntocinon infusion with prostaglandin vaginal pessaries when spontaneous rupture of the membranes occurs without labour after 34 weeks gestation. Aust NZ J Obstet Gynaecol 1992; 32: 22-4.

305. Shalev E, Schantz A, Wahlin A, et al. Management of prelabor rupture of membranes at term. A randomized study. Acta Obstet Gynecol Scand 1993; 72: 627

306. Shalev E, Peleg D, Eliyahu S, Nahum Z. Comparison of 12- and 72-hour expectant management of premature rupture of membranes in term pregnancies. Obstet Gynecol 1995; 85: 766-8.

307. Wagner MV, Chin VP, Peters CJ, Drexler B, Newman LA. A comparison of early and delayed induction of labor with spontaneous rupture of membranes at term. Obstet Gynecol 1989; 74: 93-7.

308. Zlatnik FJ. Management of premature rupture of membranes at term. Obstet Gynecol Clin North Am 1992; 19: 353-64.

309. Tan BP, Hannah ME. Oxytocin for prelabour rupture of membranes at or near term. Cochrane Database Syst Rev 2000: CD000157.

310. Lin MG, Nuthalapaty FS, Carver AR, Case AS, Ramsey PS. Misoprostol for labor induction in women with term premature rupture of membranes: a meta-analysis. Obstet Gynecol 2005; 106: 593-601.

311. Hannah ME, Hodnett ED, Willan A, et al. Prelabor rupture of the membranes at term: expectant management at home or in hospital? The TermPROM Study Group. Obstet Gynecol 2000; 96: 533-8. 


\section{Sonia S. Hassan, Roberto Romero, Francesca Gotsch, Lorraine Nikita, and Tinnakorn Chaiworapongsa}

\section{INTRODUCTION}

The uterine cervix has a central role in the maintenance of pregnancy and in normal parturition. Preterm cervical ripening may lead to cervical insufficiency or preterm delivery. Moreover, delayed cervical ripening has been implicated in a prolonged latent phase of labor at term. This chapter will review the anatomy and physiology of the uterine cervix during pregnancy and focus on the diagnostic and therapeutic challenges of preterm cervical ripening and the role of cerclage in obstetrics.

\section{ANATOMY AND FUNCTION OF THE UTERINE CERVIX}

The uterus is composed of three parts: corpus, isthmus, and cervix. The corpus is the upper segment of the organ and predominantly contains smooth muscle (myometrium). The isthmus lies between the anatomical internal os of the cervix and the histologic internal os and, during labor, gives rise to the lower uterine segment. The anatomical internal os refers to the junction between the uterine cavity and the cervical canal, while the histologic internal os is the region where the epithelium changes from endometrial to endocervical (1). The term "fibromuscular junction" was introduced by Danforth, who identified the boundary between the connective tissue of the cervix and the myometrium. The fibromuscular junction is in close proximity to the histologic internal os (2) (Figures 1A-D) (3).

\section{Function of the Uterine Cervix}

The main function of the uterine cervix is to serve as a barrier to the expulsion of the conceptus. The endocervical glands generate mucous, which forms the mucous plug, an anatomical and biochemical barrier to microorganisms. "Cervical ripening" is a term used to describe the changes in cervical dilatation, effacement, and consistency, which generally precede the onset of spontaneous labor. This process is associated with complex changes in the extracellular matrix aimed at increasing cervical compliance. The conventional view has been that uterine contractions lead to cervical change, a concept based on the relationship between increased uterine contractility and cervical dilatation during spontaneous labor at term. However, the process of cervical ripening begins weeks before the onset of labor. Similarly, preterm cervical ripening can occur without a demonstrable increase in uterine contractility. Experimental evidence indicates that cervical changes can occur even if the cervix is transected from the myometrium; therefore, these two components of the uterus (fundus and cervix) can undergo changes in the preparation for labor, which are independent from each other.

\section{A Brief Summary of the Biology of Cervical Ripening and Remodeling}

The uterine cervix is essentially a connective tissue organ. Smooth muscle accounts for $<8 \%$ of the distal part of the cervix (4). Cervical competency, defined as the ability of the cervix to retain the conceptus during pregnancy, is unlikely to depend upon a traditional muscular sphincteric mechanism. Experiments in which strips of human cervix have been incubated with vasopressin (a hormone that induces smooth muscle contractility) indicate that the contractile response of the cervix is substantially lower than that of the tissue obtained from the isthmus of the uterine fundus (5). It is now well established that the normal function of the cervix during pregnancy depends upon collagen synthesis and extracellular matrix.

The connective tissue remodeling of the uterine cervix during pregnancy has been proposed to occur in four stages: (i) softening, (ii) ripening, (iii) dilatation, and (iv) repair (6). These phases are overlapping and cannot be sharply separated during gestation (Fig. 2). The interested reader is referred to the reviews and original work by numerous authors $(2,6-31)$ for a detailed discussion of the biochemical and cellular events underlying cervical remodeling during pregnancy and labor.

Word et al. have proposed that early in pregnancy, tensile strength of the softened cervix is maintained by increasing collagen synthesis and cervical growth (6). Collagens type I and III confer tensile strength. During cervical ripening, the cervix becomes thin and pliable, and the collagen concentrations are decreased. This decrease is due to a relative increase in hydrophilic glycosaminoglycans and non-collagenous proteins. The increased expression of aquaporin water channels leads to tissue hydration; this, in turn, disperses collagen fibers and increases collagen solubility and its susceptibility to endogenous proteases. The primary glycosaminoglycan involved is decorin, which protects collagen fibers; however, later in gestation, decorin decreases and hyaluronan increases. The latter can weaken the interaction between collagen and fibronectin, contributing to collagen dispersal (6). Hyaluronan has been found in human endocervical mucous (32). Mahendroo's laboratory has demonstrated that, in mice, the hyaluronan content of the cervix is increased with advancing gestational age along with the expression of the

This work is based on several chapters that were published previously by the authors in other textbooks. This work has been modified and adapted for this textbook. The original chapters are referenced and contain a more extensive discussion of the subject. This chapter has a clinical emphasis. The work has been primarily done by Roberto Romero, who is a government employee, and therefore, is not subject to copyright. 


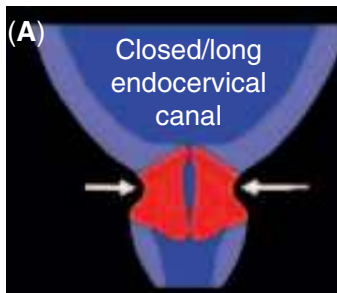

(A)

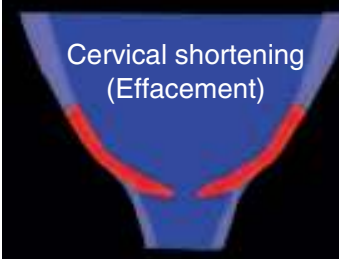

(C)
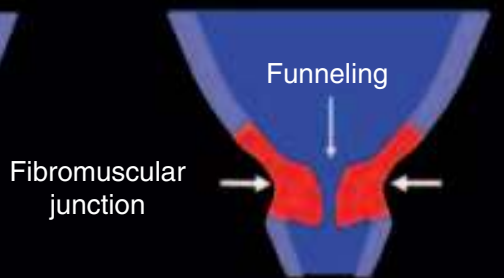

(B)

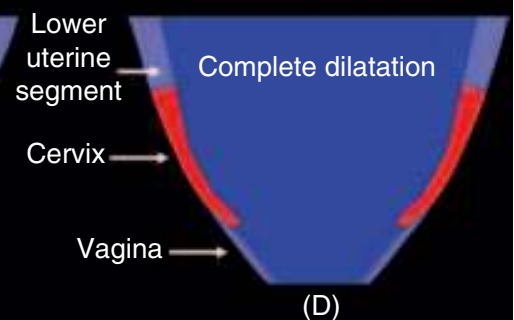

(D)

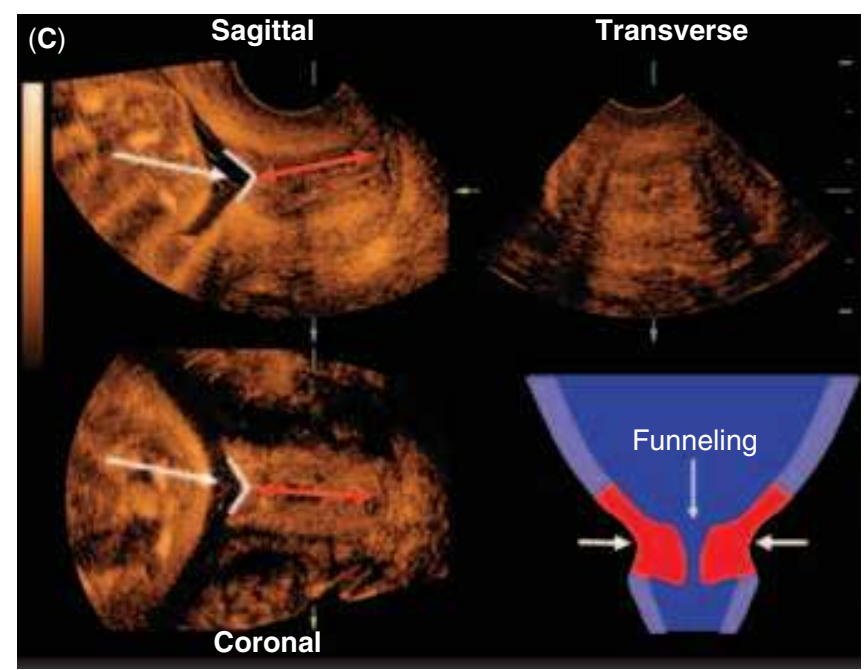

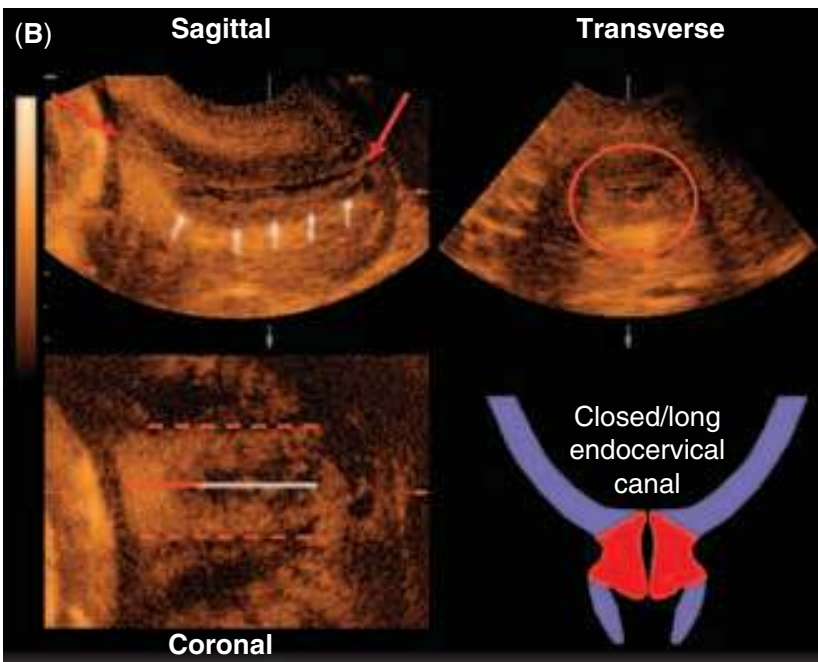

(D) Sagittal Transverse

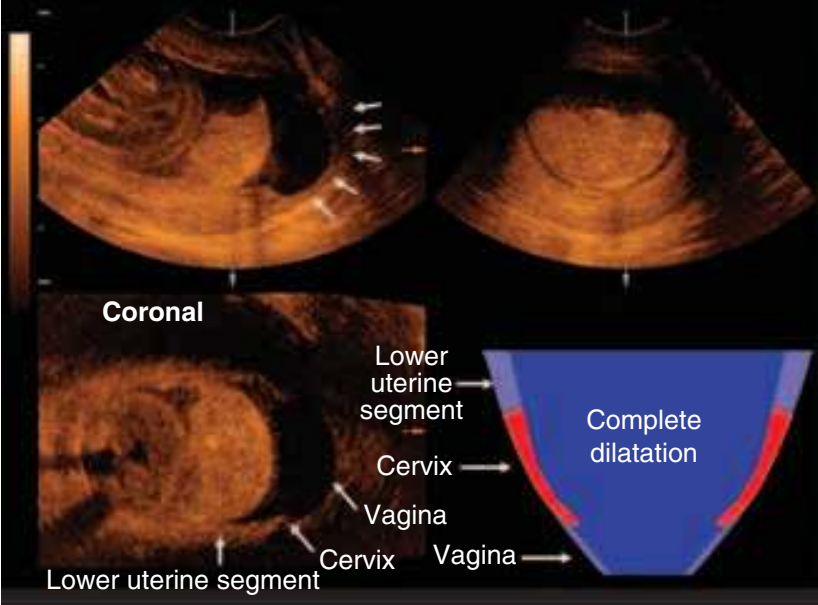

Figure 1(A-D) Ultrasound images and schematics representing the location of the fibromuscular junction and the cervical changes seen throughout pregnancy and labor. Source: From Ref. 3.

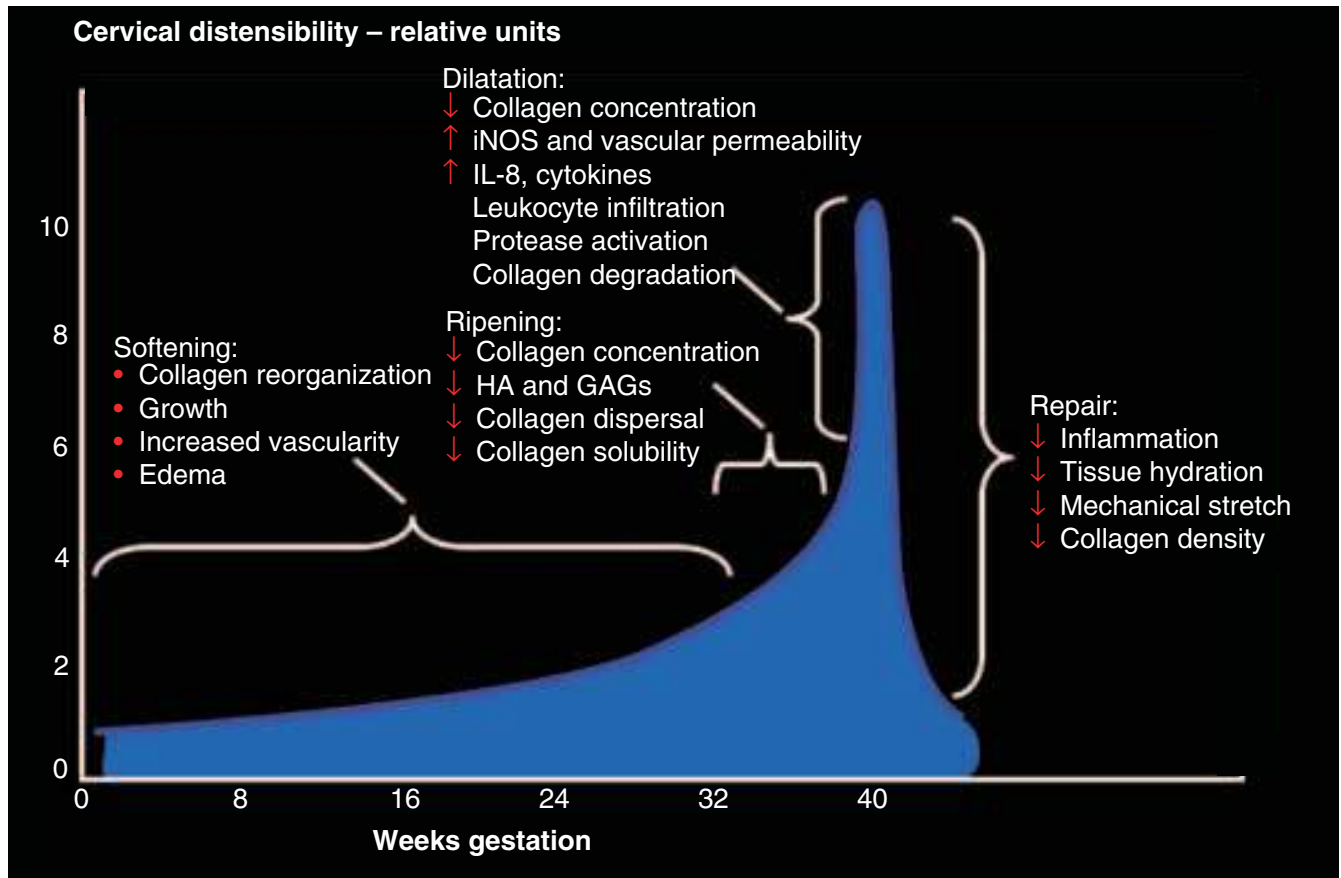

Figure 2 Stages of cervical function during pregnancy and the puerperium as proposed by R. Ann Word et al. (6). Abbreviations: GAG, glycosaminoglycan; HA, hyaluronan; IL-8, interleukin-8; iNOS, inducible nitric oxide synthase. Source: From Ref. 6. 
enzyme hyaluronan synthase 2 (28). Low-molecular weight hyaluronan can bind CD44 and activate macrophages to produce chemokines that attract inflammatory cells (33). Thus, the current understanding is that once collagen is solubilized, an inflammatory cascade is initiated. Studies in humans are necessary to determine the biochemistry of these processes. Strong evidence suggests that a suspension of progesterone action can lead to cervical ripening $(34,35)$.

\section{ORIGIN OF THE CONCEPT OF CERVICAL INCOMPETENCE/INSUFFICIENCY}

One of the first descriptions of "cervical incompetence" has been attributed to Cole, Culpepper, and Rowland in 1658 (36), who wrote in a chapter on the state of being barren: "the second fault in women which hindered conception is when the seed is not retained or the orifice of the womb is so slack that it cannot rightly contract itself to keep in the seed; which is chiefly caused by abortion or hard labor and childbirth, whereby the fibers of the womb are broken in pieces one from another and the inner orifice of the womb overmuch slackened" (37). The term "cervical incompetence" was mentioned by Gream in an article published in the Lancet in 1865 (38). Interestingly, the earliest observations were made nearly 300 years before surgical treatment was developed by Shirodkar (39) and, subsequently, McDonald (40). Although the term "cervical incompetence" has been used for many years (41), this condition is now referred to as "cervical insufficiency" to avoid the negative connotation that the term "incompetence" may imply to patients (42).

\section{Defining Cervical Insufficiency}

Definitions of cervical insufficiency have been proposed by many authors and vary slightly. Such definitions need to be examined critically, particularly in light of recent observations with ultrasound and results of studies that have reframed the concept of cervical insufficiency.

The clinical diagnosis of cervical insufficiency has been traditionally applied to patients with a history of recurrent midtrimester spontaneous abortions and/or early preterm deliveries in which "the basic process is thought to be the failure of the cervix to remain closed during pregnancy" (41). The assumption is that cervical dilatation and effacement have occurred in the absence of increased uterine contractility (41). The presenting symptom is sometimes considered to be a feeling of vaginal pressure, possibly caused by protruding membranes in the midtrimester of pregnancy. Sometimes the membranes rupture. Typically, there is no vaginal bleeding, the fetuses are often born alive, and the labor is short $(40,41,43)$.

We find it difficult to establish a causal relationship between the clinical presentation outlined above and its attribution to a primary cervical disease (i.e., "insufficiency") (42). Clearly, a condition exists in which patients present before 24 weeks of gestation with a dilated and effaced cervix in which the membranes protrude into the vagina (Fig. 3). This condition can often be visualized on ultrasound as illustrated in Figure 4.

Harger, in a review of the literature, defined "cervical insufficiency" as "the inability of the uterine cervix to retain a pregnancy in the absence of contractions or labor" (44). However, the use of this definition in clinical practice is problematic. How can an obstetrician identify "the inability of

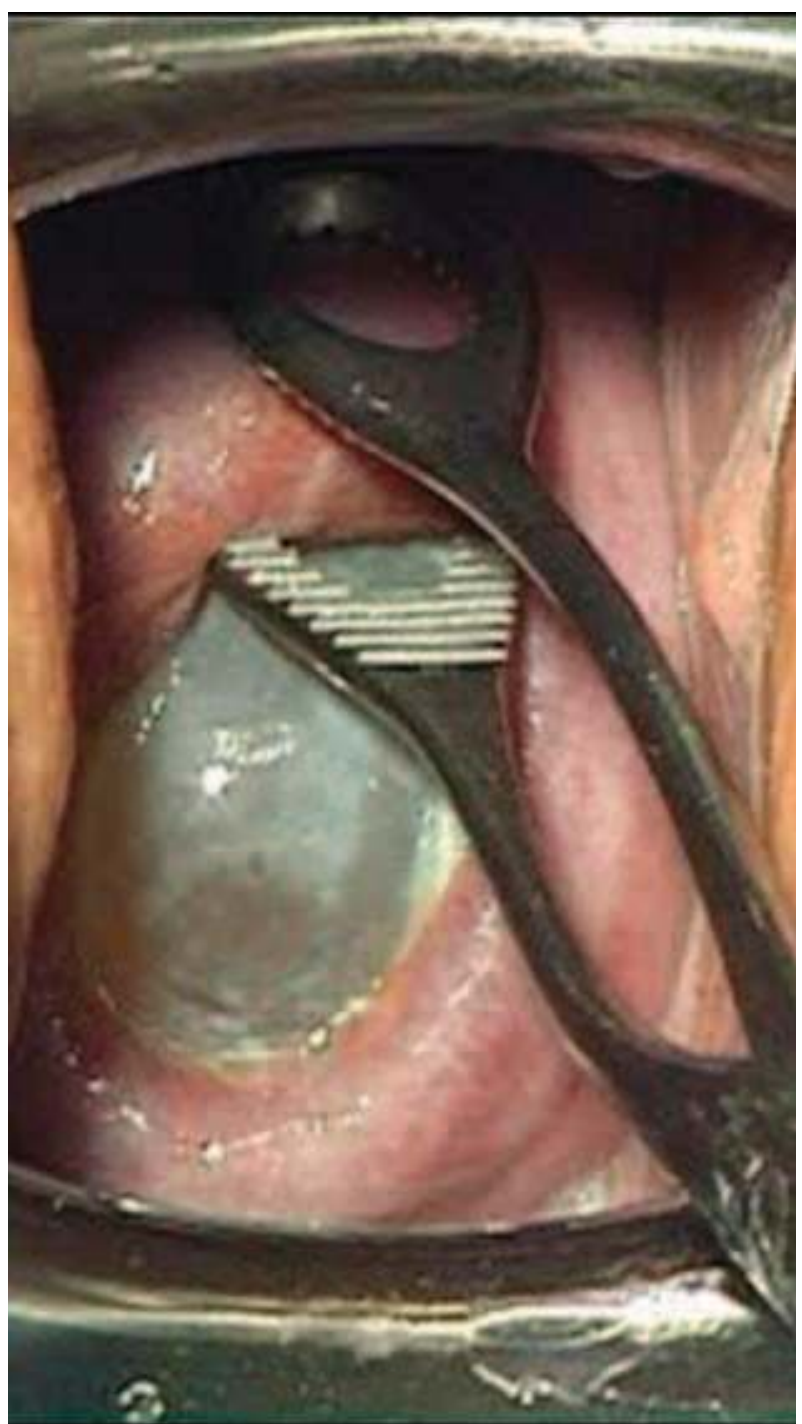

Figure 3 A patient who presented prior to 24 weeks of gestation with a dilated and effaced cervix in which the membranes are protruding into the vagina. Roberto Romero, MD and Karim Kalache, MD. Cover Image - American Journal of Obstetrics and Gynecology, Jan 2006 - Dec 2006 (Vol 194.1-195.6).

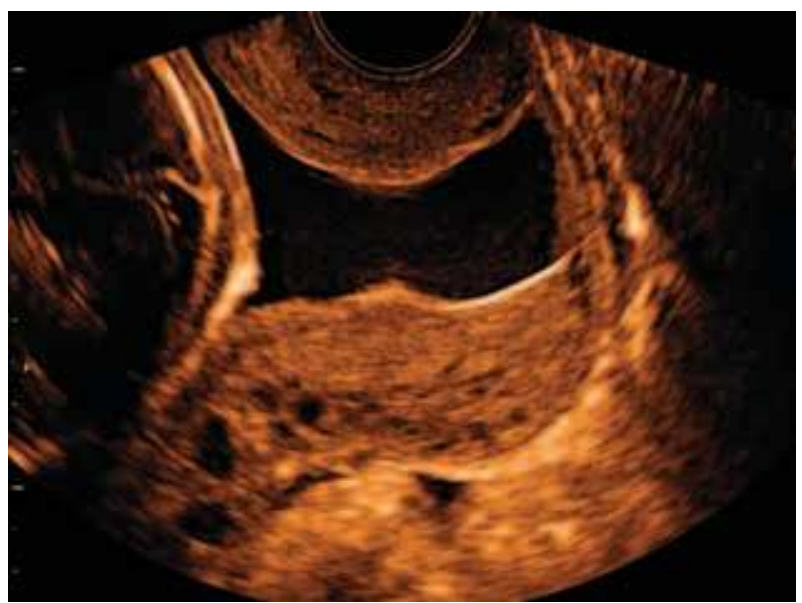

Figure 4 An ultrasound image demonstrating a dilated cervix in a patient with suspected cervical insufficiency.

the cervix to retain the pregnancy"? There is no clinical test that would allow this determination. Some have proposed that the response to treatment offers criteria for the diagnosis. 
Namely, a patient with a history of recurrent midtrimester pregnancy losses who is subsequently treated with a cerclage and delivers at term could be considered to have cervical insufficiency, and hence, the claim that such patient needs a cerclage in every subsequent pregnancy; yet, observational studies do not support this conclusion.

One approach by some authors to examine the accuracy of the diagnosis of cervical insufficiency is to determine pregnancy outcome in patients with this diagnosis who did not have treatment in subsequent pregnancies. In 1961, Dunn and Dans (45) reported the outcome of 30 patients who had two or more consecutive midtrimester abortions without an intervening first-trimester abortion. Interestingly, 13 of the 30 patients had three consecutive midtrimester abortions. The authors reasoned that if cervical insufficiency was the operative mechanism for pregnancy failure, it would be expected that all subsequent untreated pregnancies would result in pregnancy loss; however, this was not the case. Indeed, in $50 \%$ of cases, patients delivered neonates who exceeded 28 weeks of gestation and weighed $>1000 \mathrm{~g}$. Thus, the authors proposed that the spontaneous "cure rate" of this entity would be $50 \%$. The editor of Obstetrical and Gynecological Survey published a commentary (46) in which he argued that a more appropriate endpoint for spontaneous cure would be delivery at term in the subsequent pregnancy. Although he recalculated the spontaneous "cure rate" to be $20 \%$, a clear message of the study is that even in patients with two and sometimes three midtrimester abortions, term pregnancy is possible without a cerclage.

In 1984, Socol et al. (47) reported the outcome of a group of patients who had previously delivered a live-born infant between 20 and 32 weeks of gestation. In the subsequent pregnancy, some patients were treated with a cervical cerclage and others were not (at the discretion of the practitioner). The rate of preterm delivery was $36 \%(5 / 14)$ in patients who had a cerclage and $38 \%(10 / 26)$ in those managed expectantly. One interpretation of this data is that delivery at term was frequently possible in patients with a previous midtrimester pregnancy loss or early preterm delivery without a cervical cerclage.

Yet obstetricians often face the clinical dilemma of how to manage a patient who had a previous successful pregnancy with a cervical cerclage. Was the first cerclage truly necessary? Would the patient have delivered at term regardless of the placement of the cerclage? These questions are pervasive because of the subjective nature of the diagnosis. For example, some obstetricians place a cerclage after a midtrimester pregnancy loss with rupture of membranes, while others consider that this is not enough evidence of cervical insufficiency to justify the operation.

In a 1994 article entitled "Once a cerclage, not always a cerclage," Fejgin et al. (48) reported the pregnancy outcome of patients who had a history of at least one pregnancy in which a McDonald cerclage had been placed and the physician caring for the subsequent pregnancy was uncertain as to whether the procedure was necessary. A committee of three obstetricians reviewed the history, a hysterosalpingogram when available, and the results of a pelvic examination. There were 35 patients who had 58 pregnancies with a cerclage, and 52 subsequent pregnancies without the procedure. The outcome of pregnancies without a cerclage was better than those with a cerclage.
Collectively, these retrospective studies question the accuracy of the diagnosis of cervical insufficiency and/or the effectiveness of cerclage. The results of these studies (and others) are at the root of the controversy about the nature of this clinical entity, its diagnosis, and the precise role of cerclage in clinical medicine.

There is no objective diagnostic test for cervical insufficiency. The diagnosis is often made in a patient in the midtrimester who presents with dilatation of the cervix and different degrees of membrane prolapse. The evaluation of the nonpregnant patient represents an unsolved challenge. Several methods have been proposed for the identification of the patient at risk for cervical insufficiency, including (i) the passage of Hegar dilators $(6-8 \mathrm{~mm})$ or Pratt dilators through the internal cervical os (49-51), (ii) the use of a balloon test (52), or (iii) the ability of the cervix to hold an inflated Foley catheter during hysterosalpingography (53). However, there is a paucity of scientific evidence to support the value of these tests in predicting subsequent pregnancy outcome (44).

\section{SONOGRAPHIC CERVICAL LENGTH}

Digital examination of the cervix was the method used to determine cervical status (effacement, dilatation, position, and consistency) before the introduction of ultrasound. Bishop developed his cervical scoring system primarily to predict when spontaneous labor at term would occur and found that the higher the score, the sooner the labor would start (54). Wood et al. (55) were the first to report that a short cervix was a risk factor for preterm labor and delivery. A large study reported by Papiernik consisted of serial digital examinations in 8303 women, where dilatation of the internal os was the strongest risk factor for preterm delivery. A short cervix $(\leq 1 \mathrm{~cm})$ also increased the risk (56). These findings have been confirmed by others (57-62). However, digital examination is subjective and has limitations. For example, the coefficient of variation for effacement has been reported to be $26 \%$ (63). Moreover, evaluation of effacement requires placing the examining finger in close proximity to the fetal membranes. Sonographic imaging of the cervix is less invasive and more objective in assessing cervical length as well as changes in the anatomy of the internal os (64).

Transvaginal sonography is superior to transabdominal for examination of the cervix $(64,65)$. Numerous studies have proven that the shorter the sonographic cervical length in the midtrimester, the higher the risk of spontaneous preterm labor/delivery (66-70). However, there is no agreement concerning what constitutes a sonographic short cervix. For example, Iams et al. (67) proposed that a cervix of $26 \mathrm{~mm}$ or shorter at 24 weeks of gestation increases the risk of spontaneous preterm delivery (Relative Risk (RR) 6.19, 95\% CI 3.84-9.97). The prevalence of spontaneous preterm delivery (defined as $<35$ weeks) in this study was $4.3 \%$, and the positive predictive value was $17.8 \%$ for a cervical length $\leq 25 \mathrm{~mm}$ at 24 weeks of gestation (67). Thus, most women with a short cervix (defined as $25 \mathrm{~mm}$ or less) will not deliver a preterm neonate. Other investigators have proposed a cut-off of $15 \mathrm{~mm}$, because a cervical length of $15 \mathrm{~mm}$ or less is associated with nearly a $50 \%$ risk of spontaneous preterm delivery at 32 weeks of gestation or less when neonatal morbidity is substantial $(68,70)$.

It is important to stress that sonographic cervical length is not a screening test for spontaneous preterm delivery, because 
only a fraction of all patients who will have a spontaneous preterm birth have a short cervix in the midtrimester. Sonographic cervical length is only a method for risk assessment for spontaneous preterm delivery and not a screening test. Cervical length can modify the a priori risk for preterm delivery. For example, a woman with a history of preterm delivery or one with a twin or triplet gestation will have a higher risk for preterm delivery than a patient with the same cervical length, but without such history (71-80). It is now possible to provide women with an individualized estimation of risk for preterm delivery based upon cervical length and whether they have a history of preterm birth (81).

\section{CERVICAL SUFFICIENCY/INSUFFICIENCY AS A CONTINUUM}

The hypothesis that cervical competence or sufficiency represents a spectrum was studied by Parikh and Mehta, who used digital examination of the cervix and concluded that degrees of cervical competence did not exist (82). Iams et al., (83) using sonographic examination of the cervix, concluded that cervical competence was a continuum. The authors reported a strong relationship between cervical length in pregnancy and previous obstetrical history. This relationship is nearly linear. However, patients with a typical history of an incompetent cervix appear to represent a different group than those who delivered preterm (83). Similar results have been reported by Guzman et al. (84). Collectively, these studies suggest that there is a relationship between a history of preterm delivery and the cervical length in a subsequent pregnancy. Inasmuch as patients with a short cervix are at increased risk for a midtrimester pregnancy loss or spontaneous preterm delivery with intact or ruptured membranes, a short cervix could be considered as the expression of a spectrum of cervical diseases or functions. However, it is noteworthy that some women with a short cervix have an adverse pregnancy outcome, while others have an uncomplicated term delivery (65-70,83-97). Indeed, approximately $50 \%$ of women with a cervix of $15 \mathrm{~mm}$ or less deliver after 32 weeks (70). This indicates that cervical length is only one of the factors determining the degree of cervical competence and that a short cervix should not be equated with "cervical insufficiency."

\section{CERVICAL INSUFFICIENCY IS A SYNDROME}

In a manner similar to preterm labor, pre-eclampsia, small for gestational age, fetal death, and preterm premature rupture of membranes (PROM), the clinical conditions that describe cervical insufficiency can be considered "an obstetrical syndrome" (98). Cervical ripening in the midtrimester may be the result of (i) the loss of connective tissue after a cervical operation such as conization (99-101) or loop electrosurgical excision procedure (LEEP) (101), (ii) a congenital disorder such as cervical hypoplasia after diethylstilbestrol (DES) exposure (102-105), (iii) intrauterine infection (106-108), (iv) a suspension of progesterone action $(34,35)$ : there is experimental evidence that progesterone can reverse cervical compliance induced by the administration of dexamethasone to pregnant sheep (109). Moreover, recent studies have indicated that progesterone administration to women with a short cervix can reduce the rate of preterm birth $(110,111)$, or (v) a cervical disorder. Each of these different causes of the syndrome could be affected by genetic or environmental

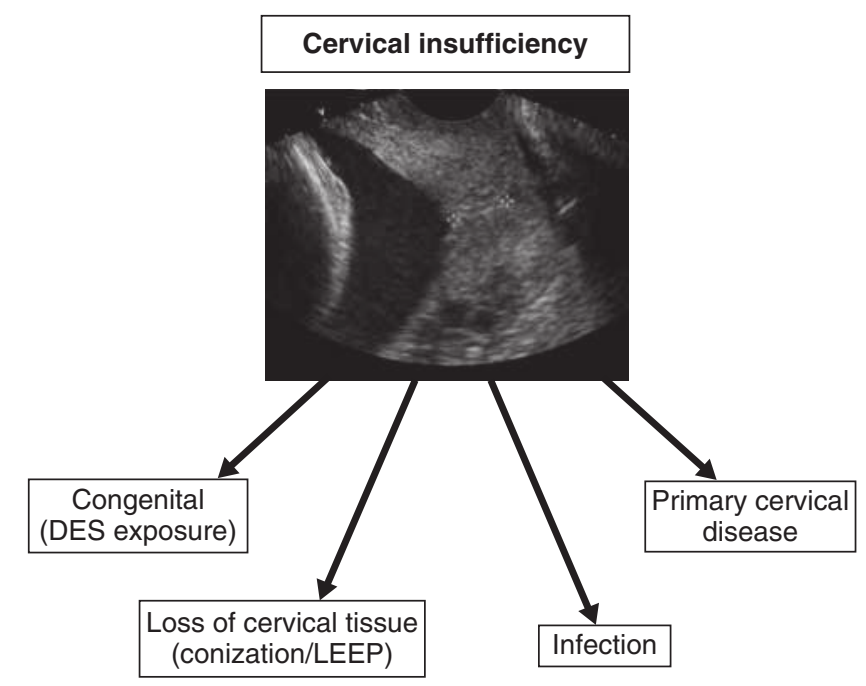

Figure 5 The proposed causes of the "cervical insufficiency syndrome." Abbreviations: DES, diethylstilbestrol; LEEP, loop electrosurgical excision procedure. Source: From Ref. 42.

factors (Figure 5) (42). Moreover, more than one mechanism of disease may be operative in a specific patient. The possibility of novel and yet to be discovered mechanisms of disease playing a role must also be considered.

\section{PREVIOUS TRAUMA AS A CAUSE FOR CERVICAL INSUFFICIENCY}

Procedures such as laser ablation, LEEP, and cold-knife conization may increase the risk of a preterm birth $(100,112-118)$. In a recent retrospective study by Shin et al., the rate of preterm delivery was significantly higher in women with a history of a conization when compared with those without one [32.1\% (18/56) vs. $15.2 \%(3355 / 22,070), p<0.001]$. Yet the use of a McDonald cerclage $(\mathrm{n}=25)$ was not associated with a reduction in the rate of preterm delivery when compared with those without cerclage $(\mathrm{n}=31)$ (expectantly managed group vs. cerclage group; $<28$ week, $6.5 \%$ vs. $8.0 \%$, $p=1.000 ;<34$ week, $19.4 \%$ vs. $20.0 \%, p=1.000 ;<37$ week, $29.0 \%$ vs. $36.0 \%, p=0.579)(119)$.

\section{CONGENITAL CERVICAL INSUFFICIENCY}

Exposure to DES in utero has been reported to increase the risk of second-trimester pregnancy loss fivefold (6.3\% in DES exposed vs. $1.6 \%$ in the control group) (120).

There is evidence of familial aggregation in cervical insufficiency. In one study, approximately $25 \%$ of patients with cervical insufficiency had a first-degree relative with a similar diagnosis (121). This has been attributed to genetic factors involved in the regulation of extracellular matrix, specifically, polymorphisms in the genes encoding for collagen 1 alpha 1 (COLIAI) and transforming growth factor beta (TGF $\beta$ ) (121), and recently IL-10 (122). A genetic predisposition to cervical insufficiency has been reported in women with Ehlers-Danlos syndrome $(123,124)$ and Marfan syndrome (125-128).

\section{"CERVICAL INSUFFICIENCY" AS A CLINICAL MANIFESTATION OF INTRAUTERINE INFECTION}

A proportion of patients presenting with asymptomatic cervical dilatation in the midtrimester have microbial invasion of the amniotic cavity (MIAC) $(106,107)$ that can be as high as 
$51.5 \%$ (106). MIAC may be due to premature cervical dilatation with the exposure of the chorioamniotic membranes to the microbial flora of the lower genital tract. Microorganisms may gain access to the amniotic cavity by crossing intact membranes (106). Under these circumstances, infection would be a secondary phenomenon to primary cervical disease. An alternative is that intrauterine infection or one caused by activation of microorganisms present within the uterine cavity (129) in the second trimester of pregnancy produces myometrial contractility and cervical ripening. Since uterine contractions are usually clinically silent in the midtrimester of pregnancy, the clinical picture of an infection-induced spontaneous abortion may be indistinguishable from that of an incompetent cervix $(106,130)$. The most frequently isolated organisms from the amniotic fluid in women with suspected cervical insufficiency include Ureaplasma urealyticum and Ureaplasma parvum $(106,131,132)$. We have established that $9 \%(5 / 57)$ of women with a short endocervix $(<25 \mathrm{~mm})$ have microbiologically proven intra-amniotic infection (108), suggesting that these infections are subclinical and may precede the development of the clinical picture of acute "cervical insufficiency" (dilated and effaced cervix with bulging membranes).

\section{CERCLAGE}

Cervical cerclage was introduced in 1955 by V. N. Shirodkar, Professor of Midwifery and Gynecology at the Grant Medical College in Bombay, India (39). The procedure was developed in response to his observation that "some women abort repeatedly between the fourth and seventh months and no amount of rest and treatment with hormones seemed to help them in retaining the product of conception" (39). Shirodkar referred to a group of 30 women who had had at least four abortions (some between 9 and 11 weeks). He stated that in his opinion, "95\% of cases were due to a weak cervical sphincter and the other few to an underdeveloped or malformed uterus, etc" (39). Shirodkar emphasized that his work was confined to women in whom he could prove the existence of weakness of the internal os by "repeated internal examinations" (39). Ian McDonald, from the Royal Melbourne Hospital, reported in 1957 his experience with 70 patients who had a suture of the cervix for inevitable miscarriage (40). The history of this procedure is relevant since 50 years after its introduction: cerclage is being used for indications different from those originally intended, and there is conflicting evidence about its efficacy for the new indications (e.g., prevention of preterm birth in women with a sonographic short cervix) (39,40,90-92,95-97,133-154).

There are several approaches to cervical cerclage: (i) the Shirodkar method (39), (ii) the McDonald method (40), (iii) the Wurm procedure (155), and (iv) transabdominal (156). The latter has been performed using a laparotomy approach and later described, laparoscopically $(157,158)$. The most widely used procedure is the McDonald cerclage.

\section{Complications of Cerclage}

The risks associated with the use of cerclage include those that can occur intra-operatively (anesthetic complications, blood loss, rupture of membranes), in the post-operative period (subclinical or clinical chorioamnionitis, preterm PROM, cervical laceration, preterm contractions), and at the time of labor and delivery (cervical laceration, cervical dystocia, sepsis, uterine rupture) (159-170). The rate of each complication varies as a function of the indication for the cerclage, type of cerclage, and the state of the cervix and amniotic membranes at the time of surgery $(161,164,167)$. Complications commonly reported include preterm PROM (elective 2.8-36.3\%, emergency 2.8-52.2\%) (162,167-170), preterm contractions (36-39\%) (168), cervical lacerations (elective 0-14\%, emergency 0-23.8\%) (166-169, $171)$, uterine rupture $(<0.1 \%)(172)$, chorioamnionitis (elective 5.2-14.9\%, emergency 4-39.1\%) (162,167,169,170,172), sepsis $(0.34 \%)$ (166), and bleeding (14.9\%) (168).

Harger (169) reported the outcome of 251 patients; 202 who underwent a cerclage placed based upon history and 49 in which cerclages were placed due to cervical dilation. The mean blood loss was $30 \mathrm{ml}$ in those patients who underwent a McDonald cerclage and $44 \mathrm{ml}$ in those who had a Shirodkar procedure. Two patients who had undergone an emergency cerclage lost $150 \mathrm{ml}$ of blood. The risk of pregnancy loss "apparently caused by elective procedures" was 2\% (4/202). Acute chorioamnionitis occurred in $1.2 \%$ of patients. Cervical lacerations occurred more frequently at the time of labor in women who had a Shirodkar or McDonald cerclage than in those without a cerclage $(\mathrm{n}=55,688)$ (Shirodkar: $11 \%$ and McDonald: $14 \%$ vs. control group: $2.18 \%, p<0.001)$. Furthermore, the authors argued that some cesarean sections in women who underwent cerclage (McDonald or Shirodkar) may be attributed to cervical scarring as a result of the surgery.

Charles and Edwards noted an increased risk of complications in patients undergoing cerclage after 19 weeks of gestation. The rate of rupture of the membranes within 5 days of the procedure was $19.5 \%$, preterm PROM (26-34 weeks of gestation) $52.2 \%$, and chorioamnionitis $39.1 \%$ in these patients (162).

In a retrospective review of 482 singleton pregnancies who underwent cerclage placement [McDonald cerclage $(\mathrm{n}=377)$, Shirodkar $(\mathrm{n}=104)]$ over a 6 -year period, Treadwell et al. (167) reported that PROM occurred in 38\% of patients and was the most frequent complication. The prognostic factors determining gestational age at delivery were gestational age at the time of cerclage, cervical dilation, and number of prior pregnancy losses before 24 weeks of gestation. The rate of cervical laceration was $6.7 \%$ and was similar for both emergency and elective cerclages. Of interest, the primary cesarean delivery rate of patients who had undergone a Shirodkar cerclage was higher than those who had undergone a McDonald cerclage ( $31 \%$ vs. $17 \%, p<0.005)$.

\section{Cerclage in Patients with Acute Cervical Insufficiency}

Only one randomized clinical trial has tested the effect of cerclage in patients with acute cervical insufficiency. Patients were identified if they presented with a dilated cervix and membranes at or below a dilated external os before 27 weeks of gestation. Patients were treated either with emergency cerclage and indomethacin $(\mathrm{n}=13)$, or bed rest only $(\mathrm{n}=10)$. Indomethacin was only given to one group. Preterm delivery before 34 weeks of gestation was significantly less frequent in patients allocated to have an emergency cerclage and indomethacin than in those managed expectantly $[54 \%$ $(7 / 13)$ vs. $0 \%(0 / 10) ; p=0.02]$. Antibiotics were administered to both groups of patients (173). Acute cervical insufficiency, however, can present with different degrees of severity. A patient with a dilated cervix in whom the membranes 
are visible but within the uterine cavity is not the same as the patient who has prolapsed "hourglass" membranes in the vagina with a fetal presenting part. The latter group represents a greater surgical challenge and is often associated with subclinical intrauterine infection. One recommendation is to place the patient in the Trendelenburg position and to allow reduction of the membranes to the amniotic cavity. Since patients with intra-amniotic inflammation/infection have a poor prognosis if a cerclage is placed, an amniocentesis is performed to look for infection/inflammation. The workup includes an amniotic fluid Gram stain, white blood cell count, glucose, and culture for aerobic and anaerobic bacteria as well as genital mycoplasmas. If a cerclage is placed, patients are often followed by transvaginal ultrasound. The length of the cervix after a cerclage has some prognostic value about the likelihood of success (174-177).

\section{Cerclage to Prevent Preterm Delivery in Women with a Short Cervix without a History of Preterm Delivery}

The largest randomized clinical trial in which cerclage was used in patients with a sonographic cervical length $\leq 15 \mathrm{~mm}$ was conducted by the Fetal Medicine Foundation of the United Kingdom (150). Cervical length was measured in lowrisk patients at a median gestational age of 23 weeks, and those with a cervix $\leq 15 \mathrm{~mm}$ were randomized to either the expectant management $(n=126)$ or cerclage group $(n=127)$. The rate of preterm delivery at $<33$ weeks of gestation was not significantly different [expectant management group $26 \%(33 / 126)$ vs. cerclage group $22 \%(28 / 127)]$. The conclusion of this study is that cerclage placement in patients with a short cervix without risk factors for preterm delivery does not reduce the rate of preterm birth, neonatal morbidity, or the rate of perinatal death (150).

\section{Prophylactic Cerclage and Cerclage in Patients with a History of Preterm Delivery and a Short Cervix}

The role of prophylactic cerclage in high-risk patients without a sonographic short cervix for the prevention of preterm delivery/midtrimester abortion (by history) is unclear $(144,152,165,178,179)$. While the largest trial conducted prior to the introduction of ultrasound evaluation of the cervix suggested a modest beneficial effect (179), other trials $(165,178)$ and systematic reviews (41) prior to the use of ultrasound have indicated that the evidence of effectiveness for prophylactic cerclage is either weak or nonexistent.

In the Cervical Incompetence Prevention Randomized Cerclage Trial (CIPRACT) study, Althuisius et al. $(135,180)$ randomized 73 pregnant women at $<15$ weeks of gestation with risk factors for "cervical incompetence" to have a prophylactic cerclage $(n=23)$ or be observed $(n=44)$. Four patients had a spontaneous abortion during the first trimester and two were lost for follow-up. The risk factors of "cervical incompetence" included the history of preterm delivery before 34 weeks of gestation, previous preterm PROM before 32 weeks of gestation, history of cold-knife conization, DES exposure, and a Müllerian duct abnormality. Prophylactic cerclages (i.e., McDonald) were generally placed between 10 and 12 weeks (later if enrolled at a later gestational age) using a braided polyester thread. In both groups, cervical length was evaluated every 2 weeks after randomization. The rate of preterm delivery $(<34$ weeks $)$ was similar in both groups [prophylactic cerclage $13 \%$ (3 of 23 ) vs. observation $14 \%$ (6 of 44); $p>0.05$ ] as well as the neonatal survival [prophylactic cerclage $91 \%$ (21 of 23) vs. observation $93 \%$ (41 of 44); $p>0.05$ ]. Patients allocated to the observation group were followed with serial sonography. If the cervical length shortened $(<25 \mathrm{~mm})$ before the 27 th week of gestation, patients were randomized to both therapeutic cerclage and indomethacin with bed rest $(n=20)$ or bed rest alone $(n=16)$. Patients who received a cerclage and indomethacin had a lower rate of preterm delivery at $<34$ weeks and composite neonatal morbidity (neonatal intensive care unit admission or neonatal death) [0\% vs. $44 \%$ (7 of 16), $p=0.002$; and $5 \%$ ( 1 of 19 ) vs. $50 \%$ (8 of 16), $p=0.005$ ].

By contrast, Rust et al. $(91,181)$ randomized 113 patients presenting with a short cervix $(<25 \mathrm{~mm})$ or funneling $(\geq 25 \%)$ between 16 and 24 weeks into the therapeutic cerclage group $(\mathrm{n}=55)$ and the no-cerclage group $(\mathrm{n}=58)$. The population included patients with and without risk factors for preterm birth. All patients underwent amniocentesis to exclude intraamniotic infection and received 48 hours of therapy with indomethacin and antibiotics. There were no significant differences between the two groups with respect to the rate of preterm delivery at $<34$ weeks of gestation ( $35 \%$ vs. $36.2 \%$ ), readmission for preterm labor (52\% vs. $53 \%)$, placental abruption ( $11 \%$ vs. $14 \%$ ), chorioamnionitis ( $20 \%$ vs. $10 \%)$, and the perinatal death rate (13\% vs. $12 \%)$.

The CIPRACT study $(135,152)$ enrolled only patients at risk for preterm delivery, while in the trial of Rust et al. $(91,181)$, $13 \%$ of patients were at low risk. The positive predictive value for a short cervix to predict preterm delivery is higher in patients with a history of preterm birth. The fact that the rate of preterm delivery in the control group of the CIPRACT study was higher than that of the study of Rust et al. (43.8\% vs. $36.2 \%$ ) may explain, at least in part, the different results between these two studies.

Berghella et al. (146) conducted a randomized clinical trial of the use of McDonald cerclage in women with one or more risk factors for preterm birth (one or more prior deliveries at $<35$ weeks, two or more curettages, history of DES exposure, cone biopsy, Müllerian anomaly, or twin gestation). Sixty-one patients with a cervical length $<25 \mathrm{~mm}$ or funneling $>25 \%$ were randomized to cerclage or bed rest. The authors reported that 47 pregnancies $(77 \%)$ were high-risk singleton gestations.

There was no significant difference in the rate of preterm birth prior to 35 weeks in women who underwent cerclage (14 of 31; 45\%) compared with those in the bed rest group (14 of $30 ; 47 \%$ ) (RR $0.94 ; 95 \%$ CI $0.34-2.58$ ). Similarly, patients with a singleton gestation and a prior preterm birth at $<35$ weeks of gestation and a cervical length $<25 \mathrm{~mm}(\mathrm{n}=31$ women) also had no benefit from cerclage placement ( $40 \%$ vs. 56\%; RR 0.52; 95\% CI 0.12-2.17) (146).

Owen et al. (153) reported a randomized clinical trial in which women with a prior spontaneous preterm birth before 34 weeks of gestation and a short cervix (defined as a cervical length $<25 \mathrm{~mm}$ ) were randomly assigned to have a cerclage or to be managed expectantly. The primary endpoint for the trial was birth at $<35$ weeks of gestation. Of the 302 patients randomized, 148 were allocated to the cerclage group and 153 to the non-cerclage group. There was a non-significant decrease 
in the primary endpoint (delivery at $<35$ weeks of gestation) in women allocated to the cerclage group than in the non-cerclage group (32\% vs. 42\%: OR 0.67; 95\% CI 0.42-1.07; $p=0.09$ ). A post hoc analysis demonstrated that the rate of preterm delivery at $<35$ weeks was significantly lower in women with a cervical length below $15 \mathrm{~mm}$, and there was no demonstrable effect in women with a cervical length between 16 and $24 \mathrm{~mm}$. This study reported that cerclage reduced the rate of previable birth and perinatal mortality. The results of the secondary analysis $(<15 \mathrm{~mm})$ are considered to be hypothesis-generating.

A meta-analysis of five trials in which women had singleton gestations, previous spontaneous preterm birth, and cervical length $<25 \mathrm{~mm}$ before 24 weeks of gestation demonstrated that placement of a cerclage was associated with a lower rate of preterm birth before 35 weeks of gestation [28.4\% (71/250) vs. 41.3\% (105/254); RR 0.70, 95\% CI 0.55-0.89]. Moreover, an index of perinatal mortality and morbidity (composite index) was significantly reduced in patients who had a cerclage (15.6\% in the cerclage group vs. $24.8 \%$ in patients without cerclage, RR 0.64, 95\% CI 0.45-0.91). Subgroup analysis demonstrated that in women with a cervical length of $15.9 \mathrm{~mm}$ or less, cerclage was associated with significant prevention of preterm birth at $<37,35,32$, and 28 weeks. The authors also examined the effect of cerclage as a function of cervical length. In patients with a sonographic cervical length of 16-24.9 mm, cerclage was associated with a significant reduction in the rate of preterm birth at $<37$ and $<24$ weeks of gestation (154). The authors stated that, based on their findings, "the effect of this intervention is important but clearly not the solution to the whole problem of preterm birth" and call for "further understanding of the pathophysiology of spontaneous preterm birth." Thus, cerclage may be effective in reducing the rate of preterm birth in a subset of patients.

\section{PESSARY IN WOMEN WITH CERVICAL INSUFFICIENCY AND/OR A SHORT CERVIX}

The first report of the use of a pessary for the treatment of cervical "insufficiency" was in 1959 (182). Advocates for pessaries to prevent preterm delivery argued that their use should be considered in women with suspected cervical insufficiency because cerclage had not been proven to be efficacious in the prevention of preterm birth, and pessaries would not have the complications known to occur with cerclage. Vaginal pessaries are inexpensive, can be readily placed and removed, and allow outpatient management (without anesthesia) (183).

Several studies have evaluated the use of a pessary to prevent preterm delivery in women with suspected cervical insufficiency based upon history. Most of these reports consist of small case series (183-191).

Arabin et al. (187) reported sonographic cervical length measurements on patients with a prior spontaneous preterm birth before 36 weeks of gestation or early symptoms of preterm labor (pressure or contractions) and twin pregnancies. Patients with a cervical length $\leq 15 \mathrm{~mm}$ between 22 and 28 weeks of gestation were offered the use of a silicone pessary (Arabin pessary). The outcome of 11 patients who had a pessary was compared with a gestational age and cervical length-matched group. The control group consisted of 12 singleton pregnancies and 23 twin gestations. Placement of a pessary was associated with a higher gestational age at delivery in both the singleton and twin gestations [for singletons, the mean gestational age at delivery was 38 (36-6/7 to 41$)$ vs. 33-4/7 weeks (26-38), $p=0.02$; for twins, 35-6/7 (33 to 37-4/ $7)$ vs. $33-2 / 7$ weeks $(24-4 / 7$ to $37-2 / 7), p=0.02]$. None of the 12 singleton gestations with a pessary delivered prior to 36 weeks of gestation [pessary $0 \%(0 / 12)$ vs. control 50\% (6/12), $p<0.001]$ (187). Based on these results, several trials are now in progress to determine whether a pessary can reduce the rate of preterm birth in women with a short cervix in both singleton and twin gestations (Carreras, Nicolaides, Palacio, Moratonas, Nizard). At this time, there is no evidence that cerclage is effective in twin gestations.

\section{PROGESTERONE FOR THE PREVENTION OF PRETERM BIRTH IN WOMEN WITH A SONOGRAPHIC SHORT CERVIX}

Vaginal progesterone reduces the rate of preterm delivery at $<28$, $<33,<34,<35$ weeks of gestation $(110,111)$, as well as the rate of respiratory distress syndrome and neonatal morbidity (111). The role of vaginal progesterone in the prevention of preterm birth in women with a sonographic short cervix is discussed further in the chapter focusing on preterm labor in this book (Chapter 1).

\section{ACKNOWLEDGMENTS}

This work was supported (in part) by the Perinatology Research Branch, Division of Intramural Research, Eunice Kennedy Shriver National Institute of Child Health and Human Development, National Institutes of Health, Department of Health and Human Services.

\section{REFERENCES}

1. Calder A. The human cervix in pregnancy: a clinical perspective. In: Ellwood D, Anderson A, eds. The cervix in pregnancy and labor. Churchill Livingstone, 1981.

2. Danforth DN, Buckingham JC, Roddick JW Jr. Connective tissue changes incident to cervical effacement. Am J Obstet Gynecol 1960; 80: 939-45.

3. Danforth DN, Hendricks CH. Obstetrics and gynecology. Harper and Row, 1977.

4. Schwalm H, Dubrauszky V. The structure of the musculature of the human uterus-muscles and connective tissue. Am J Obstet Gynecol 1966; 94: 391-404.

5. Danforth DN. The distribution and functional activity of the cervical musculature. Am J Obstet Gynecol 1954; 68: 1261-71.

6. Word RA, Li XH, Hnat M, Carrick K. Dynamics of cervical remodeling during pregnancy and parturition: mechanisms and current concepts. Semin Reprod Med 2007; 25: 69-79.

7. Danforth DN. The fibrous nature of the human cervix, and its relation to the isthmic segment in gravid and nongravid uteri. Am J Obstet Gynecol 1947; 53: 541-60.

8. Maillot KV, Zimmermann BK. The solubility of collagen of the uterine cervix during pregnancy and labour. Arch Gynakol 1976; 220: 275-80.

9. Junqueira LC, Zugaib M, Montes GS, et al. Morphologic and histochemical evidence for the occurrence of collagenolysis and for the role of neutrophilic polymorphonuclear leukocytes during cervical dilation. Am J Obstet Gynecol 1980; 138: 273-81.

10. Liggins G, Elwood DA, Anderson ABM. Cervical ripening as an inflammatory reaction. The cervix in pregnancy and labour: clinical and biochemical investigations. Edinburgh: Churchill-Livingstone, 1981.

11. Leppert PC, Keller S, Cerreta J, Mandl I. Conclusive evidence for the presence of elastin in human and monkey cervix. Am J Obstet Gynecol 1982; 142: 179-82.

12. Ekman G, Uldbjerg N, Malmstrom A, Ulmsten U. Increased postpartum collagenolytic activity in cervical connective tissue from women treated with prostaglandin E2. Gynecol Obstet Invest 1983; 16: 292-8. 
13. Leppert PC, Keller S, Cerreta J, Hosannah Y, Mandl I. The content of elastin in the uterine cervix. Arch Biochem Biophys 1983; 222: 53-8.

14. Uldbjerg N, Carlstedt I, Ekman G, et al. Dermatan sulphate and mucin glycopeptides from the human uterine cervix. Gynecol Obstet Invest 1983; 16: 199-209.

15. Uldbjerg N, Ekman G, Malmstrom A, Olsson K, Ulmsten U. Ripening of the human uterine cervix related to changes in collagen, glycosaminoglycans, and collagenolytic activity. Am J Obstet Gynecol 1983; 147: 662-6.

16. Timpl R, Fujiwara S, Dziadek M, et al. Laminin, proteoglycan, nidogen and collagen IV: structural models and molecular interactions. Ciba Found Symp 1984; 108: 25-43.

17. Leppert PC, Yu SY. Apoptosis in the cervix of pregnant rats in association with cervical softening. Gynecol Obstet Invest 1994; 37: 150-4.

18. Hwang JJ, Macinga D, Rorke EA. Relaxin modulates human cervical stromal cell activity. J Clin Endocrinol Metab 1996; 81: 3379-84.

19. Mahendroo MS, Cala KM, Russell DW. 5 alpha-reduced androgens play a key role in murine parturition. Mol Endocrinol 1996; 10: 380-92.

20. Rechberger T, Abramson SR, Woessner JF Jr. Onapristone and prostaglandin E2 induction of delivery in the rat in late pregnancy: a model for the analysis of cervical softening. Am J Obstet Gynecol 1996; 175: 719-23.

21. Sennstrom MK, Brauner A, Lu Y, et al. Interleukin-8 is a mediator of the final cervical ripening in humans. Eur J Obstet Gynecol Reprod Biol 1997; 74: 89-92.

22. Westergren-Thorsson G, Norman M, Bjornsson S, et al. Differential expressions of mRNA for proteoglycans, collagens and transforming growth factor-beta in the human cervix during pregnancy and involution. Biochim Biophys Acta 1998; 1406: 203-13.

23. Mahendroo MS, Porter A, Russell DW, Word RA. The parturition defect in steroid 5alpha-reductase type 1 knockout mice is due to impaired cervical ripening. Mol Endocrinol 1999; 13: 981-92.

24. Uldbjerg N, Forman A, Reece EA, Hobbins JC. Biomechanical and biochemical changes of the uterus and cervix during pregnancy. Medicine of the fetus and mother, vol. 2. Philadelphia, PA: Lippincott-Raven Publishers, 1999.

25. Osman I, Young A, Ledingham MA, et al. Leukocyte density and proinflammatory cytokine expression in human fetal membranes, decidua, cervix and myometrium before and during labour at term. Mol Hum Reprod 2003; 9: 41-5.

26. Sakamoto Y, Moran P, Searle RF, Bulmer JN, Robson SC. Interleukin-8 is involved in cervical dilatation but not in prelabour cervical ripening. Clin Exp Immunol 2004; 138: 151-7.

27. Stjernholm-Vladic Y, Stygar D, Mansson C, et al. Factors involved in the inflammatory events of cervical ripening in humans. Reprod Biol Endocrinol 2004; 2: 74.

28. Straach KJ, Shelton JM, Richardson JA, Hascall VC, Mahendroo MS Glycobiology. 2005; 15: 55-65.

29. Elovitz MA, Gonzalez J. Medroxyprogesterone acetate modulates the immune response in the uterus, cervix and placenta in a mouse model of preterm birth. J Matern Fetal Neonatal Med 2008; 21: 223-30.

30. Hassan SS, Romero R, Tarca AL, et al. The transcriptome of cervical ripening in human pregnancy before the onset of labor at term: identification of novel molecular functions involved in this process. J Matern Fetal Neonatal Med 2009; 22: 1183-93.

31. Hassan SS, Romero R, Tarca AL, et al. The molecular basis for sonographic cervical shortening at term: identification of differentially expressed genes and the epithelial-mesenchymal transition as a function of cervical length. Am J Obstet Gynecol 2010; 203: 472 e1-14.

32. Obara M, Hirano H, Ogawa M, et al. Changes in molecular weight of hyaluronan and hyaluronidase activity in uterine cervical mucus in cervical ripening. Acta Obstet Gynecol Scand 2001; 80: 492-6.

33. Ruscheinsky M, De la Motte C, Mahendroo M. Hyaluronan and its binding proteins during cervical ripening and parturition: dynamic changes in size, distribution and temporal sequence. Matrix Biol 2008; 27: 487-97.

34. Chwalisz K. The use of progesterone antagonists for cervical ripening and as an adjunct to labour and delivery. Hum Reprod 1994; 9(Suppl 1): 131-61.

35. Clark K, Ji H, Feltovich H, et al. Mifepristone-induced cervical ripening: structural, biomechanical, and molecular events. Am J Obstet Gynecol 2006; 194: 1391-8.

36. Anonymous. In: Culpeper N, Cole A, Rowland W, eds. The practice of physick. London, UK: George Sawbridge, 1678.
37. Althuisius S. Cervical incompetence, you better believe it. Department of Obstetrics and Gynecology, Division of Maternal-Fetal Medicine. Amsterdam, The Netherlands: VU Medical Center, 2001.

38. Gream GT. Dilatation or division of the cervix uteri. The Lancet 1865; 85: 381.

39. Shirodkar VN. A new method of operative treatment for habitual abortions in the second trimester of pregnancy. Antiseptic 1955; 52: 299-300.

40. McDonald IA. Suture of the cervix for inevitable miscarriage. J Obstet Gynaecol Br Emp 1957; 64: 346-50.

41. Grant A. Cervical cerclage to prolong pregnancy. In: Chalmers I, Enkin M, Keirse MJNC, eds. Effective care in pregnancy and childbirth. New York, NY: Oxford University Press, 1989.

42. Romero R, Espinoza J, Erez O, Hassan S. The role of cervical cerclage in obstetric practice: can the patient who could benefit from this procedure be identified? Am J Obstet Gynecol 2006; 194: 1-9.

43. Bengtsson LP. Cervical insufficiency. Acta Obstet Gynecol Scand 1968; 47: 7-35.

44. ACOG Practice Bulletin. Cervical insufficiency. Obstet Gynecol 2003; 102: 1091-9.

45. Dunn LJ, Dans P. Subsequent obstetrical performance of patients meeting the historical criteria for cervical incompetence. Bull Sloane Hosp Women 1961; 7: 43-5.

46. Dunn LJ, Dans P. Subsequent obstetrical performance of patients meeting the historical criteria for cervical incompetence. Bull Sloane Hosp Women 1961; 7: 43-5; Editorial Comment in Obstetrical and Gynecological Survey, p. 797.

47. Socol ML, Dooley SL, Tamura RK, Depp OR. Perinatal outcome following prior delivery in the late second or early third trimester. Am J Obstet Gynecol 1984; 150: 228-31.

48. Fejgin MD, Gabai B, Goldberger S, Ben Nun I, Beyth Y. Once a cerclage, not always a cerclage. J Perinat Med 1994; 39: 880-2.

49. Page EW. Incompetent internal os of the cervix causing late abortion and premature labor; technic for surgical repair. Obstet Gynecol 1958; 12: 509-15.

50. Toaff R, Toaff ME, Ballas S, Ophir A. Cervical incompetence: diagnostic and therapeutic aspects. Isr J Med Sci 1977; 13: 39-49.

51. Kiwi R, Neuman MR, Merkatz IR, Selim MA, Lysikiewicz A. Determination of the elastic properties of the cervix. Obstet Gynecol 1988; 71: 568-74.

52. Zlatnik FJ, Burmeister LF, Feddersen DA, Brown RC. Radiologic appearance of the upper cervical canal in women with a history of premature delivery. II. Relationship to clinical presentation and to tests of cervical compliance. J Reprod Med 1989; 34: 525-30.

53. Bergman P, Svennerud S. Traction test for demonstrating incompetence of the internal os of the cervix. Int J Fertil 1957; 2: 163-7.

54. Bishop EH. Pelvic scoring for elective induction. Obstet Gynecol 1964; 24: $266-8$.

55. Wood C, Bannerman RH, Booth RT, Pinkerton JH. The prediction of premature labor by observation of the cervix and external tocography. Am J Obstet Gynecol 1965; 91: 396-402.

56. Papiernik E, Bouyer J, Collin D, Winisdoerffer G, Dreyfus J. Precocious cervical ripening and preterm labor. Obstet Gynecol 1986; 67: 238-42.

57. Anderson AB, Turnbull AC. Relationship between length of gestation and cervical dilatation, uterine contractility, and other factors during pregnancy. Am J Obstet Gynecol 1969; 105: 1207-14.

58. Bouyer J, Papiernik E, Dreyfus J, et al. Maturation signs of the cervix and prediction of preterm birth. Obstet Gynecol 1986; 68: 209-14.

59. Leveno KJ, Cox K, Roark ML. Cervical dilatation and prematurity revisited. Obstet Gynecol 1986; 68: 434-5.

60. Stubbs TM, Van Dorsten JP, Miller MC III. The preterm cervix and preterm labor: relative risks, predictive values, and change over time. Am J Obstet Gynecol 1986; 155: 829-34.

61. Holbrook RH Jr, Falcon J, Herron M, et al. Evaluation of the weekly cervical examination in a preterm birth prevention program. Am J Perinatol 1987; 4: 240-4.

62. Catalano PM, Ashikaga T, Mann LI. Cervical change and uterine activity as predictors of preterm delivery. Am J Perinatol 1989; 6: 185-90.

63. Holcomb WL Jr, Smeltzer JS. Cervical effacement: variation in belief among clinicians. Obstet Gynecol 1991; 78: 43-5.

64. Gomez R, Galasso M, Romero R, et al. Ultrasonographic examination of the uterine cervix is better than cervical digital examination as a 
predictor of the likelihood of premature delivery in patients with preterm labor and intact membranes. Am J Obstet Gynecol 1994; 171: 956-64.

65. Andersen HF. Transvaginal and transabdominal ultrasonography of the uterine cervix during pregnancy. J Clin Ultrasound 1991; 19: 77-83.

66. Andersen HF, Nugent CE, Wanty SD, Hayashi RH. Prediction of risk for preterm delivery by ultrasonographic measurement of cervical length. Am J Obstet Gynecol 1990; 163: 859-67.

67. Iams JD, Goldenberg RL, Meis PJ, et al. The length of the cervix and the risk of spontaneous premature delivery. National Institute of Child Health and Human Development Maternal Fetal Medicine Unit Network. N Engl J Med 1996; 334: 567-72.

68. Heath VC, Southall TR, Souka AP, Elisseou A, Nicolaides KH. Cervical length at 23 weeks of gestation: prediction of spontaneous preterm delivery. Ultrasound Obstet Gynecol 1998; 12: 312-17.

69. Taipale P, Hiilesmaa V. Sonographic measurement of uterine cervix at 18-22 weeks' gestation and the risk of preterm delivery. Obstet Gynecol 1998; 92: 902-7.

70. Hassan SS, Romero R, Berry SM, et al. Patients with an ultrasonographic cervical length $<$ or $=15 \mathrm{~mm}$ have nearly a $50 \%$ risk of early spontaneous preterm delivery. Am J Obstet Gynecol 2000; 182: 1458-67.

71. Goldenberg RL, Iams JD, Miodovnik M, et al. The preterm prediction study: risk factors in twin gestations. National Institute of Child Health and Human Development Maternal-Fetal Medicine Units Network. Am J Obstet Gynecol 1996; 175: 1047-53.

72. Souka AP, Heath V, Flint S, Sevastopoulou I, Nicolaides KH. Cervical length at 23 weeks in twins in predicting spontaneous preterm delivery. Obstet Gynecol 1999; 94: 450-4.

73. Guzman ER, Walters C, O'Reilly-Green C, et al. Use of cervical ultrasonography in prediction of spontaneous preterm birth in twin gestations. Am J Obstet Gynecol 2000; 183: 1103-7.

74. Guzman ER, Walters C, O'Reilly-Green C, et al. Use of cervical ultrasonography in prediction of spontaneous preterm birth in triplet gestations. Am J Obstet Gynecol 2000; 183: 1108-13.

75. To MS, Skentou C, Cicero S, Liao AW, Nicolaides KH. Cervical length at 23 weeks in triplets: prediction of spontaneous preterm delivery. Ultrasound Obstet Gynecol 2000; 16: 515-18.

76. Yang JH, Kuhlman K, Daly S, Berghella V. Prediction of preterm birth by second trimester cervical sonography in twin pregnancies. Ultrasound Obstet Gynecol 2000; 15: 288-91.

77. Maymon R, Herman A, Jauniaux E, et al. Transvaginal sonographic assessment of cervical length changes during triplet gestation. Hum Reprod 2001; 16: 956-60.

78. Owen J, Yost N, Berghella V, et al. Mid-trimester endovaginal sonography in women at high risk for spontaneous preterm birth. JAMA 2001; 286: 1340-8.

79. Skentou C, Souka AP, To MS, Liao AW, Nicolaides KH. Prediction of preterm delivery in twins by cervical assessment at 23 weeks. Ultrasound Obstet Gynecol 2001; 17: 7-10.

80. Vayssiere C, Favre R, Audibert F, et al. Cervical length and funneling at 22 and 27 weeks to predict spontaneous birth before 32 weeks in twin pregnancies: a French prospective multicenter study. Am J Obstet Gynecol 2002; 187: 1596-604.

81. Celik E, To M, Gajewska K, Smith GC, Nicolaides KH. Cervical length and obstetric history predict spontaneous preterm birth: development and validation of a model to provide individualized risk assessment. Ultrasound Obstet Gynecol 2008; 31: 549-54.

82. Parikh MN, Mehta AC. Internal cervical os during the second half of pregnancy. J Obstet Gynaecol Br Emp 1961; 68: 818-21.

83. Iams JD, Johnson FF, Sonek J, et al. Cervical competence as a continuum: a study of ultrasonographic cervical length and obstetric performance. Am J Obstet Gynecol 1995; 172: 1097-103.

84. Guzman ER, Mellon R, Vintzileos AM, et al. Relationship between endocervical canal length between 15-24 weeks gestation and obstetric history. J Matern Fetal Med 1998; 7: 269-72.

85. Kushnir O, Vigil DA, Izquierdo L, Schiff M, Curet LB. Vaginal ultrasonographic assessment of cervical length changes during normal pregnancy. Am J Obstet Gynecol 1990; 162: 991-3.

86. Okitsu O, Mimura T, Nakayama T, Aono T. Early prediction of preterm delivery by transvaginal ultrasonography. Ultrasound Obstet Gynecol 1992; 2: 402-9.
87. Guzman ER, Pisatowski DM, Vintzileos AM, et al. A comparison of ultrasonographically detected cervical changes in response to transfundal pressure, coughing, and standing in predicting cervical incompetence. Am J Obstet Gynecol 1997; 177: 660-5.

88. Guzman ER, Vintzileos AM, McLean DA, et al. The natural history of a positive response to transfundal pressure in women at risk for cervical incompetence. Am J Obstet Gynecol 1997; 176: 634-8.

89. Guzman ER, Mellon C, Vintzileos AM, et al. Longitudinal assessment of endocervical canal length between 15 and 24 weeks' gestation in women at risk for pregnancy loss or preterm birth. Obstet Gynecol 1998; 92: 31-7.

90. Berghella V, Daly SF, Tolosa JE, et al. Prediction of preterm delivery with transvaginal ultrasonography of the cervix in patients with highrisk pregnancies: does cerclage prevent prematurity? Am J Obstet Gynecol 1999; 181: 809-15.

91. Rust OA, Atlas RO, Jones KJ, Benham BN, Balducci J. A randomized trial of cerclage versus no cerclage among patients with ultrasonographically detected second-trimester preterm dilatation of the internal os. Am J Obstet Gynecol 2000; 183: 830-5.

92. Hassan SS, Romero R, Maymon E, et al. Does cervical cerclage prevent preterm delivery in patients with a short cervix? Am J Obstet Gynecol 2001; 184: 1325-9.

93. Macdonald R, Smith P, Vyas S. Cervical incompetence: the use of transvaginal sonography to provide an objective diagnosis. Ultrasound Obstet Gynecol 2001; 18: 211-16.

94. To MS, Skentou C, Liao AW, Cacho A, Nicolaides KH. Cervical length and funneling at 23 weeks of gestation in the prediction of spontaneous early preterm delivery. Ultrasound Obstet Gynecol 2001; 18: 200-3.

95. Berghella V, Haas S, Chervoneva I, Hyslop T. Patients with prior second-trimester loss: prophylactic cerclage or serial transvaginal sonograms? Am J Obstet Gynecol 2002; 187: 747-51.

96. Williams M, Iams JD. Cervical length measurement and cervical cerclage to prevent preterm birth. Clin Obstet Gynecol 2004; 47: 775-83.

97. Althuisius SM. The short and funneling cervix: when to use cerclage? Curr Opin Obstet Gynecol 2005; 17: 574-8.

98. Romero R. Prenatal Medicine: the child is the father of the man. Prenat Neonatal Med 1996;1: 8-11.

99. Moinian M, Andersch B. Does cervix conization increase the risk of complications in subsequent pregnancies? Acta Obstet Gynecol Scand 1982; 61: 101-3.

100. Kristensen J, Langhoff-Roos J, Wittrup M, Bock JE. Cervical conization and preterm delivery/low birth weight. A systematic review of the literature. Acta Obstet Gynecol Scand 1993; 72: 640-4.

101. Raio L, Ghezzi F, Di Naro E, Gomez R, Luscher KP. Duration of pregnancy after carbon dioxide laser conization of the cervix: influence of cone height. Obstet Gynecol 1997; 90: 978-82.

102. Craig CJ. Congenital abnormalities of the uterus and foetal wastage. S Afr Med J 1973; 47: 2000-5.

103. Mangan CE, Borow L, Burtnett-Rubin MM, et al. Pregnancy outcome in 98 women exposed to diethylstilbestrol in utero, their mothers, and unexposed siblings. Obstet Gynecol 1982; 59: 315-19.

104. Ludmir J, Landon MB, Gabbe SG, Samuels P, Mennuti MT. Management of the diethylstilbestrol-exposed pregnant patient: a prospective study. Am J Obstet Gynecol 1987; 157: 665-9.

105. Levine RU, Berkowitz KM. Conservative management and pregnancy outcome in diethylstilbestrol-exposed women with and without gross genital tract abnormalities. Am J Obstet Gynecol 1993; 169: 1125-9.

106. Romero R, Gonzalez R, Sepulveda W, et al. Infection and labor. VIII Microbial invasion of the amniotic cavity in patients with suspected cervical incompetence: prevalence and clinical significance. Am J Obstet Gynecol 1992; 167: 1086-91

107. Mays JK, Figueroa R, Shah J, et al. Amniocentesis for selection before rescue cerclage. Obstet Gynecol 2000; 95: 652-5.

108. Hassan S, Romero R, Hendler I, et al. A sonographic short cervix as the only clinical manifestation of intra-amniotic infection. J Perinat Med 2006; 34: 13-19.

109. Stys SJ, Clewell WH, Meschia G. Changes in cervical compliance at parturition independent of uterine activity. Am J Obstet Gynecol. 1978 Feb. 15; 130: 414-8.

110. Fonseca EB, Celik E, Parra M, Singh M, Nicolaides KH. Progesterone and the risk of preterm birth among women with a short cervix. N Engl J Med 2007; 357: 462-9. 
111. Hassan SS, Romero R, Vidyadhari D, et al. Vaginal progesterone reduces the rate of preterm birth in women with a sonographic short cervix: a multicenter, randomized, double-blind, placebo-controlled trial. Ultrasound Obstet Gynecol. 2011 Jul. 38: 18-31.

112. Sadler L, Saftlas A. Cervical surgery and preterm birth. J Perinat Med 2007; 35: 5-9.

113. Albrechtsen S, Rasmussen S, Thoresen S, Irgens LM, Iversen OE. Pregnancy outcome in women before and after cervical conisation: population based cohort study. BMJ 2008; 337: a1343.

114. Arbyn M, Kyrgiou M, Simoens C, et al. Perinatal mortality and other severe adverse pregnancy outcomes associated with treatment of cervical intraepithelial neoplasia: meta-analysis. BMJ 2008; 337: a1284.

115. Jakobsson M, Gissler M, Paavonen J, Tapper AM. Loop electrosurgical excision procedure and the risk for preterm birth. Obstet Gynecol 2009; 114: 504-10.

116. Nam KH, Kwon JY, Kim YH, Park YW. Pregnancy outcome after cervical conization: risk factors for preterm delivery and the efficacy of prophylactic cerclage. J Gynecol Oncol 2010; 21: 225-9.

117. Ortoft G, Henriksen T, Hansen E, Petersen L. After conisation of the cervix, the perinatal mortality as a result of preterm delivery increases in subsequent pregnancy. BJOG 2010; 117: 258-67.

118. Armarnik S, Sheiner E, Piura B, et al. Obstetric outcome following cervical conization. Arch Gynecol Obstet. 2011; 283: 765-9.

119. Shin MY, Seo ES, Choi SJ, et al. The role of prophylactic cerclage in preventing preterm delivery after electrosurgical conization. J Gynecol Oncol. 2010; 21: 230-6.

120. Kaufman RH, Adam E, Hatch EE, et al. Continued follow-up of pregnancy outcomes in diethylstilbestrol-exposed offspring. Obstet Gynecol 2000; 96: 483-9.

121. Warren JE, Silver RM, Dalton J, et al. Collagen 1Alpha1 and transforming growth factor-beta polymorphisms in women with cervical insufficiency. Obstet Gynecol 2007; 110: 619-24.

122. Warren JE, Nelson LM, Stoddard GJ, et al. Polymorphisms in the promoter region of the interleukin-10 (IL-10) gene in women with cervical insufficiency. Am J Obstet Gynecol 2009; 201: 372 e1-5.

123. Leduc L, Wasserstrum N. Successful treatment with the Smith-Hodge pessary of cervical incompetence due to defective connective tissue in Ehlers-Danlos syndrome. Am J Perinatol 1992; 9: 25-7.

124. De Vos M, Nuytinck L, Verellen C, De Paepe A. Preterm premature rupture of membranes in a patient with the hypermobility type of the Ehlers-Danlos syndrome. A case report. Fetal Diagn Ther 1999; 14: 244-7.

125. Paternoster DM, Santarossa C, Vettore N, Dalla Pria S, Grella P. Obstetric complications in Marfan's syndrome pregnancy. Minerva Ginecol 1998; 50: 441-3.

126. Rahman J, Rahman FZ, Rahman W, al-Suleiman SA, Rahman MS. Obstetric and gynecologic complications in women with Marfan syndrome. J Reprod Med 2003; 48: 723-8.

127. Meijboom LJ, Drenthen W, Pieper PG, et al. Obstetric complications in Marfan syndrome. Int J Cardiol 2006; 110: 53-9.

128. Tzialidou I, Oehler K, Scharf A, et al. Marfan syndrome in pregnancy: presentation of four cases and discussion. Z Geburtshilfe Neonatol 2007; 211: 36-41.

129. Romero R, Espinoza J, Mazor M. Can endometrial infection/ inflammation explain implantation failure, spontaneous abortion, and preterm birth after in vitro fertilization? Fertil Steril 2004; 82: 799-804.

130. Romero R, Mazor M, Gomez R, et al. Cervix, incompetence and premature labor. The Fetus 1993; 3: 1-10.

131. Lee SE, Romero R, Park CW, Jun JK, Yoon BH. The frequency and significance of intraamniotic inflammation in patients with cervical insufficiency. Am J Obstet Gynecol 2008; 198: 633 e1-8.

132. Oh KJ, Lee SE, Jung $\mathrm{H}$, et al. Detection of ureaplasmas by the polymerase chain reaction in the amniotic fluid of patients with cervical insufficiency. J Perinat Med 2010; 38: 261-8.

133. Quinn MJ. Vaginal ultrasound and cervical cerclage: a prospective study. Ultrasound Obstet Gynecol 1992; 2: 410-16.

134. Heath VC, Souka AP, Erasmus I, Gibb DM, Nicolaides KH. Cervical length at 23 weeks of gestation: the value of Shirodkar suture for the short cervix. Ultrasound Obstet Gynecol 1998; 12: 318-22.

135. Althuisius SM, Dekker GA, van Geijn HP, Bekedam DJ, Hummel P. Cervical incompetence prevention randomized cerclage trial
(CIPRACT): study design and preliminary results. Am J Obstet Gynecol 2000; 183: 823-9.

136. Hibbard JU, Snow J, Moawad AH. Short cervical length by ultrasound and cerclage. J Perinatol 2000; 20: 161-5.

137. Blair O, Fletcher H, Kulkarni S. A randomised controlled trial of outpatient versus inpatient cervical cerclage. J Obstet Gynaecol 2002; 22: 493-7.

138. Groom KM, Shennan AH, Bennett PR. Ultrasound-indicated cervical cerclage: outcome depends on preoperative cervical length and presence of visible membranes at time of cerclage. Am J Obstet Gynecol 2002; 187: 445-9.

139. Harger JH. Cerclage and cervical insufficiency: an evidence-based analysis. Obstet Gynecol 2002; 100: 1313-27.

140. To MS, Palaniappan V, Skentou C, Gibb D, Nicolaides KH. Elective cerclage vs. ultrasound-indicated cerclage in high-risk pregnancies. Ultrasound Obstet Gynecol 2002; 19: 475-7.

141. Belej-Rak T, Okun N, Windrim R, Ross S, Hannah ME. Effectiveness of cervical cerclage for a sonographically shortened cervix: a systematic review and meta-analysis. Am J Obstet Gynecol 2003; 189: 1679-87.

142. Drakeley AJ, Roberts D, Alfirevic Z. Cervical cerclage for prevention of preterm delivery: meta-analysis of randomized trials. Obstet Gynecol 2003; 102: 621-7.

143. Drakeley AJ, Roberts D, Alfirevic Z. Cervical stitch (cerclage) for preventing pregnancy loss in women. Cochrane Database Syst Rev 2003: CD003253.

144. Odibo AO, Elkousy M, Ural SH, Macones GA. Prevention of preterm birth by cervical cerclage compared with expectant management: a systematic review. Obstet Gynecol Surv 2003; 58: 130-6.

145. Owen J, Iams JD, Hauth JC. Vaginal sonography and cervical incompetence. Am J Obstet Gynecol 2003; 188: 586-96.

146. Berghella V, Odibo AO, Tolosa JE. Cerclage for prevention of preterm birth in women with a short cervix found on transvaginal ultrasound examination: a randomized trial. Am J Obstet Gynecol 2004; 191: 1311-17.

147. Groom KM, Bennett PR, Golara M, Thalon A, Shennan AH. Elective cervical cerclage versus serial ultrasound surveillance of cervical length in a population at high risk for preterm delivery. Eur J Obstet Gynecol Reprod Biol 2004; 112: 158-61.

148. Higgins SP, Kornman LH, Bell RJ, Brennecke SP. Cervical surveillance as an alternative to elective cervical cerclage for pregnancy management of suspected cervical incompetence. Aust NZ J Obstet Gynaecol 2004; 44: 228-32.

149. Pramod R, Okun N, McKay D, et al. Cerclage for the short cervix demonstrated by transvaginal ultrasound: current practice and opinion. J Obstet Gynaecol Can 2004; 26: 564-70.

150. To MS, Alfirevic Z, Heath VC, et al. Cervical cerclage for prevention of preterm delivery in women with short cervix: randomised controlled trial. Lancet 2004; 363: 1849-53.

151. Baxter JK, Airoldi J, Berghella V. Short cervical length after historyindicated cerclage: is a reinforcing cerclage beneficial? Am J Obstet Gynecol 2005; 193: 1204-7.

152. Althuisius SM, Dekker GA, Hummel P, Bekedam DJ, van Geijn HP. Final results of the Cervical Incompetence Prevention Randomized Cerclage Trial (CIPRACT): therapeutic cerclage with bed rest versus bed rest alone. Am J Obstet Gynecol 2001; 185: 1106-12.

153. Owen J, Hankins G, Iams JD, et al. Multicenter randomized trial of cerclage for preterm birth prevention in high-risk women with shortened midtrimester cervical length. Am J Obstet Gynecol 2009; 201: 375 e1-8.

154. Berghella V, Rafael T, Szychowski J, Rust O, Owen J. Cerclage for short cervix on ultrasonography in women with singleton gestations and previous preterm birth a meta-analysis. Obstet Gynecol 2011; 117: 663-71.

155. Hefner JD, Patow WE, Ludwig JM Jr. A new surgical procedure for the correction of the incompetent cervix during pregnancy. The Wurm procedure. Obstet Gynecol 1961; 18: 616-20.

156. Benson RC, Durfee RB. Transabdominal cervico uterine cerclage during pregnancy for the treatment of cervical incompetency. Obstet Gynecol 1965; 25: 145-55.

157. Lesser KB, Childers JM, Surwit EA. Transabdominal cerclage: a laparoscopic approach. Obstet Gynecol 1998; 91: 855-6.

158. Scibetta JJ, Sanko SR, Phipps WR. Laparoscopic transabdominal cervicoisthmic cerclage. Fertil Steril 1998; 69: 161-3. 
159. Kuhn R, Pepperell R. Cervical ligation: a review of 242 pregnancies. Aust NZ J Obstet Gynecol 1977; 17: 79-83.

160. Aarnoudse JG, Huisjes HJ. Complications of cerclage. Acta Obstet Gynecol Scand 1979; 58: 255-7.

161. Peters WA III, Thiagarajah S, Harbert GM Jr. Cervical cerclage: twenty years' experience. South Med J 1979; 72: 933-7.

162. Charles D, Edwards WR. Infectious complications of cervical cerclage. Am J Obstet Gynecol 1981; 141: 1065-71.

163. Harger JH, Archer DF, Marchese SG, Muracca-Clemens M, Garver KL. Etiology of recurrent pregnancy losses and outcome of subsequent pregnancies. Obstet Gynecol 1983; 62: 574-81.

164. Magrina JF, Kempers RD, Williams TJ. Cervical cerclage: 20 years' experience at the Mayo Clinic. Minn Med 1983; 66: 599-602.

165. Rush RW, Isaacs S, McPherson K, et al. A randomized controlled trial of cervical cerclage in women at high risk of spontaneous preterm delivery. Br J Obstet Gynaecol 1984; 91: 724-30.

166. Chryssikopoulos A, Botsis D, Vitoratos N, Loghis C. Cervical incompetence: a 24-year review. Int J Gynaecol Obstet 1988; 26: 245-53.

167. Treadwell MC, Bronsteen RA, Bottoms SF. Prognostic factors and complication rates for cervical cerclage: a review of 482 cases. Am J Obstet Gynecol 1991; 165: 555-8.

168. Audu BM, Chama CM, Kyari OA. Complications of cervical cerclage in women with cervical incompetence. Int J Gynaecol Obstet 2003; 83: 299-300.

169. Harger JH. Comparison of success and morbidity in cervical cerclage procedures. Obstet Gynecol 1980; 56: 543-8.

170. Kurup M, Goldkrand JW. Cervical incompetence: elective, emergent, or urgent cerclage. Am J Obstet Gynecol 1999; 181: 240-6.

171. Smith SG, Scragg WH Jr. Premature cervical dilatation and the McDonald cerclage. Obstet Gynecol 1969; 33: 535-40.

172. Lauersen NH, Fuchs F. Experience with Shirodkar's operation and postoperative alcohol treatment. Acta Obstet Gynecol Scand 1973; 52: 77-81.

173. Althuisius SM, Dekker GA, Hummel P, van Geijn HP. Cervical incompetence prevention randomized cerclage trial: emergency cerclage with bed rest versus bed rest alone. Am J Obstet Gynecol 2003; 189: 907-10.

174. Rana J, Davis SE, Harrigan JT. Improving the outcome of cervical cerclage by sonographic follow-up. J Ultrasound Med 1990; 9: 275-8.

175. Guzman ER, Houlihan C, Vintzileos A, et al. The significance of transvaginal ultrasonographic evaluation of the cervix in women treated with emergency cerclage. Am J Obstet Gynecol 1996; 175: 471-6.

176. Kikuchi A, Kozuma S, Marumo G, et al. Local dynamic changes of the cervix associated with incompetent cervix before and after Shirodkar's operation. J Clin Ultrasound 1998; 26: 371-3.
177. Dijkstra K, Funai EF, O’Neill L, et al. Change in cervical length after cerclage as a predictor of preterm delivery. Obstet Gynecol 2000; 96: 346-50.

178. Lazar P, Gueguen S, Dreyfus J, et al. Multicentred controlled trial of cervical cerclage in women at moderate risk of preterm delivery. $\mathrm{Br}$ J Obstet Gynaecol 1984; 91: 731-5.

179. Final report of the Medical Research Council/Royal College of Obstetricians and Gynaecologists multicentre randomised trial of cervical cerclage. MRC/RCOG Working Party on Cervical Cerclage. Br J Obstet Gynaecol 1993; 100: 516-23.

180. Althuisius S, Dekker G, Hummel P, et al. Cervical Incompetence Prevention Randomized Cerclage Trial (CIPRACT): effect of therapeutic cerclage with bed rest vs. bed rest only on cervical length. Ultrasound Obstet Gynecol 2002; 20: 163-7.

181. Rust OA, Atlas RO, Reed J, van Gaalen J, Balducci J. Revisiting the short cervix detected by transvaginal ultrasound in the second trimester: why cerclage therapy may not help. Am J Obstet Gynecol 2001; 185: 1098-105.

182. Cross R. Treatment of habitual abortion due to cervical incompetence. The Lancet 1959; 2: 127.

183. Kimber-Trojnar Z, Patro-Malysza J, Leszczynska-Gorzelak B, Marciniak B, Oleszczuk J. Pessary use for the treatment of cervical incompetence and prevention of preterm labour. J Matern Fetal Neonatal Med 2010; 23: 1493-9.

184. Vitsky M. The incompetent cervical os and the pessary. Am J Obstet Gynecol 1963; 87: 144-7.

185. Oster S, Javert CT. Treatment of the incompetent cervix with the Hodge pessary. Obstet Gynecol 1966; 28: 206-8.

186. Vitsky M. Pessary treatment of the incompetent cervical os. Obstet Gynecol 1968; 31: 732-3.

187. Arabin B, Halbesma JR, Vork F, Hubener M, van Eyck J. Is treatment with vaginal pessaries an option in patients with a sonographically detected short cervix? J Perinat Med 2003; 31: 122-33.

188. Acharya G, Eschler B, Gronberg M, et al. Noninvasive cerclage for the management of cervical incompetence: a prospective study. Arch Gynecol Obstet 2006; 273: 283-7.

189. Dharan VB, Ludmir J. Alternative treatment for a short cervix: the cervical pessary. Semin Perinatol 2009; 33: 338-42.

190. Sieroszewski P, Jasinski A, Perenc M, Banach R, Oszukowski P. The Arabin pessary for the treatment of threatened mid-trimester miscarriage or premature labour and miscarriage: a case series. J Matern Fetal Neonatal Med 2009; 22: 469-72.

191. Abdel-Aleem H, Shaaban OM, Abdel-Aleem MA. Cervical pessary for preventing preterm birth. Cochrane Database Syst Rev 2010: CD007873. 


\section{Recurrent pregnancy loss Christine E. Ryan and Danny J. Schust}

\section{INTRODUCTION}

Recurrent pregnancy loss (RPL) can be devastating to families involved. It is defined as three or more spontaneous or two or more consecutive losses prior to 20 weeks of gestation. It can be subdivided into preclinical losses at less than 6 weeks or clinical losses at greater than or equal to 6 weeks of gestation. Clinical losses can be further subclassified as embryonic (crown-rump length is at least $5 \mathrm{~mm}$ with no cardiac activity) or fetal (10-20 weeks without cardiac activity). Other experts have instead subdivided the losses into early (prior to 12 weeks of gestation) or late (between 12 and 20 weeks). Primary RPL exists if a couple has never carried a pregnancy to viability, whereas secondary loss is present if at least one viable term pregnancy has been achieved.

In considering the incidence of this disorder, one must keep in mind the baseline risk of miscarriage: fetal viability is achieved in only $30 \%$ of all conceptions and at least $50 \%$ of pregnancies are lost before a missed period (1). Among clinically recognized pregnancies, $10 \%$ to $15 \%$ still undergo sporadic loss during the first or early second trimester (2). RPL occurs in $1 \%$ to $4 \%$ of couples, with a definite cause identified in no more than $40 \%$. Risk of subsequent loss, stratified by the number of prior losses, is detailed in Table 1.

It follows that the workup of couples with recurrent loss can justifiably be undertaken after two or more consecutive losses as the risk of recurrence is similar to that after three losses (2). Furthermore, the frequency of identifiable causative factors is similar after two or three losses. Other experts have suggested proceeding with workup for couples only after three consecutive losses at less than 10 weeks, unless the couple has had a previous euploid loss or experiences concomitant infertility or advanced maternal age (3). This latter approach can be justified by the fact that $25 \%$ of women aged 30 or less will have a subsequent loss whereas $50 \%$ to $60 \%$ of women at or beyond age 40 will experience a repeated loss $(4,5)$.

\section{PRECONCEPTION EVALUATION}

Upon presentation to the clinic, it is imperative to have an organized diagnostic approach in order to avoid overwhelming either the affected couple or the provider. We recommend evaluation after a second consecutive loss without prior liveborn child, a third loss if the couple has had a prior live-born child, or after two losses in the presence of infertility or advanced maternal age.

History should include age; past medical history and medications; characteristics, pattern, and gestational age of prior losses; exposure to toxins or recent travel; previous gynecologic or obstetrical infections; previous workup; family history [cycle regularity, spontaneous losses, diethylstilbestrol (DES) exposure, genetic syndromes, and thromboses]; and a thorough review of systems (e.g., rheumatic diseases). Karyotypes of prior losses should be recorded if available. A general overall physical examination should then be performed including a pelvic examination.

Testing can then be offered. Keep in mind when ordering tests that nocturnal gonadotropin-releasing hormone pulses do not resume until 4 weeks postpartum, so some endocrine evaluations [prolactin (PRL), follicle-stimulating hormone (FSH), if indicated] should not be pursued until at least one month after a loss. Anticardiolipin antibodies, homocysteine, and protein $S$ levels are altered by pregnancy and may not return to baseline until 6 to 12 weeks postpartum, further supporting a slight delay before initiating the workup (Table 2).

As a final note on the general evaluation, it is important that prenatal vitamin supplementation is recommended to all preconceptual patients. Poor dietary intake of vitamins has been associated with spontaneous pregnancy loss. For example, a folate level of $2.2 \mathrm{ng} / \mathrm{mL}(4.9 \mathrm{mmol} / \mathrm{L})$ is associated with an increased rate of sporadic loss with an odds ratio of 1.47 (95\% CI 1.01-2.14) (6). A recent Cochrane review of randomized trials, however, showed that vitamin supplementation does not prevent miscarriage or stillbirth. They do, however, lessen the likelihood of developing pre-eclampsia and have been shown to increase the likelihood of multiple gestations (7). Other benefits include lesser likelihood of neural tube defects, anemia, or other vitaminassociated deficiencies that may be common in pregnancy.

The remainder of this chapter will focus on the evidence supporting or refuting various proposed etiologies for RPL and their respective evidence-based management strategies.

\section{ETIOLOGY AND MANAGEMENT Uterine Abnormalities}

With the exception of the septate uterus and possible acquired anomalies, uterine abnormalities are more commonly associated with second rather than first-trimester losses. These abnormalities include congenital anomalies of fusion or resorption, fibroids and polyps, synechiae or Asherman syndrome, and DES exposure. The diagnostic modalities for appropriate diagnosis include hysterosalpingogram (HSG), hysteroscopy, sonohysterography, laparoscopy, MRI, or three-dimensional ultrasonography. Each modality has advantages and disadvantages. For example, the sensitivity and specificity of standard two-dimensional transvaginal ultrasonography in diagnosing a uterine septum are $100 \%$ and $80 \%$, respectively, while sensitivity and specificity using MRI are both $100 \%$ (8). Three-dimensional ultrasonography can be added after an abnormal two-dimensional ultrasound to improve the aforementioned specificity. Threedimensional sonography is preferred over hysterosalpingography as the former test is less expensive and less invasive and allows for improved visualization of the endometrial cavity and myometrium. Three-dimensional ultrasonography can reliably delineate between septate and bicornuate uteri $(9,10)$. 


\section{Anomalies}

Overall, the presence of a congenital uterine anomaly appears to almost double the risk of RPL: anomalies have been identified in $6.3 \%$ of recurrent loss patients versus $3.8 \%$ of fertile women $(p<0.05)$. In one investigation, the breakdown of specific anomalies among RPL patients was $1.9 \%$ bicornuate (vs. $0.4 \%$ among fertile women, $p<0.01$ ), $2 \%$ septate, and $1 \%$ arcuate uteri, with latter two not holding a statistically significant difference when compared with fertile women (Table 3) (11).

One common current theory holds that the causative factor for loss in patients with a septate uterus is implantation on the septum itself. This is thought to result in inadequate placentation secondary to poor septal vascularity and resultant early demise. This theory is supported by the timing of losses: early first trimester with septate uteri versus second-trimester losses or preterm deliveries with bicornuate and unicornuate uteri (13). In addition, although the vascular density of septa is similar to normal myometrium (14), a study of 12 pregnancies in women with septate uteri revealed that the only four successful pregnancies were those that had implanted away from the septum (15). Furthermore, several uncontrolled studies have shown that septum resection leads to an improved rate of successful subsequent pregnancy. A retrospective case series showed that $70 \%$ to $85 \%$ of RPL patients who underwent surgical correction of bicornuate and septate uteri delivered viable infants in the next pregnancy (16). Hysteroscopic resection of uterine septa

\section{Table 1 Risk of Subsequent Pregnancy Loss}

$\begin{array}{ll}\text { After one successful pregnancy } & 12 \% \\ \text { After two consecutive losses } & 24-26 \% \\ \text { After three consecutive losses } & \\ \text { With at least one previous live birth } & 30 \% \\ \text { Without previous live birth } & 32-45 \% \\ \text { After four consecutive losses } & 40-50 \%\end{array}$

leads to comparable subsequent pregnancy rates with those seen after metroplasty (16), leading the American College of Obstetricians and Gynecologists (ACOG) to make a compendium recommendation supporting hysteroscopic resection. No prospective controlled studies have been undertaken. Extensive surgical procedures, such as Strassman unification, are rarely undertaken.

The contribution of the arcuate uterus to RPL is more controversial. One study of 61 RPL patients identified an arcuate uterus in $15 \%$ via three-dimensional ultrasound and hysteroscopy versus 3\% of 1000 general gynecologic patients $(17,18)$. Others, however, have argued against the presence of an association $(19,20)$.

\section{Leiomyomata and Polyps}

Theories concerning the contribution of leiomyomata or endometrial polyps as an etiology of RPL have centered on their disruption of endometrial vasculature, the intrauterine cavity, or tubal patency. Either polyps or fibroids may act as foreign bodies, similar to intrauterine devices, and thus disrupt pregnancy. The direct association between submucosal fibroids and RPL has had some controversy in the past although uncontrolled studies do reveal an association. Intramural fibroids have been proven to interfere with implantation of in vitro fertilization pregnancies, though little data are available relative to intramural fibroids and RPL. The rate of spontaneous loss decreases after removal of fibroids over $5 \mathrm{~cm}$ in diameter, regardless of their location (21) and, therefore, resection is recommended. Hysteroscopic approaches for fibroid removal are preferred when possible.

\section{Synechiae}

Uterine synechiae may occur after uterine infection, intrauterine curettage, or other uterine instrumentation. Synechiae have been found in $5 \%$ of patients with RPL. Hysteroscopy is typically recommended to lyse these adhesions, although strong data to support this treatment are lacking.

\section{Table 2 Diagnostic Evaluation}

$\begin{array}{ll}\text { Anatomic } & \text { Sonohysterogram or hysteroscopy } \\ & \text { MRI if indicated } \\ \text { Genetic } & \text { Parental karyotype and conceptus cytogenetic results } \\ & \text { Genetic counseling if indicated } \\ \text { Thrombophilias } & \text { (Consider full testing if }>10 \text {-week loss, history of VTE, family history of thrombosis, or non-Caucasian) } \\ & \text { All patients: factor V Leiden, PT G20210A mutations, homocysteine level } \\ & \text { APC resistance } \\ & \text { Consider AT III, PC, and PS deficiency testing in all Asian patients } \\ & \text { (Low or unsustained, moderately positive values are not clinically significant) } \\ \text { Immunologic } & \text { Russel's viper venom time (RVVT) (=lupus anticoagulant) } \\ & \text { Anticardiolipin antibodies (IgG or IgM) } \\ & \text { Anti- } \beta 2 \text { glycoprotein I antibodies (IgG and IgM) } \\ & \text { Consider antithyroid antibodies } \\ & \text { Luteal phase duration (positive ovulation kit test till day } 1 \text { menses; <13 days indicative of deficiency) } \\ & \text { Day } 3 \text { FSH (for ovarian reserve), TSH, PRL (if menses irregular or with galactorrhea) } \\ \text { Endocrine } & \text { If menses irregular with signs of PCOS, consider androgens, SHBG, 17OHP, and insulin sensitivity testing }\end{array}$




\section{DES Exposure}

While isolated spontaneous loss is more common with a history of DES exposure (22), little data exist regarding its relation to RPL. No approach to the correction of anatomic abnormalities related to DES exposure has been successful. It does, however, seem warranted to seek this history, as the implications go beyond maintenance of pregnancy.

\section{Cervical Incompetence}

A Cochrane review of six trials, including 2175 women, found there to be no benefit for either prophylactic or therapeutic cervical cerclage among patients with presumed cervical incompetence and any adverse pregnancy outcomes (including RPL). For prophylactic cerclage, no reduction was seen in overall pregnancy loss and preterm delivery rates in the overall analysis, although a small decrease in births prior to 33 weeks was reported in one of the included trials (RR 0.75, 95\% CI 0.58-0.98). Additionally, the use of therapeutic cerclage upon ultrasound findings of decreased cervical length failed to positively alter total pregnancy loss, early pregnancy loss, or preterm delivery rates (23).

\section{Genetic Factors}

Parental chromosomal anomalies are identified in $4 \%$ of couples with RPL versus $0.2 \%$ of couples in the general population. Maternal anomalies are identified in a 3:1 ratio relative to paternal anomalies. Of spontaneous abortions, $60 \%$ to $75 \%$ have an abnormal fetal karyotype (24). These fetal chromosomal abnormalities have a more prominent role in single losses than recurrent losses. In fact, as the number of pregnancy losses increases for any given couple, the risk of fetal chromosomal aberrations decreases. In other words, loss of a chromosomally abnormal fetus carries a higher chance of subsequent live birth than loss of a euploid embryo $(25,26)$. To this point, if the karyotype of the first abortus is aneuploid, then the karyotype of the second successive loss is abnormal in $50 \%$ to $70 \%$; if the first is chromosomally normal, then the incidence of a karyotypic abnormality in the second loss is decreased to $20 \%(27,28)$.

At least half of clinically recognized, isolated spontaneous abortions have a chromosomal anomaly. Of these, $56 \%$ are trisomic, $20 \%$ are polyploid, $18 \%$ exhibit monosomy $\mathrm{X}$, and $4 \%$ carry unbalanced translocations (29). For comparison, $70 \%$ of preclinical spontaneous losses are chromosomally abnormal whereas only $5.6 \%$ of second- and third-trimester losses carry these abnormalities (30,31). It has been suggested that the majority of these losses may be secondary to maternal

Table 3 Reproductive Performance with Different Uterine Malformations

$\begin{array}{lccc} & \begin{array}{c}\text { Spontaneous } \\ \text { abortion (\%) }\end{array} & \begin{array}{c}\text { Preterm } \\ \text { delivery (\%) }\end{array} & \begin{array}{c}\text { Live } \\ \text { birth (\%) }\end{array} \\ \text { Didelphys } & 20 & 53.3^{\mathrm{b}, \mathrm{c}} & 40^{\mathrm{f}} \\ \text { Unicornuate } & 37.5^{\mathrm{b}} & 25^{\mathrm{b}} & 43.7^{\mathrm{f}} \\ \text { Bicornuate } & 25 & 25^{\mathrm{b}} & 62.5^{\mathrm{e}} \\ \text { Septate } & 25.5 & 14.5^{\mathrm{b}, \mathrm{d}} & 62^{\mathrm{e}} \\ \text { Arcuate } & 12.7^{\mathrm{a}} & 4.5^{\mathrm{a}} & 82.7^{\mathrm{e}}\end{array}$

Note: Statistical significance by column: ${ }^{\mathrm{a} / \mathrm{b}} p<0.05 ;{ }^{\mathrm{c} / \mathrm{d}} p<0.01 ;{ }^{\mathrm{e} / \mathrm{f}} p<0.001$. Source: Adapted from Ref. 11,12. aging rather than nonrandom events (32,33). Preimplantation genetic diagnosis (PGD) studies have irrefutably demonstrated that $50 \%$ of embryos from RPL couples have aneuploidy $(34,35)$.

The ACOG recommends karyotype screening of all RPL couples. This recommendation is based (i) on the couple's desire to identify a cause, (ii) on the fact that the couple may benefit from prenatal genetic testing if a balanced chromosomal abnormality is identified, and (iii) on the fact that normal appearing offspring may carry the abnormality and thus have potential reproductive complications of their own (2).

\section{Aneuploidy}

One aneuploid loss increases the risk of a subsequent aneuploid loss (36). Trisomies are the most common anomaly as a group, led by trisomy 16 . Still, the single most common aneuploidy found in first-trimester pregnancy loss specimens is $45 \mathrm{X}, 0$. Some experts therefore suggest that it would be prudent to perform cytogenetic analysis of second and subsequent losses, understanding that a trisomic loss does not increase the risk of recurrent loss (33). In fact, recurrent aneuploidy or polyploidy, though seen with sporadic loss, has not been proven to be associated with recurrent loss when stratified for age (37). If the karyotype of an abortus is found to be aneuploid, a maternal cause for loss can be effectively excluded. The discovery of a legitimate cause for the loss may also be a source of comfort for the grieving family.

A recent study examined 420 losses from 285 couples and compared the frequency of chromosomal anomalies among recurrent loss patients with the frequency among patients without such a history. Significant differences were found between the two groups in the distribution of cytogenetic abnormalities, but the difference dissipated after stratification for maternal age. In younger women (i.e., less than 36 years of age), more spontaneous losses were euploid (37), suggesting other etiologies for RPL.

It is important to note that generalized screening for aneuploidy (as opposed to screening for a specific genetic abnormality using PGD) among embryos from chromosomally normal couples with a history of RPL is not currently recommended. Once considered promising, a recent review article concluded that preimplantation genetic screening (PGS) does not increase pregnancy rates in patients at risk of aneuploidy, including couples experiencing RPL (38). This is not surprising, however, given the current limitations on the numbers of chromosomes that can be screened using PGS. The outcomes of this intervention among recurrent loss patients should, therefore, be revisited when techniques for simultaneously screening all 23 chromosomes, such as complete genomic hybridization, become widely available.

\section{Chromosomal Rearrangements}

Overall, $2 \%$ to $4 \%$ of RPL couples have a partner with a chromosomal rearrangement (2). This is less common if there is no history of prior stillbirth or anomalous infant: $2.4 \%$ of females and $1.6 \%$ of males with no such history versus $4.6 \%$ of females and $1.7 \%$ of males with the aforementioned history (39). In these situations, family history may be telling as the translocation may have been in the lineage for multiple generations prior to detection. Although the carrier parents 
Table 4 Thrombophilias and Recurrent Pregnancy Loss

\begin{tabular}{|c|c|c|}
\hline Study & Associations supported & Associations refuted \\
\hline Rey 2003 (meta-analysis of 31 studies) & $\begin{array}{l}\mathrm{RPL}<13 \text { weeks associated with FVL }(\mathrm{G} 1691 \mathrm{~A}), \\
\text { Activated protein C resistance, PT gene } \\
\text { mutation }(20210 \mathrm{~A}), \text { and PS deficiency }\end{array}$ & $\begin{array}{l}\text { MTHFR, PC deficiency, and ATIII } \\
\text { deficiency were not associated }\end{array}$ \\
\hline
\end{tabular}

are balanced in their chromosomal content, segregation during meiosis results in duplication or deficiency in the chromosomal constitution of some of the gametes. The incidence of de novo translocations is $1 / 2000$ for reciprocal translocations (which makes up 60\% of anomalies and yields normal gametes one-half of the time) and 1/9000 for Robertsonian translocations (40\% of anomalies and yields normal gametes one-third of the time) (40). A natural selection of sorts appears to occur among sperm from men with balanced translocations, explaining why female carriers generally have a $10 \%$ risk of a subsequent abnormal live birth whereas the risk for male carriers is only $1 \%$.

Therapy for the couples with RPL and balanced translocations relies on genetic counseling and discussion of options for prenatal diagnosis, donor gametes for the affected partner, or PGD with implantation of unaffected embryos. For fully evaluated RPL couples in whom one partner carries a structural chromosomal rearrangement, the subsequent livebirth rate is greater than $70 \%$ after evidence-based treatment, close monitoring, and supportive care (41).

\section{Other}

Other chromosomal anomalies associated with pregnancy loss tend to involve the X-chromosome. Women with Turner mosaicism who become pregnant are more susceptible to spontaneous loss. Of 160 pregnancies in these mosaic patients, $29 \%$ ended in spontaneous loss, 20\% delivered children with malformations, and $7 \%$ resulted in perinatal death (42). Additionally, highly skewed X-chromosome inactivation may be responsible for otherwise unexplained recurrent losses (43). However, as commercial tests are generally unavailable for $\mathrm{X}$-inactivation and other rare genetic abnormalities, testing is generally not available, performed, or recommended.

\section{Thrombophilias}

Thrombophilias include a diffuse group of inherited hypercoagulable states. The most often discussed include Factor V Leiden (FVL), prothrombin (PT) G20210A promoter mutation, deficiencies of proteins $\mathrm{C}$ and $\mathrm{S}$ (PC and PS, respectively), antithrombin III deficiency (ATIII), and hyperhomocysteinemia [including abnormalities in methyltetrahydrofolate reductase (MTHFR)] function. The antiphospholipid syndrome (APS) is another hypercoagulable state associated with RPL, but it is acquired and will be discussed separately under the Immunology section. It has been suggested that patients to be tested for familial hypercoagulability should include those with unexplained fetal death beyond 10 weeks, personal history of venous thromboembolism, a suggestive family history, and those with at least three first-trimester losses.

The presence of two or more heritable thrombophilic defects has been associated with adverse outcomes, including pre-eclampsia, thrombosis, intrauterine growth restriction (IUGR), and even fetal death (44). The association between RPL and hypercoagulable states continues to be somewhat controversial with some research supporting (45-48) and other reports refuting (49-53) an association. The results of two recent meta-analyses and one review have been summarized in Table 4. Since controversy continues, it is useful to discuss the individual defects separately.

First, consider FVL and the PT promoter gene mutation. These mutations are common and carry low thrombotic risk in the heterozygotic state. They are reported to be present in approximately 9\% and 3\% of Caucasian women in the United States, respectively. These two thrombophilias have been associated with $25 \%$ of isolated thromboses and $50 \%$ of familial thromboses. Among RPL patients (with at least three losses and no successful pregnancies), the prevalence of FVL is 9\% (OR 2.6, 95\% CI 1-6.7, $p=0.048$ ) (48). Furthermore, a prospective case-control study of FVL patients has shown lower pregnancy success rate among women with FVL who were not treated with anticoagulants (54).

The autosomal-dominant deficiencies in ATIII, PC, and PS are much less common in Caucasians, but carry a significantly greater thrombotic risk. These three mutations double the relative risk of abortion and stillbirth in carriers when compared with women who test negative (RR 2, 95\% CI 1.2-3.3) (55). Diagnosis, however, can be affected by many factors. Oral contraceptive pills increase levels of both PC and PS while decreasing ATIII. Cigarette smoking decreases levels of PC and PS, while pregnancy decreases only the level of PS. Protein C and S testing can evaluate protein function or antigen levels. Protein $\mathrm{S}$ functional screening is non-diagnostic if less than $60 \%$ and antigenic levels should be performed.

Hyperhomocysteinemia may be caused by MTHFR mutation (heritable) and/or low levels of vitamin B6 or B12, or folate (acquired). Random homocysteine levels are considered mildly elevated at 16 to $24 \mu \mathrm{mol} / \mathrm{L}$, moderately elevated at 25 to $100 \mu \mathrm{mol} / \mathrm{L}$, and severely elevated at $>100 \mu \mathrm{mol} / \mathrm{L}$. Dietary supplementation with vitamins $\mathrm{B}_{6}, \mathrm{~B}_{12}$, and folate can 
Table 5 Diagnostic Criteria for Antiphospholipid Syndrome

Patients should have at least one clinical and one laboratory criteria

Clinical criteria

- $\geq 1$ clinical episodes of arterial, venous, or small-vessel thrombosis

- $\geq 1$ unexplained pregnancy loss of a morphologically normal fetus of at least 10 weeks of gestation

- $\geq 1$ preterm births of a morphologically normal newborn at $<34$ weeks of gestation due to severe pre-eclampsia or placental insufficiency

- $\geq 3$ unexplained consecutive spontaneous abortions before 10 weeks of gestation, with anatomic, hormonal, and parental structural genetic factors excluded

Laboratory criteria: the same antibody must be present on two separate occasions $\geq 12$ weeks apart

- Anticardiolipin IgG and/or IgM, present in medium or high titers ( $>40$ GPL or MPL)

- Anti $\beta_{2}$-glycoprotein-1 IgG and/or IgM, present in titers $>99$ th percentile

- Lupus anticoagulant, detected according to the guidelines of the International Society on Thrombosis and Hemostasis

Source: Adapted from Ref. 65.

normalize homocysteine levels, but has not yet been show to decrease thrombotic risk (56).

To summarize, evidence is mounting that supports the association between heritable and acquired thrombophilias and RPL as well as other adverse pregnancy outcomes. Our best data to date are from Caucasian populations and support to testing for FVL, PT promoter gene mutations, activated protein $\mathrm{C}$ resistance, homocysteine elevations, and the APS (dealt with in more detail in the next section). In nonCaucasian patients, the data are more limited and additional testing may be prudent. For instance, protein $\mathrm{C}$, protein $\mathrm{S}$, and ATIII deficiencies are significantly more common among patients of Asian descent than among Caucasian patients.

Ideal treatment for patients with heritable thrombophilias and RPL remains to be determined. A recent Cochrane review of anticoagulants for the treatment of RPL in the absence of APS noted that when 20 females with fetal loss after the 20th week and a thrombophilic defect were randomized to enoxaparin or aspirin, enoxaparin increased the live-birth rate (RR 10, 95\% CI 1.56-64.2). Live-birth rates were unchanged, however, in controlled studies of aspirin versus placebo. The review's summary recommendation was that "data are too limited to recommend use of anticoagulants" in the setting of two or more spontaneous losses or one late intrauterine fetal death without apparent causes other than inherited thrombophilias except for anticardiolipin antibodies (57). The formal ACOG statement agrees that "whether antithrombotic treatment improves subsequent pregnancy outcome is uncertain" (2). Further trials are therefore needed.

\section{Immunologic}

APS

APS or Hughes syndrome is present in and felt to be responsible for $5 \%$ of RPL. In this syndrome, losses more commonly occur beyond 10 weeks of gestation, generally during the second or even early third trimesters $(58,59)$. The mechanisms for APS-related losses are thought to be related to impaired vascular flow to the fetus. Since blood flow in placental vessels is not established until at least 9 to 10 weeks of gestation, losses at less than 10 weeks of gestation may be the result of impaired gestational nutrition, which occurs via diffusion prior to the establishment of intervillous blood flow. Thereafter, theories propose that thrombosis and vasoconstriction may occur due to antiphospholipid antibody binding to platelets and vascular endothelial membranes (60).
Other researchers purport that the antibodies in APS cause inhibition of cytotrophoblastic fusion, invasion, and differentiation and that this inhibition in turn leads to recurrent pre-embryonic or embryonic pregnancy loss (61-63).

Diagnosis of APS is based on an international consensus that was most recently updated in their criteria in 2006 (Table 5). The syndrome may be subdivided into primary (no other related disease present) or secondary (systemic lupus erythematosus (SLE), valvular heart disease, or other rheumatic diseases coexist). ACOG recommends testing all RPL patients for APS laboratory criteria as outlined in Table 5. Although there exists evidence linking antiphosphatidylserine antibody positivity to early RPL, its role in diagnosis, treatment, and prognosis remains controversial $(64,65)$.

Numerous interventional studies have been performed in patients with RPL. The most promising approaches involve various regimens of aspirin and heparin. In controlled studies, combinations of aspirin, low molecular-weight heparin (LMWH), and human chorionic gonadotropin (hCG) as well as unfractionated heparin and aspirin led to $70 \%$ to $75 \%$ successful pregnancy outcome versus $50 \%$ in untreated controls $(66,67)$. Heparin clearly lends benefit, as shown in Empson's study, where addition of heparin to aspirin when compared with aspirin alone resulted in a $54 \%$ decreased rate of pregnancy loss (RR 0.46, CI 0.29-0.71) (68). Heparin also proved more effective than intravenous immunoglobulin (IVIG) in randomized trials (69). A low-dose aspirin versus placebo study is currently underway. LMWH has also been suggested as an effective alternative to unfractionated heparin $(67,70-72)$ although not all investigators agree. Steroids should not be given if no other clinical indication is present, as they have been shown to decrease the live-birth rate and increase the rate of fetal and maternal complications $(73,74)$.

Current recommendations for the treatment of patients with APS and RPL (but no prior thrombotic episode) support initiation of heparin at the time of a positive pregnancy test. If given LMWH, these patients are maintained on prophylactic doses through approximately 36 weeks of gestation when it is recommended to convert to unfractionated heparin to decrease the risk of hematoma with epidural placement or hemorrhage at the time of delivery. Anticoagulation prior to conception is discouraged due to the risk of hemorrhage with ovulation. Therapeutic rather than prophylactic doses of LMWH or unfractionated heparin should be used if a history of thrombosis is present. Close maternal and fetal surveillance 
should be performed instituted in all patients with a history of APS due to the increased risk of significant perinatal morbidity including pre-eclampsia, IUGR, and preterm labor. Finally, since the risk of thrombosis continues to be increased during the first 6 weeks postpartum, anticoagulation should be continued after delivery bridge this time period.

\section{Thyroid Antibodies}

Associations have been made between the presence of thyroid antibodies and spontaneous abortion. An increased rate of spontaneous pregnancy loss is seen with autoantibodies to thyroglobulin or thyroid peroxidase (TPO) if identified in early pregnancy or immediately before conception $(75,76)$. In other studies, the presence of antithyroperoxidase antibodies increased the risk of spontaneous loss from 23\% to 50\% (77). A meta-analysis published in 2004 found the odds ratio for spontaneous miscarriage in the presence of thyroid antibodies to be 2.73 (95\% CI 2.2-3.4) using 8 case-control studies and 2.3 (95\% CI 1.8-2.95) using 10 longitudinal studies (78).

Antimicrosomal and antithyroglobulin antibodies have been identified with increased frequency among females experiencing RPL. An investigation of RPL patients in 2000, however, concluded that if the patient is euthyroid, the presence of antithyroid antibodies does not affect pregnancy outcomes (Rushworth 2000). Nevertheless, much additional work has been done since 2000 that suggests otherwise and, although not specific to the recurrent loss population, is worthy of discussion. Studies have shown that patients with subclinical hypothyroidism (antibody status not delineated) do experience significantly increased rates of adverse obstetric events (Abalovich 2002, Casey 2005). A more recent study prospectively divided 984 euthyroid pregnant women into three groups: those with positive TPO antibodies to whom they gave levothyroxine (group A), those with antibodies that did not receive levothyroxine (group $\mathrm{B}$ ), and controls who were negative for antibodies and untreated (group C). These researchers found a similar miscarriage rates for groups A and C (3.5 and 2.4\%, respectively), but both $\mathrm{A}$ and $\mathrm{C}$ had significantly lower miscarriage rates than women in group $\mathrm{B}$ at $13.4 \%[p<0.05$, RR 1.72 (95\% CI 1.13-2.25) and $p<0.01$, RR 4.95 (95\% CI 2.59-9.48), respectively]. Additionally, untreated antibodypositive women (group B) had a higher preterm delivery rate $(22.4 \%)$ than women in group A $(7 \%)(p<0.05$, RR $1.66(95 \%$ CI 1.18-2.34)) or group C (8.2\%) $(p<0.01$, RR 12.18 (95\% CI 7.93-18.7)) (79). These data support treatment of euthyroid pregnant women who test positive for TPO antibodies with levothyroxine to decrease the rates of both spontaneous abortion and preterm delivery. Further study in patients with a history of RPL is certainly warranted. It is, however, important to note that in the presence of anti-TPO antibodies, the risk of developing hypothyroidism during pregnancy or the postpartum period is increased. Therefore, it is recommended that the thyroidstimulating hormone be checked during each trimester and after delivery in antibody positive women $(80,81)$.

\section{Antinuclear Antibodies}

It has been reported that $7.5 \%$ of RPL females, compared with less than $2 \%$ of controls, test positive for antinuclear antibodies (ANA) (82). Still, testing for ANA among RPL patients does not appear to be helpful in the absence of co-existent rheumatic autoimmune disorders. Subsequent pregnancy outcomes among RPL patients with an untreated, isolated, positive ANA is no different from those who test negative. In a randomized trial, patients with positive autoantibodies (including ANA) had no difference in outcomes when treated with prednisone and aspirin versus placebo (74). Data, therefore, do not support testing for ANA or treating positive results.

\section{SLE}

Patients with SLE have increased rates of both spontaneous and recurrent pregnancy loss in all trimesters. Not surprisingly, the most sensitive marker for poor pregnancy outcome among this group is the presence of antiphospholipid antibodies, which are seen in $37 \%$ of SLE patients $(83,84)$. Additional predictors of poor prognosis include preexisting renal disease and the number and severity of pre-pregnancy lupus flares $(85,86)$. Patients who meet the criteria for the APS should be treated with heparin and low-dose aspirin (see above).

\section{Alloimmune Disorders}

Alloimmune disorders are purported to cause pregnancy loss via an abnormal cell-mediated maternal immune response to placental or fetal antigens. One hypothesis for alloimmune RPL holds that in normal pregnancies, maternal production of a blocking factor prevents rejection of the fetus and that these factors are missing in women experiencing RPL (87). Evidence used to support this theory includes (i) the tendency for RPL partners to share human leukocyte antigens, (ii) the fact that females experiencing RPL often do not produce purported serum blocking factors, and (iii) the fact that women with a history of RPL commonly produce anti-leukocytotoxic antibodies against paternal leukocytes (2). The blocking factor hypothesis has been widely studied, but is poorly supported.

Among RPL patients, there is a proposed dysregulation of normal immune mechanisms at the maternal-fetal interface. Theories suggest that a $\mathrm{T}$ helper cell type 2 (Th-2) cytokine predominance is necessary for successful pregnancy and that $\mathrm{T}$ helper cell type 1 (Th-1) cytokines (IFN- $\lambda$ and TNF- $\alpha$ ) negatively affect embryo and trophoblast viability (88-90). One investigator demonstrated that production of an embryotoxic factor (possibly like IFN- $\lambda$ ) from RPL patient leukocytes cocultured with placental extracts predicted subsequent pregnancy failure (88). These findings have not been successfully reproduced in other studies (91). Testing for embryotoxic factor and treatment based on such testing is not currently recommended.

Decidual NK-like cells that secrete transforming growth factor may also be required for successful pregnancy (92). Studies show both increased (93) and decreased $(92,94)$ NK-like cell number in luteal phase endometrial biopsies from recurrent loss patients. Subsequent pregnancy outcomes may be worse with increased decidual NK cell numbers (95). Furthermore, higher levels of circulating NK cells have also been shown to predict poor outcome with the next pregnancy $(96,97)$. The relationship of peripheral NK cell numbers and activity of the NK-like cells present at the site of implantation is unclear. 
To date, no specific testing regimens or treatments for abnormal alloimmune responses among RPL patients have been definitively shown to predict future pregnancy outcomes $(3,98,99)$. Flow cytometric assays to document maternal antibodies to paternal leukocytes have been proposed and do offer biologic plausibility. However, investigations into the role of these allo-antibodies have lacked controls and no efficacious therapies have resulted. Randomized controlled trials of mononuclear cell immunization for patients with a history of RPL have yielded conflicting results. Although initial investigations appeared promising (100), further studies have concluded that immunotherapy to stimulate tolerance is not effective. Rather, alloimmune mononuclear cell immunization increases the rate of pregnancy loss when compared with placebo while carrying the infectious disease and allergic risks of transfusions with several other blood products (101). Trials using IVIG in patients with RPL have been fairly extensive with five randomized trials demonstrating a benefit with intervention (102) while other investigations and two meta-analyses have shown no benefit (103-107). A recent Cochrane review of this topic concluded that "paternal cell immunization, third party donor leukocytes, trophoblast membranes, and IVIG provide no significant benefit over placebo in improving the live birth rate" (108). In light of this body of evidence, a recent ACOG practice bulletin stated that HLA typing, measurement of maternal anti-leukocyte antibodies against paternal leukocytes, and measurement of maternal serum blocking factors do not predict the outcome of subsequent pregnancies. Furthermore, luteal phase biopsy for the presence of decidual NK cells, testing for percentage of NK cells in maternal peripheral blood, and testing for embryotoxic factor are not indicated in patients with a history of RPL. In short, alloimmune testing is not indicated and no beneficial immunologic treatment options are currently available.

\section{Maternal Infection}

Although no single infectious agent has been clearly linked as causative in recurrent loss, several infectious agents have been linked to sporadic losses. These include vaginal and cervical culture-proven infections with Toxoplasma gondii, rubella, herpes simplex virus, measles, cytomegalovirus, coxsackievirus, Listeria monocytogenes, Chlamydia trachomatis, and Mycoplasma hominis. RPL patients do exhibit increased rates of endometrial colonization with Ureaplasma urealyticum (109). In light of the large number of possible infectious agents, it could be cost-effective to treat each partner empirically with azithromycin $1 \mathrm{~g}$ or doxycycline $100 \mathrm{mg}$ twice daily for 10 days rather than performing multiple repeat cultures. The risks associated with this approach are low. It is also reasonable to completely omit diagnostic testing and treatment for infectious etiologies in RPL as ACOG recommends that tests for these agents are generally not useful and empiric antibiotics not warranted.

\section{Endocrine}

Before considering individual endocrine abnormalities that have been associated with RPL, it is important to highlight that no current evidence exists to support that any asymptomatic endocrine or metabolic diseases directly cause RPL.

\section{Luteal Phase Defect}

Luteal phase defect (LPD) is one of the most scrutinized of the proposed endocrine causes of RPL. It is hypothesized that with LPD, a defective corpus luteum produces insufficient amounts of progesterone, preventing implantation or impairing pregnancy maintenance. It is also hypothesized that poor follicular-phase oocyte development often precedes and causes LPD due to disordered estrogen secretion and dysfunction of the corpus luteum. This may also lead to increased luteinizing hormone levels and a hyperandrogenic decidual environment.

The diagnostic criteria for LPD require a histologic lag of more than 2 days between dating of an endometrial specimen and the documented day of the menstrual cycle. This lag must be documented in two cycles. This definition introduces the first line of criticism concerning the relationship of LPD with RPL: there is considerable inter-observer (110-112) and intraobserver $(113,114)$ variations in histologic interpretation of the endometrial biopsy. It has been estimated that had a different pathologist read an individual biopsy, the "in-phase" or "out-of-phase" classifications would change in 20\% to $40 \%$ of cases $(112,115)$. Although LPD has been more often associated with RPL than with subfertility, the utility of the test is called into question by the fact that no histologic differences can be documented in the biopsy specimens from fertile and infertile women (116). Furthermore, LPD is common in all women tested. Females without a history of pregnancy loss show histologic LPD in up to 50\% of single biopsies and 25\% of sequential cycles (117).

Proposed treatments for LPD in patients with RPL have included artificial insemination, ovarian stimulation with clomiphene citrate or gonadotropins, administration of hCG, and progesterone supplementation. Administration of hCG to support the corpus luteum was tested in an international, multicentered randomized trial of 75 women. In this trial, injection of 10,000 IU of hCG at the diagnosis of pregnancy followed by $5000 \mathrm{IU}$ weekly was compared with injection of placebo. No significant differences were noted in pregnancy outcomes (118). Abnormal expression of endometrial progesterone receptors or endometrial adhesion molecules during the window of implantation have been detected in women with impaired fertility and/or pregnancy outcomes and these can sometimes be reversed using supplemental progesterone or clomid (119). This restoration of endometrial protein expression may be a biomarker for resumption of normal endometrial function. Further support for the use of progesterone in the treatment of patients with RPL can be found in a recent Cochrane review. This meta-analysis of 15 trials including 2118 women showed no statistically significant difference when progesterone was used for the prevention of isolated spontaneous miscarriage (OR 0.98, 95\% CI 0.78-1.24). By contrast, among women with three or more consecutive pregnancy losses, a benefit of progesterone supplementation was noted (OR 0.38 , 95\% CI 0.2-0.7), regardless of the route of administration and with no adverse effects for mother or baby (120). Although the utility of progesterone supplementation in women with otherwise unexplained RPL is clear, it remains unclear whether the mechanism of progesterone action is through correction of LPD, through immune modulation, or through alternative or additional activities. 


\section{Thyroid Disease}

While inadequately treated hyper-, hypo-, and subclinical hypothyroidism have been associated with an increased rate of isolated spontaneous pregnancy loss and preterm delivery, no causal relationship has been established with RPL (121). Clinical thyroid dysfunction has also been linked with pregnancy complications such as low birth weight, placental abruption, reduced childhood intelligence quotients, and fetal death (122). Due to these associations, it is recommended that patients receive treatment for thyroid disorders and become euthyroid prior to attempting pregnancy. For additional discussion of this topic, please refer to the previous section on thyroid auto-antibodies and RPL.

\section{Diabetes Mellitus, Insulin Resistance, and Polycystic Ovarian Syndrome}

Diabetes mellitus is a risk factor for many adverse pregnancy outcomes. It is well established that poor diabetes control leads to an increased rate of spontaneous loss, while improved glucose control using insulin leads to decreased loss rates (123-125). Tight preconceptional glucose control is essential among RPL patients with diabetes mellitus.

The prevalence of insulin resistance is also higher among RPL patients than controls (27\% compared with 9.5\%; OR 3.55, 95\% CI 1.4-9.01) (126). This may indicate causality. In fact, results from small retrospective studies support this in that treatment of insulin-resistant RPL patients with insulin-sensitizing agents appears to improve pregnancy outcomes (127-129).

Obesity itself has also been proven to carry an increased risk for pregnancy loss. A body mass index of over $30 \mathrm{~kg} / \mathrm{m}^{2}$ is associated with a 1.2 odds ratio of first-trimester loss (95\% CI 1.01-1.46) and a 3.5 odds ratio for RPL (95\% CI 1.03-12.01) (130).

Among patients with RPL, ultrasonographic evidence of polycystic-appearing ovaries has been found more commonly than in the general population (131-134). The presence of polycystic ovaries itself did not predict worse outcomes than the finding of normal ovaries (131-134), although among women with polycystic ovarian syndrome, those who suffer pregnancy loss tend to have higher levels of circulating androgens than those who have successful pregnancies (134).

In summary, while no single treatment has definitively proven to improve pregnancy outcomes among patients with disorders of glucose metabolism, evidence is mounting that weight loss and improved glucose control using insulin sensitizers or optimized insulin regimens will probably increase pregnancy maintenance.

\section{Decreased Ovarian Reserve}

The prevalence of elevated FSH levels among RPL patients is similar to that of an infertility population (135). Women with unexplained RPL, however, have a greater incidence of elevated day 3 FSH levels than RPL patients with an identified etiology. Among 57 women with three or more spontaneous pregnancy losses, $31 \%$ of those with unexplained losses $(\mathrm{n}=36)$ had day 3 FSH levels greater than $10 \mathrm{mIU} / \mathrm{mL}$ compared with only $5 \%$ of women with explained losses (136). Testing for ovarian reserve among older RPL patients may help to determine the level of urgency in diagnostic testing and interventions.
Table 6 Nonsteroidal Anti-inflammatory Drug Use and Risk of Pregnancy Loss

Hazard ratio $(95 \% \mathrm{CI})$

$\begin{array}{ll}\text { Users overall } & 1.8(1.0-3.2) \\ \text { Gestational age at first use: } & \\ \quad \text { At conception } & 5.6(2.3-13.7) \\ \quad \text { After conception } & 1.2(0.5-2.6) \\ \text { Duration of use: } & \\ \quad \leq 1 \text { week } & 1.3(0.7-2.6) \\ >1 \text { week } & 8.1(2.8-23.4)\end{array}$

Abbreviation: CI, confidence interval. Source: Adapted from Ref. 138.

\section{Hyperprolactinemia}

Elevated levels of PRL induce LPDs and may decrease progesterone production by the corpus luteum. Hyperprolactinemia is also thought to interact with the hypothalamicpituitary-ovarian axis to cause inadequate follicular development and oocyte maturation. After evaluation for intracranial lesions, treatment of elevated serum PRL levels in women experiencing RPL can be safely undertaken with bromocriptine or cabergoline. Pharmacologic normalization of the PRL levels has been proven to improve pregnancy success rates among RPL patients (137).

\section{Environmental/Occupational Factors and Habits}

No associations have been clearly established between RPL and environmental factors, occupational exposures, or work itself. Furthermore, exercise has not been proven to increase the rate of sporadic loss, though no studies have been conducted involving recurrent loss patients.

It is important to recognize, however, that some environmental exposures lead to a slight, but statistically significant, increase in the rate of isolated spontaneous pregnancy losses including nonsteroidal anti-inflammatory drugs (Table 6), tobacco, alcohol, and heavy caffeine use. These may act in a dose-dependent fashion or synergistically. Their discussion with RPL patients must be carefully approached to avoid generating guilt. It may be useful to discuss these factors as methods to maximize desirable pregnancy outcomes.

\section{Tobacco Abuse}

Smokers incur a 1.4- to 1.8-fold increase in their risk of pregnancy loss $(139,140)$, and secondhand smoke leads to a 1.52 - to 2.18 -fold increase in the risk (141). It remains important to encourage both partners to attempt smoking cessation.

\section{Alcohol}

Drinking more than one alcoholic beverage per day carries an adjusted odds ratio for spontaneous pregnancy loss of 4.84 (95\% CI 2.87-8.16) (142). Additionally, the average number of daily drinks is directly proportional to the risk of secondtrimester pregnancy loss, irrespective of age, parity, race, marital status, smoking, or the number of previous spontaneous losses or induced abortions (143) (Table 7).

\section{Coffee and Caffeine}

Excessive caffeine intake has an established link to an increase in the risk of isolated spontaneous pregnancy loss of fetuses with normal karyotypes among nonsmokers (144). 
Table 7 Alcohol Use and Age-Adjusted Relative Risk of Pregnancy Loss

\begin{tabular}{lcc}
$\begin{array}{l}\text { Average no. } \\
\text { of drinks }\end{array}$ & $\begin{array}{c}\text { First-trimester } \\
\text { loss rate }\end{array}$ & $\begin{array}{c}\text { Second-trimester } \\
\text { loss rate }\end{array}$ \\
\hline$<1 /$ day & $1.12(\mathrm{~ns})$ & $1.03(\mathrm{~ns})$ \\
$1-2 /$ day & $1.15(\mathrm{~ns})$ & $1.98(p<0.01)$ \\
$\geq 3$ day & $1.15(\mathrm{~ns})$ & $3.53(p<0.01)$ \\
\hline
\end{tabular}

Abbreviation: ns, non-significant $p$ value. Source: Adapted from Ref. 143.
Table 8 Caffeine Intake and Risk of Pregnancy Loss

Caffeine intake
$100-299 \mathrm{mg} / \mathrm{d}$
$300-499 \mathrm{mg} / \mathrm{d}$
$\geq 500 \mathrm{mg} / \mathrm{d}$
$\geq 375 \mathrm{mg} / \mathrm{d}$

Pregnancy loss risk

RR 1.3 (95\% CI 0.9-1.8)

RR 1.4 (95\% CI 0.9-2)

RR 2.2 (95\% CI 2.3-3.8)

Adjusted OR 2.21

(95\% CI 1.53-3.18) (142)

Abbreviations: RR, relative risk; OR, odds ratio.

Table 9 Etiology-Specific Interventions for Patients with Recurrent Pregnancy Loss

\begin{tabular}{|c|c|}
\hline Disorder & Intervention \\
\hline \multicolumn{2}{|l|}{ Anatomic } \\
\hline Müllerian anomalies & - Hysteroscopic resection of septa, adhesions, submucosal fibroids, and polyps \\
\hline Intrauterine synechiae & - Myomectomy for intramural and subserosal fibroids $>5 \mathrm{~cm}$ \\
\hline \multicolumn{2}{|l|}{ Asherman's Syndrome } \\
\hline \multicolumn{2}{|l|}{ Leiomyomata } \\
\hline \multicolumn{2}{|l|}{ Endometrial polyps } \\
\hline \multicolumn{2}{|l|}{ Genetic } \\
\hline \multirow[t]{3}{*}{ Parental balanced translocations } & - Genetic counseling \\
\hline & - IVF with pre-implantation genetic diagnosis \\
\hline & - Donor gametes \\
\hline \multicolumn{2}{|l|}{ Thrombophilic (heritable) } \\
\hline & - Combined thrombophilic defects-therapeutic anticoagulation \\
\hline & $\begin{array}{l}\text { - Isolated defect and no personal or strong family history of thrombotic } \\
\text { complications_-prophylactic anticoagulation }\end{array}$ \\
\hline & $\begin{array}{l}\text { - Hyperhomocysteinemia-supplemental folic acid }(0.4-1.0 \mathrm{mg} / \text { day }) \text {, vitamin } \mathrm{B}_{6} \\
(6 \mathrm{mg} / \text { day }) \text {, and possibly vitamin } \mathrm{B}_{12}(0.025 \mathrm{mg} / \mathrm{day})\end{array}$ \\
\hline \multicolumn{2}{|r|}{ (0) } \\
\hline Antiphospholipid antibody syndrome & $\begin{array}{l}\text { - Low-dose aspirin plus prophylactic heparin in women without a history of a } \\
\text { systemic autoimmune disease like SLE or a history of thrombosis }\end{array}$ \\
\hline \multirow{2}{*}{\multicolumn{2}{|c|}{ ( }} \\
\hline & \\
\hline PCOS & - Metformin \\
\hline Hypothyroidism & - Thyroid-hormone replacement \\
\hline Luteal phase defect/unexplained & - Progesterone supplementation \\
\hline Diabetes mellitus & - Appropriate management of diabetes, insulin if indicated \\
\hline \multicolumn{2}{|r|}{ НP TP } \\
\hline Environmental exposures & - Limit exposures to maximize pregnancy outcomes (e.g., tobacco, alcohol, caffeine) \\
\hline \multirow[t]{2}{*}{ All patients/unexplained } & - Progesterone supplementation \\
\hline & - Daily aspirin (81-100 mg), if losses at greater than 13 weeks of gestation \\
\hline
\end{tabular}

The half-life of caffeine is halved in smokers and is doubled in users of oral contraceptives. See Table 8 for the caffeine doserelated increases in the risk of spontaneous pregnancy loss.

\section{Irradiation}

The incidence of chromosomal anomalies among spontaneously expelled ova is higher among couples in which either the female or the male was exposed to irradiation prior to conception (145). These data provide validity to the generally accepted recommendation that both partners avoid irradiation when attempting pregnancy.

\section{Environmental Toxins}

Anesthetic gases, tetrachloroethylene (a dry-cleaning chemical), lead, mercury, and isotretinoin (Accutane) have all been associated with an increased rate of spontaneous pregnancy loss. Organic solvents have rarely been linked to sporadic loss.

\section{Unexplained}

Much research has been devoted to possible therapeutic options for couples with unexplained RPL. Two recent studies have evaluated the role of preimplantation genetic screening for couples with idiopathic RPL. They have yielded conflicting results. Munne et al. studied 58 couples with unexplained RPL among whom the female partner was 35 years of age and older. The authors performed PGS for chromosomes X, Y, 13, 15, 16, $17,18,21$, and 22 and transferred up to four embryos (euploid for these particular chromosomes). The subsequent miscarriage rate after identification of a gestational sac was $15.7 \%$, which was lower than expected. They also found the "takehome" baby rate per retrieval to be $36 \%$ (146). Work by Platteau and colleagues (147) included 49 women with idiopathic RPL in a prospective cohort study. Their embryos were tested for abnormalities in chromosomes X, Y, 13, 16, 18, 21 , and 22. For women less than age 37, the pregnancy rate per cycle initiated was $23 \%$, whereas for women at or beyond 
37 years of age, the rate dropped drastically to $3 \%$, with $44 \%$ and $67 \%$ aneuploidy rates, respectively. Like Shahine and colleagues (38), these researchers concluded that no therapeutic evidence currently exists to support the use of in vitro fertilization with or without PGS for idiopathic RPL patients. Oocyte donation may be effective (148). Randomized controlled trials are needed.

Other studies have supported or refuted the benefit of various other treatment options. In considering the following, keep in mind that a meta-analysis from 1995 (149) concluded that $60 \%$ to $70 \%$ of unexplained RPL couples will have success with the next pregnancy in the absence of intervention; this provides both a frame of reference for evaluation of treatment success and an encouraging fact with which to counsel patients. After negative testing with karyotyping, HSG or hysteroscopy, and antiphospholipid antibodies for second-trimester losses, patients with recurrent loss beyond 13 weeks had an improved live-birth rate with daily low-dose aspirin (OR 1.88; 95\% CI 1.04-3.37) (150). Providing supportive care to idiopathic first-trimester RPL couples is perhaps one of the best interventions, leading to a live-birth rate near $75 \%$ (vs. $50 \%$ of those not attending an early pregnancy clinic; $p=0.002$ ) (4). Appropriately timed intercourse may also improve outcomes as intercourse remote from ovulation has been associated with an increase spontaneous loss rate due to chromosomal anomalies $(145,151)$. Requiring bed rest for RPL patients during pregnancy is not supported by evidence (RR 1.54, 95\% CI 0.92-2.58), although bed rest alone did perform better when compared with supplemental hCG without bed rest in a Cochrane review (RR 2.5, 95\% CI 1.22-5.11) (152). An additional Cochrane review has been performed to evaluate the utility of hCG administration to treat women with a history of unexplained RPL. Randomized trials comparing the use of hCG in early pregnancy versus placebo or no treatment for women with two or more miscarriages $(n=180)$ showed a decreased risk of pregnancy loss among hCG-treated patients (OR 0.26, 95\% CI $0.14-0.52$ ) (153). Readers are encouraged by the authors of this study to interpret the data cautiously due to a large influence on the data by the two weakest studies. These authors therefore conclude that "there is not enough evidence to evaluate the use of hCG during pregnancy to prevent miscarriage" in patients with unexplained RPL (153).

It has been shown in meta-analyses that treatment of unexplained RPL with progesterone supplementation is useful (see above). We recommend the use of vaginal progesterone, typically $100 \mathrm{mg}$ twice daily, starting 3 days after ovulation during cycles in which the couple is attempting conception. Progesterone supplementation is thought to have implications in treating both immune and luteal phase aberrancies though specific mechanisms are unclear.

\section{POSTCONCEPTION EVALUATION}

Close monitoring of subsequent pregnancies leads to an increased pregnancy success rate among patients with a history of RPL (154). These couples are prone to heightened senses of anger, depression, anxiety, and even psychoses. Careful evaluation for signs and symptoms of these disorders is warranted. The availability of counseling services, with or without the use of support groups and/or a reproductive psychiatrist, is strongly encouraged.

\section{SUMMARY}

The assessment and management of patients with RPL continue to be somewhat controversial and both are likely to remain so. The disorder itself is multifactorial and the baseline rate of isolated pregnancy losses secondary to spontaneous fetal aneuploidy is high. The prognosis for most patients, even in the absence of a defined etiology, is remarkably good, making assessment of the utility of a given diagnostic test or intervention difficult in the absence of very large trials.

Evidence does exist for etiologies that should be excluded and effective treatment regimes for most diagnosed etiologies are available. After a thorough evaluation, if the resulting diagnosis is unexplained RPL, interventions such as progesterone supplementation, aspirin therapy (in selected patients), conception timing, and close clinical follow-up during early pregnancy have merit. Table 2 lists our recommendations for useful diagnostic testing in patients with a history of RPL. Table 9 lists etiology-specific interventions.

\section{REFERENCES}

1. Edmonds DK, Lindsay KS, Miller JF, et al. Early embryonic mortality in women. Fertil Steril 1982; 4: 447-53.

2. American College of Obstetricians and Gynecologists. Management of recurrent early pregnancy loss. ACOG Practice Bulletin 2001; 24: 1-12.

3. Stephenson M, Kutteh W. Evaluation and management of recurrent early pregnancy loss. Clin Obstet Gynecol 2007; 1: 132-45.

4. Clifford K, Rai R, Regan L. Future pregnancy outcome in unexplained recurrent first trimester miscarriage. Hum Reprod 1997; 2: 387-9.

5. Quenby S, Farquharson RG. Human chorionic gonadotropin supplementation in recurring pregnancy loss: a controlled trial. Fertil Steril 1994; 4: 708-10.

6. George L, Mills JL, Johansson AL, et al. Plasma folate levels and risk of spontaneous abortion. JAMA 2002; 15: 1867-73.

7. Rumbold A, Middleton P, Crowther CA. Vitamin supplementation for preventing miscarriage. Cochrane Database Syst Rev 2005; 2: CD004073.

8. Pellerito JS, McCarthy SM, Doyle MB, et al. Diagnosis of uterine anomalies: relative accuracy of MR imaging, endovaginal sonography and hysterosalpingography. Radiology 1992; 3: 795-800.

9. Andreotti RF, Fleischer AC, Mason LE Jr. Three-dimensional sonography of the endometrium and adjacent myometrium: preliminary observations. J Ultrasound Med 2006; 10: 1313-19.

10. Wu MH, Hsu CC, Huang KE. Detection of congenital mullerian duct anomalies using three-dimensional ultrasound. J Clin Ultrasound 1997; 9: 487-92.

11. Raga F, Bauset C, Remohi J, et al. Reproductive impact of congenital Mullerian anomalies. Hum Reprod 1997; 10: 2277-81.

12. Schust DJ. Recurrent pregnancy loss. In: Lebovic DI, Gordon JD, Taylor RN, eds. Reproductive Endocrinology and Infertility-Handbook for Clinicians 1st. Arlington: Scrub Hill Press, 2005.

13. Lin PC. Reproductive outcomes in women with uterine anomalies. J Womens Health (Larchmt) 2004; 1: 33-9.

14. Dabirashrafi H, Bahadori M, Mohammad K, et al. Septate uterus: new idea on the histologic features of the septum in this abnormal uterus. Am J Obstet Gynecol 1995; 1(Pt 1): 105-7.

15. Fedele L, Dorta M, Brioschi D, et al. Pregnancies in septate uteri: outcome in relation to site of uterine implantation as determined by sonography. AJR Am J Roentgenol 1989; 4: 781-4.

16. March CM, Israel R. Hysteroscopic management of recurrent abortion caused by septate uterus. Am J Obstet Gynecol 1987; 4: 834-42.

17. Jurkovic D, Geipel A, Gruboeck K, et al. Three-dimensional ultrasound for the assessment of uterine anatomy and detection of congenital anomalies: a comparison with hysterosalpingography and two-dimensional sonography. Ultrasound Obstet Gynecol 1995; 4: 233-7. 
18. Jurkovic D, Gruboeck K, Tailor A, et al. Ultrasound screening for congenital uterine anomalies. Br J Obstet Gynaecol 1997; 11: 1320-1.

19. Maneschi F, Zupi E, Marconi D, et al. Hysteroscopically detected asymptomatic mullerian anomalies. Prevalence and reproductive implications. J Reprod Med 1995; 10: 684-8.

20. Sorensen SS, Trauelsen AG. Obstetric implications of minor mullerian anomalies in oligomenorrheic women. Am J Obstet Gynecol 1987; 5: $1112-18$.

21. Bajekal N, Li TC. Fibroids, infertility and pregnancy wastage. Hum Reprod Update 2000; 6: 614-20.

22. Kaufman RH, Adam E, Hatch EE, et al. Continued follow-up of pregnancy outcomes in diethylstilbestrol-exposed offspring. Obstet Gynecol 2000; 4: 483-9.

23. Drakeley AJ, Roberts D, Alfirevic Z. Cervical stitch (cerclage) for preventing pregnancy loss in women. Cochrane Database Syst Rev 2003; 1: CD003253.

24. Fritz B, Aslan M, Kalscheuer V, et al. Low incidence of UPD in spontaneous abortions beyond the 5th gestational week. Eur J Hum Genet 2001; 12: 910-16.

25. Christiansen M, Olesen Larsen S. An increase in cost-effectiveness of first trimester maternal screening programmes for fetal chromosome anomalies is obtained by contingent testing. Prenat Diagn 2002; 6: 482-6.

26. Ogasawara M, Aoki K, Okada S, et al. Embryonic karyotype of abortuses in relation to the number of previous miscarriages. Fertil Steril 2000; 2: 300-4.

27. Daniely M, Aviram-Goldring A, Barkai G, et al. Detection of chromosomal aberration in fetuses arising from recurrent spontaneous abortion by comparative genomic hybridization. Hum Reprod 1998; 4: 805-9.

28. Hassold TJ. A cytogenetic study of repeated spontaneous abortions. Am J Hum Genet 1980; 5: 723-30.

29. Jacobs P, Hassold TJ. Chromosome abnormalities: origin and etiology in abortions and livebirths. In: Human Genetics. Berlin: Springer-Verlag, 1987; 233-44.

30. Ohno M, Maeda T, Matsunobu A. A cytogenetic study of spontaneous abortions with direct analysis of chorionic villi. Obstet Gynecol 1991; 3: $394-8$.

31. Simpson JL. Incidence and timing of pregnancy losses: relevance to evaluating safety of early prenatal diagnosis. Am J Med Genet 1990; 2: $165-73$.

32. Warburton D, Fraser FC. Spontaneous abortion risks in man: data from reproductive histories collected in a medical genetics unit. Am J Hum Genet 1964; 16: 1-25.

33. Warburton D, Kline J, Stein Z, et al. Does the karyotype of a spontaneous abortion predict the karyotype of a subsequent abortion? Evidence from 273 women with two karyotyped spontaneous abortions. Am J Hum Genet 1987; 3: 465-83.

34. Simon C, Rubio C, Vidal F, et al. Increased chromosome abnormalities in human preimplantation embryos after in-vitro fertilization in patients with recurrent miscarriage. Reprod Fertil Dev 1998; 1: 87-92.

35. Vidal F, Gimenez C, Rubio C, et al. FISH preimplantation diagnosis of chromosome aneuploidy in recurrent pregnancy wastage. J Assist Reprod Genet 1998; 5: 310-13.

36. Golbus MS. The influence of strain, maternal age, and method of maturation on mouse oocyte aneuploidy. Cytogenet Cell Genet 1981; 2: 84-90.

37. Stephenson MD, Awartani KA, Robinson WP. Cytogenetic analysis of miscarriages from couples with recurrent miscarriage: a case-control study. Hum Reprod 2002; 2: 446-51.

38. Shahine LK, Cedars MI. Preimplantation genetic diagnosis does not increase pregnancy rates in patients at risk for aneuploidy. Fertil Steril 2006; 1: 51-6.

39. Simpson JL, Martin AO. Prenatal diagnosis of cytogenetic disorders. Clin Obstet Gynecol 1976; 4: 841-53.

40. Warburton D. De novo balanced chromosome rearrangements and extra marker chromosomes identified at prenatal diagnosis: clinical significance and distribution of breakpoints. Am J Hum Genet 1991; 5: 995-1013.

41. Stephenson MD, Sierra S. Reproductive outcomes in recurrent pregnancy loss associated with a parental carrier of a structural chromosome rearrangement. Hum Reprod 2006; 4: 1076-82.
42. Tarani L, Lampariello S, Raguso G, et al. Pregnancy in patients with Turner's syndrome: six new cases and review of literature. Gynecol Endocrinol 1998; 2: 83-7.

43. Lanasa MC, Hogge WA, Kubik C, et al. Highly skewed X-chromosome inactivation is associated with idiopathic recurrent spontaneous abortion. Am J Hum Genet 1999; 1: 252-4.

44. Preston FE, Rosendaal FR, Walker ID, et al. Increased fetal loss in women with heritable thrombophilia. Lancet 1996; 9032: 913-16.

45. Brenner B, Mandel H, Lanir N, et al. Activated protein C resistance can be associated with recurrent fetal loss. Br J Haematol 1997; 3: 551-4.

46. Foka ZJ, Lambropoulos AF, Saravelos H, et al. Factor V leiden and prothrombin G20210A mutations, but not methylenetetrahydrofolate reductase $\mathrm{C677T}$, are associated with recurrent miscarriages. Hum Reprod 2000; 2: 458-62.

47. Rai R, Regan L, Hadley E, et al. Second-trimester pregnancy loss is associated with activated C resistance. Br J Haematol 1996; 2: 489-90.

48. Ridker PM, Miletich JP, Buring JE, et al. Factor V Leiden mutation as a risk factor for recurrent pregnancy loss. Ann Intern Med 1998; 12(Pt 1): $1000-3$.

49. Balasch J, Reverter JC, Fabregues F, et al. First-trimester repeated abortion is not associated with activated protein C resistance. Hum Reprod 1997; 5: 1094-7.

50. Carp H, Dolitzky M, Tur-Kaspa I, et al. Hereditary thrombophilias are not associated with a decreased live birth rate in women with recurrent miscarriage. Fertil Steril 2002; 1: 58-62.

51. Dizon-Townson DS, Meline L, Nelson LM, et al. Fetal carriers of the factor $\mathrm{V}$ Leiden mutation are prone to miscarriage and placental infarction. Am J Obstet Gynecol 1997; 2: 402-5.

52. Kutteh WH. Report from the Society for Gynecologic Investigation, Atlanta, Georgia, March 11-14, 1998. J Reprod Immunol 1998; 2: $175-82$.

53. Pauer HU, Neesen J, Hinney B. Factor V Leiden and its relevance in patients with recurrent abortions. Am J Obstet Gynecol 1998; 3: 629.

54. Rai R, Backos M, Elgaddal S, et al. Factor V Leiden and recurrent miscarriage-prospective outcome of untreated pregnancies. Hum Reprod 2002; 2: 442-5.

55. Sanson BJ, Friederich PW, Simioni P, et al. The risk of abortion and stillbirth in antithrombin-, protein C-, and protein S-deficient women. Thromb Haemost 1996; 3: 387-8.

56. Makris M. Hyperhomocysteinemia and thrombosis. Clin Lab Haematol 2000; 3: 133-43.

57. Di Nisio M, Peters L, Middeldorp S. Anticoagulants for the treatment of recurrent pregnancy loss in women without antiphospholipid syndrome. Cochrane Database Syst Rev 2005; 2: CD004734.

58. Oshiro BT, Silver RM, Scott JR, et al. Antiphospholipid antibodies and fetal death. Obstet Gynecol 1996; 4: 489-93.

59. Simpson JL, Carson SA, Chesney C, et al. Lack of association between antiphospholipid antibodies and first-trimester spontaneous abortion: prospective study of pregnancies detected within 21 days of conception. Fertil Steril 1998; 5: 814-20.

60. Jaffe R, Jauniaux E, Hustin J. Maternal circulation in the first-trimester human placenta-myth or reality? Am J Obstet Gynecol 1997; 3: 695-705.

61. Adler RR, Ng AK, Rote NS. Monoclonal antiphosphatidylserine antibody inhibits intercellular fusion of the choriocarcinoma line, JAR. Biol Reprod 1995; 4: 905-10.

62. Katsuragawa $\mathrm{H}$, Kanzaki $\mathrm{H}$, Inoue $\mathrm{T}$, et al. Monoclonal antibody against phosphatidylserine inhibits in vitro human trophoblastic hormone production and invasion. Biol Reprod 1997; 1: 50-8.

63. Quenby S, Mountfield S, Cartwright JE, et al. Antiphospholipid antibodies prevent extravillous trophoblast differentiation. Fertil Steril 2005; 3: 691-8.

64. Franklin RD, Kutteh WH. Antiphospholipid antibodies (APA) and recurrent pregnancy loss: treating a unique APA positive population. Hum Reprod 2002; 11: 2981-5.

65. Miyakis S, Lockshin MD, Atsumi T, et al. International consensus statement on an update of the classification criteria for definite antiphospholipid syndrome (APS). J Thromb Haemost 2006; 2: 295-306.

66. Kutteh WH. Antiphospholipid antibody-associated recurrent pregnancy loss: treatment with heparin and low-dose aspirin is superior to low-dose aspirin alone. Am J Obstet Gynecol 1996; 5: 1584-9. 
67. Rai R, Cohen H, Dave M, et al. Randomised controlled trial of aspirin and aspirin plus heparin in pregnant women with recurrent miscarriage associated with phospholipid antibodies (or antiphospholipid antibodies). BMJ 1997; 70761: 253-7.

68. Empson M, Lassere M, Craig JC, et al. Recurrent pregnancy loss with antiphospholipid antibody: a systematic review of therapeutic trials. Obstet Gynecol 2002; 1: 135-44.

69. Triolo G, Ferrante A, Ciccia F, et al. Randomized study of subcutaneous low molecular weight heparin plus aspirin versus intravenous immunoglobulin in the treatment of recurrent fetal loss associated with antiphospholipid antibodies. Arthritis Rheum 2003; 3: 728-31.

70. Greer IA. Exploring the role of low-molecular-weight heparins in pregnancy. Semin Thromb Hemost 2002; Suppl 3: 25-31.

71. Noble LS, Kutteh WH, Lashey N, et al. Antiphospholipid antibodies associated with recurrent pregnancy loss: prospective, multicenter, controlled pilot study comparing treatment with low-molecular-weight heparin versus unfractionated heparin. Fertil Steril 2005; 3: 684-90.

72. Stephenson MD, Ballem PJ, Tsang P, et al. Treatment of antiphospholipid antibody syndrome (APS) in pregnancy: a randomized pilot trial comparing low molecular weight heparin to unfractionated heparin. J Obstet Gynaecol Can 2004; 8: 729-34.

73. Cowchock FS, Reece EA, Balaban D, et al. Repeated fetal losses associated with antiphospholipid antibodies: a collaborative randomized trial comparing prednisone with low-dose heparin treatment. Am J Obstet Gynecol 1992; 5: 1318-23.

74. Laskin CA, Bombardier C, Hannah ME, et al. Prednisone and aspirin in women with autoantibodies and unexplained recurrent fetal loss. N Engl J Med 1997; 3: 148-53.

75. Lejeune B, Grun JP, de Nayer P, et al. Antithyroid antibodies underlying thyroid abnormalities and miscarriage or pregnancy induced hypertension. Br J Obstet Gynaecol 1993; 7: 669-72.

76. Stagnaro-Green A, Roman SH, Cobin RH, et al. Detection of at-risk pregnancy by means of highly sensitive assays for thyroid autoantibodies. JAMA 1990; 11: 1422-5.

77. Poppe K, Glinoer D. Thyroid autoimmunity and hypothyroidism before and during pregnancy. Hum Reprod Update 2003; 2: 149-61.

78. Prummel MF, Wiersinga WM. Thyroid autoimmunity and miscarriage. Eur J Endocrinol 2004; 6: 751-5.

79. Negro R, Formoso G, Mangieri T, et al. Levothyroxine treatment in euthyroid pregnant women with autoimmune thyroid disease: effects on obstetrical complications. J Clin Endocrinol Metab 2006; 7: 2587-91.

80. Abalovich M, Amino N, Barbour LA, et al. Management of thyroid dysfunction during pregnancy and postpartum: an Endocrine Society Clinical Practice Guideline. J Clin Endocrinol Metab 2007; 8(Suppl): S1-47.

81. Esplin MS, Branch DW, Silver R, et al. Thyroid autoantibodies are not associated with recurrent pregnancy loss. Am J Obstet Gynecol 1998; 6(Pt 1): 1583-6.

82. Harger JH, Archer DF, Marchese SG, et al. Etiology of recurrent pregnancy losses and outcome of subsequent pregnancies. Obstet Gynecol 1983; 5: 574-81.

83. Kutteh WH. Antiphospholipid antibodies and reproduction. J Reprod Immunol 1997; 2: 151-71.

84. Lockshin MD, Druzin ML, Goei S, et al. Antibody to cardiolipin as a predictor of fetal distress or death in pregnant patients with systemic lupus erythematosus. N Engl J Med 1985; 3: 152-6.

85. Bobrie G, Liote F, Houillier P, et al. Pregnancy in lupus nephritis and related disorders. Am J Kidney Dis 1987; 4: 339-43.

86. Hayslett JP, Lynn RI. Effect of pregnancy in patients with lupus nephropathy. Kidney Int 1980; 2: 207-20.

87. Sargent IL, Wilkins T, Redman CW. Maternal immune responses to the fetus in early pregnancy and recurrent miscarriage. Lancet 1988; 8620: 1099-104.

88. Ecker JL, Laufer MR, Hill JA. Measurement of embryotoxic factors is predictive of pregnancy outcome in women with a history of recurrent abortion. Obstet Gynecol 1993; 1: 84-7.

89. Hill JA, Polgar K, Anderson DJ. T-helper 1-type immunity to trophoblast in women with recurrent spontaneous abortion. JAMA 1995; 24: 1933-6.
90. Hill JA, Polgar K, Harlow BL, et al. Evidence of embryo- and trophoblast-toxic cellular immune response(s) in women with recurrent spontaneous abortion. Am J Obstet Gynecol 1992; 4: 1044-52.

91. Hewitt MJ, Pratten MK, Regan L, et al. The use of whole rat embryo culture as a technique for investigating potential serum toxicity in recurrent miscarriage patients. Hum Reprod 2000; 10: 2200-4.

92. Clark DA, Vince G, Flanders KC, et al. CD56+ lymphoid cells in human first trimester pregnancy decidua as a source of novel transforming growth factor-beta 2-related immunosuppressive factors. Hum Reprod 1994; 12: 2270-7.

93. Clifford K, Flanagan AM, Regan L. Endometrial CD56+ natural killer cells in women with recurrent miscarriage: a histomorphometric study. Hum Reprod 1999; 11: 2727-30.

94. Lachapelle MH, Miron P, Hemmings R, et al. Endometrial T, B, and NK cells in patients with recurrent spontaneous abortion. Altered profile and pregnancy outcome. J Immunol 1996; 10: 4027-34.

95. Quenby S, Bates M, Doig T, et al. Pre-implantation endometrial leukocytes in women with recurrent miscarriage. Hum Reprod 1999; 9: 2386-91.

96. Aoki K, Kajiura S, Matsumoto Y, et al. Preconceptional natural-killer-cell activity as a predictor of miscarriage. Lancet 1995; 8961: 1340-2.

97. Coulam CB, Goodman C, Roussev RG, et al. Systemic CD56+ cells can predict pregnancy outcome. Am J Reprod Immunol 1995; 1: 40-6.

98. Coulam CB. Immunologic tests in the evaluation of reproductive disorders: a critical review. Am J Obstet Gynecol 1992; 6: 1844-51.

99. Cowchock FS, Smith JB. Predictors for live birth after unexplained spontaneous abortions: correlations between immunologic test results, obstetric histories, and outcome of next pregnancy without treatment. Am J Obstet Gynecol 1992; 5: 1208-12.

100. Mowbray JF, Gibbings C, Liddell H, et al. Controlled trial of treatment of recurrent spontaneous abortion by immunisation with paternal cells. Lancet 1985; 8435: 941-3.

101. Ober C, Karrison T, Odem RR, et al. Mononuclear-cell immunisation in prevention of recurrent miscarriages: a randomised trial. Lancet 1999; 9176: 365-9.

102. Coulam CB, Krysa L, Stern JJ, et al. Intravenous immunoglobulin for treatment of recurrent pregnancy loss. Am J Reprod Immunol 1995; 6: 333-7.

103. Christiansen OB, Mathiesen O, Husth M, et al. Placebo-controlled trial of treatment of unexplained secondary recurrent spontaneous abortions and recurrent late spontaneous abortions with i.v. immunoglobulin. Hum Reprod 1995; 10: 2690-5.

104. Daya S, Gunby J, Clark DA. Intravenous immunoglobulin therapy for recurrent spontaneous abortion: a meta-analysis. Am J Reprod Immunol 1998; 2: 69-76.

105. Daya S, Gunby J, Porter F, et al. Critical analysis of intravenous immunoglobulin therapy for recurrent miscarriage. Hum Reprod Update 1999; 5: 475-82.

106. Perino A, Vassiliadis A, Vucetich A, et al. Short-term therapy for recurrent abortion using intravenous immunoglobulins: results of a double-blind placebo-controlled Italian study. Hum Reprod 1997; 11 : 2388-92.

107. Stephenson MD, Dreher K, Houlihan E, et al. Prevention of unexplained recurrent spontaneous abortion using intravenous immunoglobulin: a prospective, randomized, double-blinded, placebo-controlled trial. Am J Reprod Immunol 1998; 2: 82-8.

108. Porter TF, LaCoursiere Y, Scott JR. Immunotherapy for recurrent miscarriage. Cochrane Database Syst Rev 2006; 2: CD000112.

109. Kundsin RB, Driscoll SG, Pelletier PA. Ureaplasma urealyticum incriminated in perinatal morbidity and mortality. Science 1981; 4506: 474-5.

110. Duggan MA, Brashert P, Ostor A, et al. The accuracy and interobserver reproducibility of endometrial dating. Pathology 2001; 3: 292-7.

111. Scott RT, Snyder RR, Strickland DM, et al. The effect of interobserver variation in dating endometrial histology on the diagnosis of luteal phase defects. Fertil Steril 1988; 6: 888-92.

112. Smith S, Hosid S, Scott L. Endometrial biopsy dating. Interobserver variation and its impact on clinical practice. J Reprod Med 1995; 1: 1-3.

113. Daya S. Efficacy of progesterone support for pregnancy in women with recurrent miscarriage. A meta-analysis of controlled trials. Br J Obstet Gynaecol 1989; 3: 275-80. 
114. Scott RT, Snyder RR, Bagnall JW, et al. Evaluation of the impact of intraobserver variability on endometrial dating and the diagnosis of luteal phase defects. Fertil Steril 1993; 4: 652-7.

115. Gibson M, Badger GJ, Byrn F, et al. Error in histologic dating of secretory endometrium: variance component analysis. Fertil Steril 1991; 2: $242-7$.

116. Coutifaris C, Myers ER, Guzick DS, et al. Histological dating of timed endometrial biopsy tissue is not related to fertility status. Fertil Steril 2004; 5: 1264-72.

117. Davis OK, Berkeley AS, Naus GJ, et al. The incidence of luteal phase defect in normal, fertile women, determined by serial endometrial biopsies. Fertil Steril 1989; 4: 582-6.

118. Harrison RF. Human chorionic gonadotrophin (hCG) in the management of recurrent abortion; results of a multi-centre placebo-controlled study. Eur J Obstet Gynecol Reprod Biol 1992; 3: 175-9.

119. Lessey BA. Two pathways of progesterone action in the human endometrium: implications for implantation and contraception. Steroids 2003; 68: 809-15.

120. Haas DM, Ramsey PS. Progestogen for preventing miscarriage. Cochrane Database Syst Rev 2008; 2: CD003511.

121. Abalovich M, Gutierrez S, Alcaraz G, et al. Overt and subclinical hypothyroidism complicating pregnancy. Thyroid 2002; 1: 63-8.

122. Casey BM, Dashe JS, Wells CE, et al. Subclinical hypothyroidism and pregnancy outcomes. Obstet Gynecol 2005; 2: 239-45.

123. Dorman JS, Burke JP, McCarthy BJ, et al. Temporal trends in spontaneous abortion associated with Type 1 diabetes. Diabetes Res Clin Pract 1999; 1: 41-7.

124. Hanson U, Persson B, Thunell S. Relationship between haemoglobin $\mathrm{A} 1 \mathrm{C}$ in early type 1 (insulin-dependent) diabetic pregnancy and the occurrence of spontaneous abortion and fetal malformation in Sweden. Diabetologia 1990; 2: 100-4.

125. Mills JL, Simpson JL, Driscoll SG, et al. Incidence of spontaneous abortion among normal women and insulin-dependent diabetic women whose pregnancies were identified within 21 days of conception. $\mathrm{N}$ Engl J Med 1988; 25: 1617-23.

126. Craig LB, Ke RW, Kutteh WH. Increased prevalence of insulin resistance in women with a history of recurrent pregnancy loss. Fertil Steril 2002; 3: 487-90.

127. Glueck CJ, Phillips H, Cameron D, et al. Continuing metformin throughout pregnancy in women with polycystic ovary syndrome appears to safely reduce first-trimester spontaneous abortion: a pilot study. Fertil Steril 2001; 1: 46-52.

128. Jakubowicz DJ, Iuorno MJ, Jakubowicz S, et al. Effects of metformin on early pregnancy loss in the polycystic ovary syndrome. J Clin Endocrinol Metab 2002; 2: 524-9.

129. Sills ES, Perloe M, Palermo GD. Correction of hyperinsulinemia in oligoovulatory women with clomiphene-resistant polycystic ovary syndrome: a review of therapeutic rationale and reproductive outcomes. Eur J Obstet Gynecol Reprod Biol 2000; 2: 135-41.

130. Lashen H, Fear K, Sturdee DW. Obesity is associated with increased risk of first trimester and recurrent miscarriage: matched case-control study. Hum Reprod 2004; 7: 1644-6.

131. Clifford K, Rai R, Watson $\mathrm{H}$, et al. An informative protocol for the investigation of recurrent miscarriage: preliminary experience of 500 consecutive cases. Hum Reprod 1994; 7: 1328-32.
132. Liddell HS, Sowden K, Farquhar CM. Recurrent miscarriage: screening for polycystic ovaries and subsequent pregnancy outcome. Aust NZ J Obstet Gynaecol 1997; 4: 402-6.

133. Rai R, Backos M, Rushworth F, et al. Polycystic ovaries and recurrent miscarriage-a reappraisal. Hum Reprod 2000; 3: 612-15.

134. Tulppala M, Stenman UH, Cacciatore B, et al. Polycystic ovaries and levels of gonadotrophins and androgens in recurrent miscarriage: prospective study in 50 women. Br J Obstet Gynaecol 1993; 4: 348-52.

135. Hofmann GE, Khoury J, Thie J. Recurrent pregnancy loss and diminished ovarian reserve. Fertil Steril 2000; 6: 1192-5.

136. Trout SW, Seifer DB. Do women with unexplained recurrent pregnancy loss have higher day 3 serum FSH and estradiol values? Fertil Steril 2000; 2: 335-7.

137. Hirahara F, Andoh N, Sawai K, et al. Hyperprolactinemic recurrent miscarriage and results of randomized bromocriptine treatment trials. Fertil Steril 1998; 2: 246-52.

138. Li DK, Liu L, Odouli R. Exposure to non-steroidal anti-inflammatory drugs during pregnancy and risk of miscarriage: population based cohort study. BMJ 2003; 7411: 368.

139. Kline J, Stein ZA, Susser M, et al. Smoking: a risk factor for spontaneous abortion. N Engl J Med 1977; 15: 793-6.

140. Ness RB, Grisso JA, Hirschinger N, et al. Cocaine and tobacco use and the risk of spontaneous abortion. N Engl J Med 1999; 5: 333-9.

141. Venners SA, Wang X, Chen C, et al. Paternal smoking and pregnancy loss: a prospective study using a biomarker of pregnancy. Am J Epidemiol 2004; 10: 993-1001.

142. Rasch V. Cigarette, alcohol, and caffeine consumption: risk factors for spontaneous abortion. Acta Obstet Gynecol Scand 2003; 2: 182-8.

143. Harlap S, Shiono PH. Alcohol, smoking, and incidence of spontaneous abortions in the first and second trimester. Lancet 1980; 8187: 173-6.

144. Cnattingius S, Signorello LB, Anneren G, et al. Caffeine intake and the risk of first-trimester spontaneous abortion. N Engl J Med 2000; 25: 1839-45.

145. Boue J, Boue A. Spontaneous abortion in women: cytogenetic and epidemiological studies. Rev Fr Gynecol Obstet 1973; 11: 625-43.

146. Munne S, Chen S, Fischer J, et al. Preimplantation genetic diagnosis reduces pregnancy loss in women aged 35 years and older with a history of recurrent miscarriages. Fertil Steril 2005; 2: 331-5.

147. Platteau P, Staessen C, Michiels A, et al. Preimplantation genetic diagnosis for aneuploidy screening in patients with unexplained recurrent miscarriages. Fertil Steril 2005; 2: 393-7; quiz 525-6.

148. Remohi J, Gallardo E, Levy M, et al. Oocyte donation in women with recurrent pregnancy loss. Hum Reprod 1996; 9: 2048-51.

149. Jeng GT, Scott JR, Burmeister LF. A comparison of meta-analytic results using literature vs individual patient data. Paternal cell immunization for recurrent miscarriage. JAMA 1995; 10: 830-6.

150. Rai R, Backos M, Baxter N, et al. Recurrent miscarriage-an aspirin a day? Hum Reprod 2000; 10: 2220-3.

151. Boue J, Boue A. Chromosomal analysis of two consecutive abortuses in each of 43 women. Humangenetik 1973; 3: 275-80.

152. Aleman A, Althabe F, Belizan J, et al. Bed rest during pregnancy for preventing miscarriage. Cochrane Database Syst Rev 2005; 2: CD003576.

153. Scott JR, Pattison N. Human chorionic gonadotrophin for recurrent miscarriage. Cochrane Database Syst Rev 2000; 2: CD000101.

154. Stray-Pedersen B, Stray-Pedersen S. Etiologic factors and subsequent reproductive performance in 195 couples with a prior history of habitual abortion. Am J Obstet Gynecol 1984; 2: 140-6. 


\section{Multiple gestation Isaac Blickstein}

\section{INTRODUCTION}

The human female is clearly programmed to ovulate a single oocyte, to carry a singleton fetus, and to nurse one neonate at a time. Consequently, multiple pregnancies overwhelm the uterine (maternal) capacity to adequately nurture the fetuses as animal and human models have repeatedly confirmed the inverse relationship between birth weight and gestational age at delivery and litter size. This natural sequence is relatively rare (about 1 twin pair per $80-100$ births) and is extremely rare in the case of high-order multiple pregnancies.

The infrequency of high-order multiple gestations might be understood from the quasi-mathematical relationship, known as the Hellin-Zellany rule for twins, triplets, and quadruplets (1). This rule states that if the frequency of twins in a given population is $1 / \mathrm{N}$, then the frequency of triplets will be $1 / \mathrm{N}^{2}$ and that of quadruplets $1 / \mathrm{N}^{3}$. The Hellin-Zellany relationship appears quite accurate as long as the population remained homogeneous and enjoyed natural procreation. However, deviations from the rule were observed already in the thirties of the 20th century mainly because of racial differences in the frequency of dizygotic (DZ) twinning. A second, and much more dramatic deviation from the HellinZellany relationship, occurred during the emergence of effective treatment of infertility. Indeed, within a very small fraction in human history, the natural order of multiples has been profoundly changed. Physician-made (i.e., iatrogenic) multiple gestations are now seen in most developed countries with frequencies approaching 50\% of twins and more than $75 \%$ of high-order sets.

Iatrogenic multiple gestations, the major contributors to the epidemic of multiple pregnancies, did not arise ex vacuo. Women nowadays are able to rely on efficient modern fertility treatment when deciding on postponing childbirth. Advanced maternal age, by itself an established risk for natural multiples, is thus a significant risk factor for age-related reduced fecundity and for the increased need for fertility treatment. It appears that social trends act in concert with available assisted procreation techniques to increase the risk of multiple pregnancy. Consequently, all developed countries exhibited a dramatic increase in multiple births. For example, the ratio of induced to spontaneous twins in the East Flanders (Belgium) over the last two decades changed dramatically from 1:46 into 1 in every 2-3 twin pairs (1). This population-based trend might be even more accentuated in hospital-based data.

Data from England and Wales and the United States demonstrate decreasing rates of higher order multiple births and represent a striking and optimistic change in trend when compared with the previous steep fourfold increase since the early 1980s (2). At the same time, however, the incidence of other and by far more frequent multiples_-twins - continued to escalate on both sides of the Atlantic Ocean. Regardless of the probable reasons for this change (2), twins are predictably associated with significantly increased likelihood of perinatal morbidity and mortality and, therefore, the implications of the ever-increasing multiple birth rates is a major public health issue. For example, the contribution of infertility treatment can be appreciated from as yet unpublished data of the Israel Neonatal Network on very low-birth weight (VLBW) infants $(<1500 \mathrm{~g})$. The data indicate that roughly one-third of all VLBW infants are from multiple pregnancies, three-fourth of which are iatrogenic multiples (i.e., about one-fourth of all VLBW infants are iatrogenic multiples). Interestingly, the proportion of iatrogenic twins conceived by assisted reproductive technology (ART) to those conceived by ovulation induction increased over the period 1996-2005, but at the same time, this proportion decreased for iatrogenic triplets.

\section{BIOLOGY}

Most human conceptions ( $>99.2 \%$ ) result from a single zygote [i.e., monozygotic (MZ)] following the fertilization of a single egg by a single spermatozoon. In the remaining cases, more than one egg is ovulated and fertilized, resulting in polyzygotic conceptions [DZ, trizygotic, etc.]. The polyzygotic conception occurs more often in taller, older, parous, heavier, and black women, pointing to a genetic predisposition. However, the exact mechanism whereby the ovary is naturally stimulated to release more than one egg per cycle is basically unknown. By contrast, all infertility treatments are associated with ovarian stimulation, multiple ripe ovarian follicles, polyovulation, and multiple conceptions.

The vast majority of $\mathrm{MZ}$ conceptions result in singleton birth. In only a small fraction of the cases $(0.4 \%$ of all natural conceptions), the zygote splits to form an MZ twin gestation. Available hypotheses proposed to explain the mechanism of zygotic splitting fail to explain why MZ twins are more prevalent after all methods of assisted reproduction (3) and which structure is likely to control this phenomenon. It was hypothesized (4) that a small proportion of oocytes might have an inborn propensity to undergo splitting upon fertilization leading to the constant prevalence of spontaneous MZ twins among different populations. Ovarian stimulation-the common denominator of all infertility treatments-would then predictably increase the number of available splitting-prone oocytes and as a result would increase the chance for such oocytes to develop upon fertilization into MZ twins. An intact zona pellucida seems to be required to control embryonic division into two distinct cell lines and to prevent ill-timed hatching. Human fertilized oocytes are able to undergo binary fissions, just as in the nine-banded armadillo (the only other mammal that produces $M Z$ quadruplets), and to produce a variety of 


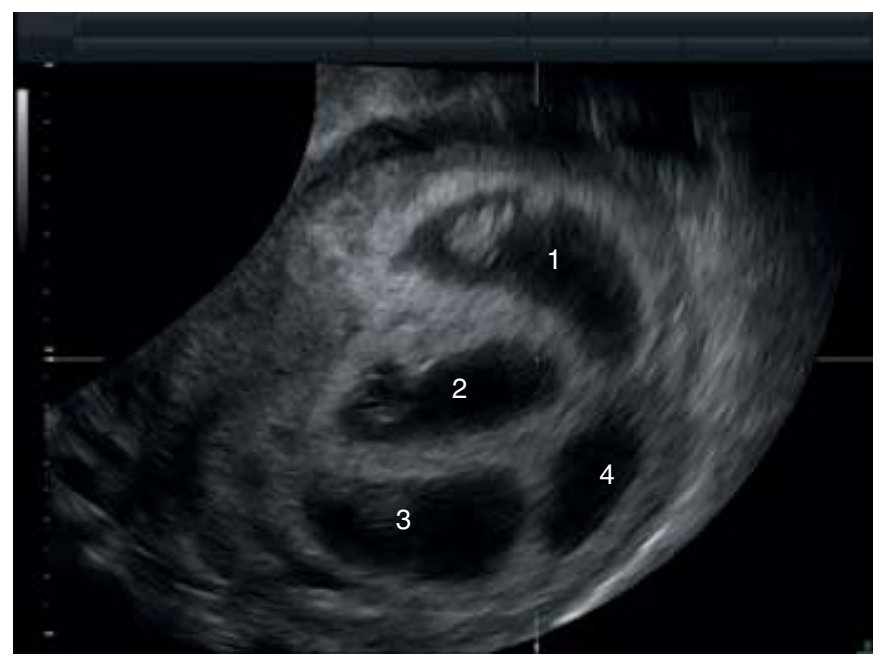

Figure 1 Quadrochorionic quadruplets. This first-trimester scan demonstrates the thick inter-twin membranes (several lambda signs).

combinations of MZ pregnancies. Importantly, this hypothesis does not negate previous genetically sound hypotheses, but places them into a broader perspective in light of observations from modern infertility treatment (4).

The increased frequencies of iatrogenic multiples changed the proportion of $\mathrm{DZ}$ and $\mathrm{MZ}$ twinning. In a population comprising mainly spontaneous gestations, the usual quoted frequency of $\mathrm{MZ}$ twinning is about one-third of the twin population, whereas in a population comprising a large proportion of iatrogenic conceptions, the expected $\mathrm{MZ}$ rate is 1 in 10 to 14 twin gestations.

\section{ZYGOSITY, CHORIONICITY, AND PLACENTATION}

DZ twins have two placentas (separate or fused), each with its chorion and amnion, forming the so-called dichorionic (DC) placenta. The inter-twin membrane comprises four layers (one chorion and one amnion from each twin) and usually appears as a thick septum during the optimal time of sonographic diagnosis at 11 to 14 weeks of gestation (Fig. 1). However, the triangle of decidual tissue between the reflections of the membranes, known as the "lambda" sign, is more easily identified and is pathognomonic of poly-chorionicity (Fig. 1). After birth, a DC placenta is confirmed by simply counting the placental discs and/or the number of layers in the inter-twin membrane.

MZ placentation, however, is presumably depended on the stage of embryonic development at which the split occurs. Early splits (about one-third) would result in DC placentas, whereas later splits result in monochorionic (MC) placentas. Moreover, if the amnion has not yet differentiated, the MC placenta includes two amniotic sacs: the MC-diamniotic placenta (about two-thirds of the cases). If the split occurs after differentiation of the amnion (i.e., $>8$ days after fertilization), an MC-monoamniotic placenta develops. Finally, even later splits are believed to result in all varieties of conjoined twins.

The sonographic diagnosis of an MC placenta is usually initiated by the absence of sonographic signs of DC placentation. In such a case, one should look for the tau sign, i.e., reflection of a thin inter-twin membrane (Fig. 2). If a firsttrimester sonographic diagnosis is not available, a subsequent

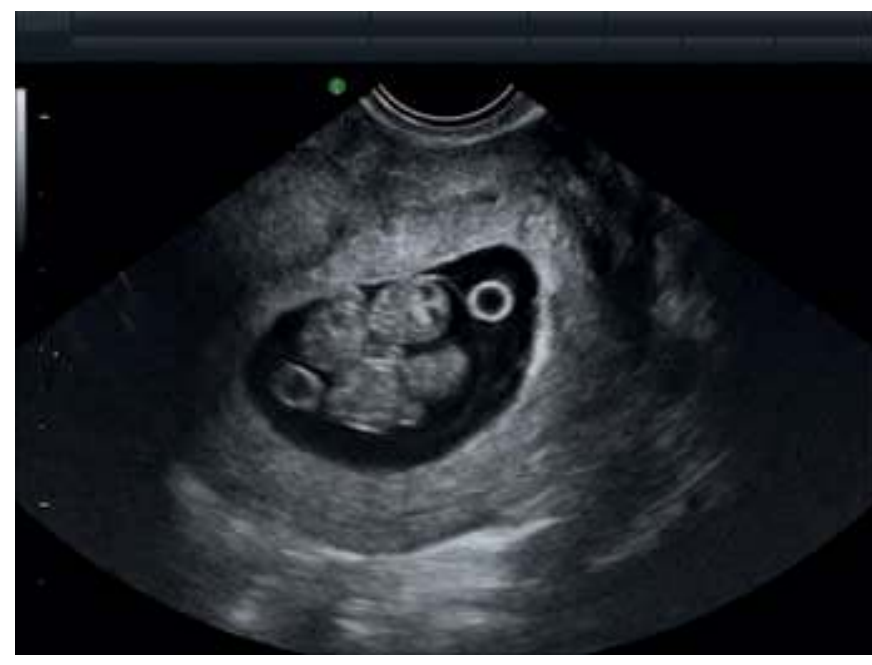

Figure 2 Monochorionic twins. At this stage, the twins are very close to each other and an inter-twin membrane is not visualized.

scan might become less accurate, but a methodical assessment of chorionicity is nevertheless required.

The early diagnosis of chorionicity does not require hightech imaging and is of utmost clinical importance. At times, however, complex cases of high-order multiples and/or illdefined chorionicity-amnionicity arrangements might be a diagnostic challenge.

A distinction should always be made between zygosity and chorionicity. Because MZ twins with a DC placenta cannot be differentiated clinically from same-sex DZ twins (half of all DZ pairs) who also have a DC placenta, zygosity can be determined with certainty only in the DC-unlike-sex twins (all must be DZ twins) and in twins with an MC placenta (all must be MZ pairs). Simple calculation reveals that we are blind to zygosity in about $45 \%$ of the cases, and zygosity determination must be performed by DNA testing. Importantly, nothing should be said about zygosity to parents of same-sex twins with a DC placenta.

\section{THE FIRST-TRIMESTER SONOGRAPHY}

As suggested in Figures 1 and 2, a "window of opportunity" exists between 10 and 14 weeks for the best sonographic assessment of chorionicity. Admittedly, not all first-trimester pregnancies are scanned and therefore early losses of one or more embryos of a multiple pregnancy are missed (the so-called vanishing twin syndrome, VTS) and the true incidence of multiples is thus underestimated. The firsttrimester scan should count the number of gestational sacs, the number of embryos, and the chorionic and amniotic arrangements. Ideally, the relative position of each embryo should be noted in a general "map" of the pregnancy, designating each embryo/fetus as being right/left, upper/lower, etc. Such "mapping" seems useful to provide and enhance some form of in utero individualized follow-up of the multiples.

Although each member of the multiple gestations has the same maternal-age-dependent risk for chromosomal anomalies, pure probability calculations show that the risk for a mother that one of her twins will have trisomy 21 is greater than that of a mother of a singleton. Roughly, this risk is 
increased by $5 / 3$, thus a 32 -year-old mother of twins has the same risk of one infant with trisomy 21 as a 35 -year-old mother of a singleton (5-7).

Because multiples are more commonly seen in older mothers (5) and invasive cytogenetic procedures (amniocentesis or chorionic villus sampling) carry a greater risk of pregnancy loss when performed in multiples, there is a genuine utility to noninvasive screening of aneuploidy to minimize the need for invasive procedures in these premium pregnancies (7). Regrettably, screening tests like the triple test [second-trimester maternal serum human chorionic gonadotropin (hCG) or free beta-hCG, alpha-fetoprotein, and unconjugated estriol] have a significantly lower predictivity for trisomy 21 in multiples compared with singletons (7). Thus, sonographic screening by measuring nuchal translucency (NT) thickness in each fetus should be offered to women who require genetic counseling. Flow in the ductus venosus (DV) might be measured to reduce the false-positive rate associated with NT measurement.

\section{MATERNAL CONSEQUENCES}

The changes in women's role in Western societies witnessed after World War II were facilitated by effective contraception, allowing ample time to achieve education and a career. This, in turn, resulted in increased maternal age at first delivery. Regrettably, age and fecundity are inversely related and infertility treatment to achieve a pregnancy often becomes inevitable for the older woman. Because all infertility treatments carry an increased risk of multiple gestations, the end result of these socio-medical trends is an increased age of the cohort of mothers of multiples. U.S. data clearly show that the increase in maternal age is more marked in high-order multiple pregnancies than in twins and in twins than in singletons, with a net result of multiples being more often delivered to older mothers in whom chronic disease conditions have already accumulated (5).

It goes without saying that a multiple pregnancy overwhelms the maternal homeostasis. Considering that the average fetus has a similar birth weight until 28 weeks (around $1000 \mathrm{~g}$ ) irrespective of plurality, it appears that by 28 weeks, the mother of twins and the mother of triplets has accumulated twice and three times the fetal mass of singletons, respectively. This excess of fetal mass comes from either existing maternal resources or from supplemental energy that must be added throughout early gestation. It is also clear why all maternal systems are overwhelmed and some may be only a step away from clinical insufficiency in the advanced multiple pregnancy.

The overwhelmed maternal homeostasis is vividly demonstrated by the increased frequency of clinically significant anemia as a result of either depleted maternal iron stores or inadequate iron supplementation. Also the increased cardiac output has been estimated to increase in certain clinical situations to as much as two to three times the normal value. It is therefore understandable why cardiac function so easily turns into dysfunction when additional load, such as betasympathomimetic tocolysis, fever, premature contractions, and so on, is created.

Regardless of the inherent changes in maternal physiology due to the multiple pregnancy, some maternal disease conditions are more frequent in multiple gestations. The best example is the two to three times increased incidence of hypertensive disorders $(8,9)$ and their most dangerous complication-eclampsia—is six times more frequent among mothers of multiple gestations (10). Moreover, pre-eclampsia occurs earlier in multiples than in singletons and often occurs in a more severe form. It appears that a plurality-dependent risk of pre-eclampsia exists. For example, with the current epidemic dimensions of multiple gestations, it has been shown that the risk of hypertensive disorders in triplets is higher than that in twins, and the risk in twins is higher than that in singletons (8). In addition, numerous reports exist on the resolution of severe pre-eclampsia following intrauterine demise of a twin. Interestingly, the reason for the increased risk of hypertensive disorders among multiples is still unclear, but data seem to support an association with the increased placental mass, that is, hyperplacentosis $(8,11)$. At this point it is important to mention that several rare but potentially pre-eclampsia-related conditions such as acute fatty liver of pregnancy are also more frequent in multiple gestations and seem to demonstrate the plurality-dependent risk pattern.

The other hyperplacentosis-related condition-gestational diabetes-seems also to be increased among mothers of multiples. Critical reading of the literature suggests that most stimulation tests to detect glucose intolerance of various degrees showed a diabetogenic effect of multiple gestations, whereas demographic analyses failed to show increased rates of gestational diabetes (12).

However, the latter studies were conducted in the era before the epidemic of iatrogenic multiples and before the effect of older maternal age could be documented (12). It is also possible that in the multiple gestations, an equilibrium is created between the increased glucose levels produced by hyperplacentosis and the glucose pumping effect created by the growing mass of the multiples. It has been shown that the risk of gestational diabetes is also plurality dependent, as is the case for hypertensive disorders (13).

All mothers of multiples are at considerably greater risk of preterm labor and delivery. Quite often, preterm contractions with or without cervical changes necessitate tocolytic treatment. Many prophylactic measures, including progesterone, cervical sutures (cerclage), beta-sympathomimetics, bed rest, and hospitalization, were proposed to reduce the preterm birth rates, but failed to demonstrate a significant reduction of this common complication of multiple pregnancy. Nevertheless, expecting mothers of multiples are frequently asked to leave work and to conduct a more sedentary lifestyle. Two promising prophylactic measures are at an empirical stage: early maternal weight gain $(14,15)$ and further studies on progesterone derivatives (16). The former approach is based on the contention that multiple pregnancy is a state of magnified nutritional requirements (14). An adequate amount and pattern of gestational weight gain, including BMI-specific weight gain goals by 20 and 28 weeks of gestation, as well as other nutritional interventions, has been associated with significantly better fetal growth and longer gestations in twin pregnancies (14). In this context, it is unknown how many of normal or overweight woman will turn obese at the end of pregnancy and remain obese thereafter, and if the beneficial 
effect of weight gain is equally important in women with highcompared with low pre-gravid BMI (15).

Although the results of earlier trials were disappointing (16), many randomized controlled trials are currently ongoing to find the usefulness of progesterone and progestogens as prophylactic measures against preterm birth in multiples. This interest seems to suggest that many researchers believe that progesterone might have an effect on multiples as it has on singletons, but the best derivative, route of administration, and dose are still unknown.

Table 1 lists the most common maternal complications during multiple gestations (17).

\section{FETAL NEONATAL CONSEQUENCES}

All animals, including the Homo sapiens, demonstrate an inverse relationship between litter size and both gestational age and birth weight. In the human, the average gestational age at

Table 1 Maternal Complications More Frequently Seen in Multiple Pregnancies

Hypertensive diseases

- Pre-eclamptic toxemia

- HELLP syndrome

- Acute fatty liver

- Pregnancy-induced hypertension

- Chronic hypertension

- Eclampsia

Anemia

Gestational diabetes mellitus (?)

Premature contractions and labor

- Complications associated with tocolysis

Delivery-associated complications

- Cesarean section

- Operative delivery

- Premature rupture of membranes

- Postpartum endometritis

- Placental abruption

Abbreviation: HELLP, hemolysis, elevated liver enzymes, low platelets.

\section{Table 2 Categories of Structural Defects in Twins}

$\begin{array}{ll}\begin{array}{l}\text { Category } \\ \text { Malformations more } \\ \text { common in twins than } \\ \text { in singletons }\end{array} & \begin{array}{l}\text { Neural tube defects } \\ \text { Hydrocephaly } \\ \text { Congenital heart disease } \\ \text { Esophageal and anorectal atresias } \\ \text { Intersex } \\ \text { Genitourinary tract anomalies }\end{array} \\ & \begin{array}{l}\text { Amniotic band syndrome } \\ \text { TRAP sequence }\end{array} \\ \text { Malformations unique to } & \begin{array}{l}\text { Conjoined twins } \\ \text { monozygotic twins }\end{array} \\ & \begin{array}{l}\text { Twin embolization syndrome } \\ \text { Single umbilical artery }\end{array} \\ \text { Placental malformations } & \text { Twin-twin transfusion syndrome } \\ & \text { Velamentous cord insertion } \\ & \text { Skeletal (postural) abnormalities } \\ \text { Deformations due to } & \\ \text { intrauterine crowding } & \\ \text { Abbreviation: TRAP, twin reverse arterial perfusion. Source: Adapted from } \\ \text { Ref. 18. }\end{array}$

birth is around 40 weeks for singletons, 36 weeks for twins, 32 weeks for triplets, and 29 weeks for quadruplets. Although multiple pregnancies exhibit many specific complications, the consequences of prematurity are by far the most common and are the most important public health concern.

\section{Malformations}

It is customary to cite a two- to threefold increased risk of malformations among multiples. Arguments against this view suggest that twins are more closely examined and therefore a selection bias exists when describing malformations in a twin population. Regardless, the increased risk seems to be primarily related to $\mathrm{MZ}$ twinning whereas the malformation rates of DZs are similar to that of singletons (18). The higher malformation rate among MZs is hypothetically related to a common teratogen: the one that causes the split of the zygote is also responsible for the malformation.

Malformations among multiples are commonly grouped into four types (Table 2) (18). The first includes malformations that are more frequent among multiples, in particularly those affecting the central nervous and cardiovascular systems. The second type involves malformations related to MZ twinning such as twin reverse arterial perfusion (TRAP) sequence and the various forms of conjoined twins. The third type relates to consequences of placental malformations, in particular the MC placenta, resulting in twin-twin transfusion syndrome (TTTS) and/or selective severe discordance. Finally, the fourth type involves skeletal (postural) deformities such as clubfoot and dolichocephaly that are caused by intrauterine fetal crowding.

Certain malformations might have a major impact on the normal co-twin. For instance, in the TRAP sequence, the circulation of the severely anomalous acardiac-acephalic twin is entirely supported by the normal (pump) twin. Sooner or later, this cardiac overload will lead to cardiac insufficiency. Similarly, both twins in the TTTS are completely normal but the anomalous transplacental shunt of blood might cause serious morbidity in both twins. The most striking example is the case of single fetal demise in MC twins, whereby the surviving fetus frequently dies or becomes seriously damaged in utero soon after the death of the first twin.

Most structural anomalies can be detected by a comprehensive sonographic scan aided by echocardiography and Doppler velocimetry. Once a serious anomaly is detected, the patient might be counseled and offered selective reduction of the anomalous twin. In multichorionic multiples, selective reduction is accomplished by ultrasound-guided intracardiac injection of potassium chloride. However, because of the risk to the survivor in MC sets, highly invasive procedures (selective cord occlusion) are used to interrupt the umbilical circulation of the anomalous twin. When indicated and permitted, selective termination of an anomalous twin in an MC set is best accomplished by bipolar cord occlusion. At times, the umbilical cord of the twin affected by the TRAP sequence is too edematous and an intrafetal radiofrequency technique might be used.

Because of the increased frequency of fetal malformations, all MC twins, and ideally all twins, should undergo a detailed anatomical scan at 18 to 22 weeks of gestation. If the cardiac assessment shows a normal "four-chamber" view and outflow 
tract, it is believed that an anatomical echocardiography seems unnecessary.

All invasive procedures in multiple pregnancies (amniocentesis, chorionic villus sampling, and the reduction methods) are associated with the risk of $5 \%$ to $10 \%$ of membrane rupture and loss of the entire pregnancy. When an invasive procedure is considered during the second trimester, the risk of extremely preterm birth of the normal twin is apparent. Thus when one twin has a lethal anomaly such as anencephaly, the risk of reducing this twin should be weighed against the risk of endangering the normal fetus by preterm birth. Conversely, the risk of preterm delivery because of a large polyhydramnios surrounding an anomalous twin might be reduced after selective reduction.

\section{Embryonic and Fetal Demise}

From the early days of ultrasound, it was apparent that there are more twin pregnancies than twin deliveries. The early loss of one twin, the VTS, is considered by many as the natural equivalent of intentional multifetal pregnancy (numerical) reduction (MFPR). Obviously, the true frequency of VTS is unknown, as many twin pregnancies are unnoticed unless first-trimester sonography is performed (19). One estimate of VTS frequency comes from iatrogenic conceptions. Spontaneous reduction of one or more gestational sacs or embryos occurred before the 12th week of gestation in 36\% of twin, $53 \%$ of triplet, and $65 \%$ of quadruplet pregnancies (20). Dickey also pointed out that spontaneous reduction of multiple gestational sacs occurs less frequently in iatrogenic pregnancies compared with spontaneously conceived multiples (21). La Sala and his co-workers (22) as well as Matias and her associates (23) demonstrate that loss rates of twin pregnancies after assisted reproduction have a two to five times lower miscarriage rate of the entire pregnancy compared with singletons. The source of this advantage of ART twins is unknown. Importantly, Pinborg et al. found that 1 in 10 in vitro fertilization (IVF) singletons originates from a twin gestation and is one reason for the higher risk of adverse obstetric outcome in IVF singletons (24).

Single fetal death beyond the first trimester is also said to be more common in multiples. In DC twins, it is believed that the risk to the surviving twin is extremely low and present only when an external insult such as maternal disease is the cause of death. By contrast, fetal death in MC twins is entirely different. In the past, some ill-defined thromboplastinlike material was assumed to be transfused from the dead to the live fetus-leading to the so-called twin embolization syndrome. The theory was that these emboli might either cause fetal death or result in end-organ damage, such as brain and kidney lesions. In the early 1990s, and following painstaking postmortem examinations of brains of survivors of the twin embolization syndrome, the embolic theory was abandoned because no emboli were found and the lesions were rather ischemic in nature.

The embolic theory was soon replaced by the ischemic theory, which postulates that blood is acutely shunted from the live twin to the low-resistance circulation of the demised fetus, causing acute hypovolemia, ischemia, and end-organ damage in the survivor. A meta-analysis found that $12 \%$ of the cotwins die very soon after death of a twin, $18 \%$ survive but with a significant damage, and 70\% enjoy an intact survival (25). Death of a co-twin in an MC pair is rarely noticed in real time. However, when singe fetal death is a recent event (e.g., after laser photocoagulation), the peak systolic velocity of the middle cerebral artery (PSV-MCA) might demonstrate acute fetal anemia (associated with other signs of fetal anemia). In such a case, prompt delivery is a valid option when gestational age permits. Alternatively, percutaneous umbilical cord (rescue) blood transfusion can be offered. However, it is unknown whether rescue transfusion actually decreases brain damage because not enough evidence exists to suggest improving outcomes after such procedures. When the timing of fetal death is unknown, as is more often the case, the diagnosis of single fetal death is made some time after the event. Data indicate that acute blood loss probably occurs just before the time of death of the surviving twin, and therefore it is unlikely that immediate delivery of the surviving twin could decrease the associated high mortality and morbidity rates associated with severe premature birth (26). It is therefore prudent to suggest conservative management in such cases, especially remote from term, and to use ultrasound and magnetic resonance imaging (MRI) to exclude brain lesions. The latter is ideally performed after the end of neuromigration at 30 to 32 weeks, unless diffusion weighted MRI, where available, might demonstrate white matter lesions earlier after the event.

\section{Twin-Twin Transfusion}

One of the most interesting consequences of MC twinning is the TTTS. TTTS is seen mainly (or only) in the diamniotic variety. The extensive literature on TTTS may lead to the erroneous impression that the syndrome is very frequent. In fact, TTTS occurs in about $10 \%$ of MC twins, and about half are of mild severity. Nonetheless, early onset (before 20 weeks of gestation) severe TTTS, unless intensively treated, is associated with $100 \%$ mortality of both twins.

It is a transplacental arteriovenous anastomosis that enables blood shunting from one twin (the donor) to the other (the recipient).Since all MC placentas have inert-twin anastomoses, the syndrome probably occurs because of fewer compensating veno-venous and arterio-arterial connections. The hypovolemia of the donor is manifested by poor micturition (absent bladder and oligohydramnios on sonographic scan). Conversely, the hypervolemic recipient is surrounded by polyhydramnios and manifests signs of cardiac overload ranging from tricuspid regurgitation to cardiac insufficiency and hydrops fetalis (27).

TTTS should be excluded on each antenatal visit of an MC pregnancy by demonstrating adequate amount of amniotic fluid. By definition, the first sign of TTTS is the twin oligopolyhydramnios sequence (TOPS) corresponding to combination of largest pocket $>8 \mathrm{~cm}$ and $<2 \mathrm{~cm}$ in the respective sacs before 20 weeks and $>10 \mathrm{~cm}$ and $<2 \mathrm{~cm}$ after 20 weeks. TOPS in MC twins is defined a Quintero's stage I and should initiate a series of targeted sonographic evaluations, including Doppler velocimetry of the umbilical artery, DV, and middle cerebral artery (MCA-PSV).

In general, TTTS means serious morbidity, but the specific outcome is related to the gestational age when TTTS developed and to the severity of the syndrome. Quintero's stages are 
useful in comparing management of TTTS. At present, selective laser photocoagulation of the inter-twin anastomoses is the preferable treatment modality and should be performed in TTTS occurring between 16 and 28 weeks of gestation. A decision of "no treatment" after 28 weeks would thus invariably lead to very preterm delivery of affected twins and it seems just logical that preterm delivery of treated TTTS might be better than delivering an untreated pair with TTTS. However, laser interventions after 28 weeks are still at an investigational stage. Selective laser photocoagulation, when available, is indicated in Quintero's stage II (invisible urinary bladder), stage III (pathologic umbilical artery Doppler values), and stage IV (hydrops fetalis). Selective laser photocoagulation might also be indicated in "symptomatic" stage I (TOPS, with uterine contractions and/or short cervix). Alternatively, Quintero's stage I might be followed very closely, because a proportion of stage I cases do not progress and remain asymptomatic. Induction of labor is reasonable in cases with Quintero's stage V and a double fetal death, whereas follow-up of the survivor seems appropriate after a single fetal demise.

Cervical length before selective laser photocoagulation bears a negative correlation with outcome following the procedure and should be considered during preoperative counseling.

Amnioreduction (amniodrainage), that is, removal of a large quantity of amniotic fluid, was used to treat symptomatic mothers suffering from respiratory problems related to TOPS. The best technique regarding how fast should the amniotic fluid be drained, how much fluid should be removed, and how frequent should the technique be repeated is not established.

Since it is currently unknown how amnioreduction works, many hold that this procedure should be reserved to symptomatic patients only and for indications such as refusal or when laser photocoagulation is not available, when laser photocoagulation is contraindicated or technically difficult to perform, or after 28 weeks of gestation in order to buy time to increase gestational age. Amnioreduction might impede laser photocoagulation thus decreasing success rates and should not be performed as an interval procedure. Other treatment methods such as creating a hole in the inter-twin septum (septostomy) are not indicated for the treatment of TTTS.

\section{Discordance in MC Twins}

In the classic definition of TTTS (28), the twins are supposed to be discordant in size (at least 20-25\%) and in hemoglobin levels (at least $5 \mathrm{~g} / \mathrm{dL}$ ). This concept changed over time, and at present, neither hemoglobin difference nor size discordance is part of the definition of TTTS. At times, however, a difference in hemoglobin is present-the so-called twin anemiapolycythemia sequence. The anemic twin can manifest all signs of anemia, especially by measuring the MCA-PSV. At birth, the donor is usually pale and anemic, whereas the recipient is polycythemic. The donor twin might be acutely distressed, with severe anemia and hypovolemic shock necessitating transfusion or exchange of blood products, or both. The recipient occasionally requires partial dilution exchange and support for cardiac failure.

Size discordance, however, seems to develop more slowly. When a significant size disparity exists, the smaller twin is at risk of intrauterine death. At present, follow-up with serial Doppler velocimetry of the umbilical artery is indicated. Three patterns are recognized: type I, normal (positive diastolic) Doppler flow; type II, absent/reversed-end diastolic flow; and type III, intermittent absent/reversed-end diastolic flow-in the same or in the subsequent sessions $(29,30)$. Deterioration of the growth-restricted fetus occurred in 90\% of type II cases, significantly higher compared with $0 \%$ and $10.8 \%$ of types I and III, respectively.. Unexpected intrauterine fetal death of the smaller twin occurred in $15.4 \%$ of type III cases, significantly higher compared with $2.6 \%$ and $0 \%$ of types I and II respectively. And finally, parenchymal brain lesions in the larger twin were observed in $19.7 \%$ of type III cases and significantly less than $5 \%$ in the other groups (30).

\section{Fetal Growth}

As noted earlier, multiples grow in utero to the same extent as singletons until about 28 weeks. Thereafter, during the third trimester, a decelerating trend of the growth curves of multiples is clearly seen. Not surprisingly, the limited uterine capacity to nurture multiples leads to growth aberrations (31). The higher risk of delivering low-birth weight (LBW) infants in a multiple birth is well known, as is the advantage for the multiparous patient. For instance, analysis of populationbased data related to 12,567 live-born twin pairs found that, overall, the risk of having at least one very LBW (VLBW, $<1500 \mathrm{~g}$ ) infant was 1:5 among nulliparous women and 1:12 among multiparous women. The risk of having two VLBW twins among nulliparas (1:11) was double that of multiparas (1:22) (32). A similar trend and similar frequencies, but for extremely LBW $(<1000 \mathrm{~g})$ babies, were found in the analysis of triplets (33).

The most common growth aberration in multiples is birth weight discordance, defined as a difference between the larger and the smaller infant of a multiple pregnancy set. In fact, it is quite rare to find that all members of the set have the same birth weight, and it is quite normal to find some variation between siblings. Discordance is usually defined by the percent difference, whereby the birth weight disparity is calculated as a percentage of the larger infant. However, this definition does not refer to the actual size of the twins, and the same degree of discordance (e.g., 20\%) can be found in a twin pair weighing 1500 and $1200 \mathrm{~g}$ as well as in a pair weighing 3000 and $2400 \mathrm{~g}$. The cumulative frequency (34) suggests that about $75 \%$ of twins exhibit less than $15 \%$ discordance, about $20 \%$ are $15 \%$ to $25 \%$ discordant, and about $5 \%$ are more than $25 \%$ discordant.

Birth weight discordance is even more complex in triplets. In a new approach, in which the relative birth weight of the middle triplet was defined, the set was considered symmetrical, low, or high skew (35). The frequencies of different types of triplet discordance (average values: 57\%, 30\%, and 13\%, respectively) did not change with gestational age, suggesting three distinct types of discordant growth in triplets that are independent of gestational age (35).

Several important related issues should be considered. First, it has been determined that at lower levels of discordance, either twin can be the smaller but the likelihood of the secondborn twin being the smaller increases with increasing discordance levels (36). At discordance levels greater than 
$25 \%$, the smaller twin was three to six times more often the second born (36). Data also suggest that there are three levels of discordance whereby in the lowest levels (probably at less than $25 \%$ ), discordance seems to be related to the normal variation expected from the natural dissimilarities between siblings whereas in the highest level (probably greater than $35 \%$ ), discordance seems to be related to the exhausted uterine environment and reflects growth restriction (37). Consequently, observation or intervention is indicated for the lowest and highest degrees of discordance, respectively.

Mortality is 11 times higher among highly discordant smaller twins $(>30 \%)$ compared with nondiscordant smaller twins. Risk estimates ranged from 1.1 among 15\% to 19\% discordant twins to 2.0 among highly discordant twins. After accounting for the association between fetal growth and discordance, mortality risk was substantially higher among smaller and larger twins who were highly discordant (30\% or more) (38). After controlling for fetal growth, smaller and larger twins affected by higher levels of birth weight discordance ( $>25 \%)$ remain at disproportionate risk for neonatal mortality (38). Finally, when neonatal mortality rates were compared among three groups of discordant twins $(>25 \%)$, distinguished by the birth weight of the smaller twin being in the lowest 10th percentile [i.e., small for gestational age (SGA), 62.4\%], in the 10 th to 50 th percentile $(32.9 \%)$, or above the 50 th percentile $(4.7 \%)$, the rate was significantly higher among pairs in which the smaller win's birth weight was in the lowest 10th percentile (29\% vs. 11.1\% and 11/1000) (39). This difference results from the higher mortality rates among the smaller but not among the larger twins. Thus, in severely discordant twin pairs, about $40 \%$ do not comprise a growth-restricted fetus and identification of this group is an imperative step in the management of birth weight discordance in twin gestations and in avoiding unnecessary interventions that may lead to iatrogenic prematurity.

Controversy exists as to whether to use singleton or twin growth curves for assessing multiple pregnancies. Because most multiple pregnancies weigh less than singletons of the same gestational age, it seems that multiples grow differently than singletons do and therefore are frequently and erroneously defined as SGA by singleton standards. Before birth, sonographic assessment of individual fetal growth cannot establish a pattern of growth restriction unless the pregnancy is longitudinally followed by repeated scans. In addition, despite the relative accuracy of sonographic estimations of the individual fetal weight, the "plus or minus" situation that exists for each estimation can cause significant under- and overestimation of the weight difference between fetuses and to low positive predictive values of discordance (40).

Growth restriction in twins - genuine or relative-is usually managed conservatively unless signs of fetal compromise are seen. At times, especially with higher levels, discordant growth and discordant fetal well-being go hand in hand. Remote from term, a decision to save the ailing fetus is in clear conflict with the potential risks of extreme preterm birth imposed on the healthy fetus. Thus, whenever the clinical circumstance of one twin jeopardizes the other, care should be exercised to select a management plan that would optimize the outcome of both fetuses. The pregnant woman should be involved in such decision making. Obviously, when the risk for the unaffected neonates is lower than the expected risk for the affected fetus, delivery is a clear option, as would be the case at greater than 32 weeks.

\section{FETAL ASSESSMENT}

Fetal surveillance before and during labor should be carried out on all fetuses. It follows that each fetus should be adequately and appropriately monitored. In fact, assessment in multiples is no different from assessment in singletons, although it might be more complicated. For instance, fetal heart rate should be traced for both twins at the same time and intrapartum dual tracing is as important as during pregnancy. Once the membranes are ruptured, the presenting twin might be traced with a scalp electrode while the nonpresenting twin is followed with an external Doppler electrode. The fetal biophysical profile is similarly assessed individually although the amniotic fluid volume is sometimes difficult to assess. Sophisticated technology, like Doppler velocimetry of the umbilical artery, DV, and MCA, three-dimensional or fourdimensional ultrasound, and MRI of the fetal brain should be reserved for special indications.

\section{DELIVERY CONSIDERATIONS}

Most twins and practically all high-order multiple pregnancies go into spontaneous labor before 38 weeks of gestation. Circumstantial evidence suggests that, at least for twins, delivery after 37 completed weeks might carry an increased risk of neonatal mortality (41) and morbidity (42), and that fetal systems of the multiple pregnancy are mature by this date.

Many reasons for a cesarean section could be indicated in most twins and all high-order multiple pregnancies (43). Because twin gestations often involve some complications and are considered "premium" pregnancies, many clinicians would deliver twins by cesarean section for many subtle reasons other than clear-cut, evidence-based indications. Vaginal birth is allowed in twins whenever the first twin is in vertex presentation. Breech delivery of the second twin or internal podalic version of a transverse-lying second twin is still permitted and clinicians await a Canadian randomized trial initiated in 2004, to evaluate the safety of such births. Otherwise, all pairs with a nonvertex presenting twin are likely to undergo a cesarean section. Nuances on this construct consider fetal size, discordance, prior uterine surgery, andmainly-the experience and dexterity of the obstetrician. Delivery considerations should include the welfare of all fetuses and the mode of delivery should be based on medical considerations pertaining to each fetus, as well as on maternal health and preferences. Delivery of multiples should ideally take place in a center that is equipped with neonatal intensive care facilities available simultaneously for each infant, where a medical care provider certified in neonatal resuscitation is present for each neonate, and with facilities for a high-risk delivery (such as availability of an experienced obstetrician, 24-hour anesthesia coverage, blood availability, etc). If this is not possible, in utero transfer may be preferable to postpartum transfer.

When a multiple birth is expected, the main neonatal problem is logistic, rather than medical, because immediate neonatal treatment of an infant of a multiple pregnancy is not significantly different from treating a singleton, except that 
twins come in pairs, and triplets come in sets of three. In practical terms, this means more staff in the delivery suite, more cribs available in the nursery, and more stations ready in the neonatal intensive care unit (NICU). At times, delivery of several very preterm sets in a short period may occupy the entire NICU for a relatively long period. It is clear that if the availability of NICU cribs lags behind the increased production of multiples, a serious public health situation may be created.

\section{OUTCOME}

Given the much-increased risk of maternal and fetal complications during a multiple pregnancy, the overall outcome for multiples is worse compared with the outcome for singletons. For example, the increased risk of cerebral palsy among multiples is clear: 28 to 45 for triplets, 7.3 to 12.6 for twins, and 1.6 to 2.3 for singletons per 1000 survivors, indicating that the greater the number of fetuses, the greater the prevalence of cerebral palsy. Moreover, the increase in cerebral palsy with the number of fetuses seems to be exponential (44). It is however unclear whether outcomes of multiples are poorer than outcomes of singletons matched for birth weight or gestational age. For example, the usual prophylactic dose of corticosteroids given to singletons does not have the same effect in multiples in terms of prevention of neonatal respiratory distress syndrome and intraventricular hemorrhage $(45,46)$. A multivariate logistic regression analysis of a cohort of VLBW infants with similar background characteristics was done on 3717 singletons, 1394 twins, and 483 triplets. Respiratory distress syndrome was significantly more common in twins and triplets despite increased exposure to antenatal steroids (47). In addition, VLBW triplets were at increased risk of death, but VLBW twins and triplets had no increased risk of chronic lung disease or adverse neurologic findings compared with matched singletons (47).

\section{PREVENTION VS. CURE}

The epidemic dimensions of multiple births, and especially of high-order multiple pregnancies, became evident toward the end of the 1980s as an aftershock of effective infertility treatment. To amend this untoward consequence of infertility treatment, clinicians proposed to reduce the number of embryos during pregnancy (MFPR) (48). MFPR, considered by many as the ultimate paradox of medicine, soon became a popular "cure" of the side effects of infertility treatment. This procedure, performed during the early second trimester via the transvaginal or transabdominal route, carries a risk of about $5 \%$ to $8 \%$ total loss. However, MFPR is certainly associated with better outcomes simply because a lower plurality will always do better than a higher plurality pregnancy.

Interestingly, and somewhat disturbingly, clinicians were more busy in refining and mastering the technique of MFPR than in trying to reduce the number of iatrogenic high-order multiples. At present, it is believed that MFPR should be seriously considered with quadruplets or more and should rarely be considered with twins. The controversy remains regarding MFPR of triplets (49).

As always in medicine, prevention is better than cure. In terms of infertility treatment, this means transferring only one embryo in ART and canceling ovulation induction cycles when more than one ripe follicle is visualized. Such preventive measures might somewhat reduce the overall success rates, although it is rarely debatable if a multiple pregnancy with three or four severely premature infants constitutes success. Put differently, the author of this chapter truly believes that if single-embryo transfer would have been the standard from the outset, it would never be possible to obtain ethical approval of a randomized trial for more than one embryo transfer.

\section{REFERENCES}

1. Blickstein I, Keith LG. Iatrogenic multiple pregnancy. Semin Neonatol 2002; 7: 169-76.

2. Blickstein I, Keith LG. The decreased rates of triplet births: temporal trends and biologic speculations. Am J Obstet Gynecol 2005; 193: 327-31.

3. Blickstein I, Jones C, Keith LG. Zygotic splitting rates following single embryo transfers in in-vitro fertilization. N Engl J Med 2003; 348: 2366-7.

4. Blickstein I, Keith LG. On the possible cause of monozygotic twinning: lessons from the 9-banded armadillo and from assisted reproduction. Twin Res Hum Genet 2007; 10: 394-9.

5. Blickstein I. Motherhood at or beyond the edge of reproductive age. Int J Fertil Womens Med 2003; 48: 17-24.

6. Meyers C, Adam R, Dungan J, Prenger V. Aneuploidy in twin gestations: when is maternal age advanced? Obstet Gynecol 1997; 89: 248-51.

7. Matias A, Montenegro N, Blickstein I. Down syndrome screening in multiple pregnancies. Obstet Gynecol Clin North Am 2005;32: 81-96.

8. Skupski DW, Nelson S, Kowalik A, et al. Multiple gestations from in vitro fertilization: successful implantation alone is not associated with subsequent preeclampsia. Am J Obstet Gynecol 1996; 175: 1029-32.

9. Sibai BM, Hauth J, Caritis S, et al. Hypertensive disorders in twin versus singleton gestations. National Institute of Child Health and Human Development Network of Maternal-Fetal Medicine Units. Am J Obstet Gynecol 2000; 182: 938-42.

10. Knight M, UKOSS. Eclampsia in the United Kingdom 2005. BJOG 2007; 114: 1072-8.

11. Bdolah Y, Lam C, Rajakumar A, et al. Twin pregnancy and the risk of preeclampsia: bigger placenta or relative ischemia? Am J Obstet Gynecol 2008; 198: 428.e1-6.

12. Hazan Y, Blickstein I. Diabetes and multiple pregnancies. In: Hod M, Jovanovic L, Di Renzo GC, De Leiva A, Langer O, eds. Textbook of Diabetes and Pregnancy, 2nd edn. London: Martin Dunitz, 2009: 338-42.

13. Sivan E, Maman E, Homko CJ, et al. Impact of fetal reduction on the incidence of gestational diabetes. Obstet Gynecol 2002; 99: 91-4.

14. Luke B. Nutrition and multiple gestation. Semin Perinatol 2005; 29: 349-54.

15. Flidel-Rimon O, Rhea DJ, Shinwell ES, Keith LG, Blickstein I. Early weight gain does not decrease the incidence of low birth weight and small for gestational age triplets in mothers with normal pre-gestational body mass index. J Perinat Med 2006; 34: 404-8.

16. Rouse DJ, Caritis SN, Peaceman AM, et al.; National Institute of Child Health and Human Development Maternal-Fetal Medicine Units Network. A trial of 17 alpha-hydroxyprogesterone caproate to prevent prematurity in twins. N Engl J Med 2007; 357: 454-61.

17. Blickstein I, Keith LG. Multi-fetal gestations. In: Gronowski A, ed. Handbook of Clinical Laboratory Testing During Pregnancy. Totowa, NJ: Humana Press, 2004: 351-8.

18. Blickstein I, Smith-Levitin M. Multifetal pregnancy. In: Petrikovsky BM, ed. Fetal disorders: diagnosis and management. New York: John Wiley and Sons, 1998: 223-47.

19. Landy HJ, Keith LG. The vanishing twin: a review. Hum Reprod Update 1998; 4: 177-83.

20. Dickey RP, Taylor SN, Lu PY, et al. Spontaneous reduction of multiple pregnancy: incidence and effect on outcome. Am J Obstet Gynecol 2002; 186: 77-83.

21. Dickey RP. Embryonic loss in iatrogenic multiples. Obstet Gynecol Clin North Am 2005; 32: 17-27. 
22. La Sala GB, Nucera G, Gallinelli A, et al. Spontaneous embryonic loss following in vitro fertilization: incidence and effect on outcomes. Am J Obstet Gynecol 2004; 191: 741-6.

23. Matias A, La Sala GB, Blickstein I. Early loss rates of entire pregnancies after assisted reproduction are lower in twin than in singleton pregnancies. Fertil Steril 2007; 88: 1452-4.

24. Pinborg A, Lidegaard O, la Cour Freiesleben N, Andersen AN. Consequences of vanishing twins in IVF/ICSI pregnancies. Hum Reprod 2005; 20: 2821-9.

25. Ong SS, Zamora J, Khan KS, Kilby MD. Prognosis for the co-twin following single-twin death: a systematic review. BJOG 2006; 113: 992-8.

26. Nicolini U, Pisoni MP, Cela E, Roberts A. Fetal blood sampling immediately before and within 24 hours of death in monochorionic twin pregnancies complicated by single intrauterine death. Am J Obstet Gynecol 1998; 179: 800-3.

27. Wee LY, Fisk NM. The twin-twin transfusion syndrome. Semin Neonatol 2002; 7: 187-202.

28. Blickstein I. The twin-twin transfusion syndrome. Obstet Gynecol 1990; 76: 714-22.

29. Lewi L, Cannie M, Blickstein I, et al. Placental sharing, birthweight discordance, and vascular anastomoses in monochorionic diamniotic twin placentas. Am J Obstet Gynecol 2007; 197: 587.e1-8.

30. Gratacós E, Lewi L, MuÑoz B, et al. A classification system for selective intrauterine growth restriction in monochorionic pregnancies according to umbilical artery Doppler flow in the smaller twin. Ultrasound Obstet Gynecol 2007; 30: 28-34.

31. Blickstein I. Normal and abnormal growth of multiples. Semin Neonatol 2002; 7: 177-85

32. Blickstein I, Goldman RD, Mazkereth R. Risk for one or two very low birth weight twins: a population study. Obstet Gynecol 2000; 96: 400-2.

33. Blickstein I, Jacques DL, Keith LG. The odds of delivering one, two or three extremely low birth weight $(<1000 \mathrm{~g})$ triplet infants: a study of 3288 sets. J Perinat Med 2002; 30: 359-63.

34. Blickstein I, Kalish RB. Birth weight discordance in multiple pregnancy. Twin Res 2003; 6: 526-31.

35. Blickstein I, Jacques DL, Keith LG. A novel approach to intertriplet birth weight discordance. Am J Obstet Gynecol 2003; 188: 1026-30.
36. Blickstein I, Goldman RD, Smith-Levitin M, et al. The relation between intertwin birth weight discordance and total twin birth weight. Obstet Gynecol 1999; 93: 113-16.

37. Blickstein I, Goldman RD, Mazkereth R. Adaptive growth restriction as a pattern of birth weight discordance in twin gestations. Obstet Gynecol 2000; 96: 986-90.

38. Branum AM, Schoendorf KC. The effect of birth weight discordance on twin neonatal mortality. Obstet Gynecol 2003; 101: 570-4.

39. Blickstein I, Keith LG. Neonatal mortality rates among growth discordant twins, classified according to the birth weight of the smaller twin. Am J Obstet Gynecol 2004; 190: 170-4.

40. Blickstein I, Manor M, Levi R, Goldchmit R. Is intertwin birth weight discordance predictable? Gynecol Obstet Invest 1996; 42: 105-8.

41. Minakami H, Sato I. Reestimating date of delivery in multifetal pregnancies. JAMA 1996; 275: 1432-4.

42. Luke B, Bigger HR, Leurgans S, Sietsema D. The cost of prematurity: a case-control study of twins vs singletons. Am J Public Health 1996; 86: 809-14.

43. Blickstein I. Cesarean section for all twins? J Perinatol 2000; 28: 169-74.

44. Blickstein I. Fetal brain injury and multiple pregnancies. In: Levene MI, Chervenak FA, eds. Fetal and neonatal neurology and neurosurgery, 4th edn. Edinburgh: Churchill Livingstone, 2009: 375-84.

45. Blickstein I, Shinwell ES, Lusky A, Reichman B, Israel Neonatal Network. Plurality-dependent risk of respiratory distress syndrome among verylow-birth-weight infants and antepartum corticosteroid treatment. Am J Obstet Gynecol 2005; 192: 360-4.

46. Blickstein I, Reichman B, Lusky A, Shinwell ES, Israel Neonatal Network. Plurality-dependent risk of severe intraventricular hemorrhage among very low birth weight infants and antepartum corticosteroid treatment. Am J Obstet Gynecol 2006; 194: 1329-33.

47. Shinwell ES, Blickstein I, Lusky A, Reichman B. Excess risk of mortality in very low birth weight triplets: a national, population-based study. Arch Dis Child Fetal Neonatal Ed 2003; 88: F36-40.

48. Evans MI, Hume RF Jr, Yaron Y, Kramer RL, Johnson MP. Multifetal pregnancy reduction. Baillieres Clin Obstet Gynaecol 1998; 12: 147-59.

49. Blickstein I, Keith LG. Outcome of triplets and higher order multiple pregnancies. Curr Opin Obstet Gynecol 2003; 15: 113-17. 


\section{Gestational hypertension and pre-eclampsia Erol Amon and Erin Dickert}

Hypertensive disorders are the most common medical complications of pregnancy. Approximately $7 \%$ to $10 \%$ of all pregnancies are complicated by hypertension. The two most common forms of hypertension are pregnancy-associated hypertensive disease, which accounts for $70 \%$ of hypertension during pregnancy, and pre-existing chronic hypertension, which is responsible for the remaining cases (1). Hypertensive disorders are associated with increased maternal and perinatal mortality and present as a wide spectrum of disorders, ranging from minimal elevation of blood pressure alone to severe hypertension with multiple organ dysfunction.

\section{CLASSIFICATIONS}

Multiple classifications have been proposed for the hypertensive disorders of pregnancy. In 2001, the National Institutes of Health Working Group on Hypertension in Pregnancy (2) defined five disorders (Table 1).

Gestational hypertension may be defined as elevated blood pressure appearing after the 20th week of pregnancy or in the first 24 hours postpartum without edema or proteinuria, and with a return to normotension within 10 days of delivery. In order to diagnose hypertension, blood pressures must be at least $140 \mathrm{mmHg}$ systolic or $90 \mathrm{mmHg}$ diastolic on at least two occasions 6 hours apart. This is the most frequent cause of hypertension during pregnancy, affecting $6 \%$ to $17 \%$ of nulliparous women and $2 \%$ to $4 \%$ of multiparous women. The majority of cases of gestational hypertension develop after 37 weeks of pregnancy. Outcomes are similar to normotensive controls if only mild elevations in blood pressure. Increased morbidities if severe blood pressure elevations.

Pre-eclampsia is defined as new-onset hypertension in the second half of pregnancy with new-onset proteinuria. In order to diagnose proteinuria, greater than $300 \mathrm{mg}$ must be present on a 24 -hour sample or $0.1 \mathrm{~g} / \mathrm{L}$ in at least two random urine samples more than 4 hours apart. Often evidence of end-organ dysfunction is present. Development of convulsions or coma in patients with signs and symptoms or pre-eclampsia in the absence of other causes of convulsions is diagnostic of eclampsia.

Chronic hypertensive disease includes patients with pregestational hypertension, patients with pre-existent elevation of blood pressure to at least $140 / 90 \mathrm{mmHg}$ on two occasions before 20 weeks of gestation, and patients with hypertension that persists for more than 6 weeks postpartum. Superimposed pre-eclampsia or eclampsia is defined as the development of pre-eclampsia or eclampsia in patients with diagnosed chronic hypertension. About $15 \%$ to $30 \%$ of chronically hypertensive women will develop superimposed pre-eclampsia.

The classifications and definitions of the hypertension disorders of pregnancy are frequently confusing. It is often difficult to differentiate between pre-eclampsia, chronic hypertension, and chronic hypertension with superimposed pre-eclampsia. The normal mid-trimester fall in blood pressure may conceal the presence of underlying chronic hypertension; therefore, unless the patient presents in the first trimester or has a well-documented history of chronic hypertension, accurate classification may be impossible.

\section{PRE-ECLAMPSIA}

Pre-eclampsia is a disorder specific to human pregnancy. Reported incidences range from $2 \%$ to $10 \%$ depending on the diagnostic criteria used and the population studied. It is principally a disease of young primigravidas. The incidence is about $6 \%$ to $7 \%$ of all nulliparous pregnancies in the United States (3).

Geographic and racial differences in incidence have been reported. Several risk factors have been identified as predisposing to the development of pre-eclampsia in varied populations (4-11) (Table 2). For unknown reasons, the incidence appears reduced in women who smoke during pregnancy $(12,13)$, as well as in those who have had previous abortions or miscarriages (14-16).

Pre-eclampsia may be classified as either mild or severe. Severe pre-eclampsia is diagnosed when any of the criteria listed in Table 3 is present; additional markers of organ dysfunction may indicate severe disease.

\section{PHYSIOLOGY}

In normal pregnancies, the diameters of the uterine spiral arteries increase in size. The endothelium and the internal elastic lamina are replaced by invading trophoblast tissue. These changes begin in the decidual layer and extend into the myometrium. These vascular changes are thought to be associated with increased perfusion. In women with preeclampsia, these changes may be localized to the decidual layer or absent altogether. The diameter of the spiral arteries in affected pregnancies is $40 \%$ of the diameter in normal pregnancies (17). This is a disease of poor perfusion and vasospasm. Placental changes associated with pre-eclampsia may be seen weeks to months prior to the development of symptoms of the disorder. The symptoms of pre-eclampsia are associated with maternal endothelial dysfunction. Abnormalities in the endothelial control of vascular tone lead to increased permeability across vessel walls leading to edema and proteinuria.

\section{DIAGNOSIS}

A spectrum of clinical signs and symptoms, presenting either alone or in combination, makes the diagnosis of pre-eclampsia a subject of great controversy. Historically, diagnosis included a relative increase in blood pressure as well as presence of edema. Neither of these criteria has proven to be reliable for 
Table 1 Classification (2)

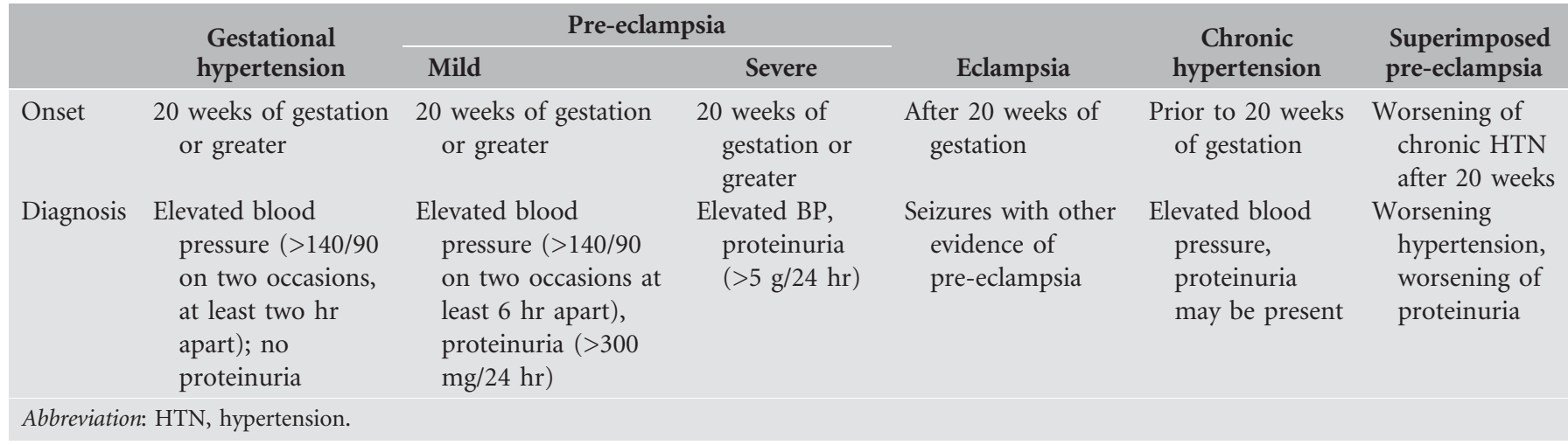

\section{Table 2 Risk Factors for Pre-eclampsia}

\section{Nulliparity}

Multiple gestation

Family history of pre-eclampsia/eclampsia

Pre-existing hypertension/renal disease/vascular disease

Personal history of pre-eclampsia/eclampsia

Pre-existing diabetes (class B-F)

Non-immune hydrops fetalis

Molar pregnancy

Obesity

diagnosis (2). A finding of abnormal blood pressure is subject to many errors; several variants may influence the readings: faulty equipment, cuff size, race, obesity, smoking, position, patient anxiety, or duration of the resting period (18).

The presence of proteinuria is usually determined by the use of dipsticks on random urine samples. The concentration of urinary protein is highly variable. It is influenced by several factors, including contamination with vaginal secretions, blood, or bacteria; urine specific gravity and $\mathrm{pH}$; and exercise and posture. Moreover, studies have found that urinary dipstick determinations correlate very poorly with the amount of proteinuria found in 24-hour urine determinations in hypertensive pregnancies (19-21). Dipstick measurements in urine samples $(+3)$ are not adequate for the diagnosis of severe preeclampsia (20). In addition, urinary protein-to-creatinine excretion is highly variable in patients with pre-eclampsia (22). Spot urinary protein-to-creatinine ratios are useful as a screening tool for pre-eclampsia. A result of less than 0.21 correlates to less than $300-\mathrm{mg}$ protein on a 24 -hour timed urine protein with a negative predictive value of $83.3 \%$ and sensitivity of $86.8 \%$ (23). Nevertheless, the definitive test for diagnosing proteinuria is a quantitative measurement of total protein excretion over a 24-hour period. An effort should be made to ensure an accurate collection by concurrently measuring the total 24-hour urine creatinine excretion. Significant proteinuria in pregnancy should be defined as $>300 \mathrm{mg}$ per 24-hour urine sample. For making the diagnosis of severe pre-eclampsia based on proteinuria only, it is recommended that a 24 -hour urine sample with protein of $>5 \mathrm{~g}$ be documented.

\section{PREVENTION OF PRE-ECLAMPSIA}

The likelihood of recurrence in subsequent pregnancies is such that numerous reports and clinical trials have focused on the

\section{Table 3 Diagnostic Criteria for Severe Pre-eclampsia}

\begin{tabular}{|c|c|}
\hline Blood pressure (3) & $\begin{array}{l}160 \mathrm{mmHg} \text { systolic or } 110 \mathrm{mmHg} \\
\text { diastolic on two occasions at least } \\
6 \mathrm{hr} \text { apart with the patient at rest }\end{array}$ \\
\hline Proteinuria (3) & $5 \mathrm{~g}$ in a $24-\mathrm{hr}$ urine collection \\
\hline Oliguria & $<400 \mathrm{~mL}$ in $24 \mathrm{hr}$ \\
\hline $\begin{array}{l}\text { Severe, persistent } \\
\text { epigastric pain }\end{array}$ & $\begin{array}{l}\text { Liver function test abnormalities } \\
\text { may exist }\end{array}$ \\
\hline $\begin{array}{l}\text { Pulmonary edema or } \\
\text { cyanosis }\end{array}$ & \\
\hline Thrombocytopenia & $<100,000 / \mathrm{mm}^{3}$ \\
\hline $\begin{array}{l}\text { Persistent cerebral } \\
\text { symptoms }\end{array}$ & Headache, visual changes \\
\hline Fetal growth restriction & $<10$ th percentile \\
\hline
\end{tabular}

use of various methods to prevent or reduce the incidence of pre-eclampsia. In 2006, recurrence rates were examined in 896 Icelandic women as described in Table 4 (24). Almost threefourths of women with a hypertensive disorder in a first pregnancy had a recurrence in the following pregnancy.

Since the etiology of the disease is unknown, various methods were used to correct the pathophysiologic abnormalities in the hopes of ameliorating the course of, or preventing, the disease. Some of the methods used are summarized in Table 5. To date, none of these methods have been shown conclusively to be effective in preventing the disease.

Alternations in diets (low salt, high protein, as well as restricted caloric intake) have been suggested as prevention for pre-eclampsia. In addition, the use of diuretics has been recommended to prevent pre-eclampsia (25). However, the efficacy of these measures has never been proven. Several epidemiologic studies and a few clinical supplementation trials have suggested a relationship between calcium, magnesium, zinc intake, and pregnancy-induced hypertension. The relationship between dietary calcium intake and hypertension has been the subject of several experimental and observational studies (26). It has been shown that there is an inverse association between calcium intake and maternal blood pressure and the incidences of pre-eclampsia and eclampsia in epidemiologic studies (27). The blood pressure-lowering effect of calcium was thought to be mediated by alterations in plasma renin activity and parathyroid hormone. In addition, calcium supplementation during pregnancy was shown to 
Table 4 Recurrence Rates

\begin{tabular}{|c|c|c|c|c|c|c|}
\hline \multirow[b]{2}{*}{ First pregnancy } & \multicolumn{6}{|c|}{ Second pregnancy } \\
\hline & Normal & $\begin{array}{c}\text { Gestational } \\
\text { HTN }\end{array}$ & $\begin{array}{c}\text { Pre- } \\
\text { eclampsia }\end{array}$ & $\begin{array}{l}\text { Chronic } \\
\text { HTN }\end{array}$ & $\begin{array}{l}\text { Superimposed } \\
\text { pre-eclampsia }\end{array}$ & $\begin{array}{c}\text { All } \\
\text { recurrences }\end{array}$ \\
\hline $\begin{array}{l}\text { Gestational HTN } \\
\quad(n=511)\end{array}$ & $153(29.9 \%)$ & $239(46.8 \%)$ & $25(4.9 \%)$ & $82(16 \%)$ & $12(2.3 \%)$ & $358(70.1 \%)$ \\
\hline $\begin{array}{l}\text { Pre-eclampsia/ } \\
\text { eclampsia } \\
(n=151)\end{array}$ & $63(41.7 \%)$ & $52(34.4 \%)$ & $\begin{array}{c}17 \\
(11.3 \%)\end{array}$ & $16(10.6 \%)$ & $3(2 \%)$ & $88(58.3 \%)$ \\
\hline $\begin{array}{l}\text { Chronic HTN } \\
\quad(n=200)\end{array}$ & $24(12 \%)$ & $69(34.5 \%)$ & $\begin{array}{c}17 \\
(11.3 \%)\end{array}$ & $16(10.6 \%)$ & $3(2 \%)$ & $68(58.3 \%)$ \\
\hline $\begin{array}{l}\text { Superimposed } \\
\text { pre-eclampsia } \\
(n=34)\end{array}$ & $2(5.9 \%)$ & $10(29.4 \%)$ & $\begin{array}{c}4 \\
(11.8 \%)\end{array}$ & $14(41.2 \%)$ & $4(11.8 \%)$ & $32(94 \%)$ \\
\hline Total $(n=896)$ & $242(27 \%)$ & $370(41.3 \%)$ & $52(5.8 \%)$ & $\begin{array}{l}203 \\
(22.7 \%)\end{array}$ & $29(3.2 \%)$ & $654(73 \%)$ \\
\hline
\end{tabular}

Table 5 Prevention of Pre-eclampsia

$\begin{array}{lll}\text { Dietary manipulation } & \text { Pharmacologic manipulation } & \text { Personal habit changes } \\ \text { Low-calorie diet } & \text { Diuretics } & \text { Frequent prenatal care } \\ \text { High-protein diet } & \text { Anti-hypertensives } & \text { Daily rest in lateral position } \\ \text { Low-salt diet } & \text { Beta-sympathomimetics } & \text { Keeping same partner } \\ \text { Nutritional supplementation } & \text { Anti-thrombotic agents } & \text { Avoiding/reducing coffee } \\ \text { Calcium } & \text { Low-dose aspirin } & \\ \text { Magnesium } & \text { Dipyridamole } & \\ \text { Zinc } & \text { Dazoxiben } & \text { Heparin } \\ \text { Fish oil } & & \\ \text { Evening primrose oil } & & \\ \text { Vitamins C and E } & & \end{array}$

reduce angiotensin II vascular sensitivities in such pregnancies $(28,29)$.

The findings of clinical studies comparing the use of calcium versus no treatment or placebo in pregnancy were reviewed in two meta-analyses (30-40). These trials differed by the population studied (low risk or high risk for hypertensive disorders of pregnancy), study design (randomization, double blind, or use of a placebo), gestational age at enrollment (20-32 weeks of gestation), sample size in each group (range 22-588), and dose of elemental calcium used (156-2000 mg/day). In addition, these studies differed regarding the definition of the hypertensive disorders of pregnancy. The findings suggest that calcium supplementation reduces the overall incidence of hypertensive disorders of pregnancy, with a trend toward reducing the incidence of pre-eclampsia.

A large, multicenter, randomized controlled trial to evaluate the benefits and side effects of calcium supplementation to prevent pre-eclampsia was performed in the United States. This trial randomized over 4500 healthy nulliparous women who were enrolled prior to 20 weeks of gestation. The results of this trial demonstrated no difference between those receiving $2 \mathrm{~g}$ of calcium as opposed to those receiving placebo in the incidence of either pre-eclampsia or gestational hypertension. In addition, calcium supplementation had no effect on maternal blood pressure throughout gestation or birth weight (40).
Pre-eclampsia is associated with vasospasm and an activation of the coagulation-hemostasis system. Enhanced platelet activation plays a central role in the above process with resultant abnormality in the thromboxane/prostacyclin balance. Aspirin inhibits the synthesis of prostaglandins by irreversibly acetylating and inactivating cyclooxygenase. In vitro, platelet cyclooxygenase is more sensitive to inhibition by very low doses of aspirin $(<80 \mathrm{mg})$ than vascular endothelial cyclooxygenase. Therefore, treatment with low doses of aspirin could alter the balance between prostacyclin (vasodilation) and thromboxane (vasoconstriction) (41).

More than 35,000 women have participated in a variety of randomized control trial describing the effects of low-dose aspirin (60-81 mg/day) on pre-eclampsia (42). Some of these studies were conducted in healthy nulliparous women $(43,44)$, whereas others included patients with various obstetric and medical complications (45-49).

Sibai and associates (44) reported a multicenter study in 2985 health nulliparous women who were randomized at 13-26 weeks of gestation to either receive $60 \mathrm{mg}$ aspirin daily or a placebo. The incidence of pre-eclampsia was reduced by only $26 \%$ in the aspirin-treated women; however, there were no differences in mean gestational age at delivery, birth weight, or the frequency of fetal growth retardation or preterm delivery. Moreover, there was a significantly higher incidence of abruptio placentae in women receiving aspirin $(0.7 \%)$ compared with those receiving placebo $(0.1 \%)$. 
Viinikka and colleagues (45) studied 197 women with preexisting chronic hypertension or a history of severe preeclampsia in their previous pregnancies who were randomized at 15 weeks of gestation to receive $50 \mathrm{mg} /$ day aspirin or a placebo. The incidences of exacerbation of hypertension as well as pre-eclampsia were similar in the two groups. In addition, the two groups had similar mean gestational age at delivery. However, patients receiving low-dose aspirin had a higher mean birth weight and lower frequency of fetal growth retardation.

An Italian multicenter trial evaluated the use of $50 \mathrm{mg} /$ day of aspirin to no treatment at 16 to 32 weeks in a large group of pregnant women considered at risk for pre-eclampsia (46). There were no differences between the two groups regarding the incidences of hypertension only, pre-eclampsia, fetal growth retardation, preterm delivery, abruptio placentae, or perinatal death.

The CLASP (Collaborative Low-Dose Aspirin Study in Pregnancy) trial (47) was a multinational randomized trial of low-dose aspirin for the prevention and treatment of preeclampsia and fetal growth retardation. In the trial, 9364 women at risk for pre-eclampsia between 12 and 32 weeks took part. There were no differences between the two study groups regarding the incidences of pre-eclampsia, fetal growth retardation, abruptio placentae, or perinatal deaths. However, the aspirin-treated group had a lower incidence of preterm delivery. In addition, there was a significant trend $(p=0.004)$ toward progressively greater reductions in pre-eclampsia (more in preterm delivery). The study group suggested that low-dose aspirin administration may be justified in women judged to be at risk for early-onset pre-eclampsia. In such women, the researchers recommended that low-dose aspirin be started before 20 weeks of gestation.

In a large multicenter clinical trial, pregnant women at 13 to 26 weeks of gestation with high-risk characteristics (previous pre-eclampsia-eclampsia, chronic hypertension, class B-F diabetes, or multifetal gestation) were randomized to either aspirin $60 \mathrm{mg} /$ day or matching placebo. This trial was conducted at 11 centers in the United States and was sponsored by the National Institute of Child Health and Human Development. The results of this trial demonstrated minimal to no benefit from the use of low-dose aspirin in such women (50).

An additional meta-analysis of individual data the Perinatal Antiplatelet Review of International Studies attempted to differentiate the success of aspirin by maternal diagnosis, timing of initiation of therapy, and dosage of aspirin (51). A reduction in preterm birth and incidence of pre-eclampsia by $10 \%$ was noted in those treated with aspirin. No subgroups of women were found for which aspirin therapy was more effective. In addition, the number needed to treat was 500 lowrisk pregnancies to prevent one preterm birth, while 50 highrisk pregnancies needed to be treated.

Oxidative stress has been suggested to play a role in the development of pre-eclampsia. Previous trials of the benefits of vitamins $\mathrm{C}$ and $\mathrm{E}$ have had disparate results. A recent trial evaluated the impact of antioxidant therapy on the prevention of pre-eclampsia (52). In the trial, 10,154 women were enrolled to participate, with $40 \%$ beginning while still in the first trimester. No differences were noted between women
Table 6 Hospital Management of Patients with Gestational HTN and Mild Pre-eclampsia

\begin{tabular}{|c|c|c|c|}
\hline & $\begin{array}{c}\text { Gilstrap } \\
\text { et al. } \\
(53)\end{array}$ & $\begin{array}{c}\text { Sibai et al. } \\
\text { (54) }\end{array}$ & $\begin{array}{l}\text { Sibai et al. } \\
\quad(56)\end{array}$ \\
\hline Patients $(n)$ & 576 & 200 & 200 \\
\hline $\begin{array}{l}\text { Mean hospitalization } \\
\text { (days) }\end{array}$ & 24 & 21 & 12 \\
\hline $\begin{array}{l}\text { Pregnancy prolongation } \\
\text { (days) }\end{array}$ & 24 & 21 & 22 \\
\hline $\begin{array}{l}\text { Preterm delivery } \\
\quad(<37 \text { weeks })\end{array}$ & 23 & - & $41-49$ \\
\hline Perinatal deaths & $9 / 1000$ & $5 / 1000$ & $0 / 1000$ \\
\hline Abruptio placentae (\%) & 0.9 & 1.7 & \\
\hline
\end{tabular}

receiving vitamin supplementation and those receiving the placebo for the primary outcomes of severe pregnancyassociated hypertension or pre-eclampsia. Rates of adverse perinatal outcomes were similar between the groups.

Based on the data available at this time, no one specific therapy appears to be more efficacious in the prevention of pre-eclampsia in high-risk patients than no therapy.

\section{MANAGEMENT OF MILD GESTATIONAL HYPERTENSION/PRE-ECLAMPSIA}

The optimal management of mild pre-eclampsia remote from term is controversial. In general, there is considerable disagreement regarding the need for hospitalization as opposed to ambulatory management. Management of gestational hypertension-pre-eclampsia by hospitalization was based largely on clinical experience rather than being the result of controlled randomized trials. In theory, hospital admission for bed rest could delay or prevent progression to severe hypertension $(53,54)$. Historically, admission to a hospital was an accepted practice worldwide and its rationale was to reduce eclampsia (55) and improve perinatal outcome (53). In some centers in the United States, management of these patients has included relative bed rest in the hospital for the duration of pregnancy. This approach has been reported to diminish the frequency of progression to severe disease and to enhance fetal survival $(15,53)$. In fact, several studies (Table 6) noted the benefits of prolonged hospitalization for patients with mild gestational hypertension remote from term.

This practice was challenged by Matthews and associates (57), who found no differences in perinatal mortality in women with gestational hypertension between those who were hospitalized for the duration and those who were managed as outpatients. Further, Matthews and colleagues (58) studied 135 patients with gestational hypertension in a randomized trial. They showed that complete bed rest appeared to have no advantage over "ambulation as desired" in controlling the severity of maternal disease. Similarly, Crowther and coworkers (59) conducted a randomized controlled trial on 218 patients with gestational hypertension. They showed that bed rest in pregnancies complicated by gestational hypertension was not associated with an overall improvement in fetal growth or reduced neonatal morbidity. Instead, the recording of fetal kick count at home and continued outpatient antenatal care provided a safe alternative to hospital admission. 


\section{Management Alternatives}

Obstetricians are increasingly utilizing outpatient management of gestational hypertension, especially with the increasing pressure to decrease medical expenses and use outpatient therapy. Outpatient management was addressed by the American College of Obstetricians and Gynecologists Practice Bulletin Number 33 (60), which noted that "Hospitalization is often initially recommended for women with new-onset preeclampsia. After maternal and fetal conditions are serially assessed, subsequent management may be continued in the hospital, at a day-care unit, or at home on the basis of the initial assessment... Hospitalization is recommended for noncompliant patients, those with logistical barriers, and those who show progressive disease as outpatients."

Randomized trials assessing the value of admission to hospital for bed rest found no benefits over outpatient management in women with mild gestational hypertension $(56,61)$. With the introduction of outpatient management alternatives, evaluation of maternal and fetal status was similar to inpatient therapy. In fact, the incidence of both eclampsia and placental abruption and the perinatal mortality rates were almost the same in both hospitalized and non-hospitalized patients.

\section{Home-Care Programs}

In an attempt to provide a safe and less expensive alternative to hospital care for women with pre-eclampsia, some home-care programs were initiated in the mid-1980s. Helewa and associates (62) reported their experience with one such program. Patients eligible for home-care management included those with blood pressure of $<150 / 100 \mathrm{mmHg}$, protein level of $<0.6 \mathrm{~g}$ in a 24-hour collection of urine, absence of symptoms of severe pre-eclampsia, platelet count of $>120,000$ / $\mathrm{mm}^{3}$ and liver enzymes of $<50 \mathrm{U} / \mathrm{L}$. Enrolled patients were visited at home by a trained nurse. The nurse measured the blood pressure and fetal heart rate, reviewed fetal movement counts, tested for the presence and amount of protein in the urine, and checked for signs and symptoms of pre-eclampsia. An assessment of the fetal biophysical profile and biochemical profile and a visit to the attending physician were carried out on a weekly basis. The program nurse contacted the physician if clinical deterioration was noted. Labor was induced electively at term when the patient had a favorable cervix or whenever pre-eclampsia became severe or there were signs of fetal distress. In this study, 321 patients met the criteria and were enrolled in the home-care program. A total of 141 patients $(44 \%)$ were admitted to the antepartum unit for inpatient monitoring: nine had severe pre-eclampsia. The mean length of enrollment in the home-care program was 11.5 days (1-42 days). Hospital stay was reduced from 5.7 days to 3.7 days, and cost was reduced by $74 \%$.

\section{Day-Care Programs}

Similarly, and in an attempt to decrease hospital bed occupancy, a fetal surveillance unit or day-care unit established within the hospital was described by some authors (63-67). The unit was established within the hospital and run by a nurse-midwife and staffed by an attending physician. Every visit included monitoring of maternal blood pressure, urine testing, with assessment of fetal heart rate, biophysical profile, and uteroplacental circulation, as needed. The patient remained under the care of the referring physician unless there was need for immediate intervention. Soothill and coworkers (63) reported a decrease in the rate of antenatal bed occupancy by $22 \%$ with no significant change in the stillbirth rate. Similarly, Tuffnell and associates (64) reported an 80\% reduction in hospital inpatient stay, and a reduction in the number of medical interventions. In addition, Dawson and colleagues (65) showed that telephonic monitoring of the fetal heart rate in such women was also associated with fewer hospital admissions and a smaller percentage of time spent in hospital. Turnbull and associates (67) reported no significant difference in fetal or maternal morbidities when compared with in-patient care.

\section{Perinatal Outpatient Monitoring Service}

There are outpatient monitoring service designed to monitor and record information associated with the major signs of obstetric hypertensive disease (blood pressure determination, weight, sleep/rest documentation, fetal movement count, and urinalysis) (57). Outpatient evaluation includes four times daily automated blood pressure and pulse measurement and daily assessment of weight, fetal kick counts, duration of rest/ sleep periods, and proteinuria. Each data element is dated and time coded. Objective and subjective data are then transmitted by phone to a perinatal center daily or immediately (if elevated blood pressure or decreased fetal movements are observed). Patients receive twice weekly antenatal evaluation with nonstress testing and frequent amniotic fluid assessment. Barton and colleagues (57) evaluated the perinatal and maternal outcomes of this outpatient monitoring service in 592 patients with mild gestational hypertension at 24-36 weeks of gestation. The mean pregnancy prolongation was $27.4 \pm 3.3$ days, whereas the mean antepartum hospitalization for management of similar patients was only 1.7 days. Maternal and perinatal outcome were similar to those reported by investigators using hospitalization for management of similar patients. In a subsequent report, Barton and associates (68) found that a similar program was safe and effective, even in young teenage patients with mild gestational hypertension remote from term.

The success rate of outpatient management depends primarily on maternal status (presence or absence of proteinuria, diastolic blood pressure, and gestational age) at the time of enrollment. Pregnancies complicated by pre-eclampsia are associated with a lower gestational age at delivery, shorter pregnancy prolongation, and an increased requirement for antepartum hospitalization as compared with pregnancies with gestational hypertension without proteinuria. Those women with gestational hypertension or preeclampsia at an earlier gestational age tend to have earlier gestational age at delivery and a worsening of the disease status and more unfavorable fetal outcome than those who develop the disease at term $(56,61)$.

\section{Management Protocol}

Management plan for patients with mild gestational hypertension/pre-eclampsia is dependent on gestational age as well as maternal and fetal findings. A recent multicenter, randomized clinical trial described by Sibai compared induction of labor 
with continued expectant management for patients with gestational hypertension/pre-eclampsia at 36 to 41 weeks (69). Once 37-week gestation was reached, there is an overall reduction in relative risk of maternal morbidity. Induction of labor was not associated with increased risk of cesarean delivery or neonatal morbidity. The principal maternal benefit of delivery is decreased disease progression. Additionally, HYPITAT study described by Koopmans (70) assessed whether induction of labor at term with mild hypertensive disease reduces maternal morbidity. This multicenter randomized trial divided patients with mild hypertensive disease at term into groups for induction of labor or expectant management. Those undergoing induction of labor had a relative risk 0.71 of worsening maternal disease. There was no statistical difference in cesarean rates or neonatal complications between the groups. An economic analysis of the HYPITAT study performed by Vijgen and colleagues demonstrated decreased cost with induction of labor as opposed to expectant management for patients with mild hypertensive disease at term (71). Based on these data, patients with gestational age beyond 37 weeks can reasonably be managed with induction of labor.

Patients who are less than 37 weeks of gestation are assigned to either ambulatory or inpatient management based on certain criteria: those who are unreliable, or have a diastolic blood pressure of $>100 \mathrm{mmHg}$, proteinuria of $>500 \mathrm{mg}$, abnormal laboratory tests, abnormal fetal testing, preterm labor, or bleeding are usually hospitalized. They are allowed to eat regular hospital diet without salt restriction, and their activity is not restricted to complete bed rest. Initially, antihypertensive drugs are not prescribed. Patients undergoing outpatient care are instructed to have daily urine dipstick measurements of proteinuria, blood pressure monitoring, daily assessment of weight gain, and daily recording of fetal kick counts. They are also educated to report any symptoms of impending eclampsia. The patient is then evaluated in the antepartum testing area for maternal and fetal well-being at least twice a week for patients with proteinuria ( $1+$ ) and once a week for those without proteinuria (0 or trace). If there is any evidence of disease progression and if acute severe hypertension develops, then hospitalization is indicated. During expectant management, these women have periodic laboratory evaluation of platelet count and liver enzymes (one to two times a week), serial ultrasound examinations for estimated fetal weight (every 2-3 weeks), antenatal testing with the nonstress test (two times a week), and weekly umbilical artery Doppler studies, as well as amniotic fluid volume assessment.

In summary, management of women with mild gestational hypertension-pre-eclampsia must always put safety of the mother first and then aim at the delivery of a live, mature newborn that will not require intensive and prolonged neonatal care. The goal of management of mild gestational hypertension-pre-eclampsia should be to identify the development of severe disease prior to maternal seizure or fetal jeopardy. Therefore, requirements for outpatient management must include well-motivated patients, a perinatal outpatient monitoring service or other management alternative for close maternal and fetal evaluation, and guidelines for contacting the health-care provider (Table 7).

\section{Table 7 Guidelines for Outpatient Management}

Well-motivated patients

Well-trained nurses

Perinatal outpatient monitoring service or other management alternative

Close maternal and fetal evaluation

Daily

- Urine dipstick for proteinuria

- Blood pressure

- Weight

- Fetal kick count

Two times per week

- Platelet count and liver enzymes (1-2 times per week)

- Fetal antenatal testing with nonstress test or biophysical profile

One time per week

- Measure of amniotic fluid volume

- Platelet count and liver enzymes (1-2 times per week)

- Physician visit

Every two to three weeks

- Serial ultrasound examinations for estimated fetal growth

Guidelines for contacting health-care provider

- Labor or rupture of membranes

- Vaginal bleeding

- Decreased fetal movement

- Symptoms of impending eclampsia (CNS, visual disturbances)

- Symptoms of impending HELLP (epigastric pain, vomiting)

Abbreviation: HELLP, hemolysis, elevated liver enzymes, and low platelet count.

\section{Antihypertensive Drugs in Mild Gestational Hypertension/Pre-eclampsia}

There are several retrospective and prospective studies that have described the use of various antihypertensive drugs in women with mild gestational hypertension/mild preeclampsia (56,72-80). Drugs used in these studies included hydralazine, methyldopa, nifedipine, prazosin, diuretics, and beta-blockers. These studies included women with gestational hypertension (proteinuria absent) and mild pre-eclampsia. The purpose of using antihypertensive drugs in this group of women was not to improve blood pressure, but rather to improve perinatal outcome by prolonging the pregnancy in those who were remote from term. Some of these studies found that labetalol had some beneficial effects on the disease process (progression to severe hypertension, development of proteinuria), whereas other studies reported detrimental effects (higher frequency of fetal growth retardation) in the treated group. In general, none of these studies have reported a better perinatal outcome than those reported in studies using hospitalization alone. In summary, there are currently few data to support the use of antihypertensive drugs in the management of mild gestational hypertension-pre-eclampsia remote from term (81).

\section{MANAGEMENT OF SEVERE PRE-ECLAMPSIA}

Pre-eclampsia affects two patients-the mother and the fetus. Traditionally, women with severe pre-eclampsia have been delivered without delay, regardless of fetal considerations. Although delivery is appropriate therapy for the mother, aggressive management with immediate delivery of a fetus remote from term leads to high neonatal mortality and morbidity resulting from prematurity. Recent studies have 
Table 8 Maternal Complications During Expectant Management of Severe Pre-eclampsia

\begin{tabular}{|c|c|c|c|c|}
\hline Study & HELLP syndrome (\%) & Pulmonary edema (\%) & Renal failure (\%) & Eclampsia (\%) \\
\hline \multicolumn{5}{|l|}{ Randomized trials } \\
\hline Odendaal et al. (85) $(n=18)$ & Not reported & 0 & 5.5 & 0 \\
\hline Sibai et al. (83) $(n=49)$ & 4.1 & 0 & 0 & \\
\hline Sibai et al. (86) $(n=54)$ & 13.0 & 0 & 0 & 5.6 \\
\hline Olah et al. (87) $(n=28)$ & 14.3 & 0 & 3.6 & 0 \\
\hline Hall et al. (82) $(n=340)$ & 5.2 & 2.1 & 1.7 & 1.2 \\
\hline Chammas et al. (89) $(n=47)$ & $17.0^{\mathrm{a}}$ & 8.5 & $17.0^{\mathrm{a}}$ & 0 \\
\hline Haddad et al. $(90)(n=239)$ & 14.2 & 3.8 & 0 & 0 \\
\hline Oettle et al. (91) $(n=131)$ & 4.6 & 0.8 & 2.3 & 2.3 \\
\hline Shear et al. (92) $(n=155)$ & 27.1 & 3.9 & Not reported & 1.9 \\
\hline
\end{tabular}

demonstrated favorable neonatal outcomes after conservative management of severe pre-eclampsia. Since 1990, randomized trials and several observational studies have evaluated expectant management of severe pre-eclampsia at less than 34 weeks of gestation. These studies included greater than 1700 women, with gestational age between 24 and 34 weeks of gestation. The results suggested that expectant treatment in a select group of women with severe pre-eclampsia between 24-0/7 and 32-6/7 weeks of gestation in a suitable hospital is safe and improves neonatal outcome (82). Candidates for conservative management should be carefully selected and managed with intensive maternal and fetal monitoring at a tertiary perinatal center.

The timing of delivery in women with severe pre-eclampsia in the second trimester is a difficult decision to make for mother and obstetrician. Aggressive management with immediate delivery will result in extremely high neonatal mortality and morbidity resulting from prematurity. Consequently, hospitalization in a neonatal intensive care unit is prolonged, and surviving infants may have long-term disabilities (83). Attempts to prolong pregnancy may improve perinatal outcome, but may expose the mother to potential morbidity (84).

Therefore, concerning expectant management, one must weigh risks and benefits to the mother and the fetus. Fetal gestational age, fetal condition, and maternal condition play important roles in reaching such a decision (83).

\section{Maternal Indications}

Because the pathophysiology of pre-eclampsia involves diffuse endothelial injury, patients may present with multiple-system involvement and differing degrees of severity. Some women with severe disease have marked improvement in blood pressure, protein excretion, and urine output shortly after hospitalization. However, others require immediate delivery because of signs of deterioration as seen in Table 8. Careful selection of patients with severe disease is important (84). Patients judged suitable for expectant management have one or more of the following clinical findings: controlled hypertension, proteinuria of $>5 \mathrm{~g} /$ day, oliguria that resolves with routine fluid or food intake, elevated liver enzymes (aspartate aminotransferase or alanine aminotransferase greater than two times the upper normal value) without epigastric pain or without right upper quadrant tenderness (84).

Visser and Wallenburg (93) rejected the general recommendation of prompt termination of pregnancy in preeclamptic patients with hemolysis, elevated liver enzymes, and low platelets [hemolysis, elevated liver enzymes, and low platelet count (HELLP) syndrome] in the late second or early third trimester. In their study, 128 pre-eclamptic patients with HELLP syndrome were matched for gestational age with 128 pre-eclamptic patients without HELLP. Both groups were treated conservatively with volume expansion and pharmacologic vasodilatation with the aim of prolonging gestation and enhancing fetal maturity. Complete reversal of HELLP occurred in $43 \%$ of patients. Perinatal mortality was $14.1 \%$ in HELLP patients and $14.8 \%$ in patients without HELLP. Similarly, Magann and coworkers (94) demonstrated significant improvement in maternal platelet count, urinary output, lactic dehydrogenase, alanine aminotransferase, and postponement of delivery in women with HELLP syndrome who received high-dose antenatal dexamethasone (10 mg intravenously every 12 hours until delivery; average time of 41 hours).

\section{Fetal Indications}

Severe pre-eclampsia may affect the fetus in a negative way. Inadequate trophoblastic invasion causing abnormal placentation is one of the main features of pre-eclampsia. This can lead to decreased uteroplacental perfusion with a resulting increased incidence of intrauterine growth retardation, fetal hypoxia, and perinatal death. Therefore, careful selection and intensive monitoring of the fetus is essential. Patients judged suitable for conservative management have one or more of the following: biophysical profile $>4$, largest vertical amniotic fluid pocket of $>2$, ultrasound-estimated fetal weight above the 5 th percentile.

It is commonly believed that maternal pre-eclampsia has a protective effect on neonatal outcome after delivery before 34 weeks of gestation. This protective effect is thought to be caused by the fact that fetuses of pre-eclamptic mothers are subjected to stress in utero and therefore have accelerated maturation and consequently a better prognosis than other preterm infants. However, in a well-designed retrospective study, Schiff and colleagues (95) studied fetal lung maturity by amniocentesis in 127 pre-eclamptic patients and a matched 
Table 9 Indication for Delivery

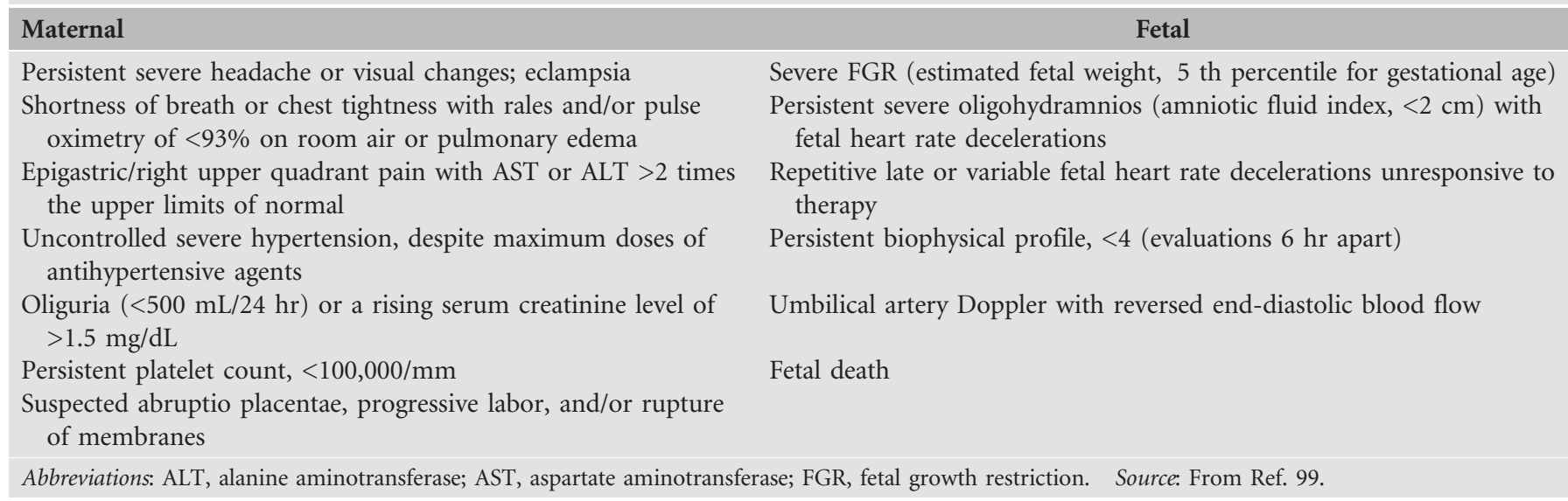

control group of women with preterm labor. They found no significant difference in the incidence of an immature laboratory result between pre-eclamptic patients and their matched controls $(39.4 \%$ vs. $38.6 \%)$. The incidence of respiratory distress syndrome was slightly, but not significantly, higher in the pre-eclamptic group. In addition, Friedman and associates (96) found no beneficial effect from pre-eclampsia on the postnatal course of infants born at 24-35 weeks of gestation.

The poor perinatal outcome associated with severe preeclampsia is believed to be related primarily to prematurity. However, severe pre-eclampsia is associated with increased vascular resistance and a decreased uteroplacental perfusion leading to an increased incidence of intrauterine growth retardation, fetal hypoxia, and perinatal death. Studies have demonstrated the efficacy of umbilical artery velocimetry in identifying fetal acidosis. Doppler ultrasound scanning is now common in the management of pregnancies complicated by hypertension and has become an indirect tool to assess fetal well-being. The absence or reversal of end-diastolic velocity in the umbilical artery has proved to be highly predictive of low birth weight or fetal death $(97,98)$. Torres and coworkers (98) retrospectively investigated the relationship between abnormal umbilical artery velocimetry and fetal outcome in 172 hypertensive pregnant women. In their study, the absence of end-diastolic velocity predicted low birth weight in $100 \%$ of pregnancies and fetal death in $66.6 \%$. All stillbirths in this study had absence of end-diastolic velocity (sensitivity 100\%). However, the value of abnormal umbilical artery Doppler results (reversed or absent end-diastolic flow) in predicting poor fetal outcome in hypertensive pregnancies has been studied in only a small number of patients.

Table 9 provides a summary of guidelines for delivery. These indications should not be considered absolute, as exceptions may exist in allowing for individualization of patient care.

\section{Management According to Gestational Age}

In general, management of severe pre-eclampsia is dependent on gestational age. Patients with fetuses at a gestational age greater than 34 weeks are generally delivered after admission. Patients at 33 to 34 weeks of gestation with immature fetal lung maturity studies may receive steroids to accelerate fetal lung maturity and may be delivered 24 hours after the last dose of steroids in the presence of any significant adverse change in maternal or fetal conditions. However, patients found eligible for conservative management should be counseled about the fact that gaining 2 more weeks could make the difference between having an infant admitted to the neonatal intensive care unit or having an infant admitted to the well-baby nursery.

Patients at 28 to 32 weeks of gestation are managed according to their clinical status during the observation period. Sibai and colleagues (83) published data from a randomized and well-controlled study comparing aggressive versus expectant management in patients with severe pre-eclampsia between 28 and 32 weeks of gestation. In this study, it was possible to prolong pregnancy by an average of 15.4 days in women assigned to expectant management without a significant increase in maternal morbidity. Additionally, the expectant management group had a lower incidence of admissions to the neonatal intensive care unit (76\% vs. $100 \%$ ), lower average number of days spent in that unit (20.2 vs. 36.6 days), reduced incidence of respiratory distress syndrome $(22.4 \%$ vs. $50.0 \%)$, and a reduced incidence of necrotizing enterocolitis ( $0 \%$ vs. $10.9 \%$ ). Similar findings were observed by Odendaal and coworkers (85) and by Fenakel and colleagues (100).

Patients with severe pre-eclampsia at a gestational age of $<27$ weeks should receive extensive counseling regarding the risks and benefits of expectant management (Table 10). Sibai (86) reported a perinatal survival rate in conservative management of severe pre-eclampsia between 25 and 27 weeks of gestation of $76 \%$ compared with $35.5 \%$ in the group that had immediate delivery. In addition, the infants in the conservative management group had a lower incidence of intraventricular hemorrhage $(41 \%$ vs. $71 \%)$ and a shorter length of stay in the neonatal intensive care unit (70 vs. 115 days). In the same study, a perinatal survival rate of $6.7 \%$ and a $27 \%$ incidence of maternal morbidity were reported in conservative management of severe pre-eclampsia at $\leq 24$ weeks of gestation. Therefore, expectant management of patients between 25 and 27 weeks of gestation had a significantly higher perinatal survival and a lower acute and long-term neonatal morbidity compared with aggressive management. Maternal complications were infrequent in both 
Table 10 Expectant management of severe pre-eclampsia at $<25$ weeks of gestation (101)

$\begin{array}{lccc} & \text { Patients } & \begin{array}{c}\text { Merinatal } \\ \text { death (\%) }\end{array} & \begin{array}{c}\text { Maternal } \\ \text { complications } \\ (\%)\end{array} \\ \text { Sibai et al. (83) } & 15 & 93 & 27 \\ \text { Moodley et al. (102) } & 10 & 100 & 50 \\ \text { Visser and } & 25 & 84 & \text { Not reported } \\ \quad \text { Wallenberg (103) } & 26 & 82 & 65 \\ \quad \text { Gaugler-Senden } & & & \\ \quad \text { et al. (104) } & 8 & 88 & 36 \\ \text { Hall et al. (82) } & 31 & 71 & 71 \\ \text { Budden et al. (105) } & & & \end{array}$

groups. Perinatal survival was low, morbidity was high, and maternal complications were also elevated. Therefore, for patients between 24 and 25 weeks of gestation, a gain of 1 or 2 weeks will markedly improve perinatal survival and reduce neonatal morbidity.

Any deterioration in the status of either the mother or the fetus may necessitate urgent delivery. Early detection of maternal or fetal complications or deterioration in their clinical status and prompt therapy and delivery may be responsible for infrequent maternal complications. Such management with frequent and daily monitoring of maternal and fetal status is essential and should be practiced only at a tertiary care center. Chari and colleagues (106) studied 68 women with severe pre-eclampsia remote from term who underwent expectant management with daily fetal testing until delivery. In this study, patients who had non-reassuring fetal testing were delivered. Neither stillbirths nor fetal compromise at birth occurred in patients undergoing daily antenatal testing; thus the authors recommended daily testing in patients with severe pre-eclampsia managed expectantly. Odendaal and associates (85) recommended performing the nonstress test at least three times daily in this group of patients. However, such evaluation must be individualized.

\section{INTRAPARTUM MANAGEMENT}

Once a decision for delivery is made, vaginal delivery is preferable if possible, and a trial of labor may be warranted.

It is generally accepted that pre-eclamptic women are at increased risk for convulsions during labor, compared with normotensive pregnant women. The risk for convulsions depends on the severity of the pre-eclamptic process. Women with gestational hypertension (absent proteinuria) are at a lower risk than those with proteinuric hypertension. The highest risk is usually in women with severe pre-eclampsia, particularly those remote from term, those with cerebral manifestations, and those with HELLP syndrome. The use of magnesium sulfate in patients with pre-eclampsia has been questioned for a long time. The incidence of eclampsia in preeclamptic women receiving magnesium sulfate is $0.15 \%$ to $0.3 \%$ vs. $1.2 \%$ in pre-eclamptic women not receiving magnesium sulfate (107). As a result, all women who are diagnosed to have pre-eclampsia should receive parental magnesium sulfate during labor. Magnesium sulfate has been the drug of choice for the prophylaxis of eclamptic seizures in the United States. Other agents (diazepam, clomethiazole, barbiturates, and phenytoin) or no agent at all have been used for the same purpose in Europe and Australia. The most commonly used regimens of magnesium sulfate administration are the standard intramuscular regimen of Pritchard ( $4 \mathrm{~g}$ IV with $10 \mathrm{~g}$ IM load, $5 \mathrm{~g}$ IM every 4 hours) and the intravenous regimens of Zuspan (4 g load, 1-2 g/hr) and Sibai (6 g load, $2 \mathrm{~g} / \mathrm{hr}$ ). Phenytoin has proved to be an effective anticonvulsant in the setting of pre-eclampsia and eclampsia, and was considered by some to be a desirable alternative to magnesium sulfate, especially because phenytoin possesses certain advantages (more rapid cervical dilatation and a smaller drop in hematocrit) and fewer side effects (108). Lucas and colleagues (109) conducted a randomized study comparing the use of magnesium sulfate with phenytoin in the prevention of eclampsia. They found that magnesium sulfate was clearly superior to phenytoin when given prophylactically for eclamptic seizures to women with peripartum hypertension. In fact, 10 of the 1089 women randomly assigned to the phenytoin regimen had eclamptic convulsions, compared with none of the 1049 women assigned to magnesium sulfate. Phenytoin is an acceptable alternative to magnesium sulfate in patients with renal insufficiency, myasthenia gravis, or magnesium failure.

Patients should receive close monitoring during labor and delivery and postpartum, with special attention to fluid intake and output. Patients with severe disease and those with the HELLP syndrome are at an increased risk for the development of pulmonary edema from fluid overload and from acute renal failure from blood loss during delivery or from hemolysis, or from a combination of both. Urinary output needs to be monitored frequently, and fluid administration should not exceed $150 \mathrm{~mL} / \mathrm{hr}$. If the patient develops oliguria $(<100 \mathrm{~mL} /$ $4 \mathrm{hr}$ ), both the rate of fluid administered and the dose of magnesium sulfate may be adjusted appropriately.

\section{Antihypertensive Drugs in Severe Pre-eclampsia}

Empirically, antihypertensive therapy is recommended for systolic blood pressure exceeding $160 \mathrm{mmHg}$ and diastolic blood pressure of $>110 \mathrm{mmHg}$ in order to protect the patient from the complications of severe hypertension, especially stroke. The aim of therapy is to maintain the blood pressure within the range of 140 to $150 \mathrm{mmHg}$ systolic and 90 to $100 \mathrm{mmHg}$ diastolic or to initially reduce mean arterial blood pressure by no more than $20 \%$ from baseline values. Antepartum diuretics are not needed, except in the presence of pulmonary edema. Irrespective of the antihypertensive drug used, care must be taken not to lower blood pressure excessively, as this may exacerbate maternal cerebral ischemia, decrease renal function, or jeopardize fetal well-being by decreasing cerebral, renal, and placental blood flow. Attempts should be made to reduce the blood pressure to a safe range within 4 hours of diagnosis. The doses are usually titrated to blood pressure response. Comparative trials have not shown one agent to be superior in the acute management of severe hypertension in pregnancy $(101,110)$. (Table 11)

Hydralazine is given in sequential intravenous boluses of 5, 5 , and $10 \mathrm{mg}$ at intervals of 15 to 20 minutes until a satisfactory response is achieved. Hydralazine causes a direct relaxation of arterial smooth muscle, resulting in stimulation of the sympathetic nervous system and causing an increased reflex 
Table 11 Treatment of Severe Hypertension

\begin{tabular}{llccc} 
Class & \multicolumn{1}{c}{ Drug } & Onset $(\mathbf{m i n})$ & Peak $(\mathbf{m i n})$ & Dose \\
Arterial dilator & Hydralazine & $10-20$ & 60 & $5-10 \mathrm{mg} \mathrm{IV} \mathrm{every} \mathrm{15-30} \mathrm{min}$ \\
Calcium channel blocker & Nifedipine & 10 & 60 & $10-20 \mathrm{mg}$ orally every 30 min, max 120 mg/d \\
Alpha/beta-blocker & Labetalol & 5 & 60 & $20-40-80 \mathrm{mg} \mathrm{IV} \mathrm{every} \mathrm{10-20} \mathrm{min} \mathrm{up} \mathrm{to} \mathrm{300} \mathrm{mg}$ \\
Arterial/venous dilator & Sodium nitroprusside & $0.5-5$ & 5 & $0.2-5.0 \mathrm{mg} / \mathrm{kg} / \mathrm{min}$
\end{tabular}

heart rate and contractility. Maternal side effects include flushing, headache, palpitations, nausea, and vomiting. Uteroplacental perfusion may decrease following parenteral administration leading to fetal heart rate decelerations. The onset of drug effect may be 20 to 30 minutes, reaching a peak at 60 minutes from administration. Therefore, the drug may accumulate after continuous intravenous infusion, resulting in overshoot hypotension.

Nifedipine is started at an initial oral dose of $10 \mathrm{mg}$. It is then repeated in 20 to 30 minutes as necessary $(10,10,20 \mathrm{mg})$. The total dose of short-acting nifedipine should not exceed $120 \mathrm{mg}$ over a 24-hour period.

In a randomized clinical trial, Fenakel and colleagues (100) compared the effects of nifedipine with hydralazine in patients with severe pre-eclampsia. Nifedipine produced a more predictable reduction in blood pressure without being associated with sudden or excessive falls in blood pressure. This characteristic is an important factor in the avoidance of acute fetal distress from sudden hypotension in pre-eclamptic patients who frequently have compromised uteroplacental blood flow. Only one of the 24 nifedipine subjects developed signs of acute fetal distress, as opposed to 11 of the 25 hydralazine patients. The longer duration of action by nifedipine resulted in fewer doses necessary to achieve blood pressure control.

Labetalol is administered in repeated bolus intravenous injections. The starting dose is $20 \mathrm{mg}$, and if no improvement in blood pressure is noted, repeated doses of 20, 40, and $80 \mathrm{mg}$ may be given every 10-20 minutes to a maximum total dose of $300 \mathrm{mg}$. When compared with hydralazine, labetalol has fewer side effects including less headache, quicker onset of action, and smoother reduction in blood pressure (101).

Sodium nitroprusside is a potent arterial and venous dilator. It is given in a continuous intravenous infusion beginning at a rate of $0.2 \mathrm{mg} / \mathrm{kg} / \mathrm{min}$. It has a rapid action, with short duration. Because of the potent hypotensive properties of the drug, invasive arterial pressure monitoring is recommended. It should be used only in extreme emergencies in pregnancy, because of the potential risks of fetal cyanide poisoning and metabolic acidosis (111).

\section{Anesthesia}

There is controversy about the use of epidural analgesia/ anesthesia in women with pre-eclampsia. Some authors believe that the administration of epidural anesthesia in women with severe pre-eclampsia is detrimental to both the mother and the fetus, owing to possible profound hypotension. Other studies have demonstrated, however, that administration of epidural anesthesia in women with pre-eclampsia has a favorable effect on maternal hemodynamics, decreases catecholamine levels, and improves intervillous as well as umbilical blood flow (112-115). Ramanathan and colleagues
(115) prospectively compared the effects of lumbar epidural $(n=11)$ and general $(n=10)$ anesthesia on the hemodynamic and neuroendocrine stress response to cesarean delivery in 21 women with severe pre-eclampsia. The authors found that, in the epidural group, there was a decrease in mean arterial pressure, and the concentrations of adrenocorticotropic hormone, beta-endorphin, and catecholamine decreased or remained unchanged during the procedure. By contrast, patients receiving general anesthesia demonstrated a significant increase in both mean blood pressure and the concentration of the various hormones studied. In addition, neonatal outcomes were similar in both groups. It is our opinion that the epidural is the anesthetic of choice in women with preeclampsia. Care must be taken to avoid fluid overload during pre-epidural fluid boluses. However, its use is contraindicated in patients with coagulopathy and/or acute fetal distress. For patients with the HELLP syndrome, the use of pudendal block is contraindicated.

\section{Postpartum Management}

Following delivery, the patient should continue to be closely monitored for 12 to 24 hours, during which time maternal vital signs including reflexes, and intake and output, should be monitored hourly. Twenty-five percent of cases of eclampsia are reported to occur postpartum; therefore, observation is mandatory. Salt restriction and diuretics are most often not needed. Most patients will show evidence of resolution of the disease process within 24 hours. The best sign of pre-eclampsia resolution is spontaneous diuresis.

Some, however, mainly those with severe disease in the midtrimester, and those with HELLP syndrome, require intensive observation for greater than 24 hours. In such patients, magnesium sulfate may be needed for an extended period of time. In addition, these patients are at increased risk for the development of pulmonary edema from fluid overload, fluid mobilization, and compromised renal function (116).

If the patient develops severe hypertension during this time, the blood pressure may be controlled with oral nifedipine. In a double-blind study, Barton (117) evaluated the effect of nifedipine in postpartum patients with severe pre-eclampsia before delivery. They found oral nifedipine to be effective in the control of blood pressure as well as improving urine output during the 24 hours after delivery. In a subsequent report, Barton (118) studied the pharmacokinetic and pharmacodynamic parameters of oral nifedipine use in the immediate postpartum period. Based on the clinical pharmacology in this setting, they suggested that dosing should be every 3-4 hours. Most patients will be normotensive at the time of discharge from the hospital. A few patients may continue to have significant hypertension, and this can be controlled with additional oral blood pressure medications. Prophylactic anticonvulsive drugs such as phenobarbital are 
not needed. The patient is then seen at weekly intervals until her blood pressure is in the normal range without the use of medication. If this change does not occur by 6 weeks, a workup to assess hypertension should be performed.

\section{LONG-TERM IMPACT}

The long-term effects of pre-eclampsia and gestational hypertension on maternal and fetal well-being are only now being realized.

\section{Maternal}

Multiple studies have demonstrated an increase in maternal cardiovascular morbidity following hypertensive disorders of pregnancy. Recently, Magnussen and colleagues (119) described a prospective study of 15,065 women to assess impact of hypertensive disease in pregnancy on maternal cardiovascular risk. The association of increased cardiovascular risk was stronger with hypertensive disease in more than one pregnancy or in whom it developed late in their reproductive career. For example, those with two episodes of pre-eclampsia were 10 times more likely to require medication to control blood pressure at follow-up. In addition, women with a history of pre-eclampsia were more likely to develop diabetes (adjusted odds ratio 2.8 after adjustment for current BMI.) In a 2010 case-control study comparing three groups of women (history of HELLP, history of pre-eclampsia, and normal pregnancy), Strobl (120) noted that 13 to 18 years after the affected pregnancy, those women in the HELLP and preeclampsia groups were more likely to have impaired cardiac function on echocardiogram. Additionally, there were more women in these groups on anti-hypertensive medications.

\section{Child}

The impact of hypertensive disease in pregnancy on the child is now a focus of increasing research. A prospective case-control trial by Tenhola and colleagues (121) compared 57 children of pre-eclamptic pregnancies with 57 born from gestational agematched unaffected pregnancies. When comparing blood pressure as well as plasma catecholamines, those exposed to pre-eclampsia had higher blood pressure. Those children with hypertension were more likely to have been born small-forgestational age. Additionally, a small increase in plasma epinephrine levels was noted in these children. There was no difference in the norepinephrine levels between groups. Palmsten and colleagues (122) examined a cohort that was followed from pregnancy into adulthood as a part of the Collaborative Perinatal Project and the New England Family Study. Adults born from hypertensive disorders of pregnancy were more likely to have increased blood pressure as adults. These findings remained after adjustment for gestational age at birth and birth weight. Exposure in utero of the fetus to oxidative stress is one possible explanation for these results.

The Helsinki Birth Cohort Study described by Kajantie and colleagues (123) included 6410 individuals born in the period 1934 to 1944 . Of these pregnancies, 284 were complicated by pre-eclampsia and an additional 1592 complicated by gestational hypertension. While it was found that individuals born of these pregnancies are more likely to be at an increased risk for stroke (relative risk 1.4-1.9), no association with coronary heart disease was found. The elevation in risk of stroke is hypothesized to be secondary to disordered local response of blood vessels as a result of impaired in utero growth.

\section{REFERENCES}

1. Sibai BM, Usta IM. Chronic hypertension in pregnancy. In: Sciarra JJ, ed. Gynecology and Obstetrics. Service 60. Philadelphia: JB Lippincott, 1995: $1-19$.

2. National High Blood Pressure Education Program Working Group on High Blood Pressure in Pregnancy. Report of the National High Blood Pressure Education ProgramWorking Group on High Blood Pressure in Pregnancy. Am J Obstet Gynecol 2000; 183: S1-22.

3. Sibai BM. Hypertension in pregnancy. Chapter 28. In: SGabbe SG, Niebyl JR, Simpson JL, eds. Obstetrics: Normal and Problem Pregnancies, 3rd edn. New York: Churchill Livingstone, 1996.

4. Long P, Oats J. Preeclampsia in twin pregnancy-severity and pathogenesis. Aust NZ J Obstet Gynaecol 1987; 27: 1-5.

5. Coonrod DV, Hickok D, Zhu K, et al. Risk factors for preeclampsia in twin pregnancies: a population-based cohort study. Obstet Gynecol 1995; 85: 645-50.

6. Sutherland A, Cooper DW, Howie PW, et al. The incidence of severe preeclampsia among mothers and mothers-in-law of preeclamptics and controls. Br J Obstet Gynaecol 1981; 88: 785-91.

7. Sibai BM, El-Nazer A, Gonzalez-Ruiz A. Severe preeclampsia-eclampsia in young primigravidas: subsequent pregnancy outcome and remote prognosis. Am J Obstet Gynecol 1986; 1555: 1011-16.

8. Sibai BM. Preeclampsia-eclampsia. Curr Prob Obstet Gynecol Fertil 1990; 13: 1-45.

9. Chesley LC. History and epidemiology of preeclampsia-eclampsia. Clin Obstet Gynecol 1984; 27: 801-20.

10. Eskenazi B, Fenster L, Sidney S. A multivariate analysis of risk factors of preeclampsia. J Am Med Assoc 1991; 266: 237-41.

11. Easterling TR, Benedetti TJ, Schmucker BC, Millard SP. Maternal hemodynamics in normal and preeclamptic pregnancies: a longitudinal study. Obstet Gynecol 1990; 76: 1061-9.

12. Sibai BM, Gordon T, Thom E, et al. and the National Institute of Child Health and Human Development Network of Maternal-Fetal Medicine Units. Risk factors for preeclampsia in healthy nulliparous women: a prospective multicenter study. Am J Obstet Gynecol 1995; 172: 642-8.

13. Marcoux S, Brisson J, Fabia J. The effect of cigarette smoking on the risk of preeclampsia and gestational hypertension. Am J Epidemiol 1989; 130: 950-7.

14. Seidman DS, Ever-Hadani P, Stevenson DK, Gale R. The effect of abortion on the incidence of preeclampsia. Eur J Obstet Gynecol Reprod Biol 1989; 33: 109-14.

15. Campbell DM, MacGillivray I. Preeclampsia in second pregnancy. Br J Obstet Gynaecol 1985; 92: 131-40.

16. Strickland DM, Guzick DS, Cox K, et al. The relationship between abortion in the first pregnancy and development of pregnancy-induced hypertension in the subsequent pregnancy. Am J Obstet Gynecol 1986; 154: 146-8.

17. Khong TY, De Wolf F, Robertson WB, et al. Inadequate maternal vascular response to placentation in pregnancies complicated by preeclampsia and by small-for-gestational age infants. BJOG 1986; 93: 1049-59.

18. Sibai BM. Pitfalls in the diagnosis and management of preeclampsia. Am J Obstet Gynecol 1988; 159: 1-5.

19. Meyer NL, Mercer BM, Friedman SA, Sibai BM. Urinary dipstick protein: a poor predictor of Absent or severe proteinuria. Am J Obstet Gynecol 1994; 170: 137-41.

20. Kuo VS, Koumantakis G, Gallery EDM. Proteinuria and its assessment in normal and hypertensive pregnancy. Am J Obstet Gynecol 1992; 167: 723-8.

21. Brown MA, Buddle ML. Inadequacy of dipstick proteinuria in hypertensive pregnancy. Aust NZ J Obstet Gynaecol 1995; 35: 366-9.

22. Lindow SW, Davey DA. The variability of urinary protein and creatinine excretion in patients with gestational proteinuric hypertension. Br J Obstet Gynaecol 1992; 99: 869-73.

23. Wheeler TL 2nd, Blackhurst DW, Dellinger EH, et al. Usage of spot urine protein to creatinine ratios in the evaluation of preeclampsia. Am J Obstet Gynecol 2007; 196: 465.e1-4. 
24. Hjartardottir S, Leifsson BG, Geirsson RT, et al. Recurrence of hypertensive disorder in second pregnancy. Am J Obstet Gynecol 2006; 194; 916-20.

25. Collins R, Yusuf S, Peto R. Overview of randomized trials of diuretics in pregnancy. Br Med J 1985; 190: 17-23.

26. Hatton DC, McCarron DA. Dietary calcium and blood pressure in experimental models of hypertension: a review. Hypertension 1994; 23: 513-30.

27. Belizan JM, Villar J, Repke J. The relationship between calcium intake and pregnancy-induced hypertension: up-to-date evidence. Am J Obstet Gynecol 1988; 158: 898-902.

28. Kawasaki N, Matsui K, Nakamura T, et al. Effect of calcium supplementation on the vascular sensitivity to angiotensin II in pregnant women. Am J Obstet Gynecol 1985; 153: 576-82.

29. Sanchez-Ramos L, Briones DK, Kaunitz AM, et al. Prevention of pregnancy-induced hypertension by calcium supplementation in angiotensin-II sensitive patients. Obstet Gynecol 1994; 84: 349-53.

30. Marya RK, Rathee S, Manrow M. Effect of calcium and vitamin D supplementation on toxaemia of pregnancy. Gynecol Obstet Invest 1987; 24: $38-42$.

31. Villar J, Repke J, Belizan JM, et al. Calcium supplementation reduces blood pressure during pregnancy: results from a randomized clinical trial. Obstet Gynecol 1987; 70: 317-22.

32. Lopez-Jaramillo P, Narvaez M, Weigel RM, et al. Calcium supplementation reduces the risk of pregnancy-induced hypertension in an Andes population. Br J Obstet Gynaecol 1987; 96: 648-55.

33. Villar J, Repke JT. Calcium supplementation during pregnancy may reduce preterm delivery in high-risk populations. Am J Obstet Gynecol 1990; 163: 124-31.

34. Montanaro D, Boscutti G, Mioni G, et al. Calcium supplementation decreases the incidence of pregnancy-induced hypertension (PIH) and preeclampsia (Abstract 91). Proceedings of the VIIth World Congress of Hypertension in Pregnancy. Perugia, Italy, 1990.

35. Belizan JM, Villar J, Gonzalez L, et al. Calcium supplementation to prevent hypertensive disorders of pregnancy. N Engl J Med 1991; 325: 1399-405.

36. Lopez-Jaramillo P, Narvaez M, Felix C, Lopez A. Dietary calcium supplementation and prevention of pregnancy hypertension, letter. Lancet 1990; 335: 293.

37. Carroli G, Duley L, Belizan JM, Villar J. Calcium supplementation during pregnancy: a systemic review of randomized controlled trials. $\mathrm{Br} \mathrm{J}$ Obstet Gynaecol 1994; 101: 753-8.

38. Bucher HC, Guyatt GH, Cook RJ, et al. Effect of calcium supplementation on pregnancy-induced hypertension and preeclampsia. A metaanalysis of randomized controlled trials. J Am Med Assoc 1996; 275: 1113-17.

39. Levine RJ, Raymon E, Der Simonian R, Clemens JD. Preeclampsia prevention with calcium supplementation. Clin Appl Nutr 1992; 2: 30.

40. Levine RJ, Esterlitz JR, Raymond EG, et al. National Institute of Health/ Maternal-Fetal Medicine Units Network, The trial of calcium for preeclampsia prevention (CPEP): rationale, design, and methods. Control Clin Trials 1996; 17: 442-69.

41. Spitz B, Magness RR, Cox SM, et al. Low-dose aspirin. I. Effect on angiotensin II pressor responses and blood prostaglandin concentration in pregnant women sensitive to angiotensin II. Am J Obstet Gynecol 1988; 159: 1035-43.

42. Duley L, Henderson-Smart DJ, Knight M, et al. Anti-platelet agents for preventing pre-eclampsia and its complications. Cochrane Database Syst Rev 2004: CD004659.

43. Hauth JC, Goldenberg RL, Parker R Jr, et al. Low-dose aspirin therapy to prevent preeclampsia. Am J Obstet Gynecol 1993; 168: 1083-93.

44. Sibai BM, Caritis SN, Thom E, et al.; and the National Institute of Child Health and Human Development Network of Maternal-Fetal Medicine Units. Prevention of preeclampsia with low-dose aspirin in healthy, nulliparous pregnant women. N Engl J Med 1993; 329: 1213-18.

45. Viinikka L, Hartikainen-Sorri A-L, Lumme R, et al. Low dose aspirin in hypertensive pregnant women: effect on pregnancy outcome and prostacyclin-thromboxane balance in mother and newborn. Br J Obstet Gynaecol 1993; 100: 809-15.
46. Italian Study of Aspirin in Pregnancy. Low-dose aspirin in prevention and treatment of intrauterine growth retardation and pregnancy-induced hypertension. Lancet 1993; 341: 396-400.

47. CLASP. A randomized trial of low-dose aspirin for the prevention and treatment of preeclampsia among 9364 pregnant women. Lancet 1994; 343: 619-29.

48. Hamid R, Robson M, Pearch JM. Low-dose aspirin in women with raised maternal serum alpha-fetoprotein and abnormal Doppler waveform patterns from the uteroplacental circulation. Br J Obstet Gynaecol 1994; 101: 481-4.

49. Estudo Colaborativo para Prevencao da Pre-eclampsia com Aspirina (ECPPA). ECPPA: randomised trial of low dose aspirin for the prevention of maternal and fetal complications in high risk pregnant women. Br J Obstet Gynaecol 1996; 103: 39-47.

50. Caritis S, Sibai BM, Hauth JC. Low dose aspirin does not prevent preeclampsia in high risk women. N Engl J Med 1998; 338: 701-5.

51. Askie LM, Duley L, Henderson-Smart DJ, et al. Anti-platelet agents for preventing pre-eclampsia: a meta-analysis of individual patient data. Lancet 2007; 369: 1791-8.

52. Roberts JM, Myatt L, Spong CY, et al. Eunice Kennedy Shriver National Institute of Child Health and Human Development Maternal-Fetal Medicine Units Network: Vitamins C and E to prevent complications of pregnancy-associated hypertension. N Engl J Med 2010; 362: 1282-91.

53. Gilstrap LC, Cunningham GF, Whalley PJ. Management of pregnancy induced hypertension in the nulliparous patient remote from term. Semin Perinatol 1978; 2: 73-81.

54. Sibai BM, Gonzalez AR, Mabie WC, et al. A comparison of labetalol versus hospitalization alone in the management of preeclampsia remote from term. Obstet Gynecol 1987; 70: 323-7.

55. Hamlin RHJ. The prevention of eclampsia and preeclampsia. Lancet 1952; 1: 64-8.

56. Sibai BM, Barton JR, Akl S, et al. A randomized prospective comparison of nifedipine and bed rest versus bed rest alone in the management of preeclampsia remote from term. Am J Obstet Gynecol 1992; 167: 879-84.

57. Matthews DD, Patel IR, Sengupta SM. Outpatient management of toxaemia. J Obstet Gynaecol 1971; 78: 610-19.

58. Matthews DD, Agarwal V, Shuttleworth TP. The effect of rest and ambulation on plasma urea levels in pregnant women with proteinuric hypertension. Br J Obstet Gynaecol 1980; 87: 1095-8.

59. Crowther CA, Bouwmeester AM, Ashurst HM. Does admission to hospital for bed rest prevent disease progression or improve fetal outcome in pregnancy complicated by non-proteinuric hypertension? $\mathrm{Br}$ J Obstet Gynaecol 1992; 99: 13-17.

60. American College of Obstetricians and Gynecologists. Diagnosis and Management of Preeclampsia. Practice Bulletin No. 33. Washington, DC: American College of Obstetricians and Gynecologists, 2002.

61. Barton JR, Stanziano GJ, Sibai BM. Monitored outpatient management of mild gestational hypertension remote from term. Am J Obstet Gynecol 1994; 170: 765-9.

62. Helewa M, Heaman M, Robinson MA, Thompson L. Community based home-care program for the management of pre-eclampsia: an alternative. Can Med Assoc J 1993; 149: 829-34.

63. Soothill PW, Campbell S, Gibbs J, et al. Effect of a fetal surveillance unit on admission of antenatal patients to hospital. Br Med J 1991; 303: 269-71.

64. Tuffnell DJ, Lilford RJ, Buchan PC, et al. Randomized controlled trial of daycare for hypertension in pregnancy. Lancet 1992; 339: 224-7.

65. Dawson AJ, Middlemiss C, Coles EC, et al. A randomized study of a domiciliary antenatal care scheme: the effect on hospital admissions. $\mathrm{Br}$ J Obstet Gynaecol 1989; 96: 1319-22.

66. Twaddle S, Harper V. An economic evaluation of day care in the management of hypertension in pregnancy. Br J Obstet Gynaecol 1992; 99: 459-63.

67. Turnbull DA, Wilkinson C, Gerard K, et al. Clinical, psychosocial, and economic effects of antenatal day care for three medical complications of pregnancy: a randomized controlled trial of 393 women. Lancet 2004; 353: 1104-9.

68. Barton JR, Stanziano GJ, Jacques DL, et al. Monitored outpatient management of mild gestational hypertension remote from term in teenage pregnancies. Am J Obstet Gynecol 1995; 73: 1865-8. 
69. Sibai BM, Barton JR. Expectant management of severe preeclampsia remote from term: patient selection, treatment, and delivery indications. Am J Obstet Gynecol 2007; 196: 514.e1-9.

70. Koopmans CM, Bijlenga D, Groen H, et al.; HYPITAT study group. Induction of labour versus expectant monitoring for gestational hypertension or mild pre-eclampsia after 36-weeks' gestation (HYPITAT): a multi-centre, open-label randomized, controlled trial. Lancet 2009; 374: 979-88.

71. Vijgen SMC, Koopmans CM, Opmeer BC, et al. An economic analysis of induction of labor and expectant monitoring in women with gestational hypertension or pre-eclampsia at term (HYPITAT trial). BJOG 2010; 117: 1575-6.

72. Rubin PC, Clark DM, Summer DJ, et al. Placebo-controlled trial of atenolol in the treatment of pregnancy associated hypertension. Lancet 1983; 1: 431-4.

73. Wichman K, Ryden G, Karlberg BE. A placebo controlled trial of metoprolol in the treatment of hypertension in pregnancy. Scand J Clin Lab Invest 1984; 44: 90-5.

74. Plouin PF, Breart GL, Maillard F, et al. Comparison of antihypertensive efficacy and perinatal safety of labetalol and methyldopa in the treatment of hypertension in pregnancy: a randomized controlled trial. Br J Obstet Gynaecol 1988; 95: 868-76.

75. Pickles CJ, Symonds EM, Broughton-Pipkin F. The fetal outcome in a randomized double-blind controlled trial of labetalol versus placebo in pregnancy-induced hypertension. Br J Obstet Gynaecol 1989; 96: 38-43.

76. Plouin F, Beart G, Llado J, et al. A randomized comparison of early with conservative use of antihypertensive drugs in the management of pregnancy-induced hypertension. Br J Obstet Gynaecol 1990; 97: $134-41$.

77. Phippard AF, Fisher WE, Horvath JS, et al. Early blood pressure control improves pregnancy outcome in primigravid women with mild hypertension. Med J Aust 1991; 154: 378-82.

78. Cruikshank DJ, Robertson AA, Campbell DM, MacGillivray I. Does labetalol influence the development of proteinuria in pregnancy hypertension? A randomized controlled study. Eur J Obstet Gynecol Reprod Biol 1992; 45: 47-51.

79. Pickles CJ, Broughton-Pipkin F, Symonds EM. A randomized placebo controlled trial of labetalol in the treatment of mild to moderate pregnancy induced hypertension. Br J Obstet Gynaecol 1992; 99: 964-8.

80. Jannet D, Carbonne B, Sebban E, Milliez J. Nicardipine versus metoprolol in the treatment of hypertension during pregnancy: a randomized controlled trial. Obstet Gynecol 1994; 84: 354-9.

81. Sibai BM. Treatment of hypertension in pregnant women. N Engl J Med 1996; 335: 257-65.

82. Hall DR, Odendaal HJ, Kristen GF, et al. Expectant management of early onset, severe preeclampsia: perinatal outcome. BJOG 2000; 107: 1258-64.

83. Sibai BM, Mercer BM, Schiff E, Friedman SA. Aggressive versus expectant management of severe pre-eclampsia at 28 to 32 weeks' gestation: a randomized controlled trial. Am J Obstet Gynecol 1994; 171: 818-22.

84. Schiff E, Friedman SA, Sibai BM. Conservative management of severe preeclampsia remote from term. Obstet Gynecol 1994; 84: 626-30.

85. Odendaal HJ, Pattison RC, Bam R, et al. Aggressive or expectant management for patients with severe preeclampsia between 28-34 week' gestation: a randomized controlled trial. Obstet Gynecol 1990; 76: $1070-5$.

86. Sibai BM, Akl S, Fairlie F, Moretti M. A protocol for managing severe preeclampsia in the second trimester. Am J Obstet Gynecol 1990; 163: $733-8$.

87. Olah KS, Redman WG, Gee H. Management of severe, early preeclampsia: is conservative management justified? Eur J Obstet Gynecol Reprod Biol 1993; 51: 175-80.

88. Vigil-DeGarcia P, Montufar-Rueda C, Ruiz J. Expectant management of severe preeclampsia between 24 and 34 weeks' gestation. Eur J Obstet Gynecol Reprod Biol 2003; 107: 24-7.

89. Chammas MF, Nguyen TM, Li MA, et al. Expectant management of severe preterm preeclampsia: Is intrauterine growth restriction an indication for immediate delivery? Am J Obstet Gynecol 2000; 183: 853-8.
90. Haddad B, Deis S, Goffinet F, et al. Maternal and perinatal outcomes during expectant management of 239 severe preeclamptic women between 24 and 33 weeks' gestation. Am J Obstet Gynecol 2004; 190: $1590-5$.

91. Oettle C, Hall D, Roux A, Grove D. Early onset severe preeclampsia: expectant management at a secondary hospital in close association with a tertiary institution. BJOG 2005; 112: 84-8.

92. Shear RM, Rinfret D, Leduc L. Should we offer expectant management in cases of severe preterm preeclampsia with fetal growth restriction? Am J Obstet Gynecol 2005; 192: 1119-25.

93. Visser W, Wallenburg H. Temporizing management of severe preeclampsia with and without the HELLP syndrome. Br J Obstet Gynaecol 1995; 102: 111-17.

94. Magann EF, Bass D, Chauhan SP, et al. Antepartum corticosteroids: disease stabilization in patients with the syndrome of hemolysis, elevated liver enzymes, and low platelets (HELLP). Am J Obstet Gynecol 1994; 171: 1148-53.

95. Schiff E, Friedman SA, Mercer BM, Sibai BM. Fetal lung maturity is not accelerated in preeclamptic patients. Am J Obstet Gynecol 1993; 169: 1096-101.

96. Friedman SA, Schiff E, Kao L, Sibai BM. Neonatal outcome after preterm delivery for preeclampsia. Am J Obstet Gynecol 1995; 172: 1785-92.

97. Yoon BH, Lee CM, Kim SW. An abnormal umbilical artery waveform: a strong and independent predictor of adverse perinatal outcome in patients with preeclampsia. Am J Obstet Gynecol 1994; 171: 713-21.

98. Torres PJ, Gratacos E, Alonso PL. Umbilical artery doppler ultrasound predicts low birthweight and fetal death in hypertensive pregnancies. Acta Obstet Gynecol Scand 1995; 74: 352-5.

99. Sibai M, Barton JR. Expectant management of severe pre-eclampsia remote from term: patient selection, treatment, and delivery indications. Am J Obstet Gynecol 2007; 196: 514.e1-9.

100. Fenakel K, Fenakel G, Appelman Z, et al. Nifedipine in the treatment of severe preeclampsia. Obstet Gynecol 1991; 77: 331-7.

101. Mabie WC, Gonzales AR, Sibai BM, Amon E. A comparative trial of labetalol and hydralazine in the acute management of severe hypertension complicating pregnancy. Obstet Gynecol 1987; 70: 328-33.

102. Moodley J, Koranteng SA, Rout C. Expectant management of early onset of severe preeclampsia in Durban. S Afr Med J 1993; 83: 584-7.

103. Visser W, Wallenburg HCS. Maternal and perinatal outcome of temporizing management in 254 consecutive patients with severe preeclampsia remote from term. Eur J Obstet Gynecol Reprod Biol 1995; 63: 147-54.

104. Gaugler-Senden IPM, Huijssoon AG, Visser W, Steegers EAP, deGroot CJM. Maternal and perinatal outcome of preeclampsia with onset before 24 weeks' gestation: audit in a tertiary referral center. Eur J Obstet Gynecol Reprod Biol 2006; 128: 216-21.

105. Budden A, Wilkinson L, Buksh MJ, Mc-Cowan L. Pregnancy outcome in women presenting with pre-eclampsia at less than 25 weeks' gestation. Aust NZ J Obstet Gynaecol 2006; 46: 407-12.

106. Chari RS, Friedman SA, O’Brien JM, Sibai BM. Daily antenatal testing in women with severe pre-eclampsia. Am J Obstet Gynecol 1995; 173: 1207-10.

107. Sibai BM, Ramanathan J. The case for magnesium sulfate in preeclampsia-eclampsia. Int J Obstet Anesth 1992; 1: 167-75.

108. Friedman SA, Lim K, Baker CA, Repke J. Phenytoin versus magnesium sulfate in preeclampsia: a pilot study. Am J Perinatol 1993; 10: 233-8.

109. Lucas MJ, Leveno KJ, Cunningham FG. A comparison of magnesium sulfate with phenytoin for the prevention of eclampsia. N Engl J Med 1995; 333: 201-5.

110. Ashe RE, Moodley J, Richards AM, Philpott RH. Comparison of labetalol and dihydralazine in hypertensive emergencies of pregnancy. S Afr Med J 1987; 71: 354-6.

111. Shoemaker CT, Meyers M. Sodium nitroprusside for control of severe hypertensive disease of pregnancy: a case report and discussion of potential toxicity. Am J Obstet Gynecol 1984; 149: 171-3.

112. Abboud T, Artal R, Sarkis F, et al. Sympathoadrenal activity, maternal, fetal and neonatal responses after epidural anesthesia in the preeclamptic patient. Am J Obstet Gynecol 1982; 144: 915-18.

113. Jouppila P, Jouppila R, Hollmen A, et al. Lumbar epidural analgesia to improve intervillous blood flow during labor in severe preeclampsia. Obstet Gynecol 1982; 59: 158-61. 
114. Miles GJ, Dempster J, Patel NB, Taylor DJ. Epidural analgesia and its effect on umbilical artery flow velocity waveform patterns in uncomplicated labour and labour complicated by pregnancy-induced hypertension. Eur J Obstet Gynaecol Reprod Biol 1990; 36: 35-41.

115. Ramanathan J, Coleman P, Sibai BM. Anesthetic modification of hemodynamic and neuroendocrine stress responses to cesarean delivery in women with severe preeclampsia. Anesth Analg 1991; 73: 772-9.

116. Sibai BM, Mabie BC, Harvey CJ, Gonzalez AR. Pulmonary edema in severe preeclampsia-eclampsia: analysis of 37 consecutive cases. Am J Obstet Gynecol 1987; 156: 1174-9.

117. Barton JR, Hiett AK, Conover WC. The use of nifedipine during the postpartum period in patients with severe preeclampsia. Am J Obstet Gynecol 1990; 162: 788-92.

118. Barton JR, Prevost RR, Wilson DA, et al. Nifedipine pharmacokinetics and pharmacodynamics during the immediate postpartum period in patients with pre-eclampsia. Am J Obstet Gynecol 1991; 165: 91-4.
119. Magnussen EB, Vatten LJ, Smith GD, Romundstad PR. Hypertensive disorders in pregnancy and subsequently measured cardiovascular risk factors. Obstet Gynecol 2009; 114: 961-70.

120. Strobl J, Windbichler G, Strasak A, et al. Left ventricular function many years after recovery from pre-eclampsia. BJOG 2011; 118: 76-83.

121. Tenhola S, Rahiala E, Halonen P, et al. Maternal preeclampsia predicts elevated blood pressure in 12-year-old children: evaluation by ambulatory blood pressure monitoring. Pediatr Res 2006; 59: $320-4$.

122. Palmsten K, Buka SL, Michels KB. Maternal pregnancy-related hypertension and risk for hypertension in offspring later in life. Obstet Gynecol 2010; 116: 858-64.

123. Kajantie E, Eriksson JG, Osmond C, et al. Pre-eclampsia is associated with increased risk of stroke in adult offspring, The Helsinki Birth Cohort Study. Stroke 2009; 40: 1176-80. 


\section{Placenta previa and placental abruption Ann K. Lal and William J. Watson}

Antepartum hemorrhage can be divided into obstetric and non-obstetric causes. Frequent non-obstetric causes of antepartum vaginal hemorrhage include cervicitis, cervical polyps, cervical cancer, or vaginal lacerations. In the third trimester, the main clinically important causes of antepartum hemorrhage are placenta previa and placental abruption. When the clinician is evaluating a patient with antepartum hemorrhage, a precise diagnosis is needed so that proper management can be expeditiously implemented.

\section{PLACENTA PREVIA}

Placenta previa is defined as abnormal placental implantation over the cervical os. There are several variants of this definition. A low-lying placenta occurs when the placenta implants in the lower uterine segment; the placental edge lies near, but not over the cervical os. A low-lying placenta is not considered a category of placenta previa, but it is a form of irregular placentation, which may cause vaginal bleeding when the lower uterine segment develops during labor. When the edge of the placenta lies exactly at the internal cervical os, a marginal placenta previa is present. A complete placenta previa occurs when the internal cervical os is completely covered by the placenta. Clinicians refer to a "central previa" when the center of the placenta is covering the internal os of the cervix (Figs. 1 and 2). The term "partial placenta previa" in which part of the placenta covers the internal os of the cervix is not clinically useful, given the above definitions.

\section{Epidemiology}

Placenta previa is thought to be caused by an implantation abnormality. With increasing gravity and parity, for example, the underlying endometrium may be damaged, and in subsequent pregnancies, the embryo may implant in the lower uterine segment because it is a potentially more suitable site (1). The incidence of placenta previa complicating pregnancy is $0.26 \%$ to $1 \%(2,3)$. In gravidas with parity of five or greater, the incidence increases to $2.2 \%$ (4).

There are many known risk factors for placenta previa. One of the most significant risk factors is prior cesarean delivery. In a patient with two prior cesarean deliveries, the risk of previa is approximately $1.9 \%$; this increases to $4.1 \%$ in those with three or more previous cesareans (5). If a patient with a prior cesarean section has a placenta previa in a subsequent pregnancy, the probability that the previa will persist until the term is increased (Table 1) (6).

A history of placenta previa confers an eightfold increased risk of previa in a subsequent pregnancy (7). A history of prior uterine curettage is a risk factor for previa, most likely due to the endometrial scarring from the surgical procedure (8). Advanced maternal age also increases the risk for the development of placenta previa. One report found that in women of age 19, the risk of previa was $1 / 1500$, but the incidence of previa increased to $1 / 100$ in gravidas greater than 35 years old (3). Smoking poses a twofold elevated risk for the development of placenta previa. This is thought to be secondary to carbon monoxide-induced hypoxemia, which causes placental hypertrophy and impaired decidual vascularization (9). Likewise, an amplified risk is noted in patients who live at higher altitudes, most likely due to an increased placental surface area (10).

\section{Clinical Presentation}

The classic presentation of placenta previa is painless sudden vaginal bleeding. The first episode of bleeding is called the "sentinel bleed." Most sentinel bleeds occur after the 28th week of gestation. About $70 \%$ of patients with placenta previa experiencing an antepartum hemorrhage will have this occur prior to 34 weeks of gestation (11). This painless hemorrhage usually has an onset without previous signs or symptoms. Fortunately, most initial bleeds are small and stop spontaneously. In patients with marginal placenta previa, it is more likely that the sentinel bleed will occur with the onset of labor. The cause of this bleeding is the development, or thinning, of the lower uterine segment and dilation of the cervix, which can cause dissociation of the placental connection to the uterus. This bleeding is augmented by the fact that the lower uterine segment has less contractile activity and is therefore impaired in its ability to constrict and stop ongoing hemorrhage. Bhide et al. studied 121 patients with low-lying placenta and placenta previa and noted that in cases of partial or marginal placenta previa, 57\% had antepartum hemorrhage (12). The risk of bleeding with low-lying placenta was inversely related to the distance of the placental edge from the cervical os.

\section{Diagnosis}

The diagnosis of placenta previa is clinically important when a patient presents with antepartum hemorrhage. Among patients with placenta previa, specifically those with antepartum hemorrhage, the average gestational age at diagnosis is 32 weeks. Classical teaching is that pelvic exams are contraindicated in a patient with antepartum hemorrhage and unknown placental location. Before the availability of accurate real-time ultrasound, if a pelvic exam was to be performed in a patient with vaginal bleeding in the latter half of pregnancy, a double setup was required. A double setup is a digital cervical exam performed in the operating room with the necessary staff and equipment ready to perform an immediate cesarean delivery should heavy bleeding occur. Current ultrasound technology has made the double setup exam obsolete and relegated this procedure to the history books.

Initially, transabdominal ultrasound was used for diagnosis. Transabdominal ultrasonography, however, may be limited by 


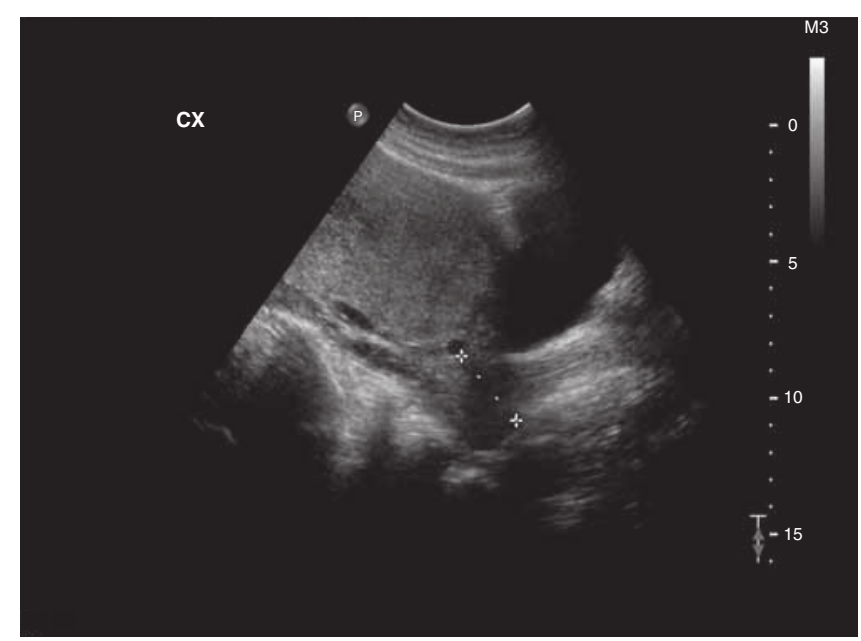

Figure 1 Abdominal ultrasound image of complete placenta previa, with a cervical length of $3.24 \mathrm{~cm}$.

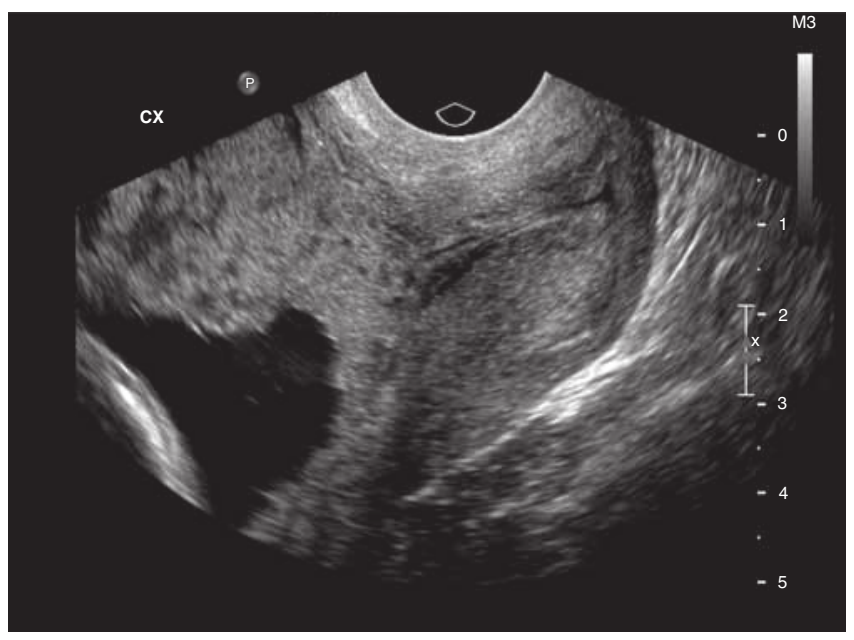

Figure 2 Transvaginal ultrasound image of complete placenta previa.

\section{Table 1 Risk Factors for Placenta Previa}

Prior cesarean delivery

Prior uterine curettage

Cigarette smoking
Prior placenta previa Multiparity Advanced maternal age difficulty visualizing a posterior placenta, difficulty visualizing the lower uterine segment secondary to fetal positioning, and poor visualization in cases of maternal obesity (13). Although pelvic examination is contraindicated, clinical experience has shown that transvaginal ultrasound is safe to perform in patients with placenta previa (14). It has also been proven to be superior to abdominal ultrasound as diagnostic tool (15). Translabial ultrasounds can be of diagnostic assistance, but this technique does not appear to offer an advantage over transvaginal sonography. With the improved diagnostic accuracy of transvaginal ultrasound, in cases with a low-lying placenta, the ultrasound report should state the actual distance of the placental edge from the cervical os (13).

\section{Natural History}

If placenta previa is diagnosed at less than 24 weeks of gestation in an asymptomatic patient, a repeat ultrasound should be performed at 32 weeks. This is done because $90 \%$ of placenta previas diagnosed at mid-trimester will resolve by term (16). The chance that the placenta previa will continue until term depends on whether it is marginal or complete. Zelop and colleagues found that complete placenta previa will persist at delivery $26 \%$ of the time, while a marginal placenta previa will persist only $2.5 \%$ of the time (17). If the placental edge is not touching the internal os at mid-trimester scan, the chance of a placenta previa at term is remote $(15,18)$. Mustafa and colleagues, in a study of 337 gravidas examined by transvaginal ultrasound, found that in the general population, $42.3 \%$ had placenta previa at 11 to 14 weeks, 3.9\% had previa at 20 to 24 weeks, and $1.9 \%$ had previa at term (19).

Because of the clinical observation that placenta previa diagnosed early in pregnancy often resolves later in pregnancy, the question of placental migration must be addressed. The phenomenon of placental migration can be attributed to two underlying causes (20). The first is trophotropism, which states that the placenta migrates toward areas of better endometrial vascularity. The second is that the placenta appears to migrate because it undergoes atrophy of the areas implanted over the cervical os, due to inadequate blood supply. Another hypothesis is that the placenta moves away from the cervical os as the lower uterine segment develops. The lower uterine segment extends during pregnancy and has been shown to change its total length from $0.5 \mathrm{~cm}$ at 20 weeks to $5 \mathrm{~cm}$ at term (21). It is noteworthy that the rate of placental migration appears to be variable (18).

\section{Clinical Care}

In patients with placenta previa, delivery occurs at a gestational age less than 36 weeks over $50 \%$ of the time. Because of this, careful management decisions must be made both in patients with a known diagnosis of previa and in those who present symptomatically with vaginal bleeding. The clinician must consider both maturity of the infant and maternal well-being.

In present-day obstetric practice, most cases of placenta previa are diagnosed early in pregnancy, and thus, management and delivery plans are started very early in pregnancy. No activity restrictions appear to be necessary in a stable patient without clinical symptoms prior to 28 weeks of gestation. Some clinicians advise against intercourse after the diagnosis is made early in pregnancy, but there is no objective data to guide the clinician. After 28 weeks of gestation in an asymptomatic patient, there are a few basic principles which are followed. Patients are counseled to present to the labor and delivery unit early with any sign of hemorrhage, and they are advised to avoid strenuous physical activity and sexual intercourse (8). Follow-up should include ultrasounds to assess the placental location. If the placenta previa resolves during this time of follow-up, no further evaluation is needed and the patient is free from activity restrictions. If, however, the placenta previa persists to the third trimester, the clinician should continue to follow serial ultrasounds and the patient should continue with activity restrictions.

In the clinical management of gravidas who present with preterm bleeding, the initial management should consist of admission to the labor and delivery unit with continuous fetal heart monitoring and monitoring of maternal vital signs. Largebore intravenous access should be established in the mother. 
Initial laboratory evaluation includes hemoglobin, hematocrit, platelet count, type and screen, and coagulation studies; Rhimmune globulin should be administered to Rh-negative gravidas. If the gestational age is $<34$ weeks, antenatal corticosteroids should be administered. Depending on the location of the institution and availability of neonatal care, transfer to a larger facility should be considered. Transfer of an actively bleeding patient may not be possible because of the maternal risk during transport. Of gravidas who present with an initial bleeding episode greater than $500 \mathrm{~mL}, 50 \%$ will not require immediate delivery (10). Only $5 \%$ who present initially with profuse vaginal bleeding need immediate delivery (22).

When patients with placenta previa present with a sentinel bleed, many experience uterine contractions or uterine irritability, which may augment the bleeding. Tocolytic therapy may decrease uterine irritability and decrease bleeding that is occurring secondary to uterine contractions (23). Depending on the clinical presentation, tocolysis can be utilized. Magnesium sulfate may be a better choice when compared with beta-mimetics, due to a lesser effect on the maternal cardiovascular status (24). In one retrospective study by Towers et al., $72 \%$ of patients diagnosed with placenta previa received tocolytic agents. The mean birth weight of infants born to mothers who had received tocolytics was 2276 versus $2076 \mathrm{~g}$ for those who did not receive tocolytic therapy (25). Neither the need for blood transfusion was increased in patients who received tocolytic therapy nor the maternal morbidity. Besinger et al. found that in the group of patients who received tocolysis, the mean birth weight was 2520 versus $2124 \mathrm{~g}$ in the no tocolytic group (26). Additionally, delivery was 39.2 days from admission in the group that received tocolytics versus the group that did not, where it was 26.9 days. In this study, both short-term and long-term tocolyses were used. In patients receiving only short-term intravenous magnesium sulfate treatment, the time until delivery was 15.3 days. In patients receiving longer term tocolysis, the delay until delivery was 40.9 and 47.4 days, respectively. This was statistically significant from the shortterm application of tocolytics only (26). Similarly, a study by Sharma and colleagues using ritodrine as a tocolytic showed prolonged time to delivery, higher mean birth weight, no difference in bleeding episodes between the two groups, and an acceptable maternal and fetal safety profile (27).

The use of any tocolytic is controversial in modern obstetric practice. Specifically in the case of a stable patient with placenta previa and contractions, there are no prospective studies to guide the clinician. Tocolysis is appropriate to use during maternal transport. We think that tocolysis with a nonbeta-mimetic is reasonable during the 48-hour window of steroid administration. There is no convincing evidence that use of long-term tocolysis in this clinical circumstance is beneficial. The use of a prophylactic tocolytic agent, such as 17-alpha-hydroxyprogesterone, to prevent the development of the lower uterine segment and possibly delay the onset of the sentinel bleed has not been investigated.

The conservative management of patients who are carrying preterm infants and are clinically stable after an initial antepartum hemorrhage includes expectant management with in-hospital bed rest. This in-hospital management includes daily fetal monitoring, fluid management, maternal blood transfusion, if needed, and fetal lung maturity tests (25). Lam et al. assessed patients with known placenta previa and found that the overall incidence was $0.9 \%$, with $60 \%$ of the patient experiencing antepartum hemorrhage (11). Between the patients who experienced an antepartum hemorrhage and those who did not, there was no difference in their age, parity, initial hemoglobin level, previous elective abortions, previous spontaneous abortions, previous dilation and curettages, prior cesarean sections, and history of placenta previa. In the group of patients presenting with antepartum hemorrhage, there was an increase in antenatal corticosteroid use, tocolytic administration, and an increase in emergency cesarean sections. With conservative management, this same study showed that the mean interval between bleeding and delivery via cesarean section was 3 weeks. Less than $25 \%$ of women had cesarean sections within 1 week of the initial presentation with bleeding.

In patients with previa who present with an initial bleeding episode and remain clinically stable without bleeding for 48 hours of observation, some might be eligible for outpatient management (20). For a patient to be eligible for outpatient care, she must understand her diagnosis and its implications. She should also live in close proximity to the hospital and have constant access to a telephone and transportation. In a randomized controlled trial by Wing and colleagues evaluating the significant differences in outcome between inpatient and outpatient management, there was no significant difference in mean time interval from enrollment to delivery, mean gestational age of the infant at delivery, birth weight, neonatal intensive care unit (NICU) admissions, and neonatal morbidity (22). Only 5 of 26 patients initially randomized to outpatient care had to be admitted for a third episode of bleeding, which was the criterion for changing groups in this study. Similar numbers of inpatients and outpatients had a second episode of vaginal bleeding (22). In Love's retrospective study assessing management strategy for placenta previa, the need for urgent delivery, defined as delivery within 4 hours of the presenting episode of vaginal bleeding, was unpredictable and showed no association with the number of bleeding incidents, the type of placenta previa, or outpatient management (28). Moreover, in women with placenta previa managed on an outpatient basis who needed an immediate delivery, there was no increase in maternal or neonatal morbidity and mortality (28). The decision for outpatient management needs to be individualized, with an emphasis on careful patient selection and education prior to discharge.

In most all patients with placenta previa, the mode of delivery must be cesarean section. Timing of delivery is a delicate balance between the risk of maternal bleeding and fetal prematurity. Without acute bleeding, delivery can be accomplished at 36 weeks with or without an amniocentesis. In a patient with any bleeding at a gestational age $>34$ weeks, it is reasonable to deliver her. In patients with a marginal placenta previa, greater than $2 \mathrm{~cm}$ from the internal cervical os, a vaginal delivery can be attempted; however, the facility and clinician must be prepared for immediate cesarean delivery. Additionally, there is an increased risk for postpartum hemorrhage in these patients (15).

During the cesarean delivery, two to four units of packed red blood cells should be readily available. The clinician should be prepared for significant postpartum hemorrhage. Ideally, 
the placenta should not be disrupted with the hysterotomy. However, a transverse incision will most often extend through the placenta and, nonetheless, maternal and fetal outcomes remain good. The fetus should be delivered expeditiously when going through the placenta after hysterotomy. During the delivery, risk factors for blood transfusion include use of general anesthesia, history of cesarean delivery, and the need to perform a cesarean hysterectomy (29). In the case of previa with prior cesarean and suspected accreta, the incision should be made vertically, high on the uterus, above the placental implantation site.

Postpartum hemorrhage is a known risk that may occur after cesarean section for placenta previa. Hemostasis can be attempted with intravenous or intramuscular oxytocin, methylergonovine, carboprost, or uterine massage. These techniques may not be effective because the bleeding is coming from the lower uterine segment, which may not contract down well after delivery. If bleeding continues, the physician can try uterine artery ligation, bilateral ligation of the internal iliac artery, or cesarean hysterectomy as a last resort. Additional procedures to try during surgery include circular isthmic-cervical suture placement and parallel vertical compression sutures. Cervical isthmic sutures obstruct the site where the placenta was abnormally attached by interrupting the uterine blood flow below the placental implantation site (30). Dedes reported no complications from the suture placement; hysterectomy was not needed, and at ultrasonography performed 6 weeks after delivery, no abnormalities were demonstrated. Vertical compression sutures placed in the lower uterine segment can also help to achieve control of postpartum hemorrhage in patients with abnormal placentation (31). These sutures were found to effectively control bleeding in 14 women in whom they were placed, without intraoperative complications. Additionally, all women had a normal ultrasound 1 month postpartum and resumed normal menstrual flow after the conclusion of breastfeeding. If all other means for obtaining hemostasis have been attempted, and bleeding continues despite abnormal maternal coagulation status, cesarean hysterectomy can be performed. In one report, the most common cause for cesarean hysterectomy was placenta accreta (51.1\%), followed by placenta previa at $26.7 \%$ (32).

\section{PLACENTA ACCRETA}

A significant risk associated with placenta previa is placenta accreta. Placenta accreta is abnormal attachment of the placenta, with an absence of deciduas basilas and an incomplete development of the fibrinoid layer. More severe variants are placenta increta and percreta, with placental extension into the myometrium and through the myometrium, respectively. Two significant risk factors for placenta accreta formation are history of a prior cesarean section and a diagnosis of placenta previa. In a patient with a placenta previa and no prior uterine scar, the risk of an accreta is $3 \%$. However, in patients with one cesarean section and a previa, the accreta risk is $11 \%$ and increases to $67 \%$ in those with four prior cesareans (33). In the absence of a placenta previa, placenta accreta is rare (34). Because of the significant risk associated with placenta accreta, investigators have attempted to elucidate ultrasonographic signs to accurately diagnose this condition.
Multiple irregular vascular spaces in the placenta have been associated with accrete (Figure 3) (15). Other ultrasonographic signs include loss of the normal hypoechoic zone behind the placenta, focal exophytic masses in the placental bed, and a disturbed bladder-uterine connection $(33,35)$. Doppler may also be helpful in making this diagnosis $(33,35)$. MRI can also be used as an adjuvant tool to assess placental invasion. In one study when the placenta was implanted anteriorly overlying a previous cesarean section scar, $29 \%$ of cases were accreta (34). The major morbidity associated with accreta is postpartum hemorrhage and maternal blood loss; $55 \%$ of cases require blood transfusion, and in $15 \%$ of cases, there can be more than 5 liters of blood loss (34). Other surgical complications include ureteral transection, cystotomy, disseminated intravascular coagulation, hypotensive shock, and enterotomy.

\section{VASA PREVIA}

Vasa previa is defined as fetal blood vessels running through the membranes over the maternal cervix, unprotected by either the umbilical cord or the placenta (Figure 4). It is associated with velamentous umbilical cord insertion. With

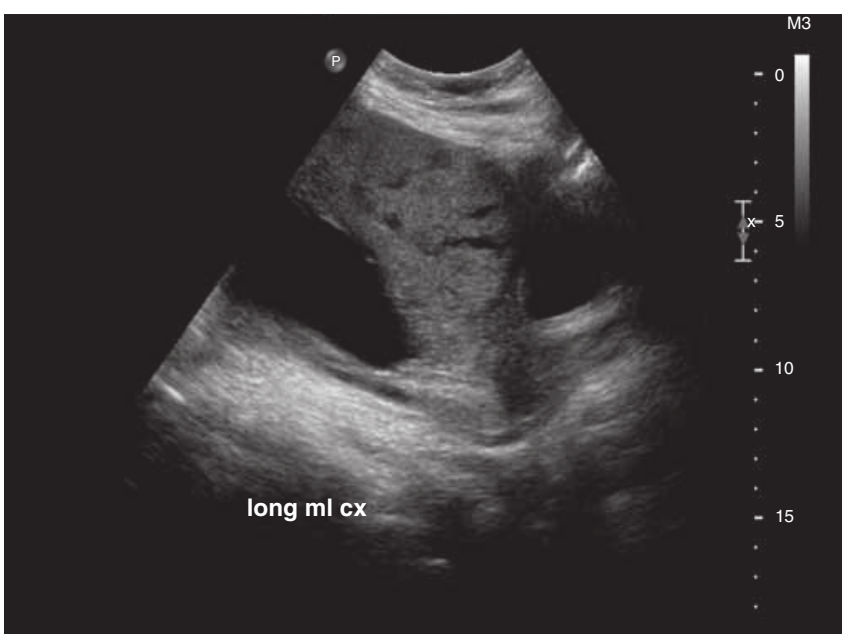

Figure 3 Placenta previa and accreta seen on ultrasound with multiple vascular lacunae present.

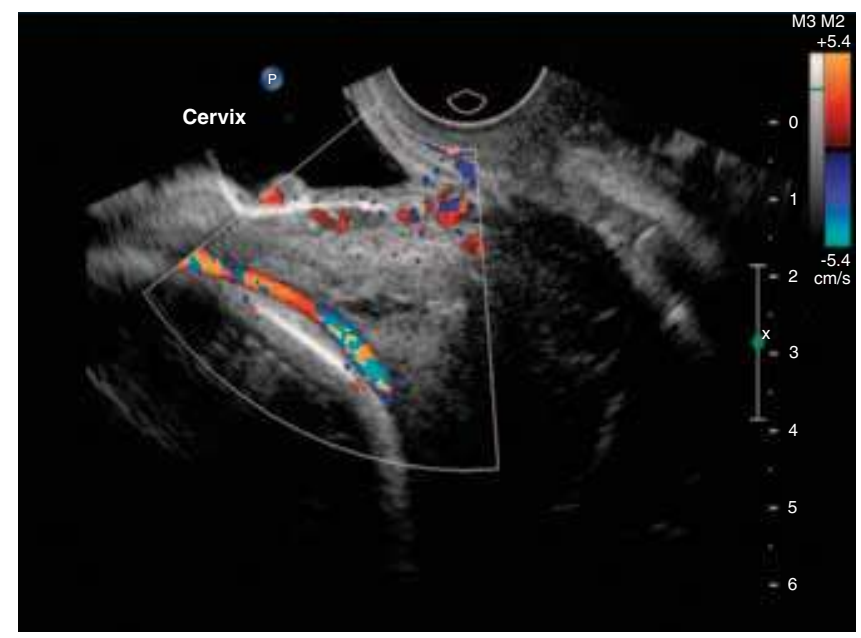

Figure 4 Ultrasonographic depiction of vasa previa with fetal vessels, unprotected by umbilical cord, evident by Doppler ultrasound between fetal head and cervix. 
the rupture of membranes, these fetal vessels can rupture and result in significant fetal bleeding. The fetus can lose a large portion of its blood volume; therefore, this condition is associated with a high fetal mortality rate (8). It is also noteworthy that vasa previa can be present after a low-lying placenta is visualized on a second-trimester ultrasound (15).

\section{POST-DELIVERY COMPLICATIONS}

The clinician also needs to be aware of post-delivery complications. Placenta previa is rarely associated with coagulopathy. Massive blood loss per se can be associated with coagulopathy (see chapter on postpartum hemorrhage). However, in a study by Wing and colleagues, fibrinogen was abnormal in only $1.1 \%$ of the cases, and prothrombin times were normal in all of the women with symptomatic placenta previa who were studied (36). In patients with placenta previa, there is a significant increased risk of postpartum hemorrhage, probably due to the poor contractility of the lower uterine segment. In one report, $8.3 \%$ of women with previa had postpartum hemorrhage, even without a diagnosis of placenta accreta (12). Hemoglobin and hematocrit are most likely to be abnormal of the initial laboratory studies (36). After massive blood loss, complications from hypotension can occur, including acute tubular necrosis and Sheehan syndrome.

\section{FETAL RISKS}

The most significant fetal complication is prematurity. The mean gestational age at delivery is 34 to 35 weeks of gestation. Lam et al. found that $60 \%$ of infants were born at less than 37 weeks, in $26 \%$ the infant was born at less than 34 weeks, and in $5 \%$ delivery occurred at less than 30 weeks (11). Because of this increase in premature birth rates, the neonatal mortality rate is increased threefold. Importantly, however, when controlled for gestational age at delivery, there is no association with intrauterine growth restriction. There is not a difference in the birth weight of term infants (11). There is a slightly elevated risk of fetal anemia. In women with placenta previa who experience antepartum hemorrhage, neonatal risks include respiratory distress syndrome, lower birth weight, lower Apgar scores, and an increase in NICU admission (11).

\section{SPECIAL CIRCUMSTANCES}

First-trimester bleeding is a common problem for an obstetrician. Typically, placenta previa is not associated with first-trimester bleeding; it has been shown, however, that threatened abortion is a 2.5 -fold increased risk factor for placental abruption and placenta previa (37). Varma found that if a placenta previa is detected with ultrasound in the first trimester or early second trimester, it is linked to an increase in vaginal bleeding during pregnancy, an increased rate of spontaneous abortion, and an increased chance of a placenta previa persisting to a later gestational age (38). Obed and colleagues examined 374 patients with threatened abortion; they found that $2 \%$ had placenta previa, compared with a control group rate of $1 \%$ for the same diagnosis (37).

While second-trimester bleeding is less common than firsttrimester bleeding, if bleeding does occur, it is necessary to elucidate the cause of the bleeding. Two common causes are placenta previa and non-previa bleeding that is most likely associated with placental abruption (39). Towers and Burkhart found that in gravidas who bleed between 16 and 24 weeks, the best outcomes were associated with light bleeding, whether or not the patient was diagnosed with a placenta previa. The worst outcome was associated with a patient who presented with heavy vaginal bleeding, not attributed to a placenta previa; $50 \%$ of these pregnancies were lost prior to viability (39).

A patient with a known placenta previa and either an intrauterine fetal demise or a fetus with chromosomal or structural abnormalities diagnosed in the second trimester presents a special problem for the clinician. In these patients, the safest approach for termination of the pregnancy needs to be decided. Halperin retrospectively studied 306 gravidas; $2.6 \%$ had a complete placenta previa and underwent laminaria placement and subsequent dilation and evacuation (40). This study found no difference in operative time, the need for packed red blood cells, postoperative morbidity, duration of hospital stay or bleeding with laminaria placement, when comparing those with and without placenta previa. In Thomas's retrospective study, there was slight increase in blood loss in patients with placenta previa; however, there was no increase in blood transfusion, infection, or postoperative complications (41). Most patients with placenta previa have a previous cesarean section; however, it has been shown that even in patients with prior uterine surgery who undergo laminaria placement and subsequent dilation and evacuation, there is no increase in anesthetic complications, uterine perforation, mean operative time, or cervical lacerations (42).

In pregnancy complicated by placenta previa with fetal demise or fetal anencephaly after 22 weeks of gestation, hysterotomy is the generally accepted practice for delivery. It may be possible in rare cases, after fetal demise has occurred, to attempt vaginal delivery by expectantly managing the patient, allowing the vascularity of the placenta to decrease. There are risks of conservative management in such cases, which include bleeding and coagulopathy. Delivery must occur in a facility where immediate cesarean can be performed. In such cases, maternal care must be individualized.

\section{PLACENTAL ABRUPTION}

Placental abruption is defined as separation of the placenta from the maternal endometrium that occurs before birth. This leads to bleeding at the site of separation from local maternal blood vessels. Most often, the maternal blood then tracks downward between the fetal membranes and uterine wall and comes out of the cervix. If this bleeding remains trapped behind the placenta and is not clinically evident, then the abruption is "concealed." This occurs in approximately $10 \%$ of cases. This placental separation may occur secondary to an acute process, such as maternal trauma, or from a chronic process, such as maternal smoking.

The etiology of abruption is unknown. Some cases are associated with rapid changes in uterine size or shape. This is the case with maternal trauma or abruption that can occur after delivery of one fetus in a multifetal pregnancy or after reduction amniocentesis. Other cases may be related to acute vascular changes in the mother, such as that which may occur after maternal cocaine use. Abruption may also occur in association with chronic placental disease. It is known that 
Table 2 Risk Factors for Placental Abruption

$\begin{array}{ll}\text { Maternal trauma } & \text { Cigarette smoking } \\ \text { Maternal cocaine use } & \text { Hypertension during pregnancy } \\ \begin{array}{c}\text { Abruption in a previous } \\ \text { pregnancy }\end{array} & \text { Premature rupture of membranes } \\ \text { Reduction amniocentesis } & \begin{array}{l}\text { Polyhydramnios } \\ \text { Chorioamnionitis }\end{array} \\ \begin{array}{c}\text { Disorders of maternal } \\ \text { fibrinogen }\end{array} & \text { Advanced maternal age }\end{array}$

abruption is increased, for example, in gravidas with elevated maternal serum alpha fetoprotein levels. Low Papp-A levels are similarly associated with abruption. Serum levels of placental growth factor are decreased in gravida who subsequently develop placental abruption (43). Thrombophilia disorders are common, and some studies have noted an association between thrombophilia and placental abruption (44-47). This association, however, does not establish thrombophilia as a cause of abruption. Newer prospective studies are finding little association between inherited thrombophilias and pregnancy complication such as placental abruption. Currently, there is no evidence that patients with a history of abruption and known thrombophilia should be treated with heparin during pregnancy for the prevention of abruption recurrence (48). It is reasonable to use folate to treat hyperhomocysteinemia in patients with prior abruption. Risk factors for abruption are shown in Table 2 (49-53).

Clinical evidence of abruption occurs in approximately $1 \%$ of pregnancies (49). The frequency is higher than this if one considers abnormal placental findings in asymptomatic gravidas. The spectrum of clinical disease ranges from mild bleeding with a stable mother and baby to fetal death with heavy maternal bleeding and coagulopathy. It is estimated that an abruption of $50 \%$ of the placental surface volume will cause fetal mortality. Abruption severe enough to cause fetal loss is estimated to occur with a frequency of 1 in 830 births (54). Clinical observation indicates that abruption occurs most frequently during the gestational age of 24 to 26 weeks, with a decreasing frequency as the pregnancy progresses to term (49). Abruption is important clinically because it can be a cause of premature birth, with consequent increased perinatal mortality.

The diagnosis of abruption is clinical. Clinical findings include uterine hypertonus with pain and vaginal bleeding. This helps the clinician to distinguish abruption from previa; with placenta previa, painless vaginal bleeding is most commonly seen. The diagnosis can be confounded because, as mentioned above, in some cases of abruption, bleeding is concealed and pain may be insignificant. Abruption should be suspected in a gravida who presents with abdominal or back pain, with vaginal bleeding, or even with symptoms of preterm labor. Typically the uterine contractions are described as high frequency/low amplitude. This pattern may occur as a focal uterine response to limit bleeding at the point of placental separation. The amount of vaginal bleeding observed may not correlate with the extent of abruption. Furthermore, the rate of progression or extension of abruption is unpredictable. Clinicians have noted "port-wine"-colored amniotic fluid in some cases of placental abruption.
The main usefulness of ultrasound lies in finding the placenta location and excluding placenta previa. It is well known that abruption may not be visible on ultrasound study, because the blood may be similar in appearance to the placenta. A retroplacental blood collection may also be hyperechoic or hypoechoic relative to the placenta. If a hyperor hypoechoic mass is seen in a pregnant women with the classical presentation of abruption, then the sonographic accuracy for diagnosis of abruption is high. Sonography, however, cannot be used to exclude abruption. Nyberg et al. reviewed 57 cases of placental abruption sonographically and noted that acute hemorrhage was difficult to distinguish from the adjacent placenta and may be isoechoic with the placenta (55). Resolving hematomas became hypoechoic over 1 to 2 weeks of time period. Some cases were confused with other mass lesions. Glantz reported ultrasound findings on 149 patients with vaginal bleeding after 24 weeks of gestation and confirmed the poor sensitivity of ultrasound in the detection of placental abruption (56).

\section{CLINICAL MANAGEMENT}

Abruption can present as an acute or a chronic process. Acute abruption typically presents clinically with pain and bleeding. In a patient who presents with abdominal pain and vaginal bleeding after the period of fetal viability, the clinician must quickly assess maternal and fetal status. It is necessary to obtain IV access and to send blood for type and crossmatch, as well as coagulation studies. A Kleihauer-Betke test, to assess for fetal cells in the maternal circulation, may be indicated only in the Rh-negative mother. The most important parameter in the coagulation panel is maternal fibrinogen level. In normal pregnancy, fibrinogen levels increase. A fibrinogen level below $250 \mathrm{mg} \%$ is low during pregnancy and levels below $150 \mathrm{mg} \%$ are critically low. A clot (red top) tube can be used to rapidly estimate maternal coagulation status (see chapter on postpartum hemorrhage). If the fetus is alive (less than $50 \%$ abruption) and at a viable gestation age, then electronic fetal monitoring (EFM) is used to assess fetal status. After a bedside ultrasound excludes previa, a vaginal exam to assess cervical status is safe to do and is clinically indicated to assess the status of labor.

If fetal demise has occurred, the goal is to accomplish vaginal delivery while minimizing maternal morbidity. Maternal anemia and coagulopathy, if present, can be corrected by transfusion of packed red blood cells and fresh frozen plasma. Coagulopathy is more commonly seen with large abruptions, and it may be seen in up to $30 \%$ of cases where abruption causes fetal demise. Clinically, labor in most cases is rapid, and vaginal delivery can be accomplished despite maternal bleeding. In the case of massive bleeding in a mother remote from delivery, cesarean delivery may be needed for maternal indications, despite the fetal demise. When performing a cesarean section on a mother with coagulopathy, blood products to correct coagulopathy (fresh frozen plasma, platelets) should be infusing at the time of the initial skin incision, if possible.

Cesarean is also indicated in cases of abruption with a living fetus at a viable gestational age, if there is evidence of abnormal EFM. Ominous findings may include sustained fetal bradycardia or recurrent late decelerations. Rapid delivery in cases of fetal bradycardia may improve fetal outcome in pregnancy 
complicated by abruption. The uterine incision at the time of cesarean is dictated by obstetric indications. A vertical incision may be needed if the fetus is a premature breech or back-down transverse lie. Bleeding will usually abate after delivery of the placenta and correction of coagulopathy, if present.

At the time of cesarean section, the obstetrician may note a dark purple discoloration of the uterus. This is called a Couvelaire uterus; it is caused by extravasation of blood into the myometrium. This was described by Alexandre Couvelaire, a French obstetrician, who wrote a classic paper on the subject in 1911 (57). He termed the condition "uterine apoplexy" and noted that blood from abruption could extravasate not only into the uterine muscle but also into the serosa of the uterus, the broad ligament, and the pelvic peritoneum. Initially it was thought that Couvelaire uterus was an indication for hysterectomy, but with the current therapy for coagulopathy, Couvelaire uterus per se is not considered an indication for hysterectomy.

Following delivery, bleeding may continue due to coagulopathy or uterine atony, and these conditions can be managed medically, as they would in a patient without abruption (see chapter on postpartum hemorrhage). After surgery, the patient should be monitored closely, and general parameters include transfusing packed red cells to keep the hematocrit at or near $30 \%$, platelets above $50,000 / \mathrm{ml}$, and fibrinogen above $150 \mathrm{mg} \%$. Fluids are given to maintain urine output at $>30 \mathrm{cc} / \mathrm{hr}$. Serial assessments of complete blood count and coagulation parameters are needed.

Chronic abruption presents a clinical dilemma because there is no known way to predict recurrent bleeding or extension of abruption. Second-trimester bleeding in the absence of previa most commonly is from marginal placental abruption, and perinatal mortality is increased. In cases managed conservatively after fetal viability, steroids should be given to the mother to enhance fetal pulmonary maturity. If bleeding abates for 48 hours and the fetus has reassuring testing, the patient may be discharged to close outpatient follow-up. Since abruption is associated with growth restriction, it is important to perform serial ultrasound evaluation for growth.

\section{RECURRENCE RISKS}

Previous abruption is a known risk factor for recurrence in a subsequent pregnancy. The recurrence risk is estimated to be $5 \%$ to $15 \%$. If the initial abruption was from a temporary risk factor such as trauma, then the recurrence risk may be lower. In abruption associated with modifiable risk factors, such as maternal smoking or cocaine use, quitting these activities will decrease the recurrence incidence. An interesting observation is that quitting smoking before pregnancy may not reduce the abruption recurrence risk to that of the general population. The abruption risk of former smokers remains elevated (58). In gravidas whose prior pregnancy was complicated by abruption, there is an increase in growth restriction, preeclampsia, and preterm birth in subsequent pregnancies, in addition to abruption. This suggests a common etiologic process of vasculopathy in these conditions.

\section{REFERENCES}

1. Hull AD, Resnik R. Placenta previa, placenta accreta, abruptio placentae, and vasa previa. In: Creasy R, Resnik R, Iams J, Lockwood C, Moore T, eds.
Maternal Fetal Medicine: Principles and Practice, 6th edn. Philadelphia: Saunders Elsevier, 2009: 725.

2. Babinski A, Kerenyi T, Torok O. Perinatal outcome in grand and greatgrand multiparity: effects of parity on obstetrics risk factors. Am J Obstet Gynecol 1999; 181: 669-74.

3. Cunningham FG, Leveno KL, Bloom SL, et al. Obstetrical hemorrhage. In: Cunningham FG, Leveno K, Bloom S, Hauth J, Gilstrap L, Wenstrom K, eds. Williams Obstetrics, 22nd edn. Chicago: McGraw Hill Medical Publishing Division, 2005: 820.

4. Kay HH. Placenta previa and abruption. In: Gibbs R, Karlan B, Haney A, Nygaard I, eds. Danforth's Obstetrics and Gynecology, 10th edn. Philadelphia: Lippincott Williams and Wilkins, 2008: 385-6.

5. Miller DA, Diaz FG, Paul RH. Incidence of placenta previa with previous Cesarean. Am J Obstet Gynecol 1996; 174: 345.

6. Dashe JS, McIntire DD, Ramus RM. Persistence of placenta previa according to gestational age at ultrasound detection. Obstet Gynecol 2002; 99: 692-7.

7. Monica G, Lilja C. Placenta previa, maternal smoking and recurrence risk. Acta Obstet Gynaecol 1995; 74: 341-5.

8. Francois KE, Foley MR. Antepartum and postpartum hemorrhage. In: Gabbe S, Niebyl J, Simpson JL, eds. Obstetrics: Normal and Problem Pregnancies, 5th edn. Philadelphia: Churchill Livingstone Elsevier, 2007: $463,465$.

9. Williams MA, Mittendorf R, Lieberman E, et al. Cigarette smoking during pregnancy in relation to placenta previa. Am J Obstet Gynecol 1991; 165: $28-32$.

10. Cotton, DB, Read JA, Paul RH, Quilligan EJ. The conservative aggressive management of placenta previa. Am J Obstet Gynecol 1980; 137: 687-95.

11. Lam CM, Wong SF, Chow KM, Ho LC. Women with placenta previa and antepartum haemorrhage have a worse outcome than those who do not bleed before delivery. J Obstet Gynaecol 2000; 20: 27-31.

12. Bhide A, Prefumo F, Moore J, et al. Placental edge to internal os distance in the late third trimester and mode of delivery in placenta previa. $\mathrm{Br}$ J Obstet Gynaecol 2003; 110: 860-4.

13. Oppenheimer L. Diagnosis and management of placenta previa. J Obstet Gynaecol Can 2007; 189: 261-6.

14. Timor-Tritsch IE, Yunis RA. Confirming the safety of transvaginal sonography in patients suspected of placenta previa. Obstet Gynecol 1993; 81: 742-4.

15. Bhide A, Thilaganathan B. Recent advanced in the management of placenta previa. Curr Opin Obstet Gynecol 2004; 16: 447-51.

16. Farine D, Peisner DB, Timor-Tritsch IE. Placenta previa-is the traditional diagnostic approach satisfactory? J Clin Ultrasound 1990; 18: 328-30.

17. Zelop CC, Bromley B, Frigoletto FD Jr, Benacerraf BR. Second trimester sonographically diagnosed placenta previa: prediction of persistent placenta previa at birth. Int J Gynaecol Obstet 1994; 44: 207.

18. Oppenheimer L, Holmes P, Simpson N, Dabrowski A. Diagnosis of lowlying placenta: can migration in the third trimester predict outcome? Ultrasound Obstet Gynecol 2001; 18: 100-2.

19. Mustafa SA, Brizot ML, Carvalho MHB, et al. Transvaginal ultrasonography at delivery: a longitudinal study. Ultrasound Obstet Gynecol 2002; 20: $356-9$.

20. Oyelese Y. Placenta previa and related placenta disorders. In: Queenan JT, Spong C, Lockwood CJ, eds. Management of High-Risk Pregnancy, 5th edn. Malden: Blackwell Publishing, 2007: 364.

21. Lavery JP. Placenta previa. Clin Obstet Gynecol 1990; 33: 414-21.

22. Wing D, Paul R, Millar L. Management of the symptomatic placenta previa: a randomized controlled trial of inpatient versus outpatient expectant management. Am J Obstet Gynecol 1996; 175: 806-11.

23. Silver R, Depp R, Sabbagha RE, et al. Placenta previa: aggressive expectant management. Am J Obstet Gynecol 1984; 150: 15-22.

24. Watson WJ, Cefalo RC. Magnesium sulfate tocolysis in selected patients with symptomatic placenta previa. Am J Perinatol 1990; 7: 251-3.

25. Towers C, Pircon R, Heppard M. Is tocolysis safe in the management of third trimester bleeding? Am J Obstet Gynecol 1999; 180: 1572-8.

26. Besinger RE, Moniak CW, Paskiewicz LS, et al. The effect of tocolytic use in the management of symptomatic placenta previa. Am J Obstet Gynecol 1995; 172: 1770-8.

27. Sharma A, Suri V, Gupta I. Tocolytic therapy in conservative management of symptomatic placenta previa. Int J Gynacol Obstet 2004; 84: 109-13. 
28. Love C, Fernando K, Sargent L, Hughes R. Major placenta previa should not preclude outpatient management. Eur J Obstet Gynecol 2004; 117: 24-9.

29. Frederiksen MC, Glassenberg R, Stika CS. Placenta previa: a 22 year analysis. Am J Obstet Gynecol 1999; 180: 1432-7.

30. Dedes I, Ziogas V. Circular isthmic-cervical sutures can be an alternative method to control peripartum haemorrhage during cesarean section for placenta previa accreta. Arch Gynecol Obstet 2008; 278: 555-7.

31. Hwu Y, Chen C, Chen H, Su T. Parallel vertical compression sutures: a technique to control bleeding from placenta previa or accreta during cesarean section. Br J Obstet Gynecol 2005; 112: 1420-3.

32. Daskalakis G, Anastasakis E, Papantoniou N, et al. Emergency obstetric hysterectomy Acta Obstet Gynecol Scand 2007; 86: 223-7.

33. Silver RM, Landon MB, Rouse DJ, et al. Maternal morbidity associated with multiple repeat cesarean deliveries. Obstet Gynecol 2006; 107: 122632.

34. Miller D, Chollet J, Goodwin TM. Clinical risk factors of placenta previaplacenta accreta. Am J Obstet Gynecol 1997; 177: 210-14.

35. Finberg H, Williams J. Placenta accreta: prospective sonographic diagnosis in patients with placenta previa and prior cesarean section. J Ultrasound Med 1992; 11: 333-43.

36. Wing DA, Paul RH, Millar LK. The usefulness of coagulation studies and blood banking in the symptomatic placenta previa. Am J Perinatol 1997; 14: 601-4.

37. Obed JY, Adewole IF. Antepartum hemorrhage: the influence of first trimester uterine bleeding. West Afr J Med 1997; 16: 24-6.

38. Varma TR. The implication of a low implantation of the placenta detected by ultrasonography in early pregnancy. Acta Obstet Gynecol Scand 1981; 60: 265-8.

39. Towers CV, Burkhart AE. Pregnancy outcomes after a primary antepartum hemorrhage between 16 and 24 weeks' gestation. Am J Obstet Gynecol 2008; 198: 684.e1-5.

40. Halperin R, Vaknin Z, Langer R, et al. Late midtrimester pregnancy termination in the presence of placenta previa. J Reprod Med 2003; 48: 175-8.

41. Thomas AG, Alvarez M, Friedman F Jr, et al. The effect of placenta previa on blood loss in second trimester pregnancy termination. Obstet Gynecol 1994; 84: 58-60.

42. Schneider D, Bukovsky I, Caspi E. Safety of midtrimester pregnancy termination by laminaria and evacuation in patients with previous cesarean section. Am J Obstet Gynecol 1994; 171: 554-7.
43. Signore C, Mills JL, Qian C, et al. Circulating angiogenic factors and placental abruption. Obstet Gynecol 2006; 108: 338-44.

44. Roger MA, Paidas M. Do thrombophilias cause placenta-mediated pregnancy complications? Semin Thromb Hemost 2007; 33: 597-603.

45. Prochazka M, Lubuusky M, Slavik L, et al. Frequency of selected thrombophilias in women with placental abruption. Aust NZ J Obstet Gynaecol 2007; 47: 297-301.

46. Prochazka M, Happach C, Marsal K, et al. Factor V Leiden in pregnancies complicated by placental abruption. BJOG 2003; 110: 462-6.

47. Eskes TK. Clotting and placental abruption: homocysteine-a new risk factor. Eur J Obstet Gynecol Reprod Biol 2001; 95: 206-12.

48. Roger MA, Paidas M, McLintock C, et al. Inherited thrombophilia and pregnancy complications revisited. Obstet Gynecol 2008; 112: 320-4.

49. Oyelese Y, Ananth CV. Placental abruption. Obstet Gynecol 2006; 108: 1005-16.

50. Kramer MS, Usher RH, Pollack R, et al. Etiologic determinants of abruptio placentae. Obstet Gynecol 1997; 89: 221-6.

51. Ananth CV, Oyelese Y, Srinivas N, et al. Preterm premature rupture of membranes, intrauterine infection, and oligohydramnios: risks factors of placental abruption. Obstet Gynecol 2004; 104: 71-7.

52. Nath CA, Ananth CV, Smulian JC, et al. Histologic evidence of inflammation and risk of placental abruption. Am J Obstet Gynecol 2007; 1297: 319.e1-6.

53. Slutsker L. Risks associated with cocaine use during pregnancy. Obstet Gynecol 1992; 79: 778-89.

54. Pritchard JA, Mason R, Corley M, Pritchard S. Genesis of severe placental abruption. Am J Obstet Gynecol 1970; 108: 22-7.

55. Nyberg DA, Mack LA, Benedetti TJ, et al. Placental abruption and placental hemorrhage: correlation of sonographic finding with fetal outcome. Radiology 1987; 164: 357-61.

56. Glantz C, Purnell L. Clinical utility of sonography in the diagnosis and treatment of placental abruption. J Ultrasound Med 2002; 21: 837-40.

57. Couvelaire A. Traitment chirurgical des hemorrhagies utero-placentaires avec décollement du placenta normalement insere. Ann de Gynec 1911; 8: 591-608.

58. Ananth CV, Cnattingius S. Influence of maternal smoking on placental abruption in successive pregnancies: a population-based prospective cohort study in Sweden. Am J Epidemiol 2007; 166: 289-95. 


\section{Postpartum hemorrhage Wade D. Schwendemann and William J. Watson}

While the incidence of postpartum hemorrhage has not changed significantly in the past several years, the maternal mortality rate has decreased in developed countries because of better education regarding the issue and new treatment modalities $(1,2)$. New therapies developed for the treatment of postpartum hemorrhage hold considerable promise in expanding the arsenal of the physician in conservatively treating cases refractory to standard therapy.

Developing countries still see a significant maternal mortality rate from this condition, enough to make hemorrhage the most common cause of maternal death worldwide $(1,2)$. In addition to mortality, significant maternal morbidity can result.

\section{DEFINITION AND EPIDEMIOLOGY}

There are several different definitions of postpartum hemorrhage. The first is an estimated blood loss greater than $500 \mathrm{cc}$ with vaginal delivery or greater than $1000 \mathrm{cc}$ with a cesarean delivery (3). These definitions are the standard for most clinicians. However, obstetric texts quote a mean of $500 \mathrm{cc}$ of blood loss for vaginal delivery and $1000 \mathrm{cc}$ of blood loss for cesarean delivery. This confuses the issue, because the average blood loss cannot represent a pathologic condition. We believe that a more reasonable definition of a postpartum hemorrhage is $1000 \mathrm{cc}$ or more with vaginal delivery or greater than $1500 \mathrm{cc}$ with cesarean delivery.

A second definition is a $10 \%$ decrease in the hematocrit when comparing pre- and postdelivery blood counts. This definition has several limitations as well. In our current environment of medical cost containment, a predelivery hematocrit might not be obtained in low-risk cases. Furthermore, following acute blood loss, the hematocrit value may take hours to equilibrate.

These definitions also present a problem because clinicians have been shown to be very poor at estimating actual blood loss, consistently underestimating it (4-7). This difficulty is increased in the obstetric setting due to the presence of amniotic fluid at the time of delivery. Training and graded drapes have been shown to improve the accuracy of blood loss estimates $(8,9)$ and should be implemented, particularly in areas where postpartum hemorrhage is more common.

Further subclassification of postpartum hemorrhage divides cases into early and late hemorrhage. Early postpartum hemorrhage is any hemorrhage that occurs up to 24 hours postdelivery. Postpartum hemorrhage that occurs from 24 hours to 12 weeks postdelivery is considered a late postpartum hemorrhage. This distinction is important to understand because the likelihood of a given diagnosis as a cause for the hemorrhage differs based on the timing.
The incidence of postpartum hemorrhage will vary based on the criteria used to define the condition. The best estimates available indicate that somewhere between $1 \%$ and $5 \%$ of deliveries will be complicated by postpartum hemorrhage (10).

\section{DIFFERENTIAL DIAGNOSIS}

The differential diagnosis of postpartum hemorrhage includes retained placental tissue, uterine atony, lacerations, uterine inversion, and defects in maternal coagulation. Women are at an increased risk of hemorrhage due to uterine atony if they have multiple gestation or polyhydramnios or have recently been administered any medications to reduce frequency of uterine contractions, such as terbutaline (11-14). Hemorrhage caused by laceration is increased in cases of operative vaginal delivery, large for gestational-age infants, and in women with a history of previous lacerations (11-14). Obstetric hemorrhage can also be the presenting symptom in women with a previously undiagnosed clotting disorder (15). Women with the HELLP (hemolysis, elevated liver enzymes, low platelets) syndrome are also at an increased risk for blood loss due to coagulation defects (16).

\section{ASSESSMENT OF BLOOD LOSS/COAGULATION STATUS}

The initial assessment of the status of the patient is crucial. This involves clinical examination, including vital signs, urine output, and laboratory testing. The signs and symptoms of clinically significant blood loss include vertigo, tachycardia, and hypotension. Other vasovagal syndromes can also be confused based on symptomatology alone. Immediate postpartum hemorrhage is rarely misdiagnosed, but a late hemorrhage, particularly in the setting of a cesarean delivery, can be missed if the clinician does not have a high index of suspicion. Blood loss of approximately $10 \%$ to $15 \%$ of the circulating blood volume (500-1000 cc) can cause tachycardia, while it generally takes at least $25 \%$ to $35 \%(1500-2000 \mathrm{cc})$ to cause hypotension (17). This is, of course, dependent on maternal body size and the rate of blood loss.

Changes in vital signs can provide some information regarding the degree of hemorrhage, but as the majority of obstetric patients are young and healthy, there can be a considerable amount of blood loss with compensation that can occur prior to a significant change in vital signs.

Initial laboratory evaluation should include a complete blood count (CBC) and coagulation studies including fibrinogen, prothrombin time, international normalized ratio, and activated partial thromboplastin time. It is important to remember that the initial $\mathrm{CBC}$ value may not reflect the true degree of anemia because it may not have yet equilibrated. In the delivery room, one method of rapid assessment of the maternal coagulation status is to collect a red top (nonheparinized) tube of the mother's blood. This blood should 
clot within 6 minutes. If it does not, a diagnosis of a coagulation defect is suspected, and treatment can be instituted if clinically indicated without waiting for the results of a full coagulation panel.

\section{THERAPY}

Initial treatment after all deliveries includes removal of placental tissue, either expectantly or through the use of the Crede maneuver (18), as well as fundal massage. This also allows for assessment of uterine contraction. A fundus that is not firm should be vigorously massaged both abdominally and vaginally to increase uterine tone.

Further initial prophylaxis involves treatment with oxytocin (19), either 20 units in one liter of IV fluid or 10 units intramuscularly. The timing of this therapy remains a matter of some debate, and local practice methods often govern whether it is administered after cord clamp or following delivery of the placenta (20-22).

Upon recognition of an immediate (early) postpartum hemorrhage, several factors must be assessed virtually simultaneously. To accomplish this, it is recommended that help be requested immediately. Initial assessment should include an evaluation of uterine tone through bimanual examination, evaluation of the placenta and membranes for any evidence of incomplete removal, and inspection of the cervix, vagina, perineum, and rectum to evaluate for laceration.

Treatment for atony involves uterine massage, IV fluid resuscitation, and administration of uterotonic agents. In addition to the oxytocin recommended for prophylaxis, further oxytocin can be given. Doubling the previously mentioned dose is a recommended first step. Other medications can be provided as well. These are summarized in Table 1.

Treatment for retained placenta involves extraction of remaining placental tissue. This can be accomplished either through manual exploration or through surgical intervention. Prior to either intervention, care should be taken to ensure adequate anesthesia for the patient. Either IV narcotic medications or re-dosage of a previously placed epidural catheter can be safely performed in the patient with postpartum hemorrhage. Manual exploration and removal of retained placental tissue through curettage with a banjo curette can be safely performed in the delivery room. Bedside ultrasound can be invaluable in this clinical situation. Realtime ultrasound is used to assess for retained tissue and as a guide for surgical procedures. In curettage for suspected retained placenta, the clinician should select the largest curette that can be safely passed through the cervix in order to minimize the risk of uterine perforation. Generally, a banjo curette can be easily passed through the dilated cervix in the immediate postpartum period.

A thorough inspection of all vaginal, cervical, and perineal tissues should be performed as quickly as possible. Lacerations should be repaired with absorbable suture, typically in a running, locked fashion. Repair of lacerations should be performed only under excellent visualization. Such visualization may require transport to an operating room for better lighting and equipment. Transfer to the operating suite should not be delayed due to hemorrhage. In addition, inspection can reveal the presence of a vaginal hematoma. Unless this hematoma is large, or expanding, it should not be opened and inspected due to the possibility of significant blood loss with the minimal chance for identification of a discrete source.

Rare causes of postpartum hemorrhage should not be discounted. During the pelvic examination following delivery, uterine inversion can be diagnosed. This condition often presents as a beefy-red mass at the introitus. Failure to visualize the cervix around this mass should arouse the clinical suspicion for this condition. The clinician may mistake this for a prolapsed leiomyoma. Once inversion is recognized, attempts at manual replacement should be made first $(23,24)$. Should this fail to alleviate the problem, IV nitroglycerine can be administered in hopes of relaxing the uterus. If attempts at medical therapy also fail, a laparotomy should be performed; the uterus can then be reverted by progressive traction on the round ligaments or, if this fails, by incising the uterine contraction ring posteriorly. The vertical incision should then be repaired in layers, similar to the repair of any other vertical uterine incision (25).

If rare causes of postpartum hemorrhage have been ruled out and standard medical therapies have failed to relieve the bleeding, further intervention is required. Which method one chooses will be largely based on the method of delivery. In a vaginal delivery, if the patient has not been transported to the operating suite, this should be done. The room should be prepared for the possibility of an emergent laparotomy. Consideration should be given to the idea of uterine tamponade prior to surgical intervention. Packing of the uterus is one method (26-28), although newer techniques

Table 1 Medications Commonly used in the Treatment of Postpartum Hemorrhage Due to Uterine Atony, Including Dosage and Contraindications

\begin{tabular}{|c|c|c|}
\hline Medication & $\begin{array}{l}\text { Dosage and route of } \\
\text { administration }\end{array}$ & $\begin{array}{l}\text { Contraindicated in } \\
\text { patients with }\end{array}$ \\
\hline Pitocin (oxytocin) & $\begin{array}{l}20-40 \text { Units IV } \\
\text { Or } 10 \text { Units IM (including uterine injection } \\
\text { during } \mathrm{C} / \mathrm{S} \text { or laparotomy) }\end{array}$ & \\
\hline $\begin{array}{l}\text { Methergine } \\
\text { (methylergonovine) }\end{array}$ & $\begin{array}{l}0.2 \mathrm{mg} \text { IM (including uterine injection during } \\
\mathrm{C} / \mathrm{S} \text { or laparotomy) every } 2-4 \text { hours }\end{array}$ & Hypertension \\
\hline $\begin{array}{l}\text { Hemabate }(15 \text {-methyl } \\
\text { prostaglandin } \mathrm{F} 2 \alpha)\end{array}$ & $\begin{array}{l}0.25 \mathrm{mg} \text { IM (including uterine injection during } \\
\mathrm{C} / \mathrm{S} \text { or laparotomy) every } 15-90 \text { minutes }\end{array}$ & Asthma \\
\hline $\begin{array}{l}\text { Cytotec (misoprostol, } \\
\text { prostaglandin E1) }\end{array}$ & Protocols vary, 800 mcg PR hourly & None, but monitor for maternal fever \\
\hline Recombinant factor VIIA & Doses of $20-90 \mathrm{mcg} / \mathrm{kg}$ IV & $\begin{array}{l}\text { Used for refractory coagulopathy. } \\
\text { No known contraindication, but cost is a factor }\end{array}$ \\
\hline
\end{tabular}


have replaced it to a large extent. The Bakri balloon was developed specifically for this purpose (29). The balloon must be inserted completely into the uterine cavity and inflated with 300 to $500 \mathrm{cc}$ of saline. Care should be taken to ensure that the balloon does not cross the cervical os, as this placement will not be effective and could cause cervical trauma.

In the clinical setting of ongoing bleeding refractory to standard medical therapy, after lacerations have been repaired and retained products ruled out, factor VIIa may be a useful adjunct to therapy. It has been shown to be effective in approximately $80 \%$ of cases during one recent review (30). Other case reports have also demonstrated its effectiveness as an adjunct therapy (31-34). There is not a known method to measure the effectiveness of factor VIIa therapy.

Some institutions will have the ability to perform a rapid arterial embolization for emergent treatment of postpartum hemorrhage. Embolization may be most appropriate for cases of postpartum hemorrhage secondary to laceration, if blood loss is ongoing. If an interventional radiologist is available and the patient is stable, she can be transferred to the radiology suite for evaluation and treatment as an acceptable alternative (35-37). Occasionally, a discrete vessel can be identified and embolized. Angiography of both uterine arteries can be performed and an embolization of a feeder vessel can be performed. The availability of this technique is limited, and its use should not delay definitive therapy in cases where the patient is not stable for transport. It is not a standard therapy for cases in which uterine atony is the primary cause of bleeding. Patients with high risk of bleeding, such as those with a suspected accreta or known previa with previous cesarean section, could have catheter sheaths placed prophylactically prior to a scheduled procedure to facilitate the embolization should it become necessary.

Should tamponade fail, and interventional radiology either fail or be unavailable, the next step is laparotomy. Subsequent steps can also be performed to treat hemorrhage at the time of cesarean section. Hemorrhage can be treated by ligature of the vessels supplying the uterus. First to be secured should be the uterine arteries bilaterally. Originally described by O'Leary (38-40), this involves placing an absorbable suture into the lateral aspect of the lower segment of the uterine corpus, then around the uterine vessels and secured lateral to these vessels. Care must be taken to identify and avoid the ureter. This procedure should be completed bilaterally. If this procedure does not completely cease bleeding, additional sutures can be placed to secure the uterine branches supplied by the ovarian arteries (41). The purpose of these therapies is to decrease the pulse pressure through their respective vessels and to decrease overall flow to the affected area in hopes of controlling the hemorrhage.

If these therapies are unsuccessful, the anterior division of the internal iliac (hypogastric) artery can be ligated (38,42-45). This dissection is considerably more complicated than either of the previous two described above. This procedure will ideally be performed with the assistance of a surgeon experienced in operating in the retro peritoneum. Care should be taken to avoid injury to local structures, particularly the ureter and the hypogastric veins.

Given the technical difficulties associated with ligature of the hypogastric arteries, techniques have been developed to treat uterine atony with compression rather than via decreased blood flow (46-49). The B-Lynch suture provides a degree of compression similar to that done manually. This allows the practitioner to determine its effectiveness prior to placement. If manual compression does not decrease the bleeding to an acceptable level, then the suture is unlikely to do so. If manual compression does provide adequate hemostatic control, then the suture can be placed with a reasonable chance of success. Placement of the suture involves use of chromic gut placed entering the body of the uterus inferior to the hysterotomy and exiting superior to the incision. The suture is carried to the posterior aspect of the uterus (over the fundus) where it enters on one side of the lower uterine segment and exits on the other side. It is then carried over the fundus once more and entered into the uterus superior to the incision, then exits parallel to the initial suture placement. The suture is secured to compress the uterus (46-49). This is made considerably easier with surgical assistance. Case reports of pregnancy following compression sutures have been published, although there are no data to suggest any subfertility or increased rate of spontaneous pregnancy loss (49-51).

Hysterectomy should be employed only as a last resort, but should not be delayed in cases where it is necessary. The blood loss from a delay can result in significant maternal morbidity or even mortality and definitive treatment should be readily available. The technique does not differ significantly from the nonpregnant patient, but distortion of the normal anatomy of the pelvis presents challenges. The gravid uterus obstructs the operative view, and the increased blood flow to the area represents a potential for further blood loss. Physiologic dilation of the ureter can make this structure more difficult to avoid. The soft consistency of the cervix and its effacement can also make identification of the cervix more difficult and result in excess vaginal tissue being removed, leading to a foreshortening of the vagina. These changes, and others, make the procedure one fraught with morbidity (52). Surgeons with experience in this procedure should be consulted for assistance whenever available.

Late postpartum hemorrhage is much more likely to be related to retained products of conception than to any other cause (53). Ultrasound of the uterus can often identify these products $(54,55)$. Curettage should be performed once these products are identified. Endomyometritis can also cause a delayed postpartum hemorrhage. In these cases, bleeding should be treated as necessary, primarily with medical therapy, but antibiotic therapy should be instituted once the diagnosis is made.

\section{BLOOD AND BLOOD PRODUCT REPLACEMENT}

The clinician must also pay continued attention to the hematologic status of the patient. Initial efforts at control should not be withheld during the attempts to obtain laboratory analysis on the patient, but once available, these laboratory values, particularly any abnormalities, must be addressed quickly. First among concerns in the bleeding patient is the red blood cell transfusion. Patients should receive two units of packed red blood cells as soon as deemed clinically appropriate by the attending clinician. Ideally this blood will be typed and crossmatched to the patient, but in cases where hemorrhage was not expected, uncrossmatched $\mathrm{O}$ negative blood may need to be infused. The degree of bleeding that 
Table 2 Expected Results from Blood Product Transfusion

\begin{tabular}{|c|c|c|}
\hline Blood product & Contains & Expected result \\
\hline $\begin{array}{l}\text { Packed red blood cells } \\
\qquad(1 \text { unit }=200-250 \mathrm{~mL})\end{array}$ & Red blood cells only & $\begin{array}{l}\text { Increase of } 1 \mathrm{gm} / \mathrm{dL} \text { hemoglobin } \\
\text { or } 3 \% \text { hematocrit }\end{array}$ \\
\hline Platelets ( 1 unit $=50 \mathrm{~mL}$ ) & Platelets only & Increase of $5-10,000 / d L$ \\
\hline Cryoprecipitate $(1$ unit $=10-15 \mathrm{~mL})$ & Fibrinogen, factors V, VIII, XIII, and vWF & Increase fibrinogen $\sim 7 \mathrm{mg} / \mathrm{dL}$ \\
\hline
\end{tabular}

would necessitate the transfusion of uncrossmatched units depends upon the assessment of the patient and the timing of the availability of type-specific crossmatched blood. Clinicians should consider red cell transfusion in cases where the estimated blood loss exceeds $1000 \mathrm{cc}$ during a vaginal delivery or $1500 \mathrm{cc}$ during a cesarean delivery. The transfusion of one unit of packed red blood cells can be expected to raise the hemoglobin concentration by $1 \mathrm{gm} / \mathrm{dL}$ or the hematocrit by $3 \%$. The decision to transfuse a patient depends on the amount of blood loss, any maternal comorbidities, and the risk of recurrent bleeding (Table 2).

During the initial treatment of hemorrhage, infusion of intravenous fluids should not be overlooked. Approximately 3 liters of IV fluid is required to replace 1 liter of blood loss. This fluid does not contain the necessary clotting factors or the oxygen-carrying capacity of the blood lost, so treatment with fluid does not replace treatment with blood products.

Once coagulation studies are available, or sooner if there is an abnormal clot tube, or if clinically indicated, clotting factors should be replaced as well. In order to replace platelets, platelet transfusion is performed. Most conventional blood banks will perform transfusion in terms of units of platelets, but some will still use the term "six pack," which refers to six units of platelets. Transfusion of each unit of platelets can be expected to raise the platelet count by 5 to $10 \mathrm{~K} / \mathrm{dL}$. Correction of the underlying cause of platelet consumption is critical or these platelets may undergo the same consumption that initially caused the thrombocytopenia.

Fibrinogen should be replaced to keep the level above $100 \mathrm{mg} / \mathrm{dL}$ (56). Hemostasis can generally be achieved when the activity of coagulation factors is at least $25 \%$ of normal, and when the level of fibrinogen is $100 \mathrm{mg} / \mathrm{dL}$. Since the plasma volume in adults is approximately $40 \mathrm{~mL} / \mathrm{kg}, 10-15 \mathrm{~mL} / \mathrm{kg}$ of fresh frozen plasma may be needed. This dose is approximately three to five units of FFP for adult patients with BMI in the normal range, and may lead to volume overload. Replacement of fibrinogen can be accomplished in two ways. Transfusion of FFP, one unit (200-300 mL), can be expected to increase the fibrinogen level 7 to $10 \mathrm{mg} / / \mathrm{dL}$. FFP also has the advantage of containing virtually all clotting factors, making its administration ideal in the treatment of disseminated intravascular coagulation. Cryoprecipitate contains fibrinogen as well as factors V, VIII, XIII, and vWF. One bag of cryoprecipitate will raise the fibrinogen of the patient by $7 \mathrm{mg} / \mathrm{dL}$. The advantage of cryoprecipitate is the smaller volume that must be infused. This presents an advantage in patients where fluid overload is a concern, such as those with severe pre-eclampsia/HELLP syndrome.

In addition to the above, care must be taken to pay particular attention to the electrolyte component of blood loss. Replacement of four units of packed red blood cells should be accompanied by one ampule of calcium intravenously to prevent derangements in calcium balance, particularly as it relates to the citrate binding of calcium and the potential for metabolic acidosis due to the citrate. Hepatic function should also be considered, as liver derangements common in preeclampsia may result in a decreased ability to process the citrate, leading to a greater risk for hypocalcemia in these patients. Older blood may contain a higher percentage of lysed red blood cells, and care must be taken to follow the patient's potassium level in such cases and hyperkalemia should be treated if necessary. Transfused blood and blood products should be warmed to prevent hypothermia.

\section{RECURRENCE}

Risks for recurrent postpartum hemorrhage may be as high as $10 \%$, depending on the previous cause (57). Patients with a history of postpartum hemorrhage should have a type and screen performed prior to scheduled induction of labor or cesarean delivery, and all obstetric team members should be aware of the patient's history and should be prepared in case of a recurrent bleeding.

\section{REFERENCES}

1. Khan KS, Wojdyla D, Say L, Gulmezoglu AM. WHO analysis of causes of maternal death: a systematic review. Lancet 2006; 367: 1066-74.

2. Betran AP, Wojdyla D, Posner SF, Gulmezoglu AM. National estimates for maternal mortality: an analysis based on the WHO systematic review of maternal mortality and morbidity. BMC Public Health 2005; 5: 131.

3. Ratnoff OD, Pritchard JA, Colopy JE. Hemorrhagic states during pregnancy. N Engl J Med 1955; 253: 97-102.

4. Stafford I, Dildy GA, Clark SL, Belfort MA. Visually estimated and calculated blood loss in vaginal and cesarean delivery. Am J Obstet Gynecol 2008; 199: 519 e1-7.

5. Gahres EE, Albert SN, Dodek SM. Intrapartum blood loss measured with Cr 51-tagged erythrocytes. Obstet Gynecol 1962; 19: 455-62.

6. Dildy GA 3rd, Paine AR, George NC, Velasco C. Estimating blood loss: can teaching significantly improve visual estimation? Obstet Gynecol 2004; 104: 601-6.

7. Duthie SJ, Ven D, Yung GL, et al. Discrepancy between laboratory determination and visual estimation of blood loss during normal delivery. Eur J Obstet Gynecol Reprod Biol 1991; 38: 119-24.

8. Toledo P, McCarthy RJ, Hewlett BJ, Fitzgerald PC, Wong CA. The accuracy of blood loss estimation after simulated vaginal delivery. Anesth Analg 2007; 105: 1736-40, table of contents.

9. Patel A, Goudar SS, Geller SE, et al. Drape estimation vs. visual assessment for estimating postpartum hemorrhage. Int J Gynaecol Obstet 2006; 93: 220-4.

10. Carroli G, Cuesta C, Abalos E, Gulmezoglu AM. Epidemiology of postpartum haemorrhage: a systematic review. Best Pract Res Clin Obstet Gynaecol 2008; 22: 999-1012.

11. Al-Zirgi I, Vangen S, Forsen L, Stray-Pedersen B. Prevalence and risk factors of severe obstetric haemorrhage. BJOG 2008; 115: 1265-72.

12. Stones RW, Paterson CM, Saunders NJ. Risk factors for major obstetric haemorrhage. Eur J Obstet Gynecol Reprod Biol 1993; 48: 15-18.

13. Sheiner E, Sarid L, Levy A, Seidman DS. Obstetric risk factors and outcome of pregnancies complicated with early postpartum hemorrhage: 
a population-based study. J Matern Fetal Neonatal Med 2005; 18: 149-54.

14. Magann EF, Evans S, Hutchinson M, et al. Postpartum hemorrhage after vaginal birth: an analysis of risk factors. South Med J 2005; 98: 419-22.

15. Kadir RA, Kulkarni AA, Lee CA. Pregnancy in women with von Willebrands disease or factor XI deficiency. Br J Obstet Gynaecol 1998; 105: 314-21.

16. Roberts WE, Perry KG Jr, Woods JB, et al. The intrapartum platelet count in patients with HELLP (hemolysis, elevated liver enzymes, and low platelets) syndrome: is it predictive of later hemorrhagic complications? Am J Obstet Gynecol 1994; 171: 799-804.

17. Bonnar J. Massive obstetric haemorrhage. Baillieres Best Pract Res Clin Obstet Gynaecol 2000; 14: 1-18.

18. Shaw WF. Credes expression of the placenta. J Obstet Gynaecol Br Emp 1948; 55: 502-4.

19. Nordstrom L, Fogelstam K, Fridman G, Larsson A, Rydhstroem H. Routine oxytocin in the third stage of labour: a placebo controlled randomised trial. Br J Obstet Gynaecol 1997; 104: 781-6.

20. Jackson KW Jr, Allbert JR, Schemmer GK, et al. A randomized controlled trial comparing oxytocin administration before and after placental delivery in the prevention of postpartum hemorrhage. Am J Obstet Gynecol 2001; 185: 873-7.

21. Huh WK, Chelmow D, Malone FD. A double-blinded, randomized controlled trial of oxytocin at the beginning versus the end of the third stage of labor for prevention of postpartum hemorrhage. Gynecol Obstet Invest 2004; 58: 72-6.

22. Zamora LA. A randomized controlled trial of oxytocin administered at the end of the second stage of labor versus oxytocin administered at the end of the third stage of labor in the prevention of postpartum hemorrhage. Philipp J Obstet Gynecol 1999; 23: 125-33.

23. Moldavsky LF. Management of inversion of the uterus; report of 4 cases. Obstet Gynecol 1967; 29: 488-94.

24. Campbell J, Pash J, Walters WA. Acute inversion of the uterus and its management. Med J Aust 1972; 2: 475-6.

25. Essid DM, Chelli H, Chelli M. 3 cases of uterine inversion. Description of a new technic for the management of this complication. J Gynecol Obstet Biol Reprod (Paris) 1983; 12: 287-90.

26. Goldrath $\mathrm{MH}$. Uterine tamponade for the control of acute uterine bleeding. Am J Obstet Gynecol 1983; 147: 869-72.

27. Maier RC. Control of postpartum hemorrhage with uterine packing. Am J Obstet Gynecol 1993; 169(2 Pt 1): 317-21; discussion 321-3.

28. Wax JR, Channell JC, Vandersloot JA. Packing of the lower uterine segment-new approach to an old technique. Int J Gynaecol Obstet 1993; 43: 197-8.

29. Bakri YN, Amri A, Abdul Jabbar F. Tamponade-balloon for obstetrical bleeding. Int J Gynaecol Obstet 2001; 74: 139-42.

30. Alfirevic Z, Elbourne D, Pavord S, et al. Use of recombinant activated factor VII in primary postpartum hemorrhage: the Northern European registry 2000-2004. Obstet Gynecol 2007; 110: 1270-8.

31. Nohira T, Osakabe Y, Suda S, et al. Successful management by recombinant activated factor VII in a case of disseminated intravascular coagulopathy caused by obstetric hemorrhage. J Obstet Gynaecol Res 2008; 34(4 Pt 2): 623-30.

32. Laird R, Carabine U. Recombinant factor VIIa for major obstetric haemorrhage in a Jehovahs Witness. Int J Obstet Anesth 2008; 17: 193-4.

33. Bouma LS, Bolte AC, Van Geijn HP. Use of recombinant activated factor VII in massive postpartum haemorrhage. Eur J Obstet Gynecol Reprod Biol 2008; 137: 172-7.

34. Hamaekers AE, van Mook WN, Offermans JP, Marcus MA. Successful use of recombinant factor VIIa for treatment of severe postpartum hemorrhage. Am J Crit Care 2006; 15: 399-401.
35. Ratnam LA, Gibson M, Sandhu C, et al. Transcatheter pelvic arterial embolisation for control of obstetric and gynaecological haemorrhage. J Obstet Gynaecol 2008; 28: 573-9.

36. Mushtaq S, Kurdi W, Al-Shammari M. Prophylactic catheters placement and intraoperative internal iliac artery embolisation in a patient with placenta accreta. J Obstet Gynaecol 2007; 27: 853-5.

37. Ornana D, White R, Pollak J, Tal M. Pelvic embolization for intractable postpartum hemorrhage: long-term follow-up and implications for fertility. Obstet Gynecol 2003; 102(5 Pt 1): 904-10.

38. OLeary JA. Uterine artery ligation in the control of postcesarean hemorrhage. J Reprod Med 1995; 40: 189-93.

39. OLeary JL, OLeary JA. Uterine artery ligation for control of postcesarean section hemorrhage. Obstet Gynecol 1974; 43: 849-53.

40. OLeary JL, OLeary JA. Uterine artery ligation in the control of intractable postpartum hemorrhage. Am J Obstet Gynecol 1966; 94: 920-4.

41. Cruikshank SH, Stoelk EM. Surgical control of pelvic hemorrhage: bilateral hypogastric artery ligation and method of ovarian artery ligation. South Med J 1985; 78: 539-43.

42. Clark SL, Phelan JP, Yeh SY, Bruce SR, Paul RH. Hypogastric artery ligation for obstetric hemorrhage. Obstet Gynecol 1985; 66: 353-6.

43. Sziller I, Hupuczi P, Papp Z. Hypogastric artery ligation for severe hemorrhage in obstetric patients. J Perinat Med 2007; 35: 187-92.

44. Hebisch G, Huch A. Vaginal uterine artery ligation avoids high blood loss and puerperal hysterectomy in postpartum hemorrhage. Obstet Gynecol 2002; 100: 574-8.

45. Joshi VM, Otiv, SR, Majumder R, Nikam YA, et al.Internal iliac artery ligation for arresting postpartum haemorrhage. BJOG 2007; 114: 356-61.

46. Ferguson JE, Bourgeois FJ, Underwood PB. B-Lynch suture for postpartum hemorrhage. Obstet Gynecol 2000; 95(6 Pt 2): 1020-2.

47. Hayman RG, Arulkumaran S, Steer PJ. Uterine compression sutures: surgical management of postpartum hemorrhage. Obstet Gynecol 2002; 99: 502-6.

48. Holtsema H, Nijland R, Huisman A, Dony J, van den Berg PP. The B-Lynch technique for postpartum haemorrhage: an option for every gynaecologist. Eur J Obstet Gynecol Reprod Biol 2004; 115: 39-42.

49. Tsitlakidis C, Alalade A, Danso D. B-Lynch C ten year follow-up of the effect of the B-Lynch uterine compression suture for massive postpartum hemorrhage. Int J Fertil Womens Med 2006; 51: 262-5.

50. Api M, Api O, Yayla M. Fertility after B-Lynch suture and hypogastric artery ligation. Fertil Steril 2005; 84: 509.

51. Sentilhes L, Gromez A, Trichot C, et al. Fertility after B-Lynch suture and stepwise uterine devascularization. Fertil Steril 2009. Mar; 91: 934.

52. Smith J, Mousa HA. Peripartum hysterectomy for primary postpartum haemorrhage: incidence and maternal morbidity. J Obstet Gynaecol 2007; 27: 44-7.

53. Rome RM. Secondary postpartum haemorrhage. Br J Obstet Gynaecol 1975; 82: 289-92.

54. Wolman I, Gordon D, Yaron Y, et al. Transvaginal sonohysterography for the evaluation and treatment of retained products of conception. Gynecol Obstet Invest 2000; 50: 73-6.

55. Hertzberg BS, Bowie JD. Ultrasound of the postpartum uterus. Prediction of retained placental tissue. J Ultrasound Med 1991; 10: 451-6.

56. Friedman K, Menitove JE. Preparation and clinical use of plasma and plasma fractions. In: Lichtman M, Coller BS, et al. eds. Williams Hematology, 6th edn. New York: McGraw-Hill, 2001.

57. Ford JB, Roberts CL, Bell JC, Albert CS, Morris JM. Postpartum haemorrhage occurrence and recurrence: a population-based study. Med J Aust 2007; 187: 391-3. 


\section{Septic shock \\ Bryan E. Freeman and Michael R. Foley}

\section{INTRODUCTION/EPIDEMIOLOGY}

The idea that infectious etiologies underlie many complications related to illness and medical and surgical procedures is a relatively young area of interest and understanding in the medical profession. Throughout time, scientists have considered that toxins, miasmas, and "bad humors" may be the culprits behind various disease processes, but it was not until the last 300 years that a viable link between bacteria and infection, including sepsis, has been made.

Sir John Pringle's work in the 1700s relating the ideas of septic and antiseptic conditions to systemic diseases such as fever and dysentery in jails and on the battlefield as relating was a step in the right direction, despite an incorrect mechanism of infection. Although he blamed "putrid air" as the source of disease, awareness of his ideas led to a decrease in the incidence of sepsis (1).

Further advances in medical understanding of the etiology behind infectious diseases were made in the 1800s. The French chemist, Louis Pasteur, discovered that microorganisms were responsible for disease, and Joseph Lister, the English surgeon, was responsible for the introduction of sterilization of surgical instruments and sterile technique. The 1900s brought Alexander Fleming's discovery of penicillin to combat grampositive bacteria.

Shock, also known as physiologic or circulatory shock, is an alteration in normal physiology that results in severe and potentially life-threatening changes in the circulatory system. There are various categories of shock, but the most common are cardiogenic, hypovolemic (hemorrhagic), and septic shock. While the etiologies behind these categories are different, the effect on the body is essentially the same. The result of shock is a cascade of events that includes hypoperfusion of tissues, cellular hypoxia, metabolic acidosis, and eventually cellular death.

The incidence of sepsis is difficult to determine due to a lack of use of appropriate terminology as well as inaccurate reporting. However, recent data in the United States regarding severe sepsis have shown an overall rate of three cases per 1000 population, with a substantial, directly proportional increase with increasing age. Outcomes are variable based upon the etiology and underlying comorbidities of the individual, as well as by location of infection. Annual national costs relating to sepsis are estimated to be $\$ 16.7$ billion per year in the United States alone (2).

Sepsis remains one of the most significant causes for medical morbidity and mortality worldwide. It was responsible for $9.3 \%$ of all deaths in the United States in 1995 and has been shown to have a $30 \%$ mortality rate, although this varies depending upon the patient population (2).

Septic shock affects an estimated one in 8338 deliveries (3). However, much of the data relating to sepsis and septic shock deals with a non-obstetric population. Generally, since women of childbearing age are young, healthy women, without the serious comorbidities of the elderly populations that are usually studied, rates are significantly lower. In fact, a review of the percentage of total cases of sepsis in the United States demonstrated that pregnant patients comprised approximately $0.6 \%$ of all cases in 1979 to 1984 , but $0.3 \%$ of cases in 1995 to 2000 (4).

Despite the fact that sepsis is such a concern worldwide, a surprising number of people have not heard of, much less understand, what sepsis is. A study performed using telephone interviews of 6021 citizens in Italy, Spain, the United Kingdom, France, and the United States revealed that public knowledge of simply the term "sepsis" was astonishingly low and that $58 \%$ of those surveyed did not know that sepsis is a leading cause of death (5). The implications of this alone may indicate why early sepsis and its significance may present challenges in the treatment of less severe disease.

\section{ETIOLOGY}

Causes of bacteremia in the obstetric population may be due to a single or multiple pathogens and may be made in patients with different illnesses. Blanco et al. found that the most common pathogens were Escherichia coli (32\%), group B streptococcus (16\%), and Bacteriodes spp. (15\%), each of which led their respective categories of gram-negative, grampositive, and anaerobic bacteria, respectively (6). The conditions that these infections were associated with were variable, but have been shown to be the most prominent etiologies in other studies. Of 176 patient studies, diagnoses included endometritis (70\%), pyelonephritis (16\%), chorioamnionitis (8\%), and various other causes (6). Therefore, sepsis in the obstetric population is a concern both antepartum as well as postpartum.

Septic shock has been shown to be associated with the same conditions, but in a slightly different order of frequency according to recent studies. Mabie et al. in 1997 published an article that examined the outcomes of 18 women with septic shock in pregnancy. Their definition of septic shock conforms to the definition set forth by the 1992 American College of Chest Physicians/Society of Critical Care Medicine (ACCP/ SCCM) Consensus Conference (7). The mortality rate was $28 \%$, with etiologies for septic shock ranging from pyelonephritis $(33 \%)$, chorioamnionitis $(17 \%)$, toxic shock $(11 \%)$, and postpartum endometritis (11\%). The remaining causes included septic abortion, ruptured appendix, ruptured ovarian abscess, necrotizing fasciitis, and bacterial endocarditis (approximately 5\% each). Etiology varied due to the clinical history of the patient and the presence of significant comorbidities. Other significant findings were that depressed left ventricular function was noted in $64 \%$ of survivors and 
$80 \%$ of those that died. Organisms isolated from cultures included Escherichia coli, group A streptococcus, group B streptococcus, Klebsiella pneumoniae, Streptococcus viridans, Staphylococcus epidermidis, and others. Of note, E. coli was present in $61 \%$ of patients, and there was one patient who was culture negative (3).

\section{PATHOPHYSIOLOGY}

Despite the continual discovery of new cytokines, intercellular relationships, and systemic changes that accompany the sepsis spectrum of diseases, the precise mechanisms behind severe sepsis and septic shock are not completely understood. Under normal conditions, the site of bacterial infection is targeted by the body's natural immune response. This involves an increase in inflammatory cytokines, activation of the clotting cascade, and decreased fibrinolysis in a localized fashion. In the setting of a normally functioning immune system, this leads to the body being able to effectively eliminate the invading pathogen, after which damage caused by the insult may be repaired.

Sepsis and septic shock, on the other hand, are exaggerations of the body's natural response to infection. Pro-inflammatory and anti-inflammatory mediators are upregulated, along with the natural immune response, but on a systemic scale, with effects far beyond the area in which it is needed. The precise mechanisms behind the regulation of a normal immune response and the lack of regulation in sepsis-related conditions are an area of ongoing research and interest. Understanding of the signaling pathways and resultant mediators produced has advanced greatly over recent years.

An extensive summary of current knowledge about the molecular biology behind inflammation and sepsis was published by Cinel and Opal in early 2009. The following are selections from this article that shed light on the mechanisms involved in these processes (8).

One popular area of sepsis research is the examination and elucidation of mechanisms behind the action of toll-like receptors (TLRs). Essentially, TLRs function to activate immune responses once the body's natural barriers (skin, vascular system, etc.) have been breached. Current research has shown that binding of TLRs causes activation of intracellular transcription activators including interferon regulator factors, phosphoinositide 3-kinase (PI3K), and cytosolic nuclear factor-kappa $\beta$ (NF- $\beta$ ). NF- $\beta$ then enters the nucleus and ultimately causes the activation of genes for acute phase proteins, inducible nitric oxide synthase, coagulation factors, and pro-inflammatory cytokines, among others (8).

The significance of PI3K is that it can either positively or negatively regulate TLRs in order to influence proinflammatory cytokine production. For instance, positive regulation of TLRs by $\mathrm{PI} 3 \mathrm{~K}$ leads to increased production of pro-inflammatory cytokines interleukin-1 $\beta$ (IL-1 $\beta$ ), IL-6, and IL-8 (8).

Sepsis also leads to increased neutrophil activation. Normally, these proapoptotic neutrophils are responsible for the effective clearance of the invading pathogen while remaining well controlled so as not to damage healthy host cells. In sepsis, neutrophils have been found to evade the normal clearance by macrophages, which usually regulate their number and effect. This lack of regulation leads to increased numbers of circulating neutrophils, which have a proinflammatory effect (8).

Along these same lines, macrophage migration inhibitory factor (MIF) is induced by glucocorticoids in sepsis and is probably involved in sepsis pathogenesis. Experiments in mice have found that inhibition of MIF leads to attenuation of inflammatory mediators such as TNF- $\alpha$ and IL-1 $\beta$ (8).

Tumor necrosis factor $\alpha$ (TNF- $\alpha$ or cachectin), which is produced primarily by macrophages, and secondarily released from damaged endothelial cells, is a pro-inflammatory cytokine. It acts with other cytokines, such as IL-1 and IL-6, to cause various alterations in the function of multiple organs. In the setting of sepsis, insulin resistance and fever increased.

Activation of the clotting cascade is another mechanism by which the body fights infection and its effects. The formation of a clot (which serves as an intravascular barrier) and initiation of inflammation are attempts to isolate the area of infection and prevent systemic spread of the pathogen. However, this is not always held in check, resulting in sepsis and related conditions. In sepsis, the extrinsic (tissue factor) pathway is activated, leading to increased thrombin production. This is the target of action of activated protein $C$, which will be discussed later (8).

\section{DIAGNOSIS}

Initial diagnosis of septic shock and related conditions include a thorough history and physical examination, baseline laboratory tests, and blood cultures. The history can help to elucidate the etiology of the septic shock and to direct treatment. This is important as a simple clinical history can help elucidate a probable etiology for the condition.

In general, baseline laboratory tests that need to be obtained include a complete blood count, at least two blood cultures, coagulation studies, a complete metabolic panel, and any other cultures that are deemed clinically applicable. Specific recommendations are outlined in the following paragraphs.

In order to properly diagnose which entity is involved, understanding of the currently accepted definitions is essential. The terminology surrounding the sepsis spectrum of disorders has been inconsistently and incorrectly utilized, leading to misunderstanding of the disease process involved. This has led to a variety of terms being used for a spectrum of conditions related to sepsis such as bacteremia and septicemia. Other etiologies for conditions resembling sepsis exist, and it has not been until the past two decades that an attempt has been made to clarify the differences between these entities.

Therefore, it is more appropriate to think of sepsis and related conditions as a continuum of conditions that overlap to various degrees. They share the same etiologies, but have more serious implications the further they exist along this continuum.

In recognition of this, the ACCP/SCCM has established more firm definitions. In 1992, the members of the ACCP/ SCCM Consensus Conference made four recommendations to create more precise terminology for sepsis-related entities. Along with this, they introduced the term "systemic inflammatory response syndrome" (SIRS). The goal of these formal definitions was to give clinicians the ability to 
differentiate between illnesses in order to better direct patient care. The disease categories now include sepsis, severe sepsis, SIRS, septic shock, sepsis-induced hypotension, and multiple organ dysfunction syndrome (7).

SIRS can be the result of a variety of injuries (trauma, infection, burns, or sterile inflammatory processes such as pancreatitis) that lead to a series of complex findings. Assigning the diagnosis by 1992 criteria initially required at least two of the following:

1. Body temperature $>38^{\circ} \mathrm{C}$ or $<36^{\circ} \mathrm{C}$

2. $\mathrm{HR}>90 \mathrm{bpm}$

3. Tachypnea ( $\mathrm{RR}>20 / \mathrm{min}$ ) or hyperventilation $\left(\mathrm{PaCO}_{2}<32 \mathrm{mmHg}\right)$

4. WBC count $>12,000$ cells $/ \mu \mathrm{L}$ or $<4000$ cells $/ \mu \mathrm{L}$, or presence of bands (7)

Sepsis is the systemic inflammatory response that is due to an infection. More simply, it is SIRS that has occurred due to an infectious process. The clinical and laboratory criteria are therefore the same as listed for SIRS (7). This allows a differentiation to be made between the other potential etiologies that may lead to SIRS and clearly identifies a specific subdivision of SIRS.

In order to clarify other terms that are commonly used with sepsis, they proposed the following definitions. Infection is the invasion of a normally sterile host by an organism that leads to an inflammatory response. Bacteremia refers to viable bacteria in the blood. If the invading pathogen is not bacteria, the appropriate corresponding term should be employed (viremia, fungemia, etc.) (7).

Severe sepsis is sepsis complicated by organ dysfunction, hypoperfusion abnormality (lactic acidosis, oliguria, or altered mental status), or sepsis-induced hypotension (7). Sepsis-induced hypotension is systolic blood pressure $<90 \mathrm{mmHg}$ or a decrease in systolic blood pressure of $\geq 40 \mathrm{mmHg}$ from baseline, without any other identifiable cause for hypotension (7).

Septic shock is a subdivision of severe sepsis and is defined as sepsis-induced hypotension (as defined above) that is unresponsive to fluid resuscitation, accompanied by hypoperfusion abnormalities or organ dysfunction. This definition still applies to patients who have been treated with vasopressors, as hypoperfusion abnormalities and organ dysfunction may not occur until after treatment (7).

Septicemia has been determined to be an inaccurate term, and the members of the ACCP/SCCM Consensus Conference have recommended its removal from sepsis vocabulary (7).

The definitions set forth at the 1992 ACCP/SCCM Consensus Conference were reevaluated after almost a decade of use. Representatives from Europe and the United States met at the 2001 SCCM/ESICM/ACCP/ATS/SIS International Sepsis Definitions Conference to discuss the efficacy and potential need for modification of the previously mentioned definitions.

Regarding the definition of SIRS, the committee felt that since the clinical signs of inflammation may be quite variable, a specific definition for SIRS was too difficult to formulate. The definition established by conference in 1992 remained unchanged, but with the acknowledgement that it is "overly sensitive and nonspecific" (9). Subcommittees had been assigned to look at common markers found in sepsis, as well as other laboratory derangements brought about by this condition. Increased levels of IL-6, adrenomedullin, soluble CD14, soluble endothelial cell/leukocyte adhesion molecule 1, macrophage inflammatory protein $1 \alpha$, extracellular phospholipase $A_{2}$, procalcitonin, and C-reactive protein had been noted by committee members. Although these and other markers may be elevated during times of inflammation, there are no laboratory panels to create from these to function as a diagnostic tool for SIRS. However, understanding of the significance of the individual markers may aid in targeting specific treatment to a given organ, the coagulation cascade, and so on (9).

The definition of septic shock was expanded minimally to include a mean arterial pressure $(\mathrm{MAP})<60$. The definitions for infection, sepsis, and severe sepsis remained essentially unchanged (9).

Aside from the reevaluation of definitions for the spectrum of sepsis conditions, the 2001 Committee developed a prototype classification tool for sepsis, called PIRO. This mnemonic represents

1. predisposing conditions,

2. insult (the nature and extent of the insult),

3. response (the nature and magnitude of the host response), and

4. organ (the degree of organ dysfunction).

Based upon the utility derived from this in subsequent studies, this tool may be of use in the future for assigning a prognosis or determining the appropriate treatment protocol for patients with sepsis (9).

Although pregnant women were excluded from the study, a group of researchers recently explored the possibility of utilizing a biomarker panel to predict serious outcomes for patients with suspected sepsis who were admitted through the emergency department. The primary outcome was to predict the probability of development of sepsis and the secondary outcomes included septic shock and inpatient mortality. Nine biomarkers were studied in all, but the combination of protein C, IL-1 receptor antagonist (IL-1ra), and neutrophil gelatinase-associated lipocalin (NGAL) was found to be the most predictive of identifying both primary and secondary outcomes. Protein $\mathrm{C}$ is a marker of activation of coagulation, IL-1ra indicates inflammation, and NGAL reflects renal dysfunction. This may be an area of future research to help direct physician intervention at the time of initial assessment. The results of these tests were used by the authors to arrive at a Sepsis Score (10). When this has undergone further testing, there may be utility for treating the obstetric population as well.

\section{MANAGEMENT}

Management recommendations are largely based upon work that has been done in the non-obstetric population. Therefore, there are not established protocols to deal with the pregnant patient with septic shock or that take into account the significant physiologic changes that accompany pregnancy and how these changes may alter the effectiveness of a given therapy. The basic goals of therapy, which have been used successfully, are based upon early detection and diagnosis of 
the problem, aggressive fluid resuscitation and use of vasopressors, glycemic control, corticosteroids, and, more recently, the utilization of activated protein C (11).

Expanding upon this, in 2008, the Surviving Sepsis Campaign Committee published updated guidelines for the management of severe sepsis and shock. Extensive evaluation of each step of severe sepsis management was performed, and a grading system was then used to assign which interventions were either recommended or simply suggested, according to the strength of supporting evidence. Initial management was divided into 10 categories: initial resuscitation, diagnosis, antibiotic therapy, source control, fluid therapy, vasopressors, inotropic therapy, corticosteroids, activated protein $\mathrm{C}$, and use of blood products, which are summarized below (12).

The Surviving Sepsis Campaign protocol for initial resuscitation should be initiated as soon as tissue hypoperfusion (persistent hypotension despite fluid administration or serum lactate concentration $\geq 4 \mathrm{mmol} / \mathrm{L}$ ) is recognized. The goals during the first 6 hours of therapy are as follows:

1. Central venous pressure $=8-12 \mathrm{mmHg}$

2. $\mathrm{MAP} \geq 65 \mathrm{mmHg}$

3. Urine output $\geq 0.5 \mathrm{~mL} / \mathrm{kg} / \mathrm{hr}$

4. Central venous oxygen saturation $\geq 70 \%$ or mixed venous oxygen saturation $\geq 65 \%$

If these criteria are not able to be met within 6 hours, the committee suggests transfusing packed red blood cells until a hematocrit is $\geq 30 \%$ is reached and/or administering dobutamine up to $20 \mu \mathrm{g} / \mathrm{kg} / \mathrm{min}$ (12).

Diagnostic studies should be performed quickly. If possible, prior to the administration of antibiotics, at least two blood cultures should be obtained, in addition to cultures of intravascular devices (and each lumen of these devices) greater than 48 hours old. Other areas representing potential sources of infection, such as urine, wounds, respiratory tract, and so on, should be cultured according to clinical suspicion. If, and only if, obtaining any of these cultures risks serious delay of initiation of antibiotic administration, antibiotics should be started first. The cultures must be collected soon thereafter to avoid the risk of sterilization of blood cultures, which may occur within a few hours of antibiotic administration. Additionally, imaging studies should be performed as soon as possible to help confirm a diagnosis (12).

Antibiotic therapy should be initiated as soon as possible with intravenous medications within the first hour of recognition of severe sepsis and septic shock. As previously stated, collection of cultures should ideally be performed prior to antibiotic administration, if possible. Since etiologies of sepsis and septic shock vary depending upon each patient's clinical history and underlying conditions, the choices of antibiotic are also variable and will be dictated by these factors. Recently used antibiotics should be avoided and the choice of a broad-spectrum antibiotic that would cover pathogens associated with the presumed source of infection should be chosen. Other considerations include awareness of the increasing prevalence of methicillin- and oxacillin-resistant Staphylococcus aureus (MRSA and ORSA) or whether candidemia is suspected, which would require appropriate antifungal coverage (12).

Additionally, loading doses of antibiotics should be used, but with the awareness of organ damage and large changes in volume due to fluid resuscitation that may alter serum levels of any medication. Therefore, physicians and pharmacists need to carefully monitor serum levels to avoid toxicity. The recommended course of antibiotics should be at least 7 to 10 days and needs to be tailored to reflect the culture and sensitivity results (12).

Source control needs to be achieved within 6 hours of treatment. This includes establishing a diagnosis, locating the etiology of the infection, and taking steps to appropriately deal with the cause. In the case of an intravascular device being the culprit, it should be removed immediately after alternate intravascular access has been achieved (12).

Fluid therapy should include administration of either colloids or crystalloids. The SAFE study demonstrated no difference in efficacy between these two types (13). Goals of fluid resuscitation are initially to achieve a central venous pressure of $\geq 8 \mathrm{mmHg}$ (or $12 \mathrm{mmHg}$ if the patient is mechanically ventilated). Fluid challenges should be administered in the form of either at least $1000 \mathrm{~mL}$ of crystalloids or 300 to $500 \mathrm{~mL}$ of colloids over 30 minutes. As long as the patient responds to fluid challenges, they should continue. However, if central venous pressures or pulmonary artery pressures increase without simultaneous hemodynamic improvement, the rate of fluid administration should be substantially reduced (12).

Vasopressors should be utilized to maintain MAPs of at least $65 \mathrm{mmHg}$. The committee recommends that first-line vasopressor therapy should include norepinephrine or dopamine administered via a central line. Epinephrine, phenylephrine, and vasopressin should not be used as first-line therapy, although epinephrine may be used if initial efforts with norepinephrine or dopamine were unsuccessful in achieving the desired MAP. Additionally, low-dose dopamine for renal protection is not recommended (12).

Inotropic therapy with dobutamine may be utilized in patients with evidence of myocardial dysfunction (high cardiac filling pressures and low cardiac output).

Recommendations relating to the utility of corticosteroid treatment in septic shock are largely based upon results from the Corticosteroid Therapy of Septic Shock Study Group. Routine use of corticoid therapy as adjuvant treatment should not be employed in patients with septic shock. The ACTH test is not recommended to determine which patients should receive hydrocortisone treatment. Corticosteroid treatment should be reserved for patients with septic shock only if their hypotension is non-responsive to fluid therapy and vasopressors $(12,14)$. Additionally, since etomidate is frequently used for sedation in the intensive care unit, including care for patients with severe sepsis, care should be taken to avoid performing an ACTH test for any reason in patients who have received etomidate, as it can adversely impact their survival (15).

Activated protein $\mathrm{C}$ therapy [also known as recombinant human activated protein $\mathrm{C}$ (rhAPC), drotrecogin alfa] should be based upon the diagnosis of the patient and their Acute Physiology and Chronic Health Evaluation (APACHE) II score. The APACHE II score is a tool used to assess the severity of disease in adults admitted to intensive care units and is based upon physical assessment of patients during the first 24 hours of their admission. Patients with severe sepsis, an 
APACHE II score $>25$ or multiple organ failure, and who are deemed to be at high risk of death should receive rhAPC, assuming that they have no contraindications. On the other hand, patients with severe sepsis, an APACHE II score $<20$ or single organ failure, and who are at low risk for death should not receive rhAPC therapy (12). These recommendations are consistent with conclusions from the Administration of Drotrecogin Alfa (Activated) in Early Stage Severe Sepsis (ADDRESS) Study Group, published in 2005 (16).

Although the exact mechanism is unknown, activated protein $\mathrm{C}$ possesses both anti-thrombotic and anti-inflammatory properties. As mentioned before, sepsis involves activation of the extrinsic (tissue factor) pathway of the clotting cascade, which leads to overproduction of thrombin. This is the target of APC. Normally protein C, the zymogen of APC, is activated by thrombin. However, there are two aspects of sepsis that cause disruptions in this system. The first problem is that circulating levels of protein C, APC, and protein $S$ (the cofactor for protein $C$ ) are decreased. The other issue is that the thrombin:thrombomodulin complex that is usually responsible for the conversion of protein $\mathrm{C}$ to APC is also disrupted, which leads to the unregulated production of thrombin. Administration of rhAPC compensates for the lack of naturally created APC and exerts an anti-thrombotic effect by blocking the conversion of factors VIIIa and Va to factors VIII and V, as well as by inhibiting plasminogen activator inhibitor-1 (PAI-1), thereby allowing fibrinolysis to occur. Other mechanisms may be involved as well (17).

After resolution of hypoperfusion, and in the absence of complicating factors such as myocardial infarction, acute hemorrhage, and so on, administration of blood products (not covered in recommendations for initial resuscitation) may be considered. Packed red blood cells should be transfused for hemoglobin levels $<7.0 \mathrm{~g} / \mathrm{dL}$, with a target hemoglobin of 7.0 to $9.0 \mathrm{~g} / \mathrm{dL}$. The use of erythropoietin is not recommended in the treatment of severe sepsis unless there is another reason for its administration, such as renal failure (12).

Other blood products, such as fresh frozen plasma, may be utilized to correct coagulopathy. Platelet administration should be performed in severe sepsis when platelet counts are $<5000 / \mathrm{mm}^{3}$, whether or not the patient is bleeding. For platelet counts in the range of 5000 to $30,000 / \mathrm{mm}^{3}$, platelet transfusion should be considered. Goals of platelet therapy are dependent upon future interventions that are to be made. In the event of anticipated surgery, platelet transfusion should be performed to reach a count of $>50,000 / \mathrm{mm}^{3}$ (12).

One final area of management that has received recent attention is that of glucose control in patients with severe sepsis and septic shock. The Efficacy of Volume Substitution and Insulin Therapy in Severe Sepsis (VISEP) is the most recent study to address this issue and their findings coincide with prior studies. The investigators randomized patients into groups of either conventional or intensive insulin therapy. The conventional insulin group was treated via insulin drip if blood glucose levels exceeded $200 \mathrm{mg} / \mathrm{dL}$ and were maintained in the range of 180 to $200 \mathrm{mg} / \mathrm{dL}$. The intensive insulin therapy group received insulin via insulin drip once blood glucose levels exceeded $110 \mathrm{mg} / \mathrm{dL}$ and were maintained in the range of 80 to $110 \mathrm{mg} / \mathrm{dL}$. No benefit was found to intensive insulin therapy. In fact, this treatment was terminated early by the data and safety monitoring board at the time of the first safety analysis due to the high incidence of hypoglycemia (18).

The Surviving Sepsis Campaign Committee recommends that following patient stabilization, insulin therapy should be initiated. The goal is to maintain blood glucose levels $<150 \mathrm{mg} / \mathrm{dL}$, with levels checked every 1 to 2 hours until stable, then every 4 hours (12).

\section{CONCLUSIONS}

Fortunately, sepsis and septic shock in pregnancy is a rare entity. Normal pregnancy involves many physiologic changes that would present problems for any other patient due to the increased demands on the cardiovascular and other systems. As noted, the work and recommendations that currently exist for the treatment of the continuum of sepsis conditions are for non-obstetric populations and do not take into account the changes associated with pregnancy. Given the paucity of data that exist strictly in regard to septic shock in pregnancy, it is reasonable to proceed with septic shock management according to these guidelines. Definitions that specify the exact entity within the spectrum of sepsis-related diseases can help focus management and facilitate communication with colleagues. Until specific studies in the pregnant prove otherwise, quick assessment and diagnosis of this condition followed by critical care and emergency protocols should be followed.

\section{REFERENCES}

1. Milne I. Sir John Pringle's observations on the diseases of the army - an early scientific account of epidemiology and the prevention of cross infection. J Epidemiol Commun Health 2005; 59: 966.

2. Angus DC, Linde-Zwirble WT, Lidicker J, et al. Epidemiology of severe sepsis in the United States: analysis of incidence, outcome, and associated costs of care. Crit Care Med 2001; 29: 1303-10.

3. Mabie WC, Barton JR, Sibai B. Septic shock in pregnancy. Obstet Gynecol 1997; 90(4 pt 1): 553-61.

4. Martin GS, Mannino DM, Eaton S, Moss M. The epidemiology of sepsis in the United States from 1979 through 2000. N Engl J Med 2003; 348: 1546-54.

5. Rubulotta FM, Ramsay G, Parker MM, et al. An international survey: public awareness and perception of sepsis. Crit Care Med 2009; 37: 167-70.

6. Blanco JD, Gibbs RS, Castaneda YS. Bacteremia in obstetrics: clinical course. Obstet Gynecol 1981; 58: 621-5.

7. Bone RC, Balk RA, Cerra FB, et al. American college of chest physicians/ society of critical care medicine consensus conference committee: Definitions for sepsis and organ failure and guidelines for the use of innovative therapies in sepsis. Crit Care Med 1992; 20: 864-74.

8. Cinel I, Opal SM. Molecular biology of inflammation and sepsis: a primer. Crit Care Med 2009; 37: 291-304.

9. Levy MM, Fink MP, Marshall JC, et al. 2001 SCCM/ESICM/ACCP/ATS/ SIS international sepsis definitions conference. Crit Care Med 2003; 29: 530-8.

10. Shapiro NI, Trzeciak S, Hollander JE, et al. A prospective, multicenter derivation of a biomarker panel to assess risk of organ dysfunction, shock, and death in emergency department patients with suspected sepsis. Crit Care Med 2009; 37: 96-104.

11. Martin SR, Foley MR. Intensive care in obstetrics: an evidence based review. Am J Obstet Gynecol 2006; 195: 673-89.

12. Dellinger RP, Levy MM, Carlet JM, et al. Surviving sepsis campaign: International guidelines for management of severe sepsis and septic shock: 2008. Crit Care Med 2008; 36: 296-327.

13. Finfer S, Bellomo R, Boyce N, et al. A comparison of albumin and saline for fluid resuscitation in the intensive care unit. N Engl J Med 2004; 350: 2247-56.

14. Sprung CL, Annane D, Keh D, et al. Hydrocortisone therapy for patients with septic shock. N Engl J Med 2008; 358: 111-24. 
15. Lipiner-Friedman D, Sprung CL, Laterre PF, et al. Adrenal function in sepsis: the retrospective Corticus cohort study. Crit Care Med 2007; 35: 1012-18.

16. Abraham E, Laterre PF, Garg R, et al. Drotrecogin alfa (activated) for adults with severe sepsis and a low risk of death. N Engl J Med 2005; 353: 1332-41.
17. Bernard GR, Vincent JL, Laterre PF, et al. Efficacy and safety of recombinant human activated protein $\mathrm{C}$ for severe sepsis. $\mathrm{N}$ Engl J Med 2001; 344: 699-709.

18. Brunkhorst FM, Engel C, Bloos F, et al. Intensive insulin therapy and pentastarch resuscitation in severe sepsis. N Engl J Med 2008; 358: 125-39. 


\section{Invasive hemodynamic monitoring in obstetrics Luis D. Pacheco, Shannon Clark, and Gary D. V. Hankins}

\section{CENTRAL VENOUS ACCESS}

Approximately $0.17 \%$ to $1.1 \%$ of all pregnant patients require admission to the intensive care unit (ICU) at some point during their pregnancies (1). Obstetric complications, including hemorrhage and hypertensive disorders (primarily preeclampsia and eclampsia), account for most of these ICU admissions comprising $47 \%$ to $93 \%$ of patients (2). Understanding the main hemodynamic changes associated with pregnancy is crucial when invasive monitoring is applied to the critically ill pregnant patients (Table 1). Central venous access is more commonly obtained through internal jugular (IJ) vein or subclavian vein catheterization, as seen in the nonpregnant population as well.

The main indications for central venous catheter (CVC) placement include administration of vasoactive medications such as norepinephrine or dopamine, infusion of caustic or hypertonic solutions such as potassium chloride or parenteral alimentation, administration of incompatible medicines through a multi-lumen catheter, requirement of acute or subacute renal replacement therapy, necessity of hemodynamic monitoring or transvenous cardiac pacing, and rapid infusion of fluids $(3,4)$. Due to the emergent nature of most CVC placements and their lifesaving indications, there are truly no absolute contraindications to their placement. A few general contraindications do exist and include infection of the area overlying the target vein, thrombosis of the target vein, and coagulopathy (4).

Recent statistics show that approximately 5 million CVCs are placed each year in the United States with one or more complications occurring in $>15 \%$ of catheter placements $(5,6)$. The risk of complications increases with operator inexperience, emergent placement, patient obesity, history of difficult cannulation, and coagulopathy. Common mechanical complications include arterial puncture, hematoma formation, pneumothorax, hemothorax, arrhythmias, and improper location of the catheter $(7,8)$. Venous thrombosis is another complication that occurs less frequently in the subclavian vein and more frequently with cannulation of the femoral vein $(5,6)$. The risk of pneumothorax during CVC insertion increases with hyperinflation of the lungs associated with mechanical ventilation with large tidal volumes or with increased positive end-expiratory pressure. Inadvertent arterial puncture can usually be distinguished by the pulsatile nature of the flow and the red color of arterial blood (4). If in doubt, the operator may insert a small 18-gauge catheter over the guidewire and determine the waveform of the vessel to distinguish arterial from venous cannulation (4). Alternatively, the 18-gauge catheter may be connected to a regular intravenous tubing that is allowed to fill with blood, and when raised, the column of blood should drop if the catheter is inside a vein. Finally, a chest $\mathrm{x}$-ray to confirm proper placement should be obtained after central venous catheterization regardless of insertion site (except for the femoral vein placement).

In addition to mechanical complications, catheter-related infections pose a significant risk to the patient. Infection of the CVC may occur locally at the insertion site, from hub colonization and subsequent infection through the catheter lumen, or through hematogenous seeding of the catheter (5). The Centers for Disease Control and Prevention recommend the subclavian vein as the site of choice to reduce the risk of catheter-related sepsis (9). The Institute for Healthcare Improvement recommends five steps to reduce central-line infections: hand washing, maximal barrier precautions, chlorhexidine skin antisepsis, selection of an optimal catheter site, and prompt removal of the CVC when no longer needed. Antiseptic-containing hubs (chlorhexidine) and antimicrobialimpregnated catheters (minocycline and rifampin) have been shown to decrease the rate of catheter-related bloodstream infections when compared with non-impregnated catheters and should be considered in all CVC insertions when the institutional infection rate exceeds $2 \%(4,5)$.

The use of ultrasound guidance has been shown in various prospective/randomized trials and two meta-analyses since 1990 to reduce mechanical complications of CVC insertion into the IJ and improve first-pass success (10). Twodimensional ultrasound can be used indirectly or directly. With indirect ultrasound guidance, the ultrasound is used for vessel localization, and the puncture site is identified. After this, puncture of the vein is performed without real-time ultrasound guidance. Direct ultrasound guidance allows visualization of the needle puncture in real time after localization of the vessel and can employ one or two operators.

In 2003, Hind et al. evaluated data from 18 studies in a meta-analysis that excluded Doppler studies (11). They found that two-dimensional ultrasound was significantly better for central venous access when compared with the traditional landmark methods and was more effective in avoiding failures in the first attempt of venous cannulation.

Particularly for IJ catheter placements, ultrasound guidance is now considered the standard of care.

\section{Internal Jugular Vein}

IJ cannulation has a lower risk of pneumothorax as compared with the subclavian approach. However, the risks of infection and thrombosis are higher with the IJ.

The anatomical landmarks commonly used to place a central line in the IJ consist of the triangle formed by the clavicle and the sternal and clavicular heads of the sternocleidomastoid muscle. The apex of this triangle corresponds to the separation of the two heads of the muscle. In the apex, the carotid pulse is felt, and the artery is gently retracted to the 
Table 1 Hemodynamic Changes in Pregnancy

$\begin{array}{lcc} & \text { Nonpregnant } & \text { Pregnant } \\ \text { Cardiac output }(\mathrm{L} / \mathrm{min}) & 4.3 \pm 0.9 & 6.2 \pm 1.0 \\ \text { Heart rate }(\text { beats/min) } & 71 \pm 10.0 & 83 \pm 10.0 \\ \text { Stroke volume }(\mathrm{mL}) & 65 & 75-85 \\ \text { Systemic vascular resistance }\left(\text { dynes } \times \mathrm{s} \times \mathrm{cm}^{-5}\right) & 1530 \pm 520 & 1210 \pm 266 \\ \text { Pulmonary vascular resistance }\left(\text { dynes } \times \mathrm{s} \times \mathrm{cm}^{-5}\right) & 119 \pm 47.0 & 78 \pm 22.0 \\ \text { Colloid oncotic pressure }(\mathrm{mmHg}) & 14.5 \pm 2.5 & 10.5 \pm 2.7 \\ \text { Mean arterial pressure }(\mathrm{mmHg}) & 86.4 \pm 7.5 & 90.3 \pm 5.8 \\ \text { Pulmonary capillary wedge pressure }(\mathrm{mmHg}) & 6.3 \pm 2.1 & 7.5 \pm 1.8 \\ \text { Central venous pressure }(\mathrm{mmHg}) & 3.7 \pm 2.6 & 3.6 \pm 2.5 \\ \text { Left ventricular stroke work index }\left(\mathrm{g} \times \mathrm{min} \times \mathrm{m}^{2}\right) & 41 \pm 8 & 48 \pm 6 \\ \text { Blood volume }(\mathrm{L}) & 4.5 & 6.5 \\ \text { Plasma volume }(\mathrm{L}) & 2.5 & 3.5-4.6\end{array}$

midline (the IJ usually is located lateral to the carotid pulse). The needle should be inserted at the apex level just lateral to the carotid pulse. The site is prepared and draped in a sterile fashion. The operator is gowned and gloved for gaining venous access and for positioning the catheter. The patient is positioned, and the head is tilted to the side opposite to the vessel selected for cannulation. The Trendelenburg position aids in distending the venous caliber. With the use of $1 \%$ xylocaine and a 21- or 22-gauge 2-inch needle, the skin is injected and a wheal is made over the planned cannulation site. The needle is then advanced with the bevel up at a 45' angle toward the ipsilateral nipple. Turning the patient's head to the opposite side will straighten the course of the vein and thus facilitate the procedure. The right side is usually preferred since it has a straighter course toward the superior vena cava. In most cases, the vein will be found at a depth of less than $2 \mathrm{~cm}$. Often the vein walls will collapse against each other by the pressure of the needle, and the vessel is completely penetrated without blood return. Slow retraction of the needle while maintaining negative pressure on the syringe will overcome this problem, as during retraction, the needle will re-enter the vessel and blood return will be obtained. Once the vessel is located with this "finding needle," an 18-gauge needle is used to puncture the vein following the tract established with the initial small needle. After this, the syringe is detached from the needle (which should stay in place), and a guidewire is advanced through it. After the wire is passed into the vessel, the needle is withdrawn over the wire. A scalpel is used to make a $5-\mathrm{mm}$ cut in the skin, following which the larger venous dilator is inserted to dilate the tract. After this, the dilator is removed, and the catheter is advanced over the guidewire. The wire is then withdrawn, and the catheter remains in place. This same principle (Seldinger) is applied for the placement of lines in the subclavian or femoral veins.

When using ultrasound guidance for IJ catheter insertion, blood is hypoechoic and appears dark on the ultrasound screen with the IJ typically seen anterior and lateral to the carotid artery. The IJ vein can be distinguished from the artery because it is compressible, nonpulsatile, and distended by Trendelenburg position or the Valsalva maneuver (10). There are several factors that must be considered when using the ultrasound for IJ catheter insertion: (i) excessive pressure applied to the transducer decreases IJ vein diameter, making it harder to access; (ii) extreme contralateral head rotation can decrease IJ vein diameter and increase overlap onto the carotid artery; (iii) employing the Trendelenburg position and using correct head positioning can significantly increase IJ vein diameter and facilitate placement; and (iv) patient intravascular volume status can affect the size of the IJ.

Passage of a needle into the IJ can be done with either a transverse (short axis) view or a longitudinal (long axis) view. The transverse view is associated with a shorter learning curve and makes it easier to visualize smaller vessels (10). Using the longitudinal (long axis) view, as recommended by the American College of Emergency Physicians, allows better visualization of the advancing needle tip, which may reduce perforation of the posterior wall of the vessel and potential entry into the artery (10).

Figure 1 illustrates the placement of a CVC in the IJ site.

\section{Subclavian Vein}

Due to the low risk of thrombosis and infection, in addition to the patient's comfort and easy access, the subclavian vein is the preferred site for CVC placement in the ICU setting.

Coagulopathy is a relative contraindication since in the event of bleeding, the vessels (subclavian vein or artery) are not directly accessible for compression.

The subclavian vein lies just beneath the clavicle. Exposure of the vein may be improved by placing a small rolled towel between the shoulder blades. The right side is also preferred since the right lung apex is lower than the left, diminishing the chances of a pneumothorax when this side is chosen. Also injuries to the thoracic duct may happen (albeit infrequently) when attempting to access the left subclavian vein.

The head of the patient is turned to the opposite side, and Trendelenburg positioning is accomplished. The entry site is usually 2 to $3 \mathrm{~cm}$ caudal to the midpoint of the clavicle. The 18-gauge introducer needle is advanced with the bevel up toward the sternal notch and beneath the clavicle. Care should be taken to avoid directing the needle too caudally (risk of pneumothorax) or too cephalad (risk of subclavian artery puncture). Once blood flow is obtained, the bevel should be rotated 90' clockwise so that the wire, when advanced, will follow the direction toward the superior vena cava. The Seldinger technique is then followed to advance the catheter.

When considering the subclavian vein, several recent articles have suggested that ultrasound can increase the likelihood of successful placement of a subclavian catheter 


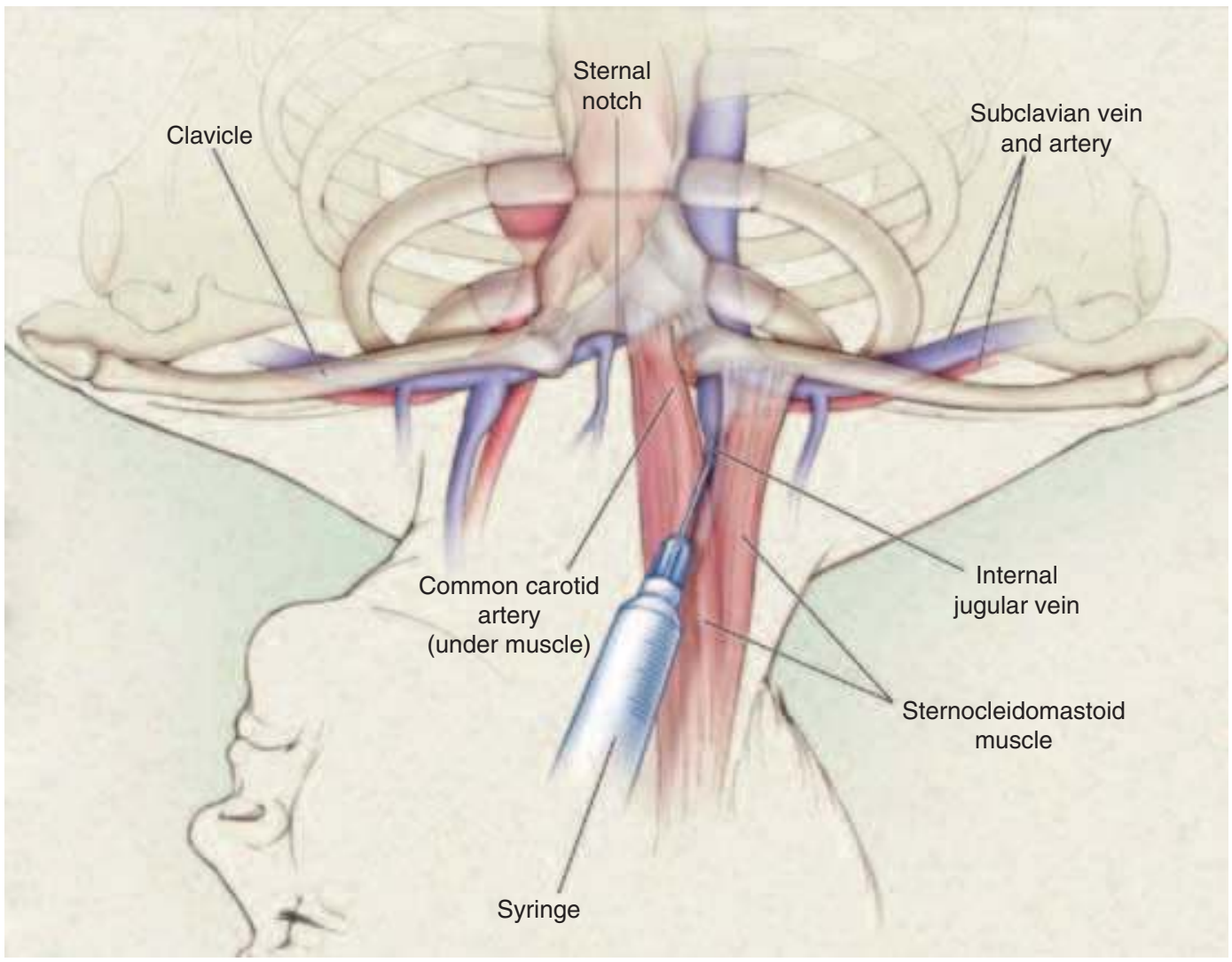

Figure 1 Placement of a central venous catheter in the right internal jugular vein. Source: With permission from New England Journal of Medicine.

despite the presence of the clavicle (12). In addition, Doppler can also be used to distinguish artery from vein due to the difficulty in identifying the vein by compression alone (13).

Figure 2 illustrates the placement of a CVC in the subclavian site.

\section{Femoral Vein}

Placement of the femoral catheter begins with optimal exposure of the femoral region by externally rotating and abducting the leg away from the midline. The femoral vein is 1 to $2 \mathrm{~cm}$ medial to the artery and runs distal to the inguinal ligament. The vein can be located by palpating the pulsating femoral artery. The site must be prepped and draped and the insertion site must be anesthetized with local anesthesia. The introducer needle should then be inserted at a $45^{\prime}$ angle directed along the course of the vein $2 \mathrm{~cm}$ below the inguinal ligament $(2 \mathrm{~cm}$ below an imaginary line that connects the symphysis pubis and the superior-anterior iliac crest). Avoid puncturing above the inguinal ligament since retroperitoneal hemorrhage may ensue. The femoral artery should be palpated at all times while introducing and advancing the needle in an attempt to avoid femoral artery puncture.

When compared with the IJ and subclavian vein cannulations, femoral catheters are associated with higher risk of infection, thrombosis, and arterial puncture (5). Inadvertent puncture of the femoral artery can be resolved with direct pressure. As with the IJ and subclavian veins, ultrasound has been shown to increase success and reduce complications in femoral catheter placement (11).

\section{ARTERIAL LINES}

The preparation for arterial line (A-line) placement begins with sterile preparation of the site, local anesthesia of the insertion site, and positioning of the patient's wrist on a hand board in the dorsiflexed position (14). The radial artery is located 1 to $2 \mathrm{~cm}$ from the wrist, between the bony head of the distal radius and the flexor carpi radialis tendon. The two techniques for A-line placement (over-the-wire and over-theneedle) begin with palpation of the artery and location of the radial pulse followed by entry of the needle at a $30^{\prime}-45^{\prime}$ angle. In the over-the-wire technique, the catheter is advanced through the artery until a flash of blood is noted. At this point, both the needle and the catheter are advanced $1 \mathrm{~cm}$ deeper in order to completely transect the artery. After this, the needle is removed and the catheter is lowered until it is almost parallel with the skin. The catheter is pulled gently until pulsatile blood is noted. At this point, the guidewire is introduced, and then the catheter is advanced into the artery through the guidewire. Finally, pressure should be placed proximal to the catheter and the wire removed. For the over-the-needle technique, the needle is advanced slowly through the skin until a flash of blood is seen. The needle is then lowered $10^{\prime}-15^{\prime}$, and the catheter is advanced into the artery. The catheter is then secured in place.

Continuous blood pressure monitoring is mandatory in patients requiring vasopressors.

Use of the radial artery is commonly employed due to the dual arterial supply of the hand by the ulnar artery (7). However, the radial artery is susceptible to vasospasm and hematoma formation after multiple cannulation attempts. In a 


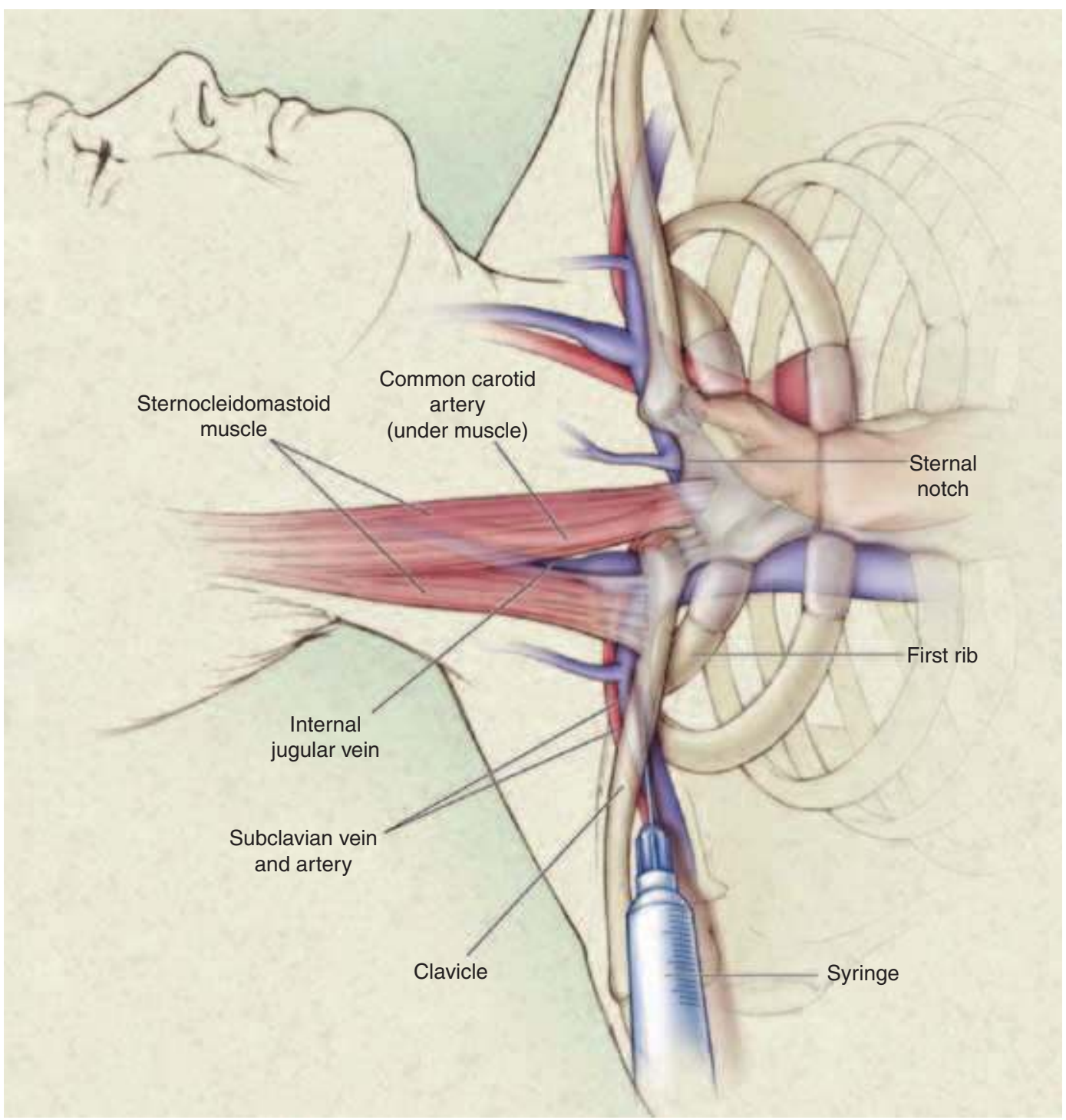

Figure 2 Placement of a central venous catheter in the right subclavian vein. Source: With permission from New England Journal of Medicine.

prospective randomized study by Levin et al. comparing ultrasound-guided radial artery cannulation versus the traditional palpation technique, two-dimensional ultrasoundguided catheterization was superior to palpation for first insertion attempt and overall total number of attempts (15).

\section{CENTRAL VENOUS PRESSURE}

One of the main goals of hemodynamic monitoring is to predict which patients will improve their hemodynamic conditions when a fluid bolus is given. This is of outmost importance since excessive fluid administration may lead to unwanted consequences such as cerebral edema, pulmonary edema, heart edema with diastolic dysfunction, bowel edema and intra-abdominal hypertension, and poor wound and anastomotic healing, among others. Obstetricians are faced with this challenge frequently when deciding to administrate fluids to a pre-eclamptic patient.

Central venous pressure (CVP) is the pressure measured through a CVC placed with its distal tip at the junction of the superior vena cava and the right atrium. Such a catheter is attached to a fluid-filled system and a pressure transducer, producing a characteristic waveform in the monitor (Fig. 3).
Clinical use of CVP requires careful attention in order to achieve proper measurements (16). The anatomical site to "zero" the line should be $5 \mathrm{~cm}$ vertically below the sternal angle, which corresponds to the midpoint of the right atrium.

If one desires to estimate cardiac preload, the CVP measurement should be done at the base of the " $\mathrm{c}$ " wave (Fig. 3). This corresponds to the last pressure estimate prior to ventricular contraction, thus yielding the best estimate of cardiac preload. In cases where the " $\mathrm{c}$ " wave of the CVP tracing cannot be identified, the base of the "a" wave may be used. Alternatively, this point may be identified by tracing a vertical line from the $\mathrm{Q}$ wave of the continuous EKG tracing in the monitor (16). All estimates should be performed at the end of expiration (17). Simply reading the CVP value displaced in the monitor is inaccurate since this is an average of pressures taken over time irrespective of the diastolic cycle.

CVP variations during the respiratory cycle have classically been associated with hypovolemia. In spontaneously breathing patients and in mechanically ventilated patients with triggered efforts, an inspiratory fall in CVP (measured at the base of the "c" or "a" wave) greater than $1 \mathrm{mmHg}$ may indicate fluid responsiveness. 


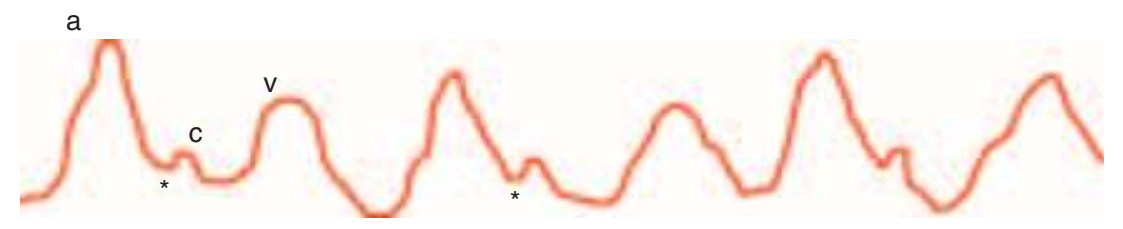

Figure 3 Central venous pressure waveform. Wave "a" corresponds to atrial contraction and wave "c" is secondary to the closure of the tricuspid valve and the isovolumetric contraction of the right ventricle, which displaces the tricuspid leaflets upward, causing an increase in pressure in the right atrium. Wave " $\mathrm{v}$ " corresponds to passive right atrium filling during early diastole. The correct place to measure CVP is shown by the asterisks, which approximate the base of wave "c"

A CVP tracing with a prominent " $y$ " descent usually indicates that cardiac output $(\mathrm{CO})$ will not increase with fluid administration (16).

CVP has been used as a monitor of central blood volume and thus preload to the heart. This latter concept has been challenged during the last years as CVP is affected by multiple factors such as right ventricular compliance, tricuspid valve regurgitation, positive pressure ventilation, pericardial disease, vascular tone, and arrhythmias (18).

For example, patients with decreased right-sided $\mathrm{CO}$ secondary to a myocardial infarction or pulmonary hypertension or an acute pulmonary embolism will all have elevated CVP, and this will show no correlation with the overall volume status. The same situation is typical in patients with cardiac tamponade, where despite elevated CVP values, initial therapy includes aggressive fluid resuscitation.

The major pitfall of CVP in predicting fluid responsiveness is that it is a static preload measurement. The latter means that a specific value of CVP will not necessarily guarantee an increase in $\mathrm{CO}$ after a fluid challenge. A recent trial showed that among patients with a CVP of less than $8 \mathrm{mmHg}$, the positive predictive value for fluid responsiveness, defined as an increase in cardiac index of $\geq 15 \%$, was only $47 \%$ (19).

A systematic review of the literature showed that there was no association between CVP values and circulating blood volume and that CVP does not predict fluid responsiveness (20). Furthermore, the receiver operator characteristic curve for CVP was only 0.56 . This means that a low CVP will predict an increase in CO in only $56 \%$ of the cases. This is similar to flipping a coin!

Thus, a patient is equally likely to respond to fluid with a low or a high CVP (20). Despite lack of scientific evidence, the 2008 Surviving Sepsis Campaign still recommends utilizing CVP as an end point for fluid resuscitation in the setting of septic shock (21).

During early sepsis (first 6 hours of presentation), the use of a CVP-guided protocol of resuscitation as shown by Rivers et al. did improve outcomes (22). We believe that the use of CVP in these first hours of sepsis management is acceptable.

Most of the recent literature concurs in that CVP should not be used to guide fluid management. However, CVP may still be useful as a marker of right ventricular performance in cases of right-sided myocardial infarction, post heart transplant, pulmonary hypertension, and acute pulmonary embolism (20).

\section{PULMONARY ARTERY OCCLUSION PRESSURE}

The pulmonary artery catheter (PAC) allows one to measure multiple hemodynamic variables. The catheter is $110 \mathrm{~cm}$ long and contains a variable amount of ports. An infusion port is usually present. The proximal port connects to the proximal lumen, which is located $30 \mathrm{~cm}$ proximal to the distal end of the catheter. This port, when connected to a fluid-filled system and a pressure transducer, permits continuous measurement of the CVP. The distal port allows continuous measurements of pulmonary artery pressures. An additional port allows a syringe (with $1.5 \mathrm{~mL}$ of air) to be connected to it. By inflating this port, one inflates the balloon present in the distal part of the catheter. When this happens, blood flow after that point will be interrupted due to vascular occlusion secondary to the inflated balloon. The pressure immediately distal to the balloon, when inflated, is the pulmonary artery occlusion pressure (PAOP). PAOP is also measured at the end of expiration. This, theoretically, is a surrogate of the left ventricular filling pressure at the end of diastole since a "column" of blood will be present between the site of balloon inflation (in a branch of the pulmonary arterial system), the pulmonary venous system, the left atrium, the mitral valve, and, finally, the left ventricle (LV).

The catheter also allows measuring the $\mathrm{CO}$ by the thermodilution method. Once the $\mathrm{CO}$ is calculated, the systemic vascular resistances (SVR) are also calculated as follows: SVR $=(\mathrm{MAP}-\mathrm{CVP} / \mathrm{CO}) \times 80$, where MAP is the mean arterial pressure.

The PAC is advanced through a larger introducer, which is placed as any other central line with the Seldinger technique. The two preferred approaches to float ${ }^{\circ}$ a Swan-Ganz catheter are the right IJ vein and the left subclavian vein. As the catheter is advanced, characteristic waveform changes will occur as its tip enters the right atrium, right ventricle, and pulmonary artery as well as when the inflated balloon occludes the vessel completely (PAOP). Figure 4 depicts these waveform changes.

Complications with the use of the PAC include pneumothorax, hemothorax, arrhythmias, pulmonary infarcts and hemorrhages, and heparin-induced thrombocytopenia, among others. Extreme care is recommended when obtaining a PAOP value in the patient with severe pulmonary hypertension, where pulmonary hemorrhage may occur while obstructing blood flow with the inflated balloon.

Recent literature has questioned the benefit of the PAC in clinical practice. A recent trial involving patients with acute lung injury and adult respiratory distress syndrome (ARDS) showed that the PAC did not improve outcomes as compared with patients managed with a conventional CVP line (23). Another prospective cohort study including patients with severe sepsis also showed no benefit from the PAC (24). A randomized multicenter control study including patients with shock, ARDS, or both similarly showed no benefit from the PAC (25). Similar findings have been reported in the setting of 


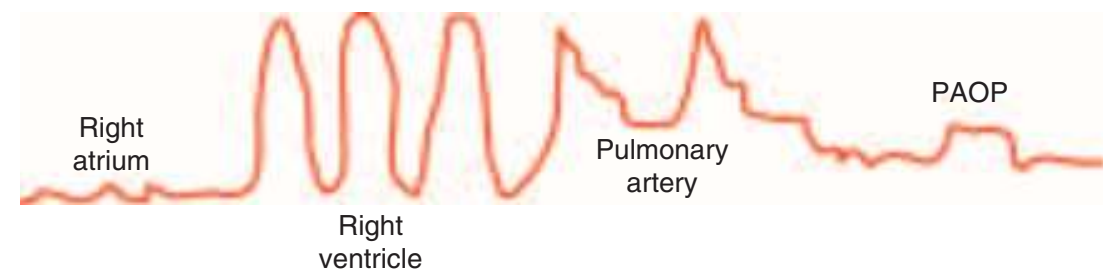

Figure 4 As the pulmonary artery catheter is advanced, the waveform analysis in the monitor will display typical patterns according to the anatomical site, where the tip is located. When the tip of the pulmonary artery catheter (PAC) is in the superior vena cava and right atrium, the waveform corresponds to the central venous pressure tracing. Entrance to the right ventricle leads to a sudden increase in the systolic pressure (15-30 mmHg) with the same diastolic pressure as on the right atrium $(0-8 \mathrm{mmHg})$. As the PAC is advanced more deeply, the pulmonary artery is entered. The latter leads to an increase in diastolic pressure (6-12 $\mathrm{mmHg}$ ) with the same previous systolic pressure seen in the right ventricle. Also the entrance to the pulmonary artery may be noticed by the appearance of a notch in the waveform caused by the closure of the pulmonary valve. If one keeps advancing the catheter, one will notice in the monitor the tracing of the pulmonary artery occlusion pressure (PAOP) when the balloon obstructs the flow distal to its position. This pressure should generally be less than the pulmonary artery diastolic pressure and is always measured at the end of expiration.

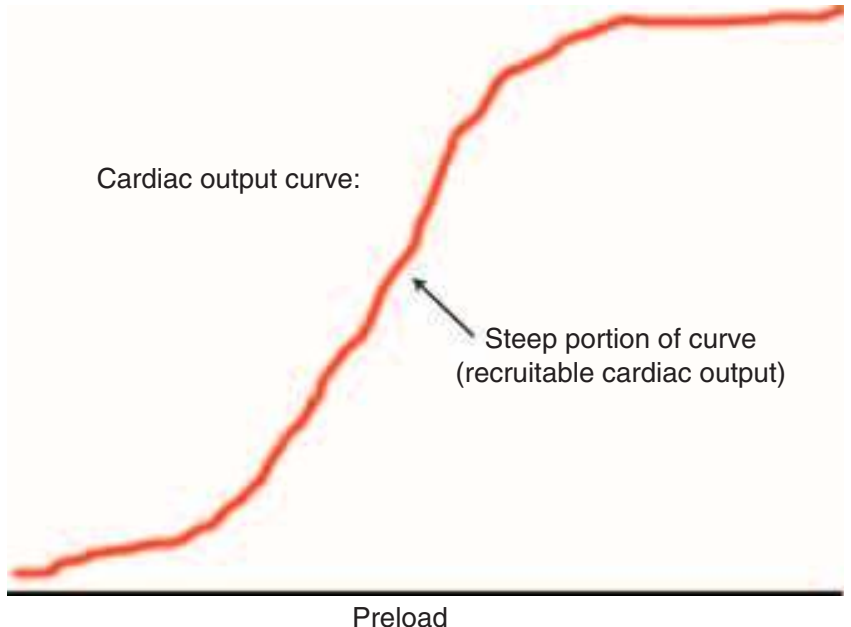

Preload

Figure 5 Frank-Starling curve. When the heart operates in the steep portion of this curve, it will respond by increasing stroke volume in the setting of a fluid bolus.

congestive heart failure, general critical illness, and high-risk perioperative patients (26). In trauma patients, most trials have shown no benefit with the use of the PAC to manage resuscitation. Only one trial, to our knowledge, showed a decrease in mortality in a subset of trauma patients (severely injured with severe shock and elderly patients with moderate shock) (27).

The use of PAOP to predict fluid responsiveness has the same limitations discussed previously for the CVP. It is a static measurement of preload and consequently does not predict fluid responsiveness (28). A PAOP of $<12 \mathrm{mmHg}$ predicted fluid responsiveness (defined as an increase in cardiac index of at least $15 \%$ ) in only $54 \%$ of critically ill septic patients (19). This is not surprising since PAOP is not a reliable surrogate of the left ventricular filling pressure; it only reflects the internal pressure of the pulmonary veins relative to atmospheric pressure (18). This measurement is also widely affected by multiple factors. Positive end-expiratory pressure increases PAOP. Of particular concern in obstetrics, pre-eclamptic patients frequently have left ventricular diastolic dysfunction. The latter means that the ventricle will be unable to accommodate significant amounts of fluid. This will cause retrograde accumulation of blood, providing high values in PAOP despite a hypovolemic state.
Significant increases in afterload will diminish left ventricular stroke volume. This will also elevate PAOP despite no changes in total blood volume.

Again, the adequacy of intravascular volume cannot be assessed by one CVP or PAOP (29).

Classic obstetrical literature has attempted to provide a list of clinical scenarios where the PAC is usually indicated (e.g., severe pre-eclampsia not responsive to anti-hypertensive therapy, difficult to manage pulmonary edema, acute kidney injury in the setting of pre-eclampsia); however, it is our opinion that most of these patients can be managed safely without this device.

We favor dynamic measurements of preload responsiveness, where the hemodynamic effect after a fluid challenge can be assessed at the bedside, as will be discussed later in this chapter.

\section{ECHOCARDIOGRAPHY AND FLUID STATUS}

The use of transthoracic echocardiography (TTE) may be useful in evaluating hemodynamics. This is an attractive option due to the noninvasiveness of the technique.

Simple subjective assessment of the left ventricular volume by estimating the left ventricular size may aid in guiding fluid therapy (30). A small hyperdynamic LV is typical of hypovolemic patients. Also systolic obliteration of the left ventricular cavity is very suggestive of decreased blood volume (30).

Obviously, certain limitations exist. A large LV end-diastolic area does not always guarantee adequate preload as this may be secondary to LV dilation in the setting of heart failure. On the contrary, a small LV end-diastolic area (frequently suggestive of hypovolemia) may be present in patients with a significant drop in SVR (e.g., sepsis), which favor LV emptying.

Circulating volume status may also be evaluated indirectly by assessing the change in diameter of the inferior vena cava (IVC) during the respiratory cycle (31). During spontaneous breathing, the negative pressure generated in the thoracic cavity during inspiration will favor preload return to the right heart; this will decrease the caliber of the IVC. The more pronounced the respiratory variation in the IVC diameter, the more likely right atrial pressures will be low (and theoretically, the more likely the patient will respond to a fluid bolus). A dilated IVC (diameter more than $2 \mathrm{~cm}$ ) that does not decrease caliber during spontaneous inspiration is indicative of high right atrial pressures (30). 


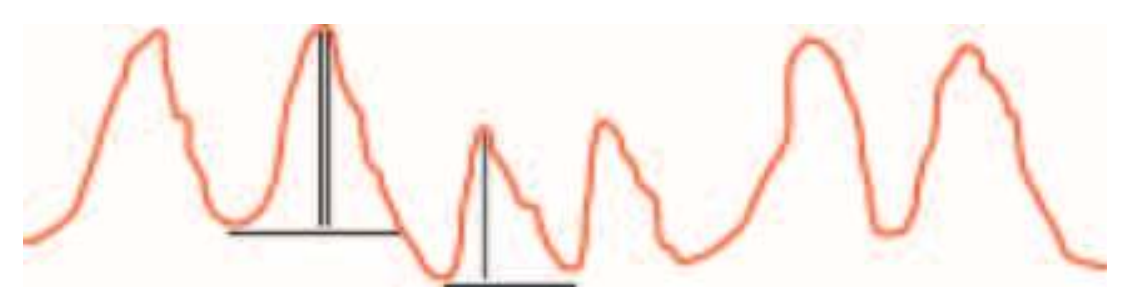

Figure 6 Example of pulse pressure (PP) variation in an arterial line tracing. In this situation, the double line is the maximal PP, while single line corresponds to the minimal PP. This variation in PP in patients on mechanical ventilation predicts fluid responsiveness.

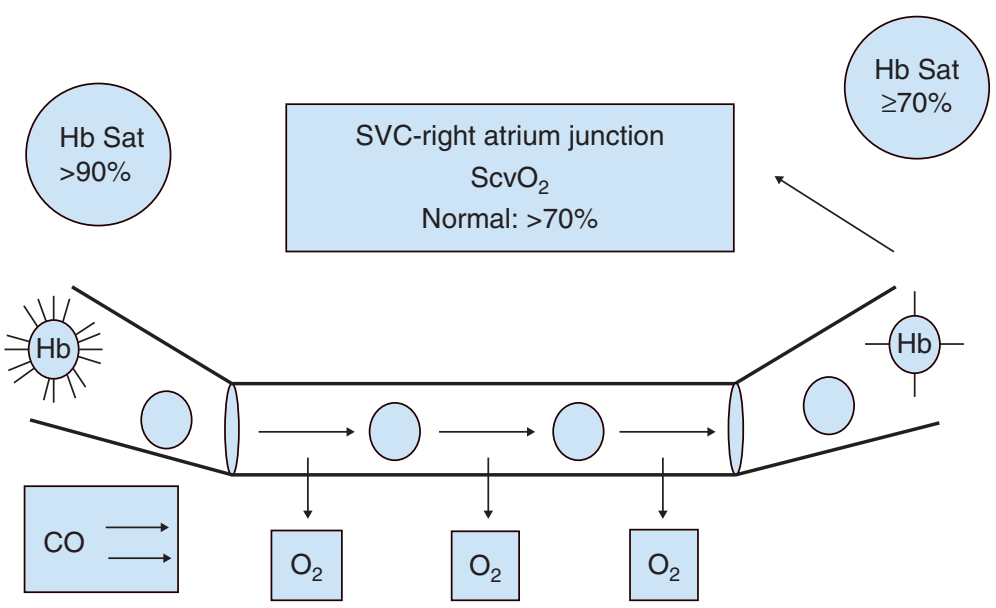

Figure 7 When oxygen delivery $\left(\mathrm{DO}_{2}\right)$ is compromised, tissues will increase extraction of $\mathrm{O}_{2}$ from hemoglobin. As a consequence, evaluation in the central venous circulation will reveal low hemoglobin oxygen saturation. Abbreviations: $\mathrm{CO}$, cardiac output; $\mathrm{Hb}$, hemoglobin; SVC, superior vena cava.

Some data suggest that using a $12 \%$ cutoff in IVC diameter variation with respiration will predict fluid responsiveness with a positive predictive value of $93 \%$ (32).

The accuracy of the IVC diameter in predicting fluid responsiveness in patients mechanically ventilated is significantly decreased, as the increased intrathoracic pressures may prevent IVC collapse. Also the application of this method during pregnancy is unknown.

Other methods used to asses LV volume include left ventricular end-diastolic measurements. A left ventricular end-diastolic diameter smaller than $2.5 \mathrm{~cm}$ or an LV enddiastolic area less than $55 \mathrm{~cm}^{2}$ is typically associated with hypovolemia (33).

Just like CVP and PAOP, most of these echocardiographicbased measurements are static measures of volume status and, as such, poorly predict fluid responsiveness (34).

\section{PULSE PRESSURE VARIATION}

Since static preload measurements (CVP, PAOP, LV enddiastolic measurements) are no better than random chance at predicting fluid responsiveness, other dynamic approaches have come into clinical practice.

Pulse pressure (PP) is the difference between systolic and diastolic pressures. The use of pulse pressure variation (PPV) to predict fluid responsiveness applies only to patients on mechanical ventilation, not triggering the ventilator, with tidal volumes of 8 to $10 \mathrm{~mL} / \mathrm{kg}$, and in sinus heart rhythm.

A recent prospective observational study found that PPV was still an adequate tool to predict fluid responsiveness despite the use of lower tidal volumes $(6 \mathrm{~mL} / \mathrm{kg}$ in setting of ARDS) (34,35).
During conventional mechanical ventilation, each time the ventilator delivers the tidal volume, right atrial pressure will increase. This increase in pressure will decrease preload to the right ventricle in patients who are preload responsive (28). By achieving this, there will be less leftward displacement of the interventricular septum, allowing more filling of the LV. Tidal volume delivery also squeezes ${ }^{\circ}$ the lungs, increasing volume return to the LV through the pulmonary venous system. In addition, during this inspiratory phase, the left ventricular afterload will be significantly decreased. All of the above will lead to an increase in LV stroke volume during inspiration (reversed pulsus paradoxus) (36). This means that during inspiration, PP will increase. If the variation in PP during inspiration and expiration is larger than $13 \%$, patients will likely respond to fluids. Ventilator-induced cyclic changes in preload to both right and left ventricles are more pronounced when the ventricles operate on the steep portion of the FrankStarling curve (Fig. 5).

In patients with an arterial line in place, the latter is relatively easy to identify at the bedside. The arterial line tracing is evaluated over a period of 30 to 60 seconds, and the maximal and minimal PPs are recorded (Fig. 6). The PPV is then calculated by using the following formula: $\mathrm{PPV}=[\mathrm{Max}$ $\mathrm{PP}-\min \mathrm{PP} /(\max \mathrm{PP}+\min \mathrm{PP}) 0.5] \times 100$. If $\mathrm{PPV}$ is $>13 \%$, patients will respond to a fluid bolus (28).

The latter concept may also be applied to the pulse oximetry plethysmographic curve (36). A sharp waveform with a clear dicrotic notch indicates adequate peripheral perfusion, while a sinus-shaped waveform reflects poor perfusion. Actually, the height of the pulse oximetry curve correlates well with PP (36). Consequently, in mechanically ventilated patients who meet 
the same criteria cited above for PPV evaluation, respiratoryinduced changes in the plethysmographic waveform is a useful method to predict fluid responsiveness.

\section{PASSIVE LEG RAISING}

Passive leg raising (PLR) is a useful method to predict fluid responsiveness. Unlike PPV, PLR may be used in patients who are spontaneously breathing or triggering the ventilator. It may also be used despite the presence of cardiac arrhythmias (34).

This test simply involves elevation of the lower extremities at a $30^{\prime}$ to $45^{\prime}$ angle for 1 minute. The maneuver causes a 300-mL blood bolus that will persist for up to 3 minutes before intravascular volume redistribution happens (28). Ideally, while doing PLR, some form of real-time measurement of blood flow should be available (e.g., esophageal Doppler monitoring allowing continuous measurement of both diameter and blood velocity in the aorta) in order to evaluate the immediate cardiovascular response to the maneuver. Patients who will respond to fluids (by increasing $\mathrm{CO}$ ) will display an increase in aortic blood flow and diameter.

The latter technology is not widely available. A recent trial utilized TTE to evaluate response to PLR. A stroke volume increase higher than $13 \%$ after PLR predicted fluid responsiveness with a sensitivity of $77 \%$ and a specificity of $100 \%$ (37).

During pregnancy, mechanical compression by the uterus to the IVC may alter the response to this maneuver. No data are available about this issue.

\section{$\mathrm{SVO}_{2}$ AND SCVO}

$\mathrm{SvO}_{2}$ is the saturation of hemoglobin measured from a sample of blood obtained from the pulmonary circulation. This may be accomplished by sampling through the distal lumen of a PAC. The normal value is $>65 \%$.

$\mathrm{ScvO}_{2}$ is the hemoglobin saturation of a sample obtained at the junction of the superior vena cava and the right atrium. Collecting this sample only requires the presence of a regular CVC. The normal value is $>70 \%$.

During pregnancy, there is an increase in basal metabolic rate of $20 \%$ as compared with an increase in CO of $40 \%$ to $50 \%$. The higher the CO, the faster the red blood cell travels through the distal tissues. The latter will decrease the available time of perfused tissues to extract oxygen from hemoglobin, leading to higher hemoglobin saturation in the venous circulation. Consequently, one would expect that during pregnancy, $\mathrm{SvO}_{2}$ (and probably $\mathrm{ScvO}_{2}$ ) would be higher than nonpregnant values. However, Hankins and colleagues reported an $\mathrm{SvO}_{2}$ of $72 \%$ in a group of normal pregnant women who received a PAC during the third trimester (38). This is not different from the values reported in healthy nonpregnant individuals.

Decreased values associate with poor oxygen delivery since hypoperfused tissues will extract more oxygen from hemoglobin, leading to low hemoglobin saturations in the venous pool (Fig. 7).

The clinician may attempt to correct low values by increasing resuscitation efforts including fluid resuscitation, blood transfusions, inotropic support, and decreasing oxygen consumption through mechanical ventilation and sedation.

$\mathrm{ScrO}_{2}$ monitoring has decreased mortality when used to guide resuscitation during early sepsis management (22).

No data exist on the use of these parameters to guide resuscitation during pregnancy.

\section{REFERENCES}

1. Baskett T, Sternadel J. Maternal intensive care and near-miss mortality in obstetrics. BJOG 1998; 105: 981-4.

2. Martin SR, Foley MR. Intensive care in obstetrics: an evidence-based review. Am J Obstet Gynecol 2006; 195: 673-89.

3. Braner DAV, Lai S, Eman S, Tegtmeyer K. Videos in clinical medicine. Central venous catheterization-subclavian vein. N Engl J Med 2007; 357: e26.

4. Taylor RW, Palagiri AV. Central venous catheterization. Crit Care Med 2007; 35: 1-7.

5. McGee DC, Gould MK. Preventing complications of central venous catheterization. N Engl J Med 2003; 348: 1123-33.

6. Merrer J, De Jonghe B, Golliot F, et al. Complications of femoral and subclavian venous catheterization in critically ill patients: a randomized controlled trial. JAMA 2001; 286: 700-7.

7. Maecken T, Grau T. Ultrasound imaging in vascular access (review). Crit Care Med 2007; 35: S178-85.

8. Graham AS, Ozment C, Tegtmeyer K, et al. Videos in clinical medicine. Central venous catheterization. N Engl J Med 2007; 356: e21.

9. O'Grady NP, Alexander M, Dellinger EP, et al. Guidelines for the prevention of intravascular catheter-related infections. MMWR Morb Mortal Wkly Rep 2002; 51: 1-29.

10. Feller-Kopman D. Ultrasound-guided internal jugular access: a proposed standardized approach and implications for training and practice. Chest 2007; 132: 302-9.

11. Hind D, Calvert N, McWilliams R, et al. Ultrasonic locating devices for central venous cannulation: meta-analysis. BMJ 2003; 327: 361.

12. Orihashi K, Imai K, Sato K, et al. Extrathoracic subclavian venipuncture under ultrasound guidance. Circ J 2005; 69: 1111-15.

13. Tegtmeyer K, Brady G, Lai S, et al. Videos in clinical medicine: placement of an arterial line. N Engl J Med 2006; 354: e13.

14. Levin PD, Sheinin O, Gozal Y. Use of ultrasound guidance in the insertion of radial artery catheters. Crit Care Med 2003; 31: 481-4.

15. Magder S. Central venous pressure: a useful but not so simple measurement. Crit Care Med 2006; 34: 2224-7.

16. Lodato RF. Use of the pulmonary artery catheter. Semin Respir Crit Care Med 1999; 20: 29-42.

17. Polanco PM, Pinsky MR. Principals of hemodynamic monitoring. In: Ronco C, Bellomo R, Kellum JA, eds. Acute Kidney Injury, 1st edn. Basel: Karger, 2007: 133-57. (Contributions to Nephrology; vol 156).

18. Osman D, Ridel C, Ray P, et al. Cardiac filling pressures are not appropriate to predict hemodynamic response to volume challenge. Crit Care Med 2007; 35: 64-8.

19. Marik PE, Baram M, Vahid B. Does central venous pressure predict fluid responsiveness? Chest 2008; 134: 172-8.

20. Dellinger RP, Levy MM, Carlet JM, et al. Sepsis surviving campaign: International guidelines for management of severe sepsis and septic shock: 2008. Crit Care Med 2008; 36: 296-327.

21. Rivers E, Nguyen B, Haystand S, et al. Early goal-directed therapy in the treatment of severe sepsis and septic shock. N Engl J Med 2001; 345: 1368-77.

22. The National Heart, Lung, and Blood Institute Acute Respiratory Distress Syndrome (ARDS) Clinical Trials Network. Pulmonary-artery versus central venous catheters to guide treatment of acute lung injury. N Engl J Med 2006; 354: 2213-24.

23. Yu DT, Platt R, Lanken PN, et al. Relationship of pulmonary artery catheter use to mortality and resource utilization in patients with severe sepsis. Crit Care Med 2003; 31: 2734-41.

24. Richard C, Warszawski J, Anguel N, et al. Early use of the pulmonary artery catheter and outcomes in patients with shock and acute respiratory distress syndrome. JAMA 2003; 290: 2713-20. 
25. Harvey S, Welch CA, Harrison DA, et al. Post hoc insights from PACMan-The UK pulmonary artery catheter trial. Crit Care Med 2008; 36: $1714-21$.

26. Friese RS, Shafi S, Gentilello LM. Pulmonary artery catheter use is associated with reduced mortality in severely injured patients: A National Trauma Data Bank analysis of 53,312 patients. Crit Care Med 2006; 34: 1597-601.

27. Pinsky MR. Hemodynamic evaluation and monitoring in the ICU. Chest 2007; 132: 2020-9.

28. Vincent JL, Weil MH. Fluid challenge revisited. Crit Care Med 2006; 34: 1333-7.

29. Beaulieu Y. Bedside echocardiography in the assessment of the critically ill. Crit Care Med 2007; 35: S235-49.

30. Kircher BJ, Himelman RB, Schiller NB. Non-invasive estimation of right atrial pressure from the inspiratory collapse of inferior vena cava. Am J Cardiol 1990; 66: 493-6.

31. Feissel M, Michard F, Faller JP, Teboul JL. The respiratory variation in inferior vena cava diameter as a guide to fluid therapy. Intensive Care Med 2004; 30: 1834-7.
32. Balachundhar S, Talmor D. Echocardiography for management of hypotension in the intensive care unit (review). Crit Care Med 2007; 35: S401-7.

33. Monnet X, Teboul JL. Volume responsiveness. Curr Opin Crit Care 2007; 13: 549-53.

34. Huang $\mathrm{C}, \mathrm{Fu} \mathrm{JY}, \mathrm{Hu} \mathrm{H}$, et al. Prediction of fluid responsiveness in acute respiratory distress syndrome patients ventilated with low tidal volume and high positive end-expiratory pressure. Crit Care Med 2008; 36: 2810-16.

35. Charron C, Caille V, Jardin F, Vieillard-Baron A. Echocardiographic measurement of fluid responsiveness. Curr Opin Crit Care 2006; 12: 249-54.

36. Bendjelid K. The pulse oximetry plethysmographic curve revisited (review). Curr Opin Crit Care 2008; 14: 348-53.

37. Lamia B, Ochagavia A, Monnet X, et al. Echocardiographic prediction of volume responsiveness in critically ill patients with spontaneously breathing activity. Intensive Care Med 2007; 33: 1125-32.

38. Hankins GVD, Clark SL, Uckan E, Van Hook JW. Maternal oxygen transport variables during the third trimester of normal pregnancy. Am J Obstet Gynecol 1999; 180: 406-9. 


\section{Shoulder dystocia \\ Randall C. Floyd and James S. Smeltzer}

One of the most frightening experiences in obstetrics is the failure of the shoulders to deliver spontaneously. This sequence, the cardinal sign of its occurrence, and the usual methods and frequently tragic consequences of its inappropriate management were described most vividly by Morris (1):

\begin{abstract}
Antenatally, a careful observer may have recognized that the child was unusually large, though this is notoriously difficult to judge ... it may have been necessary to intervene in the second stage ... The hairy scalp slides out with reluctance. When the forehead has appeared it is necessary to press back the perineum to deliver the face. Fat cheeks eventually emerge. A double chin has to be hooked over the vulval commissure, to which it remains tightly opposed [Turtle sign] ... Time passes. The child's face becomes suffused ... Abdominal efforts by the mother or by her attendants produce no advance, gentle head traction is equally unavailing. Usually equanimity forsakes the attendants. They push, they pull. Alarm increases. Eventually "by greater strength of muscle or by some infernal juggle" the difficulty appears to be overcome ... It dawns on the attendants that their anxiety was not illfounded, the baby lies limp and voiceless, and only too often remains so.
\end{abstract}

Such a disaster, not only the more common but also tragic result of fetal brachial plexus injury, can usually be averted by appropriate management. This management is based on a solid understanding of the biomechanics of normal shoulder delivery and of shoulder dystocia and the pathophysiology of excessive fetal growth. The steps in this management are prevention of the condition when it can be prevented prenatally, detection of the possibly large infant, and avoidance of operative vaginal delivery, preparation for the possible occurrence of shoulder dystocia when a large infant is anticipated, prompt recognition of the dystocia when it occurs, and employment of maneuvers with proven effectiveness, which are least likely to harm the fetus or mother.

\section{THE MECHANISM OF SHOULDER DYSTOCIA}

The mechanism of normal shoulder delivery can be partly verified by pelvic examination of a normal patient after delivery of the fetal head, if there is a generous episiotomy. During delivery of the head, one shoulder has entered the pelvis obliquely and is resting in the sciatic notch or hollow of the sacrum. This is the posterior shoulder on restitution. The identity of this shoulder, not any "memory" of previous attitude, determines the direction of "restitution" $(1,2)$. This position in the hollow of the sacrum or sacrosciatic notch provides room for the anterior shoulder to slide under the pubis or rotate from the obturator foramen. The turtle sign of fetal head retraction after delivery indicates that the above sequence has not occurred and that a shoulder dystocia exists.

The position of the shoulders in shoulder dystocia is anterior-posterior. In the more common high dystocia, the posterior shoulder is lodged at the inlet on the sacral promontory and the anterior shoulder is wedged against the pubis $(1,3,4)$ (Fig. 1). The turtle sign is produced by traction between the delivered head and the posterior shoulder, and this traction locks the shoulders in an unfavorable position. As observed by Jacquemier (5) and by Woods (6) and demonstrated conclusively by Schwartz and Dixon (3), direct traction and pressure applied to such a "locked" position are likely to fail both to deliver the infant and to produce injury. This position will not let the posterior shoulder enter the pelvis, will not provide room for the anterior shoulder to slip under the pubis, and will stretch the cervical brachial plexus roots of the posterior arm, causing a greater chance of injury to this plexus (7).

This ominous situation occurs because the shoulders do not normally fit through the pelvic inlet together, and the shoulder destined to be the posterior must enter first. This fact is ignored by most writers on the subject, but is ascertainable by episiotomy and pelvic examination before performing maneuvers when the turtle sign is present. Most term infants have a minimum shoulder diameter that is greater than the maximum diameter of the normal maternal pelvic inlet and cannot traverse it together (8). The gynecoid pelvis, perfectly adapted to the delivery of the fetal head, has a higher relative risk for shoulder dystocia than other types, and maternal pelvic capacity is not an absolute factor in shoulder dystocia and fetal brachial plexus injury (9).

This situation can be made worse by ignorance and panic. The parturient wants to push the baby out, perhaps with the "help" from others by fundal pressure, further wedging the shoulders at the inlet. Attempting to push the anterior shoulder through the inlet with suprapubic pressure would further impact the posterior shoulder. The inclination of the novice accoucheur is to overcome the impaction by force, pulling the fetal head posteriorly, which stretches the brachial plexus of the anterior arm, or inferiorly, which further stretches the plexus of the posterior arm. The fetus is lucky if dystocia is mild or the obstruction is overcome by the fracture of a clavicle, either spontaneously or by the use of suprapubic pressure. The inclination to use suprapubic pressure is reinforced by many articles and texts on shoulder dystocia. It is usually ineffective in high dystocia (10) and should not be used until it is verified that the posterior shoulder is in the pelvis or in an attempt to fracture the clavicle. Once the 
posterior shoulder is definitely in the pelvis, suprapubic pressure becomes rational, indicated, and likely to work.

The pelvic inlet, like the walls of Troy, cannot be breached by force without unacceptable loss $(3,5,11,12)$. The posterior shoulder alone, like the Trojan horse, is small and mobile and can enter easily if permitted to do so. Once the posterior shoulder is in the pelvis, the anterior usually has room to traverse the pubis or can be persuaded to do so by other means. The mechanism of successful treatment of the normal high shoulder dystocia is to break the tie that occurs at the pelvic inlet and emulate the natural sequence of shoulder delivery. Successful treatments involve the following principles (Table 1):

1. Relieve the traction that is locking the posterior shoulder in an undeliverable position. This is the opposite of the pushing and pulling that is sometimes employed. Traction is relieved by a large episiotomy, McRoberts maneuver or supine squat (12), and the Hibbard maneuver or partial cephalic replacement (4).

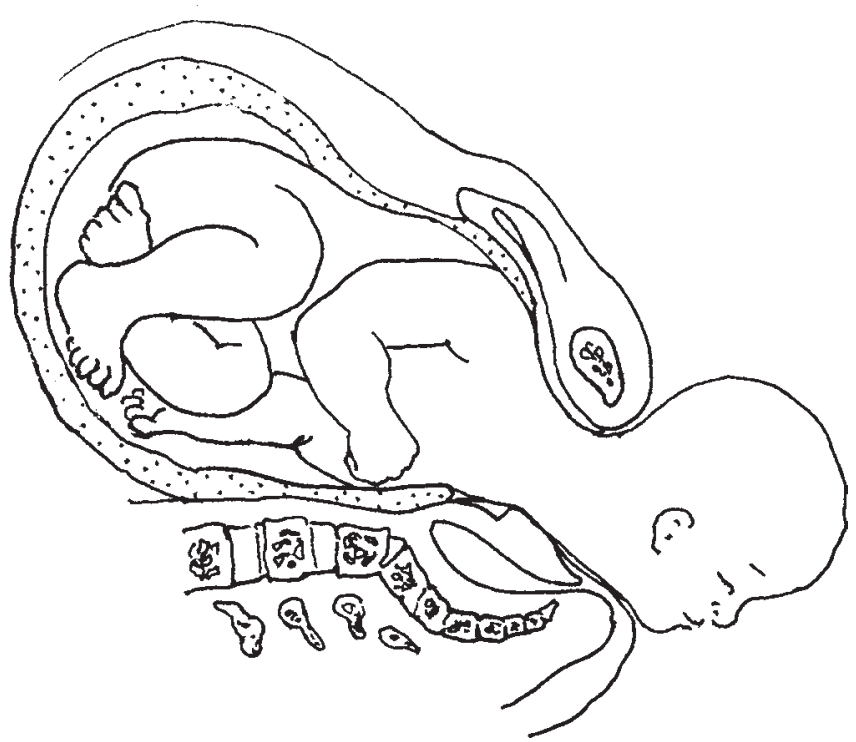

Figure 1 Classic shoulder dystocia. The head is delivered, but retracted by the tension between the chin and the shoulder, which are at the inlet. Further progress for the anterior shoulder or fetus is not safe until the posterior enters the pelvis.
2. Rotate the posterior shoulder into an oblique diameter. Rock the anterior shoulder abdominally or push the posterior forward from behind (Rubin maneuver) (8), push the distal clavicle back from in front (Woods maneuver) (6), or do this while pushing the anterior shoulder in the opposite direction from behind above the pubis with the opposite hand (DeLee maneuver). If the shoulders rotate in either direction, the posterior shoulder will enter the pelvis.

3. If the posterior shoulder cannot be rotated, it will enter the pelvis over the sacral promontory when the posterior arm is delivered (Jacquemier maneuver) $(3,5)$.

4. When there is insufficient room to deliver the anterior shoulder with the posterior shoulder in the pelvis (either turtle sign absent or after delivery of the posterior shoulder into the pelvis), the anterior arm can be delivered (Couder maneuver), which brings the anterior shoulder out under the pubis. This is the authors' choice. Alternatively, the shoulders can be rotated until the posterior is anterior and delivered under the pubis. This requires the posterior shoulder to be inferior to the spines $(2,6)$. Once the posterior shoulder is delivered under the pubis, the anterior shoulder is now posterior, is in the pelvis, and can be spontaneously delivered.

5. If all these fail, relax the uterus, replace the head and perform a cesarean section (Zavanelli maneuver) (13), or perform a symphysiotomy (14).

6. If shoulder dystocia should occur during an in-bed delivery outside the delivery room, these maneuvers will be difficult or impossible in the supine position. If the McRoberts maneuver cannot be applied, or fails, the hands-and-knees position (15), the kneechest position, or the squat (15) should be used. The first two positions give good access to the posterior shoulder and arm if necessary. These all give a favorable angulation of the pelvis, and gravity aids rather than impedes release of the posterior shoulder from the inlet.

\section{Table 1 Sequence of Action When Shoulder Dystocia is Encountered}

1 Recognize turtle sign: posterior shoulder is arrested at inlet or high in pelvis. Anterior shoulder is not deliverable and do not attempt to deliver it

2 Obtain help for McRoberts maneuver, neonatal resuscitation, and anesthesia

3 Perform McRoberts maneuver. Insure adequate flexion of hips (knees to shoulders). If it fails, reapply and continue

4 If turtle sign is released, attempt gentle delivery of anterior shoulder

5 If turtle sign is not released or anterior does not progress, cut generous episiotomy

6 Examine patient vaginally: Where is posterior shoulder? Is there a fetal tumor obstructing delivery?

7 Unlock locked shoulders with partial cephalic replacement

8 Rotate posterior shoulder off symphysis or under pubis symphysis if in pelvis

9 Deliver posterior arm across chest (Jacquemier maneuver) if posterior shoulder not in pelvis. This delivers posterior shoulder into pelvis

10 Deliver anterior arm (Couder maneuver) or use suprapubic pressure if posterior shoulder is in pelvis and anterior not delivered.

This delivers the anterior shoulder under pubis symphysis

11 If this is not possible, rotate posterior shoulder to anterior (delivered position). Anterior will now be in mid-pelvis and deliverable (Woods maneuver)

12 Relax uterus, replace head, and perform cesarean section (Zavanelli maneuver) if above measures cannot be performed for some reason or fetus has a tumor obstructing delivery 


\section{PREVENTION}

The incidence of shoulder dystocia is $0.2 \%$ to $0.8 \%$ and this risk increases directly with birth weight, especially among macrosomic infants. There is no way to predict with any confidence that a shoulder dystocia will occur in a particular delivery, however (16-19).

Shoulder dystocia cannot be strictly prevented because normal term fetuses, with shoulders wider than the fetal head, are at some risk for shoulder dystocia, and most infants of mothers without diabetes with shoulder dystocia are not macrosomic (16-20). The risk is increased by factors that increase the discrepancy between fetal body and shoulder size and head size. About two-thirds of fetuses with shoulder dystocia are male. The risks for macrosomia and shoulder dystocia are increased by a history of prior shoulder dystocia, prior large infant, and a large maternal birth weight. The mnemonic DOPE_-diabetes, obesity, prolonged pregnancy, and excessive fetal size or maternal weight gain-indicates current pregnancy factors that should alert the clinician to an increased risk for shoulder dystocia (Table 2).

\section{PROLONGED PREGNANCY}

As body growth continues and head growth diminishes at term, shoulder dystocia risk increases with prolonged pregnancy and is negligible before term. The incidence of shoulder dystocia in pregnancies beyond 41 weeks is $1.5 \%$, but the risks of macrosomia $(5.2 \%>4500 \mathrm{~g})$, birth trauma $(0.7 \%)$, and shoulder dystocia were not reduced by routine induction of labor at 41 weeks versus observation and antenatal testing in one randomized study of 3407 pregnancies. However, cesarean section for fetal distress was reduced by routine induction using prostaglandin gel at 41 weeks (21), thus induction at term may be of use.

Fetal body and bone growth, unlike brain growth, are sensitive to fetal insulin, which in turn responds to a maternalfetal glucose load. Maternal conditions such as diabetes, gestational glucose intolerance, excessive weight gain, and obesity can cause fetal overnutrition and are associated with an increased risk for macrosomia, operative delivery, and shoulder dystocia (16-20).

\section{OBESITY}

Maternal obesity is not preventable but is treatable. Past blanket proscriptions against excessive weight gain were obviously misguided. A woman who starts pregnancy $50 \mathrm{~kg}$ overweight already has caloric reserves that are the equivalent of 200 days of a normal diet. Her ideal weight gain is in the range of $5-10 \mathrm{~kg}$. Moreover, fat exacerbates the normal gestational resistance to insulin and thus provides the fetus excess glucose. A balanced diet emphasizing complex carbohydrates, moderate portion size, distribution of calories throughout the day, and high fiber intake (22) would seem prudent. These women should know that the objective is not to lose weight, but to limit heavy intake of simple sugars and provide the fetus with appropriate nutrition. The urine should be checked for ketones if weight gain is not adequate, and calories should be increased or distributed more favorably if ketonuria is present.

\section{EXCESSIVE MATERNAL WEIGHT GAIN}

Increased maternal weight gain is associated with shoulder dystocia, especially when the mother is already obese. Overall perinatal mortality is generally lower when weight gain during pregnancy is 10 to $20 \mathrm{~kg}$ for normal-weight women or over $20 \mathrm{~kg}$ for slim women. Caloric limitations would not be indicated for these women. Increased maternal weight gain and fundal height suggest the possibility of fetal macrosomia, however.

\section{DIABETES}

Infants of diabetic mothers have a greater risk for macrosomia and a greater risk of excess shoulder size (23) and shoulder dystocia for a given fetal weight $(20,24)$. Glucose is the primary energy source for the fetus. It is absorbed by facilitated diffusion, with a rate regulated only by maternal serum glucose concentration. Fetal insulin responds to the fetal absorbed glucose, and it in turn acts as a trophic hormone on fetal liver, bone, muscles, and fat. Prevention of fetal overnutrition and macrosomia requires maternal glucose homeostasis.

Tight glucose control, with target fasting and preprandial glucose of $60-80 \mathrm{mg} / \mathrm{dL}$ and 2-hour postprandial glucose of $<120 \mathrm{mg} / \mathrm{dL}$, is associated with a significant reduction of the risk for macrosomia, shoulder dystocia, operative delivery, and, most importantly, perinatal mortality among diabetic women (25). It is also clear that recognition and control of hyperglycemia prior to 32 weeks is more successful than late control for reducing the risk of macrosomia (26). Obese women have insulin resistance, high insulin production, and delayed insulin secretion, rather than a lack of insulin. Thus, they may require large doses of insulin to achieve euglycemia, but have considerable range between adequate and oversupply of exogenous insulin because the endogenous insulin production moderates their response to exogenous insulin. Excellent glucose control can be readily accomplished with a balanced regular diet high in fiber and complex carbohydrates, with calories and carbohydrates distributed throughout the day.

The problem of hypoglycemia may limit the ability to meet these objectives in Type 1 diabetics who have little endogenous insulin production. For these women, "tight" control requires

\section{Table 2 Prevention of Shoulder Dystocia and Injury}

1 Offer cesarean section for high-risk vaginal deliveries: extremely large fetuses (over $5 \mathrm{~kg}$ ), very large fetuses (over $4.5 \mathrm{~kg}$ ) with maternal diabetes, large fetuses (over $4 \mathrm{~kg}$ ) with a history of shoulder dystocia, second-stage protraction with large fetus

2 Identify and treat diabetes mellitus

3 Be prepared with a plan

4 Recognize the presence of dystocia before maternal pushing, staff assistance (fundal and suprapubic pressure), and traction have produced or contributed to injury

5 Note times and get help when shoulder dystocia is recognized. Help includes assistants for McRoberts, obstetric back-up, neonatal resuscitation, and anesthesia 
close attention to regularization of calories, carbohydrates, and exercise; fiber is even more important. The risks of miscarriage and congenital anomalies are increased if maternal glucose is not controlled in the first trimester. In addition, early treatment of maternal hyperglycemia would better reduce the risk of macrosomia. Regular insulin or the newer rapidonset insulins (humalog, novalog) administered at abdominal sites has more rapid and predictable absorption and can be taken before each meal, with small doses of long-acting insulin (NPH, Lantus, Levemir) provided at appropriate intervals to meet basal needs.

Women with possible gestational glucose intolerance, as evidenced by family history of diabetes, prior large infant, prior shoulder dystocia or stillbirth, should have a preconceptual glucose screen of fasting and $75 \mathrm{~g}$ 2-h determinations. These women should also have mid-pregnancy screening to exclude the possibility of overt diabetes. Nonobese patients without a suggestive history should undergo the regular early third-trimester screening (27).

The patient with type 2 diabetes controlled with oral agents or those pregnant with abnormal 3-hour glucose tolerance testing with normal fasting glucose but elevated postprandial glucose levels may benefit from treatment with appropriate doses of glyburide (28). The goals for fasting and postprandial capillary blood glucose levels are the same as those noted above. The use of metformin during pregnancy is under investigation but cannot be recommended at this time.

Untreated women with an abnormal screen but only one abnormal value on glucose tolerance test are at higher risk for macrosomia than women identified and treated as gestational diabetics (29). These patients should follow the same diet as those with an abnormal glucose tolerance test and have periodic screening for overt diabetes.

Optimal glycemic control reduces but cannot eliminate the risk of macrosomia. Maintaining maternal serum glucose at normal fasting levels during labor may alleviate fetal asphyxia if a difficult labor or delivery occurs $(30,31)$ and decrease the risk of neonatal hypoglycemia.

Kjos and colleagues (32) found that induction of labor routinely at 38 to 39 weeks of gestation in insulin-requiring diabetes reduced the risk for macrosomia and shoulder dystocia versus conservative management and did not increase the cesarean section rate. It is currently our practice to deliver all patients with glucose intolerance at no later than 40 weeks of gestation. Those with poorly controlled diabetes as indicated by suspected macrosomia and/or persistently elevated capillary blood glucose monitoring are offered amniocentesis for evaluation of fetal lung maturity with delivery if maturity is documented secondary to concerns about increasing risk of fetal mortality and morbidity.

\section{DETECTING FETAL MACROSOMIA AND PREDICTING SHOULDER DYSTOCIA}

Most fetuses who experience shoulder dystocia but are not macrosomic have nondiabetic mothers, while most macrosomic fetuses with shoulder dystocia have diabetic mothers $(17,20,23,24)$. Accurate prediction of the fetal weight could identify fetuses at increased risk for shoulder dystocia in this group. Unfortunately, error in predicting birth weight from ultrasonography is $10 \%$ to $20 \%$ of the actual birth weight with the greater error seen at the extremes of estimated fetal weights. More babies are born at normal weights as opposed to very large weights; an infant predicted to be over $4000 \mathrm{~g}$ is as likely to be less than $4000 \mathrm{~g}$ as over $4000 \mathrm{~g}$ (20). With a predicted birth weight over $5000 \mathrm{~g}$, the fetus is likely to be at least over $4000 \mathrm{~g}$. This also means that some large fetuses will be missed.

Elective cesarean section for prevention of fetal injury has been proposed for macrosomic infants of nondiabetic and diabetic mothers with estimated fetal weights of above $5000 \mathrm{~g}$ and above $4500 \mathrm{~g}$ (or even above $4000 \mathrm{~g}$ ), respectively. As large infants of diabetic mothers have a higher risk for shoulder dystocia and fetal trauma, weight for weight, elective cesarean section may be indicated if estimated fetal weight could be accurately identified (20).

Elliott and colleagues (33) described a sonographic finding of abdominal minus biparietal diameter difference of greater than $1.4 \mathrm{~cm}$ as predictive of an infant over $4000 \mathrm{~g}$ in diabetics, with a sensitivity of $87 \%$ and specificity of $72 \%$. Using a similar difference measure for fetuses with an estimated weight of 3800 to 4200 also in diabetic mothers, Cohen and associates (34) found that a cutoff value of $2.6 \mathrm{~cm}$ identified 20 fetuses with a $30 \%$ incidence of shoulder dystocia.

Kitzmiller and coworkers (35) described detection of macrosomia by measurement of shoulder diameter in diabetics by computed tomography scan, with $14.5 \mathrm{~cm}$ as the cutoff level for macrosomia. Sensitivity was 100\% and specificity was $87 \%$ for fetal weight over $4200 \mathrm{~g}$.

The reported incidences of shoulder dystocia with a fetus over 4500 g vary from $2 \%$ to $35 \%$, with most studies reporting incidences of $20 \%$ to $40 \%$ among infants of diabetic mothers delivering vaginally $(20,24,36)$ and less than half that risk among nondiabetic patients $(20,24)$. The risk for permanent brachial plexus neurologic injury arising from shoulder dystocia varies from $0.6 \%$ (19) to $27.5 \%$ (37). The risk for perinatal mortality varies between zero (36) and 28\% (21). There appears to be considerable variation in the ability to diagnose and safely manage shoulder dystocia. The advocacy for elective cesarean section based on estimated weight by the various authors may be directly proportional to their own morbidity and mortality rates.

No rational cesarean section policy can completely prevent shoulder dystocia $(9,11,12,16-20)$. Centers with high neonatal morbidity and mortality rates from shoulder dystocia would probably prevent more neonatal morbidity and mortality by meticulous treatment of diabetes in pregnancy and provision of formal training of staff in safe management of shoulder dystocia, rather than expending these resources on performing more elective cesarean sections.

Several studies have shown the benefit of simulation training with measurable improvement in outcomes and appropriate use of management techniques documented $(15,21,25,38,39)$. These improvements have been demonstrated in providers at all levels of training. The use of simulation training appears demonstrably more effective than that of didactic training or didactic training combined with case review. Current simulation training with computerized simulators (such as the NOELLE) allows the provider the ability to practice various interventions in an extremely realistic situation with monitoring of the effectiveness of the 
maneuvers. Various scenarios may be programmed by the proctor of the simulation and instantaneous feedback provided regarding the success or failure of the interventions performed.

The risk for a brachial plexus injury sustained at birth to become permanent is about $5 \%$, based on the collaborative perinatal project data (9).

The recognition of the possibility of a large infant is definitely beneficial for management during labor. Labor disorders of all types are more common in pregnancies destined for shoulder dystocia $(9,24,38)$. All studies with a significant number of operative vaginal delivery cases clearly indicate that there is a three- to sixfold increased risk for shoulder dystocia in association with abnormal labor curves. Infants over $4000 \mathrm{~g}$ with operative vaginal delivery, especially mid-pelvic delivery, have a several-fold increased risk $(23 \%)$ for shoulder dystocia $(9,38)$. Macrosomic infants of diabetic mothers have even a greater risk of shoulder dystocia (50\%) and subsequent traumatic injury (24). It is uncertain whether operative vaginal delivery causes birth of the head when the shoulders are too large to follow or fails to give the shoulders time to follow the proper mechanism for birth. In any case, nonemergent operative vaginal delivery should be avoided in those patients with anticipated large infants. The mnemonic for the above becomes DODOPE (disordered labor, operative vaginal delivery, diabetes, obesity, prolonged pregnancy, and excessive fetal size or maternal weight gain), an association that is, hopefully, becoming extinct. The exceptions are cases of fetal distress and severe maternal disease, in which the actual risks of additional time to delivery or cesarean section must be weighed against the potential risk of shoulder dystocia individually.

\section{PREPARATION}

The delivery team has time physically, mentally, and psychologically to prepare themselves and the patient for the possibility of shoulder dystocia when a large infant is suspected. This permits review of maneuvers to be used and effective coordination of treatment if the problem is encountered. This physical and mental preparation is the antidote for the panic that can arise in a difficult shoulder delivery. It is helpful to have an anesthetist and a physician experienced in the treatment of shoulder dystocia available, and two people familiar with the McRoberts maneuver in the room. It may also help to have someone experienced in neonatal evaluation and resuscitation.

\section{RECOGNITION}

Usually the diagnosis is obvious, when the "turtle sign" of head retraction after final extension is found. Rarely, shoulder dystocia occurs when the posterior shoulder is in the pelvis and the turtle sign may not be present. These cases will become apparent when the anterior shoulder does not deliver easily with normal effort. Either situation calls for assistance, including all experienced help, anesthesia, and neonatal care.

Considerable force is often applied to the fetal head prior to the recognition and appropriate treatment of shoulder dystocia (40). Traction, neck twisting, and lateral head force are not only ineffective, but all stretch the cervical brachial plexus roots and can avulse them leading to permanent damage.

\section{TREATMENT}

\section{The McRoberts Maneuver}

The McRoberts maneuver should be employed immediately. Assistants should be coached to use and continue the McRoberts maneuver. This accomplishes delivery in most cases and aids in delivery in others (41). It also provides assistants with a constructive alternative to fundal and downward suprapubic pressure, which only impacts the shoulders further. The McRoberts maneuver or supine squat flexes the lumbar spine and retracts the perineum (Fig. 2). The maneuver releases the traction that is locking and depressing the posterior shoulder against the sacral promontory. This spontaneous traction by itself can cause an Erb's palsy, especially if the parturient spontaneously extends rather than flexes the pelvis $(42,43)$. The McRoberts maneuver also straightens out the lumbosacral lordosis and the sacral promontory and removes the compressive force of the delivery table on the sacrum. Radiologic studies evaluating the changes in the pelvis that occur have documented a significant increase in the size of the pelvic inlet with flattening of the sacral prominence (44). In addition, it pushes the anterior shoulder superiorly and places the pelvic inlet perpendicular to the axis of the fetus. These actions together usually place the posterior shoulder of the fetus in the maternal pelvis as the maneuver is being applied; it reduces the force necessary for delivery by $30 \%$ and produces less risk for injury of a simulated fetus (45).

As the posterior shoulder enters the pelvis, the baby's neck appears to get longer, usually as the maneuver is being applied. This "lengthening" should not be confused with the moderate release of tension that occurs with the McRoberts maneuver when the posterior shoulder is still at the inlet. If there is a good regional block and generous episiotomy, this maneuver should be applied with a hand in the pelvis to feel it work. If this succeeds, easy delivery of the anterior shoulder is to be anticipated without further maneuvers. If this fails, the legs can be relaxed somewhat and the maneuver repeated once, in conjunction with pressure on the fetal head toward the sacral promontory to free the shoulders (Hibbard maneuver) (4).

When the McRoberts maneuver is applied correctly, the patient's knees almost or actually touch her shoulders. An excited patient can make the maneuver difficult, as the hamstrings, gluteus, and low back muscles of a large patient are quite strong. Maternal pelvic extension should be avoided, however, as it can injure the fetus.

An average-sized assistant can effectively apply the maneuver with correct application of force and mass to mechanical advantage in the following fashion: (i) face the patient's head from near the stirrup, (ii) place her knee on your inboard shoulder, and (iii) lock your hands or forearms around the upper thigh and pull the upper thigh snugly against your hip and bend forward, using your mass to flex the hip. The McRoberts maneuver works most of the time if it is used in experienced hands (41) and over $80 \%$ of the time at many institutions. 
(A)

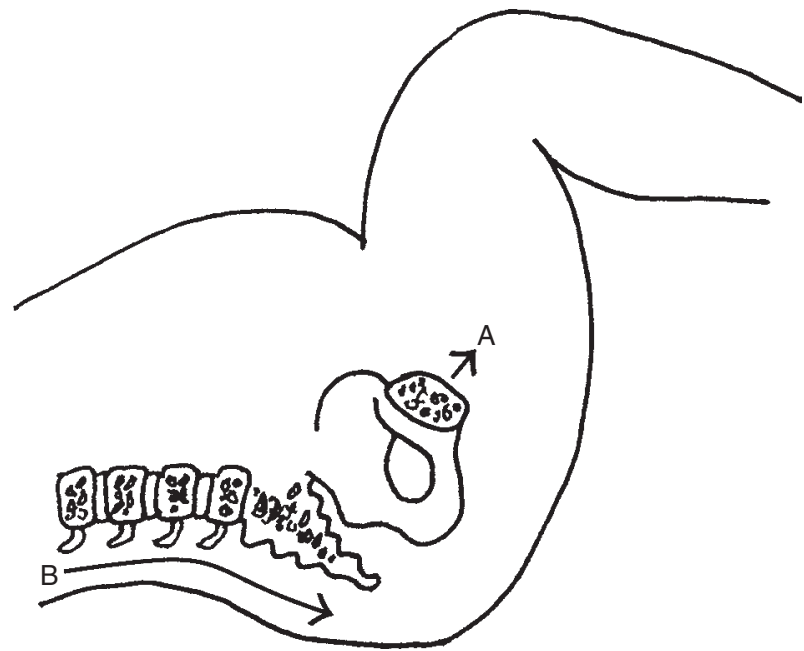

(B)

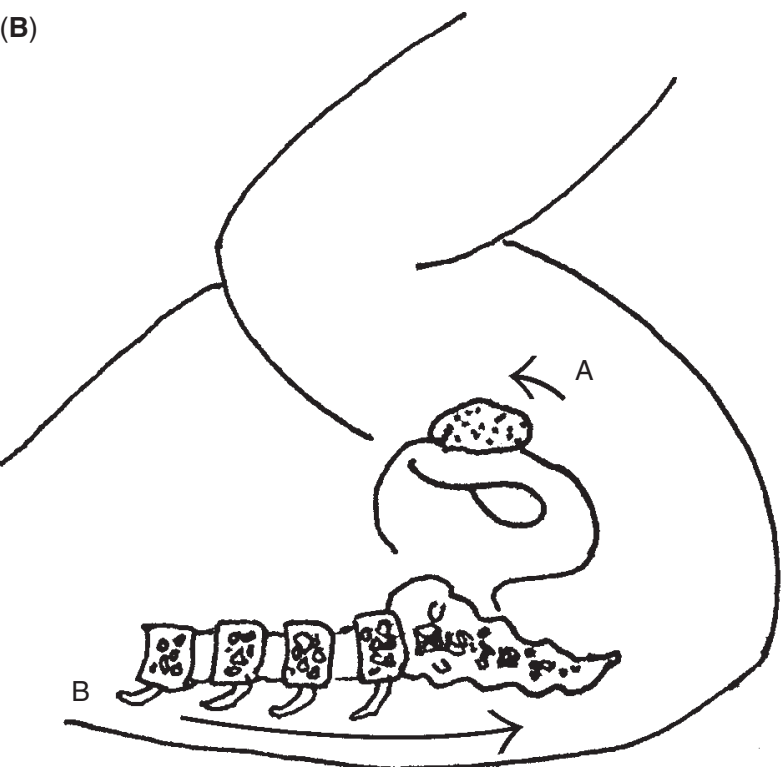

Figure 2 The McRoberts maneuver (A and B) rotates the pelvis cephalad, straightens the lumbosacral lordosis, pushes the anterior shoulder back, and lifts the posterior off the sacral promontory. It also relieves the tension between the posterior shoulder and soft tissues by retracting them.

\section{Episiotomy}

If the McRoberts maneuver position has not affected release of the posterior shoulder and delivery, it can be continued through the other maneuvers, as it facilitates their action. This position should be relaxed during induction of anesthesia if general anesthesia is required at this time. It is imperative to determine the actual cause of the dystocia and to determine the position of the posterior shoulder and take appropriate action for a safe delivery. It is essential to release the tension between the fetal head and shoulders with minimum impediment and risk for serious fetal injury and extensive maternal vaginal lacerations. Some recommend a large mediolateral episiotomy. Others prefer a complete perineotomy. The simplest way to achieve the latter is a proctoepisiotomy. This is usually not necessary (19). The proper episiotomy depends on the clinical setting, the size of the fetus, and the size of the operator's hand. The only "wrong" episiotomy is the one that is not sufficient to permit the maneuvers needed to effect delivery without lasting fetal injury.

\section{Examination}

The next step is to verify the location of the posterior shoulder and to confirm the absence of dorsal tumors that would make further attempt at vaginal delivery either fruitless or dangerous. This can be accomplished with the hand toward the infant's back after restitution. This leads immediately to the Rubin maneuver.

\section{The Rubin Maneuver}

In the Rubin maneuver (8), the posterior shoulder is located, usually on the sacral promontory. The shoulder is rotated forward from behind, either by direct pressure or by hooking the scapula. The anterior shoulder can be rocked laterally to free it or rotated in the opposite direction with the heel of the free hand suprapubically. If the posterior shoulder is at the inlet, rotation will permit it to enter the pelvis and rest in the sacrosciatic notch. If it is already in the pelvis while the anterior shoulder is undeliverable, rotation of the posterior shoulder should be continued until it is anterior and can be delivered under the pubis. Rotation using the fetal head has been described, but this may increase stretching of the brachial plexus, similar to direct and lateral traction on the fetal head (1). If the posterior shoulder does not rotate with moderate pressure, the operator should proceed immediately to the next maneuver.

\section{The Woods Maneuver}

In the Woods maneuver (6), the hand toward the fetal back is withdrawn, and the opposite hand is placed in the pelvis in front of the fetus. Although anterior fetal tumors are rarer than dorsal ones, if they obstruct further delivery, the operator should proceed to the Zavanelli maneuver. The posterior shoulder is rotated backward by pressure on the distal clavicle. This may be assisted by pressure with the opposite hand on the anterior shoulder to the front suprapubically (DeLee maneuver). If no rotation occurs with moderate pressure, the operator should proceed immediately to the next maneuver.

\section{Delivery of the Posterior Arm (Jacquemier Maneuver)}

The most effective maneuver is delivery of the posterior arm $(3,5,9,11,12)$. The main risks are vaginal lacerations and fetal humeral fracture. The latter risk can be minimized in the following way (Fig. 3): The operator's hand is extended further into the uterus to find the humerus attached to the posterior shoulder. The humerus is brought to the front along the side of the fetus. The elbow is flexed so that the forearm is brought across the body. The forearm is firmly grasped by the operator's entire hand and pulled out in a reverse Pinard sequence. The delivery of the arm causes the posterior shoulder to enter the pelvis. The anterior shoulder should now be easily deliverable under the pubis. Otherwise, an identical maneuver is performed on the anterior arm, except 
(A)

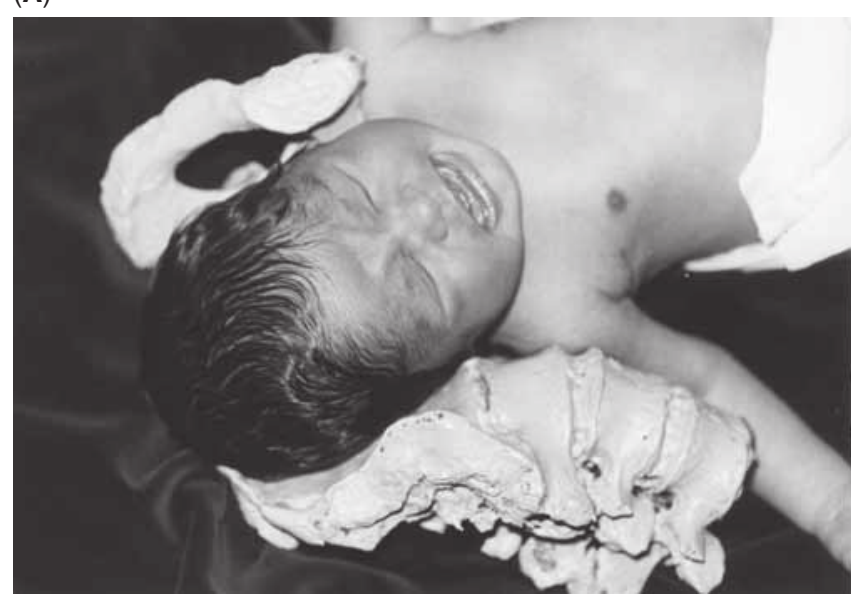

(C)

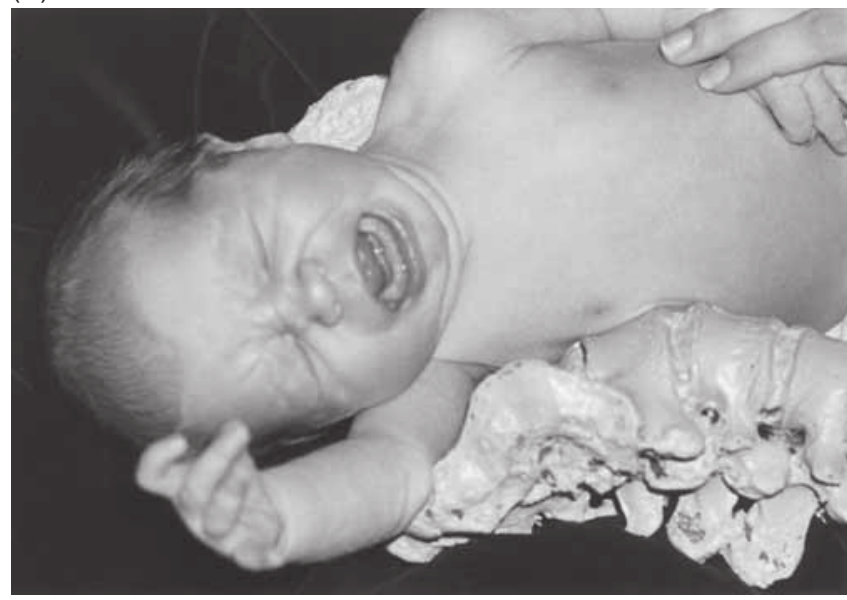

(B)

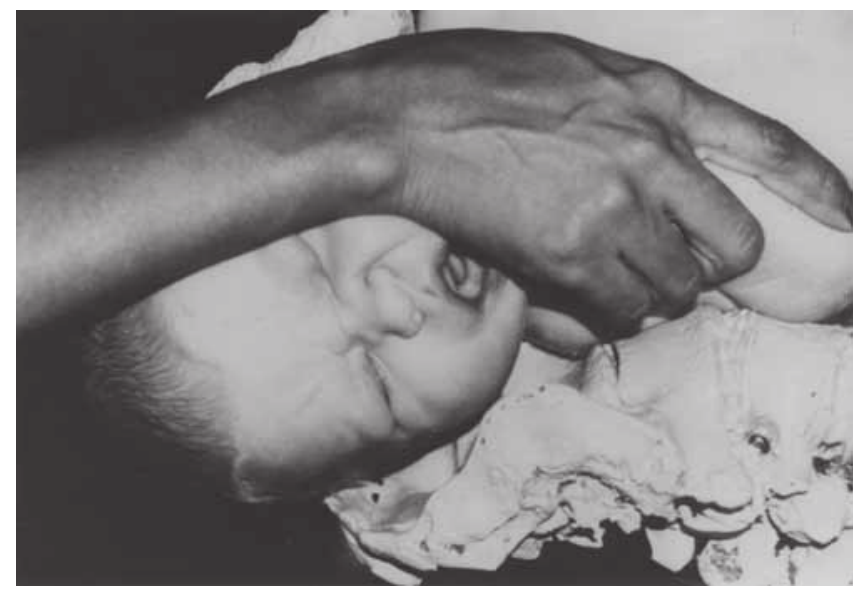

(D)

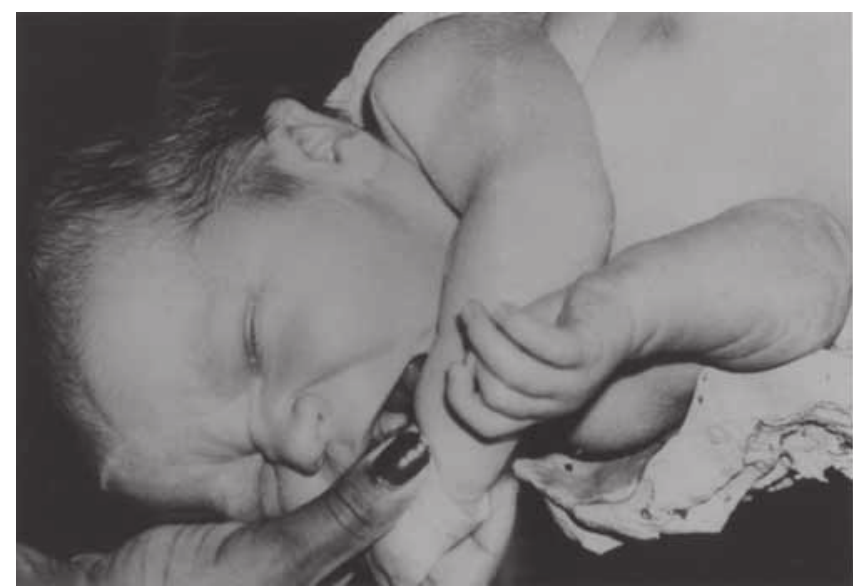

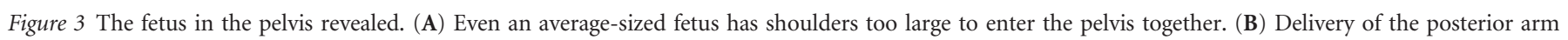

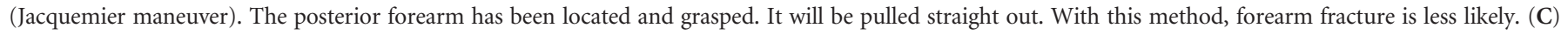

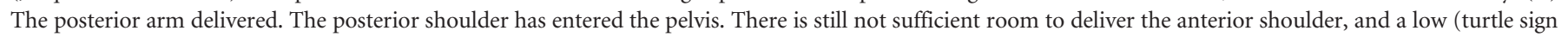

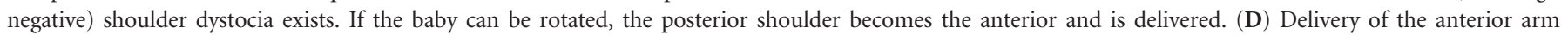
(Couder maneuver) relieves the impaction of the anterior shoulder. This time the pull is more downward.

that the traction on the forearm is more toward the mother's posterior (42) (Fig. 3C,D). This sequence of advance of the shoulders-the posterior one into the pelvis, the anterior one under the pubis, and the posterior one out of the pelvis-is exactly that of a normal delivery. The only difference is that this advance is accomplished by sequential delivery of the arms.

As an alternative, the posterior shoulder can be rotated forward to turn the fetus until it is anterior (6). The formerly anterior shoulder will be the posterior and in the pelvis. Unlike the sequential arm delivery, these may increase traction on the cervical brachial plexus with manipulation.

Potential problems encountered with arm delivery are (i) failure to get the operator's hand into the pelvis from inadequate episiotomy; (ii) difficulty in getting the operator's hand through the inlet, which can be facilitated by working the flat hand against the chest (which is compressible) and bringing the arm out through the deltopectoral triangle of the opposite shoulder; (iii) difficulty in finding the posterior arm, which can be corrected by starting at the shoulder; and (iv) difficulty in flexing the elbow, which can be corrected by freeing the forearm from the umbilical cord, the knees, and so on. Like the Pinard maneuver, it may be necessary to start flexion by pushing the elbow toward the fetal back.

\section{Hands-and-Knees Position}

This position is difficult in the delivery room, but should be tried immediately during a delivery in bed if the McRoberts maneuver fails or immediately if the head is delivered with the mother not supine. It can be atraumatic and highly effective (15). It partially flexes the maternal pelvis, especially if the patient rocks back into a knee-chest position. Gravity aids rather than impedes maneuvers because it brings the fetal body in line with the pelvic inlet and disimpacts the anterior (now dependent) shoulder from the pubis, freeing the posterior from the sacrum. The position provides excellent access for episiotomy, examination, and delivery of the posterior arm from a position of comfort, if needed, in bed. The McRoberts maneuver brings the buttocks out of the mattress but requires the operator to assume truly awkward positions to try other maneuvers.

\section{Anesthesia}

All of these maneuvers can usually be accomplished within 5 minutes with no local or regional anesthesia and calm 
instructions with the patient's cooperation. If things are not in order, then induction of general anesthesia, if immediately available, can provide time to restore calm, relax the patient, and get expert assistance.

\section{The Zavanelli Maneuver}

If the above maneuvers fail or cannot be performed, the fetal head can be replaced in the pelvis, with or without relaxing the uterus (with terbutaline, nitroglycerine, magnesium sulfate, or inhalation anesthetics). Replacement is accomplished by depressing the perineum and reversing the fetal head extension. Delivery can be completed by cesarean section, at a time indicated by fetal monitoring. This usually results in an intact infant, but possibilities of hypoxic ischemic encephalopathy or stillbirth and permanent brachial plexus injury still exist (13). The risks may be comparable with those associated with forceful vaginal delivery.

\section{Symphysiotomy and Other Maneuvers}

The reader can find many other maneuvers that have been described for the treatment of shoulder dystocia, and other variations of those presented. The ones presented here have either been observed by the authors to be effective without injury or are theoretically likely to be effective biomechanically (hands-and-knees position) and have been reported to be safe and effective. The other maneuvers that may be safe and effective are (i) fracture of the clavicle, often the result of sharply applied suprapubic pressure but usually much more difficult to accomplish than anticipated and (ii) symphysiotomy, which is simple, rapid, and effective (14) though usually outside of the scope of practice of most providers.

\section{Prognosis and Medicolegal Concerns}

Stillbirth may occur if delivery is greatly prolonged. Unlike survivors of the Zavanelli maneuver, survivors of shoulder dystocia, even with brachial plexus injury, do not have excessive risk for cognitive deficit when compared with national norms (7) or appropriate controls (9).

Effective delivery with shoulder dystocia is therefore a lifesaving treatment for the fetus. The most frequent severe disability for the fetus is Erb's palsy, usually of the posterior arm (7). This will resolve spontaneously in about $90 \%$ to $95 \%$ of cases $(9,11,18,44)$. There may be some increase in speech dysfunction (7).

Many cases of neonatal brachial plexus injury are not associated with recognized shoulder dystocia, some not even with vaginal delivery $(9,11,12,46,47)$. Indeed, Gherman et al. have published a report of six documented cases of brachial plexus injury in infants delivered by cesarean, five of whom were delivered for labor abnormalities but one in which delivery occurred in the absence of active labor (48). Brachial plexus injury has been observed by some $(42,49)$ in the absence of any traction to effect delivery. It is likely that the traction between the locked head and posterior shoulder at the pelvic inlet is sufficient at times to cause harm, even when resolved spontaneously or by McRoberts maneuver.

Malpractice suits have been brought, by parents of children saved by the effective treatment of shoulder dystocia, for the brachial plexus injury sustained during that birth, which may or may not have actually resulted from the treatment. Most shoulder dystocia suits have been successfully defended. Avoiding injury to the extent possible is the most effective prevention.

A fetus without previous compromise has at least 10 minutes from the time shoulder dystocia occurs until there is any risk of death from asphyxia. Previously compromised infants, or those exposed to high maternal serum glucose levels in the hours prior to delivery, may die or develop cerebral compromise after a shorter period of anoxia $(30,31)$.

The best way to prevent potential lawsuits is a sincere attempt to render the best care possible, honest communication with the family, and adequate documentation of exactly what happened.

\section{CONCLUSION}

Shoulder dystocia risk increases with increasing fetal body size. There is no effective way to predict with confidence the occurrence of shoulder dystocia or even macrosomia. The only way to decrease the risk for shoulder dystocia may be early and adequate glucose screening and appropriate diet and insulin therapy.

An estimated weight of over $5 \mathrm{~kg}$ in nondiabetics, a bisacromial diameter of $14.5 \mathrm{~cm}$ by computed tomography or estimated fetal weight of $4.5 \mathrm{~kg}$ in diabetics, or estimated weight of over $4 \mathrm{~kg}$ with a history of shoulder dystocia (50) are situations that warrant a discussion of shoulder dystocia and the potential risks and benefits of elective cesarean section with the mother. When dystocia is encountered, planned orchestrated action is undertaken to unlock the locked fetal position and to deliver the fetus with procedures involving minimal invasion and least risk. These should emulate the natural mechanism of shoulder delivery. These are the McRoberts or hands-and-knees position, generous episiotomy and partial cephalic replacement to unlock the shoulders, delivery of the posterior shoulder into the pelvis by fetal rotation or delivery of the posterior arm, followed, if necessary, by delivery of the anterior shoulder by delivery of the anterior arm or rotation of the posterior shoulder into the anterior position.

Simulation training of obstetric residents in training and providers in active practice may significantly improve the approach to the management of shoulder dystocia. The opportunity to practice the required maneuvers in a simulated situation with immediate feedback can improve the ability to perform them in the stress of the real situation. This can significantly decrease the risk of injury to both the parturient and the infant during these deliveries.

It is important to have a health-care provider who is proficient in neonatal resuscitation present in the delivery room. Neurologic evaluation of the infant is indicated when maternal injury occurs. The delivery should be clearly and completely documented and discussed with the patient.

\section{REFERENCES}

1. Morris W. Shoulder dystocia. J Obstet Gynaecol Br Emp 1955; 62: 302.

2. Beer E, Folghera MG. La distocia della spalle: considerazioni su un caso risolto conla manovra di McRoberts. Arch Obstet Ginecol 1994; 1: 3.

3. Schwartz BC, Dixon DM. Shoulder dystocia. Obstet Gynecol 1958; 11: 468.

4. Hibbard LT. Shoulder dystocia. Obstet Gynecol 1969; 34: 424-9.

5. Jacquemier J. Distocie par volume exagere et absence de rotation des epaules. Gaz Hebd 1860; 7: 661-4, 692-775. 
6. Woods CE. A principle of physics as applicable to shoulder delivery. Am J Obstet Gynecol 1962; 83: 1486-90.

7. McCall JO Jr. Shoulder dystocia: a study of aftereffects. Am J Obstet Gynecol 1943; 45: 769-804.

8. Rubin A. Management of shoulder dystocia. J Am Med Assoc 1964; 1989: 835-7.

9. Gordon M, Rich H, Deutschberger J, Green M. The immediate and longterm outcome of obstetric birth trauma 1. Brachial plexus paralysis. Am J Obstet Gynecol 1973; 117: 51-6.

10. Lurie S, Ben-Arie A, Hagay Z. The ABC of shoulder dystocia management. Asia Oceana J Obstet Gynecol 1994; 20: 195-7.

11. Gross SJ, Shime J, Farine D. Shoulder dystocia: predictors and outcome. A five-year review. Am J Obstet Gynecol 1987; 156: 334-6.

12. Gonik B, Hollyer VL, Allen R. Shoulder dystocia recognition: differences in neonatal risk for injury. Am J Perinatol 1991; 8: 31-4.

13. Sandberg EC. The Zavanelli maneuver extended: progression of a revolutionary concept. Am J Obstet Gynecol 1988; 158: 1347-53.

14. Maharry J. Symphysiotomy for shoulder dystocia. Am J Obstet Gynecol 1988; 158: 1352-3.

15. Meenan AL, Gaskin IM, Hunt P, Ball CA. A new (old) maneuver for the management of shoulder dystocia. J Fam Prac 1991; 32: 625-9.

16. American College of Obstetricians and Gynecologists. Fetal Macrosomia, ACOG Technical Bulletin 159. Washington, DC: ACOG, 1991.

17. Gross TL, Sokol RJ, Williams T, Thompson K. Shoulder dystocia: a fetalphysical risk. Am J Obstet Gynecol 1987; 156: 1408-18.

18. Nocon JJ, McKenzie DK, Thomas LJ, Hansell RS. Shoulder dystocia: an analysis of risks and obstetric maneuvers. Am J Obstet Gynecol 1993; 168: 1732-7.

19. Sandmire HF. Whither ultrasonic prediction of fetal macrosomia? Obstet Gynecol 1993; 82: 860-2.

20. Langer O, Berkus MD, Huff RW, Samueloff A. Shoulder dystocia: should the fetus weighing $4000 \mathrm{~g}$ be delivered by Cesarean section. Am J Obstet Gynecol 1991; 165: 831-7.

21. Hannah ME, Hannah WJ, Hellmann J, et al. Induction of labor as compared with serial antenatal monitoring in post-term pregnancy. A randomized controlled trial. The Canadian Multicenter Post-term Pregnancy Trial Group. N Engl J Med 1992; 326: 1587-92.

22. Fraser RB, Ford FA, Milner RDG. A controlled trial of a high dietary fibre intake in pregnancy - effects on a plasma glucose and insulin levels. Diabetologia 1983; 25: 238-41.

23. Modanlou HD, Komatsu G, Dorchester W, et al. Large-for-gestationalage neonates: anthropometric reasons for shoulder dystocia. Obstet Gynecol 1982; 60: 417.

24. Acker DB, Sacha BP, Friedman EH. Risk factors for shoulder dystocia. Obstet Gynecol 1985; 66: 762-8.

25. Langer O, Ridriguez DA, Xenakis EM, et al. Intensified versus conventional management of gestational diabetes. Am J Obstet Gynecol 1994; 170: 1036-46.

26. Lin C-C, River J, River P, et al. Good diabetic control early in pregnancy and favorable fetal outcome. Obstet Gynecol 1986; 67: 51-6.

27. Coustan DR, Imarah J. Prophylactic insulin treatment of gestational diabetes reduces the incidence of macrosomia, operative delivery and birth trauma. Am J Obstet Gynecol 1984; 150: 836-42.

28. Langer O, Conway D, Berkus M, et al. A comparison of glyburide and insulin in women with gestational diabetes mellitus. N Engl J Med 2000; 343: 1134 .
29. Langer O, Brustman L, Anyaegbunam A, Mazze R. The significance of one abnormal glucose tolerance test value on adverse outcome in pregnancy. Am J Obstet Gynecol 1987; 157: 758-63.

30. Myers RE, Wagner KR, deCourten-Myers GM. Brain metabolic and pathologic consequences of asphyxia. Adv Perinat Med 1983; 3: 67.

31. Mimouni F, Miodovnik M, Siddiqi TA, et al. Perinatal asphyxia in infants of insulin-dependent diabetic mothers. J Pediatr 1988; 113: 345-53.

32. Kjos SL, Henry OA, Montoro M, et al. Insulin-requiring diabetes in pregnancy: a randomized trial of active induction of labor and expectant management. Am J Obstet Gynecol 1993; 169: 611-15.

33. Elliot JP, Garite TJ, Freeman RK, et al. Ultrasonic prediction of fetal macrosomia in diabetic patients. Obstet Gynecol 1982; 1982: 159-62.

34. Cohen B, Penning S, Major C, et al. Sonographic prediction of shoulder dystocia in infants of diabetic mothers. Obstet Gynecol 1996; 88: 10-13.

35. Kitzmiller JL, Mall JC, Gin GD, et al. Measurement of fetal shoulder width with computed tomography in diabetic women. Obstet Gynecol 1987; 70: 941-5

36. Keller JD, Lopez-Zeno JA, Dooley SL, Socol ML. Shoulder dystocia and birth trauma in gestational diabetes: a five-year experience. Am J Obstet Gynecol 1991; 165: 928-30.

37. Bahar AM. Risk factors and fetal outcome in cases of shoulder dystocia compared with normal deliveries of a similar birthweight. Br J Obstet Gynaecol 1996; 103: 868-72.

38. Benedetti TJ, Gabbe SG. Shoulder dystocia: a complication of fetal macrosomia and prolonged second stage of labor with midpelvic delivery. Obstet Gynecol 1978; 52: 526-9.

39. Deering S, Poggi S, Macedonia C, Gherman R, Satin A. Improving resident competency in the management of shoulder dystocia with simulation training. Obstet Gynecol 2004; 103: 1224-8.

40. Allen R, Sorab J, Gonik B. Risk factors for shoulder dystocia: an engineering study. Obstet Gynecol 1991; 77: 352-5.

41. O'Leary JA, Pollack NB. McRoberts maneuver for shoulder dystocia: a survey. Int J Gynecol Obstet 1991; 35: 129-31.

42. Smeltzer JS. Prevention and management of shoulder dystocia. Clin Obstet Gynecol 1986; 29: 299-306.

43. Hankins GD, Clark SL. Brachial plexus palsy involving the posterior shoulder at spontaneous vaginal delivery. Am J Perinatol 1995; 12: 44-5.

44. Gherman R, Tramont J, Muffley P, Goodwin T. Analysis of McRoberts' maneuver by x-ray pelvimetry. Obstet Gynecol 2000; 95: 43-7.

45. Gonik B, Allen R, Sorab J. Objective evaluation of the shoulder dystocia phenomenon: effect of maternal pelvic orientation on force reduction. Obstet Gynecol 1989; 74: 44-8.

46. Jennett RJ, Tarby TJ, Kreinick CJ. Brachial plexus palsy: an old problem revisited. Am J Obstet Gynecol 1992; 166: 1673-6.

47. Lerner $\mathrm{H}$, Salamon E. Permanent brachial plexus injury following vaginal delivery without physician traction or shoulder dystocia. Am J Obstet Gynecol 2008; 198: e7-8.

48. Gherman R, Goodwin T, Ouzounian J, Miller D, Paul R. Brachial plexus palsy associated with cesarean section: an in utero injury? Am J Obstet Gynecol 1997; 177: 1162-4.

49. Lerner H, Salamon E. Permanent brachial plexus injury following vaginal delivery without physician traction or shoulder dystocia. Am J Obstet Gynecol 2008; 198: e7-8.

50. Lewis DF, Raymond RD, Perkins MD, et al. Recurrence rate of shoulder dystocia. Am J Obstet Gynecol 1995; 172: 1369-71. 


\section{Breech presentation \\ Randall C. Floyd and Martin L. Gimovsky}

Breech presentation is a significant obstetric event associated with dramatic increases in perinatal morbidity and mortality $(1,2)$. The three areas of greatest risk for these infants are the often overlapping problems of preterm delivery, congenital anomalies, and birth trauma. More than one quarter of breechpresenting fetuses are premature. Concomitantly, severe or lethal anomalies further complicate up to $20 \%$ of such preterm deliveries and fully $6 \%$ to $7 \%$ of term breech deliveries (a relative risk of three- to fivefold), depending on gestational age. The outcome of vaginal breech delivery has always been noted to be associated with a higher risk of neonatal injury with both shortterm and long-term morbidity as well as an increased risk of neonatal death. These risks have led to numerous attempts to evaluate the risk of vaginal breech delivery versus that of elective cesarean delivery for the persistent breech at term. The results of studies published prior to the Term Breech Trial (TBT) (3) in 2000 were inconclusive with some showing no increased risk of vaginal breech delivery and some showing an increased risk of fetal injury and death in those infants delivered vaginally. These studies were, for the most part, small and retrospective, which left room for error in their conclusions. In 2000 the publication of the TBT, a randomized, multinational multicenter trial that incorporated all levels of care in both advanced and developing countries, demonstrated a clear benefit to the delivery of the term fetus in a breech presentation by elective cesarean.

The TBT gathered information from 121 participating centers in 26 countries and included 2088 women randomized into two arms: planned vaginal delivery or planned cesarean delivery. The details of the study will not be reviewed here, but it is a publication of significant importance and should be read by all of those who are actively involved in the delivery of obstetric care. Other studies have supported the conclusions of the TBT (4-6). The study is not without its critics and there have been numerous reviews written questioning the methodology and the interpretation of the results (7-11); however, the study appears to be able to withstand the test of both time and these critics. Indeed, the American College of Obstetricians and Gynecologists, in a committee opinion, initially recommended that all deliveries of term infants from breech presentation be performed by cesarean; however, this has since been revised to state that the decision regarding mode of delivery should depend upon the experience of the health-care provider $(12,13)$. Perhaps the decrease in vaginal breech deliveries is due to the willingness of those currently practicing obstetrics to accept a philosophy that is, to them, so apparent or possibly it is based upon a self-fulfillment of the decreasing numbers of providers with sufficient training to develop those skills required to perform an elective vaginal breech delivery (14). For whatever reason, the incidence of elective vaginal breech deliveries continue to decrease and the option will likely fade into rarity.

The need to learn the maneuvers necessary to perform a breech delivery remains, however. Delivery of the breech infant by cesarean requires similar maneuvers in order to successfully extract the infant. Delivery of the nonvertexpresenting second twin following delivery of the cephalicpresenting first twin in an otherwise uncomplicated twin gestation concordant for weight also requires the ability to perform these maneuvers. Finally the rare patient who presents in active labor with the breech on the perineum would likely be poorly served by emergent cesarean delivery and providers should possess knowledge of the maneuvers necessary to successfully complete the delivery. The use of simulation training in teaching the skills necessary for vaginal breech delivery has been shown to be effective (15).

Any consideration of breech management involving either a trial of labor or cesarean delivery is bedeviled by issues of birth injury and medicolegal risk. External cephalic version has been championed as a means of avoiding the issue by converting breech into cephalic presentations. Unfortunately, the results of these major shifts in methodology of delivery have not resulted in the substantial reductions in fetal/neonatal injuries that were originally confidently predicted. Utilizing perinatal mortality as an endpoint, a review of the obstetric literature from the 1930s to the 1960s demonstrates a rough linear relationship - that is, the perinatal death rate for term-sized neonates was reduced as the cesarean delivery rate increased from $3 \%$ to $30 \%$ or more (Fig. 1). Fetal/neonatal injuries still occur at cesarean delivery to a surprising degree (2). In 1981, Green and coworkers (16) reported that, in a relatively controlled setting, that is, one department over a period of time, increasing the use of cesarean delivery for breech from $22 \%$ to $94 \%$ did not eliminate preventable trauma pointing up the need for the obstetric provider to have a fundamental understanding of those maneuvers necessary to decrease the risk of injury of the breech-presenting infant. The problem for the profession is to define which maneuvers can and should be taught, how to maintain a high level of competence in these, and how to present the options of trial of labor or cesarean delivery fairly to our patients.

\section{SPECIAL ISSUES}

\section{External Cephalic Version}

A potential technique for reducing the incidence of breech presentation is external cephalic version. External cephalic version performed at 37 weeks to term has decreased the rate of intrapartum breech presentation from $3-4 \%$ to $1-2 \%$ (17-21). Earlier groups of investigators had attempted version at 28 to 32 weeks (6) or 30 to 32 weeks (20), but failed to effect any change in the intrapartum rate of breech presentation. These 


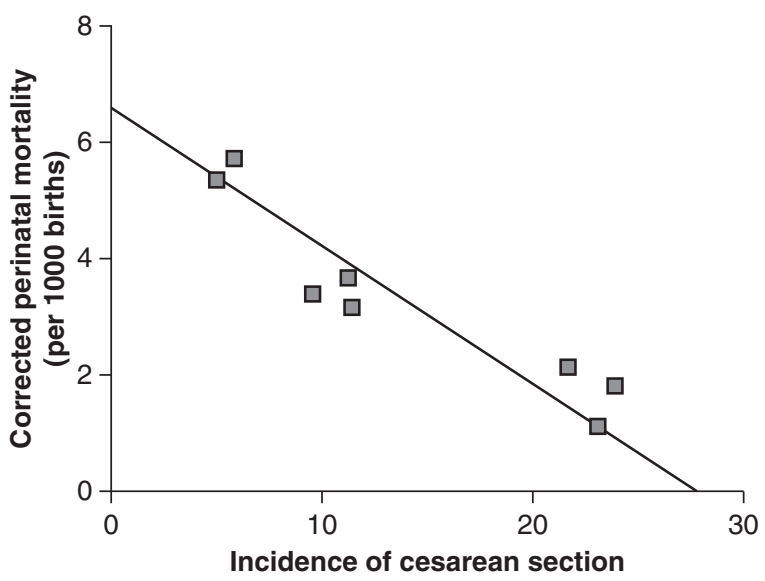

Figure 1 Correlation between cesarean delivery rates and decreased perinatal mortality in term breech infants in the 1940s and 1950s.

failures erroneously led clinicians to believe that the only versions that succeeded were those that would have occurred spontaneously. These "early" versions were probably unsuccessful because of the relatively large amount of amniotic fluid with respect to the size of the fetus, which therefore reverted to breech after the original procedure. Also anesthesia and analgesia were employed in a number of these trials. This allowed more forceful procedures, leading to occasional transplacental hemorrhage and isoimmunization, a substantial risk in the era prior to the availability of rhesus immune globulin.

Even in modern practice, version is not without risk, albeit small. Both umbilical cord complications and placental abruption have been described in conjunction with external version (22). The most common problem associated with version is failure to alter the fetal lie, despite manipulation. Currently, external cephalic version at 36-37 weeks gestation performed under tocolysis (terbutaline, IV nitroglycerine or oral glyceryl trinitrate spray $(23,24)$, nifedipine $(25)$, or intravenous magnesium sulfate) has a reasonable success rate (50-80\%). Also the concomitant use of nonstress testing, realtime ultrasound, and the avoidance of analgesia/anesthesia has contributed to the overall safety of the procedure. An exception to the rule of no analgesia/anesthesia is the occasional use of epidural anesthesia for intrapartum version, a special application for selected cases $(26,27)$.

There is a place for external cephalic version even in early labor. Depending on the stage of labor at which the patient is admitted and the time when breech presentation is diagnosed, immediate $B$-mimetic tocolysis is often successful in permitting manipulations. However, after rupture of the membranes and the onset of true labor, any attempt at version becomes both difficult and inherently dangerous, owing to the descent of the presenting part and the potential risk of cord prolapse.

Continued efforts at refinement of external cephalic version have revolved around the issues of tocolysis and anesthesia. Robertson and coworkers (27) found that the use of tocolytics for adjunctive treatment after a trial of external cephalic version without tocolytics did not improve success rates. Samuels (26) has described the successful use of regional anesthesia instead of tocolysis for version. No difficulty with sequelae of excessive force was described in his original report. Despite these interesting reports, brief treatment with tocolytics prior to attempting manipulations remains popular, because of the inherent safety, minimal expense and ease of administration of these drugs, and their perceived utility. Most clinicians have found that the use of a tocolytic before version (usually 0.125 or $0.250 \mathrm{mg}$ of terbutaline administered subcutaneously) reduces patient discomfort as well as the external force required for the procedure.

\section{CLINICAL ISSUES}

Despite a decision to submit all breeches to cesarean delivery, some instances of vaginal breech delivery cannot be avoided, owing to unforeseen events such as undiagnosed breech presentation or breech presentation diagnosed too late in labor. Regardless of their view toward vaginal trials or cesarean delivery, it is vital for all clinicians to develop experience in the management of both breech extraction at cesarean section and vaginal breech delivery. The routine resort to cesarean delivery does neither guarantee a perfect infant nor obviate the clinician's need for great care and substantial skill as an accoucheur in the operating room. Not surprisingly, measurable rates of the entire range of birth injuries occur in cesarean sections, although the incidence is low. The occurrence of these injuries at cesarean section underscores the need for full understanding of the manual maneuvers utilized for breech delivery. In the performance of safe cesarean section for breech presentation, the surgeon must possess facility with total breech extraction. Even with the exposure possible at laparotomy, breech extraction remains an inherently more dangerous procedure than the assisted breech delivery performed in the majority of vaginal breech births (28). Such procedures require thoughtful and judicious application of force. Commensurate with the increased use of abdominal operative delivery, maternal morbidity, both immediate and remote, is also increased, although the majority of these complications prove to be of trivial clinical consequence (29).

\section{Selective Trial of Labor}

Protocols for vaginal delivery of breech infants need critical review. An important consideration in determining best management is the type of breech presentation. The largest group of breech fetuses at term is in frank breech position. The best data reflect management protocols for these most favorable cases. Collea and coworkers (29) conducted a prospective randomized study that compared elective cesarean section with protocol-managed labor for fetuses with frank breech presentation at term. They reported significant maternal morbidity among patients delivered by cesarean section, without any dramatic improvement in neonatal outcome. Among 60 infants delivered vaginally, there were two instances of brachial plexus injury. O'Leary (30) performed a similar study at the University of South Alabama. Among term frank breech presentations screened by a protocol similar to that described by Collea and coworkers, O'Leary described one injury in 81 vaginal deliveries, and this occurred during a breech extraction performed for fetal distress. In this study, the comparison group was a cohort of breech deliveries managed without the specific requirements of protocol. In this non-protocol group, a similar number of infants were stillborn; however, one infant died of trauma, eight had low Apgar scores, 
Table 1 Results by Delivery Type, Presentation, and Outcome

\begin{tabular}{|c|c|c|c|c|}
\hline & Protocol group & Non-protocol group & Control group 1 & Control group 2 \\
\hline Type of delivery & Vaginal & Vaginal & Spontaneous vaginal & Elective cesarean section \\
\hline Presentation & Breech & Breech & Vertex & Vertex $(95 \%)$, breech $(5 \%)$ \\
\hline Mean birth weight (g) & 3125 & 3025 & 3170 & 2980 \\
\hline Number of deliveries & 130 & 78 & 130 & 130 \\
\hline Ward/private ratio & $59 / 41$ & $62 / 38$ & $60 / 40$ & $58 / 42$ \\
\hline Mean 5-min Apgar score & 8.6 & 8.1 & 8.7 & 8.6 \\
\hline Intrapartum mortality & 0 & 1 & 0 & 0 \\
\hline Perinatal mortality $^{\mathrm{a}}$ & 0 & 12.8 & 0 & 0 \\
\hline
\end{tabular}

and four experienced birth injuries. The author concluded that the use of the protocol had significantly improved the outcome for the breech infants in the study.

In a study of all types of breech presentation (including non-frank or complete, incomplete, and footling breeches) at the Sloane Hospital for Women in New York, a protocol similar to that of O'Leary was followed by Gimovsky and coworkers (31). The authors found that selective management by protocol of term breech fetuses, even those non-frank in presentation, gave acceptable immediate neonatal performance in comparison with elective cesarean section (Table 1). The outcome for protocol-managed breech fetuses delivered vaginally was essentially identical to that of all patients delivered by elective cesarean section. The protocol in use at Columbia (32) has since been revised. In 1983, Gimovsky and coworkers (33) reported preliminary results of the new protocol that confirmed a similar outcome between patients with elective cesarean section and selected protocol-managed patients. In that prospective study of non-frank breech presentations at term, a rigorous protocol was employed for intrapartum management (Fig. 2). One fetus in the study group of 105 patients died during the intrapartum period, owing to an unusual complication that emphasized the complex problems of clinical medicine. This occurred when a woman became hysterical during a trial of vaginal delivery. Efforts to place her under anesthesia to affect safe vaginal delivery were prolonged and the infant eventually delivered could not be resuscitated, despite intensive efforts. The discussion of complex protocols to select appropriate candidates for a trial of breech vaginal delivery is likely to become moot. The results of the TBT will likely make the use of any protocol for the evaluation of the patient presenting with a persistent breech presentation outdated with the exception of the attempt at external version.

\section{Informed Consent}

In an area such as management of breech presentation that is rife with controversy, informed patient consent for any clinical approach is not only prudent, but it is also mandatory. The mother (and her spouse) must be advised of the facts necessary to make an informed, independent choice of either undergoing a trial of labor or of proceeding with cesarean delivery. This explanation needs to be simple, factual, balanced, and complete. Despite the complexities that attend such assessment and obstetric decision making, the obstetrician has an obligation to make the essential and pivotal factors that are central to a clinical choice understandable to the patient. Ideally, the actual decision should be made by the patient and not simply surrendered to the obstetrician. To do otherwise invites the risk that a patient who experiences a sad or untoward result will respond with blame and feelings of betrayal.

The focus of the obstetrician's explanation should emphasize a detailed comparison of risks attendant on the alternatives: specifically trial of labor versus prompt cesarean delivery once labor begins or term has been reached. The issue of possible version should also be carefully discussed. The nature, purpose, and risks (to both mother and infant) should be outlined for both approaches. The obstetrician must explain and discuss the following.

1. The nature of breech presentation. In this review, the clinician explains how breech differs from cephalic presentation and factors that influence perinatal morbidity and mortality in breech delivery.

2. A comparison of the mechanics of cesarean section with vaginal delivery after a trial of labor, with emphasis on the increased risk of fetal injuries secondary to the dynamics of the delivery process. This explanation should include the observations that, with cephalic presentation, the largest and most unyielding part comes first and then the softer, more malleable parts follow. With breech presentation, the softer more malleable parts are received first, followed by the relatively larger and unyielding head. If the head proves too big for the pelvic outlet, and the rest of the baby is already delivered, there is a substantially increased risk for significant injury to the infant.

3. The nature of specific risks of cesarean section to the mother from hemorrhage, infection, or unanticipated lacerations versus the potential for genital tract lacerations and hemorrhage associated with breech delivery with or without the use of forceps.

4. Data on potential fetal injury, including the risks to the infants of intracranial hemorrhage and significant long-term neurologic injury, either by cesarean section or by vaginal incidental to breech delivery. 
1. Verify breech presentation (fetal ultrasound)

Estimated gestational age: $36-42$ weeks

Estimated fetal weight: $2-4 \mathrm{~kg}$

2. Labor active? Advanced past $7 \mathrm{~cm}$ ?

Consider tocolysis with terbutaline $0.25 \mathrm{mg}$ subcutaneously

3. Prepare for expenditious cesarean section

4. Discuss potential alternatives, risks and benefits of each, with patient and family

5. Fetal and pelvic X-ray (CT scan where possible)

Rule out fetal hyperextension at the cervical spine $\left(105^{\circ}\right)$

Maternal pelvic measurements:

inlet of the pelvis:

$>11 \mathrm{~cm}$ in the anteroposterior diameter

$>12 \mathrm{~cm}$ in the greatest transverse diameter midpelvis

$>10 \mathrm{~cm}$ at the interspinous diameter

6. Patient elects trial of labor

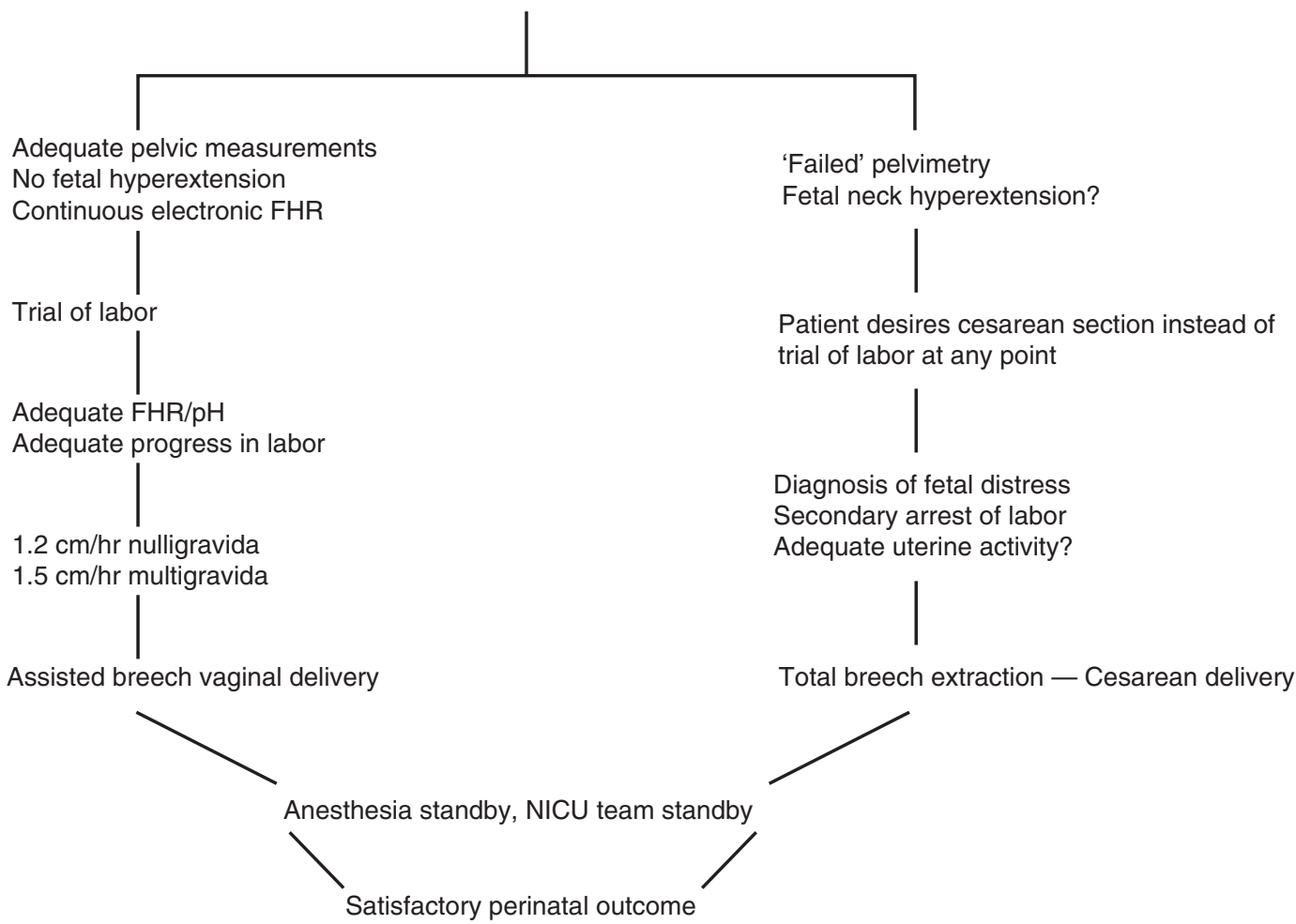

Figure 2 Selective management protocol for intrapartum breech management. Abbreviations: FHR, fetal heart rate; NICU, neonatal intensive care unit. Source: From Ref. 57.

5. The role of version. This discussion critically reviews the advantages versus the risks of external cephalic version and why this procedure should or should not be attempted in the patient's specific case.

Because there is controversy surrounding the decision to recommend a trial of labor with breech presentation, as opposed to proceeding immediately to cesarean section, the obstetrician is well advised to make a balanced presentation of both views. The clinician must be prepared to explain that those who favor cesarean delivery in all cases rely on statistics, which suggest that three times as many fetal injuries occur with vaginal/breech delivery as compared with cesarean section. Those advocating trial of labor with a breech presentation will want to explain how a meticulously implemented protocol of surveillance may render the statistical difference between these procedures negligible. In making such explanations, the obstetrician should resist favoring the decision he/she wants by either exaggerating or underrating the risks of one management plan over another. The use of dramatic overstatement or florid language will undoubtedly be long remembered by an apprehensive mother or an expectant father. If the decision is to be truly that of the patient, based upon information, the facts and just the facts should be presented in a logical and unemotional manner, as free from bias as possible. At the end of this discussion, an obstetrician is well advised to invite any questions the patient might have and make every effort to ensure that both she and her partner are satisfied with the explanation given.

In cases in which the breech presentation has been recognized prior to the onset of labor, such discussions will be likely to have occurred outside the hospital. It is prudent to review the same data briefly with the woman/family following admission for labor/delivery. These in-hospital conversations are best conducted in the presence of a nurse, who can then independently document in the medical record that the pertinent consent discussions have taken place. 


\section{Pelvic Measurement and Fetal Evaluation}

When selecting patients as possible candidates for a trial of labor, careful, objective evaluation of the fetopelvic size is mandatory. Imaging studies can provide this information and also document that the fetal skull is normal in both general appearance and attitude. Ultrasound scanning has also been extensively employed in describing fetal size, structural normality, and head attitude and is useful in acute evaluation of the baby $(34,35)$.

Ultrasound or other imaging studies are particularly useful in the diagnosis of hyperextension of the fetal head, a condition that occurs when an angle of more than $105^{\circ}$ exists between the fetal mandible and the main axis of the cervical spine. This is an absolute contraindication to an attempt at vaginal delivery (36). Even at cesarean delivery with an adequate incision, meticulous attention to minimal force is required for safe delivery of a breech infant with cranial hyperextension. Experience suggests that up to 5\% of term breech infants have this additional risk. Early diagnosis is important, as prolonged labor with hyperextended head may also result in cervical spine injury, even when delivery is ultimately accomplished by cesarean section (37).

The additional purpose of imaging assessment of the mother is to diagnose women with moderate degrees of pelvic disproportion prior to attempting delivery of the aftercoming head. Entrapment of the fetal head and/or difficult delivery may be largely avoided if such cases are taken to cesarean section without a trial of labor. Imaging studies can define patients with potential borderline pelves; however, these studies are neither guarantors of perfect safety nor do they substitute for clinical expertise.

Radiographic pelvimetry first appeared in Europe in the late 19th century following Roentgen's discovery of the X-ray. Over its long history, the popularity of radiographic pelvimetry has waxed and waned, owing, in part, to its risks and to questions regarding its accuracy. Subsequently, there has been a shift to pelvic measurement by computerized tomography (CT). A pilot study in 1985 indicated the applicability of such management (38). In 1986, Kopelman and coworkers (39) assessed the reliability of this method in a breech protocol and reported favorable results. Standard pelvimetry techniques in use up to that time, with the radiation exposure to maternal and fetal gonads, were compared with the equally useful single CT scan (40). CT generates more accurate and more reproducible data than other techniques (38) (Fig. 3). Magnetic resonance imaging has also been used to evaluate obstetric pelvimetry and has the advantage of avoiding the use of ionizing radiation (41).

Taken alone, the measurement of pelvic diameters is not capable of reliably directing management, especially for women in labor. Experience has shown that minimal pelvic inlet measurements of $11 \mathrm{~cm}$ in the anteroposterior diameter and $12 \mathrm{~cm}$ at the transverse of the inlet, coupled with $10 \mathrm{~cm}$ at the mid-pelvic plane of the intraspinous diameter, are consistent with a normal bony pelvis $(10,11,12$ rule) $(31-33,42)$. These recommended measurements should be used as a starting point in considering the labor of an average-sized fetus at term. A clinical consideration of fetal size is obviously a major issue in deciding upon the appropriateness of a labor trial. It may be helpful for the attending obstetrician to review the pelvimetry directly. The use of the fetal head circumference obtained by ultrasonography in addition to the CT-derived pelvic inlet measurements has been evaluated as a more definitive guide to fetopelvic disproportion (43).

As maternal position during labor affects pelvic diameters, careful maternal placement and positioning during attempted vaginal delivery are important. Thorough evaluation of the fetus for structural abnormalities and position by real-time scanning is also critical prior to making decisions regarding management. When circumstances dictate an expeditious delivery by cesarean section, brief tocolysis lessens both operative and anesthetic risks by relaxing the uterus and permitting sufficient time for a proper discussion of informed consent (44). It should be noted that clinical pelvic examination is still important. Soft tissue resistance, which can create a significant problem for vaginal delivery, is not directly measurable but can be assessed by an experienced attendant. Although it is difficult to quantify, the slope of the pelvic sidewalls and the position of the sacrum also play a role in the estimated pelvic capacity and the likelihood of an uncomplicated vaginal delivery.

\section{Fetal Surveillance}

Continuous fetal surveillance is essential in intrapartum management of breech labor, as umbilical cord prolapse is a potential hazard in all breech presentations. Cord complications are most likely to occur in the second stage of labor, which should be conducted in the delivery room. In 1963, in the pre-electronic fetal monitoring era, Rovinsky and coworkers (45) showed that vertex fetuses had the same risk of intrapartum demise from overt cord prolapse as did frank breeches. It is of interest that both these presentations subject the fetus to a greater risk for compromise from cord accidents than do footling and complete breech presentations, probably because of the close clinical attention paid to the non-frank breech labor.

The usual criteria for interpreting intrapartum electronic fetal monitoring apply, regardless of fetal position. Nishijimi and colleagues (46) compared fetal heart rate patterns of nulliparas and multiparas in labor for breech and vertex presentations and found no essential difference.

Wheeler and Greene (47) have suggested that periodic fetal heart rate accelerations are more common in breech babies, presumably because of a difference in vaginal stimulation. Our experience, based upon umbilical cord gas studies at birth, confirms that the fetal acid-base status is normal when fetal heart rate patterns are normal during labor.

Vaginal breech delivery results in cord respiratory blood gas values and Apgar scores at birth that are similar to those observed at cesarean delivery. These data are consistent with the previously described findings concerning the similarity between both fetal heart rate monitoring patterns and capillary $\mathrm{pH}$ in breech-presenting fetuses, independent of the mode of delivery $(46,47)$.

\section{Anesthesia}

Skilled anesthetic support is essential for safe breech management. In the past, practitioners commonly used pudendal nerve blocks in trials of labor, with a general anesthetic 
(A)

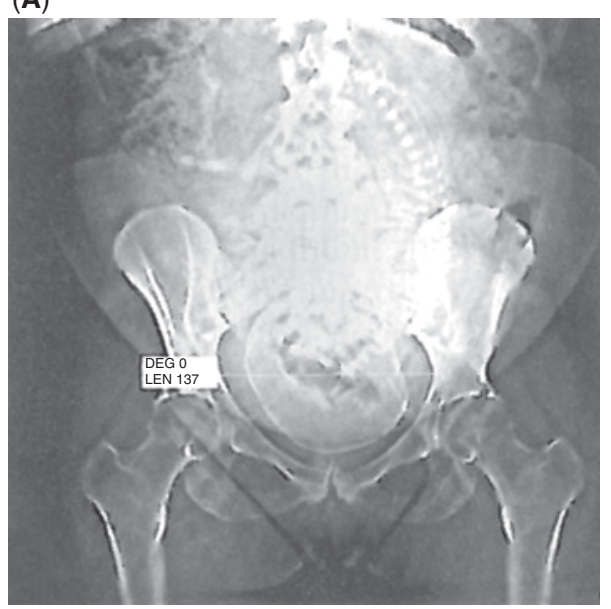

(C)

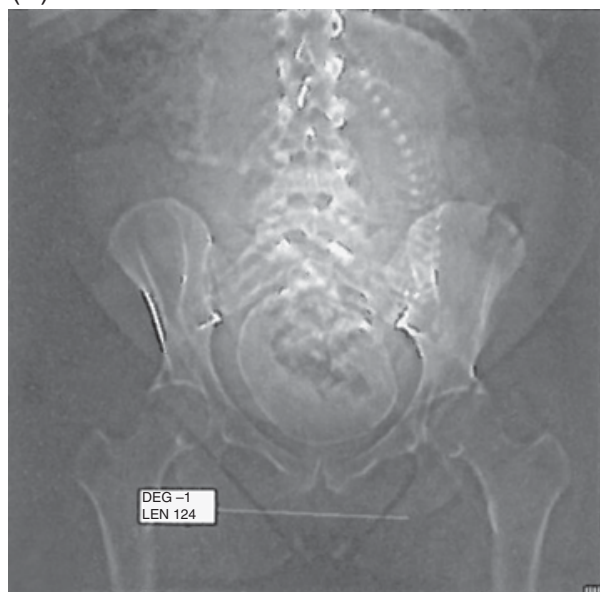

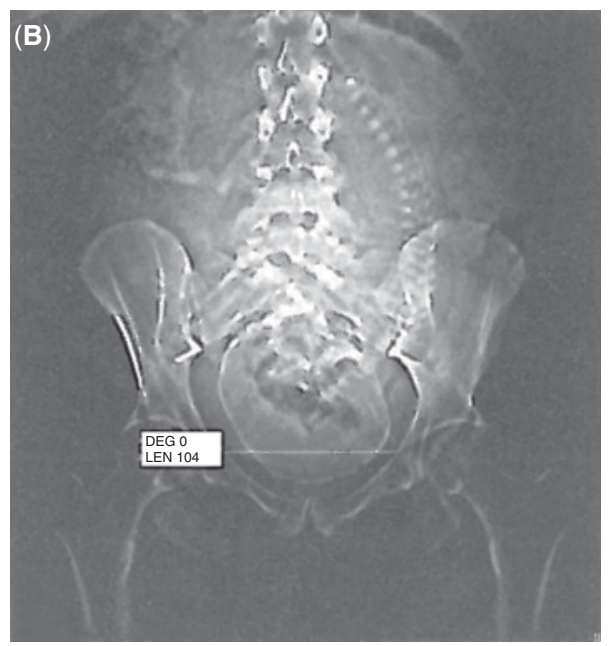

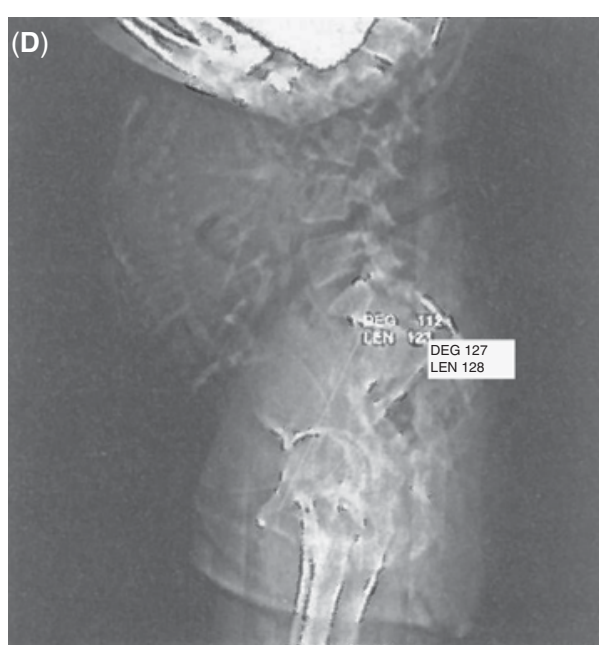

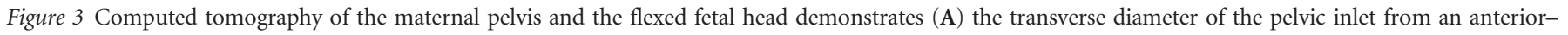

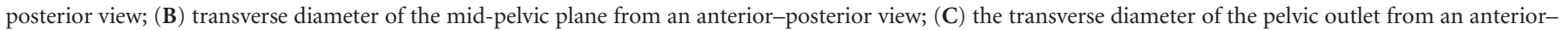
posterior view; and (D) the anterior-posterior diameters of the pelvic outlet (123) and mid-pelvis (128) from a lateral view.

supplement in about $10 \%$ of cases, if required (2). Epidural anesthesia is by far the superior method and is the current first choice for many clinicians (48). For the small breech fetus or the footling breech presentation, adequate anesthesia helps to dampen maternal bearing-down efforts, retarding spontaneous membrane rupture and involuntary pushing. This also decreases the risk of umbilical cord or fetal body prolapse through an incompletely dilated cervix (48). The absence of such involuntary bearing-down efforts is also desirable when the clinician's intent is to deliver a markedly premature fetus in caul.

The anesthesiologist should be immediately available. Adequate anesthesia for either emergency cesarean section, should it be necessary, or vaginal manipulations is essential to a successful and safe trial of labor. Neonatal support is also important for a good outcome. Regardless of the delivery route, the breech presentation is a high-risk situation and neonatal resuscitation, when required, must be prompt, proper, and expeditious.

\section{Dynamics of Labor}

Parity plays little or no role in the outcome of trials of labor. In fact, despite the beliefs of many clinicians, there may actually be an increase in perinatal morbidity among multiparas undergoing vaginal breech delivery (31). In multiparas, the aftercoming fetal head can deliver so quickly that acute and unpredictable fluctuations occur in fetal intracranial pressure, which presumably lowers Apgar scores.

The judicious use of oxytocin in selected cases is permissible and does not prejudice outcome $(1,29,30,33)$. In their experience at Moffitt Hospital, Flanagan and coworkers (49) reported no difference in the perinatal outcome seen among patients with breech presentation given a trial of labor with or without the administration of oxytocin. In this study, almost $20 \%$ of the patients received oxytocin, primarily for labor induction.

Therefore, if the breech is engaged, the fetus is of normal size, the fetal head is flexed, and the radiographic measurements show the pelvis to be adequate and induction of labor by oxytocin is acceptable (42). However, oxytocin augmentation of breech labor is rarely indicated, given both the potential risks and the current medicolegal atmosphere. The experience at Los Angeles County, University of Southern California Medical Center, indicates that oxytocin augmentation rarely achieves safe vaginal delivery of a breech infant if a secondary arrest of active-phase labor has occurred (2). Therefore, great care is needed if normal progression in labor does not occur. These data tend to confirm an earlier observation of Friedman (50) 
that fetopelvic disproportion is a frequent cause of secondary arrest in breech labor. There is also an association between larger infants and abnormalities in labor. Approximately three-fourths of women who deliver breech infants weighing more than $3500 \mathrm{~g}$ develop a dysfunctional labor pattern. An abnormal labor pattern is therefore a critical clue that a decision for vaginal trial should be carefully reasoned and that cesarean delivery may be indicated.

The authors consider that $3500-4000 \mathrm{~g}$ is the upper limit considered as a safe fetal size for vaginal breech delivery. Therefore, the practitioner must be careful to avoid "forcing" a trial of labor in the presence of a known or suspected large breech fetus. Both ultrasound and clinical weight estimates should be combined in judging fetal size. The lower limits of safe progress in labor are (16) $1.2 \mathrm{~cm} / \mathrm{hr}$ for nulliparas and (16) $1.5 \mathrm{~cm} / \mathrm{hr}$ for multiparas. In the presence of normal uterine activity, women failing to dilate at this rate should be taken to cesarean section without an attempt at oxytocin augmentation of labor.

\section{Accouchement}

If a vaginal trial is elected, the second stage of labor should be carried out in the delivery room with continuous fetal monitoring. Perhaps more than in any other obstetric setting, vaginal delivery of a breech fetus requires both watchful waiting and gentle manipulation. An experienced, gowned, and gloved assistant and a scrub nurse should be present for the delivery.

A safe assisted breech delivery requires that the accoucheur understands the judicious application of force. An $80-\mathrm{kg}$ obstetrician can easily overpower a 3000-g neonate. However, excessive force alone is not necessarily the major cause of injury. Acute rotation of the fetal body for delivery of the arms or head exceeding $90^{\circ}$ may compromise the vertebral blood supply to the central nervous system. In addition, maintaining flexion of the aftercoming head during the actual birth process is crucial. Gentle but firm and continuous suprapubic force needs to be judiciously applied by an assistant to the aftercoming head as the delivery proceeds. The accoucheur must also be prepared either to apply forceps or to reach for the face of the aftercoming fetal head during delivery. It is well to recall that Piper forceps were designed to act as a lever and not as a tractor in aiding the delivery of the head. Good results with forceps to the aftercoming head have been described by Milner (51) (Table 2). If forceps are not applied, the Mauriceau-Smellie-Veit (MSV) maneuver is the procedure of choice in completing the breech delivery. The purpose of the suprapubic pressure is not to effect delivery by accouchement forcé but simply to maintain cranial flexion during descent, thus facilitating the entry of the fetal skull into the pelvis by continuously presenting the smallest diameter. If the clinician fails to "follow" the fetal head by exerting gentle but continuous transabdominal pressure on the uterus, cranial extension can occur, resulting in a difficult delivery as a larger diameter of the cranium is presented to the maternal pelvis. Haste is dangerous in the management of breech delivery and almost always unnecessary. Slow, gentle manipulations are always best. Normal cord gases are seen even after a 6-min delay from emergence of the umbilicus to final delivery.
Table 2 Vaginal Breech Delivery: Impact of Forceps to Aftercoming Head

\begin{tabular}{|lcc|} 
& \multicolumn{2}{c}{ Neonatal deaths $(\%)$} \\
\cline { 2 - 3 } Birth weight $(\mathrm{g})$ & No forceps & Forceps \\
$1500-1999$ & 22.3 & 11.0 \\
$2000-2499$ & 7.8 & 0.0 \\
$2500-2999$ & 3.5 & 0.5 \\
Source: From Ref. 36. & & \\
\hline
\end{tabular}

A comparison of duration between the vaginal route of delivery and cesarean section is illuminating. We have found that, in two-thirds of cases, the duration of time during vaginal delivery is shorter than the incision-to-delivery time at cesarean section (52).

\section{Documentation}

Complete and accurate records are essential in the conduct of breech management, regardless of the mode of delivery chosen. Within the progress notes, the obstetrician should detail the discussions that are the foundation of patient informed consent. As has already been discussed, the obstetrician is well advised to have a knowledgeable labor and delivery nurse present at the time of these interactions who can, in his/her own nursing notes, confirm the nature and extent of the discussions.

The obstetrician should record in the progress notes the basis for the clinical judgments made in support of the decision to conduct a trial of labor. This note should stress the clinical and diagnostic factors that favor the choice that was made. If a trial of labor is planned, the note should make a statement of the specifics of the protocol to be followed, with emphasis on the elements of meticulous observation during the progress of labor, ongoing assessment of adequacy of descent, continuous fetal monitoring, and preparedness for immediate emergency cesarean delivery. The progress note recorded during the course of labor itself should set forth in detail the basis for all clinical decisions in response to significant changes in maternal/fetal status. This process may at first appear to be excessive, but it is nothing more than a plan for strict adherence to the usual standards for documentation of high-risk labor.

\section{COMPLICATIONS}

\section{Prematurity and Low Birth Weight}

The low-birth weight breech fetus, between 25 and 36 weeks of gestation, presents a clinical challenge $(36,53,54)$. These fetuses have up to a one in five chance of having a major congenital malformation (2). Furthermore, such pregnancies are commonly complicated by significant obstetric problems including but not limited to placenta previa, abruptio placentae, and multiple gestations (55). Owing to the relative sizes of head and abdomen, the risk of cord prolapse (or body prolapse) through an incompletely dilated cervix is high.

The limits of ultrasound measurement are also a problem. Underestimation of fetal weight results in fetuses that are essentially dismissed as being too small to survive (56). Less likely is the overestimation of fetal weight, which can lead to heroic efforts for fetuses with limited chance for survival. In the growth-retarded fetus, the abdomen can be significantly 
smaller than the head and thus more likely to prolapse through a partially dilated cervix, resulting in entrapment of the fetal head.

For the fetus between 34 and 38 weeks of gestation, there may be a crucial difference, based on the estimates of gestational age and fetal weight, in the likelihood of success and safety of a trial of labor versus cesarean delivery. By insisting on a clinically estimated fetal weight of more than $2000 \mathrm{~g}$ and a gestational age of $>36$ weeks, a reasonable margin of error is built into the protocol.

\section{Untoward Results}

In the event of an untoward result following either a vaginal trial or a cesarean delivery, the facts of the occurrence must be carefully documented. If the obstetrician has appropriately documented adherence to the originally decided protocol, and clinical decision making throughout the course of the trial of labor or cesarean delivery has followed this plan, there should be little need for lengthy commentary or explanation in the progress note. Protracted retrospective explanations entered into the medical record with the hope that subsequent medical/legal reviewers will be persuaded to exonerate a physician's conduct seldom prove effective, unless the remainder of the chart adequately documents a coherent prospective plan. In fact, many physicians find themselves compromised by their own "explanatory remarks," because such hastily formulated notations are frequently either incomplete or poorly articulated.

Initial discussions with the mother concerning the injury or death of her infant ought to be prompt. In these conversations, the clinician should frankly discuss the infant's status and/or prognosis, if it is accurately known. Imparting either falsely hopeful or falsely negative information is more harmful than simply stating that the baby's condition is uncertain or under evaluation. Because an atmosphere of strong emotion often attends this initial communication, it is sometimes wise for the obstetrician to defer detailed discussion of the circumstances which led to the unpleasant result (if they are known) until a later time. Once the mother/ family is calm and reasonably receptive to such discussion, the physician should explain what happened. Obstetricians who regularly pride themselves on their skill, knowledge, and professional acumen are at that moment met with a singularly demanding challenge: embracing their own human limitations and explaining in plain language why they were powerless to prevent this untoward result. Honest acknowledgment of both human and technical limitations is necessary and appropriate. If done properly, this should simply reinforce what the patient should have known before the delivery was ever undertaken. The gift of simple humanity on the part of the physician at this juncture will go a long way toward healing the loss experienced by both the patient and the doctor. It is good practice to have another observer (nurse, colleague, etc.) present during these discussions. Obviously, it is prudent to notify the hospital risk management service of such complications, as it is always uncertain whether formal legal proceedings will result from any complication and the lag time between the event and the legal complaint can be long indeed.

\section{CONCLUSIONS}

The results of the TBT have changed the approach to breech vaginal delivery by the majority of obstetric providers. This will eventually lead to the loss of the experience to provide safe vaginal delivery to those who present with the persistent breech-presenting infant. There currently remain some centers that provide vaginal breech delivery with a management program consisting of radiologic confirmation of pelvic adequacy in conjunction with careful assessment of labor and continuous fetal monitoring, which usually produces

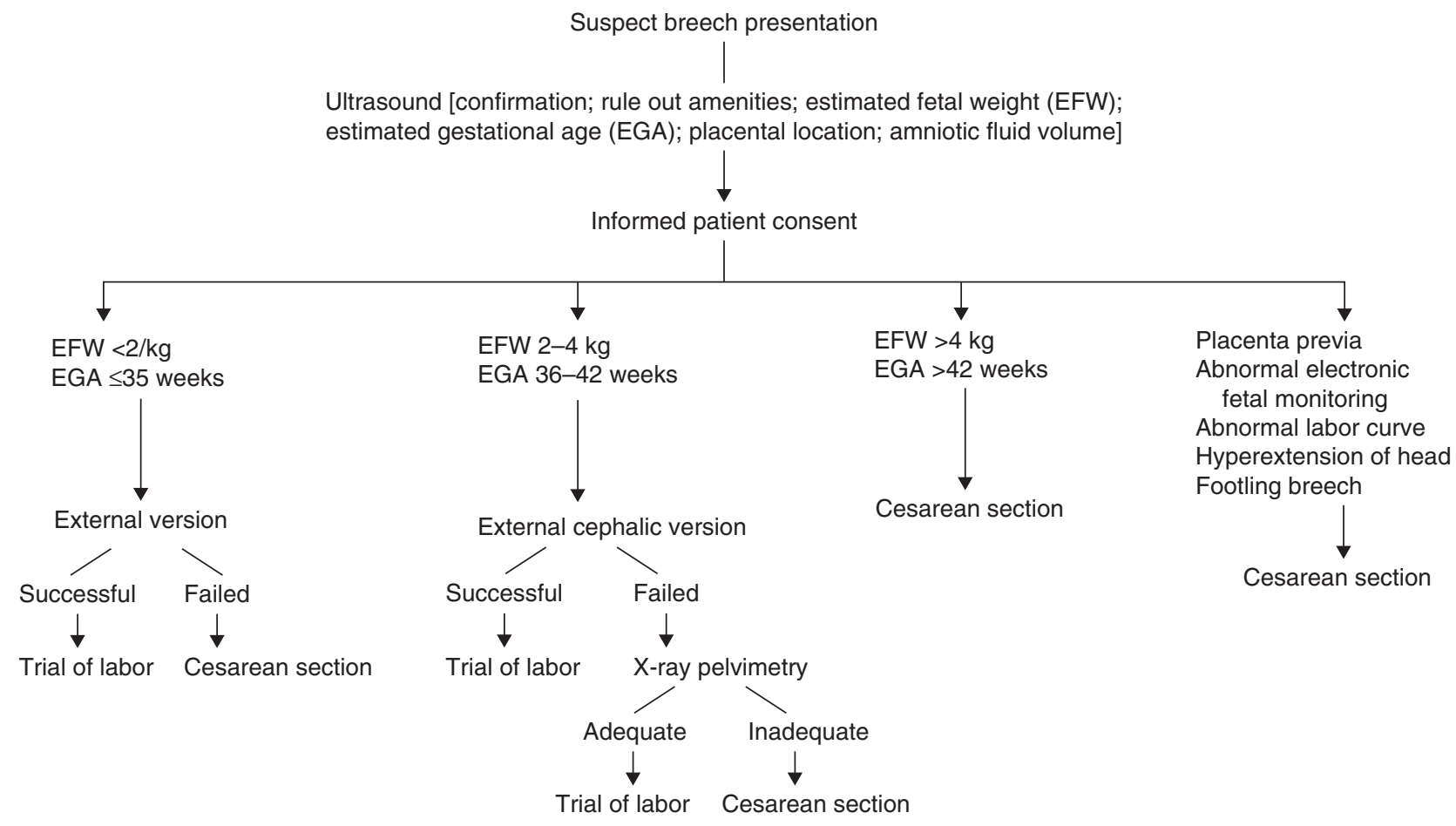

Figure 4 Overview and flow diagram of the management of breech presentation in early labor. Source: From Ref. 58. 
outcomes in neonates born vaginally that are comparable with those delivered abdominally (29-33) (Fig. 4).

The liberal use of cesarean section reduces the potential for trauma and hypoxia in breech delivery, but it by no means eliminates it. In establishing any protocol and ultimately in reaching a management plan for any individual patient, it is necessary to balance the risks and benefits for both mother and neonate from cesarean section and vaginal delivery and to counsel families accordingly. Where facilities allow for expeditious cesarean section and a stringent protocol for evaluation, selected patients may safely be offered a trial of labor. However, to assure maternal and fetal safety, the obstetrician must be committed to thorough evaluation of the maternal pelvis and close attention to the course of labor. Furthermore, the clinician must know the mechanics of safe breech delivery, regardless of the delivery route initially chosen. At cesarean section, although the infant is being delivered through incisions in the abdominal wall, the mechanism of delivery by extraction has the potential for greatly traumatizing the infant unless extreme care is exercised. Even at cesarean section, peripheral nerve and bony injuries to the fetus are not only possible, but seen to occur with regularity (57). The obstetrician can minimize the risk of trauma to the breech fetus born by cesarean section or vaginally after a labor trial by close attention to detail with the application of the standard obstetric techniques for assisted delivery. The same approaches to delivery apply to both cesarean section and vaginal delivery: minimal force, careful pressure to the aftercoming head to prevent the loss of flexion, and judicious use of the MSV maneuver and/or Piper forceps for cranial extraction.

Based on the cumulative experience of many clinicians on multiple obstetric services, traumatic birth injuries to both the parturient and her fetus can often be avoided and always be minimized in the management of breech presentation. A program of antepartum external cephalic version offers the advantage of decreasing the incidence of breech presentation in labor, thus avoiding these dilemmas for the clinician. Cesarean section will be used to deliver the vast majority of breech infant, but a selective trial of labor employing strict protocol adherence can be satisfying to both physician and parturient and result in an equally good outcome.

\section{REFERENCES}

1. Kauppila D. The perinatal mortality in breech deliveries and observation of affecting factors. Acta Obstet Gynecol Scand (Suppl) 1975; 39: 1-145.

2. Gimovsky M, Paul R. Singleton breech presentation in labor-experience in 1980. Am J Obstet Gynecol 1982; 143: 733-80.

3. Hannah M, Hannah W, Hewson S, et al. Planned caesarean section versus planned vaginal birth for breech presentation at term: a randomised multicentre trial. Term Breech Trial Collaborative Group. Lancet 2000; 356: 1375-83.

4. Roman J, Bakor O, Cnattingius S. Pregnancy outcomes by mode of delivery among term breech births: Swedish experience 1987-1993. Obstet Gynecol 1998; 92: 945-50.

5. Gilbert W, Hicks S, Boe N, Danielsen B. Vaginal versus cesarean delivery for breech presentation in California: a population-based study. Obstet Gynecol 2003; 102(5 Pt 1): 911-17.

6. Krebs L, Langhoff-Roos J. Elective cesarean delivery for term breech. Obstet Gynecol 2003; 101: 690-6.

7. Goffinet F, Carayol M, Foidart J, et al. Is planned vaginal delivery for breech presentation at term still an option? Results of an observational prospective survey in France and Belgium. Am J Obstet Gynecol 2006; 194: 1002-11.

8. Glezerman M. Five years to the term breech trial: the rise and fall of a randomized controlled trial. Am J Obstet Gynecol 2006; 194: 20-5.

9. Kotaska A. Inappropriate use of randomised trials to evaluate complex phenomena: case study of vaginal breech delivery. BMJ 2004; 329: 1039-42.

10. Alarab M, Regan C, O'Connell M, et al. Singleton vaginal breech delivery at term: still a safe option. Obstet Gynecol 2004; 103: 407-12.

11. Giuliani A, Scholl W, Basver A, Tamussino K. Mode of delivery and outcome of 699 term singleton breech deliveries at a single center. Am J Obstet Gynecol 2002; 187: 1694-8.

12. Committee on Obstetric Practice. ACOG committee opinion: number 265, December 2001. Mode of term single breech delivery. Obstet Gynecol 2001; 98: 1189-90.

13. ACOG Committee on Obstetric Practice. ACOG Committee Opinion No. 340. Mode of term singleton breech delivery. Obstet Gynecol 2006; 108: 235-7.

14. Chinnock M, Robson S. Obstetric trainees’ experience in vaginal breech delivery: implications for future practice. Obstet Gynecol 2007; 110: 900-3.

15. Deering S, Brown J, Hodor J, Satin A. Simulation training and resident performance of singleton vaginal breech delivery. Obstet Gynecol 2006; 107: 86-9.

16. Green J, McLean F, Smith L, et al. Has an increased Cesarean section rate for term breech delivery reduced the incidence of birth asphyxia, trauma and deaths? Am J Obstet Gynecol 1981; 142: 643-8.

17. Van Dorsten J, Schifrin B, Wallace R. Randomized controlled trial of external cephalic version with tocolysis in late pregnancy. Am J Obstet Gynecol 1981; 141: 417-21.

18. Hotmyer G. Effect of external cephalic version in late pregnancy on breech presentation and Cesarean section rate: a controlled trial. $\mathrm{Br} \mathrm{J}$ Obstet Gynaecol 1983; 90: 392-4.

19. Brosset A. The value of prophylactic external cephalic version in cases of breech presentation. Acta Obstet Gynecol Scand (Suppl) 1956; 35: 555-7.

20. Kasule J, Chimbria T, Brown I. Controlled trial of external cephalic version. Br J Obstet Gynaecol 1985; 92: 14-17.

21. Fortinato S, Mercer L, Guzick D. External cephalic version with tocolysis factors associated with success. Obstet Gynecol 1988; 72: 59-61.

22. Gimovsky M, O'Grady J, Keroack E. Immediate neonatal outcome of vaginal breech delivery at term in oxytocin stimulated labor (abstract). Am J Obstet Gynecol 1993; 168: 436.

23. Hilton J, Allan B, Swaby C, et al. Intravenous nitroglycerin for external cephalic version: a randomized controlled trial. Obstet Gynecol 2009; 114: 560-7.

24. Yanny H, Johanson R, Balwin K, et al. Double-blind randomised controlled trial of glyceryl trinitrate spray for external cephalic version. BJOG 2000; 107: 562-4.

25. Kok M, Bais J, van Lith J, et al. Nifedipine as a uterine relaxant for external cephalic version: a randomized controlled trial. Obstet Gynecol 2008; 112(2 Pt 1): 271-6.

26. Samuels P. Epidural facilitates external version. Obstet Gynaecol News 1990; $25: 1$.

27. Robertson A, Kopelman J, Read J, et al. External cephalic version at termis a tocolytic necessary Obstet Gynecol 1987; 70: 896-7.

28. Kerr M. Breech presentation. In: Myciscorh P, Mori J, eds. Munro Kerr's Operative Obstetrics, 8th edn. Baltimore: Williams \& Williams, 1971.

29. Collea J, Chein C, Quillgean E. The randomized management of the term frank presentation-a study of 208 cases. Am J Obstet Gynecol 1980; 137: 235-41.

30. O'Leary J. Vaginal delivery of the term breech. Obstet Gynecol 1979; 53:341-5.

31. Gimovsky M, Petrie R, Todd W. Neonatal performance of the selected term vaginal breech delivery. Obstet Gynecol 1980; 56: 687-91.

32. Todd W, Steer C. Term breech: review of 1006 term breech deliveries. Obstet Gynecol 1963; 22: 583-91.

33. Gimovsky M, Wallace R, Schifrin B, et al. Randomized management of the nonfrank breech presentation at term-a preliminary report. Am J Obstet Gynecol 1983; 146: 34-41.

34. Caterini H, Langer A, Sanra J, et al. Fetal risk in hypertension of the fetal head in breech presentation. Am J Obstet Gynecol 1983; 123: 632-5.

35. Phelan J, Bethel M, Gimovsky M, et al. Use of ultrasound in breech presentation with hyperextension of the fetal head. J Ultrasound Med 1983; 2: 373-4. 
36. Myers S, Glescher N. Why the dilemmas Am J Obstet Gynecol 1987; 156: 6-10.

37. Queenan J. Newborn's neck broken during breech delivery. Contemp Obstet Gynecol 1991; 36: 102.

38. Gimovsky M, Willark K, Neglio M, et al. X-ray pelvimetry in a breech protocol-a comparison of digital radiography and conventional method. Am J Obstet Gynecol 1985; 153: 887-91.

39. Kopelman J, Duff P, Karl R, et al. Computed tomographic pelvimetry in the evaluation of breech presentation. Obstet Gynecol 1986; 68: 455-61.

40. Federle M, Cohen H, Rosenwein M, et al. Pelvimetry by digital radiography-a low dose examination. Radiology 1982; 143: 733-6.

41. Michel S, Rake A, Treiber K, et al. MR obstetric pelvimetry: effect of birthing position on pelvic bony dimensions. AJR Am J Roentgenol 2002; 179: $1063-7$.

42. Gimovsky M, O'Grady J, Morris B, et al. An assessment of CT pelvimetry within a selective breech management protocol. J Reprod Med 1994; 39: 489-91.

43. Gimovsky M, Petrie R. Intrapartum ultrasound as an adjunct in the selective management approach to breech presentation. 10th Annual Meeting of the Society of Perinatal Obstetricians, 1990.

44. Gimovsky M. Short term tocolysis adjunctive to intrapartum term breech management. Am J Obstet Gynecol 1985; 153: 233.

45. Rovinsky J, Miller J, Kaplan S. Management of breech presenting at term. Am J Obstet Gynecol 1973; 115: 497-505.

46. Nishijimi N, Tatsuni H, Amano K, et al. Differences of FHR patterns between cephalic and breech presentations at term. J Perinat Med (Suppl) 1981; 9: 129-34.

47. Wheeler T, Greene K. FHR monitoring during breech labor. Br J Obstet Gynaecol 1975; 82: 208-14.
48. Crawford B. An appraisal of lumbar epidural blockade in patients with singleton fetus presenting by the breech. J Obstet Gynaecol Br Commonw 1974; 81: 867-8.

49. Flanagan T, Mulclahey K, Korenbrot C, et al. Management of term breech presentation. Am J Obstet Gynecol 1987; 156: 1492-9.

50. In: Friedman E, ed. Clinical Management of Labor, 2nd edn. New York: Appleton, Century, Crofts, 1978.

51. Milner R. Neonatal mortality of breech deliveries with and without forceps to the after-coming head. Br J Obstet Gynaecol 1975; 82: 783-5.

52. Gimovsky M, Nishiyama M, Halle J. Fetal respiratory status at birth as reflected by umbilical cord acid-base parameters. 9th Annual Meeting of the Society of Perinatal Obstetricians. San Francisco, CA, 1989.

53. Cruckshank D, Pitten R. Delivery of the premature breech. Obstet Gynecol 1977; 50: 367-9.

54. Ingemarson I, Westgven M, Svennignsen N. Long term follow-up of preterm infants in breech presentation delivered by Cesarean section. Lancet 1978; 2: 172-4.

55. Kauppila O, Gronross M, Aro P, et al. Management of low birth weight breech delivery. should Cesarean section be routine? Obstet Gynecol 1981; 57: 289-94.

56. Paul R, Koh K, Monfared A. Obstetric factors influencing the outcome in infants weighing from 1000 to 1500 grams. Am J Obstet Gynecol 1979; 133: 503.

57. Gimovsky M, Petrie R. The intrapartum management of the breech presentation. Clin Perinatol 1989; 16: 975-86.

58. Gimovsky ML. Diagnostic difficulty after failed external cephalid version under tocolysis. J Perinatol 1985; 4: 59. 


\section{Cesarean section and vaginal birth after cesarean section Jessica Winn and Hung N. Winn}

\section{INTRODUCTION}

In the United States, cesarean section was the most common surgical operation in 2006 (1) and about $31.8 \%$ of approximately 4.3 million infants born in 2007 were delivered by cesarean section (2). The rates of cesarean section increased dramatically from an annual rate of $5.5 \%$ in 1970 to $24.1 \%$ in 1986 , stabilized between 1986 and 1989 , then gradually declined to $21 \%$ in 1996 , and finally steadily rose again to $31.8 \%$ in 2007 (2-5). Between 1996 and 2007, the overall cesarean section rate increased for all pregnant women, regardless of the maternal age, race, and the state of residence (5). The overall rate of cesarean section increased or decreased with the fall or rise in the rate of vaginal birth after previous cesarean section (VBAC), respectively. The overall rate of cesarean section increased by $53 \%$ between 1996 and 2007 while the rate of VBAC dramatically declined during the same period $(5,6)$.

Cragin's dictum “once a cesarean, always a cesarean" dates back to 1916, when he suggested that uterine rupture following vaginal delivery after previous cesarean birth was so catastrophic an event that a repeat cesarean should be done prior to the onset of labor (7). This opinion was then cited in the fourth edition of Williams' Obstetrics (8) and the attitudes favoring repeat cesarean sections began to prevail. All this dates back to a pre-antibiotic, pre-transfusion era when cesarean section involved a classical uterine incision. In 1980, the National Institutes of Health (NIH) Consensus Development Conference pointed out that repeat cesarean was one of the major reasons for the rising cesarean rate. The literature was reviewed and the Consensus Development Task Force on Cesarean Childbirth concurred that labor following a low transverse uterine incision was associated with lower maternal morbidity and mortality, with equivalent infant outcomes. They concluded that VBAC was an appropriate option (9). Subsequently, the rate of VBAC steadily increased from $5 \%$ to $28.3 \%$ while the overall rate of cesarean section decreased from 25\% to 21\% between 1985 and 1996 (4). After peaking in 1996, the trend for VBAC was reversed with the rates of VBAC plummeted to $12.6 \%$ in 2002 and to $8.5 \%$ in 2006 with a concomitant rise of overall cesarean section to $31.1 \%$ in 2006 (10). The reverse trend in VBAC could be attributed to (i) the increased number of reports of maternal complications and perinatal morbidities and mortalities associated with VBAC (11-15) and (ii) professional liability concerns (16). The overall success rates of VBAC is about $74 \%$ with a range from $54 \%$ to $89 \%$ (5,6,17-19). The indications for the primary cesarean sections such as failure to progress, breech presentation, fetal distress, and genital herpes infection do not appear to influence the high success rate of VBAC delivery (17-19). Factors that are associated with a higher rate of successful
VBAC include spontaneous labor, birth weight of less than $4000 \mathrm{~g}$, and a history of prior vaginal delivery especially a prior successful VBAC $(1,5,6,20-23)$.

\section{RISK OF UTERINE RUPTURES Oxytocin}

The risk of uterine rupture in patients undergoing trial of labor after cesarean section (TLCS) ranges from $0.4 \%$ to $0.5 \%$ with spontaneous labor and $0.8 \%$ to $1.1 \%$ with oxytocin induction of labor $(24,25)$. The higher the maximum dose of oxytocin, the higher the risk of uterine rupture (26). Similarly, overuse of oxytocin to augment labor for prolonged latent phase in patients undergoing TLCS is associated with a risk of uterine rupture 2.7 times that of a control group $(26,27)$. The risk of uterine rupture is not increased if uterine pressure is carefully monitored, preferably with an intrauterine pressure catheter (28).

\section{Prostaglandin Use}

The risk of uterine rupture in patients undergoing TLCS ranges from $0.4 \%$ to $0.5 \%$ with spontaneous labor and $1.4 \%$ to $2.2 \%$ with prostaglandin induction of labor $(24,25)$. The increased risk of uterine rupture seems to exist only in the setting of sequential process of cervical ripening with prostaglandin $\mathrm{E}_{2}$ then induction of labor with intravenous oxytocin (29). In addition, the use of prostaglandin $E_{1}$ (misoprostol) in patients with previous cesarean sections is consistently associated with a much higher risk of uterine rupture (30-33). Limited data suggest that cervical ripening with prostaglandin (PGE2) gel alone in patients with a previous low transverse cervical cesarean section appears to be safe, without the increased risk of uterine rupture of maternal perinatal morbidity or mortality (29). Careful monitoring of uterine contraction and fetal heart rates (FHRs) is strongly recommended.

Induction of labor with prostaglandins including misoprostol during the second trimester for fetal demise in patients with previous cesarean sections is a reasonable option since it carries a uterine rupture risk of less than $1 \%$ that is comparable with that of unscarred uterus (34-36).

\section{Epidural Anesthesia}

The initial concern of epidural anesthesia for analgesia in masking the symptoms and signs of uterine rupture such as pain or hypotension in patients undergoing TLCS has not been supported by clinical data. Epidural anesthesia is not associated with an increased risk of uterine rupture in patients undergoing TLCS $(27,37)$. The use of epidural anesthesia allows adequate pain relief during labor and ready access to safe anesthesia when cesarean section is required. 


\section{Fetal Macrosomia}

Fetal macrosomia is defined as a fetus having an estimated weight of $4000 \mathrm{~g}$ or more (38). Trial of labor in patients with fetal macrosomia is not associated with an increased risk of uterine rupture and perinatal or maternal morbidity or mortality $(27,39)$. It is uncertain whether fetal macrosomia affects the success rates of VBAC (40). One study showed VBAC success rates of $78 \%$ with infants having birth weights of $<4000$ g, $58 \%$ with birth weights between 4000 and 4499 g, and $26 \%$ with birth weights of $>4500 \mathrm{~g}$ (41). Another study showed a lower VBAC success rate if the neonatal birth weight of the index pregnancy is larger than the one associated with a prior cesarean section done for cephalopelvic dystocia (42). Trial of labor should not be discouraged because of fetal macrosomia, in view of its safety and the inaccuracy of predicting fetal weight in macrosomic fetuses.

\section{Breech Presentation}

External version can be safely performed in patients with a previous cesarean section, followed by a trial of labor and vaginal delivery (43). Success rates of $82 \%$ for external cephalic version and $65 \%$ for subsequent vaginal delivery have been reported (44).

\section{Twins}

The management of patients with multiple gestations remains a topic of debate. There are inadequate data on the management of patients with previous cesarean sections who have multiple gestations (45-48).

\section{Amnioinfusion}

Amnioinfusion has been performed for indications such as oligohydramnios, meconium-stained amniotic fluid, and variable FHR decelerations in patients with previous cesarean sections. The success rates of VBAC range from 58\% to $83 \%$ without a significant increase in the incidences of uterine rupture and maternal or perinatal morbidity/mortality $(49,50)$. The incidence of uterine rupture is $0.8 \%$ (49). Amnioinfusion does not appear to be a contraindication for a trial of labor in patients with previous cesarean sections.

\section{Multiple Previous Cesarean Sections}

Patients undergoing a trial of labor after having more than one previous cesarean section have VBAC success rates ranging from $64 \%$ to $77 \%$ that were comparable with those with one previous cesarean section $(17,18,51-56)$. Uterine wound separation appeared to be increased, even though not statistically significantly in patients with multiple previous cesarean sections compared with those with a single previous cesarean section (52). There was no increased perinatal morbidity or mortality that could be attributed to uterine separation or rupture $(17,52)$. More recent studies demonstrated that the risk of uterine rupture was inconsistent, either about the same $(0.9 \%$ for one previous cesarean section vs. $0.7 \%$ for multiple ones) or increased $(0.9 \%$ for one previous cesarean section vs. $1.8 \%$ for two previous ones) $(53,54)$. Thus, a trial of labor for patients with two previous cesarean sections is still a reasonable option in the presence of other favorable factors for successful VBAC.

\section{A Previous Low Uterine Vertical Cesarean Section}

Patients who undergo a trial of labor after a previous low uterine vertical cesarean section have VBAC success rates ranging from $83 \%$ to $85 \%$, risks of uterine rupture ranging from $1.1 \%$ to $1.3 \%$, and no maternal deaths. There is no significantly increased incidence of maternal or perinatal morbidity/mortality due to uterine rupture (57-59). Therefore, a trial of labor could be attempted in patients who had a previous low vertical uterine cesarean section with close maternal and fetal monitoring.

\section{Unknown Uterine Scar}

Documentation of the uterine incision in the previous cesarean section is one of the main criteria for undergoing a trial of labor. A dilemma occurs when the type of uterine incision is unknown. Limited data reveal that the rate of uterine rupture is $2.2 \%$ without a significant increase in maternal or perinatal mortality or morbidity (60). Augmentation of labor during the latent phase with intravenous oxytocin increased the incidence of uterine separation compared with that of patients who were managed expectantly without oxytocin (61).

In 1984, Beall and coworkers (62) reported on 451 women with unknown types of uterine scar. Ninety-seven patients labored with an $87 \%$ vaginal delivery rate. The rate of scar dehiscence in the women who labored was 1\% that did not significantly differ from the one in women with unknown scar who did not labor. Perinatal mortality was similar in both groups.

\section{CONCLUSIONS}

The risk of uterine rupture in patients undergoing TLCS varies with the type of previous uterine incision and the number of previous cesarean sections. The risks of uterine rupture range from $0.6 \%$ to $0.9 \%$ with one previous low transverse uterine incision $(19,28,53,54,63-66), 0.7 \%$ to $1.8 \%$ with more than one previous low transverse uterine incision $(53,54,56,57)$, and is about $12 \%$ with a previous classical uterine incision (59). Other predisposing factors include excessive amount of intravenous oxytocin for augmentation or induction of labor, and dysfunctional labor. Fetal macrosomia and epidural anesthesia do not appear to increase the risk of uterine rupture (27). Signs of uterine rupture include abdominal pain, vaginal bleeding, abnormal labor, and abnormal FHR patterns such as sudden onset of prolonged fetal bradycardia and severe variable decelerations $(18,67)$. Trial of labor in patients with previous cesarean sections does not increase maternal mortality compared with those undergoing elective cesarean section (59).

Despite the reassuring data, VBAC has been steadily decreased during the last decade. Undue concern from both obstetricians and patients about the potential maternal, fetal complications and legal issues associated with uterine rupture and the convenience of scheduled cesarean sections have contributed to the low rate of trial of labor. Patients with a previous low transverse cervical cesarean section should be encouraged to undergo a trial of labor. In addition to the low risk of uterine rupture, patients who have successful VBAC have significantly lower maternal morbidity in comparing with those undergoing elective repeat cesarean section $(19,68)$. Trial 
of labor may be offered to patients who have two previous low transverse cervical cesarean sections. Patients with a low vertical uterine cesarean section or unknown uterine scar should not be discouraged from a trial of labor after being informed of the slightly higher risk of uterine ruptures (up to $2 \%$ ) and potential-associated maternal or perinatal complications. Fetal macrosomia is not a contraindication for a trial of labor. Epidural anesthesia for pain control may be offered to patients who undergo VBAC. Fetal heart rate, maternal labor and medical condition should be closely monitored during labor. The American College of Obstetricians and Gynecologists has recommended that the institution's resources and the medical staff should be immediately available to respond to maternal and perinatal emergencies including emergency cesarean section delivery if a trial of labor after a previous cesarean section is undertaken (69).

\section{REFERENCES}

1. Rageth JC, Juzi C, Grossenbacher H. Delivery after previous cesarean: a risk evaluation. Swiss Working Group of Obstetric and Gynecologic Institutions. Obstet Gynecol 1999; 93: 332-7.

2. Hamilton BE, Martin JA, Ventura SJ. Births: preliminary data for 2007. Natl Vital Stat Rep 2009; 57: 1-23.

3. Clarke SC, Taffel S. Changes in Cesarean delivery in the United States, 1988 and 1993. Birth 1995; 22: 63-7.

4. Menacker F, Declercq E, Macdorman MF. Cesarean delivery: background, trends, and epidemiology. Semin Perinatol 2006; 30: 235-41.

5. Landon MB, Leindecker S, Spong CY, et al. The MFMU Cesarean Registry: factors affecting the success of trial of labor after previous cesarean delivery. National Institute of Child Health and Human Development Maternal-Fetal Medicine Units Network. Am J Obstet Gynecol 2005; 193: 1016-23.

6. National Institutes of Health. NIH Consensus Development Conference: vaginal birth after cesarean: new insights. Consensus Development Conference statement. Bethesda (MD): NIH, 2010. [Available from: http://consensus.nih.gov/2010/images/vbac/vbac_statement.pdf. Retrieved April 23, 2010].

7. Craigin EB. Conservation in obstetrics. NY Med J 1916; 104: 1.

8. Williams JM. Williams Obstetrics. New York: S Appleton, 1917.

9. Rosen M, et al. The Cesarean Birth Task Force: National Institutes of Health consensus development statement on Cesarean childbirth. Obstet Gynecol 1981; 57: 37.

10. Martin JA, Hamilton BE, Sutton PD, et al. Births: final data for 2006. Natl Vital Stat Rep 2009; 57: 1-104.

11. Sachs BP, Kobelin C, Castro MA, Frigoletto F. The risks of lowering the cesarean-delivery rate. N Engl J Med 1999; 340: 54-7.

12. McMahon MJ, Luther ER, Bowers WA, Olshan AF. Comparison of a trial of labor with an elective second cesarean section. N Engl J Med 1996; 335: 689-95.

13. Mozurkewich EL, Hutton EK. Elective repeat cesarean delivery versus trial of labor: a meta-analysis of the literature from 1989 to 1999. Am J Obstet Genecol 2000; 183: 1187-97.

14. Lydon-Rochelle M, Holt V, Easterling T, Martin D. Risk of uterine rupture during labor among women with a prior cesarean delivery. N Engl J Med 2001; 345: 3-8.

15. Greene M. Vaginal delivery after Cesarean Section - is the risk acceptable? N Engl J Med 2001; 345: 55.

16. Yang YT, Mello MM, Subramanian SV, Studdert DM. Relationship between malpractice litigation pressure and rates of cesarean section and vaginal birth after cesarean section. Med Care 2009; 47: 234-42.

17. Rosen MG, Dickinson JC. Vaginal birth after Cesarean: a meta-analysis of indicators for success. Obstet Gynecol 1990; 76: 865-9.

18. Cowan RK, Kinch RAH, Ellis B, Anderson R. Trial of labor following Cesarean delivery. Obstet Gynecol 1994; 83: 933-6.

19. Flamm BL, Goings JR, Liu Y, Wolde-Tsadik G. Elective repeat Cesarean delivery versus trial of labor. A prospective multicenter study. Obstet Gynecol 1994; 83: 927-32.
20. Macones GA, Hausman N, Edelstein R, Stamilio DM, Marder SJ. Predicting outcomes of trials of labor in women attempting vaginal birth after cesarean delivery: a comparison of multivariate methods with neural networks. Am J Obstet Gynecol 2001; 184: 409-13.

21. Sims EJ, Newman RB, Hulsey TC. Vaginal birth after cesarean: to induce or not to induce. Am J Obstet Gynecol 2001; 184: 1122-4.

22. Zelop CM, Shipp TD, Repke JT, Cohen A, Lieberman E. Outcomes of trial of labor following previous cesarean delivery among women with fetuses weighting 4000 g. Am J Obstet Gynecol 2001; 185: 903-5.

23. Elkousy MA, Sammel M, Stevens E, Peipert JF, Macones G. The effect of birth weight on vaginal birth after cesarean delivery success rates. Am J Obstet Gynecol 2003; 188: 824-30.

24. Landon MB, Hauth JC, Leveno KJ, et al. Maternal and perinatal outcomes associated with a trial of labor after prior cesarean delivery. National Institute of Child Health and Human Development MaternalFetal Medicine Unites Network. N Engl J Med 2004; 351: 2581-9.

25. Lydon-Rochelle M, Holt VL, Easterling TR, Martin DP. Risk of uterine rupture during labor among women with a prior cesarean delivery. N Engl J Med 2001; 345: 3-8.

26. Cahill AG, Waterman BM, Stamilio DM, et al. Higher maximum doses of oxytocin are associated with an unacceptably high risk for uterine rupture in patients attempting vaginal birth after cesarean delivery. Am J Obstet Gynecol 2008; 199: 32.e1-5.

27. Leung AS, Farmer RM, Leung EK, et al. Risk factors associated with uterine rupture during trial of labor after Cesarean delivery: as casecontrol study. Am J Obstet Gynecol 1993; 168: 1358-63.

28. Rosen MG, Dickinson JC, Westhoff CL. Vaginal birth after Cesarean: a meta-analysis of morbidity and mortality. Obstet Gynecol 1991; 77: 465-70.

29. Macones GA, Peipert J, Nelson DB, et al. Maternal complications with vaginal birth after cesarean delivery: a multicenter study. Am J Obstet Gynecol 2005; 193: 1656-62.

30. Bennett BB. Uterine rupture during induction of labor at term with intravaginal misoprostol. Obstet Gynecol 1997; 89: 832-3.

31. Wing DA, Lovett K, Paul RH. Disruption of prior uterine incision following misoprostol for labor induction in women with previous cesarean delivery. Obstet Gynecol 1998; 91: 828-30.

32. Plaut MM, Schwartz ML, Lubarsky SL. Uterine rupture associated with the use of misoprostol in the gravid patient with a previous cesarean section. Am J Obstet Gynecol 1999; 180: 1535-42.

33. Aslan H, Unlu E, Agar M, Ceylan Y. Uterine rupture associated with misoprostol labor induction in women with previous cesarean delivery. Eur J Obstet Gynecol Reprod Biol 2004; 113: 45-8.

34. Hammond C. Recent advances in second-trimester abortion: an evidence-based review. Am J Obstet Gynecol 2009; 200: 347-56.

35. Goyal V. Uterine rupture in second-trimester misoprostol-induced abortion after cesarean delivery: a systematic review. Obstet Gynecol 2009; 113: 1117-23.

36. Berghella V, Airoldi J, O’Neill AM, Einhorn K, Hoffman M. Misoprostol for second trimester pregnancy termination in women with prior caesarean: a systematic review. BJOG 2009; 116: 1151-7.

37. Kieser KE, Baskett TF. A 10-year population-based study of uterine rupture. Obstet Gynecol 2002; 100: 749-53.

38. The American College of Obstetricians and Gynecologists. Fetal Macrosomia. ACOG Technical Bulletin 159. Washington, DC: ACOG, 1991.

39. Leung AS, Farmer RM, Leung EK, et al. Risk factors associated with uterine rupture during trial of labor after Cesarean delivery: a casecontrol study. Am J Obstet Gynecol 1993; 168: 1358-63.

40. Elkousy MA, Sammel M, Stevens E, Peipert JF, Macones G. The effect of birth weight on vaginal birth after cesarean delivery success rates. Am J Obstet Gynecol 2003; 188: 824-30.

41. Flamm BL, Goings JR. Vaginal birth after Cesarean section: is suspected fetal macrosomia a contraindication? Obstet Gynecol 1989; 74: 694-7.

42. Peaceman AM, Gersnoviez R, Landon MB, et al. The MFMU Cesarean Registry: impact of fetal size on trial of labor success for patients with previous cesarean for dystocia. National Institute of Child Health and Human Development Maternal-Fetal Medicine Units Network. Am J Obstet Gynecol 2006; 195: 1127-31. 
43. Sela HY, Fiegenberg T, Ben-Meir A, Elchalal U, Ezra Y. Safety and efficacy of external cephalic version for women with a previous cesarean delivery. Eur J Obstet Gynecol Reprod Biol 2009; 142: 111-14.

44. Flamm BL, Fried MW, Lonky NM, Giles WS. External cephalic version after previous Cesarean section. Am J Obstet Gynecol 1991; 165: 370-2.

45. Gilbert L, Saunders N, Sharp F. The management of multiple pregnancy in women with a lower-segment Cesarean scar. Is a repeat Cesarean section the 'safe' option? Br J Obstet Gynaecol 1988; 95: 1312-16.

46. Strong TH, Phelan JP, Ahn MO, Sarno AP. Vaginal birth after Cesarean delivery in the twin gestation. Am J Obstet Gynecol 1989; 161: 29-32.

47. Cahill A, Stamilio DM, Pare E, et al. Vaginal birth after cesarean (VBAC) attempt in twin pregnancies: is it safe? Am J Obstet Gynecol 2005; 193: 1050-5.

48. Varner MW, Thom E, Spong CY, et al. Trial of labor after one previous cesarean delivery for multifetal gestation. National Institute of Child Health and Human Development (NICHD) Maternal-Fetal Medicine Unites Network (MFMU) Obstet Gynecol 2007; 110: 814-19.

49. Strong TH, Vega JS, O’Shaughnessy MJ, et al. Amnioinfusion among women attempting vaginal birth after Cesarean delivery. Obstet Gynecol 1992; 79: 673-4.

50. Ouzounian JG, Miller DA, Paul RH. Amnioinfusion in women with previous Cesarean births: a preliminary report. Am Obstet Gynecol 1996; 174: 783-6.

51. Farmakides G, Duviver R, Schulman H, et al. Vaginal birth after two or more previous Cesarean sections. Am J Obstet Gynecol 1987; 156: 565-6.

52. Phelan JP, Ahn MO, Diaz F, et al. Twice a Cesarean, always a Cesarean? Obstet Gynecol 1989; 73: 161-5.

53. Asakura H, Myers SA. More than one precious Cesarean delivery: a 5-year experience with 435 patients. Obstet Gynecol 1995; 85: 924-9.

54. Landon MB, Spong CY, Thom E, et al. Risk of uterine rupture with a trial of labor in women with multiple and single prior cesarean delivery. National Institute of Child Health and Human Development MaternalFetal Medicine Units Network. Obstet Gynecol 2006; 108: 12-20.

55. Macones GA, Cahill A, Pare E, et al. Obstetric outcomes in women with two prior cesarean deliveries: is a vaginal birth after cesarean delivery a viable option? Am J Obstet Gynecol 2005; 192: 1223-8.
56. Pruett KM, Kirshon B, Cotton DB, Poindexter AN 3rd. Is vaginal birth after two or more cesarean sections safe? Obstet Gynecol 1988; 72: 163-5.

57. Naef RW, Ray MA, Chaunan SP, et al. Trial of labor after Cesarean delivery with a lower-segment, vertical uterine incision: Is it safe? Am J Obstet Gynecol 1995; 172: 1666-74.

58. Adair CD, Sanchez-Ramos L, Whitaker D, et al. Trial of labor in patients with a previous lower uterine vertical Cesarean section. Am J Obstet Gynecol 1996; 174: 966-70.

59. Martin JN Jr, Perry KG Jr, Roberts WE, Meydrech EF. The case for trial of labor in the patient with a prior low-segment vertical cesarean incision. Am J Obstet Gynecol 1997; 177: 144-8.

60. Rosen MG, Dickenson JC, Westhoff CL. Vaginal birth after Cesarean: a meta-analysis of morbidity and mortality. Obstet Gynecol 1991; 77: 465-70.

61. Grubb DK, Kjos SL, Paul RH. Latent labor with an unknown uterine scar. Obstet Gynecol 1996; 88: 351-5.

62. Beall M, Eglinton GS, Clark SL, Phelan JP. Vaginal delivery after Cesarean section in women with unknown types of uterine scar. J Reprod Med 1984; 29: 31-5.

63. Lavin J, Stephens R, Miodovnik M, Barden T. Vaginal delivery in patients with a prior Cesarean section. Obstet Gynecol 1982; 59: 135.

64. Nielsen TF, Ljungblad U, Hagberg H. Rupture and dehiscence of Cesarean section scar during pregnancy and delivery. Am J Obstet Gynecol 1989; 160: 569-73.

65. Horowitz B, Edelstein S, Lippman L. Once a Cesarean, always a Cesarean. Obstet Gynecol Surv 1981; 36: 592-8.

66. Farmer R, Kirschbaum T, Potter D, et al. Uterine rupture during trial of labor after previous Cesarean section. Am J Obstet Gynecol 1991; 1651: 996-1001.

67. Scott JR. Mandatory trial of labor after Cesarean delivery: an alternative viewpoint. Obstet Gynecol 1991; 77: 811-14.

68. Rossi AC, D’Addario V. Maternal morbidity following a trial of labor after cesarean section vs elective repeat cesarean delivery: a systematic review with metaanalysis. Am J Obstet Gynecol 2008; 199: 224-31.

69. American College of Obstetricians and Gynecologists. ACOG Practice Bulletin no. 115: Vaginal birth after previous cesarean delivery. Obstet Gynecol 2010; 116(2 pt 1): 450-63. 


\section{Diabetes in pregnancy Donald. R. Coustan}

\section{EPIDEMIOLOGY OF DIABETES IN PREGNANCY}

Preexisting diabetes mellitus complicates approximately $0.3 \%$ of all pregnancies in the United States (1), while gestational diabetes mellitus (GDM) complicates 5\% to 10\% (2). Type 2 diabetes, characterized by the typical clinical presentation of obesity and insulin resistance, is roughly 10 times more prevalent in the American population than type 1 diabetes; in recent years, as the nationwide epidemic of obesity and type 2 diabetes has impacted younger and younger individuals, type 2 diabetes is becoming proportionally more common in pregnancy. Individuals with type 1 diabetes are prone to ketoacidosis whereas those with type 2 diabetes rarely manifest this complication.

\section{ETIOLOGY}

Diabetes mellitus is a heterogeneous group of disorders all characterized by hyperglycemia. Type 2 diabetes, as described above, is commonly related to obesity and appears to be inherited in a polygenic manner. There are a number of less common forms of type 2 diabetes, formerly called "MODY" (maturity onset of diabetes in youth), which are inherited as single gene defects in an autosomal dominant manner (3). It is not yet clear whether insulin resistance alone is sufficient to cause type 2 diabetes or whether there must also be an element of relative insulinopenia. Type 1 diabetes, on the other hand, is an autoimmune disorder in which the immune system is directed against the islet cells in the pancreas. There is clearly a genetic predisposition toward such pancreatic destruction, as evidenced by the preponderance of specific HLA antigens in individuals with type 1 diabetes. However, the genetic predisposition is not sufficient to bring about the disease, as evidenced by the less than $50 \%$ concordance of identical twins for this disorder (3).

GDM is a condition of "carbohydrate intolerance of variable severity with onset or recognition during pregnancy" (4). Women with GDM are at increased risk, compared with the general population, for the subsequent development of type 2 diabetes, with $60 \%$ developing this condition within 20 years of the index pregnancy (5). One possible explanation is that the metabolic changes brought about by pregnancy induce transient carbohydrate intolerance in individuals whose metabolism is at some point along a continuum ultimately leading to diabetes (6).

\section{PATHOPHYSIOLOGY}

Pregnancy has profound effects on maternal metabolism. It is useful to think of these effects as being designed to assure a plentiful supply of fuel (glucose, gluconeogenic amino acids) and structural material (various amino acids) for the building of a fetus. When the gravida has fasted, circulating glucose and amino acid levels are lower than those in nonpregnant fasting women (7). While it is tempting to speculate that this relative hypoglycemia is secondary to fetal consumption of fuel, the change occurs relatively early in pregnancy, when the fetus cannot yet utilize enough fuel to account for the observed phenomenon. The fall in glucose levels during fasting is associated with a rise in circulating levels of ketone bodies, to an extent greater than that observed in the nonpregnant state. This fall in glucose and rise in ketones has been termed "accelerated starvation," which is a useful concept. Insulin levels after an overnight fast are significantly higher than those in the nonpregnant individual, despite lower glucose values. Such apparent insulin resistance is believed by many to be the underlying cause of GDM, as will be discussed below.

When the pregnant woman ingests glucose, particularly after an overnight fast, her circulating glucose level increases somewhat more slowly, but to a greater amplitude, than in the nonpregnant state, despite the fact that her insulin level is quite a bit higher than that in nonpregnant women (7). This "insulin resistance" is present in both the liver and the periphery. Nevertheless, in the context of the usual day-to-day ingestion of mixed meals, it appears that normal pregnant women maintain their circulating glucose levels within a rather narrow range $(7,8)$.

The insulin resistance mentioned above is most probably the cause of the appearance of GDM in 5\% to $10 \%$ of women in the United States. Whether there is also a relative deficiency of insulin production in such individuals remains to be determined.

Individuals with preexisting diabetes who become pregnant are impacted dramatically by the increase in insulin resistance brought about by the pregnancy. Those with type 2 diabetes are likely to require markedly increased doses of insulin in order to maintain relative euglycemia. Those with type 1 diabetes generally experience a two- to threefold increase in insulin requirements as pregnancy progresses (9).

Most importantly, despite a tendency toward hypoglycemia during the first trimester, individuals with type 1 diabetes are significantly more likely to experience diabetic ketoacidosis (DKA) during the second half of pregnancy than at other times. This proclivity toward DKA appears to be related to the insulin resistance of pregnancy. The most important causes of DKA are absolute or relative insulinopenia and glucagon excess, resulting in hyperglycemia from hepatic glycogenolysis and gluconeogenesis and hyperketonemia due to uninhibited lipolysis and increased hepatic production of ketone bodies (10). When DKA is present, hepatic glucose production is increased as much as threefold, accounting for marked hyperglycemia even in patients who have not eaten for a considerable length of time. The maternal effects of hyperglycemia during DKA include osmotic diuresis, which may result in dehydration. The resultant hypovolemia can cause hypotension, renal dysfunction, mental 
aberrations, and shock. Electrolytes, particularly total body sodium and potassium, become depleted. Because ketone bodies are moderately strong acids, the maternal $\mathrm{pH}$ falls, the serum bicarbonate level falls, and an anion gap [(serum sodium)(serum bicarbonate + serum chloride) $>10-15 \mathrm{mEq} / \mathrm{L}]$ results, indicative of metabolic acidosis. The acidosis is the cause of the characteristic tachypnea and increased tidal volume known as Kussmaul's respirations. Nausea and vomiting frequently accompany the acidosis, further exacerbating the hypovolemia and electrolyte depletion. The fetus is particularly at risk, with fetal mortality rates historically reported to be in the range of $50 \%$ to $90 \%$ when maternal DKA occurs. Explanations for this high rate of fetal death include hypoxia due to uterine hypoperfusion resulting from maternal hypovolemia and the possible fetal effects of the rapid transport of hydrogen ions across the placenta. More recent studies (10) report lower perinatal mortality rates of between $9 \%$ and 35\%, suggesting some improvement in the management of this complication over the years. During pregnancy, common causes of DKA appear to be similar to those seen in the nonpregnant state: intercurrent infection and omission of insulin injections. However, in addition, iatrogenic causes may contribute, including the use of betamimetic agents for tocolysis and corticosteroids for the enhancement of fetal pulmonary maturity. DKA is not caused by the administration of exogenous glucose unless there is simultaneous underinsulinization.

Even in the absence of DKA, hyperglycemia is associated with perinatal morbidity and mortality in diabetic pregnancy. In fact, according to the "Pedersen hypothesis" (11), all of the problems comprising "diabetic fetopathy" are related to fetal hyperinsulinemia, which results from hyperglycemia transmitted from the maternal to the fetal compartment. This concept was extended by Freinkel (12) as the "modified Pedersen hypothesis" to acknowledge the probable role of non-glucose secretagogues in stimulating fetal pancreatic insulin production and release.

There is a body of experimental evidence to support the Pedersen hypothesis with regard to both preexisting and GDM. Although a randomized, prospective trial of loose versus strict metabolic control in diabetic pregnancy has not been carried out, existing data on human pregnancy support the concept that near-normalization of maternal circulating glucose levels strongly predicts favorable perinatal outcome. Karlson and Kjellmer (13) demonstrated that diabetic women whose average blood glucose level in the third trimester exceeded $150 \mathrm{mg} / \mathrm{dL}$ manifested a $24 \%$ perinatal mortality rate, compared with $4 \%$ in those whose average third-trimester glucose level was less than $100 \mathrm{mg} / \mathrm{dL}$. Virtually every series published since that time in which nearnormalization of glucose levels was attempted has reported a perinatal mortality rate of less than $5 \%$. The pathophysiology of the relationship between maternal hyperglycemia and perinatal death is yet to be determined. However, studies in laboratory animal models have demonstrated that fetal hyperinsulinemia can lead to hypoxia, acidosis, and fetal death (14). Morbidity, such as fetal macrosomia, neonatal hypoglycemia, respiratory distress syndrome, plethora, and jaundice, has likewise been linked to fetal hyperinsulinemia (14).
It has long been appreciated that infants of diabetic mothers suffer from birth defects at a rate of two to three times that in the general population. Because virtually all structural anomalies in infants of diabetic mothers have taken place by 8 to 10 weeks after the last menstrual period (15), the cause(s) of such anomalies in humans has been difficult to study. Laboratory investigations have demonstrated that maternal hyperglycemia during organogenesis can induce fetal anomalies in rodent embryos (16). In human pregnancies, firsttrimester glycosylated hemoglobin levels have been positively correlated with the likelihood of anomalies, raising the possibility of a cause-and-effect relationship between hyperglycemia (or some other aspect of poor metabolic control) and birth defects (17-19). In the Diabetes in Early Pregnancy (DIEP) study, an observational study in which women were asked to perform multiple daily self glucose monitoring tests, an approximate halving of the rate of congenital anomalies $(4.9 \%)$ was noted in diabetic individuals registering for care prior to conception or during very early pregnancy compared with those enrolled after 8 weeks (9\%), even though no specific goals for glucose control were imposed (20). In this study, offspring of diabetic women registering early for care still had an approximate doubling of the malformation rate compared with control children of nondiabetic mothers. Furthermore, no correlation could be found between the level of glucose control and malformation rate, a finding possibly attributable to the paucity of pregnancies with either very good or very poor control in the series. In an interventional study, Fuhrmann and coworkers (21) demonstrated a malformation rate of $2 \%$ to $3 \%$ in the offspring of diabetic mothers who were given intensive prepregnancy and early pregnancy care, a rate similar to that in the general population and considerably lower than the $8 \%$ rate among offspring of mothers registering for care after the eighth week of gestation. Kitzmiller and colleagues (22) reported a major malformation rate of $1.2 \%$ among the offspring of 84 diabetic women who entered a preconception care program, compared with $10.9 \%$ among the offspring of 110 diabetic women presenting for prenatal care at 6 weeks of gestation or later. Average ambient glucose levels during organogenesis were considerably lower in the preconception care group than in the women participating in the DIEP study cited above. Poor maternal metabolic control has also been linked to spontaneous abortion in diabetic pregnancy $(19,23,24)$. It therefore seems likely that there exists a continuum of reproductive damage related to aberrations of metabolism in early diabetic pregnancy. This ranges from spontaneous abortion when poor control is very early and/or severe, to anomalies when it comes somewhat later, is less severe, or happens at a critical time. As described above, aberrations of metabolic control occurring later in pregnancy, after organogenesis has been completed, appear capable of inducing a wide range of problems which may be called, collectively, "diabetic fetopathy."

The above pathophysiologic mechanisms appear to be operative in gestational diabetic pregnancy, as well as in pregnancy complicated by overt diabetes, albeit to a lesser degree. Because the metabolic alterations seen in GDM are generally, but not always, less severe than those of preexisting diabetes, perinatal mortality is distinctly less likely with the milder forms of diabetes, particularly if the condition is 
identified and treated in some way. However, studies of undiagnosed and untreated GDM suggest a marked increase in the perinatal mortality rate $(25,26)$. Even in gestational diabetic pregnancies maintained in reasonably good metabolic control, there is an excess of fetal macrosomia (27). Furthermore, childhood and young adult obesity has been reported in infants of gestational diabetic mothers (28-30), suggesting the possibility that the intrauterine milieu may have profound and long-lasting effects on adipose tissue formation. Strengthening this hypothesis is the finding of a significant relationship between maternal response to a glucose challenge during pregnancy and the likelihood of the offspring developing diabetes and of the female offspring developing GDM (31).

\section{DIAGNOSIS}

By definition, preexisting or overt diabetes has already been diagnosed prior to pregnancy; it is GDM, therefore, that is the subject of this section. The diagnostic criteria currently accepted in the United States utilize a 100-g, 3-hour oral glucose tolerance test (OGTT) and are based on the work of O'Sullivan and Mahan (32), in which normative data were collected for 752 consecutive prenatal patients. Various thresholds derived from these data were then applied to a separate group of subjects who underwent the test during pregnancy and then were followed for 7 years. The criteria recommended were the most efficient predictors of subsequent diabetes. According to these criteria, GDM is diagnosed if at least two of the four glucose tolerance test values meet or exceed thresholds two standard deviations above the means for their respective specific time intervals. In O'Sullivan's original study (32), venous samples of whole blood were utilized, and the Somogyi-Nelson technology was applied for glucose measurement. The resultant thresholds were fasting $90 \mathrm{mg} / \mathrm{dL}, 1$ hour $165 \mathrm{mg} / \mathrm{dL}, 2$ hours $143 \mathrm{mg} / \mathrm{dL}$, and 3 hours $127 \mathrm{mg} / \mathrm{dL}$. These were rounded off to the nearest $5 \mathrm{mg} / \mathrm{dL}$ for ease of recall.

In the 1970s most clinical laboratories switched to venous plasma or serum, which yield values approximately 15\% higher than whole blood samples. Accordingly, in 1979, the National Diabetes Data Group (NDDG) (33) attempted to convert the O'Sullivan and Mahan criteria to plasma values by adding approximately $15 \%$ to each threshold. The resultant criteria were at one time recommended by the American Diabetes Association (ADA) (34) and the American College of Obstetricians and Gynecologists (ACOG) (35) and were widely utilized in the United States. The recommended thresholds for plasma or serum glucose samples were fasting $105 \mathrm{mg} / \mathrm{dL}, 1$ hour $190 \mathrm{mg} / \mathrm{dL}, 2$ hours $165 \mathrm{mg} / \mathrm{dL}$, and 3 hours $145 \mathrm{mg} / \mathrm{dL}$ for the 100-g, 3-hour OGTT; GDM was diagnosed if two or more samples meet or exceed the above thresholds.

In addition to the change from whole blood to plasma or serum samples, another alteration in glucose testing occurred in the 1970s. The Somogyi-Nelson method, which measures approximately $5 \mathrm{mg} / \mathrm{dL}$ of reducing substances other than glucose, was replaced by more specific enzymatic methods such as glucose oxidase and hexokinase. Consequently, in 1982 (36), our group published suggested thresholds to take into account both methodological advances: fasting $95 \mathrm{mg} / \mathrm{dL}, 1$ hour $180 \mathrm{mg} / \mathrm{dL}, 2$ hours $155 \mathrm{mg} / \mathrm{dL}$, and 3 hours $140 \mathrm{mg} / \mathrm{dL}$. One study (37), in which the original methodology of
O'Sullivan and Mahan was compared directly with the current technology, found that the NDDG criteria were above the 95\% confidence limits of the O'Sullivan criteria at three of the four glucose tolerance testing intervals, whereas the criteria of Carpenter and Coustan were within such confidence intervals at all times. Subsequently, the ADA (38) recommended the use of these lower thresholds, and ACOG (39) recommends using either set of thresholds.

It is important to point out that the relationship between glucose intolerance and perinatal morbidity and mortality is, in all likelihood, continuous. Magee and colleagues (40) found a similar level of perinatal morbidity in pregnancies in which GDM diagnosed by the Carpenter and Coustan conversion of O'Sullivan and Mahan's criteria, but not the NDDG conversion criteria, were met; both groups manifested significantly more morbidity than did the general population. Naylor and associates (41) compared patients known to their caregivers to have GDM (NDDG criteria), patients whose caregivers were blinded to the diagnosis of GDM (Carpenter and Coustan criteria), and normoglycemic controls. Both groups of patients with GDM had a greater likelihood of delivering by cesarean section than did normal controls. However, in the patients with undiagnosed, milder GDM, cesarean section was related to an increased likelihood of macrosomia. In the patients with known GDM, the treatment apparently lowered the macrosomia rate to that of the normal controls, but high cesarean section rates persisted, possibly because of the caregivers' expectations of macrosomia (42).

The 3-hour, 100-g OGTT was considered too time consuming and expensive to be universally applied. Therefore, a screening procedure was deemed necessary in order to choose which patients should be tested. It was at one time traditional to use the taking of a history as the screening test. Patients who reported the presence of certain risk factors (e.g., family history of diabetes, previous macrosomic baby or stillbirth, etc.) would undergo glucose tolerance testing. Unfortunately, this method of screening is too insensitive for clinical use, identifying only approximately $50 \%$ of women with GDM (43). For this reason, both the ADA (38) and the ACOG (39) have recommended the use of a 50-g, 1-hour glucose challenge, administered without respect to the time of the last meal, as a screening test for GDM. The test is administered at 24 to 28 weeks, and a plasma or serum glucose level at or above 130 or $140 \mathrm{mg} / \mathrm{dL}$ is considered by these two organizations to be an indication for the full 3 -hour, $100-\mathrm{g}$ OGTT. In our center, we use a threshold of $130 \mathrm{mg} / \mathrm{dL}$, since we have determined that $10 \%$ of women with GDM manifest screening test values of 130 to $139 \mathrm{mg} / \mathrm{dL}$ (43). Currently, the ACOG (39) acknowledges that the benefits to a population of screening for GDM have not been proven, but suggests that if screening is to be performed, it be done universally. Exceptions would be populations in which the prevalence is unusually high or low. In high-prevalence populations, such as the Pima Indians, it may make the most sense to omit the screening test and go right to the full 3-hour OGTT, since the likelihood of having GDM by virtue of being a Pima is higher than that imparted by a positive screening test. In low-prevalence populations, such as attendees to an adolescent pregnancy clinic, screening based on risk factors may be appropriate. The ADA made similar recommendations (44). 
The original O'Sullivan criteria for GDM, as well as the NDDG and Carpenter and Coustan adaptations of those criteria to more modern methodology, were validated by their predictive value for subsequent diabetes in the mother. It would be much preferable to use criteria that are based upon pregnancy outcomes. Furthermore, the criteria described above are used in the United States, whereas other sets of criteria and other glucose challenges, such as 50 and $75 \mathrm{~g}$, are in use at various places around the world. How much better it would be if there were diagnostic criteria accepted worldwide. Recently, the Hyperglycemia and Adverse Pregnancy Outcomes study was carried out in an attempt to define the relationship between hyperglycemia less severe than diabetes and adverse pregnancy outcomes (45). Over 23,000 pregnant women in 15 centers in nine countries participated in a blinded 75-g, 2-hour OGTT. Each of the three OGTT values (fasting, 1 and 2 hours) was linearly and significantly related to the four primary outcomes studied (birth weight above the 90th centile, primary cesarean section, cord blood C-peptide above the 90th centile, and clinical neonatal hypoglycemia) as well as a number of secondary outcomes such as preterm delivery and pre-eclampsia. Based upon these results, the International Association of Diabetes and Pregnancy Study Group (IADPSG) convened a consensus panel to make recommendations for the diagnosis and classification of hyperglycemia in pregnancy (46). That group recommended that a 75-g, 2-hour OGTT be used and that GDM be diagnosed if any one or more of the following thresholds are met or exceeded: fasting plasma glucose $92 \mathrm{mg} / \mathrm{dL}$, 1-hour plasma glucose $180 \mathrm{mg} / \mathrm{dL}$, and 2-hour plasma glucose $153 \mathrm{mg} / \mathrm{dL}$. If these recommendations are adopted by professional organizations in various countries, the goal of universal diagnostic thresholds based upon pregnancy outcomes will have been realized. The IADPSG consensus panel also recommended that if women are considered to be at high risk for preexisting diabetes, they may be tested at the first prenatal visit with either a fasting plasma glucose (values at or above $126 \mathrm{mg} / \mathrm{dL}$ indicate overt diabetes), hemoglobin $A_{1 c}$ (values of $6.5 \%$ or more indicate overt diabetes), or random plasma glucose (values of $200 \mathrm{mg} / \mathrm{dL}$ or more indicate overt diabetes if confirmed by fasting plasma glucose or $\mathrm{Hb}_{\mathrm{Ac}}$ ). The American Diabetes Association endorsed these recommendations in January 2011 (92).

\section{MANAGEMENT}

The management of diabetes in pregnancy brings together advances in internal medicine, obstetrics, pediatrics, physiology, and metabolism. Although there are marked similarities in the management of pregnancies complicated by preexisting diabetes and those complicated by GDM, the differences in the degree of risk and maternal glycemic stability make it most convenient to consider each separately.

\section{Preexisting Diabetes-Medical Management Preconception Care}

As discussed above, diabetic pregnancy presents a rare opportunity to prevent congenital anomalies. Preconception care should begin at the time diabetes mellitus is diagnosed in any female prior to or during the age of reproduction.
Pediatricians, internists, family doctors, and others caring for such patients should educate young diabetic women about the need for planning prior to pregnancy, and the increased risk of congenital anomalies when a pregnancy begins prior to the institution of reasonable metabolic control. Various approaches to contraception should be discussed (47) in order to minimize the likelihood of inadvertent pregnancy.

Once the individual with diabetes has decided to attempt pregnancy, a thorough evaluation of her medical status should be undertaken $(48,49)$. This should include renal function tests, cardiac assessment, hemogram, immunity testing for hepatitis and rubella, blood type, $\mathrm{Rh}$ and antibody testing, and HIV testing. A thorough physical examination is appropriate, as is an expert ophthalmologic examination in patients whose diabetes is not of recent onset. Once risk factors have been assessed, the patient should be counseled as to their significance. The patient's list of medications should be reviewed, since drugs such as statins, angiotensin converting-enzyme inhibitors, and angiotensin-II receptor blockers are contraindicated in pregnancy. If pregnancy is to be initiated, intensive management aimed at near-normalization of circulating glucose levels should be instituted. There is not widespread agreement as to the most appropriate goals for glycemic control during the preconception period and during the time of organogenesis in early pregnancy, but the ADA recommends that hemoglobin $\mathrm{A}_{1 \mathrm{c}}$ be reduced to $1 \%$ or less above the normal range prior to conception, in order to reduce the risk of congenital malformations (49). In our center, we attempt to maintain fasting and 2-hour postprandial glucose levels, measured at least four times a day, at under $150 \mathrm{mg} / \mathrm{dL}$ during this time period. Although we strive for tighter glucose control later in pregnancy, the periconceptional period is of uncertain duration and hypoglycemic reactions appear to be more likely than that in the second half of pregnancy. Glycosylated hemoglobin levels are followed at 1- to 2 -month intervals. Once they fall within $1 \%$ above the normal range for the method being employed, and glucose control appears to be stable, we inform the patient that it is reasonable to discontinue contraception and attempt pregnancy. Preconception care has been shown to be cost effective (50), and an outline of its contents has been published by the ADA $(49,51)$.

\section{Care During Early Pregnancy}

Unfortunately, the majority of diabetic women do not present for care until after conception. Furthermore, most of those presenting when already pregnant are not in optimum metabolic control. Because organogenesis has been virtually completed by the 8 th to 10th week after the last menstrual period (15), patients who register prior to this time may benefit from attempts to normalize glycemia as rapidly as possible. Often, this means a brief period of hospitalization. Patients who commence care after organogenesis has been completed will also benefit from near-normalization of circulating glucose levels, but the degree of urgency is less and outpatient management can usually accomplish the needed improvement in glycemia without unnecessarily interrupting the patient's life style.

If the patient has not had the benefit of preconception care, the baseline evaluation described earlier should be carried out at the time of the first visit in pregnancy. Patients with preexisting diabetes will benefit from a team approach to the 
management of diabetic pregnancy, including physicians with special expertise in diabetes management and high-risk pregnancy management, as well as dieticians, nurse educators, social workers, and subspecialists as needed. Frequent contact with the health-care team is essential in order to respond to problems as they arise.

\section{Blood Glucose Monitoring}

Glycemic control can best be assessed by daily self blood glucose monitoring, using glucose oxidase-impregnated test strips and reflectance meters. There are a number of different approaches, including measuring fasting and preprandial glucose prior to each meal, 1-hour postprandial glucose measurements, and 2-hour postprandial measurements. In one study of gravidas with type 1 diabetes, postprandial glucose values were more predictive of fetal macrosomia than preprandial values (52). A randomized trial of preprandial versus postprandial glucose measurements in insulin-requiring gestational diabetic women demonstrated that preprandial measurements were more effective in preventing adverse outcomes (53). In our center, we advise our patients to measure glucose in the morning prior to breakfast and 2 hours after each meal, at a minimum. Depending upon the clinical circumstances, glucose may be measured at other times, including the middle of the night if the Somogyi effect or the "dawn phenomenon" is suspected (54).

Goals for metabolic control utilized in our center include the maintenance of fasting values below $95 \mathrm{mg} / \mathrm{dL}$, 1-hour values below 130-140 $\mathrm{mg} / \mathrm{dL}$, and 2-hour postprandial values below $120 \mathrm{mg} / \mathrm{dL}$, with glucose levels under $60 \mathrm{mg} / \mathrm{dL}$ being viewed as unnecessarily low. All pregnant patients with preexisting diabetes, and their significant support persons, should be familiar with the appropriate responses to hypoglycemia, including the use of glucagon injections. While hypoglycemia has not yet been demonstrated to be clearly harmful to the fetus, it carries risk enough for the mother that its frequency and severity should be minimized to the extent possible, compatible with the achievement of good metabolic control during pregnancy.

\section{Insulin Management}

A number of different approaches to insulin administration have been used successfully (55) during pregnancy. Rarely, if ever, does a single daily injection of intermediate-acting insulin suffice to establish and maintain near-normoglycemia. In our center, we tend to start with simpler regimens and increase the complexity if the need arises. Based upon the insulin secretion patterns of normal pregnant women in the third trimester (56), we begin with twice-daily injections of mixed intermediate- and short-acting insulins. The morning total dose, administered before breakfast, is twice the evening total dose, administered before dinner. The proportion of intermediate- to short-acting insulins is 2:1 in the morning and $1: 1$ in the evening (57). Once a patient has been started on this "split, mixed" regimen, the various doses can be adjusted to improve the appropriate glucose measurement. Many patients require the evening dose to be divided so that the intermediate insulin is given at bedtime to avoid morning hyperglycemia. Another approach is the use of short-acting insulin before each meal, with long-acting insulin given daily at bedtime, or in the morning, or at both times. In some centers, the subcutaneous insulin infusion pump is used by pregnant women with diabetes, although a randomized trial has demonstrated no distinct advantage for this approach compared with intensive conventional therapy (58). The specific regimen is probably less important than the degree of familiarity, understanding, and comfort of both the caregiver and the patient.

A number of insulin analogs have come on the market. Insulin lispro and insulin aspart are short-acting insulins, with a more rapid onset of action than that of regular insulin. Each differs from natural human insulin by a single amino acid. They can be given immediately before a meal, which increases flexibility of lifestyle. Insulin lispro does not cross the placenta to any great extent $(59)$, and insulin aspart appears to have similar properties $(60,61)$. Insulin glargine and insulin detemir are long-acting insulins with at least 24 hours of duration of action. Their absorption curve appears to be flat with no peak, and so their use can mimic basal insulin production by the pancreas or that of a continuous subcutaneous insulin infusion pump. In isolated placental perfusion models, insulin glargine at therapeutic concentrations on the maternal side did not reach detectable levels in the fetus, and even at very high levels, the rate of transfer was low (62). No published studies regarding transplacental passage of insulin detemir were found. If long-acting insulin analogs are used in pregnancy, insulin glargine is preferred to insulin detemir at the present time.

\section{Dietary Management}

Approaches to dietary management of diabetic pregnancy have varied greatly over the years (63), and there is no single generally accepted dietary prescription. Research is ongoing as to the advisability of caloric restriction in obese women with type 2 diabetes in pregnancy, but there is concern about the possible adverse fetal effects of ketonemia induced by relative starvation (64). The ADA has published a set of recommendations for dietary management of diabetes during pregnancy that emphasize individualization of the prescription for medical nutrition therapy (65). The most important aspect of the diet is the consistency with which it is followed. Dieticians should take into account the ethnic and cultural food preferences of their patients.

\section{Vascular Complications}

Women with diabetes are at risk for a number of vascular complications, including nephropathy, retinopathy, neuropathy, coronary heart disease, and macrovascular disease. A detailed discussion of these specific problems is beyond the scope of the present chapter, but may be found elsewhere (66-68). It is not clear whether pregnancy has an independent effect on vascular complications of diabetes. Although one prospective study has demonstrated an independent effect of pregnancy on worsening retinopathy (69), it is possible that the adverse effect was due not to pregnancy itself, but to the rapid institution of tight metabolic control coincident with pregnancy. It is important that these complications be diagnosed and managed promptly and effectively even in the nonpregnant individual and appropriate care becomes even more important when pregnancy coexists. For example, 
patients with diabetic nephropathy generally manifest increasing hypertension and almost invariably develop increasing proteinuria as pregnancy progresses (70). It may be exceedingly difficult to distinguish nephropathy from preeclampsia. In our center, the worsening of hypertension, or its new appearance, are considered grave complications in diabetic pregnancy and necessitate hospitalization and intensive monitoring of mother and fetus. While proliferative retinopathy does not pose a particular threat to the fetus, it poses a serious risk of maternal blindness. Laser photocoagulation is safe to perform during pregnancy and should be applied using the usual ophthalmologic indications.

\section{Preexisting Diabetes-Obstetric Management Obstetric and Neonatal Complications}

In addition to perinatal mortality as described earlier, a number of obstetric complications occur with greater frequency in diabetic than nondiabetic pregnancies $(14,71)$. Among these are hypertensive disorders, urinary tract infections, hydramnios, and preterm delivery. Particular care should be exercised in treating diabetic women with betamimetic agonist tocolysis and/or corticosteroids because of the possibility of severe hyperglycemia and ketoacidosis developing (72). Cesarean section is more common in diabetic pregnancies, and respiratory distress syndrome occurs with greater frequency at any given gestational age, possibly related to poor metabolic control. Neonatal hypoglycemia, plethora, jaundice, and hypocalcemia are also more common than in the offspring of nondiabetic mothers.

\section{Fetal Surveillance}

Because of the increased perinatal mortality and morbidity risks described above, a number of different modalities of fetal surveillance are brought to bear on diabetic pregnancy. The increased risk for congenital malformations, particularly in cases registering for care after pregnancy has been established and among those whose metabolic control is suboptimal, merits screening for birth defects. Maternal serum multiple marker testing is generally recommended in diabetic pregnancy. The laboratory performing the alphafetoprotein and other screening tests should be informed of the patient's diabetic status, as adjustments in interpretation are sometimes necessary. Furthermore, directed ultrasound examination is appropriate, particularly when metabolic control during organogenesis has been poor or undocumented, even in the presence of normal alpha-fetoprotein levels. Many of the major malformations can be diagnosed (73), and families can be offered options for the management of affected pregnancies.

Serial ultrasound examinations for fetal growth and amniotic fluid volume may be helpful during the third trimester. Antepartum testing is usually performed, but there is no agreement as to which test is ideal, what gestational age is appropriate for the commencement of testing, and with what frequency testing should be carried out (74). In our center, we use the modified biophysical profile (nonstress test plus amniotic fluid index) as our primary approach because of its simplicity of application. In patients with clear high risk for fetal death, such as those with vascular disease and known growth restriction and oligohydramnios, we may commence testing as early as 24 to 26 weeks, although the danger of a false-positive test is magnified in such early gestations in which delivery has a high inherent perinatal mortality and morbidity risk. Patients with new or worsening hypertensive disorders likewise may be started on early testing. On the other hand, patients with excellent metabolic control and no other complications may delay the onset of testing until as late as 34 to 35 weeks, particularly if daily fetal movement determinations are made. We generally perform surveillance twice weekly or even more often, depending upon the circumstances.

\section{Timing of Delivery}

Although diabetic pregnancies formerly were routinely delivered as early as 4 to 6 weeks prior to term, owing to concern about the possibility of unanticipated fetal death, current surveillance practices have improved the identification of fetuses in jeopardy. In addition, improvements in metabolic control appear to be effective in preventing fetal death (75). Therefore, automatic early delivery is no longer necessary. However, it may be appropriate to deliver those patients whose metabolic control is poor or undocumented prior to term. In most cases, we await the spontaneous onset of labor. If the cervix is "ripe," consideration may be given to inducing labor at 39 weeks or beyond in order to diminish the inconvenience and expense of continued testing, as well as the anxiety inherent in such high-risk pregnancies. This timing is based upon the increasing evidence that delivery prior to 39 weeks of gestation is associated with greater neonatal morbidity (76). In our center, we attempt to document pulmonary maturity by amniocentesis on those rare occasions when totally elective delivery by induction of labor or cesarean section is planned prior to 39 weeks, provided that the amniotic fluid is abundant and accessible (77). We have allowed diabetic pregnancies to proceed past 40 weeks, but only rarely.

\section{Mode of Delivery}

Maternal diabetes is not an indication for cesarean section, but its complications may be (78). Complications such as hypertensive disorders may require early delivery, increasing the likelihood of unsuccessful labor. Fetal macrosomia may also make cesarean section more likely. Problems such as shoulder dystocia appear to occur more frequently in diabetic pregnancies than in others. Although there is no general agreement on the subject, we tend to deliver by primary cesarean section without a trial of labor in cases of diabetic pregnancy in which the estimated fetal weight is $4500 \mathrm{~g}$ or more (78). If the estimate is between 4000 and $4499 \mathrm{~g}$, we individualize the decision based upon past obstetric history, clinical pelvimetry, and events during labor. Unfortunately, our ability to estimate fetal weight by ultrasound is less than ideal, rendering any rigid protocol problematic.

\section{Management of GDM}

The maternal and fetal implications of GDM differ from those of preexisting diabetes only by degree. There has been controversy as to whether GDM should be treated at all. As recently as 2008, the U.S. Preventive Services Task Force stated that the evidence is insufficient to recommend for or 
against the screening for GDM (79). However, a randomized trial of screening for and treatment of GDM has now demonstrated a benefit in terms of decreased perinatal mortality and morbidity (80). Furthermore, a randomized double-blind trial of intervention for mild GDM versus standard obstetric care also demonstrated a reduction in morbidity with identification and treatment (81). Glucose control is generally accomplished more easily in GDM, and many patients can be managed with dietary intervention alone. The recommendations of ACOG (39) are that fasting glucose values be maintained below $95 \mathrm{mg} / \mathrm{dL}$ and 2-hour postprandial values be below $120 \mathrm{mg} / \mathrm{dL}$. Most women with GDM perform self glucose monitoring. In our center, each woman with GDM tests her blood glucose fasting and 2 hours after breakfast, lunch, and dinner. If the fasting values exceed $95 \mathrm{mg} / \mathrm{dL}$ or the postprandial value exceeds $120 \mathrm{mg} / \mathrm{dL}$ during the third trimester, insulin therapy is begun in a dose of 20 units NPH mixed with 10 units short acting insulin each morning prior to breakfast. A number of studies $(52,82)$, including one randomized trial $(53)$, have demonstrated that postprandial glucose measurements are better predictors of macrosomia and perinatal morbidity than are fasting or preprandial values.

Oral antidiabetic agents are gaining favor in the treatment of GDM. While first-generation sulfonylureas were originally considered to be contraindicated because of potential fetal effects, initial placental perfusion studies of glyburide, a second-generation sulfonylurea, demonstrated that very little reached the fetal circulation (83). Subsequently, a randomized trial of glyburide, in doses up to $20 \mathrm{mg} /$ day, compared with insulin, in patients with GDM who required treatment, demonstrated similar outcomes in both groups (84). Following publication of this report, glyburide was increasingly utilized in patients with GDM. In June 2009, a report from the National Institute of Child Health \& Human Development Obstetric-Fetal Pharmacology Research Units Network demonstrated that fetal levels of glyburide average $70 \%$ of maternal levels (85). Although no harmful effects of glyburide on the fetus have been demonstrated to date, little is known about the possible long-term effects of fetal exposure to a drug that increases pancreatic insulin secretion. Metformin is a biguanide insulin sensitizer, which was demonstrated to be similarly effective to insulin in a randomized trial among gestational diabetic women needing treatment (86). However, close to $50 \%$ of patients randomized to metformin required supplemental insulin to control their blood glucose levels. Of greater concern, metformin has been shown to cross the placenta to a significant degree (87). For these reasons, insulin remains the first-line drug for treating GDM when medical nutrition therapy is not effective enough. If oral agents are prescribed to pregnant women, there should be thorough counseling and documentation of the unknown risk of these drugs for the fetus.

Although there are data to suggest that women with uncomplicated diet treated GDM do not require fetal surveillance other than fetal movement determinations until term (88), in our center, we institute weekly nonstress testing at 36 weeks in order to avoid inadvertently missing gestational diabetic women with risk factors being discovered retrospectively. We prefer to document fetal pulmonary maturity prior to elective delivery at less than 39 weeks, as in preexisting diabetes.
It should be remembered that fetal macrosomia can complicate gestational diabetic pregnancy despite apparently near-normal blood glucose levels (27). A number of possible explanations exist, primarily centering around the likelihood that our definitions of "near-normoglycemia" are not really normal, but rather approximately two standard deviations above the mean. Various approaches to preventing macrosomia have been developed (89). One randomized trial (90) demonstrated that ultrasonographic demonstration of a large fetal abdominal circumference at 29 to 33 weeks could select fetuses that would benefit from maternal insulin therapy, decreasing the likelihood of neonatal macrosomia, even though the maternal fasting serum glucose level was below $105 \mathrm{mg} / \mathrm{dL}$. A randomized trial (91) of induction versus expectant management at 38 weeks of gestation in women with insulin-treated GDM demonstrated similar cesarean section rates, but fewer large-for-date infants with induction of labor.

As mentioned early in this chapter, GDM is clearly a risk factor for the subsequent development of diabetes. For this reason, it is important that each patient with GDM be counseled to undergo a 75-g, 2-hour glucose tolerance test for the diagnosis of diabetes in the nonpregnant state at around the time of the 6-week postpartum checkup and ideally annually thereafter. Such women should attempt to avoid obesity as they mature. There is no reason to counsel women with GDM not to have further pregnancies, although they should be informed of the approximately 50\% risk of recurrence.

\section{REFERENCES}

1. Buchanan TA. Pregnancy in preexisting diabetes. In: Harris ML, et al., eds. Diabetes in America, 2nd edn. NIH Publication 95-1468. Washington, DC: National Diabetes Data Group, 1995: 719-33.

2. Ferrara A. Increasing prevalence of gestational diabetes mellitus. Diabetes Care 2007; 30(Suppl 2): S141-6.

3. Metzger BE, Cho NH, Brickman WJ. The rising tide of diabetes mellitus: implications for women of all ages. In: Reece EA, Coustan DR, Gabbe SG, eds. Diabetes Mellitus in Women. Chapter 2. 3rd edn. Philadelphia: Lippincott Williams and Wilkins, 2004: 9-26.

4. Metzger BE. Third International Workshop Conference on Gestational Diabetes Mellitus: summary and recommendations. Diabetes 1991; 40 (Suppl 2): 197-201.

5. O'Sullivan JB. Subsequent morbidity among gestational diabetic women. In: Sutherland HW, Stowers JM, eds. Carbohydrate Metabolism in Pregnancy and the Newborn. New York: Churchill Livingstone, 1984: 174-80.

6. Damm P, Hornnes P, Kühl C, Mølsted-Pedersen L. A longitudinal study of plasma insulin and glucagon in women with previous gestational diabetes. Diabetes Care 1995; 18:654-65.

7. Catalano P, Buchanan TA. Metabolic changes during normal and diabetic pregnancies. In: Reece EA, Coustan DR, Gabbe SG, eds. Diabetes Mellitus in Women. Chapter 10. 3rd edn. Philadelphia: Lippincott Williams and Wilkins, 2004: 129-46.

8. Reece EA, Homko CJ. Glucose evaluation and control. In: Reece EA, Coustan DR, Gabbe SG, eds. Diabetes Mellitus in Women. Chapter 18. 3rd edn. Philadelphia: Lippincott Williams and Wilkins, 2004: 243-56.

9. Rudolf MCJ, Coustan DR, Sherwin RS, et al. Efficacy of the insulin pump in the home treatment of pregnant diabetics. Diabetes 1981; 30: 891-5.

10. Herman WH, Kitzmiller JL. Metabolic disturbances. In: Kitzmiller JL, Jovanovic L, Brown F, Coustan D, Reader DM, eds. Part 2. Management of diabetic/medical complications in pregnancy in Managing Preexisting Diabetes and Pregnancy: Technical Reviews and Consensus Recommendations for Care. Alexandria, Virginia: American Diabetes Association, 2008: 270-5. 
11. Pedersen J. Pathogenesis of the characteristic features of newborn infants of diabetic women. In The Pregnant Diabetic and Her Newborn. Baltimore: Williams and Wilkins, 1967: 128-37.

12. Freinkel N. Banting lecture 1980: of pregnancy and progeny. Diabetes 1980; 29: 1023-35.

13. Karlson K, Kjellmer I. The outcome of diabetic pregnancies in relation to the mother's blood sugar level. Am J Obstet Gynecol 1972; 112: 213-20.

14. Coustan DR. Hyperglycemia-hyperinsulinemia: effect on the infant of the diabetic mother. In: Jovanovic L, Peterson CM, Fuhrmann K, eds. Diabetes and Pregnancy: Teratology, Toxicity and Treatment. New York: Praeger, 1986: 131-56.

15. Mills JL, Baker L, Goldman AS. Malformations in infants of diabetic mothers occur before the seventh gestational week. Diabetes 1979; 28: 292-3.

16. Reece EA, Pinter E, Homko C, et al. The yolk sac theory: closing the circle on why diabetes-associated malformations occur. J Soc Gynecol Invest 1994; 1: 3-13.

17. Leslie RDG, Pyke DA, John PN, White JM. Haemoglobin $A_{1}$ in diabetic pregnancy. Lancet 1978; 2: 958-9.

18. Miller E, Hare JW, Cloherty JP, et al. Elevated maternal hemoglobin $A_{1 c}$ in early pregnancy and major congenital anomalies in infants of diabetic mothers. N Engl J Med 1981; 304: 1331-4.

19. Greene MF, Hare JW, Cloherty JP, et al. First-trimester hemoglobin $A_{1}$ and risk for major malformation and spontaneous abortion in diabetic pregnancy. Teratology 1989; 39: 225-31.

20. Mills JL, Knopp RH, Simpson JL, et al. Lack of relation of increased malformation rates in infants of diabetic mothers to glycemic control during organogenesis. N Engl J Med 1988; 318: 671-6.

21. Fuhrmann K, Reiher H, Semmler K, Glockner E. The effect of intensified conventional insulin therapy during and before pregnancy on the malformation rate in offspring of diabetic mothers. Exp Clin Endocrinol 1984; 83: 173-7.

22. Kitzmiller JL, Gavin LA, Gin GD, et al. Preconception care of diabetes: glycemic control prevents congenital anomalies. J Am Med Assoc 1991; 265: 731-6.

23. Mills JL, Simpson JL, Driscoll SG, et al. Incidence of spontaneous abortion among normal women and insulin-dependent diabetic women whose pregnancies were identified within 21 days of conception. N Engl J Med 1988; 319: 1617-23.

24. Hanson U, Persson B, Thunell S. Relationship between haemoglobin $A_{1 c}$ in early type I (insulin-dependent) diabetic pregnancy and the occurrence of spontaneous abortion and fetal malformation in Sweden. Diabetologia 1990; 33: 100-4.

25. O’Sullivan JB, Charles D, Mahan CM, Dandrow RV. Gestational diabetes and perinatal mortality rate. Am J Obstet Gynecol 1973; 116: 901-4.

26. Pettitt DJ, Knowler WC, Baird HR, Bennett PH. Gestational diabetes: infant and maternal complications of pregnancy in relation to thirdtrimester glucose tolerance in Pima Indians. Diabetes Care 1980; 3: 458-64.

27. Widness JA, Cowett RM, Coustan DR, et al. Neonatal morbidities in infants of mothers with glucose intolerance in pregnancy. Diabetes 1985; 34(Suppl 2): 61-5.

28. Vohr BR, Lipsitt LP, Oh W. Somatic growth of children of diabetic mothers with reference to birth size. J Pediatr 1980; 97: 196-9.

29. Pettitt DJ, Knowler WC, Bennett PH, et al. Obesity in offspring of diabetic Pima Indian women despite normal birth weight. Diabetes Care 1987; 10: 76-80.

30. Green OC, Winter RJ, Depp R, et al. Fuel-mediated teratogenesis: prospective correlations between anthropometric development in childhood and antepartum maternal metabolism. Clin Res 1987; 35: 657A.

31. Pettitt DJ, Bennett PH, Saad MF, et al. Abnormal glucose tolerance during pregnancy in Pima Indian women: long-term effects on offspring. Diabetes 1991; 40(Suppl 2): 126-30.

32. O'Sullivan JB, Mahan CM. Criteria for the oral glucose tolerance test in pregnancy. Diabetes 1964; 13: 278-85.

33. National Diabetes Data Group. Classification and diagnosis of diabetes mellitus and other categories of glucose intolerance. Diabetes 1979; 28: 1039-57.

34. American Diabetes Association. Position statement on gestational diabetes mellitus. Diabetes Care 1996; 19(Suppl 1): S29.
35. American College of Obstetricians and Gynecologists. Management of Diabetes Mellitus in Pregnancy, Technical Bulletin no. 92. Washington, DC: ACOG, 1986.

36. Carpenter MW, Coustan DR. Criteria for screening tests for gestational diabetes. Am J Obstet Gynecol 1982; 144: 768-73.

37. Sacks DA, Abu-Fadil S, Greenspoon JS, Fotheringham N. Do the current standards for glucose tolerance testing in pregnancy represent a valid conversion of O'Sullivan's original criteria? Am J Obstet Gynecol 1989; 161: 638-41.

38. American Diabetes Association. Diagnosis and classification of diabetes mellitus. Diabetes Care 2009; 329(Suppl 1): S62-7.

39. American College of Obstetricians and Gynecologists: Gestational Diabetes. ACOG Practice Bulletin \#30, 2001 (reaffirmed 2008). Obstet Gynecol 2001; 98: 525-38.

40. Magee MS, Walden CE, Benedetti TJ, Knopp RH. Influence of diagnostic criteria on the incidence of gestational diabetes and perinatal morbidity. J Am Med Assoc 1993; 269: 609-15.

41. Naylor CD, Sermer M, Chen E, Sykora K. Cesarean delivery in relation to birth weight and gestational glucose tolerance: pathophysiology or practice style? J Am Med Assoc 1996; 275: 1165-70.

42. Coustan DR. Management of gestational diabetes: a self-fufilling prophecy? J Am Med Assoc 1996; 275: 1199-200.

43. Coustan DR, Nelson C, Carpenter MW, et al. Maternal age and screening for gestational diabetes: a population-based study. Obstet Gynecol 1989; 73: 557-61.

44. American Diabetes Association. Report of the Expert Committee on Diagnosis and Classification of Diabetes Mellitus. Diabetes Care 1997; 20: 1183-97.

45. The HAPO Study Cooperative Research Group. Hyperglycemia and adverse pregnancy outcomes. N Engl J Med 2008; 358: 1991-2002.

46. International Association of Diabetes and Pregnancy Study Groups Consensus Panel. IADPSG recommendations on the diagnosis and classification of hyperglycemia in pregnancy. Diabetes Care 2010; 33: 676-82.

47. Kjos SL, Buchanan TA. Postpartum management, lactation and contraception. In: Reece EA, Coustan DR, Gabbe SG, eds. Diabetes Mellitus in Women. Chapter 32. 3rd edn. Philadelphia: Lippincott Williams and Wilkins, 2004: 441-50.

48. Miodovnik M, Rosenn B. Preconceptional care of women with diabetes. In: Reece EA, Coustan DR, Gabbe SG, eds. Diabetes Mellitus in Women. Chapter 6. 3rd edn. Philadelphia: Lippincott Williams and Wilkins, 2004: 73-90.

49. American Diabetes Association. Preconception care of women with diabetes (Position Statement). Diabetes Care 2004; 27(Suppl 1): S76-8.

50. Elixhauser A, Wechsler JM, Kitzmiller JL, et al. Cost- benefit analysis of preconception care for women with established diabetes mellitus. Diabetes Care 1993; 16: 1146-57.

51. Kitzmiller JL, Buchanan TA, Kjos S, et al. Technical review of preconception care of diabetes, congenital malformations and spontaneous abortions. Diabetes Care 1996; 19: 514-41.

52. Jovanovic-Peterson L, Peterson CM, Reed GF, et al. Maternal postprandial glucose levels and infant birth weight: the Diabetes in Early Pregnancy study. Am J Obstet Gynecol 1991; 164: 103-11.

53. de Veciana M, Major CA, Morgan MA, et al. Postprandial versus preprandial glucose monitoring in wsen with gestational diabetes requiring insulin therapy. N Engl J Med 1995; 333: 1237-41.

54. Coustan DR. Management of the pregnant diabetic. In: Olefsky JM, Sherwin RS, eds. Diabetes Mellitus: Management and Complications. New York: Churchill Livingstone, 1985: 311-30.

55. Landon MB, Gabbe SG. Insulin treatment of the pregnant patient with diabetes mellitus. In: Reece EA, Coustan DR, Gabbe SG, eds. Diabetes Mellitus in Women. Chapter 19. 3rd edn. Philadelphia: Lippincott Williams and Wilkins, 2004: 257-71.

56. Lewis SB, Wallin JD, Kuzuya H, et al. Circadian variation of serum glucose, C-peptide immunoreactivity and free insulin in normal and insulin-treated diabetic pregnant subjects. Diabetologia 1976; 12: 343-7.

57. Lewis SB, Murray WK, Wallin JD, et al. Improved glucose control in non-hospitalized pregnant diabetic patients. Obstet Gynecol 1976; 48: $260-5$. 
58. Coustan DR, Reece EA, Sherwin RS, et al. A randomized clinical trial of the insulin pump vs intensive conventional therapy in diabetic pregnancies. J Am Med Assoc 1986; 255: 631-6.

59. Jovanovic L, Pettitt D. Treatment with insulin and its analogs in pregnancies complicated by diabetes. Diabetes Care 2007; 30(Suppl 2): S220-4.

60. McCance DR, Damm P, Mathiesen ER, et al. Evaluation of insulin antibodies and placental transfer of insulin aspart in pregnant women with type 1 diabetes mellitus. Diabetologia 2008; 51: 2141-3.

61. Mathiesen ER, Kinsley B, Amiel SA, et al. Maternal glycemic control and hypoglycemia in type 1 diabetic pregnancy: a randomized trial of insulin aspart versus human insulin in 322 pregnant women. Diabetes Care 2007; 30: 771-6.

62. Pollex EK, Feig DS, Lubetsky A, Yip PM, Koren G. Insulin glargine safety in pregnancy: a transplacental transfer study. Diabetes Care 2010; 33: 29-33.

63. Luke B. Dietary management. In: Reece EA, Coustan DR, Gabbe SG, eds. Diabetes in Women: Adolescence, Pregnancy and Menopause. Chapter 20. 3rd edn. Philadelphia: Lippincott Williams and Wilkins, 2004: 273-81.

64. Phelps RL, Metzger BE. Caloric restriction in gestational diabetes mellitus: when and how much? J Am Coll Nutr 1992; 11: 259-62.

65. Reader DM, Thomas AM, Gunderson EP. Medical nutrition therapy goals for pregnancy complicated by preexisting diabetes mellitus. In: Kitzmiller JL, Jovanovic L, Brown F, Coustan D, Reader DM, eds. Managing Preexisting Diabetes in Pregnancy. Alexandria, VA: American Diabetes Association, 2008: 15-89.

66. Jovanovic L. Diabetic retinopathy. In: Reece EA, Coustan DR, Gabbe SG, eds. Diabetes in Women: Adolescence, Pregnancy and Menopause. Chapter 28. 3rd edn. Philadelphia: Lippincott Williams and Wilkins, 2004: 371-82.

67. Kitzmiller JL. Diabetic nephropathy. In: Reece EA, Coustan DR, Gabbe SG, eds. Diabetes in Women: Adolescence, Pregnancy and Menopause. Chapter 29. 3rd edn. Philadelphia: Lippincott Williams and Wilkins, 2004: 383-424.

68. Leguizamón GF, Reece EA. Diabetic neuropathy and coronary heart disease. In: Reece EA, Coustan DR, Gabbe SG, eds. Diabetes in Women: Adolescence, Pregnancy and Menopause. Chapter 30. 3rd edn. Philadelphia: Lippincott Williams and Wilkins, 2004: 425-32.

69. Klein BEK, Moss SE, Klein R. Effect of pregnancy on progression of diabetic retinopathy. Diabetes Care 1990; 13: 34-40.

70. Reece EA, Coustan DR, Hayslett JP, et al. Diabetic nephropathy: pregnancy performance and fetomaternal outcome. Am J Obstet Gynecol 1988; 159: 56-66.

71. Cousins L. Obstetric complications in diabetic pregnancies. In: Reece EA, Coustan DR, Gabbe SG, eds. Diabetes in Women: Adolescence, Pregnancy and Menopause. Chapter 26. 3rd edn. Philadelphia: Lippincott Williams and Wilkins, 2004: 351-62.

72. Montoro MN. Diabetic ketoacidosis in pregnancy. In: Reece EA, Coustan DR, Gabbe SG, eds. Diabetes in Women: Adolescence, Pregnancy and Menopause. Chapter 25. 3rd edn. Philadelphia: Lippincott Williams and Wilkins, 2004: 345-50.

73. Gabrielli S, Pilu G, Reece EA. Prenatal diagnosis and management of congenital malformations in pregnancies complicated by diabetes. In: Reece EA, Coustan DR, Gabbe SG, eds. Diabetes in Women: Adolescence, Pregnancy and Menopause. Chapter 22. 3rd edn. Philadelphia: Lippincott Williams and Wilkins, 2004: 299-319.

74. Rosenn BM, Miodovnik M. Fetal biophysical and biochemical testing. In: Reece EA, Coustan DR, Gabbe SG, eds. Diabetes in Women: Adolescence,
Pregnancy and Menopause. Chapter 24. 3rd edn. Philadelphia: Lippincott Williams and Wilkins, 2004: 335-44.

75. Coustan DR. Perinatal mortality and morbidity. In: Reece EA, Coustan DR, Gabbe SG, eds. Diabetes in Women: Adolescence, Pregnancy and Menopause. Chapter 14. 3rd edn. Philadelphia: Lippincott Williams and Wilkins, 2004: 205-10.

76. Tita AT, Landon MB, Spong CY, et al.; for the Eunice Kennedy Shriver NICHD Maternal-Fetal Medicine Units Network. Timing of elective repeat cesarean delivery at term and neonatal outcomes. N Engl J Med 2009; 360: 111-20.

77. Ojomo EO, Coustan DR. Absence of evidence of pulmonary maturity at amniocentesis in term infants of diabetic mothers. Am J Obstet Gynecol 1990; 163: 954-7.

78. Coustan DR. Delivery: timing, mode and management. In: Reece EA, Coustan DR, Gabbe SG, eds. Diabetes in Women: Adolescence, Pregnancy and Menopause. Chapter 31. 3rd edn. Philadelphia: Lippincott Williams and Wilkins, 2004: 433-40.

79. U.S. Preventive Services Task Force. Screening for gestational diabetes mellitus: USPSTF recommendation statement. Ann Int Med 2008; 148 : 759-65.

80. Crowther CA, Hiller JE, Moss JR, et al. Effect of treatment of gestational diabetes mellitus on pregnancy outcomes. N Engl J Med 2005; 352: 2477-86.

81. Landon MB, Spong CY, Thom E, et al. A multicenter, randomized trial of treatment for mild gestational diabetes. N Engl J Med 2009; 361: 1339-48.

82. Combs CA, Gunderson E, Kitzmiller JL, et al. Relationship of fetal macrosomia to maternal postprandial glucose control during pregnancy. Diabetes Care 1992; 15: 1251-7.

83. Elliott BD, Langer O, Schenker S, Johnson RF. Insignificant transfer of glyburide occurs across the human placenta. Am J Obstet Gynecol 1991; 165: 807-12.

84. Langer O, Conway D, Berkus MD, et al. A Comparison of glyburide and insulin in women with gestational diabetes mellitus. N Engl J Med 2000; 343: 1134-8.

85. Hebert MF, Ma X, Naraharisetti SB, et al. Are we optimizing gestational diabetes treatment with glyburide? Clin Pharmacol Ther 2009; 85: 607-14.

86. Rowan JA, et al.; for the MiG Trial Investigators. Metformin versus insulin for the treatment of gestational diabetes. N Engl J Med 2008; 358: 2003-15.

87. Vanky E, Zahlsen K, Spigset O, Carlsen SM. Placental passage of metformin in women with polycystic ovary syndrome. Fertil Steril 2005; 83: $1575-8$.

88. Landon MB, Gabbe SG. Antepartum fetal surveillance in gestational diabetes mellitus. Diabetes 1985; 34(Suppl 2): 50-4.

89. Coustan DR. Maternal insulin to lower the risk of fetal macrosomia in diabetic pregnancy. Clin Obstet Gynecol 1991; 34: 288-95.

90. Buchanan TA, Kjos SL, Montoro MN, et al. Use of fetal ultrasound to select metabolic therapy for pregnancies complicated by mild gestational diabetes. Diabetes Care 1994; 17: 275-83.

91. Kjos S, Henry OA, Montoro M, et al. Insulin-requiring diabetes in pregnancy: a randomized trial of active induction of labor and expectant management. Am J Obstet Gynecol 1993; 169: 611-15.

92. American Diabetes Association. Standards of medical care in diabetes2011. Diabetes Care 2011; 34(Suppl 1): S15. 


\section{Chronic hypertension and acute hypertensive crisis William F. Rayburn and Lauren Plante}

\section{INTRODUCTION}

Chronic hypertension occurs in up to $5 \%$ of pregnant women $(1,2)$. Rates vary, however, according to the population studied and the criteria used for confirming the diagnosis. Terminology and criteria used to diagnose this complication can be confusing, as well as the benefit and potential harm of treatment during pregnancy. Chronic hypertension may result in maternal, fetal, or neonatal morbidity or mortality. These complications have significant economic impact, including costs of treating sick mothers and neonates and costs of intensive antenatal monitoring. The purposes of this chapter are to review the effects of chronic hypertension on pregnancy, clarify the terminology and criteria used to define and diagnose it during pregnancy, and review evidence about treatment options.

\section{HYPERTENSION IN REPRODUCTIVE-AGE WOMEN}

Young hypertensive women who are not pregnant are generally active individuals with increased sympathetic tone and elevated plasma renin activity. Extracellular fluid volumes are usually normal or decreased. More than $80 \%$ of hypertensive adults have primary or essential hypertension of unknown etiology, that is, without an identifiable cause (3). Most have mild hypertension, defined as a diastolic blood pressure of 90 to $104 \mathrm{mmHg}$.

Arguments have been made both for and against treating mild elevations of diastolic pressure in young adults. Antihypertensive therapy reduces morbidity and mortality from stroke, coronary artery disease, heart failure, and renal failure for all degrees of hypertension, but the benefit is modest for mild hypertension and is concentrated primarily on those with hypercholesterolemia, glucose intolerance, left ventricular hypertrophy, and cigarette smoking (4).

In the United States, nonpregnant patients with systolic pressure above $140 \mathrm{mmHg}$ or diastolic pressures above $90 \mathrm{mmHg}$ who have not responded to lifestyle modifications are considered to be the candidates for drug intervention. Current European recommendations for drug therapy suggest that both blood pressure and total cardiovascular risk be factored into the decision to begin pharmacotherapy (5). These specific cardiovascular risk factors include abdominal obesity, smoking, dyslipidemia, diabetes, or family history of premature cardiovascular disease.

In the past, a traditional "step-care" approach to pharmacologic treatment of hypertension involved the patient being first given a single commonly used drug such as a beta blocker, calcium channel antagonist, angiotensin-converting enzyme (ACE) inhibitor, or diuretic. The blood pressure response usually is consistent within a given class of agents; therefore, if a drug fails to control blood pressure, another agent from the same class is unlikely to be effective. At times, however, a change within drug class may be useful in reducing adverse effects. The lowest possible effective dosage should be used to control blood pressure, adjusted every 1 to 3 months as needed. Many patients with stage 1 (mild) hypertension can attain adequate blood pressure control with single-drug therapy (3). The Antihypertensive and Lipid-Lowering Treatment to Prevent Heart Attack (ALLHAT) trial suggested that a diuretic was the best initial choice of agent for nonpregnant adults, being equally efficacious and less expensive than the alternatives (6).

When a second drug is needed, it can generally be chosen from among the other first-line agents. A diuretic should be added first, as doing so may enhance effectiveness of the first drug, yielding more than a simple additive effect (7). In considering a modification of therapy because of inadequate response to the current regimen, the physician should investigate other possible contributing factors. Poor patient compliance, use of antagonistic drugs (i.e., sympathomimetics, antidepressants, steroids, nonsteroidal anti-inflammatory drugs, caffeine, thyroid hormones, and cocaine), inappropriately high sodium intake, or increased alcohol consumption should be considered before antihypertensive drug therapy is modified (3).

\section{Preconception Counseling and Early Prenatal Care}

Generally, isolated elevated blood pressure or, less commonly, headaches are the only signs or symptoms in women with chronic hypertension. The history should seek to discover secondary causes of hypertension and note the presence of medications that can affect blood pressure (see above.)

Ideally, hypertensive women considering pregnancy should be counseled before conception. They should be informed of the potential for superimposed pre-eclampsia and worsening of renal disease or systemic illness. Valuable baseline data are provided from laboratory tests performed when hypertensive patients are first seen before conception or in early gestation. Tests to help identify target-organ damage and provide a baseline for assessing adverse effects of therapy would include the following: urinalysis, hematocrit, plasma glucose, serum potassium, serum creatinine, calcium uric acid, fasting serum cholesterol and triglyceride level, and electrocardiogram. Baseline determination of renal function (24-hour proteinuria and creatinine clearance), uric acid, transaminase levels, and platelet count are useful for comparison with values in later pregnancy to help determine if increases in blood pressure during pregnancy represent usual physiologic increases or the onset of pre-eclampsia.

Because of the risk of fetal growth restriction, an early sonogram for accurate dating is encouraged. Depending on the circumstance, other various specialized clinical tests at the initial visit may include echocardiography (for left ventricular 
hypertrophy), ophthalmologic examination, and renal ultrasonography. Sleep apnea is increasingly recognized as a treatable cause of hypertension, and a screening questionnaire or sleep study may be appropriate.

Normotensive patients with intrinsic renal disease but minimal renal dysfunction usually do well in pregnancy (8). Women with significant renal disease (serum creatinine $>1.4 \mathrm{mg} / \mathrm{dL}$ ) may experience deterioration of renal function. Those with a serum creatinine $>2.0 \mathrm{mg} / \mathrm{dL}$ and hypertension should be advised of a high incidence of superimposed preeclampsia, perinatal morbidity and mortality, and deteriorating renal function $(8,9)$.

Many women with chronic hypertension are under the care of a primary care physician and already have been evaluated for causes of secondary hypertension, such as primary aldosteronism, pheochromocytoma, or Cushing's disease (3). Women with paroxysmal hypertension, frequent "hypertensive crisis," seizure disorders, or anxiety attacks should be evaluated for pheochromocytoma with measurements of 24-hour urine vanillylmandelic acid, metanephrines, or unconjugated catecholamines (10). Primary aldosteronism is rare in pregnancy, but may present with hypokalemia. Imaging studies (magnetic resonance imaging or computed tomography) may be helpful in demonstrating or localizing an adrenal tumor. Doppler flow studies or magnetic resonance angiography can reveal renal artery stenosis (3).

\section{Nonpharmacologic Therapy}

Borderline or mildly elevated blood pressures can often be controlled without medication. Diastolic blood pressures from 90 to $99 \mathrm{mmHg}$ are not difficult to attain, because pressures fall during the first and second trimesters in most pregnant women (1). Lifestyle modifications should be encouraged in all hypertensive patients regardless of whether they require medication. These changes may have beneficial effects on other cardiovascular risk factors. Some standard methods of treating hypertension must be changed in pregnancy. For example, weight reduction is not encouraged during gestation, although it is advisable before pregnancy for those who are obese. Saturated fats, alcohol, and smoking should be avoided. Data are insufficient to routinely recommend additional dietary calcium supplementation for the treatment of pregnant women with chronic hypertension.

The severity of hypertension correlates with the extent of plasma volume contraction and that pregnant women with chronic hypertension have lower plasma volumes than normotensive women (11). Thus, sodium is generally not restricted during pregnancy, except for the rare pregnant woman with chronic hypertension who is salt sensitive or whose creatinine clearance is reduced.

Although exercise has not been sufficiently studied in hypertensive pregnant women, theoretic concerns exist about the role of uteroplacental blood flow in the pathogenesis of pre-eclampsia. Strict bed rest is seldom necessary, but despite a lack of formal research on the subject, rest is often prescribed to lower blood pressure and promote diuresis (1). Adjustments may be necessary in child care and employment.

We encourage patients to take their blood pressures outside the physician's office and keep a record of readings (12). Selfmonitoring of blood pressure is often helpful in determining whether borderline or mildly elevated blood pressures remain elevated at home or in the work setting and whether limitation of activity is necessary to better control the blood pressures. Adjustments of drug therapy or withdrawal are more plausible with the knowledge of frequent blood pressure recordings. The patient should have formal instruction on the correct technique for applying the appropriately sized cuff, measuring the blood pressures while sitting. The patient is also to be instructed to notify the office if the blood pressure rises significantly before the next scheduled visit.

Two readings should be taken, separated by 2 minutes. Systolic blood pressure should be noted with the appearance of Korotkoff sounds (phase I) and diastolic blood pressure with the disappearance of sounds (phase V). In certain patients, the Korotkoff sounds do not disappear but are present to $0 \mathrm{mmHg}$. In this case, the initial muffling of Korotkoff sounds (phase IV) should be taken as the diastolic blood pressure. In order to reduce inaccurate readings, an appropriately sized cuff should be used (length 1.5 times upper arm circumference or a cuff with a bladder that encircles $80 \%$ or more of the arm). The appropriate position in which to measure blood pressure is sitting, with the arm at the level of the heart. Measuring BP in the "up" arm with the patient side lying gives an artifactually lower reading, which does not represent a true decrease in BP.

\section{Mild Chronic Hypertension}

The goal of treating a pregnant woman with chronic hypertension is to reduce cardiovascular risks to the mother while avoiding compromise of fetal well-being (13). Since most pregnant women with chronic hypertension have only borderline or mild elevations in blood pressure, the risk of acute cardiovascular complication is extremely low.

Pharmacotherapy of mild hypertension at diastolic levels of 90 to $99 \mathrm{mmHg}$ must be undertaken cautiously because of the possibilities that medication may reduce placental blood flow or cross the placenta to directly affect the fetus $(1,13)$. Although the literature is conflicting, many obstetricians do not prescribe drugs for chronic hypertension in pregnancy unless the mother's blood pressure remains above 150/ $100 \mathrm{mmHg}$ or her hypertension was well controlled by medication before the pregnancy (13). Clinical trials and experience have not shown whether long-term treatment of chronic hypertension prevents pre-eclampsia.

Pharmacologic management of chronic hypertension during pregnancy is too small to either prove or disprove moderate to large benefits ( $>20 \%$ improvements). A paucity of scientific data exists regarding the most appropriate management of women with well-controlled or mild hypertension already taking antihypertensive medications at the time of pregnancy. Although therapy may offer long-term benefits to the mother, such therapy is of unproven short-term benefit and could interfere with uteroplacental blood flow and fetal growth. In a meta-analysis of 623 women with mild chronic hypertension from seven trials comparing antihypertensives with no therapy, drug treatment was associated with a decrease in the incidence of severe hypertension but no improvement in perinatal outcomes (14).

A systematic review of chronic hypertension management during pregnancy revealed that this condition alone doubles 
the risk for placental abruption (OR, 2.1; 95\% CI, 1.1, 3.9) and triples the risk for perinatal mortality (OR, 3.4; 95\% CI, 3.0, 3.7) (13). Several studies in this review demonstrated an association between chronic hypertension and pre-eclampsia (variously defined) and preterm, small-for-gestational age (SGA), or low-birth weight infants when compared with normotensive women or the general obstetric population. The risk of these complications was increased even in the absence of superimposed pre-eclampsia, although the absolute increased risk from mild hypertension could not be calculated (13). In the largest clinical trial, which demonstrated a reduction in perinatal deaths, most women took methyldopa beginning before or during the second trimester. In a review of 263 women with mild hypertension randomized to methyldopa, labetalol, or no treatment at 6 to 13 weeks of gestation, treatment with antihypertensive medications did not decrease the incidence of complications such as intrauterine growth restriction, superimposed pre-eclampsia, placental abruption, or perinatal mortality (13).

Data are inconclusive about the benefits and potential adverse fetal effects from treatment of mild chronic hypertension during pregnancy. It would seem reasonable to defer antihypertensive therapy in women with mild hypertension who become pregnant unless they have other complicating factors (e.g., cardiovascular or renal disease) and to either stop or reduce medication in women who are already taking antihypertensive therapy (1). Adjusting or withdrawing any drug therapy should be undertaken cautiously. A conservative approach is necessary to avoid excessive reductions or rises in blood pressure. As suggested by the National High Blood Pressure Education Program Working Group on High Blood Pressure in Pregnancy, therapy could be increased or reinstituted for women with blood pressures exceeding 150 to $160 \mathrm{mmHg}$ systolic or 100 to $110 \mathrm{mmHg}$ diastolic (15).

Since the evidence for the efficacy of antihypertensive treatment for mild hypertension during pregnancy is lacking, safety considerations are paramount for clinicians who elect to prescribe antihypertensive medication in this indication. The following areas have been suggested by Ferrer et al. for further research: (i) a better understanding of current practice, (ii) benefits and harms of commonly-used, unproven therapies to be tested in large collaborative multicenter and populationbased studies among women with clearly established mild chronic hypertension, (iii) comparison between pharmacologic therapy begun early in the course of pregnancy with a placebo and commonly used alternative therapies, (iv) better surveillance systems that routinely monitor adverse events, and (v) large trials that compare alternative strategies and use clinically important outcomes to establish appropriate and cost-effective methods for monitoring (13).

\section{DRUG THERAPY DURING PREGNANCY}

Table 1 lists drugs commonly used to treat chronic hypertension before or during pregnancy (3). Standard oral dose regimens and adverse effects to mother and fetus are shown for each medication. The prescribing clinician should know the efficacy of at least a few of these drugs and their acute and long-range effects on fetal well-being.

Nearly all nonpregnant women can achieve adequate blood pressure control with many of these medications. Considerations about safety during pregnancy are as important as the patient's hemodynamic status. Experience has shown that, except for the ACE inhibitors, the drugs currently prescribed do not alone pose any additional risk of perinatal morbidity or mortality. A detailed description of the different classes of antihypertensive drugs is presented elsewhere, but experience with their use during pregnancy is summarized below (3).

\section{Centrally Acting Adrenergic Inhibitors}

Methyldopa was the first antihypertensive used during pregnancy and remains the most commonly prescribed central adrenergic antagonist for pregnant women. Because of its long-term safety, methyldopa is considered by many authorities to be the initial drug of choice for pregnant women with chronic hypertension and the standard by which other drugs are measured (15). Long-term follow-up evaluations of outcomes are reassuring, and studies of children exposed in utero to methyldopa reported normal mental and physical development at $7 \frac{1}{2}$ years of age (16).

\section{Beta-adrenergic Blocking Agents}

Many women with chronic hypertension are treated with labetalol (an alpha and beta blocker) or a pure beta ${ }_{1}$ adrenergic blocker (e.g., atenolol, metoprolol). Most studies of these drugs include relatively small numbers of cases with gestational ages at entry usually being at 29 to 33 weeks (17). Beta-blocking agents cross the placenta easily; they may interfere with interpretation of the fetal heart rate (FHR) pattern and, in theory, may compromise the fetus's ability to withstand hypoxic stress. A search for growth restriction is recommended after long-term exposure to beta blockers.

Labetalol causes a decrease in maternal blood pressure without diminishing blood flow through the fetal aorta, umbilical vein, or intervillous space $(18,19)$. Based on experimental animal studies and human reports, labetalol therapy does not appear to increase the risk of congenital anomalies (20). This agent compares favorably with other antihypertensive medications for use during pregnancy.

In a meta-analysis of beta-receptor blockers prescribed for pregnancies complicated by hypertension, there was an increase in SGA infants born to those women who took oral beta blockers for mild hypertension (OR, 2.46; 95\% CI, 1.02, 5.92) (21). In randomized trials comparing oral atenolol with metoprolol, no adverse fetal effects were demonstrated with either drug (22).

\section{Calcium Channel Blockers}

The most experience with calcium channel blockers has been during the second half of pregnancy as a treatment for hypertension or arrhythmias (23-26) or for tocolysis (27-29). Nifedipine, the best known calcium channel blocker, exerts a greater effect on vascular smooth muscle than on the myocardium (30,31). A review on 102 women who received oral nifedipine for tocolysis concluded that therapy appeared safe for mother and fetus (32). A human investigation that utilized Doppler ultrasound techniques to assess fetal and uteroplacental circulation did not uncover any significant effects after short-term nifedipine therapy (33). Oral nifedipine is rapid acting and has been used without complications; however, long-term trials of calcium channel blockers during 
Table 1 Standard Oral Doses and Maternal and Fetal Adverse Effects of Antihypertensive Drugs

\begin{tabular}{|c|c|c|c|}
\hline Drug & Initial oraldose (mg)(usual range) & Maternal adverse effects ${ }^{a}$ & Fetal adverse effects \\
\hline \multicolumn{4}{|l|}{$\begin{array}{l}\text { Central adrenergic } \\
\text { antagonists }\end{array}$} \\
\hline Methyldopa & 250 bid $(250-2000)$ & $\begin{array}{l}\text { Sedation, decreased mental } \\
\text { acuity, fatigue, sodium } \\
\text { retention, hepatic dysfunction, } \\
\text { drug fever, positive antinuclear } \\
\text { antibody Coombs test, } \\
\text { hemolytic anemia, } \\
\text { galactorrhea and postural } \\
\text { hypotension }\end{array}$ & None reported \\
\hline \multicolumn{4}{|l|}{ Beta blocker agents } \\
\hline Metoprolol & 50-100 (50-450) & Sodium retention, aggravation of & Intrauterine growth retardation, \\
\hline Atenolol & $50(25-100)$ & heart failure, bradycardia, & hypoglycemia, respiratory \\
\hline Labetalol & 100 bid $(200-1200)$ & $\begin{array}{l}\text { depression, aggravation of } \\
\text { bronchoconstrictor disease, } \\
\text { vivid dreams, interference of } \\
\text { diabetic control, CNS } \\
\text { disturbances (headache, } \\
\text { tremulous) }\end{array}$ & depression, bradycardia \\
\hline \multicolumn{4}{|l|}{ Vasodilators } \\
\hline Hydralazine & 10 qid (30-300) & $\begin{array}{l}\text { Tachycardia, palpitations, } \\
\text { flushing, headache, sodium } \\
\text { retention, aggravation of } \\
\text { angina, lupus syndrome, } \\
\text { neuropathy, dizziness }\end{array}$ & $\begin{array}{c}\text { None reported on long-term } \\
\text { use; fetal heart rate changes } \\
\text { when given acutely at term }\end{array}$ \\
\hline \multicolumn{4}{|c|}{ Calcium channel blockers } \\
\hline Nifedipine & 10 tid $(30-120)$ & $\begin{array}{l}\text { Palpations, flushing, headache, } \\
\text { orthostatic hypotension }\end{array}$ & $\begin{array}{l}\text { None reported but chance for } \\
\text { reduced placental perfusion }\end{array}$ \\
\hline \multicolumn{4}{|c|}{$\mathrm{cos}$} \\
\hline
\end{tabular}

pregnancy are necessary (29). A comparison of nifedipine and atenolol reported no differences with either agent (34). Fetoplacental hemodynamics, birth weights, and Apgar scores were similar between the two groups.

\section{ACE Inhibitors and Angiotension II Receptor Blockers}

Angiotensin-converting enzyme (ACE) inhibitors, such as captopril and enalapril, are commonly prescribed to reproductive-age women. Side effects are minimal, and the drugs have considerable appeal for patients with renal disease. First-trimester effects were suggested by one study but have not been established (35). Women who inadvertently become pregnant on these medications can be switched to an alternative antihypertensive agent.

The U.S. Food and Drug Administration has issued a Public Health Advisory about ACE inhibitors in pregnancy. Use in the second and third trimesters is discouraged because of multiple risks to the fetus, including oligohydramnios, hypoplasia of the calvarium, possible death, and patent ductus arteriosus or anuria in the neonate. Hypoplasia of the fetal calvarium may result from uterine pressure, oligohydramnios, or decreased perfusion to the developing head (36). The teratogenic risk appears to be similar for the entire drug class (37).
Angiotensin receptor blockers are not used in pregnancy by extrapolation from the effects of ACE inhibitors.

\section{Vasodilators}

Hydralazine, a peripheral vasodilator, is ineffective when used orally as a sole agent (3). In pregnant women with chronic hypertension, it should be limited to use as a second- or thirdline drug. Hydralazine is most often prescribed with methyldopa in treating preexisting hypertension in pregnancy. When hydralazine is combined with a beta-adrenergic blocking agent, reflex tachycardia is lessened and blood pressures are reduced.

It is considered safe for both mother and fetus, although one survey in Scandinavia reported fetal thrombocytopenia (38). Use of the parenteral form is discussed in the section on hypertensive crisis.

\section{Diuretics}

Diuretics reduce intravascular volume, and women who use diuretics from early in pregnancy do not develop the expanded blood volume normal in pregnancy (39). Based on experimental animal studies and human experience, hydrochlorothiazide use during pregnancy is not expected to 
increase the risk of congenital malformations (37). Theoretically, use during pregnancy might produce fetal biochemical abnormalities and decreased uteroplacental perfusion, but these problems are not believed to occur in women treated chronically with thiazide diuretics beginning prior to conception.

A meta-analysis of nine randomized trials involving more than 7000 patients revealed a tendency toward less edema or hypertension with no increase in adverse fetal effects (40). The National High Blood Pressure Education Program Working Group on High Blood Pressure in Pregnancy concluded, "If diuretics are indicated, they are safe and efficacious agents that can markedly potentiate the response to other antihypertensive agents and are not contraindicated in pregnancy except in settings in which uteroplacental perfusion is already reduced" (15).

\section{Aspirin}

Small doses of aspirin $(60-100 \mathrm{mg})$ by women at high risk of pre-eclampsia may reduce the incidence of pregnancy-induced hypertension (41). Conclusions from a multinational study of more than 90,000 women indicated that low-dose aspirin begun before 20 weeks reduced fetal morbidity in a select population (42). These women had preexisting disorders, such as chronic hypertension or renal disease, or had developed pre-eclampsia before 32 weeks of gestation in a previous pregnancy.

No fetal complications attributable to the aspirin have been identified. Low-dose aspirin therapy during pregnancy does not alter fetal cardiovascular wave forms, decrease fetal urine excretion, or increase neonatal bleeding complications (43-45).

\section{WORSENING HYPERTENSION}

Risks to women with diastolic blood pressures of $100 \mathrm{mmHg}$ or greater are sufficient to warrant drug therapy. Hospitalization is sometimes necessary. Methyldopa and labetalol are commonly the initial drugs of choice (46). If neither is successful or the patient does not tolerate it, reasonable alternatives are dual therapy, diuretics, or calcium channel blockers.

No evidence exists to show that antihypertensive therapy improves fetal well-being or reduces risks of placental abruption, disseminated intravascular coagulation, seizures, or other maternal complications: (15) with or without drug therapy, both fetus and mother should receive frequent assessments, with special alertness for signs of renal disease or superimposed pre-eclampsia.

It is often difficult, if not impossible, to distinguish worsening chronic hypertension from superimposed preeclampsia. The prevalence of superimposed pre-eclampsia averages $15 \%$ to $25 \%$ among women with chronic hypertension (1). Some studies have shown that infants of chronically hypertensive women who do not develop superimposed pre-eclampsia do as well as those born to normotensive women (1). However, other studies reported higher perinatal loss rates in otherwise uncomplicated hypertensive pregnancies than those without hypertension. Coexisting conditions such as renal disease, diabetes, and obesity also affect the risk of perinatal loss.
Signs of superimposed pre-eclampsia may mimic worsening primary renal disease. Conversely, pre-eclampsia may be disguised by pre-existing proteinuria or antihypertensive medications. Some investigators suggest that the diagnosis can be improved by monitoring for decreasing platelet counts and increasing serum uric acid levels. A sudden rise in blood pressure complicated by proteinuria or symptoms of central nervous system irritability should strongly raise the suspicion of superimposed pre-eclampsia. With either superimposed pre-eclampsia or eclampsia, the outlook for both infant and mother can be grave, unless the pregnancy is terminated.

\section{INDICATIONS FOR DELIVERY}

Delivery is recommended, even preterm, if superimposed pre-eclampsia develops, renal function deteriorates, severe fetal growth restriction is confirmed, or fetal compromise is suspected on biophysical assessment. The most common techniques of antepartum fetal surveillance are fetal movement counting, nonstress testing, and biophysical profiles. Daily fetal movement charting can begin early in the third trimester. Weekly FHR testing is typically recommended, beginning by the 32nd gestational week. Earlier or more intensive surveillance is probably appropriate if hypertension is more difficult to control or if placental insufficiency is suspected (47).

Serial ultrasound examinations to assess fetal growth are commonly recommended every 3 to 4 weeks during the second half of pregnancy. As long as the fetus continues to grow appropriately and the quantity of amniotic fluid is adequate, it can be inferred that placental function and uterine blood flow are adequate at that time. Oligohydramnios may signify less effective placental perfusion and is associated with umbilical cord compression before and during labor. Doppler assessment of the fetal arterial or venous system is often used as an adjunct.

Hospitalization is indicated when systolic and diastolic values are persistently above 170 and $110 \mathrm{mmHg}$ respectively (despite medication adjustment), the clinician suspects superimposed pre-eclampsia or fetal growth restriction, or antenatal fetal surveillance warrants. Hospitalization allows for intensified maternal and fetal surveillance, for ancillary therapies such as antenatal steroids, and for additional consultation if needed. If maternal condition deteriorates rapidly or fetal compromise persists in the hospital, delivery is indicated. The predictive value of fetal surveillance testing may be invalidated by rapid changes in the maternal health. Evidence of reduced perfusion should be sought periodically in the brain, kidneys, liver, and placenta.

Fetal considerations often dictate the timing of delivery. The physician should reconsider delivery as soon as fetal lungs are documented mature, if control of blood pressure remains problematic, if fetal growth is suboptimal, or if maternal or fetal complications could be ameliorated by delivery. When delivery is anticipated before 34 weeks, corticosteroids are to be given to enhance fetal lung maturity if the mother's condition is stable, and delivery can be delayed at least 24 hours (48). Amniocentesis may be done if determination of pulmonary maturity would influence decision-making.

Whether these plans of action improve perinatal outcome is unclear. Despite good intentions, the approach toward 
delivering a very premature but surviving perinate who requires intensive care and faces the attendant risk of longrange developmental disability is open to debate. Such an approach is probably best attempted at tertiary care centers staffed by specialized personnel.

\section{INTRAPARTUM CARE}

Vaginal delivery is preferable to cesarean delivery, regardless of gestational age. If the condition permits, an induction of labor should be attempted regardless of cervical condition. A clear endpoint is encouraged from the onset, with delivery usually being expedited within 24 to 48 hours after the decision to induce labor (49). If vaginal delivery cannot be effected within a reasonable time, a cesarean may be preferable. Regional anesthesia is preferred to general anesthesia because of the risk of provoking uncontrolled hypertension when the airway is instrumented.

Although intrapartum analgesia with narcotics may be used, attempting to manage or prevent eclampsia with profound maternal sedation is dangerous and ineffective. Epidural anesthesia is permissible when an experienced anesthesiologist is available and no coagulopathies are present. Hazards include the possibility of extensive sympatholysis with resultant decreased cardiac output, maternal hypotension, and impairment of an already compromised uteroplacental perfusion (1). The advantage, of course, is that epidural anesthesia blunts the sympathetic response to pain, which may be exaggerated in women with preexisting hypertension. Women with chronic hypertension complicated by significant cardiovascular or renal disease require special attention to intake and urine output, because they may be susceptible to fluid overload with resultant pulmonary edema.

\section{POSTPARTUM CONSIDERATIONS}

Acute hypertensive changes usually dissipate within the first few days after delivery. The likelihood of underlying chronic hypertension is greatly increased if hypertension persists more than 3 days after delivery. In such cases, oral antihypertensive therapy is often begun before discharge and the women are reevaluated in the next week. Hypertension that persists longer than the postpartum period ( 12 weeks post delivery) is classified as chronic (1).

Reinstituting the prepregnancy regimen treatment is often appropriate for women who were previously well controlled. As an alternative, either oral nifedipine or labetalol is commonly prescribed, using the lower doses shown in Table 1. Self-blood pressure monitoring at home or work is helpful in determining the need to continue any antihypertensive agent. If prepregnancy blood pressures were normal or unknown, it is reasonable to stop medications after 3 to 4 weeks and observe.

Many chronically hypertensive women wish to breastfeed. When hypertension is mild, medication may be withheld and the blood pressure should be observed closely. For a breastfeeding mother with more elevated recordings, the clinician should continue drug therapy at the lowest effective dose. All agents have low but measurable amounts in the milk, and no clinical trials have studied the cardiovascular effects of an antihypertensive agent on the breast-fed infant (50). Most antihypertensive drugs, including ACE inhibitors, are considered "compatible with breastfeeding" by the American Academy of Pediatrics.

Postpartum contraception is advisable to reduce the risk of an unplanned pregnancy. Oral contraceptives, depot medroxyprogesterone acetate (Depo Provera), and other hormonal contraception are not contraindicated in chronically hypertensive patients who are under 35 and do not smoke, as long as the blood pressure is controlled and monitored (51).

\section{HYPERTENSIVE CRISIS}

Hypertensive emergencies still occur, albeit less frequently: in the years before the availability of antihypertensive drugs, 7\% of individuals with hypertension could expect to develop a hypertensive emergency (with an associated 1-year mortality risk nearly $80 \%$ ); the figure is now $1 \%$ to $2 \%(52,53)$. Most patients who develop a hypertensive crisis have a history of hypertension, often inadequately treated. This may not be the case in pregnancy, however, where hypertensive crisis may manifest against a background of either chronic hypertension or pre-eclampsia.

\section{Incidence and Definition}

Hypertensive crisis can occur at any time before or after delivery. There are no reliable data on the incidence of hypertensive crisis complicating pregnancy. A query of the National Hospital Discharge Survey for severe maternal morbidity included 50 million deliveries and reported 36,000 cases of eclampsia and 6000 cerebrovascular accidents in the United States across the time period from 1991 to 2003 (54). This excludes those pregnant women whose hypertensive crisis did not result in one of those diagnoses, but it would appear that in the United States at least 3500 women per year develop a hypertensive crisis during pregnancy, which is roughly 1 per 1000 live births. Figures from Canada appear to be about half of that (55).

The clinical entity of hypertensive crisis is poorly defined, but this term has replaced earlier terms such as "malignant hypertension" and "accelerated hypertension." While it seems intuitively obvious that it refers to a state of severe hypertension, there is no consensus as to the degree of hypertension involved. The Joint National Committee (JNC) on Detection, Evaluation, and Treatment of High Blood Pressure (JNC) reports of 1993 and 1997 divided acute severe hypertension into categories of "hypertensive emergency" and "hypertensive urgency" $(56,57)$. The distinction between the two related not to blood pressure measurement but to the presence of end-organ damage. The most recent iteration, JNC 7, does not address hypertensive emergency specifically but refers to stage 1 (systolic BP 140-159 mmHg or diastolic 90-99 $\mathrm{mmHg}$ ) and stage 2 (systolic $\geq 160 \mathrm{mmHg}$ or diastolic $\geq 100 \mathrm{mmHg}$ ) hypertension, largely for the purposes of initiating outpatient drug therapy (7). JNC 8 is scheduled for release in 2010 and may redress this flaw (58).

\section{Pathophysiology}

The pathophysiology of hypertensive crisis is still inadequately understood. Because blood pressure is determined by cardiac output and peripheral vascular resistance, and cardiac output itself is a product of heart rate and stroke volume, there are several possible triggers, but it appears that an abrupt increase 
in systemic vascular resistance is most commonly involved (53). Mechanical stress causes vascular endothelial damage, which in turn provokes increased permeability, activation of the coagulation cascade, and fibrin deposition. Fibrinoid necrosis of the arterioles can further decrease vascular perfusion and thus activate the mediators commonly associated with ischemia, further worsening injury. In many (but not all) hypertensive crises, the renin-angiotensin system is activated, forcing further vasoconstriction and production of inflammatory cytokines (59). Because of pressure natriuresis, effective circulating volume is decreased: this stimulates the kidney to produce vasoconstrictive mediators. A feed-forward loop or vicious circle becomes difficult to break.

\section{Hypertensive Crisis: Emergency Vs. Urgency}

Individuals with systolic blood pressure $\geq 180 \mathrm{mmHg}$, or diastolic $\geq 110 \mathrm{mmHg}$, are commonly considered to be in a state of hypertensive crisis (53). If evidence exists for acute target-organ involvement (Table 2), then pressures must emergently be reduced, within the next 1 to 2 hours, to limit damage or prevent further organ failure: this qualifies as a hypertensive emergency. In the absence of end-organ findings, while it is certainly prudent to begin measures to lower blood pressures, the process can be allowed to take 24 hours or longer, and the situation is hypertensive urgency.

This cutoff point, admittedly arbitrary, is even less well defended in the case of hypertension in pregnancy. Current guidelines from the American College of Obstetricians and Gynecologists allow a diagnosis of severe pre-eclampsia if a systolic pressure of $\geq 160 \mathrm{mmHg}$ is achieved or any of the criteria in Table 3 is met (60). Comparing these with the standard list of clinical syndromes, which defines hypertensive emergency, CNS findings, pulmonary edema, and thrombocytopenia or microangiopathic hemolytic anemia are common to both. Martin et al. have suggested that systolic blood pressure in pre-eclampsia is far more important than diastolic and reported that in $96 \%$ cases of stroke related to preeclampsia, systolic pressure was greater than $160 \mathrm{mmHg}$ immediately preceding the event (61).

While nonpregnant patients with hypertensive crisis most commonly present to emergency care with symptoms related to target-organ involvement, this may not be true for pregnant patients, who have many interactions with the health-care system during pregnancy. The pregnant patient with hypertensive crisis (e.g., hypertensive urgency rather than emergency) may be asymptomatic and be admitted for evaluation after hypertension is noted in the office. As a rule, the patient with abnormally high blood pressure but no signs or symptoms of target-organ damage is considered to have a hypertensive urgency rather than emergency. Unlike true hypertensive emergency, hypertensive urgency allows 24 to 48 hours to normalize blood pressure: oral agents are acceptable. It is prudent to lower mean arterial pressure gradually so that the limits of autoregulation are not exceeded.

\section{Initial Evaluation and Management}

If a hypertensive crisis is suspected, then diagnosis, assessment, and therapy should proceed at equal pace. In the setting of hypertensive emergency, speed of intervention is important in limiting complications. A history should be obtained, focusing
Table 2 Hypertensive Emergency: Organ System and Clinical Manifestation

$\begin{array}{ll}\text { Cerebral } & \begin{array}{l}\text { Hypertensive encephalopathy } \\ \text { Stroke } \\ \text { Cardiovascular }\end{array} \\ & \begin{array}{l}\text { Acute aortic dissection } \\ \text { Acute myocardial infarction } \\ \text { Acute left ventricular failure }\end{array} \\ \text { Renal } & \text { Acute renal failure } \\ \text { Hematologic } & \text { Microangiopathic hemolytic anemia } \\ \text { Placental } & \text { Severe pre-eclampsia (?) } \\ & \text { Eclampsia } \\ & \text { HELLP syndrome }\end{array}$

Abbreviation: HELLP, hemolysis, elevated liver enzymes, and low platelet count.

Table 3 Criteria for Diagnosis of Severe Pre-eclampsia (One or More of the Following)

BP $\geq 160 \mathrm{mmHg}$ systolic or $\geq 110$ diastolic

(two occasions, at least $6 \mathrm{hr}$ apart)

Proteinuria $\geq 5 \mathrm{~g}$ in $24 \mathrm{hr}$

Oliguria $(<500 \mathrm{ml}$ in $24 \mathrm{hr})$

Cerebral or visual disturbances

Pulmonary edema or cyanosis

Epigastric or right upper quadrant pain

Impaired liver function

Thrombocytopenia

Fetal growth restriction

on any known history of hypertension, medications (prescribed, over-the-counter, and illicit), obstetric history, and symptoms. Blood pressure should be measured in each arm, with the patient sitting, using the correctly sized cuff. The remainder of the physical examination should incorporate assessment for pulmonary edema, peripheral pulses, and neurologic evaluation. In the nonpregnant patient with hypertensive crisis, fundoscopic examination of the retinas and auscultation of the flanks for bruits in the renal arteries are recommended as well (53), but in the hands of an obstetrician-gynecologist inexperienced in these examinations, they are likely to be of low yield.

Blood should be sent for electrolytes, blood urea nitrogen, serum creatinine, hepatic function, complete blood count, and coagulation profile. If a specific concern exists (illicit drug use, pheochromocytoma), additional laboratory testing should be ordered as relevant. Fetal assessment is important. Fetal cardiac activity should be confirmed or excluded immediately upon admission. Ultrasound assessment for fetal growth, amniotic fluid, Doppler, and the like should be deferred until after the maternal condition is stabilized. The utility of electronic fetal monitoring is discussed below.

Once diagnosed with a hypertensive emergency (abnormally high BP plus evidence of target-organ damage), the patient must be treated immediately with the goal of reducing BP within 1 to 2 hours. This will require parenteral medication and high-acuity nursing care. It will often require minute-tominute evaluation, so the threshold for intra-arterial monitoring should be low. There are no good clinical trials that could 
Table 4 Drug Therapy for Acute Hypertensive Crisis

\begin{tabular}{|c|c|c|c|c|c|c|c|c|}
\hline Drug & Drug classification & $\begin{array}{l}\text { FDA } \\
\text { pregnancy } \\
\text { category }\end{array}$ & Dose (initial) & Dose adjustment & Onset & Duration & Side effect profile & Notes \\
\hline Clevidipine & $\begin{array}{l}\text { Calcium channel } \\
\text { blocker } \\
\text { (dihydropyridine) }\end{array}$ & $\mathrm{C}$ & $\begin{array}{l}\text { 1-2 mg/hr iv } \\
\text { infusion }\end{array}$ & $\begin{array}{l}\text { Initially, double dose } \mathrm{q} \\
90 \mathrm{sec} \text {; as target } \mathrm{BP} \\
\text { approached, lengthen } \\
\text { interval to } 5-10 \mathrm{~min} \text {, } \\
\text { decrease to less than } \\
\text { doubling, based on } \\
\text { patient response; max } \\
16 \mathrm{mg} / \mathrm{hr}\end{array}$ & $2-4 \min$ & $\begin{array}{l}\text { 5-15 min } \\
\text { after } \\
\text { stopping } \\
\text { infusion }\end{array}$ & $\begin{array}{l}\text { Atrial fibrillation, } \\
\text { nausea, headache, } \\
\text { tachycardia }\end{array}$ & $\begin{array}{l}\text { Lipid emulsion; cannot } \\
\text { use if allergic to soy } \\
\text { or eggs; adverse } \\
\text { effects on lipid } \\
\text { profile }\end{array}$ \\
\hline Enalaprilat & $\begin{array}{l}\text { Angiotensin- } \\
\text { converting enzyme } \\
\text { inhibitor }\end{array}$ & $\begin{array}{l}\mathrm{C} \text { (first } \\
\text { trimester) }\end{array}$ & $1.25 \mathrm{mg}$ iv & Repeat q $6 \mathrm{hr}$, max $5 \mathrm{mg}$ & & & $\begin{array}{l}\text { Nausea, vomiting, } \\
\text { headache }\end{array}$ & \\
\hline Esmolol & Beta $_{1}$ blocker & $\mathrm{C}$ & $\begin{array}{l}500 \mathrm{mcg} / \mathrm{kg} / \mathrm{min} \\
\text { load over } \\
1 \text { minute, } \\
\text { followed by } \\
50 \mathrm{mcg} / \mathrm{kg} / \\
\mathrm{min} \times 4 \mathrm{~min}\end{array}$ & $\begin{array}{l}\text { May repeat loading dose } \\
\text { ( } 500 \mathrm{mcg} / \mathrm{kg} / \mathrm{min}) \text {, } \\
\text { then increase infusion } \\
\text { rate by } 50 \mathrm{mcg} / \mathrm{kg} / \\
\text { min, q } 5 \mathrm{~min} \text {; as target } \\
\text { approached, lengthen } \\
\text { interval or decrease } \\
\text { increment }\end{array}$ & Immediate & $<10 \min$ & $\begin{array}{l}\text { Bradycardia, injection } \\
\text { site pain, nausea }\end{array}$ & $\begin{array}{l}\text { Metabolized by red } \\
\text { blood cell esterases; } \\
\text { independent of } \\
\text { hepatic or renal } \\
\text { function }\end{array}$ \\
\hline Fenoldopam & $\begin{array}{l}\text { Dopamine }(\mathrm{D} 1) \\
\text { receptor agonist }\end{array}$ & B & $\begin{array}{l}\text { No bolus dose; } \\
\text { begin infusion } \\
\text { at } 0.03- \\
0.1 \mathrm{mcg} / \mathrm{kg} / \\
\text { min }\end{array}$ & $\begin{array}{l}\text { Increase q } 15 \mathrm{~min} \text { by } \\
0.05-0.1 \mathrm{mcg} / \mathrm{kg} / \mathrm{min} \text {, } \\
\max \text { dose } 1.6 \mathrm{mcg} / \mathrm{kg} / \\
\min \end{array}$ & & $5 \mathrm{~min}$ & $\begin{array}{l}\text { Flushing, nausea, } \\
\text { headache, } \\
\text { tachyarrhythmia }\end{array}$ & \\
\hline Hydralazine & $\begin{array}{l}\text { Peripheral vasodilator } \\
\text { (arteriolar) }\end{array}$ & $\mathrm{C}$ & $5 \mathrm{mg}$ IV bolus & $10 \mathrm{mg}, 20 \mathrm{mg}$ & $15-20 \mathrm{~min}$ & $3-6 \mathrm{hr}$ & $\begin{array}{l}\text { Tachyarrhythmia, } \\
\text { headache, nausea, } \\
\text { dyspnea }\end{array}$ & $\begin{array}{l}\text { Rarely: hepatotoxicity, } \\
\text { lupus. Promotes } \\
\text { salt/water retention. } \\
\text { Tachyphylaxis } \\
\text { common }\end{array}$ \\
\hline Ketanserin & $\begin{array}{l}\text { Serotonin receptor } \\
\text { blocker }\end{array}$ & $\mathrm{n} / \mathrm{a}$ & $\begin{array}{l}\text { 5-10 mg IV } \\
\text { bolus } \\
\text { followed by } \\
4 \mathrm{mg} / \mathrm{hr}\end{array}$ & $\begin{array}{l}\text { Repeat bolus, increase } \\
\text { infusion rate by } 2 \mathrm{mg} / \\
\text { hr; dose may be } \\
\text { adjusted at } 20 \text {-min } \\
\text { intervals, max } \\
\text { infusion rate } 14 \mathrm{mg} / \mathrm{hr}\end{array}$ & $10-20 \mathrm{~min}$ & $13-16 \mathrm{hr}$ & $\begin{array}{l}\text { May inhibit uterine } \\
\text { contractions. Failure } \\
\text { rate appears to be } \\
\text { high. Q-T } \\
\text { prolongation }\end{array}$ & $\begin{array}{l}\text { Not FDA approved; } \\
\text { used in Europe, } \\
\text { South Africa }\end{array}$ \\
\hline
\end{tabular}




\begin{tabular}{|c|c|c|c|c|c|c|c|c|}
\hline Labetalol & $\begin{array}{l}\text { Mixed alpha and beta } \\
\text { blocker (beta } \\
\text { predominates, } 7: 1 \text { ) }\end{array}$ & $\mathrm{C}$ & $\begin{array}{l}\text { 10-20 mg IV } \\
\text { bolus }\end{array}$ & $\begin{array}{l}\text { Repeat q } 5-10 \mathrm{~min} \text {, } \\
\text { doubling the dose to } \\
\text { max bolus } 80 \mathrm{mg} \text {. } \\
\text { Continuous infusion } \\
1-2 \mathrm{mg} / \mathrm{min} \text { is an } \\
\text { alternate to bolus } \\
\text { dosing }\end{array}$ & $2-5 \min$ & $3-6 \mathrm{hr}$ & $\begin{array}{l}\text { Bradycardia, dizziness, } \\
\text { GI effects, abnormal } \\
\text { liver function, } \\
\text { hypoglycemia }\end{array}$ & \\
\hline Nicardipine & $\begin{array}{l}\text { Calcium channel } \\
\text { blocker } \\
\text { (dihydropyridine) }\end{array}$ & $\mathrm{C}$ & $\begin{array}{l}\text { Initial rate } \\
5 \mathrm{mg} / \mathrm{hr}\end{array}$ & $\begin{array}{l}\text { Increase rate by } 2.5 \mathrm{mg} / \\
\text { hr q 5-15 min; max } \\
15 \mathrm{mg} / \mathrm{hr}\end{array}$ & $5-10 \mathrm{~min}$ & $1-4 \mathrm{hr}$ & $\begin{array}{l}\text { Tachycardia, dizziness, } \\
\text { headache, edema }\end{array}$ & \\
\hline Nitroglycerin & $\begin{array}{l}\text { Nitrate, vasodilator } \\
\text { (venous and } \\
\text { arteriolar) }\end{array}$ & $\mathrm{C}$ & $\begin{array}{l}\text { Initial rate } \\
\qquad 5 \mathrm{mcg} / \mathrm{min}\end{array}$ & $\begin{array}{l}\text { Increase rate by } 5 \mathrm{mcg} / \\
\min \mathrm{q} 3-5 \mathrm{~min}, \max \\
100 \mathrm{mcg} / \mathrm{min}\end{array}$ & $2-5 \min$ & $3-5 \min$ & $\begin{array}{l}\text { Tachyarrhythmia, } \\
\text { headache, nausea, } \\
\text { dizziness, visual } \\
\text { disturbance; rare } \\
\text { hematologic }\end{array}$ & \\
\hline $\begin{array}{l}\text { Sodium } \\
\text { nitroprusside }\end{array}$ & $\begin{array}{l}\text { Vasodilator } \\
\text { (venous and } \\
\text { arteriolar) }\end{array}$ & $\mathrm{C}$ & $\begin{array}{l}\text { No bolus; begin } \\
\text { at } 0.2- \\
0.3 \mathrm{mcg} / \mathrm{kg} / \\
\text { min }\end{array}$ & $\begin{array}{l}\text { Increase by } 0.2-0.5 \mathrm{mcg} / \\
\mathrm{kg} / \mathrm{min} \text { (q } 5 \mathrm{~min} \text { ) to } \\
\text { usual max } 2 \mathrm{mcg} / \mathrm{kg} / \\
\mathrm{min} \text {; rarely as high as } \\
5 \mathrm{mcg} / \mathrm{kg} / \mathrm{min} \text {. Use } \\
\text { lowest } \\
\text { dose possible, shortest } \\
\text { time possible }\end{array}$ & Instant & $\sim 1 \mathrm{~min}$ & $\begin{array}{l}\text { Bradyarrhythmia, } \\
\text { tachyarrhythmia, } \\
\text { rash, sweating, } \\
\text { dizziness, headache, } \\
\text { altered mental } \\
\text { status, restlessness, } \\
\text { azotemia }\end{array}$ & $\begin{array}{l}\text { Metabolic acidosis, } \\
\text { cyanide toxicity, } \\
\text { thiocyanate toxicity. } \\
\text { Therapeutic and } \\
\text { toxic ranges may } \\
\text { overlap. No quick } \\
\text { lab test for cyanide } \\
\text { level exists }\end{array}$ \\
\hline Phentolamine & Alpha blocker & $\mathrm{C}$ & $1-5 \mathrm{mg}$ iv bolus & & $1-2 \min$ & $5-15 \min$ & $\begin{array}{l}\text { Dysrhythmia, nasal } \\
\text { congestion }\end{array}$ & \\
\hline Trimethaphan & Ganglionic blocker & $\mathrm{D}$ & $\begin{array}{l}\text { Initial rate } 0.5- \\
1 \mathrm{mg} / \mathrm{min}\end{array}$ & $\begin{array}{l}\text { Increase by } 0.5-1 \mathrm{mg} / \\
\min , \max 15 \mathrm{mg} / \mathrm{min}\end{array}$ & $1-5 \min$ & $10 \mathrm{~min}$ & $\begin{array}{l}\text { Tachyarrhythmia, } \\
\text { hyperglycemia, } \\
\text { hypokalemia, GI } \\
\text { symptoms, diplopia, } \\
\text { pupillary dilation, } \\
\text { paralytic ileus, } \\
\text { urinary retention }\end{array}$ & \\
\hline
\end{tabular}


form the basis for the management of hypertensive emergencies, let alone hypertensive emergencies in the setting of pregnancy. Management as currently recommended is driven largely by expert and consensus opinion. Given these caveats, the recommendation is to aim for a reduction in mean arterial pressure of $15 \%$ to $25 \%$ over 1 to 2 hours $(53,62,63)$. While the presence of symptoms is a clear indicator that blood pressure is dangerously high, a too-rapid or too-acute lowering risks worsening the ischemia, which already accompanies hypertensive crisis and may exceed the threshold of autoregulation, particularly in the cerebral circulation. At this time, it seems appropriate to remark upon the sensitivity of the uteroplacental circulation to perfusion pressure and to note that an exaggerated drop in blood pressure is frequently reflected in decelerations in the FHR tracing.

\section{Drug Therapy}

The ideal agent for use would be easily administered, have a rapid time to both onset and offset, be easily titratable, free of side effects, and well tested in pregnancy. Unfortunately, none of the agents currently available meet these criteria. Hydralazine and labetalol are the drugs most commonly used in obstetrics for hypertensive crises. Hydralazine, contrary to popularly held opinion, has a number of factors that render it unsuitable in the setting of hypertensive crisis (53). Its onset of action can be delayed by as long as 20 minutes after bolus injection, its effect is unpredictable (especially in the common scenario of volume depletion), and its duration is unacceptably long (up to 6 hours). It has been shown in a meta-analysis to produce far higher rates of maternal hypotension than other antihypertensives, more adverse effects on the FHR, and more maternal side effects: these side effects are commonly headache and nausea, which are easily confused with deterioration of the clinical condition (64). While labetalol shares with hydralazine a long duration of effect, the response to labetalol is typically seen within 5 minutes, so the risk of overshoot is lower.

Other drugs that have been used to treat hypertensive crisis include sodium nitroprusside, nitroglycerin, nicardipine, esmolol, clevidipine, fenoldopam, phentolamine, trimethaphan, enalaprilat, ketanserin, and diazoxide (Table 4). Because of their immediate effects, they are best given when the patient has an arterial line in place. Most of these are given by continuous infusion and must be titrated to effect. Depending on local protocol, the use of vasoactive infusions may require the patient be admitted to an intensive care unit. The intravenous form of diazoxide is no longer manufactured, and so it is of historical interest only. Enalaprilat has occasionally been used for hypertensive crisis in nonpregnant patients, but like all ACE inhibitors, it should not be used in pregnancy.

Data in pregnancy are limited for most of these drugs: nicardipine, nitroglycerin, sodium nitroprusside, and esmolol have been reported. Nicardipine is developing a track record in pregnancy. It has been used successfully as a second-line treatment for pre-eclampsia after failure of ketanserin, labetalol, or hydralazine (65). In a small randomized trial, intravenous nicardipine was just as efficacious as labetalol for controlling blood pressure in hypertensive emergency during pregnancy (66). It has definite advantages over hydralazine, being a more predictable and titratable drug, with a much shorter time to onset and quicker disappearance once discontinued.
Trimethaphan and phentolamine have largely been replaced by other drugs of better efficacy and greater predictability, and ketanserin is not available in the United States. There are, as yet, no data on fenoldopam or clevidipine in pregnancy.

Whichever drug is chosen, the goal is to reduce mean arterial pressure by $15 \%$ to $25 \%$ within 1 to 2 hours and then gradually lower BP into the range of $160 / 100$ over the next 2 to 6 hours (67). During this period of time, judicious volume repletion should also occur, since the majority of patients in hypertensive crisis will be significantly volume depleted because of pressure natriuresis. Precipitating factors should be corrected if identified (i.e., conditions of catecholamine excess, drugs that may contribute to hypertension, etc.). The FHR should be monitored both as a sign of uteroplacental perfusion and as a sign of fetal status: placental abruption is an ongoing concern in severe hypertension, and fetal growth restriction, a common complication of pre-eclampsia, signifies a tenuous fetal status that may require intervention. It is inappropriate, however, to rush to delivery before maternal status is stabilized. In cases in which fetal compromise is thought to exist, if delivery is contemplated, it will usually be by cesarean, and both anesthesia and surgery entail risks in the unstable patient. Stroke is an especially feared complication when a patient with inadequately controlled hypertension requires laryngoscopy and intubation for a general anesthetic.

Once blood pressure control has been achieved with parenteral agents, oral medications can generally be started while the parenteral drug is being tapered (68). However, in case of pregnancy, hypertensive crisis often means a commitment to delivery. Absorption of oral medications may be unpredictable in labor, and is not feasible during abdominal surgery, and therefore the plan is often to defer oral agents until after delivery, whether vaginal or cesarean. Oral agents should be started at a low dose and increased as necessary to the desired effect. Agents that have been used in pregnancy include nifedipine, clonidine, and labetalol; postpartum, the ACE inhibitors can also be used. There is no good role for methyldopa or oral hydralazine as a follow-up agent after hypertensive crisis in pregnancy, because adequate blood pressure control is crucial in preventing another episode.

\section{REFERENCES}

1. American College of Obstetricians and Gynecologists. Chronic hypertension in pregnancy. ACOG Practice Bulletin 2001; 29: 1-8.

2. Martin JA, Hamilton BE, Sutton PD, et al. Births: final data for 2005. Natl Vital Stat Rep 2007; 56.

3. Kannel WB. Hypertension and other risk factors in coronary disease. Am Heart J 1987; 114: 918-24.

4. Morrison A, Vijayan A. Hypertension. In: Cooper D, Krainik A, Lubner S, Reno H, eds. The Washington Manual of Medical Therapeutics, 32nd edn. Philadelphia: Lippincott Williams and Wilkins', 2007: 102-18.

5. Mancia G, De Backer G, Dominiczak A, et al. 2007 ESH-ESC practice guidelines for the management of arterial hypertension. J Hypertension 2007; 25: 1751-62.

6. The ALLHAT Officers and Coordinators for the ALLHAT Collaborative Research Group. Major outcomes in high risk hypertensive patients randomized to angiotensin-converting inhibitor or calcium channel blocker versus diuretic. JAMA 2002; 288: 2981-97.

7. Chobanian AV, Bakris GL, Black HR, et al. National High Blood Pressure Education Program Coordinating Committee. The Seventh Report of the Joint National Committee on Prevention, Detection, Evaluation, and Treatment of High Blood Pressure. The JNC 7 Report. JAMA 2003; 289: 2560-72. 
8. Cunningham FG, Cox SM, Harstad TW, et al. Chronic renal disease and pregnancy outcome. Am J Obstet Gynecol 1990; 163: 453-9.

9. Jones DC. Pregnancy complicated by chronic renal disease. Clin Perinatol 1997; 24: 483-96.

10. Botchan A, Hauser R, Kupfermine M, et al. Pheochromocytoma in pregnancy: case report and review of the literature. Obstet Gynecol Surv 1995; 50: 321-7.

11. Gallery ED, Hunyor SN, Gyory AZ. Plasma volume contraction: a significant factor in both pregnancy-associated hypertension (preeclampsia) and chronic hypertension in pregnancy. Q J Med 1979; 48: 593-602.

12. Zuspan F, Rayburn W. Self blood pressure monitoring during pregnancy: practical considerations. Am J Obstet Gynecol 1990; 164: 2-6.

13. Ferrer RL, Sibai BM, Mulrow CD, et al. Management of mild chronic hypertension during pregnancy: a review. Obstet Gynecol 2000; 96: 849-60.

14. Magee LA, Ornstein MP, von Dadelszen P. Fortnightly review: management of hypertension in pregnancy. BMJ 1999; 318: 1332-6.

15. Report of the National High Blood Pressure Education Program Working Group on High Blood Pressure in Pregnancy. Am J Obstet Gynecol 2000; 183: S1-22.

16. Ounsted M, Cockburn J, Moar VA, et al. Maternal hypertension with superimposed pre-eclampsia: effects of child development at $7 \frac{1}{2}$ years. $\mathrm{Br}$ J Obstet Gynaecol 1983; 90: 644-9.

17. Lamming GD, Pipkin FB, Symonds EM. Comparison of the alpha and beta blocking drug, labetalol, and methyldopa in the treatment of moderate and severe pregnancy-induced hypertension. Clin Exp Hypertens 1980; 2: 865-95.

18. Nylund L, Lunell R, Lewander R, et al. Labetalol: pharmacokinetics and effects on the utero placental blood flow. Acta Obstet Gynecol Scand 1984; 118: 710-13.

19. Jouppila P, Kirkinen P, Koivula A, et al. Labetalol does not alter the placental and fetal blood flow or maternal prostanoids in pre-eclampsia. Br J Obstet Gynaecol 1986; 93: 543-7.

20. Macpherson M, Pipkin F, Rutter N. The effect of maternal labetalol on the newborn infant. Br J Obstet Gynaecol 1986; 93: 5239-42.

21. Magee LA, Elran E. Bull SB, et al. Risks and benefits of beta-receptor blockers for pregnancy hypertension: overview of the randomized trials. Eur J Obstet Gynecol Reprod Biol 2000; 88: 15-26.

22. Wichman K, Ryden G, Karlberg B. A placebo controlled trial of metoprolol in the treatment of hypertension in pregnancy. Scand J Clin Lab Invest 1984; 169: 80-7.

23. Walters BNJ, Redman CWG. Treatment of severe pregnancy-associated hypertension with the calcium antagonist nifedipine. Br J Obstet Gynecol 1984; 91: 330-3.

24. Constantine G, Beevers DG, Reynolds AL, et al. Nifedipine as a second line antihypertensive drug in pregnancy. Br J Obstet Gynecol 1987; 94: $1136-42$.

25. Klein V, Repke JT. Supraventricular tachycardia in pregnancy: cardioversion with verapamil. Obstet Gynecol 1984; 63(Suppl): 16S-18S.

26. Rotmensch HH, Elkaya U, Frishman W. Antiarrhythmic drug therapy during pregnancy. Ann Intern Med 1983; 98: 487-97.

27. Read MD, Welby DE. The use of calcium antagonist (nifedipine) suppress preterm labor. Br J Obstet Gynaecol 1986; 93: 933-7.

28. Childress CH, Katz VL. Nifedipine and its indications in obstetrics and gynecology. Obstet Gynecol 1994; 83: 616-24.

29. Magee L, Schick B, Donnerfeld A, et al. The safety of calcium channel blockers in human pregnancy: a prospective, multi-center cohort study. Am J Obstet Gynecol 1996; 174: 823-8.

30. Constantine G, Beevers DG, Reynolds AL, et al. Nifedipine as a secondline antihypertensive drug I pregnancy. Br J Obstet Gynaecol 1987; 94: $1136-42$.

31. Ulmsted U. Treatment of normotensive and hypertensive patients with preterm labor using oral nifedipine, a calcium antagonist. Arch Gynecol 1984; 236: 69-72.

32. Waisman G, Mayorga L, Amer M, et al. Magnesium plus nifedipine: potentiation of hypotensive effect I pre-eclampsia? Am J Obstet Gynecol 1989; 159: 308-9.

33. Ferguson JE, Dyson D, Holbrook H, et al. Cardiovascular and metabolic effects associated with nifedipine and ritodrine tocolysis. Am J Obstet Gynecol 1989; 161: 788-95.
34. Montan S. Drugs used in hypertensive diseases in pregnancy. Curr Opin Obstet Gynecol 2004; 16: 111-15.

35. Hanssens M, Keirse M, Vankelecom F, et al. Fetal and neonatal effects of treatment with angiotensin-converting enzyme inhibitors. Obstet Gynecol 1991; 78: 128-33.

36. Barr M Jr, Cohen MM Jr. ACE inhibitor fetopathy and hypocalvaria: the kidney-skull connection. Teratology 1991; 44: 485-95.

37. Briggs GG, Freeman RK, Yaffe SJ. Drugs in pregnancy and lactation: a reference guide to fetal and neonatal risk, 7th edn. Baltimore: Williams \& Wilkins, 2005.

38. Widerlov E, Karlman I, Storsater J. Hydralazine-induced neonatal thrombocytopenia (letter). N Engl J Med 1980; 301: 1235.

39. Sibai BM, Grossman RA, Grossman HG. Effects of diuretics on plasma in pregnancies with long-term hypertension. Am J Obstet Gynecol 1984; 150: 831-5.

40. Collins R, Yususf S, Peto R. Overview of randomized trials of diuretics in pregnancy. Br Med J 1985; 290: 17-23.

41. Sibai BM, Caritis SN, Thom E, et al. Prevention of pre-eclampsia with low dose aspirin in healthy, nulliparous pregnant women. N Eng J Med 1993; 329: 1213-18.

42. CLASP Collaborative Group. CLASP: a randomized trial of low-dose aspirin for the prevention and treatment of pre-eclampsia among 9364 pregnant women. Lancet 1994; 343: 619-29.

43. Veille JC, Hanson R, Sivakoff M, et al. Effects of maternal ingestion of low-dose aspirin on the fetal cardiovascular system. Am J Obstet Gynecol 1993; 168: 1430-7.

44. DiSessa TG, Moretti ML, Khoury A, et al. Cardiac function in fetuses and newborns exposed to low-dose aspirin during. Am J Obstet Gynecol 1994; 171: 892-900.

45. Maher JE, Owen J, Goldenberg R, et al. The effect of low-dose aspirin on fetal urine output and amniotic fluid volume. Am J Obstet Gynecol 1993; 169: $885-8$.

46. Sibai BM, Mabie WC, Villar M, et al. A comparison of no medication versus methyldopa or labetalol in chronic hypertension during pregnancy. Am J Obstet Gynecol 1990; 162: 960-6.

47. Pircon RA, Lagrew DC, Towers CV, et al. Antepartum testing in the hypertensive patient: when to begin. Am J Obstet Gynecol 1991; 164: 1563-70.

48. Fetal Lung Maturity. ACOG Practice Bulletin No. 97. American College of Obstetricians and Gynecologists. Obstet Gynecol 2008; 112: 717-26.

49. Berkley E, Meng C, Rayburn WF. Success rates with low dose misoprostol before induction of labor for nulliparas with severe preeclampsia. J Matern Fetal Neonatal Med 2007; 20: 825-31.

50. White WB. Management of hypertension during lactation. Hypertension 1984; 6: 297-300.

51. ACOG practice bulletin \#73, June 2006. Use of hormonal contraception in women with coexisting medical conditions. In: Compendium of Selected Publications, American College of Obstetricians and Gynecologists, Washington DC, 2008.

52. Laragh J. Laragh's lessons in pathophysiology and clinical pearls for treating hypertension. Am J Hypertens 2001; 14: 837-54.

53. Marik PE, Varon J. Hypertensive crises: challenges and management. Chest 2007; 131: 1949-62.

54. Callaghan WM, MacKay AP, Berg CJ. Identification of severe maternal morbidity during delivery hospitalizations, United States, 1991-2003. Am J Obstet Gynecol 2008; 199: 133.e1-8.

55. Wen SJ, Huang L, Liston R, et al.; for the Maternal Health Study Group, Canadian Perinatal Surveillance System. Severe maternal morbidity in Canada, 1991-2001. CMAJ 2005; 173: 7.

56. The Fifth Report of the Joint National Committee on Detection, Evaluation, and Treatment of High Blood Pressure. Arch Intern Med 1993; 153: 154-83.

57. The Sixth Report of the Joint National Committee on Prevention, Detection, and Treatment of High Blood Pressure. Arch Intern Med 1997; 157: 2413-46.

58. National Heart Lung and Blood Institute. Hypertension guidelines update, JNC 8. [Available from: http://www.nhlbi.nih.gov/guidelines/ cvd_adult/background.htm]. Accessed 11/16/08. 
59. Blumenfeld JD, Laragh JH. Management of hypertensive crises: the scientific basis for treatment decisions. Am J Hypertens 2001; 14: 1154-67.

60. American College of Obstetricians and Gynecologists. ACOG Practice Bulletin no. 33, January 2002. Diagnosis and management of preeclampsia and eclampsia. Obstet Gynecol 2002; 99: 159-67.

61. Martin JN, Thigpen BD, Moore RC, et al. Stroke and severe preeclampsia and eclampsia: a paradigm shift focusing on systolic blood pressure. Obstet Gynecol 2005; 105: 246-54.

62. Varon J. Treatment of acute severe hypertension: current and newer agents. Drugs 2008; 68: 283-97.

63. Vaughan CJ, Delanty N. Hypertensive emergencies. Lancet 2000; 356: 411-77.
64. Magee LA, Cham C, Waterman EJ, et al. Hydralazine for treatment of severe hypertension in pregnancy: meta-analysis. BMJ 2003; 327: 955-60.

65. Hanff LM, Vulto AG, Bartels PA, et al. Intravenous use of the calciumchannel blocker nicardipine as second-line treatment in severe, earlyonset pre-eclamptic patients. J Hypertens 2005; 23: 2319-26.

66. Elatrous S, Nouira S, Ouanes Besbes L, et al. Short-term treatment of severe hypertension of pregnancy: prospective comparison of nicardipine and labetalol. Intens Care Med 2002; 28: 1281-6.

67. Varon J. Treatment of acute severe hypertension: current and newer agents. Drugs 2008; 68: 283-97.

68. Tuncel M, Ram VCS. Hypertensive emergencies: etiology and management. Am J Cardiovasc Drugs 2003; 3: 21-31. 


\section{Rheumatologic diseases and antiphospholipid syndrome Thomas J. Santoro, Michiyo Tomita, and Alfonse T. Masi}

Rheumatic diseases and associated autoimmune phenomena occur frequently in women of childbearing age (1). Many of these disorders occur in women who are pregnant or desire to become so. In pregnancy, complications arising from coexisting autoimmune diseases may be minor (e.g., exacerbations of low back pain in women with HLA-B27-related spondyloarthropathy syndromes) or life-threatening [e.g., progressive glomerulonephropathy, in patients with flare of renal involvement from systemic lupus erythematosus (SLE) or systemic sclerosis (SSc), or serious pregnancy-related thromboembolic events in patients with antiphospholipid (aPL) syndromes (APS)] (2-4).

\section{GENERAL CONSIDERATIONS}

Strategies for managing certain of these patients are controversial. Although all pregnancies in women with diffuse connective tissue disorders (CTDs), e.g., SLE or SSc, should be considered high risk, little effect from pregnancy may occur in such patients with remitted or mild disease. The obstetrician and internist/rheumatologist team should construct a wellplanned assessment and management strategy in anticipation of pregnancy in the setting of autoimmunity. The plan should be aimed at

1. surveillance for disease activity,

2. provision of fully adequate therapy for maternal disease, and

3. assessment of fetal growth and well-being.

Diligent search for indications of maternal or fetal compromise is required, if prompt and effective therapy is to be provided.

Management decisions should consider both the effects of pregnancy on maternal disease and the effects of the disease on the developing fetus. Active management of the pregnancy might include

1. monitored progression to delivery (5),

2. interruption by early delivery and immediate specialized neonatal care (6), or

3. therapeutic abortion, if maternal survival is jeopardized.

The optimal goal is to attain a healthy term outcome for both mother and baby.

EFFECTS OF ANTIRHEUMATIC MEDICATIONS ON THE FETUS AND PREGNANCY

Ethical considerations mandate that most of the information that is used to assess the relative risk of maternal use of a drug on the viability of a pregnancy and/or fetus is based on developmental toxicity and reproductive studies done in animal models. The results of such studies may contribute significantly to placing a drug in U.S. Food and Drug Administration Pregnancy Category A, B, C, D, or X. Such classification exists despite the fact that extrapolating data derived from teratogenicity studies done in animals to the human situation frequently requires assumptions that may not, in certain cases, be entirely valid. "Hard" human pregnancy safety data are available for only approximately $20 \%$ of the drugs currently marketed in the United States (7). With these limitations in mind, this section will provide a summary of our current understanding of the risks associated with the use of the more common antirheumatic drugs (i.e., anti-inflammatory agents, corticosteroids, small molecules, and biologics) in the setting of pregnancy.

Nonsteroidal Anti-inflammatory Agents (reviewed in ref. (8)) Nonsteroidal anti-inflammatory agents (NSAIDs), such as celecoxib, diclofenac, and ketorolac, are FDA category $\mathrm{C}$ drugs. This category derives from animal reproduction studies in which an adverse effect was shown on the fetus, and there are no adequate and well-controlled studies in humans, but potential benefits may warrant the use of the drug in pregnant women despite potential risks. However, all other NSAIDs, including ibuprofen, are category B drugs. For these drugs, animal reproduction studies have failed to demonstrate a risk to the fetus and there are no adequate and well-controlled studies in pregnant women. The $\mathrm{D}$ category drugs have shown positive evidence of human fetal risk based on adverse reaction data from investigational or marketing experience or studies in humans. However, potential benefits may warrant the use of the drug in pregnant women despite potential risks.

Results from a number of studies have indicated that NSAIDs may, although rarely, be associated with congenital malformations $(9,10)$ and, possibly, increase the risk of spontaneous abortions $(11,12)$. These untoward effects discourage the use of NSAIDS during the third trimester and just prior to delivery (10). Whether maternal use of selective cyclooxygenase (COX) inhibitors such as celecoxib reduces the potential risk of congenital defects in the fetus compared with NSAIDS that target both COX-1 and COX-2 is currently unclear.

\section{Glucocorticoids}

Fetal regulation of glucocorticoid exposure is achieved by the placental glucocorticoid "barrier," which involves glucocorticoid inactivation within the labyrinth zone by 11-betahydroxysteroid dehydrogenase 2 (11beta-HSD2) (13). Data from Murphy et al. (14) indicate that placental 11beta-HSD2 also has the capacity to metabolize a number of synthetic corticosteroids, including beclomethasone dipropionate, betamethasone, dexamethasone, and prednisolone, but not others, such as budesonide and fluticasone propionate. In a 
series of 34 pregnancies in 30 women, prednisolone use was associated with increased placental insufficiency, fetal distress, and/or stillbirths (15). Overall, however, the available data suggest that the risk associated with prenatal exposure to corticosteroids, although real, is relatively small.

\section{Small Molecules (Slow-Acting Antirheumatic Drugs; Disease-Modifying Agents)}

Among the commonly used slow-acting antirheumatic drugs (SAARDs), or small molecules, methotrexate and leflunomide have been classified as FDA pregnancy category $\mathrm{X}$ drugs. Animal or human studies of these drugs have demonstrated fetal abnormalities and/or investigational or marketing data have indicated human fetal risk. The risk involved in use of these drugs in pregnancy clearly outweighs the potential benefits.

Methotrexate is considered the "anchor" drug in the treatment of rheumatoid arthritis (RA). It is an abortifacient (16) that may induce a pattern of congenital abnormalities, referred to as the fetal aminopterin/methotrexate syndrome (17). This condition consists of growth retardation, and craniofacial, limb, and neurologic developmental anomalies, which may be dose dependent. In planning conception, it is important to realize that it takes approximately 20 weeks for cell-associated methotrexate to become undetectable (18). In contrast to methotrexate, there are minimal data on the effects of leflunomide on human pregnancy, and the FDA's pregnancy category $\mathrm{X}$ classification of this small molecule is largely based on the drug's known inhibitory effects on transcription and translation (19) and its demonstrated teratogenicity and embryotoxicity in animal reproductive studies. Woman who become pregnant while undergoing treatment with leflunomide are advised to reduce fetal exposure to the drug by undergoing cholestyramine elimination procedure. In a study of 64 pregnant women with RA who were treated with leflunomide during pregnancy (61 of whom received cholestyramine), no increase in adverse pregnancy outcomes was found relative to 108 pregnant women with RA not treated with leflunomide and 78 healthy pregnant women (20).

The antimalarial, hydroxychloroquine, a pregnancy category C drug, has not been associated with growth or developmental abnormalities. However, it has known potential untoward effects of retinal or ototoxicity following maternal exposure to the drug in the first trimester for the treatment of a variety of connective tissue diseases $(21,22)$. In a randomized, controlled trial of nonpregnant lupus patients, discontinuation of hydroxychloroquine doubled the risk of an SLE flare within the subsequent 6 months (23). Taken together, the data suggest that hydroxychloroquine use in pregnant patients with connective tissue diseases is relatively safe, although not without risk. It may be prudent to continue such therapy in some SLE patients who unexpectedly become pregnant.

D-penicillamine traverses the placental barrier and can probably decrease molecular cross-linking of collagen in the fetus. Two reports describe congenital cutis laxa and associated connective tissue birth defects, probably due to D-penicillamine treatment during pregnancy $(24,25)$. Discontinuation of this drug is recommended prior to conception as well as during pregnancy and lactation $(26,27)$.

Sulfasalazine is FDA approved for the treatment of RA. Isolated case reports of fetal malformations exist in the setting of maternal sulfasalazine use (28). However, a populationbased case-control study (29) together with two observational studies $(30,31)$ revealed no evidence of teratogenicity in the offspring of pregnant women with inflammatory bowel disease who had been treated with sulfasalazine. Similarly, no increase in the rate of birth defects compared with the general population was found in two more recent studies when sulfasalazine was used during pregnancy (29,32). Accordingly, sulfasalazine enjoys an FDA pregnancy category B classification. If used in a pregnant patient with an autoimmune disease (e.g., RA), it should be administered with folic acid supplementation, since this drug impairs folate absorption and metabolism $(29,32)$.

\section{Immunosuppressive Agents}

Chlorambucil and cyclophosphamide are FDA pregnancy category D drugs. They should be discontinued during pregnancy and lactation based on their teratogenic potential $(33,34)$. Mycophenolate mofetil is used in the treatment of lupus and in refractory RA. Although initially given pregnancy category $\mathrm{C}$ status, use of this drug has been associated with recurrent pregnancy loss and congenital malformations and it is now classified as a category D drug (available from: http:// www.fda.gov/downloads/Safety/MedWatch/SafetyInformation/SafetyAlertsforHumanMedicalProducts/ucm093675. pdf). Cyclosporin A is FDA approved for the treatment of RA and is used as an immunosuppressive agent in a variety of other autoimmune diseases as well. Based on the transplantation literature, the observed rate of birth defects and neonatal deaths after cyclosporine A treatment in doses of 2.5 to $5 \mathrm{mg}$ / $\mathrm{kg} /$ day during pregnancy does not exceed that seen in the general population $(35,36)$.

Azathioprine was not infrequently employed in the treatment of refractory RA prior to the widespread use of biologics. Azathioprine is currently used in the treatment of pregnant patients with active SLE, where toxicity concerns for the fetus obviate the use of cyclophosphamide. Solid evidence linking congenital abnormalities to azathioprine and its more active metabolite, 6-mercaptopurine (6-MP), does not exist $(16,37,38)$. In this connection, a recent study showed no increase in the rate of birth defects in 160 children of women who were exposed to azathioprine in doses of 50 to $100 \mathrm{mg} /$ day (39). This notwithstanding, it is prudent to discontinue the use of azathioprine, 6-MP, or cyclosporine A prior to conception and to avoid the use of these drugs during pregnancy, if possible, especially in the first trimester and during lactation $(16,37,38)$.

\section{Biologics}

Inhibitors of tumor necrosis factor-alpha (TNF- $\alpha$ ) have become a mainstay in the treatment of aggressive and refractory RA. Over 300 women have been exposed to the anti-TNF- $\alpha$ agents, infliximab, etanercept, and adalimumab during pregnancy, most during the first trimester (40). Approximately $10 \%$ of such women were treated with TNF- $\alpha$ inhibitors throughout pregnancy. Overall, the numbers are 
too small to draw definitive conclusions. Two prospective controlled studies have shown comparable rates of malformation and miscarriage rates in women treated or not with antiTNF- $\alpha$ agents (41). However, the monoclonal anti-TNF- $\alpha$ agents (infliximab, adalimumab) do cross the placenta and achieve significant plasma level in cord blood (42), and the role of TNF- $\alpha$ in embryogenesis remains incompletely understood. As of 2005, 61 congenital anomalies in 41 children born to mothers taking TNF- $\alpha$ inhibitors have been reported to the FDA (43). The data provided by the FDA do not permit drawing a conclusion of the actual rate of birth defects with the use of such agents, but tend to argue against the use of TNF- $\alpha$ inhibitors in pregnancy.

Rituximab is a monoclonal antibody, which is directed against a receptor expressed on a broad lineage of $B$ cells that is FDA approved for the treatment of RA. Anecdotal reports indicated that rituximab can suppress neonatal B cell development and the manufacturer recommends the drug be discontinued 12 months before pregnancy (44).

\section{Bisphosphonates}

Bisphosphonates are frequently used to prevent glucocorticoid-induced osteoporosis in a number of autoimmune diseases. In a small study $(n=21)$, exposure to these drugs during the first trimester did not increase the risk of abortion, birth defects, or growth retardation relative to controls (45). This notwithstanding, bisphosphonates have a half-life of approximately 10 years (in bone), may be mobilized during pregnancy, and have been shown in experimental studies to cross the placenta and accumulate in the fetus. Given the limited amount of data available on this class of drugs, use of bisphosphonates in pregnancy is not advised.

DRUGS DURING LACTATION (REVIEWED IN REF. (38)) Effects of NSAIDs on the infant are largely unknown. Aspirin is generally considered safe for nursing mothers. However, the American Academy of Pediatrics recommends that aspirin should be used cautiously by the mother during lactation because of potential adverse effects in the nursing infant (38). The SAARDs, particularly cytotoxic agents, biologics, and bisphosphonates, are not recommended during lactation $(16,38,46)$.

\section{RA AND PREGNANCY}

\section{Clinical Profile of RA}

RA is a chronic systemic disease characterized by inflammatory changes in the synovial membranes that primarily affect small joints of the extremities in a symmetrical fashion (47). The untreated pathologic process often extends beyond the synovial tissue and can erode adjacent joint cartilage and bone, leading to deformities and disability. The underlying mechanisms preceding symptomatic onset that predispose to this disease are unknown (48), although inflammatory pathways predominate in the clinical phase. Women are affected about three times as often as men or to an even greater preponderance during childbearing ages. This sex predisposition and increasing incidence with age in adults are important clues to predisposing mechanisms (48).

\section{Course of RA in Pregnancy}

The course of this disease often improves during pregnancy, usually beginning in the first trimester, although a full remission occurs only in the minority of patients $(49,50)$. About a quarter of patients do not improve or can worsen during the course of pregnancy. Clinically, the features of pain, objective swelling, and systemic inflammatory markers tend to diminish. Aggravating or stressor factors can increase both symptoms and objective manifestations of RA in pregnancy, as with other patients. The ameliorating influence usually diminishes within weeks following delivery and the course may even rebound, unless actively treated $(49,50)$.

\section{Theories of Pregnancy-Amelioration of RA}

Multiple hypotheses have been advanced to explain gestational improvement in RA, as recently reviewed $(49,50)$. Interactions in the core hormonal, immunologic, and microvascular endothelial (MVE) systems are believed to be important determinants of RA (51). These alterations may be favorably influenced during pregnancy, but reverse postpartum (52). The glucocorticoid and reproductive hormone physiology of normal pregnancy and postpartum have been described (53). However, their influences upon the neuroendocrine immune (NEI) and MVE systems have been less well documented (47). During pregnancy, the placenta produces increasing amounts of corticotrophin-releasing hormone (CRH) (54,55). A state of physiologic hypercortisolism with increased production and secretion of cortisol thereby results during pregnancy from placental CRH-induced hypothalamic-pituitary-adrenal (HPA) activation (53-55). The placental CRH induces HPA stimulation and also causes adrenal gland hypertrophy during pregnancy (55).

Generalized immunosuppression is not evident during pregnancy, as recently reviewed $(49,50)$. However, the increased adrenal and ovarian hormones produced during pregnancy are believed to suppress $\mathrm{T}$ helper type 1 (Th1) activity, which is a pathway implicated in the pathogenesis of RA $(56,57)$. Moreover, concentrations of estradiol (E2), as found during pregnancy, promoted proliferation of $\mathrm{T}$ regulatory (Treg) cells exposed to an activating stimulus (58).

The above NEI interactions favor a shift from Th1 to Th2 immunity during pregnancy that is believed to have a salutary effect on RA activity $(49,50)$. However, recent research has called greater attention to the roles of natural killer $(\mathrm{NK})$ and NK-T cells in local immune responses of pregnancy and in the production of pro-inflammatory cytokines $(49,50,59)$. Insufficient studies have been performed of cytokine levels during normal pregnancy or in those with RA. Critical sequential (repeated) multivariate analytic techniques are needed to uncover independent predictors of inflammatory mediators and clinical features of RA activity status during pregnancy (49).

In normal pregnancies, syncytiotrophoblast microvilli, fetal cells, and cell-free fetal nucleic acids are found circulating in maternal blood and in even significantly higher levels in preeclamptic states $(60,61)$. These microparticles are scavenged by antigen-presenting cells and are believed to influence cytokine production (59). Serum levels of fetal DNA correlated with arthritis improvement during pregnancy in one study (62). Microchimerism, that is, two genetically distinct and 
separately derived populations of cells, can occur during pregnancy from placental release of fetal cells into the maternal circulation. Incompatibility of HLA-class II antigens between mother and fetus is suspected to influence the maternal course of RA (63). This area of HLA antigens, maternal-fetal incompatibility, and microchimerism were recently reviewed $(49,50)$.

\section{RA and Fertility, and Fetal Outcome in Patients}

Biologic fertility is not believed to be diminished in RA, but data are few. Before the onset of RA, the length of time of unprotected intercourse until first pregnancy (fecundity) was reported to be longer in one study (64), but not in a more recent investigation (65). Fertility following the onset of RA may be influenced by lessened sexual desire in active disease, but firm data are lacking. Fetal outcome of RA patients is believed to be comparable with healthy controls (66).

\section{Effects of Pregnancy on Risk of Developing RA}

The timing of pregnancy history was studied in relation to RA onset compared with age-matched normal control women (67). The risk of developing RA was about $30 \%$ of expected incidence during the pregnancy period, but was about fivefold greater during the first 3 months postpartum. These data mirror the previously described suppression of disease activity during pregnancy and postpartum flare in women with RA. The postpartum flare was suspected to be explained by breastfeeding during that period (68), but the risk ratio of developing RA while breast-feeding for 3 months or less was not excessive in another study (69).

\section{ANKYLOSING SPONDYLITIS AND PREGNANCY Clinical Profile of AS}

Ankylosing spondylitis (AS) mainly affects the spine and sacroiliac joints (SIJs) and also those of the girdles as well as lower extremities to a lesser degree (70). A striking (circa 80fold) association occurs with the genetic factor HLA-B27 and familial aggregation is strong. The typical spinal and peripheral lesions are localized at attachments of ligaments, tendons, or joint capsules to bone, that is, enthesopathy sites. The SIJ lesions in AS are also unique, characterized by joint space narrowing, erosions, bony bridging, and late-stage intraarticular bony fusion. Underlying disease mechanisms remain obscure, but the enthesopathy feature and SIJ pathology suggest that biomechanical stresses could predispose to onset and also activate inflammatory pathways in its physiopathogenesis (70). Unlike RA, AS occurs in opposite male to female preponderance (circa $2-3 \mathrm{M}: 1 \mathrm{~F}$ ), and has disease onset mainly during adolescence or early adulthood (70).

In early disease, pain and stiffness of the spine and other affected areas are the typical symptoms. In later disease, symptomatology is accompanied by restricted motion or spinal deformities and arthritis of the hips or other lower extremity joints. Disease course tends to be less progressive in women than men, and fertility is not adversely affected.

\section{Course of AS in Pregnancy and Outcomes}

Pregnancy does not improve manifestations of primary AS, that is, without associated ulcerative colitis or psoriasis (71-73). However, postpartum flares can occur during the first 3 months after delivery up to about $90 \%$ of the AS pregnancies (73). Nevertheless, no substantial change was observed in the overall course of disease $(71,72)$. Rather, the chronic low back, sacroiliac area, or other pain sites were aggravated during a flare. Available data indicate that AS does not adversely affect pregnancy $(71,72)$. Worsening of symptoms in AS patients during pregnancy or postpartum may require additional rest or physical measures, for example, non-straining and stretching activities, hot showers or baths, and avoidance of impacts or undue fatigue. Lowest effective dosages of antiinflammatory medication are advisable for limited time periods, until discontinuation in the last month of pregnancy (72).

\section{POLYMYOSITIS DERMATOMYOSITIS AND PREGNANCY Clinical Profile of PM-DM}

Polymyositis (PM) and dermatomyositis (DM) are acquired degenerative and inflammatory myopathies of striated muscles that belong to a spectrum of CTDs $(74,75)$. Proximal, rather than distal, muscle weakness is the typical early feature, with skin involvement of the face, neck, and extensor articular surfaces occurring in dermatomyositis. The onset of these disorders is bimodal in age, with peaks occurring in the juvenile and middle ages. Associated malignancy occurs in a minority of adults, particularly in those older than 40 years, circa $10 \%$ to $15 \%$.

Active PM-DM is rarely seen in association with pregnancy $(76,77)$, due to the low incidence of this disease in childbearing ages, less than one-tenth of SLE. However, a greater number of remitted patients with prior history of juvenile-onset PM-DM may present in pregnancy or for prepregnancy counseling $(76,77)$. Only rarely does PM-DM first develop during pregnancy. Overall, the prognosis for the mother is good (78), and the chances of fetal loss reflect the level of maternal disease $(76,77)$. The pregnancy with PM-DM should be considered high risk for both the mother and the baby, with monitoring of disease activity in the mother and of the growth and well-being of the fetus (79).

\section{Onset of PM-DM in Pregnancy}

As stated, onset of PM-DM during pregnancy is rare, but has the poorest prognosis for the fetus $(77,78,80,81)$. Increasing corticosteroid dosage is a standard treatment for active dermatomyositis in pregnancy, but can have deleterious maternal effects. Intravenous immunoglobulin monotherapy was successful for the outcome of mother and infant in managing a primigravida who presented with dermatomyositis in the first trimester of pregnancy (81).

\section{PM-DM Antedating Pregnancy}

A preceding history of PM-DM occurs more frequently than new disease in pregnant patients, due to the juvenile onset of this disease $(78,80)$. One study $(78)$ reported 10 pregnancies in 6 juvenile-onset PM-DM women. The six pregnancies without exacerbation of PM-DM resulted in term births, whereas the four pregnancies with exacerbations were associated with two abortions. Although the disease is often controlled before the onset of pregnancy, management of such patients is guided by the activity of the PM-DM. The dosage of corticosteroids should be sufficient to control disease, since exacerbation may increase fetal loss or prematurity $(77,78)$. No neonatal effects 
of PM-DM were reported in one study of surviving children nor have placental abnormalities been reported (78)).

\section{SSC AND PREGNANCY}

\section{Clinical Profile of Systemic Sclerosis}

SSc or scleroderma is an uncommon multisystem CTD that encompasses a broad spectrum of clinical entities (82). The spectrum of skin involvement ranges from the most limited form of tightening and hardening that affects only the fingers (sclerodactyly) to a diffuse form that affects proximal extremities and the trunk. Raynaud's phenomenon, or the discoloration of skin on exposure to cold or increased emotion, occurs typically before or during the earliest skin manifestations of scleroderma. An example of a limited form of SSc is the CREST syndrome (calcinosis cutis, Raynaud's phenomenon, esophageal dysmotility, sclerodactyly, and telangiectasias).

The systemic manifestations are also broad and depend upon the degree of the three main pathologic features of this disease: (i) increased deposition of collagen in tissues, which contributes to the skin hardening; (ii) microvascular damage and dysfunction, which contribute to skin atrophy and ischemia of parenchymal tissues of the gastrointestinal tract and circulatory organs (heart, lungs, and kidneys); and (iii) immune activation leading to multiple autoantibodies $(83,84)$. A number of other scleroderma-like fibrosing conditions can also present with clinically hard skin and tissue fibrosis, which should be differentiated from SSc (85). Such conditions and subsets of SSc can be differentiated by their clinical features and occurrence of specific autoantibodies $(83,84)$.

\section{Effect of Pregnancy on SSc}

Pregnancy in SSc may be uneventful, with good outcomes in mother and fetus (86). Those women who have diffuse scleroderma could be at higher risk for developing cardiopulmonary or renal problems for which they should be carefully monitored. In one study of 59 women with SSc who had 91 pregnancies (87), miscarriage was increased only in those with long-standing diffuse scleroderma. Three cases of renal crisis during pregnancy occurred in that subset. Preterm births occurred in almost one-third (29\%) of pregnancies, and all but one of the infants survived.

\section{Effect of SSc on Pregnancy and Fertility}

In a case-control study of women with self-reported scleroderma, spontaneous abortion was twice as high in the cases than controls (88). Pregnancy loss was also high (44\%) in another series of $28 \mathrm{SSc}$ patients, even though the majority (78\%) of the 81 gestations had occurred before clinical disease onset (89). In another case-control study of 46 women with SSc, the odds ratio of pregnancy (abortive or completed) was lower than that in controls (90), suggesting lower fertility. In another study of 100 consecutive women with SSc (91), those with limited disease had significantly more children than those with diffuse disease, again suggesting subfertility in the latter group.

\section{SLE AND PREGNANCY}

\section{Clinical Profile of SLE}

SLE, or lupus, is distinguished from other diffuse CTD by its characteristic multiple organ system and serologic abnormal- ities $(1,4,92)$. Erythematosus malar rash, discoid skin lesions, photosensitivity, and painless oral or nasopharyngeal ulcers are characteristic mucocutaneous features. The arthritis of SLE is non-erosive, but may show deformities, such as ulnar deviation of the fingers. Pleuritis, pericarditis, and abnormalities in renal (proteinuria or cellular casts), neurologic (seizures or psychosis), and hematologic (hemolytic anemia, leukopenia, or thrombopenia) function are also clinically typical of SLE and contribute to its classification criteria (92).

Lupus predominantly affects younger adult women, having a F:M sex ratio in the child-bearing ages of approximately 8:1. The disease is more common in non-Caucasians (1). The clinical presentation, degree of severity, and course of disease are all highly variable (4).

Diagnosis depends upon characteristic clinical manifestations together with confirmation by typical laboratory abnormalities $(1-4,93)$. The antinuclear antibody (ANA) test is positive in essentially all SLE patients. However, it also occurs frequently in other CTD. The ANA has greater diagnostic import when either absent (tending to exclude SLE) or when present in high titers, especially with homogeneous or peripheral-staining nuclear patterns (typical of SLE). An isolated low-titer ANA lacks association with SLE or adverse pregnancy outcome, compared with high-titer ANA or the more specific antibodies that correlate with this disorder. The specific and confirmatory immunologic abnormalities of SLE include anti-native (double-stranded) DNA (nDNA) or anti-Smith (Sm) antibody. Positive lupus erythematosus (LE) cell preparation, a test that is rarely performed today, or chronic (at least 6 months) biologic false-positive test for syphilis supports, but does not confirm the diagnosis, since these serologic findings are typical but are not specific for SLE (1).

\section{Course of SLE in Pregnancy}

The outcome for pregnant women with SLE has substantially improved over the last four decades as evidenced by the decline in fetal loss from $40 \%$ in 1960 to 1965 to $17 \%$ in 2000 to 2003 (94). However, pregnancy in women with lupus can still be associated with significant maternal and fetal complications. A recent study reported that the incidence of spontaneous abortion, stillbirth, intrauterine growth retardation (IUGR), and prematurity are increased at least twofold among SLE patients compared with the normal population (95). Following the diagnosis of SLE, a greater than fourfold increase was observed in pregnancy loss compared with an individual's reproductive history before the diagnosis of lupus was made (96). Similarly, a cross-sectional analysis of adverse outcomes in 1029 pregnancies of Afro-Caribbean women in Trinidad found that SLE pregnancies were more than twice as likely to end in fetal death than non-SLE pregnancies (97). In a study of 16.7 million pregnancies, which included 13,555 lupus patients, an increased maternal mortality of more than 20 -fold, 325 per 100,000 live births, was observed in SLE patients (98). The latter rate is lower than that reported in the Hopkins Cohort Study (1130 maternal deaths per 100,000 live births) (99).

There is general agreement that increased lupus activity at the time of conception increases the likelihood of a flare during gestation (99-102). Hence the recommendation: pregnancy 
should be planned when SLE is in remission. In support of this recommendation, a number of reports indicate that pregnancy may not increase the risk of lupus flares in the inactive or wellcontrolled patient $(94,98,99)$ and that frequency of flares may not be greater in the well-controlled lupus patient in pregnant state than in the nonpregnant state $(4,93,103-105)$.

Exacerbation of disease has been reported in up to $50 \%$ of SLE pregnancies. In a recent case-control study, flares were observed in $18 \%$ of SLE pregnancies (106), a rate that agrees favorably with another recently published report in Argentina (107). Apart from glomerulonephritis and, less commonly, neuropsychiatric SLE, flares during pregnancy and in the postpartum period are generally mild or moderate, with a predominance of cutaneous, articular, and minor hematologic manifestations $(98,100,107)$. Of the 309 pregnancies cited in the review by Moroni and Ponticelli (102), only 12\% of SLE flares were associated with renal insufficiency. Active lupus nephritis was found to result in greater fetal loss compared with pregnancies in women without lupus nephritis and in more frequent preterm delivery in the study by Wagner et al. (108). By contrast, active renal disease had no effect on pregnancy outcome (save for low birth rate) in the study by Gladman et al. (109).

A high rate of adverse fetal events can occur in pregnant woman with lupus independent of the presence of lupus nephritis $(2-4,110,111)$. Published data have identified several risk factors for poor pregnancy outcomes, including the presence of hypertension, the presence of lupus nephritis, the presence and degree of proteinuria $(112,113)$, and the presence of high-titer aPL antibodies. Most American physicians do not believe that routine "prophylactic" steroid therapy to suppress disease activity during pregnancy reduces risk for maternal flare or decreases fetal wastage, unless needed to control intercurrent disease flare.

Decidual vasculopathy with necrotizing inflammatory lesions and "acute atherosis" occurs in placentas from lupus patients $(114,115)$ as well as in those with the aPL syndrome $(115,116)$, irrespective of whether the pregnancies resulted in fetal deaths (115) or in live-born SGA infants (117). Placental size is reduced in SLE patients compared with both healthy and diabetic controls (118). Most later pregnancy losses may be attributable to placental dysfunction (114-116,118).

\section{NEONATAL LUPUS SYNDROME AND CONGENITAL HEART BLOCK}

Neonatal lupus is characterized by transient hematologic and cutaneous disorders and permanent incomplete or complete congenital heart block (CCHB). It occurs in less than $1 \%$ of the offspring of SLE patients (3,4,119-123) and only in pregnancies associated with maternal antibodies to SSA (i.e., Sjögren's syndrome A/Ro) or SSB/La (124). These autoantibodies are present in $25 \%$ to $32 \%$ of pregnant SLE patients (122). In one prospective study of sequential pregnancies, none of 38 infants of lupus mothers with antiSSA (Ro) antibody had children with heart block (122). In another prospective study of 100 women with anti-Ro/SSA antibodies, 2 had infants who developed CCHB in utero. Among the 53 lupus mothers who harbored such antibodies, no case of CCHB occurred (125). Thus, most infants with this syndrome are born of asymptomatic mothers with such antibodies who rarely develop a lupus-like disorder later in life $(121,124,126)$.

\section{CCHB}

Substantial morbidity and mortality are associated with CCHB (123). This rare, potentially fatal complication $(120,122,124,126,127)$ is more problematic than the noncardiac transient abnormalities in the neonatal lupus syndrome. The CCHB is associated with myocarditis and with simultaneously present maternal anti-Ro (SSA) and anti-La (SSB) antibodies (120-122,124,126,127). If cardiac conduction defects are detected by antepartum fetal heart rate monitoring, a fetal echocardiogram should be obtained for detection of other cardiac anomalies (120-122,128). Appropriate fetal monitoring and management procedures should be employed (128).

\section{Effects of SLE on Fertility}

There are insufficient data to support a deleterious effect of SLE on fertility (129-132). Generally, fertility is not considered to be impaired in women with SLE $(127,133)$ except in those with severe renal disease (i.e., creatinine clearance $<50 \mathrm{ml} /$ min) $(3,119)$ or those exposed to cyclophosphamide or chlorambucil.

\section{MANAGEMENT OF PREGNANT WOMEN WITH SLE Initial Assessment and Monitoring}

Management of pregnant women with SLE should be a collaborative effort between the obstetrician and the rheumatologist. The initial intake should consist of a careful history, including duration of lupus, an assessment of the patient's current disease activity, the patient's past obstetric history, and the timing of past pregnancies with regard to onset of lupus. If the patient is in remission, the duration of remission should be noted. Physical examination should include blood pressure measurement, cardiovascular assessment, and accurate pregnancy dating with confirmation by early obstetrical sonogram. Laboratory testing is suggested to include a complete blood count with quantitative assessment of platelets; serum creatinine (and measurement of creatinine clearance if indicated); complement 3 (C3), C4, and total complement levels; the presence of anti-dsDNA, anticardiolipin antibodies (aCLs), lupus anticoagulant (LAC), anti-SSA (Ro), and antiSSB (La) (96). In pregnancy, baseline values of C3 and C4 are higher secondary to the permissive effect of estrogen on complement synthesis. Thus, normal levels of C3 and/or C4 do not necessarily mean that a pregnant SLE patient's disease is quiescent.

\section{Monitoring During Pregnancies}

The complete blood count, creatinine, serum complements, anti-dsDNA, and urine with microscopic analysis should be repeated at 1 - to 2-month intervals, as appropriate, during pregnancy and for 3 months postpartum (112). Beginning at 20 weeks of gestation, obstetrical visits are increased to every 2 weeks, because pre-eclampsia may occur at this point. At 28 weeks, visits should be weekly (96). aCL Should be repeated if initially negative, each trimester. The other laboratory parameter to indicate lupus flares are increases in erythrocyte sedimentation rate, lower hemoglobin, and increased activity 
indices such as Systemic Lupus Erythematosus Disease Activity Index (SLEDAI), European Consensus Lupus Activity Measure (ECLAM), and Lupus Activity Index (LAI). The SLEDAI and LAI have been validated as indicators of flares in pregnant SLE patients with a sensitivity of $93 \%$ and a specificity of $98 \%$ (134). Clinical and laboratory measures of disease activity are used to distinguish kidney involvement in lupus from superimposed pre-eclampsia. Early detection and aggressive management of lupus activity is the approach recommended by most rheumatologists, although determination of lupus activity during the pregnancy may be challenging $(96,101,112)$.

\section{Fetal Surveillance}

Surveillance for fetal growth can be conducted by serial obstetrical sonography $(96,135-138)$. This is especially important in patients with chronic hypertension in whom preeclampsia has developed, and in SLE mothers with aPL antibodies and a history of prior unexplained pregnancy losses $(139,140)$. Interestingly, although spontaneous abortions were somewhat higher among those with $(20.6 \%)$ than without (11.3\%) renal involvement, the presence of nephropathy did not significantly alter fetal outcome overall among patients in the Mexico City study (110).

In those patients at risk for fetal congenital heart block associated with anti-SSA (Ro) and anti-SSB (La) antibodies, additional testing is required (127). This fetal condition occurs after 16 weeks of gestation. Screening by m-mode echocardiography should be started at 16 weeks and should continue weekly until delivery (127).

\section{Treatment}

(See also Effects of Antirheumatic Medications on the Fetus and Pregnancy above). The reader is referred to a number of recent reviews for a discussion of treatment of SLE in pregnancy $(96,101,102)$.

\section{Route and Timing of Delivery}

For SLE patients, route and timing of delivery should be based upon results of fetal studies with attention to the maternal condition, especially renal and blood pressure status. The need for neonatologist attendance should be determined prior to labor by both maternal and fetal statuses. If necessary, transport should be arranged to a facility equipped for care of complicated premature infants.

\section{APS}

\section{Definition of APS}

Antibodies to anionic (negatively charged) phospholipids or phospholipid-binding proteins (aPL antibodies) are associated with recurrent, usually mid-trimester, spontaneous abortions, autoimmune thrombocytopenia, arterial and deep venous thromboses, and livedo reticularis (141-145). The definition of recurrent miscarriage has varied in the literature, with implications as to prognosis of the untreated pregnancy. The standard obstetrical definition is three or more consecutive losses. However, the definition used for most rheumatologic patients has been two or more losses (sometimes even one). Incidence of pregnancy loss increases with greater number of preceding miscarriages, which has implications in evaluating efficacy of one or another therapeutic approach.

\section{aPL Antibodies and Laboratory Tests for Measuring aPL Antibodies}

The aPL antibodies are heterogeneous, polyclonal and may vary in titer over the course of follow-up. LACs are aPL antibodies that block phospholipid surfaces and reduce the coagulant potential of plasma, thereby prolonging the clotting time in coagulation tests based on the activated partial thromboplastin time (146). The presence of LAC is determined functionally by (i) a failure to correct the prolonged clotting time after mixing with normal platelet-free plasma and (ii) the dilute Russell's viper venom time (147). aCL Are IgG, IgM, or IgA antibodies that bind to the phospholipid cardiolipin. They do not prolong functional assessments of clotting (i.e., coagulation assays). Detection of aCL is done using an enzyme-linked immunosorbent assay (148). It is generally believed that aCL antibodies are significant only when present in the context of a thrombotic event and that anti-IgG antibodies carry greater pathogenetic significance than do those of the IgM isotype (149). The aCL antibodies are sensitive, being present in $\sim 80 \%$ of patients with APS, but not as specific as that of LAC and have been reported in numerous infectious diseases (150-153). False-positive tests for syphilis occur with increased frequency in individuals with LAC and aCL activities using a variety of tests (e.g., the rapid plasma reagin and Venereal Disease Research Laboratories tests). These tests are not reliable indicators of clinically important outcomes. In patients with false-positive syphilis tests secondary to aPL antibodies, the fluorescent treponema antibody test remains negative (154).

Beta-2-glycoprotein I (beta2GPI) (155) is a naturally occurring anticoagulant and potential antigenic target for some aPL antibodies that are detected by solid-phase assay (156). It is thought that aPL-associated thrombosis occurs when anti-beta2GPI antibodies bind and promote dimerization of beta2GPI, which increases binding affinity for phospholipids and induces activation of complement, platelets, endothelial cells, and monocytes (157). The revised Sapporo criteria (158) for diagnosing APS (159) include the presence of LAC, or IgG or IgM aCL, or anti-beta2GPI antibodies (measured on two occasions 12 weeks apart) together with vascular thrombosis or pregnancy morbidity.

\section{Primary and Secondary APS}

aPL Antibodies without clinical symptoms may be found in otherwise healthy persons (144). Those with symptoms who do not have SLE or previously recognized clinical disorders are designated as having a "primary" APS (160). Persons with SLE or other rheumatic disorders $(143-145,161,162)$ are designated as having "secondary" APS. Close to $50 \%$ of patients who have APS also have SLE; however, less than 10\% of SLE patients have an APS (163). In lupus, APS seems to correlate with increased fetal loss $(115,139,140,164)$ and appears to be part of the disease, rather than a separate, associated condition (162). That said, there may be a negative correlation of aPL antibodies with active lupus (115), nephrotic syndrome, and certain cutaneous forms of lupus (162). Secondary and primary APS patients have similar overall clinical and 
laboratory profiles (165), although lupus patients with APS have more hemolytic anemia, endocardial valve disease, neutropenia, and lower C4 levels (165). In pregnancy, lupus disease activity and associated APS, when present, behave independently. A patient with remitted lupus and secondary aPL syndrome behaves like one with primary APS. A patient with active lupus and APS develops complications of, and needs treatment for, both diseases.

\section{Do aPL Antibodies Predispose to Fetal Wastage?}

A literature review concluded that there was an association between the prevalence of aPL antibodies in women with SLE and history of fetal loss $(60 \%)$, but remained inconclusive about the relationship between aPL antibodies and miscarriages in women without SLE (4-13\% losses) (144). Most studies, however, do find an association between high-level aPL antibodies and repeated, but not first, fetal loss $(4,139,140,161,164,166)$. In one study, aCL titers remained high in aborting patients (167), which is not uncommon $(142,164,168)$. aPL Antibodies have been detected in 5\% to $15 \%$ of otherwise healthy women with a history of unexplained recurrent abortions (144).

Frequencies of aCL antibodies are low $(<5 \%)$ in consecutive samples of healthy pregnant women without prior pregnancy losses, and there is no significant correlation with fetal or maternal complications (169). Moderate to high levels of aCL antibodies, of the IgG isotype, do correlate with fetal wastage, in referral, high-risk $(142,164)$ and general obstetric $(168,170)$ populations.

Antibodies to beta2GPI-CL complex were significantly associated with fetal complications in a large cohort of apparently healthy pregnant women followed prospectively in Japan (171). It is not known whether women with positive titers have other primary risk factors for increased fetal wastage such as clinically inactive lupus (114-116,118,166).

\section{Fetal Complications Reported with aPL Antibodies}

APS is the most common association of recurrent miscarriage syndrome, a condition that affects more than 500,000 women in the United States annually $(172,173)$. Recurrent fetal losses due to intrauterine death at any stage of pregnancy, but particularly in the second and third trimesters of gestation, and fetal growth restriction, are characteristic complications associated with aPL antibodies $(168,170,174)$. Recurrent, late first-trimester fetal loss after detection of fetal heart activity has also been associated with aPL antibodies (175). No fetal pathology can explain the spontaneous abortions and stillbirths. Instead, the placentas invariably show scattered areas of infarction, intervillous thrombosis, and fibrin deposition (114-118,174).

Few published data are available on the aPL antibody status of live-born infants of mothers positive for these antibodies. Most infants in whom antibody can be detected are well, except for the effects of prematurity and IUGR, although reports are anecdotal.

\section{Maternal Complications Reported with aPL Syndromes}

Maternal complications of pregnancies associated with APS can be life-threatening or fatal $(172,173)$ and include severe pre-eclampsia (176), arterial or venous thromboses $(177,178)$, pulmonary hypertension (179), and the syndrome of hemolysis, elevated liver enzymes, and low platelets (HELLP) (163). Although aPL antibodies are not present in the majority of individuals with pre-eclampsia (180), a systematic review indicates that positivity for aPL antibodies is a highly significant risk factor for the development of pre-eclampsia (181). Over half of women presenting with obstetrical problems in the setting of APS have not had a previously recognized autoimmune disease (166) although a minority go on to develop SLE. The majority experience thrombotic vascular complications of APS (165). There may also be an increased risk of cardiopulmonary complications associated with delivery $(159,160,166,167)$ and an increased risk of stroke in the postpartum period (176).

\section{Treatment of Pregnancy-Related Complications Associated with aPL Antibodies}

Patients with aPL antibodies should have consultations with maternal-fetal specialists, either on a preconception basis or immediately following verification of pregnancy. Patients with prior complicated aPL-related gestations should be managed by a maternal-fetal medicine team familiar with the illness and skilled in critical care. Infants of such gestations should be delivered in facilities experienced in the care of premature small infants (500-1500 g birth weight). Careful monitoring of fetal growth and uteroplacental function (5), beginning as early as the second trimester, may detect impending fetal compromise and necessitate early delivery (6).

\section{Anticoagulation plus Low-Dose Aspirin}

In an earlier uncontrolled study, fetal wastage was prevented with heparin therapy in most women with aCL and LAC antibodies who had recurrent losses or previous abnormal outcomes (182). However, successful pregnancy has been observed in SLE patients with untreated LAC (183). Full-dose heparin plus low-dose aspirin was found to provide significantly better pregnancy outcome than low-dose aspirin alone in women who had at least three consecutive pregnancy losses, without causing increased complications (184). By contrast, ongoing studies (185) indicate that treatment of aPL antibody-associated pregnancy loss with lower dose heparin and aspirin is equivalent to full-dose anticoagulation (184). As recently pointed out (186), the optimal dose of heparin to maximize benefit and minimize harm is currently unknown.

Based on controlled clinical trials $(184,185,187,188)$, aspirin (81 $\mathrm{mg} /$ day) plus heparin, either unfractionated heparin or low-molecular weight heparin, is the preferred treatment for repeated fetal losses associated with aPL antibodies (172). However, a Cochrane collaboration review on the therapy of miscarriage in women with aPL recommends aspirin $(81 \mathrm{mg} /$ day) and unfractionated heparin (186). Therapy with aspirin and heparin should be begun immediately postconception and should be taken throughout the pregnancy (172).

\section{Prednisone Suppression of Maternal LAC}

Initial reports in the 1980s (166) suggested that high-dose prednisone therapy (e.g., 40-60 mg/day, orally) promotes fetal survival in pregnancies of women with recurrent abortions associated with LAC; however, more recent experience argues against glucocorticoid prophylaxis $(4,139,189)$ in favor of 
anticoagulation therapy as noted above (184-188,190). Importantly, lupus patients will likely need to be treated with sufficient prednisone for suppression of disease activity in pregnancy. However, there is no compelling evidence to indicate that higher doses of steroids intended for immunosuppression of aPL antibodies per se are beneficial. Furthermore, since pre-eclampsia may be associated with the presence of $\operatorname{LAC}(176,191)$, the indication of fluid retention and hypertension argues against the use of high-dose prednisone $(189,192)$. Finally, the use of highdose prednisone increases the risk of long-term complications, especially osteonecrosis and osteoporosis.

\section{Intravenous Gamma-globul in Therapy}

Anecdotal reports comprising a small number of patients have suggested improved pregnancy outcomes with experimental gamma-globulin therapy $(193,194)$ in patients with recurrent miscarriages due to APS. However, The Pregnancy Loss Study Group reported that gamma-globulin therapy had no beneficial effect in such patients in a double-blinded randomized trial (195). Also it has been shown that immunoglobulin therapy is inferior to aspirin plus heparin in the treatment of recurrent fetal loss associated with aPL antibodies (196).

\section{Low-Dose Aspirin}

Although mini-dose aspirin (e.g., 60-100 mg daily) has been recommended for treatment in pregnant women with primary APS and history of recurrent abortions (190), recent studies suggest that aspirin therapy in the absence of anticoagulation is not sufficient to prevent fetal loss in high-risk pregnancies (184).

\section{ACKNOWLEDGMENTS}

The authors wish to acknowledge the expert technical assistance of Lori Orton and Jessica Debis.

\section{REFERENCES}

1. Masi AT, Medsger TA Jr. Epidemiology of the rheumatic diseases. In: McCarty DJ, ed. Arthritis and Allied Conditions, 11th edn. Philadelphia: Lea \& Febiger, 1989: 16-54.

2. Black CM, Stevens WM. Scleroderma. Rheum Dis Clin North Am 1989; 15: 193-212.

3. Dombroski RA. Autoimmune disease in pregnancy. Med Clin North Am 1989; 73: 605-21.

4. Lockshin M, Druzin M. Rheumatic diseases. In: Barron W, Lindheimer MD, eds. Medical Disorders During Pregnancy, 2nd edn. St Louis: Mosby-Year Book, Inc., 1995: 307-37.

5. Trudinger BJ, et al. Monitoring lupus anticoagulant[c1]-positive pregnancies with umbilical artery flow velocity waveforms. Obstet Gynecol 1988; 72: 215-18.

6. Druzin ML, et al. Second-trimester fetal monitoring and preterm delivery in pregnancies with systemic lupus erythematosus and/or circulating anticoagulant. Am J Obstet Gynecol 1987; 157: 1503-10.

7. Lo WY, Friedman JM. Teratogenicity of recently introduced medications in human pregnancy. Obstet Gynecol 2002; 100: 465-73.

8. Masi A, Feigenbaum SL, Lockshin M. Rheumatological diseases and antiphospholipid syndrome. In: Winn HN, Hobbins JC, eds. Clinical Maternal-Fetal Medicine. Chapter 45. London: Parthenon Publishing, 2000: 515-36.

9. Aselton P, et al. First-trimester drug use and congenital disorders. Obstet Gynecol 1985; 65: 451-5.

10. Ericson A, Kallen BA. Nonsteroidal anti-inflammatory drugs in early pregnancy. Reprod Toxicol 2001; 15: 371-5.

11. Nielsen GL, et al. Risk of adverse birth outcome and miscarriage in pregnant users of non-steroidal anti-inflammatory drugs: population based observational study and case-control study. BMJ 2001; 322: 266-70.
12. Li DK, Liu L, Odouli R. Exposure to non-steroidal anti-inflammatory drugs during pregnancy and risk of miscarriage: population based cohort study. BMJ 2003; 327: 368.

13. Dy J, et al. Placental 11beta-hydroxysteroid dehydrogenase type 2 is reduced in pregnancies complicated with idiopathic intrauterine growth restriction: evidence that this is associated with an attenuated ratio of cortisone to cortisol in the umbilical artery. Placenta 2008; 29: 193-200.

14. Murphy VE, et al. Metabolism of synthetic steroids by the human placenta. Placenta 2007; 28: 39-46.

15. Warrell DW, Taylor R. Outcome for the foetus of mothers receiving prednisolone during pregnancy. Lancet 1968; 1: 117-18.

16. Bermas BL, Hill JA. Effects of immunosuppressive drugs during pregnancy. Arthritis Rheum 1995; 38: 1722-32.

17. Del Campo M, et al. Developmental delay in fetal aminopterin/ methotrexate syndrome. Teratology 1999; 60: 10-12.

18. Dalrymple JM, et al. Pharmacokinetics of oral methotrexate in patients with rheumatoid arthritis. Arthritis Rheum 2008; 58: 3299-308.

19. Prakash A, Jarvis B. Leflunomide: a review of its use in active rheumatoid arthritis. Drugs 1999; 58: 1137-64.

20. Chambers CD, et al. Birth outcomes in women who have taken leflunomide during pregnancy. Arthritis Rheum 2010; 62: 1494-503.

21. Costedoat-Chalumeau N, et al. Safety of hydroxychloroquine in pregnant patients with connective tissue diseases. Review of the literature. Autoimmun Rev 2005; 4: 111-15.

22. Costedoat-Chalumeau N, et al. Safety of hydroxychloroquine in pregnant patients with connective tissue diseases: a study of one hundred thirty-three cases compared with a control group. Arthritis Rheum 2003; 48: 3207-11.

23. The Canadian Hydroxychloroquine Study Group. A randomized study of the effect of withdrawing hydroxychloroquine sulfate in systemic lupus erythematosus. The Canadian Hydroxychloroquine Study Group. N Engl J Med 1991; 324: 150-4.

24. Mjolnerod OK, et al. Congenital connective-tissue defect probably due to D-penicillamine treatment in pregnancy. Lancet 1971; 1: 673-5.

25. Solomon L, et al. Neonatal abnormalities associated with D-penicillamine treatment during pregnancy. N Engl J Med 1977; 296: 54-5.

26. Roubenoff R, et al. Effects of antiinflammatory and immunosuppressive drugs on pregnancy and fertility. Semin Arthritis Rheum 1988; 18: $88-110$.

27. Endres W. D-penicillamine in pregnancy-to ban or not to ban? Klin Wochenschr 1981; 59: 535-7.

28. Hoo JJ, Hadro TA, Von Behren P. Possible teratogenicity of sulfasalazine. N Engl J Med 1988; 318: 1128.

29. Norgard B, et al. Population-based case control study of the safety of sulfasalazine use during pregnancy. Aliment Pharmacol Ther 2001; 15 : 483-6.

30. Willoughby CP, Truelove SC. Ulcerative colitis and pregnancy. Gut 1980; 21: 469-74.

31. Mogadam M, et al. Pregnancy in inflammatory bowel disease: effect of sulfasalazine and corticosteroids on fetal outcome. Gastroenterology 1981; 80: 72-6.

32. Moskovitz DN, et al. The effect on the fetus of medications used to treat pregnant inflammatory bowel-disease patients. Am J Gastroenterol 2004; 99: 656-61.

33. Shotton D, Monie IW. Possible Teratogenic Effect of Chlorambucil on a Human Fetus. JAMA 1963; 186: 74-5.

34. Clowse ME, Magder L, Petri M. Cyclophosphamide for lupus during pregnancy. Lupus 2005; 14: 593-7.

35. Lamarque V, et al. Analysis of 629 pregnancy outcomes in transplant recipients treated with Sandimmun. Transplant Proc 1997; 29: 2480.

36. Bar $\mathrm{Oz} \mathrm{B}$, et al. Pregnancy outcome after cyclosporine therapy during pregnancy: a meta-analysis. Transplantation 2001; 71: 1051-5.

37. Ostesen M. Optimisation of antirheumatic drug treatment in pregnancy. Clin Pharmacokinet 1994; 27: 486-503.

38. Briggs G, Freeman R, Yaffe S. Reference Guide to Fetal and Neonatal Risk, Drugs in Pregnancy and Lactation, 4th edn. Baltimore: Williams \& Wilkins, 1995.

39. Goldstein LH, et al. Pregnancy outcome of women exposed to azathioprine during pregnancy. Birth Defects Res A Clin Mol Teratol 2007; 79: 696-701. 
40. Ostensen M, Forger F. Management of RA medications in pregnant patients. Nat Rev Rheumatol 2009; 5: 382-90.

41. Chambers CD, Johnson DL, Jones KL. Pregnancy outcome in women who were exposed to adalimumab: the OTIS autoimmune diseases in pregnancy project (abstract 514). Arthritis Rheum 2006; 54(Suppl): S251.

42. Vasiliauskas EA, et al. Case report: evidence for transplacental transfer of maternally administered infliximab to the newborn. Clin Gastroenterol Hepatol 2006; 4: 1255-8.

43. Carter JD, et al. A safety assessment of tumor necrosis factor antagonists during pregnancy: a review of the food and drug administration database. J Rheumatol 2009; 36: 635-41.

44. Klink DT, et al. Rituximab administration in third trimester of pregnancy suppresses neonatal B-cell development. Clin Dev Immunol, 2008; 2008: 271363.

45. Levy S, et al. Pregnancy outcome following in utero exposure to bisphosphonates. Bone 2009; 44: 428-30.

46. Lee P. Anti-inflammatory therapy during pregnancy and lactation. Clin Invest Med 1985; 8: 328-32.

47. Masi A, Aldag JC. Rheumatic disorders and stress. In: Fink G, ed. Encyclopedia of Stress. Chapter 459. 2nd edn. Oxford: Elsevier, 2007.

48. Masi AT, Aldag JC. Integrated neuroendocrine immune risk factors in relation to rheumatoid arthritis: should rheumatologists now adopt a model of a multiyear, presymptomatic phase? Scand J Rheumatol 2005; 34: 342-52.

49. Masi A, Santoro T, Aldag JC. Pregnancy and postpartum influences on rheumatoid arthritis activity: Nature's Model to Investigate Systemic Biological Mechanisms in the Disease. Curr Rheum Rev 2007; 3: 217-24.

50. Ostensen M, Villiger PM. The remission of rheumatoid arthritis during pregnancy. Semin Immunopathol 2007; 29: 185-91.

51. Masi AT, et al. Integrated hormonal-immunological-vascular (H-I-V triad) systems interactions in the rheumatic diseases. Clin Exp Rheumatol 1995; 13: 203-16.

52. Kanik KS, Wilder RL. Hormonal alterations in rheumatoid arthritis, including the effects of pregnancy. Rheum Dis Clin North Am 2000; 26: 805-23.

53. Braunstein G. Williams Textbook of Endocrinology. In: Larsen P, et al., eds. Endocrine changes in pregnancy. 10th edn. Philadelphia: W. B Saunders, 2003: 795-810.

54. Chrousos GP, Torpy DJ, Gold PW. Interactions between the hypothalamic-pituitary-adrenal axis and the female reproductive system: clinical implications. Ann Intern Med 1998; 129: 229-40.

55. Mastorakos G, Ilias I. Maternal hypothalamic-pituitary-adrenal axis in pregnancy and the postpartum period. Postpartum-related disorders. Ann NY Acad Sci 2000; 900: 95-106.

56. Ostensen M. Sex hormones and pregnancy in rheumatoid arthritis and systemic lupus erythematosus. Ann N Y Acad Sci 1999; 876: 131-43; discussion 144.

57. Wilder RL, Elenkov IJ. Hormonal regulation of tumor necrosis factoralpha, interleukin-12 and interleukin-10 production by activated macrophages. A disease-modifying mechanism in rheumatoid arthritis and systemic lupus erythematosus? Ann NY Acad Sci 1999; 876: 14-31.

58. Prieto GA, Rosenstein Y. Oestradiol potentiates the suppressive function of human CD4 CD25 regulatory $\mathrm{T}$ cells by promoting their proliferation. Immunology 2006; 118: 58-65.

59. Sargent IL, Borzychowski AM, Redman CW. NK cells and human pregnancy-an inflammatory view. Trends Immunol 2006; 27: 399-404.

60. Knight M, et al. Shedding of syncytiotrophoblast microvilli into the maternal circulation in pre-eclamptic pregnancies. Br J Obstet Gynaecol 1998; 105: 632-40.

61. Hahn S, Huppertz B, Holzgreve W. Fetal cells and cell free fetal nucleic acids in maternal blood: new tools to study abnormal placentation? Placenta 2005; 26: 515-26.

62. Yan Z, et al. Prospective study of fetal DNA in serum and disease activity during pregnancy in women with inflammatory arthritis. Arthritis Rheum 2006; 54: 2069-73.

63. Van Der Horst-Bruinsma IE, et al. Influence of HLA-class II incompatibility between mother and fetus on the development and course of rheumatoid arthritis of the mother. Ann Rheum Dis 1998; 57: 286-90.

64. Yoshino S, Uchida S. Sexual problems of women with rheumatoid arthritis. Arch Phys Med Rehabil 1981; 62: 122-3.
65. Van Dunne FM, et al. Miscarriage but not fecundity is associated with progression of joint destruction in rheumatoid arthritis. Ann Rheum Dis 2004; 63: 956-60.

66. Siamopoulou-Mavridou A, et al. Outcome of pregnancy in patients with autoimmune rheumatic disease before the disease onset. Ann Rheum Dis 1988; 47: 982-7.

67. Silman A, Kay A, Brennan P. Timing of pregnancy in relation to the onset of rheumatoid arthritis. Arthritis Rheum 1992; 35: 152-5.

68. Brennan P, Silman A. Breast-feeding and the onset of rheumatoid arthritis. Arthritis Rheum 1994; 37: 808-13.

69. Karlson EW, et al. Do breast-feeding and other reproductive factors influence future risk of rheumatoid arthritis? Results from the Nurses' Health Study. Arthritis Rheum 2004; 50: 3458-67.

70. Masi A, Benjamin M, Vleeming A. Movement, Stability and Lumbopelvic Pain: integration of research and therapy. In: Vleeming A, Mooney V, Stoeckart R, eds. Anatomical, biochemical, and clinical perspectives on sacroiliac joints: an integrative synthesis of biodynamic mechanisms related to ankylosing spondylitis. 2nd edn. Edinburgh: Elsevier Limited, 2007: 205-27.

71. Ostensen M, Husby G. A prospective clinical study of the effect of pregnancy on rheumatoid arthritis and ankylosing spondylitis. Arthritis Rheum 1983; 26: 1155-9.

72. Ostensen M, Husby G. Ankylosing spondylitis and pregnancy. Rheum Dis Clin North Am 1989; 15: 241-54.

73. Ostensen M. The effect of pregnancy on ankylosing spondylitis, psoriatic arthritis, and juvenile rheumatoid arthritis. Am J Reprod Immunol 1992; 28: 235-7.

74. Pipitone N, Salvarani C. Established and new treatments of the idiopathic inflammatory myopathies: dermatomyositis and polymyositis. Clin Exp Rheumatol 2007; 25: 896-906.

75. Greenberg SA. Inflammatory myopathies: evaluation and management. Semin Neurol 2008; 28: 241-9.

76. Silva CA, Sultan SM, Isenberg DA. Pregnancy outcome in adult-onset idiopathic inflammatory myopathy. Rheumatology (Oxford) 2003; 42: $1168-72$.

77. Vancsa A, et al. Pregnancy outcome in idiopathic inflammatory myopathy. Rheumatol Int 2007; 27: 435-9.

78. Mintz G. Dermatomyositis. Rheum Dis Clin North Am 1989; 15: 375-82.

79. Gutierrez G, Dagnino R, Mintz G. Polymyositis/dermatomyositis and pregnancy. Arthritis Rheum 1984; 27: 291-4.

80. Rosenzweig BA, et al. Primary idiopathic polymyositis and dermatomyositis complicating pregnancy: diagnosis and management. Obstet Gynecol Surv 1989; 44: 162-70.

81. Williams L, et al. Successful treatment of dermatomyositis during pregnancy with intravenous immunoglobulin monotherapy. Obstet Gynecol 2007; 109(2 Pt2): 561-3.

82. Masi A. Clinical-epidemiologic perspective of systemic sclerosis (scleroderma). In: Jayson MIV, Black CM, eds. Systemic SclerosisScleroderma. Sussex, England: Wiley and Sons Ltd., 1988: 7-31.

83. Roufosse FE, De Bellefon LM. Systemic sclerosis (scleroderma). Acta Clin Belg 2007; 62: 323-9.

84. Steen VD. The many faces of scleroderma. Rheum Dis Clin North Am 2008; 34: 1-15; v.

85. Boin F, Hummers LK. Scleroderma-like fibrosing disorders. Rheum Dis Clin North Am 2008; 34: 199-220; ix.

86. Steen VD. Pregnancy in scleroderma. Rheum Dis Clin North Am 2007; 33: 345-58, vii.

87. Steen VD. Pregnancy in women with systemic sclerosis. Obstet Gynecol 1999; 94: 15-20.

88. Silman AJ, Black C. Increased incidence of spontaneous abortion and infertility in women with scleroderma before disease onset: a controlled study. Ann Rheum Dis 1988; 47: 441-4.

89. McHugh NJ, Reilly PA, McHugh LA. Pregnancy outcome and autoantibodies in connective tissue disease. J Rheumatol 1989; 16: 42-6.

90. Pisa FE, et al. Reproductive factors and the risk of scleroderma: an Italian case-control study. Arthritis Rheum 2002; 46: 451-6.

91. Launay D, et al. Relationship between parity and clinical and biological features in patients with systemic sclerosis. J Rheumatol 2001; 28: 509-13. 
92. Peaceman AM, Ramsey-Goldman R. Autoimmune connective tissue disease in pregnancy. Gynecology and Obstetrics 1998; 3: 1-12.

93. Bobrie G, et al. Pregnancy in lupus nephritis and related disorders. Am J Kidney Dis 1987; 9: 339-43.

94. Clark CA, Spitzer KA, Laskin CA. Decrease in pregnancy loss rates in patients with systemic lupus erythematosus over a 40-year period. J Rheumatol 2005; 32: 1709-12.

95. Smyth A, Garovic VD. Systemic lupus erythematosus and pregnancy. Minerva Urol Nefrol 2009; 61: 457-74.

96. Witter FR. Management of the high-risk lupus pregnant patient. Rheum Dis Clin North Am 2007; 33: 253-65, v-vi.

97. Molokhia M, et al. Cross-sectional analysis of adverse outcomes in 1,029 pregnancies of afro-caribbean women in Trinidad with and without systemic lupus erythematosus. Arthritis Res Ther 2007; 9: R124.

98. Clowse ME, et al. A national study of the complications of lupus in pregnancy. Am J Obstet Gynecol 2008; 199: 127 e1-6.

99. Clowse ME, et al. The impact of increased lupus activity on obstetric outcomes. Arthritis Rheum 2005; 52: 514-21.

100. Clowse ME. Lupus activity in pregnancy. Rheum Dis Clin North Am 2007; 33: 237-52, v.

101. Doria A, Tincani A, Lockshin M. Challenges of lupus pregnancies. Rheumatology (Oxford) 2008; 47(Suppl 3): iii9-12.

102. Moroni G, Ponticelli C. Pregnancy after lupus nephritis. Lupus 2005; 14: 89-94.

103. Hayslett JP, Lynn RI. Effect of pregnancy in patients with lupus nephropathy. Kidney Int 1980; 18: 207-20.

104. Lockshin MD. Does lupus flare during pregnancy? Lupus 1993; 2: 1-2.

105. Lockshin M, Druzin M. Rheumatic disease. In: Barron W, Lindheimer MD, eds. Medical Disorders During Pregnancy. St Louis: Mosby-Year Book, Inc., 1991: 366-99.

106. Yan Yuen S, et al. Pregnancy Outcome in Systemic Lupus Erythematosus (SLE) is improving: results from a case control study and literature review. Open Rheumatol J 2008; 2: 89-98.

107. Cavallasca JA, et al. Maternal and fetal outcomes of 72 pregnancies in Argentine patients with systemic lupus erythematosus (SLE). Clin Rheumatol 2008; 27: 41-6.

108. Wagner SJ, et al. Maternal and foetal outcomes in pregnant patients with active lupus nephritis. Lupus 2009; 18: 342-7.

109. Gladman DD, et al. The effect of lupus nephritis on pregnancy outcome and fetal and maternal complications. J Rheumatol 2010; 37: 754-8.

110. Mintz G, Rodriguez-Alvarez E. Systemic lupus erythematosus. Rheum Dis Clin North Am 1989; 15: 255-74.

111. Lockshin MD, et al. Lupus pregnancy. Case-control prospective study demonstrating absence of lupus exacerbation during or after pregnancy. Am J Med 1984; 77: 893-8.

112. Lockshin MD. Pregnancy does not cause systemic lupus erythematosus to worsen. Arthritis Rheum 1989; 32: 665-70.

113. Urowitz MB, et al. Lupus and pregnancy studies. Arthritis Rheum 1993; 36: $1392-7$.

114. Abramowsky CR, et al. Decidual vasculopathy of the placenta in lupus erythematosus. N Engl J Med 1980; 303: 668-72.

115. Samaritano LM, M, Kaplan C. A prospective study of clinical features and placental pathology in systemic lupus erythematosus (SLE) with and without antiphospholipid antibodies (aPL). Arthritis Rheum 1995; 38: S218(abst).

116. Nayar R, Lage JM. Placental changes in a first trimester missed abortion in maternal systemic lupus erythematosus with antiphospholipid syndrome; a case report and review of the literature. Hum Pathol 1996; 27: 201-6.

117. Althabe O, Labarrere C, Telenta M. Maternal vascular lesions in placentae of small-for-gestational-age infants. Placenta 1985; 6: 265-76.

118. Hanly JG, et al. Lupus pregnancy. A prospective study of placental changes. Arthritis Rheum 1988; 31: 358-66.

119. Kitridou RC, Mintz G. Dubois' Lupus Erythematosus. In: Wallace DJ, Hahn BH, eds. The mother in systemic lupus erythematosus, 5th edn. Baltimore: Williams \& Wilkins, 1997: 967-1021.

120. Watson RM, et al. Neonatal lupus erythematosus. A clinical, serological and immunogenetic study with review of the literature. Medicine (Baltimore) 1984; 63: 362-78.
121. Buyon J, Szer I. Passively acquired autoimmunity and the maternal fetal dyad in systemic lupus erythematosus. Springer Semin Immunopathol 1986; 9: 283-304.

122. Lockshin MD, et al. Neonatal lupus risk to newborns of mothers with systemic lupus erythematosus. Arthritis Rheum 1988; 31: 697-701.

123. Friedman DM, Rupel A, Buyon JP. Epidemiology, etiology, detection, and treatment of autoantibody-associated congenital heart block in neonatal lupus. Curr Rheumatol Rep 2007; 9: 101-8.

124. Waltuck J, Buyon JP. Autoantibody-associated congenital heart block: outcome in mothers and children. Ann Intern Med 1994; 120: 544-51.

125. Brucato A. Prevention of congenital heart block in children of SSApositive mothers. Rheumatology (Oxford) 2008; 47(Suppl 3): iii35-7.

126. Press J, et al. Long-term outcome of mothers of children with complete congenital heart block. Am J Med 1996; 100: 328-32.

127. Boumpas DT, et al. Systemic lupus erythematosus: emerging concepts. Part 2: dermatologic and joint disease, the antiphospholipid antibody syndrome, pregnancy and hormonal therapy, morbidity and mortality, and pathogenesis. Ann Intern Med 1995; 123: 42-53.

128. Copel JA, Buyon JP, Kleinman CS. Successful in utero therapy of fetal heart block. Am J Obstet Gynecol 1995; 173: 1384-90.

129. Bertsias G, et al. EULAR recommendations for the management of systemic lupus erythematosus. Report of a task force of the EULAR standing committee for international clinical studies including therapeutics. Ann Rheum Dis 2008; 67: 195-205.

130. Balasch J, et al. Antiphospholipid antibodies and human reproductive failure. Hum Reprod 1996; 11: 2310-5.

131. Geva E, et al. Undiagnosed systemic lupus erythematosus in a cohort of infertile women. Am J Reprod Immunol 2004; 51: 336-40.

132. Silva CA, et al. Gonadal function in adolescents and young women with juvenile systemic lupus erythematosus. Lupus 2002; 11: 419-25.

133. Doria A, et al. Th2 immune deviation induced by pregnancy: the two faces of autoimmune rheumatic diseases. Reprod Toxicol 2006; 22: 234-41.

134. Ruiz-Irastorza G, et al. Measuring systemic lupus erythematosus activity during pregnancy: validation of the lupus activity index in pregnancy scale. Arthritis Rheum 2004; 51: 78-82.

135. Williams KP, et al. Screening for fetal well-being in a high-risk pregnant population comparing the nonstress test with umbilical artery doppler velocimetry: a randomized controlled clinical trial. Am J Obstet Gynecol 2003; 188: 1366-71.

136. Johnstone FD, et al. The effect of introduction of umbilical doppler recordings to obstetric practice. Br J Obstet Gynaecol 1993; 100: 733-41.

137. Tyrrell SN, et al. Randomized comparison of routine vs highly selective use of Doppler ultrasound and biophysical scoring to investigate high risk pregnancies. Br J Obstet Gynaecol 1990; 97: 909-16.

138. Ewigman B, LeFevre M, Hesser J. A randomized trial of routine prenatal ultrasound. Obstet Gynecol 1990; 76: 189-94.

139. Englert HJ, et al. Pregnancy and lupus: prognostic indicators and response to treatment. Q J Med 1988; 66: 125-36.

140. Kutteh WH, et al. Association of anticardiolipin antibodies and pregnancy loss in women with systemic lupus erythematosus. Fertil Steril 1993; 60: 449-55.

141. Nilsson IM, et al. Intrauterine death and circulating anticoagulant (“antithromboplastin"). Acta Med Scand 1975; 197: 153-9.

142. Harris EN, et al. Thrombosis, recurrent fetal loss, and thrombocytopenia. Predictive value of the anticardiolipin antibody test. Arch Intern Med 1986; 146: 2153-6.

143. Harris EN, Pierangeli SS. Antiphospholipid antibodies and the antiphospholipid syndrome. Springer Semin Immunopathol 1994; 16: 223-45.

144. Love PE, Santoro SA. Antiphospholipid antibodies: anticardiolipin and the lupus anticoagulant in systemic lupus erythematosus (SLE) and in non-SLE disorders. Prevalence and clinical significance. Ann Intern Med 1990; 112: 682-98.

145. Sammaritano LR, Gharavi AE, Lockshin MD. Antiphospholipid antibody syndrome: immunologic and clinical aspects. Semin Arthritis Rheum 1990; 20: 81-96.

146. Triplett DA. Antiphospholipid antibodies. Arch Pathol Lab Med 2002; 126: 1424-9.

147. Baker WF Jr, Bick RL, Fareed J. Controversies and unresolved issues in antiphospholipid syndrome pathogenesis and management. Hematol Oncol Clin North Am 2008; 22: 155-74, viii. 
148. Harris EN, et al. Anticardiolipin antibodies: detection by radioimmunoassay and association with thrombosis in systemic lupus erythematosus. Lancet 1983; 2: 1211-14.

149. Harris EN, Pierangeli SS. Revisiting the anticardiolipin test and its standardization. Lupus 2002; 11: 269-75.

150. Mouritsen S, et al. The specificity of anti-cardiolipin antibodies from syphilis patients and from patients with systemic lupus erythematosus. Clin Exp Immunol 1989; 76: 178-83.

151. Harris EN, et al. Use of an enzyme-linked immunosorbent assay and of inhibition studies to distinguish between antibodies to cardiolipin from patients with syphilis or autoimmune disorders. J Infect Dis 1988; 157: 23-31.

152. Intrator L, et al. Anticardiolipin antibodies in HIV infected patients with or without immune thrombocytopenic purpura. Br J Haematol 1988; 68 : 269-70.

153. Galvez J, et al. Thrombophlebitis in a patient with acute Q fever and anticardiolipin antibodies. Med Clin (Barc) 1997; 108: 396-7.

154. Koskela P, et al. Significance of false positive syphilis reactions and anticardiolipin antibodies in a nationwide series of pregnant women. J Rheumatol 1988; 15: 70-3.

155. McNeil HP, et al. Anti-phospholipid antibodies are directed against a complex antigen that includes a lipid-binding inhibitor of coagulation: beta 2-glycoprotein I (apolipoprotein H). Proc Natl Acad Sci USA 1990; 87: 4120-4.

156. Lim W, Crowther MA. Antiphospholipid antibodies: a critical review of the literature. Curr Opin Hematol 2007; 14: 494-9.

157. De Laat B, et al. Pathogenic anti-beta2-glycoprotein I antibodies recognize domain I of beta2-glycoprotein I only after a conformational change. Blood 2006; 107: 1916-24.

158. Solano C, et al. Comparison of the 1999 Sapporo and 2006 revised criteria for the classification of the antiphospholipid syndrome. Clin Exp Rheumatol 2009; 27: 914-19.

159. Miyakis S, et al. International consensus statement on an update of the classification criteria for definite antiphospholipid syndrome (APS). J Thromb Haemost 2006; 4: 295-306.

160. Asherson RA, et al. The "primary" antiphospholipid syndrome: major clinical and serological features. Medicine (Baltimore) 1989 68: 366-74.

161. Lockshin M. The Antiphospholipid Syndrome. In: Asherson RA, et al, ed. New perspectives in the study and treatment of the antiphospholipid syndrome. Boca Raton: CRC Press, 1996: 323-9.

162. Alarcon-Segovia D, et al. Preliminary classification criteria for the antiphospholipid syndrome within systemic lupus erythematosus. Semin Arthritis Rheum 1992; 21: 275-86.

163. Salmon JE, Girardi G, Lockshin MD. The antiphospholipid syndrome as a disorder initiated by inflammation: implications for the therapy of pregnant patients. Nat Clin Pract Rheumatol 2007; 3: 140-7; quiz 1 p following 187.

164. Lockshin MD, et al. Antibody to cardiolipin as a predictor of fetal distress or death in pregnant patients with systemic lupus erythematosus. N Engl J Med 1985; 313: 152-6.

165. Vianna JL, et al. Comparison of the primary and secondary antiphospholipid syndrome: a European Multicenter Study of 114 patients. Am Med 1994; 96: 3-9.

166. Branch DW, et al. Obstetric complications associated with the lupus anticoagulant. N Engl J Med 1985; 313: 1322-6.

167. Triplett DA, et al. The relationship between lupus anticoagulants and antibodies to phospholipid. JAMA 1988; 259: 550-4.

168. Lockwood CJ, et al. The prevalence and biologic significance of lupus anticoagulant and anticardiolipin antibodies in a general obstetric population. Am J Obstet Gynecol 1989; 161: 369-73.

169. Harris E, Spinnato J. Should sera of healthy pregnant women be screened for anticardiolipin antibodies? Arthritis Rheum 1990; 33: S28 (abstr).

170. Silver RM, et al. Anticardiolipin antibodies: clinical consequences of "low titers." Obstet Gynecol 1996 87: 494-500.

171. Katano K, et al. Beta 2-Glycoprotein I-dependent anticardiolipin antibodies as a predictor of adverse pregnancy outcomes in healthy pregnant women. Hum Reprod 1996; 11: 509-12.

172. Bick RL. Antiphospholipid syndrome in pregnancy. Hematol Oncol Clin North Am 2008; 22: 107-20, vii.
173. Ruiz-Irastorza G, Khamashta MA. Antiphospholipid syndrome in pregnancy. Rheum Dis Clin North Am 2007; 33: 287-97, vi.

174. Lubbe WF, et al. Lupus anticoagulant in pregnancy. Br J Obstet Gynaecol 1984 91: 357-63.

175. Rai RS, et al. High prospective fetal loss rate in untreated pregnancies of women with recurrent miscarriage and antiphospholipid antibodies. Hum Reprod 1995; 10: 3301-4.

176. Branch DW, et al. The association of antiphospholipid antibodies with severe preeclampsia. Obstet Gynecol 1989; 73: 541-5.

177. Bendon RW, et al. A maternal death due to thrombotic disease associated with anticardiolipin antibody. Arch Pathol Lab Med 1987; 111: 370-2.

178. Rallings P, Exner T, Abraham R. Coronary artery vasculitis and myocardial infarction associated with antiphospholipid antibodies in a pregnant woman. Aust N Z J Med 1989; 19: 347-50.

179. McMillan E, et al. Management of pregnancy in women with pulmonary hypertension secondary to SLE and anti-phospholipid syndrome. Lupus 2002; 11: 392-8.

180. Branch DW, Khamashta MA. Antiphospholipid syndrome: obstetric diagnosis, management, and controversies. Obstet Gynecol 2003; 101 1333-44.

181. Milne F, et al. The pre-eclampsia community guideline (PRECOG): how to screen for and detect onset of pre-eclampsia in the community. BMJ 2005; 330: 576-80.

182. Rosove $\mathrm{MH}$, et al. Heparin therapy for pregnant women with lupus anticoagulant or anticardiolipin antibodies. Obstet Gynecol 1990; 75: $630-4$.

183. Stafford-Brady FJ, Gladman DD, Urowitz MB. Successful pregnancy in systemic lupus erythematosus with an untreated lupus anticoagulant Arch Intern Med 1988; 148: 1647-8.

184. Kutteh WH. Antiphospholipid antibody-associated recurrent pregnancy loss: treatment with heparin and low-dose aspirin is superior to lowdose aspirin alone. Am J Obstet Gynecol 1996; 174: 1584-9.

185. Kutteh WH, Ermel LD. A clinical trial for the treatment of antiphospholipid antibody-associated recurrent pregnancy loss with lower dose heparin and aspirin. Am J Reprod Immunol 1996; 35: 402-7.

186. Empson, M., et al. Prevention of recurrent miscarriage for women with antiphospholipid antibody or lupus anticoagulant. Cochrane Database Syst Rev 2005: CD002859.

187. Cowchock FS, et al. Repeated fetal losses associated with antiphospholipid antibodies: a collaborative randomized trial comparing prednisone with low-dose heparin treatment. Am J Obstet Gynecol 1992; 166: 1318-23.

188. Silver RK, et al. Comparative trial of prednisone plus aspirin versus aspirin alone in the treatment of anticardiolipin antibody-positive obstetric patients. Am J Obstet Gynecol 1993; 169: 1411-17.

189. Lockshin MD, Druzin ML, Qamar T. Prednisone does not prevent recurrent fetal death in women with antiphospholipid antibody. Am J Obstet Gynecol 1989; 160: 439-43.

190. Balasch J, et al. Low-dose aspirin for prevention of pregnancy losses in women with primary antiphospholipid syndrome. Hum Reprod 1993; 8 : 2234-9.

191. Shibata S, et al. Risk factors in the pregnancy of patients with systemic lupus erythematosus: association of hypocomplementaemia with poor prognosis. Ann Rheum Dis 1992; 51: 619-23.

192. Boey ML, et al. Thrombosis in systemic lupus erythematosus: striking association with the presence of circulating lupus anticoagulant. Br Med J (Clin Res Ed) 1983; 287: 1021-3.

193. Scott JR, et al. Intravenous immunoglobulin treatment of pregnant patients with recurrent pregnancy loss caused by antiphospholipid antibodies and Rh immunization. Am J Obstet Gynecol 1988; 159: 1055-6.

194. Spinnato JA, et al. Intravenous immunoglobulin therapy for the antiphospholipid syndrome in pregnancy. Am J Obstet Gynecol 1995; 172(2 Pt 1): 690-4.

195. The Pregnancy Loss Study Group. A multi-center randomized double blind pilot study of IVIG therapy during pregnancy in women with antiphospholipid syndrome. Lupus 1998; 7(Suppl 2): S196 (abst).

196. Triolo G, et al. Randomized study of subcutaneous low molecular weight heparin plus aspirin versus intravenous immunoglobulin in the treatment of recurrent fetal loss associated with antiphospholipid antibodies. Arthritis Rheum 2003; 48: 728-31. 


\section{Sickle cell disease \\ Marc R. Parrish and John C. Morrison}

\section{INTRODUCTION}

Hemoglobinopathies complicating pregnancy number in the hundreds, but fortunately most of them are very rare (1). These inherited disorders usually result from structural abnormalities such as additions, deletions, or substitutions in the amino acids that comprise the two pairs of polypeptide chains common to each hemoglobin molecule (Table 1). These amino acid linkages contain either 141 or 147 amino acids, are responsible for the nomenclature, and comprise the common types of hemoglobin such as $\mathrm{A}, \mathrm{A}_{2}, \mathrm{~F}$, and so on (2).

Sickle cell disease (SCD) refers to a group of autosomal recessive disorders involving abnormal hemoglobin (hemoglobin S). Hemoglobin S differs from the normal $\mathrm{Hb} \mathrm{A}$ because of a single nucleotide substitution of thymine for adenine in the globin gene. This alteration causes a substitution of valine for glutamic acid in the number six position of the globin polypeptide. Asymptomatic individuals with heterozygous $\mathrm{Hb} \mathrm{S}$ genotypes (carriers) are said to have sickle cell trait. The most severe form of the disease, Hb S-S (homozygous Hb S), is called sickle cell anemia (3).

Sickle cell crisis occurring at any time during gestation is a medical emergency (4). Vaso-occlusive sickle cell crisis is the most common maternal complication noted in gestations complicated by sickle hemoglobinopathies (5). Crisis is associated with increased maternal and perinatal morbidity and mortality (6). Severity as well as frequency of crises among pregnant women is extremely variable and may occur during the prenatal or intrapartum period as well as the puerperium (7). Improvements in management over the last two decades have resulted in improved outcome for such women and their progeny. Nevertheless, providers who care for these high-risk patients must be prepared to practice preventive medicine, recognize the early signs of crisis, and respond rapidly with a well-founded perinatal management plan.

\section{EPIDEMIOLOGY}

In the United States, sickle cell anemia affects about 70,000 people (8). It mainly affects African-Americans. The disease occurs in about 1 out of every 500 African-American births. Sickle cell anemia also affects Hispanic Americans. The disease occurs in 1 out of every 36,000 Hispanic American births $(8,9)$. Patients with $\mathrm{Hb} \mathrm{S}-\mathrm{S}$, as well as with other variants, such as hemoglobin S-C (Hb S-C) and hemoglobin S- $\beta$ thalassemia ( $\mathrm{Hb}$ S- $\beta$ Thal), are said to have SCD and this is most often responsible for adverse effects on the mother, the fetus, and the newborn (3). These are also the disorders most likely to be associated with sickle cell crisis. Other sickle hemoglobinopathies such as $\mathrm{Hb} \mathrm{S}-\mathrm{D}, \mathrm{Hb} \mathrm{A}-\mathrm{S}$, and $\mathrm{Hb} \mathrm{S}-\mathrm{E}$ are unlikely to result in sickling episodes.

\section{SICKLE CELL TRAIT}

About 2 million Americans have sickle cell trait. The condition occurs in about 1 in 12 African-Americans (8). Sickle cell trait has been considered to be an essentially benign condition during pregnancy, with few exceptions $(10,11)$. A common problem tends to be an increased rate of urinary tract infections, thought to be associated with an inability to concentrate urine and asymptomatic bacteriuria among patients with sickle cell trait. There is some literature that contradicts the benign nature of the carrier state revealing an increased risk of intrauterine fetal demise, spontaneous miscarriage, and intrauterine growth restriction $(12,13)$. However, due to a lack of sufficient evidence, routine obstetrical management with monthly urinalysis is the only recommendation in this group of patients at this time.

\section{MORBIDITY AND MORTALITY}

Patients with $\mathrm{Hb} \mathrm{S}-\mathrm{S}$ or $\mathrm{Hb} \mathrm{S}-\mathrm{C}$ have no hemoglobin $\mathrm{A}$, therefore the predominant hemoglobin is $\mathrm{Hb} \mathrm{S}, 90 \%$ to $95 \%$, with the remainder being $\mathrm{Hb} \mathrm{F}$. Patients with this disorder have been shown to have a shorter life span, more common infections, and when pregnant, a higher maternal as well as perinatal mortality/morbidity (1). The fertility rate does not appear to be decreased unless there are frequent crises and disseminated disease affecting many systems. Due to improvements in modern medicine, the maternal death rate has been reduced to less than $2 \%$, and many women are now electing to proceed with pregnancies (14).

Women with SCD are at an increased risk to experience both medical complications (such as infections, thromboembolic events, and stroke) and pregnancy-related complications (such as pre-eclampsia, eclampsia, preterm labor, placental abruption, and fetal growth restriction). The risk for experiencing these complications is significantly higher than that in other women $(15,16)$. Decreased placental blood flow due to sickling in the tortuous, arcuate vessels of the myometrium has been associated with poor perinatal outcomes (2). More than one-third of pregnancies in women with SCD terminate in miscarriage, stillbirth, or neonatal death, according to the National Institutes of Health Cooperative Study of Sickle Cell Disease (17). The neonatal mortality rates are not increased in patients with SCD per se but may appear to increase due to preterm deliveries.

These women are also more likely to experience pneumonia, pyelonephritis, postpartum infection, sepsis, and systemic inflammatory response syndrome compared with women without SCD. At the time of delivery, these women are also more likely to have asymptomatic bacteriuria and genitourinary infections (15). Severe cardiopulmonary ailments may be observed in those women with long-standing disease. Cardiovascular sequelae are often a consequence of chronically 
Table 1 Normal/Abnormal Hemoglobin

\begin{tabular}{|c|c|c|c|c|}
\hline Hemoglobin classification & Globin defect & Chain present & Common name & Hematologic destination \\
\hline Adult hemoglobin ${ }^{\mathrm{a}}$ & None & $\begin{array}{l}2 \mathrm{a} \\
2 \mathrm{~b}\end{array}$ & $\mathrm{Hb} \mathrm{A}$ & $\mathrm{a}_{2}{ }^{\mathrm{A}} \mathrm{d}_{2}{ }^{\mathrm{A}}$ \\
\hline Adult hemoglobin ${ }^{a}$ & None & $\begin{array}{l}2 \mathrm{a} \\
2 \mathrm{~d}\end{array}$ & $\mathrm{Hb} \mathrm{A} \mathrm{A}_{2}$ & $\mathrm{a}_{2}{ }^{\mathrm{A}} \mathrm{d}_{2}{ }^{\mathrm{A}}$ \\
\hline Adult fetal hemoglobin ${ }^{\mathrm{a}}$ & None & $\begin{array}{l}2 \mathrm{a} \\
2 \mathrm{~g}\end{array}$ & $\mathrm{Hb} \mathrm{F}$ & $\mathrm{a}_{2}{ }^{\mathrm{A}} \mathrm{g}_{2} \mathrm{~F}$ \\
\hline Sickle hemoglobin ${ }^{\mathrm{b}}$ & b-chain substitution & $\begin{array}{l}2 \mathrm{a} \\
2 \mathrm{a}\end{array}$ & $\mathrm{Hb} \mathrm{S}$ & $\mathrm{a}_{2}{ }^{\mathrm{A}} \mathrm{b}_{2}{ }^{\mathrm{S}}\left(6 \mathrm{glu}{ }^{\circledR} \mathrm{val}\right)$ \\
\hline C-hemoglobin ${ }^{\mathrm{b}}$ & b-chain substitution & $\begin{array}{l}2 \mathrm{a} \\
2 \mathrm{~b}\end{array}$ & $\mathrm{Hb} \mathrm{C}$ & $\mathrm{a}_{2}{ }^{\mathrm{A}} \mathrm{b}_{2}{ }^{\mathrm{C}}$ (6 glu ${ }^{\circledR}$ lys $)$ \\
\hline Methemoglobin ${ }^{\mathrm{b}}$ & a-chain substitution & $\begin{array}{l}2 a \\
2 b\end{array}$ & Hb M Boston & $\mathrm{a}_{2}{ }^{\mathrm{M}}\left(58\right.$ his $\left.{ }^{\circledR} \mathrm{tyr}\right) \mathrm{b}_{2}{ }^{\mathrm{A}}$ \\
\hline b-Thalassemia ${ }^{a}$ & None (decreased synthesis) & $\begin{array}{l}2 \mathrm{a} \\
2 \mathrm{~b}\end{array}$ & Hb B Thal & $a_{2}{ }^{A} b_{2}$ Thal \\
\hline
\end{tabular}

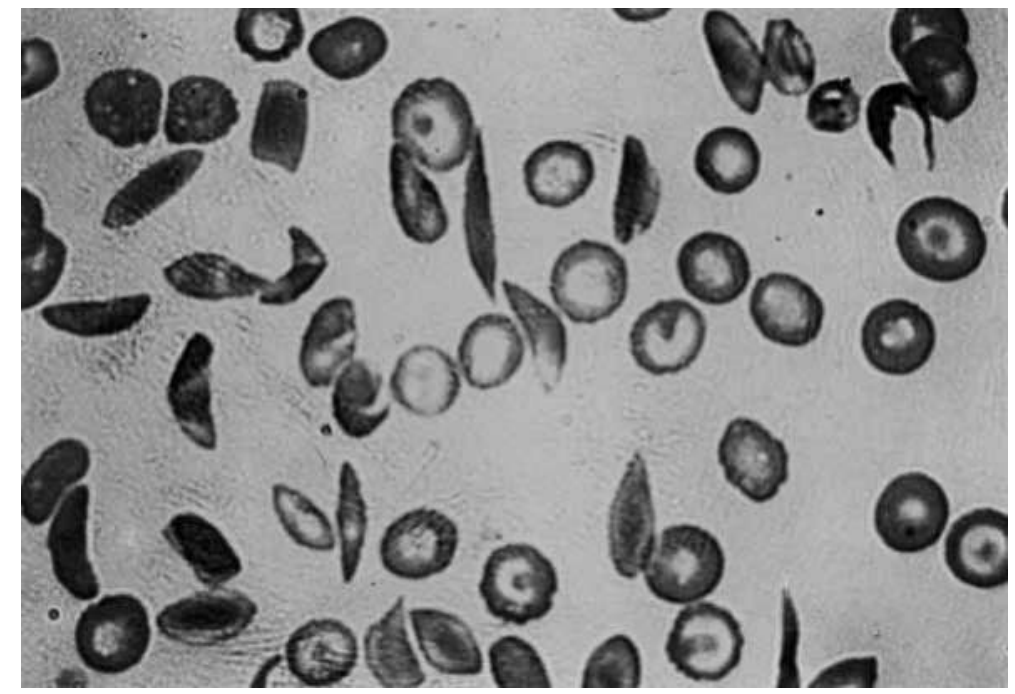

Figure 1 Blood film in patient with sickle cell crisis.

high cardiac output due to anemia, although these women rarely die of heart disease, but almost all eventually have some degree of ventricular dysfunction from hypertrophy and diastolic dysfunction. These underlying changes make affected women less tolerant of the additional increase in cardiac output noted in pregnancy (18). Pulmonary hypertension is acknowledged increasingly as a complication of SCD. It has been described in up to $30 \%$ of this population and is associated with a high mortality rate (19). The statistically significant presence of pulmonary hypertension at delivery supports the previous observation and warrants the need for appropriate evaluation and management.

Although neonates are asymptomatic at birth because of a higher concentration of hemoglobin $\mathrm{F}$, the incidence of neonatal mortality increases after about 6 months with $20 \%$ of overall deaths from SCD occurring in the first 2 years, $10 \%$ between the ages of 2 and 5 years, and the remainder between 5 and 30 years (20). During pregnancy, severe anemia is the rule among patients with SCD and rarely does the hematocrit rise above $25 \%$. Infections are also common and usually involve the urinary tract, pulmonary system, and orthopedic system.

Diminished oxygenation in the intravascular compartment in patients with SCD converts the normal oval red blood cells into the classic sickle-shaped erythrocytes noted in subjects undergoing crisis (Fig. 1). These sickle cells lodge in the microvasculature of many organs and result in a decreased life span of red blood cells as well as the maternal and perinatal complications mentioned above. Any event leading to deoxygenation can initiate a sickle cell crisis. For instance, infections from viruses or bacteria, acidosis, dehydration, trauma, blood loss, severe stress, strenuous physical activity, high altitude, and hypothermia have been incriminated.

There are several different kinds of sickle cell crisis. The most common is the vaso-occlusive crisis, which is most frequently noted in pregnant women and adults. The less common hematologic type of crisis is more often seen, but not exclusively so, in children (Table 2). The clinical expressions of the vaso-occlusive crises are extremely variable in their presentation but usually follow a characteristic pattern in each patient. These recurrent and sudden attacks of pain most often involve the abdomen, extremities, central nervous system, and chest although no body area is immune from these attacks (21). Some patients exhibit generalized pain and malaise, while others have more localized syndromes involving a compromised microcirculation in various areas of the body. 
Table 2 Sickle Cell Crisis Classification

Vaso-occlusive
Abdominal
Chest
Joints
Central nervous system
Hematologic
Aplastic
Hemolytic
Megaloblastic
Splenic sequestration

On the other hand, hematologic crises are infrequent among pregnant women, but if they occur, aplastic crisis is the most common type. Fortunately, this type of crisis is usually self-limited and most often associated with infection. Hemolytic crises, in general, are most commonly related to concomitant spherocytosis or glucose-6-phosphate dehydrogenase deficiency. Megaloblastic crises are usually secondary to folate depletion and are remedial to supplementation. Finally, splenic sequestration crises are usually limited to childhood. As a group, hematologic crises are characterized by sudden anemia without icterus, pale conjunctiva, malaise, reticulocytopenia, and cardiopulmonary failure.

\section{ETIOLOGY}

In a patient with SCD, the symptom constellation of the chronic, severe anemia and frequent pain leads one to suspect a vaso-occlusive crisis. Most commonly, this event in the pregnant adult is associated with apparent or occult infection (20-33\%) (21). Pneumonia, urinary tract infection, and puerperal endomyometritis, as well as osteomyelitis, are the infectious sites commonly encountered with sickle cell crisis. Pneumococcal pneumonia or meningitis is particularly frequent in adolescent pregnant patients with SCD (22). Gram-negative bacteria, such as Escherichia coli or Salmonella, are the most common organisms noted in the third decade of life (4). These organisms are particularly likely to attack the renal and gastrointestinal (gallbladder) systems, respectively. During the second decade of life, Mycoplasma pneumoniae is also common.

Factors other than infection have been associated with vasoocclusive crisis in these patients. Acidosis from dehydration and hypotension has been noted to initiate such crises (1). Strenuous activity, as well as drug overdose and hypothermia, have likewise been associated with these events (2). Therefore, when confronted with a suspected vaso-occlusive crisis in a parturient with SCD, one should suspect infection first but rule out other factors during intensive assessment.

\section{PATHOPHYSIOLOGY}

The variable clinical symptoms with which these patients present is not fully explained. There is evidence to suggest that this variability may be related to the presence of linked and unlinked genes, which modify the disease expression (23). Another theory has been that of incomplete penetrance. As previously noted, the molecular basis for sickle cell crisis can be traced to the single amino acid substitution in the sickle hemoglobin molecule, which distinguishes it from hemoglobin A. During deoxygenation or under conditions of acidosis as well as hypoxia, the molecules of $\mathrm{Hb} \mathrm{S}$ aggregate in a process called nucleation.

Hydrophobic bonding occurs between the abnormally substituted valine, owing to the change in electromagnetic charge. These hydrophobic bonds lead to the formation of polymerized hemoglobin strands, which become tactoids and microcables that eventually transform the cell into the half-moon or crescent shape (Fig. 1) (24). This overall process of gelation of the sickle hemoglobin is enhanced by increasing levels of sickle hemoglobin and/or 2,3-diphosphoglycerate levels. It is reduced by lowering the oxygenation or $\mathrm{pH}$ (25).

Repetitive cycles of gelation during the red blood cell life cycle distort the cellular membrane, with resultant potassium and water reduction as well as calcium accumulation and membrane fragmentation (24). This leads to an increase in the intracellular hemoglobin concentration with a concomitant increase in membrane rigidity to form discocytes or irreversibly sickled (26). The irreversibly sickled cells retain the half-moon shape despite oxygenation. These irreversibly sickled cells constitute up to $50 \%$ of the red cell mass in such parturients. However, the index of reversible/irreversible sickle cells does not appear to correlate well with vaso-occlusive crisis or its severity, but does have a positive relationship to the degree of anemia.

These abnormalities of the red cells themselves do not, alone, explain the pathophysiology of the sickle cell crisis. It is currently felt that there exists a combination of the erythrocyte abnormalities, plasma flow alterations, and vessel wall interactions that are associated with sequestration and destruction of abnormal red cells in the microcirculation (Fig. 2) (27). This would explain patient variability noted in the clinical expression of a sickle crisis. Another factor involved in the pathophysiology of sickle cell crisis appears to be the large oscillation in blood flow in the microcirculation (28). With the use of laser-Doppler velocimetry, rather large changes in blood flow in factors of 7-10 s with peak/trough magnitudes measured at $50 \%$ of mean flow were noted. These findings would suggest that during sickle cell crisis, there is periodic flow in the microcirculation with synchronized rhythmic oscillations versus the continuous even-flow pattern observed in patients with normal hemoglobin.

Therefore, although the environmental factors may stimulate deoxygenation resulting in aggregation, polymerization, and subsequent formation of sickled red cells, flow dynamics and also microcirculation factors are important determinants of the crisis cycle (Fig. 2). These factors combine to increase whole blood viscosity and, thus, alter vasomotion in the microcapillaries. This would lead to sludging and destruction of red cells in the microcirculation. This promotes further deoxygenation, which leads to vascular injury via ischemic necrosis, which results in pain and the other symptoms expressed during vaso-occlusive crises.

The marked susceptibility of parturients with sickle hemoglobinopathies to infection is related to functional hyposplenism, defective activation of complement fixation, decreased serum IgM, impaired serum opsonizing capacity, and defective granulocyte phagocytosis (29). There does not appear to be any difference in the percentage of helper or suppressor T cells or B cells in subjects in crisis (30). This finding is compatible with the observation that patients with sickle hemoglobinopathies did not show increased susceptibility to those infections that are normally associated with depressed cell-mediated immunity (31). 


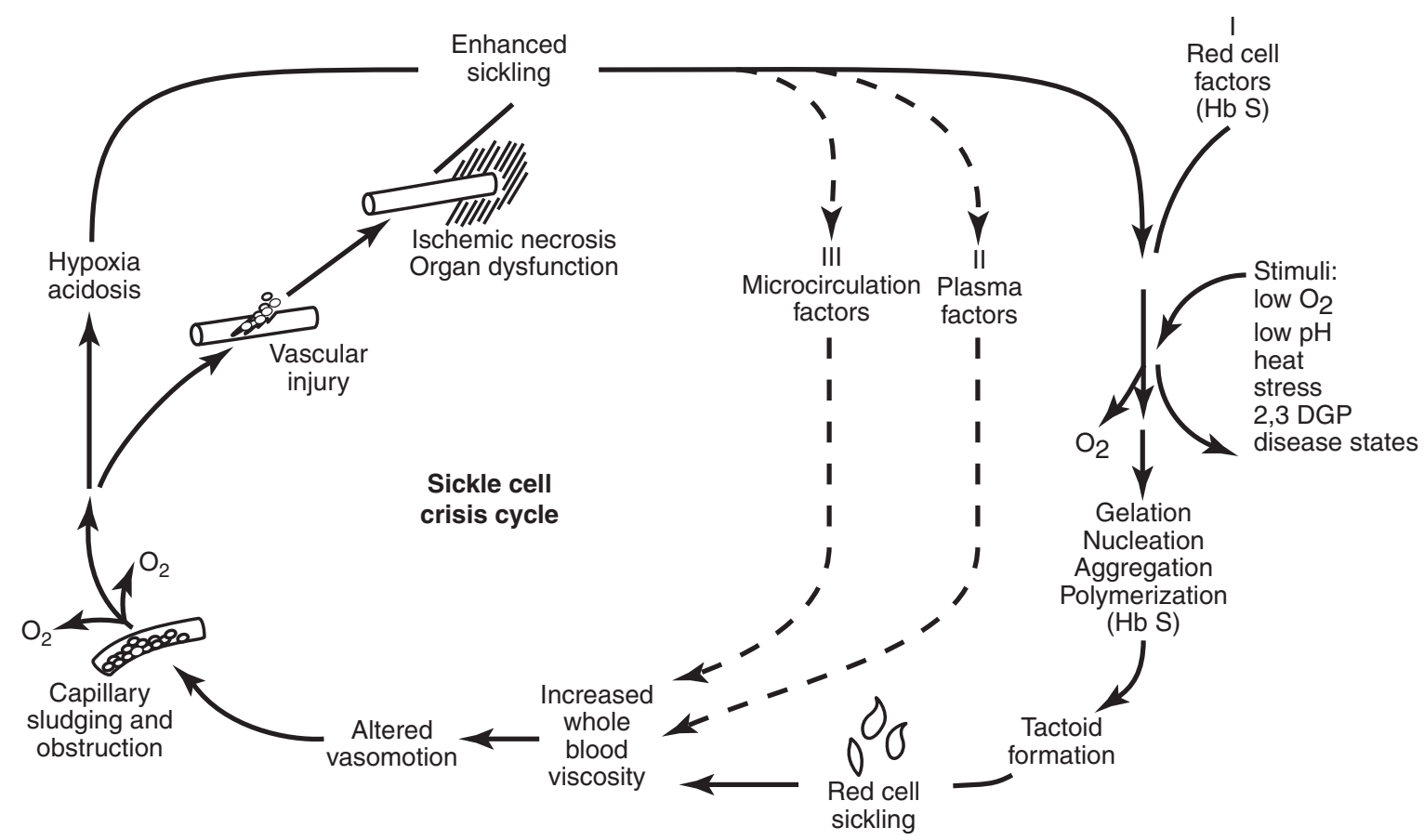

Figure 2 Vicious cycle of sickle cell crisis. Abbreviation: 2,3 DGP, 2,3-diphosphoglycerate.

\section{DIAGNOSIS}

The diagnosis of hemoglobinopathies, including sickle cell disorders, is made by hemoglobin electrophoresis. In the homozygous form, nearly all the hemoglobin is $\mathrm{Hb} \mathrm{S}$ with small amounts of $\mathrm{Hb} \mathrm{A}_{2}$ and $\mathrm{Hb} \mathrm{F}$. Heterozygous sickle cell trait ( $\mathrm{Hb} \mathrm{AS})$ is identified by a larger percentage of $\mathrm{Hb} \mathrm{A}$ and an asymptomatic course. Solubility tests (Sickledex) alone are inadequate for the diagnosis of sickle cell disorders because they cannot distinguish between the heterozygous AS and homozygous SS genotypes. In addition, they fail to detect other pathologic variants such as $\mathrm{Hb} \mathrm{C}$ trait, thalassemia trait, $\mathrm{Hb} \mathrm{E}$ trait, $\mathrm{Hb} \mathrm{B}$ trait, and $\mathrm{Hb} \mathrm{D}$ trait (3).

Although some tests for crisis are helpful, the diagnosis is largely a clinical experience and basically one of exclusion. Fortunately, most women with sickle hemoglobinopathies are known prior to pregnancy. For patients with SCD, the first step is to confirm the diagnosis with hemoglobin electrophoresis. Once the diagnosis is documented, surgical/medical/ obstetric conditions associated with a similar symptom complex or even malingering must be ruled out (Table 3 ). Because a parturient may have any one of many medical, surgical, or obstetric complications, pain in a pregnant woman with SCD does not always result from vaso-occlusive crisis.

Infection and crisis are usually associated with an increase in the segmented neutrophil counts as well as the presence of bands. A hemolytic crisis with anemia is presumed in the presence of an elevated lactate dehydrogenase, a reticulocyte count of $3 \%$ to $15 \%$, an elevation in unconjugated bilirubin, and a low serum haptoglobin.

\section{MANAGEMENT}

\section{General}

Pregnant patients with SCD need increased prenatal folic acid supplementation. The standard $1 \mathrm{mg}$ of folate in prenatal vitamins is not adequate for patients with hemoglobinopa-
Table 3 Differential Diagnosis of Sickle Cell Anemia

\section{Obstetric factors}

Threatened abortion

Preterm labor

Placental abruption

Uterine rupture

Ectopic pregnancy

Degenerating leiomyomata

Ovarian torsion

Pregnancy-induced hypertension

Medical factors

Renal/ureteral colic

Cystitis

Pyelonephritis

Other factors

Pneumonia

Pulmonary embolism

Arthritis

Surgical factors

Cholecystitis

Cholelithiasis

Appendicitis

Pancreatitis

Peptic ulcer disease

Perforated viscus

Intestinal obstruction

Volvulus

Ischemic colitis

Malingering

Drug addiction

thies; $4 \mathrm{mg}$ /day of folic acid should be prescribed because of the continual turnover of red blood cells (3). Supplemental iron is not usually recommended due to the risk of iron overload that accompanies chronic hemolysis and transfusions. Its administration can be considered if serum iron and 
ferritin are found to be diminished. Routine prenatal labs including evaluation for the hepatitis B antigen and HIV should be performed at the patient's initial visit. Other labs to consider would be serum uric acid, creatinine, liver function tests, and a 24-hour urine collection for protein and creatinine to assess for the presence of preexisting vascular disease. If not already accomplished, the patient should be immunized with both the pneumococcal and meningococcal vaccines due to the likelihood of autosplenectomy during childhood and their susceptibility to encapsulated organisms. Neither vaccine is contraindicated in pregnancy.

Intrauterine growth restriction, reduced birth weight, and lower placental weights are common with this disease and may be due to vascular changes within the uteroplacental unit as well as preterm delivery $(32,33)$. It is likely that chronic vascular occlusion, even in the absence of clinically overt pain crisis or acute chest syndrome, contributes to placental injury and adversely affects fetal growth. Altered vascular flow rather than anemia may account for low birth weights in infants of women with $\mathrm{Hb} \mathrm{S}-\mathrm{S}$, which does not appear to improve with transfusion support (14). Starting at 24 to 28 weeks, monthly ultrasonography is recommended to assess fetal growth, and patients should be instructed in daily fetal movement counts. Nonstress testing and biophysical profile testing should be considered weekly beginning at 32 to 34 weeks. Doppler ultrasound, performed at 28 to 30 weeks to assess umbilical artery flow and systolic/diastolic ratios as a predictor of intrauterine growth restriction, may be a useful adjunct.

Despite advances during the past 20 years and the appreciation of the molecular basis of sickle cell crisis, there is no specific therapy available. However, an understanding of the pathophysiology involved in vaso-occlusive crisis allows the provider to address those aspects of this event that can be treated. Such therapeutic regimens generally include (i) adding fluids to reduce intracellular hemoglobin concentration, (ii) correcting acidosis when present, (iii) treating infection, and (iv) increasing oxygen concentration.

\section{Antepartum}

During the prenatal period when the patient in crisis is not in labor and is remote from term, hospitalization in a quiet area where bed rest, analgesia, and hydration can be carried out is optimal. Because of the risk of fetal loss, health assessment via biophysical profile and/or fetal heart rate monitoring is carried out during crisis treatment if gestational age is sufficient to consider intervention for fetal distress. In addition, continuous fetal heart rate monitoring is helpful, because resolution of worrisome signs, such as late decelerations, has been demonstrated during the treatment of sickle cell crisis with blood infusion (34).

Initial medical assessment should focus on detection of the following medical complications requiring specific therapy: infection, dehydration, acute chest syndrome (fever, tachypnea, chest pain, hypoxia, radiologic chest infiltrates), severe anemia, cholecystitis, splenic enlargement, abdominal crisis, and neurologic events (cerebral infarct, cerebral hemorrhage, transient ischemic attack, seizure). Vigorous rehydration is useful, particularly in crisis patients who are febrile (4). In the absence of cardiopulmonary disease, infusion of a liter of Ringer's lactate or isotonic saline during the first 2 -hour period with continuing replacement at $125 \mathrm{~mL} / \mathrm{hr}$ is undertaken.
Careful monitoring of intake and output is essential, but invasive hemodynamic monitoring and urinary catheterization are usually avoided, because of the risk of infection. The administration of alkali during the hydration process has not been found to be helpful (35). For analgesia, opiates are superior to nonsteroidal agents in the presence of severe pain. Opiates are not associated with teratogenicity, congenital malformations, or toxic effects other than transient suppression of movement and variability in fetal heart tones. Chronic exposure to opiates, such as that in women who require methadone or other medications to control severe chronic pain, can result in neonatal abstinence syndrome after birth. Acetaminophen is preferable if mild analgesia is required. As soon as the pain begins to regress, nonnarcotic agents are recommended. It is important to recognize that assessment of fetal well-being during a pain episode may be complicated by the use of opiates to treat sickle cell crisis pain, which transiently affect nonstress testing and biophysical profile scores (36). Caution must be used when interpreting the results of these tests during an acute pain episode, because they may not be predictive of increased perinatal morbidity and mortality in the absence of other findings (14).

Although the utility of oxygen therapy is unproven, it is often employed during crisis. Oxygen is administered at 3-6 L/min by tight face mask or nasal cannula. Arterial blood gas assessment is usually recommended if hypoxemia is suspected. Because infection is present in up to one-third of patients with vaso-occlusive crisis and since it is frequently associated with maternal death occurring in sickle cell crisis, attempts to detect occult infection are undertaken immediately. If an infection is suspected, then broad-spectrum antibiotic therapy is begun after appropriate cultures are obtained. Prophylactic antibiotic use, however, is not generally recommended.

\section{BLOOD TRANSFUSION}

Controversy exists regarding the role of prophylactic blood transfusion in the management of SCD in pregnancy (37-41). In the only randomized controlled trial published to date, prophylactic transfusion was associated with a decreased risk for painful crisis and severe anemia, but no difference was observed for pregnancy outcome (38). By limiting transfusion to situations in which it is clinically indicated, patients are not subjected to the increased risk for blood-borne infections, iron overload, and alloimmunization. The rate of alloimmunization in sickle cell patients is estimated to be between $18 \%$ and $36 \%$ (42). It is estimated that six to eight deaths per year in the United States occur as a result of delayed transfusion reactions (43). It appears from the available evidence that the reduction in morbidity and mortality of SCD in pregnancy may be attributable to improvements in general management of pregnancy rather than prophylactic transfusion per se (3).

However, prophylactic transfusions may be indicated in patients who have particularly severe disease manifestations or for symptomatic patients who are unresponsive to conservative management (44). Major complications (e.g., worsening anemia; intrapartum complications such as hemorrhage, septicemia, and cesarean delivery; painful crisis; chest syndrome) may require intervention with an exchange transfusion. There is no consensus regarding the exact hematocrit value below which transfusion should be considered. However, when a transfusion is clinically indicated in the patient with SCD, the 


\section{Table 4 Manual Partial Exchange Transfusion}

Measure hemoglobin A level and hematocrit

Type and match six units packed red blood cells

Start intravenous administration of $1 \mathrm{~L}$ normal saline

(200-400 mL in the first hour) then $150-250 \mathrm{~mL} / \mathrm{hr}$

Phlebotomize $500 \mathrm{~mL}$ blood from opposite arm over $30 \mathrm{~min}$

Give two units leukocyte-poor washed red cells (under pressure

and warmed) over 1-2 hours

Repeat procedure after 4 hours

If hematocrit 35\% and hemoglobin A 50\%, discharge home

If hemoglobin $A<50 \%$, repeat procedure

objective is to lower the percentage of $\mathrm{Hb} \mathrm{S}$ to approximately $40 \%$ while simultaneously raising the total hemoglobin concentration to about $10 \mathrm{~g} / \mathrm{dL}$ (3).

Exchange transfusion, also known as erythrocytapheresis, allows the Hb S-containing cells and irreversibly sickled cells to be removed by extracorporeal, differential centrifugation. It affords the simultaneous return by venous access in the other arm of the patient's own plasma, leukocytes, platelets, and clotting factors along with donor, leukocyte-poor washed $\mathrm{Hb}$ A-containing red cells. This type of transfusion occurs via a machine that allows for automated continuous erythrocytapheresis. In addition, this method maintains isovolemia at all times and allows the provider to accurately monitor the patient's hematologic indices such as hemoglobin levels and hematocrit. It also has the advantage of administration on an outpatient basis. If such a device is not available, the manual "push-pull" mechanism can be considered (Table 4) (45).

Generally, six units of donor packed red cells are exchanged by this process and there is rapid resolution of crisis symptomatology and ongoing sickling. In addition to being more isovolemic, automated continuous erythrocytapheresis requires less transfusion time than the manual method (46).

Obviously, most of the complications associated with this approach are related to the risk of the blood products. Blood products given to these patients should be from cytomegalovirus-seronegative donors or leukoreduced. Careful crossmatching to minimize minor blood incompatibilities and alloimmunization is critical in avoiding problems later for these patients who may need blood products at various points during their life. The use of blood from family members or friends matched for recipient and donor red cell antigens are clinically helpful in reducing the number of post-transfusion crises. Such episodes are known as delayed hemolytic transfusion reactions. The exact mechanism of hemolytic transfusion reaction is not well understood, and the pathophysiology seems to be far more complex because it involves the destruction of both patient and donor RBCs. A subcategory of this problem is known as a delayed transfusion reaction. It is classically characterized by a clinical triad of fever, hyperbilirubinemia, and anemia occurring 3-10 days after transfusion. Laboratory tests that aid diagnosis include increased reticulocyte count, free hemoglobin in the urine, fragmented RBCs on peripheral smear, decreased post-transfusion $\mathrm{Hb} \mathrm{A}$ on electrophoresis, or discovery of previously undetected alloantibodies (47).

\section{Intrapartum}

The occurrence of a vaso-occlusive crisis during labor offers additional challenges to the provider. Obviously, with painful uterine contractions, the diagnosis of a vaso-occlusive crisis may be more difficult. If delivery is expected within a short time, a simple transfusion of two units of leukocyte-reduced washed red cells can be considered. During labor, patients should remain in the lateral recumbent position and receive oxygen by tight-fitting face mask. Careful monitoring of maternal and fetal vital signs is essential. Usually, when crisis has been diagnosed during labor and late decelerations appear, infusion of blood products has been associated with resolution of suspected fetal hypoxemia. Maternal blood gas assessment, if necessary, is carried out, but invasive hemodynamic monitoring is avoided unless other concomitant disease processes such as preeclampsia, and so on, are present. Urinary catheters, as well as intrauterine catheters, are discouraged, because of their association with increased infection.

No consensus exists concerning the anesthetic care of parturients with SCD (48-50). It appears that the use of general anesthesia can result in a significant increase in postpartum sickling complications (51). We feel that epidural analgesia/ anesthesia is ideal if the vaso-occlusive crisis is mild or can be aborted with blood infusion. If not, intermittent intravenous analgesia with pudendal block for vaginal delivery may be appropriate. Continuous epidural morphine has also been utilized with success in these patients. If cesarean delivery is indicated, the choice of regional or general anesthesia depends on the patient's general condition and the preference of the anesthesiologist. Careful monitoring of the patient's vital signs is important during anesthesia so that hypoxemia and acidosis are obviated. Avoidance of hypothermia and hypoxia during labor, as well as in the delivery room, is critical. Persons skilled at neonatal resuscitation should be present at delivery. Consultation with anesthesia and neonatology during labor is important. The use of oxytocic agents for induction/augmentation or an ecbolic agent during the third stage of labor is not contraindicated.

\section{Postpartum}

Crises in Hb S-S women after delivery usually do not develop unless hypovolemia, acidosis, or infection occurs. On the other hand, patients with $\mathrm{Hb} \mathrm{S-C}$ are more likely to experience crises postpartum than during pregnancy. The same pitfalls of management applied during the antepartum period are also prudent after birth. Both urinary tract infection and endometritis are common and are frequently associated with crises. There is also a substantial increased risk of pulmonary edema and thromboembolic disease in these patients. Maintenance of isovolemia and high $\mathrm{Hb}$ A levels (>20\%) appear to be important points in management.

\section{SUMMARY}

Overall, pregnant women with SCD appear to be more likely to experience antepartum, intrapartum, and postpartum complications when compared with unaffected women. Access to high-risk obstetric care, patient education, and close follow-up are important to minimize maternal morbidity and mortality.

In the absence of consistent data supporting a reduction in adverse obstetric or fetal outcomes, the use of transfusion therapy is generally limited to patients who experience a significant increase in SCD activity with severe complications, including acute chest syndrome, acute severe anemia ( $\mathrm{Hb}$ $<5.0-6.0 \mathrm{~g} / \mathrm{dL}$ ), severe, or refractory pre-eclampsia, and those 
who are in anticipation of surgery $(14,52)$. A high index of suspicion and good diagnostic acumen is necessary to obtain optimal results in the pregnant patient with SCD.

\section{REFERENCES}

1. Laros RK Jr. The hemoglobinopathies. In: Laros RK Jr, ed. Blood Disorders in Pregnancy. Philadelphia: Lea \& Febiger, 1986: 37-61.

2. Palmer SM, Sherrill J, Morrison JC. Disease of the blood. In: Danforth DN, Scott JR, eds. Obstetrics and Gynecology, 5th edn. Philadelphia: Lippincott, 1986.

3. ACOG Committee on Obstetrics. ACOG Practice Bulletin No. 78: Hemoglobinopathies in pregnancy. Obstet Gynecol 2007; 109: 229-37.

4. Klauser C, Morisson JC. Sickle cell disease. In: Queenan JT, Hobbins JC, Spong CY, eds. Protocols for high risk pregnancies, 4th edn. Malden, Massachusetts: Blackwell Publishing Inc, 2005: 171-5.

5. Morrison JC. Red blood cell disorders. In: Gleicher N, ed. Principles and practice of medical therapy in pregnancy, 2nd edn. Norwalk: Appleton and Lange, 1992: 997.

6. Pritchard JA, Scott DE, Whalley PJ, et al. The effect of maternal sickle cell hemoglobinopathies and sickle trait on reproductive performances. Am J Obstet Gynecol 1973; 117: 662-70.

7. Ogedenbe OK, Akinyanju OO. The hemoglobinopathies and pregnancy in Lagos. Int J Gynecol Obstet 1988; 26: 229-33.

8. National Heart, Lung and Blood Institute, National Institute of Health. Sickle cell anemia: Who is at risk? [Available from: www.nhlbi.nih.gov/health/dci/ Diseases/Sca/SCA_WhoIsAtRisk.html] Accessed 06 October 2008.

9. Sun P, Wilburn W, Raynor D, Jamieson D. Sickle cell disease in pregnancy: twenty years of experience at Grady Memorial Hospital, Atlanta, Georgia. Am J Obstet Gynecol 2001; 184: 1127-30.

10. Stamilio DM, Harish MS, Macones GA. Pregnant women with the sickle cell trait are not at increased risk for developing preeclampsia. Am J Perinat 2003; 20: 41-8.

11. Tita AT, Biggio JR, Chapman V, et al. Perinatal and maternal outcomes in women with sickle or hemoglobin C trait. Obstet Gynecol 2007; 110: 1113-19.

12. Taylor MY, Wyatt-Ashmead J, Gray J, et al. Pregnancy loss after first trimester viability in women with sickle cell trait: time for a reappraisal? Am J Obstet Gynecol 2006; 194: 1604-8.

13. Taylor MY, Wyatt-Ashmead J, Gray J, et al. Pregnancy loss after first trimester viability in women with sickle cell trait: a preliminary report. South Med J 2008; 101: 150-1.

14. Hassell K. Pregnancy and sickle cell disease. Hematol Oncol Clin North Am 2005; 19: 903-16.

15. Villers MS, Jamison MG, De Castro LM, James AH. Morbidity associated with sickle cell disease in pregnancy. Am J Obstet Gynecol 2008; 199: 125.e1-5.

16. James AH, Bushnell CD, Jamison MG, Myers ER. Incidence and risk factors for stroke in pregnancy and the puerperium. Obstet Gynecol 2005; 106: 509-16.

17. Smith J, Espeland M, Bellevue R, et al. Pregnancy in sickle cell disease: Experience of the Cooperative Study of Sickle Cell Disease. Obstet Gynecol 1996; 87: 199-204.

18. Veille J, Hanson R. Left ventricular systolic and diastolic function in pregnant patients with sickle cell disease. Am J Obstet Gynecol 1994; 170: 107-10.

19. Ataga KI, Sood N, De Gent G, et al. Pulmonary hypertension in sickle cell disease. Am J Med 2004; 117: 665-9.

20. Perry KG Jr, Martin JN Jr, Morrison JC. Hematologic and hemorrhagic diseases. In: Sweet AY, Brown EG, eds. Fetal and Neonatal Effects of Maternal Disease. St Louis: Mosby Year Book, 1991: 224-40.

21. Martin JN Jr, Martin RW, Morrison JC. Acute management of sickle cell crisis in pregnancy. Clin Perinatol 1986; 13: 853-68.

22. Bjornson AB, Lobel JS, Beatrice C, Lampkin BC. Humoral components of host defense in sickle cell disease during painful crisis and asymptomatic periods. J Pediatr 1980; 96: 259-62.

23. Nagel RL, Fabry ME, Pagnier J, et al. Hematologically and genetically distinct forms of sickle cell anemia in Africa: the Senegal type and Benin type. N Engl J Med 1985; 312: 880-4.

24. Dean J, Schechter AN. Sickle-cell anemia. Molecular and cellular basis of therapeutic approaches. N Engl J Med 1978; 752: 804, 863.

25. Iammatteo MD, Ramnano CJ, Riva HL, et al. Managing the pregnant patient with sickle cell disease. Female Patient 1989; 14: 30.
26. Rodgers GP, Noguchi CT, Schecter AN. Irreversibly sickled erythrocytes in sickle cell anemia: a quantitative reappraisal. Am J Hematol 1985; 20: 17-23.

27. Wautier J, Galacteros F, Wautier MP, et al. Clinical manifestations and erythrocyte adhesion to endothelium in sickle cell syndrome. Am J Hematol 1985; 19: 121-30.

28. Rodgers GP, Schechter AN, Noguchi CT, et al. Periodic microcirculatory flow in patients with sickle-cell disease. N Engl J Med 1984; 311: 1534-8.

29. Pearson HA. Sickle cell anemia and severe infection due to encapsulated bacteria. J Infect Dis 1977; 136: S25-30.

30. Venkataraman M, Westerman MP. B-cell changes occur in patients with sickle cell anemia. Am J Clin Pathol 1985; 84: 153-8.

31. De Ceulaer K, Pagliuca A, Forbes M, et al. Recurrent infections in sickle cell disease: hematological and immune studies. Clin Chim Acta 1985; 148: $161-5$.

32. Rathod KB, Jaiswal KN, Shrivastava AC, Shrikhande AV. Study of placenta in sickle cell disorders. Indian J Pathol Microbiol 2007; 50: 698-701.

33. Thame M, Lewis J, Trotman H, et al. The mechanisms of low birth weight in infants of mothers with homozygous sickle cell disease. Pediatrics 2007; 120: 686-93.

34. Morrison JC, Pryor JA. Hematologic disorders. In: Eden R, Boehm F, eds. Assessment and Care of the Fetus: Physiologic, Clinical and Medicolegal Principles. Norwalk, CT: Appleton \& Lange, 1990.

35. Morrison JC, Ruvinsky ED, Nicolls ET. Sickling and pregnancy: a worrisome combination. Contemp Obstet Gynecol 1981; 18: 125-35.

36. Anyaegbunam A, Gauthier Morel M, Merkatz I. Antepartum fetal surveillance tests during sickle cell crisis. Am J Obstet Gynecol 1991; 165: 1081-3.

37. Tuck SM, James CE, Brewster EM, et al. Prophylactic blood transfusion in maternal sickle cell syndromes. Br J Obstet Gynaecol 1987; 94: 121-5.

38. Koshy M, Burd L, Wallace D, et al. Prophylactic red-cell transfusions in pregnant patients with sickle cell disease. A randomized cooperative study. N Engl J Med 1988; 319: 1447-52.

39. Morrison JC, Schneider JM, Whybrew WD, et al. Prophylactic transfusions in pregnant patients with sickle cell hemoglobinopathies: benefit versus risk. Obstet Gynecol 1980; 56: 274-80.

40. Brumfield CG, Huddleston JF, DuBois LB, Harris BA Jr. A delayed hemolytic transfusion reaction after partial exchange transfusion for sickle cell disease in pregnancy: a case report and review of literature. Obstet Gynecol 1984; 63: 13s-15s.

41. Ricks P Jr. Exchange transfusion in sickle cell anemia in pregnancy. Obstet Gynecol 1965; 25: 117-19.

42. Aygun B, Padmanabhan S, Paley C, Chandrasekaran V. Clinical significance of RBC alloantibodies and autoantibodies in sickle cell patients who received transfusions. Transfusion 2002; 42: 37-43.

43. Proudfit CL, Atta E, Doyle NM. Hemolytic transfusion reaction after preoperative prophylactic blood transfusion for sickle cell disease in pregnancy. Obstet Gynecol 2007; 110: 471-4.

44. Koshy M, Burd L. Management of pregnancy in sickle cell syndromes. Hematol Oncol Clin North Am 1991; 5: 585-96.

45. Morrison JC, Whybrew WD, Bucovaz ET. Use of partial exchange transfusion preoperatively in patients with sickle hemoglobinopathies. Am J Obstet Gynecol 1978; 132: 59-63.

46. Cabibbo S, Fidone C, Garozzo G, et al. Chronic red blood cell exchange to prevent clinical complications in sickle cell disease. Transfus Apher Sci 2005; 32: 315-21.

47. National Institutes of Health, National Heart, Lung, and Blood Institute, and Division of Blood Diseases and resources. The Management of Sickle Cell Disease, 4th edn. Bethesda: NIH Publication No 02-2117, 2002.

48. Esseltine DW, Baxter MR, Bevan JC. Sickle cell states and the anaesthetist. Can J Anaesth 1988; 35: 385-403.

49. Danzer BI, Birnbach DJ, Thys DM. Anesthesia for the parturient with sickle cell disease. J Clin Anesth 1996; 8: 598-602.

50. Firth PG, Head CA. Sickle cell disease and anesthesia. Anesthesiology 2004; 101: 766-85.

51. Camous J, Nda A, Etienne-Julan M, Stepahn F. Anesthetic management of pregnant women with sickle cell disease-effect on postnatal sickling complications. Can J Anaesth 2008; 55: 276-83.

52. Rogers Z. Review: clinical transfusion management in sickle cell disease. Immunohematology 2006; 22: 126-31. 


\section{Cardiac diseases in pregnancy Saravanan Kuppuswamy and Sudarshan Balla}

\section{INTRODUCTION}

Major hemodynamic alterations in the cardiovascular system occur during normal pregnancy, labor, and delivery. With advances in surgical techniques and improved diagnostic and therapeutic alternatives, greater numbers of women with congenital and acquired heart disease are surviving to the childbearing years. Therefore, management of the pregnant patient, especially those with underlying cardiovascular disease, requires a thorough understanding of both the normal cardiovascular adjustments to pregnancy and the predicted response of the abnormal cardiovascular system to the hemodynamic stresses of pregnancy.

\section{NORMAL CARDIOPULMONARY ADJUSTMENTS DURING PREGNANCY \\ Symptoms and Signs}

Table 1 summarizes the cardiovascular symptoms and signs in normal pregnancy and findings that need further investigations.

\section{Cardiovascular Hemodynamics in Pregnancy}

Table 1 Normal and Pathologic Findings in Pregnancy

\section{Normal findings seen in pregnancy}

Fatigue, dyspnea, and decreased exercise tolerance

Peripheral edema

Apical impulse will be laterally displaced

First heart sound (S1) will have an increased amplitude and a wide splitting of the two components (mitral and tricuspid) due to early closure of the mitral valve

The second heart sound (S2) is little affected

Majority of patients (84\%) will develop a third heart sound (S3)

Continuous murmurs due to cervical venous hum and mammary soufflé

Pathologic findings warranting evaluation (1)

Paroxysmal nocturnal dyspnea

Hemoptysis

Diastolic murmurs

Continuous murmurs

Loud systolic murmurs (louder than grade 2 on the six-point scale)

Pansystolic murmurs

Murmurs associated with symptoms or an abnormal electrocardiogram

Table 2 Cardiovascular Changes in Pregnancy

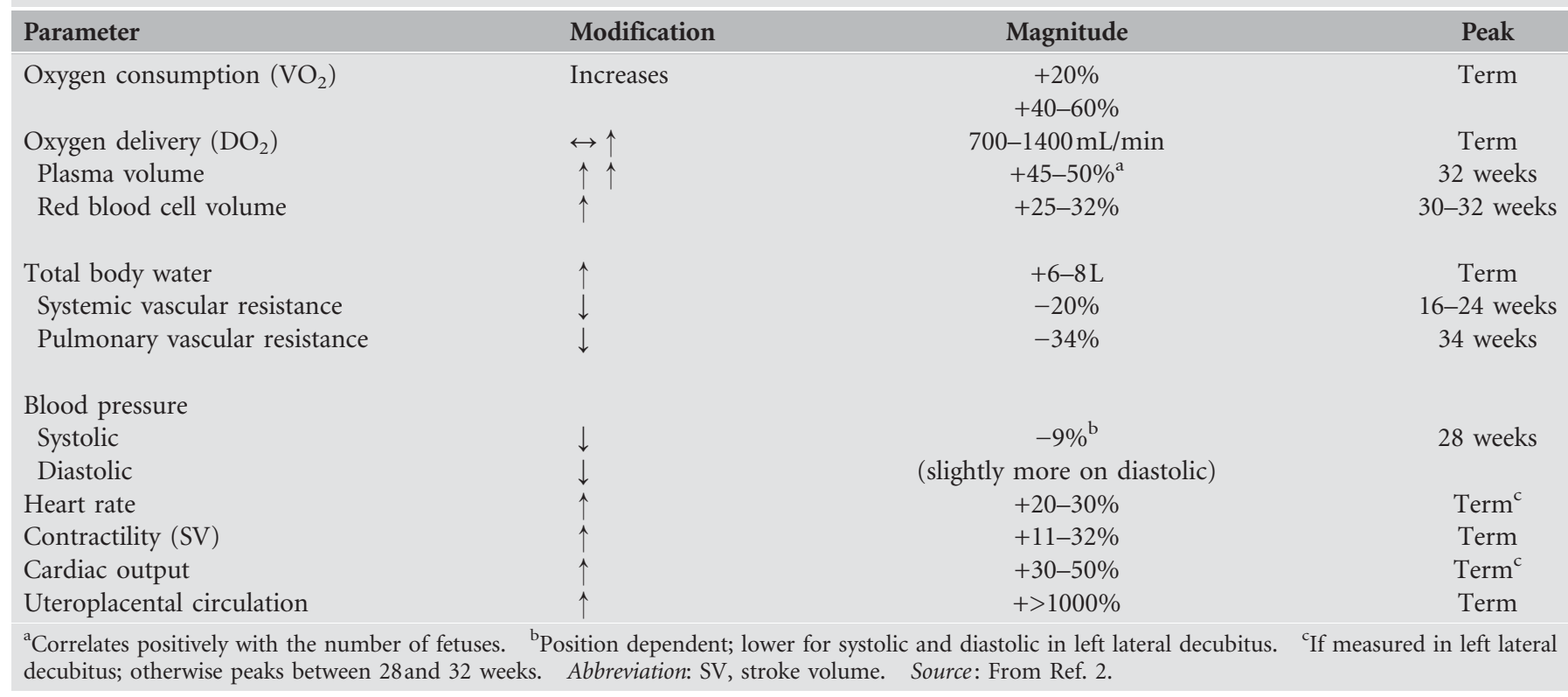

\section{Alterations During Normal Labor and Delivery}

Numerous cardiovascular changes take place during labor and delivery, and these changes are affected by a wide range of variables including anesthesia, analgesia, pain sensation, and mode of delivery.
During labor, transient increases in cardiac output occur with each uterine contraction, as well as increases in blood pressure, both systolic and diastolic. These changes are attenuated by anesthesia, with the minimum alterations occurring with spinal or epidural anesthesia, and with the greatest alterations seen during balanced general anesthesia. 
Changes in blood volume during delivery are primarily a function of blood loss, with greater loss occurring after cesarean section rather than vaginal delivery. After delivery of the fetus, preload may increase dramatically due to the decompression of the vena cava and the return of uterine blood into the systemic circulation.

The point at which cardiac changes regress to preconception levels is debatable; normal levels have been reported from 5 to over 12 weeks postpartum $(3,4)$.

\section{PRECONCEPTIONAL CARE}

The woman with heart disease should be considered a highrisk patient even before she becomes pregnant (5). Thorough planning, beginning even before conception, is the key for a successful pregnancy. Ideally, the timing of pregnancy should be a decision that the woman should decide after a detailed discussion with her obstetrician and the cardiologist. The following are the key points to be discussed before conception:

1. The natural history of the patient's disease. Pregnancy may be postponed for the medical treatment of a condition, for example, remission in the case of lupus or acute phase of myocarditis or for the surgical repair of structural abnormalities, such as valvular disease or shunts (2).

2. The expected outcomes of pregnancy in terms of maternal and perinatal morbidity and possible mortality of these conditions $(6,7)$. Table 3 summarizes the maternal risk in commonly encountered heart diseases in pregnancy and can serve as a guide for counseling.

3. The frequency of office visits, multiple specialty consultations, medications, and peripartum management should also be discussed. This discussion prepares the patient or the couple for economic and other social issues (10).

4. The risk for transmitting the cardiac condition or syndrome to offspring (11-13). Accurate estimates of the risk for cardiovascular defects in the offspring of affected parents are difficult to obtain. Frequently, the risk is quoted as approximately $5 \%$, which is six times higher than the risk in the general population (14). More recent studies have observed risks in the range of $13 \%$ to $16 \%$ (Table 4) $(12,15,16)$.

\section{Preconceptional Risk Stratification}

Risk stratification is based on three main factors: (i) the cardiac lesion, (ii) the baseline functional compromise, and (iii) the possibility and probability of complications during gestation based on the published literature. The spectrum of risk ranges from small to prohibitive. Table 5 summarizes some of the conditions that are indications for strong consideration of termination of pregnancy, and patients with these conditions should be strongly advised against pregnancy.

A thorough discussion and informed decision before conception minimize the need for a difficult decision like termination of pregnancy. After conception, the decision to terminate or continue the pregnancy is that of the woman. For some of these patients, there may be only one chance for a successful pregnancy (19). The team should provide the patient with the best information possible and to continue to assist her regardless of her decision (20).

\section{PRENATAL CARE}

Management of the pregnant woman with heart disease should be coordinated by an obstetrician experienced in high-risk cases, with a multidisciplinary team of cardiology and aanesthesia (21).

Most patients with cardiac disease are aware of their cardiac condition, but in some, cardiac disease is suspected and confirmed subsequently (22). Between $16 \%$ and $25 \%$ of the patients referred for the suspicion of cardiac disease have significant structural heart disease (23). The reasons for referrals are for the evaluation of murmurs (43\%), arrhythmias (35\%), and congenital (15.6\%) and acquired heart disease $(7 \%)(23,24)$. A previous successful obstetric outcome does not exclude a significant cardiac pathology, for example, rheumatic heart disease may not manifest until second or third pregnancy (25). In addition, patients with already diagnosed heart disease may have acquired new lesions that may complicate their usual symptomatology (Table 6) (26).

A targeted ultrasound to evaluate for fetal congenital anomalies (the fetal heart and major vessels) should be scheduled between 16 and 20 weeks of gestation in women with defined clinical syndromes at risk for complications and in women with a history of congenital heart disease $(16,27)$.

\section{Prenatal Risk Stratification}

Assessing the risk of maternal and fetal mortality and morbidity is a continuous process throughout the pregnancy. A clinically relevant tool to assess the risk would be to use Cardiac Disease in Pregnancy (CARPREG) risk index. A retrospective study examined the risks and predictors of pregnancy-related cardiac complications in women with heart disease (28). The findings were then validated by the CARPREG investigators in a prospective study (29).

Four predictors of cardiac events were identified as follows:

- Poor functional class [New York Heart Association (NYHA) class III or IV] or cyanosis

- Previous cardiac event (e.g., heart failure (HF), transient ischemic attack, stroke) or arrhythmia

- Left heart obstruction (mitral valve area of $<2 \mathrm{~cm}^{2}$, aortic valve area of $<1.5 \mathrm{~cm}^{2}$, peak left ventricular outflow gradient $>30 \mathrm{mmHg}$ )

- Left ventricular systolic dysfunction (ejection fraction $<40 \%$ )

The presence of none, one, or more than one risk factor was associated with $3 \%, 30 \%$, and $66 \%$ rates of cardiac events (pulmonary edema, arrhythmia requiring treatment, stroke, cardiac arrest, or death) during gestation, respectively. In the presence of one or more risk factors, the score was $91 \%$ sensitive and $61 \%$ specific for the occurrence of cardiac events (28).

\section{GENERAL PRINCIPLES FOR MANAGEMENT OF} CARDIAC DISEASE DURING PREGNANCY

A multidisciplinary approach is key to a successful outcome. The frequency of assessment and the types of investigations needed depend on individual conditions. High-risk pregnancies 
Table 3 Maternal Mortality Risk Groups of Cardiac Disease Associated with Pregnancy

\begin{tabular}{|c|c|c|}
\hline Risk group & Conditions & Mortality \\
\hline Low & $\begin{array}{l}\text { Atrial septal defect } \\
\text { Ventricular septal defect (uncomplicated) } \\
\text { Patent ductus arteriosus (uncomplicated) } \\
\text { Pulmonic/tricuspid disease } \\
\text { Corrected tetralogy of Fallot } \\
\text { Bioprosthetic valve } \\
\text { Asymptomatic AS with low mean gradient (less than } 25 \mathrm{mmHg} \text { and valve area greater than } 1.5 \mathrm{~cm}^{2} \text { ) } \\
\text { in presence of normal LV systolic function (EF greater than } 0.50 \text { ) } \\
\text { NYHA functional class I or II AR with normal LV systolic function } \\
\text { NYHA functional class I or II MR with normal LV systolic function } \\
\text { MVP with no MR or with mild to moderate MR with normal LV systolic function } \\
\text { Mild MS (MVA greater than } 1.5 \mathrm{~cm}^{2} \text {, gradient less than } 5 \mathrm{mmHg} \text { ) without severe pulmonary } \\
\text { hypertension } \\
\text { Mild to moderate pulmonary valve stenosis }\end{array}$ & $<1 \%$ \\
\hline Moderate & $\begin{array}{l}\text { Mitral stenosis with atrial fibrillation } \\
\text { Artificial valve } \\
\text { Coarctation of the aorta (uncomplicated) } \\
\text { Uncorrected tetralogy of Fallot } \\
\text { Previous myocardial infarction }\end{array}$ & $5-15 \%$ \\
\hline High & $\begin{array}{l}\text { Mitral stenosis, NYHA II, III, and IV } \\
\text { Severe aortic stenosis with or without symptoms } \\
\text { AR with NYHA functional class III-IV symptoms } \\
\text { MR with NYHA functional class III-IV symptoms } \\
\text { Aortic and/or mitral valve disease with severe LV dysfunction (EF less than 0.40) }\end{array}$ & \\
\hline & $\begin{array}{l}\text { Marfan syndrome with normal aorta } \\
\text { Pulmonary hypertension } \\
\text { Coarctation of the aorta } \\
\text { Marfan syndrome with aortic involvement }\end{array}$ & $25-50 \%$ \\
\hline
\end{tabular}

should be managed in a tertiary care center, where immediate access to specialists is readily available. The following pages summarize the management of the different conditions.

\section{SPECIFIC CARDIOVASCULAR ABNORMALITIES OF LOW RISK TO THE PREGNANT PATIENT (FIG 1) Atrial Septal Defect}

Ostium secundum atrial septal defect (ASD) is the most common congenital cardiovascular defect seen in pregnancy. Most women are asymptomatic and pregnancy is usually well tolerated in the pregnant patient with an ASD.

Numerous reports suggest that pregnancy is safe (30), although there are reports of the development of congestive $\mathrm{HF}$ and arrhythmias in these patients (31). If circumstances allow, ASDs should be closed before pregnancy. For a secundum defect, catheter device closure can be performed during pregnancy if necessary (with transesophageal or intracardiac echocardiographic guidance). In most instances, in pregnant women diagnosed with an ASD during pregnancy, closure can be deferred for 6 months after delivery (32).

A small proportion of patients with an ASD may develop Eisenmenger syndrome and should be considered as high-risk pregnancy. The management of high-risk patients is discussed below.

\section{Ventricular Septal Defect}

Women who reach childbearing years with an uncorrected ventricular septal defect (VSD) generally have smaller defects that are well tolerated, since the clinical picture is largely determined by the size of the defect; larger defects often require repair in childhood. Physical exam is characterized by a harsh holosystolic murmur.

Pregnancy is usually very well tolerated in women with a VSD (33). Morbidity and mortality is related to the presence of pulmonary hypertension, with a higher complication rate in those patients with Eisenmenger's physiology (31). Management of Eisenmenger syndrome is discussed below.

\section{Patent Ductus Arteriosus}

With improved pediatric surgical techniques, most patients with a patent ductus arteriosus (PDA) are surgically corrected during infancy and childhood, so it is a rare finding in pregnancy. Many patients are asymptomatic unless there is associated pulmonary hypertension. The physical examination is characterized by a "machinery" or continuous murmur in the second intercostal space. As with other shunt lesions, echocardiography is useful in defining chamber dimensions and shunt detection.

The patient with a PDA generally tolerates pregnancy well, whether surgically corrected (34) or uncorrected (30,31), but the presence of pulmonary hypertension increases the morbidity and mortality.

\section{Mitral Regurgitation}

The causes of mitral regurgitation (MR) are myriad, but in young women of childbearing age, the most common 
Table 4 Maternal and Fetal Outcomes in Cardiac Lesions

\begin{tabular}{|c|c|c|c|c|c|}
\hline Maternal lesion & $\begin{array}{l}\text { Number of } \\
\text { pregnancies }\end{array}$ & $\begin{array}{l}\text { Number of } \\
\text { abortions }\end{array}$ & $\begin{array}{c}\text { Maternal } \\
\text { mortality (\%) }\end{array}$ & $\begin{array}{c}\text { Maternal ccardiovascular } \\
\text { complications }(\%)\end{array}$ & $\begin{array}{c}\text { Live } \\
\text { births (\%) }\end{array}$ \\
\hline Mitral stenosis & $\begin{array}{c}408 \\
339^{\mathrm{a}}\end{array}$ & $\begin{array}{l}0 \\
0\end{array}$ & $\begin{array}{c}1.5 \\
0.3 \\
(0-0.5)\end{array}$ & $\begin{array}{l}16 \\
-\end{array}$ & $\begin{array}{l}- \\
-\end{array}$ \\
\hline Aortic stenosis & $\begin{array}{l}38^{\mathrm{b}} \\
59\end{array}$ & $\begin{array}{l}9 \\
0\end{array}$ & $\begin{array}{c}11 \\
3.4 \\
(0-5.3)\end{array}$ & $\begin{array}{l}- \\
-\end{array}$ & 68 \\
\hline $\begin{array}{l}\text { Aortic coarctation (unrepaired) } \\
\text { Aortic coarctation (repaired) } \\
\text { Fallot (corrected) }\end{array}$ & $\begin{array}{l}32 \\
21 \\
62\end{array}$ & $\begin{array}{c}18 \\
2 \\
14\end{array}$ & $\begin{array}{l}0 \\
0 \\
0\end{array}$ & $\begin{array}{l}50 \\
4.8 \\
8.1\end{array}$ & $\begin{array}{c}93 \\
100 \\
100\end{array}$ \\
\hline Transplanted heart & 30 & 5 & 0 & $>40$ & 100 \\
\hline $\begin{array}{l}\text { Pulmonary hypertension } \\
\text { (primary and secondary } \\
\text { excluding Eisenmenger) }\end{array}$ & 27 & Up to 50 & $\begin{array}{l}30(14-50) \\
56(35-76)\end{array}$ & $20-66$ & $\begin{array}{l}75-95 \\
86-91\end{array}$ \\
\hline Eisenmenger syndrome & $\begin{array}{l}70 \\
53\end{array}$ & $\begin{array}{l}14 \\
35\end{array}$ & $\begin{array}{c}33 \\
5.7 \\
(5.3-5.9)\end{array}$ & $\begin{array}{l}- \\
-\end{array}$ & $\begin{array}{l}63 \\
84\end{array}$ \\
\hline
\end{tabular}

\section{Table 5 Indications for Pregnancy Termination}

\section{Pulmonary arterial hypertension of any cause}

Severe ventricular systolic dysfunction

NYHA III-IV symptom class or LVEF $<30 \%$

Previous peripartum cardiomyopathy with any residual impairment of left ventricular function

Severe left heart obstruction defined as mitral stenosis: mitral valve area $<1.0 \mathrm{~cm}^{2}$ Aortic stenosis: aortic valve area $<1.0 \mathrm{~cm}^{2}$ or (nonpregnant) mean gradient $>50 \mathrm{mmHg}$

Marfan syndrome with aorta dilated $>40 \mathrm{~mm}$

Abbreviations: LVEF, left ventricular ejection fraction; NYHA, New York Heart Association. Source: From Ref. 17,18.

etiologies are rheumatic (almost always seen in association with mitral stenosis) and as a component of Barlow's syndrome (click-murmur or mitral valve prolapse syndrome).

The characteristic finding on examination is a holosystolic murmur at the apex, which radiates to the axilla. Echocardiography provides a comprehensive assessment of the etiology and the severity of the MR. In general, pregnancy is very well tolerated. Mitral valve surgery, preferably repair for symptomatic MR, may be indicated before conception (35). However, any associated left ventricular dysfunction is unlikely to improve after surgery (36) and will increase maternal risk during pregnancy (37). Severe MR may lead to HF during pregnancy or labor. The management of HF is discussed separately.

\section{Aortic Insufficiency}

Aortic insufficiency is seen infrequently in women of childbearing age, and the usual etiology is rheumatic, bicuspid aortic valve, previous endocarditis, and Marfan syndrome.

Physical findings include widened pulse pressure, and a diastolic murmur (blow) heard at the upper sternal border and the diagnosis is confirmed by echo. Aortic insufficiency without HF is generally well tolerated during pregnancy, and these patients remain essentially asymptomatic. Patients with NYHA class III-IV are at increased risk.

\section{Tricuspid and Pulmonary Valve Lesions}

Tricuspid regurgitation is an extremely common finding in normal pregnancy (38) and is rarely clinically important. The 
Table 6 Initial Evaluation for Known or Suspected Cardiac Disease During Pregnancy

\section{History}

Previous surgeries

Previous pregnancies (symptoms, admissions, outcomes)

Previous admissions (centers, physicians' names, medical records, evaluations)

History of rheumatic fever or of monthly prophylactic antibiotic injections

Drug and alcohol abuse

Functional class assessment

Physical examination

General appearance including specific facies and cyanosis

Funduscopic examination

Heart examination: rhythm, rate, precordial thrill, S1 and S2 characteristics, presence of S3 or S4, presence of murmurs and characteristics

Evaluation of pulses in all extremities

Presence and grading of edema

Cardiac diagnostics

12-lead electrocardiogram

Holter evaluation

Chest radiograph

Echocardiogram

Source: From Ref. 2. exception is tricuspid regurgitation seen in association with Ebstein's anomaly, which increases the morbidity of pregnancy. Tricuspid stenosis and pulmonary insufficiency are uncommon findings in pregnancy, and there are few published data on the outcome of pregnancy in these patients.

Pulmonary stenosis (PS) represents a common congenital cardiac defect and is seen both alone and as part of the syndrome of tetralogy of Fallot. Isolated PS is usually detected in childhood; both surgical and nonsurgical (with balloon valvuloplasty) repairs are often curative. Patients with all but the severest forms of PS often tolerate pregnancy well $(31,39,40)$. The incidence of inherited cardiac defects in the offspring of these patients appears to be high (41). Balloon valvuloplasty is recommended before conception if the gradient across the right ventricular outflow track is $>50 \mathrm{mmHg}$ at rest (42) or if the patient is symptomatic. The procedure is rarely indicated during pregnancy in patients who are either asymptomatic or mildly symptomatic before pregnancy. Vaginal delivery is tolerated well and can be permitted in the great majority of patients with PS.

\section{Mitral Valve Prolapse}

Mitral valve prolapse (MVP) is a common cardiac condition, seen in up to $2.4 \%$ of the general population (43).

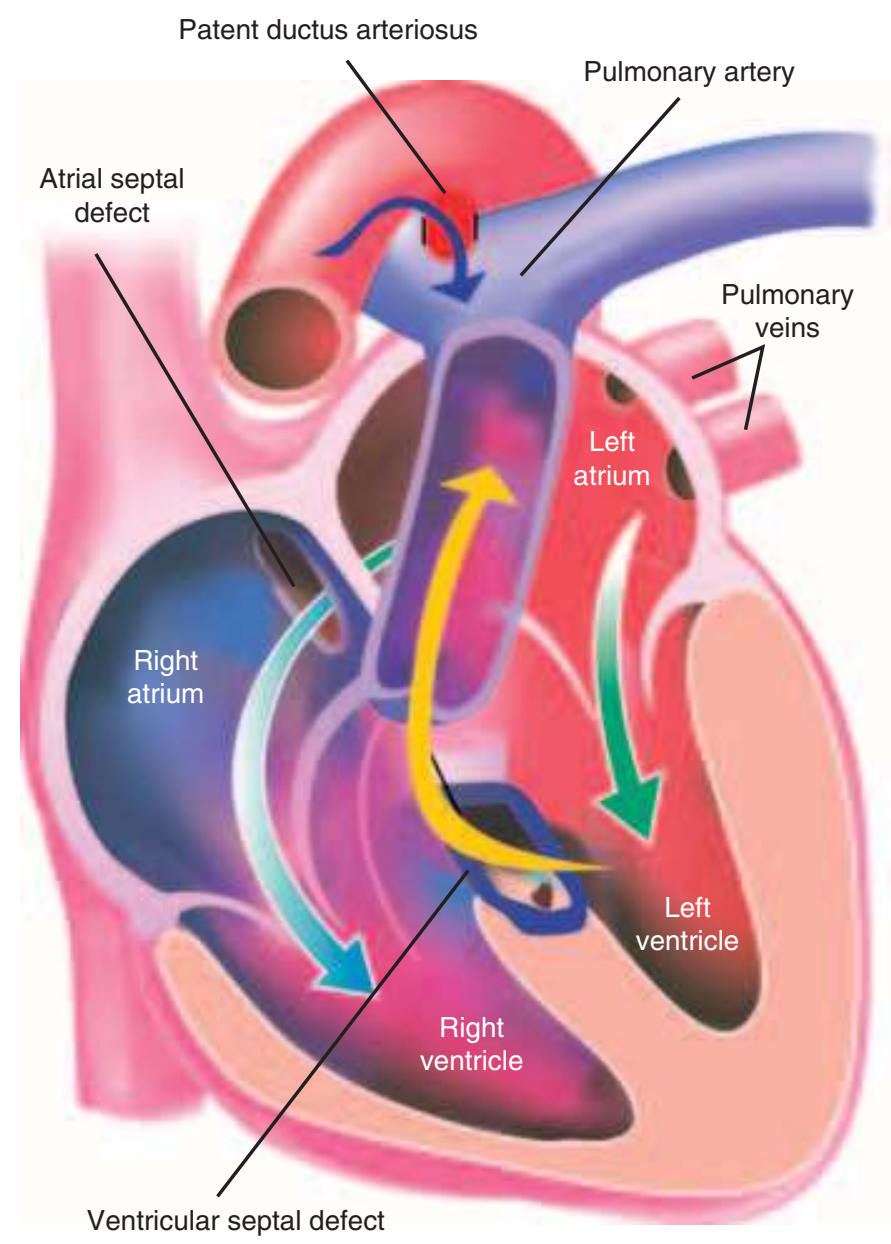

Figure 1 Anatomic locations of left-to-right shunts. 
Although the majority of patients with mitral valve prolapse are asymptomatic, some patients may experience palpitations, fatigue, and chest pain. On physical examination, mid-systolic ejection click and a murmur of MR may be heard. Management of MVP is similar to patients with MR. Infective endocarditis prophylaxis is no longer recommended (44) (see section on antibiotic prophylaxis below).

\section{SPECIFIC CARDIOVASCULAR ABNORMALITIES OF MODERATE RISK TO THE PREGNANT PATIENT (FIG. 2) Mitral Stenosis}

A common etiology for mitral stenosis in a woman of childbearing years is rheumatic heart disease, and mitral stenosis represents the most common rheumatic valvular lesion. Physiologic changes in pregnancy and atrial fibrillation can result in pulmonary edema.

These patients may have symptoms of fatigue and dyspnea. Physical examination may reveal evidence of pulmonary congestion and a diastolic murmur at the apex associated with an opening snap. Echo can assess the severity, suggest etiology, and guide in the management of mitral stenosis.

It is not uncommon for women with occult mitral stenosis to become symptomatic during pregnancy, usually by midpregnancy, when the pregnancy-induced hemodynamic changes are at the maximum. The risk of pulmonary edema exists from this period until several days after delivery.

Predictors of adverse maternal outcomes in these patients include a reduced mitral valve area (less than $1.5 \mathrm{~cm}^{2}$ ) and the NYHA functional class before pregnancy (45). Mortality among pregnant women with minimal symptoms is less than $1 \%$ (15). Severe mitral stenosis with NYHA class III-IV symptoms has a $4 \%$ to $5 \%$ maternal mortality and $30 \%$ fetal mortality (46). The mortality is even higher if atrial fibrillation is present (47). Improved diagnostic techniques, better prenatal management, and effective cardiac drugs have improved the outcome of these patients. Development of atrial fibrillation requires prompt treatment, including cardioversion. Beta blockers and digoxin can be safely used for rate control. Antiarrhythmic drugs preferred are beta blockers (sotalol, atenolol) or flecainide. Procainamide is another safe alternative, while mexiletine and amiodarone have also been used with success in small numbers of patients in the acute setting with success (48). Anticoagulant therapy is indicated in patients with mitral stenosis and atrial fibrillation is indicated to decrease the risk of systemic embolism $(49,50)$. Anticoagulation in pregnancy is discussed separately. In patients who present with severe symptoms during pregnancy, successful percutaneous balloon mitral valvuloplasty, performed during the second trimester, has been associated with normal subsequent deliveries and excellent fetal outcomes (51). The maternal and perinatal benefits of the procedure probably outweigh the small risk of fetal exposure to the radiation. Open cardiac surgery has been performed during pregnancy for severe mitral stenosis. Maternal outcomes are approximately the same as those among nonpregnant, but there is fetal loss in $10 \%$ to $30 \%$ of cases (52). See also Figure 3.

\section{Aortic Stenosis}

Aortic stenosis is not a commonly seen valvular abnormality in pregnancy, because it is usually prevalent in an older population. However, patients with aortic stenosis who have a bicuspid aortic valve may become symptomatic in their twenties and thirties. Patients may present with symptoms of angina, syncope, and HF. The physical examination reveals a crescendo-decrescendo systolic murmur at the upper sternal border, and in severe cases, a single or absent second heart

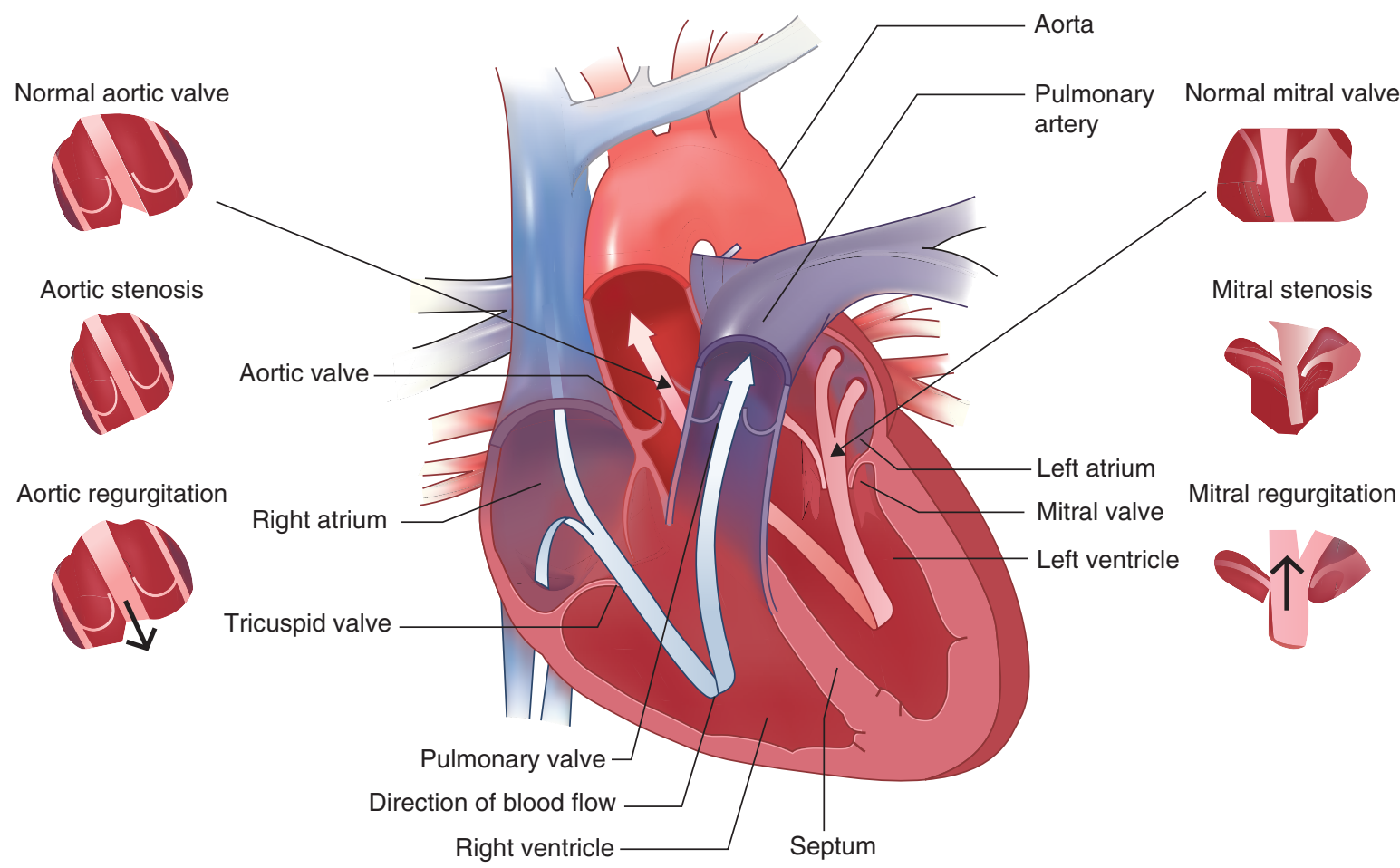

Black arrows indicate abnormal direction of flow

Figure 2 Normal and diseased aortic and mitral valve states. 
sound may be present. EKG may show left ventricular hypertrophy. Echo assesses the severity of stenosis and left ventricular (LV) systolic function.

Pregnancy can usually be successfully carried to term in all but the most severe cases of aortic stenosis; reported maternal mortality in severe aortic stenosis is 17\% (53). A more recent review of 12 studies demonstrated an average maternal mortality of $6.6 \%$ and an average perinatal mortality of $4 \%$ (54). The fetal risks of congenital heart diseases range from $17 \%$ to $26 \%(12,55)$. Complications arise when the already low forward output is compromised further by reduced preload from either hypovolemia or peripheral vasodilatation.

Patients who are symptomatic or who have a peak outflow gradient of more than $50 \mathrm{mmHg}$ are advised to delay conception until after surgical correction (56). Termination of pregnancy should be strongly considered if the patient is symptomatic before the end of the first trimester. Aortic valve replacement and palliative aortic balloon valvuloplasty have been performed during pregnancy with some associated maternal and fetal risk (52,57-59).

\section{Coarctation of the Aorta, Uncomplicated}

Coarctation of the aorta is a stenosis of the aorta, usually located just distal to the left subclavian artery at the ligamentum arteriosum (fetal ductus arteriosus). The lesion may be associated PDA, congenital bicuspid aortic valve, and aneurysms of the circle of Willis.

The hallmark of coarctation is an asymmetry of upper and lower limb blood pressures with a markedly decreased pressure in the lower extremities. Patients who have had coarctation of the aorta corrected prior to pregnancy and no other associated anomalies usually tolerate the pregnancy well. These patients, however, are still at a higher risk for cardiovascular compromise compared with nonpregnant patients.

Complications associated with uncorrected coarctation of the aorta include hypertension, HF, and aortic dissection. The mainstay of therapy is control of hypertension and avoidance of hypovolemia during the peripartum period. In cases of

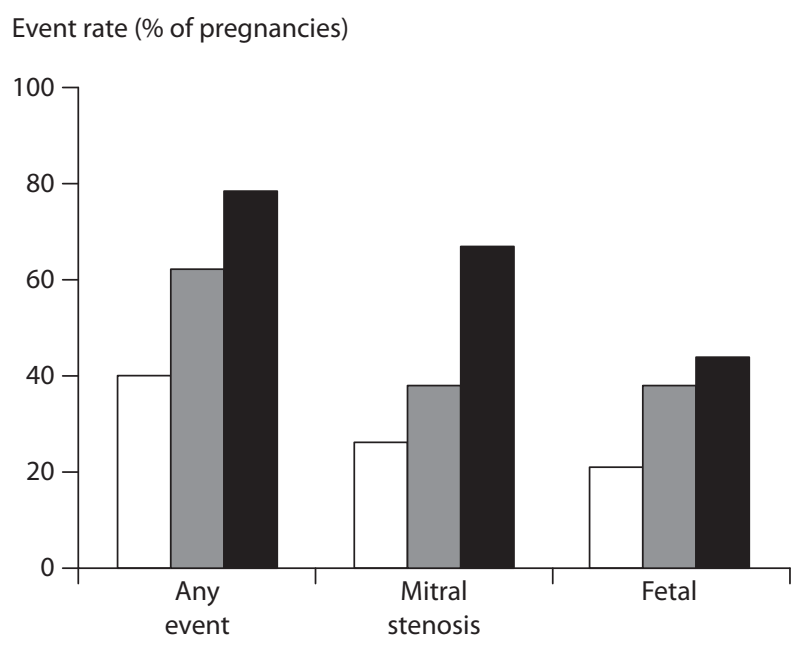

Figure 3 Relation between severity of multiple sclerosis (MS) and frequency of adverse maternal cardiac, fetal, or neonatal events. Any event refers to cardiac, fetal, or neonatal events. White bars indicate pregnancies in women who have mild MS; gray bars indicate pregnancies in women who have moderate MS, and black bars indicate pregnancies in women who have severe MS. Source: From Ref. 138. aortic dissection or severe HF unresponsive to medical therapy, surgical repair has been successfully attempted during pregnancy $(60,61)$. Fetal morbidity and mortality are high because of decreased placental blood flow from hypertension or reduced cardiac output and increased incidence of fetal cardiac anomalies (34).

\section{Marfan Syndrome, Normal Aortic Root}

Marfan syndrome is an autosomal dominant condition with defective collagen synthesis affecting the ocular, skeletal, and cardiovascular abnormalities with variable severity. The cardiovascular manifestations include mitral valve prolapse with MR and aneurysmal dilatation of the proximal aorta; the latter may be occasionally associated with aortic regurgitation and dissection.

Pregnancy seems to increase the risk of aortic rupture in patients with Marfan syndrome. The maternal morbidity and mortality depends on whether there are aortic root dilatation or valvular abnormalities $(62,63)$. Maternal mortality from aortic rupture or dissection may be up to $50 \%$ when the aortic root diameter is greater than $40 \mathrm{~mm}$ (63). On the other hand, pregnancy in patients who have aortic root diameter less than $40 \mathrm{~mm}$ and no valvular involvements can be successfully carried to term with minimal maternal mortality or morbidity. Patients should be advised of the risk and should be closely followed for signs and symptoms of aortic dissection. Serial echocardiograms during pregnancy are recommended to assess the size of the aortic root and the presence of valvular regurgitation. In selected patients, beta beta blockers may lower the risk of progressive aortic dilatation $(64,65)$.

\section{Prior History of Myocardial Infarction}

Coronary artery disease is rare in women of childbearing years, and there are only a few reports of pregnancies in women who have sustained myocardial infarctions prior to pregnancy. The pregnancy outcome of these patients is related to left ventricular function. Myocardial infarction during pregnancy poses a much higher risk (see section below).

\section{SPECIFIC CARDIOVASCULAR ABNORMALITIES OF HIGH RISK TO THE PREGNANT PATIENT}

Considering the high morbidity and mortality of pregnancy in patients with lesions in this category, counseling should be directed toward the avoidance of pregnancy. If pregnancy occurs, elective termination during the first trimester may be offered. If the pregnancy has to be continued, it is imperative that the patient is followed up closely in a tertiary care center with a multidisciplinary team including obstetrician, cardiologist, anesthesiologist, and neonatologist with experience in high-risk pregnancies (66-68). Frequent visits are recommended, and hospitalization during the third trimester may facilitate management. Detailed plans including timing and mode of delivery should be discussed with the patient in advance (66). Maternal safety needs to be weighed against the risk of premature delivery, intrauterine growth retardation, and the need for neonatal care, when decisions about timing and type of delivery are made.

These conditions are associated with a very high maternal mortality (23-50\%), which may occur during the pregnancy or the postpartum period $(33,69)$. The patient should be 
informed about the risk and offered the option of termination of pregnancy.

If the patient continues the pregnancy, careful monitoring of arterial oxygen saturation, cardiac rhythm, and blood pressure is recommended in the peripartum period. The management also includes oxygen supplementation to prevent fetal hypoxemia, invasive hemodynamic monitoring in select cases, and shortening of the second stage of labor with low or outlet forceps delivery. Hospitalization is necessary. Since the incidence of intrauterine growth retardation and fetal death is high (69), close fetal monitoring with serial ultrasound examinations and nonstress tests and/or biophysical profiles is recommended.

\section{Eisenmenger's Syndrome}

In patients with left-to-right shunts (e.g., PDA, VSD, ASD), worsening pulmonary hypertension, approaching systemic levels, results in a reversal of shunting (Eisenmenger physiology) and causes hypoxemia and death. These patients may present with peripheral cyanosis, HF, and hemoptysis.

General principles of management are discussed above. Other measures include maintaining maternal $\mathrm{PaO}_{2}$ above $70 \%$ to ensure adequate fetal oxygenation (70). There is no standard thromboprophylaxis for pregnant women with Eisenmenger (71). However, anticoagulation is needed if patient has documented pulmonary artery thrombosis.

The peripartum period is a precarious time, owing to a rapid shift in blood volume and chance of hemorrhage. Measures should be taken to avoid a reduction in preload such as hypotension or hemorrhage, since severe hypoxemia and death may ensue from decreased cardiac output. The patients should be closely monitored in the hospital setting for at least several days after delivery, since the significantly increased risk of maternal mortality still persists during this period.

\section{Idiopathic Pulmonary Artery Hypertension}

Idiopathic pulmonary artery hypertension (IPAH) is a disease of unknown etiology, primarily seen in young women and results in a progressive increase in pulmonary artery pressures. Presenting symptoms include dyspnea, fatigue, palpitations, and occasional syncope.

Right ventricular failure (elevation of jugular venous pressure, hepatomegaly, and peripheral edema) may be present.

Echocardiography remains the key diagnostic test in pulmonary hypertension, and a right heart catheterization may be needed to confirm the diagnosis of pulmonary arterial hypertension and, if needed, for the assessment of response to therapy. Right heart catheterization can be done without exposure to any radiation.

Pregnancy is poorly tolerated in patients with IPAH, with maternal mortality in excess of $40 \%$ (72). This excess mortality appears to be present even in patients who were asymptomatic or only mildly symptomatic prior to becoming pregnant. In addition, there appears to be a high incidence of fetal and neonatal death associated with maternal IPAH. Patients with IPAH often come to clinical attention during the second trimester, when the hemodynamic changes of pregnancy are maximum and the presenting symptoms are often those of right ventricular failure.
General principles of management are discussed above. Over the past decade, advanced therapies (prostacyclin analogs, phosphodiesterase inhibitors, and endothelin-receptor antagonists) have revolutionized $\mathrm{PAH}$ management considered before patients decompensate or thrombotic complications ensue. The effect of advanced therapies in pregnancy with $\mathrm{PAH}$ is unknown.

Bosentan may have teratogenic effects (73) and is, thus, contraindicated during pregnancy; limited data on sildenafil suggest no deleterious effects on the mother or offspring (74). Calcium channel blockers are safe during pregnancy (75) and may prevent preterm labor (76), a common complication in patients with PAH. However, calcium channel blockers may be beneficial only in responders to vasoreactivity tests, and only $6.8 \%$ are reported to have a long-term benefit (77).

Inhaled nitric oxide (NO) has been shown to decrease the pulmonary vascular resistance in IPAH, in Eisenmenger syndrome, and in chronic pulmonary thromboembolic disease. The effect of NO during pregnancy or labor on outcomes remains uncertain (78).

Low-dose subcutaneous heparin prophylaxis is generally recommended in pregnant women with $\mathrm{PAH}$, but some patients may require higher levels of anticoagulation (e.g., patients with a history of thromboembolic events or atrial fibrillation) (68).

The optimal mode of delivery (vaginal vs. caesarean section) in patients with $\mathrm{PAH}$ remains a matter of debate (29). Invasive pulmonary arterial pressure monitoring remains controversial and the lack of evidence that it improves outcomes in these patients (79).

\section{Complex Cyanotic Congenital Heart Disease}

Many children with complex cyanotic congenital heart disease die during infancy and childhood, and do not reach childbearing age, but with greatly improved surgical techniques, a larger number of these patients are surviving into adulthood. Specific congenital lesions of interest include (i) tetralogy of Fallot (PS, VSD, overriding aorta, and right ventricular hypertrophy); (ii) Ebstein's anomaly (displacement of the tricuspid valve into the right ventricular cavity, resulting in a small right ventricle and poor forward output, often associated with right-to-left shunting through an ASD; (iii) truncus arteriosus (single outflow tract and outflow valve distal to both ventricles, often associated with a VSD); (iv) transposition of the great vessels (separate pulmonary and systemic circulations operating in parallel with communication via a VSD; and (v) tricuspid atresia (absent tricuspid orifice, small nonfunctional right ventricle, and a connection between the pulmonary and systemic circulations). These lesions represent many of the important cyanotic congenital heart lesions, and individual lesions may be variable in severity.

In general, patients with uncorrected cyanotic congenital heart defects do not survive long enough to become pregnant; those who do survive should be counseled against pregnancy. Despite the high morbidity and mortality of pregnancy in these patients, there are case reports of successful completion of pregnancy in patients with these congenital lesions (72). If the cardiac abnormality has been surgically corrected, maternal morbidity and mortality during pregnancy appears to be related to residual cardiac defects, with a marked improvement 
in prognosis for those who have undergone successful complete correction. Infective endocarditis prophylaxis is discussed below.

\section{Marfan Syndrome Associated with an Abnormal Aortic Root}

Marfan syndrome, discussed earlier in this chapter, markedly increases maternal and fetal mortality if there is evidence of a dilated aortic root, valvular regurgitation, or dissection. Transesophageal echocardiography is superior to transthoracic echocardiography in diagnosing aortic root dilatation and aortic dissection. Preventive replacement of the ascending aorta with a graft has been recommended in patients with the aortic root diameter of at least $60 \mathrm{~mm}$ prior to pregnancy (80). Patients who contemplate pregnancy may consider preventive replacement of the ascending aorta when the aortic root dilatation is progressive and approaching $55 \mathrm{~mm}$ in diameter (64). If pregnancy occurs in patients with significant aortic root dilatation, therapeutic termination during the first trimester may be offered. If the patient chooses to continue the pregnancy, careful assessment of the patient throughout pregnancy should include evaluation for aortic dissection and use of beta blockers during pregnancy to reduce the incidence of dissection. The patient should be encouraged to avoid strenuous physical activity. Urgent delivery and vascular repair should be undertaken if clinical evidence of dissection occurs, regardless of the viability of the fetus.

\section{Myocardial Infarction During Pregnancy}

The epidemiology of coronary artery disease is such that myocardial infarction is extremely rare in women of childbearing years, estimated to occur in 1 in 10,000 pregnancies (81). Risk factors for coronary heart disease in pregnant women are the same as those for the general population and include cigarette smoking, hyperlipidemia, diabetes mellitus, and hypertension. The causes of occlusion or stenosis of the coronary arteries include thrombus and atherosclerosis; uncommon etiology includes dissection and vasospasm (82-86).

In ST elevation myocardial infarction (STEMI) in the general population, primary PCI (percutaneous coronary intervention) is the recommended therapy, if the infarct-related artery can be opened within 90 minutes of presentation (commonly referred to as door to balloon time). Treatment with thrombolytics is relatively contraindicated in pregnancy (87).

In selected group of pregnant patients with myocardial infarction, including patient with STEMI and high-risk non-ST elevation myocardial infarction (NSTEMI), PCI may be the therapy of choice during pregnancy. However, before completion of major organogenesis (before 15 weeks after menses), radiation exposure must, whenever possible, be avoided.

Cardiac catheterization offers the advantage of diagnosing and treating, in addition to thromboembolic disease, other causes such as coronary artery dissection.

All reported stenting during the acute phase of MI during pregnancy were performed with bare metal stents. The safety of drug-eluting stents in pregnant woman is therefore still unknown. Because drug-eluting stents require prolonged antiplatelet therapy with aspirin and thienopyridine, and the incidence of cesarean section deliveries in patients with heart disease is relatively high, the use of drug-eluting stent during pregnancy should be avoided if possible. Although animal experiments do not demonstrate teratogenic effect, there are no studies in pregnant women and should be used in pregnancy only if clearly needed.

Successful vaginal deliveries without further myocardial injury have been reported $(82,84-86)$. Therefore, myocardial infarction is not a contraindication for a vaginal delivery.

\section{HEART FAILURE (HF) SYNDROMES \\ Peripartum Cardiomyopathy}

This entity involves the development of HF during the last month of pregnancy or in the first five postpartum months, without obvious etiology. In the United States, the incidences vary from 1 in 4000 deliveries to 1 in 1500 deliveries, peak during the second postpartum month and increase among older, multiparous black females. The overall maternal mortality ranges from $25 \%$ to $50 \%(88,89)$.

Although the etiology of peripartum cardiomyopathy is not known, various factors have been suggested to play a role in its development, including hypertension (90), viral illness (91), an immunologic reaction (92), and vitamin deficiency (93).

Presenting clinical symptoms include those of left-sided HF (orthopnea, dyspnea, weakness) as well as peripheral edema, palpitations, and occasionally hemoptysis. On physical examination, these patients may have pulmonary rales, cardiomegaly, a gallop rhythm, distended neck veins, and peripheral edema. Cardiomegaly and pulmonary venous congestion are typical chest X-ray findings.

The diagnosis of PPCM has been based upon four clinical criteria (94-96):

- Development of cardiac failure in the last month of pregnancy or within 5 months of delivery.

- Absence of an identifiable cause for the cardiac failures (see "Causes of dilated cardiomyopathy").

- Absence of recognizable heart disease prior to the last month of pregnancy.

- LV systolic dysfunction (e.g., $\mathrm{LVEF}<45 \%$ or a reduced shortening fraction).

Echocardiography is helpful in ruling out a valvular or pericardial etiology for the clinical presentation, assessing cardiac chamber size and overall left ventricular function.

Treatment is directed toward managing HF, which is discussed below. These patients are at risk for thromboembolic disease, so prophylactic anticoagulation with heparin should be considered. If HF is refractory to medical management, early delivery should be considered.

The prognosis appears to be related to the course following pregnancy. Patients who have persistent cardiomegaly have a poor prognosis (97), whereas a return to normal heart size within 6-12 months postpartum predicts a more favorable outcome (94). Women with persistent LV dysfunction should be advised to avoid pregnancy due to the risk of HF progression and death (98). Successful subsequent pregnancies have been reported in patients who have a return to normal heart size $(99,100)$. For patients who have a refractory downhill course following the diagnosis of peripartum cardiomyopathy, cardiac transplantation should be considered. Case reports of successful completion of pregnancy following 
cardiac transplantation have been published (101-105). Patients in whom left ventricular dysfunction develops as a result of peripartum cardiomyopathy require echocardiographic follow-up of ventricular function. Improvement in systolic function beyond that assessed at 6 months after delivery is uncommon.

\section{Heart Failure}

The etiology of the HF could be ischemic or non-ischemic. Ischemia is an uncommon etiology in this population. Nonischemic causes include peripartum cardiomyopathy, viral illness, and valvular heart disease among the many. Presenting symptoms include fatigue, dyspnea, orthopnea, and pedal edema. Prognosis depends on the etiology for congestive HF (Table 7).

In patients with stable $\mathrm{HF}$, initial evaluation includes careful history and physical examination, assessment of NYHA functional class, and echocardiogram. In addition, medication adjustments and serial echocardiography during each trimester is recommended. The goals of therapy include relief of symptoms and continuation of the chronic therapy if possible, and treat precipitating causes such as anemia, arrhythmia, and thyroid disorders.

Angiotensin-converting enzyme inhibitors (ACEI), and angiotensin receptor blockers (ARB), and aldosterone antagonists like spironolactone are contraindicated throughout pregnancy. Due to the risks of embryopathy associated with ACE inhibitor use in the first trimester, ACEI should be discontinued when the woman with chronic stable $\mathrm{HF}$ is attempting conception. Beta blockers are generally safe and effective during pregnancy, although some adverse effects have been reported (106).

In patients with moderate symptoms of $\mathrm{HF}$, in addition to sodium restriction, diuretics such as furosemide and afterloadreducing agents like hydralazine should be considered. Digitalis is safe during pregnancy and may be used to maximize contractility and control heart rate, but improved survival rates have not been shown.

In decompensated $\mathrm{HF}$, positive inotropes like dobutamine and dopamine and, in select patients, intravenous nitroglycerin may be needed.

Table 7 New York Heart Association Classification of Heart Failure

\section{NYHA}

class

I No limitation of physical activity. Ordinary physical activity does not cause undue fatigue, palpitation, or dyspnea (shortness of breath)

II Slight limitation of physical activity. Comfortable at rest, but ordinary physical activity results in fatigue, palpitation, or dyspnea

III Marked limitation of physical activity. Comfortable at rest, but less than ordinary activity causes fatigue, palpitation, or dyspnea

IV Unable to carry out any physical activity without discomfort. Symptoms of cardiac insufficiency at rest. If any physical activity is undertaken, discomfort is increased

\section{Hypertrophic Cardiomyopathy}

Hypertrophic cardiomyopathy has an autosomal dominant inheritance pattern with a widely variable penetrance, but sporadic cases occur as well.

The clinical presentation is characterized by dyspnea, chest pain, angina, dizziness, and syncope, although many patients may remain asymptomatic. Occasionally, sudden death is the presenting symptom. In patients with outflow obstruction, a loud systolic murmur and thrill can be detected along the left sternal border. Maneuvers that increase venous return or chamber size (handgrip, squatting) decrease the intensity of the murmur and Valsalva maneuver increases the intensity of the murmur.

The echocardiogram provides comprehensive assessment of the thickness of the left ventricular wall, dynamic outflow tract obstruction with different maneuvers, and valvular regurgitation.

In a study of 82 pregnancies, Kumar and Elkayam (107) concluded that the outcome of pregnancy is generally favorable, although worsening of symptoms may occur.

In asymptomatic patients, management involves avoiding situations that increase the outflow tract obstruction such as hypovolemia, vasodilatation, and medications like digoxin, inotropes, and diuretics (Table 8). For symptomatic pregnant patients, treatment should be aimed at alleviating symptoms with beta blockers or non-dihydropyridine calcium channel blockers. Arrhythmias, both atrial and ventricular, occur frequently in patients with hypertrophic cardiomyopathy. General measures during labor and delivery in these patients include laboring in the left lateral decubitus position to maximize venous return to the heart, avoidance of hypovolemia, and a shortened second stage of labor. Prostaglandins, such as $\mathrm{PGE}_{2}$ (because of their vasodilatory effect), and betaadrenergic stimulants should be avoided in these patients.

\section{OTHER CONDITIONS WITH VARIABLE RISKS: ARRHYTHMIAS}

\section{Tachyarrhythmias}

Cardiac arrhythmias may be classified as supraventricular or ventricular and may occur during pregnancy either spontaneously or related to preexisting heart disease. Sinus tachycardia is a frequent occurrence during pregnancy, as are premature atrial and ventricular contractions. The hormonal and hemodynamic changes of pregnancy may predispose women, even those with normal cardiovascular systems, to frequent ectopic beats. Although the essentials of treatment of the arrhythmias in pregnant women are similar to those for nonpregnant women, the treatment has to be individualized depending on the stage of pregnancy and the risk-benefit ratio of exposing the fetus to the drugs. The principle is to avoid maternal hypotension and fetal hypoperfusion.

Atrial fibrillation in pregnancy is usually seen in the setting of rheumatic mitral stenosis. Two important considerations in pregnancy are the following: (i) If the ventricular rate is rapid, the diastolic filling time shortens, resulting in a fall in both stroke volume and forward output. This can cause decreased placental perfusion and fetal compromise. For this reason, women who are at risk for developing atrial fibrillation during pregnancy should be maintained on prophylactic digoxin or a beta blocker to prevent the rapid ventricular response, should atrial fibrillation develop. (ii) Atrial fibrillation in mitral 
Table 8 Cardiovascular Drugs in Pregnancy

\begin{tabular}{|c|c|c|c|c|}
\hline Drug & Use in pregnancy & Potential side effects & Breast feeding & Risk factors \\
\hline Adenosine & Maternal and fetal arrhythmias & $\begin{array}{l}\text { No side effects reported; data on } \\
\text { use during first trimester are } \\
\text { limited }\end{array}$ & Data NA & $\mathrm{C}$ \\
\hline Amiodarone & Maternal arrhythmias & $\begin{array}{l}\text { IUGR, prematurity, congenital } \\
\text { goiter, hypothyroidism and } \\
\text { hyperthyroidism, transient } \\
\text { bradycardia, and prolonged } \\
\text { QT in the newborn }\end{array}$ & Not recommended & C \\
\hline $\begin{array}{l}\text { Angiotensin- } \\
\text { converting } \\
\text { enzyme } \\
\text { inhibitors }\end{array}$ & Hypertension & $\begin{array}{l}\text { Oligohydramnios, IUGR, } \\
\text { prematurity, neonatal } \\
\text { hypotension, renal failure, } \\
\text { anemia, death, skull } \\
\text { ossification defect, limb } \\
\text { contractures, patent ductus } \\
\text { arteriosus }\end{array}$ & Compatible & $\begin{array}{l}\text { First trimester: C } \\
\text { Second and third } \\
\text { trimester: D }\end{array}$ \\
\hline Aldomet & Hypertension & & & C \\
\hline Beta blockers & $\begin{array}{l}\text { Hypertension, maternal arrhythmias, } \\
\text { myocardial ischemia, mitral stenosis, } \\
\text { hypertrophic cardiomyopathy, } \\
\text { hyperthyroidism, Marfan syndrome }\end{array}$ & $\begin{array}{l}\text { Fetal bradycardia, low placental } \\
\text { weight, possible IUGR, } \\
\text { hypoglycemia, no information } \\
\text { on carvedilol }\end{array}$ & $\begin{array}{l}\text { Compatible, monitoring of } \\
\text { infant's heart rate } \\
\text { recommended }\end{array}$ & $\begin{array}{l}\text { Acebutolol: B } \\
\text { Labetalol: C } \\
\text { Metoprolol: C } \\
\text { Propranolol: C } \\
\text { Atenolol: D }\end{array}$ \\
\hline Digoxin & $\begin{array}{l}\text { Maternal and fetal arrhythmias, heart } \\
\text { failure }\end{array}$ & $\begin{array}{l}\text { No evidence for unfavorable } \\
\text { effects on the fetus }\end{array}$ & Compatible & $\mathrm{C}$ \\
\hline Diltiazem & Myocardial ischemia, tocolysis & $\begin{array}{l}\text { Limited data; increased incidence } \\
\text { of major birth defects }\end{array}$ & Compatible & $\mathrm{C}$ \\
\hline Disopyramide & Maternal arrhythmias & $\begin{array}{l}\text { Limited data; may induce uterine } \\
\text { contraction and premature } \\
\text { delivery }\end{array}$ & Compatible & $\mathrm{C}$ \\
\hline Diuretics & Hypertension, congestive heart failure & $\begin{array}{l}\text { Hypovolemia leads to reduced } \\
\text { uteroplacental perfusion, fetal } \\
\text { hypoglycemia, } \\
\text { thrombocytopenia, } \\
\text { hyponatremia, hypokalemia; } \\
\text { thiazide diuretics can inhibit } \\
\text { labor and suppress lactation }\end{array}$ & Compatible & C \\
\hline Flecainide & Maternal and fetal arrhythmias & $\begin{array}{l}\text { Limited data; two cases of fetal } \\
\text { death after successful } \\
\text { treatment of fetal SVT } \\
\text { reported, but relation to } \\
\text { flecainide uncertain }\end{array}$ & Compatible & $\mathrm{C}$ \\
\hline
\end{tabular}




\begin{tabular}{|c|c|c|c|c|}
\hline Drug & Use in pregnancy & Potential side effects & Breast feeding & Risk factors \\
\hline Heparin & Anticoagulation & None reported & Compatible & $\mathrm{C}$ \\
\hline Hydralazine & $\begin{array}{l}\text { Hypertension, hypertensive } \\
\text { emergencies }\end{array}$ & None reported & Compatible & $\mathrm{C}$ \\
\hline Lidocaine & Local anesthesia, maternal arrhythmias & $\begin{array}{l}\text { No evidence for unfavorable fetal } \\
\text { effects; high serum levels may } \\
\text { cause central nervous } \\
\text { depression at birth }\end{array}$ & Compatible & $\mathrm{C}$ \\
\hline Mexiletine & Maternal arrhythmias & Limited data & Not recommended & $\mathrm{C}$ \\
\hline Nifedipine & Hypertension, tocolysis & $\begin{array}{l}\text { Fetal distress related to maternal } \\
\text { hypotension reported }\end{array}$ & Compatible & $\mathrm{C}$ \\
\hline Nitrates & $\begin{array}{l}\text { Myocardial infarction and ischemia, } \\
\text { hypertension, pulmonary edema, } \\
\text { tocolysis }\end{array}$ & $\begin{array}{l}\text { Limited data; use is generally } \\
\text { safe, few cases of fetal heart } \\
\text { rate deceleration and } \\
\text { bradycardia have been } \\
\text { reported }\end{array}$ & Data NA & $\mathrm{C}$ \\
\hline Procainamide & Maternal and fetal arrhythmias & $\begin{array}{l}\text { Limited data; no fetal side effects } \\
\text { reported }\end{array}$ & Compatible & $\mathrm{C}$ \\
\hline Propafenone & Fetal arrhythmias & $\begin{array}{l}\text { Limited data; fetal death reported } \\
\text { after direct intrauterine } \\
\text { administration in fetuses with } \\
\text { fetal hydrops }\end{array}$ & Data NA & $\mathrm{C}$ \\
\hline Quinidine & Maternal and fetal arrhythmias & $\begin{array}{l}\text { Minimal oxytocic effect, high } \\
\text { doses may cause premature } \\
\text { labor or abortion; transient } \\
\text { neonatal thrombocytopenia } \\
\text { and damage to eighth nerve } \\
\text { reported }\end{array}$ & Compatible & $\mathrm{C}$ \\
\hline $\begin{array}{l}\text { Sodium } \\
\text { nitroprusside }\end{array}$ & $\begin{array}{l}\text { Hypertension, aortic dissection, } \\
\text { hypertensive emergencies }\end{array}$ & $\begin{array}{l}\text { Limited data; potential } \\
\text { thiocyanate fetal toxicity, fetal } \\
\text { mortality reported in animals }\end{array}$ & Data NA & $\mathrm{C}$ \\
\hline Sotalol & $\begin{array}{l}\text { Maternal arrhythmias, hypertension, } \\
\text { fetal tachycardia }\end{array}$ & $\begin{array}{l}\text { Limited data; two cases of fetal } \\
\text { death and two cases of } \\
\text { significant neurologic } \\
\text { morbidity in newborns } \\
\text { reported, as well as } \\
\text { bradycardia in newborns }\end{array}$ & $\begin{array}{l}\text { Compatible, monitoring of } \\
\text { infant's heart rate } \\
\text { recommended }\end{array}$ & B \\
\hline Verapamil & $\begin{array}{l}\text { Maternal and fetal arrhythmias, } \\
\text { hypertension, tocolysis }\end{array}$ & $\begin{array}{l}\text { Limited data; other than a single } \\
\text { case of fetal death of uncertain } \\
\text { cause, no adverse fetal or } \\
\text { newborn effects have been } \\
\text { reported }\end{array}$ & Compatible & $\mathrm{C}$ \\
\hline
\end{tabular}




\begin{tabular}{|c|c|c|c|c|}
\hline Warfarin & Anticoagulation & $\begin{array}{l}\text { Crosses placental barrier; fetal } \\
\text { hemorrhage in utero, } \\
\text { embryopathy, central nervous } \\
\text { system abnormalities }\end{array}$ & Compatible & $\mathrm{X}$ \\
\hline Aspirin & Antiplatelet & $\begin{array}{l}\text { Increased maternal and fetal } \\
\text { hemorrhage, increased } \\
\text { perinatal mortality, } \\
\text { intrauterine growth } \\
\text { retardation, and premature } \\
\text { closure of the ductus } \\
\text { arteriosus. Chronic high-dose } \\
\text { use not recommended. Low- } \\
\text { dose use }(<150 \mathrm{mg} / \text { day }) \text { safe }\end{array}$ & $\begin{array}{l}\text { Cautious use of aspirin during } \\
\text { lactation }\end{array}$ & $\begin{array}{l}\text { High dose in third } \\
\text { trimester: } \mathrm{D}\end{array}$ \\
\hline $\begin{array}{c}\text { Thienopyridine } \\
\text { derivatives }\end{array}$ & & Very limited data & Not recommended & B \\
\hline $\begin{array}{l}\text { Glycoprotein } \\
\text { IIb/IIIa } \\
\text { inhibitors }\end{array}$ & & $\begin{array}{l}\text { Data NA, cesarean section should } \\
\text { be considered as the method of } \\
\text { delivery to avoid the risk of } \\
\text { fetal intracranial hemorrhage }\end{array}$ & Data NA & $\begin{array}{l}\text { (Risk category B: } \\
\text { eptifibatide, tirofiban; } \\
\text { risk category C: } \\
\text { abciximab) }\end{array}$ \\
\hline $\begin{array}{l}\text { HMG-CoA } \\
\text { rireductase } \\
\text { inhibitors } \\
\text { (statins) }\end{array}$ & & $\begin{array}{l}\text { Skeletal abnormalities maternal, } \\
\text { fetal, and neonatal mortality in } \\
\text { animal studies. Post marketing } \\
\text { surveillance-no adverse } \\
\text { outcome }\end{array}$ & Not recommended & $\mathrm{X}$ \\
\hline
\end{tabular}

fetal, and neonatal mortality i

ance-no adverse

outcome utious use of aspirin during

clardation, and premature

rteriosus. Chronic high-dose

dose use $(<150 \mathrm{mg} /$ day) safe

Glycoprotein

IIb/IIIa

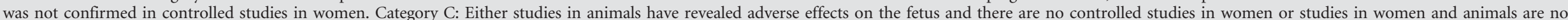

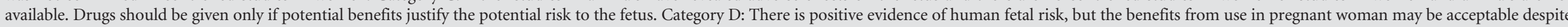
who are or may become pregnant. Abbreviations: NA, not available; SVT, supraventricular tachycardia; IUGR, intrauterine growth retardation. Source: From Refs. 9,87. 
stenosis carries a higher risk of systemic embolization. Anticoagulation in pregnancy is described below.

Supraventricular tachycardias can be terminated by vagal maneuvers such as carotid sinus massage, splashing the face with cold water, or a Valsalva maneuver. If these vagal maneuvers are unsuccessful, intravenous adenosine or verapamil should be tried. Electrical cardioversion has been used successfully during pregnancy for reversion to sinus rhythm (108).

Ventricular tachycardia is rare during pregnancy and often accompanies structural heart disease. If the ventricular tachycardia is slow $(<100-120$ beats/min) or non-sustained (self-terminating within 30 seconds), and there is no apparent structural heart disease, the prognosis is excellent and further therapy with antiarrhythmic drugs is usually not necessary. Patients with ventricular tachycardia who have structural heart disease or those with symptomatic episodes of ventricular tachycardia warrant treatment. The urgency of treatment depends on the presenting symptoms. Rapid ventricular tachycardia in the pregnant patient who is demonstrating significant hemodynamic compromise should be treated urgently with electrical cardioversion, to maintain the fetal blood supply. Patients who are hemodynamically stable can be treated with intravenous procainamide or lidocaine, both of which have been shown to be safe for the fetus. Once sinus rhythm has been reestablished, a thorough evaluation should be undertaken to determine the cause of the ventricular tachycardia before long-term therapy is initiated.

\section{Bradyarrhythmias, Conduction Blocks}

Despite the fact that sinus bradycardia and Wenckebach atrioventricular (AV) block (type I second-degree AV block) have been observed during pregnancy, labor, and delivery (109), conduction blocks are not a normal finding in pregnancy. Type II second-degree AV block and third-degree AV block are associated with an increased incidence of sudden death, and permanent pacemaker should be considered. The exception to this is congenital complete heart block, which is generally well tolerated and, because it carries a good prognosis, usually does not need to be treated with a pacemaker, unless it is symptomatic.

\section{MANAGEMENT DURING LABOR AND DELIVERY}

As discussed elsewhere in this chapter, a number of profound cardiovascular changes take place during labor and delivery, and these changes are affected by numerous variables including type of delivery (vaginal vs. cesarean), level of pain, and anesthesia.

\section{Labor and Delivery}

Timing of Delivery

The main factors considered in deciding the time for delivery are as follows:

- The current NYHA functional status (hemodynamic compensation)

- The presence or absence of fetal compromise (intrauterine growth restriction, oligohydramnios, or nonreassuring fetal testing)

- The functional status trend over time
- The possibility of improving or relieving the current functional status through optimization of treatment (e.g., evaluation of compliance, adjustment of medication doses or additional medications, additional rest)

Women in functional classes NYHA I and II and without fetal indications for delivery are allowed to go into labor spontaneously. Women in functional classes III and IV or in high risk are electively delivered. If the maternal and fetal conditions allow, the induction of labor is scheduled between 38 and 39 weeks of gestation (110). Patients in functional class III or IV are best managed in an obstetric intensive care unit, if available, or in an alternative intensive care unit.

\section{Route of Delivery}

Considering the hemodynamic changes of an uncomplicated vaginal delivery in comparison with an abdominal delivery, the vast majority of these women better tolerate a vaginal delivery $(12,64,110,111)$. Cesarean delivery increases the risk of hemorrhage, postpartum infection, pulmonary morbidity, and the fluid shifts and metabolic demands of these women during the period of recovery (13).

The indications for cesarean delivery are the same for women with cardiac disease and the general population (112). There are a few exceptions to this principle. Some women with heart disease may benefit from proceeding directly to cesarean delivery including women with endocarditis needing an emergency valve replacement at or near term; women with severe intrauterine growth restriction and Eisenmenger's reaction and unripe cervical conditions; and women with severe aortic stenosis, aortic dilatation, or dissection (2). Similarly, acute severe cardiovascular decompensation during labor might prompt the performance of a cesarean delivery (2).

\section{Induction and Augmentation}

General guidelines for the management of pregnant cardiac patients include induction of labor at term with favorable cervix and laboring in the lateral position. Women in higher functional classes may need cervical ripening procedures before induction. Mechanical methods (e.g., Foley catheter) have the advantage of avoiding hemodynamic effects and are associated with lower rates of uterine hyperstimulation and fetal bradycardia. They are the method of choice in higher functional classes and patients with cyanotic lesions. Prostaglandin-based ripening can be used in patients with lower functional classes without tachycardia or a history of asthma.

\section{Maternal and Fetal Monitoring Considerations}

Continuous EKG and pulse oximetry monitoring is recommended to monitor for hypotension, hypoxemia, myocardial ischemia, and arrhythmias. The role of pulmonary artery catheter is controversial and should be decided on a case-bycase basis. Women in functional class IV and selected cases in functional class III, pulmonary hypertension, may benefit from a pulmonary artery catheter insertion before the process of labor and delivery is started (8). In some conditions, such as mitral stenosis, controlling the heart rate with a beta blocker infusion may be indicated to avoid tachycardia and decrease in cardiac output (113). 
Continuous fetal heart rate monitoring is indicated throughout labor. The fetus is an excellent parameter of maternal perfusion. If cervical conditions allow, internal monitoring is preferable. In the presence of nonreassuring fetal patterns (e.g., nonreactive fetal strip, fetal tachycardia, repetitive late decelerations), prompt evaluation of the maternal hemodynamic parameters and oxygenation is indicated.

\section{Analgesic and Anesthetic Considerations}

Anaesthesia can alleviate anxiety and pain, which are associated with tachycardia, increased oxygen consumption and increase in cardiac workload. Cardiovascular hemodynamics is also affected by the type and amount of anesthesia.

An anesthesiologist with experience in managing these patients is pivotal to a successful maternal and fetal outcome. The combination of intrathecal and epidural analgesia (combined spinal-epidural technique or coaxial anesthesia) provides excellent analgesic control, minimizing the patient's anxiety and tachycardia. Epidural anesthesia should be used with caution in patients with hypertrophic cardiomyopathy, in other conditions with low or fixed cardiac outputs such as aortic stenosis, and in potential or actual right-to-left shunts (114). Epidural anesthesia may be contraindicated in patients with Eisenmenger's physiology because it can increase the right-to-left shunt (115). Alternatively, parenteral administration of meperidine or morphine can be used to achieve patient comfort, with the combined use of a pudendal block for analgesic relief of the second stage (114). For vaginal delivery, the second stage of labor is routinely shortened with outlet forceps or vacuum extractor (2).

\section{Postpartum Considerations}

It is important to remember that the majority of deaths in this group of patients occur during the puerperium (110). Specifically, the risks of pulmonary edema persist 24 to 72 hours postpartum (2).

Some studies have reported a significantly higher incidence of postpartum hemorrhage among patients with Marfan syndrome, higher functional classes (NYHA III or IV), or cyanosis (2). Postpartum hemorrhage should be managed promptly. Patients who have Eisenmenger syndrome may sustain an increase in the right-to-left shunt during the immediate postpartum period secondary to a decrease in systemic vascular resistance due to blood loss or relief of aortic compression by the fetus (13). In this subset of patients, vasopressors like norepinephrine may be started at the time of delivery to prevent the increase in right-to-left shunting (16).

Early assisted ambulation, use of elastic support stockings, and prophylactic anticoagulation, if needed, will minimize the risk of thromboembolism and are encouraged. If the patient has a need for prolonged anticoagulation, warfarin can be started the day after delivery with an overlap with heparin for 1 or 2 more days after reaching goal INR (116).

Prolonged postpartum admissions are recommended for patients with Eisenmenger syndrome and those with IPAH because most deaths occur during the first month after delivery (68).

\section{Breastfeeding}

No specific contraindications for breastfeeding exist provided that an adequate state of hydration is maintained. It is important to consider the fetal risks from the drugs that may be excreted in the breast milk.

\section{Follow-Up}

Cardiovascular responses do not completely normalize until 7 months after delivery. These patients should be closely followed by the cardiologists, in addition to the obstetrician.

\section{SPECIAL CONSIDERATION \\ Cardiac Surgery}

Cardiac Surgery During Pregnancy

Certain patients with heart disease may develop refractory symptoms during pregnancy that necessitate cardiac surgical procedures. In some cases, surgery may be lifesaving for the mother, albeit resulting in increased fetal mortality. The outcome of cardiovascular surgery performed during pregnancy is affected by numerous variables, such as the indication, maternal condition, the timing of the surgery relative to the duration of the pregnancy, the interval between admission and surgery, and the use of duration of cardiopulmonary bypass. These factors should be reviewed with the patient on an individual basis.

With the advances in knowledge and technology, the maternal and fetal outcome has improved significantly. Numerous pregnant patients with mitral stenosis have undergone valve replacement for both aortic and mitral valve diseases $(92,117,118)$. Although this procedure has been shown to be relatively safe and effective, it carries a high fetal mortality and should be reserved for patients refractory to medical management. In selected patients, closed mitral valvotomy offers excellent results, comparable with those of nonsurgical treatments (119). Other successful intracardiac procedures during pregnancy include pulmonary valvuloplasty, ASD and VSD repair, excision of left atrial myxomas, and, as an emergency, repair of an aortic dissection. In addition, there have been several successful coronary artery bypass graft procedures carried out during pregnancy $(92,117)$. The chief concerns in the optimal management of pregnant patients who are undergoing cardiopulmonary bypass ( $\mathrm{CPB}$ ) are the control of temperature, perfusion pressure, and the nature of the bypass flow. Current evidence favors maintaining normothermic CPB instead of the hypothermic CPB. The role of off pump coronary artery bypass grafting is a safe and accepted technique for coronary revascularization; however, its role in pregnancy needs further evaluation.

\section{Pregnancy Following Cardiac Surgery}

The outcome of pregnancy in women with corrected congenital heart disease depends on many factors including the success of the surgical repair, the extent of residual cardiac lesions, and the need for cardiovascular medications such as Coumadin. In addition, some maternal cardiac defects are associated with an increased incidence of congenital fetal cardiac and noncardiac defects that may affect fetal prognosis. Pregnancies following a heart transplant are usually not complicated $(101,102,104,105)$. Peripartum cardiomyopathy may not recur after the heart transplant (102).

Patients with successfully corrected cardiac lesions without sequelae have a good prognosis during pregnancy and can 
often be managed in the same way as a noncardiac patient. Patients with residual stenosis, or residual pulmonary hypertension, are at an increased risk.

Women who have had valve replacement with a mechanical prosthesis pose a special problem, owing to the need for anticoagulation. Anticoagulation in pregnancy is discussed below. Management of the pregnant patient with a bioprosthetic valve is not markedly different from that of patients with native valves, because the valve does not require anticoagulation.

\section{Devices}

With the expanding indications for implantable cardioverter defibrillators (ICDs), there are increasing number of pregnant patients with ICD. The mere presence of an ICD should not deter women from becoming pregnant and that pregnancy does not increase the risk of major ICD-related complications or result in high number of ICD discharges (120). For those pregnant women with ongoing malignant arrhythmias despite pharmacologic treatment, an ICD may be a safe alternative (48). It is recommended that ICD should remain activated during labor and should be switched off during cesarean section due to the interference with electrocautery and promptly reprogrammed after the surgery.

\section{Medications \\ Anticoagulation}

Certain medical conditions such as venous thrombosis of the proximal venous system, or certain types of prosthetic valves, necessitate anticoagulation during pregnancy. As with other therapy during pregnancy, the risks arising from the untreated condition must be weighed against the risks and side effects of the treatment. For conditions such as mechanical prosthetic valve, anticoagulation is a necessity.

\section{Warfarin}

Warfarin, a vitamin $\mathrm{K}$ antagonist, crosses the placenta and has been associated with increased risk of spontaneous abortion and "warfarin embryopathy." This condition is characterized by hypoplasia of the nasal bone and a wide variety of central nervous system abnormalities including optic atrophy, mental retardation, deafness, and microcephaly (121-123). The risk is higher in the second half of first trimester. In addition, warfarin predisposes both the mother and her fetus to hemorrhagic complications including fetal intracranial hemorrhage (124-127).

\section{Heparin}

Unfractionated heparin (UFH) is a large molecule that does not cross the placenta and has little effect on the fetus. Heparin can be administered by continuous intravenous infusion or by intermittent subcutaneous doses (every 8-12 hours).

\section{Low-Molecular Weight Heparins}

Low-molecular weight heparins (LMWHs) have potential advantages over UFH during pregnancy. They do not cross the placenta and are safe for the fetus (128). Plasma anti-Xa levels 4 to 6 hours after the morning dose may be used to guide therapy with LMWH. Dosage of LMWH is adjusted to achieve an anti-Xa level of approximately 0.7 to 1.2 units/LmL (129).
Although LMWHs have been used successfully to treat deep venous thrombosis in pregnant patients, there are no data to guide their use in the management of patients with mechanical heart valves (130). Reports of LMWH use in pregnant women with prosthetic heart valves are becoming more frequent, and many physicians now prescribe these agents during pregnancy in women with mechanical valves, but treatment failures have been reported.

The 2008 ACCP Guidelines for antithrombotic therapy recommended one of three approaches for anticoagulation during pregnancy: (131)

- Adjusted-dose bid LMWH throughout pregnancy. Dosing to be adjusted to achieve the manufacturer's peak anti-Xa LMWH 4 hours after subcutaneous injection.

- Adjusted-dose UFH throughout pregnancy administered subcutaneously q12 hours in doses adjusted to keep the mid-interval activated partial thromboplastin time at least twice control or attain an anti-Xa heparin level of 0.35 to $0.70 \mathrm{U} / \mathrm{mL}$.

- UFH or LMWH (as above) until the 13th week with warfarin substitution until close to delivery when UFH or LMWH is resumed.

\section{Antibiotic Prophylaxis}

Per most recently updated American Heart Association guidelines (44), prophylactic antibiotics solely to prevent endocarditis are not recommended for patients who undergo gastrointestinal (GI) or genitourinary (GU) tract procedures. In general, the incidence of infective endocarditis is relatively low during uncomplicated vaginal delivery and antibiotic prophylaxis is not recommended.

Patients with infections of the GI or GU tract may have intermittent or sustained enterococcal bacteremia. For patients with the conditions listed in Table 9, with an established GI or GU tract infection, or for those who receive antibiotic therapy to prevent wound infection or sepsis associated with a GI or GU tract procedure, it may be

Table 9 Conditions Requiring Infective Endocarditis (IE) Prophylaxis

Prosthetic cardiac valve or prosthetic material used for cardiac valve repair

Previous IE

Congenital heart disease (CHD) ${ }^{\mathrm{a}}$

Unrepaired cyanotic CHD, including palliative shunts and conduits

Completely repaired congenital heart defect with prosthetic material or device, whether placed by surgery or by catheter intervention, during the first 6 months after the procedure ${ }^{b}$

Repaired CHD with residual defects at the site or adjacent to the site of a prosthetic patch or prosthetic device (which inhibit endothelialization)

Cardiac transplantation recipients who develop cardiac valvulopathy

${ }^{a}$ Except for the conditions listed above, antibiotic prophylaxis is no longer recommended for any other form of CHD. ${ }^{b}$ Prophylaxis is reasonable because endothelialization of prosthetic material occurs within 6 months after the procedure. Prevention of infective endocarditis: guidelines from the American Heart Association (44). 
reasonable that the antibiotic regimen includes an agent active against enterococci, such as penicillin, ampicillin, piperacillin, or vancomycin (44).

\section{Cardiac Diagnostic Testing During Pregnancy}

\section{Tests Not Involving Ionizing Radiation}

Electrocardiogram

The EKG can be enormously helpful in diagnosing a wide variety of abnormalities, including arrhythmias, and poses no risk to either the mother or the fetus. Premature atrial and ventricular complexes are usually benign and seen frequently, and a wide variety of brady- and tachyarrhythmias have been documented during labor (132).

\section{Echocardiography}

Echocardiography has been widely used and poses no risk to either the mother or the fetus. An echocardiographic evaluation would be appropriate in instances of systolic murmurs in the presence of symptoms, systolic murmurs with associated thrill, pansystolic murmurs, diastolic murmurs, or murmurs accompanied by arrhythmias or gallop.

Transesophagealechocardiographyhasbeenreportedtobewell tolerated during pregnancy and is helpful to further elucidate structuralandfunctionalchangesnotwelldefinedontransthoracic echocardiography, such as endocarditis vegetations, prosthetic valves,andtheseverityofvalvulardisease $(16,64,133)$.

\section{Magnetic Resonance Imaging}

Magnetic resonance imaging (MRI) has been studied in detail and no apparent long-term adverse effects have been demonstrated on the fetuses of pregnant mice. The safety of fetal MRI has not been fully evaluated. Fetal MRI using 1.5-T magnets may be performed in any trimester (134). However, because of limited data, MRI in the first trimester is currently avoided whenever feasible.

\section{Cardiac Diagnostic Tests Involving Ionizing Radiation}

Ionizing radiation, depending on the dose and the gestational age, may pose risks to the fetus. During the first few weeks after conception, a maternal dose of $0.1 \mathrm{~Gy}$ results in a $0.1 \%$ increase in abortions over the naturally occurring spontaneous abortion rate of $25 \%$ to $50 \%$. The period of organogenesis (2-8 weeks) is critically important. A dose of $0.1 \mathrm{~Gy}$ is estimated to produce a $1 \%$ increase in the frequency of congenital fetal abnormalities (135). After 8 weeks of gestation, the primary effect of radiation exposure is an increased incidence of childhood leukemia. A dose of 0.01 Gy may increase the frequency of malignant disease by up to 11 cases per 1,000,000 live births (135).

Fetal exposure of 0.1 Gy or greater may increase the rise of childhood leukemia and fetal abnormalities such as microcephaly (136). Some limit the safe fetal dose to $5 \mathrm{mGy}$. Occasionally, the need arises during pregnancy for cardiac diagnostic testing that involves ionizing radiation. If the information obtained is crucial for the management of the patient, the test should be performed in such a way as to minimize exposure to the fetus.

\section{Chest Radiograph}

A chest X-ray delivers approximately $0-0.05 \mathrm{mGy}$ to the fetus, which is considered a negligible amount. In addition, the abdomen of the pregnant patient can often be shielded during the test to minimize exposure by scattered radiation. An inadvertent chest X-ray during early pregnancy poses little, if any, risk to the fetus, but should be performed only if the benefits outweigh the very small risk.

\section{Myocardial Perfusion Imaging and Radionuclide Ventriculogram} Radionuclide imaging using ${ }^{99 \mathrm{~m}}$ technetium-labeled sestamibi or ${ }^{201}$ thallium is expected to yield $<1 \mathrm{rad}$ of radiation to the conceptus (87). These tests are rarely needed in pregnancy and can be substituted by echocardiography if indicated.

\section{Cardiac Catheterization}

There are cardiac events during pregnancy that require cardiac catheterization and obligate exposure to ionizing radiation. The classic example would be acute myocardial infarction, where the risk of fetal exposure to ionizing radiation may be less than the benefits of immediate revascularization and prevention of complications.

The amount of fetal exposure to radiation during chest radiography in PCI results in a mean exposure of $0.02 \mathrm{mSv}$ and a maximum of $0.1 \mathrm{mSv}$ in difficult PCI procedures (National Council of Radiation Protection and Measurements 1998). Radiation that scattered from the directly irradiated area reaches the fetus. Shielding the abdomen and pelvis will not intercept the scattered irradiation. Catheterization through the radial artery will keep fetal radiation exposure to a minimum.

As with other tests, catheterization should be avoided unless the critical information cannot be obtained in other ways. Before completion of major organogenesis (before 15 weeks after menses), radiation exposure must, whenever possible, be avoided. Doses in excess of 50 to $100 \mathrm{mSv}$ increase the incidence of fetal malformation and may warrant termination of pregnancy (137).

Pregnancy in cardiac patients poses multitude of challenges to the multidisciplinary team taking care of these patients. With meticulous planning, education, counseling, and advances in technology, the maternal and fetal outcome continues to improve.

\section{REFERENCES}

1. Mishra M, Chambers JB, Jakson G. Murmurs in pregnancy: an audit of echocardiography. BMJ 1992; 304: 1413-14.

2. Gei AF, Hankins GD. Cardiac disease and pregnancy. Obstet Gynecol Clin North Am 2001; 28: 465-512.

3. Capless EL, Clapp JF. When do cardiovascular parameters return to their preconception values? Am J Obstet Gynecol 1991; 165: 883-6.

4. Cole PL, Plappert T, Saltzman DH, St John Sutton M. Changes in left ventricular geometry, load and function following pregnancy. J Am Coll Cardiol 1987; 9: 43A.

5. Easby MH. The early recognition of cardiac insufficiency in the presence of pregnancy. 1937. Cardiol Clin 1998; 16: 115-20.

6. Elkayam U, Ostrzega E, Shotan A, Mehra A. Cardiovascular problems in pregnant women with the Marfan syndrome. Ann Intern Med 1995; 123: 117-22.

7. Thilen U, Olsson SB. Pregnancy and heart disease: a review. Eur J Obstet Gynecol Reprod Biol 1997; 75: 43-50.

8. Clark SL, Cotton DB, Hankins GDV, et al. In: Belfort MA, Saade GR, eds. Critical Care Obstetrics, 3 edn. Malden, MA: John Wiley \& Sons, 2010 .

9. Bonow RO, Carabello BA, Chatterjee K, et al. 2008 focused update incorporated into the ACC/AHA 2006 guidelines for the management of patients with valvular heart disease: a report of the American College of 
Cardiology/American Heart Association Task Force on Practice Guidelines (Writing Committee to revise the 1998 guidelines for the management of patients with valvular heart disease). Endorsed by the Society of Cardiovascular Anesthesiologists, Society for Cardiovascular Angiography and Interventions, and Society of Thoracic Surgeons. J Am Coll Cardiol 2008; 52: e1-142.

10. Smedstad KG, Cramb R, Morison DH. Pulmonary hypertension and pregnancy: a series of eight cases. Can J Anaesth 1994; 41: 502-12.

11. Murphy EA, Pyeritz RE. Assessment of genetic risk in congenital heart disease. J Am Coll Cardiol 1991; 18: 338-40.

12. Whittemore R, Hobbins JC, Engle MA. Pregnancy and its outcome in women with and without surgical treatment of congenital heart disease. Am J Cardiol 1982; 50: 641-51.

13. Mendelson MA. Congenital cardiac disease and pregnancy. Clin Perinatol 1997; 24: 467-82.

14. O'Riordan AC. Genetic counseling and congenital heart disease. Resident Staff Phys J 1993; 29: 37.

15. Clark SL. Cardiac disease in pregnancy. Crit Care Clin 1991; 7: 777-97.

16. Webb GD, Harrison DA, Connelly MS. Challenges posed by the adult patient with congenital heart disease. Adv Intern Med 1996; 41: 437-95.

17. Thorne S, MacGregor A, Nelson-Piercy C. Risks of contraception and pregnancy in heart disease. Heart 2006; 92: 1520-5.

18. Thorne SA, Nelson-Piercy C, MacGregor A, et al. Pregnancy and contraception in heart disease and pulmonary hypertension. J Fam Plann Reprod Health Care 2006; 32: 74-81.

19. Somerville J. The denolin lecture: the woman with congenital heart disease. Eur Heart J 1998; 19: 1766-75.

20. Ogendo SW. Pregnancy in open heart surgical patients at Kenyatta National Hospital. East Afr Med J 1999; 76: 19-22.

21. Cole BK. Cardiac disease in pregnancy. Ohio State Med J 1970; 66: 924-6.

22. McFaul PB, Dornan JC, Lamki H, Boyle D. Pregnancy complicated by maternal heart disease. A review of 519 women. Br J Obstet Gynaecol 1988; 95: 861-7.

23. Tan J, de Swiet M. Prevalence of heart disease diagnosed de novo in pregnancy in a West London population. Br J Obstet Gynaecol 1998; 105: 1185-8.

24. Northcote RJ, Knight PV, Ballantyne D. Systolic murmurs in pregnancy: value of echocardiographic assessment. Clin Cardiol 1985; 8: 327-8.

25. Teerlink JR, Foster E. Valvular heart disease in pregnancy. A contemporary perspective. Cardiol Clin 1998; 16: 573-98, x.

26. Devlin EG, Lavery GG, Lewis MA. Peripartum cardiomyopathy in a parturient with a ventricular septal defect. Eur J Anaesthesiol 1994; 11: 241-4.

27. Clark SL. Cardiac disease in pregnancy. Obstet Gynecol Clin North Am 1991; 18: 237-56.

28. Siu SC, Sermer M, Harrison DA, et al. Risk and predictors for pregnancy-related complications in women with heart disease. Circulation 1997; 96: 2789-94.

29. Siu SC, Sermer M, Colman JM, et al. Prospective multicenter study of pregnancy outcomes in women with heart disease. Circulation 2001; 104: 515-21.

30. Metcalfe J, McAnulty JH, Ueland K. Heart disease and pregnancy, physiology and management. Boston: Little, Brown, 1986.

31. Schaefer G, Arditi LI, Solomon HA, Ringland JE. Congenital heart disease and pregnancy. Clin Obstet Gynecol 1968; 11: 1048.

32. Webb G, Gatzoulis MA. Atrial septal defects in the adult: recent progress and overview. Circulation 2006; 114: 1645-53.

33. Gleicher N, Midwall J, Hochberger D, Jaffin H. Eisenmenger's syndrome and pregnancy. Obstet Gynecol Surv 1979; 34: 721.

34. Whittenmore R, Hobbins JC, Engle MA. Pregnancy and its outcome in women with and without surgical treatment of congenital heart disease. Am J Cardiol 1982; 50: 641

35. Enriquez-Sarano M, Schaff HV, Orszulak TA, et al. Valve repair improves the outcome of surgery for mitral regurgitation. A multivariate analysis. Circulation 1995; 91: 1022-8.

36. Tribouilloy CM, Enriquez-Sarano M, Schaff HV, et al. Impact of preoperative symptoms on survival after surgical correction of organic mitral regurgitation: rationale for optimizing surgical indications. Circulation 1999; 99: 400-5.

37. Elkayam U, Gleicher N. Cardiac problems in pregnancy: diagnosis and management of maternal and fetal disease. New York: Wiley-Liss, 1998

38. Limacher MC, Ware JA, O'Meara ME, et al. Tricuspid regurgitation during pregnancy: two dimensional and pulsed Doppler echocardiogram observations. Am J Cardiol 1985; 55: 1059.

39. Neilson G, Galea EG, Blunt A. Congenital heart disease and pregnancy. Med J Aust 1970; 1: 1806

40. Mendelson CL. Cardiac disease in pregnancy. Philadelphia: F.A. Davis, 1960.

41. Nora JJ, Nora AH. The evolution of specific genetic and environmental counselling in congenital heart diseases. Circulation 1978; 57: 205.

42. Therrien J, Gatzoulis M, Graham T, et al. Canadian Cardiovascular Society Consensus Conference 2001 update: Recommendations for the Management of Adults with Congenital Heart Disease-Part II. Can J Cardiol 2001; 17: 1029-50.

43. Freed LA, Levy D, Levine RA, et al. Prevalence and clinical outcome of mitral-valve prolapse. N Engl J Med 1999; 341: 1-7.

44. Wilson W, Taubert KA, Gewitz M, et al. Prevention of infective endocarditis: guidelines from the American Heart Association: a guideline from the American Heart Association Rheumatic Fever, Endocarditis, and Kawasaki Disease Committee, Council on Cardiovascular Disease in the Young, and the Council on Clinical Cardiology, Council on Cardiovascular Surgery and Anesthesia, and the Quality of Care and Outcomes Research Interdisciplinary Working Group. Circulation 2007; 116: 1736-54.

45. Barbosa P, LA, Feotpsa G, et al. Prognostic factors of rheumatic mitral stenosis during pregnancy and puerperium. Arq Bras Cardiol 2000; 75: $220-4$.

46. Brady K, Duff P. Rheumatic heart disease in pregnancy. Clin Obstet Gynecol 1989; 32: 21-40.

47. al Kasab SM, Sabag T, al Zaibag M, et al. Beta-adrenergic receptor blockade in the management of pregnant women with mitral stenosis. Am J Obstet Gynecol 1990; 163: 37-40.

48. Adamson DL, Nelson-Piercy C. Managing palpitations and arrhythmias during pregnancy. Heart 2007; 93: 1630-6.

49. Chan WS. What is the optimal management of pregnant women with valvular heart disease in pregnancy? Haemostasis 1999; 29: 105-6.

50. Robin F, Lecuru F, Desfeux P, Boucaya V, Taurelle R. Anticoagulant therapy in pregnancy. Eur J Obstet Gynecol Reprod Biol 1999; 83: 171-7.

51. Fawzy ME, Kinsara AJ, Stefadouros M, et al. Long-term outcome of mitral balloon valvotomy in pregnant women. J Heart Valve Dis 2001; 10: $153-7$.

52. Bernal JM, Miralles PJ. Cardiac surgery with cardiopulmonary bypass during pregnancy. Obstet Gynecol Surv 1986; 41: 1-6.

53. Arias F, Pineda J. Aortic stenosis and pregnancy. J Reprod Med 1978; 20: $229-32$.

54. Lao TT, Sermer M, MaGee L, et al. Congenital aortic stenosis and pregnancy - a reappraisal. Am J Obstet Gynecol 1993; 169: 540-5.

55. Rose V, Gold RJM, Lindsay G, Allen M. A possible increase in the incidence of congenital heart defects among the offspring of affected parents. J Am Coll Cardiol 1985; 6: 376-82.

56. ACC/AHA guidelines for the management of patients with valvular heart disease. A report of the American College of Cardiology/American Heart Association. Task Force on Practice Guidelines (Committee on Management of Patients with Valvular Heart Disease). J Am Coll Cardiol 1998; 32: 1486-588.

57. Reimold SC, Rutherford JD. Valvular heart disease in pregnancy. N Engl J Med 2003; 349: 52-9.

58. Sullivan HJ. Valvular heart surgery during pregnancy. Surg Clin North Am 1995; 75: 59-75.

59. Parry AJ, Westaby S. Cardiopulmonary bypass during pregnancy. Ann Thorac Surg 1996; 61: 1865-9.

60. Goodwin JF. Pregnancy and coarctation of the aorta. Lancet 1958; 1: $16-20$.

61. Wachtel HL, Czarnecki SW. Coarctation of the aorta and pregnancy. Am Heart J 1966; 72: 251.

62. Pyeritz RE. Maternal and fetal complications of pregnancy in the Marfan syndrome. Am J Med 1981; 71: 784. 
63. Pyeritz RE. The Marfan syndrome. Am Fam Physician 1986; 34 : 83.

64. Elkayam UE, Ostrzega E, Shotan A, Mehra A. Cardiovascular problems in pregnant women with the Marfan syndrome. Ann Intern Med 1995; 123: 117-22.

65. Pyeritz RE. Propranolol retards aortic root dilatation in the Marfan syndrome. Circulation 1983; 68: 111.

66. Kiely DG, Elliott CA, Webster VJ, Stewart P. Pregnancy and pulmonary hypertension: new approaches to the management of life-threatening condition. In: Steer PJ, GM, Baker P, eds. Heart Disease and Pregnancy. London: RCOG Press, 2006: 211-29.

67. Warnes CA. Pregnancy and pulmonary hypertension. Int J Cardiol 2004; 97(Suppl 1): 11-13.

68. Weiss BM, Hess OM. Pulmonary vascular disease and pregnancy: current controversies, management strategies, and perspectives. Eur Heart J 2000; 21: 104-15.

69. Avila WS, Grinberg M, Snitcowsky R, et al. Maternal and fetal outcome in pregnant women with Eisenmenger's syndrome. Eur Heart J 1995; 16: 460-4.

70. Sobervilla LA, Cassinelli MT, Carcelen A, et al. Human fetal and maternal oxygen tension and acid base status during delivery at high altitude. Am J Obstet Gynecol 1971; 111: 1111.

71. Pitts JA, Crosby WM, Basta LL. Eisenmenger's syndrome in pregnancy: does heparin prophylaxis improve the maternal mortality rate? Am Heart J 1977; 93: 321-6.

72. Elkayam U, Gleicher N. Cardiac Problems in Pregnancy, 2nd edn. New York: Alan R Liss, 1990.

73. Madsen KM, Neerhof MG, Wessale JL, Thaete LG. Influence of ET(B) receptor antagonism on pregnancy outcome in rats. J Soc Gynecol Investig 2001; 8: 239-44.

74. Villanueva-Garcia D, Mota-Rojas D, Hernandez-Gonzalez R, et al. A systematic review of experimental and clinical studies of sildenafil citrate for intrauterine growth restriction and pre-term labour. J Obstet Gynaecol 2007; 27: 255-9.

75. Magee LA, Schick B, Donnenfeld AE, et al. The safety of calcium channel blockers in human pregnancy: a prospective, multicenter cohort study. Am J Obstet Gynecol 1996; 174: 823-8.

76. Simhan HN, Caritis SN. Prevention of preterm delivery. N Engl J Med 2007; 357: 477-87.

77. Sitbon O, Humbert M, Jais X, et al. Long-term response to calcium channel blockers in idiopathic pulmonary arterial hypertension. Circulation 2005; 111: 3105-11.

78. Bedard E, Dimopoulos K, Gatzoulis MA. Has there been any progress made on pregnancy outcomes among women with pulmonary arterial hypertension? Eur Heart J 2009; 30: 256-65.

79. Shah MR, Hasselblad V, Stevenson LW, et al. Impact of the pulmonary artery catheter in critically ill patients: meta-analysis of randomized clinical trials. JAMA 2005; 294: 1664-70.

80. Gott VL, Pyeritz RE, Magovern GJ Jr, et al. Surgical treatment of aneurysms of the ascending aorta in the Marfan syndrome. Results of composite-graft repair in 50 patients. N Engl J Med 1986; 314: 1070-4.

81. Ascarelli MH, Grider AR, Hsu HW. Acute myocardial infarction during pregnancy managed with immediate percutaneous transluminal coronary angioplasty. Obstet Gynecol 1996; 88: 655-7.

82. Fujito $\mathrm{T}$, Inoue $\mathrm{T}$, Mizoguchi $\mathrm{K}$, et al. Acute myocardial infarction during pregnancy. Cardiology 1996; 87: 361-4.

83. Glazier JJ, Eldin AM, Hirst JA, et al. Primary angioplasty using a urokinase-coated hydrogel balloon in acute myocardial infarction during pregnancy. Cathet Cardiovasc Diagn 1995;36: 216-19.

84. Taylor GW, Moliterno DJ, Hillis LD. Peripartum myocardial infarction. Am Heart J 1993; 126: 1462-3.

85. Shalev Y, Ben-Hur H, Hagay Z, et al. Successful delivery following myocardial ischemia during the second trimester of pregnancy. Clin Cardiol 1993; 16: 754-6.

86. Sheikh AU, Harper MA. Myocardial infarction during pregnancy: management and outcome of two pregnancies. Am J Obstet Gynecol 1993; 169: 279-84.

87. Roth A, Elkayam U. Acute myocardial infarction associated with pregnancy. J Am Coll Cardiol 2008; 52: 171-80.

88. Homans DC. Peripartum cardiomyopathy. N Engl J Med 1985; 312: 1432.
89. Veille JC. Peripartum cardiomyopathies: a review. Am J Obstet Gynecol 1984; 148: 805.

90. Brockington IF. Postpartum hypertensive heart failure. Am J Cardiol 1971; 27: 650 .

91. Faruque AA. Acute fulminating puerperal myocarditis. Br Heart J 1965; 27: 139.

92. Becker RM. Intracardiac surgery in pregnant women. Ann Thorac Surg 1983; 36: 453.

93. Blegen SD. Postpartum congestive heart failure: Beri-beri heart disease. Acta Med Scand 1965; 178: 515.

94. Demakis JF, Rahimtoola SH, Sutton GC, et al. Natural course of peripartum cardiomyopathy. Circulation 1971; 44: 1053.

95. Hibbard JU, Lindheimer M, Lang RM. A modified definition for peripartum cardiomyopathy and prognosis based on echocardiography. Obstet Gynecol 1999; 94: 311-16.

96. Pearson GD, Veille JC, Rahimtoola S, et al. Peripartum cardiomyopathy: National Heart, Lung, and Blood Institute and Office of Rare Diseases (National Institutes of Health) workshop recommendations and review. JAMA 2000; 283: 1183-8.

97. Walsh JJ, Burch GE, Black WC, et al. Idiopathic myocardiopathy of the puerperium (postpartal heart disease). Circulation 1965; 32: 19.

98. Elkayam U, Tummala PP, Rao K, et al. Maternal and fetal outcomes of subsequent pregnancies in women with peripartum cardiomyopathy. N Engl J Med 2001; 344: 1567-71.

99. Cole P, Cook F, Plappert T, et al. Longitudinal changes in left ventricular architecture and function in peripartum cardiomyopathy. Am J Cardiol 1987; 60: 871.

100. MSt J Sutton, Cole P, Plappert M, et al. Effects of subsequent pregnancy on left ventricular function in peripartum cardiomyopathy. Am Heart J 1991; 121: 1776-8.

101. Camann WR, Goldman GA, Johnson MD, et al. Cesarean delivery in a patient with a transplanted heart. Anesthesiology 1989; 71: 618-20.

102. Carvalho AC, Almeida D, Cohen M, et al. Successful pregnancy, delivery and puerperium in a heart transplant patient with previous peripartum cardiomyopathy. Eur Heart J 1992; 13: 1589-91.

103. Hedon B, Montoya F, Cabrol A. Twin pregnancy and vaginal birth after heart transplantation. Lancet 1990; 335: 476.

104. Key TC, Resnik R, Dittrich HC, Reisner LS. Successful pregnancy after cardiac transplantation. Am J Obstet Gynecol 1989; 160: 367-71.

105. Lowenstein BR, Vain NW, Perrone SV, et al. Successful pregnancy and vaginal delivery after heart transplantation. Am J Obstet Gynecol 1988; 158: 589-90.

106. Bayliss H, Churchill D, Beevers M, Beevers DG. Anti-hypertensive drugs in pregnancy and fetal growth: evidence for "pharmacological programming" in the first trimester? Hypertens Pregnancy 2002; 21: 161-74.

107. Kumar A, Elkayam U. Hypertrophic cardiomyopathy in pregnancy. In: Elkayam U, Gleicher N, eds. Cardiac Problems in Pregnancy. Volume 129. New York: Alan R Liss, 1990: 129.

108. Schroeder JS, Harrison DC. Repeated cardioversion during pregnancy. Am J Cardiol 1971; 27: 445.

109. Copeland GD, Stern TN. Wenckebach periods in pregnancy and puerperium. Am Heart J 1958; 56: 291.

110. Freeman RK. Intrartum management of the pregnant patient with heart disease. Clin Obstet Gynecol 1975; 18: 75-9.

111. Manning PR, Mestman JH, Lau FY. Management of the pregnant patient with mitral stenosis. Clin Obstet Gynecol 1975; 18: 99-106.

112. Mane SV, Gharpure VP, Merchant RH. Maternal heart disease and perinatal outcome. Indian Pediatr 1993; 30: 1407-11.

113. Oakley CM. Cardiovascular disease in pregnancy. Can J Cardiol 1990; 6(Suppl B): 3B-9B.

114. Morgan M. Anesthetic choice for the cardiac obstetric patient. Middle East J Anesthesiol 1990; 10: 621-32.

115. Oakley CM. Pregnancy and heart disease. Br J Hosp Med 1996; 55: 423-6.

116. Tiede DJ, Nishimura RA, Gastineau DA, et al. Modern management of prosthetic valve anticoagulation. Mayo Clin Proc 1998; 73: 665-80.

117. Pomini F, Mercogliano D, Cavatelli C, et al. Cardiopulmonary bypass in pregnancy. Ann Thorac Surg 1996; 61: 259-68. 
118. Vosa C, Renzulli A, Festa M, et al. Cardiac valve replacement during pregnancy-Report of two cases. Ital J Surg Sci 1988; 18: 175.

119. Aggarwal N, Suri V, Goyal A, et al. Closed mitral valvotomy in pregnancy and labor. Int J Gynaecol Obstet 2005; 88: 118-21.

120. Natale A, Davidson T, Geiger MJ, Newby K. Implantable cardioverterdefibrillators and pregnancy: a safe combination? Circulation 1997; 96: 2808-12.

121. Baillie M, Allen ED, Elkington AR. The congenital warfarin syndrome: a case report. Br J Ophthalmol 1980; 64: 633-5.

122. Hall JG, Pauli RM, Wilson KM. Maternal and fetal sequelae of anticoagulation during pregnancy. Am J Med 1980; 68: 122-40.

123. Harrod MJE, Sherrod PS. Warfarin embryopathy in siblings. Obstet Gynecol 1981; 57: 673-6.

124. Chen WWC, Chan CS, Lee PK, et al. Pregnancy in patients with prosthetic heart valves: an experience with 45 pregnancies. Q J Med 1982; 51: 358-65.

125. Michiels JJ, Stibbe J, Vellenga E, Van VHHDM. Prophylaxis of thrombosis in antithrombin III-deficient women during pregnancy and delivery. Eur J Obstet Gynecol Reprod Biol 1984; 18: 149-53.

126. Oakley C. Pregnancy in patients with prosthetic heart valves. Br Med J 1983; 286: 1680-3.

127. Vallenga E, Van Imhoff GW, Aarnoudse JG. Effective prophylaxis with oral anticoagulants and low dose heparin during pregnancy in an antithrombin III deficient woman. Lancet 1983; 2: 224.

128. Ginsberg JS, Chan WS, Bates SM, Kaatz S. Anticoagulation of pregnant women with mechanical heart valves. Arch Intern Med 2003; 163: 694-8.
129. Bates SM, Greer IA, Hirsh J, Ginsberg JS. Use of antithrombotic agents during pregnancy: the Seventh ACCP Conference on Antithrombotic and Thrombolytic Therapy. Chest 2004; 126: 627S-44S.

130. Elkayam UR. Anticoagulation in pregnant women with prosthetic heart valves: a double jeopardy. J Am Coll Cardiol 1996; 27: 1704-6.

131. Bates SM, Greer IA, Pabinger I, Sofaer S, Hirsh J. Venous thromboembolism, thrombophilia, antithrombotic therapy, and pregnancy*. Chest 2008; 133: 844S-86S.

132. Upshaw CB. A study of maternal electrocardiograms recorded during labor and delivery. Am J Obstet Gynecol 1970; 107: 17.

133. Stoddard MF, Longaker RA, Vuocolo LM, Dawkins PR. Transesophageal echocardiography in the pregnant patient. Am Heart J 1992; 124: $785-7$.

134. Shellock FG, Crues JV. MR procedures: biologic effects, safety, and patient care. Radiology 2004; 232: 635-52.

135. Bushong SC. Management of the pregnant employee and the pregnant patient. Radiol Manage 1984; 6: 8.

136. Swartz HM, Reichling BA. Hazards of radiation exposure for pregnant women. J Am Med Assoc 1978; 239: 1907.

137. Colletti PM, Lee K. Cardiovascular imaging in the pregnant patient. In: Gleicher UEAN, ed. Cardiac Problems in Pregnancy. New York, NY: Wiley-Liss, 1998: 33-6.

138. Silversides CK, Colman JM, Stermer M, Siu SC. Cardiac risk in pregnant women with rheumatic mitral stenosis. Am J Cardiol 2003; 91: $1382-5$. 


\section{Dermatologic diseases and pregnancy Holly Edmonds, Dana Ward, Ann G. Martin, and Susana Leal-Khouri}

\section{DERMATOLOGIC DISEASES AND PREGNANCY}

The skin during pregnancy undergoes profound changes that are associated with multiple endocrine, metabolic, and immunologic adjustments to this state. Pregnant patients develop physiologic or endocrine-induced skin changes, dermatoses specifically related to pregnancy, and skin diseases seen in the nonpregnant state. The physiologic changes occur with such regularity during pregnancy that they are considered virtually normal, but these changes sometimes become pathologic when severe. The physiologic changes often occur in the general population but are increased in frequency among pregnant women, and most regress postpartum. The most common physiologic changes affect pigmentation, striae formation, the vascular system, the pilosebaceous unit, oral mucosa, and sweat glands (Table 1).

The specific dermatoses of pregnancy include pemphigoid gestationis (herpes gestationis), pruritic urticarial papules and plaques of pregnancy (PUPPP), pustular psoriasis of pregnancy (impetigo herpetiformis), cholestasis of pregnancy (CP; prurigo gravidarum), prurigo of pregnancy (PP; papular dermatitis of pregnancy), and pruritic folliculitis of pregnancy (PFP) (Table 2). These diseases are discussed later in the chapter.

\section{PHYSIOLOGIC CHANGES ASSOCIATED WITH PREGNANCY}

Pigmentary Changes

Ninety percent of pregnant patients develop hyperpigmentation, usually mild and generalized. There is accentuation of normally hyperpigmented regions such as the areolae, nipples, axillae, genitalia, perineum, anus, and inner thighs (1-3). Generally, hyperpigmentation appears during the first trimester, progresses until delivery, and regresses postpartum. However, anatomic sites already hyperpigmented (areola, nipples, and genitalia) usually do not return to their previous color. The cause of hyperpigmentation is controversial. Most investigators agree that an increase in the production of pituitary, placental, and ovarian hormones is responsible (4-6). Melanocyte-stimulating hormone (MSH), estrogen, and progesterone are strong melanogenic stimulants and may play an important role. MSH is increased during pregnancy and decreases postpartum (7-9). Studies have also shown that the placenta contains other bioactive molecules that stimulate melanocytes by upregulating tyrosinase activity (10).

Pigmentary changes during pregnancy include darkening of the areolae, producing what is known as secondary areolae. This is usually seen as pigmentation between the two areolae. Areolar pigmentation is readily distinguished from nevoid hyperkeratosis of the nipple and areola, a rare condition which also develops during pregnancy (11). The linea alba often darkens and is renamed the linea nigra; this is most notable in dark-skinned women. Linea nigra is often accompanied by the shifting of the umbilicus to the right side of the patient at term, known as the "ligamentum teres sign," which persists postpartum until the abdominal muscles redevelop normal tone $(11,12)$. Pigmentary demarcation lines, seen as borders of abrupt transition between more deeply pigmented skin and that of lighter pigmentation, may occur on the lower limbs during pregnancy (13). Severe generalized hypermelanosis is rare and its occurrence suggests hyperthyroidism.

Melasma (chloasma), or the "mask of pregnancy," presents as blotchy, irregularly shaped, usually sharply demarcated patches of light to dark brown pigmentation of the face. It occurs in greater than $50 \%$, with some studies reporting up to $75 \%$, of pregnant women and in up to $34 \%$ of women taking oral contraceptives $(14,15)$. Melasma usually begins during the second trimester and is more common in dark-skinned (skin types IV-VI) women (16). The most commonly affected sites are the nose and cheeks followed by the upper lips, forehead, chin, and eyebrows (17). Three clinical patterns exist, which can be distinguishable by examination with the Wood's lamp. The causative agent for melasma is unknown. Contributing factors, aside from pregnancy and oral contraceptives, include genetics, skin type, exposure to UV radiation, nutrition, hepatic diseases, thyroid dysfunction, drugs, and cosmetics (16). Preventive measures such as avoidance of sun, usage of broad-spectrum sunscreens, and non-allergenic cosmetics are recommended. Treatment is frequently deferred until after delivery, since gestational melasma usually regresses within a year postpartum but may persist in less than $10 \%$ of patients (11). If melasma does persist, treatment options (in addition to consistent daily use of broad-spectrum SPF 30 sunscreen and sun protective hats) include topical hydroquinone products, or hydroquinone in combination with tretinoin and topical steroid creams, azelaic acid topical preparations, laser therapy, and chemical peels.

Freckles, melanocytic nevi, and recent scars may also darken. Although melanocytic nevi may appear somewhat darker during pregnancy, an increase in size, significant change, or variation in color, or irregular shape of a nevus is always of concern and therefore warrants an excisional biopsy. Data support the finding that women with previously diagnosed dysplastic nevus syndrome demonstrate an increased rate of changing nevi during pregnancy compared with their nonpregnant counterparts (18). Other studies have demonstrated that women who are pregnant or on oral contraceptives have an increased number of estrogen and progesterone receptors on their nevi cells similar to those observed in malignant melanoma and severely dysplastic nevi (19). Histopathologic evaluation of malignant melanoma diagnosed during pregnancy shows an increased tumor thickness. This may be associated with growth factor accelerating tumor growth 
Table 1 Physiologic Skin Changes of Pregnancy

Pigmentary changes
Hyperpigmentation
Melasma
Hair changes
Hirsutism
Telogen effluvium
Male pattern baldness
Nail changes
Transverse grooving
Distal onycholysis
Striae distensae
Vascular and hematologic changes
Spider angiomas
Palmar erythema
Varicosities
Non-pitting edema
Mucous membrane changes
Cutaneous tumors
Granuloma gravidarum
Hemangiomas
Molluscum gravidarum
Glandular activity

\section{Table 2 Dermatoses of Pregnancy}

Pemphigoid gestationis

Pruritic urticarial papules and plaques of pregnancy

Pustular psoriasis of pregnancy

Cholestasis of pregnancy

Prurigo of pregnancy

Pruritic folliculitis of pregnancy

or may reflect a delay in diagnosis. However, there has been no study that has shown a difference in anatomic location or histologic type when comparing malignant melanoma in the pregnant and nonpregnant patients (18).

\section{Striae Distensae}

Striae distensae, or stretch marks, are linear violaceous bands, which progress over time to pink or white atrophic lines. Striae develop opposite to skin tension lines on the abdomen, breast, upper arms, lower back, buttocks, thighs, and groin and are reported to occur in $55 \%$ to $90 \%$ of pregnant women during the 6th and 7th months (1-3,20-22).

The exact etiology is unknown, but several contributing factors include hormonal changes (adrenocorticotropins, relaxin, and estrogens) affecting connective tissue formation, maternal weight gain, degree of abdominal distension, and genetic predisposition (11). A recent study by Atwal et al. showed that the most significant risk factor for developing striae is low maternal age (due to increased fragility of fibrillin), followed by BMI greater than 26, maternal weight gain greater than $15 \mathrm{~kg}$, and high neonatal birth weight (23). Postpartum, the purplish striae improve cosmetically, becoming flesh-colored or hypopigmented, although striae generally do not disappear entirely. No treatment has proven definitive to prevent or decrease the occurrence of striae; topical agents have been tried (11) with minimal success.

\section{Hair and Nail Changes}

Some degree of hirsutism develops in many women early in pregnancy and is most pronounced in women with preexisting abundant body hair or dark hair. Excessive hair growth is most pronounced on the face (upper lip, chin, and cheeks); however, the arms, legs, back, and suprapubic areas may be affected. Within 6 months postpartum, excess vellus-type hairs disappear, but coarse terminal hairs may remain.

The cause of hirsutism is unknown, but it is thought to be due to increased ovarian androgen secretion or increased adrenocorticotropic hormone secretion $(24,25)$. Patients with marked persistent hirsutism should have an appropriate evaluation to exclude virilizing tumors. Therapy consists of reassuring the patient, although when hirsutism persists more than 6 months postpartum, cosmetic treatment (hair removal lasers or electrolysis) may be considered.

Lynfield reported an increased proportion of anagen hair follicles (growth phase) during pregnancy, which is thought to be secondary to a decreased rate of conversion of anagen to telogen hairs (resting phase), producing a thicker than normal growth (26). This increased proportion of anagen to telogen hairs is due to the increased estrogen levels during pregnancy, which prolong the anagen phase (4). The mean percentage of anagen hairs in the first trimester is comparable with the nonpregnant state at $85 \%$ and is increased to $95 \%$ by the second trimester (26). Postpartum, a greater than usual number of hairs enter the telogen phase manifesting clinically as a sudden increased shedding of telogen hairs, known as telogen effluvium. Telogen effluvium begins classically 3 months after delivery, but may start 1 to 5 months postpartum and usually persists for approximately 1 year with complete regrowth by 15 months. The causes of telogen effluvium include the stress of delivery and changes in postpartum hormone balance. There is an abrupt decrease in estrogen after delivery probably leading to the dramatic shift from anagen to telogen phase. Lynfield found the percentage of anagen hairs dropped from $95 \%$ to $76 \%$ shortly after delivery. Therapy involves reassurance, with most patients returning to baseline hair growth and thickness within 1 to 2 years.

A mild degree of frontoparietal recession in a male pattern distribution may develop toward the end of pregnancy. This may not be fully reversible postpartum (11). Diffuse thinning of the hair during the last trimester is also seen. The causes of male pattern frontal recession and hypotrichosis are unknown, but inhibition of gonadotropic activity secondary to high steroid levels occurring late in pregnancy may play an important role.

Nail changes seen during pregnancy include Beau's lines (transverse grooving), brittleness or softening, distal onycholysis, subungual keratosis, and accelerated nail growth (1-3, $24,27)$. The pathogenesis of these pregnancy nail changes is unclear. It is important to consider nail changes associated with psoriasis, lichen planus, and onychomycosis. Treatment consists of careful nail trimming and avoidance of external sensitizers and infections.

\section{Vascular and Hematologic Changes}

Vascular changes occur during pregnancy and are related to distention, fragility, and proliferation of vessels. Elevated 
levels of circulating estrogens and angiogenesis factors are believed to be important in the pathogenesis of "spider" angiomas, palmar erythema, hemangiomas, and pyogenic granulomas.

A spider angioma is a bright red macule or papule, with a telangiectatic puncta, radiating branches, and surrounding erythema, easily blanchable with pressure. Spider angiomas appear most commonly in areas drained by the superior vena cava (neck, throat, periorbital, and arms) (28). These changes appear in $67 \%$ of white and $11 \%$ of black women between the 2nd and 5th months of pregnancy and regress within 3 months postpartum in $75 \%$ of cases (29). Persistent spider angiomas may be best treated for cosmetic purposes with electrodesiccation or vascular lasers (28). Treatment is usually deferred until after delivery.

Palmar erythema may be localized to the thenar or hypothenar area or can present as a diffuse mottling of the hand with cyanosis and pallor. It is seen in $66 \%$ of white and $33 \%$ of black women beginning in the first trimester (1-3). The erythema may be accompanied by a burning sensation and therefore mistaken for erythromelalgia (12). Palmar erythema usually resolves 1 week postpartum. Diffuse palmar erythema is indistinguishable from that seen in hyperthyroidism and hepatic cirrhosis.

Varicosities involving the saphenous, vulvar, and hemorrhoidal veins appear in $40 \%$ of women starting from the $3 \mathrm{rd}$ month of pregnancy $(2,3,21)$. A familial tendency for increased elastic tissue fragility in conjunction with increased venous pressure may account for varicosities. However, because most varicosities develop toward the end of the first trimester when intrapelvic pressure is not significant, vascular fragility may be the more important factor (28). Partial regression tends to occur postpartum. Prevention or minimizing the risk of development of varicosities is the focus of therapy. Leg elevation, lying in the left lateral decubitus position, sleeping in Trendelenburg position, compression stockings, and avoidance of tight-fitting clothes are recommended but no studies definitively show their efficacy (28).

Other vascular changes occur less commonly in pregnancy. Cutis marmorata and livedo reticularis of the legs, facial flushing, and pallor are presumably due to vasomotor instability secondary to increased estrogens. Patients with preexisting Raynaud's phenomena may improve because of vasomotor relaxation. Non-pitting edema of the eyelids, face, hands, and feet is common during the third trimester. The edema tends to decrease during the day and is due to sodium and water retention in conjunction with increased capillary permeability. Small purpuric lesions on the legs secondary to increased capillary permeability are common during the last half of pregnancy.

\section{Mucous Membrane Changes}

Gingival hyperemia is present in up to $80 \%$ of pregnant women with an increased incidence of gingivitis beginning in the 2 nd month of pregnancy $(1-3,30)$. The gingivae enlarge, darken, become red, swollen, and may bleed spontaneously. The causes are unclear, although poor oral hygiene, local gingival irritation, nutritional deficiencies, and progesteroneinduced vascular proliferation are thought to be important in the pathogenesis.

\section{Cutaneous Tumors}

Proliferation of capillaries in hypertrophied gingivae results in granuloma gravidarum (pyogenic granuloma of pregnancy or pregnancy tumor). In $27 \%$ of women, it appears as a deep-red friable nodule arising from the gingiva during the 2 nd to 5 th month of pregnancy (30). Clinically and histologically, it is identical to pyogenic granuloma in nonpregnant women, but tends to regress postpartum. If regression does not occur, surgical removal of the nodule is a reasonable therapy.

Molluscum gravidarum (skin tags) may appear on the neck, chest, axillae, and inframammary areas in the latter stages of pregnancy and may spontaneously regress after delivery. Persistent bothersome skin tags are easily removed by simple scissoring, cryotherapy, or electrodessication if desired.

Hemangiomas, hemangioendotheliomas, glomangiomas, glomus tumors, neurofibromas, dermatofibromas, and leiomyomas may arise during gestation. Except for neurofibromas, these tumors tend to regress postpartum, but may recur during subsequent pregnancies. Keloids may grow rapidly and desmoid tumors may also develop during pregnancy. Desmoid tumors should be treated by wide local excision if there is rapid enlargement and resultant local tissue destruction.

\section{Glandular Activity}

Eccrine sweating progressively increases during the third trimester accounting for occasional hyperhidrosis and increased incidence of miliaria rubra (heat rash). The cause is unknown, but it is believed that these changes are due to increased thyroid activity. By contrast, palmar sweating is decreased, which may be secondary to increased adrenocortical activity. If eccrine sweating is significantly increased, topical treatment with a solution of $20 \%$ aluminum chloride hexahydrate in ethyl alcohol applied every night for 1 week may be helpful and then as necessary. Careful application to dry skin reduces potential irritation.

Apocrine gland activity decreases during pregnancy, improving preexisting Fox-Fordyce disease and hidradenitis suppurativa, but there may be a rebound postpartum. Sebaceous gland activity increases in some patients during the third trimester. Acne and oily skin may develop, but the effects of pregnancy on acne are unpredictable. Sebaceous glands are associated with lactiferous ducts on the areolae hypertrophy in $30 \%$ to $50 \%$ of women, beginning during the 6th week of gestation, and appear as elevated brown papules called Montgomery's tubercles $(1-3,13,25)$. The cause of the glandular changes is not well understood, but estrogen, progesterone, and cortisol are implicated. Breast changes during pregnancy include enlargement, tenderness, erect nipples, hyperpigmentation of nipples and areolae, prominence of veins, striae, and Montgomery's tubercles. Most of these changes regress postpartum.

\section{DERMATOSES OF PREGNANCY}

The dermatoses of pregnancy can be divided into two main categories: dermatoses without fetal risk and dermatoses with fetal risk. Terminology of the disease processes continues to be an ongoing controversy. The newest terminology will be used to discuss each entity. Fetal risk is associated with pemphigoid gestationis, pustular psoriasis of pregnancy, and CP. 


\section{Pemphigoid Gestationis}

Pemphigoid gestationis is the least common, but best characterized disease of pregnancy and the puerperium. It is an autoimmune disease characterized by an intensely pruritic, urticarial eruption, which rapidly progresses to bullae. The disease is most commonly associated with pregnancy, although rare occurrences have been reported with hydatidiform mole and choriocarcinoma $(31,32)$. The incidences of pemphigoid gestationis range from 1:1700 deliveries to 1:50,000 deliveries (33-35). The older term "herpes gestationis" is a misnomer, reflecting the "herpetic" clinical appearance reported by JL Milton in 1872 (36), although the condition is not related to any known viral infection. Mayou and colleagues have proposed that the term "pemphigoid gestationis" is more appropriate given its clinical and histologic resemblance to bullous pemphigoid (37). Both names can be found in the literature.

The etiology of pemphigoid gestationis appears to be autoimmune in pathogenesis, and it is associated with $\mathrm{C} 4 \mathrm{Q} 0$ allele in $90 \%$ of affected patients. However, the clinical relevance of the presence of a $\mathrm{C} 4 \mathrm{Q} 0$ allele remains to be elucidated (38). All women with a history of pemphigoid gestationis have anti-HLA antibodies, with $50 \%$ exhibiting both HLA-DR3 and HLA-DR4 (38-40). Circulating maternal autoantibodies react with an "antigenic" constituent of the cutaneous basement membrane as well as the chorionic epithelium and amnion of the placenta. This antigen is a normal constituent of the basement membrane and may be found in the placenta as early as the second trimester. Subepidermal blisters develop from tissue damage incurred by this antigen-antibody reaction with resultant complement activation. A recent finding of abnormal expression of paternal major histocompatibility complex (MHC) II region molecules in the placenta suggests that the maternal immune system evokes an allogeneic reaction (41). This has been theorized to be the etiology for the production of maternal antibodies to the amniotic basement membrane. The amniotic basement membrane is antigenically similar to skin thus leading to a cross-reaction causing maternal and occasional newborn disease (38).

The pemphigoid gestationis antigen has been characterized as a $180-\mathrm{kDa}$ glycoprotein found in the lamina lucida of the basement membrane of skin and placenta $(42,43)$. It is an interesting observation that pemphigoid gestationis antigen may first appear in the placenta during the second trimester of pregnancy, coinciding with the time course of the development of pemphigoid gestationis (44). This $180-\mathrm{kDa}$ antigen is also known as bullous pemphigoid antigen 2 and most recently as type XVII collagen. This is a transcellular glycoprotein in the hemidesmosome with both a cytoplasmic and extracellular domain. The function of the hemidesmosome is to anchor the basal cells of the epidermis to the basement membrane thus keeping it attached to the dermis. The NC16A segment of the $180-\mathrm{kDa}$ antigen is the immunoreactive site in the majority of affected patients (38).

Pemphigoid gestationis presents as an extremely pruritic, urticarial, and bullous eruption that may begin at any time during the pregnancy, but most commonly occurs during the second and third trimesters. It frequently begins during the first pregnancy and has a tendency to recur earlier and with greater severity in subsequent pregnancies. Initial onset in the postpartum period has also been well documented in up to $25 \%$ of patients and is often explosive, occurring within hours of delivering. A frequent observation is a relative remission in the weeks prior to delivery followed by a postpartum flare.

Early lesions of pemphigoid gestationis begin as erythematous, edematous papules and plaques, which rapidly develop into vesicles and tense bullae (Fig. 1). The vesicles and bullae may be grouped, hence the historical description of "herpetic," and develop into erosions with crusting. The eruption begins in and around the umbilicus in the majority (50\%) of patients and then spreads to involve the abdomen and thighs (Fig. 2). The back, breast, palms, and soles are frequently affected. The head and mucosa are involved less frequently $(<20 \%)$. A constant feature of pemphigoid gestationis is pruritus. Itching is often extreme and may begin days or weeks prior to the appearance of clinical lesions.

Two skin biopsies are needed to establish the diagnosis: one punch biopsy of an intact bullous lesion for routine $\mathrm{H}$ \& $\mathrm{E}$ histology and a second punch biopsy taken from perilesional skin for direct immunofluorescence. Histology of an early urticarial lesion shows a perivascular infiltrate consisting of lymphocytes and many eosinophils. Papillary dermal edema with spongiosis and intracellular edema of the overlying

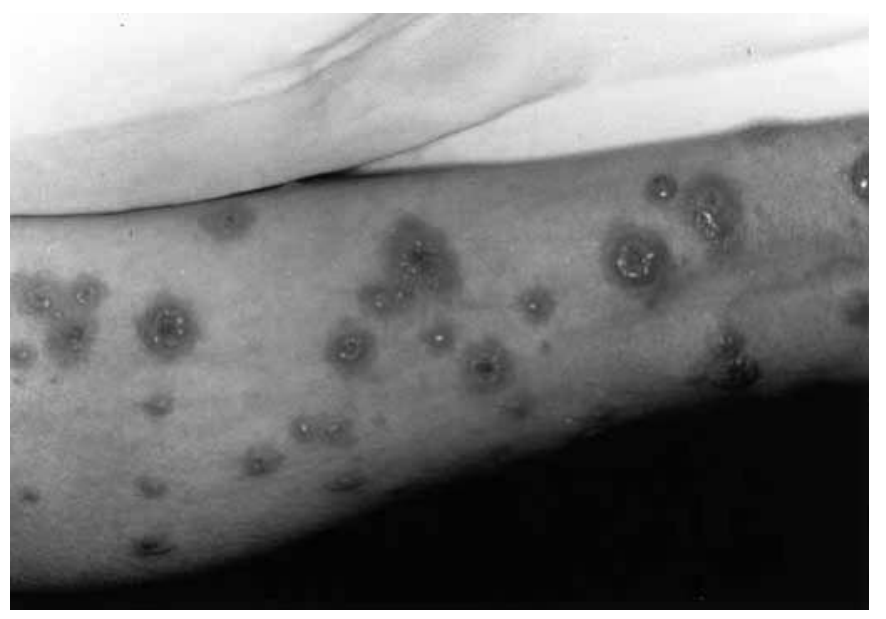

Figure 1 Early lesions of herpes gestationis.

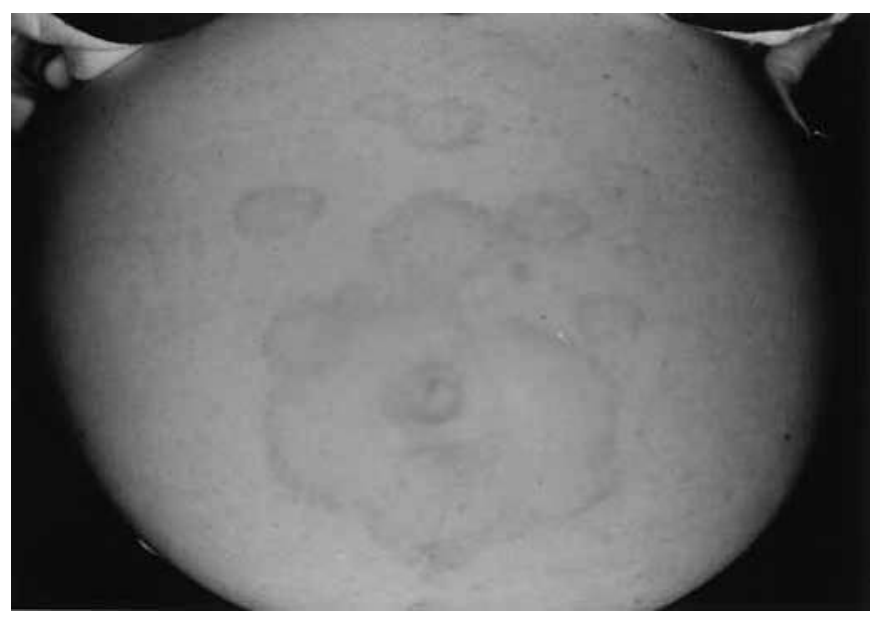

Figure 2 Periumbilical distribution of herpes gestationis. 
epidermis are seen. Histology of a bulla reveals basal cell necrosis with a resultant subepidermal bulla. There is a pronounced inflammatory infiltrate containing many eosinophils. The presence of eosinophils is the most constant feature of pemphigoid gestationis (45).

Using a direct immunofluorescence assay, patients with pemphigoid gestationis have a linear deposition of complement 3 (C3) along the basement membrane zone in clinically normal and involved skin (46). This finding traditionally is the hallmark of the disease, remaining the most sensitive and specific test for pemphigoid gestationis (45). Immunoglobulin $\mathrm{G}(\mathrm{IgG})$ deposition is seen as well (30-40\% of patients), and less frequently IgA and IgM.

The sera of patients with pemphigoid gestationis contain an antibody to a basement membrane constituent of normal skin. This antibody, known as "herpes gestationis factor," avidly binds complement and is an IgG1 molecule (47). Using conventional indirect immunofluorescence, only $25 \%$ of patients show evidence of "herpes gestationis factor," and usually in low titers. However, complement added indirect immunofluorescence reveals the circulating anti-basement membrane zone $\operatorname{IgG}$ in nearly all patients, thus this technique is much more sensitive (38). Salt split skin studies reveal the immunoreactants to localize in the epidermal side of the lamina lucida, a finding similar to that seen in bullous pemphigoid (48). Placental studies reveal a similar deposition of $\mathrm{C} 3$ and immunoglobulin along the amniotic basement membrane. As in lesional skin and plasma, IgG1 is the most frequently found immunoglobulin (49).

Laboratory evaluation is not necessary for the diagnosis of pemphigoid gestationis, although there is an increase in incidence of antithyroid antibodies detected in affected patients but clinically apparent thyroid dysfunction is rare $(38,50)$. ELISA testing is now available and will potentially replace direct immunofluorescence for diagnosis in the future.

The primary disease entity in the differential diagnosis of pemphigoid gestationis is PUPPP. There is clinical overlap between the two diseases in that they are both pruritic dermatoses of pregnancy presenting with urticarial plaques, wheals, and vesicles. Pemphigoid gestationis is distinctive, however, in developing grouped bullae (PUPPP occasionally is vesicular), in its initial periumbilical distribution (PUPPP classically arises in abdominal and thigh striae), and its earlier appearance within the second trimester (PUPPP usually begins in the third trimester). On $\mathrm{H} \& \mathrm{E}$ histology, one cannot definitively differentiate them. However, direct immunofluorescence of pemphigoid gestationis is consistently positive for linear deposits of $\mathrm{C} 3$ at the basement membrane, while PUPPP shows nonspecific direct immunofluorescence. Indirect immunofluorescence studies in PUPPP are negative (51).

Other vesiculobullous diseases, such as dermatitis herpetiformis, bullous pemphigoid, and bullous erythema multiforme, are easily differentiated from pemphigoid gestationis by clinical presentation and routine immunofluorescence. Bullous pemphigoid has many similarities to pemphigoid gestationis both in its clinical appearance and in its ultrastructural findings. However, bullous pemphigoid is a disease of the elderly and would rarely be seen in the reproductive years. The dominant Ig subclass in bullous pemphigoid is IgG4 compared with IgG1 in pemphigoid gestationis (45).
Systemic steroids are the mainstay of therapy for moderate to severe pemphigoid gestationis. Recommended prednisone dosing is $0.5 \mathrm{mg} / \mathrm{kg} /$ day. One can frequently taper to a daily prednisone dose of $10 \mathrm{mg}$. Fortunately, the use of low-dose systemic steroids in the second and third trimester of pregnancy is not associated with increased risk of congenital anomalies, although careful monitoring is needed for gestational diabetes and hypertension (52). Mild cases are often treated with mid- to high-potency topical glucocorticoids and oral antihistamines (some studies have shown these medications to be ineffective, but they may offer symptomatic relief) (38). Alternative therapies include dapsone, cyclosporine, plasmapheresis, and cyclophosphamide, each variably effective at best, and all with significant potential for morbidity $(38,53,54)$.

The usual course of pemphigoid gestationis is extremely variable. Many experience complete remission several weeks after delivery and mild flaring with resumption of menses. Rarely, severe cases of pemphigoid gestationis will persist far beyond the postpartum period and require corticosteroid therapy. Recurrent disease flares are reported from 2 weeks up to 12 years postpartum $(38,45)$. Monthly perimenstrual flares, ovulatory flares, and flares with the use of birth control pills or estrogen therapy have all been reported in up to $25 \%$ of patients. These reports support the contention that once pemphigoid gestationis is initiated, it is hormonally mediated. Once a patient has pemphigoid gestationis, there is a clear tendency for it to recur in subsequent pregnancies (there are also reports of "skip pregnancies" in 5-10\% of cases) with an earlier onset and more severe disease, but there are no good data to support this $(38,55)$.

The impact of pemphigoid gestationis on perinatal morbidity and mortality is controversial. While Lawley and colleagues noted an increase in fetal morbidity and mortality, Shornick and coworkers found no increase in maternal or fetal complications $(34,56)$. More recent data suggested an increase in preterm delivery and fetal growth retardation $(57,58)$. No studies show that treatment with systemic steroids alters the risk of preterm delivery in these patients (38). In any case, good obstetrical and perinatal care gives a favorable outcome in the great majority of cases. Rarely do infants born to mothers with pemphigoid gestationis have vesicles or bullae $(<10 \%)$. When present, the eruption is mild and transient. There appear to be no ill effects on the child nor evidence of increased incidence of other autoimmune diseases (46). Women with a history of pemphigoid gestationis have an increased risk for the development of Grave's disease (38).

\section{Pruritic Urticarial Papules and Plaques of Pregnancy}

PUPPP is the most common of the dermatoses of pregnancy with an incidence varying from 1 in 160 to 1 in 300 pregnancies $(50,59)$. In the first report of PUPPP, a woman was described with all the clinical features: a pruritic, urticarial eruption developing within abdominal striae during the third trimester and generalizing to the thighs and buttocks. While it was termed toxemic rash of pregnancy, there is no association of PUPPP with pre-eclampsia, and this terminology was not accepted (60). In 1979, Lawley and colleagues (61) reported seven cases in which they carefully defined the clinical features of PUPPP and gave it its widely accepted, yet controversial, name of pruritic urticarial papules and plaques of pregnancy. 
Later Holmes and Black suggested the name polymorphic eruption of pregnancy based on the varying morphology of the lesions (62). Both names are now found in the literature.

While the etiology remains unknown, there are several current theories, one of which involves an inflammatory reaction to connective tissue damaged within striae secondary to late abdominal wall distension $(63,64)$. Hormonal modulation may be involved as evidenced by patients with PUPPP having higher prevalence of multiple gestations compared with the general population. One study found a $13 \%$ incidence of PUPPP in multiple gestational pregnancies compared with $1 \%$ incidence in single gestational pregnancies (65). Other theories include maternal weight gain, fetal weight, infant sex (males more common), and fetal DNA $(66,67)$.

The onset of PUPPP varies from as early as 17 weeks to 1 week postpartum, with the majority of patients presenting within the third trimester (mean onset 35 weeks) (38). The mean duration of the disease is 6 weeks, but it may persist as long as 16 weeks. Severe symptoms usually last 1 week. PUPPP classically presents with erythematous urticarial plaques on the abdomen usually in association with striae and always sparing the periumbilical region (Fig. 3) (38,65). Other presentations include vesicular, purpuric, targetoid, or polycyclic lesions, hence the polymorphous nature. This cutaneous eruption then spreads rapidly to involve the thighs, buttocks, breasts, and arms while the face, palms, and soles are rarely involved. Pruritus is localized to the involved skin.

Histopathologic findings of an early urticarial lesion reveal epidermal and upper dermal edema with a superficial lymphohistiocytic perivascular infiltrate. Eosinophils often are present in the infiltrate. However, blood eosinophilia is a less common feature. Vesicles are present in the most florid examples, in which case intense focal spongiosis is seen in the epidermis. Direct immunofluorescence studies are nonspecific, and indirect immunofluorescence studies are negative, which helps differentiate PUPPP from pemphigoid gestationis.

Treatment of PUPPP is symptomatic due to its self-limiting course and lack of serious outcomes. Management of pruritus, often very intense and unremitting, is usually accomplished with medium-potency topical steroid preparations and oral antihistamines. Systemic corticosteroids are sometimes used. Reassurance to the patient about this common cutaneous

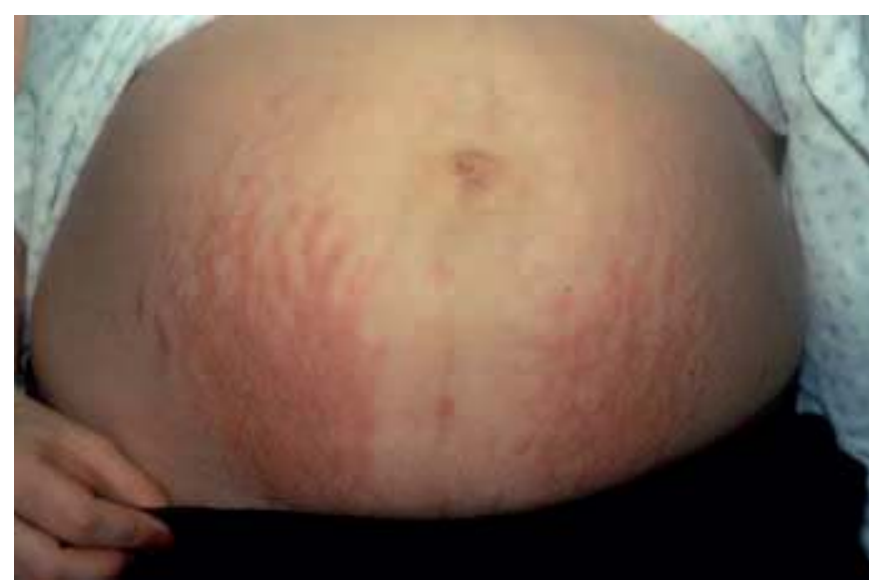

Figure 3 Pruritic urticarial papules and plaques of pregnancy developing in striae. disease of pregnancy is important, because it usually resolves within a few weeks without risk to the fetus.

Maternal and fetal prognosis is excellent (68). Recurrence during subsequent pregnancies, with menses, or oral contraceptives is uncommon. If it does recur, the disease tends to be attenuated.

\section{Pustular Psoriasis of Pregnancy (Impetigo Herpetiformis)}

Pustular psoriasis of pregnancy is rare, with fewer than 350 cases reported. It was first described by Von Hebra in 1872, and it consists of an acute pustular eruption resembling pustular psoriasis arising suddenly in a patient without prior history of psoriasis (69). Pustular psoriasis of pregnancy continues to be debated as to whether this disease is a variant of pustular psoriasis or a distinct clinical entity truly specific to pregnancy. Several factors favor a pregnancy-specific dermatosis. These include the absence of a positive family history of psoriasis, abrupt cessation of symptoms after delivery, and the tendency for recurrence with subsequent pregnancies and oral contraceptives (38).

The onset of pustular psoriasis of pregnancy is usually during the last trimester of pregnancy, but it may occur as early as the third month. Skin lesions begin as circinate erythematous macules or plaques, with 1 to $3 \mathrm{~mm}$ pustules advancing along the peripheral edge, preferentially occurring in intertriginous areas. It may spread to mucous membranes as well as to the entire integument, sparing only the face, hands, and feet. Pruritus is usually not present or very mild. The lesions heal with non-scarring post-inflammatory hyperpigmentation. Systemic symptoms are common including fever, chills, malaise, nausea, diarrhea, and arthralgias. Rarely does the patient present with tetany, delirium, and seizures secondary to hypocalcemia. Other potential lab abnormalities include leukocytosis with neutrophilia, elevated erythrocyte sedimentation rate, hypoalbuminemia, and iron deficiency anemia (70-72).

Histologically, pustular psoriasis of pregnancy is identical to pustular psoriasis and is characterized by parakeratosis, elongation of the rete ridges, and subcorneal collections of neutrophils. Immunofluorescence studies are all negative. It is important to remember that these pustules are sterile unless the skin becomes secondarily bacterially infected.

Early recognition of pustular psoriasis of pregnancy is crucial, with both maternal and fetal morbidity and mortality increased (73). Maternal complications may include profound hypocalcemia and the risk of bacterial sepsis, due to failure of skin barrier function. Fetal complications include placental insufficiency with sequelae such as miscarriage, fetal growth restriction, and possible stillbirth $(73,74)$. Fetal well-being should be monitored with nonstress tests, biophysical profiles, and ultrasound studies as indicated (70).

First-line treatment during pregnancy is oral corticosteroids. A prednisone dose as high as $60 \mathrm{mg} /$ day may be required, with a careful slow taper to prevent a rebound phenomenon. Therapeutic consideration may be given to cyclosporine (class C), depending on the severity of the clinical situation $(38,73)$. After delivery, treatment options are broader with oral retinoids (isotretinoin or etretinate), narrowband ultraviolet $\mathrm{B}$ phototherapy, and methotrexate more commonly used (71). 


\section{CP}

$\mathrm{CP}$, also known as prurigo of pregnancy and prurigo gravidarum, is characterized by sudden onset of intense pruritus in the absence of primary cutaneous lesions, although secondary excoriations may occur (Fig. 4). It classically presents during the third trimester in $80 \%$ of cases (with $10-20 \%$ during first and second trimesters). Symptoms remit within 2 to 4 weeks after delivery but can recur with successive pregnancies $(38,51)$. The incidence of this disorder ranges from $0.02 \%$ to $3 \%$ depending on the region, with the highest incidence in Chile at $14 \%$ to $16 \%(4,38,75)$.

The etiology of CP is secondary to maternal intrahepatic bile secretory dysfunction with the pathogenesis remaining unclear. Hormonal alterations most likely play a large role in CP. Increased levels of estrogen and progesterone during pregnancy have been shown to inhibit the enzyme glucuronyl transferase, resulting in impaired bilirubin conjugation and excretion leading to intrahepatic cholestasis. Disease onset and resolution correlating with elevation and reduction of hormones also favor hormonal alterations. However, several other factors including genetic, environmental, and alimentary etiologies may play a role in inducing cholestasis, which results in elevated levels of bile acids in serum and skin in genetically predisposed women (76).

Clinical presentation is moderate to severe pruritus, which may be intermittent initially, occurring only at night. Pruritus may be localized to the palms and soles, but tends to become generalized. Occasionally, patients may have anorexia, nausea, vomiting, and diarrhea. The progression to jaundice occurs in approximately one in five patients (38). Jaundice is usually mild and may be associated with dark urine and light-colored stools. It becomes detectable 1 to 4 weeks after the onset of pruritus. The symptoms resolve rapidly postpartum (within 2 to 4 weeks), but may recur in subsequent pregnancies or with the use of oral contraceptives in $45 \%$ to $70 \%$ of patients (38).

CP should be highly suspected in a patient who presents in the third trimester with the complaint of pruritus, but with few or absent skin findings. Skin biopsy is of little benefit. Laboratory evaluation is necessary for diagnosis, with elevation of bile acid the most sensitive indicator of CP (levels greater than $11.0 \mathrm{M}$ are significant) (77). The degree of pruritus tends to correlate with the bile acid level. Other liver function tests may be elevated including transaminases, alkaline phosphatase, and bilirubin (76). It is also important to rule out other causes of liver abnormalities, jaundice, and pruritus.

Maternal and fetal risks are associated with CP. Maternal outcomes are generally favorable, but severe cases may lead to postpartum hemorrhage secondary to low vitamin $\mathrm{K}$ (38). Other complications include an increased tendency toward cholelithiasis and cholecystitis. Fetal risk includes fetal distress in $20 \%$ to $30 \%$ of cases, premature birth in $20 \%$ to $60 \%$ of cases, and stillbirth in $1 \%$ to $2 \%$ of cases. Fetal risks are thought to be due to the result of placental anoxia secondary to decreased fetal elimination of toxic bile acids $(38,51,76)$.

Medical management of CP is twofold: fetal monitoring and treating maternal symptoms. Fetal monitoring should begin around the 34th week of gestation. Delivery may need to be induced depending on the severity of fetal distress. Topical therapy with steroid preparations, emollients, and oral antihistamines provides some relief to mild pruritus. In patients with intense pruritus, ursodeoxycholic acid has replaced cholestyramine as first-line therapy. Ursodeoxycholic acid treatment is the only medical therapy shown to decrease both maternal pruritus and fetal mortality $(38,51,78)$. Doses range from 450 to $1200 \mathrm{mg} /$ day (38).

\section{PP and PFP}

In the past, these two dermatoses of pregnancy were viewed as separate entities by the majority, but recently AmbrosRudolph et al. have proposed the term "atopic eruption of

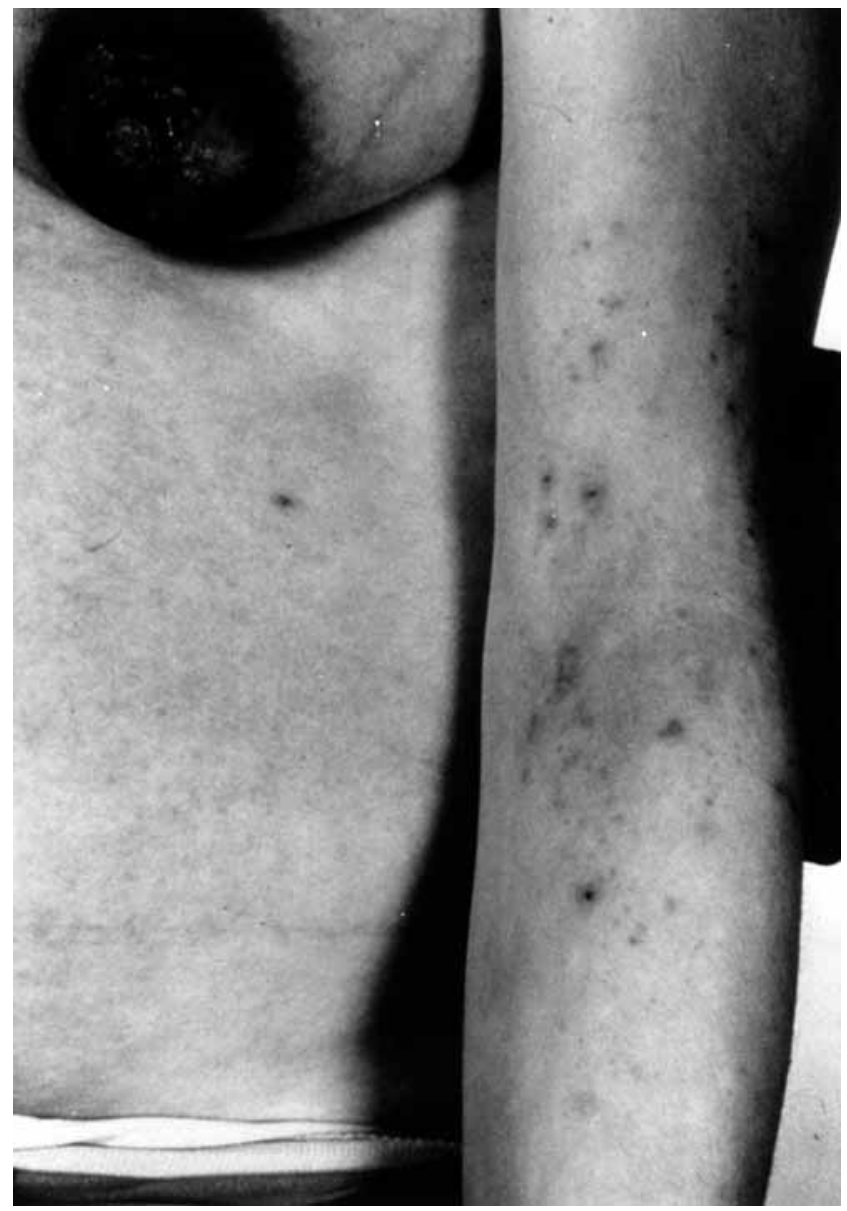

Figure 4 Excoriations in pruritus gravidarum.

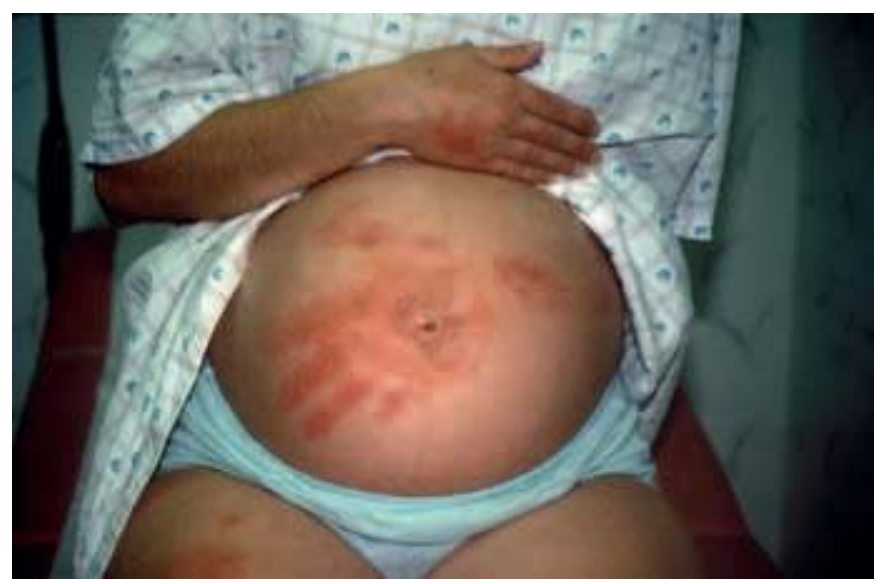

Figure 5 Allergic contact dermatitis, a common dermatosis, which also occurs in pregnant patients. 
pregnancy" (51). This term encompasses PP, PFP, and eczema in pregnancy. Though discussed separately, PP and PFP may best be considered part of a spectrum.

PP has an incidence of 1 in 450. It tends to manifest during the second or third trimester. Clinically, the patient presents with erythematous, excoriated papules or nodules along the extensor surfaces most commonly. The lesions can appear eczematous or crusted. This is more common in patients with a history of atopy (79). A laboratory workup is usually not necessary. PP resolves postpartum, but may persist for up to 3 months. Fetal and maternal prognoses are excellent. It may recur in subsequent pregnancies (79). Treatment for pruritus is symptomatic including topical steroids and antihistamines.

PFP has an incidence of approximately 1 in 3000 (79). Clinical presentation occurs during the second or third trimester with pruritic, follicular, monomorphous papules and occasionally pustules. The skin findings tend to be on the trunk but may become generalized. PFP is a sterile folliculitis; however, evaluation to rule out other infectious etiologies is warranted. Fetal and maternal outcomes appear to be unaltered. One study reported decreased birth weight and a male to female ratio of 2:1 (80). Treatment is aimed at symptomatic relief. Topical medium potency steroids and narrowband ultraviolet $\mathrm{B}$ phototherapy have proven beneficial (38).

\section{CONCLUSION}

Pregnancy affects the majority of the biologic systems, and the skin is not an exception. It is important to remember that many common dermatoses also occur in pregnant patients (Fig. 5) as well as significant dermatoses specific to pregnancy. Risks to both mother and fetus, associated with specific dermatoses of pregnancy, may be identified quickly and treated appropriately with accurate dermatologic diagnosis.

\section{REFERENCES}

1. Wong RC, Ellis CN. Physiologic skin changes in pregnancy. J Am Acad Dermatol 1984; 10: 929-40.

2. Winton GB, Lewis CW. Dermatoses of pregnancy. J Am Acad Dermatol 1982; 6: 977-98.

3. Hellreich PD. The skin changes of pregnancy. Cutis 1974; 13: 82-6.

4. Wade TR, Wade SL, Jones HE. Skin changes and diseases associated with pregnancy. Obstet Gynecol 1978; 52: 233-42.

5. Sasseville D, Wilkinson RD, Schnader JY. Dermatoses of pregnancy. Int J Dermatol 1981; 20: 223-41.

6. Callen JP. Pregnancy's effects on the skin: common and uncommon changes. Postgrad Med 1984; 75: 138-45.

7. Clark D, Thody AJ, Shuster S, et al. Immunoreactive alpha-MSH in human plasma in pregnancy. Nature 1978; 273: 163-4.

8. Thody AJ, Plummer NA, Burton JL. Plasma beta-melanocyte-stimulating hormone levels in pregnancy. J Obstet Gynaecol Br Commonw 1974; 81: 875-7.

9. McGuinness BW. Melanocyte-stimulating hormone: a clinical and laboratory study. Ann NY Acad Sci 1963; 100: 640-57.

10. Mallick S, Singh SK, Sarker C, et al. Human placental lipid induces melanogenesis by increasing the expression of tyrosinase and its related proteins in vitro. Pigment Cell Res 2005; 18: 25-33.

11. Muallem MM, Rubeiz NG. Physiological and biological skin changes in pregnancy. Clin Dermatol 2006; 24: 80-3.

12. Tunzi M, Gray GR. Common skin conditions during pregnancy. Am Fam Physician 2007; 75: 211-18

13. James WD, Meltzer MS, Guill MA, et al. Pigmentary demarcation lines associated with pregnancy. J Am Acad Dermatol 1984; 11: 438-40.
14. Sanchez NP, Pathak MA, Sato S, et al. Melasma: a clinical, light microscopic, ultrastructural, and immuno-fluorescence study. J Am Acad Dermatol 1981; 4: 698-710.

15. Smith AG, Shuster S, Thody AJ, et al. Chloasma, oral contraceptives, and plasma immunoreactive beta-melanocyte stimulating hormone. J Invest Dermatol 1976; 68: 367-76.

16. Grimes P. Melasma: etiologic and therapeutic considerations. Arch Dermatol 1995; 131: 1453-7.

17. Muzaffar F, Hussain I, Haroon TS. Physiologic skin changes during pregnancy: a study of 140 cases. Int J Dermatol 1998; 37: 429-31.

18. Driscoll MS, Grant-Kels JM. Hormones, nevi, and melanoma: an approach to the patient. J Am Acad Dermatol 2007; 57: 919-31.

19. Ellis DL, Wheeland RG. Increased nevus estrogen and progesterone ligand binding related to oral contraceptives or pregnancy. J Am Acad Dermatol 1986; 14: 25-31.

20. Demis DJ, Dolson RL. Disorders of pregnancy. In: Demis DJ, ed. Clinical Dermatology. Volume 4. New York: Harper \& Row, 1987: 1-15.

21. Benson RC. Current obstetric and gynecologic diagnosis and treatment. Los Altos: Lange Medical Publications, 1982: 841.

22. Liu DTY. Striae gravidarum. Lancet 1974; 1: 625

23. Atwal GSS, Manku LK, Griffiths CEM, Polson DW. Striae gravidarum in primiparae. Br J Dermatol 2006; 155: 965-9.

24. Cumming K, Derbes VJ. Dermatoses associated with pregnancy. Cutis 1967; 3: 120-6.

25. Demis DJ. Skin conditions during pregnancy. In: Demis DJ, ed. Clinical Dermatology. Volume 2. New York: Harper and Row, 1987: 1-9.

26. Lynfield YL. Effect of pregnancy on the human pair cycle. J Invest Dermatol 1960; 35: 323-7.

27. Benson RC. Current obstetric and gynecologic diagnosis and treatment. Los Altos: Lange Medical Publications, 1982: 581.

28. Nussbaum R, Benedetto AV. Cosmetic aspects of pregnancy. Clin Dermatol 2006; 24: 133-41.

29. Barnhill RS, Wolf JE Jr. Angiogenesis and the skin. J Am Acad Dermatol 1987; 16: 1226-42.

30. Goldman HS, Marder MZ. Physician's guide to the diseases of the oral cavity. New Jersey: Medical Economics Co., 1982: 118-20.

31. Dupont C. Herpes gestationis with hydatidiform mole. Trans St Johns Hosp Dermatol Soc 1974; 60: 103.

32. Slazinski L, Degefu S. Herpes gestationis associated with choriocarcinoma. Arch Dermatol 1982; 118: 425-8.

33. Kolodny RC. Herpes gestationis: a new assessment of incidence, diagnosis, and fetal prognosis. Am J Obstet Gynecol 1969; 104: 39-45.

34. Shornick JK, Bangert JL, Freeman RG, et al. Herpes gestationis: clinical and histologic features of twenty-eight cases. J Am Acad Dermatol 1983; 8: 214-24.

35. Roger D, Vaillant L, Fignon A, et al. Specific pruritic diseases of pregnancy. A prospective study of 3192 pregnant women. Arch Dermatol 1994; 130: 734-9.

36. Milton JL. The pathology and treatment of diseases of the skin. London: Robert Hardwicke, 1872: 201-9.

37. Mayou SC, Black MM, Holmes RC. Pemphigoid (herpes) gestationis Semin Dermatol 1988; 7: 104-10.

38. Karen JK, Pomeranz MK. Skin changes and diseases in pregnancy. In: Wolff K, Goldsmith LA, Katz SI, Gilchrest BA, Paller AS, Leffell DJ, eds. Fitzpatrick's Dermatology in General Medicine, 7th edn. McGraw Hill Companies, Inc, 2008: 955-62.

39. Shornick JK, Artlett CM, Jenkins RE, et al. Complement polymorphism in herpes gestationis: association with C4 null allele. J Am Acad Dermatol 1993; 29: 545-9.

40. Shornick JK, Jenkins RE, Briggs DC, et al. Anti-HLA antibodies in pemphigoid gestationis (herpes gestationis). Br J Dermatol 1993; 129: 257-9.

41. Borthwick GM, Holmes RC, Stirrat GM. Abnormal expression of class II MHC antigens in placenta from patients with pemphigoid gestationis: analysis of class II MHC subregion product expression. Placenta 1988; 9: 81-94.

42. Kelly SE, Bohogal BS, Black MM, et al. Western blot analysis of the antigen pemphigoid gestationis. J Invest Dermatol 1988; 91: 397.

43. Morrison LH, Labib RS, Zone JJ, et al. Herpes gestationis autoantibodies recognize a $180 \mathrm{kD}$ human epiderma antigen. J Clin Invest 1988; 81: 2023-6. 
44. Kelly SE, Black MM. Pemphigoid gestationis: placental interactions. Semin Dermatol 1989; 8: 12-17.

45. Castro LA, Lundell RB, Krause PK, et al. Clinical experience in pemphigoid gestationis: report of 10 cases. J Am Acad Dermatol 2006; 55: 823-8.

46. Shornick JK. Herpes gestationis. J Am Acad Dermatol 1987; 17: 539-56.

47. Kelly SE, Cerio R, Bhogal BS, et al. The distribution of IgG subclass in pemphigoid gestationis. PG factor is an IgG autoantibody. J Invest Dermatol 1989; 92: 695-8.

48. Yaoita H, Gullino M, Katz SI. Herpes gestationis: ultrastructure and ultrastructural localization of in vivo-bound complement: modified tissue preparation processing for horseradish peroxidase staining of skin. J Invest Dermatol 1976; 66: 383-8.

49. Fine JD, Smith LT, Holbrook KA, et al. The appearance of four basement membrane zone antigens in developing human fetal skin. J Invest Dermatol 1984; 83: 66-9.

50. Shornick JK. Dermatoses of pregnancy. Semin Cutan Med Surg 1998; 17: 172.

51. Ambros-Rudolph CM, Mullegger RR, Vaughan-Jones SA, et al. The specific dermatoses of pregnancy revisited and reclassified: results of a retrospective two-center study on 505 pregnant patients. J Am Acad Dermatol 2006; 54: 395-403.

52. Schatz M, Patterson R, Zeitz S, et al. Corticosteroid therapy for the pregnant asthmatic patient. J Am Med Assoc 1975; 233: 804.

53. Van De Weil A, Hart HC, Flinterman J, et al. Plasma exchange in herpes gestationis. Br Med J 1980; 281: 1041-2.

54. Castle SP, Mather-Mondrey M, Bennion S, et al. Chronic herpes gestationis and antiphospholipid antibody syndrome successfully treated with cyclophosphamide. J Am Acad 1996; 34: 333-6.

55. Jenkins RE, Hern S, Black MM. Clinical features and management of 87 patients with pemphigoid gestationis. Clin Exp Dermatol 1999; 24: 255.

56. Lawley TJ, Stingl G, Katz SI. Fetal and maternal risk factors in herpes gestationis. Arch Dermatol 1978; 114: 552-5.

57. Holmes RC, Black MM. The fetal prognosis in pemphigoid gestationis (herpes gestationis). Br J Dermatol 1984; 110: 67-72.

58. Shornick JK, Black MM. Fetal risks in herpes gestationis. J Am Acad Dermatol 1992; 26: 63-8.

59. Vaughan-Jones SA, Black MM. Pregnancy dermatoses. J Am Acad Dermatol 1999; 40: 233

60. Bourne G. Toxemic rash of pregnancy. Proc R Soc Med 1962; 55: 462-4.

61. Lawley TJ, Hertz KC, Wade TR, et al. Pruritic urticarial papules and plaques of pregnancy. J Am Acad Dermatol 1984; 10: 473-80.

62. Holmes RC, Black MM. The specific dermatoses of pregnancy. J Am Acad Dermatol 1983; 8: 405-12.

63. Holmes RC. Polymorphic eruption of pregnancy. Semin Dermatol 1989; 8: $18-22$.
64. Cohen LM, Capeless EL, Krusinski PA, et al. Pruritic urticarial papules and plaques of pregnancy and its relationship to maternal-fetal weight gain and twin pregnancy. Arch Dermatol 1989; 125: 1534-6.

65. Rudolph CM, Al-Fares S, Vaughan-Jones SA, et al. Polymorphic eruption of pregnancy: clinicopathology and potential trigger factors in 181 patients. Br J Dermatol 2006; 154: 54-60.

66. Aractingi S, Berkane N, Bertheau $\mathrm{P}$, et al. Fetal DNA in skin of polymorphic eruptions of pregnancy. J Am Acad Dermatol 2008; 58: 63.

67. Vaughan-Jones SA, Dunnill MG, Black MM. Pruritic urticarial papules and plaques of pregnancy (polymorphic eruption of pregnancy): two unusual cases. Br J Dermatol 1996; 135: 102-5.

68. Matz H, Orion E, Wolf R. Pruritic urticarial papules and plaques of pregnancy: polymorphic eruption of pregnancy (PUPPP). Clin Dermatol 2006; $24: 105-8$.

69. Von Hebra F. On some affections of the skin occurring in pregnant and puerperal women. Wien Med Wchnschr 1872; 48: 1197; abstracted Lancet 1872; 1: 399 Am J Syph Dermatol 1873; 4: 156.

70. Ekuma-Nkama EN, Garg VK, Al Khars M. Impetigo herpetiformis: an obstetric concern. Ann Saudi Med 1998; 18: 537-8.

71. Cravo M, Vieira R, Tellechea O, Figueiredo A. Recurrent impetigo herpetiformis successfully treated with methotrexate. J Eur Acad Dermatol Venereol 2009; 23: 336-7.

72. Lim KS, Tang MBY, Ng PPL. Impetigo herpetiformis- a rare dermatosis of pregnancy associated with prenatal complications. Ann Acad Med 2005; 34: 565-8

73. Oumeish OY, Parish JL. Impetigo herpetiformis. Clin Dermatol 2006; 24: 101-4.

74. Bukhari IA. Impetigo herpetiformis in a primigravida: successful treatment with etretinate. J Drugs Dermatol 2004; 3: 449.

75. Dacus JV, Muram D. Pruritis in pregnancy. South Med J 1987; 80: 614-17.

76. Orr B, Usatine RP. Pruritus in pregnancy. J Fam Pract 2007; 56: 913-16.

77. Brites D, Rodrigues CMP, Van-Zeller H, et al. Relevance of serum bile acid profile in the diagnosis of intrahepatic cholestasis of pregnancy in an high incidence area: Portugal. Eur J Obstet Gynecol Reprod Biol 1998; 80: 31-8.

78. Davies MH, da Silva RCMA, Jones SR, et al. Fetal mortality associated with cholestasis of pregnancy and the potential benefit of therapy with ursodeoxycholic acid. Gut 1995; 37: 580-4.

79. Roger D, Vaillant L, Fignon A, et al. Specific pruritic diseases of pregnancy: a prospective study of 3192 pregnant women. Arch Dermatol 1994; 130: 734.

80. Vaughan Jones SA, Hern S, Nelson-Piercy C, et al. A prospective study of 200 women with dermatoses of pregnancy correlating clinical findings with hormonal and immunopathological profiles. Br J Dermatol 1999; 141: 71 . 


\section{Endocrine diseases and pregnancy Stephen A. Brietzke}

\section{INTRODUCTION}

The epidemiology and natural history of endocrine diseases are such that they commonly occur in women of childbearing age. While major endocrinopathies often adversely affect fertility, and thereby in effect prevent pregnancy, the innate resilience of the human reproductive system, and the fundamental good health of most endocrine patients, assures that incidental pregnancies will frequently be encountered in patients with established endocrine disease. Pregnancy profoundly affects many of the endocrine systems and the laboratory testing upon which confirmation of disease relies; thus, physicians who treat pregnant patients are well advised to have familiarity with these alterations. Endocrine diseases may have adverse consequences for maternal, fetal, and neonatal health; likewise, the pregnancy may alter the expression of endocrine disease and as well may alter the interpretation of diagnostic laboratory testing commonly performed in nongravid patients. Additionally, pregnancy alters choice of pharmacotherapy and diagnostic modalities and reagents.

\section{THYROID DISORDERS}

\section{Physiologic Adaptations of Pregnancy}

In the normal woman, serum thyroxine (T4) and triiodothyronine (T3) concentrations increase by approximately $50 \%$ because of expansion of the protein-bound pool of thyroid hormones. This in turn is due to marked increase in plasma thyroxine-binding globulin (TBG), a consequence of reduced hepatic clearance owing to estrogen-induced sialylation of TBG (1). In addition to the expansion of the total circulating pool of thyroid hormones, renal clearance of iodide increases, due to the increase in glomerular filtration rate accompanying pregnancy-related volume expansion. Finally, early in gestation, maternal thyroid hormones are passed via the placenta to the fetus, and throughout pregnancy, iodide is transferred to the fetus transplacentally (2). To meet the expanded requirement for total serum thyroxine and to offset increased loss of iodide in the urine and to the fetus, maternal thyroid synthesis and secretion of T4 and T3 increases throughout gestation. Accurately assessing the adequacy of circulating maternal thyroid hormones is challenging. Clinical laboratory measurement of free $\mathrm{T} 4$ is increasingly available and has largely supplanted older methodology wherein total T4 ("free" plus TBG-bound T4) was measured. However, at this time, free T4 normal ranges have not been benchmarked well for each trimester of pregnancy, and there are inconsistent data reporting values of free T4 lower, the same, or higher than that in nonpregnant patients $(1,3)$. At the present time, it is prudent to request measurement of total serum T4 and adjust the normal reference range to 1.5 times the nonpregnant adult normal range (4).

Thyroidal iodide transport, synthesis of thyroid hormone, and release of stored thyroid hormone are all controlled by the secretion of thyroid-stimulating hormone (TSH) from the pituitary gland. In turn, TSH is stimulated by hypothalamic thyrotropin-releasing hormone (TRH). TRH and TSH secretion is inhibited in negative feedback fashion by rise in serum T4 and T3 to the equilibrium set point. During the first trimester of pregnancy, TSH levels consistently fall below the non-gravid baseline. Since this change accompanies the rise in serum human chorionic gonadotropin (beta-hCG), and since hCG has considerable structural homology to TSH, it has been assumed that in the first trimester, hCG transiently supplants TSH as the primary stimulator of maternal thyroid function. As hCG concentrations wane from gestational weeks 10 to 20, serum TSH returns toward its usual baseline value (1). In the first trimester, to allow for partial replacement of TSH function by hCG, it has been suggested that the normal upper limit for maternal TSH be adjusted to no more than $2.5 \mathrm{mU} / \mathrm{L}$ (5). With this caveat, TSH is an excellent screening test for thyroid disease in pregnancy and is generally recommended as the test of first choice.

\section{Normal Fetal and Neonatal Thyroid Physiology}

The fetal thyroid begins to take up iodine and synthesize T4 at 10 weeks of gestation. Fetal T3 is low throughout gestation (6). Normally, only a small amount of maternal thyroid hormone traverses the placenta to reach the fetal circulation, but may be vital for fetal brain development (7), particularly in athyrotic fetuses (8). Iodides, antithyroid drugs, and thyroid-stimulating immunoglobulins (TSIs) cross the placenta maternal-to-fetus readily and affect fetal thyroid function. In the immediate postpartum period, neonatal plasma TSH peaks at approximately $30 \mathrm{mU} / \mathrm{mL}$ several hours after birth, accompanied by increased plasma T4 and T3. Thereafter, plasma TSH falls to the adult reference range within a few days, and plasma $\mathrm{T} 4$ and T3 fall to the adult reference range within 1 month.

\section{Hypothyroidism}

Primary hypothyroidism is defined by elevated serum TSH above the normal reference range, and this finding occurs in about $2.5 \%$ of pregnancies $(3,9)$. Severity of the thyroid deficiency is further characterized by the magnitude of TSH elevation ("mild" disease = TSH 2.5-10mU/L; "moderate" disease $=$ TSH 10 to $<20 \mathrm{mU} / \mathrm{L}$; "severe" disease $=\mathrm{TSH}>20 \mathrm{mU} / \mathrm{L}$ and T4 below the lower limit of the pregnancy-adjusted reference range). Secondary hypothyroidism due to pituitary insufficiency is far less common (unless the patient is known to have pituitary or hypothalamic disease), since anterior pituitary insufficiency commonly adversely affects pulsatile gonadotropin secretion to a profound degree, with the result that unassisted conception is unusual in such patients. Secondary hypothyroidism is defined by subnormal plasma T4 without an appropriate degree of TSH elevation.

Unless the patient has a history of radioactive iodine treatment or thyroid surgery, primary hypothyroidism is 
overwhelmingly due to chronic lymphocytic thyroiditis (Hashimoto's thyroiditis). Postablation hypothyroidism or post-thyroidectomy hypothyroidism, stemming from radioactive iodine or surgical treatment of hyperthyroidism, goiter, or thyroid nodular disease (including thyroid cancer), is the other principal cause of hypothyroidism encountered in pregnant women. In most instances, the patient will be known hypothyroid prior to pregnancy, although of course exceptions will be encountered in clinical practice.

Symptoms of hypothyroidism in pregnant women are subtle and often attributed to the pregnancy itself. Cold intolerance, mild weight gain, fatigue, somnolence, depression, dry skin, constipation, and myalgias are some of the "classic" symptoms. There may have been a history of anovulation, dysfunctional uterine bleeding, or infertility pre-dating the pregnancy. Physical signs offering clues to diagnosis include goiter (Hashimoto's thyroiditis glands are often of palpably firm consistency), pretibial myxedema (rare), and delayed return phase of deep tendon reflexes, particularly at the ankle. Untreated maternal hypothyroidism increases the risks of gestational hypertension (up to 36\%) (10), caesarean section due to fetal distress (up to 56\%) (11), placental abruption (threefold increased risk vs. euthyroid pregnancies) (12), and pre-term labor (approximately twofold increased risk vs. euthyroid pregnancies) (12). Neonatal consequences of maternal hypothyroidism include a two- to threefold increased risk for low birth weight and delayed mental and motor developmental milestone attainment. There is also concern that ultimate cognitive attainment of offspring of hypothyroid mothers is slightly less than offspring of euthyroid mothers (5).

Hypothyroidism in pregnancy should be treated with levothyroxine (13). Owing to the increased TBG-bound pool of circulating T4, higher levothyroxine doses are prescribed for pregnant than for nonpregnant patients. For the patient newly diagnosed and not previously treated, if the serum TSH is $>10 \mathrm{mU} / \mathrm{L}$, a starting levothyroxine dose of $2 \mathrm{mcg} / \mathrm{kg} /$ day is prudent (4), whereas for TSH $<10 \mathrm{mU} / \mathrm{L}$, a starting levothyroxine dose of $1 \mathrm{mcg} / \mathrm{kg} / \mathrm{day}$ may be sufficient (2). For women already receiving levothyroxine treatment at the time of conception, the dose requirement increases predictably by $25 \%$ to $50 \%$. Functionally athyrotic patients (those with postablation hypothyroidism or post-thyroidectomy hypothyroidism) have a larger dose increase than patients with primary hypothyroidism due to Hashimoto's thyroiditis, probably because in the latter condition, the thyroid is still capable of some T4 generation albeit to a subnormal extent (14). Conservatively, a serum $\mathrm{TSH}<2.5 \mathrm{mU} / \mathrm{L}$ should be targeted as goal for therapy; dose of levothyroxine should be increased for values $>2.5 \mathrm{mU} / \mathrm{L}$ and TSH retested monthly with further dose titration until stable. A suggested formula for dose increase based on TSH is to increase levothyroxine by $50 \mathrm{mcg} / \mathrm{day}$ for $\mathrm{TSH}<10 \mathrm{mU} / \mathrm{L}$; by $75 \mathrm{mcg} / \mathrm{day}$ for TSH 10 to $20 \mathrm{mU} / \mathrm{L}$; and by $100 \mathrm{mcg} /$ day for TSH $>20 \mathrm{mU} / \mathrm{L}(2,14)$.

\section{Congenital Hypothyroidism}

Approximately 1 in 5000 live births is associated with congenital hypothyroidism, most usually due to thyroid agenesis or dysgenesis (15). Other causes include genetic defects of hormone synthesis, maternal treatment with antithyroid drugs or radioactive iodine, and placental transfer of maternal TSH receptor inhibitory antibodies. Antithyroid drugs and iodides may induce fetal goiter large enough to produce tracheal obstruction (16). Hypothyroid infants appear normal at birth, but develop mental retardation due to the syndrome of cretinism unless treated soon after birth. Neonatal screening of TSH is essential for early diagnosis and prevention of irreversible neurologic damage and has become a universal practice in the United States.

\section{Thyrotoxicosis (Hyperthyroidism)}

The term "thyrotoxicosis" distinguishes excessive serum T4 and/or T3 from all causes (including, importantly, excessive ingestion of levothyroxine, liothyronine, or dessicated thyroid hormone), from "hyperthyroidism," which specifically implies excessive active production of thyroid hormones by the native thyroid gland. In practice, the term "hyperthyroidism" is generally used interchangeably with "thyrotoxicosis." Hormonally, the syndrome is defined by suppressed serum TSH (usually $<0.1 \mathrm{mU} / \mathrm{L}$ ); if T4 and/or T3 are not elevated beyond the normal range but the TSH is suppressed, the condition is referred to as "subclinical hyperthyroidism." Prevalence of hyperthyroidism in pregnancy is in the range of $0.1 \%$ to $0.5 \%$, and $85 \%$ to $90 \%$ of cases are due to diffuse toxic goiter (Graves' disease). As the name suggests, Graves' disease is usually accompanied by a fleshy-consistency goiter ranging from minimally enlarged to three to four times that of the normal thyroid. Graves' disease is caused by an autoantibody, TSI, which binds to and activates the thyroidal TSH receptor, usurping the normal function of TSH in unregulated fashion (17). Other than Graves' disease, possible causes of hyperthyroidism in pregnancy include toxic adenoma (a solitary, usually large, thyroid nodule with autonomous, $\mathrm{TSH}$-independent production of T4 and/or T3), toxic multinodular goiter (typically with multiple thyroidal nodules or regions autonomously producing $\mathrm{T} 4$ and/or T3), subacute thyroiditis (painless lymphocytic thyroiditis), and excessive ingestion of thyroid hormones either prescribed or obtained surreptitiously. Trophoblastic tumors associated with very high levels of beta-hCG (typically $>100,000 \mathrm{IU} / \mathrm{L}$ ) can cause hyperthyroidism due to activation of the TSH receptor by hCG $(18,19)$. In the first trimester, some patients with hyperemesis gravidarum have elevation of $\mathrm{T} 4$ and nonsuppression of $\mathrm{T} 3$, in contrast to other conditions with nausea, vomiting, and poor oral intake, wherein T3 levels are invariably low (20). Sixty percent of patients with hyperemesis have serum $\mathrm{TSH}<0.4 \mathrm{mU} / \mathrm{L}$ and approximately half have a serum free T4 elevated above the normal range. TSH and T4 normalize as hCG serum levels decline beyond week 20 of gestation.

Symptoms of thyrotoxicosis include heat intolerance, weight loss, anxiety, palpitations, tachycardia, and excessive sweating, along with poor exertional tolerance and easy fatiguability. Not surprisingly, in many instances, the symptoms will be erroneously attributed to those of normal pregnancy. Physical clues to the possibility of thyrotoxicosis include goiter, proptosis, lid lag, stare, a fine resting hand tremor, warm moist palmar skin, and generalized hyperreflexia. 


\section{Maternal-Fetal Risks of Hyperthyroidism}

Untreated maternal hyperthyroidism is associated with several adverse outcomes of the pregnancy. Preterm labor occurs in up to $88 \%$ of untreated mothers, compared with $8 \%$ whose hyperthyroidism is optimally treated (21). Pre-eclampsia in untreated or poorly controlled hyperthyroid mothers occurs at approximately twice the frequency as in well-controlled hyperthyroid mothers (22), and stillbirth may occur in up to $50 \%$ of untreated mothers, compared with a $0 \%$ to $16 \%$ risk in optimally treated mothers (21). Small-for-gestational age and various congenital malformations may occur at increased frequency in the neonates of untreated or poorly controlled hyperthyroid mothers (17).

\section{Treatment}

Thionamide drugs are the only treatment, which can be recommended with respect to both maternal and fetal health during the course of pregnancy and can be used to treat hyperthyroidism due to Graves' disease, toxic adenoma, or toxic multinodular goiter. Due to the mechanism of action of this class of drug and the pathophysiology of subacute thyroiditis (passive leakage of pre-formed T4 and T3 from a damaged gland, rather than excess production), thionamides are ineffective in the treatment of subacute thyroiditis. Propylthiouracil (PTU) and methimazole (MMI) are the available thionamides in the United States. The mechanism of action of thionamides is interference with the key steps of thyroid hormone synthesis (thyroidal organification of iodine and coupling of iodotyrosines), by serving as a competitive substrate for the thyroid peroxidase enzyme. Accumulation of thionamide in the thyroid gland, rather than serum levels of drug, is required for optimal effect. Thus, 7 to 8 weeks of treatment is usually required before a euthyroid state can be expected clinically (23). Both drugs cross the placenta and have potential to cause fetal hypothyroidism and, for this reason, can be used to treat fetal hyperthyroidism. Historically, PTU is preferred over MMI in pregnancy because MMI is thought to be associated with a rare congenital scalp defect, aplasia cutis. The association is, however, very weak and has not been observed in several well-done case-control series. Therefore, for patients who do not tolerate PTU, MMI could be considered as alternative therapy and is probably far preferable to the alternative of no treatment of severe maternal hyperthyroidism (2). PTI is given in a dose range of 50 to $150 \mathrm{mg} /$ day orally three times; doses $>600 \mathrm{mg}$ /day pose high risk of fetal/neonatal hypothyroidism. Even at a maternal dose of PTU $<100 \mathrm{mg} /$ day, about one in five neonates have above-normal serum TSH at birth (24). To minimize the risk of significant neonatal hypothyroidism, the general recommendation is to titrate treatment of maternal hyperthyroidism so as to minimize symptoms, but maintain T4 levels in the upper third of the normal range for pregnancy and not necessarily normalize serum TSH during the pregnancy (25). The lowest possible dose of PTU, which attains these goals of treatment, should be maintained if at all possible. Adequacy of treatment can be reassessed at 2- to 4-week intervals throughout gestation, with adjustment of the PTU dose at about that frequency (2). Most authorities recommend not exceeding a dose of 450 to $600 \mathrm{mg} /$ day PTU. If MMI is used as an alternative to PTU, the dose should not exceed 30 to $40 \mathrm{mg} /$ day.
Unlike PTU, MMI can be effectively administered as a single daily dose, which may be of value in patients who have difficulty fully adhering to a thrice-daily medication schedule. Adverse effects of thionamides include, commonly, skin rashes, most characteristically, urticaria. Serious drug-related hepatitis (due to PTU) and cholestasis (due to MMI) are possible, and periodic monitoring of hepatic transaminases (aspartate aminotransferase and alanine aminotransferase) is recommended. Approximately 1 in 200 users of PTU or 1 in 300 users of MMI develop agranulocytosis, which usually declares as neutropenic fever. Standard recommendation to patients is to seek prompt care and have a complete blood count with differential for any febrile illness, while they are taking a thionamide drug (26).

A beta blocker drug can be used during pregnancy as an adjunct to thionamide therapy and is especially useful to quickly gain control of tachycardia, extreme nervousness, and tremor in extremely symptomatic patients. The best-studied drug in pregnancy is propranolol, which can be given in a dose range of $10-40 \mathrm{mg} /$ day orally four times. The dose is titrated to subjective symptomatic improvement and to heart rate $<90$ beats/min. Curtailing the dose to the minimum necessary to attenuate symptoms is prudent, as beta blockers may cause fetal growth retardation in dose-dependent fashion. If possible, delaying the initiation of propranolol until the second trimester may also be prudent, because of evidence from one study that combined use of thionamide and propranolol was associated with a $24 \%$ risk of first-trimester miscarriage, versus $5.5 \%$ miscarriage rate when thionamide alone was used (27). Beyond the first trimester, the only significant risk of propranolol has been low birth weight.

Stable low-dose iodide used for 1 to 2 weeks in conjunction with thionamide therapy appears safe, as long as the dose is limited to 6 to $40 \mathrm{mg} /$ day of potassium iodide or equivalent (28). The major practical usage of iodide therapy would be in shortterm preparation of a patient for thyroid surgery in pregnancy or to rapidly reduce circulating $\mathrm{T} 4$ and $\mathrm{T} 3$ in a patient with severe hyperthyroid illness. Radioactive iodine, which is a popular treatment for Graves' disease, toxic adenoma, and toxic multinodular goiter in non-gravid patients, is strictly contraindicated in pregnancy, due to concern not only for fetal thyroid ablation but also for possible induction of various congenital defects.

For very severe maternal hyperthyroidism that cannot be controlled by PTU or MMI, or for patients unable to take thionamides due to serious adverse drug reactions, or for large maternal goiter threatening airway obstruction, subtotal thyroidectomy prior to gestational week 24 in the second trimester is a reasonable consideration (29). Preparation of the patient for surgery can include PTU or MMI, if tolerated, plus a beta blocker and potassium iodide 6 to $40 \mathrm{mg} /$ day beginning 10 to 14 days preoperatively. The role of iodide in this setting is to reduce the vascularity of the thyroid prior to surgery, thereby limiting operative blood loss (2).

\section{Fetal and Neonatal Hyperthyroidism}

Fetal and neonatal hyperthyroidism is caused by placental transfer of maternal TSI to the fetus (30). Fetal disease is heralded by tachycardia $(>160 / \mathrm{min})$, growth retardation, goiter, craniosynostosis, and increased perinatal mortality (31). Fetal hyperthyroidism can be treated in utero by prescribing 
PTU 50 to $150 \mathrm{mg} /$ day orally three times to the mother, anticipating that the drug will cross the placenta and be incorporated into fetal thyroid, thereby inhibiting synthesis of T4 and T3. Active previously untreated Graves' disease obligates PTU treatment in the mother as well as in the fetus. However, a common dilemma involves a mother previously treated with radioactive iodine (less commonly, with subtotal thyroidectomy) prior to gestation for Graves' disease who now has postablation hypothyroidism, is euthyroid on replacement levothyroxine, but who continues to have high-titer TSI. In such pregnancies, there is still a finite risk of fetal and neonatal hyperthyroidism due to placental transmission of TSI to the fetus. A strategy for the detection and treatment of fetal disease, and prevention of neonatal disease, is to measure maternal TSI titer during the second trimester in mothers who have a history of Graves' disease. A highly positive TSI test leads to careful surveillance of fetal growth and heart rate (2). For poor growth or excessive tachycardia, the mother continues to receive her usual levothyroxine dose but is concurrently treated with PTU 50 to $150 \mathrm{mg}$ /day three times, titrating the dose to fetal heart rate and goiter size as assessed by ultrasound at 2-week intervals (31). Neonatal hyperthyroidism complicates $1 \%$ to $2 \%$ of pregnancies in women with Graves' disease (32) and manifests as irritability, tachycardia, poor weight gain, goiter, and, potentially, heart failure and death. T4 and TSH should be measured in cord blood if the mother has a history of Graves' disease. Neonatal hyperthyroidism is treated with PTU, iodine, and propranolol and usually resolves within $1-3$ months, as TSIs are cleared from plasma.

\section{POSTPARTUM THYROID DISORDERS}

Graves' disease commonly spontaneously improves during the third trimester and flares in the postpartum period, and may first become evident then. The differential diagnosis of postpartum thyrotoxicosis is principally between Graves' disease and postpartum subacute thyroiditis (also known as painless lymphocytic thyroiditis), which affects about $5 \%$ of postpartum women (33). It occurs within the first 3 postpartum months and produces mild or moderate thyrotoxicosis that lasts up to 3 months, and then either resolves or is followed by transient hypothyroidism, which lasts for several months and then spontaneously resolves. In up to one-third of affected patients, permanent hypothyroidism ensues; such cases are difficult to distinguish from Hashimoto's thyroiditis and are usually accompanied by high levels of thyroid peroxidase autoantibody. Distinguishing postpartum Graves' disease from postpartum thyroiditis can be difficult. Graves' disease is very likely to be associated with a significant goiter, which may be accompanied by a bruit over the thyroid or supraclavicular fossa, while postpartum thyroiditis is associated with normal thyroid or minimal goiter, and no bruit. Proptosis is not seen in postpartum thyroiditis, so if present implies Graves' disease. Measurement of radioactive iodine uptake (RAIU) can be performed if the patient is not nursing; uptake is always nearly undetectable ( $<5 \% 24$-hour RAIU) in thyroiditis and is elevated (>30\% 24-hour RAIU) in Graves' disease. TSI would only be positive in Graves' disease, but is an expensive and usually unnecessary test. The pathophysiology of postpartum thyroiditis involves leakage of T4 and T3 from a damaged gland; thus, thyroid hormones are not being actively synthesized, and antithyroid drug therapy with thionamides has no rational role. Treatment of postpartum thyroiditis is limited to beta blocker for control of symptoms, including heart rate, nervousness, and tremor. Close follow-up with measurement of TSH and T4 every 1 to 2 months is desirable. If hypothyroidism occurs and is bothersome to the patient, treatment with levothyroxine can be entertained for up to 6 months. Hypothyroidism persisting beyond 6 months postpartum is likely to be permanent, and levothyroxine titrated to normalization of TSH is desirable at that time. Women who experience postpartum thyroiditis during one pregnancy are likely to experience recurrences with subsequent pregnancy and can be counseled and monitored accordingly (33).

\section{Thyroid Nodules}

Solitary thyroid nodules occur in up to $15 \%$ of women in the first trimester and in up to $24 \%$ of women in the first 3 months postpartum (34). Upward of $90 \%$ of such nodules represent benign disease (follicular or colloid adenomas), but deserve cytologic diagnosis to exclude the possibility of thyroid carcinoma. As in non-gravid patients, women in the first two trimesters of pregnancy can be evaluated by ultrasoundguided fine needle biopsy (35). If cytology confirms papillary thyroid carcinoma, the patient could undergo thyroidectomy during the second trimester. Alternatively, the size of the lesion may be followed by repeat ultrasound at midtrimester. A known cancer, which has enlarged significantly, can be referred for surgery; if it has remained stable in size, surgery is deferred until the patient is postpartum (2). Conversely, if cytology is read as "consistent with follicular neoplasm" (which conveys an approximately 20\% risk of malignancy), it is a reasonable practice to delay thyroidectomy until the postpartum period. Conclusively benign cytologic findings in euthyroid patients provide excellent reassurance, and simple long-term clinical follow-up is sufficient.

\section{ANTERIOR PITUITARY DISORDERS \\ Normal Physiology}

Thyrotropin (TSH), corticotropin (ACTH), the gonadotropins [luteinizing hormone ( $\mathrm{LH}$ ) and follicle-stimulating hormone $(\mathrm{FSH})]$, prolactin, and growth hormone $(\mathrm{GH})$ comprise the hormonal products of the anterior pituitary gland. With one key exception, the secretion of anterior pituitary hormones is governed by secretion of stimulatory hypothalamic releasing hormones, which traverse the pituitary stalk via the portal veins. The known hypothalamic releasing hormones specific for each of the anterior pituitary are TRH, corticotrophin-releasing hormone $(\mathrm{CRH})$, gonadotropinreleasing hormone $(\mathrm{GnRH}$, for both $\mathrm{LH}$ and $\mathrm{FSH})$, and growth hormone-releasing hormone (GHRH) for GH. Control of prolactin secretion, in contrast to the other trophic hormones, is primarily inhibitory, with the inhibitory signal hormone being dopamine of hypothalamic origin, circulated to the anterior pituitary via the hypothalamic-hypophyseal portal veins in the pituitary stalk. Knowledge of the normal hypothalamic stimulatory and inhibitory control of anterior pituitary hormone secretion explains common patterns of disease. Hypothalamic lesions are frequently heralded by deficiency of GH, ACTH, TSH, LH, and FSH and concurrently 
by hyperprolactinemia. Anatomically large secretory tumors of one discrete cell type of anterior pituitary origin usually overexpress one hormonal product (ACTH, TSH, or GH) producing a clinically recognizable syndrome; compression of the pituitary stalk by the tumor may impede communication of releasing hormones and dopamine, and therefore such large tumors commonly are accompanied by partial hypopituitarism and modest hyperprolactinemia.

\section{Pituitary Adaptations in Normal Pregnancy}

Beginning soon after conception, under the influence of rising estrogen concentration, hypertrophy and hyperplasia of lactotroph cells result in an approximately $40 \%$ increase in pituitary volume by term $(36,37)$. In accord with the gain in lactotroph cell numbers, serum prolactin increases, usually peaking at approximately $200 \mathrm{ng} / \mathrm{mL}$ during the third trimester of pregnancy. Pituitary LH and FSH secretion are inhibited by prolactin, due to feedback inhibition of GnRH secretion by hyperprolactinemia (38).

Placental production of a variant $\mathrm{GH}$ stimulates elevated serum levels of somatomedin-C during the third trimester, which in turn inhibits GHRH and native GH of pituitary origin secretion in the third trimester $(39,40)$.

Throughout gestation, serum cortisol levels progressively increase, reaching two to three times the non-gravid baseline value by term. Some of the increase is accounted for by the expansion of the serum pool of protein-bound, biologically inactive cortisol due to estrogen-stimulated hepatic synthesis of cortisol-binding globulin. However, plasma free cortisol is also approximately three times elevated versus the non-gravid baseline value and is reflected by 24-hour urinary free cortisol values of the same magnitude.

Because rising concentrations of human chorionic gonadotropin early in gestation transiently usurp control of thyroid hormone secretion from TSH, TSH levels normally decline in the first trimester, gradually returning to baseline values by the third trimester $(3,40)$.

\section{Pituitary Tumors}

Pituitary tumors, which are most frequently benign adenomas of a single cell type, are classified as microadenomas if $<1 \mathrm{~cm}$ in diameter, and as macroadenomas if $>1 \mathrm{~cm}$ in diameter. They may be either clinically nonfunctional (in which case, immunostaining is commonly positive for FSH), or secretory, with prolactin, $\mathrm{GH}$, or ACTH excess. Microadenomas produce clinical effects only through hormone excess or hormone deficiency caused by feedback signaling, whereas macroadenomas can cause hypopituitarism by either stalk compression or displacement of normal anterior pituitary cells and can produce symptoms due to mass effect, with headaches due to dural stretch, and/or visual field defects due to encroachment of optic chiasm $(40,41)$.

Clinically important pituitary hormone deficiencies during pregnancy are secondary hypothyroidism and secondary adrenal insufficiency, caused respectively by TSH and ACTH deficiency. Prolactin deficiency poses no significant hazard to the viability of the pregnancy or to maternal-fetal health; however, prolactin deficiency would declare in the postpartum period as failure to lactate. Evaluation of suspected hypopituitarism or pituitary tumor during pregnancy should include measurement of serum free T4 measurement, assessment of cortisol response to injected cosyntropin (synthetic ACTH), and magnetic resonance imaging (MRI) (without gadolinium contrast) of the sellar region. If there is suspicion of visual field compromise based on clinical exam, or if the MRI shows encroachment of the optic chiasm, formal visual field assessment (Goldman fields) by an ophthalmologist is recommended (40). If adenoma resection is required, the vast majority can be removed by a transsphenoidal approach (31).

\section{Hyperprolactinemia}

Hyperprolactinemia is a common endocrine disorder in young women and may be responsible for up to one-third of cases of infertility. Because hyperprolactinemia suppresses GnRH, which in turn results in diminished gonadotropin pulsatility, disruption of ovulation and consequent anovulatory bleeding, amenorrhea, or luteal phase defects can all occur, and the hyperprolactinemia may also produce galactorrhea. Significant and persistent hyperprolactinemia may be due to either microor macro-sized prolactinomas. Modest hyperprolactinemia (serum prolactin, $20-50 \mathrm{ng} / \mathrm{mL}$ ) may be due to microprolactinoma, pituitary stalk compression by a non-prolactinoma pituitary tumor, hypothalamic lesions, or a variety of dopamine antagonist medications (40).

Prolactin levels $>200 \mathrm{ng} / \mathrm{mL}$ are virtually certainly due to prolactinoma; however, since physiologic hyperprolactinemia of pregnancy approaches $200 \mathrm{ng} / \mathrm{mL}$, and since lactotroph hyperplasia and hypertrophy of normal pregnancy may mimic pituitary adenoma, it is rare that prolactinoma would be suspected or confirmed during pregnancy (40).

Because medical therapy or surgical therapy of prolactinoma can be highly effective in restoring ovulatory function to formerly infertile hyperprolactinemic women, intended or unintended pregnancy in such patients is an entirely likely outcome. Drugs used to treat prolactinoma are bromocriptine, pergolide, quinagolide (not approved for use in the United States), and cabergoline; commonly, when pregnancy is recognized, the patient with prolactinoma will be receiving one of these agents. What is known about this class of drugs during pregnancy is largely reassuring. Bromocriptine and cabergoline are classified by the U.S. Food and Drug Administration as class B (no definite evidence of harm; available information and reported experience indicates probably safe for use) in pregnancy. Once pregnancy is recognized, a decision about how to manage dopamine agonist drug therapy for the duration of pregnancy should be based on the tumor size, symptoms, and the natural history of prolactinomas during pregnancy. Pooled reported experience with 376 patients suggests that only $1.3 \%$ of microprolactinomas enlarge sufficiently during pregnancy so as to produce clinical symptoms of mass effect (visual compromise or intractable headache) $(40,42)$. Given the favorable prognosis, recommendation is that drug therapy for microprolactinoma be discontinued as soon as pregnancy is confirmed. Neuroimaging during the pregnancy would be recommended only if symptoms of visual field defect or intractable headache were to occur. Monitoring of serum prolactin during pregnancy is not helpful and not advised. Of the previously untreated patients with macroprolactinoma, $20 \%$ to $25 \%$ may develop symptomatic tumor enlargement during pregnancy, but if 
the macroprolactinoma has been previously treated with surgery, the risk of symptomatic enlargement is only $2 \%$ to $3 \%$. For the patient who has previously undergone transsphenoidal resection of macroprolactinoma, and who is not receiving medication therapy at the time of conception, careful follow-up during the pregnancy, with monthly visits emphasizing symptoms of headache or visual compromise, is a prudent recommendation. Women with macroprolactinoma treated with primary medication treatment (no surgery) often respond with considerable size reduction of the tumor, are able to conceive, and constitute the most problematic group of hyperprolactinemic patients in pregnancy for whom generic recommendations are made. Consideration is given to simply continuing bromocriptine or cabergoline throughout the pregnancy, as rapid enlargement of macroadenomas is a risk when medication therapy is discontinued. At a minimum, careful surveillance and monthly office visits to elicit symptoms, and reinstitution of medical therapy or transsphenoidal tumor debulking should be pursued if symptomatic tumor enlargement occurs. Formal testing of visual fields in each trimester of pregnancy is commonly advised $(40,42)$.

\section{Acromegaly}

Maternal Effects

Infertility or hypogonadotropic hypogonadism frequently accompany acromegaly, and pregnancy is an unusual enough occurrence that there have been fewer than 100 cases of pregnant acromegalics reported in the literature. Acromegaly is caused by $\mathrm{GH}$ excess and is most commonly due to a pituitary adenoma. Approximately $80 \%$ of recognized acromegalic pituitary tumors are $>1 \mathrm{~cm}$ in size (macroadenomas), with the remainder being microadenomas. Characteristic clinical findings include enlargement of hands (particularly broadening of the digits) and feet, with increased glove, ring, and shoe size often reported by patients. Characteristic facial features include wide spacing between teeth and prognathism, consequent to enlargement of the mandible, frontal bossing consequent to the enlargement of frontal sinuses, thick lips and broad fleshy nose consequent to soft-tissue expansion, and prominent nasolabial folds. Carpal tunnel syndrome, hypertension, and diabetes mellitus occur with increased frequency in actively acromegalic patients (40).

Confirmation of acromegaly suspected for the first time during pregnancy is complicated by the normal increase in serum IGF-1 in late pregnancy, and by the GH variant of placental origin, which cannot be easily distinguished from $\mathrm{GH}$ of pituitary origin. Early in pregnancy, a very high serum IGF-1 would be suggestive of acromegaly, and the diagnosis can be confirmed by documenting failure of GH suppressibility ( $<2 \mathrm{ng} /$ $\mathrm{mL}$ ) during a 75-g oral glucose suppression test. MRI of the sella and suprasellar region should be performed to identify and assess the size of the responsible pituitary adenoma. By the third trimester, definitive diagnosis of acromegaly may reasonably be deferred until after delivery (40).

\section{Fetal Effects}

The limited reported clinical experience with acromegalic pregnancies suggests no ill effect on fetal development directly attributable to maternal GH and IGF-1 excess. Rather, the major fetal risks are likely related to acromegaly-linked complications of diabetes mellitus (which can occur in up to $20 \%$ of acromegalic pregnancies) and hypertension (up to $35 \%$ of acromegalic pregnancies) (43). If observed, these complications are managed in usual fashion for the duration of the pregnancy.

\section{Management of the Pregnant Acromegalic Woman}

The most likely clinical scenario involving acromegaly and pregnancy is pregnancy in a woman previously diagnosed and treated for the disease. She may in fact have been cured by transsphenoidal resection of an adenoma; confirmation of cure rests with sustained suppressibility of GH to oral glucose challenge and normalization of serum IGF-1. If so, pregnancy should be managed as a normal pregnancy. For patients not surgically cured, the most conventional and effective longterm management involves use of a long-acting somatostatin analog, either octreotide or lanreotide. Frequently, treatment with a somatostatin analog normalizes IGF-1 and restores fertility; consequently, pregnancy can occur. The available information about pregnancies occurring in treated women identifies no adverse fetal consequences (44-46). However, because octreotide and lanreotide do cross the placenta (47) and because experience with continuation of these drugs throughout pregnancy is extremely limited, a conservative and mainstream recommendation is to discontinue the somatostatin analog once pregnancy is recognized (40). Symptomatic enlargement of acromegalic pituitary adenomas during pregnancy is an uncommon complication (one reported case) (48); should this be encountered in the first two trimesters, consideration could be given to transsphenoidal resection or to reinstitution of somatostatin analog therapy.

\section{Lymphocytic Hypophysitis}

Late in the third trimester or early in the postpartum period, lymphocytic hypophysitis represents a rare cause of hypopituitarism or visual field defects. Anatomically, the disorder may be suspected by thickening of the pituitary stalk or by sellar mass, which cannot be distinguished from a pituitary tumor. The diagnosis is definitively established by surgical resection or biopsy demonstrating absence of tumor cells and diffuse lymphocytic infiltration (40). Clinically, lymphocytic hypophysitis is distinguished from Sheehan's syndrome by the absence of obstetric hemorrhage in the former and its presence in the latter (49). If surgical biopsy is not undertaken, spontaneous resolution may occur $(50,51)$, and attention should be given to treatment of hypopituitarism. The limited available experience suggests that ACTH deficiency is especially common (49), so assessment of the hypothalamicpituitary-cortisol axis is especially important; identified deficiency should be treated with a cortisol agonist in standard physiologic replacement dose (e.g., hydrocortisone $20 \mathrm{mg}$ orally in the morning and $10 \mathrm{mg}$ in the late afternoon or equivalent).

\section{Sheehan's Syndrome}

Postpartum pituitary necrosis (Sheehan's syndrome) results from an ischemic insult to the pituitary as a complication of peripartum hemorrhage (52) and is distinctly rare in modern obstetric practice (53). Physiologic enlargement of the pituitary in late gestation, consequent to the physiologic 
expansion of lactotrophs, seems to render the gland especially sensitive to hemodynamic insult. In the setting of known obstetric hemorrhage or maternal hypotension during the labor and delivery process, Sheehan's syndrome is suspected if postpartum lactation fails to occur. Lactation failure heralds possible loss of other critical pituitary hormonal function, including ACTH and TSH, which should be assessed through appropriate testing. Diagnosis is confirmed by MRI, typically demonstrating the absence of a pituitary tumor and possibly demonstrating an edematous or hemorrhagic gland. Frequently, deficiencies of ACTH and TSH are not evident for years, so long-term follow-up of affected women is essential (40).

\section{POSTERIOR PITUITARY DISORDERS}

In normal pregnancy, the osmotic thresholds for vasopressin [arginine vasopressin (AVP), also known as antidiuretic hormone $(\mathrm{ADH})]$ release and thirst are lowered, resulting in plasma osmolality about $10 \mathrm{mOsm} / \mathrm{kg}$ lower than that in nonpregnant women (54). The metabolic clearance of AVP is markedly accelerated by mid-pregnancy, probably owing to circulating placental vasopressinase, which degrades AVP.

\section{Diabetes Insipidus}

Diabetes insipidus (DI) is the inability to concentrate urine, owing to AVP deficiency [central or neurogenic diabetes insipidus (CDI)] or renal tubular resistance to AVP (nephrogenic diabetes insipidus) (55). Causes of CDI include head trauma and hypothalamic tumors and other anatomic lesions; many cases are idiopathic. Transient forms of CDI may occur in pregnancy, owing to unmasking of subclinical disease by circulating vasopressinase (56) or to excessive circulating vasopressinase in the setting of hepatic dysfunction (57). Other causes of polyuria should be excluded by appropriate and pragmatic patient assessment. Diabetes mellitus causes polyuria, but if so, it is always accompanied by glycosuria. Urine dipstick consistently negative for glucose excludes this possibility. So long as thirst sensation is intact, plasma osmolality remains normal if patients are able to drink and have access to water. In the presence of CDI or nephrogenic DI, nonavailability of water or inability of the patient to drink because of nausea, vomiting, or impaired cognition will quickly result in dehydration and hyperosmolar hypernatremia. Various forms of dipsogenic DI also occur, but since AVP secretion and response are typically normal, a strong clinical clue suggestive of dipsogenic DI is significant hypoosmolar hyponatremia (as opposed to slight hypernatremia or normal serum sodium concentration, which is expected in either CDI or nephrogenic DI). Dipsogenic DI is commonly due to a primary psychiatric disorder-notably, schizophrenia-or may be due to hypothalamic disorders (e.g., neurosarcoidosis), which reset the "osmostat" for thirst.

Cardinal symptoms of DI are polyuria and polydipsia. Polyuria must not be confused with increased frequency of voiding, which is a common symptom of normal pregnancy. Actual polyuria is defined by increased urine volume (more than 2.5liters/24hours), in the absence of glycosuria. A water deprivation test (55), which includes hourly measurement of urine volume and osmolality, body weight, and vital signs, and measurement of plasma sodium every 3-4hours, is the "gold standard" traditional method of establishing the diagnosis of DI, but may be unnecessary if matched serum and urinary sodium, osmolality, and urine volumes reconcile with the clinical history. If a water deprivation test is performed, the patient should be seated or standing throughout the test, since urinary concentrating capacity is compromised during pregnancy by the lateral recumbent position. Water deprivation is achieved by enforced withholding of beverages and food, until a clinical endpoint is reached during the test. Endpoints justifying termination of water deprivation include (i) 3\% decrease in baseline (time 0) body weight, (ii) urine osmolality rise $<30 \mathrm{mOsm} / \mathrm{kg} / \mathrm{hr}$ for 3 consecutive hours, or (iii) plasma sodium $>$ upper limit of normal reference range. Upon attainment of an endpoint, the AVP analog desmopressin (DDAVP) $0.1 \mathrm{~mL}$ is administered intranasally, and urine osmolality is measured again after 1 to 2 hours. In severe CDI, maximal urine osmolality increases more than $50 \%$ after administration of DDAVP. In nephrogenic DI, maximal urine osmolality increases significantly less than 50\%, and often not at all following administration of DDAVP.

Pregnant women with severe CDI may be treated with maintenance dosing of DDAVP, which is not degraded by placental vasopressinase. The usual dose is 0.1 to $0.2 \mathrm{~mL}$ (10-20 mg) intranasally once daily at bedtime or else twice daily. The major clinical risk of DDAVP treatment is excessive dosing, with the potential for seizures due to water intoxication (severe plasma hypoosmolar hyponatremia). In the authors' experience, this risk is minimized by limiting therapy to once-daily dosing, administered at bedtime, deliberately allowing a late-day period of breakthrough polyuria. Some patients with preexisting CDI may require an increase in the dose of DDAVP during pregnancy; therapy can be titrated by clinical response of symptoms and further guided by matched urine and serum sodium and osmolality.

\section{ADRENAL DISORDERS}

\section{Normal Glucocorticoid Physiology}

The adrenal cortex produces two hormones of biologic importance, cortisol and aldosterone. If cortisol or aldosterone production is biologically inefficient due to defective steroidogenic enzymes, androgenic precursor steroids may accumulate to a physiologically significant degree. Important roles of cortisol include sustainment of blood glucose under physiologically stressful or fasting conditions via facilitation of gluconeogenesis, modulation of the systemic inflammatory response, sustainment of blood pressure, and facilitation of renal free water excretion and promotion of renal sodium conservation and potassium excretion. Cortisol circulates in plasmas in both free and protein-bound forms; the free form is biologically active and the protein-bound form represents a slow-release reservoir pool. Cortisol-binding globulin (CBG) is the predominant binding protein for cortisol, and $C B G$ production can be reduced or increased in disease states and under a variety of physiologic conditions. Cortisol secretion is controlled by circadian pulses of ACTH (in synergy with $\mathrm{ADH}$ ). Normal circadian ACTH fluctuation results in peak secretion during the nocturnal hours after midnight, resulting in peak serum cortisol between 0600 and 0800hours. Throughout the day, ACTH secretion wanes toward nadir between 2200 hours and midnight, at which time serum 
cortisol normally does not exceed $5 \mathrm{mcg} / \mathrm{dL}$. Signals for ACTH release include hypothalamic $\mathrm{CRH}$ as well as $\mathrm{ADH}$. $\mathrm{CRH}$ and $\mathrm{ADH}$ secretion is stimulated by physiologic stress and hypoglycemia, and CRH and ACTH secretion is inhibited by rising concentrations of serum cortisol (negative feedback inhibition).

The other major adrenal cortical steroid hormone, aldosterone, is a mineralocorticoid promoting renal sodium reabsorption and potassium excretion, and maintenance of extracellular fluid volume and blood pressure. Although ACTH stimulates aldosterone secretion, the primary regulation of aldosterone secretion is via the renin-angiotensin signal pathway. Decreased delivery of sodium to glomeruli via afferent arterioles in the nephron stimulates renin secretion by juxtaglomerular cells. Renin in turn activates the angiotensinconverting enzyme, resulting to conversion of inactive angiotensin- 1 to active angiotensin- 2 in the lung; angioten$\sin -2$ is the major stimulant of aldosterone synthesis and secretion by the adrenal. Aldosterone in turn promotes renal sodium conservation and extracellular fluid volume expansion, resulting in rising arterial pressure and increasing delivery of water and sodium to the proximal nephron. Renin secretion is inhibited by increased sodium delivery to the afferent renal arteriole, thereby completing the feedback loop of the renin-angiotensin-aldosterone axis.

In pregnancy, total plasma cortisol is increased, not only because of estrogen-stimulated increased CBG but also because of increased free cortisol. These changes occur early and progressively during gestation, increase further during labor, and persist for several days postpartum. Suppression of $\mathrm{CRH}$ and ACTH by exogenous glucocorticoids such as dexamethasone is impaired during pregnancy (58). It is possible that tissue responsiveness to cortisol is reduced, as normal pregnant women do not show physiologic consequences of cortisol excess. Maternal plasma renin and aldosterone are increased throughout pregnancy. The fetal adrenal gland (with a large fetal zone) contributes substantial dehydroepiandrosterone sulfate, which is converted by the placenta to estrogens (59).

\section{Adrenal Insufficiency}

Adrenal insufficiency in pregnancy has an estimated frequency of 1 per 300 births (60). Recognition and treatment of adrenal insufficiency has virtually eliminated maternal mortality, and the miscarriage rate may be as low as $15 \%$ (61). However, primary adrenal insufficiency due to autoimmune adrenalitis (Addison's disease) is frequently associated with other autoimmune disorders, including antiphospholipid antibody syndrome and systemic lupus erythematosus, which independently confer hazard to the pregnancy (62).

Adrenal failure can result in either disease of the adrenocortical tissue itself (primary adrenal insufficiency), due to autoimmune-mediated destruction of adrenocortical tissue (Addison's disease), or replacement of normal adrenocortical elements by metastatic tumor or granulomatous tissue, or from loss of trophic stimulation of the adrenal cortex by ACTH (secondary adrenal insufficiency). Secondary adrenal failure may be caused by any anatomic hypothalamic or pituitary lesion, including lymphocytic hypophysitis (49). In most cases, adrenal insufficiency will have been diagnosed prior to pregnancy, which will then usually be uneventful provided maternal therapy is not interrupted. New-onset adrenal insufficiency in a pregnant woman may be heralded by symptoms such as malaise, salt-craving, orthostatic faintness, persistent vomiting, and weight loss (63). Significant hyponatremia is present in over $90 \%$ of cases, but may be difficult to distinguish from the "physiologic" hyponatremia of normal pregnancy. Hyperkalemia, present in about two-thirds of nongravid patients with untreated primary adrenal insufficiency, is usually absent in the pregnant patient (64).Definitive diagnosis of adrenal insufficiency rests with measurement of plasma ACTH and cortisol, and performance of a cosyntropin (synthetic ACTH; Cortrosyn) stimulation test. A very low early morning (0600-0800 hr) plasma cortisol $(\leq 3.0 \mathrm{mcg} / \mathrm{dL})$ accompanied by a plasma $\mathrm{ACTH}>100 \mathrm{pg} / \mathrm{mL}$ is adequate to establish the diagnosis of primary adrenal insufficiency at any stage of gestation $(65,66)$. After drawing basal blood samples for ACTH and cortisol, the cosyntropin stimulation test is performed by administering cosyntropin $250 \mathrm{mcg}$ intramuscularly or intravenously, and drawing blood samples for cortisol 30 and 60 minutes post-cosyntropin. While cutoff points for "normal" and "abnormal" have not been formally established for pregnant patients, there is general agreement that maximum stimulated cortisol $>30 \mathrm{mcg} / \mathrm{dL}$, even in the late third trimester, is adequate to exclude the diagnosis of adrenal insufficiency (66). Likewise, a maximum stimulated cortisol $<18 \mathrm{mcg} / \mathrm{dL}$ is adequate to establish the diagnosis of adrenal insufficiency with confidence (63). Additional testing of patients found to have adrenal insufficiency may include measurement of serum 21-hydroxylase autoantibodies (positive in $90 \%$ of patients with Addison's disease), and plasma renin-to-aldosterone ratio, which will be markedly elevated in primary adrenal insufficiency (63). Patients with "normal" or "suppressed" ACTH can be evaluated with pituitary imaging via non-gadolinium-enhanced MRI, whereas those with primary adrenal insufficiency who test negative for 21hydroxylase antibodies can be assessed with MRI of the adrenals. Adrenal insufficiency caused by neoplastic or granulomatous disease is most usually associated with adrenal enlargement, whereas autoimmune disease causes adrenal atrophy.

Treatment of adrenal insufficiency is straightforward and largely empiric. Primary adrenal insufficiency obligates hormone replacement for both cortisol and aldosterone, whereas secondary adrenal insufficiency obligates replacement only for cortisol (since the renin-angiotensin-aldosterone axis is preserved). For cortisol replacement, a physiologically sound surrogate regimen is hydrocortisone $20 \mathrm{mg}$ orally in the morning and $10 \mathrm{mg}$ orally in the late afternoon; a pharmacologically equivalent alternative glucocorticoid such as cortisone acetate or prednisone may be substituted. As aldosterone surrogate, the synthetic mineralocorticoid fludrocortisone (Florinef) is used in a dose averaging $0.1 \mathrm{mg}$ (range $0.05-0.2$ $\mathrm{mg}$ ) orally each morning (67). The dose of fludrocortisone can be adjusted to maintain blood pressure and plasma potassium within the normal range. During labor and delivery, surgery, or severe intercurrent illness, supplemental glucocorticoid in doses intended to mimic natural stress-stimulated hypercortisolemia is prescribed. A traditional "stress" regimen is hydrocortisone $50 \mathrm{mg}$ intravenously every 8 hours (63). At 
this dosage, hydrocortisone exhibits sufficient mineralocorticoid agonism that fludrocortisones is unnecessary.

\section{Cushing's Syndrome}

Cushing's syndrome is the consequence of hypercortisolism (68). When diagnosed in pregnancy, the predominant cause $(60 \%$ of total) is an adrenal tumor (benign or malignant) (69). Other causes include ACTH-dependent Cushing's syndrome from a pituitary adenoma or from an ectopic source, or hypercortisolism of an iatrogenic or surreptitious source from exogenous glucocorticoids.

Clinical manifestations of Cushing's syndrome include central obesity, moon-like plethoric face, dorsal cervical and supraclavicular fat pads, thin skin with easy bruising and wide, deeply recessed, reddish or violaceous striae, proximal muscle wasting and weakness, osteoporosis, and hirsutism. Hypokalemia, hypertension, and diabetes mellitus commonly complicate Cushing's syndrome, but are not invariably present. Anovulatory oligomenorrhea and infertility frequently prevent pregnancy, so Cushing's syndrome during pregnancy is an unusual occurrence. However, the presence of gonadotropin receptors in pigmented nodular adrenal hyperplasia and in some adrenal adenomas may account for first expression of Cushing's syndrome manifestations during pregnancy (69). When pregnancy does accompany Cushing's syndrome, pre-eclampsia, hypertension, miscarriage, premature labor, intrauterine growth retardation, and stillbirth are distinct risks $(58,70)$. However, neonatal ACTH suppression and adrenal insufficiency are rare, and fetal virilization does not occur. Thus, most therapeutic attention and diagnostic efforts are directed toward maternal health during the pregnancy.

Accurate diagnosis of Cushing's syndrome during pregnancy is difficult based on physiologic changes in cortisol secretion in normal pregnancy. Ascertaining whether or not the hypercortisolism is ACTH-dependent is an important first step, and plasma ACTH $<10 \mathrm{pg} / \mathrm{mL}$ establishes ACTH independence (63). In the first trimester, daily cortisol production is comparable with the non-gravid state, and 24-hour urine free cortisol reflects this. Thus, in the first trimester, 24-hour urine free cortisol values distinctly above the normal range establish the diagnosis with some confidence. As pregnancy progresses into the second and third trimesters, cortisol production normally increases, peaking at about three times the non-gravid normal range in the third trimester; thus, to confirm hypercortisolism in the third trimester, one would need to observe 24-hour urine free cortisol well in excess of three times the normal upper limit to diagnose Cushing's syndrome with confidence. Salivary cortisol can be collected at home by patients and reflects production of cortisol on an hour-to-hour basis. Cushing's syndrome eliminates the normal diurnal fluctuation of cortisol production, such that the secretory trough normally observed at 2200 to 2400 hours is lost. Hence, salivary cortisol at midnight significantly above the upper limit of the normal range confirms loss of diurnal fluctuation and is consistent with Cushing's syndrome. The combination of 24-hour urine free cortisol $>$ three times the upper limit of the normal range and elevated midnight salivary cortisol possibly represents the optimal means of confirming Cushing's syndrome in patients in the second and third trimesters $(63,71)$.
If Cushing's syndrome is confirmed by 24-hour urine free cortisol and midnight salivary cortisol, appropriate adrenal or pituitary imaging is performed, based on whether plasma ACTH is suppressed or non-suppressed. If the ACTH is $<10$ $\mathrm{pg} / \mathrm{mL}$, the adrenals should be imaged by non-gadoliniumenhanced MRI. For non-suppressed ACTH, the pituitary should be assessed by MRI (63).

Because of the hazards posed by hypercortisolism to the pregnancy and to maternal health, treatment during the pregnancy is recommended. Adrenal tumors can be resected by flank incision, since up to $20 \%$ may be malignant. For patients with ACTH-producing pituitary adenoma (Cushing's disease), transsphenoidal adenoma resection has been successfully performed in pregnancy (72). If imaging does not localize the site of disease with confidence, medical therapy can be entertained, although experience with effective drugs in pregnancy is very limited. Metyrapone and ketoconazole are categorized as FDA class $\mathrm{C}$ agents and represent the best available alternative treatments for patients whose Cushing's syndrome cannot be treated surgically $(63,73)$.

\section{Fetal Virilizing Disorders}

Fetal virilization from maternal androgen excess is uncommon in pregnancy, since hyperandrogenemia suppresses GnRH and gonadotropins and thus is far more likely to cause oligomenorrheic infertility or amenorrhea. Rarely, virilizing adrenal (74) or ovarian tumors (75) can occur and are generally heralded by signs of maternal virilization, including hirsutism, male-pattern balding, increasing skin seborrhea, and deepening of the voice.

A more commonly encountered scenario in pregnancy is in couples with a prior history of a virilized newborn with 21hydroxylase deficiency, the most common form of virilizing congenital adrenal hyperplasia. For couples with a personal or family history of 21-hydroxylase deficiency, genetic screening is available through GYP21 genotyping. The couple genetically at risk may elect prenatal treatment of the mother with dexamethasone, at a dose of $20 \mathrm{mcg} / \mathrm{kg} / \mathrm{day}$ of non-gravid baseline maternal weight. Chorionic villous sampling performed at 9 to 11 weeks of gestation is performed to establish fetal sex and presence of fetal CYP21 gene (76). Maternal treatment is unnecessary for a male fetus or if fetal CYP21 is negative. Dexamethasone treatment of the pregnant woman is not without risk, as excessive weight gain, edema, impaired glucose tolerance or frank diabetes mellitus, or hypertension occurs in up to $50 \%$ of treated patients (63).

\section{Primary Hyperaldosteronism}

Just over 30 cases of primary aldosteronism in pregnancy have been reported in the past 40 years (77). Aldosteronism is characterized by suppressed plasma renin accompanied by autonomous adrenal secretion of aldosterone, with clinical consequences of hypertension, metabolic alkalosis, and frequently (but not invariably) hypokalemia. Plasma renin activity increases in the first trimester, and steadily throughout gestation, reaching levels three to seven times non-gravid baseline values by the late third trimester. Likewise, plasma aldosterone increases 5- to 20 -fold during pregnancy (63). Normally, despite high levels of these hormones, maneuvers such as plasma volume expansion with saline or administra- 
tion of synthetic mineralocorticoid result in appropriate suppression of the renin-angiotensin-aldosterone axis. Rising plasma progesterone throughout gestation is an important physiologic antagonist of mineralocorticoids and offers protection against maternal hypertension, despite markedly elevated levels of renin, angiotensin, and aldosterone. Very low levels of plasma renin (approaching undetectable), accompanied by elevated plasma aldosterone in a pregnant woman with hypertension or hypokalemia, would establish the diagnosis of primary aldosteronism with some accuracy. Adrenal imaging with non-gadolinium-enhanced MRI would then represent the next step in evaluation; identification of a unilateral adrenal nodule would be consistent with aldosterone-producing adenoma. In nonpregnant patients, catheterization of adrenal veins for measurement of aldosterone and cortisol is frequently performed. Such invasive testing would be difficult and largely contraindicated in a pregnant patient, so consideration may be given to medical management until after delivery (78). Amiloride is an FDA class C antagonist of aldosterone and may be the drug of choice for treatment of a pregnant patient with hyperaldosteronism (79).

\section{Pheochromocytoma}

Pheochromocytoma is a tumor of the adrenal medulla or extra-adrenal chromaffin tissue that hypersecretes catecholamines (80). Prevalence in pregnancy is estimated to be approximately 1 in 54,000, and over 200 cases have been reported in the literature (81). Typical clinical manifestations include sustained or paroxysmal hypertension, pallor, headache, palpitations, and diaphoresis. Hypertensive crisis can occur and is a feared complication. Serious sequelae include chest or abdominal pain, which can mimic acute myocardial infarction or acute surgical abdomen, seizures, hemodynamic shock (due to volume contraction induced by the excessive vasoconstricted state, when catecholamine secretory troughs occur), and death. Approximately 10\% of pheochromocytomas are familial, including the multiple endocrine neoplasia (MEN) $2 \mathrm{a}$ and $2 \mathrm{~b}$ syndromes (with pheochromocytoma about half as prevalent as medullary thyroid carcinoma), von Hippel-Lindau, and neurofibromatosis syndromes. Timely evaluation and definitive treatment of pheochromocytoma in pregnancy is essential, as untreated maternal mortality may exceed $15 \%$, and if treated, mortality may be as low as $1 \%(82)$. Abdominal palpation and labor are known provocateurs of catecholamine secretion by pheochromocytoma tumors, and hypertensive crisis may accompany a secretory surge of adrenomedullary hormones.

Pheochromocytoma is generally suspected based on the presence of maternal symptoms, hypertension, or identification of a family history of MEN, von Hippel-Lindau, or neurofibromatosis. Elevated plasma catecholamines and metanephrines are strikingly elevated and are reflected in 24hour urinary samples for catecholamines (epinephrine and norepinephrine) and metanephrines (metanephrine and normetanephrine). Most surgically confirmed pheochromocytomas have been associated with 24-hour urinary secretion three or more times the upper limit of normal for one or more of these hormones (83). When clinical suspicion is heightened by the finding of one or more extremely elevated urinary catecholamines or metanephrines (epinephrine, norepinephrine, metanephrine, or normetanephrine), adrenal imaging with non-gadolinium-enhanced MRI is accomplished, to investigate whether there is a unilateral adrenal tumor. Characteristically, pheochromocytomas image very bright on T2-weighted MRI and may be very helpful in the pregnant patient, for whom imaging modality options are limited (84).

Confirmation of a pheochromocytoma prior to the third trimester should lead to expedited surgical evaluation, to optimally perform adrenalectomy during the second trimester. In the third trimester, surgery is usually deferred until postpartum, but the patient is treated medically for the pheochromocytoma, and caesarian section is usually planned so that the hypertensive crises associated with vaginal delivery can be avoided (63). Prior to surgery, or otherwise if the patient cannot be treated surgically, drug treatment emphasizes alpha blocker and later beta blockers. The traditional alpha blocker drug is phenoxybenzamine 10 to $20 \mathrm{mg} /$ day orally twice; the drug may be titrated to a maximum dose of 1 $\mathrm{mg} / \mathrm{kg} /$ day until symptoms and hypotension are controlled (82). After several days of phenoxybenzamine therapy, a beta blocker is added, titrating to heart rate control, with two caveats. First, because pheochromocytoma hypertension is a profoundly vasoconstricted state, successful vasodilation may unmask clinical hypovolemia with orthostatic hypotension. Orthostatic blood pressure and symptoms should be routinely checked in the drug-treated patient. Second, alpha blocker drugs are always used first, for several days, to avoid theoretically catastrophic unopposed beta blockade, exacerbating vasoconstriction and potentially provoking a hypertensive crisis (63). After successful surgical resection of a pheochromocytoma, 24-hour urinary metanephrines and catecholamines should normalize, and medication therapy can be gradually tapered to complete withdrawal.

\section{CALCIUM DISORDERS}

\section{Physiologic Adaptations of Maternal Calcium Metabolism in Pregnancy}

Approximately $50 \%$ of circulating plasma calcium is ionized (free), and the remainder is complexed with plasma protein, most significantly albumin. Parathyroid hormone (PTH) is the most important determinant of plasma calcium, directly or indirectly controlling all routes of calcium intake and egress. Direct effects of PTH are on bone, the body's largest endogenous reservoir of calcium, and on kidney, which can up- or downregulate calcium egress. In bone, PTH induces osteoblasts to release cytokines stimulating osteoclastic resorption of calcium and phosphorus from bone, into the circulation. In the proximal nephron, $\mathrm{PTH}$ induces active reclamation of calcium from glomerular filtrate to interstitial fluid and from thence to the circulation. Indirectly, $\mathrm{PTH}$ also promotes absorption of calcium and phosphorus from the gut, by inducing the 1-alpha-hydroxylase enzyme in renal epithelium to convert comparatively inactive calcidiol (25-hydroxyvitamin D3) to calcitriol (1,25 dihydroxy-vitamin D3), which directly activates gut absorption of calcium and phosphorus. PTH is exquisitely sensitive to serum-ionized calcium; slight decrement in ionized plasma calcium rapidly results in increased PTH secretion; conversely, slight increment in ionized plasma calcium inhibits PTH synthesis and secretion. 
Mineralization of the fetal skeleton requires approximately $30 \mathrm{~g}$ of calcium by term, about $80 \%$ of which accrues during the third trimester of pregnancy. This need is met by increased maternal absorption of calcium from the gut, medicated by calcitriol (1,25 dihydroxy-vitamin D3), as opposed to hyperresorption of calcium salts from the maternal skeleton. Compared with the non-gravid state, maternal adaptations of calcium homeostasis to pregnancy include decreased serum $\mathrm{PTH}$, increased PTH-related peptide (PTHrP), and increased calcitriol. It is presumed that PTHrP is largely responsible for increased calcitriol production. Maternal serum calcitonin also increases, but its physiologic significance is unknown (85).

\section{Hypercalcemia}

Primary hyperparathyroidism (PHP) is the most common cause of hypercalcemia and is rare in pregnancy, with about 150 reported cases $(86,87)$. The only entirely satisfactory treatment for this disease is surgical resection of a solitary parathyroid adenoma (the most common culprit) or debulking of diffuse parathyroid hyperplastic glands; about $0.8 \%$ of large surgical series of parathyroidectomies were performed in pregnant patients (88). While PHP in pregnancy is rare, recognition and treatment are important, as maternal complications occur in about $67 \%$ and fetal or neonatal complications in $80 \%$ (89). Approximately $80 \%$ of pregnant women with PHP will have at least one symptom; these include nausea, vomiting, abdominal pain, renal colic, muscle weakness, mental sluggishness, depression, and bone pain (90). Additionally, hyperemesis gravidarum, weight loss, seizures, and pancreatitis may occur more frequently in women with PHP than in unaffected women $(87,88)$. Fetal complications of maternal PHP include stillbirth (approximately $2 \%$ ), neonatal death (approximately $2 \%$ ), and neonatal tetany (approximately 15\%) (91). Neonatal tetany results from profound suppression of the neonatal parathyroid function by maternal hypercalcemia, which is unmasked at the time of delivery, when the previous continuous milieu of hypercalcemia is rapidly lost. The condition commonly persists for 3 to 5 months, but permanent neonatal hypoparathyroidism can rarely be permanent (91).

Optimal management of PHP recognized during pregnancy is parathyroidectomy performed during the second trimester (89). Risk of all neonatal complications was reduced by $53 \%$ as compared with surgically untreated patients, in one large reported series (87). Premature labor is a distinct risk when parathyroidectomy is delayed until the third trimester, occurring in four of seven treated cases in one reported series (92). Influencing the decision of whether or not to perform surgery should be the awareness that there are no evidence-based medical therapies to offer patients as a surgical alternative; treatments would be limited to saline hydration and high-dose magnesium infusions (93). Neonates suffering from hypocalcemic tetany are treated with oral or parenteral calcium, and calcitriol, as needed until the condition resolves.

Hypercalcemic disorders other than PHP are very rarely encountered in pregnancy. Familial hypocalciuric hypercalcemia, also known as familial benign hypercalcemia, was first characterized in the early 1970s as an autosomal dominant genetic disorder notable for mild to moderate hypercalcemia accompanied by extremely low fractional excretion of calcium (ratio of calcium to creatinine clearance $<0.01$ ). It is now known to result from a mutation of the calcium-sensing receptor in both parathyroid and renal tubular tissues. There are no maternal consequences of this disorder, but neonatal hypocalcemic tetany can occur, with management similar as in maternal hyperparathyroidism (85). It is important to recognize the disorder for what it is so that maternal parathyroidectomy is not attempted. Malignancy-related hypercalcemia has only been reported in two pregnant patients, with maternal demise occurring within 4 months postpartum in both cases $(94,95)$.

\section{Hypocalcemic Disorders}

Apparent hypocalcemia in pregnant patients may simply represent physiologic dilutional hypoalbuminemia. If necessary, measurement of serum ionized calcium can confirm this possibility, which is otherwise usually inferred by the concurrent findings of slight hypocalcemia and hypoalbuminemia. The traditional estimate of total serum calcium for hypoalbuminemia is to add $0.8 \mathrm{mg} / \mathrm{dL}$ to measured serum calcium for every decrement of serum albumin of $1.0 \mathrm{mg} / \mathrm{dL}$ below the lower limit of the normal laboratory range. True hypocalcemia (low serum ionized calcium) is most often consequent to hypoparathyroidism (which can be idiopathic, but is more often secondary to a complication of thyroid or parathyroid surgery) or pseudohypoparathyroidism (hereditary resistance to PTH). Most often, a pregnant woman with these disorders will have come to clinical attention prior to becoming pregnant and is likely to already be receiving chronic therapeutic intervention. Maternal consequences of severe hypocalcemia include oral and acral paresthesias, carpopedal spasms, and potentially fatal laryngospasm; fetal consequences include skeletal demineralization and possibly hypercalcemia due to neonatal hyperparathyroidism. Because maternal PTH normally declines during pregnancy, and calcitriol increases under the influence of PTHrP, maternal hypocalcemia is sometimes ameliorated by pregnancy, and need for oral calcium supplements and exogenous calcitriol is reduced $(85,96)$. However, this is by no means universal, and careful monitoring of maternal serum calcium is important throughout the pregnancy.

Emergent therapy of symptomatic hypocalcemia (seizures or carpopedal or laryngospasm) should be treated with intravenous infusion of $10 \%$ calcium gluconate, $20 \mathrm{~mL}$ intravenously over 10 minutes, followed by continuous intravenous drip infusion of calcium 0.5 to $2 \mathrm{mg} / \mathrm{kg} / \mathrm{hr}$, adjusted to keep the plasma calcium level at 8 to $9 \mathrm{mg} / \mathrm{dL}$ (97). Oral maintenance therapy of chronic hypocalcemic disorders is usually accomplished with synthetic calcitriol (Rocaltrol), 0.25 to $0.5 \mathrm{mg}$ orally daily to three times daily, and calcium carbonate 1 to $2 \mathrm{~g}$ orally three times a day with meals. The dose of calcitriol is titrated as needed at 2- to 4 -week intervals to maintain plasma serum calcium of 8 to $9 \mathrm{mg} / \mathrm{dL}$. As noted, pregnancy may either decrease or increase the nongravid maintenance dose, and frequent (every 2-4weeks) measurement of serum calcium throughout the gestational period is recommended. 


\section{REFERENCES}

1. Glinoer D, de NP, Bourdoux P, et al. Regulation of maternal thyroid during pregnancy. J Clin Endocrinol Metab 1990; 71: 276-87.

2. LeBeau SO, Mandel SJ. Thyroid disorders during pregnancy (review). Endocrinol Metab Clin North Am 2006; 35: 117-36, vii.

3. Glinoer D. The regulation of thyroid function in pregnancy: pathways of endocrine adaptation from physiology to pathology (review). Endocr Rev 1997; 18: 404-33.

4. Mandel SJ, Spencer CA, Hollowell JG. Are detection and treatment of thyroid insufficiency in pregnancy feasible? Thyroid 2005; 15: 44-53.

5. Haddow JE, Palomaki GE, Allan WC, et al. Maternal thyroid deficiency during pregnancy and subsequent neuropsychological development of the child. N Engl J Med 1999; 341: 549-55.

6. Burrow GN, Fisher DA, Larsen PR. Maternal and fetal thyroid function (review). N Engl J Med 1994; 331: 1072-8.

7. Vulsma T, Gons MH, de Vijlder JJ. Maternal-fetal transfer of thyroxine in congenital hypothyroidism due to a total organification defect or thyroid agenesis. N Engl J Med 1989; 321: 13-16.

8. deZegher F, Pernasetti F, Vanhole C, et al. The prenatal role of thyroid hormone evidenced by fetomaternal Pit-1 deficiency. J Clin Endocrinol Metab 1995; 80: 3127-30.

9. Klein RZ, Haddow JE, Faix JD, et al. Prevalence of thyroid deficiency in pregnant women. Clin Endocrinol 1991; 35: 41-6.

10. Leung AS, Millar LK, Koonings PP, Montoro M, Mestman JH. Perinatal outcome in hypothyroid pregnancies. Obstet Gynecol 1993; 81: 349-53.

11. Wasserstrum N, Anania CA. Perinatal consequences of maternal hypothyroidism in early pregnancy and inadequate replacement. Clin Endocrinol 1995; 42: 353-8.

12. Casey BM, Dashe JS, Wells CE, et al. Subclinical hypothyroidism and pregnancy outcomes. Obstet Gynecol 2005; 105: 239-45.

13. Roti E, Minelli R, Salvi M. Clinical review 80: management of hyperthyroidism and hypothyroidism in the pregnant woman (review). J Clin Endocrinol Metab 1996; 81: 1679-82.

14. Kaplan MM. Monitoring thyroxine treatment during pregnancy. Thyroid 1992; 2: 147-52.

15. Fisher DA. Clinical review 19: management of congenital hypothyroidism (review). J Clin Endocrinol Metab 1991; 72: 523-9.

16. Davidson KM, Richards DS, Schatz DA, Fisher DA. Successful in utero treatment of fetal goiter and hypothyroidism. N Engl J Med 1991; 324: 543-6.

17. Burrow GN. Thyroid function and hyperfunction during gestation (review). Endocr Rev 1993; 14: 194-202.

18. Rajatanavin R, Chailurkit LO, Srisupandit S, Tungtrakul S, Bunyaratvej S. Trophoblastic hyperthyroidism: clinical and biochemical features of five cases. Am J Med 1988; 85: 237-41.

19. Yoshimura M, Hershman JM. Thyrotropic action of human chorionic gonadotropin (review). Thyroid 1995; 5: 425-34.

20. Goodwin TM, Montoro M, Mestman JH. Transient hyperthyroidism and hyperemesis gravidarum: clinical aspects. Am J Obstet Gynecol 1992; 167: 648-52.

21. Davis LE, Lucas MJ, Hankins GD, Roark ML, Cunningham FG Thyrotoxicosis complicating pregnancy. Am J Obstet Gynecol 1989; 160: 63-70.

22. Millar LK, Wing DA, Leung AS, et al. Low birth weight and preeclampsia in pregnancies complicated by hyperthyroidism. Obstet Gynecol 1994; 84: 946-9.

23. Wing DA, Millar LK, Koonings PP, Montoro MN, Mestman JH. A comparison of propylthiouracil versus methimazole in the treatment of hyperthyroidism in pregnancy. Am J Obstet Gynecol 1994; 170(1 Pt 1): 90-5.

24. Momotani N, Noh JY, Ishikawa N, Ito K. Effects of propylthiouracil and methimazole on fetal thyroid status in mothers with Graves' hyperthyroidism. J Clin Endocrinol Metab 1997; 82: 3633-6.

25. Momotani N, Noh J, Oyanagi H, Ishikawa N, Ito K. Antithyroid drug therapy for Graves' disease during pregnancy. Optimal regimen for fetal thyroid status. N Engl J Med 1986; 315: 24-8.

26. Cooper DS. Antithyroid drugs (review). N Engl J Med 2005; 352: 905-17.

27. Sherif IH, Oyan WT, Bosairi S, Carrascal SM. Treatment of hyperthyroidism in pregnancy. Acta Obstet Gynecol Scand 1991; 70: 461-3.

28. Momotani N, Hisaoka T, Noh J, Ishikawa N, Ito K. Effects of iodine on thyroid status of fetus versus mother in treatment of Graves' disease complicated by pregnancy. J Clin Endocrinol Metab 1992; 75: 738-44.
29. Mestman JH. Hyperthyroidism in pregnancy (review). Best Pract Res Clin Endocrinol Metab 2004; 18: 267-88.

30. Cove DH, Johnston P. Fetal hyperthyroidism: experience of treatment in four siblings. Lancet 1985; 1: 430-2.

31. Mestman JH, Goodwin TM, Montoro MM. Thyroid disorders of pregnancy (review). Endocrinol Metab Clin North Am 1995; 24: 41-71.

32. McKenzie JM, Zakarija M. Fetal and neonatal hyperthyroidism and hypothyroidism due to maternal TSH receptor antibodies (review). Thyroid 1992; 2: 155-9.

33. Learoyd DL, Fung HY, McGregor AM. Postpartum thyroid dysfunction (review). Thyroid 1992; 2: 73-80.

34. Kung AW, Chau MT, Lao TT, Tam SC, Low LC. The effect of pregnancy on thyroid nodule formation. J Clin Endocrinol Metab 2002; 87: 1010-14.

35. Tan GH, Gharib H, Goellner JR, van Heerden JA, Bahn RS. Management of thyroid nodules in pregnancy. Arch Intern Med 1996; 156: 2317-20.

36. Scheithauer BW, Sano T, Kovacs KT, et al. The pituitary gland in pregnancy: a clinicopathologic and immunohistochemical study of 69 cases. Mayo Clin Proc 1990; 65: 461-74.

37. Elster AD, Sanders TG, Vines FS, Chen MY. Size and shape of the pituitary gland during pregnancy and post partum: measurement with MR imaging. Radiology 1991; 181: 531-5.

38. Prager D, Braunstein GD. Pituitary disorders during pregnancy (review). Endocrinol Metab Clin North Am 1995; 24: 1-14

39. Caufriez A, Frankenne F, Hennen G, Copinschi G. Regulation of maternal insulin-like growth factor I by placental growth hormone in pregnancy. Possible action of maternal IGF-I on fetal growth (review). Horm Res 1994; 42: 62-5.

40. Molitch ME. Pituitary disorders during pregnancy (review). Endocrinol Metab Clin North Am 2006; 35: 99-116, vi.

41. Kupersmith MJ, Rosenberg C, Kleinberg D. Visual loss in pregnant women with pituitary adenomas. Ann Intern Med 1994; 121: 473-7.

42. Molitch ME. Pregnancy and the hyperprolactinemic woman (review). N Engl J Med 1985; 312: 1364-70.

43. Molitch ME. Clinical manifestations of acromegaly (review). Endocrinol Metab Clin North Am 1992; 21: 597-614.

44. deMenis E, Billeci D, Marton E, Gussoni G. Uneventful pregnancy in an acromegalic patient treated with slow-release lanreotide: a case report. J Clin Endocrinol Metab 1999; 84: 1489.

45. Mozas J, Ocon E, Lopez de la TM, et al. Successful pregnancy in a woman with acromegaly treated with somatostatin analog (octreotide) prior to surgical resection. Int J Gynaecol Obstet 1999; 65: 71-3.

46. Neal JM. Successful pregnancy in a woman with acromegaly treated with octreotide. Endocr Pract 2000; 6: 148-50.

47. Caron P, Gerbeau C, Pradayrol L. Maternal-fetal transfer of octreotide. N Engl J Med 1995; 333: 601-2.

48. Kupersmith MJ, Rosenberg C, Kleinberg D. Visual loss in pregnant women with pituitary adenomas. Ann Intern Med 1994; 121: 473-7.

49. Thodou E, Asa SL, Kontogeorgos G, et al. Clinical case seminar: lymphocytic hypophysitis: clinicopathological findings. J Clin Endocrinol Metab 1995; 80: 2302-11.

50. Leiba S, Schindel B, Weinstein R, et al. Spontaneous postpartum regression of pituitary mass with return of function. JAMA 1986; 255: 230-2.

51. McGrail KM, Beyerl BD, Black PM, Klibanski A, Zervas NT. Lymphocytic adenohypophysitis of pregnancy with complete recovery. Neurosurgery 1987; 20: 791-3.

52. Kelestimur F. Sheehan's syndrome (review). Pituitary 2003; 6: 181-8.

53. Feinberg EC, Molitch ME, Endres LK, Peaceman AM. The incidence of Sheehan's syndrome after obstetric hemorrhage. Fertil Steril 2005; 84: 975-9.

54. Lindheimer MD, Barron WM. Water metabolism and vasopressin secretion during pregnancy (review). Baillieres Clin Obstet Gynaecol 1994; 8: 311-31.

55. Robertson GL. Diabetes insipidus (review). Endocrinol Metab Clin North Am 1995; 24: 549-72.

56. Williams DJ, Metcalfe KA, Skingle L, et al. Pathophysiology of transient cranial diabetes insipidus during pregnancy. Clin Endocrinol 1993; 38: 595-600.

57. Durr JA, Hoggard JG, Hunt JM, Schrier RW. Diabetes insipidus in pregnancy associated with abnormally high circulating vasopressinase activity. N Engl J Med 1987; 316: 1070-4. 
58. Aron DC, Schnall AM, Sheeler LR. Cushing's syndrome and pregnancy (review). Am J Obstet Gynecol 1990; 162: 244-52.

59. Hadden DR. Adrenal disorders of pregnancy (review). Endocrinol Metab Clin North Am 1995; 24: 139-51.

60. Albert E, Dalaker K, Jorde R, Berge LN. Addison's disease and pregnancy. Acta Obstet Gynecol Scand 1989; 68: 185-7.

61. Ambrosi B, Barbetta L, Morricone L. Diagnosis and management of Addison's disease during pregnancy (review). J Endocrinol Invest 2003; 26: 698-702.

62. Grottolo A, Ferrari V, Mariano M, et al. Primary adrenal insufficiency, circulating lupus anticoagulant and anticardiolipin antibodies in a patient with multiple abortions and recurrent thrombotic episodes. Haematologica 1988; 73: 517-19.

63. Lindsay JR, Nieman LK. Adrenal disorders in pregnancy (review). Endocrinol Metab Clin North Am 2006; 35: 1-20, v.

64. Gradden C, Lawrence D, Doyle PM, Welch CR. Uses of error: Addison's disease in pregnancy. Lancet 2001; 357: 1197.

65. Grinspoon SK, Biller BM. Clinical review 62: laboratory assessment of adrenal insufficiency (review). J Clin Endocrinol Metab 1994; 79: 923-31.

66. McKenna DS, Wittber GM, Nagaraja HN, Samuels P. The effects of repeat doses of antenatal corticosteroids on maternal adrenal function. Am J Obstet Gynecol 2000; 183: 669-73.

67. Clutter WE. Endocrine disease. In: Ewald GA, McKenzie CR, eds. Manua of Medical Therapeutics, 28th edn. Little-Brown, 1995

68. Orth DN. Cushing's syndrome (review). N Engl J Med 1995. 332: 791803. [Erratum appears in N Engl J Med 1995; 332: 1527].

69. Lindsay JR, Jonklaas J, Oldfield EH, Nieman LK. Cushing's syndrome during pregnancy: personal experience and review of the literature. J Clin Endocrinol Metab 2005; 90: 3077-83.

70. Aron DC, Schnall AM, Sheeler LR. Cushing's syndrome and pregnancy (review). Am J Obstet Gynecol 1990; 162: 244-52.

71. Putignano $\mathrm{P}$, Toja $\mathrm{P}$, Dubini A, et al. Midnight salivary cortisol versus urinary free and midnight serum cortisol as screening tests for Cushings syndrome. J Clin Endocrinol Metab 2003; 88: 4153-7.

72. Casson IF, Davis JC, Jeffreys RV, et al. Successful management of Cushing's disease during pregnancy by transsphenoidal adenectomy (review). Clin Endocrinol 1987; 27: 423-8.

73. Berwaerts J, Verhelst J, Mahler C, Abs R. Cushing's syndrome in pregnancy treated by ketoconazole: case report and review of the literature (review). Gynecol Endocrinol 1999; 13: 175-82.

74. Kirk JM, Perry LA, Shand WS, et al. Female pseudohermaphroditism due to a maternal adrenocortical tumor (review). J Clin Endocrinol Metab 1990; 70: 1280-4.

75. Shortle BE, Warren MP, Tsin D. Recurrent androgenicity in pregnancy: a case report and literature review. Obstet Gynecol 1987; 70(3 Pt 2): 462-6.

76. New MI, Carlson A, Obeid J, et al. Prenatal diagnosis for congenital adrenal hyperplasia in 532 pregnancies. J Clin Endocrinol Metab 2001; 86: 5651-7.

77. Okawa T, Asano K, Hashimoto T, et al. Diagnosis and management of primary aldosteronism in pregnancy: case report and review of the literature. Am J Perinatol 2002; 19: 31-6.
78. Hammond TG, Buchanan JD, Scoggins BA, Thatcher R, Whitworth JA. Primary hyperaldosteronism in pregnancy. Aust NZ J Med 1982; 12: 537-9.

79. Deruelle P, Dufour P, Magnenant E, Courouble N, Puech F. Maternal Bartter's syndrome in pregnancy treated by amiloride. Eur J Obstet Gynecol Reprod Biol 2004; 115: 106-7.

80. Cryer PE. Pheochromocytoma (review). West J Med 1992; 156: 399-407.

81. Botchan A, Hauser R, Kupfermine M, et al. Pheochromocytoma in pregnancy: case report and review of the literature (review). Obstet Gynecol Surv 1995; 50: 321-7.

82. Harper MA, Murnaghan GA, Kennedy L, Hadden DR, Atkinson AB Phaeochromocytoma in pregnancy. Five cases and a review of the literature (review). Br J Obstet Gynaecol 1989; 96: 594-606.

83. Lenders JW, Pacak K, Eisenhofer G. New advances in the biochemical diagnosis of pheochromocytoma: moving beyond catecholamines (review). Ann NY Acad Sci 2002; 970: 29-40.

84. Ilias I, Pacak K. Current approaches and recommended algorithm for the diagnostic localization of pheochromocytoma (review). J Clin Endocrinol Metab 2004; 89: 479-91.

85. Kovacs CS, Fuleihan G. Calcium and bone disorders during pregnancy and lactation (review). Endocrinol Metab Clin North Am 2006; 35: 21-51, v.

86. Haenel LC, Mayfield RK. Primary hyperparathyroidism in a twin pregnancy and review of fetal/maternal calcium homeostasis (review). Am J Med Sci 2000; 319: 191-4.

87. Kelly TR. Primary hyperparathyroidism during pregnancy (review). Surgery 1991; 110: 1028-33; discussion 1033-4.

88. Kort KC, Schiller HJ, Numann PJ. Hyperparathyroidism and pregnancy (review). Am J Surg 1999; 177: 66-8.

89. Schnatz PF, Curry SL. Primary hyperparathyroidism in pregnancy: evidencebased management (review). Obstet Gynecol Surv 2002; 57: 365-76.

90. Kristoffersson A, Dahlgren S, Lithner F, Jarhult J. Primary hyperparathyroidism in pregnancy. Surgery 1985; 97: 326-30.

91. Shangold MM, Dor N, Welt SI, Fleischman AR, Crenshaw MC Jr. Hyperparathyroidism and pregnancy: a review (review). Obstet Gynecol Surv 1982; 37: 217-28.

92. Carella MJ, Gossain VV. Hyperparathyroidism and pregnancy: case report and review (review). J Gen Intern Med 1992; 7: 448-53.

93. Dahan M, Chang RJ. Pancreatitis secondary to hyperparathyroidism during pregnancy (review). Obstet Gynecol 2001; 98(5 Pt 2): 923-5.

94. Illidge TM, Hussey M, Godden CW. Malignant hypercalcaemia in pregnancy and antenatal administration of intravenous pamidronate. Clin Oncol (R Coll Radiol) 1996; 8: 257-8.

95. Usta IM, Chammas M, Khalil AM. Renal cell carcinoma with hypercalcemia complicating a pregnancy: case report and review of the literature. Eur J Gynaecol Oncol 1998; 19: 584-7.

96. Kovacs CS, Kronenberg HM. Maternal-fetal calcium and bone metabolism during pregnancy, puerperium, and lactation (review). Endocr Rev 1997; 18: 832-72.

97. Dagogo-Jack S, Clutter WE. Mineral and bone disease. In: Ewald GA, McKenzie CR, eds. Manual of Medical Therapeutics, 28th edn. LittleBrown, 1995. 


\section{Gastrointestinal diseases and pregnancy Murtaza Arif, Anjana Sathyamurthy, Jessica Winn, and Jamal A. Ibdah}

\begin{abstract}
INTRODUCTION
Pregnancy has a significant effect on the normal physiology of the gastrointestinal tract, and gut-related symptoms are extremely common during gestation. In this chapter, a review is presented of the common gastrointestinal disorders in pregnancy, which may present difficult management problems. The treatment of these disorders in the nonpregnant as well as the pregnant patient is discussed, so that the reader will become familiar with the special diagnostic and therapeutic considerations during pregnancy.
\end{abstract}

\section{GASTROESOPHAGEAL REFLUX DISEASE AND REFLUX ESOPHAGITIS}

Gastroesophageal reflux disease (GERD) results from the inappropriate backwash of gastric contents into the esophagus. Although this process occurs frequently in asymptomatic individuals, it can also result in heartburn and regurgitation. Reflux esophagitis is defined as the presence of typical symptoms of GERD in conjunction with endoscopic and pathologic changes in the esophagus.

\section{Epidemiology}

The prevalence of symptoms of GERD is quite high in the general population. Large-scale surveys have revealed that approximately $33 \%$ to $44 \%$ of adult Americans experience reflux symptoms on a twice weekly to monthly basis (1). However, the great majority of this group does not seek medical attention. Of those individuals who go to a doctor, $40 \%$ to $70 \%$ will show endoscopic evidence of esophagitis. Gastroesophageal reflux symptoms are extremely common during pregnancy and are an important cause of morbidity in pregnancy. Reflux symptoms are reported by $40 \%$ to $85 \%$ women during pregnancy $(2,3)$. The incidence of true reflux esophagitis is difficult to determine, because large-scale endoscopic studies of pregnant women have not been performed.

\section{Defense Mechanisms for Prevention of Gastroesophageal Reflux}

There are several defense mechanisms that act to limit contact with and damage to the esophagus by gastric contents (Table 1).

1. Lower esophageal sphincter (LES): The LES is probably the major protective barrier, preventing reflux of food, acid, and secretions from the stomach into the esophagus. The LES relaxes in response to hormones, foods, and drugs. Some patients with reflux esophagitis have decreased resting LES pressures or a completely incompetent
LES; reflux events also occur in patients with normal LES pressures, presumably owing to transient LES relaxation $(4,5)$.

2. Esophageal motility and gravity: Contact of the esophageal mucosa with damaging substances is limited by esophageal peristalsis and gravity, which promote clearing. Aperistalsis, as found in scleroderma, contributes substantially to the development of esophagitis in these patients (in addition to an incompetent LES).

3. Saliva: Saliva has also been shown to neutralize acid.

4. Intrinsic epithelial defense: The esophageal epithelial layer has intrinsic defense systems including cell membrane and intercellular components, which prevent influx of hydrogen ions as well as intracellular proteins, which function as buffers.

\section{Pathophysiology}

The pathophysiology of GERD and reflux esophagitis in pregnant patients is incompletely understood. It may be multifactorial, involving both mechanical and hormonal factors.

1. LES: Some analyses have shown that resting LES pressure decreases in pregnancy, in both early and late gestations $(6,7)$. In other studies, LES pressure was normal in early pregnancy but hypofunction of the LES was detected, as measured by decreased responsiveness to drugs that increase sphincter pressure (8). Decreased LES pressure probably results from elevated serum estrogen and especially progesterone levels during pregnancy (6).

2. Intra-abdominal pressure: Although increased intra-abdominal pressure has been postulated to produce reflux, a study of men with tense ascites as a model of pregnancy (9) showed an increase in LES pressure and no demonstrable acid reflux. Thus, the contribution of increased abdominal girth in reflux esophagitis is unclear, but the relevance of this model may be questioned. Intragastric pressures appear to be increased even in early pregnancy, and patients with heartburn have lower mean barrier pressures (LES pressuregastric pressure) than asymptomatic pregnant individuals (10).

3. Esophageal motility: Normal esophageal motility, which helps clear potentially damaging material from the esophagus, is altered in pregnancy; peristaltic wave speed and amplitude are both reduced (11). 
Table 1 Esophageal Mucosal Defense Mechanisms

\begin{tabular}{ll} 
Mechanism & \multicolumn{1}{c}{ Effect } \\
Lower esophageal sphincter & Barrier \\
Esophageal motility & Promotes clearing \\
Gravity (when upright) & Promotes clearing \\
Saliva & Acid neutralization \\
Intrinsic epithelial defense & Cell membrane, inter- and \\
& intracellular components
\end{tabular}

\section{Clinical Features}

The clinical features of GERD during pregnancy do not differ from those in the general adult population. Classic symptoms of GERD include heartburn (retrosternal burning pain) and regurgitation. In patients with long-standing reflux esophagitis, strictures may result, producing dysphagia or difficulty swallowing. Severe reflux esophagitis may produce painful swallowing (odynophagia), although this is more likely to be associated with infectious causes of esophagitis. Acid reflux may lead to esophageal dysmotility and atypical noncardiac chest pain. Extra-esophageal symptoms may include hoarseness, chronic cough, laryngitis, asthma, and recurrent aspiration.

\section{Complications}

A variety of complications can result from reflux esophagitis:

1. Stricture formation

2. Bleeding

3. Pulmonary aspiration

4. Barrett's esophagus_Chronic esophageal inflammation is thought to lead to Barrett's esophagus, in which the normal squamous esophageal epithelium is replaced by specialized columnar epithelium. This diagnosis is made by endoscopy with biopsy; routine surveillance with periodic endoscopic examination and biopsies is indicated because of an increased risk of esophageal cancer.

\section{Diagnosis}

During pregnancy, initial diagnosis of GERD is based on symptoms, and an empiric trial of therapy may be initiated.

1. Barium radiography: Barium studies are less sensitive compared with upper endoscopy for diagnosis of reflux esophagitis. They should be avoided during pregnancy because of risk of radiation exposure to the fetus.

2. Upper endoscopy: Endoscopy allows direct visualization of the epithelium, and biopsies can be taken for histologic analysis. Endoscopy can be safely performed during pregnancy for evaluation of intractable symptoms refractory to a trial of standard medical therapy or complications such as gastrointestinal bleeding. Please refer to the section on "Endoscopy and procedural sedation in pregnancy" for further discussion.

3. Esophageal manometry and $p H$ monitoring: These studies are rarely used in patients with atypical or refractory symptoms to detect reflux and correlate symptoms with reflux events.
Table 2 Medications Used in Pregnancy for Gastroesophageal Reflux Disease

\begin{tabular}{lc} 
Drug name & FDA category \\
\hline $\begin{array}{l}\text { Antacids } \\
\text { Aluminum-, calcium-, or }\end{array}$ & None \\
$\quad$ magnesium-containing antacids & \\
H2RAs & \\
Cimetidine & $\mathrm{B}$ \\
Ranitidine (preferred) & $\mathrm{B}$ \\
Famotidine & $\mathrm{B}$ \\
$\begin{array}{l}\text { Nizatidine } \\
\text { Mucosal protectant }\end{array}$ & $\mathrm{B}$ \\
$\begin{array}{l}\text { Sucralfate } \\
\text { Proton pump inhibitors }\end{array}$ & \\
$\begin{array}{l}\text { Omeprazole } \\
\text { Lansoprazole (preferred) }\end{array}$ & $\mathrm{B}$ \\
$\begin{array}{l}\text { Rabeprazole } \\
\text { Pantoprazole }\end{array}$ & $\mathrm{C}$ \\
$\begin{array}{l}\text { Esomeprazole } \\
\text { Promotility agents }\end{array}$ & $\mathrm{B}$ \\
Metoclopramide & $\mathrm{B}$ \\
$\begin{array}{l}\text { Abbreviations: FDA, Food and Drug Administration; H2RAs, histamine-2 } \\
\text { receptor antagonists. }\end{array}$ & $\mathrm{B}$ \\
\end{tabular}

\section{Management with Maternal-Fetal Consideration}

1. Lifestyle modifications: Initial management of GERD usually consists of conservative measures such as lifestyle modifications and dietary changes. Avoidance of smoking, late-night meals, and substances that decrease LES pressure such as high-fat foods, caffeine, alcohol, and chocolate often provide some relief. Patients should remain upright after meals to minimize reflux of acidic contents, allowing gravity to aid esophageal clearance. Elevation of the head of the bed by shock blocks helps maintain the beneficial effect of gravity at night.

2. Medications: Various medications are used for the management of GERD (Table 2).

Antacids: Antacids are generally considered safe in pregnancy (12) and are used by $30 \%$ to $50 \%$ of women for relief of reflux symptoms during pregnancy (13). Magnesium-, aluminum-, or calcium-containing antacids are not teratogenic in animal studies (13). There are limited data on the safety and efficacy of antacids during pregnancy. A large retrospective study showed an increased incidence of birth defects in women who used antacids along with many other medications in the first trimester (14); however, no particular antacid was specified, and these data have not been verified in another study. More recent analyses of pregnant women in both the United States and the Netherlands $(15,16)$ indicate that antacid use is common; although no obvious increase in rare birth defects has been noted, small increases in common abnormalities such as spontaneous abortion, cleft palate, or low birth weight would be difficult to detect (15). The major immediate side effects of large doses of antacids include constipation induced by aluminum-containing antacids and diarrhea caused by magnesium-based preparations. The use of a combination of these medications usually resolves 
bowel-related problems. Antacids containing sodium bicarbonate should be avoided because it can precipitate metabolic alkalosis and volume overload (17).

Sucralfate: Sucralfate is FDA pregnancy category B. It is a polysulfated salt of sucrose, which binds to exposed proteins found in the base of ulcerated mucosa and produces a protective coating of the ulcer site. In a randomized controlled study, sucralfate-treated pregnant patients $(1 \mathrm{~g}$ three times daily) had a higher frequency of remission of heartburn $(90 \%$ vs. $43 \%)$ and regurgitation ( $83 \%$ vs. $27 \%$ ) compared with lifestyle and dietary modifications (18). In animal studies, sucralfate was not teratogenic with doses up to 50 times those used in humans (19).

Histamine-2 receptor antagonists: All histamine-2 receptor antagonists (H2RAs) including cimetidine, ranitidine, famotidine, and nizatidine are classified as FDA pregnancy category B. H2RAs have been recently replaced by proton pump inhibitors for treatment of reflux esophagitis in nonpregnant patients; however, in pregnant patients with GERD, H2RAs are the most commonly used and safest medications for those not responding to lifestyle modifications and antacids. Generally, 8 ormore weeks of therapy is required to produce complete healing, and recurrences are frequent when medication is discontinued. In a study, efficacy of ranitidine once or twice daily was compared with placebo in pregnant women (20). Only ranitidine $150 \mathrm{mg}$ twice daily demonstrated significant relief in symptoms and reduction in the use of antacids. No adverse pregnancy or fetal outcomes were noted. A surveillance study in the United States found no increase in the incidence of congenital anomalies among infants exposed to various H2RAs including ranitidine, cimetidine, and famotidine (21). Ranitidine is usually the preferred H2RA during pregnancy because of the absence of teratogenicity in animal studies and documented efficacy in pregnancy.

Proton pump inhibitors: Proton pump inhibitors (PPIs) including lansoprazole, rabeprazole, pantoprazole, and esomeprazole are classified as FDA pregnancy category B drugs. Omeprazole is the only PPI, which is classified as FDA pregnancy category $\mathrm{C}$ because of dose-related embryonic and fetal mortality observed in animal studies. PPIs act as inhibitors of parietal cell $\mathrm{H}^{+} / \mathrm{K}^{+}$ATPase (the proton pump responsible for hydrogen ion secretion) and are the most effective inhibitors of gastric acid secretion. These are the drugs of choice in nonpregnant patients for treatment of severe erosive reflux esophagitis and can heal esophageal lesions that are refractory to treatment with H2RAs. However, PPIs have not been extensively used in pregnancy and limited safety data are available for their use during pregnancy. A recent meta-analysis showed no significant increase in the risk of congenital anomalies in pregnant women exposed to various PPIs (22). PPIs should be used only in pregnant women with refractory symptoms who are not responding to H2RAs. Lansoprazole may be the preferred PPI because of its lack of teratogenicity in animal studies and limited safety reports during pregnancy.

\section{Conclusion}

GERD and reflux esophagitis in pregnancy should be managed in a step-up approach starting with lifestyle modifications. Medical therapy should be initiated for those who do not respond to lifestyle and dietary modifications. Antacids and sucralfate are considered as first-line medical agents for GERD and/or reflux esophagitis. H2RAs should be introduced if symptoms persist despite the use of antacids. PPIs should be reserved for pregnant patients with refractory symptoms or complications secondary to GERD. Antireflux surgery is avoided during pregnancy because the symptoms usually resolve after delivery.

\section{PEPTIC ULCER DISEASE}

An ulcer is an injury to or interruption of the mucosal lining of the gastrointestinal tract extending through the muscularis mucosa. Peptic ulcer disease (PUD) is an important cause of morbidity and mortality in the general population; however, severity of symptoms and frequency of complications associated with PUD decrease during pregnancy (23-25).

\section{Epidemiology}

The incidence of PUD is low in pregnant women (23-25). It is difficult to ascertain the true incidence of PUD in pregnancy because diagnostic testing is generally avoided. Symptoms of PUD may be attributed to GERD or nausea and vomiting of pregnancy with resolution of symptoms upon treatment, thereby precluding further testing (26). Patients may also avoid medical attention by self-treatment with nonprescription medications for mild to moderate gastrointestinal symptoms.

A number of hypotheses have been proposed for the low incidence of PUD in pregnancy. Plasma levels of histaminases are increased during pregnancy due to placental synthesis. The histaminases can metabolize maternal histamine leading to decreased gastric acid secretion during pregnancy (27). Increased estrogen levels can result in hypochlorhydria, and gestational progesterone may increase synthesis of the protective gastric mucous layer (28). Immunologic tolerance during pregnancy may permit growth and colonization of Helicobacter pylori without immunologic response and mucosal injury (29,30). Maternal avoidance of ulcerogenic factors, such as smoking, alcohol, and nonsteroidal anti-inflammatory drug (NSAID) use, and reduced psychologic stress may also play a role in the decreased incidence of PUD during pregnancy $(31,32)$.

\section{Etiology and Pathophysiology}

The etiology of PUD is complex and multifactorial. The two most common causes of PUD are H. pylori infection and use of NSAIDs. H. pylori has been implicated in the pathogenesis of PUD. Patients who are colonized with $H$. pylori have histologic evidence of antral gastritis, and eradication of infection leads to resolution of gastritis. $H$. pylori survives in the harsh gastric environment by the production of urease, which neutralizes acid by the generation of ammonia. H. pylori also produces protease, which thins the mucous layer and allows penetration and damage of the initial layer of mucosal defense. Almost all patients with duodenal ulcers, and gastric ulcers unassociated with the use of NSAIDs, are infected with $H$. pylori. The healing of ulcers occurs when $H$. pylori is eradicated and, most importantly, relapse of ulcer disease is prevented (33).

Gastric mucosal defenses, including a thick mucous layer and the ability of the mucosa to secrete bicarbonate, can 
resist damage by acid, pepsin, and biliary reflux. Impairment or disruption of these protective mechanisms occurs because of inhibition of cyclooxygenase, prostaglandin synthesis, and mucosal mucous and bicarbonate secretion, leading to superficial gastric mucosal injury or gastric ulcer, predominantly in the gastric antrum. NSAIDs, including aspirin, increase the risk of PUD by inhibiting cyclooxygenase and a direct toxic effect on the gastric mucosa. There are several factors that influence the risk of developing PUD in patients using NSAIDs. The risk is more in patients taking higher doses of NSAIDs for a prolonged duration. Other risk factors for PUD include cigarette smoking and alcohol consumption (34). Cigarette smoking has been shown to slow ulcer healing and is associated with a higher rate of ulcer recurrence (35) in smokers compared with nonsmokers.

\section{Clinical Features}

Typical symptoms of PUD include epigastric pain, which is relieved by food or antacids. Patients often describe a dull "gnawing" or burning sensation, which may awaken them at night or may occur in the morning before eating or after meals. Because the abdominal pain is often relieved by eating, patients with PUD may gain weight. Other symptoms arise when duodenal or gastric ulcer is complicated by hemorrhage, perforation, penetration, or obstruction (Table 3). If the ulcer bleeds, the patient may develop black or tarry stools (melena) or hematemesis. Ulcer penetration into an arterial blood vessel can lead to massive, brisk bleeding with rapid transit of blood through the gastrointestinal tract and bright red blood per rectum (hematochezia). Abdominal pain that radiates to the back indicates possible penetration of the ulcer posteriorly through the wall of the stomach or duodenal bulb into surrounding organs. Duodenal bulb ulcers may penetrate into the pancreas, whereas gastric ulcers can erode into the liver or colon. The sudden onset of severe pain in conjunction with physical findings of an acute abdomen (rebound tenderness, guarding, absent bowel sounds, and distention) is associated with free perforation of the ulcer, which is a surgical emergency. Finally, prolonged nausea and vomiting can result from gastric outlet obstruction caused by edema and inflammation surrounding an ulcer crater located in the prepyloric or pyloric region. It is important to note that complicated ulcer disease may present with no antecedent history of abdominal pain or other symptoms.

In pregnancy, the signs and symptoms of PUD and its complications may be obscured. First, symptoms that are consistent with PUD may also be associated with GERD, which is quite common in pregnancy. Nausea and vomiting indicating the presence of an obstructing ulcer may be mistakenly considered to be "normal" pregnancy-related symptoms. Because of the morbidity and mortality associated with complicated PUD during gestation, a high index of suspicion should be maintained for those patients who develop nausea, vomiting, and abdominal pain in the second and third trimesters. Finally, in the immediate postdelivery period, signs of perforation such as abdominal rebound or guarding may be absent or decreased due to muscular relaxation, leading to delayed or misdiagnosis.

\section{Diagnosis}

During pregnancy, barium radiography is contraindicated because of risk of radiation teratogenesis. Upper endoscopy (esophagogastroduodenoscopy, EGD) is preferred for the diagnosis of PUD in both pregnant and nonpregnant patients because of a higher sensitivity and specificity when compared with barium radiography (30). EGD also has the ability to obtain histologic specimens to test for $H$. pylori infection and to exclude malignant gastric ulcer. In pregnant patients, EGD should be reserved only for patients with symptoms refractory to intensive medical therapy or PUD-related complications such as hemorrhage or gastric outlet obstruction. Since duodenal ulcers are benign, follow-up endoscopy to document complete healing is unnecessary. A significant although small percentage of gastric ulcers are malignant. Therefore, EGD and biopsy of gastric ulcers with follow-up until complete healing is documented is recommended.

In all patients with documented PUD, the presence of H. pylori infection should be determined. Upper endoscopy with gastric biopsy for histologic examination is considered the "gold standard" for the detection of active $H$. pylori infection. Histologic examination may also reveal changes in the gastric mucosa indicative of $H$. pylori infection, such as intestinal metaplasia or dysplasia. Gastric biopsy for detection of urease activity (Clotest), an enzyme produced in abundance by $H$. pylori, can also be used for the detection of active infection. Noninvasive methods include serologic detection of IgG antibodies to $H$. pylori, stool antigen assay for $H$. pylori, and urease breath test. Antibiotics, bismuth, and PPIs can affect the sensitivity of the test results. It is recommended to discontinue antibiotics for 4 weeks and hold PPIs for at least 2 weeks prior to testing for $H$. pylori infection.

\section{Management of PUD}

Acid secretion by the parietal cell is controlled by neural, paracrine, and endocrine influences. Receptors for histamine ( $\mathrm{H} 2$ receptors) and gastrin are present on the surface of the parietal cell; exposure to either histamine or gastrin leads to acid secretion. Cholinergic neural signals interact with muscarinic receptors to produce acid secretion. In response to these and other stimuli, vesicles containing the proton pump, or $\mathrm{H}^{+} / \mathrm{K}^{+}$ATPase, fuse to the cell membrane and $\mathrm{H}^{+}$ ions are secreted into the gastric lumen. Treatment of PUD in the general population is primarily directed at blocking the interaction of histamine with its receptor. The treatment of PUD focuses on (i) healing of the ulcer crater and (ii) permanently eradicating $H$. pylori if present. Healing of the ulcer crater can be achieved by 4 to 8 weeks of therapy with antacids, H2RAs, or PPIs.

\section{Table 3 Complications of Peptic Ulcer Disease}

Gastrointestinal bleeding (melena, hematemesis, hematochezia if brisk bleeding)

Perforation (acute abdomen with abdominal distention, rebound, guarding, absent bowel sounds)

Penetration (intractable abdominal pain, pancreatitis, gastrocolic fistula)

Obstruction (nausea, vomiting, early satiety) 


\section{Management with Maternal-Fetal Consideration}

Antacids are preferred as the first line of therapy for PUD during gestation. Most antacids are considered safe in pregnancy, particularly those containing magnesium or aluminum, because they are not well absorbed systemically after oral ingestion. Recommended doses of antacids are 15 to $30 \mathrm{~mL} 1$ hour after meals and at bedtime. Iron malabsorption may result from antacids, and because iron deficiency is common during pregnancy, antacids should be administered at a different time than iron supplements to avoid this possible drug complication (36).

Sucralfate is FDA pregnancy category B and is a good alternative to antacids in pregnancy. It is a preferred drug for PUD during pregnancy because it is not absorbed systemically and has not been shown to be teratogenic in animal studies (12).

H2RAs are highly effective in the treatment of PUD. About $80 \%$ of duodenal ulcers in the general population heal after treatment with H2RAs (37). All H2RAs including cimetidine, ranitidine, famotidine, and nizatidine are classified as FDA pregnancy category B. A recent meta-analysis revealed that H2RAs are not associated with an increased risk of congenital malformations (38). Use of H2RAs in pregnancy is recommended for refractory PUD, which does not respond to antacids or sucralfate, and for complicated ulcer disease (e.g., gastrointestinal bleeding, obstruction). Ranitidine is the preferred H2RA because of a better documented safety profile in humans.

PPIs effectively suppress gastric acid secretion by inhibiting the $\mathrm{H}^{+} / \mathrm{K}^{+}$ATPase on the surface of the parietal cell. PPIs are highly effective in the treatment of PUD. Omeprazole is rated as category $\mathrm{C}$ during pregnancy because of embryotoxicity without teratogenicity in animal studies (19). All other PPIs, including lansoprazole, rabeprazole, pantoprazole, and esomeprazole, are classified as FDA pregnancy category B drugs. Lansoprazole is the preferred PPI during pregnancy because of lack of clinical data regarding the safety of newer PPIs. Treatment with PPIs during pregnancy should be reserved only for patients with refractory or complicated PUD, which does not respond to alternative medical therapies, including H2RAs.

\section{Eradication of $\boldsymbol{H}$. pylori Infection}

Treatment of $H$. pylori infection is recommended in the general population because the risk of ulcer recurrence is lower after $H$. pylori eradication (39). Triple drug regimen consisting of antibiotics and PPI therapy is usually used for treatment of H. pylori infection. The recommended antibiotics for eradication of $H$. pylori infection during pregnancy include amoxicillin (FDA category B), clarithromycin (FDA category C), and metronidazole (FDA category B). However, administration of antibiotics for treatment of $H$. pylori infection should be avoided during pregnancy. Therapy is usually deferred until postpartum because of low risk of complications from untreated $H$. pylori infection during pregnancy and to avoid potential fetal risk from exposure to antibiotics.

\section{HYPEREMESIS GRAVIDARUM}

\section{Definition}

Hyperemesis gravidarum ( $\mathrm{HG}$ ) is a severe and disabling condition with potentially life-threatening complications. One of the most widely quoted definitions of $\mathrm{HG}$ is that proposed by Fairweather (40) in 1968, who described this syndrome as "vomiting occurring in pregnancy, appearing for the first time before the twentieth week of gestation and of such severity as to require the patient's admission to the hospital, the vomiting being unassociated with such coincidental conditions as appendicitis, pyelitis, etc." HG is defined as nausea and vomiting in pregnancy, typically in the first trimester, resulting in dehydration, weight loss, and electrolyte imbalances severe enough to interfere with the nutritional and metabolic status of the patient and requiring admission.

\section{Epidemiology}

Nausea or vomiting is a common symptom occurring in up to $80 \%$ of women during pregnancy (41). HG is a much rarer disorder, affecting only $0.3 \%$ to $1.5 \%$ of pregnancies (42). HG is the most common cause of hospitalization during the first half of pregnancy. There is an increased risk of recurrence, with the risk of $\mathrm{HG}$ about $15 \%$ in a woman with a previous history of HG during pregnancy, compared with $0.7 \%$ with no prior history of HG (43). Several epidemiologic risk factors have been identified (Table 4).

\section{Pathophysiology}

Although nausea and vomiting in pregnancy and hyperemesis have been studied for many years, the etiology of these disorders remains obscure. A variety of pathophysiologic mechanisms for HG have been proposed. Etiology of HG is probably multifactorial, with both physiologic and psychologic components.

\section{Hormonal}

Because of its presentation early in the first trimester, a hormonal etiology has been suggested. Serum and urinary human chorionic gonadotropin (hCG) levels have been compared in emetic, hyperemetic, and non-emetic pregnancies; the results of these studies are inconsistent. Some groups have found no correlation between hCG levels and nausea and vomiting $(44,45)$, whereas others report higher levels in emetic vs. non-emetic patients (46). However, increased free beta subunit of hCG has been found in the sera of hyperemetic patients compared with normal pregnant controls (47).

Analyses of serum estradiol levels also conflict. Depue and coworkers (44) found that estradiol levels and sex hormone binding globulin capacity were elevated in hyperemetic patients in the first trimester. Others did not show a correlation between

Table 4 Epidemiologic Risk Factors and Associated Conditions in Hyperemesis Gravidarum (HG)

HG is more common in Western countries

Ethnicity: Higher incidence in Asians and Africans

Young age ( $<20$ years): HG is more common in younger maternal age

Family history of HG: Increased likelihood of HG

Parity and multiple gestations: HG is more common in nulliparous and multiple pregnancy

Body mass index (BMI): Low prepregnancy BMI is associated with HG

Coexisting medical conditions: HG is associated with molar pregnancy, hyperthyroidism, psychiatric illness, diabetes, and asthma

Prior history of HG: 15\% recurrence risk 
estradiol levels and nausea and vomiting, but these analyses did not include many hyperemetic patients (46).

There is a strong association between HG and abnormal thyroid function tests. Transient hyperthyroidism is frequently observed in hyperemetic patients (48). In one series, $73 \%$ of hyperemetic pregnant patients showed an increased free thyroxine (T4) index (48). Hyperthyroxinemic patients demonstrated tachycardia and weight loss but no other symptoms of Graves' disease. The hyperthyroid state tends to be short-lived, resolving by the third trimester, and usually does not require treatment. The mechanisms underlying transient hyperthyroidism in patients with hyperemesis are unknown. hCG has been implicated due to the structural similarity between hCG and thyroid-stimulating hormone (TSH), which may lead to hCG stimulating thyroid activity. Thyroxine and TSH levels in early pregnancy have been correlated with the severity of HG symptoms and electrolyte abnormalities (49).

\section{Gastric Dysmotility}

Decreased gastric motility is found to cause nausea. Measurement of gastric myoelectric activity in women with and without nausea in pregnancy revealed a much higher incidence of abnormal electrical patterns in nauseated patients compared with non-nauseated pregnant women (50); the physiologic significance of these findings is unclear.

\section{Psychogenic}

Psychologic factors have been considered for many years in the pathogenesis of hyperemesis. Analyses of the maternal relationships of pregnant hyperemetic women have revealed a variety of disturbances including over-strong maternal attachments (51) or negative maternal relationships (52). Persistence of hyperemesis into the third trimester was significantly associated with psychiatric symptoms (52).

\section{Clinical Signs and Symptoms}

Although vomiting may begin before the first missed menstrual period, persistent vomiting requiring hospitalization usually peaks between 8 and 12 weeks of gestation $(40,51)$. Majority of the symptoms resolve by 20 weeks. Patients usually describe typical "morning sickness," which then becomes more severe and persists throughout the day. Intractable vomiting may lead to development of complications including dehydration and oliguria. Weight loss of more than $5 \%$ of prepregnancy weight is usually one of the diagnostic criteria. Physical examination may reveal signs of dehydration with poor skin turgor and tachycardia. Jaundice is occasionally present and the odor of ketones may be noted in the patient's breath. Neurologic symptoms include changes in mental status ranging from drowsiness to coma, and evidence of peripheral neuropathy due to vitamin $\mathrm{B}_{6}$ or $\mathrm{B}_{12}$ deficiency.

\section{Diagnosis}

The diagnosis of HG is based on the presence of symptoms of intractable nausea and vomiting, and any of the several indicators of severity including weight loss of more than 5\%, ketonuria, signs of dehydration, and serum electrolyte imbalances. Evidence of major organ dysfunction may be present in more severe cases, including neurologic symptoms (Wernicke's encephalopathy (53) and peripheral neuropathy (54)), renal failure, liver dysfunction, or retinal hemorrhage.

Elevated levels of blood urea nitrogen and creatinine, abnormal thyroid and liver function tests, ketosis and ketonuria, hyponatremia, hypochloremia, and hypokalemia are common laboratory abnormalities. HG is a diagnosis of exclusion. It is important to exclude other causes of nausea and vomiting such as pyelonephritis, gastroenteritis, and acute intra-abdominal conditions including pancreatitis, appendicitis, and cholecystitis (51).

\section{Management}

Treatment for HG is supportive because the condition is mostly self-limiting. The principles of management of HG include control of nausea and vomiting, correction of dehydration and electrolyte abnormalities, psychologic support, and prevention and treatment of complications.

\section{Control of Nausea and Vomiting}

Because of reluctance to use medications, especially in the first trimester, patients with uncomplicated nausea and vomiting are generally advised lifestyle modifications including small meals and avoid ingesting large amounts of liquid; specific therapy is usually not required. By contrast, the management of HG is challenging and often frustrating. Recurrent admissions are common and occur more often in nulliparous women (55).

Antiemetics are commonly used for the management of nausea and vomiting during pregnancy, but there are little data regarding their safety and efficacy in HG (Table 5). Antihistamines and phenothiazines are considered the most effective antiemetic medications. In general, antihistamines are considered to have the lowest teratogenic risk. A metaanalysis showed no evidence of teratogenicity or other serious maternal or fetal adverse outcomes associated with the use of antihistamines for nausea and vomiting during pregnancy (56). Phenothiazines include prochlorperazine and chlorpromazine. Isolated case reports have reported cleft palate, skeletal, limb, and cardiac abnormalities in association with

Table 5 Common Antiemetics Used in Nausea and Vomiting of Pregnancy

\begin{tabular}{|c|c|}
\hline Drug class & Dosage \\
\hline \multicolumn{2}{|l|}{ Antihistamines } \\
\hline Promethazine & $\begin{array}{l}12.5-25 \mathrm{mg} \text { orally, i.m., or rectally } \\
\text { every } 4 \text { hours }\end{array}$ \\
\hline Dimenhydrinate & $\begin{array}{l}50-100 \mathrm{mg} \text { orally every } 4-6 \text { hours or } \\
50 \mathrm{mg} \text { i.m. or i.v. every } 4-6 \text { hours } \\
\text { (total dose should not exceed } \\
400 \mathrm{mg} / \text { day, } 200 \mathrm{mg} / \text { day if also } \\
\text { taking doxylamine) }\end{array}$ \\
\hline Doxylamine & $12.5 \mathrm{mg}$ orally three to four times daily \\
\hline \multicolumn{2}{|l|}{ Dopamine antagonists } \\
\hline Prochlorperazine & $\begin{array}{l}\text { 5-10 mg orally or i.v. every } 6 \text { hours } \\
\text { (Max dose } 40 \mathrm{mg} / \text { day) }\end{array}$ \\
\hline Metoclopramide & $\begin{array}{l}5-10 \mathrm{mg} \text { orally, i.m., or i.v. every } \\
8 \text { hours }\end{array}$ \\
\hline \multicolumn{2}{|l|}{$5 H T 3$ antagonist } \\
\hline Ondansetron & $4-8 \mathrm{mg}$ orally or i.v. every 8 hours \\
\hline
\end{tabular}


the use of phenothiazines; however, there is no evidence of adverse fetal effects in clinical studies (57). No adverse fetal outcomes have been found with the use of metoclopramide in animals or humans (57). Recently 5HT3 antagonists (ondansetron) have been shown to have efficacy for nausea and vomiting during pregnancy, and their use seems to be increasing (58). No adverse effects in animal studies and no reported increase in birth defects have been found with the use of ondansetron during pregnancy (58).

\section{Correction of Dehydration and Electrolyte Abnormalities}

Initial therapy should focus on repletion of fluids, usually by an intravenous route, and resolution of electrolyte imbalances. Chronic or severe hyponatremia must be corrected slowly and cautiously to avoid the complication of cerebral pontine myelinolysis $(54,59)$. An increase in serum sodium of no more than $12 \mathrm{mmol} / \mathrm{L} /$ day is recommended.

Repletion of vitamin deficiencies including thiamine is critical since prolonged vomiting may lead to severe depletion. Thiamine deficiency resulting in Wernicke's encephalopathy should be considered in pregnant patients with mental status changes and other neurologic symptoms. All women with HG symptoms should be prescribed thiamine supplementation (thiamine $50 \mathrm{mg}$ orally or $100 \mathrm{mg}$ intravenously daily) if their symptoms are prolonged for 3 weeks or more. Women with HG should take folic acid $5 \mathrm{mg}$ daily, once oral intake has resumed because of deficiency induced by vomiting.

When nausea and vomiting have resolved after treatment with intravenous fluids, oral hydration is begun slowly and diet is advanced from liquid to solid as tolerated $(51,60)$. The cessation of vomiting and commencement of normal weight gain are the therapeutic endpoints to be achieved. In severe cases, in-hospital and even home-based parenteral hyperalimentation can be used if nutrition cannot be maintained orally (61).

\section{Psychologic Support}

Psychologic support is offered by nursing staff and physicians depending upon the needs of the patient. Intervention by a psychiatrist is recommended if there is evidence of an obvious psychiatric disorder (51) or occasionally for patients with third-trimester vomiting, which may indicate more serious psychologic problems (52).

\section{Management of Complications}

In the past, HG was regarded as a dreaded complication of pregnancy. Maternal mortality was high and primarily caused by severe metabolic disturbances. Improvements in fluid and electrolyte management have dramatically reduced the mortality rate of HG to virtually zero (51). Although uncomplicated nausea and vomiting in pregnancy may be an indicator of a favorable fetal outcome (62), the effects of hyperemesis on the fetus are unclear. A study of hyperemetic pregnancies associated with abnormal thyroid function indicated a higher than expected rate of premature labor and an increased incidence of low birth weight (49). Whether these effects were due to hyperemesis or hyperthyroxinemia is unclear. Analyses of the birth weights of babies born to hyperemetic mothers have been confounded by a varied patient population. Fairweather (40) found no change in birth weight when he reviewed birth records of a large group of women with hyperemesis. However, others $(62,63)$ have subclassified hyperemetic patients into mild versus severe disease based on maternal weight loss, heavy ketonuria, elevated urea nitrogen or creatinine, and electrolyte disturbances; those with severe hyperemesis gave birth to babies with significantly lower weights. Also more growth-retarded babies were found in the groups of mothers with hyperemesis and weight loss, or hyperemesis requiring multiple admissions (55). The risk of congenital anomalies may also be increased. One study suggested an increase in minor integumentary defects including webbed toes and an extra finger, and skin tags. However, the number of patients was small and the possible influence of anti-emetic medication was not clarified.

\section{INFLAMMATORY BOWEL DISEASE}

The inflammatory bowel diseases (IBDs) include two disorders, Crohn's disease (CD) and ulcerative colitis (UC). Both illnesses are characterized by inflammation of the gastrointestinal tract. Clinical manifestations commonly include diarrhea and abdominal pain, and patients may suffer multiple exacerbations and remissions. In $\mathrm{CD}$, a transmural inflammatory process occurs, which can affect any part of the gastrointestinal tract from mouth to anus. On the other hand, the inflammatory process of UC is mucosal and is limited to the colon and rectum. IBD commonly affects women during the childbearing years; therefore, problems in the management of these disorders during pregnancy are frequently encountered by both the obstetrician and the gastroenterologist. In this section, the effect of IBD on fertility and pregnancy outcome, and the impact that pregnancy has on the course of IBD are discussed, and an approach to the management of these disorders in pregnancy is presented.

\section{Epidemiology}

The incidence of $\mathrm{CD}$ is now approximately equivalent to $\mathrm{UC}$ in North America and Europe. In North America, incidence rates range from 2.2 to 14.3 cases per 100,000 person years for UC and 3.1 to 14.6 cases per 100,000 person years for CD (64). Combined prevalence of CD and UC is approximately 100 per 100,000 population. The incidence of IBD has a bimodal distribution, with the peak age of onset between 15 and 30 years of age (65). A second peak in the incidence of IBD occurs between the ages of 50 and 80 . There is marked geographic variation in the incidence of IBD. IBD is more common in developed nations compared with countries with poor sanitation. The regions with the highest incidence of UC include the United States, Northern Europe, and Israel. There is no gender specificity; however, within the United States, there is racial and ethnic variation. Incidence rates are increased in Jews and in white versus non-white populations, although the incidence in black populations may in fact be increasing (66).

\section{Etiology}

The etiology of IBD is at present unknown, but is thought to be multifactorial (67). A number of infectious agents have been postulated to be pathogenic. A bacterium similar to Mycobacterium paratuberculosis was isolated from the intestine of patients who underwent surgical resection for active $C D$, yet in the majority of patients, this organism cannot be cultured. Other potential agents involved in eliciting an immune 
response have been postulated to include cow's milk proteins, other foods, water, viruses, and smoking, yet none has been definitively linked to either disease. Intestinal permeability has been shown to be altered in $\mathrm{CD}$; a "leaky bowel" may allow translocation of antigens that elicit an immune response. Although the antigen that triggers the gut's immunologic response is unknown, there is active research directed at understanding the intestinal mucosal immune system in normal and affected individuals. The contribution of autoimmunity, cytokines, mucosal $\mathrm{B}$ and $\mathrm{T}$ cells, as well as neutrophil function is being actively examined in IBD.

Genetic factors play an important role as indicated by family studies. First-degree relatives of patients with IBD are approximately 3 to 20 times more likely to develop the disease than the general population (68). In one report, children in whom both parents had IBD (CD or UC) had up to a $33 \%$ chance of developing IBD by age 28 (68). Stronger familial association has been shown for CD than for UC. Twin studies showing disease concordance in monozygotic but not dizygotic twins further strengthen the evidence that genetic influences play a role in the pathogenesis of CD (67). In a study that included 80 twins with IBD, the concordance rate for monozygotic twins was markedly higher in $\mathrm{CD}$ than UC (58\% vs. $6 \%)(69)$.

The inflammatory process of UC is confined to the colon. This disease may affect the rectum alone, the rectum plus the left colon, or it may be pancolonic. By contrast, in CD, inflammation can be found anywhere throughout the gastrointestinal tract, from mouth to anus. The commonest sites of involvement are small bowel alone (predominantly distal ileum) $(30 \%)$, colon alone (15-25\%), or ileocolon (40-55\%). Perianal and perirectal disease is commonly encountered, manifested by fissures, fistulae, and abscesses.

In UC, mucosal lesions predominate; the muscularis propria is rarely involved. Crypt abscesses, in which collections of neutrophils invade crypt epithelium and may even be present in the crypt lumen, are characteristic of UC, yet may also be seen in CD. Evidence of chronic inflammation is also apparent with lymphocytic, plasma cell, and eosinophilic involvement of the lamina propria. Marked edema, hemorrhage, and pseudopolyps are often found in active UC. By contrast, the presence of granulomas and transmural inflammation with deep ulcerations that extend to the muscularis propria are more characteristic of CD. "Skip areas" (intervening normal mucosa) or rectal sparing are consistent with CD.

\section{Clinical Signs and Symptoms}

There are both similarities and differences in the presentation of UC and CD. Patients afflicted with either disorder may present with abdominal pain, diarrhea, weight loss, fevers, and malaise. Multiple extraintestinal manifestations accompany both illnesses.

\section{$U C$}

The inflammatory process characteristic of UC is confined to the colon. Some patients initially present with inflammation confined to the rectum (ulcerative proctitis); the disease may progress during subsequent exacerbations to other areas of the colon or may remain in the rectum. Other patients suffer from more extensive colitis and may have disease up to and including the cecum. Due to rectal involvement, patients often complain of urgency and frequency of bowel movements as well as tenesmus. Rectal bleeding and diarrhea are the hallmarks of this disease. Abdominal pain is usually mild but may be severe in fulminant colitis. In mild cases, physical examination may be unremarkable, whereas patients with more active disease develop fevers, tachycardia, diffuse abdominal tenderness, and occasionally distention. A serious complication of acute UC, which may be heralded by the appearance of abdominal distention, is toxic megacolon. Massive dilatation of the inflamed colon may progress to perforation; this is a surgical emergency and requires immediate colectomy. A significant long-term complication of UC is the development of colon cancer. Patients are at an increased risk for carcinoma after 8 to 10 years of disease and are screened with yearly colonoscopy.

\section{$C D$}

$\mathrm{CD}$ is a chronic indolent disorder characterized by multiple remissions and exacerbations. Patients commonly present with abdominal pain, diarrhea, weight loss, anal pain, and drainage. On physical examination, abdominal tenderness and inflammatory masses particularly in the right lower quadrant and pelvis can be palpated. Because the inflammatory lesion is transmural, perianal fissures, internal fistulae between the bowel and skin, bladder, or vagina, as well as free perforation with abscess formation are common complications.

\section{Extraintestinal Manifestations}

Both UC and CD are associated with a wide variety of extraintestinal manifestations. The "colitis-related" manifestations include skin, eye, and joint diseases, which present in parallel with recrudescent disease activity. Skin manifestations such as erythema nodosum and pyoderma gangrenosum, and oral lesions including diffuse aphthous ulcerations may be severe enough to require treatment with corticosteroids. Arthritic complaints include ankylosing spondylitis, sacroiliitis, and peripheral large joint arthritis. Ocular diseases such as uveitis, iritis, and episcleritis may be present in approximately $15 \%$ to $20 \%$ of patients and may be the initial symptom that prompts a visit to a physician. Nonspecific complications of CD include a variety of liver diseases such as fatty liver, pericholangitis, primary sclerosing cholangitis, hepatic granulomas, and abscesses. In patients who have had extensive small bowel resection, malabsorption may result in deficiency of vitamin $\mathrm{D}$ and calcium, leading to osteoporosis. Increased absorption of oxalate from the gut lumen may lead to enhanced urinary excretion and the development of oxalate stones.

\section{Diagnosis in the Nonpregnant Patient}

In the nonpregnant patient, endoscopy has a pivotal role in establishing a diagnosis of IBD. The presence and extent of disease tend to be underestimated when radiographic studies are compared with direct endoscopic visualization, since subtle mucosal abnormalities can only be detected with endoscopy. The addition of biopsy adds considerably to the sensitivity and specificity of endoscopy in the diagnosis of IBD. Radiographic studies are now considered an adjunctive rather than a primary diagnostic tool. Small bowel barium radiograms may demonstrate terminal ileal narrowing, strictures, and enteric fistulae. 
This may be useful in the evaluation of stricturing or fistulizing $\mathrm{CD}$, and if patients are unable to undergo colonoscopy due to medical comorbidities. However, other imaging modalities such as small bowel wireless capsule endoscopy, CT, or MRI have supplanted the barium radiographic studies for evaluation of small bowel involvement in CD.

Endoscopic evaluation including upper endoscopy (for patients who have upper abdominal symptoms suggestive of $\mathrm{CD}$ ), flexible sigmoidoscopy, and colonoscopy provides direct visualization of the gut's mucosa. Characteristic findings of ulcerative and Crohn's colitis include erythema, edema, granularity, ulceration, and friability of the colonic epithelium. Strictures may result from chronic inflammation. However, it is important to emphasize that these findings, while consistent with IBD, are not diagnostic, since they may also be found in a variety of infectious and other colitides. Abnormalities such as aphthous ulcerations and erythema may also be found in the upper gastrointestinal tract in $\mathrm{CD}$. Biopsies taken during endoscopy may show the presence of granulomas or crypt abscesses and may help confirm the clinical impression.

\section{Diagnosis in Pregnancy}

There are limited data regarding the safety of endoscopic procedures during pregnancy. In those patients with undiagnosed abdominal pain, diarrhea, or rectal bleeding in whom IBD is suspected, flexible sigmoidoscopy under either minimal or no sedation appears to be safe (70). Complete colonoscopy usually requires a greater degree of sedation and is avoided when possible, although it is safe with appropriate monitoring of vital signs and oxygenation. In the pregnant patient, all efforts should of course be made to avoid fetal exposure to radiation, and radiologic procedures are not recommended. However, in emergent situations (when bowel perforation, abscess, or toxic megacolon are being considered), radiologic examinations may be necessary; there is some evidence to suggest that a very low dose of irradiation may have no long-term harmful effects (71).

Stool studies for routine bacterial pathogens (including Clostridium difficile) are extremely important in the initial evaluation of a patient in whom IBD is suspected. Infectious colitides such as those caused by salmonella, shigella, yersinia, Campylobacter fetus, Giardia lamblia, and amebae may present with an identical picture and demonstrate similar endoscopic findings. In patients infected with human immunodeficiency virus, organisms such as Mycobacterium avium-intracellulare, cryptosporidiosis, isospora, and cytomegalovirus can be ruled out with stool cultures and histologic examination of biopsies.

\section{Management with Maternal-Fetal Consideration}

Critical issues in the management of IBD during pregnancy include (i) the effects of pregnancy on the course of IBD and (ii) the impact of IBD and its therapy on fertility, pregnancy, and fetal outcome.

\section{Effects of Pregnancy on the Course of IBD}

Early studies in the 1950s and 1960s indicated that pregnancy had an adverse effect on the course of UC, yet more recent studies dispute these findings. Part of the reason for this discrepancy is the realization that the spontaneous risk of exacerbation of UC in the nonpregnant patient is similar to the rate of relapse reported for pregnant patients with this illness. Pregnant women with IBD are as likely to flare (34\% per year) as nonpregnant women with UC (32\% per year) (72). With the advent of more effective medical therapy for UC, it has become apparent that pregnancy does not adversely affect the course of this disease. Those patients with quiescent disease will usually remain inactive $(73,74)$, whereas those with active colitis will demonstrate persistent disease or worsen during pregnancy (75).

Patients with quiescent $\mathrm{CD}$ are probably not at increased risk for precipitating active disease when pregnant $(76,77)$. Again, in those patients who are already active, approximately two out of three will continue to be ill or will worsen during pregnancy $(75,77,78)$. Therefore, patients with IBD are usually counseled to avoid pregnancy when in a phase of moderate to severe disease activity.

\section{Effects of IBD on Fertility, Pregnancy, and Fetal Outcome}

Patients with UC demonstrate fertility rates that are comparable with those of the general population $(76,78)$, although there are few case-controlled studies. In considering CD, there are conflicting reports; some groups have shown that as many as $50 \%$ of women of child-bearing age are subfertile, correlating with disease activity, whereas others have shown no difference in fertility rates when compared with the general population (76,79-81). Many studies did not account for disease activity, the patient's desire to become pregnant, or other risk factors that could decrease fertility. In fact, a recent report revealed that fertility may not be impaired in women who wished to conceive; there was a voluntary decrease in the number of pregnancies, arising from a desire to avoid pregnancy because of illness (79). Men with IBD who take sulfasalazine may show diminished fertility due to direct toxic effect on sperms (82).

The risk of spontaneous abortion in patients with quiescent $\mathrm{CD}$ or UC is probably no higher than that in the general population (75), but in several studies it has been shown to be increased as much as twofold in those patients with active disease $(72,83,84)$. Reports indicate that IBD predisposes patients to a significant risk of preterm delivery (79), as defined by birth before 37 weeks of gestation. Therefore, vigilant monitoring of patients in the third trimester is recommended. Fortunately, once pregnancy occurs, the likelihood of a normal fetal outcome is identical to that in the general population. In almost all studies, the incidence of small-for-gestational-age babies and congenital defects was not increased, even in those patients on multiple medications such as steroids and sulfasalazine.

\section{Management of IBD in Pregnancy}

To improve the likelihood of a favorable outcome, patients are generally encouraged to avoid pregnancy during periods of disease activity if this is at all possible. Numerous retrospective analyses have ascertained that patients with IBD can be successfully treated without adverse effect to the mother or fetus. Medications should be reduced to the minimal amount required. In reports in which low birth weight or congenital anomalies were detected, it was often difficult to ascertain whether the severity of the bowel disease or the medications used were the major contributor. 
Aminosalicylates are one of the cornerstones of medical therapy of IBD and are considered low risk for use in pregnancy. Most are pregnancy category B (sulfasalazine, mesalamine, and balsalazide) with the exception of olsalazine, which is category C. Sulfasalazine contains a sulfapyridine residue linked to 5-aminosalicylic acid (5-ASA), the active moiety. Its mechanism of action is unknown, but it has been shown to inhibit production of leukotriene $\mathrm{B}_{4}$, an important mediator of inflammation, block prostaglandin production, and inhibit immunoglobulin secretion by mononuclear cells. Sulfasalazine crosses the placenta (85) and is found in breast milk, although in low concentrations. Serum levels of sulfasalazine in newborns are the same as in the mother (86). This drug is thought to be safe during pregnancy (87). There are a few case reports of congenital defects in infants of treated patients; however, a population-based study did not find a significant increase in congenital abnormalities in children of women treated with sulfasalazine (88). Its efficacy in maintaining remission in UC makes its continuation during pregnancy an important consideration. Patients are usually tapered to the lowest dose that is effective in suppressing disease activity. Sulfasalazine has anti-folate effects, and it is recommended that women take folate supplementation of at least $2 \mathrm{mg}$ daily during the prenatal period and pregnancy.

Because of multiple side effects associated with the presumably inactive sulfapyridine moiety, both topical and oral agents that contain only 5-ASA have been developed. Topical therapy with 5-ASA (mesalamine) enemas is an effective treatment for acute proctitis or proctitis with leftsided colitis. In addition, several oral forms of 5-ASA $\left(\right.$ Pentasa $^{\circledR}$, Asacol $^{\circledR}$, and Dipentum ${ }^{\circledR}$ ) have been released for treatment of both CD and UC. These drugs contain 5-ASA in formulations that prevent their premature absorption from the gastrointestinal tract. A number of studies have established the safety of oral and topical aminosalicylates in pregnancy (89-91). A meta-analysis of mesalamine formulations used during pregnancy demonstrated no increased risk for congenital malformations, stillbirths, spontaneous abortions, preterm delivery, or low birth weight with the use of mesalamine (92). Thus sulfasalazine and 5-ASA drugs can be used during pregnancy as in nonpregnant women with IBD.

Corticosteroids, including budesonide, are pregnancy category C. Steroid therapy is frequently used in the treatment of moderate to severe and fulminant UC, and moderate to severe active CD. In severely ill, hospitalized patients, high doses of intravenous steroids are employed to control bowel inflammation. Corticosteroid enemas are important topical agents for the treatment of proctitis and left-sided colitis. The major side effects associated with long-term corticosteroid therapy (such as cataracts, osteoporosis, diabetes, and hypertension) make it imperative that patients be tapered off this medication as soon as possible. Unfortunately, some patients with IBD require long-term corticosteroid use to treat persistently active disease; in these patients, a trial of an immunosuppressive agent such as 6-mercaptopurine (6-MP) or azathioprine (AZA) may be used to facilitate steroid tapering.

Evidence suggests that steroids should be used with caution in the first trimester because of an association with an increased risk of oral clefts (93). A number of studies showed no adverse effects on fetal outcome in women who took corticosteroids during pregnancy $(94,95)$. A meta-analysis of five studies of oral corticosteroid use did not reveal a major teratogenic risk of major malformations in the offspring of exposed patients at therapeutic doses (95). A significant increase was noted in risk of oral clefts (OR 3.69; 95\% CI, 2.15-6.32); however, the increased risk appeared to be in women taking steroids for non-IBD diseases such as asthma. A case series of eight patients receiving budesonide during pregnancy demonstrated no increase in maternal adverse events (96). Corticosteroids also appear to be safe during breast-feeding (97). As in their nonpregnant counterparts, pregnant patients should be taken off steroids if at all possible, yet treatment during pregnancy can be safely initiated or continued as needed to control disease activity.

Immunosuppressive agents such as 6-MP and its prodrug AZA are primarily used to maintain remission in UC and CD. These agents are often used as steroid-sparing agents to facilitate discontinuation or dose reduction of steroids. 6-MP and AZA are pregnancy category D. Animal studies show teratogenicity with increased rates of cleft palate and skeletal anomalies (98). However, in these studies, animals were exposed to significantly higher doses than those used in humans. Limited data exist regarding the long-term adverse effects that may result from the use of these medications in IBD. However, regarding usage in pregnancy, there is a large body of data from the transplant population, which indicate that treatment with these agents is surprisingly well tolerated. The doses used for treatment of UC and $\mathrm{CD}$ are much lower than that used in transplant patients. Few studies also indicate their relative safety in pregnant IBD patients $(99,100)$. It is reasonable to continue these drugs during pregnancy in patients who cannot be managed with other alternative medications.

AZA and 6-MP are detectable at low levels in breast milk. 6MP excretion in breast milk occurs within the first 4 hours after intake (101). Mothers taking these medications should be discouraged from nursing; however, there is no absolute contraindication to breast-feeding. Lactating mothers are advised to wait 4 hours after dosing to feed.

Methotrexate is a pregnancy category $\mathrm{X}$ drug. Methotrexate is a folic acid antagonist and should not be used in women considering conception. It is contraindicated in pregnancy because of its mutagenic and teratogenic effects. Methotrexate is associated with multiple congenital anomalies including craniofacial malformations and central nervous system abnormalities, including anencephaly and mental retardation (102).

Antibiotics are frequently used in the treatment of $\mathrm{CD}$. Particularly, metronidazole is useful in the treatment of perianal complications such as fistulae or abscesses. Metronidazole is pregnancy category B. A number of studies have demonstrated that metronidazole is not associated with an increased risk of birth defects or cancer in humans $(103,104)$. However, long-term use in pregnancy remains controversial due to its teratogenic and carcinogenic potential. Breastfeeding should be withheld for 12 to 24 hours if a single dose of metronidazole is given. Long-term use of metronidazole should be avoided during breast-feeding due to potential toxic effects.

Biologic therapy has opened a new era in the treatment of IBD. All three biologic agents including infliximab, adalimumab, and certolizumab are pregnancy category B. These agents inhibit 
the activity of tumor necrosis factor and are used for induction and maintenance of remission in IBD patients. Data on the safety of infliximab in pregnancy are limited; however, no maternal toxicity, embryotoxicity, or teratogenicity to infliximab has been observed. The two largest studies are the Crohn's Therapy, Resource, Evaluation, and Assessment Tool (TREAT) registry and the Infliximab Safety Database $(105,106)$. The TREAT registry is a prospective, multicenter study of $\mathrm{CD}$ patients. Of more than 6200 patients enrolled, 117 of 168 pregnancies were exposed to infliximab. No fetal malformations were seen. Miscarriage and neonatal complication rates were not statistically significant between the infliximab-exposed and unexposed group (105). The Infliximab Safety Database is a retrospective database that records voluntary adverse event reports associated with infliximab use. The expected versus observed outcomes among women exposed to infliximab were reported no different than those of the general population (106). In a recent case series, infliximab remained undetectable in breast milk while maternal levels remained in the therapeutic range (107). Case reports have also described successful use of adalimumab in women with CD and pregnancy (108-110).

Special Considerations-Surgical Management, Pregnancy with Ileostomy, or Ileoanal Anastomosis

Despite advances in the medical management of IBD and improved therapy during pregnancy, surgical treatment is occasionally necessary. Emergent colectomy may be indicated for fulminant colitis unresponsive to medical therapy or for complications of UC such as toxic megacolon and colonic perforation. Morbidity and mortality rates for both fetus and mother are unknown; although there are scattered case studies of successful surgical intervention, fetal deaths have been reported.

Fertility and fecundity (rate of conception per menstrual cycle) are decreased after surgery for CD and colectomy with ileal pouch anal anastomosis for UC $(111,112)$. Ileostomy may predispose to intestinal obstruction, which may be difficult to recognize, since abdominal pain may be mistakenly attributed to uterine contractions. An increased frequency of nocturnal stooling was noted in patients with ileoanal pouches (113). Patients may undergo vaginal or cesarean delivery and fetal outcome seems unaffected by the presence of an ileostomy or ileoanal anastomosis.

\section{PANCREATITIS}

Acute inflammation of the pancreas, or pancreatitis, is rare in pregnancy, ranging in incidence from 1 case per 1000 to 1 in 10,000 pregnancies occurring usually late in the third trimester or early in the postpartum period (114-116). Acute pancreatitis can cause significant fetal and maternal morbidity and mortality including premature delivery, shock, sepsis, and multiorgan dysfunction syndrome. Management of this disorder in pregnancy is often complicated and requires careful monitoring of the mother and fetus.

\section{Etiology and Pathogenesis}

The etiology and pathogenesis of acute pancreatitis are similar to those in the nonpregnant population (Table 6). Cholelithiasis is the most frequent cause of acute pancreatitis during pregnancy accounting for more than $70 \%$ cases (117). Weight gain and hormonal changes during pregnancy predispose to

\section{Table 6 Etiologies of Pancreatitis}

Gallstones (most common)

Hypertriglyceridemia

Alcohol

Obstruction (tumors, strictures)

Drugs (diuretics, azathioprine, sulfonamides, metronidazole,

estrogens, valproic acid)

Trauma (blunt or penetrating abdominal injury, ERCP, surgery)

Viruses (mumps, hepatitis B, Coxsackie)

Metabolic (hypercalcemia, hyperparathyroidism)

Pre-eclampsia/eclampsia

Idiopathic (20\% cases)

gallstone formation (118). Cholesterol secretion in bile increases during pregnancy leading to a supersaturated bile. Changes in serum hormonal levels may also result in bile stasis, which in turn favor stone development. Acute pancreatitis may be precipitated when gallstones become impacted at the ampulla of Vater or pass into the duodenum initiating premature activation of intracinar trypsinogen to trypsin (119). Release of activated digestive enzymes into the pancreatic parenchyma may result in autodigestion and injury.

Hypertriglyceridemia (serum triglyceride levels $>1000 \mathrm{mg} / \mathrm{dL}$ ) is the second most common cause of acute pancreatitis during pregnancy. Hyperlipidemic pancreatitis accounts for $4 \%$ to $6 \%$ of cases with the majority of cases being associated with either type I or V familial hyperlipoproteinemia (117). During pregnancy, triglyceride levels elevate due to increase in estrogen-induced synthesis (120). In women with familial hyperlipidemias, further elevation of serum lipid levels during pregnancy leads to an even higher risk of pancreatitis. In patients with hypertriglyceridemia, it is postulated that pancreatic lipase becomes activated and releases fatty acids into the pancreas, producing inflammation, or that vascular obstruction results from blockage of vessels by fat globules (121).

Alcohol causes pancreatitis probably due to a direct toxic effect. Patients who have pancreatic duct obstruction from strictures, tumors, or other lesions may develop pancreatitis because of backflow of pancreatic juices into the gland, although the mechanism of enzyme activation in the pancreas is unclear. Pre-eclampsia or eclampsia is a rare cause of pancreatitis with its microvascular abnormalities that may affect the hepatic, splanchnic, renal, and placental circulation (117).

\section{Clinical Signs and Symptoms}

Acute pancreatitis can be divided into mild and severe or necrotizing pancreatitis. The presenting signs and symptoms of pancreatitis in pregnant patients are similar to those in the nonpregnant population. Characteristic symptoms include acute abdominal pain radiating to the back and associated with nausea and vomiting. Abdominal pain may worsen while lying flat or improve after assuming a forward or fetal position. Obstruction of the common bile duct as it courses through the head of the pancreas can lead to jaundice. Fever, pleuritic pain, and shock may result from peripancreatic fluid collection and pancreatic parenchymal hemorrhage. Physical examination may reveal epigastric tenderness, guarding and rebound, decreased, or absent bowel sounds, ecchymosis of the flank (Grey-Turner's sign) or periumbilical region (Cullen's sign) and ascites. Several other diseases such as duodenal ulcer 
perforation, cholecystitis, hepatitis, bowel obstruction, diabetic ketoacidosis, and pre-eclampsia may share a similar presentation (122).

\section{Diagnosis}

Typical symptoms of severe acute epigastric pain radiating to the back, nausea, and vomiting in conjunction with elevated serum amylase and/or lipase levels strongly suggest the diagnosis of pancreatitis. Pregnancy does not affect serum amylase or lipase levels; therefore, marked elevations in amylase and lipase levels are reliable markers of acute pancreatitis during pregnancy (117). However, there are many other pathologic states in which these enzymes may be elevated, since organs other than the pancreas synthesize and release amylase. Bowel ischemia or obstruction, perforated viscus, ovarian/tubal diseases such as salpingitis or ruptured ectopic pregnancy, cholecystitis, and a variety of salivary gland and pulmonary diseases (mumps, pneumonia) may also lead to elevations in serum amylase and lipase. Elevation of serum alanine aminotransferase to more than three times the upper limit of normal is a high sensitivity for biliary pancreatitis (123).

The severity of pancreatitis can be predicted to a certain extent by using Ranson's criteria, a series of biochemical parameters (124). Poor prognostic signs include (at presentation) age greater than 55 years, white blood cell count greater than $16,000 / \mathrm{mm}^{3}$, glucose concentration greater than $200 \mathrm{mg} / \mathrm{dL}$, lactate dehydrogenase level greater than $350 \mathrm{IU} / \mathrm{L}$, and serum glutamic-oxaloacetic transaminase level greater than 250IU/L. Within 48 hours of presentation, a decrease in hematocrit of more than $10 \%$, a drop in serum $\mathrm{Ca}^{++}$level below $8 \mathrm{mg} / \mathrm{dL}$, arterial oxygen pressure less than $60 \mathrm{mmHg}$, base deficit greater than $4 \mathrm{mEq} / \mathrm{L}$, rise in blood urea nitrogen level of greater than $5 \mathrm{mg} / \mathrm{dL}$, or fluid sequestration of more than 6liters may also indicate more severe disease.

Ultrasonography, without fetal radiation, is the initial test of choice to detect gallstones with a high accuracy during pregnancy. However, if common bile duct stones or biliary sludge are suspected based on ultrasound examination or biochemical abnormalities, a more invasive test may be required such as endoscopic ultrasonography (EUS) or magnetic resonance cholangiopancreatography (MRCP), which can provide an adequate view of the bilio-pancreatic duct anatomy. EUS and MCRP do not involve radiation and are often the tests of choice for detecting CBD stones. Computer tomography (CT) scan is not recommended during pregnancy as it carries a small radiation risk to the fetus. Endoscopic retrograde cholangiopancreatography (ERCP) is no longer widely used as a diagnostic study due to its postprocedure pancreatitis risk, radiation exposure, and the availability of newer and safer techniques such as EUS and MRCP (119). Performing diagnostic EUS or MRCP helps to identify patients who require therapeutic ERCP.

\section{Complications}

\section{Maternal Complications}

Patients with pancreatitis may develop hypovolemia and shock due to fluid sequestration and prolonged nausea and vomiting. Hypocalcemia may occur, possibly owing to entrapment of calcium in necrotic pancreatic tissue. Other metabolic abnormalities include hypochloremic alkalosis resulting from vomiting. Severe pancreatitis may result in disseminated intravascular coagulation and adult respiratory distress syndrome.

Pancreatic pseudocyst is a collection of pancreatic fluid within a fibrous cavity; its presence may be heralded by persistent abdominal pain or chronically elevated amylase levels. Infected pancreatic necrosis must be considered if patient develops fever and leukocytosis. A CT scan or ultrasonogram (the latter is preferable in the pregnant patient) may reveal the presence of fluid and necrosis in the pancreas, but cannot determine whether or not there is concomitant infection. The diagnosis of infected pancreatic necrosis is therefore usually made on clinical grounds. Hemorrhagic pancreatitis due to necrosis and blood vessel rupture may lead to massive bleeding. Pancreatic ascites results from rupture of pancreatic ducts with leakage of amylase-rich fluid into the peritoneum.

Over the years, the maternal mortality rate associated with pancreatitis has significantly decreased. Older reviews reported a very high mortality rate of up to $37 \%$ (125); however, more recent studies have shown no maternal mortality attributed to acute pancreatitis $(126,127)$. The improvement in the outcome of pregnant patients with pancreatitis is mainly due to earlier diagnosis with better imaging and less invasive therapeutic options including ERCP and laparoscopic cholecystectomy.

\section{Fetal Complications}

Premature labor is the main cause of adverse fetal outcomes in pancreatitis during late pregnancy (116). The rates of preterm delivery ranged between $15 \%$ and $19 \%$ (128). Fetal growth restriction can occur but may in part be avoided by the use of total parenteral nutrition (TPN). Fetal loss rate of $4.7 \%$ has also been reported (119).

\section{Management with Maternal-Fetal Consideration}

The treatment of acute pancreatitis in pregnancy follows the same guidelines as in the nonpregnant patient. The management is primarily supportive and aimed at correcting any underlying predisposing factors. Close observation is imperative, keeping in mind that multiple organ systems may be affected. The treatment of gallstone and hyperlipidemic pancreatitis in pregnancy deserves special consideration and is discussed separately.

\section{General Measures}

The general management of uncomplicated acute pancreatitis is mainly supportive including bowel rest, fluid resuscitation, correction of electrolyte and metabolic abnormalities, and pain control. Volume depletion is frequently encountered due to vomiting and fluid sequestration into the retroperitoneum. Aggressive intravenous fluid replacement is necessary to prevent pancreatic necrosis and systemic complications. Patients are given nothing by mouth for bowel rest. Fluid and electrolyte balance is closely observed; in particular, correction of hypocalcemia and hypomagnesemia may be necessary. Abdominal pain is a prominent feature in acute pancreatitis and may be severe requiring analgesics. Intravenous opiates may be used cautiously for pain control during pregnancy. Meperidine and fentanyl are the preferred analgesics during pregnancy (129). 
Prophylactic antibiotics are not routinely recommended in uncomplicated pancreatitis if there is no evidence of infection (130). Although therapeutic delivery has been considered in the past, recent literature suggests that termination of pregnancy does not affect the outcome of acute pancreatitis and is not recommended (131).

\section{Nutrition}

In mild pancreatitis, oral intake should be initiated when pain subsides and advanced as tolerated. Patients with a prolonged course due to severe pancreatitis or its complications may be unable to eat even after several days of hospitalization. To ensure adequate fetal growth and maintenance of maternal nutrition, TPN can be instituted (132-134). Most of the reported complications of TPN in pregnancy are the same as those found in the nonpregnant patients and are mainly catheter related, including sepsis and thrombosis. The rate of maternal complications such as septic complications secondary to centrally inserted central venous catheters for TPN is greater than that in the nonpregnant patients. Thus, peripherally inserted central venous catheters may be preferable for parenteral nutrition during pregnancy $(119,129)$. There are no reports of adverse fetal outcomes related to TPN in pregnant patients. Enteral nutrition (EN) via nasojejunal feeding is preferable compared with TPN (129). EN has been shown to be associated with fewer infectious complications, shorter hospital stay, and reduced surgical interventions in patients with acute pancreatitis (135). EN helps to maintain intestinal barrier and prevents bacterial translocation. Monitoring fetal growth with serial ultrasonograms is generally recommended (132) to ensure that fetal nutritional demands are met.

\section{Management of Complications of Acute Pancreatitis}

Patients with pancreatic pseudocysts should be monitored with serial ultrasonograms to document resolution of the cysts. Patients should be closely monitored for new symptoms such as abdominal pain, fever, chills, gastric outlet obstruction, or obstructive jaundice. Drainage of pseudocysts should be considered in patients who are symptomatic or have an infected pseudocyst. Endoscopic, percutaneous, or surgical drainage of the pseudocysts may be considered.

The occurrence of infected pancreatic necrosis is a leading cause of morbidity and mortality in severe pancreatitis. Infected pancreatic necrosis should be suspected if patient develops unexplained fever, leukocytosis, and/or signs of sepsis in conjunction with a CT scan, which reveals necrosis in the pancreas. Antibiotic therapy with broad-spectrum antibiotics such as imipenem should be initiated if pancreatic infection is suspected. Radiologic-guided fine-needle aspiration of the necrotic area is recommended to determine whether it is sterile or infected. Sterile necrosis is usually managed conservatively; however, infected pancreatic necrosis requires surgical debridement (necrosectomy).

\section{Gallstone Pancreatitis}

Because of the risk of recurrent pancreatitis due to cholelithiasis, indications for cholecystectomy during pregnancy should be the same as in the nonpregnant patient. Once pancreatitis resolves, laparoscopic cholecystectomy is ideally performed in the second trimester because during this period organogenesis is complete and technical problems due to an enlarging uterus are limited (131). A laparoscopic approach is preferable over open cholecystectomy because it is associated with shorter hospital stay, decreased narcotic use, and an earlier resumption of oral intake without affecting maternal or fetal mortality (119). In patients with onset of pancreatitis in the third trimester, postpartum cholecystectomy may be performed. ERCP with sphincterotomy may be useful in patients with severe acute pancreatitis, cholangitis, persistent biliary obstruction, and in those who are not ideal surgical candidates during the first and third trimesters of pregnancy (119). Fetal radiation exposure during ERCP can be kept below the teratogenic doses by limiting fluoroscopy times and shielding the pelvis.

\section{Hyperlipidemic Pancreatitis}

The management of hyperlipidemic pancreatitis poses a challenging problem. Attempts at refeeding patients after symptoms of pancreatitis subside may be difficult since even low-fat diets may lead to elevation of triglycerides and recurrent symptomatology (133). In these situations, particularly in patients who are not near term, adequate nutrition must be maintained. TPN has proven to be an efficacious method for delivering an appropriate calorie load without supplementation with fats, leading to improved regulation of triglyceride levels. Other supportive therapies include low-fat diet, antihyperlipidemic therapy, insulin, heparin, and plasmapheresis (134). Planned delivery of patients at term who have pancreatitis due to hyperlipidemia is recommended by some authors (136), and preterm delivery may be considered only in patients whose clinical status deteriorates, since lipid abnormalities are clearly worsened by pregnancy.

\section{ENDOSCOPY AND PROCEDURAL SEDATION IN PREGNANCY}

Endoscopy during pregnancy appears to be safe provided the indication for endoscopy is appropriate and adequate precautions are taken. A multidisciplinary team involving an endoscopist, obstetrician, and an anesthesiologist may be required for the management of gastrointestinal diseases in pregnancy requiring endoscopic diagnosis and intervention. Potential risks associated with endoscopy during pregnancy include the following:

1. Fetal hypoxia: Caused by maternal hypotension and hypoxia due to oversedation and may lead to fatal consequences.

2. Exposure of the fetus to potentially teratogenic drugs and radiation.

3. Maternal positioning during endoscopy can cause inferior vena caval obstruction by the pregnant uterus, which can lead to decreased uterine blood flow and fetal hypoxia.

According to the guidelines issued by the American Society for Gastrointestinal Endoscopy for endoscopy in pregnant women (137):

1. Always have a strong indication, particularly in high-risk pregnancies, 
2. Defer endoscopy to the second trimester whenever possible,

3. Use the lowest dose of sedative medication,

4. Minimize procedure time,

5. Position the pregnant patient in the left pelvic tilt or left lateral position to avoid vena caval or aortic compression,

6. Presence of fetal heart sounds should be confirmed before sedation is begun and after the endoscopic procedure is completed,

7. Obstetric support should be available in the event of a pregnancy-related complication, and

8. Endoscopy is contraindicated in obstetric complications such as placental abruption, imminent delivery, ruptured membranes, or pre-eclampsia.

\section{EGD}

EGD appears to be safe during pregnancy. The indications for considering EGD during pregnancy include significant upper gastrointestinal bleed, dysphagia, odynophagia, severe abdominal pain, and refractory nausea and vomiting. In one study, the diagnostic yield of EGD for upper gastrointestinal bleed was reported to be $95 \%$ (138). There were no cases of premature labor or congenital fetal malformation.

\section{Flexible Sigmoidoscopy/Colonoscopy}

The common indications for flexible sigmoidoscopy and/or colonoscopy during pregnancy are rectal bleeding, diarrhea with negative evaluation, and strong indication for a colon mass. Sigmoidoscopy may establish diagnosis in majority of patients and should be considered first. Colonoscopy may be required if refractory lower gastrointestinal symptoms persist and sigmoidoscopy was not diagnostic. The safety and efficacy of flexible sigmoidoscopy and colonoscopy were reported in a study in which 48 sigmoidoscopies (46 patients) and 8 colonoscopies (8 patients) were safely performed during pregnancy (70). There were no adverse fetal outcomes, and no evidence that sigmoidoscopy or colonoscopy precipitated the onset of labor. The safety of polyethylene glycol (PEG) electrolyte solutions has not been studied in pregnancy. PEG solutions are classified as category C. Tap water enemas may be used for bowel preparation for flexible sigmoidoscopy. Removal of majority of colon polyps should be deferred until the postpartum period.

\section{ERCP}

Pregnancy is associated with an increased risk of gallstone formation due to alterations in the bile composition (139). Therefore, the usual indications for ERCP in pregnant patients include complications related to gallstones including choledocholithiasis, cholangitis, and gallstone pancreatitis. Several studies have reported safety of ERCP during pregnancy with no adverse fetal effects $(140,141)$; however, ERCP should be performed only by an experienced endoscopist preferably at a higher volume tertiary care center. ERCP should be avoided during the first trimester due to radiation exposure. Fluoroscopy and procedure times should be minimized. Radiation exposure should be kept to a minimum by placing lead sheets under the pelvis and lower abdomen. Other diagnostic modalities that do not involve radiation such as MRCP and
Table 7 Common Sedation Medications for Endoscopic Procedures

\begin{tabular}{lc} 
Drug name & FDA category \\
\hline Meperidine & B \\
Fentanyl & C \\
Midazolam & D \\
Propofol & B \\
Glucagon (antiperistaltic agent) & B \\
Naloxone (opioid reversal agent) & B \\
Flumazenil (benzodiazepine reversal agent) & C
\end{tabular}

endoscopic ultrasonography (EUS) should be considered if probability of choledocholithiasis requiring therapeutic intervention is low.

\section{General Precautions}

Patient Positioning

Pregnant patients should not be placed in the decubitus or prone position during endoscopic procedures. Position pregnant patients in left pelvic tilt or left lateral position to avoid vena caval or aortic compression. External abdominal pressure should be avoided. If pressure is required, it should be minimal and directed away from the uterus.

\section{Medications for Sedation During Pregnancy and Lactation}

EGDs and flexible sigmoidoscopies may be performed without sedation. However, if sedation is required, it should be kept to a minimum. For procedural sedation during pregnancy, meperidine (category B) is preferred. Small doses of midazolam (category D) may be used as needed. If deep sedation is required, then propofol administered by an anesthesiologist should be the preferred choice (Table 7).

Fentanyl is preferred to meperidine for procedural sedation in women who are breast-feeding. The concentrations of fentanyl in breast milk are low, and lactation may be continued after fentanyl administration. Infants should not be breast-fed for at least 4 hours after midazolam administration. After 4 hours, breast milk should be pumped and discarded before reinitiating breast-feeding. Continued breast-feeding after propofol exposure is not recommended, although the period of prohibition is not known (137).

\section{REFERENCES}

1. Sontag SJ. The medical management of reflux esophagitis: role of antacids and acid inhibition. Gastrointest Clin North Am 1990; 19: 683-712.

2. Olans LB, Wolf JL. Gastroesophageal reflux in pregnancy. Gastrointest Endosc Clin N Am 1994; 4: 699-713.

3. Torbey CF, Richter JE. Gastrointestinal motility disorders in pregnancy. Semin Gastrointest Dis 1995; 6: 201-16.

4. Dodds WJ, Dent J, Hogan WJ. Pregnancy and the lower esophageal sphincter. Gastroenterology 1978; 74: 1334-6.

5. Mittal RK, McCallum RW. Characteristics and frequency of transient relaxations of the lower esophageal sphincter in patients with reflux esophagitis. Gastroenterology 1988; 95: 593-9.

6. Van Thiel DH, Gavaler JS, Joshi SN, et al. Heartburn of pregnancy. Gastroenterology 1977; 72: 666-8.

7. Bainbridge ET, Nicholas SD, Newton JR, Temple JG. Gastrooesophageal reflux in pregnancy: altered function of the barrier to reflux in asymptomatic women during early pregnancy. Scand J Gastroenterol 1984; 19: 85-9. 
8. Fisher RS, Roberts GS, Grabowski CJ, Cohen S. Altered lower esophageal sphincter function during early pregnancy. Gastroenterology 1978; 74: 1233-7.

9. Van Thiel DH, Wald A. Evidence refuting a role for increased abdominal pressure in the pathogenesis of the heartburn associated with pregnancy. Am J Obstet Gynecol 1981; 140: 420-2.

10. Brock-Utne JG, Dow TGB, Dimopoulos GE, et al. Gastric and lower oesophageal sphincter (LOS) pressures in early pregnancy. Br J Anaesth 1981; 53: 381-4.

11. Ulmsten U, Sundstrom G. Esophageal manometry in pregnant and nonpregnant women. Am J Obstet Gynecol 1978; 132: 260-4.

12. Lewis $\mathrm{JH}$, Weingold $\mathrm{AB}$. The use of gastrointestinal drugs during pregnancy and lactation. Am J Gastroenterol 1985; 80: 912-23.

13. Ching C, Lam S. Antacids: indications and limitations. Drugs 1994; 47: 305-17.

14. Nelson MM, Forfar JO. Associations between drugs administered during pregnancy and congenital abnormalities of the fetus. Br Med J 1971; 1: 523-7.

15. de Jong PC, Nijdam WS, Zielhuis GA, Eskes TK. Medication during low-risk pregnancy. Eur J Obstet Gynecol Reprod Biol 1991; 41: 191-6.

16. Buitendijk S, Bracken MB. Medication in early pregnancy: prevalence of use and relationship to maternal characteristics. Am J Obstet Gynecol 1991; 165: 33-40.

17. Hill LM, Kleinberf F. Effects of drugs and chemicals on the fetus and newborn. Mayo Clin Proc 1984; 59: 707-16.

18. Ranchet G, Gangemi O, Petrone M. Sucralfate in the treatment of gravid pyrosis. G Ital Ostericia Ginecol 1990; 12: 1-16.

19. Brigg GG, Freeman RY, Yaffe SJ. Drugs in pregnancy and lactation: a reference guide to fetal and neonatal risk. Baltimore: Williams and Wilkins, 2002.

20. Larson JD, Patatanian E, Miner PB, et al. Double-blind, placebo controlled study of ranitidine for gastroesophageal reflux symptoms during pregnancy. Am J Obstet Gynecol 1997; 90: 83-7.

21. Schwethelm B, Margolis LH, Miller C, et al. Risk status and pregnancy outcome among Medicaid recipients. Am J Prev Med 1989; 5: 157-63.

22. Nikfar S, Abdollahi M, Moretti ME, et al. Use of proton pump inhibitors during pregnancy and rates of major malformations. A meta-analysis. Dig Dis Sci 2002; 47: 1526-9.

23. Cuningham FG, Gant NF, Leveno KJ, et al. Gastrointestinal disorders. In: William's obstetrics, 21st edn. New York: McGraw-Hill, 2001: 1273-306.

24. Hess LW, Morrison JC, Hess DB. General medical disorders during pregnancy. In: DeCherney AH, Pernoll ML, eds. Current obstetric and gynecologic diagnosis and treatment, 8 th edn. Norwalk (CT): Appleton \& Lange, 1994: 479.

25. Jones PF, McEwan AB, Bernard RM. Hemorrhage and perforation complicating peptic ulcer in pregnancy. Lancet 1969; 2: 350-1.

26. Singer AJ, Brandt LJ. Pathophysiology of the gastrointestinal tract during pregnancy. Am J Gastroenterol 1991; 86: 1695-712.

27. Tornqvist A. The effects of exogenous histamine on the forearm blood flow in pregnant and nonpregnant women before and after inhibition of histaminases. Acta Obstet Gynecol Scand 1968; 47: 391-403.

28. Montoneri C, Drago F. Effects of pregnancy in rats on cysteamineinduced peptic ulcers: role of progesterone. Dig Dis Sci 1997; 42: 2572-5.

29. Feinberg BB, Gonik B. General precepts of the immunology of pregnancy. Clin Obstet Gynecol 1991; 34: 3-16.

30. Cappell MS. Gastric and duodenal ulcers during pregnancy. Gastroeterol Clin North Am 2003; 32: 263-308.

31. Michaletz-Onody PA. Peptic ulcer disease in pregnancy. Gastroenterol Clin North Am 1992; 21: 817-26.

32. Rubenstein M, Taylor M. The acute abdomen in pregnancy. In: Taylor MB, Gollan JB, Steer ML, et al. eds. Gastrointestinal emergencies, 2nd edn. Baltimore: Williams \& Wilkins, 1997: 755-63.

33. Graham DY, Lew GM, Klein PD, et al. Effect of treatment of Helicobacter pylori infection on the long term recurrence of gastric or duodenal ulcers: a randomized controlled study. Ann Intern Med 1992; 116: 705-8.

34. Kurata JH. Epidemiology: peptic ulcer risk factors. Semin Gastrointest Dis 1993; 4: 2-12.

35. Sontag S, Graham DY, Belsito A, et al. Cimetidine, cigarette smoking, and recurrence of duodenal ulcer. N Engl J Med 1984; 311: 689-93.
36. Broussard CN, Richter JE. Treating gastro-oesophageal reflux disease during pregnancy and lactation: what are the safest therapy options? Drug Saf 1998; 19: 325-7.

37. Jones DB, Howden CW, Burget DW, et al. Acid suppression in duodenal ulcer: a metaanalysis to define optimal dosing with antisecretory drugs. Gut 1987; 28: 1120-7.

38. Gill KS, O'Brien L, Koren G. The safety of histamine 2 (H2) blockers in pregnancy: a meta-analysis. Dig Dis Sci 2009; 54: 1835-8.

39. Hopkins RJ, Girardi LS, Turney EA. Relationship between Helicobacter pylori eradication and reduced duodenal and gastric ulcer recurrence: a review. Gastroenterology 1996; 110: 1244-52.

40. Fairweather DV. Nausea and vomiting in pregnancy. Am J Obstet Gynecol 1968; 102: 135-75.

41. Jarnfelt-Samsioe A, Samsioe G, Velinder GM. Nausea and vomiting in pregnancy - a contribution to its epidemiology. Gynecol Obstet Invest 1983; 16: 221-9.

42. Bailit JL. Hyperemesis gravidarum: epidemiologic findings from a large cohort. Am J Obstet Gynecol 2005; 193: 811-14.

43. Trogstad LI, Stoltenberg C, Magnus P, et al. Recurrence risk in hyperemesis gravidarum. BJOG 2005; 112: 1641-45.

44. Depue RH, Bernstein L, Ross RK, et al. Hyperemesis gravidarum in relation to estradiol levels, pregnancy, and other maternal factors: a seroepidemiologic study. Am J Obstet Gynecol 1987; 156: 1137-4.

45. Soules MR, Hughes CL, Garcia JA, et al. Nausea and vomiting of pregnancy: role of human chorionic gonadotropin and 17-hydroxyprogesterone. Obstet Gynecol 1980; 55: 696-700.

46. Masson GM, Anthony F, Chau E. Serum chorionic gonadotrophin (hCG), schwangerschafts protein 1 (SP1), progesterone and oestradiol levels in patients with nausea and vomiting in early pregnancy. Br J Obstet Gynaecol 1985; 92: 211-15.

47. Goodwin TM, Hershman JM, Cole L. Increased concentration of the free b-subunit of human chorionic gonadotropin in hyperemesis gravidarum. Acta Obstet Gynecol Scand 1994; 73: 770-2.

48. Bouillon R, Naesens M, Van Assche FA, et al. Thyroid function in patients with hyperemesis gravidarum. Am J Obstet Gynecol 1982; 143: 922-6.

49. Tan JY, Loh KC, Yeo GS, et al. Transient hyperthyroidism of hyperemesis gravidarum. BJOG 2002; 109: 683-8.

50. Koch KL, Stern RM, Vasey M, et al. Gastric dysrhythmias and nausea of pregnancy. Dig Dis Sci 1990; 35: 961-8.

51. Fairweather DV. Nausea and vomiting during pregnancy. Obstet Gynecol Annu 1978; 7: 91-105.

52. Fitzgerald CM. Nausea and vomiting in pregnancy. Br J Med Psychol 1984; 57: 159-65.

53. Mumford CJ. Papilloedema delaying diagnosis of Wernicke's encephalopathy in a comatose patient. Postgrad Med J 1989; 65: 371-3.

54. Fraser D. Central pontine myelinolysis as a result of treatment of hyperemesis gravidarum. Case report. Br J Obstet Gynaecol 1988; 95 : $621-3$.

55. Godsey RK, Newman RB. Hyperemesis gravidarum: a comparison of single and multiple admissions. J Reprod Med 1991; 36: 287-90.

56. Seto A, Einarson T, Koren G. Pregnancy outcome following first trimester exposure to antihistamines: meta-analysis. Am J Perinatol 1997; 14: 119-24.

57. Gill SK, Einarson A. The safety of drugs for the treatment of nausea and vomiting of pregnancy. Expert Opin Drug Saf 2007; 6: 685-94.

58. Einarson A, Maltepe C, Navioz Y, et al. The safety of ondansetron for nausea and vomiting of pregnancy: a prospective comparative study. BJOG 2004; 111: 940-3.

59. Castillo RA, Ray RA, Yaghmai F. Central pontine myelinolysis and pregnancy. Obstet Gynecol 1989; 73: 459-6.

60. Schulman PK. Hyperemesis gravidarum: an approach to the nutritional aspects of care. J Am Diet Assoc 1982; 80: 577-8.

61. Levine MG, Esser D. Total parenteral nutrition for the treatment of severe hyperemesis gravidarum: maternal nutritional effects and fetal outcome. Obstet Gynecol 1988; 72: 102-7.

62. Gross S, Librach C, Cecutti A. Maternal weight loss associated with hyperemesis gravidarum: a predictor of fetal outcome. Am J Obstet Gynecol 1989; 160: 906-9.

63. Chin RK, Lao TT. Low birth weight and hyperemesis gravidarum. Eur J Obstet Gynecol Reprod Biol 1988; 28: 179-83. 
64. Loftus EV Jr. Clinical epidemiology of inflammatory bowel disease: incidence, prevalence, and environmental influences. Gastroenterology 2004; 126: 1504-17.

65. Andres PG, Friedman LS. Epidemiology and the natural course of inflammatory bowel disease. Gastroenterol Clin North Am 1999; 28: 255-81.

66. Calkins BM, Lilienfeld AM, Garland CF, Mendeloff AI. Trends in incidence rates of ulcerative colitis and Crohn's disease. Dig Dis Sci 1984; 29: 913-20.

67. Gitnick G. Etiology of inflammatory bowel diseases: where have we been? Where are we going? Scand J Gastroenterol 1990; 175: 93-6.

68. Laharie D, Debeugny S, Peeters M, et al. Inflammatory bowel disease in spouses and their offspring. Gastroenterology 2001; 120: 816-19.

69. Tysk C, Lindber E, Jarnerot G, et al. Ulcerative colitis and Crohn's disease in an unselected population of monozygotic and dizygotic twins. A study of heritability and the influence of smoking. Gut 1988; 29: 990-6.

70. Cappell MS, Sidhom OA, Colon V. A study at ten medical centers of the safety and efficacy of 48 flexible sigmoidoscopies and 8 colonoscopies during pregnancy with follow-up of fetal outcome and with comparison to control groups. Dig Dis Sci 1996; 41: 2353-60.

71. Hanan MI, Kirsner JB. Inflammatory bowel disease in the pregnant woman. Clin Perinat 1985; 12: 669-81.

72. Nielsen $\mathrm{OH}$, Andreasson B, Bondesen S, Jarnum S. Pregnancy in ulcerative colitis. Scand J Gastroenterol 1983; 18: 735-42.

73. Korelitz BI. Pregnancy, fertility, and inflammatory bowel disease. Am J Gastroenterol 1985; 80: 365-70.

74. Willoughby CP, Truelove SC. Ulcerative colitis and pregnancy. Gut 1980; 21: 469-74.

75. Mogadam M, Korelitz BI, Ahmed SW, et al. The course of inflammatory bowel disease during pregnancy and postpartum. Am J Gastroenterol 1981; 75: 265-9.

76. Vender RJ, Spiro HM. Inflammatory bowel disease and pregnancy. J Clin Gastroenterol 1982; 4: 231-49.

77. Miller JP. Inflammatory bowel disease in pregnancy: a review. J R Soc Med 1986; 79: 221-5.

78. Weterman IT. Fertility and pregnancy in inflammatory bowel disease. Neth J Med 1989; 35: S67-75.

79. Baird DD, Narendranathan M, Sandler RS. Increased risk of preterm birth for women with inflammatory bowel disease. Gastroenterology 1990; 99: 987-4.

80. Khosla R, Willoughby CP, Jewell DP. Crohn's disease and pregnancy. Gut 1984; 25: 52-6.

81. Mayberry JF, Weterman IT. European survey of fertility and pregnancy in women with Crohn's disease: a case control study by European collaborative group. Gut 1986; 27: 821-5.

82. Toovey S, Hudson E, Hendry WF, Levi AJ. Sulphasalazine and male infertility: reversibility and possible mechanism. Gut 1981; 22: 445-51.

83. Nielsen $\mathrm{OH}$, Andreasson B, Bondesen S, et al. Pregnancy in Crohn's disease. Scand J Gastroenterol 1984; 19: 724-32.

84. Woolfson K, Cohen Z, McLeod RS. Crohn's disease and pregnancy. Dis Colon Rectum 1990; 33: 869-73.

85. Khan AK, Truelove SC. Placental and mammary transfer of sulphasalazine. Br Med J 1979; 2: 1553.

86. Jarnerot G, Into-Malmberg MB, Esbjorner E. Placental transfer of sulphasalazine and sulphapyridine and some of its metabolites. Scand J Gastroenterol 1981; 16: 693-7.

87. Mogadam M, Dobbins WO, Korelitz BI, Ahmed SW. Pregnancy in inflammatory bowel disease: effect of sulfasalazine and corticosteroids on fetal outcome. Gastroenterology 1981; 80: 72-6.

88. NorgÅrd B, Czeizel AE, Rockenbauer M, et al. Population-based case control study of the safety of sulfasalazine use during pregnancy. Aliment Pharmacol Ther 2001; 15: 483-6.

89. Habal FM, Hui G, Greenberg GR. Oral 5-aminosalicylic acid for inflammatory bowel disease in pregnancy: safety and clinical course. Gastroenterology 1993; 105: 1057-60.

90. Bell CM, Habal FM. Safety of topical 5-aminosalicylic acid in pregnancy. Am J Gastroenterol 1997; 92: 2201-2.

91. Diav-Citrin O, Park YH, Veerasuntharam G, et al. The safety of mesalamine in human pregnancy: a prospective controlled cohort study. Gastroenterology 1998; 114: 23-8.
92. Rahimi R, Nikfar S, Rezaie A, Abdollahi M. Pregnancy outcome in women with inflammatory bowel disease following exposure to 5aminosalicylic acid drugs: a meta-analysis. Reprod Toxicol 2008; 25: 271-5.

93. Carmichael SL, Shaw GM. Maternal corticosteroid use and risk of selected congenital anomalies. Am J Med Genet 1999; 86: 242-4.

94. Mogadam M, Dobbins WO, Korelitz BI, Ahmed SW. Pregnancy in inflammatory bowel disease: effect of sulfasalazine and corticosteroids on fetal outcome. Gastroenterology 1981; 80: 72-6.

95. Park-Wyllie L, Mazzotta P, Pastuszak A, et al. Birth defects after maternal exposure to corticosteroids: prospective cohort study and meta-analysis of epidemiological studies. Teratology 2000; 62: 385-92.

96. Beaulieu DB, Ananthakrishnan AN, Issa M. Budesonide induction and maintenance therapy for Crohn's disease during pregnancy. Inflamm Bowel Dis 2009; 15: 25-8.

97. Ost L, Wettrell G, Bjorkhem I, Rane A. Prednisolone excretion in human milk. J Pediatr 1985; 106: 1008-11.

98. Polifka JE, Friedman JM. Teratogen update: azathioprine and 6mercaptopurine. Teratology 2002; 65: 240-61.

99. Alstead EM, Ritchie JK, Lennard-Jones JE, et al. Safety of azathioprine in pregnancy in inflammatory bowel disease. Gastroenterology 1990; 99: 443-637.

100. Francella A, Dyan A, Bodian C, et al. The safety of 6-mercaptopurine for childbearing patients with inflammatory bowel disease: a retrospective cohort study. Gastroenterology 2003; 124: 9-17.

101. Christensen LA, Dahlerup JF, Nielsen MJ. Azathioprine treatment during lactation. Aliment Pharmacol Ther 2008; 28: 1209-13.

102. Milunsky A, Graef JW, Gaynor MF Jr. Methotrexate induced congenital malformations. J Pediatr 1968; 72: 790-5.

103. Czeize AE, Rockenbauer M. A population based case-control teratologic study of oral metronidazole treatment during pregnancy. Br J Obstet Gynaecol 1998; 105: 322-7.

104. Caro-Paton T, Carvajal A, Martin de Diego I, et al. Is metronidazole teratogenic? A meta-analysis. Br J Clin Pharmacol 1997; 44: 179-82.

105. Lichtenstein GR, Feagan BG, Cohen RD, et al. Serious infections and mortality in association with therapies for Crohn's disease: TREAT registry. Clin Gastroenterol Hepatol 2006; 4: 621-30.

106. Katz JA, Antoni C, Keenan GF, et al. Outcome of pregnancy in women receiving infliximab for the treatment of Crohn's disease and rheumatoid arthritis. Am J Gastroenterol 2004; 99: 2385-92.

107. Kane S, Ford J, Cohen R, Wagner C. Absence of infliximab in infants and breast milk from nursing mothers receiving therapy for Crohn's disease before and after delivery. J Clin Gastroenterol 2009; 43: 613-16.

108. Coburn LA, Wise PE, Schwartz DA. The successful use of adalimumab to treat active Crohn's disease of an ileoanal pouch during pregnancy. Dig Dis Sci 2006; 51: 2045-7.

109. Mishkin DS, Van Deinse W, Becker JM, et al. Successful use of adalimumab (Humira) for Crohn's disease in pregnancy. Inflamm Bowel Dis 2006; 12: 827-8.

110. Vesga L, Terdiman JP, Mahadevan U. Adalimumab use in pregnancy. Gut 2005; 54: 890 .

111. Hudson M, Flett G, Sinclair TS, et al. Fertility and pregnancy in inflammatory bowel disease. Int J Gynaecol Obstet 1997; 58: 229-37.

112. Waljee A, Waljee J, Morris AM, Higgins PD. Threefold increased risk of infertility: a meta-analysis of infertility after ileal pouch anal anastomosis in ulcerative colitis. Gut 2006; 55: 1575-80.

113. Nestor H, Dozois RR, Kelly KA, et al. The effect of pregnancy and delivery on the ileal pouch-anal anastomosis functions. Dis Colon Rectum 1989; 32: 384-8.

114. Corlett RC, Mishell DR. Pancreatitis in pregnancy. Am J Obstet Gynecol 1972; 113: 281-90.

115. Hasselgren PO. Acute pancreatitis in pregnancy. Acta Chir Scand 1980; 146: 297-9.

116. Ellsbury KE. Abdominal pain in pregnancy. J Fam Pract 1986; 22: 365-71

117. Augustin G, Majerovic M. Non-obstetrical acute abdomen during pregnancy. Eur J Obstet Gynecol Reprod Biol 2007; 131: 4-12.

118. Stampfer MJ, Maclure KM, Colditz GA, et al. Risk of symptomatic gallstones in women with severe obesity. Am J Clin Nutr 1992; 55 : 652-8. 
119. Pitchumoni CS, Yegneswaran B. Acute pancreatitis in pregnancy. World J Gastroenterol 2009; 15: 5641-6.

120. Lippi G, Albiero A, Montagnana M, et al. Lipid and lipoprotein profile in physiological pregnancy. Clin Lab 2007; 53: 173-7.

121. Lykkesfeldt G, Bock JE, Pedersen FD, et al. Excessive hypertriglyceridemia and pancreatitis in pregnancy: association with deficiency of lipoprotein lipase. Acta Obstet Gynecol Scand 1980; 60: 79-82.

122. Sharp H. The acute abdomen during pregnancy. Clin Obstet Gynecol 2002; 45: 405-13.

123. Wang SS, Lin XZ, Tsai YT, et al. Clinical significance of ultrasonography, computed tomography, and biochemical tests in the rapid diagnosis of gallstone-related pancreatitis: a prospective study. Pancreas 1988; 3: 153-8.

124. Ranson JH, Rifkind KM, Turner JW. Prognostic signs and non-operative peritoneal lavage in acute pancreatitis. Surg Gynecol Obstet 1976; 143: 209-19.

125. Wilkinson EJ. Acute pancreatitis in pregnancy: a review of 98 cases and a report of 8 new cases. Obstet Gynecol Surv 1973; 28: 281-303.

126. Hernandez A, Petrov MS, Brooks DC, et al. Acute pancreatitis and pregnancy: a 10-year single center experience. J Gastrointest Surg 2007; 11: 1623-7.

127. Date RS, Kaushal M, Ramesh A. A review of the management of gallstone disease and its complications in pregnancy. Am J Surg 2008; 196: 599-608.

128. Gideonson EJ, Grobman WA, O’Halloran P. Pancreatitis in pregnancy. Obstet Gynecol 2008; 112: 1075-81.

129. Jain P. Acute pancreatitis in pregnancy: an unresolved issue. World J Gastroenterol 2010; 16: 2065-6.

130. Steer ML. Acute pancreatitis. In: Yamada T, ed. Textbook of Gastroenterology. Philadelphia, PA: JP Lippincott, 1991: 1859-74.
131. Young KR. Acute pancreatitis in pregnancy: two case reports. Obstet Gynecol 1982; 5: 653-7.

132. Gineston JL, Capron JP, Delcenserie R, et al. Prolonged total parenteral nutrition in a pregnant woman with acute pancreatitis. J Clin Gastroenterol 1984; 6: 249-52.

133. Weinberg RB, Sitrin MD, Adkins GM, Lin CC. Treatment of hyperlipidemic pancreatitis in pregnancy with total parenteral nutrition. Gastroenterology 1982; 83: 1300-5.

134. Mao EQ, Tang YQ, Zhang SD. Formalized therapeutic guideline for hyperlipidemic severe acute pancreatitis. World J Gastroenterol 2003; 9: 2622-6.

135. Marik PE, Zaloga GP. Meta-analysis of parenteral nutrition versus enteral nutrition in patients with acute pancreatitis. BMJ 2004; 328: 1407.

136. Nies BM, Dreiss RJ. Hyperlipidemic pancreatitis in pregnancy: a case report and review of the literature. Am J Perinatol 1990; 7: 166-9.

137. Qureshi WA, Rajan E, Adler DG, et al. ASGE Guideline: guidelines for endoscopy in pregnant and lactating women. Gastrointest Endosc 2005; 61: 357-62.

138. Cappell MS, Colon V, Sidhom O. A study at eight medical centers of the safety and clinical efficacy of esophagogastroduodenoscopy in 83 pregnant females with follow-up of fetal outcome and with comparison to control groups. Am J Gastroenterol 1996; 91: 348-54.

139. Maringhini A, Ciambra M, Baccelliere $\mathrm{P}$, et al. Biliary sludge and gallstones in pregnancy: incidence, risk factors and natural history. Ann Intern Med 1993; 119: 116-20.

140. Jamidar PA, Beck GJ, Hoffman BJ, et al. Endoscopic retrograde cholangiopancreatography in pregnancy. Am J Gastroenterol 1995; 90: 1263-7.

141. Tham TC, Vandervoort J, Wong RC, et al. Safety of ERCP during pregnancy. Am J Gastroenterol 2003; 98: 308-11. 


\section{Hepatic disorders in pregnancy Ghassan M. Hammoud and Jamal A. Ibdah}

Liver disease in pregnancy is uncommon, and in the majority of cases, there is no need for a change in the management of liver disorder till pregnancy ceases. Most often, abnormalities in liver tests are related to conditions not specific to pregnancy. The liver is affected both physiologically and anatomically during the course of pregnancy. As pregnancy progresses, the liver is squeezed toward the chest and, therefore, a palpable liver below the right subcostal region is indicative of undergoing hepatic disorder.

Liver diseases in pregnancy are classified into those not specific to pregnancy and those unique to pregnancy. Furthermore, liver diseases unique to pregnancy are classified according to their onset during pregnancy and their association with pre-eclampsia. In this chapter, we will review changes in liver anatomy and function during pregnancy, liver diseases not specific, and liver diseases unique to pregnancy.

\section{LIVER ANATOMY, HISTOLOGY, AND FUNCTION IN PREGNANCY}

Anatomically, the liver is generally not palpable below the right costal margin secondary to the enlarging gravid uterus. Both liver size and gross appearance do not change during pregnancy. Furthermore, minimal histologic changes occur in pregnancy. These include cellular and nuclear pleomorphism, increased numbers of binucleate cells, steatosis, increased cellular and nuclear glycogen, hypertrophied Kupffer cells, and some lymphocytic infiltration of the portal tracts. During normal pregnancy, plasma volume is increased by $50 \%$ to $70 \%$ and reaches its maximum by 32 to 34 weeks of pregnancy. This results in hemodilution of all serum protein concentrations. Serum albumin concentration decreases, but the rates of albumin synthesis and catabolism are not affected. Hypoalbuminemia can occur in the third trimester regardless of liver disease. However, prolongation of prothrombin time (PT) and hyperbilirubinemia would raise the concern of significant liver disease. Other serum protein concentrations such as alpha-2-macroglobulin, alpha-1antitrypsin, and ceruloplasmin are increased. Pregnancy is characterized by a hypercoagulable state and increased risks of deep venous thrombosis. Almost all coagulation factors increase during pregnancy except protein S. Moreover, fibrinolysis is inhibited. This physiologic state tends to decrease the risk of bleeding during delivery; however, it increases the risk of maternal venous thrombosis. Indeed, PT is generally not affected during pregnancy and any changes in PT should be considered abnormal and justify further investigation. Serum lipid concentrations increase in late pregnancy secondary to increased levels of estrogen and progesterone. Elevations in estrogen and progesterone levels enhance cholestasis and production of lithogenic bile and reduce gallbladder motility. These alterations predispose pregnant women for gallstone and biliary sludge formation. Measurement of serum lipids in pregnancy is rarely useful unless in the setting of acute pancreatitis. During pregnancy, the total red cell mass is actually increased but rapidly decreases as pregnancy ceases. The cardiac output increases but the absolute hepatic blood flow remains unchanged. Plasma volume increases specially during the second trimester resulting in falling hemoglobin levels due to hemodilution. Serum aminotransferases [alanine aminotransferase (ALT) and aspartate aminotransferase (AST)] are not altered by pregnancy and are considered the most useful test indicative of hepatocellular injury. Serum alkaline phosphatase activity levels rise during the third trimester as a result of increased production of placental and bone isoenzymes. Indeed, measurement of serum alkaline phosphatase activity is not a suitable test for the diagnosis of cholestasis during pregnancy. Total and free bilirubin concentrations are decreased during pregnancy partly due to hemodilution (Table 1). Gilbert's syndrome is characterized by an increase in unconjugated hyperbilirubinemia with absolute normal aminotransferases. The management of pregnant women with Gilbert's syndrome is not any different than those who are not pregnant, and medical reassurance should be provided. The syndrome of hemolysis, elevated liver enzymes, and low platelets (HELLP) is associated with indirect hyperbilirubinemia and should be differentiated from Gilbert's syndrome. Pregnancy is also associated with mild increase in serum bile acids secondary to reduced bile salt transport. Measurement of serum bile acid concentrations may be useful for the diagnosis of cholestasis during pregnancy.

\section{PHYSICAL EXAMINATION DURING NORMAL PREGNANCY}

Spider naevi and palmar erythema are commonly observed during pregnancy and in oral contraceptive receivers. Spider naevi consist of a central arterial vessel from which small capillaries lead off radially. These are found in $53 \%$ by the 9 months of pregnancy (1) and tend to be more common among white women. Palmar erythema is frequently observed in patients with chronic liver disease and in pregnancy. It manifests itself as a diffuse patchy erythema of the palms up to the start of the wrist joint. Occasionally, the soles of the feet are also affected, hence the name plantar erythema. Palmar erythema is observed in $63 \%$ of white women and $35 \%$ of black women during pregnancy (2). Scratch marks and pruritus are found in cases with cholestasis and biliary cirrhosis. Pregnancy is considered a pruritogenic state. The cause of this unbearable itching is deemed to be due to a decrease in bile salt transport secondary to a reduction in both sinusoidal and canalicular bile salt transporters. The concentration of bile salts in blood is within normal range in most pregnant women, but levels of unconjugated chenodeoxycholic acid are higher in late pregnancy. Anatomically, the liver 
is displaced toward the chest by an enlarging uterus and palpable liver is considered abnormal in pregnancy. It is important to screen for cirrhosis in the physical examination of pregnant women with viral hepatitis. Detection of cirrhosis may not always be easy because spider angioma and palmar erythema can be seen in both cirrhotic and pregnant states. However, evidence for splenomegaly is suggestive of cirrhosis.

\section{LIVER DISEASE NOT SPECIFIC TO PREGNANCY Viral Hepatitis}

Viral hepatitis is the most common cause of jaundice in pregnancy. The hepatotropic viruses (hepatitis A, B, C, D, E, non- $\mathrm{A}$, and non-B) are responsible for the majority of these infections and accounts for $40 \%$ of jaundice in pregnant women in the United States (3). The course and outcome of viral hepatitis is no different in pregnant patients than in other individuals except in the case of hepatitis $\mathrm{E}$, which has been associated with a higher morbidity and mortality in pregnant women in the third trimester (4).

\section{Table 1 Biochemical Changes During Normal Pregnancy}

$\begin{array}{ll}\text { Test } & \\ \text { White cell count } & \uparrow \\ \text { Hemoglobin } & \downarrow \\ \text { Platelets } & - \\ \text { Albumin } & \downarrow \\ \text { Aminotransferases } & - \\ \text { Alkaline phosphatase } & \uparrow \\ \text { GGT } & - \\ \text { Bilirubin } & -/ \downarrow \\ \text { Prothrombin time } & - \\ \text { Fibrinogen } & \uparrow \\ \text { Globulins } & \uparrow \text { in } \alpha \text { and } \beta \text { globulins and } \downarrow \text { in } \gamma \text { globulin } \\ \text { Glucose } & - \\ \text { Creatinine } & - \\ \text { Uric acid } & - \\ \text { Bile acids } & - \\ \text { Cholesterol } & \uparrow \\ \text { Triglycerides } & \uparrow \\ \text { Alpha-fetoprotein } & \uparrow \\ \text { Ceruloplasmin } & \uparrow \\ \text { Ferritin } & \uparrow \\ \text { Abbreviation: GGT, gamma glutamyl transpeptidase. }\end{array}$

Herpes hepatitis is an extremely rare disease in immunocompetent adults with an associated mortality of 39\% (5). Most infections occur in the third trimester of pregnancy. Altered CD4/CD8 lymphocyte ratio and impaired natural killer cell function are blamed for the development of systemic herpes virus infection. Hepatitis manifests with coagulopathy, markedly elevated liver transaminases (>1000IU), and low bilirubin. Liver biopsy reveals coagulative necrosis, multinucleated cells with ground-glass intranuclear inclusions, and peripheral chromatin condensation (6). Early treatment with intravenous acyclovir is lifesaving.

The diagnosis of viral hepatitis is usually made on the basis of serologic testing (Table 2). Although viral hepatitis is not associated with teratogenicity, transmission of viral infection to the fetus may occur, which may result in premature labor. Transmission of infection is believed to occur primarily during the peripartum. For some infections, measures can be taken to prevent or lessen the severity of hepatitis in the newborn.

\section{Hepatitis A}

Hepatitis A virus (HAV) is a small, spherical RNA virus that usually causes a self-limiting clinical hepatitis. The virus is transmitted by the fecal-oral route and replicates exclusively within the hepatocytes and is excreted in bile. HAV infection does not lead to chronic hepatitis but relapsing cholestatic hepatitis have been described (7). The average incubation period is about 28 days (range 15-50 days). Fecal shedding occurs for about 2 weekes after infection. The overall casefatality ratio is less than $1 \%$. Intrauterine transmission of HAV is very rare but perinatal infection could occur. Acute HAV infection during pregnancy is associated with high risk of maternal complications and preterm labor (8). Serologic diagnosis of acute HAV is made by the presence of anti-HAV IgM. Anti-HAV IgM is typically present for several weeks but may be present for up to 1 year. The presence of anti-HAV IgG antibody denotes immunity either by previous infection or vaccination and confers long-lasting immunity. Intrauterine transmission of HAV is very rare; however, perinatal transmission can occur. Neonates of mothers who acquire HAV in the last trimester should receive immune globulin (IG) at the time of delivery (9). Pregnant women who travel to an endemic area should receive prophylactic IG. Individuals who are exposed to acute HAV should receive IG within 10 days of exposure. Studies show that post-exposure prophylaxis can prevent

Table 2 Serologic Diagnosis of Common Viral Hepatitis in Pregnancy

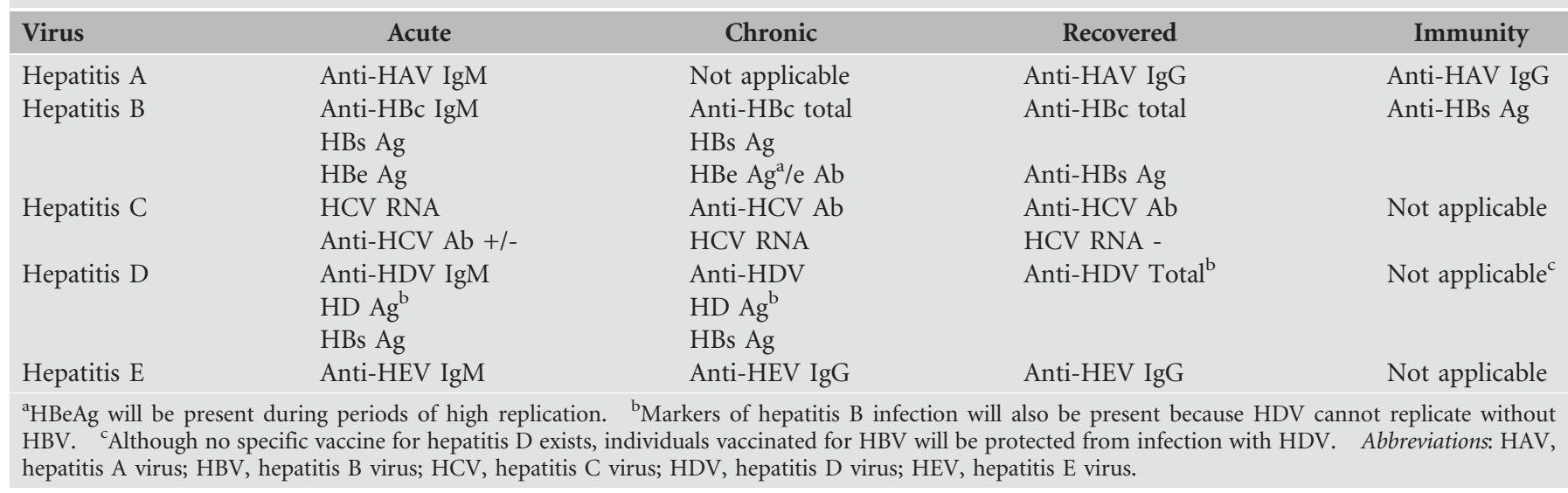


infection or lessen its severity in those who become infected. Mothers infected with HAV are encouraged to breast-feed and HAV infection is not a contraindication for breast-feeding (10).

\section{Hepatitis B}

Hepatitis B virus $(\mathrm{HBV})$ is a $3.2 \mathrm{~kb}$, circular, partially doublestranded deoxyribonucleic acid (DNA) virus belongs to the Hepadnavirus group. HBV can result in both acute and chronic infections. Carriers with chronic HBV are at risk for the development of liver cirrhosis, hepatic decompensation, and hepatocellular carcinoma (HCC) (11). A person is said to be a hepatitis B surface antigen (HBsAg) carrier if serum HBsAg has persisted for more than 6 months; if the clinical and chemical findings fail to show any signs of acute hepatitis; and if no or minimal histologic changes are detectable. HBV is generally noncytolytic but liver injury is attributed to host immune response. In contrast to $\mathrm{HCV}$ that replicates in the cytoplasm of the hepatocytes, HBV (dsDNA) enters into the nucleus and is converted to covalently closed circular form known as cccDNA (12). This is remarkably a stable species from which all viral mRNAs are transcribed and is resistant to antiviral therapy. The virus is transmitted by perinatal, percutaneous, and sexual exposure, as well as by close person-to-person contact. Vertical transmission is very infrequent in the first and second trimesters, yet prevails in the third trimester. With the coexistence of HBsAg, HBeAg, and high HBV DNA in the third trimester, the child is nearly always infected. Perinatal transmission of $\mathrm{HBV}$ infection represents the single largest cause of chronically infected individuals worldwide (13). An estimated 350 million persons worldwide are chronically infected with HBV (14). North America, Northern Europe, and the Oceanic areas are low prevalence areas $(<2 \%)(15,16)$. In the United States, there are an estimated 1.25 million hepatitis B carriers, defined as persons positive for HBsAg for more than 6 months (16). The incubation period is 25 to 180 days. The age at which HBV infection occurs is an important factor affecting the outcome. The earlier the infection occurs, the higher is the risk for chronicity. The risk of developing chronic HBV infection after acute exposure ranges from $90 \%$ in newborns of $\mathrm{HBeAg}$ positive mothers to $25 \%$ to $30 \%$ in infants and children under 5 and to less than $5 \%$ in adults. The diagnosis of acute HBV infection is established by the presence of positive serum HBsAg and the IgM fraction of the antibody to hepatitis B core (anti-HBc IgM). Pregnant women who had an immediate exposure to hepatitis $\mathrm{B}$ virus may be given injection of hepatitis B immunoglobulin (HBIG) ideally within 72 hours of exposure and hepatitis B vaccine within 7 days of exposure (17). Hepatitis $B$ vaccine is generally safe in all trimesters of pregnancy. The current HBV screening guidelines from the Centers for Disease Control and Prevention, the American College of Obstetrics and Gynecology, and the Immunization Practices Advisory Committee recommend that all pregnant women be tested for HBsAg during each pregnancy and that women at high risk for chronic hepatitis B be screened on admission for delivery, even if they previously have been tested or vaccinated (18). HBsAgpositive women who are pregnant should be counseled and make sure that their providers are informed so hepatitis $B$ immune globulin (HBIG) and hepatitis $B$ vaccine can be administered to their newborn immediately after delivery.
Immunization with HBIG and vaccine starting at birth reduces the risk of transmission to less than $10 \%$ among infants born to HBsAg/HBeAg-positive mothers (19). One such immunization schedule includes three intramuscular injections, the first one given between the time of birth and before the hospital discharge, and the second and third injections given at 1 and 6 months of age, respectively (18). The standard vaccination strategy for infants born to mothers who test positive for HBsAg includes the administration of 100IU HBIG (human hepatitis B Immunoglobulin-VF, CSL Bioplasma) and hepatitis $B$ vaccine [Recombivax HB (5mg), Merck, or Engerix-B (10mg), GlaxoSmithKline] within 12 hours of birth (20). HBIG is given intramuscularly and at a different site from that used for vaccination. Immediate household contacts should also be given HBIG and vaccination. The current vaccines contain noninfectious HBsAg and should cause no potential risk to the fetus.

The current (2009) Federal Drug Administration (FDA)approved therapy for HBV in nonpregnant patients include subcutaneous injection of interferon/pegylated interferon alpha and orally administered nucleoside/tide analogs such as Lamivudine, Adefovir, Entecavir, Telbivudine, and Tenofovir (21). Interferon is considered FDA category C drug in pregnancy and is not recommended. Lamivudine, an oral nucleoside analog, classified as FDA category $\mathrm{C}$ drug in terms of safety, is given to mothers with high HBV DNA during the second and third trimesters to reduce the risk of transmission at the time of delivery. It is generally not recommended for use against $\mathrm{HBV}$ in the first trimester of pregnancy. However, potential benefits may justify the potential risk. The data on adefovir, entecavir, telbivudine, and tenofovir safety are limited in pregnancy. Although HBV may be found in breast milk, a study comparing breast-fed versus non-breast-fed babies of seropositive mothers demonstrated no difference in the infection rate.

\section{Hepatitis $C$}

Hepatitis C virus (HCV) is a hepatotropic positive singlestranded RNA virus of the Hepacivirus genus and a member of the Flaviviridae family. HCV infection is one of the major causes for development of liver cirrhosis and end-stage liver disease in the world. The prevalence of HCV antibody in pregnant women is equivalent to that of the general population (0.5\%) (22). The most remarkable feature of hepatitis $\mathrm{C}$ virus is its ability to establish chronic infection in $55 \%$ to $85 \%$ of acute cases. Approximately $20 \%$ of chronically infected patients go on to develop cirrhosis, hepatic decompensation, and HCC after 20 to 30 years of infection (23). Chronic HCV infection is estimated to affect 180 million people worldwide, including 2 to 3 million Americans (1.6\% of the general population) (24). There are six HCV genotypes $(1,2,3,4,5$, and 6$)$, and a large number of subtypes (1a, 1b, 1c, etc.) have been identified (25). Currently, injection drug use is the primary mode of $\mathrm{HCV}$ transmission in the United States. Other risk factors include clotting factor therapy prior to the year 1987, blood transfusion prior to the year 1992, contaminated therapeutic equipment, occupational needlestick, and sexual and perinatal transmission. The average rate of perinatal transmission is $5 \%$ to $6 \%$ (26). Infants born to mothers with HCV and human immunodeficiency virus coinfection have increased rate of HCV transmission (14-17\%) (27). The risk of perinatal HCV transmission is related to HCV RNA titer and is 
unrelated to delivery mode (vaginal vs. cesarean). Factors associated with increased risk of perinatal transmission are prolonged rupture of the membrane for more than 6 hours, amniocentesis, and perineal lacerations. Although HCV can be transmitted intrapartum, prevention by caesarean delivery is not generally indicated. Studies have evaluated breast-feeding of infants born to HCV-infected women, which indicate that the average rate of infection is $4 \%$ in both breast-fed and bottle-fed infants (28), and most experts do not consider breast-feeding a mode of transmission. The prevalence of hepatitis $\mathrm{C}$ in pregnancy does not differ significantly from that of hepatitis $\mathrm{C}$ in the ageand sex-matched general population. Routine prenatal $\mathrm{HCV}$ screening is not recommended; however, women with significant risk factors for infection should be offered antibody screening.

Fetal outcomes, such as birth weight, American Pediatric Gross Assessment Record score, and umbilical pH, have not been found to be different in children born to hepatitis C-infected mothers. Similarly, maternal outcome and obstetric complications are not greater in mothers chronically infected with hepatitis $\mathrm{C}$ in the absence of cirrhosis and portal hypertension. The diagnosis of acute or chronic HCV infection generally requires testing of serum for both antibody to HCV (anti-HCV) and for HCV RNA with a sensitive quantitative HCV RNA assay (Table 3). Anti-HCV is passively acquired by the infant and may persist for up to 18 months. Moreover, the rate of spontaneous clearance in the perinatal transmission of $\mathrm{HCV}$ is high. Infants born to mothers with HCV should not be tested for HCV till 18 months of age (29).

The course of hepatitis $\mathrm{C}$ can be affected by pregnancy. Some studies have reported an increase in HCV RNA levels in the third trimester of pregnancy, but others have not. On the contrary, serum ALT levels tend to be lower in the third trimester and return to normal by 6 months after delivery.

The currently recommended therapy of chronic HCV infection in nonpregnant patients is the combination of a pegylated interferon-alfa and ribavirin. This regimen has been shown to be effective in $40 \%$ to $70 \%$ of patients (30-32). In mid 2011, two new direct-acting antiviral agents have been approved in combination with pegylated interferon and ribavirin for hepatitis $\mathrm{C}$ therapy in non-pregnant genotype 1 patients. HCV treatment success is measured by sustained virologic response (SVR) (defined as undetectable HCV RNA by a sensitive assay 6 months following discontinuation of treatment). Interferon is classified by the FDA as a category $\mathrm{C}$ drug while ribavirin is a category $\mathrm{X}$ product, indicating that its use is contraindicated in women who are pregnant. Interferon monotherapy is far less effective than combined therapy with ribavirin and is associated with low SVR rates, and hence the risk of HCV therapy outweighs its benefit during pregnancy.

\section{Hepatitis $E$}

Hepatitis E virus (HEV) is a positive-stranded RNA virus that belongs to the Hepeviridae family. The virus is transmitted via the fecal-oral route, commonly by contaminated water supplies. Vertical transmission of hepatitis $\mathrm{E}$ virus ranged between $33 \%$ and $50 \%(33,34)$. The virus is endemic in the Middle East, India, Southeast Asia, Central Asia, Central America, and South America. Hepatitis E is an acute disease that does not progresses to chronicity, although reports of chronic HEV have been described in liver transplant recipients $(35,36)$. The clinical presentation is basically the same as that for HAV. $\mathrm{HEV}$ infection is known to cause severe hepatitis, fulminant liver failure, preterm labor, and increased mortality in pregnant women especially in their third trimester with reported maternal mortality rates as high as $20 \%$ to $31.1 \%(4,37)$. The incubation period is 3-8 weekes, with a mean of 40 days. Cholestatic hepatitis is common. Acute hepatitis E infection is diagnosed by detection of IgM anti-HEV or a rising titer of IgG anti-HEV. The treatment is supportive therapy. A proved effective vaccine against $\mathrm{HEV}$ is currently not available. There is no current evidence that HEV infection is transmitted in breast milk.

\section{Autoimmune Hepatitis}

Autoimmune hepatitis (AIH) is a syndrome of progressive hepatocellular inflammation and necrosis of unknown cause, frequently leading to cirrhosis (38). The disease is characterized by loss of tolerance to hepatic autoantigens. AIH occurs predominantly among women, particularly in their younger years, and may influence their fertility and pregnancy. Pregnant women with AIH have a reduced fertility rate secondary to amenorrhea and anovulation, which might be related to hypothalamic-pituitary dysfunction. Serious maternal complications can occur in $9 \%$ of cases with a high rate (52\%) of postpartum flares (39). In one large review by Candia et al. analyzing 101 pregnancies, fetal death rate was observed in $19 \%$ with a perinatal mortality of $4 \%(40)$. Most fetal deaths occurred before the 20th week of pregnancy.

In the United States, AIH affects 100,000 to 200,000 patients with an incidence of 1.9 per 100,000 per year and a point prevalence of 16.9 per 100,000 persons per year (41). AIH occurs at two age peaks, one in adolescence and young adulthood and the other in the sixth or seventh decade. About $70 \%$ of cases involve females, and there is a frequent association with other autoimmune disorders such as thyroiditis.

The diagnosis is based on histologic abnormalities (portal tract inflammation with moderate infiltration with lymphocytes, plasma cells, and piecemeal necrosis), characteristic clinical and laboratory findings (absence of other causes of

Table 3 Interpretation of Serum Hepatitis C Virus (HCV) Antibody and HCV RNA

$\begin{array}{llr}\text { Anti-HCV } & \text { HCV RNA } & \text { Interpretation } \\ \text { Negative } & \text { Negative } & \text { No previous or current exposure to HCV } \\ \text { Positive } & \text { Positive } & \text { Acute or chronic HCV infection } \\ \text { Positive } & \text { Negative } & \begin{array}{r}\text { Resolution of HCV either spontaneously or after } \\ \text { response to treatment, false positive ELISA test }\end{array} \\ \text { Negative } & \text { Positive } & \text { Acute HCV infection; chronic HCV in setting of } \\ & & \text { immunosuppressed state; false-positive HCV RNA test }\end{array}$


liver injury and abnormal aminotransferases), abnormal levels of serum globulins, and the presence of one or more characteristic autoantibodies (42). Immunosuppressive therapy with azathioprine (AZA) and corticosteroids are the principal therapy of AIH. Corticosteroid therapy induces clinical, laboratory, and histologic improvements in $80 \%$ of patients with AIH (43). Corticosteroids and AZA are generally safe during pregnancy but birth defects have been described (44). Pregnant women with AIH need careful monitoring during pregnancy and for several months postpartum.

\section{Wilson's Disease}

Wilson's disease (WD) is a rare autosomal recessive disorder of hepatic copper metabolism caused by defective copper transporting ATPase in the liver causing a decrease in biliary excretion of copper and abnormal accumulation of copper in many tissues, notably the liver, brain, kidneys, and cornea. The most common gene mutation described in WD is ATP7B. WD is a rare disorder with a worldwide prevalence of 1:30,000. Clinical manifestations depend on the affected organs and include acute or chronic hepatitis, cirrhosis, hemolysis, chorea, tremors, spasticity, and psychiatric changes. A Kayser-Fleischer ring is often observed once neurologic or psychiatric symptoms develop. The diagnosis is made by a low serum level of ceruloplasmin and an elevated concentration of copper in the liver tissue. Women with WD have high rates of infertility due to menstrual irregularities and recurrent miscarriages $(45,46)$. The former is the result of hormonal changes caused by hepatic failure and copper toxicity and the latter is caused probably by increased copper deposition in the uterus. Hemolysis can be a presenting feature in some cases.

The current available anticopper agents are penicillamine, zinc, trientine, and tetrathiomolybdate. The main therapy is penicillamine, a copper-chelating agent. Although this agent has been used in pregnancy without teratogenic effect, transient goitrous hypothyroidism in infants born to mothers with WD on penicillamine has been reported (47). Cessation of therapy can lead to fulminant liver failure and death during pregnancy. Zinc intake at a dose of 25 to $50 \mathrm{mg}$ three times daily in pregnancy appears to be safe with very minimal teratogenicity (48). Since penicillamine has an antipyridoxine effect, supplementation with vitamin $B_{6}$ is recommended.

\section{Primary Biliary Cirrhosis}

Primary biliary cirrhosis (PBC) is an autoimmune cholestatic liver disease characterized by progressive destruction of intrahepatic bile ducts, predominantly seen in middle-aged women. Typical symptoms are fatigue, pruritus, and abdominal pain. Jaundice develops in advanced liver disease. At presentation, about $40 \%$ of the patients are asymptomatic, but $30 \%$ to $50 \%$ already have hepatomegaly and $15 \%$ present with splenomegaly. $\mathrm{PBC}$ is associated with a large variety of other diseases, such as Sjogren's syndrome, arthropathy, CREST (calcinosis cutis, Raynaud's phenomenon, esophageal dysfunction, sclerodactyly, and telangiectasia) syndrome, and autoimmune thyroiditis. The characteristic serologic hallmark of $\mathrm{PBC}$ is the anti-mitochondrial antibody, a highly disease-specific autoantibody found in $90 \%$ to $95 \%$ of patients (49). Up to now, it is not known whether pregnancy was harmful in PBC patients. Previous reports suggested increased rates of infertility and preterm labor. Ursodeoxycholic acid (UDCA, $10-15 \mathrm{mg} / \mathrm{kg} /$ day) is regarded as the firstline treatment for PBC. UDCA appears to be safe and is considered FDA class $B$ in pregnancy. UDCA may improve pruritus and serum liver tests.

\section{Portal Vein Thrombosis}

Portal vein thrombosis (PVT) is a rare occurrence during pregnancy. Malignant tumors, cirrhosis, and other causes of intra-abdominal inflammation such as pancreatitis, appendicitis, cholecystitis, duodenal ulcer, and inflammatory bowel disease are the leading local risk factors (50). Management differs in acute and chronic PVT in pregnancy. The goal of treatment of acute PVT is to recanalize the obstructed veins, which may prevent intestinal infarction and subsequent portal hypertension. Correction of the causal factors should be achieved as soon as possible. Anticoagulation should be given for at least 3 months and that a permanent anticoagulation therapy is considered for patients with prothrombotic conditions (51). Pregnant patients with chronic PVT should be offered screening for gastroesophageal varices. In patients with extrahepatic portal hypertension without gastroesophageal varices, labor and vaginal delivery are preferable and less morbid, with a passive second stage of labor in order to avoid increased intra-abdominal pressure. Elective cesarean section is advisable in patients with gastroesophageal varices. Portal vein cavernoma is a rare disease resulting from extrahepatic PVT and development of collateral venous circulation (51). This disease is often accompanied by splenomegaly, portal hypertension, esophageal, gastric, or jejunal varices, and thrombocytopenia. Early diagnosis and treatment of gastroesophageal varices before pregnancy are recommended, which can be accomplished by screening upper endoscopy and therapy with sclerotherapy or esophageal band ligation of esophageal varices and/or institution of nonselective -beta blocker therapy is warranted.

\section{Budd-Chiari Syndrome}

Budd-Chiari syndrome (BCS), also known as hepatic venous outflow tract obstruction, is characterized by occluded hepatic venous outflow tract at various levels from small hepatic veins to inferior vena cava, resulting from thrombosis or its fibrous sequelae $(51,52)$. Most patients with BCS have a "primary" disorder and share one or more prothrombotic risk factors, mainly myeloproliferative disorders (MPD), oral contraceptive use, factor V Leiden mutation, and antiphospholipid syndrome. MPD, particularly polycythemia vera, are observed in approximately half of the cases of BCS, but fewer than $10 \%$ of the subjects with overt MPD develop BCS. The characteristic clinical triad of acute BCS is right upper quadrant pain, hepatomegaly, and ascites $(53,54)$. The diagnosis of BCS is established with Doppler sonography of the hepatic vessels. MRI can provide detailed examination of the hepatic and splanchnic vessels. Chronic thrombosis may evolve to precipitate portal hypertension with the formation of ascites, porto-systemic encephalopathy, and gastroesophageal varices. Women with BCS who desire to become pregnant should be screened for gastroesophageal varices with upper endoscopy, and if found, treatment should be offered preferably with esophageal band ligation or sclerotherapy. Most patients are placed on long-term warfarin therapy if nonpregnant and on 
low-molecular weight heparin if pregnant as soon as possible, and preferably before conception.

\section{Cirrhosis and Portal Hypertension}

Pregnancy is uncommon in the setting of cirrhosis possibly due to altered sex hormones and hepatocellular injury. The rates of spontaneous abortion, premature birth, and perinatal death are all increased in women with cirrhosis. The incidence of cirrhosis in pregnancy is estimated to be approximately 1 in 5950 pregnancies (55). Maternal morbidity and mortality is high during pregnancy in cirrhotic patients (10.3-18\%). Maternal deaths are due primarily to gastrointestinal hemorrhage from varices (56). During pregnancy, a hypervolemic state develops leading to an increase in portal flow and elevation of portal pressure transmitted to the collateral veins with increased risk of variceal bleeding. Esophageal variceal bleeding has been reported in $18 \%$ to $32 \%$ of pregnant women with cirrhosis and in up to $50 \%$ of those with known portal hypertension. Those with preexisting varices, $78 \%$ will have gastrointestinal bleeding during pregnancy, with a mortality rate of $18 \%$ to $50 \%$ (57). The outcome of pregnancy in women with cirrhosis is influenced by the underlying etiology of liver disease. AIH and alcoholic liver disease tend to have a favorable outcome in comparison with those with viral hepatitis. Endoscopic surveillance and banding of esophageal varices are recommended during pregnancy. Upper endoscopy in general appears to be safe during pregnancy. Primary prophylaxis with nonselective beta blockers is recommended. Propranolol and/or nadolol are considered FDA category C. The risks of nonselective beta blockers include fetal bradycardia, hypotension, hypoglycemia, and intrauterine growth retardation. Endoscopic band ligation seems to be a safe procedure in pregnancy. Termination of pregnancy is warranted in the presence of progressive hepatic decompensation. Vaginal delivery is usually safe and early forceps delivery or vacuum extraction should be considered to prevent any rise in portal pressure due to prolonged straining during labor. Cesarean section with general anesthesia is preferable in high-risk patients to avoid increase in portal pressure during labor.

\section{Gall Stones and Biliary Tract Disease}

The incidence of gallstones during pregnancy is estimated to be $3 \%$ to $12 \%$; however, symptomatic gallstones occur in only $1.2 \%$ of these pregnancies (58). Hormonal changes during pregnancy, altered gallbladder motility, and increased cholesterol secretion are major predisposing factors for the development of gallstones and biliary sludge. The commonest clinical presentations are biliary pain and gallstone pancreatitis and the least common is acute cholecystitis. The management of symptomatic uncomplicated biliary tract disease is usually conservative. In patients with symptomatic choledocholithiasis, endoscopic management using endoscopic retrograde cholangiopancreatography (ERCP) is an alternative option. Since fluoroscopy poses a radiation risk to the fetus, abdominal shield to the pregnant patient should be provided. Laparoscopic cholecystectomy can be performed safely during pregnancy with no maternal mortality, although spontaneous abortions and preterm labor have been reported.
Table 4 Classification of Liver Diseases Unique to Pregnancy According to Their Onset in Pregnancy and Association with Pre-eclampsia/Eclampsia

$\begin{array}{cc}\begin{array}{l}\text { Liver diseases associated } \\ \text { with pre-eclampsia/eclampsia }\end{array} & \begin{array}{c}\text { Liver diseases not associated } \\ \text { with pre-eclampsia/eclampsia }\end{array} \\ \begin{array}{cc}\text { HELLP syndrome (occurs in } \\ \text { second/third trimester) }\end{array} & \begin{array}{c}\text { Hyperemesis gravidarum (occurs } \\ \text { mainly in first trimester) }\end{array} \\ \begin{array}{c}\text { Acute fatty liver disease of } \\ \text { pregnancy (occurs mainly }\end{array} & \begin{array}{c}\text { Intrahepatic cholestasis of } \\ \text { pregnancy (occurs in second } \\ \text { in second/third trimester) }\end{array} \\ \text { abbreviation: HELLP, hemolysis, elevated liver enzymes, and low platelets. }\end{array}$

\section{LIVER DISEASES UNIQUE TO PREGNANCY}

Four unique disorders of liver dysfunction have been recognized during pregnancy. These include (i) hepatic involvement in hyperemesis gravidarum (HG), (ii) intrahepatic cholestasis of pregnancy (ICP), (iii) the syndrome of HELLP, and (iv) acute fatty liver of pregnancy (AFLP) (Table 4).

\section{Hyperemesis Gravidarum}

During pregnancy, nausea and vomiting are common; however, in some instances, the entity is protracted and may result in hospitalization. Intense nausea and vomiting in the first trimester associated with 5\% weight loss, dehydration, ketonuria and electrolytes imbalance, and metabolic alkalosis are highly suggestive of HG. Risk factors include previous history of HG, previous molar pregnancy, preexisting diabetes, gastrointestinal disorders, asthma, singleton female pregnancy, pregnancy with multiple male fetuses, multiple gestations, hyperthyroid disorders, psychiatric illness, and small prepregnancy body weight (59). Maternal smoking prior to pregnancy appears to be protective (60). The most common hepatic abnormality associated with $\mathrm{HG}$ is mild elevation of liver transaminases (usually $<300 \mathrm{IU}$ ), and it is thought to be associated with a temporary impairment of mitochondrial fatty acid oxidation (61). Jaundice is infrequent and total bilirubin is generally $<4 \mathrm{mg} / \mathrm{dL}$. These liver abnormalities are seen only in $20 \%$ to $30 \%$ of patients. Liver biopsy is generally not indicated unless to rule out other etiologies and, if performed, may show bland cholestasis. Maternal complications include Mallory-Weiss tears, preterm labor, and low birth weight (62). Treatment is supportive and directed at symptomatic relief with intravenous fluid and electrolytes replacement, thiamine and pyridoxine supplementation, and use of antiemetics. Hyperemesis with hepatic abnormalities has been reported to recur in subsequent pregnancies.

\section{Intrahepatic Cholestasis of Pregnancy}

ICP is characterized by pruritus and biochemical cholestasis typically presenting in the third trimester. This condition accounts for approximately $20 \%$ of jaundice during pregnancy. Although this is an uncommon disorder, it frequently recurs with subsequent pregnancies or the administration of synthetic estrogen compounds. Pruritus gravidarum is a term applied to patients with pruritus and biochemical cholestasis, whereas cholestasis of pregnancy is generally reserved for those patients with jaundice. The prevalence of ICP in the United States is estimated to be around $1 / 1000$ to $1 / 10,000$ pregnancies. 
The highest prevalence is seen among the Araucanian (27.6\%) and Aymara (11.8\%) Indians of Chile (63). Although the exact etiology is unknown, genetic and hormonal factors play a role. Genetic factors have been implicated owing to the frequent history of cholestasis in female family members of index patients. Heterozygous non-sense mutation of the multidrug resistance-associated protein 3 (MDR3) gene has been implicated in familial ICP (64). Estrogens produce lithogenic bile because estrogens decrease bile salt-independent flow and increase diffusion of water from bile to plasma. Pruritus generally involves the palms of the hands and the soles of the feet. Skin examination should be performed to rule out other skin disorders of pruritus. Jaundice may or may not be present. Some patients will have subclinical steatorrhea that may affect nutritional status. The diagnosis is established by the presence of typical history of generalized intractable pruritus in the second or third trimester of pregnancy with elevated maternal serum bile acids in the absence of radiologic evidence of biliary obstruction. Laboratory abnormalities are cholestatic with elevations in alkaline phosphatase up to 10-fold and modest hyperbilirubinemia, typically less than $5 \mathrm{mg} / \mathrm{dL}$. Serum aminotransferases are usually elevated but $<1000 \mathrm{U} / \mathrm{L}$. Cholestatic enzymes such as alkaline phosphatase are also elevated; however, serum gamma glutamyl transpeptidase is normal or slightly elevated. Elevated maternal serum bile acids are the hallmark of cholestasis and are usually $10 \mu \mathrm{mol} / \mathrm{L}$ (65). A cutoff level of serum bile acids $40 \mu \mathrm{mol} / \mathrm{L}$ is associated with impaired fetal outcome (66). Currently, genetic tests are performed in research laboratories only and are not applicable for diagnosis or risk stratification. However, mutation analysis of ABCB4 might be considered. Maternal outcome is favorable with prompt resolution of symptoms postpartum. Women with ICP have an increased incidence of gallstones due to lithogenic bile. Liver biopsy is unnecessary for diagnosis; however, if performed, it shows canalicular bile plugs with centrilobular cholestasis and intact parenchymal architecture. ICP recurs in about $70 \%$ of patients. Fetal outcome is not so favorable. Premature delivery occurs in $10 \%$ to $20 \%$, meconium staining in $35 \%$ to $60 \%$, and fetal distress in $19 \%$ to $60 \%$.

Treatment of ICP is largely symptomatic. UDCA, a tertiary bile acid, is the main treatment to alleviate pruritus and cholestasis. The recommended dose is $15 \mathrm{mg} / \mathrm{kg} /$ day in divided doses. UDCA improves liver transaminases, lower serum bile acids, and improve pruritus $(67,68)$. Other treatment such as hydroxyzine, Ondansetrone, and S-adenosyl-L-methionine (SAMe) has shown to be of some benefit (69). Fat-soluble vitamins should also be administered. In addition to the symptomatic relief of pruritus, a strict fetal monitoring must be carried out especially during the last few weeks of pregnancy, where rates of fetal morbidity and mortality are increased.

\section{HELLP}

Pre-eclampsia develops in $5 \%$ to $11 \%$ of all pregnancy and is characterized by the triad of proteinuria (greater than $300 \mathrm{mg}$ in 24 hours), edema, and hypertension (>140/90 $\mathrm{mmHg}$ ) after 20 weeks of gestation and/or within 48 hours after delivery. If the central nervous system becomes involved, for example, seizures or coma, the disorder is termed eclampsia. Hepatic involvement is a sign of advanced pre-eclampsia/eclampsia and may manifest as HELLP syndrome or hepatic rupture. The incidence of HELLP syndrome is approximately $0.6 \%$ of pregnancies and $3.1 \%$ to $12 \%$ of patients with pre-eclampsia (70). HELLP syndrome occurs mainly in the antepartum period in $70 \%$ to $92 \%$ of cases and postpartum in $8 \%$ to $30 \%$.

The symptoms include right upper quadrant abdominal pain, headache, malaise, nausea, and vomiting. Concomitant signs and symptoms of pre-eclampsia (hypertension, proteinuria, and edema) may or may not be present. Jaundice and bleeding, due to thrombocytopenia, are a very uncommon mode of presentation. Severe right upper quadrant abdominal pain that is radiated to the neck or shoulder may herald impending hepatic rupture or presence of hepatic hematoma. Serum aminotransferases range from 300 to $3000 \mathrm{U} / \mathrm{L}$ and serum bilirubin is usually less than $5 \mathrm{mg} / \mathrm{dL}$, but higher values have been reported. Hepatic parenchymal injury results from microangiopathic hemolytic anemia, thrombocytopenia, and localized segmental vasospasm and may lead to hepatic infarction, hematoma, and rupture resulting in maternal death. Disseminated intravascular coagulation (DIC) is found in $8 \%$ to $21 \%$ of patients. If hepatic parenchymal injury is severe, hypoglycemia may be observed. Liver biopsies or autopsy specimens show periportal fibrin deposition and hemorrhage; portal tracts are usually involved but in some cases lesions may be focal. More recently, hepatic microvesicular fat similar to that seen in AFLP has been observed in histologic specimens, leading to speculation that pre-eclampsia and AFLP may be different presentations of the same process. Physicians should be aware of other causes that may resemble HELLP such as acute viral hepatitis, hemolytic uremic syndrome, thrombotic thrombocytopenic purpura, antiphospholipid syndrome, and AFLP. Both HELLP syndrome and AFLP occur in the third trimester and have similar presentations, but liver dysfunction is usually more pronounced in the latter and is more frequently associated with coagulopathy, hypoglycemia, and renal failure (Table 5). The coagulopathy of the AFLP is due to liver failure, whereas in HELLP syndrome, coagulopathy develops as a part of DIC syndrome.

Initial therapy is directed at control of hypertension and prevention of seizures, and optimal management includes prompt delivery. Frequent monitoring of serum glucose is warranted and intravenous dextrose solution is infused as needed. Maternal outcomes are generally favorable with mortality ranges from $2 \%$ to $4 \%$ in reported cases. Corticosteroids have been shown to improve maternal laboratory values but did not affect overall outcome. Platelet transfusion is recommended in the case of bleeding or severe thrombocytopenia (platelet count $<20 \times 10^{9} / \mathrm{L}$ ).

Liver rupture is a rare, life-threatening complication of HELLP that carries a very high maternal mortality. It is usually preceded by an intraparenchymal hemorrhage progressing to a contained subcapsular hematoma in the right hepatic lobe in patients with severe thrombocytopenia. Hepatic rupture usually requires immediate laparotomy with evacuation of the hematoma with pressure packing and drainage, followed by consideration of hepatic artery embolization or ligation, partial hepatectomy, or oversewing of the laceration.

\section{AFLP}

AFLP is a rare disorder ( 1 per 13,000 deliveries) unique to pregnancy, which occurs in the third trimester or peripartum (71). 
Table 5 Features of Liver Diseases Unique to Pregnancy

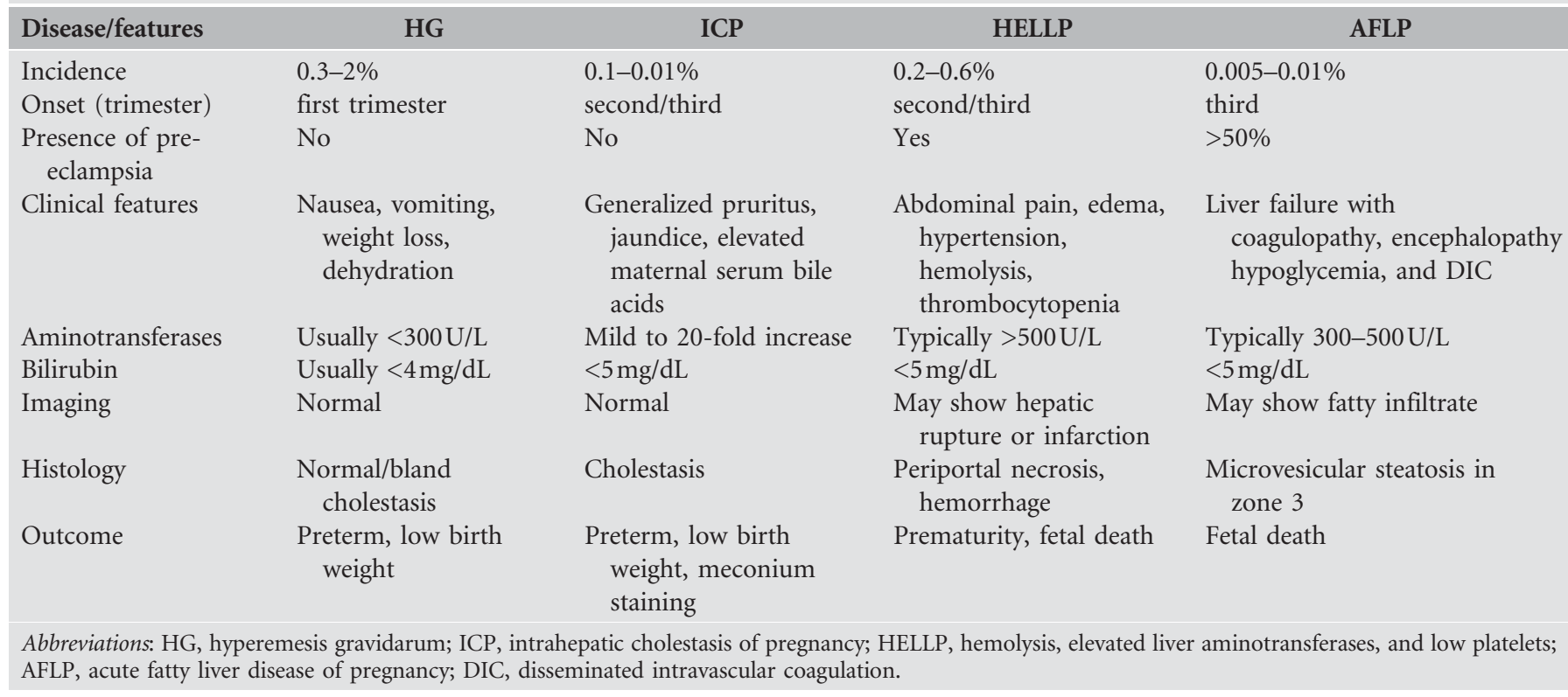

This was first described by Sheehan in 1940, who termed it obstetric acute yellow atrophy. The disorder is more common in primagravidas, twin gestations, and those with a male fetus. The disease occurs at any age in the childbearing period. Characteristically, it develops in the last 10 weekes and usually in the last 4 weeks of pregnancy.

Typically patients present with nausea and vomiting for several days, followed by jaundice and then progressive encephalopathy. Right upper quadrant pain and epigastric abdominal pain are common (50-80\%) (72). Fever, headache, diarrhea, back pain suggestive of acute pancreatitis, and myalgias are occasionally initial presentation. Hepatic failure may ensue. Approximately $50 \%$ of patients may also have features of preeclampsia. Physical examination may reveal only right upper quadrant tenderness. Laboratory abnormalities include elevated serum aminotransferases usually 300 to $500 \mathrm{U} / \mathrm{L}$, moderate increase in serum bilirubin $5 \mathrm{mg} / \mathrm{dL}$ and variable degrees of leukocytosis (73), DIC, hypoglycemia, hyperuricemia, and bacteremia. Patients may develop gastrointestinal hemorrhage, pancreatitis, and renal failure. Radiographic evaluations may suggest the diagnosis from steatosis seen by ultrasound, computerized tomography, or magnetic resonance imaging. However, this finding may not be universally present and normal studies do not exclude the diagnosis.

AFLP is characterized by a microvesicular fatty infiltration of the hepatocytes involving perivenular and mid-zones (74). Extensive hepatocellular necrosis, a common histologic feature of HELLP syndrome, is absent in AFLP, while microvesicular fatty infiltration, the predominant feature of AFLP, is generally absent in HELLP syndrome (72).

There is a strong association between AFLP and a deficiency of the enzyme long-chain 3-hydroxyacyl-CoA dehydrogenase (LCHAD) in the fetus, a disorder of mitochondrial fatty acid beta-oxidation (75). In a study by Ibdah and colleagues, 79\% of women who carried LCHAD-deficient fetuses developed AFLP (76). Infants born to mothers with AFLP may exhibit immediate or delayed nonketotic hypoglycemia, hepatic encephalopathy, cardiomyopathy, slowly progressing peripheral neuropathy, skeletal myopathy, or sudden unexpected death $(77,78)$. Other studies reported involvement of fetal hepatic carnitine palmitoyltransferase (CPT I) deficiency and maternal medium-chain acyl-CoA dehydrogenase deficiency in AFLP $(79,80)$. It is recommended that neonates born to pregnancies complicated by AFLP be tested for the common LCHAD G1528C mutation (81) and that this testing when done early after birth can be lifesaving as it may identify LCHAD-deficient children before they manifest the disease allowing early dietary intervention by institution of a diet low in fat and high in carbohydrate, and by substitution of the long-chain fatty acids with medium-chain fatty acids. This strong association between AFLP and the common G1528C mutation in the fetus is significant $(82,83)$, and hence screening the offspring of women who develop AFLP at birth for this mutation can be lifesaving.

Successful management of AFLP is dependent upon prompt delivery and supportive care. Overall previous reported maternal and fetal mortality is $26 \%$ and $32 \%$ respectively, but more recent series suggest improving maternal and fetal mortality with prompt delivery. Fetal mortality is believed to occur as a result of rapid maternal decompensation, a high rate of premature delivery, and the maternal DIC resulting in fibrin deposition in the placenta, culminating in uteroplacental insufficiency, infarct, and fetal asphyxia (84). Immediate delivery would therefore be expected to improve fetal outcome, since basic organ systems are developed. AFLP may recur in subsequent pregnancy, particularly in mothers with a fatty acid oxidation defect in the offspring (72).

\section{Liver Transplantation and Pregnancy}

Since the first pregnancy in a transplant recipient in 1958, pregnancy in recipients of solid organ transplants has become increasingly common. The first successful pregnancy in a liver graft recipient was reported in 1978 (85). Given the improving success of liver transplantation over the past two decades and decreasing levels of immunosuppression, most organ transplant recipients lead happy and healthy lives with an average 
1-year survival rate of $>85 \%$ at better transplant programs. As of January 2007, there were 1595 pregnancies in female transplant recipients reported to the National Transplantation Pregnancy Registry (NTPR). In 131 liver transplant recipients in the NTPR, 219 pregnancies and 223 outcomes were reported $(86,87)$. Children born to female liver recipients have a greater risk of prematurity and low birth weight than the general population; however, no congenital malformation patterns have been observed. Majority of women after liver transplantation will deliver a healthy baby. In a large single-center experience that evaluated the outcome of 71 pregnancies after liver transplantation, pregnancyinduced hypertension was seen in $20 \%$, pre-eclampsia in $13 \%$, acute cellular rejection in $17 \%$, and renal impairment in $11 \%(88)$. Pregnancies occurring within 1-year posttransplantation had an increased incidence of prematurity (58\% vs. $29 \%$ ), low birth weight, and acute cellular rejection (33\% vs. $14 \%$ ) compared with those occurring later than 1 year (88). Although no statistically significant differences in outcome were identified, rates of acute rejection and graft loss are similar to nonpregnant liver recipients. An NTPR analysis of liver recipients noted that those who reported rejection during pregnancy had poorer newborn outcomes and graft survival (89). If desired, pregnancy should be deferred for 1 year after liver transplantation. This recommendation is supported by NTPR data. Immunosuppression regimens typically include cyclosporin (CyA) or FK506, mycophenolate mofetil, and prednisone with or without AZA. Additional medications for liver transplant recipients may include prophylaxis for Pneumocystis carinii infection with trimethoprim/sulfamethoxazole or pentamidine, and for herpes virus infection with acyclovir. Every effort should be exercised to avoid teratogenic medications during pregnancy. Family planning and multidisciplinary team involvement are optimum. Breast-feeding is discouraged while women are on immunosuppressant medication.

\section{REFERENCES}

1. Esteve E, Saudeau L, Pierre F, et al. Physiological cutaneous signs in normal pregnancy: a study of 60 pregnant women. Ann Dermatol Venereol 1994; 121: 227-31.

2. Bean WB, Cogswell R. Vascular changes of the skin in pregnancy; vascular spiders and palmar erythema. Surg Gynecol Obstet 1949; 88: $739-52$.

3. Hay JE. Liver disease in pregnancy. Hepatology 2008; 47: 1067-76.

4. Boccia D, Guthmann JP, Klovstad H, et al. High mortality associated with an outbreak of hepatitis E among displaced persons in Darfur, Sudan. Clin Infect Dis 2006; 42: 1679-84.

5. Kang AH, Graves CR. Herpes simplex hepatitis in pregnancy: a case report and review of the literature. Obstet Gynecol Surv 1999; 54: 463-8.

6. Jacques SM, Qureshi F. Herpes simplex virus hepatitis in pregnancy: a clinicopathologic study of three cases. Hum Pathol 1992; 23: 183-7.

7. Sjogren MH, Tanno H, Fay O, et al. Hepatitis A virus in stool during clinical relapse. Ann Intern Med 1987; 106: 221-6.

8. Elinav E, Ben-Dov IZ, Shapira Y, et al. Acute hepatitis A infection in pregnancy is associated with high rates of gestational complications and preterm labor. Gastroenterology 2006; 130: 1129-34.

9. Rustgi VK, Hoofnagle JH. Viral hepatitis during pregnancy. Semin Liver Dis 1987; 7: 40-6.

10. Letson GW, Shapiro CN, Kuehn D, et al. Effect of maternal antibody on immunogenicity of hepatitis A vaccine in infants. J Pediatr 2004; 144: 327-32.

11. Beasley RP. Hepatitis B virus. The major etiology of hepatocellular carcinoma. Cancer 1988; 61: 1942-56.
12. Locarnini S. Molecular virology and the development of resistant mutants: implications for therapy. Semin Liver Dis 2005; 25(Suppl 1): 9-19.

13. Alter MJ. Epidemiology of hepatitis B in Europe and worldwide. J Hepatol 2003; 39(Suppl 1): S64-9.

14. Lavanchy D. Hepatitis B virus epidemiology, disease burden, treatment, and current and emerging prevention and control measures. J Viral Hepat 2004; 11: 97-107.

15. McMahon BJ. Epidemiology and natural history of hepatitis B. Semin Liver Dis 2005; 25(Suppl 1): 3-8.

16. McQuillan GM, Coleman PJ, Kruszon-Moran D, et al. Prevalence of hepatitis B virus infection in the United States: the National Health and Nutrition Examination Surveys, 1976 through 1994. Am J Public Health 1999; 89: 14-18.

17. Edlich RF, Diallo AO, Buchanan L, et al. Hepatitis B virus: a comprehensive strategy for eliminating transmission in the United States. J Long Term Eff Med Implants 2003; 13: 117-25.

18. Mast EE, Weinbaum CM, Fiore AE, et al. A comprehensive immunization strategy to eliminate transmission of hepatitis B virus infection in the United States: recommendations of the Advisory Committee on Immunization Practices (ACIP) Part II: immunization of adults. MMWR Recomm Rep 2006; 55: 1-33; quiz CE1-4.

19. Recommendations of the Immunization Practices Advisory Committee (ACIP). Hepatitis B virus: a comprehensive strategy for eliminating transmission in the United States through universal childhood vaccination. MMWR Recomm Rep 1991; 40: 1-25.

20. Buchanan C, Tran TT. Management of chronic hepatitis B in pregnancy. Clin Liver Dis 2010; 14: 495-504.

21. Lok AS, McMahon BJ. Chronic hepatitis B: update 2009. Hepatology 2009; 50: 661-2.

22. Hutchinson SJ, Goldberg DJ, King M, et al. Hepatitis C virus among childbearing women in Scotland: prevalence, deprivation, and diagnosis. Gut 2004; 53: 593-8.

23. Alter MJ, Margolis HS, Krawczynski K, et al. The natural history of community-acquired hepatitis $\mathrm{C}$ in the United States. The Sentinel Counties Chronic non-A, non-B Hepatitis Study Team. N Engl J Med 1992; 327: 1899-905.

24. Armstrong GL, Wasley A, Simard EP, et al. The prevalence of hepatitis C virus infection in the United States, 1999 through 2002. Ann Intern Med 2006; 144: 705-14.

25. Bukh J, Miller RH, Purcell RH. Genetic heterogeneity of hepatitis C virus: quasispecies and genotypes. Semin Liver Dis 1995; 15: 41-63.

26. Airoldi J, Berghella V. Hepatitis C and pregnancy. Obstet Gynecol Surv 2006; 61: 666-72.

27. Marcellin P, Bernuau J, Martinot-Peignoux M, et al. Prevalence of hepatitis $\mathrm{C}$ virus infection in asymptomatic anti-HIV1 negative pregnant women and their children. Dig Dis Sci 1993; 38: 2151-5.

28. Alter MJ, Kruszon-Moran D, Nainan OV, et al. The prevalence of hepatitis C virus infection in the United States, 1988 through 1994. N Engl J Med 1999; 341: 556-62.

29. Ferrero S, Lungaro P, Bruzzone BM, et al. Prospective study of motherto-infant transmission of hepatitis C virus: a 10-year survey (1990-2000). Acta Obstet Gynecol Scand 2003; 82: 229-34.

30. Manns MP, McHutchison JG, Gordon SC, et al. Peginterferon alfa-2b plus ribavirin compared with interferon alfa- $2 \mathrm{~b}$ plus ribavirin for initial treatment of chronic hepatitis C: a randomised trial. Lancet 2001; 358: 958-65.

31. Fried MW, Shiffman ML, Reddy KR, et al. Peginterferon alfa-2a plus ribavirin for chronic hepatitis C virus infection. N Engl J Med 2002; 347: 975-82.

32. Hadziyannis SJ, Sette H Jr, Morgan TR, et al. Peginterferon-alpha2a and ribavirin combination therapy in chronic hepatitis C: a randomized study of treatment duration and ribavirin dose. Ann Intern Med 2004; 140: 346-55.

33. Kumar A, Beniwal $\mathrm{M}$, Kar $\mathrm{P}$, et al. Hepatitis $\mathrm{E}$ in pregnancy. Int J Gynaecol Obstet 2004; 85: 240-4.

34. Singh S, Mohanty A, Joshi YK, et al. Mother-to-child transmission of hepatitis E virus infection. Indian J Pediatr 2003; 70: 37-9.

35. Pischke $\mathrm{S}$, Wedemeyer $\mathrm{H}$. Chronic hepatitis $\mathrm{E}$ in liver transplant recipients: a significant clinical problem? Minerva Gastroenterol Dietol 2010; 56: 121-8.

36. Haagsma EB, van den Berg AP, Porte RJ, et al. Chronic hepatitis E virus infection in liver transplant recipients. Liver Transpl 2008; 14: 547-53. 
37. Aggarwal R, Krawczynski K. Hepatitis E: an overview and recent advances in clinical and laboratory research. J Gastroenterol Hepatol 2000; 15: 9-20.

38. McFarlane IG. Autoimmune hepatitis: diagnostic criteria, subclassifications, and clinical features. Clin Liver Dis 2002; 6: 605-21.

39. Schramm C, Herkel J, Beuers U, et al. Pregnancy in autoimmune hepatitis: outcome and risk factors. Am J Gastroenterol 2006; 101: 556-60.

40. Candia L, Marquez J, Espinoza LR. Autoimmune hepatitis and pregnancy: a rheumatologist's dilemma. Semin Arthritis Rheum 2005; 35: 49-56.

41. Cooper GS, Stroehla BC. The epidemiology of autoimmune diseases. Autoimmun Rev 2003; 2: 119-25.

42. Czaja AJ. Autoimmune hepatitis. Clin Liver Dis 2002; 6: xi-xii.

43. Czaja AJ. Current and future treatments of autoimmune hepatitis. Expert Rev Gastroenterol Hepatol 2009; 3: 269-91.

44. Heneghan MA, Norris SM, O’Grady JG, et al. Management and outcome of pregnancy in autoimmune hepatitis. Gut 2001; 48: 97-102.

45. Chin RK. Pregnancy and Wilson's disease. Am J Obstet Gynecol 1991; 165: 488-9.

46. Tarnacka B, Rodo M, Cichy S, et al. Procreation ability in Wilson's disease. Acta Neurol Scand 2000; 101: 395-8.

47. Hanukoglu A, Curiel B, Berkowitz D, et al. Hypothyroidism and dyshormonogenesis induced by D-penicillamine in children with Wilson's disease and healthy infants born to a mother with Wilson's disease. J Pediatr 2008; 153: 864-6.

48. Brewer GJ, Johnson VD, Dick RD, et al. Treatment of Wilson's disease with zinc. XVII: treatment during pregnancy. Hepatology 2000; 31: 364-70.

49. Invernizzi $\mathrm{P}$, Lleo A, Podda M. Interpreting serological tests in diagnosing autoimmune liver diseases. Semin Liver Dis 2007; 27: 161-72.

50. Kumar S, Sarr MG, Kamath PS. Mesenteric venous thrombosis. N Engl J Med 2001; 345: 1683-8.

51. de Franchis R. Revising consensus in portal hypertension: report of the Baveno $\mathrm{V}$ consensus workshop on methodology of diagnosis and therapy in portal hypertension. J Hepatol 2010; 53: 762-8.

52. Janssen HL, Garcia-Pagan JC, Elias E, et al. Budd-Chiari syndrome: a review by an expert panel. J Hepatol 2003; 38: 364-71.

53. Singh V, Sinha SK, Nain CK, et al. Budd-Chiari syndrome: our experience of 71 patients. J Gastroenterol Hepatol 2000; 15: 550-4.

54. Slakey DP, Klein AS, Venbrux AC, et al. Budd-Chiari syndrome: current management options. Ann Surg 2001; 233: 522-7.

55. Aggarwal N, Sawnhey H, Suril V, et al. Pregnancy and cirrhosis of the liver. Aust NZ J Obstet Gynaecol 1999; 39: 503-6.

56. Pajor A, Lehoczky D. Pregnancy in liver cirrhosis. Assessment of maternal and fetal risks in eleven patients and review of the management. Gynecol Obstet Invest 1994; 38: 45-50.

57. Tan J, Surti B, Saab S. Pregnancy and cirrhosis. Liver Transpl 2008; 14: 1081-91.

58. Ko CW, Beresford SA, Schulte SJ, et al. Incidence, natural history, and risk factors for biliary sludge and stones during pregnancy. Hepatology 2005; 41: 359-65.

59. Matsuo K, Ushioda N, Nagamatsu M, et al. Hyperemesis gravidarum in Eastern Asian population. Gynecol Obstet Invest 2007; 64: 213-16.

60. Kallen B, Lundberg G, Aberg A. Relationship between vitamin use, smoking, and nausea and vomiting of pregnancy. Acta Obstet Gynecol Scand 2003; 82: 916-20.

61. Outlaw WM, Ibdah JA. Impaired fatty acid oxidation as a cause of liver disease associated with hyperemesis gravidarum. Med Hypotheses 2005; 65: 1150-3.

62. Dodds L, Fell DB, Joseph KS, et al. Outcomes of pregnancies complicated by hyperemesis gravidarum. Obstet Gynecol 2006; 107(2 Pt 1): 285-92.

63. Reyes H, Gonzalez MC, Ribalta J, et al. Prevalence of intrahepatic cholestasis of pregnancy in Chile. Ann Intern Med 1978; 88: 487-93.

64. Jacquemin E, Cresteil D, Manouvrier S, et al. Heterozygous non-sense mutation of the MDR3 gene in familial intrahepatic cholestasis of pregnancy. Lancet 1999; 353: 210-11.

65. Brites D. Intrahepatic cholestasis of pregnancy: changes in maternal-fetal bile acid balance and improvement by ursodeoxycholic acid. Ann Hepatol 2002; 1: 20-8.
66. Glantz A, Hanns-Ulrich M, Lars-Ake M. Intrahepatic cholestasis of pregnancy: Relationships between bile acid levels and fetal complication rates. Hepatology 2004; 40: 467-74.

67. Palma J, Reyes H, Ribalta J, et al. Ursodeoxycholic acid in the treatment of cholestasis of pregnancy: a randomized, double-blind study controlled with placebo. J Hepatol 1997; 27: 1022-8.

68. Zapata R, Lorena S, Joaqũ̃-n P, et al. Ursodeoxycholic acid in the treatment of intrahepatic cholestasis of pregnancy. A 12-year experience. Liver Int 2005; 25: 548-54.

69. Almasio P, Bortolini M, Pagliaro L, et al. Role of S-adenosyl-L-methionine in the treatment of intrahepatic cholestasis. Drugs 1990; 40(Suppl 3): 111-23.

70. Araujo AC, Leao MD, Nobrega MH, et al. Characteristics and treatment of hepatic rupture caused by HELLP syndrome. Am J Obstet Gynecol 2006; 195: 129-33.

71. Knox TA, Olans LB. Liver disease in pregnancy. N Engl J Med 1996; 335: 569-76.

72. Ibdah JA. Acute fatty liver of pregnancy: an update on pathogenesis and clinical implications. World J Gastroenterol 2006; 12: 7397-404.

73. Mjahed K, Charra B, Hamoudi D, et al. Acute fatty liver of pregnancy. Arch Gynecol Obstet 2006; 274: 349-53.

74. Rolfes DB, Ishak KG. Acute fatty liver of pregnancy: a clinicopathologic study of 35 cases. Hepatology 1985; 5: 1149-58.

75. Pons R, Roig M, Riudor E, et al. The clinical spectrum of long-chain 3-hydroxyacyl-CoA dehydrogenase deficiency. Pediatr Neurol 1996; 14: 236-43.

76. Ibdah JA, Bennett MJ, Rinaldo P, et al. A fetal fatty-acid oxidation disorder as a cause of liver disease in pregnant women. N Engl J Med 1999; 340: 1723-31.

77. Ibdah JA, Tein I, Dionisi-Vici C, et al. Mild trifunctional protein deficiency is associated with progressive neuropathy and myopathy and suggests a novel genotype-phenotype correlation. J Clin Invest 1998; 102: 1193-9.

78. Rinaldo P, Raymond K, al-Odaib A, et al. Clinical and biochemical features of fatty acid oxidation disorders. Curr Opin Pediatr 1998; 10: 615-21.

79. Browning MF, Levy HL, Wilkins-Haug LE, et al. Fetal fatty acid oxidation defects and maternal liver disease in pregnancy. Obstet Gynecol 2006; 107: 115-20.

80. Santos L, Patterson A, Moreea SM, et al. Acute liver failure in pregnancy associated with maternal MCAD deficiency. J Inherit Metab Dis 2007; 30: 103.

81. Yang Z, Yamada J, Zhao Y, et al. Prospective screening for pediatric mitochondrial trifunctional protein defects in pregnancies complicated by liver disease. JAMA 2002; 288: 2163-6.

82. Sims HF, Brackett JC, Powell CK, et al. The molecular basis of pediatric long chain 3-hydroxyacyl-CoA dehydrogenase deficiency associated with maternal acute fatty liver of pregnancy. Proc Natl Acad Sci USA 1995; 92: 841-5.

83. IJIst L, Wanders RJ, Ushikubo S, et al. Molecular basis of long-chain 3-hydroxyacyl-CoA dehydrogenase deficiency: identification of the major disease-causing mutation in the alpha-subunit of the mitochondrial trifunctional protein. Biochim Biophys Acta 1994; 1215: $347-50$.

84. Moise KJ Jr, Shah DM. Acute fatty liver of pregnancy: etiology of fetal distress and fetal wastage. Obstet Gynecol 1987; 69(3 Pt 2): 482-5.

85. Walcott WO, Derick DE, Jolley JJ, et al. Successful pregnancy in a liver transplant patient. Am J Obstet Gynecol 1978; 132: 340-1.

86. Armenti VT, Constantinescu S, Moritz MJ, et al. Pregnancy after transplantation. Transplant Rev (Orlando) 2008; 22: 223-40.

87. Armenti VT, Daller JA, Constantinescu S, et al. Report from the National Transplantation Pregnancy Registry: outcomes of pregnancy after transplantation. Clin Transpl 2006: 57-70.

88. Christopher V, Al-Chalabi T, Richardson PD, et al. Pregnancy outcome after liver transplantation: a single-center experience of 71 pregnancies in 45 recipients. Liver Transpl 2006; 12: 1138-43.

89. Armenti VT, Radomski JS, Moritz MJ, et al. Report from the National Transplantation Pregnancy Registry (NTPR): outcomes of pregnancy after transplantation. Clin Transpl 2000: 123-34. 


\section{Neurologic disorders in pregnancy \\ Robert Burger, Terry Rolan, David Lardizabal, Upinder Dhand, Aarti Sarwal, and Pradeep Sahota}

From headache to neuromuscular disease to stroke and movement disorders, neurologic disorders are common. They occur in men and women. Pregnancy is no exception. In fact, pregnancy may have an effect on neurologic condition; in turn, neurologic disorders may have an effect on pregnancy or outcome of pregnancy. Treatment of neurologic disorders is another important aspect whereby knowledge of teratogenicity is important for the physician treating a pregnant woman. While this is an extensive area, an attempt will be made to cover some key areas. The coverage will include headache, multiple sclerosis (MS), neuromuscular disorders (NMDs), epilepsy, movement disorders, and stroke.

\section{HEADACHES IN PREGNANCY}

Headache is perhaps the most common complaint in medicine and is a common complaint during pregnancy. Most headaches during pregnancy are due to primary headache disorders (migraine, tension-type, and cluster headaches). Although these may be debilitating, they are almost always benign. Secondary headache disorders are those in which an underlying cause can be identified for the headache. These include headaches due to infection, trauma, vascular disorders, pre-eclampsia, or cerebrospinal fluid (CSF) derangement. This chapter will attempt to look at the diagnosis and treatment of the major primary and secondary headaches during pregnancy.

\section{Primary Headaches}

\section{Migraine}

Migraine headaches are usually a moderate or severe unilateral or bilateral throbbing or pulsating headache with associated increased sensitivity to light, noise, or physical movement and may be accompanied by nausea and vomiting. Migraine pain also commonly involves the neck and medial shoulders in addition to the head. The usual duration of a migraine headache is 4 to 72 hours. Up to $25 \%$ of migraine headaches may be preceded or accompanied by an aura. The aura is due to neuronal dysfunction that is thought to move through the cortex and is accompanied by decreased regional blood flow (1).

The most common migraine auras are visual and sensory. Visual auras may produce positive visual phenomena such as seeing lights, stars, shimmering lights (scintillating scotoma), and pixilated or mosaic vision. The visual dysfunction may gradually spread across the visual field. Negative visual phenomena may also occur such as dark visual scotoma. Somatosensory migraine auras may produce numbness in the face or arm and hand that may slowly travel over the affected area. Less commonly, patients may report dysarthria or aphasia as a form of aura accompanying a migraine attack.

The typical migraine aura builds up over 10 to 20 minutes and usually completely resolves in 60 minutes. The conclusion of the migraine attack usually coincides with the onset of the migraine headache.

In most women, migraine usually improves during pregnancy. Migraine improves in up to $50 \%$ to $80 \%$ of women during pregnancy (2,3). Migraine may recur in the first postpartum week in up to $50 \%$ of migraineurs (4).

\section{Treatment of Migraine During Pregnancy}

Migraine headaches affect up to $18 \%$ of women during their lifetime and are common during the childbearing years. Treatment of migraine during pregnancy is geared toward providing headache relief for the mother and avoiding potential adverse effects on the developing fetus. Acetaminophen (650-1000 mg po), codeine (30-60 mg po with acetaminophen), and hydrocodone (5.0-7.5 mg with acetaminophen) are considered safe to use for acute pain relief. Narcotics should be avoided in the third trimester because of the risk of neonatal dependence and withdrawal following delivery. Narcotic use should be restricted to several days a week to avoid development of medication overuse headache. Nonsteroidal anti-inflammatory drugs (NSAIDs) such as ibuprofen and naproxen are considered safe (pregnancy class B) in the first and second trimesters but should be avoided in the third trimester due to potential risks of inhibiting labor or premature closure of the ductus arteriosus. The jury is still out on use of triptans (pregnancy class C) such as sumatriptan during pregnancy (5). At this time, it is difficult to recommend triptan usage during pregnancy unless felt absolutely essential for the well-being of the mother.

Ergotamine and dihydroergotamine (pregnancy class X) are contraindicated in pregnancy as they are considered potential abortifacients.

Antiemetics such as prochlorperazine (10 mg IV), metoclopramide (10 mg IV or PO), or ondansetron (4mg IV) are considered relatively safe in pregnancy and are particularly useful in women who have significant nausea, vomiting, and dehydration accompanying their migraines. Metoclopramide and ondansetron are pregnancy class B.

Women who have prolonged, recurrent, or severe migraines, particularly when resistant to acute treatment, should be considered for preventative migraine treatment. Beta blockers (atenolol, nadolol, propranolol, and timolol), some tricyclic antidepressants (doxepin and protriptyline), fluoxetine [a selective serotonin reuptake inhibitor (SSRI)], verapamil (a calcium channel blocker) are all class C 
pregnancy drugs. Tricyclic antidepressants should be stopped at least 2 weeks before delivery due to the potential of neonatal respiratory distress.

There is inadequate information regarding use of the newer anticonvulsants [lamotrigine, gabapentin (GBP), topiramate (TPM), oxcarbazepine and levetiracetam (LEV)] for migraine prophylaxis in pregnancy (6). Valproic acid (VPA; pregnancy category D) should be avoided in women actively seeking to become pregnant or in early pregnancy due to reports of risks of neural tube defects (NTDs) when taken early in pregnancy (7).

The safest migraine treatment in pregnancy encompasses good headache hygiene-regular meals and sleep, avoidance of known migraine triggers for a particular patient, and the avoidance of stress when possible. Behavioral therapy such as focused relaxation techniques and stress management when available can also provide safe alternatives to pharmacologic treatment. The same modalities can be beneficial for tensiontype headache sufferers.

\section{Tension-Type Headaches}

Tension-type headaches are the most common recurring headaches during pregnancy. The headache pain is usually described as mild to moderate (less severe than the usual moderate to severe pain of a migraine headache). The pain quality is dull and aching and located diffusely throughout the head or in a band-like encircling pattern. They are not typically associated with increased sensitivity to light, noise, or physical movement and are not accompanied by nausea and vomiting. The duration of tension-type headaches is variable, but most last less than a day. Like migraines, they may be triggered by stress. The most common variety of tension-type headaches is those that occur episodically (1-10 episodes less than 15 days a month). Chronic tension-type headaches (more than 15 days a month) occur in a small percentage of patients and may be associated with medication overuse.

Tension-type headaches in pregnancy can be treated pharmacologically with acetaminophen or NSAIDs (ibuprofen or naproxen in the first of second trimesters). Mild tensiontype headaches often respond to rest, relaxation, cold or hot packs, physical therapy, or even acupuncture. Chronic tension-type headaches, especially if associated with depression, can be treated with an SSRI such as fluoxetine.

\section{Cluster Headaches}

Cluster headaches are more common in men than women (3-5:1) and are seen uncommonly in pregnancy. The typical cluster headache is a unilateral orbital, supraorbital, or temporal head pain of rapid onset (5-15 minutes to reach peak severity) that lasts from 15 to 180 minutes and subsides rapidly and completely. The pain is very severe and is usually described as boring, claw-like, or exploding. Cluster headaches are usually accompanied by ipsilateral autonomic symptoms with some combination of ptosis, conjunctival injection, tearing, rhinorrhea, or forehead sweating. Cluster headaches may occur several times a day and may be triggered by the onset of rapid eye movement sleep, awakening patients within an hour after going to sleep. Patients are usually very restless during a cluster attack and frequently rock back and forth or pace. Lying down (as opposed to migraine) may aggravate the pain. Patients may have one or more attacks a day for 1 to 3 months during a cluster period and then have a period of complete remission for one to several years. Cluster headaches can usually be treated effectively with $\mathrm{O}_{2}$ inhalation at $7 \mathrm{~L} / \mathrm{min}$ for 10 minutes by a non-rebreathing face mask. Up to two of three patents may obtain significant relief in 5 to 10 minutes (8).

\section{Breast-Feeding Following Delivery}

Drugs that are safe during pregnancy are safe to use in breastfeeding mothers. Acetaminophen and NSAIDs are considered safe for acute migraine and tension-type headaches. Shortacting triptans such as sumatriptan (half-life 2.5 hours) can be used by women who pump and store breast milk prior to triptan use. Beta blockers, verapamil, and narcotics (codeine and morphine) are also relatively safe.

\section{Secondary Headaches}

Secondary headaches in pregnancy include a number of disease states with potentially serious morbidity of the mother. These include subarachnoid hemorrhage, cerebral venous thrombosis (CVT) and stroke, pre-eclampsia, central nervous system (CNS) infections, and pseudotumor cerebri.

\section{Subarachnoid Hemorrhage}

Subarachnoid hemorrhage usually presents with a sudden onset, severe headache that patients frequently describe as the "worst of their life." Pregnancy does appear to increase the risk of aneurysmal and arteriovenous malformation (AVM) rupture with the greatest risk later in pregnancy, at the time of delivery, and in the puerperium (9). Most subarachnoid hemorrhages can be diagnosed with the use of MRI or CT scanning. A small percentage however may have negative cranial imaging and require analysis of CSF for detection of the hemorrhage. The risk of subarachnoid hemorrhage is estimated at 1 to 2 per 10,000 pregnancies.

\section{CVT and Stroke}

The risk of CVT is increased both during pregnancy and in the puerperium. Many conditions such at thrombophilias (factor $\mathrm{V}$ Leiden and prothrombin gene mutations, deficiencies of protein $\mathrm{C}$ or $\mathrm{S}$, or presence of high titers of antiphospholipid antibodies), red blood cell disorders such as sickle-cell anemia or polycythemia, local infections (meningitis, cerebral abscess, sinus or inner ear infections), and dehydration among others can predispose to CVT. When the superior sagittal sinus is involved by thrombosis, the intracranial pressure may be raised producing headaches and papilledema. If the cerebral cortical veins are involved, cerebral hemorrhages, strokes, and seizures may occur. Diagnosis of CVT is made by magnetic resonance imaging (MRI) and MR venography (MRV). Treatment of CVT is anticoagulation with heparin. Warfarin is contraindicated in pregnancy due to the risk of fetal hemorrhage and malformation.

The risk of stroke appears to be increased in pregnancy. The exact risk is not known but is estimated to be at least several fold (10). Most strokes in pregnancy are due to arterial occlusions and be accompanied by headache in up to $29 \%$ of patients (11). Hemorrhagic strokes can also occur in pregnancy and may be accompanied by headache in up to 
$57 \%$ of patients (11). Pregnancy itself appears to be accompanied by a hypercoagulable state predisposing to both arterial and venous thrombosis. Cardiac embolic disease, systemic or cerebral vasculitis, hematologic disorders, and thrombophilias are the common causes of secondary strokes, although in up to a third of cases, no clear cause can be identified. A reasonable diagnostic approach to a stroke in pregnancy includes cerebral imaging with an MRI of the brain, a carotid and cerebral MR angiogram, a transthoracic echocardiogram with saline contrast injection to look for a right to left cardiac shunt, and blood work to help rule out hematologic, metabolic, infectious, and inflammatory causes. Treatment of stroke in pregnancy must be individualized.

\section{Pre-eclampsia}

Pre-eclampsia usually occurs in the third trimester and may be seen in up to $7 \%$ of pregnancies (12). Pre-eclampsia may present with headache and may at times be the only symptom of the condition. Other signs and symptoms of pre-eclampsia include hypertension (BP of 140/90 or greater), proteinuria of greater than $300 \mathrm{mg} / \mathrm{dL}$, and blurred vision or non-formed visual hallucinations. If left untreated, pre-eclampsia may lead to a seizure (eclampsia), due to small cortical or subcortical hemorrhages. Treatment of pre-eclampsia consists of antihypertensives to control the hypertension, and if seizures are present, use of anticonvulsants (fosphenytoin or possibly LEV) to control the seizures.

\section{CNS Infections}

CNS infections may present with headache, fever, and neck stiffness, but are relatively uncommon during pregnancy. Suspected CNS infections should be evaluated with MRI brain imaging, followed by an analysis of spinal fluid obtained by lumbar puncture. CSF analysis should include cultures for bacteria, fungi, and acid-fast bacillus, and herpes simplex virus polymerase chain reaction.

\section{Idiopathic Intracranial Hypertension (Pseudotumor Cerebri)} Women may present with raised intracranial pressure (idiopathic intracranial hypertension- $\mathrm{IIH}$ ) during pregnancy. The risk is higher in obese women. The usual clinical manifestations are pressure like or dull, throbbing, generalized headache, blurred vision, or tinnitus. The optic disc margins may be blurred or frank papilledema may be present. Following an MRI brain, a lumbar puncture shows an opening pressure of 20 to $25 \mathrm{~cm}$ or higher. Treatment consists of serial lumbar punctures or treatment with acetazolamide $(500-2000 \mathrm{mg}$ a day). Acetazolamide may be used in the second half of pregnancy. Occasionally optic nerve sheath decompression may be necessary to help preserve vision (13).

\section{Diagnostic Evaluation of Headache in Pregnancy}

Evaluation of a pregnant patient with the complaint of headache starts with a detailed history and thorough physical examination. The history should include a full characterization of the headache, any history of previous headaches, any history of recent trauma, a general medical history, and a history of concomitant drug use (including prescription, nonprescription, and illicit drugs). Both a careful neurologic examination and a physical examination should be performed with attention to vital signs, the cranial nerves (especially the eyes), the motor and sensory systems, and the cardiovascular system. If the fetus is of viable age (24 weeks or greater), fetal monitoring should be performed. Appropriate blood work would include a complete blood count and differential, comprehensive metabolic profile, erythrocyte sedimentation rate, and thyroid function studies. MRI procedures (MRI brain, MR angiogram of the cerebral and cervical circulation, and MRV) for visualization of the venous sinuses with gadolinium are the procedures of choice for suspected cerebral or cerebrovascular pathology. If MRI is not readily available, noncontrast CT scanning with abdominal shielding poses very limited risk to the developing fetus. As noted above, lumbar puncture should be considered for pregnant patients with suspected CNS infection, suspected subarachnoid hemorrhage with negative neuroimaging, or idiopathic intracranial hypertension.

In summary, the diagnosis and treatment approach to headaches during pregnancy should be directed toward providing relief and comfort for the mother and safety for the developing fetus.

\section{MS IN PREGNANCY}

MS is an immune-mediated demyelinating disease of the CNS. Up to twice as many women are affected by MS compared with men. Up to $80 \%$ of women affected by MS develop clinical evidence of the disease between the ages of 20 and 40, the prime childbearing years.

\section{Epidemiology of MS}

There is extensive literature on the epidemiology and genetics of MS, which can be briefly summarized as follows:

- Gender: As noted above, MS is more common in females by a 2:1 margin. Women tend to present earlier than men. Women also tend to have less severe disease course overall compared with men.

- Race: Caucasians have the highest rates of MS, particularly those of Northern European ancestry (especially those of Scandinavian descent). The risk of African-Americans is about half of that of Caucasians in the same geographic area. In the United States, Asians and Native Americans have the lowest rates of MS.

- Geographic distribution: The prevalence of MS is the greatest in temperate climates and increases with increasing distance from the equator. The areas of greatest prevalence contain a proportionally higher Caucasian population.

- Migration: Epidemiologic studies have shown migration affects the development of MS, especially if immigration occurs from a low-risk to a high-risk area before the age of 15 . When migration occurs after the age of 15 , people tend to maintain the risk of their native country. Children of immigrants from low-risk to highrisk areas (or vice versa) tend to acquire the risk of their new homeland (14).

- Familial occurrence: First-degree family members of MS patients have a significantly increased risk (1015 times) of development of the disease. The risk of 
development of MS for children with one affected parent is $1.8 \%$. The concordance rate for the development of MS is at least six times higher in monozygotic twins compared with dizygotic twins (31\% compared with 5\%) (15).

- Genetics: Numerous genes have been evaluated as possible causes or markers for MS. The strongest association with MS has been found in individuals who possess certain HLA (human leukocyte antigens) genes found on chromosome 6. Specifically, the presence of HLA-DW2 haplotype is found in up to $50 \%$ to $70 \%$ of northern Europeans with MS. The presence of these genes is currently thought to confer an increased susceptibility for the development of MS. Multiple other sites on the human genome involved in immune function and modulation are being examined for their possible role in MS (16).

\section{Types of MS}

Several different subtypes of MS have been identified.

- Relapsing-remitting MS (RRMS): The most common type of MS accounting for up to $60 \%$ of patients. RRMS patients have discrete attacks of neurologic dysfunction (relapses or exacerbations) lasting weeks to a few months. Recovery from relapses may be incomplete and patients may accumulate increasing disability over time. Patients tend to have relative stability of disease between relapses.

- Secondary progressive MS (SPMS): Many patients with RRMS develop a gradually progressive course although they may continue to have occasional relapses. SPMS may account for up to $30 \%$ of MS cases.

- Primary progressive MS (PPMS): Accounting for up to $9 \%$ to $10 \%$ of MS cases, patients with PPMS have a continuous downhill course from onset of the disease without relapses.

- Progressive relapsing MS (PRMS): These patients have a progressive course from onset with intermittent relapses with continuing progression between relapses. This pattern can be seen in up to $5 \%$ of MS cases.

\section{Clinical Features of MS}

The clinical manifestations of MS are due to lesions in the CNS, from lesions in the brain, or spinal cord. Although the initial lesions are due to demyelination and may be reversible, demyelinating lesions may progress to axonal loss and gliosis producing permanent neurologic deficits. MS can produce a wide range of neurologic dysfunction. The most common clinical manifestations are reviewed below.

- Motor symptoms: Muscle weakness is common in MS and may be a major source of disability. Muscle weakness in MS usually involves the legs to a greater degree than the arms or hands and may be the greatest in the proximal legs. Hyperreflexia, ankle clonus, and extensor plantar responses (Babinski signs) are frequently seen in the legs. Spasticity (increased muscle tone) is common in the legs and patients may have both flexor and extensor spasms in the legs. The motor symptoms of MS are frequently worsened after exposure to increased environmental temperature and worsen later in the day.

- Sensory symptoms: Patients with MS often complain of numbness, tingling, or burning sensations. The distribution of symptoms usually does not correspond to a particular nerve root or dermatomal pattern. Shock-like or electric-type sensations may occur in the neck or back with neck flexion (Lhermitte's symptom). On examination, patients may have areas of impaired sensation to light touch, temperature, vibration, or position sense. Bilateral leg pain is another common complaint in MS.

- Brainstem symptoms: Cranial nerve dysfunction is commonly seen in MS. Patients may complain of bluffed vision or diplopia and, on examination, may have horizontal, vertical, or rotary nystagmus. Many patients have an internuclear ophthalmoplegia due to a lesion of the medial longitudinal fasciculus in the brainstem. In this condition, examination reveals an ipsilateral adduction paralysis with nystagmus of the contralateral eye. Dysarthria and dysphagia may be seen in MS, particularly late in the disease. Although hearing loss is unusual in MS, vertigo is a fairly common complaint. Trigeminal neuralgia and both central and peripheral (Bell's palsy) facial palsy may also be seen.

- Fatigue: Fatigue is one of the most common complaints in MS patients and at times may be a major source of disability (17). Sleep complaints are also common in MS and poor-quality sleep may contribute to fatigue.

- Cognitive and psychiatric dysfunction: Many patients with MS complain of difficulty with memory, thinking, and concentration. Formal neuropsychologic testing may reveal deficits that patients are unaware of. Depression may be seen in up to $75 \%$ of patients at some time during the disease (18).

- Autonomic disturbance: Many MS patients complain of difficulty with bowel and bladder or sexual dysfunction. Most patients have constipation but bowel incontinence and fecal urgency may occur. Bladder frequency and urgency is also common in MS. Urodynamic studies may be necessary to define bladder dysfunction. Up to $50 \%$ of female MS patients may report sexual dysfunction, which may be due to decreased libido, vaginal dryness, or impaired perineal sensation.

\section{Diagnosis of MS}

The diagnosis of MS can be challenging. As with all medical conditions, a careful history is mandatory and should focus on current neurologic symptoms, any history of previous neurologic dysfunction (possibly indicating a previous unrecognized relapse), and inquiry as to other significant medical 
conditions. A thorough physical examination should be performed with a full neurologic examination with emphasis on the mental status, visual system, motor and sensory systems, gait and balance, and reflexes.

- Neuroimaging: MRI of the CNS is the most sensitive test for determining the presence of demyelinating lesions. As up to $10 \%$ of MS lesions occur in the spinal cord, the cervical and thoracic spinal cord should be imaged in addition to the brain. Unless contraindicated, MRI imaging should be performed with gadolinium enhancement to look for evidence of active or acute demyelinating lesions. MRI is positive in at least $95 \%$ of patients with clinically definite MS. Typical MRI lesions in MS are seen in the periventricular regions, in the corpus callosum, and in the brainstem. Spinal cord MRI lesions in MS are often ovoid and involve two or less spinal segments. Spinal cord atrophy may also be seen.

- CSF: CSF analysis provides important information in MS and should be considered to help confirm a diagnosis of MS. The CSF may be abnormal in up to at least $80 \%$ of MS patients, although a normal CSF examination does not rule out the disease. Immunoglobulin $\mathrm{G}(\mathrm{IgG})$ in the CSF can be compared with levels in the serum and may be elevated in the CSF of patients with MS (IgG index and IgG synthesis rate). CSF electrophoresis may also reveal the presence of oligoclonal bands, which are due to IgG synthesis in the CNS. Elevated levels of myelin basic protein indicating myelin damage may also be seen in MS. None of the above CSF abnormalities are specific for MS although their presence in the setting of a typical clinical history can aid in making a diagnosis.

- Evoked potentials: Visual evoked potential (VEP) and brainstem auditory evoked potentials (BAEP) studies are useful at times in the diagnosis of MS as they can show subclinical lesions in the visual (VEP) and auditory (BAEP) pathways.

Ultimately, the diagnosis of MS is clinically based and usually rests on a clinical history of attacks of neurologic dysfunction in different areas of the brain or spinal cord at different times supported by evidence of positive findings on MRI, in the CSF, or on evoked potential testing.

\section{Treatment of MS}

Currently, a number of drugs have been shown to modify the natural history of MS and are approved to treat both the relapsing-remitting and the secondary progressive form of the disease. The interferons, glatiramer acetate, and natalizumab are approved for the relapsing forms of the disease. These drugs alter immune responses and have been shown to decrease relapse rates by up to $30 \%$ to $50 \%$ and are thought to retard disease progression over the long term. None of the drugs are indicated for use during pregnancy, and it is recommended that these drugs be stopped when a woman becomes pregnant or ideally 3 months prior to attempting to become pregnant.

\section{The Course of MS in Pregnancy}

Currently, pregnancy is not thought to impact the long-term progression of MS. Multiple studies have shown no adverse effects on pregnancy outcome in women with MS $(19,20)$. Studies have also shown a significantly reduced rate of relapses during pregnancy (by 60-70\%). Following pregnancy, there appears to be an increase in the relapse rate up to 3 months postpartum (21). Additionally, breast-feeding has not been shown to have a significant impact on exacerbation rates (22). In short, pregnancy does not appear to influence long-term disability in MS and a woman with MS who desires pregnancy should not be discouraged from pregnancy if her general health is adequate.

Finally, a woman who does have a significant MS exacerbation during pregnancy can be considered for treatment with glucocorticoids (methylprednisolone-pregnancy class $\mathrm{C}$ ) only if the potential benefit to the mother outweighs potential risk to the fetus. Mild or moderate exacerbations are probably left best untreated in pregnancy.

In summary, women with MS who are contemplating pregnancy or who have become pregnant should be reassured that pregnancy does not adversely affect their long-term course of the disease.

\section{NEUROMUSCULAR DISEASES (NMDs) IN PREGNANCY}

NMDs are traditionally divided into diseases of anterior horn cell, peripheral nerves, neuromuscular junction, and skeletal muscles. In a pregnant woman, these may be approached as two categories: (i) pregnancy-related NMD including disorders with increased incidence or occurring as specific complications during pregnancy, delivery, or postpartum period, and (ii) chronic NMD that may influence maternal morbidity and mortality as well as fetal outcome. Following discussion focuses on the common NMDs encountered during pregnancy and puerperium.

\section{Pregnancy-Related NMDs \\ Carpal Tunnel Syndrome}

Incidence of carpal tunnel syndrome (CTS) is two to three times in pregnant women as compared with nonpregnant women of childbearing age (23). Gestational CTS is more common during third trimester. Various factors such as edema, weight gain, and abnormal hand position with need to sleep on side may contribute to increased incidence $(24,25)$. Symptoms of burning pain and paresthesia in median nerve distribution or whole hand are typically worse at night and may be bilateral in $60 \%$ of patients. Loss of sensation or weakness in median nerve distribution, and Tinel or Phalen signs may be seen. Electrodiagnostic testing helps to confirm the diagnosis and distinguish it from nonspecific paresthesia of hands during pregnancy. Spontaneous resolution of symptoms occurs in majority in the immediate postpartum period, and therefore conservative treatment with wrist brace, physical therapy, and modification of activity is recommended $(23,24)$. Patients with severe symptoms may benefit from corticosteroid injection. Women with the onset of symptoms in early part of pregnancy may be more likely to fail conservative treatment and need decompressive surgery on follow-up $(24,26)$. 


\section{Lumbar Radiculopathy}

Low back pain affects about 50\% of pregnant women. Incidence of sciatica-type pain, however, is only $1 \%$ and clinical signs of radiculopathy are rare, occurring in 1:10,000 pregnancies (27). Women with prior history of back pain and sciatica are more prone to have recurrence during pregnancy. Lumbar lordosis, laxity of ligaments due to relaxin hormone, and pressure of gravid uterus may contribute to high incidence of lumbar pain (24). Lateral disc herniation commonly affects L5 or S1 nerve root and central disc herniation may cause cauda equina compression. MRI without the use of contrast agent is the investigation of choice, but care should be taken to interpret MRI abnormality in relation to clinical deficits. Most women respond to conservative treatment with analgesics and physical therapy $(24,27)$. Decompressive surgery may be indicated for uncontrolled pain and neurologic deficits, and has been safely performed during pregnancy (28).

\section{Meralgia Paresthetica}

Lateral femoral cutaneous nerve is prone to entrapment or stretch during third trimester due to expanding abdominal wall and lordosis $(23,29)$. Symptoms of pain, tingling, and numbness over lateral aspect of thigh may be unilateral or bilateral. Compression may also occur due to thigh flexion during delivery or pressure from the retractor during caesarian section. Treatment consists of reassurance for spontaneous resolution, and topical Lidoderm patches or local anesthetic injection in severe cases (24). Use of GBP or amitriptyline is preferably avoided during pregnancy. Intercostal neuralgias may also occur due to stretch injury and lead to chest or abdominal wall pain in a dermatomal pattern (23).

\section{Bell's Palsy}

Risk for Bell's palsy is three times higher during late pregnancy and early puerperium, probably related to increased tendency for reactivation of herpes virus infection. Corresponding incidence is lower in early pregnancy and therefore overall frequency of Bell's palsy is unaffected during pregnancy (30). Typical presentation is unilateral acute facial weakness, which progresses over 3 to 7 days. Increased lacrimation, alteration of taste, or hyperacusis may be present. There is no increase in recurrence rate during subsequent pregnancies. Gestational facial palsy is associated with increased occurrence of hypertension and pre-eclampsia, and close monitoring of affected women is recommended $(30,31)$. Standard treatment with short course of prednisone, $1 \mathrm{mg} / \mathrm{kg}$ for 5 days and acyclovir $400 \mathrm{mg}$ five times daily for 10 days, is recommended except in the first trimester of pregnancy. Lubricating eye drops and eye patching are used to prevent corneal injury. Prognosis for recovery is excellent in patients with incomplete facial weakness; however, worse prognosis during pregnancy, noted in some reports, may reflect higher chance of severe facial palsy in third trimester.

\section{Neuralgic Amyotrophy}

Predilection for occurrence of brachial neuritis (neuralgic amyotrophy or Parsonage-Turner syndrome) in immediate postpartum period is well recognized (32). Clinically, acute onset of shoulder/upper arm pain is followed by patchy weakness and wasting commonly in the distribution of upper and middle trunks. The disorder may be sporadic or hereditary (autosomal dominant) and has possible immunologic basis. Bilateral and recurrent episodes are common in familial type. The onset may be within a few hours to weeks after the childbirth. Mode of delivery does not seem to impact the susceptibility. Spontaneous improvement with good motor function over 1 to 2 years is reported in $80 \%$ (27). Management in acute phase includes pain control, range of movement, and active physical therapy as tolerated. Intravenous methylprednisolone or intravenous human immunoglobulin (IVIG) may reduce the intractable pain but do not affect the course of the disease (33).

\section{Guillain-Barré Syndrome}

Guillain-Barré syndrome (GBS) is an acute or subacute predominantly motor, immune-mediated demyelinating polyneuropathy. Increase in risk for GBS is noted in postpartum period although overall incidence during pregnancy is similar to general population (27). Diagnosis is based on clinical features of generalized weakness, areflexia, elevated protein in CSF without pleocytosis, and abnormal electrophysiologic studies. Antecedent infection is seen in about two-thirds of patients. Significant association with Campylobacter jejuni, cytomegalovirus, and human immunodeficiency virus is described, and recognition of these infections in pregnancy may have important implications for maternal and fetal health. Pregnant women with GBS may be more prone to respiratory compromise due to elevated diaphragm and about 35\% may need ventilatory support (34). Preterm labor may be more frequent, and caution should be exercised in the use of tocolytic agents to avoid autonomic complications. Vaginal delivery is possible, and caesarian section is performed for obstetric indications only (24). Efficacy and safety of plasmapheresis and intravenous immunoglobulin therapy for GBS during pregnancy are similar to nonpregnant population. General management includes respiratory and hemodynamic monitoring, prevention of thromboembolic complications, adequate nutrition, and physical therapy.

\section{Maternal Obstetric Nerve Injuries}

Lumbosacral plexus and its branching nerves are susceptible to injury during labor and delivery. The overall incidence of intrapartum nerve injuries has declined with present-day obstetric care, but is still fairly significant at $1 \%$ of live births (29). The predisposing factors include primiparity, short stature, dystocia, prolonged labor, and improper positioning (29). Injury to the components of lower plexus (L4-5, S1) presents as postpartum foot drop, while injury to the upper plexus (L2-4) causes mainly femoral and/ or obturator nerve palsy. Pudendal nerve injuries are related to pelvic floor trauma.

\section{Lumbosacral Plexus Injury}

Lumbosacral trunk is unprotected as it crosses the pelvic brim to enter the true pelvis and is prone to compression by the fetal head or forceps. Rarely sciatic nerve itself may be compressed. Injury to lumbosacral plexus or sciatic nerve predominantly affects the peroneal nerve component. Foot drop is noted immediately postpartum. There is weakness of dorsiflexion, inversion and eversion of the foot, and 
sensory impairment corresponding to L5 dermatome. Ankle reflex is preserved with L4-5 trunk lesion but is absent with sciatic nerve injury. Differential diagnosis is from lumbar radiculopathy and peroneal nerve compression at the fibular neck. Electrophysiologic studies help distinguish by findings of denervation in paraspinal muscles in lumbar radiculopathy and focal nerve conduction abnormality at fibular head in peroneal neuropathy. When difficult to distinguish from lumbar radiculopathy, MRI of lumbar spine is warranted.

The lesion is usually neurapraxic with variable axonal involvement. Significant spontaneous improvement is seen within 3 to 6 months. Patients with more than 50\% axonal injury show slower and incomplete recovery. Treatment is symptomatic with physical therapy and ankle-foot orthosis. Caesarian section should be considered in subsequent pregnancies to avoid further compromise $(23,29)$. Rarely, idiopathic or inherited lumbosacral plexopathy akin to brachial neuritis may occur in postpartum period.

\section{Peroneal Neuropathy}

External compression of peroneal nerve at fibular head is uncommon during labor and may be due to improper positioning in stirrups, manual pressure, or prolonged squatting. Women who receive epidural anesthesia may be more prone as they are not aware of pain and fail to change position to prevent prolonged pressure (35). Patients report pain and paresthesia over the anterior-lateral aspect of leg and dorsum of foot. Foot drop, weakness of dorsiflexion, eversion, and toe extension are noted. Normal inversion strength distinguishes from L5 radiculopathy and lumbosacral plexopathy. Nerve conduction demonstrates focal slowing and conduction block of peroneal nerve at the fibular head. Prognosis for recovery is good.

\section{Femoral and Obturator Neuropathy}

Incidence of femoral neuropathy in parturients has considerably declined with modern obstetric care. Branches of the lumbar plexus (L2-4) do not enter the true pelvis; the compression of femoral nerve therefore usually occurs at the inguinal ligament due to prolonged lithotomy position (29). Findings include difficulty walking and knee buckling, weakness of quadriceps femoris, absent patellar reflex, and impaired sensation in saphenous nerve distribution. Weakness of iliopsoas when present indicates a more proximal lesion due to nerve stretch from abduction and external rotation of hip or injury from instrumentation. Proximal part of femoral nerve has poor blood supply and is also prone to ischemia. Prognosis is favorable in majority of patients consistent with neurapraxic injury.

Obturator neuropathy is much less frequent as the nerve is deep within the pelvis and medial thigh. There is weakness of thigh adduction and sensory deficit over the medial thigh. A combined lesion of femoral and obturator nerves points to lumbar plexus involvement. Imaging of pelvis with computed tomography or MRI should be performed if intrapelvic pathology such as hematoma is suspected.

Various strategies that may help prevent nerve injuries in a parturient include avoiding prolonged thigh flexion and extreme abduction and external rotation, frequent changing of the position of lower extremities, and reducing the active pushing time $(29,35)$.

\section{Pregnancy and Chronic NMDs}

\section{Motor Neuron Diseases}

Late-onset spinal muscular atrophy (SMA), poliomyelitis/ post-polio syndrome, and amyotrophic lateral sclerosis (ALS) may affect women of childbearing age. Pregnancy is rare in ALS, but severe exacerbation of ALS and respiratory failure are likely during pregnancy. Respiratory function should be closely monitored. Patients with severe SMA may be advised to avoid pregnancy. Presence of kyphoscoliosis and respiratory compromise are the major concerns in pregnant SMA patients. There may be increased incidence of premature labor in SMA and ALS, but fetal outcome is reported to be satisfactory $(24,36,37)$.

For female survivors of poliomyelitis, spine deformity and cephalopelvic disproportion pose risks in pregnancy and parturition. Study of a large cohort of women with old poliomyelitis showed higher risk of complications during pregnancy and delivery, higher rate of caesarian section, and low birth weight of infants (38).

\section{Peripheral Neuropathies}

Chronic Inflammatory Demyelinating Polyneuropathy Chronic inflammatory demyelinating polyneuropathy (CIDP) is autoimmune acquired demyelinating polyneuropathy presenting as relapsing or chronic progressive forms. CIDP is three times more likely to relapse in pregnancy particularly during third trimester and early postpartum period $(23,27)$. This is attributed to relative suppression of cellular immunity and stimulation of humoral immunity during gestation. Fetal outcome is not affected (39). Treatment with prednisone can be maintained, but other immunosuppressive agents should be avoided. Exacerbation is treated with plasmapheresis or IVIG.

\section{Hereditary Neuropathy}

Charcot-Marie-Tooth disease (CMT) is the most common hereditary NMD. Worsening of disease is noted in $50 \%$ of affected pregnant women, especially in those with childhood onset of CMT. Endoneurial edema and local pressure from gravid uterus may be the contributory factors (24). Some resolution of worsening may occur in about one-third of patients after delivery, but remaining continue to have progressive course. Women with CMT may have increased risk for fetal malpresentation, postpartum bleeding, and higher incidence of assisted delivery or caesarian section (40).

\section{Myasthenia Gravis}

Myasthenia gravis (MG) occurs predominantly in women of reproductive years with peak incidence in third decade (41). Management of MG in a pregnant woman is a unique challenge needing many therapeutic decisions at different stages. The course of MG during pregnancy is unpredictable with exacerbation in about $40 \%$, remission in $30 \%$, and no change in the remaining $(24,42,43)$. The risk of worsening is the greatest during first trimester and early puerperium, and in 
women with recent diagnosis of MG (31). Women with MG who are considering pregnancy should be instructed on optimal disease control, minimizing oral immunosuppressants, and risks to mother and fetus. Elective thymectomy if being considered should be done before pregnancy as the benefit of thymectomy is delayed $(24,41)$. Treatment with acetylcholinesterase inhibitors (pyridostigmine, edrophonium) and prednisone is safe during pregnancy. Immunosuppressive agents such as azathioprine and mycophenolate mofetil are contraindicated $(41,43)$. Aggressive treatment is needed in patients with bulbar or respiratory involvement. Plasmapheresis or intravenous immunoglobulin therapy can be used for control of severe symptoms and in myasthenic crisis (41). Maintenance IVIG therapy can help to avoid the use of immunosuppressive agents. There is higher risk of maternal mortality in myasthenic women especially within 1 year of diagnosis (42). It is advisable for MG women to delay childbirth for 2 years after the onset of illness.

MG has no significant effect on fertility, course of pregnancy, or mode of delivery $(31,43)$. During labor, MG does not affect the uterine contraction; however, myasthenic weakness may limit active pushing necessitating assisted delivery. Parenteral cholinesterase inhibitors can be used during labor to tide over the myasthenic fatigue. Neostigmine $1.5 \mathrm{mg}$ intramuscularly or $0.5 \mathrm{mg}$ intravenously is equivalent to $60 \mathrm{mg}$ oral dose of pyridostigmine. Magnesium sulfate as well as neuromuscular blocking agents should be avoided as these can aggravate weakness or precipitate myasthenic crisis (43). Care is also needed while managing pain, infection, and hypertension to avoid medications that may potentiate muscle weakness in MG.

Fetal/neonatal complications in myasthenic pregnancies include higher perinatal mortality (6-8\%), stillbirth (2\%), pulmonary hypoplasia, polyhydramnios, neonatal MG (10$20 \%$ ), and arthrogryposis multiplex congenita (AMC) $(31,41,42)$. Neonatal MG is related to placental transfer of acetylcholine receptor antibody. The neonate may develop hypotonia, poor sucking and swallowing, and respiratory difficulties. The onset is within 1 to 4 days and weakness resolves over 4 to 6 weeks. Intrauterine exposure to acetylcholine receptor antibody is also the basis for poor fetal movement and consequent AMC (27). There is a high risk of recurrence of AMC in subsequent pregnancies.

\section{Muscle Diseases}

Myotonic Dystrophy Type 1

An autosomal dominant, trinucleotide (CTG) repeat disorder is the most common muscular dystrophy of adulthood (24). Pregnancy in DM1 carries multiple risks for mother and fetus. The course of disease may be accelerated with increasing muscle weakness, congestive hear failure, and respiratory compromise. There is increased risk of spontaneous abortion, polyhydramnios, preterm labor, uterine atony, and postpartum hemorrhage $(27,44)$. Perinatal loss may be up to $15 \%$. Congenital myotonic dystrophy is a maternally transmitted condition and is reported in $6 \%$ to $20 \%$ of infants $(24,44)$. Prenatal diagnostic testing is available. At times, the mother is first diagnosed with DM1 when she delivers an affected child. During pregnancy and delivery, use of tocolytic agents, pentobarbital, and neuromuscular blocking agents should be avoided.

\section{DM Type 2}

This follows a more benign course than DM1 $(45,46)$. Pregnancy may unmask or exacerbate the symptoms of DM2 in affected women. There may be predisposition to pre-term labor but no increase in fetal or perinatal loss is reported. Congenital myotonic dystrophy does not occur with DM2.

\section{Other Rare Inherited Muscle Diseases}

Some of these are likely to be encountered in a pregnant woman: facioscapulohumeral muscular dystrophy and various forms of congenital and metabolic myopathies. Vaginal delivery is possible in majority of the affected women. Increased muscle weakness during pregnancy, low birth weight, need for operative delivery, and presence of AMC are variably reported $(24,27,47)$. Inhaled anesthetics are contraindicated as these may precipitate malignant hyperthermia (48).

\section{Inflammatory Myopathy}

Polymyositis and Dermatomyositis

This can affect women of childbearing age. Pregnancy may worsen the disease course. Women in clinical remission of polymyositis/dermatomyositis (PM/DM) carry no increased risk for obstetric complications. Active disease during pregnancy, however, is linked to poor outcome with almost $50 \%$ chance of fetal death and 33\% incidence of intrauterine growth retardation (49). Corticosteroids are the mainstay of treatment. IVIG has been used for exacerbation or as maintenance treatment (50). Caution is needed while using chemotherapeutic agents. Assisted vaginal delivery or caesarian section is recommended to avoid maternal exhaustion and to reduce the risk of rhabdomyolysis.

\section{EPILEPSY AND PREGNANCY}

Epilepsy is a neurologic disorder that causes recurrent unprovoked seizures. There are an estimated 50 million people with epilepsy worldwide. Many issues in the treatment of epilepsy are sex specific. In women, this is not only related to maternity issues such as pregnancy and delivery, but also to puberty, menstruation, and contraception. The care of women with epilepsy (WWE) requires an understanding of antiepileptic drug (AED) pharmacology, and the neuroendocrine changes during the menstrual cycle and pregnancy. This review will be divided into three parts: WWE before pregnancy (preconceptual), pregnancy and epilepsy, and postpartum care of WWE.

\section{WWE Before Pregnancy (Preconceptual)}

Every woman with childbearing capability should receive preconceptual care and counseling with her neurologist. The woman should be asked about her seizure type and frequency and response to AED therapy, as well as side effects. Additional information should include the menstrual cycle, method of contraception, plans for pregnancy, and history of birth defects or major malformation. This section will discuss the basic principles of AED therapy before pregnancy, oral 
contraception, the interaction of AEDs, sex steroid hormones and seizures, and teratogenicity of AEDs.

\section{Seizure Management Before Pregnancy}

In every epilepsy patient, the first goal is the optimization of AED treatment with no seizures and side effects (51-53). It is advisable to give the lowest possible AED dose or sub-toxic dose. The appropriate drug is based on the epilepsy syndrome and seizure type (generalized vs. partial seizures). Each epilepsy patient is different in their dosage requirement, tolerance, and side effects. Preferably, AED monotherapy should be used. When seizures are controlled, it is important to obtain a baseline preconceptual (or prepregnancy) AED level. A trough or pre-dose AED level, which is before the first morning dose, should be measured. This AED serum level will serve the individual's therapeutic level and this may be within or outside the therapeutic range reported by the laboratory. This can also serve as an important marker for drug compliance, and to monitor serum drug fluctuations during menstruation, pregnancy, or hormonal contraception. Poorly controlled seizures despite multiple AED therapy should prompt a referral to an epilepsy center for comprehensive evaluation.

If a woman with epilepsy plans to become pregnant and is taking a drug with high teratogenic potential, attempts can be made to switch her to a drug with a more favorable teratogenic profile. However, these medication changes should be completed at least 6 months before a planned conception, if possible, to avoid fetal damage from a breakthrough seizure. When the seizures are controlled, the woman should be advised not to discontinue or lower the dose of the AED if she desires to become pregnant or if she discovers to be pregnant. WWE are also advised not to switch an AED indiscriminately without notifying their neurologist or family physician. Discontinuation of AEDs is appropriate if the patient is seizure free for 2 to 5 years, has a normal EEG, normal neurologic examination, and with a single seizure type. Some women who have not satisfied all these conditions may also wish to try discontinuing AEDs because of concerns about teratogenic effects or interference with hormonal contraception. Before doing so, the risk of seizures must be carefully considered. The risk of seizure recurrence is the greatest within the first 6 months after discontinuation (51).

\section{Oral Contraception and Antiepileptic Medications}

Beginning in adolescence, it is important to be sure that all female patients have full information regarding effective contraception (54-56). Contraceptive management requires careful consideration and is closely tied to epilepsy management. However, only $3 \%$ of pregnant women saw an obstetrician in the 3 months before conception, whereas another survey found that one-third had never been counseled regarding the issues of pregnancy and seizures. A number of safe and effective methods of contraception are available. Like all women, WWE must carefully consider their best contraceptive option; however, they need additional information regarding the possible interaction of their epilepsy medication and contraceptive choice. Most studies have shown that estrogen-based contraceptives do not affect seizure frequency. Most current guidelines suggest that the combination oral contraceptive (COC) pills give a sufficient dose of estrogen to inhibit ovulation, generally $35 \mu \mathrm{g}$ or greater. It is the progestin component that prevents breakthrough bleeding and is only indirectly involved in contraceptive efficacy. In the ideal situation, oral contraceptive (OC) is highly reliable with an annual failure rate of $<1 \%$. The annual failure rate of $\mathrm{OC}$ with AED use is about 6\%. Enzyme-inducing AEDs (EIAEDs) affect the efficacy of COC. The EIAEDs include phenytoin (PHT), primidone (PMD), phenobarbital $(\mathrm{PB})$, carbamazepine (CBZ), TPM, felbamate, and oxcarbazepine. They do this by inducing enzymes, that is, CYP3A4 component of the hepatic cytochrome P450, leading to faster clearance of steroid hormones and by increasing the production of sex hormonebinding globulin (SHBG) $(51,55)$. For WWE taking EIAEDs, COCs containing $1 \mathrm{mg}$ of norethindrone, $0.150 \mathrm{mg}$ of levonorgestrel, or $0.300 \mathrm{mg}$ of norgestrel are recommended. COCs with at least $50 \mu \mathrm{g}$ of the estrogen component are recommended. If there is breakthrough bleeding, the estrogen dose may be increased to 75 to $100 \mu \mathrm{g}$ daily. Other methods of contraception are barrier methods (e.g., condom, diaphragm, cervical cap). EIAEDs reduce the contraceptive efficacy of levonorgestrel implant but not with intramuscular medroxyprogesterone. It is recommended that injections be administered every 10 weeks instead of the usual 12 weeks for WWE. If the "morning after pill" containing levonorgestrel postcoital contraception is used after unprotected sexual activity, some practitioners recommend that the first dose should be doubled to two pills. IUDs that contain progestin or Mirena coil that secrete levonorgestrel hormone locally and copper IUDs are not known to be affected by EIAEDs. The rhythm method is not a reliable method of birth control for WWE. EIAEDs also affect the efficacy of transdermal contraception. There is no additional risk of OC failure with non-EIAEDs. AEDs that do not induce cytochrome P450 3A4 enzymes include VPA, GBP, LEV, tiagabine (TG), vigabatrin $(\mathrm{VG})$, zonisamide (ZON), and pregabalin (PG). Vagal nerve stimulators, which are used in refractory epilepsy, have no effect on sex hormone metabolism.

There is a special situation where seizure exacerbation may occur among women taking lamotrigine and OCs (55). It has been found that lamotrigine levels, when adjusted for dose and body weight, were found to be reduced by $50 \%$ in a group of 22 women taking lamotrigine and an OC that combined ethinyl estradiol and levonorgestrel compared with women taking lamotrigine alone. Further evaluation of this interaction has shown that it is the ethinyl estradiol component that produces the interaction; the progestin component has no effect on lamotrigine levels. The mechanism of this interaction is the induction of uridine diphosphate-glucuronyl-transferase $1 \mathrm{~A} 4$ by ethinyl estradiol, which is the main metabolic enzyme for lamotrigine. The lamotrigine-OC interaction is further complicated by the risk that lamotrigine levels will increase during the OC placebo or pill-free week, which in most regimens occurs every fourth week. There are reported increase in lamotrigine levels of $27 \%$ on the 3 rd placebo day, $63 \%$ on the 5 th, and $116 \%$ on the 7 th placebo day relative to lamotrigine levels during OC administration. There is also a bidirectional interaction for OCs and lamotrigine, in which lamotrigine was associated with a small decrease in levels of progestin, levonorgestrel, by $12 \%$ (54-56). 


\section{Sex Hormones, Reproductive Function, and Seizures}

To understand the far-reaching effects of epilepsy and AEDs on hormones and vice versa, the reader must review the normal physiologic mechanisms of the hypothalamicpituitary-gonadal axis (HPGA). In the female, the gonadotropin-releasing hormone $(\mathrm{GnRH})$ is secreted by the hypothalamus. This stimulates the anterior lobe of the pituitary leading to the release of the follicle-stimulating hormone (FSH) and the luteinizing hormone (LH). FSH and $\mathrm{LH}$, in turn, stimulate the formation of ovarian follicles, hence stimulating the release of estrogen (estradiol). This phase is the follicular or preovulatory phase; the phase occurs when LH stimulates the preovulatory follicular theca cells to secrete androstenedione, which aromatizes into estradiol. Estradiol binds to the SHBG, a glycoprotein that acts as a carrier in the bloodstream. This feeds back to the hypothalamus with high levels of estrogen; the hypothalamus increases the release of $\mathrm{GnRH}$, which increases the amount of LH and FSH. A spike of FSH and LH from the pituitary results in ovulation and the follicle transforms into the corpus luteum. This marks the luteal phase in which the corpus luteum produces both estrogen and progesterone. Progesterone feeds back to the hypothalamus and inhibits GnRH secretion and thus the decrease in FSH and $\mathrm{LH}$ from the anterior pituitary. As the corpus luteum breaks down, the inhibition of the hypothalamus lessens and the cycle begins again (57).

There is an interaction between steroid sex hormones (estradiol, progesterone) and neurons. Estrogen is known as a proconvulsant and progesterone, a potent anticonvulsant. Overall, the ovarian sex steroids act at the level of the neuron in the CNS and alter the frequency and severity of seizures. It has been shown in WWE that intravenous administration of conjugated estrogens activates epileptiform discharges and may result in seizures. Estradiol also potentiates glutamate receptor-mediated excitatory post-synaptic potentials and excitatory post-synaptic currents on hippocampal CA1 pyramidal cells. Progesterone exerts an inhibitory effect via metabolites such as allopregnanolone, which is a GABA-Areceptor-modulating neurosteroid. Progesterone increases seizure threshold and also has anti-anxiety and sedating effect. The effect of sex hormones and neuronal excitability may explain the pattern of seizure exacerbation during menstruation. Seizures have been reported to occur in a cyclic pattern in between 5\% and 70\% of WWE, for example, catamenial epilepsy. About 35\% of WWE will have significant ( $>$ two times) increase in seizure frequency around menstruation. Women with localization-related epilepsies may have an increase in seizures at the time of menarche. Treatment with progesterone may be helpful and the average decline in seizure frequency is about $55 \%$. The possible side effects of progesterone treatment include sedation, depression, weight gain, breast tenderness, and breakthrough vaginal bleeding. This is managed by discontinuing the progesterone or lowering the dose (57).

WWE are at risk for altered neuroendocrine regulation $(56,57)$. Epilepsy can affect the various aspects of sexual and reproductive functioning. The most common symptoms are hyperandrogenism, menstrual disorders, ovarian failure, polycystic ovary syndrome (PCOS) with or without divalproex (VPA) therapy, hyperinsulinemia, and weight gain especially with VPA therapy. An unusually high number of WWE will have some degree of sexual or reproductive dysfunction, which can include difficulty with libido, arousal, orgasm, and menstrual irregularities. Menstrual disturbances are more frequent with epilepsy as compared with women without epilepsy. This is particularly common with temporal lobe epilepsy. About $30 \%$ to $50 \%$ of women will have menstrual irregularities, reduced fertility, irregular bleeding, and anovulation. Aside from catamenial epilepsy, another reproductive hormone-related disorder in WWE is PCOS. It is characterized by polycystic ovaries on ultrasound, hirsutism, obesity, acne, endocrine disturbance, elevated LH/FSH ratio, chronic anovulation, and increased risk of endometrial cancer and breast cancer (54). The prevalence of PCOS in the general population is approximately $17 \%$ to $22 \%$ of women. Up to $15 \%$ of females with primary generalized or partial seizures may exhibit features of PCOS. With the frequent disruption of the normal function of the HPGA caused by ictal and interictal epileptiform discharges, a whole cascade of events happens. The LH/FSH rates are elevated, and follicular maturation is disrupted leading to elevation in androgens, leading to hyperandrogenism. The fertility rate of WWE is about $60 \%$ to $80 \%$ of the normal population, the major contributing factor being PCOS. Anovulatory cycles are more frequent in women with idiopathic generalized epilepsy and those taking VPA. The management of PCOS should be symptom focused and individualized, such as encouraging weight loss if body mass index $>25 \mathrm{~kg} / \mathrm{m}^{3}$ or fasting glucose tolerance is above normal and the insulin level is elevated. Hirsutism can be managed by laser treatments, electrolysis, waxing, and bleaching to improve hair growth. Hyperandrogenism can be treated with a combination of estrogen only and combined contraceptives, which are used in the first 21 days of the menstrual cycle and antiandrogen acetate $(50-100 \mathrm{mg})$, used for the first 10 days of menstruation. OCs are useful in adolescents to prevent unwanted pregnancy but may worsen insulin resistance associated with PCOS. Spironolactone with antiandrogenic properties can be tried in young women where oral contraception is contraindicated (54).

\section{AEDs and Teratogenicity}

Rates of congenital malformations range from $2.3 \%$ to $18.6 \%$ (combined risk about 7\%) in infants of WWE versus 2\% to 3\% in the general population (58-65). Estimates of risk vary, but a recent study indicates $4.5 \%$ frequency for AED monotherapy in utero exposure and $8.6 \%$ for AED polytherapy. The most common major congenital malformations are heart malformations (e.g., ventricular septal defect), urologic defects Z (e.g., hypospadia), skeletal abnormalities (e.g., radial ray defects, phalangeal hypoplasia), and NTDs (e.g., spina bifida) (58-65). Congenital malformations are physical defects that warrant medical or surgical intervention and cause major functional problems. Most major and minor malformations have been reported with the older AEDs such PHT, CBZ, PB, and VPA largely because they are the most widely used AEDs globally. Exposure in the first trimester poses the highest risk. Barbiturates have been found to be associated with major congenital malformations. PHT has been associated with facial clefts, congenital heart defects, and urogenital defects. There have also been reports of dysmorphic facial features and distal phalangeal hypoplasia with PHT. CBZ has been associated 
with NTDs, heart defects, hypospadias, hip dislocations, and inguinal hernia. PHT, CBZ, and PB are folic acid antagonist, so their teratogenicity may be attributable to their folate deficiency. VPA has been shown to carry a high risk of spina bifida aperta, cardiovascular complications, urogenital malformations, and skeletal defects. Association of VPA with congenital defects is dose dependent and is most likely when VPA is used with polytherapy and monotherapy above the doses of $1000 \mathrm{mg} /$ day. Minor anomalies are deviations from the normal morphology, but do not threaten health, impair function, or require intervention. Examples include hypertelorism (widely spaced eyes), low set ears, and distal phalangeal hypoplasia. Minor anomalies occur at a rate of up to $30 \%$ in the offspring of WWE taking AEDs versus 15\% in newborns of matched controls without epilepsy.

Knowledge of the effects of newer generation AEDs is incomplete. Data on GBP, OXC, PG, TG, and TPM are very limited. One study did find an association between OXC and a major malformation. LTG has the most data available at this time, primarily from the Lamotrigine Registry and the UK Epilepsy and Pregnancy Registry. When LTG and VPA are given together, there is a higher frequency of major malformations. Emerging data from a pregnancy registry suggest an association between LTG and nonsyndromic oral cleft lip/palate 24 times greater than expected. Data on ZON are quite limited. Very few pregnancy outcomes have been reported with LEV, but it has been speculated that LEV may have a favorable profile for pregnant women because it has $<10 \%$ protein binding and is eliminated primarily via renal excretion and extrahepatic hydrolysis. Renal blood flow increases in early pregnancy and persists throughout; LEV elimination has been found to be enhanced with this increased renal blood flow. Preliminary studies have noted a good pregnancy safety profile with LEV; however, more data are needed before it can be said to be non-teratogenic.

There are different pregnancy registries in epilepsy with varying results in the rate of major malformations (58). The North American Registry with over 3000 women enrolled while having AED during pregnancy has reported that $\mathrm{PB}$ $(6.5 \%)$ and valproate $(10.7 \%)$ have increased rates for major malformations. There is also a specific risk for cleft lip/palate (0.89\%) for lamotrigine and CBZ (0.57\%). The UK registry with 3607 pregnancy outcomes found higher major malformation rate for valproate $(6.2 \%)$ and increased risk for lamotrigine at higher dose. LEV was associated with major malformation in $2.7 \%$. The Australian Registry with 555 outcomes reported malformations in 5\% of live births and additional $1 \%$ in induced abortions. Valproate monotherapy in the first trimester at doses above $1100 \mathrm{mg} /$ day was associated with significantly higher risk (38.5\%) of malformations than other AEDs, which were $3.8 \%$ for CBZ, $0 \%$ for lamotrigine, and $5.9 \%$ for PHT. Both the Swedish and Finnish Birth Registries reported higher malformation rates for valproate. Valproate effect was dose dependent in the Finnish study. The International Lamotrigine Pregnancy Registry reported 2.9\% risk for malformation, which was not dose dependent.

Animal studies have demonstrated that in utero AED exposure can produce behavioral defects at dosages lower than those required to produce somatic malformation $(59,61,63,65)$. Cognitive studies in humans are less clear.
There has been no evidence that children exposed to CBZ produced IQ impairment or autistic spectrum disorder. By contrast, valproate in utero exposure was associated with lower IQ scores and impairment of verbal/language skills. The effect of valproate was dose dependent. Prospective populationbased studies give no definite evidence that CBZ or PHT fetal exposure impair intelligence. Data on fetal $\mathrm{PB}$ exposure are insufficient. AED polytherapy in utero exposure results in higher risk for cognitive/behavioral teratogenesis. There is no information to suggest whether some combination would be safer or more hazardous than others, except that polytherapies with valproate appear to be at higher risk. Overall, polytherapy is associated with more negative effects on cognitive functioning than monotherapy.

The mechanism underlying adverse AED effects on development is uncertain. Teratogens interact with genotype to produce both anatomic and behavioral defects (59). Whether a defect occurs depends on susceptible genotype and may involve interaction of multiple liability genes. Proposed mechanisms underlying teratogenicity of AEDs include folate, ischemia, neuronal suppression, reactive intermediates (e.g., epoxides or free radicals), and AEDinduced neuronal apoptosis. Neuronal dysfunction short of apoptosis or migration disorders may also contribute. Mechanisms of anatomic and behavioral teratogenesis may well differ since it appears that anatomic defects result from first-trimester AED exposure while behavioral defects arise from third-trimester exposure. A leading hypothesis of anatomic teratogenesis involves oxidative macromolecular damage from free radicals formed as reactive intermediates of AED metabolism. AED-induced apoptosis has been proposed as a possible mechanism for the behavioral deficits. Widespread neuronal apoptosis in animals occurs as a result of exposure to clonazepam, diazepam, PB, PHT, VG, or valproate in neonatal rats. The effect is dose dependent, occurs at therapeutically relevant blood levels, requires only a relatively brief exposure, and can be synergistic suggesting possible increased polytherapy risk. Similar apoptotic effects were not seen at therapeutic dosages for CBZ, LEV, lamotrigine, or TPM monotherapy. Further, fetal exposure of GABAergic AEDs such as valproate and VG can result in hippocampal and cortical dysplasia secondary to impaired migration and neuronal death.

\section{Folate Supplementation}

Folate is a water-soluble coenzyme that occurs naturally in food (e.g., green leafy vegetable, dried beans, fruits). In addition, fortified foods like ready-to-eat cereals are fortified with $100 \%$ of the recommended daily allowance for folate. Even so, most women do not consume enough folate that is provided in supplements and added to fortified foods. Folate helps produce and maintain new blood cells and is necessary for proper CNS development. This is especially important during periods of rapid cell division and growth as early in the first trimester of pregnancy. Folate-deficient women who become pregnant are at greater risk of giving birth to lower birth weight premature infants. Folate deficiencies have been implicated in the development of certain birth defects, particularly NTDs. Neural tube closure occurs at about day 26 of pregnancy, often before a woman is aware that she is 
pregnant. Neural tube and cardiac defects occur in the first 28 days following conception, so folate supplementation should begin before conception is attempted and continue throughout the pregnancy. If folate supplement is begun more than 30 days after conception, it will not provide protection against NTD. About $40 \%$ to $50 \%$ of pregnancies with epilepsy are not planned and, hence, it is reasonable to recommend routine use of folate supplement for any women of childbearing age considered to be at risk for pregnancy. Evidence is mounting that folic acid protects developing fetuses against NTDs. There have been studies that have failed to demonstrate a neuroprotective effect for periconceptual folate supplementation. Folic acid supplementation may enhance PHT metabolism and thus lower PHT blood levels, placing women at risk for breakthrough seizures. EIAEDs have been shown to decrease serum folic acid levels by increasing the metabolism of circulating folic acid. The appropriate dosage for folate is probably dependent on a variety of factors including AED/no AED therapy, whether AED is taken, maternal age, and other concurrent medication. For any sexually active woman of childbearing potential, recommended daily allowances of folic acid have been increased to $400 \mu \mathrm{g} /$ day for nonpregnant women, $600 \mu \mathrm{g} /$ day for pregnant women, $600 \mu \mathrm{g} /$ day for pregnant women and those contemplating pregnancy, and $500 \mu \mathrm{g} /$ day for lactating women. Many epileptologists recommend higher doses $(800 \mu \mathrm{g} /$ day to $4 \mathrm{mg} /$ day $)$ for WWE. In most cases, folate intake should not exceed $1000 \mu \mathrm{g}$ daily to avoid provoking seizures or masking the hematologic effects of $\mathrm{B}_{12}$ deficiency and pernicious anemia. However, for women with a family history of NTDs, $4 \mathrm{mg} /$ day is the recommended dosage and vitamin $B_{12}$ levels should be checked (54).

\section{Epilepsy Care During Pregnancy}

WWE can be reassured that having epilepsy should not prevent them from having children. About 1 million WWE in the United States are of childbearing age, and about 20,000 babies are born to WWE each year. It is probably not helpful to change an AED during pregnancy with the goal of using a less teratogenic drug, because of the critical phases of embryologic development occur very early in pregnancy. Despite the increased risk of maternal and fetal complications, around $90 \%$ of pregnant WWE deliver healthy newborns. Close medical care, however, is essential. A multidisciplinary approach is recommended, involving a patient's primary care, an obstetrician who specializes in high-risk pregnancies, and a neurologist (60).

\section{EPILEPTIC SEIZURES DURING PREGNANCY}

Epilepsy per se does not pose a teratogenic risk. Most WWE have no change in seizure frequency during pregnancy or even develop fewer seizures. Only $15 \%$ to $33 \%$ have more seizures during pregnancy $(51,66-68)$. AED pharmacokinetics is altered during pregnancy. Serum drug concentrations are reduced by increasing maternal blood volume, a phenomenon that reaches its nadir at term $(69,70)$. However, seizure frequency increases as early as the first trimester and not necessarily around term, so reduced AED levels only partially explain the phenomenon. Hormonal changes may also contribute: the ratio of estrogen (which lowers seizure threshold) to progesterone (which raises seizure threshold) increases during pregnancy reaching its peak between weeks 8 and 16. Other factors that may contribute to a lower seizure threshold include stress, anxiety, and sleep deprivation. Also some women stop therapy because of the fear of teratogenic effects. Generalized tonic clonic seizure during pregnancy can result in fetal intracranial hemorrhage, transient fetal bradycardia, heart beat variability, fetal hypoxia, and stillbirth. Status epilepticus resulted in 30\% maternal mortality and 50\% infant mortality. There is no evidence that non-convulsive seizures during pregnancy adversely affect outcome apart from trauma.

\section{Therapeutic Drug Monitoring During Pregnancy}

Several published guidelines recommend monitoring serum AED concentration at the beginning of every trimester and 1 month after delivery $(69,70)$. Monitoring free drug concentration has been recommended for both highly and moderately protein bound drugs such as PHT, PB, CBZ, and sodium valproate. Additional monitoring should be considered if adverse effects or poor seizure control are seen, or if nonadherence is suspected. In view of the large inter- and intraindividual variation of drug concentrations, some have recommended monthly serum AED concentration monitoring, particularly for lamotrigine. Since well-defined therapeutic ranges have not been established for the newer AEDs, including lamotrigine, it is suggested that an optimal therapeutic range should be established for each particular individual before conception and that this be used to guide dosage adjustment during pregnancy. Identifying an individual therapeutic range prior to pregnancy is similarly useful for older AEDs, as the clinical response to similar doses and serum drug concentrations can be highly variable. If AED doses have been increased during pregnancy, they should be readjusted after delivery, as concentrations normalize quickly in the postpartum period. At this time, AED toxicity may occur and serum concentrations may be a useful guide for reestablishing the correct dose.

By contrast, the unpredictability and variability of serum drug concentration have prompted others to recommend a more conservative approach, with alterations in dosage being made on clinical grounds. The practical limitations of free drug concentration monitoring and lack of a population target range make adjustments made on clinical grounds a more attractive and pragmatic approach. In fact, current evidence has failed to show that routine adjustment of AED doses during pregnancy based on serum concentration improves seizure control. There are no studies comparing seizure control in pregnancy in those with serum monitoring and those without. Additionally, increases in dose may potentially harm the fetus. While most changes in the dose are made in the second and third trimesters of pregnancy, when organogenesis is complete, there is concern that the potential longer term adverse effects of AED exposure in utero may be more likely to be related to exposure in the latter half of pregnancy. Women should have the opportunity to discuss the pros and cons of routine AED dose adjustment, and their views of the relative risk of seizures and potential adverse fetal outcomes should be taken into account. In practical terms, routine monitoring of serum AED concentration in pregnancy is not useful unless directed at answering a specific clinical question. 
For example, if seizures increase or are likely to increase, serum AED concentrations may be useful to plan or anticipate the extent of the change (69).

\section{Prenatal Screening for Fetal Malformations}

All WWE should be offered prenatal screening for fetal malformation: maternal serum alpha-fetoprotein testing at 14 to 16 weeks of gestation and structural ultrasonography at 16 to 20 weeks of gestation (51). Performed together, these tests have more than $95 \%$ sensitivity in detecting open NTDs. Patients with equivocal results should undergo amniocentesis, which increases the sensitivity to more than $99 \%$. Cardiac anomalies can also be diagnosed prenatally with detailed sonographic imaging of the fetal heart (18-20weeks of gestation), which is $85 \%$ sensitive. The accuracy of ultrasonography for the prenatal diagnosis of cleft lip is less well established. The screening test has inherent ethical implications and may lead to difficult choices if a problem appears to be present.

\section{Vitamin K Supplementation}

Some case series have found that newborns exposed to EIAEDs have a higher risk of hemorrhagic disease of the newborn, a condition that has a mortality rate of up to $30 \%$. When it occurs, bleeding usually happens within the first day of life and affects internal organs such as the lungs, abdomen, and brain. It is not clear whether the alteration of vitamin $\mathrm{K}$ metabolism caused by some AEDs is the only mechanism at work.

It is suggested that oral vitamin $\mathrm{K}$ supplementation at 10 to $20 \mathrm{mg} /$ day be prescribed during the last month of pregnancy and $0.5 \mathrm{mg}$ administered intramuscularly immediately after delivery for WWE. This is particularly important for women taking EIAEDs. Vitamin $\mathrm{K}$ is recommended for all neonates at birth; however, neonates born to WWE should be monitored for bleeding. The recommended dosage for neonates is $1 \mathrm{mg}$ intramuscularly or intravenously at birth. If a woman has not taken vitamin $\mathrm{K}$ before beginning labor, it can still be administered during labor $(51,53)$.

\section{Labor and Delivery}

About 95\% of WWE have a safe vaginal delivery, without a seizure during, or in the 24 hour following, delivery $(51,53)$. Women with generalized epilepsy are more at risk for seizures during delivery than are those with partial epilepsy. It is important to remind WWE to bring their AEDs to the hospital during labor and to take regular doses during this period under the supervision of hospital staff. Subtherapeutic or barely therapeutic AED concentrations can be a cause of seizures during labor and delivery. Intravenous or intramuscular AEDs may be needed if the woman is not able to keep down oral medication. Sleep deprivation can provoke a seizure, so in some cases, obstetric anesthesia may be indicated prior to delivery. WWE are more likely than the general population to have labor induced, although epilepsy is not an indication for induction. Cesarean sections and mechanically assisted delivery are also more common in WWE.

\section{Epilepsy Care After Pregnancy}

\section{Postpartum Care and Breast-Feeding}

New mothers with epilepsy should be encouraged to breastfeed their babies. Most experts believe that the benefits to the mother and baby outweigh the risk $(54,67)$. All AEDs cross into breast milk, but only the free fraction-that which is not bound to maternal serum binding proteins-is available to cross. Breast-feeding conveys substantial benefits to the infant, and these benefits to the mother and infant outweigh any risk from AED exposure. AED concentrations in breast milk are far less than maternal serum levels; however, serum half-life of AEDs is longer in the neonate than in the mother. Nevertheless, the American Academy of Neurology supports breastfeeding for infants whose mothers are taking AEDs. Caution is advised if the mother is treated with PB, ESM, or PRM. For infants of women taking high-dose LTG, drug levels and adverse events in the infant should be monitored. Parents should alert their pediatricians if increased lethargy is noted. When the baby is born, an appointment for a neurologic evaluation should be made for age 4 to 6 weeks (67).

AED levels should be monitored closely in the weeks following delivery. During this period, AED levels may increase gradually. The increase in postpartum plasma levels of EIAEDs starts about 10 to 14 days postpartum. The levels of drugs that are excreted renally such as LEV, OXC, and LTG increase within days of delivery. Thus, some women require dose adjustment immediately following delivery.

\section{Parenting Issues and Safety}

WWE need to organize their lives to minimize fatigue in the weeks following delivery in order to minimize the occurrence of seizures precipitated by fatigue. They may also have an increased risk for breakthrough seizures due to stress. Napping is encouraged and asking help from other adults to do nighttime feedings can help with fatigue. For women who are breast-feeding, this can be accomplished either with expressed breast milk or with supplementary formula.

Each woman needs to assess her particular seizure pattern and to make appropriate arrangements for her own safety and that of her baby. For example, if her seizures make her fall, she may wish to rely on a stroller or carriage in the house rather than carrying the infant. If she tends to drop things, perhaps a baby harness is appropriate. Baby care such as changing diapers and clothing can be done on a blanket on the floor rather than on a high changing table, especially during the immediate postpartum period when AED levels fluctuate. A second person should be present during baths to avoid the danger than even a momentary lapse in alertness can pose (54).

\section{MOVEMENT DISORDERS AND PREGNANCY}

Disorders of human motor control are common neurologic problems. Although the majority of these disorders are more common in the older postmenopausal age groups, a few can be seen in women of childbearing age. Human motor control is achieved through complex interactions of many CNS regions but intimately involve regions of the brain known as the basal ganglia. The basal ganglia utilize a variety of neurotransmitters to achieve the desired sequencing of motor activity and 
production of normal movement. The effects of progesterone and estrogen on the basal ganglia are unclear as experiments with rat models have produced improvement in dopamine function, where as in humans either no effect or deleterious effects have been reported $(71,75)$.

Rarely, abnormal movements known as choreas appear during gestation, typically following the first trimester known as chorea gravidarum. The chorea is generally seen only on half of the body and is known as a hemichorea. The incidence is said to be 1:139,000 pregnancies (76). It was previously associated with rheumatic fever; however, in the United States, the virtual elimination of this disorder has made this association less likely. More often now, it is linked to systemic lupus erythematosus or anti-phospholipid antibody syndrome $(77,78)$. The chorea typically resolves spontaneously, often after delivery. Rarely, the movements are so severe as to induce rhabdomyolysis and hyperthermia (79). Treatment is typically by using dopamine receptor blockers. Due to concerns involving the fetus, haloperidol is preferred since of all of the medications available, it is the least likely to cause harm (80). Similar choreiform movements may occur following institution of birth control pills as well. These typically resolve after discontinuation of OC pills.

\section{Dystonias}

Dystonias are uncommon disorders that involve sustained muscle contraction and abnormal posturing. They can be associated with twisting and repetitive movements as well (81). Only very rare cases of dystonias being induced by pregnancy exist (authors' personal experience). More often, a preexisting dystonia may be seen to complicate pregnancy or delivery. The majority of medications used to treat dystonias have a high probability of being associated with fetal malformation; however, the exception to this is doparesponsive dystonias treated with levodopa. Although experience with this levodopa in pregnancy is limited, small series and case reports published to date do not suggest any fetal malformations occurring while the mother is taking this medication (75). More recently, botulinum toxin injections have been used, and occasional case reports (82) suggest that this treatment is effective for the mother, and not harmful to the fetus.

\section{Essential Tremor}

Essential tremor is the most common movement disorder in the general population and is frequently seen in younger patients. This movement disorder more likely than any others to be encountered by a treating obstetrician. It involves action tremors of the hands, head, voice, and occasionally legs or trunk, and frequently runs in families. The tremor occurs when the patient is using the extremity; however, the head tremor occurs with any upright posture. There is no other impairment of neurologic function or cognition. The disorder follows a benign course and is generally only socially disabling; however, in more severe cases, it can be very difficult to eat or write. The most effective treatment for this disorder involves the use of PMD and propranolol. Other medications have been used with varying success. Given the unclear danger to the fetus of these medications, as well as the benign nature of the disorder, it is probably wise to counsel the mother to take no medication for this condition while she is pregnant or breastfeeding. If the mother's tremor is so severe that it precludes reasonable normal daily function, the choice of medications should be discussed between the obstetrician and the neurologist to determine which medication is felt to be least likely, at that time, to cause harm to the fetus and most effective for that patient.

\section{Tourette's Syndrome}

Tourette's syndrome is also quite common, and since it begins in childhood, this disorder will be frequently seen in the obstetric population. Tourette's syndrome involves the combination of motor and vocal tics, which evolve and fluctuate over time. A tic is a brief un-voluntary movement or sound that can include throat clearing, coughing, sighing, or making sounds or words. The tics can be suppressed voluntarily but take a great deal of effort from the patient to do so. For this reason, they are called un-voluntary rather than involuntary. Patients with Tourette's syndrome also frequently have attention deficit disorder and obsessive-compulsive disorder. The tics typically respond to dopamine receptor blockade, and haloperidol is a very effective treatment. As discussed previously, this medication is felt to be the safest choice for the fetus (80).

\section{Huntington's Disease}

Huntington's disease occasionally presents during childbearing years, although classically presents in the patients' 30 s or 40 s at a time when many women have already had children. Although not caused or necessarily exacerbated by pregnancy, the Huntington's patient presents several unique challenges. The first is treatment of a pregnant patient with significant symptoms. The symptoms of Huntington's disease include chorea, dementia, and psychiatric disorders. All treatment is symptomatic, as no neuroprotective or preventive therapies currently exist. The safest option for treatment of chorea or psychosis is dopamine blockade using haloperidol and the treatment for depression felt to be least harmful for the fetus is fluoxetine (80). A more common scenario is that a woman who has already delivered children will later develop the symptoms of Huntington's disease. The disorder is an autosomal dominant genetic disease that can be predicted by an expanded CAG repeat in the region that codes for the Huntington protein on chromosome four. Adult patients with symptomatic parents have the option of genetic testing to determine whether they will develop this disease, although not all will choose to do so. Genetic testing should not be carried out lightly, and the physician ordering this test must be prepared to properly counsel the asymptomatic adult patient. This responsibility is usually best carried out by a neurologist with expertise in Huntington's disease or a geneticist. The children of an affected parent have a 50\% chance of developing the disorder, and some parents choose to proceed with pregnancy whether or not they expect to develop the disease themselves (83). For the affected patients who choose not to conceive naturally, techniques for artificial insemination or in vitro fertilization of donor ova could be considered as appropriate. 


\section{Parkinson's Disease}

Because Parkinson's disease is more common over age 50, it is only rarely found in the age group likely to conceive children. Very little experience exists with this condition in the reproductive age group, although a small series (84) of patients suggests that levodopa is not associated with any significant fetal malformations or birth problems. Other antiparkinsonian medications appear to be associated with variable risks to the fetus in many cases and probably should be avoided if at all possible (80). Parkinson's disease is a neurodegenerative disorder that generally begins with increased muscle tone or resting tremor on one side of the body progressing to the other side over a variable length of time. Gait problems and significant problems with activities of daily living and self-care are later findings. Young people with Parkinson's disease often have a more aggressive progression of disease, but hopefully would be able to be adequately maintained throughout pregnancy on levodopa monotherapy. Consultation with a movement disorder neurologist would be very helpful with this patient group.

\section{Wilson's Disease}

Wilson's disease is a very rare disorder involving disordered copper metabolism. Clinically, there is a wide variability in the age of onset as well as presentation. In children, it more commonly presents with symptoms of liver disease, but in young adults psychiatric or movement disorders tend to predominate. The clinical picture consists of liver cirrhosis, a wide variety of abnormal involuntary movements, and psychiatric illnesses. The most common treatment for this disorder is the copper chelator penicillamine, which is known to be teratogenic. Trientine is another chelating agent sometimes used, but may also be associated with fetal malformations. More recently, the therapy with zinc acetate has been shown to be effective in the treatment of Wilson's disease (85), and a small case series suggests only a mildly elevated birth defect rate compared with the general population (86). The authors of that paper conclude that zinc acetate is a preferable treatment for Wilson's disease in pregnant women.

In this section, we have reviewed movement disorders that appear to have a direct link with sex hormones as well as those that are preexisting and may change their manifestations during pregnancy or otherwise complicate pregnancy in some fashion. Movement disorders induced by pregnancy are rarely seen, and the majority of experience is from cumulative case reports from many authors. The rarity of these disorders in the childbearing age group makes it unlikely that any single physician will have much experience with the management of these conditions during pregnancy, and referral to a movement disorder neurologist would be appropriate.

\section{STROKE AND PREGNANCY}

Cerebrovascular disorders, which include ischemic and hemorrhagic stroke, are an uncommon but feared complication of pregnancy contributing to $5 \%$ to $12 \%$ of all maternal deaths (87). Eclampsia occasionally shares presentation similar to stroke but importance of prompt recognition of either cannot be overemphasized. For the sake of clinical management, the issue of stroke in pregnancy has been divided into cerebrovascular risk factors before pregnancy, during pregnancy, and in immediate puerperium.

\section{Stroke Risk Before Pregnancy}

\section{Contraception in Relation to Stroke}

Contraceptives containing more than $80 \mu \mathrm{g}$ of estrogen are linked to increased risk of stroke. This risk increases for hemorrhagic stroke if smoking is combined with estrogen intake.

\section{Stroke Risk During Pregnancy}

Incidence: Though studies have shown variable rates, most agree (88) that risk of cerebral infarction is the same in pregnancy as compared with age-matched controls though risk of intracranial hemorrhage is about 2.5 times higher during pregnancy. Witlin et al. reviewed the incidence of stroke in pregnancy and only reported 20 strokes in 130,000 women who delivered over a period of 10 years (88). Overall, for either type of stroke during or within 6 weeks after pregnancy, the adjusted relative risk is about 2.4 (95\% confidence interval, 1.6 to 3.6), and the attributable, or excess, risk is 8.1 strokes per 100,000 pregnancies (95\% confidence interval, 6.4 to 9.7$)$. The prevalence of hypertension, diabetes mellitus, and current smoking was found to be lower among pregnant women with stroke than among nonpregnant women with stroke in Baltimore-Washington Cooperative Young Stroke Study (89).

\section{Pregnancy As a Risk Factor}

Pregnancy is a relatively hypercoagulable state with markedly increased levels of fibrinogen, total body, and plasma levels of factors VII, X, and XII. The increased activity of factors of hemostasis is accompanied by an increase in activity and concentration of ATIII and acquired activated protein C resistance predisposing to thromboembolic events (90).

Many other physiologic changes occur in pregnancy that may impact the pathogenesis and management of stroke during pregnancy (91). Blood pressure decreases during the second and third trimesters due to decreased peripheral vascular resistance. Cardiac output, systemic blood pressure, and vascular resistance contribute to cerebral perfusion. Dramatic shifts in hemodynamics coupled with alterations in coagulation profile underscore the clinical significance of the physiologic adaptations in cerebral endothelial permeability and pressure. Middle cerebral artery perfusion pressure has been shown to increase steadily during gestation.

\section{Presentation}

The clinical features of arterial ischemic strokes during pregnancy are not unique, except for those of eclampsiaassociated ischemic strokes, which comprise seizures and/or impairment of consciousness, in addition to focal deficits. CVT presents with a wide spectrum of symptoms and signs and can be confused with other diseases, in particular with eclampsia, encephalitis, intraparenchymal hemorrhage, and even with post-lumbar puncture headache. The differential diagnosis of hemorrhagic strokes includes eclampsia, pituitary necrosis, and CVT. It is noteworthy that hypertension and albuminuria, two main features of eclampsia, have been observed in about a third of intracranial hemorrhage caused by a ruptured vascular malformation during pregnancy (92). 
Table 1 Causes of stroke in pregnancy

Generic causes of stroke during pregnancy

Premature atherosclerosis

Hypertension

Cardiac disease

Hyperlipidemia

Diabetes

Arterial dissection

Takayasu's disease

Vasculitis

Antiphospholipid antibody syndrome

Systemic lupus erythematosus

Sickle cell disease

Thrombotic thrombocytopenic purpura

Coagulopathies

\section{Tobacco}

Cocaine or drug abuse

Thrombophilic states

Protein $\mathrm{C}$ and $\mathrm{S}$ deficiencies

Antithrombin III deficiency

Factor V Leiden mutation

Hyperhomocysteinemia

Mutations in the prothrombin gene

Lupus anticoagulant

Anticardiolipin antibody
Causes of stroke unique to pregnancy

Eclampsia

Caesarean delivery (88)

Choriocarcinoma

Postpartum cerebral angiopathy

Postpartum cardiomyopathy

Amniotic fluid embolus during labor

Associations of stroke in pregnancy

Migraine headache

Blood transfusion

Anemia

Age more than 35 years

Non-white race

Bromocriptine mesylate (used in postpartum for suppression of lactation)

Methylergonovine maleate (used in postpartum hemorrhage caused by uterine atony)

\section{Etiology of Stroke}

Causes of stroke in pregnant females are usually the same as causes in nonpregnant females. Though associations of migraine headaches and blood transfusion have been suggested, their exact etiologic role is unclear (87). Transfusion increased the risk of stroke more than 10-fold. Although the need for transfusion is increased in conditions that place a woman at an increased risk of stroke, such as severe preeclampsia, postpartum hemorrhage, and sickle cell anemia, transfusion itself may be a risk factor for stroke. Storage and preservation of red blood cells increases their aggregability, possibly increasing the risk of thrombosis (87). Age greater than 35 years and African-American race have also been found to be significant risk factors. Eclampsia is the main pregnancyspecific cause, which may be associated with focal neurologic deficits of sudden onset, consistent with a clinical diagnosis of stroke. The two other pregnancy-specific conditions (choriocarcinoma and amniotic fluid embolism) are rarely responsible for focal cerebral ischemia. Table 1 lists the causes of stroke in pregnancy grouped under generic causes, pregnancy specific causes, and associations of stroke in pregnancy that may not necessarily have an etiological role.

\section{Pregnancy-Related Complications}

Gestational diabetes and hypertension are both associated with increased risk of stroke (93).

Complications of pregnancy such as severe pre-eclampsia, eclampsia, infection, hyperemesis, and fluid and electrolyte imbalance are significant risk factors for pregnancy-related stroke (87).

\section{Pre-eclampsia and Eclampsia}

Pre-eclampsia is known to be an important risk factor for pregnancy-associated stroke. One-fourth of the pregnancy-related infarcts are associated with eclampsia or pre-eclampsia. The hypercoagulable state of normal pregnancy is further potentiated in pre-eclampsia. There is increased expression of procoagulant proteins, such as tissue plasminogen activator, PAI1, von Willebrand factor, fibronectin, homocysteine, and thrombomodulin, and reduced levels of anticoagulant proteins, including antithrombin III, protein C, and protein $S$ in women with pre-eclampsia (93). Beyond increasing risk of stroke during pregnancy, women with preeclampsia were $60 \%$ more likely to have a stroke in the months and years that followed their pregnancy.

Neurologic features of the pre-eclamptic-eclamptic syndrome include headaches, visual abnormalities owing to retinal or occipital lobe lesions, impairment of consciousness, seizures, or focal deficits of sudden onset (92). The clinical and neuroimaging findings are more consistent with edema, which is thought to result from a rapid increase in blood pressure with vasospasm of cerebral arterioles, subsequent loss of autoregulatory capacity, and disruption of the blood-brain barrier. Angiograms in patients with eclampsia have also shown segmental narrowing consistent with vasospasm of large and medium-sized cerebral arteries. Several causes of altered vascular reactivity, including increased sensitivity to normally circulating pressor agents, deficiency of vasodilating prostaglandins, and endothelial cell dysfunction, have been reported in eclampsia. MRI in preeclampsia or eclampsia may show nonspecific hyposensitives in posterior regions, finding often labeled as posterior reversible encephalopathy syndrome.

\section{Ischemic Stroke}

During pregnancy, risk of cerebral infarction is the same as age-matched control subjects. Exacerbations of systemic lupus 
erythematosus and Takayasu's disease may occur during pregnancy and may manifest as ischemic strokes. Pregnancy is classically associated with an increased risk of rheumatic disease recurrence.

Antibiotic prophylaxis has been advocated for all pregnant patients with a history of rheumatic fever, especially those with a history of rheumatic heart disease. The high risk of thromboembolism in gravid patients with prosthetic heart valves emphasizes the need for effective anticoagulation throughout the pregnancy, despite the potential fetal and maternal adverse effects of this treatment. Bioprosthesis do not seem to be associated with an increased risk of thromboembolism during pregnancy and may not require anticoagulant therapy.

\section{Hemorrhagic Stroke}

Eclampsia and ruptured vascular malformations are the most frequent causes of intraparenchymal hemorrhage (92). The risk of hemorrhagic stroke during pregnancy is more than the risk of ischemic stroke though it increases further 6 weeks postpartum. Common associations of intracranial hemorrhage in pregnancy are eclampsia, bleeding diathesis, cocaine, bacterial endocarditis, sickle cell disease, and metastatic choriocarcinoma. Aneurysmal subarachnoid hemorrhage may be life threatening and warrants urgent correction.

\section{AVMs}

The increased tendency of an aneurysm to bleed with advancing gestational age suggests that hemodynamic, hormonal, or other physiologic changes of pregnancy may play a role in aneurysmal rupture (92).

Risk of hemorrhage from an unruptured AVM is estimated to be around $3.5 \%$. The optimal time to wait to conceive after surgical correction is controversial. For previously ruptured AVMs, risk of hemorrhage outweighs risk from surgical corrections. Surgical treatment might be considered before planning pregnancy. Women with repaired AVMs can undergo vaginal delivery under epidural, caesarean may be preferred for partially repaired or untreated AVMs. Stereotactic radiotherapy is not used in pregnant women. No data are available concerning embolization of AVM in pregnancy (92).

\section{Neuroimaging Modalities}

Computed tomography may harm the fetus or embryo both by exposure to ionizing radiation and by administration of iodine-based contrast material, if contrast is used. During head CT examination, only head in directly irradiated. Fetus is exposed only to radiation scattered throughout the body and shielding the abdomen does not significantly reduce the minimal fetal radiation dose. The teratogenic risk linked to radiation exposure is limited to the embryogenesis period, which runs from the second to fourth week of gestation. Iodinated contrast though not shown to have adverse effects except neonatal hypothyroidism is categorized as B class by FDA. Digital angiography procedures decrease the X-ray exposure and the amount of administered iodine if deemed necessary. Pregnancy does not modify the maternal tolerance to iodine. Good maternal hydration is needed to avoid fetal dehydration. After 6 months, there is a risk of fetal hypothyroidism, which can be diagnosed and treated at birth with an excellent prognosis (92).
Though longitudinal studies are lacking, MRI is generally considered safe during pregnancy. American College of Obstetricians and Gynecologists have reported no adverse fetal effects but still advise against the use of MRI in first trimester. Risk of gadolinium to fetus is not known; it is classified as category C by FDA. Magnetic resonance angiography might avoid the risk of radiation associated with computed tomography and angiography while evaluating blood vessel in stroke or AVM.

Pregnancy does not contraindicate radiologic procedures if they are deemed necessary. In case of acute stroke, CT head noncontrast may not detect acute strokes less than 6 to 8 hours in onset. MRI sequences including diffusion weight imaging (DWI) are currently the most accurate imaging test available for acute ischemia with sensitivity and specificity approaching $100 \%$. Intravenous contrast is not required for DWI or magnetic resonance angiography.

\section{Treatment Modalities}

The potential benefits of antithrombotic drugs should be weighted against the potential risks to the mother and the fetus. Aspirin should be used for same indications as for stroke as in nonpregnant female. It has not been reported to cause any harmful effects in the second and third trimesters. Lowdose aspirin (60-150 mg/day) have been largely administered by obstetricians during the second and third trimesters of pregnancy in women at risk for pregnancy-induced hypertension without evidence of fetal or maternal adverse effects. The potential adverse effects of aspirin consumption late in pregnancy are maternal and fetal hemorrhage, premature closure of the ductus arteriosus, prolongation of labor, and delay in the onset of labor (92).

Warfarin is known to be teratogenic and causes congenital malformations in fetus by causing micro-hemorrhages in the fetal brain. Fetal warfarin syndrome is a well-reported entity. When therapeutic anticoagulation is warranted, heparin (unfractionated of low molecular weight) should be used. It does not cross the placenta and its short half-life makes dose adjustment easier in case of overdose, bleeding, or premature delivery. Heparin therapy may cause a persistent anticoagulant effect (for up to $28 \mathrm{~h}$ after the last injection of heparin) at the time of delivery, which increases the risk of bleeding and contraindicates epidural analgesia.

When anticoagulants are indicated during pregnancy, two schemes of anticoagulant treatment have been recommended: (i) heparin throughout pregnancy or (ii) heparin until week 13 followed by warfarin until the middle of the third trimester followed by heparin. Heparin should be discontinued immediately before delivery. Anticoagulation treatment is started again after delivery (92).

\section{Partum-Postpartum Period}

Kittner et al. in Baltimore-Washington Cooperative Young Stroke Study registry found that the risks of both cerebral infarction and intracerebral hemorrhage are increased in the 6 weeks after delivery but not during pregnancy itself (89). The extremely high relative risk of stroke during the postpartum period suggests a causal role for the large decrease in blood volume or the rapid changes in hormonal status that follow a live birth or stillbirth, perhaps by means of hemodynamic, 
coagulative, or vessel-wall changes. A causal role for preeclampsia and eclampsia does not fully explain the much stronger associations with stroke found for the postpartum state than for pregnancy itself.

\section{Postpartum Cerebral Angiopathy}

Postpartum cerebral angiopathy is characterized by the rapid occurrence of severe headaches, nausea or vomiting, seizures, and less often focal neurologic signs with a delay varying from a few hours to 1 month after a normal pregnancy. This picture may suggest the diagnosis of a subarachnoid hemorrhage, but the CSF is normal or shows a moderate pleocytosis. Eclampsia may present with similar features, but in postpartum angiopathy patients do not necessarily have hypertension or proteinuria. Rapid spontaneous clinical recovery occurs in the vast majority of cases. Angiography shows transient focal vasospasm. In most reports cases, ergot alkaloids or other sympathomimetic drugs had been given in the preceding days or hours, hence inclusion of the disease in the category of CallFleming syndrome (92). Outcomes are variable though rapid spontaneous clinical recovery has been generally reported.

\section{CVT}

CVT presents with headaches, seizures, stroke-like symptoms, or mental status changes. Peak incidence is in immediate postpartum period though its occurrence during pregnancy is also known. CVT may lead to ischemic infarction or to intraparenchymal hemorrhage. MRI usually shows hemorrhagic infarcts and magnetic resonance venography may show thrombus in major sinuses or cerebral veins. Conditions that may present with similar symptoms like eclampsia, meningitis, or cerebral mass should be excluded.

Caesarian section and age greater than 25 are risk factors for CVT. Hypercoagulable state of pregnancy, preexisting coagulopathy like protein $\mathrm{C}$ or $\mathrm{S}$ deficiency, factor $\mathrm{V}$ Leiden mutation or antithrombin III antibodies, dehydration, or infection are usual causes. Heparin anticoagulation should be considered with similar indications as in nonpregnant female. Efficacy of venous thrombolysis or venous stenting is not established in pregnancy.

Traumatic damage to the endothelial lining of cerebral sinuses and veins during labor, hypercoagulable state, and stasis of intracerebral blood flow are the most likely explanations for the occurrence of most CVT in the puerperium. Puerperal infections and dehydration may contribute to the high frequency of CVT in developing countries.

\section{Mode of Delivery and Outcomes}

Risk of stroke in relation to the full range of outcomes of pregnancy (spontaneous or induced abortion, stillbirth, and live birth) has not been adequately studied. Caesarean delivery by itself is a risk factor for stroke accounting to the risk of thromboembolism from surgery (88).

Mode of delivery has not been adequately shown to affect outcomes in the history of gestational stroke though series have found higher incidence of caesarian deliveries in such patients (88). James et al. (87) reported several complications associated with pregnancy-related stroke-postpartum hemorrhage (OR 1.8), pre-eclampsia and gestational hyper- tension (OR 4.4), fluid and electrolyte imbalance (OR 7.2), transfusion (OR 10.3), and the highest, pregnancy-related infection (OR 25.0). Case fatality rate for pregnancy-related stroke have been reported to be anywhere between $4.5 \%$ and $24 \%$ (87). Stroke during one pregnancy by itself is not a risk factor for stroke during subsequent gestation.

\section{CONCLUSION}

Stroke during pregnancy or shortly thereafter is etiologically heterogeneous and, as with stroke in any young patient, requires systematic evaluation (88). Prompt neuroimaging and neurologic consultation are recommended in any pregnant or postpartum female who presents with new neurologic symptoms suggestive of stroke.

\section{ACKNOWLEDGEMENT}

We want to acknowledge Ms Betty March for her work and assistance with this manuscript.

\section{REFERENCES}

1. Olesen J, Friberg 1, Skyhot-Olsen T, et al. Timing and topography of cerebral blood flow, aura, and headache during migraine attacks. Ann Neurol 1990; 27: 791-8.

2. Granella F, Sances G, Zinferrari D, et al. Migraine without aura and reproductive life events: a clinical epidemiological study in 1,300 women. Headache 1993; 33: 385-9.

3. Chen TC, Leviton A. Headache recurrences in pregnant women with migraine. Headache 1994; 34: 107-10.

4. Stein GS. Headaches in the first postpartum week and their relationship to migraine. Headache 1981; 21: 201-5.

5. Loder E. Safety in sumatriptan in pregnancy: a review of the data so far. CNS Drugs 2003; 17: 1-7.

6. Morrell MJ. The new anti-epileptic drugs in women: efficacy, reproductive health, pregnancy and fetal outcome. Epilepsia 1996; 37: 534-44.

7. Silberstein SD. Headaches and women: treatment of the pregnant and lactating migraneur. Headache 1993(b); 33: 533-40.

8. Kudrow L. Response of cluster headache attacks to oxygen inhalation. Headache 1981; 21: 1-4.

9. Stoodley MA, MacDonald L, Weir BK. Pregnancy and intracranial aneurysm. Neurosurg Clin N Am 1998; 9: 549-56.

10. Wiebers D, Whisnant J. The incidence of stroke in pregnant women in Rochester, Minnesota, 1955 through 1979. JAMA 1985; 254: 3055-7.

11. Portenoy RK, Abissi CJ, Lipton RB, et al. Headache in cerebrovascular disease. Stroke 1984; 15: 1009-12.

12. Pleasure Jr. Neurologic problems in pregnancy. In: Rosenberg RN Pleasure DE, eds. Compr Neurol, 2nd edn. New York: John Wiley, 1998: 925-847.

13. Kelmanse SE, Heaps R, Wolf A, et al. Optic nerve decompression surgery improves visual function in patients with psuedotumor cerebri. Neurosurgery 1992; 30: 391-5.

14. Kurtzke JF. The epidemiology of multiple sclerosis. In: Raine SC, McFarland H, Tourtellotte WW, eds. Multiple Sclerosis: Clinical and Pathogenetic Basis. London: Chapmans Hall, 1997: 91-139.

15. Sadovnick AD, Armstrong H, Rice GPA, et al. A population based study of multiple sclerosis in twins: update. Ann Neurol 1993; 33: 281-5.

16. The genetics of multiple sclerosis. In: Cook SD, ed. Handbook of Multiple Sclerosis, 3rd edn. New York: Marcel Dekker, Inc., 2001: 33-65.

17. Freal JE, Kraft GH, Coryell SK. Symptomatic fatigue in multiple sclerosis. Arch Phys Med Rehabil 1984; 65: 135-8.

18. Whitlock FA, Siskind MM. Depression as a major symptom of multiple sclerosis. J Neurol Neurosurg Psychiatry 1980; 43: 861-5.

19. Poser S, Poser W. Multiple sclerosis and gestation. Neurology 1983; 33: $1422-7$.

20. Worthington J, Jones R, Crawford M, Forti A. Pregnancy and multiple sclerosis: a three year prospective study. J Neurol 1994; 241: 228-33. 
21. Roullet E, Verdier-Taillefer M-H, Amarenco P, et al. Pregnancy and multiple sclerosis: a longitudinal study of 125 remittent patients. J Neurol Neurosurg Psychiatry 1993; 56: 1062-5.

22. Nelson L, Franklin G, Jones M, the Multiple Sclerosis Study Group. Risk of multiple sclerosis exacerbation during pregnancy and breast-feeding. JAMA 1988; 258: 3441-3.

23. Massey EW, Stolp KA. Peripheral neuropathy in pregnancy. Phys Med Rehabil Clin N Am 2008; 19: 149-62.

24. Sax TW, Rosenbaum RB. Neuromuscular disorders in pregnancy. Muscle Nerve 2006; 34: 559-71.

25. McCabe SJ, Uebele AL, Pihur V, et al. Epidemiologic associations of carpal tunnel syndrome and sleep position: is there a case for causation? Hand 2007; 2: 127-34.

26. Finsen V, Zeitlmann H. Carpal tunnel syndrome during pregnancy. Scand J Plast Reconstr Surg Hand Surg 2006; 40: 41-5.

27. Briemberg HR. Neuromuscular diseases in pregnancy. Semin Neurol 2007; 27: 460-6.

28. Brown MD, Levi ADO. Surgery for lumbar disc herniation during pregnancy. Spine 2001; 26: 440-3.

29. Wong CA, Scavone BM, Smith DS, et al. Incidence of postpartum lumbosacral spine and lower extremity nerve injuries. Obstet Gynecol 2003; 101: 279-82.

30. Vrabec JT, Isaacson B, Van Hook JW. Bell's palsy and pregnancy. Otolaryngol Head Neck Surg 2007; 137: 858-61.

31. Shehata HA, Okosun H. Neurological disorders in pregnancy. Curr Opin Obstet Gynecol 2004; 16: 117-22.

32. Wilbourn AJ, Lederman RJ. Postpartum neuralgic amyotrophy. Neurology 1996; 47: 1213-19.

33. Dhand UK. Brachial neuritis. In: Gilman S, ed. MedLink Neurology. San Diego: MedLink Corporation. [Available from: www.medlink.com] Accessed 12 August 2008.

34. Chan LY, Tsui MH, Leung TN. Guillain-Barré syndrome in pregnancy. Acta Obstet Gynecol Scand 2004; 83: 319-25.

35. Tsen LC. Neurologic complications of labor analgesia and anesthesia. Int Anesthesiol Clin 2002; 40: 67-88.

36. Chiò A, Calvo A, Di Vito N, et al. Amyotrophic lateral sclerosis associated with pregnancy: report of four new cases and review of the literature. Amyotroph Lateral Scler Other Motor Neuron Disord 2003; 4: 45-8.

37. Pugh CP, Healey SK, Crane JM, Young D. Successful pregnancy and spinal muscular atrophy. Obstet Gynecol 2000; 95: 1034.

38. Veiby G, Daltveit AK, Gilhus NE. Pregnancy, delivery and perinatal outcome in female survivors of polio. J Neurol Sci 2007; 258: 27-32.

39. Nwosu EC, Tandon S, Breze C, et al. Chronic inflammatory demyelinating polyneuropathy treated with intravenous immunoglobulin. $\mathrm{Br}$ J Obstet Gynaecol 1999; 106: 174-6.

40. Hoff JM, Gilhus NE, Daltveit AK. Pregnancies and deliveries in patients with Charcot-Marie-Tooth disease. Neurology 2005; 64: 459-62.

41. Ciafaloni E, Massey JM. Myasthenia gravis and pregnancy. Neurol Clin 2004; 22: 771-82.

42. Hoff JM, Daltveit AK, Gilhus NE. Myasthenia gravis: consequences for pregnancy, delivery and the newborn. Neurology 2003; 61: 1362-6.

43. Kalidindi M, Ganpot S, Tahmesebi F, et al. Myasthenia gravis and pregnancy. J Obstet Gynaecol 2007; 27: 30-2.

44. Rudnick-Schoneborn S, Zerres K. Outcome in pregnancies complicated by myotonic dystrophy: a study of 31 patients and review of literature. Eur J Obstet Gynaecol Reprod Biol 2004; 114: 44-53.

45. Rudnick-Schoneborn S, Schneider-Gold C, Raabe U, et al. Outcome and effect of pregnancy in myotonic dystrophy type 2. Neurology 2006; 66: 579-80.

46. Day JW, Ricker K, Jacobsen JF, et al. Myotonic dystrophy type 2: molecular diagnostic and clinical spectrum. Neurology 2003; 60: 657-64.

47. Ciafaloni E, Pressman EK, Loi AM, et al. Pregnancy and birth outcomes in women with facioscapulohumeral muscular dystrophy. Neurology 2006; 67: 1887-9.

48. Eskandar OS, Eckford SD. Pregnancy in a patient with nemaline myopathy. Obstet Gynecol 2007; 109: 501-4.

49. Silva CA, Sultan SM, Isenberg DA. Pregnancy outcome in adult-onset idiopathic inflammatory myopathy. Rheumatol (Oxford) 2003; 42: $1168-72$.
50. Vánsca A, Ponyi A, Constantin T, et al. Pregnancy outcome in idiopathic inflammatory myopathy. Rheumatol Int 2007; 27: 435-9.

51. Jeha LE, Morris HH. Optimizing outcomes in pregnant women with epilepsy. Cleve Clin J Med 2005; 72: 938-40, 942-5.

52. Pennell PB. 2005 AES annual course: evidence used to treat women with epilepsy. Epilepsia 2006; 47(Suppl 1): 46-53.

53. Røste LS, Taubøll E. Women and epilepsy: review and practical recommendations. Expert Rev Neurother 2007; 7: 289-300.

54. Cramer JA, Gordon J, Schachter S, Devinsky O. Women with epilepsy: hormonal issues from menarche through menopause. Epilepsy Behav 2007; 11: 160-78.

55. Harden CL, Leppik I. Optimizing therapy of seizures in women who use oral contraceptives. Neurology 2006; 67(12 Suppl 4): S56-8.

56. Noe KH. Gender-specific challenges in the management of epilepsy in women. Semin Neurol 2007; 27: 331-9.

57. Opaleke A, Helmers SL. Hormonal consequences of epilepsy. Semin Pediatr Neurol 2007; 14: 189-95.

58. Meador KJ, Pennell PB, Harden CL, et al.; HOPE Work Group, Pregnancy registries in epilepsy: a consensus statement on health outcomes. Neurology 2008; 71: 1109-17.

59. Meador KJ, Baker G, Cohen MJ, Gaily E, Westerveld M. Cognitive/ behavioral teratogenetic effects of antiepileptic drugs. Epilepsy Behav 2007; 11: 292-302.

60. Kaplan PW, Norwitz ER, Ben-Menachem E, et al. Obstetric risks for women with epilepsy during pregnancy. Epilepsy Behav 2007; 11: 283-91.

61. Holmes GL, Harden C, Liporace J, Gordon J. Postnatal concerns in children born to women with epilepsy. Epilepsy Behav 2007; 11: 270-6.

62. Prakash, Prabhu LV, Nasar MA, et al. Lamotrigine in pregnancy: safety profile and the risk of malformations. Singapore Med J 2007; 48: 880-3.

63. Sankar R. Teratogenicity of antiepileptic drugs: role of drug metabolism and pharmacogenomics. Acta Neurol Scand 2007; 116: 65-71.

64. Lateef TM, Nelson KB. In utero exposure to antiepileptic drugs: teratogenicity and neonatal morbidity. Curr Neurol Neurosci Rep 2007; 7: 133-8.

65. Marsh ED, Brooks-Kayal AR, Porter BE. Seizures and antiepileptic drugs: does exposure alter normal brain development? Epilepsia 2006; 47: 1999-2010.

66. Kälviäinen R, Tomson T. Optimizing treatment of epilepsy during pregnancy. Neurology 2006; 67(12 Suppl 4): S59-63.

67. Pennell PB, Gidal BE, Sabers A, Gordon J, Perucca E. Pharmacology of antiepileptic drugs during pregnancy and lactation. Epilepsy Behav 2007; 11: 263-9.

68. Harden CL. Pregnancy and epilepsy. Semin Neurol 2007; 27: 453-9.

69. Adab N. Therapeutic monitoring of antiepileptic drugs during pregnancy and in the postpartum period: is it useful? CNS Drugs 2006; 20: 791-800.

70. Tomson T, Battino D. Pharmacokinetics and therapeutic drug monitoring of newer antiepileptic drugs during pregnancy and the puerperium. Clin Pharmacokinet 2007; 46: 209-19.

71. Kompoliti K, Comella CL, Jaglin RN, et al. Menstrual-related changes in motoric function in women with Parkinson's disease. Neurology 2000; 55: $1572-4$.

72. Ohtania H, Nomotob M, Douchia T. Chronic estrogen treatment replaces striatal dopaminergic function in ovariectomized rats. Brain Res 2001; 900: $163-8$.

73. Dluzen D, Ramireza V. Progesterone enhances 1 DOPA-stimulated dopamine release from the caudate nucleus of freely behaving ovariectomized estrogen-primed rats. Brain Res 1989; 494: 122-8.

74. Shulman L, Minagar A, Weiner W. The Effect of pregnancy in Parkinson's disease. Mov Disord 2000; 15: 132-5.

75. Golbe L. Parkinson's disease and pregnancy. Neurology 1987; 37: 1245-9.

76. Zegart KN, Schwarz RH. Chorea gravidarum. Obstet Gynecol 1968; 32: $24-7$.

77. Wolf RE, McBeath JG. Chorea gravidarum in systemic lupus erythematosus. J Rheumatol 1985; 12: 992-3.

78. Khamashta MA, Gil A, Anciones B, et al. Chorea in systemic lupus erythematosus: association with antiphospholipid antibodies. Ann Rheum Dis 1988; 47: 681-3.

79. Cardoso F, Seppi K, Mair KJ, Poewe W. Seminar on choreas. Lancet 2006; 5: 589-602. 
80. Ward RM, Bates BA, McCarver GD, Notterman DA, Walson PD. Use of psychoactive medication during pregnancy and the possible effects on the fetus and newborn. Pediatrics 2000; 105: 880-7.

81. Lim EC, Seet RC, Wilder-Smith EP, Ong BK. Dystonia gravidarum: a new entity? Mov Disord 1997; 12: 261.

82. Newman W, Davis T, Padaliya B, et al. Botulinum toxin type A therapy during pregnancy. Mov Disord 2004; 19: 1384-5.

83. Lesca G, Goizet C, Durr A. Predictive testing in the context of pregnancy: experience in Huntington's disease and autosomal dominant cerebellar ataxia. J Med Genet 2002; 39: 522-55.

84. Hagell P, Odin P, Vinge E. Pregnancy in Parkinson's disease: a review of the literature and a case report. Mov Disord 1998; 13: 34-8.

85. Brewer G, Dick R, Johnson V, et al. Treatment of Wilson's disease with zinc: XV long-term follow-up studies. J Lab Clin Med 1998; 132: 264-78.

86. Brewer G, Johnson V, Dick R, et al. Treatment of Wilson's disease with zinc: XVII: treatment during pregnancy. Hepatology 2000; 31: 364-70.
87. James AH, Bushnell CD, Jamison MG, Myers ER. Incidence and risk factors for stroke in pregnancy and the puerperium. Obstet Gynecol 2005; 106: 509-16.

88. Witlin AG, Mattar F, Sibai BM. Postpartum stroke: a twenty-year experience. Am J Obstet Gynecol 2000; 183: 83-8.

89. Kittner SJ, Stern BJ, Feeser BR, et al. Pregnancy and the risk of stroke. N Engl J Med 1996; 335: 768-74.

90. Borrelli AL, De Lucia D, Bernacchi M, et al. Haemocoagulative modifications correlated with pregnancy. Minerva Ginecol 2006; 58: 315-22.

91. Feske SK. Stroke in pregnancy. Semin Neurol 2007; 27: 442-52.

92. Mas JL, Lamy C. Stroke in pregnancy and the puerperium. J Neurol 1998; 245: 305-13.

93. Garovic VD, Hayman SR. Hypertension in pregnancy: an emerging risk factor for cardiovascular disease. Nature 2007; 3: 613-22.

94. Tomson T, Hiilesmaa V. Epilepsy in pregnancy. BMJ 2007; 335: 769-73. 


\section{Sleep disorders and pregnancy Pradeep Sahota and Sanjay Jain}

\section{INTRODUCTION}

Pregnancy is associated with several hormonal, physiologic, physical, and behavioral changes that can cause alterations in sleep architecture, quality, and duration. As a result, it is not surprising that a majority of women report sleep disruption during pregnancy. In addition, pregnancy can affect preexisting sleep disorders such as insomnia, sleep-disordered breathing, and restless legs syndrome (RLS). The intimate relation between sleep and pregnancy has been well recognized and has, especially within the last few years, seen an increased interest in this field. This chapter aims to review changes occurring during pregnancy, which may have a bearing on sleep, and sleep disorders during pregnancy.

\section{HORMONAL CHANGES DURING PREGNANCY THAT MAY AFFECT SLEEP}

Pregnancy is characterized by hormonal changes that are aimed toward successful maturation of the fetus. These changes can affect normal sleep patterns directly and indirectly. There are increases in levels of circulating estrogen and progesterone throughout pregnancy. Estrogen production progressively increases in pregnancy, and levels during the last few weeks prior to delivery may be 1000 -fold higher than that in ovulatory premenopausal women (1). Level of estrogen decreases abruptly after delivery of the fetus and placenta. Estrogen has been shown to reduce rapid eye movement (REM) sleep in animal studies. This may be due to its ability to increase brainstem norepinephrine (2). Progesterone levels gradually increase from $25 \mathrm{ng} / \mathrm{mL}$ at 6 weeks to $150 \mathrm{ng} / \mathrm{mL}$ at 37 weeks of pregnancy (3). Exogenously administered progesterone is associated with decreasing wakefulness, shortened latency to non-REM sleep, and increased duration of non-REM sleep (4). The effects of progesterone appear to be mediated by the action of its metabolites on brain $\mathrm{GABA}_{\mathrm{A}}$ receptors $(4,5)$.

Levels of other hormones that can influence sleep patterns such as prolactin and cortisol are also elevated during pregnancy. Overall, the effect of hormonal changes on sleep during pregnancy is unpredictable due to the wide variation in circulating levels of different hormones as well as the effect of other physical and physiologic changes that occur during pregnancy.

\section{PHYSIOLOGIC CHANGES IN PREGNANCY THAT MAY} HAVE A BEARING ON SLEEP

The expectant mother undergoes a variety of physiologic and physical changes that may lead to alteration of sleep during pregnancy. Majority of these changes occur as a result of the hormonal changes described above, but other factors play a role as well. Renal blood flow increases to a level of 50\% to $85 \%$ above baseline throughout the first and second trimesters (6).
Dilatation of the renal pelvis and ureters occurs due to hormonal changes and mechanical effects of the enlarged uterus (7). These changes predispose to frequent micturition and therefore to several nocturnal awakenings to go to the bathroom.

Lower esophageal sphincter pressure begins to decrease in the first trimester of pregnancy, reaching a nadir in the 36th week. Gastrointestinal reflux symptoms are common, particularly in late pregnancy (8), and may be associated with frequent arousals from sleep.

Upper airway hyperemia, mucosal edema, hypersecretion, and increased friability may occur as a result of increased estrogen levels (3). Progesterone reduces the integrity of the vascular endothelium leading to fluid leakage from the intravascular to the extravascular space, causing tissue edema (9). Additionally, during pregnancy, there is an increase in the level of relaxin, an insulin-like growth factor hormone. It is both a muscle relaxant and a powerful vasodilator (10), properties that increase airway collapsibility and decrease airway caliber. Thus the increased levels of these hormones can potentially reduce the size of the upper airway significantly. Furthermore, progesterone increases phrenic nerve activity with consequent increase in diaphragmatic drive (11), and its property as a powerful respiratory stimulant has been recognized since the 1940s (12). An increase in metabolic $\mathrm{CO}_{2}$ production and the increase in respiratory drive cause a significant increase in minute ventilation (13). This ventilatory augmentation, which is in excess of the increased $\mathrm{CO}_{2}$ production, produces a respiratory alkalosis. The reduced arterial $\mathrm{CO}_{2}$ of pregnancy may be a potential source of respiratory instability during sleep (14). Moreover, augmented respiratory drive may induce obstructive sleep-disordered breathing by increasing negative suction pressure on narrowed upper airway structures (15). In addition to changes in respiratory drive, pulmonary function in pregnancy is also affected by changes in the airways and thoracic cage. Chest wall and total respiratory compliance are reduced in late pregnancy (16). The expanding uterus produces a cephalad displacement of the diaphragm, causing a progressive $10 \%$ to $25 \%$ decrease in functional residual capacity (FRC) toward term $(3,17)$. As a result of increased fetal demands and metabolic alterations in the mother, oxygen consumption increases by $20 \%$ to $33 \%$ by the third trimester (18). The combination of a reduced FRC and increased oxygen consumption results in a lowered oxygen reserve. Conditions that may cause further lowering of this reserve may potentially affect fetal oxygenation with adverse consequences.

\section{SLEEP ARCHITECTURE CHANGES DURING PREGNANCY}

Nonpregnant women of childbearing age have total sleep times on average of 7 to 9 hours per night, with $55 \%$ of the time 
spent in light sleep, 20\% in slow wave sleep (SWS), and 20\% to $25 \%$ in REM sleep (19).

Numerous studies have examined changes in sleep architecture during pregnancy with variable findings (20-24). Most of these studies were however consistent in noting lighter and more disrupted sleep as pregnancy progresses. Wake after sleep onset increased from the second to third trimester, where as REM sleep decreased from the first trimester to second $(24,25)$. Brunner et al. also found, by spectral analysis, a progressive decrease in EEG power density in non-rapid eye movement sleep (25).

\section{SLEEP DISORDERS AND PREGNANCY}

The mother-to-be is predisposed to a number of sleep disorders due to the hormonal and consequently the physical and physiologic changes occurring during pregnancy. Insomnia, parasomnias, sleep-related movement disorders, sleeprelated breathing disorders, and hypersomnolence have all been reported in pregnant patients.

\section{INSOMNIA}

The American Academy of Sleep Medicine, in its diagnostic and coding manual, defines insomnia as repeated difficulty with sleep initiation, duration, consolidation or quality that occurs despite adequate time, and opportunity for sleep and results in some form of daytime impairment. Insomnia is very commonly reported during pregnancy. In a survey of 192 pregnant women, $88 \%$ reported alteration in sleep as compared with their usual experience (21). The most frequent reasons for sleep disruption during pregnancy are urinary frequency (26), heartburn, and backache or ache in hips (21). In addition, there may be disruption of sleep due to nausea and vomiting during the first trimester and because of fetal movements during the third trimester of pregnancy.

Maternal factors such as maternal age $(27)$, parity $(28,29)$, altered circadian rhythm (30), and pregnancy-related disorders such as pre-eclampsia could also influence sleep during pregnancy. Parity may have a bearing on sleep quality and architecture. Compared with primiparas, multiparas had reduced sleep efficiency at all time points (19).

Nonpregnancy-related factors such as maternal smoking, inadequate sleep hygiene, and adjustment disorder may contribute to insomnia independent of the pregnant state.

Ohida and colleagues (31) reported that women exposed to passive smoking were likely to have sleep disturbances, such as subjective insufficient sleep, difficulty in initiating sleep, short sleep duration, and snoring loudly/breathing uncomfortably. Smoking pregnant women had an even greater prevalence of same sleep disturbances and also experienced excessive daytime sleepiness and early morning awakening (31).

\section{SLEEP-DISORDERED BREATHING DURING PREGNANCY}

Although snoring and apneas occur in pregnancy (32), there is lack of data regarding incidence and prevalence of sleep apnea in pregnant women. Most studies investigating sleepdisordered breathing were in the form of questionnaires with variable results (33-37). Despite conflicting results, there is agreement regarding higher prevalence of snoring in pregnant women as opposed to nonpregnant women.
Loube et al. (34), in their survey of 350 pregnant women and 110 age-matched nonpregnant women, found that snoring was more frequent $(14 \%)$ in pregnant women as opposed to nonpregnant women $(4 \%)$. They concluded that snoring was not associated with fetal compromise or adverse outcome. By contrast, the study by Franklin and Holmgren (35) suggests that snoring may be independently predictive of maternal hypertension and fetal growth retardation. Of the 502 pregnant women, $23 \%$ in their study reported snoring every night during the last week of pregnancy. Hypertension and pre-eclampsia were more common, and the incidence of infants born with Apgar score less than or equal to 7 was higher in women who habitually snored.

Obesity has been shown to have a causal association with sleep apnea $(38,39)$. Pregnancy is associated with weight gain, which may exceed $20 \%$ or more by term (40). A recently published study suggests that the prevalence of pre-gravid obesity (defined as BMI $>29 \mathrm{~kg} / \mathrm{m}^{2}$ ) in the United States has increased significantly from 13\% immediately before pregnancy in 1993 to $22 \%$ immediately before pregnancy in 2002/ 2003 (41). Rapid gain in weight is known to be associated with a higher incidence of obstructive sleep apnea (OSA). It has not, however, been established by sleep studies whether excessive weight gain during pregnancy causes sleep apnea. In 1992, Feinsilver et al. (42) reviewed the physiologic adaptations in pregnancy and suggested an increased likelihood of sleepdisordered breathing in pregnant women. Upper airway changes, as described earlier, would predispose the patient to snoring and OSA. In addition, the respiratory alkalosis resulting from augmented minute ventilation and the enhanced sensitivity of the respiratory center to $\mathrm{CO}_{2}$, as occurs in pregnancy, could predispose to central sleep apnea. On the other hand, there are physiologic adaptations, such as increased minute ventilation and pharyngeal muscle tone, due to elevated progesterone levels during pregnancy (43), which may be protective against sleep-disordered breathing. There is reduction in REM sleep (22) during pregnancy, which may protect against sleep apnea. Additionally, in late pregnancy, there is a greater tendency for women to sleep on their sides, thereby reducing the tendency to manifest severe sleep apnea.

The increased possibility of OSA occurring in pregnancy and its possible adverse effect on the fetoplacental unit has been hypothesized (44). There is evidence that pregnant women with preexisting obesity have a higher apneahypopnea index (45) and they may be at increased risk of OSA. In pregnant women who do have sleep apnea, it is felt that due to the lowered oxygen reserve in these patients, occurrence of OSA with oxygen desaturation can have undesirable consequences for the fetus. There are isolated reports of increase in incidence of babies with intrauterine growth retardation in pregnant women diagnosed with OSA $(45,46)$. Other case reports suggest that treatment of the mother to be resulted in delivery of babies with normal birth weight (47). There are, however, no systematic studies of adverse outcomes of OSA during pregnancy.

Despite a lack of conclusive evidence of poor outcomes of pregnancy in women with OSA, pregnant women, especially those with preexisting obesity, and those giving a history of loud snoring, excessive daytime somnolence, or witnessed apneas should be considered to be at increased risk for sleep 
apnea with possibility of adverse consequences for the fetus. There should, for this reason, be a low threshold for obtaining an overnight sleep study and initiation of treatment with nasal continuous positive airway pressure (CPAP) even if the patient has mild sleep apnea.

\section{SLEEP-DISORDERED BREATHING AND PRE-ECLAMPSIA}

Pre-eclampsia is a condition arising in the third trimester of pregnancy. It affects approximately $7 \%$ of all pregnancies and is characterized by abrupt hypertension, proteinuria, and edema of the hands, feet, and face. Risk factors for preeclampsia include family history, primiparity, advanced maternal age, multiple gestation, obesity, chronic hypertension, and renal disease (48). Sleep architecture is altered in women with pre-eclampsia, with a markedly increased time spent in SWS as compared with primigravidas with normal pregnancies (49). Women with pre-eclampsia were noted to have upper airway narrowing in both upright and supine postures as compared with nonpregnant and pregnant women (50). This predisposes women with pre-eclampsia to snoring and as many as $75 \%$ of women with pre-eclampsia were reported to snore by Iczi et al. Sleep studies in a subgroup of pregnant women with pre-eclampsia revealed higher prevalence of inspiratory flow limitation in these patients without associated oxygen desaturation (51). It is unclear whether this may have implications in the pathophysiology and treatment of this disorder. There is evidence that snoring is associated with increased blood pressure in women who suffer from pre-eclampsia, and it has been suggested that using a nasal mask to deliver CPAP may reduce obstruction and improve nocturnal blood pressure control.

\section{RLS}

RLS is a sensorimotor disorder characterized by a strong, nearly irresistible, urge to move the legs. This urge to move is often accompanied by other uncomfortable paresthesias felt deep inside the legs. The urge to move, or the unpleasant sensations, begin or worsen during periods of rest and are partially or totally relieved with movement. Symptoms typically occur in the evening or night.

Pregnancy is a known cause of secondary RLS. In a study by Nikkola, 4 out of 10 women were diagnosed with RLS after becoming pregnant (52). Sleep studies in these patients showed periodic limb movements in all 10 mothers-to-be, with an average frequency of 21.7 movements per hour. In another study, $23 \%$ women were noted to develop symptoms of RLS after becoming pregnant (Lee).

\section{PARASOMNIAS}

Parasomnias are undesirable physical events or experiences that occur during entry into sleep, within sleep, or during arousals from sleep. These events are manifestations of central nervous system activation transmitted into skeletal muscle and autonomic nervous system channels, often with experiential concomitants. Parasomnias, such as sleepwalking, and night terrors occurring during pregnancy and exacerbated by the condition have been reported. Hedman and colleagues, however, found that the frequency of most parasomnias decreases during pregnancy. They found a significant decrease in sleepwalking, sleep talking, bruxism, and hypnagogic hallucinations from the prepregnant state. Prevalence of sleep paralysis was noted to decrease during the first trimester, but it increased during later pregnancy from $5.7 \%$ to $13.3 \%$ in the second trimester.

\section{NARCOLEPSY}

Narcolepsy is a condition characterized by excessive daytime sleepiness, with or without cataplexy. Other features such as hypnagogic hallucinations, sleep paralysis, and nocturnal sleep disruption may commonly occur in these patients. Narcolepsy is usually diagnosed in patients during adolescence or young adulthood. There is a paucity of data regarding effect of pregnancy on the course of narcolepsy. It is likely that symptoms of narcolepsy may be exacerbated by the sleep disturbances associated with pregnancy and also due to the fact that medicines typically used to treat narcolepsy have to be stopped, causing a withdrawal effect. Cataplexy, in severe cases, may be disabling, predisposing the pregnant patient to injury and jeopardizing the fetus.

\section{MANAGEMENT OF SLEEP DISORDERS IN THE PREGNANT PATIENT}

General management consists of advice regarding regular sleep schedules and practice of adequate sleep hygiene. Patients with insomnia should be counseled regarding stimulus control and relaxation techniques. Recognition of etiologic factors is important and every attempt should be made to optimally manage any underlying medical condition. For example, patients with gastrointestinal reflux symptoms may benefit from life style modification changes such as sleeping with the head end of bed elevated and by limiting portion sizes as well as avoiding oral intake at least 2 hours prior to bedtime.

Understandably, there are no clinical trials of pharmacologic treatment modalities for sleep disorders during pregnancy. Pharmacologic options therefore must be viewed with caution because of safety issues associated with use of medicines during pregnancy. Short-term zolpidem or diphenhydramine may be considered in patients with severe insomnia not responding to other measures.

Patients with narcolepsy should discontinue treatment with stimulants such as dextroamphetamine or methamphetamine. They may have to accept less stringent control of excessive daytime sleepiness and allow time for more frequent daytime naps. REM sleep suppressing medicines such as paroxetine or fluoxetine, which are pregnancy category B agents, may be used for control of cataplexy.

Similarly, medicines for RLS such as dopaminergic agents and benzodiazepines should ideally be discontinued. Patients may benefit from avoidance of caffeinated beverages and heavy exercise too close to scheduled bedtime. Administration of iron and folate supplements as is usually the practice during pregnancy may be helpful.

Patients with prior history of sleep apnea or those newly diagnosed with sleep apnea should be advised to use CPAP regularly. Despite theoretical concerns regarding diminished cardiac output and placental flow, there are no reports to suggest adverse effects from CPAP to the mother or the fetus. There may, however, be difficulty in tolerating CPAP due to factors such as intolerance to pressure, nasal congestion, and 
stuffiness, which may decrease adherence to CPAP regimen. This may be overcome by measures such as saline nasal rinses and use of topical nasal steroids, lowering of pressure to a more tolerable level, and sleeping with the head end of bed elevated.

\section{CONCLUSION}

Sleep disturbance is frequently reported during pregnancy (53). This is due to the several hormonal, physiological, physical, and behavioral changes that occur during the pregnant state. As a result of these changes, the pregnant woman may develop or have worsening of pathologic sleep conditions such as insomnia, sleep-disordered breathing, sleep-related movement disorders. Many of these conditions may be effectively treated, and lack of treatment may have adverse consequences for the mother and the fetus. Sleep evaluation should, therefore, be a part of routine prenatal checkup. Particular attention should be given to obese pregnant women and those who gain excessive weight during pregnancy, and to women who develop gestational hypertension or pre-eclampsia. There should be a low threshold for obtaining an overnight polysomnogram in these patients and treatment with CPAP should be considered even in patients who have mild sleep apnea. Treatment should also focus on adequate sleep hygiene techniques.

There is need for larger, multicenter studies with clinical and polysomnographic evaluation to improve our understanding of association between pregnancy and sleep disorders and to determine whether (i) sleep disorders influence the outcome of pregnancy and (ii) pregnancy influences the course of certain sleep disorders such as narcolepsy.

\section{REFERENCES}

1. Cunningham GF, Macdonald PC, Gant NF. Williams Obstetrics, 18th edn. Appleton \& Lange, 1989: 67-85.

2. Heritage AS, Stumpf WE, Sar M, et al. Brainstem catecholamines are target sites for sex hormones. Science 1980; 207: 1377-9.

3. Elkus R, Popovich J. Respiratory physiology in pregnancy. Clin Chest Med 1992; 13: 555-65.

4. Lancel M, Faulhaber J, Holsboer F, et al. Progesterone induces changes in sleep comparable to those of agnositic gabaa receptor modulators. Am J Physiol 1996; 271: E 763-72.

5. Edgar DM, Seidel WF, Gee KW, et al. Ccd-3693: an orally bioavailable analog of the endogenous neuroactive steroid, pregnanolone, demonstrates potent sedative hypnotic actions in the rat. J Pharmacol Exp Ther 1997; 282: 420-9.

6. Davison JM, Dunlop W. Renal hemodynamics and tubular function in normal human pregnancy. Kidney Int 1980; 18: 152-61.

7. Merchant DJ. Alterations in anatomy and function of the urinary tract during pregnancy. Clin Obstet Gynaecol 1978; 21: 855-62.

8. Van Thiel DH, Gavaler JS, Joshi SN, et al. Heartburn of pregnancy. Gastroenterology 1978; 72: 666-8.

9. Stachenfield NS, Taylor HS. Effects of estrogen and progesterone administration on extracellular fluid. J Appl Physiol 2004; 96: 1011-18.

10. Sherwood OD. Relaxin's physiological roles and other diverse actions. Endocr Rev 2004; 25: 205-34.

11. Bayliss DA, Millhorn DE, Gallman EA, et al. Progesterone stimulates respiration through a central nervous system steroid receptor-mediated mechanism in cat. Proc Natl Acad Sci USA 1987; 84: 7788-92.

12. Dempsey J, Olson EB, Skatrud J. Hormones and neurochemicals in the regulation of breathing, Vol. 2. Washington, DC: American Physiological Society, 1986.

13. Lapinsky SE, Kruczynski K, Slutsky A. Critical care in the pregnant patient. Am J Respir Crit Care Med 1995; 152: 427-55.

14. Skatrud JB, Dempsey JA. Interaction of sleep state and chemical stimuli sustaining rhythmic ventilation. J Appl Physiol 1983; 55: 813-22.
15. Remmers JE, DeGroot WJ, Sauerland EK, Anch AM. Pathogenesis of upper airway occlusion during sleep. J Appl Physiol 1978; 44: 931-8.

16. Marx GF, Moorthi PK, Orkin LR. Static compliance before and after vaginal delivery. Br J Anaesth 1970; 42: 1100-4.

17. Alaily AB, Carrol KB. Pulmonary ventilation in pregnancy. Br J Obstet Gynaecol 1978; 85: 518-24.

18. Artal R, Wiswell R, RomemY, et al. Pulmonary responses to exercise in pregnancy. Am J Obstet Gynecol 1986; 154: 378-83.

19. Sloan EP. Sleep disruption during pregnancy. Sleep Med Clin 2008; 3: 73-80.

20. Schweiger MS. Sleep Disturbances in Pregnancy. A subjective survey. Am J Obstet Gynecol 1972; 114: 879-82.

21. Suzuki S, Dennerstein L, greenwood KM, et al. Sleeping patterns during pregnancy in Japanese women. J Psychosom Obstet Gynecol 1994; 15: 19-26.

22. Driver HS, Shapiro CM. A longitudinal study of sleep stages in young women during pregnancy and postpartum. Sleep 1992; 15: 449-53.

23. Karacan I, Wayne H, Harman AW, et al. Characteristics of sleep patterns during late pregnancy and the postpartum periods. Am J Obstet Gynecol 1968; 101: 579-86.

24. Hertz G, Fast A, Feinsilver S, et al. Sleep in normal late pregnancy. Sleep 1992; 15: 246-51.

25. Brunner DP, Munch M, Biedermann, et al. Changes in sleep and sleep electroencephalogram during pregnancy. Sleep 1994; 17: 576-82.

26. Baratte-Beebe KR, Lee K. Sources of midsleep awakenings in childbearing women. Clin Nurs Res 1999; 8: 386-97.

27. Worth J, Oneyeije CI, Ferber A, et al. The associations between fetal and maternal sleep patterns in third trimester pregnancies. Am J Obstet Gynecol 2002; 186: 924-5.

28. Waters MA, Lee KA. Differences between primigravidae and multigravidae mothers in sleep disturbances, fatigue and functional status. J Nurse Mid-wifery 1996; 41: 364-7.

29. Oga M, Shono H, Kohara M, et al. Chronological changes in subjective symptoms during pregnancy in nulliparous and multiparous women. Acta Obstet Gynecol Scand 1995; 74: 784-7.

30. Labyak S, Lava S, Turek F, et al. Effects of shift work on sleep and menstrual function in nurses. Health Care Women Int 2002; 23: 703-14.

31. Ohida T, Kaneita Y, Osaki Y, et al. Is passive smoking associated with sleep disturbance among pregnant women? Sleep 2007; 30: 1155-61.

32. Kowall J, Clark G, Nini-Murcia G, et al. Precipitation of obstructive sleep apnea during pregnancy. Obstet Gynecol 1989; 74: 453-5.

33. Schutte S, Gross A, Segal J, et al. Self reported snoring and sleep in highrisk pregnancies. Sleep Res 1995; 24: 342.

34. Loube DI, Poceta JS, Morales MC, et al. Self-reported snoring in pregnancy. Association with fetal outcome. Chest 1996; 109: 885-9.

35. Franklin KA, Holmgren PA, Jonsson F, et al. Snoring, pregnancy-induced hypertension, and growth retardation of the fetus. Chest 2000; 117: 137-41.

36. Hedman C, Pohjasvaara T, Tolonen U, et al. Effects of pregnancy on mother's sleep. Sleep Med 2002; 3: 37-42.

37. Pien GW, Fife D, Pack AI, et al. Serial assessment of symptoms of sleepdisordered breathing in pregnant women using the multivariable apnea prediction index. Sleep 2002; 25: A 467-8.

38. Dixon JB, Schachter LM, O'Brien PE. Predicting sleep apnea and excessive daytime sleepiness in the severely obese: Indications for polysomnography. Chest 2003; 123: 1134-41.

39. Kyzer S, Charuzi I. Obstructive sleep apnea in the obese. World J Surg 1998; 22: 998-1001.

40. Gunderson EP, Abrams B. Epidemiology of gestational weight gain and body weight changes after pregnancy. Epidemiol Rev 1999; 21: 261-75.

41. Kim SY, Dietz PM, England L, et al. Trends in pre-pregnancy obesity in nine states, 1993-2003. Obesity (Silver Spring) 2007; 15: 986-93.

42. Feinsilver SH, Hertz G. Respirations during sleep in pregnancy. Clin Chest Med 1992; 13: 637-44.

43. Popovic RM, White DP. Upper airway muscle activity in normal women: influences of hormonal status. J Applied Physiol 1998; 84: 1055-62.

44. Schoenfeld A, Ovadia Y, Neri A, et al. Obstructive sleep apnea (OSA) implications in maternal-fetal medicine. A hypothesis. Med Hypotheses 1989; 30: 51-4.

45. Maasilta P, Bachour A, Teramo K, et al. Sleep-related disordered breathing during pregnancy in obese women. Chest 2001; 120: 1448-54. 
46. Joel-Cohen SJ, Schoenfeld. Fetal response to periodic sleep apnea: a new syndrome in obstetrics. Eur J Obstet Gynecol Reprod Biol 1978; 8: 77-81.

47. Lewis DF, Chesson AL, Edwards MS, et al. Obstructive sleep apnea during pregnancy resulting in pulmonary hypertension. South med J 1998; 91: $761-2$.

48. Zhang J, Zeisler J, Hatch MC, et al. Epidemiology of pregnancy induced hypertension. Epidemiol Rev 1997; 19: 218-32.

49. Edwards N, Blyton DM, Kesby GJ, et al. Pre-eclampsia is associated with marked alteration in sleep architecture. Sleep 2000; 23: 619-25.
50. Izci B, Riha RL, Martin SE, et al. The upper airway in pregnancy and preeclampsia. Am J Respir Crit Care Med 2003; 167: 137-40.

51. Connolly G, Razak AR, havanga A, et al. Inspiratory flow limitation during sleep in pre-eclampsia: comparison with normal pregnant and nonpregnant women. Eur Respir J 2001; 18: 672-6.

52. Nikkola E, Ekblad U, Ekholm E, et al. Sleep in multiple pregnancy: breathing patterns, oxygenation, and periodic leg movements. Am J Obstet Gynecol 1996; 174: 1622-5.

53. Sahota P, Jain S, Dhand R. Sleep Disorders in Pregnancy. Current Opinion in Pulm Crit Care Med 2003; 9: 477-83. 


\section{Pulmonary diseases in pregnancy Leah Lande, Abraham Sanders, and Dana Zappetti}

\section{INTRODUCTION}

Pregnancy induces significant changes in normal pulmonary physiology while also causing increased susceptibility to the effects of certain respiratory diseases. In the following chapter, the changes in pulmonary function and respiratory physiology that occur during normal pregnancy will be described, followed by a review of specific pulmonary disorders during pregnancy. Obstructive and restrictive lung diseases, pulmonary vascular disease, pulmonary infections, respiratory failure, and other topics will be reviewed in the context of pregnancy. In addition, the challenge of distinguishing between the normal physiologic dyspnea of pregnancy and an underlying pulmonary or cardiac disease will be discussed.

Respiratory disorders account for a small, but significant percentage of maternal morbidity and mortality $(1,2)$. These are sometimes due to preexisting conditions that may be exacerbated by pregnancy such as pulmonary hypertension $(\mathrm{PH})$, cystic fibrosis $(\mathrm{CF})$, or restrictive lung disease or due to acute illnesses such as pulmonary embolism (PE), pneumonia, or adult respiratory distress syndrome. Particularly with respect to chronic progressive pulmonary diseases, physicians must be familiar with the potential maternal and fetal complications that can occur and be prepared to counsel women regarding these issues.

\section{Changes in Lung Function During Pregnancy \\ Chest Wall and Diaphragm}

Early in pregnancy, the overall diameter of the chest wall increases, possibly due to relaxation of the ligamentous attachments between the ribs and the sternum. More specifically, the subcostal angle increases by approximately $35^{\circ}$, the transverse diameter of the chest increases by approximately $2 \mathrm{~cm}$, and the chest circumference expands by 5 to $7 \mathrm{~cm}$. In later pregnancy, as the uterus enlarges, the level of the diaphragm rises approximately $4 \mathrm{~cm}$, but this does not affect diaphragmatic excursion (3).

\section{Pulmonary Function}

Overall, respiratory muscle function is not altered by pregnancy. Maximum inspiratory and expiratory pressures remain unchanged. However, due to the changes in chest wall configuration as noted above, static lung volumes are altered. The total lung capacity is minimally reduced and the functional residual capacity (the amount of air remaining in the lungs at end expiration) is more significantly reduced due to elevation of the diaphragm in later stages of gestation. By contrast, due to the decrease in functional residual capacity, the inspiratory capacity (the maximum amount of air that can be inspired at end expiration in the resting state) is increased, and steady-state tidal volumes may be increased up to $30 \%$ to 40\% (Table 1).
Airway function remains unaffected by pregnancy, with no change in forced expiratory volume in one second, forced vital capacity (FVC), or peak expiratory flow rates. Therefore, the use of a peak flowmeter in pregnant asthmatics that are experiencing respiratory symptoms can be invaluable in distinguishing between worsening of underlying airway disease and alternative causes for their symptoms, including the normal dyspnea of pregnancy. However, it is important that peak flows be performed in the upright position, as lying supine can result in small alterations in peak flow rates.

\section{Gas Exchange}

As gestation progresses, a state of chronic hyperventilation ensues. This is probably due to progressively increasing levels of progesterone, which is a known stimulant of respiratory drive, from approximately $25 \mathrm{ng} / \mathrm{mL}$ at 6 weeks to $150 \mathrm{ng} / \mathrm{mL}$ at term (4). By 8 weeks of gestation, there is already an increase in tidal volume as noted above. This increased tidal volume results in increased alveolar ventilation, with subsequent decreased arterial carbon dioxide $\left(\mathrm{PaCO}_{2}\right) \cdot \mathrm{PaCO}_{2}$ levels typically decrease from normal levels of 37 to $40 \mathrm{mmHg}$ to 27 to $32 \mathrm{mmHg}$. This decrease in maternal $\mathrm{PaCO}_{2}$ creates a favorable gradient for $\mathrm{CO}_{2}$ transfer from the fetus across the placenta. The maternal $\mathrm{pH}$ is maintained within the normal range through increased renal excretion of bicarbonate with a subsequent mild metabolic acidosis, with typical serum bicarbonate levels of 18 to $21 \mathrm{mEq} / \mathrm{L}$.

Maternal oxygenation $\left(\mathrm{PaO}_{2}\right)$ usually increases early in pregnancy $(106-108 \mathrm{mmHg})$ as a result of decreased $\mathrm{PaCO}_{2}$, but as the uterus enlarges in the third trimester, the $\mathrm{PaO}_{2}$ decreases slightly $(101-104 \mathrm{mmHg})$, with a slight increase in alveolar to arterial oxygen gradient from 14 to $20 \mathrm{mmHg}$. This decrease in $\mathrm{PaO}_{2}$ is even more pronounced in the supine position.

Oxygen consumption increases by approximately $20 \%$ during pregnancy to meet the increased metabolic demands of maternal organs, the fetus, and the placenta (5). These oxygen requirements are more pronounced during exercise and labor (6). Due to the decreased functional residual capacity, maternal oxygen reserve is lower, and pregnant women can develop a more rapid onset of hypoxia during periods of apnea or during attempted endotracheal intubation.

In the setting of slightly elevated maternal $\mathrm{pH}$ and elevated 2,3 DPG during pregnancy, the oxygen-hemoglobin dissociation curve is shifted to the right, resulting in a decreased affinity of hemoglobin for oxygen (Fig. 1). This not only favors an increased transfer of oxygen to the fetus, but also results in a decreased oxygen saturation of hemoglobin for any given level 
Table 1 Pulmonary Function Changes During Pregnancy

\begin{tabular}{|c|c|c|}
\hline Measurement & Definition & Change in pregnancy \\
\hline Respiratory rate & Number of breaths per minute & None \\
\hline Tidal volume & Amount of air inspired and expired with normal breathing & Increased $30-40 \%$ \\
\hline Total lung capacity & Amount of air in lungs at maximal inspiration & Decreased $5 \%$ \\
\hline Residual volume & Amount of air remaining in lungs after maximal expiration & Decreased $20-25 \%$ \\
\hline Functional residual capacity & Amount of air remaining in lungs at resting expiratory level & Decreased $20 \%$ \\
\hline Inspiratory capacity & Amount of air that can be inspired from resting expiratory level & Increased $5-10 \%$ \\
\hline Expiratory reserve volume & Amount of air that can be expired from resting expiratory level & Decreased $15-20 \%$ \\
\hline
\end{tabular}

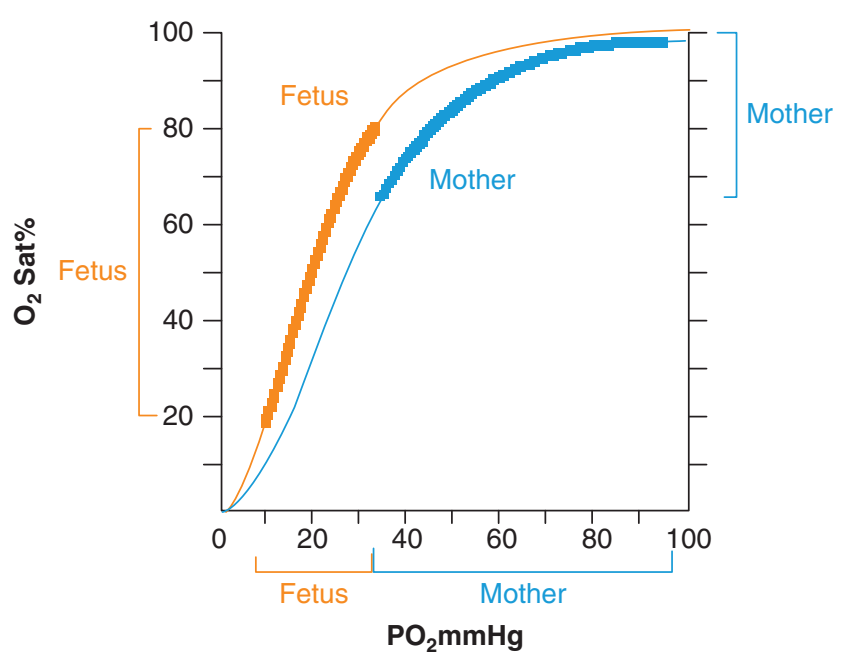

Figure 1 Oxygen dissociation curves for human maternal and fetal blood, indicating the physiologic range of $\mathrm{PO}_{2}$ and $\mathrm{O}_{2}$ for mother and fetus. Source: From Ref. 7.

of $\mathrm{PaO}_{2}$ (8). This also causes the pregnant women to be more susceptible to hypoxemia. Although fetal partial pressure of oxygen is much lower $(25-35 \mathrm{mmHg})$, the saturation is relatively higher than that in the adult due to displacement of the fetal oxygen-hemoglobin dissociation curve to the left. The fetus can be very sensitive to a small decrease in maternal $\mathrm{PaO}_{2}$ due to the steep slope of the fetal oxygen dissociation curve in the $50 \%$ saturation range (9).

\section{Dyspnea of Pregnancy}

The increasing levels of progesterone throughout the course of gestation as well as the changes in lung function noted above are thought to be the main mechanisms responsible for the physiologic dyspnea of pregnancy. During the course of normal pregnancy, $60 \%$ to $70 \%$ of women experience a sensation of dyspnea (10). This sensation of breathlessness usually begins during the first trimester and gradually increases throughout the second and third trimesters. At 28 weeks of gestation, approximately $50 \%$ of women experience dyspnea walking up hills or climbing stairs, and 20\% have dyspnea walking on flat ground (11). Distinguishing between the physiologic dyspnea of pregnancy and the presence of underlying cardiac or pulmonary disease can be a diagnostic challenge. The dyspnea of pregnancy is typically gradual in onset, is not associated with cough, wheezing, or chest pain, and is characterized by a clear lung exam and normal spirometry.

\begin{abstract}
AIRWAY DISEASES

\section{Asthma}

Epidemiology

Asthma is the most common pulmonary disorder in pregnancy, affecting $8 \%$ of pregnant women (12). The National Asthma Education Program Working Group on Asthma in Pregnancy estimated that $10 \%$ of pregnant asthmatics have an acute exacerbation of asthma during labor, and $0.2 \%$ experience status asthmaticus during the course of their pregnancy $(13,14)$. Approximately one-third of asthmatics have an improvement in asthma control during pregnancy, one-third have worsening during pregnancy, and one-third experience no change (13).
\end{abstract}

\section{Maternal and Fetal Effects}

Over the last 30 years, multiple studies have investigated the effect of asthma on maternal and fetal outcomes, with varying results. There have been reports of adverse outcomes, including low infant birth weight, congenital malformations, increased incidence of preterm labor, and increased incidence of maternal pre-eclampsia and hypertension, particularly in poorly controlled asthmatics (15). A recent case-control study of over 3000 women over 10 years showed only an increase in pre-eclampsia and maternal hypertension in poorly controlled asthmatic patients (16).

\section{Symptoms and Triggers}

Asthma is characterized by either partial or completely reversible airway obstruction, with airway inflammation, bronchospasm, and mucous hypersecretion. Typical symptoms of asthma include dyspnea, cough, wheezing, and chest tightness. These may be worse at night and, with exercise, may occur in the setting of an upper respiratory tract infection or after exposure to environmental triggers such as hot or cold air, dust or pollen, house dust mites, cockroach antigens, pets, or other inhaled irritants. Asthma symptoms can also be triggered by gastroesophageal reflux that is estimated to occur in up to $50 \%$ of pregnant women (17) or by postnasal drip that is precipitated by hyperemia and glandular hyperactivity in the upper respiratory mucosa during pregnancy.

\section{Diagnosis and Monitoring}

The diagnosis of asthma is made based on the clinical history as noted above with or without supporting physical exam findings of expiratory wheezing or rhonchi. The diagnosis is confirmed by spirometry demonstrating a reduced forced expiratory volume in one second (FEV1) with a reduced ratio 
Table 2 Assessing Asthma Severity

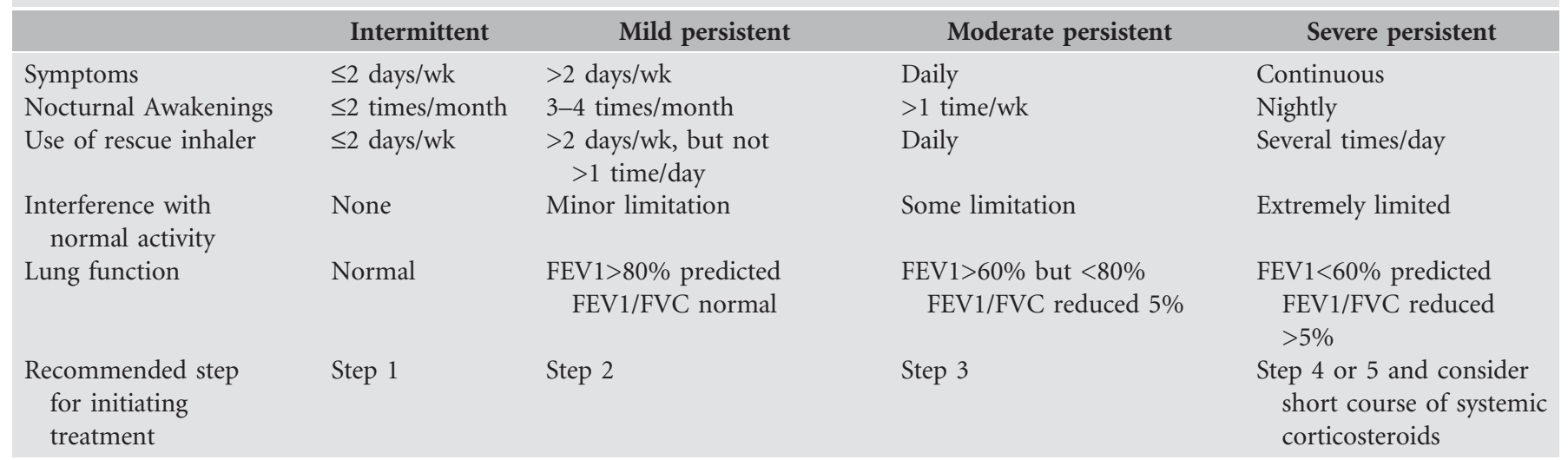

of FEV1 to FVC, with further confirmation if airway reversibility is shown by an improvement by $12 \%$ and $200 \mathrm{cc}$ in either the FEV1 or the FVC after the administration of an inhaled bronchodilator. The sensitivity, however, of spirometry in the diagnosis of asthma is limited (18). Methacholine challenge testing, which can be used to diagnose asthma in patients with normal spirometry, is contraindicated in pregnant women due to a lack of safety data (19).

Once the diagnosis of asthma has been established, the cornerstone of asthma assessment is patient self-monitoring with a portable peak flowmeter. Patients should be instructed to measure their peak expiratory flow on a regular basis at different times of day to establish a baseline, and then to use the meter when experiencing new or changing respiratory symptoms, in order to facilitate early detection of an acute asthma exacerbation. The peak flowmeter is a particularly helpful objective assessment of pulmonary function in distinguishing between the normal dyspnea of pregnancy and worsening of asthma symptoms.

Physician assessment of asthma control involves tracking the frequency and severity of asthma symptoms, including the presence of nocturnal awakenings due to asthma and the use of short-acting inhaled beta-agonist rescue therapy. Most importantly, patients should be asked about their consistent use of controller medications and avoidance of environmental triggers. Pregnant asthmatics requiring regular controller medications should undergo monthly physician visits.

\section{Treatment}

The first step in the treatment of asthma is to identify and counsel patients on the avoidance of asthma triggers. In addition, assessment should be performed for the presence of other conditions that can exacerbate asthma such as allergic rhinitis, sinusitis, and gastroesophageal reflux disease and these conditions should be treated if present.

It is important to note that the risks of uncontrolled asthma far outweigh any risks from the medications used to control asthma (20).

Airway inflammation is treated with inhaled corticosteroids and bronchial hyper-responsiveness is treated with short- or long-acting bronchodilators. In addition, leukotriene receptor antagonists, theophylline, and cromolyn appear to be safe during pregnancy and may be considered in select patients (21). Most data on the safety of asthma medications in pregnancy are observational, with the most extensively studied being albuterol and inhaled budesonide (21-23). Albuterol appears to be safe based on a review of six published studies including 1599 women and a prospective study including 1828 women, in whom no significant relationship was found between the use of inhaled beta- 2 agonists and adverse pregnancy outcomes $(21,24)$. The National Asthma Education and Prevention Program Working Group reviewed 10 studies, including 6113 patients who were treated with inhaled corticosteroids during pregnancy and found no increases in congenital malformations or adverse perinatal outcomes in these patients (24).

The pharmacologic treatment of asthma depends on the severity of the asthma symptoms and degree of physiologic impairment. Patients with mild, intermittent asthma (symptoms $\leq 2$ days/wk and $\leq 2$ nights/month with normal FEV1) do not require daily controller medications and can be managed with a short-acting inhaled bronchodilator as needed. Patients with persistent asthma (symptoms $>$ two times/wk or $>2$ nights/month) require daily treatment with inhaled corticosteroids at increasing dosages depending on the severity and frequency of their asthma symptoms, with the addition of inhaled long-acting beta agonists in steps 4 to $6(19,24,25)$ (Tables 2, 3, and 4).

Pregnant patients who had good asthma control prior to pregnancy should generally be instructed to continue on their prepregnancy regimen. In nonpregnant patients, it is recommended that if their asthma has been well controlled for 3 months, then they be considered for a step-down in their therapy. This may also be considered in pregnant patients requiring high-dose inhaled corticosteroids with long-acting inhaled beta agonists, with the goal of first eliminating the long-acting beta agonist if possible, as data are lacking regarding the safety of long-acting beta agonists during pregnancy. The safety of short-acting beta agonists and the accumulating clinical experience with these medications during pregnancy are reassuring. Therapy should be increased by one step (Table 3) in patients with poorly controlled asthma. Patients using their rescue inhalers more than twice a week should be considered for a step-up in therapy. If patients have very poor asthma control, therapy should be increased by two steps or a short course of systemic corticosteroids should be prescribed. A typical regimen would be oral prednisone 40 to $60 \mathrm{mg}$ daily for 1 week, followed by a taper over 7 to 10 days. 
Table 3 Steps of Asthma Therapy During Pregnancy

\begin{tabular}{|c|c|c|}
\hline Step & Preferred controller medication & Alternative controller medication \\
\hline 1 & None & - \\
\hline 2 & Low-dose inhaled corticosteroid & LTRA, cromolyn, theophylline \\
\hline 3 & Medium-dose inhaled corticosteroid & $\begin{array}{l}\text { Low-dose inhaled corticosteroid plus LABA, } \\
\text { LTRA, or theophylline }\end{array}$ \\
\hline 4 & Medium-dose inhaled corticosteroid plus LABA & $\begin{array}{l}\text { Medium-dose inhaled corticosteroid plus either } \\
\text { LTRA or theophylline }\end{array}$ \\
\hline 5 & High-dose inhaled corticosteroid plus LABA & - \\
\hline
\end{tabular}

Table 4 Controller Medications for the Management of Asthma During Pregnancy

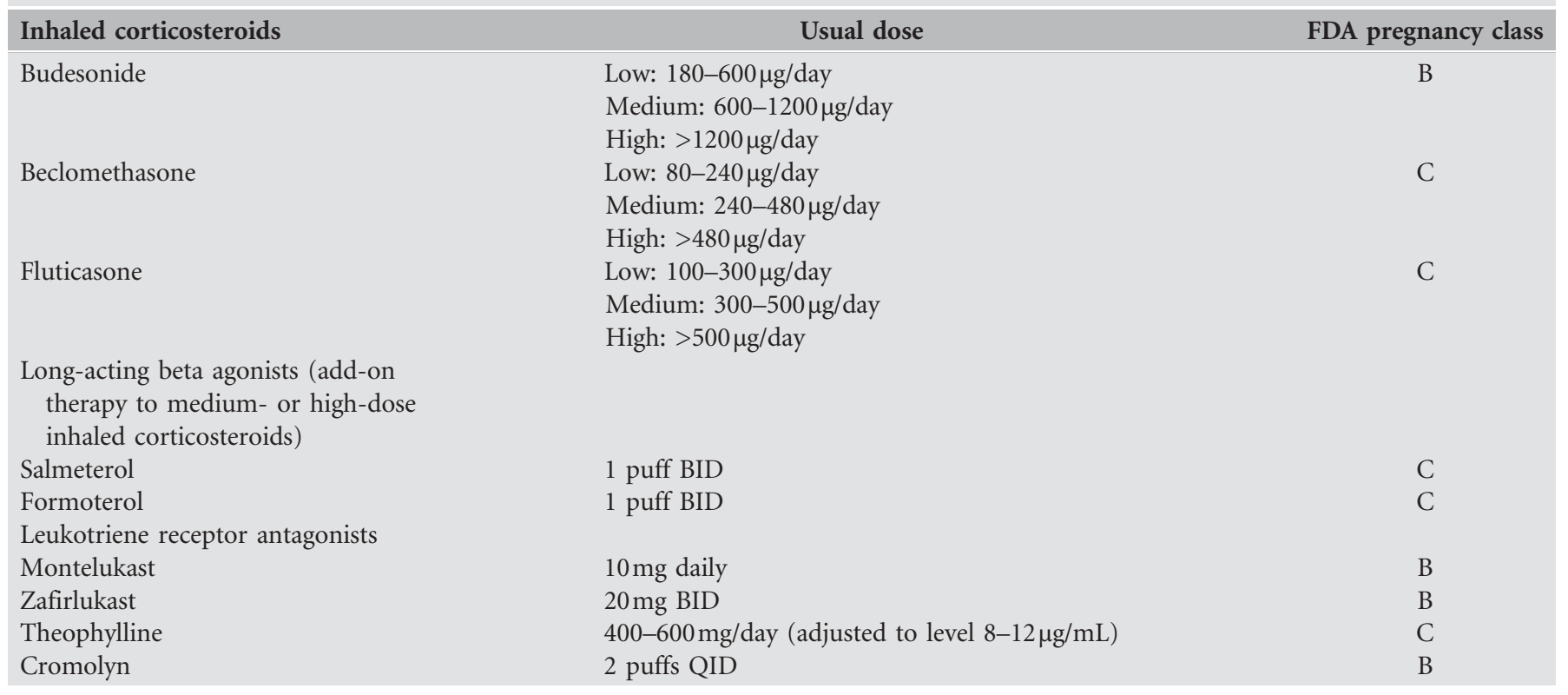

\section{Labor and Delivery Management}

Acute exacerbations of asthma are rare during labor and delivery. Adequate analgesia with the use of epidural anesthesia may reduce the risk of bronchospasm and has the added benefit of reducing oxygen consumption and minute ventilation during labor. The ultimate goal of asthma management during labor and delivery is maintaining adequate oxygenation of the fetus by prevention of hypoxic episodes in the mother.

\section{Cystic Fibrosis}

Cystic Fibrosis occurs in about 1 in 1500 whites and 1 in 17,000 blacks (26). With improved therapy, median survival is now 33.4 years $(26,27)$. The Cystic Fibrosis Foundation found that in 2004, 191 American women with CF were pregnant. Though many women with CF, particularly those with more mild disease, are able to undergo pregnancy without significant problems, pregnancy in CF patients has been associated with adverse fetal and maternal outcomes. Close management by a multidisciplinary team is required throughout the pregnancy. Overall, $25 \%$ of patients had preterm delivery, and the 2-year perinatal death rate was $14 \%$. The mortality risk appears to be associated with more severe disease, with the suggestion that an FEV $1<60 \%$ of predicted, colonization with Burkholderia cepacia, poor nutrition, and diabetes mellitus are poor prognostic factors for both maternal and fetal outcomes (28-30). Women with CF with good pulmonary function prior to pregnancy do develop an increased frequency of pulmonary exacerbations and hospitalizations throughout the course of gestation, but there is no change in long-term survival compared with controls (30).

\section{Bronchiectasis}

There is little information known about the impact of bronchiectasis on pregnancy. Based on case reports in the literature, it appears that there is no change in pulmonary function during pregnancy and no significant effects on fetal outcomes if normal oxygenation is maintained (31,32). In cases in which maternal hypoxia was present, fetal growth retardation has been reported (31).

\section{DISORDERS OF THE PULMONARY VASCULATURE Pulmonary Hypertension}

Pulmonary Hypertension is defined as an elevated mean pulmonary artery pressure of greater than $25 \mathrm{mmHg}$ and a pulmonary capillary wedge pressure of greater than 
$15 \mathrm{mmHg}$, measured with a right heart catheter at rest (33). It can be primary, caused by a vasculopathy in the pulmonary arteries, or secondary, due to diseases affecting the lungs such as pulmonary emboli, interstitial lung diseases of many etiologies, emphysema, cardiac disease, or extra pulmonary diseases such as HIV, liver failure, or sleep apnea, with resultant hypoxemia (34).

It becomes relevant for obstetricians to learn about $\mathrm{PH}$ because women of childbearing age are more likely to suffer from primary pulmonary arterial hypertension (PAH) or congenital heart disease, and the hemodynamic demands of pregnancy can lead to increased cardiac output and hemodynamic or respiratory decompensation. With maternal mortality estimated between $30 \%$ and $50 \%$, historically, the recommendation has been, for women with $\mathrm{PAH}$, to avoid pregnancy with effective contraception and to terminate pregnancy should it occur (35). More recently, medications have become available that can lower pulmonary artery pressures, thereby improving exercise tolerance, quality of life, and survival. These medications, however, do not cure the condition $(35,36)$. Use of these therapies, such as IV epoprostenol, inhaled nitric oxide, inhaled iloprost, oral calcium channel blockers, endothelin receptor antagonists (bosentan, ambrisentan), and sildenafil, have been tried and, in review, have resulted in lower but still high mortality, over $25 \%(37-40)$.

We would continue the recommendation to avoid pregnancy, but should the woman wish to proceed, consultation with expert cardiologists, pulmonologists, and anesthesiologists with experience with these new therapies and complications during delivery should be obtained. Termination of pregnancy should be considered if $\mathrm{PH}$ worsens, which is reported most often between 20 and 24 weeks, when most hemodynamic changes have occurred $(37,40)$.

\section{Pulmonary Embolism}

\section{Overview}

Deep venous thrombosis and its manifestation, pulmonary embolism, (DVT/PE) are the leading cause of maternal death in the developed world. Estimated deaths in the United States and Europe are 1.1 to 1.5 per 100,000 deliveries, with 5 to 12 events of DVT/PE per 100,000 pregnancies, which is 7 to 10 times higher than age-matched nonpregnant women (41).

Two-thirds of DVT occur antepartum and are equally distributed among the trimesters. PEs occur more commonly post delivery, $43 \%$ to $60 \%$, and the risk of DVT/PE is elevated for 6 weeks following delivery. Pregnancy is thus considered a hypercoagulable or thrombophilic state, increasing the risk of DVT/PE (42).

Pregnancy is associated with hypercoagulable changes in blood, venous stasis with decreased blood flow, compression of iliac veins by the uterus, the crossing of the right iliac artery over the left iliac vein, and immobilization. Trauma from the delivery or from interventions to aid in delivery can lead to vascular damage and therefore all variables of Virchow's triad: hypercoagulability, venous stasis, and vascular damage (41-43).

Other factors increasing the risk for DVT/PE include underlying thrombophilias (found in approximately 50\% of episodes in pregnancy), previous DVT or family history of
DVT/PE, elevated BMI, immobilization, assisted reproduction, hemorrhage, infection, Caesarean delivery (especially emergent multiple pregnancies), age over 35 , and pre-eclampsia (42).

\section{Diagnosis}

The diagnosis of DVT/PE in pregnancy is more complicated than that in the nonpregnant population, as the symptoms are nonspecific, overlap with those of normal pregnancy, and require the use of radiation-associated imaging, which patients and physicians wish to avoid due to negative effects on the fetus. Recommendations are extrapolated from studies in nonpregnant populations from which pregnant women were specifically excluded (41-45).

Most patients with DVT/PE will complain of shortness of breath, pleuritic chest pain, hemoptysis, syncope, or leg swelling occurring over minutes to hours. They may have tachypnea, tachycardia, hypotension, and shock. While these symptoms and signs are sensitive, they are not specific to confirm the diagnosis, and further objective testing, such as imaging, is required.

Consideration of the possibility of DVT/PE is the most important factor leading to a correct diagnosis and requires that one recognize the signs and symptoms while screening for risk factors in addition to pregnancy. Examination of the patient should focus on temperature, heart rate, respiratory rate, blood pressure, oxygen saturation, the presence of jugular venous distention, wheezing, rales, pleural rubs, heart murmurs or gallops, leg swelling, tenderness, and/or warmth. The differential diagnosis often includes pulmonary congestion, heart failure, atelectasis, asthma, pneumonia, and pneumothorax in addition to DVT/PE. A clinical probability for DVT/PE should be made. Clinical prediction rules and algorithms exist and have been evaluated in the nonpregnant population, but have not been similarly developed for the pregnant group. Therefore, if DVT/PE is suspected, further studies are required (41-43).

Electrocardiograms and arterial blood gases have not been demonstrated to be useful in excluding or making the diagnosis of PE, although they can aid in the evaluation of other conditions, such as myocardial infarction. d-Dimer testing remains problematic, as the levels increase in the blood through each trimester and often exceed the upper range of normal, which has been established in nonpregnant subjects (46). Furthermore, levels have not been developed to allow the treating physician to discriminate DVT/PE from normal pregnancy. By contrast, however, threshold levels have been developed in the nonpregnant patient. Therefore, the combination of a low suspicion for DVT/PE and a normal level of d-dimer in the blood effectively rules out the diagnosis. However, in those who have an intermediate or high clinical suspicion, a d-dimer study will neither diagnose nor exclude DVT and, therefore, it is not recommended.

Once the diagnosis is considered, and further testing is in progress, treatment should begin. The next test should be compression ultrasonography of the lower extremities. It is noninvasive and does not require radiation. If DVT is seen, PE can be diagnosed and treatment continues as it is the same for DVT and PE. Limitations to this study are its decreased accuracy in those without symptoms or signs of DVT or with isolated calf, iliac vein, or pelvic vein thromboses. If DVT is 
not seen, further testing is indicated. Available studies include chest radiography, ventilation-perfusion scintigraphy (V/Q), computed tomography pulmonary angiography, pulmonary angiography, and CT or conventional venography $(41,42,45)$.

While chest radiography can suggest an alternate diagnosis, such as fluid overload, pneumonia, or pneumothorax, it cannot definitively exclude or make a diagnosis of DVT/PE. V/Q scanning has been validated in the diagnosis of $\mathrm{PE}$, in combination with pretest clinical probability determinations. A normal or near-normal scan and low clinical probability practically excludes the diagnosis in nonpregnant patients. A high clinical suspicion with a high probability scan confirms the diagnosis. All other combinations of clinical probability and scan interpretation do not confirm or exclude the diagnosis. The radiation dose from a complete V/Q scan is from 0.00028 to $0.00051 \mathrm{~Gy}$; doses in humans of $0.01 \mathrm{~Gy}$ during gestation are estimated to be a threshold above which increased congenital abnormalities are seen. Fetal exposure to radiation of $0.01 \mathrm{~Gy}$ above normal background radiation may increase the probability of cancer before age 20 by 0.017 (41).

Results of V/Q scanning show that 70\% are interpreted as normal and 25\% are non-diagnostic. These results suggest that V/Q scanning may be a good first test to exclude PE, but many patients will require further testing (47).

CT scanning delivers 0.00003 to 0.00013 Gy to the fetus, similar to V/Q scanning; however, more radiation is received by the mother's breasts than the fetus. The incidence of breast cancer may be increased with chest CT. CT scans are reported either as positive or negative and are accurate in lobar vessels (97\% accurate). In smaller segmental and subsegmental vessels, accuracy declines and both false-positive (reported clot where none exists) or false-negative results are noted. However, a negative scan can reliably exclude the diagnosis, and follow-up of those with negative scans show good outcomes without treatment. An advantage of CT is that other diagnoses may be made. The problem of isolated pelvic vein thrombosis can be associated with abdominal and back pain, and swelling of the leg may require CT scanning of the iliac veins and pelvis $(41,48,49)$.

In studies of nonpregnant patients who undergo full diagnostic testing for DVT/PE, up to $25 \%$ of patients will have the diagnosis confirmed. In pregnant women, $10 \%$ to $15 \%$ will be found to have DVT/PE. Despite studying many patients who do not have DVT/PE, it is felt that with a mortality of $30 \%$ when untreated, compared with $8 \%$ when treated, evaluation leading to a diagnosis is required if a pregnant patient presents with signs or symptoms suggestive of possible DVT/PE (41).

If the V/Q scan and chest CT are non-diagnostic, conventional angiography can be done. This choice requires trained personnel both to perform and to interpret the study. It delivers more radiation than other studies, but offers the most certain diagnosis. However, with the widespread availability of CT scans, this test is less frequently done.

A suggested approach to the evaluation of DVT/PE is to consider the diagnosis in those who complain of acute dyspnea, pleuritic chest pain, hemoptysis, or syncope. A chest radiograph, electrocardiogram, and ultrasound of the lower extremities should be obtained. Blood studies can include a blood count and d-dimer. If an alternative diagnosis is not made, or the ultrasound does not reveal DVT, then further imaging is needed. We recommend a CT angiogram, as this will be diagnostic in most cases. A CT angiogram requires informed consent from the patient and should include a discussion of radiation exposure to the patient and fetus, including a possible slightly elevated risk of cancer. If available, a discussion with radiology personnel and the application of breast shields can be attempted $(41,50)$.

\section{Therapy}

The initial treatment of DVT/PE in both pregnant and nonpregnant patients is heparin. Low-molecular weight heparin (LMWH) (enoxaparin $1 \mathrm{mg} / \mathrm{kg}$ up to $100 \mathrm{mg}$ twice daily, dalteparin using weight-based dosing of 5000-10,000 units twice daily, or tinzaparin 175 units $/ \mathrm{kg}$ once daily at all weights) is preferred $(41,44)$. Unfractionated heparin, given intravenously, was the standard historically, but studies have shown that the subcutaneous use of LMWH has therapeutic equivalence, better bioavailability and predictable dose response, a reduced risk of bleeding, weight-based dosing without the need for monitoring of coagulation, reduced risk of heparin-induced osteoporosis and fractures, and reduced risk of heparin-induced thrombocytopenia. Heparins neither cross the placenta nor are they excreted in breast milk. Allergic reactions are uncommon and include itching, urticaria, and erythematous plaques (44).

Full anticoagulation in the absence of other factors, such as an underlying thrombophilia or prior DVT/PE, must continue for at least 6 months, throughout the pregnancy, and at least 6 weeks postpartum.

In patients with massive $\mathrm{PE}$, mortality with aggressive therapies exceeds $50 \%$. These patients are hemodynamically unstable, with a large clot burden causing obstructive shock, right ventricular failure, hypoxemia, and hypotension. In the nonpregnant patient, the use of thrombolytics, such as tissue plasminogen activating factor, or streptokinase, are used. Thrombolysis, as well as surgical embolectomy, has been used in pregnant patients with successful outcomes $(41,44,51)$.

For patients receiving heparin, some clinicians have recommended a reduction in dose to $75 \%$ of a full treatment dose after 30 days, extrapolating from studies in nonpregnant patients. This approach has not been confirmed with studies in pregnant patients but is not unreasonable (44).

If pregnant patients are receiving anticoagulation therapy and are approaching delivery, they can be switched from subcutaneous LMWH to IV unfractionated heparin 24 to 36 hours prior to elective induction of labor or caesarean section. If, however, spontaneous labor begins while the patient is receiving subcutaneous LMWH, epidural anesthesia cannot be administered.

A special situation involves women with a recent (less than 1 week previously) DVT/PE receiving full anticoagulation therapy near delivery. There is a higher rate of recurrence during this period. Options include insertion of an inferior vena cava filter and induction of labor with reinstitution of anticoagulation following delivery when hemostasis is 
obtained, or switching from LMWH heparin subcutaneously to IV unfractionated heparin and cessation 4 to 6 hours prior to expected delivery (44).

\section{Prevention}

If a patient with known risk factors for DVT/PE, such as a history of prior idiopathic or pregnancy-associated DVT/PE or a known thrombophilia, becomes pregnant, their risk for recurrent DVT/PE during pregnancy will be increased. As a preventative strategy, low-dose subcutaneous LMWH should be given throughout the pregnancy and postpartum for 6 weeks. If women with known thrombophilia have not had DVT in the past, with the exception of antithrombin deficiency, they should receive low-dose prophylactic therapy throughout the pregnancy and for 6 weeks after delivery $(34,44)$.

\section{AMNIOTIC FLUID EMBOLISM SYNDROME}

A rare but catastrophic situation is amniotic fluid embolism syndrome (also called anaphylactoid syndrome of pregnancy). It is rare, unpredictable, unpreventable, and has a high mortality rate between $20 \%$ and $90 \%$. Survivors are often left with anoxic encephalopathy. After pulmonary thromboembolism, it is the next leading cause of unexpected maternal death. Women who undergo a difficult labor, who are older, who have a caesarean or instrumental delivery, and who have placenta previa or abruptio are at an increased risk (52).

The clinical presentation is the sudden onset of hypoxemia, hypotension, seizures, and coagulopathy during labor or within 30 minutes of delivery. The condition appears as a systemic process with hypoxemia due to ventilation perfusion mismatching followed by non-cardiac pulmonary edema, hypotension, decreased left ventricular function, and, within minutes, disseminated intravascular coagulation with bleeding. Occasional cases are reported following abortion, amniocentesis, or trauma. There are no specific tests that confirm the diagnosis. The differential diagnosis includes sepsis, acute PE, anaphylaxis, air embolism, anesthetic complications, transfusion reactions, eclampsia, heart failure, hemorrhage, or aspiration and all of these must be considered $(52,53)$.

Treatment is supportive with supplemental oxygen, most likely with intubation and mechanical ventilation, trying to maintain a maternal $\mathrm{PO}_{2}$ greater than $65 \mathrm{mmHg}$. Blood pressure should be maintained with norepinephrine or dopamine and fluids, while central access is obtained, and evaluation of fluid status is undertaken. Blood and blood products may be necessary, and delivery might be necessary as well, but bleeding must be controlled first $(20,21)$.

The etiology of this syndrome is unknown, but it is felt to be inflammatory-a response to antigens in amniotic fluid in susceptible hosts (52-54).

\section{RESTRICTIVE LUNG DISEASES}

There are a number of chronic lung diseases affecting women of childbearing years that are characterized by reduced lung volumes and normal expiratory airflow. Their physiology is described as restrictive, as compared with the "obstructive" diseases such as asthma and CF. These diseases can cause increased respiratory distress in pregnant women, as the normal physiology of pregnancy progresses with its increase in minute ventilation and oxygen consumption, changes in rib cage displacement, and intravascular volume, which add to baseline pulmonary dysfunction. We will discuss some of the more common disorders and their response to pregnancy $(55,56)$.

\section{Kyphoscoliosis}

Kyphoscoliosis is a deformity of the spine with excessive curvature either posterior (kyphosis) or lateral (scoliosis). Women are more commonly affected than men, with a prevalence of $10.7 \%$ in women 25 to 74 years, documented in one study. Kyphoscoliosis, by causing a reduction in lung volumes, can lead to respiratory failure and death. The deformity of the chest wall compresses the lungs and leads to ventilation perfusion mismatching, hypoxemia, and, occasionally, hypercarbia. PH and heart failure can occur (57).

Despite these findings, and worsening respiratory function, reported outcomes of pregnancies appear to be good. Patients should have their pulmonary function tests measured, and oxygen saturation followed throughout pregnancy, at rest and with exercise, and should be supplemented when oxygen saturations drop below 95\% (58).

\section{Lymphangioleiomyomatosis}

Lymphangioleiomyomatosis (LAM) is a rare lung disease more common in women. It can occur sporadically or can be associated with tuberous sclerosis. Pathologically, cysts and a multifocal nodular proliferation of immature smooth and perivascular epithelioid cells are found. Women may complain of shortness of breath, cough, or hemoptysis and can be found to have a pneumothorax or a chylous effusion in the chest or abdomen. The condition is associated with angiomyolipomas of the kidneys and meningiomas.

In the lungs, chest CT scanning shows multiple (more than 10) thin-walled air-filled cysts with preserved lung volumes and no other lung involvement. Pulmonary function tests may show expiratory airflow obstruction and/or restrictive abnormalities with hypoxemia and normal $\mathrm{PCO}_{2}$. Prognosis is variable with median survival of 8 to 10 years following diagnosis and has been reported to be up to 20 years. No treatment is of proven benefit. Treatment is supportive with oxygen, bronchodilators, and occasionally lung transplantation.

It is believed that estrogens are related to the progression of the disease and should therefore be avoided. Pregnancy in these patients has been associated with an increased risk of pneumothorax and chylothorax. Those who are pregnant prior to the diagnosis of LAM appear to do well, while those whose diagnosis is made during pregnancy have an increased incidence of premature births and can have greater progression of the disease.

Therefore, women with LAM should be evaluated prior to pregnancy and advised of possible complications. Should they elect to become pregnant, or proceed with pregnancy, they should be evaluated for the progression of disease and pneumothoraces (59). 


\section{Sarcoidosis}

Sarcoidosis is a multisystem granulomatous disorder of unknown etiology that results in noncaseating granulomas in involved organs. It most commonly affects people under 40 years of age and, while affecting all races, is more common in blacks than whites. The lung is the most commonly involved organ (over 90\%), and patients present with dyspnea, cough, fever, malaise, and weight loss. Other organs such as the heart, eyes, and skin are frequently involved. The diagnosis is made with a consistent clinical picture and a consistent biopsy. Many patients will spontaneously improve, while others will require treatment, usually glucocorticoids. Patients can progress to respiratory failure due to interstitial lung disease and fibrosis.

Sarcoidosis does not seem to affect fertility or increase the incidence of maternal or fetal complications. Corticosteroids may be administered before and during pregnancy. However, with the use of systemic steroids during pregnancy, there is a reported increase in the incidence of cleft palate in fetuses and women should be made aware of this possible side effect. Some women improve during pregnancy as endogenous steroid levels rise. If respiratory function deteriorates, supplemental oxygen might be needed (60).

\section{INFECTIOUS DISEASES}

\section{Allergy and Upper Respiratory Tract Infections}

Upper respiratory tract infections are common disorders for which patients, pregnant and nonpregnant, seek therapy. These disorders can be challenging in the pregnant patient because many of the relieving medications must be avoided and because noninfectious rhinitis is a common symptom during normal pregnancy.

Rhinitis of pregnancy occurs in 20\% to $30 \%$ of women, and although it does not appear to affect outcome, it can certainly affect sleep, nutrition, and increase stress (61). Rhinitis of pregnancy is defined as nasal congestion and/or rhinorrhea lasting more than 6 weeks in the absence of signs of infection or a known allergen. Usually it is not necessary to treat pharmacologically and measures such as exercise (nasal vasoconstriction), saline spray or irrigation, and head of the bed elevation can be very effective (62). Intranasal glucocorticoid sprays do not seem to be beneficial, and when necessary, pseudoephedrine can be used after the first trimester and if there is no maternal hypertension (63).

Much like asthma, allergic rhinitis that is present prior to pregnancy may remain stable, worsen, or improve (64). Skin testing to identify an allergen is avoided during pregnancy given the possibility of a severe systemic allergic response during testing. Intranasal cromolyn sodium is safe and efficacious, and unlike in non-allergic rhinitis of pregnancy, intranasal glucocorticoids are effective and considered safe (65). Antihistamines are generally less effective and the intranasal formulations should be avoided. Second-generation antihistamines such as loratadine and cetirizine are the formulations of choice if more than topical therapy is needed. Decongestants have the added benefit of having vasoconstrictive properties, but because of this effect, they should be avoided in the first trimester. Nasal oxymetolazone, as in nonpregnant patients, should be used only for a short duration of 2 to 3 days to avoid rebound symptoms. If necessary, pseudoephedrine can be used after the first trimester and if there is no maternal hypertension $(65,66)$. Allergen immunotherapy is often successful in controlling symptoms of allergy_both rhinitis and asthma. While it is not recommended to start immunotherapy during pregnancy, for fear of systemic allergic reaction, patients who are tolerating a stable dose and who are benefiting from the therapy prior to pregnancy should continue their injections throughout pregnancy (67).

The practitioner must have a low threshold for suspecting bacterial sinusitis, as this entity is a common upper respiratory tract complication of pregnancy. Often the symptoms of fever and purulent discharge are absent. Radiologic evaluation with $\mathrm{X}$-ray, computed tomography, or magnetic resonance imaging should be reserved for those not improving on antibiotics. Pathogens are identical to those in all bacterial sinusitis: Haemophilus influenza, Moraxella catarrhalis, and Streptococcus pneumoniae. Treatment should be initiated with high-dose amoxicillin, amoxicillin-clavulanate, or one of the oral second- or third-generation cephalosporins. A penicillin allergic patient may receive trimethoprim-sulfamethoxazole or azithromycin but the fluoroquinolones, tetracycline, and doxycycline should all be avoided (68).

Just as in the nonpregnant host, it is estimated that $90 \%$ of acute bronchitis - productive cough due to airway inflammation with a clear X-ray-is viral in origin. Rhinovirus, influenza, parainfluenza, respiratory syncytial virus, and adenovirus are the usual implicated viral pathogens. Without a chest radiograph, it can be difficult to determine whether a lower respiratory tract infection is bronchitis or pneumonia. The presence of high fever, lack of upper respiratory tract viral symptoms, and leukocytosis might suggest pneumonia, and a chest X-ray and/or a course of antibiotics is probably warranted (69). Humidified steam can be more helpful than prescription or over-the-counter cough medication and should be encouraged.

\section{Bacterial Pneumonia}

Pneumonia in pregnant patients is rarely fatal and has the same incidence as in non-gravid individuals. However, there is both maternal and fetal morbidity associated with the condition such as hypoxemia and low birth weight (69). While a young, otherwise healthy pregnant, woman may not have a classic presentation, fever, rigors, dyspnea, productive cough, and occasionally pleurisy are common presenting signs and symptoms of bacterial pneumonia. A chest radiograph is required and the risk of an undiagnosed pneumonia far outweighs the risk of fetal radiation if the usual shielding precautions are taken. Causative organisms are those common in community acquired pneumonia such as S. pneumoniae, $H$. influenza, Staphylococcus aureus, Legionella, Mycoplasma, and Chlamydia. Treatment with azithromycin and, in severe cases, ceftriaxone, is recommended. As with sinusitis, doxycycline, clarithromycin, and the fluoroquinolones should be avoided. Aspiration is common during pregnancy, and when pneumonia develops around the time of delivery or after an aspiration event, anaerobic organisms should be considered and treated (70). 


\section{Influenza}

There have been three major influenza pandemics in the past 100 years-1918, 1957, and the H1N1 pandemic of 2009. In all three pandemics, there was higher morbidity and mortality among pregnant women. Seasonal influenza also takes a toll on the gravid patient with smokers, asthmatics, and women with other comorbid medical conditions having a higher rate of serious complications and hospitalization. This is especially true in the third trimester. Why influenza infection affects this pregnant group of young patients more seriously than their nonpregnant peers is unclear - theories suggest some difference in the immune system, the propensity of pregnant patients to have pulmonary edema due to hypoalbuminemia, and decreased oncotic pressure or their higher baseline oxygen consumption. Pregnant patients with known or suspected seasonal influenza should be observed closely so that if respiratory symptoms appear, they can be hospitalized. Otherwise, the usual treatment of rest, fluids, and acetaminophen for fever and myalgias applies (71).

The 2009 pandemic H1N1 influenza virus caused serious disease requiring hospitalization in many pregnant patients. Especially in the third trimester, infected women experienced respiratory failure, miscarriage, and preterm births. This led to the gravid patient being a priority for vaccination that season. In fact, influenza vaccination is recommended for all pregnant patients as experience has shown no risk to the fetus and a favorable benefit (72). Oseltamivir should be given to all hospitalized gravid patients with the H1N1 strain even if the conventional 48 hours of symptoms has passed. As with most viral respiratory infections, the bacterial superinfection rate is high and concomitant antibiotics should be considered (73).

\section{Tuberculosis}

Over 11,000 active cases of tuberculosis were reported in the United States in 2009 (74). Pregnancy does not appear to change the pathogenesis or disease progression of tuberculosis (75). Skin testing is safe and the results are not altered by pregnancy. However, routine testing for latent tuberculosis is not recommended unless the woman has a specific risk of quick progression from latent to active disease such as HIV infection or a recent significant exposure. Skin testing during pregnancy indicates that the practitioner has an intention to treat during pregnancy. In that setting, a positive skin test warrants a chest X-ray and evaluation to rule out active disease and then prophylactic therapy with isoniazid. In everyone other than those with immunosuppression or a recent exposure, testing and/or latent tuberculosis therapy should be deferred until at least the third month postpartum, since the risk of isoniazid hepatotoxicity is increased during pregnancy and the early postpartum period (76-78).

Active tuberculosis occurring during pregnancy may be initially hard to diagnose, as fatigue and malaise might be misinterpreted and weight loss can be mild. The workup for tuberculosis is the same in a pregnant patient as for a nonpregnant patient, and a new diagnosis of active tuberculosis should always prompt an HIV test. Active disease should be treated, as the risk of untreated disease is certainly higher than that of antituberculosis drug therapy
(77). First-line therapy is isoniazid, rifampin, and ethambutol, but pyrazinamide should be avoided because of limited safety data in pregnancy. However, in those patients with HIV, drug resistance, or extensive disease, its use should be considered. Streptomycin is a known teratogen and causes congenital deafness, so it should be avoided. Kanamycin, amikacin, and capreomycin are to be avoided because all cause eighth nerve damage and can cause renal toxicity. Generally any patient with multidrug resistant disease or who fails first-line therapy should be seen by an expert in tuberculosis. All patients are monitored using directly observed therapy and for hepatotoxicity (78).

\section{Pneumocystis Jiroveci Pneumonia due to the Human Immunodeficiency Virus/Acquired Immunodeficiency Syndrome}

With the implementation of highly active antiretroviral therapy, the last decade has seen a remarkable decrease in both the morbidity and the mortality associated with HIV and AIDS. However, the percentage of AIDS cases in women continues to grow annually. By 2007, 27\% of adult AIDS patients were woman, and of these, $83 \%$ were women from communities of color (79). Heterosexual contact is the most common mode of acquisition in women and therefore issues surrounding the management of HIV/AIDS during pregnancy have become increasingly important.

In addition to an increased rate of viral and bacterial infections of the upper and lower airway, AIDS patients with a CD4 count $<200$ are susceptible to infection with $P$. jiroveci. Limited review of pregnant patients with $P$. jiroveci pneumonia during pregnancy suggests that mortality is higher in this group $(50 \%)$ than in nonpregnant subjects and that risk is higher in the second and third trimesters of gestation (80). However, only a small number of cases have been reported in the literature and the women reported were almost all unaware of their HIV/AIDS status at the time of pneumonia diagnosis. Therefore, they were not receiving prophylaxis against this infection. Trimethoprim-sulfamethoxazole prophylaxis should be initiated using the same guidelines as for nonpregnant women (CD4 count less than 200). Generally, pentamidine should be used during the first trimester rather than trimethoprim-sulfamethoxazole. While the fetus is most susceptible to drug teratogenicity during the first 10 weeks of gestation, if the mother's health is poor, the benefit of therapy during this early gestational period likely outweighs the risk to the fetus (81-85). For active $P$. jiroveci infection at any time during pregnancy, trimethoprim-sulfamethoxazole remains the standard of care and steroid therapy should be considered in severe cases (80).

\section{MISCELLANEOUS DISORDERS Pulmonary Edema}

Cardiogenic pulmonary edema is uncommon during pregnancy, but can occur late in pregnancy and early postpartum, usually due to heart disease, valvular problems such as mitral stenosis, or idiopathic cardiomyopathy. Patients complain of dyspnea and chest tightness, and may have bilateral infiltrates on chest radiograph. Therapy is similar to nonpregnant patients and includes diuresis and oxygen. However, 
non-invasive positive pressure ventilation via mask is best not used due to the increased incidence of aspiration (86).

Non-cardiogenic pulmonary edema occurs in women receiving beta sympathomimetic agents for tocolysis. Usually, this condition occurs during administration of the drug, but can occur within 24 hours after discontinuation. Women complain of dyspnea and chest tightness, and rales may be present, as well as bilateral alveolar infiltrates on chest radiograph. Therapy is supportive with discontinuation of the beta agonist, oxygen, and diuresis. Resolution is expected within 24 hours (87).

\section{Pleural Effusion}

While there has been no specific study that identifies the disease processes causing pleural effusion in pregnancy, likely up to $25 \%$ of pregnant patients have small, asymptomatic pleural effusions visible on ultrasound. When pleural effusions are symptomatic, they cause dyspnea, cough, and possibly chest pain-if significant, there may be room air hypoxia due to shunting through underlying atelectatic lung. Symptomatic effusions in early pregnancy are either due to ovarian hyperstimulation syndrome or the same processes that cause effusions in nonpregnant patients: infection, cardiomyopathy, and PE. Neither pre-eclampsia nor the HELLP syndrome is associated with pleural effusion. Thoracentesis with drainage is indicated for diagnosis and/or the relief of symptoms due to a significant amount of pleural fluid. The small bilateral effusions described above that are asymptomatic generally are self-limiting and do not require intervention (88).

\section{Sleep Disorders}

Sleep-disordered breathing is prevalent in the general population and sleep disturbance is one of the most common complaints during pregnancy. However, there are limited data to elucidate the causes for sleep disturbance during pregnancy-difficulty lying in the supine position, nasal congestion, weight gain, and decreased time spent in rapid eye movement sleep have all been implicated $(89,90)$. There is a suggestion that obese women who become pregnant have more sleep-disordered breathing, even in the first trimester, and this may be important if there is nighttime hypoxia that can affect the growth of the fetus. More work is needed in this area of sleep medicine (90).

\section{Aspiration}

Aspiration into the lung of stomach contents occurs most often during or immediately after labor. The combination of delayed gastric emptying, a relaxed lower esophageal sphincter, and increased intra-abdominal pressure with recumbency and sedation or analgesia increase the risk of vomiting and aspiration. Acutely, aspiration can lead to a chemical pneumonitis or bronchospasm, and aspiration of upper airway organisms such as anaerobes and streptococci can ultimately lead to a bacterial pneumonia. Chemical pneumonitis, while hard to distinguish from pneumonia, generally has an acute onset with dyspnea, cough, and low-grade fever. Radiographs reveal multifocal infiltrates with basilar predominance and treatment is supportive. Bronchospasm can be indistinguishable from asthma with dyspnea and wheezing and is treated with bronchodilators $(91,92)$.

\section{Respiratory Failure and Mechanical Ventilation}

Respiratory failure, defined as a $\mathrm{PaO}_{2}$ of $<60 \mathrm{mmHg}$ or $\mathrm{pCO}_{2}>50 \mathrm{mmHg}$, is a rare complication of pregnancy. The patient in respiratory failure will be distressed, tachypneic, and with shallow breaths despite the use of accessory muscles. If supplemental oxygen by nasal cannula or face mask fails to keep the oxyhemoglobin saturation above 95\% (required for adequate fetal oxygenation), intubation and mechanical ventilation should be considered (93). Oral intubation of the pregnant patient can be difficult, with the decreased functional residual capacity and increased oxygen consumption causing more rapid hypoxia and upper airway edema obscuring adequate visualization during direct laryngoscopy. Intubation should be performed by experts in airway management. While receiving treatment tailored to the cause of the respiratory failure, mechanical ventilation should be managed to maintain a $\mathrm{pCO}_{2}$ in the 27 to 32 range, as this is the usual $\mathrm{pCO}_{2}$ during pregnancy. Severe respiratory alkalosis (potentially causing decreased uterine blood flow), hypoxia, and acidosis should be avoided (93).

\section{REFERENCES}

1. Berg CJ, Mackay AP, Qin C, Callaghan WM. Overview of maternal morbidity during hospitalization for labor and delivery in the United States: 1993-1997 and 2001-2005. Obstet Gynecol 2009; 113: 1075-81.

2. Panchal S, Arria A, Labhsetwar S. Maternal mortality during hospital admission for delivery: A Retrospective Analysis Using a State-Maintained Database, Anesth Analg. 2001; 93: 1134-41.

3. Obstetrics: Normal and problem pregnancies, 2010 Elsevier.

4. Bonica, JJ. Maternal respiratory changes during pregnancy and parturition. Clin Anesth 1972; 10: 1 .

5. Prowse CM, Gaensler EA. Respiratory and acid base changes during pregnancy. Anesthesiology 1965; 26: 381.

6. Crapo R. Normal cardiopulmonary physiology during pregnancy. Clin Obstet Gynecol 1996; 39: 3.

7. Towell ME. Fetal respiratory physiology in perinatal medicine. In: Goodwin JW, Chance GW, eds. Longman: Toronto, Canada, 1976.

8. Maternal F, et al. Neonatal Physiology: A Clinical Perspective 2007, Saunders Elesevier.

9. McClure JH, James JM. Oxygen administration to the mother and its relation to blood oxygen in the newborn infant. Am J Obstet Gynecol 1960; 80: 554-6.

10. Simon PM, Schwartzstein RM, Weiss JW, et al. Distinguishable types of dyspnea in patients with shortness of breath. Am Rev Respir Dis 1990; 142: 1009

11. Zeldis SM. Dyspnea during pregnancy. Distinguishing cardiac from pulmonary causes. Clin Chest Med 1992; 13: 567.

12. Kwon HL, Triche EW, Belanger K, Bracken MB. The epidemiology of asthma during pregnancy: prevalence, diagnosis, and symptoms. Immunol Allergy Clin North Am 2006; 26: 29-62.

13. National Asthma Education program. National Heart, Lung, and Blood Institute, National Institutes of Health: Report of the Working Group on Asthma and Pregnancy. Executive Summary: Management of Asthma During Pregnancy, NIH Publication No. 93-3279A, 1993.

14. Mabie WC, Barton JR, Wasserstrum N, et al. Clinical observations on asthma in pregnancy. J Maternal Fetal Med 1992; 1: 45-50.

15. Murphy VE, Gibson PG, Smith R, Clifton VL. Asthma during pregnancy: mechanisms and treatment implications. ERJ 2005; 25: 731-50.

16. Martel MJ, Rey E, Beauchesne MF, et al. Use of inhaled corticosteroids during pregnancy and risk of pregnancy induced hypertension: nested case-control study. BMJ 2005; 330: 230. 
17. Malfertheiner MV, Mönkemüller K, Röhl FW, Malfertheiner P, Costa SD. Gastroesophageal reflux disease and management in advanced pregnancy: a prospective survey. Digestion 2009; 79: 115-20.

18. Hunter CJ, Brightling CE, Woltmann G, Wardlaw AJ, Pavord ID. A comparison of the validity of different diagnostic tests in adults with asthma. Chest 2002; 121: 1051-7.

19. ACOG practice bulletin: clinical management guidelines for obstetriciangynecologists number 90, February 2008: asthma in pregnancy. Obstet Gynecol 2008; 111: 457-64.

20. Dombrowski MP. Asthma and pregnancy. Obstet Gynecol 2006; 108: 667-81.

21. Schatz M, Dombrowski MP, Wise R, et al. The relationship of asthma medication use to perinatal outcomes. J Allergy Clin Immunol 2004; 113: 1040-5.

22. Kallen B, Rydhstroem H, Aberg A. Congenital malformations after the use of inhaled budesonide in early pregnancy. Obstet Gynecol 1999; 93: 392-5.

23. Norjavaara E, De Verdier MG. Normal pregnancy outcomes in a population based study including 2,968 pregnant women exposed to budesonide. J Allergy Clin Immunol 2003; 111: 736-42.

24. National Asthma Education and Prevention Program expert panel report: managing asthma during pregnancy; recommendations for pharmacologic treatment - 2004 update. J Allergy Clin Immunol 2005; 115: 34-46.

25. National Asthma Education and Prevention Program. Expert Panel Report 3: guidelines for the diagnosis and management of asthma: full report 2007. [Available from: www.nhlbi.nih.gov/guidelines/asthma/ asthgdln.pdf].

26. Davis PB. Cystic Fibrosis since 1938. Am J Respir Crit Care Med 2006; 173: 475-82.

27. Ratjen F, Doring G. Cystic fibrosis. Lancet 2003; 361: 681-9.

28. Edenborough FP, Stableforth DE, Webb AK, Mackenzie WE, Smith DL. Outcome of pregnancy in women with cystic fibrosis. Thorax 1995; 50: 170-4.

29. Gilljam M, Antoniou M, Shin J, et al. Pregnancy in cystic fibrosis: fetal and maternal outcome. Chest 2000; 118: 85-91.

30. McMullen AH, Pasta DJ, Frederick PD, et al. Impact of pregnancy on women with cystic fibrosis. Chest 2006; 129: 706-11.

31. Bhatia P, Bhatia K, Pregnancy and the Lungs. Postgrad Med J 2000; 76: 683-9.

32. Howie AD, Milne JA. Pregnancy in patients with bronchiectasis. $\mathrm{Br}$ J Obstet Gynecol 1978; 85: 197-200.

33. Rich S, Dantzker DR, Ayres SM, et al. Primary pulmonary hypertension: a national prospective study. Ann Int Med 1987; 107: 216-23.

34. Rubin LJ. Diagnosis and management of pulmonary arterial hypertension: ACCP evidence-based clinical practice guidelines. Chest 2004; (Suppl 126/1): 7S-10S.

35. Badesch DB, Abman SH, Ahern GS, et al. Medical therapy for pulmonary arterial hypertension. Chest 2004; (Suppl): 35S-62S.

36. Weiss BM, Zemp L, Seifert B, et al. Outcome of pulmonary vascular disease in pregnancy: a systemic overview from 1978 through 1996. J Am Coll Cardiol 1998; 31: 1650-7.

37. Stewart R, Tuazon D, Olson G, et al. Pregnancy and pulmonary hypertension: successful outcome with epoprostenol therapy. Chest 2001; 119/3: 973-5.

38. Fischer LG, Aken HV, Burkle H. Management of pulmonary hypertension: physiological and pharmacological considerations for anesthesiologists. Anesth Analg 2003; 96; 1603-16.

39. Bonnin M, Mercier FJ, Sitbon O. Severe pulmonary hypertension during pregnancy: mode of delivery and anesthetic management of 15 consecutive cases. Anesthesiology 2005; 102, 6; 1133-7.

40. Bedard E, Dimopoulos K, Gatzoulis MA. Has there been any progress made on pregnancy outcomes among women with pulmonary arterial hypertension? European Heart Journal 2009; 30: 256-65.

41. Bourjeily G, Paidas N, Khalil H, et al. Pulmonary embolism in pregnancy. Lancet 2010; 375: 500-12.

42. Marik PE, Plante LA. Venous Thromboembolic Disease and Pregnancy. New Eng J Med 359, 19: 2025-33.

43. Rosenberg VA, Lockwood CJ. Thromboembolism in pregnancy. Obstet Gynecol Clin N Am 2007; 34: 481-500.

44. Bates SM, Greer IA, Pabinger I, et al. Venous thromboembolism, thrombophilia, antithrombotic therapy and pregnancy. American college of chest physicians evidence based-clinical practice guidelines (8th edition). Chest 2008; 133: 844S-886S.

45. PIOPED Investigators. Value of the Ventilation/Perfusion Scan in Acute Pulmonary Embolism. Results of the Prospective Investigation of Pulmonary Embolism Diagnosis (PIOPED). JAMA 1990; 263; 2753-9.

46. Kline JA, Williams GW, Hernandez-Nino J. D-Dimer Concentrations in Normal Pregnancy: New Diagnostic Thresholds Are Needed; Clin Chem 2005; 5: 825-9.

47. Chan WS, Ray JG, Murray S, et al. Suspected pulmonary embolism in pregnancy: Clinical presentation, Results of lung scanning, and subsequent maternal and pediatric outcomes. Arch Intern Med 2002; 162: $1170-5$.

48. Stein PD, Fowler SE, Goodman LR, et al. Multidetector computer tomography for acute pulmonary embolism. N Eng J Med 2006; 354: 2317-27.

49. Winer-Muram HT, Boone JM, Brown HL, et al. Pulmonary embolism in pregnant patients: Fetal radiation dose with helical CT. Radiology 2002; 224: 487-92.

50. Agnelli G, Becattini C. Acute pulmonary embolism 2010. N Eng J Med 363: 3, 266-74.

51. Leonhardt G, Gaul C, Nietsch HH, et al. Thrombolytic therapy in pregnancy. J Thromb Thrombolysis 2006; 21: 271-6.

52. Baldisseri MR. Amniotic fluid embolism syndrome, 2010. [Available from: www.uptodate.com].

53. Moore J, Baldisseri MR. Amniotic fluid embolism. Crit Care Med 2005: 33 (Suppl 10): 5279-85.

54. Moore J. Amniotic fluid embolism. Lancet 2006: 368; 1399-401.

55. King TE. Restrictive lung disease in pregnancy. Clin Chest Med 1992; 4: 607-22.

56. Bogess KA, Easterling TR, Raghu G. Management and outcome of women with interstitial and restrictive lung disease. Am J Obstet Gynecol 1995; 173, 4: 1007-14.

57. Siegler D, Zorab PA. Pregnancy in thoracic scoliosis. Brit J Dis Chest 1981; 75; 4, 363-70.

58. Reddy R, Evans E, Khoo O. Pregnancy in kyphoscoliosis: Benefit of noninvasive ventilator support. J Obstet Gynaecol 2005; 25; 267-8.

59. Johnson SR, Cordier JF Lazor R, et al. European respiratory society guidelines for the diagnosis and management of lymphangioleiomyomatosis. Eur Respir J 2010; 35: 14-26.

60. Statement on Sarcoidosis. Joint Statement of the American Thoracic Society (ATS), European Respiratory Society (ERS) and the World Association of Sarcoidosis and other Granulomatous Diseases (WASOG) adopted by the ATS Board of Directors and by the ERS Executive Committee. Am J Resp Crit Care Med 1999; 160: 736-55.

61. Somoskovi A, Bartfai Z, Tamasi L, et al. Population-based case-control study of allergic rhinitis during pregnancy for birth outcomes. Eur J Obstet Gynecol Reprod Biol 2006.

62. Ellegard EK. The etiology and management of pregnancy rhinitis. Am J Respir Med 2003; 2: 469-75.

63. Ellegard EK, Hellgren M, Karlsson NG. Fluticasone propionate aqueous nasal spray in pregnancy rhinitis. Clin Otolaryngol Allied Sci 2001; 26: 394-400.

64. Kircher S, Schatz M, Long L. Variables affecting asthma course during pregnancy. Ann Allergy Asthma Immunol 2002; 89: 463-6.

65. Busse WW. NAEPP Expert Panel Report: Managing asthma during pregnancy: Recommendations for pharmacologic treatment - 2004 update. J Allergy Clin Immunol 2005; 115: 34.

66. Kallen B. Use of antihistamine drugs in early pregnancy and delivery outcome. J Matern Fetal Neonatal Med 2002; 11: 146-52.

67. ACOG Practice Bulletin No. 90: Asthma in Pregnancy. Obstet Gynecol 2008; 111: 457.

68. Laibl V, Sheffield J. The management of respiratory infections during pregnancy. Immunol Allergy Clin North Am 2006; 26: 155.

69. Lim WS, Macfarlane JT, Colthorpe CL. Treatment of communityacquired lower respiratory tract infections during pregnancy. Am J Respir Med 2003; 2: 221.

70. Sheffield JS, Cunningham FG. Community Acquired pneumonia in pregnancy. Obstet Gynecol 2009; 114: 915. 
71. Hartert TV, Neuzil KM, Shintani AK, et al. Maternal morbidity and perinatal outcomes among pregnant women with respiratory hospitalizations during influenza season. Am J Obstet Gynecol 2003; 189: 1705.

72. Harper SA, Fukuda K, Uyeki TM, et al. Prevention and control of influenza: recommendations of the Advisory Committee on Immunization Practices (ACIP). MMWR Recomm Rep 2004; 53: 1.

73. Jamieson DJ, Honein MA, Rasmussen SA, et al. H1N1 2009 influenza virus infection during pregnancy in the USA. Lancet 2009; 374: 451

74. [Available from: www.CDC.gov/tb/statististics].

75. Carter EJ, Mates S. Tuberculosis during pregnancy. The rhode island experience, 1987-1991. Chest 1994; 106: 1466.

76. Starke JR. Tuberculosis in childhood and pregnancy. In: Tuberculosis: Current concepts and treatment, 2nd edn, Friedman LN ed. CRC Press: Boca Raton; 2000

77. Davidson PT. Managing Tuberculosis during pregnancy. Lancet 1995; 346: 199.

78. Saukkonen J, Cohn DL, jasmer RM, et al. An Official ATS Statement: hepatotoxicity in antituberculosis therapy. Am J Resp Crit Care Med 2006: 174: 935.

79. Centers for Disease Control and Prevention. HIV AIDS Surveill Rep 2007; 19: 2009.

80. Ahmad $\mathrm{H}$, et al. Pneumocystis carinii pneumonia in pregnancy. Chest 2001; 120: 666-71.

81. Cozzi Lepri A, Pezzotti P, Dorrucci M, Phillips AN, Rezza G. HIV disease progression in 854 women and men infected through injecting drug use and heterosexual sex and followed for up to nine years from seroconversion. Italian Seroconversion Study. BMJ 1994; 309: 1537-42.

82. Clark RA, Brandon W, Dumestre J, Pindaro C. Clinical manifestations of infection with the human immunodeficiency virus in women in Louisiana. Clin Infect Dis 1993; 17: 165-72.
83. Minkoff $\mathrm{H}$. Human immunodeficiency virus infection in pregnancy. Obstet Gynecol 2003; 101: 797-810.

84. Public Health Service Task Force Recommendations for use of Antiretroviral Drugs in Pregnant HIV-Infected Women for Maternal Health and Interventions to Reduce Perinatal HIV Transmission in the United States. [Available from: www.AIDSInfo.nih.gov].

85. Perinatal HIV Guidelines Working Group. Public Health Service Task Force Recommendations for Use of Antiretroviral Drugs in Pregnant HIV-Infected Women for Maternal Health and Interventions to Reduce Perinatal HIV Transmission in the United States. July 8, 2008: 1-98. [Available from http://aidsinfo.nih.gov/ContentFiles/PerinatalGL.pdf].

86. Sciscione AC, Ivester T, Largoza M, et al. Acute pulmonary edema in pregnancy. Obst Gynecol 2003; 101: 511-15.

87. Pisani RJ, Rosenow III, EC. Pulmonary edema associated with tocolytic therapy. Ann Int Med 1989; 9: 714-18.

88. Light RW. Pleural disease. Pleural disease in Obstetrics and Gynecology. Lippincott Williams and Wilkins, Philly, 2007: 272-5.

89. Feinsilver SH, Hertz G. Respiration during sleep in pregnancy. Clin Chest Med 1992; 13: 637-44.

90. Maasilta $\mathrm{P}$, et al. Sleep related disordered breathing during pregnancy in Obese women. Chest 2001; 120: 1448-54.

91. Bynum LJ, Pierce AK. Pulmonary Aspiration of Gastric Contents. Am Rev Respir Dis 1976; 114: 1129-36.

92. Finegold SM, Aspiration Pneumonia. Rev Infect Dis 1991; 13 (suppl 9): S737-742.

93. Cole DE, Taylor TL, et al. ARDS in Pregnancy. Crit Care Med 2005; 33 (suppl 10): S269-278. 


\section{Psychiatric disorders in pregnancy Robin Kopelman and Scott Stuart}

Pregnancy and childbirth are naturally occurring life events accompanied by momentous neuroendocrine and psychosocial changes. These transitions are often accompanied by changes in mood and psychological functioning, and these changes in turn may have an impact on the developing fetus and/or infant.

One of the most unique aspects of perinatal medicine is its subject matter: it addresses the life and well-being of both the mother and her offspring. Likewise, perinatal psychiatry must address the suffering of the individual pregnant woman and her safety while being mindful of the significant potential effects of maternal mental illness on early child development $(1,2)$.

Pregnancy and childbirth has historically been describedincorrectly - as a time of well-being, calm, and inner peace for women. Elevated gonadotropin levels, the sense of fulfillment, and the responsibility of caring for the young were believed to confer protection from mental disorders $(3,4)$. Carefully designed studies, however, found no such decrease in the incidence of perinatal mental disorders $(5,6)$. The current empirically based position is that events in women's reproductive cycles such as menstruation, pregnancy, childbirth, and menopause can be understood as biological (7) and psychological risk factors (8) for mood disorders.

Psychiatric disorders in the obstetric setting are frequent, with up to $40 \%$ of women having an identifiable mental disorder, most commonly depression $(9,10)$. In the first several months postpartum, there is an increased risk for psychiatric admission or outpatient contact $(4,11,12)$. It appears that the perinatal period places a subset of women at increased risk, and the overall prevalence and impact of the perinatal psychiatric disorders, as well as the unique treatment considerations for this population, make these disorders a topic of great significance for perinatal healthcare providers. This chapter provides a current overview of the most common psychiatric disorders during pregnancy and the postpartum period.

\section{MOOD DISORDERS IN PREGNANCY AND THE PUERPERIUM}

An occasional low mood is a normal reaction to the disappointments, setbacks, and losses inherent in everyday life. It is usually transient and not associated with significant functional impairment. A depressed mood, however, may be a part of a serious, potentially disabling disorder such as major depressive disorder (MDD), bipolar disorder (depressive episodes alternate with periods of abnormally elevated or irritable mood), or dysthymic disorder (characterized by lowgrade, more persistent depressive mood that has been present for at least 2 years). Epidemiologic data from around the world show that MDD is twice as common in women as in men (13).
Because low mood can be a normal experience, it is important for the clinician to be familiar with the diagnostic criteria for clinical depression. The Diagnostic and Statistical Manual of Mental Disorders (14) (DSM-IV) requires the presence of depressed mood or loss of interest and at least four of the following symptoms over the same 2-week period to diagnose major depression: weight loss or gain, insomnia or hypersomnia nearly every day, psychomotor agitation or retardation, fatigue or loss of energy, difficulties with concentration and memory, and guilt or suicidal thoughts.

Despite the serious consequences, detection rates for perinatal depression remain low, with only a small proportion of depressed women identified by health professionals $(10,15)$. Depression in pregnant and recently delivered women may be underdiagnosed because of the common presence of symptoms that are also associated with normal perinatal changes, such as fatigue, insomnia, weight or appetite changes, and changes in libido $(16,17)$.

Ultimately, less than one-third of depressed perinatal women receive treatment $(10,15)$. Financial issues and concerns about safety of medications during pregnancy may play a significant role (18). Often, women discontinue medication on their own once they become aware that they are pregnant (19). Relapse rates for those women who discontinue antidepressants during pregnancy are high, with higher relapse rates in women with depression for more than 5 years, more than four episodes, and of younger age (20).

Rates of treatment can be improved by accurately identifying women with perinatal depression. A self-report questionnaire to screen for depression should be used with a standard interview in the obstetric setting to facilitate this (21). The Edinburgh Postnatal Depression Scale (22) is a commonly used screening measure, which is both sensitive and specific for perinatal depression. It focuses on psychologic symptoms, rather than the somatic symptoms associated with new parenthood, and has been validated for use in pregnancy (23). Additionally, it is brief (10 items) and is an easy-to-use score.

The treatment and therapeutic responses for depression during pregnancy and the postpartum period are similar to those outside of the perinatal period (24). The treatments of choice are psychotherapy and antidepressant medications (25-29).

\section{DEPRESSION IN PREGNANCY}

The incidence of major and minor depression during pregnancy is approximately $10 \%$ (30), which is similar to the nonpregnant female population (5). However, it may be as high as $27.6 \%$ among women in poor urban communities (31). Risk factors for perinatal depression are similar to those for depression at other times and consist primarily of a 
personal or family history of depression and poor social support, particularly from the partner $(8,32)$.

The risks of untreated depression during pregnancy are significant. Antenatal depression has been associated with an increased risk for pre-eclampsia (33), preterm delivery (34), and fetal growth retardation (35). Depressed pregnant women obtain less adequate prenatal care (36) and have more negative health behaviors (e.g., tobacco use) (37), both of which also negatively impact birth outcomes.

\section{POSTPARTUM MOOD DISORDERS}

The spectrum of postpartum mood disorders includes postpartum blues, postpartum depression (PPD), and postpartum psychosis.

\section{Postpartum Blues}

Postpartum blues is a transient experience characterized by insomnia, anxiety, mood lability, irritability, and tearfulness, occurring exclusively within the first 2 weeks after delivery. It occurs in up to $75 \%$ of postpartum women (38), making it so common that most practitioners believe that it should not be considered a disorder. It has a minor functional impact and usually resolves spontaneously. Management is limited to reassurance and support without medications. However, close follow-up is indicated; women experiencing severe blues symptoms are at higher risk to develop a postpartum depressive episode (39).

\section{PPD}

Prevalence estimates for PPD range from $6.8 \%$ to $13 \%$ $(8,17,40-42)$ and up to $26 \%$ among adolescent mothers (43).

Risk factors for PPD include the following:

1. Personal or family history of depression $(17,44)$.

2. Presence of depression during pregnancy (45).

3. Psychosocial factors such as marital conflict, being a single mother, lack of social/family support, the stress of caring for the infant (disrupted sleep and sleep deprivation), multiple births, complications during pregnancy (especially if necessitating prolonged bed rest), and other stressful life events during the year preceding the birth $(30,44,46)$.

The onset of PPD occurs in up to $70 \%$ of patients within the first 3 months postpartum $(41,47)$. The depressive episode may last from 3 months to 14 months (48), though women may have significant depression lasting much longer (49). The clinical picture of PPD is similar to that of major depression occurring in the nonpuerperal period $(50,51)$.

Unrecognized or untreated depression may impair function and adversely affect the new mother's ability to care for her infant. Depression may also interfere with the new mother's ability to respond optimally to the infant and consequently may have adverse effects on early child development $(1,2,52)$. Women with PPD are also less likely to engage in positive and healthy parenting practices (53).

Suicide and infanticide are the most serious complications of PPD. While approximately $15 \%$ of depressed individuals in the general population end their lives by suicide $(54,55)$, having living children may offer some protection from suicide in the postpartum period (3). Women with perinatal depression may nonetheless have thoughts of harming themselves or the infant (56) and the risk for suicide is a particular concern for these women (55). In the United Kingdom, suicide related to psychiatric disorder was found to be the leading cause of maternal death over the year following delivery (57).

Risk factors for suicide include a history of depression, prior suicide attempts, psychosis, and several psychosocial risk factors such as living alone, lack of family or social support, and feelings of guilt and hopelessness. Obstetricians should be aware of the risk and ask women about suicide and self-harm, as well as infanticide, with such questions as "Have you been feeling like life is not worthwhile?," "Have you had thoughts of harming yourself (or your baby), wanting to die or wanting to end your life?," "Have you thought about how you might harm yourself (or your baby) or end your life?," and "How likely is it that you might actually do something like that?" When suicidal or infanticidal ideation is present, emergency psychiatric consultation is warranted.

The pathogenesis of PPD remains undetermined. The belief that hormonal factors are the greatest contributors to PPD persists even though the empirical support that serum levels of gonadal hormones account for postpartum mood symptoms is equivocal at best (7). Likewise, there has not been clear evidence to support the hypothesis that a rapid decline of gonadal hormones and cortisol and/or the effect of their withdrawal on neurotransmitter activity trigger PPD $(46,58,59)$.

There is, however, some recent research that suggests that there may be a subset of women who are susceptible to the abrupt hormonal withdrawal in the postpartum $(60,61)$. Epidemiologic data demonstrating a high degree of overlap between the diagnosis of premenstrual dysphoric disorder and PPD have supported this theory $(62,63)$. Several investigators have suggested that serotonin dysregulation in particular might play in PPD due to its role in depression at other times and its interaction with gonadal steroids (especially estradiol) $(17,64-66)$. To date, treatment with selective serotonin reuptake inhibitors (SSRIs) have mixed results in preventing the onset of PPD (67). There are small amount of data suggesting prophylactic effects (58) and possible treatment benefit in PPD patients being treated with estradiol $(68,69)$.

\section{Postpartum Psychosis}

The incidence of postpartum psychosis is $0.1 \%$ to $0.2 \%$ or 1 to 2 per 1000 births (70). In most cases, the onset occurs during the first 2 weeks postpartum. It is frequently heralded by one to two nights of insomnia and irritability (7). The most characteristic clinical presentation consists of confusion and delirium-like psychosis with disorganized behavior, mood lability, and hallucinations and delusions that are often focused on childbirth themes. While infanticide is quite rare, women with postpartum psychosis are more likely to experience thoughts of harming their infants than women with nonpsychotic PPD and are at risk for neglecting their infant $(71,72)$.

Differential diagnoses for postpartum psychosis include bipolar disorder presenting as a manic episode, schizoaffective disorder, and major depression with psychotic features. The cognitive disturbance associated with postpartum psychosis 
also makes thorough evaluation for delirium essential, with possible causes including cerebrovascular incidents (e.g., due to eclampsia), medications used in delivery, and metabolic or infectious etiologies (73). Research data suggest, however, that postpartum psychosis is most often a manifestation of bipolar disorder $(74,75)$.

A personal or family history of postpartum psychosis as well as bipolar disorder $(70,76)$ increases a woman's risk. The risk of recurrence in subsequent pregnancies is estimated to be $40 \%$ to $60 \%(77-79)$.

In addition to postpartum psychosis, psychotic illnesses in the puerperium also include schizophrenia. The lifetime prevalence of schizophrenia is about $1.5 \%$ spread equally across both genders, and women with schizophrenia get pregnant and give birth. Given that its peak age of onset is between 15 and 35 years of age, it is of particular clinical importance during women's reproductive years. However, the incidence of schizophrenia does not appear to be impacted by pregnancy and childbirth $(70,80)$.

Schizophrenia and other psychotic disorders manifest a variable course ranging from acute episodes to a protracted disturbance of thought and behavior with gradual decline. Clinical course and prognosis are significantly improved by the availability of family and social support and psychiatric care. Patients may show negative symptoms (such as apathy or flat affect), disorganization, eccentric thinking and behavior, or acute psychotic manifestations, such as hallucinations or delusions. These symptoms may have a devastating impact on the mother and fetus or infant; women with schizophrenia have increased rates of a number of adverse pregnancy outcomes $(81,82)$ and are at risk for suicide and other selfdestructive behaviors, as well as infanticide (83).

As noted, any new-onset psychosis during pregnancy or the postpartum should be promptly evaluated for an identifiable medical (e.g., drug-induced psychosis, temporal lobe epilepsy, stroke, systemic lupus erythematosus, acute intermittent porphyria) or psychiatric (e.g., brief reactive psychosis, manic or depressive phase of bipolar disorder) cause. Treatment in the perinatal period includes control of the symptoms of disorganized thought and behavior through the administration of the lowest effective dose of antipsychotic medication (preferably from the high potency subgroup, such as haloperidol) and addressing psychosocial needs.

\section{ANXIETY DISORDERS IN THE PERINATAL PERIOD}

The course of anxiety disorders in the perinatal period, including obsessive-compulsive disorder (OCD), posttraumatic stress disorder (PTSD), panic disorder, and generalized anxiety disorder, is variable. The relationship between the perinatal period and both obsessive compulsive disorder and PTSD makes additional discussion of these important.

Clinically, OCD presents with obsessions (intrusive thoughts or images) and/or compulsions (actions and rituals that the individual feels compelled to perform to reduce intense anxiety). Perinatal patients often report a fear of accidentally or intentionally harming the fetus or baby $(84,85)$. The clinician should keep in mind that some patients may feel guilty because of their strange and frightening thoughts and may not report them spontaneously. It is helpful, therefore, for the clinician to inquire in a tactful and nonjudgmental way whether the mother has any of these fears. These thoughts are not seen exclusively in OCD, as over $40 \%$ of women with PPD $(86,87)$ and over $20 \%$ of new parents without a mental disorder (88) report these types of thoughts.

The prevalence of OCD during the perinatal period ranges from $0.2 \%$ to $3.9 \%$ and may be more common in the postpartum (89). Some women may be asymptomatic during one pregnancy, yet develop OCD in subsequent pregnancies (90). Pregnancy is believed to be a potential trigger for the onset of OCD (91) and the postpartum is considered to be a period for worsening of OCD (92). The onset of OCD may in fact be neurohormonally modulated. The possible role of estrogen, progesterone, oxytocin, and the serotonergic system has been investigated $(93,94)$, but none of these hormones has been definitively linked to perinatal OCD.

PTSD develops subsequent to exposure to a traumatic event involving actual or threatened death or serious injury or a threat to the physical integrity of self or others. The response to the perceived threat is usually fear, helplessness, or horror. A typical triad of symptoms include reexperiencing of the event (e.g., nightmares, flashbacks), avoidance of stimuli associated with the event, and signs of hyperarousal such as insomnia or irritability (14). The prevalence of PTSD symptoms in perinatal women varies widely $(0-7.7 \%)(95,96)$, with PTSD being most prevalent in a group of economically disadvantaged pregnant women.

In contrast to OCD, it is the events associated with the perinatal period rather than the hormonal changes associated with pregnancy and childbirth that are speculated to act as triggers for the development or exacerbation of PTSD symptoms. For example, deliveries that women appraise as traumatic, particularly those involving difficulties with pain control, powerlessness, or lack of control, are associated with PTSD (89). For women with a history of sexual abuse, procedures such as internal examination and delivery may trigger memories of the abuse $(95,97)$.

Untreated anxiety disorders have been associated with negative outcomes such as preterm delivery (98). Anxiety in pregnancy, like perinatal depression, may have adverse effects on the emotional and behavioral well-being of children (99). More research is needed in this area as it is increasingly recognized that untreated anxiety and depression are likely to have a deleterious effect on fetal development.

Women with a history of anxiety disorders who plan to conceive should be informed of the variable course and the potential treatments. Treatment options for anxiety include psychotherapy and medication. Anxiety disorders are frequently associated with interpersonal difficulties such as fears of expressing anger, isolation, and excessive concern of being judged by others. Psychotherapy may be effective for both the anxiety and the interpersonal difficulties these patients have.

Medication may be necessary if symptoms interfere significantly with daily functions and/or psychotherapy is not accessible. As with other mental disorders, the use of medication should be assessed within a framework that balances the risks associated with nontreatment of the illness and the contextual and clinical factors that impact the woman's preferences, including her ability to access treatment, the risks to her personally from the illness, and the risks to the fetus or breast-feeding infant $(100,101)$. 
SSRI antidepressants, or the tricyclic antidepressants (TCAs), such as nortriptyline, may be helpful. Benzodiazepines have a role in the treatment of anxiety in the general population, but use of antidepressants is preferred in the perinatal period because they have better safety profiles in pregnancy and lactation (102).

\section{TREATMENT: PSYCHOTHERAPY AND MEDICATIONS}

Depression during pregnancy, PPD, and psychosis are potentially severe and disabling. Untreated, they may carry a significant risk to both the mother and to her offspring. The clinician needs to carefully weigh both the maternal benefits from the medications and the potential fetal risks $(26,29,100,103)$. Medications during the first trimester, pregnancy, or the postpartum might be avoided if the depression is not severe and psychotherapy could be undertaken $(26,27)$. This decision should be made in partnership with the patient. Again, careful assessment of the patient's experiences and clinical history, as well as the acceptability and accessibility of treatments is essential $(18,100,101)$.

Psychotherapy can be an effective option for some women with perinatal depression. For women with milder symptoms who prefer psychosocial treatment, it should be considered first line. Psychologic treatments like interpersonal psychotherapy (IPT) are efficacious for antenatal depression (104). At present, there are not efficacy studies demonstrating the benefit of cognitive behavioral therapy (CBT) for antenatal depression. For postpartum women with milder symptoms, various psychosocial treatments delivered by nonmental health or mental health professionals have been shown to improve depressive symptoms (27). For more severe depression, benefit has only been shown in studies with well-defined treatments, such as IPT or CBT, delivered by highly trained professionals (27).

Psychotropic medication will be the first treatment choice for many women, particularly those with more severe illness or for whom other treatments are inaccessible. All psychotropic medications cross the placenta and their potential effects on the developing fetus must be considered (29). Three areas of potential impact of medications given during pregnancy are teratogenicity, if the medication is administered during the period of organogenesis; neonatal toxicity or withdrawal if the medication is given close to the time of birth; and later behavioral sequelae (103). There are few longitudinal studies examining behavioral changes in children exposed to antidepressants or depression during pregnancy (105), so subsequent discussion is focused on teratogenicity and withdrawal effects.

\section{Antidepressants}

TCAs have been available in the United States since 1963. Extensive cumulative experience has revealed no increased risk of major congenital malformations with their use in the first trimester $(17,106)$. Use of TCAs in pregnancy has been associated with perinatal complications such as jitteriness and irritability in the newborn $(103,107)$. Most investigators recommend the use of the secondary amine TCAs (e.g., nortriptyline) over the tertiary amines (amitriptyline).

While the SSRI antidepressants have been used for a shorter period of time, they have significant advantages over the TCAs and are undoubtedly the most commonly prescribed (108). They have a more benign side effect profile, lacking cardiac toxicity or anticholinergic side effects, and have a much better safety profile compared with the tricyclics in cases of overdose (109).

The majority of studies have not found an increased rate of major malformations with the use of SSRIs in pregnancy (110 112). Some studies have demonstrated an increased, but small, risk of select anomalies (e.g., omphalocele, septal defects) with exposure to certain SSRIs (111-113). Concern has been raised about paroxetine, in particular, as two studies suggest that it confers an increased risk of cardiac defects with first-trimester exposure. The manufacturer subsequently changed product warning labels (114). Findings remain controversial (115), but at present, one should avoid initiating new treatment with paroxetine in women of childbearing age and consider fetal echocardiography in women exposed during early pregnancy. Third-trimester exposure to SSRIs has also been associated with a possible increased risk for persistent pulmonary hypertension of the newborn (116).

Peripartum effects of exposure include an association between SSRIs and premature delivery and low birth weight (34), though most studies have been unable to control for the possible impact of depression itself on pregnancy outcome. Furthermore, the slight decrease in birth weight and gestational age is not considered by most experts to be clinically significant. Both SSRIs and TCAs have been associated with symptoms secondary to discontinuation of medication exposure in the neonate $(103,107,117)$. To minimize the risk of neonatal toxicity and withdrawal after delivery, the maternal dose of medication could be reduced during the 2 weeks prior to delivery (17).

Given the risk for recurrence of depression, psychiatric follow-up and maintenance treatment is considered the standard of care for perinatal disorders (118). Overall, a reasonable therapeutic goal for treating depression during pregnancy or PPD is to control symptoms and to recover function at a minimum effective dose of medication (17). When making a decision to reduce or temporarily discontinue medication, a discussion of the potential for relapse during pregnancy or postpartum is essential.

Other antidepressants are less commonly used, but may be encountered in perinatal women. The studies examining mirtazapine (119), bupropion $(120,121)$, venlafaxine (122), trazodone, and nefazodone (123) do not suggest an increased risk of fetal anomalies. Monoamine oxidase inhibitors are generally not recommended for use in the perinatal period due to the lack of data and potential for hypertensive crisis (124).

In the immediate time period surrounding delivery, several additional issues should be considered. First, hepatic immaturity in the newborn may affect the metabolism of psychotropic medications, thus leading to potentially higher blood levels in the newborn (17). The infant's pediatrician should be informed of maternal medications to prevent possible adverse drug interactions with other medications that may be prescribed for the infant. Second, women who are not symptomatic in pregnancy or the postpartum, but who have a history of depression, especially PPD, may benefit from a preventive intervention with psychotherapy or antidepressant medication, though research data are limited at this time. 
A woman might elect to initiate prophylactic treatment approximately 48 hours after delivery with an SSRI or start a preventive counseling intervention spanning pregnancy and postpartum $(67,125)$.

All psychotropic medications are found in the breast milk at various concentrations. Infants' absorption rates vary and significant drug levels are rarely detected in infants' serum. These medications are not found in quantifiable amounts in breast milk and have not caused any adverse neonatal effect (126). In a pooled analysis, levels were particularly low for sertraline, paroxetine, and nortriptyline, making these medications preferred. Elevated infant levels occurred with fluoxetine and citalopram (127).

There are case reports of adverse events with breast-feeding exposure to all types of antidepressants. Most reports involve fluoxetine (105), probably due to its long half-life and resultant increased blood levels, making it generally not recommended for use in nursing mothers (126). Few longterm studies exist and clear evidence of developmental problems has not been described in infants exposed to antidepressant medications in breast milk (128). Prescription of an antidepressant for a breast-feeding woman is a casespecific, risk-benefit decision (101).

\section{Mood Stabilizers}

The mainstay of treatment and prevention of recurrence of bipolar disorder, including that during the perinatal period, is a mood stabilizer such as lithium carbonate, valproic acid, carbamazepine, or lamotrigine (129). Women who discontinue mood stabilizer treatment in the perinatal period have high rates of relapse (130). During an acute depressive or manic episode, an antidepressant or an antipsychotic medication may be necessary. Sleep hygiene in bipolar patients has been shown to be very important, and sleep deprivation itself may trigger relapse (131).

The association of cardiac malformations with lithium exposure in the first trimester is widely documented by the Lithium Baby Register. The risks of major congenital anomalies, cardiovascular abnormalities, and Ebstein's anomaly in fetuses who were exposed to lithium during the first trimester have been reported as $8 \%(15 / 183)$ and $3 \%$, respectively $(132,133)$. The relative risk seems to be smaller than initially reported and is estimated to be 1.5 to 3.5 . Women who are on lithium maintenance and contemplate pregnancy should have preconception counseling.

Patients who have had a single episode of mood disorder could taper their lithium before conception and restart it if necessary after the first trimester. Lithium levels should be monitored closely during pregnancy. Current guidelines recommend taper 48 hours before delivery to avoid toxicity in the newborn (17) and restarting after delivery at prepregnancy dosing (134).

Patients with bipolar disorder who have had multiple episodes run a high risk of relapse without the medication. The maternal risk from recurrence may outweigh the fetal risk from exposure to lithium $(17,135)$. Lithium is generally considered to be contraindicated during breast-feeding because its concentration in breast milk is about $40 \%$ of maternal serum (136-140). Both valproic acid and carbamazepine are considered compatible with breast-feeding
$(17,140)$. Both should be avoided during pregnancy if possible because of their association with neural tube defects $(17,141,142)$. An ultrasound examination of the fetal anatomy at 18 to 20 weeks is advisable. Lamotrigine may be another option for treatment during pregnancy and breast-feeding as it appears to have a more favorable safety profile than the other anticonvulsant mood stabilizers (143).

\section{Antipsychotics}

All antipsychotic medications (neuroleptics) cross the placenta and are secreted in breast milk. The atypical antipsychotics (clozapine, olanzapine, and risperidone) lack sufficient data with respect to potential teratogenic effects. Though they are increasingly used for psychosis and bipolar disorder due to improved medication tolerance and effectiveness in treating negative symptoms in schizophrenia, caution in use should be exercised.

Typical antipsychotic drugs have a larger amount of reproductive safety data available, especially haloperidol. The high-potency antipsychotics (e.g., haloperidol) are preferable to the low-potency drugs. Several of the typical antipsychotics (chlorpromazine, haloperidol) have been previously prescribed during pregnancy for hyperemesis with safety data in pregnancy obtained in these studies $(144,145)$.

\section{CONCLUSION}

Psychiatric disorders are very common during pregnancy and the postpartum, and have a great deal of morbidity for both mother and infant. Many women are not recognized as having a psychiatric disorder, leading to a strong recommendation that screening instruments be used routinely in all obstetric settings. Both medications and psychotherapy are effective treatments, and all decisions regarding treatment must be made with a careful risk-benefit analysis that enlists the woman collaboratively in her care.

\section{REFERENCES}

1. Beck CT. The effects of postpartum depression on child development: a meta-analysis. Arch Psychiatr Nurs 1998; 12: 12-20.

2. Murray L, Cooper PJ. Postpartum depression and child development. Psychol Med 1997; 27: 253-60.

3. Appleby L. Suicidal behavior in childbearing women. Int Rev Psychiatry 1996; 8: 107-15.

4. Appleby L, Mortensen PB, Faragher EB. Suicide and other causes of mortality after post-partum psychiatric admission. Br J Psychiatry 1998; 173: 209-11.

5. O’Hara MW, Zekoski EM, Philipps LH, Wright EJ. Controlled prospective study of postpartum mood disorders: comparison of childbearing and nonchildbearing women. J Abnorm Psychol 1990; 99: 3-15.

6. Cohen LS, Sichel DA, Faraone SV, et al. Course of panic disorder during pregnancy and the puerperium: a preliminary study. Biol Psychiatry 1996; 39: 950-4.

7. Wisner KL, Stowe ZN. Psychobiology of postpartum mood disorders. Semin Reprod Endocrinol 1997; 15: 77-89.

8. O’Hara M, Swain A. Rates and risk of postpartum depression: a metaanalysis. Int Rev Psychiatry 1996; 8: 37-54.

9. Kelly RH, Zatzick DF, Douglas F, Anders TF. The detection and treatment of psychiatric disorders and substance use among pregnant women cared for in obstetrics. Am J Psychiatry 2001; 158: 213-19.

10. Smith MV, Rosenheck RA, Cavaleri MA, et al. Screening for and detection of depression, panic disorder, and PTSD in public-sector obstetric clinics. Psychiatr Serv 2004; 55: 407-14. 
11. Kendell RE, Rennie D, Clarke JA, Dean C. The social and obstetric correlates of psychiatric admission in the puerperium. Psychol Med 1981; 11: 341-50.

12. Munk-Olsen T, Laursen TM, Pedersen CB, Mors O, Mortensen PB. New parents and mental disorders: a population-based register study. JAMA 2006; 296: 2582-9.

13. Weissman MM, Olfson M. Depression in women: implications for health care research. Science 1995; 269: 799-801.

14. American Psychiatric Association Diagnostic and statistical manual of mental disorders. Washington, D.C.: APA, 2000.

15. Flynn HA, Blow FC, Marcus SA. Rates and predictors of depression treatment among pregnant women in hospital-affiliated obstetrics practices. Gen Hosp Psychiatry 2006; 28: 289-95.

16. Affonso DD, Lovett S, Paul SM, Sheptak S. A standardized interview that differentiates pregnancy and postpartum symptoms from perinatal clinical depression. Birth 1990; 17: 121-30.

17. Stowe ZN, Strader J, Nemeroff CB, Schatzberg AF, Nemeroff CB. Psychopharmacology during pregnancy and lactation. Essentials of clinical psychopharmacology. C.B. Nemeroff. Washington, DC: American Psychiatric Association, 2001: 659-80.

18. Kopelman RC, Moel J, Mertens C, et al. Barriers to care for antenatal depression. Psychiatr Serv 2008; 59: 429-32.

19. Marcus SM, Flynn HA, Blow F, Barry K. A screening study of antidepressant treatment rates and mood symptoms in pregnancy. Arch Womens Ment Health 2005; 8: 25-7.

20. Cohen LS, Altshuler LL, Nonacs R, et al. Relapse of major depression during pregnancy in women who maintain or discontinue antidepressant treatment. JAMA 2006; 295: 499-507.

21. Gaynes BN, Gavin N, Meltzer-Brody S, et al. Perinatal depression: prevalence, screening accuracy, and screening outcomes. Evidence Report/Technology Assessment No. 119. AHRQ. Rockville, MD: AHRQ, 2005.

22. Cox JL, Holden JM, Sagovsky R. Detection of postnatal depression. Development of the 10-item Edinburgh Postnatal Depression Scale. Br J Psychiatry 1987; 150: 782-6.

23. Murray L, Carothers AD. The validation of the Edinburgh Post-natal Depression Scale on a community sample. Br J Psychiatry 1990; 157: 288-90.

24. Dean C, Kendell RE. The symptomatology of puerperal illnesses. Br J Psychiatry 1981; 139: 128-33.

25. Stuart S. Evidence-guided treatment of postpartum depression. Psychiatr Ann 2005; 35: 530-1.

26. Yonkers KA, Wisner KL, Stewart DE, et al. The management of depression during pregnancy: a report from the American Psychiatric Association and the American College of Obstetricians and Gynecologists. Gen Hosp Psychiatry 2009; 31: 403-13.

27. Kopelman R, Stuart S. Psychological treatments for postpartum depression. Psychiatr Ann 2005; 35: 556-65.

28. Abreu A, Stuart S. Medical treatments for postpartum depression. Psychiatr Ann 2005; 35: 568-75.

29. Altshuler LL, Cohen LS, Moline ML, et al. The Expert Consensus Guideline Series. Treatment of depression in women. Postgrad Med 2001; (Spec No): 1-107.

30. O'Hara MW. Social support, life events, and depression during pregnancy and the puerperium. Arch Gen Psychiatry 1986; 43: 569-73.

31. Hobfoll SE, Ritter C, Lavin J, Hulsizer MR, Cameron RP. Depression prevalence and incidence among inner-city pregnant and postpartum women. J Consult Clin Psychol 1995; 63: 445-53.

32. Gjerdingen DK, Froberg DG, Fontaine P. The effects of social support on women's health during pregnancy, labor and delivery, and the postpartum period. Fam Med 1991; 23: 370-5.

33. Kurki T, Hiilesmaa V, Raitasalo R, Mattila H, Ylikorkala O. Depression and anxiety in early pregnancy and risk for preeclampsia. Obstet Gynecol 2002; 95: 487-90.

34. Wisner KL, Sit DKY, Hanusa BH, et al. Major depression and antidepressant treatment: impact on pregnancy and neonatal outcomes. Am J Psychiatry 2009; 166: 557-66.

35. Hofman S, Hatch MC. Depressive symptomatology during pregnancy: evidence for an association with decreased fetal growth in pregnancies of lower social class women. Health Psychol 2000; 19: 535-43.
36. Kelly RH, Danielsen BH, Golding JM, et al. Adequacy of prenatal care among women with psychiatric diagnoses giving birth in California in 1994 and 1995. Psychiatr Serv 1999; 50: 1584-90.

37. Zuckerman B, Amaro H, Bauchner H, Cabral H. Depressive symptoms during pregnancy: relationship to poor health behaviors. Am J Obstet Gynecol 1989; 160: 1107-11.

38. Flynn HA. Epidemiology and phenomenology of postpartum mood disorders. Psychiatr Ann 2005; 35: 544-51.

39. Henshaw C, Foreman D, Cox J. Postnatal blues: a risk factor for postnatal depression. J Psychosom Obstet Gynaecol 2004; 25: 267-72.

40. Gotlib IH, Whiffen VE, Mount JH, Milne K, Cordy NI. Prevalence rates and demographic characteristics associated with pregnancy and the postpartum. J Consult Clin Psychol 1989; 57: 269-74.

41. Kumar R, Robson KM. A prospective study of emotional disorders in childbearing women. Br J Psychiatry 1984; 144: 35-47.

42. Beck CT. Screening methods for postpartum depression. J Obstet Gynecol Neonatal Nurs 1995; 24: 308-12.

43. Troutman BR, Cutrona CE. Nonpsychotic postpartum depression among adolescents. J Abnorm Psychol 1990; 99: 69-78.

44. O'Hara MW, Schlechte JA, Lewis DA, Wright EJ. Prospective study of postpartum blues: biologic and psychosocial factors. Arch Gen Psychiatry 1991; 48: 801-6.

45. Beck CT. Predictors of postpartum depression: an update. Nurs Res 2001; 50: 275-85.

46. O’Hara MW, Schlechte JA, Lewis DA, Varner MW. Controlled prospective study of postpartum mood disorders: psychological, environmental, and hormonal variables. J Abnorm Psychol 1991; 100: 63-73.

47. O'Hara MW. The nature of postpartum depressive disorders. In: Murray L, Cooper PJ, eds. Postpartum depression and child development. New York, NY: The Guilford Press, 1997: 3-31.

48. Cox JL, Murray D, Chapman G. A controlled study of the onset, duration, and prevalence of postnatal depression. Br J Psychiatry 1993; 92: 27-31.

49. Campbell SB, Cohn JF. The timing and chronicity of postpartum depression: implications for infant development. In: Murray L, Cooper PJ eds. Postpartum Depression and Child Development. New York, NY: The Guilford Press, 1997: 3-31.

50. Purdy D, Frank E. Should postpartum mood disorders be given a more prominent or distinct place in the DSM-IV? Depression 1993; 1: 59-70.

51. O'Hara M. Postpartum "blues," depression, and psychosis: a review. J Psychosom Obstet Gynaecol 1987; 7: 205-27.

52. Murray L, Cooper P. Effects of postnatal depression on infant development. Arch Dis Child 1997; 77: 99-101.

53. Paulson JF, Dauber S, Leiferman JA. Individual and combined effects of postpartum depression in mothers and fathers on parenting behavior. Pediatrics 2006; 118: 659-68.

54. Gotlib IH, Whiffen VE, Wallace PM, Mount JH. Prospective investigation of postpartum depression: factors involved in onset and recovery. J Abnorm Psychol 1991; 100: 122-32.

55. Appleby L. Suicide during pregnancy and in the first postnatal year. $\mathrm{Br}$ Med J 1991; 302: 137-40.

56. Berggren-Clive K. Out of the darkness and into the light: women's experiences with depression after childbirth. Can J Commun Ment Health 1998; 17: 103-20.

57. Oates M. Deaths from suicide and other psychiatric causes. Why mothers die. Report on confidential enquiries into maternal deaths in the United Kingdom. Confidential Enquiry into Maternal and Child Health (CEMACH). 2004. [Available from: www.cemach.org.uk/ publications/WMD2000_2002/content.htm] Retrieved 26 April 2005.

58. Sichel DA, Cohen LS, Robertson LM, Ruttenberg A, Rosenbaum JF. Prophylactic estrogen in recurrent postpartum affective disorder. Biol Psychiatry 1995; 38: 814-18.

59. Klier CM, Muzik M, Dervic K, et al. The role of estrogen and progesterone in depression after birth. J Psychiatr Res 2007; 41: 273-9.

60. Bloch M, Schmidt PJ, Danaceau M, et al. Effects of gonadal steroids in women with a history of postpartum depression. Am J Psychiatry 2000; 157: 924-30.

61. Bloch M, Daly RC, Rubinow DR. Endocrine factors in the etiology of postpartum depression. Compr Psychiatry 2003; 44: 234-46. 
62. Pearlstein TB, Frank E, Rivera-Tovar A, Thoft JS. Prevalence of axis I and axis II disorders in women with late luteal phase dysphoric disorder. J Affect Disord 1990; 20: 129-34.

63. Sugawara M, Toda MA, Shima S, Mukai T. Premenstrual mood changes and maternal mental health in pregnancy and the postpartum period. J Clin Psychol 1997; 53: 225-32.

64. Harris B, Lovett L, Newcombe RG, et al. Maternity blues and major endocrine changes: cardiff puerperal mood and hormone study II. BMJ 1994; 308: 949-53.

65. Owens MJ, Nemeroff CB. Role of serotonin in the pathophysiology of depression: focus on the serotonin transporter. Clin Chem 1994; 40: 288-95.

66. Rubinow DR, Schmidt PJ, Roca CA. Estrogen-serotonin interactions: implications for affective regulation. Biol Psychiatry 1998; 44: 839-50.

67. Wisner KL, Perel JM, Peindl KS, et al. Prevention of postpartum depression: a pilot randomized clinical trial. Am J Psychiatry 2004; 161: 1290-2.

68. Henderson AF, Gregoire AJ, Kumar RD, Studd JW. Treatment of severe postnatal depression with oestradiol skin patches. Lancet 1991; 338: 816-17.

69. Gregoire AJP, Kumar R, Everitt B, Henderson AF, Studd JW. Transdermal estrogen for treatment of severe postnatal depression. Lancet 1996; 347: 930-3.

70. Kendell RE, Chalmers JC, Platz C. Epidemiology of puerperal psychoses. Br J Psychiatry 1987; 150: 662-73.

71. Kumar R, Marks M, Platz C, Yoshida K. Clinical survey of a psychiatric mother and baby unit: characteristics of 100 consecutive admissions. J Affect Disord 1995; 33: 11-22.

72. Wisner KL, Peindl K, Hanusa BH. Symptomatology of affective and psychotic illnesses related to childbearing. J Affect Disord 1994; 30: 77-87.

73. Sit D, Rothschild AJ, Wisner KL. A review of postpartum psychosis. J Womens Health 2006; 15: 352-68.

74. Brockington I. Postpartum psychiatric disorders. Lancet 2004; 363 : 303-10.

75. Wisner KL, Peindl KS, Hanusa BH. Psychiatric episodes in women with young children. J Affect Disord 1995; 34: 1-11.

76. Jones I, Craddock N. Familiality of the puerperal trigger in bipolar disorder: results of a family study. Am J Psychiatry 2001; 158: 913-17.

77. Cohen LS, Sichel DA, Robertson LM, Heckscher E, Rosenbaum JF. Postpartum prophylaxis for women with bipolar disorder. Am J Psychiatry 1995; 152: 1641-5.

78. Reich T, Winokur G. Postpartum psychosis in patients with manic depressive disease. J Nerv Ment Disord 1970; 151: 60-8.

79. Marks MN, Wieck A, Checkley SA, Kumar R. Life stress and postpartum psychosis: a preliminary report. Br J Psychiatry Suppl 1991: 45-9.

80. Davies A, McIvor RJ, Kumar RC. Impact of childbirth on a series of schizophrenic mothers: a comment on the possible influence of oestrogen on schizophrenia. Schizophr Res 1995; 16: 25-31.

81. Nilsson E, Lichtenstein P, Cnattingius S, Murray RM, Hultman CM. Women with schizophrenia: pregnancy outcome and infant death among their offspring. Schizophr Res 2002; 58: 221-9.

82. Jablensky AV, Morgan V, Zubrick SR, Bower C, Yellachich LA. Pregnancy, delivery, and neonatal complications in a population cohort of women with schizophrenia and major affective disorders. Am J Psychiatry 2005; 162: 79-91.

83. Bourget D, Grace J, Whitehurst L. A review of maternal and paternal filicide. J Am Acad Psychiatry Law 2007; 35: 74-82.

84. Buttolph LH, Holland AD. Obsessive-compulsive disorder in pregnancy and childbirth. Obsessive-Compulsive Disorder: Theory and Management. Chicago, Ill, Yearbook Medical Publishers, 1990: 89-95.

85. Sichel DA, Cohen LS, Dimmock JA, Rosenbaum JF. Postpartum obsessive compulsive disorder: a case series. J Clin Psychiatry 1993; 54: 156-9.

86. Donahue Jennings K, Ross S, Popper S, Elmore M. Thoughts of harming infants in depressed and nondepressed mothers. J Affect Disord 1999; 54: 21-8.

87. Wisner KL, Peindl KS, Gigliotti T, Hanusa BH. Obsessions and compulsions in women with postpartum depression. J Clin Psychiatry 1999; 60: 176-80.
88. Leckman JF, Mayes LC, Feldman R, et al. Early parental preoccupations and behaviors and their possible relationship to the symptoms of obsessive-compulsive disorder. Acta Psychiatr Scand 1999; 100: 1-26.

89. Ross LE, McLean LM. Anxiety disorders during pregnancy and the postpartum period: a systematic review. J Clin Psychiatry 2006; 67: 1285-98.

90. Williams KE, Koran LM. Obsessive-compulsive disorder in pregnancy, the puerperium, and the premenstruum. J Clin Psychiatry 1997; 58: 330-4; quiz 335-6.

91. Neziroglu F, Anemone R, Yaryura-Tobias JA. Onset of obsessivecompulsive disorder in pregnancy. Am J Psychiatry 1992; 149: 947-50.

92. Labad J, Menchón JM, Alonso P, et al. Female reproductive cycle and obsessive-compulsive disorder. J Clin Psychiatry 2005; 66: 428-35.

93. Biegon A, Reches A, Snyder L, McEwen BS. Serotonergic and noradrenergic receptors in the rat brain: modulation by chronic exposure to ovarian hormones. Life Sci 1983; 32: 2015-21.

94. Leckman JF, Goodman WK, North WG, et al. The role of central oxytocin in obsessive compulsive disorder and related normal behavior. Psychoneuroendocrinology 1994; 19: 723-49.

95. Loveland Cook CA, Flick LH, Homan SM, et al. Posttraumatic stress disorder in pregnancy: prevalence, risk factors, and treatment. Obstet Gynecol 2004; 103: 710-17.

96. Wenzel A, Haugen EN, Jackson LC, Brendle JR. Anxiety symptoms and disorders at eight weeks postpartum. J Anxiety Disord 2005; 19: 295-311.

97. Cromptom J. Post-traumatic stress disorder and childbirth. Br J Midwifery 1996; 4: 7-14.

98. Diane H-D, Bartlett TR, Blickman AL, Miles MS. Posttraumatic stress symptoms in mothers of premature infants. J Obstet Gynecol Neonatal Nurs 2003; 32: 161-71.

99. O’Connor TG, Heron J, Glover V; Alspac Study Team. Antenatal anxiety predicts child behavior/emotional problems independently of postnatal depression. J Am Acad Child Adolesc Psychiatry 2002; 41: 1470-7.

100. Wisner K, Zarin D, Holmboe ES, et al. Risk-benefit decision making for treatment of depression during pregnancy. Am J Psychiatry 2000; 157: 1933-40.

101. Sit DKY, Wisner KL. Decision making for postpartum depression treatment. Psychiatr Ann 2005; 35: 577-85.

102. Briggs G, Freeman R, Yaffe S. Drugs in pregnancy and lactation. Philadelphia: Williams and Wilkins.

103. Altshuler LL, Cohen L, Szuba MP, et al. Pharmacologic management of psychiatric illness during pregnancy: dilemmas and guidelines. Am J Psychiatry 1996; 153: 592-606.

104. Stuart S, O’Hara MW. Psychosocial treatments for mood disorders in women. In: Steiner M, Yonkers KA, Eriksson E, eds. Mood Disorders in Women. London: Martin Dunitz, Ltd, 2000: 521-42.

105. Pearlstein T. Perinatal depression: treatment options and dilemmas. J Psychiatry Neurosci 2008; 33: 302-18.

106. Simon GE, Cunninghma ML, Davis RL. Outcomes of prenatal antidepressant exposure. Arch Gen Psychiatry 2002; 159: 2055-61.

107. Kallen B. Neonate characteristics after maternal use of antidepressants in late pregnancy. Arch Pediatr Adolesc Med 2004; 158: 312-16.

108. Cooper WO, Willy ME, Pont SJ, Ray WA. Increasing use of antidepressants in pregnancy. Am J Obstet Gynecol 2007; 196: 544. e1-5.

109. Stowe ZN, Casarella J, Landry J, Nemeroff CB. Sertraline in the treatment of women with postpartum major depression. Depression 1995; 3: 49-55.

110. Bengt AJK, Petra Otterblad O. Maternal use of selective serotonin reuptake inhibitors in early pregnancy and infant congenital malformations. Birth Defects Res A Clin Mol Teratol 2007; 79: 301-8.

111. Alwan S, Reefhuis J, Rasmussen SA, Olney RS, Friedman JM. Use of selective serotonin-reuptake inhibitors in pregnancy and the risk of birth defects. N Engl J Med 2007; 356: 2684-92.

112. Louik C, Lin AE, Werler MM, Hernández-Díaz S, Mitchell AA. Firsttrimester use of selective serotonin-reuptake inhibitors and the risk of birth defects. N Engl J Med 2007; 356: 2675-83.

113. Pedersen LH, Henriksen TB, Vestergaard M, Olsen J, Bech BH. Selective serotonin reuptake inhibitors in pregnancy and congenital malformations: population based cohort study. BMJ 2009; 339: b3569. 
114. GlaxoSmithKline. Use of paxil or paxil CR during pregnancy. [Available from: http://US.GSK.COM/DOCS-PDF/MEDIA-news/mi_letter_paroxetine_pregnancy.pdf] Retrieved 9 June 2009.

115. Einarson A, Pistelli A, DeSantis M, et al. Evaluation of the risk of congenital cardiovascular defects associated with use of paroxetine during pregnancy. Am J Psychiatry 2008.

116. Chambers CD, Hernandez-Diaz S, Van Marter LJ, et al. Selective serotonin-reuptake inhibitors and risk of persistent pulmonary hypertension of the newborn. N Engl J Med 2006; 354: 579-87.

117. Moses-Kolko EL, Bogen D, Perel J, et al. Neonatal signs after late in utero exposure to serotonin reuptake inhibitors: literature review and implications for clinical applications. JAMA 2005; 293: 2372-83.

118. American PA. Practice guidelines for the treatment of patients with major depression. Am J Psychiatry 2000; 157: 1-45.

119. Djulus J, Koren G, Einarson TR, et al. Exposure to mirtazapine during pregnancy: a prospective, comparative study of birth outcomes. J Clin Psychiatry 2006; 67: 1280-4.

120. Cole J, Modell JG, Haight BR, et al. Bupropion in pregnancy and the prevalence of congenital malformations. Pharmacoepidemiol Drug Saf 2007; 16: 474-84.

121. Chun-Fai-Chan B, Koren G, Fayez I, et al. Pregnancy outcome of women exposed to bupropion during pregnancy: a prospective comparative study. Am J Obstet Gynecol 2005; 192: 932-6.

122. Einarson A, Fatoye B, Sarkar M, et al. Pregnancy outcome following gestational exposure to venlafaxine: a multicenter prospective controlled study. Am J Psychiatry 2001; 158: 1728-30.

123. Einarson A, Bonari L, Voyer-Lavigne S, et al. A multicentre prospective study to determine the safety of trazodone and nefazodone use during pregnancy. Can J Psychiatry 2003; 48: 106.

124. Gracious BL, Wisner KL. Phenelzine use throughout pregnancy and the puerperium: case report, review of the literature, and management recommendations. Depress Anxiety 1997; 6: 124-8.

125. Zlotnick C, Miller IW, Pearlstein T, Howard M, Sweeney P. A preventive intervention for pregnant women on public assistance at risk for postpartum depression. Am J Psychiatry 2006; 163: 1443-5.

126. Wisner KL, Perel JM. Antidepressant treatment during breast-feeding. American Journal of Psychiatry 1996; 153: 1132-1137.

127. Weissman AM, Levy BT, Hartz AJ, et al. Pooled analysis of antidepressant levels in lactating mothers, breast milk, and nursing infants. Am J Psychiatry 2004; 161: 1066-78.

128. Gentile S. SSRIs in pregnancy and lactation: emphasis on neurodevelopmental outcome. CNS Drugs 2005; 19: 623-33.
129. Yonkers KA, Wisner KL, Stowe Z, et al. Management of bipolar disorder during pregnancy and the postpartum period. Am J Psychiatry 2004; 161: $608-20$.

130. Viguera AC, Whitfield T, Baldessarini RJ, et al. Risk of recurrence in women with bipolar disorder during pregnancy: prospective study of mood stabilizer discontinuation. Am J Psychiatry 2007; 164: 1817-24.

131. Wehr TA. Sleep loss: a preventable cause of mania and other excited states. J Clin Psychiatry 1989; 50(Suppl): 8-16.

132. Rane A, Tomson G, Bjarke B. Effects of maternal lithium therapy in a newborn infant. J Pediatr 1978; 93: 296-7.

133. Arnon RG, Marin-Garcia J, Peeden JN. Tricuspid valve regurgitation and lithium carbonate toxicity in a newborn infant. Am J Dis Child 1981; 135: 941-3.

134. Newport DJ, Viguera AC, Beach AJ, et al. Lithium placental passage and obstetrical outcome: implications for clinical management during late pregnancy. Am J Psychiatry 2005; 162: 2162-70.

135. Pariser SF, Nasrallah HA, Gardner DK. Postpartum mood disorders: clinical perspectives. J Womens Health 1997; 6: 421-34.

136. Schou M, Goldfield MD, Weinstein MR, Villeneuve A. Lithium and pregnancy, I: report from the register of lithium babies. Br Med J 1973; 2: $135-6$.

137. Schou M, Amdisen A, Steenstrup OR. Lithium and pregnancy. II. Hazards to women given lithium during pregnancy and delivery. Br Med J 1973; 2: 137-8.

138. Schou M, Amdisen A. Lithium and pregnancy. 3. Lithium ingestion by children breast-fed by women on lithium treatment. Br Med J 1973; 2: 138.

139. Schou M. What ever happened to the lithium babies? A follow-up study of children born without malformations. Acta Psychiatr Scandinavia 1976; 54: 193-7.

140. Committee on Drugs. American Academy of Pediatrics. The transfer of drugs and other chemicals into human milk. Pediatrics 1994; 93: 137.

141. Centers for Disease Control. United States Department of Health and Human Services. Valproic acid and spina bifida: a preliminary report. MMWR 1982; 31: 565-6.

142. Lammer EJ, Sever LE, Oakley GP Jr. Teratogen update: valproic acid. Teratology 1987; 35: 465-73.

143. Cunnington $\mathrm{M}$, Tennis $\mathrm{P}$, et al. Lamotrigine and the risk of malformations in pregnancy. Neurology 2005; 64: 955-60.

144. Van Waes A, Van de Velde E. Safety evaluation of haloperidol in the treatment of hyperemesis gravidum. J Clin Pharmacol 1969; 9: 224-37.

145. Miklovich L, van den Berg BJ. An evaluation of the teratogenicity of certain antinauseant drugs. Am J Obstet Gynecol 1976; 125: 244-8. 


\section{Renal diseases in pregnancy Dana Negoi and Khaled Mohamed}

\section{INTRODUCTION}

Caring for pregnant women with renal disease is usually a difficult task for both the obstetrician and the nephrologist. These pregnancies are viewed as high risk, due to concerns of increased maternal and perinatal morbidity and mortality. It is estimated that at least $4 \%$ of the women in the childbearing age carry a diagnosis of chronic kidney disease (CKD) (1) of different etiologies: diabetic nephropathy, lupus nephritis, polycystic kidney disease, reflux nephropathy, or different forms of glomerulonephritis. Although the frequency of conception decreases as the impairment in kidney function progresses, with levels as low as $0.5 \%$ per year in dialysis patients (2), it is not unheard of patients on either hemodialysis or peritoneal dialysis (PD) to get pregnant. When end-stage renal disease patients get a kidney transplant, the return of fertility is usually the rule and caring for these pregnancies is even more challenging due to the continued need for immunosuppression, with its associated fetal risks for congenital anomalies, and small-for-gestational-age babies (2).

This chapter is intended to provide information needed to take care of these patients, and will start by reviewing normal renal anatomy and physiology during pregnancy. The interplay between CKD, acute renal failure (ARF) or acute kidney injury, hypertension (HTN) and pregnancy will be further explored.

\section{RENAL ANATOMY AND PHYSIOLOGY DURING PREGNANCY}

There are marked changes in the renal hemodynamics and function during normal pregnancy, associated with significant anatomical changes such as increased renal size and dilatation of the collecting system. They occur in concert with multiple other physiologic alterations that take place during pregnancy, including significant expansion of the blood volume, increased cardiac output, and enhanced blood flow in several regions of the body (3) (Table 1).

Blood volume can increase almost $50 \%$ in a normal pregnancy, compared with the nonpregnant state. This represents roughly $1500 \mathrm{~mL}$ increase, of which about $500 \mathrm{~mL}$ is in red blood cell volume. Volume expansion starts in the first trimester, but is more marked in the second trimester and the rise is much less in late pregnancy. It is believed to be caused by the increased metabolic demands of the enlarged uterus with its increased vascularity, placenta and fetus, the need to protect the fetus against the harmful effects of decreased venous return when in supine and upright position, and the need to protect the mother against blood loss during delivery (3). Aldosterone levels are elevated, but it is not known whether this contributes to hypervolemia. It is accompanied by vasodilatation, especially in the pelvis. There are no clinical signs of volume overload in a normal pregnancy (3). Expansion of the blood volume may be greater in multiple gestations or smaller in pre-eclampsia (4).

Other cardiovascular changes include increase in cardiac output and significant decrease in systemic vascular resistance with resultant decrease in blood pressure in normal pregnancy (4).

\section{Dilatation of the Collecting System}

Dilatation of the collecting system seems to be the result of a combined progesterone-induced ureteral smooth muscle relaxation and compression of the ureter by the enlarged uterus and iliac vessels. A pelvicaliceal diameter of up to $2 \mathrm{~cm}$ is usually allowed as "physiologic hydronephrosis" of pregnancy. Hydronephrosis is most commonly seen on the right, due to anatomical reasons $(5,6)$. By ultrasound criteria, hydronephrosis is defined as at least $5 \mathrm{~mm}$ (5) dilatation of the calyceal diameter. This dilatation of the urinary collecting system starts developing during the second trimester, is seen only above the linea terminalis, and resolves in few weeks after delivery (6). Symptomatic hydronephrosis is not a frequent event, occurring in about $0.2 \%$ to $3 \%$ of pregnant women (7), but those who are symptomatic frequently have associated urinary tract infections (6).

The dilated collecting system can retain some of the urine meant to be saved for the twenty-four hour urine collections. As a result, creatinine clearance may be underestimated, unless the collection is done carefully and the patient is volume repleted before the test. If in the last few hours of the collection the patient is maintained in the left lateral recumbent position, the chances of obtaining a full collection are also increased.

\section{Increase in Renal Volume}

During pregnancy, the kidneys increase in volume, probably due to increase in blood flow and interstitial volume. A study of humans that excluded dilatation of the renal pelvicaliceal system demonstrated that renal volume can increase by $30 \%$ in normal pregnancy (8).

\section{GFR}

Renal hemodynamics undergo significant changes: renal plasma flow (RPF) increases by $50 \%$ to $80 \%$, while GFR increases by $50 \%$ (9). Systemic and renal hemodynamics adaptations occur early in pregnancy, before complete placentation takes place, demonstrating that they are dependent mainly on maternal factors for their development (10). They are also present in pseudopregnancy, a condition where the fetoplacental unit is absent (11), and different studies have suggested roles for relaxin, an ovarian hormone, and also for nitric oxide synthase, prostaglandins, and endothelin (9).

The physiologic increase in GFR is the result of expansion in the renal plasma blood flow, associated with characteristic 
renal vasodilatation resulting from both afferent and efferent arteriolar relaxation (11). The parallel reduction in preglomerular (afferent) and postglomerular (efferent) arteriolar resistance explains why single nephron GFR increases without an increase in the glomerular capillary pressure (11), which is an important phenomenon, and explains further why normal pregnancy is not followed by loss of kidney function. This last aspect was demonstrated in animal studies on rats (12), where it was shown that repetitive pregnancies were not associated with increased risk of deterioration of kidney function. Rats provide a good animal model to study this particular problem since they have a gestation of only 22 days. After six repetitive pregnancies,

$\begin{array}{ll}\text { Table } 1 \text { Physiologic and Anatomic Changes in Normal } \\ \text { Pregnancy } \\ \text { Blood volume } & \text { Increased } 50 \%(1500 \mathrm{cc}) \\ \text { Cardiac output } & \text { Mild increase } \\ \text { Net sodium retention } & 900 \text { mmol } \\ \text { Systemic vascular resistance } & \text { Decreased } \\ \text { Systemic blood pressure } & \text { Decreased } \\ \text { Systolic } & 15 \text { mmHg } \\ \text { Diastolic } & 10 \text { mmHg } \\ \text { RPF } & \text { Increased } 50-80 \% \\ \text { GFR } & \text { Increased } 50 \% \\ \text { Glomerular capillary pressure } & \text { Unchanged } \\ \text { Serum osmolality } & \text { Decreased } 10 \text { mmOsm } \\ \text { Urine concentration and } & \text { No change } \\ \text { dilution } & \\ \text { Proteinuria } & 200-300 \text { mg/day } \\ \text { Glucosuria } & \text { Present in the absence of } \\ \text { Serum creatinine, uric acid, } & \text { diabetes } \\ \text { BUN } & \text { Decreased } \\ \text { Serum cystatin C } & \text { Increased } \\ \text { Acid-base } & \text { Compensated respiratory } \\ \text { Kidney volume } & \text { alkalosis } \\ \text { Urinary collecting system } & \text { Increased } 30 \% \\ \text { Abbreviations: RPF, renal plasma flow; } & \text { GFR, glomerular filtration rate; } \\ \text { BUN, blood urea nitrogen. } & \\ \end{array}$

the study rats had no renal changes compared with neverpregnant controls (12).

GFR increases to a maximum of about $50 \%$ by midpregnancy (13), remains elevated during the pregnancy, and returns to baseline values 3 months after delivery (1), while RPF starts to fall toward the end of pregnancy (13) (Fig. 1). The increase in GFR is not accompanied by an increase in the production of urea and creatinine: their serum concentration will decrease in pregnancy. Serum creatinine concentration falls by about $0.4 \mathrm{mg} / \mathrm{dL}$, so the normal pregnancy range is 0.4 to $0.8 \mathrm{mg} / \mathrm{dL}$ (14).

Estimation of GFR using the modification of diet in renal disease (MDRD) formula has been recently evaluated in pregnant women. In a study that looked at three groups of pregnant women-healthy pregnant volunteers, women with pre-eclampsia, and pregnant women with previously known CKD-the MDRD formula significantly underestimated kidney function although performed better in pre-eclampsia and CKD group (15). Alper et al. found that the MDRD formula, along with the Cockcroft-Gault formula, was inaccurate in predicting GFR in pre-eclamptic patients (16).

Cystatin C is a low-molecular weight (MW) protein, which has been recently studied in the general population in an attempt to find a better endogenous marker that could be used for more accurate estimation of GFR (17). Studies have found that while serum creatinine levels decrease in pregnancy, cystatin $\mathrm{C}$ levels were higher for pregnant women as compared with healthy nonpregnant women (18) and also significantly higher in twin as compared with singleton pregnancies (18). Cystatin $\mathrm{C}$ levels increase during pregnancy in relationship to gestational age, being highest in the third trimester (19). Its actual role in pregnancy is very interesting, as cystatin $\mathrm{C}$ is a member of the cystatin superfamily of cysteine protease inhibitors. Proteinases and their inhibitors regulate the trophoblast invasion, and the serum concentration of cystatin C has been found in several studies to be increased not only in late pregnancy but also in pre-eclampsia (18-20). Based on the available data, it is unlikely that cystatin $\mathrm{C}$ will have any utility in monitoring kidney function in pregnancy but might prove useful in the early detection of patients who will develop pre-eclampsia (21).

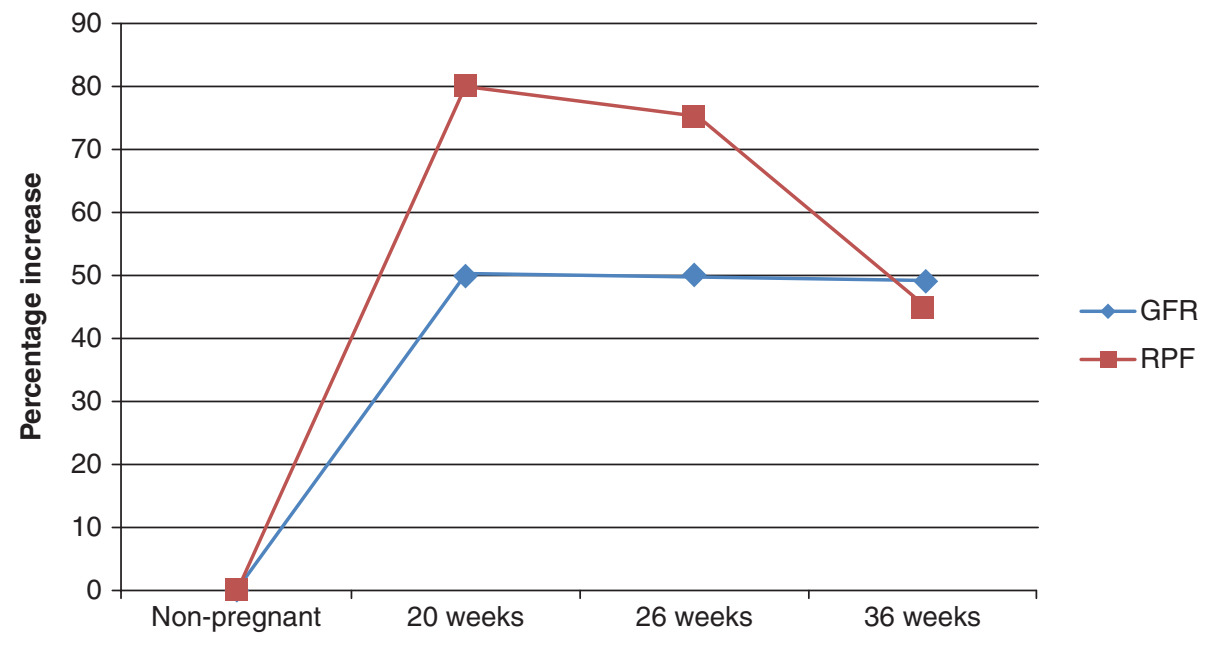

Figure 1 Renal hemodynamics during normal pregnancy. Source: From Ref. 13. Abbreviations: GFR, glomerular filtration rate; RPF, renal plasma flow. 


\section{Renal Tubular Function in Pregnancy}

\section{Uric Acid}

Uric acid after being freely filtered at the glomerular level is extensively reabsorbed, mainly in the proximal tubule, so that only $10 \%$ of the filtered load is excreted (13). Plasma concentration decreases early on in pregnancy by at least $25 \%$, largely due to the significant rise in GFR, but also due to decrease in tubular reabsorption (13). In late pregnancy, the fractional excretion of uric acid decreases and the plasma levels trend toward nonpregnant levels (13).

\section{Glucose}

The presence of glucose in the urine may rather be a sign of tubular dysfunction than diabetes mellitus. Proximal and distal tubules have a decreased capacity to reabsorb filtered glucose due to an unknown mechanism. The increase in GFR causes an increase in filtered load of glucose on one hand. On the other hand, the increase in plasma volume may inhibit the tubular reabsorption of sodium and hence glucose (13).

\section{Sodium}

There is a gradual retention of sodium during pregnancy, reaching $900 \mathrm{mmol}$, due to a positive sodium balance and an increase in tubular reabsorption of sodium (22).

\section{Water Metabolism}

Although the kidneys' abilities to maximally dilute and concentrate urine are intact, the serum sodium concentration and serum osmolality concentration are about $5 \mathrm{mEq} / \mathrm{L}$ and $10 \mathrm{mOsm} / \mathrm{kg}$, respectively, lower throughout most of gestation than in the nonpregnant state. This is mainly due to a decrease in osmotic threshold for thirst and arginine vasopressin (AVP) release (23). In addition, a transient increase in AVP clearance during mid-gestation may produce a diuresis, the so-called transient diabetes insipidus of pregnancy.

\section{Proteins}

Proteins present in the urine are a combination of plasma proteins that are normally filtered at the glomerular capillary level, and escape reabsorption in the proximal tubule, and proteins produced by the urogenital tract: $40 \%$ are represented by albumin, $15 \%$ to $20 \%$ are immunoglobulins, and $55 \%$ to $60 \%$ is Tamm-Horsfall glycoprotein, the latter being of tubular origin (24).

Albumin, MW 69,000 daltons (D), is a plasma protein usually filtered to a limited degree due to its $\mathrm{MW}$ and anionic charge. Below MW $60,000 \mathrm{D}$, there is a progressive increase in filtration at the glomerular level so that in healthy, nonpregnant subjects, low-MW proteins and only small amounts of albumin are normally filtered, then almost entirely reabsorbed and catabolized in the proximal tubule.

Normal urinary protein excretion averages 20 to $80 \mathrm{mg} /$ day in normal nonpregnant women, with an accepted upper limit of normal of $150 \mathrm{mg} /$ day. Albuminuria is considered to be a more sensitive marker for glomerular damage and CKDs than total proteinuria (25), and the upper limit of normal for daily albuminuria is considered to be $30 \mathrm{mg}$ (26).

In pregnancy, proteinuria is considered to be abnormal if exceeds $300 \mathrm{mg} /$ day (27). Studies that have looked at proteinuria and albuminuria in normal pregnancy have found a maximum total daily urinary protein excretion of $260 \mathrm{mg}$ /day (28), or even a little lower, at $200 \mathrm{mg}$ /day (29), with an absolute amount of albumin, which does not seem to be higher than that in normal, nonpregnant state (30). Higby (28) found that total daily albumin excretion in normal pregnancy was $29 \mathrm{mg}$ and Beetham (30) showed that there is a tendency for albuminuria to increase as the normal pregnancy progresses, being significantly higher in the third trimester compared with the first, but the absolute amount will remain less than $30 \mathrm{mg} /$ day. The available evidence suggests that the diminished tubular reabsorption of low-MW proteins play a significant role for the increase in proteinuria during normal pregnancy $(30,31)$.

The gold standard for total protein quantification is the 24-hour urine collection with its well-known limitations: can be inaccurate due to undercollection, is cumbersome for the patient, and the results are delayed pending collection. Screening for proteinuria using the dipstick (most sensitive to albumin) is fast and convenient in the clinic, where a random urine sample can be used. Unfortunately, the accuracy of the dipstick urinalysis in predicting significant proteinuria is poor (32), but the use of a protein to creatinine ratio in a spot urine seems to be a practical and reasonable choice (14).

\section{Acid-Base Regulation}

Plasma bicarbonate decreases during pregnancy, ranging from 18 to $22 \mathrm{mEq} / \mathrm{L}$. It is believed that progesterone-induced hyperventilation occurs during pregnancy, leading to respiratory alkalosis with decrease in $\mathrm{PCO}_{2}$ close to $27 \mathrm{mmHg}$ and increase in $\mathrm{pH}$ to about 7.44. There is no apparent defect in renal handling of acid (33).

\section{PREGNANCY IN WOMEN WITH CKD}

The influence of pregnancy on the course of renal disease and the effect of renal disease on the outcome of pregnancy are important concerns in treating and advising patients with renal disease. The available published data on pregnancy in women with CKD have limitations due to small sample size, single-center and retrospective studies, using varied definitions of kidney disease, and adverse maternal and fetal outcomes $(1,14,34)$. Given the low prevalence of CKD in women of childbearing age and difficulties related to randomization, it is easy to understand the complexity of conducting good quality trials in this population. In spite of these imperfections, factors that have consistently emerged, and are widely agreed upon, as important in influencing both maternal and fetal outcomes are advanced CKD, preexistent HTN, and proteinuria. Pregnant women who have normal or only mildly impaired kidney function, normal blood pressure, and little or no proteinuria before conception are expected to have an uneventful pregnancy, without maternal complications (worsening kidney function, HTN, and preeclampsia) and without fetal complications (prematurity, 
small-for-gestational-age, fetal loss, etc.). On the other hand, those who have significantly impaired kidney function and/or HTN and proteinuria before conception are at increased risk for maternal and fetal complications. With respect to the type of the renal disease, the current consensus is that in primary nephropathies (IgA nephropathy, focal and segmental glomerulosclerosis, membranous glomerulonephritis, etc.), the abovementioned factors dictate the maternal and fetal outcomes irrespective of the type of the disease; in systemic diseases with renal involvement such as diabetes mellitus (DM) and systemic lupus erythematosus (SLE), there are additional diseasespecific features that will affect prognosis $(1,14,34)$.

\section{Effects of Pregnancy on Kidney Disease}

In a study (35) involving CKD patients with serum creatinine levels lower than $1.4 \mathrm{mg} / \mathrm{dL}$, it was shown that GFR, creatinine clearance, and effective RPF increase during gestation, similar to the response observed in women without underlying renal disease. It is then expected to see a decline in serum creatinine levels in these patients during pregnancy and a return to baseline values after delivery. Women with more advanced renal dysfunction will not always have the physiologic increase in GFR seen in women without or only with mild renal insufficiency, and their pregnancies are more frequently complicated with worsening kidney function $(2,11,36)$. A potential mechanism for inducing permanent kidney damage during pregnancy could be an increase in intraglomerular pressure, but animal studies (11) have showed that even in the presence of preexistent renal damage, pregnancy does not lead to glomerular HTN.

Proteinuria usually increases during pregnancy, then declines after delivery (11). Among others, Katz et al. (35) analyzed 121 pregnancies in 89 women with previously known CKD. De novo or increased proteinuria was noted in almost $50 \%$ of the pregnancies, and $60 \%$ of those who were proteinuric during pregnancy had regression of proteinuria at follow-up.

When counseling patients regarding the effects of pregnancy on their kidney disease, prognosis is based, in general, on three categories of renal function, which have been described in the literature as mild, moderate, and severe (1).

Mild Decrease in Kidney Function (Serum Creatinine Lower Than $1.4 \mathrm{mg} / \mathrm{dL}$ ) Before Conception

There is a general consensus that pregnancy usually does not modify the natural course of kidney disease in this setting. However, some patients with normal kidney function can have a temporary decline in renal function during pregnancy $(35,37)$. Rarely, there can be an unexpected and irreversible loss of kidney function during pregnancy $(38,39)$, which usually happens in more aggressive histologic forms of glomerular and/or tubulointerstitial disease (34). Jungers et al. (37) have shown that in women with reflux nephropathy, pregnancy had no negative impact on their kidney disease if they had normal kidney function at conception. Multiple other authors report similar positive results of pregnancies in women with serum creatinine less than $1.4 \mathrm{mg} / \mathrm{dL}(35,40,41)$.

The presence of HTN and proteinuria becomes major predictive factors for worsening kidney function in this clinical scenario.
Preexistent or untreated HTN has been found to be an independent risk factor for loss of renal function in few studies $(41,42)$. The role of proteinuria was convincingly demonstrated by Hemmelder (43) in a retrospective analysis of 30 pregnancies in 19 patients with primary glomerular disease and normal kidney function and blood pressure at conception. This group was compared with a control group of 31 patients who did not become pregnant after the diagnosis of kidney disease. Patients were followed for 11 years, and at the end of follow-up, patients who became pregnant had higher serum creatinine levels than controls. Pregnancy and initial proteinuria were found to be independently related with the rate of loss of kidney function. Of the 30 pregnancies that were preceded and complicated with nephrotic-range proteinuria, 10 were followed by an accelerated rate of loss of kidney function compared with the other 20 pregnancies.

Jungers (41) also found that in patients with primary chronic glomerulonephritis and normal kidney function, proteinuria had a predictive role for the progression of kidney disease on pregnancy, although not as strong as HTN.

\section{Moderate Decrease in Kidney Function (Serum Creatinine $1.4-1.8 \mathrm{mg} / \mathrm{dL}$ )}

Moderate decrease in kidney function (serum creatinine 1.4$1.8 \mathrm{mg} / \mathrm{dL}$ ) is associated with a higher risk of further decline during pregnancy. In a retrospective review (37), 19\% of the patients with moderate decline in kidney function had irreversible further loss of maternal renal function. Proteinuria and HTN are more frequent in this population and additive risk factors for further decline in renal function $(1,44)$.

\section{Severe Decrease in Kidney Function (Serum Creatinine Greater Than $1.9 \mathrm{mg} / \mathrm{dL}$ )}

Severe decrease in kidney function (serum creatinine greater than $1.9 \mathrm{mg} / \mathrm{dL}$ ) has been shown to be a higher risk factor for renal function loss during pregnancy. The landmark study by Jones and Hayslett (44), published in 1996, showed that the risk of accelerated progression to end-stage renal disease was the highest when the serum creatinine concentration was above $2 \mathrm{mg} / \mathrm{dL}$ at the beginning of the pregnancy. With an initial serum creatinine of at least $1.4 \mathrm{mg} / \mathrm{dL}$ in 67 women and 82 pregnancies, they reported a pregnancy-related loss in renal function in $43 \%$ of the cases. The risk is even higher when uncontrolled HTN is present (34).

The only prospective study that analyzed the outcomes of pregnancies in patients with serum creatinine levels greater than $1.4 \mathrm{mg} / \mathrm{dL}$ (36) found an accelerated loss of kidney function only in the subgroup of patients with both estimated GFR $<40 \mathrm{~mL} / \mathrm{min} / 1.73 \mathrm{~m}^{2}$ and proteinuria $>1 \mathrm{~g} /$ day before pregnancy.

Women with serum creatinine levels $>3 \mathrm{mg} / \mathrm{dL}$ are usually infertile (45) due to amenorrhea or anovulatory menstrual cycles.

Based on the available information, women with CKD who contemplate to become pregnant should be advised about the possible risk of accelerated loss of kidney function if serum creatinine level is greater than $1.4 \mathrm{mg} / \mathrm{dL}$ before pregnancy. Proteinuria exceeding $1 \mathrm{~g} /$ day portends a higher risk for accelerated loss of kidney function and, in some cases, even a twofold higher incidence of end-stage renal 
disease (1). HTN control should be a major goal of pregnancy. Even if they are normotensive before pregnancy, these patients are at higher risk of becoming hypertensive during pregnancy $(1,35)$.

Pregnancy should probably be discouraged in patients with multiple risk factors: moderate to severe kidney disease, proteinuria, and HTN.

\section{Effect of Kidney Disease on Fetal Outcomes}

Mild Decrease in Kidney Function (Serum Creatinine Lower Than $1.4 \mathrm{mg} / \mathrm{dL}$ ) Before Conception

Katz (35) observed in a retrospective study that there were more fetal complications in patients with serum creatinine lower than $1.4 \mathrm{mg} / \mathrm{dL}$ than in general population, but at acceptable levels. Ninety-four percent of the pregnancies resulted in live births. Abe (40) has also noted same positive results in women with GFR $>70 \mathrm{~mL} / \mathrm{min}$ and blood pressure less than 140/90 mmHg. In women with autosomal dominant polycystic kidney disease (42) and normal kidney function before conception (serum creatinine less than $1.2 \mathrm{mg} / \mathrm{dL}$ ), preexistent HTN was the most important risk factor for the development of poor fetal outcomes, including prematurity and fetal loss.

Munkhaugen (46) found in a very recent study that women with estimated GFR 60 to $89 \mathrm{~mL} / \mathrm{min} / 1.73 \mathrm{~m}^{2}$ were not at increased risk of pre-eclampsia, small-for-gestational-age, or preterm delivery unless they were also hypertensive.

\section{Moderate and Severe Decrease in Kidney Function (Serum Creatinine $>1.4 \mathrm{mg} / \mathrm{dL}$ )}

Moderate and severe decrease in kidney function (serum creatinine $>1.4 \mathrm{mg} / \mathrm{dL}$ ) translates in higher risk for fetal loss, prematurity, and small birth weight. Jones and Hayslett (44) reported moderately reduced fetal loss (93\% survival rate), but significantly increased preterm delivery (59\% compared with the expected rate of $10 \%$ in general population) and growth retardation (37\% of infants were below the 10th percentile for birth weight).

Abe (40) also found that in women with moderately decreased kidney function, the rate of live births was relatively high, except for cases where HTN was present before pregnancy and/or was not controlled during pregnancy.

Imbasciati (36) found that in women with GFR $<40 \mathrm{~mL} / \mathrm{min} /$ $1.73 \mathrm{~m}^{2}$ and proteinuria $>1 \mathrm{~g} /$ day, pregnancy was associated with poor fetal outcomes: increased perinatal death and low birth weight.

Jungers (34) also found a high risk of fetal death when serum creatinine was 1.8 to $2.0 \mathrm{mg} / \mathrm{dL}$. HTN and nephroticrange proteinuria, when present early in pregnancy, were found to be additive risk factors for poor fetal prognosis.

In summary, when counseling women with CKD regarding fetal outcomes, the following recommendations could be made:

- Fetal survival rate seems to higher than $90 \%$, but decreases when uncontrolled HTN and/or proteinuria are also present.

- Preterm delivery and growth retardation are more commonly found in women with at least moderate impairment in kidney function.
- HTN and proteinuria are additional risk for poor outcomes in this category, too.

\section{MANAGEMENT}

Over the years, the benefits of progress in obstetrics in neonatal care, including improved access to medical care, improved technology for fetal surveillance and antihypertensive medications available to treat high blood pressure in pregnant women, have translated in improved maternal and fetal outcomes in pregnancies that occur in women afflicted with CKD and HTN $(37,42)$.

Pregnant women with CKD should be followed in collaboration with a nephrologist and with an obstetrician familiar with the effects of renal disease on pregnancy. Their prenatal visits should occur more frequently, ideally every 2 weeks until the third trimester and then weekly. Maternal renal function should be followed very closely, at least at monthly intervals, and asymptomatic bacteriuria should be promptly treated. Likewise, fetal surveillance with ultrasound and fetal heart monitoring should be done very closely. Maternal HTN requires special attention and needs to be treated efficiently. Preterm delivery needs to be offered when maternal renal function deteriorated rapidly, if fetal distress occurs, or pre-eclampsia is severe.

\section{LUPUS NEPHRITIS}

It remains questionable whether pregnancy exacerbates lupus. Earlier studies suggest that pregnant women with SLE clinically flare their disease three times more frequently during pregnancy and seven times more frequently postpartum compared with nonpregnant controls (47). More recently, it became apparent that the incidence of a lupus flares during pregnancy is more likely correlated with the disease activity before conception. Hayslett (48) showed that exacerbations or relapse occur in a wide range of patients during pregnancy or in the immediate period after delivery, namely in $25 \%$ to $60 \%$ of pregnancies. Relapses or exacerbations occur only in onethird of the patients if they had no signs of clinical activity 6 months before conception, as opposed to two-thirds of the patients when they had signs of clinical activity at onset of pregnancy. Activity of the disease also influenced fetal survival, which was $85 \%$ to $95 \%$ in patients with inactive disease and $50 \%$ to $80 \%$ in patients with active disease at conception. The presence of circulating anticardiolipin antibodies and lupus anticoagulant factors appears to worsen the perinatal outcomes. Fetal loss rate increases from $25 \%$ to about $60 \%$, and the risk of maternal thromboembolism increases to about $50 \%(49,50)$.

The risk of prematurity and pre-eclampsia is increased even if patients have stable, but established renal disease and controlled HTN in the 6 months prior to conception (51). The risk of pre-eclampsia is high especially if the women have a history of antiphospholipid syndrome (52).

Flares cannot be prevented with routine increase in dose or prophylactic use of steroids, which are not recommended for this purpose either before pregnancy or after delivery (53). Therefore, women with lupus nephritis should be encouraged to postpone pregnancy for at least 6 months after the last flare. 
The diagnosis of a lupus flare in pregnancy is not always straightforward, and the most challenging scenario is to make a distinction between pre-eclampsia and lupus flare, as they can both present with HTN, proteinuria, thrombocytopenia, and kidney injury (53). A renal biopsy is rarely needed, because a rising anti-dsDNA titer, and low complement levels are in favor of lupus flare, as opposed to hyperuricemia and abnormal liver function tests that favor pre-eclampsia. Flares are more common in women who had more than three flares before pregnancy, and the type of flare follows the previous known pattern of disease specific to the individual patient $(51,53)$.

In pregnant women with active lupus nephritis, the maternal and perinatal outcomes are improved if a remission is achieved. Corticosteroids are the drugs of choice $(51,53)$, given either as an increased oral form or intravenous methylprednisolone $500 \mathrm{mg}$ daily for 3 days. Azathioprine can be used safely in pregnancy and during lactation $(51,53)$. Hydroxychloroquine, often used to prevent flares, seems to be safe for use in pregnancy and should not be stopped in early pregnancy (51-53). Antiphospholipid-positive patients should be started on at least low-dose heparin to prevent thrombosis (52). The use of low-dose aspirin has been advocated for prevention of pre-eclampsia in patients at risk.

\section{DIABETIC NEPHROPATHY}

About $5 \%$ of pregnant women with type $1 \mathrm{DM}$ can have diabetic nephropathy (54). Fortunately, most of them have preserved kidney function and the progression of their diabetic nephropathy does not seem to be affected by pregnancy in this situation. As is true with all patients with moderate to severe $\mathrm{CKD}$, diabetic patients also will have higher risk of irreversible deterioration of kidney function during pregnancy, which has been estimated in some series to be as high as $40 \%$ (55); the risk of HTN and pre-eclampsia are also increased in diabetics. Proteinuria increases during pregnancy, an effect that could also be attributed to the cessation of ACE inhibition therapy due to potential teratogenic effects (54).

Women with diabetic nephropathy often have diabetic retinopathy, which should not go untreated during pregnancy. Diabetic retinopathy can progress during pregnancy and laser treatments are sometimes needed to prevent vision loss (54).

Perinatal outcomes are generally good with tight glycemic and blood pressure control.

Perinatal survival exceeds $95 \%(56,57)$, but there is a high risk of preterm delivery (50\%) and small babies (15\%) (56, $58-60$ ). There is concern that prematurity can predispose the infants of diabetic mothers to long-term morbidity: severe psychomotor retardation and mild developmental retardation have been described in this setting (61).

\section{ARF}

The definition of ARF in pregnancy varies across studies. ARF has been defined as a creatinine level above $0.8 \mathrm{mg} / \mathrm{dL},>25 \%$ decrement in GFR over the level documented before the beginning of pregnancy, or the need for dialysis (62-64). Since the 1960s, there has been a consistent decrease in the incidence of the ARF in pregnancy, which has been attributed to a steady decrease in the septic abortions in most developed countries due to legalization of abortions and due to better access to care $(63,64)$. The incidence of ARF in pregnancy has decreased from $20 \%$ to $40 \%$ in the 1960 s to $2 \%$ to $10 \%$ in the 1980 s (63).

Despite the decreased incidence, the development of ARF in pregnancy remains associated with significant morbidity and mortality.

ARF in pregnancy can be induced by any of the disorders leading to renal failure in the nonpregnant women: volume depletion, nephrotoxins, obstruction, interstitial nephritis, and so on. Pregnancy complications characteristic of each trimester can result in ARF. It is believed that the most common cause of ARF in pregnancy is pregnancy-related HTN $(62,64,65)$.

\section{Pre-eclampsia}

Pre-eclampsia is a disorder specific of pregnancy, complicates around $5 \%$ of all pregnancies (66), and is characterized by new-onset HTN and proteinuria in excess of $300 \mathrm{mg} / \mathrm{day}$, usually after 20 weeks of gestation. Blood vessels throughout the body are affected, and recently identified antiangiogenic substances of placental origin seem to be the cause of the diffuse endothelial dysfunction. The characteristic glomerular lesion, "endotheliosis," appears histologically as endothelial swelling and occlusion of the capillary lumens, and although it is a variant of thrombotic microangiopathy, intraglomerular thrombosis is unusual (66). ARF is rare, and only $1.5 \%$ to $2 \%$ of the pre-eclamptic patients will have impairment of the kidney function (62). Patients with pre-eclampsia have an overall decrease in GFR and RPF, but not significant enough to cause ARF $(62,63)$. They also have vasoconstriction and intravascular volume depletion, this way being at increased risk for the development of ARF when another obstetrical complication like hemorrhage or abruptio placentae is present. Superimposed volume depletion in these clinical scenarios will commonly lead to acute tubular necrosis and in the most severe cases to cortical necrosis (63). Treatment of preeclampsia-related ARF requires delivery of the fetus and placenta (63).

\section{HELLP Syndrome}

HELLP (hemolysis, elevated liver enzymes, low platelets) syndrome occurs in $10 \%$ to $20 \%$ of the cases of severe preeclampsia (67). It has been reported that ARF can occur in $7 \%$ to $36 \%$ of the cases (67). Renal biopsy studies most commonly reported acute tubular necrosis or acute cortical necrosis in more severe cases (68) same as in less severe preeclampsia. Associated thrombotic microangiopathy has also been described, but with acute tubular necrosis as the dominant lesion (69).

\section{Acute Fatty Liver of Pregnancy}

Acute fatty liver of pregnancy (AFLP) is not a common complication of pregnancy, but represents an obstetric emergency of the third trimester $(70,71)$. The patients present with abdominal pain, nausea, vomiting, headache, malaise, anorexia, jaundice, and rarely with hepatic encephalopathy. The etiology could be related to a familial genetic defect in fatty acid metabolism (70). Up to $90 \%$ of the women with AFLP can develop ARF (70). Disseminated intravascular coagulopathy is usually present. Serum transaminases are generally not as 
severely elevated as they are in hepatitis. Most patients with renal failure have evidence of decreased renal perfusion similar to hepatorenal syndrome or acute tubular necrosis. Treatment again involves delivery of the baby.

\section{Thrombotic Microangiopathies}

Thrombotic microangiopathies are thrombotic thrombocytopenic purpura (TTP) and hemolytic-uremic syndrome (HUS), pathologies that are very rarely seen in pregnancy, occurring in less than 1 in 100,000 pregnancies (71). HUS was initially described as occurring in the postpartum period, between 1 day and 3 months after delivery, but it has become clear that it can occur during pregnancy also (70). In patients with a severe form of familial deficiency of ADAMTS13, TTP can also present during pregnancy (72). ADAMTS13 is a metalloprotease, which normally binds the von Willebrand factors secreted by the endothelial cells. When ADAMTS13 is deficient, abnormally large multimers of von Willebrand factors accumulate and trigger platelet adhesion and aggregation inside the blood vessels with microthrombi formation (72). Familial forms of HUS have been also described, with both autosomal dominant and recessive form of inheritance and pregnancy as an eliciting event. A functionally abnormal factor $\mathrm{H}$ protein could be the cause of HUS in the autosomal dominant transmitted familial cases $(72,73)$. Normally functional factor $\mathrm{H}$ protects endothelial cells from the damaging effects of the activated complement (72). The primary pathologic disorder in both TTP and HUS will be systemic or intrarenal microvascular aggregation of platelets, causing microangiopathic hemolytic anemia. These changes will be manifested by decrease in hemoglobin and platelets, presence of fragmented red blood cells on the peripheral blood smear (schistocytes), increase in lactic dehydrogenase (LDH), and decrease in haptoglobin. Liver enzymes may be normal or elevated, and coagulation studies are frequently normal (71). The classic clinical pentad of TTP consists of thrombocytopenia, microangiopathic hemolytic anemia, neurologic abnormalities, fevers, and renal dysfunction. In HUS, renal failure is the predominant feature and sometimes it is difficult to make a clear distinction between the two entities (72). Renal involvement is associated with hematuria and proteinuria (71). Management involves plasma exchanges (plasmapheresis) until platelet counts and LDH levels normalize. Sometimes immunosuppressive therapy is required. Platelet transfusions should be discouraged, due to risk of microvascular thrombosis. Maternal morbidity and mortality are high in pregnancies complicated with TTP/HUS (71).

\section{Obstructive Uropathy}

Obstructive uropathy is uncommon in pregnancy. "Physiologic hydronephrosis" of pregnancy is an expected phenomenon, present in $80 \%$ to $90 \%$ of the normal pregnancies $(5,6)$. Right-sided hydronephrosis occurs more commonly than left-sided one. The diagnosis of pathologic urinary tract obstruction during pregnancy can be difficult and can be recognized in some cases by the normalization of renal function in the lateral recumbent position, which relieves pressure on the ureters by the uterus, and its recurrence when supine (6). In some cases, however, either insertion of a ureteral catheter or delivery of the fetus is required.

\section{Renal Cortical Necrosis}

Renal cortical necrosis can be considered a severe form of ATN, often associated with disseminated coagulopathy and usually occurring in a setting of septic shock, septic abortion, or severe abruptio placentae. Bilateral renal cortical necrosis (BRCN) is estimated to occur in about $2 \%$ of the adult patients with ARF, but the incidence is the highest in obstetric patients, especially in the last part of pregnancy and postpartum (74). The risk of BRCN seems to be higher with prolonged anuria, usually longer than 2 weeks. Patients require dialysis and some will become dialysis dependent. Kidney biopsy provides the definitive diagnosis and prognostic information. Imaging techniques could be employed to show decreased cortical blood flow. CT angiogram or renal angiography shows decreased cortical nephrogram.

\section{DIALYSIS AND TRANSPLANTATION}

Multiple mechanisms that interfere with the hypothalamicpituitary-ovarian axis cause marked decrease in fertility in young women with advanced CKD and those requiring dialysis (75). Recent publications report fertility rates ranging between $1 \%$ and $7 \%$, which is much improved compared with older literature (76-78). These improved trends could be due to improved CKD management translated in increased dialysis population, better dialysis techniques, and improved care of the high-risk pregnancies.

Pregnancy in dialysis patients remains a very challenging clinical situation, where even the diagnosis of pregnancy itself may be a difficult task. Serum markers like $\beta$-HCG may be falsely elevated in dialysis patients due to poor renal excretion, and ultrasound evaluation is needed to confirm and date the pregnancy (79). Spontaneous abortion remains common, occurring in $21 \%$ of pregnancies reaching second trimester (80). Overall, it has been reported that only $50 \%$ of the pregnancies result in surviving infants (77). Premature delivery is very common, occurring in $80 \%$ of pregnant dialysis women (77). Other common fetal complications include polyhydramnios and low birth weight $(77,81,82)$. Maternal risks include acid-base abnormalities, HTN, volume overload, and pre-eclampsia. Due to these increased complication rates, most centers do recommend contraception in women of childbearing age on dialysis. When pregnancy does occur, it should be managed by a multidisciplinary team comprising nephrologists, obstetricians, dialysis staff, and neonatologists.

Although in most reported cases pregnant patients have been on hemodialysis, successful pregnancies have also been reported in PD (77,82-84). Whether one dialysis modality is superior to other in pregnant patients is unclear due to lack of randomized controlled studies. Feasibility of intensifying hemodialysis, limitations of PD volumes due to the gravid uterus, and prevalent practices among nephrologists may play a role in the choice of a particular renal replacement therapy over another.

The precise details of the dialysis management in pregnant patients are beyond the scope of this textbook, but some of the principles suggested by experts in this field are enumerated in Table 2.

Prolonged or intensive dialysis during pregnancy results in longer gestational periods, higher fetal birth weights, and 
Table 2 Recommendations for Dialysis Management in a Pregnant Patient

\begin{tabular}{|c|c|}
\hline Treatment factor & Recommendation \\
\hline Frequency of treatments & $\begin{array}{l}\text { Intensifying HD four to six times/wk after first trimester (goal }>20 \mathrm{hr} / \mathrm{wk} \text { ) } \\
\text { Increasing no. of PD dwells ( } 8-12 \mathrm{~L} / \text { day) }\end{array}$ \\
\hline Predialysis BUN & $<45-50 \mathrm{mg} / \mathrm{dL}$ \\
\hline Dialysate & $\begin{array}{l}\text { Bicarbonate- } 25 \mathrm{mEq} / \mathrm{L} \\
\text { Potassium } 3-4 \mathrm{mEq} / \mathrm{L} \text { (adjust based on HD frequency and serum values) }\end{array}$ \\
\hline Anemia & $\begin{array}{l}\text { Hgb target } 10-11 \mathrm{~g} / \mathrm{dL} \\
\text { May need increase in Erythropoietin dose } \\
\text { Iron saturation }>30 \% \text {, use IV iron to maintain levels } \\
\text { Folate } 1 \mathrm{mg} / \text { day }\end{array}$ \\
\hline Blood pressure/hemodynamics & $\begin{array}{l}\text { No clear guidelines. Diastolic BPs below } 80-90 \\
\text { Avoid maternal volume depletion and hypotension during dialysis } \\
\text { Avoid ACEI, ARBs } \\
\text { Ultrafiltration goals to be adjusted as pregnancy progresses accommodating placental and } \\
\text { fetal growth (approx. } 1 \mathrm{lb} / \mathrm{wk} \text { ) } \\
\text { Fetal monitoring during HD once fetus viable }\end{array}$ \\
\hline
\end{tabular}

greater fetal survivability $(76,77,80)$. Home hemodialysis and nocturnal hemodialysis are emerging modes of intensive HD that have shown to offer multiple clinical advantages in terms of HTN, volume status, and mineral metabolism. A retrospective study from Canada prescribing nocturnal HD $(>36 \mathrm{hr}$ dialysis) for women in childbearing age group showed not only improved fertility rates (15\%) but also more successful live births and fewer maternal-fetal complications (85).

With successful kidney transplantation, fertility seems to be restored to near normal within a few months. The U.S. National Transplant Pregnancy Registry (NTPR) reported a total of 1208 pregnancies in 781 kidney transplant recipients (86). Live births occurred in approx. $75 \%$ of the patients based on NTPR and the U.K. registry data $(87,88)$.

Despite improved outcomes following transplantation, a different set of complexities entail kidney transplant recipients who become pregnant. Impact of pregnancy on the kidney allograft, premature delivery, pre-eclampsia, and the longterm effects of immunosuppressive medications on the offspring remains some challenges in this group. Counseling about pregnancy should start in the pre-transplant period, continued following transplantation, and reliable contraception is recommended until patient is ready to embark on a planned pregnancy (89-91). The optimal timing of pregnancy after transplantation is considered to be 1 to 2 years. Analyses of the NTPR data have suggested greater percentage of nonviable outcomes in female transplant recipients who became pregnant less than 6 months after transplantation (86). Several individual factors should be considered when offering advice for the timing of pregnancy including adequacy of allograft function (serum creatinine $<1.5 \mathrm{mg} / \mathrm{dL}$ ), recent episodes of acute rejection, and risk of opportunistic infections (86).

These pregnancies should also be considered high risk and preferably managed by both transplant physicians and specialists in high-risk pregnancies. Major goals during pregnancy are to optimize maternal health, including allograft function, maintain a normal metabolic environment (normoglycemia), minimize complications associated with preterm births, detect and manage hypertensive complications especially pre-eclampsia, and ensure adequate fetal growth. Pre-eclampsia occurs in approximately one-third of pregnant women with kidney transplant, and diagnosing it can be challenging as many transplant patients can have preexisting proteinuria and an increasing trend in blood pressures after 20 weeks of gestation (92).

Urinary tract infections are the most common complication in pregnancies following kidney transplantation probably due to an immunosuppressed state and the increased likelihood of reflux. Most investigators recommend screening in all pregnant women and treating any positive cultures (93).

Rejection of the kidney allograft is unusual and the risk is considered to be no higher than in the nonpregnant transplant patients if pregnancy is properly planned (90). Anemia can frequently complicate pregnancy post-transplantation even in the absence of kidney function deterioration (90). Compared with normal pregnancies, transplant recipients have lower erythropoietin levels (94).

The anatomic location of the allograft in the iliac fossa does not interfere with vaginal delivery and most transplant patients can deliver vaginally unless there are some obstetric indications (90). One of the challenges in pregnancies posttransplantation is the choice and dosing of immunosuppressive medications. Transplant recipients should be maintained on immunosuppressive medications during pregnancy with some modifications to avoid allograft rejection and consequently fetal loss (90). Cell counts, renal parameters, liver function tests, and drug levels should be frequently monitored to avoid significant toxicity.

Most immunosuppressive medications are considered risky by the Food and Drug Administration; however, the U.S. registry data do not show any consistent pattern of congenital anomalies associated with the use of calcineurin inhibitors (cyclosporine and tacrolimus), azathioprine, or steroids (89). These are still considered as mainstay therapy for pregnant transplant patients by most centers given their relatively safer profile. Cyclosporine can be associated with low birth weight and also may exacerbate HTN during pregnancy (90). Tacrolimus has been associated with an increased incidence of DM and hyperkalemia in the newborns (95). Azathioprine has been shown to be teratogenic 
in higher doses in animals; however, these effects were not reproduced in humans (96). Case reports have described fetal adrenal insufficiency and thymic hypoplasia when corticosteroids are used in higher doses but these rarely occurred in doses of $15 \mathrm{mg} /$ day or less (97-99).

Mycophenolate mofetil and sirolimus are contraindicated and should be stopped 6 to 12 weeks prior to conception due to risks of teratogenicity $(86,100)$.

Most transplant immunosuppressive medications are excreted in the breast milk although in small amounts and no adverse effects have been reported (86). Although recommendations are evolving in this area, most transplant centers currently advise against breast-feeding (101).

In the absence of any guidelines for the management of pregnant dialysis patients and increased fetal complications, it may be prudent to advise effective contraception and delaying pregnancy until after transplantation. Improved outcomes have been reported in the transplant literature; however, the use of immunosuppressive medications adds another dimension and makes pregnancies more difficult to deal with in the post-transplant period.

\section{REFERENCES}

1. Fischer MJ. Chronic kidney disease and pregnancy: maternal and fetal outcomes. Adv Chronic Kidney Dis 2007; 14: 132-45.

2. Hou S. Pregnancy in chronic renal insufficiency and end-stage renal disease. Am J Kidney Dis 1999; 33: 235-52.

3. Pritchard JA. Changes in the blood volume during pregnancy and delivery. Anesthesiology 1965; 26: 393-9.

4. Yeomans ER, Gilstrap LC 3rd. Physiologic changes in pregnancy and their impact on critical care. Crit Care Med 2005; 33: S256-8.

5. Peake SL, Roxburgh HB, Langlois SL. Ultrasonic assessment of hydronephrosis of pregnancy. Radiology 1983; 146: 167-70.

6. Rasmussen PE, Nielsen FR. Hydronephrosis during pregnancy: a literature survey. Eur J Obstet Gynecol Reprod Biol 1988; 27: 249-59.

7. Fainaru O, Almog B, Gamzu R, Lessing JB, Kupferminc M. The management of symptomatic hydronephrosis in pregnancy. BJOG 2002; 109: 1385-7.

8. Christensen T, Klebe JG, Bertelsen V, Hansen HE. Changes in renal volume during normal pregnancy. Acta Obstet Gynecol Scand 1989; 68: 541-3.

9. Lindheimer MD, Davison JM, Katz AI. The kidney and hypertension in pregnancy: twenty exciting years. Semin Nephrol 2001; 21: 173-89.

10. Chapman AB, Abraham WT, Zamudio S, et al. Temporal relationships between hormonal and hemodynamic changes in early human pregnancy. Kidney Int 1998; 54: 2056-63.

11. Baylis C. Glomerular filtration rate in normal and abnormal pregnancies. Semin Nephrol 1999; 19: 133-9.

12. Baylis C, Rennke HG. Renal hemodynamics and glomerular morphology in repetitively pregnant aging rats. Kidney Int 1985; 28: 140-5.

13. Davison JM, Dunlop W. Renal hemodynamics and tubular function normal human pregnancy. Kidney Int 1980; 18: 152-61.

14. Maynard SE, Thadhani R. Pregnancy and the kidney. J Am Soc Nephrol 2009; 20: 14-22.

15. Smith MC, Moran P, Ward MK, Davison JM. Assessment of glomerular filtration rate during pregnancy using the MDRD formula. BJOG 2008; 115: 109-12.

16. Alper AB, Yi Y, Webber LS, et al. Estimation of glomerular filtration rate in preeclamptic patients. Am J Perinatol 2007; 24: 569-74.

17. Coll E, Botey A, Alvarez L, et al. Serum cystatin C as a new marker for noninvasive estimation of glomerular filtration rate and as a marker for early renal impairment. Am J Kidney Dis 2000; 36: 29-34.

18. Strevens H, Wide-Swensson D, Torffvit O, Grubb A. Serum cystatin C for assessment of glomerular filtration rate in pregnant and nonpregnant women. Indications of altered filtration process in pregnancy. Scand J Clin Lab Invest 2002; 62: 141-7.

19. Akbari A, Lepage N, Keely E, et al. Cystatin-C and beta trace protein as markers of renal function in pregnancy. BJOG 2005; 112: 575-8.
20. Kristensen K, Larsson I, Hansson SR. Increased cystatin C expression in the pre-eclamptic placenta. Mol Hum Reprod 2007; 13: 189-95.

21. Thilaganathan B, Ralph E, Papageorghiou AT, Melchiorre K, Sheldon J. Raised maternal serum cystatin $\mathrm{C}$ : an early pregnancy marker for preeclampsia. Reprod Sci 2009; 16: 788-93.

22. Brown MA, Gallery ED. Volume homeostasis in normal pregnancy and pre-eclampsia: physiology and clinical implications. Baillieres Clin Obstet Gynaecol 1994; 8: 287-310.

23. Lindheimer MD, Barron WM, Davison JM. Osmotic and volume control of vasopressin release in pregnancy. Am J Kidney Dis 1991; 17: 105-11.

24. Kumar S, Muchmore A. Tamm-Horsfall protein-uromodulin (19501990). Kidney Int 1990; 37: 1395-401.

25. Eknoyan G, Hostetter T, Bakris GL, et al. Proteinuria and other markers of chronic kidney disease: a position statement of the national kidney foundation (NKF) and the national institute of diabetes and digestive and kidney diseases (NIDDK). Am J Kidney Dis 2003; 42: 617-22.

26. Shihabi ZK, Konen JC, O’Connor ML. Albuminuria vs urinary total protein for detecting chronic renal disorders. Clin Chem 1991; 37: 621-4.

27. ACOG practice bulletin. Diagnosis and management of preeclampsia and eclampsia. Number 33, January 2002. American College of Obstetricians and Gynecologists. Int J Gynaecol Obstet 2002; 77: 67-75.

28. Higby K, Suiter CR, Phelps JY, Siler-Khodr T, Langer O. Normal values of urinary albumin and total protein excretion during pregnancy. Am J Obstet Gynecol 1994; 171: 984-9.

29. Kuo VS, Koumantakis G, Gallery ED. Proteinuria and its assessment in normal and hypertensive pregnancy. Am J Obstet Gynecol 1992; 167: 723-8.

30. Beetham R, Dawnay A, Menabawy M, Silver A. Urinary excretion of albumin and retinol-binding protein during normal pregnancy. J Clin Pathol 1988; 41: 1089-92.

31. Bernard A, Thielemans N, Lauwerys R, van Lierde M. Selective increase in the urinary excretion of protein 1 (Clara cell protein) and other low molecular weight proteins during normal pregnancy. Scand J Clin Lab Invest 1992; 52: 871-8.

32. Waugh JJ, Clark TJ, Divakaran TG, Khan KS, Kilby MD. Accuracy of urinalysis dipstick techniques in predicting significant proteinuria in pregnancy. Obstet Gynecol 2004; 103: 769-77.

33. Lim VS, Katz AI, Lindheimer MD. Acid-base regulation in pregnancy. Am J Physiol 1976; 231: 1764-9.

34. Jungers P, Houillier P, Forget D, Henry-Amar M. Specific controversies concerning the natural history of renal disease in pregnancy. Am J Kidney Dis 1991; 17: 116-22.

35. Katz AI, Davison JM, Hayslett JP, Singson E, Lindheimer MD. Pregnancy in women with kidney disease. Kidney Int 1980; 18: 192-206.

36. Imbasciati E, Gregorini G, Cabiddu G, et al. Pregnancy in CKD stages 3 to 5: fetal and maternal outcomes. Am J Kidney Dis 2007; 49: 753-62.

37. Jungers P, Houillier P, Chauveau D, et al. Pregnancy in women with reflux nephropathy. Kidney Int 1996; 50: 593-9.

38. Becker GJ, Fairley KF, Whitworth JA. Pregnancy exacerbates glomerular disease. Am J Kidney Dis 1985; 6: 266-72.

39. Packham DK, North RA, Fairley KF, et al. Primary glomerulonephritis and pregnancy. Q J Med 1989; 71: 537-53.

40. Abe S. An overview of pregnancy in women with underlying renal disease. Am J Kidney Dis 1991; 17: 112-15.

41. Jungers $\mathrm{P}$, Houillier $\mathrm{P}$, Forget $\mathrm{D}$, et al. Influence of pregnancy on the course of primary chronic glomerulonephritis. Lancet 1995; 346: 1122-4.

42. Chapman AB, Johnson AM, Gabow PA. Pregnancy outcome and its relationship to progression of renal failure in autosomal dominant polycystic kidney disease. J Am Soc Nephrol 1994; 5: 1178-85.

43. Hemmelder MH, de Zeeuw D, Fidler V, de Jong PE. Proteinuria: a risk factor for pregnancy-related renal function decline in primary glomerular disease Am J Kidney Dis 1995; 26: 187-92.

44. Jones DC, Hayslett JP. Outcome of pregnancy in women with moderate or severe renal insufficiency. N Engl J Med 1996; 335: 226-32.

45. Hayslett JP. Interaction of renal disease and pregnancy. Kidney Int 1984; 25: $579-87$.

46. Munkhaugen J, Lydersen S, Romundstad PR, et al. Kidney function and future risk for adverse pregnancy outcomes: a population-based study from HUNT II, Norway. Nephrol Dial Transplant 2009; 24: 3744-50. 
47. Garsenstein M, Pollak VE, Kark RM. Systemic lupus erythematosus and pregnancy. N Engl J Med 1962; 267: 165-9.

48. Hayslett JP. Maternal and fetal complications in pregnant women with systemic lupus erythematosus. Am J Kidney Dis 1991; 17: 123-6.

49. Kincaid-Smith P, Fairley KF, Kloss M. Lupus anticoagulant associated with renal thrombotic microangiopathy and pregnancy-related renal failure. Q J Med 1988; 68: 795-815.

50. Loizou S, Byron MA, Englert HJ, et al. Association of quantitative anticardiolipin antibody levels with fetal loss and time of loss in systemic lupus erythematosus. Q J Med 1988; 68: 525-31.

51. Williams DJ. Renal disease in pregnancy. Curr Obstet Gynaecol 2004; 14: $166-74$.

52. Kong NC. Pregnancy of a lupus patient-a challenge to the nephrologist. Nephrol Dial Transplant 2006; 21: 268-72.

53. Germain S, Nelson-Piercy C. Lupus nephritis and renal disease in pregnancy. Lupus 2006; 15: 148-55.

54. Landon MB. Diabetic nephropathy and pregnancy. Clin Obstet Gynecol 2007; 50: 998-1006.

55. Purdy LP, Hantsch CE, Molitch ME, et al. Effect of pregnancy on renal function in patients with moderate-to-severe diabetic renal insufficiency. Diabetes Care 1996; 19: 1067-74.

56. Gordon M, Landon MB, Samuels P, Hissrich S, Gabbe SG. Perinatal outcome and long-term follow-up associated with modern management of diabetic nephropathy. Obstet Gynecol 1996; 87: 401-9.

57. Grenfell A, Brudenell JM, Doddridge MC, Watkins PJ. Pregnancy in diabetic women who have proteinuria. Q J Med 1986; 59: 379-86.

58. Kitzmiller JL, Brown ER, Phillippe M, et al. Diabetic nephropathy and perinatal outcome. Am J Obstet Gynecol 1981; 141: 741-51.

59. Reece EA, Coustan DR, Hayslett JP, et al. Diabetic nephropathy: pregnancy performance and fetomaternal outcome. Am J Obstet Gynecol 1988; 159: 56-66.

60. Biesenbach G, Zazgornik J. Incidence of transient nephrotic syndrome during pregnancy in diabetic women with and without pre-existing microalbuminuria. BMJ 1989; 299: 366-7.

61. Kimmerle R, Zass RP, Cupisti S, et al. Pregnancies in women with diabetic nephropathy: long-term outcome for mother and child. Diabetologia 1995; 38: 227-35.

62. Galvagno SM Jr, Camann W. Sepsis and acute renal failure in pregnancy. Anesthesia \& Analgesia 2009; 108: 572-5.

63. Gammill HS, Jeyabalan A. Acute renal failure in pregnancy. Crit Care Med 2005; 33: S372-84.

64. Nzerue CM, Hewan-Lowe K, Nwawka C. Acute renal failure in pregnancy: a review of clinical outcomes at an inner-city hospital from 1986-1996. J Natl Med Assoc 1998; 90: 486-90.

65. Silva GB Jr, Monteiro FA, Mota RM, et al. Acute kidney injury requiring dialysis in obstetric patients: a series of 55 cases in Brazil. Arch Gynecol Obstet 2009; 279: 131-7.

66. Stillman IE, Karumanchi SA. The glomerular injury of preeclampsia. J Am Soc Nephrol 2007; 18: 2281-4.

67. Haram K, Svendsen E, Abildgaard U. The HELLP syndrome: clinical issues and management. A review. BMC Pregnancy Childbirth 2009; 9: 8.

68. Sheikh IA, Shaheen FA. Acute renal failure and HELLP syndrome: a single center's experience. Saudi J Kidney Dis Transpl 1998; 9: 290-3.

69. Abraham KA, Kennelly M, Dorman AM, Walshe JJ. Pathogenesis of acute renal failure associated with the HELLP syndrome: a case report and review of the literature. Eur J Obstet Gynecol Reprod Biol 2003; 108: 99-102.

70. Hou S, Peano C. Acute renal failure in pregnancy. Saudi J Kidney Dis Transpl 1998; 9: 261-6.

71. Sibai BM. Imitators of severe pre-eclampsia. Semin Perinatol 2009; 33: 196-205.

72. Moake JL. Thrombotic microangiopathies. N Engl J Med 2002; 347: 589-600.

73. Caprioli J, Bettinaglio P, Zipfel PF, et al. The molecular basis of familial hemolytic uremic syndrome: mutation analysis of factor $\mathrm{H}$ gene reveals a hot spot in short consensus repeat 20. J Am Soc Nephrol 2001; 12: 297-307.
74. Kleinknecht D, Grunfeld JP, Gomez PC, Moreau JF, Garcia-Torres R. Diagnostic procedures and long-term prognosis in bilateral renal cortical necrosis. Kidney Int 1973; 4: 390-400.

75. Palmer BF. Sexual dysfunction in uremia. J Am Soc Nephrol 1999; 10: 1381-8.

76. Levy DP, Giatras I, Jungers P. Pregnancy and end-stage renal diseasepast experience and new insights. Nephrol Dial Transplant 1998; 13: 3005-7.

77. Reddy SS, Holley JL. Management of the pregnant chronic dialysis patient. Adv Chronic Kidney Dis 2007; 14: 146-55.

78. Hou SH. Frequency and outcome of pregnancy in women on dialysis. Am J Kidney Dis 1994; 23: 60-3.

79. Davison JM. Dialysis, transplantation, and pregnancy. Am J Kidney Dis 1991; 17: 127-32.

80. Hou S. Pregnancy in dialysis patients: where do we go from here? Semin Dial 2003; 16: 376-8.

81. Reister F, Reister B, Heyl W, et al. Dialysis and pregnancy-a case report and review of the literature. Ren Fail 1999; 21: 533-9.

82. Okundaye I, Abrinko P, Hou S. Registry of pregnancy in dialysis patients. Am J Kidney Dis 1998; 31: 766-73.

83. Shemin D. Dialysis in pregnant women with chronic kidney disease. Semin Dial 2003; 16: 379-83.

84. Chan WS, Okun N, Kjellstrand CM. Pregnancy in chronic dialysis: a review and analysis of the literature. Int J Artif Organs 1998; 21: 259-68.

85. Barua M, Hladunewich M, Keunen J, et al. Successful pregnancies on nocturnal home hemodialysis. Clin J Am Soc Nephrol 2008; 3: 392-6.

86. Armenti VT, Constantinescu S, Moritz MJ, Davison JM. Pregnancy after transplantation. Transplant Rev (Orlando) 2008; 22: 223-40.

87. Armenti VT, Radomski JS, Moritz MJ, et al. Report from the National Transplantation Pregnancy Registry (NTPR): outcomes of pregnancy after transplantation. Clin Transpl 2004: 103-14.

88. Sibanda N, Briggs JD, Davison JM, Johnson RJ, Rudge CJ. Pregnancy after organ transplantation: a report from the UK Transplant pregnancy registry. Transplantation 2007; 83: 1301-7.

89. McKay DB, Josephson MA, Armenti VT, et al. Reproduction and transplantation: report on the AST Consensus Conference on Reproductive Issues and Transplantation. Am J Transplant 2005; 5: 1592-9.

90. Josephson MA, McKay DB. Considerations in the medical management of pregnancy in transplant recipients. Adv Chronic Kidney Dis 2007; 14: 156-67.

91. Ross LF. Ethical considerations related to pregnancy in transplant recipients. N Engl J Med 2006; 354: 1313-16.

92. Stratta P, Canavese C, Giacchino F, et al. Pregnancy in kidney transplantation: satisfactory outcomes and harsh realities. J Nephrol 2003; 16: 792-806.

93. Lindheimer M, Katz A. The normal and diseased kidney in pregnancy. In: Schrier RW, ed. Diseases of the kidney and urinary tract, 7th edn. Philadelphia: Lippincott Williams \& Wilkins, 2001.

94. Magee LA, von Dadelszen P, Darley J, Beguin Y. Erythropoiesis and renal transplant pregnancy. Clin Transplant 2000; 14: 127-35.

95. Jain A, Venkataramanan R, Fung JJ, et al. Pregnancy after liver transplantation under tacrolimus. Transplantation 1997; 64: 559-65.

96. Use of immunosuppressive drugs in pregnancy and lactation. UpToDate., 2007. [Available from: http://www.uptodateonline.com].

97. Blanford AT, Murphy BE. In vitro metabolism of prednisolone, dexamethasone, betamethasone, and cortisol by the human placenta. Am J Obstet Gynecol 1977; 127: 264-7.

98. Muirhead N, Sabharwal AR, Rieder MJ, Lazarovits AI, Hollomby DJ. The outcome of pregnancy following renal transplantation-the experience of a single center. Transplantation 1992; 54: 429-32.

99. Penn I, Makowski EL, Harris P. Parenthood following renal transplantation. Kidney Int 1980; 18: 221-33.

100. Sifontis NM, Coscia LA, Constantinescu S, et al. Pregnancy outcomes in solid organ transplant recipients with exposure to mycophenolate mofetil or sirolimus. Transplantation 2006; 82: 1698-702.

101. McKay DB, Adams PL, Bumgardner GL, et al. Reproduction and pregnancy in transplant recipients: current practices. Prog Transplant 2006; 16: 127-32. 


\section{Acute abdomen in pregnancy Nicole Fearing and William L. Holcomb}

One of the most anxiety-provoking patients to deal with is the pregnant patient with acute abdominal pain. It is widely quoted that 1/500-635 pregnancies will be complicated by an acute abdomen requiring surgery. With two patients to think about, mother and child, and the complications that can go along with a delayed or missed diagnosis, it is no wonder it leads to anxiety for all involved. To deal with the complexities that go along with diagnosing an acute abdomen in a pregnant patient, one needs to understand not only the differential diagnosis but also the physiologic changes of pregnancy that will affect the evaluation and diagnosis.

\section{PHYSIOLOGIC CHANGES OF PREGNANCY}

Physical findings in the pregnant patient with an acute abdomen are different from that of the nonpregnant one. Much of the decreased prominence of abdominal signs is due to the expanding uterus. In the patient who is not pregnant, the average weight of the uterus is $70 \mathrm{~g}$. Over 40 weeks of pregnancy, it can expand to $1110 \mathrm{~g}$ and can have an intrauterine volume of 5 liters (1-3). As this enlargement occurs, the intestine and omentum are pushed into the upper abdomen. The omentum is less able to be the watchman of the abdomen and wall off areas of peritonitis due to encroachment of the uterus. The ovaries will come to lie posterior to the uterus (1). This makes assessment of ovarian pathology more difficult especially by transabdominal ultrasound. With the enlargement of the uterus also comes stretching of the abdominal wall. As the abdominal wall stretches, it shows fewer signs of direct peritoneal irritation. Classic signs, such as rebound and guarding, are less prominent.

Previously a paper by Baer et al. in 1932 reported that with the enlargement of the uterus, the appendix moves upward and laterally from its usual position at McBurney's point so that by late pregnancy it is actually closer to the gallbladder. He showed this by performing barium enemas to show the position of the appendix and cecum (4). In 2003, a paper by Hodjati and Kazerooni in the International Journal of Gynecology and Obstetrics reevaluated the anatomical location of the appendix with pregnancy. In their prospective study, three groups of women were compared. The first was 165 women in their 37 th to 40 th week of pregnancy who were undergoing elective c-sections. The second group was 26 women between 19 and 39 weeks of pregnancy with acute appendicitis. The final group was 100 nonpregnant females with acute appendicitis. Each was evaluated for appendix location and normal was noted to be within $2 \mathrm{~cm}$ of McBurney's point. In their study, $15 \%$ of the first group had an abnormal position, $23 \%$ of the second group, and $17 \%$ of the third. The study showed no significant difference between the normal control and those who were pregnant (5).
Other physiologic changes may be attributed to hormonal changes associated with pregnancy such as the elevation in estrogen and progesterone levels. Several studies have shown that as levels of these hormones increase throughout pregnancy, orocecal motility slows. A study by Wald et al. showed that the gastrointestinal motility slowed by one-third during the third trimester of pregnancy compared with postpartum rates $(6,7)$. It has also been shown that levels of the hormone motilin decrease. This affects the migrating motor complex also leading to slowed orocecal motility (8). The slowed transit can compound problems with constipation or other underlying abdominal pathology making diagnosis again more difficult. Progesterone has also been linked to decreased lower esophageal sphincter pressure and affects the urologic system. Dilation and slowing of peristalsis of the ureters have been associated with increase in progesterone. This may lead to urinary tract infection from urinary stasis $(9,10)$.

There are normal changes that take place in pregnancy, which change the results of laboratory tests. This can make looking for bleeding or infection more difficult. Laboratory test results expected in pregnancy are shown in Table 1. For a few tests, the lab results can still have significant diagnostic value especially in the case of pyelonephritis, hepatitis, or pancreatitis.

\section{IMAGING AND THE PREGNANT PATIENT}

With the fetal effects of radiation to consider, it is important to understand what imaging modalities are available and safe. While there may be anxiety in using some radiologic modalities, it is important to remember that the greatest danger to both fetus and mother may be delay in diagnosis.

\section{Ultrasound}

Ultrasound may be used as both a diagnostic and a therapeutic modality. It is the imaging modality of choice due to its ease of use, low cost, and safety. It may be used to evaluate mother and fetus at the same time providing evaluation of amniotic fluid and size of the fetus while possibly diagnosing relevant pathology in the mother.

\section{Magnetic Resonance Imaging}

Magnetic resonance imaging (MRI) is a safe modality to use providing diagnostic information on the mother with little risk to the fetus at any stage of pregnancy. MRI is beneficial compared with computed tomography (CT) in that it is capable of giving large field-of-view images in any plane with excellent soft-tissue contrast (11). There is no exposure of the mother or the fetus to any ionizing radiation. Often no contrast is needed to establish a diagnosis. Gadolinium, a contrast agent used in MRIs, does cross the placenta and its 
Table 1 Changes in Laboratory Test Results Expected in Pregnancy

\begin{tabular}{ll} 
Test & \multicolumn{1}{c}{ Result } \\
Hemoglobin & Decreased \\
Hematocrit & Decreased \\
White blood cell count & Increased \\
Differential white blood cell count & Increased neutrophils \\
Platelet count & Unchanged \\
Electrolytes (serum) & Decreased $\mathrm{CO}_{2}$ \\
Glucose (fasting) & Decreased \\
Creatinine (serum) & Decreased \\
Amylase & Slightly increased \\
Aspartate aminotransferase & Unchanged \\
Alanine aminotransferase & Unchanged \\
Alkaline phosphatase & Increased \\
g-Glutamyl transpeptidase & Unchanged \\
Urinalysis & Unchanged \\
Arterial blood gas & Decreased pCO $;$ \\
& slightly increased pH
\end{tabular}

effects on the fetus are not well understood. However, there is general consensus that it should not be used in the pregnant patient unless there is high likelihood that benefits of its use outweigh the risk to the fetus. In the acute abdominal setting, MRI is beneficial for evaluation of the biliary tree for stones with sensitivity greater than $90 \%$ (11). It may be used to diagnose acute appendicitis, bowel obstruction, and areas of abscess formation, inflammation, and hemorrhage.

\section{CT}

CT use is often feared due to the risk of ionizing radiation to the fetus. Guidelines for surgical problems during pregnancy from the Society of American Gastrointestinal and Endoscopic Surgeons (SAGES) state that expeditious and accurate diagnosis should take precedence over concerns for ionizing radiation. Radiation dosage should be limited to 5 to 10 rads in the first 25 weeks of pregnancy (12). Modern CT scanners deliver doses of radiation below this as a single test. A CT of the abdomen-pelvis delivers about 2 to 4 rads (13-16). However, cumulative radiation exposure must be considered: other tests that have been done may affect the decision to use CT. Consider also that IV contrast should not be used and try to minimize cuts of the CT.

\section{Nuclear Medicine}

Fetal exposure with nuclear medicine studies is quoted to be less than 0.5 rads. This again is below the limit of 5 to $10 \mathrm{rads}$ quoted. In light of this, the radionucleotides may be administered including technetium-99 $(13,17)$.

Once workup is completed, decision for treatment will depend on the specific diagnosis. However, there are some tenants for surgery that hold true for all patients. Indications for emergency laparotomy are no different in the pregnant patient than that in a nonpregnant patient. If possible, elective procedures should wait until after the pregnancy is completed unless that is a compelling reason that it benefits the patient or the pregnancy. Elective procedures include cholecystectomy for uncomplicated gallstone disease and inguinal hernia repair for asymptomatic hernia. The second trimester of pregnancy is the best time for performance of any elective or semi-elective procedure. The decision to perform a c-section delivery should be made outside of the decision for an exploratory laparotomy. Successful vaginal deliveries can be performed postoperatively.

\section{Perioperative Considerations}

Perioperative consideration should be given to fetal heart monitoring. It is difficult to keep fetal heart monitors on sterilely during an abdominal surgery and monitoring may create a significant distraction. There are no definitive studies demonstrating that intraoperative fetal monitoring makes a difference in fetal outcome. The value of intraoperative fetal monitoring must be determined on a case by case basis. Preand postoperative fetal and contraction monitoring are recommended at a viable gestational age.

Preterm labor is also a concern with this population. The use of tocolytics is somewhat controversial and no consensus exists. The SAGES guidelines recommend no prophylactic use of tocolytics (13). Their use is also not recommended in the presence of maternal infection (9). Tocolytic benefits must be weighed against risks and obstetric consultation is warranted.

\section{Laparoscopy and Pregnancy}

At one time, it was felt that laparoscopy could only be used safely in the first and second trimesters of pregnancy. However, there are several published reports of use of laparoscopy in the third trimester without detriment to the patient or fetus. The SAGES guidelines now state that laparoscopy may be performed in any trimester and indications are the same in the pregnant and nonpregnant patients (13).

\section{ACUTE APPENDICITIS}

Appendicitis is the most common indication for nonobstetric surgery and occurs in $1 / 766$ to $1 / 3000$ pregnancies $(18,19)$. There is no increased risk of appendicitis associated with pregnancy, but there is credible concern that the severity is increased in the pregnant patient. This may be due to the difficulty and delay of diagnosis during pregnancy. There are many common symptoms of pregnancy that may mimic appendicitis, such as nausea, vomiting, and round ligament pain. The leukocytosis of pregnancy may also obscure evaluation. It has also been previously reported that the anatomic location of the appendix changes with the growing uterus. More recent studies as noted previously in this chapter have refuted this idea and overall the appendix remains in its normal position. The diagnosis of appendicitis preoperatively in the pregnant patient has been reported in only one-half to threefourth of the cases taken to the operating room (OR) $(18,20,21)$. Finally due to the difficulty in diagnosis and the subjective reluctance of some to take a pregnant patient to the OR, the incidence of perforation is about $25 \%$ to $40 \%(18,21,22)$. This is even more concerning in light of the risk of fetal demise, which is $7 \%$ to $10 \%$ in the nonperforated appendicitis case and up to $24 \%$ if the appendix is perforated $(18,22-24)$.

\section{Signs}

Direct abdominal tenderness is the most constant physical finding among pregnant patients with appendicitis. Only rarely is this finding absent $(25,26)$. In the first trimester, tenderness is usually well localized in the right lower quadrant. With advancing gestation, there is still some thought that 
tenderness may be found in the right periumbilical area or right upper quadrant; however, in the study by Hodjati et al., this was not necessarily true. About $55 \%$ to $75 \%$ of patients have demonstrable rebound tenderness (21,25-28). One-half to twothirds of patients have abdominal muscle rigidity (21,25-29). Rovsing's sign is present approximately equally in pregnant as in nonpregnant patients with appendicitis. Psoas irritation is less frequently observed in pregnancy (30). Rectal examination is often overlooked in the pregnant patient with appendicitis, but one group has found that tenderness is usually present, especially in the first trimester (25). Fever and tachycardia are variably present and are not sensitive signs for appendicitis in pregnancy.

\section{Diagnostic Aids}

The physiologic leukocytosis (as high as $15,000 / \mathrm{mm}^{3}$ ) during pregnancy and the wide range of normal values limit the usefulness of the leukocyte count for the diagnosis of appendicitis (21). Severe disease may be present when the leukocyte count is normal (20). Greater than $80 \%$ polymorphonuclear leukocytes are often present when appendicitis complicates pregnancy $(21,25)$. Again, however, the test is not sufficiently sensitive to rule out disease and it discriminates poorly between those patients with and those without appendicitis. Ten to twenty percent of pregnant patients with appendicitis have an abnormal urinalysis, usually showing pyuria alone $(25,27)$. Bacteriuria indicates a urinary tract infection. Asymptomatic bacteriuria is common enough in pregnancy that it may well occur coincidentally with appendicitis (31). Ultrasound may be the initial radiologic evaluation tool for diagnosis of appendicitis. However, it may be variable in its results due to operator performance, the gravid uterus, or a retrocecal appendix. MRI and CT are gaining popularity as evaluation tools. A recent systematic review from 2009 showed good sensitivity and specificity of both when ultrasound was normal or inconclusive (18).

Treatment for appendicitis is either open or laparoscopic appendectomy. Choice should be made depending on the skill and comfort level of the surgeon and clinical picture. No longer is laparoscopy limited to the first and second trimesters of the pregnancy. Reports now show the safety of laparoscopy in the third trimester as well (32). Advantages reported for laparoscopy over the open approach include better intraabdominal visualization, less uterine manipulation, less postoperative pain, early return of GI function, and earlier ambulation postoperatively $(32,33)$.

Technique for laparoscopic entrance into the abdomen is variable. Veress needle, and open Hassan-type techniques are both reported and are safe in those experienced in the techniques (32). Veress needle insertion usually is done in the midclavicular line about $2 \mathrm{~cm}$ below the costal margin. Once performed, the port may be placed blindly or better yet with an optical trocar. Open surgery should be performed over the point of maximum tenderness or at McBurney's point.

\section{CHOLELITHIASIS}

Although laparoscopic cholecystectomy is reported to be the most common laparoscopic procedure in pregnancy, pregnancy itself does not increase the risk of acute cholecystitis. It is reported to occur in about 1 in 1600 to 10,000 pregnancies (34-36). During pregnancy, the formation of gallstone and gallbladder sludge is common, but most women remain asymptomatic.

\section{Signs and Symptoms}

As in the nonpregnant patient, the most reliable symptom is right upper quadrant pain. Previous studies have shown that about $28 \%$ present with pain $(9,37)$.

About half of the patients have vomiting and a minority are febrile (38). Patients will often give a history of previous similar episodes. Right upper quadrant direct tenderness is usually present, but in the absence of complications, rebound tenderness is rare. The pain may radiate to the back. In the occasional patient, there is a palpable gallbladder.

Cholecystitis may be confused with appendicitis, particularly in the third trimester. Landers and colleagues (38) reported a woman at 30 weeks of gestation who underwent laparotomy to rule out appendicitis. The appendix was normal, but she developed Salmonella sepsis postoperatively. The gallbladder was found to be the source of the organism when she subsequently returned to the OR for cholecystectomy.

\section{Diagnostic Aids}

Gallstones are present in more than $95 \%$ of patients with cholecystitis. Ultrasonography has been found to be extremely accurate in the diagnosis of cholelithiasis. With cholecystitis, it may show not only gallstones but also gallbladder wall thickening and pericholecystic fluid. Ultrasound can also show common bile duct dilation. Since this modality does not require ionizing radiation, it is preferred in pregnancy. If additional diagnostic information is needed for clinical management, the radiation dose of a radionucleotide scan of the gallbladder is not prohibitive.

Blood tests are of limited diagnostic value for the patient suspected to have cholecystitis. Leukocytosis may be present, but it is neither a sensitive nor a specific marker. Serum alkaline phosphatase is normally elevated in pregnancy. A high value should not be taken as evidence of cholestasis. Other hepatic enzymes, such as aspartate transferase and alanine transferase, may be helpful in distinguishing cholecystitis from hepatitis. Transient elevation of the serum amylase level may be seen in up to one-third of patients $(39,40)$. A markedly elevated serum amylase level suggests pancreatitis, which may complicate the course of gallbladder disease. Occasionally, a patient may be jaundiced with an elevated bilirubin level. If the patient has had persistent vomiting, evaluation of electrolyte status is essential.

\section{Treatment}

Treatment of symptomatic cholelithiasis, otherwise known as biliary colic, can be controversial. Many people recommend nonoperative treatment for biliary colic. However, as shown in their review of the disease, Steinbrook et al. reported recurrence of symptomatic gallstones in $92 \%$ of women managed nonoperatively in their first trimester, $64 \%$ in their second, and 44\% in their third $(13,41)$. A $57 \%$ symptom recurrence during pregnancy and a $23 \%$ complication rate of acute cholecystitis and gallstone pancreatitis are reported $(13,42)$. 
Many favor initial treatment for the pregnant patient with uncomplicated acute cholecystitis to be supportive. Intravenous fluids should be given to prevent dehydration and the patient is made nothing-by-mouth. Nasogastric suction is appropriate if there has been significant vomiting. Analgesia may be given as needed. Meperidine is preferred to morphine, as the latter may produce spasm of the sphincter of Oddi. All narcotics may affect the sphincter of Oddi, but it appears to be more so with morphine. Since early cholecystitis is a sterile process, antibiotics are often unnecessary. If symptoms are persistent, or if systemic or local signs are prominent, broadspectrum antibiotic coverage is warranted (43). Patients who fail to respond to conservative measures, or who have recurrent bouts of illness, are candidates for surgery. Selected patients may be considered for a percutaneous drainage procedure, that is, cholecystostomy tube, in order to defer definitive therapy (44).

The patient who responds promptly to supportive therapy may be observed, with the hope of avoiding an operation during pregnancy, or may be scheduled for an elective cholecystectomy. A prospective comparison of these treatment strategies is unavailable. Many authors favor the former approach, but the latter has proponents $(45,46)$. Medical therapy is associated with a high risk of relapse, often requiring hospitalization prior to delivery (46). If there is choice about the timing of cholecystectomy, the second trimester seems to be the interval when fetal complications are minimized.

Though once considered contraindicated, there is a growing body of literature supporting the safety of laparoscopic cholecystectomy during pregnancy $(47,48)$. SAGES guidelines list laparoscopic cholecystectomy as the procedure of choice in the pregnant patient with gallbladder disease regardless of trimester (13). The procedure is usually performed in the second trimester, but many cases have been reported in the first and third trimesters. Guidelines published by SAGES note that intraoperative cholangiography exposes both the mother and the fetus minimally and may be used selectively intraoperatively during cholecystectomy (13). Pelvic shielding is recommended. Endoscopic retrograde cholangiopancreatography with papillotomy and stone extraction may also be performed during pregnancy (49-51). This may be done pre-, post-, or intraoperatively (13). Candidates for this procedure would be those with known or suspected calculous obstruction of the biliary duct. Fetal irradiation can be minimized by uterine shielding and limitation of fluoroscopy time.

\section{Course}

Acute cholecystitis may be complicated by empyema, perforation, pancreatitis, or failure to respond to medical management. As stated previously, management is supportive unless the patient has pancreatitis, cholangitis, or common bile duct obstruction (9). Among patients managed conservatively during pregnancy, $62 \%$ to $84 \%$ may be carried through to delivery without operation $(38,39,40)$. In the series reported by Landers and coworkers (38), medical therapy was deemed to have failed if symptoms persisted beyond the 4th day in the hospital, or if there were more than three recurrences in the same trimester. Pleading against excessive conservatism, Dixon and colleagues (45) cited two cases in which patients required total parenteral nutrition for 27 and 30 days, respectively. One of these patients developed pancreatitis. Of 26 patients in their series, 15 had recurrent episodes of biliary colic during pregnancy (45). Data from published series are often incomplete, but perinatal outcome appears to be favorable if disease is uncomplicated or if surgical treatment is effected in the second trimester $(31,39,40,45,52)$.

\section{PANCREATITIS}

\section{Occurrence}

Pancreatitis is an unusual, but potentially devastating, complication of pregnancy that may present with acute abdominal pain. In more recent series, case:delivery ratios range from 1:1289 to 1:3333 (53-56). Pregnancy may predispose women to the development of pancreatitis, but this notion is poorly substantiated and controversial $(53,54,57,58)$. Recognized risk factors for pancreatitis include cholelithiasis, alcohol use, hyperlipidemia, hyperparathyroidism, abdominal trauma, viral infections, and certain drugs (particularly diuretics). The presence of gallstones is the most common etiology among pregnant patients; they are found in up to $90 \%$ of cases $(55-57,59)$.

\section{Symptoms and Signs}

The presentation of pancreatitis is similar in the pregnant and nonpregnant patients. Among cases reviewed by Wilkinson (55), 75\% presented with acute abdominal pain. Typically, the pain is of sudden onset and located in the epigastrium. Nausea and vomiting are usually present and may be severe. Very ill patients may present in shock. Generally, the patient is in acute distress. There may be a low-grade fever and a few patients are jaundiced. Epigastric tenderness is the most reliable physical finding. Peritoneal signs are absent or minimal. Bowel sounds are diminished. With severe hemorrhagic pancreatitis, blood may infiltrate the flanks (Grey Turner's sign) or the umbilicus (Cullen's sign).

\section{Laboratory Aids}

Serum amylase and lipase should be evaluated in pregnant patients with concern for pancreatitis as would be done for those who were not pregnant. Karsenti et al. in 2001 performed a prospective case-controlled study of serum amylase and lipase controls in normal pregnancy. Their study showed that serum amylase levels are the same in both pregnant and nonpregnant patients. Lipase is different in pregnant patient during the first trimester only and is lower at that time than in nonpregnant patients. They found that during the final two trimesters, there are no significant differences. Their final conclusion was that both amylase and lipase levels should be evaluated in the pregnant patient. They also concluded that serum lipase levels correlated better with acute pancreatitis that did amylase levels $(35,60)$. Elevations of serum amylase also may be seen with intestinal perforation, infarction, or obstruction, and a variety of other conditions.

Other laboratory findings may be hypoglycemia (sometimes severe), hyperglycemia, hyperbilirubinemia, hypocalcemia, hemoconcentration, and electrolyte derangements. Imaging procedures are of limited usefulness for the patient with suspected pancreatitis in pregnancy. Upper abdominal ultrasonography may yield information about concomitant gallbladder disease, but the pancreas is often not well seen. CT or 
MRI may be of benefit in severely ill patients with a doubtful diagnosis.

\section{Treatment}

The initial treatment of pancreatitis in pregnancy is supportive and does not differ from treatment for the nonpregnant patient. Corrections of hypovolemia, as well as the electrolyte, glucose, and calcium disturbances, are necessary. Oral intake is withheld. If the patient has moderate or severe disease, continuous nasogastric suctioning is often instituted. Total parenteral nutrition may be used if the course of disease is prolonged (61). If gallbladder disease is causative, cholecystectomy or endoscopic papillotomy may be considered once the patient's condition is stable (49).

\section{Course}

The mean duration of acute symptoms is about 6 days, but recovery may be prolonged and complicated (55). Estimates of maternal mortality vary widely from $0 \%$ to $37 \%$, probably reflecting bias in case selection and improvements in diagnosis and therapy $(53,55-57,59)$. In one compilation of reported cases, the perinatal mortality associated with maternal pancreatitis was estimated to be $38 \%$ (55). More recent series indicate a better outlook with perinatal mortality of $11 \%$ or less $(53,57,59)$. In a recent review of 43 pregnant patients at one institution from 1983 to 1993 , there were no maternal deaths, two stillbirths, and one neonatal death due to prematurity (56). As expected, the risk of perinatal death increased with severity of maternal disease.

\section{INTESTINAL OBSTRUCTION}

\section{Occurrence}

An increasing frequency of intestinal obstruction during pregnancy has been attributed to a higher rate of intraabdominal operations, with subsequent adhesions, among women of reproductive age. Rates of occurrence are from $1 / 1500$ to $1 / 16,000(35,62)$. Despite the fact that $50 \%$ of pregnant women experience nausea and $33 \%$ have problems with emesis, cases rarely occur during the first 4 months of pregnancy $(35,63)$. They are fairly evenly distributed thereafter and into the puerperium. A previous laparotomy, most often appendectomy or an adnexal operation, seems to be the most important risk factor. About one-half of simple intestinal obstruction during pregnancy is due to postoperative adhesions. Volvulus is the next most common condition (9). It, too, may be caused by adhesions. Intussusception is less common. Incarcerated inguinal or femoral hernias, or obstruction resulting from carcinoma, are exceedingly rare among pregnant patients (64).

A special note should be made about pregnant patients who have had gastric bypass surgery. A known risk of this surgery is internal herniation, which can lead to acute bowel obstruction. This may be due to a defect in the mesentery at the jejunaljejunostomy or a Peterson's defect behind the Roux limb in a Roux En Y gastric bypass. This complication can put a patient and the fetus at serious risk of morbidity and mortality. Any patient who has had bariatric surgery should undergo immediate evaluation when showing signs of obstruction, and surgery should not be delayed if suspicion is high for internal herniation.

\section{Symptoms and Signs}

Abdominal pain, vomiting, and constipation are symptoms expected with intestinal obstruction. Almost $90 \%$ of affected pregnant patients will have abdominal pain (66). The pain may be constant or periodic. In the third trimester, it may be confused with the pain of labor or placental abruption. Pain may sometimes radiate to the flank, imitating that of pyelonephritis (65). The severity of the pain may not reflect the severity of the disease (67). Patients are often anxious and apprehensive (68). Emesis is a highly variable symptom among patients with intestinal obstruction during pregnancy. With more proximal obstruction, vomiting appears earlier in the course of disease and is more frequent. There may be considerable delay in resumption of emesis after the first episode. Severe obstruction may be present without any vomiting (66). Whereas constipation is a common symptom in pregnancy, complete cessation of stool and flatus is not. Such a report should strengthen suspicion for the diagnosis. With proximal obstruction, bowel movements may continue for a time following the onset of disease. Obstruction may lead to significant dehydration associated with low urine output, mild-to-moderate tachycardia, and hypotension.

Early in the course of disease, physical findings may be subtle. The classic picture of direct abdominal tenderness, distension, and high-pitched tinkling bowel sounds is the exception rather than the rule. Abdominal tenderness may be entirely absent (67). Pressure on the uterus may cause pain when transmitted to distended bowel, misleading the clinician to suspect a uterine process. Abdominal distension is difficult to assess in late pregnancy when the risk for bowel obstruction is the greatest. At presentation, bowel sounds are often described as normal (66). In some cases, particularly with cecal volvulus, a tender cystic mass may be palpable $(69,70)$. Rebound tenderness, fever, tachycardia, and shock are late findings (67).

\section{Diagnostic Aids}

Leukocytosis may occur, but is of little help in the diagnosis of intestinal obstruction. Electrolyte abnormalities and hemoconcentration may be present if there has been prolonged emesis or if fluid has accumulated within distended bowel. There may be an elevation of serum amylase levels. Radiographic studies are often essential for diagnosis and should not be avoided out of concern for fetal effects. An upright plain film of the abdomen is the best initial study. Other studies, with or without contrast media, may also be appropriate and should not be delayed if findings on plain films are not typical and suspicion is present. Sequential films may be necessary for proper evaluation of initially normal or equivocal radiologic findings (67).

\section{Treatment and Course}

Treatment of intestinal obstruction in pregnancy is the same as that for the nonpregnant patient. Clinical management includes prompt correction of fluid and electrolyte deficits, decompression of bowel, relief of obstruction, and resection of nonviable tissue. Prompt fluid resuscitation is especially important in pregnancy, since uterine blood flow depends upon a normal maternal blood volume. If patients are in shock or significantly impaired, a foley catheter may be required to 
monitor urine output. As fetus mortality is high with bowel obstruction, early surgery is warranted. Adequate operative exposure and thorough inspection of the full length of the bowel should not be compromised. Usually, a midline abdominal laparotomy incision is optimal. In some cases, it may be necessary to empty the uterus to accomplish satisfactory surgical therapy. However, this can most often be avoided (71). As a rule, the treatment of intestinal obstruction is surgical. Selected cases of early sigmoid volvulus have been successfully treated with colonoscopy, long-tube and rectal-tube decompression alone (72). In the case of bowel obstruction, it may not be optimal to attempt a laparoscopic exploration in a pregnant patient. There will be limited exposure due not only to the enlarged uterus but also to the dilated bowel. The bowel may also be friable and easily torn with manipulation causing enterotomies.

The obstetrician should be aware of the entity called colonic pseudo-obstruction, or Ogilvie's syndrome, which tends to occur following cesarean section (73). The clinical presentation is that of large bowel obstruction, but no mechanical stricture or volvulus is present. Radiographs will show marked gaseous distension of the cecum that usually extends to involve the ascending colon and transverse colon. The distal colon and rectum are gasless. Typically, there is little, if any, fluid in the distended bowel and contrast studies reveal no site of mechanical obstruction. If massive distension goes unrelieved, cecal perforation, a life-threatening complication, may occur. Treatment consists of bowel decompression by either colonoscopy or operative cecostomy (74). Neostigmine is an alternative treatment. This agent must be used in a monitored setting with atropine available as it may cause significant bradycardia.

Intestinal obstruction is a serious complication of pregnancy associated with maternal mortality in $10 \%$ to $20 \%$ and perinatal mortality in $20 \%$ to $30 \%$ of cases $(68,71,75)$. There are several recent reviews of the topic (76-78). Perdue et al. noted in their review of 66 cases of obstruction that time from the patients' admission to surgery was about 48 hours. Twenty-three percent of patients in that review were found to have dead bowel requiring resection; maternal mortality was $6 \%$ and fetal mortality was $26 \%$ (78). Intrauterine fetal death and preterm delivery are the most common perinatal complications. Diagnostic vigilance and timely therapy afford the best prospects for a favorable outcome.

\section{URETERAL CALCULI}

A case:delivery ratio of approximately 1:1600 has been estimated for symptomatic urolithiasis in pregnancy $(79,80)$. Stone passage seems to occur as often during pregnancy as outside of pregnancy. The causes for urolithiasis are varied. Among 78 women of reproductive age who were evaluated for urolithiasis, $42 \%$ had idiopathic hypercalciuria, 13\% had hyperuricuria, $10 \%$ had primary hyperparathyroidism, 13\% had infection stone, 3\% had cystinuria, and 19\% had idiopathic lithiasis (81).

Pain, usually in the flank but sometimes in the abdomen, is almost always the presenting complaint. Nausea, vomiting, dysuria, urgency, fever, or gross hematuria may be associated. About one patient in four will give a past history of urolithiasis $(79,80)$. Costovertebral angle tenderness is almost always present. In one series, abdominal tenderness was elicited in 6 of 20 patients (79). Concomitant urinary tract infection may obscure the diagnosis of ureteral calculi.

Microscopic hematuria is found in about $75 \%$ of cases. The absence of hematuria at presentation does not rule out a stone. The patient suspected of urolithiasis should be instructed to strain her urine in search of a stone until the diagnosis is clarified. Ultrasonographic imaging of the urinary tract may be helpful in the evaluation of the patient with suspected urinary calculi; the stone, or evidence of ureteral obstruction, may be apparent. Transvaginal ultrasound examination may reveal a distal ureteral calculus (82).

The urinary collecting system, particularly the right side, is often physiologically dilated in the latter half of pregnancy (83). This effect should not be confused with ureteral obstruction. Duplex Doppler waveforms of the intrarenal arteries may help distinguish obstructed from non-obstructed collecting systems, obstruction being associated with a high resistivity index (84). Intrarenal resistivity in pregnancy appears to be no different from that outside of pregnancy, even in the presence of physiologic pelvicaliectasis (85). Radiographic evaluation, if needed, may be accomplished with minimal radiation exposure by obtaining a plain abdominal film and a film 20 to 30 minutes after injection of contrast dye. Additional delayed films may be necessary in some cases. If urolithiasis is confirmed, a metabolic evaluation is warranted.

In planning treatment, relevant factors include the size and location of the stone, the degree of obstruction, the severity of symptoms, and the presence of infection. Most calculi will pass spontaneously with hydration. If necessary, minimally invasive treatment options, such as ureteral stent placement, ureteroscopic retrieval, or percutaneous nephrostomy, may be considered (86). These procedures have been safely performed throughout pregnancy $(87-89)$. Open ureterolithotomy is rarely required. Extracorporeal shock-wave lithotripsy has not been approved for use during pregnancy. Unless severe infection complicates the course of urolithiasis, a good perinatal outcome is expected.

\section{ADNEXAL TORSION}

Among women requiring laparotomy for adnexal torsion, about $20 \%$ are pregnant $(90,91)$. About one-half of the cases of adnexal torsion are associated with an ovarian neoplasm, most often a dermoid cyst. In the reproductive-age group, approximately $2 \%$ of cases are associated with ovarian malignancy (90). Gonadotropin-induced ovarian hyperstimulation is a risk factor for torsion and about $16 \%$ of affected pregnancies are complicated by adnexal torsion (92). Symptomatic torsion occurs usually in the first, sometimes in the second, but rarely in the third trimester. The right side is involved more frequently than the left (90).

Pain is almost always the presenting symptom, but the nature of the pain is highly variable. The onset may be sudden or gradual. The character may be sharp and intermittent, or dull and constant. In about two-thirds of cases, the pain is unilateral in the lower abdomen. It may be generalized or, uncommonly, it may radiate to the back or flank. Nausea and/ or vomiting are present in about half of the patients. Various urinary symptoms are less often reported $(91,92)$. Abdominal 
tenderness is the most constant physical finding. Peritoneal signs are variably present. There may be adnexal tenderness or a mass. Leukocytosis may be present, but a normal white blood cell count does not rule out the diagnosis. Ultrasonographic imaging may aid in the identification and characterization of an adnexal mass. Color Doppler sonography may show absent arterial flow in the central ovarian parenchyma. Absent central ovarian venous flow may be a more sensitive finding in cases of adnexal torsion (93). Comparison with the uninvolved contralateral ovary may aid in sonographic diagnosis. Given the nonspecific clinical presentation, it is not surprising that the preoperative diagnosis is often erroneous $(90,91)$.

Torsion of the ovary and/or the Fallopian tube leads to venous congestion, edema, and, ultimately, arterial compromise. The course may be complicated by ovarian rupture or hemorrhage. Some patients report previous episodes of transient pain, indicating that spontaneous reversal of torsion may, at times, occur.

Surgery may be done laparoscopically and is recommended by SAGES guidelines for both diagnosis and treatment of the torsed ovary unless clinical severity warrants a laparotomy (13). The conventional surgical therapy of adnexal torsion is extirpation of the involved tissues, particularly if they appear ischemic or necrotic. If it is necessary to remove the corpus luteum prior to 10 weeks of gestation, there is justification for progesterone supplementation in an effort to maintain the pregnancy. If tissue distal to the site of torsion appears viable, unwinding of the adnexa is an alternative therapy. One group has advocated unwinding, regardless of the appearance of the adnexa, in cases of ovarian hyperstimulation syndrome (94). If the ovary is conserved, the surgeon must be assured that it does not contain a neoplasm. There is little information available to quantify the fetal risk following adnexal torsion. Generally, the pregnancy outcome is good (92).

\section{HERNIAS}

Hernias of the abdominal wall or groin are not uncommon in the general population today. Repair of a hernia is one the most common surgical procedures in the country. Hernias during pregnancy, however, are uncommon, and much less is written about them in the literature. Whenever a pregnant patient has a hernia that is symptomatic, prompt consultation with a general surgeon is prudent.

\section{GROIN HERNIAS}

The two most common types of groin hernia are inguinal and, less commonly, femoral. The incidence in women is about $8 \%$ to $9 \%$ of all groin hernias (95-98). In a retrospective analysis of 21,318 pregnant patients by Ochsenbein-Kolble et al., it was found that there was an incidence of inguinal hernia during pregnancy of $1 / 2000$. Seventy-five percent of those women were multiparas (99).

\section{Diagnosis}

Diagnosis of a groin hernia may be more difficult in a female than a male patient. The standard exam is for palpation of bulge in the inguinal region on valsalva that is either reducible or irreducible. If a bulge is difficult to feel, consideration for an ultrasound should be made. Ultrasound of the groin for hernia evaluation may be more difficult in the obese or pregnant patient. Occasionally, MRI or CT may be used if clinically warranted to evaluate the groin region. The differential diagnosis for a groin mass includes round ligament varicosity, enlarged lymph node, lipoma, abscess, vascular aneurysm, and of course direct, indirect, or femoral hernias $(95,97,99,100)$.

\section{Treatment}

Treatment for an inguinal hernia in a pregnant female varies little from that in a nonpregnant female. If the patient has an acute problem such as incarceration or strangulation of that hernia, emergent treatment should be done. An open inguinal herniorrhaphy may be performed, and if the bowel is found to be nonviable, conversion to an open procedure may be warranted. Mesh may be used for a repair if there is no infection or nonviable bowel. If there is concern for infection, a tissue repair should be performed. In the event that it is not an urgent situation, watchful waiting would be prudent with the repair of the hernia after the pregnancy is complete (97). Buch et al. in their review of 12 patients with inguinal or umbilical hernias during pregnancy showed that repair could be done postpartum with no significant increase in recurrence. Surgery on these patients was done on average at 22 weeks postpartum (97). Reports of inguinal hernia repair at time of c-section have been written. A study by Ochsenbein-Kolble showed that in eight women who developed an inguinal or umbilical hernia during pregnancy, a tissue repair as a combined procedure with cesarean section was not only feasible but also safe with no recurrences at 56 months (99).

\section{VENTRAL HERNIA}

Ventral hernias encompass umbilical, epigastric, and incisional. Incidence of umbilical hernias in pregnancy has been reported to be between $2 \%$ and $5 \%$. The incidence of incisional hernias in pregnancy is unknown $(95,101,102)$. Herniation of abdominal contents, uterine fibroids, or the gravid uterus through the hernia defect can be a concern in the pregnant female with a ventral hernia $(95,101)$. If the gravid uterus is incarcerated in the hernia defect, consideration for immediate surgery must be taken due to risk not only of intrauterine growth retardation but also of strangulation of the uterus. In this case, cesarean section is performed with tissue repair of the hernia if possible.

\section{DIAPHRAGMATIC HERNIA}

A rare type of hernia that can complicate pregnancy is the diaphragmatic hernia. This type of hernia may be congential, such as a posterolateral Bochdalek hernia or an anteriomedial hernia of Morgagni, or the more common acquired hiatal hernias.

\section{Signs and Symptoms}

Patients with these types of hernias may not know that they have a hernia of the diaphragm until symptoms appear during pregnancy $(103,104)$.

Symptoms may include upper abdominal or chest pain, nausea, and vomiting all of which may worsen as the pregnancy progresses $(103,105,106)$. Patients may also present acutely obstructed with a toxic appearance due to dehydration and herniation of abdominal contents into the chest. These patients may be tachycardic and or have dyspnea (103). 


\section{Diagnosis}

Diagnosis may be made by an upright anteroposterior and lateral chest X-ray. This may show abdominal contents in the chest with air fluid levels noted in the chest.

\section{Treatment}

Management will depend on the acuity of the situation. Due to the increased intra-abdominal pressure exerted by the growing uterus, there is concern that if not repaired during the pregnancy, there may be high morbidity and possibly mortality for the mother and child during the pregnancy or labor. Kurzel et al. in 1988 were emphatic about surgery for this condition stating that in their review of 18 reported cases of this type of hernia, there were 8 maternal and 6 fetal deaths. They felt that repair should be performed prior to labor regardless of whether the hernia was symptomatic or not. Their recommendations for treatment that if there were signs of obstruction, immediate repair should be undertaken regardless of fetal stage of development. If the patient was in the first or second trimester, repair should be done to prevent intra-abdominal contents from pushing into the chest cavity and possibly becoming strangulated. Furthermore, if the patient was asymptomatic in her third trimester, the baby should be followed for fetal maturity. When deemed mature, then elective c-section should be performed with hernia repair done at that time (105).

\section{CONCLUSION}

There are many sources of acute abdominal pain in the pregnant patient. Prompt evaluation and coordination between the obstetrician and the general surgeon may make the difference between an excellent outcome and a catastrophe.

\section{REFERENCES}

1. Sivanesaratnam V. The acute abdomen and the obstetrician. Baillieres Clin Obstet Gynaecol 2000; 14: 89-102.

2. Hytten FE. Weight changes in pregnancy. In: Hytten F, Chamberlain G, eds. Clinical Physiology in Obstetrics, 2nd edn. London: Blackwell Scientific, 1991: 173-203.

3. Cunningham FG, MacDonald PC, Grant NF, et al. Williams' Obstetrics, 19th edn. Norwalk, CT: Appleton and Lange, 1993.

4. Baer JL, Feis RA, Arens RA. Appendicitis in pregnancy with changes in position and axis of the normal appendix in pregnancy. JAMA 1932; 98 : 1359-64.

5. Hodjati H, Kazerooni T. Location of the appendix in the gravid patient: a re-evaluation of the established concept. Int J Gynaecol Obstet 2003; 81: 245-7.

6. Wald A. Constipation, diarrhea and symptomatic hemorrhoids during pregnancy. Gastroenterol Clin 2003; 32: 309-22.

7. Wald A, Van Thiel DH, Hoechstetter L, et al. Effect of pregnancy on gastrointestinal transit. Dig Dis Sci 1982; 27: 1015-18.

8. Christofides ND, Ghateri MA, Bloom SR, Borberg C, Gillmer MD. Decreased plasma motilin concentrations in pregnancy. BMJ 1982; 285: 1453-4.

9. Kilpatrick CC, Monga M. Approach to the acute abdomen in pregnancy. Obstet Gynecol Clin N Am 2007; 34: 389-402.

10. Cormier CM, Canzoneri BJ, Lewis DF, et al. Urolithiasis in pregnancy: current diagnosis, treatment, and pregnancy complications. Obstet Gynecol Surv 2006; 61: 733-41.

11. Leyendecker JR, Gorengaut V, Brown JJ. MR imaging of maternal diseases of the abdomen and pelvis during pregnancy and the immediate postpartum period. Radiographics 2004; 24: 1301-16.

12. ACOG committee opinion Number 299. Guidelines for diagnostic imaging during pregnancy. Am Coll Obstet Gynecol 2004; 104: 647-51.
13. Society of American Gastrointestinal and Endoscopic Surgeons (SAGES). Guidelines for diagnosis, treatment and use of laparoscopy for surgical problems during pregnancy. Los Angeles, CA: SAGES, 2007.

14. Kennedy A. Assessment of acute abdominal pain in the pregnant patient. Semin Ultrasound CT MR 2000; 21: 64-77.

15. Hurwitz LM, Yoshizumi T, Reiman RE, et al. Radiation dose to the fetus from body MDCT during early gestation. AJR Am J Roentgenol 2006; 186: 871-6.

16. Forsted DH, Kalbhen CL. CT of pregnant women for urinary tract calculi, pulmonary thromboembolism and acute appendicitis. AJR Am J Roentgenol 2002; 178: 1285.

17. Adelstein SJ. Administered radionuclides in pregnancy. Teratology 1999; 59: 236-9.

18. Basaran A, Basaran M. Diagnosis of acute appendicitis during pregnancy. A systematic review. Obstet Gynecol Surv 2009; 64: 481-8.

19. Kort B, Katz VL, Watson WJ. The effect of nonobstetric operation during pregnancy. Surg Gynecol Obstet 1993; 177: 371-6.

20. Anderson B, Nielsen TF. Appendicitis in pregnancy: diagnosis, management and complications. Acta Obstet Gynecol Scand 1999; 78: 758-62.

21. Bailey LE, Finley RK Jr, Miller SF, et al. Acute appendicitis during pregnancy. Am Surg 1986; 52: 218-21.

22. Yilmaz HG, Akgun Y, Bac B, et al. Acute appendicitis in pregnancy-risk factors associated with principal outcomes: a case control study. Int J Surg 2007; 5: 192-7.

23. Firstenberg MS, Malangoni MA. Gastrointestinal surgery during pregnancy. Gastroenterol Clin North Am 1998; 27: 73-88.

24. Ueberrueck T, Koch A, Meyer L, et al. Ninety-four appendectomies for suspected appendicitis during pregnancy. World J Surg 2004; 28: 50811.

25. Cunningham F, McCubbin J. Appendicitis complicating pregnancy. Obstet Gynecol 1975; 45: 415-20.

26. Babaknia A, Hossein P, Woodruff J. Appendicitis during pregnancy. Obstet Gynecol 1977; 50: 40-4.

27. Gomez A, MacDonald W. Acute appendicitis during pregnancy. Am J Surg 1979; 137: 180-3.

28. Finch D, Lee E. Acute appendicitis complicating pregnancy in the Oxford region. Br J Surg 1974; 61: 129-32.

29. Brant H. Acute appendicitis in pregnancy. Obstet Gynecol 1967; 29: 130-8.

30. Richards C, Daya S. Diagnosis of acute appendicitis in pregnancy. Can J Surg 1989; 32: 358-60.

31. McGee T. Acute appendicitis in pregnancy. Aust NZ J Obstet Gynaecol 1989; 29: 378-85.

32. Barnes SL, Shane MD, Schoemann MB, Bernard AC, Boulanger BR. Laparoscopic appendectomy after 30 weeks pregnancy: report of two cases and description of technique. Am Surg 2004; 70: 733-6.

33. Al-Fozan H, Tulandi T. Safety and risks of laparoscopy in pregnancy. Curr Opin Obstet Gynecol 2002; 14: 375-9.

34. Rollins MD, Chan KJ, Price RR. Laparoscopy for appendicitis and cholelithiasis during pregnancy. Surg Endosc 2004; 18: 237-41.

35. Augustin G, Majerovic M. Non-obstetrical acute abdomen during pregnancy. Eur J Obstet Gynecol Reprod Biol 2007; 131: 4-12.

36. Kammerer W. Nonobstetric surgery during pregnancy. Med Clin North Am 1979; 63: 1157-64.

37. Maringhini A, Ciambra M, Baccelliere $P$, et al. Biliary sludge and gallstones in pregnancy: incidence, risk factors and natural history. Ann Intern Med 1993; 119: 116-20.

38. Landers D, Carmona R, Crombleholme W, Lim R. Acute cholecystitis in pregnancy. Obstet Gynecol 1987; 69: 131-3.

39. Hiatt J, Hiatt J, Williams R, Klein S. Biliary disease in pregnancy: strategy for surgical management. Am J Surg 1986; 151: 263-5.

40. Friley M, Douglas G. Acute cholecystitis in pregnancy and the puerperium. Am Surg 1972; 38: 314-17.

41. Steinbrook RA, Brooks DC, Datta S. Laparoscopic cholecystectomy during pregnancy. Review of anesthetic management, surgical considerations. Surg Endosc 1996; 10: 511-15.

42. Glasgow RE, Visser BC, Harris HW, et al. Changing management of gallstone disease during pregnancy. Surg Endosc 1988; 12: 241-6.

43. Malet P, Soloway R. Diseases of the gallbladder and bile ducts. In: Wyngaarden J, Smith LJ, eds. Cecil Textbook of Medicine, 18th edn. Philadelphia: WB Saunders, 1988: 859-72. 
44. Allmendinger N, Hallisey M, Ohki S, Straub J. Percutaneous cholecystostomy treatment of acute cholecystitis in pregnancy. Obstet Gynecol 1995; 86: 653-4.

45. Dixon N, Faddis D, Silberman H. Aggressive management of cholecystitis during pregnancy. Am J Surg 1987; 154: 292-4.

46. Swisher S, Schmit P, Hunt K, et al. Biliary disease during pregnancy. Am J Surg 1994; 168: 576-9.

47. Lanzafame R. Laparoscopic cholecystectomy during pregnancy. Surg 1995; 118: 627-33.

48. Soper N, Hunter J, Petrie R. Laparoscopic cholecystectomy during pregnancy. Surg Endosc 1992; 6: 115-16.

49. Nesbitt T, Kay H, McCoy M, Herbert W. Endoscopic management of biliary disease during pregnancy. Obstet Gynecol 1996; 87: 806-9.

50. Friedman R, Friedman I. Acute cholecystitis with calculous biliary duct obstruction in the gravid patient. Surg Endosc 1995; 9: 910-13.

51. Jamidar P, Beck G, Hoffman B, et al. Endoscopic retrograde cholangiopancreatography in pregnancy. Am J Gastroenterol 1995; 90: 1263-7.

52. Hill L, Johnson C, Lee R. Cholecystectomy in pregnancy. Obstet Gynecol 1975; 46: 291-3.

53. Corlett R, Mishell D. Pancreatitis in pregnancy. Am J Obstet Gynecol 1972; 113: 281-90.

54. Jouppila P, Mokka R, Larmi T. Acute pancreatitis in pregnancy. Surg Gynecol Obstet 1974; 139: 879-82.

55. Wilkinson E. Acute pancreatitis in pregnancy: a review of 98 cases and a report of 8 new cases. Obstet Gynecol Surv 1973; 28: 281-300.

56. Ramin K, Ramin S, Richey S, Cunningham F. Acute pancreatitis in pregnancy. Am J Obstet Gynecol 1995; 173: 187-91.

57. Block P, Kelly T. Management of gallstone pancreatitis during pregnancy and the postpartum period. Surg Gynecol Obstet 1989; 168: 426-8.

58. DeVore G. Acute abdominal pain in the pregnant patient due to pancreatitis, acute appendicitis, cholecystitis, or peptic ulcer disease. Clin Perinatol 1980; 7: 349-69.

59. McKay A, O’Neill J, Imrie C. Pancreatitis, pregnancy and gallstones. Br J Obstet Gynaecol 1980; 87: 47-50.

60. Karsenti D, Bacq Y, Brechot JF, et al. Serum amylase and lipase activities in normal pregnancy: a prospective case-control study. Am J Gastroenterol 2001; 96: 697-9.

61. Kirby D, Fiorenza V, Craig R. Intravenous nutritional support during pregnancy. J Pen 1988; 12: 72-80.

62. Ballantyne GH, Brouder MD, Beart RWJ, et al. Volvulus of the colon: incidence and mortality. Am Surg 1985; 202: 83-92.

63. Wax J, Christie T. Complete small bowel volvulus complication the second trimester. Obstet Gynecol 1993; 83: 689-91.

64. Pratt AT, Donaaldson RC, Evertson LR, et al. Cecal volvulus in pregnancy. Obstet Gynecol 1981; 57(Suppl): 37S-40S.

65. Hill L, Symmnonds R. Small bowel obstruction in pregnancy: a review and report of four cases. Obstet Gynecol 1976; 49: 170-3.

66. Morris E. Intestinal obstruction and pregnancy. J Obstet Gynaecol $\mathrm{Br}$ Commonw 1965; 72: 36-44.

67. Davis M, Bohon C. Intestinal obstruction in pregnancy. Clin Obstet Gynecol 1983; 26: 832-42.

68. Goldthorp W. Intestinal obstruction during pregnancy and the puerperium. Br J Clini Pract 1966; 20: 367-76.

69. Pratt A, Donaldson R, Evertson L, Yon J. Cecal volvulus in pregnancy. Obstet Gynaecol 1981; 57: 37S-40S.

70. Fanning J, Cross C. Post-cesarean cecal volvulus. Am J Obstet Gynecol 1988; 158: 1200-2.

71. Harer W, Harer W. Volvulus complicating pregnancy and puerperium. Obstet Gynecol 1958; 12: 399-406.

72. Malkasian G, Welch J, Hallenbeck G. Volvulus associated with pregnancy: a review and report of 3 cases. Am J Obstet Gynecol 1959; 78: 112.

73. Reece E, Petrie R, Hutcherson H. Ogilvie's syndrome in the postCesarean section patient. Am J Obstet Gynecol 1982; 144: 849-50.

74. Rodriguez-Ballesteros R, Torres-Bautista A, Torres-Valdez F, RuizMoreno J. Ogilvie's syndrome in the post-Cesarean section patient. Int J Gynecol Obstet 1989; 28: 185-7.

75. Beck W. Intestinal obstruction in pregnancy. Obstet Gynecol 1974; 43: 374-8.

76. Connolly M, Unti J, Nora P. Bowel obstruction in pregnancy. Surg Clin N Am 1995; 75: 101-13.
77. Meyerson S, Holtz T, Ehrinpreis M, Dhar R. Small bowel obstruction in pregnancy. Am J Gastroenterol 1995; 90: 299-302.

78. Perdue $\mathrm{P}$, Johnson $\mathrm{H}$, Stafford $\mathrm{P}$. Intestinal obstruction complicating pregnancy. Am J Surg 1992; 164: 384-8.

79. Jones W, Correa R, Ansell J. Urolithiasis associated with pregnancy. J Urol 1979; 122: 333-5.

80. Strong D, Murchison R, Lynch D. The management of ureteral calculi during pregnancy. Surg Gynecol Obstet 1978; 146: 604-8.

81. Coe F, Parks J, Lindheimer M. Nephrolithiasis during pregnancy. N Engl J Med 1978; 298: 324-6.

82. Loughlin K. Management of urologic problems during pregnancy. Urology 1994; 44: 159-69.

83. Schulman A, Herlinger $\mathrm{H}$. Urinary tract dilation in pregnancy. $\mathrm{Br} \mathrm{J}$ Radiol 1975; 48: 638-45.

84. Platt J, Rubin J, Ellis J, DiPietro M. Duplex Doppler US of the kidney: differentiation of the obstructive from nonobstructive dilation. Radiology 1989; 171: 515-7.

85. Hertzberg B, Carroll B, Bowie J, et al. Doppler US assessment of maternal kidneys: analysis of intrarenal resistivity indexes in normal pregnancy and physiologic pelvicaliectasis. Radiology 1993; 186: 689-92.

86. Hendricks S, Ross S, Krieger J. An algorithm for diagnosis and therapy of management and complication of urolithiasis during pregnancy. Surg Gynecol Obstet 1991; 172: 49-54.

87. Peer A, Strauss S, Witz E, et al. Use of percutaneous nephrostomy in hydronephrosis of pregnancy. Eur J Radiol 1992; 15: 220-3.

88. Scarpa R, de Lisa A, Usai E. Diagnosis and treatment of ureteral calculi during pregnancy with rigid ureteroscopes. J Urol 1996; 155: 875-7.

89. Ulvik N, Bakke A, Hoisaeter P. Ureteroscopy in pregnancy. J Urol 1995; 154: 1660-3.

90. Hibbard L. Adnexal torsion. Am J Obstet Gynecol 1985; 152: 456-61.

91. Lomano J, Trelford J, Ullery J. Torsion of the uterine adnexa causing an acute abdomen. Obstet Gynecol 1970; 35: 221-5.

92. Mashiach S, Goldenberg M, Bider D, et al. Adnexal torsion of hyperstimulated ovaries in pregnancies after gonadotropin therapy. Fertil Steril 1990; 53: 76-80.

93. Fleischer A, Stein S, Cullinan J, Warner M. Color Doppler sonography of adnexal torsion. J Ultrasound Med 1995; 14: 523-8.

94. Bider D, Ben-Rafael Z, Godenberg M, et al. Pregnancy outcome after unwinding of twisted ischaemic-haemorrhagic adnexa. $\mathrm{Br} \mathrm{J}$ Obstet Gynaecol 1989; 96: 428-30.

95. Augustin G, Matosevic P, Kekez T, Majerovic M, Delmis J. Abdominal hernias in pregnancy. J Obstet Gynaecol 2009; 35: 203-11.

96. Primatesta P, Goldacre MJ. Inguinal hernia repair: incidence of elective and emergency surgery, readmission and mortality. Int J Epidemiol 1996; 25: 835-9.

97. Buch KE, Tabrizian P, Divino CM. Management of hernias in pregnancy. J Am Coll Surg 2008; 207: 539-42.

98. Nilsson E, Kald A, Anderberg B, et al. Hernia surgery in a defined population: a prospective three year audit. Eur J Surg 1997; 163: 823-9.

99. Ochsenbein-Kolble N, Demartines N, Ochsenbein-Imhof N, Zimmermann R. Cesarean section and simultaneous hernia repair. Arch Surg 2004; 139: 893-5.

100. Cheng D, Lam H, Lam C. Round ligament varices in pregnancy mimicking inguinal hernia: an ultrasound diagnosis. Ultrasound Obstet Gynecol 1997; 9: 198-9.

101. Dales RD, Rane A. Extreme uterine herniation in pregnancy through an umbilical defect. Aust NZ Obstet Gynaecol 2001; 41: 232.

102. Beischer NA, Mackay EV, Colditz P. Obstetrics and the newborn: an illustrated textbook. Volume 57. 3rd edn. London: WB Saunders, 1997: 77 .

103. Sano A, Kato H, Hamatani H, et al. Diaphragmatic hernia with ischemic bowel obstruction in pregnancy: report of a case. Surg Today 2008; 38: 836-40.

104. Chai Y, Zhang G, Shen G. Adult Bochdalek hernia complicated with a perforated colon. J Thoracic Cardiovasc Surg 2005; 130: 1729-30.

105. Kurzel RB, Naunheim KS, Schwartz RA. Repair of symptomatic diaphragmatic hernia during pregnancy. Obstet Gynecol 1988; 71: 869-71.

106. Palanivelu C, Rangarajan M, Maheshkumaar GS, Parthasarathi R. Laparoscopic mesh repair of a Bochdalek diaphragmatic hernia with acute gastric volvulus in a pregnant patient. Singapore Med J 2008; 49: e26-8. 


\section{Medical evaluation and management of pregnant patients undergoing non-obstetrical surgery John G. Gianopoulos}

The approach to the pregnant patient undergoing nonobstetric surgery requires thorough knowledge of the normal maternal physiologic and anatomic adaptation to pregnancy, potential fetal effects of diagnostic tests or ancillary pharmaceutical therapies, and the potential for any obstetric complications.

\section{MATERNAL ADAPTATION OF PREGNANCY}

There is a significant alteration in both the physiology and the anatomy of many maternal organ systems in response to the hormonal changes of pregnancy. A thorough understanding of these alterations is needed in establishing the diagnosis, monitoring during a procedure, and conducting the operative procedure for a safe maternal and fetal outcome (Table 1).

\section{Cardiovascular System}

The cardiovascular system undergoes significant alteration under the influence of the altered hormonal milieu of pregnancy. Progesterone inhibits distal tubular sodium reabsorption leading to natriuresis. The juxtaglomerular cells of the kidney in response to this secrete renin to stimulate aldosterone release from the adrenals, maintaining sodium homeostasis (1-3). Renin is converted into angiotensin and catecholamines are released from the adrenal gland. Pregnancy is a state of maternal hyper-catecholaminism from its early stages. These catecholamines stimulate both inotropic and chronotropic effects on the heart, leading to an increase in cardiac output. Cardiac output begins to rise in the first trimester and continues on a steady increase to peak at $30 \%$ to $50 \%$ of the preexisting levels by approximately 32 weeks of gestation (3-7). Both heart rate and stroke volume increase. Peripheral systemic vascular resistance is reduced secondary to progesterone, exerting a direct effect to relax the intimal smooth muscle in the precapillary resistance vessels $(2,3)$. The resulting vasodilatation leads to a decreased vascular resistance. There is a slight decrease in mean arterial pressure in the second trimester of a normal pregnancy due to the reduction in peripheral resistance. Blood volume increases with pregnancy, peaking at approximately $50 \%$ of prepregnancy levels at around 32 weeks of gestation. As the pregnant woman approaches term, mean arterial pressure normalizes as the increase in blood volume compensates for the decreased resistance and fills the capacitance of the vasculature $(8,9)$.

The pulmonic and systemic circulations undergo similar alterations. There is vasodilatation with an increased volume of capacitance. However, in the pulmonic circulation, the volume and the capacitance change almost equal each other. Therefore, there are no changes in pulmonic pressures. When the pulmonic circulation is evaluated by central catheterization, no changes in pulmonary artery pressures or wedge pressures are seen due to pregnancy $(6,7)$. The change with increased pulmonic blood volume and increased capacitance fills the pulmonic circulation. This renders the pregnant women susceptible to fluid overload and pulmonary edema (10). Pulmonary edema will occur much more readily in pregnancy, secondary to this maternal adaptation.

In the third trimester, the enlarged uterus may compress the vena cava (especially in the supine position). This may lead to a decrease in cardiac venous return and a drop in cardiac output with a drop in systemic blood pressure. While positioning for operative procedures, the pregnant patient is best positioned with the uterus displaced to the left, allowing adequate vena cava flow and cardiac return to avoid hypotension. Increased cardiac output leads to an increased flow across the aortic valve, thus producing a soft aortic flow murmur. This murmur is auscultated from approximately the mid-second trimester through term in many pregnant patients. Diastolic murmurs or harsh crescendo-decrescendo murmurs in the systolic range are always considered pathologic. However, a soft continuous flow murmur heard in the aortic value region may be physiologic due to the increased flow in pregnancy.

\section{Respiratory Adaptations}

Progesterone has a direct effect on the hypothalamic apneustic center, altering its $\mathrm{CO}_{2}$ sensitivity. The hypothalamic response to a lower level of $\mathrm{CO}_{2}$ drives an increased tidal volume and respiratory rate. This reduces $\mathrm{CO}_{2}$, and $\mathrm{pH}$ begins to rise. The kidney secretes bicarbonate equalizing the system and acidbase balance. The pregnant woman is in a chronic state of compensated respiratory alkalosis. The normal blood gases of pregnancy are compensated respiratory alkalosis (11). The normal $\mathrm{pH}$ is 7.44 and bicarbonate levels decrease by $4 \mathrm{meg} / \mathrm{L}$. This loss of bicarbonate buffer renders the pregnant women more susceptible to metabolic acidosis. Arterial $\mathrm{PO}_{2}$ rises by approximately 5 to $10 \mathrm{~mm}$ of mercury. However, in the supine position, arterial $\mathrm{PO}_{2}$ decreases by at least 6 to $10 \mathrm{~mm}$ of mercury. Positioning the pregnant women with the uterus displaced to the left reduces this positional effect on oxygenation in the blood. Vital capacity and maximum voluntary ventilation are not altered. Functional residual capacity is reduced as the diaphragm is elevated by the rising uterus $(11,12)$.

\section{Hematologic Adaptations}

The hematologic system undergoes marked change secondary to pregnancy. Plasma volume increases by $50 \%$ over prepregnancy levels. There is a stimulation of the bone marrow by renal erythropoietin, which increases the red cell mass by approximately $30 \%$, over prepregnancy levels $(9,13)$. As the plasma volume increases more than the red cell mass, a 
Table 1 Physiologic Maternal Adaptation to Pregnancy

\begin{tabular}{|c|c|}
\hline System & Alterations \\
\hline Cardiovascular system & $\begin{array}{l}\text { Cardiac output } \mathrm{HR} \times \mathrm{SV}=\mathrm{CO} \\
\text { Increased } 20-30 \% \\
\text { Both heart rate and stroke volume } \\
\text { increased }\end{array}$ \\
\hline $\begin{array}{l}\text { Peripheral vascular } \\
\text { resistance }\end{array}$ & $\begin{array}{l}\text { Decreased as resistance vessels with } \\
\text { vasodilatation }\end{array}$ \\
\hline Blood flow & $\begin{array}{l}\text { Increased to } \\
\text { Uterus } \\
\text { Skin } \\
\text { Kidneys } \\
\text { Breasts }\end{array}$ \\
\hline Pulmonic circulation & $\begin{array}{l}\text { Blood volume increases equal } \\
\text { capacitance increase } \\
\text { No change in pulmonary artery } \\
\text { pressures }\end{array}$ \\
\hline Pulmonary system & $\begin{array}{l}\text { Tidal volume increased } \\
\text { Respiratory rate increased } \\
\text { Functional residual capacity reduced } \\
\text { Compensated respiratory alkalosis }\end{array}$ \\
\hline Renal system & $\begin{array}{l}\text { Renal artery perfusion increased } \\
\text { Glomerular filtration rate increased } \\
\text { Creatinine clearance increased } \\
\text { BUN, serum creatinine, serum uric } \\
\text { acid decreased } \\
\text { Renal clearance of drugs increased } \\
\text { Bladder muscularis relaxation } \\
\text { Urinary stasis infection risk } \\
\text { Dilated renal pelvises and ureters }\end{array}$ \\
\hline Gastrointestinal system & $\begin{array}{l}\text { Decreased gastric motility } \\
\text { Aspiration risk with anesthesia } \\
\text { Decreased colonic motility } \\
\text { Constipation complaints }\end{array}$ \\
\hline Hematologic system & $\begin{array}{l}\text { Plasma volume increases } 40-50 \% \\
\text { Red cell mass increases } 20-30 \% \\
\text { "Physiologic anemia" } \\
\text { Leukocytosis } \\
\text { Increased liver-produced clotting } \\
\text { factors } \\
\text { Increased fibrinogen } \\
\text { Hypercoagulable state }\end{array}$ \\
\hline
\end{tabular}

delusional effect occurs decreasing hemoglobin concentration (lower normal 10.5-11 g/dL), and hematocrit levels decrease to $30 \%$ to $35 \%$ (13). This phenomenon has been termed the "physiologic anemia" of pregnancy.

White blood cell (WBC) count increases by 5000 to 10,000 cells $/ \mathrm{mL}$ in the pregnant women. This is due to the stimulation of demargination of white cells from the endothelial wall by catecholamines and steroids. These cells are all mature polymorphogranular leukocytes. Care must be taken in interpreting the increased WBC counts in pregnancy $(9,13)$. The differential count is very helpful since if marrow elements are observed (bands or blasts), pregnancy adaptation is not the etiology of the leukocytosis and infection or inflammatory etiologies should be pursued.

Estrogen has a direct effect on the hepatocyte endoplasmic reticulum, leading to increased protein production. All liverproduced clotting factors (II, VII, IX, and X) are increased in pregnancy. Fibrinogen also increases by $20 \%$ to $30 \%$ with an average level of fibrinogen of approximately $400 \mathrm{mg}$ in normal pregnancy (14). The pregnant woman is in a hypercoagulable state from the first trimester of pregnancy. Pregnant woman undergoing prolonged procedures or placed immobile at bed rest requires venous thromboembolism prophylaxis to prevent thromboembolic events. Sequential pneumatic compression devices or heparin are used as prophylaxis. Unfractionated heparin due to its reversibility with protamine and ease of monitoring with a partial thromboplastin time is preferred over fractionated heparins in acute surgical situations. Subcutaneous heparin 5000 to 10,000 units BID are recommended as a prophylaxis.

\section{Renal Adaptations}

There is a significant increase in renal blood flow in pregnancy both to the glomeruli and to the renal parenchyma. Renal plasma blood flow and glomerular filtration rate increase by approximately $30 \%$ to $50 \%$ over prepregnant levels (15). This results in an increased creatinine, urea, and uric acid clearance, with a resultant decrease in serum creatinine (normal 0.5$0.9 \mathrm{mg} / \mathrm{dL}$ ), blood urea nitrogen (normal $10-15 \mathrm{mg} / \mathrm{dL}$ ), and uric acid (normal $2.5-3.5 \mathrm{mg} / \mathrm{L}$ ) (13-15). Drugs with renal clearance will have an increased clearance resulting in lower plasma and blood levels. Therefore, these doses need to be adjusted to account for this to maintain therapeutic blood and plasma levels during pregnancy (14). The muscularis of the bladder is relaxed by progesterone, and urinary stasis occurs. Urinary tract infections are common in pregnancy as the urethral vaginal angle is altered and vaginal bacteria may enter the bladder. If bladder catheterization is needed for more than 12 hours following a surgical procedure, antibiotic prophylaxis should be considered to prevent urinary tract infection.

\section{Gastrointestinal Adaptations}

Progesterone produces smooth muscle relaxation throughout the body. Gastric motility, gastric emptying, and small bowel transit time are all reduced in pregnancy. Pregnant women undergoing emergency surgery are at significant risk for the aspiration of gastric contents $(8,10,16)$. Whenever possible, regional anesthetic techniques are preferred over general inhalational anesthesia to reduce the risk of aspiration of gastric contents. It is recommended to premedicate gravida patients undergoing surgery with antacid preparations to reduce gastric $\mathrm{pH}(16-18)$. Agents to stimulate gastric emptying, such as metoclopramide, are also useful to reduce aspiration risk. Patients who require emergency general inhalational anesthesia may benefit from nasogastric aspiration of stomach contents prior to extubation. Premedication with antacids is also a recommended technique to reduce gastric $\mathrm{pH}$ and prevent possible pulmonary injury from aspiration of gastric contents.

\section{FETAL CONSIDERATIONS \\ Diagnostic Radiation Exposure}

Diagnostic radiologic procedures are often necessary as a preoperative assessment in patients needing nonobstetric surgery during their pregnancy. These procedures may be undertaken with care. Adverse fetal effects from ionizing radiation have been reported with fetal exposure of greater than $10 \mathrm{cGy}$. 
Microcephaly, intrauterine growth restriction, poor fetal development, and death have all been reported (19-21). Direct fetal exposure of $5 \mathrm{cGy}$ or less has not been shown to increase fetal malformation rates. There is a very small risk of increased childhood malignancies, particularly leukemias, with the level of radiation of $5 \mathrm{cGy}$ or less. Direct fetal exposure of $1 \mathrm{cGy}$ or less has not been shown to produce any significant fetal effect (19-21). Single-shot direct images such as chest X-rays, abdominal images, or images of long bones expose the fetus to little or no risk. Fluoroscopic examinations expose the fetus to significant radiation risk and should be avoided if possible in pregnancy. Computed tomography (CT) exams of the cranium and thorax expose the pelvis to very little direct radiation (0.05-0.1 cGy). These exams, if clinically indicated, may be undertaken with relative safety (22).

Abdominal and pelvic CT exams expose the fetus from 3 to $10 \mathrm{cGy}$ and should be avoided if possible during the first trimester. In the second and third trimesters, these exams may be performed, but care must be taken in making the decision to do these as the fetus will be exposed to a significant radiation dose $(22,23)$. If a significant alteration in the management will result from the information gained from abdominal and pelvic CT examinations performed during pregnancy, then the potential fetal risk is warranted $(22,23)$.

Magnetic resonance imaging (MRI) has not been extensively studied in pregnancy. This technology has been considered safe, to date, with no untoward fetal effects reported. MRI scanning may be considered a safe alternative to CT scan in the first trimester $(24,25)$. MRI exams have been shown to be helpful in the second and third trimesters to clarify diagnosis in fetal cranial anomalies and in determining aberrant placental implantations (accreta, increta, percreta). Gadolinium contrast agents should be avoided throughout the course of pregnancy, if possible.

Radionuclide procedures may be done in pregnancy; generally, the radiation dose to the fetus is low. Most radionuclide contrast agents are renal cleared. Indwelling bladder catheterization will reduce total fetal exposure by avoiding retained urine in the maternal bladder containing radioactive agents from increasing the total radiation dose to the fetus.

If the fetus is inadvertently exposed to significant amounts of ionizing radiation, it is important to calculate a fetal isodose radiation curve. Exposure of $10 \mathrm{cGy}$ or greater may produce significant fetal effect. Table 2 outlines the potential fetal effect from in utero radiation exposure (26) Tables 2,3.

\section{Anesthetic Effects}

Inhalation anesthetic agents (nitrous oxide, cyclopropane, diethyl ether, and halothane) are lipid soluble and readily cross the placenta. Animal models have raised some concern, as these agents have had teratogenicity in some animal models. The human data regarding these agents are not robust. However, anecdotal data, risk assessments, and epidemiologic assessments point to the relative safety of these agents $(14,16,17,27,28)$. Exposure to inhalation anesthetic agents in the first trimester of pregnancy (especially during the period of organogenesis 3-8 weeks) is estimated to produce a relative risk of malformation of approximately $0.2 \%$ to $0.5 \%$. Neural tube, limb, cardiac, and gastrointestinal abnormalities
Table 2 Estimated Radiation Dose to Pelvis at Ovarian Level from Common Radiologic Procedures

$\begin{array}{lc}\text { Procedure } & \text { Pelvic dose (cGy) } \\ \text { Chest radiography } & 8 \\ \text { Chest fluoroscopy } & 71 \\ \text { Abdominal radiography_series } & 289 \\ \text { Lumbar spine_-series } & 275 \\ \text { X-ray pelvimetry } & 40 \\ \text { Intravenous pyelogram } & 407 \\ \text { Barium enema } & \\ \quad \text { X-ray series } & 439 \\ \quad \text { Fluoroscopy } & 366 \\ \quad \text { Total } & 805 \\ \text { Upper GI } & \\ \quad \text { X-ray series } & 360 \\ \quad \text { Fluoroscopy } & 198 \\ \quad \text { Total } & 558 \\ \text { Source: From Ref. 112. Abbreviation: GI, gastrointestinal. } & \end{array}$

Table 3 Radiation Dose and Fetal Effect

\begin{tabular}{ll}
$\begin{array}{l}\text { Radiation dose } \\
\text { to fetus }\end{array}$ & \multicolumn{1}{c}{ Theoretical or actual fetal effect } \\
$0-5 c G y$ & $\begin{array}{c}\text { No reported malformation. Potential for } \\
\text { oncogenesis and increased cancer risk } \\
\text { Potential for oncogenesis, Potential for IUGR }\end{array}$ \\
$\begin{array}{l}\text { 5-10cGy } \\
10-20 \mathrm{cGy} \\
20-50 \mathrm{cGy}\end{array}$ & $\begin{array}{l}\text { Microcephaly, IUGR, 2.4\% mental retardation } \\
\text { Microcephaly, IUGR, fetal death, mental } \\
\text { retardation } \\
\text { Microcephaly, IUGR, 18\% mental retardation, } \\
\text { fetal death }\end{array}$ \\
Source: From Ref. 112. ${ }^{*}$ Abbreviation: IUGR, intrauterine growth restriction.
\end{tabular}

have all been reported. Regional block anesthetic techniques are recommended in the first trimester. If surgery is semielective and can be safely delayed until at least 12 weeks, the fetus will not suffer exposure to these agents during a critical organ-forming period. If, however, the clinical situation requires a general anesthetic technique in the first trimester, the physician and the patient can be reassured that overall fetal risks are small, then these agents should not be withheld if clinically indicated (29). A few studies have critically assessed anesthetic drugs in pregnancy and no consistent pattern of human malformation has been observed (28-30).

\section{Analgesic Agents}

Pain control in both the pre- and postoperative period may be obtained with the use of opiate narcotic agents. Morphine, meperidine, hydromorphine, and fentanyl administered orally intramuscular or intravenously in patient-controlled pumps or by delivery patch have demonstrated no adverse fetal effects in the short term $(29,31,32)$. Chronic opiate use in pregnancy has been associated with deleterious fetal effects. Intrauterine growth restriction and intrauterine and postnatal fetal/neonatal withdrawal have all been reported (29). In utero fetal withdrawal has been linked to intrauterine fetal demise (29). Chronic oral opiate use for pain control in pregnancy should be used with extreme caution. Codeine-containing compounds have been shown to have a small teratogenic effect and should be avoided if possible in the first trimester (29). In the second and third trimesters, these compounds may be used for short 


$\begin{array}{lc}\text { Table } 4 \text { Antibiotics in Pregnancy } & \\ \begin{array}{l}\text { Penicillin/cephalosporin } \\ \text { No adverse effect in } \\ \text { nonallergic patient }\end{array} & \begin{array}{c}\text { Sulfa drugs } \\ \text { Avoid first trimester }\end{array} \\ \begin{array}{cc}\text { Aminoglycosides } & \text { Third-trimester use } \\ \text { with bilirubin } \\ \text { displacement }\end{array} \\ \begin{array}{l}\text { Renal toxicity and ototoxicity } \\ \text { Use in life-threatening infections }\end{array} & \begin{array}{c}\text { Chlornicterus } \\ \text { Tetracycline } \\ \text { Contraindicated }\end{array} \\ \text { Staining of teeth } & \text { Gray baby syndrome } \\ \text { Bone demineralization } & \text { Fluoroquinolones } \\ \text { Source: From Ref. } 112 . & \text { Fetal effect-avoid use }\end{array}$

intervals with little fetal effect. Nonsteroidal inflammatory agents should be avoided, if possible. They have been shown to decrease fetal renal blood flow, which has resulted in significant oligohydramnios. After 32 weeks of gestation, these agents may cause permanent closure of the ductus arteriosus in utero with resultant fetal pulmonary hypertension (29). Prior to 32 weeks of gestation, intermittent ductal constriction occurs and may also lead to fetal pulmonary hypertension. As a tocolytic agent, short courses of indomethacin have been shown to be effective without profound significant fetal effect. Benzodiazipam compounds may be used and have not been shown to be associated with adverse fetal outcomes. At or near the time of delivery, these agents have been associated with significant neonatal depression and should be avoided during the parturient period $(18,30,33)$.

\section{Antibiotics}

Adjunctive antibiotic therapy is often indicated in nonobstetric surgery during pregnancy. Penicillin, penicillin derivatives, and first- and second-generation cephalosporins have no reported fetal adverse effects (29). Erythromycin base, clindamycin, and azithromycin are considered safe in pregnancy. Vancomycin, although considered relatively safe, has had some case reports of fetal renal toxicity. Aminoglycosides, although used when needed, have been associated with fetal ototoxicity and real toxicity $(18,29,34)$. Streptomycin and kanamycin have been reported to have both ototoxicity and renal toxicity. Gentamicin has not shown significant fetal ototoxicity and may be used with careful monitoring of maternal blood levels in life-threatening maternal infections (34).

Sulfonamides displace bilirubin from protein binding and if given near delivery (especially in the late third trimester) may lead to neonatal hyperbilirubinemia and kernicterus. These agents may be used for infections remote from delivery especially in the second trimester $(18,29,34)$. Tetracyclines need to be avoided throughout pregnancy as they lead to permanent staining of the teeth and abnormal long bone development $(18,29,34)$. Fluoroquinolones have been shown to disrupt fetal cartilage production leading to skeletal abnormalities and they are best avoided throughout pregnancy (Table 4).

\section{Preoperative, Inoperative, and Postoperative Evaluation and Management}

The preoperative evaluation of the pregnant patient should include all the elements one would perform if the patient were not pregnant plus an antenatal assessment of the fetus and pregnancy to assess for the effect of the condition needing the surgery on the outcome of the pregnancy. Determination of gestational age, fetal well-being by nonstress test or biophysical profile assessment, assessment of the maternal cervical status, and an assessment for contractions should be carried out. Laboratory assessment, imaging studies, and preoperative assessments must take into account the maternal adaptations of pregnancy and gestational age. Ionizing radiation studies should be limited and fetal status should be considered before any imaging studies are performed.

If elective or semi-elective procedures are needed, it is best to wait until the second trimester of pregnancy if possible. If emergency surgery is needed, it can be done in any trimester. In the first trimester, if regional anesthesia is a choice, it is preferable over general anesthesia due to the small teratogenic risk of general anesthesia during the first trimester of pregnancy. Before proceeding to the operating room, the patient should be given $30 \mathrm{~mL}$ of $0.3 \mathrm{~m}$ sodium citrate (bicitra) to help neutralize gastric $\mathrm{pH}$ and decrease the risk of chemical pneumonitis if aspiration of gastric contents were to occur (35).

\section{Intraoperative Management}

In the second and third trimesters, if non-abdominal surgery is being performed, the patient should be positioned with the uterus displaced to the maternal left side, to improve the vena cava return to the heart. This is best accomplished with a wedge placed under the maternal right hip. It is important to maintain maximal oxygenation status keeping maternal $\mathrm{O}_{2}$ saturation above $90 \%$ to $95 \%$ to meet fetal oxygen needs $(36,37)$. Maternal blood pressure should be kept in the normal range to continue normal uterine blood flow, and if pressors are needed during a procedure, dopamine or isoproterenol at lower doses do not disrupt uterine artery flow. Hypotension secondary to regional anesthesia may be corrected with phenylepherine with little adverse effect to the fetus. After viability, 23 to 24 weeks of gestation, in nonabdominal surgery, continuous fetal monitoring is indicated. If excessive blood loss occurs, transfusion of packed RBCs is needed to maintain maternal hemoglobin above $8 \mathrm{~g} / \mathrm{dL}$ to insure adequate uteroplacental perfusion.

If intra-abdominal procedures are being performed either laparoscopically or via open technique, care must be taken to avoid excessive manipulation of the pregnant uterus to prevent the stimulation of uterine contractions.

\section{Postoperative Management}

The patient should be recovered in an obstetric unit with continuous fetal monitoring. Contraction monitoring with external tocodynamometer should be done. If preterm contractions are observed, hydration with IV fluids often will alleviate them, as most often maternal dehydration is the etiology of these contractions. If tocolytic medications are needed, terbutaline $0.25 \mathrm{mg}$ subcutaneously or nifedipine $10 \mathrm{mg}$ PO every 20 minutes for three doses often will stop these contractions (38). Precipitation of frank labor and subsequent fetal delivery is a rare event following nonobstetric surgery in pregnancy. If ovarian cysts are removed prior to 15 weeks of gestation, particularly if the corpus luteum is felt to have been removed, progesterone supplementation may be considered to 
reduce fetal loss (38). Analgesia for pain can be accomplished with the use of opiate narcotics. Codeine or opiate narcotics combined with acetaminophen as discussed earlier are often safe in pregnancy. Nonsteroidal anti-inflammatory agents should be avoided due to their risk of oligohydramnios and disruption of ductus arteriosus flow. All ancillary pharmacologic agents needed, such as antiemetics, antibiotics, antihypertensive medications, should be reviewed for their fetal safety profile before administration. If labor ensues, a recent abdominal incision does not contraindicate valsalva in the second stage of labor and vaginal birth should be performed. Cesarean section should be reserved for routine obstetric indications (39).

\section{Laparoscopic Surgery in Pregnancy}

Laparoscopic surgical procedures may be performed in the first and second trimesters of pregnancy with relative fetal and maternal safety. These procedures offer the advantage of shorter recovery time, less blood loss, and shorter hospital length of stay. A theoretic risk to the fetus exists with insufflations of the abdominal cavity (40-44). As intraabdominal pressure rises, uterine blood flow may be compromised. This could potentially lead to fetal hypoxemia, acidosis, and injury. To prevent this, it is best to keep the intraabdominal pressure as low as possible (43). A maximum intraabdominal pressure of $15 \mathrm{mmHg}$ has been advocated (43). There is also the potential risk of perforating the uterus with the veres needle or trochar, which could directly injure the fetus or lead to ruptured membranes and preterm delivery (45). Veres needle insertion under ultrasonic guidance can reduce this risk. The trochar should be positioned at least $6 \mathrm{~cm}$ above the uterine fundus (45). This may require a supraumbilical right or left upper quadrant insertion site. Tenting of the abdominal wall away from the uterus has also proven effective. In the mid-to-late second-trimester open laparoscopic techniques have proven safer than traditional veres needle and trochar insertions $(41,43)$. Maternal respiratory functions need to be closely monitored. Arterial or transcutaneous carbon dioxide monitoring has been recommended. Maternal end-tidal volume carbon dioxide determination may not be as informative a measure due to the carbon dioxide absorption that is changed because of pregnancy. As with all proposed surgical procedures if the laparoscopic procedure can be safely delayed until after the pregnancy is completed, it should be done at that time $(41,43)$.

\section{SPECIFIC SURGICAL PROCEDURES}

Any surgical procedure performed during pregnancy can pose some risk for the mother and the fetus. As pointed out in the previous sections of this chapter, maternal adaptation to pregnancy may increase the risk of thromboembolism, organ injury, hemorrhage, and the onset of labor. Fetal risks due to pharmacologic agents, anesthesia, diagnostic radiographic procedures, and the risk of preterm birth are low but remain significant. Therefore, any surgical procedure performed during pregnancy needs to be carefully considered. Purely elective procedures for nonthreatening conditions are best delayed until pregnancy is completed. However, there are some conditions that require intervention during pregnancy. Some urgent but not emergent procedures are best delayed until after the first trimester; however, emergency conditions that require surgical intervention should not be delayed despite the maternal pregnancy.

\section{Breast Disease in Pregnancy}

Breast malignancies are not an uncommon occurrence in pregnancy; approximately 10,000 pregnancies per year are associated with breast cancer (46). The prognosis of pregnant patients with breast carcinoma is poorer than those without pregnancy, not due to blood flow changes or hormonal milieu but due to a delay in diagnosis. Physiologic and anatomic alterations of the breast function and anatomy may mask parenchymal breast lesions and self-discovery of a mass or discovery by physical exam may be delayed until a mass has grown to a larger size $(47,48)$. Breast engorgement, proliferation of glands and ducts, lactation, and increased vascularity all contribute to this phenomenon $(48,49)$.

The incidence of breast carcinoma in pregnancy is 1 in 3300 to 5000 pregnant women (47-49). The prognosis is poorer in pregnant women due to delay in diagnosis with approximately $75 \%$ of pregnant patients with breast carcinoma having positive axillary lymph nodes at the time of diagnosis. Fiveyear survival rates have been reported as low as $17 \%$ in this group of patients $(49,50)$. The key to improved outcome is early diagnosis. There should be no reluctance to biopsy of any suspicious mass due to the patient's pregnant state. Needle aspiration, fine needle biopsy, or open biopsy all may be performed. Milk abscess, galactorrhea at the biopsy site, and infection are more common with biopsies performed in pregnancy (50). Imaging lesions with mammography is less sensitive in pregnancy due to increased radio density of the breast in pregnancy and suspicious lesions may be missed (48-50). Ultrasound and MRI are less affected by the anatomic alterations of pregnancy and are recommended as imaging techniques in pregnancy.

The management of a breast carcinoma in pregnancy is similar to the nonpregnant state. Mastectomy is more often performed, as radio therapy is relatively contraindicated in pregnancy as there is significant spread of radiation to the pelvis even with shielding $(50,51)$. Chemotherapy may be used in pregnancy in the second and third trimesters with little fetal effect. Agents such as methotrexate or taxol may cause fetal death and are best avoided (50-53). Alkylating agents such as cytoxan, 5-fluorouracil, and adriamycin also may be used. Chemotherapy should be avoided in the first trimester if possible. Breast carcinoma may metastasize to the placenta, but there have been no reported case of fetal metastases to date. Pregnancy termination has not been shown to effect maternal outcome. The decision to terminate a pregnancy, if first trimester chemotherapy is needed, for fetal indication, should be discussed with the patient. A full explanation of fetal and maternal risks should be undertaken (50-53).

\section{Appendicitis in Pregnancy}

The most common general surgical condition complicating pregnancy is appendicitis. Acute appendicitis occurs in approximately 1 in 1500 gestations (54). The clinical symptoms of appendicitis are similar in pregnancy to the nonpregnant state: right lower quadrant pain, nausea, vomiting, pyrexia, anorexia, and malaise. In the past, the 
location of the pain in acute appendicitis was felt to change with the changing gestational age and the enlarging uterus moving the cecum higher into the abdomen with the thirdtrimester acute appendicitis pain found in the right upper quadrant. However, it has now been shown that most patients with appendicitis in pregnancy will have right lower quadrant pain regardless of their gestational age $(55,56)$. Some patients, however, will have the anatomic location of the appendix change and appendicitis needs to be considered when patients present with right upper quadrant pain or even flank pain in the case of retrocecal appendix.

Clinical symptoms of nausea, vomiting, and anorexia are common in early pregnancy and sometimes delay in the diagnosis of appendicitis occurs $(55,56)$. Leukocytosis is often associated with appendicitis. However, there is a physiologic leukocytosis of pregnancy as outlined earlier. The leukocytosis of pregnancy may be as high as 20,000 to $30,000 \mathrm{cell} / \mathrm{cm}^{3}$ (57). All of these cells are mature polymorphogranular leukocytes. If bands or marrow elements are observed, infection and inflammation should be suspected. The differential count, therefore, is very helpful in establishing this diagnosis.

Physical findings of acute peritonitis such as guarding and rebound may not be found in the pregnant women with acute appendicitis. The gravid uterus may tent the parietal peritoneum and omentum away from the inflamed appendix, thus producing less peritoneal inflammation and less abdominal wall symptoms (58-60). Imaging may be performed. Ultrasound of the appendix with graded compression has been shown to be very sensitive $(86 \%)$ and specific $(81 \%)(61,62)$. Ultrasound due to its fetal safety should be the image modality of choice. MRI has also been shown to be very sensitive and specific for the diagnosis of appendicitis in pregnancy (96\% and $93.6 \%$ respectively) and should be used in cases were ultrasound is inconclusive (63-66). CT exams are also very sensitive (94\%) and specific (95\%) in the diagnosis of acute appendicitis in pregnancy. Although the risk is low, there is concern for fetal exposure to ionizing radiation and these exams should be used if ultrasound is inconclusive and MRI is not available $(67,68)$.

Once a diagnosis is made or strongly considered, laparoscopy or laparotomy and appendectomy should be performed. Delay of more than 24 hours increases the risk of perforation, which will occur in $14 \%$ to $43 \%$ of patients. Preterm labor and delivery are much higher if appendiceal perforation occurs (36\% vs. 15\%) (69,70). In the third trimester, cesarean section at the time of appendectomy is not indicated unless there is an obstetric indication. After the appendectomy procedure, in the third trimester, if the patient goes into labor, vaginal delivery may occur and the risk of rupture of the abdominal wall repair is low. In the late second and throughout the third trimesters, laparotomy is performed with relative maternal and fetal safety. In the first and early second trimesters, laparoscopic approach may be entertained with care in technique as described earlier.

\section{Biliary Disease in Pregnancy}

The maternal adaptations to pregnancy predispose to the formation of cholesterol, cholelithiasis in pregnancy (71). Biliary disease requiring surgery occurs in 0.2 to 0.5 per 1000 pregnant women (72). Biliary sludge or small gallstones have been found on ultrasound in $5 \%$ to $8 \%$ of pregnant women in the late second and early third trimesters (71-73). Symptoms of acute cholecystitis in pregnancy are similar to the nonpregnant state: nausea, vomiting, right upper quadrant pain, and anorexia. Imaging with ultrasound will show stones, sludge, and wall thickening (73-75). Management is usually conservative. Most patients will improve with supportive care, nothing-by-mouth, IV hydration, analgesics, and low-fat diet. If cholecystitis or cholangitis is suspected, antibiotics may be added (76). If there are progressive symptoms unresponsive to supportive therapy, surgery is indicated. The best time period to perform these surgeries, with the least pregnancy effect, is the second trimester. However, cases that require immediate intervention such as perforation, gangrene, progressive fever, or intractable pain should have surgery regardless of the gestational age. In the first and early second trimesters, laparoscopic techniques can be performed (75-77). There have been case reports pointing to the safety of these techniques in early pregnancy. In the late second and third trimesters, laparotomy is needed. Occasionally, with cholecystectomy, uterine contractions have been produced and tocolytic agents are needed. Most patients who require cholecystectomy in pregnancy do well.

\section{Ovarian Masses}

Pathologic adnexal masses have been estimated to complicate $0.2 \%$ to $2 \%$ of all pregnancies (78). As many as $10 \%$ of pregnant women will have a cyst of at least $5 \mathrm{~cm}$ in the first trimester of pregnancy. By 10 to 11 weeks of gestation, only $1.3 \%$ of pregnant patients have a persistent significant ovarian cyst $(79,80)$. The appearance of the mass on ultrasound is very important in differentiating between benign and malignant lesions. Solid components, excrescences into the cyst, abnormal blood flow patterns, and Doppler assessments are suggestive of malignancy $(79,80)$. Fortunately, most ovarian masses diagnosed during pregnancy are not malignant. Leiserowitz and colleagues reported on 9000 pregnant women with ovarian masses in 2006 . Only $0.93 \%$ of these masses were malignant, and more than half (57\%) of these malignant masses were of low malignant potential (78).

Most ovarian lesions are found inadvertently on an ultrasound. Occasionally, symptoms of pain may prompt on ultrasonic investigation. The most common neoplasm found in pregnancy is cystadenoma (33\%), followed by mature teratoma (dermoid; 27\%). Biochemical tumor markers such as CA125 and alpha-fetoprotein are elevated in normal pregnancy and are not very useful in the management of these lesions in pregnant women (79).

Most ovarian lesions found in pregnancy may be treated conservatively. Even if they are found to be malignant at a later time, most are slow growing and no further spread would have occurred due to the delay $(81,82)$. There are however some ovarian masses that must be surgically removed in pregnancy. These are ruptured hemorrhagic cysts, acute ovarian torsions, and those masses that are highly suspicious for malignancy. It is reported that approximately 1 in 1300 pregnancies will require surgery for an ovarian mass during pregnancy $(81,82)$.

The mass that is growing during pregnancy or presents at a size greater than $6 \mathrm{~cm}$ into the second trimester will require surgical evaluation. Patients who present with acute pain or an 
acute abdomen due to torsion or ovarian cyst rupture also require acute surgical intervention (81-83). Highly suspicious findings on ultrasound, for malignancy in an ovarian mass such as size $>10 \mathrm{~cm}$, nodularity, cystic and solid components, internal septation, abdominal ascites, or pelvic fixation also require surgical exploration. If the patient is near term, exploration for these masses may be delayed until the postpartum period. If found in the second and early third trimesters, surgical exploration should not be delayed $(83,84)$. First-trimester masses that do not present with acute symptoms could have surgical exploration delayed until at least 11 to 12 weeks of gestation (84-88).

If a functional ovarian cyst (corpus luteum) is removed before 12 weeks of pregnancy, the patient should be given progesterone supplementation. This may be administered as an IM injection of $50 \mathrm{mg}$ of progesterone in oil daily or via vaginal suppository $100 \mathrm{mg}$ twice daily. This supplementation may be discontinued after 12 to 14 weeks of gestation (86).

An adnexal mass incidentally encountered during a c-section should be removed at the time of the c-section by cystectomy. Simple aspiration is not recommended as seeding of malignancy to the peritoneal cavity may occur if the mass is malignant.

\section{Cardiac Thoracic Surgery During Pregnancy}

Cardiac disease complicates approximately $1 \%$ to $1.5 \%$ of pregnancies. In the past, rheumatic heart disease once was considered the most common cardiac condition complicating pregnancy. However, as more patients with congenital heart disease are now surviving to reach childbearing age, congenital heart disease, either surgically corrected or uncorrected, has become the most common condition complicating pregnancy (86). Most cardiac conditions do not require corrective surgery during pregnancy. Congenital cardiac lesions are best corrected prior to attempting pregnancy or postponed until pregnancy has been completed. If patients however decompensate during pregnancy with pulmonary edema, hemoptysis, or heart failure unresponsive to medical interventions, then either discontinuation of the pregnancy or cardiac surgery should be entertained $(7,89)$.

The approach to any planned cardiothoracic surgical intervention during pregnancy must account for the significant physiologic and anatomic alterations produced in pregnancy. These were reviewed earlier in this chapter. The diagnostic assessment of a pregnant patient with cardiac disease does not differ from the nonpregnant patient. These include a careful history and physical assessment and ancillary diagnostic studies. The ancillary diagnostic studies include electrocardiogram, echocardiogram, hemoglobin/ hematocrit, chest X-ray, and rarely cardiac catheterization or stress electrocardiogram or echocardiograms. The optimal gestational age to perform cardiac surgery during pregnancy is 14 to 20 weeks of gestation $(89,90)$. After 28 to 32 weeks of gestation, the peak cardiovascular effects at pregnancy occur increasing the potential operative morbidity. However, regardless of the gestational age, if the maternal condition warrants intervention, it should be undertaken. After 30 to 32 weeks of gestation, consideration for preterm delivery of the fetus should be entertained prior to maternal cardiac surgery.
If necessary, cardiopulmonary bypass procedure can be performed in pregnant patients, but care must be taken to prevent hypotension and decreased placental profusion $(89,90)$. Alteration in bypass technique to adjust flow ratios to avoid these systemic volume shifts is needed to prevent disruption of adequate placental perfusion and fetal oxygenation $(89,90)$. Continuous fetal monitoring is helpful in monitoring the adequacy of fetal placental perfusion while the patient is on cardiopulmonary bypass. It is also vital to maintain maternal temperature to prevent maternal hypothermic response, which can also disrupt placental blood flow $(91,92)$.

Valvuloplasty with catheterization balloon technique has been shown to be safe during pregnancy and may be an alternative to open valvuloplasty during pregnancy $(93,94)$. There have been reports of fetal decelerations and bradycardia during balloon inflation. There have been rare reports of placental abruption due to maternal hypotension. Therefore, these procedures should be undertaken only if there is immediate availability of cesarean operation with personal resources available $(93,94)$.

Rarely, coronary bypass surgery is necessary during pregnancy. There have been case series reports of successful outcomes for pregnancy in these patients (92). However, due to the length of time needed on bypass, these procedures are best delayed until after pregnancy, if possible. Robotic bypass surgery with beating heart technique, with no cardiopulmonary bypass, may offer a safer alternative to coronary artery bypass perfusion if coronary artery bypass is needed during pregnancy. These procedures are relatively new and little data of these during pregnancy exist.

\section{Neurosurgery During Pregnancy}

Brain neoplasms requiring neurosurgical intervention in pregnancy is an uncommon event. There are approximately 90 to 100 cases reported annually in pregnant patients in the United States (95). Most benign lesions will not require surgical interventions during pregnancy and definitive treatment may sometimes be delayed until pregnancy has been completed $(95,96)$. If malignant neoplasm is suspected and is potentially operable, surgery should not be delayed and intervention is warranted. Benign lesions that cause significant symptoms such as increased intracranial pressure, intractable headaches, or disk disease may require surgical intervention during pregnancy.

Agents to treat cerebral edema such as mannitol and dexamethasone may be used during pregnancy. There are little data regarding the use of stereotactic radiosurgery to treat deep inoperable lesions during pregnancy and fetal safety has not been established. However, if the risk-benefit ratio for the mother warrants these procedures, they can be entertained with full explanation of the small but potential fetal risks discussed $(19,97,98)$.

Patients with intracranial lesions or increased intracranial pressure do not require cesarean section. Vaginal delivery with avoidance of maternal valsalva maneuvers is the preferred route of delivery. The second stage of labor is shortened by employing vacuum or forceps delivery techniques (99).

\section{Traumatic Injury in Pregnancy}

Traumatic injury in pregnancy is the most common cause of maternal mortality not related to obstetric factors. 
Approximately $6 \%$ to $7 \%$ of all pregnant patients will suffer a traumatic injury during pregnancy, but only about $1 \%$ of these patients are seriously injured enough to require hospitalization (100). The pregnant patient is much less likely to demonstrate signs of hypovolemia and shock after acute blood loss due to the increased maternal blood volume that manifests during gestation. However, intravascular volume shifts decreasing uterine blood flow occur early in hypovolemia maternal states, leading to fetal compromise. The abdominal position at the uterus in the third trimester makes this organ much more susceptible to traumatic injury especially in cases of penetrating traumatic injury.

The most common traumatic injury seen in pregnancy are motor vehicle accidents accounting for $60 \%$ of traumatic injuries in pregnant women. Pregnancy outcome is directly related to the severity of the maternal injuries. The most common cause of fetal loss is maternal death $(100,101)$. The most common obstetric complication following blunt of deceleration injury is placental abruption occurring in approximately $2 \%$ to $4 \%$ of patients suffering these injuries (101-103). Most abruptions will occur within the first 4 to 8 hours following the injury. Ultrasound has shown poor positive predictive value for the diagnosis of abruption (103).

Uterine contraction monitoring has a high negative but low positive predictive value for the diagnosis of posttraumatic abruption. The absence of uterine contractions following blunt trauma or deceleration is highly predictive of no abruption having occurred $(104,105)$. All Rh-negative patients should receive Rhogam, $300 \mathrm{mg} / \mathrm{IM}$. Delayed abruption, up to 48 hours post-injury, has been observed although there is no test to accurately predict delayed abruption (104). Patients with significant fetal-maternal hemorrhage are at greater risk. A positive Kleheiur-Betkie test for fetal-maternal hemorrhage should warrant a longer period of post-injury observation (104).

Penetrating injuries to pregnant women are most commonly knife or firearm wounds. As the large muscular uterus protects the maternal intra-abdominal organs, pregnant patients have a better prognosis from penetrating abdominal trauma. However, the fetus is at significant risk due to the anterior and central location of the uterus in the maternal abdomen. The fetus sustains injury in over $60 \%$ of those cases with a $40 \%$ to $70 \%$ fetal mortality rate (106-108). Both conservative and surgical management in these cases have been advocated. The best approach is to individualize with surgical exploration performed when fetal or maternal conditions warrant $(107,108)$.

\section{Burn Injuries in the Pregnant Patient}

Patients who suffer burn injuries in pregnancy should not have their management altered due to the pregnancy (109). Antibiotics, fluid replacement, and oxygen administration remain the recommended therapeutic approach. The severity of the maternal injury and any secondary maternal complications will dictate the fetal outcome. When the pregnant patient suffers a greater than $50 \%$ body surface burn injury, fetal mortality rate approaches $100 \%$. Where there is a $50 \%$ or greater burn injury to the pregnant patient in the third trimester, delivery of the fetus is indicated (110). With lesser degrees of surface area involvement (30\% or less), fetal survival approaches $80 \%$ (110). Steroids to enhance fetal pulmonary maturity are indicated in these pregnant patients with significant burn injury who are remote from term. Maternal septic complications may lead to labor or the development of chorioamnionitis. Mainstays of burn therapy such as antibiotics, immunoglobulin therapy, and tetanus toxoid are not contraindicated in pregnancy and should be given to these patients as needed (111). The optimal pregnancy outcome will occur when therapy is early and aggressive and not delayed due to the patient's pregnant state.

\section{SUMMARY}

Nonobstetric surgical procedures may be undertaken during pregnancy, when indicated, with relative fetal and maternal safety. A thorough understanding of the maternal adaptations of pregnancy and potential fetal risks of diagnostic studies or pharmacologic agents, as well as any potential effect to the pregnancy, is necessary before proceeding with any procedure. In most cases, this will result in a good outcome for both the mother and the fetus.

\section{REFERENCES}

1. Christianson RE. Studies on blood pressure during pregnancy. I. Influence of parity and age. Am J Obstet Gynecol 1976; 125: 509-13.

2. Hill LM. Prophylactic use of hydroxyprogesterone caproate in abdominal surgery during pregnancy: a retrospective evaluation. Obstet Gynecol Clin North Am 1975; 46: 287

3. Metcalfe J, Ueland K. Maternal cardiovascular adjustments to pregnancy. Prog Cardiovasc Dis 1974; 16: 363-74.

4. Adams JQ, Alexander AM Jr. Alterations in cardiovascular physiology during labor. Obstet Gynecol 1958; 12: 542-9.

5. Bader RA, Bader ME, Rose DJ, et al. Hemodynamics at rest and during exercise in normal pregnancy as studied by cardiac catheterization. J Clin Invest 1955; 34: 1524.

6. Elkayam UG. Cardiovascular physiology of pregnancy. Cardiac Problems in Pregnancy. New York: Alan R. Liss, 1982.

7. Ueland K, Novy MJ, Peterson EN, Metcalfe J. Maternal cardiovascular dynamics. IV. The influence of gestational age on the maternal cardiovascular response to posture and exercise. Am J Obstet Gynecol 1969; 104: 856-64.

8. Caton WL, Roby CC, Reid DE, et al. The circulating red cell volume and body hematocrit in normal pregnancy and the puerperium by direct measurement using radioactive red cells. Am J Obstet Gynecol 1951; 61: 1207-17.

9. Lund CJ, Donovan JC. Blood volume during pregnancy. Significance of plasma and red cell volumes. Am J Obstet Gynecol 1967; 98: 394-403.

10. Barton WM. The pregnant surgical patient: medical evaluation and management. Ann Intern Med 1987; 101: 683.

11. Weinberger SE, Weiss ST, Cohen WR, Weiss JW, Johnson TS, Pregnancy and the lung. Am Rev Respir Dis 1980; 121: 559-81.

12. Awe RJ, Nicotra MB, Newsom TD, Viles R. Arterial oxygenation and alveolar-arterial gradients in term pregnancy. Obstet Gynecol 1979; 53: 182-6.

13. Pritchard JA, Rowland RC. Blood volume changes in pregnancy and the puerperium. Am J Obstet Gynecol 1964; 88: 391.

14. Barron WM. Medical evaluation of the pregnant patient requiring nonobstetric surgery. Clin Perinatal 1985; 12: 481.

15. Barron WM, Lindheimer MD. Renal sodium and water handling in pregnancy. Obstet Gynecol Annu 1984; 13: 35-69.

16. Brodsky JB. Anesthesia and surgery during early pregnancy and fetal outcome. Clin Obstet Gynecol 1983; 26: 449-57.

17. Otake M, Schull WD. In utero exposure to A-band radiation and mental retardation. Br J Anaesth 1984; 129: 611.

18. Pedersen H, Finster M. Anesthetic risk in the pregnant surgical patient. Anesthesiology 1979; 51: 439.

19. Brent RL. The effects of embryonic and fetal exposure to X-ray, microwaves, and ultrasound. Clin Obstet Gynecol 1983; 26: 484-510. 
20. Mossman KL, Hill LT. Radiation risks in pregnancy. Obstet Gynecol 1982; 60: 237-42.

21. Houston CS. Diagnostic irradiation of women during the reproductive period. Can Med Assoc J 1977; 117: 648-51.

22. Wagner LK, Archer BR, Zeck OF. Conceptus dose from two state-ofthe-art CT scanners. Radiology 1986; 159: 787-92.

23. Forested DH, Kalbhon CL. CT of pregnant women for urinary tract calculi, pulmonary thromboembolism and acute appendicitis. AJR Am J Roentgenol 2002; 178: 1285.

24. Weinreb JC, Lowe TW, Santos-Ramos R, Cunningham FG, Parkey R. Magnetic resonance imaging in obstetric diagnosis. Radiology 1985; 154: $157-61$.

25. Shellock FG. Bioeffects and safety of MRI procedures, in Edelman. In: Edelman RR, Hesselink JR, Zlatkin MB, eds. Clinical Magnetic Resonance Imaging, 4th edn. WB Saunders, 2000: 935.

26. Smith EM, Warner GG. Estimates of radiation dose to the embryo from nuclear medicine procedures. J Nucl Med 1976; 17: 836-9.

27. Kammerer WD. Non-obstetric surgery in pregnancy. Med Clin North Am 1987; 71: 551.

28. Smith BE. Fetal prognosis after anesthesia during gestation. Anesth Analg 1963; 42: 521.

29. Briggs GG, Bodendorfer TW, Freeman RK, et al. Drugs in pregnancy and lactation: a reference guide to fetal and neonatal risk. Williams \& Wilkins, 1994: 190

30. Mazze RI, Kallen B. Reproductive outcome after anesthesia and operation during pregnancy: a registry study of 5405 cases. Am J Obstet Gynecol 1989; 161: 1178-85.

31. Kalter H, Warkany J. Congenital malformation. N Engl J Med 1983; 308: 491.

32. Abboud TK, Raya J, Noueihed R, Daniel J. Intrathecal morphine for relief of labor pain in a parturient with severe pulmonary hypertension. Anesthesiology 1983; 59: 477-9.

33. Knill-Jones RP, Newman BJ, Spence AA. Anesthetic practice and pregnancy. Lancet 1975; 2: 807.

34. Shepard TH. Human teratogenicity. Adv Pediatr 1986; 33: 225-68.

35. Gibbs CP, Banner TC. Effectiveness of Bicitra as a preoperative antacid. Anesthesiology 1984; 61: 97-9.

36. Barcroft J. On anoxaemia. Lancet 1920; 2: 485.

37. Sidi D, Kuipers JR, Teitel D, Heymann MA, Rudolph AM. Developmental changes in oxygenation and circulatory responses to hypoxemia in lambs. Am J Physiol 1983; 245: H674-82.

38. Podratz KC, Field CS. Ovarian tumors during pregnancy. In: Cibilis La, ed. Surgical Disease in Pregnancy. New York: Springer-Veritag, 1990.

39. Mazze RI, Kallen B. Appendectomy during pregnancy: a Swedish registry study of 778 cases. Obstet Gynecol 1991; 77: 835-40.

40. Morrell DG, Mullins JR, Harrison PB. Laparoscopic cholecystectomy during pregnancy in symptomatic patients. Surgery 1992; 112: 856-9.

41. Curet MJ, Allen D, Josloff RK, et al. Laparoscopy during pregnancy. Arch Surg 1996; 131: 546-50; discussion 550-1.

42. Gurbuz AT, Peetz ME. The acute abdomen in the pregnant patient. Is there a role for laparoscopy? Surg Endosc 1997; 11: 98-102.

43. Nezhat FR, Tazuke S, Nezhat CH, et al. Laparoscopy during pregnancy: a literature review. JSLS 1997; 1: 17-27.

44. Affleck DG, Handrahan DL, Egger MJ, Price RR. The laparoscopic management of appendicitis and cholelithiasis during pregnancy. Am J Surg 1999; 178: 523-9.

45. Yuen PM, Ng PS, Leung PL, Rogers MS. Outcome in laparoscopic management of persistent adnexal mass during the second trimester of pregnancy. Surg Endosc 2004; 18: 1354-7.

46. White TT. Prognosis of breast cancer for pregnant and nursing women; analysis of 1,413 cases. Surg Gynecol Obstet 1955; 100: 661-6.

47. Deemarsky LJ, Nieshtadt EL. Breast cancer and pregnancy. Breast 1981; 7: 17.

48. Dupont WD, Page DL. Breast cancer risk associated with proliferation disease, age at first birth, and a family history of breast cancer. Am J Epidemiol 1987; 125: 769.

49. Dupont WD, Page DL. Risk factors for breast cancer in women with proliferative breast disease. N Engl J Med 1985; 312: 146-51.

50. Hubay CA, Barry FM, Marr CC. Pregnancy and breast cancer. Surg Clin North Am 1978; 58: 819-31.

51. Zinns JS. The association of pregnancy and breast cancer. J Reprod Med 1979; 22: 297-301.
52. Wilkinson EJ, Schuettke CM, Ferrier CM. Fine needle aspiration of breast masses: analysis of 276 aspirates. Acta Cytol 1989; 33: 613.

53. Harvey JC, Rosen PP, Ashikari R, Robbins GF, Kinne DW. The effect of pregnancy on the prognosis of carcinoma of the breast following radical mastectomy. Surg Gynecol Obstet 1981; 153: 723-5.

54. Tamir IL, Bongard FS, Klein SR. Acute appendicitis in the pregnant patient. Am J Surg 1990; 160: 571-5; discussion 575-6.

55. Mourad J, Elliott JP, Erickson L, Lisboa L. Appendicitis in pregnancy: new information that contradicts long-held clinical beliefs. Am J Obstet Gynecol 2000; 182: 1027-9.

56. Hodjati $\mathrm{H}$, Kazerooni T. Location of the appendix in the gravid patient: a re-evaluation of the established concept. Int J Gynaecol Obstet 2003; 81: $245-7$.

57. Cunningham FG, McCubbin JH. Appendicitis complicating pregnancy. Obstet Gynecol 1975; 45: 415-20.

58. Sharp HT. Gastrointestinal surgical conditions during pregnancy. Clin Obstet Gynecol 1994; 37: 306-15.

59. McGee TM. Acute appendicitis in pregnancy. Aust NZ J Obstet Gynaecol 1989; 29: 378-85.

60. Bailey LE, Finley RK Jr, Miller SF, Jones LM. Acute appendicitis during pregnancy. Am Surg 1986; 52: 218-21.

61. Lim HK, Bae SH, Seo GS. Diagnosis of acute appendicitis in pregnant women: value of sonography. AJR Am J Roentgenol 1992; 159: 539-42.

62. Barloon TJ, Brown BP, Abu-Yousef MM, Warnock N, Berbaum KS. Sonography of acute appendicitis in pregnancy. Abdom Imaging 1995; 20: $149-51$

63. Cobben LP, Groot I, Haans L, Blickman JG, Puylaert J. MRI for clinically suspected appendicitis during pregnancy. AJR Am J Roentgenol 2004; 183: 671-5.

64. Pedrosa I, Levine D, Eyvazzadeh AD, et al. MR imaging evaluation of acute appendicitis in pregnancy. Radiology 2006; 238: 891-9.

65. Birchard KR, Brown MA, Hyslop WB, Firat Z, Semelka RC. MRI of acute abdominal and pelvic pain in pregnant patients. AJR Am J Roentgenol 2005; 184: 452-8.

66. Oto A, Ernst RD, Shah R, et al. Right-lower-quadrant pain and suspected appendicitis in pregnant women: evaluation with MR imaging-initial experience. Radiology 2005; 234: 445-51.

67. Ames Castro M, Shipp TD, Castro EE, et al. The use of helical computed tomography in pregnancy for the diagnosis of acute appendicitis. Am J Obstet Gynecol 2001; 184: 954.

68. Wagner LK, Huda W. When a pregnant women with suspected appendicitis is referred for a CT scan, what should a radiologist do to minimize potential radiation risk? Pediatr Radiol 2004; 3: 589.

69. Bickell NA, Aufses AH Jr, Rojas M, Bodian C. How time affects the risk of rupture in appendicitis. J Am Coll Surg 2006; 202: 401-6.

70. Yilmaz HG, Akgun Y, Bac B, Celik Y. Acute appendicitis in pregnancyrisk factors associated with principal outcomes: a case control study. Int J Surg 2007; 5: 192-7.

71. Stauffer RA, Adams A, Wygal J, Lavery JP. Gallbladder disease in pregnancy. Am J Obstet Gynecol 1982; 144: 661-4.

72. Landers D, Carmona R, Crombleholme W, Lim R. Acute cholecystitis in pregnancy. Obstet Gynecol 1987; 69: 131-3.

73. Maringhini A, Marceno MP, Lanzarone F, et al. Sludge and stones in gallbladder after pregnancy. Prevalence and risk factors. J Hepatol 1987; 5: 218-23.

74. Valdivieso V, Covarrubias C, Siegel F, Cruz F. Pregnancy and cholelithiasis: pathogenesis and natural course of gallstones diagnosed in early puerperium. Hepatology 1993; 17: 1-4.

75. Ko CW, Beresford SA, Schulte SJ, Matsumoto AM, Lee SP. Incidence, natural history, and risk factors for biliary sludge and stones during pregnancy. Hepatology 2005; 41: 359-65.

76. Swisher SG, Schmit PJ, Hunt KK, et al. Biliary disease during pregnancy. Am J Surg 1994; 168: 576-9; discussion 580-1.

77. Printen KJ, Ott RA. Cholecystectomy during pregnancy. Am Surg 1978; 44: 432-4.

78. Leiserowitz GS, Xing G, Cress R, et al. Adnexal masses in pregnancy: how often are they malignant? Gynecol Oncol 2006; 101: 315-21.

79. Hoffman MS, Sayer RA. Adnexal masses in pregnancy. OBG Manage 2007; 19: 27. 
80. Csapo AI, Pulkkinen MO, Ruttner B, Sauvage JP, Wiest WG. The significance of the human corpus luteum in pregnancy maintenance. I. Preliminary studies. Am J Obstet Gynecol 1972; 112: 1061-7.

81. Whitecar MP, Turner S, Higby MK. Adnexal masses in pregnancy: a review of 130 cases undergoing surgical management. Am J Obstet Gynecol 1999; 181: 19-24.

82. Hess LW, Peaceman A, OBrien WF, Winkel CA. Adnexal mass occurring with intrauterine pregnancy: report of fifty-four patients requiring laparotomy for definitive management. Am J Obstet Gynecol 1988; 158: 1029.

83. Schmeler KM, Mayo-Smith WW, Peipert JF, et al. Adnexal masses in pregnancy: surgery compared with observation. Obstet Gynecol 2005; 105(5 Pt 1): 1098-103.

84. Curtin JP. Management of the adnexal mass. Gynecol Oncol 1994; 55 (3 Pt 2): S42-6.

85. Zanetta G, Mariani E, Lissoni A, et al. A prospective study of the role of ultrasound in the management of adnexal masses in pregnancy. BJOG 2003; 110: 578 .

86. Bernhard LM, Klebba PK, Gray DL, Mutch DG. Predictors of persistence of adnexal masses in pregnancy. Obstet Gynecol 1999; 93: 585-9.

87. Thornton JG, Wells M. Ovarian cysts in pregnancy: does ultrasound make traditional management inappropriate? Obstet Gynecol 1987; 69: 717-21.

88. Ueland K. Rheumatic heart disease and pregnancy. In: Elkayam V and Gleicher N, eds. Cardiac Problems in Pregnancy. New York, NY: Alan R. Liss Inc., 1990.

89. Bernal JM, Miralles PJ. Cardiac surgery with cardiopulmonary bypass during pregnancy. Obstet Gynecol Surv 1986; 41: 1-6.

90. Becker RM. Intracardiac surgery in pregnant women. Ann Thorac Surg 1983; 36: 453-8.

91. Koh KS, Friesen RM, Livingstone RA, Peddle LJ. Fetal monitoring during maternal cardiac surgery with cardiopulmonary bypass. Can Med Assoc J 1975; 112: 1102-4.

92. Rossouw GJ, Knott-Craig CJ, Barnard PM. Intracardiac operation in seven pregnant women. Ann. Thoracic Surg 1993; 55: 1172-4.

93. Loya YS, Desai DM, Sharma S. Mitral and pulmonary balloon valvotomy in pregnant patients. Indian Heart J 1993; 45: 57-9.

94. Glantz JC, Pomerantz RM, Cunningham MJ, et al. Percutaneous balloon valvuloplasty for severe mitral stenosis during pregnancy: a review of therapeutic options. Obstet Gynecol Surv 1993; 1: 503.

95. Simon RH. Brain tumors in pregnancy. Semin Neurol 1988; 8: 214-21.
96. Peter CM. Neurologic surgery in pregnancy. In: Barber HRK, Graber EA, eds. Surgical Disease in Pregnancy. Philadelphia, PA: Saunders Company.

97. Sternberg J. Irradiation and radiocontamination during pregnancy. Am J Obstet Gynecol 1970; 108: 490-513.

98. Friedman WA. LINAC radiosurgery. Neurosurg Clin N Am 1990; 1: 991-1008.

99. Finfer SR. Management of labour and delivery in patients with intracranial neoplasms. Br J Anaesth 1991; 67: 784-7.

100. Drost TF, Rosemurgy AS, Sherman HF, Scott LM, Williams JK. Major trauma in pregnant women: maternal/fetal outcome. J Trauma Injury Infect Crit Care 1990; 30: 574-8.

101. Rothenberger D, Quattlebaum FW, Perry JF Jr, Zabel J, Fischer RP. Blunt maternal trauma: a review of 103 cases. J Trauma Injury Infect Crit Care 1978; 18: 173-9.

102. Goodwin T, Breen M. Pregnancy outcome and fetal maternal hemorrhage after non-catastrophic trauma. Am J Obstet Gynecol 1990; 162: 665.

103. Dahmus MA, Sibai BM. Blunt abdominal trauma: are there any predictive factors for abruptio placentae or maternal-fetal distress? Am J Obstet Gynecol 1993; 169: 1054-9.

104. Pearlman MD, Tintinalli JE, Lorenz RP. Blunt trauma during pregnancy. N Engl J Med 1990; 323: 1609-13.

105. Pearlman MD, Tintinallli JE, Lorenz RP. A prospective controlled study of outcome after trauma during pregnancy. Am J Obstet Gynecol 1990; 162: 1502-7; discussion 1507-10.

106. Buchsbaum H. Penetrating injury of the abdomen. Trauma in pregnancy. Philadelphia: Saunders, 1979: 82.

107. Awwad JT, Azar GB, Seoud MA, Mroueh AM, Karam KS. High-velocity penetrating wounds of the gravid uterus: review of 16 years of civil war. Obstet Gynecol 1994; 83: 259-64.

108. Kuhlmann RS, Cruikshank DP. Maternal trauma during pregnancy. Clin Obstet Gynecol 1994; 37: 274-93.

109. Amy BW, McManus WF, Goodwin CW, Mason A Jr, Pruitt BA Jr. Thermal injury in the pregnant patient. Surg Gynecol Obstet 1985; 161: 209-12.

110. Rayburn W, Smith B, Feller I, Varner M, Cruikshank D. Major burns during pregnancy: effects on fetal well-being. Obstet Gynecol 1984; 63: 392-5.

111. Lavery JP, Staten-McCormick M. Management of moderate to severe trauma in pregnancy. Obstet Gynecol Clin North Am 1995; 22: 69-90.

112. Gianopoulos JG. Management of the obstetric patient in the intensive care setting. In: Irwin R. and Rippe J, eds. Maternal-Fetal Medicine, 2nd edn. London: Informa Health, 2009; 1821-1830. 


\section{Anesthesia and analgesia in pregnancy \\ Steven T. Fogel}

\section{INTRODUCTION}

The first public demonstration of the use of ether for anesthesia in 1846 was quickly followed by its application to obstetrics in January 1847 by James Young Simpson of Scotland. Almost immediately, controversy surrounded its use. In the United States, Charles D Meigs, Professor of Obstetrics at Jefferson Medical College, denounced attempts to ameliorate the pain of labor, claiming that it was inseparable from the effectiveness of uterine contractions. Simpson, on the other hand, claimed that the pain of labor was of no physiologic value (1). Eventually, then, as today, public demand for effective relief of labor pain settled the argument. By the latter half of the 20th century, there was nearly full agreement within the medical profession that relief of labor pain should not be withheld from a woman in active labor. This position was formally recognized in 1992 when the American College of Obstetricians and Gynecologists and the American Society of Anesthesiologists published a joint statement underscoring the importance of the availability of pain relief for the parturient:

"Labor results in severe pain for many women. There is no circumstance where it is considered acceptable for a person to experience untreated severe pain, amenable to safe intervention, while under a physician's care. In the absence of a medical contraindication, maternal request is a sufficient medical indication for pain relief during labor. Pain management should be provided whenever medically indicated" (Joint ASA-ACOG Statement, American Society of Anesthesiologists, Park Ridge, IL: October 21, 1992; amended October 1997) (2). While many women may choose to labor without any pharmacologic intervention, the provision of pain relief during childbirth has become an integral part of obstetric practice.

\section{NOCICEPTIVE PATHWAYS OF PARTURITION}

During the first stage of labor, pain signals from nociceptors in the myometrium and cervix are transmitted via the paracervical and hypogastric plexi, and, traveling with sympathetic fibers of the lumbar sympathetic chain, enter the spinal cord at segments T10 to L1. These fibers synapse with secondary neurons in the Rexed laminae of the dorsal horns and are modulated by mu receptors in the substantia gelatinosa, also located in the dorsal horns of the spinal cord. The pain is perceived primarily as visceral pain. Pain signals in the second stage of labor are transmitted via the pudendal nerves, which enter the spinal cord via nerve roots S2-S4. From there, pain signals are transmitted via secondary neurons to the contralateral spinothalamic tract and on to the thalamus. Signals from the thalamus are then projected to cognitive cortical centers, where they are perceived primarily as somatic pain. Pain can be modulated or interrupted with blockade at several levels during its transmission. Paracervical block provides pain relief during the first stage of labor as does epidural and/or spinal blockade with local anesthetics or with spinal opiates either alone or in combination with local anesthetics. Pain during the second stage of labor can be managed with pudendal block, spinal (saddle) blockade, or extension of epidural blockade. Since the pain of the second stage of labor is somatic, it is not as responsive to spinal opiates as is the pain of the first stage of labor.

\section{NONPHARMACOLOGIC METHODS OF PAIN RELIEF}

Psychoprophylaxis is probably the most common method of nonpharmacologic management of labor pain. Its premise is that knowledge, education, and preparation reduce the severity of the fear-tension-pain cycle. The process usually begins with childbirth education classes that inform the pregnant patient and her partner of the normal progress of pregnancy, labor, and delivery as well as the expectations. In addition, relaxation and distraction techniques are taught as an adjunct to the management of labor pain. These include breathing exercises, focal points, and massage. The patient's partner plays an important role in the process. Emotional support decreases the level of isolation the patient may feel. Nonfamily individuals trained to provide support (doulas) have been shown to have a beneficial effect on the patient's perception of pain and the progress of labor (3). Touch and massage are frequent components used to alleviate the pain of labor. These techniques may include effleurage, light touch, and/or counterpressure applied to the low back. The ultimate goals are for the parturient to take an active role in the management of her labor and labor pain. Indeed, studies have shown that patients who have successfully completed a program of childbirth education and utilized the above techniques have a lesser need for pharmacologic treatment as well as a lower incidence of obstetric interventions $(4,5)$. Other non-pharmacologic techniques that have been used include transcutaneous electrical nerve stimulation (TENS), acupuncture, and hypnosis.

TENS has had no discernible adverse fetal effects. Its efficacy has been reported to be essentially of minimal benefit to possibly shorter labors (6-9). Acupuncture requires knowledgeable and skilled practitioners, which probably explains the varied results that have been reported (10-13). Because of the need for additional training as well as for cultural reasons, this technique is not likely to be widely practiced in the foreseeable future. Like acupuncture, hypnosis requires a trained practitioner. It is time consuming and at least partially depends on the susceptibility of the patient to this technique. Patients who are susceptible to hypnosis have been shown to require less systemic analgesia than patients in a control group as well as experience shorter labors. Patients who are not susceptible to hypnosis do not experience much benefit (14). 


\section{SYSTEMIC ANALGESIA}

Systemic analgesics have been widely used throughout the latter half of the 20th century. The mainstay of this technique is the use of opioids, both naturally occurring and synthetic. Morphine is derived from opium and has a very long history of use. As with all opioids, it binds to mu receptors in the CNS and, in addition to providing analgesia, may also cause respiratory depression, nausea, vomiting, and sedation. Systemically administered morphine can induce histamine release and cause pruritus (15). An associated skin rash may also develop. When given intravenously (IV), its onset of action is within 3 to 5 minutes with peak effect in 20 minutes. The pharmacologic effect of morphine does not correlate well with plasma concentrations following intravenous administration. This is believed to be secondary to a delay in transfer across the blood-brain barrier since a large percentage of morphine is ionized at physiologic $\mathrm{pH}$ (16-18). An intramuscular (IM) injection will have an onset of 15 to 30 minutes with a peak effect of 45 to 90 minutes. Morphine undergoes hepatic metabolism. With the availability of more potent synthetic opioids such as fentanyl, remifentanil, and the agonists/antagonists nalbuphine and butorpanol, morphine is more commonly used during prelabor or the very early stages of labor and less so during active labor.

Meperidine is a widely used opioid that has the benefit of low cost and a long history of successful administration. Usual doses are 25 to $50 \mathrm{mg}$ IV/IM. Like most opioids, it has the potential of causing respiratory depression in both the mother and the neonate. Onset is within 5 minutes of intravenous administration. The neonatal half-life of maternally administered meperidine is considerably longer than maternal half life, approximately 18 versus 3 hours $(19,20)$. A limiting factor in the administration of meperidine is the accumulation of the metabolically active metabolite normeperidine, which can cause seizures when levels are sufficiently high (17). Normeperidine can remain in the neonate for as long as 60 hours (21). The pharmacokinetics of meperidine is such that the optimal time for delivery is either with in 1 hour after maternal administration or more than 2 to 4 hours following administration, depending on the dose $(22,23)$.

Fentanyl is a potent synthetic opioid. The potency relative to morphine is 100 to 1 . It is most often administered IV and has a rapid onset of less than 1 minute. It has a relatively short duration of action and requires frequent dosing (18). Because of its short duration of action, it is titratable and hence it is attractive when administered as patientcontrolled analgesia (PCA). Because its potency is much greater than morphine and meperidine, it has a greater potential for causing severe respiratory depression. Patients receiving fentanyl should be closely monitored. Nevertheless, studies have demonstrated a lesser degree of neonatal effects with the use of fentanyl than with the use of meperidine or morphine $(24,25)$.

The agonists/antagonists nalbuphine and butorphanol have also been widely used in many institutions for labor analgesia. These drugs act primarily at the kappa receptor and more weakly at the mu receptor (18). Their advantage over pure opiates is a ceiling effect on respiratory depression. When compared with meperidine, nalbuphine causes less maternal nausea and vomiting, but lower neonatal neurobehavioral scores at 2 to 4 hours of age (26). Butorphanol, when compared with meperidine, affords similar degrees of pain relief (27). Like nalbuphine, there is less maternal nausea and vomiting compared with meperidine. Fetal and neonatal effects are similar when comparing butorphanol and meperidine (28).

In the general patient population, PCA has been found to provide better pain relief than intermittent, nurse-administered analgesia (29). Nalbuphine has been shown to provide better labor analgesia when administered in a PCA mode, while PCA administration of meperidine does not demonstrate any significant improvement $(30,31)$. Opioids that are shorter acting may lend themselves well to PCA administration. Fentanyl has been used in a PCA mode, but neonatal depression has been reported. In one retrospective study, the total dose of fentanyl received by mothers of infants who required naloxone was significantly higher than the group of mothers whose infants did not require naloxone (32). Remifentanil is a synthetic opioid with a very short duration of action. It is rapidly metabolized by blood esterases with a half-life of 3.5 minutes. There are no active metabolites, no drug accumulation, and metabolism is not organ dependent (33). Remifentanil has been administered in a PCA mode successfully for labor analgesia. Problems have been reported with respect to maternal respiratory depression and decreasing oxygen saturation, but adverse neonatal effects are few (34-38). Remifentanil administered via PCA may be the best opioid analgesic alternative when epidural analgesia is contraindicated.

Ketorolac is a nonsteroidal anti-inflammatory agent that has good analgesic efficacy for various types of painful conditions. It is attractive in that it causes no sedation and no respiratory depression (39). There are concerns about its use in obstetrics in that it may decrease uterine contractions and/or close the fetal ductus arteriosus. In one study comparing the analgesic effect of ketorolac with meperidine, ketorolac was not found to be superior (40). Another study demonstrated potential adverse effects on neonatal platelet function (41). At present, there appears to be no benefit from the use of ketorolac for labor analgesia.

\section{Placental Transfer}

Virtually, all drugs administered maternally have the potential to transfer to the fetus. Several mechanisms are involved in the placental transfer of drugs from the maternal to fetal circulation, the more common of which being simple diffusion, facilitated diffusion, and active transport. Simple diffusion is governed by the Fick equation:

$$
\mathrm{Q} / \mathrm{T}=\left[\mathrm{kA}\left(\mathrm{C}_{\mathrm{m}}-\mathrm{C}_{\mathrm{f}}\right)\right] / \mathrm{D}
$$

Where $\mathrm{Q} / \mathrm{T}$ is the rate of diffusion of a given drug and $\mathrm{k}$ is a diffusion constant determined by the lipid solubility, molecular configuration, and molecular weight. $\mathrm{A}$ is the area available for diffusion, $\mathrm{C}_{\mathrm{m}}$ is the maternal blood concentration, and $\mathrm{C}_{\mathrm{f}}$ is the fetal blood concentration. $\mathrm{D}$ is the thickness of the diffusion membrane. Thus the size and thickness of the placental membrane along with variations in the distribution of vascular flow determine the rate of placental drug transfer. Other determinants include drug ionization, protein binding, and maternal and 
fetal metabolism. Protein binding can be an important factor in drug transfer. Differences in protein binding in the maternal and fetal circulations may either inhibit or facilitate the degree of transfer. Variations in maternal blood concentrations of protein as may occur in various disease states may also influence drug transfer. Substances of smaller molecular size (less than 500 daltons) are more likely to transfer than larger molecules. Large polar compounds such as muscle relaxants that are used in general anesthesia transfer only in small amounts.

The rate and degree of drug transfer are measured by comparing umbilical vein (UV) and maternal vein (MV) concentrations. UV drug concentration reflects the amount of drug transferred to the fetal circulation via the placenta. A UV/MV ratio close to 1 indicates a high degree of placental transfer. The degree of fetal drug distribution and metabolism is reflected by comparing the umbilical artery (UA) and UV concentrations of a drug. Thus a UA/UV ratio of 1 will indicate equilibrium between fetal drug delivery and fetal distribution. A UA/UV ratio of less than 1 will indicate continued fetal drug uptake and a UA/UV ratio greater than 1 will indicate fetal elimination of drug via distribution into the maternal circulation.

The placental transfer of morphine is rapid. UV to MV ratio is close to 1 at 5 minutes. Fetal heart rate (FHR) variability is often decreased following maternal administration of morphine; however, fetal metabolism of morphine is high. Studies of morphine metabolism in the fetal baboon show that it is limited primarily by hepatic blood flow (42-44). Because the fetal exposure to morphine is short, it may actually be more beneficial than meperidine when used for systemic labor analgesia.

Maternally administered intravenous meperidine appears in the fetal plasma within 2 minutes and reaches equilibrium in 6 minutes. The UV/MV ratio increases with greater time following IM administration $(21,22,45,46)$. Like morphine and other opioids, a decrease in FHR variability can be expected (47). However, variability is not effected during fetal movements or uterine contractions (48).

Fentanyl rapidly crosses the placenta and is detectable in fetal blood within 1 minute (49). Depending on total dose, UV/MV ratios of 0.05 to 0.76 have been reported. A decrease is FHR variability has been noted with a duration of approximately 30 minutes (50).

The UV/MV ratio for nalbuphine is reported to be 0.61 to $0.74(26,51,52)$. Fetal effects include decreases in frequency of accelerations, decreased FHR variability, and the appearance of sinusoidal patterns $(53,54)$. In spite of these changes, the fetus will still respond to fetal acoustic stimulation (55).

Butorphanol rapidly crosses the placenta. In fetal sheep, butorphanol can be detected within 1 minute of maternal administration (56). The UV to MV concentration ratio is 0.84 . When compared with meperidine, there were no differences in effect on FHR (27).

\section{PARACERVICAL AND PUDENDAL BLOCKS}

Up until the mid-1970s, paracervical and pundendal blocks were frequently used in many centers for analgesia for labor and vaginal delivery. Paracervical block interrupts nerve transmission at the paracervical plexus. It provides several hours or more of pain relief during the first stage of labor, depending on the local anesthetic used. During long labors, it may need to be repeated. Paracervical block is useful only during the first stage of labor. It is of no value during the second stage of labor. In the 1970s, multiple reports of fetal bradycardia began to appear. The mechanism was believed to be a transfer of large amounts of local anesthetic to the fetal circulation via the uterine artery. Other mechanisms that have been proposed are uterine artery vasospasm, UA vasospasm, and uterine hypertonus (57-60). Currently, paracervical block for labor analgesia is rarely used because of the concerns regarding adverse fetal effects. It may have some utility in special circumstances such as in patients with intrauterine fetal death who, in addition, have contraindications to epidural analgesia.

Pudendal anesthesia is achieved by blocking the pudendal nerve as it exits the pelvis at the ischial spine. It provides anesthesia to the perineum and lower one-third of the vagina. It is useful for late second-stage labor and vaginal delivery. It is of no value during the first stage of labor or for intrauterine manipulation. Because of the flexibility and reliability of epidural analgesia for both the first and second stages of labor, the use of pudendal block has been declining. It is rarely used at the University of Missouri, and the Mayo Clinic recently reported declining usage as well (61). Nevertheless, it is a valuable option when neuraxial analgesia is contraindicated or unavailable.

\section{NEURAXIAL ANALGESIA}

Epidural analgesia has become a very successful and widely used technique for managing the pain of labor in the United States. In 1992, epidural analgesia was used in slightly greater than $50 \%$ of laboring women delivering in hospitals with greater than 1500 deliveries per year (62). Today, rates of $75 \%$ or greater are not uncommon. Continuous lumbar epidural analgesia provides pain relief that is superior to any other method of labor analgesia. Compared with parenteral opioids, epidural analgesia provides superior pain relief, greater maternal satisfaction, and less neonatal depression (63). Epidural analgesia has been shown to significantly decrease the stress response to labor. Stress indicators such as epinephrine, cortisol, and beta-endorphins decrease following epidural analgesia (64-68). This can be important when dealing with patients with cardiac disease (see below).

Epidural analgesia involves the placement of local anesthetics often combined with low-dose opioids in the epidural space via a small-gauge catheter. The posterior epidural space is bound by the ligamentum flavum posteriorly and the dura anteriorly. Typically, the patient is placed in a sitting or lateral decubitus position. The lumbar approach is commonly used, most often L2 to L5. Following an aseptic technique with local anesthetic injected at the chosen interspace, an epidural needle is inserted into the epidural space utilizing a "loss of resistance" technique with a syringe filled with saline or air. An epidural catheter is then threaded into the epidural space and the needle is removed. The catheter is then secured and local anesthetic infused until an appropriate level of analgesia is achieved. Since the epidural catheter can be placed intravascularly or in the subarachnoid space, a test dose is 
performed to rule out placement at these sites. A small dose of local anesthetic with epinephrine is injected via the catheter following careful aspiration. Typical test doses include $45 \mathrm{mg}$ of lidocaine and $15 \mathrm{mcg}$ of epinephrine. If injected IV the small dose of epinephrine will cause a transient maternal tachycardia. If injected into the subarachnoid space, the parturient will develop a limited motor block. Neither of these responses will occur if the catheter is in the epidural space.

Utilizing an epinephrine containing test dose as a marker of intravascular catheter, placement is not always accurate, however. Colonna-Romano and colleagues showed that the epinephrine test dose has a $100 \%$ negative predictive value, but a $55 \%$ to $73 \%$ positive predictive value. They calculated that as many as $27 \%$ to $45 \%$ of epidural catheters with a positive epinephrine test dose would be unnecessarily removed (69). Other authors have questioned the value of an epinephrine containing test dose as a method of detecting an intravascularly placed epidural catheter $(70,71)$. The catheter design may also affect the positive and negative predictive value of test doses. Closed-tip multiport catheters, which are popular among many anesthesiologists because of improved local anesthetic spread, may present problems with multi-compartment placement (72). Because of these limitations, anesthesiologists have taken the position that every dose of a local anesthetic administered via an epidural catheter should be considered a test dose. Local anesthetics are therefore administered in small aliquots and the patient is observed for signs of toxicity (intravascular placement) or unexpected levels of motor block (subarachnoid placement).

Once the epidural catheter is placed, secured, and tested, it is dosed with a low concentration of local anesthetic alone or in combination with an opioid to achieve a T10 level. This usually requires a bolus loading dose of volumes ranging from 8 to $16 \mathrm{~mL}$ given in 5 - $\mathrm{mL}$ aliquots. While intermittent single "top up" doses were given in the past for maintenance, continuous infusions are now almost universal. Patientcontrolled epidural analgesia (PCEA) has gained popularity in a number of centers. Usually a background infusion of dilute local anesthetic with or without opioid is maintained and the patient is allowed to self-administer a "top up" dose with a predetermined lockout and hourly maximum volume programmed into a pump device. PCEA has been shown to improve patient satisfaction and decrease the number of anesthetic interventions with lower total volumes of local anesthetic than conventional continuous infusion techniques (73-75). A number of regimens for both continuous and PCEA techniques have been proposed, each with varying results. At the University of Missouri, PCEA is used almost exclusively.

A modification of conventional epidural analgesia, the combined spinal-epidural (CSE) technique, has also been gaining usage at major centers over the past decade. Following placement of the epidural needle in the epidural space, a smallgauge pencil-point spinal needle is passed through the epidural needle and the dura is punctured. A small dose of preservativefree opioid or opioid local anesthetic combination is injected intrathecally. The epidural catheter is then passed through the epidural needle and tested. Analgesia is achieved rapidly and there is no need for a bolus loading dose of local anesthetic via the epidural catheter $(76,77)$. Following placement and testing of the epidural catheter, a maintenance infusion is begun. By the time the intrathecal dose has dissipated, the epidural infusion has reached a sufficient level to maintain satisfactory analgesia.

The CSE technique has several distinct advantages. If an opiate alone is administered intrathecally, then there is no motor block and the patient is able to ambulate for a period of time, usually 90 to 120 minutes (the so-called walking epidural). Some institutions will follow the intrathecal dose with an ultralow concentration of local anesthetic with opioid and epinephrine adjuvants to allow continued ambulation $(78,79)$. The CSE technique allows confirmation of epidural placement of the epidural needle, which may decrease the need for replacement of failed epidural catheters $(80,81)$. A recent retrospective study suggested that the quality of analgesia obtained with a CSE technique is better than that obtained with a traditional epidural technique (82). Because of the rapid onset of analgesia when the CSE technique is used, patients with advanced labor may still be able to benefit from neuraxial analgesia. At the University of Missouri, patients rarely fail to receive neuraxial labor analgesia because it is "too late." Comparisons of outcome of labor and complications between CSE and conventional epidural techniques have shown the two to be similar (83-85). A more recent study showed that when an epidural catheter is dosed for cesarean delivery, those catheters placed via a CSE technique are more likely to be successful than those placed via a traditional epidural technique (86). CSE is therefore used almost routinely at the University of Missouri.

While epidural and CSE analgesia for labor have distinct advantages over other methods of labor pain management, absolute and relative contraindications exist. Patient refusal, untreated sepsis, frank coagulopathy, uncorrected hypovolemia/hypotension, and infection at the injection site are the generally accepted absolute contraindications. Relative contraindications may include uncooperative patient, neurologic disease, severe obstructive cardiac lesions, thrombocytopenia, and prior spine surgery. Fortunately, absolute contraindications are rare and the greatest majority of parturients can benefit from neuraxial analgesia.

\section{Complications of Neuraxial Analgesia and Anesthesia}

Serious complications from epidural analgesia are rare, but include local anesthetic toxicity, high or total spinal anesthesia, epidural hematoma or abscess, and permanent neurologic injury. Less serious but more troublesome complications are hypotension and post-dural puncture headache.

\section{Local Anesthetics and Local Anesthetic Toxicity}

The local anesthetics most commonly used in obstetric anesthesia are bupivacaine, lidocaine, 2-chloroprocaine, ropivacaine, and levobupivacaine. Local anesthetics are composed of a lipophilic aromatic moiety and a hydrophilic amine moiety linked by either an amide or an ester intermediate chain. They are therefore classified as either amides or esters. Except for 2-chloroprocaine, all of the local anesthetics commonly used in obstetrics are in the class of amide local anesthetics. 2-Chloroprocaine is an ester local anesthetic. Of the above local anesthetics, bupivacaine is the most potent, most lipophilic, longest lasting and slowest in onset. Lidocaine 
is intermediate in these characteristics and 2-chloroprocaine is the fastest in onset, least potent, least toxic, and shortest in duration. Bupivacaine has been popular as a local anesthetic for epidural labor analgesia because of its capacity to develop a "differential block," meaning that it can provide greater sensory block with less associated motor block at low doses. Bupivacaine, however, has greater cardiotoxic potential than does lidocaine and has been the limiting factor in its use. Generally, seizures will occur during a local anesthetic toxic reaction prior to the onset of cardiac arrhythmias or arrest. However, the cardiotoxic to central neurotoxic plasma ratio for bupivacaine is higher than that for lidocaine, and with a rapid intravascular injection of bupivacaine, cardiac toxicity may be the first event. In addition, bupivacaine has a very slow washout from cardiac tissue and makes resuscitation difficult (87). Indeed, cardio-pulmonary bypass may be needed as a last ditch heroic measure (88). More recently, lipid emulsions have been used successfully to treat toxicity from local anesthetics, specifically bupivacaine (89). Some anesthesia units are now keeping intralipid on their resuscitation carts. It has been thought that pregnancy confers a greater sensitivity to the cardiotoxic effects of bupivacaine (90). This, however, has not been borne out in more recent studies (91). Ropivacaine and levobupivacaine are newer local anesthetics that are related to bupivacaine. While potencies may vary from bupivacaine, they are less cardiotoxic (91). As a result, ropivacaine has become popular in obstetric anesthesia. Concentration of $0.1 \%$ or $0.2 \%$ ropivacaine with additional opiate is used almost exclusively for labor epidural analgesia at the University of Missouri.

In the event of a toxic reaction, seizures should be controlled with either propofol, midazolam, or thiopental; the airway should be controlled with endotracheal intubation; ventilation should be supported if necessary; and the parturient should be placed in left lateral uterine displacement position. Supplemental oxygen should be administered and fluids be begun. Blood pressure should be supported with additional fluids and vasopressors. Further therapy depends on the results of initial treatment. The administration of intralipid in the face of bupivacaine toxicity is worthy of serious consideration. Cardio-pulmonary resuscitation should be performed in the left lateral position to facilitate venous return and maintain uteroplacental blood flow.

\section{Hypotension}

Epidural analgesia has the potential of creating a sympathetic block and subsequent hypotension. This is primarily dependent on the level of block and the rate of administration. It is therefore common practice that patients are prehydrated with 500 to $1000 \mathrm{~mL}$ of crystalloid immediately prior to initiation of epidural analgesia. In some cases, a colloid such as hetastarch may be used. The need for routine prehydration with currently used epidural and CSE techniques, however, has been questioned (92). This may be important when caring for patients with conditions mandating limited fluid administration. Clinically significant hypotension that is otherwise asymptomatic to the mother may manifest itself as late decelerations of the FHR or fetal bradycardia. Treatment includes left uterine displacement to increase venous return, cardiac output and uterine blood flow, intravenous fluids, and judicious administration of vasopressors and oxygen. Ephedrine is the vasopressor of choice. It is both an alpha and a beta receptor agonist and has the potential of causing maternal tachycardia. When maternal tachycardia preexists or is undesirable, then phenylephrine, a pure alpha agonist, can be used.

\section{FHR Changes}

Not long following the introduction of CSE analgesia for labor, reports of sudden fetal bradycardia appeared. This was believed secondary to the rapid onset of analgesia with a subsequent drop in circulating catecholamines, resulting in uterine disinhibition and markedly increased uterine activity (93). Subsequent studies comparing the fetal effect of CSE analgesia versus conventional epidural analgesia had varying results. A systematic review in 2002, however, demonstrated that there was indeed an increased risk of fetal bradycardia following CSE analgesia compared with convention epidural analgesia. Surprisingly, there was no increased risk of cesarean section with CSE, nor was there an increased risk of cesarean delivery in yet another large study of the use of CSE $(94,95)$. Another study in 2004, however, compared the incidence of fetal bradycardia among three groups of patients. Group 1 received a conventional epidural, group 2 received a CSE regimen consisting of low-dose intrathecal bupivacaine and low-dose opioid, and Group 3 received a CSE regimen of only high-dose intrathecal opioid. There was no difference in the incidence of fetal bradycardia between groups 1 and 2, but there was a higher incidence of fetal bradycardia in group 3. The authors recommended limiting the amount of opioid administered intrathecally (96). At the University of Missouri, we therefore use a combination of $3 \mathrm{~mL}$ of isobaric ropivacaine $0.1 \%$ and $6 \mu \mathrm{g}$ of fentanyl intrathecally in our CSE regimen. When fetal bradycardia does occur following CSE secondary to uterine hyperactivity, we treat with either terbutaline or sublingual nitroglycerin, both of which have been reported to be effective $(93,97,98)$.

\section{Accidental Dural Puncture}

Accidental dural puncture (ADP) is not an uncommon complication of epidural analgesia. The rate may vary from $0.6 \%$ to $4.2 \%$ in training institutions $(83,99)$. ADP would not necessarily present a problem were it not for the $70 \%$ postdural puncture headache rate associated with the use of 18-gauge epidural needles (100,101). Post-dural puncture headache (spinal headache) typically presents approximately 24 to 48 hours following dural puncture. It is rare for symptoms to appear before 24 hours. Patients typically present with a frontooccipital headache that may be described as throbbing and severe with associated posterior cervical pain or stiffness. The headache will be totally or nearly totally gone with the supine position and reappear within minutes of obtaining an erect position. It cannot be overly stressed that the hallmark of a post-dural puncture headache is its positional nature. Almost universally, the relief of headache is very noticeable when taking the supine position. The headache is believed to be secondary to low cerebrospinal fluid (CSF) pressure when erect causing traction on meninges and/or reactive cerebral vasodilation $(102,103)$. 
Post-dural puncture headache can mimic other more serious conditions, which may include intracranial hemorrhagic disorders, mass lesions and cortical venous thrombosis (104-106). Not all postpartum headache is a post-dural puncture headache. Patients who do not present with classic features of a post-dural puncture headache should be evaluated with further studies such as MRI or CT scanning of the central nervous system.

Post-dural puncture headache can be very debilitating. While the nonpregnant patient recovering from surgery or illness may tolerate long periods of bed rest, the parturient must be able to care for an infant. Inability to maintain an erect posture may interfere with breast-feeding. Treatment of post-dural puncture headache in the obstetric patient population may therefore carry greater importance than that in the rest of the population.

Typical conservative measures of treatment include bed rest, hydration, caffeine, opioids, and nonsteroidal antiinflammatory analgesics. Caffeine has vasoconstrictive properties that counteract the presumed vasodilation that occurs reactively to low CSF pressures. Sumatriptan, another medication with vasoconstrictor properties used to treat migraine headache, has been shown to be effective in one study but ineffective in another $(107,108)$. Generally, it has not been used as a first-line drug for post-dural puncture headache in the obstetric population.

Epidural blood patch is considered the definitive treatment for post-dural puncture headache. The technique involves the injection of 10 to $20 \mathrm{~mL}$ of autologous blood in the epidural space near the original epidural puncture site. Its effectiveness has been reported to be as high as $97 \%$ (109). A long-term follow-up study of nonobstetric patients, however, showed that only $61 \%$ of patients had a permanent resolution of headache despite an initial success rate of $88 \%$ to $96 \%$ (110). More recently, a study in an obstetric population showed that only $15 \%$ required a repeat blood patch (111). Epidural blood patch has a long history of effectiveness and safety. Nevertheless, if a patient should require more than two blood patches, a search for another cause of headache should be made.

\section{Other Complications}

High spinal anesthesia is a potentially lethal complication. Large volumes of local anesthetics are required to establish adequate labor analgesia for both the first and second stages of labor. An accidental intrathecal injection of a large volume of local anesthetic has the potential of causing respiratory arrest, hypotension, and even cardiac arrest. Treatment is supportive and requires respiratory assistance including tracheal intubation, fluids, and vasopressors until the block recedes. Careful use of an appropriate test dose prior to dosing the epidural catheter will markedly decrease the likelihood of this complication.

Epidural abscess and epidural hematoma are very rare complications that can be devastating if not diagnosed in a timely manner. Both present as mass spinal lesions with pain, sensory and/or motor loss, and, in the event of abscess, fever. Both almost always require surgical drainage. The risk of abscess can be decreased by paying close attention to sterile technique and by avoiding neuraxial analgesia/anesthesia in the face of untreated bacteremia or septicemia. Epidural hematoma can be minimized by avoiding neuraxial regional analgesia/anesthesia in the patient at risk for coagulopathy.

Peripheral neuropathies are not rare in the obstetric patient population. When these problems occur in a patient who has had epidural analgesia, the anesthesiologist is often consulted. Lower extremity peripheral neuropraxia can result from stretch or compression lesions that occur during labor and delivery. Foot drop, quadriceps weakness, weak hip adduction, and meralgia paresthetica can result from lumbosacral trunk or peroneal nerve, femoral nerve, obturator nerve, and lateral femoral cutaneous nerve injury, respectively. Peroneal nerve injury can result from nerve compression at the knee due to positioning equipment. Femoral and lateral femoral nerve injury can result from prolonged hip flexion during the second stage of labor. If the patient has a dense epidural block at that time, she may not complain of pain or numbness with overzealous hip flexion. Detailed and conscientious evaluation will help differentiate peripheral obstetric neuropathies from direct nerve root or spinal cord injuries (112). Early neurology consultation and spine imaging may elucidate the etiology and direct appropriate treatment in a timely manner.

\section{Epidural Analgesia and the Progress of Labor}

The effect of epidural analgesia on the progress and outcome of labor has, in the past, been controversial. Retrospective studies have demonstrated an increased risk of cesarean delivery when epidural analgesia was utilized (113). Subsequent prospective randomized studies, however, did not show any significant difference in the cesarean delivery rate when patients were randomized to receive either epidural or parenteral analgesia $(114,115)$. While prospective randomized studies provide good data, problems still exist. Patients do not always stay in their assigned group, leading to "intent to treat" analyses. Retrospective "sentinel" event studies overcome this confounding factor. These studies examine the cesarean delivery rate before and after the institution of an "on demand" epidural analgesia service (116). While retrospective in nature, there are no selection biases and no opportunity for patients to drop out of a study group. Results of these studies show that there is no increase in cesarean delivery rate when there is a sudden increase in the utilization of epidural analgesia. One study in particular did offer an interesting insight. While there was no change in cesarean delivery rate with a marked increase in epidural analgesia overall, it was noted that following the institution of epidural analgesia on demand, those women who went on to cesarean delivery for dystocia were approximately eight times more likely to have had an epidural. The authors concluded that dystocia is more painful and women who request epidural analgesia are already at risk for cesarean delivery for dystocia (117). This result may explain why retrospective studies that examined cesarean delivery rates in institutions where epidural analgesia services were well established showed higher rates of cesarean delivery in association with epidural analgesia. Patients who request epidural analgesia are different from those who do not. Epidural analgesia is therefore a risk marker, not a risk factor.

While epidural analgesia does not increase the risk of cesarean delivery for dystocia, it does slow labor. A metaanalysis of randomized trials published in 1998 concluded 
that, overall, the first stage of labor increased by 42 minutes and the second stage of labor by 14 minutes $(114,118)$. A more recent study concluded that while the first stage of labor is not prolonged, the second stage is (119). Not only does epidural analgesia not increase the incidence of cesarean delivery for dystocia but neither does the timing of epidural placement. A study published in 2005 examined the cesarean delivery rate of early (less than $4 \mathrm{~cm}$ dilatation) versus late epidural placement and found no difference in the cesarean delivery rate for dystocia. Indeed, patients who had early CSE analgesia had shorter labors (120). At the University of Missouri, women are not required to reach a preset cervical dilatation before epidural analgesia is administered. This position is supported by the American College of Obstetricians and Gynecologists, which has recently stated:

... the decision of when to place epidural analgesia should be made individually with each patient, with other factors, such as parity, taken into consideration. Women in labor should not be required to reach $4-5 \mathrm{~cm}$ of cervical dilatation before receiving epidural analgesia (121).

\section{Epidural Analgesia and Maternal Fever}

A rise in maternal temperature has been associated with the use of epidural analgesia (122). Studies that are retrospective and/or nonrandomized are likely to be biased because factors that lead to increased requests for epidural analgesia such as long labors complicated by dystocia are also likely to lead to maternal fever. Nevertheless, prospective randomized trials have shown that a rise in maternal temperature can occur in association with epidural use in the absence of infection (123). The reasons are unclear and have been attributed to alterations in vascular responsiveness and thermoregulatory control. Parturients who shiver immediately following induction of epidural analgesia are more likely to develop an earlier and greater rise in temperature than those who do not (124). While mild to modest increases in maternal temperature may not have significant physiologic significance, it has led to increases in neonatal sepsis evaluations (125). When criteria for neonatal sepsis evaluations are better refined, however, there are no increases in sepsis evaluations of neonates born to women with epidural-associated fevers (126). Currently, the wide use of epidural analgesia does not seem to be negatively effected by concerns regarding the association of epidural use and maternal fever (119).

\section{ANESTHESIA FOR CESAREAN DELIVERY}

The cesarean delivery rate in the United States has steadily increased during the past several decades. In high-risk tertiary care institutions, rates have climbed higher than 30\% (127).

In 1981, general anesthesia was used more often than either epidural or spinal anesthesia in larger institutions $(>1500$ deliveries per year). By 2001, only 3\% of elective cesarean deliveries were performed under general anesthesia, the remainder utilizing either spinal or epidural anesthesia (128). These data underscore the preference for regional anesthesia for cesarean deliveries. Significant advantages exist over general anesthesia: an awake mother with spontaneous respirations and ability to control the airway with a lower risk of aspiration, contracted gut, more complete muscular relaxation, and an opportunity to provide excellent postoperative analgesia with the use of spinal opiates.

From 1979 to 1990, the maternal mortality rate from anesthesia-related causes for patients undergoing cesarean delivery in the United States steadily decreased for regional anesthesia but remained approximately the same for general anesthesia. By 1990, the relative risk of death from anesthesiarelated causes in cesarean delivery patients was 16.7 for general versus regional anesthesia (129). By 1996, the relative risk decreased to 6.7 (130). The major risks from general anesthesia are primarily inability to intubate, inability to ventilate and aspiration. Pregnant patients at term and in labor are considered to be individuals with "full stomachs" due to increased gastric emptying time. In addition, pregnant patients at term are more likely to present with "difficult airways" that cannot be intubated by conventional means or ventilated (131). Because term pregnant patients are considered to have "full stomachs," general anesthesia must be administered via a "rapid sequence" induction technique. With patients who are not at risk for aspiration, anesthesia is generally induced with an intravenous agent that usually renders the patient apneic. An attempt at mask ventilation is made and, if successful, a muscle relaxant is administered and mask ventilation is continued until the patient is ready for intubation. During this time, aspiration is possible since airway reflexes are obtunded and the airway is unprotected. This event is extraordinarily rare, however, in patients who have no risk factors for aspiration such as morbid obesity, full stomachs or trauma. In patients who are at risk of aspiration, such as pregnant women, an induction agent is administered and immediately followed with a rapidly acting muscle relaxant and intubation without first testing the airway with mask ventilation. Cricoid pressure is applied to reduce the chance of regurgitation. This technique, known as the rapid sequence induction, diminishes the time that the airway is unprotected and is therefore the standard technique used for patients who are at risk of aspiration. Patients who are unexpectedly found to have an airway that cannot be intubated or ventilated at this time, however, become susceptible to hypoxic injury or death. For this reason, all pregnant patients at risk for cesarean delivery should have their airways evaluated. At the University of Missouri, all patients admitted in labor are examined regardless of the anticipated need for analgesia/anesthesia. This allows the anesthesiologist to identify in advance patients who may have a "difficult airway." Plans to avoid or limit the likelihood of general anesthesia for a "stat" cesarean delivery can then be made in advance for these at risk patients.

\section{General Anesthesia}

Despite the increased risks of general anesthesia over regional anesthesia, it still has indications and some advantages under specific circumstances. It can be administered quickly and reliably. Uterine relaxation responds to potent inhalation anesthetics (e.g., sevoflurane, isoflurane, and desflurane) in a dose-dependent manner. When marked uterine relaxation is required such as with in utero fetal surgery, intrauterine manipulations, and placental extractions, general anesthesia with potent inhalational anesthetics is reliable and effective. General anesthesia is most often chosen either because of 
coexisting contraindications to regional anesthesia or because circumstances do not allow time to establish a sufficient level of regional anesthesia.

The usual technique for general anesthesia for cesarean delivery is to administer a non-particulate clear antacid shortly prior to induction. The patient is then placed in supine position with left uterine displacement, standard monitors are applied, and the patient is pre-oxygenated with four or five vital capacity breaths of $100 \%$ oxygen. Cricoid pressure is then applied just prior to induction with an intravenous agent such as propofol or thiopental and followed immediately with the administration of the rapidonset, short-acting muscle relaxant succinylcholine. Following approximately 60 seconds, the trachea is orally intubated. Once intubation is confirmed via breath sounds and endtidal $\mathrm{CO}_{2}$, the cricoid pressure can be released and incision made. Potent inhalational agents are usually continued at low concentrations with or without the addition of nitrous oxide. Pregnancy confers a greater sensitivity to anesthetics, which is fortuitous because inhalational agents cause uterine relaxation in a dose-dependent manner. At low concentrations of inhalational agents, oxytocin can still be effective in providing uterine contractility and hemostasis. When necessary, the potent inhalational agent can be discontinued. Nitrous oxide does not cause uterine relaxation and can be initiated or continued with the addition of narcotics, benzodiazepines, and muscle relaxants. In order to decrease the risk of aspiration upon emergence, the patient should be extubated when sufficiently awake such that airway protective reflexes are intact and the risk of laryngospasm is low.

A misconception that many obstetricians have is that time is of the essence in performing a cesarean delivery under general anesthesia. Uterine incision to delivery time is more important in neonatal outcome than induction to delivery time. With regional anesthesia, neonates do not become acidotic until greater than 3 minutes have elapsed following uterine incision (132). Induction agents used for general anesthesia do not anesthetize the neonate when usual doses are given. The reasons for this are multifactorial and include dilution of the induction agent with blood from the fetal vena cava, first-pass fetal hepatic metabolism, and fetal shunting. Furthermore, in sheep models, a period of 15 to 20 minutes is required for isoflurane administered to the ewe to reach full anesthetizing levels in the fetal circulation (133). Even if a prolonged induction to delivery time is required, as long as maternal blood pressure and oxygenation are maintained, an anesthetized but not severely acidotic neonate is likely to be delivered as long as fetal distress was not present at the time of induction and uterine incision to delivery time was less than 3 minutes. It is probably better for the obstetrician to take sufficient time to perform a careful operation than risk maternal injury for fear of delivering an anesthetized infant.

\section{Spinal Anesthesia}

Spinal anesthesia is more correctly termed intrathecal or subarachnoid anesthesia as the local anesthetic drug is injected through the dura-arachnoid into the CSF below the terminal end of the spinal cord. The most common interspaces chosen are the L3-4 or L4-5 levels. Hyperbaric bupivacaine is most commonly used in the United States. A dosage of $12 \mathrm{mg}$ reliably offers a sufficient level of anesthesia to keep the patient comfortable, although some practitioners may use slightly more or less than $12 \mathrm{mg}(134,135)$. This dosage may be less than what is commonly used in the nonobstetric patient population as pregnancy confers greater sensitivity to local anesthetics. In addition, engorged epidural vasculature may lead to lower spinal CSF volumes. While a lower abdominal incision is utilized in cesarean deliveries, a T4 anesthetic level is required for lower abdominal intraperitoneal operations. Pain from peritoneal traction may be referred to mid or upper thoracic levels. Testing the level of anesthesia at the lower abdomen prior to incision will therefore not guarantee a sufficient level of anesthesia once the peritoneal cavity is entered. A single injection spinal anesthetic utilizing $12 \mathrm{mg}$ of hyperbaric bupivacaine will provide 90 to 120 minutes of anesthesia.

Hypotension is the most common side effect of spinal anesthesia and can be limited but not completely prevented by prehydration with 1000 to $1500 \mathrm{~mL}$ of crystalloid administered immediately prior to induction. The use of colloid may be more effective (136). When fluid administration is insufficient to prevent or treat hypotension, vasopressors are often required. In the past, ephedrine had been the drug of choice. Ephedrine has both alpha and beta agonist properties. Phenylephrine, however, is a pure alpha vasoconstrictor and was once considered to be potentially harmful as it was shown in sheep models that potent vasoconstrictors decrease uterine blood flow (137). More recently, it has been determined that, clinically, phenylephrine does not have deleterious fetal/ neonatal effects when administered judiciously. Indeed, infants born to mothers receiving phenylephrine have higher umbilical cord arterial blood $\mathrm{pH}$ levels than those treated with ephedrine (138). Another troublesome side effect from spinal anesthesia is post-dural puncture headache. The use of smallgauge pencil-point needles, however, has reduced the incidence of post-dural puncture headache to an acceptable rate of $0.5 \%$ to $1 \%$ (139). In a 2001 survey, spinal anesthesia was the most commonly used anesthetic technique for elective cesarean delivery. Even for emergent cesarean deliveries, spinal anesthesia was used $45 \%$ to $59 \%$ of the time (128).

\section{Epidural Anesthesia}

Epidural anesthesia for cesarean deliveries is most often used for women who have been in labor with an active epidural analgesic already in place. The level of the block can be intensified and extended by dosing the catheter with $2 \%$ lidocaine with epinephrine, 3\% 2-chloroprocaine, $0.5 \%$ bupivacaine, or $0.5 \%$ ropivacaine. Epidural anesthesia has the advantage of titration of the level of block, slower onset with greater ability to manage hypotension, and flexibility. The epidural catheter can be re-dosed as needed for procedures that end up being longer than anticipated. Lidocaine 2\% with epinephrine $1: 400,000$ or $1: 200,000$ is probably the drug most often used. It is relatively rapid in onset and of intermediate duration lasting 90 to 120 minutes. 2-Chloroprocaine 3\% has an onset time within 8 minutes or less in laboring patients with preexisting epidural catheters and can be used for urgent cesarean deliveries (140). It is rapidly metabolized in maternal serum with little transfer and accumulation in an acidotic fetus (141). 2-Chloroprocaine has a shorter duration of action, 
however, lasting 40 to 50 minutes. When used, it must be followed up with additional local anesthetic, most often $2 \%$ lidocaine. 2-Chloroprocaine may interfere with the effectiveness of subsequently administered bupivacaine or epidurally administered opioids $(142,143)$.

Epidural anesthesia for cesarean delivery may not be as reliable as spinal anesthesia. Incomplete or "patchy" block may limit its usefulness. This may require extra time to allow further distribution of the local anesthetic. The addition of epidural adjuvants such as fentanyl may be helpful. Intravenous additives such as opioid, small judicious doses of propofol, or ketamine may also be required. The anesthesiologist must be careful not to obtund the patient's airway reflexes. Ketamine in small doses is often useful under these circumstances as it provides profound amnesia and analgesia. It can cause dysphoria and therefore requires the addition of midazolam to limit this unpleasant side effect. When epidural anesthesia is ineffective, general anesthesia may be necessary. From the standpoint of airway protection, general endotracheal anesthesia is the most conservative approach to take. Ineffective or incomplete epidural anesthesia during cesarean delivery can be avoided if the effectiveness of the labor epidural is carefully evaluated prior to cesarean delivery. If there is any question, then proceeding to spinal anesthesia or repeat placement of the epidural catheter is in order (144).

If spinal anesthesia is chosen immediately following failed or unsuccessful epidural anesthesia, then caution must be taken to avoid a high spinal block, which may render the patient hypotensive and/or apneic. The reasons for this may be related to an already existing partial block or compression of the intrathecal sac from epidurally administered fluid. A downward adjustment of local anesthetic dose may therefore be necessary (145). If the anesthesiologist chooses to replace the epidural catheter, the potential for local anesthetic toxicity must be kept in mind as large doses of local anesthetic are typically used.

\section{CSE Anesthesia}

CSE anesthesia offers the advantages of both the techniques for cesarean delivery. Spinal anesthesia provides a more rapid onset and more reliable block than does epidural anesthesia (146). Because of its rapid onset, however, maternal hypotension may be problematic. In addition, spinal anesthesia provides a fixed time period of anesthesia. Should the operative time run beyond the length of the spinal anesthetic, then general anesthesia may be required for the remainder of the procedure. CSE anesthesia allows for the use of lower doses of spinal anesthetic, minimizing maternal hypotension, and has the flexibility to extend and prolong the duration of the block via epidural "top-ups" during the procedure $(147,148)$. At the University of Missouri, we often use CSE anesthesia when a longer than usual procedure is anticipated such as multiple prior cesarean deliveries, morbid obesity, or anticipated cesarean hysterectomy. The more favorable hemodynamics and reliability of CSE anesthesia have allowed regional anesthesia to be utilized in patients with severe pre-eclampsia and obstructive cardiac lesions (149-151).

\section{Post-cesarean Pain Management}

The increasing use of regional anesthesia for cesarean delivery has been paralleled with an increasing use of spinal opioids for the management of postoperative pain. Postoperative pain following cesarean delivery is unique in that the parturient must care for a newborn infant as well as for herself. Parenteral opioids have a long history of effectiveness, but can be associated with troublesome side effects, particularly somnolence. Spinal opioids administered epidurally or intrathecally can provide effective pain relief with a minimum degree of somnolence. Preservative free morphine is probably the most commonly used drug in the United States. It can be administered intrathecally at the time of spinal anesthesia or via the epidural catheter during or immediately following cesarean delivery. A single dose of morphine, 2 to $5 \mathrm{mg}$, administered via an epidural catheter will provide pain relief for up to 24 hours (152). While a portion of the morphine administered into the epidural space will be absorbed into the vasculature and redistributed to the CNS, the remainder will transfer directly to the CSF and bind to mu receptors at the spinal cord level. Stimulation of mu receptors in turn blocks the transmission of nociceptive signals to higher levels (153). Administered intrathecally at the time of spinal anesthesia, small doses of morphine, ranging from 0.1 to $0.25 \mathrm{mg}$, will provide analgesia for 18 to 24 hours $(154,155)$. Unlike local anesthetics, spinal opiates do not cause motor block and allow for early ambulation.

Other opiates have been used in place of or in addition to morphine. Meperidine and hydromorphone have also been used successfully $(156,157)$. Fentanyl is more lipid soluble than morphine. It quickly binds to mu receptors at the spinal cord level and is of much shorter duration lasting only a few hours. It is usually used as an adjunct to local anesthetics during cesarean delivery. If it is to be used at all for postoperative pain relief, it is usually added to a continuous epidural infusion of low-concentration local anesthetic.

While effective in providing pain relief, spinal opiates are not without troubling side effects. Nausea and vomiting, pruritus, and respiratory depression can complicate an otherwise excellent method of postoperative analgesia. These side effects are primarily mediated by $\mathrm{mu}$ receptors. $\mathrm{Mu}$ antagonists can therefore be used for treatment when side effects become particularly bothersome. It is important to note that pruritus from spinal opiates is not primarily histamine mediated. Antihistamines are therefore less likely to be effective in treating pruritus than mu antagonists. Low-dose naloxone either as a bolus or continuous intravenous infusion can effectively treat side effects without diminishing analgesia (158). Agonists-antagonists such as nalbuphine have also been used successfully (159).

Of the side effects that can occur with the use of spinal opiates, respiratory depression is the most concerning. Fortunately, most obstetric patients are young and healthy with a very low incidence of significant respiratory depression following the use of spinal opiates (152). Nevertheless, it is prudent to follow these patients closely with hourly nursing checks and/or continuous pulse oximetry. It is important to note that with spinal morphine, respiratory depression may be delayed by up to 12 hours following administration. Most anesthesiologists therefore wish to be consulted before any sedative drugs are administered to patients who have received spinal morphine during the first 24 postoperative hours. While side effects and the fear of respiratory depression may be 
disconcerting, spinal opiates have become the mainstay of postoperative pain management following cesarean delivery in the United States.

\section{ANESTHESIA FOR THE HIGH-RISK PATIENT}

Of the myriad complications of pregnancy that have anesthetic implications, pre-eclampsia and deteriorating fetal status (fetal distress) are among the more common. In prior decades, patients with congenital heart disease did not often survive to reproductive age. Currently, however, more and more patients are living to childbearing age and it is becoming more common to encounter patients with corrected or partially corrected congenital heart lesions. With the obesity epidemic making its way into an ageing obstetric population, we can expect to see an increase in the frequency of cardiovascular diseases complicating pregnancy.

\section{Pre-eclampsia/Eclampsia}

Hypertensive disorders complicate $5 \%$ to $10 \%$ of pregnancies. While pre-eclampsia affects multiple systems, the cardio-pulmonary, hepatic, coagulation, and airway systems are of particular interest to the anesthesiologist. Patients with severe pre-eclampsia are intravascularly volume depleted even though there may be a positive water and sodium balance. Intravascular volume depletion may present a challenge to the anesthesiologist providing regional analgesia for labor or anesthesia for cesarean delivery. In addition, most patients with severe pre-eclampsia have some degree of increased systemic vascular resistance with varying degrees of cardiac function ranging from hyperdynamic to normal to impaired (160). In the past, epidural analgesia for labor was controversial in patients with severe pre-eclampsia because of the concern for marked reductions in blood pressure and utero-placental blood flow. Epidural analgesia, however, has been shown to provide superior pain relief, decrease blood pressure, decrease catecholamine levels, and improve utero-placental blood flow (161,162). Marked drops in blood pressure can be prevented with judicious use of crystalloids and or colloids for prehydration and treated with small doses of ephedrine. Indeed, in a 2001 study, the incidence of hypotension requiring treatment was only $11 \%$ (163). In patients whose severe pre-eclampsia is complicated by pulmonary edema and/or oliguria, further studies including invasive central monitoring and/or echocardiography may be indicated before initiating epidural analgesia.

General anesthesia for cesarean delivery in patients with severe pre-eclampsia can be particularly hazardous. An edematous airway can make intubation difficult or impossible, posing a serious threat to maternal safety. Tracheal intubation during general anesthesia is very stimulating and can be associated with dangerously high blood pressures placing the patient at risk for intracranial hemorrhage. For these reasons, regional anesthesia is the preferred method of anesthesia in patients with severe pre-eclampsia. When general anesthesia is necessary, blood pressure should be controlled with additional doses of antihypertensives. The response to intubation can be blunted with opioids and/or intravenous lidocaine just prior to induction. Awake intubation may be necessary to avoid the very dangerous cannot-intubate/cannot-ventilate scenario.
In the past, spinal anesthesia was not recommended in patients with severe pre-eclampsia undergoing a cesarean delivery. Many anesthesiologists believed that spinal anesthesia would lead to difficult-to-treat hypotension. More recent studies however have shown that the incidence or severity of hypotension is no greater in severe pre-eclamptic patients having spinal anesthesia than epidural anesthesia (164). It is interesting to note that a study as far back as 1950 demonstrated that severe pre-eclamptic patients were more resistant to hypotension following spinal anesthesia than were normal patients (165). At the University of Missouri, spinal anesthesia is frequently administered to patients with severe pre-eclampsia undergoing cesarean delivery.

The coagulopathy that may accompany severe pre-eclampsia is another major concern to the anesthesiologist. Uncorrected severe coagulopathy is an absolute contraindication to regional analgesia/anesthesia for fear of traumatizing the epidural vasculature, which in turn may lead to the formation of an epidural hematoma. Most anesthesiologists will place an epidural catheter or perform a spinal anesthetic with platelet counts above $100,000 / \mathrm{mm}^{3}$ in the absence of any clinical evidence of abnormal bleeding. There is no absolute cutoff for platelet counts below which regional anesthesia is contraindicated. Regional analgesia/anesthesia has been safely performed in parturients with platelet counts as low as $50,000 / \mathrm{mm}^{3}$ to $69,000 / \mathrm{mm}^{3}$ when other coagulation indices are normal and there is no clinical evidence of abnormal bleeding $(166,167)$. The decision to perform regional analgesia/anesthesia in a patient with thrombocytopenia, however, must always be made on an individual basis keeping the risk/benefit ratio in mind. It is also worthwhile to keep in mind that epidural hematomas are very rare in the pregnant patient population. Difficult airways, unfortunately, are not.

Early anesthesia consultation is recommended when the obstetrics service admits a patient with severe pre-eclampsia. This is particularly true in patients who are at risk for an emerging coagulopathy. Many obstetric anesthesiologists are now advising early epidural placement when the patient is committed to delivery. As the disease progresses, regional anesthesia may not be possible further in labor if a coagulopathy is already well established. An epidural catheter placed early in labor can be continued even if the platelet count drops to very low levels. Not only would this allow the patient to receive the benefit of epidural analgesia for labor, but in the event of cesarean delivery, the epidural catheter can also be dosed to a surgical level of anesthesia and avoid the much greater hazards of general anesthesia.

\section{Deteriorating Fetal Status}

The term "fetal distress" is imprecise and nonspecific. Its use can often lead to unnecessary haste, placing both the mother and the fetus at risk for complications and medical errors. The American College of Obstetricians and Gynecologists has recommended that the term "fetal distress" be replaced with "non-reassuring fetal status" with further descriptors clarifying the abnormality or presumed etiology (168). Either regional anesthesia or general anesthesia can be used for cesarean deliveries performed for non-reassuring fetal status with comparable outcomes (169). In instances of non-reassuring fetal status, the degree of urgency and time limitations often 
determine the choice of anesthesia. Some practitioners have the perception that general anesthesia can be administered more rapidly than regional anesthesia and have encouraged its use when "fetal distress" is diagnosed. It must be kept in mind, however, that the risk of maternal mortality from anesthetic causes is significantly greater for general anesthesia than regional anesthesia (130). Recognizing the increased maternal risks of general anesthesia versus regional anesthesia, the American College of Obstetricians and Gynecologists has recently stated:

The significant added morbidity of general anesthesia over regional anesthesia for cesarean delivery suggests that regional anesthesia is the preferred method of pain control and should be used unless a contraindication to regional anesthesia is present. Although general anesthesia may be indicated in some cases of fetal heart rate abnormality, the severity of the abnormality should be considered before incurring the excess risk of maternal morality associated with general anesthesia (170).

Inability to intubate and aspiration of gastric contents present the greatest risk of general anesthesia to the parturient. The incidence of failed intubation has been estimated to be as high as 1 in 250 patients (171). When patients at risk for failed intubation are identified, close communication among obstetric and anesthesia team members is critical. These patients may benefit from early epidural catheter placement. When anesthesia team members are notified early in the process of initiating a "stat" cesarean delivery, dosing a well-established labor epidural catheter for cesarean delivery can be performed nearly as rapidly as general anesthesia (172). When an unexpected difficult airway is encountered, a decision must be made to proceed without the benefit of endotracheal intubation, incurring the risk of aspiration, or allowing the patient to awaken and proceeding with an awake intubation technique, which will usually require much more time to accomplish than the standard rapid sequence induction. Newer ventilation and intubation methods such as the laryngeal mask airway (LMA), intubating LMA, and Pro-Seal $\mathrm{LMA}^{\mathrm{TM}}$ have been recognized as valuable techniques for managing these very stressful situations $(173,174)$. If ventilation cannot be established, then either the patient must be awakened or a surgical airway be established (175).

The importance of close communication between obstetrician and anesthesiologist cannot be stressed more. The highest risk to the patient occurs when an anesthesiologist first encounters a patient already in the operating room prepped and draped for "stat" cesarean delivery. The anesthesiologist is given very little time to evaluate the patient and prepare for anticipated difficulties. Conversely, the obstetrician must be apprised as early as possible when the anesthesiologist anticipates difficulty. At the University of Missouri, when patients are brought to the operating room for "stat" cesarean delivery for rapidly deteriorating fetal status, FHR monitoring is continued. This allows the establishment of regional anesthesia to continue if the FHR returns to normal or to stable levels. If an internal electrode is in place, it is not removed until just prior to incision or may be kept in place if general anesthesia is required. The real-time information gained with this approach may help guide the management of a failed intubation. In the event of a failed intubation, if fetal status has improved and remains stable, the patient can be awakened and a different course of action can be taken. If there is continued fetal deterioration, the anesthetic can then be continued by mask or LMA as noted above, with continuous cricoid pressure (176).

\section{Cardiac Disease}

The cardiac adaptations to pregnancy are well known. Blood volume and cardiac output increase significantly during gestation and are tolerated very well in healthy individuals. Women with cardiac disease who otherwise may be sufficiently compensated in the nonpregnant state, however, may deteriorate significantly when faced with the cardiac demands of pregnancy.

\section{Congenital Heart Disease}

In 2001, the majority of pregnant women presenting with cardiac disease were those with congenital heart disorders (177). This is not surprising since a greater number of women with congenital heart disease are living to childbearing age than that in earlier decades. Patients with fully corrected lesions with no residual dysfunction do well during pregnancy and do not require special attention other than prophylactic antibiotics. Those with partially corrected lesions or residual dysfunction, however, may require special considerations.

Individuals with shunts must maintain a balance between right- and left-sided pressures. Increasing left-sided pressures such as systemic hypertension may worsen preexisting left-to-right shunting. A right-to-left shunt will worsen if peripheral vascular resistance drops as it may with regional anesthesia. Patients with obstructive lesions who are preload depended may decompensate if there is a significant drop in venous return. In the past, regional anesthesia was considered relatively contraindicated in these individuals. More recently, however, regional anesthesia has been shown to be of benefit in many patients and is recommended when it is advisable to avoid the high catecholamine levels of pain and stress, which may lead to maternal tachycardia, systemic hypertension and pulmonary hypertension (178). Patients with obstructive lesions and pulmonary hypertension who do not tolerate drops in venous return or systemic vascular resistance may still significantly benefit from epidural analgesia, provided that it is dosed slowly while maintaining preload and systemic vascular resistance. Patients with regurgitant lesions and left-to-right shunts may benefit from the decreased systemic vascular resistance that epidural analgesia provides as it promotes forward flow. The increased demands on cardiac output, workload, and oxygen consumption present during the second stage of labor can be ameliorated with a carefully managed epidural analgesic allowing for a passive second stage of labor. Patients with these lesions may require invasive monitoring as well as continuous oxygen saturation monitoring to optimize cardiac function and avoid the hemodynamic perturbations that regional anesthesia may cause. 


\section{Valvular Lesions}

Patients with stenotic valvular lesions and dynamic obstructive cardiomyopathy do not tolerate tachycardia and decreased systemic vascular resistance well, nor do they tolerate the tachycardia and stress associated with the pain of labor. Epidural analgesia is beneficial in these individuals as long as preload and systemic vascular resistance are maintained. This is particularly true during the second stage of labor. When treating hypotension in these patients, beta agonists are to be avoided.

Epidural analgesia is the preferred pain management technique in patients with regurgitant valvular lesions. The decreased systemic vascular resistance that results from regional anesthesia decreases afterload, reduces the regurgitant fraction, and improves forward flow. In addition, it prevents the increase in systemic vascular resistance that results from the pain of labor.

\section{Ischemic Cardiac Disease}

Ischemic cardiac disease in pregnancy is rare. Nevertheless, as the pregnant patient population ages, particularly in association with assisted reproductive techniques used in an aging population, we can expect to see increasing numbers of patients with coronary artery disease. Epidural analgesia is well suited to this patient population. The sympathectomy associated with epidural analgesia decreases catecholamine levels and cardiac workload. A passive second-stage labor can be achieved, which will further decrease myocardial oxygen demand. Perfusion pressures must be maintained, however, but epinephrine-containing local anesthetic and beta agonists should be avoided as they may lead to troublesome tachycardia. Intensive cardiac monitoring may be necessary in patients who have significant myocardium at risk.

The successful management of the pregnant cardiac patient requires an interdisciplinary approach. Obstetrician, cardiologist, and anesthesiologist should be involved early in the patient's pregnancy. Clinical data, particularly results of echocardiograms and stress tests, should be shared among all involved prior to the patient's arrival on labor and delivery. It does not serve the patient well when the anesthesiologist is first consulted at the time that the patient is already in active labor or presents for cesarean delivery. Early consultation will provide for a coordinated team approach leading to the best results possible (178).

\section{CONCLUSION}

Obstetric anesthesia has made great strides over the past 50 years. Maternal mortality from anesthetic causes has declined dramatically, primarily due to increasing usage of regional anesthesia. Regional analgesia for labor is effective, safe, and has little effect on the course of labor or neonatal outcome. Newer techniques have led to the expansion of regional analgesia to allow for its application to a wide range of patients with pregnancy complications. In contemporary labor and delivery units, virtually all patients are able to enjoy the benefits of regional analgesia should they choose to do so.

Currently, a national effort is underway to improve patient safety. As part of this effort, anesthesiologists are becoming more and more proactive in perioperative and obstetric care. At the University of Missouri, strategies have been developed and are continuing to be refined, to help reduce general anesthesia-related morbidity and mortality. An attempt is made for an anesthesiologist to evaluate all patients admitted to labor and delivery regardless of the anticipated need for anesthesia services. Patients who may present with potential anesthetic complications are referred for anesthesia consultation during gestation, prior to admission. This includes patients who are morbidly obese, have had prior anesthetic difficulties or a family history of anesthesia complications. This is also true for patients with heart disease, prior spine surgery, neurologic, hematologic, or significant pulmonary disorders. Patients with an anticipated or known difficult airway are encouraged to have an epidural catheter placed early in labor. This is also true for patients at risk of developing a coagulopathy, such as with severe pre-eclampsia or hemolysis, elevated liver enzymes, and low platelet count syndrome.

It cannot be overstressed that continuous close communication with all members of the obstetric team is vital to improving patient outcome.

\section{REFERENCES}

1. Caton D. Obstetric anesthesia: the first ten years. Anesthesiology 1970; 33: 102-9.

2. ACOG Committee Opinion: Committee on Obstetrics: Maternal and Fetal Medicine Number 118-January 1993. Pain relief during labor. Int J Gynaecol Obstet 1993; 42: 73.

3. Keenan P. Benefits of massage therapy and use of a doula during labor and childbirth. Altern Ther Health Med 2000; 6: 66-74.

4. Hetherington SE. A controlled study of the effect of prepared childbirth classes on obstetric outcomes. Birth 1990; 17: 86-90.

5. Scott JR, Rose NB. Effect of psychoprophylaxis (Lamaze preparation) on labor and delivery in primiparas. N Engl J Med 1976; 294: 1205-7.

6. Harrison RF, Woods T, Shore M, Mathews G, Unwin A. Pain relief in labour using transcutaneous electrical nerve stimulation (TENS). A TENS/TENS placebo controlled study in two parity groups. Br J Obstet Gynaecol 1986; 93: 739-46.

7. Vincenti E, Cervellin A, Mega M, et al. Comparative study between patients treated with transcutaneous electric stimulation and controls during labour. Clin Exp Obstet Gynecol 1982; 9: 95-7.

8. Robson JE. Transcutaneous nerve stimulation for pain relief in labour. Anaesthesia 1979; 34: 357-60.

9. Bundsen P, Ericson K, Peterson LE, Thiringer K. Pain relief in labor by transcutaneous electrical nerve stimulation. Testing of a modified stimulation technique and evaluation of the neurological and biochemical condition of the newborn infant. Acta Obstet Gynecol Scand 1982; 61: 129-36

10. Umeh BU. Sacral acupuncture for pain relief in labour: initial clinical experience in Nigerian women. Acupunct Electrother Res 1986; 11: 147-51.

11. Wallis L, Shnider SM, Palahniuk RJ, Spivey HT. An evaluation of acupuncture analgesia in obstetrics. Anesthesiology 1974; 41: 596-601.

12. Pei DE, Huang YL. Use of acupuncture analgesia during childbirth. J Tradit Chin Med 1985; 5: 253-5.

13. Abouleish E, Depp R. Acupuncture in obstetrics. Anesth Analg 1975; 54: 82-8.

14. Harmon TM, Hynan MT, Tyre TE. Improved obstetric outcomes using hypnotic analgesia and skill mastery combined with childbirth education. J Consult Clin Psychol 1990; 58: 525-30.

15. Stellato C, Cirillo R, de Paulis A, et al. Human basophil/mast cell releasability. IX. Heterogeneity of the effects of opioids on mediator release. Anesthesiology 1992; 77: 932-40.

16. Aitkenhead AR, Vater M, Achola K, et al. Pharmacokinetics of singledose i.v. morphine in normal volunteers and patients with end-stage renal failure. Br J Anaesth 1984; 56: 813-19.

17. Stoelting R. Opioid agonists and antagonists, pharmacology and physiology in anesthetic practice, 2nd edn. Philadelphia: J.B. Lippincott, 1991: 70-101. 
18. Coda BA. In: Barash PG, Cullen BF, Stoelting RK, eds. Opioids, Clinical Anesthesia, 5th edn. Philadelphia: Lippincott Williams \& Wilkins, 2006: 353-83.

19. Kuhnert BR, Kuhnert PM, Tu AS, et al. Meperidine and normeperidine levels following meperidine administration during labor. I. Mother. Am J Obstet Gynecol 1979; 133: 904-8.

20. Kuhnert BR, Kuhnert PM, Prochaska AL, Gross TL. Plasma levels of 2chloroprocaine in obstetric patients and their neonates after epidural anesthesia. Anesthesiology 1980; 53: 21-5.

21. Caldwell J, Wakile LA, Notarianni LJ, et al. Maternal and neonatal disposition of pethidine in childbirth-a study using quantitative gas chromatography-mass spectrometry. Life Sci 1978; 22: 589-96.

22. Kuhnert BR, Philipson EH, Kuhnert PM, Syracuse CD. Disposition of meperidine and normeperidine following multiple doses during labor. I. Mother. Am J Obstet Gynecol 1985; 151: 406-9.

23. Belfrage $\mathrm{P}$, Boreus LO, Hartvig $\mathrm{P}$, et al. Neonatal depression after obstetrical analgesia with pethidine. The role of the injection-delivery time interval and of the plasma concentrations of pethidine and norpethidine. Acta Obstet Gynecol Scand 1981; 60: 43-9.

24. Rayburn WF, Smith CV, Parriott JE, Woods RE. Randomized comparison of meperidine and fentanyl during labor. Obstet Gynecol 1989; 74: 604-6.

25. Smith CV, Rayburn WF, Allen KV, et al. Influence of intravenous fentanyl on fetal biophysical parameters during labor. J Matern Fetal Med 1996; 5: 89-92.

26. Wilson CM, McClean E, Moore J, Dundee JW. A double-blind comparison of intramuscular pethidine and nalbuphine in labour. Anaesthesia 1986; 41: 1207-13.

27. Maduska AL, Hajghassemali M. A double-blind comparison of butorphanol and meperidine in labour: maternal pain relief and effect on the newborn. Can Anaesth Soc J 1978; 25: 398-404.

28. Hodgkinson R, Huff RW, Hayashi RH, Husain FJ. Double-blind comparison of maternal analgesia and neonatal neurobehaviour following intravenous butorphanol and meperidine. J Int Med Res 1979; 7: 224-30.

29. Ballantyne JC, Carr DB, Chalmers TC, et al. Postoperative patientcontrolled analgesia: meta-analyses of initial randomized control trials [see comment]. J Clin Anesth 1993; 5: 182-93.

30. Podlas J, Breland BD. Patient-controlled analgesia with nalbuphine during labor. Obstet Gynecol 1987; 70: 202-4.

31. Rayburn W, Leuschen MP, Earl R, et al. Intravenous meperidine during labor: a randomized comparison between nursing- and patientcontrolled administration. Obstet Gynecol 1989; 74: 702-6.

32. Morley-Forster PK, Weberpals J. Neonatal effects of patient-controlled analgesia using fentanyl in labor. Int J Obstet Anesth 1998; 7: 103-7.

33. Balki M, Kasodekar S, Dhumne S, et al. Remifentanil patient-controlled analgesia for labour: optimizing drug delivery regimens. Can J Anaesth 2007; 54: 626-33.

34. Evron S, Ezri T. Options for systemic labor analgesia [review]. Curr Opin Anaesthesiol 2007; 20: 181-5.

35. Evron S, Glezerman M, Sadan O, et al. A novel systemic analgesic for labor pain. Anesth Analg 2005; 100: 233-8.

36. Owen MD, Poss MJ, Dean LS, Harper MA. Prolonged intravenous remifentanil infusion for labor analgesia. Anesth Analg 2002; 94: 918-19.

37. Jones R, Pegrum A, Stacey RG. Patient-controlled analgesia using remifentanil in the parturient with thrombocytopaenia. Anaesthesia 1999; 54: 461-5.

38. Kan RE, Hughes SC, Rosen MA, et al. Intravenous remifentanil: placental transfer, maternal and neonatal effects. Anesthesiology 1998; 88: 1467-74.

39. Nuutinen LS, Laitinen JO, Salomaki TE. A risk-benefit appraisal of injectable NSAIDs in the management of postoperative pain [review]. Drug Saf 1993; 9: 380-93.

40. Walker JJ, Johnston J, Fairlie FM, et al. A comparative study of intramuscular ketorolac and pethidine in labour pain. Eur J Obstet Gynecol Reprod Biol 1992; 46: 87-94

41. Greer IA, Johnston J, Tulloch I, Walker JJ. Effect of maternal ketorolac administration of platelet function in the newborn. Eur J Obstet Gynecol Reprod Biol 1988; 29: 257-60

42. Gerdin E, Salmonson T, Lindberg B, Rane A. Maternal kinetics of morphine during labour. J Perinat Med 1990; 18: 479-87.
43. Garland M, Abildskov KM, Taylor S, et al. Fetal morphine metabolism and clearance are constant during late gestation. Drug Metab Dispos 2006; 34: 636-46.

44. Garland M, Abildskov KM, Kiu TW, et al. The contribution of fetal metabolism to the disposition of morphine. Drug Metab Dispos 2005; 33: $68-76$.

45. Crawford JS, Rudofsky S. The placental transmission of pethidine. $\mathrm{Br}$ J Anaesth 1965; 37: 929-33.

46. Szeto HH, Mann LI, Bhakthavathsalan A, et al. Meperidine pharmacokinetics in the maternal-fetal unit. J Pharmacol Exp Ther 1978; 206: 448-59.

47. Petrie RH, Yeh SY, Murata Y, et al. The effect of drugs on fetal heart rate variability. Am J Obstet Gynecol 1978; 130: 294-9.

48. Zimmer EZ, Divon MY, Vadasz A. Influence of meperidine on fetal movements and heart rate beat-to-beat variability in the active phase of labor. Am J Perinatol 1988; 5: 197-200.

49. Craft JB, Levinson G, Shnider SM. Anaesthetic considerations in caesarean section for quadruplets. Can Anaesth Soc J 1978; 25: 236-9.

50. Rayburn W, Rathke A, Leuschen MP, et al. Fentanyl citrate analgesia during labor. Am J Obstet Gynecol 1989; 161: 202-6.

51. Wilson SJ, Errick JK, Balkon J. Pharmacokinetics of nalbuphine during parturition. Am J Obstet Gynecol 1986; 155: 340-4.

52. Nicolle E, Devillier P, Delanoy B, et al. Therapeutic monitoring of nalbuphine: transplacental transfer and estimated pharmacokinetics in the neonate. Eur J Clin Pharmacol 1996; 49: 485-9.

53. Giannina G, Guzman ER, Lai YL, et al. Comparison of the effects of meperidine and nalbuphine on intrapartum fetal heart rate tracings. Obstet Gynecol 1995; 86: 441-5.

54. Wakefield ML. In: Chestnut DH, ed. Obstetric Anesthesia Principles and Practice, 3rd edn. Philadelphia: Elsevier Moby, 2004: 315.

55. Poehlmann S, Pinette M, Stubblefield P. Effect of labor analgesia with nalbuphine hydrochloride on fetal response to vibroacoustic stimulation. J Reprod Med 1995; 40: 707-10.

56. Maduska AL, Pittman KA, Ahokas RA, et al. Placental transfer and other physiologic studies with intravenous butorphanol in the anesthetized pregnant ewe. Res Commun Chem Pathol Pharmacol 1980; 29: 229-41.

57. Vasicka A, Robertazzi R, Raji $M$, et al. Fetal bradycardia after paracervical block. Obstet Gynecol 1971; 38: 500-12.

58. Miller FC, Quesnel G, Petrie RH, et al. The effects of paracervical block on uterine activity and beat-to-beat variability of the fetal heart rate. Am J Obstet Gynecol 1978; 130: 284-8.

59. Shnider SM, Asling JH, Holl JW, Margolis AJ. Paracervical block anesthesia in obstetrics. I. Fetal complications and neonatal morbidity. Am J Obstet Gynecol 1970; 107: 619-25.

60. Greiss FC Jr, Still JG, Anderson SG. Effects of local anesthetic agents on the uterine vasculatures and myometrium. Am J Obstet Gynecol 1976; 124: 889-99.

61. Amols MH, Traynor KD, Creedon DJ. Where have all the pudendals gone? Anesthesiology 2007; 106(3 Suppl 1): A-41.

62. Hawkins JL, Gibbs CP, Orleans M, et al. Obstetric anesthesia work force survey, 1981 versus 1992. Anesthesiology 1997; 87: 135-43.

63. Halpern SH, Leighton BL, Ohlsson A, et al. Effect of epidural vs parenteral opioid analgesia on the progress of labor: a meta-analysis. JAMA 1998; 280: 2105-10.

64. Buchan PC, Milne MK, Browning MC. The effect of continuous epidural blockade on plasma 11-hydroxycorticosteroid concentrations in labour. J Obstet Gynaecol Br Commonw 1973; 80: 974-7.

65. Thornton CA, Carrie LE, Sayers L, et al. A comparison of the effect of extradural and parenteral analgesia on maternal plasma cortisol concentrations during labour and the puerperium. Br J Obstet Gynaecol 1976; 83: 631-5.

66. Abboud TK, Sarkis F, Hung TT, et al. Effects of epidural anesthesia during labor on maternal plasma beta-endorphin levels. Anesthesiology 1983; 59: 1-5.

67. Shnider SM, Abboud TK, Artal R, et al. Maternal catecholamines decrease during labor after lumbar epidural anesthesia. Am J Obstet Gynecol 1983; 147: 13-15.

68. Falconer AD, Powles AB. Plasma noradrenaline levels during labour. Influence of electric lumbar epidural blockade. Anaesthesia 1982; 37: 416-20. 
69. Colonna-Romano P, Lingaraju N, Godfrey SD, Braitman LE. Epidural test dose and intravascular injection in obstetrics: sensitivity, specificity, and lowest effective dose. Anesth Analg 1992; 75: 372-6.

70. Norris MC, Fogel ST, Dalman H, et al. Labor epidural analgesia without an intravascular "test dose." Anesthesiology 1998; 88: 1495-501.

71. Norris MC, Ferrenbach D, Dalman H, et al. Does epinephrine improve the diagnostic accuracy of aspiration during labor epidural analgesia? Anesth Analg 1999; 88: 1073-6.

72. Leighton BL, Katsiris SE, Halpern SH, et al. Multiport epidural catheters: can orifice location be tested? Anesthesiology 2000; 92: 1840-2.

73. Gambling DR, McMorland GH, Yu P, Laszlo C. Comparison of patientcontrolled epidural analgesia and conventional intermittent "top-up" injections during labor. Anesth Analg 1990; 70: 256-61.

74. Gambling DR, Yu P, Cole C, et al. A comparative study of patient controlled epidural analgesia (PCEA) and continuous infusion epidural analgesia (CIEA) during labour. Can J Anaesth 1988; 35: t-54.

75. Purdie J, Reid J, Thorburn J, Asbury AJ. Continuous extradural analgesia: comparison of midwife top-ups, continuous infusions and patient controlled administration. Br J Anaesth 1992; 68: 580-4.

76. Grieco WM, Norris MC, Leighton BL, et al. Intrathecal sufentanil labor analgesia: the effects of adding morphine or epinephrine. Anesth Analg 1993; 77: 1149-54.

77. Kuczkowski KM. Labor pain and its management with the combined spinal-epidural analgesia: what does an obstetrician need to know? Arch Gynecol Obstet 2007; 275: 183-5.

78. Kuczkowski KM. Ambulation with combined spinal-epidural labor analgesia: the technique. Acta Anaesthesiol Belg 2004; 55: 29-34.

79. Nageotte MP, Larson D, Rumney PJ, et al. Epidural analgesia compared with combined spinal-epidural analgesia during labor in nulliparous women. N Engl J Med 1997; 337: 1715-9.

80. Norris MC. Are combined spinal-epidural catheters reliable? Int J Obstet Anesth 2000; 9: 3-6.

81. Albright GA, Forster RM. The safety and efficacy of combined spinal and epidural analgesia/anesthesia (6,002 blocks) in a community hospital. Reg Anesth Pain Med 1999; 24: 117-25.

82. Miro M, Guasch E, Gilsanz F. Comparison of epidural analgesia with combined spinal-epidural analgesia for labor: a retrospective study of 6497 cases. Int J Obstet Anesth 2008; 17: 15-19.

83. Norris MC, Grieco WM, Borkowski M, et al. Complications of labor analgesia: epidural versus combined spinal epidural techniques. Anesth Analg 1994; 79: 529-37.

84. Norris MC, Fogel ST, Conway-Long C. Combined spinal-epidural versus epidural labor analgesia. Anesthesiology 2001; 95: 913-20.

85. Miro M, Gussch E, Gilsanz F. Comparison of epidural analgesia with combined spinal-epidural analgesia for labor: a retrospective study of 6497 cases. Int J Obstet Anesth 2008; 17: 15-19.

86. Lee S, Lew E, Lim Y, Sia AT. Failure of augmentation of labor epidural analgesia for intrapartum cesarean delivery: a retrospective review. Anesth Analg 2009; 108: 252-4.

87. de Jong RH, Ronfeld RA, DeRosa RA. Cardiovascular effects of convulsant and supraconvulsant doses of amide local anesthetics. Anesth Analg 1982; 61: 3-9.

88. de Jong RH. Lipid infusion for cardiotoxicity: promise? Yes-panacea? Not. Anesthesiology 2007; 106: 635-6.

89. Rosenblatt MA, Abel M, Fischer GW, et al. Successful use of a $20 \%$ lipid emulsion to resuscitate a patient after a presumed bupivacaine-related cardiac arrest [see comment]. Anesthesiology 2006; 105: 217-8.

90. Santos AC, Pedersen H, Harmon TW, et al. Does pregnancy alter the systemic toxicity of local anesthetics? Anesthesiology 1989; 70: 991-5.

91. Santos AC, DeArmas PI. Systemic toxicity of levobupivacaine, bupivacaine, and ropivacaine during continuous intravenous infusion to nonpregnant and pregnant ewes. Anesthesiology 2001; 95: 1256-64.

92. Hofmeyr G, Cyna A, Middleton P. Prophylactic intravenous preloading for regional analgesia in labour. [update of Cochrane Database Syst Rev. 2002; (2): CD000175; PMID: 12076382]. Cochrane Database Syst Rev 2004: CD000175.

93. Clarke VT, Smiley RM, Finster M. Uterine hyperactivity after intrathecal injection of fentanyl for analgesia during labor: a cause of fetal bradycardia? Anesthesiology 1994; 81: 1083.
94. Mardirosoff C, Dumont L, Boulvain M, Tramer MR. Fetal bradycardia due to intrathecal opioids for labour analgesia: a systematic review. BJOG 2002; 109: 274-81.

95. Albright GA, Forster RM. Does combined spinal-epidural analgesia with subarachnoid sufentanil increase the incidence of emergency cesarean delivery? Reg Anesth 1997; 22: 400-5.

96. Van de Velde $M$, Teunkens A, Hanssens $M$, et al. Intrathecal sufentanil and fetal heart rate abnormalities: a double-blind, double placebo-controlled trial comparing two forms of combined spinal epidural analgesia with epidural analgesia in labor. Anesth Analg 2004; 98: 1153-9.

97. Bell E. Nitroglycerin and uterine relaxation. Anesthesiology 1996; 85: 683.

98. Friedlander JD, Fox HE, Cain CF, et al. Fetal bradycardia and uterine hyperactivity following subarachnoid administration of fentanyl during labor. Reg Anesth 1997; 22: 378-81.

99. Stride PC, Cooper GM. Dural taps revisited. A 20-year survey from Birmingham Maternity Hospital. Anaesthesia 1993; 48: 247-55.

100. Norris MC, Leighton BL, DeSimone CA. Needle bevel direction and headache after inadvertent dural puncture. Anesthesiology 1989; 70: 729-31.

101. Reynolds F. Dural puncture and headache. BMJ 1993; 306: 874-6.

102. Kalbach LR. Spinal headache: cause and care. Orthop Nurs 1989; 8: $51-5$.

103. Kuczkowski KM. The management of accidental dural puncture in pregnant women: what does an obstetrician need to know? Arch Gynecol Obstet 2007; 275: 125-31.

104. Stocks GM, Wooller DJ, Young JM, Fernando R. Postpartum headache after epidural blood patch: investigation and diagnosis. $\mathrm{Br} \mathrm{J}$ Anaesth 2000; 84: 407-10.

105. Alfery DD, Marsh ML, Shapiro HM. Post-spinal headache or intracranial tumor after obstetric anesthesia. Anesthesiology 1979; 51: 92-4.

106. Kardash K, Morrow F, Beique F. Seizures after epidural blood patch with undiagnosed subdural hematoma. Reg Anesth Pain Med 2002; 27: 433-6.

107. Carp H, Singh PJ, Vadhera R, Jayaram A. Effects of the serotoninreceptor agonist sumatriptan on postdural puncture headache: report of six cases. Anesth Analg 1994; 79: 180-2.

108. Connelly NR, Parker RK, Rahimi A, Gibson CS. Sumatriptan in patients with postdural puncture headache. Headache 2000; 40: 316-9.

109. Abouleish E, Vega S, Blendinger I, Tio TO. Long-term follow-up of epidural blood patch. Anesth Analg 1975; 54: 459-63.

110. Taivainen T, Pitkanen M, Tuominen M, Rosenberg PH. Efficacy of epidural blood patch for postdural puncture headache. Acta Anaesthesiol Scand 1993; 37: 702-5.

111. Van de Velde M, Schepers R, Berends N, et al. Ten years of experience with accidental dural puncture and post-dural puncture headache in a tertiary obstetric anaesthesia department. Int J Obstet Anesth 2008; 17: 329-35.

112. Wong CA, Scavone BM, Dugan S, et al. Incidence of postpartum lumbosacral spine and lower extremity nerve injuries. Obstet Gynecol 2003; 101: 279-88.

113. Thorp JA, Parisi VM, Boylan PC, Johnston DA. The effect of continuous epidural analgesia on cesarean section for dystocia in nulliparous women. Am J Obstet Gynecol 1989; 161: 670-5.

114. Leighton BL, Halpern SH. The effects of epidural analgesia on labor, maternal, and neonatal outcomes: a systematic review. Am J Obstet Gynecol 2002; 186(5 Suppl): S69-77.

115. Halpern SH, Leighton BL, Ohlsson A, et al. Effect of epidural vs parenteral opioid analgesia on the progress of labor: a meta-analysis. JAMA 1998; 280: 2105-10.

116. Segal S, Su M, Gilbert P. The effect of a rapid change in availability of epidural analgesia on the cesarean delivery rate: a meta-analysis. Am J Obstet Gynecol 2000; 183: 974-8.

117. Fogel ST, Shyken JM, Leighton BL, et al. Epidural labor analgesia and the incidence of cesarean delivery for dystocia [see comment]. Anesth Analg 1998; 87: 119-23.

118. Halpern SH, Leighton BL, Ohlsson A, et al. Effect of epidural vs parenteral opioid analgesia on the progress of labor: a meta-analysis JAMA 1998; 280: 2105-10. 
119. Leighton BL, Halpern SH. Epidural analgesia: effects on labor progress and maternal and neonatal outcome. Semin Perinatol 2002; 26: 122-35.

120. Wong CA, Scavone BM, Peaceman AM, et al. The risk of cesarean delivery with neuraxial analgesia given early versus late in labor. $\mathrm{N}$ Engl J Med 2005; 352: 655-65.

121. American College of Obstetricians and Gynecologists. ACOG Practice Bulletin Number 36, ACOG 2008 Compendium of Selected Publications. Washington DC: The American College of Obstetricians and Gynecologists, 2008: 727-40.

122. Yancey MK, Zhang J, Schwarz J, et al. Labor epidural analgesia and intrapartum maternal hyperthermia. Obstet Gynecol 2001; 98: t-70.

123. Fusi L, Steer PJ, Maresh MJ, Beard RW. Maternal pyrexia associated with the use of epidural analgesia in labour. Lancet 1989; 1: 1250-2.

124. Gleeson NC, Nolan KM, Ford MR. Temperature, labour, and epidural analgesia. Lancet 1989; 2: 861-2.

125. Lieberman E, Lang JM, Frigoletto F Jr, et al. Epidural analgesia, intrapartum fever, and neonatal sepsis evaluation. Pediatrics 1997; 99: 415-9.

126. Kaul B, Vallejo M, Ramanathan S, Mandell G. Epidural labor analgesia and neonatal sepsis evaluation rate: a quality improvement study. Anesth Analg 2001; 93: 986-90.

127. Michelson KA, Carr DB, Easterling TR. The impact of duration of labor induction on cesarean rate. Am J Obstet Gynecol 2008; 199: 299-4.

128. Bucklin BA, Hawkins JL, Anderson JR, Ullrich FA. Obstetric anesthesia workforce survey: twenty-year update. Anesthesiology 2005; 103: 645-53.

129. Hawkins JL, Koonin LM, Palmer SK, Gibbs CP. Anesthesia-related deaths during obstetric delivery in the United States, 1979-1990. Anesthesiology 1997; 86: 277-84.

130. Hawkins JL. Anesthesia-related maternal mortality. Clin Obstet Gynecol 2003; 46: 679-87.

131. Ezri T, Szmuk P, Evron S, et al. Difficult airway in obstetric anesthesia: a review. Obstet Gynecol Surv 2001; 56: 631-41.

132. Datta S, Ostheimer GW, Weiss JB, et al. Neonatal effect of prolonged anesthetic induction for cesarean section. Obstet Gynecol 1981; 58: 331-5.

133. Biehl DR, Yarnell R, Wade JG, Sitar D. The uptake of isoflurane by the foetal lamb in utero: effect on regional blood flow. Can Anaesth Soc J 1983; 30: 581-6.

134. Norris MC. Height, weight, and the spread of subarachnoid hyperbaric bupivacaine in the term parturient. Anesth Analg 1988; 67: 555-8.

135. De Simone CA, Leighton BL, Norris MC. Spinal anesthesia for cesarean delivery. A comparison of two doses of hyperbaric bupivacaine. Reg Anesth 1995; 20: 90-4.

136. Ueyama H, He YL, Tanigami $\mathrm{H}$, et al. Effects of crystalloid and colloid preload on blood volume in the parturient undergoing spinal anesthesia for elective Cesarean section. Anesthesiology 1999; 91: 1571-6.

137. Ralston DH, Shnider SM, DeLorimier AA. Effects of equipotent ephedrine, metaraminol, mephentermine, and methoxamine on uterine blood flow in the pregnant ewe. Anesthesiology 1974; 40: 354-70.

138. Lee A, Ngan Kee WD, Gin T. A quantitative, systematic review of randomized controlled trials of ephedrine versus phenylephrine for the management of hypotension during spinal anesthesia for cesarean delivery. Anesth Analg 94: 920-6.

139. Herbstman CH, Jaffee JB, Tuman KJ, Newman LM. An in vivo evaluation of four spinal needles used for the combined spinal-epidural technique. Anesth Analg 1998; 86: 520-2.

140. Bjornestad E, Iversen OL, Raeder J. Similar onset time of 2-chloroprocaine and lidocaine + epinephrine for epidural anesthesia for elective Cesarean section. Acta Anaesthesiol Scand 2006; 50: 358-63.

141. Philipson EH, Kuhnert BR, Syracuse CD. Fetal acidosis, 2-chloroprocaine, and epidural anesthesia for cesarean section. Am J Obstet Gynecol 1985; 151: 322-4.

142. Corke BC, Carlson CG, Dettbarn WD. The influence of 2-chloroprocaine on the subsequent analgesic potency of bupivacaine. Anesthesiology 1984; 60: $25-7$.

143. Grice SC, Eisenach JC, Dewan DM. Labor analgesia with epidural bupivacaine plus fentanyl: enhancement with epinephrine and inhibition with 2-chloroprocaine. Anesthesiology 1990; 72: 623-8.
144. Portnoy D, Vadhera RB. Mechanisms and management of an incomplete epidural block for cesarean section. Anesthesiol Clin North Am 2003; 21: 39-57.

145. Furst SR, Reisner LS. Risk of high spinal anesthesia following failed epidural block for cesarean delivery. J Clin Anesth 1995; 7: 71-4.

146. Bray JK, Fernando R, Patel NP, Columb MO. Suprasternal Doppler estimation of cardiac output: standard versus sequential combined spinal epidural anesthesia for cesarean delivery. Anesth Analg 2006; 103 : 959-64.

147. Birnbach DJ, Soens MA. Hotly debated topics in obstetric anesthesiology 2008: a theory of relativity. Minerva Anestesiol 2008; 74: 409-24.

148. Van de Velde M, Van Schoubroeck D, Jani J, et al. Combined spinalepidural anesthesia for cesarean delivery: dose-dependent effects of hyperbaric bupivacaine on maternal hemodynamics. Anesth Analg 2006; 103: 187-90.

149. Boso EB. A case for combined spinal-epidural anesthesia for Cesarean section in a patient with aortic stenosis. W V Med J 2008; 104: 20-1.

150. Guasch E, Dominguez A, Alsina E, Gilsanz F. Combined spinal-epidural anesthesia with very low dose hyperbaric levobupivacaine for cesarean section in a preeclamptic patient. Int J Obstet Anesth 2007; 16: 91-3.

151. Van de Velde M, Berends N, Spitz B, et al. Low-dose combined spinalepidural anaesthesia vs. conventional epidural anaesthesia for Caesarean section in pre-eclampsia: a retrospective analysis. Eur J Anaesthesiol 2004; 21: 454-9.

152. Fuller JG, McMorland GH, Douglas MJ, Palmer L. Epidural morphine for analgesia after caesarean section: a report of 4880 patients. Can J Anaesth 1990; 37: 636-40.

153. Cousins MJ, Mather LE. Intrathecal and epidural administration of opioids. Anesthesiology 1984; 61: 276-310.

154. Abouleish E, Rawal N, Rashad MN. The addition of $0.2 \mathrm{mg}$ subarachnoid morphine to hyperbaric bupivacaine for cesarean delivery: a prospective study of 856 cases. Reg Anesth 1991; 16: 137-40.

155. Abboud TK, Dror A, Mosaad P, et al. Mini-dose intrathecal morphine for the relief of post-cesarean section pain: safety, efficacy, and ventilatory responses to carbon dioxide. Anesth Analg 1988; 67: 137-43.

156. Ngan Kee WD, Lam KK, Chen PP, Gin T. Epidural meperidine after cesarean section: the effect of diluent volume. Anesth Analg 1997; 85: 380-4.

157. Halpern SH, Arellano R, Preston R, et al. Epidural morphine vs hydromorphone in post-caesarean section patients. Can J Anaesth 1996; 43: 595-8.

158. Luthman JA, Kay NH, White JB. Intrathecal morphine for post caesarean section analgesia: does naloxone reduce the incidence of pruritus? Int J Obstet Anesth 1992; 1: 191-4.

159. Cohen SE, Ratner EF, Kreitzman TR, et al. Nalbuphine is better than naloxone for treatment of side effects after epidural morphine. Anesth Analg 1992; 75: 747-52.

160. Cotton DB, Lee W, Huhta JC, Dorman KF. Hemodynamic profile of severe pregnancy-induced hypertension. Am J Obstet Gynecol 1988; 158: $\mathrm{t}-9$.

161. Head BB, Owen J, Vincent RD Jr, et al. A randomized trial of intrapartum analgesia in women with severe preeclampsia. Obstet Gynecol 2002; 99: 452-7.

162. Hollmen AI, Jouppila R, Jouppila P, et al. Effect of extradural analgesia using bupivacaine and 2-chloroprocaine on interfiles blood flow during normal labour. Br J Anaesth 1982; 54: 837-42.

163. Lucas MJ, Sharma SK, McIntire DD, et al. A randomized trial of labor analgesia in women with pregnancy-induced hypertension. Am J Obstet Gynecol 2001; 185: 970-5.

164. Hood DD, Curry R. Spinal versus epidural anesthesia for cesarean section in severely preeclamptic patients: a retrospective survey. Anesthesiology 1999; 90: 1276-82.

165. Assail NS, Prystowsky H. Studies on autonomic blockade. I. Comparison between the effects of tetraethylammonium chloride (TEAC) and high selective spinal anesthesia on blood pressure of normal and toxemic pregnancy. J Clin Invest 1950; 29: 1354-66.

166. Beilin Y, Zahn J, Comerford M. Safe epidural analgesia in thirty parturients with platelet counts between 69,000 and $98,000 \mathrm{~mm}(-3)$. Anesth Analg 1997; 85: 385-8.

167. Frenk V, Camann W, Shankar KB. Regional anesthesia in parturients with low platelet counts. Can J Anaesth 2005; 52: 114. 
168. Committee on Obstetric Practice ACOG Committee Opinion. Number 326, December 2005. Inappropriate use of the terms fetal distress and birth asphyxia. Obstet Gynecol 2005; 106: 1469-70.

169. Marx GF, Luykx WM, Cohen S. Fetal-neonatal status following caesarean section for fetal distress. Br J Anaesth 1984; 56: 1009-13.

170. Goetzl LM, ACOG Committee on Practice Bulletins-Obstetrics. ACOG Practice Bulletin. Clinical Management Guidelines for ObstetricianGynecologists Number 36, July 2002. Obstetric analgesia and anesthesia. Obstet Gynecol 2002; 100: 177-91.

171. Bernard PD, Jenkins JG. Failed tracheal intubation in obstetrics: a 6-year review in a UK region. Anaesthesia 2000; 55: 690-4.

172. Pop ham P, Better A, Mendoza M. Anaesthesia for emergency caesarean section, 2000-2004, at the Royal Women's Hospital, Melbourne. Anaesth Intensive Care 2007; 35: 74-9.
173. Cook TM, Brooks TS, Van der Westhuizen J, Clarke M. The Proseal LMA is a useful rescue device during failed rapid sequence intubation: two additional cases. Can J Anaesth 2005; 52: 630-3.

174. Kuczkowski KM, Reisner LS, Benumof JL. Airway problems and new solutions for the obstetric patient. J Clin Anesth 2003; 15: 552-63.

175. Vasdev GM, Harrison BA, Keegan MT, Burkle CM. Management of the difficult and failed airway in obstetric anesthesia. J Anesth 2008; 22: 38-48.

176. Chestnut DH. Anesthesia for fetal distress. In: Chestnut DH, ed. Obstetric Anesthesia Principles and Practice, 3rd edn. Philadelphia: Elsevier Mosby, 2004: 447-59.

177. Siu SC, Colman JM. Heart disease and pregnancy. Heart 2001; 85: 710-15.

178. Ray P, Murphy GJ, Shutt LE. Recognition and management of maternal cardiac disease in pregnancy. Br J Anaesth 2004; 93: 428-39. 


\section{Breast cancer and pregnancy Beth Kliethermes, Dani Stramer, and Edward R. Sauter}

\section{EPIDEMIOLOGY}

Three percent of all cases of breast carcinoma occur in women aged 35 or younger. Breast cancer is the leading cause of cancer death for women 15 to 29 years of age (1) with approximately 11,000 new cases of in situ or invasive breast cancer and 1100 breast cancer deaths in women younger than age 40 (2).

Young women with breast cancer are more likely to present with more advanced disease and have worse outcomes than older women (3). This is likely due to a variety of factors, including the biology of the disease and the challenges to early detection in young women. Young women with breast cancer are more likely to have hormone-insensitive, higher grade disease, with a higher proliferative rate (4) and a higher likelihood of bone marrow metastases (5).

Studies investigating how reproductive factors influence the development of breast cancer are increasing our understanding of possible reasons why carcinoma presents at an advanced stage among this group of women. Specifically, concurrent or recent pregnancy is associated with increased aggressiveness of the tumor and poorer survival. While the occurrence of breast cancer at the time of pregnancy or lactation is 3\% or less of all women who develop breast cancer (6), the prevalence increases to over $15 \%$ among women before age 40 . Pregnancy-associated breast cancer $(\mathrm{PABC})$ is defined as breast cancer diagnosed during pregnancy or within the first 12 months postpartum. Currently, the average age of women with PABC is between 32 and 38 years (7), and the median gestational age at the time of diagnosis is 17 to 25 weeks (8). The incidence of PABC may increase as a growing number of women are choosing to postpone childbearing until their mid-to-late 30 s.

\section{ETIOLOGY}

Reproductive factors such as age at menarche, parity, and age at first birth are well-established risk factors for breast cancer incidence due to their influence on the length of hormonal exposure to estrogen and progesterone (9). Epidemiologic studies confirm that pregnancy has a dual effect on breast cancer risk (10). Childbearing reduces the lifetime risk of developing breast cancer, although risk increases during the time surrounding pregnancy (11).

Women who have their first full-term pregnancy at an early age (defined variously as before 20 up to age 26) have a decreased lifetime risk of developing breast cancer. When compared with nulliparous women, the risk of breast cancer increases in women whose first full-term pregnancy occurred after 35 years of age (12). Nonetheless, for women who develop PABC, early age at first full-term pregnancy has been associated with more aggressive breast cancers. A recent study found that a woman with a first full-term pregnancy before age 20 had a 3.2 increased odds of having an aggressive tumor (13).
Studies have also shown that a diagnosis of breast cancer shortly after pregnancy is associated with reduced chance of survival (14). The transient increase in breast cancer risk for 3 to 15 years after childbirth is followed by a decrease in long-term risk (15).

The relationship between age at first full-term pregnancy and risk of breast cancer is likely related to physiologic and hormonal changes that occur during and after pregnancy (14). An early first full-term pregnancy imprints a specific genomic signature on the breast tissue, with differences in RNA expression and protein concentration compared with those with a later age at first childbirth (16). First full-term pregnancy appears to have a lasting effect on the hormonal environment of the mother and on her metabolism of estrogen (17). One of the mechanisms suggested to account for the incidence of breast cancer in general is exposure to high endogenous estrogen levels. Estrogen levels peak during pregnancy. Endogenous estradiol can initiate the transformation of premalignant to malignant breast cells or accelerate the progression of existing tumors (18). In addition to estrogen, a full-term pregnancy alters the concentration of other hormones, providing another possible way in which pregnancy might influence breast cancer risk. As a result, specific changes in the levels of hormones during and following pregnancy may affect the breast tissue by increasing the risk of mutations, by altering the tissue's susceptibility to hormones, or by modulating its levels of gene expression (19).

Studies specifically addressing the issue of breast differentiation and involution after pregnancy and lactation may shed light on the possible mechanisms of PABC, including changes in the pattern of breast lobular development and differentiation, cell growth, and gene expression (20). The mammary gland reaches full lobular development late during a woman's first full-term pregnancy in preparation for lactation. If this occurs at a young age, the chance for mutational events to have occurred in the breast epithelium prior to pregnancy are less than if a woman's first pregnancy is at an older age. It is therefore less likely that the stimulatory effects of pregnancy on the breast will drive abnormal breast epithelial cells to progress to cancer. Moreover, during weaning, breast epithelial differentiation and involution occurs leading to a long-term breast cancer risk reduction. Differentiated cells do not divide or proliferate under normal conditions and are less susceptible to carcinogenic transformation long term (21). Breast remodeling following lactation resembles the microenvironment that occurs with inflammation. The activated cells secrete proteins, growth factors, tissue-remodeling enzymes, and provisional extracellular matrix, all of which facilitate the return of the breast to the prepregnancy state. This involution microenvironment may promote the growth and development of transformed tumor cells if present in the 
tissue (22). Furthermore, metastatic spread is facilitated by the increased vascularity and lymphatic drainage from the gravid and lactating breast (23). These observations suggest that women with precancerous or cancerous epithelial cells within the breast at the time of pregnancy may be at a high risk for tumor promotion and metastasis.

\section{PATHOPHYSIOLOGY}

Overall, women with PABC have similar pathologic features to age-matched controls. The most common histologic subtype is invasive ductal carcinoma, which is usually high grade (24). Tumor size, degree of nodal involvement, and stage at diagnosis are usually more advanced among women with PABC compared with age-matched nonpregnant women with breast cancer $(25,26)$. Controlling for disease stage, tumor size, hormone receptor status, age, race, and type of surgery, PABC is associated with approximately a $14 \%$ increase in risk of death compared with nonpregnant controls (27). The reason(s) for this is not clear, since overall rates of treatment with radiation and chemotherapy are similar. Theories proposed include the relative immune suppression of pregnancy and differences in tumor biology due to elevated estrogen and progesterone levels during pregnancy (27). Other studies do not indicate that termination of pregnancy improves the prognosis of PABC (28).

Hormone receptor status in PABC tumors is commonly negative, more often than their age-matched controls with breast cancer (29). Between $54 \%$ and $80 \%$ of PABCs are ERnegative, possibly due to the downregulation of receptors in pregnancy (30). Using a variety of antibodies, HER2 positivity has been observed in $28 \%$ to $58 \%$ of PABCs. Sample sizes in these studies are too small to conclude whether the frequency of HER2 positivity is higher than that seen in age-matched controls (31).

\section{DIAGNOSIS}

Clinical examination of the breast is more difficult during pregnancy and nursing because the breasts are hypervascular, engorged, and nodular. Pregnancy-related changes can conceal otherwise palpable tumors in the breast. PABC most commonly presents as a painless mass or thickening. A discreet mass is often difficult to palpate during lactation and involution, and malignancy may be mistaken for mastitis (32).

On average, diagnosis of PABC is delayed 5 to 15 months (7). Difficulty in evaluating mass lesions in the pregnant and lactating breast, a reluctance to perform biopsy, false diagnosis as an inflammatory mass, and inadequate follow-up by patient and physician have all been reported as possible reasons for this delay (33). Mammography with abdominal shielding poses a low risk of radiation to the fetus in the second and third trimesters, but is not recommended in pregnant or postpartum women because the high density of the breast leads to a high rate of false negatives (34). Ultrasonography is a more sensitive alternative for pregnant and lactating women and can distinguish between cystic and solid lesions (35). Magnetic resonance imaging offers high sensitivity but low specificity for the diagnosis of breast cancer. This imaging technique is not recommended during the first trimester until more information becomes available (8). Gadolinium should be avoided if possible since it crosses the placenta and its effects on the fetus are unknown (36). Available evidence indicates that fetal exposure to less than $0.05 \mathrm{~Gy}$ ionizing radiation is not associated with a detectable increase in deleterious effects (37). CT scans to assess disease extent, especially those of the abdomen and pelvis, are generally not recommended during pregnancy due to the relatively high mean doses to the fetus of $0.0036 \mathrm{~Gy}$ for abdominal and 0.089Gy for pelvic irradiation (38).

Since PABC often presents as a palpable mass, core or open biopsy is an effective diagnostic procedure. Lactation should be suppressed prior to biopsy in nursing women to reduce the risk of abscess and milk fistula formation (39). Fine-needle aspiration is less reliable during pregnancy and lactation due to the hyperproliferative features in the tissue of the pregnant, lactating, or involution breast (40).

\section{MANAGEMENT}

Patients with PABC should be treated as soon as possible and according to the latest clinical guidelines, carefully weighing the risk to the fetus. The first treatment for most breast cancer patients is surgery. General anesthesia is safe if precautions are taken to compensate for physiologic changes induced by pregnancy (41). During the first and second trimesters of pregnancy, modified radical mastectomy is recommended to eliminate the need for postoperative radiation therapy and chemotherapy for patients with early-stage disease. For accurate staging, axillary evaluation is necessary to determine nodal status. Nodal metastases are relatively common in PABC, and disease extent affects the need for adjuvant chemotherapy (42). For women diagnosed with PABC late in pregnancy or after delivery, lumpectomy with axillary dissection can be performed, with radiation treatment after childbirth (43). A small series that used Tc-99m and/or blue dye in women of gestational ages ranging from 4 to 32 weeks suggests that lymphatic mapping followed by sentinel lymph node biopsy is safe (44). Therapeutic radiotherapy is unsafe for the fetus and is contraindicated throughout all stages of pregnancy.

Adjuvant or neoadjuvant chemotherapy is potentially teratogenic to the fetus in the first trimester of pregnancy (45), with an estimated risk of fetal malformations of up to $17 \%$ when administered during the first trimester (8). If it is felt unsafe to delay adjuvant therapy until after delivery, chemotherapy can be considered in the second and third trimesters. The anthracyclines doxorubicin (46) and epirubicin (47), as well as taxanes (48), appear relatively safe, with the few series available reporting a $0 \%$ to $6 \%$ death rate (intrauterine, stillbirth, or neonatal) after treatment. It is postulated that placental P-glycoprotein may limit the transplacental transfer of these agents. Chemotherapy should be administered at least 3 weeks before delivery to avoid sepsis and bleeding in a mother with low red blood cell, white blood cell, and platelet counts (28). Trastuzumab use to treat HER-2positive $\mathrm{PABC}$ was associated with anhydramnios in three of six women and should be avoided during pregnancy since it appears to interfere with the normal development of the fetal kidney (48). Lactation should be suppressed during chemotherapy to prevent agent transmission to the infant (49). The long-standing recommendation to avoid pregnancy for 2 years after a diagnosis of breast cancer is being questioned, since median time to relapse is more than 5 years, even for 
patients with stage II node-positive disease who receive standard-of-care regimens of surgery and adjuvant therapy (50).

There are few reports documenting the long-term effects of chemotherapy. One study that assessed physical, neurologic, and psychologic development of 84 children with a median age of 18.7 years born to mothers who received chemotherapy during pregnancy found normal development, and no reports of malignancy (51). Two large prospective studies, one initiated by the German Breast Group and another by the Breast International Group (BIG-03), should provide more information on long-term sequelae after in utero exposure to chemotherapy (8).

For hormone-sensitive tumors, tamoxifen, a selective estrogen-receptor modulator, may be used as adjuvant treatment after surgery or as treatment for advanced cancer. Due to possible teratogenic effects, tamoxifen is not recommended during pregnancy (52). Bisphosphonates are often used to manage the complications of breast cancer skeletal metastases. Reports of their use during the third trimester of pregnancy suggest that postpartum, neonates of mothers treated with the bisphosphonate pamidronate were hypocalcemic but otherwise developed normally, suggesting that calcium levels should be closely monitored in neonates of mothers treated with these agents (53).

\section{REFERENCES}

1. Di Nubila B, Cassano E, Urban LA, et al. Radiological features and pathological-biological correlations in 348 women with breast cancer under 35 years old. Breast 2006; 15: 744-53.

2. Society AC. Breast cancer facts and figures 2005-2006. Atlanta: 2006.

3. Axelrod D, Smith J, Kornreich D, et al. Breast cancer in young women. J Am Coll Surg 2008; 206: 1193-203.

4. Chung M, Chang HR, Bland KI, Wanebo HJ. Younger women with breast carcinoma have a poorer prognosis than older women. Cancer 1996; 77: 97-103.

5. Braun S, Vogl FD, Naume B, et al. A pooled analysis of bone marrow micrometastasis in breast cancer. N Engl J Med 2005; 353: 793-802.

6. White TT. Carcinoma of the breast and pregnancy; analysis of 920 cases collected from the literature and 22 new cases. Ann Surg 1954; 139: 9-18.

7. Institute NC. Breast cancer and pregnancy. In cancer topics. National Cancer Institute, 2004.

8. Ring AE, Smith IE, Ellis PA. Breast cancer and pregnancy. Ann Oncol 2005; 16: 1855-60.

9. Kelsey JL, Gammon MD, John EM. Reproductive factors and breast cancer. Epidemiol Rev 1993; 15: 36-47.

10. Pathak DR. Dual effect of first full term pregnancy on breast cancer risk: empirical evidence and postulated underlying biology. Cancer Causes Control 2002; 13: 295-8.

11. Chie WC, Hsieh C, Newcomb PA, et al. Age at any full-term pregnancy and breast cancer risk. Am J Epidemiol 2000; 151: 715-22.

12. Medina D. Breast cancer: the protective effect of pregnancy. Clin Cancer Res 2004; 10: 380S-4S.

13. Veneroso C, Siegel R, Levine PH. Early age at first childbirth associated with advanced tumor grade in breast cancer. Cancer Detect Prev 2008; 32: 215-23.

14. Daling JR, Malone KE, Doody DR, Anderson BO, Porter PL. The relation of reproductive factors to mortality from breast cancer. Cancer Epidemiol Biomarkers Prev 2002; 11: 235-41.

15. Albrektsen G, Heuch I, Kvale G. The short-term and long-term effect of a pregnancy on breast cancer risk: a prospective study of 802,457 parous Norwegian women. Br J Cancer 1995; 72: 480-4.

16. Ginger MR, Rosen JM. Pregnancy-induced changes in cell-fate in the mammary gland. Breast Cancer Res 2003; 5: 192-7.
17. Musey VC, Collins DC, Brogan DR, et al. Long term effects of a first pregnancy on the hormonal environment: estrogens and androgens. J Clin Endocrinol Metab 1987; 64: 111-18.

18. Trivers KF, Gammon MD, Abrahamson PE, et al. Association between reproductive factors and breast cancer survival in younger women. Breast Cancer Res Treat 2007; 103: 93-102.

19. Oestreicher N, White E, Malone KE, Porter PL. Hormonal factors and breast tumor proliferation: do factors that affect cancer risk also affect tumor growth? Breast Cancer Res Treat 2004; 85: 133-42.

20. Balogh GA, Heulings R, Mailo DA, et al. Genomic signature induced by pregnancy in the human breast. Int J Oncol 2006; 28: 399-410.

21. Russo J, Mailo D, Hu YF, et al. Breast differentiation and its implication in cancer prevention. Clin Cancer Res 2005; 11: 931s-6s.

22. McDaniel SM, Rumer KK, Biroc SL, et al. Remodeling of the mammary microenvironment after lactation promotes breast tumor cell metastasis. Am J Pathol 2006; 168: 608-20.

23. Schedin P. Pregnancy-associated breast cancer and metastasis. Nat Rev Cancer 2006; 6: 281-91.

24. Middleton LP, Amin M, Gwyn K, Theriault R, Sahin A. Breast carcinoma in pregnant women: assessment of clinicopathologic and immunohistochemical features. Cancer 2003; 98: 1055-60.

25. Smith RA, Saslow D, Sawyer KA, et al. American Cancer Society guidelines for breast cancer screening: update 2003. CA Cancer J Clin 2003; 53: 141-69.

26. Kroman N, Wohlfahrt J, Andersen KW, et al. Time since childbirth and prognosis in primary breast cancer: population based study. BMJ 1997; 315: 851-5.

27. Rodriguez AO, Chew H, Cress R, et al. Evidence of poorer survival in pregnancy-associated breast cancer. Obstet Gynecol 2008; 112: 71-8.

28. Psyrri A, Burtness B. Pregnancy-associated breast cancer. Cancer J 2005; 11: 83-95.

29. Aziz S, Pervez S, Khan S, et al. Case control study of novel prognostic markers and disease outcome in pregnancy/lactation-associated breast carcinoma. Pathol Res Pract 2003; 199: 15-21.

30. Colditz GA, Rosner BA, Chen WY, Holmes MD, Hankinson SE. Risk factors for breast cancer according to estrogen and progesterone receptor status. J Natl Cancer Inst 2004; 96: 218-28.

31. Elledge RM, Ciocca DR, Langone G, McGuire WL. Estrogen receptor, progesterone receptor, and HER-2/neu protein in breast cancers from pregnant patients. Cancer 1993; 71: 2499-506.

32. Moore HC, Foster RS Jr. Breast cancer and pregnancy. Semin Oncol 2000; 27: 646-53.

33. Lambe M, Ekbom A. Cancers coinciding with childbearing: delayed diagnosis during pregnancy? BMJ 1995; 311: 1607-8.

34. Hogge JP, De Paredes ES, Magnant CM, Lage J. Imaging and management of breast masses during pregnancy and lactation. Breast J 1999; 5: 272-83.

35. Liberman L, Giess CS, Dershaw DD, Deutch BM, Petrek JA. Imaging of pregnancy-associated breast cancer. Radiology 1994; 191: 245-8.

36. Shellock FG, Kanal E. Safety of magnetic resonance imaging contrast agents. J Magn Reson Imaging 1999; 10: 477-84.

37. Stovall M, Blackwell CR, Cundiff J, et al. Fetal dose from radiotherapy with photon beams: report of AAPM Radiation Therapy Committee Task Group No. 36. Med Phys 1995; 22: 63-82.

38. Osei EK, Faulkner K. Fetal doses from radiological examinations. Br J Radiol 1999; 72: 773-80.

39. Woo JC, Yu T, Hurd TC. Breast cancer in pregnancy: a literature review. Arch Surg 2003; 138: 91-8; discussion 9.

40. Novotny DB, Maygarden SJ, Shermer RW, Frable WJ. Fine needle aspiration of benign and malignant breast masses associated with pregnancy. Acta Cytol 1991; 35: 676-86.

41. Duncan PG, Pope WD, Cohen MM, Greer N. Fetal risk of anesthesia and surgery during pregnancy. Anesthesiology 1986; 64: 790-4.

42. Petrek JA, Dukoff R, Rogatko A. Prognosis of pregnancy-associated breast cancer. Cancer 1991; 67: 869-72.

43. Jacobs IA, Chang CK, Salti GI. Coexistence of pregnancy and cancer. Am Surg 2004; 70: 1025-9.

44. Khera SY, Kiluk JV, Hasson DM, et al. Pregnancy-associated breast cancer patients can safely undergo lymphatic mapping. Breast J 2008; 14: 250-4.

45. Meirow D, Schiff E. Appraisal of chemotherapy effects on reproductive outcome according to animal studies and clinical data. J Natl Cancer Inst Monogr 2005: 21-5. 
46. Germann N, Goffinet F, Goldwasser F. Anthracyclines during pregnancy: embryo-fetal outcome in 160 patients. Ann Oncol 2004; 15: 146-50.

47. Mir O, Berveiller P, Rouzier R, et al. Chemotherapy for breast cancer during pregnancy: is epirubicin safe? Ann Oncol 2008; 19: 1814-5.

48. Mir O, Berveiller P, Ropert S, et al. Emerging therapeutic options for breast cancer chemotherapy during pregnancy. Ann Oncol 2008; 19: 607-13.

49. Eedarapalli P, Jain S. Breast cancer in pregnancy. J Obstet Gynaecol 2006; 26: 1-4.

50. Early Breast Cancer Trialists' Collaborative Group. Systemic treatment of early breast cancer by hormonal, cytotoxic, or immune therapy. 133 randomised trials involving 31,000 recurrences and 24,000 deaths among 75,000 women. Lancet 1992; 339: 71-85.

51. Aviles A, Neri N. Hematological malignancies and pregnancy: a final report of 84 children who received chemotherapy in utero. Clin Lymphoma 2001; 2: 173-7.

52. Isaacs RJ, Hunter W, Clark K. Tamoxifen as systemic treatment of advanced breast cancer during pregnancy-case report and literature review. Gynecol Oncol 2001; 80: 405-8.

53. Dunlop DJ, Soukop M, McEwan HP. Antenatal administration of aminopropylidene diphosphonate. Ann Rheum Dis 1990; 49: 955. 


\section{Neoplasia in pregnancy Nora T. Kizer and David G. Mutch}

Cancer complicates approximately 1 in 1000 pregnancies (1). The Third National Cancer Survey found that $12.8 \%$ of all cancers in women occur during the reproductive years, and the three most common cancers in decreasing order of frequency are thyroid, cervical, and melanoma of the skin (2). The most common malignancies actually encountered during pregnancy include cervical, breast, and hematologic malignancies (3).

Malignancy in pregnancy is a rare and distressing condition, which requires consideration of mother and fetus. Specific issues that complicate medical management of malignancy in pregnancy include the possible benefits of pregnancy termination to allow aggressive treatment, the effect of therapy on the developing fetus, the timing of delivery or termination with ongoing treatment, and the role of altered immune and hormonal status during pregnancy, which may affect the prognosis of the coexistent cancer. Most malignancies in females occur outside the reproductive age group; therefore, there are few large studies that adequately examine these issues in detail. However, in reviewing the composite literature, several general guidelines have emerged. First, the anatomic and physiologic changes of pregnancy can obscure the signs and symptoms of early malignancy. Patient complaints are often dismissed as simply symptoms of pregnancy, thereby delaying the performance of timely examination and diagnostic studies. Second, although immunosurveillance may be altered in pregnancy, stage-for-stage prognosis and survival data for malignancy in pregnancy when properly treated are not significantly different than those for the nonpregnant population. Finally, the risk of an anomalous fetus resulting from treatment depends upon the gestational age of the fetus, as well as the mode and intensity of therapy. Many cancers arising during pregnancy can be treated aggressively with surgery, chemotherapy, and even radiation, without necessarily requiring therapeutic abortion.

\section{GYNECOLOGIC MALIGNANCIES}

The following section details the gynecologic malignancies by disease site. Staging for the major gynecologic malignancies is presented in Tables 1-5 (4). Overall, endometrial cancer has the highest incidence among gynecologic malignancies for all age groups, followed by cervical and ovarian cancers. The incidence of gynecologic malignancies in the reproductive age group is altered, as cervical cancer far outranks ovarian cancer while endometrial carcinoma is the rarest, unless one considers gestational trophoblastic disease (GTD) in this group. A brief outline of etiology, epidemiology, diagnosis, and treatment of each disease site is presented, with specific reference to pregnancy.

\section{Human Papillomavirus/Warts of Lower Genital Tract}

The human papillomavirus (HPV) is the causative agent of genital warts, is related to a variety of skin diseases in humans, and plays a role in the tumorigenesis of a variety of malignant tumors of the head, neck, anus, and lower genital tract. The discovery of its predominant role in the development of cervical cancer has allowed the development of a variety of screening and prevention protocols. To date, more than 100 HPV types have been identified. The prevalence of HPV in pregnancy has been reported anywhere from $6 \%$ to $70 \%$ (5) with the highest prevalence in women under the age of 26 . It has also been suggested that the pregnant state may activate HPV expression, the mechanism of which is unclear, but likely involves a change in the immune status of the mother. HPV is of concern during pregnancy for several reasons. First, although it remains unclear the frequency of perinatal transmission, consensus holds that neonates can be exposed to maternal cervical HPV during pregnancy and birth. Studies have shown HPV to be causally related to recurrent laryngeal papillomatosis in children $(6,7)$. Maternal history of genital warts in pregnancy has been shown to be the strongest predictive factor for respiratory papillomatosis in the child (8). A second concern is that the papillary lesions can proliferate and cause soft-tissue dystocia or potential hemorrhage upon attempted vaginal delivery.

Treatment during pregnancy is problematic, but when the lesions are occluding, the introitus and/or vagina are large and friable, treatment with trichloroacetic acid, and rarely laser or cryotherapy, may be attempted. Podophyllin and interferon are contraindicated in pregnancy. Without adequate resolution of obstructive or hemorrhagic papillomas, cesarean section is indicated. When smaller lesions or subclinical infections are present, the decision as to the route of delivery can be problematic. Recent evidence suggests that perinatal transmission of HPV may not be avoided by abdominal delivery, since transplacental in utero infection may be possible with intact membranes $(9,10)$. Additionally, there is a lack of concordance of HPV types detected in newborns compared with their mothers, suggesting other routes of transmission that may occur postnatally (11). Other theories include acquisition via semen at fertilization, hematogenously, or via an ascending maternal infection. Overall, further studies are required to elucidate the mode of transmission and the potential benefits of cesarean section delivery over vaginal delivery to avoid maternal-fetal transmission.

Recently, investigative work has focused on the development of HPV vaccines as primary prevention of HPV infections and the subsequent development of cervical cancer (12-15). At this time, a quadrivalent vaccine is approved and a bivalent vaccine is undergoing phase III clinical trials both for both primary prevention and therapeutic benefits $(14,15)$. As both vaccines target HPV 16 and 18 strains, they could potentially prevent $70 \%$ of future cervical cancer cases. At this time, controversy still exists as to what age groups should 
Table 1 International Federation of Obstetrics and Gynecology Staging of Vulvar Carcinoma (3)

$\begin{array}{ll}\text { Stage 0 Tis } & \begin{array}{c}\text { Carcinoma in situ, intraepithelial } \\ \text { carcinoma } \\ \text { Tumor confined to the vulva and/or } \\ \text { perineum-2 cm or less in greatest } \\ \text { dimension. No nodal metastasis }\end{array} \\ \text { Stage I T1 N0 M0 } & \begin{array}{c}\text { Tumor confined to the vulva and/or } \\ \text { perineum-more than } 2 \mathrm{~cm} \text { in } \\ \text { greatest dimension. No nodal } \\ \text { metastasis }\end{array} \\ \text { Stage III T3 N0 M0 } & \begin{array}{l}\text { Tumor of any size with } \\ \text { 1. adjacent spread to the lower urethra } \\ \text { and/or the vagina, or the anus; and/or }\end{array} \\ \text { Stage T1 N1 M0 } & \begin{array}{l}\text { 2. unilateral regional lymph node } \\ \text { metastasis }\end{array} \\ \text { Stage T2 N1 M0 } & \begin{array}{l}\text { Tumor invades any of the following: } \\ \text { Upper urethra, bladder mucosa, rectal } \\ \text { Stage IVA T1 N2M0 }\end{array} \\ \text { Stage T2 N2 M0 } & \begin{array}{l}\text { mucosa, pelvic bone, and/or bilateral } \\ \text { regional node metastasis }\end{array} \\ \text { Stage T3 N2 M0 } & \end{array}$

Table 2 International Federation of Obstetrics and Gynecology Staging for Vaginal Carcinoma (3)

\begin{tabular}{|c|c|}
\hline Stage 0 & Carcinoma in situ, intraepithelial carcinoma \\
\hline Stage I & The carcinoma is limited to the vaginal wall \\
\hline Stage II & $\begin{array}{l}\text { The carcinoma has involved the subvaginal tissue, } \\
\text { but has not extended to the pelvic wall }\end{array}$ \\
\hline Stage III & The carcinoma has extended to the pelvic wall \\
\hline Stage IB & $\begin{array}{l}\text { The carcinoma has extended beyond the true } \\
\text { pelvis or has involved the mucosa of the bladder } \\
\text { or rectum; bullous edema as such does not } \\
\text { permit a case to be allotted to stage IV }\end{array}$ \\
\hline Stage IVA & $\begin{array}{l}\text { Spread of growth to adjacent organs and/or } \\
\text { direct extension beyond the true pelvis }\end{array}$ \\
\hline Stage IVB & Spread to distant organs \\
\hline
\end{tabular}

receive the vaccine, what methods should be employed to implement international public health policies regarding its use, and as to what marketing strategies against a sexually transmitted infection would be acceptable. Furthermore, these vaccines are currently contraindicated during pregnancy, and long-term data are necessary to evaluate their impact on overall mortality.

\section{Cervical Neoplasia}

The most common gynecologic neoplasia encountered during pregnancy arises from the cervix, including both invasive and preinvasive diseases. Although the mean age of diagnosis for carcinoma of the cervix is 50 years, this disease process has a bimodal peak incidence with age ranges of 35 to 39 years and 60 to 64 years (16). Clearly, this early peak corresponds with the reproductive age group. The incidence of preinvasive and invasive cancer of the cervix is estimated to be 1.3 and 0.45 to 1.0 per 1000 pregnancies, respectively (17).

\section{Preinvasive Disease}

Cervical intraepithelial neoplasia (CIN) arises in the transformation zone between squamous-lined ectocervix and columnar-lined endocervix and has a peak age in the mid to late
Table 3 International Federation of Obstetrics and Gynecology Staging for Cervical Carcinoma (3)

Stage $0 \quad$ Carcinoma in situ, intraepithelial carcinoma

Stage I The carcinoma is strictly confined to the cervix (extension to the corpus should be disregarded)

Stage IA Preclinical carcinoma of cervix; that is, those diagnosed only by microscopy

Stage IA1 Minimal microscopically evident stromal invasion

Stage IA2 Clinical lesions detected microscopically that can be measured; depth $65 \mathrm{~mm}$ from the base of the epithelium, either surface or glandular, from which it originates and horizontally spread

Stage IB Clinical lesions confined to the cervix of preclinical lesions greater than IA

Stage IB1 Clinical lesions no greater than $4.0 \mathrm{~cm}$ in size

Stage IB2 Clinical lesions greater than $4.0 \mathrm{~cm}$ in size

Stage II Carcinoma extends beyond the cervix, but not to pelvic wall. The carcinoma involves the vagina, but not as far as the lower third

Stage IIA No obvious parametrial involvement

Stage IIB Obvious parametrial involvement

Stage III Carcinoma has extended to the pelvic wall or tumor involves the lower third of the vagina. Hydronephrosis or nonfunctioning kidneys are stage III, unless due to other causes

Stage IIIA No extension to the pelvic wall

Stage IIIB Extension to the pelvic wall and/or hydronephrosis or nonfunctioning kidney

Stage IV

Stage IVA Spread of growth to adjacent organs (i.e., bladder or rectal mucosa)

Stage IVB Spread to distant organs

Table 4 International Federation of Obstetrics and Gynecology Staging for Endometrial Carcinoma (3)

\begin{tabular}{|c|c|}
\hline Stage IA, G123 & $\begin{array}{l}\text { Tumor limited to endometrium or invasion } \\
\text { to less than one-half of the myometrium }\end{array}$ \\
\hline Stage IB, G123 & $\begin{array}{l}\text { Tumor invasion to more than one-half of } \\
\text { the myometrium }\end{array}$ \\
\hline Stage II & Cervical stromal invasion \\
\hline Stage IIIA, G123 & Tumor invasion of serosa and/or adnexa \\
\hline Stage IIIB, G123 & Vaginal metastases \\
\hline Stage IIIC1 & Metastases to pelvic lymph nodes \\
\hline Stage IIIC2 & $\begin{array}{l}\text { Metastases to para-aortic lymph nodes with } \\
\text { or without positive pelvic lymph nodes }\end{array}$ \\
\hline Stage IVA & $\begin{array}{l}\text { Tumor invasion of bladder and/or bowel } \\
\text { mucosa }\end{array}$ \\
\hline Stage IVB & $\begin{array}{l}\text { Distant metastases, including intra- } \\
\text { abdominal and/or inguinal lymph nodes }\end{array}$ \\
\hline Stage G1 & $\begin{array}{l}5 \% \text { or less of a non-squamous or } \\
\text { non-morular solid growth pattern }\end{array}$ \\
\hline Stage G2 & $\begin{array}{l}6-50 \% \text { of a non-squamous or non-morular } \\
\text { solid growth pattern }\end{array}$ \\
\hline Stage G3 & $\begin{array}{l}>50 \% \text { of a non-squamous or non-morular } \\
\text { solid growth pattern }\end{array}$ \\
\hline
\end{tabular}

twenties. Over recent years, the integral role of that HPV plays in the genesis of CIN and subsequent transformation into malignant cells has been further elucidated. The progression of CIN to invasive disease does not appear to be accelerated by 
Table 5 International Federation of Obstetrics and Gynecology Staging for Ovarian Carcinoma (3)

Stage I

Stage IA

Growth limited to ovaries containing malignant cells; no tumor on external surfaces; capsule intact

Growth limited to one ovary; no ascites present

Stage IB Growth limited to both ovaries; no ascites present containing malignant cells; no tumor on external surfaces, capsule intact

Stage IC Tumor involving one or both the ovaries either with tumor on external surfaces, or with capsule ruptured, or with ascites containing malignant cells, or with positive peritoneal washings

Stage II Growth involving one or both the ovaries with pelvic extension

Stage IIA Extension and/or metastases to uterus and/or tubes Stage IIB Extension to other pelvic tissues

Stage IIC Tumor either stage IIA or IIB, but with tumor on surface of one or both the ovaries, or with capsule(s) ruptured, or with ascites present containing malignant cells, or with positive peritoneal washings

Stage III Tumor involving one or both the ovaries with peritoneal implants outside the pelvis and/or positive retroperitoneal or inguinal nodes. (Superficial liver metastasis equals stage III. Tumor is limited to the true pelvis but with histologically proven malignant extension to small bowel or omentum.)

Stage IIIA Tumor grossly limited to the true pelvis with negative nodes, but with histologically confirmed microscopic seeding of abdominal peritoneal surfaces

Stage IIIB Tumor of one or both the ovaries with histologically confirmed implants of abdominal peritoneal surfaces, none exceeding $2 \mathrm{~cm}$ in diameter; negative nodes

Stage IIIC Abdominal implants greater than $2 \mathrm{~cm}$ in diameter; negative and/or positive retroperitoneal or inguinal nodes

Stage IV Growth involving one or both the ovaries, with distant metastasis. (If pleural effusion is present, there must be positive cytology to allot a case to stage IV. Parenchymal liver metastasis equals stage IV.)

pregnancy, despite the possibility that pregnancy represents an immunocompromised state and may even activate HPV infections. As in the nonpregnant state, the screening method of choice for CIN is the Papanicolaou test, which should be performed at the first prenatal visit. Although not approved by the manufacturer for use in pregnancy, studies have shown that use of the standard cytobrush is safe and effective during pregnancy and can be routinely employed when obtaining cervical cells for evaluation. Although the pregnant cervix differs from the nonpregnant cervix with the appearance of greater inflammation, larger ectropion, and the presence of decidual cells (Arias-Stella reaction) that mimic atypical cells, evidence demonstrates that the accuracy of the Pap test in pregnancy remains unchanged. LaPolla and colleagues (18) reported a cytologic and histologic concordance rate of $84 \%$ in 248 pregnant patients with abnormal Pap tests who underwent colposcopically directed biopsies and found no false-negative results after rigorous postpartum follow-up. Furthermore, in pregnancy, the transformation zone is expanded and, as such, a Pap test obtained during pregnancy that lacks endocervical cells should be repeated.

\section{Abnormal Papanicolaou Results}

The management of an abnormal cervical Pap test is a clinical dilemma often encountered during pregnancy. The algorithm for management of an abnormal Pap test is shown in Figure 1. Key points include that HPV triage of a Pap test showing atypical squamous cells of undetermined significance is acceptable in pregnancy. However, colposcopy may be deferred until the 6-week postpartum visit. Similarly, women with a result of a low-grade squamous intraepithelial lesion (LSIL) on Pap test may also defer colposcopy until the postpartum period. Those women who do undergo colposcopy antenatally and receive a histologic diagnosis of CIN I should have follow-up postpartum without any further treatment. To do otherwise is considered unacceptable. Patients with a high-grade squamous intraepithelial lesion by Pap test have a 1 in 100 chance of an invasive lesion. Most will have persistence of the high-grade lesion on postpartum follow-up without progression. Colposcopy with directed biopsies during pregnancy is recommended and should be performed by an experienced clinician. A result of atypical glandular cells on a Pap test in pregnancy should be managed in a fashion identical to that of a nonpregnant woman except that endometrial biopsy and endocervical curettage are contraindicated, and cold-knife conization is not advisable unless invasion is otherwise suspected. Postpartum evaluation may be negative as a high-grade lesion may commonly resolve after delivery. However, a thorough postpartum evaluation is necessary to determine whether the lesion is still present. Of note, patients with normal Pap tests but who are HPV positive for high-risk strains should have repeat testing 6 weeks postpartum as HPV infections are often transient during pregnancy.

\section{Diagnostic Procedures in Pregnancy}

In determining which diagnostic and therapeutic interventions to utilize for the assessment of an abnormal Pap test in pregnancy, maternal risk in the form of underdiagnosed invasive cancer, and thus inadequate or delayed treatment, must be balanced against fetal risk, in the form of premature rupture of membranes, chorioamnionitis, and spontaneous abortion from conization. As mentioned earlier, conservative management should be exercised with low-grade cervical lesions as these patients are highly unlikely to have an invasion lesion on pathologic examination (19). Additionally, Patsner (20) used serial evaluation of the Pap test, colposcopy, and directed biopsy to rule out occult or new malignancy in a large group of pregnant patients with mild dysplasia; the conclusion of this study was that patients with mild dysplasia require at most one antepartum colposcopy prior to thorough postpartum reevaluation, without significant increased risk of developing invasive cervical cancer. Some authors now suggest that it is neither cost-effective nor necessary to perform colposcopy on every patient with LSIL on Pap test (19). Advocacy of conservative management assumes that 


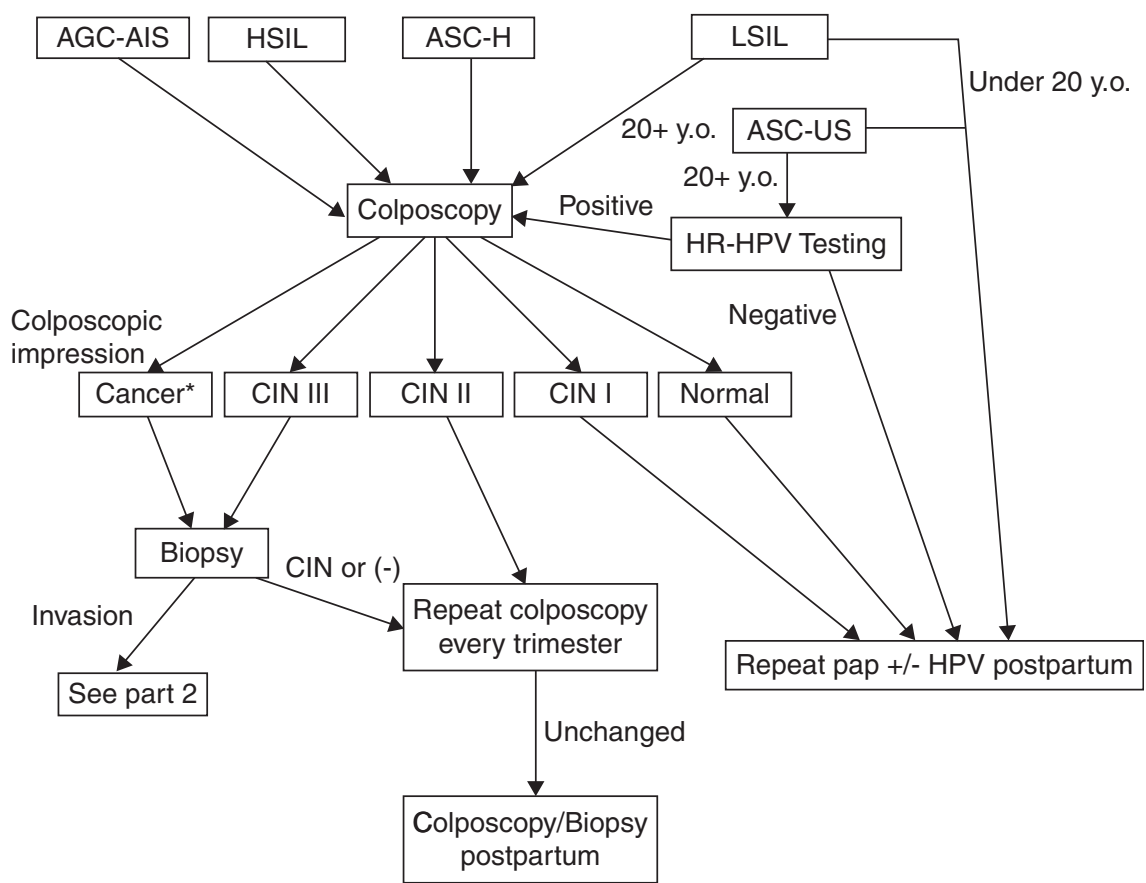

Figure 1 Algorithm for the management of the abnormal Pap smear and cervical intraepithelial neoplasia (CIN) in pregnancy. Abbreviations: AGC, atypical glandular cells; AIS, adenocarcinoma in situ; ASC-H, atypical squamous cells favoring high grade; ASC-US, atypical squamous intraepithelial lesion of undetermined significance; HR-HPV, high-risk human papillomavirus testing; HSIL, high-grade squamous intraepithelial lesion; LSIL, lowgrade SIL. Source: From Ref. 42.

colposcopy is performed by an experienced operator, while cytology and histology are reviewed by a reliable laboratory. If these standards are not met, more frequent colposcopy and closer follow-up are probably necessary. Colposcopy with biopsies should always be performed for high-grade lesions and any lesion on repeat examination that appears to have progressed or is suspicious for invasive carcinoma.

\section{Colposcopy During Pregnancy}

The pregnant cervix is actually quite amenable to colposcopy, as the transformation zone tends to evert as pregnancy progresses and allows for optimal colposcopic visualization of the cervix. However, given the changes that occur within a gravid cervix compared with the nonpregnant state, it is imperative that the colposcopy be performed by a skilled physician with experience and training in advanced colposcopy. Colposcopic appearance of the pregnant cervix is highlighted by edematous and hyperemic changes secondary to increased vascularity and an underlying decidual stromal reaction. Increased friability with brisk bleeding upon cervical contact may be encountered during pregnancy and, if persistent, is an indication for evaluation. Acetic acid staining is often more intense during pregnancy, causing some minor abnormalities to be interpreted falsely as significant lesions. Nonetheless, aceto-white areas, especially those that have more opaque changes with mosaicism, punctuation, or frankly atypical vessels, deserve histologic evaluation by biopsy. Any gross lesion and the worst representative abnormal areas by colposcopic visualization should be biopsied regardless of gestational age unless delivery is imminent, whereby biopsy may be delayed up to several weeks. Cervical biopsy during pregnancy may cause increased bleeding, which is generally well controlled by silver nitrate sticks, ferric sulfate solution, or, rarely, suture placement. In a series of 100 pregnant patients, Hacker and associates (17) reported a diagnostic accuracy of $99.5 \%$ and a complication rate of $0.6 \%$ for colposcopy with biopsies. No endocervical curettage should be performed on any patient with known or suspected pregnancy. The overall goal of a thorough colposcopic cervical examination in pregnancy is to exclude invasive disease, thus averting unnecessary conizations and allowing safe postponement of ablative therapy for CIN, including carcinoma in situ, until after delivery. Thus, the treatment of all forms of CIN in pregnancy is conservative, with required follow-up postpartum. It is imperative that all patients with histologically proven CIN 2 and 3 antenatally have repeat colposcopy with biopsies at the 6-week postpartum visit.

\section{Large Loop Excision of the Transformation Zone}

Inadequate colposcopic visualization of either the transformation zone or the entire proximal extent of a lesion is generally delayed during pregnancy and performed postpartum. In addition, endocervical curettage is not performed during pregnancy; thus, the primary indications for excisional procedures during pregnancy are histologic microinvasive disease or marked discordance between a Pap test suggestive of carcinoma and a histologic specimen with minimal abnormalities. Every effort should be made to reconcile such discordance by review of the cytologic interpretation and even repeat colposcopic biopsies to avoid unnecessary excisional procedures during pregnancy. Overall, a diagnostic excisional procedure should be considered only if the cytology, colposcopic impression, or cervical biopsies are suspicious for invasive carcinoma only. Treatment during pregnancy is otherwise unacceptable. When excision by large loop excision of the transformation zone (LLETZ) is required, it is best performed in the first or early second trimester when the risk of spontaneous abortion and bleeding from the increased 
vascularity of the cervix are reduced. Current evidence demonstrates mixed results in regard to the impact of LLETZ on risk for premature rupture of membranes, preterm labor, and cesarean section (21-23). As such, it is considered acceptable to perform LLETZ with reasonable maternal safety if indicated. However, it should be performed by an experienced surgeon and ideally during the previable stage of pregnancy and when invasive cancer is highly suspected. These procedures should always be performed in the operating room with sufficient appropriately trained staff.

\section{Cone Biopsy During Pregnancy}

Averette and coworkers (24) collected the largest series of conizations during pregnancy with 180 patients and reported that $9.4 \%$ required a blood transfusion and $63 \%$ underwent a spontaneous abortion. Others have described less frequent but significant complications associated with cone biopsy in pregnancy, including cervical infection, stenosis, chorioamnionitis, preterm labor, thrombophlebitis, pulmonary embolism, and cervical laceration during labor. In an attempt to minimize bleeding and violation of the endocervical canal, DiSaia and colleagues (16) and others (25) have advocated excising a shallow "coin"-shaped biopsy rather than a "cone"shaped specimen. Multiple hemostatic sutures can be placed at the vaginal reflection of the cervix and a vasoconstrictive agent can be used to infiltrate the cervical stroma in an attempt to decrease blood loss. Overall, the standard cone biopsy is not recommended during pregnancy due to the high complication rate associated with its use.

The so-called coin biopsy has been described as a safe method for performing diagnostic conization during pregnancy (26). The biopsy shape is usually smaller and shallower, resulting in an increased risk of residual disease with positive margins. The compilation of seven studies by Hacker and colleagues (17) showed $43.4 \%$ of cones for carcinoma in situ have residual disease upon repeat conization or hysterectomy. As such, this technique is considered diagnostic rather than therapeutic.

\section{Invasive Cervical Carcinoma}

More than $40 \%$ of cervical cancer cases occur in women of childbearing age, and up to $3 \%$ of all cases of cervical cancer are diagnosed during pregnancy (27-29). The incidence of invasive disease varies from 1 to 13 cases per 10,000 pregnancies $(16,17)$. Pregnancy is not thought to play an etiologic role in the development of cervical carcinoma, since the age-adjusted incidence is the same in pregnant as in nonpregnant women. Despite increases in estrogen and progesterone levels, the natural history of cervical cancer is likewise unaffected by pregnancy, as the prognosis is the same in pregnant and nonpregnant patients $(16,17,30)$. Precise survival data by stage for pregnancy complicated by invasive cervical carcinoma are difficult to ascertain, because more than 15 series reported a span greater than 30 years and their treatment, as well as staging, vary (31). Nonetheless, stage-forstage, 5-year survival rates are the same for pregnant and nonpregnant patients, with rates approaching $100 \%$ for stage IA, $85 \%$ for IB, and $60 \%$ to $70 \%$ for stage II using aggregate data from more recent studies $(17,32,33)$. The average age of patients with cervical cancer in pregnancy is 30 years, while the average parity is four, but neither variable has been shown to
Table 6 Cervical Carcinoma Associated with Pregnancy

\section{Trimester}

First Second Third Postpartum Total Average

\begin{tabular}{lcccccc}
$\begin{array}{l}\text { Time of } \\
\quad \text { diagnosis }\end{array}$ & 19.3 & 14.2 & 14.9 & 51.6 & 100 & N/A \\
$\begin{array}{l}\text { 5-year } \\
\quad \text { survival }\end{array}$ & 68.6 & 62.7 & 51.7 & 46.3 & N/A & 51.3 \\
$\begin{array}{l}\text { Stage } \\
\text { IB }\end{array}$ & 64.3 & 60.6 & 50.0 & 46.5 & N/A & 55.4 \\
II & 28.0 & 30.8 & 20.7 & 29.4 & N/A & 27.2 \\
III/IV & 7.7 & 8.6 & 29.3 & 24.1 & N/A & 17.4 \\
\multicolumn{5}{l}{ Abbreviations: N/A, not applicable. Source: From Ref. 9. }
\end{tabular}

be of prognostic value (17). Table 6 shows the breakdown by trimester (stage and 5-year survival) in cases of cervical cancer associated with pregnancy. Highlighted is the trend of decreased survival and increased likelihood of advanced stage at presentation as pregnancy progresses. Selection bias may influence the data. Patients who defer prenatal care and present later in pregnancy, or even during delivery, are more likely to ignore early symptoms of cervical cancer and not comply with regular cytologic screening. The distribution of histology is similar to the nonpregnant state; $95 \%$ or more are squamous cell carcinomas and 3\% are adenocarcinomas.

The cornerstone of detection remains the Pap test. Biopsy is the requisite for diagnosis. Vaginal bleeding or abnormal discharge is the most frequent presenting symptom. Earlystage disease is often asymptomatic, but advanced disease may present with symptoms of urinary retention, pelvic pain, lymphedema, or weight loss. Once the diagnosis is made, thorough staging should be performed and may include cystoscopy, proctoscopy, and sigmoidoscopy. Imaging studies should be used judiciously; magnetic resonance imaging (MRI) may be the study of choice, as excellent soft-tissue delineation is obtained without ionizing radiation. Positron emission tomography (PET) imaging has newly recognized value in both the pretreatment and posttreatment management of nonpregnant cervical cancer patients (34-41). However, as the side effects the radioisotope required for the study may have on the developing fetus remains unclear, PET is considered contraindicated at this time during pregnancy. Single-exposure intravenous pyelography can be used and renal ultrasound may also be of value. Cervical malignancies tend to be understaged during pregnancy, because proper workup is oftentimes not performed and parametrial induration may be less prominent and more difficult to detect (32).

Although cervical cancer is not uncommonly found during pregnancy, the data regarding its treatment are limited secondary to the unique ethical considerations surrounding pregnancy that prevent many research methods. Treatment for invasive forms of cervical carcinoma depends on the stage of pregnancy and disease, as well as upon the wishes of the mother. It is also important to remember that most preinvasive lesions found during pregnancy do not progress and that oftentimes both high-grade lesions and low-grade lesions will regress during pregnancy and delivery to either low-grade lesions or normal appearance, respectively. As such, it is necessary to procure histologic confirmation of invasive carcinoma whenever feasible. 
Treatment recommendations during pregnancy should be modified to the individual characteristics of the tumor and the patient's desires, and should involve multidisciplinary input. A thorough ultrasound should also be preformed to rule out any fetal anomalies that could influence treatment choice. In general, the patient may be offered the same standard of treatment outlined for all nonpregnant cervical cancer patients with the understanding that only treatment of very early lesions allows future fertility, and significant fetal risks are involved that would require termination of the pregnancy. However, some suggest that other treatment options exist based on a lower level of evidence (42). The limit of 20 weeks of gestation is often used as a cutoff for deciding treatment timing, with termination and immediate treatment reserved for a confirmed gestational age of less than 20 weeks.

Microinvasive tumors confirmed by conization are considered to be either stage IA1 or IA2 with invasion less than $3 \mathrm{~mm}$ below the basement membrane and negative margins, no greater than $7 \mathrm{~mm}$ of lateral spread and the absence or presence of lymphovascular space involvement as defining criteria. Previous data exist exploring fertility-sparing treatment in nonpregnant patients with microinvasive disease and demonstrates low incidence of recurrence with conization (43-45). However, rarely is an excisional conization procedure currently recommended during pregnancy given the high rate of complications. If conservative treatment is desired, it should be reserved for those patients with stage IA1 disease and squamous cell histology. Conservative treatment would involve repeat cytology and colposcopy to exclude regrowth of the tumor after conization is indicated (17). Patients with stage IA2 cervical cancer are not considered to be the candidates for the same fertility-sparing excisional procedures offered to IA1 patients. Outside of pregnancy, standard of care is radical hysterectomy. These patients may be offered the aforementioned purposeful delayed management strategy versus pregnancy termination with immediate treatment. New data in the literature also offer the possibility of radical trachelectomy as a new fertility-sparing treatment option in this particular group of patients (46). If treatment is delayed until after delivery, route of delivery should be based primarily on obstetric indications. If cesarean section is indicated, a cesarean extrafascial or radical hysterectomy can be performed at delivery. Close postpartum follow-up without surgery is advocated by some when future childbearing is desired in selected patients with minimal microinvasive disease (16).

Frankly, invasive tumors of the cervix demand prompt treatment, although slight delay of up to 6-8 weeks has been shown not to alter prognosis $(17,33)$. For stage IB1 and selected IIA tumors early in pregnancy, treatment options include radiation therapy and chemotherapy or radical hysterectomy with bilateral pelvic lymphadenectomy. Surgery does allow ovarian preservation, but the patient should be counseled prior that certain risk factors could lead to adjuvant radiation or chemotherapy. This option can be effective therapy with the fetus in situ up to 18-20 weeks (47); thereafter, hysterotomy and uterine evacuation should be performed. Radiation is equally efficacious, and no hysterotomy is necessary prior to 20 weeks of gestation with radiation therapy. In general, spontaneous abortion occurs rapidly after exposure to radiation in early pregnancy $(48,49)$.
Some authors now recommend using misoprostol to promote expulsion of the pregnancy if it does not occur within an expected time frame to avoid further complications. If the pregnancy is advanced, hysterotomy is required prior to intracavitary radiation. For IB and IIA tumors found in the third trimester, classical cesarean section can be followed by radical hysterectomy with pelvic and para-aortic lymph node dissection. If radiation is planned, lymph node dissection alone can be performed, which may guide subsequent therapy.

With cervical cancer beyond stage IIA, radiation is the treatment of choice with chemosensitization now being routinely performed with radiosensitizing chemotherapy $(50,51)$. Commencement of treatment is immediate, with the exception of late second- or third-trimester pregnancies when treatment may be delayed until after the documentation of fetal pulmonary maturity and delivery. It must be emphasized that the impact of treatment delay on maternal and fetal health is uncertain. Overall evidence suggests that some amount of delay is reasonable without greatly affecting maternal outcome $(49,51-59)$ and allowing potential small but significant gains in fetal outcomes. The literature suggests that prognosis is unchanged over many weeks and patients can be counseled as such allowing the opportunity to delay treatment if so desired. Clearly, these patients must be closely followed for any evidence of disease progression.

In patients with advanced-stage cervical cancer who strongly desire delay of treatment, neoadjuvant chemotherapy is considered a reasonable option. Limited data suggest that platinum agents in association with either bleomycin or paclitaxel when administered in the second and third trimesters appear to have no fetal toxicity or long-term sequelae (60-62).

Route of delivery has not been shown to be of prognostic significance. Recent studies suggest that there may be a slight advantage to delivery by cesarean section (63-66). However, at this point, choice of route should be based on obstetric indications, extent of cervical tumor involvement, and therapy planned. In general, early lesions allow for vaginal delivery if otherwise indicated, while advanced disease favors abdominal delivery because of the risk of cervical laceration and hemorrhage or infection. Additionally, it is important to note that vaginal delivery through a malignant cervix has been associated with cancer implantation and recurrence at previous episiotomy scar sites (67-75).

\section{Vulvar Carcinoma}

Carcinoma of the vulva represents only about $4 \%$ of gynecologic malignancies and is quite rare in pregnancy, because the vast majority of vulvar malignancies occur in postmenopausal patients. Approximately $15 \%$ of vulvar cancers are found in women less than 40 years of age. Of these, about $90 \%$ of the cases are squamous cell cancers. Other cell types include melanoma, adenocarcinoma, basal cell carcinoma, and sarcoma. Presenting symptoms are similar to those of the nonpregnant state and include itching, irritation, discharge, and occasionally bleeding. The etiology of vulvar cancer is unknown, but common patient characteristics include obesity, diabetes, hypertension, and perhaps lessthan-optimal vulvar hygiene. Recent evidence suggests that $\mathrm{HPV}$ infection is a causative or associative factor in preinvasive 
vulvar intraepithelial neoplasia as well as in invasive vulvar cancer. Examination of the vulva will demonstrate areas of ulceration, exophytic growth, hyper- or hypopigmentation, or red or white discoloration. The key to early diagnosis is biopsy. All abnormal areas of the vulva, including raised, depressed, discolored, or warty lesions, deserve consideration for biopsy. Biopsy may be performed in the office under local anesthesia by simple excision or by utilizing the Keyes punch biopsy instrument. Toluidine blue staining and colposcopy may be used as adjunctive procedures, but should not substitute for biopsies, even in lieu of pregnancy.

A review of pregnancy-associated vulvar cancers in the literature by Barclay (76) revealed only 31 reported cases. Since this review, several other small series have been added. The youngest reported patient was 17 years old, while the average age was 27. Such a small number of patients presenting with vulvar carcinoma during pregnancy limits the development of guidelines for prognosis and treatment; nonetheless, certain conclusions can be drawn. Prognostically, these patients appear to do as well stage-for-stage with proper treatment as their nonpregnant counterparts. Treatment for vulvar intraepithelial neoplasia should be conservative with multiple biopsies to rule out invasion and then close follow-up, including colposcopy, until definitive therapy can be carried out postpartum. Invasive disease should be treated as in the nonpregnant state during the first and second trimesters, with radical or modified radical resection and groin node dissection. Lesions less than $2 \mathrm{~cm}$ in diameter and with invasion of $1 \mathrm{~mm}$ or less that are confined to the vulva with no evidence of nodal involvement may be considered for radical local excision. Bilateral or multiple ipsilateral positive groin nodules require adjunctive radiation to the groin and pelvis. Even if radiation is given after the period of organogenesis, the risk of spontaneous pregnancy loss is great. Thus, depending on the wishes of the patient, radiation treatment may be delayed until after delivery $(3,77)$. Alternatively, in this unusual circumstance, consideration may be given to a pelvic node dissection, which, if negative, might allow omission of pelvic irradiation. Positive or close margins of resection, while also treatable with radiation, may be considered for re-excision during pregnancy to avoid the deleterious effects of radiation.

Invasive lesions presenting late in pregnancy (third trimester) may be widely excised and definitive treatment delayed until after delivery. These patients must be informed that these alternative forms of therapy during pregnancy deviate from the standard of treatment and may carry an increased risk of recurrence. The route of delivery is generally not altered by treatment, including radical vulvectomy and groin node dissection. Marked vaginal constriction and perineal fibrosis rarely necessitate cesarean section delivery. After treatment for vulvar cancer, there is no contraindication to future pregnancies. Past studies have showed successful pregnancies following treatment for vulvar carcinoma, with surgery-pregnancy intervals ranging from 6 months to $4 \frac{1}{2}$ years $(76,78)$.

\section{Vaginal Carcinoma}

The occurrence of vaginal cancer has limited demographic overlaps with the reproductive age group, as the vast majority of cases are diagnosed in patients beyond 50 years of age (25). Less than $2 \%$ of gynecologic malignancies are of primary vaginal origin. Metastatic disease from the cervix or vulva is far more common and must always be excluded. Up to $95 \%$ of cases are of squamous cell origin, while melanomas, sarcomas, and adenocarcinomas, including clear cell histologies, are also found.

The incidence of squamous cell carcinoma of the vagina during pregnancy is very rare, with fewer than 20 reported cases (1). Symptoms of vaginal cancers are bleeding or discharge. More advanced disease may present with symptoms of bladder or bowel dysfunction, including frequent urinary tract infections, urinary retention, constipation, or tenesmus. While a persistently abnormal Papanicolaou (Pap) smear may rarely provide initial evidence of vaginal cancer, thorough speculum examination with biopsy of suspicious gross lesions provides definitive diagnosis. Preinvasive disease, vaginal intraepithelial neoplasia, which is far more common than invasive disease in the pregnant population, is best diagnosed with the use of colposcopic-guided biopsies. If biopsy confirms preinvasive disease, treatment with laser, 5-FU, or other ablative therapy can be postponed until completion of the pregnancy.

In the exceptional event that invasive cancer of the vagina is discovered during pregnancy, treatment is generally unaltered, except possibly during the late second or third trimester, when postponement of treatment until after delivery may be considered. Therapy is dependent upon the location and stage of disease, but the primary mode of treatment is radiation. Surgery is a viable option when early-stage disease is confined to the upper one-third of the vagina and is thus resectable with radical hysterectomy and upper vaginectomy. Disease localized to the distal portion of the vagina has been reported as resectable with lower vaginectomy. Utilizing radiation or surgery, fetal loss is inevitable. However, there is one case report of stage I squamous cell carcinoma of the vagina treated by wide local excision at 23 weeks (79). The patient then underwent cesarean section at 33 weeks with pelvic lymphadenectomy and a second wide local excision with no evidence of recurrence.

Surgery allows preservation of ovarian function in this young population with postoperative radiation indicated only for close or positive margins (80). Advanced-stage disease of any histology requires prompt treatment, which may necessitate hysterotomy for removal of products of conception, thus allowing initiation of radiation therapy. It is important to note that even in patients with early-stage disease (I-II), the risk of pelvic lymph node metastases is high (20-35\%) (81).

Several histologic types of vaginal cancer, although rare, tend to occur more commonly than squamous cell carcinoma in women of reproductive age. Adenocarcinoma, usually of the clear cell variety, may develop after in utero exposure to diethylstilbestrol. The link between diethylstilbestrol exposure and clear cell adenocarcinoma of the lower genital tract, including the cervix, was well established by 1971, after which the use of diethylstilbestrol during pregnancy was discontinued. The risk of developing adenocarcinoma of the vagina or cervix is actually less than 1 in 1000 for those exposed to the transplacental carcinogen diethylstilbestrol. Approximately one-third of diethylstilbestrol-exposed patients develop adenosis, which may be a precursor to clear cell 
carcinoma. Such diethylstilbestrol-linked malignancies rarely occur before 14 years of age or after 30 years of age. Thus, frequent cervical and vaginal cytology with careful colposcopic evaluation for abnormal findings are indicated beginning at menarche. Most clear cell adenocarcinomas are diagnosed early (stage I) and thus have a good prognosis with an $80 \%$ 5-year survival. In pregnancy, treatment is largely unaltered, as surgery is performed without fetal regard, unless in the final trimester, in which case delay until after delivery may be contemplated.

Melanomas are very rare as primary tumors of the vagina. The lower posterior one-third of the vagina is the most frequent site of origin. Sarcoma botryoides of the vagina generally occurs in infants and children and presents as a group of cystic masses. The occurrence of both vaginal melanomas and sarcomas is exceedingly rare and reportable in pregnancy. Both tumors have a very poor prognosis, and management must be individualized according to the stage of disease, desires of the patient, and gestational age.

\section{Endometrial Carcinoma}

Endometrial carcinoma in pregnancy is extremely rare. There are fewer than 10 well-documented cases in the world literature and great care is necessary to exclude misdiagnosis secondary to an intense Arias-Stella phenomenon. Diagnosis is usually made at dilatation and curettage for spontaneous or therapeutic abortion. Treatment is unaltered by pregnancy.

\section{Ovarian Neoplasia}

\section{Adnexal/Pelvic Mass}

Pelvic masses in pregnancy present difficult management problems, because one must also consider the fetus as well as the patient. Therefore, the proper treatment of this problem is not always clear and requires clinical judgment. Pregnancy complicated by a pelvic mass has been reported with an incidence varying from 1 in 79 pregnancies to 1 in 2489; the average of 21 publications in the literature is 1 in 800 (82-103). The wide range of reported incidences is likely due to different detection methods, study populations, and types of masses reported. Of those publications, approximately 1 in 16,000 pregnancies is complicated by a malignant adnexal mass.

Prior to the advent of ultrasound, fibroids, ectopic pregnancies, appendicitis, and pelvic kidneys were frequently included in the diagnosis of pelvic mass. These entities are now diagnosed more accurately with ultrasound, avoiding unnecessary laparotomy (104-106). In general, simple, unilateral ovarian masses smaller than $5 \mathrm{~cm}$ during the first trimester are usually functional luteal cysts and oftentimes will resolve on ultrasound by 14 weeks of gestation. Those adnexal masses that are bilateral, greater than $5 \mathrm{~cm}$, growing rapidly or persisting into the second and third trimesters, with solid, septate, or multicystic components need further imaging and/or evaluation (107). Bernhard et al. showed that out of 423 adnexal masses found in pregnancy, only 25 persisted and were surgically treated of which 2 were confirmed malignancies by histologic diagnosis. Ultrasound has been shown to be less accurate in characterizing malignant lesions (102). As such, MRI is useful for followup imaging on patients with concerning adnexal masses initially identified by ultrasound $(108,109)$. Computed tomography should probably be avoided during pregnancy.

It is important to note that most masses found during pregnancy are benign (110). Only up to $5 \%$ of pelvic masses during pregnancy are malignant versus $20 \%$ in the nonpregnant state (16). The most common masses found during gestation are serous or mucinous cystadenoma, mature teratoma, or a miscellaneous array of simple cysts, fibroids, and theca luteal cysts. Masses over $15 \mathrm{~cm}$ identified during pregnancy are seldom malignant (111-113). Of malignant pelvic massed found during pregnancy, the most common are germ cell tumors (45\%), epithelial tumors (37.5\%), sex cordstromal tumors (10\%), and other pathologies (7.5\%) (114).

Masses discovered during pregnancy can be managed expectantly or surgically. Expectant management is frequently complicated by acute rupture, torsion, tumor previa, or malignancy. Grimes (115) reported that these complications occurred most commonly when masses were greater than $5 \mathrm{~cm}$. Acute rupture or torsion resulting in an acute abdomen occurred in $34 \%$ of patients expectantly managed. Tumor previa occurred about $5 \%$ of the time, while malignancy occurred only in about $4.5 \%$ of cases. Surgical management seeks to avoid these complications and removes the mass under more controlled and less emergent conditions. If possible, surgery should be postponed to 16 to 18 weeks, as this timing is associated with a reduced risk of spontaneous abortion, hormonal independence of the corpus luteum of pregnancy, and the resolution of functional cysts.

\section{Ovarian Cancer}

Although rare, ovarian malignancies are the second most common gynecologic cancer diagnosed during pregnancy after cervical cancer (116). They are usually found as an incidental finding during routine prenatal care via ultrasound (117-119) and, as such, are generally found at an earlier stage than in nonpregnant women $(116,117,120)$. Once a malignant adnexal mass is suspected, extensive preoperative discussion with the patient and family is required prior to laparotomy, and management should be multidisciplinary in conjunction with a high-risk obstetrician, gynecologic oncologist, and medical oncologist specialized in gynecologic cancers. If a malignancy is found, the patient should be staged and treated as if she were not pregnant. Pelvic washings should be obtained, extent of disease be assessed, and appropriate surgery be performed. Conservative surgery to preserve fertility and the current pregnancy is attempted where appropriate. However, the patient may always alter her treatment based on her desire to preserve the fetus. Of note, ovarian tumors do not spread to the fetus, in contrast to certain other malignancies.

Germ cell tumors, both benign and malignant, occur primarily in young women (121). The two most common malignant types are dysgerminomas and endodermal sinus (yolk sac) tumors. As dysgerminomas are the most common malignant germ cell tumors and most often occur in young women, it is not surprising that they are the most common malignant adnexal mass found in pregnancy. Approximately $10 \%$ to $15 \%$ of dysgerminomas occur bilaterally. A review of 27 reported cases of dysgerminomas reported all patients presented with large-size tumors ranging from 12 to $28 \mathrm{~cm}$ and most were stage IA (122). Because of their large size, obstetric 
complications have been reported in association with dysgerminomas including obstructed labor and cesarean section (122). Surgical treatment is required as an initial management for all patients regardless of pregnancy status. Oftentimes, if appropriate, surgery will be postponed until 16 to 18 weeks when the risk for spontaneous abortion is minimized $(83,123)$. Unilateral salpingo-oophorectomy with preservation of the contralateral ovary and of the uterus is adequate for early-stage disease of both germ cell and epithelial cancers.

Treatment of more advanced stages is controversial. It has been suggested that if the uterus and other ovary appear free of metastatic disease, then adequate staging can be performed with omental and peritoneal biopsies and sampling of suspicious lymph nodes (122). Biopsy of the contralateral ovary is recommended only if it seems to be affected by disease (124). Ipsilateral lymph node dissection may be considered as this is the most common site of first metastases. Adjuvant chemotherapy is considered unnecessary for stage IA and possibly stage IB, as the risk for recurrence is low, and there is a high cure rate for recurrent disease $(123,125)$. Of note, serum lactate dehydrogenase levels are unaffected by pregnancy and can be used for initial diagnosis and follow-up response to treatment.

Endodermal sinus tumors comprise approximately onefifth of the ovarian germ cell tumors and are the second most common $(126,127)$. They are much less frequently encountered during pregnancy, but may be suspected when a serum alpha fetoprotein level is much higher than expected. Treatment for these tumors is more aggressive as the 5-year survival rate is only $13 \%$ (128). The best treatment strategy is early surgical resection followed by multiagent adjuvant chemotherapy. Adequate surgical treatment can also be achieved usually with the preservation of the contralateral ovary and uterus. The most common chemotherapy regimen used consists of bleomycin, etoposide, and cisplatin (BEP). There are several reports of pregnant women treated with combination chemotherapy who delivered normal infants (122). However, fetal complications have been reported as well (129). Some suggest that, if the mother desires, chemotherapy can be postponed until after early delivery with confirmed fetal lung maturity (130).

Ovarian epithelial tumors are rare in pregnancy as they are usually a disease of the elderly. They are categorized into three groups: benign, borderline, or invasive cancer. Borderline tumors or low malignant potential tumors are a disease of younger women and are more likely to be encountered in pregnancy. Standard of treatment for invasive cancers is upfront surgical cytoreduction followed by adjuvant chemotherapy. Unfortunately, this surgical approach precludes continuation of pregnancy. There have been reports in the literature of patients with invasive epithelial ovarian tumors of at least a stage III who underwent initial unilateral salpingooophorectomy with the completion of the surgical debulking postponed until after the delivery of the fetus (122,131-133). These women received platinum-based chemotherapy during pregnancy. All infants were delivered without any gross abnormalities or significant complications. One woman died of recurrence 29 months later, and others were disease free with follow-up periods ranging from 6 to 36 months. Overall, management must be discussed with the patient with the understanding of the limited evidence in regard to the delay of treatment. The overall prognosis for advanced-stage ovarian cancer is low at approximately $30 \%$ at 5 years (134).

Borderline tumors are distinguished from their invasive counterparts by the histologic findings of papillary epithelial proliferation and atypia, but lack of stromal invasion $(135,136)$. Treatment is primarily surgical. However, given their favorable prognosis, surgery to preserve fertility can oftentimes be achieved. Adjuvant chemotherapy is usually given only in the presence of confirmed invasive implants and, as such, can frequently be avoided during pregnancy.

Other less commonly found ovarian cancers include sexcord stromal tumors. These tumors usually present with abdominal pain, torsion, and increased abdominal girth. Most patients are diagnosed with stage I disease and require only conservative surgical intervention due to their low-malignant potential. In cases of more advanced disease, adjuvant chemotherapy should be considered and usually consists of BEP (137-141). Another tumor rarely found in pregnancy is carcinosarcoma of the ovary also known as a "malignant mixed mesodermal tumor." The prognosis is poor even with aggressive treatment $(142,143)$ and only three cases have been reported in the literature (144-146). Given the aggressive nature of carcinosarcomas and the fact that an optimal debulking procedure at the time of initial surgery is an important determinant of future outcomes, there is no role for conservative surgery in their management plan (147). Lastly, metastatic tumors to the ovary are always a consideration in the differential for malignant adnexal masses. They are not uncommon and the most frequently identified primary sites include colon, breast, and gastric cancers $(123,148)$. Treatment is based on the type of primary malignancy and is usually individualized based on different factors such as histologic type, gestational week, and the patient's wishes.

In general, several principles can help guide the management of ovarian malignancies in pregnancy. Hysterectomy should be performed during pregnancy only if it contributes significantly to tumor debulking $(116,149)$ and overall prognosis. Additionally, chemotherapy is contraindicated in the first trimester due to high rate of abortion (150) and abnormal fetal development (151), but it is compatible in the second or third trimester $(150,152)$. Platinum-based chemotherapy regimens have the most scientific data supporting their safe use in pregnancy (153). An interval of 3 to 4 weeks between the last chemotherapy administration and the delivery is optimal due to both maternal and fetal hematologic risks from treatment (3). The majority of adverse fetal outcomes are likely due to prematurity with few reports of congenital anomalies $(150,154,155)$. In the postpartum period, breast-feeding should be discouraged while receiving cytotoxic agents $(156,157)$. As always, treatment strategy must be case dependent, and the patient must remain fully informed at all times.

\section{GTD}

The term "gestational trophoblastic disease" comprises a wide variety of pathologic entities, from the benign hydatidiform mole to the highly malignant choriocarcinoma. Hydatidiform mole has been observed since the era of Hippocrates. 
Table 7 International Federation of Obstetrics and Gynecology Prognostic Scoring System for Gestational Trophoblastic Neoplasia

Score $^{\mathrm{a}}$

\begin{tabular}{|c|c|c|c|c|}
\hline Risk factor & $\mathbf{0}$ & 1 & 2 & 4 \\
\hline Age & $<39$ & $>39$ & & \\
\hline Prior pregnancy & Mole & Abortion & Term & \\
\hline Interval (months) ${ }^{\mathrm{b}}$ & 4 & $4-6$ & $7-12$ & $>12$ \\
\hline Pretreatment hCG $(\log )$ & $<3$ & $<4$ & $<5$ & $>5$ \\
\hline Largest tumor $(\mathrm{cm})$ & $<3$ & $3-4$ & $\geq 5$ & \\
\hline Site of metastases & & Spleen, kidney & Gastrointestinal tract & Brain, liver \\
\hline Number of metastases & 0 & $1-4$ & $5-8$ & $>8$ \\
\hline Prior failed chemotherapy agents & & & Single & Multiple \\
\hline
\end{tabular}

Richardson and Hertig provided the first account of a hydatid mole in 1638, while in 1827 the midwife Bolvin was the first to ascribe moles as products of gestational origin. In 1895, Felix Morchand finally demonstrated that hydatidiform moles and their malignant sequelae derived from trophoblast, but his concept was not widely accepted until $1903(158,159)$.

Trophoblast may be regarded as the first organ of the developing embryo. Normal trophoblast is a unique tissue that bears several characteristics of cancer. Among these is the ability to invade the myometrium and to embolize into the maternal circulation. Whether the trophoblast is normal or neoplastic, it contains both syncytiotrophoblast and cytotrophoblast and secretes human chorionic gonadotropin (hCG) in proportion to its volume. This fact allows clinicians to monitor this disease and predict eradication of trophoblastic tissue from the host (160).

Abnormal trophoblastic proliferation is manifested in two entities: partial and complete hydatidiform mole. The complete mole consists of abnormal trophoblast of paternal origin with a karyotype of $46, \mathrm{XX}$. This entity presents with abnormal bleeding $95 \%$ of the time between the 6 th and 16th week gestational age. Other manifestations of molar gestation include pre-eclampsia, hyperemesis, and hyperthyroidism. Rarely, patients can present with trophoblastic embolization and symptoms of congestive heart failure or pulmonary edema. hCG levels are usually markedly elevated above those appropriate for the gestational age of the pregnancy. The diagnosis is generally made with ultrasound. Histologically, complete hydatid moles are characterized by hydropic villi, proliferation of both syncytiotrophoblast and cytotrophoblast, absence of fetal vessels, and no definitive fetal structures $(161,162)$.

By contrast, the diagnosis of partial mole is usually made at the time of curettage for a presumed missed or incomplete abortion. The clinical features are similar to those of a complete mole, but less severe. In addition, partial moles are usually of polyploid karyotype and associated with fetal structures.

The incidence of complete hydatidiform mole varies throughout the world from a high of 1 in 450 in Japan to 1 in 2500 in the United States (163). These data are based on population-based studies. The treatment of choice for a complete mole is suction curettage. Blood products, such as packed red blood cells, should be made available preoperatively,
Table 8 International Federation of Obstetrics and Gynecology Staging of Gestational Trophoblastic Neoplasia

I Disease confined to the uterus

II Disease extends outside the uterus but is limited to the genital structures (adnexa, vagina, broad ligament)

III Disease extends to the lungs with or without genital tract involvement

IV Disease involves other metastatic sites

(1) Good prognosis

(a) Serum hCG $<40,000 \mathrm{mIU} / \mathrm{mL}$ (random) or urinary hCG $<100,000$ IU (24 hr)

(b) Symptoms present less than 4 months

(c) No brain or liver metastases

(d) No prior chemotherapy

(e) Prior pregnancy: a mole or abortion of ectopic pregnancy

(2) Poor prognosis

(a) Serum hCG $>40,000 \mathrm{mIU} / \mathrm{mL}$ (random) or urinary hCG $>100,000 \mathrm{IU}(24 \mathrm{hr})$

(b) Symptoms present more than 4 months

(c) Brain or liver metastases

(d) Failure of prior chemotherapy

(e) Antecedent-term pregnancy

as blood loss can be unexpectedly high. Furthermore, a substantial risk of post-evacuation pulmonary edema from trophoblastic deportation or rapid fluid replacement is possible. The incidence of malignant sequelae of complete moles is about $15 \%$. The incidence of malignancy following a partial mole is much less. All moles require careful follow-up with weekly hCG levels. A rising level or one that plateaus requires immediate attention to staging and initiation of cytotoxic therapy $(164,165)$.

The malignant sequelae of molar gestation, categorized as gestational trophoblastic neoplasia (GTN), include invasive mole, choriocarcinoma, and placental site trophoblastic tumors. The prognosis for these entities is excellent, exceeding $90 \%$, with proper treatment. Patients diagnosed with GTN are categorized into risk groups based on the International Federation of Obstetrics and Gynecology (FIGO) staging and scoring system $(166,167)$ (Tables 7,8$)$. The scoring system is based on a modified version of the World Health Organization (WHO) prognostic factor scoring system. Once malignant trophoblastic disease is diagnosed, staging studies consisting of computed tomography scans of the head, abdomen, and 
Table 9 Chemotherapy Regimens for Low-Risk Gestational Trophoblastic Neoplasia

1 MTX, $0.4 \mathrm{mg} / \mathrm{kg}$ (maximum $25 \mathrm{mg}$ )/day, IV or IM for 5 days. Repeat every 14 days

2 MTX, 30-50 mg/m², IM weekly ${ }^{\mathrm{a}}$

3 MTX, $1 \mathrm{mg} / \mathrm{kg}$, IM days 1, 3, 5, and 7; folinic acid, $0.1 \mathrm{mg} / \mathrm{kg}$, IM days $2,4,6$, and 8 . Repeat every 15-18 days or as needed

4 MTX, $100 \mathrm{mg} / \mathrm{m}^{2}$, IVP, then $200 \mathrm{mg} / \mathrm{m}^{2}$ in $500 \mathrm{~mL}$ of $\mathrm{D}_{5} \mathrm{~W}$ over 12 hours. Folinic acid, $15 \mathrm{mg}$ IM or orally every 12 hours for four doses beginning 24 hours after starting MTX. Repeat every 18 days or as needed ${ }^{a}$

5 Act-D, $10-12 \mu \mathrm{g} / \mathrm{kg}$, IV every day for 5 days. Repeat every 14 days

6 Act-D, $1.25 \mathrm{mg} / \mathrm{m}^{2}$, IV every 2 weeks ${ }^{\mathrm{a}}$

7 Alternating MTX/Act-D regimens 1 and 5

${ }^{a}$ Should not be used for metastatic or resistant disease or choriocarcinoma. Abbreviations: Act-D, actinomycin-D; $\mathrm{D}_{5} \mathrm{~W}, 5 \%$ dextrose in water; IM, intramuscularly; IV, intravenously; IVP, intravenous push; MTX, methotrexate.

Table 10 Etoposide, Methotrexate, Actinomycin-D, Cyclophosphamide, and Vincristine Chemotherapy for High-Risk Gestational Trophoblastic Neoplasia

\begin{tabular}{llc} 
& \multicolumn{1}{c}{ Drug } & \multicolumn{1}{c}{ Dosing } \\
Day 1 & Etoposide & $100 \mathrm{mg} / \mathrm{m}^{2}$ IV over \\
& & $30 \mathrm{~min}$ \\
& Actinomycin-D & $0.5 \mathrm{mg} \mathrm{IVP}$ \\
& Methotrexate & $100 \mathrm{mg} / \mathrm{m}^{2}$ IVP, then \\
& & $200 \mathrm{mg} / \mathrm{m}^{2}$ in $500 \mathrm{~mL}$ \\
& & of $\mathrm{D}_{5} \mathrm{~W}$ over $12 \mathrm{hours}$ \\
Day 2 & Etoposide & $100 \mathrm{mg} / \mathrm{m}^{2}$ IV over \\
& & $30 \mathrm{~min}$ \\
& Actinomycin-D & $0.5 \mathrm{mg} \mathrm{IVP}$ \\
& Folinic acid & $15 \mathrm{mg}$ IM or orally every \\
& & $12 \mathrm{hours}$ for four \\
& & doses starting 24 hours \\
& & after starting MTX \\
Day 8 & Cyclophosphamide & $600 \mathrm{mg} / \mathrm{m}^{2}$ IV \\
& Vincristine & $1.0 \mathrm{mg} / \mathrm{m}^{2}$ IVP \\
Abbreviations: & $\mathrm{D}_{5} \mathrm{~W}, 5 \%$ dextrose in water; IM, intramuscularly; IV, \\
intravenously; IVP, intravenous push; MTX, methotrexate.
\end{tabular}

pelvis, serum chemistries including liver and renal function studies, and quantitative beta-HCG need to be performed. A chest X-ray is mandatory. Computed tomography of the chest may identify low-risk patients who are likely to fail primary single-agent therapy (168). Cure for patients with nonmetastatic (stage I) and low-risk metastatic GTN (stages II and III, score $<7$ ) disease approaches $100 \%$ in published studies (167). Patients classified as having high-risk metastatic GTN should receive multiagent chemotherapy plus adjuvant radiation or surgery to achieve survival rates of $80 \%$ to $90 \%$ (Tables 8,9). Of note, prior reports from the Brewer Center in Chicago, Illinois, have demonstrated the importance of having centralized trophoblastic disease centers to provide expertise in managing these patients. They noted that their successful secondary GTN treatment rates increased from 59\% during 1962 to 1978 to $93 \%$ from 1979 to 2006 (169). They also noted that the most common reasons for unsuccessful GTN treatment were using single-agent chemotherapy for high-risk disease and inappropriately using weekly methotrexate to treat patients with metastatic disease, FIGO scores $\geq 7$, and/or nonpostmolar choriocarcinoma. This highlights the importance of adhering to the FIGO staging and scoring system to administer chemotherapy regimens accordingly (Tables 9 and 10).

\section{NONGYNECOLOGIC MALIGNANCIES \\ Breast Cancer}

One in nine women will develop breast cancer during her lifetime, representing one-third of all cancers in women (170). Approximately $15 \%$ of breast cancers occur in women under the age of 41 years; 1 in 3000 pregnancies is complicated by breast cancer, with $3 \%$ of all breast cancer being diagnosed during pregnancy or lactation (1). The incidence of breast cancer in association with pregnancy is expected to continue to rise, owing to the trend toward delayed childbearing. It currently is the second most common malignancy diagnosed in pregnancy after cervical cancer (171).

Breast engorgement and hypertrophy during pregnancy may delay the detection of breast cancer. Some cases of inflammatory cancer, as well as some masses, can be confused with mastitis or other pregnancy-related changes. Most cases present with a painless lump often noted by the patient upon self-examination. If the woman is breast-feeding, she may note a "milk rejection sign" in which the infant refuses the breast affected by cancer (172). The initial prenatal visit should include a thorough breast examination with detailed instructions on breast self-examination.

More recent studies report an average delay in diagnosis of 1 to 2 months (173-177) although older studies noted delays of 6 months or greater (178-180). A 1-month delay in primary tumor treatment increases the risk of axillary metastases by $0.9 \%$ and a 6 -month delay increases the risk by $5.1 \%(181)$. The presence of axillary metastases is a poor prognostic factor, and early detection despite pregnancy is essential.

Mammography should be limited during pregnancy to minimize radiation exposure. In addition, pregnancy changes, such as elevated water density, increase the rates of falsepositive and false-negative mammographic examinations (182). Ultrasound is a recommended, good, inexpensive first step in the workup of a breast mass as it can distinguish between cystic and solid lesions in $97 \%$ of patients $(175,176,183)$. For the diagnosis of metastases, MRI is preferred to ultrasonography especially for hepatic and brain imaging $(184,185)$. Of note, gadolinium used during MRIs crosses the placenta, is associated with fetal abnormalities in rats, and its use during pregnancy should be limited to those cases where the benefits outweigh the risks to the fetus. Radioisotope bone scan, generally avoided during pregnancy, may be indicated only when metastases are clinically suspected. Adequate maternal hydration may increase fetal exposure to radiation.

For definitive diagnosis, any palpable mass should be biopsied. Stopping lactation beforehand reduces the risk for milk fistula formation. Fine-needle aspiration (FNA) has a specificity and sensitivity of about $95 \%$, and no harmful effects upon the pregnancy have been reported (186). However, the accuracy of FNA is highly dependent on the pathologist's experience with pregnancy-associated breast cancer and is 
important to avoid a misdiagnosis of hyperproliferative changes $(185,187)$. If FNA is unsuccessful, open biopsy, preferably under local anesthesia, is performed. Core needle biopsy may also be a more cost-effective method as studies in nonpregnant women have shown it to also have a high sensitivity and specificity (188-191).

In the nonpregnant state, wide local excision with axillary node dissection and radiation is the preferred treatment for small unifocal masses. This alternative is less attractive during pregnancy, as radiation with boost doses to the tumor bed would cause internal scatter, resulting in fetal radiation exposure above acceptable levels (186). As such, mastectomy with axillary node dissection is traditionally the procedure of choice for stage I or II disease during pregnancy, as nodal metastases are commonly found in pregnancy-associated breast cancers, and the procedure may eliminate the need for postoperative irradiation. Treatment may be modified by delaying radiation until after delivery if the diagnosis is made in the late second trimester or beyond, while carrying out lumpectomy and axillary dissection during pregnancy at the time of initial diagnosis.

Patients with either node-positive disease or node-negative disease, but tumor greater than $1 \mathrm{~cm}$, should receive 4 to 6 months of chemotherapy. If the woman is far from term, breast-conserving surgery can be followed by chemotherapy after the first trimester and irradiation after delivery (185). Chemotherapy is usually withheld until the second trimester, as at least half of infants have no complications when their mothers are treated after the first trimester (150,192-194). The most common side effects of chemotherapy during pregnancy are preterm delivery, low birth weight, transient infant leukopenia, transient tachypnea of the newborn, and intrauterine growth restriction. Long-term effects of chemotherapy on the newborn are unknown.

Advanced disease calls for careful individualization of treatments. Delay of palliative treatment until achievement of fetal viability is a reasonable option when the diagnosis is made late in pregnancy. Otherwise, aggressive palliative treatment should be instituted without delay. Such treatment, which includes radiation, multiagent chemotherapy, and hormonal therapy, separately or in combination, poses considerable risk to the fetus. If such advanced disease is diagnosed early in pregnancy, termination may be considered. Therapeutic abortion does not appear to enhance maternal remission or survival in early-stage disease; however, it does allow for prompt initiation of therapy in advanced disease.

Prognosis for pregnancy-associated breast cancer has been traditionally deemed dismal based on early reports. More recent studies have shown that the prognosis for breast cancer, stage-for-stage, is unchanged by pregnancy. Prognosis is most influenced by maternal age with 5-year survival rates of $75 \%$ for women over 40 years of age, but only $55 \%$ for those under 40 years of age $(195,196)$. Pregnancy-associated breast cancer tends to present at later stages with twice the rate of lymph node involvement ( $74 \%$ vs. $37 \%$ ), even when controlled for age (195). This worse prognosis may be due to delayed diagnosis and treatment of pregnant patients. In addition, pregnancy-induced hormonal changes may alter the behavior of tumors. Over $70 \%$ of women with pregnancy-associated breast cancer had estrogen-receptor-negative tumors thought to be secondary to the downregulation of receptor status during pregnancy (195).

The traditional recommendation to wait 2 years after breast cancer treatment prior to a subsequent pregnancy is arbitrary as little scientific evidence exists in regard to this topic. The prognosis, both maternal and fetal, appears unaltered by subsequent pregnancy (196). However, some data now show that those women who do establish future pregnancies tend to demonstrate a "healthy mother effect" with better 10-year survival rates $(197,198)$. A recent large population-based study of over 10,000 women demonstrated a reduced risk of dying in women who had a full-term pregnancy subsequent to breast cancer treatment (199). There was no evidence that a pregnancy after treatment for breast cancer has a negative influence on prognosis. Overall, future pregnancies appear to be safe unless the mother has an ER-positive cancer that has not been cured (197, 200, 201, 202). By contrast, there have also been studies evaluating the prognosis of a new breast cancer diagnosed after a pregnancy $(203,204)$. The authors suggest that a new diagnosis of breast cancer within 2 years of a prior pregnancy is associated with decreased survival. The negative effect no longer persisted if the diagnosis was made greater than 10 years after the last pregnancy (204).

\section{Colorectal Carcinoma}

Colorectal carcinoma (CRC), one of the leading causes of cancer death in females, occurs only in 1 in 50,000 to 1 in 100,000 pregnancies, with approximately 300 total reported cases. Most originate distally below the level of the peritoneal reflection $(205,206)$. The mean age of diagnosis is 32 years (207). CRC represents a serious threat not only to the mother, but also to the fetus as one prior study showed that only 25 of 32 women diagnosed with CRC during pregnancy went on to have healthy live born infants (208). Additionally, CRC is uncommon in young women and, when diagnosed within this age group, is oftentimes associated with predisposing factors such as hereditary nonpolyposis CRC (Lynch syndrome), familial adenomatous polyposis, Gardner syndrome, and chronic history of ulcerative colitis (209).

Presenting symptoms include constipation, obstipation, abdominal pain, rectal or abdominal mass, rectal bleeding, nausea, and vomiting. Many of these signs and symptoms represent common complaints of normal pregnancy and therefore a delay in diagnosis until it has progressed to advanced-stage disease is common. Rectal bleeding of guaiacpositive stool is an alarming symptom, which requires full evaluation. Routine digital examination should be performed during prenatal evaluation. Distribution of lesions appears to be similar to that in the nonpregnant population, with $57 \%$ arising in the rectum, $20 \%$ in the sigmoid colon, and $23 \%$ in the remainder (207).

Workup of a colorectal lesion normally requires colonoscopy with biopsy, a serum carcinoembryonic antigen level, and abdominal imaging (210,211). However, little data exist for colonoscopy during pregnancy and is still considered experimental. As such, risks and benefits should be fully disclosed to the patient and oftentimes only a partial colonoscopy will be performed to confirm the diagnosis while minimizing risk. Barium enema should be avoided if possible. Levels of the serum tumor marker, carcinoembryonic antigen, are generally 
Table 11 Dukes Staging for Colorectal Carcinoma

$\begin{array}{ll}\text { A } & \text { Penetration into, but not beyond, the bowel wall } \\ \text { B } & \text { Penetration through the bowel wall } \\ \text { C } & \text { Positive lymph node involvement } \\ \text { D } & \text { Distant metastasis }\end{array}$

not affected in normal pregnancy and provide prognostic value $(212,213)$. In regard to abdominal imaging during pregnancy, ultrasonography or MRI is the modality of choice.

Treatment during the first two trimesters is generally immediate surgery with limited regard for the pregnancy, although frequently the pregnancy is unharmed if cancer had not metastasized to other pelvic structures. Even distal tumors may be resected with primary re-anastomosis using automatic staplers and abdominoperineal or low anterior resection procedures. If optimal resection is prevented by the gravid uterus, hysterectomy may at times be necessary; such a possibility must be addressed with the mother prior to surgery. If tumor is not resectable, diverting colostomy for decompression and palliation is the treatment of choice to allow the pregnancy to progress toward term. During the third trimester, one can await fetal maturity and perform cesarean section with subsequent tumor excision or diverting colostomy, depending on the extent of the disease. If the tumor is discovered near term, vaginal delivery may be considered, provided that tumor is not obstructing the pelvic outlet. With advanced-stage and surgically unresectable tumor identified at any stage of pregnancy, consideration may be given to awaiting fetal maturity, thus delaying palliative treatment until after delivery (207). Irradiation for colorectal cancer during pregnancy has a limited role due to the colon's close proximity to the uterus, with no evidence of prolonging survival. Chemotherapy is recommended for Dukes C and D CRC. Several studies have shown a survival advantage if chemotherapy is administered within 5 weeks of resection of Dukes C CRC (213). Recently, research into new chemotherapeutic agents has also demonstrated survival benefits for Dukes D CRC (213).

Pregnancy has not been shown to affect maternal prognosis or the natural source of colorectal cancer. The prognosis of pregnant women with CRC is dismal likely due to its delayed diagnosis. There are no reported 5-year survivors for colon cancer. Few with rectal carcinoma are reported, owing to the advanced stage at presentation. Over one-half of pregnant patients with CRC present with Dukes stage C or a more advanced stage (see Table 11 for staging) (206) and 25\% of pregnant women with CRC have ovarian metastases compared with $3 \%$ to $8 \%$ in nonpregnant women $(214,215)$. Theoretically, the estrogen-binding capacity of cells derived from CRCs may indicate accelerated cancer growth during pregnancy. However, survival data between pregnant and nonpregnant patients are comparable stage-for-stage. Information regarding fertility after CRC is limited and has focused on those cancers treated with 5-fluorouracil. The general consensus is to wait at least 1 to 2 years before pursuing a subsequent pregnancy to allow the body sufficient time to recover.

\section{Melanoma}

Melanoma represents $1 \%$ of all cancers diagnosed in the United States, with approximately $30 \%$ occurring in women of reproductive age (216). It complicates pregnancy with an estimated incidence of 0.14 to 2.8 cases per 1000 pregnancies (217). In general, incidence rates have increased dramatically over recent decades in the United States (218) and, as such, could increase the incidence rates during pregnancy.

The majority of patients present with a change in the size and color of a preexisting nevus. More advanced melanomas may present with ulceration and bleeding. Excisional biopsy of the entire suspicious pigmented lesion is the diagnostic procedure of choice. Microscopic measurement of the actual tumor thickness, as outlined by Breslow, most accurately reflects the prognostic index and is of utmost importance in the clinical management of patients (219). The Clark level, which describes the anatomic level of invasion, should also be assessed. Following histopathologic evaluation of the biopsy, the patient should be clinically staged with a thorough history and complete physical examination. The most common clinical staging system defines stage I as local disease without clinically palpable regional lymph nodes, stage II as having suspiciously palpable regional lymph nodes, and stage III as having distant metastases (220). Further tests, such as serum liver function studies, or radionucleotide or computed tomography scans of the liver, bone, or brain, should be performed only for specific signs or symptoms suggesting metastatic disease.

Local excision with wide margins is the only effective cure for melanoma and this approach should not be influenced by pregnancy (221). Regional lymphadenectomy has not proven to be beneficial when lymph nodes are not palpable, but clinically involved lymph nodes should be excised. Distant metastatic disease may be treatable with systemic chemotherapy with a response rate of $20 \%$ to $25 \%$. Metastatic melanoma is resistant to most forms of therapy. Immunotherapy or radiotherapy are unproven modalities during pregnancy.

Pregnancy-associated increases in pigmentation and melanocyte-stimulating hormone, presence of estrogen receptors on $46 \%$ of melanomas, reports of rapidly fatal courses in pregnancy, and reports of tumor regression postpartum or with hormonal therapy have led to the speculation that the course of malignant melanoma may be adversely affected by pregnancy (222). Additionally, incidence rates are higher in females compare with males only during the first four decades of life. However, two population-based studies showed that the number of incident melanoma cases diagnosed during pregnancy did not differ from the number expected based on current estimated prevalence rates $(223,224)$. Overall, research has failed to demonstrate a clear association between estrogen and melanoma or between pregnancy-associated factors and subsequent development of melanoma (225).

Melanoma is one of the most unpredictable cancers. Extensive research had failed to provide conclusive evidence for either a beneficial or deleterious effect of pregnancy on its clinical course. Most recent studies that control for prognostic factors have demonstrated similar survival between pregnant and nonpregnant patients. When controlling of tumor site, age, Breslow thickness, and Clark level, an overall mortality of $25 \%$ in the pregnancy group was found during a 7 -year mean follow-up period (226). For all patients, melanoma prognosis is closely related to Breslow thickness (the depth of the lesion from the granular cell layer of the epidermis to the deepest 
identifiable tumor cells) $(227,228)$ as well as anatomic site and presence of tumor ulceration (229).

Early-stage disease is managed surgically while more advanced-stage disease is often treated with combination chemotherapy. Although Reintgen and colleagues (216) reported no difference in survival for patients who developed melanoma during pregnancy or for those who became pregnant within 5 years of diagnosis, most clinicians advise patients to avoid a subsequent pregnancy for 2 to 5 years after diagnosis, since $60 \%$ to $86 \%$ of melanomas recur during this period $(216,221)$.

In addition, when melanoma complicates pregnancy, both the fetus and the placenta should be examined closely after delivery because melanoma is the most common tumor metastasizing to the fetus and the placenta. The presence of metastases to the placenta is classified as stage IV disease, and all patients with placental metastases, except one, have died within 7 months (230). Overall, this phenomenon is exceedingly rare, with fewer than 20 reported cases. Termination of the pregnancy is not warranted, as infant survival, even with metastatic melanoma, is $75 \%(221,222)$. Of note, no cases of fetal metastases have been reported when the placenta did not demonstrate malignant transformation (225). Such information may prove useful in counseling couples after delivery.

\section{Hematologic Malignancies}

Hematologic malignancies are the third most common cancer complicating pregnancy (25\% of cases) after cervical cancer and breast cancer (231-233). The three most common include Hodgkin's lymphoma, non-Hodgkin's lymphoma, and leukemia.

\section{Leukemia}

The development of leukemia during pregnancy is rare, with an incidence of 1 in 75,000 pregnancies (234). Acute and chronic leukemias occur with equal frequency, although acute leukemias are more frequently diagnosed during pregnancy $(235,236)$. Among the acute forms, acute myelogenous leukemia (AML) is more common than acute lymphocytic leukemia (ALL). Ninety percent of the chronic leukemia cases are myelocytic, with the remainder being chronic lymphocytic leukemia (237).

The majority of pregnant women found to have leukemia are diagnosed during routine prenatal care. Signs and symptoms of acute leukemia include anemia, granulocytopenia, thrombocytopenia, splenomegaly, fatigue, fever, infection, and evidence of a bleeding diathesis. Physical exam may demonstrate pallor, petechiae, or ecchymoses. The diagnosis of leukemia oftentimes is confirmed by bone marrow examination. However, it can be initially suspected when a peripheral blood smear demonstrates a normocytic, normochromic anemia with a mild to severe thrombocytopenia. Blast cells are virtually always present despite a normal or low white blood cell count (238). Additionally, if the patient presents with a leukocytosis, flow cytometry and cytogenetics of a peripheral blood sample can be sufficient for diagnosis.

ALL in adults is seen in the 30- to 40-year-old age group. While anemia and splenomegaly are common, only $25 \%$ of patients will have white blood cell counts greater than
$50,000 / \mathrm{mm}^{3}$ (239). By contrast, AML most commonly occurs in 30- to 60 -year-old women and $75 \%$ will have white blood cell counts greater than $50,000 / \mathrm{mm}^{3}$. The usual age of presentation of acute leukemia during pregnancy ranges from 23 to 28 years (237). As such, it is not unexpected that AML is more commonly encountered during pregnancy (240).

Chronic leukemia usually presents with lymphadenopathy, splenomegaly, leukocytosis, and constitutional symptoms. Chronic myelocytic leukemia (CML) accounts for the majority of chronic leukemias complicating pregnancies, with a median age of 35 years (241). CML is generally indolent, with a median age onset of 60 years, which accounts for its relative rarity during gestation.

Treatment of acute leukemia with chemotherapy is based on the ultimate goal of eradicating all leukemic clone cells from the bone marrow with subsequent prevention of future emergence of resistant clone cells. As such, the treatment strategy is based on divided phases classified as induction, consolidation, and maintenance $(242,243)$. For those patients who do not achieve remission, allogeneic hematopoietic stem cell transplant is an option. Common chemotherapy induction regimens for ALL include anthracyclines, cytarabine, and vincristine in combination with steroids and L-asparaginase. AML is treated with anthracyclines and cytarabine only. Additionally, induction-induced morbidities must be prevented and treated as needed, such as cotrimoxazole for pneumocystis carinii prophylaxis and fluconazole for prevention of Candida albicans infections.

Experience in the treatment of leukemia during pregnancy is limited. Without treatment, the median survival time for patients with acute leukemia is 2 months. With treatment, $65 \%$ to $75 \%$ of adults with ALL will gain complete remission; however, recurrence is common and only one-third of patients with ALL will be long-term survivors. A similar proportion of individuals less than 60 years of age treated for AML will achieve complete remission, with $40 \%$ of these experiencing eventual cure (241). Chronic leukemia is more indolent and can be successfully controlled with chemotherapy for a long period of time.

The patient diagnosed with acute leukemia during pregnancy poses a difficult problem. Due to the poor prognosis and rapid progression of this disease, immediate treatment is needed. Since there is no evidence that pregnancy alters the natural course of leukemia, terminating the pregnancy will not improve survival of the patient. Additionally, expert opinion holds that the outcome of pregnant patients with acute leukemia is adversely affected only when appropriate therapy is withheld for more than a few weeks (244).

Combination chemotherapy has become the therapeutic management of choice in patients with acute leukemia. Children who were exposed to chemotherapeutic agents in utero showed normal growth and development from 1 to 17 years of age (235). If given after the second trimester, chemotherapy is not associated with an increased rate of fetal malformations (240). Remission rates of pregnant women treated with combination chemotherapy compare favorably with those in comparable nonpregnant women. Standard antileukemic agents, such as cytarabine and anthracyclines, can be safely administered during the second and third trimesters. However, antifolates should be avoided during the first 
trimester, owing to a $10 \%$ to $20 \%$ risk of congenital anomalies (245). Use of L-asparaginase during pregnancy should always been done with caution as it has been shown to decrease the levels of certain thrombosis inhibitors and is associated with a significantly increased risk of thromboembolism (246-249). It is important to note that evidence shows that delaying appropriate chemotherapy for more than a few weeks at any time other than the latter part of the third trimester is associated with excessive fetal mortality $(235,250,251)$.

Intrathecal chemotherapy and whole-brain radiation may safely be performed during any trimester of pregnancy (252). There is an increased risk of infection, stillbirth, and spontaneous abortion associated with leukemia treatment (250). Patients diagnosed with leukemia during the first half of pregnancy have only a $50 \%$ to $60 \%$ chance of producing a healthy live infant. Intrauterine growth restriction is seen in approximately $8 \%$ of infants of mothers treated for leukemia during pregnancy (244).

Chronic leukemia is generally a more indolent disease and treatment should be withheld until after delivery if possible. As seen with acute leukemia, pregnancy does not appear to alter the natural course of chronic leukemia. Maternal and fetal outcomes are generally excellent, with survival rates of $96 \%$ and $84 \%$, respectively (253), and are not significantly different from that expected for nonpregnant women. Interferon- $\alpha$ has been used in at least 10 cases of chronic leukemia-complicated pregnancy without adverse maternal effects with only one case of transient neonatal thrombocytopenia $(254,255)$. The main goal of treating chronic leukemias during pregnancy is to control splenomegaly, leukocytosis, and constitutional symptoms.

\section{Lymphoma}

Lymphoma is a relatively common malignancy presenting during the reproductive years. It occurs in 1 in 1000 to 1 in 6000 pregnancies. Approximately 25\% to $40 \%$ of lymphomas are Hodgkin's type, with the average age of onset being 32 years. Hodgkin's disease is the most common type of lymphoma associated with pregnancy (256).

The majority of women with lymphoma are asymptomatic. On physical examination, $80 \%$ will have superficial lymphadenopathy. Those with Hodgkin's disease are more likely to have localized lymphadenopathy, most often involving cervical, submaxillary, or axillary nodes. A small percentage of patients have fever, night sweats, weight loss, or pruritus, which are associated with poor prognosis. Biopsy is the "gold standard" of diagnosis. The histology of Hodgkin's lymphoma often shows multinucleated Reed-Sternberg cells with a pattern that is most commonly nodular sclerosing or lymphocytic. Histology is no longer thought to contribute to overall prognosis, as recent evidence demonstrates that the two most important prognostic factors are stage of disease and patient's age (244). Hodgkin's lymphoma is staged by the Ann Arbor staging system.

Patients with non-Hodgkin's lymphoma commonly present with high- or intermediate-grade diffuse histologic patterns and have a poorer prognosis than those with a nodular pattern (53). They are histologically distinguished from Hodgkin's lymphoma by the absence of Reed-Sternberg cells. They comprise a heterogeneous group of lymphoid malignancies and differ in their presentation, stage at diagnosis, and prognosis. The classification system now used is based on the WHO classification system and uses morphologic, genetic, immunophenotypic, and clinical features. This has been shown to provide a higher degree of diagnostic accuracy and reproducibility (257).

The treatment of lymphoma depends on accurate staging, and minimal evaluation requires examination by the physician, chest radiographs, bone marrow biopsy with aspirate and core, and imaging of the abdomen usually with MRI. Abdominal ultrasound examination for retroperitoneal adenopathy or hydronephrosis may be helpful in staging. Lymphangiograms are rarely used in the current evaluation of lymphoma.

Once staging is complete, treatment generally should not be compromised because of the pregnancy. In the nonpregnant woman, nodal radiotherapy and combination chemotherapy result in cures for $70 \%$ of patients with Hodgkin's disease and $50 \%$ of patients with non-Hodgkin's lymphoma (256). Patients in the third trimester with localized Hodgkin's disease can be allowed to deliver prior to therapy (245). Treatment during the first and second trimesters and in patients with advanced disease or high-grade tumors is more complex. Localized disease above the diaphragm can be treated with radiotherapy with abdominal shielding. More extensive disease is best treated with combination chemotherapy to reduce the large fetal radiation exposure. The same considerations concerning combination chemotherapy as discussed with leukemia apply to lymphoma.

Placental metastases have been reported in both leukemia and lymphoma patients and, as such, the placenta should be closely examined after delivery as well as be sent to pathology for histologic evaluation. As a group, they account for 19\% of the malignancies metastatic to the products of conception and $50 \%$ of those metastatic to the fetus $(232,258)$. Postpartum contraception is important as many patients often require prolonged maintenance therapy. Breast-feeding should be avoided as little data exist regarding the specific chemotherapy agents used to treat these malignancies and their potential for neonatal adverse effects.

\section{Thyroid Cancer}

Thyroid cancer is the most frequently encountered cancer in the reproductive age group, with $50 \%$ of cases occurring in women of 15 to 44 years of age. In the general population, it is the most common endocrine cancer, with an estimated incidence of 9 per 100,000 (259). Approximately $10 \%$ of thyroid cancers that occur during the reproductive years are diagnosed during pregnancy or in the first year after birth $(260,261)$. Some case-control studies suggest that hormonal factors may play an etiologic role in the development of thyroid cancer (262). This theory is supported by isolated studies showing an increased incidence in pregnancy and a decreased incidence, equal to that in males, following menopause. Clinically detectable thyroid nodules are three to four times more likely in pregnant women, and although most are benign, there are reports that up to $39 \%$ to $43 \%$ of these nodules are malignant (261). Thyroid stimulation is thought to be caused by the high levels of beta-hCG, which also has thyroid-stimulating activity. However, there is currently no 
consensus about the impact of pregnancy on thyroid cancer, and the precise incidence of thyroid cancer in pregnancy is unknown (263).

The most common histologic type is papillary, followed by follicular and mixed papillary-follicular. These histologies have a favorable prognosis, whereas the less commonly occurring medullary and anaplastic patterns do not. The usual clinical presentation is that of a single thyroid nodule that has up to a $20 \%$ chance of being malignant. Rapid growth and fixation of the nodule to the surrounding structures or cervical lymphadenectomy are common findings for advanced disease. Risk factors for developing thyroid cancer are prior head or neck irradiation and a family history of multiple endocrine neoplasms.

Diagnosis is often made by palpation, but must be confirmed by biopsy. FNA has been shown to be a safe and effective technique in pregnant women, with false-positive results being exceedingly rare and false-negative results occurring in only $5 \%$ to $10 \%$ of cases (252). Some advocate fine-needle aspiration of all thyroid nodules prior to 20 weeks of gestation, and after 20 weeks, only those that grow during suppressive therapy should be sampled (264). Radioisotope nuclear scanning is contraindicated in pregnancy, because radioactive isotopes are taken up by the fetus. Other methods, such as ultrasound, can be used to distinguish solid-form cystic nodules. Thyroid function tests may also be helpful and can diagnose unsuspected thyroiditis. If medullary thyroid cancer is suspected, a calcitonin level should be checked as well. Normally, pregnancy witnesses a hypertrophy of the thyroid gland from follicular cell hyperplasia, and total triiodothyronine and thyroxine levels are elevated while triiodothyronine resin uptake is decreased secondary to increased thyroid-binding globulin. Thyroid-stimulating hormone is in the normal to slightly elevated range.

Treatment of choice for all primary carcinomas is surgery. Thyroidectomy is safe in the midtrimester and does not appear to increase the risk of adverse perinatal outcomes (265). Cancer discovered during the first or early second trimester should prompt immediate surgery (263). In the third trimester, a brief delay to allow fetal pulmonary maturity may be acceptable, and even malignant nodules diagnosed at any time after 20 weeks may be followed unless noted to be rapidly growing or of undifferentiated histology $(261,266)$. Similarly, surgery for indeterminate findings on FNA can be delayed until after delivery as well unless the nodule is increasing in size (261). Well-differentiated forms of papillary and follicular histology are treated with subtotal thyroidectomy. Extensive neck dissection performed for cervical lymphadectomy carries an increased incidence of spontaneous abortion. Poor prognostic medullary or anaplastic forms may present at very advanced stages, in which case regard for fetal development may predominate. Treatment with radioactive iodine, which is often used for adjuvant therapy in follicular thyroid cancers, is contraindicated, since it crosses the placenta and is trapped by the fetal thyroid.

Overall, prognosis during pregnancy appears unchanged when controlling for stage and histology; thus, abortion or prevention of subsequent pregnancy is unwarranted. When surgery is performed, vigilance for hypothyroidism and hypoparathyroidism should be maintained throughout pregnancy as well as postpartum. After surgery and iodine treatment, hormonal replacement therapy is suggested to suppress thyroid-stimulation hormone. Thyroid hormone replacement is safe and effective in pregnancy. Subsequent pregnancy after treatment with radioactive iodine is safe as the gonadal impairment is only temporary and reversible, and there have been no reports of stillbirths, malformations, early deaths, or malignancies (267). In general, it is recommended to wait 1 year after treatment prior to pursuing another pregnancy to avoid all potential adverse side effects.

\section{Bone and Soft-Tissue Sarcomas}

Bone and soft-tissue sarcomas are relatively common malignancies in the reproductive age group. Their presence in association with pregnancy, however, is very rare, as there are less than 50 such recorded cases in the literature (268). The majority of these cases are osteosarcomas, followed by Ewing's sarcomas. Genital sarcomas of the cervix, vagina, and vulva include liposarcomas, histiocytomas, myosarcomas, and leiomyosarcomas, with and without peritoneal dissemination.

The majority of patients present with symptoms of localized pain or a mass. Diagnostic procedures commonly used to assess sarcomas include radiographs with abdominal shielding during pregnancy. Osteogenic sarcomas may require radioisotope bone scanning, which is thought to be of low risk to the fetus (269). Definitive diagnosis of any type of sarcoma requires biopsy. Although pregnancy has no proven deleterious effects on the natural course of sarcomas, delayed diagnosis results in postponed treatment. These tumors present at advanced stages, which may explain isolated reports of worsened prognosis for sarcomas in pregnancy (270). The aggressive biologic behavior of pelvic sarcomas may be enhanced by hypervascularity or hormonal stimulus; however, an impaired prognosis for most sarcomas associated with pregnancy remains unsubstantiated.

Owing to the diversity of histologic types, anatomic locations, stages of disease, and forms of treatment for different types of sarcomas, it is difficult to generalize treatment options. Since maternal prognosis for all types of sarcomas is poor, a combination of surgery, irradiation, and aggressive multiagent chemotherapy is warranted (271). Most clinicians recommend that the pregnant woman should be managed without regard for the pregnancy during the first two trimesters of gestation. In cases presenting in the third trimester, the fetus may be delivered vaginally or by cesarean section for obstetric indications as soon as pulmonary maturity is documented, so that aggressive treatment can be initiated. Although based on limited experience, more recent reports advocate the initiation of multiagent chemotherapy for control of systemic disease during any stage of gestation. Such therapy is based on the rationale that mortality in sarcomas often results from distant metastases and not local recurrence, and chemotherapy is relatively well tolerated by the fetus with the exception of the first trimester.

\section{Tumors of the Central Nervous System}

The Third National Cancer Study indicates that $21 \%$ of brain tumors affect women in the childbearing years (2); however, only 220 cases of central nervous system (CNS) tumors occurring during pregnancy have been reported in the world's 
literature over the past 90 years (272). Intracranial tumors occur in pregnancy with a reported frequency of 3.6 per 10 (25) births (273). The most common neoplasms are glial neoplasms, followed by meningiomas and acoustic neuromas (274) and have similar frequencies in pregnant and nonpregnant women. Certain CNS tumors, such as meningiomas, pituitary adenomas, and vascular neoplasms, have a slightly increased incidence during pregnancy.

Although not well established, the fact that meningiomas predominate in women, enlarge rapidly during pregnancy, and express estrogen and progesterone receptors suggests a causal relationship between the tumors and pregnancy (275). Pregnancy normally results in pituitary enlargement secondary to hyperplasia of prolactin-secreting acidophilic cells and it also appears to stimulate the growth of pituitary microadenomas, making them clinically apparent (276). The relative frequency of CNS vascular tumors is also increased during pregnancy, with hemangioblastomas and spinal hemangiomas predominating. This increased incidence is attributed to retrograde engorgement of vascular channels in the tumor secondary to increased venous pressure from compression of the inferior vena cava in the gravid uterus, as well as an overall increased blood volume (277). These factors permit occult vascular tumors to become clinically apparent. Additionally, growth of residual lesions or recurrence is possible during pregnancy in previously treated patients (274).

Symptoms of brain tumors manifesting during pregnancy, such as nausea and vomiting, can be mistakenly attributed to the pregnancy. In addition, headache and visual disturbances commonly caused by brain tumors may be misinterpreted as signs of pre-eclampsia or optic neuritis. Spinal cord tumors cause symptoms of spinal cord compression, depending on the level involved. Early symptoms, such as fatigue, backache, and nerve-root irritation, may also be confused with common pregnancy-related complaints.

The diagnosis of CNS tumors can be established by radiographic studies, such as computed tomography scans, MRI, myelography, and angiography. Prolactin levels are useful in assessing pituitary tumors, and examination of the cerebrospinal fluid is helpful with spinal cord tumors. Definitive diagnosis of any tumor requires histologic examination. The coexistence of a CNS tumor and pregnancy should not preclude diagnostic studies.

The management of CNS tumors must be considered on an individual basis. In general, immediate treatment is necessary when symptoms are severe or progressive. Once the diagnosis of a CNS tumor is established, the patient should be closely observed for sudden increase in intracranial pressure, loss of visual fields, or progressive neurologic deficits. The treatment of choice for most CNS tumors, including meningiomas and spinal cord tumors, is surgery. Other tumors may respond to radiation therapy, which is not contraindicated during pregnancy provided the tumor is above the thoracic region and the fetus can be appropriately shielded. Prolactinomas can be treated with bromocriptine even during pregnancy, but surgical management is still preferred. When symptoms are mild or occur during the third trimester, careful observation with conservative medical treatment aimed at reducing intracranial pressure until fetal maturity is suggested (278). When symptoms are severe, treatment is mandatory without regard to the pregnancy to prevent further neurologic deficits or death. Neurosurgery is particularly safe in the third trimester. The best mode of delivery in the presence of increased intracranial pressure remains to be determined.

\section{CHEMOTHERAPY IN PREGNANCY}

Oftentimes, chemotherapy treatment cannot be delayed until after delivery for pregnant women diagnosed with certain cancers. Most chemotherapeutic agents have been associated with adverse fetal side effects (279). However, safe use of chemotherapy has also been reported in the second and third trimesters with little evidence for adverse neonatal outcomes. The risks regarding chemotherapy use during pregnancy depend on the timing of exposure, the dose, and the characteristics affecting placental transfer. Those factors that most readily affect placental transfer from mother to fetus are high lipid solubility, low molecular weight, and loose binding to plasma proteins. It is also thought that genetic predisposition to teratogenicity might explain why some women have increased susceptibility compared with others (279). Risks of chemotherapy vary depending on the gestational age. Use of chemotherapy in the first trimester carries the risk of spontaneous abortion, fetal death, and major malformations. The gestational weeks 2 to 8 comprise the period of organogenesis and, as such, represent the time when the fetus is most vulnerable to teratogenic insults. Chemotherapy administered during the second and third trimesters increases the risk for intrauterine growth restriction and low birth weight. Unfortunately, transplacental studies have been unsuccessful in effectively monitoring fetal exposure to the administration of chemotherapeutic agents to the mother (280).

Pharmacokinetic changes associated with pregnancy are largely unknown, and pregnant women receive similar weightbased doses as those who are not pregnant. Given the increased blood volume and renal clearance that occurs with pregnancy, these women may receive decreased doses of the active agent. There is also a thought that a faster hepatic mixed-function oxidase system combined with decreased plasma albumin levels may also affect drug absorption. Currently, no pharmacokinetic studies have been done in pregnant women receiving chemotherapy.

Overall, delivery should be avoided during the maternal nadir period, which occurs 2 to 3 weeks after treatment. Chemotherapy should not be administered after 35 weeks of gestation as spontaneous delivery could occur before the bone marrow has recovered. The ability of an agent to cross the placenta correlates poorly with its ability to pass into breast milk, and breast-feeding is considered contraindicated while undergoing chemotherapy. As always, the decision to use chemotherapy in pregnancy must be weighed against the effect of treatment delay on maternal survival and the overall risk to the fetus.

\section{REFERENCES}

1. Donegan WL. Cancer and pregnancy. Cancer 1983; 33: 194-214.

2. Third National Cancer Survey. Incidence data NCI. Monogr 1975; 41: 108-11.

3. Amant F, Calsteren KV, Vergote I, Ottevanger N. Gynecologic oncology in pregnancy. Crit Rev Oncol Hematol 2008; 67: 187-95. 
4. Creasman WT. New gynecologic cancer staging. Obstet Gyencol 1990; 75: 287.

5. Arena S, Marconi M, Ubertosi M, et al. HPV and pregnancy: diagnostic methods, transmission, and evolution. Minerva Gynecol 2002; 54: 225-37.

6. Wood C. Laryngeal papillomas in infants and children. Relationship to maternal venereal warts. J Nurse Mid 1991; 36: 297-302.

7. Lee JH, Smith RJ. Recurrent respiratory papillomatosis: pathogenesis to treatment. Curr Opin Otolaryngol Head Neck Surg 2005; 13: 354-9.

8. Silverberg MJ, Thorsen P, Lindeberg H, Grant LA, Shah KV. Condyloma in pregnancy is strongly predictive of juvenile-onset recurrent respiratory papillomatosis. Obstet Gynecol 2003; 101: 645-52.

9. Rombaldi RL, Serafini EP, Mandelli J, Zimmermann E, Losquiavo KP. Transplacental transmission of human papillomavirus. Virol J 2008; 5: 106.

10. Deng D, Wen L, Chen W, Ling X. Asymptomatic genital infection of human papillomavirus in pregnant women and the vertical transmission route. J Huazhong Univ Sci Technolog Med Sci 2005; 25: 343-5.

11. Syrjänen S, Puranen M. Human papillomavirus infection in children: the potential role of maternal transmission. Crit Rev Oral Biol Med 2000; 11: 259-74.

12. Nardelli-Haefliger D, Wirthner D, Schiller JT, et al. Specific antibody levels at the cervix during the menstrual cycle of women vaccinated with human papillomavirus 16 virus-like particles. J Natl Cancer Inst 2003; 95: 1128-37.

13. Koutsky LA, Ault KA, Wheeler CM, et al.; Proof of Principle Study Investigators. A controlled trial of a human papilloma virus type 16 vaccine. NEJM 2002; 347: 1645-51.

14. Jansen KU, Shaw AR. Human papilloma virus vaccines and prevention of cervical cancer. Annu Rev Med 2004; 55: 319-31.

15. Billich A. HPV vaccine MedImmune/GlaxoSmithKline. Curr Opin Ivestig Drugs 2003; 4: 210-13.

16. DiSaia PJ, Creasman WT. Cancer in pregnancy. In: DiSaia PJ, Creasman WT, eds. Clinical gynecologic oncology. St. Louis, MO: CV Mosby, 1989.

17. Hacker NF, Berek JS, Lagasse LD. Carcinoma of the cervix associated with pregnancy. Obstet Gynecol 1982; 59: 735-46.

18. LaPolla JP, O’Neill C, Wetrich D. Colposcopy management of abnormal cervical cytology in pregnancy. J Reprod Med 1988; 33: 301-6.

19. Jain AG, Higgins RV, Boyle MJ. Management of low-grade squamous intraepithelial lesions during pregnancy. Am J Obstet Gynecol 1997; 177: 298-302.

20. Patsner B. Management of low grade cervical dysplasia during pregnancy. South Med J 1990; 83: 1405-12.

21. Robinson WR, Webb S, Tirpack J, Degefu S, O’Quinn AG. Management of cervical intraepithelial neoplasia during pregnancy with LOOP excision. Gynecol Oncol 1997; 64: 153-5.

22. Mitsuhashi A, Sekiya S. Loop electrosurgical excision procedure (LEEP) during first trimester of pregnancy. Int J Gynaecol Obstet 2000; 71: 237-9.

23. Dunn TS, Ginsburg V, Wolf D. Loop-cone cerclage in pregnancy: a 5-year review. Gynecol Oncol 2003; 90: 577-80.

24. Averette HE, Nasser N, Yankow SL, Little WA. Cervical conization in pregnancy. Am J Obstet Gynecol 1970; 106: 543-8.

25. Lutz MH, Underwood PB, Rozier JC, Putney FW. Genital malignancy in pregnancy. Am J Obstet Gynecol 1977; 129: 536-42.

26. Goldberg GL, Ataras MM, Block B. Cone cerclage in pregnancy. Obstet Gynecol 1991; 77: 315-17.

27. Smith LH, Dalrymple JL, Leiserowitz GS, Danielsen B, Gilbert WM. Obstetrical deliveries associated with maternal malignancy in California 1992 through 1997. Am J Obstet Gynecol 2001; 184: 1504-12; discussion 1512-13.

28. Smith H, Danielsen B, Allen ME, Cress R. Cancer associated with obstetric delivery: results of linkage with the California cancer registry. Am J Obstet Gynecol 2003; 189: 1128-35.

29. Demeter A, Sziller I, Caspo Z, Szantho A, Papp Z. Outcome of pregnancies after cold-knife conization of the uterine cervix during pregnancy. Eur J Gynaecol Oncol 2002; 23: 207-10.

30. Jacob JH, Stringer CA. Diagnosis and management of cancer during pregnancy. Semin Perinatol 1990; 14: 79-87.

31. Zemlickis D, Lishner M, Degendorfer $\mathrm{P}$, et al. Maternal and fetal outcome after invasive cervical cancer in pregnancy. J Clin Oncol 1991; 9: 1956-61.
32. Yagizi R, Cunningham FG. Cancer and pregnancy. Williams Obstet 1990; 4(Suppl): 1-15.

33. Lee RB, Neglia W, Park RC. Cervical carcinoma in pregnancy. Obstet Gynecol 1981; 58: 584-9.

34. Grigsby PW, Siegel BA, Dehdashti F. Lymph node staging by positron emission tomography in patients with carcinoma of the cervix. J Clin Oncol 2001; 19: 3745-9.

35. Grigsby PW, Dehdashti F, Siegel BA. FDG-PET evaluation of carcinoma of the cervix. Clin Pos Imag 1999; 2: 105-9.

36. Rose PG, Adler LP, Rodriguez M, et al. Positron emission tomography for evaluating para-aortic nodal metastasis in locally advanced cervical cancer before surgical staging: a surgicopathologic study. J Clin Oncol 1999; 17: 41-5.

37. Wong TZ, Jones IL, Coleman RE. Positron emission tomography with 2-deoxy-2-[(18)F]fluoro-D-glucose for evaluating local and distant disease in patients with cervical cancer. Mol Imag Biol 2004; 6: 55-62.

38. Miller TR, Grigsby PW. Measurement of tumor volume by PET to evaluate prognosis in patients with advanced cervical cancer treated by radiation therapy. Int J Radiat Oncol Biol Phys 2002; 53: 353-9.

39. Miller TR, Pinkus E, Dehdashti F, Grigsby PW. Improved prognostic value of $18 \mathrm{~F}-\mathrm{FDG}$ PET using a simple visual analysis of tumor characteristics in patients with cervical cancer. J Nucl Med 2003; 44: 192-7.

40. Grigsby PW, Siegel BA, Dehdashti F, Rader J, Zoberi I. Posttherapy $\left[{ }^{18} \mathrm{~F}\right]$ fluorodeoxyglucose positron emission tomography in carcinoma of the cervix: response and outcome. J Clin Oncol 1999; 17: 41-5.

41. Brooks RA, Rader JS, Dehdashti F, et al. Surveillance FDG-PET detection of asymptomatic recurrences in patients with cervical cancer. Gynecol Oncol 2009; 112: 104-9.

42. Hunter MI, Tewari K, Monk BJ. Cervical neoplasia in pregnancy. Part 2: current treatment of invasive disease. Am J Obstet Gynecol 2008; 199: 10-18.

43. Diakomanolis E, Haidopoulos D, Rodolakis A, et al. Laser $\operatorname{CO}(2)$ conization: a safe mode of treating conservatively microinvasive carcinoma of the uterine cervix. Eur J Obstet Gynecol Reprod Biol 2004; 113: 229-33.

44. Itsukaichi M, Kurata H, Matsushita M, et al. Stage Ial cervical squamous cell carcinoma: Conservative management after laser conization with positive margins. Gynecol Oncol 2003; 90: 387-9.

45. Okamoto Y, Ueki K, Ueki M. Pathological indications for conservative therapy in treating cervical cancer. Acta Obstet Gynecol Scand 1999; 78: 818-23.

46. Van De Nieuwenhof HP, Van Ham MAPC, Lotgering FK, Massugar LFAG. First case of vaginal radical trachelectomy in a pregnant patient. Int J Gynecol Cancer 2008; 18: 1381-5.

47. Sivanesaratnam V, Jayalakshmi P, Loo C. Surgical management of early invasive cancer of the cervix associated with pregnancy. Gynecol Oncol 1993; 48: 68-75.

48. Sood AK, Sorosky JI, Mayr N, et al. Radiotherapeutic management of cervical carcinoma that complicates pregnancy. Cancer 1997 80: 1073-8.

49. Prem KA, Makowski EL, McKelvey JL. Carcinoma of the cervix associated with pregnancy. Am J Obstet Gynecol 1966; 95: 99-108.

50. Rose PG, Bundy BN, Watkins EB, et al. Concurrent cisplatin-based radiotherapy and chemotherapy for locally advanced cervical cancer. N Engl J Med 1999; 340: 1144-53.

51. Morris M, Eifel PJ, Lu J, et al. Pelvic radiation with concurrent chemotherapy compared with pelvic and para-aortic radiation for highrisk cervical cancer. N Engl J Med 1999; 340: 1137-43.

52. Duggan B, Muderspach LI, Roman LD, et al. Cervical cancer in pregnancy: Reporting on planned delay in therapy. Obstet Gynecol 1993; 82: 598-602.

53. Sorosky I, Squatrito R, Ndubisi BU, et al. Stage I squamous cell cervical carcinoma in pregnancy: planned delay in therapy awaiting fetal maturity. Gynecol Oncol 1995; 59: 207-10.

54. Monk BJ, Montz FJ. Invasive cervical cancer complicating intrauterine pregnancy: treatment with radical hysterectomy. Obstet Gynecol 1992; 80: 199-203.

55. Sood AK, Sorosky JI, Krogman S, et al. Surgical management of cervical cancer complicating pregnancy: a case-control study. Gynecol Oncol 1996; 63: 294-8. 
56. Takushi M, Moromizato H, Sakumoto K. Kanazawa K. Management of invasive carcinoma of the uterine cervix associated with pregnancy: outcome of intentional delay in treatment. Gynecol Oncol 2002; 87: 185-9.

57. Tewari K, Cappuccini F, Gambino A, et al. Neoadjuvant chemotherapy in the treatment of locally advanced cervical carcinoma in pregnancy: a report of two cases and review of issues specific to the management of cervical carcinoma in pregnancy including planned delay of therapy. Cancer 1998; 82: 1529-34.

58. Greer BE, Easterling TR, McLennan DA, et al. Fetal and maternal considerations in the management of stage I-B cervical cancer during pregnancy. Gynecol Oncol 1989; 34: 61-5.

59. Lee RB, Neglia W, Park RC. Cervical carcinoma in pregnancy. Obstet Gynecol 1981; 58: 584-9.

60. Marana HR, de Andrade JM, da Silva Mathes AC, et al. Chemotherapy in the treatment of locally advanced cervical cancer and pregnancy. Gynecol Oncol 2001; 80: 272-4.

61. Sood AK, Shahin MS, Soroski JI. Paclitaxel and platinum chemotherapy for ovarian carcinoma during pregnancy. Gynecol Oncol 2001; 83: 599-600.

62. Mendez LE, Mueller A, Salom E, Gonzales-Quintero VH. Paclitaxel and carboplatin chemotherapy administered during pregnancy for advanced epithelial ovarian cancer. Obstet Gynecol 2003; 5: 1200-2.

63. Barber HR, Brunschwig A. Gynecologic cancer complicating pregnancy. Am J Obstet Gynecol 1963; 85: 156-64.

64. Mikuta JJ. Invasive carcinoma of the cervix in pregnancy. South Med J 1967; 60: 843-7.

65. Cliby WA, Dodson MK, Podratz KC. Cervical cancer complicated by pregnancy: episiotomy site recurrences following vaginal delivery. Obstet Gynecol 1994; 84: 179-82.

66. Gordon AN, Jensen R, Jones HW 3rd. Squamous carcinoma of the cervix complicating pregnancy: recurrence in episiotomy after vaginal delivery. Obstet Gynecol 1989; 73: 850-2.

67. Baloglu A, Uysal D, Aslan N, Yigit S. Advanced stage of cervical carcinoma undiagnosed during antenatal period in term pregnancy and concomitant metastasis on episiotomy scar during delivery: a case report and review of the literature. Int J Gynecol Cancer 2007; 17: 1131-71.

68. Burgess SP, Waymont B. Implantation of a cervical carcinoma in an episiotomy site. Br J Obstet Gynaecol 1987; 94: 598-9.

69. Copeland LJ, Saul PB, Sneige N. Cervical adenocarcinoma: tumor implantation in the episiotomy sites of two patients. Gynecol Oncol 1987; 28: 230-5.

70. Gordon AN, Jensen R, Jones HW 3rd. Squamous carcinoma of the cervix complicating pregnancy: recurrence in episiotomy after vaginal delivery. Obstet Gyencol 1989; 73: 850-2.

71. Van Dam PA, Irvine L, Lowe DG, et al. Carcinoma in episiotomy scars. Gynecol Oncol 1992; 44: 96-100.

72. Khalil AM, Khatib RA, Mufarrij AA, Tawil AN, Issa PY. Squamous cell carcinoma of the cervix implanting in the episiotomy site. Gyencol Oncol 1993; 51: 408-10.

73. Cliby WA, Dodson MK, Podratz KC. Cervical cancer complicated by pregnancy: episiotomy site recurrences following vaginal delivery. Obset Gynecol 1994; 84: 179-82.

74. Goldman NA, Goldberg GL. Late recurrence of squamous cell cervical cancer in an episiotomy site after vaginal delivery. Obstet Gyencol 2003; 101: 1127-9.

75. Amanie J, Pearcey RG, Honore L, Sloboda R. Metastatic adenocarcinoma of the cervix in a delivery-induced traumatic lower vaginal tear. Gynecol Oncol 2005; 96: 857-9.

76. Barclay DL. Surgery of the vulva, perineum, and vagina in pregnancy. In: Barber HRK, Braber EA, eds. Surgical Disease in Pregnancy. Philadelphia: WB Saunders, 1974.

77. Arena S, Marconi M, Ubertosi M, et al. HPV and pregnancy: diagnostic methods, transmission, and evolution. Minerva Gynecol 2002; 54: 225-37.

78. Monaghan JM, Lindeque G. Vulvar carcinoma in pregnancy [editorial]. Br J Obstet Gynaecol 1986; 93: 785-6.

79. Fujita K, Aoki Y, Tanaka K. Stage I squamous cell carcinoma of vagina complicating pregnancy: successful conservative management. Gynecol Oncol 2005; 98: 513-15.

80. Stock RG, Chen ASJ, Seski J. A 30-year experience in the management of primary carcinoma of the vagina; analysis of prognostic factors and treatment modalities. Gynecol Oncol 1995; 56: 43-52.
81. Davis KP, Stanhope CR, Garton GR, Atkinson EJ, O’Brien PC. Invasive vaginal carcinoma: analysis of early-stage disease. Gynecol Oncol 1991; 42: $131-6$.

82. Grimes WH, Bartholomew RA, Colvin ED, Fish JS, Lester WM. Ovarian cyst complicating pregnancy. Am J Obstet Gynecol 1954; 68: 594-605.

83. Struyk APHB, Treffers PE. Ovarian tumors in pregnancy. Acta Obstet Gyencol Scand 1984; 63: 421-4.

84. Booth RT. Ovarian tumors in pregnancy. Obstet Gynecol 1963; 21: 189-92.

85. White KC. Ovarian tumors in pregnancy. Am J Obstet Gynecol 1973; 116: 544-50.

86. Buttery BW, Beisher NA, Fortune DW, Macafee CA. Ovarian tumors in pregnancy. Med J Aust 1973; 1: 345-9.

87. Hess W, Peaceman A, O'Brien WF, et al. Adnexal mass occurring with intrauterine pregnancy: report of fifty-four patients requiring laparotomy for definitive management. Am J Obstet Gynecol 1988; 158: 1029-34.

88. Thornton JG, Wells M. Ovarian cysts in pregnancy: does ultrasound make traditional management inappropriate? Obstet Gynecol 1987; 69: 717-21.

89. Tawa K. Ovarian tumors in pregnancy. Am J Obstet Gynecol 1964; 90: 511-15.

90. Hill LM, Johnson CE, Raymond AL. Ovarian surgery in pregnancy. Am J Obstet Gynecol 1975; 122: 565-9.

91. Hasan A, Amr S, Issa A, Bata M. Ovarian tumors complicating pregnancy. Int J Gynaecol Obstet 1983; 21: 279-82.

92. Ballard CA. Ovarian tumors associated with pregnancy termination patients. Am J Obstet Gynecol 1984; 149: 384-7.

93. Hopkins MP, Duchon MA. Adnexal surgery in pregnancy. J Reprod Med 1986; 31: 1035-7.

94. Nelson MJ, Cavalieri R, Graham D, Sanders RC. Cysts in pregnancy discovered by sonography. J Clin Ultrasound 1986; 14: 509-12.

95. Askenazy M, Kessler I, Czernobilsky B, Nahshoni A, Lancet M. Ovarian tumors in pregnancy. Int J Gynaecol Obstet 1988; 27: 79-83.

96. Tchabo JG, Stay EJ, Limaye NS. Ovarian tumors in pregnancy: a community hospital's fie year experience. Int Surg 1987; 72: 227-9.

97. Koonings PP, Platt LD, Wallace R. Incidental adnexal neoplasms at cesarean section. Obstet Gynecol 1988; 72: 767-9.

98. Sunoo CS, Terada KY, Kamemoto LE, Hale RW. Adnexal masses in pregnancy: occurrence by ethnic group. Obstet Gynecol 1990; 75: 38-40.

99. el-Yahia AR, Rahman J, Rahman MS, al-Suleiman SA. Ovarian tumours in pregnancy. Aust NZ J Obstet Gynaecol 1991; 31: 327-30.

100. Platek DN, Henderson CE, Goldberg GL. The management of a persistent mass in pregnancy. Am J Obstet Gynecol 1995; 173: 1236-40.

101. Ueda M, Ueki M. Ovarian tumors associated with pregnancy. Int J Gynaecol Obstet 1996; 55: 59-65.

102. Bromley B, Benacerraf B. Adnexal masses during pregnancy: accuracy of sonographic diagnosis and outcome. J Ultrasound Med 1997; 16: 447-52.

103. Whitecar MP, Turner S, Higby MK. Adnexal masses in pregnancy: a review of 130 cases undergoing surgical management. Am J Obstet Gynecol 1999; 181: 19-24.

104. Gustafson GW, Gardener SH, Stout FE. Ovarian tumors complicating pregnancy: a review of 45 surgically proved cases. Am J Obstet Gynecol 1954; 67: 1210-23.

105. Levine W, Diamond B. Surgical procedures during pregnancy. Am J Obstet Gynecol 1961; 81: 1046-52.

106. Child CG, Douglas RG. Surgical problems arising during pregnancy. Am J Obstet Gynecol 1943; 47: 213-28.

107. Bernhard LM, Klebba PK, Gray DL, Mutch DG. Predictors of persistence of adnexal masses in pregnancy. Obstet Gynecol 1999; 93: 585-9.

108. Curtis M, Hopkins MP, Zarlingo T, et al. Magnetic resonance imaging to avoid laparotomy in pregnancy. Obstet Gynecol 1993; 82: 833-6.

109. Chiang G, Levine D. Imaging of adnexal masses in pregnancy. J Ultrasound Med 2004; 23: 805-19.

110. Beischen HA, Buttery BW, Fortune DW, McCafee CAJ. Growth and malignancy of ovarian tumors in pregnancy. Aust NZ J Obstet Gynecol 1971; 11: 208-20.

111. Dougherty CM, Lund CJ. Solid ovarian tumors complicating pregnancy: a clinicopathologic study. Am J Obstet Gynecol 1950; 60: 261-72.

112. Chung A, Birnbaum SJ. Ovarian cancer associated with pregnancy. Obstet Gynecol 1977; 41: 211-14. 
113. Bitson JR, Golden ML. Cancer and pregnancy. Am J Obstet Gynecol 1961; 85: 345-54.

114. Copeland LJ, Landon MB. Malignant disease in pregnancy. In: Gabbe SG, Niebyl JR, Simpson JL, eds. Obstetrics, normal and problems pregnancies, 3rd edn. New York: Churchill Livingstone, 1996: 115-1181.

115. Grimes DA. Epidemiology of gestational trophoblastic disease. Am J Obstet Gynecol 1984; 150: 309-18.

116. Zanotti KS, Belinson JL, Kennedy AW. Treatment of gynecologic cancers in pregnancy. Semin Oncol 2000; 27: 686-98.

117. Zhao XY, Huang HF, Lian LJ, Lang JH. Ovarian cancer in pregnancy: a clinicopathologic analysis of 22 cases and review of the literature. Int J Gynecol Cancer 2006; 16: 8-15.

118. Hermans RH, Fischer DC, Van Der Putten HW, et al. Adnexal masses in pregnancy. Onkologie 2003; 26: 167-72.

119. Takeuchi T, Suzuki S, Hayashi Z, Shinagawa T, Araki T. Primary ovarian tumor undergoing surgical management during pregnancy. J Nippon Med Sch 2002; 69: 39-42.

120. Partridge EE, Phillips JL, Menck HR. The National Cancer Data Base report on ovarian cancer treatment in the United States hospitals. Cancer 1996; 78: 2236-46.

121. Zalel Y, Piura B, Elchalal U, et al. Diagnosis and management of malignant germ cell ovarian tumors in young females. Int J Gynaecol Obstet 1996; 55: 1-10.

122. Sayar H, Lhomme C, Verschraegen CF. Malignant adnexal masses in pregnancy. Obstet Gynecol Clin N Am 2005; 32: 569-93.

123. Disaia PJ, Creasman WT. Clinical gynecologic oncology, 6th edn. St. Louis, Missouri: Mosby, 2002.

124. Buller RE, Darrow V, Manetta A, Porto M, Disaia PJ. Conservative surgical management of dysgerminoma concomitant with pregnancy. Obstet Gynecol 1992; 79: 887-90.

125. Oehler M, Wain G, Brand A. Gynaecological malignancies in pregnancy: a review. Aust NZ J Obstet Gynaecol 2003; 43: 414-20.

126. Kumar V, Fausto N, Abbas A. Pathologic basis of disease, 7th edn. St. Louis, Missouri: WB Saunders/Elsevier, 2005.

127. Fujita $M$, Inoue $M$, Tanizawa $O$, et al. Retrospective review of 41 patients with endodermal sinus tumor of the ovary. Int J Gynecol Cancer 1993; 3: 329-35.

128. Tewari K, Cappuccini F, Disaia PJ, et al. Malignant germ cell tumors of the ovary. Obstet Gynecol 2000; 95: 128-33.

129. Elit L, Bocking A, Kenyon C, Natale R. An endodermal sinus tumor diagnosed in pregnancy: case report and review of the literature. Gynecol Oncol 1999; 72: 123-7.

130. Shimizu Y, Komiyama S, Kobayashi T, Nakata K, Iida T. Successful management of endodermal sinus tumor of the ovary associated with pregnancy. Gynecol Oncol 2003; 88: 447-50.

131. Ferrandina G, Distefano M, Testa A, de Vincenzo R, Scambia G. Management of an advanced ovarian cancer at 15 weeks of gestation: case report and review of the literature. Gynecol Oncol 2005; 97: 693-6.

132. Modares Gilani M, Karimi Zarchi M, Behtash N, et al. Preservation of pregnancy in a patient with advanced ovarian cancer at 20 weeks of gestation: case report and review of the literature. Int J Gyncol Cancer 2007; 17: 1131-71

133. Mantovani G, Mais V, Parodo G, Carrucciu GM. Use of chemotherapy for ovarian cancer during human pregnancy: case report and literature review. Eur J Obstet Gynecol Rep Biol 2007; 131: 235-45.

134. Picone O, Lhomme C, Tournaire M, et al. Preservation of pregnancy in a patient with a stage IIIB ovarian epithelial carcinoma diagnosed at 22 weeks of gestation and treated with initial chemotherapy: case report and literature review. Gynecol Oncol 2004; 94: 600-4.

135. Russell P. Surface epithelial-stromal tumors of the ovary. In: Kurman RJ, ed. Blaustein's pathology of the female genital tract, 4th edn. New York: Springer Verlag, 1994: 705.

136. Morice P, Camatte S, Wicart-Poque F, et al. Results of conservative management of epithelial malignant and borderline ovarian tumours. Hum Reprod Update 2003; 9: 185-92.

137. Schumer ST, Cannistra SA. Granulosa cell tumor of the ovary. J Clin Oncol 2003; 21: 1180-9.

138. Williams SD, Birch R, Einhorn LH, et al. Treatment of disseminated germ-cell tumors with cisplatin, bleomycin, and either vinblastine or etoposide. N Engl J Med 1987; 316: 1435-40.

139. Gershenson DM, Morris M, Burke TW, et al. Treatment of poor prognosis sex-cord stromal tumors of the ovary with combination of bleomycin, etoposide, and cisplatin. Obstet Gynecol 1996; 87: 527-31.

140. Savage P, Constenla D, Fisher C, et al. Granulosa cell tumors of the ovary: demographics survival and the management of advanced disease. Clin Oncol (R Coll Radiol) 1998; 10: 242-5.

141. Homesley HD, Bundy BN, Hurteau JA, Roth LM. Bleomycin, etoposide and cisplatin combination therapy of ovarian granulose cell tumors and other stromal malignancies: a Gynecologic Oncology Group study. Gynecol Oncol 1999; 72: 131-7.

142. Hanjani P, Petersen RO, Lipton SE, Nolte SA. Malignant mixed mesodermal tumors and carcinosarcoma of the ovary: report of eight cases and review of the literature. Obstet Gynecol Surv 1983 38: 37-545.

143. DiSilvestro PA, Gajewski WH, Ludwig ME, et al. Malignant mixed mesodermal tumors of the ovary. Obstet Gyencol 1995; 86: 780-2.

144. Kowalski MS. Case of primary sarcoma of the ovary in pregnancy. Pol Tyg Lek (Wars) 1854; 9: 12-14.

145. Schroder H. Sarcoma and carcinoma of ovary in pregnancy. Zentralbl Gynakol 1966; 88: 1047-51.

146. Bonevat N, Sophonn S, San LK, Samban M. Ovarian sarcoma and pregnancy (apropos of a personal case). Bull Fed Soc Gynecol Obstet Lang Fr 1967; 19: 214-15.

147. Brown E, Stewart M, Rye T, et al. Carcinosarcoma of the ovary: 19 years prospective data from a single center. Cancer 2004; 100: 2148-53.

148. Yada-Hashimoto N, Yamamoto T, Kamiura S, et al. Metastatic ovarian tumors: a review of 64 cases. Gynecol Oncol 2003; 89: 314-17.

149. Boulay R, Podczaski E. Ovarian cancer complicating pregnancy. Obstet Gynecol Clin N Am 1998; 25: 385-99.

150. Zemlicks D, Lishner M, Degendorfer P, et al. Fetal outcome after in utero exposure to cancer chemotherapy. Arch Intern Med 1992; 152: 573-6.

151. Randall T. National registry seeks scarce data on pregnancy outcomes during chemotherapy. JAMA 1993; 269: 323.

152. Doll DC, Ringenberg QS, Yarbro JW. Antineoplastic agents and pregnancy. Semin Oncol 1989; 16: 337-46.

153. Otton G, Higgins S, Phillips KA, Quinn M. A case of early-stage epithelial ovarian cancer in pregnancy. Int J Gynecol Cancer 2001; 11: 413-17.

154. Bakri YN, Ezzat A, Akhtar M, Dohami H, Zahrani A. Malignant germ cell tumors of the ovary. Pregnancy considerations. Eur J Obstet Gynecol Reprod Biol 2000; 90: 87-91.

155. Karlen JR, Akbari A, Cook WA. Dysgerminoma associated with pregnancy. Obstet Gynecol 1979; 53: 330-5.

156. Briggs GG, Freeman RK, Yaffe SJ. A Reference Guide to Fetal and Neonatal Risk: Drugs in pregnancy and lactation. Baltimore: Williams and Wilkins, 1994.

157. Committee on Drugs, American Academy of Pediatrics. The transfer of drugs and other chemicals into human milk. Pediatrics 1994; 93: 137.

158. Obner WB. Choriocarcinoma: historical notes. In: Szulman AE Buschsbaum J, eds. Gestational Trophoblastic Disease. New York: Springer Verlag, 1987.

159. Dehner LP. Gestational and nongestational trophoblastic disease. Am J Surg Pathol 1980; 4: 43-58.

160. Vaitukaitis JL, Braunstein GD, Ross GT. A radioimmunoassay which specifically measures human chorionic gonadotropin in the presence of human luteinizing hormone. Am J Obstet Gynecol 1972; 113: 751-8.

161. Voss IP, Riottmanm G, Kazii T. Hydatidiform mole: two entities. Am J Obstet Gynecol 1977; 127: 167-70

162. Lawler SD, Fisher RA, Dent J. A prospective genetic study of complete and partial hydatidiform moles. Am J Obstet Gyencol 1991; 164: 1270-7.

163. Grimes DA. Epidemiology of gestational trophoblastic disease. Am J Obstet Gynecol 1984; 150: 309-18.

164. Kahan EI. Clinical management and the neoplastic sequelae of trophoblastic embolization associated with hydatidiform mole. Obstet Gynecol Surv 1987; 42: 484-8.

165. Berkowitz RJ, Goldstein DP, DuBeshten B, Bernstein MR. Management of complete molar pregnancy. J Reprod Med 1987; 32: 634-9.

166. Bageshawe KD. Risk and prognostic factors in trophoblastic neoplasm. Cancer 1976; 58: 1373-85.

167. Hammond CB, Berchert LG, Tyrey L, Creasman WT, Parker RT. Treatment of metastatic trophoblastic disease: good and poor prognosis. Am J Obstet Gynecol 1973; 115: 451-7. 
168. Mutch DG, Soper JT, Baker ME, et al. Role of computer axial tomography of the chest in staging patients with nonmetastatic gestational trophoblastic disease. Obstet Gynecol 1986; 68: 348-52.

169. Morgan JM, Lurain JR. Gestational trophoblastic neoplasia: an update. Curr Oncol Rep 2008; 10: 497-504.

170. American College of Obstetricians and Gynecologists. Carcinoma of the Breast. Technical bulletin no. 158. ACOG, 1991.

171. Antonelli NM, Dotters DJ, Katz VL, Kuller JA. Cancer in pregnancy: a review of the literature: part I. Obstet Gynecol Surv 1996; 51: 125-34.

172. Gallenberg M, Loprinzi C. Breast cancer and pregnancy. Semin Oncol 1989; 16: 369-76.

173. King R, Welch J, Martin JJ, Coulam C. Carcinoma of the breast associated with pregnancy. Surg Gynecol Obstet 1985; 160: 228-32.

174. Tretli S, Kvalheim G, Thoresen S, Host H. Survival of breast cancer patients diagnosed during pregnancy or lactation. Br J Cancer 1988; 58: 382-4.

175. Ishida T, Yokoe T, Kasumi F, et al. Clinicopathological characteristics and prognosis of breast cancer patients associated with pregnancy and lactation: analysis of case-control study in Japan. Jpn J Cancer Res 1992; 83: 1143-9.

176. Liberman L, Giess C, Dershaw D, Deutch B, Petrek J. Imaging of pregnancy-associated breast cancer. Radiology 1994; 191: 245-8.

177. Bonnier P, Romain S, Dilhuydy J, et al. Influence of pregnancy on the outcome of breast cancer: a case-control study. Int J Cancer 1997; 72: 720-7.

178. Byrd BJ, Bayer D, Robertson J. Treatment of breast cancer associated with pregnancy and lactation. Ann Surg 1962; 155: 940-7.

179. Bunker M, Peters M. Breast cancer associated with pregnancy or lactation. Am J Obstet Gynecol 1963; 85: 312-21.

180. Applewhite R, Smith L, DiVincenti F. Carcinoma of the breast associated with pregnancy and lactation. Am Surg 1973; 39: 101-4.

181. Nettleton J, Long J, Kuban D, et al. Breast cancer during pregnancy: quantifying the risk of treatment delay. Obstet Gynecol 1996; 87: 414-18.

182. Gallenberg MM, Loprinzi CL. Breast cancer and pregnancy. Semin Oncol 1989; 16: 369-76.

183. Samuels T, Liu F-F, Yaffe M, Haider M. Gestational breast cancer. Can Assoc Radiol J 1998; 49: 172-80.

184. Nicklas A, Baker M. Imaging strategies in pregnant cancer patients. Semin Oncol 2000; 27: 623-32.

185. Gwyn K, Theriault R. Breast cancer during pregnancy. Oncology (Huntingt) 2001; 15: 39-46.

186. Hoover HC. Breast cancer during pregnancy and lactation. Surg Clin North Am 1990; 70: 1151-63.

187. Novotny D, Maygarden S, Shermer R, Frable W. Fine needle aspiration of benign and malignant breast masses associated with pregnancy. Acta Cytol 1991; 35: 676-86.

188. Westenend PJ, Sever AR, Beekman-De Volder JH, Leim SJ. A comparison of aspiration cytology and core needle biopsy in the evaluation of breast lesions. Cancer 2001; 93: 146-50.

189. Shannon J, Douglas-Jones AG, Dallimore NS. Conversion of core biopsy in preoperative diagnosis of breast lesions: is it justified by results? J Clin Pathol 2001; 54: 762-5.

190. Poniecka AW, Krasuski P, Gal E, et al. Granulomatous inflammation of the breast in a pregnant woman: report of a case with fine needle aspiration diagnosis. Acta Cytol 2001; 45: 797-801.

191. Brenner RJ, Bassett LW, Fajardo LL, et al. Stereotactic core-needle breast biopsy: a multi-institutional prospective trial. Radiology 2001; 218: 866-72.

192. Glacalone P-L, Laffargue F, Benos P. Chemotherapy for breast carcinoma in pregnancy: a French national survey. Cancer 1999; 86: 2266-72.

193. Ebert U, Loffler H, Kirch W. Cytotoxic therapy and pregnancy. Pharmacol Ther 1997; 74: 207-20.

194. Nicholson HO. Cytotoxic drugs in pregnancy: review of reported cases. J Obstet Gynaecol Br Commonw 1968; 75: 307-12.

195. Nugent P, O’Connell TX. Breast cancer and pregnancy. Arch Surg 1985; 120: 1221-4.

196. Petrek JA, Dukoff R, Rogatko A. Prognosis of pregnancy-associated breast cancer. Cancer 1991; 61: 869-72.

197. Gelber S, Coates A, Goldhirsch A, et al.; Effect of pregnancy on overall survival after the diagnosis of early-stage breast cancer. J Clin Oncol 2001; 19: 1671-5.
198. Sankila R, Heinvaara S, Hakulinen T. Survival of breast cancer patients after subsequent term pregnancy: healthy mother effect. Am J Obstet Gynecol 1994; 170: 818-23.

199. Kroman N, Jensen M-B, Wohlfahrt J, Ejlertsen B. Pregnancy after treatment of breast cancer - a population-based study on behalf of Danish Breast Cancer Cooperative Group. Acta Oncol 2008; 47: 545-9.

200. Ariel I, Kempner R. The prognosis of patients who become pregnant after mastectomy for breast cancer. Int Surg 1989; 74: 185-7.

201. Petrek J. Pregnancy safety after breast cancer. Cancer 1994; 74: 528-31.

202. Malamos N, Stathopoulos G, Keramopoulos A, Papadiamantis J, Vassilaros S. Pregnancy and offspring after the appearance of breast cancer. Oncology 1996; 53: 471-5.

203. Whiteman MK, Hillis SD, Curtiss KM, et al. Reproductive history and mortality after breast cancer diagnosis. Obstet Gynecol 2004; 1: 146-54.

204. Kroman N, Mouridsen HT. Prognostic influence of pregnancy before, around, and after diagnosis of breast cancer. Breast 2003; 12: 516-21.

205. Gonsoulin W, Mason B, Carpenter RS. Colon cancer in pregnancy with elevated maternal serum a-fetoprotein level at presentation. Am J Obstet Gynecol 1990; 163: 1172-3.

206. Nesbitt JC, Moise KJ, Sawyers JL. Colorectal carcinoma in pregnancy. Arch Surg 1985; 12: 636-40.

207. Hill JA, Kassam SH, Talled OE. Colonic cancer in pregnancy. South Med J 1984; 77: 375-8.

208. Woods JB, Martin JN Jr, Ingram FH, et al. Pregnancy complicated by carcinoma of the colon above the rectum. Am J Perinatol 1992; 9: 102-10.

209. Minter A, Malik R, Ledbetter L, et al. Colon cancer in pregnancy. Cancer Control 2005; 12: 196-202.

210. Cappell MS. Colon cancer during pregnancy. Gastroenterol Clin North Am 1998; 27: 225-56.

211. Balthazer EJ, Megibow AJ, Hulnick D, Naidich DP. Carcinoma of the colon: Detection and preoperative staging by CT. AJR Am J Roentgenol 1988; 150: 301-6.

212. Fletcher RH. Carcinoembryonic antigen. Ann Intern Med 1986; 104 : 66-73.

213. Saif MW. Management of colorectal cancer in pregnancy: a multimodality approach. Clin Colorectal Cancer 2005; 5: 247-56.

214. Knoepp LF Jr, Ray JE, Overby I. Ovarian metastases from colorectal carcinoma. Dis Colon Rectum 1973; 16: 305-11.

215. Mason MH, Kovalcik PJ. Ovarian metastases from colon carcinoma. J Surg Oncol 1981; 17: 33-8.

216. Reintgen DS, McCarty KS, Vollmer R, Cox E, Seigler HF. Malignant melanoma and pregnancy. Cancer 1985; 55: 1340-4.

217. Allen HH, Nisker JA. Cancer in Pregnancy: Therapeutic Guidelines. Mt. Kisko, NY: Futura, 1986.

218. Ries LAG, Eisner MP, Kosary CL, et al., eds. SEER cancer statistics review, 1975-2002. National Cancer Institute, Bethesda, MD. [Available from: http://seer.cancer.gov/csr/1975_2002/, based on November 2004 SEER data submission, posted to the SEER website 2005]. Accessed 1 May 2005.

219. Balch CM, Murad TM, Soong SJ, et al. A multifactorial analysis of melanoma: prognostic histopathological features comparing Clark's and Breslow's staging methods. Ann Surg 1978; 188: 732-42.

220. Ho VC, Sober AJ. Therapy for cutaneous melanoma: an update. J Am Acad Dermatol 1990; 22: 159-76.

221. Wong DJ, Strassner HT. Melanoma in pregnancy. Clin Obstet Gynecol 1990; 33: 782-90.

222. Colbourn DS, Nathanson L, Belilos E. Pregnancy and malignant melanoma. Semin Oncol 1989; 16: 377-87.

223. Lambe M, Ekbom A. Cancers coinciding with child bearing: delayed diagnosis during pregnancy? BMJ 1995; 311: 1607-8.

224. Haas JF. Pregnancy in association with a newly diagnosed cancer: a population-based epidemiologic assessment. Int J Cancer 1984; 34: 229-35.

225. Wiggins CL, Berwick M, Newton Bishop JA. Malignant melanoma in pregnancy. Obstet Gynecol Clin N Am 2005; 32: 559-68.

226. Slingliff LL, Reintgen DS, Vollmer RT. Malignant melanoma arising during pregnancy: a study of 100 patients. Ann Surg 1990; 211: 552-9.

227. Balch CM, Soong SJ, Gershenwald JE, et al. A prognostic factors analysis of 17, 600 melanoma patients: validation of the American Joint 
Committee on Cancer melanoma staging system. J Clin Oncol 2001; 19: 3622-34.

228. Breslow A. Thickness, cross-sectional areas and depth of invasion in the prognosis of cutaneous melanoma. Ann Surg 1970; 172: 902-8.

229. Stadelmann WK, Rapaport DP, Soong SJ, et al. Prognostic, clinical, and pathologic features. In: Balch CM, Houghton AN, Sober AJ, et al., eds. Cutaneous melanoma, 3rd edn. St. Louis: Quality Medical Publishing, 1998: 11-35.

230. Altman JF, Lowe L, Redman B, et al. Placental metastases of maternal melanoma. J Am Acad Dermatol 2003; 49: 1150-3.

231. Smith LH, Danielsen B, Allen ME, Cress R. Cancer associated with obstetric delivery: results of linkage with the California cancer registry. Am J Obstet Gynecol 2003; 189: 1128-35.

232. Dildy GA, Moise KJ, Carpenter RJ Jr, Klima T. Maternal malignance metastatic to the products of conception: a review. Obstet Gynecol Surv 1989; 44: 535-40.

233. Pavlidis NA. Coexistence of pregnancy and malignancy. Oncologist 2002; 7: 279-87.

234. McLain CR. Leukemia in pregnancy. Clin Obstet Gynecol 1974; 17: 185-94.

235. Reynoso EE, Shepherd FA, Messner HA, et al. Acute Leukemia during pregnancy: the Toronto Leukemia Study Group experience with longterm follow-up of children exposed in utero to chemotherapeutic agents. J Clin Oncol 1987; 5: 1098-106.

236. Kuzel TM, Benson AB. Non-Hodgkin's lymphoma. In: Gleicher N, ed. Principles and practice of medical therapy in pregnancy, 2nd edn. East Norwalk (CT): Appleton and Lang, 1992: 1078-80.

237. Juarez S, Cuarado Pastor JM, Feliu J, et al. Association of leukemia and pregnancy: clinical and obstetric aspects. J Clin Oncol 1988; 11: 159-65.

238. Hoelzer D, Gokbuget N. Acute lymphocytic leukemia and Klinefelter's syndrome. Pediatr Hematol Oncol 1992; 9: 81-5.

239. Wiernek PH. Acute leukemia in adults. In: DeVita VT, ed. Cancer: Principles and Practice of Oncology, 3rd edn. Philadelphia: JB Lippincott, 1989.

240. Chelghoum Y, Vey N, Raffoux E, et al. Acute leukemia during pregnancy: a report on 37 patients and a review of the literature. Cancer 2005; 104: 110-17.

241. Koren G, Weiner L, Lishner M, Zemelickes D, Finegen J. Cancer in pregnancy: identification of unanswered questions on maternal and fetal risks. Obstet Gyencol Surv 1990; 45: 509-14.

242. Hoelzer D, Gokbuget N. Acute lymphocytic leukemia in adults. In: Hoffman R, Benz EJ, Shattil SJ, eds. Hematology: basic principles and practice, 4th edn. Philadelphia: Elsevier, 2005: 1177

243. Todeschini G, Tecchio C, Meneghini V, et al. Estimated 6-year eventfree survival of $55 \%$ in 60 consecutive adult acute lymphoblastic leukemia patients treated with an intensive phase II protocol based on high induction dose of daunorubicin. Leukemia 1998; 12: 144-9.

244. Hurley TJ, McKinnell JV, Irani MS. Hematologic malignancies in pregnancy. Obstet Gynecol Clin N Am 2005; 32: 595-614.

245. Williams SF, Bitran JD. Cancer and pregnancy. Clin Perinatol 1985; 12: 609-23.

246. Elliot MA, Wolf RC, Hook CC, et al. Thromboembolism in adults with acute lymphoblastic leukemia during induction with L-asparaginasecontaining multi-agent regimens: incidence, risk factors, and possible role of antithrombin. Leuk Lymphoma 2004; 45: 1545-51.

247. Beinant G, Damon L. Thrombosis associated with L-asparaginase therapy and low fibrinogen levels in adult acute lymphoblastic leukemia. Am J Hematol 2004; 77: 331-5.

248. Mauz-Körholz C, Junken R, Göbel U, Nowak-Göttl U. Prothrombotic risk factors in children with acute lymphoblastic leukemia treated with delayed E. coli asparaginase (CCOALL-92 and 97 protocols). Thromb Haemost 2000; 83: 840-3.

249. Jaime-Perez JC, Gomez-Almaguer D. The complex nature of the prothrombotic state in acute lymphoblastic leukemia of childhood. Haematology 2003; 88: ELT 25.

250. Caligiuri MA, Mayer RJ. Pregnancy and leukemia. Semin Oncol 1989; 16: 338-96.

251. Greenlund LJS, Letendne L, Tefferi A. Acute leukemia during pregnancy: a single institutional experience with 17 cases. Leuk Lymphoma 2001; 41: $571-7$.
252. Jacob JH, Stringer CA. Diagnosis and management of cancer during pregnancy. Semin Perinatol 1990; 14: 79-87.

253. Sadural E, Smith LG. Hematologic malignancies during pregnancy. Clin Obstet Gynecol 1995; 38: 525-46.

254. Mesquita M, Pestana A, Mota A. Successful pregnancy occurring with interferon-alpha therapy in chronic myeloid leukemia. Acta Obstet Gynecol 2005; 84: 300-1.

255. Mubarak AA, Kakil ER, Awidi A, et al. Normal outcome of pregnancy in chronic myeloid leukemia treated with interferon-alpha in 1st trimester: report of 3 cases and review of the literature. Am J Hematol 2002; 69: 115-18.

256. DeVita VT, Hellman S, Rosenberg SA. Hodgkin's disease and the nonHodgkin's lymphomas. In: DeVita VT, ed. Cancer: Principles and Practice of Oncology, 3rd edn. Philadelphia: JB Lippincott, 1989.

257. Weinshel EL, Peterson BA. Hodgkin's disease. CA Cancer J Clin 1993; 43: 327-45.

258. Walker JW, Reinisch JF, Monforte HL. Maternal pulmonary adenocarcinoma metastatic to the fetus: first recorded case report and literature review. Pediatr Pathol Mol Med 2002; 21: 57-69.

259. Yasmeen S, Cress R, Romano PS, et al. Thyroid cancer in pregnancy. Int J Gynecol Obstet 2005; 91: 15-20.

260. SEER cancer statistics review. National Cancer Institute Surveillance, Epidemiology, and End Results; 1975-2000. [Available from: http://seer. cancer.gov/csr/1975_2000/results/merged/sect_16_thyroid cancer.pdf Accessed January 2005.

261. Morris PC. Thyroid cancer complicating pregnancy. Obstet Gynecol Clin 1998; 25: 401-6.

262. Preston-Martin S, Bernstein L, Pike MC, Maldonado AA, Henderson BE. Thyroid cancer among young women related to prior thyroid disease and pregnancy history. Cancer 1987; 55: 191-5.

263. Rosen IB, Walfish PG. Pregnancy as a predisposing factor in thyroid neoplasia. Arch Surg 1986; 121: 1287-90.

264. Mazzaferi E. Evaluation and management of common thyroid disorders in women. Am J Obstet Gynecol 1997; 176: 507.

265. Hamer CL, McCready VR. Thyroid cancer: differentiated carcinoma. Cancer Treat Rev 1996; 22: 161-77.

266. Doherty C, Shindo M, Rice D, Montero M, Mestman JH. Management of thyroid nodules during pregnancy. Laryngoscope 1995; 105: 251-5.

267. Casara D, Rubello D, Saladini G, et al. Pregnancy after high therapeutic doses of iodine-131 in differentiated thyroid cancer: potential risks and recommendations. Eur J Nucl Med 1993; 20: 192-4.

268. Yutaka K, Kenji I, Tadayuki A. Sarcoma associated with pregnancy. Am J Obstet Gynecol 1989; 161: 94-6.

269. Simon MA, Phillips WA, Bonfiglio M. Pregnancy and aggressive or malignant bone tumors. Cancer 1984; 53: 2564-9.

270. Huvos AG, Butler A, Bretsky SS. Osteogenic sarcoma in pregnant women. Cancer 1985; 56: 2326-31.

271. Haerr RW, Pratt AT. Multiagent chemotherapy for sarcoma diagnosed during pregnancy. Cancer 1985; 56: 1028-33.

272. Roelvink NC, Kamphorst W, van Alphen H, Rao BR. Pregnancy related primary brain and spinal tumors. Arch Neurol 1987; 44: 209-15.

273. Haas JF, Janisch W, Staneczed W. Newly diagnosed primary intracranial neoplasms in pregnant women: a population based assessment. J Neurol Neurosurg Psychiatry 1986; 49: 874-80.

274. Johnson N, Sermer M, Lausman A, Maxwell C. Obstetric outcomes of women with intracranial neoplasms. Int J Gyencol Obstet 2009, doi: 10.1016/j.jigo.2008.11.037 105: 56-9.

275. Wan WL, Geller JL, Feldon SE, Sadun AA. Visual loss caused by rapidly progressive intracranial meningiomas during pregnancy. Opthalmology 1990; 97: 18-21.

276. Magyar DM, Marshall JR. Pituitary tumors and pregnancy. Am J Obstet Gyencol 1978; 132: 739-49.

277. Apuzzio J, Pelioso MA, Ganesh VV, Caterini H, Iffy L. Spinal cord tumors during pregnancy. Int J Gynecol Obstet 1980; 17: 608-10.

278. Simon RH. Brain tumors in pregnancy. Semin Neurol 1988; 8: 214-21.

279. Cardonick E, Iacobucci A. Use of chemotherapy during human pregnancy. Lancet Oncol 2004; 5: 283-91.

280. Roboz J, Gleicher N, Wuk K, et al. Does doxorubicin cross the placenta? Lancet 1979; 2: 1382-3. 


\section{Chemotherapy in pregnancy Mark I. Hunter}

\section{INTRODUCTION}

Fortunately, both the diagnosis of cancer during pregnancy and the diagnosis of pregnancy during the active treatment of a previously diagnosed cancer are rare occurrences, with cancer affecting approximately 1 in every 1000 pregnancies (1). However, with such a large population of females within the reproductive age range, the number of patients and providers having to face such difficult treatment decisions is not insignificant. Fortunately, there are a growing number of options besides pregnancy termination, based on an increasing understanding of the effects of various chemotherapeutic agents on the growing fetus, at different stages of development.

The cancers occurring most commonly in pregnancy include cancers of the breast, uterine cervix, ovary, colon, and the lymphomas (2). Alternatively, the cancers most likely to occur in a woman of reproductive age include thyroid cancer and cervical cancer. As more women are waiting until into their 30 s and 40 s to have children, age-dependent cancers occurring in pregnancy may be of increasing importance. Regardless of the type of cancer diagnosed, patients are often left with difficult choices. They frequently have the incorrect assumption that they cannot simultaneously make the decision to save their own life, while preserving the life and health of their unborn child. Naturally, a team of support staff and counselors should be available to work through these issues as patients are educated and decisions are made.

\section{COMMON CANCERS IN PREGNANCY \\ Breast Cancer}

It is estimated that breast cancer affects between 19 and 33 per 100,000 pregnancies (3-5). The pathologic distribution of these tumors appears to coincide with other young nonpregnant patients and is predominantly ductal invasive carcinoma (6). They tend to be less likely to be estrogen or progesterone receptor positive, and there are mixed data on whether the pregnant state alters the likelihood of Her-2/neu positivity $(7,8)$. The prognosis for women with breast cancer diagnosed in pregnancy is similar to that of other nonpregnant young women $(9,10)$.

Treatment for patients with breast cancer may include surgery, chemotherapy, and radiation. The use of radiation treatment is best avoided whenever possible at any time during pregnancy. Doses lower than 1 Gy have been associated with increases in the rate of mental retardation and are most pronounced when the fetus is exposed in the first trimester (11). Surgery, however, is thought to be safe and can be performed at any time during the pregnancy. As with all cancers, it is generally preferred to wait until after 12 weeks of gestation to perform surgery, avoiding the coincident association with a spontaneous abortion.
The use of cytotoxic chemotherapy in pregnancy has been reported for a number of different tumor types. While most of these studies have a limited number of patients, the data would suggest that many of these agents are quite safe in pregnancy, particularly in the second and third trimesters. In breast cancer, the use of a combination of 5-fluorouracil (5-FU), doxorubicin, and cyclophosphamide was found to be without complication to the fetus or infant when given in the second or third trimester (12). Taxanes have been used to treat patients with breast cancer. However, published studies at this time are limited to a small number of case reports $(13,14)$. Other drugs used to treat breast cancer include vinorelbine, capecitabine, gemcitabine, trastuzumab, and tamoxifen, many of which are discussed subsequently.

\section{Cervical Cancer}

Owing to the adoption of a revolutionary screening program, most cervical cancers are caught early. However, there are many women who either fail to participate in cervical cancer screening or are failed by the inherent false-negative rate of modern screening techniques. Indeed, as many as $40 \%$ of women diagnosed with cervical cancer are of childbearing age, with the incidence of cervical cancer in pregnancy estimated to be between 1.5 and 12 per $100,000(5,15,16)$.

Surgical options for the treatment of women with cervical cancer include cold knife conization and extrafascial hysterectomy for microinvasive disease, and radical hysterectomy for more advanced stages. Although conization has been performed safely in pregnancy, it has also been associated with severe bleeding and preterm labor, and is generally discouraged (17). It may be appropriate to delay treatment until after the completion of pregnancy for patients with microinvasive disease. It is recommended that these patients then undergo cesarean section, with or without hysterectomy. Hysterectomy can be performed at the time of diagnosis with the fetus in utero if pregnancy termination is desired.

In cases where cervical cancer is diagnosed early in pregnancy and termination is not an acceptable option, investigators have described the use of neoadjuvant chemotherapy. Significant responses have been reported in some cases, with reductions in tumor volume, enabling both delays in delivery and effective postpartum treatments $(18,19)$. Agents used include combinations of cisplatin with vincristine or bleomycin $(19,20)$. Delays in definitive treatment of up to 32 weeks have been reported, with patients who have gone on to achieve surgical resection without further recurrence (21).

\section{Ovarian Cancer}

As the use of ultrasound has become exceedingly common in the evaluation of early pregnancies and is, in fact, the standard of care in the midtrimester, an increasing number of ovarian 
masses are being incidentally discovered. Although the majority of these masses are benign, a reported $2 \%$ to $3 \%$ are found to be malignant (22). The estimated incidence of ovarian cancer is 7 to 8 per 100,000 pregnancies $(22,23)$. If reasonable, it is best to delay the surgical evaluation of an adnexal mass until after the rate of spontaneous abortion has dropped and the pregnancy has become independent of the corpus luteum, with an optimal target window of 16 to 18 weeks of gestation. If the mass is found to be cancer on frozen section, then surgical staging and optimal cytoreduction can be performed without significant risks to the fetus.

Adjuvant chemotherapy for the treatment of ovarian cancer currently includes the use of a platinum and a taxane, such as carboplatin and paclitaxel. The use of a platinum with paclitaxel has been reported in pregnancy with normal growth and development in infants and children at follow-up $(24,25)$.

\section{Malignant Melanoma}

Unlike most other cancers developing in pregnancy, transplacental spread of malignant melanoma from mother to fetus has been described (26). Treatment of early-stage lesions is focused on surgical excision and can be performed safely in pregnancy as described previously. It is now quite common to perform sentinel lymph node mapping on patients at risk of having regional metastasis. Overall, it is thought that the use of radioactive tracers in pregnancy exposes the fetus to a very low dose of radiation $(<0.5 \mathrm{cGy})$ and can be used safely (27). Alternatively, for lesions excised later in the pregnancy, lymph node mapping and excision can be delayed until the postpartum period.

For patients with distant metastatic spread of melanoma, the use of chemotherapy has not met with impressive results. Agents administered during pregnancy include dacarbazine, temozolomide, cisplatin, vinblastine, and interferon alpha2b $(28,29)$.

\section{Lymphoma}

Lymphoma, including both Hodgkin's lymphoma (HL) and non-Hodgkin's lymphoma (NHL), has been estimated to affect 8 to 16 per 100,000 pregnancies $(5,30)$. Patients with NHL tend to have more aggressive histologic subtypes and advanced-stage disease. Staging for the pregnant patient with lymphoma may include history and physical examination, blood tests, bone marrow biopsies, and chest X-ray with abdominal shielding. Although CT scans are well below the levels that might be considered teratogenic, such scans may be replaced with ultrasound or MRI where appropriate.

The use of radiation in the treatment of lymphoma is fairly routine. Although for reasons discussed previously, it may be omitted in the treatment of a pregnant patient. Furthermore, pregnancy termination should be considered at least for the patient diagnosed with aggressive NHL in the first trimester, when immediate treatment is critical.

$\mathrm{HL}$ is commonly treated with regimens such as ABVD (adriamycin, bleomycin, vinblastine, dacarbazine). Other regimens include BEACOPP (bleomycin, etoposide, doxorubicin, cyclophosphamide, vincristine, procarbazine, and prednisone), the Stanford V protocol (mechlorethamine, doxorubicin, vinblastine, vincristine, bleomycin, etoposide, and prednisone), and MOPP (mechlorethamine, vincristine, procarbazine and prednisone). Rituximab with combined chemotherapy has also been described (31).

\section{Thyroid Cancer}

While thyroid cancer is not the most commonly diagnosed malignancy occurring in pregnancy, it is more likely to be diagnosed during pregnancy because of its predilection for females of reproductive age. Imaging studies commonly used in the evaluation of a possible thyroid cancer include CT scans to evaluate both the extension of the tumor into adjacent tissues and the presence of cervical lymphadenopathy, and ultrasound, to delineate lesion size, and its cystic and solid characteristics. Fine-needle aspiration is of varying accuracy depending on histologic type, although it can generally be performed safely in pregnancy.

Surgery remains the most important treatment modality for patients with differentiated thyroid cancer, and total thyroidectomy is preferred to hemithyroidectomy (32). However, surgical timing must be weighed against risks to the fetus and the inherent and often coincidental rate of spontaneous miscarriage in the early trimester, as previously discussed. Unique to thyroidectomy, however, is the careful consideration of neurologic development in a fetus exposed to improper levels of thyroid hormone following complete removal of the thyroid. After about 18 weeks of gestation, fetal thyroid production begins, and the effects of a maternal thyroidectomy become less important. However, it should be noted that several retrospective studies have failed to demonstrate a significant difference in disease progression or outcomes for patients in whom thyroidectomy was delayed until after delivery, when compared with those performed during the second trimester (32). Radioactive iodine is a commonly used adjuvant therapy for patients with thyroid cancer. It is frequently given 3 to 6 months after thyroidectomy and is not recommended during pregnancy.

\section{SYSTEMIC CHEMOTHERAPY \\ Introduction}

The overall incidence of congenital malformations after the use of cytotoxic drugs during pregnancy has been reported to be approximately $3 \%$, which does not differ significantly from the baseline risk of malformations in the general population of $2 \%$ to $3 \%$ (33). Naturally, the risk of exposure to the fetus from these agents differs depending on the stage of organogenesis, with the risk of malformations following the first-trimester exposure as high as $10 \%$ to $20 \%$, in contrast to $1 \%$ to $3 \%$ for the second and third trimesters (34-36).

The pharmacokinetics of standard chemotherapies administered during pregnancy would be expected to depart from well-investigated standards due to changes in renal clearance, large increases in circulating blood volumes, lower serum albumin, and third spacing of many chemotherapeutic compounds into the amniotic fluid. Overall, drug clearances are higher, although there have been few studies that have investigated the differences in chemotherapy pharmacokinetics in pregnancy and it is thus unclear what proper dose adjustments are necessary in this population (37). Most chemotherapeutic compounds are lipid soluble and can easily cross the placenta. As a result, it is expected that the fetus or amniotic fluid will have a similar or even a higher 
concentration of the drug, or its active metabolite, than that in the maternal circulation.

The risk of exposure to chemotherapy can be stratified based on the age of the gestation, with the greatest risk of physical defects coinciding with the exposure during the period of organogenesis, from 2 to 8 weeks. For practical purposes, most report on the timing of exposure as being based on the first, second, or third trimester. As noted previously, there is some suggestion that second-trimester exposure has been linked to low birth weight. Chemotherapy given in the third trimester can predictably cause side effects in the newborn, which are similar to that expected in the adult, such as neutropenia, anemia, and thrombocytopenia.

\section{Taxanes}

Taxanes, including agents such as paclitaxel, docetaxel, ortataxel, and tesetaxel, are compounds originally derived from the bark of yew trees. They affect their antineoplastic properties by stabilizing tubulin, which blocks the function of microtubules essential for cell division. These agents are considered first-line therapy for the treatment of many cancers including those of the lung, ovary, and breast.

In rabbit and rat studies, fetal exposure to both docetaxel and paclitaxel have been associated with an increase in intrauterine deaths, increased fetal resorption, reduced fetal weight, and delayed ossification. Human studies have been limited to a scant number of case reports. In one report, docetaxel, cisplatin, and gemcitabine were given to a patient in the first and second trimesters of an unrecognized pregnancy (38). A healthy infant was delivered by cesarean section at 33 weeks, without congenital malformations. Sood et al. reported on the use of paclitaxel and cisplatin in a patient diagnosed with stage IIIC ovarian cancer at 27 weeks. After surgical debulking, she was given three cycles of chemotherapy prior to delivery. The child was 30 months of age, at the time of the publication, without any known abnormalities or developmental delays (24). Morris et al. reported on a patient with recurrent metastatic breast cancer diagnosed at 29 weeks of gestation. She received a total of six cycles of weekly paclitaxel and delivered a normal infant by elective cesarean section at 38 weeks (39). Additionally, a normal infant was delivered after three 21-day cycles of docetaxel given to a patient diagnosed with recurrent breast cancer at 15 weeks of gestation (13). Other reports have further confirmed that taxanes can be used safely in the second and third trimesters of pregnancy without significant risk of fetal malformations $(14,40,41)$.

\section{Alkylating Agents}

Alkylating agents include the subclass of nitrogen mustards, of which the compounds in most common use today include cyclophosphamide, ifosfamide, and melphalan, and chlorambucil. To exhibit their antineoplastic properties, many of these agents, such as cyclophosphamide, must be converted to an active metabolite in the liver. The compounds then attach to the alkyl groups in DNA, causing cross-linking between double-helix strands and preventing the uncoiling of DNA that is necessary for replication. Alkylating agents are used in several different tumor types including, but not limited to, breast cancer, NHL, chronic lymphocytic leukemia, ovarian cancer, bone and soft-tissue sarcomas, and rhabdomyosarcomas.

Cyclophosphamide has been demonstrated to have significant absorption through the skin and mucous membranes, and great care should be taken by fertile women when administering this drug (42). Exposure to cyclophosphamide in the first trimester has been associated, in a small number of reports, with growth deficiency, developmental delay, craniosynostosis, blepharophimosis, flat nasal bridge, abnormal ears, and distal limb defects including hypoplastic thumbs and oligodactyly (43). Zemlickis et al. reported on a patient with acute lymphocytic leukemia who received cyclophosphamide daily in the first trimester of a twin gestation (44). Interestingly, the male twin was diagnosed with papillary thyroid cancer at 11 years of age and neuroblastoma at 14 years of age. The female twin was unaffected. While no fetal deformities have been noted from exposure in the second and third trimesters, growth restriction, microcephaly, and neonatal pancytopenia have been described (45).

There have been several detailing the use of ifosfamide in the second and third trimesters, most commonly in multiagent combinations (46-48). No fetal anomalies have been noted and the infants appeared to be developing normally. One report, however, described the development of fetal growth restriction and anhydramnios after the first cycle of combination chemotherapy including ifosfamide, which was initiated in the second trimester for a maternal diagnosis of rhabdomyosarcoma of the face (49). The effects of ifosfamide given to humans during the first trimester are unknown. However, animal studies have demonstrated increased resorption and lethal anomalies when the drug has been given at high doses early in the pregnancy.

In animal studies, there has been some suggestion that there may be inheritable chromosomal aberrations caused by exposure of germ cells to chlorambucil and melphalan (50). In humans, cardiac and renal defects, including renal agenesis, have been reported with fetal exposure to chlorambucil in the first trimester $(51,52)$. Still, others have described the use of chlorambucil throughout pregnancy with delivery of normal infants (51).

\section{Platinum Agents}

In a manner similar to the alkylating agents, platinum compounds, including among others cisplatin, carboplatin, and oxaliplatin, cross-link DNA and prevent uncoiling, however, without the use of an alkyl group. They have been described as being alkylating-like. They have been approved for the treatment of many cancers including those of the ovary, uterus, bladder, head and neck, esophagus, lung, lymphoma, leukemia, and choriocarcinoma. Representing one of the only reports of a platinum compound being administered in the first trimester, $\mathrm{Li}$ et al. described a single case of microphthalmos following the use of the Dartmouth regimen (dacarbazine, carmustine, cisplatin, and tamoxifen) to treat a patient with metastatic melanoma (53).

Tabata et al. reported on the use of carboplatin as a single agent for four cycles, with delivery of a normal infant at 33 weeks, who demonstrated normal development at 1 year (54). Another child exhibited normal development at 18 months following two cycles of single-agent carboplatin in the 
treatment of stage III ovarian cancer diagnosed at 22 weeks of gestation (55). Carboplatin has also been combined with paclitaxel, resulting in normal development at 6 months of a child following three cycles of the combination prior to delivery (56). Mir et al. reviewed more than three dozen reports on the use of cisplatin or carboplatin, alone or in combination, to treat malignancies in the second and third trimesters (57). They report no fetal malformations in any patient treated with either compound when started after the first trimester, with the exception of one case of ventriculomegaly, diagnosed in a fetus in close proximity to chemotherapy administration.

\section{Antimetabolites}

Antimetabolites include methotrexate and 5-FU. Methotrexate, a folic acid antagonist, is indicated for use in the treatment of many cancers, including breast cancer, head and neck cancer, acute lymphocytic leukemia, NHL, bladder cancer, colorectal cancer, and gestational trophoblastic disease. It is an abortifacient and should not be given at any time during pregnancy. When given in the first trimester, this class of agents is associated with a constellation of severe fetal anomalies known as "aminopterin syndrome." The syndrome includes growth deficiency, patent cranial sutures, craniosynostosis, unusual skull shapes, dysmorphic facies, hydrocephalus, mental retardation, and skeletal defects (35).

There are few reports on the use of methotrexate in the second and third trimesters. One patient presented at 25 weeks of gestation with metastatic breast cancer and was treated with two cycles of doxorubicin, methotrexate, and vincristine prior to delivery of a female without anomalies, who was developing normally at 2 years (58). Nabers et al. reported on the use of methotrexate initiated at 27 weeks in a patient with choriocarcinoma metastatic to the lung, with a coincident normal pregnancy (59). Additionally, Ring et al. reported on 12 patients treated with cyclophosphamide, methotrexate, and fluorouracil in the second and third trimesters, with no adverse fetal outcomes (60).

Much like methotrexate, 5-FU, a pyrimidine analog, has been associated with multiple congenital abnormalities when it has been used during the first trimester, including radial dysplasia, absent digits, and hypoplasias of the lungs, aorta, esophagus, duodenum, and ureters (61). However, Berry et al. reported on 24 patients treated for breast cancer in pregnancy. They noted no fetal abnormalities or neonatal complications with the use of a combination of fluorouracil, doxorubicin, and cyclofosfamide (12). Fluorouracil is also used in topical form to treat conditions of the vulva and vagina. Several patients have been inadvertently treated with this preparation in the first trimester, without any report of deformities (62).

\section{Antibiotics}

The most common antineoplastic antibiotics include dactinomycin-D and bleomycin. The former exerts antitumor activity by inhibiting DNA synthesis and function, and promotes the accumulation of intracellular free radicals that further damage DNA. It is approved for the treatment of Wilms' tumor, rhabdomyosarcoma, germ cell tumors, gestational trophoblastic disease, and Ewing's sarcoma. Exposure of the fetus to this compound has been limited to a few case reports in the second and third trimesters, resulting in intact infants with normal development (63-65).

Bleomycin is a peptide that binds to iron and creates oxygen free radicals, damaging DNA. It is indicated for cancers of the head and neck, squamous cell carcinomas of the skin, vulva, and cervix, germ cell tumors, HL, and NHL. As with dactinomycin-D, no case reports of malformations from the first-trimester exposure have been reported for this drug. Second- and third-trimester exposures have been associated with the delivery of infants without deformities, and normal development has been reported $(66,67)$. Nantel et al. reported on the use of 12 weekly cycles of methotrexate, doxorubicin, cyclophosphamide, vincristine, prednisone, and bleomycin in the treatment of NHL in a patient. The patient went into preterm labor and delivered twins without congenital abnormalities (68). Another patient with an endodermal sinus tumor diagnosed at 18 weeks was treated with three cycles of cisplatin, vinblastine, and bleomycin and delivered a healthy infant without abnormalities at 31 weeks (69).

\section{Nucleoside Analogs}

Nucleoside analogs, including cytarabine and gemcitabine, exhibit their antineoplastic properties by causing incorporation of either the drug or its metabolite into DNA and terminating replication. Cytarabine is indicated in the treatment of multiple types of leukemias, as well as in leptomeningeal carcinomatosis. The compound has been associated with otic and auditory canal anomalies, digital anomalies, lobster claw hand, and lower limb defects $(70,71)$. Unlike most other chemotherapies, cytarabine has been associated with in utero fetal death, when given in the later trimesters, and should be avoided in pregnancy altogether, when alternative agents are available (72).

Gemcitabine is indicated in the treatment of cancers of the ovary, pancreas, bladder, lung, and in sarcomas. There are little data regarding the intrapartum use of gemcitabine in the literature. Studies in mice have demonstrated teratogenicity including decreased fetal weight, cleft palette, and increased mortality (73). Kim et al. reported on a woman with non-small cell lung cancer, treated with palliative docetaxel, gemcitabine, and cisplatin from weeks 9 to 22 of an unrecognized pregnancy. A healthy infant was delivered at 33 weeks with no recognizable anomalies (38). Hematologic toxicity is commonly dose limiting and the compound should be used with caution toward the end of the third trimester. In the absence of more case reports, or perhaps a series of patients, other compounds might be considered prior to choosing gemcitabine during pregnancy.

\section{Topoisomerase Inhibitors}

Topoisomerase I and II are involved in the cleaving of DNA strands for the purpose of replication. Topoisomerase inhibitors bind to the DNA-topoisomerase complex and prevent the advancement of the replication fork. Topotecan and irinotecan are examples of compounds specific to topoisomerase I, while etoposide binds specifically to the topoisomerase II-DNA complex.

Both topotecan and irinotecan are derivatives from the Camptotheca acuminata tree. As with the nonpregnant population, the most pronounced maternal toxicity associated 
with topotecan is myelosuppression. To date, there have been no human studies evaluating the teratogenicity of this compound, and unlike most other antineoplastic agents, there are no reports of any pregnant patients receiving this drug. However, animal studies have been concerning. At levels equivalent to human doses, topotecan has been associated with reduced fetal body weight, fetal demise, fetal resorption, and brain, skull, and vertebrae malformations. Most commonly, malformations of the eyes were noted, including microphthalmia, anophthalmia, retinal defects, and ectopic orbits. Granted, most fetal effects are seen by early pregnancy exposure, in the absence of any human data, it is not recommended that topotecan be used at any time during pregnancy.

Irinotecan has also demonstrated animal embryotoxicity at doses equivalent to recommended human levels. Teratogenic effects include visceral and skeletal abnormalities, as well as some external defects. Furthermore, rats administered irinotecan after organogenesis and through weaning had decreased learning ability and decreased fetal weights in the female offspring (74). Unlike topotecan, there has been a single report of the use of irinotecan in pregnancy. A 34-year-old patient was diagnosed with recurrent colon cancer, metastatic to a single ovary at 15 weeks of gestation. She underwent surgical resection and, starting at 18 weeks, was treated with 10 courses of 5-FU and irinotecan over the next 5 months. She delivered a healthy infant at 37 weeks, with no appreciated teratogenic or developmental effects at 4 months (75).

Unlike the topoisomerase I inhibitors, there are dozens of published reports detailing the use of etoposide in pregnancy. Many of these patients have been diagnosed with endodermal sinus tumors, although a variety of other tumors have also been treated with this agent in pregnancy (76). Animal studies have demonstrated multiple fetal abnormalities when the drug is administered during organogenesis, including fetal loss, exencephaly, encephalocele, hydrocephalus, gastroschisis, microphthalmia or anophthalmia, dextrocardia, and axial skeleton defects (77). Etoposide is most frequently given with other agents such as bleomycin and cisplatin (BEP), and the reports occurring in the pregnant patients are no exception. One fetus developed ventriculomegaly and the child now has cerebral atrophy following a single course of BEP (78). Another patient had two cycles of BEP starting at 18 weeks and delivered a normal infant during laparotomy for metastatic disease at 31 weeks. Yet another patient, diagnosed at 26 weeks of gestation with Burkitt's lymphoma, was treated with cyclophosphamide, vincristine, doxorubicin, cytarabine, etoposide, ifosfamide, mesna, and intrathecal cytarabine and delivered a healthy baby at 32 weeks (46). Another patient was treated with BEP at 26 weeks for a metastatic tumor of unknown primary. She developed septicemia and delivered prematurely. The infant has moderate sensorineural hearing loss (79). Several other reports describe the delivery of normal, healthy infants following etoposide administration.

Doxorubicin is indicated in the treatment of many cancers, including, but not limited to, breast cancer, HL, and NHL, soft-tissue sarcoma, ovarian cancer, lung cancer, bladder cancer, thyroid cancer, and gastric cancer. The drug is an anthracycline antibiotic isolated from Streptomyces species. It intercalates into DNA, which inhibits DNA synthesis. Doxorubicin also inhibits transcription, by inhibiting DNAdependent RNA polymerase, and the function of topoisomerase II. Myelosuppression is dose limiting, but both acute and chronic cardiotoxicity have been well described. When given in the first trimester, in combination with other agents, doxorubicin has been associated with imperforate anus, rectovaginal fistula, and microcephaly (80). In the largest series to date, Hahn et al. reported on the use of doxorubicin in combination with 5-FU, and cyclophosphamide in 57 pregnant women (81). They reported three congenital anomalies, including Down syndrome, clubfoot, and bilateral ureteral reflux. Several others have reported the use of doxorubicin alone or in combination with other therapies, starting as early as the second trimester, without obvious effects on the fetus. There have been no reports of fetal or infant cardiotoxicity associated with doxorubicin use in pregnancy $(67,82,83)$.

Epirubicin is sometimes used instead of doxorubicin as it can have faster elimination and fewer side effects. It has been used in breast cancer, ovarian cancer, lymphomas, gastric cancer, and lung cancer. There have been a few larger series describing the use of epirubicin in pregnancy. Azim et al. described the second-trimester administration of either doxorubicin or epirubicin to a total of 3 and 23 patients, respectively (84). They reported two preterm deliveries, no fetal anomalies, and normal development in all children.

\section{Vinca Alkaloids}

Vincristine is a vinca alkaloid antimicrotubule agent derived from the periwinkle Catharanthus roseus plant. It is indicated for the treatment of choriocarcinoma, brain tumors, thyroid cancer, leukemia, lymphoma, sarcomas, and several other malignancies. As noted above, vincristine has been used in combination with bleomycin and other agents, delivering infants without abnormalities. Tewari et al. reported on the neoadjuvant treatment of cervical cancer with cisplatin and vincristine in two pregnant women, both of whom experienced significant reductions in tumor volume (19). No adverse fetal effects were noted. Bader et al. also reported on the use of cisplatin and vincristine in a patient for the neoadjuvant treatment of a cervical cancer beginning in the 23rd week of pregnancy (85). At 33 weeks, the patient then underwent a cesarean radical hysterectomy, delivering a normal infant. There have been some sporadic reports of atrial septal defects, renal hypoplasia, and pancytopenia following the use of vincristine in pregnancy $(45,86,87)$. Several other authors have reported the successful use of vincristine, most frequently in combination with other agents, leading to the delivery and normal development of healthy infants $(88,89)$.

Vinblastine is also a vinca alkaloid derived from the periwinkle plant. It is indicated for the treatment of HL and NHL, breast cancer, Kaposi's sarcoma, and renal cell carcinoma. It exerts its antitumor effect by inhibiting tubulin polymerization and disrupting microtubules during the M-phase of the cell cycle. As with most agents described here, animal studies in early pregnancy have demonstrated increased resorption, spontaneous abortion, and gross fetal abnormalities. However, some human studies have reported 
no teratogenic effects from first-trimester exposure (90). At 18 weeks of gestation, a patient was diagnosed with an endodermal sinus tumor of the ovary, and following laparotomy, she received three courses of cisplatin, vinblastine, and bleomycin (69). She delivered a healthy and normal infant at 31 weeks. To date, there have been more than half a dozen other patients who have received vinblastine in combination regimens, followed by the delivery of normal, healthy infants $(91,92)$.

Vinorelbine is a synthetic derivative of vinblastine. It is indicated in the treatment of non-small cell lung cancer, breast cancer, ovarian cancer, and HL. When administered to a patient with lung cancer from the 27th to 39th week of pregnancy, a healthy and normal infant resulted (93). Several other patients have reportedly received vinorelbine during pregnancy, with therapy being initiated between 15 and 29 weeks of gestation. In this setting, the drug has most commonly been administered as part of a combination of chemotherapies, being paired with 5-FU, cisplatin, or trastuzumab. All infants have been delivered without congenital abnormalities, had normal birth weights, and have been developing normally (94-96).

\section{Biologic and Growth Factor Agents}

Trastuzumab is an antibody indicated for the treatment of patients with HER2 overproducing breast cancers. The antibody exerts its effect on cancer cells both by activating the body's immune response against the cells and by shutting down the signal that tells the cancer cells to grow and divide uncontrollably. Trastuzumab was given to six pregnant patients for the treatment of breast cancer. Anhydramnios developed in three out of the six patients treated (97). Another patient with recurrent metastatic breast cancer was treated with trastuzumab for the first 23 weeks of an undiagnosed pregnancy (98). The patient was delivered by cesarean section at 27 weeks due to severe vaginal bleeding. This pregnancy was also noted to have oligohydramnios. The infant was noted to have uncommonly strong capillary leak syndrome, persisting infections, and necrotizing enterocolitis. The baby died due to multiple organ failure 21 weeks after delivery. Other patients have received trastuzumab in the second and third trimesters without complication to the fetus (96).

Bevacizumab inhibits tumor angiogenesis by binding directly to vascular endothelial growth factor (VEGF). It is currently indicated for the treatment of glioblastoma, renal cell carcinoma, breast cancer, lung cancer, and colon cancer. Rabbit studies at human doses have demonstrated teratogenicity including gross and skeletal malformations, as well as low fetal body weights and increased resorption. Reports on the use of this compound in human pregnancies are very limited. Petrou et al. described two early pregnancy losses at 6 weeks of gestation 7 and 10 days following intravitreal injection of bevacizumab (99). Rosen et al., however, reported the use of the therapy in the second trimester without significant fetal effects (100).

Rituximab is a monoclonal antibody against CD20, which exerts its effect by selectively depleting CD20+ B cells. It is indicated in the use of rheumatoid arthritis and in lymphomas. Kimby et al. reported on the unintentional use of rituximab in a patient with recurrent NHL in the first trimester of pregnancy (101). She delivered a normal, healthy infant without evidence of hematologic or immunologic abnormalities. Other reports of first-trimester administration have also demonstrated no teratogenic or immunologic effects on the fetus or neonate (102). Pellkofer et al. reported on the use of rituximab for the treatment of neuromyelitis optica 1 week prior to conception (103). The patient delivered a healthy infant with normal development at 15 months. Another patient with idiopathic thrombocytopenic purpura was treated with rituximab weekly for 4 weeks in the third trimester (104). A healthy infant was born 1 month after the last administration of the drug. However, the child had no detectable B lymphocytes. Six months following delivery, her B-lymphocyte counts were normal, and at 10 months, she had normal vaccination titers. Other authors have confirmed this finding of significant rituximab concentrations in the neonate, transient B-lymphocyte depletion, quick recovery, and an absence of immunologic or other neonatal consequences (105-107).

Imatinib is a specific inhibitor of a number of tyrosine kinase enzymes and is indicated for the treatment of chronic myelogenous leukemia. It has been associated with a high rate of teratogenicity (108). However, Skoumalova et al. reported on its administration throughout the first trimester of pregnancy, followed by the use of interferon for the remainder of the gestation, with the delivery of a normal and healthy child (108). Neither the drug nor its active metabolite appears to cross the placenta in significant concentrations (109). Nonetheless, Pye et al. reported on the evaluation of 125 pregnancies complicated by the use of imatinib (110). They reported that 3 of the 35 patients who terminated their pregnancies did so because of detected fetal malformations. Of the remaining infants delivered, 12 had malformations, and 3 of them had similar complex malformations including renal and vertebral anomalies.

Sunitinib is an oral inhibitor of multiple receptor tyrosine kinases including VEGF receptors. It is indicated for the treatment of refractory metastatic renal cell carcinoma and gastrointestinal stromal tumors. Rats and rabbits were given the drug during the periods of organogenesis and demonstrated malformations including thoracic and lumbar vertebral alterations, cleft lip and palate, and increased resorption (111). To date, there have been no reports of this drug administered during a human pregnancy.

Several interferon compounds have now been developed and marketed for the treatment of many kinds of diseases and malignancies. A thorough discussion of the fetal and neonatal effects of each of these drugs is beyond the scope of this chapter. However, there have been several reviews and case reports suggesting that interferon alpha appears to be associated with the delivery of normal and healthy infants (112-114). Mubarak et al. reported on the use of interferon alpha for the treatment of chronic myeloid leukemia beginning in the first trimester (115). Although one child was born with transient thrombocytopenia, all three infants were without abnormalities and developing appropriately. It should be noted that there have been reports of maternal postpartum cardiomyopathy developing in women who have been treated with interferon prior to pregnancy (116). 


\section{Birth Weights, Development, and Long Term Follow Up}

Some authors have suggested that birth weights for infants born to mothers with breast cancer were significantly lower than those of healthy controls (117). However, in one of the largest series to date, Aviles et al. reported on the long-term follow-up of 84 children and grandchildren of patients treated with chemotherapy for hematologic malignancies during pregnancy (118). The median follow-up was 18 years and ranged from 6 to 29 years. They reported normal birth weights, normal learning and educational performance, no congenital abnormalities, and no cancer or leukemia, with a median follow-up of 18 years and a range of 6 to 29 years.

\section{Infant Hemotoxicities}

Some authors suggest that patients should not be treated after 35 weeks of gestation, as many infants may be born with neutropenia, thrombocytopenia, and profound anemia under these circumstances (117). Indeed, infants have been born with severe anemia requiring prolonged ICU admissions, following chemotherapy administered during pregnancy (119). Progenitor studies in these infants have revealed severe bone marrow suppression. Infants should be monitored following delivery for such effects for a prolonged period of time.

\section{SUMMARY}

With such a large and growing population of women of reproductive range, the absolute number of women who will be diagnosed with a pregnancy during cancer treatment or diagnosed with a cancer during the course of her pregnancy is far from negligible. The contrast that can occur when the happiest time in a woman's young life is shattered by the finding of often metastatic disease is universally devastating for the patient, her family, and all of those who are involved with her care. Significant moral and ethical dilemmas arise, and questions regarding teratogenicity, pregnancy termination, and disease prognosis have to be approached and considered by a team of physicians, nurses, social workers, counselors, and loved ones. Fortunately, it is clear that there are a number of such malignancies that are curable, and it is further understood that most chemotherapies can be well tolerated in the second and third trimesters without effects on the fetus or on the future development of the child.

With a growing number of chemotherapeutic compounds available, and at least some differences among their effects in pregnancy, multiagent and single-agent combinations should be custom-tailored to provide patients with the best possible treatment of their neoplasm, while exposing the fetus to the least teratogenic, growth restricting, and hemotoxic regimens. Fortunately, it is evident that at least some patients will emerge from this difficult situation disease free and raise healthy and developmentally normal children.

\section{REFERENCES}

1. Potter JF, Schoeneman M. Metastasis of maternal cancer to the placenta and fetus. Cancer 1970; 25: 380-8.

2. Donegan WL. Cancer and pregnancy. CA Cancer J Clin 1983; 33: 194-214.

3. White TT. Prognosis of breast cancer for pregnant and nursing women; analysis of 1,413 cases. Surg Gynecol Obstet 1955; 100: 661-6.
4. Anderson JM. Mammary cancers and pregnancy. Br Med J 1979; 1: 1124-7.

5. Smith LH, Danielsen B, Allen ME, Cress R. Cancer associated with obstetric delivery: results of linkage with the California cancer registry. Am J Obstet Gynecol 2003; 189: 1128-35.

6. Parente JT, Amsel M, Lerner R, Chinea F. Breast cancer associated with pregnancy. Obstet Gynecol 1988; 71(6 Pt 1): 861-4.

7. Middleton LP, Amin M, Gwyn K, Theriault R, Sahin A. Breast carcinoma in pregnant women: assessment of clinicopathologic and immunohistochemical features. Cancer 2003; 98: 1055-60.

8. Elledge RM, Ciocca DR, Langone G, McGuire WL. Estrogen receptor, progesterone receptor, and HER-2/neu protein in breast cancers from pregnant patients. Cancer 1993; 71: 2499-506.

9. Petrek JA. Breast cancer during pregnancy. Cancer 1994; 74(Suppl 1): 518-27.

10. Petrek JA, Dukoff R, Rogatko A. Prognosis of pregnancy-associated breast cancer. Cancer 1991; 67: 869-72.

11. International Commission on Radiological Protection. Pregnancy and medical radiation. Ann ICRP 2000; 30: iii-viii, 1-43.

12. Berry DL, Theriault RL, Holmes FA, et al. Management of breast cancer during pregnancy using a standardized protocol. J Clin Oncol 1999; 17: 855-61.

13. De Santis M, Lucchese A, De Carolis S, Ferrazani S, Caruso A. Metastatic breast cancer in pregnancy: first case of chemotherapy with docetaxel. Eur J Cancer Care (Engl) 2000; 9: 235-7.

14. Gadducci A, Cosio S, Fanucchi A, et al. Chemotherapy with epirubicin and paclitaxel for breast cancer during pregnancy: case report and review of the literature. Anticancer Res 2003; 23: 5225-9.

15. Smith LH, Dalrymple JL, Leiserowitz GS, Danielsen B, Gilbert WM. Obstetrical deliveries associated with maternal malignancy in California, 1992 through 1997. Am J Obstet Gynecol 2001; 184: 1504-12; discussion 1512-3.

16. Demeter A, Sziller I, Csapo Z, Szantho A, Papp Z. Outcome of pregnancies after cold-knife conization of the uterine cervix during pregnancy. Eur J Gynaecol Oncol 2002; 23: 207-10.

17. Klein M, Rosen A, Vavra N, Gitsch G, Beck A. Conization in pregnancy and its significance for the further course of pregnancy. Geburtshilfe Frauenheilkd 1991; 51: 990-2.

18. Giacalone PL, Laffargue F, Benos P, Rousseau O, Hedon B. Cisplatinum neoadjuvant chemotherapy in a pregnant woman with invasive carcinoma of the uterine cervix. Br J Obstet Gynaecol 1996; 103: 932-4.

19. Tewari K, Cappuccini F, Gambino A, et al. Neoadjuvant chemotherapy in the treatment of locally advanced cervical carcinoma in pregnancy: a report of two cases and review of issues specific to the management of cervical carcinoma in pregnancy including planned delay of therapy. Cancer 1998; 82: 1529-34.

20. Marana HR, de Andrade JM, da Silva Mathes AC, et al. Chemotherapy in the treatment of locally advanced cervical cancer and pregnancy. Gynecol Oncol 2001; 80: 272-4.

21. Hunter MI, Tewari K, Monk BJ. Cervical neoplasia in pregnancy. Part 2: current treatment of invasive disease. Am J Obstet Gynecol 2008; 199: 10-18.

22. Zhao XY, Huang HF, Lian LJ, Lang JH. Ovarian cancer in pregnancy: a clinicopathologic analysis of 22 cases and review of the literature. Int J Gynecol Cancer 2006; 16: 8-15.

23. Sayedur Rahman M, Al-Sibai MH, Rahman J, et al. Ovarian carcinoma associated with pregnancy. A review of 9 cases. Acta Obstet Gynecol Scand 2002; 81: 260-4.

24. Sood AK, Shahin MS, Sorosky JI. Paclitaxel and platinum chemotherapy for ovarian carcinoma during pregnancy. Gynecol Oncol 2001; 83: 599-600.

25. Mendez LE, Mueller A, Salom E, Gonzalez-Quintero VH. Paclitaxel and carboplatin chemotherapy administered during pregnancy for advanced epithelial ovarian cancer. Obstet Gynecol 2003; 102(5 Pt 2): 1200-2.

26. Trumble ER, Smith RM, Pearl G, Wall J. Transplacental transmission of metastatic melanoma to the posterior fossa. Case report. J Neurosurg 2005; 103(Suppl 2): 191-3.

27. Adelstein SJ. Administered radionuclides in pregnancy. Teratology 1999; 59: 236-9.

28. Harkin KP, Drumm JE, O’Brien P, Daly A. Metastatic malignant melanoma in pregnancy. Ir Med J 1990; 83: 116-17. 
29. Beyeler M, Hafner J, Beinder E, et al. Special considerations for stage IV melanoma during pregnancy. Arch Dermatol 2005; 141: 1077-9.

30. Pereg D, Koren G, Lishner M. The treatment of Hodgkin's and nonHodgkin's lymphoma in pregnancy. Haematologica 2007; 92: 1230-7.

31. Rey J, Coso D, Roger V, et al. Rituximab combined with chemotherapy for lymphoma during pregnancy. Leuk Res 2009; 33: e8-9.

32. Fanarjian N, Athavale SM, Herrero N, Fiorica J, Padhya TA. Thyroid cancer in pregnancy. Laryngoscope 2007; 117: 1777-81.

33. Kalter H, Warkany J. Medical progress. Congenital malformations: etiologic factors and their role in prevention (first of two parts). N Engl J Med 1983; 308: 424-31.

34. Doll DC, Ringenberg QS, Yarbro JW. Antineoplastic agents and pregnancy. Semin Oncol 1989; 16: 337-46.

35. Ebert U, Loffler H, Kirch W. Cytotoxic therapy and pregnancy. Pharmacol Ther 1997; 74: 207-20.

36. Woo JC, Yu T, Hurd TC. Breast cancer in pregnancy: a literature review. Arch Surg 2003; 138: 91-8; discussion 99.

37. Cardonick E, Iacobucci A. Use of chemotherapy during human pregnancy. Lancet Oncol 2004; 5: 283-91.

38. Kim JH, Kim HS, Sung CW, et al. Docetaxel, gemcitabine, and cisplatin administered for non-small cell lung cancer during the first and second trimester of an unrecognized pregnancy. Lung Cancer 2008; 59: 270-3.

39. Morris PG, King F, Kennedy J. Cytotoxic chemotherapy for pregnancyassociated breast cancer: single institution case series. J Oncol Pharm Pract 2009; 15: 241-7.

40. Potluri V, Lewis D, Burton GV. Chemotherapy with taxanes in breast cancer during pregnancy: case report and review of the literature. Clin Breast Cancer 2006; 7: 167-70.

41. Gonzalez-Angulo AM, Walters RS, Carpenter RJJ, et al. Paclitaxel chemotherapy in a pregnant patient with bilateral breast cancer. Clin Breast Cancer 2004; 5: 317-19.

42. Hirst M, Tse S, Mills DG, Levin L, White DF. Occupational exposure to cyclophosphamide. Lancet 1984; 1: 186-8.

43. Enns GM, Roeder E, Chan RT, et al. Apparent cyclophosphamide (cytoxan) embryopathy: a distinct phenotype? Am J Med Genet 1999; 86: $237-41$

44. Zemlickis D, Lishner M, Erlich R, Koren G. Teratogenicity and carcinogenicity in a twin exposed in utero to cyclophosphamide. Teratog Carcinog Mutagen 1993; 13: 139-43.

45. Pizzuto J, Aviles A, Noriega L, et al. Treatment of acute leukemia during pregnancy: presentation of nine cases. Cancer Treat Rep 1980; 64: $679-83$.

46. Lam MS. Treatment of Burkitt's lymphoma during pregnancy. Ann Pharmacother 2006; 40: 2048-52.

47. Nakajima W, Ishida A, Takahashi M, et al. Good outcome for infant of mother treated with chemotherapy for Ewing sarcoma at 25 to 30 weeks' gestation. J Pediatr Hematol Oncol 2004; 26: 308-11.

48. Merimsky O, Le Chevalier T, Missenard G, et al. Management of cancer in pregnancy: a case of Ewing's sarcoma of the pelvis in the third trimester. Ann Oncol 1999; 10: 345-50.

49. Fernandez H, Diallo A, Baume D, Papiernik E. Anhydramnios and cessation of fetal growth in a pregnant mother with polychemotherapy during the second trimester. Prenat Diagn 1989; 9: 681-2.

50. Generoso WM, Witt KL, Cain KT, et al. Dominant lethal and heritable translocation tests with chlorambucil and melphalan in male mice Mutat Res 1995; 345: 167-80.

51. Jacobs C, Donaldson SS, Rosenberg SA, Kaplan HS. Management of the pregnant patient with Hodgkin's disease. Ann Intern Med 1981; 95: $669-75$.

52. Steege JF, Caldwell DS. Renal agenesis after first trimester exposure to chlorambucil. South Med J 1980; 73: 1414-15.

53. Li RH, Tam WH, Ng PC, et al. Microphthalmos associated with Dartmouth combination chemotherapy in pregnancy: a case report. J Reprod Med 2007; 52: 575-6.

54. Tabata T, Nishiura K, Tanida K, et al. Carboplatin chemotherapy in a pregnant patient with undifferentiated ovarian carcinoma: case report and review of the literature. Int J Gynecol Cancer 2008; 18: 181-4.

55. Picone $\mathrm{O}$, Lhomme $\mathrm{C}$, Tournaire $\mathrm{M}$, et al. Preservation of pregnancy in a patient with a stage IIIB ovarian epithelial carcinoma diagnosed at 22 weeks of gestation and treated with initial chemotherapy: case report and literature review. Gynecol Oncol 2004; 94: 600-4.
56. Modares Gilani M, Karimi Zarchi M, Behtash N, et al. Preservation of pregnancy in a patient with advanced ovarian cancer at 20 weeks of gestation: case report and literature review. Int J Gynecol Cancer 2007; 17: $1140-3$.

57. Mir O, Berveiller P, Ropert S, Goffinet F, Goldwasser F. Use of platinum derivatives during pregnancy. Cancer 2008; 113: 3069-74.

58. Willemse PH, Van Der Sijde R, Sleijfer DT. Combination chemotherapy and radiation for stage IV breast cancer during pregnancy. Gynecol Oncol 1990; 36: 281-4.

59. Nabers J, Splinter TA, Wallenburg HC, et al. Choriocarcinoma with lung metastases during pregnancy with successful delivery and outcome after chemotherapy. Thorax 1990; 45: 416-18.

60. Ring AE, Smith IE, Jones A, et al. Chemotherapy for breast cancer during pregnancy: an 18-year experience from five London teaching hospitals. J Clin Oncol 2005; 23: 4192-7.

61. Stephens JD, Golbus MS, Miller TR, Wilber RR, Epstein CJ. Multiple congenital anomalies in a fetus exposed to 5-fluorouracil during the first trimester. Am J Obstet Gynecol 1980; 137: 747-9.

62. Van Le L, Pizzuti DJ, Greenberg M, Reid R. Accidental use of low-dose 5-fluorouracil in pregnancy. J Reprod Med 1991; 36: 872-4.

63. Gililland J, Weinstein L. The effects of cancer chemotherapeutic agents on the developing fetus. Obstet Gynecol Surv 1983; 38: 6-13.

64. Haerr RW, Pratt AT. Multiagent chemotherapy for sarcoma diagnosed during pregnancy. Cancer 1985; 56: 1028-33.

65. Weed JC, Roh RA, Mendenhall HW. Recurrent endodermal sinus tumor during pregnancy. Obstet Gynecol 1979; 54: 653-6.

66. van der Zee AG, de Bruijn HW, Bouma J, et al. Endodermal sinus tumor of the ovary during pregnancy: a case report. Am J Obstet Gynecol 1991; 164: 504-6.

67. Schaffer M, Hegenbart U, Klostermann K, Stepan H. Hodgkin's disease in pregnancy-case report and literature review. Z Geburtshilfe Neonatol 2003; 207: 71-3.

68. Nantel S, Parboosingh J, Poon MC. Treatment of an aggressive nonHodgkin's lymphoma during pregnancy with MACOP-B chemotherapy. Med Pediatr Oncol 1990; 18: 143-5.

69. Motegi M, Takakura S, Takano H, Tanaka T, Ochiai K. Adjuvant chemotherapy in a pregnant woman with endodermal sinus tumor of the ovary. Obstet Gynecol 2007; 109(2 Pt2): 537-40.

70. Wagner VM, Hill JS, Weaver D, Baehner RL. Congenital abnormalities in baby born to cytarabine treated mother. Lancet 1980; 2: 98-9.

71. Schafer AI. Teratogenic effects of antileukemic chemotherapy. Arch Intern Med 1981; 141: 514-15.

72. Volkenandt M, Buchner T, Hiddemann W, Van de Loo J. Acute leukaemia during pregnancy. Lancet 1987; 2: 1521-2.

73. Eudaly JA, Tizzano JP, Higdon GL, Todd GC. Developmental toxicity of gemcitabine, an antimetabolite oncolytic, administered during gestation to CD-1 mice. Teratology 1993; 48: 365-81.

74. Camptosar (irinotecan) [package insert]. Pharmacia and a.U. Company, Editors. 2006: Kalamazoo (MI).

75. Taylor J, Amanze A, Di Federico E, Verschraegen C. Irinotecan use during pregnancy. Obstet Gynecol 2009; 114(2 Pt 2): 451-2.

76. Malhotra N, Sood M. Endodermal sinus tumor in pregnancy. Gynecol Oncol 2000; 78: 265-6.

77. Sieber SM, Whang-Peng J, Botkin C, Knutsen T. Teratogenic and cytogenetic effects of some plant-derived antitumor agents (vincristine, colchicine, maytansine, VP-16-213 and VM-26) in mice. Teratology 1978; 18: 31-47.

78. Elit L, Bocking A, Kenyon C, Natale R. An endodermal sinus tumor diagnosed in pregnancy: case report and review of the literature. Gynecol Oncol 1999; 72: 123-7.

79. Raffles A, Williams J, Costeloe K, Clark P. Transplacental effects of maternal cancer chemotherapy. Case report. Br J Obstet Gynaecol 1989; 96: 1099-100.

80. Murray CL, Reichert JA, Anderson J, Twiggs LB. Multimodal cancer therapy for breast cancer in the first trimester of pregnancy. A case report. JAMA 1984; 252: 2607-8.

81. Hahn KM, Johnson PH, Gordon N, et al. Treatment of pregnant breast cancer patients and outcomes of children exposed to chemotherapy in utero. Cancer 2006; 107: 1219-26.

82. Meyer-Wittkopf M, Barth H, Emons G, Schmidt S. Fetal cardiac effects of doxorubicin therapy for carcinoma of the breast during pregnancy: 
case report and review of the literature. Ultrasound Obstet Gynecol 2001; 18: 62-6.

83. Toki H, Okabe K, Kamei $\mathrm{H}$, et al. Successful chemotherapy on a pregnant non-Hodgkin's lymphoma patient. Acta Med Okayama 1990; 44: 321-3.

84. Azim HAJ, Peccatori FA, Scarfone G, et al. Anthracyclines for gestational breast cancer: course and outcome of pregnancy. Ann Oncol 2008; 19: 1511-12.

85. Bader AA, Petru E, Winter R. Long-term follow-up after neoadjuvant chemotherapy for high-risk cervical cancer during pregnancy. Gynecol Oncol 2007; 105: 269-72.

86. Thomas PR, Biochem D, Peckham MJ. The investigation and management of Hodgkin's disease in the pregnant patient. Cancer 1976; 38: 1443-51.

87. Mennuti MT, Shepard TH, Mellman WJ. Fetal renal malformation following treatment of Hodgkin's disease during pregnancy. Obstet Gynecol 1975; 46: 194-6.

88. Reimer P, Rudiger T, Muller J, et al. Subcutaneous panniculitis-like Tcell lymphoma during pregnancy with successful autologous stem cell transplantation. Ann Hematol 2003; 82: 305-9.

89. Rodriguez JM, Haggag M. VACOP-B chemotherapy for high grade nonHodgkin's lymphoma in pregnancy. Clin Oncol (R Coll Radiol) 1995; 7: 319-20.

90. Garrett MJ. Letter: teratogenic effects of combination chemotherapy. Ann Intern Med 1974; 80: 667.

91. Christman JE, Teng NN, Lebovic GS, Sikic BI. Delivery of a normal infant following cisplatin, vinblastine, and bleomycin (PVB) chemotherapy for malignant teratoma of the ovary during pregnancy. Gynecol Oncol 1990; 37: 292-5.

92. Malone JM, Gershenson DM, Creasy RK, et al. Endodermal sinus tumor of the ovary associated with pregnancy. Obstet Gynecol 1986; 68(Suppl 3): 86S-89S.

93. Garrido M, Clavero J, Huete A, et al. Prolonged survival of a woman with lung cancer diagnosed and treated with chemotherapy during pregnancy. Review of cases reported. Lung Cancer 2008; 60: 285-90.

94. Cuvier C, Espie M, Extra JM, Marty M. Vinorelbine in pregnancy. Eur J Cancer 1997; 33: 168-9.

95. Janne PA, Rodriguez-Thompson D, Metcalf DR, et al. Chemotherapy for a patient with advanced non-small-cell lung cancer during pregnancy: a case report and a review of chemotherapy treatment during pregnancy. Oncology 2001; 61: 175-83.

96. Fanale MA, Uyei AR, Theriault RL, Adam K, Thompson RA. Treatment of metastatic breast cancer with trastuzumab and vinorelbine during pregnancy. Clin Breast Cancer 2005; 6: 354-6.

97. Mir O, Berveiller P, Ropert S, et al. Emerging therapeutic options for breast cancer chemotherapy during pregnancy. Ann Oncol 2008; 19: $607-13$.

98. Witzel ID, Muller V, Harps E, Janicke F, Dewit M. Trastuzumab in pregnancy associated with poor fetal outcome. Ann Oncol 2008; 19: $191-2$.

99. Petrou P, Georgalas I, Giavaras G, et al. Early loss of pregnancy after intravitreal bevacizumab injection. Acta Ophthalmol 2010; 88: e136.

100. Rosen E, Rubowitz A, Ferencz JR. Exposure to verteporfin and bevacizumab therapy for choroidal neovascularization secondary to punctate inner choroidopathy during pregnancy. Eye (Lond) 2009; 23: 1479.

101. Kimby E, Sverrisdottir A, Elinder G. Safety of rituximab therapy during the first trimester of pregnancy: a case history. Eur J Haematol 2004; 72: $292-5$.
102. Ojeda-Uribe M, Gilliot C, Jung G, Drenou B, Brunot A. Administration of rituximab during the first trimester of pregnancy without consequences for the newborn. J Perinatol 2006; 26: 252-5.

103. Pellkofer HL, Suessmair C, Schulze A, Hohlfeld R, Kuempfel T. Course of neuromyelitis optica during inadvertent pregnancy in a patient treated with rituximab. Mult Scler 2009; 15: 1006-8.

104. Klink DT, van Elburg RM, Schreurs MW, van Well GT. Rituximab administration in third trimester of pregnancy suppresses neonatal Bcell development. Clin Dev Immunol 2008; 2008: 271-363.

105. Friedrichs B, Tiemann M, Salwender H, et al. The effects of rituximab treatment during pregnancy on a neonate. Haematologica 2006; 91: $1426-7$.

106. Decker M, Rothermundt C, Hollander G, Tichelli A, Rochlitz C. Rituximab plus CHOP for treatment of diffuse large B-cell lymphoma during second trimester of pregnancy. Lancet Oncol 2006; 7: 693-4.

107. Scully M, Starke R, Lee R, et al. Successful management of pregnancy in women with a history of thrombotic thrombocytopaenic purpura. Blood Coagul Fibrinolysis 2006; 17: 459-63.

108. Skoumalova I, Vondrakova J, Rohon P, et al. Successful childbirth in a patient with chronic myelogenous leukemia treated with imatinib mesylate during early pregnancy. Biomed Pap Med Fac Univ Palacky Olomouc Czech Repub 2008; 152: 121-3.

109. Russell MA, Carpenter MW, Akhtar MS, Lagattuta TF, Egorin MJ. Imatinib mesylate and metabolite concentrations in maternal blood umbilical cord blood, placenta and breast milk. J Perinatol 2007; 27: 241-3.

110. Pye SM, Cortes J, Ault P, et al. The effects of imatinib on pregnancy outcome. Blood 2008; 111: 5505-8.

111. Patyna S, Haznedar J, Morris D. Evaluation of the safety and pharmacokinetics of the multi-targeted receptor tyrosine kinase inhibitor sunitinib during embryo-fetal development in rats and rabbits. Birth Defects Res B Dev Reprod Toxicol 2009; 86: 204-13.

112. Sakata H, Karamitsos J, Kundaria B, DiSaia PJ. Case report of interferon alfa therapy for multiple myeloma during pregnancy. Am J Obstet Gynecol 1995; 172(1 Pt 1): 217-9.

113. Mesquita MM, Pestana A, Mota A. Successful pregnancy occurring with interferon-alpha therapy in chronic myeloid leukemia. Acta Obstet Gynecol Scand 2005; 84: 300-1.

114. Egberts F, Lischner S, Russo P, Kampen WU, Hauschild A. Diagnostic and therapeutic procedures for management of melanoma during pregnancy: risks for the fetus? J Dtsch Dermatol Ges 2006; 4: 717-20.

115. Mubarak AA, Kakil IR, Awidi A, et al. Normal outcome of pregnancy in chronic myeloid leukemia treated with interferon-alpha in 1st trimester: report of 3 cases and review of the literature. Am J Hematol 2002; 69: $115-8$.

116. Razaq W, Hyde P. Reversible peripartum cardiomyopathy in a patient with prior exposure to interferon. Am J Ther 2006; 13: 373-5.

117. Zemlickis D, Lishner M, Degendorfer P, et al. Maternal and fetal outcome after breast cancer in pregnancy. Am J Obstet Gynecol 1992; 166: $781-7$

118. Aviles A, Neri N. Hematological malignancies and pregnancy: a final report of 84 children who received chemotherapy in utero. Clin Lymphoma 2001; 2: 173-7.

119. Murray NA, Acolet D, Deane M, Price J, Roberts IA. Fetal marrow suppression after maternal chemotherapy for leukaemia. Arch Dis Child Fetal Neonatal Ed 1994; 71: F209-10. 


\section{Maternal obesity}

\section{Yvette LaCoursiere and Thomas R. Moore}

\section{EPIDEMIOLOGY}

Obesity is recognized as a major increasing health threat in the United States. In the year 2000, 117 billion dollars in healthcare costs and 300,000 deaths were attributed to obesity $(1,2)$. In 2003 to $2004,62 \%$ of women were overweight or obese, $33 \%$ were obese, and $7 \%$ were severely obese (3). Women suffer a disproportionate burden of disease compared with men as a result of the increases in morbidity (4).

Regrettably, the obesity epidemic has spilled over into the obstetric population. In fact, recent statistics have shown a marked increase in the prevalence of pregravid overweight and/or obesity over the past few decades $(5,6)$. In a study of 66,000 women in nine states, $22 \%$ of women were obese prepregnancy, a $70 \%$ increase in the study period (6). As a testament to the pervasiveness of maternal obesity, no subgroup had less than $43 \%$ increase in obesity over the interval. The greatest increases in obesity were seen in those who were $>20$ years of age, black, parous (three or more children), with a high school education, nonsmokers, and enrolled in WIC.

The rising prevalence would not be problematic if it were not for the sequelae for mother and baby. Obesity has been associated with infertility, spontaneous abortion (SAB), intrauterine fetal demise (IUFD), inaccurate fetal imaging, gestational diabetes mellitus (GDM), pre-eclampsia, cesarean delivery, anesthetic complications, deep vein thrombosis/ pulmonary embolus (DVT/PE), wound complications, mood disorders, breast-feeding difficulties, contraceptive failures, and longer-term issues such as excess gestational weight gain (GWG), later-life obesity, diabetes, and heart disease (5,7-16). Likewise, the fetus incurs risks of macrosomia, associated complications, and pediatric obesity $(11,17)$ (Table 1$)$.

Increasing maternal obesity has potential public health implications as well. As an example, a study in Utah revealed a $40 \%$ increase in overweight and obesity between 1991 and 2001 (5). While this study described a smaller overall increase in body mass index (BMI) compared with Kim's data, it described the impact to the community as a whole. An estimate of the attributable fraction of cesarean delivery in the state revealed that one in seven were attributable to obesity after controlling for other factors. Additionally, after assessing trends among potential confounders including maternal age, ethnicity, chronic hypertension, and renal disease, the study described a parallel rise in pre-eclampsia among nulliparous women from $5 \%$ to $8 \%$ in the same interval in which obesity increased (5).

\section{ETIOLOGY}

The etiology of maternal obesity is complex and multifactorial. While not classically discussed in perinatal textbooks, the ecologic context in which a patient lives is important to include in a chapter on maternal obesity, given that some management is dependent on lifestyle interventions. Access to healthy foods and physical activity (PA) opportunities have a potential effect on maternal and fetal well-being. Living more than 4 miles from a supermarket doubles the likelihood of having the lowest quartile of food quality (18). This type of research has recently been linked to adverse birth outcomes. Lane and colleagues documented that mothers living near markets without fresh produce and dairy were at significant risk of delivering low-birth weight infants (19). Also living in areas with social spaces lowers the risk of low or excess GWG (20). Inquiring about the environment may guide counseling for obese women.

\section{PATHOPHYSIOLOGY}

Complex alterations in glucose and lipid metabolism, inflammation, adipokines, and vascular dysfunction occur in obese women. Significant endocrinopathies are associated with obesity, and examples from nonpregnant populations are relevant as they describe the milieu into which a fetus is exposed. Obese individuals are more likely to develop insulin resistance and hyperinsulinemia (21). Adult twins who were discordant with respect to weight were studied and the obese twin of the dyad had significantly higher insulin levels and less insulin sensitivity to maintain normal glucose levels (21). At a cellular level, obesity causes post-receptor binding defects, impaired second messengers, and enzymatic defects, which diminish the ability of the cells to import glucose into the cell. This is exacerbated by the production of human placental lactogen during pregnancy. Additionally, obesity causes abnormal lipogenesis and protein synthesis and increases lipolysis, which then increases free fatty acids (FFAs). At the muscle, this results in FFA oxidation and decreased glucose use leading to hyperglycemia, and at the liver, the increased FFA oxidation increases gluconeogenesis also resulting in hyperglycemia. Thus, at least three mechanisms result in hyperglycemia associated with obesity. These pathophysiologic changes in the overweight and obese translate into increased relative risks of developing diabetes mellitus both during pregnancy and outside of pregnancy (22). In a meta-analysis of over 20 studies, increasing BMI increased the odds of having GDM. The unadjusted ORs of developing GDM were 2.14 (95\% CI 1.82-2.53), 3.56 (3.05-4.21), and 8.56 (5.07-16.04) among overweight, obese, and severely obese compared with normal-weight pregnant women, respectively (23). Likewise, obese women with GDM were twice as likely to develop type 2 after pregnancy.

Hypertensive consequences also increase with overweight and obesity. Nurse's Health Study data show a 1.7-fold increase in the risk of hypertension in overweight women compared with normal-weight women (24). A fivefold 
increase is seen in obese women and abdominal obesity doubles the risk. Likewise, both weight gain and loss modify these risks. Thus, obese women entering pregnancy are at risk for chronic hypertension. The literature supports the association between obesity and pre-eclampsia, even lacking chronic hypertension. Sibai et al. reported a threefold increase in preeclampsia to $12 \%$ of women with a BMI of $\geq 34 \mathrm{~kg} / \mathrm{m}^{2}$ compared with lean women (25).

Unlike hyperinsulinemia and hyperglycemia, the mechanism remains to be elucidated. The vascular volume is increased in obesity and in pregnancy, but peripheral resistance is only slightly elevated in obesity and decreased in pregnancy. Other theories include increased renal sodium and water, activation of the sympathetic system, alteration in the function of sodium-hydrogen ATPases and changes to the vascular wall, and subclinical inflammation $(22,26)$.

Overall, the data regarding preterm birth (PTB) and obesity are conflicting. In a large European cohort, the rate of PTB was increased (aOR 1.6) (10), others have reported a decrease (7), and yet some report that postdate pregnancy is increased by obesity (27). Nohr and colleagues suggest that actually indicated PTB is increased as a result of maternal medical conditions, not spontaneous PTB (28).

Obesity increases multiple aspects of parturition including induction, duration of labor, oxytocin requirement, and cesarean delivery $(29,30)$. Cesarean delivery is performed in up to half of obese women and increases as BMI rises. As an example, cesarean delivery rates are shown in Figure 1 for women with and without the risk factors of diabetes and hypertension (31). Obesity has been shown to similarly decrease the success of women undergoing a trial of labor after cesarean. Juhasz and colleagues demonstrated that overweight and obese women were 50\% less likely to have a successful vaginal birth after cesarean (VBAC), and a weight gain of over $40 \mathrm{lb}$ decreased a woman's chance of VBAC $40 \%$ when compared with underweight women (32). Similar failure rates with trial of labor were described by Durnwald et al. even after controlling for other additional factors (normal BMI 70.5\%, obese $54.6 \%, p=0.003)(33)$.

\section{Psychosocial Factors and Obesity in Pregnancy (Table 2)}

When undiagnosed depression has potential to take a severe and prolonged course for the mother resulting in behavioral

Table 1 Adverse Maternal Outcomes Associated with Obesity

\section{Infertility}

SAB/IUFD

Inaccurate imaging

Gestational diabetes

Pre-eclampsia

Cesarean delivery

Anesthetic complications

DVT/PE

Wound complications

Mood disorders

Breast-feeding difficulties

Contraceptive failures

Abbreviations: SAB, spontaneous abortion; IUFD, intrauterine fetal demise; DVT/PE, deep vein thrombosis/pulmonary embolus. issues in their children, this makes identifying and treating those at greatest risk essential $(34,35)$. Obesity is one of the factors that has recently been found to be a risk marker for depressed mood during pregnancy and postpartum.

The link between BMI, eating attitudes, and depression and anxiety during pregnancy and at 4 and 14 months postpartum was first described by Carter et al. (8). A recent analysis showed that depression, one of six negative psychologic states assessed, was associated with pregravid BMI. While the relationship was seen in all overweight and obese women, a BMI of $>40$ was strongly associated with depressive and anxiety symptoms, locus of control, perceived stress, powerful others, restrained eating and dieting, and weight cycling (9).

In a 2010 study of over 1000 predominantly Caucasian women, recruited immediately postpartum, prepregnancy BMI was associated with depressive symptoms (12). The percentage of women screening positive for postpartum depression was $14.4 \%$ in normal-weight women with an increase in all other BMI strata: underweight, $18.0 \%$; overweight, $18.5 \%$; obese class $1,18.8 \%$; obese class $2,32.4 \%$; and obese class 3, 40.0\%. In a regression model, class 2 and class 3 obesity were strongly associated with screening positive for depression.

Not all studies have found this association. A secondary analysis of overweight and obese women enrolled in a postpartum weight loss intervention did not find a link between obesity and depressed mood. However, by virtue of the recruitment into an intense trial, obese women with depression could have been excluded (36).

Table 2 Psychosocial Factors Linked to Obesity in Women

Psychosocial stressors

Early traumatic events

Socioeconomic/workforce

Depression/anxiety/suicide

Stigmatization

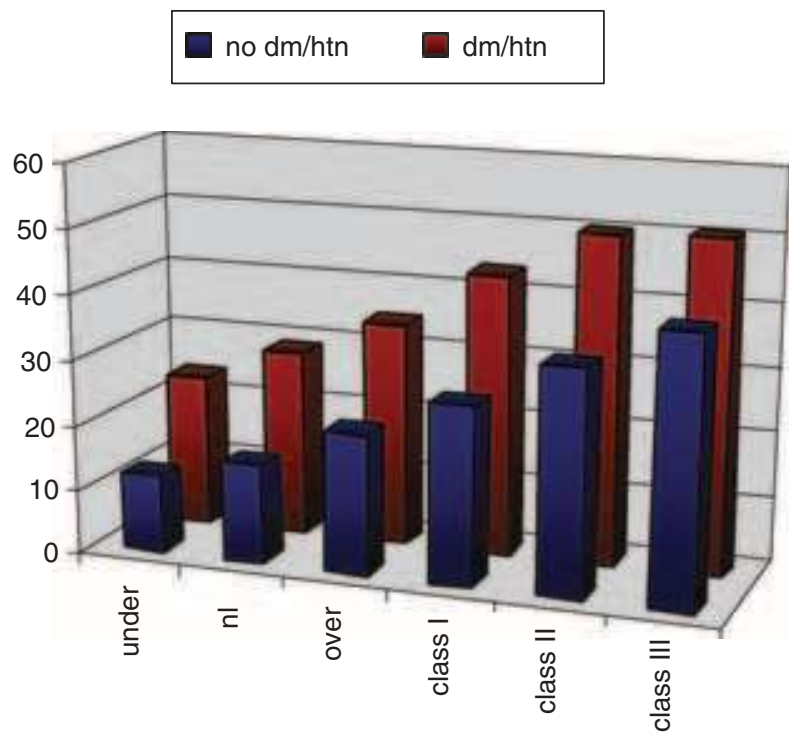

Figure 1 Cesarean delivery by body mass index strata and risks of diabetes and hypertension. Abbreviations: DM, diabetes mellitus; HTN, hypertension; nl, normal. 


\section{Long-Term Consequences}

While an increasing number of studies have focused attention on the immediate maternal and neonatal complications associated with prepregnancy overweight and obesity, less attention has been paid to the long-term consequences of excess maternal weight gain and postpartum weight retention, which may contribute to midlife obesity and subsequent development of diabetes and heart disease $(5,14,15)$. Olson demonstrated that only $42 \%$ of women had attained their prepregnancy weight and $26 \%$ of women had retained over $10 \mathrm{lb} 1$ year after delivery (37). They estimated that $69 \%$ of incident cases of obesity could have been prevented by decreasing weight gain during pregnancy. This excess weight gain and postpartum retention translated into an increase in long-term obesity and subsequent development of obesityrelated illnesses. One decade after delivering, women who gained less than recommended during pregnancy were $4 \mathrm{~kg}$ heavier on average, compared with 6 and $8 \mathrm{~kg}$ in women gaining the recommended and greater-than-advised, respectively. A weight gain of $16 \mathrm{~kg}$ during pregnancy was a significant predictor of long-term weight retention $(14,15,38)$.

\section{Bariatric Surgery}

Between 1998 and 2005, bariatric surgery has increased 800\% with the majority of the procedures performed on women (39). Overall, women who have successfully lost weight after bariatric surgery appear to have lower rates of GDM, hypertension, cesarean delivery, and macrosomia (40). Maggard and colleagues reported a GDM rate of 22\% in obese women and $0 \%$ in the post-bariatric surgery group. Preeclampsia rates were lower as well (3\% obese women vs. $0 \%$ post-bariatric surgery group). There was no evidence of increased growth restriction or preterm deliveries in the postbariatric surgery group compared with the obese controls (39).

Some women after bariatric surgery experience deficiencies of iron, folate, vitamin $B_{12}$, and calcium, particularly in those with malabsorptive procedures. Additionally, there are reports in the literature of complications during pregnancy after bariatric surgery including anastomotic leaks, internal hernias, intestinal obstruction, gastrointestinal bleeding and erosion, band migration, and maternal death (41). Population-level data comparing the benefits and risks of bariatric surgery compared with pregnancy in the severely obese are not readily available.

\section{Breast-Feeding}

Interesting work reveals an association between obesity and breast-feeding failures even after controlling for socioeconomic status $(42,43)$. Increased prepregnancy BMI is associated with shorter breast-feeding duration (42). This is unfortunate as both maternal obesity and short duration of breast-feeding are independent risk factors for childhood overweight (43). Recently, biologic data support this epidemiologic association between obesity and short duration of breast-feeding. Increased prepregnancy BMI predicts a lower prolactin response to suckling at 48 hours. Prolactin is responsible for stimulating milk production and thus a decrease in responsiveness could lead to a diminished ability to make milk and perhaps contribute to breast-feeding discontinuation (44). Perhaps interventions that improve prepregnancy BMI and or maternal weight gain might improve a woman's ability to breast-feed.

\section{Effects on the Offspring (Table 3)}

Fetal anomalies are reportedly increased in fetuses of obese women. Specifically, neural tube, cleft palate, and cardiac defects are increased, and concomitantly the ability to detect defects is impaired in this population by at least 20\% (45). Stothard and colleagues attempted to determine how the decreased detection rate might contribute to the overall increase in anomalies by a decrease in pregnancy terminations in obese women. They included pregnancy terminations in their analyses and found no change in the odds (46).

\section{IUFD and Infant Mortality}

An increasing number of studies have described an increase in IUFD among obese women. Denison and colleagues reported a threefold increase in IUFD in women with a $\mathrm{BMI} \geq 35$ (aOR 3.90, CI 2.44-6.22) (27) (Table 3). In a study of 24,000 women, after adjusting for antepartum complications, the association was attenuated but still present for IUFD and neonatal death (47). Mortality after delivery is also increased in neonates born to obese women (48). It is unclear whether this increase in IUFD warrants aggressive antepartum monitoring, given the rising prevalence of obesity and the relatively small risk of fetal demise.

\section{Macrosomia and Childhood Obesity}

Birth weight increases with maternal obesity. In a secondary analysis of the hyperglycemia and adverse pregnancy outcomes, Metzger reported that $13.6 \%$ nondiabetic obese women developed macrosomia ( $>4000 \mathrm{~g}$ birth weight) compared with $6.7 \%$ of non-obese normal-weight women (49) (Table 3). In a study of 116,976 macrosomic infants, after controlling for antepartum risk factors, those born to obese mothers were more likely to experience hyaline membrane disease (aOR 2.14, CI 1.73-2.66), extended assisted ventilation (aOR 1.71, CI 1.44-2.04), birth injury (aOR 1.58, CI 1.37-1.84), and meconium aspiration (aOR 1.42, CI 1.09-1.89) (50). The consequences of maternal obesity persist: large-for-gestational-age (LGA) babies and maternal obesity have been shown to have a twofold increase in the risk of metabolic syndrome in children (hazard ratios LGA 2.15, CI 1.25-3.82, maternal obesity 1.81, CI 1.03-3.19) (51). GWG likewise increases macrosomia and is worse in overweight and obese women. It is hypothesized that GWG and maternal obesity lead to

Table 3 Fetal, Neonatal, and Pediatric Consequences of Maternal Obesity and Excess Gestational Weight Gain (GWG)

$\begin{array}{lc}\text { Maternal obesity } & \text { Excess GWG } \\ \text { Birth defects } & \text { Macrosomia } \\ \text { SAB/IUFD } & \text { Childhood obesity } \\ \text { Infant mortality } & \\ \text { Macrosomia } & \\ \text { Childhood obesity } & \\ \text { Abbreviations: SAB, spontaneous abortion; IUFD, intrauterine fetal demise. }\end{array}$


maternal insulin resistance and glucose intolerance, which increase fetal glucose, insulin, steroid and growth hormones, amino acids, and lipids (Fig. 2). The resultant increase in fetal fat deposition leads to accelerated birth weights, neonatal complications, and childhood obesity (52).

\section{DIAGNOSIS}

\section{Defining Obesity and Measuring GWG}

In nonpregnant adults, BMI is commonly used as a measure of adiposity. The World Health Organization defines normal BMI as a BMI between 18.5 and 24.9, underweight as $<18.5$, overweight as 25 to 29.9 , and obesity as $>30$. For more detailed assessments and risk stratification, it defines class 1 obesity as 30 to 34.9 , class 2 obesity as 35 to 39.9 , and class 3 obesity as $>40$. In obstetrics, it is the practice to use a woman's prepregnancy BMI for risk assessment and to follow their GWG. Using a measured prepregnancy BMI is preferable. Alternatively, a first-trimester measured BMI or self-reported prepregnancy BMI can serve as a proxy. Notably, a selfreported pregravid BMI will underestimate a woman's actual BMI category. Table 4 provides formulas for calculation and two online references. BMI can be calculated by (i) dividing

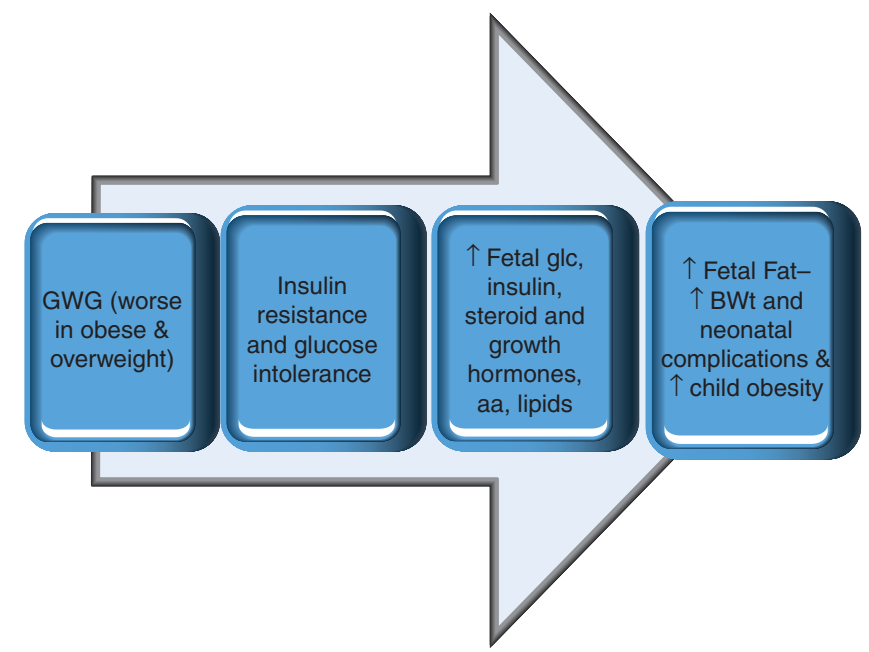

Figure 2 Effect of excess gestational weight gain (GWG) on the fetus. Abbreviations: aa, amino acids; BWt, birth weight.

Table 4 BMI Formulas, Websites-Calculator and Table

Formulas

$\mathrm{kg} / \mathrm{m}^{2}$

$\mathrm{lb} \times 703 /$ in. $^{2}$

Websites

Calculator http://www.nhlbisupport.com/bmi/

Table http://www.nhlbi.nih.gov/guidelines/obesity/bmi_tbl.htm the individual's weight in kilograms by height in meters squared or (ii) multiplying their weight in pounds by 703 and dividing this by the height in inches squared. Waist circumference is measured in the horizontal plane at the level of the iliac crest at the end of inspiration.

After extensive review, new recommendations for weight gain during pregnancy were released in 2009 by the Institute of Medicine (52). The document states that "Although the guidelines ... are not dramatically different ... fully implementing them would represent a radical change in the care of women of childbearing age." In fact, the recommendations are not significantly different. Only women in the obese category saw a reduction in the recommended weight gain to 11 to $20 \mathrm{lb}$. The Institute of Medicine (IOM) based the limited changes on the fact that there are limited data on metabolic profile, diet patterns, and eating behaviors of those without weight gain. The IOM cautioned that insufficient gain $(<15 \mathrm{lb})$ would imply mobilization of adipose and protein stores and could result in ketonemia with the potential to result in neurologic deficits in the infant (52). The guidelines presented in Table 5 drew some frank criticism from prominent obstetricians who suggested that this decrease in GWG was insufficient.

The IOM modified its recommendations for special populations. Women of short stature, adolescents, and all race/ethnicities are encouraged to gain the standard amount by their prepregnancy BMI category. Additionally, they changed the weight gain recommendations for twins to 37 to $54 \mathrm{lb}$ for normal-weight women, 31 to $50 \mathrm{lb}$ for overweight women, and 25 to $42 \mathrm{lb}$ for obese women. They were unable to stratify the recommendations for obese women carrying singletons or twins by class 1 , class 2 , or class 3 obesity citing insufficiency of representative published data.

\section{MANAGEMENT \\ Logistical Considerations}

In order to provide compassionate care, clinicians should recognize the challenges obese women face in obtaining medical care. Simple strategies to make the clinical setting more hospitable will facilitate effective communication and development of rapport. While the following recommendations may seem straightforward and perhaps inconsequential, they in fact set the stage for effective and compassionate communication. Office staff can assist clinicians in making the office more comfortable by evaluating a few key pieces of the patient's visit (53).

- Chairs with armrests may be constraining to overweight and obese women. Consider making benches available or having access to seating without armboards or wider seats.

Table 5 IOM Recommendations for Total and Rate of Weight Gain During Pregnancy, by Prepregnancy BMI

$\begin{array}{llcr} & \text { BMI } & \begin{array}{c}\text { Total weight } \\ \text { gain (lb) }\end{array} & \begin{array}{c}\text { Rates of weight gain in } \\ \text { and third trimesters (lb/week) }\end{array} \\ \text { Underweight } & <18.5 & 28-30 & 1(1-1.3) \\ \text { Normal weight } & 18.5-24.9 & 25-35 & 1(0.8-1) \\ \text { Overweight } & 25.0-29.9 & 15-25 & 0.6(0.5-0.7) \\ \text { Obese (includes all classes) } & \geq 30.0 & 11-20 & 0.5(0.4-0.6) \\ \text { Source: Institute of Medicine, 2009. } & & & \end{array}$


- Weight is considered a private issue by many women. Obese women may be embarrassed by having their weight measures in a public location, especially if they are heavier than the maximum capacity of the scale. Weigh women in a private location.

- Large blood pressure cuffs should be available at each location where blood pressures are taken to avoid unnecessary embarrassment to the patient. Have larger sized gowns and sheets in each examination room.

- Limited mobility may make it difficult for obese women to get onto an examination table or place their feet securely on the footrests. Provide adequate assistance for accessing the table and consider performing the pelvic examination without footrests.

- Large speculums should be available in each examination room.

\section{Lifestyle Interventions-Nutrition, PA, and Successful Interventions}

Currently, attempts are being made to identify interventions improving nutrition and PA during pregnancy. Clearly, there is room for improvement. There are no nationally representative data available on pregnant women, thus using reproductive-aged women as a proxy, women are not eating a diet rich in fruits and vegetables. Less than $2 \%$ of women aged 19 to 30 eat the recommended amounts of fruits and vegetables. Even when the requirement is lowered to the essential five-a-day servings of fruits and vegetables, less than $30 \%$ of women aged 19 to 30 and less than $40 \%$ of women aged 31 to 50 meet the target (54).

We perform no better in the realm of PA. When measured by accelerometry, only $5 \%$ of adults meet the recommended 30 minutes of moderate PA per day, 5 days per week (55). Data in pregnancy are limited; however, DiNallo and colleagues reported that $<12 \%$ of women meet PA recommendations in pregnancy (56). This is despite more recent data supporting that moderate PA in pregnancy is not associated with preterm labor, low birth weight, or pregnancy loss, and most studies show an inverse association between PA and GWG (57). Lifestyle interventions in overweight and obese women are limited, but none yet in the United States has been completely successful. Of the four intensive interventions, one showed no effect on GWG in obese women, one lowered GWG in lowincome women, the other in normal weight, and the last showed that dietary and lifestyle changes resulted in a 7\# decrease in GWG. The Europeans have more consistent success with 2- to 3-kg improvements in GWG and improvements in dietary practices. Currently, interventionists are attempting to determine what works and in which populations.

These research interventions clearly include an entire curriculum and consume a significant amount of resources that are not available to most clinicians. If available, clinicians should consider referrals to nutritionists in early pregnancy and each trimester. Alternatively, free online resources for nutritional plans are available for pregnant mothers on mypyramid.gov (Fig. 3).

Assessing current level of PA and encouraging continued exercise and active choices is a simple intervention a physician can perform in clinic. Tables 6 and 7 describe the American College of Obstetricians and Gynecologists recommendations and contraindications and precautions.

\section{Management Plan}

A management plan for obese pregnant women is included in Table 8. Acknowledging that half of pregnancies are unintended, the discussion about appropriate weight during pregnancy and consequences of obesity should begin at the well-woman examination. Currently, only one-quarter of

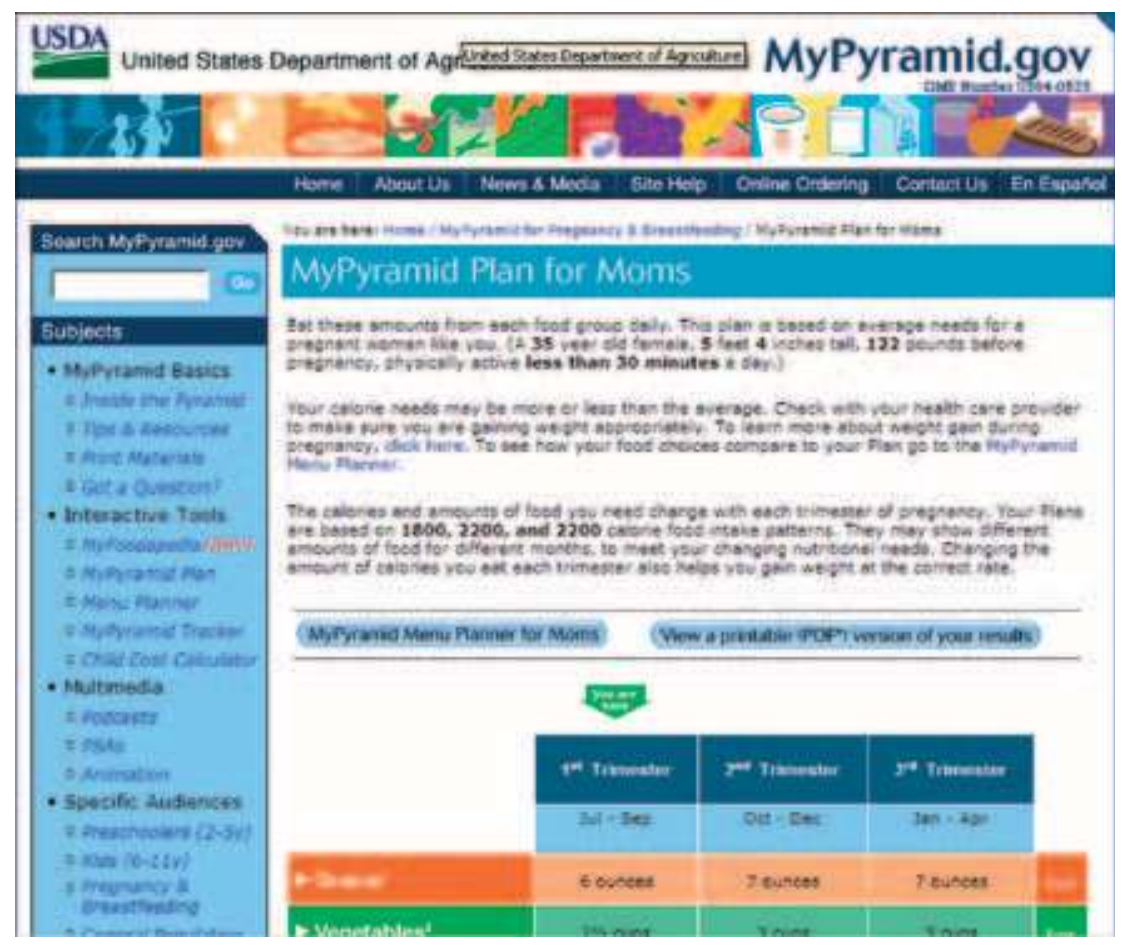

Figure 3 "MyPyramid Plan for Moms." Source: From MyPyramid.gov. 


\section{Table 6 Physical Activity Recommendations in Pregnancy}

$\geq 30 \mathrm{~min} /$ day moderate exercise 5 days/week

Avoid supine position with exercise

Avoid trauma, fall risk, and scuba

Source: ACOG, 2006.

Table 7 Contraindications and Precautions for Physical Activity in Pregnancy

Absolute contraindications
Significant heart disease or restrictive lung disease
Incompetent cervix
Multiple gestation
Second- and third-trimester bleeding
Previa, PROM, PTL, or pre-eclampsia
Relative contraindications
Anemia
Arrhythmias
Poorly controlled DM 1
BMI>40
Underweight
IUGR
Poorly controlled HTN or thyroid disease
Precautions
Loss of fluid, vaginal bleeding, cramping, regular contractions,
chest pain, shortness of breath dizziness, decreased fetal
movement

Abbreviations: PROM, premature rupture of membranes; PTL, preterm labor; DM 1, diabetes mellitus 1; BMI, body mass index; IUGR, intrauterine growth restriction; HTN, hypertension. Source: ACOG, 2006.

providers address weight among overweight and obese women at annual examinations (58). Documentation of BMI should be performed and overweight and obese patients should be referred to available PA and nutrition counseling. Women who are contemplating pregnancy should be encouraged to take folic acid as obesity is a risk factor for inadequate folic acid supplementation. Also they should be educated regarding the increased risks that obese women and their infants experience and screened for diabetes.

In early pregnancy, BMI should be calculated, recorded, and used to assess risks and set a GWG target. Throughout prenatal care, the GWG should be described and patient be given appropriate feedback, an important point being that clinicians and ancillary services should be providing consistent messaging. Folic acid supplementation should be encouraged. The Royal College of Obstetricians and Gynaecologists recommends increasing folic acid supplementation to $5 \mathrm{mg}$; however, the IOM concludes that the increased rate of neural tube defects may result from inadequate folate use not inadequate dosing $(59,60)$. Serum screening and first-trimester ultrasonographic screening should be encouraged, as secondtrimester screening is less efficacious at detecting anomalies. It is unclear whether fetal surveillance for obese women is costeffective; however, consideration should be made for antenatal testing in the severely obese as they are at greatest risk for IUFD.

Intrapartum care should include anesthesia consultation and assessment of an early epidural, given that a $1-\mathrm{cm}$ increase in neck circumference translates to a 1.13 increase in unsuccessful or complicated intubations (61). VBAC counseling should be modified to include the decrease in success.

\section{Table 8 Management Plan}

Preconception care (including gynecologic care and well-woman examinations)

Measure height and weight, calculate BMI

Address weight, physical activity, and nutrition (referral)

If considering conception

Folic acid $400 \mathrm{mcg}$

Weight loss before conception

Risk counseling by prepregnancy BMI

Screen for diabetes

Antepartum

Calculate prepregnancy or early pregnancy BMI

Set weight gain goal and review risks (2009 IOM recommendations)

Monitor gain and provide feedback

Consistent messaging-GWG, nutrition, and PA Nutrition website, nutrition referral $400 \mathrm{mcg}$ folic acid

Serum screening - first, second, and integrated screens are BMI corrected

Ultrasonographic screening Offer first $\Delta$ US for NT (no $\downarrow$ in detection rate)

Second $\Delta$ scan $20-30 \% \downarrow$ common anomalies

Antenatal testing 36 weeks if $\mathrm{BMI}>40^{*}$

Intrapartum

Anesthesia consult, consider early CLE (BMI $>35)$

Avoid elective induction

Modify VBAC counseling $\downarrow 50 \%$

$>40 \#$ GWG $\downarrow 40 \%$

Vaginal delivery

Low threshold to deliver in $\mathrm{OR}-\mathrm{BMI}>35$

Steps

Cesarean delivery

Table limit

Air mattress and sufficient staff

Incision placement

Surgical incise drape or tape (for cephalad retraction of pannus)

360-degree protected edge

In situ uterine closure

Subcutaneous layer 3.0 vicryl closure (if $>2 \mathrm{~cm}$ )

Compression stockings or prophylactic heparin

Immediately postpartum

Early ambulation

Lactation consultation

Early outpatient visit (7-14 days)

Wound

Breast-feeding

Edinburgh Postnatal Depression Scale (EPDS)

Postpartum visit (6 weeks)

Weight management

Mood

Contraception

Abbreviations: BMI, body mass index; IOM, Institute of Medicine; GWG, gestational weight gain; PA, physical activity; NT, nuchal translucency; VBAC, vaginal birth after cesarean. ${ }^{*}$ Consider

The gynecologic, general surgery, and anesthesia literature supports the increased risk of surgical morbidity. Surgical complications include increases in operative time, blood loss, transfusions, length of stay, pressure sores, nerve injuries, deep vein thromboses, wound complications, difficult intubations, and associated pulmonary complications (62-65). 


\section{Table 9 Key Points for Women after Bariatric Surgery}

Post-op counseling regarding increased fertility

Delay contraception for 12-18 months after bariatric surgery

Assess for vitamin and mineral deficiency (Fe, Folate, $\mathrm{B}_{12}$, calcium, vitamin D)

High suspicion for band/bypass complication

Co-manage with general surgery

Source: ACOG 2009, Kirshtein 2010.

For cesarean deliveries, the provider must know the weight limit of the OR table and prepare accordingly; table extensions must be available. Sufficient staff and/or air mattress lift devices should be available. Incision placement requires assessment of the location of the anterior superior iliac spine and the pannus. In severely obese women, the pannus tends to deviate the abdominal wall caudally and as such vertical periand supra-umbilical incisions might be required to access the lower uterine segment. Two additional techniques that may be useful include taping the pannus to a bar that resides at shoulder level with tape or a surgical incise drape to retract the pannus cephalad and using a cesarean o-ring retractor. Often, it is not possible to exteriorize the uterus and an in situ closure is performed. To decrease the likelihood of these complications, surgeons should be aware of the operating table weight limit, administer an adequate dose of preoperative antibiotics (if BMI $>35$ or $>220$ \# double the Ancef dose to $2 \mathrm{~g}$ ), and close the subcutaneous tissue (66). Early ambulation, sequential compression devices, or prophylactic heparin should be used to protect against deep vein thromboses.

Immediately, postpartum women should be encouraged to ambulate and be provided with lactation assistance. Inviting women to return to clinic 1 to 2 weeks postpartum provides an opportunity to assess wounds, support breast-feeding, and ensure that mood is stable. Clinicians should begin a weight management dialogue at 6 weeks postpartum, evaluate mood, and discuss the importance of adequate contraception.

\section{Bariatric Surgery}

Even if a woman has a history of infertility, she should be counseled that spontaneous conception is possible after bariatric surgery. It is important that women acknowledge this and delay contraception for the first 12 to 18 months to avoid pregnancy in the rapid weight loss phase to protect the fetus from growth restriction. When pregnancy does occur, women should be screened for vitamin and mineral insufficiency each trimester and supplemented as needed using rapid release or oral solutions in lieu of extended release medications. Some women may not tolerate a 1-hour glucose tolerance test and may require fasting and 1 to 2 hours postprandial glucose levels for 1 week at 25 to 28 weeks. Clinicians should have a high index of suspicion for band or bypass complications when women present with abdominal pain, nausea/vomiting, and anorexia. Consultation with bariatric or general surgeons should be sought readily and early (Table 9) $(40,41)$.

\section{Future Directions}

Unanswered questions abound in the care of the obese pregnant patients. Well-designed prospective studies are needed to answer these research questions. Potential topics include identifying (i) whether modest decreases in weight (5-10\%) preconception elicit significant improvement in obstetric outcomes, (ii) appropriate GWG recommendations by class of obesity, (iii) successful interventions to improve GWG, and (iv) the effects of PA on obstetric and psychologic outcomes. Future research should inform best practices.

\section{REFERENCES}

1. Prevention Center for Disease Control \& Prevention. Preventing chronic diseases: investing wisely in health. Preventing obesity and chronic diseases through good nutrition and physical activity. In. www.cdc.gov/ nccdphp/Publications/factsheets/prevention/pdf/obesity.pdf.

2. Allison DB, Fontaine KR, Manson JE, Stevens J, VanItallie TB. Annual deaths attributable to obesity in the United States. JAMA 1999; 282: 1530-8.

3. Ogden CL, Carroll MD, Curtin LR, et al. Prevalence of overweight and obesity in the United States, 1999-2004. JAMA 2006; 295: 1549-55.

4. Muennig P, Lubetkin E, Jia H, Franks P. Gender and the burden of disease attributable to obesity. Am J Public Health 2006; 96: 1662-8.

5. LaCoursiere DY, Bloebaum L, Duncan JD, Varner MW. Populationbased trends and correlates of maternal overweight and obesity, Utah 1991-2001. Am J Obstet Gynecol 2005; 192: 832-9.

6. Kim SY, Dietz PM, England L, Morrow B, Callaghan WM. Trends in prepregnancy obesity in nine states, 1993-2003. Obesity (Silver Spring) 2007; 15: 986-93.

7. Sebire NJ, Jolly M, Harris JP, et al. Maternal obesity and pregnancy outcome: a study of 287,213 pregnancies in London. Int J Obes Relat Metab Disord 2001; 25: 1175-82.

8. Carter AS, Baker CW, Brownell KD. Body mass index, eating attitudes, and symptoms of depression and anxiety in pregnancy and the postpartum period. Psychosom Med 2000; 62: 264-70.

9. Laraia BA, Siega-Riz AM, Dole N, London E. Pregravid weight is associated with prior dietary restraint and psychosocial factors during pregnancy. Obesity (Silver Spring) 2009; 17: 550-8.

10. Cnattingius S, Bergstrom R, Lipworth L, Kramer MS. Prepregnancy weight and the risk of adverse pregnancy outcomes. N Engl J Med 1998; 338: 147-52.

11. Lu GC, Rouse DJ, DuBard M, et al. The effect of the increasing prevalence of maternal obesity on perinatal morbidity. Am J Obstet Gynecol 2001; 185: 845-9.

12. LaCoursiere DY. BJOG, 2010; 117: 1011-8.

13. Lacoursiere DY, Baksh L, Bloebaum L, Varner MW. Maternal body mass index and self-reported postpartum depressive symptoms. Matern Child Health J 2006; 10: 385-90.

14. Rooney BL, Schauberger CW. Excess pregnancy weight gain and longterm obesity: one decade later. Obstet Gynecol 2002; 100: 245-52.

15. Rooney BL, Schauberger CW, Mathiason MA. Impact of perinatal weight change on long-term obesity and obesity-related illnesses. Obstet Gynecol 2005; 106: 1349-56.

16. Amorim AR, Rossner S, Neovius M, Lourenco PM, Linne Y. Does excess pregnancy weight gain constitute a major risk for increasing long-term BMI? Obesity (Silver Spring) 2007; 15: 1278-86.

17. Dubois L, Girard M. Early determinants of overweight at 4.5 years in a population-based longitudinal study. Int J Obes (Lond) 2006; 30: 610-17.

18. Laraia BA, Siega-Riz AM, Kaufman JS, Jones SJ. Proximity of supermarkets is positively associated with diet quality index for pregnancy. Prev Med 2004; 39: 869-75.

19. Lane SD, Keefe RH, Rubinstein R, et al. Structural violence, urban retail food markets, and low birth weight. Health Place 2008; 14: 415-23.

20. Laraia B, Messer L, Evenson K, Kaufman JS. Neighborhood factors associated with physical activity and adequacy of weight gain during pregnancy. J Urban Health 2007; 84: 793-806.

21. Ronnemaa T, Koskenvuo M, Marniemi J, et al. Glucose metabolism in identical twins discordant for obesity. The critical role of visceral fat. J Clin Endocrinol Metab 1997; 82: 383-7.

22. Pi-Sunyer F. The obesity epidemic: pathophysiology and consequences of obesity. Obes Res 2002; 10: 97S-103S.

23. Chu SY, Callaghan WM, Kim SY, et al. Maternal obesity and risk of gestational diabetes mellitus. Diabetes Care 2007; 30: 2070-6. 
24. Field AE, Coakley EH, Must A, et al. Impact of overweight on the risk of developing common chronic diseases during a 10-year period. Arch Intern Med 2001; 161: 1581-6.

25. Sibai BM, Ewell M, Levine RJ, et al. Risk factors associated with preeclampsia in healthy nulliparous women. The Calcium for Preeclampsia Prevention (CPEP) Study Group. Am J Obstet Gynecol 1997; 177: 1003-10.

26. Wolf M, Kettyle E, Sandler L, et al. Obesity and preeclampsia: the potential role of inflammation. Obstet Gynecol 2001; 98(5 Pt 1): 757-62.

27. Denison FC, Price J, Graham C, Wild S, Liston WA. Maternal obesity, length of gestation, risk of postdates pregnancy and spontaneous onset of labour at term. BJOG 2008; 115: 720-5.

28. Nohr EA, Bech BH, Vaeth M, et al. Obesity, gestational weight gain and preterm birth: a study within the Danish National Birth Cohort. Paediatr Perinat Epidemiol 2007; 21: 5-14.

29. Pevzner L, Powers BL, Rayburn WF, Rumney P, Wing DA. Effects of maternal obesity on duration and outcomes of prostaglandin cervical ripening and labor induction. Obstet Gynecol 2009; 114: 1315-21.

30. Stotland NE, Washington AE, Caughey AB. Prepregnancy body mass index and the length of gestation at term. Am J Obstet Gynecol 2007; 197: 378 e1-5.

31. LaCoursiere DY. Population-based trends in maternal obesity. J Soc Gynecol Investig 2004; 11: 191a.

32. Juhasz G, Gyamfi C, Gyamfi P, Tocce K, Stone JL. Effect of body mass index and excessive weight gain on success of vaginal birth after cesarean delivery. Obstet Gynecol 2005; 106: 741-6.

33. Durnwald CP, Ehrenberg HM, Mercer BM. The impact of maternal obesity and weight gain on vaginal birth after cesarean section success. Am J Obstet Gynecol 2004; 191: 954-7.

34. Miller LJ. Postpartum depression. JAMA 2002; 287: 762-5.

35. Grace SL, Evindar A, Stewart DE. The effect of postpartum depression on child cognitive development and behavior: a review and critical analysis of the literature. Arch Womens Ment Health 2003; 6: 263-74.

36. Krause KM, Ostbye T, Swamy GK. Occurrence and correlates of postpartum depression in overweight and obese women: results from the active mothers postpartum (AMP) study. Matern Child Health J 2009; 13: $832-8$

37. Olson CM, Strawderman MS, Hinton PS, Pearson TA. Gestational weight gain and postpartum behaviors associated with weight change from early pregnancy to 1 y postpartum. Int J Obes Relat Metab Disord 2003; 27: $117-27$.

38. Linne $\mathrm{Y}$, Neovius M. Identification of women at risk of adverse weight development following pregnancy. Int J Obes (Lond) 2006; 30: 1234-9.

39. Maggard MA, Yermilov I, Li Z, et al. Pregnancy and fertility following bariatric surgery: a systematic review. JAMA 2008; 300: 2286-96.

40. ACOG. Practic Bulletin 105: Bariatric Surgery and Pregnancy. 2009.

41. Kirshtein B, Lantsberg L, Mizrahi S, Avinoach E. Bariatric emergencies for non-bariatric surgeons: complications of laparoscopic gastric banding. Obes Surg 2010; 20: 1468-78.

42. Oddy WH, Li J, Landsborough L, et al. The association of maternal overweight and obesity with breastfeeding duration. J Pediatr 2006; 149: 185-91.

43. Li C, Kaur H, Choi WS, et al. Additive interactions of maternal prepregnancy BMI and breast-feeding on childhood overweight. Obes Res 2005; 13: 362-71.

44. Rasmussen KM, Kjolhede CL. Prepregnant overweight and obesity diminish the prolactin response to suckling in the first week postpartum. Pediatrics 2004; 113: e465-71.
45. Dashe JS, McIntire DD, Twickler DM. Effect of maternal obesity on the ultrasound detection of anomalous fetuses. Obstet Gynecol 2009; 113: 1001-7.

46. Stothard KJ, Tennant PW, Bell R, Rankin J. Maternal overweight and obesity and the risk of congenital anomalies: a systematic review and meta-analysis. JAMA 2009; 301: 636-50.

47. Kristensen J, Vestergaard M, Wisborg K, Kesmodel U, Secher NJ. Pre-pregnancy weight and the risk of stillbirth and neonatal death. BJOG 2005; 112: 403-8.

48. Thompson DR, Clark CL, Wood B, Zeni MB. Maternal obesity and risk of infant death based on Florida birth records for 2004. Public Health Rep 2008; 123: 487-93.

49. Metzger. ADA presentation. 2010.

50. Salihu HM, Weldeselasse HE, Rao K, Marty PJ, Whiteman VE. The impact of obesity on maternal morbidity and feto-infant outcomes among macrosomic infants. J Matern Fetal Neonatal Med 2011; 24: 1088-94.

51. Boney CM, Verma A, Tucker R, Vohr BR. Metabolic syndrome in childhood: association with birth weight, maternal obesity, and gestational diabetes mellitus. Pediatrics 2005; 115: e290-6.

52. Medicine Io. Weight gain during pregnancy: reexamining the guidelines. Washington D.C.: National Academies Press, 2009.

53. Thomas A, Hodges B, Chapin M, et al. Obesity in women - a guide to assessment and management. Brigham and Women's Hospital, Mary Horrigan Connors Center for Women's Health.

54. Serdula MK, Gillespie C, Kettel-Khan L, et al. Trends in fruit and vegetable consumption among adults in the United States: behavioral risk factor surveillance system, 1994-2000. Am J Public Health 2004; 94: 1014-18.

55. Troiano RP, Berrigan D, Dodd KW, et al. Physical activity in the United States measured by accelerometer. Med Sci Sports Exerc 2008; 40: 181-8.

56. DiNallo JM, Le Masurier GC, Williams NI, Downs DS. Walking for health in pregnancy: assessment by indirect calorimetry and accelerometry. Res Q Exerc Sport 2008; 79: 28-35.

57. Stuebe AM, Oken E, Gillman MW. Associations of diet and physical activity during pregnancy with risk for excessive gestational weight gain. Am J Obstet Gynecol 2009; 201: 58 e1-8.

58. Scott JG, Cohen D, DiCicco-Bloom B, et al. Speaking of weight: how patients and primary care clinicians initiate weight loss counseling. Prev Med 2004; 38: 819-27.

59. Medicine IO. Weight Gain During Pregnancy: Reexamining the Guidelines: Brief Report. 2009.

60. CMACE. 2010.

61. Modesitt SC, van Nagell JR Jr. The impact of obesity on the incidence and treatment of gynecologic cancers: a review. Obstet Gynecol Surv 2005; 60: 683-92.

62. Villavicencio MA, Sundt TM 3rd, Daly RC, et al. Cardiac surgery in patients with body mass index of 50 or greater. Ann Thorac Surg 2007; 83: 1403-11.

63. Ebert TJ, Shankar H, Haake RM. Perioperative considerations for patients with morbid obesity. Anesthesiol Clin 2006; 24: 621-36.

64. Saravanakumar K, Rao SG, Cooper GM. The challenges of obesity and obstetric anaesthesia. Curr Opin Obstet Gynecol 2006; 18: 631-5.

65. Bamgbade OA, Rutter TW, Nafiu OO, Dorje P. Postoperative complications in obese and nonobese patients. World J Surg 2007; 31: 556-60.

66. Walsh C, Scaife C, Hopf H. Prevention and management of surgical site infections in morbidly obese women. Obstet Gynecol 2009; 113(2 Pt 1) 411-15. 


\section{Medicolegal considerations Erol Amon and Gilad Gross}

\section{INTRODUCTION}

It is important to initially view the professions of medicine and law from the perspective of the roles they serve in a civilized society. In many ways, the professions are quite similar and yet have important differences. We do not rely on the practice of voodoo medicine, nor do we rely on capriciousness in the practice of law. Each profession has a well-developed set of values, norms, and methods taught within a higher educational system. The final practical step is that each professional is granted a license by the state in which they practice based on personal achievement, professionalism, and meeting obligations to the public.

Analogous to the physician-patient relationship is that of the attorney-client. Both share the duty of confidentiality and to place their patients' or clients' interest before their own. In most instances, clients or patients are extremely vulnerable and in need of professional assistance. This asymmetric relationship and need for profound levels of duty call for high degrees of professionalism from members of both professions.

Alternatively, there are marked differences. Medicine employs an open, cooperative, yet tightly constrained scientific methodology invested in discovering the truth. By contrast, the American legal system uses a relatively hidden, more creative, adversarial approach in which the courts and jurors unearth the truth to best meet the needs of the justice system. The attorney's goal is to use the adversarial system to win their case for their client rather than a thorough searching for truth. While scientific studies generally advance a physician's purpose to care for and remedy his patient's illness, some of these studies may actually detract from an attorney's theory on an alleged malpractice case. This occurs because in all circumstances attorneys have the ethical duty to advocate zealously for their client. In medicine, scientific unknowns remain as inconclusive unanswered questions, whereas legal disputes are always conclusively decided upon and finality reached regardless of the quality and quantity of evidence presented. Physicians are trained in the traditional sciences and the scientific method while attorneys are generally schooled in disciplines of advocacy, debate, and political science (1).

There are multiple opportunities for the two professions to interface. Broadly, there are two major meeting grounds: (i) professional liability litigation and (ii) contractual or regulatory aspects of medical practice (2). This chapter will focus on several aspects of professional liability and its litigation.

Most physicians will be called on during their career to provide legal testimony in one context or another. They could be a party to a lawsuit either as plaintiff or defendant. They could be a fact witness providing first-hand knowledge about a patient's condition, treatment, and records. In this capacity, personal testimony relates to what the physician observed, heard, or did in the case being litigated. They may also be called upon to provide expert testimony and opine on past events relating to a malpractice lawsuit, in which they often have no first-hand knowledge about the patient's condition or care. They could also be called upon to predict future medical outcomes, including life expectancy, and in a psychiatric context to discern whether a patient is dangerous enough to be involuntarily committed (homicidal or suicidal) by a judicial decision.

\section{LITIGATION FOR ALLEGED MEDICAL MALPRACTICE}

The American legal system is known as the adversarial system having its origin in English law. This method of litigation is the foundation of the American justice system. In theory, it has potential to perform quite well. Accordingly, the logic is as follows (3):

1. if two equally matched attorneys (with intelligence, experience, and resources)

2. zealously and competently represent their clients,

3. within the bounds of ethical and legal rules,

4. with neutral judges and juries,

5. then the correct results should be reached.

It is noteworthy that judges do not often take active roles; rather, they simply are a neutral referee ruling on admissibility of evidence and other legal issues as they arise. The court will not be interested in reviewing evidence the parties do not present. Jurors are also passive participants usually unable to ask clarifying questions (3).

As a result, attorneys for each side bear an enormous responsibility. Each side is presumed to look after their own interests. They must investigate, prosecute, and defend their clients by collecting, organizing, and presenting the evidence (4). Each party to the litigation will attempt to advance its own favorable evidence (interpretation of historical facts) while attempting to limit the admissibility and credibility of its opponent's evidence. In contrast to medical practice, there is no obligation to present all the facts, only those favorable to a particular side. Opposing attorneys examine and cross-examine the witnesses and, by using the rules of evidence, attempt to convince a relatively passive court of the correctness of their client's position.

Each side defines the scope of relevant evidence. Evidence law states that evidence is relevant if it has "any tendency to make the existence of any fact that is of consequence to the determination of the action more probable or less probable than it would be without the evidence" (5). This evidence must be pertinent to the case and help the judge or jury make a final decision based on a "preponderance of the evidence." The trier of fact (judge or jury) assesses the evidence presented and unearths the "real truth." Regardless of how close the 
pro and con evidence stacks up, ultimately, a conclusion must be reached and one side must win.

To assist the court in making its final decision, attorneys will call upon expert witnesses to help explain to the lay jury the applicable standards of care, whether they were breached, whether the breach caused the injury, and the measure of the past and projected monetary damages based on the nature of the injury (6).

\section{PROVING PROFESSIONAL NEGLIGENCE}

The burden of proof rests with the plaintiff in the American judicial system; the degree to which this must be demonstrated, however, is variable. In criminal cases in which a defendant's life or liberty is at stake, the burden is to provide evidence that is "beyond a reasonable doubt," which typically translates to a 95\% likelihood. However, in professional negligence cases where the compensation for injury secondary to negligence is money, the courts apply a mere "preponderance of the evidence" standard (6). This often equates to proving only $51 \%$ likelihood or as common parlance as "more likely than not."

There are four elements that must be demonstrated in a professional negligence lawsuit. These are duty, breach, causation, and injury. Each element must be proved by the preponderance of evidence standard (2).

The first of these elements, a legal duty, is created by the physician-patient relationship. The obligation arising out of this relationship is the duty to provide the standard of medical care that would be exercised by other similarly situated physicians under similar circumstances with comparable resources (6). These standards of care refer to appropriate conduct (behavior), that is, actions or inactions by the defendant. It does not refer to a mind-set (character) to harm or help the plaintiff.

It is imperative to understand the contrast of legal duty from ethical duty. A legal duty is what must or must not be done that a court of law may or will enforce. An ethical duty is what someone should or should not do and a court may not or will not find it enforceable. Consider a physician who happens to be shopping at a mall and the person standing in front of him collapses and stops breathing. The physician ethically should assist that person. However, in the absence of a preexisting physician-patient relationship, he has no legal duty, no legal requirement, to help and cannot be hailed into a court for failing to provide medical care. However, once this physician takes action to help, he must do so nonnegligently. To encourage these selfless acts, almost all states have enacted "Good Samaritan" statutes to protect these good people from lawsuits (7).

The second element that has to be proved by the plaintiff is the defendant's breach of the standard of care (the legal duty). Here the physician's conduct is at issue. The alleged negligent (breached) conduct is either an omission of an owed duty or commission of a negligent act. To prove this element, expert witness testimony is generally required (2) to provide admissible evidence for the benefit of the court by answering two questions:

1. what is or should of been the standard of medical care and
2. whether the duty to provide that standard was breached by the defendant physician.

The legal principle of Res Ipsa Loquitur is an exception to the plaintiff's requirement for expert witness testimony (2). This legal doctrine addresses the adverse outcome that could only have come about by the defendant's negligence. It is a rule of circumstantial proof (6). A prime example is the unintentional leaving of a sponge in a patient's abdomen after a surgical procedure. Here the lay person may use their common sense and reason that this was clearly negligence.

The third element to prove is legal causation. There must be a reasonably close and causal connection between the breach of the standard care and the alleged injury. The legal question asked of and answered by the expert witness is "but for" the defendant's breach, would the plaintiff suffered the claimed injury (6). In other words, the breach must be a necessary condition that leads to the injury. Furthermore, the attorney will often be required to ask the expert witness that "within a reasonable degree of medical certainty" whether the alleged breach resulted in the alleged injury (2).

In medicine, causation is complex and depends on multiple factors. To establish scientific proof using "evidenced-based" medicine, physicians often rely on controlled clinical trials with mathematical probabilities. Scientific studies minimize the occurrence of happenstance or chance by quantifying a probability of error to $<5 \%$. Causation is further strengthened if these results are independently reproducible.

In law, however, the notion of legal cause is generally quite different than medical causation. The focus is not scientific truth. The heart of the legal matter is the attribution of responsibility (8). Therefore, by necessity, the law commences a retrospective investigation of particular conduct of particular persons and uses the somewhat complex rules of legal evidence to help the jury make a final decision. In these situations, the probability of error in malpractice litigation $(<50 \%)$ can be much higher than that in science $(<5 \%)$. Thus, the $51 \%$ standard proof (i.e., preponderance of the evidence) is invoked to persuade the jury.

The final element that requires legal proof is the development of an injury. The purpose of awarding monetary damages is to make the victim "whole" from a legal standpoint. If there is no injury, there is no recovery on which damages can be based. Compensatory damages are usually of two types: economic (measurable) and non-economic (nonmeasurable). Economic damages include case-related past, present, and future medical expenses and related loss of past, present, and future wages. An economic expert known as a "life planner" will testify as to the amount of economic damages. Noneconomic damages include those related to "pain and suffering," loss of consortium, and mental anguish. Unfortunately, these cannot be accurately quantified and thus are subject to sizable degrees of variation among juries. Many state legislatures, through tort law reforms, have enacted or are attempting to enact a hard cap on noneconomic damages to protect against runaway jury awards, to improve the predictability of judgment amounts, and to stabilize insurance premiums and maintain access to medical care (9).

Ultimately, all four elements must be linked together for the plaintiff to prevail by proving each element by a 
preponderance of the evidence (2). If a physician defendant has no legal duty to the patient, that suit will automatically fail. If the defendant can show that the standard of care was met despite an adverse outcome (maloccurrence), the defendant should prevail. Even if the defendant did breach a standard of medical care owed to the plaintiff, but the breach did not cause or was unrelated to the injury, the plaintiff should not recover any damages.

Legal duty is hardly ever litigated because in most cases there is usually a well-defined physician-patient relationship. On the other hand, evidence pertaining to the other three elements is inevitably controversial, often litigated, and most often introduced into court through the testimony of experts.

\section{GUIDELINES FOR EXPERT WITNESS TESTIMONY}

An expert witness is defined as a person who, by reason of education, special training, and/or experience possesses knowledge of a particular subject area at greater depth than the judge or lay jury. The judge will often decide on the admissibility of this evidence (expert's testimony) by assessing the proffered expert's qualifications and opposing counsel's objections. Once allowed to testify in the courtroom, the jury will weigh the expert's testimony about their interpretation (opinions) of the facts of the case (6).

Multiple experts may be used to prove each element independently. In contrast to fact witnesses, expert witnesses are permitted to reflect, opine, and pontificate in court. Initially, experts on standard of care through testimony educate the jury on what the appropriate medical practice is or should be, and whether or not the defendant's conduct falls within the standard of care. Causation experts may be used to establish whether the alleged breach "within a reasonable degree of medical certainty" caused the alleged injury. A single expert witness can provide testimony on both standard of care and causation, or alternatively and more likely, multiple experts will be utilized. Economic experts are separate individuals who make claims regarding the degree of damages, life expectancy, and projected future costs.

Expert witnesses are employed by opposing parties of a malpractice claim. Physicians who accept both plaintiff and defense cases are thought to be less biased. Depending on individualized State Tort Law, an expert may render appropriate opinions by virtue of similar practice and does not need to practice an identical discipline of medicine. Nonetheless, the expert must have knowledge of the standard of care and be familiar with the locality of the case setting. A favorable expert is one who is active clinically. While it helps to be familiar with current literature, it is very important that they have an understanding of the standards of care during the time period that the case actually took place. Oftentimes, years, and even decades, have passed before a case is filed. Also it is important that experts understand the setting by region and facility and how these factors can impact the evidence of a case.

Obstetric cases stemming from alleged neurologic birth injuries attract a relatively small group of physician experts who testify in a large portion of cases. Many witnesses tend to establish themselves as plaintiff or defendant "friendly" (10). Although specific qualifications can vary from state to state, the American College of Obstetricians and Gynecologists has
Table 1 American College of Obstetricians and Gynecologists Qualifications for the Expert (11)

1 The physician expert witness must have a current, valid, and unrestricted license to practice medicine in the state in which he or she practices

2 The physician expert witness should be currently certified by a board recognized by the American Board of Medical Specialties, as well as by experience or demonstrated competence in the subject of the case

3 The specialty, training, and experience of the physician expert witness should be appropriate to the subject matter in the case

4 The physician expert witness should be familiar with the standard of care provided at the time of the alleged occurrence. In addition, the physician expert witness should have been actively involved in the clinical practice of the specialty or the subject matter of the case within 5 years from the time the expert was retained to provide an expert opinion in the matter

5 The physician expert witness should be able to demonstrate evidence of continuing medical education relevant to the specialty or the subject matter of the case

6 The physician expert should be prepared to document the percentage of time that is involved in serving as an expert witness. In addition, the physician expert should be willing to disclose the amount of fees or compensation obtained for such activities and the total number of times the physician expert has testified for the plaintiff or defendant

offered a guide in an attempt to promote the integrity of the individual expert witness and his professional organization (11). Their recommendations are found in Table 1.

As stated previously, the expert witness who testifies on behalf of both plaintiff and defendant is considered less ethically biased (12). The expert should distinguish maloccurrence from malpractice. Maloccurrence is an adverse outcome unrelated to the quality of care provided, whereas malpractice is substandard care causing harm. It is unethical for a physician expert to accept compensation that is contingent on the outcome of litigation (13).

Additional ethical principles for expert testimony (12) include the following:

1. The physician's review of medical facts must be thorough, fair, and impartial and must not exclude any relevant information. It must not be biased to create a view favoring the plaintiff, the government, or the defendant. The goal of a physician testifying in any judicial proceeding should be to provide testimony that is complete, objective, and helpful to a just resolution of the proceeding.

2. The physician's testimony must reflect an evaluation of performance in light of generally accepted standards, neither condemning performance that falls within generally accepted practice standards nor endorsing or condoning performance that falls below these standards. Medical decisions often must be made in the absence of diagnostic and prognostic certainty.

3. The physician must make every effort to assess the relationship of the alleged substandard practice to 
the outcome, because deviation from a practice standard is not always substandard care or causally related to a bad outcome.

4. Finally, the physician must be prepared to have testimony given in any judicial proceeding subjected to peer review by an institution or professional organization to which he or she belongs.

When called upon to testify, most physicians bring a high degree of professionalism to the legal process and play a very important role in the administration of justice (13). Unequivocally, a patient injured by genuine medical malpractice deserves due compensation. When true malpractice has occurred, the individual (patient) and society (all of us) will benefit by professional testimony that assists a court in reaching that conclusion (9).

Most physicians desire the best for their patients. More often than not, unfortunate maloccurrence is the nature of an adverse outcome rather than true medical malpractice. Physicians can benefit and avoid litigation by application of the principles of risk management.

\section{SELECTED ASPECTS OF RISK MANAGEMENT}

Maloccurrence is defined as a bad or undesirable outcome that is unrelated to the quality of care provided. In some cases, these outcomes are anticipated and unavoidable. In other cases, they are unanticipated yet unavoidable. Fully informed consent goes a long way to preventing litigation in these situations. Malpractice, on the other hand, is essentially the provision of substandard medical care that causes significant harm (12).

Basic risk management principles include listening carefully to patients, documenting their complaints, comments, and responses, as well as timely documentation of how physicians respond both verbally and physically. While this communication and documentation will confer some degree of protection from claims, it remains a basic tenet that errors must be avoided.

Practicing the principles of patient safety minimizes medical errors. Open and extensive communication among caregivers is paramount to this process. It builds trust and respect through such endeavors as team training, structured sign outs, team huddles that anticipate problems, mock drills, and clinical simulations. Significant improvements in the management and outcomes of obstetric emergencies such as shoulder dystocia and hemorrhage have been reported. The concept of full disclosure to patients following adverse outcomes has also been shown to reduce the risk of malpractice claims. Physicians who demonstrate compassion by being available to discuss, answer questions, and importantly apologize as appropriate are less apt to be the target of claims. Still some physicians appear to be prone to lawsuits.

Frequently sued obstetricians demonstrate a pattern of behavior that is fairly predictable. They are often rushed and unavailable. When present, they often are poor communicators and do not take the time to listen or offer pertinent information. They seem unconcerned and show disrespect to their patients. Despite these shortcomings, the outcomes of lawsuits in the setting of these contextual risk factors still seem to favor the lawsuit-prone physicians (14). Although there may be unprofessional behavior, the alleged acts of negligence acts are often not as claimed. They may turn out not breaches of the standards of care; furthermore, the untoward outcome may be a temporary injury that resolves with minimal effects or no injury at all.

In contrast to cases that result in no payment, the greatest predictor of a professional liability payment to a patient is the severity of outcome, namely an unanticipated death or permanent disability. Being a great communicator, being personable, and being well liked by your patient is very important but does not provide complete immunity from a lawsuit. If there is a significantly bad outcome due to substandard care, and there are appropriate expert plaintiff attorneys and expert witnesses, there is a high likelihood that when litigation does happen, it will rightfully result in favor of the plaintiff.

What can institutions do? Current initiatives to reduce liability via vigorous quality improvement (QI) programs are being developed. QI endeavors can be implemented in solo locations or system-wide entities. A series of "triggers" are identified that when encountered, set off an automatic review by select representatives on the committee. Examples from the field of obstetrics include postpartum hemorrhage, a return to the operating room, and maternal readmission. These reviews are done to identify variables that contribute to adverse outcomes or near misses. Events are deemed avoidable or unavoidable and action items are created. Responsible parties are then tasked with addressing deficiencies and propagating progressive change.

\section{CONCLUSION}

In conclusion, we reviewed the four elements of medical malpractice case, the importance of expert witness testimony, the guidelines for integrity and ethical physician expert witness testimony, and some practical aspects of risk management and litigation avoidance.

\section{REFERENCES}

1. Wecht CH, Koehler SA. Book review. J Leg Med 2005; 26: 529-34.

2. Sanbar SS, ed. Legal Medicine, 6th edn. Philadelphia, PA: Mosby, 2004.

3. Gross SR. Expert evidence. 1991 Wisconsin Law Review. 1991: 1113.

4. Golan T. Laws of men and laws of nature: the history of scientific expert testimony in England and America. Cambridge, MA: Harvard Univ. Press, 2004.

5. Federal Rules of Evidence. Rule 401. Definition of "Relevant Evidence."

6. Furrow BR, Greaney TL, Johnson SH, et al. Health Law. St. Paul MN, West Publishing Co, 1995.

7. Revised Statutes of Missouri. 537.037. Emergency care, no civil liability, exceptions (Good Samaritan law).

8. Freckleton I, Mendelson D, eds. Causation in law and medicine. Burlington VT: Dartmouth Publishing Company, 2002.

9. Amon E, Winn H. Review of the professional medical liability insurance crisis: lessons from Missouri. Am J Obstet Gynecol 2004; 190: 1534-8; discussion 1538-40.

10. Kesselheim AS, Studdert DM. Characteristics of physicians who frequently act as expert witnesses in neurologic birth injury litigation. Obstet Gynecol 2006; 108: 273-9.

11. Executive Board of the American College of Obstetricians and Gynecologists. Qualifications for the physician expert witness. 2003. [Available from: http://www.acog.org/departments/dept_notice.cfm? recno $=4$ \&bulletin $=2644]$.

12. ACOG Committee Opinion Number 374. August 2007. [Available from: http://www.acog.org/departments/dept_notice.cfm?recno=4\& bulletin $=2644]$.

13. AMA Code of Medical Ethics (2002) 9. 07 Medical testimony. American Medical Association.

14. Hickson GB, Clayton EW, Entman SS, et al. Obstetricians' prior malpractice experience and patients' satisfaction with care. JAMA 1994; 272: 1583-7. 


\section{Drug abuse in pregnancy: Marijuana, LSD, cocaine, amphetamines, alcohol, and opiates Jacquelyn C. Howitt and Anita Bublik-Anderson}

\section{INTRODUCTION}

Most health professionals will at some time encounter the issue of substance abuse in pregnancy, as increasing numbers of pregnant women cope with the aid of illicit drugs or continue established substance abuse patterns despite pregnancy. The drug-abusing population includes women from every portion of society, every ethnic group, and every socioeconomic stratum. In 2007, 19.9 million Americans aged 12 or older were current (past month) drug users; 6.9 million people aged 12 and older were classified as having a dependence on, or abuse of, illicit drugs. Among pregnant women aged 15 to 44 years, 3.9\% reported using illicit drugs in the past month (1). Familiarity with drugs of abuse, recognition of the hallmarks and subtleties of drug use, and the ability to be prepared for possible complications of drug use in mother, fetus, and neonate is therefore essential for those caring for pregnant women and their offspring.

Relatively little is known about the direct effects of specific illicit drugs on human pregnancy. Since most women who abuse drugs use more than one drug, studies are rarely "pure." As polydrug use complicates even the best of human studies, and as malnutrition, anemia, and infection are frequently confounding factors, most authors caution against too narrow an interpretation of data regarding the effects of a single drug. Nevertheless, inferences can be drawn from human studies and data extrapolated from animal studies. The following pages contain a review of some of the more common drugs of abuse in pregnancy.

\section{MARIJUANA}

Marijuana is the most commonly used illicit drug; its use has increased in reproductive-aged women (1). Delta-9tetrahydrocannabinol (D9THC) is its main pharmacologically active component. Cannabidiol and cannabinol, other components of marijuana that can affect the metabolism and activity of D9THC, do not possess significant pharmacologic activity of their own (2). The primary site of marijuana metabolism is the liver; the lung also affords some metabolic action (3). The primary metabolite of D9THC (11-hydroxy D9THC), which may also be pharmacologically active, is in turn converted to more polar metabolites that are excreted through bile, feces, and urine (2). Two weeks after the marijuana use, D9THC may still be detected in urine (4). Smoking marijuana leads to rapid onset of drug action (within minutes), yielding effects that usually last less than 2 to 3 hours (3). Marijuana is lipophilic and readily distributed to organs with relatively high blood flow (liver, lung, kidney, spleen). However, the brain, which also has a good blood supply, receives comparatively small amounts of marijuana (2), which accumulates mainly in the gray matter. Physiologic effects of marijuana use include tachycardia, slight decrease in blood pressure, reddening of the conjunctiva, fine hand tremors, and a modest reduction in muscle strength. Marijuana also possesses analgesic activity, has antiemetic properties, and causes hallucinations in high doses (3). Following marijuana administration, users experience a brief stimulatory phase in which they may become anxious and restless or euphoric. They typically report enhanced perception of the five senses. A period of sedation follows in which users seem to move in and out of a "dream-like" state, during which they have a shortened attention span, an altered sense of time and distance, and impairment of short-term memory.

The placenta can accumulate significant amounts of D9THC. Placental transfer has been documented in monkeys (5), sheep, and mice and rats (2). Maternal administration of D9THC in these animals yields lower concentrations of marijuana in the fetus than in the mother (5).

The actual effect of marijuana use on human pregnancy outcome and on the fetus is controversial. Suggested effects include a higher occurrence of preterm labor and abruptio placentae (6) (possibly dose dependent), an increased risk of precipitous labor (4), an increased risk of abnormal antenatal tests (4), a higher likelihood of meconium staining of amniotic fluid at the time of delivery (4), and an increased risk of prolonged, protracted, or arrested labor (4). Few authors have confirmed the findings of an increased risk of preterm labor or precipitous labor, and the increased incidence of meconium is not a universal finding (7). The incidences of miscarriage, obstetric complications, or major fetal physical anomalies in human pregnancies complicated by marijuana use are the same as those of controls (8). There is no increased risk of perinatal death, nor is there a need for special care of human babies prenatally exposed to inhaled cannabis (9). A consistent relationship between marijuana use and the incidences of premature rupture of membranes, fetal distress, and fetal malposition has not been demonstrated (6). Frequent and regular use of marijuana throughout pregnancy is associated with a detectable, though not statistically significant, decrease in birth weight (9). Fried et al. found no effect of maternal marijuana use on infant weight, length, head circumference, or Apgar scores (8).

A significant amount of research has been done to evaluate the impact of marijuana on brain development, learning, and behavior of the prenatally exposed fetus. Cannabinoid (CB) receptors identified in the white matter of the brain of fetal rats have an influence on early brain development (10) through the expression of dopamine (11), serotonin, and glutamate (12), involving glial cells and axonal linkages and the hypothalamicpituitary axis (13). In the fetal, neonatal, and adult human 
brain, $\mathrm{CB}$ receptor-binding sites are found throughout the frontal cortex; there is an increase in receptor binding in developing brains. Rats injected with CB-1 agonists demonstrate learning disruption and changes in emotional reactivity; authors postulate that an impairment in cortical glutamatergic neurotransmission is associated with the observed reduction in cortical neurons and impaired neurite branching (12). Human fetuses exposed in utero to marijuana at 18 to 22 weeks were found to have a significant reduction of the $\mathrm{D} 2$ gene expression in the amygdala (11). Decreased D2 mRNA expression in the amygdala (linked to emotional behavior and mood states) provides a possible explanation for observed cognitive and behavioral states of older children exposed in utero to marijuana, according to Wang et al. (11).

Purported neurobehavioral effects following prenatal exposure to marijuana include an increase in fine tremors and exaggerated and prolonged startle responses in newborn infants. These infants may have a poorer habituation response to visual stimuli (8), but they show a normal response to auditory stimuli (5). By 12 and 24 months of age, infants exposed prenatally to marijuana are similar to controls when assessed for visual, mental, motor or language capabilities. At 48 months, memory and verbal parameters of children exposed to more than regular marijuana use are affected, and 3-year-olds also have poorer abstract and visual reasoning (14). Head circumference is negatively impacted at 9 to 12 years of age (14). While there is no uniform decrease in intelligence, certain processes of executive function such as complex visual-perceptual tasks, planning, and attention are affected into adolescence (14).

Although controversy remains about the effects of perinatal marijuana use on pregnancy, research supports a long-lasting effect on the developing brain. This may help guide in counseling the marijuana user about potential effects of in utero exposure. Increased vigilance in the reproductive-aged population is warranted, as marijuana use is so prevalent, concomitant use of other drugs is so common, and selfreporting is often incomplete.

\section{LYSERGIC ACID DIETHYLAMIDE}

The hallucinogen lysergic acid diethylamide (LSD) reached its peak popularity in the late 1960s. Since that time, the overall use of LSD has declined dramatically $(1,15)$. After oral administration, LSD is rapidly absorbed into the blood. Physiologic effects in humans are generally sympathomimetic in character: pupillary dilation, an increase in blood pressure, tachycardia, hyperreflexia, piloerection, and increased temperature (3). LSD induces states of altered perception. Initial symptoms, which occur within minutes following ingestion, include dizziness, weakness, nausea, and paresthesias. Within 2 to 3 hours, the user experiences visual illusions and affective symptoms. Within 4 to 5 hours, users may experience major panic attacks or merely feel detached. Drug effects may differ among users as well as with the same person on different occasions. The half-life of LSD is approximately 3 hours and symptoms begin to abate after 12 hours. There is no withdrawal syndrome, and no deaths have been attributed to the direct effects of LSD.

In 1968 and 1969, three case reports suggested human teratogenicity from prenatal LSD exposure. These involved limb defects, specifically finger abnormalities, and occasionally toe abnormalities of either or both the feet $(16,17)$. In all three reports, each fetus had also been exposed to marijuana. McGlothlin et al. (18) studied the effects of parental LSD exposure and observed an increased risk of spontaneous abortion among women exposed to LSD. Several authors have suggested a causative association between LSD intake and chromosomal damage while others have disputed this correlation $(17,19)$.

\section{COCAINE}

Cocaine is a stimulant drug derived from the coca plant, Erythroxylon coca. Cocaine use in pregnancy was widely publicized in the 1980s with reports of the urban phenomenon of "crack babies" flooding maternity wards and burdening society. While harm from cocaine to multiple organ systems is clear, and much of the risk in pregnancy is known, there are numerous confounders that make effects directly attributable to cocaine difficult to isolate; the mania of the media blitz may be unfounded $(20,21)$. A retrospective study of 139 cocaineabusing women revealed concomitant use of other drugs such as alcohol, tobacco, marijuana, diazepam, heroin, and methadone to occur in $92.8 \%$ of users (20). Cocaine use has increased, but its use still pales when compared with that of marijuana and alcohol. In the United States, 1.5 million persons over the age of 12 met the criteria for the abuse or dependence of cocaine or crack cocaine over the past year (1).

Cocaine is an alkaloid related to topical anesthetic agents. Hydrochloride can be added to the alkaloid and then heated to yield the free base known as "crack." Cocaine can be sniffed, injected, or smoked as the freebase form. Cocaine causes a "rush" of intense euphoria, which lasts 20 minutes after injection and 60 to 90 minutes following intranasal use. Mood is elevated along with energy and alertness, and the appetite is decreased. When this effect wears off, the user may "crash" and become lethargic, depressed, and somnolent. Finally in an "extinction" phase, an episodic intense craving for the drug will gradually decrease in frequency and severity, but can last months or years after last use (22).

Cocaine blocks the presynaptic reuptake of catecholamines at neuron terminals, which then increases postsynaptic levels of norepinephrine, dopamine, and epinephrine (23). The plasma half-life of cocaine is approximately 40 minutes, and it is then metabolized by the liver, plasma esterases, and the cytochrome P450 enzymes $(22,23)$ and then excreted in the urine. Cocaine can be detected for 8 hours in the urine and its metabolites detected in urine for up to 7 days.

The major physiologic effects of cocaine relate to its sympathomimetic characteristics: hypertension, tachycardia, hyperglycemia, hyperpyrexia, and mydriasis. Coronary vasoconstriction can cause spasm, angina, acute myocardial infarction, arrhythmias, and sudden death. Acute vasoconstriction can cause subarachnoid hemorrhage, ischemic or hemorrhagic stroke (24), and intestinal necrosis (23). Sniffing or snorting cocaine can cause anosmia, chronic rhinitis, septal degeneration, necrosis, and perforation (22). Smoking cocaine can cause direct lung damage, pulmonary edema, spontaneous pneumomediastinum, and pneumopericardium (22). Higher doses of cocaine can cause disinhibition, disturbances in judgment, grandiosity, impulsive behavior, hypersexuality, and paranoid psychosis (22). 
Perinatal effects associated with gestational cocaine use are fogged by confounding risk factors such as polysubstance abuse, malnutrition, and lifestyle. Associations include an increase in spontaneous abortions $(25,26)$, preterm premature rupture of membranes $(21,27)$, chorioamnionitis (21), meconium-stained amniotic fluid (20,21), and precipitous labor $(21,28)$. The most commonly cited perinatal effects of prenatal cocaine exposure are prematurity, growth restriction, low birth weight, and placental abruption. Most of these effects are thought to be related to cocaine's vasoconstrictive properties.

In 1983, Acker et al. reported two cases of abruptio placentae associated with cocaine use (29). While other investigators had similar findings $(25,26)$, some of these have low statistical significance and other reports dispute them altogether $(27,28,30)$. In one study, infants whose urine tested cocaine positive (exposure shortly before delivery) had a similar rate of abruptio placentae as infants with cocainenegative urine (no recent exposure) (21). The rate of abruption in both groups was, however, higher than that of the general population. Several case reports have noted an association of cocaine use and uterine rupture (31-33), thought to be related to the increase in contractile force of the myometrium (34).

Most authors agree that cocaine use increases the likelihood of preterm labor $(28,35)$ and of preterm birth $(20,27,28)$ although there is no significant difference when controlled for polydrug use. While prematurity and low birth weight both increase the risk of intracerebral events, the risk appears to be higher in cocaine-exposed babies, as compared with controls (36). Decreased birth weight (28), low birth weight (LBW) (less than $2500 \mathrm{~g})(27,28)$, intrauterine growth retardation (IUGR), and small-for-gestational-age (SGA) infants occur following prenatal cocaine exposure $(20,28,35)$, though there is not a statistically significant difference in IUGR if multiple drugs are used (28). Cocaine use is predictive of low birth weight even when other variables such as age, race, gravidity, socioeconomic status, and lack of prenatal care are controlled (27). The use of other drugs is additive with respect to LBW. Burkett et al. found that the combination of cocaine with tobacco and other drugs, excluding alcohol, had more effect on the incidence of SGA and LBW infants than cocaine alone (21). Zuckerman et al. found the use of marijuana and cocaine to be independently associated with impaired fetal growth (37).

Neonatal effects of cocaine exposure are varied and without a typical presentation. Cocaine has no consistent effect on 5-minute Apgar scores $(20,28)$ or umbilical artery $\mathrm{pH}$ values (28). Neonatal neurobehavioral abnormalities include tremulousness, irritability and muscular rigidity, sleeping problems, feeding difficulties, vomiting, diarrhea, fever, hypotonia, a high-pitched cry, tachypnea, hyperreflexia, tachycardia, seizures, and hyperresponsiveness (20). A poor sucking response and neurologic deficits are frequently prominent features in these infants (21). Apneic spells and abnormal pneumograms have been noted (21), and some authors report an increased incidence of sudden infant death syndrome (SIDS) (25) although this association has been debated (38).
Congenital anomalies associated with cocaine exposure have included skull defects (26), urogenital anomalies (39), urinary anomalies without genital defects (40), and congenital cardiovascular defects such as atrial septal defects, ventricular septal defects, cardiomegaly (20), transposition of the great vessels, hypoplastic right heart (26), and polydactyly (21). Many investigators have found no statistical increase in cocaine-induced congenital anomalies $(28,30)$.

Animal studies have been particularly helpful in exploring potential effects of prenatal cocaine use. Woods et al. were able to demonstrate dose-dependent cardiovascular effects in pregnant and fetal sheep following intravenous injection of cocaine (41). The authors suggest that cocaine given to the pregnant ewe may produce fetal hypoxemia via uterine artery vasoconstriction as well as direct fetal cardiovascular effects via transplacental passage of cocaine into the fetal circulation.

Webster and Brown-Woodman administered cocaine to pregnant rats as single or multiple doses, during specified gestational periods, and examined the fetuses at various times for evidence of hemorrhage or malformation (42). They found no evidence of teratogenicity when cocaine was given during the main organogenic period, even at toxic maternal doses. However, when given at later specific gestational ages, cocaine exposure was associated with damage to fetal limbs and tail, and hemorrhage in the genital tubercle. In general, cocaine administration was followed by the occurrence of edema and hemorrhage at certain sites. When involved fetuses were reinspected at a later date, reduction anomalies were identified at these sites. It appears, then, that the malformations are a direct result of hemorrhage and edema, followed by necrosis, and subsequent disruption and/or amputation. The authors postulate that the fetal vasculature must attain a certain degree of maturation prior to being able to respond to hypoxia, thereby protecting developing tissues during organogenesis.

Other investigators have been particularly interested in neurobehavioral effects of prenatal cocaine exposure. Spear et al. found that cocaine-exposed rat offspring had cognitive defects in some, but not all, conditioning situations (43). These authors have offered the suggestion, based on their work in the animal model, that chronic exposure to cocaine during gestation results in downregulation of dopaminergic systems, conferring later hyperactivity and attention deficit disorders. Fetal cocaine exposure during synaptogenesis, even at doses well below maternal toxicity, results in long-term changes in the neurochemistry of dopaminergic systems (44). The exact duration of those changes and the impact is still not fully understood.

\section{AMPHETAMINES}

Use of methamphetamine (MA) and its derivatives increased significantly in the 1990s. Approximately 10.4 million (4.3\%) of the adult population in the United States reported at least one-time use (1). The Infant Development, Environment, and Lifestyle study reported a $5 \%$ use rate in pregnant women (45).

MA, the most popular amphetamine, can be smoked, snorted, injected, orally ingested, or administered anally. Smoking and injection allow rapid uptake of the drug into the brain, and the user experiences a "rush" that lasts only a few minutes. Snorting and ingestion produce euphoria, which lasts longer. Amphetamines stimulate the release of 
neurotransmitters and interfere with their reuptake at the nerve terminal $(3,46)$. The catecholamine excess accounts for amphetamine's peripheral alpha and beta actions and central nervous system (CNS) stimulant effects of vasoconstriction and bronchodilation, increased systolic and diastolic blood pressures, and an increased respiratory rate. An enhanced perception of environmental stimuli, mood elevation, and a decrease in hunger and fatigue are thought to result from amphetamine's ability to stimulate the reticular activating system. High doses of amphetamines can cause hypertension, retinal damage, cardiac seizures, stroke, and death. Chronic use can lead to anxiety, insomnia, hallucinations, confusion, psychosis headache, pallor or flushing, palpitations, blood pressure instability, and cardiac arrhythmias (3).

"Designer drugs," synthetic analogs, and amphetamine derivatives, which share many of the effects of the parent drugs, are powerful psychostimulants with often unpredictable side effects. Illegal manufacturing of these derivative drugs may involve the inclusion of contaminants, which may pose further dangers.

Most investigators who have studied antenatal amphetamine abuse have found polydrug abuse to be the norm $(47,48)$. Because of the confounding effects of multiple drugs, the possibility of drug impurities, malnutrition, and psychosocial environments, it has been difficult to isolate adverse perinatal and pediatric effects attributable to amphetamine use during pregnancy. Amphetamines cross the placenta and directly affect the fetus. In sheep studies, MA was found to cross the placenta, with resulting fetal drug levels equilibrating with maternal levels within 20 minutes (49).

Amphetamine use during pregnancy has inconsistently been associated with preterm delivery $(47,48,50)$ and inconsistently associated with an increase in congenital heart defects and cleft lip (51). Findings of altered growth parameters including SGA (50), overall smaller birth weights and birth lengths, and smaller head circumferences (47) occur more consistently. Infant withdrawal symptoms have been reported (50). Because amphetamines directly affect the monoaminergic systems of the brain, it has been postulated that amphetamines are capable of causing long-term CNS changes to the developing fetus (46). In a prospective study of children who had been prenatally exposed to amphetamine, Eriksson et al. found the children to exhibit normal physical health parameters (including growth) and normal IQs, but they show a statistically significant increase in aggressive behavior, peerrelated problems, and an increased risk of learning disabilities lasting into school ages (48).

\section{ALCOHOL}

Alcohol use in pregnancy may well be the largest preventable cause of birth defects and developmental disorders. Alcohol use is prevalent around the world; moderate use during pregnancy was accepted behavior until very recently. In the past, intravenous drips of alcohol were even prescribed as a tocolytic for preterm contractions. Controversy exists today on the topic of what constitutes a "safe" amount of alcohol in pregnancy. In 1981, the U.S. Surgeon General recommended that pregnant women "limit the amount of alcohol they drink." This warning was updated in 2005 to the following:
"No amount of alcohol consumption can be considered safe during pregnancy. Alcohol can damage a fetus at any stage of pregnancy," and "The cognitive deficits and behavioral problems resulting from prenatal alcohol exposure are lifelong" (52). Despite warnings from the Surgeon General and massive public health campaigns, alcohol use during pregnancy continues to be a common problem. More than 1 in 5 pregnant women report alcohol use in the first trimester, 1 in 14 consume alcohol in the second trimester, and 1 in 20 in the third trimester (53). One in 30 pregnant women report highrisk drinking, defined as seven or more drinks a week or five or more drinks on any one occasion. One in nine pregnant women binge-drink in the first 3 months of pregnancy; $8.8 \%$ of pregnant women aged 15 to 17 and $3.8 \%$ of pregnant $26-$ to 44-year-olds report binge-drinking (54). Alcohol use in pregnancy is also found more frequently in women who are unmarried, over 30 years old, with a higher parity, and with co-use of tobacco and other substances $(1,54)$. Alcohol use and abuse include every ethnic group, every socioeconomic strata, every age group. The most well-known screen for alcohol use is the CAGE (Cut-down, Annoyed, Guilty, Eye-opener) questionnaire (55), which has a sensitivity of $75 \%$ in women, but does not assess tolerance. The TWEAK (Tolerance, Worried, Eye-openers, Amnesia, Cut-down) scale, with an $87 \%$ sensitivity in women, was developed as a screen for riskdrinking in pregnancy (56).

Alcohol, a small molecule that is readily absorbed from the gastrointestinal tract and distributed throughout the body, is oxidized in the liver by the enzyme alcohol dehydrogenase (3) and metabolized to acetaldehyde, acetate, and then to acetyl coenzyme A. It is further oxidized via the citric acid cycle or is utilized in various anaerobic reactions. Metabolism of alcohol results in increased production of lactate and fatty acids. The consumption of excessive alcohol can result in hypertension, slurred speech, staggering gait, ketoacidosis, myopathy, hepatomegaly, pancreatitis, other upper gastrointestinal disorders, malabsorption and vitamin deficiencies, mood swings, memory lapses, blackouts, other neurobehavioral disorders, hematologic disorders, and cardiac dysfunction $(3,57)$. Evaluation of complete blood count, red blood cell indices, liver function tests, and urine assessment for ketones is recommended.

Ethanol readily crosses the placenta and can interfere with active transport of amino acids across the placenta (58). Acetaldehyde is also a mutagen and is also capable of altering placental transport of nutrients (57).

Perinatal effects associated with alcohol consumption include an increased risk of spontaneous abortion (57), stillbirth (59), lower birth weight $(60,61)$, and IUGR (62). Because alcohol use is associated with many comorbidities, it is difficult to interpret the data. For example, the occurrence of decreased birth weight is not always statistically significant when results are controlled for smoking (59). Also the IUGR infants are born to those classified as heavy drinkers as opposed to the occasional or moderate drinkers (58). Indeed, the evidence is inconsistent when low-to-moderate use of alcohol is considered. A review article revealed that small amounts of alcohol may have a paradoxically protective effect on outcomes such as stillbirth, IUGR, and birth weight (63). The amount and timing of drinking seem to be important too. Women with three or more binge episodes had an increased 
risk of stillbirth, but there was no effect on fetal survival if alcohol intake is low (64). There is a statistically weak association with heavy alcohol use and fetal distress, placental abruption, and 5-minute Apgar scores of less than 6 (59). Antenatal testing may also be affected by alcohol as seen in a transient effect on fetal heart rate (FHR) reactivity (65). Fetal breathing may also be affected for up to 3 hours after alcohol ingestion, according to McLeod et al., but this change does not coincide with the changes in fetal movement or FHR (66).

In 1968, Lemoine, a French pediatrician, published his observations of children whose mothers drank heavily (67). These children displayed characteristic craniofacial abnormalities, growth retardation, and delayed psychomotor maturation. In 1973, Jones and Smith coined the term "fetal alcohol syndrome" (FAS) (68). FAS is characterized by maternal exposure to alcohol, prenatal and/or postnatal growth retardation (weight, length, <10th percentile), CNS involvement (deficient brain growth, structural abnormalities, microcephaly, signs of neurologic abnormality, developmental delay, or intellectual delay), and characteristic facial dysmorphology (microcephaly, microphthalmia and/or short palpebral fissures, poorly developed philtrum, or flattening of the maxillary area). Neonatal features associated with heavy prenatal alcohol exposure include cardiac abnormalities, neonatal irritability and hypotonia, hyperactivity, genitourinary abnormalities, skeletal and muscular abnormalities, ocular problems, or hemangiomas (57).

Fetal effects from alcohol are now considered to fall within a larger diagnostic category of alcohol-related birth defects (ARBD). A minority of exposed children will qualify as having FAS; most will fall into the spectrum of disorders. Birth prevalence of FAS ranges from 0.2 to 2.0 per 1000 live births in the United States with a higher incidence in certain groups (e.g., up to 9 per 1000 live births found in certain Native American groups) (54).

In a 10-year follow-up study of FAS infants in Germany, most children identified as having FAS had persistent features into adolescence (69). Growth deficiency and mental and developmental retardation persisted in patients with mild-tomoderate FAS. Children with FAS and ARBD display a large range of mental abilities in both IQ values and in the extent of developmental disability (69). In an Italian study, children diagnosed with FAS had deficiencies in nonverbal and abstract reasoning, language comprehension, and displayed greater levels of inattention and hyperactivity than their peers (70).

Certain progenitor cells that give rise to craniofacial structures are especially vulnerable in the first trimester (71). According to Sulik et al., craniofacial malformations may be caused by abnormal development of the neural plate and its derivatives, suggesting that the time of critical exposure is at approximately the third week of human gestation (Fig. 1) (72). Mice studies of alcohol have shown that a single exposure of alcohol to the developing brain can cause neuroapoptosis. Prolonged high blood levels of alcohol can cause neuronal loss on a massive scale (71). The combination of alcohol and CBs appears to be especially toxic, with disseminated and severe neuronal degeneration after exposure to mildly toxic ethanol doses (73). Peak vulnerability in humans to ethanol-induced neuroapoptosis is in the last half of gestation, with effects lasting to several years after birth (71).
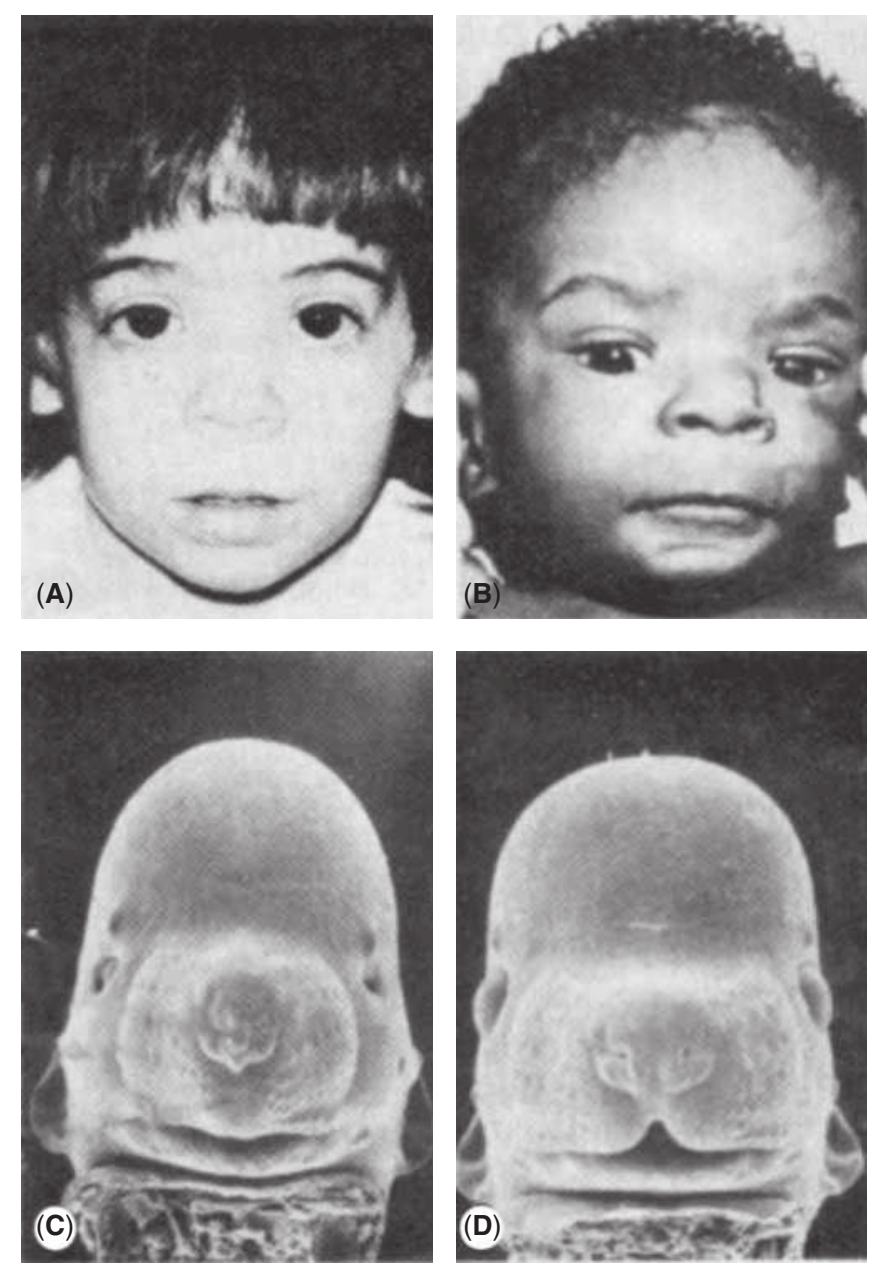

Figure 1 A and B Fetal alcohol syndrome in children. C and D Fourteen-dayold mouse fetuses from ethanol-treated and control mothers, respectively.

\section{OPIATES}

Opiates act by binding to various endogenous opioid receptors, activating euphorigenic effects. After opioid injection, a user experiences warmth and flushing of the skin and sensations described as orgasmic $(3,74)$. Physical dependence and tolerance can develop with regular use, leading the addict to increase doses to get the desired effects.

Opioid overdose causes stupor, a decrease in body temperature, flaccid muscles, eventually coma, convulsions, and cardiorespiratory arrest (74). Opioid withdrawal, on the other hand, is marked by dilated pupils, irritability and restlessness, insomnia, anorexia, tachycardia, weakness, depression, nausea, vomiting, stomach and muscular cramps, muscle twitching, "gooseflesh," chills, hypertension and tachycardia, tearing, rhinorrhea, and yawning. Withdrawal, though distinctly uncomfortable, is usually not lifethreatening (3). Opioid abuse in pregnancy has both direct and indirect effects on the fetus. There is a direct opiate effect due to the transplacental passage of opiates, while indirect effects are a result of related maternal malnutrition or infection, and other sequelae of polydrug abuse and highrisk behaviors. For example, heroin addicts have higher rates of infections, including sexually transmitted diseases $(75,76)$; intravenous drug use increases the risk of contracting such diseases as HIV and hepatitis (76). Opiates readily cross the placenta and have been identified in 
amniotic fluid, umbilical cord blood, neonatal urine, and meconium (76).

In the pregnant abuser, withdrawal may be marked by uterine irritability and increased fetal activity, which may precede other maternal withdrawal symptoms. Perinatal risks of opiate abuse include spontaneous abortion, stillbirth (75), abruptio placentae (76), preterm birth $(75,77)$, low birth weight (77-79), smaller head circumference $(78,79)$, and low birth length (77-79), although birth lengths are similar when controlled for smoking and maternal weight gain, according to at least one author (78). At the ages of 12, 18, 24, and 36 months, short stature was not more prevalent among the infants of opiate addicts $(78,79)$. Likewise, infant weights at 12 through 24 months of age did not differ from those of controls (79). Teratogenic effects following opiate abuse have not been reported $(75,80)$. Dispute remains in regard to respiratory distress syndrome occurring less frequently in the opiate-addicted neonate (75). There is a reported increased risk of SIDS in infants prenatally exposed to opiates $(81,82)$.

As many as $90 \%$ of newborns of opiate addicts will demonstrate some degree of postnatal withdrawal (74). Signs and symptoms of neonatal heroin withdrawal, which typically appear 24 to 48 hours after birth, are hyperactivity, irritability, tremors, convulsions, persistent high-pitched crying, hypertonia alternating with periods of hypotonia, gastrointestinal dysfunction, respiratory distress, sneezing, sweating, tearing, mottling, and fever (74). Untreated infants with neonatal abstinence risk death from respiratory depression.

Methadone maintenance has been the gold standard for treatment of pregnant opiate addict since the 1970s. Methadone is a synthetic mu receptor agonist that blocks the euphoric effects of heroin, decreases the cravings for heroin, and prevents the symptoms of withdrawal. Methadone programs in pregnancy offer several advantages over no treatment. Methadone-maintained women have more prenatal visits and less anemia than other drug abusers $(80,83)$; and methadone can reduce the use of nonprescribed opiates and of related criminal behavior (84). Methadone also has the advantage of providing a more steady concentration of drug, the advantage of which is prevention of recurrent fetal withdrawals.

Methadone for the treatment of opiate abuse is highly regulated: it must be administered at a specially licensed facility. Patients typically present weekly to get their medication, which is usually in the range of 60 to $150 \mathrm{mg}$. (In pregnancy, the lowest dose possible to prevent withdrawal symptoms is recommended.) Methadone is usually taken orally and has a half-life of 24 to 36 hours. It has a long duration of action and extensive, nonspecific tissue binding. Peak plasma levels occur about 4 hours after the ingestion of a maintenance dose (74). Metabolism occurs primarily in the liver, and methadone is excreted in feces and urine (85). Both the placenta and the fetus can further metabolize methadone (85). Neonatal withdrawal from methadone may be more severe than that from other opiates (83), may be prolonged $(80,83)$, and may not occur until 2 to 4 weeks after birth.

It has been suggested by some investigators that fetal opiate withdrawal may be causally related to hypoxia and to meconium passage in utero. Substantiating this idea is the increased incidence of meconium-stained fluid during labor (75). An increased likelihood of shortened gestation has been reported (75), though the clinical significance of 0.6 weeks (77) is questionable. A decrease in frequency of fetal movements and FHR accelerations after methadone ingestion has been reported (86). In a retrospective study of 30 infants of drug-abusing mothers with birth weights less than $1500 \mathrm{~g}$ (of which 15 were infants of methadone addicts), and matched drug-free controls, a lesser probability of intraventricular hemorrhage (IVH) was noted in infants having had prenatal opiate exposure as compared with infants of drug-free women with similar risk factors (87). This suggests that gestational opiate exposure may actually offer protection against IVH in these very small infants. In a study by Edelin et al., rates of occurrence of LBW (birth weights less than 2500g) were similar in the methadone group and the polydrug group ( $29.6 \%$ and $34 \%$ respectively), but they differed significantly from the rates in drug-free controls (16.7\%) (83). This finding has been confirmed by others (80).

Newborn infants of opiate addicts often exhibit neurobehavioral abnormalities $(79,88)$. It has been postulated that downregulation of opiate receptors in the fetus exposed prenatally to opiates is a likely mechanism of these neurobehavioral changes. Methadone-exposed infants are more likely than infants without opiate exposure to have affected motor behaviors at 1 day and at 1 month of age, demonstrating hypertonia, tremulousness, and jerky movements (88). These abnormalities may persist. At 1 year of age, fine motor coordination of methadone-exposed infants is significantly less than that of drug-free controls (80). As young children, those exposed to prenatal methadone may be hyperactive, with difficulty in fine motor coordination and with difficulties relating socially (88).

Data from studies of methadone exposure in the rat tend to support clinical findings. Rat pups perinatally exposed to methadone demonstrate behavioral changes also in that they are abnormally slow to respond to hot-plate stimulation (89). Prenatal methadone exposure in the rat leads to downregulation of opioid receptors in the cerebral cortex and hypothalamus and to a decrease in alpha-adrenergic receptors in the cerebral cortex (90).

Buprenorphine is a partial mu-opioid agonist and kappa antagonist, which was approved for the treatment of nonpregnant, opioid-dependent adults in 2002. Buprenorphine is labeled as an FDA pregnancy category C drug (91). Buprenorphine also crosses the placenta, but possibly to a lesser degree than does methadone (92). Data are limited on its use in detoxification and maintenance therapy in pregnant women, but it appears that buprenorphine may offer some advantages over methadone for prenatal opioid management. Buprenorphine, in contrast to methadone, can be prescribed by physicians who meet specific certification requirements, such as the completion of a training course, thereby potentially increasing access and treatment availability for the opiate dependent (91). Dosing of buprenorphine depends on the type of opioid used and the stage of treatment (91). No statistically significant differences in Apgar scores, in occurrence of neonatal abstinence (requiring treatment), or in the dose of morphine required to manage neonatal withdrawal, were apparent in a Cochrane review of available studies in which outcomes following buprenorphine therapy 
were compared with outcomes in pregnancies involving methadone treatment (93).

The care of the pregnant opioid abuser during labor can be quite complicated. Specific recommendations include continuation of usual maintenance doses of methadone or buprenorphine in labor. (Methadone is used only to prevent withdrawal; it will not provide adequate analgesia.) Methadone or other opiates (e.g., morphine) should be given when evidence of maternal withdrawal exists. Agonist-antagonist combinations should not be given, as they will provoke withdrawal. Similarly, naloxone should not be given to infants of opiate addicts in the delivery room, as it may provoke neonatal withdrawal.

\section{ACKNOWLEDGMENT}

The authors gratefully acknowledge the help of Dr. James Woods, Jr., with the preparation of a previous version of this text.

\section{REFERENCES}

1. Substance Abuse and Mental Health Services Administration (SAMHSA). Results from the 2005 National Survey on Drug Use and Health. NSDUH Series H-30. DHHS SMA06-4194. 2006. [Available from: http://www. oas.samhsa.gov/NSDUH/2k5NSDUH/2k5Results.htm\#3.4] Accessed January 2009.

2. Abel EL. Prenatal exposure to cannabis: a critical review of effects on growth, development, and behavior. Behav Neural Biol 1980; 29: 137-56.

3. Jaffe JH. Drug addiction and drug abuse. In: Gilman AG, Rall TW, Nies AS, Taylor P, eds. The Pharmacological Basis of Therapeutics, 8th edn. Elmsford, NY: Pergamon Press, Inc., 1990: 522-73.

4. Greenland S, Staisch KJ, Brown N, et al. Effects of marijuana on human pregnancy, labor, and delivery. Neurobehav Toxicol Teratol 1982; 4: 447-50.

5. Asch RH, Smith CG. Effects of D9-THC, the principal psychoactive component of marijuana, during pregnancy in the rhesus monkey. J Reprod Med 1986; 31: 1071-81.

6. Linn S, Schoenbaum SC, Monson RR, et al. The association of marijuana use with outcome of pregnancy. Am J Public Health 1983; 73: 1161-4.

7. Fried PA. Postnatal consequences of maternal marijuana use in humans. Ann NY Acad Sci 1989; 562: 123-32.

8. Fried PA. Marijuana use by pregnant women and effects on offspring: an update. Neurobehav Toxicol Teratol 1982; 4: 451-4.

9. Fergusson DM, Lynskey MT, Horwood LJ. Maternal use of cannabis and pregnancy outcome. BJOG 2002; 109: 21-7.

10. Romero J, Garcia-Palomero E, Berrendero F, et al. Atypical location of cannabinoid receptor in white matter areas during rat brain development. Synapse 1997; 26: 317-23.

11. Wang X, Dow-Edwards D, Anderson V, et al. In utero marijuana exposure associated with abnormal amygdala dopamine D2 gene expression in the human fetus. Biol Psychiatry 2004; 56: 909-15.

12. Antonelli T, Tomasini MC, Tattoli M, et al. Prenatal exposure to the CB1 receptor agonist WIN 55,212-2 causes learning disruption associated with impaired cortical NMDA receptor function and emotional reactivity changes in rat offspring. Cereb Cortex 2005; 15: 2013-20.

13. Viveros, MP, Llorente R, Moreno E, Marco EM. Behavioural and neuroendocrine effects of cannabinoids in critical developmental periods. Behav Pharmacol 2005; 16: 353-62.

14. Fried PA, Smith AM. A literature review of the consequences of prenatal marihuana exposure: an emerging theme of a deficiency in aspects of executive function. Neurotoxicol Teratol 2001; 23: 1-11.

15. NIDA Research Monograph 35. In: Richards L, ed. Demographic Trends and Drug Abuse, 1980-1995. Rockville, MD: NIDA, 1981.

16. Assemany SR, Neu RL, Gardner LI. Deformities in a child whose mother took L.S.D. Lancet 1970; 1: 1290.

17. Titus RJ. Lysergic acid diethylamide: its effects on human chromosomes and the human organism in utero. A review of current findings. Int J Addict 1972; 7: 701-4.

18. McGlothlin WH, Sparkes RS, Arnold DO. Effect of LSD on human pregnancy. JAMA 1970; 212: 1483-7.
19. Warren RJ, Rimoin DL, Sly WS. LSD exposure in utero. Pediatrics 1974; 45: 466-9.

20. Burkett G, Yasin S, Palow D. Perinatal implications of cocaine exposure. J Reprod Med 1990; 35: 35-42.

21. Mastrogiannis DS, Decavalas GO, Verma U, et al. Perinatal outcome after recent cocaine usage. Obstet Gynecol 1990; 76: 8-11.

22. Rosenak D, Diamant YZ, Yaffe H, et al. Cocaine: maternal use during pregnancy and its effect on the mother, the fetus, and the infant. Obstet Gynecol Surv 1990; 45: 348-59.

23. Plessinger MA, Woods JR Jr. A review of the cardiovascular effects of cocaine use in pregnancy. Reprod Toxicol 1991; 5: 99-113.

24. Levine SR, Brust JCM, Futrell N, et al. Cerebrovascular complications of the use of the "crack" form of alkaloidal cocaine. N Engl J Med 1990; 323: 699-704.

25. Chasnoff IJ, Burns WJ, Schnoll SH, et al. Cocaine use in pregnancy. N Engl J Med 1985; 313: 666-9.

26. Bingol N, Fuchs M, Diaz V, et al. Teratogenicity of cocaine in humans. J Pediatr 1987; 110: 93-6.

27. Chouteau M, Namerow PB, Leppert P. The effect of cocaine abuse on birthweight and gestational age. Obstet Gynecol 1988; 72: 351-4.

28. MacGregor SN, Keith LG, Chasnoff IJ, et al. Cocaine use during pregnancy: adverse perinatal outcome. Am J Obstet Gynecol 1987; 157: 686-90.

29. Acker D, Sachs BP, Tracey KJ, et al. Abruptio placentae associated with cocaine use. Am J Obstet Gynecol 1983; 146: 220-1.

30. Lutiger B, Graham K, Einarson TR, Koren G. Relationship between gestational cocaine use and pregnancy outcome: a meta-analysis. Teratology 1991; 44: 405-14.

31. Iriye BK, Bristow RE, Hsu CD, et al. Uterine rupture associated with recent antepartum cocaine abuse, Obstet Gynecol 1994; 83: 840-841.

32. Mishra A, Landzberg BR, Parente JT. Uterine rupture in association with alkaloidal (“crack") cocaine abuse. Am J Obstet Gynecol 1995; 173: 243-4.

33. Gonsoulin W, Borge D, Moise KJ Jr. Rupture of unscarred uterus in primigravid woman in association with cocaine use. Am J Obstet Gynecol 1990; 163: 526-7.

34. Monga M, Weisbrodt NW, Andres RL, et al. The acute effect of cocaine exposure on pregnant human myocontractile activity. Am J Obstet Gynecol 1993; 169: 782-5.

35. Little BB, Snell LA, Klein VR, et al. Cocaine abuse during pregnancy: maternal and fetal implications. Obstet Gynecol 1989; 73: 157-60.

36. Cohen HL, Sloves JH, Laungani S, et al. Neurosonographic findings in fullterm infants born to maternal cocaine abusers: visualization of subependymal and periventricular cysts. J Clin Ultrasound 1994; 22: 327-33.

37. Zuckerman B, Frank DA, Hingson R, et al. Effects of maternal marijuana and cocaine use on fetal growth. N Engl J Med 1989; 320: 762-8.

38. Bauchner HJ, Zuckerman B, McClain M. Risk of sudden infant death syndrome among infants with in utero exposure to cocaine. J Pediatr 1988; 113: 831-4.

39. Chasnoff IJ, Chisum GM, Kaplan WE. Maternal cocaine use and genitourinary tract malformations. Teratology 1988; 37: 201-4.

40. Chavez GF, Mulinare J, Cordero JF. Maternal cocaine use during early pregnancy as a risk factor for congenital urogenital anomalies. JAMA 1989; 262: 795-8.

41. Woods JR Jr, Plessinger MA, Scott K, et al. Prenatal cocaine exposure to the fetus: a sheep model for cardiovascular evaluation. Ann NY Acad Sci 1989; 562: 267-79.

42. Webster WS, Brown-Woodman PDC. Cocaine as a cause of congenital malformations of vascular origin: experimental evidence in the rat. Teratology 1990; 41: 689-97.

43. Spear LP, Kirstein CL, Frambes NA. Cocaine effects on the developing CNS: behavioral, psychopharmacological and neurochemical studies. Ann NY Acad Sci 1989; 562: 290-307.

44. Dow-Edwards DL. Long-term neurochemical and neurobehavioral consequences of cocaine during pregnancy. Ann NY Acad Sci 1989; 562: 280-9.

45. Arria AM, Derauf C, LaGasse LL, et al. Methamphetamine and other substance use during pregnancy: preliminary estimates from the infant development, environment, and lifestyle (IDEAL) study. Matern Child Health J 2006; 10: 293-302.

46. Middaugh LD. Prenatal amphetamine effects on behavior: possible mediation by brain monoamines. Ann NY Acad Sci 1989; 562: 308-17. 
47. Little BB, Snell LM, Gilstrap LC III. Methamphetamine abuse during pregnancy: fetal outcome and fetal effects. Obstet Gynecol 1988; 72: $541-4$.

48. Eriksson M, Billing L, Steneroth G, et al. Health and development of 8-year-old children whose mothers abused amphetamine during pregnancy. Acta Paediatr Scand 1989; 78: 944-9.

49. Burchfield DJ, Lucas VW, Abrams RM, et al. Disposition and pharmacodynamics of methamphetamine in pregnant sheep. JAMA 1991; 265: 1968-78.

50. Smith L, Yonekura ML, Wallace T, et al. Effects of prenatal methamphetamine exposure on fetal growth and drug withdrawal symptoms in infants born at term. J Dev Behav Pediatr 2003; 24: 17-23.

51. Nora JJ, Vargo TA, Nora AH, et al. Dexamphetamine: a possible environmental trigger in cardiovascular malformations. Lancet 1970; 1: 1290-1.

52. US Department of Health and Human Services. US Surgeon General Advisory on alcohol use in pregnancy. HHS Press Release 2005. [Available from: http://www.surgeongeneral.gov/pressreleases/sg05052005.html] Accessed January 2009.

53. National Household Survey on Drug Abuse. Substance abuse among pregnant women during 1999 and 2000. The NHSDA Report/SAMHSA 2002. [Available from: http://www.oas.samhsa.gov $/ 2 \mathrm{k} 2 / \mathrm{preg} / \mathrm{preg} . \mathrm{htm}$ ] Accessed January 2009.

54. May PA, Gossage JP. Estimating the prevalence of Fetal Alcohol Syndrome: a summary. Alcohol Res Health 2001; 25: 159-67.

55. Ewing JA. Detecting alcoholism: the CAGE questionnaire. JAMA 1984; 252: 1905-7.

56. Russell M, Martier SS, Sokol RJ, et al. Screening for pregnancy riskdrinking. Alcohol Clin Exp Res 1994; 18: 1156-61.

57. Jessup M, Green JR. Treatment of the pregnant alcohol-dependent woman. J Psychoactive Drugs 1987; 19: 193-202.

58. Rosett HL, Weiner L. Alcohol and pregnancy: a clinical perspective. Annu Rev Med 1985; 36: 73-80.

59. Marbury MC, Linn S, Monson R, et al. The association of alcohol consumption with outcome of pregnancy. Am J Public Health 1983; 73: 1165-8.

60. Hanson JW, Streissguth AP, Smith DW. The effects of moderate alcohol consumption during pregnancy on fetal growth and morphogenesis. J Pediatr 1978; 92: 457-60.

61. Day NL, Jasperse D, Richardson G, et al. Prenatal exposure to alcohol: effect on infant growth and morphologic characteristics. Pediatrics 1989; 84: 536-41.

62. Russell M. Intrauterine growth in infants born to women with alcoholrelated psychiatric diagnoses. Alcohol Clin Exp Res 1977; 1: 225-31.

63. Henderson J, Gray R, Brocklehurst P. Systematic review of effects of lowmoderate prenatal alcohol exposure on pregnancy outcome. BJOG 2007; 114: 243-52.

64. Strandberg-Larsen K, Nielsen NR, Gronboek M, et al. Binge drinking in pregnancy and risk of fetal death. Obstet Gynecol 2008; 111: 602-9.

65. Halmesmaki E, Ylikorkala O. The effect of maternal ethanol intoxication on fetal cardiotocography: a report of four cases. Br J Obstet Gynaecol 1986; 93: 203-5.

66. McLeod W, Brien J, Loomis C, et al. Effect of maternal ethanol ingestion on fetal breathing movements, gross body movements, and heart rate at 37 to 40 weeks' gestational age. Am J Obstet Gynecol 1983; 145: 251-7.

67. Lemoine P, Harrousseau H, Borteyru JP, et al. Les enfant de parents alcooliques. Anomalies observes. A propos de 127 cas. Quest Medicale 1968; 25: 476-82.

68. Jones KL, Smith DW. Recognition of the fetal alcohol syndrome in early infancy. Lancet 1973; 2: 999-1001.

69. Spohr HL, Willms J, Steinhausen HC. Prenatal alcohol exposure and long-term developmental consequences. Lancet 1993; 341: 907-10.

70. Kodituwakku P, Coriale G, Fiorentino D, et al. Neurobehavioral characteristics of children with fetal alcohol spectrum disorders in communities from Italy: preliminary results. Alcohol Clin Exp Res 2006; 30: 1551-61.

71. Young C, Olney JW. Neuroapoptosis in the infant mouse brain triggered by a transient small increase in blood alcohol concentration. Neurobiol Dis 2005; 22: 548-54.

72. Sulik KK, Johnston MC, Webb MA. Fetal alcohol syndrome: embryogenesis in a mouse model. Science 1981; 214: 936-8.

73. Hansen H, Krutz B, Sifringer M, Stefovska V. Cannabinoids enhance susceptibility of immature brain to ethanol neurotoxicity. Ann Neurol 2008; 64: 42-52.

74. Jaffe JH, Martin M. Drug addiction and drug abuse. In: Gilman AG, Rall TW, Nies AS, Taylor P, eds. The Pharmacological Basis of Therapeutics, 8th edn. Elmsford, NY: Pergamon Press, Inc., 1990: 485-521.

75. Wolman I, Niv D, Yovel I, et al. Opioid-addicted parturient, labor, and outcome: a reappraisal. Obstet Gynecol Surv 1989; 44: 592-7.

76. Bauer CR, Shankaran S, Bada HS, et al. The Maternal Lifestyle Study: drug exposure during pregnancy and short-term maternal outcomes. Am J Obstet Gynecol 2002; 186: 487-95.

77. Little BB, Snell LM, Klein VR, et al. Maternal and fetal effects of heroin addiction during pregnancy. J Reprod Med 1990; 35: 159-62.

78. Lifschitz MH, Wilson GS, O’Brian Smith E, et al. Fetal and postnatal growth of children born to narcotic-dependent women. J Pediatr 1983; 102: 686-91.

79. Chasnoff IJ, Burns KA, Burns WJ, et al. Prenatal drug exposure: effects on neonatal and infant growth and development. Neurobehav Toxicol Teratol 1986; 8: 357-62.

80. Wilson GS, Desmond MM, Wait RB. Follow-up of methadone-treated and untreated narcotic-dependent women and their infants: health, developmental, and social implications. J Pediatr 1981; 98: 716-22.

81. Chavez CJ, Ostrea EM Jr, Stryker JC, et al. Sudden infant death syndrome among infants of drug-dependent mothers. J Pediatr 1979; 95: 407-9.

82. Pierson PS, Howard P, Kleber HD. Sudden death in infants born to methadone-maintained addicts. JAMA 1972; 220: 1733-4.

83. Edelin KC, Gurganious L, Golar K, et al. Methadone maintenance in pregnancy: consequences to care and outcome. Obstet Gynecol 1988; 71: 399-404.

84. Kandall SR, Doberczak TM, Jantunen M, et al. The methadonemaintained pregnancy. Clin Perinatol 1999; 26: 173-83.

85. Pond SM, Kreek MJ, Tong T, et al. Altered methadone pharmacokinetics in methadone-maintained pregnant women. J Pharmacol Exp Ther 1985; 233: $1-6$.

86. Archie CL, Lee MI, Sokol RJ, et al. The effects of methadone treatment on the reactivity of the nonstress test. Obstet Gynecol 1989; 74: 254-5.

87. Cepeda EE, Lee MI, Mehdizadeh B. Decreased incidence of intraventricular hemorrhage in infants of opiate dependent mothers. Acta Paediatr Scand 1987; 76: 16-18.

88. Marcus J, Hans SL, Jeremy RJ. Differential motor and state functioning in newborns of women on methadone. Neurobehav Toxicol Teratol 1982; 4: 459-62.

89. Zagon IS, McLaughlin PJ. Analgesia in young and adult rats perinatally exposed to methadone. Neurobehav Toxicol Teratol 1982; 4: 455-7.

90. Wang C, Pasulka P, Perry B, et al. Effect of perinatal exposure to methadone on brain opioid and alpha-2-adrenergic receptors. Neurobehav Toxicol Teratol 1986; 8: 399-402.

91. US Department of Health and Human Services. Clinical guidelines for the use of buprenorphine in the treatment of opioid addiction. DHHS Publication No. 2004; (SMA) 04-3939. [Available from: http://buprenorphine.samhsa.gov/Bup_Guidelines.pdf] Accessed January 2009.

92. Nanovskaya TN, Deshmukh S, Brooks M, et al. Transplacental transfer and metabolism of buprenorphine. J Pharmacol Exp Ther 2002; 300: 26-33.

93. Minozzi S, Amato L, Vecchi S, et al. Maintenance agonist treatments for opiate dependent pregnant women. Cochrane Database Syst Rev 2008; 2: CD006318. 


\section{Tobacco and alcohol use during pregnancy Beth A. Bailey and Robert J. Sokol}

\section{INTRODUCTION}

Substance use, including the most common behaviors of smoking and drinking, is a major health issue for women, regardless of age and reproductive or pregnancy status. Health risks to women can be substantial, and during pregnancy, these risks are shared with the developing fetus. Even with the recognition of the adverse impact of these substances on maternal and fetal health, obstetric clinicians, many of us who might be said to have "surgical personalities," often have difficulty dealing effectively with these "behavioral medicine" issues. Our goal in this concise chapter is to highlight the magnitude of the problems these substances pose during pregnancy and the adverse consequences of the use of these substances on the mother and the offspring, briefly noting some plausible mechanisms for effect. We then turn our attention to the practical issues of how to identify pregnant women who are smoking or consuming alcohol and how to help her make changes in her behavior to benefit both her own health and that of her unborn child.

\section{EPIDEMIOLOGY}

\section{Cigarettes}

Nationally, approximately $11 \%$ to $13 \%$ of women report smoking throughout pregnancy $(1,2)$. However, rates of pregnancy smoking vary considerably by region. For example, many Southern states have rates of pregnancy smoking near $20 \%$ (2), with rural regions seeing rates from $30 \%$ to $40 \%$ (3-5).

Smoking during pregnancy can occur in any subpopulation; however, certain women are statistically more likely to smoke while pregnant. Research has revealed that white women are significantly more likely to smoke during pregnancy than their African-American or Hispanic counterparts $(1,6)$. Additionally, women who live in poverty, who have lower levels of education, and who receive Medicaid are more likely to be pregnancy smokers than other women (6-8).

\section{Alcohol}

Each year in the United States, approximately half a million pregnant women are thought to drink alcohol (9). This translates into a prevalence rate of nearly $13 \%$ and is consistent with the more than $10 \%$ of pregnant women who reported current alcohol use as part of the 2002 Behavioral Risk Factor Surveillance Survey (10) and the 15\% to $20 \%$ identified in a large-scale study conducted more than 15 years ago (11). While estimates vary, anywhere from $2 \%$ to $6 \%$ of women engage in heavy, frequent, or binge-drinking while pregnant $(9,10,12)$.

Drinking during pregnancy occurs across diverse populations and sociodemographic conditions. However, certain racial groups see higher rates of pregnancy drinking, including African-Americans and American Indians/Alaskan
Natives (13). Additionally, women over the age of 30, those who are unmarried, and those who have lower incomes are also at a statistically increased risk of pregnancy alcohol consumption (14). Finally, smoking and drinking often go hand in hand during pregnancy. Women who smoke more than half a pack of cigarettes per day during pregnancy are more than twice as likely as nonsmokers to consume alcohol (38\% vs. $17 \%)$ (14).

\section{ETIOLOGY AND PATHOPHYSIOLOGY Cigarettes}

A significant amount of evidence has accumulated documenting the deleterious effects of pregnancy smoking. The potential short- and long-term effects for the mother have been well reported and include significantly increased risk of respiratory, cardiovascular, and cancer-related diseases. The potential for harmful fetal effects is also profound. Tobacco use in pregnancy is associated with significantly increased rates of pregnancy complications and adverse birth outcomes (15). Ectopic pregnancy, placenta previa and abruption, stillbirth, premature rupture of membranes, and intrauterine growth restriction have all been associated with gestational exposure to cigarettes (16-19). For a smoke-exposed fetus that survives to delivery, the increased likelihood of low birth weight and preterm birth is substantial, with risk increasing with the number of cigarettes smoked per day $(6,20)$. Nationally, smoking accounts for $20 \%$ to $30 \%$ of all low birth weight births (20), and research indicates that infants born to women who smoke are 200 to $300 \mathrm{~g}$ lighter than those born to nonsmokers $(4,21)$. Smoking in pregnancy also nearly doubles the risk of preterm birth $(22,23)$, producing a significant economic cost through neonatal intensive care unit (NICU) admission and treatment. Indeed, babies born to smokers are twice as likely to be admitted to the NICU as those born to nonsmokers (4). Smoke-exposed newborns have an increased risk for respiratory dysfunction, intraventricular hemorrhage, necrotizing enterocolitis, and retinopathy (15). Finally, an increased likelihood of infant mortality is associated with heavy pregnancy smoking, with exposure accounting for $5 \%$ of perinatal deaths (24).

Prenatal exposure to cigarettes has also been shown to have long-term developmental consequences. Physical health effects well into childhood include growth restriction, abnormal neuromotor tone, increases in respiratory infections, asthma, otitis media, and obesity (25-30). Long-term effects on cognitive development, including learning problems and delayed academic achievement, have also been reported (31-33). Finally, prenatal exposure to cigarettes has been implicated in significantly increased rates of behavioral and mental health problems, including attention deficit hyperactivity disorder, conduct disorders, 
depressive and anxiety disorders, criminal behavior, and substance use, abuse, and dependence (34-44).

While not yet completely understood, the mechanisms by which maternal smoking leads to fetal harm and longer term developmental problems have been explored in both animal and human models. The most often cited compounds in cigarettes known to cause harm to the fetus are nicotine, carbon monoxide, and cyanide. Cyanide has been shown, in multiple studies, to be harmful to rapidly dividing cells (45). Carbon monoxide combines with hemoglobin to produce carboxyhemoglobin thus lowering blood oxygen tension and likely contributing to fetal hypoxia (46). In addition, nicotine and carbon monoxide both reduce placental blood flow, impacting fetal growth. A recent study suggested that maternal smoking leads to decreased concentrations of fetal insulin, insulin-like growth factor (IGF), and IGF binding protein, which all may be involved in the pathway by which prenatal cigarette exposure impacts development (47). Thus, both hypoxia and chemicals responsible for growth may be responsible for cell death, structural alterations, and consequent delayed development when prenatal cigarette exposure occurs. Still other studies have focused on the potential impact of nicotine on the developing nervous and neurochemical systems (48). Fetal exposure to smoking, and nicotine in particular, has been proposed to impact serotonin uptake, changes in dopaminergic systems, and changes in DNA and RNA synthesis in the brain during critical developmental periods $(49,50)$. Any one of these potential pathways could impact both structural and functional development, leading to the negative outcomes that have been observed in children exposed to smoking during gestation (51). However, further study is needed.

\section{Alcohol}

It has been four decades since the characteristics of children born to women who consumed alcohol during pregnancy were described in the medical literature (52). The term "fetal alcohol syndrome" (FAS) describes children with prenatal alcohol exposure and resultant growth restriction, central nervous system/neurodevelopmental delays, and facial malformations. In addition to typical or classic FAS, terms such as "alcoholrelated neurodevelopmental disorder" and "fetal alcohol spectrum disorder" characterize a range of problems associated with prenatal alcohol exposure (53). Research has clearly documented the adverse outcomes associated with alcohol exposure during gestation. Full FAS is characterized by prenatal and postnatal growth deficiency, including reduced overall height and microcephaly, and being born small for gestational age with little catch-up seen into childhood; specific facial dysmorphology including long smooth philtrum, thin upper lip, midfacial hypoplasia, small eyes, and inner epicanthal folds; and central nervous system and neurodevelopmental abnormalities including decreased cognitive abilities (52,53).

Even in the absence of diagnosable FAS, alcohol consumption during pregnancy increases the risk of poor outcomes in children. Prenatal alcohol consumption is a strong predictor of low birth weight. Early studies clearly identified a link between even moderate consumption and low birth weight (54), and recent large-scale studies have reported strong associations between exposure and birth weight (55). Consuming one or more drinks per day was associated with a fivefold increase in the likelihood of a low-birth weight delivery (56), while three or more drinks per week were associated with a nearly 150 -g reduction in birth weight (57). Recent evidence suggests that some of the lowered birth weight associated with pregnancy alcohol consumption is accounted for by an increased risk of preterm birth. One or more drinks per day have been found to more than double the risk of preterm birth (56). Even lesser levels of consumption have been linked to 10 -fold or more increases in the risk of extreme preterm birth (prior to 32 weeks of gestation) (58). Mild prematurity was also linked to pregnancy alcohol consumption, particularly in women over the age of 30 (58).

The link between prenatal alcohol exposure and low birth weight and preterm birth is of significant consequence as these are leading causes of neonatal morbidity and mortality in the United States and the strongest biologic predictors of immediate and long-term developmental outcomes (59). In addition to the pathway through birth weight and gestational age, gestational alcohol exposure, even in the absence of full FAS, appears to independently impact a multitude of outcomes into childhood. Continued growth restriction, delays in gross and fine motor development, and impairments in visual motor integration have all been reported (30,60-62). Decreases in overall cognitive performance and general intelligence quotient scores have been associated with even moderate levels of prenatal alcohol exposure (63), in addition to specific deficits including slowed mental processing (64), delayed language and reading abilities $(65,66)$, and increased learning problems (63). Studies have shown that children with prenatal alcohol exposure have problems with organizing and maintaining attention, and with impulsivity (67). Behavioral problems, including hyperactivity and delinquent behavior, have also been reported $(68,69)$. Finally, long-term studies have described associations between prenatal alcohol exposure and many specific psychiatric disorders (70).

The mechanism by which prenatal exposure to alcohol leads to adverse pregnancy, newborn, and longer term developmental outcomes is not yet thoroughly understood. As has been recently reviewed, neuroimaging studies have shown overall and region-specific surface area and volumetric reductions, structural alterations, and white and gray matter density abnormalities (71). These alterations could all be involved in the physical and neurobehavioral effects of exposure. In addition, both animal and human studies have suggested that exposure to alcohol during gestation may cause hypothalamic-pituitaryadrenal (HPA) axis hyperactivity, thereby leading to significant adverse cognitive and behavioral outcomes (72). Furthermore, animal models have shown alcohol intake to decrease blood flow to the placenta, impacting cell growth and death (73). Finally, alcohol consumption increases the production of prostaglandins during pregnancy (74), resulting in decreased cell division (75). Any one, or a combination of these processes, could explain the mechanism by which prenatal alcohol exposure impacts development and outcomes, but further study is clearly needed.

\section{DIAGNOSIS}

\section{Cigarettes}

In a 2000 bulletin (updated in 2005), the American College of Obstetricians and Gynecologists (ACOG) recommended 
screening all pregnant women for smoking (76). Recent reports suggest that prenatal care providers vary widely in rates of screening all pregnant patients for smoking. For example, one study found that $98 \%$ of Ohio obstetricians reported asking all patients about smoking (77), while another study found that only $28 \%$ of obstetricians in rural southern Appalachia reported always asking (78). Reasons providers have given for failure to screen for pregnancy smoking have included lack of time, other issues taking priority, lack of confidence in skills, lack of referral sources, and beliefs that attempts to intervene will not be successful $(77,78)$.

The most common method of determining smoking status during pregnancy has been via self-report, either by oral or written questioning. Self-report data have the advantage of being inexpensive and easy to collect, with responses immediately available (79), and a handful of studies involving pregnant women have suggested that self-reported smoking status is accurate $(80,81)$. Others have suggested, however, that self-report of smoking status during pregnancy is unreliable and results in significant misclassification and underestimation of prevalence rates (82). An examination of the numerous studies that have investigated the association between selfreport of pregnancy smoking status and biochemical verification has revealed false denial or deception rates as low as $1 \%$ and as high as 35\%. A recent review of this literature concluded that measuring smoking status during pregnancy by self-report alone significantly underestimates smoking rates and misclassifies a significant proportion of women (79).

Several factors likely contribute to inaccuracies in selfreport of smoking status during pregnancy, including faulty recall and intentional deception (79). As greater attention is given to the health effects of smoking, both in general and during pregnancy, and as efforts to regulate and reduce smoking increase, smoking becomes stigmatized and individuals are sensitized to socially desirable forms of behavior (83). As this occurs, it is likely that concealment of smoking during pregnancy in particular may increase (84). Multiple studies have shown that many factors may help reduce false denial of smoking among pregnant women, including appropriate wording, timing, and setting of inquiries of smoking status $(85,86)$. For example, we know that the use of a structured multiple choice format in a private confidential setting may increase disclosure (87). Indeed, studies have shown that use of a five-response choice question (Box 1) increases disclosure over asking "Do you smoke?" by $40 \%$ (87).

Given the drawbacks of self-report, determination of pregnancy smoking status in both clinical and research settings often relies on biochemical assessment. Cotinine, a major metabolite of nicotine, is a commonly used biomarker of exposure to tobacco smoke, and cotinine levels in saliva, blood, and urine are often considered the best measure of nicotine consumption (88). Compared with self-report, cotinine levels have been shown to be more accurate indicators of smoking (79) and to be better predictors of pregnancy outcomes (89). Biochemical assessment of smoking behavior is not without limitations, however (83). In addition to the factors of cost and inconvenience, acceptance of appropriately sensitive and specific cutoff points is far from universal. Varying cotinine cutoff levels

\section{Box 1 Pregnancy Smoking Screening Question}

Please choose the response that best describes your smoking

(A) I have never smoked or I have smoked fewer than 100 cigarettes in my lifetime

(B) I stopped smoking before I found out I was pregnant, and I am not smoking now

(C) I stopped smoking after I found out I was pregnant, and I am not smoking now

(D) I smoke some now, but I cut down on the number of cigarettes I smoke since I found out I was pregnant

(E) I smoke regularly now, about the same as before I found out I was pregnant

If the patient responds $A$, no further follow-up is needed

If the patient responds $\mathrm{B}$ or $\mathrm{C}$, reinforce her decision to quit, congratulate her on the success of quitting, and encourage her to remain smoke free

If the patient responds $\mathrm{D}$ or $\mathrm{E}$, she should be classified as a smoker. Document and intervene

Source: From Ref. 87.

have been proposed to distinguish smokers from nonsmokers and from those exposed to environmental tobacco smoke (ETS) (90-92). Given varying cut-point recommendations, the use of biochemical assessment may not ever be completely accurate in distinguishing those who smoke, especially intermittently, from those who are only exposed to ETS (93). Thus, using both self-report and biochemical assessment is likely to yield the most accurate information about pregnancy smoking status.

\section{Alcohol}

For many years, ACOG has consistently advised clinicians to question all pregnant women at their first prenatal visit about current and past alcohol use (94). A recent survey of more than 600 practicing ACOG members revealed that over $90 \%$ asked all women at their first prenatal visit about alcohol use (95). Barriers noted by those who failed to routinely screen prenatal patients for alcohol use included time limitations, patient sensitivity, lack of confidence in skills, and inadequate referral sources.

Even when providers do assess pregnancy alcohol consumption, detection is complicated by many of the same factors that impact the determination of smoking status. Pregnancy alcohol consumption has also become increasingly stigmatized as public awareness of the dangers has increased, leading to the potential for high rates of denial via self-report (96). In addition, and unlike biochemical assessment of smoking status, no reliable biologic marker is available to reveal alcohol use. Although analysis of both meconium and hair samples for fatty acid ethyl esters has been proposed, neither would practically detect alcohol use during pregnancy, and no large population-based studies have validated these methods (97). Similarly, other biochemical markers, such as ethanol metabolites, that reveal only very recent use have not yet been well validated or have not been shown to have adequate diagnostic sensitivity and/or specificity in identifying drinking in pregnant women (98). Thus, most researchers and physicians rely on self-report of maternal alcohol use during pregnancy (53). 


\section{Box 2 T-ACE Screening Tool for Pregnancy Risk Drinking ${ }^{a}$}

\section{Tolerance}

"How many drinks does it take to make you feel high?"

A positive answer, scored a 2 , is more than two cans of beer, two glasses of wine, or two mixed drinks. This suggests tolerance of alcohol and very likely a history of at least moderate-to-heavy alcohol intake

\section{Annoyed}

"Have people annoyed you by criticizing your drinking?" Cut Down

"Have you felt you ought to cut down on your drinking?" Eye-Opener

"Have you ever had a drink first thing in the morning to steady your nerves or get rid of a hangover?"

${ }^{a}$ The first question is scored 0 or 2 points. The last three questions are scored 1 point if answered affirmatively. A total score of 2 or more is considered positive for risk drinking. Source: From Ref. 100.

Studies indicate that obstetricians often obtain inaccurate alcohol consumption information from their patients. For example, in a prospective study that included high-risk women, almost twice as many admitted to drinking during a research interview compared with indications from maternal medical records (99). While tools are available to assist prenatal care providers in accurately identifying women who consume alcohol during pregnancy, a recent study revealed that fewer than one quarter who screen for pregnancy alcohol consumption use a standardized screening tool (95). The T-ACE, a modification of a traditional alcohol screening test, the CAGE, but specifically adapted for use before and during pregnancy, consists of four questions that may be asked as part of the medical history by physicians or office personnel (Box 2) (100). It typically identifies $90 \%$ or more of potential pregnancy risk drinkers, and follow-up questions can rule out false positives (101). The CAGE, also a four-item scale commonly used in obstetric practices, was developed for and validated with men and has not been shown to be valid with pregnant women (100). The TWEAK, a five-item scale, was developed for and validated with pregnant women (102). However, this tool appears to have lower sensitivity for detection of drinking among minority women and has no additional validity over the T-ACE. The AUDIT-C is a three-item scale to screen for alcohol use during pregnancy, rather than consequences of use as queried in the previously described screens. While this tool includes assessment of quantity and pattern of alcohol use, and initial data suggest good sensitivity across different samples, additional validation of the AUDIT-C is needed. Consequently, ACOG and the National Institute on Alcohol Abuse and Alcoholism have both recommended the T-ACE as the best tool for screening all reproductive-aged women and pregnant women specifically for alcohol use.

\section{MANAGEMENT}

\section{Cigarettes}

Addressing cigarette exposure with pregnant smokers should be a top priority for all prenatal care providers, as research has shown that when women quit smoking during pregnancy, the risk of adverse outcomes is nearly reduced to levels experienced by nonsmokers (103). Furthermore, growing

\section{Box 3 The 5 A's Intervention for Pregnant Smokers}

Ask

Tobacco status is queried with a multiple-choice question format and answers are documented in the patient chart Advise

Urge all tobacco users to quit in a clear, strong, personalized manner. Discuss the benefits of quitting including the potential harms of smoking to the fetus and the long-term health consequences of the mother. Provide pregnancyspecific educational materials

Assess

Determine the patient's willingness to quit in the next 30 days and encourage the patient to minimize exposure to secondhand smoke. If the patient declines smoking cessation or is ambivalent, work to increase the patient's motivation to quit

Assist

Provide aid for the patient to quit. Set a quit date. Provide counseling with skills building, pregnancy-specific selfhelp and smoking cessation materials, and social support. Develop a contract to quit smoking. Encourage a patient diary to record successes, problems, and triggers. Discuss alternate behaviors, potential problem situations, and how to handle slipups

Arrange

Provide follow-up contact, either in person or by telephone soon after the quit date, and further follow-up encounters as needed. Congratulate success during each visit. Review circumstances if relapse occurred and use it as a learning experience for the patient. Consider referral or more intensive treatment. Begin postpartum relapse prevention counseling late in pregnancy

Source: From Ref. 108.

evidence suggests that clinically proven interventions can lead to significant successes in smoking cessation (104). Increasingly, obstetric providers are implementing brief interventions (BIs) - low-cost, time-efficient, and effective self-help treatments involving counseling-with their prenatal smokers. The five A's BI method of smoking cessation counseling (ask, advise, assess, assist, and arrange), which can be administered in as few as 3 minutes, is considered best practice and has been adapted for use during pregnancy (Box 3) (105). Most physicians have found this model effective and easy to use in the course of a regular prenatal visit.

When properly implemented, the five A's method results in cessation rates up to four times higher than the rates for those who attempt to quit on their own (106), and 70\% higher than traditional physician advice (105). To date, nearly 100 published studies have detailed the effectiveness of various office-based smoking cessation interventions for pregnant women. A 2008 Cochrane Review of clinical trials showed a significant reduction in pregnancy smoking intervention groups compared with controls (107). The most effective interventions were those based on or similar to the five A's, with these "low-intensity" interventions just as effective as those of higher intensity (i.e., longer and more frequent sessions conducted by counseling professionals encompassing 
Box 4 Brief Intervention for Reducing Alcohol Use During Pregnancy_FRAMES Approach

F: Feedback of personal risk. Compare the patient's level and drinking patterns with pregnancy-recommended abstinence. The patient may not be aware that what she considers normal activity is actually risky

$\mathrm{R}$ : Responsibility for personal control. Stress to the patient that it is her responsibility to make the change for the health of her baby

A: Advice to change. Give direct advice (not insistence) to the patient to change her drinking behavior

M: Мenu of ways to reduce/stop drinking. Identify when the patient is likely to drink and solicit alternate ways that she might handle these situations

E: Empathy. Use a style of interaction that is understanding and involved. Remain positive and encouraging and avoid being judgmental and preachy

S: Self-efficacy. Elicit and reinforce self-motivating statements such as "I am confident that I can stop drinking." Encourage the patient to develop strategies, implement them, and commit to change

Source: From Ref. 111.

multiple types of efforts). In a 2005 bulletin, ACOG established the five A's as a standard component of prenatal care for all smokers (108). And in a 2009, the U.S. Preventive Services Task Force reaffirmed that the net benefit of using a five A's office-based intervention with pregnant smokers is substantial (109).

\section{Alcohol}

Once a prenatal patient had been identified as consuming alcohol, the management options available to the provider will depend upon training, expertise, and available resources. For women who drink but are not alcohol dependent, an inoffice BI may be all that is needed to reduce the risk of an alcohol-exposed pregnancy. For alcohol-dependent women, a BI, coupled with referral to specialized treatment programs, is recommended (110). BIs, including the structured five A's approach described above for use with smokers, can be delivered by health professionals who are not specialists in the treatment of substance use and dependence. Increasing numbers of physicians have obtained continuing education training in motivational interviewing for use with pregnant women who consume alcohol. Motivational interviewing, a type of BI, helps empower the patient to make lifestyle changes while minimizing resistance (111). Using this approach with pregnant alcohol users specifically, the physician can illustrate the importance of abstinence or decreased alcohol intake and the avoidance of bingedrinking, and offer encouragement and optimism about change (112). The most successful interventions follow the FRAMES approach (Box 4).

Research has suggested that women, even those identified as heavy drinkers, respond to motivational interviewing $(113,114)$. In a multicenter randomized controlled trial to reduce the risk for alcohol-exposed pregnancies, 830 at-risk women received educational information plus motivational interviewing or information alone (115). The intervention consisted of four counseling sessions and one contraceptive consultation. The intervention group had a $70 \%$ decrease in risk for alcohol-exposed pregnancies, more than twice the risk reduction for the information-only group at 3-, 6-, and 9-month follow-up (ORs 2.3, 2.2, and 2.1, respectively). In another randomized controlled trial involving only pregnant women, 255 patients who reported alcohol consumption were recruited from a community-based setting and randomly assigned to receive intervention or assessment only (116). The intervention consisted of multiple BI sessions with a nutritionist that included education and feedback, cognitive behavioral procedures, goal setting, and contracting. Compared with women in the control group, women who received the intervention were five times more likely to report abstinence by the end of the pregnancy. In addition, their newborns had significantly higher birth weights and lengths, and a three times lower fetal mortality rate. In another study, a BI administered by nurses and physicians during a prenatal care visit was evaluated with 300 alcohol-consuming women (117). The BI in this study was a single session, averaging 25 minutes, which included knowledge assessment with feedback, goal setting and contracting, behavioral modification, and tips for handling difficult situations. Although pregnancy alcohol consumption decreased for both those who received the intervention and those who received only an assessment, those who received the BI had the most significant decrease. In addition, the greatest decreases were seen among women with the highest initial consumption and those whose partners also participated in the BI. Still other studies have shown that follow-up is critical, as a series of BIs is generally more effective than a single suggestion to stop drinking (118).

\section{CONCLUSIONS}

As many as $20 \%$ of pregnancies are complicated by smoking and/or alcohol consumption. Exposure to either substance significantly increases the risk of poor pregnancy outcomes including low birth weight and preterm birth. Prenatal smoke exposure in particular is implicated in substantial health problems early in life, while both smoke and alcohol exposure can lead to long-term growth delays and cognitive and behavioral problems. While biochemical assessment can be used to identify women who smoke during pregnancy, no reliable biomarker for alcohol consumption is yet clinically applicable. Self-report of smoking and alcohol use is typically relied upon in clinical settings, and reliable and well-validated tools are available and recommended for use with all prenatal patients. Once women who smoke or drink during pregnancy are identified, brief proven interventions can aid the busy clinician in working with patients to reduce or eliminate these pregnancy risk behaviors.

\section{REFERENCES}

1. Martin JA, Hamilton BE, Sutton PD, et al. Births: final data for 2002. Natl Vital Stat Rep 2003; 52: 1-113.

2. March of Dimes. Peristats. [Available from: http://www.marchofdimes .com/peristats/]. Accessed 18 February 2008.

3. Bailey BA. Factors predicting pregnancy smoking in Southern Appalachia. Am J Health Behav 2006; 30: 413-21.

4. Bailey BA, Jones Cole LK. Rurality and birth outcomes: findings from Southern Appalachia and the potential role of pregnancy smoking. J Rural Health 2009; 25: 141-9. 
5. Missouri Monthly Vital Statistics. Focus... Maternal Smoking Trends in Missouri: 1978-1997. Jefferson City, MO: Missouri Department of Health. Center for Health Information Management and Epidemiology $1998 ; 32$.

6. Zotti ME, Replogle WH, Sappenfield WM. Prenatal smoking and birth outcomes among Mississippi residents. J MSMA 2003; 44: 3-9.

7. Jesse DE. Prenatal psychological needs: differences between a TennCare group and a privately insured group in Appalachia. J Health Care Poor Underserved 2003; 14: 535-49.

8. Phares TM, Morrow B, Lansky A, et al. Surveillance for disparities in maternal health related behaviors-selected states, pregnancy risk assessment monitoring system (PRAMS), 200-2001. MMWR 2004; 53 : $1-13$.

9. Floyd RL, Sidhu JS. Monitoring prenatal alcohol exposure. Am J Med Genet C 2004; 127C: 3-9.

10. CDC. Behavioral Risk Factor Surveillance System. Atlanta, GA: US Department of Health and Human Services, CDC. [Available from: http://www.cdc.gov/brfss] .

11. Ebrahim SH, Luman ET, Floyd RL, et al. Alcohol consumption by pregnant women in the United States, 1988-1995. Obstet Gynecol 1998; 92: $187-92$

12. Stratton K, Howe C, Battaglia F, eds. Fetal Alcohol Syndrome: Diagnosis, Epidemiology, Prevention, and Treatment. Washington, DC: National Academy Press, 1996.

13. Fetal alcohol syndrome - Alaska, Arizona, Colorado, and New York, 1995-1997. MMWR 2002; 51: 433-5.

14. Centers for Disease Control and Prevention. Sociodemographic and behavioral characteristics associated with alcohol consumption during pregnancy-United States. MMWR 1995; 44: 261-4.

15. Crawford JT, Tolosa JE, Goldenberg RL. Smoking cessation in pregnancy: why, how, and what next. Clin Obstet Gynecol 2008; 51: 419-35.

16. Dominguez-Rojas V, de Juanes-Pardo JR, Astasio-Arbiza P, et al. Spontaneous abortion in a hospital population: are tobacco and coffee intake risk factors? Eur J Epidemiol 1994; 10: 665

17. Harger JH, Hsing AW, Tuomala RE, et al. Risk factors for preterm premature rupture of fetal membranes: a multicenter case-control study. Am J Obstet Gynecol 1990; 163: 130-7.

18. Ananth CV, Savitz DA, Luther ER. Maternal cigarette smoking as a risk factor for placental abruption, placenta previa, and uterine bleeding in pregnancy. Am J Epidemiol 1996; 144: 881.

19. Spinillo A, Capuzzo E, Nicola SE, et al. Factors potentiating the smoking-related risk of fetal growth retardation. Br J Obstet Gynaecol 1994; 101: 954.

20. U.S. Department of Health and Human Services. Women and smoking: a report of the surgeon general. Rockville, MD: USDHHS, CDC, National Center for Chronic Disease Prevention and Health Promotion, Office of Smoking and Health, 2001.

21. Chiolero A, Bovet P, Paccaud F. Association between maternal smoking and low birth weight in Switzerland: the EDEN study. Swiss Med Wkly 2005; 135: 525-30.

22. Hammoud AO, Bujold E, Sorokin Y, et al. Smoking in pregnancy revisited: findings from a large population-based study. Am J Obstet Gynecol 2005; 192: 1856-62.

23. Kolas T, Nakling J, Salvesen KA. Smoking during pregnancy increases the risk of preterm births among parous women. Acta Obstet Gynecol Scand 2000; 79: 644-8.

24. No authors. Medical care expenditures attributable to cigarette smoking during pregnancy-United States. MMWR 1997; 46: 1048-50.

25. Rona RJ, China S, DuVe FC. Exposure to cigarette smoking and children's growth. Int J Epidemiol 1985; 14: 402-9.

26. Fried PA. Cigarettes and marijuana: are there long term measurable neurobehavioral teratogenic effects? Neurotoxicol 1989; 10: 577-84.

27. Fergusson DM, Horwood LJ. Parental smoking and respiratory illness during early childhood: a 6-year longitudinal study. Pediatr Pulmonol 1985; 1: 99-106.

28. Seidman DS, Laor A, Stevenson SK. Is maternal and paternal smoking during pregnancy associated with asthma in late adolescence? Pediatr Res 1997; 41: 1042A.

29. Toschke AM, Montgomery SM, Pfeiffer U, et al. Early intrauterine exposure to tobacco-inhaled products and obesity. Am J Epidemiol 2003; 158: 1068-74.
30. Covington CV, Nordstrom-Klee B, Ager J, et al. Birth to age 7 growth of children prenatally exposed to drugs: a prospective cohort study. Neurotoxicol Teratol 2002; 24: 489-96.

31. Batstra L, Hadders-Algra M, Neeleman J. Effect of antenatal exposure to maternal smoking on behavioral problems and academic achievement in childhood: prospective evidence from a Dutch birth cohort. Early Hum Dev 2003; 75: 21-33.

32. Fried PA. Adolescents prenatally exposed to marijuana: examination of facets of complex behaviors and comparisons with the influence of in utero cigarettes. J Clin Pharmacol 2002; 42: 97S-102S.

33. Sexton M, Fox NL, Hebel JR. Prenatal exposure to tobacco: II. Effects on cognitive functioning at age 3. Int J Epidemiol 1990; 19: 72-7.

34. Fergusson DM, Horwood LJ, Lynskey MT. Maternal smoking before and after pregnancy: effects on behavioral outcomes in middle childhood. Pediatr 1993; 92: 815-22.

35. Fergusson DM, Woodward LJ, Horwood LJ. Maternal smoking during pregnancy and psychiatric adjustment in late adolescence. Arch Gen Psychiatr 1998; 55: 721-7.

36. Wakschlag LS, Pickett KE, Cook E, Benowitz NL, Leventhal BL. Maternal smoking during pregnancy and severe antisocial behavior in offspring: a review. Am J Public Health 2002; 92: 966-74.

37. Nigg JT, Breslau N. Prenatal smoking exposure, low birth weight, and disruptive behavior disorders. J Am Acad Child Adolesc Psychiatr 2007; 46: 362-9.

38. Milberger S, Biederman J, Faraone SV, Jones J. Further evidence of an association between maternal smoking during pregnancy and attention deficit hyperactivity disorder: findings from a high-risk sample of siblings. J Clin Child Psychol 1998; 27: 352-8.

39. Williams GM, O’Callaghan M, Najman JM, et al. Maternal cigarette smoking and child psychiatric morbidity: a longitudinal study. Pediatrics 1998; 102: el1.

40. Rasanen $\mathrm{P}$, Hakko $\mathrm{H}$, Isohanni $\mathrm{M}$, et al. Maternal smoking during pregnancy and risk of criminal behavior among adult male offspring in the northern Finland 1966 birth cohort. Am J Psychiatr 1999; 156: 857-62.

41. Brennan PA, Grekin ER, Mortensen EL, Mednick SA. Relationship of maternal smoking during pregnancy with criminal arrest and hospitalization for substance abuse in male and female adult offspring. Am J Psychiatr 2002; 159: 48-54.

42. Weissman MM, Warner V, Wickramaratne PJ, Kandel DB. Maternal smoking during pregnancy and psychopathology in offspring followed to adulthood. J Am Acad Child Adolesc Psychiatr 1999; 38: 892-9.

43. Buka SL, Shenassa ED, Niaura R. Elevated risk of tobacco dependence among offspring of mothers who smoked during pregnancy: a 30-year prospective study. Am J Psychiatr 2003; 169: 1978-84.

44. Cornelius MD, Leech SL, Goldschmidt L, Day NL. Is prenatal tobacco exposure a risk factor for early adolescent smoking? A follow-up study Neurotoxicol Teratol 2005; 27: 667-76.

45. Andres RL, Day MC. Perinatal complications associated with maternal tobacco use. Semin Neonatal 2000; 5: 231-41.

46. Lehtovirta P, Forss M. The acute effects of smoking on intervillous blood flow of the placenta. Br J Obstst Gynaecol 1978; 85: 729-31.

47. Ingvarsson RF, Bjarnason AO, Dagbjartsson A, et al. The effects of smoking in pregnancy on factors influencing fetal growth. Acta Pediatrica 2007; 96: 383-6.

48. Mansi G, Raimondi F, Pichini S, et al. Neonatal urinary cotinine correlates with behavioral alterations in newborns prenatally exposed to tobacco smoke. Pediatr Res 2007; 61: 257-61.

49. Fergusson DM. Prenatal smoking and antisocial behavior. Arch Gen Psychiatry 1999; 56: 223-4.

50. Falk L, Nordberg A, Seiger A, Kjaeldgaard A, Hellstrom-Lindahl E. Smoking during early pregnancy affects the expression pattern of both nicotinic and muscarinic acetylcholine receptors in human first trimester brainstem and cerebellum. Neuroscience 2005; 132: 389-97.

51. Roza SJ, Verburg BO, Jaddoe VWV, et al. Effects of maternal smoking in pregnancy on prenatal brain development. The Generation R Study. Eur J Neurosci 2007; 25: 611-17.

52. Jones KL, Smith DW. Recognition of the fetal alcohol syndrome in early infancy. Lancet 1973; 2: 999-1001.

53. Sokol RJ, Delaney-Black V, Nordstrom B. Fetal alcohol spectrum disorder. JAMA 2003; 290: 2996-9. 
54. Sokol RJ, Miller SI, Reed G. Alcohol abuse during pregnancy: an epidemiological study. Alcohol Clin Exp Res 1980; 4: 135-45.

55. Bada HS, Das A, Bauer CR, et al. Low birth weight and preterm births: etiologic fraction attributable to prenatal drug exposure. J Perinatol 2005; 25: 631-7.

56. Jaddoe VWV, Bakker R, Hoffman A, et al. Moderate alcohol consumption during pregnancy and the risk of low birth weight and preterm birth. The Generation R Study. Ann Epidemiol 2007; 17: 834-40.

57. Windham GC, Fenster L, Hopkins B, et al. The association of moderate maternal and paternal alcohol consumption with birthweight and gestational age. Epidemiol 1995; 6: 591-7.

58. Sokol RJ, Janisse JJ, Louis JM, et al. Extreme prematurity: an alcoholrelated birth effect. Alcohol Clin Exp Res 2007; 31: 1031-7.

59. Hack M, Kline NK, Taylor HG. Long-term development outcomes of low birth infants. Future Child 1995; 5: 35-6.

60. Day NL, Leech SL, Richardson GA, et al. Prenatal alcohol exposure predicts continued deficits in offspring size at 14 years of age. Alcohol Clin Exp Res 2002; 26: 1584-91.

61. Barr HM, Streissguth AP, Darby BL, et al. Prenatal exposure to alcohol, caffeine, tobacco and aspirin: effects on fine and gross motor performance in 4-year-old children. Dev Psychol 1990; 26: 339-48.

62. Mattson SN, Riley FP, Gramling L, et al. Neuropsychological comparison of alcohol-exposed children with or without physical features of fetal alcohol syndrome. Neuropsychol 1998; 12: 146-53.

63. Streissguth AP, Barr HM, Sampson PD. Moderate prenatal alcohol exposure: effect on child IQ and learning problems at age 7 1/2 years. Alcohol Clin Exp Res 1990; 14: 662-9.

64. Burden MJ, Jacobson SW, Jacobson JL. Relation of prenatal alcohol exposure to cognitive processing speed and efficiency. Alcohol Clin Exp Res 2005; 29: 1473-83.

65. Russell M, Czarnecki DM, Cowan R, et al. Measures of maternal alcohol use as predictors of development in early childhood. Alcohol Clin Exp Res 1991; 15: 991-1000.

66. Stressguth AP, Barr HM, Carmichael Olson H, et al. Drinking during pregnancy decreases word attack and arithmetic scores on standardized tests: adolescent data from a population-based prospective study. Alcohol Clin Exp Res 1994; 18: 248-54.

67. Nanson JL, Hiscock M. Attention deficits in children exposed to alcohol prenatally. Alcohol Clin Exp Res 1990; 14: 656-61.

68. Bailey BN, Delaney-Black V, Covington CY, et al. Prenatal exposure to binge drinking and cognitive and behavioral outcomes at age 7 years. Am J Obstet Gynecol 2004; 191: 1037-43.

69. Carmichael Olson H, Sampson PD, Barr HM, et al. Prenatal exposure to alcohol and school problems in late childhood: a longitudinal prospective study. Dev Psychopathol 1992; 4: 341-59.

70. Barr HM, Bokstein FL, O'Malley KD, et al. Binge drinking during pregnancy as a predictor of psychiatric disorders on the Structured Clinical Interview for DSM-IV in young adult offspring. Am J Psychiatry 2006; 163: 1061-5.

71. Guerri C, Bazanet A, Riley EP. Foetal alcohol spectrum disorders and alterations in brain and behavior. Alcohol Alcohol 2009; 44: 108-14.

72. Lee S, Choi I, Kang S, Rivier C. Role of various neurotransmitters in mediating the long-term endocrine consequences of prenatal alcohol exposure. Ann NY Acad Sci 2008; 1144: 176-88.

73. Jones PJ, Leichter J, Lee M. Placental blood flow in rats fed alcohol before and during gestation. Life Sci 1981; 29: 1153-9.

74. Anton RF, Becker HC, Randall CL. Ethanol increases PGE and thromboxane production in mouse pregnant uterine tissue. Life Sci 1990; 46: 1145-53.

75. Pennigton S. Ethanol-induced growth inhabitation: the role of cyclic AMP-dependant protein kinase. Alcohol Clin Exp Res 1988; 12: 125-9.

76. American College of Obstetricians and Gynecologists. ACOG educational bulletin: Smoking cessation during pregnancy, Number 260, September 2000. Int J Gynaecol Obstet 2001; 75: 345-8.

77. Jordan TR, Dake JA, Price JH. Best practices for smoking cessation in pregnancy: do obstetrician/gynecologists use them in practice. J Womens Health 2006; 15: 400-11.

78. Bailey BA, Jones Cole LK. Are obstetricians following best-practice guidelines for addressing pregnancy smoking? Results from Northeast Tennessee. South Med J 2009; 102: 894-899.
79. Russell T, Crawford M, Woodby L. Measurements for active cigarette smoke exposure in prevalence and cessation studies: why simply asking pregnant women isn't enough. Nicotine Tob Res 2004; 6: 141-51.

80. George L, Granath F, Johansson AL, Cnattingius S. Self-reported nicotine exposure and plasma levels of cotinine in early and late pregnancy. Acta Obstet Gynecol Scand 2006; 85: 1331-7.

81. McDonald SD, Perkins SL, Walker MC. Correlation between selfreported smoking status and serum cotinine during pregnancy. Addict Behav 2005; 30: 853-7.

82. Kendrick JS, Zahniser SC, Miller N, et al. Integrating smoking cessation into routine public prenatal care: the Smoking Cessation in Pregnancy project. Am J Public Health 1995; 85: 217-22.

83. Patrick DL, Cheadle A, Thompson DC, et al. The validity of self reported smoking: a review and meta-analysis. Am J Public Health 1994; 84: 1086-93.

84. England LJ, Grauman A, Qian C, et al. Misclassification of maternal smoking status and its effects on an epidemiologic study of pregnancy outcomes. Nicotine Tob Res 2007; 9: 1005-13.

85. Dolan-Mullen P, Carbonari JP, Tabak ER, Glenday MC. Improving disclosure of smoking by pregnant women. Am J Obstet Gynecol 1991; 165: 409-13.

86. Kharrazi M, Epstein D, Hopkins B, et al. Evaluation of four maternal smoking questions. Public Health Rep 1999; 114: 60-70.

87. Mullen PD, Carbonari JP, Tabak ER, Glenday MC. Improving disclosure of smoking by pregnant women. Am J Obstet Gynecol 1991; 165: 409-13.

88. Perez-Stable EJ, Benowitz NL, Marin G. Is serum cotinine a better measure of cigarette smoking than self-report? Prev Med 1995; 24: $171-9$.

89. Haddow JE, Knight GJ, Palomaki GE, et al. Cigarette consumption and serum cotinine in relation to birth weight. Br J Obstet Gynaecol 1987; 94: 678-81.

90. Parna K, Rahu M, Youngman LD, et al. Self-reported and serum cotinine-validated smoking in pregnant women in Estonia. Matern Child Health J 2005; 9: 385-92.

91. Etter JF, Vu Duc T, Perneger TV. Saliva cotinine levels in smokers and nonsmokers. Am J Epidemiol 2000; 151: 251-8.

92. U.S. Department of Health and Human Services. Third national report on human exposure to environmental chemicals (NCEH Pub No 050570). Centers for Disease Control and Prevention, National Center for Environmental Health, 2005.

93. Boyd NR, Windsor RA, Perkins LL, Lowe JB. Quality of measurement of smoking status by self-report and saliva cotinine among pregnant women. Matern Child Health J 1998; 2: 77-83.

94. ACOG Committee Opinion. No. 422: at-risk drinking and illicit drug use: ethical issues in obstetric and gynecologic practice. Obstet Gynecol 2008; 112: 1449-60.

95. Diekman ST, Floyd RL, Decoufle P, et al. A survey of obstetriciangynecologists on their patients' alcohol use during pregnancy. Obstet Gynecol 2000; 95: 756-63.

96. Ernhart CB, Morrow-Tlucak M, Sokol RJ, Martier S. Underreporting of alcohol use in pregnancy. Alcohol Clin Exp Res 1988; 12: 506-11.

97. Klein J, Chan D, Koren G. Neonatal hair analysis as a biomarker for in utero alcohol exposure. N Engl J Med 2002; 347: 2086.

98. Cook JD. Biochemical markers of alcohol use in pregnant women. Clin Biochem 2003; 36: 9-19.

99. Stoler JM, Holmes LB. Under-recognition of prenatal alcohol effects in infants of known alcohol abusing women. J Pediatr 1999; 135: 430-6.

100. Sokol RJ, Martier SS, Ager JW. The T-ACE questions: practical prenatal detection of risk -drinking. Am J Obstet Gynecol 1989; 160: 863-8.

101. Chang G, Wilkins-Haug L, Berman S. Alcohol use and pregnancy: improving identification. Obstet Gynecol 1998; 91: 892-8.

102. Russell M. New assessment tools for risk drinking during pregnancy: T-ACE, TWEAK, and others. Alcohol Health Res World 1994; 8: $55-61$.

103. England LJ, Kendrick JS, Wilson HG, et al. Effects of smoking reduction during pregnancy on the birth weight of term infants. Am J Epidemiol 2001; 154: 694-701.

104. Melvin C, Gaffney C. Treating nicotine use and dependence of pregnant and parenting smokers: an update. Nicotine Tobacco Res 2004; 6: S107-124. 
105. Melvin CL, Dolan-Mullen P, Windsor RA, et al. Recommended cessation counseling for pregnant women who smoke: a review of the evidence. Tobacco Control 2000; 9: III80-4.

106. Fiore MC, Bailey WC, Cohen SJ, et al. Treating tobacco use and dependence: clinical practice guideline. Rockville, MD: US Dept of Health and Human Services, 2000.

107. Lumley J, Oliver SS, Chamberlain C, et al. Interventions for promoting smoking cessation during pregnancy. Cochrane Database Syst Rev 2004; 8: CD001055.

108. American College of Obstetricians and Gynecologists. ACOG educational bulletin: smoking cessation during pregnancy, Number 316, October 2005. Obstet Gynecol 2005; 106: 883-8.

109. U.S. Preventive Services Task Force. Counseling and interventions to prevent tobacco use and tobacco-caused disease in adults and pregnant women: U.S. Preventive Services Task Force reaffirmation recommendation statement. Ann Intern Med 2009; 150: 551-5.

110. Bailey BA, Sokol RJ. Pregnancy and alcohol use: evidence and recommendations for prenatal care. Clin Obstet Gynecol 2008; 51: 436-44.
111. Miller WR, Rollnick S. Motivational interviewing: preparing people to change addictive behavior. New York, NY: Guilford Press, 1991.

112. Handmaker NS, Wilbourne P. Motivational interventions in prenatal clinics. Alcohol Res Health 2001; 25: 219-29.

113. Chang G, Goetz MA, Wilkins-Haug L, et al. A brief intervention for prenatal alcohol use: an in-depth look. J Subst Abuse Treat 2000; 18: 365-9.

114. Handmaker NS, Miller WR, Manicke M. Findings of a pilot study of motivational interviewing with pregnant drinkers. J Stud Alcohol 1999; 60: 285-7.

115. Floyd RL, Sobell M, Velasquez MM, et al. Project Choices - a brief intervention with Motivational Interviewing (MI) to reduce pregnancy alcohol exposure. Am J Prev Med 2007; 32: 1-10.

116. O'Connor MJ, Whaley SE. Brief intervention for alcohol use by pregnant women. Am J Pub Health 2007; 97: 252-8.

117. Chang G, McNamara TK, Orav J, et al. Brief intervention for prenatal alcohol use: a randomized trial. Obstet Gynecol 2005; 105: 991-8.

118. Larsson G. Prevention of fetal alcohol effects: an antenatal program for early detection of pregnancies at risk. Acta Obstet Gynecol Scand 1983; 62: $171-8$. 


\section{Exercise and pregnancy Raul Artal}

The epidemic of obesity that began in 1980 continues to proliferate. The sedentary lifestyle and gestational weight gain of many pregnant women has become a major contributor to the obesity epidemics. Lifestyle factors play a significant role particularly during pregnancy when pregnant women gain additional weight over prepregnancy weight. The healthrelated benefits of exercise are well documented in the general population (1-4). Although exercise is being promoted to the general population, health providers are still reluctant to prescribe lifestyle programs to pregnant women. However, pregnancy is a unique period in a woman's and her family's life; health awareness increases and women are particularly inclined to accept lifestyle modifications of judicious diets and exercise. During their pregnancies, even previously sedentary women may seek to modify their lifestyle and initiate exercise activities. The potential health benefits, safety, and feasibility of different amounts and intensities of physical activity in pregnancy have been endorsed by several organizations. In 2002, the American College of Obstetricians and Gynecologists (ACOG) published recommendations and guidelines for exercise during pregnancies, which have been recently reaffirmed $(5,6)$; the guidelines mirror those issued to the general population by the Centers for Disease Control and Prevention. The American College for Sports Medicine (7) and the Society of Obstetricians and Gynecologists of Canada (8) have each published guidelines similar to the ACOG guidelines. Data obtained in 2000 indicate that $15.8 \%$ of pregnant women meet minimum physical activity recommendations and indications are that it has not changed (9). Studies and reviews evaluating the effects of physical activity on pregnancy outcome have identified certain beneficial trends and very few complications (9-11). Physically active pregnant women benefit by engaging in physical activities: among the benefits, they have less macrosomic babies and caesarian sections and develop significantly less gestational diabetes. For patients who require insulin, insulin requirements decrease significantly and frequently could be replaced to a diet and exercise regimen alone. Dissemination of such information could only lead to safe conduct of an active lifestyle.

It has been recognized that lifestyle modification in pregnancy can result in persistent habits that could influence close family and offspring; this has important public health implications, particularly since exercise has been recognized as an effective adjunctive to management of diabetes in pregnancy and postpartum.

Health-care providers should familiarize themselves with the general principals of exercise prescription and be cognizant of the physiologic changes associated with pregnancy and potential maternal and fetal risks $(6,12)$. Exercise guidelines for healthy pregnant women do not differ significantly from those to the general population; however, pregnancy is associated with significant physiologic alterations that could affect both mother and fetus. These alterations are in response to the increased metabolic demands for both mother and fetus.

\section{BIOMECHANICAL AND ANATOMICAL CONSIDERATIONS FOR EXERCISE GUIDELINES IN PREGNANCY}

Several changes in pregnancy affect the biomechanics of movement. Under the influence of estrogen, progesterone, and elastin, there is a generalized connective tissue relaxation leading to ligamentous laxity and joint instability. The increase in weight gain increases the load on the musculoskeletal system, producing higher reaction forces. Additional strain on the musculoskeletal system comes from the continuous change in the body center of mass or point of gravity. The immediate consequence is a combination of progressive lordosis and a limiting in the range of hip joint motion (13). The change in the point of gravity will require greater muscular effort with certain movements, for example, raising from squatting or sitting positions. One way to reduce the muscular effort of the lower extremity muscles is to utilize the upper extremity muscles when rising from the above-mentioned positions. The progressive lordosis in pregnancy frequently results in lower back pain. The above limitation could frequently result in lower back pain. This limitation could be prevented or reduced by improved muscular strength, preferably prior to pregnancy. Most significant, as it relates to exercise guidelines and prescription, is that the biomechanical changes of pregnancy could create balance problems and orthopedic injuries. In addition to back and hip pain, common orthopedic injuries in pregnancy are a consequence of accumulation of interstitial fluid that could result in nerve compression syndromes such as carpal tunnel syndrome.

\section{CARDIOVASCULAR RESPONSES TO EXERCISE IN PREGNANCY}

The major hemodynamic response to exercise is redistribution of blood flow away from the visceral organs and toward the working muscles. During exercise, the cardiac output to the brain and heart remains unchanged, while the uterine blood flow is significantly restricted depending on the intensity of the exercise.

Pregnancy is characterized by a significant increase in blood volume, compensated in part by increased venous capacitance and reduced peripheral vascular resistance (16). Early in pregnancy, the cardiac output increases in excess of the increment in uterine blood flow. Cardiac output reaches maximum values by midpregnancy and becomes highly variable in the third trimester. Increases in heart rate, stroke volume, and cardiac output are observed not only at rest but 
also during submaximal exercise until the third trimester of pregnancy (17).

During cycle ergometry in pregnancy, different kinetic adjustments to the increased maternal mass have been demonstrated $(18,19)$. The relative increases in cardiac output and stroke volume during exercise are similar in pregnant and nonpregnant subjects.

However, during strenuous exercise, the cardiac output response appears to be blunted in pregnancy, a possible reflection of impaired cardiac mechanical functions (20). These findings are significant from the clinical point of view as the preferential redistribution of blood flow during strenuous exercise does not meet the expected requirements, limiting physical performance. Anecdotal observations indicate that such responses may differ and some elite athletes are capable of overcoming such limitations. At lower exercise intensity, the increase in cardiac output is primarily due to higher heart rates, whereas at higher exercise intensities the contribution is primarily due to increased stroke volume. Hemodynamic changes during rest or exercise in the upright position in pregnancy are influenced significantly by the venous return. Venous return can be significantly impaired in the supine position in pregnancy, even at rest, and can cause symptomatic reduction in cardiac output in about $5 \%$ or more of pregnant women (21).

In one invasive study conducted in pregnant women, uterine blood flow during mild cycle exercise was reduced by $25 \%$ in healthy women, during their third trimester of pregnancy (22). A further progressive reduction in blood flow and cardiac output occurs as the intensity of exercise increases. Confirmation for such hemodynamic changes can be found in studies conducted in nonpregnant adults (23). It is plausible that most of the hemodynamic responses to exercise in pregnancy are a reflection of the hemodynamic changes of pregnancy combined with progressive detraining.

Health benefits could be derived from mild to moderate exercise routines at an intensity of training of 12 to 14 on the 20-point Borg Scale of Rating of Perceived Exertion (RPE). The RPE scale was first introduced by Borg (24). An RPE of 12 to 14 will correspond to a physical activity that will be perceived "somewhat hard" at the most by the exercising subject and could be more easily monitored than target heart rates.

\section{PULMONARY RESPONSES TO EXERCISE IN PREGNANCY}

Significant changes in pulmonary functions parallel the other anatomical and physiologic changes of pregnancy. The increase in inspiratory capacity of $300 \mathrm{~mL}$ (inspiratory volume + tidal volume) is paralleled by an increased chest circumference and an increase in the resting oxygen consumption by $10 \%$ to $20 \%$. The physiologic purpose of the pregnancy state of hyperventilation appears to be aimed at reducing the $\mathrm{pCO}_{2}$ and maintaining $\mathrm{pH}$ at 7.44. The increases in tidal volume result in an increase in ventilation, which is the cause of a common subjective feeling of dyspnea. The resulting mild maternal alkalosis facilitates placental gas exchange and prevents fetal acidosis (25). Many conditions such as strenuous exercise may alter these interactions and affect fetal oxygenation.
Comparison of pulmonary responses during mild, moderate, and $\mathrm{VO}_{2}$ max exercise (Fig. 1) indicates that only a modest increase in $\mathrm{VO}_{2}$ max is achieved in pregnancy (26). This limitation appears to be primarily related to the progressive increase in weight. It does appear that both pregnancy and exercise increase the respiratory demands in the presence of reduced maternal ventilatory reserve. Ventilatory responses and aerobic conditioning have been studied in longitudinal studies in pregnancy, and it appears that during submaximal exercise, the differences are reflected predominantly in a significant increase in tidal volume during exercise in pregnancy (26).

Another study (27) found that in recreational athletes who maintain a moderate to high level of exercise performance during and after pregnancy, pregnancy is followed by a small increase in $\mathrm{VO}_{2}$ max.

\section{FUEL UTILIZATION DURING EXERCISE IN PREGNANCY}

The combined needs of pregnancy and exercise evoke complex hormonal interactions. Stress hormones play an essential role in this process and notably release of catecholamines, glucagon, and cortisol increases while insulin secretion is suppressed (Figs. 2,3) (28,29). Changes in fuel metabolism are modulated to satisfy the requirements of pregnancy (Figs. 2,3). The heavier the workload, the higher the sympathoadrenal activity. Catecholamines promote both glycogenolysis and lipolysis. Epinephrine is recognized to play an essential role in maintaining normoglycemia. Normoglycemia is one of the most guarded physiologic functions in pregnancy. Assessing fuel utilization during exercise in pregnancy is essential

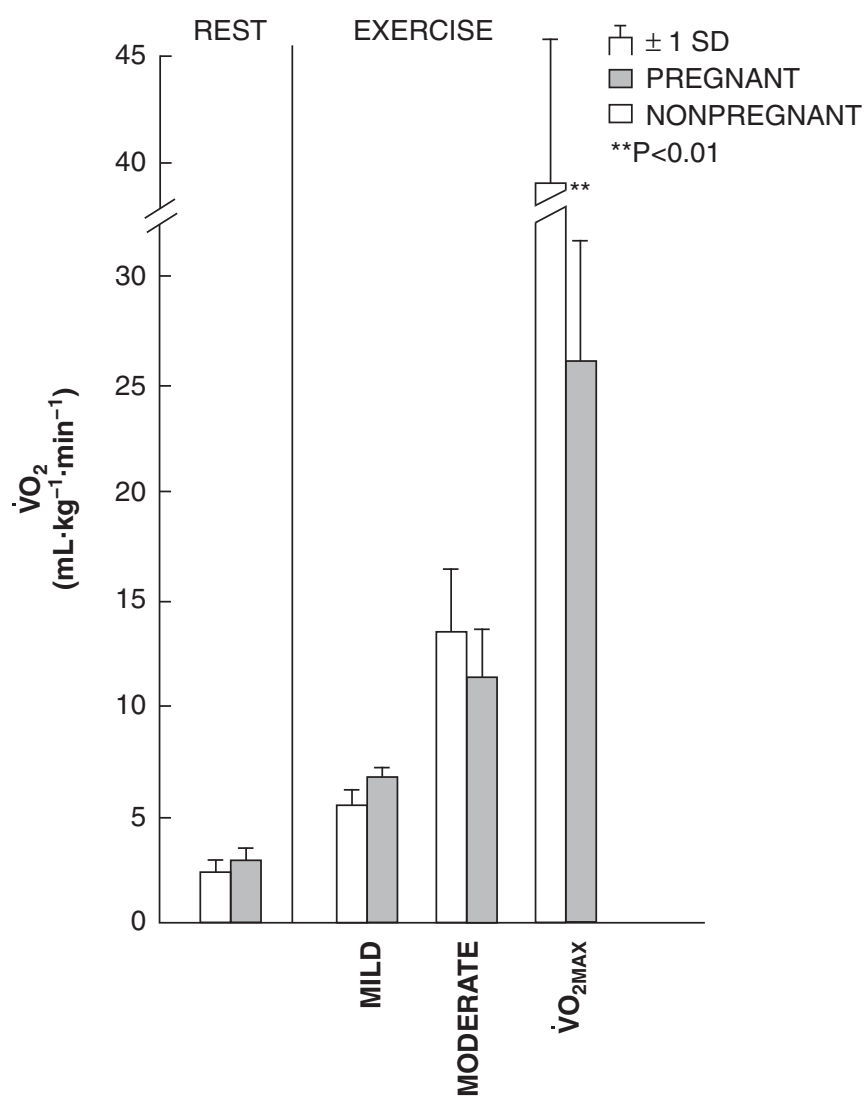

Figure 1 Oxygen consumption during mild, moderate, and $\mathrm{VO}_{2}$ max exercise. Source: From Ref. 26. 
because of the possible metabolic effects of exercise-induced maternal hypoglycemia. Such events are unlikely to occur during mild-moderate exercise, but might occur during prolonged exercise (Fig. 4) (29).

Measurements by indirect calorimetry indicate preferential use of carbohydrates during non-weight-bearing exercise in pregnancy (31). Studies have demonstrated that there is a tendency for higher respiratory exchange ratios (RERs) during pregnancy and during exercise in pregnancy, which suggests a preferential utilization of carbohydrates (31) (Fig. 5).

The RER is a variable that reflects the ratio between carbon dioxide output $\left(\mathrm{CO}_{2}\right)$ and oxygen uptake $\left(\mathrm{VO}_{2}\right)$. The RER will provide information on the proportion of substrate derived from various foodstuffs. For carbohydrates to be completely oxidized to $\mathrm{CO}_{2}$ and $\mathrm{H}_{2} \mathrm{O}$, one volume of $\mathrm{CO}_{2}$ is produced for each volume of $\mathrm{O}_{2}$ consumed. An RER value of 1.0 would indicate that only carbohydrates are being utilized.

The increases in norepinephrine that parallel the exercise intensity have clinical relevance since norepinephrine has restrictive effects on the splanchnic, renal, and uterine blood flow and could precipitate uterine activity (28).

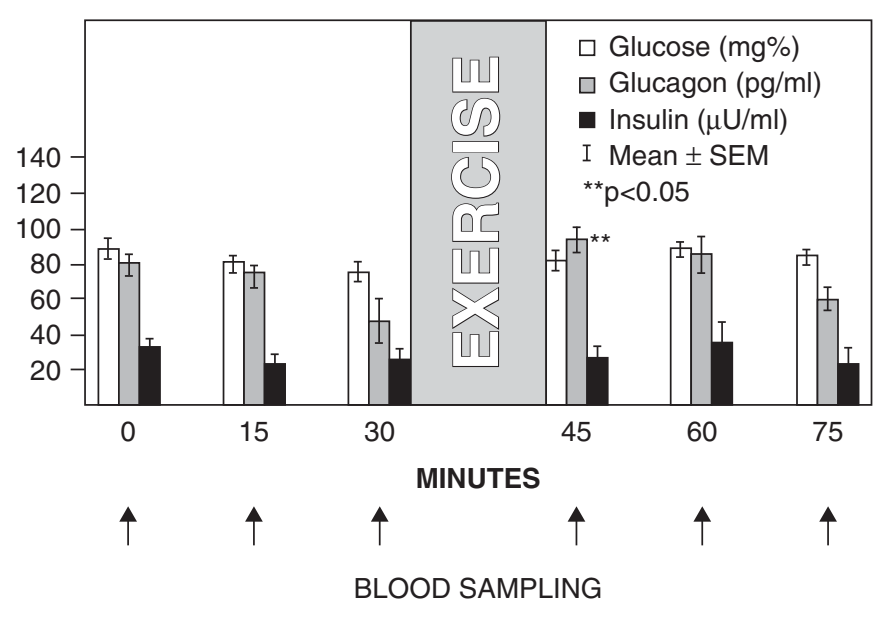

Figure 2 Effect of exercise on maternal glucose, glucagon, and insulin concentrations. The asterisks indicate a significant difference for that value when compared with the pre-exercise value. Source: From Ref. 28.
On occasions, uterine activity has been demonstrated to occur during and after physical activity in pregnancy (28); however, it could potentially have clinical significance only in patients who are at risk for premature labor. And indeed, patients at risk for premature labor are advised to refrain from exercise during pregnancy (Boxes 1-3).

\section{NUTRITION FOR THE ACTIVE PREGNANT WOMAN}

The nutritional needs of active pregnant women are not currently defined; however, the recommended dietary intake for pregnant women at large has been well researched. It has been estimated that the caloric cost of pregnancy is approximately 85,000 calories, approximately 300 extra calories per day above prepregnancy requirements. The average weight gain during pregnancy is approximately $12.5 \mathrm{~kg}$, a $20 \%$ increase in body weight for most women, with most of the weight gain occurring during the second half of pregnancy.

About $40 \%$ of the total gain is represented by the products of conception-fetus, placenta, and amniotic fluid (33). Due to methodology difficulties, it has not yet been determined what the true change in body composition during pregnancy is. However, it appears that there is a modest (less than 5\%) increase in the percentage of body fat while the remainder is interstitial accumulation of fluids (34). It is well recognized that individual weight gain in pregnancy can affect the outcome of pregnancy. Obese women (20\% above standard weight for height) more frequently deliver large infants (greater than $4.0 \mathrm{~kg}$ ); conversely, women who begin pregnancy $15 \%$ below standard weight for height frequently deliver lowbirth weight infants.

Many active women will begin pregnancy underweight. Among these women, there is an increased awareness of body image, and many of them will continue to consume inadequate low-calorie diets during their pregnancies. To compensate for these low-calorie diets, they will frequently turn to excessive use of vitamins. Excessive intake of vitamins can result in an increased incidence of congenital malformations. Excessive ingestion of vitamin D could result in a neonatal syndrome consisting of supravalvular aortic stenosis, elfin facies, and mental retardation (35). Hypervitaminosis A

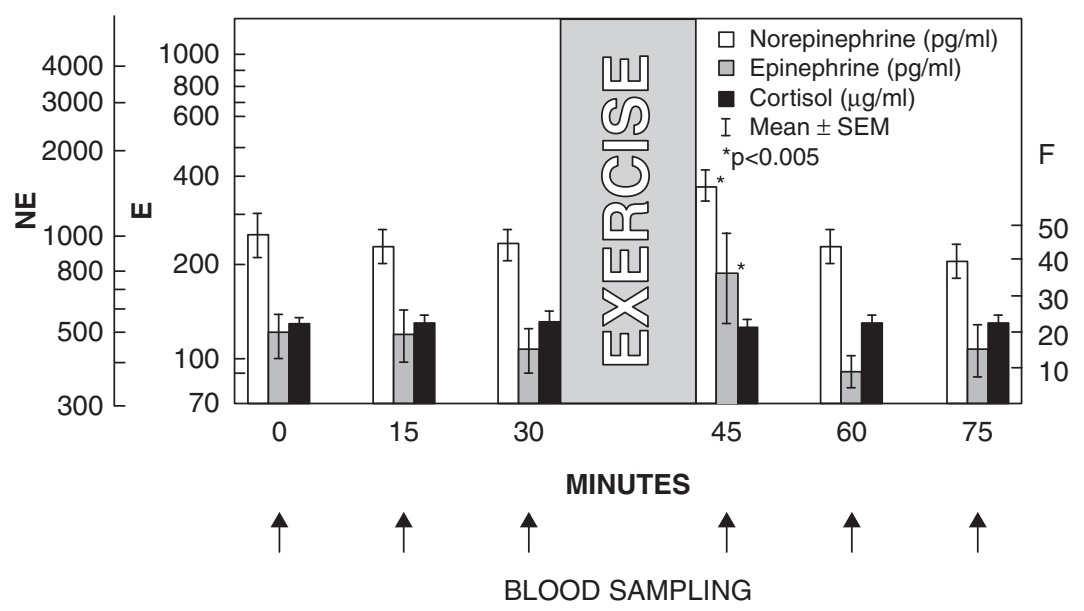

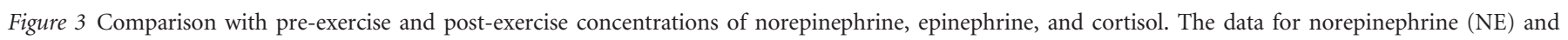

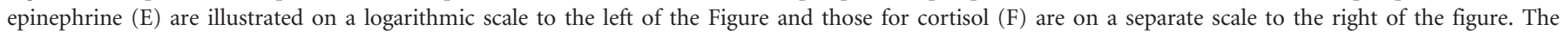
asterisks indicate a significant statistical difference for that value when compared with the pre-exercise value. Source: From Ref. 28. 


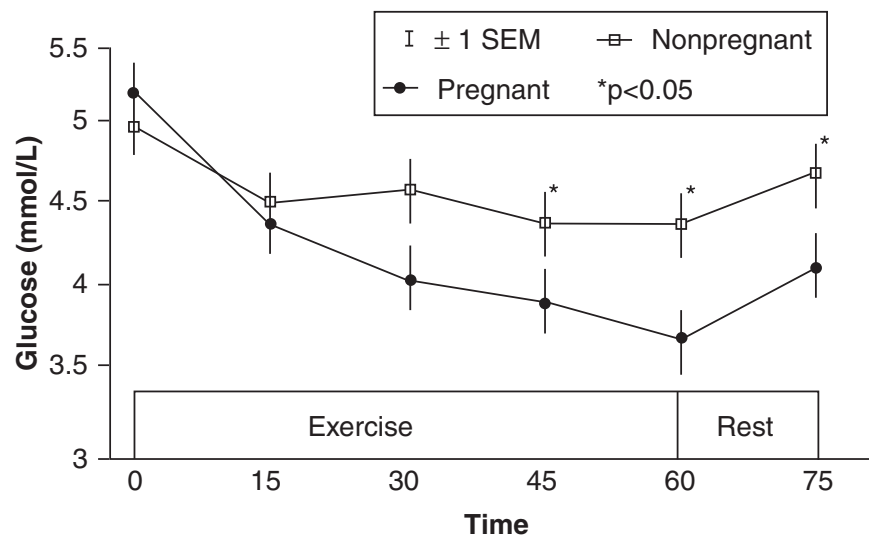

Figure 4 Glucose concentrations during prolonged exercise: pregnant versus nonpregnant. Source: From Ref. 30.

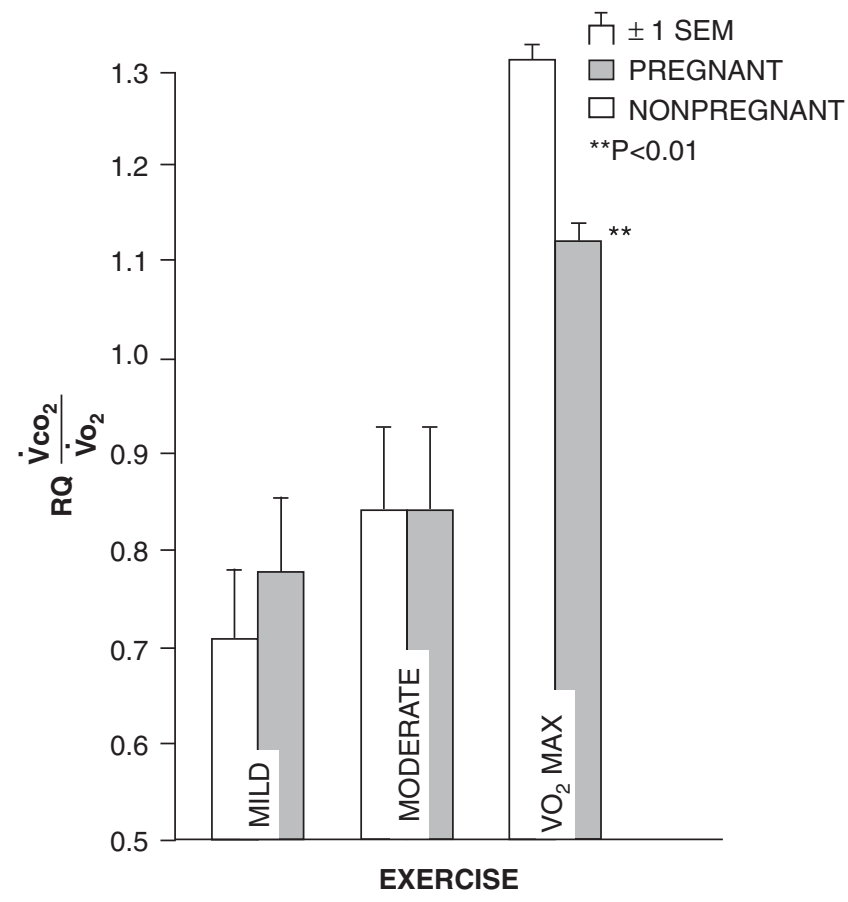

Figure 5 Comparison of respiratory exchange ratio (RQ) during mild, moderate, and $\mathrm{VO}_{2}$ max exercise. Source: From Ref. 26.

may cause urogenital anomalies, ear malformations, cleft palate, and neural tube defects (36). The iron status should be assessed since many of these women may have preexisting depleted iron stores.

The daily nutritional requirements should include an additional $300 \mathrm{cal} /$ day, which is required to provide for the increased basal metabolic needs of pregnancy. Additional calories will be needed depending on the activities conducted. Furthermore, in the postpartum period, lactating women will require an additional 400 to $600 \mathrm{cal} /$ day to meet the metabolic needs. The Institute of Developmental Biology and Molecular Medicine has published in 2009 guidelines for the rate of weight gain during pregnancy by prepregnancy BMI (37). The Institute of Medicine (IOM) recommendations of a single standard of weight gain for all obesity classes has come under criticism since higher body mass index levels combined with additional weight gain are associated with more severe pregnancy complications, such as pre-eclampsia and gestational diabetes (38). The IOM recommendations retained the 1990 focus on the theoretical association between poor gestational weight gain and low birth weight. Important to point out that most causes for low birth weight are the consequences of other conditions than poor nutrition; there is lack of evidence in developed countries that dietary supplementation increases birth weight.

\section{FETAL RESPONSES TO MATERNAL EXERCISE}

The main concerns related to exercise in pregnancy are focused on the fetus and any potential maternal benefits that could be offset by fetal injuries. In the uncomplicated pregnancy, such events are highly unlikely. It appears now that with mild-moderate exercise, the risks for pregnant women are not different from the nonpregnant.

It is well recognized that during other obstetric events, transient hypoxia could result initially in fetal tachycardia and an increase in fetal blood pressure. These fetal responses are protective mechanisms for the fetus to facilitate transfer of oxygen and decrease the $\mathrm{CO}_{2}$ tension across the placenta. Any acute alterations could result in fetal heart rate (FHR) changes, causing possible fetal distress, whereas chronic effects may result in intrauterine growth retardation. Though fetal demise may be associated with either of the above events, there has never been a report in literature to link them with maternal exercise.

The crux of the theoretical risk is related to the selective redistribution of blood flow during exercise to the exercising muscles and away from the visceral organs. Studies conducted in nonpregnant (23) and pregnant (16) subjects confirm that blood flow selectively redistributes during exercise. In experimental animal studies, a reduction in uterine blood flow by $50 \%$ or more could lead to fetal respiratory acidosis. Theoretically, such events could occur during either strenuous or prolonged exercise. Most of the studies addressing fetal responses to exercise have examined FHR changes during or after exercise (39-41). The summary and results of these studies is listed in Table 1 (39-63). Most of the studies demonstrate a minimum or moderate increase in FHR by 10 to 30 beats $/$ min over baseline during or following maternal exercise. FHR decelerations has been reported to occur spontaneously with a frequency of $8.9 \%$ and recorded on rare occasions during and after exercise (62). On few occasions when FHR was measured during exercise in labor with a fetal scalp electrode attached, no FHR abnormalities were recorded either in the presence or absence of uterine activity (60). The mechanism leading to fetal bradycardia during maternal exercise can only be speculated upon: most likely are either vagal reflex, cord compression, or fetal head malposition with no known consequences.

Due to the inaccessibility of the fetus, validation of events of FHR responses could ultimately be obtained only with direct internal FHR monitoring (60) or two-dimensional ultrasound $(63,64)$.

Several studies (58) have attempted to assess umbilical blood flow during maternal exercise with Doppler velocimetry, demonstrating various or no changes. Doppler velocimetry studies are technically difficult to conduct during exercise, so most measurements are taken before and after exercise, by which time any changes could have returned to normal.

The presence of fetal activity has often been interpreted as a reflection of well-being. The same is true for fetal breathing, 
Box 1 Absolute Contraindications of Aerobic Exercise During Pregnancy

- Hemodynamically significant heart disease

- Restrictive lung disease

- Incompetent cervix/cerclage

- Multiple gestation at risk for premature labor

- Persistent second- or third-trimester bleeding

- Placenta previa after 24 weeks of gestation

- Premature labor during the current pregnancy

- Rupture membranes

- Pregnancy-induced hypertension

Source: From Ref. 32.

Box 2 Relative Contraindications to Aerobic Exercise

During Pregnancy

- Severe anemia

- Unevaluated maternal cardiac arrhythmia

- Chronic bronchitis

- Poorly controlled type I diabetes

- Extreme morbid obesity

- Extreme underweight (body mass index $<12$ )

- History of extremely sedentary lifestyle

- Intrauterine growth restriction in current pregnancy

- Poorly controlled hypertension/pre-eclampsia

- Orthopedic limitations

- Poorly controlled seizure disorder

- Poorly controlled thyroid disease

- Heavy smoker

Source: From Ref. 32.

Box 3 Warning Signs to Terminate Exercise While Pregnant

- Vaginal bleeding

- Dyspnea before exertion

- Dizziness

- Headache

- Chest pain

- Muscle weakness

- Calf pain or swelling (need to rule out thrombophlebitis)

- Preterm labor

- Decreased fetal movement

- Amniotic fluid leakage

which is related to the stage in gestation, diurnal variations, maternal plasma glucose, and catecholamine concentration. And, indeed, a direct relationship has been demonstrated between the sympathetic activity of the mother and the frequency of occurrence of both fetal breathing movements and fetal body movements.

Numerous studies have examined the effects of exercise on pregnancy outcome since 2002. The results of these studies are summarized in Table 2 (74).

Epidemiologic studies have suggested for a long time that a link exists between strenuous work and the development of intrauterine growth retardation. Working mothers have a tendency to deliver earlier and small-for-gestational-age infants $(75,76)$. Uncontrolled studies done in elite athletes indicate conflicting evidence (76,77). Most of the studies conducted in pregnant athletes report a very low incidence of complications, but at least one study (79) found that the number of newborn babies of Olympian mothers weighing 2600 to $3000 \mathrm{~g}$ (potentially growth restricted or born prematurely) was greater in their investigations than those weighing in excess of $3500 \mathrm{~g}$. The evidence in the literature is too limited to allow risk assignment for either premature labor or fetal growth restriction for exercising mothers. However, anecdotal clinical observations indicate that patients at risk for premature labor may have labor triggered by exercise. Furthermore, women who are diet conscious often do not receive the minimum required nutrients. The combined energy requirements of pregnancy and exercise coupled with poor weight gain may lead to fetal retardation.

\section{Exercise, hyperthermia, and risk for malformations}

During exercise, the working muscles generate heat, which could increase the body core temperature to levels that are considered teratogenic. Data obtained in studies conducted in research animals indicate that hyperthermia in excess of $39^{\circ} \mathrm{C}$ could be teratogenic and frequently result in neurotubular defects (80-82).

Human data implicating hyperthermia as teratogenic include primarily case reports (83-87) that may suggest an association, but cannot prove causality. The only prospective study, which was conducted with 165 women exposed to first-trimester hyperthermia, has failed to confirm its teratogenic effects (85). Nevertheless, the theoretical risk cannot be dismissed easily, and pregnant women should be advised to avoid hyperthermia during the first weeks of gestation. The malformations implicated are the result of a failure of closure of the neural tube in the phase of embryogenesis that occurs 25 to 27 days after conception or 50 days from last menstrual period.

\section{CLINICAL APPLICATIONS FOR EXERCISE IN PREGNANCY}

Gestational diabetes occurs in $4 \%$ to $7 \%$ of the obstetric population. Insulin therapy and diet may not be the optimal treatment to attain euglycemia. The hormonal changes of pregnancy reduce insulin peripheral sensitivity and are further amplified in patients affected by gestational diabetes. Reduced insulin sensitivity may be reversed most efficiently with exercise. Exercise has been recognized for a long time as an adjunct or alternative therapeutic modality for type II diabetic patients. Pregnant diabetics have been denied this option in the past, primarily because of the potential fetal risks. Published studies on fetal responses to exercise have removed the initial concerns (Table 1).

Exercise training results in sustained insulin sensitivity and improves glucose clearance. Because these functions are altered in gestational diabetes, exercise should be considered not only optional, but also preferable, because it obviates insulin therapy. During exercise, plasma concentrations of glucose counter-regulatory hormones plan an important role in maintaining glucose homeostasis.

Studies conducted in pregnant diabetics indicate that these patients' physiologic and metabolic responses to exercise are similar to healthy nondiabetics (88). No maternal or fetal adverse responses have been reported to date in pregnant 
Table 1 Fetal Heart Rate (FHR) Responses to Maternal Exercise

\begin{tabular}{|c|c|c|c|c|c|c|c|}
\hline Author & $\begin{array}{l}\text { Sample FHR size } \\
\text { after exercise }\end{array}$ & Population & GA & Monitoring device & Type of exercise & $\begin{array}{l}\text { Intensity of } \\
\text { exercise }\end{array}$ & $\begin{array}{l}\text { FHR during } \\
\text { exercise }\end{array}$ \\
\hline Hon \&(40) & 26 & Mixed & $34-41$ & Abdominal ECG & Master step test & Moderate & NA \\
\hline Soiva et al. (41) & 24 & Mixed & $28-40$ & Phonocardiograph & Bicycle ergometer & Mild, moderate-strenuous & NA \\
\hline Hodr \& Brotanek (42) & 56 & Mixed & $29-36$ & Phonocardiograph & Master step test & Moderate & NA \\
\hline Stembera \& Hodr (43) & 67 & Mixed & $38-40$ & Phonocardiograph & Master step test & Moderate & NA \\
\hline Pokorny \& Rous (44) & 14 & Mixed & $36-40$ & Phonocardiograph & Bicycle ergometer & Mild & $=-$ \\
\hline Pomerance et al. (45) & 54 & Normal & $35-37$ & Auscultation & Bicycle ergometer & Moderate & NA \\
\hline Eisenberg de (46) & 22 & Mixed & $28-40$ & Abdominal ECG test; treadmill & Master step & Moderate & NA \\
\hline Pernoll et al. (47) & 16 & Mixed & $24-40$ & Doppler U/S & Bicycle ergometer & Mild & NA \\
\hline Sibley et al. (48) & 7 & Normal & $17-40$ & Doppler U/S & Swimming & Strenuous & $-=$ \\
\hline Dale et al. (49) & 4 & Normal & $31-37.5$ & Doppler U/S & Treadmill & Strenuous & $-=$ \\
\hline Hauth et al. (50) & 7 & Normal & $28-38$ & Doppler U/S & Jogging & Moderate & NA \\
\hline Collings et al. (51) & 20 & Normal & $22-34 \pm$ & Doppler U/S & Bicycle ergometer & Strenuous & - \\
\hline Artal et al. (39) & 15 & Normal & $35.1 \pm 5.65$ & Doppler U/S & Treadmill & Mild & - \\
\hline Artal et al. (39) & 15 & Normal & $34.7 \pm 4.31$ & Doppler U/S & Treadmill & Moderate & - \\
\hline Artal et al. (39) & 15 & Normal & $34.1 \pm 6.85$ & Doppler U/S & Treadmill & $\mathrm{VO}_{2} \max$ & \\
\hline Pipers et al. (52) & 28 & Normal & 35.6 & Doppler U/S & Semirecumbent cycling & Moderate & NA \\
\hline Clapp (53) & 6 & Normal & 20 and 32 & Doppler U/S & Treadmill & Moderate-strenuous & NA \\
\hline Jovanic et al. (54) & 6 & Normal & $36-38.5$ & Doppler U/S & Cycling & Moderate & \\
\hline Veille et al. (55) & 10 & Normal & $33 \pm 3$ & Doppler U/S & Walking & Moderate-strenuous & NA \\
\hline Veille et al. (55) & 10 & Normal & $37 \pm 1$ & Doppler U/S & Cycling & Mild-moderate & $=$ \\
\hline Rauramo (56) & 61 & Mixed & $32-40$ & Doppler U/S & Bicycle ergometer & Mild & NA \\
\hline Paolone et al. (57) & 4 & Normal & $28-34$ & M-Mode echocardiograph & $\begin{array}{l}\text { Bicycle ergometer and } \\
\text { treadmill }\end{array}$ & Mild-moderate & $=$ \\
\hline Carpenter et al. (58) & 45 & Normal & $20-34$ & Linear array two-dimension U/S & Bicycle ergometer & Mild, moderate-strenuous & $=$ \\
\hline Wolfe et al. (59) & 12 & Normal & $37.8 \pm 0.6$ & Doppler U/S & Isometric handgrip & Mild-moderate & $=$ \\
\hline Artal et al. (60) & 12 & Normal & 40 & $\begin{array}{l}\text { Direct fetal scalp monitoring } \\
\quad \text { (during labor) }\end{array}$ & Bicycle ergometer & Moderate & $=$ \\
\hline
\end{tabular}

Abbreviations: GA, gestational age; NA, not applicable, U/S, ultrasound. Source: From Ref. 61. 
Table 2 Studies Examining the Effects of Exercise on Pregnancy Outcome

\begin{tabular}{|c|c|c|c|c|c|}
\hline $\begin{array}{l}\text { Author, year, and } \\
\text { outcome }\end{array}$ & Study type & Activity type & Activity period & $\begin{array}{l}\text { Size of study } \\
\text { population }\end{array}$ & $\begin{array}{l}\text { Main findings: relative risks, odds } \\
\text { ratios, and } 95 \% \text { CI }\end{array}$ \\
\hline \multicolumn{6}{|l|}{ GDM } \\
\hline $\begin{array}{l}\text { Dempsey et al., } \\
2004(65)\end{array}$ & Case control & Recreational & $\begin{array}{l}\text { Year before pregnancy } \\
\text { First } 20 \text { weeks of } \\
\text { pregnancy } \\
\text { Both periods }\end{array}$ & $\begin{array}{l}155 \text { cases } \\
386 \text { controls }\end{array}$ & $\begin{array}{l}\text { Any vs. none } 0.45(0.28-0.74) \\
\text { Vigorous vs. none } 0.29(0.16-0.51) \\
\text { Any vs. none } 0.52(0.33-0.80) \\
\text { Vigorous vs. none } 0.34(0.19-0.63) \\
\text { Active both vs. inactive both } 0.40 \\
\quad(0.23-0.68)\end{array}$ \\
\hline $\begin{array}{l}\text { Dempsey et al., } \\
2004(65)\end{array}$ & Prospective & Recreational & $\begin{array}{l}\text { Year before pregnancy } \\
7 \text { days prior to interview } \\
\text { held at mean gestational } \\
\text { age of } 12.7 \text { weeks } \\
\text { Both periods }\end{array}$ & 909 & $\begin{array}{l}\text { Any vs. none } 0.44(0.21-0.91) \\
4.2 \mathrm{hr} / \text { wk vs. none } 0.24(0.10-0.64) \\
21.1 \mathrm{MET}-\mathrm{hr} / \text { wk vs. none } 0.26 \\
\quad(0.10-0.65) \\
\text { Any vs. none } 0.69(0.37-1.29) \\
6.0 \mathrm{hr} / \text { wk vs. none } 0.67(0.31-1.43) \\
28.0 \mathrm{MET}-\mathrm{hr} / \text { wk vs. none } 0.67 \\
\quad(0.31-1.43) \\
\text { Active both vs. inactive both } 0.31 \\
\quad(0.12-0.79)\end{array}$ \\
\hline $\begin{array}{l}\text { Zhang et al., } \\
2006(66)\end{array}$ & Prospective & $\begin{array}{l}\text { Recreational, walking, } \\
\text { stair-climbing }\end{array}$ & $\begin{array}{l}\text { Potentially } 10 \text { years } \\
\text { before pregnancy }\end{array}$ & 21,765 & $\begin{array}{l}\text { Mean weekly total activity score } \\
\text { Highest vs. lowest quintile } 0.81 \\
\quad(0.68-1.01) \\
\text { Mean weekly vigorous activity score } \\
\text { Highest vs. lowest quintile } 0.77 \\
\quad(0.69-0.94) \\
\text { Brisk/very brisk walking vs. casual } \\
\text { walking with no vigorous activity } \\
0.66 \text { ( } 0.46-0.95) \\
15 \text { flights stairs/day vs. } \leq 2 \text { flights stairs/ } \\
\text { day with no vigorous activity } 0.50 \\
\text { (0.27-0.90) }\end{array}$ \\
\hline $\begin{array}{l}\text { Oken et al., } \\
2006(67)\end{array}$ & Prospective & Recreational & $\begin{array}{l}\text { Year before pregnancy } \\
3 \text { months before } 26-28 \\
\text { weeks of gestation } \\
\text { Both periods }\end{array}$ & 1805 & $\begin{array}{l}\text { Vigorous vs. none } 0.56(0.33-0.95) \\
\text { Vigorous vs. none } 0.90(0.47-1.70) \\
\text { Vigorous before and light/ } \\
\text { moderate or } \\
\text { vigorous during vs. light/moderate } \\
\text { before and inactive during } 0.49 \\
\quad(0.24-1.01)\end{array}$ \\
\hline Dye et al., 1997 (68) & Case control & Any exercise & Entire pregnancy & $\begin{array}{l}372 \text { cases } \\
12,404 \text { controls }\end{array}$ & $\begin{array}{l}\text { None vs. any } 1.0(0.8-1.3) \\
\text { BMI }>33.0 \text { none vs. any } 1.9 \\
\quad(1.2-3.1)\end{array}$ \\
\hline \multicolumn{6}{|l|}{ Pre-eclampsia } \\
\hline $\begin{array}{l}\text { Sorensen et al., } \\
2003 \text { (69) }\end{array}$ & Case control & Recreational & $\begin{array}{l}\text { Year before pregnancy } \\
\text { First } 20 \text { weeks of } \\
\text { pregnancy } \\
\text { Both periods }\end{array}$ & $\begin{array}{l}201 \text { cases } \\
383 \text { controls }\end{array}$ & $\begin{array}{l}\text { Any vs. none } 0.67(0.42-1.08) \\
\text { Vigorous vs. none } 0.40(0.23-0.69) \\
\text { Any vs. none } 0.65(0.43-0.99) \\
\text { Vigorous vs. none } 0.46(0.27-0.79) \\
\text { Active both vs. inactive both } 0.59 \\
\quad(0.35-0.98)\end{array}$ \\
\hline $\begin{array}{r}\text { Saftlas et al., } \\
2004(70)\end{array}$ & $\begin{array}{l}\text { Nested case } \\
\text { control }\end{array}$ & $\begin{array}{l}\text { Work activity } \\
\text { Leisure-time physical } \\
\text { activity (LTPA) }\end{array}$ & $\begin{array}{l}\text { Year before pregnancy } \\
<16 \text { weeks of gestation }\end{array}$ & $\begin{array}{l}44 \text { cases } \\
2422 \text { controls }\end{array}$ & $\begin{array}{l}\text { Virtually none assessed } \\
\text { Any LTPA vs. no LTPA } 0.66 \\
\quad(0.35-1.22) \\
\text { Non-sedentary jobs vs. sedentary jobs } \\
\quad 0.71(0.37-1.36)\end{array}$ \\
\hline $\begin{array}{r}\text { Rudra et al., } \\
2005 \text { (71) }\end{array}$ & Case control & Recreational & Year before pregnancy & $\begin{array}{l}244 \text { cases } \\
470 \text { controls }\end{array}$ & $\begin{array}{l}\text { Perceived very strenuous to maximal } \\
\text { exertion vs. perceived exertion of } \\
\text { none to weak } 0.22(0.11-0.44) \\
\text { BMI }<25 \text { trend of lower risk with } \\
\text { increasing perception of exertion } \\
\quad(p<0.001) \\
\text { BMI } 25 \geq \text { trend of lower risk with } \\
\text { increasing perception of exertion } \\
\quad(p<0.001)\end{array}$ \\
\hline
\end{tabular}


Table 2 (continued) Studies Examining the Effects of Exercise on Pregnancy Outcome

\begin{tabular}{|c|c|c|c|c|c|}
\hline $\begin{array}{l}\text { Author, year, and } \\
\text { outcome }\end{array}$ & Study type & Activity type & Activity period & $\begin{array}{l}\text { Size of study } \\
\text { population }\end{array}$ & $\begin{array}{l}\text { Main findings: relative risks, odds } \\
\text { ratios, and } 95 \% \mathrm{CI}\end{array}$ \\
\hline \multicolumn{6}{|l|}{ Weight-gain restriction } \\
\hline Artal et al., 2007 (72) & Intervention & $\begin{array}{l}\text { Moderate exercise } \\
\text { Program equivalent } \\
\text { to } 60 \% \text { symptom- } \\
\text { limited to } \\
\mathrm{VO}_{2} \max \end{array}$ & $\begin{array}{l}\text { Enrolled }<33 \text { weeks } \\
\text { Gestation to delivery }\end{array}$ & $\begin{array}{l}\text { Obese with GDM } \\
\text { Exercise + diet } \\
\quad(\mathrm{ED}) n=39 \\
\operatorname{Diet}(\mathrm{D}) n=57\end{array}$ & $\begin{array}{l}\text { Percentage gaming weight during study } \\
\text { ED vs. D ( } 53.8 \% \text { vs. } 78.9 \%, p<0.01) \\
\text { Mean weight gain/week } \mathrm{ED} \text { vs. } \mathrm{D}(0.1 \\
\pm 0.4 \mathrm{~kg} \text { vs. } 0.3 \pm 0.4 \mathrm{~kg}, p<0.05) \\
\text { Percentage macrosomic infants born } \\
\text { to women gaining weight during } \\
\text { study (ED or D) vs. to women } \\
\text { losing weight or having no mean } \\
\text { weight change during study } \\
(17.9 \% \text { vs. } 4.2 \%, p=0.12)\end{array}$ \\
\hline \multicolumn{6}{|l|}{$\begin{array}{l}\text { Normalization } \\
\text { of blood } \\
\text { glucose }\end{array}$} \\
\hline
\end{tabular}

diabetics engaging in mild-to-moderate physical activities. Nor have there been reported significant complications in nondiabetic, physically active pregnant women.

To date, several studies have tested several exercise prescription regimens for pregnant diabetics. These studies have assessed maternal and fetal safety, and the efficacy of exercise prescription to improve carbohydrate tolerance and obviate insulin therapy.

The prescribed exercise regimens include the following:

1. Artal R (89) - 20 minutes of bicycle ergometry (18 subjects at $50 \% \mathrm{VO}_{2}$ max after each meal (three times/day) for at least 5 days/week for 6 weeks prior to expected date of confinement (EDC).

2. Jovanovic-Peterson (90)-20 minutes arm ergometry (10 subjects) at less than $50 \% \mathrm{VO}_{2}$ max daily for 6 weeks prior to EDC.

3. Bung $\mathrm{P}$ (73) - 45 minutes of bicycle ergometry (21 subjects) at $50 \%$ of $\mathrm{VO}_{2}$ max three to four times/week for at least 6 weeks prior to EDC.

All the above studies have demonstrated that exercise prescription is feasible in about $25 \%$ of pregnant diabetics and may be utilized as an optional therapeutic approach even in previously sedentary gestational diabetics. The rate of complications observed was similar in the study and control groups. In one study, two patients experienced premature labor of which one was successfully tocolyzed (73). The type, frequency, and intensity of exercise utilized in the above studies were sufficient to attain and maintain euglycemia. Non-weight-bearing exercise appears to be particularly suited for these types of patients.

It appears that in the absence of either medical or obstetric complications, exercise prescription can be an optional adjunct therapy for gestational diabetes. This concept has been well accepted, but not widely prescribed.

\section{SUMMARY}

Exercise in pregnancy conducted in moderation appears to be beneficial to both mother and fetus, by and large, safe. Questions remain as to the safety and potential benefits for strenuous exercise.

The current published literature includes the following consistent findings (3):

1. Women who exercised before pregnancy and continued to do so during pregnancy tend to weigh less, gain less weight, and deliver by and large normal size and fewer macrosomic babies.

2. All women, regardless of initial level of physical activity, decrease their activity as pregnancy progresses.

3. Active pregnant diabetic women can prevent or ameliorate gestational diabetes.

4. Physically active women appear to tolerate labor pain better.

5. Lifestyle modification in pregnancy has a beneficial effect on mothers, their families, and future generations.

Pregnancy should not be a state of confinement, and cardiovascular and muscular fitness can be reasonably maintained. Restriction or modification of physical activity routines should be dictated by obstetric and medical indications only.

\section{REFERENCES}

1. Blair SN, Kohl HW, Paffenberges RS, et al. Physical fitness and all-cause mortality: a prospective study of healthy men and women. J Am Med Assoc 1989; 262: 2395-401.

2. Bouchard C, Shephard RL, Stephens T, eds. Physical activity fitness and health: international proceedings and consensus statement. Champaign, III: Human Kinetics, 1994.

3. American College of Sports Medicine - Position stand. The recommended quantity and quality of exercise for developing and maintaining cardiorespiratory and muscular fitness in healthy adults, 1990. 
4. Pate RR, Pratt M, Blair SN, et al. Physical activity and public health: a recommendation from the Centers for Disease Control and Prevention and the American College of Sports Medicine. J Am Med Assoc 1995; 273: $402-7$.

5. ACOG committee opinion, Number 267, January 2002. Exercise during pregnancy and the post partum period. Obstet Gynecol 2002; 99.

6. Artal R, O'Toole M. Guidelines of the American College of Obstetricians and Gynecologists for exercise during pregnancy and the postpartum period. Br J Sports Med 2003; 37: 6-12.

7. American College of Sports Medicine: Exercise - Prescription for healthy populations and special consideration. ACSM's Guidelines for Exercise Testing and prescription. Chapter 8, 2010: 183-4.

8. SOGC/CSEP. Exercise in pregnancy and postpartum period select SOGC/ CSEC. Clinical practice guidelines, 2003: 129.

9. Petersen AM, Leet TL, Brownson RC. Correlates of physical activity among pregnant women in United States. Med Sci Sports Exerc 2005; 37: 1748-53.

10. Artal RM, Dorey FJ, Kirschbaum TH. Effect of maternal exercise on pregnancy outcome. In: Artal RM, Wiswell RA, Drinkwater BL, eds. Exercise in Pregnancy, 2nd edn. Williams and Wilkins, 1991: 225-9.

11. Melzer K, Schutz Y, Boulvain M, Kayser B. Physical Activity and Pregnancy; cardiovascular adaptations, recommendations and pregnancy outcome. Sports Med 2010; 40: 493-507.

12. Artal RM, Wiswell RA, Drinkwater BL, et al. Exercise guidelines in pregnancy. In: Artal RM, Wiswell RA, Drinkwater BL, eds. Exercise in Pregnancy. Williams \& Wilkins, 1991: 303.

13. Calganeri M, Bird HA, Wright V. Changes in joint laxity occurring during pregnancy. Ann Rheum Dis 1982; 41: 126-8.

14. Gleeson PB, Panol JA. Obstetrical physical therapy. Phys Ther 1988; 68: 1699-702.

15. Artal R, Khodiguian N, Kammula R, et al. Cardiopulmonary adaptations to graded exercise in pregnancy. Proc Soc Gynecol Investig 1986; 66.

16. Clapp JF. The effects of exercise on uterine blood flow. In: Rosenfeld CR, ed. The Uterine Circulation. Ithica: Perinatology Press, 1989: 300-10.

17. Wolfe LA, Ohtake PJ, Mottola MF, et al. Physiological interactions between pregnancy and aerobic exercise. Exerc Sport Sci Rev 1989; 17: 295-351.

18. Guzman CA, Caplan R. Cardiorespiratory response to exercise during pregnancy. Am J Obstet Gynecol 1970; 108: 600-5.

19. Khodiguian N, Jacque-Fortunato S, Wiswell RA, et al. A comparison of cross-sections and longitudinal methods of assessing the influence of pregnancy on cardiac function during exercise. Semin Perinatol 1996; 20: 232-41.

20. Jacque-Fortunateo S, Wiswell RA, Khodiguian N, et al. A comparison of the ventilatory responses to exercise in pregnant, postpartum and nonpregnant women. Semin Perinatol 1996; 20: 263-76.

21. Kerr MG. The mechanical effects of the gravid uterus in late pregnancy. J Obstet Gynaecol Br Commonw 1965; 72: 513-29.

22. Morris N, Osborn SB, Wright HP, et al. Effective uterine blood flow during exercise in normal and pre-eclamptic pregnancies. Lancet 1956; 2: 481 .

23. Rowell LB. Human circulation. In Regulation During Physical Stress. New York: Oxford University Press, 1986: 232.

24. Borg G. Physical Performance and Perceived Exertion. Lund, Sweden: Gleenup, 1962: 1-63.

25. Liboratore SM, Pistelli R, Patalano F, et al. Respiratory function during pregnancy. Respiration 1984; 46: 145-50.

26. Artal R, Wiswell R, Romem Y, et al. Pulmonary responses to exercise in pregnancy. Am J Obstet Gynecol 1986; 154: 378-83.

27. South-Paul JE, Rajagopal KR, Tenholder KB. The effect of participation in a regular exercise program upon aerobic capacity during pregnancy. Obstet Gynecol 1988; 71: 175-9.

28. Artal R, Platt LD, Sperling M, et al. Exercise in pregnancy: maternal cardiovascular and metabolic responses in normal pregnancy. Am J Obstet Gynecol 198; 140: 123-8.

29. Soultanakis H, Artal R, Wiswell R. Prolonged exercise in pregnancy: glucose homeostasis ventilatory and cardiovascular responses. Semin Perinatol 1996; 20: 315-24.

30. Soultanakis HN. Glucose homeostasis during pregnancy in response to prolonged exercise. Postdoctoral theses, University of Southern California, 1988.
31. Artal R, Masaki DI, Khodiguian N, et al. Exercise prescription in pregnancy: weight-bearing versus non-weight bearing exercise. Am J Obstet Gynecol 1989; 161: 1464-9.

32. The American College of Obstetricians and Gynecologists, ACOG Committee Opinion Number 267, Exercise During Pregnancy and the Postpartum Period, January 2002.

33. Romem Y, Masaki DI, Artal R. In: Artal R, Wiswell RA, Drinkwater BL, eds. Physiological and endocrine adjustments to pregnancy in exercise in pregnancy, 2nd edn. Williams and Wilkins, 1991.

34. Jacque-Fortunato S, Khodiguian N, Artal R, et al. Body composition in pregnancy. Semin Perinatol 1996; 20: 340-2.

35. Garcia RE, Friedman WF, Koback MM. Idiopathic hypercalcemia and supravalvular stenosis: documentation of a new syndrome. N Engl J Med 1964; 271: 117-20.

36. Bernhardt IB, Dorsey DJ. Hypervitaminosis A and congenital renal anomalies in a human infant. Obstet Gynecol 1974; 43: 750-5.

37. Rasmussen KM, Yaktine AL, eds. Weight gain during pregnancy: reexamining the recommendation. Washington, DC: The National Academies P, Press, 2009.

38. Artal R, Lockwood CJ, Brown HL. Weight gain recommendations in pregnancy and the obesity epidemic. Obstet Gynecol 2010; 115: 152-5.

39. Artal R, Romem $Y$, Wiswell R. Fetal heart responses to maternal exercise. Am J Obstet Gynecol 1986; 155: 729-33.

40. Hon EH, Wohlgemuth R. The electronic evaluation of fetal heart rate. Am J Obstet Gynecol 1961; 81: 362.

41. Soiva K, Salmi A, Gronroos M, et al. Physical working capacity during pregnancy and effect of physical tests on fetal heart rate. Ann Chir Gynaecol 1963; 53: 187.

42. Hodr J, Brotanek Y. Changes of actography and fetal heart rates in premature deliveries. In: Horsky J, Stembera ZK, eds. Intra-uterine dangers to the fetus. Amsterdam: Excerpta Medica Foundation, 1967: 343.

43. Stembera ZK, Hodr J. The exercise test as an early diagnostic aid for fetal distress. In: Horsky J, Stembera ZK, eds. Intra-uterine dangers to the fetus. Amsterdam: Excerpta Medica Foundation, 1967: 349.

44. Pokorny J, Rous J. The effect of mother's work on fetal heart sounds. In: Horsky J, Stembera ZK, eds. Intra-uterine dangers to the fetus. Amsterdam: Excerpta Medica Foundation, 1967: 359.

45. Pomerance JJ, Gluck L, Lynch VA. Maternal exercise as a screening test for uteroplacental insufficiency. Obstet Gynecol 1974; 44: 383.

46. Eisenberg de Smoler P, Krachmer SK, Ayala LC, et al. El electrocardiogram fetal durante el ejercicio materno. Ginecol Obstet Mex 1974; 35: 521.

47. Pernoll ML, Metcalf J, Paul M. Fetal cardiac response to maternal exercise. In: Longo LD, Reneau DD, eds. Fetal and newborn cardiovascular physiology. Volume 2. New York: Garland Press, 1978: 389.

48. Sibley L, Ruhling RO, Cameron-Foster J, et al. Swimming and physical fitness during pregnancy. J Nurse Midwifery 1981; $26: 3$.

49. Dale E, Mullinax KM, Bryan DH. Exercise during pregnancy: effects on the fetus. Can J Appl Sport Sci 1982; 7: 98.

50. Hauth JC, Gilstrap LC, Widmer K. Fetal heart rate reactivity before and after maternal jogging during the third trimester. Am J Obstet Gynecol 1983; 142: 545.

51. Collings CA, Curet LB, Mullin JP. Maternal and fetal responses to a maternal aerobic exercise program. Am J Obstet Gynecol 1983; 145: 702.

52. Pipers L, Wladimiroff W, McGhie Y. Effect of short-term maternal exercise on maternal and fetal cardiovascular dynamics. Br J Obstet Gynaecol 1984; 91: 1081.

53. Clapp JF III. Fetal heart rate response to running in mid-pregnancy and late pregnancy. Am J Obstet Gynecol 1985; 153: 251-2.

54. Jovanic L, Kessler A, Peterson CM. Human maternal and fetal responses to graded exercise. J Appl Physiol 1985; 58: 1719-22.

55. Veille JC, Hohimer AR, Burry K, et al. The effect of exercise on uterine activity in the last eight weeks of pregnancy. Am J Obstet Gynecol 1985; 151: $727-30$

56. Rauramo I. Effect of short-term physical exercise on fetal heart rate and uterine activity in normal and abnormal pregnancies. Ann Chir Gynaecol 1987; 76: 1-6.

57. Paolone AM, Shangold M, Paul D, et al. Fetal heart rate measurements during maternal exercise - avoidance of artifact. Med Sci Sports Exec 1987; 19: 605-9. 
58. Carpenter MW, Sady SS, Hoegsberg B, et al. Fetal heart rate response to maternal exertion. J Am Med Assoc 1988; 259: 3006-9.

59. Wolfe LA, Lowe-Wylde SJ, Tranmer JE. Fetal heart rate during maternal static exercise. Can J Sport Sci 1988; 13: 95-6P.

60. Artal R, Khodiguian N, Paul RH. Intrapartum fetal heart rate responses to maternal exercise. Unpublished.

61. Artal R, Posner M. Fetal responses to maternal exercise. In: Artal R, Wiswill R, Drinkwater B, eds. Exercise in pregnancy. Williams and Wilkins Pbl., 1991.

62. Artal R, Romem Y, Paul RH, et al. Fetal bradycardia induced by maternal exercise. Lancet 1984; 2: 258.

63. Moore DH, Jarrett JC, Bendick PJ. Exercise induced changes in uterine artery blood flow, as measured by Doppler ultrasound, in pregnant subjects. Am J Perinatol 1989; 5: 94.

64. Veille JC, Bacevice AE, Wilson B. Umbilical artery waveform during bicycle exercise in normal pregnancy. Obstet Gynecol 1989; 73: 957.

65. Dempsey JC, Sorensen TK, Williams MA, et al. Prospective study of gestational diabetes mellitus risk in relation to maternal recreational physical activity before and during pregnancy. Am J Epidemiol 2004; 159: 663-70.

66. Zhang C, Solomon CG, Manson JE, et al. A prospective study of pregravid physical activity and sedentary behaviors in relation to the risk for gestational diabetes mellitus. Arch Intern Med 2006; 166: 543-8.

67. Oken E, Ning Y, Rifas-Shiman SL, et al. Associations of physical activity and inactivity before and during pregnancy with glucose tolerance. Obstet Gynecol 2006; 108: 1200-7.

68. Dye TD, Knox KL, Artal R, et al. Physical activity, obesity, and diabetes in pregnancy. Am J Epidemiol 1997; 146: 961-5.

69. Sorensen TK, Williams MA, Lee IM, et al. Recreational physical activity during pregnancy and risk of preeclampsia. Hypertension 2003; 41: $1273-80$

70. Saftlas AF, Logsden-Sachett N, Wang W, et al. Work, leisure-time physical activity, and risk of preeclampsia and gestational hypertension. Am J Epidemiol 2004; 160: 758-65.

71. Rudra CB, Williams MA, Lee IM, et al. Perceived exertion during prepregnancy physical activity and preeclampsia risk. Med Sci Sports Exerc 2005; 37: 1836-41.

72. Artal R, Catanzaro RB, Gavard JA, et al. A lifestyle intervention of weightgain restriction: diet and exercise in obese women with gestational diabetes mellitus. Appl Physiol Nutr Metab 2007; 32: 596-601.
73. Bung P, Artal R, Khodignian N, et al. Exercise in gestational diabetes: an optional therapeutic approach. Diabetes 1991.

74. Gavard JA, Artal R. Effect of exercise on pregnancy outcome. Clin Obstet Gynecol 2008; 51: 467-80 12.

75. Fox ME, Harris RE, Brekken AL. The active-duty military pregnancy: a new high-risk category. Am J Obstet Gynecol 1984; 129: 705.

76. Taferi N, Naeye RL, Gobzie A. Effects of maternal undernutrition and heavy physical work during pregnancy on birth weight. Br J Obstet Gynaecol 1980; 87: 222.

77. Clapp JF, Dickstein S. Endurance exercise and pregnancy outcome. Med Sci Sports Exerc 1984; 16: 556.

78. Erdelyi GJ. Gynecological survey of female athletes. J Sports Med Phys Fit 1962; 2: 174-5.

79. Zaharieva E. Olympic participation by women; effects on pregnancy and childbirth. J Am Med Assoc 1972; 221: 992-5.

80. Edwards MJ. Congenital defects in guinea pigs: fetal resorptions, abortions and malformations following induced hyperthermia during early gestation. Teratology 1969; 2: 313 .

81. Skreb N, Frank Z. Developmental abnormalities in the rat induced by heat shock. J Embryol Exp Morphol 1983; 11: 445.

82. Kelham L, Ferm VH. Exencephaly in fetal hamsters exposed to hyperthermia. Teratology 1976; 14: 323.

83. Miller P, Smith DW, Shepard TH. Maternal hyperthermia as a possible cause of anencephaly. Lancet 1978; 1: 519-21.

84. Shiota K. Neural tube defects and maternal hyperthermia in early pregnancy. Epidemiology in a human embryonic population. Am J Med Genet 1982; 12: 281-8.

85. Edwards MJ. Hyperthermia as a teratogen. Teratol Carcinog Mutagen 1986; 6: 563-82.

86. Harvey MAS, McRorie MM, Smith DW. Suggested limits to the use of hot tubs and saunas by pregnant women. Can Med Assoc J 1981; 125: 50-4.

87. Clarren SK, Smith DW, Harvey MAS. Hyperthermia - a prospective evaluation of a possible teratogenic agent in man. J Pediatr 1979; 95: 81.

88. Artal R, Wiswell R, Romem Y. Hormonal responses to exercise in diabetic and nondiabetic pregnant patients. Diabetes 1985; 39: 78-80.

89. Artal R, Masaki D. Exercise in gestational diabetes. Pract Diabetol 1989; 8: $7-14$.

90. Jovanovic-Peterson L, Durak EP, Peterson CM. Randomized trial of diet versus diet plus cardiovascular conditioning on glucose levels in gestational diabetes. Am J Obstet Gynecol 1989; 161: 415-19. 


\title{
42 Ethical considerations
}

\author{
Frank A. Chervenak and Laurence B. McCullough
}

\section{INTRODUCTION}

Ethics is an essential dimension of maternal-fetal medicine (1-3). In this chapter, we develop a framework for clinical judgment and decision-making about the ethical dimensions of clinical practice and research in maternal-fetal medicine. We emphasize a preventive ethics approach that appreciates the potential for ethical conflict and adopts ethically justified strategies to prevent those conflicts from occurring. Preventive ethics helps to build and sustain a strong physician-patient relationship. We first define ethics, medical ethics, and the fundamental ethical principles of medical ethics, beneficence, and respect for autonomy. Second, we show how these two principles should interact in clinical judgment and practice in maternal-fetal medicine, with emphasis on the core concept of the fetus as a patient. Third, we set out an ethical framework for innovation and research in maternal-fetal medicine, with an emphasis on ethically justified criteria to guide the initiation and implementation of research in maternal-fetal medicine.

\section{ETHICS, MEDICAL ETHICS, AND ETHICAL PRINCIPLES}

Ethics is the disciplined study of morality. Medical ethics is the disciplined study of morality in medicine and concerns the obligations of physicians and health-care organizations to patients as well as the obligations of patients (4). It is important not to confuse medical ethics with the many sources of morality in a pluralistic society. These include, but are not limited to, law, our political heritage as free people, the world's religions (most of which now exist in our country), ethnic and cultural traditions, families, the traditions and practices of medicine (including medical education and training), and personal experience. Medical ethics since the eighteenthcentury European and American Enlightenments has been secular (5). It makes no reference to God or revealed tradition, but to what rational discourse requires and produces. At the same time, secular medical ethics is not intrinsically hostile to religious beliefs. Therefore, ethical principles and virtues should be understood to apply to all physicians, regardless of their personal religious and spiritual beliefs (6).

The traditions and practices of medicine constitute a major source of morality for physicians. They provide an important reference point for medical ethics because they are based on the obligation to protect and promote the health-related interests of the patient. This obligation tells physicians what morality in medicine ought to be, but in very general abstract terms. Providing a more concrete, clinically applicable account of that obligation is the central task of medical ethics, using ethical principles (4).

The ethical principle of beneficence in its general meaning and application requires one to act in a way that is expected reliably to produce the greater balance of benefits over harms in the lives of others (6). To put this principle into clinical practice requires a reliable account of the benefits and harms relevant to the care of the patient, and of how those goods and harms should be reasonably balanced against each other when not all of them can be achieved in a particular clinical situation, such as a request for an elective cesarean delivery (7). In medicine, the principle of beneficence requires the physician to act in a way that is reliably expected to produce the greater balance of clinical benefits clinical over harms for the patient (4).

Beneficence-based clinical judgment has an ancient pedigree, with its first expression found in the Hippocratic Oath and accompanying texts (8). Beneficence-based clinical judgment makes an important claim: to interpret reliably the health-related interests of the patient from medicine's perspective. This perspective is provided by accumulated scientific research, clinical experience, and reasoned responses to uncertainty. As rigorously evidence based (9), beneficencebased judgment is thus not the function of the individual clinical perspective of any particular physician and therefore should not be based merely on the clinical impression or intuition of an individual physician. On the basis of this rigorous clinical perspective, focused on the best available evidence, beneficence-based clinical judgment identifies the clinical benefits that can be achieved for the patient in clinical practice based on the competencies of medicine. The clinical benefits that medicine is competent to seek for patients are the prevention and management of disease, injury, handicap, and unnecessary pain and suffering, and the prevention of premature or unnecessary death. Pain and suffering become unnecessary when they do not result in achieving the other goods of medical care, for example, allowing a woman to labor without effective analgesia (4).

Nonmaleficence means that the physician should prevent causing harm and is best understood as expressing the limits of beneficence. This is also known as Primum non nocere or "first do no harm." This commonly invoked dogma is really a Latinized misinterpretation of the Hippocratic texts, which emphasized beneficence while avoiding harm when approaching the limits of medicine (4). Nonmaleficence should be incorporated into beneficence-based clinical judgment: when the physician approaches the limits of beneficence-based clinical judgment, that is, when the evidence for expected benefit diminishes and the risks of clinical harm increase, then the physician should proceed with great caution. The physician should be especially concerned to prevent serious, far-reaching, and irreversible clinical harm to the patient. It is important to note that there is an inherent risk of paternalism in beneficence-based clinical judgment. By this, we mean that beneficence-based clinical judgment, if it is mistakenly considered to be the sole source of moral responsibility and therefore moral authority in medical care, invites the unwary 
physician to conclude that beneficence-based judgments can be imposed on the patient in violation of her autonomy. Paternalism is a dehumanizing response to the patient and, therefore, should be avoided in the practice of obstetrics and gynecology.

The preventive ethics response to this inherent paternalism is for the physician to explain the diagnostic, therapeutic, and prognostic reasoning that leads to his or her clinical judgment about what is in the interest of the patient so that the patient can assess that judgment for herself. This general rule can be put into clinical practice in the following way: the physician should disclose and explain to the patient the major factors of this reasoning process, including matters of uncertainty. In neither medical law nor medical ethics does this require that the patient be provided with a complete medical education (10). The physician should then explain how and why other clinicians might reasonably differ from his or her clinical judgment. The physician should then present a well-reasoned response to this critique. The outcome of this process is that beneficence-based clinical judgments take on a rigor that they sometimes lack in maternal-fetal medicine and the process of their formulation includes explaining them to the patient. It should be apparent that beneficence-based clinical judgment will frequently result in the identification of a continuum of clinical strategies that protect and promote the patient's health-related interests, such as indications for cesarean delivery for fetal distress. Awareness of this feature of beneficence-based clinical judgment provides an important preventive ethics antidote to paternalism by increasing the likelihood that one or more of these medically reasonable, evidence-based alternatives will be acceptable to the patient. All beneficence-based clinical alternatives should be identified and explained to all patients, regardless of how the physician is paid, especially those that are well established in evidencebased obstetrics and gynecology.

One advantage for the physician in carrying out this approach to communicating with the patient would be, we believe, to increase the likelihood of compliance (11). This is an especially pertinent consideration in the management of pregnancy, where the pregnant patient often must monitor herself for clinical changes, for example, self-observation for unusual weight gain or bleeding. Another advantage would be to provide the patient with a better-informed opportunity to make a decision about whether to seek a second opinion. The approach outlined above should make such a decision less threatening to her physician, who has already shared with the patient the limitations on clinical judgment.

In contrast to the principle of beneficence, there has been increasing emphasis in the literature of medical ethics on the ethical principle of respect for autonomy (6). This principle requires one always to empower the patient in the informed consent process by proving her with information about the beneficence-based alternatives for managing her or the fetus's condition and then implement the value-based preferences of the pregnant patient, unless there is compelling ethical justification for not doing so. The pregnant patient increasingly brings to her medical care her own perspective on what is in her interest. The principle of respect for autonomy translates this fact into autonomy-based clinical judgment. Because each patient's perspective on her interests is a function of her values and beliefs, it is impossible to specify the benefits and harms of autonomy-based clinical judgment in advance. Indeed, it would be inappropriate for the physician to do so, because the definition of her benefits and harms and their balancing are the prerogative of the patient. Not surprisingly, autonomy-based clinical judgment is strongly antipaternalistic in nature (4).

To understand the demands of this principle in clinical practice, we need an operationalized concept of autonomy to make it relevant to clinical practice. To do this, we identify three sequential autonomy-based behaviors on the part of the patient: (i) absorbing and retaining information about her condition and alternative diagnostic and therapeutic responses to it, (ii) understanding that information (evaluating and rank-ordering those responses and appreciating that she could experience the risks of treatment), and (iii) expressing a value-based preference. The physician has a role to play in each of these. They are, respectively, (i) to recognize the capacity of each patient to deal with medical information (and not to underestimate that capacity), provide information (disclose and explain all beneficencebased alternatives), and recognize the validity of the values and beliefs of the patient; (ii) not to interfere with but, when necessary, to assist the patient in her evaluation and ranking of diagnostic and therapeutic alternatives for managing her condition; and (iii) to elicit and implement the patient's value-based preference (4).

The legal obligations of the physician regarding informed consent were established in a series of cases during the twentieth century. In 1914, Schloendorff v. The Society of The New York Hospital established the concept of simple consent, whether the patient says "yes" or "no" to medical intervention $(10,12)$. To this day in the medical and bioethics literature, this decision is quoted: "Every human being of adult years and sound mind has the right to determine what shall be done with his body, and a surgeon who performs an operation without his patient's consent commits an assault for which he is liable in damages" (12). The legal requirement of consent further evolved to include disclosure of information sufficient to enable patients to make informed decisions about whether to say "yes" or "no" to medical intervention (10).

There are two accepted legal standards for such disclosure. The professional community standard defines adequate disclosure in the context of what the relevantly trained and experienced physician tells patients. The reasonable person standard, which has been adopted by most states, goes further and requires the physician to disclose "material" information, what any patient in the patient's condition needs to know and the layperson of average sophistication should not be expected to know. Patients need to know what the physician thinks is clinically salient, that is, the physician's beneficence-based clinical judgment. This reasonable person has emerged as the ethical standard, and we therefore urge perinatologists to adopt it. On this standard, the physician should disclose to the patient her or the fetus's diagnosis (including differential diagnosis when that is all that is known), the beneficence-based (and therefore medically reasonable) alternatives to diagnose and manage the patient's condition, and the short-term and long-term benefits and risks of each alternative. 
THE INTERACTION OF BENEFICENCE AND RESPECT FOR AUTONOMY IN CLINICAL JUDGMENT AND

\section{PRACTICE IN MATERNAL-FETAL MEDICINE}

The ethical principles of beneficence and respect for autonomy play a complex role in obstetric clinical judgment and practice. There are obviously beneficence-based and autonomy-based obligations to the pregnant patient: the physician's perspective on the pregnant woman's health-related interests provides the basis for the physician's beneficence-based obligations to her, whereas her own perspective on those interests provides the basis for the physician's autonomy-based obligations to her. Because of an insufficiently developed central nervous system, the fetus cannot meaningfully be said to possess values and beliefs. Thus, there is no basis for saying that a fetus has a perspective on its interests. There can therefore be no autonomy-based obligations to any fetus. Hence, the language of fetal rights has no meaning and therefore no application to the fetus in obstetric clinical judgment and practice despite its popularity in public and political discourse in the United States and other countries. Obviously, the physician has a perspective on the fetus's health-related interests, and the physician can have beneficence-based obligations to the fetus, but only when the fetus is a patient. Because of its importance for obstetric clinical judgment and practice, the ethical concept of the fetus as a patient requires detailed consideration (4).

The ethical concept of the fetus as a patient is essential to obstetric clinical judgment and practice. Developments in fetal diagnosis and management strategies to optimize fetal outcome have become widely accepted, encouraging the development of this concept. This concept has considerable clinical significance because when the fetus is a patient, directive counseling, that is, recommending a form of management, for fetal benefit is appropriate, and when the fetus is not a patient, nondirective counseling, that is, offering but not recommending a form of management for fetal benefit, is appropriate. However, there can be uncertainty about when the fetus is a patient. One approach to resolving this uncertainty would be to argue that the fetus is or is not a patient in virtue of personhood or some other form of independent moral status. We now show that this approach fails to resolve the uncertainty, and we therefore defend an alternative approach that does resolve the uncertainty.

One prominent approach for establishing whether or not the fetus is a patient has involved attempts to show whether or not the fetus has independent moral status. Independent moral status for the fetus means that one or more characteristics that the fetus possesses in and of itself and, therefore, independently of the pregnant woman and physician, generate and therefore ground obligations to the fetus on the part of the pregnant woman and her physician. Many fetal characteristics have been nominated for this role, including moment of conception, implantation, central nervous system development, quickening, and the moment of birth. It should come as no surprise that there is considerable variation among ethical arguments about when the fetus acquires independent moral status. Some take the view that the fetus has independent moral status from the moment of conception or implantation. Others believe that independent moral status is acquired in degrees, thus resulting in "graded" moral status. Still others hold, at least by implication, that the fetus never has independent moral status so long as it is in utero $(13,14)$.

Despite an ever-expanding theologic and philosophical literature on this subject, there has been no closure on a single authoritative account of the independent moral status of the fetus. This is an unsurprising outcome because, given the absence of a single method that would be authoritative for all of the markedly diverse theologic and philosophical schools of thought involved in this endless debate, closure is impossible. For closure ever to be possible, debates about such a final authority within and between theologic and philosophical traditions would have to be resolved in a way satisfactory to all, an inconceivable intellectual and cultural event. The concept of the independent moral status of the fetus has no stable or clinically applicable meaning. We therefore abandon these futile attempts to understand the ethical concept of the fetus as a patient in terms of independent moral status of the fetus and turn to an alternative approach that makes it possible to identify ethically distinct senses of the fetus as a patient and their clinical implications for directive and nondirective counseling.

Our analysis of the ethical concept of the fetus as a patient begins with the recognition that being a patient does not require that one possesses independent moral status. Rather, being a patient means that one can benefit clinically from the applications of the clinical skills of the physician. Put more precisely, a human being without independent moral status is properly regarded as a patient when two conditions are met: that a human being (i) is presented to the physician and (ii) there exist clinical interventions that are reliably expected to be efficacious, in that they are reliably expected to result in a greater balance of clinical benefits over harms for the human being in question (15). This is the sense in which the ethical concept of the fetus as a patient should be understood, what we call the dependent moral status of the fetus.

The authors have argued elsewhere that beneficence-based obligations to the fetus exist when the fetus is reliably expected later to achieve independent moral status as a child and a person (4). That is, the fetus is a patient when the fetus is presented for medical interventions, whether diagnostic or therapeutic, that reasonably can be expected to result in a greater balance of goods over harms for the child and the person the fetus can later become during early childhood. The ethical significance of the concept of the fetus as a patient, therefore, depends on links that can be established between the fetus and its later achieving independent moral status.

\section{The Viable Fetal Patient}

One such link is viability. Viability, however, must be understood in terms of both biologic and technologic factors. It is only by virtue of both factors that a viable fetus can exist ex utero and thus achieve independent moral status. When a fetus is viable, that is, when it is of sufficient maturity so that it can survive into the neonatal period and achieve independent moral status given the availability of the requisite technologic support, and when it is presented to the physician, the fetus is a patient.

Viability exists as a function of biomedical and technologic capacities, which are different in different parts of the world. As a consequence, there is, at the present time, no worldwide 
uniform gestational age to define viability. In the United States, we believe, viability presently occurs at approximately 24 weeks of gestational age $(16,17)$.

When the fetus is a patient, directive counseling for fetal benefit is ethically justified. In clinical practice, directive counseling for fetal benefit involves one or more of the following: recommending against termination of pregnancy, recommending against nonaggressive management, or recommending aggressive management. Aggressive obstetric management includes interventions such as fetal surveillance, tocolysis, cesarean delivery, or delivery in a tertiary care center when indicated. Nonaggressive obstetric management excludes such interventions. Directive counseling for fetal benefit, however, must take account of the presence and severity of fetal anomalies, extreme prematurity, and obligations to the pregnant woman.

It is very important to appreciate in obstetric clinical judgment and practice that the strength of directive counseling for fetal benefit varies according to the presence and severity of fetal anomalies. As a rule, the more severe the fetal anomaly, the less directive counseling should be for fetal benefit. In particular, when lethal anomalies such as anencephaly can be diagnosed with certainty, there are no beneficence-based obligations to provide aggressive management. Such fetuses are dying patients, and the counseling, therefore, should be nondirective in recommending between nonaggressive management and termination of pregnancy, but directive in recommending against aggressive management for the sake of maternal benefit (18). By contrast, third-trimester abortion for Down syndrome, or achondroplasia, is not ethically justifiable, because the future child with high probability will have the capacity to grow and develop $(19,20)$.

Directive counseling for fetal benefit in cases of extreme prematurity of viable fetuses is appropriate. In particular, this is the case for what we term just-viable fetuses, those with a gestational age of 24 to 26 weeks, for which there are significant rates of survival but high rates of mortality and morbidity. These rates of morbidity and mortality can be increased by nonaggressive obstetric management, whereas aggressive obstetric management may favorably influence outcome. Thus, it appears that there are substantial beneficence-based obligations to just-viable fetuses to provide aggressive obstetric management. This is all the more the case in pregnancies beyond 26 weeks of gestational age. Therefore, directive counseling for fetal benefit is justified in all cases of extreme prematurity of viable fetuses, considered by itself. Of course, such directive counseling is appropriate only when it is based on documented efficacy of aggressive obstetric management for each fetal indication. For example, such efficacy has not been demonstrated for routine cesarean delivery to manage extreme prematurity.

Any directive counseling for fetal benefit must occur in the context of balancing beneficence-based obligations to the fetus against beneficence-based and autonomy-based obligations to the pregnant woman. Any such balancing must recognize that a pregnant woman is obligated only to take reasonable risks of medical interventions that are reliably expected to benefit the viable fetus or child later. A unique feature of obstetric ethics is that the pregnant woman's autonomy influences whether, in a particular case, the viable fetus ought to be regarded as presented to the physician.
Obviously, any strategy for directive counseling for fetal benefit that takes account of obligations to the pregnant woman must be open to the possibility of conflict between the physician's recommendation and a pregnant woman's autonomous decision to the contrary. Such conflict is best managed preventively through the informed consent process as an ongoing dialogue throughout a woman's pregnancy, augmented as necessary by negotiation and respectful persuasion (21).

\section{The Previable Fetal Patient}

The only possible link between the previable fetus and the child it can become is the pregnant woman's autonomy. This is because technologic factors cannot result in the previable fetus becoming a child. The link, therefore, between a fetus and the child it can become when the fetus is previable can be established only by the pregnant woman's decision to confer the status of being a patient on her previable fetus. The previable fetus, therefore, has no claim to the status of being a patient independently of the pregnant woman's autonomy. The pregnant woman is free to withhold, confer, or, having once conferred, withdraw the status of being a patient on or from her previable fetus according to her own values and beliefs. The previable fetus is presented to the physician as a function of the pregnant woman's autonomy (4).

Counseling the pregnant woman regarding the management of her pregnancy when the fetus is previable should be nondirective in terms of continuing the pregnancy or having an abortion if she refuses to confer the status of being a patient on her fetus. If she does confer such status in a settled way, at that point beneficence-based obligations to her fetus come into existence, and directive counseling for fetal benefit becomes appropriate for these previable fetuses. Just as for viable fetuses, such counseling must take account of the presence and severity of fetal anomalies, extreme prematurity, and obligations owed to the pregnant woman.

For pregnancies in which the woman is uncertain about whether to confer such status, the authors propose that the fetus be provisionally regarded as a patient. This justifies directive counseling against behavior that can harm a fetus in significant and irreversible ways, for example, substance abuse, especially alcohol, until the woman settles on whether to confer the status of being a patient on the fetus.

In particular, nondirective counseling is appropriate in cases of what we term near-viable fetuses, that is, those that are 22 to 23 weeks of gestational age, for which there are anecdotal reports of survival $(16,17,22)$. In our view, aggressive obstetric and neonatal management should be regarded as clinical investigation (a topic addressed in the next section of this chapter), not a standard of care. There is no obligation on the part of a pregnant woman to confer the status of being a patient on a near-viable fetus, because the efficacy of aggressive obstetric and neonatal management has yet to be proven (17).

\section{ETHICS OF RESEARCH IN MATERNAL FETAL MEDICINE}

\section{Definition of Research}

The definition of research in the federal regulations in the United States is the following: "an activity designed to test a hypothesis, permit conclusions to be drawn, and thereby to develop or contribute to generalizable knowledge" (23). 


\section{Three Components of Research Ethics}

The history of human experimentation appears to be coincident with the history of medicine. Concern about the scientific and ethical quality of research with human subjects begins to emerge in eighteenth-century medical ethics. One of major figures of that period, Dr. John Gregory (1724-1773) of Scotland, wrote the first modern medical ethics in the English language (24). He developed a research ethics to address the potential abuse of patients in the Royal Infirmary of Edinburgh by younger physicians anxious to establish their reputations. These physicians would pronounce Infirmary patients incurable, not to abandon them (which was the common practice), but to justify introducing experimental medicines into patient care.

Gregory condemned this practice. His first concern was that such experimentation was premature: standard remedies had not yet been attempted and shown to be ineffective in a patient's care. His second concern was that experimentation was often poorly designed. For example, compound drugs would be used without attention to the question of which elements of the compound might cause observed clinical effects. His third concern was that such physicians used the sick poor to advance their own reputational interests, subjecting them to unnecessary risk of clinical harm (a violation of the ethical principle of beneficence) out of personal self-interest. Gregory called this "sporting" with the sick poor; we now call it exploitation.

Gregory's research ethics introduced one of the key components of any adequate research ethics: the protection of research subjects. Such protection was gained by ensuring that there is a clinical justification for research and that the research is scientifically well designed. A second key component of any adequate research ethics, the consent of research subjects, was introduced in the nineteenth century.

Making a reasonable effort to prevent what is known as the therapeutic misconception is one of the major responsibilities of clinical investigators in the informed consent process. The therapeutic misconception occurs when potential subjects fail to appreciate that some aspects of what they will experience in a study are not justified by a clinical judgment of what is in the patient's health-related interest but by scientific considerations. Subjects, instead, confuse these scientific study design issues with regular medical care (25). For example, pregnant women being asked to enroll in a randomized fetal research trial should understand that intervention will be randomly selected rather than individually tailored by their physician's clinical judgment.

The need for both scientific and ethical integrity as components of research ethics was reinforced by the scientific and ethical catastrophe of the Nazi medical war crimes. A major result of the trials of the Nazi physicians was the promulgation of the Nuremberg Code. This is regarded as the founding document of contemporary research ethics and insists on sound scientific method and consent, which have become two of the three main components of research ethics globally (26).

The third and final key component of research ethics was introduced by the Declaration of Helsinki. It requires independent overview of clinical investigation, for both its scientific and its ethical integrity (26).
As a result of this centuries-long history of medical ethics, there has emerged an international consensus that there are three key components of research ethics: (27)

1. Clinical research with human subjects must be clinically justified and scientifically sound. The clinical need for research should be well established on the basis of a critical, evidence-based evaluation of current clinical practice. Clinical research should be well designed scientifically, with clearly stated research questions and testable hypotheses and a method adequate to test the hypotheses and this answers the research questions.

2. Informed consent is required. The Nuremberg Code did not allow any exceptions to this requirement, a position that is no longer part of the international consensus. It has been recognized in recent decades that there are populations of patients for whom we need to improve the quality of medical care but who cannot consent to becoming research subjects. This may be a result of the clinical circumstances of research (e.g., in emergencies where there is no time for the consent process) or the inability of the potential subject to engage in the informed consent process (e.g., fetuses).

3. Oversight of research is required. Investigators are obligated to prepare research protocols that establish clinical need, meet standards of scientific integrity, and describe the informed consent process (or justify its waiver) and submit protocols for prospective review by independent committees established for this purpose (known in the United States as Institutional Review Board and in most of the rest of the world as Research Ethics Committees).

Ethical Criteria for Innovation in Maternal-Fetal Medicine Innovation in maternal-fetal medicine should begin with the design of an intervention and its implementation in animal models, followed by a single case and then case series. This rigorous approach is required to determine the feasibility, safety, and efficacy of innovations in fetal research. It is a basic tenet of research ethics that potential subjects should be protected from potentially harmful innovation. Three criteria must be satisfied in order to conduct such preliminary investigations in fetal research in an ethically responsible fashion, by taking into account beneficence-based obligations to the fetal patient and beneficence-based obligations to the pregnant woman. The previable fetus is a patient in these cases because, as explained above, the woman has made a decision to continue her pregnancy, in order to have the opportunity to gain the potential benefits of the innovation. She remains free to withdraw that status before viability:

1. the proposed fetal intervention is reliably expected on the basis of previous animal studies either to be life-saving or to prevent serious and irreversible disease, injury, or handicap for the fetus;

2. among possible alternative designs, the intervention is designed in such a way as to involve the least risk of mortality and morbidity to the fetal patient (which is required by beneficence and will satisfy 
the U.S. research requirement of minimal risk to the fetus); (23) and

3. on the basis of animal studies and analysis of theoretical risks both for the current and for the future pregnancies, the mortality risk of the fetal intervention to the pregnant woman is reliably expected to be low and the risk of disease, injury, or handicap to the pregnant woman is reliably expected to be low or manageable (28).

The first two criteria are based on beneficence-based obligations to the fetal patient. Research on animal models should suggest that there would be therapeutic benefit without disproportionate iatrogenic fetal morbidity or mortality. If animal studies result in high rates of mortality or morbidity for the animal fetal subject, then innovation should not be introduced to human subjects until these rates improve in subsequent animal studies.

The third criterion reflects the fact that fetal intervention in the form of fetal surgery is necessarily also as maternal surgery and that ethically and clinically the fetal patient is not a separate patient. This criterion reminds investigators that the willingness of a subject, in this case, the pregnant woman, to consent to risk does not by itself establish whether the risk/ benefit ratio is favorable. Judgments about an acceptable risk/ benefit ratio should not be autonomy based, but beneficence based. This is because investigators have an independent beneficence-based obligation to protect human subjects from unreasonably risky research and should use beneficence-based, not autonomy-based, risk-benefit analyses. Phrases such as "maternal-fetal surgery" are useful if they remind investigators of the need for such comprehensive analysis. If they are used systematically to subordinate fetal interests to maternal interest and rights, and therefore to undermine the concept of the fetus as a patient in favor of the concept that the fetus is merely a part of the pregnant woman, such phrases lack ethical utility.

\section{Ethical Criteria in Randomized Trials in Maternal-Fetal Medicine}

Preliminary innovation should end and randomized clinical trials begin when there is clinical equipoise. Until recently, equipoise has meant that there is "a remaining disagreement in the expert clinical community, despite the available evidence, about the merits of the intervention to be tested" (26). Brody notes that one challenge here is identifying how much disagreement can remain for there still to be equipoise (26). Lilford has suggested that when two-thirds of the expert community, measured reliably, no longer disagree, equipoise is not satisfied (29).

This older concept of equipoise is based on the distribution of opinion in the clinical community, which may or may not be evidence based. To make judgments of equipoise more rigorous, it has been argued that it should be evidence based. This is known as normative equipoise or evidence based, which is the preferred concept (27). When evidence-based evaluation of clinical experience and the current literature supports the judgment that the experimental intervention is more harmful than nonintervention, equipoise cannot be achieved.
The satisfaction of the previous three criteria for innovation, with slight modifications, should count as equipoise in the expert community:

1. the initial case series indicates that the proposed fetal intervention is reliably expected either to be lifesaving or to prevent serious and irreversible disease, injury, or handicap;

2. among possible alternative designs, the intervention continues to involve the least risk of morbidity and mortality to the fetus; and

3. the case series indicates that the mortality risk to the pregnant woman is reliably expected to be low and the risk of disease, injury, or handicap to the pregnant woman, including for future pregnancies, is reliably expected to be low or manageable (28).

One good test for the satisfaction of the first and third criteria is significant trends in the data from the case series. When evidence-based equipoise has been achieved on the basis of these three criteria, randomized clinical trials should commence. They must have relevant and clearly defined primary and secondary endpoints and a design and sample size adequate to measure these endpoints.

The above three criteria can be used in a straightforward manner to define stopping rules for randomized clinical trials in fetal research. When the data support a rigorous clinical judgment that the first or third criterion is not satisfied, the trial should be stopped.

\section{Criteria for Making the Transition from Research to Clinical Practice}

When a clinical trial in fetal research is completed, its outcome can be assessed to determine whether the investigational fetal intervention should be introduced into clinical practice. Trial results should meet the following three criteria in order to make this important transition:

1. the fetal intervention has a significant probability of being lifesaving or preventing serious or irreversible disease, injury, or handicap for the fetus;

2. the fetal intervention involves low mortality and low or manageable risk of serious and irreversible disease, injury, or handicap to the fetus; and

3. the mortality risk to the pregnant woman is low and the risk of disease, injury, or handicap is low or manageable, including for future pregnancies (28).

Brody has underscored the value of data safety and monitoring boards to prevent investigator bias and to protect subjects (26). Such boards should be used in fetal research, especially to ensure adherence of the above-mentioned ethical criteria as a basis for monitoring such research.

\section{CONCLUSION}

Ethics is an essential dimension of clinical practice and research in maternal-fetal medicine. An understanding and clinical application of the ethical principles of beneficence and respect for autonomy and of the ethical concept of the fetus as a patient should guide clinicians and investigators, to better serve pregnant and fetal patients. 


\section{REFERENCES}

1. American College of Obstetricians and Gynecologists. Ethics in Obstetrics and Gynecology, 2nd edn. Washington, DC: American College of Obstetricians and Gynecologists, 2004.

2. Association of Professors of Gynecology and Obstetrics. Exploring Medical-legal Issues in Obstetrics and Gynecology. Washington, DC: APGO Medical Education Foundation, 1994.

3. FIGO Committee for the Study of Ethical Aspects of Human Reproduction. Recommendations of Ethical Issues in Obstetrics and Gynecology. London: International Federation of Gynecology and Obstetrics, 1997.

4. McCullough LB, Chervenak FA. Ethics in obstetrics and gynecology. New York, NY: Oxford University Press, 1994.

5. Engelhardt HT Jr. The Foundations of Bioethics, 2nd edn. New York, NY: Oxford University Press, 1995.

6. Beauchamp TL, Childress JF. Principles of Biomedical Ethics, 6th edn. New York, NY: Oxford University Press, 2008.

7. Chervenak FA, McCullough LB. An ethically justified algorithm for offering, recommending, and performing cesarean delivery and its application in managed care practice. Obstet Gynecol 1996; 87: 302-5.

8. Hippocrates. Oath of hippocrates. In: Temkin O, Temkin CL, eds Ancient Medicine: Selected Papers of Ludwig Edelstein. Baltimore, MD: Johns Hopkins University Press, 1976: 6.

9. McCullough LB, Coverdale JH, Chervenak FA. Argument-based medical ethics: a formal tool for critically appraising the normative medical ethics literature. Am J Obstset Gynecol 2004; 191: 1097-102.

10. Faden RR, Beauchamp TL. A History and Theory of Informed Consent. New York, NY: Oxford University Press, 1986.

11. Wear S. Informed Consent: Patient Autonomy and Clinician Beneficence within Health Care, 2nd edn. Washington, DC: Georgetown University Press, 1998.

12. Schloendorff V. The Society of The New York Hospital, 211 N.Y. 125, 126, 105 N.E. 92, 93 (1914).

13. Callahan S, Callahan D, eds. Abortion: understanding differences. New York, NY: Plenum Press, 1984.

14. Annas GJ. Protecting the liberty of pregnant patient. N Engl J Med 1988; 316: $1213-14$
15. Chervenak FA, McCullough LB. Ethics in obstetrics and gynecology: an overview. Eur J Obstet Gynecol Reprod Med 1997; 75: 91-4.

16. Chervenak FA, McCullough LB. The limits of viability. J Perinat Med 1997; 25: 418-20.

17. Chervenak FA, McCullough LB, Levene MI. An ethically justified clinically comprehensive approach to peri-viability: gynaecological, obstetric, perinatal, and neonatal dimensions. J Obstet Gynaecol 2007; 27: 3-7.

18. Chervenak FA, McCullough LB. An ethically justified, clinically comprehensive management strategy for third-trimester pregnancies complicated by fetal anomalies. Obstet Gynecol 1990; 75: 311-16.

19. Chervenak FA, McCullough LB, Campbell S. Is third trimester abortion justified? Br J Obstet Gynaecol 1995; 102: 434-5.

20. Chervenak FA, McCullough LB, Campbell S. Third trimester abortion: is compassion enough? Br J Obstet Gynaecol 1999; 106: 293-6.

21. Chervenak FA, McCullough LB. Clinical guides to preventing ethical conflicts between pregnant women and their physicians. Am J Obstet Gynecol 1990; 162: 303-7.

22. Lucey JF, Rowan CA, Shiono P, et al. Fetal infants: the fate of 4172 infants with birth weights of 401 to 500 grams-the Vermont Oxford Network experience (1996-2000). Pediatrics 2004; 113: 1559-66.

23. Department of Health and Human Services. Regulations for the Protection of Human Subjects. 45 CFR 46. [Available from: http:// www.hhs.gov/ohrp/] Accessed 31 January 2010.

24. McCullough LB. John Gregory and the Invention of Professional Medical Ethics and the Profession of Medicine. Dordrecht, The Netherlands: Kluwer Academic Publishers, 1998.

25. Lidz CW, Appelbaum PS, Grisso T, Renaud M. Therapeutic misconception and the appreciation of risks in clinical trials. Soc Sci Med 2004; 58 1689-97.

26. Brody BA. The Ethics of Biomedical Research: An International Perspective. New York: Oxford University Press, 1998.

27. Brody BA, McCullough LB, Sharp R. Consensus and controversy in research ethics. JAMA 2005; 294: 1411-14.

28. Chervenak FA, McCullough LB. A comprehensive ethical framework for fetal research and its application to fetal surgery for spina bifida. Am J Obstet Gynecol 2002; 187: 10-14.

29. Lilford RJ. The substantive ethics of clinical trials. Clin Obstet Gynecol 1992; 35: 837-45. 


\section{Basic genetics and patterns of inheritance Dorothy K. Grange}

\section{INTRODUCTION}

The field of medical genetics is rapidly expanding. Genetics is becoming increasingly important in the general practice of medicine. Thus, the primary care physician has a responsibility to acquire adequate knowledge of genetics to provide his or her patients with the most up-to-date medical care. In addition to advances in the understanding of the genetic basis for many of the more frequent chromosomal, single-gene, and multiple anomaly syndromes, there is greater knowledge of the role of genetics in common disorders such as hypertension, diabetes, heart disease, cancer, psychiatric disorders, and other medical problems. Medical genetics has a particularly great impact on the practice of obstetrics and on the evaluation and the management of fetal abnormalities, resulting in a need for close working relationships between clinical geneticists and specialists in maternal-fetal medicine. As the growth of information and technology continues, it is anticipated that new forms of genetic therapy, at the somatic cell level and possibly at the germ cell level, will be developed. Some of these treatments may be employed preconceptually and during pregnancy, requiring the involvement of obstetricians and gynecologists.

The human genome contains three billion base pairs of DNA, packaged into units known as chromosomes and encoding an estimated 20,000 to 25,000 genes $(1,2)$. At the present time, over 12,000 human genes have been mapped and almost 3000 single-gene disorders have been described for which the molecular genetic basis is known (3). The Human Genome Project, begun in the late 1980s and completed in 2003, produced a haploid human genome sequence, which was a composite of the DNA sequence of several individuals. As a result, new genes and new information about the genetic basis for many disorders have been discovered. This has already begun to have a huge impact on the practice of medical genetics and medicine in general.

\section{CYTOGENETICS}

Cytogenetics is the study of chromosomes and their abnormalities $(4,5)$. The field of cytogenetics is a relative newcomer in clinical laboratory medicine. It was not until the mid-1950s that chromosome analysis could be done. Soon after it was determined that the normal number of chromosomes in humans is 46, rapid discoveries in the area of human cytogenetics were made. In 1959, LeJeune identified the cytogenetic basis of Down syndrome, and around the same time, sex chromosome abnormalities were found in patients with anomalies of sexual development. Techniques for the analysis of chromosomes have continued to be improved and refined, allowing more subtle abnormalities to be identified. Newer molecular cytogenetic methods have revolutionized the identification and confirmation of microdeletions, microduplications, and cryptic translocations.
Humans have a total of 46 chromosomes, including 22 pairs of numbered chromosomes, or autosomes, and one pair of sex chromosomes. Each chromosome comprises a single length of double-stranded DNA, ranging in size from 50 million to 250 million base pairs. This genetic information is tightly compressed and packaged in the formation of the chromosomes. The DNA molecules are complexed with proteins called histones. The histones are packaged into nucleosomes, which are further compacted into a helical structure known as a solenoid. Finally, the solenoids are wound in a helical fashion and compressed to form the chromatin loops. Chromatin is packaged into chromosomes, which can be viewed microscopically during the metaphase stage of cell division.

Performing cytogenetic analysis has become an integral part of many areas of medical practice, including obstetrics, pediatrics, and oncology. Traditional chromosome analysis first involves obtaining a sample of living tissue. This can be blood, skin, amniotic fluid, products of conception, bone marrow, or any viable solid tissue. Blood is the most frequently analyzed tissue for routine chromosome analysis. For blood, the lymphocytes are isolated. For amniotic fluid, the amniocytes are obtained by spinning down the fluid and removing the cell pellet. For solid tissues, the tissue is minced and/or sonicated. For all tissue types, the cells are cultured in tissue culture for 48 to 72 hours. Cell division is arrested at metaphase by the addition of colcemid. The cells are then harvested and placed on a microscope slide. The cell nuclei are ruptured by adding a hypotonic solution, then stained to show the bands, and images of the metaphase chromosome spreads are analyzed. Each chromosome is studied by looking at the banding pattern to identify not only numerical abnormalities, but also structural problems. Newer computerized technology allows karyotype analysis by digital imaging methods. By convention, the 22 autosome pairs are arranged by size, from the largest to the smallest, in four rows, with the pair of sex chromosomes in the lower right corner (Fig. 1). Ideograms are schematic representations of banding patterns used by cytogeneticists to standardize numbering of specific bands (Fig. 2).

Chromosomes have distinctive structural appearances when viewed microscopically. There are two arms with a central narrowing called the centromere. The shorter arm is termed "p" for "petit," while the longer arm is denoted "q," because $\mathrm{q}$ comes after $\mathrm{p}$ alphabetically. The $\mathrm{p}$ arm is always shown at the top on karyotypes. There are three types of chromosomes structurally (Fig. 3). Metacentric chromosomes have p and $\mathrm{q}$ arms that are almost equal in length, with the centromere located in the middle. Submetacentric chromosomes have a shorter $\mathrm{p}$ arm with the centromere placed closer to the top of the chromosome and acrocentric chromosomes have small $\mathrm{p}$ arms called satellites with the centromere located near the top of the chromosome. The ends of chromosomes are called the telomeres. 

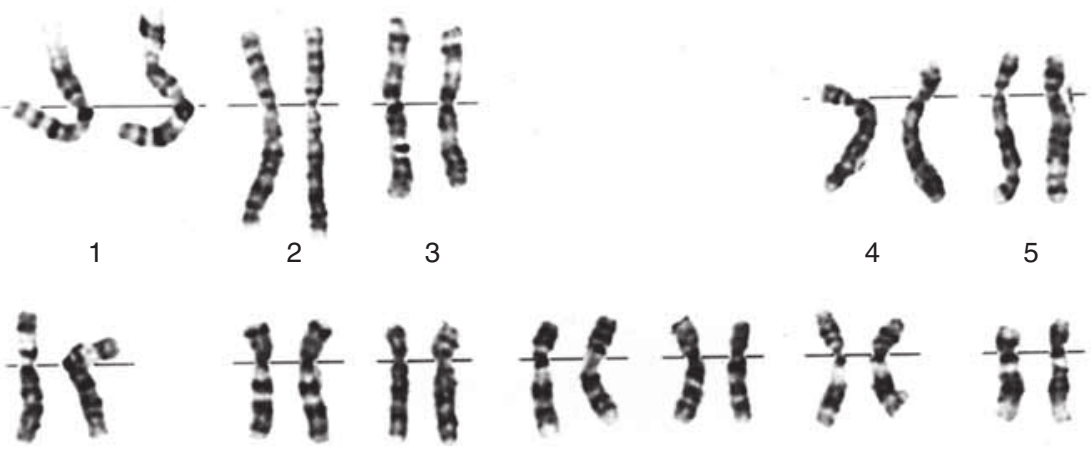

6
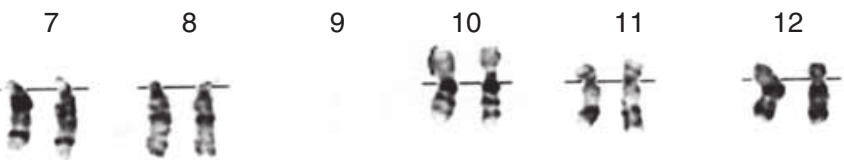

13

14

15

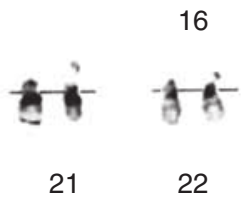

17

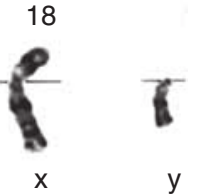

19

20

Figure 1 Normal male karyotype.
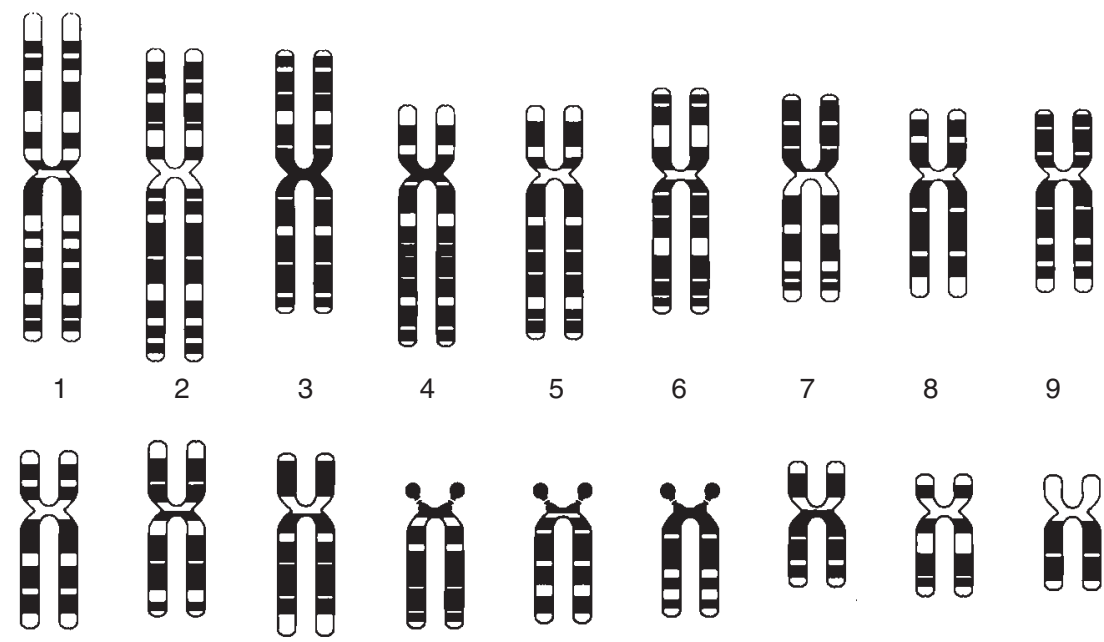

10

11

12

13

14

15

16

17

18

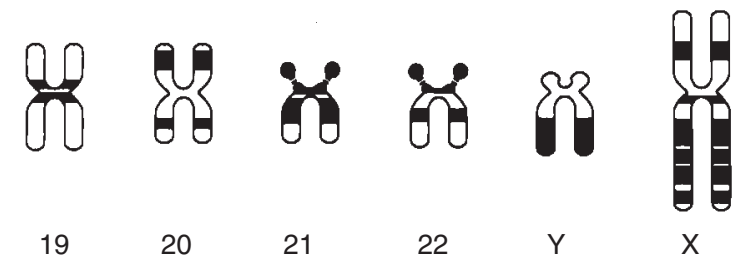

Figure 2 Ideogram of human chromosome showing Giemsa banding patterns. Source: From Ref. 6.

Various banding techniques are used to visualize the chromosomes. The most frequently used method is Giemsa banding or G-banding, which results in a specific pattern of dark and light bands on each chromosome. The older method, quinacrine banding, or Q-banding, produces the same dark and light patterns, but requires the use of a fluorescence microscope and is not used routinely. Reverse banding or R-banding, results in the opposite of the dark and light pattern seen with G-banding; this may be used to better see the ends of the chromosomes. C-banding stains the constitutive heterochromatin, which is near the centromeres and NOR stain visualizes the nucleolar organizing regions of the satellites and stalks of acrocentric chromosomes. For routine karyotype analysis, G-banding is typically used by most laboratories. Routine karyotyping cannot detect gains or losses of cytogenetic material smaller than about $4 \mathrm{Mb}$ in size and therefore can miss significant abnormalities. 


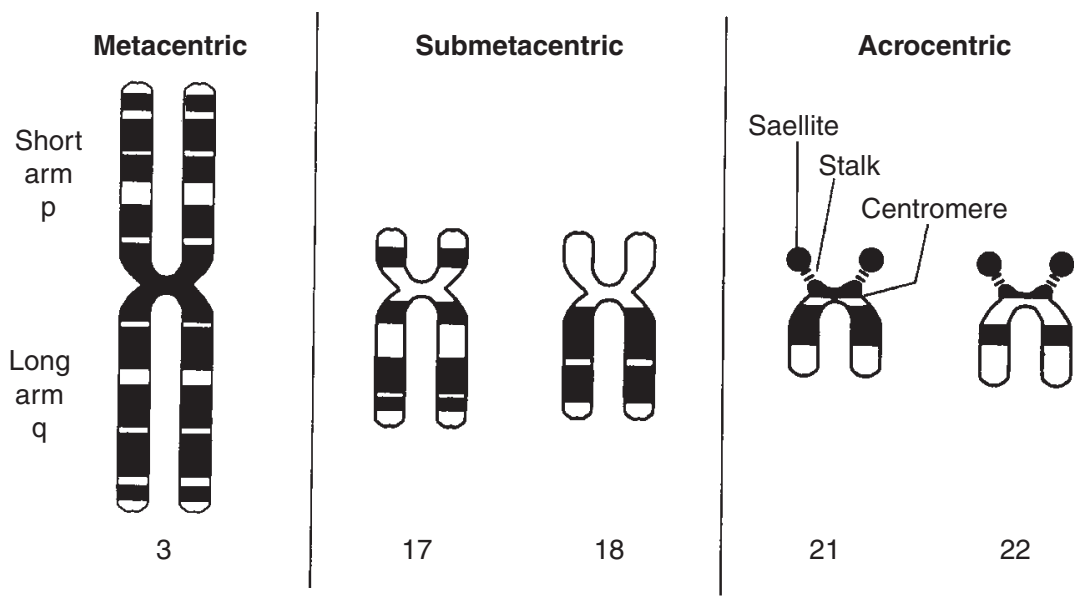

Figure 3 Human chromosome morphology as classified by variation in chromosome size and centromere position. Source: From Ref. 6.

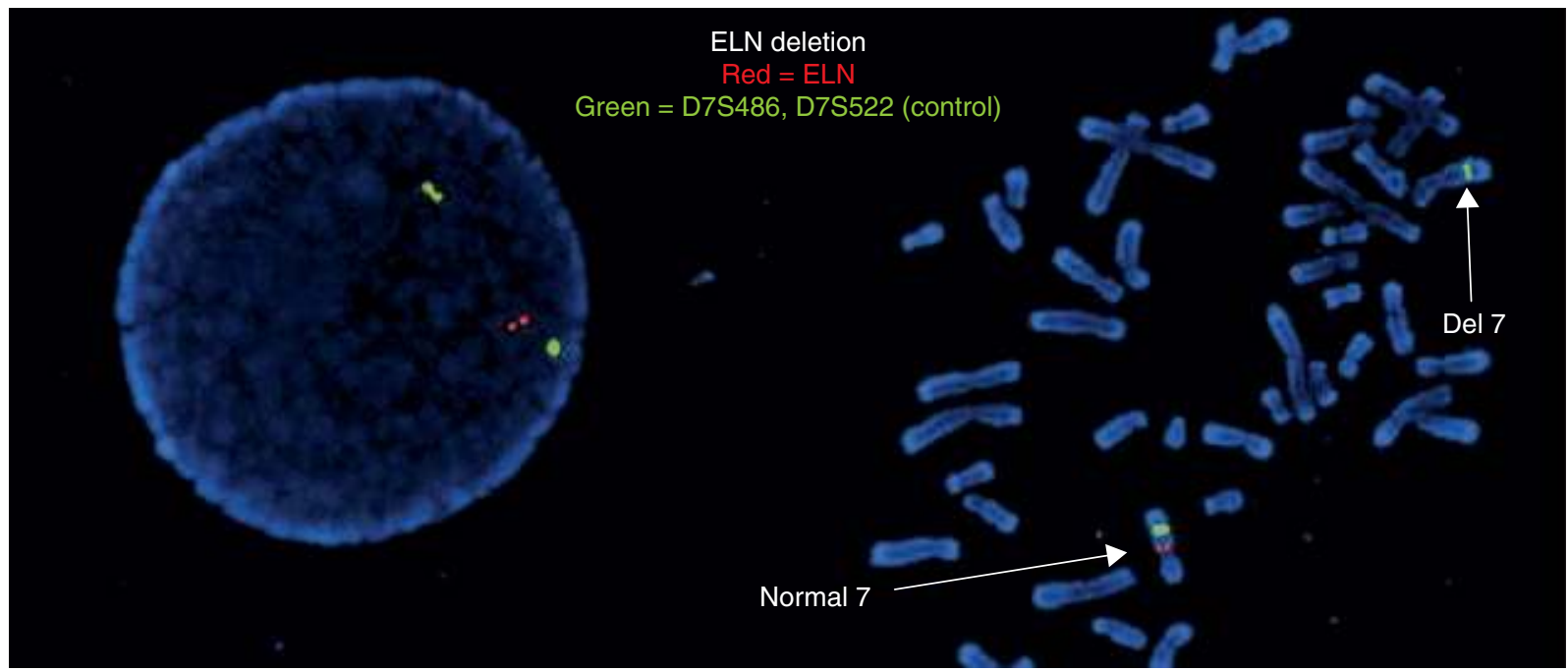

Figure 4 Example of fluorescence in situ hybridization analysis in a patient with Williams syndrome. On the left panel, an interphase cell is shown with a deletion of one copy of the elastin (ELN) gene within the critical 7q11.23 Williams syndrome region; there is only one red ELN signal and there are two green control signals. On the right panel, a metaphase cell is shown indicating a deletion on chromosome 7 with a missing red signal. Source: Courtesy of Dr. Shashikant Kulkarni.

Fluorescence in situ hybridization (FISH) is a technique in which DNA probes attached to fluorescent dyes are hybridized to specific chromosome regions from patient samples, either interphase cells or metaphase chromosome spreads, most often from lymphocytes or amniocytes. The chromosomes are then visualized by fluorescent microscopy. Probes can be designed to hybridize to whole chromosomes, specific chromosome segments of interest, centromeres, or telomeres. FISH is widely used for the diagnosis of suspected recognizable microdeletion syndromes (Fig. 4). Multicolor FISH is useful for analyzing submicroscopic structural rearrangements undetectable by classic cytogenetic techniques or for identifying marker chromosomes. FISH is also used for rapid screening for aneuploidy including trisomy 13, 18, or 21 and abnormalities of $\mathrm{X}$ and $\mathrm{Y}$.

Chromosome microarray analysis using array-based comparative genomic hybridization (aCGH) is a powerful new technique for the detection of genomic copy number alterations, including deletions and duplications (7-9). It was originally developed as a research tool, but is rapidly replacing traditional cytogenetic methods of analysis for the diagnosis of chromosomal defects. With this method, DNA from the patient's DNA and DNA from a normal control are each labeled with different tags for analysis by fluorescence (Fig. 5). The patient and control DNA are mixed together along with unlabeled human cot-1 DNA to suppress repetitive DNA sequences, and the mix is hybridized to a slide containing thousands of defined DNA probes. The (fluorescence) color ratio along the chromosomes is used to evaluate regions of DNA gain or loss in the patient sample as compared with the control DNA. Clinically available arrays include focused arrays that are designed to identify all microdeletions detectable by FISH, bacterial artificial chromosome arrays that can detect rearrangements greater than $1 \mathrm{Mb}$, oligonucleotide arrays using DNA probes that can pick up imbalances as small as 20 to $80 \mathrm{~kb}$, and single-nucleotide polymorphism arrays. Diagnosis of alterations in DNA dosage or genomic copy number that are known to be associated with a variety of syndromic and non-syndromic pediatric and adult genetic disorders can be found by aCGH analysis. Copy number variations $(\mathrm{CNV})$ or polymorphisms that are not known to be associated with an abnormal phenotype or are considered to be benign are often detected. In addition, previously undescribed deletions or duplications with unclear clinical 
(A)

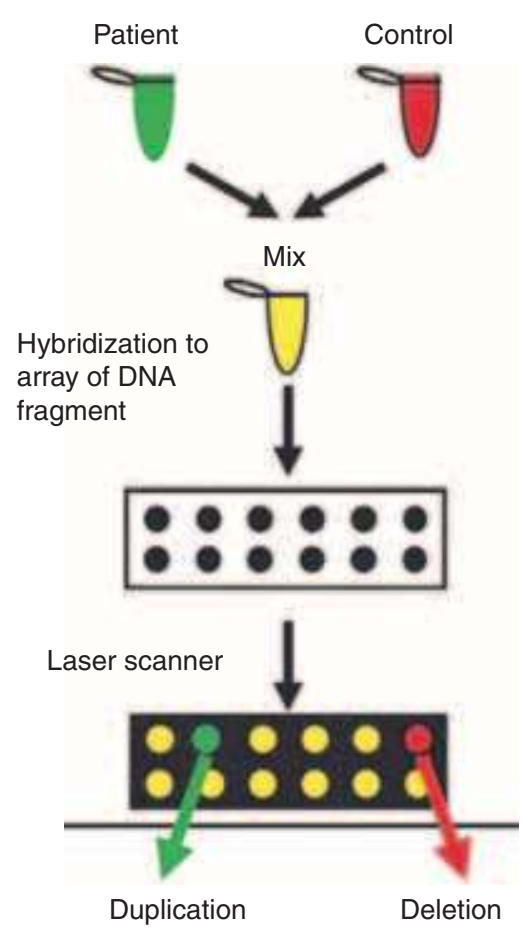

(B)

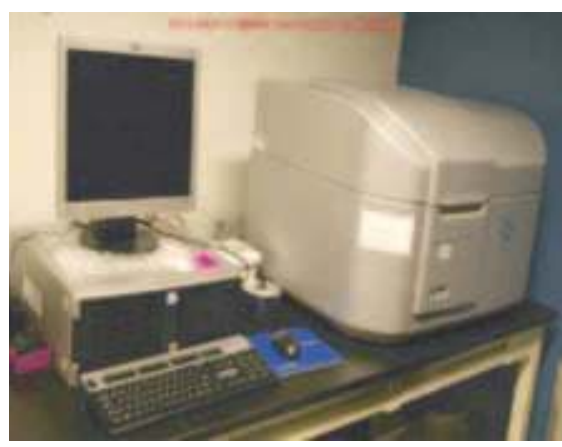

(C)

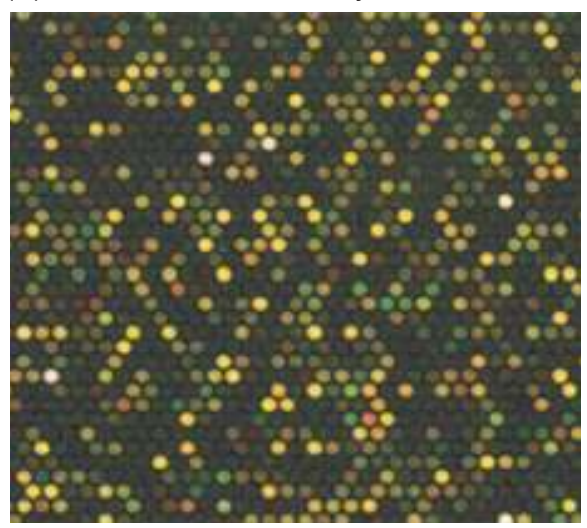

(D) Array profile

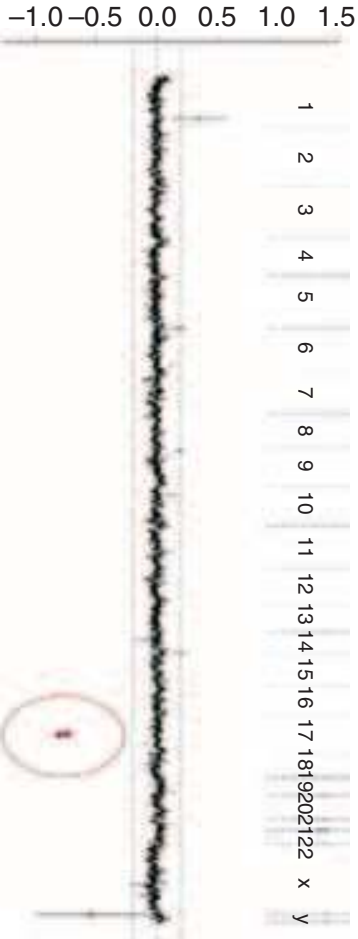

Figure 5 Array comparative genomic hybridization (chromosome microarray) technology. (A) DNA from the patient sample is labeled with a green fluorescence dye and a control DNA is labeled with a red fluorescence dye. The two samples are mixed and co-hybridized to an array of genomic DNA targets on a slide. The ratios of fluorescence intensities are proportional to the ratio of copy numbers of DNA sequences in the patient versus the control samples. Green area indicates duplication and red area indicates deletion. (B) The slides are scanned into image files using a microarray scanner. (C) The output shows hundreds of spots with different ratios of fluorescence intensities. (D) The array profile is analyzed; shown here is a patient profile indicating a deletion of three DNA markers within the neurofibromatosis type 1 critical region on chromosome 17p11.2. Source: From Ref. 7.

significance may be identified, requiring parental analysis to determine inheritance of the abnormality and often making genetic counseling for the family difficult. Another caveat of using chromosome microarray analysis is that it cannot detect balanced chromosome rearrangements such as translocations or inversions if there is no gain or loss of a chromosome segment.

\section{CHROMOSOMAL ABNORMALITIES}

Chromosome abnormalities are seen in 1 in 150 to 1 in 170 live births and thus, as a group, contribute significantly to morbidity and mortality among neonates and infants (1). Any baby that has two or more congenital anomalies should have a karyotype performed (Table 1). Some of the more frequent chromosome anomalies will be reviewed, as well as the categories of chromosome abnormalities.

\section{Aneuploidy}

The term "aneuploidy" refers to an abnormal total number of chromosomes (10). For example, an individual who has either a missing or extra copy of one of the chromosomes is said to have aneuploidy. Aneuploidy is the most common type of chromosome aberration found in humans and arises by a process known as "nondisjunction." Nondisjunction occurs when there is a failure of proper distribution of chromosome pairs into daughter cells during meiosis, leading to gametes that have either an extra copy of a given chromosome or a missing copy of that chromosome. Meiosis is the process by

\section{Table 1 Indications for Chromosome Analysis}

- Suspected recognizable chromosome abnormality

- Infant with two or more malformations

- Mental retardation or developmental delay of unknown etiology, with or without malformations

- Relatives of people with known translocation, deletion, or duplication

- Stillborn infants with malformations or no known reason for fetal demise

- Females with short stature

- Males with infertility, small testes, or gynecomastia

- Women and their partners who have experienced recurrent miscarriages

which the chromosomes in the gamete are reduced from the diploid state (46 chromosomes) to the haploid state (23 chromosomes). There are two cell divisions in meiosis, called meiosis I and meiosis II. In meiosis I cell division, which is also known as the reduction division stage, two haploid cells are formed from one diploid cell. Meiosis II division results in replication of each haploid cell. Nondisjunction can occur in either meiosis I or meiosis II, although $80 \%$ of the time it occurs during meiosis I (11). There is a clear association between advancing maternal age and an increased risk for nondisjunctional events (12). However, regardless of parental ages, it has been shown that the extra number 21 in children with trisomy 21 is of maternal origin in $95 \%$ of cases and of paternal origin in only $5 \%$ of cases (13). In general, if a zygote 

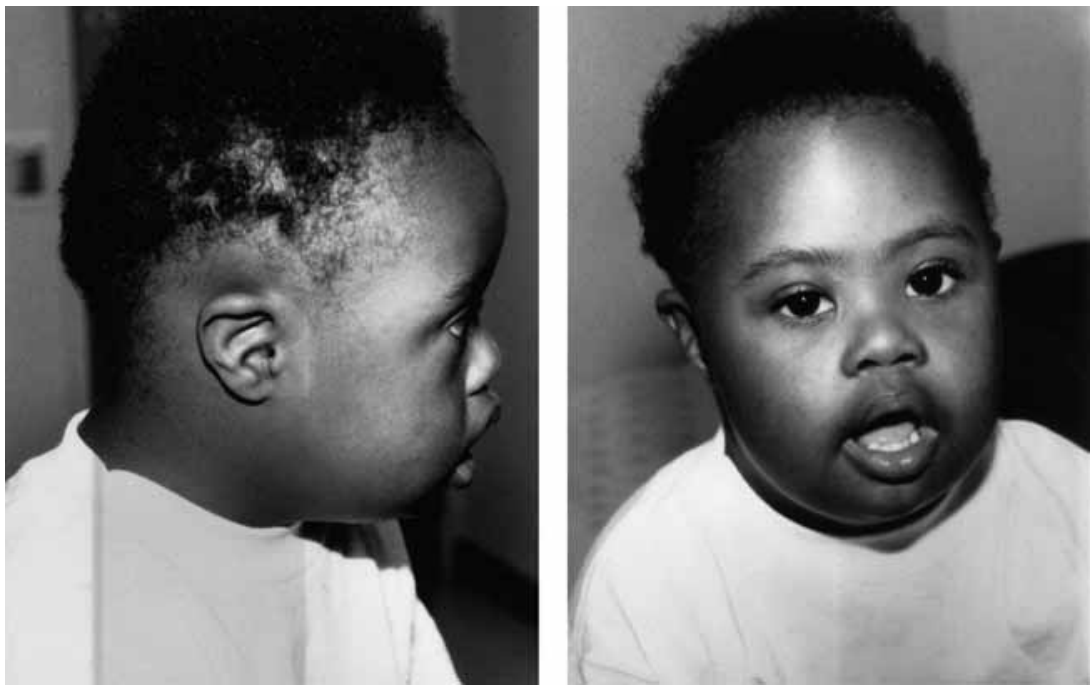

Figure 6 Two-year-old boy with Down syndrome. Note brachycephaly, dysplastic ears, epicanthal folds, and upslanting palpebral fissures.

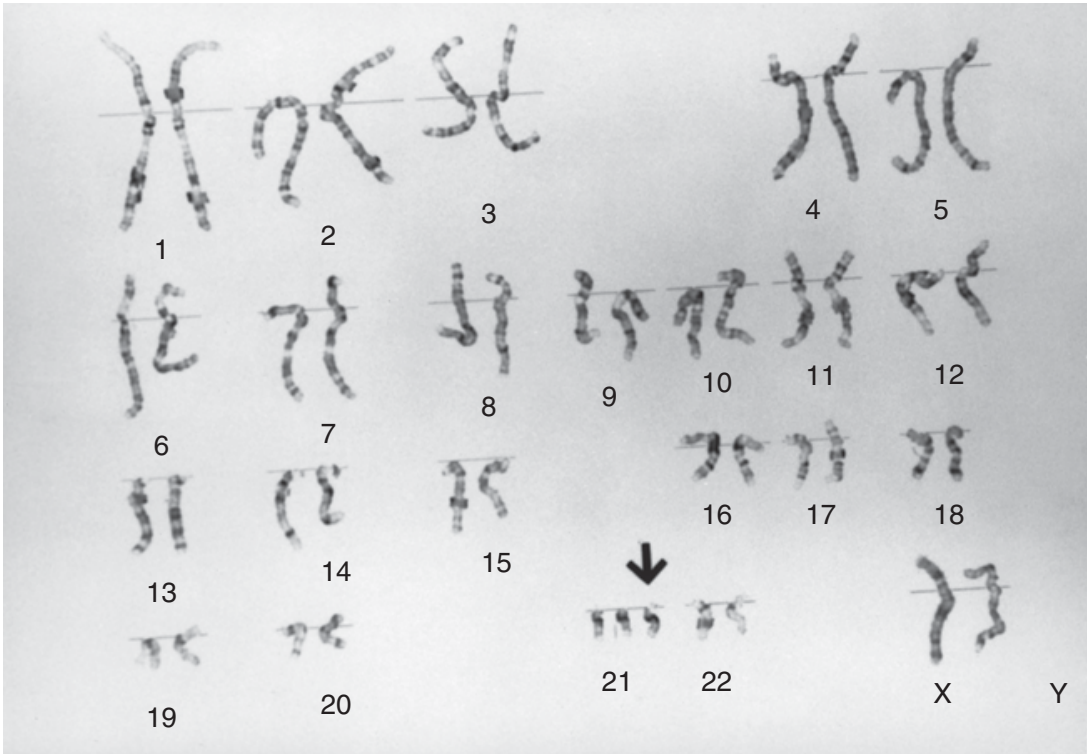

Figure 7 Karyotype of trisomy 21 karyotype. The arrow indicates extra chromosome 21.

has monosomy for an autosome, early embryogenesis will fail and a spontaneous pregnancy loss will occur, sometimes even before the first missed menstrual period. On the other hand, trisomy for an autosome may result in a longer surviving embryo or fetus, or even a liveborn infant. Trisomy 13, trisomy 18, and trisomy 21 (Down syndrome) are the only autosomal trisomies that can result in viable infants in the non-mosaic state. However, mosaicism for many different autosomal trisomies, that is, the presence of both normal and trisomic cells in one individual, has been described in viable infants. Sex chromosome aneuploidy has, in general, less deleterious effects on the affected individual.

\section{Down Syndrome}

Down syndrome, or trisomy 21 , is the most common autosomal chromosome abnormality seen in liveborn infants (Fig. 6). It occurs in approximately 1 in 700 newborns. As mentioned above, the risk of nondisjunction increases with advancing maternal age. The American College of Obstetrics and Gynecology has recommended that invasive prenatal testing for Down syndrome and other trisomies should be offered to all women, regardless of age, and not limited to those over age 35 (14). Down syndrome is caused by the presence of three copies of chromosome 21 (Fig. 7). In 95\% of cases, there is straightforward trisomy 21 (denoted $47, \mathrm{XX},+21$ in a female and $47, \mathrm{XY},+21$ in a male), with an extra 21 in every cell of the body, arising by nondisjunction. About $2 \%$ to $3 \%$ of patients have mosaicism, with some portion of cells having a normal chromosome complement; mosaicism is caused by postzygotic nondisjunction. The features of Down syndrome may be milder in the mosaic form, depending on what percentage of cells are normal. About $2 \%$ of Down syndrome cases are caused by a translocation, usually a Robertsonian translocation involving another acrocentric chromosome, such as the number $14(14 ; 21)$ or the number $21(21 ; 21)$. Karyotyping on all newborns with clinical features of Down syndrome will identify those who have the translocation form. Approximately 25\% of translocation cases are inherited from a parent, with a high risk of recurrence in future pregnancies $(15,16)$. Therefore, chromosome analysis should be done 


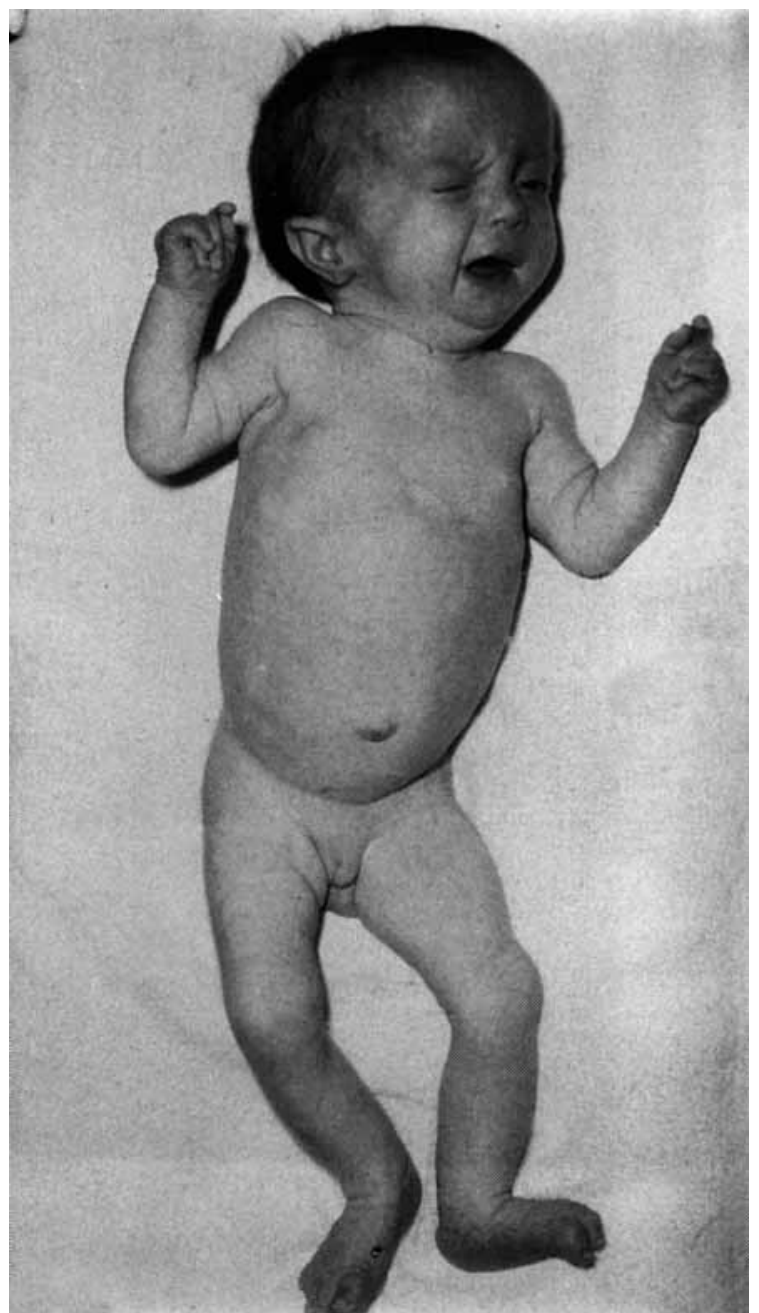

Figure 8 Newborn infant with trisomy 18, showing dysmorphic facies, flexion contracture of fingers, and rocker bottom feet.

on the parents of all cases of translocation-type Down syndrome. If a woman is a $14 ; 21$ Robertsonian translocation carrier, there is a $15 \%$ risk of Down syndrome in each pregnancy, as well as a high risk of recurrent spontaneous abortions due to the other possible unbalanced chromosome complements in the offspring. If a man is a carrier, the risk of a liveborn baby with trisomy 21 is only about $5 \%(15,16)$.

Individuals with Down syndrome usually have a moderate degree of mental retardation, with IQ in the 50 to 60 range. Some have milder mental retardation, while others fall into the severe range. The degree of mental retardation cannot be determined at birth. Early intervention, with involvement in infant stimulation and parent training programs, can improve the developmental outcome. Most people with Down syndrome are educable and many can be mainstreamed in regular classrooms in school. Increasing numbers of individuals with Down syndrome are able to live semi-independently and hold jobs in supervised settings $(17,18)$.

There are a number of associated birth defects and medical problems in Down syndrome. Approximately $40 \%$ to $50 \%$ of babies with trisomy 21 have a congenital heart defect, frequently an atrioventricular canal or septal defect (19). About $12 \%$ of these babies have a malformation of the gastrointestinal tract, most often duodenal atresia, but occasionally Hirschsprung's disease, imperforate anus, or tracheoesophageal atresia. Other birth defects include congenital cataracts $(3 \%)$, syndactyly, 11 pairs of ribs, and urinary tract anomalies (2-3\%) (20). Anomalies can be detected by prenatal ultrasound, particularly the heart defects and duodenal atresia. If either of these types of anomalies is seen, prenatal testing for Down syndrome should be considered.

Postnatal medical problems, in addition to developmental delay and mental retardation, include poor feeding and hypotonia in infancy, obesity later in life, visual impairment (myopia in 70\%), recurrent otitis media and hearing loss, hypothyroidism (20\%), atlantoaxial or atlanto-occipital instability (5-10\%), and childhood leukemia (1\%) (20).

\section{Trisomy 18}

Trisomy 18 (Edward syndrome) is a much less common autosomal trisomy, seen in approximately 1 in 7000 liveborn infants (21). It has been estimated that at least two-thirds of trisomy 18 conceptuses are lost by spontaneous abortion. Trisomy 18 is caused by the presence of three copies of the number 18 chromosome, again arising by nondisjunction in meiosis I or meiosis II.

The prognosis for trisomy 18 is extremely poor and the majority of infants die within the first 3 months of life. There are, however, a few individuals who have survived to their teens and twenties. The associated mental retardation is severe to profound. In general, the birth defects seen in trisomy 18 are more frequent and much more severe than that in trisomy 21 (Fig. 8). Most infants have significant intrauterine growth retardation, which may be identified by prenatal ultrasound. Approximately $85 \%$ of infants have a congenital heart defect, including septal defects, multivalvular dysplasia, and complex anomalies. Other anomalies are dysmorphic facial features, short sternum, joint contractures, overlapping digits, rocker bottom feet, renal abnormalities, and occasionally gastrointestinal tract anomalies and neural tube defects.

\section{Trisomy 13}

Trisomy 13 (Patau syndrome) is the third autosomal trisomy, which can be seen in liveborn infants. It is less common than trisomy 18 and occurs in about 1 in 12,000 live births (19). As in trisomy 18, the prognosis for trisomy 13 is very poor, with most infants dying within the first 3 months. Depending on the severity of the associated birth defects, longer-term survival is possible. Infants with trisomy 13 tend to be better grown than those with trisomy 18 (Fig. 9). However, they are more likely to have microcephaly, which can be seen by prenatal ultrasound. Congenital heart defects are present in $80 \%$ to $85 \%$, again often of a complex nature. Those with less significant heart defects may survive longer. Holoprosencephaly, cleft lip and palate, renal anomalies, and omphalocele are frequent abnormalities in trisomy 13, which may be detected by prenatal ultrasound.

\section{Sex Chromosome Abnormalities}

Sex chromosome abnormalities as a group are very common. Approximately 1 in 500 individuals has a sex chromosome abnormality, although it has been suggested that the true incidence may be higher. Some cases may never be ascertained due to the relatively mild signs and symptoms associated with most of the common sex chromosome problems. Many patients 


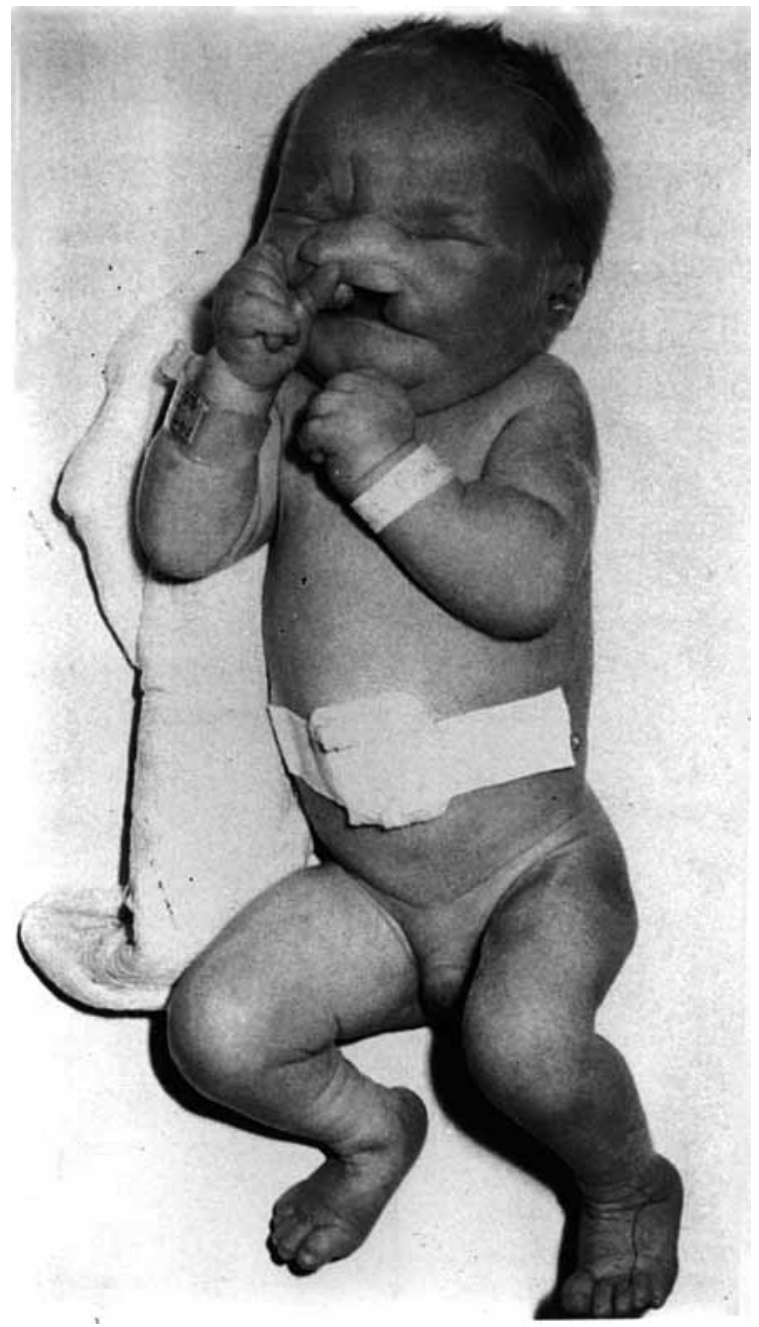

Figure 9 Newborn infant with trisomy 13, showing microphthalmia, bilateral cleft lip and palate, and postaxial polydactyly of the hands.

are not diagnosed until adulthood when they may undergo evaluation for infertility or other reproductive problems.

\section{Turner Syndrome}

Turner syndrome is one of the most familiar sex chromosome abnormalities. It is caused by the presence of only one $\mathrm{X}$ chromosome $(45, \mathrm{X})$ in phenotypic females. This classic Turner karyotype is seen in $50 \%$ of cases; other cases may be mosaic $(45, \mathrm{X} / 46, \mathrm{XX})$, or may be caused by an abnormal $\mathrm{X}$ chromosome, such as an isochromosome or ring $\mathrm{X}$ (1). There may be portions of a $\mathrm{Y}$ chromosome present or mosaicism for a 46,XY cell line in some cases. FISH testing for SRY is recommended for detection of Y chromosome material. Turner syndrome is seen in about 1 in 2500 newborn girls. However, it is well known that the incidence is much higher in early pregnancy, with more than $98 \%$ of $45, \mathrm{X}$ embryos and fetuses being lost by spontaneous miscarriage. This would suggest that the incidence among conceptuses could be as high as $1.5 \%$, an extraordinarily high frequency (5).

Features of Turner syndrome detectable by ultrasound include cystic hygroma, lymphedema, or hydrops fetalis; when these abnormalities are seen, the prognosis for survival of the pregnancy is poor (22). Smaller cystic hygromas may resolve during pregnancy, leaving a webbed neck or excess nuchal skin. Other anomalies seen in Turner syndrome include congenital heart defects, frequently coarctation of the aorta, and renal anomalies, often horseshoe kidney. Lymphedema may be seen at birth, often involving the dorsum of the hands and feet, as well as a shield-shaped chest and wide-spaced nipples. For surviving girls with Turner syndrome, the prognosis for good health and a normal lifespan is excellent. Intelligence is normal, although there may be some specific learning disabilities. However, due to the "streak" ovaries, puberty and the onset of menses will not usually occur without hormone therapy and the average adult height without growth hormone treatment is 57 inches. The long-term outcome with estrogen/progesterone replacement and growth hormone therapy is very good. A number of successful pregnancies in women with Turner syndrome have been reported, with the use of ovum donation and in vitro fertilization techniques (23).

\section{Klinefelter Syndrome}

Klinefelter syndrome is caused by the presence of an extra $\mathrm{X}$ chromosome in a phenotypic male $(47, \mathrm{XXY})$ and occurs in approximately 1 in 500 to 1 in 1000 males. If Klinefelter syndrome is detected prenatally, it is usually by chance, when an amniocentesis is being done for other reasons. There are no expected fetal anomalies in Klinefelter syndrome and most cases are not diagnosed in infancy. The diagnosis may be suspected in a boy with tall thin body habitus and mild learning disabilities. Testicular size is usually normal in prepubertal boys. However, boys with Klinefelter syndrome fail to go through puberty normally and eventually have small, firm testes and relatively hypoplastic external genitalia. They are usually sterile, and the diagnosis is sometimes made in an adult infertility or urology clinic.

\section{7, XXX Syndrome}

This chromosome abnormality is thought to occur in 1 in 1000 women, although the true incidence may be higher. Many cases are probably never ascertained because of the mild nature of the associated signs and symptoms. There is no recognizable phenotype, except perhaps tall stature and mild learning problems. Fertility is normal and the risk of sex chromosome aneuploidy in the offspring of an affected woman appears to be low.

\section{7, XYY Syndrome}

This chromosome abnormality is also fairly common, occurring in about 1 in 800 men. Again, there is no definite phenotype except taller than expected stature, slightly long facies and long digits. Most individuals have normal intelligence, but there have been reports of personality disorders and antisocial behavior. The incidence may be underestimated due to the nonspecific phenotype. This condition is most often diagnosed serendipitously.

\section{Structural Alterations}

In addition to aneuploidy, in which there is loss or gain of an entire chromosome, rearrangements, deletions, or duplications of portions of chromosomes can occur. These structural alterations can result in either balanced or unbalanced chromosome abnormalities. In unbalanced abnormalities, there is missing and/or additional chromosome material, which often causes multiple birth defects and developmental problems. Balanced chromosome abnormalities involve rearrangements of 
the chromosomes without loss or gain of chromosome material and are usually found in normal individuals.

One frequent cause of structural alteration is a translocation. There are two basic types of translocations. A reciprocal translocation occurs when two chromosomes break and the pieces exchange places (Fig. 10). The resulting abnormal chromosomes are called "derivative chromosomes." If the reciprocal translocation is balanced, the individual is usually

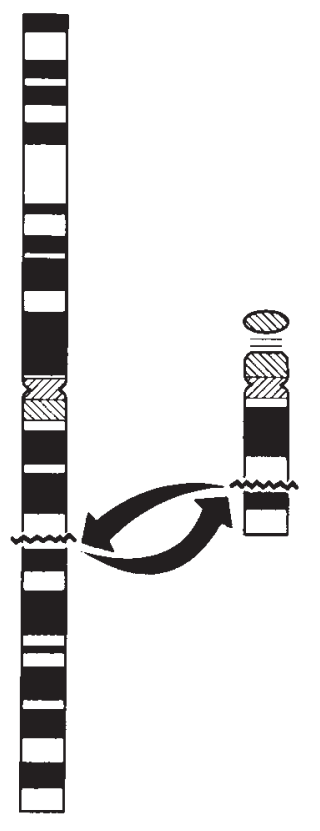

3

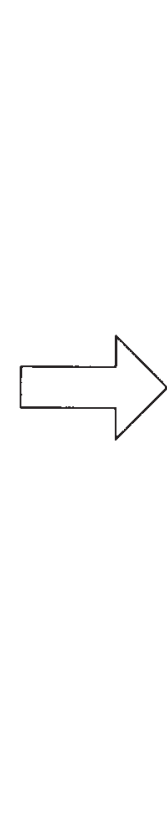

21

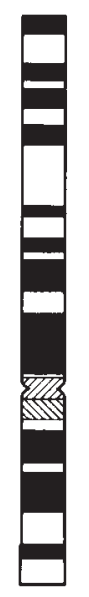

$\operatorname{der}(3)$

$t(3 ; 21)$
Figure 10 Reciprocal translocation between chromosomes 3 and 21. The break points on the long arm of each chromosome are indicated by the wavy line. Source: From Ref. 6. normal. Translocations such as these are found in approximately 1 in 500 normal individuals in the general population and are usually never detected. However, if a parent carries a balanced translocation and passes only one of his or her derivative chromosomes to an offspring, along with the other normal chromosomes, the child will have an unbalanced chromosome complement and will likely have multiple problems as a result (Fig. 11). Inheritance of a derivative chromosome in this situation causes a partial duplication (partial trisomy) of one chromosome and a partial deletion (partial monosomy) of another chromosome.

In Robertsonian translocations, the satellites of two acrocentric chromosomes are lost and the $\mathrm{q}$ arms of the chromosomes fuse at the centromeres, resulting in a single chromosome (Fig. 12). Thus, a carrier of a Robertsonian translocation has a total of only 45 chromosomes, but has a balanced chromosome complement and is a normal individual. The loss of the satellites poses no problems since these regions of chromosome material encode repetitive sequences that are not essential. A Robertsonian translocation in a parent can result in an unbalanced chromosome abnormality in his or her offspring. If the zygote inherits the translocation chromosome along with either one of the parents' corresponding normal chromosomes, the child will have trisomy for that chromosome. If the zygote receives the translocation chromosome alone, a normal carrier would be produced. If the zygote does not receive the translocation chromosome, and thus has one of the single involved chromosomes, a monosomy would be produced, which would cause a miscarriage. As previously discussed, most autosomal trisomies are lethal. The only unbalanced Robertsonian translocations seen in liveborn babies with trisomies are in Down syndrome and trisomy 13. Other trisomies for acrocentric autosomes, such as trisomy 14 , trisomy 15, or trisomy 22 are lethal abnormalities and
Reciprocal translocation

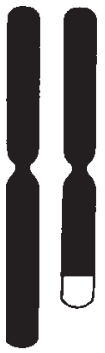

3

21

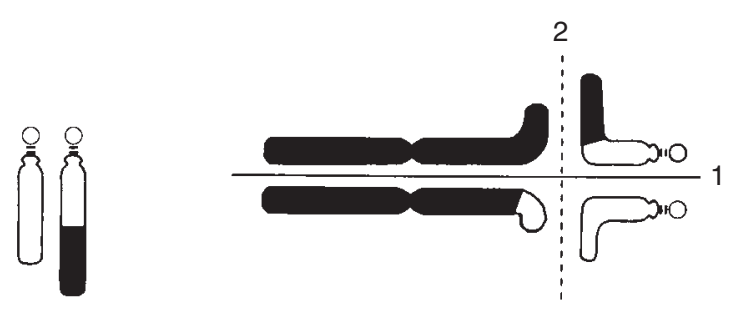

Pairing at meiosis

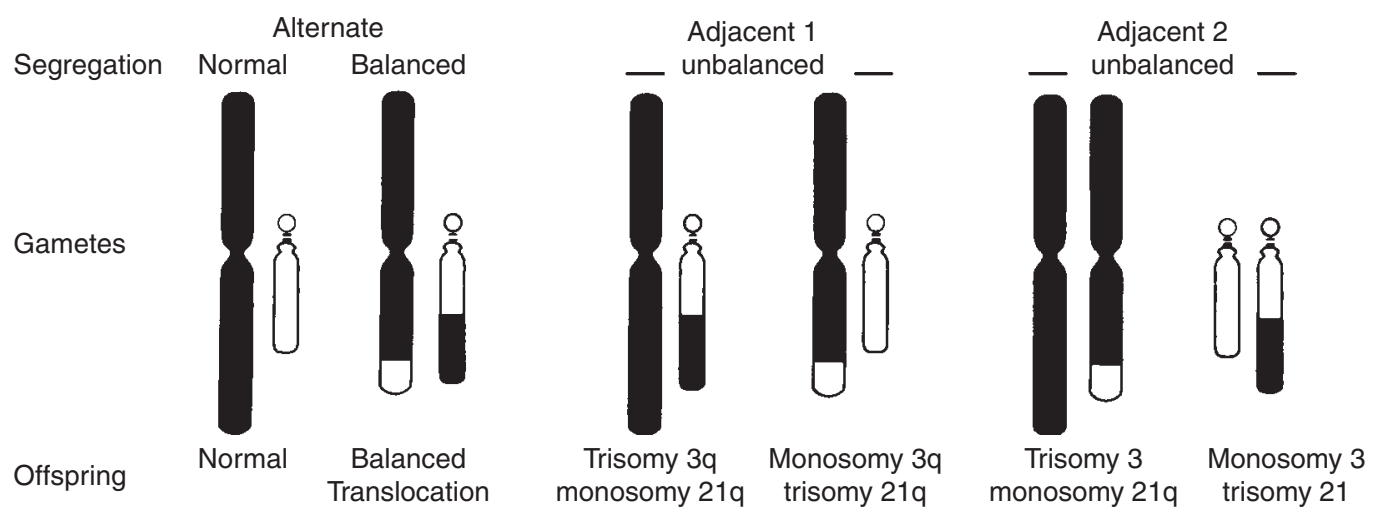

Figure 11 Meiotic segregation in a reciprocal translocation involving chromosomes 3 and 21. Source: From Ref. 6. 
would cause miscarriages. The most frequent Robertsonian translocation encountered in the general population is a $13 ; 14$ translocation, followed in frequency by a 14;21 translocation (22). The former would place a carrier at high risk for having a child with trisomy 13, while the latter would result in a high risk for trisomy 21, and both would be expected to cause a higher risk for recurrent pregnancy loss due to lethal trisomies and monosomies.

Another type of structural abnormality is a deletion, in the absence of a translocation. Deletions can be terminal, that is, at either end of the chromosome, or interstitial, within the short or long arm of the chromosome. As can be imagined, there is an almost infinite number of possible deletions that can occur as a result of accidents during meiosis. However, some specific deletions are seen more frequently. One such relatively common terminal deletion is $5 \mathrm{p}$ - or cri du chat syndrome. Cri du chat syndrome is associated with microcephaly and mental retardation in all cases, congenital heart defects in about $30 \%$, and craniofacial dysmorphic features, including a round face, epicanthal folds, hypertelorism, and dysplastic ears (19). Newborns with cri du chat syndrome have a mewing or cat-like cry, for which the syndrome was named. Another fairly frequent terminal deletion is $4 \mathrm{p}$ - or Wolf-Hirschhorn syndrome, a disorder associated with mental retardation, growth deficiency, and distinctive craniofacial dysmorphic features. Patients with Wolf-Hirschhorn have microcephaly, a prominent glabellar region, hypertelorism, strabismus, cleft lip and/or palate, and micrognathia (19). They may have associated cardiac, renal, and genital anomalies. These types of deletions are usually visible on routine karyotype analysis due to their large size. However, deletions can obviously vary widely in size; some are too small to be seen on routine studies and will require FISH analysis or chromosome microarray analysis to be detected.

There are an increasing number of recognizable microdeletion syndromes (Table 2). For example, Prader-Willi and Angelman syndromes are microdeletion syndromes, both involving a deletion at 15q11q13. Prader-Willi syndrome is a complex disorder associated with hypotonia and failure to thrive in early infancy, followed by rapid weight gain after about 2 years of age and severe obesity, mental retardation, hypogonadism, and craniofacial dysmorphic features. By contrast, the Angelman syndrome phenotype is very different, even though both syndromes are associated with the same microdeletion. Angelman syndrome, also called the "happy puppet" syndrome, presents with severe mental retardation,

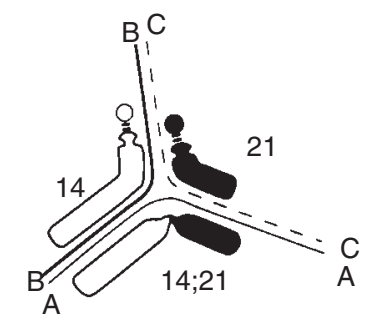

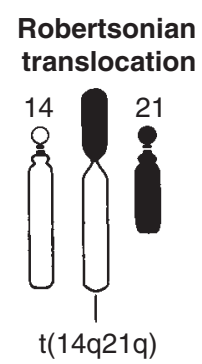

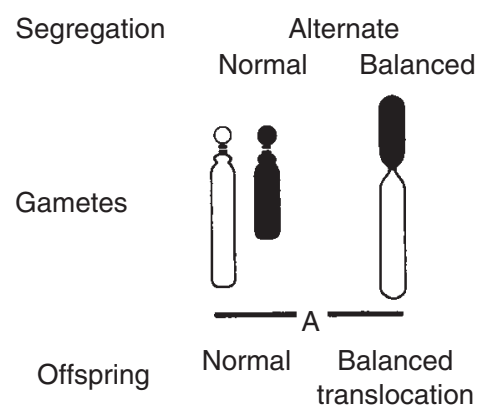

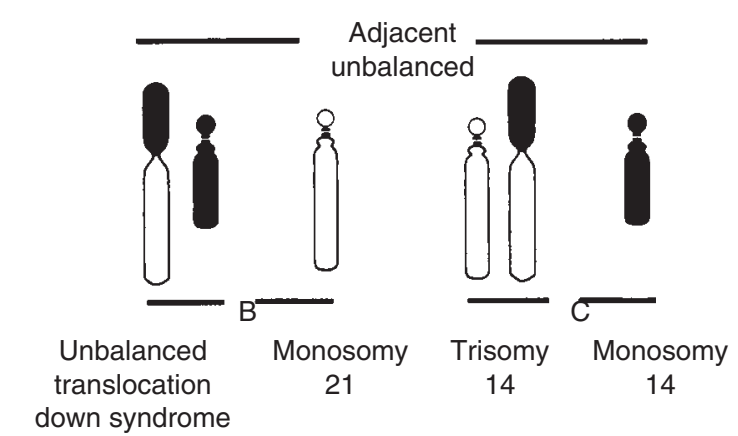

Figure 12 Meiotic segregation in Robertsonian translocation involving chromosomes 14 and 21 . Source: From Ref. 6.

Table 2 Microdeletion Syndromes

\begin{tabular}{llc} 
Syndrome & \multicolumn{1}{c}{ Clinical features } & Chromosome deletion \\
Alagille & Cholestasis, pulmonary atresia, vertebral anomalies, dysmorphic facial features & $20 \mathrm{p} 11.23$-p12.2 \\
Angelman & Mental retardation, seizures, ataxic gait & $15 \mathrm{q} 11.2 \mathrm{q} 13$ \\
DiGeorge/velocardiofacial & Characteristic facies, cleft palate, heart defect, thymic hypoplasia & $22 \mathrm{q} 11.2$ \\
Langer-Giedion & Characteristic facies, sparse hair, exostoses, mental retardation & $8 \mathrm{q} 24$ \\
Miller-Dieker & Lissencephaly, characteristic facies & $17 \mathrm{p} 13.3$ \\
Prader-Willi & Mental retardation, obesity, short stature, hypotonia, hypogonadism & $15 \mathrm{q} 11.2 \mathrm{q} 13$ \\
Smith-Magenis & Dysmorphic facies, sleep disturbance, mental retardation, self-destructive behavior & $17 \mathrm{p} 11.2$ \\
Williams & Mental retardation, supravalvular aortic stenosis, characteristic facies & $7 \mathrm{q} 11.23$ \\
Retinoblastoma & Eye tumor & $13 \mathrm{q} 14$ \\
Wilms tumor/aniridia & Mental retardation, aniridia, Wilms tumor, genital defects & $11 \mathrm{p} 13$
\end{tabular}



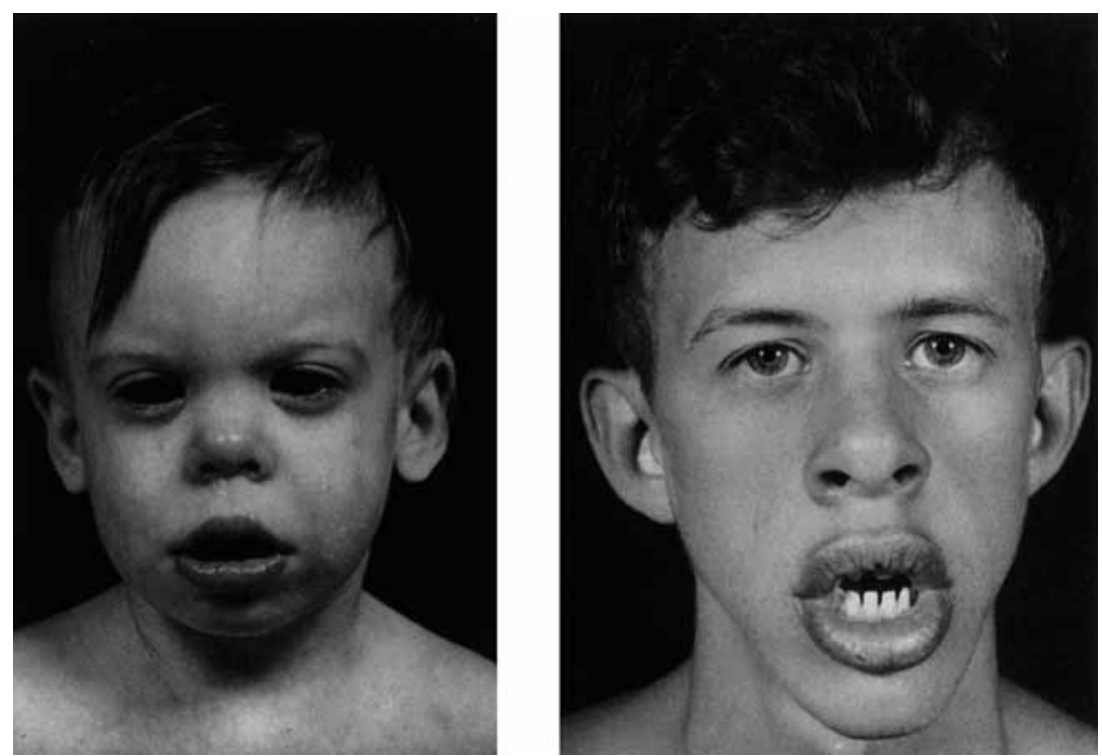

Figure 13 Two unrelated boys with Williams syndrome. Note epicanthal folds, upturned nose, and wide mouth with full lips. Source: From Ref. 25.

seizures, ataxia, and inappropriate laughter. It is now known that genomic imprinting is responsible for the phenotypic differences between these two syndromes. Genomic imprinting implies that maternally and paternally derived genetic information at the same locus is not identical and interchangeable, at least for some portions of the genome. In Prader-Willi, the deletion always occurs on the paternally derived number 15 chromosome, resulting in lack of genetic influence from the father in this region. About $70 \%$ to $75 \%$ of Prader-Willi patients have such a deletion, while the remainder have no visible abnormalities; virtually all of these remaining patients have maternal disomy, meaning that they have two copies of the mother's chromosome 15 and neither of the father's, again resulting in lack of paternal genetic influence in this region (1). The disomy can be either isodisomy, in which there are two identical copies of one of the maternal 15 chromosomes, or heterodisomy in which the two copies represent one of each of the maternal number 15 chromosomes. The mirror image situation exists in Angelman syndrome; if a deletion is found, it is always on the maternally derived number 15 , and if there is no deletion, there is paternal disomy for 15, both cases resulting in loss of maternal genetic influence in this region of 15 (1). Thus, genomic imprinting of the genes in this area at $15 \mathrm{q} 11 \mathrm{q} 13$ is taking place, since lack of influence from either parent can result in two very different syndromes.

Many other microdeletion syndromes have been identified (24). Many of these conditions were well recognized by clinical geneticists for years before the microdeletion could be detected. With the improvement of cytogenetic technology, smaller fragments of missing chromosome material can be identified using FISH. Studies in this area have been aided by the use of chromosome microarray analysis. Microdeletion syndromes have also been referred to as contiguous gene syndromes because of the loss of multiple adjacent genes. It is understandable that the phenotypes of these patients may vary depending on the size of the deletion and the number and importance of the deleted genes. One good example of this is Williams syndrome, which is readily diagnosed by FISH (Fig. 4). Williams syndrome is a well-recognized multiple anomaly syndrome characterized by moderate mental retardation, craniofacial dysmorphic features, hypercalcemia, and cardiovascular abnormalities. Facial features include epicanthal folds with periorbital fullness, stellate iris pattern, long philtrum, and wide mouth with prominent lips (Fig. 13). The cardiovascular abnormalities typically involve supravalvular aortic stenosis, but other blood vessels, such as pulmonary and renal arteries, may be affected. Most cases are sporadic, but there is autosomal dominant inheritance in some families. There is a separate, clearly autosomal dominant disorder of isolated supravalvular aortic stenosis, which is not associated with the other features of Williams syndrome. It has been discovered that the supravalvular aortic stenosis and other vascular abnormalities in both of these disorders are due to abnormalities in the gene for elastin, which is located on chromosome 7 at $7 q 11.23$ (26). In the case of Williams syndrome, one copy of the elastin gene is deleted, along with other genes in the surrounding regions. Williams syndrome patients without deletion of the elastin gene do not have arterial disease. Thus, variable expression of contiguous gene or microdeletion syndromes can be directly related to the extent of the deleted genes, but all patients share an overlapping area of involvement.

In addition to deletions, duplications of chromosome material can occur in the absence of a translocation, and again there are an increasing number of newly recognized microduplication syndromes detectable with the use of chromosome microarray analysis. Duplications can be interstitial, within the body of the chromosome, or terminal, at the end of the chromosome. Duplication of a portion of chromosome material results in partial trisomy for that region. As with deletions, there are an almost endless number of possible duplications that could result from errors in meiosis during gamete formation. Some of the common duplications have been found in regions associated with deletions of the same region, including the 22q11.2 microduplication syndrome at the same locus as the DiGeorge/velocardiofacial syndrome deletion (Fig. 14) and the $7 \mathrm{q} 11.23$ microduplication syndrome at the same locus as the Williams syndrome deletion. 
(A)

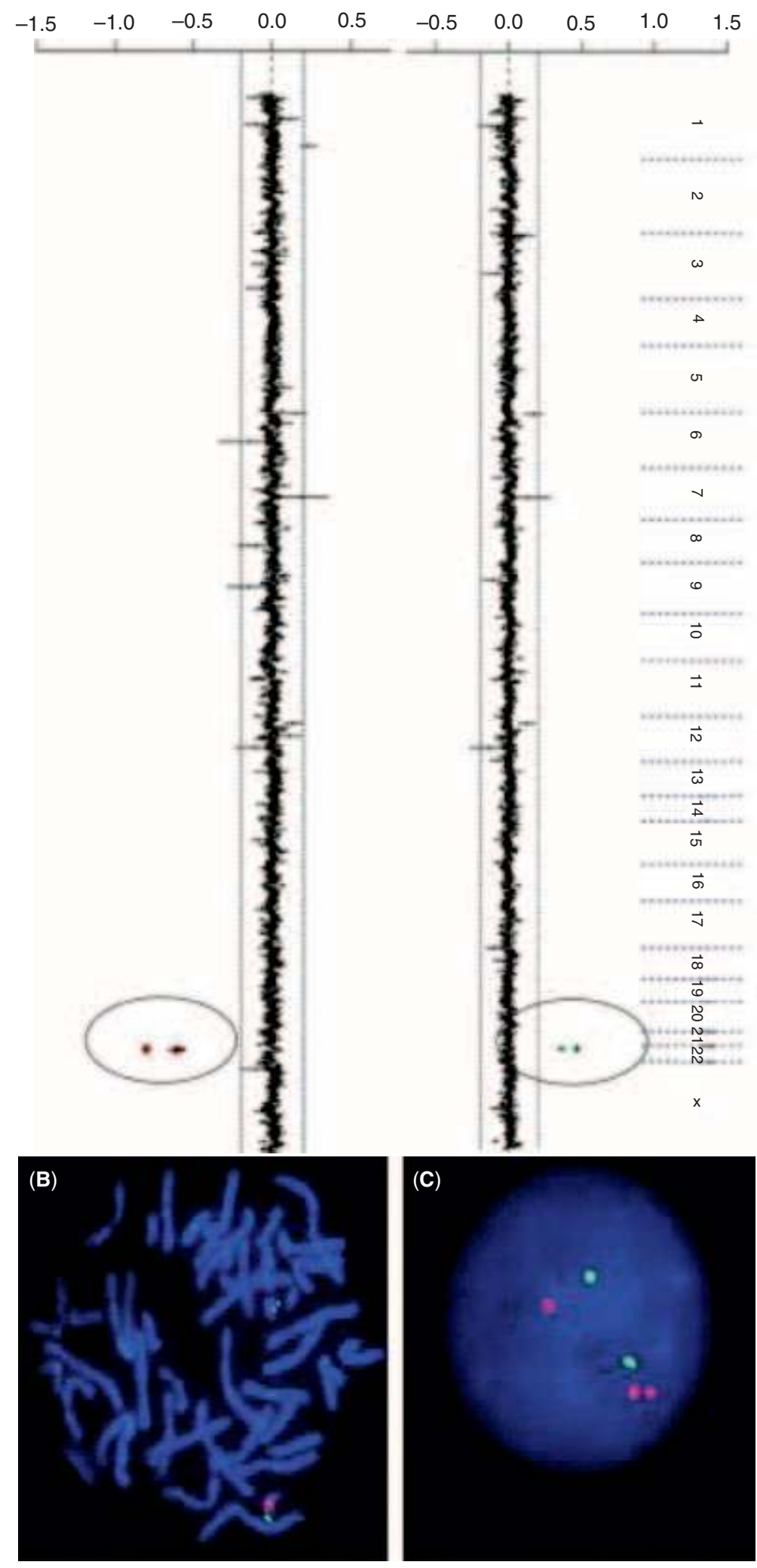

Figure 14 Array comparative genomic hybridization for 22q11.2 microdeletion and microduplication syndromes. (A) A $3 \mathrm{Mb}$ loss of chromosomal material in a patient with the 22q11.2 microdeletion syndrome (DiGeorge/velocardiofacial syndrome) is shown on the left and its reciprocal gain in a patient with the $22 \mathrm{q} 11.2 \mathrm{microduplication}$ syndrome is shown on the right. (B) Deletion confirmed by metaphase fluorescence in situ hybridization (FISH) analysis, with a single red 22q11.2 signal and two green control signals. (C) Duplication confirmed by interphase FISH showing three red signals and two control green signals. Source: From Ref. 7.

Another type of structural rearrangement is a ring chromosome. A ring occurs when the terminal $\mathrm{p}$ and $\mathrm{q}$ arms are deleted and the "sticky ends" anneal to each other. This can occur with any chromosome, perhaps most frequently with the $\mathrm{X}$ chromosome. The seriousness of the effects is related to the extent of the deletions produced.

Inversions refer to breakage of a chromosome at two places with secondary inversion and reinsertion of the 

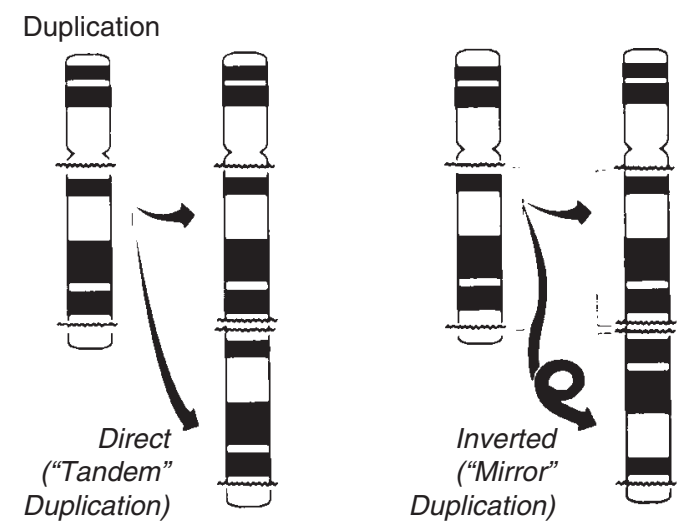

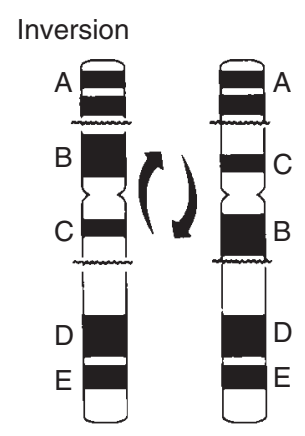

Pericentric

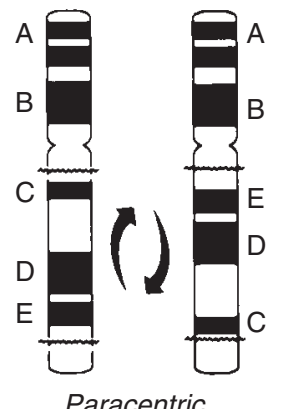

Paracentric
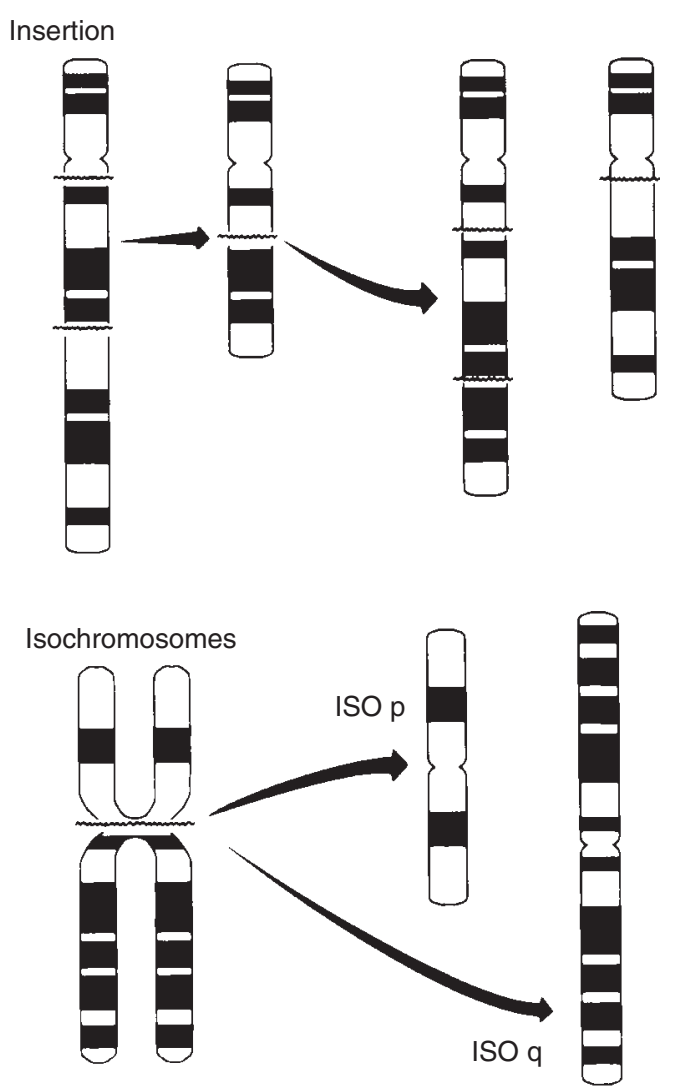

Figure 15 Structural abnormalities of chromosomes (see text for details). Source: From Ref. 6.

material back into the same chromosome. If this occurs within either the $\mathrm{p}$ arm or the $\mathrm{q}$ arm, it is said to be a paracentric inversion, since the centromere is not affected. If it occurs on either side of the centromere, it is a pericentric inversion (Fig. 15). Most individuals with inversions are normal. Sometimes, an inversion results in a deletion or duplication of chromosome material, leading to phenotypic or developmental abnormalities. If a parent has an inversion, his or her offspring could have an unbalanced chromosome constitution if proper pairing does not occur during meiosis and abnormal gametes are formed. If an inversion is present, a loop forms during chromosome pairing in meiosis I. Recombination, or crossing over, which normally occurs in meiosis I, can take place in the loop as well, potentially leading to unbalanced gametes. However, normal carriers of paracentric and pericentric inversions have different risks of producing abnormal offspring. If the inversion is paracentric, the unbalanced gametes produced are likely to be acentric or dicentric, with a very low chance of resulting in a viable liveborn child with a chromosome abnormality. If the inversion is pericentric, the centromere is not affected by recombination and abnormal gametes with duplication and deletion of material distal to the inversion can be produced, potentially leading to viable offspring with chromosome anomalies. The larger the pericentric inversion, the more likely it is that abnormal offspring can be produced, since the duplicated or deleted segments are smaller. The overall risk that a pericentric inversion carrier will have karyotypically abnormal children is estimated to be between $1 \%$ and $10 \%$ (16).

Finally, isochromosomes result from loss of either the $\mathrm{p}$ arm or the $\mathrm{q}$ arm of a metacentric or submetacentric chromosome, with duplication of the remaining arm, thus causing trisomy for the $\mathrm{p}$ or $\mathrm{q}$ arm of a pair of chromosomes and monosomy for the opposite arm. This is seen most commonly in some cases of Turner syndrome, when the $\mathrm{X}$ chromosome is involved. Isochromosomes for the autosomes would be expected to cause very severe and likely lethal abnormalities in most cases.

\section{Chromosome Abnormalities in Spontaneous Abortions}

Chromosomes examined from products of conception from pregnancy losses at various stages of gestation show an exceptionally high rate of chromosomal abnormalities. In fact, as many as $10 \%$ to $15 \%$ of early embryos have a chromosome abnormality, usually leading to a spontaneous pregnancy loss (27). Approximately $50 \%$ to $60 \%$ of spontaneous abortions are caused by a chromosome anomaly in the embryo or fetus (28). At the time of live birth, approximately 1 in 150 newborns is chromosomally abnormal. Thus, as stated by John Opitz, "it seems established that most of, 'humanity' dies before, rather than after birth, and that perhaps only a third survive from the earliest beginnings till [sic] birth..." (29). The most frequent abnormalities seen are autosomal trisomies (52\%), especially trisomy 16 (15\%), trisomy 13,18 , and 21.45 , X accounts for $18 \%$ of abnormalities and triploidy is found in $17 \%$ (30). There is a steady loss of these abnormal pregnancies throughout the first trimester. By 20 weeks, the incidence has fallen to 27 in 1000 or $2.7 \%$, and by full term, the incidence is 6 in 1000 or $0.6 \%$ (28).

In the vast majority of cases, the occurrence of a chromosomally abnormal pregnancy with spontaneous abortion does not increase the risk of recurrence in a future pregnancy. A general rule of thumb is that products of conception should be evaluated by karyotype analysis on the third pregnancy loss. Parental blood chromosomes should also be analyzed at this point, looking for a balanced translocation in 
Table 3 Common Single-Gene Disorders

\begin{tabular}{|c|c|}
\hline Disease & Incidence \\
\hline \multicolumn{2}{|l|}{ Autosomal dominant } \\
\hline $\begin{array}{l}\text { Adenomatous } \\
\text { polyposis coli }\end{array}$ & 1 in 6000 \\
\hline $\begin{array}{l}\text { Adult polycystic } \\
\text { kidney disease }\end{array}$ & 1 in 1000 \\
\hline $\begin{array}{l}\text { Familial hyper- } \\
\text { cholesterolemia }\end{array}$ & 1 in 500 \\
\hline $\begin{array}{l}\text { Hereditary nonpolyposis } \\
\text { colorectal cancer }\end{array}$ & up to 1 in 200 \\
\hline Huntington disease & 1 in 20,000 \\
\hline Marfan syndrome & 1 in 10,000 to 1 in 20,000 \\
\hline Myotonic dystrophy & 1 in 7000 to 1 in 20,000 \\
\hline Neurofibromatosis type I & 1 in 3000 to 1 in 5000 \\
\hline Osteogenesis imperfecta & 1 in 10,000 to 1 in 15,000 \\
\hline \multicolumn{2}{|l|}{ Autosomal recessive } \\
\hline A1-Antitrypsin deficiency & 1 in 2500 to 1 in 10,000 Caucasians \\
\hline Cystic fibrosis & 1 in 2000 Caucasians \\
\hline Hemochromatosis & 1 in 5000 \\
\hline Phenylketonuria & 1 in 10,000 to 1 in 20,000 \\
\hline Sickle cell disease & 1 in 400 African-Americans \\
\hline Tay-Sachs disease & 1 in 3000 Ashkenazi Jews \\
\hline Thalassemia & $\begin{array}{l}1 \text { in } 50 \text { to } 1 \text { in } 100 \text { Asians and in } \\
\text { Mediterranean populations }\end{array}$ \\
\hline \multicolumn{2}{|l|}{$X$-linked } \\
\hline $\begin{array}{l}\text { Duchenne muscular } \\
\text { dystrophy }\end{array}$ & 1 in 3500 males \\
\hline Fragile X syndrome & 1 in 1200 males; 1 in 2500 females \\
\hline Hemophilia A & 1 in 10,000 males \\
\hline Color blindness & $8 \%$ of males \\
\hline $\begin{array}{l}\text { Glucose 6-phosphate } \\
\text { dehydrogenase deficiency }\end{array}$ & $10-15 \%$ of African-American males \\
\hline
\end{tabular}

one of the partners. A translocation is found in approximately $5 \%$ to $10 \%$ of couples with recurrent miscarriages (31).

\section{MENDELIAN INHERITANCE}

In 1865, an Austrian monk named Gregor Mendel published his now-famous treatise on the inheritance of characteristics in garden peas, leading eventually to the modern study of genes and inheritance, including human genetic disorders. However, Mendel's original work was largely ignored during his lifetime. In fact, his contemporary, Charles Darwin, apparently never knew of Mendel's research and hypotheses. Mendel's work was "rediscovered" at the beginning of the 20th century. In 1902, Archibald Garrod reported the first known human Mendelian disorder, alkaptonuria. Over the last century, thousands of human single-gene traits and disorders have been described (Table 3). These conditions were catalogued over many years by Victor McKusick in his Mendelian Inheritance in Man and now in an online version OMIM (Online Mendelian Inheritance in Man) (3).

Since humans have two copies of each chromosome, we also have two copies of each gene. Alternative forms of the same gene are called "alleles." Each chromosome contains hundreds of different genes. At the time of conception, we inherit one of each pair of genes from our mother and the other of each pair from our father. Thus, we receive an equal amount of genetic information from each parent.

There are four basic inheritance patterns of human singlegene disorders. These include autosomal dominant and
Affected individual Normal individual

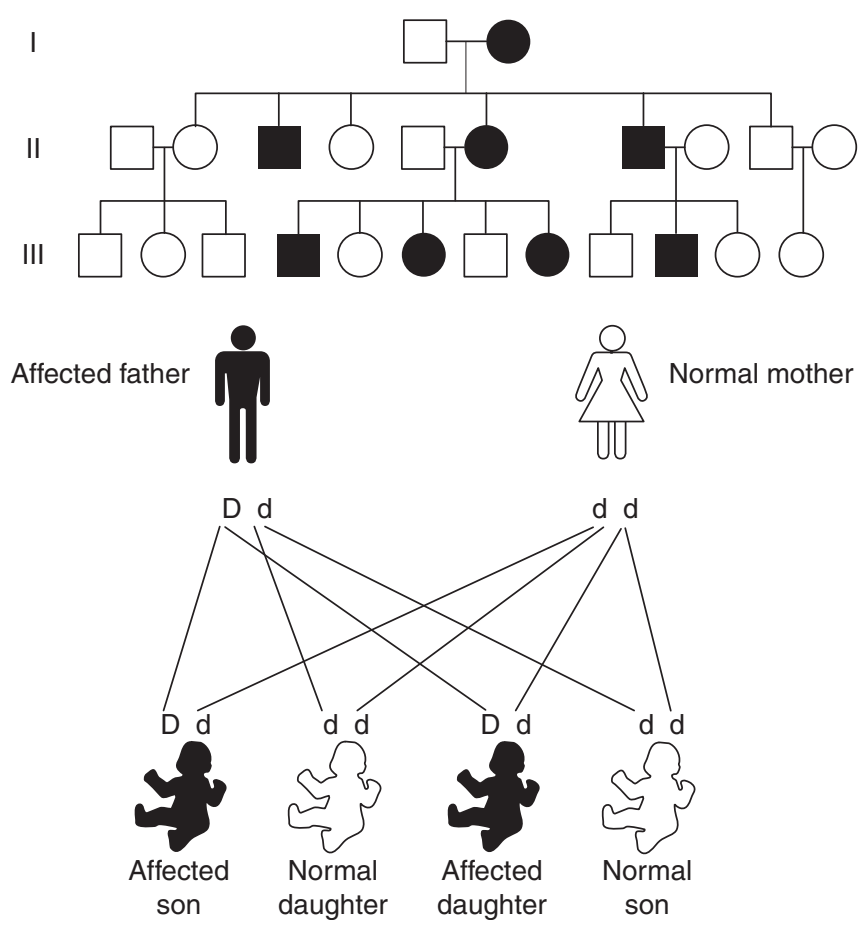

Figure 16 Autosomal dominant inheritance. Both males and females are affected and there is male-to-male transmission of the disorder. Source: From Ref. 25.

autosomal recessive inheritance, in which the mutant gene is on an autosome, and X-linked dominant and X-linked recessive inheritance, in which the mutant gene is on the $\mathrm{X}$ chromosome. Individuals can be heterozygous for a given gene, meaning that they have two different forms, or alleles, of the gene at the same locus on each of the two different chromosomes. Individuals can also be homozygous for a gene, in which the two alleles are identical. Males are said to be hemizygous with respect to genes on the $\mathrm{X}$ chromosome, since they have only one copy of the $\mathrm{X}$.

In autosomal dominant genetic disorders, only one copy of a mutant allele is necessary for expression of the disease (Fig. 16). Thus, heterozygotes will be affected. The risk of passing on the mutation to an offspring is $50 \%$ for each pregnancy. Vertical transmission of the disorder is seen on pedigree analysis, and the condition can often be traced back many generations. However, there are certain caveats to keep in mind when evaluating autosomal dominant disorders. First, there is a high degree of variability of expression of dominant conditions. Even within the same family, some individuals may be severely affected while others may have very mild and medically insignificant features. Thus, thorough review of medical histories and physical examinations on multiple family members are often parts of a genetic evaluation. Second, there may be reduced penetrance of a dominant disorder. Thus, a heterozygote may not show any manifestations of the mutant gene after a complete medical evaluation. Finally, many autosomal dominant disorders have a high new spontaneous mutation rate and a dominant condition may 

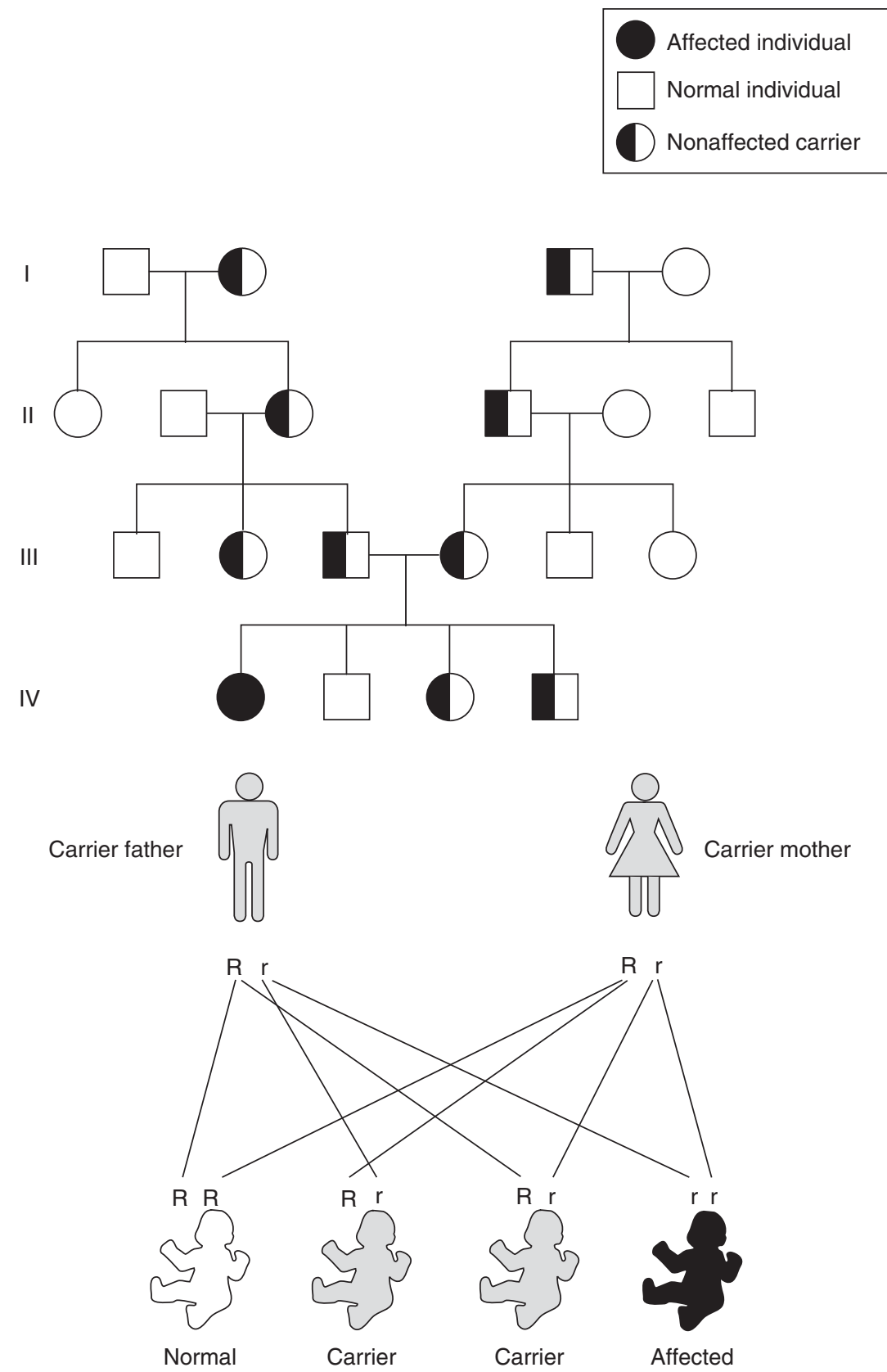

Figure 17 Autosomal recessive inheritance. Note that both parents must be carriers and the affected offspring may be male or female. Source: From Ref. 25.

appear de novo in an isolated individual in a pedigree. However, once present, it can be passed on to subsequent generations. In general, abnormal genes in autosomal dominant disorders likely encode structural proteins, such as collagen, which is abnormal in osteogenesis imperfecta, or fibrillin, which is mutant in Marfan syndrome.

In autosomal recessive conditions, two copies of a mutant allele are necessary for expression, and only homozygotes will be affected (Fig. 17). Heterozygotes for recessive genes are usually normal and do not have any manifestations of the disease, but they are carriers. Autosomal recessive disorders manifest only if both parents are carriers of the same mutant gene and the offspring receives one mutant allele from each parent. Individuals with autosomal recessive disorders are often compound heterozygotes, meaning that they have two different mutant alleles of the same gene, resulting in expression of the disorder. Recurrence risk for a recessive disorder is $25 \%$ for each pregnancy. There is a $25 \%$ chance of having a normal noncarrier child and a 50\% chance of having a carrier child. Pedigree analysis usually does not reveal other affected family members outside of the nuclear family group, unless there is consanguinity. The incidence of an autosomal recessive disorder in a population is related to the frequency of heterozygotes. The carrier rate for some conditions is high, while it is very low for others. Recessive conditions have a higher incidence in isolated or inbred populations. Recessive disorders show little variation in expression among affected members within a pedigree, although there may be interfamilial variation due to different mutant alleles. In general, recessive disorders more likely involve genes that encode enzymes or molecules important in cellular functions. Examples include inborn errors of metabolism caused by enzyme deficiencies, hemoglobinopathies, and defects in cellular membrane transport mechanisms, as in cystic fibrosis. 


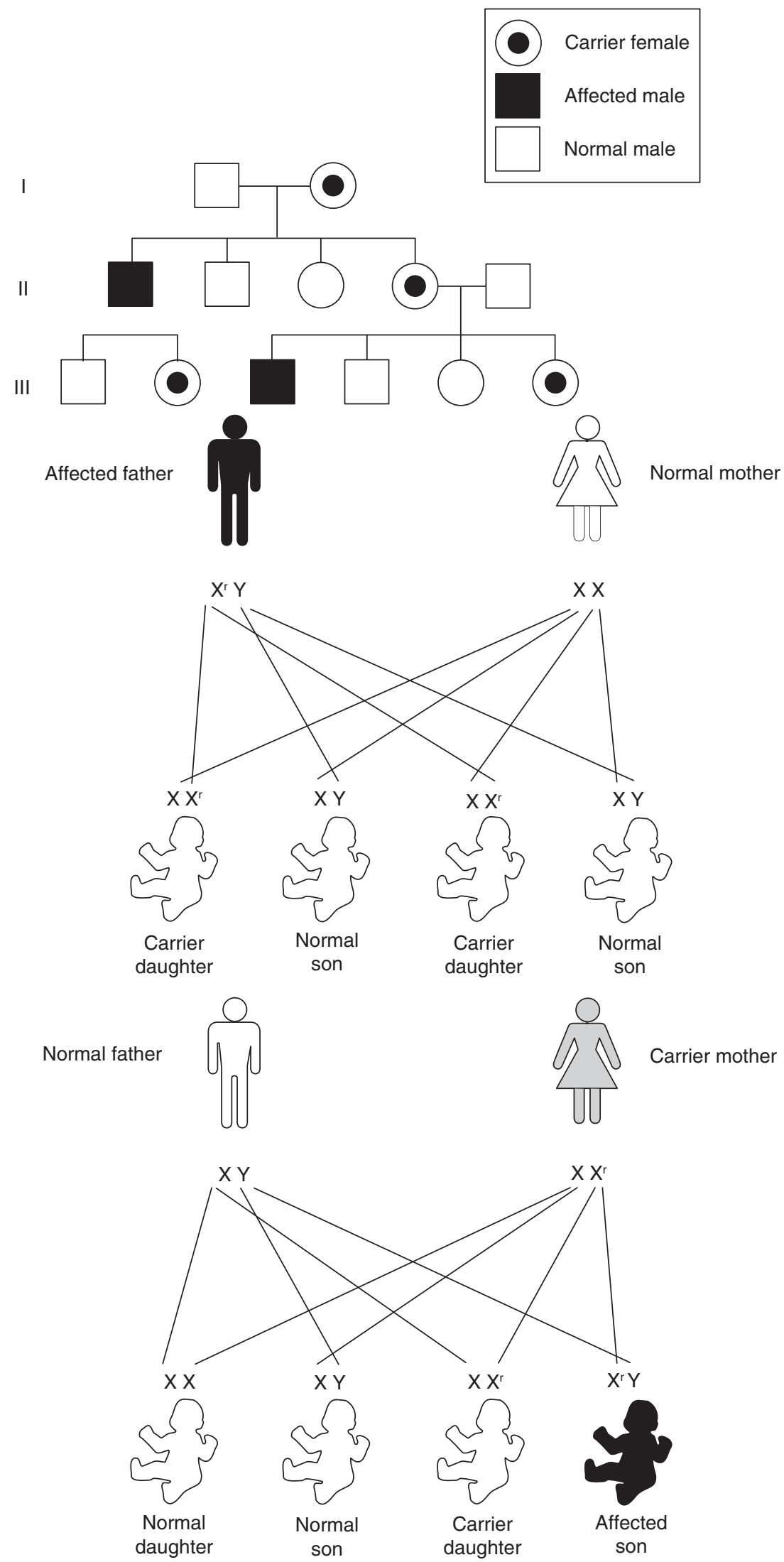

Figure 18 X-linked recessive inheritance. Males can be affected in multiple generations and they inherit the trait from their mothers. Source: From Ref. 25.

$\mathrm{X}$-linked recessive conditions are caused by mutations in genes on the $\mathrm{X}$ chromosome. Since males are hemizygous for X-linked genes, they will express such mutations, while females with a mutant recessive gene on one $\mathrm{X}$ chromosome will be carriers with a normal phenotype (Fig. 18). There are exceptions to these rules. For example, if the X-inactivation process results in a larger proportion of active $\mathrm{X}$ chromosomes carrying the mutant allele, a female may show expression or partial expression of the disorder. In X-linked dominant conditions, only one copy of the mutant allele is needed for full 

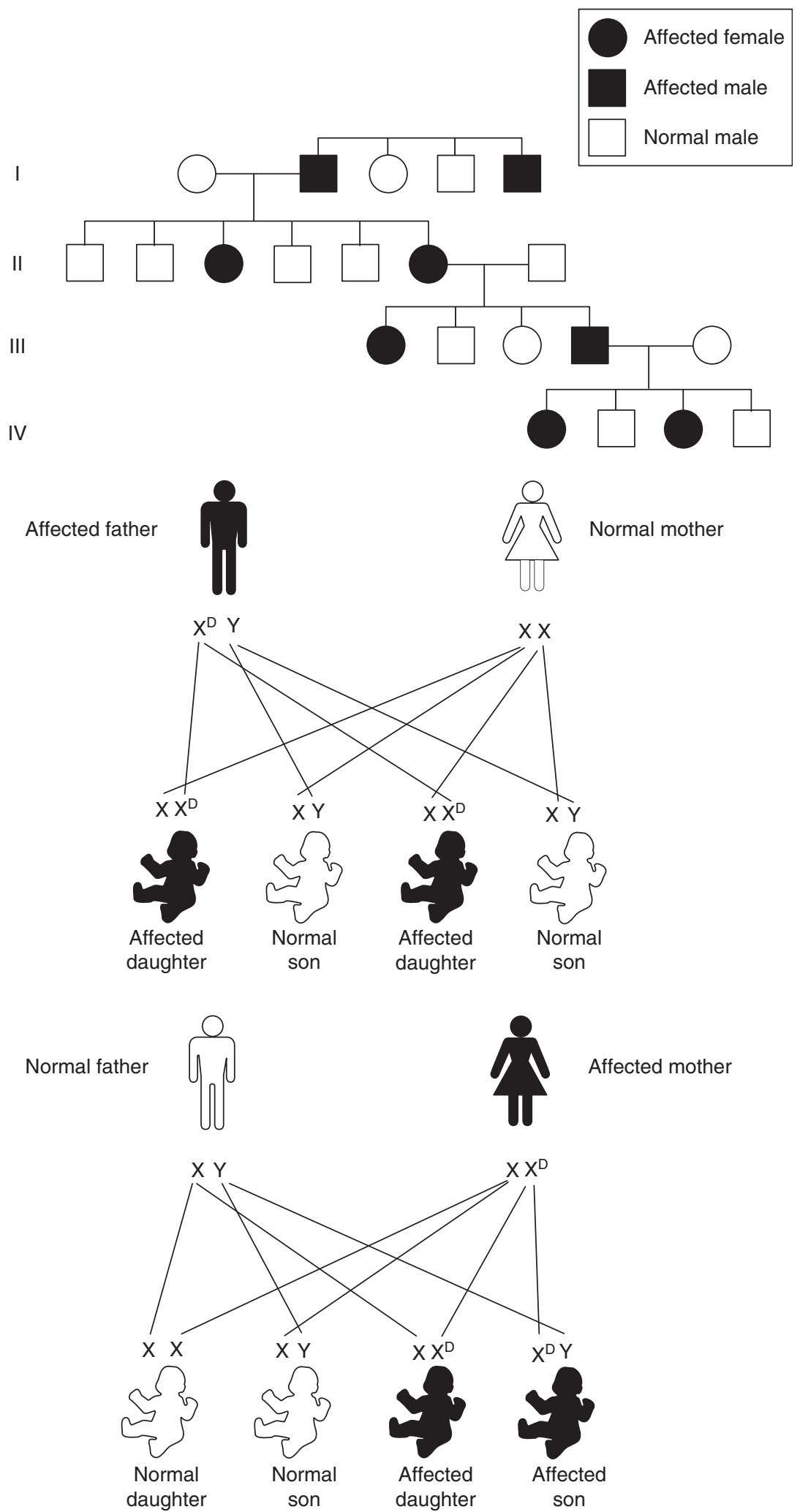

Figure $19 \mathrm{X}$-linked dominant inheritance. Males and females are affected, but male-to-male transmission does not occur.

expression of the disorder (Fig. 19). In this situation, males and females are both affected, but males will show much more severe manifestations than females, or the mutation may even be lethal in males.

There are fewer Y-linked genes known at this time. The most recent update on OMIM lists 59 genes identified on the Y chromosome, including the SRY gene, which is involved in male sex determination (3). Y-linked, or holandric, conditions, such as maleness itself, are passed exclusively from father to son.

\section{MULTIFACTORIAL AND POLYGENIC INHERITANCE}

In multifactorial inheritance, it is postulated that genetic influences from both parents, in combination with environmental factors, lead to a specific birth defect or disorder. In polygenic inheritance, multiple genetic influences in 
Table 4 Multifactorial Disorders

\begin{tabular}{ll} 
Disorder & \multicolumn{1}{c}{ Incidence } \\
$\begin{array}{l}\text { Congenital malformations } \\
\text { Cleft lip with/without cleft palate }\end{array}$ & 1 in 500 to 1 in 1000 \\
Cleft palate & 1 in 1000 \\
Club foot & 1 in 1000 \\
Congenital heart defects & 1 in 200 to 1 in 500 \\
Neural tube defects & 1 in 500 to 1 in 1000 \\
Pyloric stenosis & 1 in 300 \\
Adult diseases & 1 in 10 to 1 in 20 \\
Alcoholism & 1 in 10 \\
Alzheimer's disease & 1 in 100 to 1 in 200 \\
Bipolar affective disorder & 1 in 3 \\
Cancer & 1 in 10 \\
Diabetes (types I and II) & 1 in 3 to 1 in 5 \\
Coronary artery disease & 1 in 100 \\
Schizophrenia &
\end{tabular}

combination act together to produce a disorder. Examples of birth defects with multifactorial inheritance include isolated congenital heart defects, cleft lip with or without cleft palate, cleft palate, neural tube defects, pyloric stenosis, and congenital dislocation of the hips (Table 4). In multifactorial disorders, there is no evidence to suggest simple Mendelian inheritance. For example, the defect does not necessarily appear in sequential generations of a family, as would an autosomal dominant condition due to a single-gene mutation. However, there may be clustering of the defect in more than one member of a pedigree. The appearance of multifactorial disorders has been explained by a threshold model in which certain genes present in an individual, along with prenatal environmental factors, accumulate toward a threshold. If an individual has enough of these genes and factors, a threshold will be crossed and the defect will appear (32).

Multifactorial disorders often show an increased incidence in one sex or the other. Pyloric stenosis is much more common in males, while dislocation of the hips is more common in females. These sex differences have been explained by a shift in the threshold depending upon the sex.

Once a couple has had a child with a multifactorial condition, the risk of having a second child with the same disorder is increased over the background population risk. Recurrence risk figures can be estimated for a given defect based on population studies. For example, the incidence of cleft lip with or without cleft palate is 1 in 700 to 1 in 1000 newborns. This risk increases to approximately $4 \%$ or 1 in 25 after a family has had one child with cleft lip, representing a 40 -fold increased risk. However, the risk is still relatively small, with a $96 \%$ chance of having a normal child.

The exact environmental factors contributing to multifactorial conditions remain unknown in most cases. Regional factors must play a role, since it is well known that some areas of the world have a markedly increased risk for certain defects. For example, the incidence of neural tube defects is approximately 1 in 1000 in the general population, but in parts of Great Britain, especially Ireland and Wales, the rate is as high as 7 to 9 in 1000 births (1). Genetic influences must also play a role, since immigrants from these areas to other parts of the world still have a somewhat higher rate of neural tube defects (1). However, other
Table 5 Diseases Caused by Mitochondrial DNA Mutations

Kearns-Sayre disease, due to large mtDNA deletion

Leber hereditary optic neuropathy, due to G3460A, G11778A, or T14484C mutation in complex I subunits

Leigh syndrome (subacute necrotizing encephalopathy), due to T8993G or C mutation in complex V in $10 \%$ of cases

Mitochondrial encephalopathy, lactic acidosis, and stroke-like episodes, due to A3243G or T3271C mutations in Leucine tRNA

Mitochondrial myopathy, due to mutations in various mt-tRNA genes

Myoclonic epilepsy and ragged-red fiber disease, due to A8344G in Lysine tRNA

Neurogenic muscle weakness, ataxia, and retinitis pigmentosa, due to T8993G or C mutation in complex V

environmental variables such as diet must also be important. It has been shown that folic acid consumption in the diet of pregnant women can significantly reduce the recurrence risk of neural tube defects in subsequent pregnancies. The current recommendation by the Centers for Disease Control states that all women of childbearing age who are capable of becoming pregnant should consume $400 \mathrm{mg}$ of folic acid per day (33). All women who have had a previous child with a neural tube should take $4 \mathrm{mg}$ of folic acid per day, beginning 2 to 3 months prior to conception and continuing for the first 3 months of gestation (34). Flour and other grain products are now supplemented with folic acid in the United States to ensure proper intake for all women, since it is difficult to obtain more than $200 \mathrm{mg}$ of folic acid a day from a typical American diet.

\section{MITOCHONDRIAL INHERITANCE}

There are an increasing number of recognized human genetic disorders caused by mutations in the mitochondrial genome (Table 5) (35). Within the cytoplasm of each cell are several hundred mitochondria, which are involved in energy production for the cell. Each mitochondrion has multiple copies of its own circular DNA (mtDNA), which is $16.5 \mathrm{Mb}$ in size, encoding 37 genes for tRNA, rRNA, and some of the enzymes of oxidative phosphorylation. Because mtDNA is cytoplasmic in location, inheritance is exclusively maternal. Sperm contain very few mitochondria and, therefore, both males and females inherit essentially all of their mtDNA from their mothers. There is variable expression of mitochondrial disorders due to heteroplasmy; this term refers to the variability of the proportion of mutant mtDNA within each cell. The proportion of abnormal mtDNA at the somatic level may change over time due to replicative segregation. This can account for the apparent degenerative nature of these disorders noted clinically, which corresponds to an increase in the percentage of abnormal mitochondria $(36,37)$. A woman who has a mitochondrial DNA disorder will pass it on to all of her offspring. However, because of the highly variable expression of these conditions, not all offspring would be expected to be affected to the same degree. Offspring of men with mitochondrial DNA disorders cannot inherit the condition because all mitochondria originate from the mother. It is important to remember that there are also many disorders associated with mitochondrial dysfunction that are inherited in a Mendelian fashion, since most 
proteins active within the mitochondria are encoded by nuclear DNA genes.

\section{COMMON METABOLIC DISORDERS}

Inborn errors of metabolism are due to enzyme deficiencies that cause abnormal elevations of intermediary compounds of biochemical pathways in tissues and body fluids, with resultant medical problems. Most have an autosomal recessive inheritance pattern, but a few are X-linked recessive disorders. There are several hundred known inborn errors of metabolism, most of which are very rare (38). However, they are collectively common and affect about 1 in 1000 newborns.

One of the most common metabolic disorders is the autosomal recessive aminoacidopathy phenylketonuria (PKU), which has an incidence of 1 in 10,000 to 1 in 15,000 newborns (38). It is caused by a deficiency of the enzyme phenylalanine hydroxylase, which normally converts phenylalanine to tyrosine. Patients with PKU have elevated blood levels of phenylalanine and excretion of phenylketones in the urine. If left untreated, affected individuals will develop microcephaly and profound mental retardation due to the toxic effects of the elevated compounds. However, if diagnosed within the first few weeks of life, with institution of a low phenylalanine diet, mental retardation can be prevented. PKU is the first genetic disorder for which there has been generalized population screening. Since the 1960s, all newborns in the United States have been tested for the disorder. This has nearly eliminated PKU as a cause for mental retardation. However, a new problem has emerged; women who were allowed to stop the dietary restriction of phenylalanine after age 6 years are now of childbearing age. The elevated blood level of phenylalanine in a pregnant PKU woman has deleterious effects on the developing fetus, with increased risk for spontaneous pregnancy loss, congenital heart defects, severe microcephaly, and mental retardation. Therefore, the PKU diet must be reinstituted prior to conception and continued throughout pregnancy. New guidelines state that all people with PKU should remain on the diet for life.

The United States and most developed countries of the world perform newborn screening for many inborn errors of metabolism, endocrine disorders including hypothyroidism and congenital adrenal hyperplasia, cystic fibrosis, hemoglobinopathies, and hearing loss. All states now have significantly expanded newborn metabolic disease screening, largely due to the development of tandem mass spectrometry methods to detect many amino acid disorders, organic acid disorders, and disorders of fatty acid oxidation. For most of these disorders, it has been shown that early diagnosis in the newborn period can significantly reduce morbidity and mortality. As mentioned above for PKU, institution of dietary restriction of phenylalanine before 1 month of age can prevent mental retardation. For other inborn errors of metabolism, such as fatty acid oxidation defects, maple syrup urine disease and galactosemia, illness, mental retardation, and even death can be prevented by early detection and treatment.

\section{POPULATION SCREENING FOR GENETIC DISEASE}

Screening tests are a routine part of medical care, with the goal of early identification and treatment of specific and common diseases. For genetic diseases, population screening involves not only affected individuals, but also additional family members. Screening for genetic disease has been defined as the "search in a population for persons possessing certain genotypes that (i) are already associated with disease or predisposition to disease or (ii) may lead to disease in their descendants" (39). As outlined in the previous section, newborn screening for metabolic diseases exemplifies the first type of screening, which will result in proper identification of infants with these disorders and institution of treatment. Other examples include presymptomatic genetic testing for Huntington disease, breast cancer, or colon cancer.

The second type of screening includes testing of targeted ethnic or racial populations to identify carriers of genetic disease. Examples are cystic fibrosis in Caucasians, Tay-Sachs disease in Ashkenazi Jews, and hemoglobinopathies in African-Americans (10). On a smaller scale, genetic screening can involve karyotype analysis on family members at risk for having a chromosome translocation. Carrier testing is also available for women who are at risk to be carriers for X-linked disorders such as Duchenne muscular dystrophy or Fragile X syndrome. This type of population screening will not affect the health of the carrier, but will have a significant impact on reproductive choices for the family. It is essential that appropriate genetic counseling accompany the information given regarding carrier status.

\section{GENETIC COUNSELING}

Genetic counseling is an important part of the practice of medical genetics. The field of genetics is unique in that a diagnosis of a genetic disorder in an individual has an impact on his or her entire family. Multiple family members and their present or future offspring may be at risk for the genetic disorder as well. Genetic counseling involves the explanation of the manifestations of the disorder, the natural history and treatment, the inheritance pattern, the risks of recurrence, and the methods of prenatal and postnatal diagnosis. The genetic counselor also helps the family make the best possible adjustment to the disorder and the risks of recurrence. This often involves referral to a support organization through which the family can make contact with other affected individuals. The vast majority of genetic counselors and clinical geneticists seek to provide nondirective counseling, whereby information about the disorder and the recurrence risk is given in an unbiased and neutral way, and the family makes decisions about reproductive options in accordance with their own beliefs and values.

Reasons for referral for genetic counseling include a family history of a known or suspected single-gene disorder, chromosomal disorder, or multifactorial condition, carrier status for a genetic disease, consanguinity, advanced maternal age during pregnancy, abnormal screening tests during pregnancy, repeated pregnancy loss or infertility, or teratogen exposure during pregnancy.

In the prenatal clinic setting, the genetic counseling session often precedes any diagnostic testing. During the session, the family history is reviewed in detail, as well as the reasons for referral, the information obtainable by the prenatal testing, and the risks of the procedure. If fetal abnormalities are detected, then additional counseling sessions may be required to explain the findings to the family and to discuss options for 


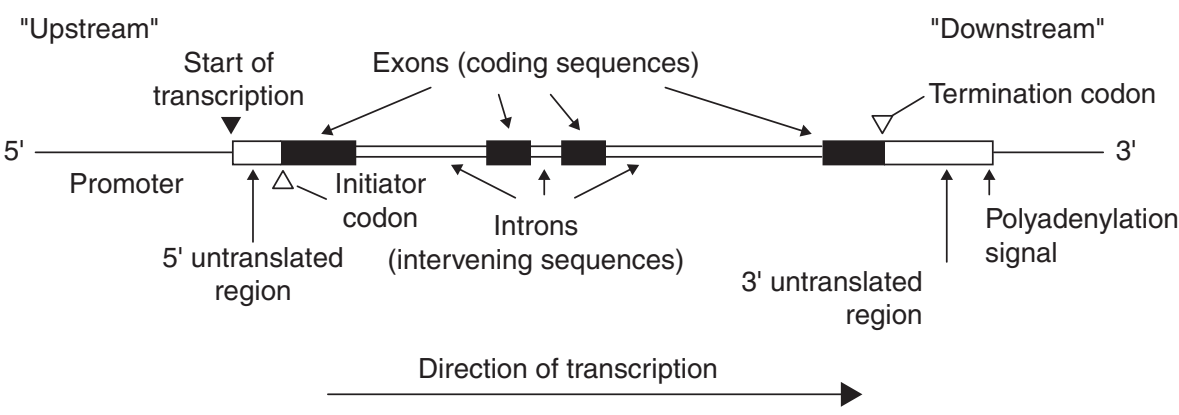

Figure 20 General structure of a typical human gene. Source: From Ref. 40.

pregnancy management. If special care or surgery will be needed for the baby immediately after birth, the genetic counselor and the obstetrician may help arrange meetings with the appropriate surgeons or pediatric specialists so that the family is optimally prepared. For cases that are likely to be lethal, it is best to introduce the idea of a postmortem examination before the birth of the baby. In the event of death, a complete autopsy is recommended to search for additional anomalies and to confirm the diagnosis. This helps ensure that accurate genetic counseling, especially with respect to recurrence risk, can be provided in subsequent pregnancies.

\section{MOLECULAR GENETICS}

Genes are the basic units of inheritance. As mentioned previously, there are several thousand single-gene diseases with a known molecular genetic cause, representing only a fraction of the estimated total 20,000 to 25,000 genes in the human genome. Over the past 5 to 10 years, there has been a dramatic increase in the number of identified, cloned, and sequenced disease genes. With this new knowledge has come increased availability and demand for both prenatal and postnatal molecular genetic testing. Molecular genetic testing is done for diagnostic purposes to confirm the presence of a disorder, as well as to identify conditions presymptomatically.

Genes are composed of deoxyribonucleic acid (DNA) and are contained on the chromosomes. Each strand of DNA has a specific sequence of four nucleotides, each containing a different base, adenine, thymine, cytosine, or guanine. Adenine pairs with thymine and cytosine pairs with guanine as two complementary strands of DNA are wound together to form a double helix. Genes have a common basic structure (Fig. 20). First, there are upstream sequences that regulate transcription, known as promoters and enhancers. Then, there is a transcription initiation site, followed by a series of alternating exons and introns. The DNA sequence serves as a template from which messenger RNA (mRNA) is made; this process is known as transcription. As transcription proceeds, a primary mRNA is made from the DNA sequence of the gene, which includes the introns. The intron sequences are then spliced out and the exons are linked together to form the mature mRNA molecule. Thus, the exons are the only portions of the gene that specify the final protein product. The mature mRNA molecule is used to make the protein product by the process of translation. Groups of three nucleotides, called codons, code for specific amino acids. Transfer RNA (tRNA) and ribosomal RNA (rRNA) interact with the mRNA to assemble the amino acids into a polypeptide chain to form the final protein molecule.
Errors in the sequence of nucleic acids of the DNA produce mutations in genes and often lead to genetic diseases. There are a variety of different kinds of mutations that can occur in genes. Some result in genetic disorders, while others may have no detrimental effect. The most common type of mutations include missense (single amino acid change), nonsense (insertion of stop codon with premature termination of translation), frameshift (insertion or deletion of nucleotides that alters downstream codons), splice site (incorrect splicing of introns), and promoter mutations (decreased transcription of mRNA). An expansion mutation occurs when there is insertion of many extra copies of a trinucleotide repeat; examples of disorders with this type of mutation are fragile $\mathrm{X}$ syndrome, Huntington disease, and myotonic dystrophy.

Diagnosis is possible for many genetic disorders using molecular genetic techniques. Genomic DNA can be obtained from peripheral blood leukocytes, solid tissues, or cultured cells. Testing can be accomplished by either direct mutation analysis or by linkage analysis (Fig. 21). In linkage analysis, information about nondisease producing variation, or polymorphisms, such as restriction fragment-length polymorphisms, variable number of tandem repeats, or microsatellite repeat polymorphisms, can trace inheritance of a chromosome containing a disease-producing gene through a family. This method of testing has disadvantages. First, multiple family members are needed to establish the phase of linkage. Second, some families may not be informative for some linked markers. Third, recombination between the marker and the disease gene may occur, causing inaccuracies in the test results.

In direct DNA testing, the disease gene must have been isolated and disease-producing mutations are identified. Specific mutations can then be sought by a variety of methods. Segments of DNA from the gene of interest is amplified by (PCR) polymerase chain reaction and then sequenced, or deletions or rearrangements of the gene can be identified by Southern blotting. Direct testing is advantageous, since it is likely to be more accurate than linkage analysis, and a single individual can be tested without requiring family information. For example, molecular genetic testing for sickle cell disease is highly accurate, since all affected individuals have the same missense mutation at position six of the beta-globin chain, which results in a substitution of valine for glutamic acid. This point mutation results in a loss of one MstII restriction enzyme site in the mutant gene, making direct DNA diagnosis very straightforward. For other genetic disorders characterized by a number of common mutations, such as cystic fibrosis, allele-specific oligonucleotide probes (ASOs) of 18 to 20 nucleotides in length complementary to known normal and 


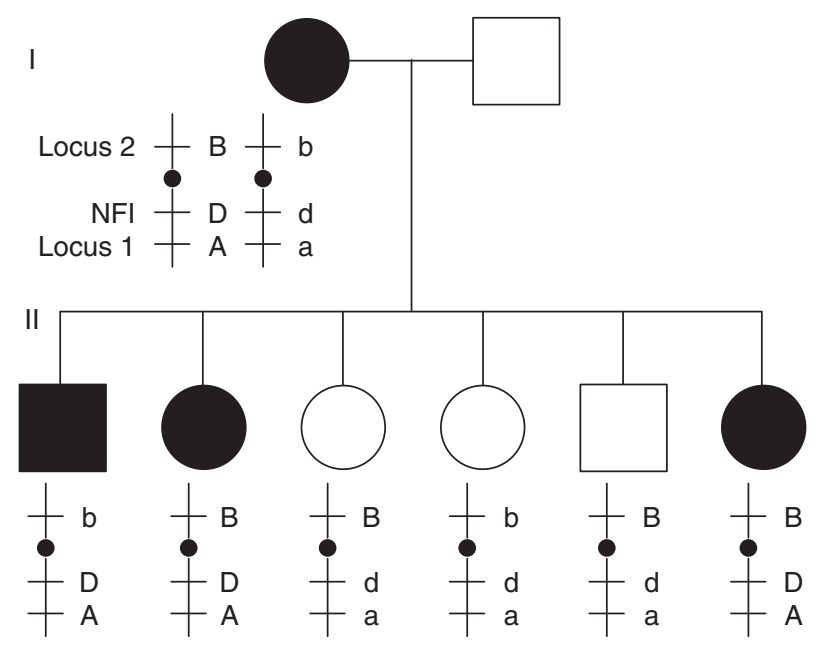

Figure 21 Linkage of the gene for neurofibromatosis 1 to a marker locus. The mother (I-1) is affected with this dominant disease and is heterozygous at the NF1 locus $(D d)$, as well as at two other loci, 1 and 2, on chromosome 17. She carries the $A$ and $B$ alleles on the same chromosome as the mutant NF1 allele $(D)$. The unaffected father is homozygous normal $(d d)$ at the NF1 locus, as well as at the two marker loci $(A A$ and $B B)$. All three affected offspring have inherited the $A$ allele at marker 1 , whereas the three unaffected offspring have inherited the $a$ allele. Thus, all six offspring are nonrecombinant for NF1 and marker locus 1 . However, individuals II-1, II-3, and II-5 are recombinant for NF1 and marker locus 2, indicating that meiotic crossing over has occurred between these two loci. Source: From Ref. 40.

mutant DNA sequences can be devised. ASOs are then hybridized to patient DNA samples to identify homozygotes or heterozygotes for specific mutations. Thus, a panel of ASOs for the most common mutations seen in a disorder can be used to rapidly screen patient DNA samples. For disorders in which there are no common mutations or when common mutations have been ruled out, the entire gene can be sequenced to look for rare or new disease-causing mutations. In the future, identification of the molecular basis for genetic defects or syndromes may be achieved by exome sequencing, in which all of the exons in the genome are sequenced to identify mutations in candidate genes, or by whole genome sequencing. Up-to-date information about genetic disorders for which molecular genetic testing is clinically available can be found at the GeneTests website (http://www.ncbi.nlm.nih.gov/ sites/GeneTests/?db=GeneTests).

\section{TREATMENT OF GENETIC DISEASES}

With the isolation of disease genes and an increasingly better understanding of the molecular genetic bases for many disorders, it has become feasible to consider treatments and potentially cures for genetic disease (41).

Gene therapy involves insertion of normal copies of genes into individuals who have genetic diseases. This can potentially be accomplished by either somatic cell or germ cell gene therapy. Most work thus far has focused on somatic cell gene therapy. There are two ways to approach somatic cell gene therapy. Ex vivo gene therapy involves removing a patient's cells from the body, inserting the normal gene copy into the cells, and then returning the cells to the body. In in vivo gene therapy, cells are treated while inside the patient's body. For successful gene therapy, the cell requiring treatment must be easily accessible and relatively long-lived. Some of the earliest human gene therapy trials were performed for severe combined immune deficiency due to adenosine deaminase deficiency, using bone marrow stem cells. Other cells under consideration for therapy have included lymphocytes, hepatocytes, muscle cells, and respiratory epithelial cells. More recently, gene therapy for Leber's congenital amaurosis has been accomplished by replacement of the defective gene locally to the retina of the eye. Types of genetic diseases that are amenable to somatic cell gene therapy are primarily autosomal recessive or X-linked disorders that result in almost total lack of normal protein. Reconstitution of even $5 \%$ to $10 \%$ of normal protein levels appears to be sufficient to treat these diseases. Dominant disorders that are caused by heterozygosity for mutant and normal genes (dominant-negative mutations) are not likely to be treatable by gene replacement; methods to block production of the mutant protein will be required.

In conditions associated with an enzyme deficiency, methods have been developed to provide systemic delivery of the missing enzyme to the patient. Enzyme replacement therapy for lysosomal storage diseases, such as Gaucher disease, Fabry disease, Hurler syndrome, and Pompe disease, has been very successful. Enzyme replacement therapy for other enzyme deficiency disorders is under development. Another approach has been to decrease the substrate for the deficient enzyme by giving a drug that inhibits an upstream enzyme in the pathway, thus decreasing accumulation of the toxic compound. This type of drug treatment has been approved for Gaucher disease and is being developed for other conditions.

With the elucidation of the molecular genetic pathways involved in certain genetic disorders, it has been possible to devise new uses for FDA-approved drugs. Examples of this include the experimental use of farnesyltransferase inhibitors in progeria, a premature aging syndrome, and an angiotensin II type 1 receptor antagonist for treatment of the dilated aortic root in Marfan syndrome. Pharmacologic chaperones are also being developed to help stabilize mutant proteins and enhance residual activity.

Finally, methods for manipulating the expression or splicing of mutant genes are being investigated. Drugs that induce read-through of a stop codon during translation of a mutant gene can result in increased expression of full-length protein. This approach has been tried experimentally with cystic fibrosis and Duchenne muscular dystrophy. In other studies, antisense oligonucleotides have been used to induce skipping 
during mRNA splicing of exons that contain mutations; this approach will produce a smaller protein, but one that might retain some residual function. This method of therapy has been investigated for Duchenne muscular dystrophy. It is anticipated that more such opportunities will become available in the future for targeted therapy of the disease manifestations of genetic disorders.

\section{REFERENCES}

1. Jorde LB, Carey JC, Bamshad MJ. Medical Genetics, 4th edn. St. Louis, MO: Mosby, 2009.

2. International Human Genome Sequencing Consortium. Finishing the euchromatic sequence of the human genome. Nature 2004; 431: 931-45.

3. Online Mendelian Inheritance in Man, OMIM (TM). McKusick-Nathans Institute of Genetic Medicine, Johns Hopkins University (Baltimore, MD) and National Center for Biotechnology Information, National Library of Medicine (Bethesda, MD), World Wide Web URL. [Available from: http://www.ncbi.nlm.nih.gov/omim/] Accessed 31 Dec, 2010.

4. Therman E. Human Chromosomes: Structure, Behavior and Effects, 2nd edn. New York: Springer-Verlag, 1986.

5. Nussbaum R, McInnes RR, Willard HF. Thompson \& Thompson Genetics in Medicine, 7th edn. Philadelphia: WB Saunders, 2007.

6. Gelehrter TD, Collins FS. Principles of Medical Genetics. Williams and Wilkins, 1990.

7. Shinawi MS, Cheung SW. The array CGH and its clinical applications. Drug Discov Today 2008; 13: 760-70.

8. Visser LELM, deVries BBA, Veltman JA. Genomic microarrays in mental retardation: from copy number variation to gene, from research to diagnosis. J Med Genet 2010; 47: 289-97.

9. Fruhman G, Van de Veyver IB. Applications of array comparative genomic hybridization in obstetrics. Obstet Gynecol Clin N Am 2010; 37: 71-85.

10. Rimoin D, Connor JM, Pyeritz R, Korf B. Emery and Rimoin's Principles and Practice of Medical Genetics, 5th edn. Elsevier, 2006.

11. Magenis RE. On the origin of chromosome anomaly. Am J Med Genet 1988; 42: 529-33.

12. Patterson D. The causes of Down syndrome. Sci Am 1987; 257: 52-60.

13. Antonarakis SE, The Down Syndrome Collaborative Group. Parental origin of the extra chromosome in trisomy 21 as indicated by analysis of DNA polymorphisms. N Engl J Med 1991; 324: 872-6.

14. ACOG Practice Bulletin No. 88, December 2007. Invasive prenatal testing for aneuploidy. Obstet Gynecol 2007; 110: 1459-67.

15. Gardner RJM, Veale AMO. De novo translocation Down's syndrome: risk of recurrence of Down's syndrome. Clin Genet 1974; 6: 160-4.

16. Harper PS. Practical Genetic Counseling, 7th edn. Oxford University Press, 2010.

17. Lister TJ, Frota-Pessoa O. Recurrence risk for Down syndrome. Hum Genet 1980; 55: 203.

18. Cooley WC, Graham JM. Down syndrome: an update and review for the primary pediatrician. Clin Pediatr 1991; 30: 233-53.

19. Jones KL. Smith's Recognizable Patterns of Human Malformation, 6th edn. Philadelphia: WB Saunders, 2005.

20. Pueschel SM. Clinical aspects of Down syndrome from infancy to adulthood. Am J Med Genet (Suppl) 1990; 7: 52-6.
21. Hennekam RCM, Allanson JE, Krantz ID. Gorlin's Syndromes of the Head and Neck, 5th edn. Oxford University Press, 2010.

22. Bernstein HS, Filly RA, Goldberg JD, Golbus MS. Prognosis of fetuses with a cystic hygroma. Prenat Diagn 1991; 11: 349-55.

23. Bondy CA, the Turner Syndrome Study Group. Care of girls and women with Turner syndrome: a guideline of the Turner Syndrome Study Group. J Clin Endocrinol Metab 2007; 92: 10-25.

24. Elias ER, Tsai AC, Manchester DK. Genetics and Dysmorphology (Chapter). In: Hay WW Jr, Levin MJ, Sonheimer JM, Deterding RR, eds. Current Diagnosis and Treatment: Pediatrics. Chapter 35. 2009: 19e. [Available from: http://www.accessmedicine.com/content.aspx? $\mathrm{aID}=3408859$ ].

25. Stevenson RE, Goodman R, Hall JG. Human Malformations and Related Anomalies. New York: Oxford University Press, 1989.

26. Ewart AK, Morris CA, Atkinson D, et al. Hemizygosity at the elastin locus in a developmental disorder; Williams syndrome. Nat Genet 1993; 5: 11-16.

27. Kalousek DK. Anatomic and chromosome anomalies in specimens of early spontaneous abortion: seven year experience. BDOAS 1987; 23: 153-68.

28. Craver RD, Kalousek DK. Cytogenetic abnormalities among spontaneously aborted previable fetuses. Am J Med Genet (Suppl) 1987; 3: 113-19.

29. Opitz JM, FitzGerald JM, Reynolds JF, et al. The Montana fetal genetic pathology program and a review of prenatal death in humans. Am J Med Genet (Suppl) 1987; 3: 83-112.

30. Hassold TJ. Chromosome abnormalities in human reproductive wastage. Trends Genet 1986; 2: 105-10.

31. DeWald GW, Michels VV. Recurrent miscarriages: cytogenetic causes and genetic counseling of affected families. Clin Obstet Gynaecol 1986; 29: 865-85.

32. Cunningham FG, Leveno KJ, Bloom SL, et al. Genetics (Chapter). In: Cunningham FG, Leveno KJ, Bloom SL, Hauth JC, Rouse DJ, Spong CY, eds. Williams Obstetrics. Chapter 12. 2010: 23e. [Available from: http://www.accessmedicine.com/content.aspx?aID=6021057].

33. U.S. Department of Health and Human Services, Centers for Disease Control. Recommendations for the use of folic acid to reduce the number of cases of spina bifida and other neural tube defects. Morb Mortal Wkly Rep 1992; 41: 1-7.

34. Centers for Disease Control. Use of folic acid for prevention of spina bifida and other neural tube defects, 1983-1991. Morb Mortal Wkly Rep 1991; 40: 513-16.

35. DiMauro S, Schon EA. Mitochondrial respiratory-chain diseases. N Engl J Med 2003; 348: 2656-68.

36. Falk MJ, Sondheimer N. Mitochondrial genetic diseases. Curr Opin Pediatr 2010; 22: 711-16.

37. Debray FG, Lambert M, Mitchell GA. Disorders of mitochondrial function. Curr Opin Pediatr 2008; 20: 471-82.

38. Valle D, Beaudet AL, Voglestein B, et al. The Online Metabolic and Molecular Bases of Inherited Disease. NewYork: McGraw-Hill, 2007.

39. National Academy of Sciences. Genetic Screening: Programs, Principles and Research. Washington, DC: National Academy of Sciences, 1975.

40. Thompson MW, McInnes RR, Willard HF. Genetics in Medicine, 5th edn. Philadelphia: WB Saunders, 1991.

41. Dietz HC. New therapeutic approaches to Mendelian disorders. N Engl J Med 2010; 363: 852-63. 


\section{Principles of teratology of drugs and radiation Frank R. Witter}

Teratology is the study of birth defects. In this chapter, the potential of drugs and radiation to produce birth defects in humans will be explored. Wilson (1) has estimated that of all human malformations, less than $1 \%$ are due to radiations and $4 \%$ to $6 \%$ are due to drugs and environmental chemicals.

Whether an agent produces a teratogenic effect is dependent on the timing of the insult. The teratologic literature is usually reported in post-conception weeks. Clinical dating is always 2 weeks greater than post-conception dating as it is based on the patient's last menstrual period or ultrasound measurements, which are reported in menstrual weeks. To avoid confusion, all timing of teratogenic exposure will be discussed in menstrual weeks. The conceptus is relatively resistant in the first 2 weeks of its existence (menstrual weeks 3 and 4) when either no effect or loss of the pregnancy is the major outcome of exposure to teratogenic agents. During this time period, the embryo has sufficient reparative powers, if not destroyed, to recover and few malformations occur. The most likely times for chemical teratogenesis are menstrual weeks 5 to 12 inclusive, which corresponds to the time of major organ differentiation. For radiation injury, the most likely times for exposure are menstrual weeks 10 to 17 inclusive (2), which correspond to the time of maximum neuronal cell proliferation and migration to the cerebral cortex. No gross malformations due to radiation have been seen in humans without the child exhibiting growth retardation, microcephaly, or gross eye malformations. After the time of gestation of maximal sensitivity, the effects of teratogens in general are to produce growth retardation (3-6). After 27 menstrual weeks, based on atomic bomb survivors, there is no proven risk of central nervous system effects, specifically severe mental retardation from the radiation $(7,8)$.

The criteria for a human teratogen are listed in Table 1 (9). In addition to these criteria, the existence of a dose-response relationship supports the contention that an agent is a teratogen. Thus, for a teratogen with increasing dose of the exposure, there should be an increase in the number or severity of defects produced. Also it should produce similar defects in experimental animal systems.

\section{DRUGS AS TERATOGENS}

Drugs that fulfill the criteria for a human teratogen may produce their effect directly, indirectly, or through a toxic intermediate. Direct-acting teratogens cause damage in their native form and require only access to the conceptus to do harm. An example of direct-acting teratogens are cytotoxic antineoplastic agents, which cause direct cell damage leading to cell death, which may result in malformation or pregnancy loss.

Indirect-acting teratogens act by perturbing maternal homeostasis to produce damage. Narcotic analgesics are not themselves teratogens; however, they can act as indirect teratogens when taken in sufficient doses to produce maternal hypoxia from respiratory depression. Hypoxia then acts directly to produce malformations by causing cell death in the developing conceptus. Therefore, by acting to compromise maternal respiration, narcotics may act as indirect teratogens.

Unlike the first two methods by which a drug can act as a teratogen, those drugs whose teratogenic potential is expressed through a toxic intermediate are not as readily predicted by animal experiments. This is because there are many pathways by which drugs may be metabolized and these may vary between species or even between individuals of the same species. The developing field of pharmacogenetics is expanding our knowledge of these differences. However, because of this variation, the toxic intermediate may not be produced in all individuals or species. A teratogen acting through a toxic intermediate may be a human teratogen and not one in animal species usually tested, or vice versa. An example of a human teratogen that would fit into this class is thalidomide, which is not a teratogen in the guinea pig.

In addition to the difficulty in extrapolating from animal experiments to man, there are other difficulties with fulfilling the criterion of Table 1. The drug in question might prevent the loss of an already malformed fetus. The condition for which the drug is used might produce the malformation. The fetal malformation might cause maternal symptoms for which the drug is taken. Finally, the drug may be commonly used in combination with other drugs that produce the malformation or only a combination of agents, not the individual agents by themselves, might produce the malformation.

Because of these difficulties and the sometimes poor quality of data, differences exist between experts on the teratogenic potential of many agents in humans. An additional reason for conflict in expert opinion on human teratogens is the reason for which the assessment was made. If one wishes to counsel a pregnant woman already exposed to an agent as to its teratogenic potential, then it is appropriate to ignore the potential therapeutic benefit of the agent and use only the information on birth defects available on it (10). If, on the other hand, one wishes to prescribe a medication for a condition during pregnancy, it is appropriate to use an assessment that balances the therapeutic benefit to the teratogenic risk. The FDA pregnancy categories (11) are an attempt at such an assessment (Table 2).

It has been reported that one in five women use FDA class C, D, or X drugs at least once during pregnancy (12). As the FDA classification system is extensively used, it is important to recognize its limitations. It is not a teratogenic risk classification. It is a fetal risk classification, which includes both teratogenic risk and the risk of fetal harm by the drug later in pregnancy. It also does not include information on the point in 
Table 1 Criteria for Recognizing a Human Teratogenic Agent (9)

1. An abrupt increase in the frequency for a particular defect or group of defects

2. Coincidence of this increase with a known environmental change, such as widespread use of a new drug or sudden exposure to a chemical or a source of radiation

3. Known exposure to the environmental change early in pregnancies yielding the characteristically defective infants

4. Absence of other factors common to all yielding infants with the characteristic defect or defects

\section{Table 2 FDA Pregnancy Categories for Drugs (11)}

Category A: Controlled studies in women fail to demonstrate a risk to the fetus in the first trimester; there is no evidence of a risk in later trimesters, and the possibility of fetal harm appears remote

Category B: Either animal reproductive studies have not demonstrated a fetal risk, but there are no controlled studies in pregnant women, or animal reproductive studies have shown an adverse effect, other than a decrease in fertility, which was not confirmed in controlled studies in women in the first trimester and there is no evidence of a risk in later trimesters

Category C: Either studies in animals have revealed adverse effects on the fetus, teratogenic or embryocidal or other, and there are no controlled studies in women, or studies in women or studies in women and animals are not available. Drugs of this class should be given only if the potential benefit justifies the potential risk to the fetus

Category D: There is positive evidence of human fetal risk, but the benefits from use in pregnant women may be acceptable despite the risk as when the drug is needed in a life-threatening situation or for a serious disease for which safer drugs cannot be used or are ineffective

Category X: Studies in animals or human beings have demonstrated fetal abnormalities or there is evidence of fetal risk based on human experience or both, and the risk of the use of the drug in pregnant women clearly outweighs any possible benefit. The drug is contraindicated in women who are or may become pregnant

pregnancy when the risk occurs or if there is a dose threshold above which the harm occurs.

An example of the problems of the system can be seen by looking at vitamin A. Vitamin A is an essential fat-soluble nutrient and a known human teratogen. Its U.S. recommended daily allowance (RDA) for pregnancy is $8000 \mathrm{IU} /$ day. Yet when taken in supplements during pregnancy of more than $10,000 \mathrm{IU} /$ day, it is teratogenic (13). According to the FDA classification system, it should be category D or X. Vitamin A in large doses or severe deficiency is harmful to the fetus and this information is not conveyed by the FDA's simple classification system. Recognizing this problem, Braggs, Freemen, and Yaffe (14) in their compendium have modified the FDA risk classes. In the case of vitamin A, they classify it as " $A$ *" with the "*»" footnote indicating category $\mathrm{X}$ if used in doses above the U.S. RDA.
There are several excellent compendiums of data on teratogenic risk (14-16) to which the reader is referred for details on specific agents. The most common human teratogens used today are listed in Table 3. These agents should be avoided in women who are pregnant or potentially pregnant. The most common agents that have teratogenic potential but whose therapeutic benefit outweighs their risk are listed in Table 4 . When assessing maternal drug therapy in pregnancy, the first consideration is whether the drug is necessary for maternal survival or well-being. Once it is established that this is the case, the most effective agent with the least toxicity and the lowest teratogenic potential should be used in the appropriate dose. Vital therapy should not be withheld from a pregnant woman because of teratogenic risk.

\section{RADIATION AS A TERATOGEN}

Unlike drugs, radiation always acts directly on the conceptus to produce birth defects or pregnancy loss. For this reason, it is easier to extrapolate from animal experiments to the human condition for radiation defects. Ionizing radiation produces gross congenital malformations, intrauterine growth retardation, and embryonic death. All of radiation's effects have a dose-response relationship and a threshold of exposure below which there is no difference between irradiated and nonirradiated populations. Only ionizing radiation has a clearly established teratogenic potential. Ionizing radiation dosage is reported in either Rads (rad) or Grays (Gy). One rad equals $0.01 \mathrm{~Gy}$. To avoid confusion, all doses will be reported in rads.

Growth retardation and central nervous system effects including microcephaly, mental retardation, and eye malformations are the most prominent manifestations of intrauterine radiation exposure in humans. Severe mental retardation has not been observed in patients who received less than 50 rads in utero, and there has been no report of radiation-induced limb or other gross anomalies in humans where growth retardation or central nervous system anomalies were not also present (17). Mental retardation has never been seen with radiation exposure prior to 10 menstrual weeks of gestation in humans (2), in spite of the fact that major organ differentiation occurs from 5 to 12 menstrual weeks of gestation. Radiation exposure prior to 10 menstrual weeks results in a high rate of loss and therefore a wide range of malformations are not seen $(2,17)$ although theoretically possible (18).

The gestational ages of 10 to 17 menstrual weeks represent the time when the maximum number of infants with mental retardation and central nervous system or eye defects are produced (2). At 18 menstrual weeks, or greater, few cases of mental retardation are seen (2); however, growth retardation continues to be produced. In humans, although malformations are not seen at doses of less than 50 rads, some disturbances of growth can occur with as little as 25 rads (17). However, at exposures in the range of 20 to 5000 mrads, which is the range of most diagnostic radiology studies, there is an extremely low risk of malformations when compared with the spontaneous rate for humans (17). The association of prenatal radiation exposure and childhood leukemia or other cancers remains to be determined (3-6). However, an increased incidence, 1.5 to 2.4 over the background rate, as a result of in utero exposure to radiation at the dosage ranging from 


\section{Table 3 Common Agents That Are Teratogens}

Anabolic steroids

Androgens

Anticancer agents

Diethylstilbestrol

Etretinate

Isotretinoin

Oral hypoglycemics

Vitamin A in large doses

Vitamin D in large doses

Table 4 Agents That Have Teratogenic Potential But Are Used in Pregnancy Because the Benefit of Their Use Outweighs the Risk of Teratogenesis

\section{Phenytoin}

Antimalarials

Propylthiouracil

Aminoglycosides

Antituberculins

1 to 4 rads, has been reported $(19,20)$. Since X-ray studies with 5 rads or less exposure to the conceptus carry a very low risk, and if medically indicated, it should be performed with the patient's informed consent (18); fetal dosages for some commonly used radiologic procedures are available through the American College of Obstetricians and Gynecologists Committee Opinion number 299 (7).

Special mention should be made of radioiodine during pregnancy. Iodine-131, if given in millicurie doses, can damage or ablate the developing fetal thyroid resulting in either congenital hypothyroidism or hypothyroidism of late onset (17). This can occur after the onset of the iodineconcentrating ability of the fetal thyroid at around menstrual weeks 10 to 12 of gestation (7). Radioiodine is therefore contraindicated in pregnancy.

\section{REFERENCES}

1. Wilson JG. Teratogenic effects of environmental chemicals. Fed Proc 1977; 36: 1690-703.
2. Otke M, Schull WJ. In utero exposure to A-bomb radiation and mental retardation; a reassessment. Br J Radiol 1984; 57: 409-14.

3. Stewart A. The carcinogenic effects of low level radiation: a re-appraisal of epidemiologists methods and observations. Health Phys 1973; 24: 223.

4. Stewart A, Kneale GW. Radiation dose effects in relation to obstetric X-rays and childhood cancers. Lancet 1970; 1: 1185.

5. Ford D, Patterson T. Fetal exposure to diagnostic X-rays and leukemia and other malignant disease in childhood. J Natl Cancer Inst 1959; 22: 1093.

6. Diamond EL, Schmerler H, Lilienfield AM. The relationship of intrauterine radiation to subsequent mortality and development of leukemia in children: a prospective study. Am J Epidemiol 1973; 97: 283.

7. The American College of Obstetricians and Gynecologists. Guidelines for Diagnostic Imaging During Pregnancy. In: 2008 Compendium of Selected publications (The American College of Obstetricians and Gynecologists). Washington DC: The American College of Obstetricians and Gynecologists, 2008: 446-50.

8. Wagner LK, Lester RG, Saldana LR. Prenatal risks from ionizing radiations, ultrasound, magnetic fields and radio frequency waves. In: Wagner LK, Lester RG, Saldana LR, eds. Exposure of the Pregnant Patient to Diagnostic Radiations. Philadelphia: Lippincott, 1985: 61-78.

9. Wilson JG. Environment and Birth Defects. New York: New York Academic Press, 1973.

10. Friedman JM, Little BB, Brent RL, et al. Potential human teratogenicity of frequently prescribed drugs. Obstet Gynecol 1990; 75: 594-9.

11. Millstein LG. FDA’s “pregnancy categories." N Engl J Med 1980; 303: 706.

12. Wen SU, Yang T, Krewshi D, et al. Patterns of pregnancy exposure to prescriptions. FDA $\mathrm{C}, \mathrm{D}$, and $\mathrm{X}$ drugs in a Canadian population. J Perinatol 2008; 28: 324-9.

13. Rothman KJ, Moore LL, Singer MR, et al. Teratogenicity of high vitamin A intake. N Engl J Med 1995; 333: 1369-73.

14. Briggs GG, Friedman RK, Yaffe JJ. Drugs in Pregnancy and Lactation: A Reference Guide to Fetal and Neonatal Risk, 8th edn. Philadelphia, Baltimore, New York, London, Buenos Aires, Hong Kong, Sidney and Tokyo: Lippincott, Williams and Wilkins, 2008.

15. Shepard TH. Catalog of Teratogenic Agents, 12th edn. Baltimore, Maryland: Johns Hopkins Press, 2007.

16. Schardein JL. Chemically Induced Birth Defects, 2nd edn. New York: Marcel Dekker, 1993.

17. Brent RL. Radiation teratogenesis. Teratology 1980; 21: 281-98.

18. De Santis M, DiGiantonio E, Straface G, et al. Ionizing radiations in the pregnancy and teratogenesis. A review of literature. Reprod Toxicol 2005; 20: 323-9.

19. Harvey EB, Boice JD, Honeyman M, et al. Prenatal X-ray exposure and childhood cancer in twins. N Engl J Med 1985; 312: 541-5.

20. Swartz HM, Reichling BA. Hazards to radiation exposure for pregnant women. J Am Med Assoc 1978; 289: 1907-8. 


\section{Fetal programming \\ Katherine E. Pelch, Jana L. Allison, and Susan C. Nagel}

\section{INTRODUCTION}

The prenatal period requires vigilance to insure a safe environment for the fetus to grow and develop appropriately. Great lengths are taken during prenatal care to avoid exposures known to have teratogenic potential. Fetal programming is the theory that environmental events in utero during sensitive windows of development can have permanent and long-lasting effects. The fetus has the capacity for developmental plasticity. That is, one genotype can lead to different phenotypes depending on environmental factors (1). In other words, the environment interacts with genes in the developing organism to permanently program gene expression in adulthood. Changes in the fetal environment can program childhood and adult response pathways. Therefore, the developmental period represents a critical opportunity for irreversible effects from the fetal environment, whether these are from pharmaceuticals, nutrition, microorganisms, the maternal hormonal milieu, and/or environmental chemicals.

There is a strong precedence for the importance of the perinatal environment. Clinicians are often among the first to recognize the deleterious effects of teratogenic pharmaceuticals. To that effect, a five-tier classification system designed to rate the safety of medications during pregnancy and lactation has greatly improved pharmaceutical counseling during the prenatal and perinatal periods. However, while many effects resulting in obvious birth defects have been documented, such as limb defects during the era of thalidomide use, the potential for pharmaceuticals to alter the fetal hormonal environment and alter adult disease susceptibility is a relatively new area of study. The long-term consequences of these types of effects are difficult to identify and are largely unknown. In addition to pharmaceuticals, maternal nutrition is known to affect fetal development. A physician, DJ Barker, was among the first to put forth the Developmental Origins of Adult Health and Disease hypothesis, highlighting the potential for maternal nutrition to impact the offspring's risk for coronary heart disease (CHD) and type II diabetes (1). Although not the focus of this chapter, an extensive body of literature has used animal models of development to confirm cause and effect relationships initially found in epidemiologic studies supporting the Developmental Origins hypothesis.

Altered fetal programming may manifest as physical, developmental, or neuropsychiatric abnormalities that often have long latencies and may not become evident until childhood or even adulthood. It is difficult to pinpoint a specific exposure during pregnancy responsible for a certain behavioral pattern or health condition that presents years after the exposure, especially when considering the vast number of potential variables, both physical and psychologic (i.e., emotional nurturing). However, a number of epidemiologic studies have found associations with altered fetal environ- ments. An area of great interest within fetal programming is the molecular changes within the fetus in response to maternal stress, medications, and exogenous hormone exposure.

We know that pharmaceuticals can program fetal development and cause effects that can be seen at birth. In addition, there is a large and growing body of literature demonstrating that the fetal environment can program the fetus and result in long-latency effects associated with increased risk of adult disease. The developmental origins of adult disease hypothesis evolved to explain this type of programming. In this chapter, we will focus on the long-term consequences of changes within the uterine environment during pregnancy, including nutritional, hormonal, pharmaceutical, and other environmental factors.

\section{BIRTH WEIGHT AND ADULT DISEASE RISK}

It is known that maternal nutrition is important for healthy fetal development; however, only recently has a link between maternal and fetal nutrition been made with adult disease (Box 1). The developing fetus is dependent on its mother to receive the proper nutrients required for normal growth and development. Low birth weight has historically been associated with an increased risk of death from CHD. Barker et al. first identified this association in a large population of men born in Hertfordshire, England, in 1911 to 1930 (3). As a baby's weight at birth or year 1 decreased, the risk of death from ischemic heart disease rose. People who were the lightest at birth ( $\leq 5.5$ pounds) and also lightest at 1 year of age ( $\leq 18$ pounds) had the highest standardized mortality ratio (SMR $=200 ; 95 \%$ CI 114-384) (3). Furthermore, Barker et al. also reported that systolic blood pressure, a risk factor for cardiovascular disease, was inversely related to birth weight in 10- and 36-year-olds born and residing in Great Britain (3).

The initial finding that birth weight was associated with CHD in adulthood led Barker to propose the Developmental Origins of Adult Health and Disease hypothesis, suggesting that changes that occurred during development that caused a baby to be born small may also impact adult disease risk. For example, low-birth weight babies are known to have increased blood pressure in adulthood $(4,5)$. It has been suggested that decreased blood flow to the kidney during key developmental periods may lead to decreased cell numbers in the glomeruli. Each glomerulus is therefore subjected to increased blood flow, and although blood pressure can initially be maintained, hyperfiltration eventually leads to glomerulosclerosis. This, in addition to the normal loss of glomeruli that occurs with aging, results in a feed-forward loop of increasing blood pressure and glomerular loss (6). Low birth weight is also associated with impaired glucose tolerance (5). In a similar mechanism, an undernourished fetus directs glucose to the developing brain at the expense of the muscles thereby resulting in less muscle mass at birth. As muscle is the primary 


Box 1 Consequences in Adult Life Associated with Low
Birth Weight
Hypertension
Type 2 diabetes
Obesity and the metabolic syndrome
Coronary heart disease
Stroke
Osteoporosis
Depression and psychosis
Age-related cognitive impairments
Chronic renal failure
Altered gonadal resposes
Altered immune responses
Overall reduced life expectancy
Source: From Ref. 2 .

location of insulin action, decreased muscle composition in adulthood results in lifelong insulin resistance (7). Another possibility is that there are fewer $\beta$-cells in the pancreas of low-birth weight babies.

Low birth weight has also been associated with diseases of the metabolic syndrome in adulthood. Curhan et al. found that low birth weight was associated with an increased risk of hypertension and diabetes in a longitudinal study of 51,529 U.S. males born from 1911 to 1946 . Compared with men born with average size (7.0-8.4lb), men born weighing less than $5.5 \mathrm{lb}$ were $26 \%$ more likely to have hypertension and $75 \%$ more likely to have diabetes (8). Curhan et al. also examined data from the Nurses' Health Studies I and II and found that low birth weight was associated with hypertension in women (8). Additionally, in a study of the Pima Indian population in Arizona, McCance et al. found that low birth weight was strongly associated with increased risk of diabetes (9). Hales et al. also found increased risk of diabetes or insulin resistance with decreasing birth weight in the Hertfordshire studies discussed above (5).

Barker further refined his Developmental Origins hypothesis with the introduction of the concept of the thrifty fetus. He hypothesized that the fetal environment could provide a "weather forecast" of the nutritional status outside of the womb. His thrifty phenotype hypothesis suggests that declines in nutritional status while in the womb can program a fetus to be "thrifty" and to store and ration energy accordingly (10). Problems arise, however, when individuals programmed in utero to be "thrifty" are introduced to an environment of plenty during early adolescence and adulthood. Being "thrifty" in development allows a fetus to conserve energy for important tissues like the brain at the expense of other tissues such as muscle and liver. Barker further suggests that the tissues and organs that were sacrificed during development have a diminished ability to properly respond to a "world of plenty" in adulthood. This hypothesis suggests that catch-up growth creates problems for those who were "thrifty" during development.

As an example of the thrifty phenotype, Barker and colleagues have extensively studied the case of people who were in utero during the Dutch Famine, which occurred from October 1944 to May 7, 1945, as a result of the German occupation in the West Netherlands during World War II. This discrete famine has allowed researchers to directly study the effects of maternal malnutrition on adult disease risk. During the famine, daily caloric intake fell to 300 to 800 calories/day. Fortunately, recovery was swift and the caloric intake rebounded to pre-famine levels by June 1945. Because of the relatively short length of the famine, researchers have been able to determine whether adult-onset diseases are linked to nutritional insufficiency during very specific times of development. Ravelli et al. examined obesity among 19-yearold military draftees who were exposed to the famine compared with those born just before or just after the famine or born during the same time but in unaffected locations. They found that men exposed to the famine during the first two trimesters of pregnancy had a significantly higher prevalence of obesity compared with men born in cities unaffected by the famine. Conversely, men exposed to famine during the third trimester of gestation through the first 3 to 5 months of postnatal life had a decreased prevalence of obesity compared with men born outside of the region of famine (11).

Another series of studies examined 297 people exposed to the Dutch famine during gestation and 444 born just before or just after the famine. Children who were exposed to the famine during mid or late gestation were lighter, shorter, and thinner with smaller heads and placentas than those unexposed and had reduced glucose tolerance following a 2-hour oral glucose test $(12,13)$. Conversely, those exposed to famine in early gestation were heavier and longer at birth than those unexposed (12). They also had a more atherogenic lipid profile, higher low-density lipoprotein to high-density lipoprotein ratio, higher body mass index (BMI), and a higher prevalence of CHD $(12,14,15)$. Since high blood pressure is related to low birth weight, it was expected that it would also be related to famine exposure, particularly late exposure that tended to produce low-birth weight babies. Researchers found, however, that while increased blood pressure was related to low birth weight, it was overall independent of famine exposure (12). They were further able to elucidate that increased systolic blood pressure was inversely related to the protein/carbohydrate ratio in the third trimester regardless of famine exposure (16).

The Dutch Famine occurred for a short, discrete period of time and was followed by a quick return to nutritional sufficiency. By contrast, the famine that was incurred during the Leningrad Siege in what is presently known as St. Petersburg, Russia, from September 1941 to January 1944 lasted much longer and those exposed remained nutritionally deprived throughout much of their early adolescence. In examining those who were exposed in utero (born January 1, 1941-June 30, 1941; $n=169$ ) or during infancy (born November 1, 1941-June 30, 1942; $n=192$ ) or those born during the same period but outside of the area of the siege, Stanner et al. found very little differences in adult disease risk factors; there was a correlation with BMI and systolic and diastolic blood pressure in females in both exposure groups and von Willebrand factor was decreased in the infant group compared with the in utero group (17). Overall, the authors concluded that there was no effect on glucose concentration or insulin-like molecules or risk for hypertensive or cardiovascular disease with exposure to the Leningrad siege famine (17). The fact that there were very little differences found in the study of those exposed to the Leningrad Siege yet there were 
extensive differences found in the study of the Dutch Famine may serve to further highlight Barker's thrifty phenotype hypothesis that catch-up or compensatory growth further attenuates the low-birth weight baby's adult disease risk.

As discussed above and demonstrated in the comparison of the two famine studies, problems arise when the postnatal environment differs from the prenatal in utero environment and catch-up growth occurs. For example, in a study of 3641 men born in Helsinki from 1924 to 1933 for which there were serial measurements of height and weight from 6 to 16 years of age, Eriksson et al. found that men who were born with the lowest ponderal index but that subsequently had the highest BMI at age 11 were the most likely to have died from $\mathrm{CHD}$ (18). Forsen et al. reported on the corresponding cohort of 3447 women born in Helsinki during the same time period (19). In contrast to the data on the men, Forsen et al. reported that the risk of CHD was not associated with childhood BMI in women. Instead, in women, an increased risk was related to length at birth and increased placental weight. Women who were shortest at birth but tallest at age 7 (i.e., had experienced "catch-up growth") had the highest risk of CHD. Low birth weight followed by catch-up growth has also been shown to be a risk factor for adult-onset type II diabetes and hypertension (20). Along with others, these studies indicate not only the importance of catch-up growth, but also the important differences between how the sexes develop, namely, that females experience slower prenatal growth than males $(21,22)$. Furthermore, recent work has shown sex-specific alterations in DNA methylation (epigenetic) patterns of genes involved in metabolism in people exposed to the Dutch Famine (23).

While the increase risk of adult disease in association to low birth weight continues to be a major concern in both developing and developed countries, an equally important association with the effects of being born large for gestational age must also be studied, especially in light of the growing obesity epidemic. Large for gestational age is typically defined as birth weight greater than the 90th percentile for gestational age and is often associated with maternal obesity and/or diabetes (24). When excess maternal glucose crosses the placenta, the fetus becomes hyperglycemic and then experiences hyperinsulinemia. As insulin is an important growth factor for the developing fetus, aberrant circulating levels negatively affect adipose, muscle, and cardiac tissues resulting in a large or macrosomic baby (24). Large babies and those born to diabetic mothers are more likely to experience complications during delivery and, as adolescents, are more likely to be obese and to develop type II diabetes $(8,9,24-27)$.

Although birth weight has historically been used as a predictor in the developmental origins of adult health and disease, several studies indicate that it might not fully explain the complex interactions that have occurred to produce a baby of any given size. The utilization of additional markers, such as maternal birth weight, third-trimester maternal weight and weight gain, maternal arm fat area, maternal height, maternal energy intake, socioeconomic status, ponderal index, birth length, placental weight, and postnatal growth rate, may help to further understand how the perinatal environment programs adult disease risks. To this end, Kuzawa and Adair have developed a novel model to address the importance of fetal growth potential (genetics) versus fetal nutrient supply by relating maternal height to the potential fetal growth demand and third-trimester maternal arm fat area to the potential maternal supply. They found that male offspring born to tall mothers with low arm fat (lowest energy reserves) had the highest risk of cardiovascular disease as measured by lipid levels at age 14 (28). In female offspring, there was no difference in lipid levels, further supporting the idea that fetal programming of CHD risk from nutrition differs between the sexes. As we move forward in our examination of how the maternal and early postnatal nutritional environment affects adult disease risk, we must continually allow for new variables that help clarify the environment in which the fetus is developing. Furthermore, as clinicians, it is important to understand all potential ramifications of counseling new mothers regarding proper nutrition, including effects that may not be seen until their children reach adulthood.

\section{PHARMACEUTICAL TERATOGENS AND BIRTH DEFECTS}

Teratogens cause fetal programming that can result in severe birth defects. Throughout history, severe malformations caused by teratogens have been documented. One of the most well-known cases is thalidomide, which was initially used as a sedative and antiemetic and resulted in 10,000 to 20,000 victims worldwide who were born with missing or severely malformed limbs. While thalidomide has both antiangiogenic and anti-inflammatory properties, it has recently been demonstrated that it is the antiangiogenic nature of the drug that prevents the development of newly forming blood vessels necessary to support limb development (29). That is, a chemical exposure permanently altered the course of development and resulted in unformed limbs in those exposed.

Another suspected teratogen, isotretinoin (trade name Accutane), is a pharmaceutical prescribed for the systemic treatment of acne and is a derivative of retinoic acid. Retinoic acid, more generally known as vitamin A, is essential during development. A concentration gradient along the anteriorposterior axis regulates the expression of homeobox genes that subsequently direct the correct timing of developmental events within a growing organ system. The timing and concentration of retinoic acid is exquisitely regulated and involved in the proper development of bone, vision, brain, face, heart, and spinal cord. Because of the molecular similarities with retinoic acid, isotretinoin exposure alters the delicate balance of retinoic acid in the fetus and can interfere with the normal patterns of development causing severe malformations. Today doctors must extensively counsel women prescribed isotretinoin on the risks of severe fetal malformations and the need to use two methods of contraception. Unfortunately, despite their best efforts, more than 2000 women have become pregnant while on isotretinoin treatment (30).

\section{LONG-LATENCY EFFECTS FROM NON-TERATOGENIC PHARMACEUTICALS}

\section{Selective Serotonin Reuptake Inhibitor Use During Pregnancy}

Treating depression in pregnancy is a difficult decision for patients and physicians; both treatment and lack of treatment are associated with increased fetal risks. Untreated maternal 
depression negatively affects secondary to neonates increased maternal tobacco use, substance abuse, inadequate prenatal care, and poor maternal-fetal bonding postnatally. Previous data also suggest an increased risk of preterm labor and fetal growth restriction in fetuses born to mothers with untreated depression. Furthermore, maternal stress and mood disorders can permanently program the fetal adaptive stress response mediated by the hypothalamic-pituitary-adrenal (HPA) axis. Exposure to stress results in an increase in corticosterone levels in humans. In animal models, exposure of fetuses to stress in the prenatal and early postnatal periods resulted in exaggerated cortisol production in response to mild stressors in adulthood (31). Maternal stress and poor mothering behaviors postnatally have also been shown to alter fetal and infant glucocorticoid metabolism through epigenetic mechanisms involving alterations of DNA methylation (32-34). Maternal stress increases cortisol within the fetal HPA axis, resulting in shorter gestations, prematurity, lower birth weights, increased congenital malformations, smaller head circumferences, and depressed neonatal neurologic functioning (35). Importantly, infant and adolescent maltreatment are associated with altered cortisol levels and impaired response to psychologic stressors (36). Specifically, maternal stress and malnutrition have been linked to increased incidence of schizophrenia in offspring (37). The decision not to treat or stop treatment during pregnancy may also increase the risk of postpartum depression (38).

On the other hand, fetal exposure to selective serotonin reuptake inhibitors (SSRIs) also carries certain risks. Certain antidepressants are linked with increased risk of congenital anomalies, such as cardiac defects, omphaloceles, and other midline defects as well as persistent pulmonary hypertension. However, the absolute risk of these conditions secondary to SSRIs (the most commonly prescribed class of antidepressants during pregnancy) is very low $(38,39)$. Although overall risk of anomalies are low, transient side effects as a result of serotonin withdrawal at the time of delivery, such as agitation, altered muscle tone, breathing, and suction problems, are commonly reported (40). These effects typically resolve spontaneously within 2 weeks and are conventionally thought to pose less severe long-term consequences to the fetus than those associated with uncontrolled maternal depression. However, subclinical effects of perinatal exposure to SSRIs on fetal neurodevelopment may be subtle and remain undiagnosed until adolescence or early adulthood. More recently, an association with fetal SSRI and autism has been suggested, as increased serotonin causes dysfunction of the amygdala, which plays a role in emotion perception, and suppressed oxytocin production, a hormone proposed to promote "pro-social" behavior (41). Furthermore, SSRIs enter placental circulation and therefore increase fetal serotonin levels, an effect that may program the fetal HPA axis to function properly only in the presence of elevated serotonin, a state removed at the time of delivery.

\section{Progestin Use During Pregnancy}

Progesterone is a necessary component to maintain a healthy pregnancy. From the earliest stages of pregnancy, progesterone assists in implantation and supports pregnancy by formation of the corpus luteum. While endogenous progesterone is essential, it is still unclear what long-term effects may occur from the use of exogenous progesterone.

Progestin is a component of oral contraceptive pills (OCP) and limited exposure during the periconceptual period occurs with failure of oral contraception. Continued use of OCPs until detection of pregnancy occurs in $2 \%$ to $5 \%$ of pregnancies and can expose fetuses to exogenous hormones during critical windows of fetal development (42). In addition, emergency contraception exposes fetuses to even higher doses of exogenous hormones and is not $100 \%$ effective at preventing implantation. Prior to the advent of modern pregnancy tests that detect human chorionic gonadotropin in urine, the major indication for progestins in pregnancy occurred from progestin administration for 3 to 5 days to induce withdrawal menstruation. Today, the major indications for progestin therapy are to prevent preterm birth and treat recurrent pregnancy loss. Progestin supplementation is also utilized in assisted reproduction protocols to promote endometrial development favorable for implantation of a transferred embryo.

Several studies have assessed the risk of hypospadias following progestin exposure, the results of which seem to be inconclusive (43). One complication is that many studies examine boys born 20 or more years ago, when different progestins were indicated during pregnancy than those in current use. However, a more recent case-control study by Carmichael et al. of children born from October 1997 to December 2000 found a 3.7-fold increased risk of second- and third-degree hypospadias when women took any progestin to help become pregnant or to prevent pregnancy complications or loss for any length of time, but not from perinatal OCP exposure (44). Furthermore, in a meta-analysis of 14 articles, Raman-Wilms et al. found no increased risk of urogenital malformations with hormonal exposure during the first trimester. In this case, exposure was from OCP, hormonal pregnancy tests, as well as treatment for threatened abortion (45).

To date, 17-hydroxyprogesterone is the formulation of greatest efficacy in preventing preterm deliveries in women with a previous spontaneous preterm birth. In a retrospective cohort study by Waters et al., women receiving weekly injections of 17-hydroxyprogesterone caproate were 3.3 times more likely to develop gestational diabetes, controlling for maternal age, BMI, and maternal race (46). Progesterone may increase insulin resistance by affecting glucose transport at the glucose transporter type 4 (GLUT-4) receptor (46). Gestational diabetes can lead to insulin resistance in utero and permanently modify expression of genes responsible for glucose transport and beta cell development, predisposing fetuses to type II diabetes mellitus in adulthood (47). As use of progesterone to prevent preterm birth continues to gain momentum, the postnatal effects will continue to be elucidated as exposed fetuses reach adolescence and adulthood.

\section{Glucocorticoid Use During Pregnancy}

At proper concentrations, glucocorticoids are essential for fetal growth and development. Typical fetal cortisol levels are much lower than maternal levels due to an enzyme within the placenta, 11-beta-hydroxysteroid dehydrogenase type 2 (11 $\beta$-HSD2), which catalyzes rapid metabolism of corticosteroids into inactive metabolites (48). Exogenous steroid administration to preterm infants is frequently utilized to 
promote lung maturity and decrease complications of preterm delivery including necrotizing enterocolitis, interventricular hemorrhage, and pulmonary hypoplasia. The two steroids most commonly used, dexamethasone and betamethasone, are utilized because they are poorly metabolized by $11 \beta-H S D 2$ and easily diffuse through the placenta, thus enhancing the protective benefits by maximizing exposure (2). However, prolonged fetal exposure to exogenous steroids has been associated with an increased incidence of growth restriction, elevated cortisol levels, and reduced placental 11 $\beta$-HSD2 activity (49). These effects are exacerbated by placental insufficiency, which can be seen with maternal hypertension and diabetes, or with modification of the 11ß-HSD2 receptor (2). Conditions that typically occur during preterm labor or preterm premature rupture of membranes, such as increased progesterone, estrogen, nitric oxide, prostaglandins, and inflammatory cytokines, can temporarily reduce activity of placental $11 \beta-\mathrm{HSD} 2$ activity, thereby increasing the susceptibility to negative side effects to the population at greatest risk (2).

While the benefits to the developing lung are obvious, epidemiologic and animal model data suggest that there are negative side effects, such as altered brain development, particularly in the HPA, following multiple steroid treatments and may be worse in dexamethasone- than betamethasonetreated individuals $(50,51)$. Neuropsychiatric disorders are also prevalent in exposed individuals, including memory impairment, increased emotionality, unsociability, avoidance, and behavioral problems (52,53). Furthermore, Drake et al. recently reported that the effects of in utero exposure of rats to dexamethasone on low birth weight and glucose dyshomeostasis are also transmitted to second-generation offspring that are not directly exposed (54). The safety of exogenous steroids given postnatally for use in respiratory distress syndrome and bronchopulmonary dysplasia has also been questioned. Metaanalyses suggest that postnatal steroid use may increase the risk of cerebral palsy by $63 \%$ and increases neurologic impairment up to $40 \%$ (51). An important question is whether fetal growth restriction is an effect of glucocorticoid exposure in utero or whether it is the primary cause in warranting steroid administration. This question will be difficult to answer unequivocally.
In laboratory rats, developmental exposure to betamethasone led to altered partner preference, sexual behavior, and reduced testosterone in adulthood (55). The authors suggest that "prenatal treatment with betamethasone, by increasing maternal corticosteroid level, may have diminished testosterone peak in male pups, a peak crucial to brain sexual differentiation."

\section{Diethylstilbestrol}

Because teratogen exposure often results in specific malformations that can be identified at birth, a cause and effect relationship between exposure and birth defect can often be established. However, linking exposure with fetal programming that results in long-latency effects is more challenging. One of the best-known examples is the case of diethylstilbestrol (DES) daughters. From 1940 to 1971, millions of women were prescribed the synthetic estrogen DES in the mistaken belief that it would prevent spontaneous abortion and promote a healthy pregnancy. The negative effects of developmental DES exposure were not known until 1971 when the physician AL Herbst published a case report of eight young girls with vaginal clear cell adenocarcinoma, a rare type of cancer that previously had only afflicted much older women. Herbst found that seven of the eight presenting cases had been exposed to DES during the first trimester (56) and later confirmed this on a larger scale (Fig. 1). Additionally, women exposed in utero to DES had increased incidence of various reproductive abnormalities including $\mathrm{T}$-shaped uteri, transverse vaginal ridges, and cervical collars (58-62). Today, it is known that both men and women exposed to DES in utero have increased risk of adult-onset diseases, several of which are discussed below. Furthermore, it appears that the risks of exposure to DES did not stop with those that were exposed in utero, but extend to a third generation providing evidence for transgenerational effects (63-67).

The phenotype seen in women from fetal programming by DES has been reproduced in laboratory mice developmentally exposed to DES $(68,69)$. In mice, the T-shaped uterus appeared to be caused by posterior shift in the expression of homeobox A genes whose sequential expression are necessary for proper tissue development (70). When the discovery that

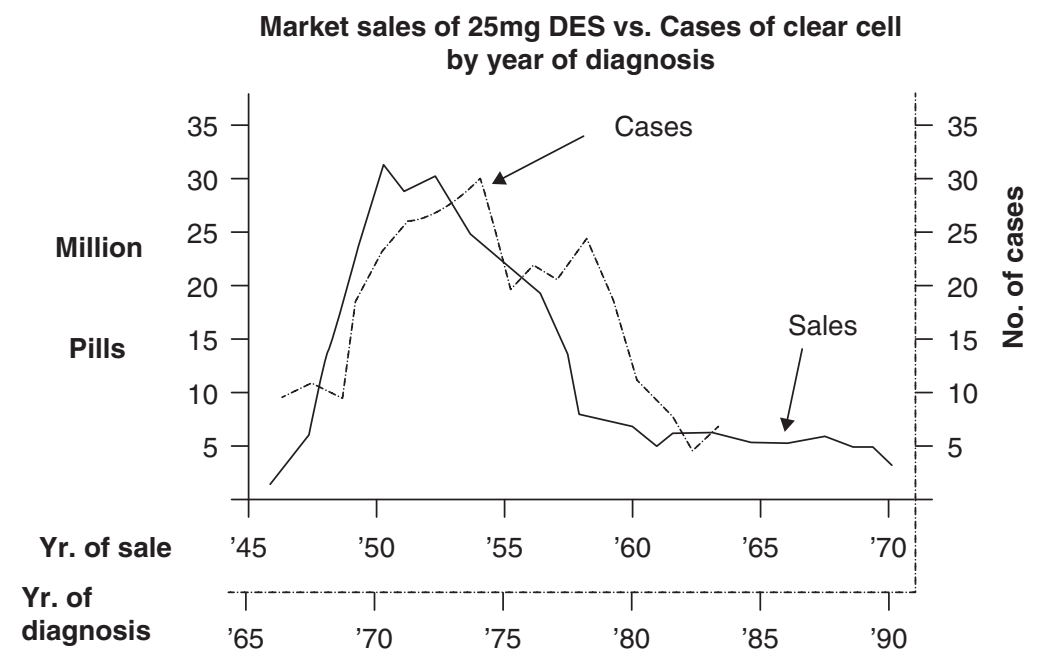

Figure 1 Market sales of $25 \mathrm{mg}$ DES versus cases of vaginal clear cell adenocarcinoma by year of diagnosis. Source: From Ref. 57 and Market share Litigation exhibit. 
developmental exposure to an estrogen pharmaceutical created long-latency developmental effects existed, it opened the door to the exploration of fetal programming. Additional sources range from natural variation in endogenous fetal estrogens to exogenous exposure to chemicals in the environment with hormonal activity, termed endocrine disruptors, among a variety of other pharmaceuticals that are just beginning to be scrutinized for potential fetal programming.

\section{ENDOGENOUS AND EXOGENOUS FETAL ESTROGEN AND ADULT DISEASE}

Altered fetal serum estrogen has been associated with fetal programming and altered risk of adult disease (Table 1). It is known that adult estrogen exposure is associated with endometriosis and breast cancer. Conditions associated with increased fetal exposure to estrogens are also associated with an increased risk of endometriosis and breast cancer in adulthood. Daughters whose mothers took the synthetic estrogen DES during pregnancy have an $80 \%$ increased risk of endometriosis and up to $300 \%$ increased risk of breast cancer (58,71). Dizygotic twins, who have two placentas, circulate $60 \%$ to $80 \%$ higher levels of estradiol than singletons (75). Dizygotic twins subsequently have approximately $70 \%$ increased risk of endometriosis and $50 \%$ increased risk of breast cancer in adulthood $(58,72)$. Monozygotic twins do not have an increased risk of breast cancer, suggesting that it is not being a twin that raises the risk but having two placentas (72). In addition, maternal estrogen concentrations are positively associated with maternal age and offspring birth weight, and breast cancer risk is also positively associated with maternal age and birth weight (reviewed in 72). High ponderal index at birth is associated with increased circulating estradiol in adulthood (76).

Conditions associated with decreased fetal exposure to estrogens are associated with reduced risk of endometriosis and breast cancer in adulthood. Smoking inhibits the estrogen-synthesizing enzyme aromatase and is associated with reduced estrogen levels (77). For example, in adult women smokers, there is a 20 -fold reduction in the incidence of the estrogen-dependent disease endometriosis $(73,74)$. Importantly, placental aromatase activity is reported to be reduced in smokers (78). Daughters born to women who smoked during pregnancy have over a fourfold reduced risk of endometriosis and have a threefold reduced risk of breast cancer $(73,74)$. Additionally, maternal estrogens are lower in pre-eclamptic patients versus controls $(79,80)$. Daughters born to pre-eclamptic mothers have a twofold decreased risk of breast cancer in adulthood (72). No data were found on the risk of endometriosis. Taken together, the fetus appears to be sensitive to permanent programming due to an altered fetal hormonal environment.

\section{ENDOCRINE-DISRUPTING CHEMICALS}

While women no longer intentionally take synthetic estrogens during pregnancy, pregnant women and their fetuses are exposed to a wide variety of environmental chemicals that may alter normal development. Many of these chemicals can surreptitiously modulate the endocrine system and have been termed endocrine disruptors or endocrine-disrupting chemicals (EDCs). Hundreds of chemicals, both natural and manmade, have been described with the ability to pirate the body's own endocrine system causing altered hormone signaling. Like teratogens, endocrine disruptors can program the fetus and result in permanently altered phenotypes (81). However, unlike teratogens, EDCs can affect both adults and developing organisms. Whereas the effects of endocrine disruptors in adulthood tend to be reversible, the effects on the developing organism tend to be permanent. For this reason, a considerable amount of research has focused on this sensitive period, where exposure can result in permanent fetal programming.

Teratogens cause severe fetal programming that results in malformations present at birth, but there are probably other persistent changes that are subtler and therefore not attributed to the exposure. Teratogens and EDCs probably represent a continuum of effects from more severe effects often with short latency to more subtle effects often with long latency. Severity can be related not only to higher exposure to teratogens but also to different mechanisms of action. Unfortunately, effects seen at high doses cannot necessarily predict those seen at low doses. The effects of endocrine disruptors most often exhibit a non-monotonic dose-response where qualitatively and/or quantitatively different effects have been described at low doses versus high doses $(82,83)$. For example, in mice developmental exposure to low doses of DES increases the adult estrogen responsiveness in the uterus while exposure to high doses decreases the responsiveness (84). This adds to the difficulty in establishing cause and effect relationships between chemical exposure and adult disease.

To date, there has been a large amount of research in the laboratory demonstrating developmental programming by EDCs. As illustrated above, conditions associated with altered hormonal environments during human pregnancies are

\section{Table 1 Altered Fetal Estrogen and Adult Disease}

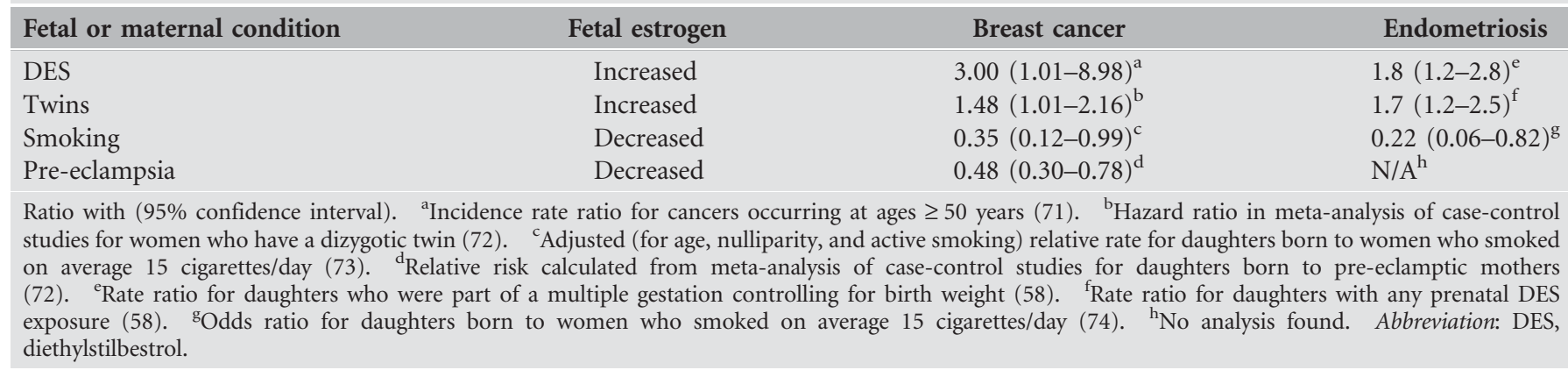


associated with altered disease risk in adulthood. While longlatency effects from hormonal chemicals are difficult to detect, humans have been shown to be sensitive to this type of fetal programming (85). Because of the prevalence of EDCs in our environment and the potential for EDCs to cause developmental programming in humans, the Endocrine Society recently released a position statement on endocrine disruptors (86): "The evidence for adverse reproductive outcomes (infertility, cancers, malformations) from exposure to endocrine disrupting chemicals is strong, and there is mounting evidence for effects on other endocrine systems, including thyroid, neuroendocrine, obesity and metabolism, and insulin and glucose homeostasis." They further suggest partnering of scientific societies to further research the effects in humans. Similar statements have been adopted by the American Medical Association, American Chemical Society, and others.

The U.S. Environmental Protection Agency (EPA) Endocrine Disruptor Screening and Testing Program was established in 1998 in response to the Food Quality Protection Act and was charged with the goal of developing screening and testing methods for endocrine-active compounds. Over 10 years later, the final selection of chemicals to be tested to validate screening and testing methods was chosen (87). Recently, the Endocrine Disruptor Prevention Act of 2009 was introduced to strengthen regulation of EDCs.

\section{MECHANISMS OF EDCS}

The U.S. EPA defines an EDC as "an exogenous agent that interferes with synthesis, secretion, transport, metabolism, binding action, or elimination of natural blood-borne hormones that are present in the body and are responsible for homeostatsis, reproduction, and developmental process." A detailed discussion of the mechanisms of EDC action is beyond the scope of this chapter but has been reviewed elsewhere $(81,86)$. Summarized below are potential consequences resulting from developmental disruption of normal estrogen, androgen or thyroid hormone signaling by EDCs in adulthood.

\section{Estrogens}

To date, the largest group of environmental chemicals with hormonal activity interferes with normal estrogen signaling. Those chemicals that bind directly to the estrogen receptor are termed xenoestrogens. They can have estrogenic, antiestrogenic, or mixed receptor activity. While hundreds of xenoestrogens have been described, a few selected ones are described below.

Bisphenol $\mathrm{A}$ is an estrogen receptor ligand and selective estrogen receptor modulator $(88,89)$. It has been shown to disrupt the endocrine system in $>100$ reports in different animal systems (82). Bisphenol A is the monomer that is polymerized to form polycarbonate plastic (hard, clear plastic) and is one of the top 50 chemicals in worldwide production, with annual production of $>6$ billion pounds (90). It is released from polycarbonate products, such as water and baby bottles $(91,92)$, the resin lining of metal food cans (93-95), some dental sealants (96), eyeglasses, CD cases, and polycarbonate laboratory animal cages and water bottles $(97,98)$. Bisphenol A was shown to comprise $84 \%$ of the estrogenic activity in landfill leachate (99).

Bisphenol $\mathrm{A}$ is an almost ubiquitous chemical in our environment and in people (100). Calafat recently reported that $>92 \%$ of Americans have detectable levels of bisphenol A in urine (101). Importantly, children had twice the level as adults ( 4.5 vs. $2.5 \mu \mathrm{g} / \mathrm{L}$ ) and women had higher levels than men. It was recently estimated that infants drinking out of polycarbonate bottles would receive a daily exposure of up to $2 \mu \mathrm{g} / \mathrm{kg}$ (102). Of particular concern, bisphenol A alters normal development at this exact dose (and lower) in animal models $(82,98,103-115)$.

Ethinyl estradiol is an orally active form of estradiol, and it is a component of OCPs. While ethinyl estradiol is not considered a teratogen, evidence from laboratory studies suggest that it is an endocrine disruptor. Millions of women use OCP, and approximately $2 \%$ to $5 \%$ will conceive while taking them due to noncompliance (116). Some of these women deliver infants exposed to 0.2 to $0.5 \mu \mathrm{g} / \mathrm{kg}$ of this potent estrogen during the critical window of sexual differentiation in the first trimester of pregnancy. This dose has been shown to alter mouse development $(105,117)$. Additionally, ethinyl estradiol has been measured in waste water influent and effluent and may be a source of human exposure (118).

Genistein is a naturally occurring phytoestrogen and the predominant soy isoflavone. Adults consuming soy-based foods, particularly vegetarians, circulate 50 to $200 \mathrm{ng} / \mathrm{mL}$ total isoflavones. Infants fed soy-based infant formula circulate approximately $700 \mathrm{ng} / \mathrm{mL}$ genistein (119). While soy-based foods have been promoted as having beneficial effects in adults, there is increasing evidence that developmental exposure during differentiation of the uterus may be detrimental (120-123).

Importantly, xenoestrogens are found in the human fetus. Pregnant women and their fetuses tend to have higher levels of bisphenol A (124-126), and bisphenol A has been found in human maternal and fetal serum at 0.2 to $10 \mathrm{ng} / \mathrm{mL}(125-127)$. Olea and colleagues recently measured total xenoestrogen bioactivity in human placentas. By separating endogenous from xenoestrogens, it was estimated that $16 \%$ of the total activity was due to xenoestrogens (128). There is a considerable amount of literature relating to EDCs: on mechanisms of action, controlled laboratory studies, and human health trends. Taken together, these strongly support fetal programming by these chemicals. Outlined below are many of these trends with supporting associations with EDCs.

\section{Puberty}

A trend toward earlier onset of puberty has recently been described, and EDCs, particularly early exposure to xenoestrogens, are thought to be a major contributing factor (129-133). Earlier puberty (defined as development of secondary sexual characteristics before 8 years of age) is a risk factor for breast and testicular cancer, polycystic ovarian syndrome, and metabolic disorders (133). Thigpen et al. found that rodent chow containing various low doses of genistein and another soy isoflavone, daidzein, advanced vaginal opening in Wistar rats and CD-1 mice (134). Other xenoestrogens including DES and bisphenol $\mathrm{A}$ and various other EDCs have also been shown to advance puberty in rodents at very low doses ( $25 \mathrm{ng}-200 \mu \mathrm{g} /$ $\mathrm{kg}$ /day) (104,105,135-138). In humans, early pubertal onset has been associated with neonatal exposure to the polybrominated biphenyl FireMaster, which was accidentally introduced into the Michigan food supply in 1973 when 
it was confused with the nutritional supplement NutriMaster $(139,140)$. Furthermore, precocious thelarche before age 2 has been associated with elevated serum levels of phthalate esters in Puerto Rico. One phthalate in particular, di-(2-ethylhexyl) phthalate, was detected in patients at levels 6.4-fold higher than controls (141). Importantly, a trend toward altered timing of pubertal onset may affect later disease risk (i.e., increased cumulative estrogen exposure increases breast cancer risk), and also how clinicians medically and pharmacologically treat children with precocious or delayed puberty.

\section{Fertility}

The rates of infertility and recurrent pregnancy loss have increased over the last 50 years. While delayed childbearing in women is a clear and strong factor in decreased fertility, it has been postulated that developmental xenoestrogen exposure may also play a role (142). McLachlan and colleagues reported reduced fertility in mice following developmental DES exposure (68). Jefferson and Newbold recently showed that developmental $500 \mu \mathrm{g} / \mathrm{kg}$ genistein exposure resulted in impaired fertility in female mice in adulthood (121). This dose of genistein is an order of magnitude lower than that consumed by infants fed exclusively soy infant formula (143).

\section{Breast Cancer}

Breast cancer is the second most fatal cancer for women and one out of eight women will be diagnosed in their lifetimes (144). While interpreting the trends in breast cancer incidence is complicated by many factors (145), it appears that incidence has increased in the second half of the twentieth century $(146,147)$. For example, in Hong Kong age-adjusted risk increased two- to threefold for women born in 1960 compared with 1900 (Fig. 2 (147)). Ravdin et al. recently reported a decline in breast cancer incidence in the United
States starting around 2002, but it is still unclear what factors have primarily accounted for the change $(146,148)$.

In humans, exposure to steroidal estrogens is associated with increased breast cancer risk, and xenoestrogen exposure has been associated with increased breast cancer risk in adulthood. As discussed above, increased fetal estrogen exposure, endogenous or exogenous, is associated with increased adult breast cancer risk, while decreased exposure is associated with reduced risk. Developmental DES exposure has clearly been associated with increased breast cancer risk (71). While it is difficult to study unknown exposures with long-latency diseases 40 to 70 years later, developmental xenoestrogen exposure has been associated with altered mammary gland development and cancer risk in laboratory animals $(107,149-161)$.

\section{Male Reproductive Tract}

There is some evidence that reproductive tract abnormalities in boys are influenced by developmental estrogen exposure. Cryptorchidism, hypospadias, and testicular cancer incidence are higher in first-born boys. Since maternal estrogens are higher in first pregnancies, these data are compatible with the hypotheses linking excessive pregnancy-estrogen exposure to conditions more common among first-born individuals (162). Additionally, cryptorchidism and testicular cancer increase in boys born to older mothers (163). However, unlike breast cancer and endometriosis, cryptorchidism does not appear to be related to maternal smoking (164).

Vegetarians tend to consume greater quantities of soy-based products than their omnivorous counterparts. Thus, vegetarians are exposed to increased levels of phytoestrogens, chemicals in plants that mimic estrogens. A prospective cohort study in the United Kingdom that sampled 7928 males born in 1991 and 1992 found an odds ratio for hypospadias of 4.99 for boys who were born to vegetarian mothers (165). An increased incidence of hypospadias has also been reported in DES grandsons (63-66).

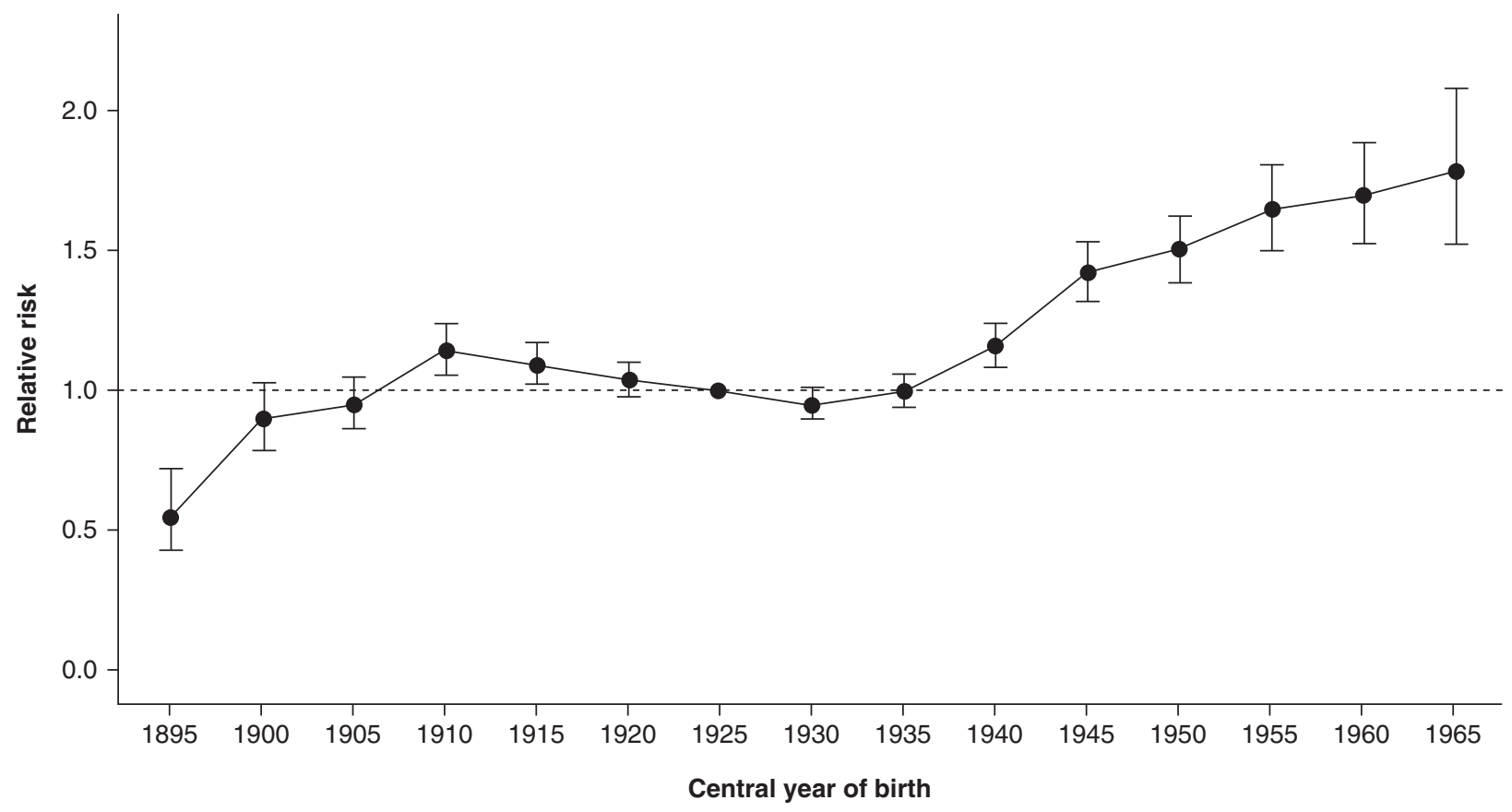

Figure 2 Relative risk estimates of incidence of breast cancer by birth cohort. Based on full age-period-cohort model with period constraints. Bars around the point estimate indicate the 95\% confidence intervals. Source: From Ref. 147. 


\section{Metabolic Programming}

Newbold and colleagues have also shown that developmental exposure to DES at a dose of $1 \mu \mathrm{g} / \mathrm{kg} /$ day but not at higher doses $(10-100 \mu \mathrm{g} / \mathrm{kg} /$ day) can cause obesity in adulthood in mice (reviewed in (166)). At 2 months of age, prior to a difference in size between DES-treated and control mice, the DES mice had elevated levels of leptin, adiponectin, IL-6, and triglycerides. By 6 months of age, densitometry studies indicated that the DES-exposed mice had increased abdominal fat, which was not likely caused by a slightly increased food intake and/or decreased activity. Furthermore, Newbold et al. have also shown that DES-exposed animals have a high level of islet cell hyperplasia, indicating that they may have problems with glucose homeostasis (reviewed in (166)).

\section{Androgens}

There are several environmental chemicals that interfere with androgen action. Chemicals that bind to the androgen receptor and prevent androgen action are known as androgen receptor antagonists, including DDE (a metabolite of the pesticide DDT), vinclozolin, a fungicide, and several phthalates. Phthalates are esters of phthalic acid and are used in hundreds of products, including plasticizers, PVC resins, and cosmetic and personal care products such as shampoos and soaps. As of 2004, manufacturers produced about 800 million pounds of phthalates each year. They contribute $10 \%$ to $60 \%$ of plastic products by weight (167).

The incidence of male reproductive tract disorders, including cryptorchidism, hypospadias, testicular cancer, and low sperm count, has increased over the last 30 to 50 years (168-174). It has been postulated that these disorders comprise a testicular dysgenesis syndrome (TDS) and may have a common etiology of deficient androgen production or action during development of the testes in fetal life (175).

Normal sexual differentiation of the male reproductive tract is androgen dependent. Exposure to antiandrogens during sexual differentiation produces malformations of the reproductive tract in humans and rodents. For example, the fungicide vinclozolin is an antiandrogen. Developmental exposure to vinclozolin during sexual differentiation in rats resulted in reduced anogenital distance, retained nipples, cleft phallus with hypospadias, cryptorchidism, a vaginal pouch, epididymal granulomas, and small-to-absent sex accessory glands (176).

\section{Anogenital Distance (AGD)}

AGD is a marker for sexual differentiation. In humans, AGD is measured from the anus to the base of the scrotum in males and from the anus to the genitals in females and is about twice as long in males as in females (177). AGD is androgen dependent and developmental exposure to antiandrogens reduces AGD. Many laboratory studies have shown that developmental exposure to EDCs with antiandrogenic activity, such as di-n-butyl phthalate, bis(2-ethylhexyl)phthalate, linuron, procymidone, and DDE (a metabolite of the pesticide DDT) can reduce AGD (176).

Phthalates have been shown to have estrogenic activity and antiandrogenic activity, and to alter androgen production. Importantly, Swan et al. prospectively examined the relationship between developmental phthalate exposure and ageadjusted AGD (anogenital index) in infant boys (178). AGI was inversely related to four phthalate metabolites in maternal circulation during pregnancy (Table 2). Boys with the highest developmental exposure were 10 times more likely to have a shorter AGD than boys with the lowest prenatal exposure (178).

\section{Testicular Cancer}

Testicular cancer incidence is associated with higher serum levels of EDCs in adulthood (179) and maternal levels of EDCs $(180,181)$. Over the past $50+$ years, the incidence of testicular cancer has risen at an alarming rate. In a number of studies from around the world, it is estimated that between approximately 1982 and 2005, incidence of testicular cancer rose $2.4 \%$ per year in Spain (182), 2.6\% per year in Australia (183), $2.3 \%$ to $3.4 \%$ per year in the Nordic countries, and by $5 \%$ in Poland and Germany (184). For men in six European countries born after 1945, the risk of testicular cancer increased in all six countries. Relative risks for those born around 1965 were 3.9 in Sweden and 11.4 in East Germany (185).

\section{Cryptorchidism}

The incidence of cryptorchidism doubled in the United States between 1968 and 1993 and has been associated with developmental exposure to several EDCs (168). During a period of peak use of the pesticide DDT in the United States between 1959 and 1967, babies born to mothers with the highest serum DDT levels had nearly a twofold increased risk of cryptorchidism when compared with those whose mothers had lowest serum DDT levels (186).

Similarly, the concentration of 21 persistent and nonpersistent EDCs in the breast milk of Finnish and Danish mothers between 1997 and 2001, as measured in a series of case reports from a prospective birth cohort, was associated with an increased risk of cryptorchidism (187-189). A combined statistical analysis for the eight most prevalent persistent EDCs ( $p, p^{\prime}$-DDE, p,p'-DDT, $\beta-\mathrm{HCH}, \mathrm{HCB}$,

\section{Table 2 Mean (Median) Phthalate Monoester Metabolite Levels by Anogenital Index Category}

\begin{tabular}{|c|c|c|c|}
\hline \multirow[b]{2}{*}{ Monoester metabolite } & \multicolumn{3}{|c|}{ AGI category [mean (median; ng/mL)] } \\
\hline & Long $^{\mathbf{a}}(n=17)$ & Intermediate $^{\mathrm{b}}(n=43)$ & Short $^{\mathrm{c}}(n=25)$ \\
\hline MBP & $13.1(11.5)$ & $22.2(13.1)$ & $38.7(24.5)$ \\
\hline MEP & $124(47.1)$ & $592(112)$ & $1,076(225)$ \\
\hline MiBP & $2.3(1.5)$ & $3.3(2.1)$ & $7.7(4.8)$ \\
\hline
\end{tabular}


$\alpha$-endosulfan, cis-HE, oxychlordane, and dieldrin) revealed higher concentrations in the breast milk of case mothers. This indicates that a mixture of low concentrations of EDCs may be more important when assessing the risk of cryptorchidism than the concentration of any single chemical (187). The concentration of the sum of seven polybrominated diphenyl ethers, which are commonly found in flame-retardants, was also significantly higher in the breast milk of mothers of cryptorchid boys compared with controls (188). Additionally, phthalate levels in the mothers' breast milk were associated with altered levels of hormones related to Leydig cell function, which might have led to decreased virilization in this cohort (189).

\section{Hypospadias}

In 1997, the CDC reported a doubling of hypospadias in the United States from 1968 to 1993 (169). European countries also reported increases in hypospadias but increases were not seen in less affluent countries (168). Exposure to industrial and agricultural EDCs during development is associated with increased risk of hypospadias as demonstrated by Dolk et al. (190) and Carbone et al. (191).

Phthalate exposure during sexual differentiation has been shown to lead to TDS in laboratory animals (192). One hypothesis for the increased incidence of male reproductive tract disorders in humans is developmental exposure to EDCs that can alter androgen signaling. Wilson et al. reviewed different groups of EDCs that converge on androgen signaling. While the exact mechanisms of these EDCs differed, the end result was a reduction in testosterone signaling through androgen receptor (193).

\section{Sperm Count}

Sperm counts have declined over the past 60 years. In a metaanalysis, Swan et al. found a twofold reduction in sperm counts from 1938 to 1990 (172). Since Swan et al. have confirmed their initial findings and expanded the scope of years examined, the number of studies examined, and further analyzed confounders and statistical methods used for approximating the decline $(171,173)$, adult and developmental exposure to environment chemicals has been linked to lower sperm counts. Swan et al. compared semen characteristics, including sperm concentration, from samples collected at four centers in the United States (Columbia, Missouri; New York, New York; Minneapolis, Minnesota; and Los Angeles, California). All samples were collected and analyzed following very strict protocols, and it was found that the men from Missouri had the lowest sperm concentrations, which in turn was associated with higher urinary concentrations of five pesticides, including alachlor and atrazine, which are commonly used herbicides $(170,194)$. The data suggest that residence in a rural community with high pesticide exposure may affect sperm concentrations.

Developmental exposure to anabolic steroids or xenobiotics in beef has been linked to low sperm count in adulthood. Fertile men whose mothers consumed seven or more beef meals per week during pregnancy had $24 \%$ lower sperm count, were more likely to have a history of infertility, and there was a threefold increase in the proportion of these men with less than 20 million sperm per mL (195).

\section{THYROID HORMONE}

Unlike xenoestrogens and environmental antiandrogens that bind directly to the estrogen and androgen receptor, EDCs that disrupt thyroid function do so without binding to the thyroid receptor. Howdeshell extensively reviewed EDCs for their potential to interfere with thyroid hormone signaling and found over 150 chemicals with the ability to disrupt thyroid hormone action (196). EDCs have been shown to disrupt nearly every step in the synthesis and metabolism of thyroid hormone (196). For example, EDCs have been shown to alter thyroid hormone synthesis, uptake of iodine by the thyroid gland, and binding to specific thyroid-binding proteins in blood.

Polychlorinated biphenyls (PCBs) are a group of chemicals used in a variety of manufacturing applications, for example, in coolants, transformers, plasticizers, oils, adhesives, and so on. PCBs are persistent organic pollutants that are highly toxic, similar in action to dioxin, and used widely until their ban in 1976 in the United States. In addition to acute toxicity, PCBs are endocrine disruptors and interfere with normal hormone action, particularly estrogen and thyroid hormone signaling.

Adequate thyroid hormone during development is essential and inadequate levels are associated with cognitive deficits in children born to pregnant women with hypothyroidism (197). PCBs have been shown in the laboratory to interfere with multiple steps in thyroid hormone physiology (reviewed in (196)). Many studies of prenatal exposure to PCBs have been undertaken in various parts of the world and have shown negative effects on neurodevelopment (198-208). For example, Jacobson et al. examined infants in the Lake Michigan area selected based on their mothers' fish consumption. Because PCBs are persistent and lipophilic, they bioaccumulate in the food chain and have been found in high concentrations in fish and fish eaters. Infants with high levels of PCBs showed impaired visual memory (204). In 4-year-olds, prenatal PCB exposure was associated with lower scores in verbal and numerical memory (203), visual discrimination processing, and impaired short-term memory scanning (209). In 11-yearolds, prenatal PCB exposure was correlated with lower fullscale and verbal IQ scores. On average, IQ scores were 6.2 points lower in the highest exposure group than their lesser-exposed peers (202). It was also estimated that PCBexposed children were up to 7.2 months behind in word comprehension (202). Since PCBs were banned in 1979, human exposure to these persistent chemicals has slowly declined. However, PCBs are very persistent chemicals and cognitive dysfunction is still reported, suggesting that environmentally relevant doses of endocrine-disrupting chemicals may alter human neurodevelopment (210).

\section{SUMMARY}

There is a great need for research to define the mechanisms of fetal programming. These mechanisms involve epigenetic changes, that is, the effects do not result from DNA mutations. Intense research centers on elucidating the mechanisms responsible for fetal programming and the epigenetic alterations regulating gene expression and adult disease. Epigenetic mechanisms include DNA and histone methylation, and histone acetylation $(211,212)$. These marks can retain fetal programming information into adult life. 
While high-dose/pharmacologic exposures have been associated with birth defects and severe long-latency effects (DES), it is likely that current human exposure to other pharmaceuticals and industrial chemicals may program the fetus and result in more subtle alterations in adult phenotype. Establishing cause and effect relationships between exposures and these subtle effects is much more difficult. Despite the difficulty in demonstrating such cause and effect relationships with subtle long-latency effects, these associations have been found in controlled experiments in human and laboratory animal studies and it is clear that subtle fetal programming occurs in people and alters adult disease risk. It is likely that only the tip of the iceberg has been described to date, and it is largely unknown the extent that the fetal environment programs lifelong phenotypes. Given this backdrop, every effort should be made to use the precautionary principle in counseling pregnant women about pharmaceutical use and minimizing exposure to environmental chemicals, such as indoor pesticides and painting while pregnant. Maternal risk of withholding medication must be considered, but when potential fetal risks outweigh maternal benefit, the precautionary principle should be employed.

\section{REFERENCES}

1. Barker DJ. The developmental origins of adult disease. Eur J Epidemiol 2003; 18: 733-6.

2. Seckl JR, Holmes MC. Mechanisms of disease: glucocorticoids, their placental metabolism and fetal 'programming' of adult pathophysiology. Nat Clin Pract Endocrinol Metab 2007; 3: 479-88.

3. Barker DJ, Winter PD, Osmond C, Margetts B, Simmonds SJ. Weight in infancy and death from ischaemic heart disease. Lancet 1989; 2: 577-80.

4. Law CM, de Swiet M, Osmond C, et al. Initiation of hypertension in utero and its amplification throughout life. BMJ 1993; 306: 24-7.

5. Hales CN, Barker DJ, Clark PM, et al. Fetal and infant growth and impaired glucose tolerance at age 64. BMJ 1991; 303: 1019-22.

6. Brenner BM, Chertow GM. Congenital oligonephropathy: an inborn cause of adult hypertension and progressive renal injury? Curr Opin Nephrol Hypertens 1993; 2: 691-5.

7. Barker DJ. Maternal nutrition, fetal nutrition, and disease in later life. Nutrition 1997; 13: 807-13.

8. Curhan GC, Chertow GM, Willett WC, et al. Birth weight and adult hypertension and obesity in women. Circulation 1996; 94: 1310-15.

9. McCance DR, Pettitt DJ, Hanson RL, et al. Birth weight and non-insulin dependent diabetes: thrifty genotype, thrifty phenotype, or surviving small baby genotype? BMJ 1994; 308: 942-5.

10. Hales CN, Barker DJ. Type 2 (non-insulin-dependent) diabetes mellitus: the thrifty phenotype hypothesis. Diabetologia 1992; 35: 595-601.

11. Ravelli GP, Stein ZA, Susser MW. Obesity in young men after famine exposure in utero and early infancy. N Engl J Med 1976; 295: 349-53.

12. Roseboom TJ, van der Meulen JH, Ravelli AC, et al. Blood pressure in adults after prenatal exposure to famine. J Hypertens 1999; 17: 325-30.

13. Ravelli AC, van der Meulen JH, Michels RP, et al. Glucose tolerance in adults after prenatal exposure to famine. Lancet 1998; 351: 173-7.

14. Roseboom TJ, van der Meulen JH, Osmond C, et al. Plasma lipid profiles in adults after prenatal exposure to the Dutch famine. Am J Clin Nutr 2000; 72: 1101-6.

15. Roseboom TJ, van der Meulen JH, Osmond C, et al. Coronary heart disease after prenatal exposure to the Dutch famine, 1944-45. Heart 2000; 84: 595-8.

16. Roseboom TJ, van der Meulen JH, van Montfrans GA, et al. Maternal nutrition during gestation and blood pressure in later life. J Hypertens 2001; 19: 29-34.

17. Stanner SA, Bulmer K, Andres C, et al. Does malnutrition in utero determine diabetes and coronary heart disease in adulthood? Results from the Leningrad siege study, a cross sectional study. BMJ 1997; 315: $1342-8$.
18. Eriksson JG, Forsen T, Tuomilehto J, et al. Catch-up growth in childhood and death from coronary heart disease: longitudinal study. BMJ 1999; 318: 427-31.

19. Forsen T, Eriksson JG, Tuomilehto J, Osmond C, Barker DJ. Growth in utero and during childhood among women who develop coronary heart disease: longitudinal study. BMJ 1999; 319: 1403-7.

20. Barker DJ, Eriksson JG, Forsen T, Osmond C. Fetal origins of adult disease: strength of effects and biological basis. Int J Epidemiol 2002; 31: 1235-9.

21. Crawford MA, Doyle W, Meadows N. Gender differences at birth and differences in fetal growth. Hum Reprod 1987; 2: 517-20.

22. Gilbert JS, Nijland MJ. Sex differences in the developmental origins of hypertension and cardiorenal disease. Am J Physiol Regul Integr Comp Physiol 2008; 295: R1941-52.

23. Tobi EW, Lumey LH, Talens RP, et al. DNA Methylation differences after exposure to prenatal famine are common and timing- and sexspecific. Hum Mol Genet 2009; 18: 4046-53.

24. Das UG, Sysyn GD. Abnormal fetal growth: intrauterine growth retardation, small for gestational age, large for gestational age. Pediatr Clin North Am 2004; 51: 639-54, viii.

25. Vohr BR, Lipsitt LP, Oh W. Somatic growth of children of diabetic mothers with reference to birth size. J Pediatr 1980; 97: 196-9.

26. Dabelea D, Knowler WC, Pettitt DJ. Effect of diabetes in pregnancy on offspring: follow-up research in the Pima Indians. J Matern Fetal Med 2000; 9: 83-8.

27. Sorensen HT, Sabroe S, Rothman KJ, et al. Relation between weight and length at birth and body mass index in young adulthood: cohort study. BMJ 1997; 315: 1137.

28. Kuzawa CW, Adair LS. A supply-demand model of fetal energy sufficiency predicts lipid profiles in male but not female Filipino adolescents. Eur J Clin Nutr 2004; 58: 438-48.

29. Therapontos C, Erskine L, Gardner ER, Figg WD, Vargesson N. Thalidomide induces limb defects by preventing angiogenic outgrowth during early limb formation. Proc Natl Acad Sci USA 2009; 106: 8573-8.

30. Meadows M. The power of Accutane. The benefits and risks of a breakthrough acne drug. FDA Consum 2001; 35: 18-23.

31. King JA. Perinatal stress and impairment of the stress response. Possible link to nonoptimal behavior. Ann NY Acad Sci 1996; 794: 104-12.

32. Weaver IC, Cervoni N, Champagne FA, et al. Epigenetic programming by maternal behavior. Nat Neurosci 2004; 7: 847-54.

33. Hager R, Cheverud JM, Wolf JB. Change in maternal environment induced by cross-fostering alters genetic and epigenetic effects on complex traits in mice. Proc Biol Sci 2009; 276: 2949-54.

34. Szyf M. Early life, the epigenome and human health. Acta Paediatr 2009; 98: 1082-4.

35. Mulder EJ, Robles de Medina PG, Huizink AC, et al. Prenatal maternal stress: effects on pregnancy and the (unborn) child. Early Hum Dev 2002; 70: 3-14.

36. Tarullo AR, Gunnar MR. Child maltreatment and the developing HPA axis. Horm Behav 2006; 50: 632-9.

37. de Weerth C, van Hees Y, Buitelaar JK. Prenatal maternal cortisol levels and infant behavior during the first 5 months. Early Hum Dev 2003; 74: 139-51.

38. Payne JL, Meltzer-Brody S. Antidepressant use during pregnancy: current controversies and treatment strategies. Clin Obstet Gynecol 2009; 52: 469-82.

39. Wisner KL, Sit DK, Hanusa BH, et al. Major depression and antidepressant treatment: impact on pregnancy and neonatal outcomes. Am J Psychiatry 2009; 166: 557-66.

40. Neonatal complications after intrauterine exposure to SSRI antidepressants. Prescrire Int 2004; 13: 103-4.

41. Hadjikhani N. Serotonin, pregnancy and increased autism prevalence: is there a link? Med Hypotheses 2010; 74: 880-3.

42. Smithells RW. Oral contraceptives and birth defects. Dev Med Child Neurol 1981; 23: 369-72.

43. Baskin LS, Himes K, Colborn T. Hypospadias and endocrine disruption: is there a connection? Environ Health Perspect 2001; 109: 1175-83.

44. Carmichael SL, Shaw GM, Laurent C, et al. Maternal progestin intake and risk of hypospadias. Arch Pediatr Adolesc Med 2005; 159: 957-62.

45. Raman-Wilms L, Tseng AL, Wighardt S, Einarson TR, Koren G. Fetal genital effects of first-trimester sex hormone exposure: a meta-analysis. Obstet Gynecol 1995; 85: 141-9. 
46. Waters TP, Schultz BA, Mercer BM, Catalano PM. Effect of 17alphahydroxyprogesterone caproate on glucose intolerance in pregnancy. Obstet Gynecol 2009; 114: 45-9.

47. Pinney SE, Simmons RA. Epigenetic mechanisms in the development of type 2 diabetes. Trends Endocrinol Metab 2010; 21: 223-9.

48. Brown RW, Chapman KE, Murad P, Edwards CR, Seckl JR. Purification of 11 beta-hydroxysteroid dehydrogenase type 2 from human placenta utilizing a novel affinity labelling technique. Biochem J 1996; 313(Pt 3): 997-1005.

49. McTernan CL, Draper N, Nicholson H, et al. Reduced placental 11betahydroxysteroid dehydrogenase type 2 mRNA levels in human pregnancies complicated by intrauterine growth restriction: an analysis of possible mechanisms. J Clin Endocrinol Metab 2001; 86: 4979-83.

50. Newnham JP, Jobe AH. Should we be prescribing repeated courses of antenatal corticosteroids? Semin Fetal Neonatal Med 2009; 14: 157-63.

51. Shinwell ES, Eventov-Friedman S. Impact of perinatal corticosteroids on neuromotor development and outcome: review of the literature and new meta-analysis. Semin Fetal Neonatal Med 2009; 14: 164-70.

52. Trautman PD, Meyer-Bahlburg HF, Postelnek J, New MI. Effects of early prenatal dexamethasone on the cognitive and behavioral development of young children: results of a pilot study. Psychoneuroendocrinology 1995; 20: 439-49.

53. French NP, Hagan R, Evans SF, Godfrey M, Newnham JP. Repeated antenatal corticosteroids: size at birth and subsequent development. Am J Obstet Gynecol 1999; 180(1 Pt 1): 114-21.

54. Drake AJ, Walker BR, Seckl JR. Intergenerational consequences of fetal programming by in utero exposure to glucocorticoids in rats. Am J Physiol Regul Integr Comp Physiol 2005; 288: R34-8.

55. Piffer RC, Garcia PC, Pereira OC. Adult partner preference and sexual behavior of male rats exposed prenatally to betamethasone. Physiol Behav 2009; 98: 163-7.

56. Herbst AL, Ulfelder H, Poskanzer DC. Adenocarcinoma of the vagina. Association of maternal stilbestrol therapy with tumor appearance in young women. N Engl J Med 1971; 284: 878-81.

57. Melnick S, Cole P, Anderson D, Herbst A. Rates and risks of diethylstilbestrol-related clear-cell adenocarcinoma of the vagina and cervix. An update. N Engl J Med 1987; 316: 514-6.

58. Missmer SA, Hankinson SE, Spiegelman D, et al. In utero exposures and the incidence of endometriosis. Fertil Steril 2004; 82: 1501-8.

59. Newbold R. Cellular and molecular effects of developmental exposure to diethylstilbestrol: implications for other environmental estrogens. Environ Health Perspect 1995; 103(Suppl 7): 83-7.

60. Swan SH. Intrauterine exposure to diethylstilbestrol: long-term effects in humans. Apmis 2000; 108: 793-804.

61. Kauffman RH, Binder GL, Gray PM, Adam E. Upper genital tract changes associated with exposure in utero to diethylstilbestrol. Am J Obstet Gynecol 1977; 128: 51-9.

62. Rennell CL. T-shaped uterus in diethylstilbestrol (DES) exposure. AJR Am J Roentgenol 1979; 132: 979-80.

63. Brouwers MM, Feitz WF, Roelofs LA, et al. Hypospadias: a transgenerational effect of diethylstilbestrol? Hum Reprod 2006; 21: 666-9.

64. Hernandez-Diaz S. Iatrogenic legacy from diethylstilbestrol exposure. Lancet 2002; 359: 1081-2.

65. Klip H, Verloop J, van Gool JD, et al. Hypospadias in sons of women exposed to diethylstilbestrol in utero: a cohort study. Lancet 2002; 359: 1102-7.

66. Pons JC, Papiernik E, Billon A, Hessabi M, Duyme M. Hypospadias in sons of women exposed to diethylstilbestrol in utero. Prenat Diagn 2005; 25: 418-19.

67. Newbold RR, Hanson RB, Jefferson WN, et al. Increased tumors but uncompromised fertility in the female descendants of mice exposed developmentally to diethylstilbestrol. Carcinogenesis 1998; 19: 1655-63.

68. McLachlan JA, Newbold RR, Shah HC, Hogan MD, Dixon RL. Reduced fertility in female mice exposed transplacentally to diethylstilbestrol (DES). Fertil Steril 1982; 38: 364-71.

69. Newbold RR, McLachlan JA. Vaginal adenosis and adenocarcinoma in mice exposed prenatally or neonatally to diethylstilbestrol. Cancer Res 1982; 42: 2003-11.

70. Block K, Kardana A, Igarashi P, Taylor HS. In utero diethylstilbestrol (DES) exposure alters Hox gene expression in the developing mullerian system. FASEB J 2000; 14: 1101-8.
71. Palmer JR, Wise LA, Hatch EE, et al. Prenatal diethylstilbestrol exposure and risk of breast cancer. Cancer Epidemiol Biomarkers Prev 2006; 15: 1509-14.

72. Xue F, Michels KB. Intrauterine factors and risk of breast cancer: a systematic review and meta-analysis of current evidence. Lancet Oncol 2007; 8: 1088-100.

73. Strohsnitter WC, Noller KL, Titus-Ernstoff L, et al. Breast cancer incidence in women prenatally exposed to maternal cigarette smoke. Epidemiology 2005; 16: 342-5.

74. Buck Louis GM, Hediger ML, Pena JB. Intrauterine exposures and risk of endometriosis. Hum Reprod 2007; 22: 3232-6.

75. Wald N, Cuckle H, Wu TS, George L. Maternal serum unconjugated oestriol and human chorionic gonadotrophin levels in twin pregnancies: implications for screening for Down's syndrome. Br J Obstet Gynaecol 1991; 98: 905-8.

76. Jasienska G, Ziomkiewicz A, Lipson SF, Thune I, Ellison PT. High ponderal index at birth predicts high estradiol levels in adult women. Am J Hum Biol 2006; 18: 133-40.

77. Barbieri RL, McShane PM, Ryan KJ. Constituents of cigarette smoke inhibit human granulosa cell aromatase. Fertil Steril 1986; 46: 232-6.

78. Kitawaki J, Inoue $\mathrm{S}$, Tamura $\mathrm{T}$, et al. Cigarette smoking during pregnancy lowers aromatase cytochrome P-450 in the human placenta. J Steroid Biochem Mol Biol 1993; 45: 485-91.

79. Garoff L, Seppala M. Toxemia of pregnancy: assessment of fetal distress by urinary estriol and circulating human placental lactogen and alphafetoprotein levels. Am J Obstet Gynecol 1976; 126: 1027-33.

80. Zeisler H, Jirecek S, Hohlagschwandtner M, et al. Concentrations of estrogens in patients with preeclampsia. Wien Klin Wochenschr 2002; 114: 458-61.

81. Pelch KE, Niebruegge BA, Beeman JM, Winkeler SM, Nagel SC. Endocrine Disruption in Mammals. In: Hormones and Reproduction of Vertebrates, Vol 5: Mammals (Norris DO, Lopez KH, eds). San Diego: Elsevier Publishing, 2010: 329-71.

82. Welshons WV, Nagel SC, vom Saal FS. Large effects from small exposures. III. Endocrine mechanisms mediating effects of bisphenol A at levels of human exposure. Endocrinology 2006; 147(6 Suppl): S56-69.

83. Welshons WV, Thayer KA, Judy BM, et al. Large effects from small exposures. I. Mechanisms for endocrine-disrupting chemicals with estrogenic activity. Environ Health Perspect 2003; 111: 994-1006.

84. Newbold RR, Jefferson WN, Padilla-Banks E, Haseman J. Developmental exposure to diethylstilbestrol (DES) alters uterine response to estrogens in prepubescent mice: low versus high dose effects. Reprod Toxicol 2004; 18: 399-406.

85. Swan SH. Prenatal phthalate exposure and anogenital distance in male infants. Environ Health Perspect 2006; 114: A88-9.

86. Diamanti-Kandarakis E, Bourguignon JP, Giudice LC, et al. Endocrinedisrupting chemicals: an Endocrine Society scientific statement. Endocr Rev 2009; 30: 293-342.

87. EPA. Final List of Initial Pesticide Active Ingredients and Pesticide Inert Ingredients to be Screened Under the Federal Food, Drug, and Cosmetic Act. Fed Regist 2009; 74: 17579-85.

88. Nagel SC, Hagelbarger JL, McDonnell DP. Development of an ER action indicator mouse for the study of estrogens, selective ER modulators (SERMs), and Xenobiotics. Endocrinology 2001; 142: 4721-8.

89. Nagel SC, vom Saal FS, Thayer KA, et al. Relative binding affinity-serum modified access (RBA-SMA) assay predicts the relative in vivo bioactivity of the xenoestrogens bisphenol A and octylphenol. Environ Health Perspect 1997; 105: 70-6.

90. Kirschner EM. Growth of top 50 chemicals slowed in 1995 from very high 1994 rate. Chem Eng News 1996: 16-22.

91. Brede C, Fjeldal P, Skjevrak I, Herikstad H. Increased migration levels of bisphenol A from polycarbonate baby bottles after dishwashing, boiling and brushing. Food Addit Contam 2003; 20: 684-9.

92. Sun Y, Wada M, Al-Dirbashi O, et al. High-performance liquid chromatography with peroxyoxalate chemiluminescence detection of bisphenol A migrated from polycarbonate baby bottles using 4-(4,5diphenyl-1H-imidazol-2-yl)benzoyl chloride as a label. J Chromatogr B Biomed Sci Appl 2000; 749: 49-56.

93. Munguia-Lopez EM, Peralta E, Gonzalez-Leon A, Vargas-Requena C, Soto-Valdez H. Migration of bisphenol A (BPA) from epoxy can coatings to jalapeno peppers and an acid food simulant. J Agric Food Chem 2002; 50: 7299-302. 
94. Kang JH, Kondo F. Bisphenol A migration from cans containing coffee and caffeine. Food Addit Contam 2002; 19: 886-90.

95. Brotons JA, Olea-Serrano MF, Villalobos M, Pedraza V, Olea N. Xenoestrogens released from lacquer coatings in food cans. Environ Health Perspect 1995; 103: 608-12.

96. Olea N, Pulgar R, Perez P, et al. Estrogenicity of resin-based composites and sealants used in dentistry. Environ Health Perspect 1996; 104: 298-305.

97. Howdeshell KL, Peterman PH, Judy BM, et al. Bisphenol A is released from used polycarbonate animal cages into water at room temperature. Environ Health Perspect 2003; 111: 1180-7.

98. Hunt PA, Koehler KE, Susiarjo M, et al. Bisphenol a exposure causes meiotic aneuploidy in the female mouse. Curr Biol 2003; 13: 546-53.

99. Kawagoshi Y, Fujita Y, Kishi I, Fukunaga I. Estrogenic chemicals and estrogenic activity in leachate from municipal waste landfill determined by yeast two-hybrid assay. J Environ Monit 2003; 5: 269-74.

100. Vandenberg LN, Hauser R, Marcus M, Olea N, Welshons WV. Human exposure to bisphenol A (BPA). Reprod Toxicol 2007; 24: 139-77.

101. Calafat AM, Ye X, Wong LY, Reidy JA, Needham LL. Exposure of the U. S. population to bisphenol A and 4-tertiary-octylphenol: 2003-2004. Environ Health Perspect 2008; 116: 39-44.

102. Maragou NC, Makri A, Lampi EN, Thomaidis NS, Koupparis MA. Migration of bisphenol A from polycarbonate baby bottles under real use conditions. Food Addit Contam 2008; 25: 373-83.

103. Gupta C. Reproductive malformation of the male offspring following maternal exposure to estrogenic chemicals. Proc Soc Exp Biol Med 2000; 224: 61-8.

104. Honma S, Suzuki A, Buchanan DL, et al. Low dose effect of in utero exposure to bisphenol A and diethylstilbestrol on female mouse reproduction. Reprod Toxicol 2002; 16: 117-22.

105. Howdeshell KL, Hotchkiss AK, Thayer KA, Vandenbergh JG, vom Saal FS. Exposure to bisphenol A advances puberty. Nature 1999; 401: 763-4.

106. Kubo K, Arai O, Omura M, et al. Low dose effects of bisphenol A on sexual differentiation of the brain and behavior in rats. Neurosci Res 2003; 45: 345-56.

107. Markey CM, Luque EH, Munoz De Toro M, Sonnenschein C, Soto AM. In utero exposure to bisphenol $\mathrm{A}$ alters the development and tissue organization of the mouse mammary gland. Biol Reprod 2001; 65: 1215-23.

108. Nagel SC, vom Saal FS, Thayer KA, et al. Relative binding affinity-serum modified access (RBA-SMA) assay predicts the relative in vivo bioactivity of the xenoestrogens bisphenol A and octylphenol. Environ Health Perspect 1997; 105: 70-6.

109. Palanza PL, Howdeshell KL, Parmigiani S, vom Saal FS. Exposure to a low dose of bisphenol A during fetal life or in adulthood alters maternal behavior in mice. Environ Health Perspect 2002; 110(Suppl 3): 415-22.

110. Schonfelder G, Flick B, Mayr E, et al. In utero exposure to low doses of bisphenol A lead to long-term deleterious effects in the vagina. Neoplasia 2002; 4: 98-102.

111. Steinmetz R, Brown NG, Allen DL, Bigsby RM, Ben-Jonathan N. The environmental estrogen bisphenol A stimulates prolactin release in vitro and in vivo. Endocrinology 1997; 138: 1780-6.

112. Steinmetz R, Mitchner NA, Grant A, et al. The xenoestrogen bisphenol A induces growth, differentiation, and c-fos gene expression in the female reproductive tract. Endocrinology 1998; 139: 2741-7.

113. Takai Y, Tsutsumi O, Ikezuki Y, et al. Preimplantation exposure to bisphenol A advances postnatal development. Reprod Toxicol 2001; 15: $71-4$.

114. vom Saal FS, Cooke PS, Buchanan DL, et al. A physiologically based approach to the study of bisphenol A and other estrogenic chemicals on the size of reproductive organs, daily sperm production, and behavior. Toxicol Ind Health 1998; 14: 239-60.

115. Ho SM, Tang WY, Belmonte de Frausto J, Prins GS. Developmental exposure to estradiol and bisphenol a increases susceptibility to prostate carcinogenesis and epigenetically regulates phosphodiesterase type 4 variant 4. Cancer Res 2006; 66: 5624-32.

116. Cerel-Suhl SL, Yeager BF. Update on oral contraceptive pills. Am Fam Physician 1999; 60: 2073-84

117. Thayer KA, Ruhlen RL, Howdeshell KL, et al. Altered prostate growth and daily sperm production in male mice exposed prenatally to subclinical doses of 17alpha-ethinyl oestradiol. Hum Reprod 2001; 16: $988-96$
118. Chimchirian RF, Suri RP, Fu H. Free synthetic and natural estrogen hormones in influent and effluent of three municipal wastewater treatment plants. Water Environ Res 2007; 79: 969-74.

119. Setchell KD, Zimmer-Nechemias L, Cai J, Heubi JE. Exposure of infants to phyto-oestrogens from soy-based infant formula. Lancet 1997; 350: 23-7.

120. Newbold RR, Banks EP, Bullock B, Jefferson WN. Uterine adenocarcinoma in mice treated neonatally with genistein. Cancer Res 2001; 61: 4325-8.

121. Jefferson WN, Padilla-Banks E, Newbold RR. Adverse effects on female development and reproduction in CD-1 mice following neonatal exposure to the phytoestrogen genistein at environmentally relevant doses. Biol Reprod 2005; 73: 798-806.

122. Jefferson WN, Couse JF, Padilla-Banks E, Korach KS, Newbold RR. Neonatal exposure to genistein induces estrogen receptor (ER)alpha expression and multioocyte follicles in the maturing mouse ovary: evidence for ERbeta-mediated and nonestrogenic actions. Biol Reprod 2002; 67: 1285-96.

123. Jefferson W, Newbold R, Padilla-Banks E, Pepling M. Neonatal genistein treatment alters ovarian differentiation in the mouse: inhibition of oocyte nest breakdown and increased oocyte survival. Biol Reprod 2006; 74: $161-8$.

124. Mahalingaiah S, Meeker JD, Pearson KR, et al. Temporal variability and predictors of urinary bisphenol A concentrations in men and women. Environ Health Perspect 2008; 116: 173-8.

125. Schonfelder G, Wittfoht W, Hopp H, et al. Parent bisphenol A accumulation in the human maternal-fetal-placental unit. Environ Health Perspect 2002; 110: A703-7.

126. Ikezuki Y, Tsutsumi O, Takai Y, Kamei Y, Taketani Y. Determination of bisphenol A concentrations in human biological fluids reveals significant early prenatal exposure. Hum Reprod 2002; 17: 2839-41.

127. Kuroda N, Kinoshita Y, Sun Y, et al. Measurement of bisphenol A levels in human blood serum and ascitic fluid by HPLC using a fluorescent labeling reagent. J Pharm Biomed Anal 2003; 30: 1743-9.

128. Lopez-Espinosa MJ, Silva E, Granada A, et al. Assessment of the total effective xenoestrogen burden in extracts of human placentas. Biomarkers 2009; 14: 271-7.

129. Anderson SE, Dallal GE, Must A. Relative weight and race influence average age at menarche: results from two nationally representative surveys of US girls studied 25 years apart. Pediatrics 2003; 111(4 Pt 1): $844-50$.

130. Freedman DS, Khan LK, Serdula MK, et al. Relation of age at menarche to race, time period, and anthropometric dimensions: the Bogalusa Heart Study. Pediatrics 2002; 110: e43.

131. Herman-Giddens ME, Slora EJ, Wasserman RC, et al. Secondary sexual characteristics and menses in young girls seen in office practice: a study from the Pediatric Research in Office Settings network. Pediatrics 1997; 99: 505-12.

132. Euling SY, Herman-Giddens ME, Lee PA, et al. Examination of US puberty-timing data from 1940 to 1994 for secular trends: panel findings. Pediatrics 2008; 121(Suppl 3): S172-91.

133. Golub MS, Collman GW, Foster PM, et al. Public health implications of altered puberty timing. Pediatrics 2008; 121(Suppl 3): S218-30.

134. Thigpen JE, Setchell KD, Padilla-Banks E, et al. Variations in phytoestrogen content between different mill dates of the same diet produces significant differences in the time of vaginal opening in CD-1 mice and F344 rats but not in CD Sprague-Dawley rats. Environ Health Perspect 2007; 115: 1717-26.

135. Ryan BC, Vandenbergh JG. Developmental exposure to environmental estrogens alters anxiety and spatial memory in female mice. Horm Behav 2006; 50: 85-93.

136. Ashby J, Tinwell H, Lefevre PA, et al. Normal sexual development of rats exposed to butyl benzyl phthalate from conception to weaning. Regul Toxicol Pharmacol 1997; 26(1 Pt 1): 102-18.

137. Rasier G, Parent AS, Gerard A, Lebrethon MC, Bourguignon JP. Early maturation of gonadotropin-releasing hormone secretion and sexual precocity after exposure of infant female rats to estradiol or dichlorodiphenyltrichloroethane. Biol Reprod 2007; 77: 734-42.

138. Nikaido Y, Yoshizawa K, Danbara N, et al. Effects of maternal xenoestrogen exposure on development of the reproductive tract and mammary gland in female CD-1 mouse offspring. Reprod Toxicol 2004; 18: $803-11$ 
139. Blanck HM, Marcus M, Hertzberg V, et al. Determinants of polybrominated biphenyl serum decay among women in the Michigan PBB cohort. Environ Health Perspect 2000; 108: 147-52.

140. Blanck HM, Marcus M, Tolbert PE, et al. Age at menarche and tanner stage in girls exposed in utero and postnatally to polybrominated biphenyl. Epidemiology 2000; 11: 641-7.

141. Colon I, Caro D, Bourdony CJ, Rosario O. Identification of phthalate esters in the serum of young Puerto Rican girls with premature breast development. Environ Health Perspect 2000; 108: 895-900.

142. Gotz F, Thieme S, Dorner G. Female infertility-effect of perinatal xenoestrogen exposure on reproductive functions in animals and humans. Folia Histochem Cytobiol 2001; 39(Suppl 2): 40-3.

143. Tuohy PG. Soy infant formula and phytoestrogens. J Paediatr Child Health 2003; 39: 401-5.

144. Overview: Breast Cancer How Many Women Get Breast Cancer?: American Cancer Society; 2009. [Available from: http://www.cancer. org/docroot/CRI/content/CRI_2__1X_How_many_people_get_breast_ cancer_5.asp]. [Available from: http://www.cancer.org/docroot/CRI/ content/CRI_2_2_1X_How_many_people_get_breast_cancer_5.asp] Accessed June 23, 2009].

145. Glass AG, Lacey JV Jr, Carreon JD, Hoover RN. Breast cancer incidence, 1980-2006: combined roles of menopausal hormone therapy, screening mammography, and estrogen receptor status. J Natl Cancer Inst 2007; 99: 1152-61.

146. Kurian AW, Clarke CA, Carlson RW. The decline in breast cancer incidence: real or imaginary? Curr Oncol Rep 2009; 11: 21-8.

147. Leung GM, Thach TQ, Lam TH, et al. Trends in breast cancer incidence in Hong Kong between 1973 and 1999: an age-period-cohort analysis. Br J Cancer 2002; 87: 982-8.

148. Ravdin PM, Cronin KA, Howlader N, et al. The decrease in breast-cancer incidence in 2003 in the United States. N Engl J Med 2007; 356: 1670-4.

149. Boylan ES, Calhoon RE. Mammary tumorigenesis in the rat following prenatal exposure to diethylstilbestrol and postnatal treatment with 7,12-dimethylbenz[a]anthracene. J Toxicol Environ Health 1979; 5: 1059-71.

150. Rothschild TC, Boylan ES, Calhoon RE, Vonderhaar BK. Transplacental effects of diethylstilbestrol on mammary development and tumorigenesis in female ACI rats. Cancer Res 1987; 47: 4508-16.

151. Munoz-de-Toro M, Markey CM, Wadia PR, et al. Perinatal exposure to bisphenol-A alters peripubertal mammary gland development in mice. Endocrinology 2005; 146: 4138-47.

152. Moral R, Wang R, Russo IH, et al. Effect of prenatal exposure to the endocrine disruptor bisphenol A on mammary gland morphology and gene expression signature. J Endocrinol 2008; 196: 101-12.

153. Vandenberg LN, Maffini MV, Wadia PR, et al. Exposure to environmentally relevant doses of the xenoestrogen bisphenol-A alters development of the fetal mouse mammary gland. Endocrinology 2007; 148: 116-27.

154. Durando M, Kass L, Piva J, et al. Prenatal bisphenol A exposure induces preneoplastic lesions in the mammary gland in Wistar rats. Environ Health Perspect 2007; 115: 80-6.

155. Hilakivi-Clarke L, Cho E, Onojafe I, Raygada M, Clarke R. Maternal exposure to genistein during pregnancy increases carcinogen-induced mammary tumorigenesis in female rat offspring. Oncol Rep 1999; 6: 1089-95.

156. Padilla-Banks E, Jefferson WN, Newbold RR. Neonatal exposure to the phytoestrogen genistein alters mammary gland growth and developmental programming of hormone receptor levels. Endocrinology 2006; 147: 4871-82.

157. Fenton SE, Hamm JT, Birnbaum LS, Youngblood GL. Persistent abnormalities in the rat mammary gland following gestational and lactational exposure to 2,3,7,8-tetrachlorodibenzo-p-dioxin (TCDD). Toxicol Sci 2002; 67: 63-74.

158. Jenkins S, Rowell C, Wang J, Lamartiniere CA. Prenatal TCDD exposure predisposes for mammary cancer in rats. Reprod Toxicol 2007; 23: 391-6.

159. Brown NM, Manzolillo PA, Zhang JX, Wang J, Lamartiniere CA. Prenatal TCDD and predisposition to mammary cancer in the rat. Carcinogenesis 1998; 19: 1623-9.

160. Lewis BC, Hudgins S, Lewis A, et al. In utero and lactational treatment with 2,3,7,8-tetrachlorodibenzo-p-dioxin impairs mammary gland differentiation but does not block the response to exogenous estrogen in the postpubertal female rat. Toxicol Sci 2001; 62: 46-53.
161. Brown NM, Lamartiniere CA. Xenoestrogens alter mammary gland differentiation and cell proliferation in the rat. Environ Health Perspect 1995; 103: 708-13.

162. Panagiotopoulou K, Katsouyanni K, Petridou E, et al. Maternal age, parity, and pregnancy estrogens. Cancer Causes Control 1990; 1: 119-24.

163. Moller H, Skakkebaek NE. Testicular cancer and cryptorchidism in relation to prenatal factors: case-control studies in Denmark. Cancer Causes Control 1997; 8: 904-12.

164. Mongraw-Chaffin ML, Cohn BA, Cohen RD, Christianson RE. Maternal smoking, alcohol consumption, and caffeine consumption during pregnancy in relation to a son's risk of persistent cryptorchidism: a prospective study in the Child Health and Development Studies cohort, 1959-1967. Am J Epidemiol 2008; 167: 257-61.

165. North K, Golding J. A maternal vegetarian diet in pregnancy is associated with hypospadias. The ALSPAC Study Team. Avon Longitudinal Study of Pregnancy and Childhood. BJU Int 2000; 85: 107-13.

166. Newbold RR, Padilla-Banks E, Snyder RJ, Phillips TM, Jefferson WN. Developmental exposure to endocrine disruptors and the obesity epidemic. Reprod Toxicol 2007; 23: 290-6.

167. Rakkestad KE, Dye CJ, Yttri KE, et al. Phthalate levels in Norwegian indoor air related to particle size fraction. J Environ Monit 2007; 9: 1419-25.

168. Paulozzi LJ. International trends in rates of hypospadias and cryptorchidism. Environ Health Perspect 1999; 107: 297-302.

169. Paulozzi LJ, Erickson JD, Jackson RJ. Hypospadias trends in two US surveillance systems. Pediatrics 1997; 100: 831-4.

170. Swan SH, Brazil C, Drobnis EZ, et al. Geographic differences in semen quality of fertile U.S. males. Environ Health Perspect 2003; 111: 414-20.

171. Swan SH, Elkin EP. Declining semen quality: can the past inform the present? Bioessays 1999; 21: 614-21.

172. Swan SH, Elkin EP, Fenster L. Have sperm densities declined? A reanalysis of global trend data. Environ Health Perspect 1997; 105: 1228-32.

173. Swan SH, Elkin EP, Fenster L. The question of declining sperm density revisited: an analysis of 101 studies published 1934-1996. Environ Health Perspect 2000; 108: 961-6.

174. Huyghe E, Matsuda T, Thonneau P. Increasing incidence of testicular cancer worldwide: a review. J Urol 2003; 170: 5-11.

175. Sharpe RM, Skakkebaek NE. Testicular dysgenesis syndrome: mechanistic insights and potential new downstream effects. Fertil Steril 2008; 89(2 Suppl): e33-8.

176. Gray LE Jr, Wolf C, Lambright C, et al. Administration of potentially antiandrogenic pesticides (procymidone, linuron, iprodione, chlozolinate, p,p'-DDE, and ketoconazole) and toxic substances (dibutyl- and diethylhexyl phthalate, PCB 169, and ethane dimethane sulphonate) during sexual differentiation produces diverse profiles of reproductive malformations in the male rat. Toxicol Ind Health 1999; 15: 94-118.

177. Salazar-Martinez E, Romano-Riquer P, Yanez-Marquez E, Longnecker MP, Hernandez-Avila M. Anogenital distance in human male and female newborns: a descriptive, cross-sectional study. Environ Health 2004; 3: 8 .

178. Swan SH, Main KM, Liu F, et al. Decrease in anogenital distance among male infants with prenatal phthalate exposure. Environ Health Perspect 2005; 113: 1056-61.

179. McGlynn KA, Quraishi SM, Graubard BI, et al. Persistent organochlorine pesticides and risk of testicular germ cell tumors. J Natl Cancer Inst 2008; 100: 663-71.

180. Hardell L, van Bavel B, Lindstrom G, et al. Increased concentrations of polychlorinated biphenyls, hexachlorobenzene, and chlordanes in mothers of men with testicular cancer. Environ Health Perspect 2003; 111: 930-4.

181. Hardell L, Van Bavel B, Lindstrom G, et al. Concentrations of polychlorinated biphenyls in blood and the risk for testicular cancer. Int J Androl 2004; 27: 282-90.

182. Llanes Gonzalez L, Lujan Galan M, Rodriguez Garcia N, Garcia Tello A, Berenguer Sanchez A. Trends in the incidence of testicular germ cell cancer in a 300.000 inhabitants Spanish population (1991-2005). Actas Urol Esp 2008; 32: 691-5.

183. Baade P, Carriere P, Fritschi L. Trends in testicular germ cell cancer incidence in Australia. Cancer Causes Control 2008; 19: 1043-9.

184. Adami HO, Bergstrom R, Mohner M, et al. Testicular cancer in nine northern European countries. Int J Cancer 1994; 59: 33-8. 
185. Bergstrom R, Adami HO, Mohner M, et al. Increase in testicular cancer incidence in six European countries: a birth cohort phenomenon. J Natl Cancer Inst 1996; 88: 727-33.

186. Bhatia R, Shiau R, Petreas M, et al. Organochlorine pesticides and male genital anomalies in the child health and development studies. Environ Health Perspect 2005; 113: 220-4.

187. Damgaard IN, Skakkebaek NE, Toppari J, et al. Persistent pesticides in human breast milk and cryptorchidism. Environ Health Perspect 2006; 114: 1133-8.

188. Main KM, Kiviranta H, Virtanen HE, et al. Flame retardants in placenta and breast milk and cryptorchidism in newborn boys. Environ Health Perspect 2007; 115: 1519-26.

189. Main KM, Mortensen GK, Kaleva MM, et al. Human breast milk contamination with phthalates and alterations of endogenous reproductive hormones in infants three months of age. Environ Health Perspect 2006; 114: 270-6.

190. Dolk H, Vrijheid M, Armstrong B, et al. Risk of congenital anomalies near hazardous-waste landfill sites in Europe: the EUROHAZCON study. Lancet 1998; 352: 423-7.

191. Carbone P, Giordano F, Nori F, et al. Cryptorchidism and hypospadias in the Sicilian district of Ragusa and the use of pesticides. Reprod Toxicol 2006; 22: 8-12.

192. Skakkebaek NE, Rajpert-De Meyts E, Main KM. Testicular dysgenesis syndrome: an increasingly common developmental disorder with environmental aspects. Hum Reprod 2001; 16: 972-8.

193. Wilson VS, Blystone CR, Hotchkiss AK, Rider CV, Gray LE Jr. Diverse mechanisms of anti-androgen action: impact on male rat reproductive tract development. Int J Androl 2008; 31: 178-87.

194. Swan SH, Kruse RL, Liu F, et al. Semen quality in relation to biomarkers of pesticide exposure. Environ Health Perspect 2003; 111: 1478-84.

195. Swan SH, Liu F, Overstreet JW, Brazil C, Skakkebaek NE. Semen quality of fertile US males in relation to their mothers' beef consumption during pregnancy. Hum Reprod 2007; 22: 1497-1502.

196. Howdeshell KL. A model of the development of the brain as a construct of the thyroid system. Environ Health Perspect 2002; 110(Suppl 3): 337-48.

197. Klein RZ, Sargent JD, Larsen PR, et al. Relation of severity of maternal hypothyroidism to cognitive development of offspring. J Med Screen 2001; 8: 18-20.

198. Budtz-Jorgensen E, Keiding N, Grandjean P, White RF. Methylmercury neurotoxicity independent of PCB exposure. Environ Health Perspect 1999; 107: A236-7.
199. Chen YC, Guo YL, Hsu CC. Cognitive development of children prenatally exposed to polychlorinated biphenyls ( $\mathrm{Yu}-\mathrm{Cheng}$ children) and their siblings. J Formos Med Assoc 1992; 91: 704-7.

200. Darvill T, Lonky E, Reihman J, Stewart P, Pagano J. Prenatal exposure to PCBs and infant performance on the fagan test of infant intelligence. Neurotoxicology 2000; 21: 1029-38.

201. Grandjean P, Weihe P, Burse VW, et al. Neurobehavioral deficits associated with PCB in 7-year-old children prenatally exposed to seafood neurotoxicants. Neurotoxicol Teratol 2001; 23: 305-17.

202. Jacobson JL, Jacobson SW. Dose-response in perinatal exposure to polychlorinated biphenyls (PCBs): the Michigan and North Carolina cohort studies. Toxicol Ind Health 1996; 12: 435-45.

203. Jacobson JL, Jacobson SW, Humphrey HE. Effects of in utero exposure to polychlorinated biphenyls and related contaminants on cognitive functioning in young children. J Pediatr 1990; 116: 38-45.

204. Jacobson SW, Fein GG, Jacobson JL, Schwartz PM, Dowler JK. The effect of intrauterine PCB exposure on visual recognition memory. Child Dev 1985; 56: 853-60.

205. Patandin S, Lanting CI, Mulder PG, et al. Effects of environmental exposure to polychlorinated biphenyls and dioxins on cognitive abilities in Dutch children at 42 months of age. J Pediatr 1999; 134: 33-41.

206. Stewart PW, Reihman J, Lonky EI, Darvill TJ, Pagano J. Cognitive development in preschool children prenatally exposed to PCBs and MeHg. Neurotoxicol Teratol 2003; 25: 11-22.

207. Walkowiak J, Wiener JA, Fastabend A, et al. Environmental exposure to polychlorinated biphenyls and quality of the home environment: effects on psychodevelopment in early childhood. Lancet 2001; 358: 1602-7.

208. Winneke G, Bucholski A, Heinzow B, et al. Developmental neurotoxicity of polychlorinated biphenyls (PCBS): cognitive and psychomotor functions in 7-month old children. Toxicol Lett 1998; 102-103: 423-8.

209. Jacobson JL, Jacobson SW, Padgett R, Brumitt G, Billings R. Effects of prenatal PCB exposure on cognitive processing efficiency and sustained attention. Dev Psychol 1992; 28: 297-306.

210. Schantz SL, Widholm JJ, Rice DC. Effects of PCB exposure on neuropsychological function in children. Environ Health Perspect 2003; 111: 357-576.

211. Reik W. Stability and flexibility of epigenetic gene regulation in mammalian development. Nature 2007; 447: 425-32.

212. Reik W, Dean W, Walter J. Epigenetic reprogramming in mammalian development. Science 2001; 293: 1089-93. 


\section{Assessment of fetal genetic disorders Teresa Martino, J. Pratt Rossiter, and Karin J. Blakemore}

\section{INTRODUCTION}

Since the first clinical use of fetal sex determination by amniocentesis in 1967, there has been a virtual explosion in both the availability and the application of prenatal diagnosis in the management of pregnancy. The rapid expansion of this field has been a result of numerous advances. The quality of ultrasonography has vastly improved, allowing detection of both gross and increasingly more subtle congenital anomalies and providing accurate guidance for diagnostic procedures including amniocentesis, chorionic villus sampling (CVS), fetal blood sampling, and fetal biopsy. The safety of these invasive methods improved due to higher resolution ultrasonography as well as progress in the development of the techniques themselves. Simultaneously, the "revolution" in molecular genetics has resulted in the identification of the genes and mutations responsible for many inherited disorders. This knowledge can, in many cases, be applied directly to prenatal diagnosis for pregnancies at risk for specific genetic diseases. All of these factors have contributed to the need for maternal-fetal medicine specialists to become experts in the field of prenatal diagnosis.

This chapter reviews the most common indications for prenatal diagnosis and the invasive methods currently used. The status of preimplantation diagnosis and of prenatal diagnosis using fetal cells or fetal DNA isolated from the maternal circulation is reviewed.

\section{INDICATIONS FOR PRENATAL DIAGNOSIS}

The most common reasons for referral for genetic counseling and prenatal diagnosis are summarized in Table 1. Currently, invasive prenatal testing is offered when the likelihood of detecting an abnormality exceeds the statistical risk of pregnancy loss related to the diagnostic procedure. Ethical issues regarding the somewhat arbitrary determination of when to offer prenatal diagnosis were recently considered and universal access to genetic diagnosis proposed (1); however, this practice is presently far from being adopted.

\section{Maternal Age}

The relationship between advancing maternal age and increasing incidence of chromosomal abnormalities due to meiotic nondisjunction is well established (2). The steadily rising risk with maternal age applies not only to trisomy 21 (Down syndrome), but to any trisomy or monosomy. The chance for a 35-year-old woman to have a live-born infant with a chromosomal abnormality is 1 in 192 (3). This risk approximates the stated 1 in 200 risk of procedure-related loss with second-trimester amniocentesis; thus, women are offered prenatal diagnosis for chromosome abnormalities when they will be 35 years of age or older at the time of delivery. Many chromosomally abnormal fetuses are lost during the course of pregnancy; therefore, the likelihood of having an aneuploid fetus at the time of amniocentesis (usually 16-20 weeks of gestation) is greater than the risk at term (4). For example, women who will be 33 years old at the time of delivery have a risk of 1 in 200 of having a chromosomally abnormal fetus at the time of amniocentesis (4). For this reason, some practitioners advocate offering prenatal diagnosis to women who are 33 years of age or older, but no consensus has yet been reached.

\section{Chromosomal Abnormalities}

Couples with a previous trisomic child are thought to have approximately a $1 \%$ risk of recurrence (5) and are thus offered prenatal diagnosis. Siblings and second-degree relatives to an individual with Down syndrome may have a slightly increased risk for having a similarly affected child (6), though most studies have not demonstrated an increased incidence of Down syndrome in second- or third-degree relatives $(7,8)$. Many people with an affected relative have a high degree of anxiety, which may justify prenatal testing.

Translocations and other chromosomal structural rearrangements can predispose to chromosomally abnormal progeny (see chapter 43 for a description of translocations). Couples in whom one partner carries a balanced reciprocal translocation are at high risk for recurrent pregnancy loss. At the time of prenatal diagnosis, approximately $10 \%$ to $12 \%$ of progeny have an unbalanced chromosome complement $(9,10)$, while this risk declines to $6 \%$ of live-born offspring because of spontaneous abortion (11). Progeny of Robertsonian translocation carriers are at risk for trisomy or monosomy of the involved chromosomes, with the risk dependent on the specific chromosomes participating in the translocation $(9,10)$. An extreme example is that of a Robertsonian 21/21 translocation carrier, whose progeny would all be predicted to be either trisomic (Down syndrome) or monosomic (lethal) for chromosome 21. Couples with a history of multiple pregnancy losses should be offered karyotypes to determine whether one member is a translocation carrier. Individuals known to be translocation carriers should be offered prenatal cytogenetic testing, as the fetal karyotype result may be normal, balanced, or unbalanced, with the proportion of each karyotype result varying, dependent on the specific translocation (10).

\section{Multiple-Marker Screening in the Second Trimester}

The history of multiple-marker screening began in 1972 when Brock and Sutcliffe described the association of fetal anencephaly and spina bifida with elevations in maternal serum alpha-fetoprotein (MSAFP) levels (12). Subsequently, studies in the United States (13) and the United Kingdom (14) proposed the establishment of screening programs to detect pregnancies complicated by fetal open neural tube defects. During the 1980s, such screening programs became widespread in the United States, where the incidence of neural tube 
Table 1 Indications for Prenatal Diagnosis

- Maternal age 33 or more at delivery

- Family history of chromosomal abnormality

- Parental translocation carrier

- Abnormal MSAFP or multiple-marker screening

- Family history of neural tube defect

- Single-gene disorder-family history or carrier detected by population screening

- Congenital malformation detected by ultrasonography

- Maternal desire

Abbreviation: MSAFP, maternal serum alpha-fetoprotein.

defects is 1 to 2 per 1000 live births (15). Elevated MSAFP values are associated not only with open neural tube defects, but also with other fetal abnormalities involving a break in skin integrity such as ventral wall defects (omphalocele, gastroschisis), teratoma, bladder exstrophy, or aplasia cutis. Numerous other fetal and placental abnormalities have been reported in association with elevated MSAFP levels $(16,17)$. In addition, elevations in MSAFP unexplained by sonographic findings are associated with an increased risk of pregnancy complications, fetal growth restriction, and fetal death $(17,18)$. The American College of Obstetricians and Gynecologists has proposed an algorithm for the evaluation of pregnancies with elevated MSAFP levels including ultrasonography, amniocentesis for amniotic fluid AFP, and acetylcholinesterase if the amniotic fluid AFP is elevated (15). Since MSAFP screening became the standard of care in the United States, elevated MSAFP has become a common indication for referral for prenatal diagnosis.

The clinical utility of MSAFP screening expanded substantially when an association was found between low MSAFP values and fetal chromosomal abnormalities (19,20). Using a combination of maternal age and MSAFP to identify pregnancies at high risk, approximately 20\% of Down syndrome pregnancies in women younger than 35 years were detected in prospective trials $(21,22)$. Subsequently, Down syndrome pregnancies were found to be associated with elevated maternal serum concentrations of human chorionic gonadotropin (hCG) (23) and decreased unconjugated estriol $\left(\mathrm{uE}_{3}\right)(24)$ as compared with normal controls. In a large prospective trial using maternal age in combination with maternal serum levels of AFP, hCG, and $\mathrm{uE}_{3}$ to assign a risk of fetal Down syndrome of $>1: 190,58 \%$ of Down syndrome pregnancies were identified, and 38 amniocenteses were performed for each confirmed case of Down syndrome (25). This trial included women ranging in age from 16 to 41 years. Since this publication in 1992, multiple-marker or "triple" screening came into more widespread use. Further applications of the triple screen have followed, with the recognition of a characteristic pattern of low values for AFP, hCG, and $\mathrm{uE}_{3}$ in association with trisomy $18(26,27)$, and a pattern similar to that for an increased risk for Down syndrome (low AFP, low $\mathrm{uE}_{3}$, high hCG) in association with Turner syndrome $(45, \mathrm{X})(28,29)$. A newer maternal serum marker, dimeric inhibin-A, also measured in the second trimester, is approximately 2.06 times the median value in fetuses affected with Down syndrome (30). The detection rate for Down syndrome with the addition of inhibin-A is approximately $80 \%$. Serum screening in the second trimester with the inclusion of
inhibin-A may be called the "quadruple screen." It is important to note that inhibin-A is not used in the calculation of trisomy 18.

Multiple-marker screening allows the detection of pregnancies that would otherwise not be considered at increased risk for either neural tube defects or chromosomal aneuploidy. Older women may opt for multiple-marker screening to readjust their age-related risk of fetal Down syndrome and trisomies 18 and 13. A recent guideline from the American College of Obstetricians and Gynecologists suggests that all women be offered aneuploidy screening before 20 weeks of gestation, regardless of maternal age (31).

One of the main disadvantages of multiple-marker screening was its timing in the second trimester. An abnormal screen, typically obtained at 16-18 weeks of gestation, sets into motion a series of events including repeat screening in some cases (if AFP is high, not for increased risk of aneuploidy), ultrasonography to confirm dating, genetic counseling, and the offer to have amniocentesis for chromosomal analysis and amniotic fluid AFP measurement. At the completion of this evaluation, most patients are beyond 18 weeks and many are beyond 20 weeks of gestation. By this time, many pregnant women have begun to feel fetal movement and are readily recognized as pregnant by casual observers. In addition, the options for termination are induction methods (prostaglandin and/or urea instillation, prostaglandin suppositories) or dilatation and evacuation at an advanced gestational age. Either method is associated with significantly greater morbidity than firsttrimester dilatation and curettage, as well as increased medical costs. For these reasons, the ability to perform screening in the first trimester provides a significant benefit over secondtrimester screening.

\section{Multiple-Marker Screening in the First Trimester}

Multiple biochemical marker screening may also be offered in the first trimester. Investigations into the first-trimester biochemical maternal serum marker screening began in the 1980s. Two useful markers emerged, free beta human chorionic gonadotropin ( $\mathrm{f} \beta \mathrm{hCG}$ ) and pregnancy-associated plasma protein A (PAPP-A). While maximum detection rates for each of these markers are at 14 completed weeks for $\mathrm{f} \beta \mathrm{hCG}$ and 9 completed weeks for PAPP-A $(32,33)$, used together (along with maternal age) at 11 to 13 weeks of gestation yields a detection rate for Down syndrome of approximately $67 \%$ with a false positive rate set at $5 \%$. Better detection rates are obtained by incorporating a sonographically acquired measurement of the sonolucent area just under the skin line on the posterior fetal neck, the nuchal translucency (NT). Using only first-trimester NT screening and maternal age (without biochemistry) has yielded a Down syndrome detection rate of $73 \%$ at the same $5 \%$ false positive rate. Today, firsttrimester "combined" screening employs maternal age, maternal serum analytes, and an NT, with or without the presence or absence of the fetal nasal bone to arrive at an individual's risk for fetal Down syndrome. In one large prospective study, in women $\geq 35$ years old, the detection rate was $89.8 \%$ for fetuses with trisomy 21 with a false positive rate of $15.25 \%$, while $100 \%$ of fetuses with trisomy 18 were detected (34). For those women $<35$ years old, first-trimester "combined" screening had a detection rate for trisomy 21 of 
$60 \%$ with a false positive rate of $5 \%$. In pregnancies complicated by fetal trisomies 13 and 18, free $\beta$ hCG and PAPP-A are decreased, while those pregnancies complicated by fetal sex chromosome abnormalities demonstrate a normal free $\beta$ hCG and PAPP-A is low (35-37).

Measurements of the NT may be taken from 10 to 14 weeks of gestation. In a prospective screening study of 1273 women mostly of advanced maternal age, Nicolaides et al., observed an NT of $\geq 3 \mathrm{~mm}$ in $86 \%$ of the trisomic and $4 \%$ to $5 \%$ of the normal fetuses. An NT of $\geq 3 \mathrm{~mm}$ was associated with a 12 -fold increase in fetal aneuploidy and was independent of maternal age (38). This study was confirmed by Chitty et al. in a group of patients who were not considered at risk for fetal aneuploidy (39). As the gestational age increases, so does the NT. Gestational age-specific multiples of the median have been derived and are used commonly.

There are several other terms in use today that describe various combinations of first-trimester screening with secondtrimester aneuploidy screening, including "integrated" screening and "sequential" screening. The "combined" firsttrimester screen includes an NT, PAPP-A, and a fBhCG. "Integrated" and "sequential" screenings both combine elements of first- and second-trimester screening and differ when the first-trimester screening results are reported. In an "integrated" test, an NT is obtained along with a PAPP-A plus a quadruple screen. The results are provided to the patient after all tests are completed. In a "stepwise sequential" test, the combined first-trimester screen is used plus a quadruple screen with results given after each test. In a "contingent sequential" test, the combined first-trimester screen is performed, and only those with a risk between 1:30 and 1:1500 have a quadruple screen (40). Not all centers offer, and not all women opt for "combined" first-trimester screening.

\section{Family History of Single-Gene Disorders}

A family history of a single-gene disorder is another common indication for referral for prenatal genetic counseling. Fetal risk assessment is dependent on many factors, such as the distance of the relationship between the affected family member and the individual seeking counseling, as well as the frequency of the disease gene in the population. Couples with a previously affected child may be obviously at higher risk for recurrence, while the risk declines with increased distance from the affected relative $\left(1^{\circ}>2^{\circ}>3^{\circ}\right)$. For example, parents of a child with an autosomal recessive disorder have a $25 \%$ risk of recurrence with each pregnancy. By contrast, the offspring to the siblings of an affected individual have a risk calculated as follows: the risk of the unaffected sibling being a carrier (2/3), times the risk that the partner is a carrier (the population carrier frequency if the family history is negative), times the risk of having an affected offspring given that both parents are carriers (1/4). Thus, for cystic fibrosis, with a carrier frequency of 1 in 25 Caucasian Americans, the risk for an unaffected sibling of an affected individual to have an offspring with cystic fibrosis is $2 / 3 \times 1 / 25 \times 1 / 4=1 / 150$. Carrier testing of the sibling, when available, may refine these odds.

Carrier screening is currently widely available for some recessive conditions, including sickle cell anemia and TaySachs disease. Carrier screening has become available for other relatively common genetic conditions, creating another group of candidates for prenatal diagnosis. In 2001, the American College of Obstetricians and Gynecologists and the American College of Medical Genetics issued guidelines that recommended cystic fibrosis carrier screening be offered to Caucasian couples who are planning a pregnancy or seeking prenatal care, and be made available to patients, regardless of ethnic background (2001 guideline). Cystic fibrosis is more common in Caucasians and in Ashkenazi Jews than in other populations, and the screening test detects a higher percentage of carriers in this population than in other groups. Due to the fact that defining a single ethnicity in many people in the United States is becoming more difficult, the guidelines for carrier screening from the American College of Obstetricians and Gynecologists were updated in 2005 and state that it is reasonable to offer cystic fibrosis carrier screening to all pregnant patients, provided that women are aware of their a priori carrier risk and the test limitations (41).

\section{Sonographic Findings}

As the resolution and therefore the sensitivity of ultrasonography improve, an increasing number of referrals for prenatal diagnosis are generated because of sonographic fetal abnormalities. Some of the more frequent findings include fetal heart defects, ventral wall defects, central nervous system abnormalities, gastrointestinal anomalies, renal anomalies, choroid plexus cysts, and nuchal fold thickening. These may be found in isolation, in conjunction with chromosomal abnormalities or as part of a genetic syndrome. As technical skills in ultrasonography as well as the resolution of the images improve, fetal abnormalities will be more frequently identified. As a result, our understanding of developmental abnormalities and what may represent normal variation will expand and thereby modify the genetic counseling engendered and what is offered in terms of prenatal diagnosis.

Not only have the indications for referral for prenatal diagnosis have expanded over the last four decades, but also the methods available to prenatally detect genetic disorders have also expanded. In addition to amniocentesis, invasive diagnostic methods currently include CVS, fetal blood sampling, and fetal biopsy for specific indications. Samples obtained by these techniques are used for cytogenetic analysis (karyotype, fluorescence in situ hybridization (FISH), array comparative genomic hybridization), molecular DNA diagnosis, and/or biochemical evaluation, dependent on the specific disorder being investigated. Each invasive procedure has risks and benefits to be considered when offering diagnostic testing.

\section{INVASIVE PRENATAL DIAGNOSTIC METHODS Midtrimester Amniocentesis}

The use of amniocentesis to prenatally detect chromosomal abnormalities was first reported in 1967 (42). Since that time, amniocentesis has become a widely accepted method for prenatal diagnosis of chromosomal abnormalities, inherited diseases, and some congenital infections. Despite about experience of three decades with midtrimester amniocentesis, determination of the risk of procedure-related pregnancy loss has been difficult. Risk estimates have ranged from $0.5 \%$ to $1 \%$ in large, prospective, multicenter trials (43-45). Total loss rates following invasive procedures are more readily accessible, 
encompassing the "background" fetal loss rate plus the procedure-related loss rate. A systematic review by Mujezinovic et al. showed a pooled pregnancy loss within 14 days after amniocentesis of $0.6 \%$ and rising to $0.9 \%$ before 24 weeks of gestation. The total pregnancy loss rate was 1.9\% (46).

While fetal loss resulting from amniocentesis is considered the most serious risk of the procedure, other fetal and maternal risks must also be considered. Fetal-needle puncture resulting in scars at birth has been reported (47), but is quite rare. Amniocentesis performed under continuous ultrasound guidance allows sampling of fluid while avoiding fetal parts, thus minimizing the risk of such an injury. Two large prospective trials found an association between midtrimester amniocentesis and neonatal respiratory difficulty $(43,44)$, while a study from the National Institute of Child Health and Human Development (NICHD) of the U.S. National Institutes of Health (43) did not corroborate this finding. An increased incidence of orthopedic postural deformities in association with amniocentesis was reported in the initial United Kingdom Medical Research Council (MRC) study (44), but was not found in two other large trials $(42,45)$.

Other complications from midtrimester amniocentesis include chorioamnionitis, leakage of amniotic fluid, and vaginal bleeding. The incidence of chorioamnionitis is $<1$ per 1000 procedures (48). Amniotic fluid leakage occurs in $1 \%$ to $2 \%$ of patients $(42,44)$, but is usually self-limited, with re-accumulation of fluid and a normal pregnancy outcome in most cases (49). The incidence of vaginal bleeding after second-trimester amniocentesis is also approximately $1 \%$ and is positively correlated with the number of needle insertions required (42).

An advantage of midtrimester amniocentesis over CVS is the ability to measure amniotic fluid levels of AFP, a sensitive marker for open neural tube defects and ventral wall defects. Normal values for amniotic fluid AFP levels in the first trimester have not been clearly established. Detection of acetylcholinesterase in the amniotic fluid is a useful adjunct to amniotic fluid AFP. Today, high-resolution ultrasound imaging with a qualified sonographer is more sensitive than MSAFP, which has an $80 \%$ sensitivity. High-resolution sonographic visualization approaches the sensitivity of amniotic fluid AFP to detect open neural tube defects. In practice, ultrasound imaging may detect some closed neural tube defects missed by AFP testing. In a study evaluating neural tube defect detection, standard ultrasound improved the sensitivity of MSAFP by correcting the errors in gestational age (50). In this study, moreover, ultrasound detection of neural tube defects was $100 \%$ (50).

\section{Chorionic Villus Sampling}

Due to the desire for prenatal diagnosis in time to allow safer termination options, increased patient privacy, and decreased emotional stress, earlier diagnostic measures were being sought. The technique of CVS entered the realm of clinical practice in the 1980s. Early attempts to obtain trophoblastic tissue had been described in the 1960s and 1970s, which were fraught with high failure and complication rates $(51,52)$. The ability to perform ultrasound guidance (53) and the development of a thin, malleable catheter for transcervical sampling (54) led to a resurgence of the technique and the naissance of first-trimester genetic diagnosis. Since the publication of these groundbreaking articles, numerous investigations, including large prospective trials to determine the risks and benefits of CVS, have been undertaken.

CVS is usually performed between 10 and 14 weeks of gestation, but can be performed transabdominally in the first trimester as well as throughout later gestation. Cytogenetic, molecular DNA analysis, and/or biochemical methods can be applied to villus tissue. These assays can detect chromosomal abnormalities, specific gene defects or linkage to at-risk loci, and/or evidence of enzymatic activity abnormalities in pregnancies at known risk for particular inherited conditions. In all of these respects, CVS generally provides the same type of information available through amniocentesis. There are several instances in which these two procedures are not equivalent in their ability to provide diagnostic information. For example, CVS is not useful in detecting a fetus with an open neural tube or ventral wall defect. Admittedly, the sonogram performed at the time of CVS might identify such a fetus; no information regarding AFP level or the presence of acetylcholinesterase, however, can be obtained through CVS. In fragile $\mathrm{X}$ testing, the methylation status may not yet be established in chorionic villi at the time of sampling, and an amniotic fluid sample may be needed if ambiguous results are obtained from villus tissue. On the other hand, some assays can be performed using chorionic villi but not amniocytes. The prenatal diagnosis of forms of osteogenesis imperfecta that are based on collagen studies cannot be completed on amniotic fluid samples (55). Thus, in contemplating the preferred method for prenatal diagnosis in a given pregnancy, both the couples' wishes and the ideal means to obtain the desired information must be considered.

The same issues that faced early laboratory investigations of cultured second-trimester amniocytes were confronted by early investigators of chorionic villus cells, including maternal contamination and chromosomal mosaicism. Maternal contamination from decidual cells poses a threat to long-term chorionic villus cell cultures. Careful dissection of the villi using a microscope with $40 \times$ magnification will minimize this risk.

Chromosomal mosaicism refers to two (or more) cell lines in the same tissue, organ, or individual. An example would be a villus sample with both a normal (e.g., 46,XX) cell line and a trisomic $(47, \mathrm{XX},+21)$ cell line. Because the placenta arises from different late progenitor cells than the embryo, mitotic errors that result in chromosomal nondisjunction can be limited to cells destined to become chorionic villi (56). This circumstance is identified as mosaicism by the laboratory at the time of CVS and is found in about $1 \%$ of chorionic villus samples (55). By contrast, mosaicism is seen in $0.25 \%$ of midtrimester amniotic fluid samples (57).

Frequently, when mosaicism is found by CVS, the fetus is chromosomally normal (58). If, however, a mitotic error occurred in a very early progenitor cell, both the villus and the embryonic descendants of this progenitor could contain the abnormality. In such a case, a chromosomal abnormality would often be revealed both at CVS and at follow-up amniocentesis. More often, follow-up amniocentesis in the midtrimester usually has normal cytogenetic results (59), with normal fetal outcomes. Though a normal karyotype on 
amniocytes provides presumptive evidence for a chromosomally normal fetus, it is not a guarantee, as mosaicism can be limited to only some fetal tissues.

As for any prenatal diagnostic method, patients are concerned about the risk of procedure-related pregnancy loss. Initial studies to assess the safety of first-trimester CVS were descriptions of clinical experience, which described total fetal loss rates from $2 \%$ to $7 \%(60,61)$ and were unable to address the procedure-related loss rate. A large proportion of the total loss rate represents the naturally occurring spontaneous abortions characteristic of an earlier gestational age. In addition, chromosomally abnormal fetuses are viable in greater proportions at 10 to 12 weeks than at 16 to 20 weeks, the time of midtrimester amniocentesis. These and other issues were subsequently addressed in several multicenter trials of CVS.

Similar rates of pregnancy loss were found between CVS and midtrimester amniocentesis in a large randomized Canadian trial (62), in which the observed difference in loss rates between eligible women with a sonographically viable fetus at 9 to 12 weeks in the CVS and midtrimester amniocentesis groups was $0.6 \%$, which was not statistically significant.

The MRC European trial of CVS (63) was less optimistic about the safety of the procedure. This multicenter, prospective study compared pregnancy outcomes after randomization with either first-trimester CVS or midtrimester amniocentesis. The study concluded that CVS "reduces the chances of a successful pregnancy outcome by $4.6 \%$ in comparison with second-trimester amniocentesis." One explanation for the increased loss rate in the MRC trial may be related to operator experience; the European study included a larger number of centers, with individual centers contributing fewer patients.

A prospective, nonrandomized trial conducted by the NICHD Collaborative Group (46) also compared pregnancy outcomes in patients undergoing first-trimester transcervical CVS with a group of patients undergoing midtrimester amniocentesis. This study showed total loss rates of desired pregnancies of $7.2 \%$ in the CVS group and $5.7 \%$ in the amniocentesis group. These rates included elective terminations for chromosomal and sonographic abnormalities, as well as spontaneous losses. After correction for differences in gestational age and maternal age between the two groups, the total excess loss rate in the CVS group was $0.8 \%$ when compared with the amniocentesis group. While this difference was not statistically significant, some centers currently quote a slightly higher procedure-related loss rate for CVS than for second-trimester amniocentesis. Many patients, nonetheless, would consider the benefits of early diagnosis in choosing to have CVS including the greater safety of pregnancy termination at an earlier gestational age. The systematic literature review cited earlier estimates a total pregnancy loss rate of $0.7 \%$ within 14 days, $1.3 \%$ before 24 weeks of gestation, and an overall pregnancy loss rate of $2 \%$ with CVS (46).

In addition to the pregnancy loss rate, the question of an increased risk of fetal limb abnormalities in association with CVS has undergone scrutiny. Firth and coworkers (46) reported an unusually high incidence of oromandibular-limb hypogenesis syndromes in pregnancies undergoing CVS at 56 to 66 days of gestation (8-9 weeks). None of the three large trials (61-63), in which CVS was usually performed at 10 to 12 weeks of gestation, found such an association. This led Firth et al. to hypothesize that an earlier gestational age at the time of CVS may predispose to limb reduction defects (64).

Subsequently, Burton and colleagues (65) reported their experience in which four transverse limb reduction defects were identified during follow-up after CVS in 436 pregnancies, an incidence far greater than the reported incidence of limb malformations of 1 in 1500 to 2000 live births (66). The procedures were performed at 9.6 to 11.3 weeks of gestation, thus the limb abnormalities cannot be explained by early timing of the CVS. This report has prompted speculation regarding the technique used in the single center involved, particularly since their spontaneous loss rate was considerably higher than that of other centers (67). A survey conducted in Taiwan (68) also found an association between CVS and limb defects, with a 100-fold increased incidence of severe limb defects after CVS exposure as compared with the general population. However, in this series, $87 \%$ of the cases involving limb defects in which the timing of CVS was known were performed prior to 63 days ( 9 weeks) of gestation. In an effort to objectively evaluate the potential etiologic role of CVS in the development of limb reduction defects she first described, Firth and colleagues reported a series of 75 infants who were exposed to CVS in utero and had limb reduction defects at birth (69). The authors found a strong correlation between increasing severity of the defects and earlier gestational age at the time of CVS, with the most severe defects following CVS at 9 weeks of gestation or less. These studies and others support postponement of CVS until 9 completed weeks of gestation to minimize the risk of fetal injury.

Operator experience may explain the lack of an association with fetal limb defects reported by the majority of U.S. centers. A publication by the World Health Organization reported an incidence of approximately $6 \mathrm{limb}$ reduction defects per 10,000 CVS cases (70), which is consistent with the background rate of this congenital anomaly (65). At this time, most centers feel that patients should be informed of the possible association, made aware of their options, and allowed to select a method of prenatal diagnosis most consistent with their medical and psychosocial needs.

Chorionic villus tissue may be obtained by one of three approaches: transcervical, transabdominal $(71,72)$, or transvaginal (73). The transcervical method is currently the most widely used, although some claim that transabdominal sampling has a lower risk of infection and is easier to perform due to the technique's similarity with amniocentesis. Several prospective randomized trials compared transabdominal and transcervical methods. Two of these studies, a collaborative one through the NICHD (74) and a single-center study by Brambati and colleagues (75), found no difference in the success of sampling, fetal loss, delivery, or neonatal outcome between groups. A third randomized trial (76) showed a significantly greater total fetal loss rate after transcervical CVS (10.9\%) when compared with transabdominal CVS (6.3\%) and midtrimester amniocentesis (6.4\%). The reasons for the differences in fetal loss rates are unclear. In all the three studies, an increased incidence of minor symptoms were found in the groups undergoing transcervical CVS. 
Common symptoms following CVS include cramping, spotting, and vaginal bleeding. Minor cramping is reported by $16 \%$ to $22 \%$ of women after transabdominal or transcervical CVS $(74,78) ; 19 \%$ to $32 \%$ reported spotting after transcervical procedures, versus $4 \%$ after transabdominal CVS (74,78); vaginal bleeding as heavy as a menstrual period was reported by up to $6 \%$ to $7 \%$ of patients after transcervical CVS and only $1 \%$ to $2 \%$ of women undergoing transabdominal CVS $(63,74)$. Amniotic fluid leakage is uncommon after CVS, occurring transiently in $1.1 \%$ and for several weeks in $0.7 \%$ of cases in the initial NICHD study (78), and less frequently in other reports. Of those women who continued to leak fluid, $20 \%$ (3 of 14 patients) went on to lose the pregnancy.

A significant concern is the risk of infection. For this reason, transcervical CVS is contraindicated in the presence of a genital tract infection, including active herpes genitalis. Case reports of septic abortion and septic shock following CVS have been published $(77,79)$; however, this complication was not reported in any of the large CVS trials.

\section{Fetal Blood Sampling}

Though amniocentesis or CVS is usually sufficient for prenatal diagnosis, fetal blood sampling can provide rapid and accurate analysis for a multitude of disorders (Table 2). For prenatal cytogenetic analysis, amniocentesis and CVS are the most widely used methods. However, patients occasionally present at late gestational ages with compelling indications for rapid fetal karyotype, such as severe intrauterine growth retardation, oligohydramnios, hydrops fetalis, or major fetal anomalies. Though CVS or amniocentesis may provide diagnostic information, the quality of a rapid cytogenetic analysis from direct preparations of villus tissue may be poor, and the time required for culture of amniocytes or villi may significantly alter obstetric management. Under such circumstances, fetal blood sampling to obtain a fetal lymphocyte karyotype, which is usually available in 2 to 3 days, may be indicated. A clinical example would be a patient in the mid-third trimester of pregnancy presenting with preterm labor, severe fetal growth retardation, and a fetal cardiac defect detected by ultrasound. A rapid diagnosis of trisomy 18 would allow that patient to undergo counseling and afford her the option of foregoing tocolysis, antepartum surveillance, cesarean section for fetal

\section{Table 2 Indications for Fetal Blood Sampling}

Chromosomal
Karyotype
Mosaicism
Intrauterine infection
Toxoplasmosis
Rubella
Varicella
Cytomegalovirus

Human parvovirus B19

Red cell isoimmunization

Platelet isoimmunization

Fetal hematologic indices

Hemoglobinopathies

Immunodeficiency syndromes

Assessment of fetal acid-base status indications, and neonatal intervention after birth. Patients may also be offered fetal blood sampling to provide rapid diagnosis at the time limits of legal pregnancy termination.

An ever-increasing number of inherited conditions can be diagnosed through molecular, cytogenetic, and biochemical techniques, as the gene alterations, chromosomal locations (for linkage analysis), and/or enzymatic derangements relevant to specific disorders are identified. As a result, fetal blood has become infrequently required for the prenatal diagnosis of coagulopathies, hemoglobinopathies, and other heritable diseases. Historically, some of the first applications of prenatal diagnosis were to detect hemoglobinopathies. This was done initially using fetoscopically obtained fetal blood, a procedure associated with higher rates of morbidity and fetal mortality $(80,81)$. Later, knowledge of specific gene defects and advancements in molecular technology allowed diagnosis using DNA extracted from amniocytes (82), and subsequently chorionic villi, methods that are now applied for the detection of numerous inherited conditions.

Other specific applications of fetal blood sampling for diagnostic and treatment purposes are considered throughout this text. Examples include fetal evaluation for viral infections, erythroblastosis fetalis, alloimmune thrombocytopenia, and assessment of fetal acid-base status. Fetal blood sampling may be performed either by cordocentesis or fetal intrahepatic vein sampling (83). The techniques and complications of fetal blood sampling are discussed at length in chapter 53 and therefore will not be considered here.

\section{NONINVASIVE METHODS FOR PRENATAL DIAGNOSIS} Preimplantation Genetic Screening and Diagnosis

Preimplantation genetic techniques have transformed our existing ideas of ascertaining genetic abnormalities in offspring. Preimplantation genetic screening (PGS) refers to the technique used to assess for the possible existence of embryonic aneuploidy. Preimplantation genetic diagnosis (PGD) refers to the technique used to rule out specific genetic conditions, by analyzing the embryonic DNA. Development of the polymerase chain reaction (PCR) (84) revolutionized molecular genetics. PCR permits millions of copies of a targeted DNA segment to be amplified from a small DNA sample in a matter of hours. This technology has been combined with in vitro fertilization in an effort to provide earlier information by PGD. For couples at risk for an inherited disorder, results obtained from amniocytes or chorionic villi may present them with a difficult decision of whether or not to continue the pregnancy. Many women would prefer to completely avoid carrying affected fetuses (85), and some would prefer a technology that allows testing of embryos prior to implantation. The technology for PGD is quite expensive, however, a factor that must be considered at a time when resources available for health care are being allocated.

The technology involved in preimplantation diagnosis includes in vitro fertilization, embryo or polar body biopsy, PCR amplification of the DNA sequences of interest, and molecular genetic techniques using the amplified DNA to determine the presence or absence of a specific allele(s) in the embryo. Only those embryos with genetic material predictive of an unaffected fetus are implanted, thus avoiding the need for pregnancy termination. 
Methods to obtain the cell(s) required for analysis include biopsy of one to several cells of the 2- to 3-day embryo, biopsy of the trophectoderm of the 5- to 6-day blastocyst, and polar body biopsy (removal of the nonfunctioning haploid product of meiosis I) (86). Each of these methods has benefits and drawbacks, including the degree of technical difficulty, variations in pregnancy rates, and errors in diagnosis due to the hazards of single-cell analysis. An analysis of various embryo biopsy techniques concluded that biopsy of a quarter of the embryo on day 3 after fertilization (approximately the 12-cell stage) may be the "most feasible" for PGS (87). Preimplantation diagnosis from polar body biopsy is in use for PGD or PGS. This technique has been performed for aneuploidy screening, $\mathrm{X}$-linked disorders, and maternal translocations. It may also be used in autosomal recessive conditions where both parents are carriers of a particular condition, and an oocyte spared of the mutant gene is used for fertilization. When polar body biopsy is used, the paternal genetic component cannot be evaluated.

Of central importance is the accuracy of diagnosis. When basing a diagnosis on DNA amplified from a single cell, problems such as contamination, PCR failure, and PCR error are of paramount importance. Navidi and Arnheim (88) considered these sources of error mathematically and suggested that blastomere analysis may be preferable in this regard to polar body analysis, while a combination of the two results in a significantly lower risk of misdiagnosis.

The clinical use of preimplantation diagnosis was first described in 1990 by Handyside and colleagues (89) for sex selection in pregnancies at risk for X-linked diseases. Since that time, this technology has been applied for the specific diagnosis of many disorders, including cystic fibrosis (90), Duchenne muscular dystrophy (91), and alloimmune thrombocytopenia (92). It is also being used to evaluate or screen for aneuploidy employing FISH, in the form of chromosomespecific probes to rapidly determine the copy number of a certain chromosome. As has been done with $\mathrm{X}$ - and Y-chromosome-specific probes for sex determination (93), PGS utilizes probes for the chromosomes most commonly involved in aneuploidy (X, Y, 18, 13, and 21) (94).

PGS is offered at a number of infertility centers in the United States. Misdiagnosis rates for PGS by single-cell blastomere vary with reporting centers. Cytogenetic FISH analysis appears to offer a positive predictive value of $83 \%$ and a negative predictive value of $81 \%$ when evaluating a single blastomere in conjunction with PGS (95). Laboratory error with FISH and early embryogenic mosaicism are primarily responsible for the inaccuracies associated with cytogenetic preimplantation testing (96). It is recommended that a confirmatory diagnosis be performed when PGS indicates a normal karyotype, or normal DNA results in the case of a single-gene disorder by PGD, in the form of an amniocentesis or a CVS, due to the risk of misdiagnosis.

As PGS is becoming readily available to women who seek diagnostic testing before the time of implantation, it will be important to determine risks, benefits, and pregnancy rates using the procedure. This is particularly true for cytogenetic PGS. Although cytogenetic PGS has been shown to lower the rate of spontaneous miscarriage in women who are $>35$ years of age $(96,97)$, studies demonstrating accuracy in diagnosing fetal aneuploidy have yielded controversial results. The number of candidate embryos eligible for transfer after PGS for aneuploidy, furthermore, is diminished. Studies examining the loss and nonuse of cytogenetically normal embryos following PGS will need to be conducted.

\section{Fetal Cells, DNA, and RNA in the Maternal Circulation}

The same molecular techniques (PCR, FISH) that have allowed the development of PGS have also played a major role in efforts to provide prenatal diagnosis based on the analysis of fetal cells obtained from maternal peripheral blood. If this type of noninvasive testing were developed to the point of cost-effective and accurate diagnostic clinical application, the risks associated with invasive prenatal diagnostic techniques (amniocentesis, CVS) could be avoided. Thus far, its potential has been evaluated more in terms of a screening test.

Earlier studies suggested that fetal cells exist in the maternal circulation $(98,99)$, and work based on PCR technology has confirmed this (100). Requirements to achieve a diagnosis based on fetal cells isolated from maternal blood would include a sensitive and specific method to identify fetal cells, a process to enrich for the infrequent fetal cells, and techniques to analyze the fetal genetic material for prenatal diagnosis. Each of these aspects is currently in the process of development, and a variety of methods have been attempted with varying success.

In order to identify fetal cells, monoclonal antibodies against various fetal cell antigens have been developed. These include antibodies to trophoblasts $(101,102)$, fetal erythrocyte cell surface antigens (103-105), and paternally derived human leukocyte antigens $(106,107)$. To enrich for fetal cells, the monoclonal antibodies have been fluorescently tagged and used in combination with flow cytometry, a method to sort individual cells based on their specific physical and chemical properties. Characteristics selected for include cell size, cell granularity, and the presence of a fluorescent tag $(104,105)$. By report, multiparameter flow cytometry applied to firsttrimester maternal peripheral blood enriches from one fetal nucleated erythrocyte in approximately $10^{7}$ to $10^{8}$ maternal cells to approximately 4 fetal cells per 1000 maternal cells (104). The proportion of fetal cells after sorting secondtrimester samples was approximately 20 fetal cells per 1000 maternal cells in this study, though other investigators have not described such high yields of fetal cells $(108,109)$. In addition to fluorescence-activated flow cytometry, isolation methods under investigation include immunospecific magnetic-activated cell sorting (110), immunomagnetic beads (111), avidin-biotin columns (112), and discontinuous density-gradient centrifugation (113). The various methods were reviewed and their advantages and disadvantages were analyzed by Holzgreve and colleagues (114).

Techniques for genetic analysis that have been applied to fetal cells isolated from the maternal circulation include PCR and in situ hybridization. PCR has been used to detect Y-chromosome-specific DNA sequences to identify male fetuses in both unsorted $(115)$ and sorted $(104,105)$ maternal peripheral blood. More recently, this technology has been applied to determine fetal rhesus antigen status $(116,117)$. FISH to detect fetal aneuploidies has also been described using fetal cells derived from the maternal circulation $(104,118,119)$. 
The ongoing research using fetal nucleated $\mathrm{RBC}$ is of critical importance. In a recent publication from Huang et al., a microfluidic device was used to isolate nucleated RBCs from pregnant women and showed a 10 - to 20 -fold enrichment of nucleated RBCs over other methods described (120).

In recent years, there has been a greater focus on using cellfree fetal DNA or RNA within the maternal plasma or serum for use in prenatal DNA diagnosis. In 1997, Lo et al. demonstrated that cell-free fetal DNA circulates within the plasma and serum of pregnant women (121). Fetal DNA can be detected within the maternal circulation as early as 7 weeks of gestation (122). In 2009, Tobias et al. reported a method of specific magnetic bead-based capture of free fetal DNA from maternal plasma. The team developed a magnetic capture hybridization technique using specific hybridization primers for the extraction of fetal DNA from maternal plasma, which is almost twice as efficient as older methods (123). Both forms of nucleic acids may prove useful for accurate fetal gender determination and fetal $\mathrm{RhD}$ blood typing. Their utility for screening and/or detection of fetal aneuploidy remains under active investigation.

\section{REFERENCES}

1. Druzin ML, Chervenak F, McCullough LB, et al. Should all pregnant patients be offered prenatal diagnosis regardless of age? Obstet Gynecol 1993; 81: 615-18.

2. Hook EB. Rates of chromosome abnormalities at different maternal ages. Obstet Gynecol 1981; 58: 282-5.

3. ACOG Technical Bulletin. Antenatal Diagnosis of Genetic Disorders (number 108). Washington, DC: American College of Obstetricians and Gynecologists, 1987.

4. Hook EB, Cross PK, Schreinemachers DM. Chromosomal abnormality rates at amniocentesis and in live-born infants. JAMA 1983; 249: 2034-8.

5. Daniel A, Stewart L, Saville T, et al. Prenatal diagnosis in 3000 women for chromosome, X-linked, and metabolic disorders. Am J Med Genet 1982; 11: 61-75.

6. Tamaren J, Spuhler K, Sujansky E. Risk of Down syndrome among second- and third-degree relatives of a proband with trisomy 21 . Am J Med Genet 1983; 15: 393-403.

7. Eunpu DL, McDonald DM, Zackai EH. Trisomy 21: rate in seconddegree relatives. Am J Med Genet 1986; 25: 361-3.

8. Berr C, Borghi E, Rethoré MO, et al. Risk of Down syndrome in relatives of trisomy 21 children: a case-control study. Ann Genet 1990; 33: $137-40$.

9. Boué A, Gallano P. A collaborative study of the segregation of inherited chromosome structural rearrangements in 1356 prenatal diagnoses. Prenat Diagn 1984; 4: 45-67.

10. Daniel A, Hook EB, Wulf G. Risks of unbalanced progeny at amniocentesis to carriers of chromosome rearrangements: data from United States and Canadian laboratories. Am J Med Genet 1989; 31: 14-53.

11. Neri G, Serra A, Campana M, Tedeschi B. Reproductive risks for translocation carriers: cytogenetic study and analysis of pregnancy outcome in 58 families. Am J Med Genet 1983; 16: 535-61.

12. Brock DJH, Sutcliffe RG. Alpha-fetoprotein in the antenatal diagnosis of anencephaly and spina bifida. Lancet 1972; ii: 197-9.

13. Macri JN, Weiss RR, Tillitt R, et al. Prenatal diagnosis of neural tube defects. JAMA 1976; 236: 1251-4.

14. U.K. Collaborative Study on Alpha-fetoprotein in Relation to Neural Tube Defects. Maternal serum-alpha-fetoprotein measurement in antenatal screening for anencephaly and spina bifida in early pregnancy. Lancet 1977; i: 1323-32.

15. ACOG Technical Bulletin Alpha-fetoprotein (number 154). Washington, DC: American College of Obstetricians and Gynecologists, 1991.

16. Thomas RL, Blakemore KJ. Evaluation of elevations in maternal serum alpha-fetoprotein: a review. Obstet Gynecol Surv 1990; 45: 269-83.
17. Burton BK. Outcome of pregnancy in patients with unexplained elevated or low levels of maternal serum alpha-fetoprotein. Obstet Gynecol 1988; 72: 709-13.

18. Evans J, Stokes IM. Outcome of pregnancies associated with raised serum and normal amniotic fluid-fetoprotein concentrations. Br Med J 1984; 288: 1494.

19. Merkatz IR, Nitowsky HM, Macri JN, Johnson WE. An association between low maternal serum a-fetoprotein and fetal chromosomal abnormalities. Am J Obstet Gynecol 1984; 148: 886-94.

20. Cuckle HS, Wald NJ, Lindenbaum RH. Maternal serum alphafetoprotein measurement: a screening test for Down syndrome. Lancet 1984; i: 926-9.

21. DiMaio MS, Baumgarten A, Greenstein RM, et al. Screening for fetal Down's syndrome in pregnancy by measuring maternal serum alphafetoprotein levels. N Engl J Med 1987; 317: 342-6.

22. New England Regional Genetics Group Prenatal Collaborative Study of Down Syndrome Screening. Combining maternal serum a-fetoprotein measurements and age to screen for Down syndrome in pregnant women under age 35. Am J Obstet Gynecol 1989; 160: 575-81.

23. Bogart $\mathrm{MH}$, Pandian MR, Jones OW. Abnormal maternal serum chorionic gonadotropin levels in pregnancies with fetal chromosome abnormalities. Prenat Diagn 1987; 7: 623-30.

24. Canick JA, Knight GJ, Palomaki GE, et al. Low second trimester maternal serum unconjugated oestriol in pregnancies with Down's syndrome. Br J Obstet Gynaecol 1988; 95: 330-3.

25. Haddow JE, Palomaki GE, Knight GJ, et al. Prenatal screening for Down's syndrome with use of maternal serum markers. N Engl J Med 1992; 327: 588-93.

26. Canick JA, Palomaki GE, Osathanondh R. Prenatal screening for trisomy 18 in the second trimester. Prenat Diagn 1990; 10: 546-8.

27. Palomaki GE, Knight GJ, Haddow JE, et al. Prospective intervention trial of a screening protocol to identify fetal trisomy 18 using maternal serum alpha-fetoprotein, unconjugated oestriol, and human chorionic gonadotropin. Prenat Diagn 1992; 12: 925-30.

28. Saller DN, Canick JA, Schwartz S, Blitzer MG. Multiple-marker screening in pregnancies with hydropic and nonhydropic Turner syndrome. Am J Obstet Gynecol 1992; 167: 1021-4.

29. Wenstrom KD, Williamson RA, Grant SS. Detection of fetal Turner syndrome with multiple-marker screening. Am J Obstet Gynecol 1994; 170: 570-3.

30. Aitken, DA, Wallace EM, Crossley J, et al. Dimeric inhibin A as a marker for Down's syndrome in early pregnancy. NEJM 1996; 334: 1231-6.

31. ACOG Practice Bulletin Screening for Fetal Chromosomal Abnormalities. Washington, DC: American College of Obstetricians and Gynecologists, 2007.

32. Spencer K, Souter V, Tul N, et al. A screening program for trisomy 21 at 10-14 weeks using fetal nuchal translucency, maternal serum free $\beta$-human chorionic gonadotropin and pregnancy-associated plasma Protein-A. Ultrasound Obstet Gynecol 1999; 13: 231-7.

33. Wald NJ, George L, Smith D, et al. Serum Screening for down syndrome between 8 and 14 weeks of pregnancy. International Prenatal Screening Research Group. Br J Obstet Gynaecol 1996; 103: 407-12.

34. Wapner R, Thom E, Simpson JL, et al. First-trimester screening for Trisomies 21 and 18. NEJM 2003; 349: 1405-13.

35. Spencer K, Tul N, Nicolaides K. Maternal serum free $\beta$ hCG and PAPP-A in fetal sex chromosome defects in the first trimester. Prenat Diagn 2000; 20: 390-4.

36. Tul N, Spencer K, Noble P, et al. screening for trisomy 18 by fetal nuchal translucency and maternal serum free $\beta$ hCG and PAPP-A at 10-14 weeks of gestation. Prenat Diagn 1999; 19: 1035-42.

37. Spencer K, Ong C, Skentou H, et al. Screening for Trisomy 13 by fetal nuchal translucency and maternal serum free $\beta$ hCG and PAPP-A at 10-14 weeks of gestation. Prenat Diagn 2000; 20: 411-16.

38. Nicolaides KH, Brizot ML, Snijders RJM. Fetal nuchal translucency: ultrasound screening for fetal trisomy in the first trimester of pregnancy. BJOG 1994; 101: 782-6.

39. Chitty L, Pandya PP. Ultrasound screening for fetal abnormalities in the first trimester. Prenat Diagn 1997; 17: 1269-81.

40. Ball RH, Caughey AB, Malone F, et al. First-and second-trimester evaluation of risk for Down syndrome. Obstet Gynecol 2007; 110: 10-17. 
41. ACOG Committee Opinion Update on Carries Screening for Cystic Fibrosis. Washington, DC: American College of Obstetricians and Gynecologists, 2005.

42. Jacobson CB, Barter RH. Intrauterine diagnosis and management of genetic defects. Am J Obstet Gynecol 1967; 99: 796-807.

43. The NICHD National Registry for Amniocentesis Study Group. Midtrimester amniocentesis for prenatal diagnosis: safety and accuracy. JAMA 1976; 236: 1471-6.

44. Medical Research Council Working Party on Amniocentesis. An assessment of the hazards of amniocentesis. Br J Obstet Gynaecol 1978; 85: 1-41.

45. Tabor A, Madsen M, Obel EB, et al. Randomised controlled trial of genetic amniocentesis in 4606 low-risk women. Lancet 1986; i: 1287-92.

46. Mujezinovic F, Alfirevic Z. Procedure-related complications of amniocentesis and chorionic villus sampling-A systematic review. Obstet Gynecol 2007; 110: 687-94.

47. Broome DL, Wilson MG, Weiss B, Kellogg B. Needle puncture of fetus: a complication of second-trimester amniocentesis. Am J Obstet Gynecol 1976; 126: 247-52.

48. Porreco RP, Young PE, Resnik R, et al. Reproductive outcome following amniocentesis for genetic indications. Am J Obstet Gynecol 1982; 143: 653-60.

49. Gold RB, Goyert GL, Schwartz DB, et al. Conservative management of second-trimester post-amniocentesis fluid leakage. Obstet Gynecol 1989; 74: 745-7.

50. Dashe J, Twickler D, Santos-Ramos R, et al. Alpha-fetoprotein detection of neural tube defects and the impact of standard ultrasound. AJOG 2006; 195: 1623-8.

51. Mohr J. Foetal genetic diagnosis: development of techniques for early sampling of foetal cells. Acta Pathol Microbiol Scand 1968; 73: 73-7.

52. Hahnemann N. Early prenatal diagnosis: a study of biopsy techniques and cell culturing from extraembryonic membranes. Clin Genet 1974; 6: 294-306.

53. Kazy Z, Rozovsky IS, Bakharev VA. Chorion biopsy in early pregnancy: a method of early prenatal diagnosis for inherited disorders. Prenat Diagn 1982; 2: 39.

54. Ward RHT, Modell B, Petrou M, et al. Method of sampling chorionic villi in first trimester of pregnancy under guidance of real time ultrasound. Br Med J 1983; 286: 1542-4.

55. Cohn DH, Byers PH. Clinical screening for collagen defects in connective tissue diseases. Clin Perinatol 1990; 17: 793-809.

56. Crane JP, Cheung SW. An embryogenic model to explain cytogenetic inconsistencies observed in chorionic villus versus fetal tissue. Prenat Diagn 1988; 8: 119-29.

57. Hsu LYF, Perlis TE. United States survey on chromosome mosaicism and pseudomosaicism in prenatal diagnosis. Prenat Diagn 1984; 4: 97-130.

58. Johnson A, Wapner RJ, Davis GH, Jackson LG. Mosaicism in chorionic villus sampling: an association with poor perinatal outcome. Obstet Gynecol 1990; 75: 573-7.

59. Vejerslev LO, Mikkelsen M. The European collaborative study on mosaicism in chorionic villus sampling data from 1986 to 1987. Prenat Diagn 1989; 9: 575-88.

60. Jackson LG, Wapner RA, Barr MA. Safety of chorionic villus biopsy. Lancet 1986; i: 674-5.

61. Hogge WA, Schoenberg SA, Golbus MS. Chorionic villus sampling: experience of the first 1000 cases. Am J Obstet Gynecol 1986; 154 1249-52.

62. Canadian Collaborative CVS - Amniocentesis Clinical Trial Group Multicentre randomised clinical trial of chorion villus sampling and amniocentesis: first report. Lancet 1989; i: 1-6.

63. MRC Working Party on the Evaluation of Chorion Villus Sampling. Medical Research Council European Trial of chorion villus sampling. Lancet 1991; 337: 1491-9.

64. Firth HV, Boyd PA, Chamberlain P, et al. Severe limb abnormalities after chorion villus sampling at 56-66 days gestation. Lancet 1991; 337: 762-3.

65. Burton BK, Schulz CJ, Burd LI. Limb anomalies associated with chorionic villus sampling. Obstet Gynecol 1992; 79: 726-30.

66. Froster-Iskenius UG, Baird PA. Limb reduction defects in over one million consecutive live births. Teratology 1989; 39: 127-35.
67. Wapner R. Is chorionic villus sampling a safe procedure? The Female Patient 1993; 18: 59-62.

68. Hsieh F-J, Shyu M-K, Shen B-C, et al. Limb defects after chorionic villus sampling. Obstet Gynecol 1995; 85: 84-8.

69. Firth HV, Boyd PA, Chamberlain PF, et al. Analysis of limb reduction defects in babies exposed to chorionic villus sampling. Lancet 1994; 343: 1069-71.

70. World Health Organization/European Regional Office. Risk of Evaluation of CVS. Copenhagen: WHO/EURO, 1992.

71. Smidt-Jensen S, Hahnemann N. Transabdominal fine needle biopsy from chorionic villi in the first trimester. Prenat Diagn 1984; 4: 163-9.

72. Elias S, Simpson JL, Shulman LP, et al. Transabdominal chorionic villus sampling for first-trimester prenatal diagnosis. Am J Obstet Gynecol 1989; 160: 879-86.

73. Shulman LP, Simpson JL, Elias S, et al. Transvaginal chorionic villus sampling using transabdominal ultrasound guidance: a new technique for first-trimester prenatal diagnosis. Fetal Diagn Ther 1993; 8: 144-8.

74. Jackson LG, Zachary JM, Fowler SE, et al. A randomized comparison of transcervical and transabdominal chorionic-villus sampling. $\mathrm{N}$ Engl J Med 1992; 327: 594-8.

75. Brambati B, Terzian E, Tognoni G. Randomized clinical trial of transabdominal versus transcervical chorionic villus sampling methods. Prenat Diagn 1991; 11: 285-93.

76. Smidt-Jensen S, Permin M, Philip J, et al. Randomised comparison of amniocentesis and transabdominal and transcervical chorionic villus sampling. Lancet 1992; 340: 1237-44.

77. Blakemore KJ, Mahoney MJ, Hobbins JC. Infection and chorionic villus sampling. Lancet 1985; ii: 339.

78. Rhoads GG, Jackson LG, Schlesselman SE, et al. The safety and efficacy of chorionic villus sampling for early prenatal diagnosis of cytogenetic abnormalities. N Engl J Med 1989; 320: 609-17.

79. Barela AI, Kleinman GE, Golditch IM, et al. Septic shock with renal failure after chorionic villus sampling. Am J Obstet Gynecol 1986; 154: $1100-2$.

80. Alter BP, Modell CB, Fairweather D, et al. Prenatal diagnosis of hemoglobinopathies: a review of 15 cases. N Engl J Med 1976; 295: 1437-43.

81. Kan YW, Trecartin RF, Golbus MS, Filly RA. Prenatal diagnosis of b-thalassemia and sickle-cell anaemia: experience with 24 cases. Lancet 1977; 1: 269-71.

82. Chang JC, Kan YW. A sensitive new prenatal test for sickle-cell anemia. N Engl J Med 1982; 307: 30-2.

83. Aina-Mumuney AJ, Holcroft CJ, Blakemore KJ, et al. Intrahepatic vein for fetal blood sampling: one center's experience. Am J Obstet Gynecol 2008; 198: 387.

84. Saiki RK, Gelfand DH, Stoffel S, et al. Primer-directed enzymatic amplification of DNA with a thermostable DNA polymerase. Science 1988; 239: 487-91.

85. Pergament E. Preimplantation diagnosis: a patient perspective. Prenat Diagn 1991; 11: 493-500.

86. Simpson JL, Carson SA. Preimplantation genetic diagnosis. N Engl J Med 1992; 327: 951-3.

87. Tarin JJ, Handyside AH. Embryo biopsy strategies for preimplantation diagnosis. Fertil Steril 1993; 59: 943-53.

88. Navidi W, Arnheim N. Using PCR in preimplantation genetic disease diagnosis. Hum Reprod 1991; 6: 836-49.

89. Handyside AH, Kontogianni EH, Hardy K, Winston RM. Pregnancies from biopsied human preimplantation embryos sexed by Y-specific DNA amplification. Nature 1990; 344: 768-70.

90. Handyside AH, Lesko JG, Tarín JJ, et al. Birth of a normal girl after in vitro fertilization and preimplantation diagnostic testing for cystic fibrosis. N Engl J Med 1992; 327: 905-9.

91. Kristjansson K, Chong SS, Van den Veyver IB, et al. Preimplantation single cell analyses of dystrophin gene deletions using whole genome amplification. Nat Genet 1994; 6: 19-23.

92. Van den Veyver IB, Chong SS, Kristjansson K, et al. Molecular analysis of human platelet antigen system 1 antigen on single cells can be applied to preimplantation genetic diagnosis for prevention of alloimmune thrombocytopenia. Am J Obstet Gynecol 1994; 170: 807-12.

93. Harper JC, Coonen E, Ramaekers FC, et al. Identification of the sex of human preimplantation embryos in two hours using an improved 
spreading method and fluorescent in situ hybridization (FISH) using directly labelled probes. Hum Reprod 1994; 9: 721-4.

94. Munne S, Lee A, Rosenwaks Z, et al. Diagnosis of major chromosome aneuploidies in human preimplantation embryos. Hum Reprod 1993; 8: 2185-91.

95. DeUgarte C, Li M, Surrey M, et al. Accuracy of FISH analysis in predicting chromosomal status in patients undergoing preimplantation genetic diagnosis. Fertil Steril 2008; 90: 1049-54.

96. Schoolcraft WB, Katz-Jaffe M, Stevens J, et al. Preimplantation aneuploidy testing for infertile patients of advanced maternal age: a randomized prospective trial. Fertil Steril 2009; 92: 157-62.

97. Munne S, Chen S, Colls P, et al. Preimplantation genetic diagnosis reduces pregnancy loss in women aged 35 years and older with a history of recurrent miscarriages. Fertil Steril 2005; 84: 331-5.

98. Walknowska J, Conte FA, Grumback MM. Practical and theoretical implication of fetal/maternal lymphocyte transfer. Lancet 1969; i: 1119-22.

99. Schroder J, de la Chapelle A. Fetal lymphocytes in the maternal blood. Blood 1972; 39: 153-62.

100. Lo YM, Wainscoat JS, Gillmer MDG, et al. Prenatal sex determination by DNA amplification from maternal peripheral blood. Lancet 1989; ii: 1363-5.

101. Covone AE, Mutton D, Johnson PM, Adinolfi M. Trophoblast cells in peripheral blood from pregnant women. Lancet 1984; 536: 841-3.

102. Mueller UW, Hawes CS, Wright AE, et al. Isolation of fetal trophoblast cells from peripheral blood of pregnant women. Lancet 1990; 336: 197-200.

103. Bianchi DW, Flint AF, Pizzimenti MF, et al. Isolation of fetal trophoblast cells from peripheral blood of pregnant women. Proc Natl Acad Sci USA 1990; 87: 3279-83.

104. Price JO, Elias S, Wachtel SS, et al. Prenatal diagnosis with fetal cells isolated from maternal blood by multiparameter flow cytometry. Am J Obstet Gynecol 1991; 165: 1731-7.

105. Wachtel S, Elias S, Price J, et al. Fetal cells in the maternal circulation: isolation by multiparameter flow cytometry and confirmation by polymerase chain reaction. Hum Reprod 1991; 6: 1466-9.

106. Herzenberg LA, Bianchi DW, Schroder J, et al. Fetal cells in the blood of pregnant women: detection and enrichment by fluorescence-activated cell sorting. Proc Natl Acad Sci USA 1979; 76: 1453-5.

107. Iverson GM, Bianchi DW, Cann HM, Herzenberg LA. Detection and isolation of fetal cells from maternal blood using the fluorescenceactivated cell sorter (FACS). Prenat Diagn 1981; 1: 61-73.

108. Bianchi DW, Shuber AP, DeMaria MA, et al. Fetal cells in maternal blood: determination of purity and yield by quantitative polymerase chain reaction. Am J Obstet Gynecol 1994; 171: 922-6.
109. Hamada H, Arinami T, Kubo T, et al. Fetal nucleated cells in maternal peripheral blood: frequency and relationship to gestational age. Hum Genet 1993; 91: 427-32.

110. Gänshirt-Ahlert D, Burschyk M, Garritsen HS, et al. Magnetic cell sorting and the transferrin receptor as potential means of prenatal diagnosis from maternal blood. Am J Obstet Gynecol 1992; 166: 1350-5.

111. Bertero MT, Camaschella C, Serra A, et al. Circulating trophoblast cells in pregnancy have maternal genetic markers. Prenat Diagn 1988; 8: 585-90.

112. Berenson RJ, Bensinger WI, Kalamasz D. Positive selection of viable cell populations using avidin-biotin immunoadsorption. J Immunol Methods 1986; 91: 11-19.

113. Bhat NM, Bieber MM, Teng NN. One step separation of human fetal lymphocytes from nucleated red blood cells. J Immunol Methods 1990; 131: $147-9$

114. Holzgreve W, Garritsen HSP, Gänshirt-Ahlert D. Fetal cells in the maternal circulation. J Reprod Med 1992; 37: 410-18.

115. Lo YMD, Patel P, Baigent CN, et al. Prenatal sex determination from maternal peripheral blood using the polymerase chain reaction. Hum Genet 1993; 90: 483-8.

116. Geifman-Holtzman O, Bernstein IM, Berry SM, Bianchi DW. Prenatal diagnosis of fetal Rhesus (Rh) C, D, E type by polymerase chain reaction (PCR) (abstract 30). Am J Obstet Gynecol 1995; 172: 265.

117. Lo YMD, Bowell PJ, Selinger M, et al. Prenatal determination of fetal $\mathrm{RhD}$ status by analysis of peripheral blood of rhesus negative mothers. Lancet 1993; 341: 1147-8.

118. Bianchi DW, Mahr A, Zickwolf GK, et al. Detection of fetal cells with 47, XY,+21 karyotype in maternal peripheral blood. Hum Genet 1992; 90: $368-70$.

119. Gänshirt-Ahlert D, Borjesson-Stoll R, Burschyk M, et al. Detection of fetal trisomies 21 and 18 from maternal blood using triple gradient and magnetic cell sorting. Am J Reprod Immunol 1993; 30: 194-201.

120. Huang R, Barber TA, Tompkins RG, et al. A microfluidics approach for the isolation of nucleated red blood cells (NRBCs) from the Peripheral blood of Pregnant Women. Prenat Diagn 2008; 28: 892-9.

121. Lo Y, Corbetta N, Chamberlain PF, et al. Presence of fetal DNA in maternal plasma and serum. Lancet 1997; 350: 485-7.

122. Sekizawa A, Kondo T, Iwasaki M, et al. Accuracy of fetal gender determination by analysis of DNA in maternal plasma. Clin Chem 2001; 10: $1856-8$

123. Tobias JL, Liu Z, Heermann KH, et al. Specific magnetic bead-based capture of free fetal DNA from maternal plasma. Transfus Apher Sci 2009; 40: 153-7. 


\section{First-trimester screening for aneuploidy Mark I. Evans and Howard S. Cuckle}

\section{INTRODUCTION}

Advanced maternal age was the be all and end all of prenatal risk assessment for aneuploidy for nearly half a century (1-3). In virtually all countries, women above a given age cutoff were regarded as being at high enough risk of aneuploidy to warrant the costs and hazards of performing an invasive, diagnostic procedure. Over the past three decades, attempts have been made to refine the assessment of an individual woman's risk using biochemical and ultrasound markers within pregnancy. These have markedly improved the sensitivity (proportion of aneuploidy pregnancies at high risk, or detection rate) and specificity (proportion of unaffected pregnancies not at high risk) (3). Using a cutoff maternal age of 35, a 30\% to $40 \%$ sensitivity and $90 \%$ to $95 \%$ specificity (or 5-10\% false-positive rate) were the best available statistics throughout the 1970s and early 1980s (4).

Over the past 25 years, there have been multiple iterations of approaches that have improved the statistics of screening $(2,3)$. Incorporation of these approaches has been haphazard with huge variability around the world and even within countries. Much has been written over the years describing the state of the art at given times and will not be repeated in extensive detail here for previous eras.

In 1984, Merkatz et al. published the association of low maternal serum alpha-fetoprotein (AFP) with an increased risk of aneuploidy in general (4), and Cuckle et al. confirmed that this holds for Down syndrome (5). In subsequent years, there was also an eventual understanding that the extent of AFP reduction differed according to the type of aneuploidy. For example, trisomy 18 has much lower values on average than Down syndrome (6).

Since AFP was already widely being used to screen for neural tube defects, at 16 to 18 weeks of gestation, it was relatively simple to include aneuploidy. This was done by the calculation of a likelihood ratio (LR) (proportion of aneuploidy pregnancies divided by proportion of unaffected pregnancies with the given AFP level) and using this to increase or decrease the maternal age-specific risk. The LR was derived from a Gaussian model of the AFP distributions in aneuploidy and unaffected pregnancies. The detailed practical mechanics of biochemical screening, such as adjustments for gestational age, race, diabetic status, multiple gestation status, and maternal weight have been published previously and will not be repeated here $(2,3)$.

Maternal serum AFP screening for aneuploidy was widely adopted and had the potential to increase the detection rate, but it was inefficient. The optimal use of a biochemical or ultrasound marker is to screen all women regardless of age and to define high risk purely on the basis of the screening result. However, many clinicians did not consider a low-risk AFP result in an older woman as sufficient grounds for not offering invasive testing. While the use of maternal serum AFP was a notable improvement over "how old are you," it left much to be desired.

The situation changed in the late 1980s when the first highly discriminatory biochemical marker was discovered, namely human chorionic gonadotropin (hCG). The molecule is a hetero-dimer consisting of $\alpha$ and $\beta$ subunits, which is not only present in maternal serum predominantly as the biologically active intact dimer, but also exists to a much lesser degree as both the free- $\alpha$ and free- $\beta$ subunits. Most modern hCG assays are actually nonspecific and measure both the intact dimer and the free- $\beta$ subunit. However, since the intact dimer is present in the maternal serum in a 200 -fold molar excess relative to the free- $\beta$ subunit, these assays primarily reflect the intact hCG concentration (3). Unfortunately, inconsistency in the terminology used to describe hCG assays has created confusion (sometimes deliberately) when comparing first-trimester screening studies. Intact hCG assays are often termed "total $\beta$ " or simply " $\beta$ hCG" assays, and some researchers have also described free $\beta$-hCG as $\beta$ hCG (7).

Both intact (or total) hCG and free $\beta$-hCG are established markers of both Down syndrome and trisomy 18, being increased on average in the former and decreased in the latter type of aneuploidy $(8,9)$.

\section{MULTIPLE BIOCHEMICAL MARKERS}

The discovery that hCG was a marker was quickly followed by another second-trimester marker, unconjugated estriol $\left(\mathrm{uE}_{3}\right)$, and later dimeric inhibin $A(3,10)$. This gave the impetus in the 1990s, for the combination of multiple second-trimester maternal serum markers $(3,10)$. As with AFP alone, an LR was calculated and used to modify the maternal age-specific risk. In this case, it was derived from a multivariate Gaussian model of the marker distributions taking into account the various correlations between markers.

Down syndrome detection rates increased to approximately $60 \%$ to $70 \%$ for a $5 \%$ false-positive rate $(11-13)$. Table 1 shows the predicted detection rate for a fixed $1 \%, 3 \%$, and $5 \%$ false positive using the principal combinations found in clinical practice (15).

While the switch over from AFP alone to two (double test), three (triple test), or four (quadruple test) marker combinations has gradually taken place in most countries, there have been continual disputes over the best combinations. There was essentially universal agreement that among the single markers in the second trimester, hCG, free $\beta$-hCG, and inhibin have the greatest discriminatory power. While $\mathrm{uE}_{3}$ has similar discriminatory power to AFP, since the latter is already used for neural tube defect screening, it is always included as the second marker. There have been disputes over whether to include $\mathrm{uE}_{3}$ as a third parameter. Some have claimed that the 
Table 1 Biochemical Marker Combinations (Second Trimester): Predicted ${ }^{\mathrm{a}}$ Detection Rate for a Given False-Positive Rate

\begin{tabular}{lccc} 
& \multicolumn{3}{c}{ False-positive rate } \\
\cline { 2 - 4 } Marker combination & $\mathbf{1 \%}$ & $\mathbf{3 \%}$ & $\mathbf{5 \%}$ \\
\hline AFP and free $\beta$-hCG & $37 \%$ & $53 \%$ & $61 \%$ \\
AFP, free $\beta$-hCG, and $\mathrm{uE}_{3}$ & $42 \%$ & $57 \%$ & $65 \%$ \\
AFP, free $\beta$-hCG, uE 3 , and inhibin & $50 \%$ & $64 \%$ & $71 \%$ \\
AFP and intact hCG & $33 \%$ & $48 \%$ & $56 \%$ \\
AFP, intact hCG, and uE & $39 \%$ & $53 \%$ & $60 \%$ \\
AFP, intact hCG, $\mathrm{uE}_{3}$, and inhibin & $46 \%$ & $60 \%$ & $67 \%$ \\
${ }^{\mathrm{a}}$ Modeling with parameters in (14). & & &
\end{tabular}

predicted marginal increase in detection rate cannot be achieved in practice. However, much of the prospective series literature did show the predicted benefit. Moreover, $\mathrm{uE}_{3}$ is of value in the detection of trisomy 18, Smith-Lemli-Opitz syndrome, and placental sulfatase deficiency, where $\mathrm{uE}_{3}$ levels are extremely low. Incidentally, levels are also slightly lowered in spina bifida and more so in anencephaly, but the changes are much less than that for AFP (16).

Like hCG, free $\beta$-hCG, and AFP, the levels of $\mathrm{uE}_{3}$ and inhibin are altered on average in trisomy 18 . For some of these markers, the direction of change is the same for both types of aneuploidy. Consequently, although the birth prevalence of trisomy 18 is 10 -fold lower than Down syndrome, it is a relatively frequent incidental diagnosis in women having invasive testing following second-trimester screening. But in order to achieve a high detection for this disorder, it is necessary to calculate a separate risk specifically (8).

Despite overwhelming evidence by the early 1990s, and recommendations of national organizations such as the American College of Obstetricians and Gynecologists, that the multiple biochemical marker combinations discussed so far be offered, even by the millennium, still nearly $20 \%$ of patients in the United States who had screening were having AFP alone (3). Such slow uptake by practicing obstetricians is worrisome precedent for the incorporation of future test combinations.

Another long promising but yet fulfilled marker was the search for fetal cells in maternal circulation. Studies throughout the 1990s and early 2000s suggested that isolation and analysis of fetal cells might, in fact, become practical and useful as a screening test $(17,18)$. Much of the 1980s and 1990s focused on ways to improve the efficacy of detection methods primarily centered on the need to increase the enrichment of fetal cells from the maternal blood circulation whose prevalence has been estimated to be approximately 1 in $10,000,000$ cells with no clear likelihood of success (19). After the failure of the first lines of methodology in detecting fetal cells, modified approaches have emerged that are being evaluated for more precise identification and isolation of fetal cells.

A more recent line of research has been the detection of fetal cell-free DNA and RNA in the maternal circulation initially suggested by Lo et al. (20). There have been three generations, to date, of technologic approaches in this area. First, the concentration of these materials in maternal blood is approximately doubled in aneuploidy, and it was thought that quantitative differences could be used as an additional marker. However, fetal DNA only makes up a small proportion of free DNA in maternal circulation such that the actual difference between a trisomic fetal contribution to overall maternal DNA levels is only a few percent. Second, Lo et al. proposed that using a placental-specific RNA such as PLAC-4 would allow a real 2:1 discrimination. His data suggested a $90 \%$ sensitivity for a $96 \%$ specificity. The methods were licensed to Sequenom whose unpublished investor presentations claimed $100 \%$ sensitivity for $99 \%$ sensitivity. Unfortunately these data were falsified, and the investigator has pleaded guilty to fraud charges setting back the approach immeasurably. Furthermore, RNA is very labile requiring stabilization within hours rendering problematic the use of the technique for mass screening. Third, the advent of the next-generation massive sequencing methods does have real scientific merit. There is significant potential for its use (21-26).

Amplification and molecular analysis of this circulating fetal product is already being used clinically for fetal gender and $\mathrm{RhD}$ determination. Other Mendelian diagnoses may probably follow in short order. It might eventually be used as a noninvasive method of prenatal diagnosis for aneuploidy, but much more research is still needed before this goal can be achieved in any reliable fashion. While the diagnosis of Mendelian disorders may be possible by fetal cells or cell-free DNA, it is very likely that aneuploidy detection will be seen as a screening test to modify risk as a predicate for invasive diagnostic procedures (26).

\section{COMBINATION OF BIOCHEMICAL WITH ULTRASOUND MARKERS}

Considerable work in the mid-1990s focused on the development of ultrasound markers to be used to modify the risk of aneuploidy. As early as 1985, Benacerraf et al. observed an increased nuchal skinfold during the second-trimester genetic ultrasound examination among aneuploid fetuses, but it was rarely used in routine screening (27). Nevertheless, in 1992, Nicolaides et al. reported on a related, but more discriminatory marker, nuchal translucency (NT) determined in the first trimester, which did enter routine practice, and results have now been reported on well over a million pregnancies scanned at 11 to 13 weeks of pregnancy (28). The literature on this development has been reviewed extensively elsewhere and will not be repeated here except to state that quality control of NT measurements is inferior to biochemical methods. Undermeasurement of NT continues to be prevalent and lowers detection rates considerably $(29,30)$. Instead, we concentrate on combinations between ultrasound NT and biochemical markers (combined test), which can be shown to yield a much higher detection rate than NT alone.

The most widely studied first-trimester biochemical markers are pregnancy-associated plasma protein (PAPP)-A and free $\beta$-hCG. The discriminatory power of PAPP-A decreases with gestation, as does NT, while that of free $\beta$-hCG increases with gestation. First-trimester multiple-marker Down syndrome screening using a combination of the biochemical and ultrasound markers has been offered clinically for nearly 15 years (3). Most centers perform the NT scan at $11 \frac{112}{2}$ to $12 \frac{1}{2}$ weeks since the NT is easier to visualize than at earlier and some fetal structural abnormalities can be seen, while by 
Table 2 Nuchal Translucency with or Without Biochemical Marker Combinations (First Trimester): Predicted ${ }^{\mathrm{a}}$ Detection Rate for a Given False-Positive Rate

\begin{tabular}{lccccc|} 
& Gestation (weeks) & \multicolumn{3}{c}{ False-positive rate } \\
\cline { 2 - 6 } Marker combination & Serum & NT & $\mathbf{1 \%}$ & $\mathbf{3} \%$ & $\mathbf{5 \%}$ \\
\hline NT alone & - & 11 & $64 \%$ & $73 \%$ & $77 \%$ \\
& - & 12 & $62 \%$ & $71 \%$ & $75 \%$ \\
& - & 13 & $57 \%$ & $66 \%$ & $71 \%$ \\
PAPP-A, free $\beta$-hCG, & 10 & 11 & $74 \%$ & $83 \%$ & $87 \%$ \\
$\quad$ and NT & 11 & 11 & $74 \%$ & $83 \%$ & $87 \%$ \\
& 11 & 12 & $73 \%$ & $82 \%$ & $86 \%$ \\
& 12 & 12 & $72 \%$ & $80 \%$ & $84 \%$ \\
& 12 & 13 & $68 \%$ & $77 \%$ & $82 \%$ \\
& 13 & 13 & $66 \%$ & $75 \%$ & $80 \%$ \\
${ }^{a}$ Modeling with parameters in (14). & & & & \\
\end{tabular}

Table 3 Predicted $^{\mathrm{a}}$ Detection Rate for a 5\% False-Positive Rate when Adding Either Intact or Free $\beta$-hCG to a PAPP-A and NT Protocol, According to Gestation when Blood Drawn (NT at 11-12 Weeks)

\begin{tabular}{|c|c|c|}
\hline Serum (weeks) & Intact hCG & Free $\beta$-hCG \\
\hline 9 & $80 \%$ & $84 \%$ \\
\hline 10 & $78 \%$ & $83 \%$ \\
\hline 11 & $75 \%$ & $81 \%$ \\
\hline 12 & $77 \%$ & $80 \%$ \\
\hline
\end{tabular}

13 weeks the discriminatory power is reduced. To maximize the effectiveness of the biochemical markers and to ensure that the risk is calculated and immediately available at the same time, the woman attends for the ultrasound examination, while the blood sample may be drawn 1 to 2 weeks before the scheduled scan date, ideally at 10 weeks of gestation $(14,31,32)$.

Table 2 shows the predicted detection rate for a fixed $1 \%$, $3 \%$, and $5 \%$ false positive using the marker combinations at different gestations and for comparison the results for NT alone. As before, the predictions are based on statistical modeling using published marker parameters derived by meta-analysis (14). The addition of biochemical markers leads to a substantial increase in the detection rate, which is considerably higher than that for the second-trimester combinations. PAPP-A levels are also altered on average in trisomy 18. Since for all the first-trimester markers the direction of change is the same for Down syndrome and trisomy 18 , unlike in the second trimester, there is little value in a separate risk calculation.

First-trimester screening not only has a yield greater than that of the second-trimester screening protocols, but also offers significant advantages including, earlier reassurance for the vast majority of patients and greater privacy and safety for patients who may decide to terminate an affected fetus (32). There is no disagreement over the utility of combining first-trimester biochemical markers and NT, but there remains controversy over the use of free $\beta$-hCG rather than intact (or total) hCG in the combination in the United States but not the rest of the world. The medical and economic implications of this choice in the United States are enormous, and we therefore dwell on this otherwise relatively minor point.

Most retrospective reports on biochemistry indicated that while free $\beta$-hCG was a very effective marker for Down syndrome in the first trimester, intact hCG was not $(3,7)$. Indeed, the discriminatory power of intact hCG is extremely low at 10 weeks and does not even reach that of the weakest second-trimester markers until 12 weeks (10). Consequently, the vast majority of prospective first-trimester screening studies have used free $\beta$-hCG in combination with PAPP-A, and NT. The Serum, Urine, and Ultrasound Screening Study tested both hCG isoforms and suggested that free $\beta$-hCG is a more discriminatory first-trimester marker than intact hCG, but there may be little difference in the overall screening performance when included in a multiple marker protocol (10). Although often described as a prospective study with results on more than 47,000 pregnancies, the biochemical results are actually based on retrospective analysis of less than 500 samples. Furthermore, we believe that a statistical correction to the analysis would have demonstrated the advantage of free $\beta$-hCG even when combined with other markers (7). Nevertheless, the study has created some uncertainty and opened a debate in the United States about which isoform of hCG to use in first-trimester screening.

This prompted us to address the issue using a meta-analysis based on a considerable body of available evidence to predict the consequence of adding either free $\beta$-hCG or intact hCG to a PAPP-A and NT protocol; the methodology was described extensively in the paper (7). At a fixed 5\% false-positive rate, when the blood sample was drawn at $9,10,11$, and 12 weeks, adding free $\beta$-hCG reduced the false-negative rate by $26 \%$, $29 \%, 33 \%$, and $35 \%$, respectively, compared with reductions of $0 \%, 5 \%, 12 \%$, and $21 \%$, respectively, adding intact hCG. Averaging across weeks, the reductions were 31\% and $9 \%$, a statistically significant difference $(p=0.01)$. Similarly, at a fixed $90 \%$ detection rate, adding free $\beta$-hCG reduced the falsepositive rate by $41 \%, 45 \%, 48 \%$, and $52 \%$ compared with $2 \%$, $8 \%, 20 \%$, and $37 \%$ for intact hCG, averages of $47 \%$ and $17 \%$ $(p=0.01)$. Overall multivariate detection rates averaged a $3 \%$ to $6 \%$ improvement using free $\beta$ as opposed to total hCG, and maximal detection was achieved by blood drawn at 9 to 10 weeks with NT at 11 to 12 weeks (Table 3) (7).

Canick et al. (33) carried out a secondary analysis of data from the First and Second Trimester Evaluation of Risk (FASTER) trial (34) and found that while the sensitivity of free $\beta$-hCG was notably higher than that for total hCG, there was only a $1 \%$ detection rate difference in combination with PAPP-A and NT and it was not statistically significant. The authors concluded that the choice of the third marker was not critical. The analysis was not based on the entire 38,000 patients in the trial, but only on statistical modeling with total hCG and free $\beta$-hCG parameters derived by retrospectively assaying stored serum samples from 79 Down syndrome and 395 unaffected pregnancies (31).

Canick's conclusions regarding screening policy are unwarranted for three reasons. First, the standard deviation of free $\beta$-hCG was much wider than generally found elsewhere, possibly due the specific reagents used to retrospectively assay the samples. The standard deviation among unaffected pregnancies, compared with values from all the 
recent large published prospective first-trimester series, and that from our own experience is shown in Table 4. This will inevitably have led to the underestimation of effectiveness for free $\beta$-hCG. Moreover, the retrospective testing was done over a short time period, reducing the standard deviation compared with routine practice. Hence, the discrepancy with routine practice is even greater. Second, decisions regarding screening policy need to take account of the totality of available evidence. Modeling with parameters derived by meta-analysis from series including several hundred Down syndrome pregnancies indicates a $2 \%$ detection advantage for free $\beta$-hCG $(7,14)$. The failure of a relatively small study to find a statistically significant effect is insufficient grounds for changing policy. Third, at the high detection rates achievable by first-trimester marker combinations, a $2 \%$ increment in detection is not small. Each marginal increase in detection is achieved by a large increase in the false-positive rate. Had the current study estimated the false-positive rate for a fixed $90 \%$ detection rate, as we have done above, this would have been apparent. Therefore, on the basis of all available evidence, there is no good reason for health planners to consider substituting intact hCG for free $\beta$-hCG in first-trimester protocols.

\section{SEQUENTIAL SCREENING METHODS}

In the last few years, considerable discussion of screening policy has been focused on carrying out screening in both the first and second trimesters, in sequence. In general, this achieves a higher yield than screening within trimester.

Three types of sequential policy have received attention. The first to be proposed was a form of nondisclosure sequential screening using first-trimester PAPP-A and NT together with

Table 4 Standard Deviation (SD) of First-Trimester Free $\beta$-hCG in Different Series of Unaffected Pregnancies

\begin{tabular}{lllc} 
Series & \multicolumn{1}{c}{ Reagents } & \multicolumn{1}{c}{$\#$} & SD $^{\mathbf{b}}$ \\
Krantz et al., 2002 & NTDLabs & 10,106 & 0.222 \\
Crossley et al., 2002 & Kryptor & 17,229 & 0.269 \\
Muller et al., 2003 & Delfia & 5634 & 0.269 \\
Spencer et al., 2003 & Kryptor and Delfia & 31,773 & 0.261 \\
Rozenberg et al., 2006 $^{\text {a }}$ & Delfia & 10,632 & 0.257 \\
Author's experience & Delfia & 28,276 & 0.266 \\
Weighted average $^{\text {c }}$ & Delfia, Kryptor, & 103,646 & 0.260 \\
& $\quad$ and NTDLabs & & \\
Canick et al., 2006 & DSL & 395 & 0.307
\end{tabular}

Delfia, Perkin-Elmer Life Sciences, and Kryptor, Brahms. ${ }^{a}$ Routine screening tests carried out in Leeds, UK. ${ }^{\mathrm{b}} \log _{10}$ units. ${ }^{\mathrm{c}}$ Weighted by the number of pregnancies. Abbreviation: DSL, Diagnostic Systems Laboratories. second-trimester $\mathrm{AFP}, \mathrm{uE}_{3}$, free $\beta$-hCG or intact $\mathrm{hCG}$, and inhibin (integrated test). Risks are not used clinically until all markers have been tested. The proponents of such "integrated" screening argue that higher sensitivities can be achieved and therefore justify the nondisclosure. However, many clinicians in the United States and elsewhere feel that it is simply not acceptable under local culture and ethical beliefs to withhold potentially serious screening results for a month when the odds of substantial change are minimal. Such an approach also has the substantial disadvantage that there is no early diagnosis or reassurance. Also there may be practical difficulties such as a considerable proportion of patients who do not present for the second half of the screening evaluation. There are also profound ethical concerns over nondisclosure of potentially considerably increased risks that could be addressed in the first trimester with its considerable advantages of privacy for the patient in difficult reproductive decisions.

A second approach (step-wise test) begins with firsttrimester PAPP-A, free $\beta$-hCG or intact hCG, and NT; those with low risk have second-trimester AFP, $\mathrm{uE}_{3}$, free $\beta$-hCG or intact hCG, and inhibin; their risk is estimated from all seven markers. It is important to use a high first-trimester cutoff than with nonsequential screening, otherwise the overall falsepositive rate will be too high. And it is essential to use all seven markers together when calculating the final risk. It is invalid to ignore the first-trimester markers at this stage although many practitioners are doing so because they do not have access to the appropriate risk calculation software. This policy restores some first-trimester diagnosis.

A third policy, more efficient than the other types, is called the contingent test. This begins with first-trimester PAPP-A, free $\beta$-hCG or intact hCG, and NT. Women with very high risk are offered immediate invasive prenatal diagnosis and only those with borderline risks are offered second-trimester AFP, $\mathrm{uE}_{3}$, free $\beta$-hCG or intact hCG, and inhibin; their risk is estimated from all seven markers. The borderline is chosen so that a large proportion of women have early assurance. This group has such a low risk that it is very unlikely that further markers will lead to a final high-risk result.

Table 5 shows the predicted detection rate for a fixed $1 \%$, $3 \%$, and $5 \%$ false positive for different sequential policies, as before based on statistical modeling using published marker parameters derived by meta-analysis. In the current predictions, the first-trimester very high cutoff for step-wise and contingent is set to obtain an early detection rate of $70 \%$, and the very low cutoff for contingent screening is set so that $85 \%$

Table 5 Sequential Screening Policies: Predicted ${ }^{\mathrm{a}}$ Detection Rate for a Given False-Positive Rate

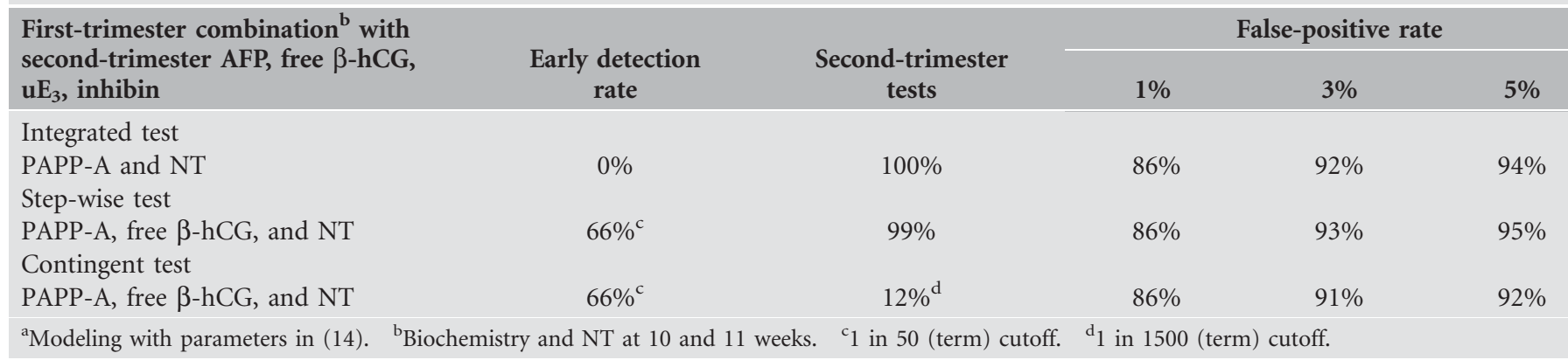


of women have early assurance. The prediction is that the contingent test is extremely efficient, achieving comparable detection rates with the other types of sequential screening but requiring second-trimester tests on just a small proportion of women. The tabulated combinations use free $\beta$-hCG, but similar analyses with intact (or total) hCG, tested in the late first trimester and/or the second trimester, reach the same conclusion.

Sequential screening can also be carried out within trimester. One contingent approach suggested by Nicolaides et al. has the potential to further increase detection while completing all screening within the first trimester (35). It is proposed that women with borderline risk based on PAPP-A, free $\beta$-hCG, and NT are offered a more specialist scan to determine, among other things nasal bone, and reassess the risk. Nasal bone hypoplasia is a very powerful marker of aneuploidy but requires appropriate training not generally available. Another approach might be to measure additional biochemical markers in a very early serum sample at about 8 weeks of gestation in addition to the 10 -week sample; one possible very early marker is ADAM12 (36).

\section{SECOND-TRIMESTER REVISITED}

Despite the clear advantages of beginning the screening process in the first trimester, some women do not present until later in pregnancy. If biochemical screening alone, even with the quadruple test, is regarded as inadequate, there is the option of combining information on the serum markers and ultrasound results to improve detection. The results of the genetic sonogram can be used to modify the risk from the biochemical markers. Provided an algorithm is used that uses a separate LR for the presence and absence of each soft marker, the detection rate can be increased by $5 \%$ to $10 \%$ (37). Moreover, there are new second-trimester ultrasound markers such as prenasal translucency, which are even more discriminatory than the traditional soft markers and can by themselves increase detection by about 15\% (38).

\section{CONCLUSIONS}

Over the past 35 years, there has been a steady evolution of biochemical and also biophysical parameters used to adjust the risk that a woman is carrying a fetus with aneuploidy. At every step, there has been controversy as to the best methods to employ in this endeavor. We have laid out many of the proposals, and our conclusions are that as of 2011:

1. A combined test in the first trimester can yield a very high detection rate for an acceptable falsepositive rate.

2. Second-trimester multiple marker biochemical screening yields a much lower detection rate and imposes a considerable emotional burden in requiring women to be very visibly pregnant, feel the baby moving, and have to undergo secondtrimester termination methods if an abnormality is found and the women choose to end the pregnancy.

3. Sequential screening in both trimesters yields even higher detection rates, and the most efficient methods is the contingent test. Centers with appropriate training and experience of newer ultrasound markers such as nasal bone hypoplasia could consider carrying out the contingent test within the first trimester.

Although comprehensive national data are not available, there is a continuing shift toward the first trimester. Only the exact components and timing remain to be determined.

\section{REFERENCES}

1. Evans MI, Cuckle HS. Biochemical screening for aneuploidy. Expert Rev Obstet Gynecol 2007; 2: 765-74.

2. Cuckle HS, Arbuzova S. Epidemiology of aneuploidy. In: Evans MI, Jonson MP, Yaron Y, Drugan A, eds. Prenatal Diagnosis. NY: McGraw Hill Publishing Co, 2006: 1932.

3. Evans MI, Galen RS, Drugan A. Biochemical screening. In: Evans MI, Jonson MP, Yaron Y, Drugan A, eds. Prenatal Diagnosis. NY: McGraw Hill Publishing Co, 2006: 277-88.

4. Merkatz IR, Nitowsky FM, Macri JN, Johnson WE. An association between low maternal serum alpha-fetoprotein and fetal chromosome abnormalities. Am J Obstet Gynecol 1984; 148: 886-94.

5. Cuckle HS, Wald NJ, Lindenbaum RH. Maternal serum alpha-fetoprotein measurement: a screening test for Down syndrome. Lancet 1984; i: 926-9.

6. Nyberg DA, Kramer D, Resta RG, et al. Prenatal monographic findings of trisomy 18: review of 47 cases. J Ultrasound Med 1993; 2: 103-13.

7. Evans MI, Krantz DA, Hallahan TW, Galen RS. Meta-analysis of First trimester Down Syndrome screening studies: free $\beta$ human chorionic gonadotropin significantly outperforms intact human chorionic gonadotropin in a multimarker protocol. Am J Obstet Gynecol 2007; 196: 198-205.

8. Benn PA, Ying J, Beazoglou T, Egan JF. Estimates for the sensitivity and false-positive rates for second trimester serum screening for Down syndrome and trisomy 18 with adjustment for cross-identification and double-positive results. Prenat Diagn 2001; 21: 46-51.

9. Wald NJ, Kennard A, Hackshaw A, McGuire A. Antenatal screening for Down's syndrome. Health Technol Assess 1998; 2: i-iv, 1-112.

10. Wald NJ, Rodeck DH, Hackshaw AK, et al. SURUSS Research Group: first and second trimester antenatal screening for Down Syndrome; the results of the Serum, urine, and ultrasound screening study (SURUSS). Health Technol Assess 2003; 7: 1-7.

11. Wald NJ, Cuckle HS, Densem JW, et al. Maternal serum screening for Down syndrome in early pregnancy. Br Med J 1988; 297: 883-7.

12. Aitken DA, McCaw G, Crossley JA, et al. First-trimester biochemical screening for fetal chromosome abnormalities and neural tube defects. Prenat Diagn 1993; 13: 681-3.

13. Rodriguez L, Sanchez R, Hernandez J, et al. Results of 12 year' combined maternal serum alpha-fetoprotein screening and ultrasound fetal monitoring for prenatal detection of fetal malformations in Havana City, Cuba. Prenat Diagn 1997; 17: 301-4.

14. Cuckle H, Benn P. Multianalyte maternal serum screening for chromosomal defects. In: Milunsky A, Milunsky JM, eds. Genetic Disorders and the Fetus: Diagnosis, Prevention and Treatment. 6th edn. Baltimore: Johns Hopkins University Press, 2010: 771-818.

15. Cuckle H, Aitken D, Goodburn S, et al. Age-standardisation for monitoring performance in Down's syndrome screening programmes. Prenat Diagn 2004; 24: 851-6.

16. Yaron Y, Hamby DD, O'Brien JE, et al. The combination of elevated maternal serum alpha-fetoprotein and low estriol is highly predictive of anencephaly. Am J Med Genet 1998; 75: 297-9.

17. Elias S, Simpson JL. Prospects for prenatal diagnosis by isolating fetal cells from maternal blood. Contemp Rev Obstet Gynecol 1995; 7: 135-9.

18. Herzenberg LA, Bianchi DW, Schroder J. Fetal cells in the blood of pregnant women: detection and enrichment by fluorescence-activated cell sorting. Proc Nat Acad Sci USA 1979; 76: 1453-5.

19. Bianchi DW, Simpson JL, Jackson LG, et al. Fetal gender and aneuploidy detection using fetal cells in maternal blood: analysis of NIFTY I data. Prenat Diagn 2002; 22: 609-15.

20. Lo YM, Corbetta N, Chamberlain PF, et al. Presence of fetal DNA in maternal plasma and serum. Lancet 1997; 350: 485-7.

21. Fan HC, Blumenfeld YJ, Chirkara U, et al. Noninvasive diagnosis of fetal aneuploidy by shotgun sequencing DNA from maternal blood. Proc Natl Acad Sci USA 2008; 105: 16266-71. 
22. Chiu RWK, Chan A, Gao Y, et al. Non invasive prenatal diagnosis of fetal chromosomal aneuploidy by massively parallel genomic sequencing of DNA in maternal plasma. Proc Natl Acad Sci USA 2008; 105: 20458-63.

23. Chiu RWK, Alolekar R, Zheng YWL, et al. non invasive prenatal assessment of trisomy 21 by multiplexed maternal plasma DNA sequencing: large scale validy study. BMJ 2011; 342c: 7401.

24. Ehrich M, Deciu C, Zwiefelhofer T, et al. Noninvasive detection of fetal trisomy 21 by sequencing of DNA in maternal blood: a study in a clinical setting Amer J Obstet Gynecol 2011; 205-e4.

25. Papageorgiou EA, Karagrigoriou A, Tsaliki E, et al. Fetal-specific DNA methylation ratio permits noninvasive prenatal diagnosis of trisomy 21 . Nature Med 2011; doi10.1038/nm.2312.

26. Lo YMD, Chan KCA, Sun H, et al. Maternal plasma DNA sequencing reveals the genome wide genetic and mutational profile of the fetus. Sci Transl Med 2010; 2: 61-91.

27. Benacerraf BR, Barss VA, Laboda LA. A sonographic sign for the detection in the second trimester of the fetus with Down's syndrome. Am J Obstet Gynecol 1985; 151: 1078-9.

28. Nicolaides KH, Bindra R, Heath V, Cicero S. One-stop clinic for assessment of risk of chromosomal defects at 12 weeks of gestation. J Matern Fetal Neonatal Med 2002; 12: 9-18.

29. Evans MI, Van Decruyes H, Nicolaides KH. Nuchal translucency (NT) measurements for 1st trimester screening: the "price" of inaccuracy. Fetal Diagn Ther 2007; 22: 401-4.

30. Evans MI, Krantz DA, Hallahan TW, Sherwin JE. Undermeasurement of nuchal translucencies: implications for screening. Obstet Gynecol 2010; 116: 815-18
31. Crossley JA, Aitken DA, Cameron AD, McBride E, Connor JM. Combined ultrasound and biochemical screening for Down's syndrome in the first trimester: a Scottish multicentre study. Br J Obstet Gynaecol 2002; 109: 667-76.

32. Evans MI, Wapner RJ, Bui TH. Future directions in genomic revolution and obstetrics and gynecology. Ballieres Obstet Gynecol 2002; 16: 757-9.

33. Canick JA, Lambert Messerlian GM, et al. Comparison of serum markers in first trimester screening for Down syndrome. Obstet Gynecol 2006; 108: 1192-9.

34. Malone FD, Canick JA, Ball RH, et al. First trimester or second trimester screening, or both, for Down syndrome. N Engl J Med 2005; 353: 2068-70.

35. Nicolaides KH, Spencer K, Avgidou F, Faiola S, Falcon O. Multicenter study of first-trimester screening for trisomy 21 in 75821 pregnancies: results and estimation of the potential impact of individual risk-orientated two-stage first-trimester screening. Ultrasound Obstet Gynecol 2005; 25: 221-6.

36. Laigaard J, Spencer K, Christiansen M, Cowans NJ, Olesen Larsen S. ADAM 12 as a first-trimester maternal serum marker in screening for Down syndrome. Prenat Diagn 2006; 26: 973-9.

37. Aagaard Tillery KM, Malone FD, Nyberg DA, et al, for the FaSTER Research Consortium. Role of second trimester genetic sonography following Down syndrome screening. Obstet Gynecol 2009; 114: 1189-96.

38. Miguelez J, Maymon R, Cuckle H, et al. Model predicted performance of second trimester Down syndrome screening with ultrasound prenasal thickness. J Ultrasound Med (In press). 


\section{Second-trimester screening for fetal abnormalities Jolene C. Muscat and Anthony M. Vintzileos}

\section{INTRODUCTION}

Screening tests to identify women at risk for fetal aneuploidy and fetal structural abnormalities are routinely offered to all women during pregnancy. A number of screening strategies are currently available, which utilize both biochemical analysis and ultrasonography to achieve high detection rates and determine whether further diagnostic testing is needed. Recently, first-trimester screening for fetal abnormalities has been utilized with increasing frequency as it offers women the opportunity of earlier testing and subsequent option for earlier diagnosis. Despite this change in practice, second-trimester biochemical and ultrasound screening remains a valuable resource in modern obstetric care as complementary to firsttrimester screening or as the only screening in women who present for prenatal care after the first trimester of pregnancy.

\section{SECOND-TRIMESTER SERUM MARKER SCREENING}

Most second-trimester serum marker screening is now performed in conjunction with first trimester-combined screening protocols that utilize either an integrated, stepwise, or contingent approach. Currently, second-trimester maternal serum screening alone is available as a triple, quadruple, or penta-marker testing modality. Whether integrated with firsttrimester serum marker testing or used in isolation for women who defer first-trimester screening or do not seek prenatal care until after 13 weeks of gestation, second-trimester serum marker screening remains a valuable tool in prenatal management.

\section{Maternal Serum Alpha-Fetoprotein}

Maternal serum alpha-fetoprotein (MSAFP) was the first maternal serum analyte utilized to detect fetal abnormalities, specifically, open neural tube and abdominal wall defects in cases of elevated MSAFP. In the mid-1980s, an association between a low MSAFP level and increased risk for Down syndrome was reported (1). MSAFP, along with other maternal serum analytes, was then incorporated into various screening protocols designed to modify maternal age-related risks for fetal aneuploidy.

\section{Elevated MSAFP}

Alpha-fetoprotein (AFP) is a normal fetal protein found in high concentrations in the fetal serum. It is present in amniotic fluid in normal pregnancies, presumably reaching the fluid through excretion in the fetal urine. From the amniotic fluid, AFP diffuses across the fetal membranes and can be detected in the maternal serum beginning at approximately 12 weeks of gestation (2). In cases of fetal anencephaly or open spina bifida, increased levels of AFP reach the amniotic fluid secondary to the concentration gradient from fetal serum to amniotic fluid. This increased AFP concentration can then be detected in the maternal serum. Ventral wall defects (i.e., omphalocele or gastroschisis) can also result in similar elevations in MSAFP through this same mechanism. Other causes of elevated MSAFP are listed in Table 1.

MSAFP screening provides an effective means for selecting patients who are at increased risk for neural tube defects and other fetal disorders and identifies patients who warrant further evaluation with diagnostic ultrasound and/or amniocentesis. Additionally, elevated MSAFP in a patient with a normal fetal anatomic survey has been associated with adverse pregnancy outcomes (3). As a matter of fact, the most frequent pathologic reason for elevated MSAFP is placental disease. Although there is no consensus regarding the management of this patient population, women in this high-risk group for obstetric complications should be followed closely for the duration of the pregnancy for stillbirth, prematurity, intrauterine growth restriction, and neonatal death. In the presence of placenta previa and elevated MSAFP, placenta accreta should be ruled out, especially in patients with previous cesarean delivery.

\section{Low MSAFP}

The association between low MSAFP and fetal chromosomal abnormalities is well described in the literature and will be discussed in further detail later in this chapter. As with elevated MSAFP levels, a low MSAFP level is associated with a variety of fetal and maternal conditions (Table 2).

Ultrasound can be used in the initial evaluation of a patient with low MSAFP. The most common explanation for a low value is incorrect pregnancy dating and a gestational age less advanced than expected. Ultrasound will also identify missed abortion or molar pregnancy as a potential cause of low MSAFP. If ultrasound reveals a viable fetus at the anticipated gestational age, targeted genetic ultrasound for evaluation of Down syndrome should be performed. Patients should be counseled regarding the availability of amniocentesis as a diagnostic procedure. Low MSAFP in a patient with normal ultrasound and amniocentesis has not consistently been associated with adverse pregnancy outcomes and at this time warrants no further investigation.

\section{Serum Screening for Trisomy 21}

An association between low MSAFP levels and fetal chromosomal abnormalities was first described by Merkatz and colleagues in 1984 (1). The initial studies focused primarily on Down syndrome detection; however, further studies have shown an association with other chromosomal abnormalities. Combined maternal serum marker panels were subsequently developed to calculate a risk for Down syndrome in the fetus for a given pregnancy. Historically, maternal age 35 years or older at the time of delivery was used to identify women at the highest risk of having a child with Down syndrome, and only these women were offered genetic counseling and amniocentesis 
Table 1 Findings Associated with Elevated Maternal Serum Alpha-Fetoprotein

Incorrect pregnancy dating

Multiple gestation

Fetal demise

Open neural tube defects

Ventral wall defects

Intestinal atresias

Skin or scalp defects

Congenital nephrosis

Cystic hygroma

Aneuploidy

Placental abnormalities or insufficiency

Partial molar pregnancy

Late pregnancy complications including stillbirth and low birth weight

Table 2 Findings Associated with Low Maternal Serum Alpha-Fetoprotein

Incorrect pregnancy dating

Missed abortion

Hydatidiform mole

Down syndrome and other aneuploidy

or chorionic villus sampling. With the introduction of biochemical serum screening for Down syndrome, the American College of Obstetricians and Gynecologists now recommends that all women should be offered invasive diagnostic testing or aneuploidy screening before 20 weeks of gestation, regardless of maternal age (4).

Triple-marker screening utilizing AFP, hCG, and unconjugated estriol was the first combined marker panel used to screen patients for trisomy 21. In the general population, triple-marker screening identifies approximately $60 \%$ to $70 \%$ of pregnancies affected by trisomy 21 with a $5 \%$ screen-positive rate (5). The quadruple screen was later developed when inhibin A was added to the triple-screen panel $(6,7)$. Penta-screen testing is now available with the addition of invasive trophoblast antigen testing (8-10). These expanded panels increase trisomy 21 detection rate at the same $5 \%$ screen-positive rate (11) (Table 3).

Maternal serum screening for trisomy 21 can be performed anytime between 15 and 22 weeks, but is most accurate when performed between 16 and 18 weeks. Serum analyte levels can vary with gestational age, maternal weight, diabetic status, race, and number of fetuses; therefore, these variables are incorporated into an individual's risk score calculation. Because the maternal age-related risk of trisomy 21 is the basis of any serum screening protocol, both the detection rate and the screen-positive rate increase with maternal age. Different laboratories have established their own reference values and screen-positive cutoffs that reflect an individual woman's reported risk. Based on this modified risk, a woman may opt to pursue invasive diagnostic procedure such as amniocentesis or further modify her risk with genetic ultrasound, which will be detailed later in this chapter.

\section{Serum Screening for Trisomy 18 and Other Fetal Aneuploidy}

In counseling patients, it is important to emphasize that multiple maternal serum screening protocols were developed
Table 3 Sensitivity and Specificity of Prenatal Screening Tests for Down Syndrome

\begin{tabular}{lcc} 
Markers & $\begin{array}{c}\text { Detection } \\
\text { rate (\%) }\end{array}$ & $\begin{array}{c}\text { False-positive } \\
\text { rate (\%) }\end{array}$ \\
\hline Age, AFP, hCG, uE3 & 72 & 5 \\
Age, AFP, hCG, uE3, DIA & 79 & 5 \\
Age, AFP, hCG, uE3, DIA, ITA & 83 & 5 \\
$\begin{array}{l}\text { Abbreviations: AFP, alpha-fetoprotein; hCG, human chorionic gonadotro- } \\
\text { pin; uE3, unconjugated estriol; DIA, dimeric inhibin A; ITA, invasive } \\
\text { trophoblast antigen. }\end{array}$
\end{tabular}

Table 4 Second-Trimester Serum Maker Levels in Various Fetal Chromosomal Abnormalities

$\begin{array}{lcccc}\text { Genetic disorder } & \text { AFP } & \text { uE3 } & \text { hCG } & \text { DIA } \\ \text { Down syndrome } & \downarrow & \downarrow & \uparrow & \uparrow \\ \text { Trisomy 18 } & \downarrow & \downarrow \downarrow & \downarrow \downarrow & \leftrightarrow \\ \text { Trisomy 13 } & \leftrightarrow & \leftrightarrow & \leftrightarrow & \leftrightarrow \\ \text { Triploidy } & \leftrightarrow & \downarrow & \downarrow \text { or } \uparrow & ?\end{array}$

Abbreviations: AFP, alpha-fetoprotein; hCG, human chorionic gonadotropin; uE3, unconjugated estriol; DIA, dimeric inhibin A.

primarily to detect trisomy 21 , and the patterns of serum markers observed in pregnancies affected by trisomy 18 and other fetal chromosomal abnormalities are different. In trisomy 18, MSAFP and unconjugated estriol are low; however, in contrast to Down syndrome, hCG value is low as well. Although these results are well correlated with trisomy 18, a positive second-trimester serum screen has poor sensitivity and poor prediction for trisomy 18 (12). By contrast, genetic ultrasound to evaluate for characteristic sonographic findings associated with this diagnosis is highly sensitive in identifying fetuses with the disorder $(13,14)$. Ultrasound evaluation of fetuses with trisomy 18 will be discussed later in this chapter. Trisomy 13 is usually associated with normal levels of all three maternal serum markers, except in cases where open fetal defects are present. In fetal triploidy, all three biochemical markers are low. Table 4 summarizes the changes in serum marker levels in various fetal chromosomal abnormalities.

\section{SECOND-TRIMESTER FETAL ANATOMY SURVEY}

The American Institute of Ultrasound in Medicine first published guidelines for the performance of obstetric ultrasound in 1994 (15). These guidelines were updated in 2007 to reflect the minimum criteria for a completion of a fetal ultrasound examination (16). A standard obstetric sonogram in the second or third trimester includes an evaluation of fetal presentation, amniotic fluid volume, cardiac activity, placental position, fetal biometry, and fetal number, plus an anatomic survey. Fetal anatomy may be adequately assessed by ultrasound after approximately 18 weeks of gestational age. Minimum requirements in the standard examination of fetal anatomy include evaluating the cerebellum, choroid plexus, cisterna magna, lateral cerebral ventricles, midline falx, cavum septum pellucidum, upper lip, nuchal fold, four-chamber view of the fetal heart, outflow tracts (if technically feasible), stomach (presence, size, and situs), kidneys, bladder, umbilical 
Table 5 American Institute of Ultrasound in Medicine

Minimum Elements of a Standard Examination of Fetal Anatomy

Head, face, and neck
Cerebellum
Choroid plexus
Cisterna magna
Lateral cerebral ventricles
Midline falx
Cavum septum pellucidum
Upper lip
Chest

Four-chamber view of fetal heart

Abdomen

Stomach

Kidneys

Bladder

Umbilical cord insertion site into the fetal abdomen

Umbilical cord vessel number

Spine

Cervical, thoracic, lumbar, and sacral spine

Extremities

Legs and arms (presence or absence)

Sex

Medically indicated in low-risk pregnancies only for evaluation of multiple gestations

cord insertion site into the fetal abdomen, umbilical cord vessel number, cervical, thoracic, lumbar, and sacral spine, extremities, and genitalia (as medically indicated in low-risk pregnancies and in the evaluation of multiple gestations) (Table 5). The placental location, appearance, and relationship to the internal cervical os should be recorded and the umbilical cord should be visualized. The maternal cervix and adnexa should be examined.

A more detailed fetal anatomic examination may be necessary if an abnormality or suspected abnormality is found on the standard examination. Multiple gestations require the documentation of additional information including chorionicity, amnionicity, comparison of fetal sizes, estimation of amniotic fluid volume on each side of the membrane, and fetal genitalia.

Fetal anatomy may be adequately assessed after 18 weeks of gestation and is ideally performed before 20 weeks to optimize assessment of anatomy while at the same time allowing time for invasive testing if necessary or desired. Factors that may limit sonographic examination include equipment quality, sonographer experience, length of time spent on examination, fetal size, position, movement, fluid abnormalities, maternal abdominal scar, and increased maternal abdominal wall thickness.

Performing a complete fetal anatomy survey should be approached in a systematic way. The examination should ideally begin with imaging of the fetal head and proceed caudally. The entire uterus should be scanned in both a transverse and longitudinal direction to determine fetal situs, fetal position, amniotic fluid volume, and placental location. The fetal anatomy is then examined in a stepwise fashion. To begin, the fetal head, face, and neck are evaluated. The calvarium should be well mineralized and elliptical. The head shape may be flattened (dolichocephaly/increased anteroposterior distance) or rounded (brachycephaly/shortened

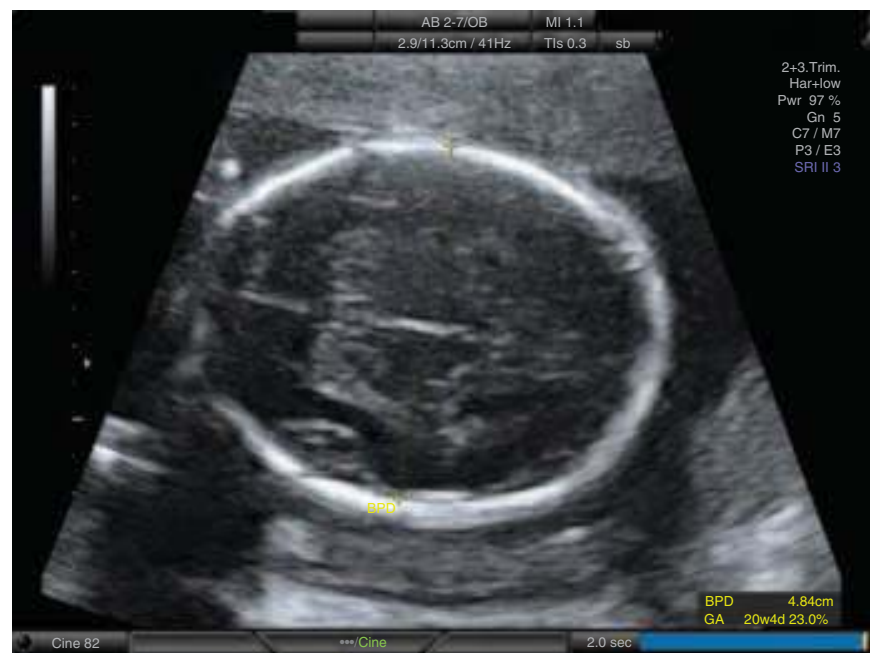

Figure 1 Axial view through fetal head at level of thalami and cavum septum pellucidum. The calipers show measurement of the biparietal diameter.

anteroposterior distance) as a normal variant or may suggest fetal aneuploidy (trisomy 21 or 18 associated with brachycephaly). A lemon-shaped head may be suggestive of a neural tube defect. The cranial sutures appear as hypoechoic areas between cranial bones and visualization excludes premature closure, craniosynostosis syndromes, and some skeletal dysplasias. The biparietal diameter is measured at the level of the thalami and cavum septum pellucidum (Fig. 1). The cerebellar hemispheres should not be visible in this transthalamic axial plane. The measurement is taken from the outer edge of the proximal skull to the inner edge of the distal skull. Head circumference is measured at the same level as the biparietal diameter, around the outer perimeter of the calvarium. The cavum septum pellucidum, midline falx, third ventricle, and frontal horns of the lateral ventricles can also be visualized in this view. The presence of a normal cavum suggests proper midline brain formation and makes diagnosis of holoprosencephaly or agenesis of the corpus collusum less likely. Next, a transventricular view should be obtained just superior to the transthalamic view (Figs. 2 and 3). This allows visualization of the lateral ventricles and bilateral choroid plexuses. The ventricle is measured through the atrium in an axial plane and is normally less than $10 \mathrm{~mm}$, and the choroid plexuses should fill the entire width of the lateral ventricles. The posterior fossa is then evaluated. This begins with obtaining a transcerebellar view to properly visualize the cerebellum, cerebellar vermis, cisterna magna, and nuchal fold (Fig. 4). Transcerebellar diameter provides a useful and accurate estimation of gestational age and is relatively spared in growth restriction $(17,18)$. Thorough evaluation of the structures in the posterior fossa is crucial to exclude spina bifida, Dandy-Walker malformation or variant, occipital encephalocele, and cerebellar agenesis or hypoplasia. The nuchal fold can be measured in this transcerebellar view, with 5 to $6 \mathrm{~mm}$ being the upper limit of normal. It is important to note that if fetal intracranial structures cannot be adequately visualized secondary to fetal positioning, transvaginal ultrasound may be utilized to complete this portion of the fetal anatomy survey.

After the fetal head is evaluated, the fetal facial structures are examined. A range of genetic syndromes and disorders are associated with characteristic facial features, and thorough 


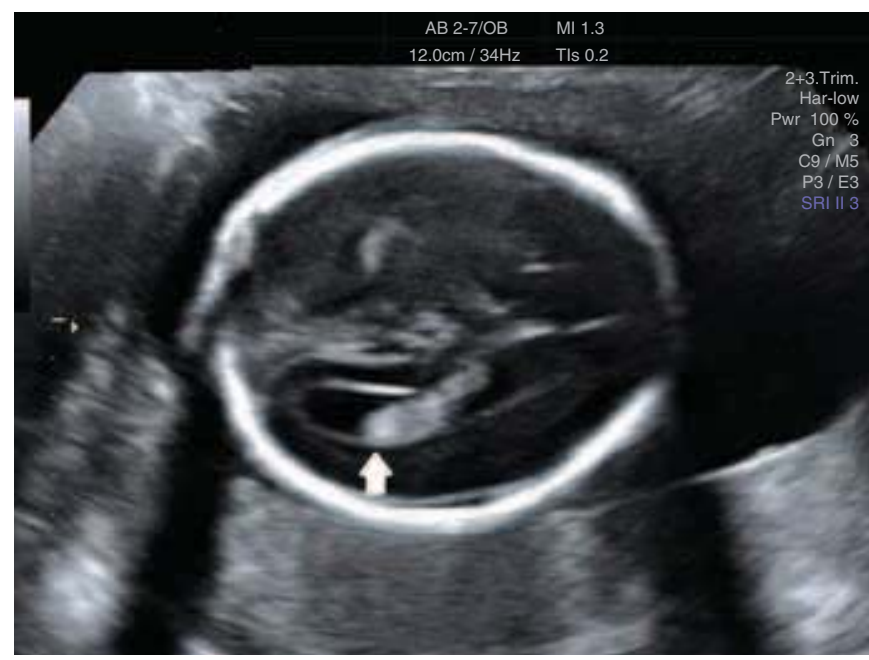

Figure 2 Transventricular view of the fetal head showing the far lateral ventricle. Note the choroid plexus filling the width of the ventricle (white arrow).

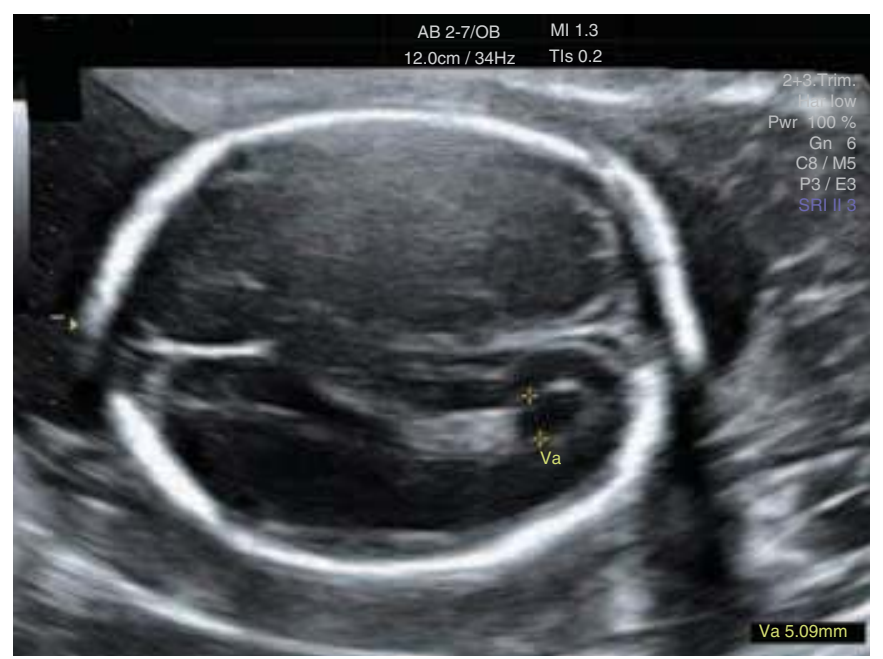

Figure 3 Transventricular view of the fetal head with calipers showing measurement of the atria of the lateral ventricle.

evaluation of the fetal face can aid in supporting or excluding their diagnosis. Obtaining a combination of coronal, sagittal, and axial views is necessary to complete the facial evaluation. To begin, a fetal profile should be obtained in the sagittal plane (Fig. 5). This allows for proper visualization of the frontal bone, nasal bone, and fetal chin. Absent or shortened nasal bone may indicate fetal aneuploidy and will be discussed later in this chapter. Micrognathia has also been associated with a variety of genetic syndromes and disorders, making characterization of the fetal chin important. The fetal nose and lips should be imaged in coronal plane and the anterior palate should be visualized to exclude cleft lip and palate.

Next, the fetal spine should be imaged and fetal skeletal survey should be performed. It is important to note that any intracranial abnormality noted in the fetal anatomy survey may be the first indication of neural tube abnormality, and the presence of any such finding warrants comprehensive review. Again, a combination of coronal, sagittal, and axial views is necessary for evaluation. The skin overlying the fetal spine should be visualized and intact to exclude open spina bifida and any mass or tumor (Fig. 6). The ossification centers

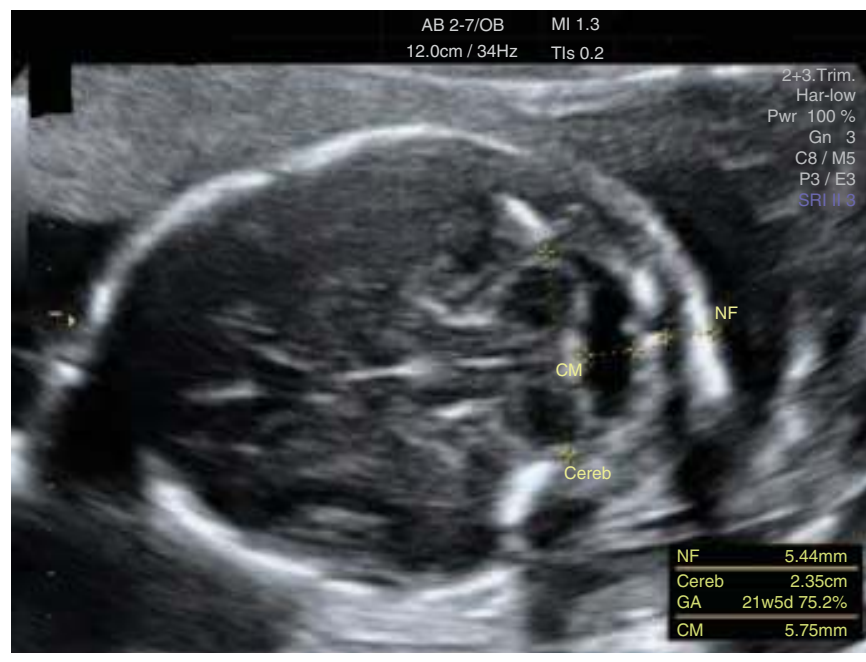

Figure 4 Transcerebellar view of the fetal head with calipers showing the cerebellar diameter (cereb), cisterna magna (CM), and nuchal fold (NF) measurements.

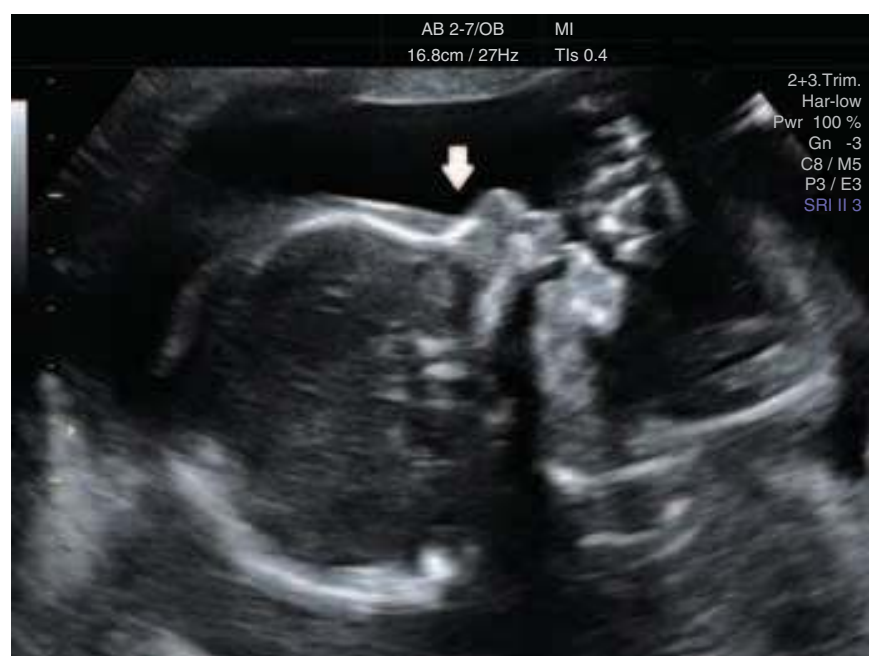

Figure 5 Sagittal profile of the second-trimester fetus. Note the presence of the nasal bone (white arrow).

of each vertebral segment should align in a symmetrical fashion to exclude hemivertebrae and neural tube defect. Notation of the spinal curvature should be made. Overall evaluation of fetal bony structures may be completed at this time, with the evaluation of fetal ribs, clavicles, and extremities. Normal echogenecity and appearance aid in the exclusion of skeletal dysplasia. Documentation of fetal extremities as present or absent is the minimum requirement included in the guidelines for routine obstetric ultrasound examination; however, more complete evaluation may provide important diagnostic information. Movement and tone should be normal. Any absence, fracture, contracture, bowing, or hypomineralization of the bones should be considered abnormal. The length of each long bone (femur, humerus, radius, ulna, tibia, and fibula) should be measured and standard nomograms are available for comparison. The hands and feet should be examined. The hands should be noted to open freely and all five fingers should extend fully, as persistently clenched hands with overlapping digits is sonographic finding often associated with trisomy 18 . The middle phalanx of the fifth digit may be imaged as its absence has been 


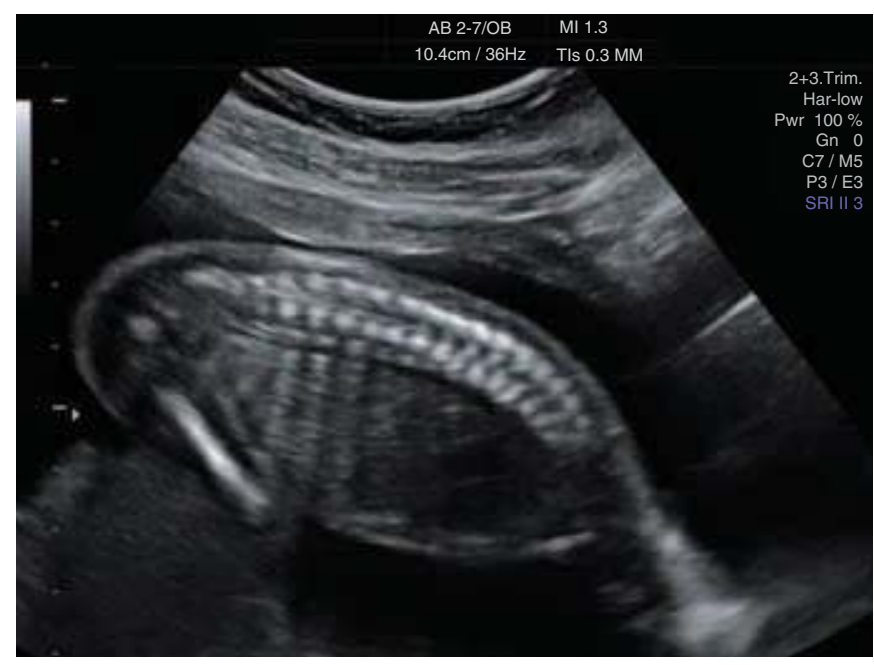

Figure 6 Normal sagittal view of the fetal spine. The skin overlying the vertebral processes is well visualized and intact.

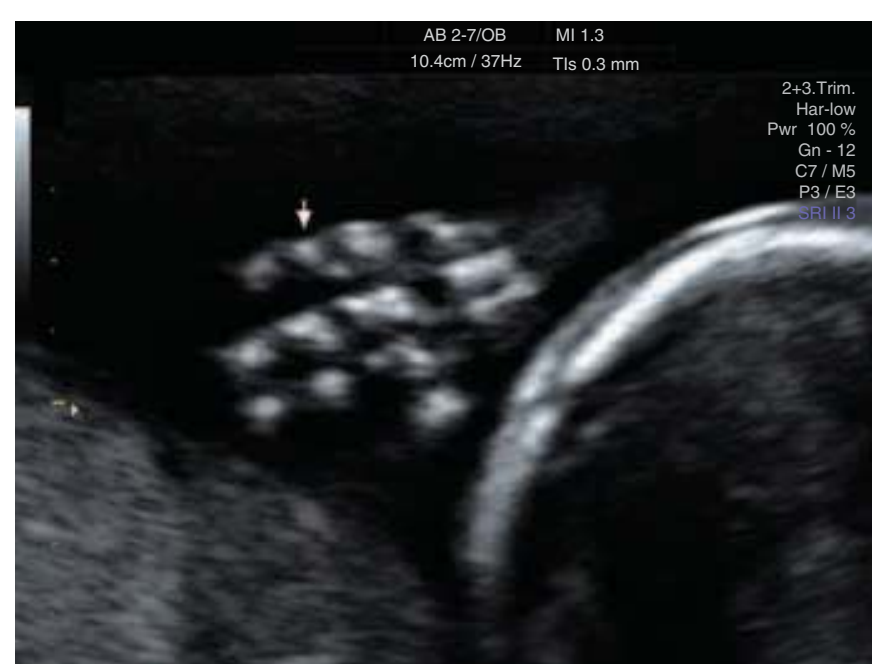

Figure 7 Fetal hand showing echogenic phalanges of four fingers, including the fifth digit (white arrow).

associated with trisomy 21 (Fig. 7). Fetal toes should be visualized and the foot length can be measured. Sagittal views of the feet should be obtained so that the plantar surface is at a right angle to the lower extremity (Fig. 8). This allows exclusion of "rocker-bottom" appearance, which also has been associated with various genetic disorders.

The fetal thorax and visceral contents are imaged next. The diaphragm is examined for evidence of diaphragmatic hernia. Fetal lung echogenecity should be homogeneous to exclude mass or tumor such as cystic adenomatoid malformation. Any pleural effusion or evidence of pulmonary hypoplasia is considered abnormal. Fetal cardiac examination should then follow. American Institute of Ultrasound in Medicine practice guidelines for basic cardiac examination include a fourchamber view of the fetal heart and, if technically feasible, views of both outflow tracts. However, a more comprehensive cardiac evaluation may be needed to exclude some fetal cardiac abnormalities. To begin, cardiac rate and rhythm should be documented. Cardiac size should be measured and is expected to be approximately one-third of the fetal thorax. The cardiac apex should point to the left at an angle of $45 \pm 20$ degrees relative to the midline. Normal situs should be established

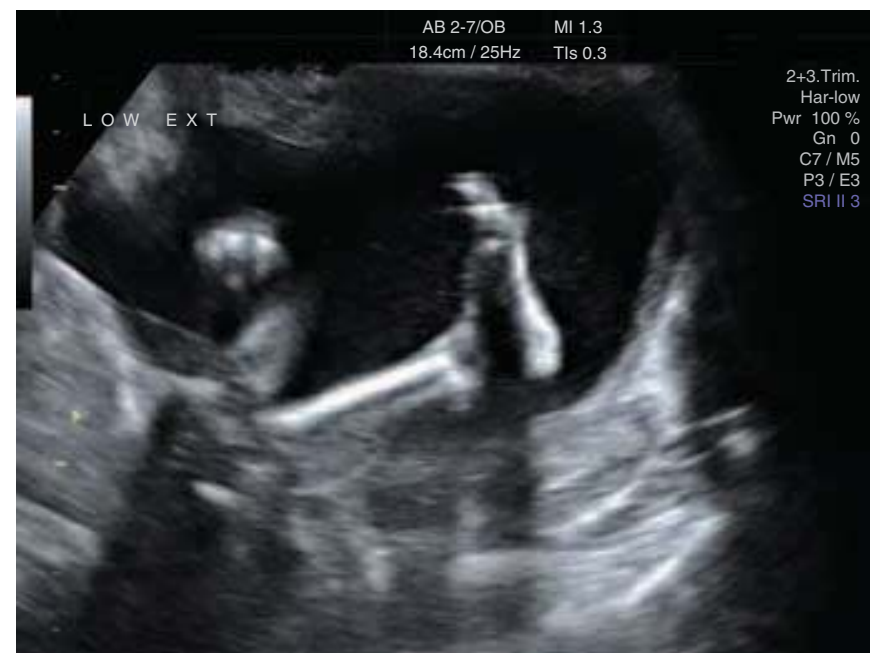

Figure 8 Fetal foot showing plantar surface at right angle with long bones of the lower extremity.

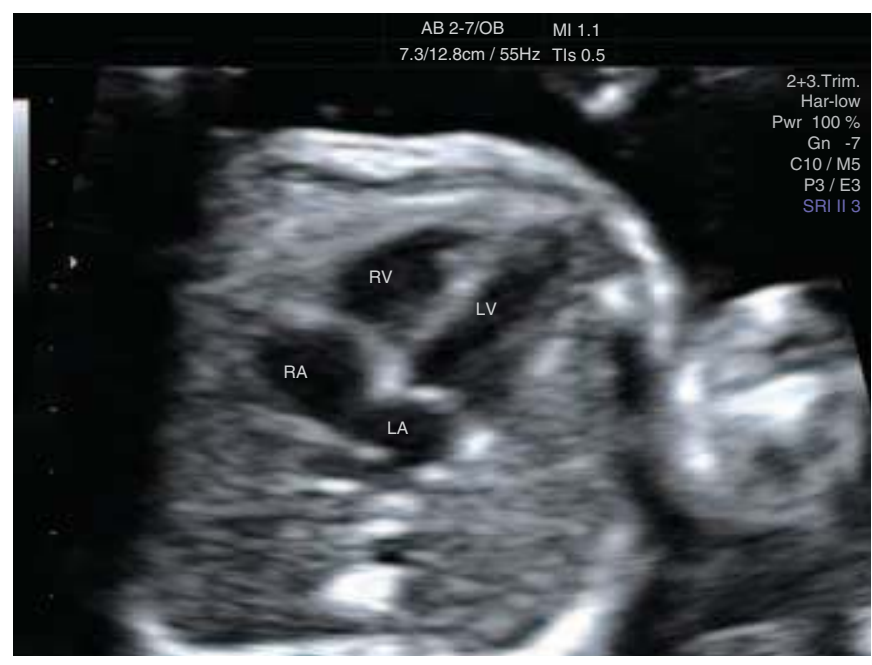

Figure 9 Apical four-chamber view of the fetal heart. Abbreviations: RA, right atrium; RV, right ventricle; LA, left atrium; LV, left ventricle.

with fetal liver on the right and stomach on the left. A fourchamber apical view should be obtained with all chambers being approximately equal in size (Fig. 9). There should be no pericardial fluid or collection. An intact intraventricular and intra-atrial septum excludes the presence of ventricular septal defect and atrial septal defect respectively. The outflow tracts, aortic arch, ductal arch, and inferior and superior vena cava should be identified. An echogenic intracardiac focus (EIF), most commonly seen in the left ventricle, can occur in up to $5 \%$ of normal pregnancies (19). It is typically caused by a specular reflection secondary to mineralization within the papillary muscles $(20,21)$. Because it is seen in approximately $13 \%$ to $18 \%$ of pregnancies complicated by Down syndrome, presence of an EIF has traditionally been utilized by some investigators as a sonographic marker to modify a woman's a priori risk of fetal aneuploidy (22-24). However, more recent literature suggests that when an EIF is seen as an isolated finding in an otherwise normal fetal anatomy survey, it is more likely to be a normal variant $(25,26)$. This will be further discussed later in the chapter.

Abdominal circumference should be determined at the skin line on a transverse view at the level of the junction of the 


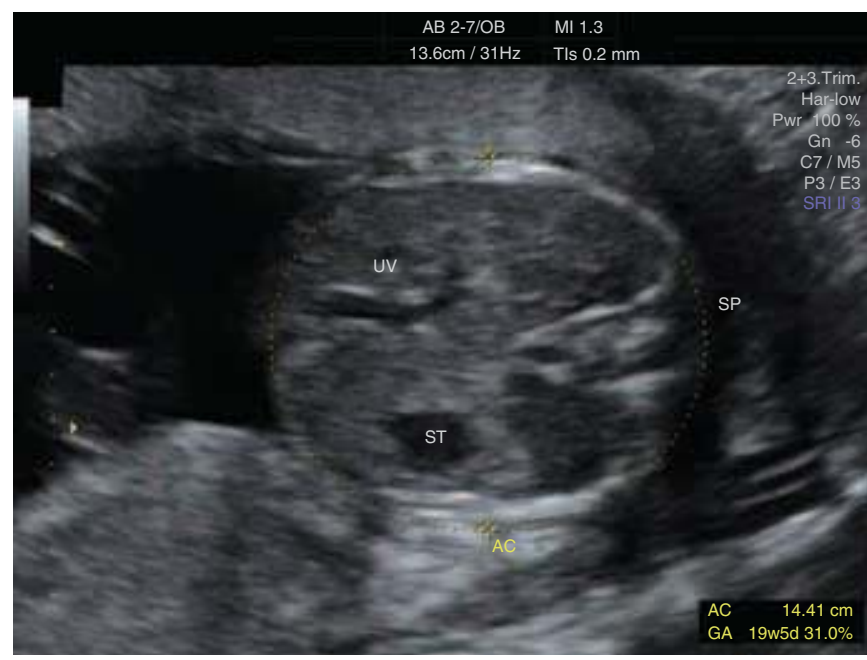

Figure 10 Appropriate plane for the measurement of abdominal circumference. Abbreviations: UV, umbilical vein; SP, spine; ST, stomach.

umbilical vein, portal sinus, and fetal stomach (Fig. 10). The umbilical vein should curve away from the fetal stomach; otherwise, persistence of the right umbilical vein is suspected. The abdominal circumference is the most sensitive fetal growth parameter and is used in conjunction with other fetal biometric measurements to determine an overall estimated fetal weight. Abdominal viscera identifiable on ultrasound include the liver, spleen, gallbladder, stomach, and bowel. The liver occupies most of the upper abdomen and should be located on the right. The gallbladder appears as a tear-shaped organ in the right upper quadrant at the inferior edge of the liver. The fetal stomach should be located on the fetal left below the level of the diaphragm. An absent or small stomach that remains unchanged throughout the course of sonographic evaluation may suggest esophageal atresia, tracheoesophageal fistula, or a diaphragmatic hernia. The appearance of the fetal bowel varies with gestational age. If fetal anatomy survey is performed in the second trimester, the bowel should appear uniformly echoic and fill the majority of the fetal abdomen. Hyperechoic bowel is associated with aneuploidy, infection, ischemia, intrauterine bleeding, and other bowel abnormalities. In the third trimester, more prominent loops of hypoechoic large bowel may be seen. More dilated loops of bowel may reflect atresia or obstruction. The umbilical cord should be identified at its insertion site into the fetal abdomen. As the umbilical vein enters the abdomen, it should course superiorly where it inters the liver to communicate with the portal vein. The umbilical vein then continues into the ductus venosus and then inferior vena cava and right atrium. The ventral abdominal wall adjacent to the umbilical cord insertion should be visualized and intact to exclude ventral wall defects including omphalocele and gastroschisis.

The genitourinary tract should next be examined for evidence of fetal anomaly. The kidneys should be identified bilaterally and appear as hypoechoic paraspinal structures. Identification of the anechoic urine-containing renal pelvis assists in their visualization (Fig. 11). Abnormal findings include enlargement or absence of the kidneys, renal masses or cysts within the parenchyma, hyperechogenicity, and dilation of the renal pelvis, calices, or ureter. When the kidneys cannot be easily identified, color or power Doppler imaging of the

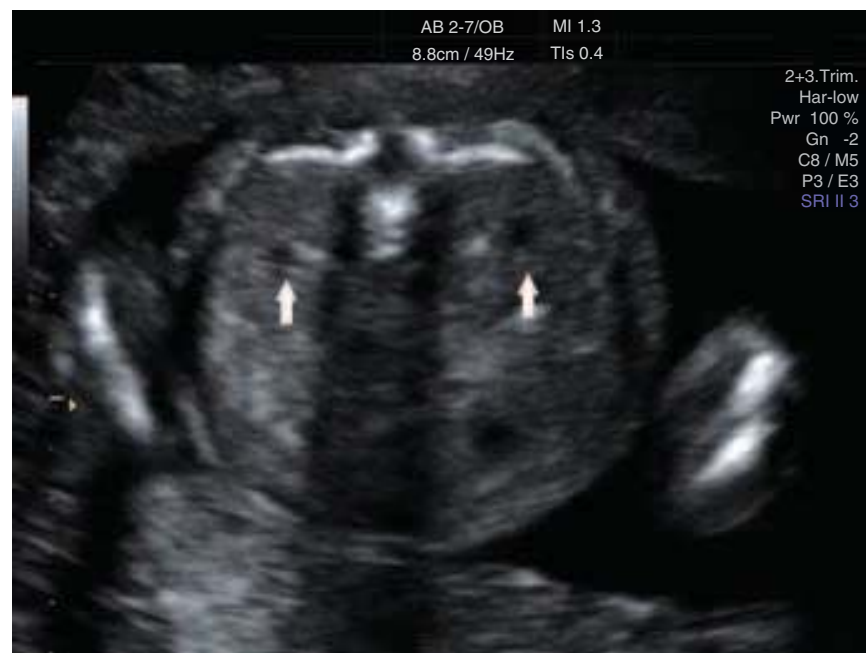

Figure 11 Transverse view through fetal abdomen at the level of fetal kidneys that appear as hypoechoic paraspinal structures. The white arrows indicate the central anechoic renal pelvis.

renal arteries can be utilized to confirm congenital absence of one or both kidneys or presence of a pelvic kidney. The bladder is an anechoic urine-filled structure located in the anterior midline of the fetal pelvis. The bladder may empty and fill during the course of the fetal anatomy survey and care should be taken to exclude bladder exstrophy or evidence of lower obstructive uropathy. The two umbilical arteries should be identified as they course around the fetal bladder to confirm the presence of a three-vessel cord. Attempt at fetal gender assignment should be made in order to exclude ambiguous genitalia. Female gender is confirmed by the identification of the labia, which may appear as three parallel linear echoes. Male gender is assigned if both the penis and scrotum are seen.

\section{SECOND-TRIMESTER GENETIC ULTRASOUND}

The genetic ultrasound is a targeted examination for fetal aneuploidy, specifically trisomy 21 . The goal of genetic ultrasound is to further modify the a priori age or serum screening risk for trisomy 21 and thus improve the selection of pregnant women who may be candidates for invasive prenatal diagnosis. First characterized in the 1990s, the combination of second-trimester serum screening with identification of ultrasound markers on genetic ultrasound improved the detection rate of fetuses affected with Down syndrome (27). At the same time, it identified patients at decreased risk of carrying a pregnancy complicated by Down syndrome if the genetic sonogram was normal $(28,29)$. With the increased use of first-trimester screening, the prevalence of fetal Down syndrome in the second trimester has decreased (30). For this reason, interpretation of second-trimester sonographic markers of Down syndrome must be interpreted in the context of first, second, and/or integrated screening results in order to achieve the most accurate risk assessment of a potentially affected fetus.

Approximately $25 \%$ of fetuses with Down syndrome have major characteristic structural abnormalities that can be identified on second-trimester ultrasound (31). For the majority of Down syndrome fetuses, no major fetal malformation will be present on sonographic evaluation. Over the past 20 years, numerous sonographic markers have been associated 
Table 6 Sonographic Aneuploidy Markers of the Genetic Sonogram

Nuchal fold thickening ( $>5-6 \mathrm{~mm}$ )

Absent or hypoplastic nasal bone

Short humerus

Short femur

Pyelectasis

Echogenic bowel

Echogenic intracardiac focus

Choroid plexus cyst(s)

Short ear length

Wide iliac angle

Sandal gap toes

Hypoplastic middle phalanx of the fifth digit

Clinodactyly

with fetal Down syndrome, which, when present, convey an increased risk for fetal aneuploidy. As with any screening modality, a useful sonographic marker Down syndrome screening should be present in a high proportion of Down syndrome pregnancies, not commonly seen in normal fetuses and amenable to imaging during a standard sonographic examination. The marker should be easily seen at a gestational age that does not preclude subsequent diagnostic testing by amniocentesis and option for pregnancy termination.

At the time of genetic ultrasound, thorough investigation for abnormal fetal biometry, fetal structural anomalies, and markers for fetal aneuploidy is performed. In this way, second-trimester ultrasound can be combined with serum biochemistry to determine which women would most benefit from amniocentesis. Additionally, a normal sonogram may be an indication to defer amniocentesis, even when a woman is at increased risk for aneuploidy based on serum screening (32). Conversely, abnormal findings on a genetic sonogram may prompt invasive testing even when a woman has normal serum biochemistry. Markers of aneuploidy include increased nuchal fold thickness, absent or hypoplastic nasal bone, shortened long bones, pyelectasis, hyperechoic bowel, EIF, choroid plexus cysts, short ear length, wide iliac angle, sandal gap toes, hypoplastic midphalanx of the fifth digit, and clinodactyly (Table 6).

When considered in isolation, each marker, except thickened nuchal fold or absent nasal bone, has a low to moderate sensitivity for trisomy 21 and may not necessarily increase the risk of aneuploidy, especially in low-risk patients (maternal age $<35$ with normal serum analyte screening) (31). The risk of fetal aneuploidy increases when two or more sonographic markers are seen in low-risk patients or when isolated markers are seen in high-risk patients.

An increased nuchal fold $(>5-6 \mathrm{~mm})$ is a highly specific marker for Down syndrome. Approximately $40 \%$ to $50 \%$ of second-trimester fetuses with Down syndrome have a thickened nuchal fold with a false-positive rate of $0.1 \%$ (33-35). To obtain this measurement, the fetal head is imaged in a transverse plane similar to that for measuring the biparietal diameter (Fig. 4). The thalami and the upper portion of the cerebellum should be in the plane of the image. The measurement is then made from the outside of the occipital bone to the distal surface of the skin edge.

The fetal nasal bone has been demonstrated to be hypoplastic or absent in up to $60 \%$ of Down syndrome

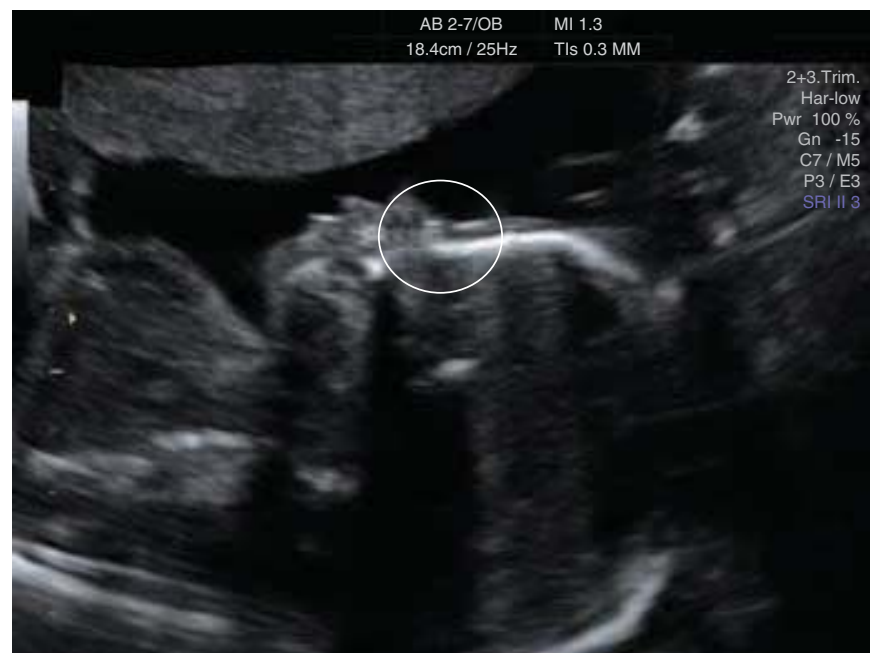

Figure 12 Sagittal profile of second-trimester fetus with absent nasal bone as indicated by circle.

pregnancies imaged in the second trimester and only about $1 \%$ to $2 \%$ of unaffected pregnancies (Fig. 12) (36,37). Initially observed at the time of first-trimester evaluation of nuchal translucency, hypoplastic or absent nasal bone ossification is being used with increased frequency as a second-trimester screen for Down syndrome (37-42). Numerous studies have been performed to determine the optimal definition of nasal bone hypoplasia as an ultrasonographic marker for aneuploidy in the second trimester. In one series, expressing multiples of the median (MoM) for the nasal bone length at each gestational age was the most efficient definition of nasal bone hypoplasia (43). Nasal bone smaller than $0.75 \mathrm{MoM}$ had a sensitivity and specificity of $49 \%$ and $92 \%$ respectively. Another series showed the positive likelihood ratio for fetal trisomy 21 increased from 17.1 with a nasal bone length of $0.7 \mathrm{MoM}$ or less to 74.1 with a nasal bone length of $0.5 \mathrm{MoM}$ or less (44). In the hands of experienced operators, nasal bone evaluation may be the best single ultrasound marker for second-trimester risk assessment. It is important to keep in mind that there is variability in the prevalence of this finding depending upon ethnicity, and this should be incorporated in to patient counseling if this finding is present on ultrasound evaluation. In general, absent nasal bone in the second trimester is seen in approximately one-third of Down syndrome fetuses (40).

Down syndrome fetuses may have a shorter humerus and femur length than expected based on biparietal diameter measurement. An observed-to-expected ratio of $\leq 0.90$ or a biparietal diameter-to-femur ratio of $>1.5$ has a reported likelihood ratio of 1.5 to 2.7 when present as an isolated finding $(45,46)$. A short humerus is more strongly associated with Down syndrome (sensitivity of 50\%, false-positive rate of $5 \%$ ) when compared with shortened femur length (sensitivity $40-50 \%$, false-positive rate of 7\%) (47-49). As with nasal bone ossification, there is variability in these long bone lengths between different ethnic groups, making these markers difficult to use as isolated findings or in a low-risk population.

Fetal pyelectasis, defined as a renal pelvis anterior-posterior diameter at least $4 \mathrm{~mm}$, is present with increased frequency in Down syndrome fetuses as compared with second-trimester euploid fetuses (Fig. 13) (50). As an isolated marker, the likelihood ratio ranges from 1.5 to 1.9 ; however, the sensitivity 


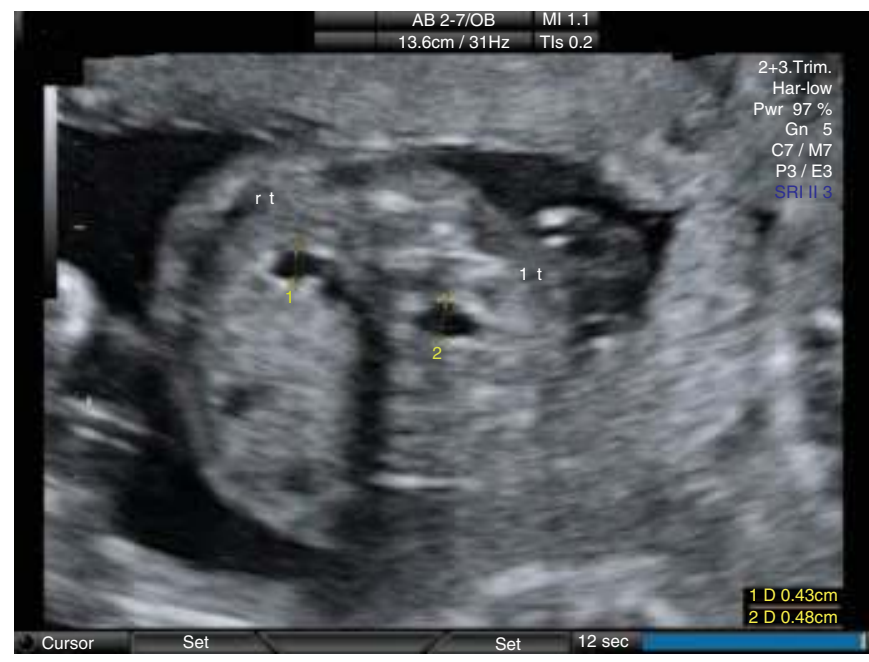

Figure 13 Transverse view at the level of fetal kidneys with calipers showing bilateral renal pyelectasis.

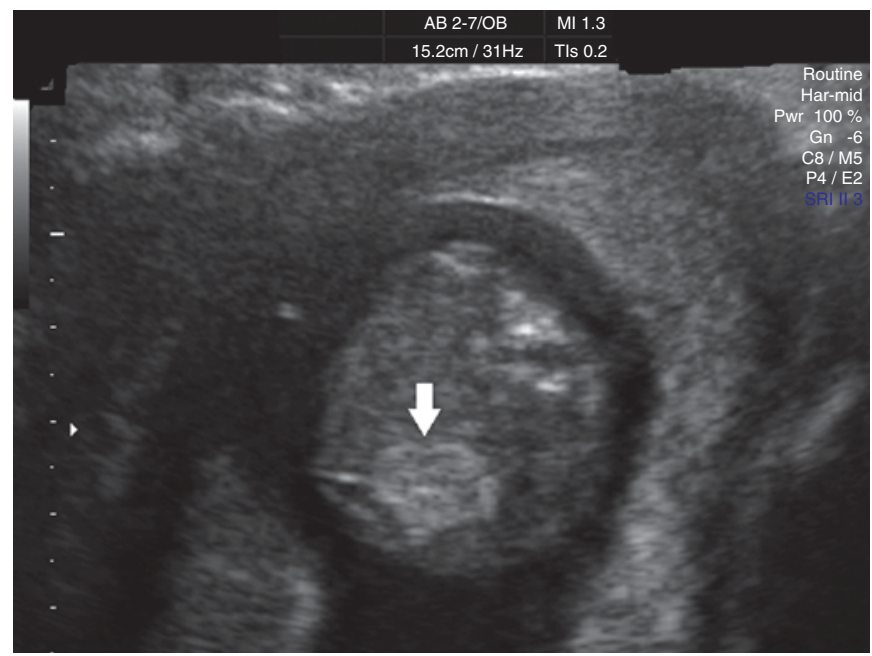

Figure 14 Fetal abdomen with increased echogenicity of the fetal bowel as indicated by white arrow.

for this marker is low (17-25\%) with a false-positive rate of $2 \%$ to $3 \%$, making this marker difficult to use as an isolated finding or in a low-risk population (49-51).

Increased echogenicity of the fetal bowel, defined as fetal bowel at least as echogenic or brighter than the surrounding bone, is a subjective marker for Down syndrome and is dependent on the machine and the operator performing the ultrasound (Fig. 14). Sensitivity of this marker ranges anywhere from $3 \%$ to $27 \%$ with a low false-positive rate of $<1 \%$ $(35,49,52-54)$. The finding of hyperechoic bowel can also be seen with fetal cystic fibrosis, cytomegalovirus infection, swallowed bloody amniotic fluid, and severe intrauterine growth restriction. This differential diagnosis list must be kept in mind when counseling patients regarding this finding.

EIFs occur in up to $5 \%$ of normal pregnancies and in approximately $16 \%$ to $18 \%$ of Down syndrome gestations (Figs. 15 and 16) (19). The risk does not seem to vary if the isolated focus is in the right or left ventricle; however, the presence of bilateral or multiple unilateral EIFs, especially in the right ventricle, may increase the risk for Down syndrome (55-57). Because an EIF is one of the most common sonographic markers of aneuploidy to be seen among normal fetuses, much recent

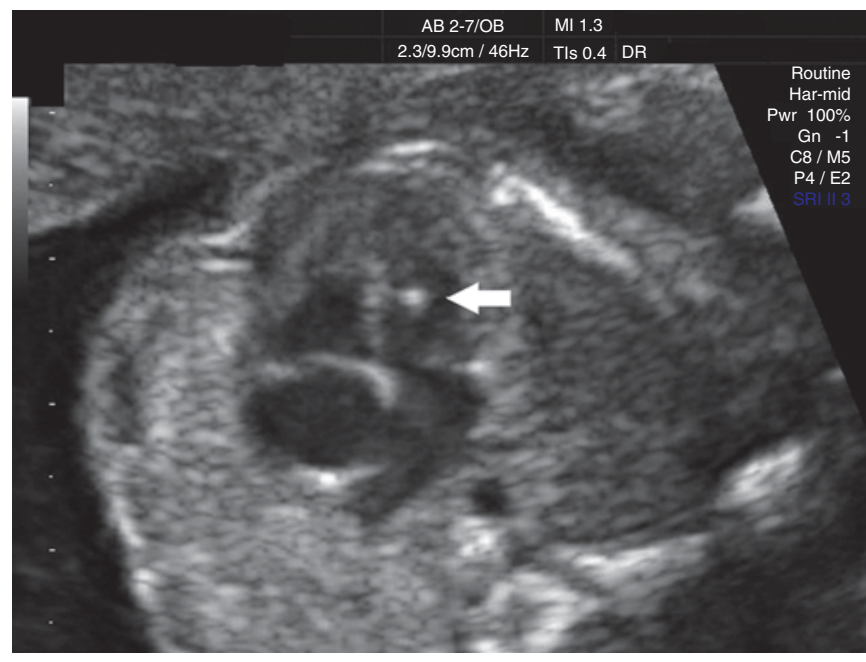

Figure 15 Apical four-chamber view of fetal heart showing single echogenic focus in the left ventricle (white arrow).

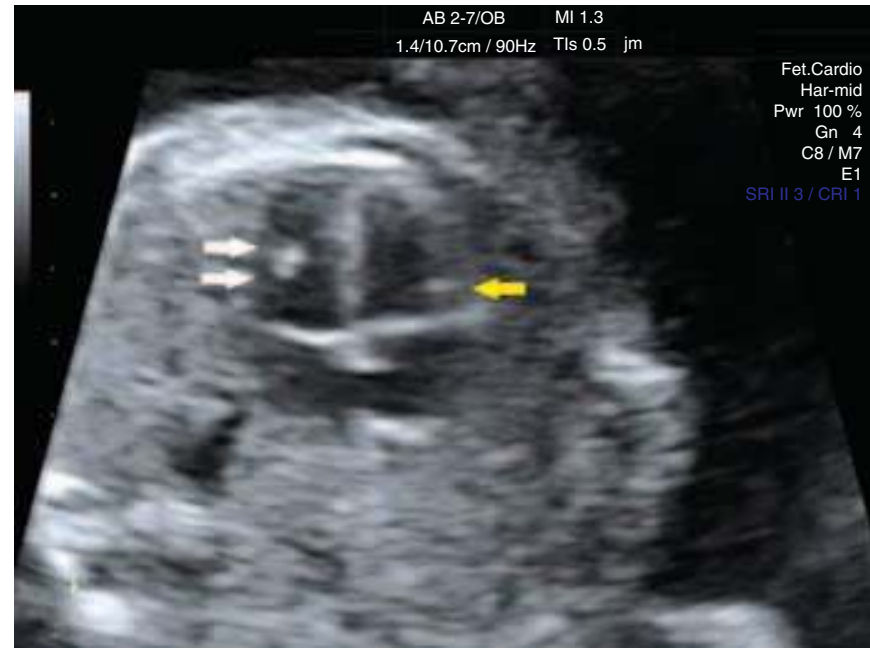

Figure 16 Apical four-chamber view of fetal heart showing multiple echogenic foci [two in the left ventricle (white arrows) and one in the right ventricle (yellow arrow)].

literature has focused on its clinical utility as a screen for Down syndrome. Recent literature has shown EIF as an isolated finding, and no additional maternal risk factor probably does not increase the risk of fetal aneuploidy and does not require invasive testing $(25,26,58)$.

Like the EIF, the presence of a choroid plexus cyst is a sonographic finding, which can also be seen in euploid fetuses (Fig. 17). Again, there has been debate in the literature regarding an association between isolated choroid plexus cyst in a low-risk woman and fetal aneuploidy, with most researchers identifying this finding as a benign variant when noted in isolation $(26,59)$.

Other minor sonographic markers associated with Down syndrome include short ears, altered foot-to-femur ratio, widened iliac wing angle, sandal gap between the first and second toes, hypoplastic fifth middle phalanx of the hand, and clinodactyly. These markers are inconsistently used because of variable operator experience and possibly high false-positive rates.

Different methods have been proposed to interpret the presence or absence of sonographic markers identified at 


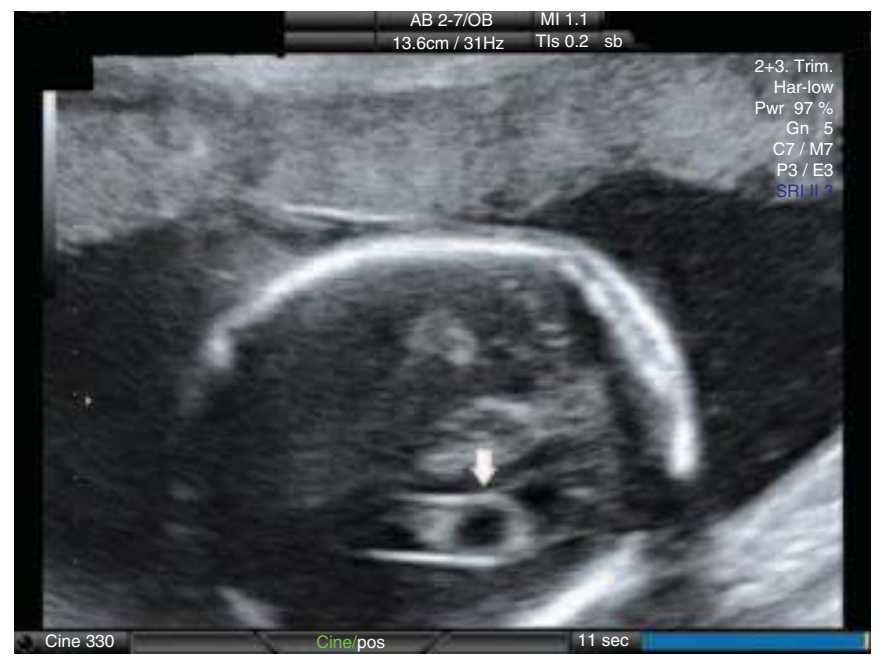

Figure 17 A single sonolucent choroid plexus cyst in the far lateral ventricle (white arrow).

the time of genetic sonogram (60). These include summing up the scores of individual markers to obtain a "Sonographic Scoring Index" (49), multiplying the likelihood ratios of individual markers (61), calculating a negative likelihood ratio in the absence of any sonographic markers (32), and using multivariate analysis to identify independent sonographic predictors $(62,63)$. A standardized methodology in applying soft markers as a screening test for Down syndrome will aid in patient counseling regarding the findings. Additionally, the development of uniform and reproducible sonographic and measurement standards will further enhance the clinical utility of the genetic sonogram. Regardless of its interpretation, the genetic sonogram plays a useful role in identifying fetuses at risk for Down syndrome or reducing the risk for Down syndrome, if normal, in high-risk women who wish to avoid amniocentesis. The reported detection rates of Down syndrome after genetic sonography when combining multiple ultrasound markers range from $50 \%$ to $90 \%$ (64-66). It is important to combine the genetic sonogram with first- and/or second-trimester maternal serum screening, as these results establish a woman's baseline or a priori risk. Additionally, combining maternal serum screening with second-trimester fetal ultrasound evaluation is more effective than either modality alone at identifying fetuses at risk for aneuploidy $(67,68)$.

Finally, obstetricians must keep in mind that although ultrasound is a low-risk and non-invasive screening tool for the evaluation of the fetus and intrauterine environment, it is not always a benign process. Pregnant women referred for targeted ultrasound because of the finding of a marker have high anxiety, with reported anxiety levels higher than women referred for ultrasound evaluation based on advanced maternal age alone and comparable with women with abnormal maternal serum screening $(69,70)$. This further supports the need for standardization in the approach to performing and interpreting sonographic aneuploidy markers and the patient counseling that accompanies this evaluation.

\section{SECOND-TRIMESTER ULTRASOUND TO DETECT} TRISOMY 18 AND 13

After Down syndrome, trisomy 18 is the second most common aneuploidy among live-born fetuses, occurring in approximately 1 in 8000 live births (71). While most of the literature detailing second-trimester ultrasound has focused on the detection for fetal structural anomalies and sonographic markers for Down syndrome, several ultrasound markers, such as choroid plexus cysts and clenched hands, have been shown to be associated with trisomy 18 (72-77). The vast majority of trisomy 18 fetuses have sonographically detectable abnormalities in the second trimester $(78,79)$. In one series of 8763 women with singleton pregnancy and serum screening significant for increased risk trisomy 18, 15\% had an abnormal genetic sonogram, including 1240 of pregnancies with normal fetuses and 50 of those with trisomy 18 (80). If the genetic sonogram was normal, a woman's risk of having a fetus with trisomy 18 was reduced by approximately 90\%. The most commonly identified abnormality was a cardiac defect, followed by skeletal, central nervous system, facial, and abdominal abnormality. Most fetuses (81\%) with trisomy 18 had an ultrasound marker present. Specifically, choroid plexus cysts and clenched hands were seen in large percentage of affected fetuses (52\% and 36\% respectively). These ultrasound markers were almost always seen in conjunction with one or more structural anomalies or other markers.

Trisomy 13 is the third most common of the autosomal trisomies to result in a live-born neonate. Prevalence of this disorder ranges between 1 in 5000 to 1 in 20,000 and it is characterized by multiple severe anomalies resulting in neonatal death (81). Severe craniofacial and midline defects are more common and severe in this trisomy when compared with the abnormalities seen in trisomy 18 or 21 fetuses. As with trisomy 18 fetuses, second-trimester sonographic examination can show many of the anomalies characteristic of trisomy 13. In one series of 28 fetuses with trisomy 13, major structural malformations were seen in 23 cases $(82.1 \%)$ and minor anomalies were detected in 16 cases $(57.1 \%)$ (82). Among major anomalies, the most common $(64.3 \%)$ were central nervous system (ventriculomegaly, holprosencephaly, posterior fossa cysts) and facial (midline clefts, microphthalmos, protrusio bulbi, hypertelorism). Cardiovascular anomalies were the next most common abnormality detected $(53.6 \%)$ followed by renal anomalies $(42.9 \%)$ and polydactyly $(7.1 \%)$. Ultrasound markers for fetal aneuploidy were also detected $(57.1 \%)$, but almost always in association with a major structural defect $(87.5 \%)$.

\section{THREE-DIMENSIONAL SONOGRAPHY}

In recent years, the use of three-dimensional (3D) ultrasound has emerged as an important imaging tool for the diagnosis of fetal abnormality. The 3D ultrasound may be utilized to further identify the nature, severity, and location of fetal defects first identified on standard ultrasound evaluation (83). It is a complementary tool that may be used to expand upon the 2D ultrasound examination. Specific applications include improved visualization of fetal facial features in the evaluation of suspected abnormal findings including micrognathia, cleft lip and palate, tumors extending to the face, and markers of trisomy 21 (84-88). The 3D ultrasound has proven useful in the assessment of limb malformation and prenatal diagnosis of skeletal abnormalities, including rib anomalies and abnormal cranial sutures (89-91). It also has been utilized to localize the severity and extent of fetal neural tube defects (92). Other 
applications include spatial presentation of blood flow and vasculature and, more recently, the ability to reconstruct a $3 \mathrm{D}$ rendered image of the fetal heart $(93,94)$.

\section{FETAL MAGNETIC RESONANCE IMAGING}

Fetal magnetic resonance imaging (MRI) has emerged as a useful complementary imaging modality to further elucidate abnormalities noted at the time of fetal ultrasound evaluation. MRI provides superior soft tissue resolution in comparison with ultrasound regardless of fetal position, gestational age, maternal body habitus, oligohydramnios, or skill of operator (95). Brain imaging is the most common application for MRI of the fetus and use of MRI for diagnosing central nervous system abnormalities is widely reported. It has been utilized in cases of unexplained hydrocephalus, suspected agenesis of the corpus collusum, abnormalities of the posterior fossa, and neural tube defects $(96,97)$. MRI has also been utilized in fetal imaging to study lung and chest anatomy, cervical masses, and urogenital abnormalities (98-101). MRI also has been shown to play a role in assessing abnormal placentation, including cases of suspected placenta accreta, increta, and percreta (102-105).

\section{REFERENCES}

1. Merkatz IR, Nitwosky HM, Macri JN, Johnson WE. An association between low maternal serum a-fetoprotein and fetal chromosomal abnormalities. Am J Obstet Gynecol 1984; 148: 886-94.

2. Tomasi TB Jr. Structure and function of alpha-fetoprotein. Annu Rev Med 1977; 28: 453-65.

3. Dugoff L. First- and second-trimester maternal serum markers for aneuploidy and adverse pregnancy outcomes. Obstet Gynecol 2010; 115: 1052-61.

4. Screening for fetal chromosomal abnormalities. ACOG Practice Bulletin No. 77. American College of Obstetricians and Gynecologists. Obstet Gynecol 2007; 109: 217-27.

5. Wald NJ, Cuckle HS, Densem JW. Maternal serum screening for Down Syndrome. Br Med J 1988; 297: 883-7.

6. Wald NJ, Densem JW, Smith D, Klee GG. Four-marker serum screening for Down syndrome. Prenat Diagn 1994; 14: 707-16.

7. Haddow JE, Palomake GE, Knight GF, et al. Second trimester screening for Down syndrome using maternal serum dimeric inhibin A. J Med Screen 1998; 5: 115-19.

8. Bahado-Singh RO, Oz UA, Shahabi S, et al. Urine hyperglycosylated hCG plus ultrasound biometry for detection of Down syndrome in the second trimester in a high-risk population. Obstet Gynecol 2000; 95: 889-94.

9. Cole LA, Shahabi S, Oz UA, et al. Hyperglycosylated human chorionic gonadotropin (invasive trophoblast antigen) immunoassay: a new basis for gestational Down syndrome screening. Clin Chem 1999; 45: 2109-19.

10. Wald NJ, Rodeck C, Hackshaw AK, et al. First and second trimester antenatal screening for Down's syndrome: the results of the Serum, Urine and Ultrasound Screening Study (SURUSS). J Med Screen 2003; 10: 56-104.

11. Palomaki GE, Neveux LM, Knight GJ, et al. Maternal serum invasive trophoblast antigen (hyperglycosylated hCG) as a screening marker for Down syndrome during the second trimester. Clin Chem 2004; 50: 1804-8.

12. Oyelese Y, Tobon L, Burton A, et al. The significance of a positive second trimester serum screen for trisomy 18. J Matern Fetal Neonatal Med 2010; 23: 633-7.

13. Cho RC, Chu P, Smith-Bindman R. Second trimester prenatal ultrasound for the detection of pregnancies at increased risk of trisomy 18 based on serum screening. Prenat Diagn 2009; 29: 129-39.

14. DeVore GR. Second trimester ultrasonography may identify 77 to $97 \%$ of fetuses with trisomy 18. J Ultrasound Med 2000; 19: 565-76.

15. American Institute of Ultrasound in Medicine: Standards for Performance of the Antepartum Obstetrical Ultrasound Examination. Laurel, MD, American Institute of Ultrasound in Medicine, 1994.
16. American Institute of Ultrasound in Medicine: Practice Guideline for the Performance of Obstetric Ultrasound Examinations. Laurel, MD, American Institute of Ultrasound in Medicine, 2007.

17. Chavez MR, Ananth CV, Smulian JC, et al. Fetal transcerebellar diameter measurement with particular emphasis in the third trimester: a reliable predictor of gestational age. Am J Obstet Gynecol 2004; 191: 979-84.

18. Chavez MR, Ananth CV, Smulian JC, Vintzileos AM. Fetal transcerebellar diameter measurement for prediction of gestational age at the extremes of fetal growth. J Ultrasound Med 2007; 26: 1167-71.

19. Bromley B, Lieberman E, Laboda LA, et al. Echogenic intracardiac focus, a sonographic sign for Down Syndrome? Obstet Gynecol 1995; 86: 998-1001.

20. Roberts DJ, Genest D. Cardiac histologic pathology characteristics of trisomies 13 and 21. Hum Pathol 1992; 23: 1130-40.

21. Brown DL, Roberts DJ, Miller WA. Left ventricular echogenic focus in the fetal heart: pathologic correlation. J Ultrasound Med 1994; 12: 613-16.

22. Bromley B, Lieberman E, Shipp TD, et al. Significance of an echogenic intracardiac focus in fetuses at high and low risk for aneuploidy. J Ultrasound Med 1998; 17: 127-31.

23. Vibhakar NI, Budorick NE, Scioscia AL, et al. Prevalence of aneuploidy with a cardiac intraventricular echogenic focus in an at-risk population. J Ultrasound Med 1999; 18: 265-8.

24. Winter TC, Anderson AM, Cheng EY, et al. Echogenic intracardiac focus in 2nd-trimester fetuses with trisomy 21: usefulness as a US marker. Radiology 2000; 216: 450-6.

25. Bradley KE, Santulli TS, Gregory KD, et al. An isolated intracardiac echogenic focus as a marker for aneuploidy. Am J Obstet Gynecol 2005; 192: 2021-8.

26. Ouzounian JF, Ludington C, Chan S. Isolated choroid plexus cyst or echogenic cardiac focus on prenatal ultrasound: is genetic amniocentesis indicated? Am J Obstet Gynecol 2007; 196: 595.e1-3.

27. Benacerraf B. The history of the second-trimester sonographic markers for detecting fetal Down syndrome, and their current role in obstetric practice. Prenat Diagn 2010; 30: 644-52.

28. Vintzileos AM, Campbell WA, Rodis JF, et al. The use of second trimester genetic sonogram in building clinical management of patients at increased risk for fetal trisomy 21. Obstet Gynecol 1996; 87: 948-52.

29. Vintzileos AM, Guzman ER, Smulian JC, et al. Choice of secondtrimester genetic sonogram for detection of trisomy 21. Obstet Gynecol 1997; 90: 187-90.

30. Egan JF, Benn PA, Zelop CM, et al. Down syndrome births in the United States from 1989 to 2001. Am J Obstet Gynecol 2004; 191: 1044-8.

31. Vintzileos AM, Egan JF. Adjusting the risk for trisomy 21 on the basis of second trimester ultrasonography. Am J Obstet Gynecol 1995; 172: 837-44.

32. Yeo L, Vintzileos AM. The use of genetic sonography to reduce the need for amniocentesis in women at high-risk for Down Syndrome. Semin Perinataol 2003; 27: 152-9.

33. Benacerraf BR, Barss BA, Laboda LA. A sonographic sign for the detection in the second trimester of the fetus with Downs syndrome. Am J Obstet Gynecol 1985; 151: 1078-9.

34. Benacerraf BR, Laboda LA, Frigoletto FD Jr. Thickened nuchal fold in fetuses not at risk for aneuploidy. Radiology 1992; 184: 239-42.

35. Nyberg DA, Resta R, Luthy DA, et al. Prenatal sonographic findings of Down syndrome: review of 94 cases. Obstet Gynecol 1990; 76: 370-7.

36. Cicero S, Curcio P, Papageorghiou A, et al. Absence of nasal bone in fetuses with trisomy 21 at 11-14 weeks of gestation: an observational study. Lancet 2001; 359: 1665-7.

37. Sonek JD, Nicolaides KH. Prenatal ultrasonographic diagnosis of nasal bone abnormalities in three fetuses with Down Syndrome. Am J Obstet Gynecol 2002; 186: 139-41.

38. Cicero S, Sonek J, McKenna D, et al. Nasal bone hypoplasia in fetuses with Trisomy 21 at 15-22 weeks' gestation. Ultrasound Obstet Gynecol 2003; 21: 15-18.

39. Bromley B, Lieberman E, Shipp T, et al. Fetal nasal bone length: a marker for Down Syndrome in the second trimester. J Ultrasound Med 2002; 21: 1387-94.

40. Vintzileos AM, Walters C, Yeo L. Absent nasal bone in the prenatal detection of fetuses with trisomy 21 in a high-risk population. Obstet Gynecol 2003; 101: 905-8. 
41. Cusick W, Provenzano J, Sullivan C, et al. Fetal nasal bone length in euploid and aneuploid fetuses between 11 and 20 weeks' gestation: a prospective study. J Ultrasound Med 2004; 23: 1327-33.

42. Odibo AO, Sehdev HM, Dunn L, et al. The association between fetal nasal bone hypoplasia and aneuploidy. Obstet Gynecol 2004; 104: 1229-33.

43. Odibo AO, Sehdev HM, Stamilio DM, et al. Defining nasal bone hypoplasia in second-trimester Down syndrome screening: does the use of multiples of the median improve screening efficacy? Am J Obstet Gynecol 2007; 197: 361.e1-4.

44. Cusik W, Shevell T, Duchan LS, et al. Likelihood ratios for fetal trisomy 21 based on nasal bone length in the second trimester: how best to define hypoplasia? Ultrasound Obstet Gynecol 2007; 30: 271-4.

45. Lockwood C, Benacerraf B, Krinsky A, et al. A sonographic screening method for Down syndrome. Am J Obstet Gynecol 1987; 157: 803-8.

46. Nyberg DA, Resta RG, Hickok DE, et al. Femur length shortening in the detection of Down syndrome: is prenatal screening feasible? Am J Obstet Gynecol 1990; 162: 1247-52.

47. Fitzsimmons J, Droste S, Shepard TH, et al. Long-bone growth in fetuses with Down Syndrome. Am J Obstet Gynecol 1989; 161: 1174-7.

48. Benacerraf BR, Neuberg D, Frigoletto FD Jr. Humeral shortening in second-trimester fetuses with Down Syndrome. Obstet Gynecol 1991; 77: 223-7.

49. Bromley B, Benacerraf BR. The genetic sonogram scoring index. Semin Perinatol 2003; 27: 124-9.

50. Benacerraf BR, Mandell J, Estroff JA, et al. Fetal pyelectasis, a possible association with Down Syndrome. Obstet Gynecol 1990; 76: 58-60.

51. Corteville JE, Dicke JM, Crane JP. Fetal pyelectasis and Down syndrome, is genetic amniocentesis warranted? Obstet Gynecol 1993; 79: 770-2.

52. Dicke JM, Crane JP. Sonographically detected hyperechoic fetal bowel: significance and implications for pregnancy management. Obstet Gynecol 1992; 80: 778-82.

53. Scioscia AL, Pretorius DH, Budorick NE, et al. Second-trimester hyperechoic bowel and chromosomal abnormalities. Am J Obstet Gynecol 1992; 167: 889-94.

54. Bromley B, Coubilet P, Frigoletto FD Jr, et al. Is fetal hyperechoic bowel on second trimester sonogram an indication for amniocentesis? Obstet Gynecol 1994; 83: 647-51.

55. Broshstein M, Jakobi P, Ofir C. Multiple fetal intracardiac echogenic foci: not always a benign sonographic finding. Prenat Diagn 1996; 16: $131-5$.

56. Wax JR, Philput C. Fetal intracardiac echogenic foci: does it matter which ventricle? J Ultrasound Med 1998; 17: 145-6.

57. Towner D, Gerscovich EO, Chiong BB, et al. Comparison of single versus multiple echogenic foci in the fetal heart regarding risk of aneuploidy. J Ultrasound Med 2010; 29: 1061-7.

58. Bradley KE, Santulli TS, Gregory KD, et al. An isolated intracardiac echogenic focus as a marker for aneuploidy. Am J Obstet Gynecol 2005; 192: 2021-6.

59. Gupta JK, Cave M, Lilford RF, et al. Clinical significance of fetal choroid plexus cysts. Lancet 1995; 346: 724-9.

60. Vergani P, Ghidini A, Weiner S, et al. Risk assessment for Down syndrome with genetic sonogram. Prenat Diagn 2008; 28: 1144-8.

61. Nyberg DA, Luthy DA, Resta BC, et al. Age-adjusted ultrasound risk assessment for fetal Downs syndrome during the second trimester: description of the method and analysis of 142 cases. Ultrasound Obstet Gynecol 1998; 12: 8-14.

62. Vergani P, Locatelli A, Piccoli MG, et al. Best second trimester sonographic markers for the detection of Trisomy 21. J Ultrasound Med 1999; 18: 469-73.

63. Bahado-Singh RO, Mendilcioglu I, Rowther M, et al. Early genetic sonogram for Down syndrome detection. Am J Obstet Gynecol 2002; 187: 1235-8.

64. Krantz DA, Hallahan TW, Macri VJ, et al. Genetic sonography after first trimester Down syndrome screening. Ultrasound Obstet Gynecol 2007; 29: 666-70.

65. Vintzileos AM. Use of genetic sonography in screening for trisomy 21. J Ultrasound Med 2001; 20: 573-5.

66. Aagaard-Tillery KM, Malone FD, Nyberg DA, et al. First and second trimester evaluation of risk research consortium. Role of second- trimester genetic sonography after Down syndrome screening. Obstet Gynecol 2009; 114: 1189-96.

67. Benn PA, Kaminsky LM, Ying J, et al. Combined second-trimester biochemical and ultrasound screening for Down syndrome. Obstet Gynecol 2002; 100: 1168-76.

68. Pinette MG, Egan JF, Wax JR, et al. Combined sonographic and biochemical markers for Down syndrome screening. J Ultrasound Med 2003; 22: 1185-90.

69. Hoskovec J, Mastrobattista JM, Johnston D, et al. Anxiety and prenatal testing: do women with soft ultrasound findings have increased anxiety compared to women with other indications for testing? Prenatal Diagn 2008; 28: 135-40.

70. Czerwinski JL, Wicklund CA, Hoskovec J. Maternal serum screening: results disclosure, anxiety, and risk perception. Am J Perinat 2010; 27: 279-84.

71. Jones KJ. Smith's Recognizable Patterns of Human Malformation. 5th edn. Philadelphia, PA: WB Saunders Co, 1997: 14-17.

72. Fitzsimmons J, Wilson D, Pascoe-Mason A. Choroid plexus cysts in fetuses with trisomy 18. Obstet Gynecol 1989; 73: 257-60.

73. Snijeder RJ, Shawa L, Nicolaides KH. Fetal choroid plexus cysts and trisomy 18: assessment of risk based on ultrasound findings and maternal age. Prenat Diagn 1994; 14: 1119-27.

74. Gross SJ, Shulman LP, Tolley EA, et al. Isolated fetal choroid plexus cysts and trisomy 18: a review and meta-analysis. Am J Obstet Gynecol 1995; 172: 83-7.

75. Demasio K, Canterino J, Ananta C. Isolated choroid plexus cyst in lowrisk women less than 35 years old. Am J Obstet Gynecol 2002; 187: 1246-9.

76. Coco C, Jeanty P. Karyotyping of fetuses with isolated choroid plexus cysts in not justified in an unselected population. J Ultrasound Med 2004; 23: 899-906.

77. Quintero RA, Johnson MP, Mendoza G, et al. Ontogeny of clenchedhand development in trisomy 18 fetuses: a serial transabdominal fetoscopic observation. Fetal Diagn Ther 1999; 14: 68-70.

78. DeVore GR. Second trimester ultrasonography may identify 77 to $97 \%$ of fetuses with trisomy 18. J Ultrasound Med 2000; 19: 565-76.

79. Watson WJ, Miller RC, Wax JR, et al. Sonographic findings of Trisomy 18 in the second trimester of pregnancy. J Ultrasound Med 2008; 27: 1033-8.

80. Cho RC, Chu P, Smith-Bindman R. Second trimester prenatal ultrasound for the detection of pregnancies at increased risk of Trisomy 18 based on serum screening. Prenat Diagn 2009; 29: 129-39.

81. Hill LM. The sonographic detection of trisomies 13, 18 and 21. Clin Obstet Gynecol 1996; 80: 349-56.

82. Papp C, Beke A, Ban Z, et al. Prenatal diagnosis of trisomy 12: analysis of 28 cases. J Ultrasound Med 2006; 25: 429-35.

83. Duckelmann AM, Kalache KD. Three-dimensional ultrasound in evaluating the fetus. Prenat Diagn 2010; 30: 631-8.

84. Timor-Tritsch IE, Platt LD. Three-dimensional ultrasound experience in obstetrics. Curr Opin Obstet Gynecol 2002; 14: 569-75.

85. Bergann A, Bamberg C, Eder K, et al. Mid-facial anthropometry in second-trimester fetuses with trisomy 21: a three-dimensional ultrasound study. Prenat Diagn 2006; 26: 158-62.

86. Faure JM, Captier G, Baumler M, Boulot P. Sonographic assessment of normal fetal palate using three-dimensional imaging: a new technique. Ultrasound Obstet Gynecol 2007; 29: 159-65.

87. Kurjak A, Azumendi G, Andonotopo W, Salihagic-Kadic A. Three- and four-dimensional ultrasonography for the structural and functional evaluation of the fetal face. Am J Obstet Gynecol 2007; 196: 16-28.

88. Avni FE, Cos T, Cassart M, et al. Evolution of fetal ultrasonography. Eur Radiol 2007; 17: 419-31.

89. Pooh RK, Pooh K. Transvaginal 3D and Doppler ultrasonography of the fetal brain. Semin Perinatol 2001; 25: 38-43.

90. Esser T, Rogalla P, Bamber C, et al. Application of the three-dimensional maximum mode in prenatal diagnosis of Apert Syndrome. Ultrasound Obstet Gynecol 2005; 193: 1743-5.

91. Esser T, Rogalla P, Sarioglu N. Three-dimensional ultrasonographic demonstration of agenesis of the 12 th rib in a fetus with trisomy 21 . Am J Obstet Gynecol 2006; 27: 714-15. 
92. Lee W, Chaiworapongsa T, Romero R, et al. A diagnostic approach for the evaluation of spina bifida by three-dimensional ultrasonography. J Ultrasound Med 2002; 21: 619-26.

93. Goncalves LF, Lee W, Espinoza J, et al. Examination of the fetal heart by four-dimensional (4d) ultrasound with spatio-temporal image correlation (STIC). Ultrasound Obstet Gynecol 2006; 27: 336-48.

94. Gindes L, Hegesh J, Weisz B, et al. Three and four dimensional ultrasound: a novel method for evaluating fetal cardiac anomalies. Prenat Diagn 2009; 29: 645-53.

95. De Wilde JP, Rivers AW, Price DL. A review of the current use of magnetic resonance imaging in pregnancy and safety implications for the fetus. Prog Biophys Mol Biol 2005; 87: 335-53.

96. Panigrahy A, Borzage M, Bluml S. Basic principles and concepts underlying recent advances in magnetic resonance imaging of the developing brain. Semin Perinatol 2010; 34: 3-19.

97. Rutherford MA. Magnetic resonance imaging of the fetal brain. Curr Opin Obstet Gynecol 2009; 21: 180-6.
98. Epelman M, Kreiger PA, Servaes S, et al. Current imaging of prenatally diagnosed congenital lung lesions. Semin Ultrasound CT MR 2010; 31: 141-57.

99. Deshmukh S, Rubesova E, Barth R. MR assessment of normal fetal lung volumes: a literature review. Am J Roentgenol 2010; 194: W212-17.

100. Kathary N, Bulas DI, Newman KD. MRI imaging of fetal neck masses with airway compromise; utility in delivery planning. Pediatr Radiol 2001; 31: 727-31.

101. Hormann M, Brugger PC, Balassy C, et al. Fetal MRI of the urinary system. Eur J Radiol 2006; 57: 303-11.

102. Gowland P. Placental MRI. Semin Fetal Neonatal Med 2005; 10: 485-90.

103. Levine D. Obstetric MRI. J Magn Reson Imaging 2006; 24: 1-15.

104. Abramovicz JS, Sheiner E. In utero imaging of the placenta: importance for diseases of pregnancy. Placenta 2007; 28(Suppl A): S14-22.

105. Bardo D, Oto A. Magnetic resonance imaging for evaluation of the fetus and the placenta. Am J Perinatol 2008; 25: 591-9. 


\section{Genetic Counseling Zoltán Papp, Valéria Váradi, and Júlia Hajdú}

Classical genetic counseling is aimed to help families with emotional support by disclosing and discussing the causes of certain "genetic" problems, the risks of recurrence, and the possibilities for prevention or other options. In many situations, no reliable figures for recurrence risk were available. The classic options in many cases included contraception, sterilization, adoption, or AID (artificial heterologous insemination by donor) (1-7).

Subsequently, a number of diagnostic techniques, applicable in pregnancy, became available, together with a much better understanding of genetically determined disease at many levels. Now, in many disorders, one can suggest undertaking further pregnancies, with the offer of prenatal genetic counseling: the phenotype may be examined (ultrasound, for malformations or growth retardation; cell biochemistry, for metabolic disorders) or the fetal genotype be examined (cytogenetic analysis, for chromosome disorders; DNA, for direct identification of mutant genes). There remain disorders for which no diagnosis is available or for which one can offer no more than "classical genetics," but it is likely that many disorders at present of unknown cause will soon be understood at the biochemical or gene level, and that many presently "unmapped" genes will be mapped within the next decades (8).

The increased availability of prenatal diagnosis (available in more centers, for more patients, for more disorders) has stimulated the development of screening for genetic disease. Subpopulations at particular risk may be easily identified (e.g., older mothers) or identified only after specific testing (e.g., for thalassemia, hemoglobinopathies, Tay-Sachs disease, and, possibly, cystic fibrosis). The screening process may take place before or during an actual pregnancy; some screening methods, for example, biochemical markers, ultrasound, can be applied only during pregnancy (8).

The goal of genetic counseling is to prevent birth defects in cooperation with the couples (9). Without prevention, at least $3 \%$ to $4 \%$ of all births are associated with major congenital defect, mental retardation, or a genetic disorder.

Clinically significant chromosomal defects occurred in $0.65 \%$ of all births before prenatal diagnosis was introduced; an additional $0.2 \%$ of infants were born with balanced structural chromosome rearrangements. Congenital malformations, disruptions, and deformations with obvious structural defects were found in about $2 \%$ to $3 \%$ of all births. More than 9000 monogenic disorders and traits have been catalogued (10). Prevalence of monogenic disorders has been estimated to be 3.6 in 1000 live births, consisting of autosomal dominant (1.4 in 1000), autosomal recessive (1.7 in 1000), and X-linked (0.5 in 1000) (8).

The indications for preconception genetic counseling should be determined at the first visit and can be considered in a few clear categories:
- advanced maternal age,

- a previous fetus or child with a genetic disorder,

- a parent with a genetic disorder, - genetic disorders that pregnancy may aggravate, - maternal genetic disorders that may threaten fetal health and survival,

- a history of infertility,

- parental carrier of a genetic trait,

- family history of a genetic disorder,

- consanguinity (11), and

- environmental exposures that threaten fetal health.

Preconception counseling identifies specific risks and attendant options, which include the following:

- decision not to have children (includes consideration of vasectomy or tubal ligation),

- adoption,

- in vitro fertilization (IVF) and other reproductive technologies,

- carrier detection tests,

- presymptomatic diagnosis and predictive genetic testing,

- preimplantation genetic diagnosis (PGD), and

- folic acid supplementation in periconceptional period.

The optimal time to initiate counseling is not during pregnancy. Counselees whose first antenatal visits occur after the second missed menstrual period miss the critical period of organogenesis, and patients referred well after conception have lost almost all their options except selective abortion.

The ideal genetic counseling is composed of (12)

- an appropriately trained professional,

- relevant and objective information,

- assurance of the counselee's understanding,

- psychologic support,

- informed consent,

- assuring autonomous decision-making,

- confidentiality of genetic information,

- considering familial implications, and

- appropriate handling of potential discrimination of testing.

The option of further pregnancies includes the option of prenatal diagnosis, which, in turn, includes the option of pregnancy termination. Many couples, even in high-risk situations, will be lucky and have a healthy child; only a few will go through the trauma of one or several terminations. Couples identified by screening as "high risk" may be at very high risk $(25 \%)$ in the cases of monogenic traits, at a lower risk $(10 \% \pm)$ if identified through positive 
biochemical tests, or at relatively low risk $(1 \% \pm)$ if identified by maternal age (13).

Decisions on pregnancy testing and termination must be the couple's own; it is the duty of the counselor to support and to inform, but not to persuade. Some couples refuse the idea of interrupting pregnancy, for example, for religious reasons. Unlike some infectious diseases, which may be controlled by immunization, compulsory screening, and therapy, genetic diseases do not endanger society directly. It is families that are affected by them (14-16).

Nevertheless, the indirect effect of genetic disease on society cannot be ignored. Unlike infectious diseases, genetic diseases do not threaten the health of other people, but they do burden society (e.g., the cost of institutional care for handicapped children). The question arises whether anybody has the right to insist on the birth of a child who will suffer from an undoubtedly incurable disease and also severely burden public finances. Some people do insist on continuing such pregnancies, and there is no doubt that the right to make individual decisions must be respected.

Often couples coming for genetic counseling are full of fears and anxiety, oppressed with the memory of one or more ill or dead children. Couples appreciate the opportunity for a counseling session and discussion, and the offer of prenatal diagnosis will be accepted by most of them $(17,18)$.

Counseling sessions may often be strongly psychologic in nature (19-24). It is essential to present a clear and full description of the relevant disorder and to answer all questions honestly and promptly. A good and harmonious relationship should develop or be developed between the counselor and the couple. The physician-patient relationship, always important in medicine, here is replaced by a physician/counselor-family relationship, which deepens in the course of counseling. This sort of relationship is necessary for the proper help and management of high-risk couples in this situation. A great deal depends on the character of the individual physician.

\section{GENETIC TESTING}

The completion of the Human Genome Project was a phenomenal accomplishment, providing researchers with the reference sequence of the human genome, which has subsequently led to the identification of many genes associated with human disease. Development of new technologies has made it possible to study these genes, search for diseasecausing mutations, and develop genetic tests (25). Genetic testing has the potential to offer dramatic benefits, both clinically and psychologically, for patients and their families. The array of benefits begins with the clarification of diagnosis and prognosis, which assists in decision-making about clinical care. Testing for familial mutations makes available predictive, carrier, and prenatal testing, all of which provide risk assessment for family members of an affected patient to assist them in making complex personal, medical, and reproductive decisions. In addition, there are a multitude of genetic tests available to those with no family history of genetic disease that can provide information about potential reproductive or future health risks.

The process of ordering genetic testing can be a complex and often bewildering process for health-care professionals. There are challenges related to test selection, laboratory choice, standards of practice, and ethical issues. Commonly, genetic counselors receive requests for genetic testing for disorders for which gene identification has only recently been reported in the scientific literature. Often these requests come from primary care providers, but increasingly they come from families who follow the research on a particular genetic disease that has been diagnosed in their family. The immediacy with which peer-reviewed research findings are available on publicly available Internet sites speeds up this process (26).

Historically, the broad categories of genetic tests were (i) cytogenetic tests (chromosome analysis), (ii) molecular tests (DNA analysis), and (iii) biochemical tests (enzyme assays, metabolites, protein analysis). Globally, cytogenetic tests detect changes in chromosome number, structure, and arrangement. Molecular tests look for changes in the DNA sequence, methylation, deletions, and duplications, and biochemical tests detect changes in the gene products, such as changes in levels of enzymes and proteins. Evolving technologies have led to considerable overlap between these categories of genetic tests as there are cytogenetic tests that actually use molecular/DNA methodologies, such as fluorescent in situ hybridization analysis and chromosomal microarray analysis. Genetic tests have different clinical applications, and these will influence the specific technology selected (26).

In genetic testing, it is often important to take into account both the genotype and the associated phenotype. The genotype is defined as the genetic makeup and the phenotype as the observable traits, characteristics, and symptoms. In an "ideal" genetic test, there is one-to-one relationship between the genotype and the phenotype. However, it is possible to have a genetic test that can accurately identify changes in genotype but can be quite limited in its ability to predict phenotype, particularly type and severity of symptoms, age of onset, and disease course. This limitation in predicting outcome can be particularly challenging and requires that genetic counselors use a wide variety of critical thinking, educational, and counseling skills in discussing these testing issues with patients.

Genetic testing is now widely available for diagnostic confirmation, predictive testing, and carrier and fetal risk assessment. Genetic counselors have a crucial role in evaluating the clinical usefulness of these tests as they work with patients and their families to assess the medical and personal benefits, as well as the risks, of undergoing testing. Critical thinking and assessment skills are needed to determine whether genetic testing is clinically indicated and in selecting the type of genetic test and the appropriate laboratory. Genetic counselors must be knowledgeable about test validation procedures and laboratory variability. Through consideration of the clinical utility of the genetic test and client-focused genetic counseling should precede the use of any genetic test. These considerations are particularly important before the utilization of new technologies, which may have a higher likelihood of inconclusive test results, or use of genetic tests whose outcomes directly influence medical interventions (27).

To appropriately select a diagnostic laboratory for their clients, genetic counselors must be aware of the rapidly evolving standards by which clinical laboratories are certified and the increasing involvement and oversight of other 
government agencies and interested entities from the private sector. When a genetic counselor is not familiar with a laboratory, the genetic counselor should inquire about the laboratory's accreditation, the additional voluntary professional guidelines the laboratory follows, and the laboratory's participation in proficiency testing, both external and internal. In addition, it is important to determine the laboratory's experience with the particular test being considered and the specific technology utilized, as well as the training and certification of the laboratory and medical directors and other staff. In general, laboratories that voluntarily participate in multiple certification programs and that adhere to guidelines provided by professional organizations provide testing at a higher standard and should be utilized whenever possible.

Genetic counselors play a critical role in ensuring that their patients receive the "ideal genetic test," one that has optimal parameters for them. Genetic counselors should continue to participate actively in research aimed at evaluating the impact of the genetic testing outcomes and work with other stakeholders in defining enhanced oversight of genetic testing and global practice guidelines for the appropriate use of genetic tests (28-34).

\section{QUESTIONS TO BE ANSWERED}

Four questions must be answered by the genetic counselor: (i) What is the disease in question (clinical and laboratory diagnosis)? (ii) How severe is it (prognosis and therapeutic possibilities)? What can be done to treat the affected child postnatally? (iii) How is it caused/inherited (risk of recurrence)? (iv) What can be done to avoid or prevent the disease (prenatal diagnosis)? (35).

\section{Diagnosis}

Knowledge of the disease in question is a prerequisite of genetic counseling. Confirmation of an exact diagnosis, which is of basic importance, may be difficult. Obtaining old or recent medical records, pedigree analysis, careful historytaking, clinical examination of relatives, special laboratory tests, and other investigations (may be involving referral to other specialist departments) can all be relevant or necessary procedures (36).

The family history and pedigree are the basis for providing clients referred for genetic evaluation and counseling with a diagnosis, risk assessment, education, and psychosocial support. Accuracy, detail, and relevance are paramount. The genetic counseling student must develop a mastery of this fundamental task and employ standardized pedigree symbols and nomenclature that enhance the utility of the information obtained. The family history and pedigree have come a long way since the era of Francis Galton. An accurate and complete family history and pedigree lay the foundation for the highest standards of patient care and genetic counseling. They are tools now clearly viewed as having an essential role in all aspects of health care (37-41).

Disease, whether genetic or nongenetic, may be due to a variety of factors. Genetic disease is particularly likely to be heterogeneous in this respect. For example, a muscular dystrophy may result from a number of mutant genes. Among the common types of muscular dystrophy, we have the facioscapulohumeral form, which is characteristically auto- somal dominant, and myotonic dystrophy, also autosomal dominant. "Limb-girdle" muscular dystrophy, a diagnosis that probably comprises a number of disorders, is autosomal recessive. Duchenne, Becker, and Emery-Dreifuss muscular dystrophies are all X-linked recessive, with fairly distinct, but overlapping, phenotypes; under some conditions, they can manifest, usually in mild degree, in females also (e.g., symptomatic carriers). Sometimes investigation of a family with "muscular dystrophy" reveals that the primary disease was not in the muscles at all but, for example, a spinal muscular atrophy, originating in the anterior horn cells, or a more generalized disorder, for example, a glycogenosis (42).

A practically identical clinical picture is characteristic of some of the commoner types of mucopolysaccharidoses. However, Hurler, Scheie, and Sanfilippo diseases are of autosomal recessive inheritance, whereas the Hunter disease is $\mathrm{X}$-linked recessive.

It should be noted that there are diseases that do not usually manifest until adulthood (e.g., Huntington disease, facioscapulohumeral muscular dystrophy), and that in connection with certain diseases of recessive (autosomal or X-linked) inheritance, heterozygotes may also show clinical symptoms, for example, axillary and pubic hair may be sparse in heterozygotes for testicular feminization, cutaneous symptoms may be seen by gene carriers for Fabry disease, and nephrolithiasis occurs in heterozygotes for cystinuria.

In some cases, medical documents may be incomplete, or unobtainable; certain tests, which are now considered essential for the diagnosis, may not have been carried out or available at that time. There may be no histologic information, no postmortem report, no roentgenograms, no photographs, no biochemical results. Genetic counseling cannot be based on a diagnosis like "mental retardation," "growth disorder," or "degenerative disease." Sometimes an exact diagnosis, like "achondroplasia," may be clearly misleading. To make a correct diagnosis, with a few exceptions, requires detailed and correct information (42).

\section{Prognosis and Therapy}

Without a correct diagnosis, the genetic counselor cannot tell the parents whether there is a high or low risk of recurrence within the family, whether tests can be carried out to detect those persons/couples at high risk of having an affected child, whether prenatal diagnosis is possible (and if so, how, where, and when), whether the disease is in any way treatable, whether the severity is highly variable within a family, whether there are other (as yet undiscussed) serious implications, or whether other "reproductive options" are available.

Although much genetic disease remains untreatable, the counselor must know the possibilities for the disease in question. $\mathrm{He} / \mathrm{she}$ must be up-to-date and informed about surgical procedures and appliances (e.g., neonatal or prenatal surgery, transplantation, artificial limbs), about medical treatment (e.g., physiotherapy and antibiotic therapy for cystic fibrosis, use of restricted/special diets-for phenylketonuria/ galactosemia, use of specific vitamins - pyridoxine for certain epilepsies, hydroxocobalamin for $\mathrm{B}_{12}$ transport disorders), and about what is available within the community (e.g., help from social services, financial help for parents with handicapped children, institutional care). He/she must be able to discuss 
Table 1 General Population Risks

\begin{tabular}{ll} 
Condition & \multicolumn{1}{c}{ Risk } \\
Fetal loss & \\
in the first trimester & $1 / 6$ \\
in the second and third trimesters & $1 / 30-1 / 100$ \\
Neonatal death & $1 / 100$ \\
Malformation detectable at birth & $1 / 33$ \\
Mental retardation & $1 / 50$ \\
Chronic disease in adulthood & $1 / 5$
\end{tabular}

more remote possibilities, such as "gene therapy," within a realistic perspective. $\mathrm{He} / \mathrm{she}$ must be able to talk to the parents in a way, and at a level, that is appropriate to their preexisting knowledge and education, and their degree of anxiety or depth of grief. He/she must be prepared to educate them further. $\mathrm{He} /$ she must be understood and supportive (42).

\section{Genetic Risk}

This term covers two concepts: the general population risk of a disorder/disease and the risk, or recurrence risk, of a disorder/ disease in a specific family situation.

Many couples have no idea at all of general population risks. They may imagine that it is extremely unusual to abort spontaneously, bear a baby with a malformation, or have a child with mental retardation. They cannot understand how such things can happen, if they are not in the family already. They do not understand why a particular disaster happened to them, as they had done nothing to deserve or provoke it.

The general population risks for a few commonplace situations are given in Table 1 . All genetic counseling has to be given, and understood, against this general "background" risk. For example, a couple may be horrified to be told there is a $1 \%$ recurrence risk for a certain malformation; they may perceive that as a very high-risk situation (although 100 to 1 odds in a gambling situation are usually perceived as far from certainty!). It may help couples realize that an additional risk of $1 \%$ for a malformation is not so enormous when compared with the starting risk of $3 \%$ to $5 \%$; they have only a $33 \%$ increase above the general risk level.

In some counseling situations, couples may be at increased risk for one or a number of problems, as compared with general population risks, yet one is not talking of "recurrence risk," the condition provoking anxiety, and the request for counseling has not yet happened. In these situations, actual risk may not be very high, though much above the general population level. For example, a woman of 40 has a risk of bearing a live-born with Down syndrome that is 20 -fold the risk she ran when she was only 20 . Yet the actual risk is only 1/100. Similar low levels of risk (yet high, compared with general population risks) may be associated for example with exposure to teratogens or radiation; slightly higher levels of risk will be run in cousin marriages.

Very high levels of risk, for a problem that has not yet appeared within the family, will seldom be met with except in connection with specific screening programs, for example, detection of Tay-Sachs heterozygotes in Ashkenazi Jews, of thalassemia carriers in Mediterranean populations; in these situations, when both (prospective) parents are heterozygotes, the risk of an affected child is $25 \%$. In, virtually, all of these very high-risk situations identified by screening, a prenatal test can be offered: but this would not be acceptable to all couples.

When a disorder has already appeared within a family, recurrence risks may be high $(>1 / 10)$, intermediate $(1 / 10-1 / 100)$, or low $(<1 / 100)$ : use of these words, high, intermediate, low, is extremely arbitrary. The counselor's use of such words may be at variance with the use, or perceptions, of the counseled couple. To the counselor a risk estimate may appear intermediate or low, yet to the counseled couple it is high. Risk figures can be "rewritten" by turning statements around; 1/100 recurrence risk, for a serious malformation may appear formidable to the threatened parents; it is more reassuring to be told there is $99 \%$ chance that the baby will be unaffected. Risk figures should be compared with general population risks. The nature of the disorder/problem itself, and the impossibility/possibility of prenatal diagnosis, will affect the way it is perceived (8).

In some situations, recurrence risk is very low. If problems with a previous pregnancy were due to teratogen exposure, and the teratogen can be avoided in further pregnancies, risks may not be much above the general population risk.

Most malformations are multifactorially determined. After a child with an isolated exomphalos, or tracheo-esophageal malformation, recurrence risk may be $1 \%$ to $2 \%$ only; although this represents a 20 - to 40 -fold increase over the general population level, it is still not a very daunting risk. After having a child with congenital heart disease, the recurrence risks are usually $2 \%$ to $5 \%$ (depending on the malformation type); the background rate, for congenital heart disease, is nearly $1 \%(8 / 1000)$.

When dealing with a malformation problem, the counselor must be sure that it is really isolated and not part of a syndrome (that may be monogenic); for example, an occipital encephalocele alone might suggest a recurrence risk, for all types of neural tube defects, of about $2 \%$; if the encephalocele was accompanied by cystic kidneys or polydactyly, the Meckel syndrome would probably be diagnosed, with a recurrence risk of $25 \%$.

In families with monogenic disorders, many relatives will run risks that are intermediate or low. For example, with a heterozygote frequency of $1 / 25$ for cystic fibrosis, risk to the child of a woman whose brother was affected is $1 / 150$, and risk to a child of a man whose brother's child was affected is $1 / 200$. In families with $\mathrm{X}$-linked recessive disease, some females, with no closely affected relatives (but with many unaffected brothers or maternal uncles), will be at very low risk for being carriers or having affected sons.

The background general population risk is not identical with the risk within a given family (specific risk), nor with the risk calculated for a given pregnancy (actual risk). For example, the background risk for a healthy person of being heterozygous for cystic fibrosis is $1 / 25$, and the risk of his/her child being affected by the disease is $1 / 2500$. However, the situation will change completely if it is revealed that the couples already have a child suffering from cystic fibrosis. In this case, the specific risk that both members of the couples are heterozygotes is $1 / 1$, and the risk for a further child being affected is $1 / 4$. If the couples undertake a pregnancy, and the pregnancy tests give normal results, the actual risk for an affected child will fall to about nearly zero.

To talk about $100 \%$ reliability of a biochemical or molecular prenatal diagnosis is, in practice, nonsense. The counselor 
should not use words like "never" or "always." Instead, expressions like "probably not," "a very high chance," and "almost certainly" are preferable and might also avoid legal complications! (42).

To explain the concept of risk, we may use analogies, for example, one coin (heads/tails) may be useful in explaining autosomal dominant inheritance and two coins may explain recessive inheritance.

Discussion of risk situations demands tact. In particular, one should avoid laying "blame" to one member of the couple: this might be difficult in the case of autosomal dominant or $\mathrm{X}$-linked recessive disorders or inherited chromosome rearrangements. It is better, if possible, to spread the "responsibility" to both members of the couple: for example, with an X-linked disorder and an affected male child, the mother gave the mutant gene, but without the paternal Y chromosome all would have been well.

In cases of multifactorial determination, it is important not to accuse anybody, e.g., the physician who prescribed a drug or the factory in which the counselee was exposed to chemicals (and which guaranteed the safety of her work). An unfortunate combination of environmental factors and predisposing genes may be stressed. The counselor should intervene to prevent any further, possibly dangerous, exposure $(43,44)$.

In many cases, the client's concerns center not on the diagnosis of an affected individual but on assessing future reproductive or personal health risk. The counselor can sometimes make such an assessment by analyzing the pedigree, taking into account the pattern of inheritance and the client's relationship to individuals with the condition. Mathematical calculations may be needed to incorporate additional information (e.g., carrier frequencies, test sensitivity and specificity, numbers of affected and unaffected individuals, the client's age) to modify the risk. Questions about carrier status may be resolved with appropriate laboratory tests. When a condition has a multifactorial basis or is genetically heterogeneous, the best risk estimates may come from epidemiologic data on other families with affected individuals. Answering concerns about potentially mutagenic or teratogenic exposures also usually relies on empirical data about the agent in question and on evaluating the timing, duration, and dose of the exposure. In some areas of genetic counseling, such as cancer risk assessment, factors such as reproductive history, hormone use, and lifestyle issues such as smoking, obesity, or alcohol use are also important variables in risk assessment.

\section{Prenatal Diagnosis}

In very many counseling situations, the specific recurrence risk is so low that apart from a general screening for malformations, further tests are superfluous. A simple reassurance may disappoint certain couples if they feel that their problem has been oversimplified.

For most couples running a higher than average risk/ recurrence risk, one can offer prenatal diagnosis. Cases require individual assessment. The reliability and the safety of the diagnostic procedures should be discussed with the parents. These should be carefully weighed, bearing in mind the nature and severity of the disease/disorder prompting the desire for diagnosis/termination. It should be explained that the test(s) will be for one problem only or a limited range of disorders, and normal results will not guarantee a child normal in every way.

The final decision, to accept or reject the offer of prenatal diagnosis, is for the couple to make. Their decision will reflect their attitude to the disorder in question, and also their feelings regarding termination of pregnancy, should the test show an "affected" fetus. They should not be required to give any consent in advance, regarding abortion in this latter eventuality.

The couples decide whether or not to undertake pregnancy and they decide whether or not to accept an offer of prenatal diagnosis. However, prenatal testing is time-consuming, laborious, and expensive; it uses human resources and materials that are, as a result, not available to others; it involves certain risks for the mother and fetus. If a couple are convinced that they would not terminate pregnancy in any event (an "affected" fetus), they will probably also be convinced that the risks, to mother and pregnancy, are not worth taking.

In some autosomal or X-linked recessive situations, prenatal diagnosis is, at present, impossible, even though the genetic risk is high. In this difficult situation, some counseled couples will decide the $25 \%$ recurrence risk is too high and will choose termination of an existing pregnancy, sterilization, conception by AID, or divorce and remarriage. Other couples regard the $75 \%$ chance of a healthy child a sufficient encouragement to make at least one more attempt to have a healthy child.

\section{Decision-Making of the Couple: Nondirective Counseling}

The physician/counselor gives information and the parents, in light of their own individual circumstances and attitudes, make the decision (45-48).

The parental decision will depend on a number of factors: of importance may be their optimistic or pessimistic attitude of mind, their ethical and religious principles, their level of education, and/or their social circumstances. Their previous experiences with the disease in question may also be of decisive importance. Many couples declare that they do not want to undergo another tragedy. This is a most convincing argument.

The personality of the counselor, too, is of great importance: the way in which information is imparted, the content and clarity of such information, the manner in which the couples' own questions, fears, and problems are discussed and dealt with. Decision-making is extremely difficult and painful for some couples, and decisions will often have lifelong consequences. The counselor should help the couple to the best of his/her ability.

All over the world, genetic counseling is primarily done by biologists, geneticists, and various medical specialists, mainly pediatricians and obstetricians (49). Pregnant women and their partners facing various genetic problems can base their decision on information and advice made available by these professionals' knowledge and expertise. Counseling can follow two principles: the more widely used nondirective genetic counseling and the so-called directive genetic counseling (50-53). Because the current era is dominated by legal claims against physicians, the nondirective method is more acceptable and more easily defendable, even though in Ethical Considerations in Prenatal Diagnosis, in many cases, patients expect and demand a decision-shaping process closely guided 
by the physicians. When applying the nondirective method, the genetic counselor is ready to share information in a nondirective manner without committing to any potential alternative. It is very important that having thoroughly described the disease in question, the consultant should also inform the patient about the risk of occurrence and/or recurrence. After that, diagnostic alternatives should be described and offered-if there are any. This has to be done in a fashion so that the patients seeking counseling can understand the basic facts, and it may vary with the given circumstances. The patient's fear and anxiety must not be worsened by giving an opinion expressed in mystical complicated sentences that are incomprehensible to ordinary people. By this point, the couples have already started the decision-making process. The physician leaves it to the patients to use the intensive interactive process to arrive at their final decision (50,53-55).

\section{PREDICTIVE TESTING AND PRESYMPTOMATIC DIAGNOSIS}

Predictive (susceptibility) testing (sometimes called "predisposition testing" in the case of mutations with less than $100 \%$ penetrancy) identifies healthy individuals who may have inherited a genetic predisposition that puts them at increased risk of developing a multifactorial disease (e.g., Alzheimer disease or cancer), but who may never develop the disease in question (56). Presymptomatic diagnosis refers the identification of healthy individuals who may have inherited a gene for a late-onset monogenically inherited disease and, if so, will develop the disorder if they live long enough (e.g., Huntington disease) $(57,58)$. DNA arrays could potentially include mutation probes for a large panel of late-onset disorders and an extended spectrum of mutations and polymorphisms for each one, dramatically complicating an already thorny genetic and social counseling problem (59). Do we have the right to inform the patient about the existence of untreatable disease before symptoms appear? Is it necessary to do so? Are we obliged to do that? Is this individual ill at all? Can or need populations be screened for certain diseases? Indeed, the development of some diseases might be slowed down if changes in lifestyle were implemented. The knowledge, however, that the development of serious disease is inevitable in a later stage of one's life could put a heavy burden on his everyday existence and might even change an individual's personality. Surviving in the knowledge that one is to expect to develop a malignant tumor by the age of 30 to 40 years is difficult (60-62). By the same token, possessing the relevant information might result in more careful diagnostic examinations, which should have a substantial effect on the life expectancy (8).

At the same time, it raises the question of who is entitled to know the information, that is, confidentiality, the individual affected or his relatives. One might think that only the individual affected should, but with the disease in question being a genetic one, are the relatives not affected too? Do they not have the right to know their risk? Is the parent obliged to tell his or her child? Can the child request the performance of a predictive test? (63) Can the parent make a decision on whether the examination should be performed for her minor child? (64). Susceptibility testing and presymptomatic diagnostics in the absence of therapeutic options should be available if certain conditions are met. It is important that the individual be provided thorough information about the limits of testing, and the information contribute to enhancing the pathography and informing the family because, in many cases, it is impossible to predict the onset and seriousness of a particular disease and its symptoms. Awareness of susceptibility could induce a change in the lifestyle that could prevent or prolong the development of a disease. If a disease is inevitable, the individual will have the chance of planning for his or her short life, as in the case of Huntington disease. Such genetic information can influence plans for marriage and having children (8). The problem is further complicated by the shortcomings of available predictive genetic tests that still carry a factor of serious uncertainty about whether a disease will develop and, if it does, when exactly and to what extent. Given the onus of this information, if there is no medical advantage concerning prevention or treatment, these examinations can best be postponed until adulthood, when the individual is able to make decisions on crucial aspects of his own life. Considering the likely rapid dissemination of predictive tests, there is an urgent need to develop a well-thought-out, detailed legal framework. Prohibition cannot be allowed because it would deprive the individual of rather important information. The proper regulation would eliminate the situation of diametrically opposed interests among the parties and would make them interested in wide-ranging examinations.

\section{TERMINATION OF PREGNANCY BECAUSE \\ OF GENETIC INDICATIONS}

Recent advances in technology have enabled the development of a wide range of methods for prenatal diagnosis (65). The demands of modern society are for a healthy "perfect baby" (66). The new techniques are aimed at early diagnosis of the abnormal fetus at a point where parents who wish to do so may terminate the pregnancy. The emphasis is not on the removal of the defective child, but rather on the provision of life for new healthy children, who may otherwise never have been born.

The most challenging moments of genetic counseling arise when a decision has to be made on the disposition of a pregnancy (67). From the consultant's point of view, those situations are the most difficult when the problem is so severe and the diagnosis is so bad that termination on a genetic basis may be proffered. Even in this case, the goal of nondirective counseling is to inform the patient about this option. The woman is about to resolve one of the most difficult situations of her life. The problems of fetal life have often been put in the center of debates, not only in professional circles, but also as a political issue. The rigid attitude of the Roman Catholic Church is well known and stirs a lot of debates even within that faith community. The church turns a deaf ear to the issue of termination and does not accept contraception as a legitimate option (with a few exceptions). Thus, it is difficult to provide the opportunities offered by the achievements of prenatal diagnosis to Catholics. In some liberal circles' view, parents must be assured of having the widest possible sphere of authority. This cannot be readily accepted by a physician or an obstetrician-geneticist. 
The weight of the decision depends on the gestational age of the pregnancy and other factors. Decisions on serious conditions detected in the first trimester are made easier (40). Given that most diagnoses are made in the second trimester, a very close relationship has sometimes developed between the fetus and the expectant mother by decision time. She may have seen its face during an ultrasound examination, and she may even know her offspring's sex. Realizing her widening waistline, the people around her may learn about the pregnancy, and this knowledge and having to wait make it even more difficult for her to cope. The necessity to make a decision presents a major state of crisis, the extent of which depends, among other things, on the obstetrical anamnesis. In such a case, the task is to give the relevant facts, help the patient to consider them thoughtfully and calmly, and encourage her to develop positive prospects for the future. It is important to outline the possible short-term and long-term consequences of the decisions, the risk of the defect being recurrent, and the details of its heredity.

If the woman opts to continue her pregnancy, she needs to be briefed on what sort of aid she can rely on from the fields of medicine and social services, as well as family care. Those deciding to terminate the pregnancy must be informed about the procedure to relieve tension and distress. In any case, this should ease the anxiety regarding the operation itself. It must be stressed that if the couple opts to terminate the pregnancy, the procedure must be initiated as early as possible. The methods used for midterm abortions vary from country to country. Third-trimester abortion also has a place in obstetrical practice. It is justified if the fetus will be unable to survive after birth, and the couple requests termination of pregnancy. Parents have a right to make this decision if the continuation of the pregnancy may increase the risk of maternal complications with no effect on fetal outcome $(68,69)$.

Fetal disease or disorder diagnosed in a twin pregnancy may be concordant or discordant. In the former situation both fetuses are affected, and termination may be carried out as for a single pregnancy. In a discordant twin pregnancy, when only one of the fetuses is affected, three outcomes are possible: (i) the couple may decide against any form of termination; (ii) the parents may decide to lose both fetuses, the affected and the healthy one; (iii) partial selective termination, bringing about death of the affected twin while preserving the normal one, may be attempted. The dead fetus develops into a fetus papyraceus, while the other fetus should develop normally (70). Multifetal pregnancy reduction also has become a well-established and integral part of infertility therapy and the attempts to deal with sequel of aggressive infertility management. Now we have very clear and precise data on the risks and benefits as well as an understanding that the risks increase substantially with the starting and finishing number of fetuses in multifetal pregnancies (71). Regardless of the method, the objective is to get the procedure over within the shortest possible time and in the least intrusive way. The patient ought to get back to her home as soon as possible to be able to deal with the tragedy with her loved ones. Access must be made available to post-termination counseling $(50,55)$.

\section{POST-TERMINATION FETOPATHOLOGY}

\section{AND COUNSELING}

Fetopathology is a postmortem specialty concerned with causes and mechanisms of reproductive loss. In late first and early second trimesters, prenatal diagnosis of fetal anatomy by ultrasound became a major factor leading to termination. Even in centers with high rate of accurate prenatal ultrasound, a high percentage of fetuses with correctly diagnosed anomalies have additional defects that are not recognized by ultrasound. At the post-termination autopsy, the fetopathologist must concentrate on small, even minute anatomic details to find these additional defects to refine the pretermination diagnosis. It cannot be emphasized enough that with all terminations and pregnancy losses, the placenta should be submitted to the pathologist and the pathologist has to evaluate it in context with the clinical history and the postmortem findings of the fetus. The precise description of a clinically suspected anomaly and fine details of dysmorphic features and a solid final diagnosis are essential to the correct estimation of the recurrence risk so that the appropriate plan for surveillance in subsequent pregnancies can be instituted. Discussion of postmortem findings at regular fetal pathology/dysmorphology meetings is also recommended for education as part of a multidisciplinary approach. In these ways, fetopathology serves to promote the quality of the care of the individual and public health $(72,73)$.

\section{PREIMPLANTATION DIAGNOSIS}

PGD is a technique devised within the last 20 years used primarily to identify genetic disorders or defects in human pre-embryos created by IVF prior to their implantation for gestation (74). PGD is intended to result in the selective destruction of extracorporeal human pre-embryos following genetic diagnosis. PGD is an attractive means of preventing genetic diseases, which, as an alternative to traditional prenatal genetic diagnosis, can avoid the need for termination of the affected fetuses. PGD is also used to detect aneuploidy and to select chromosomally normal pre-embryos for implantation, mostly in women of advanced maternal age undergoing IVF. The purpose of PGD is not simply to inform couples about the genetic nature of their pre-embryos, but the explicit purpose is also to transfer healthy pre-embryos (75). As a matter of general principle, prevention of the birth of a genetically or otherwise diseased or disabled child is plainly a morally legitimate goal. Nevertheless, strong moral objections have been made to the use of PGD to determine conditions that cannot plausibly be called "diseases," such as sex and the absence of desirable physical, mental, or social characteristics. The selection of an embryo's sex via PGD is done for two basic reasons: (i) preventing the transmission of sex-linked genetic disorders and (ii) choosing sex to achieve gender balance in a family with more than one child, to achieve a preferred order in the birth of children by sex, or to provide a parent with a child of the sex he/she prefers to raise. While little extended ethical debate exists regarding the former, sex selection for the purpose of preventing the transmission of sex-linked genetic disease, the latter is the subject of heated ethical disagreement (76). If some services become embroiled in the sex selection and in the detection of conditions having little or nothing to do with health and 
disease and some traits unrelated to health in order to satisfy patient demand or be a profitable business, its practitioners will get, and deserve, serious ethical criticism (77).

\section{SUMMARY}

Over the last four decades, the portion of the patient population that needs genetic counseling services has steadily expanded. In the early years, genetic counselors met mostly with parents of children with genetic diseases or adults who had relatively uncommon disorders. Now we are counseling patients and families who are at relative risk for cardiovascular, oncologic, neurologic, and other common diseases $(8,78)$.

The principles of genetic counseling are universal, even though the practice of genetic counseling varies nationally and internationally.

Genetic counseling is a dynamic profession, which undergoes rapid change with the discovery of new genetic information and the development of new genetic tests and treatment options.

The role of the genetic counselor is to provide the most complete information available. His/her role is not that of an advocate for society. The geneticist has continuing obligation to recontact patients when new information develops.

The 20th century witnessed revolutionary progress in the science of genetics that coincided with increasing societal demands, and therefore genetic counseling became an integral part of modern medicine and health care $(79,80)$.

\section{REFERENCES}

1. Murphy EA, Chase GA. Principles of Genetic Counseling. ChicagoLondon: Year Book Medical Publishers, 1975.

2. Kelly PT. Dealing with dilemma: a manual for genetic counselors. New York: Springer, 1977.

3. Herrmann J, Opitz JM. Genetic counseling. Postgrad Med 1980; 67: 233-43.

4. Passarge E, Vogel F. The delivery of genetic counseling services in Europe. Hum Genet 1980; 56: 1-5.

5. Reed S. Counseling in Medical Genetics. New York: Alan R Liss, 1980.

6. Hof JO, Kopinsky SM. Communication in genetic counseling. S Afr Med J 1982; 62: 758-64.

7. Harper PS. Practical Genetic Counselling, 6th edn. Oxford Press, 2004.

8. Milunsky A, ed. Genetic Disorders and the Fetus: Diagnosis, Prevention and Treatment, 5th edn. Baltimore: Johns Hopkins University Press, 2004.

9. Jones RJ. Genetic counseling and prevention of birth defects. J Am Assoc 1982; 248: 221-4.

10. McKusick VA. Mendelian Inheritance in Man, 12th edn. Baltimore: The Johns Hopkins University Press, 1998.

11. Bennett RL, Motulsky AG, Bittles AH, et al. Genetic counseling and screening of consanguineous couples and their offspring: recommendations of the National Society of Genetic Counselors. J Genet Couns 2002; 11: 97-119.

12. Rantanen E, Hietala M, Kristoffersson U, et al. What is ideal genetic counselling? A survey of current international guidelines. Eur J Hum Genet 2008; 16: 445-52.

13. Kelly TE. Clinical Genetics and Genetic Counseling. Chicago-London: Year Book Medical Publishers, 1979.

14. Abramovsky I, Godmilow L, Hirschhorn K, Smith H. Analysis of a followup study of genetic counseling. Clin Genet 1980; 17: 1-12.

15. Bringle RG, Antley RM. Elaboration of the definition of genetic counseling into a model for counselee decision-making. Soc Biol 1980; 27: $304-18$.

16. Curtis D, Johnson M, Blank CE. An evaluation of reinforcement of genetic counselling on the consultand. Clin Genet 1988; 33: 270-6.
17. Aalfs CM, Oort FJ, de Haes JCJN, Leshot NJ, Smets EMA. A comparison of counselee and counselor satisfaction in reproductive genetic counseling. Clin Genet 2007; 72: 74-8.

18. Middleton A, Crowley L, Clarke A. Reflections on the experience of counseling supervision by a team of genetic counselors from the UK. J Genet Couns 2007; 16: 143-55.

19. Kessler S, ed. Genetic counseling. Psychological dimensions. New York: Academic Press, 1979.

20. Kessler S. Psychological aspects of genetic counseling. VII. Thoughts of directiveness. J Genet Couns 1992; 1: 9-17.

21. Kessler S. Psychological aspects of genetic counseling. VIII. Suffering and counter-transference. J Genet Couns 1992; 1: 303-8.

22. Michie S, Mareau TM, Bobrow M. Genetic counseling: the psychological impact of meeting patients' expectations. J Med Genet 1996; 34: 237-41.

23. Eunpu D. Systematically-based psychotherapeutic techniques in genetic counseling. J Genet Couns 1997; 6: 1-20.

24. Weil J. Psychosocial Genetic Counseling. New York: Oxford University Press, 2000.

25. Papp Z. Ethical challenges of genomics for perinatal medicine: the Budapest Declaration. Am J Obstet Gynecol 2009; 201: 336.

26. Uhlmann WR, Schuette JL, Yashar BM, eds. A Guide to Genetic Counseling. New Jersey: Wiley-Blackwell, Hoboken, 2009.

27. Resta R, Biesecjer BB, Bennett RL, et al National Society of Genetic Counselors' Definition Task Force. A new definition of genetic counseling: National Society of Genetic Counselors' Task Force Report. J Genet Couns 2006; 15: 77-83.

28. Wertz DC, Sorenson JR. Client reactions to genetic counseling. Selfreports or influence. Clin Genet 1986; 30: 494-502.

29. Wertz DC, Fletcher JC. Attitudes of genetic counselors. A multinational survey. Am J Hum Genet 1988; 42: 592-600.

30. Walker AP, Scott JA, Biesecker BB, et al. Report of the 1989 Asilomar meeting on education in genetic counseling. Am J Hum Genet 1990; 46: 1223-30.

31. Fiddler MB, Fine BA, Baker DL, ABGC Consensus Development Consortium. A case-based approach to the development of practice-based competencies for accreditation of and training in graduate programs in genetic counseling. J Genet Couns 1996; 5: 105-12.

32. Fine BA, Baker DL, Fiddler MB, ABGC Consensus Development Consortium. Practice-based competencies for accreditations of and training in graduate program in genetic counseling. J Genet Couns 1996; 5: 113-21.

33. Heimler A. An oral history of the National Society of Genetic Counselors. J Genet Couns 1997; 6: 315-36.

34. Sahhar MA, Young MA, Sheffield LH, Aitken M. Educating genetic counselors in Australia: developing an international perspective. J Genet Couns 2005; 14: 283-94.

35. Gaff CL, Clarke AJ, Atkinson P, et al. Process and outcome in communication of genetic information within families: a systematic review. Eur J Hum Genet 2007; 5: 999-1011.

36. Quereshi N, Bethea J, Modell B, et al. Collecting genetic information in primary care: evaluating a new family history tool. Fam Pract 2005; 22: 663-9.

37. Gelehrter TD. The family history and genetic counseling. Genet Dis 1983; 73: 119-26.

38. Bennett RL, Steinhaus KA, Uhrich SB, O'Sullivan C. The need for developing standardized family pedigree nomenclature. J Genet Couns 1993; 2: 261-73.

39. Bennett RL, Steinhaus KL, Uhrich SB, et al. Recommendation for standardized human pedigree nomenclature. Am J Hum Genet 1995; 56: $745-52$.

40. Bendure WB, Mulvihill JJ. Perform a gene test on every patient: the medical family history revisited. J Okla State Med Assoc 2006; 99: 78-83.

41. Botkin JR, McMahon WM, Smith KR, Nash JE. Privacy and confidentiality in the publication of pedigrees: a survey of investigators and biomedical journals. JAMA 2008; 279: 1808-12.

42. Papp Z. Obstetric genetics. Budapest, Hungary: Academic Press, 1990.

43. Hallowell N, Statham H, Murton F, Green J, Richards M. "Talking about chance": the presentation of risk information during genetic counseling for breast and ovarian cancer. J Genet Couns 1997; 6: 269-86. 
44. Meiser B, Halliday JL. What is the impact of genetic counseling in women at increased risk of developing hereditary breast cancer? A meta-analytic review. Soc Sci Med 2002; 54: 1463-70.

45. Antley RM. The genetic counselor as facilitator of the counselee's decision process. Birth Defects Orig Articl Ser 1979; 15: 137-68.

46. Papp Z, Németi M, Papp CS, Tóth-Pál E. Reproductive decisions after genetic counseling of couples at high risk for cystic fibrosis: a perspective from the last two decades. In: Kurjak A, Chervenak FA, eds. The Fetus as a Patient. New York, London: Pathenon, 1994: 107-15.

47. Benoit LG, Veach McCarthy P, LeRoy BS. When you care enough to do your very best: Genetic counselor experiences of compassion fatigue. J Genet Couns 2007; 16: 299-312.

48. Chervenak FA, McCullough LB, Sharma G, Davis J, Gross S. Enhancing patient autonomy with risk assessment and invasive diagnosis: an ethical solution to a clinical challenge. Am J Obstet Gynecol 2008; 199: 19.e1-4.

49. Simpson JL, Elias S, Gatlin M, Martin AO. Genetic counseling and genetic services in obstetrics and gynecology: Implications for educational goals and clinical practice. Am J Obstet Gynecol 1981; 140: 70-80.

50. Papp Z, Tóth-Pál E, Papp CS. Non-directive prenatal counseling. In: Kurjak A, Chervenak FA, eds. The Fetus as a Patient. New York, London: Parthenon, 1994: 71-7.

51. Michie S, Bron F, Babrow M, Marteau TM. Nondirectiveness in genetic counseling: an empirical study. Am J Hum Genet 1997; 60: 40-7.

52. Resta RG. Eugenics and nondirectiveness in genetic counseling. J Genet Couns 1997; 6: 255-8.

53. Csaba Á, Papp Z. Genetic counseling. In: Kurjak A, Chervenak FA, eds. Textbook of Perinatal Medicine. Norfolk, UK: Informa Healthcare, 2006: 190-200.

54. Sorenson JR, Wertz DC. Couple agreement before and after genetic counseling. Am J Med Genet 1986; 25: 549-55.

55. Papp Z. Change in public demand for genetic counseling in the past 25 years. In: Chervenak FA, Kurjak A, Papp Z, eds. The Fetus as a Patient: The Evolving Challenge. Boca Ration: Parthenon Publishing Group, 2002: 130-45.

56. McAllister M. Predictive genetic testing and beyond: a theory of engagement. J Health Psychol 2002; 7: 491-508.

57. Macer DRJ. Ethics and prenatal diagnosis. In: Milunsky A, ed. Genetic Disorders and the Fetus: Diagnosis, Prevention and Treatment. Baltimore: John Hopkins University Press, 1998: 999-1024.

58. Sarangi S, Bennett K, Howell L, et al. (Mis)alignments in counseling for Huntington's disease predictive testing: clients' responses to reflective frames. J Genet Couns 2005; 14: 29-42.

59. Grody WW. Ethical issues raised by genetic testing with oligonucleotide microarrays. Mol Biotechnol 2003; 23: 127-38.

60. Meiser B. Psychological impact of genetic testing for cancer susceptibility: an update of the literature. Psychooncology 2005; 14: 1060-74.
61. Agincourt-Canning L. Genetic testing for hereditary breast and ovarian cancer: responsibility and choice. Qual Health Res 2006; 16: 97-118.

62. Berliner JL, Fay JM. Risk assessment and genetic counseling for hereditary breast and ovarian cancer: recommendations of the National Society of Genetic Counselors. J Genet Couns 2007; 16: 241-60.

63. Gaff CL, Lynch E, Spencer L. Predictive testing of eighteen year olds: counseling challenges. J Genet Couns 2006; 15: 245-51.

64. Savulescu J. Predictive genetic testing in children. Med J Aust 2001; 175: 379-81.

65. Papp Z. The history of fetal diagnosis and therapy. The Semmelweis University experience. Fetal Diagn Ther 2002; 17: 258-67.

66. Green RM. Babies by design. The ethics of genetic choice. New Haven and London: Yale University Press, 2007.

67. Papp Z. Genetic counseling and termination of pregnancy in Hungary. J Med Philos 1989; 14: 323-33.

68. Chervenak FA, McCullough LB, Campbell S. Is third trimester abortion justified? Br J Obstet Gynaecol 1995; 102: 434-5.

69. Papp Z, Gávai M, Görbe É. Is third trimester abortion justified? Br J Obstet Gynaecol 1996; 103: 187-9.

70. Kerenyi TD, Chitkara U. Selective birth in twin pregnancy with discordance for Down's syndrome. N Engl J Med 1981; 303: 1525-7.

71. Evans MI, Britt DW, Ciorica D, Fletcher JC. Selective reduction. In: Kurjak A, Chervenak FA, eds. Textbook of Perinatal Medicine. Norfolk, UK: Informa Healthcare 2006: 201-8.

72. Papp Z. Atlas of Fetal Diagnosis. Amsterdam: Elsevier, 2002.

73. Marton T, Bán Z, Papp Z. Post-termination fetopathology. In: Kurjak A, Chervenak FA, eds. Donald School of Ultrasound in Obstetrics and Gynecology. New Delhi: Jaypee Brothers Medical Publishers, 2008: 507-17.

74. Chervenak FA, McCullough LB, Rosenwaks Z. Ethical considerations in newer reproductive technologies. Semin Perinatol 2003; 27: 427-34.

75. Botkin JR. Ethical issues and practical problems in preimplantation genetic diagnosis. J Law Med Ethics 1998; 26: 17-28.

76. Pennings G. Personal desires of patients and social obligations of geneticists: applying preimplantation genetic diagnosis for non-medical sex selection. Prenat Diagn 2002; 22: 1123-9.

77. Nelson LJ. Preimplantation diagnosis. In: Kurjak A, Chervenak FA, eds. Textbook of Perinatal Medicine. Norfolk, UK: Informa Healthcare, 2006: 179-89.

78. Ledbetter DH, Faucett WA. Issues in genetic testing for ultra-rare diseases: background and introduction. Genet Med 2008; 10: 309-13.

79. Kenen RH, Smith ACM. Genetic counseling for the next years: models for the future. J Genet Couns 1995; 4: 115-24.

80. Kenen RH. Opportunities and impediments for a consolidating and expanding profession: genetic counseling in the United States. Soc Sci Med 1997; 45: 1377-86. 


\section{Assessment of fetal brain abnormalities Ritsuko K. Pooh}

\section{INTRODUCTION}

Recent advanced ultrasound imaging technologies such as high-frequency transvaginal scanning and three-dimensional (3D) sonography have been remarkably improved, and introduction of those technologies in clinical practice has contributed to prenatal evaluation of fetal central nervous system (CNS) development and assessment of CNS abnormalities in utero.

Sonographic assessment of the fetal brain in the sagittal and coronal sections requires an approach through anterior/ posterior fontanelle and/or the sagittal suture. Transvaginal sonography of the fetal brain opened a new field in medicine, "neurosonography" (1). Transvaginal approach to the normal fetal brain during the second and third trimesters was introduced in the beginning of 1990s. It was the first practical application of 3D CNS assessment by two-dimensional (2D) ultrasound (2). Transvaginal observation of the fetal brain offers sagittal and coronal views of the brain (3-6) through the fontanelles and/or the sagittal suture as ultrasound windows. Serial oblique sections (1) via the same ultrasound window reveal the intracranial morphology in detail. This method has contributed to the prenatal assessment of congenital CNS anomalies and acquired brain damage in utero. Furthermore, the brain circulation demonstrated by transvaginal power Doppler was first reported in 1996 (7,8); brain vascularity and blood supply have become clearly detectable afterward.

Three-dimensional ultrasound is one of the most attractive modality in a field of fetal ultrasound imaging. Automatic scan by dedicated 3D transducer produces motor-driven automatic sweeping and is called as a fan scan. With this method, a shift and/or angle change of the transducer is not required during scanning and scan duration needs only several seconds. After the acquisition of the target organ, multiplanar imaging analysis and tomographic imaging analysis are possible. Combination of both transvaginal sonography and 3D ultrasound (9-19) has been a great diagnostic tool for evaluation of $3 \mathrm{D}$ structure of fetal CNS. Recent advanced 3D ultrasound equipments have several useful functions as follows:

- Surface anatomy imaging

- Bony structural imaging of the calvaria and vertebrae $(13,14)$

- Multiplanar imaging of the intracranial structure

- Tomographic ultrasound imaging of fetal brain in the any cutting section (Fig. 1)

- Thick slice imaging of the intracranial structure

- Simultaneous volume contrast imaging of the same section or vertical section of fetal brain structure

- Volume calculation of target organs such as intracranial cavity, ventricle, choroid plexus, and intracranial lesions (20-25)
- Three-dimensional sono-angiography of the brain circulation (3D power Doppler) (8)

Fetal neuroimaging with advanced 3D technology is an easy, noninvasive, and reproducible method. It produces not only comprehensible images but also objective imaging data. Easy storage/extraction of raw volume data set enables easy off-line analysis and consultation to neurologists and neurosurgeons.

\section{VENTRICULOMEGALY AND HYDROCEPHALUS}

"Hydrocephalus" and "ventriculomegaly" are both the terms used to describe dilatation of the lateral ventricles. However, those two should be distinguished from each other. Hydrocephalus signifies dilated lateral ventricles resulted from increased amount of cerebrospinal fluid (CSF) inside the ventricles and increased intracranial pressure, while ventriculomegaly is the dilatation of lateral ventricles without increased intracranial pressure, due to cerebral hypoplasia or CNS anomaly such as agenesis of the corpus callosum $(26,27)$. Of course, ventriculomegaly can sometimes change into hydrocephalic state. In sonographic imaging, those two intracranial conditions can be differentiated by visualization of subarachnoid space and appearance of choroid plexus. In normal condition, subarachnoid space, visualized around both the cerebral hemispheres, is well preserved during pregnancy. Choroid plexus is a soft tissue and is easily affected by intracranial pressure. Obliterated subarachnoid space and dangling choroid plexus are observed in the case of hydrocephalus. By contrast, the subarachnoid space and choroid plexus are well preserved in cases of ventriculomegaly. It is difficult to evaluate subarachnoid space in the axial plane because the subarachnoid space is observed in the parietal side of the hemispheres. It is suggested that the evaluation of enlarged ventricles should be done in the parasagittal and coronal views by transvaginal approach to the fetal brain or 3D multidimensional analysis (Fig. 2). As a screening examination, the measurement of atrial width (AW) is useful with a cutoff value of $10 \mathrm{~mm}(28,29)$. In normal fetuses, blood flow waveforms of dural sinuses, such as the superior sagittal sinus, vein of Galen, and straight sinus have pulsatile pattern (30). However, in cases with progressive hydrocephalus, normal pulsation disappears and blood flow waveforms become flat pattern (30). Intracranial venous blood flow may be related to increased intracranial pressure.

\section{Variety of Mild Ventriculomegaly with AW 10-15 mm}

Mild ventriculomegaly is defined as of a width of the atrium of the lateral cerebral ventricles of 10 to $15 \mathrm{~mm}$. It has been reported that mild ventriculomegaly with AW 10 to $15 \mathrm{~mm}$ resolves in 29\%, remains stable in 57\%, and progresses in $14 \%$ of the cases during pregnancy (31). In cases of 


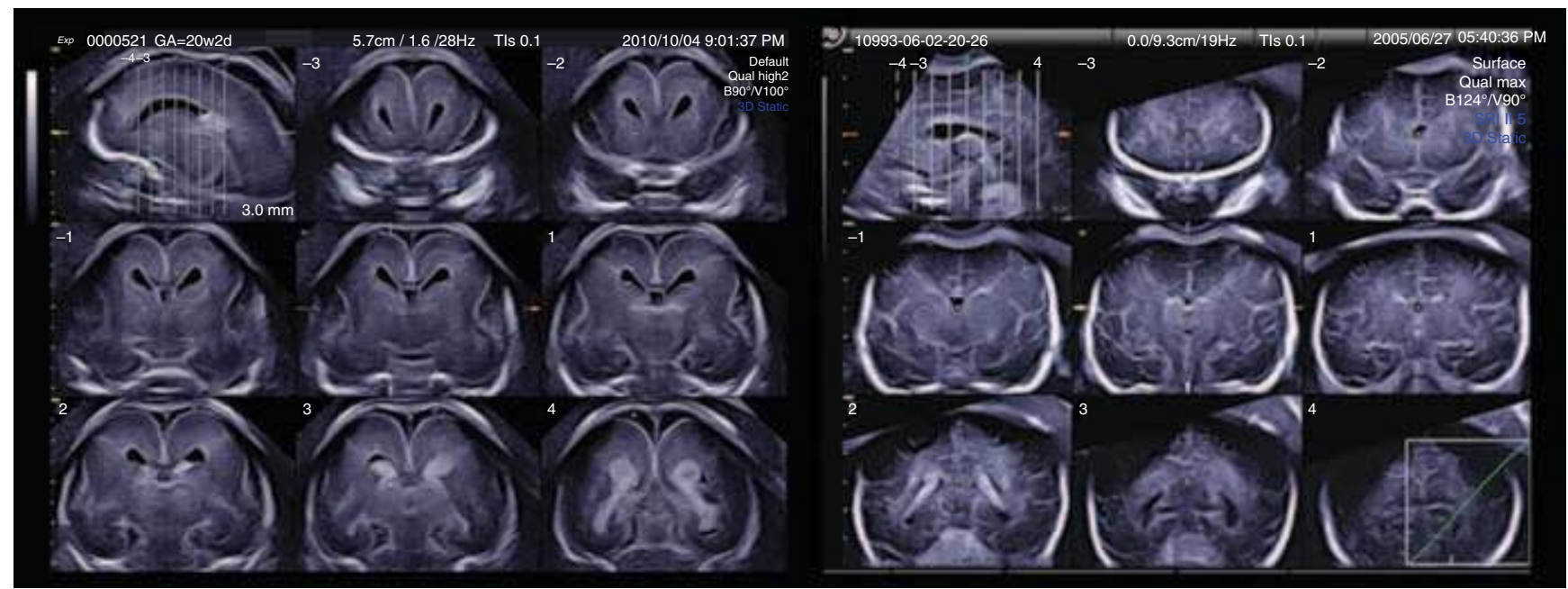

Figure 1 Tomographic ultrasound imaging by three-dimensional transvaginal sonography. Normal brain at 20 weeks (left) and 31 weeks (right) on the coronal cutting sections. Note the changing cortical development between those two different gestational stages.
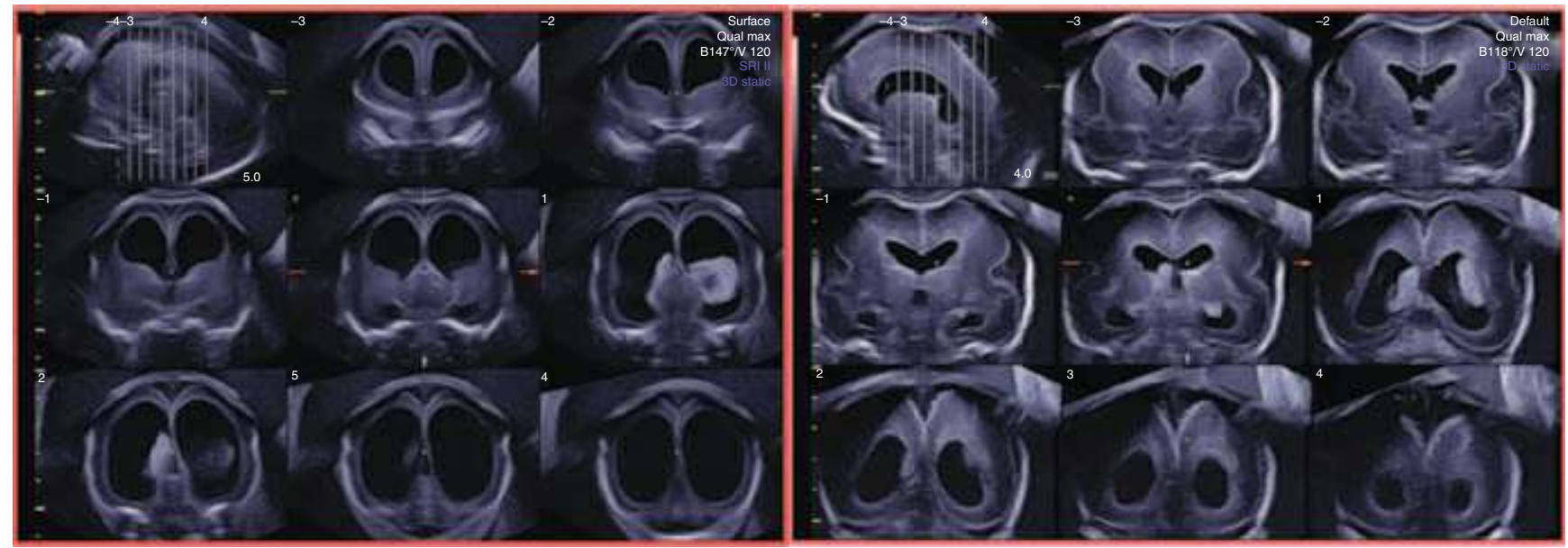

Figure 2 Hydrocephalus (left) and ventriculomegaly (right). Tomographic ultrasound imaging of X-linked hydrocephalus at 21 weeks (left) and ventriculomegaly at 25 weeks (right). Note the obliterated subarachnoid space in the hydrocephalic case compared with normal subarachnoid space in the ventriculomegaly case.

ventriculomegaly with $\mathrm{AW}$ of 10 to $13 \mathrm{~mm}$ at referral, the ultimate fetal outcome and prognosis depends on associated abnormalities.

Generally, in cases of mild fetal ventriculomegaly with a normal karyotype and an absence of malformations, the outcome appears to be favorable (32). Pilu and his colleagues (33) reviewed 234 cases of borderline ventriculomegaly including an abnormal outcome in $22.8 \%$ and concluded that borderline ventriculomegaly carries an increased risk of cerebral maldevelopment, delayed neurologic development, and, possibly, chromosomal aberrations. Isolated mild ventriculomegaly with AW of 10 to $12 \mathrm{~mm}$ may be normal variation. Signorelli and colleagues (34) described that their data of normal neurodevelopment between 18 months and 10 years after birth in cases of isolated mild ventriculomegaly (AW of $10-12 \mathrm{~mm}$ ) should provide a basis for reassuring counseling. Ouahba and colleagues (35) recently reported the outcome of 167 cases of isolated mild ventriculomegaly and concluded that in addition to associated anomalies, three criteria are often associated with an unfavorable outcome: AW greater than $12 \mathrm{~mm}$, progression of the enlargement, and asymmetrical and bilateral ventriculomegaly.
Moderate to Severe Ventriculomegaly and Hydrocephalus with $\mathrm{AW}>15 \mathrm{~mm}$

The term of "hydrocephalus" does not identify a specified disease, but is a generic term that means a group of pathologic conditions due to abnormal circulation of CSF. Treatment method of hydrocephalus should be selected according to age of onset and symptoms. Congenital hydrocephalus is classified into three categories by causes that disturb CSF circulation pathway: simple hydrocephalus, dysgenetic hydrocephalus, and secondary hydrocephalus $(15,26)$.

\section{Simple hydrocephalus}

Simple hydrocephalus, caused by developmental abnormality that is localized within CSF circulation pathway, includes aqueductal stenosis, atresia of foramen Monro, and maldevelopment of arachnoid granulation.

2. Dysgenetic hydrocephalus

Dysgenetic hydrocephalus indicates hydrocephalus as a result of cerebral developmental disorder in early developmental stage and includes hydranencephaly, holoprosencephaly, porencephaly, schizencephaly, Dandy-Walker malformation, dysraphism, and Chiari malformation. 


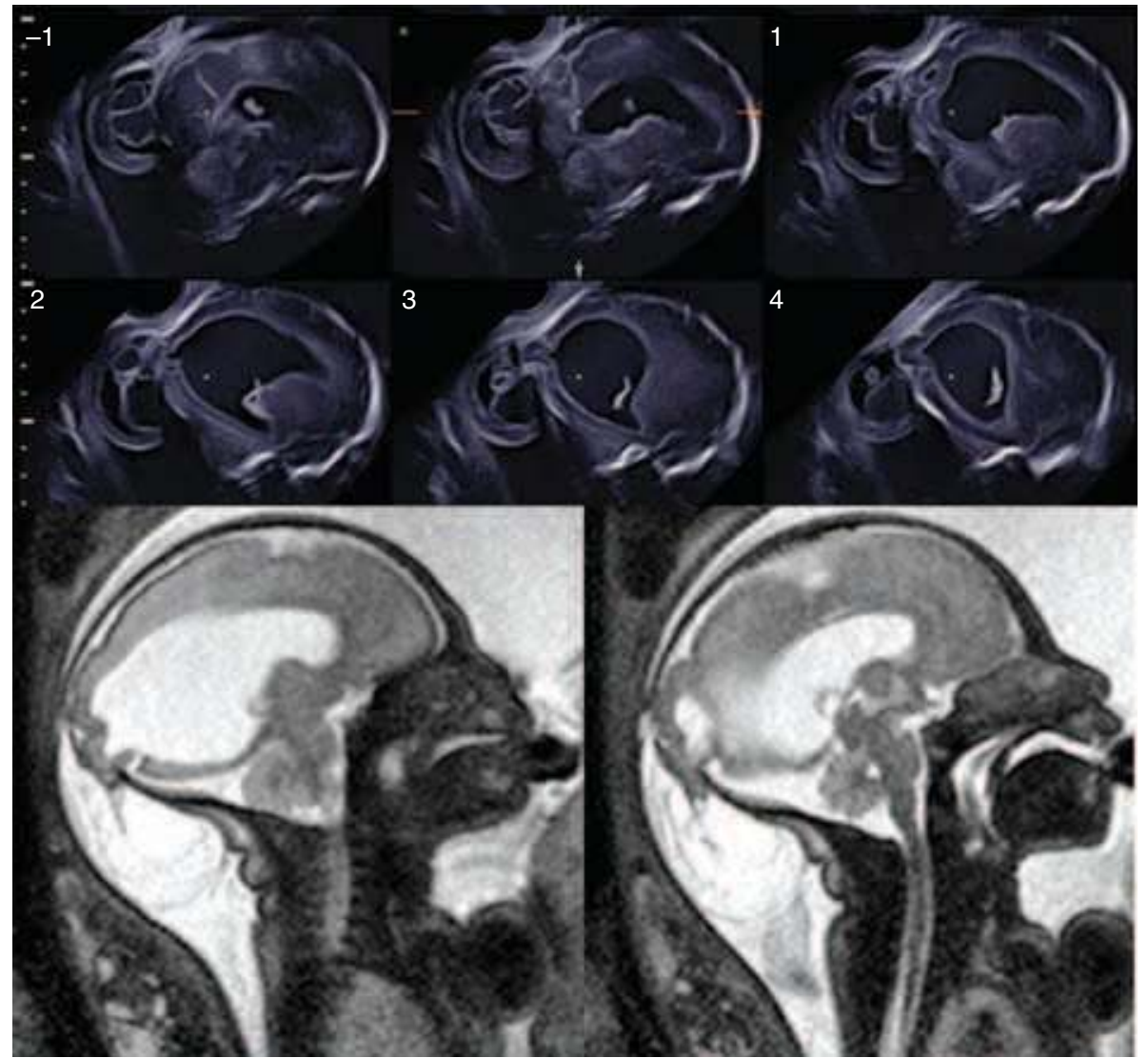

Figure 3 Encephalocele. Tomographic ultrasound imaging (upper) and MR imaging (lower) in a case of encephalocele at 28 weeks of gestation.

\section{Secondary hydrocephalus}

Secondary hydrocephalus is a generic term indicating hydrocephalus caused by intracranial pathologic condition, such as brain tumor, intracranial infection, and intracranial hemorrhage.

In cases with progressive hydrocephalus, there may be seven stages of progression: (i) increased fluid collection of lateral ventricles, (ii) increased intracranial pressure, (iii) dangling choroid plexus, (iv) disappearance of subarachnoid space, (v) excessive extension of the dura and superior sagittal sinus, (vi) disappearance of venous pulsation, and finally (vii) enlarged skull (24). In general, both hydrocephalus and ventriculomegaly are still evaluated by the measurement of biparietal diameter and AW in transabdominal axial section.

Although all cases have similar ventricular appearance, the causes of ventriculomegaly vary, such as Chiari type II malformation, aqueductal obstruction, and amniotic band syndrome (ABS). In the case of ABS, amniotic band attached to the scalp resulted in partial cranial bone defect and a small cephalocele, which may have caused Monro obstruction and enlarged ventricles.

From our data of 23 ventriculomegaly cases with $\mathrm{AW}>15$ mm (27), 9 cases (39.1\%) had no other CNS abnormality, but 2 out of those 9 were complicated with chromosomal aberration. Four cases out of seven without any complication had favorable postnatal prognosis after ventricular-peritoneal shunting procedure. Among the rest of 14 cases with other CNS abnormalities, holoprosencephaly was detected in 5 cases and myelomeningocele in 5 cases (27).

\section{NEURAL TUBE DEFECTS Cranium Bifidum}

Cranium bifidum is classified into four types of encephaloschisis (including anencephaly and exencephaly), meningocele, encephalomeningocele, encephalocystocele, and cranium bifidum occultum. Encephalocele occurs in the occipital region in $70 \%$ to $80 \%$. Acrania, exencephaly, and anencephaly are not independent anomalies. It is considered that dysraphia (absent cranial vault, acrania) occurs in very early stage and disintegration of the exposed brain (exencephaly) during the fetal period results in anencephaly. Encephalocele (Fig. 3) is often observed in the median section and in the parietooccipital part. ABS should be differentiated from acrania during early pregnancy, because ABS has completely different pathogenesis from acrania/exencephaly. In cases of ABS, cranial destruction occurs secondary to an amniotic band; similar appearance is often observed.

\section{Spina Bifida}

Spina bifida aperta, manifest form of spina bifida, is classified into four types: meningocele, myelomeningocele, myelocystocele, and myeloschisis. In myelomeningocele, the spinal cord and its protective covering (the meninges) protrude from an opening in the spine. In meningocele, the spinal cord develops normally but the meninges protrude from a spinal opening. The most common location of the malformations is the lumbar and sacral areas of the spinal cord. Chiari type II malformation and secondary hydrocephalus/ventriculomegaly are mostly, and scoliosis or kyphosis occasionally, associated with open spina bifida. Surface anatomy of the fetus and appearance of clubfoot, which occasionally manifests early in 


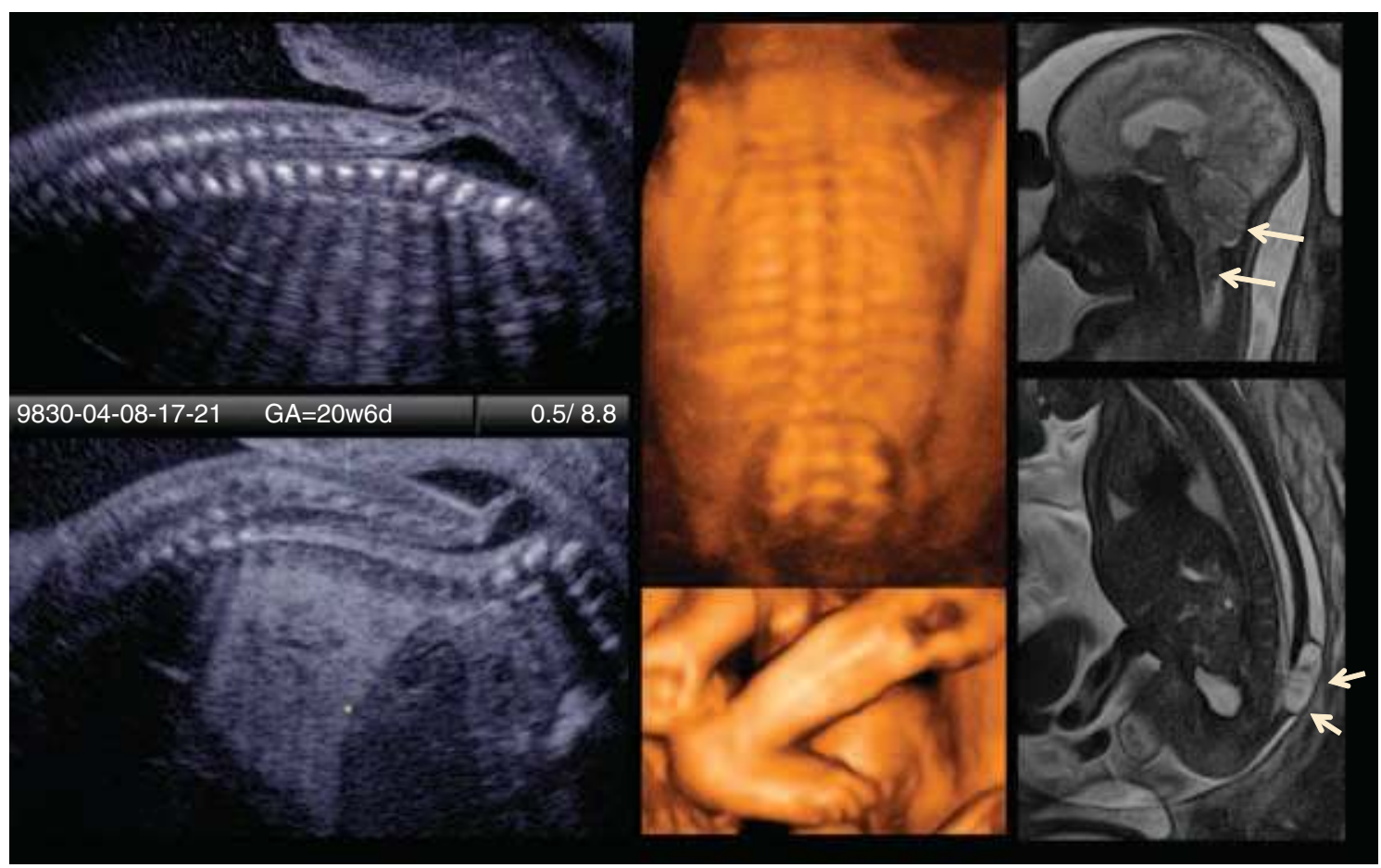

Figure 4 Myelomeningocele. Two-dimensional (2D) ultrasound of myelomeningocele (upper left) and myelomeningocele with kyphosis (lower left), 3D ultrasound (middle), and MRI (right). Right upper MRI shows Chiari type II malformation (arrows). Clear visualization of spinal cord protrusion is obtained by 2D ultrasound and MRI. The 3D ultrasound images show the bony structure that helps to determine the level of spina bifida and lower extremity appearance.

mid-gestation as a complication of spinal bifida, are easily demonstrable by using 3D ultrasound. The 3D ultrasound with maximum mode can demonstrate bony structure (Fig. 4) and is helpful to detect the spinal levels of lesion and to predict neurologic prognosis. Although most myelomeningoceles are demonstrated as a protruding swelling, fetal back appears flat in the type of myeloschisis; therefore, open spina bifida may be often overlooked. Because more than $80 \%$ of cases of open spina bifida are associated with ventriculomegaly due to Chiari type II malformation, demonstration of ventriculomegaly is usually the first observable sign leading to the detailed examination of spine and the subsequent diagnosis of spinal bifida.

\section{PROSENCEPHALIC DEVELOPMENTAL DISORDER Holoprosencephaly}

Holoprosencephalies are classified into three varieties: alobar, semilobar, and lobar types. Facial abnormalities such as cyclopia, ethmocephaly, cebocephaly, flat nose, cleft lip, and palate are invariably associated with holoprosencephaly and extracerebral abnormalities. Facial abnormalities are often associated with holoprosencephaly.

\section{Agenesis of the Corpus Callosum}

Absence of the corpus callosum (AOCC) is divided into complete agenesis, partial agenesis, or hypogenesis. Chromosomal aberration or syndromic diseases may occasionally be related to agenesis of the corpus callosum. Colpocephalic ventriculomegaly with disproportionate enlargement of trigones, occipital horns and temporal horns, and superior elongation of the third ventricle is usually observed. Interhemispheric cyst is often associated with AOCC and some cases are with pericallosal lipoma. Complete AOCC is demonstrated in the coronal and sagittal sections by sonography and fetal MRI. Typical shape of enlarged ventricles associated with AOCC is colpocephaly with large occipital horns. Typical radiated formation of brain vessels in the sagittal section is demonstrated by color Doppler study. As the corpus callosum is depicted after 17 or 18 weeks of gestation by ultrasound, it is impossible to diagnose agenesis of the corpus callosum prior to this age (36).

\section{POSTERIOR FOSSA ANOMALY \\ Chiari Malformation}

Chiari classified anomalies with cerebellar herniation in the spinal canal into three types by contents of herniated tissue: contents of type I is a lip of cerebellum; type II part of cerebellum, fourth ventricle and medulla oblongata, and pons; and type III large herniation of the posterior fossa. Thereafter, type IV with just cerebellar hypogenesis was added. However, this classification occasionally leads to confusion in neuroimaging diagnosis. Therefore, at present, the classification as below is advocated: type I not associated with myelomeningocele, type II (schematic picture is shown in Fig. 5 upper left) associated with myelomeningocele, type III associated with cephalocele or craniocervical meningocele, and type IV associated with marked cerebellar hypogenesis and posterior fossa shrinking. Chiari malformation occurs according to i) inferior displacement of the medulla and the fourth ventricle into the upper cervical canal, ii) elongation and thinning of the upper medulla and lower pons and persistence of the embryonic flexure of these structures, iii) inferior displacement of the lower cerebellum through the foramen magnum into the upper cervical region, and iv) a variety of bony defects of the foramen magnum, occiput, and upper cervical vertebra (37). Hydrocephalus is caused by 


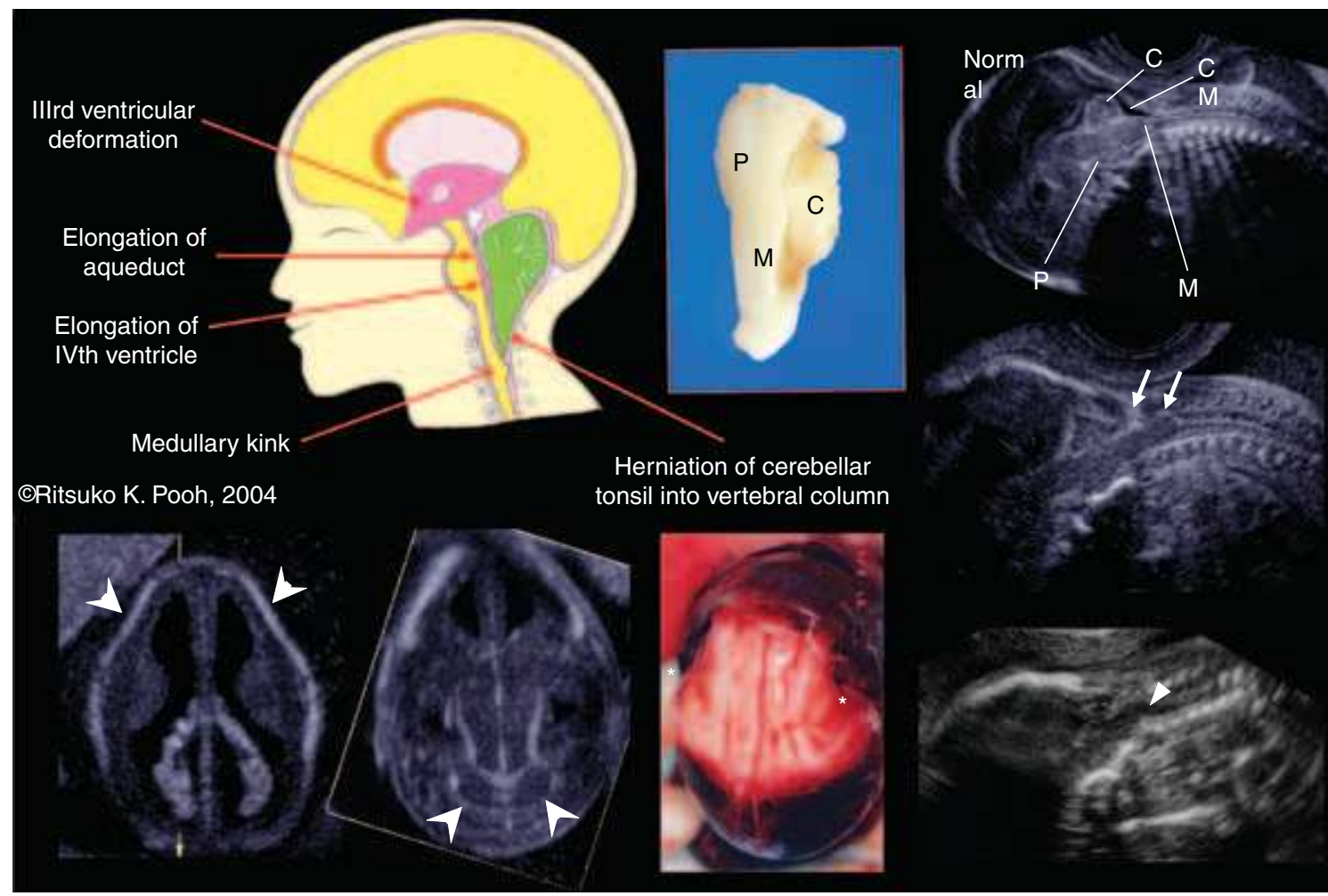

Figure 5 Chiari type II malformation. Schematic picture of Chiari type II malformation (upper left). Elongation and stenosis of the aqueduct and fourth ventricle in the specimen from aborted fetus at 21 weeks (upper middle) are shown. Lower brain images are lemon sign and banana sign from the left. Arrowheads indicate indentations of lemon-shaped skull and banana-shaped cerebellum. Lower middle picture shows the lemon sign on the autopsy of an aborted fetus. Right sagittal ultrasound images show normal cerebrospinal structure, Chiari type II malformation without kink, and Chiari II with medullary kink from above.

obstruction of fourth ventricular outflow or associated aqueductal stenosis. Eighty-eight percent of fetuses with open spina bifida develop ventriculomegaly, by 21 weeks of gestation in the majority of cases (38). As prenatal neuroimaging of Chiari malformation, lemon and banana signs (39) are circumstantial evidences of Chiari malformation, which are easily demonstrated in the early second trimester. Lemon sign indicates deformity of the frontal bone, and banana sign indicates abnormal shape of cerebellum without cisterna magna space (Fig. 5, lower). Herniation of the cerebellar tonsil and medulla oblongata and medullary kink are demonstrable (Fig. 5, right). Small clivus-supraocciput angle is seen in cases of Chiari malformation (40).

\section{DANDY WALKER MALFORMATION, DANDY WALKER VARIANT, MEGACISTERNA MAGNA}

During development of the fourth ventricular roof, a delay or total failure of the foramen of Magendie to open occurs, allowing a buildup of CSF and development of the cystic dilation of the fourth ventricle. Despite the subsequent opening of the foramina of Luschka (usually patent in Dandy-Walker malformation), cystic dilatation of the fourth ventricle persists and CSF flow is impaired. At present, the term "Dandy-Walker complex" by Barkovich et al. (41) is used to indicate a spectrum of anomalies of the posterior fossa that are classified by axial CT scans as it follows. Dandy-Walker malformation, Dandy-Walker variant, and megacisterna magna seem to represent a continuum of developmental anomalies of the posterior fossa (41). Figure 6 (upper) shows the differential diagnosis of hypoechoic lesion of the posterior fossa, and typical sonographic images of Dandy-Walker malformation are shown in the lower part of Figure 6.

- (Classic) Dandy-Walker malformation: cystic dilatation of fourth ventricle, enlarged posterior fossa, elevated tentorium, and complete or partial agenesis of the cerebellar vermis.

- Dandy-Walker variant: variable hypoplasia of the cerebellar vermis with or without enlargement of the posterior fossa.

- Megacisterna magna: enlarged cisterna magna with integrity of both cerebellar vermis and fourth ventricle.

\section{NEURONAL PROLIFERATION DISORDER Microcephaly}

Microcephaly is defined as a head circumference that is more than two standard deviations below the normal mean for age, sex, race, and gestation. Infections such as with rubella, cytomegalovirus, varicella (chicken pox) virus, and toxoplasmosis, radiation, medications, chromosome abnormalities, and genetic diseases may cause microcephaly. Occasionally, microcephaly occurs with late onset during pregnancy (42).

\section{NEURONAL MIGRATION DISORDERS}

Neuronal migration disorders are caused by the abnormal migration of neurons in the developing brain and nervous system. Neurons must migrate from the areas where they are born to the areas where they will settle into their proper neural 


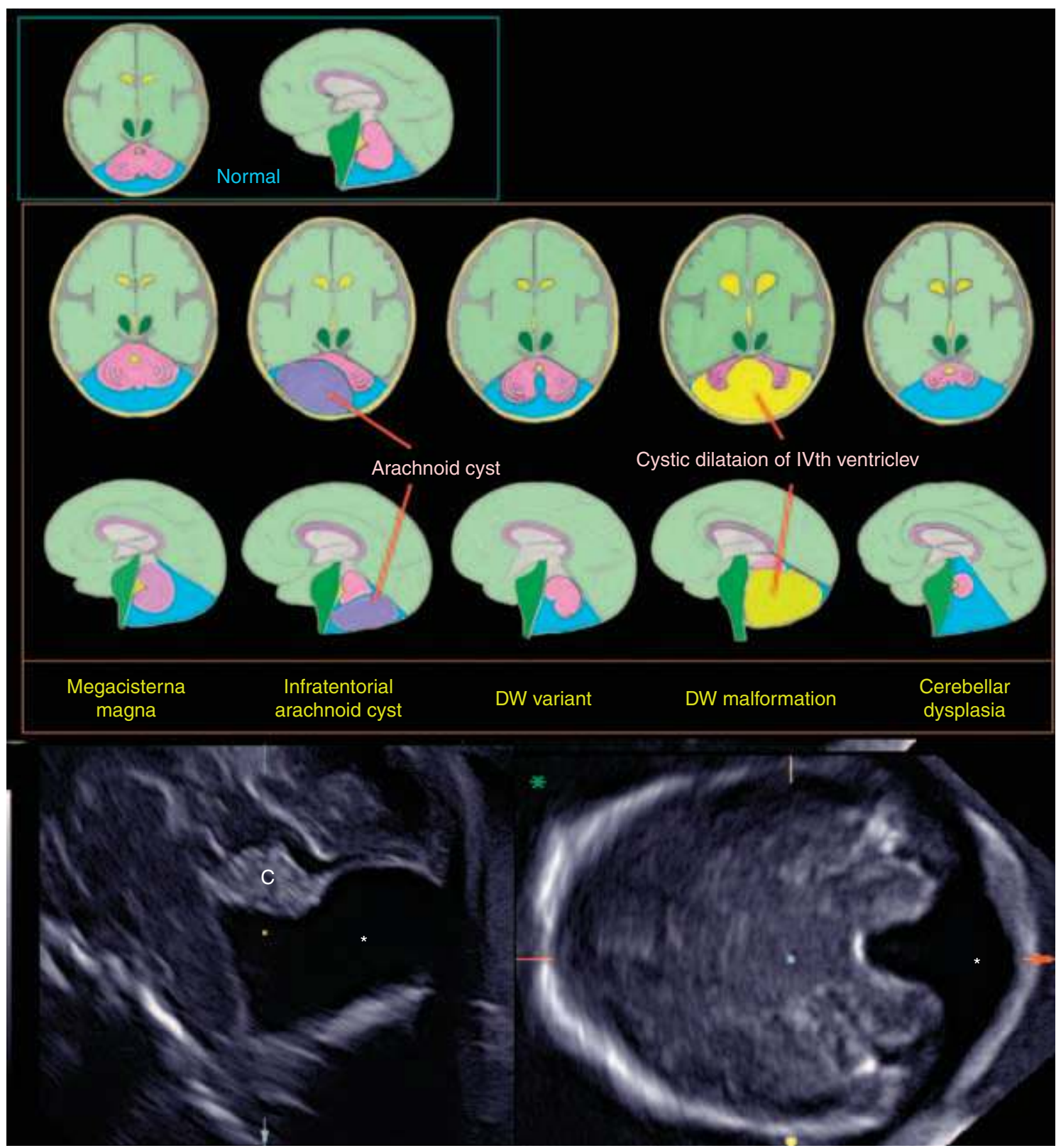

Figure 6 Differential diagnosis of hypoechoic lesion of the posterior fossa (upper) and typical sonographic images of Dandy-Walker malformation (lower). Abbreviation: C, cerebellum. Asterisks indicate cystic dilatation of the fourth ventricle.

circuits. Neuronal migration, which occurs as early as the second month of gestation, is controlled by a complex assortment of chemical guides and signals. When these signals are absent or incorrect, neurons do not end up where they belong. This can result in structurally abnormal or missing areas of the brain in the cerebral hemispheres, cerebellum, brainstem, or hippocampus, including schizencephaly, porencephaly, lissencephaly, agyria, macrogyria, pachygyria, microgyria, micropolygyria, neuronal heterotopias (including band heterotopia), agenesis of the corpus callosum, and agenesis of the cranial nerves. Symptoms vary according to the specific disorder and the degree of brain abnormality and subsequent neurologic losses, but often feature poor muscle tone and motor function, seizures, developmental delays, mental retardation, failure to grow and thrive, difficulties with feeding, swelling in the extremities, and a smaller than normal head. Most infants with a neuronal migration disorder appear normal, but some disorders have characteristic facial or skull features.

\section{Lissencephaly}

Lissencephaly is very rare and characterized by a lack of gyral development and divided into two types.

Lissencephaly type I shows a smooth surface of the brain and cerebral wall is similar to that of an approximately 12-week-old fetus (43). Isolated lissencephaly (Fig. 7) or MillerDieker syndrome is associated with additional craniofacial abnormalities, cardiac anomalies, genital anomalies, sacral dimple, creases, and/or clinodactyly. Lissencephaly type II shows cobblestone appearance. Walker-Warburg syndrome with macrocephaly, congenital muscular dystrophy, cerebellar malformation, and retinal malformation or Fukuyama congenital muscular dystrophy with microcephaly and congenital muscular dystrophy has been proven. 


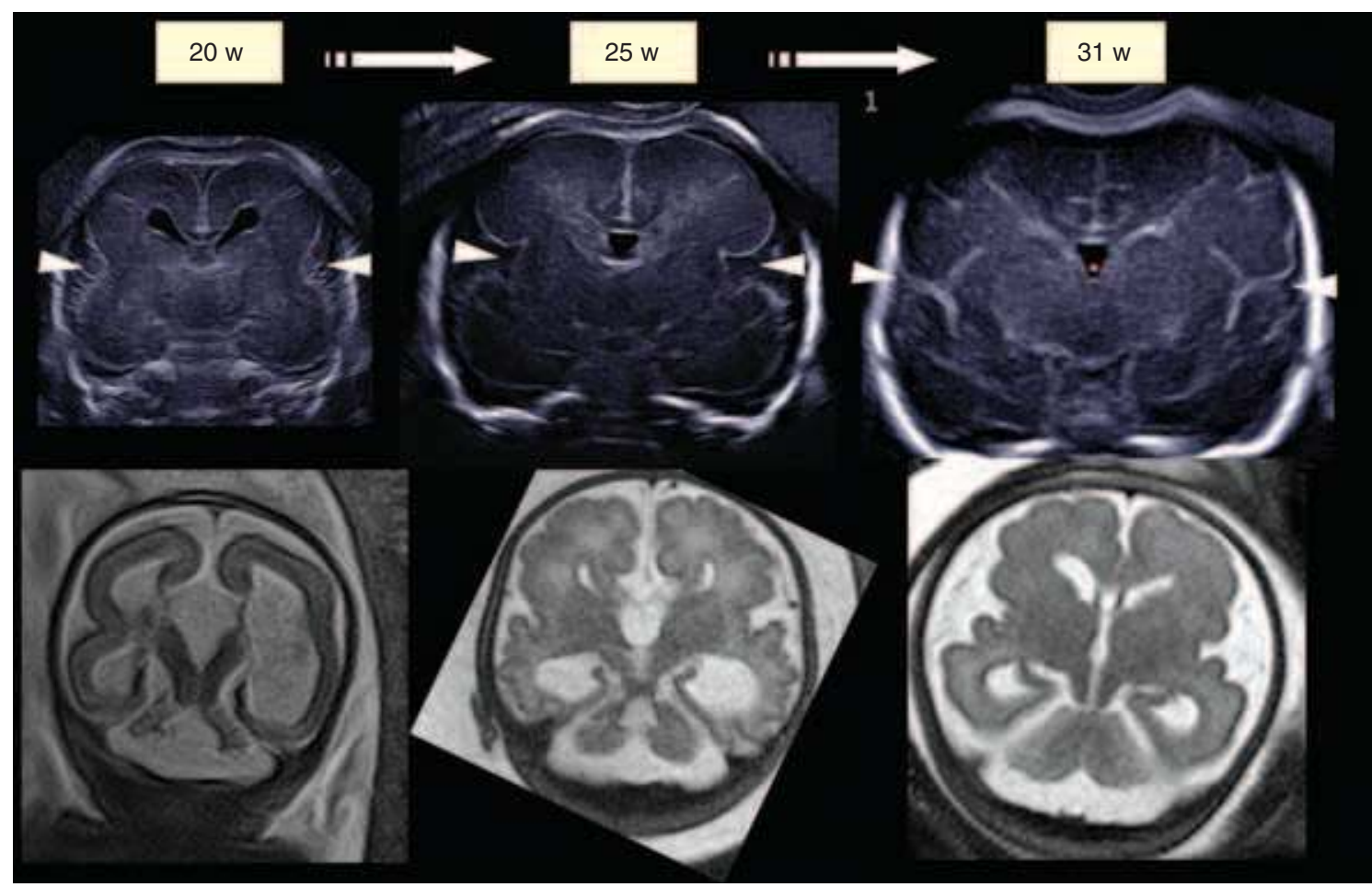

Figure 7 Changing appearance of Sylvian fissure in the anterior coronal section (upper) and abnormal Sylvian fissure in cases of migration disorder (lower). At 20 weeks of gestation, bilateral Sylvian fissures (arrowheads) appear to be indentations (left). With cortical development, Sylvian fissures are formed during the latter half of second trimester (middle) and become as lateral sulci. Sylvian fissure appearance is one of the most reliable ultrasound markers for the assessment of cortical development. Lower MR images show coronal cutting sections of various migration disorders after 30 weeks of gestation: Isolated lissencephaly, cobblestone lissencephaly, and pachygyria from left.

Recently, classification has been made based on associated malformations and etiologies.

\footnotetext{
- Classic lissencephaly (previously known as type I lissencephaly)

Lissencephaly due to LIS1 gene mutation

Type I isolated lissencephaly

Link to chromosome $17 \mathrm{p} 13.3$ and chromosome Xq24-q24

Miller-Dieker syndrome link to chromosome 17p13.3

Lissencephaly due to doublecortin gene mutation

Lissencephaly type I, isolated, without other genetic defects

- Lissencephaly X-linked with AOCC (ARX gene)

- Lissencephaly with cerebellar hypoplasia

Norman-Roberts syndrome (mutation of reelin gene)

- Microlissencephaly (lissencephaly and microcephaly)

- Cobblestone lissencephaly (previously known as type II

lissencephaly)

Walker-Warburg syndrome, HARD(E) syndrome

Fukuyama syndrome linked to chromosome 9q31,

fukutin (44)

Muscle-eye-brain disease
}

A few reports of prenatal diagnosis of lissencephaly have been published (45-47). It was described that without previous history of an affected child, lissencephaly probably cannot be reliably made until 26 to 28 weeks of gestation (48). However, from recent study in the assessment of Sylvian fissure appearance during pregnancy, there might be a potential of earlier diagnosis of migration disorders (49).

\section{Schizencephaly}

Schizencephaly is a disorder characterized by congenital clefts in the cerebral mantle, lined by pia-ependyma, with communication between the subarachnoid space laterally and the ventricular system medially. Of that $63 \%$ is unilateral and $37 \%$ bilateral. Frontal region is $44 \%$ and frontoparietal is $30 \%$ (43). Schizencephaly is associated with ventriculomegaly, microcephaly, polymicrogyria, gray matter heterotopias, dysgenesis of the corpus callosum, absence of the septum pellucidum, and optic nerve hypoplasia.

\section{OTHER CONGENITAL ANOMALIES}

Arachnoid Cyst, Interhemispheric Cyst

Congenital or acquired cyst, with prevalence of $1 \%$ of intracranial masses in newborns, is lined by arachnoid membranes and filled with fluid collection, which is of the same characteristic as the CSF. The number of cysts is mostly single, but two or more cysts can be occasionally observed. Location of arachnoid cyst is various, and it is said that approximately $50 \%$ of cysts occurs from the Sylvian fissure (middle fossa), 20\% from the posterior fossa, and $10 \%$ to $20 \%$ each from the convexity, suprasellar, interhemisphere, and quadrigeminal cistern in the pediatric field. Interhemispheric cysts are commonly associated with agenesis or hypogenesis of the corpus callosum. Callosal agenesis with interhemispheric cyst is classified as two types (50). Type I cysts appear to be an extension or diverticulum of the third or lateral ventricles, whereas type 2 cysts are loculated and do not communicate with the ventricular system. Prenatal neuroimaging examples of interhemispheric cyst, middle fossa arachnoid cyst, and suprasellar arachnoid cyst are shown in Figure 8. As intrauterine spontaneous resolution or changing cyst size is often seen during fetal period, serial scanning is important. Detection in the first trimester was 

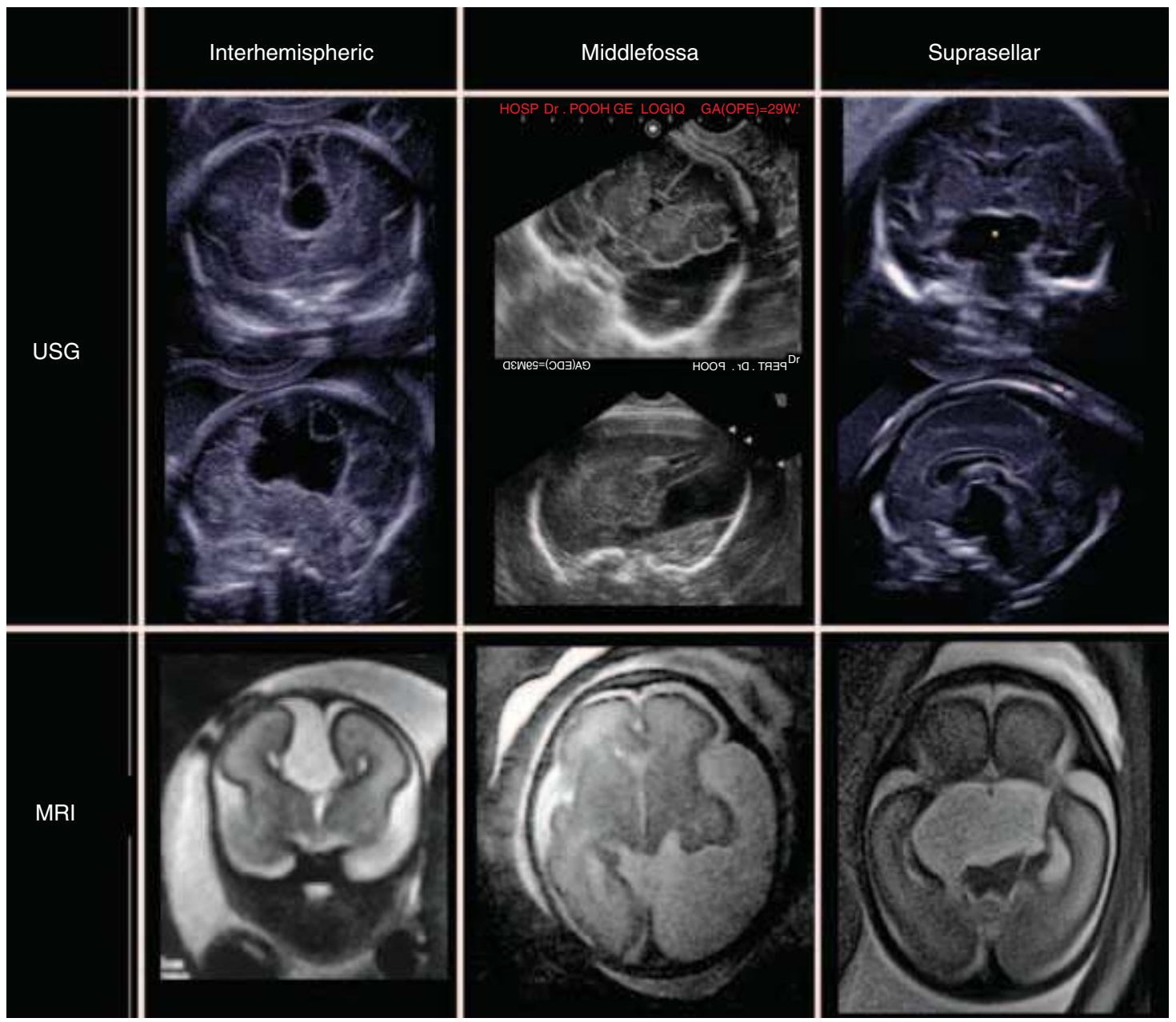

Figure 8 Prenatal neuroimaging of interhemispheric cyst, middle fossa arachnoid cyst, and suprasellar arachnoid cyst. Abbreviations: USG, ultrasonography; MRI, magnetic resonance imaging.

reported (51). Prognosis is generally good. Many are asymptomatic and remain quiescent for years, although others may expand and cause neurologic symptoms by compressing adjacent brain, development of ventriculomegaly, and/or expanding the overlying skull.

\section{Brain Tumors}

Brain tumors are divided into teratomatous, most are the commonly reported brain tumors, and nonteratomatous. Nonteratomatous tumors includes neuroepithelial tumor, such as medulloblastoma, astrocytoma, choroid plexus papilloma, choroid plexus carcinoma, ependymoma, ependymoblastoma, and mesenchymal tumor such as craniopharyngioma, sarcoma, fibroma, hemangioblastoma, hemangioma, and meningioma, and others such as lipoma of the corpus callosum, subependymal giant-cell astrocytoma associated with tuberous sclerosis (often accompanied by cardiac rhabdomyoma) (52). Depending on the site and vascularity, these tumors may lead to macrocrania or local skull swelling, epignathus, secondary hydrocephalus, intracranial hemorrhage, intraventricular hemorrhage, polyhydramnios, and heart failure by high-cardiac output (53) or hydrops. Intracranial masses with solid, cystic, or mixture pattern with or without visualization of hypervascularity can be detected by ultrasound and fetal MRI. Brain tumor should be considered in cases with unexplained intracranial hemorrhage.

\section{Craniosynostosis}

Craniosynostosis is the premature closure of cranial suture, which may affect one or more cranial sutures. Simple sagittal synostosis is most common. Various cranial shapes depend on affected suture(s).

$\begin{array}{ll}\text { Sagittal suture } & \text { Scaphocephaly or dolichocephaly } \\ \text { Bilateral coronal suture } & \text { Brachycephaly } \\ \text { Unilateral coronal suture } & \text { Anterior plagiocephaly } \\ \text { Metopic suture } & \text { Trigonocephaly } \\ \text { Lambdoid suture } & \text { Acrocephaly } \\ \text { Unilateral lambdoid suture } & \text { Posterior plagiocephaly } \\ \text { Coronal/lambdoid/metopic } & \text { Cloverleaf skull } \\ \quad \text { or squamous/sagittal suture } & \\ \text { Total cranial sutures } & \text { Oxycephaly }\end{array}$

Craniosynostosis due to specific syndromes (syndromic craniosynostosis) is usually associated with additional specific features and therefore correct differentiation between these conditions is possible. Examples include Crouzon syndrome (acrocephaly, synostosis of coronal, sagittal and lambdoid sutures and ocular proptosis, maxillary hypoplasia), Apert syndrome (brachycephaly, irregular synostosis, especially coronal suture and midfacial hypoplasia, syndactyly, broad distal phalanx of thumb, and big toe), Pfeiffer syndrome (brachycephaly, synostosis of coronal and/or sagittal sutures 


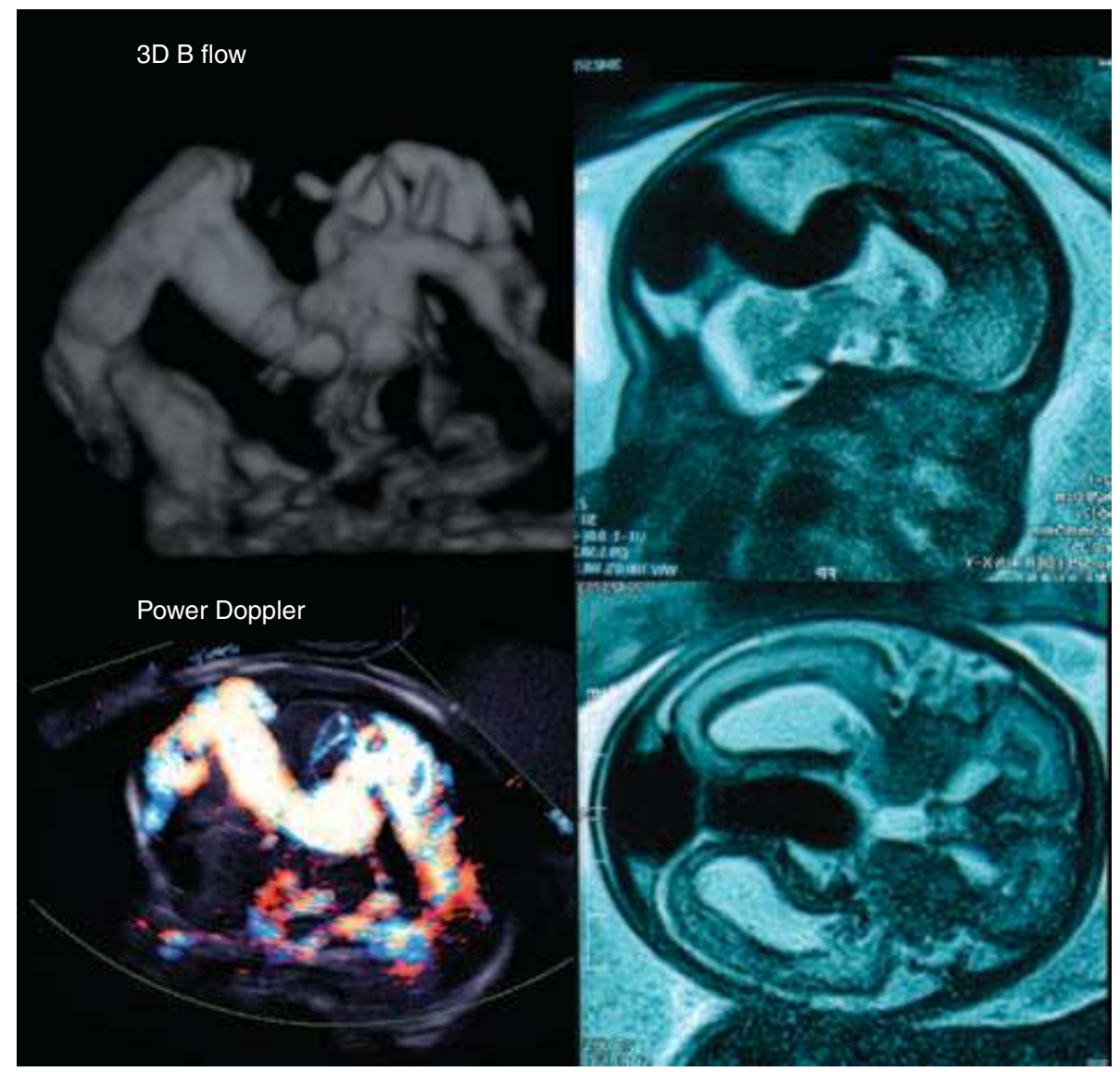

Figure 9 Three-dimensional B-flow detection, color Doppler image, and MRI of vein of Galen aneurysmal malformation.

and hypertelorism, broad thumbs and toes, and partial syndactyly), and Antley-Bixler syndrome (brachycephaly, multiple synostosis, especially of coronal suture and maxillary hypoplasia, radiohumeral synostosis, choanal atresia, arthrogryposis). Abnormal craniofacial appearance can be detected prenatally by $2 \mathrm{D} / 3 \mathrm{D}$ ultrasound $(26,54-56)$.

\section{VEIN OF GALEN ANEURYSMAL MALFORMATION}

It is a congenital malformation of blood vessels of the brain. The main structure is direct arteriovenous fistulas in which blood shunts from choroidal and/or quadrigeminal arteries into an overlying single median venous sac. Vein of Galen aneurysm is not "aneurysm" but "arteriovenous malformation (AVM)." Vein of Galen aneurysmal malformation (VGAM) is a choroidal type of AVM involving the vein of Galen forerunner. This is distinct from an AVM with venous drainage into a dilated, but already formed, vein of Galen (57). Associated anomalies are cardiomegaly, high cardiac output, secondary hydrocephalus, macrocrania, cerebral ischemia (intracranial steal phenomenon), and subarachnoid/cerebral/intraventricular hemorrhages. The 3D B-flow detection, color Doppler image, and MRI of VGAM are shown in Figure 9.

\section{Pericallosal Lipoma}

Intracranial lipomas are congenital malformations composed of mature adipocytes. They are usually located in the midline, particularly in the pericallosal region, a hemispheric location accounting for only $3 \%$ to $7 \%$ of cases. Two morphologic types of pericallosal lipoma have been described $(58,59)$.
Tubulonodular type with generally greater than $2 \mathrm{~cm}$ in diameter (often smaller than $2 \mathrm{~cm}$ in fetal period) has a high incidence of corpus callosum dysgenesis, frontal lobe anomalies, and frontal encephaloceles. Curvilinear type, which comprises thin, posteriorly situated lipomas curving around the splenium, is generally associated with a normal corpus callosum and otherwise has a low incidence of associated anomalies. High echogenic mass can be easily demonstrated by ultrasound. Several reports on prenatal diagnosis have been published (60-62).

\section{ACQUIRED BRAIN ABNORMALITIES IN UTERO}

In terms of encephalopathy or cerebral palsy, "timing of brain insult, antepartum, intrapartum, or postpartum?" is one of the serious controversial issues including medico-sociolegal-ethical problems (15). Although brain insults may relate to antepartum events in a substantial number of term infants with hypoxic-ischemic encephalopathy, the timing of insult cannot always be certain. It is a difficult task to provide a precise prediction of subsequent development of cerebral palsy after a given antepartum event or complication. Fetal heart rate monitoring cannot reveal the presence of encephalopathy, and neuroimaging by ultrasound and MR imaging is the most reliable modality for disclosure of silent encephalopathy. In many cases with cerebral palsy with acquired brain insults, especially term-delivered infants with reactive fetal heart rate tracing and good Apgar score at delivery, recent imaging studies have confirmed the presence of brain insult in utero, suggesting that the majority of cerebral palsy are of antepartum rather than intrapartum in origin. 


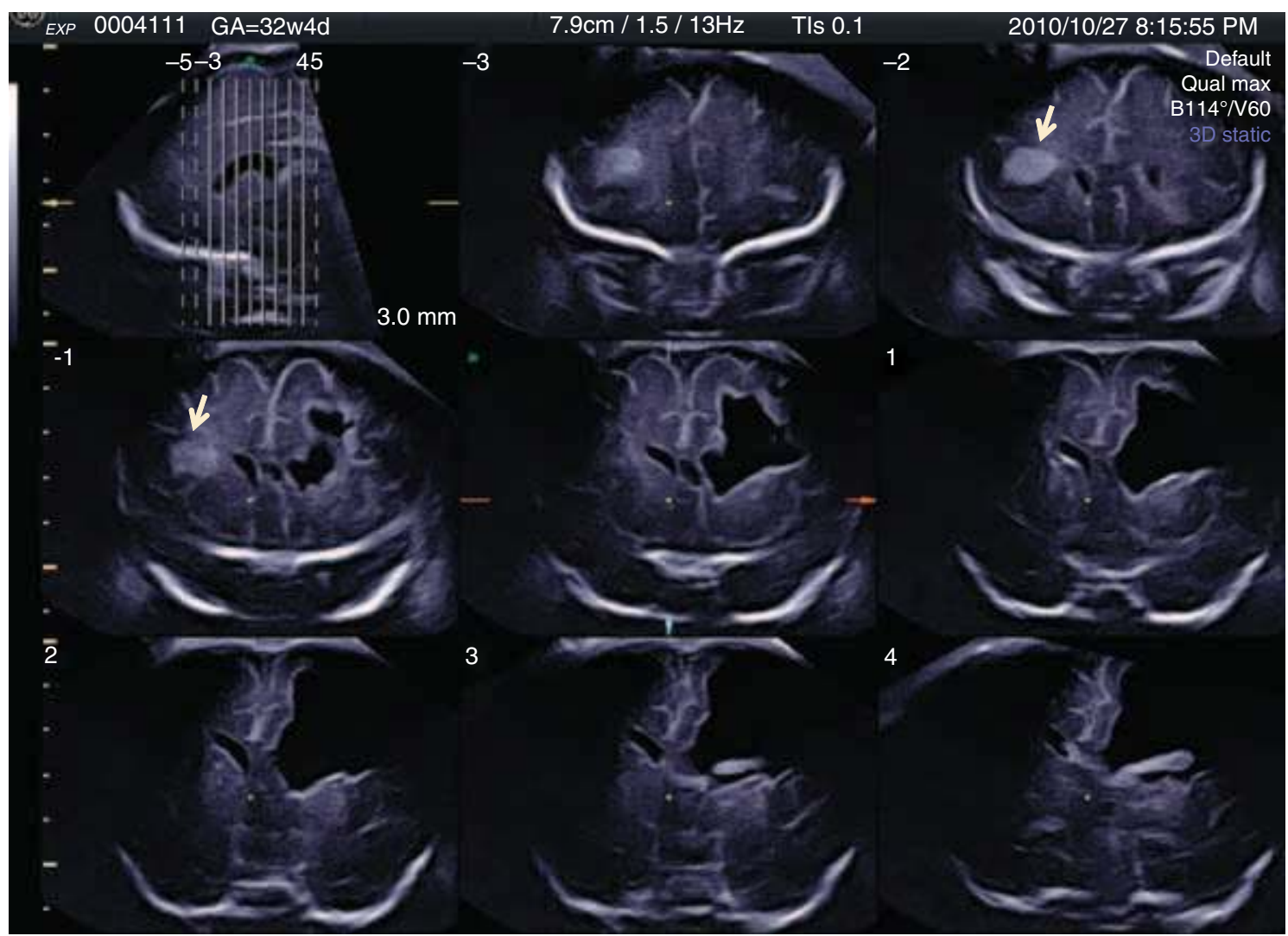

Figure 10 Intracerebral hemorrhage at 32 weeks of gestation. Transvaginal three-dimensional tomographic ultrasound images show unilateral ventriculomegaly due to cerebral hemorrhage and fresh intracerebral hemorrhage (arrows), which is going to change into porencephaly.

\section{Intracranial Hemorrhage}

Intracranial hemorrhage includes subdural hemorrhage, primary subarachnoid hemorrhage, intracerebellar hemorrhage, intraventricular hemorrhage, and intraparenchymal hemorrhage other than cerebellar hemorrhage (63). Hydrocephalus, hydranencephaly, porencephaly, and/or microcephaly are possible secondary complications, which are often detectable by imaging studies. Unilateral ventriculomegaly due to cerebral hemorrhage and fresh intracerebral hemorrhage is shown in Figure 10. The hyperechoic lesion is changing into porencephaly in a short period.

\section{Porencephaly}

Porencephaly or porencephalic cyst is defined as fluid-filled spaces replacing normal brain parenchyma and may or may not communicate with the lateral ventricles or subarachnoid space. The causes may be ischemic episode, trauma (64), demise of one twin, Intracerebral hemorrhage, and infection of cytomegalovirus (65). Some cases in utero have been reported $(66,67)$. Porencephalic cyst never causes a mass effect, which is observed in cases with arachnoid cyst or other cystic mass lesions. This condition is acquired brain insult and differentiated from schizencephaly of migration disorder.

\section{FETAL PERIVENTRICULAR LEUKOMALACIA}

Multifocal areas of necrosis are found deep in the cortical white matter, which are often symmetrical and occur adjacent to the lateral ventricles. Periventricular leukomalacia (PVL) represents a major precursor for neurologic and intellectual impairment, and cerebral palsy in later life. About
$25 \%$ to $75 \%$ of premature infants at autopsy are complicated with periventricular white matter injury. However, clinically, incidence may be much lower. About $5 \%$ to $10 \%$ of infants have less than $1500 \mathrm{~g}$ birth weight. In term infants, PVL is very rare.

\section{CONCLUSIONS}

Recent advances of imaging technology have provided us objective neuroimaging diagnosis as shown in this article. Longitudinal and careful evaluation of neurologic short-term/ long-term prognosis should be required according to precise prenatal diagnosis, for proper counseling and management based on accurate evidence.

\section{REFERENCES}

1. Timor-Tritsch IE, Monteagudo A. Transvaginal fetal neurosonography: standardization of the planes and sections by anatomic landmarks. Ultrasound Obstet Gynecol 1996; 8: 42-7.

2. Monteagudo A, Reuss ML, Timor-Tritsch IE. Imaging the fetal brain in the second and third trimesters using transvaginal sonography. Obstet Gynecol 1991; 77: 27-32.

3. Monteagudo A, Timor-Tritsch IE, Moomjy M. In utero detection of ventriculomegaly during the second and third trimesters by transvaginal sonography. Ultrasound Obstet Gynecol 1994; 4: 193-8.

4. Monteagudo A, Timor-Tritsch IE. Development of fetal gyri, sulci and fissures: a transvaginal sonographic study. Ultrasound Obstet Gynecol 1997; 9: 222-8.

5. Pooh RK, Nakagawa Y, Nagamachi N, et al. Transvaginal sonography of the fetal brain: detection of abnormal morphology and circulation. Croat Med J 1998; 39: 147-57.

6. Pooh RK, Maeda K, Pooh KH, Kurjak A. Sonographic assessment of the fetal brain morphology. Prenat Neonat Med 1999; 4: 18-38.

7. Pooh RK, Aono T. Transvaginal power Doppler angiography of the fetal brain. Ultrasound Obstet Gynecol 1996; 8: 417-21. 
8. Pooh RK. Two-dimensional and three-dimensional Doppler angiography in fetal brain circulation. In: Kurjak A, ed. 3D Power Doppler in Obstetrics and Gynecology. Carnforth: Parthenon Publishing, 1999: 105-11.

9. Pooh RK. Three-dimensional ultrasound of the fetal brain. In: Kurjak A, ed. Clinical application of 3D ultrasonography. Carnforth: Parthenon Publishing, 2000: 176-80.

10. Pooh RK, Pooh KH, Nakagawa Y, Nishida S, Ohno Y. Clinical application of three-dimensional ultrasound in fetal brain assessment. Croat Med J 2000; 41: 245-51.

11. Timor-Tritsch IE, Monteagudo A, Mayberry P. Three-dimensional ultrasound evaluation of the fetal brain: the three horn view. Ultrasound Obstet Gynecol 2000; 16: 302-6.

12. Monteagudo A, Timor-Tritsch IE, Mayberry P. Three-dimensional transvaginal neurosonography of the fetal brain: 'navigating' in the volume scan. Ultrasound Obstet Gynecol 2000; 16: 307-13.

13. Pooh RK, Nagao Y, Pooh KH. Fetal neuroimaging by transvaginal 3D ultrasound and MRI. Ultrasound Rev Obstet Gynecol 2006; 6: 123-34.

14. Pooh RK, Pooh KH. Fetal neuroimaging with new technology. Ultrasound Rev Obstet Gynecol 2002; 2: 178-81.

15. Pooh RK, Pooh KH. Antenatal assessment of CNS anomalies, including neural tube defects. In: Levene MI, Chervenak FA, eds. Fetal and Neonatal Neurology and Neurosurgery, 4th edn. Elsevier, 2008: 291-338.

16. Pooh RK, Maeda K, Pooh KH. An atlas of fetal central nervous system disease. Diagnosis and Management. London, New York: Parthenon CRC Press, 2003.

17. Pooh RK. Neuroanatomy visualized by 2D and 3D. In: Pooh RK, Kurjak A, eds. Fetal Neurology. New Delhi: Jaypee Brothers Medical Publishers, 2009: 15-38.

18. Pooh RK. Fetal central nervous system. In: Ahmed B, Adra A, Kavak ZN, eds. Donald School Basic Textbook of Ultrasound in Obstetrics and Gynecology. New Delhi: Jaypee Brothers Medical Publishers, 2008: 326-49.

19. Pooh RK, Pooh KH. Fetal neuroimaging. Fetal and Maternal Medicine Review. Volume 19. Cambridge University Press, 2008: 1-31.

20. Blaas HG, Eik-Nes SH, Berg S, Torp H. In-vivo three-dimensional ultrasound reconstructions of embryos and early fetuses. Lancet 1998; 352: 1182-6.

21. Pooh RK. Fetal brain assessment by three-dimensional ultrasound. In: Kurjak A, Kupesic S, eds. Clinical Application Of 3D Sonography. Carnforth, UK: Parthenon Publishing, 2000: 171-9.

22. Pooh RK, Pooh KH. Transvaginal 3D and Doppler ultrasonography of the fetal brain. Semin Perinatol 2001; 25: 38-43.

23. Pooh RK, Pooh KH. The assessment of fetal brain morphology and circulation by transvaginal 3D sonography and power Doppler. J Perinat Med 2002; 30: 48-56.

24. Endres LK, Cohen L. Reliability and validity of three-dimensional fetal brain volumes. J Ultrasound Med 2001; 20: 1265-9.

25. Roelfsema NM, Hop WC, Boito SM, Wladimiroff JW. Three-dimensional sonographic measurement of normal fetal brain volume during the second half of pregnancy. Am J Obstet Gynecol 2004; 190: 275-80.

26. Pooh RK. Neuroscan of congenital brain abnormality. In: Pooh RK, Kurjak A, eds. Fetal Neurology. New Delhi: Jaypee Brothers Medical Publishers, 2009: 59-139.

27. Pooh RK, Pooh KH. Fetal ventriculomegaly. Donald school. J Ultrasound Obstet Gynecol 2007; 2: 40-6.

28. Alagappan R, Browning PD, Laorr A, McGahan JP. Distal lateral ventricular atrium: reevaluation of normal range. Radiology 1994; 193: 405-8.

29. Almog B, Gamzu R, Achiron R, et al. Fetal lateral ventricular width: what should be its upper limit? A prospective cohort study and reanalysis of the current and previous data. J Ultrasound Med 2003; 22: 39-43.

30. Pooh RK, Pooh KH, Nakagawa Y, et al. Transvaginal Doppler assessment of fetal intracranial venous flow. Obstet Gynecol 1999; 93: 697-701.

31. Kelly EN, Allen VM, Seaward G, Windrim R, Ryan G. Mild ventriculomegaly in the fetus, natural history, associated findings and outcome of isolated mild ventriculomegaly: a literature review. Prenat Diagn 2001; 21: 697-700.

32. Goldstein I, Copel JA, Makhoul IR. Mild cerebral ventriculomegaly in fetuses: characteristics and outcome. Fetal Diagn Ther 2005; 20: 281-4.

33. Pilu G, Falco P, Gabrielli S, et al. The clinical significance of fetal isolated cerebral borderline ventriculomegaly: report of 31 cases and review of the literature. Ultrasound Obstet Gynecol 1999; 14: 320-6.
34. Signorelli M, Tiberti A, Valseriati D, et al. Width of the fetal lateral ventricular atrium between 10 and $12 \mathrm{~mm}$ : a simple variation of the norm? Ultrasound Obstet Gynecol 2004; 23: 14-18.

35. Ouahba J, Luton D, Vuillard E, et al. Prenatal isolated mild ventriculomegaly: outcome in 167 cases. BJOG 2006; 113: 1072-9.

36. Pilu G, Porelo A, Falco P, Visentin A. Median anomalies of the brain. In: Timor-Tritsch IE, Monteagudo A, Cohen HL, eds. Ultrasonography of the prenatal and neonatal brain, 2nd edn. New York: McGraw-Hill, 2001: 259-76.

37. Volpe JJ. Neural tube formation and prosencephalic development. Neurology of the Neuborn, 4th edn. Philadelphia: WB Saunders, 2001: 3-44.

38. Biggio JR Jr, Wenstrom KD, Owen J. Fetal open spina bifida: a natural history of disease progression in utero. Prenat Diagn 2004; 24: 287-9.

39. Nicolaides KH, Campbell S, Gabbe SG, Guidetti R. Ultrasound screening for spina bifida: cranial and cerebellar signs. Lancet 1986; 2: 72-4.

40. D'Addario V, Pinto V, Del Bianco A, et al. The clivus-supraocciput angle: a useful measurement to evaluate the shape and size of the fetal posterior fossa and to diagnose Chiari II malformation. Ultrasound Obstet Gynecol 2001; 18: 146-9.

41. Barkovich AJ, Kjos BO, Normal D, et al. Revised classification of the posterior fossa cysts and cystlike malformations based on the results of multiplanar MR imaging. AJNR 1989; 10: 977-88.

42. Schwarzler P, Homfray T, Bernard JP, Bland JM, Ville Y. Late onset microcephaly: failure of prenatal diagnosis. Ultrasound Obstet Gynecol 2003; 22: 640-2.

43. Volpe JJ. Neuronal proliferation, migration, organization and myelination. Neurology of the newborn, 4th edn. USA: W.B. Saunders, 2001: 45-99.

44. Kobayashi K, Nakahori Y, Miyake M, et al. An ancient retrotransposal insertion causes Fukuyama-type congenital muscular dystrophy. Nature 1998; 394: 388-92.

45. McGahan JP, Grix A, Gerscovich EO. Prenatal diagnosis of lissencephaly: Miller-Dieker syndrome. J Clin Ultrasound 1994; 22: 560-3.

46. Greco P, Resta M, Vimercati A, et al. Antenatal diagnosis of isolated lissencephaly by ultrasound and magnetic resonance imaging. Ultrasound Obstet Gynecol 1998; 12: 276-9.

47. Kojima K, Suzuki Y, Seki K, et al. Prenatal diagnosis of lissencephaly (type II) by ultrasound and fast magnetic resonance imaging. Fetal Diagn Ther 2002; 17: 34-6.

48. Monteagudo A, Timor-Tritsch IE. Fetal Neurosonography of congenital brain anomalies. In: Timor-Tritsch IE, Monteagudo A, Cohen HL, eds. Ultrasonography of the prenatal and neonatal brain, 2nd edn. McGrawHill, New York: 2001: 151-258.

49. Pooh RK. Fetal neuroimaging of neural migration disorder. In: Lazebnik N, Lazebnik RS, eds. Ultrasound Clinics. Volume 3. Elsevier, 2008: 541-52.

50. Barkovich AJ, Simon EM, Walsh CA. Callosal agenesis with cyst: a better understanding and new classification. Neurology 2001; 56: 220-7.

51. Bretelle F, Senat MV, Bernard JP, Hillion Y, Ville Y. First-trimester diagnosis of fetal arachnoid cyst: prenatal implication. Ultrasound Obstet Gynecol 2002; 20: 400-2.

52. Volpe JJ. Brain tumors and vein of Galen malformation. Neurology of the Neuborn, 4th edn. Philadelphia: WB Saunders, 2001: 841-56.

53. Sherer DM, Abramowicz JS, Eggers PC, et al. Prenatal ultrasonographic diagnosis of intracranial teratoma and massive craniomegaly with associated high-output cardiac failure. Am J Obstet Gynecol 1993; 168: 97-9.

54. Pooh RK, Nakagawa Y, Pooh KH, Nakagawa Y, Nagamachi N. Fetal craniofacial structure and intracranial morphology in a case of Apert syndrome. Ultrasound Obstet Gynecol 1999; 13: 274-80.

55. Benacerraf BR, Spiro R, Mitchell AG. Using three-dimensional ultrasound to detect craniosynostosis in a fetus with Pfeiffer syndrome. Ultrasound Obstet Gynecol 2000; 16: 391-4.

56. Faro C, Chaoui R, Wegrzyn P, et al. Metopic suture in fetuses with Apert syndrome at 22-27 weeks of gestation. Ultrasound Obstet Gynecol 2006; 27: 28-33.

57. Lasjaunias PL, Chng SM, Sachet M, et al. The management of vein of Galen aneurysmal malformations. Neurosurgery 2006; 59: S184-94.

58. Tart RP, Quisling RG. Curvilinear and tubulonodular varieties of lipoma of the corpus callosum: an MR and CT study. J Comput Assist Tomogr 1991; 15: 805-10. 
59. Demaerel P, Van de Gaer P, Wilms G, Baert AL. Interhemispheric lipoma with variable callosal dysgenesis: relationship between embryology, morphology, and symptomatology. Eur Radiol 1996; 6: 904-9.

60. Ickowitz V, Eurin D, Rypens F, et al. Prenatal diagnosis and postnatal follow-up of pericallosal lipoma: report of seven new cases. AJNR 2001; 22: 767-72.

61. Jeanty P, Zaleski W, Fleischer AC. Prenatal sonographic diagnosis of lipoma of the corpus callosum in a fetus with Goldenhar syndrome. Am J Perinatol 1991; 8: 89-90.

62. Malinger G, Ben-Sira L, Lev D, et al. Fetal brain imaging: a comparison between magnetic resonance imaging and dedicated neurosonography. Ultrasound Obstet Gynecol 2004; 23: 333-40.
63. Sherer DM, Anyaegbunam A, Onyeije C. Antepartum fetal intracranial hemorrhage, predisposing factors and prenatal sonography: a review. Am J Perinatol 1998; 15: 431-41.

64. Eller KM, Kuller JA. Porencephaly secondary to fetal trauma during amniocentesis. Obstet Gynecol 1995; 85: 865-7.

65. Moinuddin A, McKinstry RC, Martin KA, Neil JJ. Intracranial hemorrhage progressing to porencephaly as a result of congenitally acquired cytomegalovirus infection-an illustrative report. Prenat Diagn 2003; 23: 797-800.

66. Meizner I, Elchalal U. Prenatal sonographic diagnosis of anterior fossa porencephaly. J Clin Ultrasound 1996; 24: 96-9.

67. de Laveaucoupet J, Audibert F, Guis F, et al. Fetal magnetic resonance imaging (MRI) of ischemic brain injury. Prenat Diagn 2001; 21: 729-36. 


\section{Erythroblastosis fetalis Avinash Patil, Brian Brocato, Rebecca A. Uhlmann, and Giancarlo Mari}

\section{INTRODUCTION}

Red blood cell (RBC) alloimmunization, or isoimmunization, is the development of maternal antibodies to fetal $\mathrm{RBC}$ antigens. Hemolytic disease of the newborn/fetus is a hemolytic anemia that results from the lysis of the fetal RBCs by maternal antibodies. It is often characterized by excessive erythroblasts in the fetal bone marrow and circulation (erythroblastosis fetalis). Other features include generalized edema (hydrops fetalis) and hepatosplenomegaly. Alloimmunization and hemolytic disease of the newborn/fetus most commonly occur when maternal antibodies form against paternally derived Rhesus (D) antigens of the fetus, which is termed Rhesus (Rh) alloimmunization.

\section{HISTORY}

A midwife, Louyse Bourgeois, first described hemolytic disease of the newborn in 1609 after delivering a set of twins. One twin was a stillbirth with hydrops and the other with severe jaundice died a few days later. Sylvius, in 1679, reported cases of hydrops combined with severe jaundice, which was thought to be caused by a blockage of the "bile tract." Person and Cheyne, in 1799 and 1802, respectively, reported cases of infant fatalities with severe jaundice in which the autopsy reports showed patent bile ducts. In 1905, Auden described the features of hemolytic disease of the newborn. He noted that the first born was often unaffected and it was the subsequent pregnancies that showed hydrops and jaundice. While reporting a case of hydrops fetalis in 1912, Rautmann was the first to use the term "erythroblastosis." In 1923, Ottenburg wrote a paper in which he discussed "accidental placental transfusion of incompatible blood." This was followed by Darrow's hypothesis in 1938 that hemolysis was caused by the passage of anti-fetal hemoglobin from mother to fetus (1).

It was not until 1940 that the Rh antibody was discovered. Landsteiner and Weiner discovered the agglutination of human red cells when mixed with the antiserum of the Macacus rhesus and designated this property of human blood "Rh" (2). Wiener and Peters then demonstrated the transfusion reactions caused by giving $\mathrm{Rh}$-positive blood to Rh-negative patients (1). It was in 1941 that Levine described several cases of infants with erythroblastosis fetalis in which the hemolytic disease of the fetus was caused by maternal $\mathrm{Rh}$ isoimmunization (3).

In the 1950s and 1960s, we began to see invasive testing take place to evaluate the fetus at risk for erythroblastosis fetalis. In 1956, Bevis, using spectrophotometry, measured blood pigments in amniotic fluid and suggested that the concentration of bilirubin in the amniotic fluid was an indicator of the severity of the hemolytic process in the baby (4). In 1961, Sir William Liley, using amniocentesis and spectrophotometric measurements, described the use of spectral absorption curves to measure the degree of fetal anemia and suggested that these findings could be used to select the optimal time for delivery (5). He proposed a graph with three zones to indicate the severity of the disease from 27 weeks of gestation to term, which was known as the Liley method. In 1993, a modification of the Liley method by Queenan et al. expanded the normal spectrophotometric measurements to include 14 to 40 weeks of gestation and proposed four management zones (6). In 2000, Mari et al. proposed a noninvasive method to diagnose fetal anemia by ultrasonographic measurement of the middle cerebral artery peak systolic velocity (MCA-PSV) (7).

Before the 1960s, fetuses determined to be at significant risk for hemolytic disease could only be offered induction of labor at 34 weeks of gestation. Although perinatal mortality was greatly reduced by selective induction, there were no therapies for the severely affected fetus at less than 34 weeks of gestation (8). In 1963, after identifying an anemic fetus, Liley performed the first successful intraperitoneal fetal transfusion at 32 weeks of gestation and again at 33 weeks of gestation (8). At 34 weeks and 3 days of gestation, labor was induced and the mother eventually delivered via cesarean section a viable male infant weighing $2560 \mathrm{~g}$ (8). The boy was named Grant Liley McLeod (9). In 1964, Freda and Adamson described the first intravascular exchange transfusion at 27 weeks of gestation via hysterotomy (10). A fetal leg was delivered through a small uterine incision, a catheter was placed in the femoral artery, and the transfusion was performed. Thirty-six hours later, preterm labor ensued and the infant died from complications of prematurity (10). In 1966, Asensio performed an intravascular exchange transfusion in Puerto Rico on a severely anemic fetus at 31 weeks of gestation. The technique was similar to that described by Freda and Adamson with a few modifications. The pregnancy continued for 3 weeks postoperatively and eventually delivered via cesarean section secondary to premature rupture of membranes. A viable female infant was born weighing $2034 \mathrm{~g}$ and was discharged home 18 days after delivery (11). In 1973, Valenti used fetoscopy to perform the first aspiration of fetal blood from the umbilical cord and the blood samples were used to diagnose hemoglobinopathies (12). He proposed that the procedure could be used for intrauterine diagnosis and therapy. In 1978, Rodeck and Campbell described a technique to obtain pure fetal blood via fetoscopy in gestations ranging from 16 to 22 weeks (13). Rodeck proposed that since pure blood can be removed from the umbilical circulation via fetoscopy, transfusion should also be possible, and, in 1981, Rodeck described two cases of fetal blood transfusion directly into an umbilical vessel via fetoscopy (14). Both patients were initially transfused at 23 weeks of gestation: one fetus survived after cesarean delivery at 30 weeks of gestation, while the other underwent 
Table 1 Risk of Red Blood Cell (RBC) Alloimmunization from Different Clinical Situations

\begin{tabular}{ll} 
Clinical situation & \multicolumn{1}{c}{$\begin{array}{c}\text { Risk of RBC } \\
\text { alloimmunization (\%) }\end{array}$} \\
\hline Induced abortions & $4-5$ \\
First-trimester losses & $1-2$ \\
Chorionic villus sampling & 14 \\
Amniocentesis & $7-15$ \\
External cephalic version & $2-6$ \\
Threatened abortion & $\uparrow$ (controversial) \\
Antepartum hemorrhage & $\uparrow \uparrow$ \\
Placenta previa with bleeding & $\uparrow$ \\
Suspected abruption & $\uparrow$ \\
Blunt trauma to abdomen (including & $\uparrow$ \\
$\quad$ motor vehicle accidents) & $\uparrow$ \\
Fetal death & $\uparrow$ \\
Fetal blood sampling & $\uparrow$ \\
Fetal surgery & $\uparrow$ \\
Ectopic & $\uparrow$ \\
Partial molar pregnancy & $\uparrow$ \\
Source: From Ref. 17. &
\end{tabular}

an intrauterine demise at 26 weeks of gestation (14). And in 1982, Bang described a case of ultrasound-guided intravenous fetal transfusion in a fetus severely affected by $\mathrm{Rh}$ isoimmunization at 29 weeks of gestation. The patient delivered via cesarean section at 32 weeks of gestation and the neonatal period was unremarkable (15).

\section{PATHOPHYSIOLOGY}

Maternal alloimmunization occurs when the gravid patient develops an immune response to a paternally derived antigen found on the surface of the fetal RBC. The most common antigen to cause RBC alloimmunization is Rh (D). The majority of $\mathrm{Rh}$ (D) alloimmunization is the result of fetomaternal hemorrhage. Fetomaternal hemorrhage has been shown to affect $75 \%$ of pregnancies during or shortly after delivery (16). Less than $0.1 \mathrm{~mL}$ of fetal blood entering the maternal circulation causes most cases of alloimmunization (16). There are several clinical scenarios when a pregnancy is at risk for fetomaternal hemorrhage, the most significant of which is at the time of delivery. Other clinical scenarios that have demonstrated fetomaternal hemorrhage and the risk of RBC alloimmunization are found in Table 1.

Once $\mathrm{Rh}$ (D) alloimmunization has occurred, it is then possible for the maternal IgG antibodies to cross the placenta, attach to the fetal RBC Rh (D) antigen, and cause RBC destruction in the fetal spleen resulting in a hemolytic anemia. This fetal anemia stimulates erythropoietin and the bone marrow produces more RBCs. If the anemia is severe enough, the bone marrow alone may not be able to produce enough RBCs. In this case, erythropoiesis will take place outside of the bone marrow in sites such as the liver and spleen, causing hepatosplenomegaly. Erythropoiesis outside of the fetal bone marrow also will cause immature erythroblasts to appear in the circulation.

\section{NOMENCLATURE}

There are several systems of nomenclature used to classify the $\mathrm{Rh}$ antigens. The Fisher-Race is perhaps the most commonly used nomenclature system in obstetrics. In this system, there are five major antigens: C, c, D, E, and e (note that there is no d antigen). A group of three antigens form one gene complex, such as CDe. One gene complex is inherited from each parent, thereby forming a person's genotype (such as CDe/cDE). The most common gene complexes are CDe, cde, and cDE, which are found in $41 \%, 39 \%$, and $16 \%$ of the Caucasian population, respectively. The terms "Rh (D) positive" and "Rh (D) negative" describe the presence or absence of the $\mathrm{D}$ antigen. When a genotype includes the letter "d" (e.g., Cde), this is used to indicate the absence of the $\mathrm{D}$ antigen, which results in an $\mathrm{Rh}$ (D)-negative phenotype (18). The Rh genes are encoded on chromosome 1p34-p36. Rh (D)-negative status in Caucasians is due to a deletion of the Rh (D) gene on chromosome 1 (18).

In addition to the five major antigens in the Rh system, there are several variants. Of note is the antigen called weak D or Du. Among patients with weak $\mathrm{D}$, alloimmunization is rare and anti-D immunoglobulin is not routinely indicated for prophylaxis (19).

Other antibodies outside of the Rh system have been identified, such as Kell, Duffy, and Kidd. Anti-Lewis antibodies do not cause hemolytic disease of the fetus/newborn because the Lewis antigens do not develop on the fetal RBC until after birth and the anti-Lewis antibody is of the IgM class, making it too large to cross the placenta into the fetal circulation. Table 2 displays a more complete list of antigens known to cause alloimmunization and the associated risks of hemolytic disease.

\section{EPIDEMIOLOGY}

The $\mathrm{Rh}$ (D)-negative blood type is found in $15 \%$ of Caucasians, $8 \%$ of African-Americans, and less than $1 \%$ of Asian populations. The highest known incidence is in the Basque population, where the rate of Rh (D)-negative status is $30 \%$. In 2003, the incidence of $\mathrm{Rh}$ alloimmunization was 6.8 per 1000 live births in the United States (21). Fetal anemia from RBC alloimmunization occurs less than $1 \%$ of the time, and only $10 \%$ of those neonates will require transfusions.

\section{DIAGNOSIS}

All pregnant females should be tested for $\mathrm{ABO}$ blood group type, $\mathrm{Rh}$ (D) status, and the presence of erythrocyte antibodies at the time of their initial prenatal visit (19). This battery of tests should be repeated at the onset of every pregnancy. The intent of routine screening is to identify $\mathrm{Rh}$ (D)-negative females prior to the development of alloimmunization in order to provide Rh (D) immunoglobulin. Anti-D immunoglobulin prophylaxis, when properly administered, prevents more than $99 \%$ of cases of alloimmunization. The American Association of Blood Banks recommends reevaluation of $\mathrm{Rh}$ antibody status prior to the administration of Rh (D) immunoglobulin, postpartum, and after any traumatic events during the antepartum period. The advantage of a second screening test prior to administration of Rh (D) immunoglobulin at 28 weeks of gestation is the detection of those rare cases in which immunization occurs early in pregnancy. Unfortunately, there is no immunoglobulin available for the prevention of an $\mathrm{RBC}$ antigen reaction other than for $\mathrm{Rh}(\mathrm{D})$.

$\mathrm{Rh}$ (D)-positive females do not require further evaluation. As mentioned previously, mothers who are $\mathrm{D}$ positive, but 
Table 2 Atypical Antibodies and Their Relationship to Fetal Hemolytic Disease

\begin{tabular}{|c|c|c|c|}
\hline Blood group system & $\begin{array}{l}\text { Antigens related to } \\
\text { hemolytic disease }\end{array}$ & Hemolytic disease severity & Proposed management \\
\hline Lewis & * & & \\
\hline $\mathrm{L}$ & * & & \\
\hline \multirow[t]{7}{*}{ Kell } & $\mathrm{K}$ & Mild to severe ${ }^{\dagger}$ & Fetal assessment \\
\hline & $\mathrm{K}$ & Mild & Routine obstetric care \\
\hline & $\mathrm{K}_{0}$ & Mild & Routine obstetric care \\
\hline & $\mathrm{Kp}^{\mathrm{a}}$ & Mild & Routine obstetric care \\
\hline & $\mathrm{Kp}^{\mathrm{b}}$ & Mild & Routine obstetric care \\
\hline & Jsa & Mild & Routine obstetric care \\
\hline & Jsb & Mild & Routine obstetric care \\
\hline \multirow[t]{3}{*}{$\mathrm{Rh}$ (non-D) } & $\mathrm{E}$ & Mild to severe $^{\dagger}$ & Fetal assessment \\
\hline & $\mathrm{C}$ & Mild to severe ${ }^{\dagger}$ & Fetal assessment \\
\hline & $\mathrm{C}$ & Mild to severe $^{\dagger}$ & Fetal assessment \\
\hline \multirow[t]{3}{*}{ Duffy } & $\mathrm{Fy}^{\mathrm{a}}$ & Mild to severe $^{\dagger}$ & Fetal assessment \\
\hline & $\mathrm{Fy}^{\mathrm{b}}$ & $\ddagger$ & Routine obstetric care \\
\hline & $\mathrm{By}^{3}$ & Mild & Routine obstetric care \\
\hline \multirow[t]{3}{*}{ Kidd } & $\mathrm{Jk}^{\mathrm{a}}$ & Mild to severe & Fetal assessment \\
\hline & $\mathrm{Jk}^{\mathrm{b}}$ & Mild & Routine obstetric care \\
\hline & $\mathrm{Jk}^{3}$ & Mild & Routine obstetric care \\
\hline \multirow[t]{6}{*}{ MNSs } & M & Mild to severe & Fetal assessment \\
\hline & $\mathrm{N}$ & Mild & Routine obstetric care \\
\hline & $\mathrm{S}$ & Mild to severe & Fetal assessment \\
\hline & $\mathrm{S}$ & Mild to severe & Fetal assessment \\
\hline & $\mathrm{U}$ & Mild to severe & Fetal assessment \\
\hline & $\mathrm{Mi}^{\mathrm{a}}$ & Moderate & Fetal assessment \\
\hline \multirow[t]{5}{*}{ MSSs } & $\mathrm{Mt}^{\mathrm{a}}$ & Moderate & Fetal assessment \\
\hline & Vw & Mild & Routine obstetric care \\
\hline & Mur & Mild & Routine obstetric care \\
\hline & Hil & Mild & Routine obstetric care \\
\hline & Hut & Mild & Routine obstetric care \\
\hline \multirow[t]{2}{*}{ Lutheran } & $\mathrm{Lu}^{\mathrm{a}}$ & Mild & Routine obstetric care \\
\hline & $\mathrm{Lu}^{\mathrm{b}}$ & Mild & Routine obstetric care \\
\hline \multirow[t]{2}{*}{ Diego } & $\mathrm{D} 1^{\mathrm{a}}$ & Mild to severe & Fetal assessment \\
\hline & $\mathrm{Di}^{\mathrm{b}}$ & Mild to severe & Fetal assessment \\
\hline $\mathrm{Xg}$ & $\mathrm{Xg}^{\mathrm{a}}$ & Mild & Routine obstetric care \\
\hline $\mathrm{P}^{\circ}$ & $\mathrm{PP}_{1 \mathrm{pk}}\left(\mathrm{Tj}{ }^{\mathrm{a}}\right)$ & Mild to severe & Fetal assessment \\
\hline \multirow[t]{8}{*}{ Public antigens } & $\mathrm{Yt}^{\mathrm{a}}$ & Moderate to severe & Fetal assessment \\
\hline & $\mathrm{Yt}^{\mathrm{b}}$ & Mild & Routine obstetric care \\
\hline & Lan & Mild & Routine obstetric care \\
\hline & $\mathrm{En}^{\mathrm{a}}$ & Moderate & Fetal assessment \\
\hline & $\mathrm{Ge}$ & Mild & Routine obstetric care \\
\hline & $\mathrm{Jr}^{\mathrm{a}}$ & Mild & Routine obstetric care \\
\hline & $\mathrm{Co}^{\mathrm{a}}$ & Severe & Fetal assessment \\
\hline & $\mathrm{Co}^{1-\mathrm{b}-}$ & Mild & Routine obstetric care \\
\hline \multirow[t]{16}{*}{ Private antigens } & Batty & Mild & Routine obstetric care \\
\hline & Becker & Mild & Routine obstetric care \\
\hline & Berrens & Mild & Routine obstetric care \\
\hline & Biles & Moderate & Fetal assessment \\
\hline & Evans & Mild & Routine obstetric care \\
\hline & Gonzales & Mild & Routine obstetric care \\
\hline & Good & Severe & Fetal assessment \\
\hline & Heibel & Moderate & Fetal assessment \\
\hline & Hunt & Mild & Routine obstetric care \\
\hline & Jobbins & Mild & Routine obstetric care \\
\hline & Radin & Moderate & Fetal assessment \\
\hline & $\mathrm{Rm}$ & Mild & Routine obstetric care \\
\hline & Ven & Mild & Routine obstetric care \\
\hline & Wright $^{\mathrm{a}}$ & Severe & Fetal assessment \\
\hline & Wright $^{\mathrm{b}}$ & Mild & Routine obstetric care \\
\hline & $\mathrm{Zd}$ & Moderate & Fetal assessment \\
\hline
\end{tabular}


with $\mathrm{D}$ present in reduced quantities, formerly called $\mathrm{Du}$, do not need anti-D prophylaxis. Mothers who are partial D positive, that is, lacking some epitopes of $\mathrm{D}$, should receive anti-D immunoglobulin, since they are at risk for hemolytic disease (19). Rh (D)-negative females without alloantibodies require administration of $\mathrm{Rh}(\mathrm{D})$ immunoglobulin at defined intervals during pregnancy (see Management section). $\mathrm{Rh}$ (D)-negative females with preformed alloantibodies require further investigation to determine the potential for impact upon the fetus. The least invasive approach involves paternal testing utilizing antisera or DNA (PCR). If the father is homozygous for the $\mathrm{D}$ antigen, all his offspring will be at risk for anemia; if he is heterozygous, then $50 \%$ of the offspring will be at risk. If the father of the fetus/neonate is definitely known to be $\mathrm{Rh}(\mathrm{D})$ negative, neither antepartum nor postpartum anti-D prophylaxis needs to be administered.

Analysis of the fetal genotype is appropriate when the paternal genotype is heterozygous or uncertain. Amniocentesis with PCR of uncultured amniocytes is the most common method utilized, with a sensitivity and specificity of $98.7 \%$ and $100 \%$, respectively, and positive and negative predictive values of $100 \%$ and $96.9 \%$, respectively (22). Chorionic villus sampling (CVS) also has been utilized for this purpose, but is discouraged due to the increased risk of fetomaternal hemorrhage and alloimmunization (23). Another option is the analysis of maternal plasma or serum for fetal DNA. Greater than $90 \%$ accuracy exists in the second trimester due to the high concentrations of fetal DNA in maternal plasma (24-26).

\section{MANAGEMENT OF UNAFFECTED PREGNANCIES}

During the 1960s, efforts were made to prevent the formation of maternal antibodies. In 1961, Dr. Ronald Finn proposed that administration of antibodies to $\mathrm{Rh}(\mathrm{D})$-negative mothers could prevent a maternal immune response to $\mathrm{Rh}(\mathrm{D})$-positive fetal blood cells (27). In 1964, Dr. Vincent Freda, at Sing Sing Prison in Ossining, New York, conducted an experiment on $\mathrm{Rh}$ (D)-negative male prisoners in which he injected radiolabeled $\mathrm{Rh}(\mathrm{D})$-positive blood cells. The men were then given anti-D serum and it was noted that the radiolabeled cells were removed rapidly from their systems (28). In 1966, RhoGAM $^{\circledast}$ was first developed and found to be successful at preventing alloimmunization. It eventually was introduced in North America in 1968.

Anti-D immunoglobulin is routinely administered during pregnancy in $R h(D)$-negative women without evidence of alloimmunization. The American Association of Blood Banks recommends dosing at 28 weeks of gestation, postpartum, and after any suspected episodes of fetomaternal hemorrhage. The majority of cases of fetomaternal hemorrhage occur during uncomplicated vaginal deliveries, with an average of $0.1 \mathrm{~mL}$ of fetal blood entering the maternal circulation and causing alloimmunization (29-32). Antepartum fetomaternal hemorrhage accounts for $1 \%$ to $2 \%$ of $\mathrm{Rh}$ alloimmunization (33). Common scenarios leading to alloimmunization include spontaneous, threatened, and induced abortions, ectopic pregnancy, CVS, amniocentesis, and external cephalic version.

While a variety of $\mathrm{Rh}$ immunoglobulin is available in the United States, RhoGAM was the first approved for clinical use and is the most commonly used. Other products that are available include HyperRHO ${ }^{\circledast}$ S/D, Rhophylac ${ }^{\circledR}$, and WinRho ${ }^{\varpi}$
SDF. The primary difference in these products is the method of purification and subsequent mode of delivery. RhoGAM and HyperRHO S/D are processed by cold ethanol fractionation, resulting in a less purified product that requires intramuscular administration. By contrast, Rhophylac and WinRho SDF are prepared by chromatography, allowing for intravenous and intramuscular administration routes. Anti-D immunoglobulin is extracted from plasma of individuals with high-titer D IgG antibodies. The risk of transmission of viral infections or side effects is minimal to absent and is not a clinically significant factor.

In the United States, standard dosing of anti-D immunoglobulin for Rh (D)-negative women without evidence of alloimmunization is $300 \mu \mathrm{g}$ given at 28 weeks of gestation, and again in the postpartum period if the neonate is $\mathrm{Rh}$ (D)-positive and delivery occurs at least 3 weeks after the first administration. This practice reduces the incidence of antenatal alloimmunization from $2 \%$ to $0.1 \%(16,34)$.

An alternate anti-D immunoglobulin prophylaxis regimen, used primarily in the United Kingdom, is $100 \mu \mathrm{g}$ at 28 and 34 weeks of gestation, as well as after delivery if the neonate is $\mathrm{Rh}(\mathrm{D})$ positive. There is a trend for less immunization during pregnancy for all women [relative risk $(\mathrm{RR})=0.42$, 95\% confidence interval (CI): 0.15-1.17] and for women giving birth to an $\mathrm{Rh}(\mathrm{D})$-positive infant $(\mathrm{RR}=0.41,95 \%$ CI: 0.16-1.04) compared with no prophylaxis. Overall risk was lowered to about $0.2 \%$ without any adverse effects (35-37). Although trials have shown a nonsignificant reduction in immunization at 2 to 12 months following the birth of an Rh-positive infant in women who had received anti-D $(\mathrm{RR}=0.14,95 \% \mathrm{CI}: 0.02-1.15)$, they were significantly less likely to have a positive Kleihauer-Betke (KB) test during pregnancy $(\mathrm{RR}=0.60,95 \% \mathrm{CI}: 0.41-0.88)$ and at the birth of an $\mathrm{Rh}(\mathrm{D})$-positive infant ( $\mathrm{RR}=0.60,95 \% \mathrm{CI}$ : 0.46-0.79) (36). Similarly, in Canada, 100 to $120 \mu \mathrm{g}$ are administered at 28 and 34 weeks of gestation.

Alloimmunization can infrequently occur prior to 28 weeks of gestation, leading to the recommendation that an antibody screen be performed at the time of immunoglobulin administration to detect preexisting maternal sensitization. One dose of Rh (D) immunoglobulin is effective for 12 weeks, with a halflife of 16 to 24 days, and should be re-dosed after that interval in antepartum patients. The anti-D immunoglobulin affects the antibody screen (38). Usually, the effect of the immunoglobulin is not present until 12 weeks after its administration.

Excessive maternal-fetal hemorrhage may require more than the typical dose of $\mathrm{Rh}(\mathrm{D})$ immunoglobulin. The standard dose of $300 \mu \mathrm{g}$ can protect from maternal sensitization caused by up to $30 \mathrm{~mL}$ of fetal whole blood or $15 \mathrm{~mL}$ of fetal RBCs in the maternal circulation. Occurrence of maternal-fetal hemorrhage can be determined by the rosette test. The standard dose of Rh (D) immunoglobulin is given for negative results, while a positive result requires further testing. A KB test determines the percentage of fetal blood cells in maternal circulation by utilizing flow cytometry. This value is then multiplied by 50 to determine the volume of hemorrhage, and one unit $(300 \mu \mathrm{g})$ of immunoglobulin is provided for every $30 \mathrm{~mL}$ of hemorrhaged volume. A maximum of five units may be given by intramuscular administration in a 24-hour period; alternatively, $600 \mu \mathrm{g}$ may be provided intravenously 

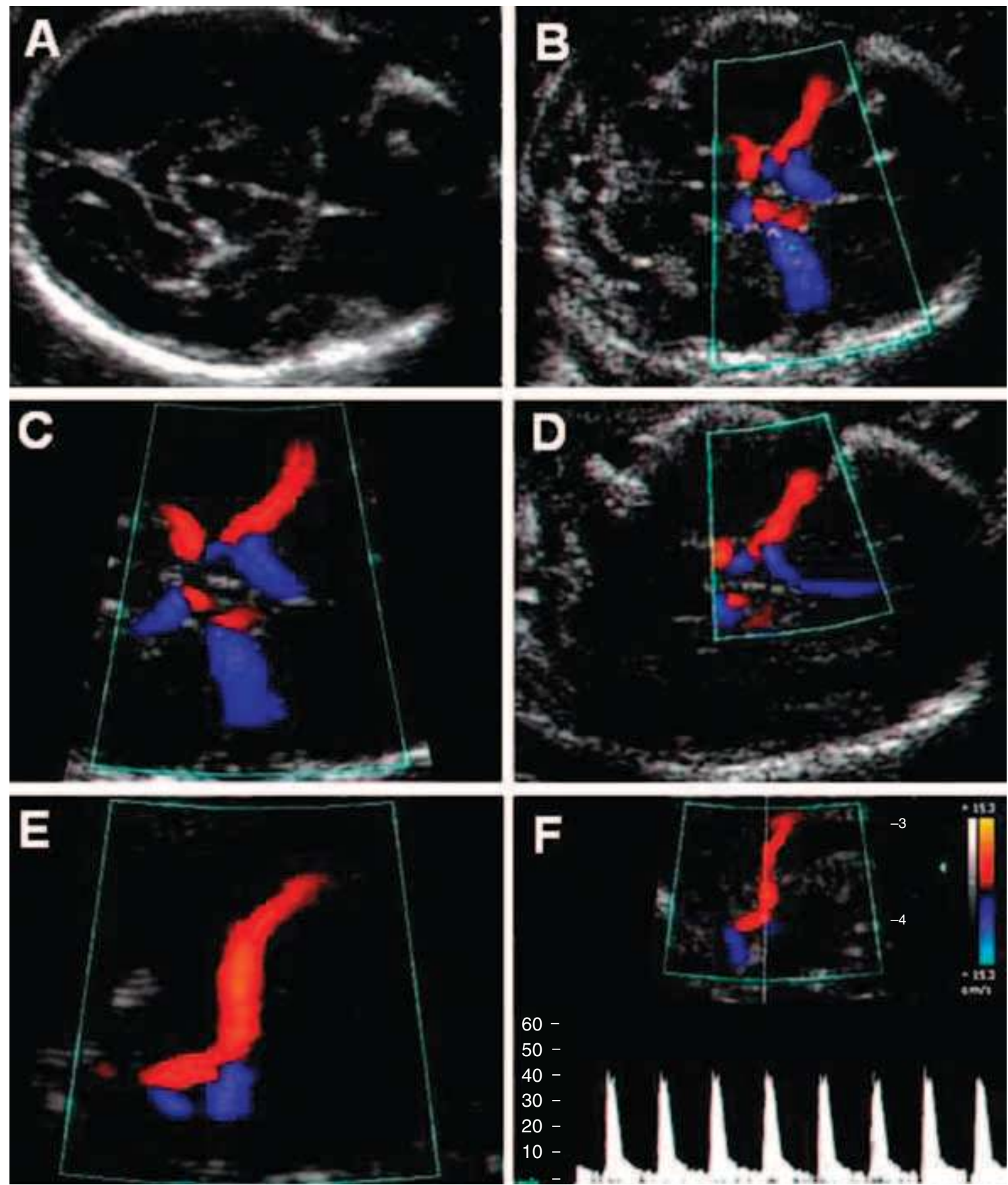

Figure 1 The steps to correctly sample the middle cerebral artery peak systolic velocity (MCA-PSV). (A) An axial section of the head is obtained at the level of the sphenoid bones; (B) color Doppler evidences the circle of Willis; (C) the circle of Willis is enlarged; (D) the color box is placed around the MCA; (E) the MCA is zoomed; and (F) the MCA flow velocity waveforms are displayed and the PSV is measured at its proximal point, after the origin from the internal carotid artery, and at a $0^{\circ}$ angle, thus avoiding angle corrections. Source: From Ref. 45.

every 8 hours until the entire dose is given. Some clinicians have advocated the KB test for all Rh (D)-negative women at delivery, since $50 \%$ of cases requiring more than the standard postpartum dose of anti-D immunoglobulin can be missed by high-risk situation screening only (39). The risk of fetomaternal hemorrhage greater than $30 \mathrm{~mL}$ is approximately $0.1 \%$ to $0.2 \%$.

Anti-D immunoglobulin given within 72 hours after childbirth is associated with a $96 \%$ decreased incidence of $\mathrm{Rh}$ (D) alloimmunization 6 months after birth and an $88 \%$ decreased incidence of $\mathrm{Rh}$ (D) alloimmunization in a subsequent pregnancy in $\mathrm{Rh}(\mathrm{D})$-negative women who have given birth to an $\mathrm{Rh}(\mathrm{D})$-positive infant (40). These benefits are seen regardless of the $\mathrm{ABO}$ status of the mother and baby. Anti-D immunoglobulin can be given as late as 28 days postpartum if indicated but previously not given. Anti-D immunoglobulin is given to all $\mathrm{Rh}(\mathrm{D})$-negative women after confirmation from cord blood of the Rh (D)-positive status of the neonate. Even when immunoglobulin is correctly administered, alloimmunization can still occur in the antepartum period in up to $2 \%$ of women with postpartum anti-D administration only. The risk of $\mathrm{Rh}$ (D) alloimmunization remains because of inadvertent antepartum or postpartum omission, failure to use the drug due to other antenatal complications, and insufficient dosing at delivery in cases of large volume fetomaternal hemorrhage. 
Table 3 Reference Ranges for Fetal Hemoglobin Concentrations as a Function of Gestational Age $\mathrm{a}^{\mathrm{a}}$

\begin{tabular}{lccccc} 
& \multicolumn{5}{c}{$\begin{array}{c}\text { Multiples of the median (MoM) grams } \\
\text { per deciliter }\end{array}$} \\
$\begin{array}{l}\text { Week of } \\
\text { gestation }\end{array}$ & $\mathbf{1 . 1 6}$ & $\mathbf{1 . 0 0}$ (median) & $\mathbf{0 . 8 4}$ & $\mathbf{0 . 6 5}$ & $\mathbf{0 . 5 5}$ \\
18 & 12.3 & 10.6 & 8.9 & 6.9 & 5.8 \\
20 & 12.9 & 11.1 & 9.3 & 7.2 & 6.1 \\
22 & 13.4 & 11.6 & 9.7 & 7.5 & 6.4 \\
24 & 13.9 & 12.0 & 10.1 & 7.8 & 6.6 \\
26 & 14.3 & 12.3 & 10.3 & 8.0 & 6.8 \\
28 & 14.6 & 12.6 & 10.6 & 8.2 & 6.9 \\
30 & 14.8 & 12.8 & 10.8 & 8.3 & 7.1 \\
32 & 15.2 & 13.1 & 10.9 & 8.5 & 7.2 \\
34 & 15.4 & 13.3 & 11.2 & 8.6 & 7.3 \\
36 & 15.6 & 13.5 & 11.3 & 8.7 & 7.4 \\
38 & 15.8 & 13.6 & 11.4 & 8.9 & 7.5 \\
40 & 16.0 & 13.8 & 11.6 & 9.0 & 7.6
\end{tabular}

${ }^{a}$ The hemoglobin values at 0.65 and 0.55 multiples of the median (cutoff points for mild and moderate anemia, respectively) are also shown. The values at 1.16 and 0.84 multiples of the median correspond to the 95th and 5th percentiles, respectively (the normal range). Source: From Ref. 7.

Table 4 Expected Peak Velocity of Systolic Blood Flow in the Middle Cerebral Artery as a Function of Gestational Age

\begin{tabular}{lcccc} 
Week of & \multicolumn{4}{c}{ Multiples of the median (MoM) centimeters } \\
gestation & $\mathbf{1 . 0 0}$ (median) & $\mathbf{1 . 2 9}$ & $\mathbf{1 . 5 0}$ & $\mathbf{1 . 5 5}$ \\
\cline { 2 - 5 } 18 & 23.2 & 29.9 & 34.8 & 36.0 \\
20 & 25.5 & 32.8 & 38.2 & 39.5 \\
22 & 27.9 & 36.0 & 41.9 & 43.3 \\
24 & 30.7 & 39.5 & 46.0 & 47.5 \\
26 & 33.6 & 43.3 & 50.4 & 52.1 \\
28 & 36.9 & 47.6 & 55.4 & 57.2 \\
30 & 40.5 & 52.2 & 60.7 & 62.8 \\
32 & 44.4 & 57.3 & 66.6 & 68.9 \\
34 & 48.7 & 62.9 & 73.1 & 75.6 \\
36 & 53.5 & 69.0 & 80.2 & 82.9 \\
38 & 58.7 & 75.7 & 88.0 & 91.0 \\
40 & 64.4 & 83.0 & 96.6 & 99.8 \\
Source: From Ref. 7. & & & &
\end{tabular}

\section{MANAGEMENT OF RBC-ALLOIMMUNIZED PREGNANCIES}

If $\mathrm{Rh}(\mathrm{D})$ antibodies are detected in the maternal circulation on the antibody screen, for example, by a positive indirect Coombs' test, the patient is considered alloimmunized. Among $\mathrm{Rh}$ (D)-alloimmunized pregnancies, mild-tomoderate hemolytic anemia and hyperbilirubinemia occur in $25 \%$ to $30 \%$ of fetuses/neonates, and $25 \%$ of those can develop hydrops (41). With correct management, the perinatal survival rate in cases of anemia is $>90 \%$; when fetal hydrops is present, survival is often $>80 \%$. There is no trial that has assessed the best management for RBCalloimmunized pregnancies, but fetal transfusion is probably the most beneficial of all fetal therapies available. Although it is reported that the risk of fetal demise is between $1 \%$ and $2 \%$ for each fetal blood sampling (FBS), there are situations in which the risk is much higher, for example, when cordocenteses and transfusions are performed at gestational ages as early as 15 to 18 weeks.
Once the fetal D antigen is known to be present in an alloimmunized pregnancy, antenatal management centers on monitoring the situation and preventing fetal hemolysis. In patients at risk, it is important to perform a first-trimester ultrasound to establish the gestational age. Assessment for the risk of fetal anemia depends on the history of Rh complications in previous pregnancies, the titer of RBC antibodies, and MCA-PSV values (22). Determination of the maternal titer using an indirect Coombs' test is the most common screening tool used to determine the degree of alloimmunization. Titer values represent the highest level of dilution that can produce an agglutination reaction. Critical titers correspond to the level of antibodies that place a fetus at significant risk for anemia/hydrops, with 1:16 $=10 \%, 1: 32=25 \%, 1: 64=50 \%$, and $1: 128=75 \%$ risk of anemia. The critical titer should be determined in each laboratory due to large differences in measurements between laboratories. In most laboratories, the critical titer for anti-D is approximately 1:16 in albumin or 1:32 using an indirect Coombs' test. For anti-Kell, the critical titer is 1:8. Maternal titers are typically drawn monthly until 24 weeks of gestation and then are repeated biweekly to detect worsening alloimmunization. Antenatal testing from 32 weeks of gestation forward and scheduled induction of labor by 38 weeks of gestation are routinely performed.

Traditionally, spectral analysis of amniotic fluid at $450 \mathrm{~nm}$ $\left(\Delta \mathrm{OD}_{450}\right)$ to detect bilirubin levels has been used as a measure of hemolytic activity. Due to its dependence upon gestational age, the $\Delta \mathrm{OD}_{450}$ is plotted on a Queenan curve (6) (14-40 weeks of gestation) or a Liley graph (8) (27 weeks of gestation to term).

Contemporary management of alloimmunized pregnancies emphasizes the noninvasive detection of worsening fetal anemia through the use of ultrasound. With fetal anemia, decreased blood viscosity leads to increased venous return, consequently increasing cardiac output with increased blood flow velocity in all vessels. Degrees of anemia correlate with corresponding changes in blood velocity. Mari et al. have previously shown that Doppler ultrasound measurement of the MCA-PSV can reliably identify fetal anemia. Compared with amniocentesis for $\triangle \mathrm{OD}_{450}$ assessment, the MCA-PSV is associated with a $70 \%$ to $80 \%$ reduction in the number of invasive tests (22). The MCA-PSV is also more accurate than amniocentesis in detecting fetal anemia $(7,42-44)$.

Screening with the MCA-PSV can be started as early as 15 weeks of gestation (8). The main advantage of the MCA is that it is easy to measure at a $0^{\circ}$ angle, which is essential for an accurate measurement of the true blood flow. The steps for the correct measurement of the MCA-PSV are (i) an axial section of the head is obtained at the level of the sphenoid bones; (ii) color Doppler evidences the circle of Willis; (iii) the circle of Willis is enlarged; (iv) the color box is placed around the MCA; (v) the MCA is zoomed; and (vi) the MCA flow velocity waveforms are displayed and the PSV is measured at its proximal point, after the origin from the internal carotid artery, and at a $0^{\circ}$ angle, thus avoiding angle corrections (Fig. 1). The waveforms should be similar. The above sequence is repeated at least three times in each fetus. Measurement at this point allows the lowest intra- and interobserver variability, as well as standardization of the measurement (46). Multiples of the median (MoM) for the hemoglobin concentration (Table 3) and the MCA-PSV (Table 4 and Fig. 2) correct for 


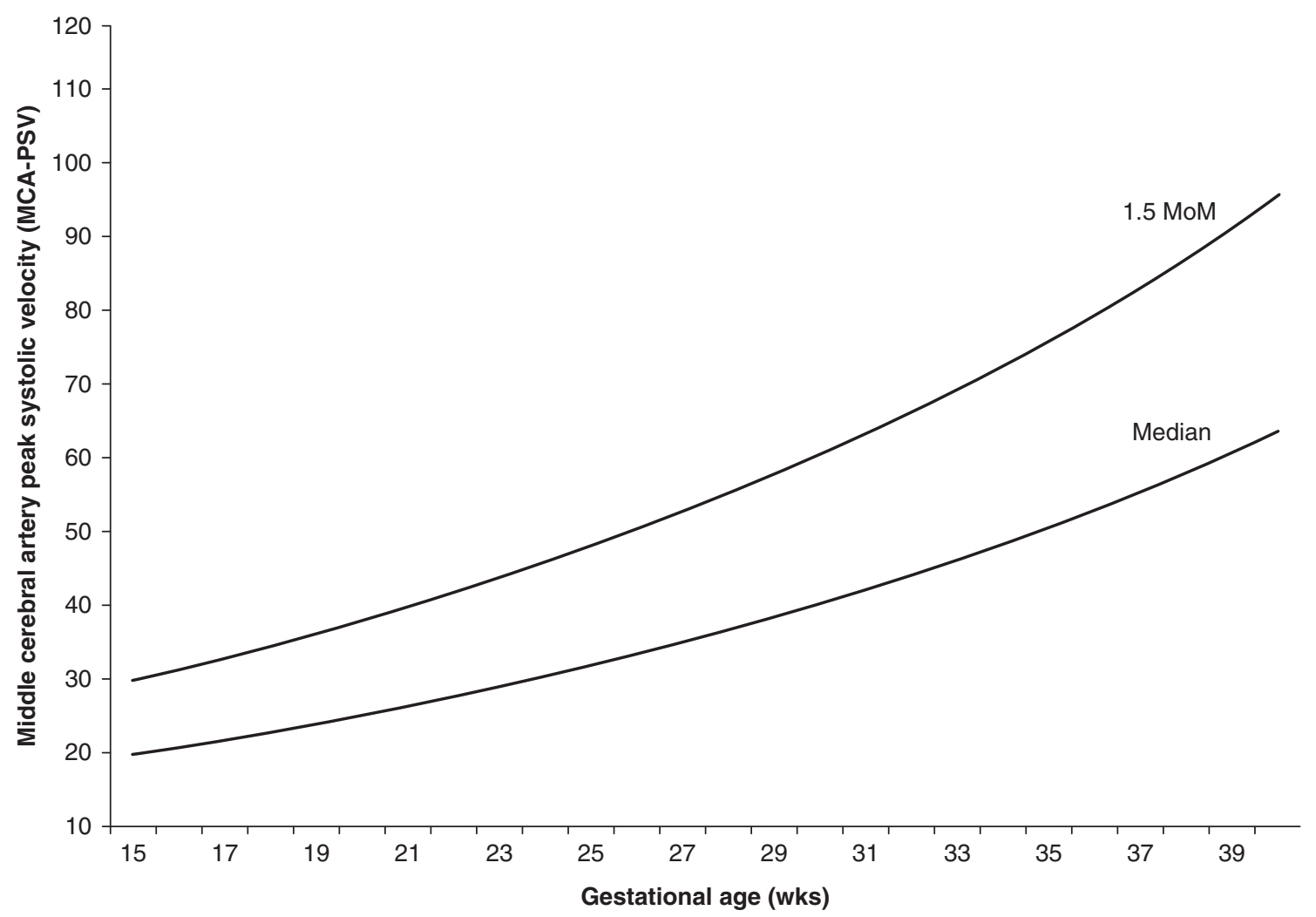

Figure 2 Middle cerebral artery peak systolic velocity (MCA-PSV) values correlated with gestational age. Abbreviation: MoM, multiples of the median. Source: From Ref. 7.

the effect of gestational age on the measurements (22). The MCA-PSV has a sensitivity of $100 \%$ (95\% CI: 0.86-1.0) for the detection of significant fetal anemia with a false positive rate of $12 \%$ at $1.50 \mathrm{MoM}$ (22). The number of false positive findings increases following 35 weeks of gestation (47). The number of false positive cases after 35 weeks of gestation may be decreased by looking at the overall trend of the MCA-PSV, rather than single values (48). An MoM > 1.5 indicates moderate-to-severe anemia and the need for invasive management.

The MCA-PSV may also be used to diagnose anemia of other etiologies, including parvovirus infection, nonimmune hydrops, fetomaternal hemorrhage, and twin-to-twin transfusion syndrome. Severe intrauterine growth restriction (IUGR) is also associated with an increased MCA-PSV (49). This should be taken into account when the MCA-PSV is used diagnostically; however, it is very unlikely that an anemic fetus is also a severe IUGR fetus. Moderate-to-severe fetal anemia may also be suggested by hydropic signs (at least two out of the following: pericardial or pleural effusion, ascites, or skin edema), an increase in size of the fetal liver or placental thickness, or by tricuspid regurgitation.

Once conservative measurements document a worsening hemolysis consistent with moderate-to-severe anemia (MCAPSV $>1.5 \mathrm{MoM}$ ), therapeutic intervention involves intrauterine fetal assessment and transfusion. This process begins with administration of a paralytic [vecuronium bromide $0.1 \mathrm{mg} / \mathrm{kg}$ of estimated fetal weight (EFW)] to stop fetal movement, followed by the acquisition of venous blood from the base of the umbilical cord by the placental insertion site. The baseline fetal hematocrit determines the volume of transfusion to yield a target hematocrit of $40 \%$ to $50 \%$. This target value takes into account an expected decline of approximately 1\% per day until a subsequent fetal transfusion. Transfusion is performed at the umbilical vein at either the placental insertion or inside the abdomen. Intraperitoneal transfusion is rarely performed and is contraindicated in the hydropic fetus because of poor absorption of blood. Corticosteroids for fetal maturation should be given before the procedure when FBS is performed after 24 weeks of gestation. Type O, Rh (D)-negative, cytomegalovirus-negative, washed, leukoreduced, irradiated packed RBCs cross-matched against maternal blood should be used. The blood usually contains $75 \%$ to $85 \%$ RBCs, allowing minimal blood volume for the transfusion (50). Transfusions are provided every 14 days until suppression of fetal erythropoiesis is documented; thereafter, the rate of declining hematocrit levels can be used to time further transfusions. Intrauterine transfusions are performed until 35 weeks of gestation, with delivery planned for 38 weeks of gestation (51).

The FBS is performed under continuous ultrasound guidance. Prophylactic antibiotics can be given, although no trial has evaluated their efficacy. Following 24 weeks of gestation, the procedure should be performed after alerting anesthesiology and in a location close to an operating room should an emergency occur. Tubing and syringes should be heparinized. Maternal skin can be anesthetized with 1\% lidocaine at the point of needle entry. A 20- or 22-gauge needle is typically used. After entering the umbilical vein, a sample of fetal blood is withdrawn and the hemoglobin concentration is immediately (within 1 or 2 minutes) determined. Fetal anemia is confirmed by a mean corpuscular volume $(\mathrm{MCV})>110 \mu \mathrm{m}^{3}$. If the hematocrit is below the fifth percentile (0.84 MoM) for gestational age, blood is transfused in a sterile fashion. A computer 
program is used to estimate the amount of blood to transfuse based on the initial fetal hematocrit, the EFW, and the concentration of the blood transfused (52,53). A final fetal blood sample is taken a few seconds after the transfusion has been completed. If the fetus is hydropic, it is better to perform another transfusion 3 to 5 days later to increase the hematocrit to the median value for the gestational age. At 24 weeks of gestation or later, the fetal heart rate should be monitored for 2 to 3 hours after the transfusion until fetal movements resume. The risk of fetal death is $1 \%$ to $2 \%$ per procedure, even with ultrasound guidance, expert operators, and accurate management (54).

Hematocrit decreases about $1 \%$ per day post-transfusion in the anemic alloimmunized fetus, and this knowledge helps to assess when to repeat the transfusion. If the fetus is nonhydropic, the second transfusion is often necessary 14 days after the first. After the third transfusion, longer intervals, such as 3 weeks, may be possible, as the fetal RBCs are replaced by donated RBCs. Following three transfusions, $99 \%$ of the fetal blood is replaced with the adult transfused blood. The value of a maternal dose of $30 \mathrm{mg}$ of phenobarbital three times per day for 7 to 10 days to enhance fetal liver maturity and the ability to conjugate bilirubin has yet to be confirmed by large studies (55).

\section{NEONATOLOGY MANAGEMENT}

Neonatology management of an infant with hemolytic disease of the newborn focuses on monitoring the level of anemia and hyperbilirubinemia. Symptoms of anemia, such as tachycardia, usually warrant $\mathrm{RBC}$ transfusions. In severe cases, exchange transfusions may be necessary to remove the maternal antibodies from the circulation of the infant. Hyperbilirubinemia clinically presents as jaundice. Bilirubin levels are monitored and controlled with phototherapy and IV hydration.

\section{REFERENCES}

1. Gold ER, Butler NR. ABO haemolytic disease of the newborn. Baltimore: The Williams and Wilkins Company, 1972.

2. Landsteiner K, Weiner AS. An agglutinable factor in human blood recognized by immune sera for Rhesus blood. Proc Soc Exp Biol Med 1940; 43: 223.

3. Levine P. Isoimmunization in pregnancy: its possible bearing on etiology of erythroblastosis fetalis. JAMA 1941; 116: 825.

4. Bevis DC. Blood pigments in haemolytic disease of the newborn. J Obstet Gynaecol Br Emp 1956; 63: 68-75.

5. Liley AW. Liquor amnil analysis in the management of the pregnancy complicated by resus sensitization. Am J Obstet Gynecol 1961; 82: 1359-70.

6. Queenan JT, Tomai TP, Ural SH, King JC. Deviation in amniotic fluid optical density at a wavelength of $450 \mathrm{~nm}$ in $\mathrm{Rh}$-immunized pregnancies from 14 to 40 weeks of gestation: a proposal for clinical management. Am J Obstet Gynecol 1993; 168: 1370-6.

7. Mari G, Deter RL, Carpenter RL, et al. Noninvasive diagnosis by Doppler ultrasonography of fetal anemia due to maternal red-cell alloimmunization. Collaborative Group for Doppler Assessment of the Blood Velocity in Anemic Fetuses. N Engl J Med 2000; 342: 9-14.

8. Liley AW. Intrauterine transfusion of foetus in haemolytic disease. Br Med J 1963; 2: 1107-9.

9. Casper MJ. The making of the unborn patient: a social anatomy of fetal surgery. New Brunswick, NJ: Rutgers University Press, 1998.

10. Adamsons K Jr, Freda VJ, James LS, Towell ME. Prenatal treatment of erythroblastosis fetalis following hysterotomy. Pediatrics 1965; 35: 848-55.

11. Asensio SH, Figueroa-Longo JG, Pelegrina IA. Intrauterine exchange transfusion. Am J Obstet Gynecol 1966; 95: 1129-33.

12. Valenti C. Antenatal detection of hemoglobinopathies. A preliminary report. Am J Obstet Gynecol 1973; 115: 851-3.

13. Rodeck CH, Campbell S. Sampling pure fetal blood by fetoscopy in second trimester of pregnancy. Br Med J 1978; 2: 728-30.
14. Rodeck CH, Kemp JR, Holman CA, et al. Direct intravascular fetal blood transfusion by fetoscopy in severe Rhesus isoimmunisation. Lancet 1981; 1: $625-7$.

15. Bang J, Bock JE, Trolle D. Ultrasound-guided fetal intravenous transfusion for severe rhesus haemolytic disease. Br Med J (Clin Res Ed) $1982 ; 284:$ 373-4.

16. Bowman JM, Pollock JM, Penston LE. Fetomaternal transplacental hemorrhage during pregnancy and after delivery. Vox Sang 1986; 51: 117-21.

17. Mari G, Hanif F, Drennan K. Hemolytic disease of the fetus/neonate. In: Berghella V, ed. Maternal-Fetal Evidence Based Guidelines. UK: Informa Healthcare, 2007: 327-36.

18. Hoffman R. Hematology: basic principles and practice, 5th edn. Philadelphia, PA: Churchill Livingston/Elsevier, 2009.

19. ACOG practice bulletin no. 75: management of alloimmunization. Obstet Gynecol 2006; 108: 457-64.

20. Weinstein L. Irregular antibodies causing hemolytic disease of the newborn: a continuing problem. Clin Obstet Gynecol 1982; 25: 321-32.

21. Hamilton BE, Martin JA, Ventura SJ, et al. Births: preliminary data for 2004. Natl Vital Stat Rep 2005; 54: 1-17.

22. Van den Veyver IB, Moise KJ Jr. Fetal RhD typing by polymerase chain reaction in pregnancies complicated by rhesus alloimmunization. Obstet Gynecol 1996; 88: 1061-7.

23. Moise KJ, Whitecar PW. Antenatal therapy for haemolytic disease of the fetus and newborn. In: Hadley A, Soothill P, eds. Alloimmune disorders in pregnancy: anaemia, thrombocytopenia and neutropenia in the fetus and newborn. Cambridge, UK: Cambridge University Press, 2002: 173-201.

24. Pertl B, Bianchi DW. Fetal DNA in maternal plasma: emerging clinical applications. Obstet Gynecol 2001; 98: 483-90.

25. Lo YM, Hjelm NM, Fidler C, et al. Prenatal diagnosis of fetal RhD status by molecular analysis of maternal plasma. N Engl J Med 1998; 339: 1734-8.

26. Gautier E, Benachi A, Giovangrandi Y, et al. Fetal RhD genotyping by maternal serum analysis: a two-year experience. Am J Obstet Gynecol 2005; 192: 666-9.

27. Finn R, Clarke CA, Donohoe WT, et al. Experimental studies on the prevention of Rh haemolytic disease. Br Med J 1961; 1: 1486-90.

28. Freda VJ, Gorman JG, Pollack W. Successful prevention of experimental $\mathrm{Rh}$ sensitization in man with an anti-Rh gamma2-globulin antibody preparation: a preliminary report. Transfusion 1964; 4: 26-32.

29. Zipursky A, Israels LG. The pathogenesis and prevention of Rh immunization. Can Med Assoc J 1967; 97: 1245-57.

30. Stedman CM, Baudin JC, White CA, Cooper ES. Use of the erythrocyte rosette test to screen for excessive fetomaternal hemorrhage in Rh-negative women. Am J Obstet Gynecol 1986; 154: 1363-9.

31. Ness PM, Baldwin ML, Niebyl JR. Clinical high-risk designation does not predict excess fetal-maternal hemorrhage. Am J Obstet Gynecol 1987; 156: 154-8.

32. Bowman JM. Suppression of Rh isoimmunization. A review. Obstet Gynecol 1978; 52: 385-93.

33. Davey M. The prevention of rhesus-isoimmunization. Clin Obstet Gynaecol 1979; 6: 509-30.

34. Siberil S, de Romeuf C, Bihoreau N, et al. Selection of a human anti-RhD monoclonal antibody for therapeutic use: impact of IgG glycosylation on activating and inhibitory Fc gamma R functions. Clin Immunol 2006; 118: 170-9.

35. Hughes RG, Craig JI, Murphy WG, Greer IA. Causes and clinical consequences of Rhesus (D) haemolytic disease of the newborn: a study of a Scottish population, 1985-1990. Br J Obstet Gynaecol 1994; 101: 297-300.

36. Cherif-Zahar B, Mattei MG, Le Van Kim C, et al. Localization of the human Rh blood group gene structure to chromosome region $1 \mathrm{p} 34.3$ 1p36.1 by in situ hybridization. Hum Genet 1991; 86: 398-400.

37. Carritt B, Kemp TJ, Poulter M. Evolution of the human RH (rhesus) blood group genes: a 50 year old prediction (partially) fulfilled. Hum Mol Genet 1997; 6: 843-50.

38. Bowman JM. The prevention of Rh immunization. Transfus Med Rev 1988; 2: 129-50.

39. Avent N. Fetal genotyping. Cambridge, UK: Cambridge University Press, 2002.

40. Bowman JM. Controversies in Rh prophylaxis. Who needs Rh immune globulin and when should it be given? Am J Obstet Gynecol 1985; 151: 289-94. 
41. Pirelli K, Pietz B, Johnson S, et al. Molecular determination of RhD zygosity. Am J Obstet Gynecol 2006; 195: S172.

42. Oepkes D, Seaward PG, Vandenbussche FP, et al. Doppler ultrasonography versus amniocentesis to predict fetal anemia. N Engl J Med 2006; 355: $156-64$.

43. Divakaran TG, Waugh J, Clark TJ, et al. Noninvasive techniques to detect fetal anemia due to red blood cell alloimmunization: a systematic review. Obstet Gynecol 2001; 98: 509-17.

44. Abel DE, Grambow SC, Brancazio LR, Hertzberg BS. Ultrasound assessment of the fetal middle cerebral artery peak systolic velocity: a comparison of the near-field versus far-field vessel. Am J Obstet Gynecol 2003; 189: 986-9.

45. Mari G, Abuhamad AZ, Cosmi E, et al. Middle cerebral artery peak systolic velocity: technique and variability. J Ultrasound Med 2005; 24: 425-30.

46. Nicolaides $\mathrm{KH}$, Rodeck $\mathrm{CH}$. Maternal serum anti-D antibody concentration and assessment of rhesus isoimmunisation. BMJ 1992; 304: $1155-6$

47. Soothill PW, Nicolaides $\mathrm{KH}$, Rodeck $\mathrm{CH}$, et al. Relationship of fetal hemoglobin and oxygen content to lactate concentration in $\mathrm{Rh}$ isoimmunized pregnancies. Obstet Gynecol 1987; 69: 268-71.
48. Geifman-Holtzman O, Grotegut CA, Gaughan JP. Diagnostic accuracy of noninvasive fetal $\mathrm{Rh}$ genotyping from maternal blood-a meta-analysis. Am J Obstet Gynecol 2006; 195: 1163-73.

49. Giannina G, Moise KJ Jr, Dorman K. A simple method to estimate volume for fetal intravascular transfusions. Fetal Diagn Ther 1998; 13: 94-7.

50. Scheier M, Hernandez-Andrade E, Fonseca EB, Nicolaides KH. Prediction of severe fetal anemia in red blood cell alloimmunization after previous intrauterine transfusions. Am J Obstet Gynecol 2006; 195: 1550-6.

51. Moise KJ Jr. Management of rhesus alloimmunization in pregnancy. Obstet Gynecol 2008; 112: 164-76.

52. Klumper FJ, van Kamp IL, Vandenbussche FP, et al. Benefits and risks of fetal red-cell transfusion after 32 weeks gestation. Eur J Obstet Gynecol Reprod Biol 2000; 92: 91-6.

53. Howe DT, Michailidis GD. Intraperitoneal transfusion in severe, earlyonset Rh isoimmunization. Obstet Gynecol 2007; 110: 880-4.

54. Oepkes D, Adama van Scheltema P. Intrauterine fetal transfusions in the management of fetal anemia and fetal thrombocytopenia. Semin Fetal Neonatal Med 2007; 12: 432-8.

55. Ruma MS, Moise KJ Jr, Kim E, et al. Combined plasmapheresis and intravenous immune globulin for the treatment of severe maternal red cell alloimmunization. Am J Obstet Gynecol 2007; 196: 138 e1-6. 


\section{Fetal alloimmune thrombocytopenia Hung N. Winn}

\section{PATHOGENESIS}

Alloimmune thrombocytopenia (AIT) is a syndrome that develops as a result of maternal sensitization to fetal platelet antigens. These women who themselves lack the specific antigen produce immunoglobulin $\mathrm{G}(\mathrm{IgG})$ antibodies directed against the platelet antigen that the fetuses inherits from their father. The antibodies are then transported across the placenta to the fetal circulation where they result in the destruction of the fetal platelets. Thus, the pathogenesis of AIT is analogous to that of hemolytic $(\mathrm{Rh})$ disease of the newborn. However, unlike $\mathrm{Rh}$ disease, prior sensitization to the platelet antigen is not necessary since approximately half of the clinically evident cases of AIT occur in the first pregnancies. This suggests that either the fetal platelets gain access to the maternal circulation more easily than red blood cells, and/or the platelet antigens are highly provocative and therefore stimulate production of IgG antibodies during the first pregnancy (1). In a family with a previously affected infant, the rate of recurrence with an antigen-positive fetus is estimated to be at least $90 \%(2,3)$. The recurrence risk of neonatal intracranial hemorrhage $(\mathrm{ICH})$ in a subsequent pregnancy after having a child with ICH due to AIT is about $77 \%$ (4). Additionally, it has been a uniform finding that the severity of thrombocytopenia in subsequent infants is either the same or worse than that of the first affected child.

\section{PLATELET SEROLOGY}

Platelets possess specific alloantigens that are expressed on the surface membrane glycoproteins. There are five major platelet alloantigen systems, all with biallelic polymorphism and inherited as codominant traits (5). The difference in alleles results from a single base substitution in the glycoprotein deoxyribonucleic acid (DNA) sequence, which ultimately results in a protein recognized as different from its allele (6). The most common antigen associated with AIT is HPA-1A, accounting for approximately $80 \%$ of reported cases (7). Other platelet antigen systems involved in AIT are shown in Table 1. The frequency of phenotypes and genotypes for each antigen differs in different ethnic groups.

\section{INCIDENCE/GENETIC ASPECTS OF MATERNAL IMMUNE RESPONSE}

Based on prospective analysis, the incidence of AIT is estimated to be between 1 per 1000 and 1 per 2000 births (7-9). The expected incidence in Caucasians based on the $98 \%$ frequency of the HPA-A1 allele is 1 to 2 per 100 births. This discrepancy between expected and observed incidence suggests that additional factors contribute to the maternal immune response. The risk of sensitization in HPAA1 negative women is correlated with the presence of specific maternal human leukocyte antigen (HLA)-associated immune response genes, namely HLA-DR3, and more specifically
HLA-DRW52 (10). The immune response markers are contributory, but not sufficient for the development of alloimmunization, as not all HPA-A1 negative women with an HLA-DRW52 haplotype will deliver thrombocytopenic infants (11). The role of immune responsiveness for other platelet antigens has not yet been established.

\section{CLINICAL PRESENTATION}

In typical cases of AIT, the mother is healthy (without thrombocytopenia) and her pregnancy, labor, and delivery are uncomplicated. The neonates who are otherwise normal present with symptomatic thrombocytopenia within several hours of birth. Affected infants often develop a generalized distribution of petechiae, as well as ecchymoses over the presenting fetal part. Visceral bleeding and bleeding during circumcision or venipuncture may be present. Of greatest consequence is the risk of $\mathrm{ICH}$, occurring in up to $30 \%$ of cases (3) and responsible for the $6.5 \%$ mortality rate (12) and the significant long-term neurologic sequelae, including hydrocephaly and porencephaly. Laboratory data are remarkable for an isolated and often severe thrombocytopenia, with normal or increased numbers of megakaryocytes found on bone marrow aspirate. The hematocrit may be decreased if significant bleeding has occurred. A definitive diagnosis can be made by phenotyping parental platelets and demonstrating antiplatelet antibodies in the maternal serum, which are specific for the antigen incompatibility identified.

Neonatal AIT is usually a self-limiting disorder, often resolving over the course of several weeks, as maternal antiplatelet antibodies disappear from the neonatal circulation. The most serious and feared complication is ICH, and until recently it had been believed that the greatest risk of ICH was at the time of labor and delivery. Hence, obstetric management had focused on the peripartum period and elective cesarean section at term was advocated $(13,14)$. In the neonatal period, the goal was to replenish the neonate's circulating platelets in order to prevent complications secondary to bleeding. These infants should receive compatible antigen-negative platelets from either the mother or compatible donors (15). If the mother's platelets are used, they must be carefully washed in order to avoid introducing additional antiplatelet antibodies into the neonatal circulation. High-dose intravenous immunoglobulin therapy $(1-2 \mathrm{~g} / \mathrm{kg}$ over $1-5$ days) may provide an additional or alternative therapy $(16,17)$ especially when matched platelets are unavailable.

\section{EVIDENCE OF ANTENATAL ICH}

These approaches, however, have no impact on fetal bleeding in utero prior to the onset of labor. In 1979, Zalneratis et al. reported two cases of AIT in which there was good evidence that ICH had occurred in utero (18). Both infants had enlarged 
Table 1 Platelet-Specific Alloantigen Systems Involved in Alloimmune Thrombocytopenia

\begin{tabular}{|c|c|c|c|}
\hline \multicolumn{2}{|c|}{ Nomenclature } & \multirow[t]{2}{*}{ Platelet glycoprotein (GP) } & \multirow[t]{2}{*}{ Alleles } \\
\hline New & Old & & \\
\hline HPA-1 & PL, Zw & GP IIIa & HPA-1a, $1 b$ \\
\hline HPA-2 & Ko & GP Ib & HPA-2a, $2 b$ \\
\hline HPA-3 & Bak & GP IIb & HPA-3a, $3 b$ \\
\hline HPA-4 & Pen, Yuk & GP IIIa & HPA- $4 a, 4 b$ \\
\hline HPA-5 & $\mathrm{Br}$ & GP Ia & HPA-5a, $5 b$ \\
\hline
\end{tabular}

heads $(>97 \%)$ at birth. The first had bifrontal and left posterior fossa cysts with obstructive hydrocephalus, and the second had a large right parieto-occipital hematoma followed by the development of hydrocephaly and a porencephalic cyst in the same location. Examination of the cyst contents obtained at the time of shunt placement revealed fluid filled with hemosiderin-laden macrophages in the first case and liquefied, "rusty" fluid in the second. The pathologic findings placed the time of bleeding at several months, and 1 to 2 weeks prior to delivery in these two cases, respectively.

Stronger confirmation that AIT could be responsible for cerebral hemorrhage in utero was presented in a series of cases where the diagnosis of porencephaly was made with ultrasound examination in the third trimester in fetuses suspected, and later confirmed, to have this disorder (19).

It is now known that up to $80.5 \%$ of ICHs secondary to AIT occur in utero (20). Fetal platelet antigens may be expressed as early as 13 weeks of gestation and $\mathrm{ICH}$ may be present by 16 weeks of gestation (21). Hence, in order to be maximally effective, the treatment of AIT must begin in utero.

\section{ANTENATAL MANAGEMENT}

Since routine screening for fetomaternal platelet incompatibility is not performed, identifying those fetuses at risk for AIT almost always consists of obtaining the history of a previously affected sibling or other positive family history. The risk to future pregnancies depends on the zygosity of the fetus's father. When the father is a heterozygote for the alloantigen, each fetus has a $50 \%$ chance of inheriting the antigen. In these cases, DNA-based testing of fetal amniocytes or chorionic villi has enabled typing of fetal platelet antigens $(6,10)$. Those fetuses who are antigen negative can be managed routinely, while those possessing the antigen are managed aggressively.

The optimal antenatal management of AIT is still debated in the literature. One approach consists of serial fetal platelet transfusions with antigen-negative platelets $(22,23)$. Evaluation of the fetal platelet counts before and after these procedures reveals that platelet transfusions are effective. However, the platelet count remains normal for less than 10 days, and hence platelet transfusions must be administered weekly. This approach subjects the pregnancy to the risk of fetal loss due to repetitive invasive procedures, which is especially significant since fetuses with AIT are at increased risk for fetal exsanguination associated with cordocentesis (24). The estimated risk for fetal loss from fetal transfusion of platelets through cordocentesis is about $6 \%$ and depends on the operator's experiences (25). A less invasive alternative involves the use of maternally administered intravenous gamma globulin (IVIG). In the pilot study of 18 patients (26), IVIG with and without steroid therapy was found to significantly increase the fetal platelet count and decrease the risk of $\mathrm{ICH}$. No cases of ICH occurred in that series, in contrast to $10 / 21$ (48\%) in untreated siblings.

A large randomized trial was designed to determine whether the addition of daily low-dose steroid therapy (1.5-mg dexamethasone) to IVIG ( $1 \mathrm{gm} / \mathrm{kg} /$ week) was more efficacious than IVIG alone in preventing severe thrombocytopenia and ICH in the antenatal management of AIT (3). Of the 54 women treated, the majority responded to therapy with success rates between $62 \%$ and $85 \%$, depending on the definition of response. The addition of low-dose dexamethasone was not found to increase the effectiveness of IVIG alone. In this series, there were no cases of ICH compared with 10 previously affected siblings, and there were no differences in response when steroids were used concomitantly. Ten initial nonresponders were entered into the "salvage arm," which consisted of $60 \mathrm{mg}$ of prednisone daily in addition to IVIG. Half of these patients responded with an increased platelet count.

The largest prospective study of antenatal treatment of 33 pregnant patients (37 fetuses) who had AIT and previous siblings with ICH demonstrated good neonatal outcomes with stratifying patients according to the timing of the previous child's ICH (4). The patients were classified as extremely high risk (EHR), very high risk (VHR), and high risk (HR) if ICH in the previous child occurred in the second trimester (less than 28 weeks of gestation), the third trimester (28-36 weeks of gestation), or during the perinatal period, respectively. The patients received primary treatment with intravenous immunoglobulin (IVIG) and salvage therapy with prednisone and/or more intravenous immunoglobulin. Only 5 of 37 fetuses developed ICH: (i) two had platelet counts greater than $100,000 / \mu \mathrm{L}$, (ii) one had grade I ICH, and (iii) two had grade III-IV ICH and were demised. The investigators recommended the following guidelines in treating patients with AIT and ICH in a previous child. First, the treatment should be started no later than 12 weeks of gestation. Second, fetal blood sampling should be delayed until 32 weeks of gestation to minimize the complications from the procedure. Third, the fetal platelet counts obtained from the fetal blood sampling would dictate the need for additional salvage therapy (prednisone or additional IVIG) or delivery. The therapeutic regimen includes (i) intravenous IgG (IVIG) $2 \mathrm{~g} / \mathrm{kg} / \mathrm{wk}$ for the EHR group or $1 \mathrm{~g} / \mathrm{kg} /$ wk for the VHR and HR groups starting at 12 weeks of gestation plus (ii) prednisone $1 \mathrm{mg} / \mathrm{kg} /$ day starting at 24 to 26 weeks of gestation for the EHR group or prednisone $1 \mathrm{mg} / \mathrm{kg} /$ day or additional IVIG $1 \mathrm{~g} / \mathrm{kg} /$ wk starting at 20 weeks of gestation for the VHR and HR groups (Table 2).

While these findings are quite promising, there have been reports of unsuccessful response (i.e., ICH in utero) to antenatal IVIG therapy $(4,27,28)$. This could be due to an inconsistent increase in fetal platelets to IVIG infusion despite of a significant increase in maternal and fetal IgG levels (29). The knowledge that (i) not all fetuses will respond to IVIG therapy and (ii) salvage therapy (high-dose prednisone, additional IVIG, or platelet transfusions) is available underscores the need to assess response to therapy with fetal blood sampling to obtain fetal platelet counts. Since fetuses with AIT are at increased risk for 
Table 2 Treatment Based on Risk Stratification

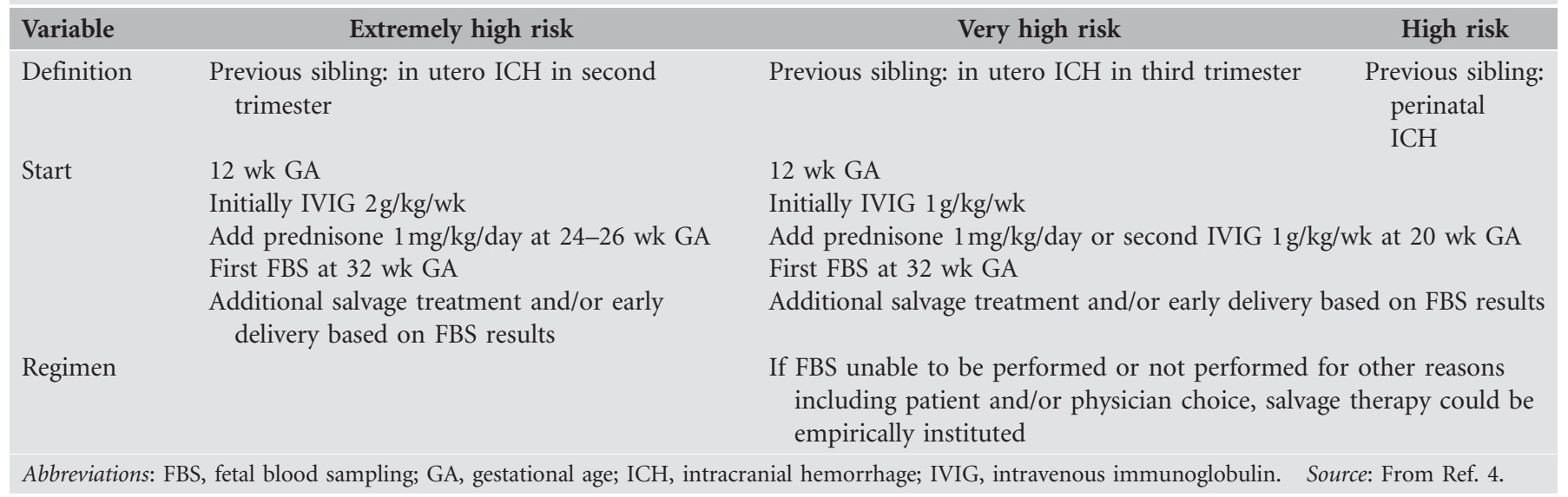

fatal bleeding at the time of cordocentesis $(24,25)$, it seems prudent to have compatible platelets available and to transfuse if the platelet count is found to be $<50,000 / \mu \mathrm{L}$.

\section{CONCLUSIONS}

At present, the prospective diagnosis of a fetus affected with AIT relies almost exclusively on the history of a previously affected sibling or other positive family history. Since this disorder can potentially result in death or permanent neurologic sequelae as a result of $\mathrm{ICH}$, the concept of routine screening of pregnant women to detect feto-maternal platelet incompatibility has been proposed. There are limitations to routine antenatal screening. All affected pregnancies may not demonstrate detectable anti-HPA-A1 antibodies and conversely the presence of anti-HPA-A1 antibodies does not invariably predict fetal thrombocytopenia. In addition, not all fetuses with AIT will develop ICH. Fetuses at greatest risk include those with severe thrombocytopenia and those with a previously affected sibling with ICH. Antenatal therapy needs to be directed at this small group of fetuses at risk for ICH in utero. A recent systemic review of the literature and analysis suggested that screening pregnant women for AIT would be cost-effective with a saving of at least 9 million euros per year in Netherlands (30). The analysis was based on i) the incidence of severe fetal AIT being about 40/100,000 pregnancies; ii) the incidence of severe ICH being about 4 children/100,000 pregnancies; and iii) including the costs of initial HPA typing, antibody detection, maternal treatment, and care for the neurologically impaired children.

The optimal antenatal management for AIT remains to be determined. Serial platelet transfusions are efficacious, but necessitate weekly invasive procedures with the additive risks of pregnancy loss. Medical management with maternally administered intravenous gamma globulin appears to be effective in the majority of, but not all, cases. The mechanism(s) of action of IVIG in preventing severe thrombocytopenia is unknown but may reflect i) decreased maternal antibody production, ii) antibody Fc receptor blockade in the placenta, and/or iii) antibody Fc receptor blockade in the fetal reticuloendothelial system. The mechanism by which IVIG prevents ICH in cases where it does not prevent severe thrombocytopenia has also not been elucidated. It is interesting to note that the HPA-A1 antigen is expressed on human endothelial cells as well as platelets. Hence, it has been proposed that HPA-A1 antibodies may damage these cells resulting in vascular injury (24) and predisposition to intracranial bleeding. IVIG may interfere with this endothelial cell-antiplatelet antibody interaction. Lastly, therapy should be initiated as early as 12 weeks of gestation given the fact that severe thrombocytopenia has been documented as early as 13 weeks of gestation.

\section{REFERENCES}

1. Shulman NR, Jordon VJ. Platelet immunology. In: Coleman RW, Hirsch J, Marder VJ, et al., eds. Hemo-stasis and Thrombosis. Philadelphia: JB Lippincott, 1982: 274-342.

2. Kaplan C, Daffos F, Forestier F, et al. Management of alloimmune thrombocytopenia: antenatal diagnosis and in utero transfusion of maternal platelets. Blood 1988; 72: 340-3.

3. Bussel JB, Berkowitz RL, Lynch L, et al. Antenatal management of alloimmune thrombocytopenia with intravenous gamma globulin: a randomized trial of the addition of low dose steroid to gamma globulin. Am J Obstet Gynecol 1996; 174: 1414-23.

4. Bussel JB, Berkowitz RL, Hung C, et al. Intracranial hemorrhage in alloimmune thrombocytopenia: stratified management to prevent recurrence in the subsequent affected fetus. Am J Obstet Gynecol 2010; 203: 135.e1-14.

5. Menell JS, Bussel JB. Antenatal management of the thrombocytopenias. Clin Perinatol 1994; 21: 591-614.

6. Khouzami A, Kickler TS, Bray PF, et al. Molecular genotyping of fetal platelet antigens with uncultured amniocytes. Am J Obstet Gynecol 1995; 173: 1202-6.

7. Mueller-Eckhardt C, Kiefel V, Grubert A, et al. 348 cases of suspected neonatal alloimmune thrombocytopenia. Lancet 1989; 1: 363-6.

8. Blanchette V, Chen L, Defreidberg Z, et al. Alloimmunization to the PLA1 platelet antigen: results of a prospective study. Br J Haematol 1990; 74: 209-15.

9. Williamson LM, Hackett G, Rennie J, et al. The natural history of fetomaternal alloimmunization to the platelet-specific antigen HPA-1a (PlA1, Zwa) as determined by antenatal screening. Blood 1998; 92: 2280-7.

10. Valentin N, Vergracht A, Bignon J, et al. HLA-DRW52 is involved in alloimmunization against PL-A1 antigen. Hum Immunol 1990; 27: 73-9.

11. Panzer S, Auerbach L, Cechova E, et al. Maternal alloimmunization against fetal platelet antigens: a prospective study. Br J Haematol 1995; 90: 655-60.

12. Murphy MF, Metcalfe P, Waters AH. Antenatal management of severe fetomaternal alloimmune thrombocytopenia: HLA incompatibility may affect responses to fetal platelet transfusions. Blood 1993; 81: 2174-9.

13. Mennuti M, Schwarz RH, Gill F. Obstetric management of isoimmune thrombocytopenia. Am J Obstet Gynecol 1974; 118: 565-6.

14. Sitarz AL, Driscoll JM, Wolff JA. Management of isoimmune neonatal thrombocytopenia. Am J Obstet Gynecol 1976; 124: 39-42. 
15. Adner MM, Fisch GR, Starobin SG, et al. Use of "compatible” platelet transfusions in treatment of congenital isoimmune thrombocytopenic purpura. N Engl J Med 1969; 280: 244-7.

16. Sidiropoulos D, Straume B. The treatment of neonatal isoimmune thrombocytopenia with intravenous immunoglobulin. Blut 1984; 48: 383-6.

17. Derycke M, Dreyfus M, Roper JC, et al. Intravenous immunoglobulin for neonatal isoimmune thrombocytopenia. Arch Dis Child 1985; 50: 667-9.

18. Zalneratis EL, Young RKS, Krishnamoorthy KS. Intracranial hemorrhage in utero as a complication of isoimmune thrombocytopenia. J Pediatr 1979; 95: 611-14.

19. Herman JH, Jumbelic MI, Ancona RJ, et al. In utero cerebral hemorrhage in alloimmune thrombocytopenia. Am J Pediatr Hematol Oncol 1986; 8: 312-17.

20. Spencer JA, Burrows RF. Feto-maternal alloimmune thrombocytopenia: a literature review and statistical analysis. Aust NZ J Obstet Gynecol 2001; 41: 45-55.

21. Johnson JM, MacFarland JG, Blanchette VS, et al. Prenatal diagnosis of neonatal alloimmune thrombocytopenia using an allele specific oligonucleotide probe. Prenat Diagn 1993; 13: 1037-42.

22. Nicolini U, Rodeck $\mathrm{CH}$, Kichenour $\mathrm{NK}$, et al. In utero platelet transfusions for alloimmune thrombocytopenia. Lancet 1988; 2: 506.
23. Murphy MF, Pullon HW, Metcalfe P, et al. Management of fetal alloimmune thrombocytopenia by weekly in utero platelet transfusions. Vox Sang 1990; 58: 45-9.

24. Paidas MJ, Berkowitz RL, Lynch L, et al. Alloimmune thrombocytopenia: fetal and neonatal losses related to cordocentesis. Am J Obstet Gynecol 1995; 172: 475-9.

25. Overton TG, Duncan KR, Jolly M, et al. Serial aggressive platelet transfusion for fetal alloimmune thrombocytopenia: platelet dynamics and perinatal outcome. Am J Obstet Gynecol 2002; 186: 826-31.

26. Lynch L, Bussel JB, McFarland JG, et al. Antenatal treatment of alloimmune thrombocytopenia. Obstet Gynecol 1992; 80: 67-71.

27. Kroll H, Kiefel V, Giers G, et al. Maternal intravenous immunoglobulin treatment does not prevent intracranial hemorrhage in fetal alloimmune thrombocytopenia. Transfusion Med 1994; 4: 293-6.

28. Bussel JB, Zabusky MR, Berkowitz RL, et al. Fetal alloimmune thrombocytopenia. N Engl J Med 1997; 337: 22-6.

29. Giers G, Wenzel F, Stockschläder $M$, et al. Fetal alloimmune thrombocytopenia and maternal intravenous immunoglobulin infusion. Haematologica 2010; 95: 1921-6.

30. Kamphuis M, Paridaans N, Porcelijin L, et al. Screening in pregnancy for fetal or neonatal alloimmune thrombocytopenia: systematic review. BJOG 2010; 117: 1335-43. 


\section{Fetal arrhythmias Júlia Hajdú, Valéria Váradi, and Zoltán Papp}

Fetal rhythm abnormalities occur in $2 \%$ of pregnancies. They are usually identified by the obstetrician or midwifes after 20 weeks.

In avian embryos, a pacemaker is first established in the inflow tract at the straight heart tube stage. When the embryonic heart first begins to contract, it has no specialized conduction system. It is thought that the cardiac conduction system is derived from independent parent cells linked together to establish the integrated conduction system of the heart. Terminal differentiation of conduction tissue occurs late in development $(1,2)$. By 6 weeks postconception, atrioventricular (AV) synchrony can be demonstrated using standard Doppler techniques. The heart rate is $150 \mathrm{bpm}$ by 14 weeks; by 20 weeks it is $140 \mathrm{bpm}$ with a gradual decrease to $130 \mathrm{bpm}$ by term (3).

There are different methods used to assess fetal arrhythmias: scalp electrodes attached to electrocardiographic recordings, fetal magnetocardiography (FMCG), fetal electrocardiographic recordings from the maternal abdomen, and fetal echocardiography (M-mode, pulsed Doppler, tissue Doppler).

The usefulness of the invasive method, scalp electrodes attached to electrocardiographic recordings, is limited because it is possible to use only at ruptured membranes. An FMCG provides a noninvasive means of analyzing complex tachyarrhythmia in utero, with efficacy approaching that of postnatal electrocardiographic rhythm monitoring (4-6). The disadvantage of FMCG is that it is expensive.

The fetal electrocardiographic recordings from the maternal abdomen are not performed routinely because of the limited quality of the surface fetal electrocardiographic signal. With newer equipment it was found that electrical PR interval was better than mechanical PR interval at differentiating between normal and prolonged PR in the fetus, and the reproducibility of the PR interval using noninvasive fetal electrocardiography was significantly better than that of the Doppler methods (7-9).

In the fetal heart, electrical activity can be inferred from the timing of the mechanical events. With M-mode method, the simultaneous recording of the movement of the right atrial wall or the opening and closing of the AV valves and of the ventricular wall or the opening and closing of the aortic valves make it possible to determine the relationship of the atrial and ventricular electric activity. The fetal M-mode echocardiogram has its limitations: inaccurate measurement of the fetal AV conduction time, the loss of clear markers of atrial and ventricular contractions in hydropic fetuses with hypocontractile myocardium, and the poor picture resolution related to factors such as maternal obesity, fetal position, or polyhydramnios.

The Doppler method was found to be superior compared with the M-mode approach when measuring fetal AV time intervals. There are different places where it is possible to measure mechanical PR interval. The MV-Ao Doppler method is recorded in five-chamber view with the septum in a vertical position, velocities generated by both inflow of blood through the mitral valve $(\mathrm{MV})$ during diastole and by the outflow through the left ventricular outlet (Ao) during systole. The PR interval is measured from the intersection of the mitral $\mathrm{E}$ and A waves to the beginning of the ventricular ejection wave (Fig. 1). With simultaneous recording of superior vena cava (SVC) and ascending aorta (AA) flow velocity waveforms (Fig. 2), pulsed-Doppler sample volume is adjusted to encompass both vessels. The AV time interval is measured from the beginning of the retrograde venous $A$ wave to the beginning of the aortic ejection wave (9-13). A PR interval of $>150 \mathrm{msec}$ on Doppler, FMCG, or postnatal ECG has been determined to be prolonged. PR intervals can vary in the fetus as they do in neonates or that at birth; changes in AV conduction or autonomic nervous system activity alter the PR interval. In cases with AV time prolongation, the SVC-Ao method seemed superior. Higher heart rates have been demonstrated to result in increasing fusion of the $\mathrm{E}$ and $\mathrm{A}$ waves in the mitral valve, overlapping the beginning of the A wave and leading to an artifactual shortening of the AV time interval. As the PR interval gets longer with no associated decrease in heart rate, the A wave will have an earlier start in relation to the $\mathrm{E}$ wave, in turn causing increasing overlapping of the E wave and an artifactual shortening of the A wave (14).

The newer technique of tissue-velocity Doppler imaging may be helpful when evaluating the fetus with borderline findings and negative maternal anti-Ro/anti-La antibodies (15). With tissue Doppler method, the best correlation was found between postnatal PR time and prenatal PR time with measurement of right ventricular Aa-IV (atrial contractionisovolumetric contraction) interval $(16,17)$.

The most common fetal rhythm anomaly is the irregular rhythm. The causes of irregular rhythm could be most often premature atrial contractions (PACs), premature ventricular contractions, or tachyarrhythmias with AV block.

Extrasystoles are most often "PACs," which are usually identified in third-trimester fetuses, and their frequency may be highly variable. They may be conducted (Fig. 3) or blocked (Fig. 4) at the AV node. These are usually benign, resolving just before or shortly after birth. The follow-up is necessary because some $(1-3 \%)$ of the affected fetuses have intermittent runs of supraventricular tachycardia (SVT). PACs identified with cardiomegaly, evidence of ventricular systolic dysfunction, AV valve regurgitation, and/or hydrops could be signs of a temporary SVT. Control of the foetal heart rate on each third day, weekly or biweekly obstetric ultrasound assessment is warranted until they have resolved or until delivery. About 2.5\% of affected fetuses have associated structural heart defect-lesions associated with significant mitral or tricuspid 


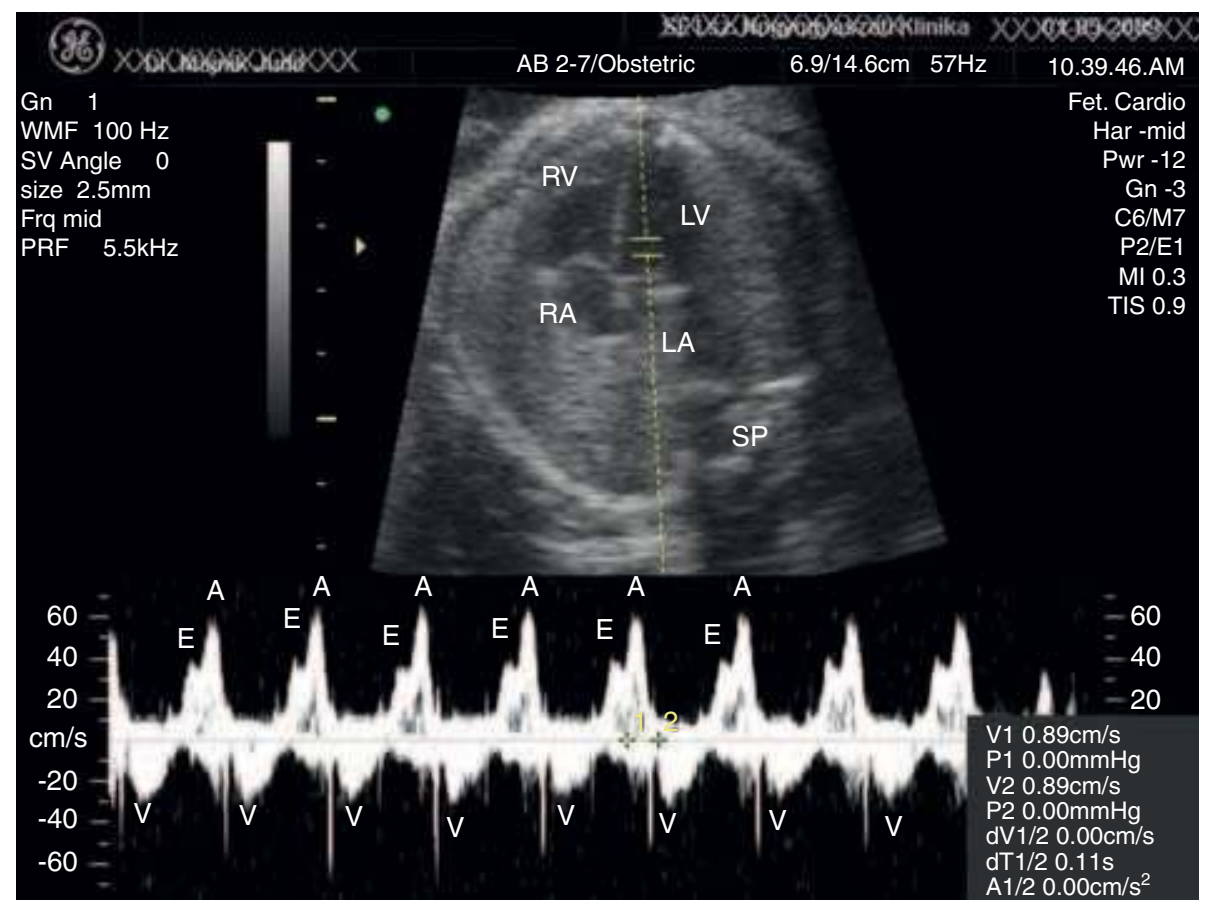

Figure $1 \mathrm{MV}$-Ao Doppler method to measure mechanical PR interval from the intersection of the mitral $\mathrm{E}$ and A waves to the beginning of the ventricular ejection wave. Abbreviations: E, E wave; A, A wave; V, aortic ejection wave; RV, right ventricle; LV, left ventricle; LA, left atrium; RA, right atrium; MV, mitral valve; mPR: 0.11."

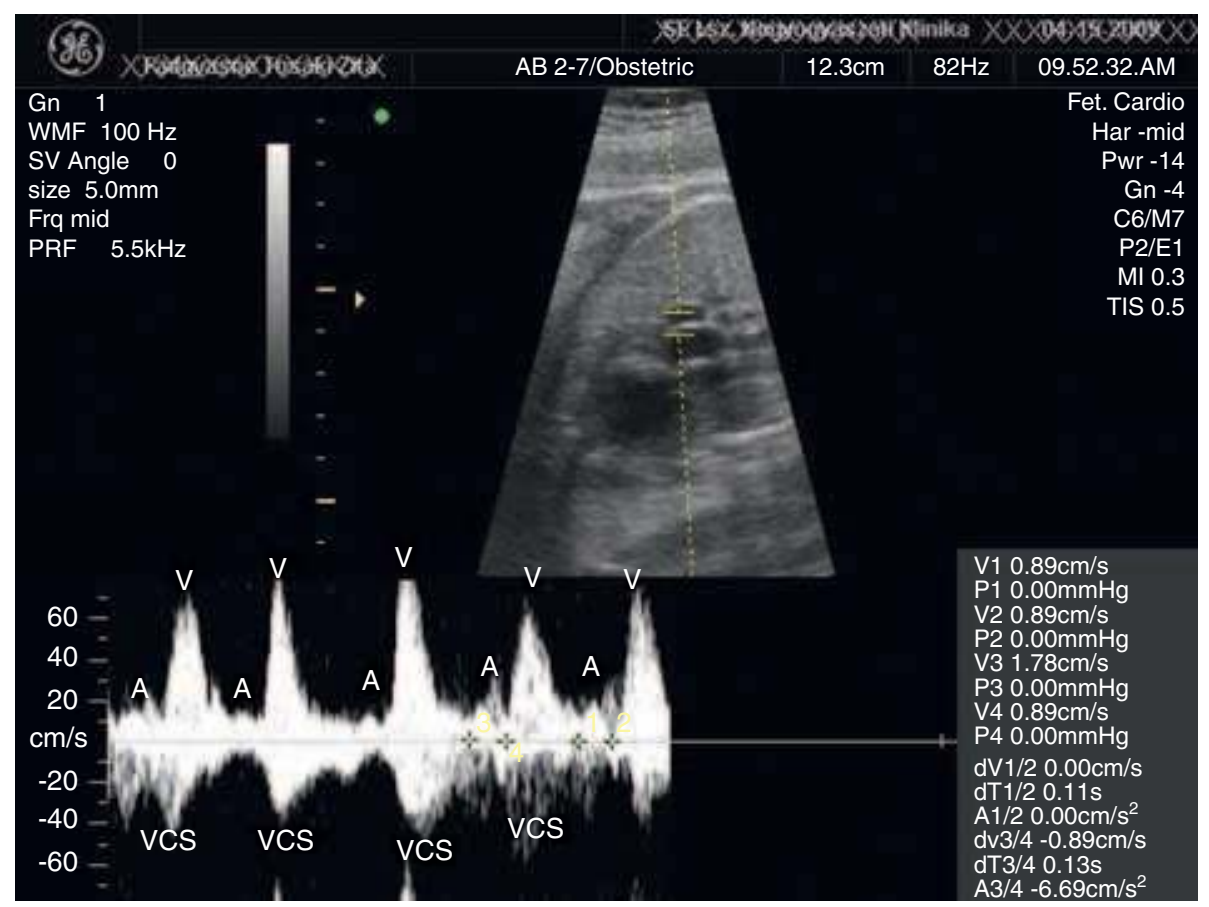

Figure 2 AA/SVC Doppler method. Abbreviations: A, A wave in the superior vena cava, retrograde wave from the right atrium; V, aortic flow; SVC, blood flow in the superior vena cava. mPR: 0.11 and mPR: 0.13 .

insufficiency, atrial dilatation, intracardiac tumors (Fig. 5), and aneurysm like dilation of the foramen ovale flap (Fig. 6). In such fetuses, the minor hemodynamic alterations that occur with the extrasystoles may not be tolerated.

Premature ventricular contractions are rare (Fig. 7). They are usually benign. It is necessary to rule out myocardial disease or intracardiac tumors. Prenatal and postnatal follow-up is always indicated to confirm the nature of the ectopy and exclude intermittent runs.
Tachycardia is defined as a fetal heart rate that exceeds $180 \mathrm{bpm}$. The origin of the tachycardia could be ventricular or supraventricular.

Ventricular tachycardia is rare during fetal life. With echocardiography, in the setting of fetal tachycardia, the findings of $\mathrm{AV}$ dissociation with a ventricular rate faster than the atrial rate suggest ventricular tachycardia (18). If there is 1:1 retrograde conduction, it is impossible to distinguish between ventricular and supraventricular tachycardias. 


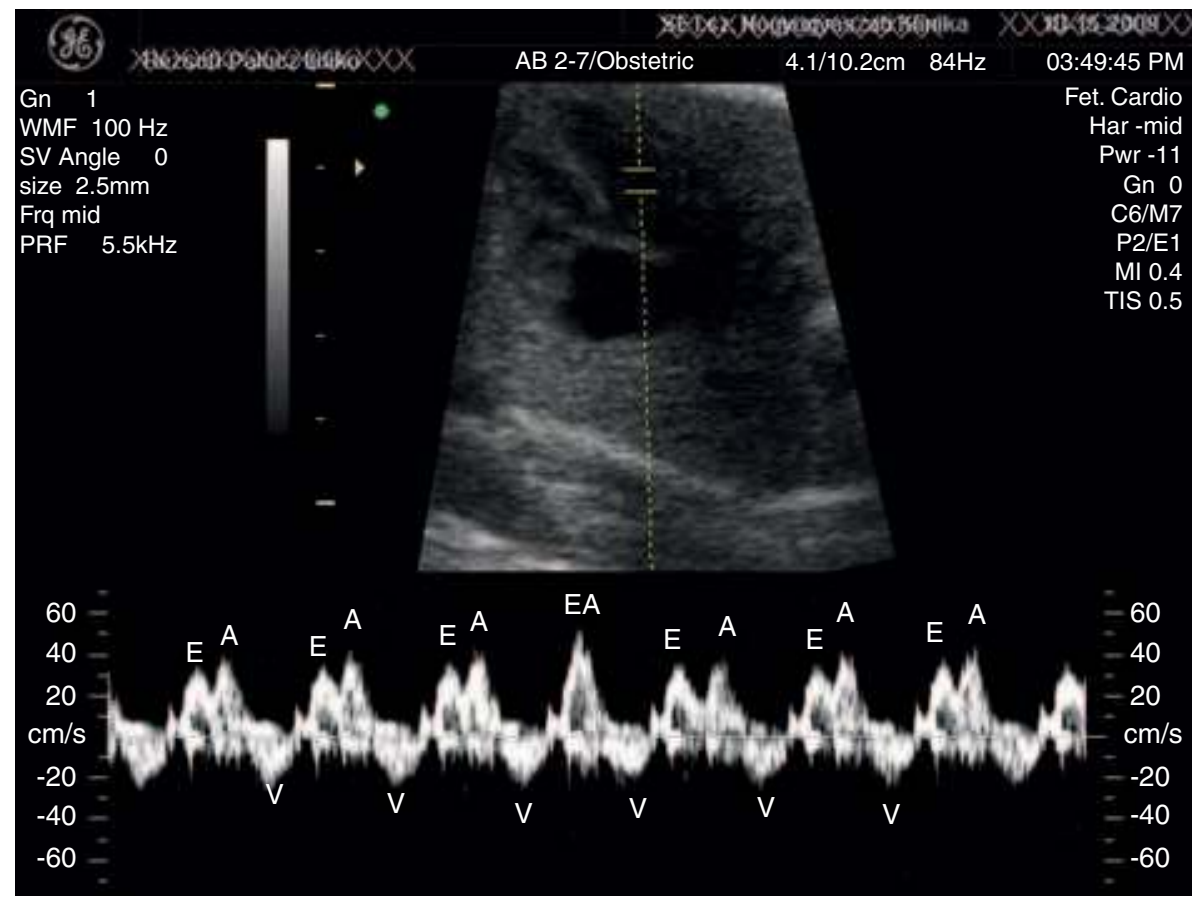

Figure 3 Conducted premature atrial beat, Doppler method. Abbreviations: E, E wave; A, A wave; EA, fused E and A waves because of premature beat; V, aortic ejection wave.

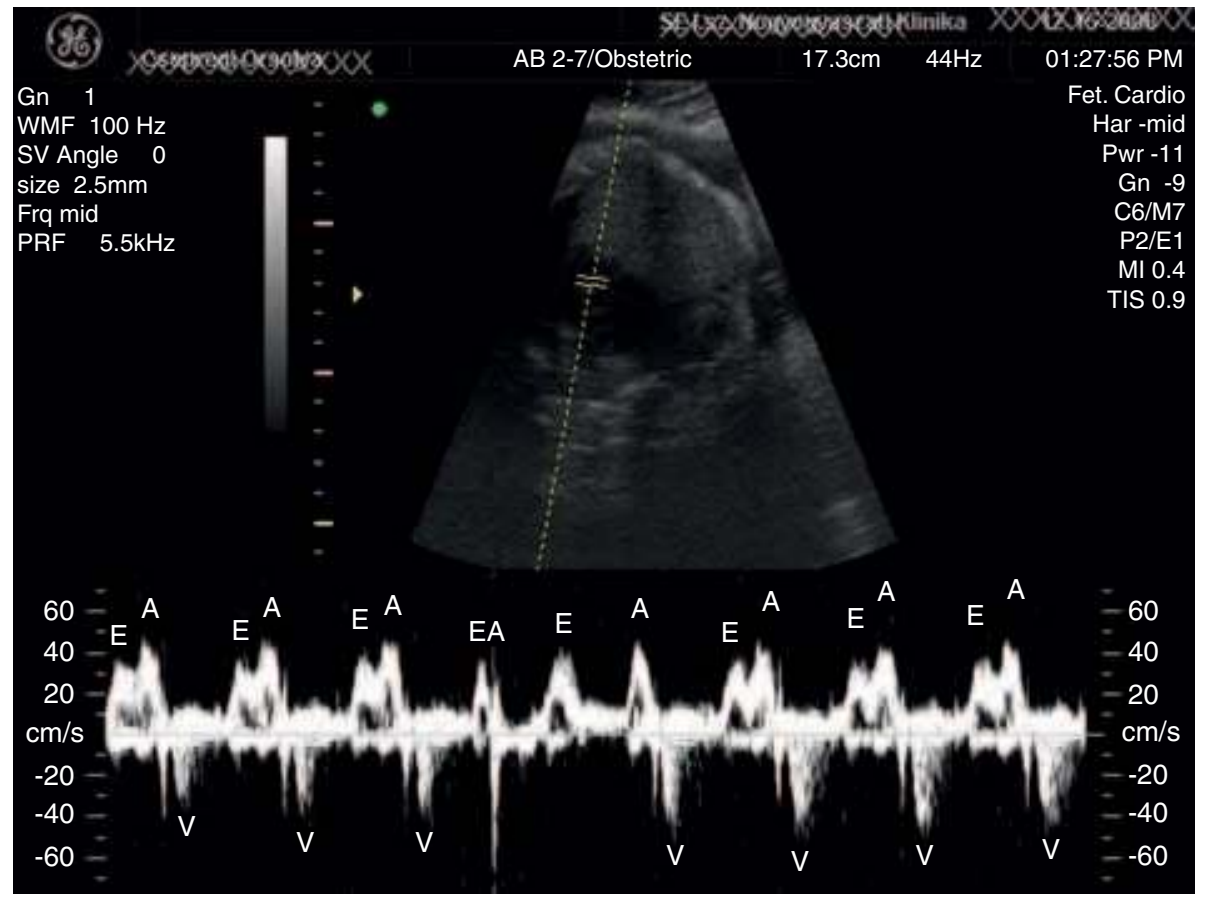

Figure 4 Blocked premature atrial beat, Doppler method. Abbreviations: E, E wave; A, A wave; EA, fused E and A waves because of premature beat; V, aortic ejection wave.

A rare presentation of fetal long QT syndrome is seconddegree AV block and ventricular tachycardia leading to congestive heart failure. The best method to diagnose QTc prolongation and torsades de pointes is the FMCG. As a sign of torsades de points, the peak-to-peak intervals of AA flow during ventricular tachycardia are markedly irregular $(14,18,19)$.

The therapy of the fetal ventricular tachycardia is difficult. In cases where it is not possible to rule out the ventricular tachycardia, digoxin therapy is contraindicated; rather, amiodarone is the drug of the choice. If the diagnosis of the ventricular tachycardia is sure, the pregnancy is before 34th week, and there is a sign of the decompensation, then therapy with lidocaine is reasonable with strong maternal control.

"SVT" is the most common cause of the fetal tachycardia. FMCG could help to clarify electrophysiologic patterns of initiation and termination of reentrant fetal SVT. Five distinct patterns of initiation and four patterns of termination were documented, with the most common patterns of initiation 


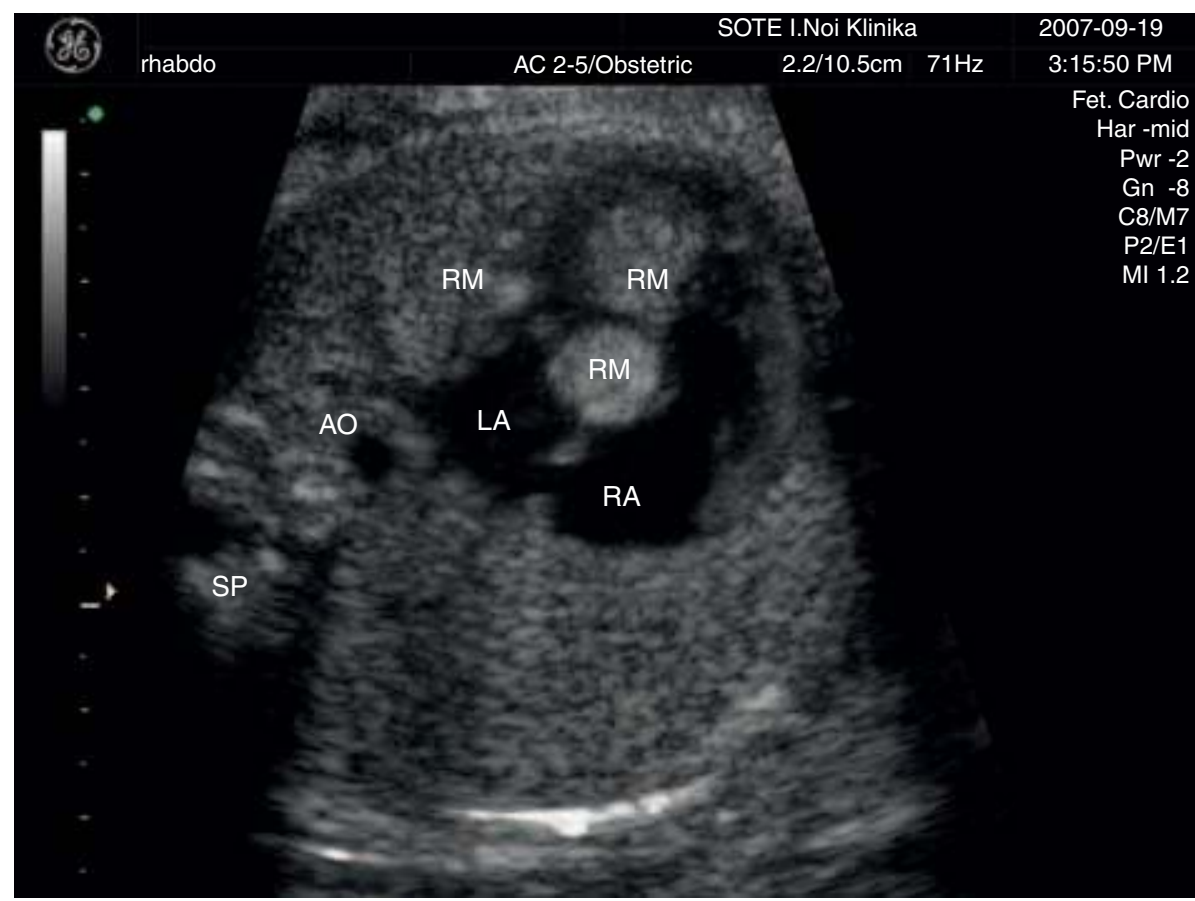

Figure 5 Multiplex rhabdomyomas in the heart. Abbreviations: RH, rhabdomyoma; LA, left atrium; RA, right atrium; SP, spine; AO, descending aorta.

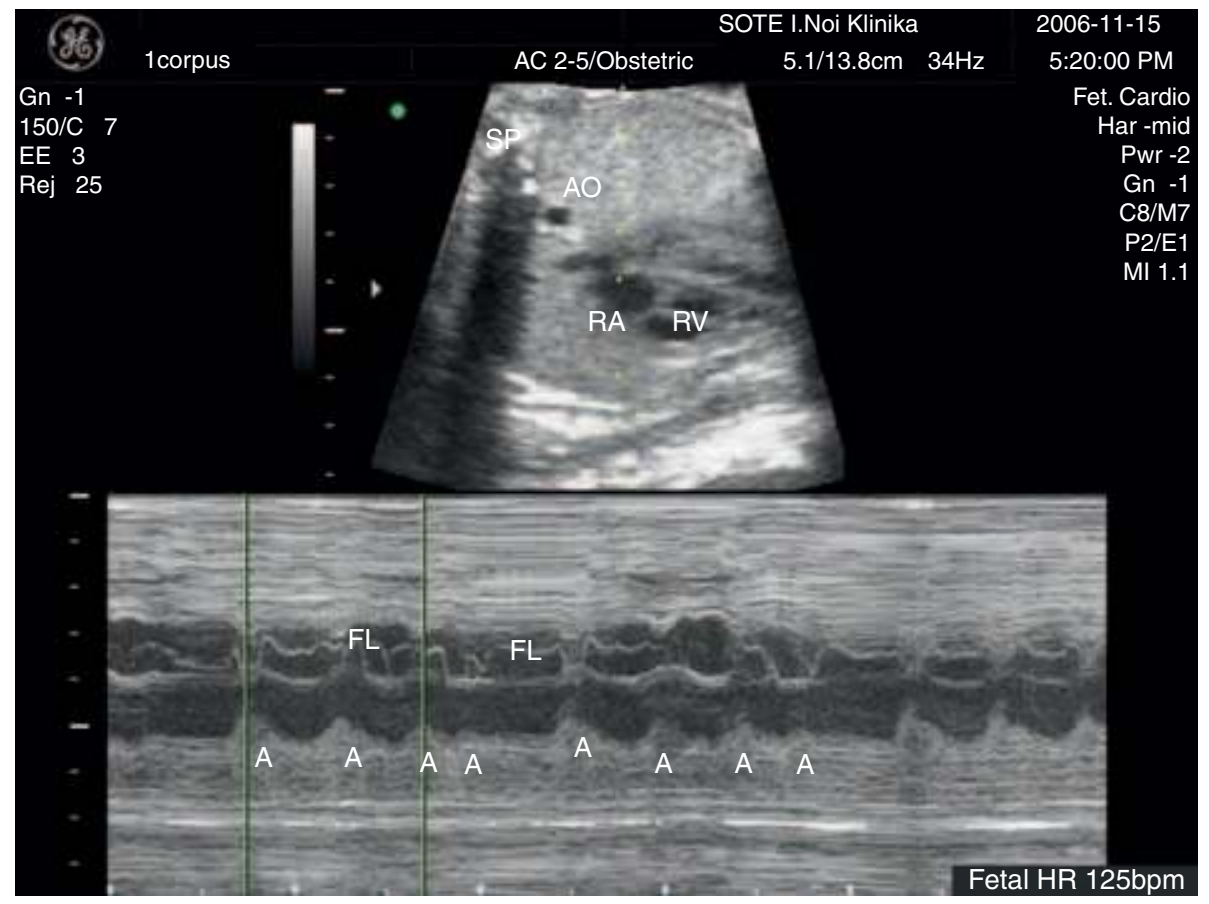

Figure 6 Premature atrial beat caused by aneurysm-like flap of the foramen ovale (M-mode). Each fourth beat is a premature atrial contraction. Abbreviations: A, atrial contraction; FL, aneurysm-like flap of the foramen ovale; RA, right atrium; RV, right ventricle.

involving reentrant PACs (Wolff-Parkinson-White syndrome, QRS aberrancy, and multiple reentrant pathways). FMCG actocardiography revealed an unexpectedly strong association between fetal trunk movement and the initiation and termination of SVT, suggesting that autonomic influences play a key role (6).

In everyday routine, it is the best to plan the management of the fetal tachyarrhythmia based on analysis of pulsed-Doppler recordings of fetal heart's blood flow. It is a short V-A (ventriculo-atrial) tachycardia, when $\mathrm{V}$-A period is shorter than
A-V (atrioventricular) period (Fig. 8). Short V-A tachycardia with 1:1 AV conduction and with ventricular rates of 230 to $280 \mathrm{bpm}$ suggests reentrant tachycardia, and digoxin is proposed as the first choice drug. Short V-A tachycardia with AV dissociation means that with high probability of junctional ectopic tachycardia, which is usually resistant to digoxin and sotalol, amiodarone could have success. The atrial ectopic tachycardia usually responds to sotalol.

Long V-A tachycardias are seen in sinus tachycardia, in permanent junctional reciprocating tachycardia, or in 


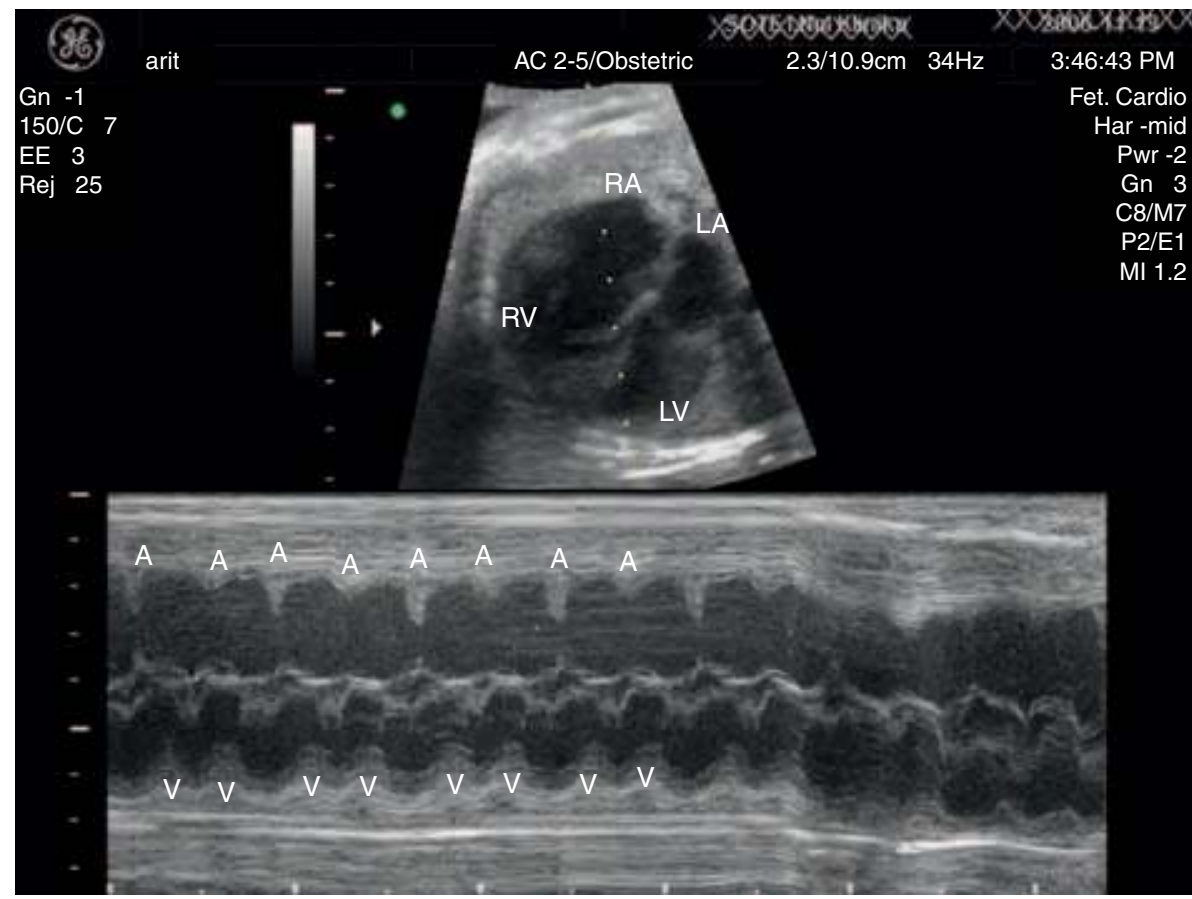

Figure 7 Ventricular extrasystole, M-Mode method. Abbreviations: A, atrial contraction; V, ventricular contraction; RA, right atrium; LA, left atrium; RV, right ventricle; $L V$, left ventricle.

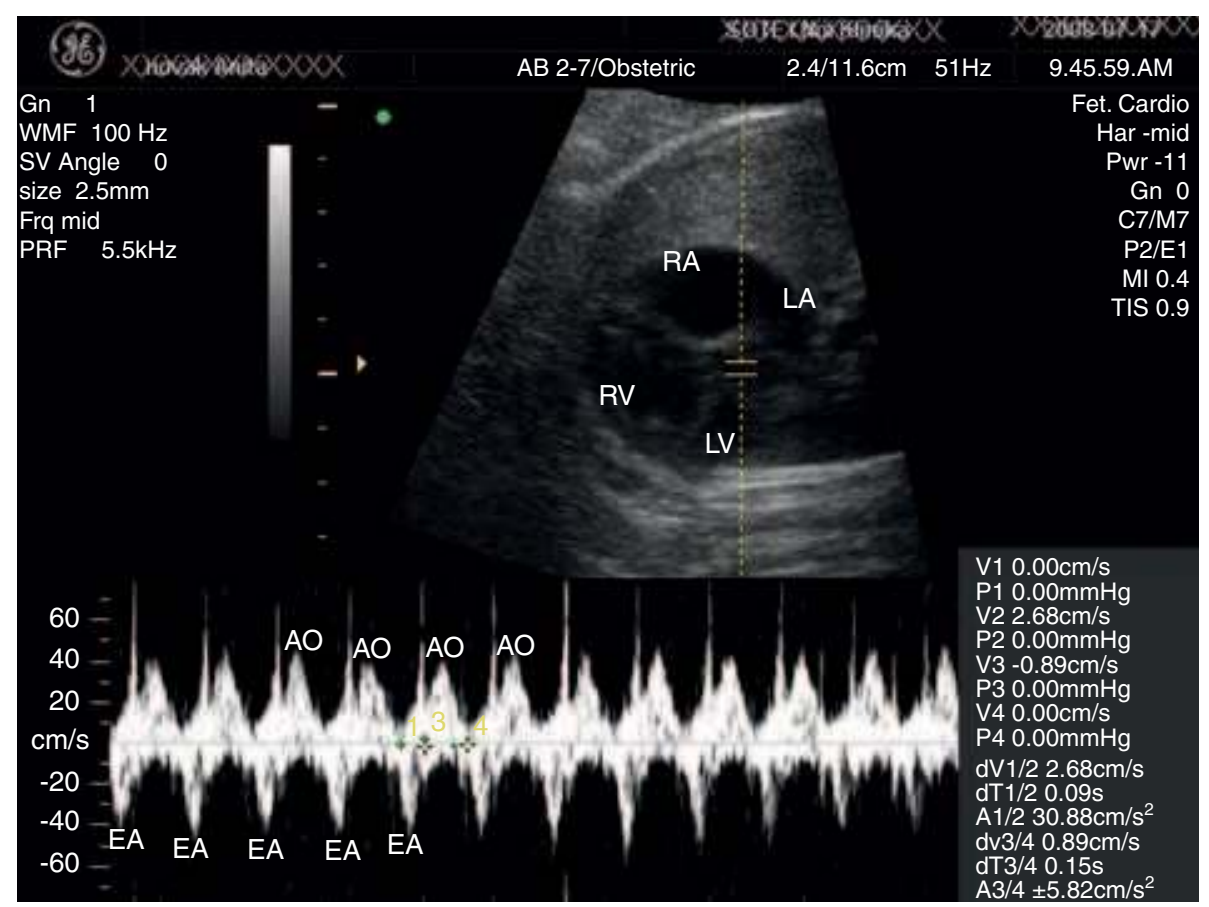

Figure 8 Long V-A tachycardia. The V-A interval is $150 \mathrm{msec}$, and the A-V interval is 90 msec. Abbreviations: EA, atrial inflow; AO, ventricular outflow; RA, right atrium; LA, left atrium; RV, right ventricle; $\mathrm{LV}$, left ventricle.

atrial ectopic tachycardia. The first-choice drug is sotalol. In intra-atrial reentrant tachycardia, the first-choice drug is digoxin $(10,20)$.

The sotalol was found to be useful in transplacental treatment of atrial flutter and SVT. The daily maternal sotalol dose is linearly related to the maternal plasma concentration. The mean fetal/maternal sotalol plasma concentration is 1.1 (range 0.67-2.87, SD 0.63), and the mean amniotic fluid/fetal blood ratio of sotalol is 3.2 (range 1.28-5.8, SD 1.4). The effectiveness of sotalol therapy could not be extrapolated from maternal blood levels. A large difference in the sensitivity of sotalol was found. As proarrhythmia is known to be dose related, low initiation doses are preferable and dosage increases should be stepwise. Close monitoring, especially during the initiation phase, is recommended. In addition, maternal potassium levels and magnesium levels should be monitored regularly and supplemented if necessary. The maternal toxic level is $2.5 \mathrm{mg} / \mathrm{L}$ at which marked QTc prolongation is noted. The F/M ratio of 1.1 shows that the fetus also stays below the "toxic" level (21). 
SVT results in reduced diastolic filling time, which, when combined with the normal limitations of relaxation and reduced compliance of the fetal myocardium, leads to increasing atrial and central venous pressures in the evolution of placental edema and dysfunction, which leads to fetal hypoxemia. Treatment of the SVT is proposed for fetuses with the risk of signs of heart failure-incessant SVT and early onset of SVT ( $<32$ weeks). Altered maternal gastric emptying and increased maternal renal clearance could necessitate the use of higher doses. It is important to control the mother, maternal heart rate, serial ECGs and telemetry, and monitoring drug concentration (22). The mortality of SVT was found to be $8 \%$ to $10 \%(23)$.

In tachycardia with a fast conducting reentrant pathway, atrial contractions occur during ventricular ejection, thus against closed AV valves. Tall reverse " $\mathrm{A}$ " waves are present in the venous system of fetuses with reentrant SVT. This type of arrhythmia could induce backward venous congestion and hydrops, even in the absence of myocardial failure. The duration of the process also plays a significant role in the development of hydrops fetalis (24). Hydropic patients have higher ventricular rates (median: $240 \mathrm{bpm}$, range 240-300 bpm) than non-hydropic fetuses (220 bpm, range 200-310), and the atrial rates are not significantly different. In cases with fetal SVT and hydrops fetalis, flecainide could control the fetal arrhythmia. The therapeutic range is 200 to $700 \mu \mathrm{g} / \mathrm{L}$. Strong maternal ECG and flecainide concentrations need to be checked out to term to avoid potential maternal and fetal toxicity. There is no evidence that flecainide is concentrated in the fetus (25). Another possibility of drug treatment for hydropic fetuses is digoxin and verapamil (26). In the absence of effective therapy, the mortality of the hydropic fetuses with supraventricular tachycardia approaches $50 \%$, and with treatment, the mortality is $10 \%$. It is recommended the maternal hyperglycemic state in the acute resuscitation of the hydropic fetus with SVT, which in providing metabolic support for the fetal myocardium, may ultimately be used coincident with initiation of antiarrhythmic treatment.

Fetal atrial flutter is usually identified late in gestation and is associated with atrial rates ranging from 300 to $550 \mathrm{bpm}$ with variable $\mathrm{AV}$ conduction and ventricular rates. The atrial rate is confirmed through M-mode or systemic venous Doppler demonstration of the atrial wave rates.

Action potential prolongation in the absence of conduction slowing seems more effective in the termination of atrial flutter than depression of the excitability. Sotalol (class III drug) seemed to be more efficacious than other drugs in fetal atrial flutter. The proposed protocol in atrial flutter is as follows: $80 \mathrm{mg}$ of sotalol twice daily, increased stepwise with $80 \mathrm{mg}$ per 3 days to a maximum of $160 \mathrm{mg}$ three times daily. Sotalol has excellent transplacental passage. Monitoring of the maternal QT interval is essential in excluding maternal proarrhythmia. Amiodarone (class III drug) has many side effects and does not cross the placenta as readily as does sotalol. The treatment with sotalol as the first drug of choice is more reasonable because transplacental passage is slower for digoxin, relapse into atrial flutter occurs more often at birth, and subjective maternal complaints are prominent with sotalol (27).

Long-standing and/or fluctuating cerebral perfusion as a result of intrauterine atrial flutter is responsible for the disturbance of the maintenance of adequate cerebral perfusion. It is exposing the brain to ischemia with increased risk of intracranial hemorrhage. It is opted to treat all fetuses with atrial flutter at time of presentation, unless immediate delivery is possible. The stress of the delivery and release of catecholamines may have an influence on retriggering the onset of atrial flutter, such seen in SVT. The early morbidity of atrial flutter is significant, but the long-term prognosis is excellent (28). The risk of neurologic morbidity must be taken into consideration. There are some case reports on probable inherited etiology of fetal atrial flutter $(29,30)$.

\section{IF THE FETAL HEART RATE IS BELOW 120/MIN, IT IS BRADYCARDIA INDEPENDENTLY FROM THE \\ GESTATIONAL AGE}

Persistent fetal sinus bradycardia is a rare condition and has been reported with central nervous system abnormalities, maternal treatment with beta blockers, excessive vagal tone, hydrops, neonatal long QT syndrome, and intrauterine growth retardation or as an atypical manifestation of congenital parvovirus B19 infection (31). Sinus bradycardia has also been associated with complex heart defects in which absence or hypoplasia of the sinus node is a typical finding (32). Sinus bradycardia has been reported recently as a possible sign of maternal anti-SSA/Ro antibodies. There are findings that in fetuses or newborns who died of congenital atrioventricular block (CAVB), the sinoatrial nodes were hypoplastic and, in some of these cases, surrounded by extensive fibrosis (33).

Prenatal sinus bradycardia or recognition of second-degree AV block may lead to early detection and treatment of long QT syndrome. QTc prolongation was found to be more common in utero than postnatally, but it does not necessarily imply that fetal QTc prolongation is a benign condition. Several studies of newborns have suggested that QT prolongation and the concomitant increased risk of ventricular tachyarrhythmias may account for the significant mortality. QT prolongation was the highest in the fetuses with the poorest outcome and was often accompanied by T-wave alternant. Common genetic polymorphisms have been shown to alter the QT interval, and prenatal molecular genetic testing for long QT syndrome has been demonstrated. The recently reported environmentgenetic interactions could result in arrhythmias or sudden infant death syndrome (4). Few cases of fetal ventricular tachycardia due to congenital long QT have been reported in the literature. Some cases presented fetal ventricular tachycardia with both bradycardia and AV block $(14,18,19)$.

"CAVB" occurred in $2.6 \%$ of a large population of fetuses evaluated for an irregular rhythm. Congenital heart defects, like L transposition (Fig. 9), left isomerism (Fig. 10), cause the AV block at about $50 \%$ of cases. If there is no cardiac malformation, the fetal CAVB is the consequence of transplacental passage of maternal autoantibodies (Figs. 11 and 12). CAVB and the neonatal lupus are the model of passively acquired autoimmunity (33). The mean histologic lesion in the heart is fibrosis of the conducting tissue and, in some cases, the surrounding myocardium. The mechanism by which maternal anti-SSA/Ro-SSB/La antibodies initiate and perpetuate inflammation and eventuate in scarring of the AV node is not yet defined. In vitro and in vivo studies suggest that one pathologic cascade leading to scarring may be initiated via apoptosis, 


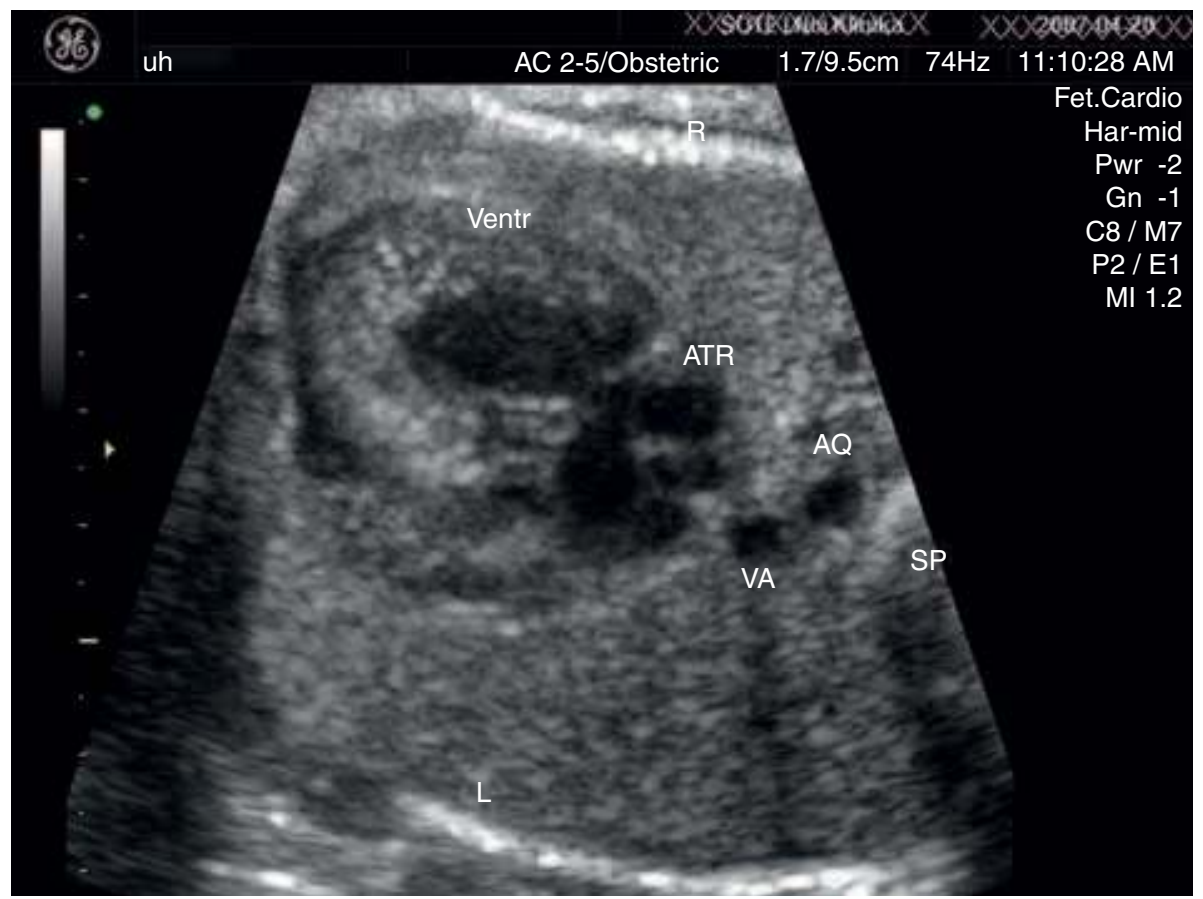

Figure 9 Horizontal cross section of the chest, with univentricular heart, dextrocardia, common atrium, interruption of the inferior caval vein. Abbreviations: Ventr, ventricle; ATR, atrium; AO, aorta; VA, azygos continuation.

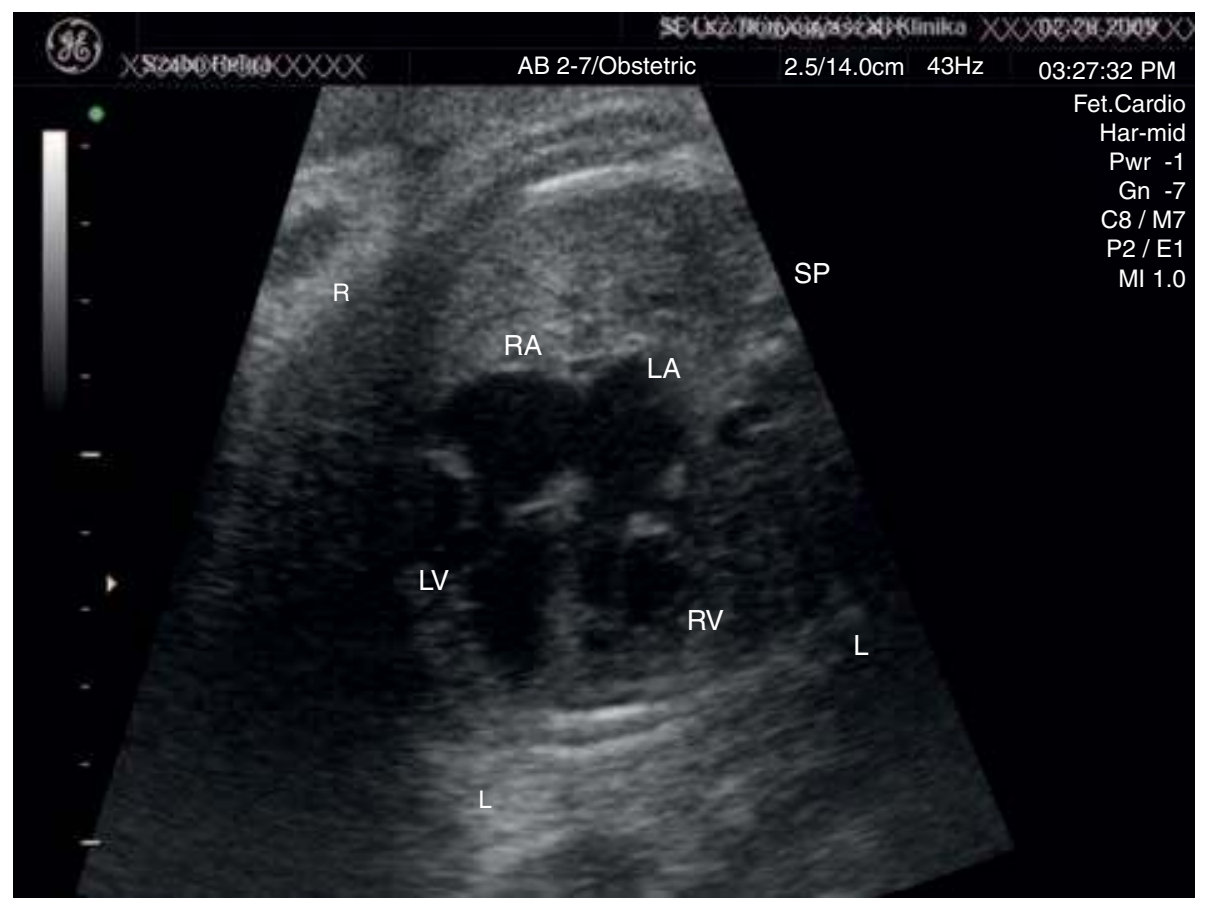

Figure 10 Horizontal cross section of the chest, with L transposition. The anatomical right ventricle (RV) is visible on the left side, below the left atrium (LA); the left ventricle (LV) on the right side, below the right atrium (RA). Abbreviations: SP, spine; R, right; L, left.

resulting in the translocation of SSA/Ro-SSB/La antigens and subsequent surface binding by maternal autoantibodies. In CAVB, the maternal anti-SSA/Ro-SSB/La antibodies result in opsonization and inflammatory/fibrotic squeal. These opsonized cardiocytes are phagocytosed by macrophages, which secrete factors those transdifferentiate fibroblasts into myofibroblasts, a scarring phenotype (34).

The development of CAVB is a two-stage process. In the first step, the maternal autoantibodies are transferred through the placenta and bind structures in the developing fetal heart, leading to first-degree AV block. This state is spontaneously reversible with further maturation of the fetus. If the second phase is entered, a progression to complete heart block commences. Factors involved in this second phase may be mainly fetal and related to the immunogenetic profile of the individual. A predisposition to an intense inflammatory reaction to the antibody deposition might lead to the reaction to the subsequent mononuclear cell infiltration and fibrosis, 


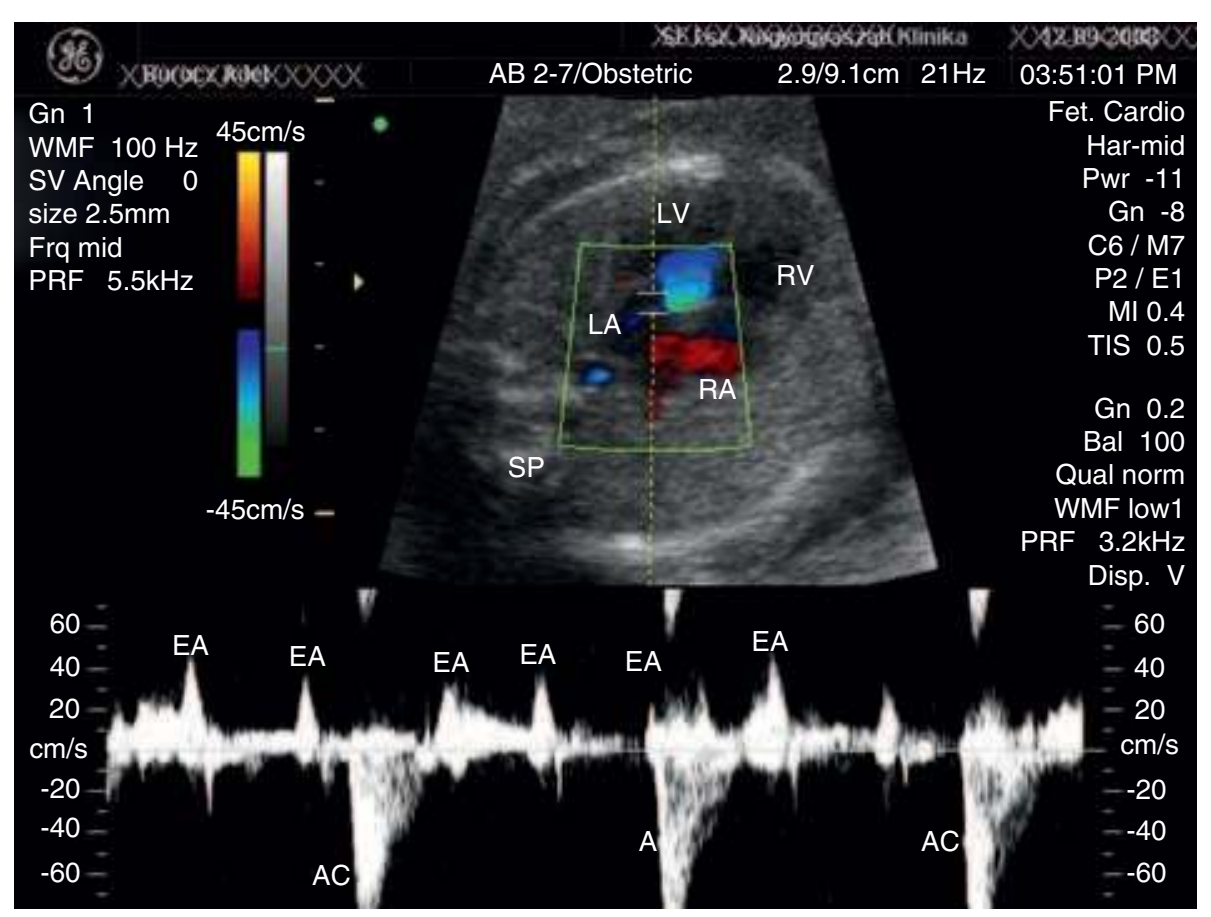

Figure 11 Complete atrioventricular dissociation. Abbreviations: EA, ventricular inflow wave; AO, ventricular ejection wave; RA, right atrium; LA, left atrium; $\mathrm{RV}$, right ventricle; $\mathrm{LV}$, left ventricle; SP, spine.

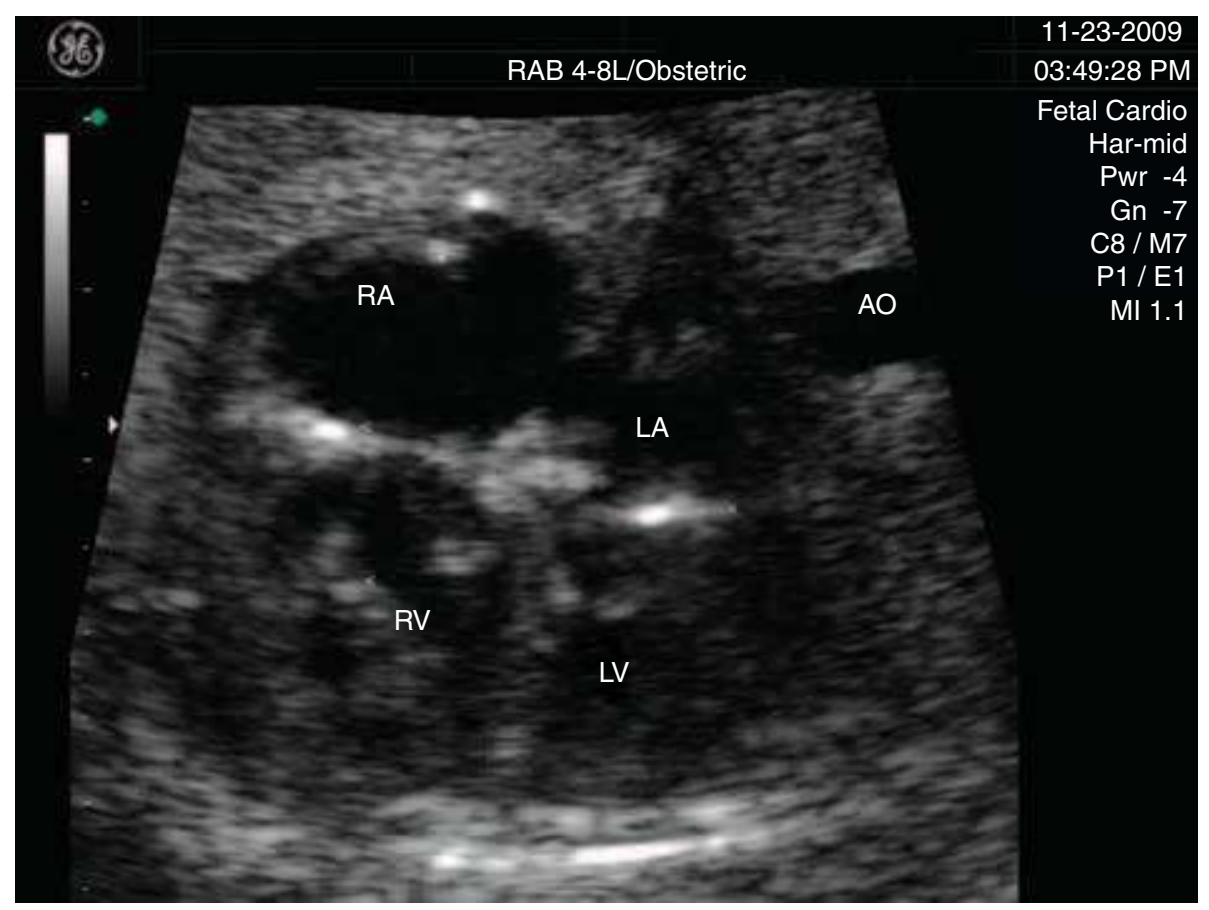

Figure 12 Cardiomegaly and focal myocardial fibrosis in a fetus with congenital atrioventricular block because of maternal autoantibodies. Abbreviations: RA, right atrium; LA, left atrium; RV, right ventricle; LV, left ventricle; < signs, fibrotic foci.

with a permanent injury as the outcome. The high prevalence of fetal first-degree AV block with normal postnatal PR intervals in a group of fetuses with maternal anti-Ro/La antibodies has been proposed due to a transient biologic response to the transplacental transfer of Ro/La antibodies (3).

If second-degree $\mathrm{AV}$ block is present, transplacental steroid treatment theoretically may limit progression. Prophylactic steroid therapy in the fetus without documented AV conduction disease is not warranted due to maternal and fetal side effects. After a high-dose steroid therapy for fetal heart block, several maternal complications could be found including diabetes, hypertension, infections, bone density loss, and cataract. Fetal risks are oligohydramnios and growth restriction, including the head and possibly central nervous system injury without clear evidence of benefit to fetal cardiac conduction (7).

Early detection of incomplete AV block successfully identifies a group at highest risk developing permanent AV block. Treating this group may incur greater benefit than risk. The anti-inflammatory effects of dexamethasone might have 
interrupted ongoing damage to the conduction system secondary to maternal autoantibodies.

The findings of a PR interval of $>150 \mathrm{msec}$ at any gestational age should prompt a search for maternal anti-Ro/anti-La antibodies and close follow-up for other complex arrhythmias $(11,12,15)$. The Doppler mechanical PR interval does not distinguish between electrical PR interval prolongation due to AV conduction delay and abnormalities of interatrial conduction. These limitations in Doppler mechanical PR interval measurements can be overcome if FMCG is available. The newer technique of tissue-velocity Doppler imaging may be helpful when evaluating the fetus with borderline findings and negative maternal anti-Ro/anti-La antibodies. May be that PR prolongation represents a variant of a normal, and only in retrospect does it have clinical significance if it is either sustained after birth or progresses to more advanced block. The prolongation of the fetal Doppler mechanical PR interval is not a definitive tool to detect early signs of anti-SSA/ Ro-associated CAVB $(34,35)$.

The risk of the third-degree heart block in pregnancies of mothers with a previous child with congenital heart block is $19 \%$ and in pregnancies in mothers without previous child with congenital heart block or rash is $4 \%$, suggesting that there must be additional maternal and/or fetal factors that influence disease development and manifestation. The maternal factors may be related to the antibody profile. The fetal factor could be viral infection or genetic predisposition (36).

Advanced block and cardiomyopathy can occur within 1 week of normal echocardiogram without initial first-degree block. This more extensive injury can occur in utero or postnatally, even as late as 9 years of age (35).

In a large study on infants whose mothers had anti-Ro antibodies, the prevalence of hydrocephalus was $8 \%$, which is significantly higher than the prevalence in the general population of $0.048 \%$ to $0.081 \%$. It seems to be possible that hydrocephalus and macrocephaly are manifestations of neonatal lupus syndrome and infants born to mothers with anti-Ro antibodies should be carefully monitored for hydrocephalus as part of their routine physical examination (37).

In patients with fetal hydrops, cholestasis and severe and irreversible liver failure could be observed. Histologically, extensive collapse of the stroma and the absence of hepatocytes (foie vide) were observed. Cholestasis has been described in patients with neonatal lupus erythematosus without $\mathrm{AV}$ block (25).

When CAVB is associated with endocardial fibroelastosis (EFE), there is a very high mortality rate. The etiology of EFE remains unclear, but it may be a result of an autoimmune process. In fetuses or infants born from mothers positive for anti-Ro/La antibodies, EFE and cardiomyopathy with clinically normal conduction systems could conceive. The EFE and CAVB are two separate disease manifestations of neonatal lupus. In the histologic examinations, an active fetal B-cell response was found in addition to the passive deposition of transplacentally passed maternal immunoglobulin. The initial myocardial damage in utero is likely secondary to the passive deposition of maternal anti-Ro and anti-La antibodies, and the progression of EFE may be the result of an ongoing fetal and postnatal autoimmune reaction. The EFE accounted for over $80 \%$ of the deaths in these patients. Some cases may go undiagnosed where there is a fetal demise. Fetal echocardiographic assessment is very subjective and often identifies only the most severe degree of EFE. Idiopathic dilated cardiomyopathy represents unrecognized maternal autoantibodyinduced EFE.

There are studies on infants with CAVB who developed late-onset dilated congestive cardiomyopathy despite early and adequate institution of cardiac pacing. At the time of clinical presentation, many infants have symptoms or signs of an upper respiratory tract infection, otitis media, or gastrointestinal infection. Myocardial biopsy or histologic examination of the explained heart revealed the following: myocyte hypertrophy, interstitial fibrosis, and myocyte degradation in two subjects. An active inflammatory infiltrate was not detected. An upper respiratory/gastrointestinal infection might have reactivated a previously dormant inflammatory process. The increased rate that followed pacemaker implantation may have provoked a form of tachycardia-induced cardiomyopathy (38). The mortality rate is as high as $25 \%$, and many patients require cardiac transplantation.

\section{THERAPY OF THE CAVB}

Although the prenatal use of corticosteroids, plasmapheresis, sympathomimetics, and either prenatal pacing or early delivery with postnatal pacing has been proposed, the true efficacy of therapy of the CAVB is not clear.

Current approach to affected pregnancies is to initiate dexamethasone therapy at diagnosis of fetal AV block $(4 \mathrm{mg} /$ day) and to add maternal sympathomimetics for ventricular rates of $<55$ beats/min. Weekly-biweekly fetal echocardiograms are used to follow the progress, and an elective delivery by caesarean section at 36 to 37 weeks is planned. If there are signs of fetal compromise, an earlier delivery with aggressive postnatal management is proposed, including early pacemaker placement. When there is oligohydramnios, maternal dexamethasone is tapered off; however, persistence of reduced amniotic fluid may prompt earlier delivery (39). (Prednisone and prednisolone are inactivated by placenta hydroxylases and only less than $10 \%$ of the mother blood level can reach fetus; dexamethasone and betamethasone cannot be inactivated.) Some data suggest that prolonged fetal exposure to dexamethasone may impair cerebral development, but there are reports on children treated with dexamethasone prenatally (cumulative dosage $20-260 \mathrm{mg}$ ), who were tested postnatally with different scales according to their age and they were found with normal intelligence quotient (40).

Intravenous immunoglobulin (IVIG) is efficient in various immune-mediated conditions. It seems conceivable to employ IVIG in cases of CAVB or neonatal lupus, either administered to the mother or directly through cordocentesis in the treatment of the fetus and neonate with reduced myocardial function suggesting more diffuse disease (41).

\section{PROGNOSIS AND FOLLOW-UP}

In an isolated AV block, diagnosed in utero, in neonatal age and in children, anti-Ro and/or anti-La are present in 95\% of fetal, $90 \%$ of neonatal, but only $5 \%$ of children's mothers tested. Patients with CAVB having fetal, neonatal, and childhood diagnosis have a mortality of $43 \%, 6 \%$, and $0 \%$, respectively, in the first two decades of life. Increased mortality 
is associated with a fetal diagnosis of CAVB, fetal hydrops $(100 \%)$, EFE (100\%), and delivery at or before 32 weeks of gestation. Timing of pacemaker implantation differ significantly among fetuses versus neonates and neonates versus children cases. At 20 years of age, only $11 \%$ and $12 \%$ of CAVB patients with neonatal or children diagnosis, respectively, are not paced. CAVB detected in utero represents a more severe spectrum of the disease than encountered postnatally (39).

The mortality decreased from $45 \%$ to $15 \%$ with close surveillance, use of maternally administered corticosteroids, delivery at 35 to 37 weeks or earlier with any evidence of fetal distress or cardiovascular compromise, neonatal cardiac intensive care management, and early permanent pacemaker therapy. Among fetuses with AV block only, the survival rate is $82 \%$, with a heart defect; the prognosis is poor, and $50 \%$ of the patients survive. Sinus bradycardia is associated with survival rates of $75 \%(33,42)$.

All fetal arrhythmias are associated with a moderately high risk for fetal distress. In cases of compromise, fetal and neonatal prognosis is poor and is an indication for perinatal medication. The risk of neurologic morbidity must be taken into consideration.

\section{REFERENCES}

1. Kirby ML. Molecular embryogenesis of the heart. Pediatr Dev Pathol 2002; 5: 516-43.

2. Gittenberger-De Groot A, Bartelings MM, Deruiter MC, et al. Basics of cardiac development for the understanding of congenital heart malformations. Pediatr Res 2005; 57: 169-76.

3. Hornberger L, Sahn DJ. Rhythm abnormalities of the fetus. Heart 2007; 93: 1294-300.

4. Zhao H, Strasburger JF, Cuneo BF, et al. Fetal cardiac repolarisation abnormalities. Am J Cardiol 2006; 98: 491-6.

5. Horigome H, Ogata K, Kandori A, et al. Standardisation of the PQRST waveform and analysis of arrhythmias in the fetus using vector magnetocardiography. Pediatr Res 2006; 59: 121-5.

6. Wakai RT, Strasburger JF, Li Z, et al. Magnetocardiographic rhythm patterns at initiation and termination of fetal supraventricular tachycardia. Circulation 2003; 107: 307-12.

7. Gardiner HM, Belmar C, Pasquini LA, et al. Fetal ECG: a novel predictor of atrioventricular block an anti-Ro positive pregnancies. Heart 2007; 93: 1454-60.

8. Taylor MJ, Smith MJ, Thomas M. Non-invasive fetal electrocardiography in singleton and multiple pregnancies. BJOG 2003; 110: 668-78.

9. Fouron JC, Proulx F, Miro J, et al. Comparison of Doppler and M-mode ultrasonography to time fetal atrial and ventricular contractions. Obstet Gynecol 2000; 96: 732-6.

10. Fouron JC, Fournier A, Proulx F, et al.: Management of fetal tachyarrhythmia based on superior vena cava/aorta Doppler flow recordings. Heart 2003; 89: 1211-1216.

11. Glickstein JS, Buyon J, Friedman D. Pulsed Doppler assessment of the fetal PR interval. Am J Cardiol 2000; 86: 236-9.

12. Adelfinger G, Fouron JC, Sonesson SE, et al. Reference values for time intervals between atrial and ventricular contractions of the fetal heart measured by two Doppler techniques. Am J Cardiol 2001; 88: 1433-636.

13. Carvalho JS, Prefumo F, Ciardelli V, et al. Evaluation of fetal arrhythmias from simultaneous pulsed wave Doppler in pulmonary artery and vein. Heart 2007; 93: 1448-53.

14. Bergman G, Jacobsson LA, Wahren-Herlenius M, et al. Doppler echocardiographic and electrocardiographic atrioventricular time intervals in newborn infants: evaluation of techniques for surveillance of fetuses at risk for congenital heart block. Ultrasound Obstet Gynecol 2006; 28: 57-62.

15. Cuneo BF, Strasburger JF, Wakai RT. Conduction system disease in fetuses evaluated for irregular cardiac rhythm. Fetal Diagn Ther 2006; 21: 307-13.

16. Nil M, Hamilton RM, Fenwik L, et al. Assessment of fetal atrioventricular time intervals by tissue Doppler and pulse Doppler echocardiography: normal values and correlation with fetal electrocardiography. Heart 2006; 92: 1830-7.

17. Rein AJT, O’Donnell C, Geva T, et al. Use of tissue velocity imaging in the diagnosis of fetal cardiac arrhythmias. Circulation 2002; 106: 1827-33.

18. Cuneo BF, Ovadia M, Strasburger JF, et al. Prenatal diagnosis and in utero treatment of torsades de pointes associated with congenital long QT syndrome. Am J Cardiol 2003; 91: 1395-8.

19. Takahash K, Shiraish H, Ohkuchi A, et al. Irregular peak-to-peak intervals between ascending aortic flows during fetal ventricular tachycardia in long QT syndrome. Ultrasound Obstet Gynecol 2009; 33: 990-2.

20. Joglar J, Page RL. Antiarrhythmic drugs in pregnancy. Curr Opin Cardiol 2001; 16: 40-5.

21. Oudjik MA, Ruskamp JM, Ververs T, et al. Treatment of fetal tachycardia with sotalol: transplacental pharmacokinetics and pharmacodynamics. J Am Coll Cardiol 2003; 4: 765-70.

22. Hornberger LK. Echocardiographic assessment of fetal arrhythmias. Heart 2007; 93: 1331-3.

23. Krapp M, Simpson JM, Sharland GK, et al. Review of diagnosis, treatment, and outcome of fetal atrial flutter compared with supraventricular tachycardia. Heart 2003; 89: 913-17.

24. Sant'Anna AM, Fouron JC, Alvarez F. Neonatal cholestasis associated with fetal arrhythmia. J Pediatr 2005; 146: 277-80.

25. Rasheed A, Simpson J, Rosenthal E. Neonatal ECG changes caused by supratherapeutic flecainide following treatment for fetal supraventricular tachycardia. Heart 2003; 89: 470.

26. Simpson JM, Sharland GK. Fetal tachycardias: management and outcome of 127 consecutive cases. Heart 1198; 79: 576-81.

27. Lisowski LA, Verheijen PM, Benatar AA, et al. Atrial flutter in the perinatal age group: diagnosis, management and outcome. J Am Coll Cardiol 2000; 35: 771-7.

28. Casey FA, McCrindle BW, Hamilton RM, et al. Neonatal atrial flutter: significant early morbidity and excellent long-term prognosis. Am Heart J 1997; 133: 302-6.

29. Tikanoja T, Kirkinen P, Nikolajev K, et al. Familial atrial fibrillation with fetal onset. Heart 1998; 79: 195-7.

30. Boldt T, Eronen M, Andersson S. Long-term outcome in fetuses with cardiac arrhythmias. Obstet Gynecol 2003; 102: 1372-9.

31. Savarese I, De Carolis MP, Costa S, et al. Atypical manifestations of congenital parvovirus B19 infection. Eur J Pediatr 2008; 167: 1463-86.

32. Qu Y, Baroundi G, Yue Y, et al. Novel molecular mechanism involving alfa 1D L-type calcium channel in autoimmune-associated sinus bradycardia. Circulation 2005; 111: 3034-41.

33. Buyon JP, Clancy RM. From antibody insult to fibrosis in neonatal lupus - the heart of the matter. Arthritis Res Ther 2003; 5: 266-70.

34. Sonesson SE, Salamonsson S, Jacobsson LA, et al. Signs of first-degree heart block occur in one-third of fetuses of pregnant women with antiSSA/Ro 52-kd antibodies. Arthritis Rheum 2004; 50: 1253-61.

35. Friedman DM, Kim MY, Copel JA, et al. Utility of cardiac monitoring in fetuses at risk for congenital heart block. Circulation 2008; 117: 485-93.

36. Nield LE, Silverman ED, Smallhorn JF, et al. Endocardial fibroelastosis associated with maternal anti-Ro and anti-La antibodies in the absence of atrioventricular block. J Am Coll Cardiol 2002; 40: 796-802.

37. Boros CA, Spence D, Blaser S, et al. Hydrocephalus and macrocephaly: new manifestations of neonatal lupus erythematosus. Arthritis Rheum 2007; 57: 261-6.

38. Moak JP, Barron KS, Hougen TJ, et al. Congenital heart block: development of late-onset cardiomyopathy, a previously underappreciated sequela. J Am Coll Cardiol 2001; 37: 238-42.

39. Jaeggi ET, Hamilton RM, Silvermann ED, et al. Outcome of children with fetal, neonatal or childhood diagnosis of isolated congenital atrioventricular block. J Am Coll Cardiol 2002, 39: 130-137.

40. Tincani A, Rebaioli CB, Frassi M, et al. Pregnancy and autoimmunity: maternal treatment and maternal disease influence on pregnancy outcome. Autoimmun Rev 2005; 4: 423-8.

41. Nussinovich U, Shoenfeld Y. Intravenous immunoglobulin - indications and mechanisms in cardiovascular diseases. Autoimmun Rev 2008; 7 : 445-52.

42. Buyon JP, Hierbert R, Copel JA, et al. Autoimmune associated congenital heart block: demographics, mortality, morbidity and recurrence rates obtained from a national neonatal lupus registry. J Am Coll Cardiol 1998; 31: $658-1666$. 


\section{Fetal growth restriction \\ Daniel L. Jackson, M. Y. Divon, and Hung N. Winn}

\section{INTRODUCTION}

Low birth weight is an important predictor of neonatal morbidity and mortality. This condition can be the result of either preterm delivery or fetal growth restriction (FGR). Fetuses whose growth has been restricted have an increased incidence of perinatal morbidity and mortality (1). Heinonen et al. have studied the first-year survival of preterm infants born at 26 to 32 weeks of gestation and concluded that FGR was associated with a threefold increase in neonatal mortality (2). Comparing with infants with normal growth, FGR is associated with a higher incidence of low Apgar scores, perinatal asphyxia, hypothermia, polycythemia, hypocalcemia, pulmonary hemorrhage, and impaired immune function (3).

\section{DEFINITION AND CLASSIFICATION OF FGR}

Fetal growth rates are determined by comparing the size of the infant at birth with an expected norm for a given gestational age. Birth weight is the most accessible and reliable measure of the newborn and is, therefore, widely used for this purpose.

Fetal size and fetal growth are often confused in clinical practice. FGR denotes a pathologic process. By contrast, smallfor-gestational age (SGA) identifies the infant whose birth weight is below an arbitrary percentile for gestational age. The 10th percentile is often used for this purpose; however, birth weights below the 25th, 15th, and 5th percentile or two standard deviations (SDs) below the mean have also been used in the past. Using birth weight criteria as the gold standard for fetal growth presents a few problems. First, the diagnosis of growth restriction is based on a single observation (birth weight) made at an end point (gestational age at delivery). Fetal growth, however, cannot be estimated from less than two measurements separated by a "reasonable" interval.

Second, not every infant whose birth weight is below the 10th percentile for gestational age is small because of a pathologic growth-restrictive process. The constitutionally small infant is indeed small but shows appropriate growth and has no other complications. The converse is also a problem. A dysmature, postdate infant may be subjected to severe growth restriction, yet its birth weight is often above the 10th percentile for gestational age. Thus, a long and very thin infant may be misclassified as appropriately grown. Third, estimation of gestational age presents recurrent problems. An infant may be assigned different gestational ages depending on the specific method used to establish this age (i.e., last menstrual period, early clinical examination, early ultrasound examination, or neonatal assessment).

Several "commonly used" norms for birth weight as a function of gestational age have been described (4). Differences between these norms are due to different geographic origin (differences in altitude are of primary importance), race, ethnicity, parity, and neonatal sex. The mean birth weight at
40 weeks of gestation reported by Lubchenco and coworkers (5) at Denver (high altitude) was $120 \mathrm{~g}$ smaller than that reported by Brenner and coworkers (6), who studied an east coast (sea-level), racially mixed population (Table 1).

Despite all of these concerns, and probably due to a lack of a more dynamic definition, FGR is often defined as birth weight below the 10th percentile for gestational age. By definition, the incidence of this condition is $10 \%$. Variations of this proportion are caused by using the wrong norm for the population under study.

Identification of FGR by the population norm as described above has inherent difficulty. The "normal" values for preterm infants are determined by infants that were themselves born preterm, a condition that is itself associated with FGR. In order to improve the sensitivity and specificity of the diagnosis of FGR, new standards for defining normal growth have been established. Figueras and Gardosi have reported on optimal weight at term calculated by using maternal height, weight, parity, and ethnic origin (7). Pathologic factors (smoking, hypertension, diabetes, and preterm delivery) are excluded. These factors are used to establish an ideal weight at term that is extrapolated back to establish a customized growth curve for the pregnancy. This method has been demonstrated to be more predictive of actual birth weight in a normal pregnancy than previously established values (7). A proportion of patients identified as associated with FGR by population percentiles are reclassified as normal by customized growth curves. These fetuses have been shown to have no increased risk of adverse outcome. The use of the customized growth curve can thus prevent unnecessary interventions in these pregnancies. Similarly, some pregnancies that fall within the normal range of population-based norms are identified as associated with FGR and at risk by customized growth curves. These pregnancies have been shown to be at risk and can benefit from more intensive prenatal care (7).

Growth and symmetry are affected by the nature and severity of the underlying growth-restrictive process as well as its timing and duration. In 1963, Gruenwald identified two distinct patterns of decreased growth-symmetric and asymmetric (8). Early-phase growth disturbance (typically first semester and early second trimester) often culminates in symmetric growth restriction. Since growth in this phase is primarily due to cell hyperplasia, restriction of growth results in a lower cell number. Thus, the newborn is symmetrically small (i.e., concomitant decrease in the size of the head, trunk, length, and all other body organs). This pattern is often seen in growth disturbance associated with chromosomal anomalies, congenital malformations, and transplacental infections. In the asymmetric type, the growth rate of the trunk is smaller than that of other organs. This is caused by relative depletion of the actively growing liver combined with decreased 
subcutaneous fat deposition. Third-trimester growth abnormalities, such as those seen with maternal vascular disease, restrict fetal growth during the hypertrophic growth phase. Due to preferential perfusion of the brain and adequate linear growth, the newborn with asymmetric growth retardation is identified by either an abnormally increased head circumference $(\mathrm{HC})$ to abdominal circumference $(\mathrm{AC})$ ratio or an abnormal ponderal index. The neonatal ponderal index has been suggested as a measure of the proportion between neonatal body mass and length (9). This index is defined as a percentage of birth weight (in grams) divided by body length (in centimeters) cubed. Theoretically, an abnormal ponderal index should differentiate the normal from the wasted and malnourished infant. Miller suggested that a low ponderal index indicates asymmetric growth restriction and that it is a more accurate predictor of fetal morbidity than birth weight alone (10). Defining FGR by an abnormal ponderal index does, however, suffer from several limitations. First, severe, early-onset growth restriction often results in a normal ponderal index due to restriction of both weight and linear growth. Second, accurate measurements of neonatal length are not easy to obtain. A small error in this measurement is cubed when calculating the ponderal index and may lead to erroneous conclusions. Because of these limitations, the ponderal index has not received widespread clinical acceptance in obstetrics. It is necessary to be aware that these symmetric and asymmetric patterns of fetal growth may merge and become indistinguishable. A maternal vascular disease beginning early enough may cause symmetric growth restriction. Likewise, a fetus with asymmetric growth retardation can become symmetric once brain sparing or linear growth is no longer maintained [this phenomenon has been identified as "late flattening of the biparietal diameter" (BPD) and is associated with increased neonatal morbidity]. It is commonly believed that "placental insufficiency" results in asymmetric growth restriction whereas chromosomal abnormalities are associated with symmetric FGR. Data presented by Nicolaides and coworkers (11) indicate, however, that fetal genetic disease may be associated with severe asymmetric growth.

Table 1 Tenth Percentile Birth Weight Values as a Function of Gestational Age

\begin{tabular}{|ccc|}
\hline \multirow{2}{*}{ Gestational age (weeks) } & \multicolumn{2}{c}{ Birth weight(g) } \\
\cline { 2 - 3 } & Brenner et al. & Lubchenco et al. \\
28 & 860 & 770 \\
29 & 970 & 900 \\
30 & 1075 & 1030 \\
31 & 1180 & 1170 \\
32 & 1290 & 1310 \\
33 & 1480 & 1490 \\
34 & 1670 & 1670 \\
35 & 1860 & 1930 \\
36 & 2050 & 2190 \\
37 & 2240 & 2350 \\
38 & 2430 & 2510 \\
39 & 2530 & 2630 \\
40 & 2630 & 2750 \\
41 & 2675 & 2790 \\
42 & 2720 & 2830 \\
\hline
\end{tabular}

In a study of 752 consecutively born, single, white, term infants, Miller (12) has noted that the ratio of asymmetric to symmetric to "mixed" types of FGR is 4.7:2.7:1.0, respectively. Basel and coworkers reported on longitudinal sonographic biometry performed on fetuses with decreased growth rates and concluded that the type of FGR is determined to a large extent by the timing and duration of the insult rather than the specific etiology (13).

\section{ETIOLOGY OF FGR}

Various disease processes may result in FGR. The final fetal size is determined by the severity, gestational age at onset, and duration of the underlying pathology.

Abrams and Newman have examined the relationship between maternal characteristics and the risk of delivering a growth-retarded neonate (birth weight $<10$ th percentile for gestational age) in 2228 women (14). These authors used multivariate analysis to show that the following variables are significant risk factors for FGR: cigarette smoking (odds ratio $=3.18)$, low maternal weight gain (odds ratio $=2.96$ ), black ethnicity (odds ratio $=2.60$ ), prepregnancy underweight (odds ratio $=2.36)$, Asian ethnicity (odds ratio $=1.88$ ), primiparity (odds ratio $=1.85$ ), and low maternal height (odds ratio $=1.63$ ). It should be noted that Asian ethnicity and low maternal height may represent predisposing factors for normal, constitutionally small infants. Maternal history of low birth weight and a previous delivery of a low-birth weight infant have been added to the above list by $\operatorname{Kramer}(15,16)$.

The etiologic factors that result in the restriction of fetal growth potential can be grouped into intrinsic and extrinsic categories (Table 2). Intrinsic fetal abnormalities, such as genetic and congenital anomalies (Tables 3 and 4), often result in a symmetric FGR. The incidence of chromosomal aberrations in growth-restricted live newborns varies from

Table 2 Etiologic Factors in Fetal Growth Restriction

\begin{tabular}{ll} 
Intrinsic factors & \multicolumn{1}{c}{ Extrinsic factors } \\
Chromosomal aberrations & $\begin{array}{c}\text { Maternal-placental-fetal } \\
\text { infections }\end{array}$ \\
$\begin{array}{l}\text { Congenital structural defects } \\
\text { Constitutional (genetic } \\
\text { heritage) }\end{array}$ & $\begin{array}{l}\text { Chronic maternal disease } \\
\text { Substrate availability and toxins }\end{array}$
\end{tabular}

Table 3 Chromosomal Anomalies Associated with Fetal Growth Restriction

\begin{tabular}{ll} 
Common & \multicolumn{1}{c}{ Rare } \\
Trisomy 13 & Trisomy 22 \\
Trisomy 18 & Turner's syndrome \\
Trisomy 21 & $4 \mathrm{p}$ and $4 \mathrm{q}$ syndromes \\
& $5 \mathrm{p}$ and $5 \mathrm{q}$ syndromes \\
& $13 \mathrm{q}$ syndrome \\
& $18 \mathrm{p}$ and $18 \mathrm{q}$ syndromes \\
& Trisomy 9 mosaic syndrome \\
& Triploidy syndrome \\
& Partial trisomy 10q syndrome \\
& Ring 1 \\
& Ring 22
\end{tabular}


$30 \%$ in severe, early-onset cases to approximately $2.2 \%$ for late onset $(17,18)$. FGR and polyhydramnios were the most common associated findings in 386 structurally anomalous fetuses (52\% and 54.5\%, respectively) reported by Wladimiroff et al. (19). Symmetric growth restriction is also seen commonly with fetal infections (Table 5) and teratogenic agents (Table 6). Other extrinsic conditions, such as maternal vascular and placental causes of FGR (Table 5), often result in an asymmetric growth restriction pattern. Structural anomalies of the placenta and the umbilical cord (Table 7) have not been associated with a particular form of growth restriction.

\section{Table 4 Fetal Structural Anomalies Associated with Fetal Growth Restriction}

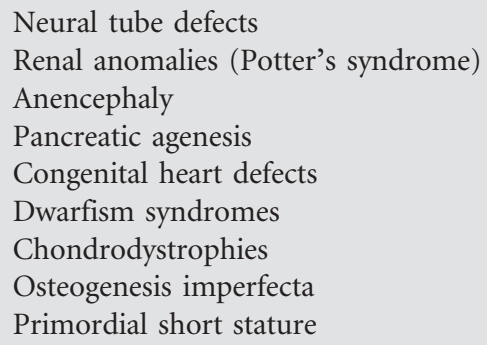

\section{Table 5 Maternal Causes of Fetal Growth Restriction}

Chronic disease
Hypertension
Collagen vascular disease
Renal disease
Cyanotic cardiopulmonary disease
Thyrotoxicosis
Advanced diabetes mellitus
Hemoglobinopathies
SS hemoglobin
Pre-eclampsia
Malnutrition
Infection
Toxoplasmosis
Malaria
Rubella
Cytomegalovirus
Herpes
Syphilis
Listeriosis

Table 6 Drugs and Medications Associated with Fetal Growth Restriction

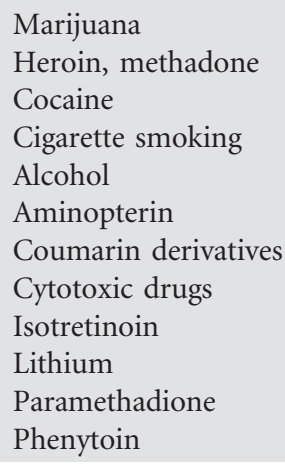

Antenatal identification of the risk factors for FGR is probably possible in as many as $50 \%$ of cases. Various etiologic factors require management decisions and have important neonatal implications. An intensive survey of maternal history (Table 8), physical examination, blood tests, and genetic studies is recommended. The astute clinician should also realize that the fetus of constitutionally small parents will most likely express its genetic heritage in its pattern of fetal growth. When growth restriction is recognized, intensive monitoring of the affected pregnancy is required, and early intervention on behalf of the compromised fetus may be advisable.

\section{SCREENING FOR FGR}

First- and second-trimester screening for FGR has not been shown to be clinically feasible. Abnormal maternal serum levels of beta hCG, pregnancy-associated plasma protein A, alphafetal protein, and inhibin-A have shown some association with the development of FGR. Unfortunately, the poor sensitivity and specificity coupled with the lack of an effective intervention strategy make this approach impossible to use as an effective and useful screening tool (20-25).

Third-trimester screening for FGR by serial fundal height and maternal weight-gain assessments should be routinely performed during prenatal care. Decreased fundal height may be associated with FGR (oligohydramnios, wrong dates, and fetal malposition should also be considered). The sensitivity of this test may be as high as 50\% (26). The false-positive rate is, however, quite high at approximately $56 \%$ (27). Beazley and Kurjak (28) reported that $25 \%$ of such assessments performed beyond 36 weeks of gestation were inaccurate by $>500 \mathrm{~g}$ and that the error increased at the extremes of the birth weight

\section{Table 7 Placental Factors in Fetal Growth Restriction}

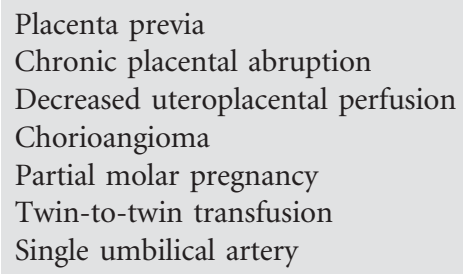

Table 8 Screening for Fetal Growth Restriction (FGR) by
History
Smoking
Altitude
Malnutrition (poor weight gain)
Previous FGR
Medications
Recreational drugs
Alcohol
Chronic maternal disease
Genetic anomalies
$\quad$ Maternal age
Family history
Habitual abortion
Alpha-fetoprotein
Environmental: lead, mercury, copper
First-trimester vaginal bleeding
Parents' size


range (when this information is most useful). Similar results were reported by Loeffler (29). Despite these inaccuracies, serial measurement of fundal height is nearly twice as sensitive as palpation for detection of FGR (30). Accuracy can be improved by using a standardized technique of measuring from the fundus to the symphysis along the fetal axis and plotting along a customized growth curve similar to that discussed previously for estimation of fetal weight (31).

Both single and serial third-trimester ultrasound examinations have been evaluated as a screening tool for FGR. Lindqvist and Molin recently demonstrated a sensitivity of $54 \%$ for a single scan at 32 weeks (32). A separate study showed a sensitivity for birth weight $<10$ th percentile to be $48 \%$ with a false-positive rate of $7 \%$ (33).

For pregnancies at high risk for FGR, there is a role for third-trimester screening. A prior history of stillbirth confers a $50 \%$ increase in the risk of FGR. Patients with diabetes during pregnancy are traditionally thought of as being at risk for macrosomia. However, these patients are also at a significantly increased risk for FGR, and screening is appropriate (7). Multiple gestation is also a risk factor for FGR. Obesity is not traditionally associated with FGR unless the patient also suffers from some other disorder such as chronic hypertension. Excessive weight gain during pregnancy is associated with macrosomia (34). The use of customized fetal growth curves has demonstrated that a subset of pregnancies in obese women who are categorized as having normal growth by populationbased norms in fact have impaired growth and are at increased risk for perinatal mortality (7). While there have been no studies showing improvements in neonatal morbidity or mortality with screening of routine pregnancies for FGR with third-trimester ultrasound examination, it is a reasonable approach in pregnancies at high risk for FGR. Pregnant women whose weight gain is inadequate or fundal height is decreased stand an increased risk of having FGR. Further evaluation by sonographic assessment should be offered to patients with risk factors and a high index of suspicion.

\section{DIAGNOSIS OF FGR}

FGR can be manifest by a variety of combinations of decreased fetal weight, body length, $\mathrm{HC}, \mathrm{AC}$, and soft tissue mass. Ultrasound is the only direct method available for assessing fetal size. It also provides for an assessment of factors associated with FGR such as fetal anomalies, oligohydramnios, and increased placental impedance. A thorough evaluation should include a detailed study of fetal, umbilical, and placental structural anomalies as well as measurements of the BPD, HC, AC, femur length (FL), and a semiquantitative assessment of amniotic fluid volume in the form of the amniotic fluid index (AFI). Morphometric ratios such as the HC:AC and FL:AC as well as a sonographic estimate of fetal weight can then be derived from these measurements. Umbilical artery (UA) Doppler velocity studies provide data regarding placental impedance to blood flow and may be constructive in patients who are at increased risk for FGR.

Several studies have evaluated the ability of both single and serial ultrasound measurements to identify FGR. The results vary widely, with sensitivities ranging from $44 \%$ to $90 \%$ (35). These measurements may not identify the symmetrically small fetus.
A strong correlation between fetal FL and neonatal crown to heel length can been demonstrated (36). The experience with this measurement as an independent predictor of FGR is limited. O'Brien and Queenan have shown that the FL helps differentiating between symmetric and asymmetric patterns of growth restriction (37). Overall, both the size of the fetal head and the length of the femur are affected late in most cases of FGR and, therefore, these measurements are too insensitive to be used as independent predictors of FGR.

Measurements of the fetal AC are commonly used as a means for identification of the growth-restrictive fetus. This measurement reflects the size of the liver (which correlates with the degree of fetal malnutrition) as well as the volume of subcutaneous fat. In addition, the AC is decreased in both symmetric and asymmetric growth restriction. Therefore, it is not surprising that sonographic measurements of the AC predict growth restriction more accurately than either the BPD or the FL. In a large study of 3616 sonographically dated pregnancies, Warsof and coworkers (38) have shown that AC measurements predicted FGR with a sensitivity of $61 \%$, specificity of $95 \%$, true-positive rate of $86 \%$, and true-negative rate of $83 \%$. Their results also indicate that the optimal gestational age to screen for FGR is approximately 34 weeks. Similarly, other investigators (39-41) have reported relatively high positive predictive values for this measurement (ranging from $84 \%$ to $100 \%$ ). Ferrazzi and coworkers (42) noted that the positive predictive value of the AC for detection of the growth-retarded fetus increases with gestational age (from $51 \%$ at $29-30$ weeks of gestation to $71 \%$ at term).

Accurate pregnancy dating is essential for the interpretation of AC measurements. However, uncertainty about gestational age occurs frequently and makes the differentiation between the appropriate-for-gestational age and the growth-restrictive fetus difficult. Because growth of the fetal abdomen is linear from 15 weeks of gestation onward, determination of the rate of growth offers a gestational age-independent parameter to identify the FGR fetus. Divon and coworkers (43) found a significant difference in the rate of growth of the fetal $\mathrm{AC}$ between FGR fetuses and fetuses that were appropriate for gestational age $(6.0 \pm 4.9 \mathrm{~mm} / 14$ days and $14.7 \pm 7.1 \mathrm{~mm} /$ 14 days, respectively). An increase in fetal AC of $<10 \mathrm{~mm} /$ 14 days had a sensitivity of $85 \%$ in identifying the FGR fetus. Thus, the use of serial measurements of AC offers an attractive method of identifying the FGR fetus, especially when gestational age is uncertain.

The comparison of growth in some body organs relative to others has led to the development of a number of morphometric ratios. These ratios are useful in the diagnosis of asymmetric FGR where the AC is smaller than expected when compared with the size of the head or the length of the body. Consequently, abnormally elevated FL/AC or $\mathrm{HC} / \mathrm{AC}$ aid in the identification of the fetus with asymmetric growth restriction. The average value of $\mathrm{HC} / \mathrm{AC}$ decreases in a linear fashion from 16 to 40 weeks of gestation. An HC/AC >2 SDs above the mean has been suggested as an abnormal value (44). Kurjak and coworkers correctly identified $80 \%$ of growthretarded fetuses with the use of this ratio (40). By contrast, Divon and coworkers (45) applied this ratio to a population of FGR fetuses of mixed etiologies and reported a relatively low sensitivity of $36 \%$, with specificity of $90 \%$, positive predictive 
value of $67 \%$, and negative predictive value of $72 \%$. These authors concluded that the $\mathrm{HC} / \mathrm{AC}$ ratio tends to be a better predictor of FGR in situations related to third-trimester "placental insufficiency" resulting in asymmetric fetal growth.

Hadlock and coworkers (41) reported on the use of the FL/AC ratio as a gestational age-independent index of fetal growth. From 21 weeks of gestation to term, the normally grown fetus has an FL/AC ratio of $22 \% \pm 2 \%( \pm \mathrm{SD})$. An FL/AC $>23.5 \%$ was associated with a sensitivity of $60 \%$ and a specificity of $90 \%$ for identification of the growth-retarded fetus. Similarly, Divon and coworkers (43) reported a sensitivity of $55 \%$ for the same cutoff value and concluded that this parameter is fairly useful in differentiating between the fetus demonstrating appropriate growth and the growthretarded fetus when gestational age is uncertain.

The most commonly used definition of FGR is a birth weight $<10$ th percentile for gestational age. Therefore, it seems reasonable to use sonographic estimation of fetal weight to diagnose FGR in the antenatal period. This parameter would also allow the clinician to evaluate changes in interval growth as compared with normative standards available throughout the latter part of pregnancy. Various equations that incorporate different variables (i.e., BPD, HC, AC, and FL) have been proposed in an attempt to accurately estimate the fetal weight (46). In general, these estimates are associated with a mean percentage absolute error of approximately $7 \%$ to $10 \%$ (47). Several investigators have evaluated the ability of fetal weight estimates to identify the fetus with growth restriction. Divon and coworkers (45) estimated fetal weight using Hadlock's formula based on BPD, AC, and FL in a population of 127 high-risk patients, 45 of whom delivered FGR neonates. They reported a sensitivity and specificity of $87 \%$, positive predictive value of $78 \%$, and negative predictive value of $92 \%$ for estimated fetal weight $<10$ th percentile for gestational age. Compared with other indices used to detect FGR, such as HC:AC ratio, FL:AC ratio, amniotic fluid volume, and Doppler velocimetry, the best predictor appeared to be an estimated fetal weight of $<10$ th percentile. Ott and Doyle (36) evaluated 595 pregnancies in which ultrasound examinations were performed within 72 hours of delivery. Fetal weight was estimated using measurements of the BPD and AC. They reported a sensitivity of $90 \%$ and a specificity of $80 \%$. All infants with severe growth restriction (birth weight $<3$ rd percentile) were correctly identified, for a sensitivity of $100 \%$.

Not surprisingly, equations that incorporate head and body proportions as well as body length seem to provide more accurate sonographic estimates of fetal weight. This has been shown to be especially true for the FGR fetus. Guidetti and coworkers (48) showed that estimates of fetal weight that include AC and FL correlated best with birth weight. Overall, $75 \%$ of the estimates using the BPD-AC-FL equation were within $10 \%$ of the actual birth weight. Clearly, as long as the definition of FGR is based on birth weight and gestational age criteria, the ability to diagnose FGR is significantly dependent on the accuracy of the estimated fetal weight.

Oligohydramnios has long been recognized as a complication of FGR. This is probably caused by decreased urination secondary to decreased renal perfusion reflecting redistribution of blood flow from non-vital to vital organs.
Manning and coworkers (49) used real-time sonography to qualitatively assess amniotic fluid volume in 120 pregnancies in which FGR was suspected. Defining oligohydramnios as the largest vertical pocket of fluid measuring $<1 \mathrm{~cm}$, they reported a sensitivity of $84 \%$, specificity of $97 \%$, positive predictive value of $90 \%$, and negative predictive value of $95 \%$. Other investigators, however, have not confirmed these results. Divon and coworkers (45) evaluated 127 patients referred with a clinical suspicion of FGR. Oligohydramnios was defined by the vertical dimension of the largest pocket of fluid measuring $<2 \mathrm{~cm}$. They reported a sensitivity of only $16 \%$ and a specificity of $98 \%$. The low sensitivity and high specificity of amniotic fluid volume as an indicator of FGR have also been shown by others (50).

An AFI of less than $5 \mathrm{~cm}$ has been suggested as a sonographic marker of oligohydramnios (51). However, the reported experience with this cutoff value and its relationship with either diagnosis or outcome in pregnancy complicated by FGR is limited. The presence of oligohydramnios assumes increased importance when gestational age is questionable. If oligohydramnios is present in the absence of ruptured membranes, congenital anomalies, or postdatism, FGR is the most likely explanation. Thus, the presence of oligohydramnios can be considered a gestational age-independent predictor of FGR (49,51-53). Its presence is considered an ominous sign with respect to fetal compromise and is generally accepted as an indication for delivery for fetuses with mature lungs or gestational age of 34 weeks or greater.

Reliable ultrasound growth assessment of the fetus continues to present a clinical challenge. It is possible that as the use of customized growth curves becomes more widely accepted, the ability of ultrasound to accurately identify infants affected by FGR and to differentiate them from those that are constitutionally small will improve. Accurate dating is obviously essential to the diagnosis of FGR using either population-based or customized growth curves. Indicators of fetal growth that are independent of gestational age such as FL: AC ratio, oligohydramnios, and serial sonographic measurements of fetal AC should be considered whenever gestational age is unknown.

\section{ANTEPARTUM FETAL TESTING}

Intensive fetal surveillance should be instituted once growth restriction is detected or suspected. The nonstress test (NST) is most commonly used for this purpose. A reactive NST indicates that the fetal central nervous system is well oxygenated and has the ability to integrate autonomic nervous system reflexes that are initiated with body movements and result in an acceleration of the fetal heart rate (FHR). A growth-retarded fetus may be moving less frequently in order to conserve energy or due to asphyxiation as a result of decreased placental function in providing adequate nutrients or respiratory exchange. Under these circumstances, the NST is likely to be nonreactive. A review (54) of NSTs has suggested that fetuses with normal growth have a lower incidence of nonreactive tests when compared with growth-retarded fetuses.

A reactive NST is highly indicative of a well-oxygenated fetus. The false-negative rate of a reactive NST performed twice weekly is approximately 2 to 3 in 1000 . The converse, 
however, is not true. The association between a nonreactive NST and fetal hypoxia is rather low. The false-positive rate of a 40-minute nonreactive NST is approximately $80 \%$ and, therefore, in addition to fetal hypoxia other causes of nonreactivity should be considered (i.e., fetal sleep-wake cycles, prematurity, congenital anomalies, maternal use of medications and drugs). A nonreactive FHR pattern with or without spontaneous decelerations may be the first sign of fetal structural anomalies associated with FGR (55). Therefore, a detailed sonographic evaluation combined with the analysis of fetal karyotype, if significant fetal anomalies are found, should be considered under these conditions. In addition, the growthretarded fetus is at an increased risk of spontaneous variable FHR decelerations due to cord decompression resultant from oligohydramnios and/or decreased Wharton's jelly. Therefore, an ultrasound assessment of the amniotic fluid volume should be performed whenever variable decelerations are detected during NST.

Several authors have suggested that the presence of severe oligohydramnios in a growth-retarded fetus should be used as an indication for delivery. Recent Doppler studies have shown that oligohydramnios in FGR fetuses is associated with increased resistance to blood flow in the fetal renal artery (56). Decreased renal perfusion in these fetuses is probably part of a compensatory mechanism resulting in redistribution of blood flow from non-vital to vital organs. As long as the central nervous system is adequately perfused, other tests of fetal well-being [such as the NST, contraction stress test (CST), or fetal breathing movements] will be normal. Most clinicians would intervene before the fetus decompensates as manifested by the presence of oligohydramnios combined with an abnormal NST, CST, or biophysical profile (BPP) score.

Chamberlain and coworkers (41) applied a 2-cm vertical "depth" rule to define oligohydramnios and concluded that its presence is an ominous sign with respect to fetal health. The corrected perinatal mortalities for fetuses with marginal and decreased amniotic fluid volume were 37.7 in 1000 and 109.4 in 1000, respectively. These authors concluded that the presence of oligohydramnios should prompt a consideration for a timely delivery.

Additional causes of oligohydramnios should be considered prior to intervention such as premature rupture of the membranes or obstructive uropathy. Differentiating renal agenesis from a structurally normal but growth-restrictive fetus may be more difficult. Fetuses with renal agenesis are often growth retarded. In addition, visualization of the fetal kidneys may be impossible in the presence of oligohydramnios. Instillation of normal saline into the amniotic cavity may improve the quality of the sonographic image.

The high false-positive rate of abnormal NST requires additional testing prior to intervention on a presumed diagnosis of fetal compromise. In the face of a nonreactive NST, fetal well-being is commonly established by BPP scoring. A BPP score of 8 to 10:10 is associated with a low risk for either fetal asphyxia or imminent fetal death. In general, as the BPP score decreases, the likelihood of fetal asphyxia increases. As with NST, BPP scores are, in general, lower in FGR fetuses when compared with other "high-risk" fetuses. Devoe and coworkers (57) noted that their FGR patients accounted for
$33 \%$ of all abnormal BPP scores, although they constituted only $20 \%$ of the tested population.

Gradual deterioration of fetal health is reflected by decreased or absent fetal activity. Therefore, BPP testing represents a rational approach for fetal surveillance. Baskett and coworkers (58) reported that growth-restrictive fetuses were more likely to have perinatal complication with the incidences of $30 \%, 23 \%$, and $21 \%$ for oligohydramnios, decreased fetal body movements, and decreased fetal tone, respectively.

Pollack and coworkers (47) have reported on 4727 patients undergoing NST followed by BPP testing when indicated. FGR was the primary indication for fetal testing in $478(10 \%)$ patients. No fetal deaths were reported in the tested FGR population. In a large study of 19,221 patients, Manning and coworkers (59) noted that 4 of 14 fetal deaths, following normal tests, occurred in FGR pregnancies. These results underscore the fact that intensive fetal testing is associated with an extremely low false-negative rate in the setting of FGR.

\section{Doppler Velocimetry}

Normal fetal growth is determined by multiple maternal-fetal variables. Continuous blood flow on either side of the placenta and adequate fetal perfusion are obviously an absolute necessity. Indeed, FGR is associated with various abnormalities of the uteroplacental, umbilical, and fetal circulations $(60,61)$. Doppler devices allow for a noninvasive evaluation of blood flow by displaying Doppler-shifted frequencies that are proportional to the velocity of red blood cells. Qualitative assessment of these waveforms is provided by angle-independent indices that are based on various ratios between the systolic and diastolic components of the waveform. Most studies concur that the simplest index (i.e., the systolic: diastolic ratio, $\mathrm{S}: \mathrm{D})$ is clinically as useful as any of the more complicated indices such as the resistance index (RI) [(Peak systolic velocity - end-diastolic velocity)/peak systolic velocity] or the pulsatility index (PI) [(peak systolic velocity - end-diastolic velocity)/average frequency shift].

Maternal and fetal blood flow velocity waveforms are influenced by the stroke volume, pressure gradient, heart rate, vessel wall elasticity, and blood viscosity. Fast heart rates result in a relatively high end-diastolic component, while increased downstream impedance causes low end-diastolic flows. Since blood flow during diastole is mostly passive, as placental impedance increases, the umbilical arterial end-diastolic flow decreases. Therefore, severe placental impedance is associated with low, absent, or reversed end-diastolic umbilical blood flow.

On the maternal side, some evidence (44) suggests that changes in uterine artery velocity waveforms (arcuate arteries) may be detected as early as the second trimester in some pregnancies, which eventually develop pre-eclampsia or FGR. Uterine artery Doppler has a sensitivity rate of $25 \%$ to $75 \%$ and a false-positive rate of $5 \%$ to $10 \%$ in detecting early onset of FGR (62-64). Newnham and coworkers (65), who evaluated uteroplacental blood flow at 14, 18, 24, 28, and 34 weeks of gestation, reported that abnormal waveforms were significantly correlated with the future development of fetal hypoxia; however, they were not predictive of subsequent development of FGR. Both Chambers and coworkers (39) and 
Jacobson and coworkers (66) concluded that Doppler studies of the uteroplacental perfusion are of a limited clinical value due to their poor sensitivity and high false-positive rate.

Many studies have reported a significant decrease in enddiastolic flow of the UA and the descending aorta of the growth-restricted fetus (61). These studies indicate that some forms of FGR may result from "placental insufficiency" defined by increased placental impedance secondary to a decreased number of intraplacental arterial channels (67-69). A decreased PI in the internal carotid arteries (70) in FGR fetuses probably reflects enhanced perfusion of the brain (brain sparing effect) from redistribution of blood flow.

Trudinger and coworkers (71) have reported on 2178 highrisk pregnancies for which UA velocity waveforms were evaluated. The incidence of FGR (birth weight $<10$ th percentile) in this population was $27 \%$; $50 \%$ of their FGR fetuses had an abnormal ( $>95$ th percentile) umbilical arterial S:D ratio. The odds ratio of a fetus with an abnormal arterial velocity waveform for being diagnosed as growth restricted by birth weight criteria was 5.9 (95\% confidence interval of 4.7-7.3). Moreover, preterm infants with abnormal S:D ratios spent twice as long in the neonatal intensive care unit as those with normal velocity waveforms. These authors concluded that "in high-risk pregnancy, Doppler UA flow velocity waveforms predict the most compromised fetuses in terms of growth restriction and requirement for neonatal intensive care."

The ability to detect FGR with Doppler velocimetry varies considerably (Table 9). Overall, the odds ratios demonstrate a significantly increased likelihood of delivering an FGR newborn once the Doppler study is abnormal. These studies are hampered by the absence of standard definitions for both FGR and abnormal velocity waveforms. In addition, most studies have preferentially evaluated high-risk populations with an abnormally high incidence of FGR. Furthermore, the positive predictive value of abnormal Doppler velocimetry is influenced by the prevalence of FGR in the population. The prevalence is in turn affected by whether FGR is defined by population-based or customized criteria. Nonetheless, Doppler ultrasound of the UA remains a mainstay of evaluation of the fetus with FGR, and abnormal values are associated with adverse fetal outcomes (72-75).

A few studies have evaluated the usefulness of screening the general population for FGR with the use of UA velocimetry. Beattie and Dornan (76) evaluated a total of 2097 singleton pregnancies at 28,34 , and 38 weeks of gestation. Less than $20 \%$ of this population was defined as "high-risk" pregnancies. The sensitivity of an abnormal S:D ratio for prediction of FGR was rather low (from $31 \%$ to $40 \%$ depending on the gestational age at the time of the study). Bruinse and coworkers (77) studied an unselected population of 405 pregnant women at 28 and 34 weeks of gestation. Doppler screening for FGR had a low sensitivity of $17 \%$ and $22 \%$, respectively. Hanretty and coworkers (78) screened uteroplacental and umbilical arteries in 543 unselected patients at 20 to 30 and 34 to 36 weeks of gestation. They found no difference in the outcome of pregnancies with normal or abnormal uteroplacental waveforms. However, birth weights were significantly lower in patients with an abnormal UA waveform at either gestational age. These studies suggest that Doppler screening of the general population is associated with a low yield. The cost: benefit ratio of such screening programs is unclear.

The combined use of Doppler velocimetry with real-time ultrasound for the diagnosis of FGR was reported by Divon and coworkers (45). The study population consisted of 127 patients referred with a clinical suspicion of FGR. Forty-five infants (35\%) were identified as having FGR by birth weight $<10$ th percentile for gestational age. These authors concluded that neither sonographic measurements nor Doppler tests were uniformly successful in identifying the growth-restricted infant. Overall, the best prediction was offered by sonographic estimates of fetal weight, which correctly identified 39 of the 45 FGR infants. An S:D ratio $>3$ was seen in $49 \%$ of the FGR fetuses. All indices similarly predicted the non-FGR infant (range of specificities: 87-98\%). Similar results were reported by Kay and coworkers (79) on 48 patients who underwent sonographic measurements and Doppler evaluations of the uterine and umbilical vasculatures. Chambers and coworkers (39) studied 145 pregnancies suspected of being small for dates and found that sonographic measurements of the fetal AC were superior to UA velocimetry for prediction of FGR with sensitivities of $73 \%$ and $47 \%$, respectively. It should, however, be emphasized that Doppler can be used as a gestational age-independent index of FGR (i.e., S:D $>3.0$ is abnormal after 30 weeks of gestation) while evaluation of the AC requires accurate gestational dating.

The utility of Doppler velocimetry in the prediction of adverse outcome in pregnancies at risk for FGR was investigated by Berkowitz and coworkers (80). These authors reported that approximately $50 \%$ of their FGR infants had

Table 9 Umbilical Artery Velocimetry for Prediction of Fetal Growth Restriction in High-Risk Pregnancies

\begin{tabular}{|c|c|c|c|c|}
\hline First author & $\begin{array}{l}\text { Study patients, } \mathbf{n} \\
\text { (prevalence of FGR) }\end{array}$ & Definition of FGR & $\begin{array}{l}\text { Definition ( } 95 \% \text { confidence } \\
\text { waveform interval) }\end{array}$ & Odds ratio \\
\hline Divon (1988) & $127(35 \%)$ & BW $<10$ th percentile & $\mathrm{S}: \mathrm{D}>3.0$ & $14.7(4.6-50.5)$ \\
\hline Berkowitz (1988) & $168(25 \%)$ & $\mathrm{BW}<10$ th percentile & $\mathrm{S}: \mathrm{D}>3.0$ & $6.6(2.7-16.4)$ \\
\hline Dempster (1989) & $205(40 \%)$ & BW $<10$ th percentile & $\mathrm{S}: \mathrm{D}>95$ th percentile & $3.3(1.6-6.5)$ \\
\hline Sijmons (1989) & $400(22 \%)$ & $\mathrm{BW}<10$ th percentile & PI $>95$ th percentile & $4.4(2.0-9.4)$ \\
\hline Lowery (1990) & $146(14 \%)$ & BW $<10$ th percentile & $\mathrm{S}: \mathrm{D}>4.0$ & $10.9(3.8-32.5)$ \\
\hline Kay (1991) & $48(37.5 \%)$ & BW $<10$ th percentile & $\mathrm{S}: \mathrm{D}>2$ standard deviations above mean & $11.3(2.1-52.0)$ \\
\hline Trudinger (1991) & $2178(27 \%)$ & BW $<10$ th percentile & S:D $>95$ th percentile & $5.9(4.7-7.3)$ \\
\hline
\end{tabular}


abnormal UA velocity waveforms and that these waveforms were valuable in identifying the growth-restrictive infant who was at increased risk for one or more of the following outcome criteria: early delivery, reduced birth weight, oligohydramnios, neonate intensive-care unit admission, and prolonged hospital stay. Reuwer and coworkers (81) reported similar results, demonstrating that Doppler velocimetry allowed accurate and early recognition of those growth-restrictive fetuses that would become compromised antenatally. Several investigators have noted that all their FGR-related neonatal deaths and a majority of their intensive-care admissions were confined to those fetuses with abnormal Doppler velocimetry results (72-75,82-85). While normal UA Doppler cannot definitively guarantee a normal fetus (86), abnormal Doppler values are associated with adverse neonatal outcomes.

Absent or reverse end-diastolic flow is also associated with fetal aneuploidy. Wenstrom and coworkers (87) studied 450 high-risk pregnancies that underwent level II sonograms after 20 weeks of gestation. Twenty-two of these patients had absent or reversed diastolic flow in the UA. Of these, 10 had FGR, 10 had either congenital malformations or were aneuploid, and in 2 cases, the etiology was not identified. Among the four cases with fetal aneuploidy, one had multiple severe anomalies including holoprosencephaly, renal agenesis, and polydactyly, and the other three had FGR. Therefore, a thorough evaluation of fetal anatomy and search for the underlying etiology, which causes absent or reversed diastolic blood flow, should be attempted when managing a patient with FGR. Whether a karyotype should also be obtained in patients with absent diastolic or reversed diastolic flow without major congenital anomalies or severe symmetric FGR needs further evaluation.

\section{MANAGEMENT}

Once the diagnosis of FGR is suspected, several management issues should be considered. These include parental counseling regarding a search for the etiology of growth restriction, fetal surveillance, timing of delivery, and neonatal outcome. In addition, the need for bed rest and adequate maternal nutrition should be discussed with the patient. The use of alcohol, tobacco or any other unnecessary toxins should be discouraged.

Evaluation of the FGR fetus with serial ultrasound examinations and Doppler blood flow is a hallmark of the management. Absent or reverse end-diastolic flow in the UA is a useful marker for potential fetal compromise. As many as $40 \%$ of acidotic fetuses will demonstrate this finding (88). Overall, absent or reverse end-diastolic flow has roughly a $60 \%$ sensitivity and specificity for adverse perinatal outcome (73). Doppler evaluation of the fetal middle cerebral artery (MCA) has also been studied. Multiple studies have demonstrated that lower values of MCA PI are associated with FGR (89-91). Chiba and Murakami (92) measured MCA RI at the time of cordocentesis. They reported that a decreasing MCA RI was associated with increasing $\mathrm{PCO}_{2}$ in the fetal circulation. Bahado-Singh et al. (93) reported that an abnormal cerebroplacental ratio (MCA PI divided by UA PI) has a statistically significant correlation with adverse perinatal outcome. This preferential shunting of blood to the brain, the so-called brain-sparing effect, can be reversed in a fetus with severe uteroplacental insufficiency. Konje and colleagues (94) reported a reversal of the brain sparing effect in eight fetuses with FGR, death of half in utero, and the death of the other half during the neonatal period. While MCA peak systolic velocity (PSV) is more classically used in the evaluation of the anemic fetus, Mari et al. have reported that an elevated MCA PSV is the best predictor of perinatal mortality in the fetus with FGR (95).

Researchers have also investigated the use of Doppler of the ductus venosus (DV) as a marker for acute fetal decompensation. Ductus venous waveforms have been shown to become abnormal about 48 to 72 hours prior to the BPP abnormality (96). The ongoing "Trial of umbilical and fetal flow in Europe" is conducted to define the optimal timing in delivering FGR fetuses using DV Doppler and traditional FHR cardiotocography (7).

FHR tracing has also been extensively used in monitoring FGR. Loss of short-term variability is temporally related with abnormal DV Doppler. Due to its high false-positive rate (as high as 50\%) (97), the use of FHR alone to monitor FGR pregnancies is inadequate. When used in concert with other monitoring, such as the BPP or DV Doppler, it can be useful. Hecher and associates demonstrated that loss of short-term FHR variability with concurrent abnormalities on DV Doppler is a particularly strong predictor of adverse perinatal outcome (98). The value of the BPP in managing FGR pregnancies is also difficult to determine. As noted above, the BPP has both a very low false-negative rate and a weak correlation between abnormal BPP and fetal acidosis (59). In addition, other studies have shown no association between abnormal BPP and cognitive function later in life (99), suggesting a higher false-positive rate than that may be recognized. Changes in amniotic fluid volume and FHR appear to be the early predictors of impending fetal decompensation, while fetal tone, breathing, and body movement appear to be associated with more advanced stages of fetal deterioration (7).

The increased incidence of perinatal morbidity and mortality among FGR pregnancies often necessitates early delivery. Preterm delivery should be considered in the growthrestrictive fetus when fetal surveillance indicates a high probability of fetal asphyxia. The best method of fetal surveillance in these pregnancies is yet to be determined. The decision regarding when to deliver is further complicated by the fact that a fetus at $<10$ th percentile on a customized growth curve is at risk of demise even with normal UA Doppler studies. Conversely, Boers and associates found no significant differences in neonatal outcome with expectant management of FGR between 36 and 39 weeks of gestation in the DIGITAT study (100). In this study, participants in the expectant management arm had twice-weekly fetal heart tone and UA Doppler evaluation. Management of the FGR pregnancy with a known fetal anomaly or an abnormal karyotype should be tailored to the specific abnormality.

Timing of the delivery of FGR fetuses is predicated on balancing the risks of prematurity with the risk of fetal demise from expectant management. The difficulty lies in the lack of optimal fetal surveillance methods. In the very preterm FGR infant, since the risks of major complications from prematurity are high, it seems prudent to delay the delivery until fetal lung maturity can be documented as long as fetal surveillance is reassuring. On the other hand, Boers and associates (100) 
Table 10 Indications for Delivery in Fetal Growth Restriction

Fetal compromise

Deteriorating maternal condition

Term pregnancy with a favorable cervix

Term pregnancy with markedly abnormal umbilical artery velocity waveform

Absence of fetal growth with documented lung maturity

showed no change in outcomes with expectant management of late preterm FGR as compared with early delivery, while Figueras et al. (7) demonstrated that FGR fetuses are at high risk for demise even with reassuring fetal surveillance.

Amniocentesis for documentation of lung maturity should be considered when FGR is present. The benefits of conservative management in the presence of documented lung maturity are probably limited. Possible indications for delivery are shown in Table 10.

\section{LONG-TERM CONSEQUENCES OF FGR}

The consequences of FGR extend beyond delivery. FGR is associated with cardiovascular disease, abnormalities of the hypothalamic pituitary axis and structural abnormalities of the cardiovascular system, insulin resistance, and the metabolic syndrome in adulthood (101).

FGR is associated with an increased risk of cardiovascular death as an adult (102). Low birth weight has also been associated with elevated cholesterol when compared with matched appropriate for gestational age controls by the age of 12 years (103). A more recent study demonstrated that FGR was associated with decreased stroke volume, abnormally globular ventricles, higher blood pressure, and increased intima-media thickness in children (104).

The "thrifty phenotype" hypothesis was originally proposed in 1992 and stated that a metabolically deprived developing fetus would become metabolically programmed for insulin resistance and impaired glucose metabolism (101). There was a strong association between FGR and elevated fasting glucose levels, elevated fasting insulin levels, prevalence of type 2 diabetes mellitus, 2-hour postprandial glucose levels, and serum insulin levels (105). Women who were born SGA have elevated dehydroepiandrosterone sulfate levels, elevated follicle-stimulating hormone levels, and a higher incidence of anovulation (106-108).

The metabolic consequences to the FGR project well beyond the womb and predispose the affected fetus to a wide range of health risks. Since there is no effective treatment to reverse FGR, controlling maternal risk factors such as smoking, alcohol consumption, illicit drug use, and preventable infection is important in reducing the perinatal morbidity and mortality and adverse long-term consequences.

\section{CONCLUSION}

Fetal growth is an important sign of fetal well-being. The clinical significance of FGR stems from its being a significant predictor of perinatal morbidity and mortality. FGR is an entity whose definition is controversial and whose etiology and natural history are poorly understood. Its antenatal diagnosis is less than optimal and many management issues remain unresolved. Nonetheless, it is a condition of paramount importance to optimize perinatal outcome by timely and accurate diagnosis coupled with appropriate intervention. Further research is needed to improve our understanding, diagnosis, and management of FGR.

\section{REFERENCES}

1. Starfield B, Shapiro S, McCormick M, et al. Mortality and morbidity in infants with intrauterine growth restriction. Pediatrics 1982; 101.

2. Heinonen K, Hakulinen A, Jokela V. Survival of the smallest. Time trends and determinants of mortality in a very preterm population during the 1980s. Lancet 1988; 2: 204-7.

3. Kazzi J, Poland RL. Neonatal risks associated with intrauterine growth retardation. In: Gross TL, ed. Intrauterine Growth Retardation-A Practical Approach. Chicago: Year Book Medical Publishers, 1989.

4. Goldenberg RL, Cutter GR, Hoffman HJ, et al. Intrauterine growth retardation: standards for diagnosis. Am J Obstet Gynecol 1989; 161:271-7.

5. Lubchenco LO, Hansman C. Intrauterine growth as estimated from liveborn birthweight data at 24 to 42 weeks of gestation. Pediatrics $1963 ; 32$.

6. Brenner WE, Edelman DA, Hendricks CH. A standard of fetal growth for the United States of America. Am J Obstet Gynecol 1976; 126: 555-64.

7. Figueras F, Gardosi J. Intrauterine growth restriction: new concepts in antenatal surveillance, diagnosis, and management. Am J Obstet Gynecol 2011; 204: 288-300.

8. Gruenwald P. Chronic fetal distress and placental insufficiency. Biol Neonat 1963; 5: 215-65.

9. Rohrer F. Der Index der Körperfulle als Mars des Ernährungszustandes. Münch Med Wochenschr 1921; 65.

10. Miller HC. Intrauterine growth retardation. An unmet challenge. Am J Dis Child 1981; 135: 944-8.

11. Nicolaides KH, Snijders R, Noble P. Cordocentesis in the study of growth retarded fetuses. In: Divon MY, ed. Abnormal Fetal Growth. New York: Elsevier Science, 1991.

12. Miller HC. Prenatal factors affecting intrauterine growth retardation. Clin Perinatol 1985; 12: 307-18.

13. Basel D, Lederer R, Diamant YZ. Longitudinal ultrasonic biometry of various parameters in fetuses with abnormal growth rate. Acta Obstet Gynecol Scand 1987; 66: 143-9.

14. Abrams B, Newman V. Small-for-gestational-age birth: maternal predictors and comparison with risk factors of spontaneous preterm delivery in the same cohort. Am J Obstet Gynecol 1991; 164: 785-90.

15. Kramer MS. Intrauterine growth and gestational duration determinants. Pediatrics 1987; 80: 502-11.

16. Kramer MS. Determinants of low birth weight: methodological assessment and meta-analysis. Bull World Health Organ 1987; 65: 663-737.

17. Chen AT, Falek A, Lester W. Chromosome aberrations in full-term low birth weight neonates. Humangenetik 1974; 21: 13-16.

18. Weiner CP, Williamson RA. Evaluation of severe growth retardation using cordocentesis - hematologic and metabolic alterations by etiology. Obstet Gynecol 1989; 73: 225-9

19. Wladimiroff JW, Sachs ES, Reuss A, et al. Prenatal diagnosis of chromosome abnormalities in the presence of fetal structural defects. Am J Med Genet 1988; 29: 289-91.

20. Dugoff L, Hobbins JC, Malone FD, et al. First-trimester maternal serum PAPP-A and free-beta subunit human chorionic gonadotropin concentrations and nuchal translucency are associated with obstetric complications: a population-based screening study (the FASTER Trial). Am J Obstet Gynecol 2004; 191: 1446-51.

21. Smith GC, Stenhouse EJ, Crossley JA, et al. Early pregnancy levels of pregnancy-associated plasma protein a and the risk of intrauterine growth restriction, premature birth, preeclampsia, and stillbirth. J Clin Endocrinol Metab 2002; 87: 1762-7.

22. Killam WP, Miller RC, Seeds JW. Extremely high maternal serum alphafetoprotein levels at second-trimester screening. Obstet Gynecol 1991; 78: 257-61.

23. Lepage N, Chitayat D, Kingdom J, Huang T. Association between second-trimester isolated high maternal serum maternal 
serum human chorionic gonadotropin levels and obstetric complications in singleton and twin pregnancies. Am J Obstet Gynecol 2003; 188: 1354-9.

24. Aquilina J, Thompson O, Thilaganathan B, Harrington K. Improved early prediction of pre-eclampsia by combining second-trimester maternal serum inhibin-A and uterine artery Doppler. Ultrasound Obstet Gynecol 2001; 17: 477-84.

25. D'Anna R, Baviera G, Corrado F, et al. Is mid-trimester maternal serum inhibin-A a marker of preeclampsia or intrauterine growth restriction? Acta Obstet Gynecol Scand 2002; 81: 540-3.

26. Rosenberg K, Grant JM, Tweedie I, et al. Measurement of fundal height as a screening test for fetal growth retardation. Br J Obstet Gynaecol 1982; 89: 447-50.

27. Welch RA, Wolfe H, Sokol RJ. The role of clinical determinants in predicting intrauterine growth restriction. In: Gross TL, ed. Intrauterine Growth Retardation. Chicago: Year Book Medical Publishers, 1989.

28. Beazley JM, Kurjak A. Prediction of foetal maturity and birthweight by abdominal palpation. Nurs Times 1973; 69: 763-5.

29. Loeffler FE. Clinical foetal weight prediction. J Obstet Gynaecol Br Commonw 1967; 74: 675-7.

30. Gardosi J, Francis A. Controlled trial of fundal height measurement plotted on customised antenatal growth charts. Br J Obstet Gynaecol 1999; 106: 309-17.

31. Morse K, Williams A, Gardosi J. Fetal growth screening by fundal height measurement. Best Pract Res Clin Obstet Gynaecol 2009; 23: 809-18.

32. Lindqvist PG, Molin J. Does antenatal identification of small-forgestational age fetuses significantly improve their outcome? Ultrasound Obstet Gynecol 2005; 25: 258-64.

33. Skovron ML, Berkowitz GS, Lapinski RH, Kim JM, Chitkara U. Evaluation of early third-trimester ultrasound screening for intrauterine growth retardation. J Ultrasound Med 1991; 10: 153-9.

34. Hillier TA, Pedula KL, Vesco KK,. et al. Excess gestational weight gain: modifying fetal macrosomia risk associated with maternal glucose. Obstet Gynecol 2008; 112: 1007-14.

35. Guidetti D, Divon MY. Sonographic detection of the IUGR fetus. In: Divon MY, ed. Abnormal Fetal Growth. New York: Elsevier Science, 1991.

36. Ott WJ, Doyle S. Ultrasonic diagnosis of altered fetal growth by use of a normal ultrasonic fetal weight curve. Obstet Gynecol 1984; 63: 201-4.

37. O'Brien GD, Queenan JT. Growth of the ultrasound fetal femur length during normal pregnancy. Part I. Am J Obstet Gynecol 1981; 141: 833-7.

38. Warsof SL, Cooper DJ, Little D, Campbell S. Routine ultrasound screening for antenatal detection of intrauterine growth retardation. Obstet Gynecol 1986; 67: 33-9.

39. Chambers SE, Hoskins PR, Haddad NG, et al. A comparison of fetal abdominal circumference measurements and Doppler ultrasound in the prediction of small-for-dates babies and fetal compromise. Br J Obstet Gynaecol 1989; 96: 803-8.

40. Kurjak A, Kirkinen P, Latin V. Biometric and dynamic ultrasound assessment of small-for-dates infants: report of 260 cases. Obstet Gynecol 1980; 56: 281-4.

41. Hadlock FP, Deter RL, Harrist RB, Roecker E, Park SK. A dateindependent predictor of intrauterine growth retardation: femur length/ abdominal circumference ratio. AJR Am J Roentgenol 1983; 141: 979-84.

42. Ferrazzi E, Nicolini U, Kustermann A, Pardi G. Routine obstetric ultrasound: effectiveness of cross-sectional screening for fetal growth retardation. J Clin Ultrasound 1986; 14: 17-22.

43. Divon MY, Chamberlain PF, Sipos L, Manning FA, Platt LD. Identification of the small for gestational age fetus with the use of gestational age-independent indices of fetal growth. Am J Obstet Gynecol 1986; 155: 1197-201.

44. Campbell S, Pearce JM, Hackett G, Cohen-Overbeek T, Hernandez C. Qualitative assessment of uteroplacental blood flow: early screening test for high-risk pregnancies. Obstet Gynecol 1986; 68: 649-53.

45. Divon MY, Guidetti DA, Braverman JJ, et al. Intrauterine growth retardation-a prospective study of the diagnostic value of real-time sonography combined with umbilical artery flow velocimetry. Obstet Gynecol 1988; 72: 611-14.

46. Woolf J, Divon MY. Microcomputer program for fetal growth assessment. In: Divon MY, ed. Abnormal Fetal Growth. New York: Elsevier Science, 1991.
47. Divon MY. Intrauterine growth retardation. In: Winn HN, Hobbins JC, eds. Clinical Maternal Fetal Medicine. Pearl River, NY: Parthenon Publishing Group, 2000.

48. Guidetti DA, Divon MY, Braverman JJ, Langer O, Merkatz IR. Sonographic estimates of fetal weight in the intrauterine growth retardation population. Am J Perinatol 1990; 7: 5-7.

49. Manning FA, Hill LM, Platt LD. Qualitative amniotic fluid volume determination by ultrasound: antepartum detection of intrauterine growth retardation. Am J Obstet Gynecol 1981; 139: 254-8.

50. Patterson RM, Prihoda TJ, Pouliot MR. Sonographic amniotic fluid measurement and fetal growth retardation: a reappraisal. Am J Obstet Gynecol 1987; 157: 1406-10.

51. Rutherford SE, Phelan JP, Smith CV, Jacobs N. The four-quadrant assessment of amniotic fluid volume: an adjunct to antepartum fetal heart rate testing. Obstet Gynecol 1987; 70(3 Pt 1): 353-6.

52. Chamberlain PF, Manning FA, Morrison I, Harman CR, Lange IR. Ultrasound evaluation of amniotic fluid volume. I. The relationship of marginal and decreased amniotic fluid volumes to perinatal outcome. Am J Obstet Gynecol 1984; 150: 245-9.

53. Philipson EH, Sokol RJ, Williams T. Oligohydramnios: clinical associations and predictive value for intrauterine growth retardation. Am J Obstet Gynecol 1983; 146: 271-8.

54. Devoe LD. Antenatal evaluation of the growth retarded fetus. In: Divon MY, ed. Abnormal Fetal Growth. New York: Elsevier Science, 1991.

55. Navot D, Mor-Yosef S, Granat M, Sadovsky E. Antepartum fetal heart rate pattern associated with major congenital malformations. Obstet Gynecol 1984; 63: 414-17.

56. Veille JC, Kanaan C. Duplex Doppler ultrasonographic evaluation of the fetal renal artery in normal and abnormal fetuses. Am J Obstet Gynecol 1989; 161(6 Pt 1): 1502-7.

57. Devoe LD, Castillo RA, Searle N, Searle JS. Prognostic components of computerized fetal biophysical testing. Am J Obstet Gynecol 1988; 158: 1144-8.

58. Baskett TF, Gray JH, Prewett SJ, Young LM, Allen AC. Antepartum fetal assessment using a fetal biophysical profile score. Am J Obstet Gynecol 1984; 148: 630-3

59. Manning FA, Morrison I, Harman CR, Lange IR, Menticoglou S. Fetal assessment based on fetal biophysical profile scoring: experience in 19,221 referred high-risk pregnancies. II. An analysis of false-negative fetal deaths. Am J Obstet Gynecol 1987; 157(4 Pt 1): 880-4.

60. Fleischer A, Guidetti D, Stuhlmuller P. Umbilical artery velocity waveforms in the intrauterine growth retarded fetus. Clin Obstet Gynecol 1989; 32: 660-8.

61. Divon MY, Hsu HW. Maternal and fetal blood flow velocity waveforms in intrauterine growth retardation. Clin Obstet Gynecol 1992; 35: 156-71.

62. Papageorghiou AT, Yu CK, Bindra R, et al. Multicenter screening for preeclampsia and fetal growth restriction by transvaginal uterine artery Doppler at 23 weeks of gestation. Ultrasound Obstet Gynecol 2001; 18: 441-9.

63. Martin AM, Bindra R, Curcio P, Cicero S, Nicolaides KH. Screening for pre-eclampsia and fetal growth restriction by uterine artery Doppler at 11-14 weeks of gestation. Ultrasound Obstet Gynecol 2001; 18: 583-6.

64. Duley L, Henderson-Smart DJ, Meher S, King JF. Antiplatelet agents for preventing pre-eclampsia and its complications. Cochrane Database Syst Rev 2007: CD004659.

65. Newnham JP, Patterson LL, James IR, Diepeveen DA, Reid SE. An evaluation of the efficacy of Doppler flow velocity waveform analysis as a screening test in pregnancy. Am J Obstet Gynecol 1990; 162: 403-10.

66. Jacobson SL, Imhof R, Manning N, et al. The value of Doppler assessment of the uteroplacental circulation in predicting preeclampsia or intrauterine growth retardation. Am J Obstet Gynecol 1990; 162: 110-14.

67. Giles WB, Trudinger BJ, Baird PJ, et al. Umbilical artery flow velocity waveforms and placental resistance: pathological correlation. Br J Obstet Gynaecol 1985; 92: 31-8.

68. McCowan LM, Mullen BM, Ritchie K. Umbilical artery flow velocity waveforms and the placental vascular bed. Am J Obstet Gynecol 1987; 157(4 Pt 1): 900-2.

69. Fok RY, Pavlova Z, Benirschke K, Paul RH, Platt LD. The correlation of arterial lesions with umbilical artery Doppler velocimetry in the placentas of small-for-dates pregnancies. Obstet Gynecol 1990; 75: 578-83. 
70. Wladimiroff JW, vdWijngaard JA, Degani S, et al. Cerebral and umbilical arterial blood flow velocity waveforms in normal and growth-retarded pregnancies. Obstet Gynecol 1987; 69: 705-9.

71. Trudinger BJ, Cook CM, Giles WB, et al. Fetal umbilical artery velocity waveforms and subsequent neonatal outcome. Br J Obstet Gynaecol 1991; 98: 378-84.

72. McCowan LM, Harding JE, Stewart AW. Umbilical artery Doppler studies in small for gestational age babies reflect disease severity. BJOG 2000; 107: 916-25.

73. Figueras F, Eixarch E, Gratacos E, Gardosi J. Predictiveness of antenatal umbilical artery Doppler for adverse pregnancy outcome in small-forgestational-age babies according to customised birthweight centiles: population-based study. BJOG 2008; 115: 590-4.

74. Valcamonico A, Danti L, Frusca T, et al. Absent end-diastolic velocity in umbilical artery: risk of neonatal morbidity and brain damage. Am J Obstet Gynecol 1994; 170: 796-801.

75. Soothill PW, Ajayi RA, Campbell S, Nicolaides KH. Prediction of morbidity in small and normally grown fetuses by fetal heart rate variability, biophysical profile score and umbilical artery Doppler studies. Br J Obstet Gynaecol 1993; 100: 742-5.

76. Beattie RB, Dornan JC. Antenatal screening for intrauterine growth retardation with umbilical artery Doppler ultrasonography. BMJ 1989; 298: 631-5.

77. Bruinse HW, Sijmons EA, Reuwer PJ. Clinical value of screening for fetal growth retardation by Doppler ultrasound. J Ultrasound Med 1989; 8: 207-9.

78. Hanretty KP, Primrose MH, Neilson JP, Whittle MJ. Pregnancy screening by Doppler uteroplacental and umbilical artery waveforms. Br J Obstet Gynaecol 1989; 96: 1163-7.

79. Kay HH, Carroll BB, Dahmus M, Killam AP. Sonographic measurements with umbilical and uterine artery Doppler analysis in suspected intrauterine growth retardation. J Reprod Med 1991; 36: 65-8.

80. Berkowitz GS, Mehalek KE, Chitkara U, et al. Doppler umbilical velocimetry in the prediction of adverse outcome in pregnancies at risk for intrauterine growth retardation. Obstet Gynecol 1988; 71: $742-6$.

81. Reuwer PJ, Sijmons EA, Rietman GW, van Tiel MW, Bruinse HW. Intrauterine growth retardation: prediction of perinatal distress by Doppler ultrasound. Lancet 1987; 2: 415-18.

82. Rochelson BL, Schulman H, Fleischer A, et al. The clinical significance of Doppler umbilical artery velocimetry in the small for gestational age fetus. Am J Obstet Gynecol 1987; 156: 1223-6.

83. McCowan LM, Erskine LA, Ritchie K. Umbilical artery Doppler blood flow studies in the preterm, small for gestational age fetus. Am J Obstet Gynecol 1987; 156: 655-9.

84. Soothill PW, Bobrow CS, Holmes R. Small for gestational age is not a diagnosis. Ultrasound Obstet Gynecol 1999; 13: 225-8.

85. Bobrow CS, Soothill PW. Fetal growth velocity: a cautionary tale. Lancet 1999; 353: 1460.

86. Drogtrop AP, Bruinse HW, Reuwer PJ. Normal umbilical artery Doppler sonography does not exclude fetal distress. Acta Obstet Gynecol Scand 1990; 69: 351-2.

87. Wenstrom KD, Weiner CP, Williamson RA. Diverse maternal and fetal pathology associated with absent diastolic flow in the umbilical artery of high-risk fetuses. Obstet Gynecol 1991; 77: 374-8.

88. Ferrazzi E, Bozzo M, Rigano S, et al. Temporal sequence of abnormal Doppler changes in the peripheral and central circulatory systems of the severely growth-restricted fetus. Ultrasound Obstet Gynecol 2002; 19: $140-6$.
89. van den Wijngaard JA, Groenenberg IA, Wladimiroff JW, Hop WC. Cerebral Doppler ultrasound of the human fetus. Br J Obstet Gynaecol 1989; 96: 845-9.

90. Rizzo G, Arduini D. Fetal cardiac function in intrauterine growth retardation. Am J Obstet Gynecol 1991; 165(4 Pt 1): 876-82.

91. Locci M, Nazzaro G, De Placido G, Montemagno U. Fetal cerebral haemodynamic adaptation: a progressive mechanism? Pulsed and color Doppler evaluation. J Perinat Med 1992; 20: 337-43.

92. Chiba Y, Murakami M. Cerebral blood flow dynamics in fetus. No To Hattatsu 1992; 24: 136-9.

93. Bahado-Singh RO, Kovanci E, Jeffres A, et al. The Doppler cerebroplacental ratio and perinatal outcome in intrauterine growth restriction. Am J Obstet Gynecol 1999; 180(3 Pt 1): 750-6.

94. Konje JC, Bell SC, Taylor DJ. Abnormal Doppler velocimetry and blood flow volume in the middle cerebral artery in very severe intrauterine growth restriction: is the occurrence of reversal of compensatory flow too late? BJOG 2001; 108: 973-9.

95. Mari G, Hanif F, Kruger M, et al. Middle cerebral artery peak systolic velocity: a new Doppler parameter in the assessment of growth-restricted fetuses. Ultrasound Obstet Gynecol 2007; 29: 310-16.

96. Baschat AA, Gembruch U, Harman CR. The sequence of changes in Doppler and biophysical parameters as severe fetal growth restriction worsens. Ultrasound Obstet Gynecol 2001; 18: 571-7.

97. Evertson LR, Gauthier RJ, Schifrin BS, Paul RH. Antepartum fetal heart rate testing. I. Evolution of the nonstress test. Am J Obstet Gynecol 1979; 133: 29-33.

98. Hecher K, Bilardo CM, Stigter RH, et al. Monitoring of fetuses with intrauterine growth restriction: a longitudinal study. Ultrasound Obstet Gynecol 2001; 18: 564-70.

99. Baschat AA, Viscardi RM, Hussey-Gardner B, Hashmi N, Harman C. Infant neurodevelopment following fetal growth restriction: relationship with antepartum surveillance parameters. Ultrasound Obstet Gynecol 2009; 33: 44-50.

100. Boers KE, Vijgen SM, Bijlenga D, et al. Induction versus expectant monitoring for intrauterine growth restriction at term: randomised equivalence trial (DIGITAT). BMJ 2010; 341: c7087.

101. Ornoy A. Prenatal origin of obesity and their complications: gestational diabetes, maternal overweight and the paradoxical effects of fetal growth restriction and macrosomia. Reprod Toxicol 2011; 32: 205-12.

102. Osmond C, Barker DJ, Winter PD, Fall CH, Simmonds SJ. Early growth and death from cardiovascular disease in women. BMJ 1993; 307: 1519-24.

103. Tenhola S, Martikainen A, Rahiala E, et al. Serum lipid concentrations and growth characteristics in 12-year-old children born small for gestational age. Pediatr Res 2000; 48: 623-8.

104. Crispi F, Bijnens B, Figueras F, et al. Fetal growth restriction results in remodeled and less efficient hearts in children. Circulation 2010; 121: 2427-36.

105. Newsome CA, Shiell AW, Fall CH, et al. Is birth weight related to later glucose and insulin metabolism?-A systematic review. Diabet Med 2003; 20: 339-48.

106. Lienhardt A, Carel JC, Preux PM, Coutant R, Chaussain JL. Amplitude of pubertal growth in short stature children with intrauterine growth retardation. Horm Res 2002; 57(Suppl 2): 88-94.

107. Ibáñez L, Potau N, Enriquez G, Marcos MV, de Zegher F. Hypergonadotrophinaemia with reduced uterine and ovarian size in women born small-for-gestational-age. Hum Reprod 2003; 18: 1565-9.

108. Ibáñez L, Potau N, Ferrer A, et al. Reduced ovulation rate in adolescent girls born small for gestational age. J Clin Endocrinol Metab 2002; 87: 3391-3. 


\section{Application of 3D and 4D ultrasound in fetal medicine Eberhard Merz}

\section{HISTORY}

In 1989, the first commercially available ultrasound scanner (Combison 330) with mechanically driven three-dimensional (3D) probes was launched by Kretztechnik AG, Austria (1). Nevertheless, many investigators with previous experience in $2 \mathrm{D}$ ultrasonography took a skeptical attitude toward the new technology.

Several questions continued to be raised by the physicians: "Is 3D ultrasound more useful in prenatal diagnosis?"; "Does it improve diagnostic accuracy, or is it only good to obtain keepsake images for the parents?"; "Does it enable the detection of a greater number of malformations?"; "Is it useful in daily routine, or is it more time-consuming?" (2).

Only since the development of significantly more userfriendly $3 \mathrm{D} / 4 \mathrm{D}$ ultrasound scanners that permit even less experienced operators to apply this technique successfully in prenatal diagnosis has 3D/4D ultrasonography found a growing number of advocates worldwide. The technology does not only furnish excellent images, but also enables the preparation of videos of the unborn baby that provide the expert with a new spectrum for fine-tuning the diagnosis while, at the same time, leaving the prospective parents with a lasting impression.

\section{TECHNICAL ASPECTS}

In essence, every 3D ultrasound procedure consists of four basic steps $(2,3)$ : (i) volume acquisition, (ii) volume display, (iii) volume manipulation/image processing, and (iv) storage of volumes, or rendered images and volume sequences.

\section{Volume Acquisition}

In contrast to conventional 2D ultrasound where only single planes are stored, all 3D ultrasound technology is based on the acquisition of multiple adjacent 2D scans that are stored at correlative sites in an electronic memory, where they are assembled to produce a volume data set (3).

Two major technologies are currently available for the acquisition of ultrasound volumes: the freehand technique and the automated technique.

In the freehand system (4), scans are obtained with a conventional 2D probe onto which an electromagnetic position sensor has been mounted. This device can sense the exact position and movements of the probe by detecting changes in a magnetic field (3).

All images acquired during the freehand movements of the probe are transmitted via the video signal of the ultrasound unit to an external workstation, which is equipped with a frame grabber. After all individual images have been digitized by the frame grabber, they are stored in the computer memory according to their known location and are finally assembled into a volume.
With an automated system a dedicated 3D transducer is matched to the ultrasound system. Volume acquisition is activated at the touch of a button, at which point the transducer element within the probe casing is automatically swept at a specified angle through a fan-shaped pattern (3).

In $3 D$ volume acquisition, individual volumes are obtained; in $4 D$ volume acquisition, multiple volumes are acquired successively in the form of a volume sequence.

\section{D/4D Volume Display}

Several grayscale and color modes are available for the demonstration of volumes:

\section{Triplanar Display}

In the tri- or multiplanar display, all three mutually perpendicular scan planes (the "orthogonal" planes) are displayed simultaneously on the monitor immediately after volume acquisition (Fig. 1) (3).

The rotation and translation controls can now be used to demonstrate not only conventional section planes but also oblique planes. Starting from a selected plane the volume can be manipulated in all three directions with millimeter precision, enabling a precise tomographic survey of a particular region.

To avoid the occurrence of spatial disorientation during rotation of the individual image planes as to left/right or top/ bottom, the application of a standardized procedure as described by Merz et al. (5) 2007 is recommended:

1. In the primary multiplanar image, the object should be displayed so that the image at the top left always corresponds to that visualized in the $2 \mathrm{D}$ image (Fig. 1). This permits to draw a valid conclusion on the position of the embryo/fetus/uterus at the time of volume acquisition.

2. In the secondary multiplanar image, the volume has to be changed by rotation maneuvers to ensure that the embryo/fetus may at all times be visualized in an upright sagittal position in the image at the top right (Figs. 2 and 3). The aim of this image is to present the object on the display screen in such a way as to allow the correct assignment of ventral and dorsal, as well as of right and left to the actual anatomy.

\section{Tomographic Ultrasound Imaging or Multi-Slice Viewing} Technique

With tomographic ultrasound imaging [TUI (General Electric)] (= multi-slice viewing technique (SamsungMedison), it is possible to view parallel 2D section planes through a volume on the monitor as in CT or MRI $(6,7)$ (Figs. 4 and 5). The selection of the direction of the volume analysis and 


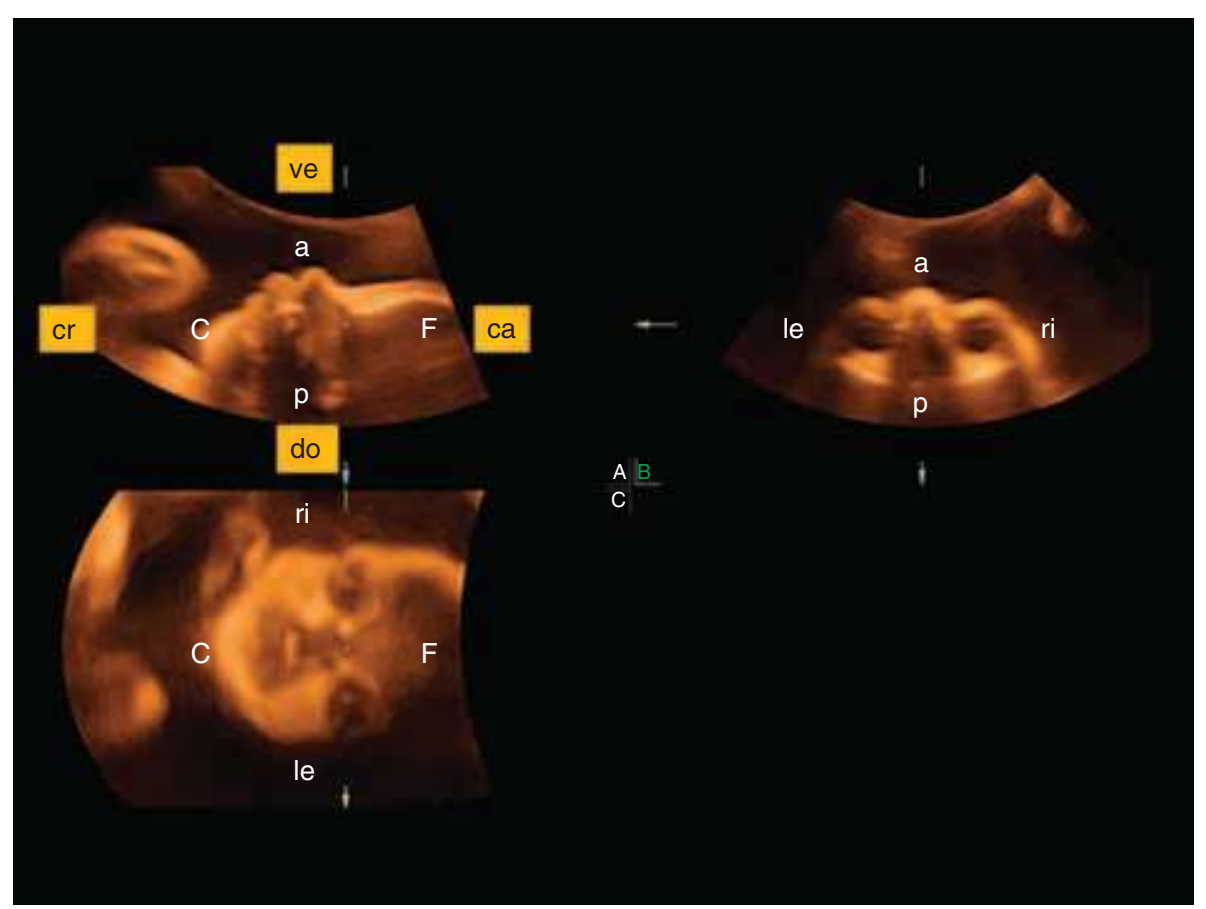

Figure 1 Triplanar display of a fetus at 35 weeks of gestation immediately after acquisition in cephalic presentation (primary image). Upper left: exact midsagittal scan (= median plane). Upper right: transverse scan at orbit level. Lower left: coronal scan at orbit level. The image at the top left always corresponds to the conventional two-dimensional image during acquisition. $\mathrm{cr}=$ cranial, $\mathrm{ca}=$ caudal, $\mathrm{ve}=$ ventral, do $=$ dorsal $(\mathrm{mother}) . \mathrm{F}=$ forehead, $\mathrm{C}=\mathrm{chin}, \mathrm{a}=\mathrm{anterior}$, $\mathrm{p}=$ posterior, $\mathrm{ri}=$ right, le $=$ left (fetus).

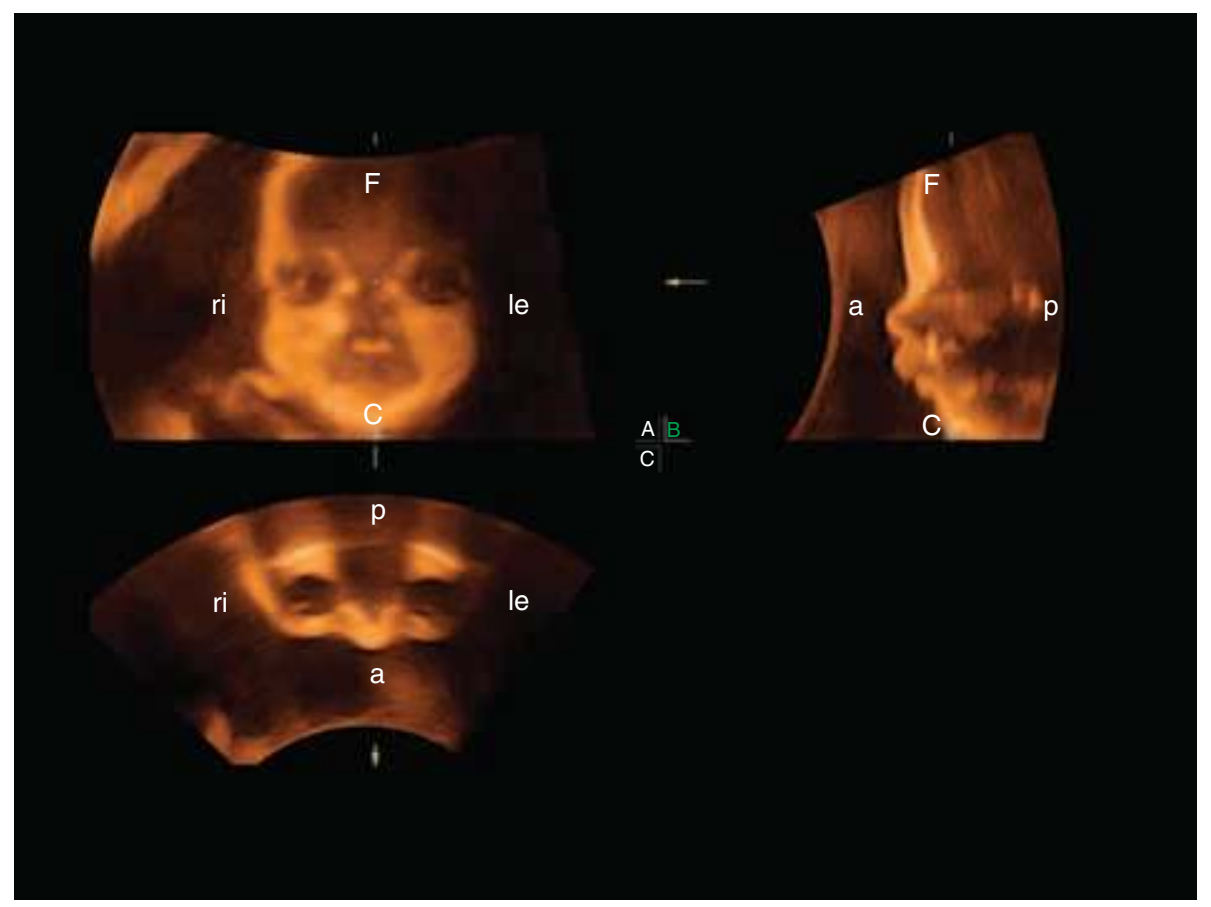

Figure 2 Fetus after rotation into an upright position (secondary image). The sagittal position of the fetus in the image at the top right guarantees a correct assignment of right and left to the actual anatomy in the coronal and the transverse scans. $\mathrm{F}=$ forehead, $\mathrm{C}=$ chin, $\mathrm{a}=$ anterior, $\mathrm{p}=$ posterior, $\mathrm{ri}=$ right, le $=$ left (fetus).

the distance of the parallel slices are optional. The choice of the distance between the individual section planes depends upon the gestational age, as well as on the location and size of the anatomic structures to be visualized (7).

\section{Surface Display}

The surface modes provide the examiner with rendered 3D images of the fetal surface (2,3) (Figs. 6-10). Various rendering algorithms can be used in surface rendering: surface mode, smooth surface mode, light mode, and gradient light mode $(2,3)$.

Prerequisites for high-quality images of the fetal surface are the presence of a sufficiently large amniotic fluid pocket in front of the structure being imaged and the absence of overlying or adjacent structures, such as the limbs, the umbilical cord, or the placenta. 


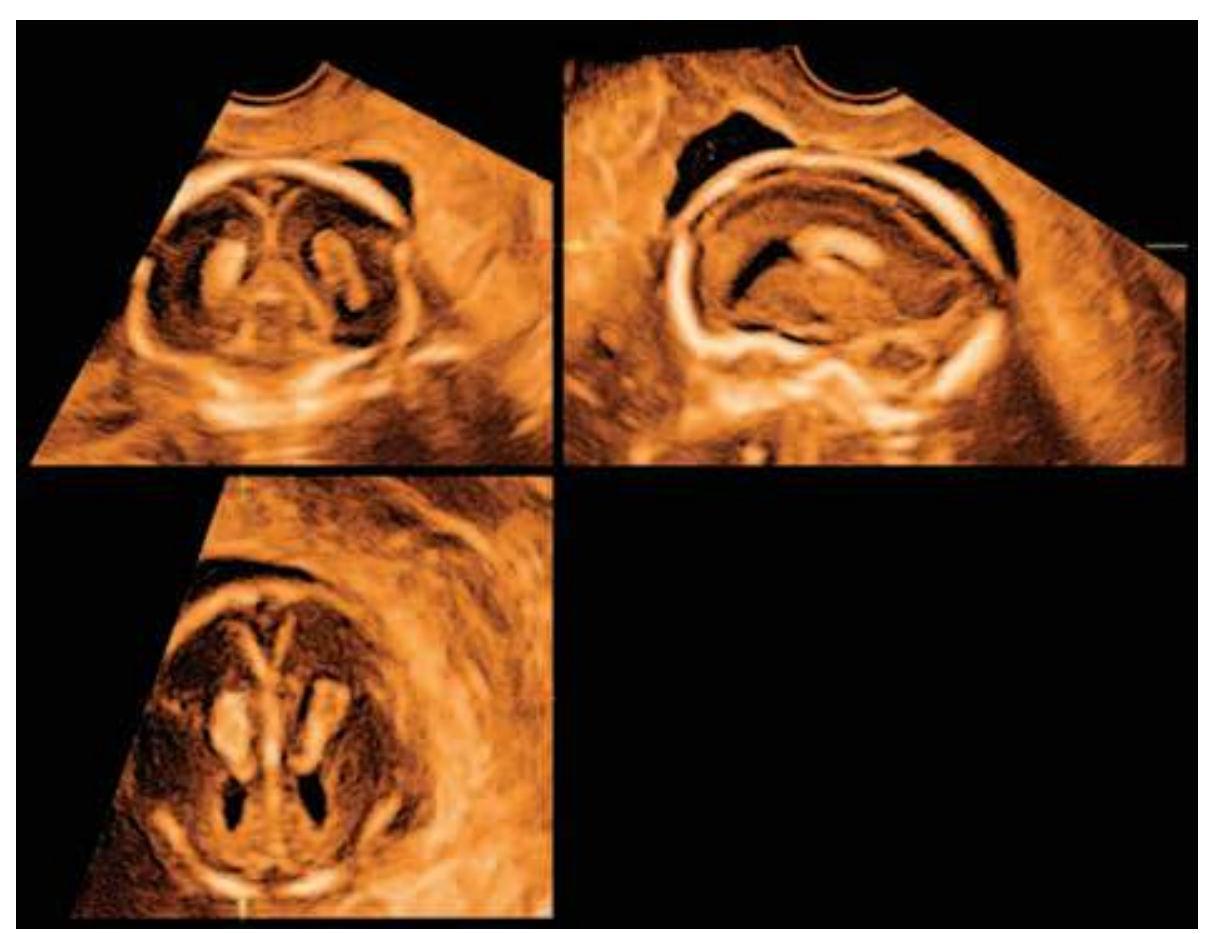

Figure 3 Triplanar demonstration of a fetal brain at 19 weeks of gestation after rotation of the fetus into an upright position. Upper left: coronal scan. Upper right: sagittal scan of the right ventricle. Lower left: Axial scan of the ventricles.

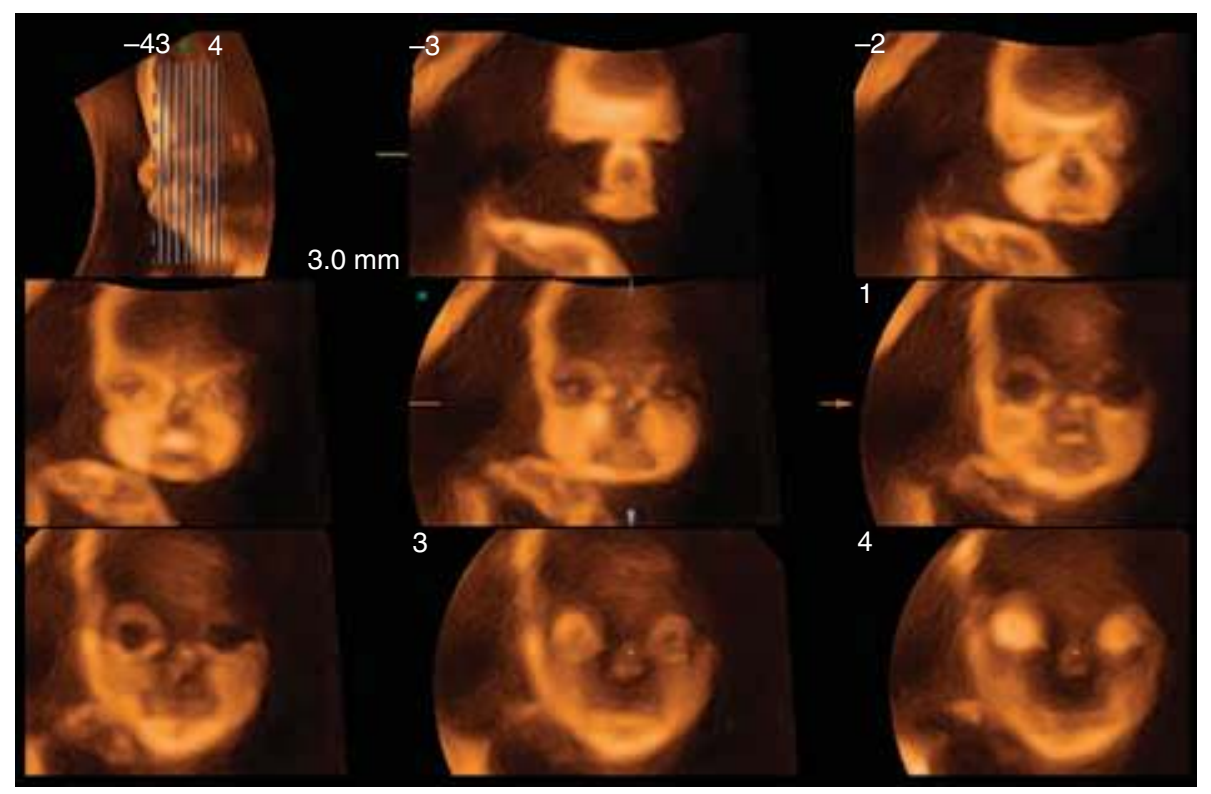

Figure 4 Tomographic ultrasound imaging showing several slices of the fetal face at distances of $6.5 \mathrm{~mm}$ (coronal views) at 35 weeks of gestation.

The surface modes can be applied not only for the demonstration of outer surfaces, but also to receive a spatial view of inner surfaces or surfaces of section planes.

In contrast to $3 \mathrm{D}$ ultrasound where only $3 \mathrm{D}$ images are acquired, the $4 \mathrm{D}$ technique (= real time $3 \mathrm{D}$ ) further provides image sequences, which allow the observation of real-time fetal movements as in a movie (2) (Fig. 11).

\section{Transparence Display}

The transparent mode (maximum mode) provides a spatial view of the fetal skeleton (Fig. 12) $(3,8)$. Unlike in surface rendering, it primarily displays hyperechoic structures while greatly attenuating less echogenic structures. This provides an overview of the fetal skeleton, with a demonstration of normal and abnormal ossification. The $4 \mathrm{D}$ transparent display may be used to demonstrate movements of the skeleton.

\section{Inversion Display}

The inversion mode converts anechoic structures into hyperechoic structures (9). This allows for the 3D display of hollow structures such as the heart chambers, vessels, the stomach, the fetal urinary bladder, or pathologic amounts of liquid in the form of opaque casts. 


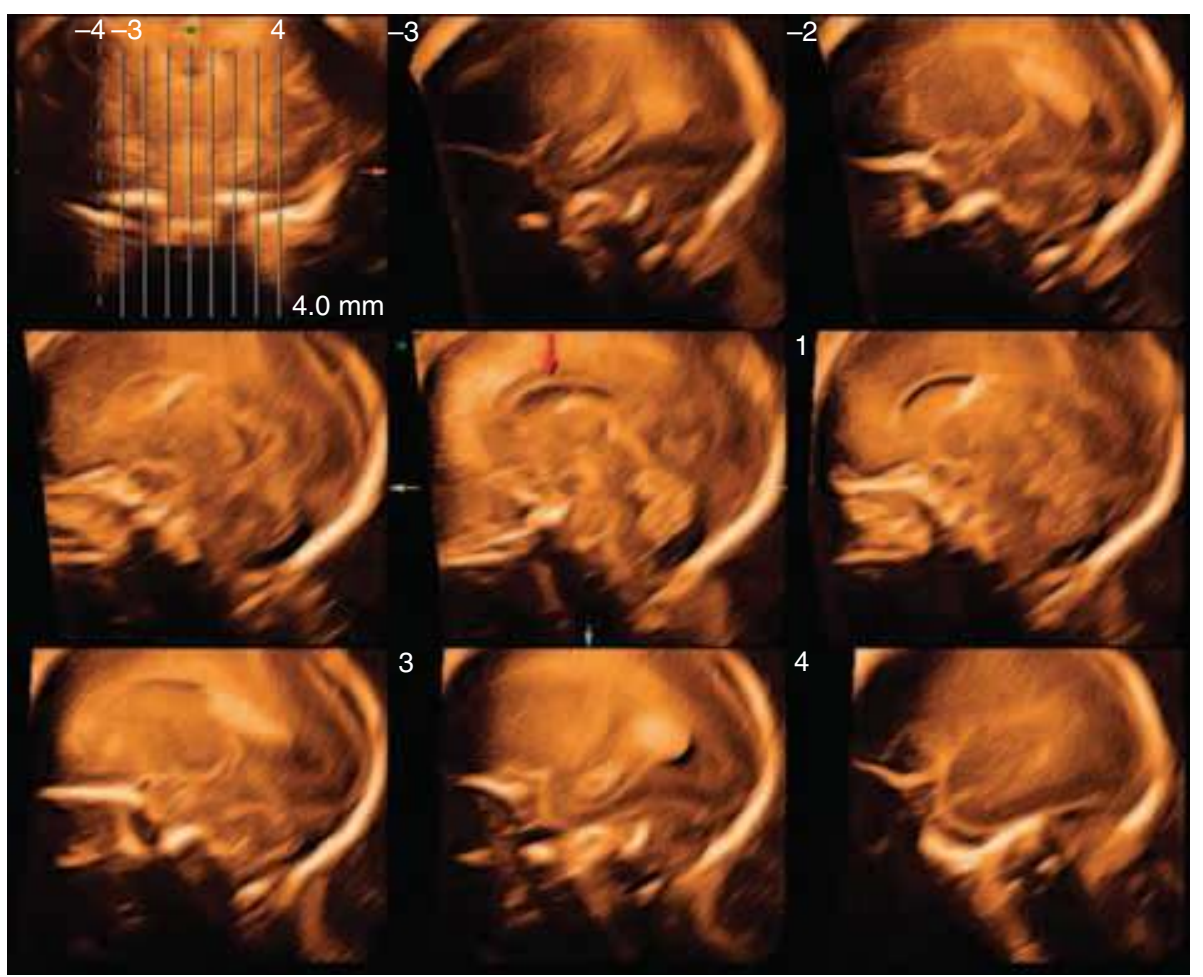

Figure 5 Tomographic ultrasound imaging of the fetal brain at distances of $6.5 \mathrm{~mm}$ (sagittal planes). The central image demonstrates the corpus callosum ( ) at

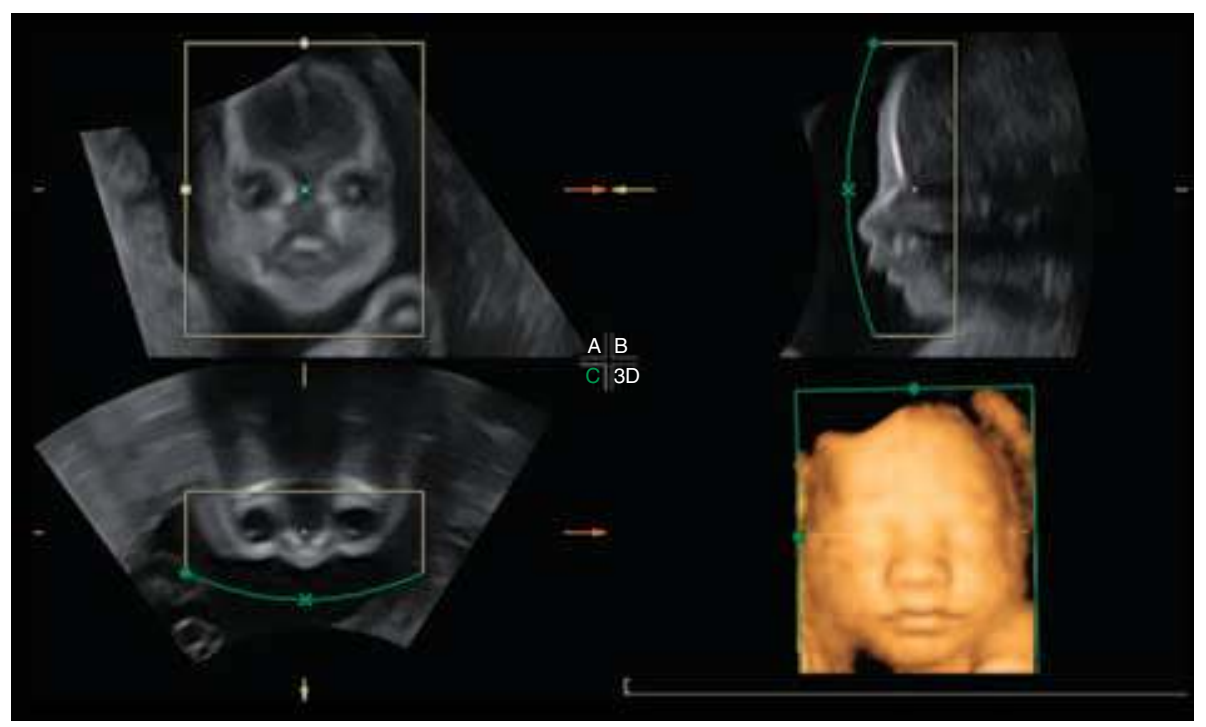

Figure 6 Multiplanar and surface-rendered frontal view of the fetal face at 28 weeks of gestation.

Glass-Body Rendering

The glass body-rendering mode generates a combined display of grayscale values and color Doppler/power Doppler signals. This offers the physician the possibility of presenting the fetal vascular system three dimensionally, as in a glass-body model (2) (Fig. 13). The deletion of the grayscale portion of the image provides a clear $3 \mathrm{D}$ color depiction of the flow of blood in fetal blood vessels.

Glass-body rendering can further be applied to visualize placental perfusion (Fig. 14).
Spatiotemporal Image Correlation

The spatiotemporal image correlation (STIC) technique $(2,3,10-12)$ is a useful tool for the depiction of the beating fetal heart in both the grayscale and the color mode (color STIC = STIC + color Doppler). In the first step, images of several cardiac cycles are acquired using a slow-motion volume scan $(7.5,10$, or $12.5 \mathrm{sec})$. The system then analyzes the volume data separately according to their temporal and spatial domains. These measures produce a $4 \mathrm{D}$ volume data set of a single cardiac cycle in an endless volume cine loop. The STIC 


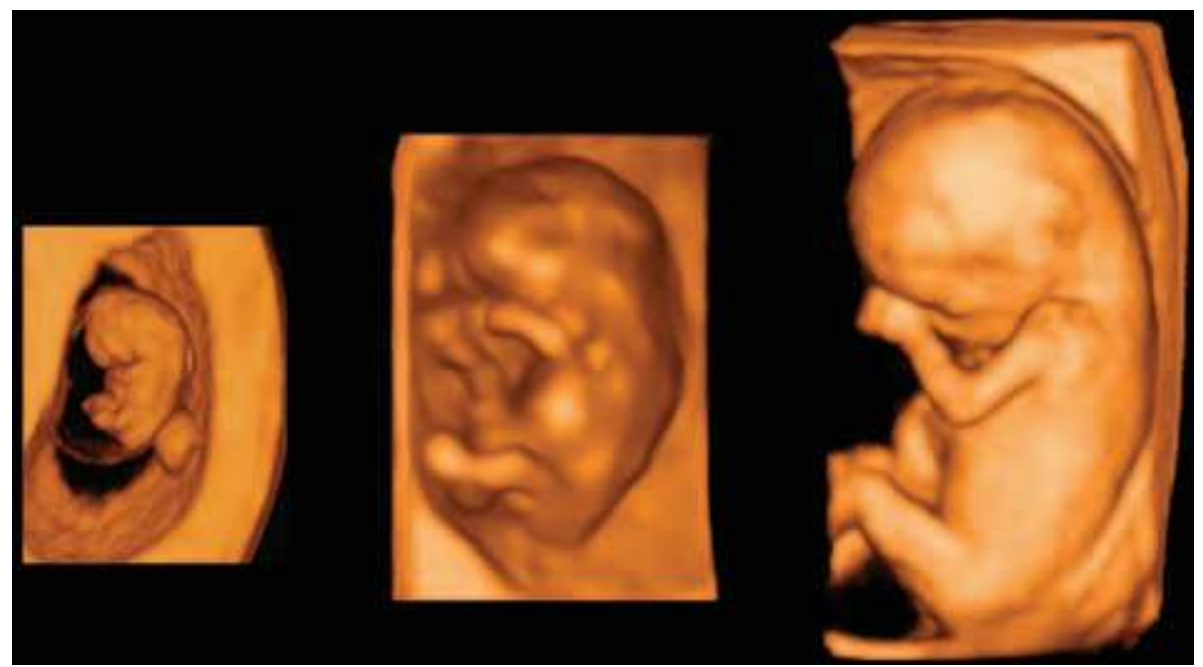

(A)

(B)

(C)

Figure 7 Surface-rendered side views of the embryo/fetus in the first trimester at 7 (A), 10 (B) and 13 (C) weeks.

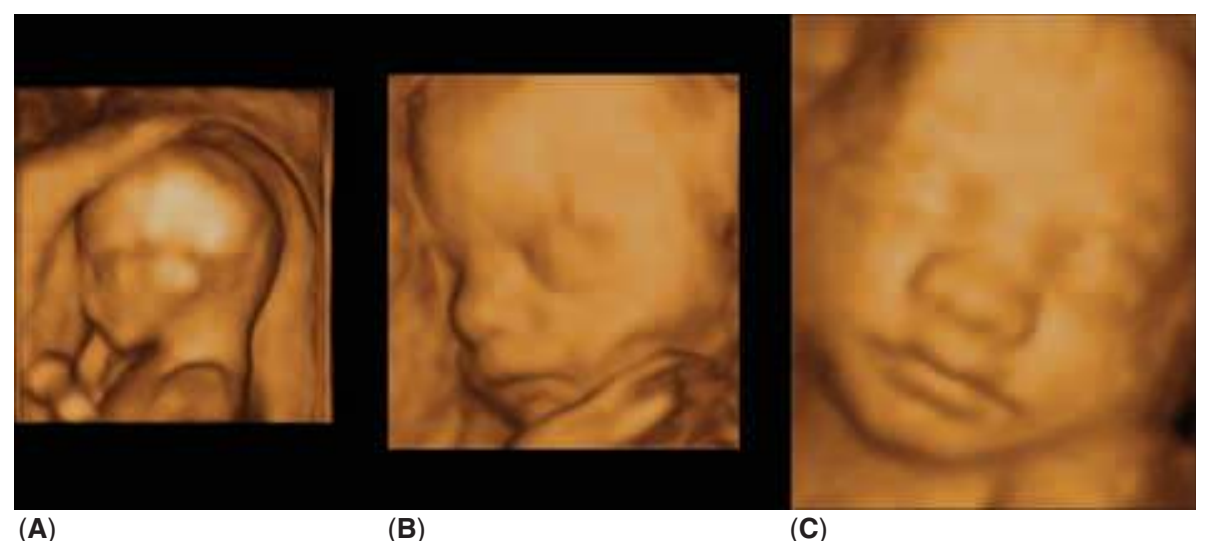

Figure 8 Surface-rendered views of the fetus in the second trimester at 14 (A), 23 (B), and 28 (C) weeks of gestation.

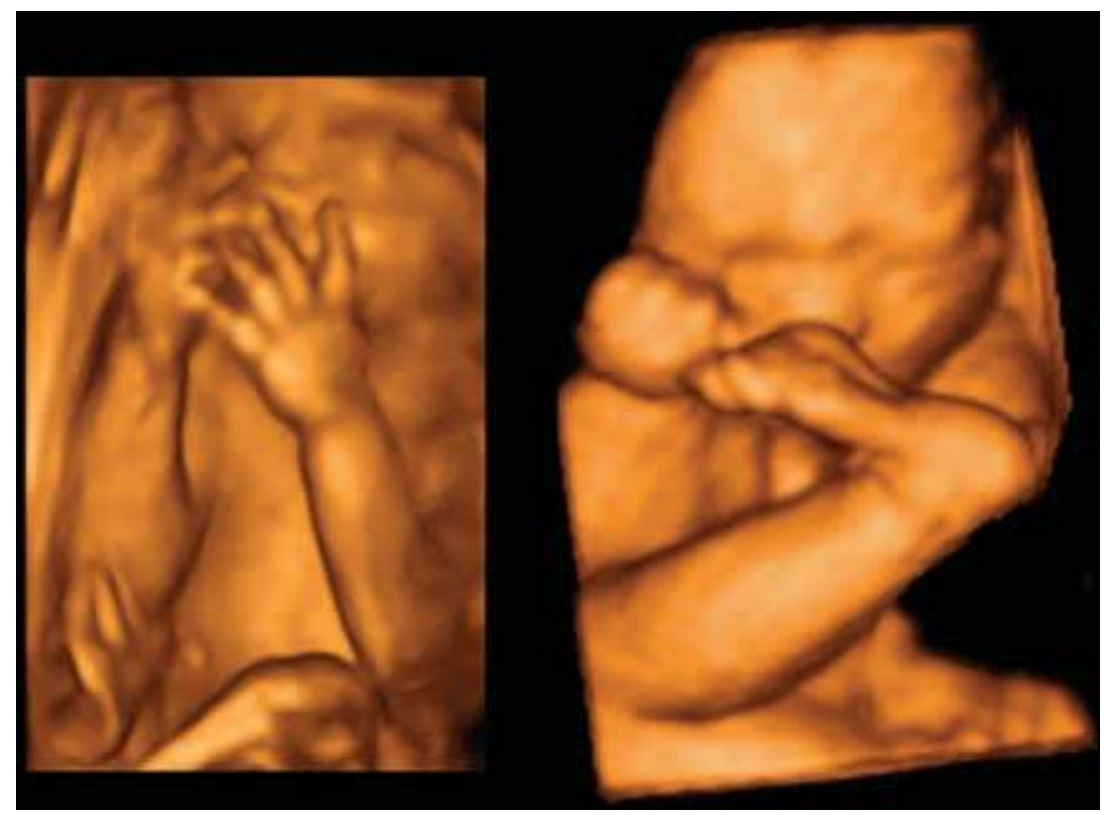

(A)

(B)

Figure 9 Surface-rendered views of the fetal hand (A) (24 weeks of gestation) and foot (B) (31 weeks of gestation). 


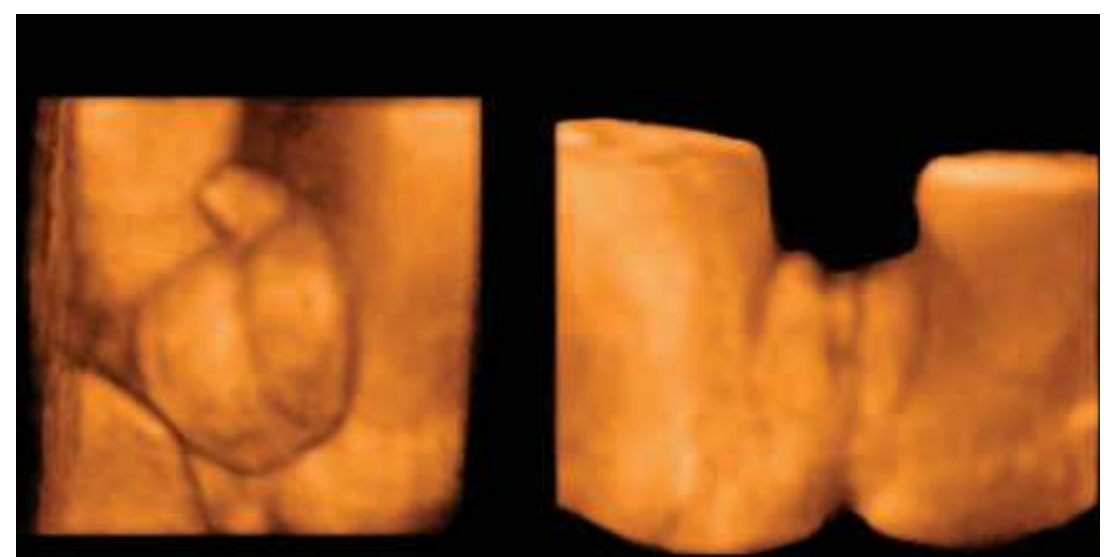

(A)

(B)

Figure 10 Surface-rendered views of male (36 weeks of gestation) (A) and female (32 weeks of gestation) gender (B).

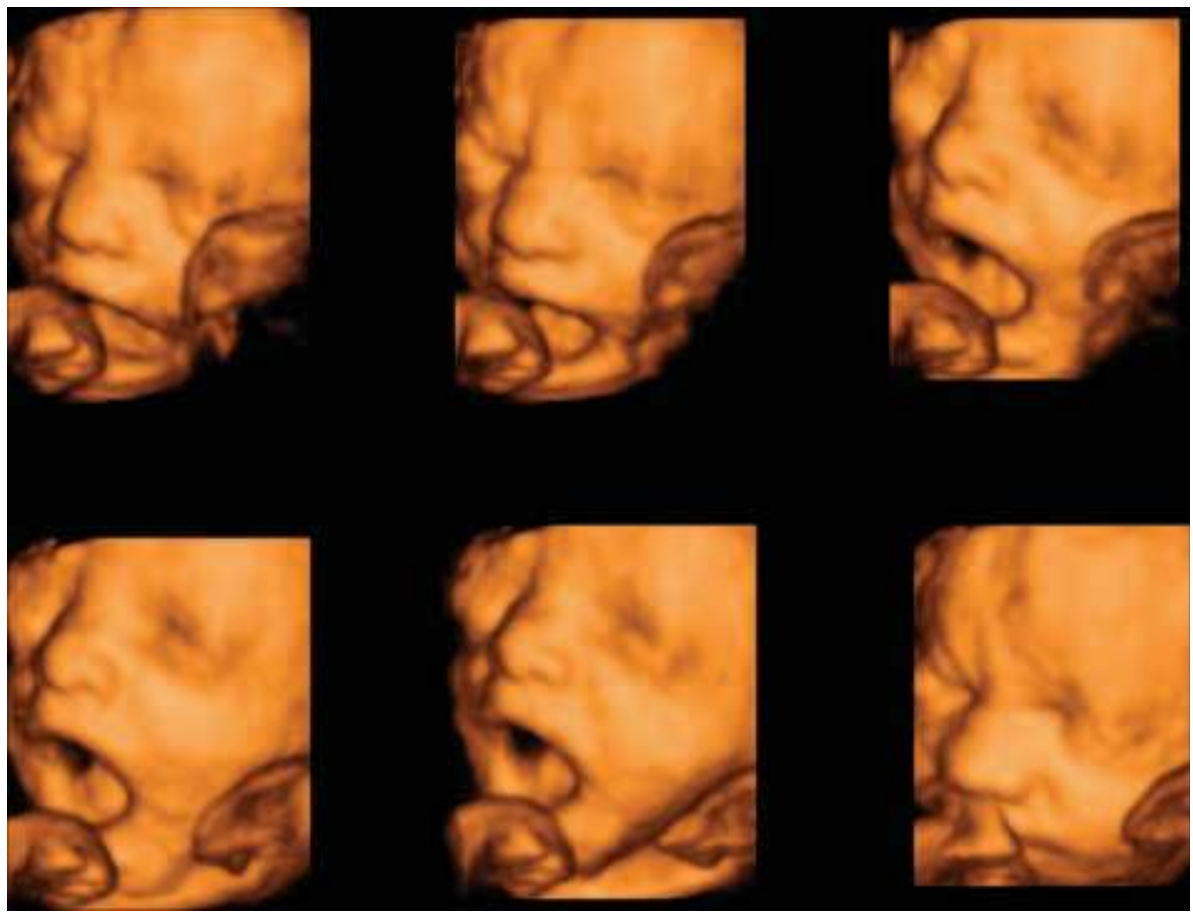

Figure 11 The 4D ultrasound: Demonstration of a yawning fetus at 24 weeks of gestation. The $4 \mathrm{D}$ display provides a real-time view of the moving face.

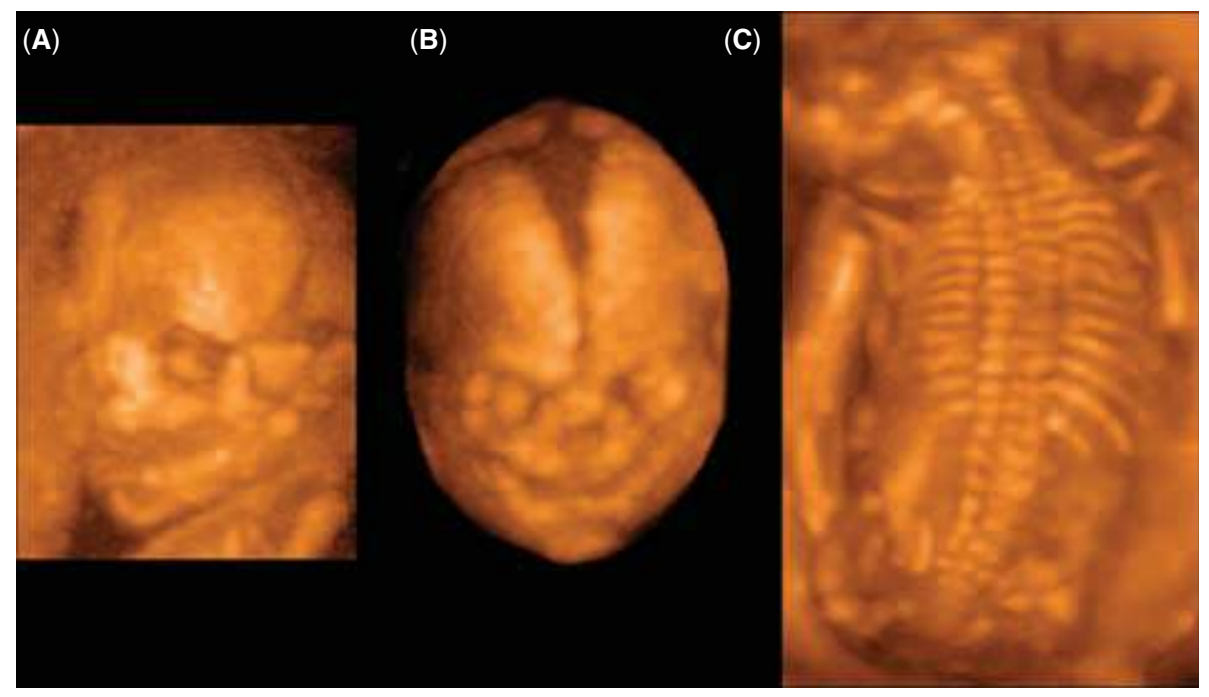

Figure 12 Transparent view (maximum mode) of the fetal skeleton. (A) Oblique side view of the ossified skull, facial bones, and the metopic and left coronal suture (23 weeks of gestation). (B) Frontal view of the ossified facial bones, the anterior fontanel and the metopic suture (21 weeks of gestation). (C) Bony thorax and ossified spine at 17 weeks of gestation. 


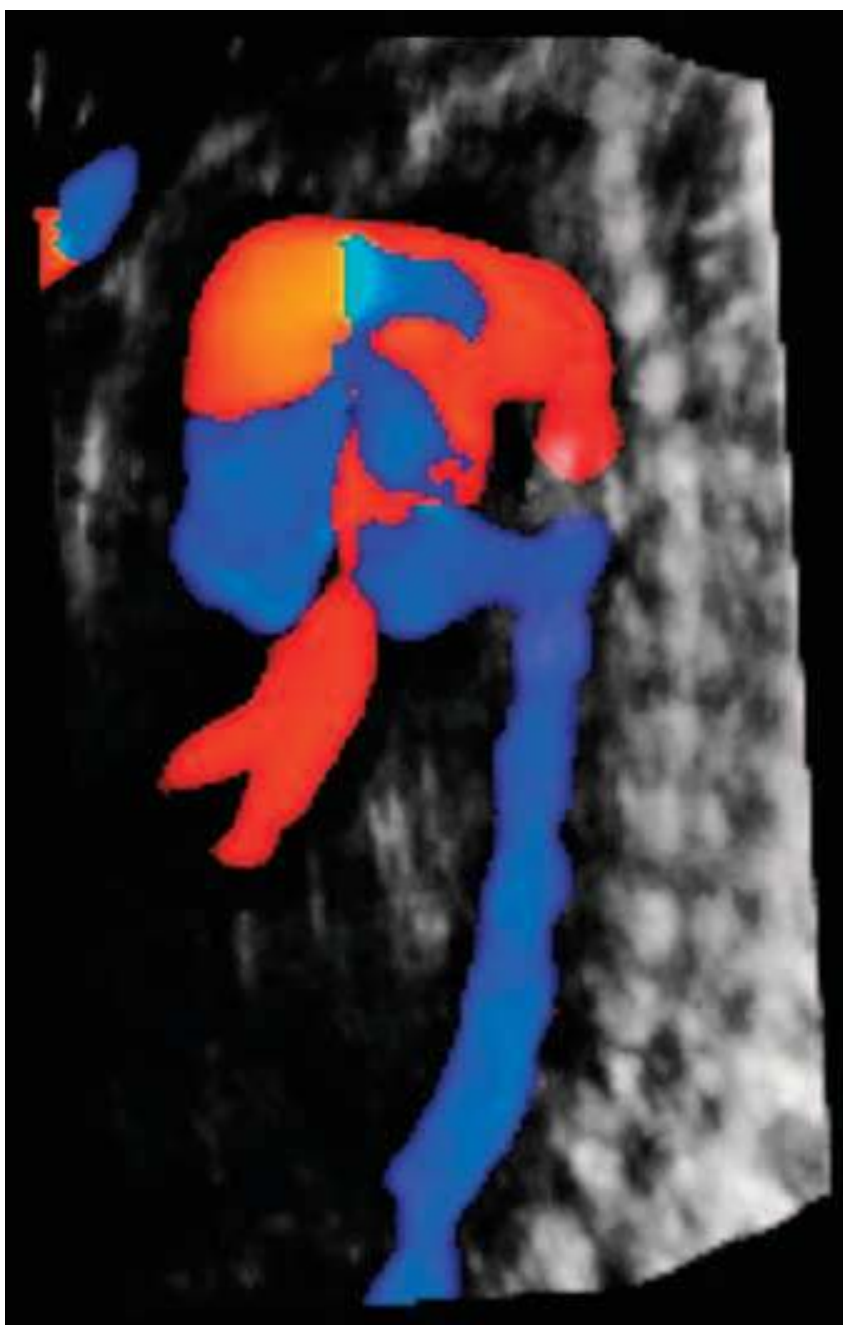

Figure 13 Glass-body rendering of the fetal heart and the aorta (25 weeks of gestation).

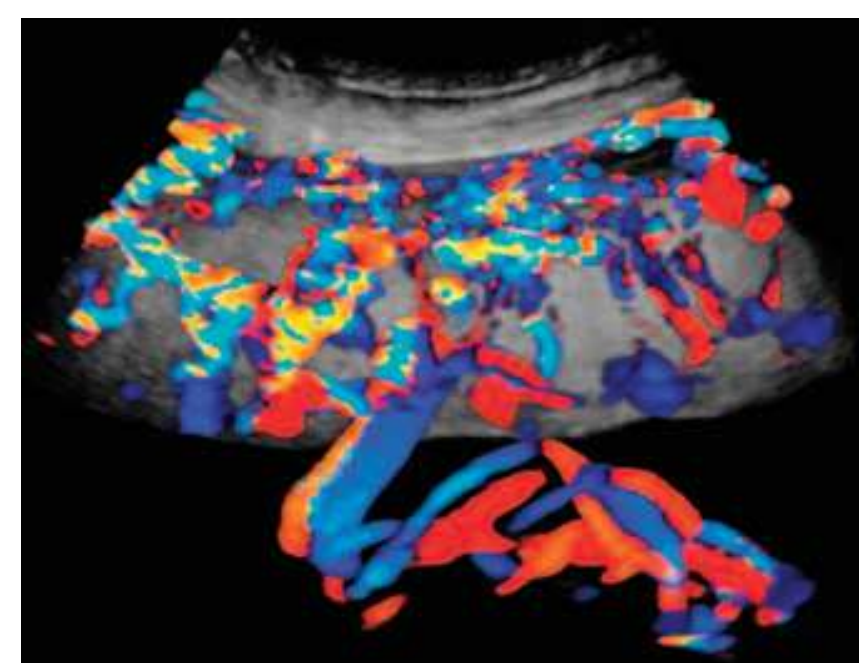

Figure 14 Glass-body rendering of the placenta and the insertion of the umbilical cord (26 weeks of gestation).

technology does not only permit the storage of a beating fetal heart in a volume and its triplanar visualization, but also aids in the detailed investigation of the heart in the endless volume cine loop following shifting and rotation of the different image planes $(2,12)$. The color STIC technology enables visualization of blood flow in the fetal heart or fetal blood vessels in the endless volume cine loop display $(2,12)$ (Fig. 15).

However, a prerequisite for the acquisition of an adequate STIC image is a sufficiently regular heart rate pattern. The application of the STIC technique is not suitable in the presence of arrhythmia.

\section{VCAD}

The automated multiplanar technique (VCAD) (13) also permits less experienced users to visualize the most important sectional planes of the fetal heart. The image of the heart acquired using the STIC technique is rotated so that the fourchamber view corresponds to the superimposed heart template. With a single keystroke, the relevant planes of the heart as, for example, the left and right outflow tract are automatically shown.

\section{Sonographic Automated Volume Count}

Sonographic automated volume count automatically detects low echogenic objects in the fetus (e.g., brain ventricles, stomach, urinary bladder) and analyzes their shape and volume. The calculated volume of the object is then listed according to size.

\section{Volume Manipulation}

Comparable with the images generated in 2D ultrasound, images acquired with 3D ultrasound can be manipulated using the following postprocessing procedures: threshold control, speckle reduction imaging (SRI), brightness and contrast controls, and the electronic scalpel.

A number of 3D images, in particular surface images, require postprocessing procedures in order to improve image quality. Because low-level echoes (speckle), for example, those found in amniotic fluid, prevent a high image quality, they should be filtered out by increasing the threshold value (3) (Fig. 16) or by using the SRI technique. This technique reduces extraneous echoes and artifacts and leads to an improved demonstration of structures and boundaries.

In surface rendering, larger interfering structures, such as loops of the umbilical cord, limbs, or the placenta, may obstruct the view of the fetal face. In approximately $70 \%$ of cases, the electronic elimination of these structures using the electronic scalpel leads to a substantial image improvement of the depicted surfaces (14) (Fig. 16). The original volume remains unaffected by the cutting procedure.

Brightness and contrast controls can be set interactively in the $3 \mathrm{D}$ image as well as in the 4D sequences; all changes are immediately visible on the monitor.

\section{Long-Term Storage of Volumes, Rendered Images, or Cine Sequences}

The 3D/4D ultrasound offers an important advantage over the conventional 2D sonography in archiving volumes, rendering images, or cine sequences of volumes instead of individual images. Once the volumes are stored in the memory of the ultrasound unit, they can be sent to various media for longterm storage (external hard drive, MOD, CD, DVD). Digital archiving of the volumes and images guarantees safe storage without quality loss. The examiner can retrieve the volumes and cine sequences at any time and navigate through them in 


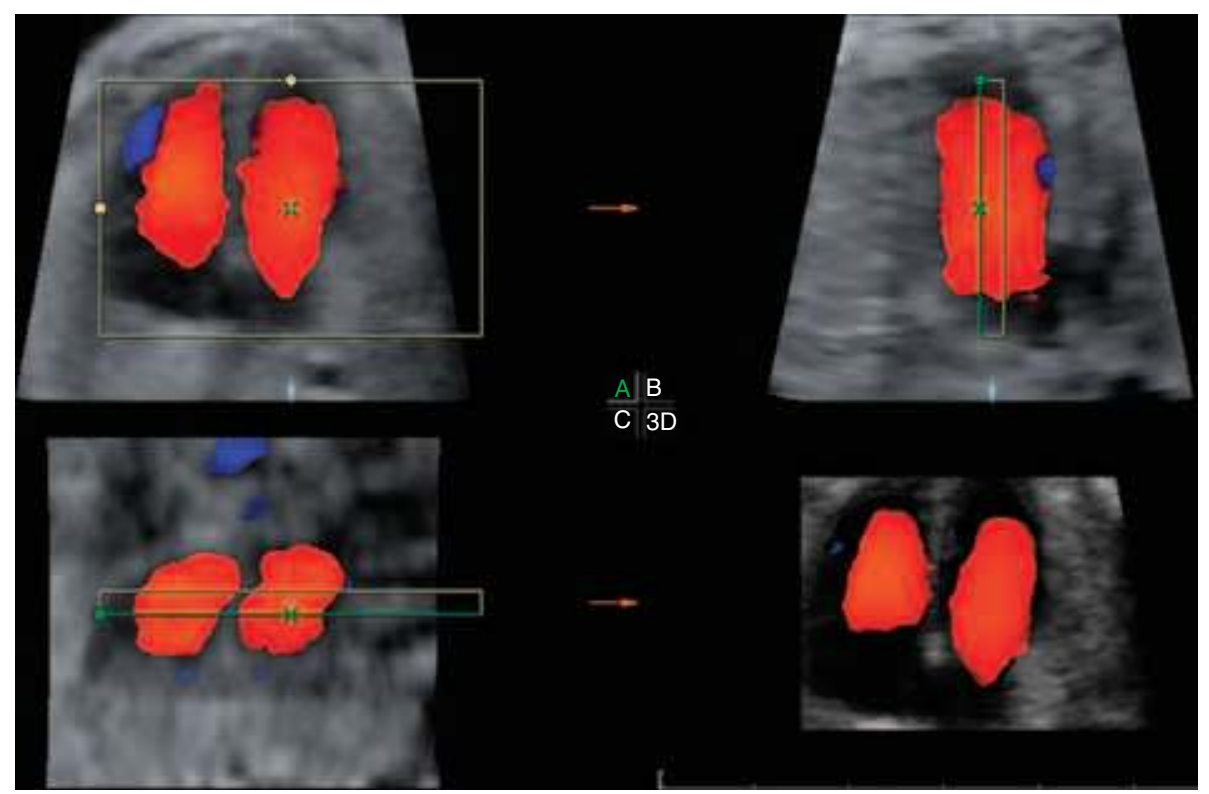

Figure 15 Triplanar spatiotemporal image correlation demonstration of the fetal heart (26 weeks of gestation). Upper left: Four-chamber view. Upper right: Sagittal scan of left chamber. Lower left: Transverse scan of left and right chambers. Lower right: Surface demonstration of the blood flow into the chambers (fourchamber view).

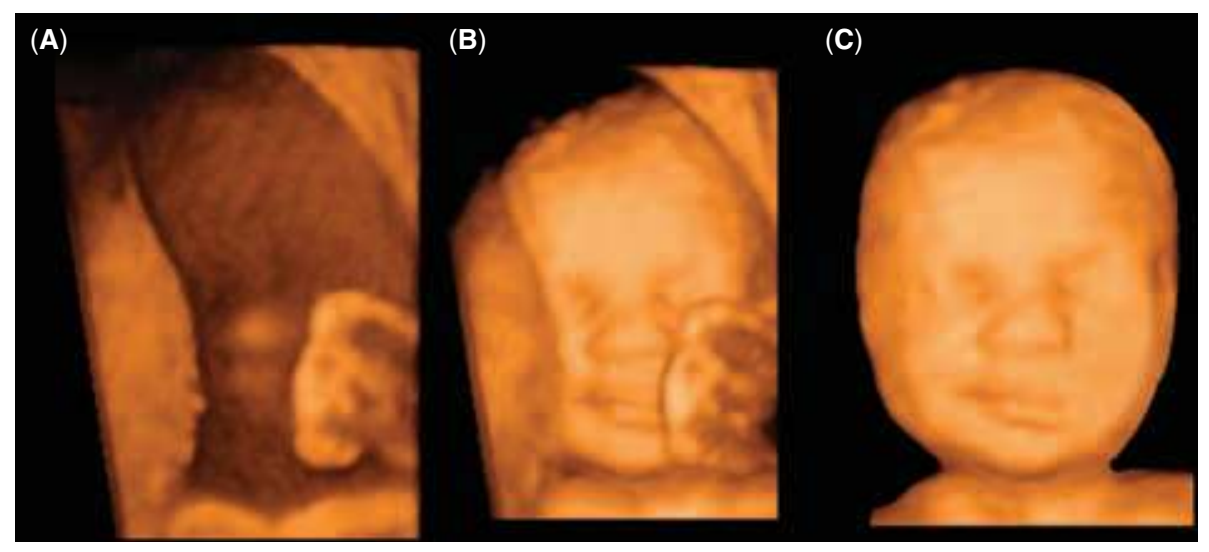

Figure 16 Surface mode of a fetal face (frontal view) (30 weeks of gestation). (A) The face is obscured by echogenic amniotic fluid (threshold 0). (B) A threshold of 50 reveals the fetal face. (C) Demonstration of the fetal face after removing adjacent gross structures with the electronic scalpel.

the absence of the patient (virtual scan) $(3,15)$. This allows the physician to scrutinize equivocal findings without a time limit or creating undue anxiety in the patient.

\section{DIAGNOSTIC CONSIDERATIONS}

The different imaging modes offered by transvaginal and transabdominal 3D/4D ultrasound technology today provide the operator with experience in the diagnosis of fetal malformations with completely new possibilities for systematic fine-tuning of the prenatal diagnosis (Figs. 17-28). This applies to both, the application of these techniques directly on the patient and the detailed assessment of stored volumes with high-quality images in the absence of the patient.

The simultaneous display of all three mutually perpendicular scan planes provided by the triplanar mode $(16,17)$ permits an accurate assessment of the fetal anatomy. It facilitates rapid targeting of a specific biometry or section plane (Fig. 20). The ability to slowly navigate through the volume data set allows the physician to demonstrate morphologic abnormalities in an optimal plane. The application of the triplanar mode is particularly useful when the left and right sides of symmetrical organs like the brain (18-22) or the orbits are to be compared.

Similarly, the TUI technique enables an exact tomographic demonstration of pathologic findings on the basis of parallel $2 \mathrm{D}$ section planes. The direction of the slices as well as the distance between the individual section planes can be changed interactively to ensure the optimal visualization of the defect (Figs. 19 and 21).

Application of the surface mode allows the reliable demonstration and exclusion of suspected pathologic findings. Conclusively demonstrated are in particular less severe surface defects as, for example, encephalocele, cleft lip/cleft palate, spina bifida, defects of the abdominal wall or the extremities, but also visualized are defects such as facial dysmorphia, external ear dysplasia, malformations of the hand, foot, or genital anomalies, as well as angular deformity of the limbs 


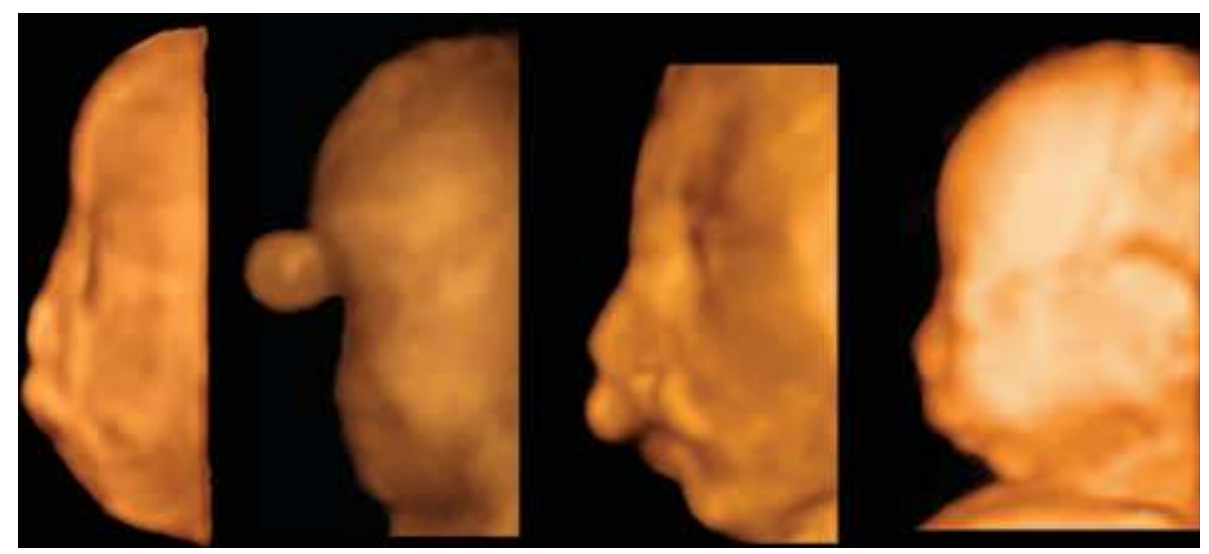

(A)

(B)

(C)

(D)

Figure 17 Surface demonstration of pathologic fetal profiles (side views). (A) Flat profile. (B) Cyclopia with proboscis. (C) Cleft lip left. (D) Retrognathia.

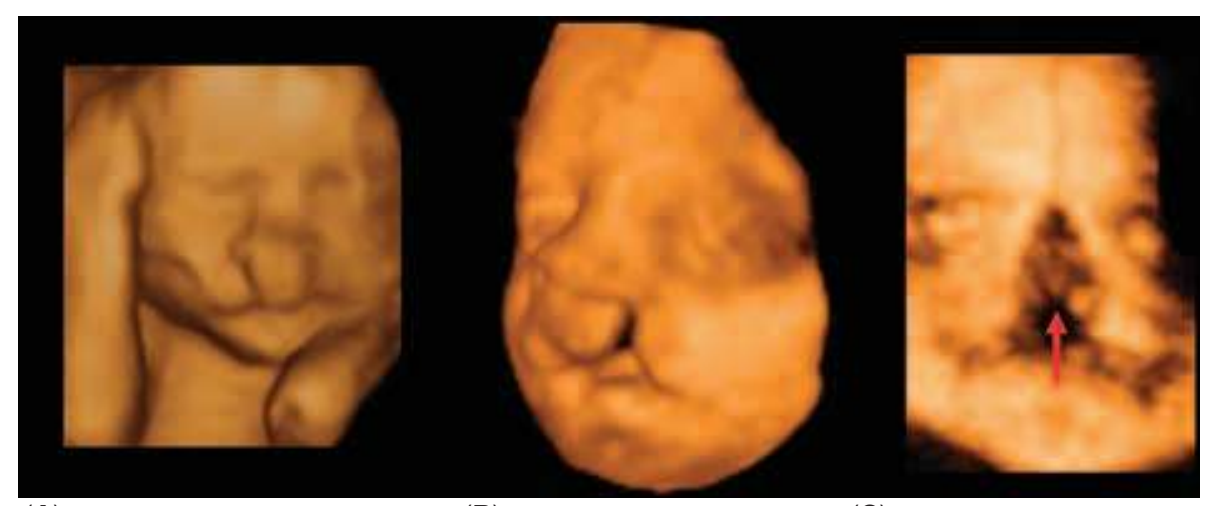

(A)

(B)

(C)

Figure 18 Surface demonstration of bilateral (A) and left-sided cleft lip (B). Surface demonstration of cleft palate ( ) after removal of the frontal part of the fetal face with the electronic scalpel (C).
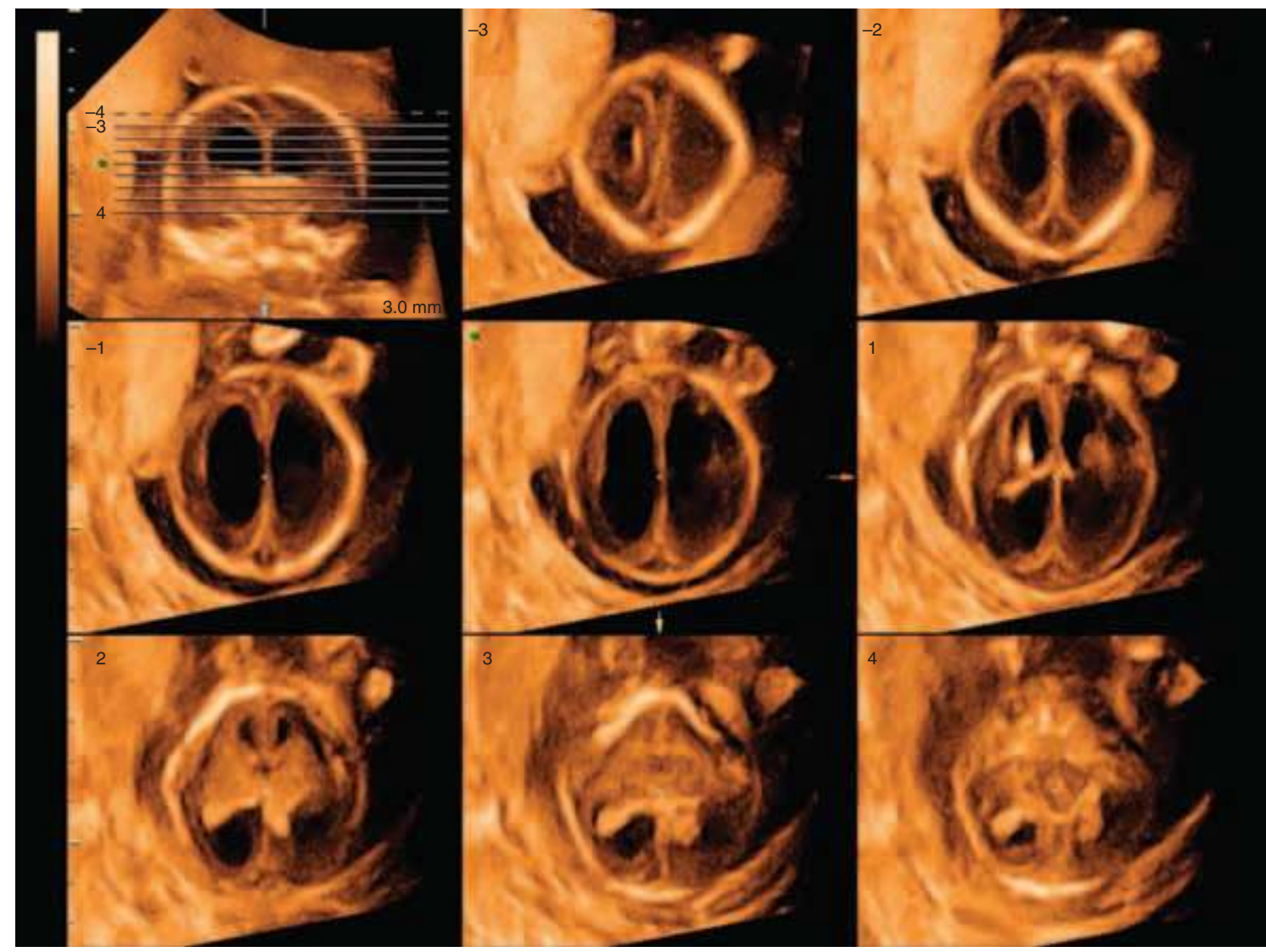

Figure 19 Tomographic ultrasound imaging of severe bilateral fetal hydrocephalus (17 weeks of gestation)—axial planes. 


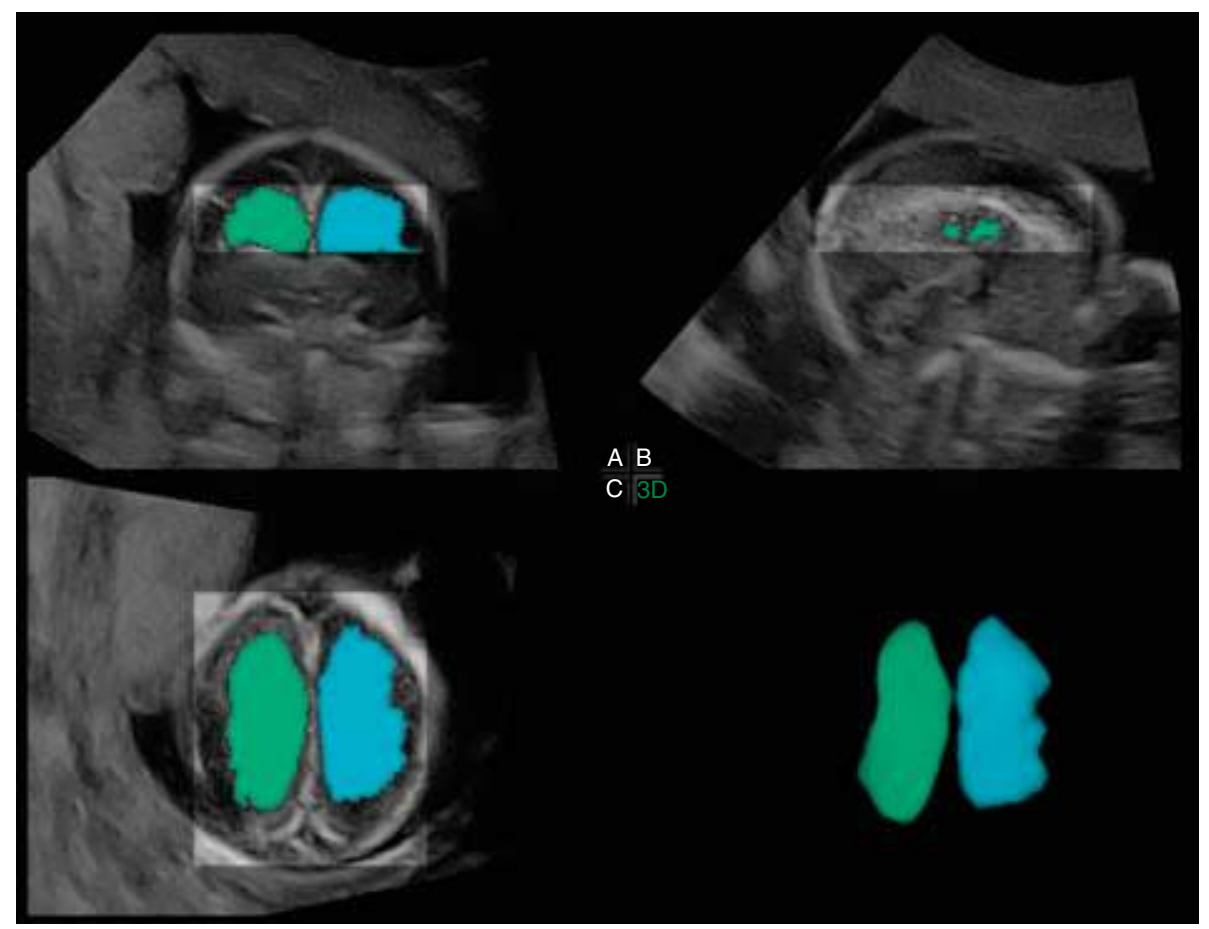

Figure 20 Sonographic automated volume count allows dyeing and volume calculation of the echogenic dilated ventricles.

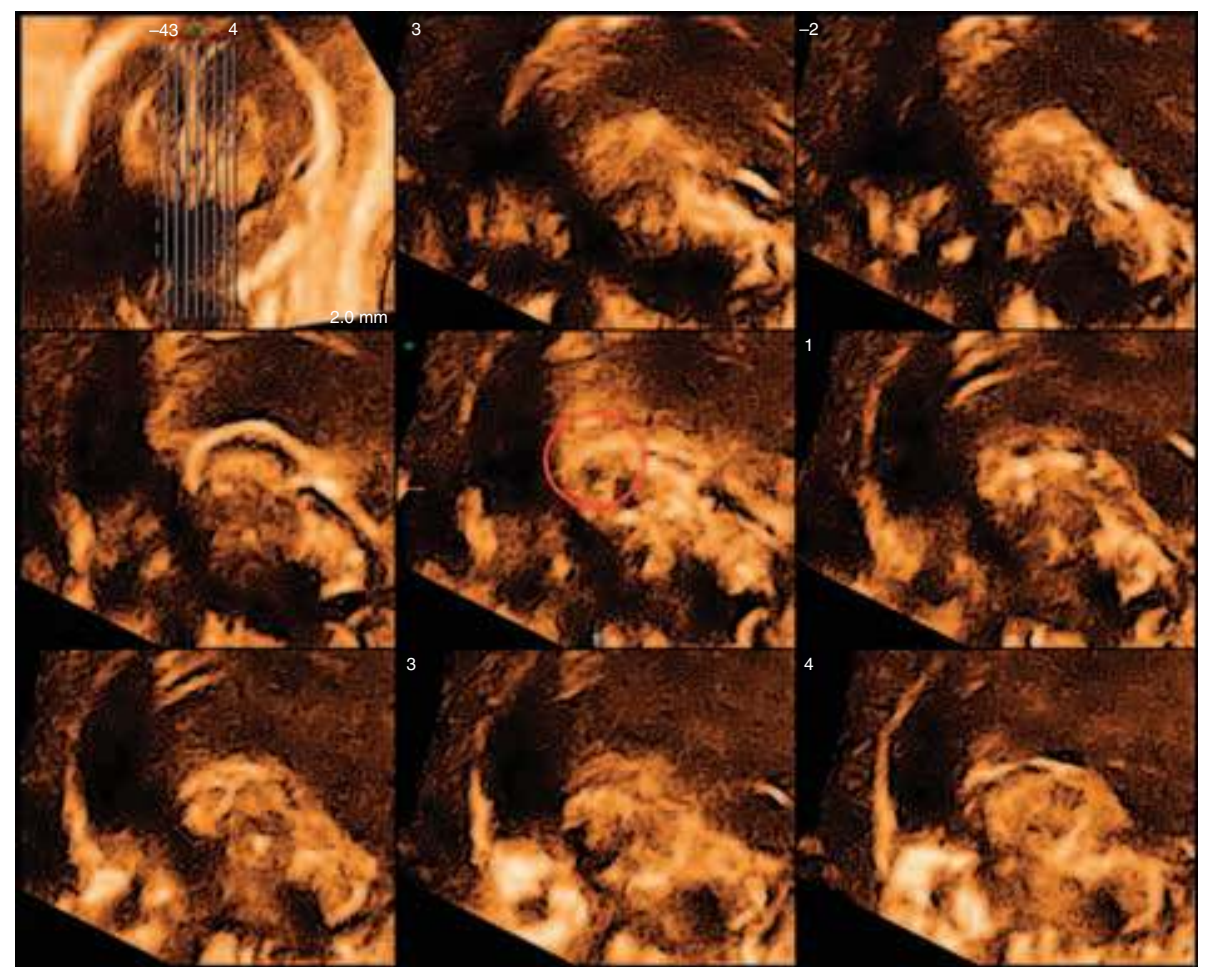

Figure 21 Tomographic ultrasound imaging of the fetal brain at distances of $6.5 \mathrm{~mm}$ (sagittal planes). The central image demonstrates absent corpus callosum at 24 weeks of gestation.

(17,23-29) (Figs. 17,18,23,25,27,28). Furthermore, internal surfaces and section planes can be rendered in the form of surface images (Figs. 18 and 26).

The transparent mode demonstrates defective ossification, osseous defects, and fractures and axial deviations of the fetal spine or the limb bones $(2,17)$ (Figs. 22 and 27).
The inversion mode is capable of converting anechoic cystic structures such as dilated lateral cerebral ventricles, renal cysts, and cystic tumors of the thorax or the abdomen into hyperechoic structures.

Glass-body rendering is a useful tool for the spatial demonstration of fetal vascular anomalies. 


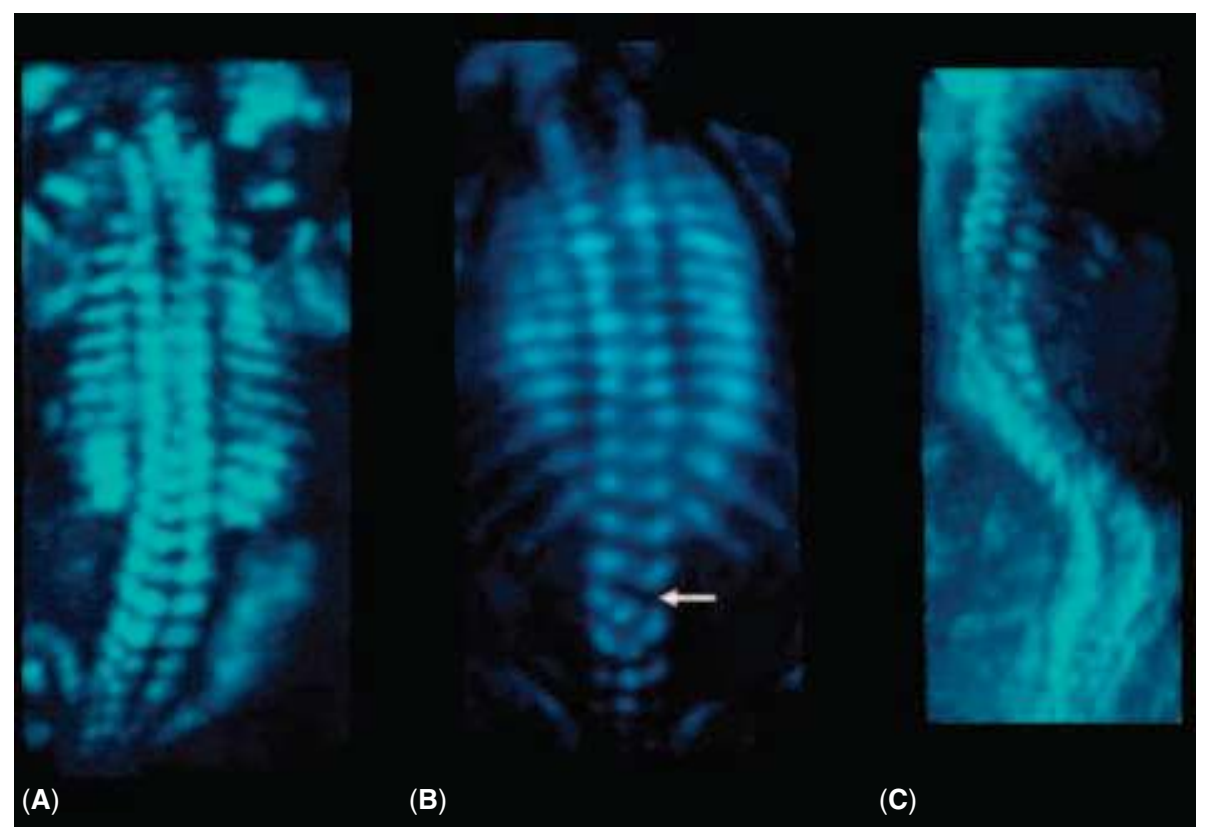

Figure 22 Transparent view (maximum mode) of the fetal skeleton. (A) Normal spine and bony thorax (20 weeks). (B) Hemivertebra ( ) (19 weeks). (C) Severe scoliosis (29 weeks).

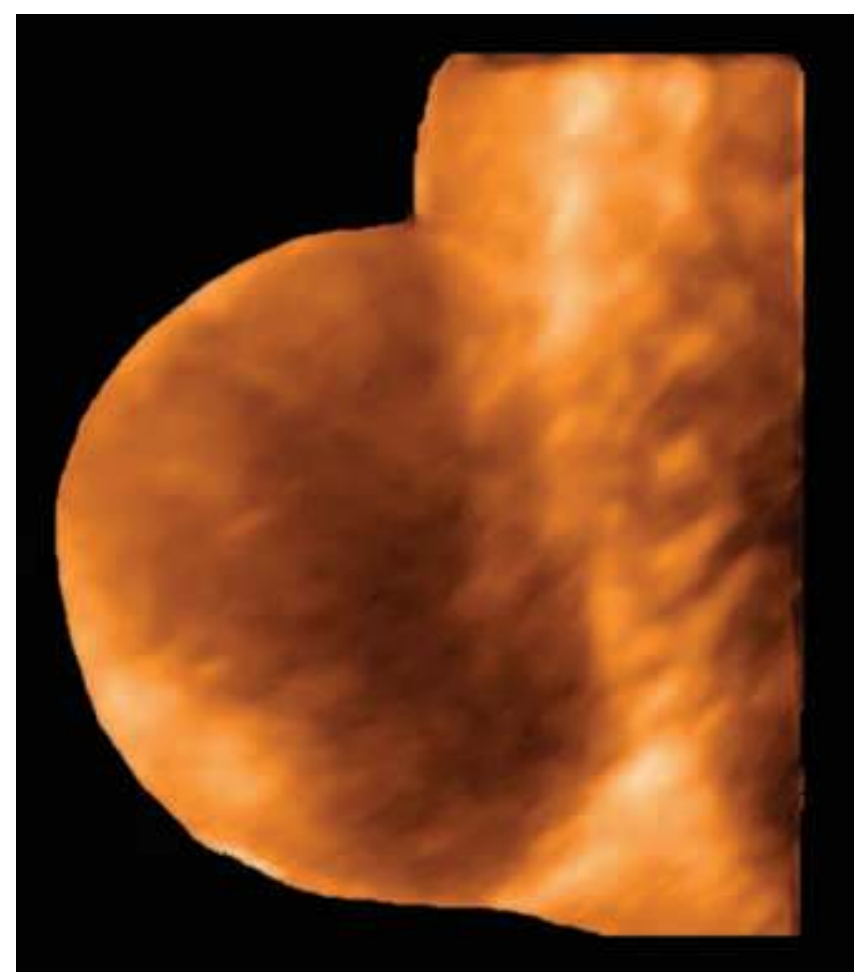

Figure 23 Surface-rendered view of myelomeningocele (31 weeks of gestation).

A detailed demonstration of cardiac defects (12) can be accomplished with the STIC technology. An acquired STIC volume data set of the heart can further be used in combination with the TUI technique to visualize a cardiac defect in parallel image planes (Fig. 22).

The $4 \mathrm{D}$ ultrasound provides the opportunity to detect abnormal fetal behavior (30). In addition, this technique can be used to record a suspicious site in the moving fetus. Once a malformation is included in the recorded volumes, the examiner can select the volume that best demonstrates the defect in 3D.

Volume sonography with its broad spectrum of different imaging modes is of particular use when a suspected finding is to be demonstrated to the pediatrician, the facial plastic surgeon or neurosurgeon, the pediatric cardiologist, the heart surgeon, or the pediatric urologist. 


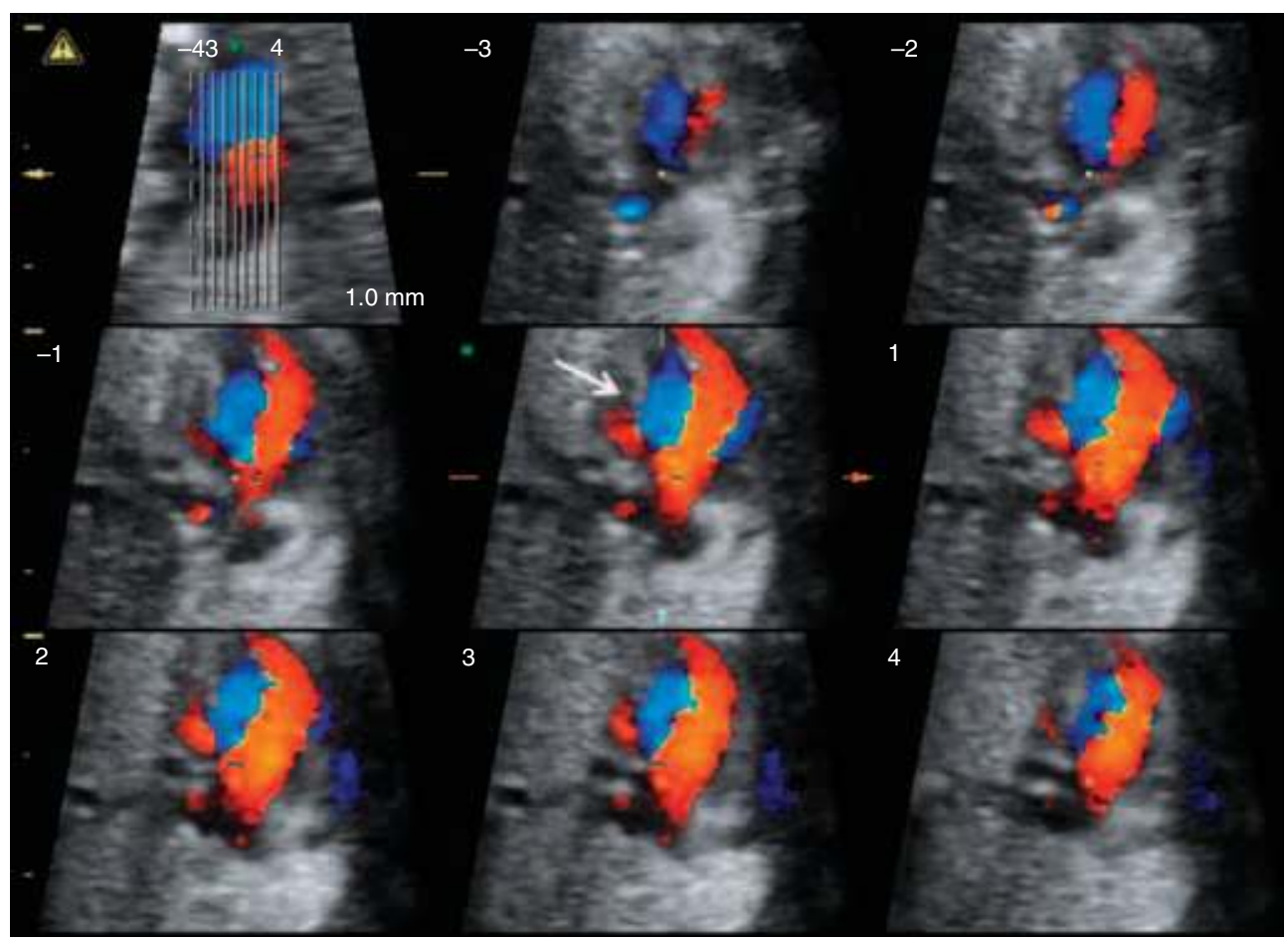

Figure 24 Tomographic ultrasound imaging of a spatiotemporal image correlation volume with hypoplastic left heart syndrome and septum defect $(\rightarrow)(21$ weeks of gestation).

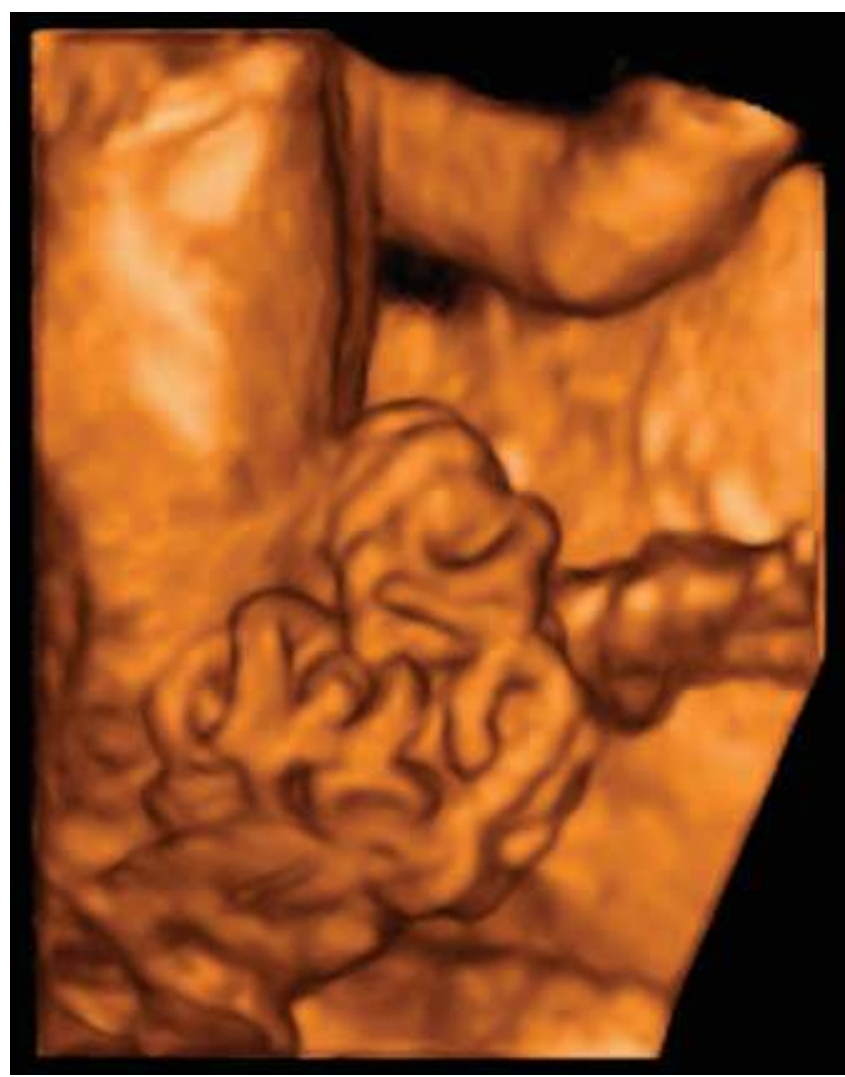

Figure 25 Surface-rendered view of gastroschisis (21 weeks of gestation).

This is of equal importance for the prospective parents, because a morphologic defect in the fetus can be more convincingly demonstrated (2), enabling them to understand the respective defect significantly better than that from a $2 \mathrm{D}$ demonstration. Conversely, in cases of an increased risk for recurrent malformation, the apprehensive parents can be reassured more impressively of the presence of a normal finding than that with 2D sonography. 


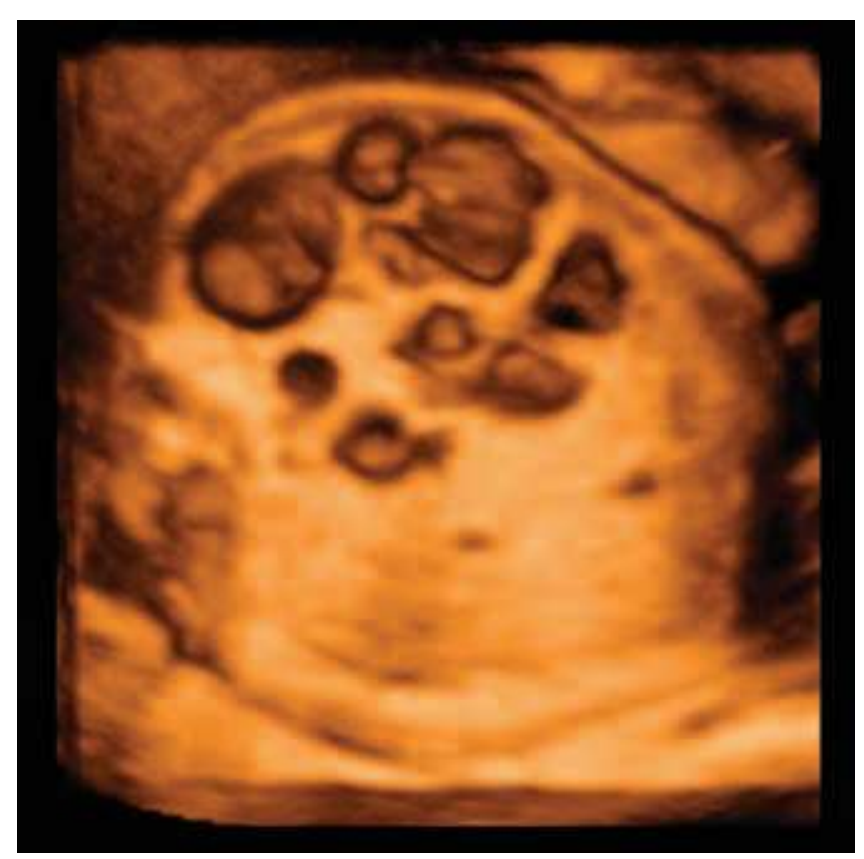

Figure 26 Surface-rendered axial view of a left-sided multicystic kidney after electronic removal of the upper part of the body (25 weeks of gestation).

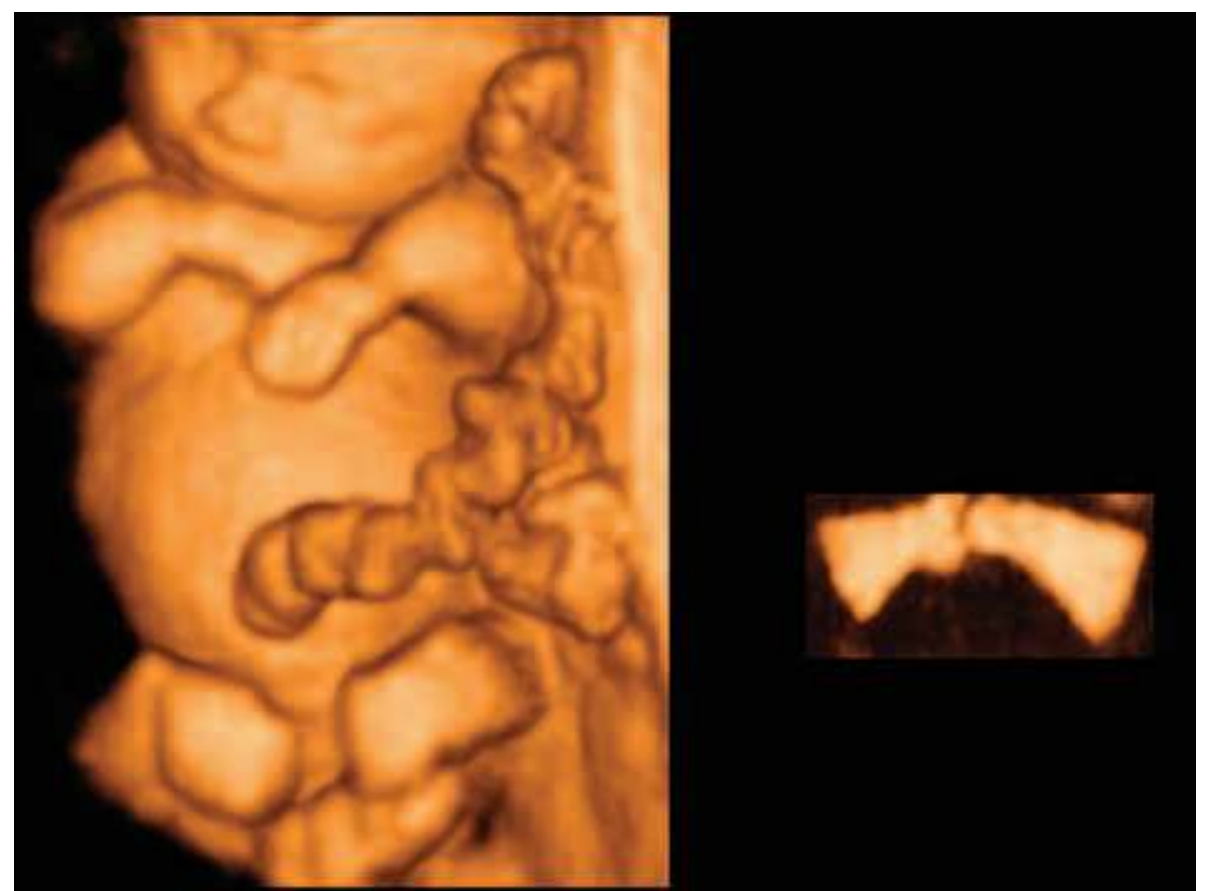

(A)

(B)

Figure 27 (A) Surface-rendered view of a fetus with osteogenesis imperfecta. Axial deviation of all four limbs due to multiple fractures (24 weeks of gestation). (B) Transparent view of the femur, revealing a fracture at the center of the bone.

KEEPSAKE IMAGES WITHOUT PRENATAL DIAGNOSIS There is no doubt that surface rendering of the fetus, and here in particular of the fetal face, represents a definite highlight for the parents and can improve maternal-fetal bonding (31). The rising demand for prenatal $3 \mathrm{D}$ or $4 \mathrm{D}$ images has lead to the development of companies in the United States that produce images and videos of the unborn baby, which are acquired outside the clinical setting. In as much as these images may be beautiful and captivating for the prospective parents and the investigator, the preparation of photos or videos of the fetus is problematic if these are not obtained in the course of a diagnostic medical procedure (32-34).

\section{SUMMARY}

The 3D/4D ultrasound technology provides both the physician and the parents with fascinating insights into the embryonic and fetal development. The 3D/4D imaging has been established as a valuable additive technique to conventional $2 \mathrm{D}$ 


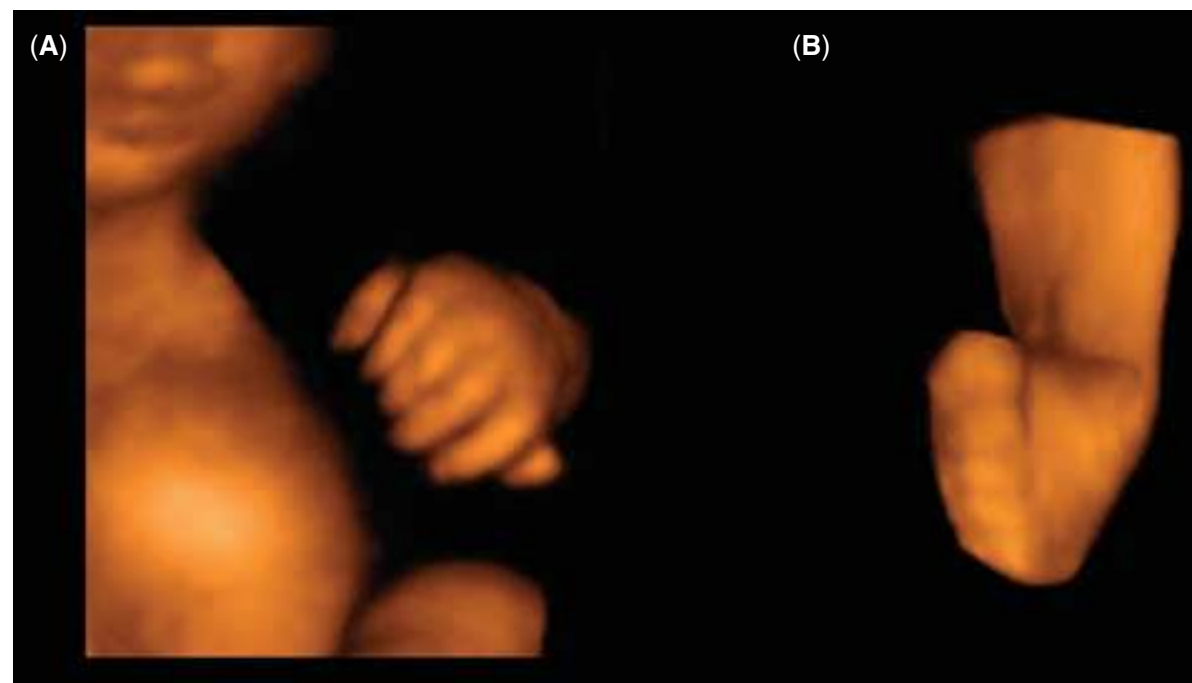

Figure 28 (A) Surface-rendered view of postaxial hexadactyly (31 weeks of gestation). (B) Club foot. The surface-rendered image shows the axial deviation of the foot of $90^{\circ}$ (31 weeks of gestation).

sonography, as it provides in particular the experienced ultrasonographist with a significant expansion of the diagnostic spectrum.

\section{REFERENCES}

1. Wiesauer F. Methodology of three-dimensional ultrasound. In: Kurjak A, Kupesic S, eds. Clinical application of 3D sonography. New York London: Parthenon Publ., 2000: 1-6.

2. Merz E. Current 3D/4D ultrasound technology in prenatal diagnosis. Eur Clin Obstet Gynaecol 2005; 1: 184-93.

3. Merz E. 3D Ultrasound in prenatal diagnosis. In: Merz E, ed. Ultrasound in Obstetrics. Stuttgart - New York: Thieme, 2005

4. Prager R, Gee A, Treece G, Berman L. Freehand 3D ultrasound without voxels: volume measurement and visualisation using the Stradx system. Ultrasonics 2002; 40: 109-15.

5. Merz E, Benoit B, Blaas HG, et al. ISUOG 3D Focus Group. Standardization of three-dimensional images in obstetrics and gynecology: consensus statement. Ultrasound Obstet Gynecol 2007; 29: 697-703.

6. Kalache KD, Bamberg C, Proquitte H, et al. Three-dimensional multislice view: new prospects for evaluation of congenital anomalies in the fetus. J Ultrasound Med 2006; 25: 1041-9.

7. Merz E. Tomographic ultrasound imaging. Ultraschall Med 2006; 27: 307-8.

8. Benoit B. The value of three-dimensional ultrasonography in the screening of the fetal skeleton. Childs Nerv Syst 2003; 19: 403-9.

9. Lee W, Goncalves L, Epinoza J, Romero R. Inversion mode. A new volume analysis tool for 3-dimensional ultrasonography. J Ultrasound Med 2005; 24: 201-7.

10. Goncalves LF, Lee W, Chaiworapongsa T, et al. Four-dimensional ultrasonography of the fetal heart with spatiotemporal image correlation. Am J Obstet Gynecol 2003; 189: 1792-802.

11. DeVore GR, Falkensammer P, Sklansky MS, Platt LD. Spatio-temporal image correlation (STIC): new technology for evaluation of the fetal heart. Ultrasound Obstet Gynecol 2003; 22: 380-7.

12. Chaoui R, Heling KS. Basics of 3D and 4D fetal echocardiography using Spatial and Temporal Image Correlation (STIC) Software. Ultraschall Med 2006; 27: 340-6.

13. Abuhamad A. Automated multiplanar imaging. A novel approach to ultrasonography. J Ultrasound Med 2004; 23: 573-6.

14. Merz E, Miric-Tesanic D, Welter C. Value of the electronic scalpel (cut mode) in the evaluation of the fetal face. Ultrasound Obstet Gynecol 2000; 16: 564-8.

15. Nelson T, Pretorius D, Lev-Toaff A, et al. Feasibility of performing a virtual patient examination using three-dimensional ultrasonographic data acquired at remote locations. J Ultrasound Med 2001; 20: 941-52.

16. Kratochwil A. Importance and possibilities of multiplanar examination in three-dimensional sonography. In: Merz E, ed. 3D ultrasound in Obstetrics and Gynecology. Philadelphia - New York - Baltimore: Lippincott, Williams and Wilkins, 1998: 105-8.

17. Merz E, Welter C. 2D and 3D ultrasound evaluation of normal and abnormal fetal anomaly in the second and third trimesters in a level III center. Ultraschall Med 2005; 26: 9-16.

18. Timor-Tritsch IE, Monteagudo A, Mayberry P. Three-dimensional ultrasound evaluation of the fetal brain: the three horn view. Ultrasound Obstet Gynecol 2000; 16: 302-6.

19. Pooh RK, Pooh KH. The assessment of fetal brain morphology and circulation by transvaginal 3D sonography and power Doppler. J Perinat Med 2002; 30: 48-56.

20. Paladini D, Volpe P. Posterior fossa and vermian morphometry in the characterization of fetal cerebellar abnormalities: a prospective threedimensional ultrasound study. Ultrasound Obstet Gynecol 2006; 27: 482-9.

21. Correa FF, Lara C, Bellver J, et al. Examination of the fetal brain by transabdominal three-dimensional ultrasound: potential for routine neurosonographic studies. Ultrasound Obstet Gynecol 2006; 27: 503-8.

22. Pilu G, Segata M, Ghi T, et al. Diagnosis of midline anomalies of the fetal brain with the three-dimensional median view. Ultrasound Obstet Gynecol 2006; 27: 522-9.

23. Merz E, Weber G, Bahlmann F, Miric-Tesanic D. Application of transvaginal and abdominal three-dimensional ultrasound for the detection or exclusion of malformations of the fetal face. Ultrasound Obstet Gynecol 1997; 9: 237-43.

24. Dyson R, Pretorius D, Budorick N, et al. Three-dimensional ultrasound in the evaluation of fetal anomalies. Ultrasound Obstet Gynecol 2000; 16 : 321-8.

25. Xu HX, Zhang QP, Lu MD, Xiao XT. Comparison of two-dimensional and three-dimensional sonography in evaluating fetal malformations. J Clin Ultrasound 2002; 30: 515-25.

26. Mangione R, Lacombe D, Carles D, et al. Craniofacial dysmorphology and three-dimensional ultrasound: a prospective study on practicability for prenatal diagnosis. Prenat Diagn 2003; 23: 810-18.

27. Mittermayer C, Blaicher W, Brugger PC, Bernaschek G, Lee A. Foetal facial clefts: prenatal evaluation of lip and primary palate by $2 \mathrm{D}$ and $3 \mathrm{D}$ ultrasound. Ultraschall Med 2004; 25: 120-5.

28. Benacerraf BR, Benson CB, Abuhamad AZ, et al. Three- and 4-Dimensional ultrasound in obstetrics and gynecology: proceedings of the American Institute of Ultrasound in Medicine Consensus Conference. J Ultrasound Med 2005; 24: 1587-97. 
29. Chaoui R, Heling KS. Three-dimensional ultrasound in prenatal diagnosis. Curr Opin Obstet Gynecol 2006; 18: 192-202.

30. Kurjak A, Azumendi G, Andonotopo W, Salihagic-Kadic A. Three- and four-dimensional ultrasonography for the structural and functional evaluation of the fetal face. Am J Obstet Gynecol 2007; 196: 16-28.

31. Ji EK, Pretorius DH, Newton R, et al. Effects of ultrasound on maternalfetal bonding: a comparison of two- and three-dimensional imaging. Ultrasound Obstet Gynecol 2005; 33: 478-90.
32. AIUM Statement: Keepsake Fetal Imaging - Approved June 22, 2005. [Available from: http://www.aium.org/publications/statements/_ statementSelected.asp?statement $=31$ ]

33. EFSUMB Newsletter. Statement on the Use of Diagnostic Ultrasound for Producing Souvenir Images or Recordings in Pregnancy. Ultraschall Med 2007; 28: 100 .

34. Merz E. 3D/4D-ultrasound in obstetrics - baby TV without diagnostics. Ultraschall Med 2008; 29: 156-8. 


\section{Prenatal diagnosis of fetal abnormality using fetal cells in maternal circulation \\ Gian Carlo Di Renzo, Elena Picchiassi, Michela Centra, and Giuliana Coata}

\section{INTRODUCTION}

The presence of rare fetal cells as well as of cell-free fetal DNA (cffDNA) and mRNA in the maternal circulation over the course of gestation has been quite recently established. The possibility of performing an early noninvasive prenatal diagnosis on fetal cells isolated from maternal peripheral blood is an important goal for modern obstetric care (1-4). Thus, fetal material can be isolated and potentially tested for genetic analysis without the need of sampling by invasive procedures as amniocentesis and chorionic villus sampling (CVS).

The types of fetal cells discovered in maternal blood are various and include lymphocytes, erythroblasts, hematopoietic stem and progenitor cells, mesenchymal cells, and trophoblasts $(5,6)$. The main advantage of using fetal cells rather than cffDNA is that they potentially offer a source of the whole fetal genome and that could be sampled in a noninvasive way for a prenatal detection of fetal aneuploidies (7). Nevertheless, the recovery and analysis of fetal cells from maternal blood is complex, and the sensitivity is still low because of the scarcity of these cells in the maternal circulation and of the lack of universal morphologic or immunocytochemical fetal markers that allow the isolation of fetal cells without contamination of maternal cells.

In this review, we summarize the current state of noninvasive prenatal diagnosis using fetal cells isolated from maternal blood during pregnancy.

\section{DISADVANTAGE OF PRENATAL DIAGNOSIS BY INVASIVE PROCEDURES}

Currently, prenatal diagnosis of fetal genetic traits relies on invasive procedures such as amniocentesis and CVS. These tests carry a small but significant risk of fetal miscarriage, and for this reason they are limited to only those women at increased risk of having a fetus with genetic abnormalities. In a recent systematic review of complications related to genetic amniocentesis and CVS, Mujezinovic et al. (8) searched on the Medline database for articles published after January 1995 reporting data for at least 100 women with singleton pregnancies with genetic amniocentesis and CVS carried out transabdominally. They found 29 articles for amniocentesis and 16 for CVS. After the statistical analysis, results demonstrated that the relative risk for fetal loss before 24 weeks was $0.6 \%$ for amniocentesis and $1.3 \%$ for CVS. They concluded that although the pregnancy loss risk is relatively low, lack of adequate controls in most studies tends to underestimate the true added risks of the procedures. Therefore, only a limited number of pregnant women with maternal age of 35 years or older and/or those having an abnormal prenatal screening should undergo invasive prenatal procedures. A recent retrospective Italian study was carried out by accessing the results of cytogenetic analysis of amniotic fluid in women with less than and greater than 35 years and performed during years 1995 to 1996 (9). This study reported results of 88,965 amniocentesis and compared the frequencies of chromosomal abnormalities in the two groups of women. The authors demonstrated that the incidence of fetal genetic abnormalities at the amniocentesis is independent from the age of the pregnant women; the results confirmed the age dependency for aneuploidies ( $>35$ years), whereas it did not confirm the age dependency for structural chromosomal abnormalities. These data provide together with other previous findings (10) important information about the actual prenatal counseling and its possible modifications due to the need to recommend noninvasive prenatal tests to all pregnant women.

For all these reasons, new noninvasive prenatal approaches are necessary.

Observation that intact fetal cells can enter and circulate within maternal blood has raised the possibility of noninvasive access to fetal genetic material that would allow the prenatal diagnosis of fetal chromosomal and fetal monogenic disorders.

\section{FETAL CELLS IN MATERNAL CIRCULATION: AN ALTERNATIVE TO NONINVASIVE PRENATAL DIAGNOSIS}

The main approaches have principally focused on the detection and genetic analysis of fetal cells and trophoblasts circulating in maternal blood and on the analysis of cffDNA and fetal mRNA in maternal plasma or serum.

\section{History of Fetal Cell Discovery in Maternal Blood}

The existence of fetal cells in maternal circulation was reported in 1893 by Schmorl (11), who first described the appearance of fetal trophoblasts in the lung of women who died of eclampsia. For many years, the existence of fetal cells in maternal blood was considered controversial. In 1959, Douglas et al. (12) showed circulating trophoblasts in maternal blood, but the proof of circulating fetal cells in maternal blood during pregnancy was obtained in 1969 from Walknowska et al. (13) reporting observations of $46, \mathrm{XY}$ metaphases in a low number of maternal blood lymphocytes isolated from women carrying a male fetus.

Despite rising interest, no consensus existed by the mid to late 1970s when Herzenberg and colleagues (14) first isolated fetal cells from maternal blood using a fluorescence-activated cell sorting (FACS) and they assumed the fetal origin of these cells for the presence of a paternal allele not present in the mother. Other investigators made similar observations until the 1990s when the fields have begun to take a more optimistic turn with the advent of several new molecular genetic 
technologies such as fluorescence in situ hybridization (FISH) and polymerase chain reaction (PCR).

For the first time, Bianchi and colleagues (15) identified fetal erythroblasts by the detection of $\mathrm{Y}$ chromosome sequences in male pregnancies after a FACS enrichment and PCR analysis. The ability to select the fetal erythroblasts and to couple it with FISH using appropriate chromosome-specific probes permitted Bianchi and other authors to identify fetal aneuploidies (16-18). Later, other successful results were obtained using trophoblasts and fetal stem progenitor cells $(19,20)$.

Finally, the discovery of cffDNA by Lo et al. (21) and more recently fetal RNA by Poon et al. (22) in maternal plasma provided other new perspectives for fetal genotyping exploitation and placental gene expression, which could have diagnostic importance for fetal pathology.

\section{Fetal Cell Trafficking in Pregnancy}

Although the placenta was traditionally seen as a barrier that separate mother and fetus, many studies have demonstrated that a variety of fetal cells and cffDNA and mRNA circulate into maternal blood throughout pregnancy and that the trafficking of this material is a frequent phenomenon occurring during every pregnancy (23). It has also been observed that the trafficking between the mother and the fetus occurs in both directions. The same bidirectional traffic seems also to take place with plasma DNA, because maternal DNA has been found in cord blood of about 30\% of samples tested (24).

The entity of the trafficking of the fetal cells depends by many factors such as complicated pregnancies and by the modes of releasing them in maternal circulation. This is due to their location inside the placenta and, as a result, different cell types are released by different modes (25).

\section{Appearance of Fetal Cells in Maternal Blood}

Data indicate that the timing appearance of fetal trafficking into maternal circulation exists $(17,26,27)$. In accordance with placental growth, the interface between fetal and maternal tissues expands, such that more fetal cells may cross the placental barrier in the early stages of gestation. Some studies provided evidences that fetal cells are present in the maternal circulation in early gestational samples. The existence of circulating fetal material was documented by the detection of $\mathrm{Y}$ chromosome-specific DNA sequences in pregnant women bearing male fetuses from 8 to 12 weeks of gestation (28). In 1995, Thomas and colleagues (29) provided evidence that Y DNA fetal cell equivalents from cellular fractions were present in peripheral blood of two pregnant women after in vitro fertilization program as early as the 5 th and 6 th weeks of gestation, respectively.

\section{Frequency of Fetal Cells in Maternal Blood}

The major limitation that continues to overwhelm the clinical use of fetal cells is their extremely low number into maternal circulation, considered not yet sufficient to perform an accurate genetic analysis.

Until now, there is no consensus about the exact number of fetal cells circulating in the maternal blood because it depends on different factors such as the fetal cell type and the form of cellular enrichment and identification techniques used, the weeks of gestation at sampling, and the presence of aneuploid pregnancies and/or pregnancy-related disorders.

Many attempts have been pursued to determine the precise number of these cells in order to individuate the best target cells for developing noninvasive prenatal testing and the best timing for their recovery. Considering their extremely low number and the absence of a specific fetal cell marker, most research groups have proceeded to apply various cellular enrichment procedures before trying to identify and count the fetal cells; consequently, only one cell category is usually isolated and examined leaving other fetal cells uncounted. Besides, it is likely that after a cellular enrichment, a large number of these rare fetal cells are lost in any case.

Therefore, few studies have striven to quantify the absolute number of all categories of fetal cells without prior enrichment. Bianchi et al. (30) determined the number of fetal-cell DNA equivalents present in the maternal whole blood samples using a quantitative PCR method aimed to identify fetal cells and Y chromosome-specific sequences as fetal marker. The mean number of male fetal-cell DNA found varied from 0.1 $91 \mathrm{Y}$-cell equivalents in $16 \mathrm{~mL}$ of maternal blood with an average of 19 Y-cell equivalents. Using a real-time PCR analysis, Ariga et al. (31) surveyed 20 pregnant women with male fetus during the entire pregnancy and found that the best time for recovering fetal cells from maternal circulation is between 10 and 12 weeks of gestation. They also reported that the level of Y signals was higher in plasma than in cells except in these two weeks of gestation where cellular amount of fetal material was higher than that in plasma. Other studies confirmed these results. In particular, Zhong et al. (32), using a novel robust real-time quantitative PCR assay, demonstrated that the number of fetal cells ranged approximately from 0.5 to 2.3 fetal cells $/ \mathrm{mL}$ of whole blood. All the data, obtained without enrichment and using quantitative PCR assays, demonstrated that it is possible to isolate approximately 1 fetal cell $/ \mathrm{mL}$ of whole maternal blood.

To actually count cells rather than cell equivalents, another strategy was used that was aimed to estimate the total fetal cell number by the fetal genetic identification using the FISH or primed in situ labeling (PRINS) analyses without enrichment procedures. The most important study in this field was conducted by Hamada et al. (26) and more recently by Krabchi et al. (33). Krabchi visualized and counted fetal nuclei using, as fetal marker, the chromosome-specific probes for $\mathrm{X}$ and $\mathrm{Y}$ chromosomes, in pregnancies bearing male fetuses. They estimated that the total number of male fetal cells $/ \mathrm{mL}$ of maternal blood varied from two to six cells with a median value of 4 fetal cells/mL of maternal blood within a group of normal pregnancies using both FISH and PRINS.

An elevated number of fetal cells in maternal blood have been observed in some pregnancy-related disorders and as pregnancies affected by fetal trisomies, pre-eclampsia, eclampsia, intrauterine growth restriction, or polyhydramnios with respect to normal pregnancies (25). Several studies have suggested that an abnormal structure of the placenta may result in an increased fetal cell trafficking to maternal circulation. Thus, an alteration of the placental barrier might explain this high number. 
It has been reported that a high number of fetal cells are present in maternal blood when the fetus is aneuploid. In two consecutive studies, Krabchi and coworkers $(34,35)$ noted a significant increase in total number of fetal cells in pregnancies carrying aneuploid fetuses. The methodological approach was similar to their previous study; the only difference was in the use of appropriate chromosome-specific probes in accordance with the chromosome to be visualized in the fetal nuclei, and aneuploidies were detected when an extra autosomal signal was observed. In the case of fetus with trisomy 21, the authors found the number of fetal cells three to five times higher than that from a normal pregnancy. The range varied from 6 to 32 fetal cells $/ \mathrm{mL}$ of whole maternal blood with an average of 12.87 fetal cells. Similar results were obtained in the second study performed by the same group (35) in pregnancies affected by fetuses with trisomies 18 and 13 . Also if the number of fetal trisomic cells was slightly less than those from fetus with trisomy 21, it has been observed as an increase of two to five times than that in normal pregnancies.

These studies, designed to detect all categories of fetal cells using cell-type-independent markers, such as aneuploidies and Y chromosome, have allowed to reach important information about the number of cells and the fact that these cells are consistently found in the blood of all women either with normal or pathologic pregnancies. Therefore, fetal cells can be reliably identified using FISH analysis in the diagnosis of fetal aneuploidies.

\section{Enrichment, Identification, and Analysis Techniques}

The absence of specific fetal antigens and the small number of fetal cells in maternal blood are the two principal caveats limiting their use in noninvasive prenatal diagnosis of fetal aneuploidies since the biologic samples to be investigated have a wide abundance of maternal cells creating a confounding background for genetic analysis.

For this reason, many attempts have been performed to isolate or enrich fetal cell populations by using numerous strategies in order to find biologic and antigenic differences between the mother and the fetus.

The most frequently used methods to enrich fetal cells from whole maternal blood are constituted by multistep procedures; usually the initial step is a density gradient separation followed by FACS or magnetic-activated cell sorting (MACS) technologies. While the first step is aspecific and allows only to reduce the abundance of maternal cells, the second step is specific since it is based on an antigen-antibody recognition using specific antibodies for a cell category of interest. Other technologies used for the enrichment of particular fetal cell population are micromanipulation based on morphologic nucleated red blood cell (NRBC) identification under microscopic observation of stained slides (36); ISET (isolation of epithelial tumor cells) technology, which allows to isolate throphoblasts on the basis of cell size by filtration (37); galactose-specific lectin-based enrichment, a method specific for isolation of fetal NRBCs, which are rich in cell-surface galactose and therefore they can be easily adsorbed to slides containing galactose sites (38); and laser microdissection and pressure catapulting, which can isolate a cell type discriminating it by morphology, fluorescence, or transfection markers (39).
Since the enrichment methods supply fetal cell samples still contaminated by maternal cells, scientific community has focused on the research of a specific fetal cell marker for their identification. Until now, fetal cell markers widely used are fetal hemoglobin, as a protein marker and $\mathrm{Y}$ chromosome, as a genetic marker. It has been shown that fetal hemoglobin is not a particularly relevant marker because it is partially present in maternal cells. Fetal cell identification based on Y chromosome by using cytogenetic (FISH) and biomolecular (PCR) techniques is an effective approach but it is limited to women carrying male fetuses.

For this reason, non-fetal gender-dependent approaches have been studied to distinguish fetal cells from maternal cells. Samura and coworkers (40) have experimented a PCR system based on analysis of microsatellite marker sequences (short tandem repeat), while the research group of Hultén (41) has detected differences in the length of telomeres in maternal versus fetal cells. The possibility of discriminating fetal and maternal cells by using these methodologies could allow the selection of fetal cells by the methods of cell isolation described above.

However, the markers used until now have shown to be rather unspecific since they are present also on a significant subpopulation of maternal blood cells.

Therefore, until now it is not possible to obtain a pure population of fetal cells, and their identification and genetic analysis are complicated by the high presence of maternal blood cells.

The genetic analysis of fetal cells has been mainly performed by PCR and FISH technologies. FISH can mainly analyze fetal gender and aneuploidies while PCR permits analysis of mutations in single-gene disorders, as well as fetal gender, rhesus blood type, and Mendelian diseases.

Since Price et al. (17) reported the first case of fetal trisomy 21 diagnosed by isolation of NRBCs from maternal blood, the FISH method has been used for noninvasive prenatal diagnosis of fetal aneuploidies, as well as fetal gender.

However, as long as new technologies for the isolation of pure fetal cell population will not be developed, the genetic analysis will be always hampered by maternal cell contamination.

\section{TARGET CELLS FOR NONINVASIVE PRENATAL DIAGNOSIS \\ Leukocytes}

Fetal leukocytes were the first type of fetal circulating material identified and enriched from maternal circulation. Fetal leukocytes were isolated by FACS with antibodies against paternally derived HLA-A2 antigens, and fetal gender and HLA type were successfully predicted (42). Despite these initial good results obtained, noninvasive clinical applications were considered unfeasible, principally due to the lack of monoclonal antibodies specific to fetal leukocyte antigens. Consequently, there is the necessity of performing HLA typing of both parents prior to flow sorting (1). Further limitation was their potential longevity. Some studies have shown that fetal leukocytes could persist in maternal blood for a period of up to 6 years postpartum, creating a state of long-term fetal microchimerism (1). This increases the risks of obtaining cells from previous pregnancy and therefore produces a confounding factor for prenatal diagnosis. 


\section{Trophoblasts}

Trophoblasts are the first fetal cells that have been documented to enter into the maternal circulation at a very early stage of gestation, approximately from the 6 th week (43).

Trophoblast invasion involves two types of cells, interstitial trophoblasts, and endovascular trophoblasts. The majority of circulating trophoblast material is constituted by interstitial trophoblasts that are deported passively into the maternal circulation by the shedding of syncytial fragments. The release of trophoblast material from placental villi is due to an apoptotic mechanism of normal turnover of this epithelium. Therefore, multinucleated syncytiotrophoblast cells cannot be recognized as circulating fetal cells because the structure is not as a cell but rather as a fragment of a multinucleated syncytium. These syncytial fragments can be detected in the uterine vein and peripheral circulation, but their isolation appears difficult since they are immediately trapped and destroyed by maternal pulmonary circulation as a consequence of their large size.

The other and predominant cell type of trophoblasts that circulates in the maternal blood is the HLA-G-positive uninucleate endovascular trophoblast. The cells, located in the lumen of spiral arteries, are in direct contact with maternal blood $(25,44)$.

Since the release of trophoblast material occurs through an apoptotic mechanism of the epithelium turnover, a major source of cffDNA circulating in maternal blood is possibly of trophoblastic origin, and although the quality of this DNA is limited (because it is apoptotic), the amount is much higher and is suitable for fetal genomic analysis by PCR-related methods (45).

Trophoblasts have been isolated from maternal blood by several different methods related to surface antigen expression, for example, HLA-G, and cell size and proof of fetal origin were accomplished with FISH and PCR-related methods (44). However, the use of these cells for noninvasive prenatal diagnosis has encountered several difficulties. Common to all circulating fetal cells studied until now is their extremely low number in maternal blood. This is principally due by the fact that multinucleated syncytiotrophoblast cells are rapidly cleared by maternal pulmonary circulation (46) and by the lack of specific monoclonal antibodies against trophoblast surface antigens that allow the isolation of a pure cellular fraction without the contamination of maternal cells. Furthermore, the syncytiotrophoblast cells are multinucleated (2-80 nuclei) and not suitable for chromosomal analysis when FISH is used. Another disadvantage of using these cells is the extra embryonic origin that could imply a discrepancy between fetal and placental karyotypes because trophoblasts could exhibit confined chromosomal mosaicism in around $1 \%$ of cases (47).

However, despite these obstacles, the HLA-G-positive uninuclear endovascular trophoblasts appear to be the most amenable kind of trophoblast cell type for the purpose of noninvasive prenatal diagnosis. The HLA-G antigen recognized by monoclonal antibody (McAb) G233 is expressed only by endovascular trophoblasts, and using this antibody for immunocytochemical identification followed by XY-FISH, van Wijk et al. (48) demonstrated the presence of these fetal cells in maternal blood. Moreover, the same authors established the frequency of these cells on average of about one fetal trophoblast expressing HLA-G per $\mathrm{mL}$ of maternal blood between 9 and 13 weeks of gestation. Furthermore, the same isolation and detection procedure has been used for prenatal diagnosis of Down syndrome and allowed confirmation of the presence of a partial hydatidiform mole (49). Hawes et al. have also accurately detected a fetal betaglobin mutation in trophoblasts present in maternal blood. Vona et al. (37) developed a strategy called ISET that is able to enrich circulating trophoblasts from maternal blood since they are larger than the peripheral blood cells. The isolated cells are individually microdissected with a laser microscope and their fetal origin was confirmed by genotyping using short tandem repeat markers. Furthermore, this method was applied to 12 pregnant women at risk of having a child affected by spinal muscular atrophy and 3 mothers were identified who were carrying an affected fetus (50).

In order to overcome the problem of the low number of these cells, Guetta et al. (19) studied the possibility to expand in vitro the trophoblasts expressing HLA-G cells in order to improve their number and possibly enable metaphase chromosome analysis. After sorting, the cells were analyzed directly or after culture. The confirmation of fetal origin was achieved by XY-FISH in samples of pregnant women carrying male fetus predicting fetal gender. The authors reached a significant fivefold increase in the yield of fetal cells compared with the sample directly analyzed. The gender prediction was successful in 93\%. This approach might represent an important progress in this field.

\section{Fetal Erythroblasts or NRBCs}

NRBCs are mononuclear and relatively well-differentiated cells (51).

They are abundant in the fetal circulation during early pregnancy since they are the first hematopoietic cell lines produced during fetal development (52). They have a short life span compared with fetal lymphocytes given their limited proliferative capacity (53), making them unlikely to persist after pregnancy.

Fetal NRBCs carry a representative complement of fetal karyotype without mosaic characteristic, unlike trophoblast cells, and are consistently present in maternal blood during pregnancy. Kleihauer et al. (54) were the first to describe this effect in 1957, by developing a staining procedure specific for fetal hemoglobin, and successively Clayton et al. (55) confirmed the observation in 1964. However, only in 1990 Bianchi et al. (15) provided the definitive proof of fetal origin of erythroblasts by isolating them from maternal blood and enriching them using FACS with antibodies specific for transferrin receptor (CD71) and by evidencing the presence of male DNA.

This last discovery was important since in that period it was thought that NRBCs were rare in adult peripheral blood (56) and consequently the most of erythroblasts present in maternal circulation should have been of fetal origin. However, cell enrichment systems developed later demonstrated that a large population of NRBCs circulate during pregnancy, but most of them are of maternal origin (57). This evidence was confirmed by others, such as Troeger et al. (58), which using single-cell PCR analysis on single micromanipulated NRBCs identified by 
May-Grunwald-Giemsa staining, demonstrated that only half of NRBCs in maternal blood were of fetal origin.

Nevertheless, the characteristics of NRBCs make them particularly suitable for the development of noninvasive prenatal diagnostic tests as proved by others, who assessed the potential of these cells by using a general setup based on enrichment, isolation, and genetic analysis as described above for the other cell types.

From the first isolation and enrichment methods developed by Bianchi et al. (15), other methods were designed based on density gradient centrifugation and on the presence of specific erythroblast surface antigens $(17,59,60)$. These approaches based on the presence of specific surface antigens such as CD71, CD36 (thrombospondin receptor), and GPA (glyphorin A) allowed fetal NRBC isolation from blood samples of all pregnancies and not only from HLA-informative couples, such as for the lymphocytes. Among these surface antigens, CD71 was the most utilized and in particular the research was focused on the development of several anti-CD71 monoclonal antibodies and on the optimization of protocols for the isolation of NRBCs from peripheral maternal blood. An attempt was conducted by Alvarez (61), who developed a specific erythroblast antibody, named $2 \mathrm{~F} 6.3$, and compared it with a commercial anti-CD71 antibody by using blood samples from pregnant women suffering from pregnancyinduced hypertension and in a control group of pregnant women without clinical features suggesting an increased risk of developing pre-eclampsia. They demonstrated that the antibody 2F6.3 succeeded in isolating a significantly higher number of erythroblasts with less maternal cell contamination than the commercial antibody in both women with pregnancy-induced hypertension and the control group.

More recent strategies of NRBC enrichment were based on the adsorption of these cells to slides containing galactose sites by galactose-bearing conjugation via soybean agglutinin, a galactose-specific lectin (62), and on microfluidic approach that consisted of microfluidic chip size-based cell separation and a magnetic device for hemoglobin-based cell isolation (63).

The possibility to enrich fetal NRBCs from maternal blood opened new perspectives of genetic analysis for noninvasive prenatal diagnosis. The main technologies used for genetic analysis were FISH (64) and PCR (65), as for the other cell types. Another molecular-cytogenetic approach employed with NRBCs was the comparative genomic hybridization (66), which allows the analysis of copy number changes (gains/ losses) in the DNA content of a given subject by using fluorescently labeled DNA in order to detect unbalanced chromosome changes.

The development of all these strategies could improve the NRBC utilization in noninvasive prenatal diagnosis, even if their genetic analysis shows considerable challenges, given their limited number.

Many attempts have been performed in order to quantify fetal erythroblasts present in maternal blood because they are considered one of the most suitable target cells for developing noninvasive prenatal tests. The first studies performed in order to establish the number of fetal erythroblasts in maternal blood were carried out by Bianchi et al. (15) and Price et al. (17) analyzing flow-sorted erythroblasts on the basis of CD71-positive cells and using PCR and FISH techniques to identify $\mathrm{Y}$ sequences. They obtained a ratio of fetal erythroblasts to maternal cells of $1: 10^{7}$ to $1: 10^{8}$ showing the reliability of isolating fetal cells from maternal blood although their number was extremely low. Moreover, the study conducted by Price et al. gave the first demonstration of the feasibility of performing prenatal detection of fetal aneuploidies on flow-sorted erythroblasts and chromosome-specific probes.

However, some recent results revealed that the analysis by FISH of fetal erythroblasts enriched from maternal blood gave low levels of diagnostic sensitivity due to the inability of detecting fetal erythroblasts by this technique. This thesis was explained by the demonstration that NRBCs have an abnormally dense nucleus with an apoptotic character, which renders it impervious to hybridization by FISH probes (62).

This finding did not dissuade the researchers from the study of the potential implications of NRBCs in noninvasive prenatal diagnosis since many clinical trials have been performed by using this kind of cells.

\section{Hematopoietic Stem Cells and Progenitor Cells}

As the other typologies of circulating fetal cells, hematopoietic stem cells and progenitor cells (HSPCs) enter into maternal circulation after accidental breakage of the villi, where it is supposed are encapsulated, or by conditions that promote exposure of the fetal endothelium and subsequent vessel breeching (25).

In reference to phenotype, stem cells express different antigenic surface markers, but the antigen mainly expressed in several stem cells lineage is the "hematopoietic" CD34 antigen. Therefore, hematopoietic CD34+ cells are the most representative cells in stem cell population. The $\mathrm{CD} 34+$ antigen, an integral membrane glycoprotein of 90 to $120 \mathrm{kD}$, functions as a regulator of hematopoietic cell adhesion to stromal cells of the hematopoietic microenvironment $(67,68)$.

First-trimester fetal blood contains a higher frequency of hematopoietic CD34+ stem cells and the number circulating in fetal blood peaks in the second trimester. Hematopoietic CD34+ stem cells together with the mesenchymal stem cells represent about $0.4 \%$ of circulating cells in fetal blood during the first trimester of pregnancy in humans (23). Several reports have demonstrated the presence of circulating fetal hematopoietic $\mathrm{CD} 34+$ stem cells (fetal CD34+ cells) in maternal blood during pregnancy. Besides, it has been demonstrated that these cells have a clonogenic capacity of colony formation, since this type of cell can be induced to divide and proliferate in vitro. The clonogenic capacity of isolated fetal CD34+ cells from maternal circulation has been demonstrated through the formation of typical colonies such as fetal colony-forming unit-erythroid (CFU-E), erythroid burst-forming units (BFU-E), and granulocyte-erythrocytemegakaryocyte-monocyte colony-forming unit (CFUGEMM) by several authors $(6,20,69)$. For this reason, among circulating fetal cells, fetal CD34+ cells appear to be one of the most promising candidate cell of fetal origin for noninvasive prenatal diagnosis. By this way, it is possible to increase the quantity of fetal material, overcoming the scarcity of the cells, for a reliable genetic analysis.

The first attempt to demonstrate the presence of these cells and their number in maternal circulation during pregnancy 
was done by Hall and Williamsons (70), who developed a purification method using an antibody directed against CD34; fetal cells were present in a range of $1 / 4.75 \times 10^{6}$ to $1 / 6 \times 10^{7}$ of the nucleated cell fraction of maternal blood. Successively, Little et al. (6) quantified fetal CD34+ cells present in maternal blood of women bearing a male fetus. After a FACS and a Y chromosome-specific probe identification, they detected a range of 0 to 7 male fetal CD34+ cells in 20 milliliter of maternal blood. More recently, other investigators $(71,72)$ established the number of these cells by using the MACS enrichment approach and the CD34 antibody coupled with the FISH analysis and the Y chromosome-specific probe. The results of the studies were very promising because they confirmed the presence of these cells in maternal blood, also if the number was low as that observed for the other categories of circulating fetal cells.

Several groups have conducted studies for identification of these cells present in maternal blood and their potential expansion.

The first evidence of feasibility of culturing fetal erythroid cells with clonal properties was achieved by Lo et al. (73). They demonstrated the selective enhancement of fetal erythroid cells with a purity rate of $0.01 \%$ to $0.25 \%$ after a coculture of male fetal liver-derived hematopoietic cells, with a ratio of fetal/ maternal cells ranged from $1 / 400$ to $1 / 9500$.

After this pioneering study, other investigators, using different antibodies against fetal clonogenic cells and semisolid medium for their expansion, confirmed the capacity of expandability of fetal cells isolated from maternal blood. Valerio et al. (69) used peripheral blood from pregnant women and enriched erythroid cells by MACS coupled with a biotin-labeled erythropoietin antibody. They identified fetal colonies after cultivation reaching a fetal colony purity rate of $18 \%$.

Later, fetal CD34+ cells in maternal blood were quantified in first-trimester samples by Little and colleagues (6). These authors studied the frequency of CD34+ cells of fetal origin in maternal blood and their in vitro proliferation. After 5 days of culture, a slight but significant expansion of fetal cells was observed. The number of cultured fetal cells ranged from 0 to 35 fetal cells in 20 milliliter of maternal blood. The fetal origin was confirmed using FISH and probes specific for chromosomes $\mathrm{X}, \mathrm{Y}$, and 21.

A further definitive confirmation of the clonogenic potential of fetal CD34+ cells was acquired by our group (20). We cultured in a semisolid culture medium, unenriched peripheral blood mononuclear cells, and enriched hematopoietic CD34+ cells by MACS from maternal blood of women bearing male fetus. Cells were then cultured for 14 days and frequencies of the expanded fetal cells were established by the use of XY-FISH. The numbers of BFU-E/CFU-E and CFU-GM colonies obtained by enriched cellular fraction were increased by 33 and 16 times respectively to unenriched cellular fraction. By FISH, we identified a suitable number of male fetal cells and a good fetal/maternal ratio even in normal pregnancies. Others studies from Tutschek et al. (74), Campagnoli et al. (75), and Guetta et al. (72) have positively raised the interest about the prospect of using fetal cell culture expansion for genetic testing. They showed, with different methodological approach, the successful clonal expansion of fetal cells combining micromanipulation and fluorescent PCR or FISH technique alone. In particular, Guetta et al. (72) demonstrated a significant 2.4-fold increase in the number of fetal cells detected by FISH, after culture with respect to samples directly analyzed after CD34+ cell sorting.

In contrast to these attempts, others have not confirmed the potential for generating pure fetal hematopoietic colonies. Chen et al. (76) cultured erythroblasts from 16 maternal blood samples in a semisolid medium with and without prior enrichment by MACS using an erythropoietin antibody. They were not able to demonstrate a conclusive fetal colony by PCR for either Y-specific sequences or autosomal microsatellite markers.

In the studies of Zimmermann et al. (77) and Manotaya et al. (78), the demonstration that maternal blood contains fetal clonogenic erythroid cells failed; they were not able to expand fetal cells because, after culture, any colonies among those isolated and analyzed by PCR and FISH technique were of fetal origin.

Unfortunately, a direct comparison of results among all these contrasting studies is not possible principally due to the differences in the methods employed for sample processing and analysis utilized by different authors.

Several authors have reported that some types of circulating fetal cells, in particular cells with stem cell-like properties, as the fetal CD34+ cells, can persist in the maternal circulation for decades after delivery, creating a state of long-term fetal microchimerism (23,79-81).

This fact has, to date, limited their use in the development of noninvasive testing, as they could still be present in the maternal circulation and potentially create a confounding factor for prenatal diagnosis. In other words, incorrect interpretation of the prenatal tests in parous women could be possibly due to circulating fetal cells from previous pregnancies.

Many researchers have focused their attention on this observation. Recently, we performed a study to verify whether the culture expansion could increase the number of these persisting CD34+ cells from previous pregnancies and whether their expanded number could interfere with a subsequent prenatal diagnosis (82). The results demonstrated that the presence of cells from previous pregnancies does not affect prenatal fetal diagnosis in current pregnancies.

\section{Clinical Trials for Detection of Fetal Aneuploidies}

The main advantage of using fetal cells enriched from maternal blood with respect to cffDNA is that they potentially offer a source of complete fetal genetic material (7) for a noninvasive prenatal diagnosis; this is an essential prerequisite for genetic diagnosis of the main fetal pathologies, as the fetal trisomies, which cannot be easily met via the analysis of cffDNA. For this reason, the use of fetal cells appears the only way for reaching the goal of the detection of fetal aneuploidies in a noninvasive manner.

Several investigators have developed and pursued strategies for detecting fetal aneuploidies within fetal cells isolated from maternal blood. For the first time, in the year 1992, Elias et al. (18) diagnosed a fetal trisomy 21. One year later Gänshirt-Ahlert et al. (83) confirmed the feasibility to diagnose fetal aneuploidies on enriched fetal erythroblasts. 
They correctly diagnosed 5 cases of trisomy 18 and 10 cases of trisomy 21. After these first observations, several other investigators confirmed this diagnostic approach and also demonstrated that it is possible to diagnose several other fetal chromosomal disorders as fetal trisomy 13 , fetal triploidy, and sex chromosome mosaicism.

Reports of identification of aneuploid fetal cells in "clinical" series are present in the scientific literature and results varied widely on the consistency with which they may be found in maternal blood (Table 1).

The National Institute of Child Health and Human Development Fetal Cell Isolation Study was the first prospective multicenter clinical project, started in 1994, with the aim of examining the feasibility of using fetal erythroblasts from maternal blood for the detection of fetal aneuploidies (84). The study was performed enrolling 2744 pregnant women considered at high risk for fetal aneuploidies. The NRBCs were isolated from maternal blood and enriched in part by FACS and in part by MACS technology and their interphase nuclei were analyzed by FISH using probes specific for 13, 18, $21 \mathrm{X}$, and $\mathrm{Y}$ chromosomes. The data of FISH analysis were compared with fetal karyotype obtained by amniocentesis or CVS. From these findings, it was evident that target cell recovery and fetal cell detection were better obtained using MACS than FACS technology. Blinded FISH assessment of samples from women carrying singleton male fetuses found at least one cell with an $\mathrm{X}$ and $\mathrm{Y}$ signal in $41.4 \%$ of cases with a false-positive rate in gender detection of $11.1 \%$. The diagnostic sensitivity in fetal aneuploidy diagnosis was $74.4 \%$, with a false-positive rate ranging between $0.6 \%$ and $4.1 \%$. The results of this clinical trial were not excellent, since the sensitivity in noninvasive prenatal diagnosis of fetal aneuploidies was comparable with that of a single marker prenatal serum screening. A possible limitation of this study could have been multiple processing protocols, which could have affected the diagnostic sensitivity in fetal aneuploidy detection.

Another study, carried out to analyze the potentiality of fetal trisomy detection by using fetal NRBCs from maternal blood (85), was performed on a large scale by testing a unique standardized protocol, overcoming in this way the limitation of the previous trial. The authors enrolled 230 pregnant women at 10 to 14 weeks of gestation, isolated NRBCs by triple-density centrifugation and by anti-CD71 magnetic cell sorting and performed FISH analysis for fetal trisomy detection. Fetal karyotype was normal in 150 cases and abnormal in 80 cases, including 36 cases with trisomy 21 . From the analysis of the study results, it was evident that the cutoff setting was of crucial importance for establishing the diagnostic performance of the protocol. In fact, while using a 21 chromosome-specific probe, three-signal nuclei were present in at least $5 \%$ of the enriched cells from $61 \%$ of the trisomy 21 pregnancies and in none of the normal pregnancies, for a cutoff of $3 \%$ of three-signal nuclei, the sensitivity for trisomy 21 was $97 \%$ with a false-positive rate of $13 \%$. Similar values were obtained in trisomies 18 and 13 using the appropriate chromosome-specific probe. Therefore, this study showed that the examination of fetal NRBCs from maternal blood could provide a noninvasive prenatal diagnostic test for trisomy 21 with the potential of identifying about $60 \%$ of affected pregnancies.

Although the research group of Al-Mufty performed a trial using a standardized protocol, it is clear that it did not reach optimal results. This fact could be explained by the poor efficiency of MACS enrichment protocol. In fact, a study carried out by Parano et al. (86) achieved promising results employing a novel procedure for NRBCs separation, which involved direct micromanipulator isolation of histochemically identified fetal hemoglobin-positive nucleated cells to obtain fetal NRBCs of high yield and purity. Using this technique, followed by cell-by-cell multicolor FISH analysis of purified fetal erythroblasts, they were able to detect Down syndrome, Klinefelter syndrome, and Patau syndrome, in 33 pregnant women. The procedure provided complete concordance with the results of amniocentesis.

The results obtained from this last study were encouraging, but clinical trials on large scale using this approach were not performed, most probably because this protocol would have been too much complex and time consuming to be introduced in clinical routine.

Therefore, until now the relative rarity of fetal NRBCs in maternal blood and the difficulty to establish their fetal origin remained the two principal problems hindering the successful spreading of noninvasive prenatal diagnosis based on fetal cells in maternal blood.

Table 1 Principal Reports Present in the Scientific Literature Focusing on Prenatal Diagnosis of Fetal Abnormalities Using Fetal Cells in Maternal Circulation

\begin{tabular}{|c|c|c|c|c|c|}
\hline Date & Author & Target cell & Methodology & $\begin{array}{l}\text { Aneuploidies } \\
\text { diagnosed }\end{array}$ & $\begin{array}{l}\text { Detection } \\
\text { rate }(\%)\end{array}$ \\
\hline 1994-2002 & Bianchi & NRBCs & MACS or FACS + FISH & Trisomies 13,18 , and 21 & 74,4 \\
\hline 1999 & Al-Mufty & NRBCs & MACS + FISH & Trisomies 13,18 , and 21 & 60 \\
\hline 2001 & Coata & $\begin{array}{l}\text { Hematopoietic } \\
\text { CD34+ cell }\end{array}$ & MACS + culture + FISH & $\begin{array}{l}\text { Trisomies } 18,21 \text {, and } \\
\text { Klinefelter syndrome }\end{array}$ & 100 \\
\hline 2006 & Di Renzo & $\begin{array}{l}\text { Hematopoietic } \\
\text { CD34+ cell }\end{array}$ & MACS + culture + FISH & Trisomies 13,18 , and 21 & $100^{\mathrm{a}}$ \\
\hline
\end{tabular}


To test the feasibility of using expanded fetal stem and progenitor cells isolated from maternal circulation in clinical practice, some researchers performed a study aimed to verify their applicability in the detection of fetal trisomies by interphase cytogenetic diagnosis. As it occurs for the other fetal cell types, detection of aneuploid cells is facilitated by the increased number of fetal cells detectable in the mother bearing a chromosomally abnormal fetus.

In several original reports, Valerio et al. $(69,87,88)$ demonstrated the practicability to sort fetal hematopoietic progenitor cells from the maternal blood of all aneuploid pregnancies and successfully expand them in culture. By this way, the authors obtained an acceptable fetal cell purity that allowed them to diagnose all the cases of aneuploid pregnancies investigated. It was possible to detect six fetal trisomy 21 , three fetal trisomy 18 , and one trisomy 13 . Fetal trisomies were documented by FISH analysis with appropriate chromosome-specific probes and confirmed by fetal karyotype.

Another study adding possibility of culturing fetal CD34+ cells was obtained by our group. We confirmed the feasibility of utilizing fetal CD34+ cells isolated from maternal blood between 11 and 16 weeks of gestation and before any invasive procedures, for the prenatal diagnosis of fetal aneuploidies $(20,71)$. In the first report we were able to diagnose two fetuses with trisomy 21 and we found a considerable number of trisomic fetal cells after expansion, with a ratio of $1 / 34$ and $1 / 12$ between fetal to maternal cells (Fig. 1). In the second
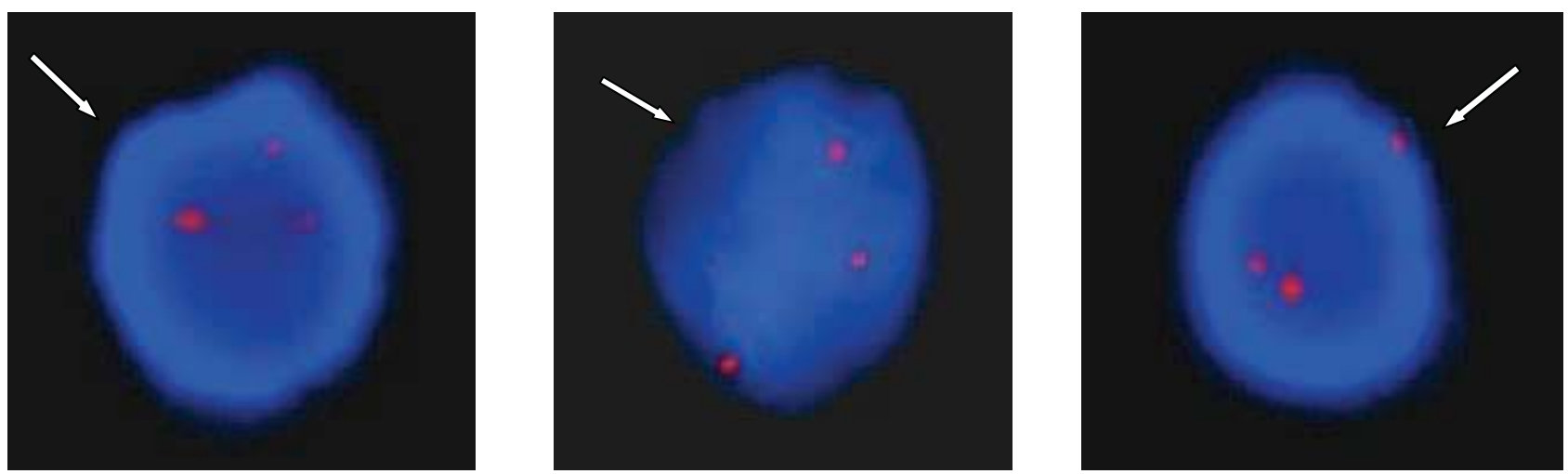

Figure 1 The figures show fetal nuclei presenting a trisomy 21 indicated by white arrows. In the nuclei, the three red signals represent chromosome 21.
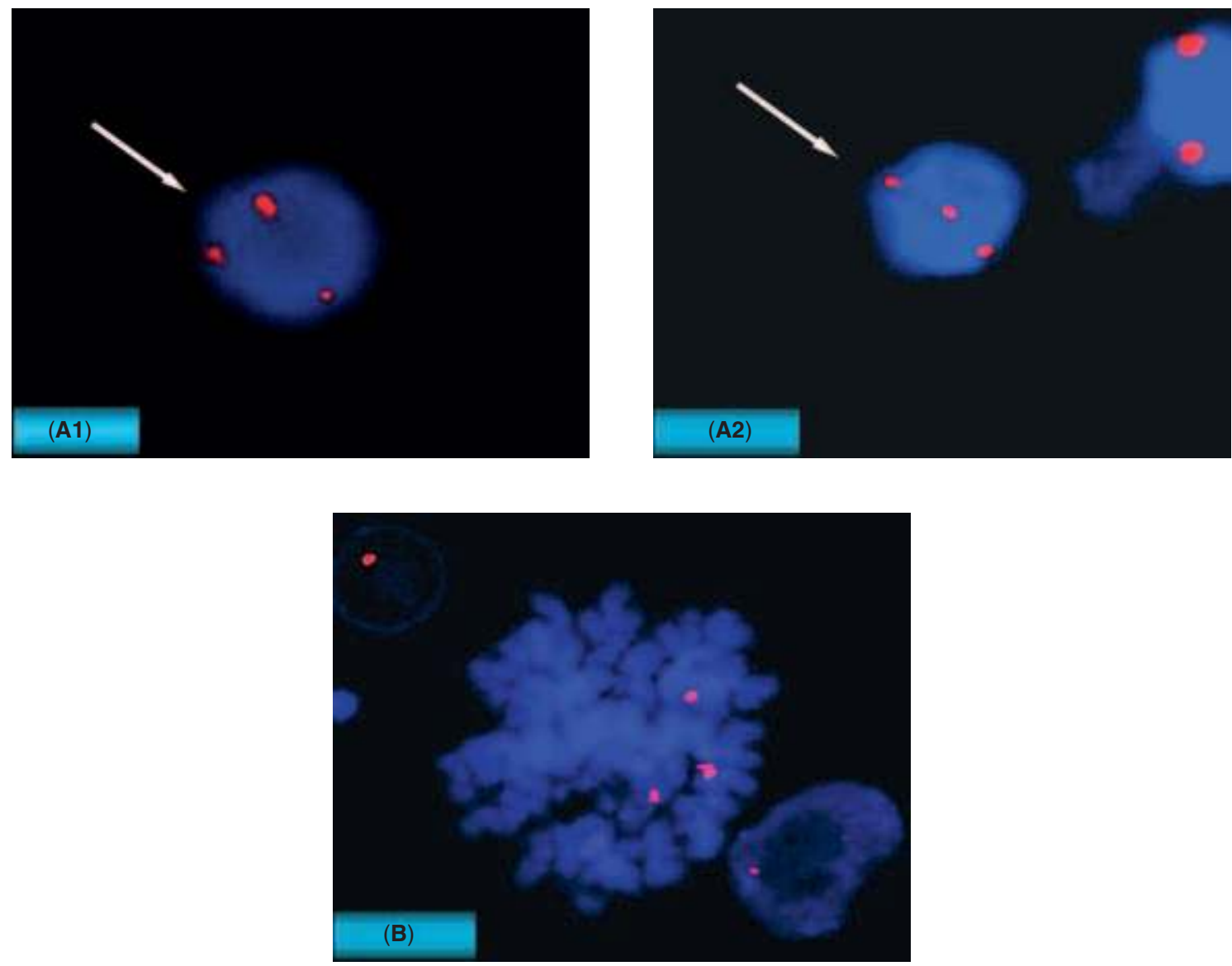

Figure 2 (A1 and A2) The figures show fetal nuclei presenting a trisomy 18 indicated by white arrows. In the nuclei, the three red signals represent chromosome 18. (B) The figure shows a metaphase presenting a trisomy 18 . 
study, we correctly detected three fetal trisomies 21 , one fetus with Klinefelter syndrome and one fetal trisomy 18 . For the first time, we were able to document the fetal origin of the expanded cells in culture by a fetal metaphase of trisomic nuclei from the fetus with a trisomy 18 (Fig. 2).

Since 2006, our group has been performing a clinical trial with estimated completion date on 2010. The purpose of this study is to validate our developed combined methodology for noninvasive prenatal identification of fetal aneuploidies and fetal gender with blood samples from pregnant women who are scheduled to undergo an amniocentesis or CVS procedure. The obtained results are then comparing with those obtained by fetal karyotype. The enrollment criteria of pregnant women are very strict and include only those women at high risk for fetal chromosomal abnormalities, providing written informed consent.

The study design involves the isolation of fetal CD34+ cells from maternal blood, the in vitro cell expansion, and FISH analysis for recognizing and counting chromosomes 13, 18, and 21 and sex chromosomes.

Our preliminary results, based on the analysis of 500 blood samples, have shown a sensibility of $100 \%$ for the identification of each studied trisomy, while the specificity have varied from $94 \%$ and $96 \%$ for trisomy 13 and 21 respectively and to $100 \%$ for the trisomy 18 .

In the same context, we evaluated whether the effect of the delay in the initial fetal cell enrichment processing of maternal blood samples from blood sampling could compromise the ability to recover fetal cells. We analyzed 45 maternal blood cells after one or more days from blood sampling. We observed a significant reduction of the CD34+ cells with respect to those samples immediately processed (usually within 1 hour). The reduction of these cells was more than $50 \%$ and this was very dangerous for our test because the fetal cells of our interest are present in this subset of cells. Consequently, we obtained a very low sensibility and specificity of the test (less than 90\%), with false-positive and false-negative results (data not yet published) in the detection of fetal trisomies. From this observation, it is essential to process immediately the maternal blood samples (within 1 hour from the sampling) in order to avoid the loss of fetal cells when we have to utilize them for the noninvasive genetic tests.

\section{CONCLUSION}

Since the demonstration of fetal cells and fetal nucleic acids in the maternal circulation, the aim of many research laboratories around the world has been to isolate fetal material for applying to genetic analysis. Nowadays, we may state that fetal nucleic acids can be reliably utilized for rapid gender detection (89-91), RhD status (92,93), and some monogenic disorders, but the application for the detection of aneuploidies although positively attempted (94-96) is still far behind. Instead the use of fetal cells and particularly erythropoietic stem cells looks as the most promising approach for the detection of aneuploidies at least limited to chromosomes 13, $18,21, \mathrm{X}$, and $\mathrm{Y}$, which represent the vast majority of fetal chromosomopathies amenable of a meaningful prenatal diagnosis. Our studies and our preliminary results are positively showing that in this direction we are pretty close to offer a reliable noninvasive diagnostic test.
However, the definitive answer will come when we will be able to develop a surface monoclonal antibody toward a fetal specific antigen, which will allow us to obtain a population of fetal cells free of maternal background to which genetic analysis can be applied.

\section{REFERENCES}

1. Bianchi DW. Fetal cells in the maternal circulation: feasibility for prenatal diagnosis. Br J Haematol 1999; 105: 574-83.

2. Holzgreve W, Hahn S. Prenatal diagnosis using fetal cells and free fetal DNA in maternal blood. Clin Perinatol 2001; 28: 353-65.

3. Jackson L. Fetal cells and DNA in maternal blood. Prenat Diagn 2003; 23: 837-46.

4. Di Renzo G, Picchiassi E. Are we facing a revolution in non-invasive prenatal genetic diagnosis? J Matern Foetal Neonatal Med 2006; 4: 195-8.

5. Simpson JL, Elias S. Isolating fetal cells from maternal blood. JAMA 1993 270: 2357-61.

6. Little MT, Langlois S, Wilson RD, Lansdorp PM. Frequency of fetal cells in sorted subpopulations of nucleated erythroid and CD34+ hematopoietic progenitor cells from maternal peripheral blood. Blood 1997; 89: 2347-58.

7. Hahn S, Zhong XY, Holzgreve W. Recent progress in non-invasive prenatal diagnosis. Semin Fetal Neonatal Med 2008; 13: 57-62.

8. Mujezinovic F, Alfirevic Z. Procedure-related complications of amniocentesis and chorionic villous sampling: a systematic review. Obstet Gynecol 2007; 110: 687-94.

9. Forabosco A, Percesepe A, Cantucci S. Incidence of non-age-dependent chromosomal abnormalities: a population-based study on 88965 amniocenteses. Eur J Hum Genet 2009; 17: 1-7.

10. Ferguson-Smith MA, Yates JR. Maternal age specific rates for chromosome aberrations and factors influencing them: report of a collaborative European study on 52965 amniocenteses. Prenat Diagn 1984; 4: 5-44.

11. Schmorl G. Pathologisch-anatomische Untersuchungen uber Publereklampsie. Leipzig: Vogel, 1893.

12. Douglas GW, Thomas L, Carr M, Cullen NM, Morris R. Trophobast in the circulating blood during pregnancy. Am J Obstet Gynecol 1959; 78: 960-73.

13. Walknowska J, Conte FA, Grumbach MM. Practical and theoretical implications of foetal-maternal lymphocyte transfer. Lancet 1969; 1: 1119-22.

14. Herzenberg LA, Bianchi DW, Schroder J, Cann HM, Iverson GM. Fetal cells in the blood of pregnant women: detection and enrichment by fluorescence-activated cell sorting. Proc Natl Acad Sci USA 1979; 76: 1453-5.

15. Bianchi DW, Flint AF, Pizzimenti MF, Knoll JH, Latt SA. Isolation of fetal DNA from nucleated erythrocytes in maternal blood. Proc Natl Acad Sci USA 1990; 87: 3279-83.

16. Bianchi DW, Mahr A, Zickwolf GK, et al. Detection of fetal cells with 47, $\mathrm{XY},+21$ karyotype in maternal peripheral blood. Hum Genet 1992; 90: 368-70.

17. Price JO, Elias S, Wachtel SS, et al. Prenatal diagnosis with fetal cells isolated from maternal blood by multiparameter flow cytometry. Am J Obstet Gynecol 1991; 165: 1731-7.

18. Elias S, Price J, Dockter M, et al. First trimester prenatal diagnosis of trisomy 21 in fetal cells from maternal blood. Lancet 1992; 340: 1033.

19. Guetta E, Gutstein-Abo L, Barkai G. Trophoblasts isolated from the maternal circulation: in vitro expansion and potential application in noninvasive prenatal diagnosis. J Histochem Cytochem 2005; 53: 337-9.

20. Tilesi F, Coata G, Pennacchi L, et al. A new methodology of fetal stem cell isolation, purification and expansion: preliminary results for non-invasive prenatal diagnosis. J Hematother Stem Cell Res 2000; 9: 583-90.

21. Lo YMD, Corbetta N, Chamberlain PF, et al. Presence of fetal DNA in maternal plasma and serum. Lancet 1997; 369: 440-2.

22. Poon LL, Leung TN, Lau TK, Lo YM. Presence of fetal RNA in maternal plasma. Clin Chem 2000; 46: 1832-4.

23. O'Donoghue K. Implication of fetal stem cell trafficking in pregnancy. Rev Gynecol Perinatal Pract 2006; 6: 87-9.

24. Lo YMD, Lau TK, Chan LY, Leung TN, Chang AM. Quantitative analysis of the bidirectional fetomaternal transfer of nucleated cells and plasma DNA. Clin Chem 2000; 46: 1301-9. 
25. Hahn S, Huppertz B, Holzgreve W. Fetal cells and cell free fetal nucleic acids in maternal blood: new tools to study abnormal placentation? Placenta 2005; 26: 515-26.

26. Hamada H, Arinami T, Kubo T, Hamaguchi H, Iwasaki H. Fetal nucleated cells in maternal peripheral blood: frequency and relationship to gestational age. Hum Genet 1993; 91: 427-32.

27. Langlois S, Wilson RD. Non-invasive prenatal fetal testing by analysis of maternal blood. Clin Invest Med 1993; 16: 333-8.

28. Lo YM, Patel P, Sampietro M, et al. Detection of single-copy fetal DNA sequence from maternal blood. Lancet 1990; 335: 1463-4.

29. Thomas MR, Tutschek B, Frost A, et al. The time of appearance and disappearance of foetal DNA from the maternal circulation. Prenat Diagn 1995; 15: 641-6.

30. Bianchi DW, Williams JM, Sullivan LM, et al. PCR quantitation of fetal cells in maternal blood in normal and aneuploid pregnancies. Am J Hum 1997; 61: 822-9.

31. Ariga $\mathrm{H}$, Ohto $\mathrm{H}$, Busch MP, et al. Kinetics of fetal cellular and cell-free DNA in the maternal circulation during and after pregnancy: implications for noninvasive prenatal diagnosis. Transfusion 2001; 41: 1524-30.

32. Zhong XY, Holzgreve W, Hahn S. Direct quantification of fetal cells in maternal blood by real-time PCR. Prenat Diagn 2006; 26: 850-4.

33. Krabchi K, Gros-Louis F, Yan J, et al. Quantification of all fetal nucleated cells in maternal blood between the 18th and 22nd weeks of pregnancy using molecular cytogenetic techniques. Clin Genet 2001; 60: $145-50$.

34. Krabchi K, Gadji M, Samassekou O, et al. Quantification of fetal nucleated cells in maternal blood of pregnant women with a male trisomy 21 fetus using molecular cytogenetic techniques. Prenat Diagn 2006; 26: 28-34.

35. Krabchi K, Gadji M, Forest JC, Drouin R. Quantification of all fetal nucleated cells in maternal blood in different cases of aneuploidies. Clin Genet 2006; 69: 145-54.

36. Takabayashi H, Kuwabara S, Ukita T, et al. Development of non-invasive fetal DNA diagnosis from maternal blood. Prenat Diagn 1995; 15: 74-7.

37. Vona G, Béroud C, Benachi A, et al. Enrichment, immunomorphological, and genetic characterization of fetal cells circulating in maternal blood. Am J Pathol 2002; 160: 51-8.

38. Kitagawa M, Sugiura K, Omi $\mathrm{H}$, et al. New technique using galactosespecific lectin for isolation of fetal cells from maternal blood. Prenat Diagn 2002; 22: 17-21.

39. Stich M, Thalhammer S, Burgemeister R, et al. Live cell catapulting and recultivation. Pathol Res Pract 2003; 199: 405-9.

40. Samura O, Pertl B, Sohda S, et al. Female fetal cells in maternal blood: use of DNA polymorphisms to prove origin. Hum Genet 2000; 107: 28-32.

41. Hultén MA, Dhanjal S. A novel assay for rapid and simple non-invasive prenatal diagnosis of genetic anomalies. In: Macek M, et al., eds. Early Prenatal Diagnosis, foetal cells and DNA in the mother. Prague, Czech Republic: Karolinum Press, 2002: 59-71.

42. Iverson GM, Bianchi DW, Cann HM, Herzenberg LA. Detection and isolation of fetal cells from maternal blood using the fluorescenceactivated cell sorter (FACS). Prenat Diagn 1981; 1: 61-73.

43. Ganshirt-Ahlert D, Garritsen HS, Hozgreve W. Fetal cells in maternal blood. Curr Opin Obstet Gynecol 1995; 7: 103-8.

44. Oudejans CB, Tjoa ML, Westerman BA, et al. Circulating trophoblast in maternal blood. Prenat Diagn 2003; 2: 111-16.

45. Alberry M, Maddocks D, Jones M, et al. Free fetal DNA in maternal plasma in anembryonic pregnancies: confirmation that the origin is the trophoblast. Prenat Diagn 2007; 5: 415-18.

46. Attwood HD, Park WW. Embolism to the lungs by trophoblast. J Opt Soc Am 1961; 68: 611-17.

47. Henderson KG, Shaw TE, Barrett IJ, et al. Distribution of mosaicism in human placentae. Hum Genet 1996; 97: 650-4.

48. van Wijk IJ, Griffioen S, Tjoa ML, et al. HLA-G expression in trophoblast cells circulating in maternal peripheral blood during early pregnancy. Am J Obstet Gynecol 2001; 184: 991-7.

49. van Wijk IJ, de Hoon AC, Griffioen S, et al. Identification of triploid trophoblast cells in peripheral blood of a woman with a partial hydatidiform molar pregnancy. Prenat Diagn 2001; 21: 1142-5.

50. Béroud C, Karliova M, Bonnefont JP, et al. Prenatal diagnosis of spinal muscular atrophy by genetic analysis of circulating fetal cells. Lancet 2003; 361: 1013-14.
51. Kelemen E, Calvo W, Fliedner TM. Atlas of human hemopoietic development. New York: Springer-Verlag, 1979.

52. Thomas DB, Yoffey JM. Human fetal hematopoiesis: the cellular composition of fetal blood. Br J Haematol 1962; 8: 290-5.

53. Pearson HA. Life-span of the fetal red blood cell. J Pediatr 1967; 70: $166-71$.

54. Kleihauer E, Braun H, Betke K. Demonstration of fetal hemoglobin in erythrocytes of a blood smear. Klin Wochenschr 1957; 35: 637-8.

55. Clayton EM Jr, Feldhaus WD, Whitacre FE. Fetal erythrocytes in the maternal circulation of pregnant women. Obstet Gynecol 1964; 23: 915-19.

56. Pembrey ME, Weatherall DJ, Clegg JB. Maternal synthesis of haemoglobin F in pregnancy. Lancet 1973; 1: 1350-4.

57. Slunga-Tallberg A, el-Rifai W, Keinänen $M$, et al. Maternal origin of nucleated erythrocytes in peripheral venous blood of pregnant women. Hum Genet 1995; 96: 53-7.

58. Troeger C, Zhong XY, Burgemeister R, et al. Approximately half of the erythroblasts in maternal blood are of fetal origin. Mol Hum Reprod 1999; 5: 1162-5.

59. Holzgreve W, Garritsen HS, Ganshirt-Ahlert D. Fetal cells in the maternal circulation. J Reprod Med 1992; 37: 410-18.

60. von Koskull H, Gahmberg N. Fetal erythroblasts from maternal blood identified with 2,3-bisphosphoglycerate (BPG) and in situ hybridization (ISH) using Y-specific probes. Prenat Diagn 1995; 15: 149-54.

61. FernÁndez A, Prieto B, Escudero A, Ladenson JH, Alvarez FV. A monoclonal antibody with potential for aiding non-invasive prenatal diagnosis: utility in screening of pregnant women at risk of preeclampsia. J Histochem Cytochem 2005; 53: 345-50.

62. Babochkina T, Mergenthaler S, De Napoli G, et al. Numerous erythroblasts in maternal blood are impervious to fluorescent in situ hybridization analysis, a feature related to a dense compact nucleus with apoptotic character. Haematologica 2005; 90: 740-5.

63. Huang R, Barber TA, Schmidt MA, et al. A microfluidics approach for the isolation of nucleated red blood cells (NRBCs) from the peripheral blood of pregnant women. Prenat Diagn 2008; 28: 892-9.

64. Pinkel D, Landegent J, Collins C, et al. Fluorescence in situ hybridization with human chromosome-specific libraries: detection of trisomy 21 and translocations of chromosome 4. Proc Natl Acad Sci USA 1988; 85: 9138-42.

65. Saiki RK, Scharf S, Faloona F, et al. Enzymatic amplification of betaglobin genomic sequences and restriction site analysis for diagnosis of sickle cell anemia. Science 1985; 230: 1350-4.

66. Yang YH, Yang ES, Kwon JY, Kim IK, Park YW. Prenatal diagnosis of trisomy 21 with fetal cells in maternal blood using comparative genomic hybridization. Fetal Diagn Ther 2006; 21: 125-33.

67. Lansdorp PM, Dogherty GJ, Humphrie SRK. CD34 epitopes. In: Knapp W, et al., eds. Leukocyte typing IV: white cell differentiation antigens. Oxford, Oxford University Press, 1989: 826-7.

68. Knapp W, Strobl H, Scheinecker C, Bello-Fernandez C, Majdic O. Molecular characterization of CD34+ human hematopoietic progenitor cells. Ann Hematol 1995; 6: 281-96.

69. Valerio D, Aiello R, Altieri V, et al. Culture of fetal erythroid progenitor cells from maternal blood for non-invasive prenatal genetic diagnosis. Prenat Diagn 1996; 16: 1073-82.

70. Hall JM, Williamsons SJ. Isolation and purification of CD34+ foetal cells from maternal blood. Am J Hum Genet 1992; 51: A257.

71. Coata G, Tilesi F, Fizzotti M, et al. Prenatal diagnosis of genetics abnormalities using fetal cells CD34+ stem cells in maternal circulation and evidence they do not affect diagnosis in later pregnancies. Stem Cells 2001; 19: 534-42.

72. Guetta E, Gordon D, Simchen MJ, Goldman B, Barkai G. Hematopoietic progenitor cells as targets for non-invasive prenatal diagnosis: detection of fetal CD34+ cells and assessment of post delivery persistence in the maternal circulation. Blood Cells Mol Dis 2003; 30: 13-21.

73. Lo YMD, Morey L, Wainscoat JS, Fleming KA. Culture of fetal erythroid cells from maternal peripheral blood. Lancet 1994; 344: 264-5.

74. Tutschek B, Reinhard J, Kögler G, Wernet P, Niederacher D. Clonal culture of fetal cells from maternal blood. Lancet 2000; 356: 1736-7.

75. Campagnoli C, Roberts IAG, Kumar S, et al. Expandability of haemopoietic progenitors in first trimester fetal and maternal blood: implications for non-invasive prenatal diagnosis. Prenat Diagn 2002; 22: 463-9. 
76. Chen H, Griffin DK, Jestice K, et al. Evaluating the culture of fetal erythroblasts from maternal blood for a non-invasive prenatal diagnosis. Prenat Diagn 1998; 18: 883-92.

77. Zimmermann B, Holzgreve W, Zhong XY, Hahn S. Inability to clonally expand fetal progenitors from maternal blood. Fetal Diagn Ther 2002; 17: 97-100.

78. Manotaya S, Elias S, Lewis DE, Simpson JL, Bischoff FZ. Evaluation of a culture system for enrichment of CD34+ hematopoietic progenitor cells present in maternal blood. Fetal Diagn Ther 2002; 17: 90-6.

79. Bianchi DW, Zickwolf GK, Weil GJ, Sylvester S, DeMaria MA. Male fetal progenitor cells persist in maternal blood for as long as 27 years postpartum. Proc Natl Acad Sci USA 1996; 93: 705-8.

80. Arlett CM. Pathophysiology of fetal microchimeric cells. Clin Chim Acta 2005; 360: 1-8.

81. Khosrotehrani K, Bianchi DW. Multi-lineage potential of fetal cells in maternal tissue: a legacy in reverse. J Cell Sci 2005; 188: 1559-63.

82. Coata G, Picchiassi E, Centra M, et al. Persistence of male hematopoietic CD34+ cells in the circulation of women does not affect prenatal diagnostic techniques. Am J Obstet Gynecol 2009; 200: 528. e1-7.

83. Gänshirt-Ahlert D, Börjesson-Stoll R, Burschyk M, et al. Detection of fetal trisomies 21 and 18 from maternal blood using triple gradient and magnetic cell sorting. Am J Reprod Immunol 1993; 30: 194-201.

84. Bianchi DW, Simpson JL, Jackson LG, et al. Fetal gender and aneuploidy detection using fetal cells in maternal blood: analysis of NIFTY I data. National Institute of Child Health and Development Fetal Cell Isolation Study. Prenat Diagn 2002; 22: 609-15.

85. Al-Mufti R, Hambley H, Farzaneh F, Nicolaides KH. Investigation of maternal blood enriched for fetal cells: role in screening and diagnosis of fetal trisomies. Am J Med Genet 1999; 85: 66-75.

86. Parano E, Falcidia E, Grillo A, et al. Noninvasive prenatal diagnosis of chromosomal aneuploidies by isolation and analysis of fetal cells from maternal blood. Am J Med Genet 2001; 101: 262-7.
87. Valerio D, Altieri V, Antonucci RF, Aiello R. Characterization of fetal haematopoietic progenitors circulating in maternal blood of seven aneuploid pregnancies. Prenat Diagn 1997; 17: 1159-69.

88. Valerio D, Altieri V, Cavallo D, Aiello R, Antonucci FR. Detection of fetal trisomy 18 by short-term culture of maternal peripheral blood. Am J Obstet Gynecol 2000; 183: 222-5.

89. Picchiassi E, Coata G, Fanetti A, et al. The best approach for early prediction of fetal gender by using free fetal DNA from maternal plasma. Prenat Diagn 2008; 28: 525-30.

90. Centra M, Picchiassi E, Bini V, et al. Diagnostic accuracy of non-invasive prenatal sex determination: a large-scale study. Clin Genet 2011; doi: 10.1111/j.1399-0004.2011.01696.x.

91. Wright CF, Burton H. The use of cell-free fetal nucleic acids in maternal blood for non-invasive prenatal diagnosis. Hum Reprod Update 2009; 15: 139-51.

92. Fanetti A, Coata G, Picchiassi E, et al. Non-invasive prenatal diagnosis of fetal RHD/SRY status using cell-free fetal DNA from maternal blood: a preliminary report. XXI European Congress of Perinatal Medicine. 10-13 September 2008. Istanbul, 2008.

93. Avent ND. Large-scale blood group genotyping: clinical implications. $\mathrm{Br}$ J Haematol 2008; 144: 3-13.

94. Lo YM, Tsui NB, Chiu RW, et al. Plasma placental RNA allelic ratio permits noninvasive prenatal chromosomal aneuploidy detection. Nat Med 2007; 2: 218-23.

95. Fan HC, Blumenfeld YJ, Chitkara U, Hudgins L, Quake SR. Non invasive diagnosis of fetal aneuploidy by shotgun sequencing DNA from maternal blood. Proc Natl Acad Sci USA 2008; 1054: 16266-71.

96. Picchiassi E, Coata G, Centra M, Pennacchi L, Bini V, Di Renzo GC. Identification of universal mRNA markers for noninvasive prenatal screening of trisomies. Prenat Diagn 2010; 30: 764-70 


\section{Recent developments in fetal therapy Gihad E. Chalouhi and Yves Ville}

The developments of ultrasound imaging prenatally as well as the implementation of ultrasound screening in pregnancy in most developed countries lead to an increasing number of fetal anomalies to be diagnosed before birth. The issue of fetal treatment is raised when the anomaly is diagnosed at an early stage and when its natural history suggests that the most likely outcome is either perinatal death or severe handicap irrespective of pediatric management. The management of fetal anomalies is ruled in each country by different laws regulating the indications for termination of pregnancy (TOP). Since many women wish to carry on with their pregnancy and TOP cannot be performed either at all or above a certain gestational age, fetal therapy has gradually developed since the early 1980s. Fetal therapy or surgery can be implemented with the aim to stabilize the effects of the anomaly on fetal development or to cure the disease itself (1). This might be either by anatomical correction of the malformation or by arresting the progression of the disease, delaying its definitive repair until after birth (2).

Only few examples fulfill these criteria (Table 1) (2,3). Since most attempts of fetal surgery concern treating rare and severe fetal conditions, they carry a large component of medical innovation, scientific research, as well as technical prowess, which unfortunately sometimes leads to procedure-related complications.

The first invasive fetal intervention began in 1963 with Liley (4). It was a blind intrauterine intraperitoneal blood transfusion. The evolution of fetal surgery has progressed since then with historical surgeries and a countless number of animal experiments. Fetal surgery is now a clinical reality (3) validated by randomized trials for a number of fetal pathologies but remains investigational for many others. The concept of fetal surgery is subjected to skepticism due to lack of clinical evidence, ethical issues, and public perception (5). Clinical trials are ethically challenging and difficult to organize and finance. This, however, should be no excuse to address all pertaining relevant issues appropriately (6).

\section{MULTIPLE MONOCHORIONIC PREGNANCIES}

Specific complications related to monochorionicity are based on hemodynamic imbalance between the twins, which are essentially normal as in twin-to-twin transfusion syndrome (TTTS) or in cases with a normal twin and its abnormal co-twin with the extreme form of the acardiac twin (7). When TTTS develops in the second trimester, the natural history leads to intra- or perinatal death in $90 \%$ of the cases, and up to $50 \%$ of the survivors show impaired neurologic development as a consequence of the death of their co-twin in utero or in the context of extreme prematurity. In all cases, intrauterine fetoscopic selective occlusion of the vascular anastomoses is attempted on the chorionic plate (Fig. 1). In more severe forms where one twin is normal and the other shows severely impaired development, selective feticide using umbilical cord occlusion of the affected twin appears to be the safest method to protect the normal twin $(8-10)$.

The technique of selective laser coagulation of placental vessels has paved the way for fetal surgery in a minimally invasive way for the pregnant woman. Initially performed using a 5-mm hysteroscope inserted through a purse-stringed hysterotomy after exposure of the uterus with a small laparotomy (11), the technique was greatly simplified by a minimally invasive percutaneous approach under ultrasound guidance and under local or epidural anesthesia that has transformed the prognosis of the disease $(8,12-15)$. An endoscope of 1.3 to $2 \mathrm{~mm}$ in diameter is introduced in a 3-mm trocar. A Nd:YAG or diode laser fiber is passed through the operative channel of the endoscope. It allows coagulation of the anastomotic vessels joining the two fetoplacental circulations (Fig. 2). This technique has been developed over 15 years and has proven to be the best firstline treatment with the highest degree of evidence, leading to survival rates of around $75 \%$ with severe morbidity in less than $10 \%$ of the survivors (9). These results are stable through to long-term follow-up and up to the age of 6 years (16).

Several techniques for selective feticide of a monochorionic fetus have been described and used, including ultrasoundguided fetal cord ligation or compression (17), bipolar coagulation, laser coagulation, transection with harmonic ultrasound scalpel (18), thermocoagulation (19), and radiofrequency ablation (20). Bipolar coagulation technique remains a gold standard for cord coagulation (21) because of the short duration of the surgery, its relative technical simplicity, and availability (21).

Bipolar umbilical cord coagulation is performed using a bipolar forceps $(2.5-\mathrm{mm}$ diameter). The procedure aims at coagulating the cord at placental or abdominal insertions, depending on their accessibility. A longitudinal portion of the cord is exposed to the bipolar forceps with its jaws perpendicular to the cord. After grasping the cord, an initial coagulation is performed using $15 \mathrm{~W}$ for 15 to 60 seconds. The efficacy of the procedure is judged by the appearance of echogenic steam bubbles and increased echogenicity of the coagulated portion of the cord. In the absence of bubbles, the power is increased in $5-\mathrm{W}$ increments up to a maximum of $50 \mathrm{~W}$. When the appropriate power is obtained, the coagulation is continued for 1 or 2 minutes, without releasing the cord. A second segment of the cord is then systematically coagulated following the same procedure. The interruption of blood flow in the coagulated cord is checked by ultrasound during the procedure. 
Table 1 Criteria for Fetal Surgery $(2,3)$

1 Accurate diagnosis and staging possible, with exclusion of associated anomalies

2 Natural history of the disease is documented, and prognosis established

3 Currently no effective postnatal therapy is available

4 In utero surgery proven feasible in animal models, reversing deleterious effects of the condition

5 Interventions performed in specialized multidisciplinary fetal treatment centers within strict protocols and approval of the local ethics committee with informed consent of the mother or parents

\section{CONGENITAL DIAPHRAGMATIC HERNIA}

Thoracic anomalies are the most illustrative to view fetal surgery in both historical and technical perspectives. These anomalies have in common to interfere with lung development through the mass effect leading to pulmonary hypoplasia that is often lethal when compression develops during the second trimester of pregnancy (22). Ultrasound screening in the second trimester depicts the majority of these severe anomalies at an early stage that is potentially amenable to fetal surgery. Thus fetuses with the most severe forms of congenital diaphragmatic hernia $(\mathrm{CDH})$ can also benefit from intrauterine fetal surgery (10).

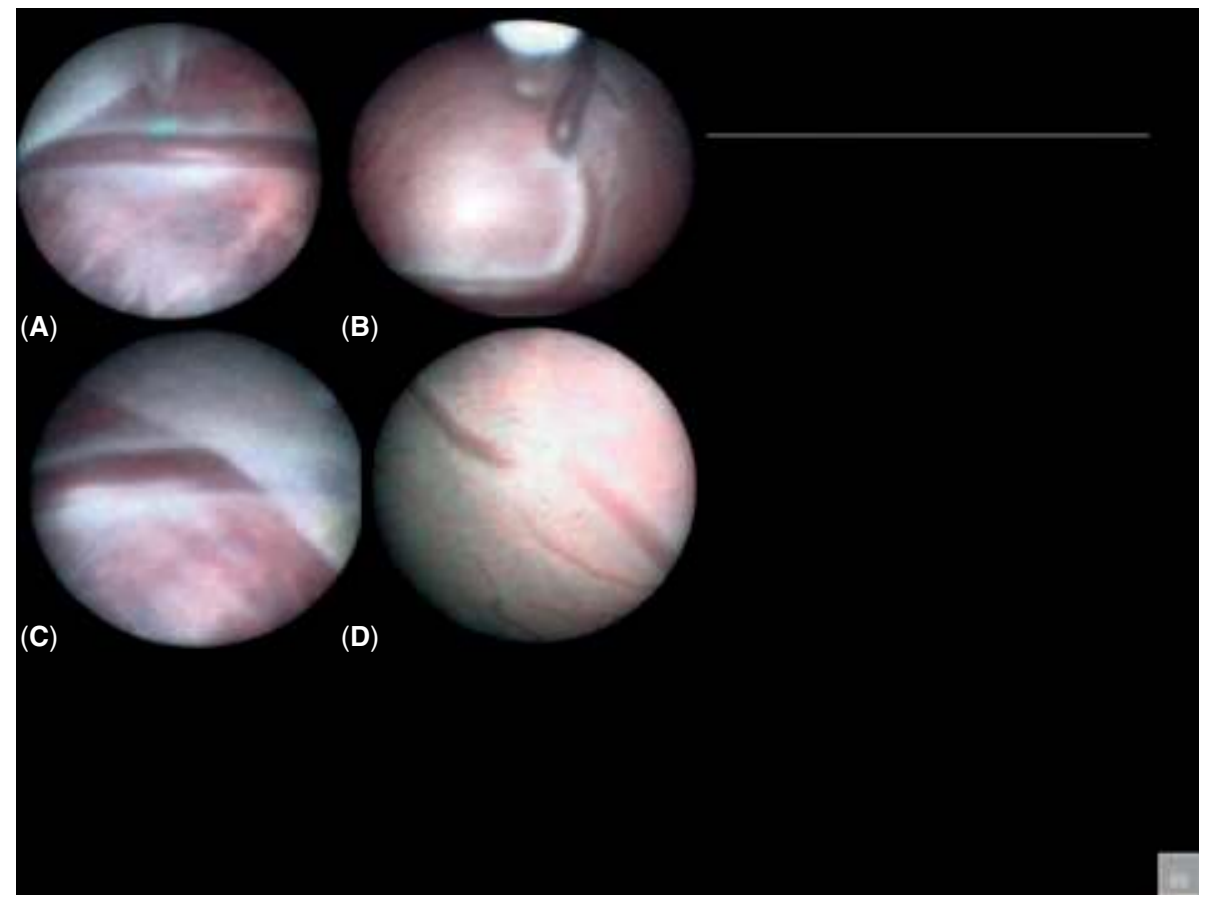

Figure 1 Vascular anastomoses between the circulations of the two twins in a monochorionic diamniotic pregnancy complicated: (A) and (C) nonselective anastomoses passing under the amniotic membrane; (B) and (D) selective. Laser fiber can be visualized at 12 o'clock in (A) and (B).

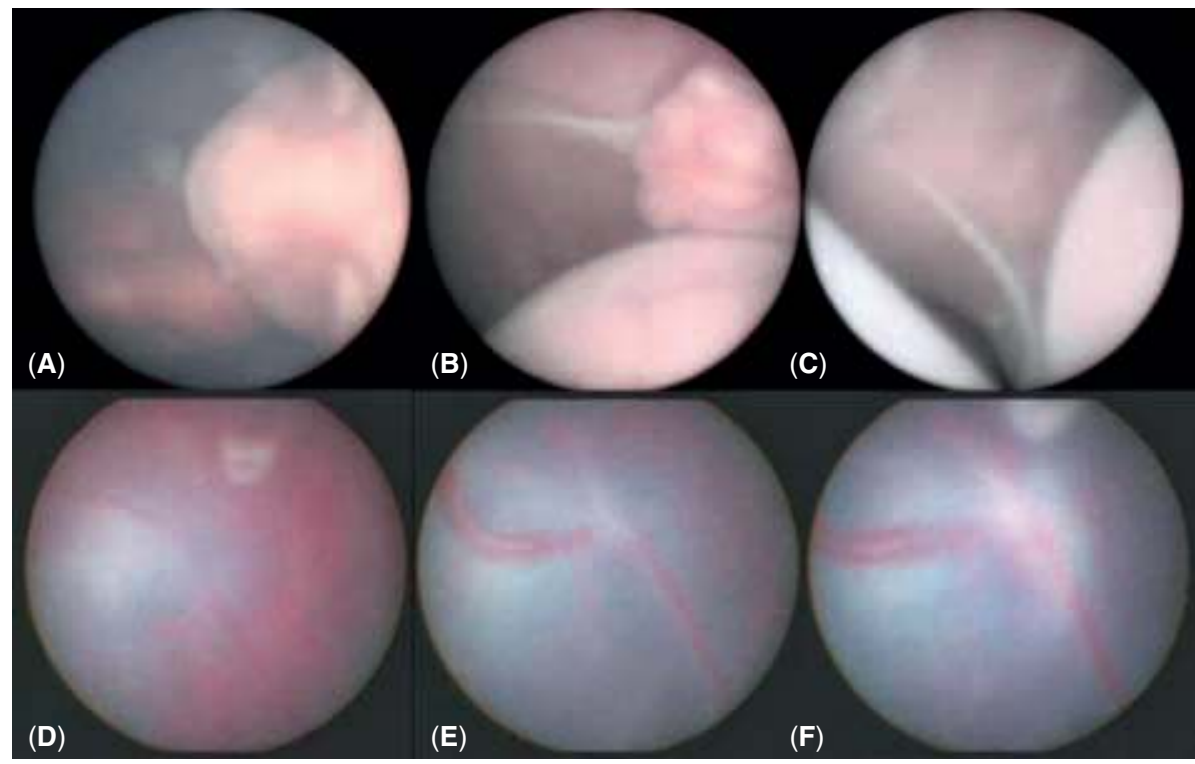

Figure 2 Selective laser coagulation of placental vessels in a twin-to-twin transfusion syndrome. Donor stuck twin in anhydramnios (A, B, C). Vascular anastomoses (D, E, F) coagulated with a laser fiber seen at 12 o'clock (D, F). 


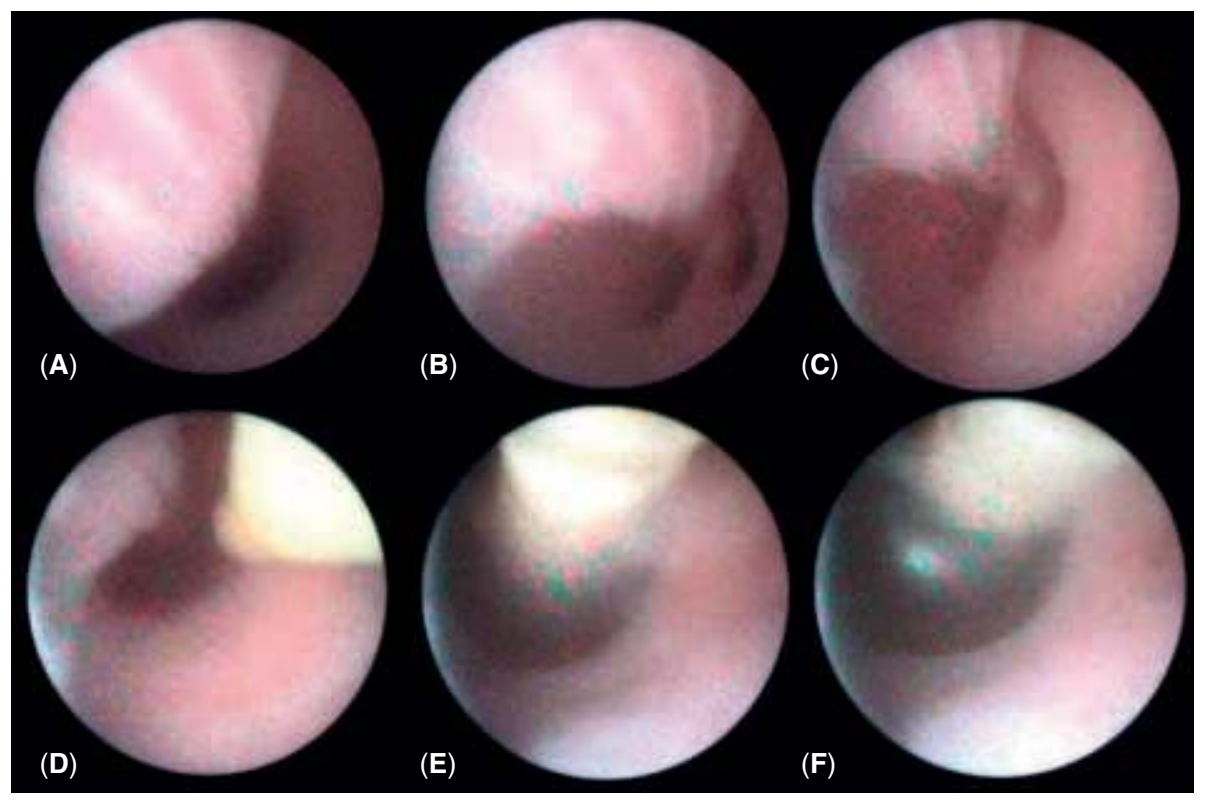

Figure 3 "Plug the lung until it grows" strategy. Fetoscopic access to the fetal trachea $(\mathbf{A}, \mathbf{B})$ reaching the carina $(\mathbf{C})$, and inflating a balloon, $1 \mathrm{~cm}$ above the carina (D, E, F).

$\mathrm{CDH}$ of intra-abdominal organs in the thorax, when it is not associated with other anomalies, allows survival between $0 \%$ and up to $85 \%$ of the cases depending upon the degree of lung development. Prenatal evaluation of the volume of the contralateral lung has been the subject of continuous research for the last 20 years. To date, the simplest evaluation of the relative size of the lung mass to the head circumference [lungto-head ratio (LHR)] has the best prognostic value. When expressed as a proportion compared with what is expected in a normal fetus (observed/expected LHR), this prediction is independent of gestational age (23). The presence of the liver in the thorax is another independent factor of poor prognosis. Fetuses with $\mathrm{CDH}$ and LHR $<1$ with liver up in the thorax are almost certainly destined to die in the perinatal period despite advanced neonatal care (24).

History of fetal $\mathrm{CDH}$ surgery begins with pediatric surgeons who operated newborns. The initial prenatal approach consisted of patch closure of the diaphragmatic defect $(25,26)$. Fetal surgery followed the rules of neonatal surgery with the aim of curing the defect through very invasive modalities for the pregnant woman, including laparotomy, hysterotomy, and aggressive tocolysis. Delivery was then doomed also to be performed by cesarean section at any gestational age, owing to the recent uterine scar, whether the child was dead or alive (27). This approach was based on successful sheep experimental surgery for 10 years but failed 20 years later for the very reason that the human uterus is far more sensitive to preterm labor triggered by uterine surgery than sheep. The natural history of yet another congenital malformation, tracheal atresia, showed the rationale for palliative intrauterine surgery in $\mathrm{CDH}$. This condition causes pulmonary hyperplasia by increasing bronchial pressure and the possibility of a temporary obstruction proved successful to stimulate the growth of hypoplastic lungs in a sheep model (28).

Tracheal occlusion was first clinically achieved by laparotomy, hysterotomy, neck dissection, and tracheal clipping (29) with impressive results on lung growth in a subset of fetuses but with a low survival rate. The first percutaneous endoluminal occlusion was reported by Quintero et al. (30). Unfortunately, the device failed to occlude and the baby died in the postnatal period.

In Europe, a clinical technique via a 3.3-mm percutaneous access was developed with balloon removal initially at the time of an ex utero intrapartum treatment (EXIT) procedure $(31,32)$. General anesthesia was used at first but was soon replaced by regional and local anesthesia with fetal sedation and immobilization. This approach is often referred to as the "plug the lung until it grows" strategy (33). This surgery consists of accessing the fetal trachea using a $1.2-\mathrm{mm}$ endoscope as described in placental surgery (see above) and inflating a balloon in the fetal trachea, $1 \mathrm{~cm}$ above the carina (Fig. 3). This is usually performed at 26 to 34 weeks and the balloon is removed 8 weeks later. Removal can either be performed following the same procedure, be deflated by needle puncture under ultrasound guidance or following neonatal intubation immediately after birth. Preliminary results in over 200 human fetuses with a poor prognosis where the expected survival xas around $10 \%$, the observed survival was $55 \%$ (24). The results depend upon estimated fetal LHR to reach $78 \%$ for LHR between 0.8 and 1 (34). However, although uterine tolerance to endoscopic fetal surgery is good, the risk of preterm rupture of the membranes in up to $20 \%$ before 32 weeks remains a challenge to the development of this surgery, which has to address at least two other issues: (1) to establish itself through a randomized study against postnatal management only. (2) to evaluate later intervention in the forms with less severe prospect based on LHR $>1$ in order to decrease prematurity-related risks. In the latter group, a previous randomized study with suboptimal design suggested that prenatal management was not beneficial, but the suboptimal design significantly biased the result of the trial (35).

Jani et al. (36) recently reported results of 210 severe CHD treated with fetal endoscopic tracheal occlusion. They concluded that their treatment is associated with a high 
incidence of preterm premature rupture of fetal membranes (PPROM) (16.7\% cases) and preterm delivery $(30.9 \%$ of patients delivered before 34 weeks) but a substantial improvement in survival. Forty-eight percent of infants were discharged from the hospital alive. They therefore increased survival in severe cases with left-sided CDH from 24.1\% to $49.1 \%$, and in right-sided $\mathrm{CDH}$ from $0 \%$ to $35.3 \%$ $(p<0.001)$ (37). The strongest predictors of survival are observed/expected LHR prior to the procedure, the absence of PPROM, and gestational age at delivery.

Although many recent nonrandomized reports of success with balloon tracheal occlusion (and release) are promising, prenatal therapy should not be widely adopted until a welldesigned prospective randomized trial demonstrating efficacy is performed (38).

This surgery has also demonstrated that the fetal airways were directly accessible to prenatal local administration of drugs opening new perspectives for gene therapy in congenital severe respiratory diseases such as cystic fibrosis.

\section{FETAL LOWER URINARY TRACT OBSTRUCTION (39)}

Lower urinary tract obstruction (LUTO) encompasses different malformations of the urethra including posterior urethral valves (PUV) (60\%), urethral agenesis, and atresia (40\%) and has a prevalence of around 2/10,000 births. The related mortality and morbidity are high and related both to progressive renal dysfunction and/or to dysplasia secondary to parenchymal compression and pulmonary hypoplasia related to oligoanhydramnios in cases with complete urethral obstruction.

Symptomatic fetal treatment should optimally rely on vesico-amniotic shunting, as it proved to work in animal models. The use of a double pigtail catheter placed into the fetal bladder as a temporary therapeutic intervention to allow obstructed urine to flow into the amniotic space has met with inconsistent success. Anhydramnios often found in LUTO presents a technical challenge when placing a vesico-amniotic shunt because the distal end of the shunt catheter needs to be placed into a pocket of amniotic fluid. Amnioinfusion allows to re-expand the amniotic cavity with fluid for successful shunt placement.

Technically, the placement of a vesico-amniotic shunt is done under local anesthesia and continuous ultrasound guidance. The two-pushrod shunt procedure, which we describe here, is the most used technique nowadays. The fetal bladder is accessed lateral to the midline below the abdominal cord insertion. Doppler is used to localize the umbilical arteries and confirm that the trocar will not injure them on passage through the abdominal and bladder walls.

First, the trocar is introduced into the amniotic space close to the lower fetal abdomen. An adequate pocket of amniotic fluid needs to be present in which to drop the distal end of the double pigtail catheter on exiting the fetal abdomen. As indicated above, an amnioinfusion is done in case of anhydramnios or insufficient fluid space. The trocar is then inserted into the bladder and positioned into a central location. The double pigtail catheter is then completely threaded into the trocar sheath. A short push rod is introduced and used to push the proximal pigtail of the catheter into the bladder. The shorter push rod is then removed and a long push rod is gently introduced until it comes in contact with the distal tip of the shunt catheter within the trocar sheath. The push rod is then held in place while the shaft of the trocar sheath is slowly pulled back about $2 \mathrm{~cm}$. At this point, the trocar sheath should lie just outside the fetal abdomen, with the straight segment of the shunt catheter traversing the region of abdomen between the bladder and amniotic space. The trocar sheath is then tilted to direct it away from the insertion site and the long push rod advanced to displace the distal pigtail of the catheter into the amniotic space (40).

Although the overall benefit of bladder shunting seems to translate into increased survival (OR 2.5; 95\% CI, 1.0-5.9; $p<0.03)$ especially in the most severe cases (OR 8; 95\% CI, $1.2-52.9 ; p<0.03)(41)$, this hardly translates into improved renal function. Several potential explanations have recently been identified:

1. Shunting is performed under local anesthesia to the mother as well as fetal analgesia and curarization. Shunts most commonly used are $2.5 \mathrm{~mm}$ in diameter and placed through a $3.5 \mathrm{~mm}$ trocar introduced percutaneously into the uterus and into the fetal bladder. It is not an easy procedure to perform and one-fourth to one-third of shunts get displaced.

2. Shunting should optimally be performed when renal function is still normal. The best single parameter seems to be beta-2-microglobulin in fetal blood since fetal urine analysis and ultrasound assessment of the kidneys have a poor predictive value.

3. The diagnosis of PUV is the only one amenable to significant improvement in survival and renal function. However, the diagnosis is rightly made in only around $60 \%$ of the cases in experienced hands.

4. Overall, it is likely that shunting is too often being placed to late in the course of the natural history of the disease.

LUTO is yet another example of failed open surgery that has been abandoned in the late eighties. The encouraging results from meta-analysis on bladder shunting are calling for a randomized controlled trial that has been initiated as the PLUTO trial (percutaneous shunting in lower urinary tract obstruction: www.pluto.bham.ac.uk). Recruitment is slow and it is hoped that 200 cases could be included over a 5 -year period to compare outcomes in fetuses with normal renal function and oligohydramnios at less than 26 weeks following either early bladder shunting or expectant management in utero.

A recent advance in this respect is in utero cystoscopy (42). Ruano et al. (43) recently reported a large series of PUV treated with fetal cystoscopy and laser fulguration. They proved it to be feasible and suggested that it might prevent renal function deterioration.

The same technique of shunt placement is also used for some compressive thoracic processes such as congenital hydrothorax or macrocystic congenital cystic adenomatoid malformation (CCAM) (40). 


\section{COMPRESSIVE FETAL PLEURAL EFFUSION AND}

\section{OTHER COMPRESSIVE LUNG MALFORMATIONS}

Fetal pleural effusions have a prevalence of $1 / 10,000$ to $1 / 15,000$ births. Irrespective of the underlying etiology, around one-fifth resolve spontaneously. However, compressive pleural effusion that is characterized by either mediastinal shift or compression in unilateral and bilateral effusions, respectively, is associated with a poor prognosis when it leads to polyhydramnios through esophageal compression and hydrops through impaired venous return and cardiac tamponade. Indeed, unilateral effusions, with spontaneous resolution and absence of hydrops, are associated with 100\% survival but progressive hydropic cases are associated with less than $50 \%$ survival. Furthermore, other malformations can be found in $25 \%$ of the cases and prolonged compression during the canalicular phase can lead to pulmonary hypoplasia $(44,45)$.

Although no randomized studies have been published, thoracocentesis or thoracoamniotic shunting are likely to benefit hydropic cases by increasing survival in hydropic fetuses from roughly $40 \%$ to over $60 \%$ (46-48). This should apply at any gestation in order to facilitate neonatal resuscitation, even late in the third trimester. For nonhydropic fetuses, the benefit of shunting is less clear $(49,50)$.

The technique is similar to that described for vesicoamniotic shunting in this chapter. Fetal anesthesia is mandatory and paralysis is optional. Ideally, the trocar should be inserted perpendicularly through the fetal chest wall into the effusion as close as possible to the mid-axillary line at the level of the base of the scapula. This should best prevent active removal of the catheter by the fetus. One half of the catheter is placed inside the effusion and the other half is left in the amniotic cavity.

In case of bilateral effusions, an attempt to rotate the fetus using the trocar loaded with a blunt stylet is made in order to avoid a second insertion in the maternal abdomen and uterus. The lower the gestational age, the easier this is (51).

Weekly ultrasound surveillance is recommended following shunt insertion. Technical failures are reported in $20 \%$ to $25 \%$ of the cases, including removal by the fetus within days after the procedure. Repeating the procedure may be justified in a large proportion of cases.

Macrocystic forms of congenital adenomatoid malformations of the lung when they are compressive are often associated with hydrops although does not usually develop when it is not present at the time of diagnosis. In these hydropic cases of CCAM, survival is around 25\% if untreated, and nonrandomized studies suggest that cyst aspiration/shunting procedures could improve survival dramatically (52-54).

Although less frequent in cases of bronchopulmonary sequestration (BPS), shunting of the compressive associated pleural effusion also seems beneficial (55). In addition, two hydropic fetuses with large BPSs have been treated by ultrasound-guided Nd:YAG laser ablation of the feeding vessels with subsequent resolution of hydrops and delivery of healthy infants at term (56).

\section{MYELOMENINGOCELE}

Intrauterine surgery requesting maternal open surgery has not taken off anywhere in the world outside the United States not only in view of the poor results of $\mathrm{CDH}$ programs but also due to the obvious conflict of interest between the pregnant woman and her fetus. This conflict relies largely on the risk of severe maternal morbidity in balance with mainly poor perinatal outcomes. It is also related to the laws regulating TOP for fetal indications in most countries. It is not surprising that this technique remains in a country where all forms of TOPs cannot be performed after 24 weeks, especially when fetal therapy has been initiated. Enrollment of women in these fetal surgery programs could be motivated by the potential fetal benefit, but the contradictory hope for the loss of a severely affected fetus cannot be entirely ruled out.

This apparent ambiguity is particularly questionable in a nonlethal condition such as spina bifida with an often-dismal neurologic motor development (57) and little prospect of improvements in postnatal management.

The rationale for in utero intervention is based on the "twohit hypothesis" $(58,59)$ that states that primary congenital abnormalities in anatomic development allow a relatively normal spinal cord to become secondarily damaged by amniotic fluid exposure, direct trauma, hydrodynamic pressure, or a combination of these factors. It is this secondary damage that may be ameliorated by early fetal surgical repair $(60)$.

The National Institute of Child Health and Human Development decided to sponsor the Management Of Myelomeningocele Study (MOMS) trial, which involves three clinical centers: the Children's Hospital of Philadelphia, Philadelphia, Pennsylvania; the University of California at San Francisco, San Francisco, California; and Vanderbilt University Medical Center, Nashville, Tennessee (61). The future of open fetal surgery is therefore largely dependent upon the results of this unblinded randomized controlled trial (www.spinabifidamoms.com). This is evaluating the benefits of palliative intrauterine surgery covering the neural tube defect in order to protect the nerves from amniotic fluid detrimental effects (62).

Preliminary studies suggested that fetal surgery results in reversal of hindbrain herniation (the Arnold-Chiari malformation), a decrease in shunt-dependent hydrocephalus, and possibly improvement in leg function, but these findings might be explained by selection bias and changing management indications. The current study is experiencing difficulties to recruit patients due to the alternative of TOP but suggests that maternal morbidity can be safely handled. However, this study is somehow challenging several ethical principles including the confusion between evaluation and direct benefit when advertising for the study and therefore influencing the emotional component of the women's decision to participate (63). If the "MOMS" trial demonstrates a decreased morbidity in survivors, open fetal surgery will inevitably be put back on the European agenda.

In brief, this fetal surgery is performed between 19 and 25 weeks of gestation. In the operating room, after laparotomy and hysterotomy, the fetus is positioned with the myelomeningocele (MMC) lesion visible through the uterine incision. The cystic membrane of the MMC is carefully excised and the attachments of the meninges to the skin and soft tissues are detached. If possible, native dura alone is closed over the spinal cord as a first layer, followed by closure of paraspinal myofascial flaps, and then the skin surrounding the lesion is 
carefully mobilized and closed over to complete the repair. When the skin cannot be closed primarily, acellular human dermis graft can be used to complete the closure (60).

As an unanticipated side effect, the MOMS trial apparently slowed down the clinical development of minimally invasive techniques to perform this operation in the United States (3). Nevertheless, endoscopic coverage of the defect is currently explored by several teams, experimentally and also clinically $(64,65)$.

\section{CRITICAL AORTIC AND PULMONARY STENOSIS}

Severe and complex cardiac malformations are among the most difficult situations to handle antenatally in making the diagnosis, in assessing the prognosis, and in the perspective of continuing the pregnancy with the prospect of difficult and often multiple surgical interventions that often lead to palliative care.

Left or right heart hypoplasias are currently associated with a $60 \%$ survival rate at 5 years with mostly univentricular cardiac function. A well-recognized common pathway leading to these conditions is the critical stenosis of the aorta or the pulmonary artery respectively. Although postnatal dilatation of the stenotic vessel improves the prognosis, the hypoplastic ventricle remains nonfunctional and a univentricular cardiac function is the rule. The hypoplasia-related hypertrophy of the affected ventricle alters the coronary circulation and pulmonary venous return, weighing heavily on the overall prognosis. The first attempt to catheterize a fetal critical aortic stenosis dates back to 1991. Since then, around 30 cases have been reported with much heterogeneity in the case-selection process as well as in their management and success rates $(66,67)$. All procedures were performed no sooner than early third trimester, which is probably too late in the natural history of most cases but is the result of a technical compromise that is required to access the affected vessel and to keep procedure-related mortality and morbidity to an acceptable level (68).

Pulmonary or aortic valve perforation or dilation can be performed under local or general maternal anesthesia. The latter performed with inhalational agents promotes uterine relaxation and facilitates fetal manipulation for positioning. The fetus is premedicated with intramuscularly administered analgesics and curarization. Under continuous ultrasound guidance, manual version is performed to optimize the fetal position. If the optimal position is not achieved or imaging is inadequate, some authors perform a limited maternal laparotomy without uterine exteriorization or incision (69). The right ventricle (RV) or left ventricle (LV) outflow tract is accessed through direct puncture, by using a 19-gauge ultrathin-walled cannula. With the cannula tip in the RV or LV outflow tract, the atretic valve is perforated with the stylet of a 19-gauge cannula. A 0.014-inch floppy-tipped guidewire is then positioned, and a coronary angioplasty balloon is inflated across the valve. After the final balloon deflation, the wire, balloon, and cannula are removed. Color Doppler ultrasound flow imaging demonstrates the success of the valvuloplasty with an antegrade jet of flow across the patent valve.

This form of minimally invasive surgery is however questioning the issue of the learning process inasmuch as that of defining clear and indisputable indications (69). Answers should ideally be reached through an exemplary collaboration between fetal medicine specialists and interventional pediatric cardiologists. This would ensure appropriate fetal access as well as adapted pediatric follow-up within an optimally run specialized perinatal network. Rather than await a proliferation of such procedures at centers that are unlikely to amass a critical volume of experience to ensure clinical competence, a prospective multicenter trial should be considered to address the nuances of technical performance of the procedure, to evaluate the short- and midterm results, and to compare these results with those of children undergoing various forms of palliation for hypoplastic left heart syndrome (70). Moreover, the perceived benefits of prenatal treatment have to be weighed against steadily improving postnatal surgical and hybrid procedures, which have been shown to reduce morbidity and mortality for these complex heart defects (71).

Furthermore, criteria to define a successful intrauterine procedure are still unclear: permeability demonstrated by Doppler flow through the valve is an immediate but incomplete one that hides somehow the significant perioperative short- and mid-term mortality and morbidity. Less than $30 \%$ of the cases reported to date following critical aortic stenosis dilatation in utero have a biventricular cardiac function. However, owing to the high incidence of TOP following prenatal diagnosis of these conditions, comparative studies are going to be difficult to set.

\section{EXIT PROCEDURES}

The EXIT procedure can somehow be considered as a fetal surgery. It is a procedure performed while the baby is still on placental support. Initially described in 1997 for the reversal of tracheal occlusion at the time of delivery in fetuses that had undergone in utero tracheal occlusion for severe $\mathrm{CDH}(72,73)$, its use has expanded to convert an emergent crisis (airway obstruction mainly) into a controlled situation (74). Its indications include now not only fetal neck masses, congenital high airway obstruction but also large thoracic lesions, $\mathrm{CDH}$, conjoined twins, unilateral pulmonary agenesis, and cardiac lesions where neonatal resuscitation may be compromised (75-78).

In brief, a multidisciplinary team is implicated in an EXIT procedure (pediatric surgeons, obstetrician, neonatologist, anesthesiologist, and nurses).

In the EXIT procedure (74), high concentrations of inhalational anesthetics and additional tocolytics, if necessary, are used to provide uterine relaxation and preserve placental perfusion. The time between induction of anesthesia and cord clamping is kept as long as it is clinically required. A low transverse maternal laparotomy incision is performed allowing a quick assessment of placental position with a sterile intraoperative ultrasound. While one assistant stabilizes fetal position, hysterotomy is performed. Only the fetal head and upper torso are delivered through the hysterotomy in order to maintain uterine volume. Amniotic fluid volume is maintained with the infusion of warm Ringer's lactate. Fetal wellbeing is continuously monitored by a pulse oximeter and by continuous echocardiography. Fetal venous access can be obtained with a peripheral intravenous line for fetal volume loading or drug administration. The procedures that can be performed on the fetus during EXIT range from direct 
laryngoscopy, rigid bronchoscopy, tracheostomy to secure the fetal airway, to resections of neck masses and thoracic masses, to the placement of extracorporeal membrane circulation cannulas. Prior to clamping of the umbilical cord and delivery of the baby, communication between the surgical and anesthesia teams is essential to prevent uterine atony and excessive maternal hemorrhage. The inhalational agent is decreased and oxytocic bolus followed by a continuous infusion is administered and titrated to uterine tone, and the umbilical cord is then clamped and divided and the newborn taken to a separate operating room for further management.

\section{OPEN FETAL SURGERY}

CCAM, bronchopulmonary sequestration (BPS), and sacrococcygeal teratoma (SCT) are most frequently benign lesions managed expectantly during the prenatal follow-up. At present, the development of early fetal hydrops is considered by some authors to be the only criterion for fetal open surgery, because it is a predictor of extremely poor prognosis (55). For the fetus with a life-threatening malformation, the risk of the procedure is small when compared with the potential benefit of salvage. The risks and benefits for the mother are more difficult to assess. We provide in the following a general description of open fetal surgery as described by Adzick (79).

Patients are admitted immediately prior to the operation for obstetric monitoring and initiation of tocolysis. Indomethacin and antibiotics are given preoperatively. Maternal general and epidural anesthesia provides anesthesia for the mother and the fetus and ensures good uterine relaxation. The uterus is exposed through a low transverse abdominal incision. Sterile intraoperative ultrasound is used to delineate the fetal position and placental location. The position and orientation of the hysterotomy is planned to stay parallel to, and at least $6 \mathrm{~cm}$ from, the placental edge and still allow exposure of the appropriate part of the fetus. For the fetal portion of the procedure, the fetus is positioned with the appropriate fetal anatomy visible through the uterine incision. Intraoperative fetal monitoring is provided by a miniaturized pulse oximeter and continuous intraoperative fetal echocardiography. The fetus is kept warm in the uterus by the continuous administration of $38-40^{\circ} \mathrm{C}$ Ringer's lactate solution. A fetal intravenous line is placed for infusion of fluid, blood, and/or medications. After the fetal procedure, a watertight two-layer uterine closure is performed with double-armed full-thickness 0 PDS interrupted stay sutures followed by a running 2-0 PDS suture. Approximately $400 \mathrm{~mL}$ of warmed Ringer's lactate containing $500 \mathrm{mg}$ of oxacillin is instilled into the amniotic cavity just prior to completing the running layer and is continued until sonography shows an adequate amniotic fluid volume (79).

In SCTs, open fetal resection of predominantly extra-pelvic tumors has been reported in five cases (80). Mean age at birth was 30 weeks and four survived long term. There is also anecdotal experience of less invasive techniques, arresting flow in feeding vessels either by fetoscopic laser (81), interstitial thermocoagulation (82), or radiofrequency ablation (83). Needle-guided intravascular embolization with alcohol or histoacryl or coils has been reported, but without measurable success (84).

\section{FETAL ANALGESIA}

The issue of fetal analgesia touches on the surgical approach itself as much as the "primum non nocere" principle (85). It is well established that very preterm neonates experience pain, and related autonomous neural connections are operating from around 22 weeks of gestation. It is therefore important and beneficial that any directly invasive fetal procedure be preceded by appropriate fetal analgesia (86). Sufentanyl is the drug of choice and can be administered under ultrasound guidance either intravascular in the umbilical cord or intramuscular. Dose regimens are established in relation to fetal estimated weight. This should be considered independently of curarization that can be requested for technical considerations but is totally ineffective in relieving fetal pain or awareness in these situations (87).

\section{REFERENCES}

1. Adzick NS, Harrison MR. Fetal surgical therapy. Lancet 1994; 343: 897-902.

2. Harrison MR, Filly RA, Golbus MS, et al. Fetal treatment. N Engl J Med 1982; 307: 1651-2.

3. Deprest J, Devlieger R, Srisupundit K, et al. Fetal surgery is a clinical reality. Semin Fetal Neonat Med 2010; 15: 58-67.

4. Liley AW. Intrauterine transfusion of fetus in haemolytic disease. Br Med J 1963; 2: 1107-9.

5. Deprest J, Flake A, Gratacos E, et al. The making of fetal surgery. Prenat Diagn 2010; 30: 653-67.

6. Chervenak FA, McCullough LB. Perinatal ethics: a practical method of analysis of obligations to mother and fetus. Obstet Gynecol 1985; 66: 442-6.

7. Ville Y. Monochorionic twin pregnancies: 'les liaisons dangereuses'. Ultrasound Obstet Gynecol 1997; 10: 82-5.

8. Ville Y, Hyett J, Hecher K, Nicolaides K. Preliminary experience with endoscopic laser surgery for severe twin-twin transfusion syndrome. N Engl J Med 1995; 332: 224-7.

9. Senat MV, Deprest J, Boulvain M, et al. Endoscopic laser surgery versus serial amnioreduction for severe twin-to-twin transfusion syndrome. N Engl J Med 2004; 351: 136-44.

10. Hecher K, Lewi L, Gratacos E, et al. Twin reversed arterial perfusion: fetoscopic laser coagulation of placental anastomoses or the umbilical cord. Ultrasound Obstet Gynecol 2006; 28: 688-91.

11. De Lia JE, Cruikshank DP, Keye WRJ. Fetoscopic neodymium:yag laser occlusion of placental vessels in severe twin-twin transfusion syndrome. Obstet Gynecol 1990; 75: 1046-53.

12. Ville Y, Hecher K, Gagnon A, et al. Endoscopic laser coagulation in the management of severe twin-to-twin transfusion syndrome. Br J Obstet Gynaecol 1998; 105: 446-53.

13. Ville Y, Hyett JA, Vandenbussche FP, Nicolaides KH. Endoscopic laser coagulation of umbilical cord vessels in twin reversed arterial perfusion sequence. Ultrasound Obstet Gynecol 1994; 4: 396-8.

14. Stirnemann JJ, Nasr B, Quarello E, et al. A definition of selectivity in laser coagulation of chorionic plate anastomoses in twin-to-twin transfusion syndrome and its relationship to perinatal outcome. Am J Obstet Gynecol 2008; 198: 62.e1-6.

15. Quintero RA, Comas C, Bornick PW, et al. Selective versus non-selective laser photocoagulation of placental vessels in twin-to-twin transfusion syndrome. Ultrasound Obstet Gynecol 2000; 16: 230-6.

16. Salomon LJ, Ortqvist L, Aegerter P, et al. Long-term developmental follow-up of infants who participated in a randomized clinical trial of amniocentesis versus laser photocoagulation for the treatment of twin-totwin transfusion syndrome (TTTS). Am J Obstet Gynecol 2010. In Press.

17. Gallot D, Laurichesse H, Lemery D. Selective feticide in monochorionic twin pregnancies by ultrasound-guided umbilical cord occlusion. Ultrasound Obstet Gynecol 2003; 22: 484-8.

18. Lopoo JB, Paek BW, Maichin GA, et al. Cord ultrasonic transection procedure for selective termination of a monochorionic twin. Fetal Diagn Ther 2000; 15: 177-9. 
19. Rodeck C, Deans A, Jauniaux E. Thermocoagulation for the early treatment of pregnancy with an acardiac twin. N Engl J Med 1998; 339: 1293-5.

20. Tsao K, Feldstein VA, Albanese CT, et al. Selective reduction of acardiac twin by radiofrequency ablation. Am J Obstet Gynecol 2002; 187: 635-40.

21. Deprest JA, Audibert F, Van Schoubroeck D, Hecher K, Mahieu-Caputo D. Bipolar cord coagulation of the umbilical cord in complicated monochorionic twin pregnancy. Am J Obstet Gynecol 2000; 182: 340-5.

22. Campandale RP, Rowland RH. Hypoplasia of the lung associated with congenital diaphragmatic hernia. Ann Surg 1995; 142: 176-81.

23. Jani J, Nicolaides KH, Keller RL, et al. Observed to expected lung area to head circumference ratio in the prediction of survival in fetuses with isolated diaphragmatic hernia. Ultrasound Obstet Gynecol 2007; 30: 67-71.

24. Deprest J, Gratacos E, Nicolaides KH, FETO Task Group. Fetoscopic tracheal occlusion (FETO) for severe congenital diaphragmatic hernia: evolution of a technique and preliminary results. Ultrasound Obstet Gynecol 2004; 24: 121-6.

25. Harrison M, Adzick N, Flake A, Jennings R. The CDH two-step: a dance of necessity. J Pediatr Surg 1993; 28: 813-16.

26. Harrison M, Adzick N, Flake A, et al. Correction of congenital diaphragmatic hernia in utero: VI: hard-earned lessons. J Pediatr Surg 1993; 28: 1411-7.

27. Jani J, Keller RL, Benachi A, et al. Antenatal-CDH-Registry Group. Prenatal prediction of survival in isolated left-sided diaphragmatic hernia. Ultrasound Obstet Gynecol 2006; 27: 18-22.

28. Fowler SF, Sydorak RM, Albanese CT, et al. Fetal endoscopic surgery: lessons learned and trends reviewed. J Pediatr Surg 2002; 37: 1700-2.

29. Flake A, Crombleholme T, Johnson $M$, et al. Treatment of severe congenital diaphragmatic hernia by fetal tracheal occlusion: clinical experience with fifteen cases. Am J Obstet Gynecol 2000; 183: 1059-66.

30. Quintero RA, Morales WJ, Bornick PW, et al. Minimally invasive intraluminal tracheal occlusion in a human fetus with left congenital diaphragmatic hernia at 27 weeks gestation via direct fetal laryngoscopy. Prenat Neonatal Med 2000; 5: 134-40.

31. Deprest J, Gratacos E, Nicolaides KH, et al. Fetoscopic tracheal occlusion (FETO) for severe congenital diaphragmatic hernia: evolution of a technique and preliminary results. Ultrasound Obstet Gynecol 2004; 24: 121-6.

32. Deprest J, Jani J, Gratacos E, et al. Deliberately delayed and shortened fetoscopic tracheal occlusion-a different strategy after prenatal diagnosis of life-threatening congenital diaphragmatic hernias. J Pediatr Surg 2006; 41: 1345-6.

33. Hedrick MH, Estes JM, Sullivan KM, et al. Plug the lung until it grows (PLUG): a new method to treat congenital diaphragmatic hernia in utero. J Pediatr Surg 1994; 29: 612-17.

34. Flageole H, Evrard VA, Piedboeuf B, et al. The plug-unplug sequence: an important step to achieve type II pneumocyte maturation in the fetal lamb model. J Pediatr Surg 1998; 33: 299-303.

35. Harrison MR, Keller RL, Hawgood SB, et al. A randomized trial of fetal endoscopic tracheal occlusion for severe fetal congenital diaphragmatic hernia. N Engl J Med 2003; 349: 1916-24.

36. Jani JC, Nicolaides KH, Gratacos E, et al. Severe diaphragmatic hernia treated by fetal endoscopic tracheal occlusion. Ultrasound Obstet Gynecol 2009; 34: 304-10.

37. Jani J, Keller RL, Benachi A, et al. Prenatal prediction of survival in isolated left-sided diaphragmatic hernia. Ultrasound Obstet Gynecol 2006; 27: 18-22.

38. Hedrick HL. Management of prenatally diagnosed congenital diaphragmatic hernia. Semin Fetal Neonatal Med 2010; 15: 21-7.

39. Lissauer D, Morris RK, Kilby MD. Fetal lower urinary tract obstruction. Semin Fetal Neonat Med 2007; 12: 464-70.

40. Mann S, Johnson MP, Wilson RD. Fetal thoracic and bladder shunts. Semin Fetal Neonatal Med 2010; 15: 28-33.

41. Clark TJ, Martin WL, Divakaran TG, et al. Prenatal bladder drainage in the management of lower urinary tract obstruction: a systematic review and meta-analysis. Obstet Gynecol 2003; 102: 367-82.

42. Welsh A, Agarwal S, Kumar S, et al. Fetal cystoscopy in the management of fetal obstructive uropathy: experience in a single European centre. Prenat Diagn 2003; 23: 1033-41.
43. Ruano R, Duarte S, Bunduki V, et al. Fetal cystoscopy for severe lower urinary tract obstruction-initial experience of a single centre. Prenat Diagn 2010; 30: 30-9.

44. Yinon Y, Kelly E, Ryan G. Perinatal outcome following fetal chest shunt insertion for pleural effusions. Best Pract Res Clin Obstet Gynaecol 2008; 22: 77-96.

45. Pettersen HN, Nicolaides KH. Pleural effusions. In: Fisk NM, Moise KJ, eds. Cambridge: Cambridge University Press, 1997: 261-72.

46. Aubard Y, Derouineau I, Aubard V, et al. Primary fetal hydrothorax: a literature review and proposed antenatal clinical strategy. Fetal Diagn Ther 1998; 13: 325-33.

47. Rustico MA, Lanna M, Coviello D, et al. Fetal pleural effusion. Prenat Diagn 2007; 27: 793-9.

48. Picone O, Benachi A, Mandelbrot L, et al. Thoracoamniotic shunting for fetal pleural effusions with hydrops. Am J Obstet Gynecol 2004; 191: 2047-50.

49. Deurloo KL, Devlieger R, Lopriore E, et al. Isolated fetal hydrothorax with hydrops: a systematic review of prenatal treatment options. Prenat Diagn 2007; 27: 893-9.

50. Sepulveda W, Galindo A, Sosa A, et al. Intrathoracic dislodgement of pleuro-amniotic shunt. Three case reports with long-term follow-up. Fetal Diagn Ther 2005; 20: 102-5.

51. Wilson RD, Hedrick HL, Liechty KW, et al. Cystic adenomatoid malformation of the lung: review of genetics, prenatal diagnosis, and in utero treatment. Am J Med Genet A 2006; 140: 151-5.

52. Adzick NS, Harrison MR, Crombleholme TM, et al. Fetal lung lesions: management and outcome. Am J Obstet Gynecol 1998; 179: 884-9.

53. Dommergues M, Louis-Sylvestre C, Mandelbrot L, et al. Congenital adenomatoid malformation of the lung: when is active fetal therapy indicated? Am J Obstet Gynecol 1997; 177: 953-8.

54. Oepkes D, Devlieger R, Lopriore E, et al. Successful ultrasound-guided laser treatment of fetal hydrops caused by pulmonary sequestration. Ultrasound Obstet Gynecol 2007; 29: 457-9.

55. Adzick NS, Harrison MR, Crombleholme TM, et al. Fetal lung lesions: management and outcome. Am J Obstet Gynecol 1998; 179: 884-9.

56. Davenport M, Warne SA, Cacciaguerra S, et al. Current outcome of antenatally diagnosed cystic lung disease. J Pediatr Surg 2004; 39: 549-56.

57. Bruner JP, Tulipan N. Intrauterine repair of spina bifida. Clin Obstet Gynecol 2005; 48: 942-55.

58. Hutchins GM, Meuli M, Meuli-Simmen C, et al. Acquired spinal cord injury in human fetuses with myelomeningocele. Pediatr Pathol Lab Med 1996; 16: 701-12.

59. Meuli M, Meuli-Simmen C, Hutchins GM, et al. The spinal cord lesion in human fetuses with myelomeningocele: implications for fetal surgery. J Pediatr Surg 1997; 32: 448-52.

60. Adzick NS. Fetal myelomeningocele: natural history, pathophysiology, and in-utero Intervention. Semin Fetal Neonatal Med 2010; 15: 9-14.

61. National Institute of Child Health and Human Development. Management of myelomeningocele study; 2010. [Available from: http:// www.spinabifidamoms.com].

62. Bruner JP. Intrauterine surgery in myelomeningocele. Semin Fetal Neonatal Med 2007; 12: 471-7.

63. Chervenak FA, McCullough LB. Ethics of maternal-fetal surgery. Semin Fetal Neonatal Med 2007; 12: 426-31.

64. Bruner JP, Tulipan NB, Richards WO, et al. In utero repair of myelomeningocele: a comparison of endoscopy and hysterotomy. Fetal Diagn Ther 2000; 15: 83-8.

65. Kohl T, Herin R, Heep A, et al. Percutaneous fetoscopic patch coverage of spina bifida aperta in the human - early clinical experience and potential. Fetal Diagn Ther 2006; 21: 185-93.

66. Marshall AC, Tworetzky W, Bergersen L, et al. Aortic valvuloplasty in the fetus: technical characteristics of successful balloon dilation. J Pediatr 2005; 147: 535-9.

67. Galindo A, Gutierrez-Larraya F, Velasco JM, de la Fuente P. Pulmonary balloon valvuloplasty in a fetus with critical pulmonary stenosis/atresia with intact ventricular septum and heart failure. Fetal Diagn Ther 2006; 21: 100-4.

68. Matsui H, Gardiner H. Fetal intervention for cardiac disease: the cutting edge of perinatal care. Semin Fetal Neonatal Med 2007; 12: 482-9.

69. Tworetzky W, McElhinney DB, Marx GR, et al. In utero valvuloplasty for pulmonary atresia with hypoplastic right ventricle: techniques and outcomes. Pediatrics 2009; 124: e510-18. 
70. Kleinman CS. Fetal cardiac intervention. Innovative therapy or a technique in search of an indication? Circulation 2006; 113: 1378-81.

71. Pavlovic M, Acharya G, Huhta JC. Controversies of fetal cardiac intervention. Early Hum Dev 2008; 84: 149-53.

72. Mychalishka GB, Bealor JF, Graf JL, et al. Operating on placental support: the ex utero intrapartum treatment (EXIT) procedure. J Pediatr Surg 1997; 32: 22-30.

73. Liechty KW, Crombleholme TM, Flake AW, et al. Intrapartum airway management for giant fetal neck masses: the EXIT procedure (ex utero intrapartum treatment). Am J Obstet Gynecol 1997; 177: 870-4.

74. Liechty KW. Ex-utero intrapartum therapy. Semin Fetal Neonatal Med 2010; 15: 34-9.

75. Hedrick HL, Flake AW, Crombleholme TM, et al. The ex utero intrapartum therapy for high-risk fetal lung lesions. J Pediatr Surg 2005; 40: 1038-44.

76. Kunisaki SM, Barnewolt CE, Estroff JA, et al. Ex utero intrapartum treatment with extracorporeal membrane oxygenation for severe congenital diaphragmatic hernia. J Pediatr Surg 2007; 42: 98-104.

77. Hedrick HL. Ex utero intrapartum therapy. Semin Pediatr Surg 2003; 10: 190-5.

78. Bouchard S, Johnson MP, Flake AW, et al. The EXIT procedure: experience and outcome in 31 cases. J Pediatr Surg 2002; 37: 418-26.
79. Adzick NS. Open fetal surgery for life-threatening fetal anomalies. Semin Fetal Neonatal Med 2010; 15: 1-8.

80. Hedrick HL, Flake AW, Crombleholme TM, et al. Sacrococcygeal teratoma: prenatal assessment, fetal intervention, and outcome. J Pediatr Surg 2004; 39: 430-8.

81. Hecher K, Hackeloer BJ. Intrauterine endoscopic laser surgery for fetal sacrococcygeal teratoma. Lancet 1996; 347: 470.

82. Makin EC, Hyett J, Ade-Ajayi N, et al. Outcome of antenatally diagnosed sacrococcygeal teratomas: single-center experience (1993-2004). J Pediatr Surg 2006; 41: 388-93.

83. Lam YH, Tang MHY, Shek TWH. Thermocoagulation of fetal sacrococcygeal teratoma. Prenat Diagn 2002; 22: 99-101.

84. Benachi A, Durin S, Maurer R, et al. Prenatally diagnosed sacrococcygeal teratoma: a prognostic classification. J Pediatr Surg 2006; 41: 1517-21.

85. Lee SJ, Ralston HJ, Drey EA, et al. Fetal pain: a systematic multidisciplinary review of the evidence. JAMA 2005; 294: 947-54.

86. White MC, Wolf AR. Pain and stress in the human fetus. Best Pract Res Clin Anaesthesiol 2004; 18: 205-20.

87. Pasquier JC, Morelle M, Bagouet S, et al. Effects of residential distance to hospitals with neonatal surgery care on prenatal management and outcome of pregnancies with severe fetal malformations. Ultrasound Obstet Gynecol 2007; 29: 271-5. 


\section{Chorionic villus sampling Giovanni Monni, Maria Angelica Zoppi, and Carolina Axiana}

\section{INTRODUCTION}

Invasive prenatal diagnosis methods have gradually seen their importance grow to advances in embryo-fetal medicine, ultrasound imaging, cytogenetic analysis, and molecular biology. Such progress has deeply impacted reproduction behavior for couples at high risk for genetic pathologies.

Information from such methods can reassure couples carrying a pregnancy or, in cases of a positive diagnosis, put the couple in a position to achieve the most appropriate decision, including voluntary pregnancy interruption, fetal therapies, delivery timing and method, and postnatal interventions.

The most widespread invasive techniques employed in prenatal diagnosis centers are chorionic villus sampling (CVS), amniocentesis, and cordocentesis. Each of the techniques has its own advantages and disadvantages, including a miscarriage risk. This risk is related to operator expertise, the method itself, the equipment employed, the sampled quantity, the gestational age at sampling, and maternal age (1-5).

\section{HISTORICAL ASPECTS OF CVS}

Research studies of the 1960s and 1970s showed that the chorion could be biopsied through a catheter and that enough placental cells could be obtained so as to allow specific genetic analyses to be done earlier in pregnancy than through amniocentesis. In 1968, in Scandinavia, Mohr made the first attempts of early antenatal genetic diagnosis using sampled chorionic villi (6). He performed a transcervical biopsy of the chorion under direct endoscopic visualization using a straight 6-mm diameter fiber optic device and a sharp blade. The sample was often mixed with amniotic membranes and blood, but it was suitable for chromosomal analysis. Despite his report of a $96 \%$ success rate in obtaining chorionic material, this approach was abandoned because of an unacceptably high miscarriage rate and the difficulty in growing chorionic villi cells. Later, fetal chromosome analysis from transcervical placental biopsy prior to termination in early pregnancies was reported by Kullander and Sandahl in 1973 and Hahnemann in 1974 (7,8). In 1975, researchers at the Tietung Hospital in Anshan, China, announced the first successful prenatal diagnostic use of CVS. In an essentially blind approach, the CVS was performed through transcervical aspiration using a 3-mm metal cannula. After its insertion, when soft resistance was encountered, a smaller internal tube was further advanced, and placental material was acquired by syringe suction. Fetal sex was established from chorionic villi cells in early pregnancy according to the presence or absence of sex chromatin (the Barr body) for the purpose of sex preselection. Prenatal losses and spontaneous abortion occurred in $4 \%$ to $5 \%$ of the cases (9).

Many researchers attempted to sample chorionic villi under endoscopic visualization for first-trimester antenatal diagnosis, but it was the arrival of real-time ultrasound and the advancement in molecular genetics that rekindled the desire for earlier and safer antenatal diagnosis of genetic diseases.

In 1980, for the first time reported in the literature, Kazy et al. described using ultrasound guidance during chorion sampling (10). Adopting either an endoscopic or an ultrasound-guided approach, they performed fetal sexing and biochemical analyses on chorion tissue biopsied at 6 to 12 weeks of gestation. Instead of cannulas, they used biopsy forceps.

Improved methods for culturing fibroblasts from trophoblast villi were reported by Niazi et al. in 1981 at the St. Mary's Hospital, London (11).

Old et al., from Great Britain, announced using realtime ultrasound and a 1.5-mm biopsy instrument for CVS for diagnosing hemoglobinopathies through molecular methods (12).

In 1983, Ward in London carried out transcervical chorionic sampling with ultrasonic guidance applying a $1.5-\mathrm{mm}$ soft polyethylene catheter and syringe suction (13). His group declared a sampling success rate of $67 \%$, including seven patients for hemoglobinopathy diagnosis through molecular methods.

The success rate of obtaining chorionic material rose from $75 \%$ to $96 \%$ under ultrasonic guidance, as was demonstrated by Brambati et al. in Milan in 1983. Brambati used a 1.5-mm polyethylene tube with a soft, stainless-steel, malleable obturator inserted into the 1-mm internal barrel. For many years, this device has been the most common design in use $(14,15)$.

Other researchers, such as Dumez in Paris, used a 2-mm rigid biopsy forceps (16).

In 1983, Simoni et al. in Milan described a technique from first-trimester chorionic villi that could be used for direct chromosome preparation and karyotyping as well as for enzyme analyses without needing preliminary culture (17). Chorionic villi were obtained by aspiration or through biopsy from the chorion frondosum, at the edge of the placental disk. With the technique they proposed, in the freshly sampled villus, the inner cytotrophoblast layer (containing actively mitotic cells) could be separated from the outer syncytiotrophoblast cells. All analyses could be performed from these fresh whole villi.

In 1984, transabdominal fine-needle villus aspiration under ultrasound guidance was introduced by Smidt-Jensen and Hahnemann in Copenhagen (18). They registered considerable success in acquiring samples at the first attempt, thus preventing further infective complications and pregnancy loss.

CVS has achieved significant improvements since then, including perfecting the instruments so that they are relatively 
easy to manipulate. In addition to advancements in sample acquisition, laboratory techniques have evolved, including those for direct karyotyping, tissue culture, and molecular and biochemical assays of chorionic villi.

The transabdominal approach instantly proved easy to learn and relatively safe. While it registered a higher detection rate, it also guaranteed comfort and privacy to the women undergoing the procedure. It could be practiced at any gestational age, thus spreading quickly in many centers and becoming today the most popular approach of chorion sampling. One particular advantage of CVS is the possibility of performing the procedure very early in pregnancy, starting from the 6th week (although it is generally recommended from the 10th to 12 th week). In this way, it is a method that is highly accepted by couples and one apt to diminish physical and psychologic issues related to late diagnosis and eventual voluntary pregnancy interruption due to genetic diseases (19).

\section{INDICATIONS}

CVS was initially applied for fetal sexing and prenatal diagnosis of hemoglobinopathies by DNA analysis. However, since then, cytogenetics and DNA analysis techniques have shown serious advances and broadened the number and types of genetic conditions detectable in the antenatal period. As a consequence, the demand for CVS in first-trimester prenatal diagnosis is accordingly making a steady increase.

At present, the primary indications of CVS are chromosomal studies, DNA analysis of genetic disorders, and the prenatal diagnosis of inborn errors of metabolism (Table 1).

Regarding chromosomal studies, CVS is primarily indicated for advanced maternal age (35 years or more), a previous pregnancy with a chromosomal abnormality, a previous pregnancy with multiple anomalies, $\mathrm{X}$-linked diseases, parents with balanced chromosome rearrangements (translocations and inversions), parents with aneuploidies, a past history of recurrent miscarriages, an abnormal ultrasound scan, and oligo-anhydramnios.

It should be noted that the demand for CVS is greater than ever now that first-trimester screening methods for the detection of fetal chromosomal anomalies are developing. As a matter of fact, the risk for fetal aneuploidies related to advanced maternal age remains a common indication for CVS, but the CVS indication has expanded to be employed for

Table 1 Main Indications for Chorionic Villus Sampling

Advanced maternal age

Positive ultrasound and biochemistry first-trimester aneuploidy screening

Parents with chromosomal anomalies

Previous child with chromosomal anomaly

Previous child with plurimalformations

High risk of single-gene disorders

Oligo/anhydramnios

Fetal anomalies detected by ultrasound

$\mathrm{X}$-linked diseases

Inborn errors of metabolism

Infectious diseases quickly confirming an abnormal karyotype in the firsttrimester fetus if a chromosomal abnormality has been suggested by ultrasound and/or biochemical screening. In the last 20 years, the screening of women for fetal Down syndrome has progressed to the evolution of numerous proficient and complex screening strategies that provide the advantages of allowing both superior detection and lower false-positive rates at earlier gestational ages. First-trimester risk assessment of chromosomal defects by the ultrasound measurement of fetal nuchal translucency and the evaluation of the nasal bone (Fig. 1), combined with a biochemical test (pregnancy-associated plasma protein-A, free beta human chorionic gonadotropin), reach a very high detection rate at more than $90 \%$ (20-26). Therefore, the choice that patients make should be based on the risk that screening tests generate and not on their age alone $(27,28)$.

In a report in January 2007, "Screening for chromosomal abnormalities," the American College of Obstetricians and Gynecologists (ACOG) Practice Bulletin advised new guidelines that seek to offer risk evaluation and invasive genetic diagnosis of fetal karyotype to all pregnant women requiring prenatal care before 20 weeks of gestation, regardless of maternal age (29). Among such less common indications for fetal karyotyping are maternal anxiety, polyabortivity, and assisted reproductive techniques by intracytoplasmic sperm injection (30).

The DNA-based diagnoses of single-gene disorders such as cystic fibrosis, hemophilia, muscular dystrophy, and hemoglobinopathies form a group that is expanding with the advance of technology and knowledge of the human genome. About $1 \%$ of live births is affected by single-gene disorders, which may be autosomal recessive, autosomal dominant, or $\mathrm{X}$-linked recessive. They imply a high risk of recurrence and are often inadequately treated so that prenatal diagnosis with termination of affected pregnancies is a significant option for couples at risk.

The purpose for prenatal diagnosis of genetic disorders is to determine the carrier status in order to help with genetic counseling of at-risk couples. Most carrier recognition and genetic counseling are retrospective, that is, after the birth of an affected child. Nonetheless, several programs related to

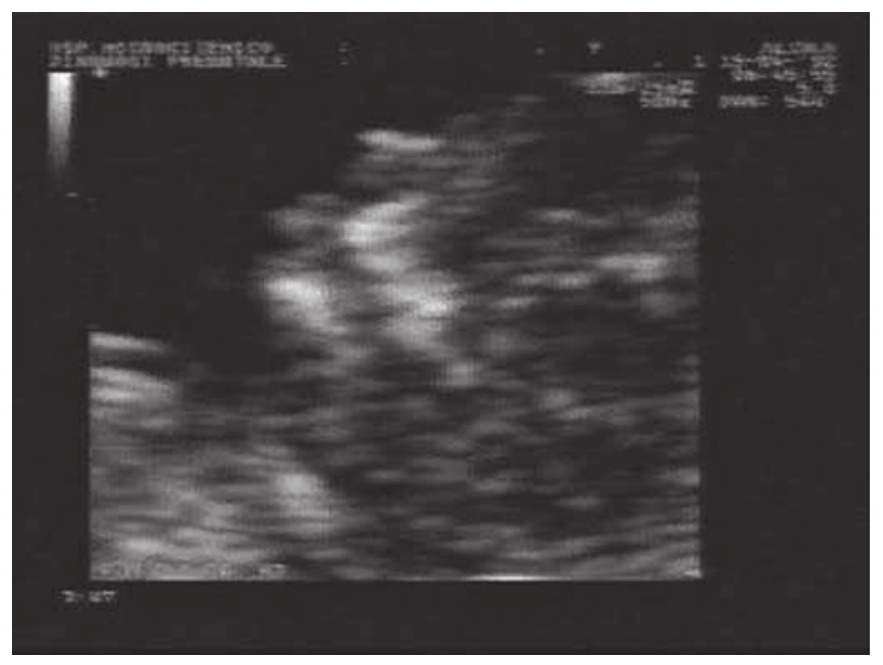

Figure 1 Fetal facial profile at 11 weeks: absent nasal bone. 
carrier screening have been launched for populations known to be at risk for specific disorders, as is the case of betathalassemia in the Mediterranean area. Beta-thalassemia is the most common single-gene disorder among Italians, where $13 \%$ of the people on the Sardinia Island are healthy carriers. Ongoing programs of screening have proved quite effective, and they have resulted in a noticeable decline in the incidence of thalassemia major. Screening programs for Sardinians are based on carrier detection by employing hematologic methods, and genetic diagnoses can be made by identifying specific mutations through DNA analysis in carrier couples. In this way, prenatal diagnosis can be accomplished by mutation analysis on PCR-amplified DNA from chorionic villi (31). Because of advances in molecular techniques, the number of single-gene disorders offered for wide-based population screening will undoubtedly grow.

Ultrasound screening of Down syndrome in the first trimester of gestation may sometimes reveal several fetal abnormalities. Holoprosencephaly, omphalocele, cystic hygroma, diaphragmatic hernia, and megacystis are widely recognized features either of aneuploidies or of certain syndromes. When these are detected in the first trimester, taking advantage of CVS can prompt fetal karyotyping or definitive DNA studies (32).

The inborn errors of metabolism form a vast group of disorders that are individually rare, yet all together embody a major source of human disease and suffering. The majority of these are caused by defects in single genes responsible for encoding enzymes that facilitate conversion of various substrates into different products. Usually, the basis of the problems is the accumulation of substances that are noxious or hamper the normal function as well as the effects of inability to synthesize essential compounds. The basic biochemical defect has been so far identified in more than 300 different inborn errors of metabolism. The greater part of these conditions are indeed rare and new prenatal diagnosis methods for specific disorders are continually being presented; therefore, it is recommended to check with a specialist referral center on the current availability and preferred prenatal diagnosis method. Usually, family history of the disorder with a well-established primary diagnosis in the index case is the indication for prenatal diagnosis but in some cases ultrasound recognizable fetal features may also prompt the prenatal diagnosis.

Sometimes, the chorion tissue must undergo various preparations because more than one analysis is required. Taking into account that chromosome abnormalities are discovered in approximately 1 in 156 live births, and in about $50 \%$ of first-trimester spontaneously aborted fetuses, fetal chromosome investigations are an important matter. However, there are cases in which the villi are sampled for other indications (33). As a matter of fact, fetal karyotyping is quite often necessary in addition to the most significant test, even in the absence of specific indications for chromosomal analysis.

Fetal HLA typing could likewise be done in pregnancies of couples at high risk of diseases amenable of treatment by stem cell transplantation, as with beta-thalassemia. In some cases, an alternative to pregnancy termination in the case of an affected fetus could be the prospect of bone marrow transplantation with an HLA well-matched sibling (34). By CVS, certain congenital infections can be detected such as rubella, toxoplasmosis, cytomegalovirus, and parvovirus (35-37).

Practicing CVS by any means should be a choice contingent on the possible benefits overcoming risks linked to the procedure.

\section{COUNSELING}

Before any CVS procedure, adequate genetic counseling is mandatory in order to inform the couple about prognosis, treatment, possible outcomes, complications, and recurrence risks associated with the genetic disease under investigation. In particular, the geneticist or obstetrician should detail advantages and disadvantages in terms of risks associated with the procedure itself, with mention of the abortivity measured in the prenatal diagnosis center providing the care and the one reported in the literature. Information should be included on diagnostic accuracy (i.e., false positives, false negatives, and misdiagnoses), timeline of diagnostic response, gestational age at sampling, success and fail rate of sampling, and all other possible complications. Consent, preferably written, will be given by a woman only after comprehensive, informative, and nondirective counseling.

\section{SAMPLING PROCEDURE}

Performing CVS must always be under direct ultrasound visualization through the vagina and the cervix (transcervical, TC-CVS), through the vagina only (transvaginal, TV-CVS), or through the maternal abdomen (transabdominal, TA-CVS) (Table 2).

\section{Transcervical Route}

A Chinese group reported success of fetal sexing achieved by transcervical withdrawal of chorionic villi, and this encouraged the development of a number catheters or cannulas for transcervical insertion, all different in material (silver or polypropylene), flexibility, and handling. The instruments employed have gone through variations and there have been alternative methods of obtaining villi; currently, the most common are under transabdominal ultrasound guidance by means of aspiration, by either a cannula or a catheter, and by means of rigid biopsy forceps (38).

For the transcervical procedure, the woman is set in the lithotomy position with comfortable leg supports. The woman's vulva, perineum, and vagina are cleansed with an antiseptic solution. A sterile speculum (or valve) is inserted into the vagina and opened, and the cervix is swabbed with the same solution. With the speculum in situ, the course of the cervical canal and a more detailed location in the sagittal and transverse view of the uterus is achieved. During the procedure, the assistant (or the operator himself) takes care

Table 2 Chorionic Sampling Approach Under Ultrasound Guidance

Transcervical Chorionic Villus Sampling

Transvaginal Chorionic Villus Sampling TC-CVS

Transabdominal Chorionic Villus Sampling
TV-CVS TA-CVS 


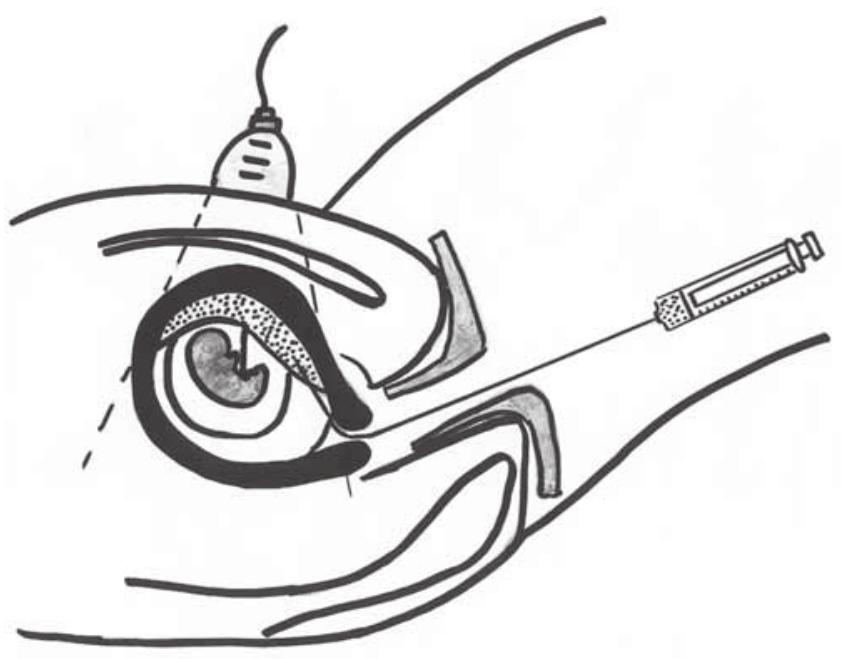

Figure 2 Transcervical chorionic villi sampling.

to establish the placental site and its relation to the cervical canal, so as to guide the instrument toward the site of sampling. Sometimes it is necessary to use a tenaculum or a vulsellum for steadying the cervix and passing through the cervical canal.

The aspiration technique represents maybe the most common method of TC-CVS (Fig. 2). It is carried out using a $1.5-\mathrm{mm}$ (16-gauge) cannula of polyethylene with a $1-\mathrm{mm}$ malleable stainless-steel or malleable stylet. To facilitate the transcervical passage into the uterus, the distal 3 to $5 \mathrm{~cm}$ of the cannula is slightly curved. The cannula must be passed through the cervix without touching the vaginal walls, and sometimes it is required to steady the cervix until any loss of resistance is perceived at the internal os. The cannula tip is visualized at this point by real-time ultrasound and is advanced under direct visualization into the chorion frondosum. The cannula is positioned parallel to the axis of the developing placenta and advanced almost to its distal end. The metallic obturator (stylet) is then removed, and a $20-\mathrm{ml}$ syringe containing $5 \mathrm{ml}$ of growth medium with heparin is attached to the end of the cannula. Chorionic villi are finally aspirated by applying negative pressure with the syringe while moving the catheter several times through the placenta in the longitudinal axis. The cannula and the attached syringe are then withdrawn. The syringe is to maintain suction throughout sampling and the withdrawal of the catheter to favor villi removal (14).

For sampling with rigid forceps, the woman should be in the same lithotomy position. After thorough local cleaning and accurate ultrasound evaluation, the forceps are introduced under direct transabdominal ultrasound monitoring. They are then gently directed to the sampling site, away from the choriodecidual junctions and margins of the chorionic plate (Fig. 3). The diameter of the forceps is only $2 \mathrm{~mm}$, so care must be taken to constantly visualize the forceps tip, the chorionic plate, and the amniotic sac in order to avoid creating a false passage. Once the site is reached, the sampling itself is performed by opening the jaws and advancing the forceps further into the placenta. They are then closed, and the forceps are slowly withdrawn. Chorion biopsy can be performed using a number of different forceps devices (15). In Europe, a 2-mm straight rigid

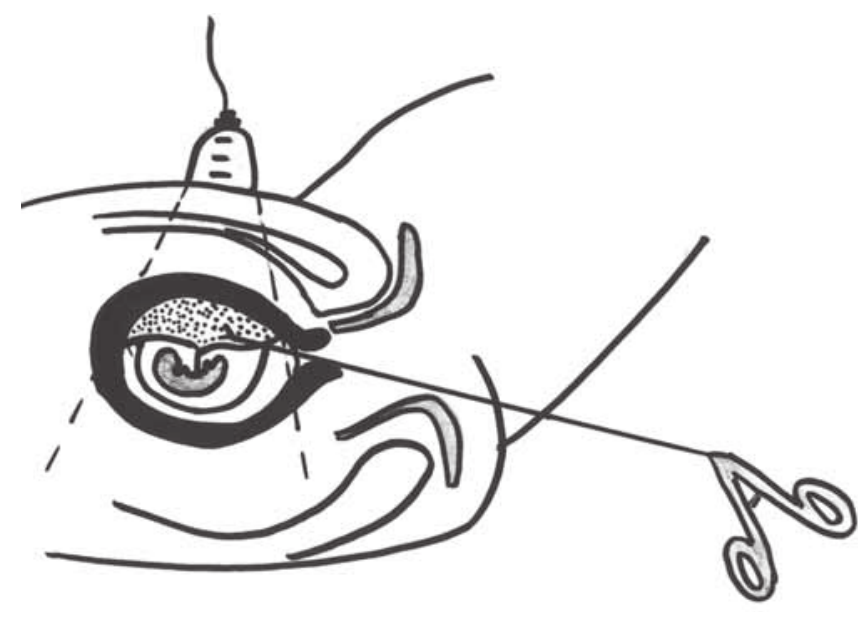

Figure 3 Chorionic villi sampling by rigid forceps.

forceps (Storz) and a thin-gauged transcervical round-tip steel biopsy forceps with a curved shank (snake forceps, $1.9 \mathrm{~mm}$ in diameter, $25 \mathrm{~cm}$ in length) are mostly employed.

\section{Transvaginal Route}

Transvaginal sampling by a needle guide attached to the vaginal ultrasound probe has also been used. The preparation of the woman is the same as for transcervical sampling. A vaginal 5- to $7.5-\mathrm{MHz}$ ultrasound probe enwrapped in a sterile envelope is inserted into the vagina and connected to a sterile needle guide. Chorionic villi sampling is then accomplished by either a 20-gauge needle with a stylet or a double-needle system, which are transvaginally and transmurally pushed forward into the chorion frondosum and moved to and fro. A target line on the ultrasound monitor sets the puncture depth and ensures reaching the desired biopsy site. Such transvaginal techniques were mostly adopted from vaginosonographically guided puncturing of follicles for oocyte harvesting. They allegedly prove more practical in cases of a fundal placenta with a retroverted uterus; however, this technique is found altogether less advantageous than TACVS and transabdominally guided TC-CVS (39).

\section{Transabdominal Route}

In the mid-1980s, the transabdominal approach was introduced using either a double- or a single-needle technique, and nowadays, it has become the most widespread technique. The woman should lie on her back on an examining table. The specific area on the woman's abdomen must be adequately cleansed with an aqueous betadine solution (antiseptic). A sterile oil is used so as to allow scanning by the transducer probe, which is commonly wrapped in a sterile drape or glove. The needles and the syringes need to be heparinized prior to skin puncture.

For the double-needle system, special needle guide adapters are necessary. They are available from ultrasound manufacturers and can be coupled with specific probes where the needle passes through a fixed path, either parallel or at an angle to the ultrasonic beam (Fig. 4) (18). First of all, a needle of $1.2 \mathrm{~mm}$ outer diameter (18 gauge) is inserted into the needle guide up to the basal plate of the placenta. After that, the stylet 


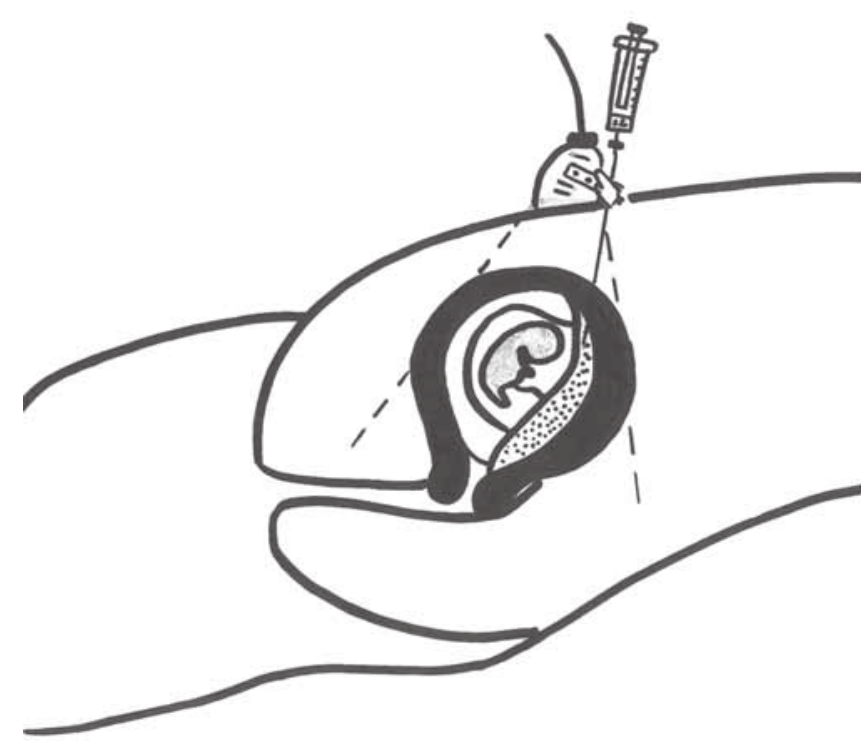

Figure 4 Transabdominal chorionic villi sampling by double-needle system.

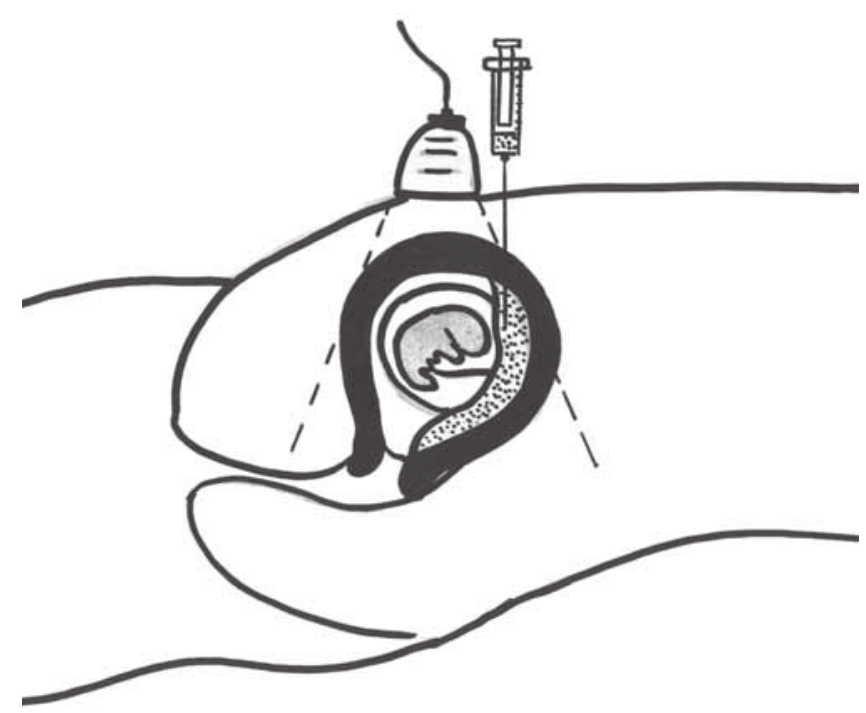

Figure 5 Transabdominal chorionic villi sampling by freehand technique. The needle is inserted longitudinally to the probe.

is removed and an aspiration needle of 0.9 or $0.8 \mathrm{~mm}$ (20 or 21 gauge) is introduced into the first needle and pushed forward into the chorion.

Regarding the single-needle technique, a suitable place of chorion must be identified on the scanner monitor. The puncture is then done freehand by a single 20-gauge spinal needle, inserted tangentially to the greater side of the probe, simultaneously visualizing the needle tip going into the placenta and straight through the shortest route so that only the needle tip is displayed (Fig. 5) (40,41). An oblique insertion, needling at the extremity of the probe, allows observing fully the needle throughout sampling but implies a lengthy course (Fig. 6). Newer needles have a special external coating and emit echo-luminance so as to enhance needle placement.

By adopting the freehand technique, after having reached the sampling site, the needle is connected to a $2.5-\mathrm{mL}$ syringe with a small amount of culture medium or sterile

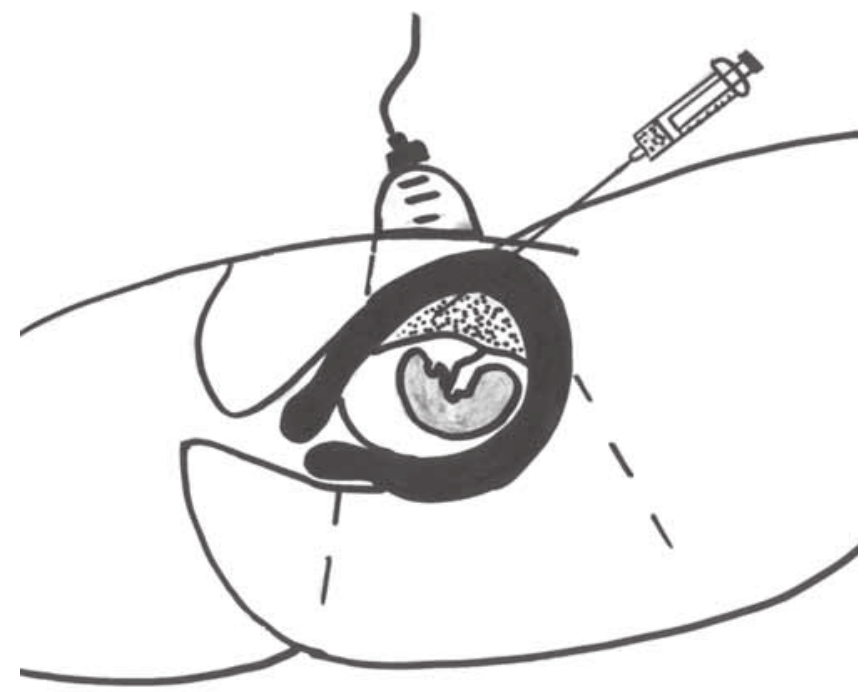

Figure 6 Transabdominal chorionic villi sampling by freehand technique. The needle is inserted tangentially to the probe.

solution. The displayed needle tip is moved up and down 5 to 10 times in order to favor detachment and suction of villi. All the while, negative pressure must be applied by manual aspiration through the syringe, which is maintained throughout sampling and withdrawing. Some operators use a $30-\mathrm{mL}$ syringe mounted on a holder as an alternative (42).

The need for the transducer probe to be manipulated by the same operator must be emphasized because it results in better hand-eye coordination and the reduction in the number of multiple insertions and the failures associated with the procedure. The single-operator freehand technique necessitates the same operator holding both the probe and the needle during insertion, thus having the sensibility to direct the proper placement of the needle. Sonographic guidance is crucial, but tactile sensation is important as well.

In fact, most procedures require at least three hands. Some operators actually need an assistant to hold the transducer during the connection of the syringe and after withdrawal, while they verify the sampling adequacy and the patient is waiting on the examination table. Other operators hold the transducer in their own hand and ask for assistance for drawing up the tissue.

If obtaining an adequate sample fails, a second insertion is performed. It is important to point out that the procedure is not to be carried out unless the operator is sure of the safe access to the intended chorion site. When difficulties are anticipated, the opinion of a more experienced physician should be sought.

Complications increase significantly if three or more insertions are attempted; therefore, no more than two needle insertions should be performed per session.

According to our own experience, a second attempt to obtain villi is rarely necessary. When the procedure seems too hard and an adequate sample is still not obtained after repeated passes, it may be wise to postpone it to the following days or week. An experienced operator should be consulted for any of these procedures if two attempts at uterine insertion fail to produce an adequate sample for analysis. 


\section{CVS Setting}

CVS, in any employed route, is generally performed in an outpatient facility or the health-care provider's office. Local anesthesia is not required since it does not deal with sources of pain in the peritoneum and the uterine wall; neither any preoperative nor postoperative medical treatment is usually necessary. Before the CVS procedure, the woman can eat and drink as usual. She may need her partner or another close contact to accompany her to the appointment and support her emotionally or to drive her home afterwards. Based on the clinical experience at our institution, the procedure proves more expeditious if the woman is at ease. The complex range of various psychologic issues related to genetic diagnosis and emotional needs of a woman experiencing CVS requires that the team who performs CVS also develops great sensitivity to the patient's apprehension and anxiety. It appears very helpful to actively involve the woman and maintain a dialogue between her and the operators during the procedure and the fine-tuning of the technical aspects of the CVS, as this minimizes the discomfort and ensures a trusting environment.

During transcervical CVS, the passage of the instrument through the cervix and the suction of villi may prove a cause for cramping, a pinching feeling, and general discomfort (nausea and anxiety). After the sampling, women may experience mild to moderate spotting for the first week.

Regarding TA-CVS, the entire procedure is completed in approximately 10 minutes. Most of the time is usually dedicated to the ultrasonographic investigation and sampling set preparation while the needle insertion itself lasts for a maximum of 10 seconds. On rare occasions, women complain of a rapidly resolving stinging sensation or cramping with the needle penetrating the skin and the uterus. A higher level of pain is likely to be experienced by patients with a thicker abdominal wall (greater than $40 \mathrm{~mm}$ ). At the end of the procedure, all symptoms are resolved. CVS may generate considerable anxiety, but most women find the pain equivalent to that of an intramuscular puncture. A recent study evaluating the woman's experience of pain during TA-CVS reports that the sampling procedure seems most painful at the approach of thicker needles, so the need to use a 19-gauge needle is questioned as acceptable results are obtained with a 20 -gauge needle as well (43).

Particular angles of access are also recommended by some experts but the data gathered so far are not exhaustive enough to guide practice. A randomized trial by van Schoubroeck et al. showed that injection of local anesthetic did not reduce pain scores as reported by women undergoing transabdominal punctures (44).

Rhesus status must be considered in every case. According to the guidelines of the ACOG (2001), Rhesus prophylaxis with anti-D immunoglobulin $(200-300 \mu \mathrm{g})$, intramuscularly, should be offered to Rh-negative unsensitized women after each procedure $(45,46)$. However, in cases of being already sensitized, the CVS is contraindicated.

Normally, in a few hours after the sampling, the woman will likely be able to resume normal activities and return home. They are counseled to contact medical staff only if they experience pain, heavy bleeding, or febrile episodes. An ultrasonographic follow-up is recommended when the diagnostic result is available so as to verify the fetus's well-being and to exclude chorionic hematomas.

\section{MULTIPLE PREGNANCIES}

Multiple pregnancies are not, as a general rule an impediment to CVS, but they require a greater amount of expertise and manual skills in order to insure that all fetuses are correctly sampled. Sampling is straightforward when placentas are well separate, but in cases of close proximity, it becomes more complex, as needles need to be serially in all placentas, possibly close to the umbilical cord insertion, in order to insure complete sampling without double passing across the same chorion point. Needles need to be replaced for each sampling $(47,48)$.

In pregnancies with high genetic risk, CVS is better suited than amniocentesis, as an eventual procedure of selective fetus reduction is much simpler and less risky when performed during the first trimester of pregnancy. Careful evaluation and documentation of gestational sacs, fetuses, placentas, and umbilical cords are mandatory when CVS is performed to correctly identify the fetuses when discordant results are obtained and the patient desires selective termination. Sampling error in multiple pregnancies is between $0 \%$ and $10 \%$ and depends critically on the expertise of the operator. Miscarriage risk is $2 \%$ to $4 \%(49-54)$.

\section{CONTRAINDICATIONS}

Absolute contraindications to TC-CVS are vaginismus and major anomalies of the cervical canal. Cervical polyps and large myomas of the lower uterine segment may represent an important obstacle to the cannula or biopsy forceps. Likewise, a fundal placenta or a posterior placentation in a retroverted uterus may be very difficult to sample by this approach. The transcervical route should be considered a risk of ascending infections in women with active genital infection, even if appropriately treated.

Conversely, it is almost always possible to obtain a sample by the transabdominal route. Actually, there are no valid contraindications to TA-CVS. An appropriate scanner pressure and an accurate bladder filling may favor reaching the sampling site and avoiding intestinal loops in front of the uterus. Myomas may be avoided by real-time guide of needle insertion. In cases of uterine retroflexion, a gentle cervicovaginal manipulation of the uterus by an assistant may be needed (Fig. 7).

Uterine contractions are occasionally seen, which can interfere with sampling; it is advisable to wait for 10 to 20 minutes for these contractions to cease, even through the help of an antispastic drug.

Some contraindications are common for either TC-CVS or TA-CVS.

In any case of active vaginal bleeding, it is advisable to reschedule any sampling procedure to a few days after the bleeding has stopped.

The occurrence of areas of retrochorionic hemorrhage at ultrasonic examination before CVS usually prompts extreme care to avoid it by the cannula or needle.

In cases of preoperative abundant sub-chorionic hemorrhage, deferral is mandatory until after a well-established resolution or to amniocentesis in the second trimester. 


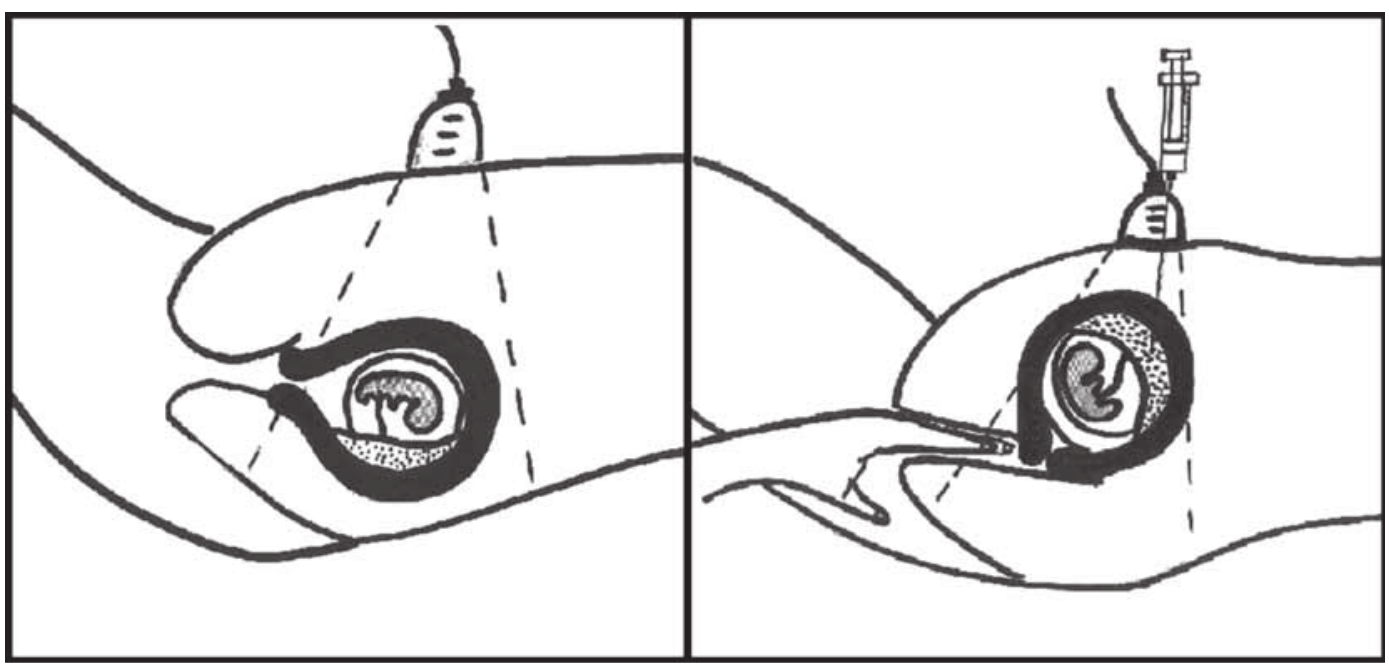

Figure 7 Cervico-vaginal manipulation in cases of uterine retroflexion.

Systemic maternal diseases do not represent a contraindication to CVS.

In the presence of inherited bleeding disorders, as in cases of hemophilia carriers with less than $50 \%$ factor VIII coagulant activity, prophylactic intravenous infusion of desmopressin (1-deamino-8-D-arginine vasopressin) just before CVS can be offered. This synthetic analog of vasopressin causes an immediate increase in the plasma concentration of factor VIII, von Willebrand factor, and platelet adhesiveness thus preventing abnormal bleeding with few side effects except mild facial flushing and headache (55).

A potential risk exists for maternal-fetal virus transmission as well as patient-clinician transmission in cases of women with blood-borne virus. The fairly small studies available for hepatitis $B$ show no evidence of a transmission risk so that the risk is expected to be very low. The "e" antigen status is likely to be significant for maternal-fetal transmission (56). Although most studies examining human immunodeficiency virus (HIV) suggest that invasive testing may be a risk factor in transmission and recommend avoidance, several authors more recently have suggested that the current risk of HIV vertical transmission associated with early invasive diagnostic techniques is lower than previously reported, provided that antiretroviral therapy is being used (57).

\section{COMPLICATIONS}

TA-CVS has a reported abortivity risk around $1 \%$, when performed by an expert hand. Randomized clinical trials have reported a similar fetal loss rate after CVS in the first trimester when compared with amniocentesis at the 16th week. In evaluating the fetal loss rate, spontaneous abortivity in the general population between the 10th and 12th week always needs to be taken into account. Post-procedural complications are highly dependent on the operator's expertise, gestational age, maternal age, the amount of chorion tissue sampled, the number and duration of needle insertions, bleeding prior to the procedure, and concomitant uterine pathologies.

Minor complications have been reported, like bleeding, spotting, intracervical hematoma, but without sizeable effects on perinatal outcome. In the early 1990s, fetal limb reduction defects have been reported after CVS performed before the 10th week in centers without a proven track record that did not employ standardized techniques. Wider studies on reference centers have however reported rates of limb reduction defects for CVS after the 9th week that are similar to the rates for the general population $(5.4 / 10,000)$. Accordingly, the WHO-sponsored committee concluded that there is little evidence to suggest a significant risk of congenital defects when CVS is carried out after the 8th completed gestational week and recommended that CVS be performed in referral centers following 9 weeks after the last menstrual period by qualified operators who are adequately trained (58-65).

\section{ANALYSIS}

Diagnostic procedure is correlated to the level of expertise of the operator, the cytogeneticist, and the molecular biologist.

The sample of chorionic villus should contain enough material for genetic analysis, generally around 20 to $30 \mathrm{mg}$. The sample of chorionic tissue should be placed in nutrient medium if cytogenetic analysis has been planned and in a sterile hydro-saline solution for other analyses. The specimen might be mailed to be analyzed but never frozen and should be examined within 24 to 48 hours. Sampled villi need to be examined under an inverted microscope to detect contamination with maternal decidua, which need to be separated so as not to affect diagnosis with unwarranted interpretations.

Two methods are currently available to fully evaluate the fetal karyotype from a CVS specimen: the direct (or shortterm) method and the indirect or culture method.

Chorionic villi consist of two cells types: cytotrophoblast cells and mesenchymal core cells. In freshly sampled chorion tissues, the villus inner cytotrophoblast layer beneath the outer syncytiotrophoblast layer contains mitotically active cells, which are rapidly dividing cells, many of which are already in metaphase. These cytotrophoblast actively mitotic cells would provide a reliable means of predicting the fetal karyotype for direct metaphase analysis, without culture. Although the direct analysis of cytotrophoblasts can provide welcome rapid results (24-72 hours), the quality of metaphases obtained is usually less than that obtained by culture.

To enhance the accuracy of CVS results, it is a good practice that villi tissue be analyzed by both the direct (or short-term) 
and the indirect (long-term or cultural) method. The first method allows analysis in a few hours, although an incubation of at least 24 hours is recommended with a diagnostic response in 3 to 5 days. The second method analysis is completed after 7 to 10 days.

Currently, confined placental mosaicism is considered the main cause of ambiguous results, which might result in falsepositive diagnoses. Following the direct analysis, the majority of discordant findings between chorionic villus samples and the fetus have been false-positive results (1-2\%) and only very few have been false negative $(1 / 3000)$.

With the use of both direct and cultured villus analyses, the incidence of true false-positive results can be greatly reduced (to $1 / 20,000$ ) and that of true false-negative results can be eliminated. However, when a mosaicism is found after CVS, follow-up amniocentesis and/or cordocentesis is required to clarify results.

Fluorescence in situ hybridization (FISH) can also be performed on noncultured chorion cells following CVS, by using chromosome-specific probes to determine the aneuploidies of chromosomes 13,18,21, X, and Y. This has allowed a rapid diagnosis of some selected chromosomal anomalies. It has also enabled the detection of subtle cytogenetic aberrations with a resolution equal to the size of the probe $(50-200 \mathrm{~Kb})$ that are associated with a clinical phenotype $(66,67)$. Despite the high sensitivity and specificity of interphase FISH for trisomy 13, 18, 21, and sex chromosome aneuploidy (99\%), culture failure or noninformative results occur in up to 5.7\% of cases and false-positive and false-negative results, although rare, have been reported. For that reason, the American College of Medical Genetics does not recommend therapeutic action on the basis of the results of FISH alone.

An implementation of the chromosomal evaluation in chorion tissue, in the 1990s, was the quantitative fluorescence polymerase chain reaction (QF-PCR), which was derived from the association of techniques of molecular biology to FISH. The technique is based on the amplification of chromosomespecific highly polymorphic DNA short tandem repeats, using fluorescence primers and PCR on chromosomes 13, 18, 21, X, and Y. The QF-PCR, which has comparable sensitivity to the FISH, consents easier identification of maternal contamination and is much less expensive because it is almost entirely automated. QF-PCR is now more common than FISH and many European laboratories consider it the method of choice for rapid aneuploidy testing complementary to full karyotype analysis and, in some specific cases, as an alternative to conventional cytogenetics analysis.

FISH and QF-PCR allow only the detection of aneuploidies involving chromosomes that are specifically tested, usually trisomies of chromosome 13, 18,21, and sex chromosomes. It means that aneuploidies involving other chromosomes and balanced chromosomal rearrangement can be missed. By using QF-PCR as a stand-alone test, the chances of missing the most common, and the only chromosome anomalies that do not increase in frequency with maternal age, are approximately 1 in 150 abnormal karyotypes, or 1 in 10 to 30,000 samples, based on age distribution; these error rates may be acceptable, although most structural anomalies will be missed (68).

Since chorionic tissue cells provide large sources of DNA suitable for DNA analyses, consequently, CVS is at present the technique of choice for prenatal diagnosis of genetic diseases with known molecular defects or when a linkage analysis is feasible (i.e., thalassemia, cystic fibrosis, Duchenne muscular dystrophy, hemophilia A, fragile X).

When a specific molecular defect is already known, the research of the defect is performed by different methods using the DNA extracted from villi and amplified by PCR (69).

For DNA-PCR diagnosis of disorders like $\beta$-thalassemia, a very small specimen of 1 to $3 \mathrm{mg}$ of chorionic tissue, supplying approximately 1 to $3 \mu \mathrm{g}$ of DNA, will suffice, but at least $3 \mathrm{mg}$ of chorionic villi is requested to reduce the risk of coamplifying the DNA from maternal decidua.

These techniques of PCR-DNA analyses, which enable reliable analysis of a small specimen of villi in 1 to 2 days, are highly efficient and accurate. Misdiagnosis and diagnostic errors are very uncommon (less than 1 in 10,000) in qualified centers when well-defined methods such as variable number tandem repeats are applied to rule out contamination by maternal decidua and blood $(31,70)$.

In utero infections can be documented for agents such as rubella, toxoplasma gondii, cytomegalovirus, varicella zoster, and parvovirus. Diagnosis can be made by extraction of DNA or RNA from chorionic villi and amplification of some wellrecognized genes of the suspected infectious agent (35-37).

\section{GUIDELINES}

In conclusion, the CVS procedure, when performed by experienced hands, is associated with an approximately $1 \%$ to $2 \%$ pregnancy loss rate. Adjusting for confounding factors such as gestational age, the procedure-related miscarriage rate following CVS at 10 weeks or later, in very experienced hands, is the same as midtrimester amniocentesis (at best 1 in 300). In less experienced hands, the risk may be higher.

CVS is presently recommended through the transabdominal route after the 9th week of pregnancy with an experienced operator and after genetic counseling. An operator is considered an expert when performing at least 100 samples per year. The training period comprises at least 50 samples under the supervision of a senior tutor. Each operator should undergo yearly audits to verify success rates of samples, fetal loss rate, diagnostic accuracy, and neonatal outcome. Data should be compared with what is reported in literature for reference centers. The analysis laboratory should also ensure an adequate number of exams in order to guarantee the highest degree of certainty (71-73).

\section{REFERENCES}

1. Monni G, Ibba RM, Zoppi MA. Prenatal genetic diagnosis through chorionic villous sampling. In: Milunsky A, Milunsky JM, eds. Genetic Disorders and the Fetus: Diagnosis, Prevention and Treatment, 6th edn. Wiley-Blackwell, Oxford, In press.

2. Wapner RJ, Evans MI, Davis G, et al. Procedural risks versus theology: chorionic villus sampling for Orthodox Jews at less than 8 weeks' gestation. Am J Obstet Gynecol 2002; 186: 1133-6.

3. Cates W Jr, Grimes DA. Morbidity and mortality of abortion in the United States. In: Hodgson JE, ed. Abortion and sterilization: medical and social aspects. London: Academic Press Inc, 1981: 155-80.

4. Bartlett LA, Berg CJ, Shulman HB, et al. Risk factors for legal induced abortion-related mortality in the United States. Obstet Gynecol 2004; 103: 729-37.

5. Nimkarn S, New MI. Prenatal diagnosis and treatment of congenital adrenal hyperplasia. Pediatr Endocrinol Rev 2006; 4: 99-105. 
6. Mohr J. Foetal genetic diagnosis: development of techniques for early sampling of foetal cells. Acta Pathol Microbiol Scand 1968; 73: 73-7.

7. Kullander S, Sandahl B. Fetal chromosome analysis after transcervical placental biopsies during early pregnancy. Acta Obstet Gynaecol Scand 1973; 52: 355-9.

8. Hahnemann N. Early prenatal diagnosis: a study of biopsy techniques and cell culturing from extra-embryonic membrane. Clin Genet 1974; 6: 294-306.

9. Tietung Hospital of Anshan Iron and Steel Company. Fetal sex prediction by sex chromatin of chorionic villi cells during early pregnancy. Chin Med J 1975; 1: 117-26.

10. Kazy Z, Rozovsky IS, Bakharev VA. Chorionic biopsy in early pregnancy. A method of early prenatal diagnosis for inherited disorders. Prenat Diagn 1982; 2: 39.

11. Niazi M, Coleman DV, Loeffler FE. Trophoblast sampling in early pregnancy. Culture of rapidly dividing cells from immature placental villi. Br J Obstet Gynaecol 1981; 88: 1081-5.

12. Old JM, Ward RH, Petrou M, et al. First-trimester fetal diagnosis for haemoglobinopathies: three cases. Lancet 1982; 2: 1413-16.

13. Ward RH, Model B, Petrou M, et al. Method of sampling chorionic villi in first trimester of pregnancy under guidance of real time ultrasound. Br Med J 1983; 286: 1542-4.

14. Brambati B, Oldrini A, Aladerun SA. Transcervical specimen of chorionic villi in the 1st trimester of pregnancy. Pathologica 1983; 75(Suppl): 179-83.

15. Monni G, Ibba RM, Olla G, et al. Chorionic villus sampling by rigid forceps: experience with 300 cases at risk for thalassemia major. Am J Obstet Gynecol 1987; 156: 912-14.

16. Dumez Y, Goossens M, Poenaru L, et al. Biopsy of chorionic villi by forceps under ultrasonic control: technic and results. J Genet Hum 1984; 32: 335-44.

17. Simoni G, Brambati B, Danesino C, et al. Efficient direct chromosome analysis and enzyme determinations from chorionic villi samples in the first trimester of pregnancy. Hum Genet 1983; 63: 349-57.

18. Smidt-Jensen S, Hahnemann N. Transabdominal fine needle biopsy from chorionic villi in the first trimester. Prenat Diagn 1984; 4: 163-9.

19. Cao A, Cossu P, Monni G, et al. Chorionic villus sampling and acceptance rate of prenatal diagnosis. Prenat Diagn 1987; 7: 531-3.

20. Pandya PP, Brizot ML, Kuhn P, et al. First-trimester fetal nuchal translucency thickness and risk for trisomies. Obstet Gynecol 1994; 84: $420-3$.

21. Cicero S, Bindra R, Rembouskos G, et al. Integrated ultrasound and biochemical screening for trisomy 21 using fetal nuchal translucency, absent fetal nasal bone, free beta-hCG and PAPP-A at 11 to 14 weeks. Prenat Diagn 2003; 23: 306-10.

22. Monni G, Zoppi MA, Ibba RM, et al. Fetal nuchal translucency test for Down's syndrome. Lancet 1997; 350: 1631; author reply 1631-2.

23. Monni G, Zoppi MA, Ibba RM. Absence of nasal bone and detection of trisomy 21. Lancet 2002; 359: 1343.

24. Zoppi MA, Putzolu M, Ibba RM, et al. First-trimester ductus venosus velocimetry in relation to nuchal translucency thickness and fetal karyotype. Fetal Diagn Ther 2002; 17: 52-7.

25. Faiola S, Tsoi E, Huggon IC, et al. Likelihood ratio for trisomy 21 in fetuses with tricuspid regurgitation at the 11 to $13+6$-week scan. Ultrasound Obstet Gynecol 2005; 26: 22-7.

26. Nicolaides KH, Bindra R, Heath V, et al. One-stop clinic for assessment of risk of chromosomal defects at 12 weeks of gestation. J Matern Fetal Neonatal Med 2002; 12: 9-18.

27. Zoppi MA, Ibba RM, Putzolu M, et al. Nuchal translucency and the acceptance of invasive prenatal chromosomal diagnosis in women aged 35 and older. Obstet Gynecol 2001; 97: 916-20.

28. Nicolaides KH, Chervenak FA, McCullough LB, et al. Evidence-based obstetric ethics and informed decision-making by pregnant women about invasive diagnosis after first-trimester assessment of risk for trisomy 21 . Am J Obstet Gynecol 2005; 193: 322-6.

29. ACOG Committee on Practice Bulletins. ACOG Practice Bulletin No. 77: screening for fetal chromosomal abnormalities. ACOG Committee on Practice Bulletins. Obstet Gynecol 2007; 109: 217-27.

30. Monni G, Cau G, Lai R, et al. Intracytoplasmic sperm injection and prenatal invasive diagnosis. Prenat Diagn 1999; 19: 390.
31. Cao A, Rosatelli MC, Monni G, et al. Screening for thalassemia: a model of success. Obstet Gynecol Clin North Am 2002; 29: 305-28, vi-vii.

32. Nicolaides KH. Nuchal translucency and other first-trimester sonographic markers of chromosomal abnormalities. Am J Obstet Gynecol 2004; 191: 45-67.

33. Nagaishi M, Yamamoto T, Iinuma K, et al. Chromosome abnormalities identified in 347 spontaneous abortions collected in Japan. J Obstet Gynaecol Res 2004; 30: 237-41.

34. Orofino MG, Argiolu F, Sanna MA, et al. Fetal HLA typing in beta thalassaemia: implications for haemopoietic stem-cell transplantation. Lancet 2003; 362: 41-2.

35. Bosma TJ, Corbett KM, Eckstein MB, et al. Use of PCR for prenatal and postnatal diagnosis of congenital rubella. J Clin Microbiol 1995; 33: 2881-7.

36. Kustermann A, Zoppini C, Tassis B, et al. Prenatal diagnosis of congenital varicella infection. Prenat Diagn 1996; 16: 71-4.

37. Dong ZW, Zhou SY, Li Y, et al. Detection of a human parvovirus intrauterine infection with the polymerase chain reaction. J Reprod Med 2000; 45: 410-12.

38. Sidransky E, Black SH, Soenksen DM, et al. Transvaginal chorionic villus sampling. Prenat Diagn 1990; 10: 583-6.

39. Monni G, Ibba RM, Lai R, et al. Transabdominal chorionic villus sampling: fetal loss rate in relation to maternal and gestational age. Prenat Diagn 1992; 12: 815-20.

40. Monni G, Ibba RM, Lai R, et al. Early transabdominal chorionic villus sampling for couples at high genetic risk. Am J Obstet Gynecol 1993; 168: 170-3.

41. Brambati B, Oldrini A, Lanzani A. Transabdominal chorionic villus sampling: a freehand ultrasound guided technique. Am J Obstet Gynecol 1987; 157: 134-7.

42. Brambati B, Lanzani A, Oldrini A. Transabdominal chorionic villus sampling. Clinical experience of 1159 cases. Prenat Diagn 1988; 8: 609-17.

43. Vandenbossche F, Horovitz J, Guyon F, et al. Pain experience during chorionic villus sampling and amniocentesis: a preliminary study. Eur J Obstet Gynecol Reprod Biol 2008; 136: 189-93.

44. Van Schoubroeck D, Verhaeghe J. Does local anesthesia at mid-trimester amniocentesis decrease pain experience? A randomized trial in 220 patients. Ultrasound Obstet Gynecol 2002; 16: 536-8.

45. Kanhai HH, Gravenhorst JB, van't Veer MB, et al. Chorionic biopsy in management of severe rhesus isoimmunisation. Lancet 1984; 2: 157-8.

46. American College of Obstetricians and Gynecologists Committee on Practice Bulletins-Obstetrics. ACOG Practice Bulletin. Clinical Management Guidelines for Obstetrician-Gynecologists. Prenatal diagnosis of fetal chromosomal abnormalities. Obstet Gynecol 2001; 97(Suppl): 1-12.

47. Monni G, Rosatelli C, Falchi AM, et al. First trimester diagnosis of $\beta$-thalassemia in a twin pregnancy. Prenat Diagn 1986; 6: 63-8.

48. Pergament E, Schulman J, Copeland K, et al. The risk and efficacy of chorionic villus sampling in multiple gestations. Prenat Diagn 1992; 12: $377-84$.

49. De Catte L, Liebaers I, Foulon W. Outcome of twin gestations after first trimester chorionic villus sampling. Obstet Gynecol 2000; 96: 714-20.

50. Brambati B, Tului L, Guercilena S, et al. Outcome of first-trimester chorionic villus sampling for genetic investigation in multiple pregnancy. Ultrasound Obstet Gynecol 2001; 17: 209-16.

51. Monni G, Zoppi MA, Cau G, et al. Importance of nuchal translucency measurement in multifetal pregnancy reduction. Ultrasound Obstet Gynecol 1999; 13: 377-8.

52. Wapner RJ, Johnson A, Davis G. Prenatal diagnosis in twin gestations: a comparison between second-trimester amniocentesis and first-trimester chorionic villus sampling. Obstet Gynecol 1993; 82: 49-56.

53. Antsaklis A, Souka AP, Daskalakis G, et al. Second-trimester amniocentesis vs chorionic villus sampling in multiple gestations. Ultrasound Obstet Gynecol 2002; 20: 476-81.

54. Van den Berg C, Braat AP, Van Opstal D, et al. Amniocentesis or chorionic villus sampling in multiple gestations? Experience with 500 cases. Prenat Diagn 1999; 19: 234-44.

55. Mannucci PM. Use of desmopressin (DDAVP) during early pregnancy in factor VIII-deficient women. Blood 2005; 105: 3382.

56. Grosheide PM, Quartero HW, Schalm SW, et al. Early invasive prenatal diagnosis in HBsAg-positive women. Prenat Diagn 1994; 14: 553-8. 
57. Somigliana E, Bucceri AM, Tibaldi C, et al. Early invasive diagnostic techniques in pregnant women who are infected with the HIV: a multicenter case series. Am J Obstet Gynecol 2005; 193: 437-42.

58. Firth HV, Boyd PA, Chamberlain P, et al. Severe limb abnormalities after chorion villus sampling at 56-66 days' gestation. Lancet 1991; 337: 762-3.

59. Monni G, Ibba RM, Lai R, et al. Limb reduction defects and chorionic villus sampling. Lancet 1991; 337: 1091.

60. Jackson LG, Wapner RJ, Brambati B. Limb abnormalities and chorionic villus sampling. Lancet 1991; 337: 1423.

61. Kuliev A, Jackson L, Froster U, et al. Chorionic villus sampling safety. Report of World Health Organization/EURO meeting in association with the Seventh International Conference on Early Prenatal Diagnosis of Genetic Diseases, Tel-Aviv, Israel, May 21, 1994. Am J Obstet Gynecol 1996; 174: 807-11.

62. Froster-Iskenius UG, Baird PA. Limb reduction defects in over one million consecutive livebirths. Teratology 1989; 39: 127-35.

63. Froster UG, Jackson L. Limb defects and chorionic villus sampling: results from an international registry, 1992-94. Lancet 1996; 347: 489-94.

64. Kuliev AM, Modell B, Jackson L, et al. Chorionic villus sampling (CVS): World Health Organization European Regional Office (WHO/EURO) meeting statement on the use of CVS in prenatal diagnosis. J Assist Reprod Genet 1992; 9: 299-302.

65. WHO/PAHO consultation on CVS. Evaluation of chorionic villus sampling safety. Prenat Diagn 1999; 19: 97-9.
66. Evans MI, Ebrahim SA, Berry SM, et al. Fluorescent in situ hybridization utilization for high-risk prenatal diagnosis: a trade-off among speed, expense, and inherent limitations of chromosome-specific probes. Am J Obstet Gynecol 1994; 171: 1055-7.

67. D'Alton ME, Malone FD, Chelmow D, et al. Defining the role of fluorescence in situ hybridisation for prenatal diagnosis of aneuploidies. Am J Obstet Gynecol 1997; 176: 769-76.

68. Nicolini U, Lalatta F, Natacci F, et al. The introduction of QF-PCR in prenatal diagnosis of fetal aneuploidies: time for reconsideration. Hum Reprod Update 2004; 10: 541-8.

69. Saiki RK, Bugawan TL, Horn GT, et al. Analysis of enzymatically amplified beta-globin and HLA-DQ alpha DNA with allele-specific oligonucleotide probes. Nature 1986; 324: 163.

70. Rosatelli C, Falchi AM, Tuveri T, et al. Prenatal diagnosis of $\beta$-Thalassemia with the synthetic oligomer technique. Lancet 1985; 2: 241-3.

71. World Health Organization Regional Office for Europe (WHO/EURO). Risk evaluation of chorionic villus sampling (CVS): report on a meeting. Copenhagen: WHO/EURO, 1992.

72. EAPM (European Association of Perinatal Medicine). Protocols and Guidelines in Prenatal Medicine. Maternal-Fetal and Neonat Medicine, 2003.

73. Monni G, Zoppi MA, Axiana C, et al. Changes in the approach for invasive prenatal diagnosis in 35,127 cases at a single center from 1977 to 2004. Fetal Diagn Ther 2006; 21: 348-54. 


\section{Amniocentesis Aris Antsaklis and Marianna Theodora}

\begin{abstract}
HISTORY
Amniocentesis is the oldest invasive procedure for prenatal diagnosis. It was first used toward the end of the 19th century for evacuative paracentesis in pregnancies complicated by hydramnion (1). At the beginning of the 1950s, it was used to determine the amniotic fluid composition in cases of Rhesus isoimmunization (2). However, amniocentesis was used for diagnostic reasons in the 1950s, as a method for sex determination by the identification of Barr bodies in the noncultured amniocytes (3). About 10 years later, the karyotype of the embryo was determined through an amniotic fluid cell culture (4). Since then, amniocentesis has been established as a basic invasive method for the prenatal diagnosis of various pregnancy-related conditions, such as fetal karyotyping, diagnosis of metabolic or enzymatic diseases, assessment of the severity of hemolytic disease, establishment of lung maturity, and diagnosis of fetal infections. Additionally, amniocentesis is used for the infusion of various drugs into the amniotic cavity, determination of the composition of the amniotic fluid, and finally for evacuation of hydramnion.
\end{abstract}

\section{INDICATIONS}

Amniocentesis is used for both prenatal diagnosis and fetal therapy (Table 1).

\section{Chromosome and DNA Analysis}

Examination of the number and structure of fetal chromosomes (fetal karyotyping) is the main indication for amniocentesis. It is offered to women in cases when the risk for fetal aneuploidies is high such us advanced maternal age (traditionally $\geq 35$ years), previous fetal aneuploidy, parental balanced translocation, positive screening test in the first or second trimester, identification of aneuploidy markers in genetic sonogram, and so on. Another indication is an ambiguous result from a previous test such as karyotype mosaicism obtained by the chorionic villus sampling (CVS). Fetal karyotype is examined either in cultured cells or with PCR technology. However, the result of the latter technique should always be confirmed with culture.

Fluorescent-labeled in situ hybridization allows for DNA study, with direct probing, and therefore the diagnosis of fetuses with gene diseases such as hemophilia, b-thalassemia, cystic fibrosis, and so on.

\section{Fetal Infection}

Diagnosis of fetal infection from Toxoplasma gondii, cytomegalovirus, and so on, in cases of maternal seroconversion is possible by examination of amniotic fluid with PCR-based technologies.

\section{Lung Maturity}

Fetal lung maturity can be assessed indirectly using both qualitative and quantitative characteristics of amniotic fluid.
Pulmonary maturity can be assessed by the measurement of lecithin/sphingomyelin ratio or the concentration of phosphatidylglycerol in amniotic fluid. Similarly, the detection of lamellar bodies and the study of other characteristics of the amniotic fluid may give information about lung maturity. However, the improvement in neonatal care and the accurate confirmation of gestational age with ultrasound early in pregnancy have diminished the need for examination of amniotic fluid and thus amniocentesis in order to confirm lung maturity.

\section{Chorioamnionitis}

Premature rupture of the membranes (PROM) and preterm labor have been associated with amniotic fluid bacterial colonization (5). Amniocentesis and gram stain, microscopy, and culture of the amniotic fluid have been advocated in cases of premature rupture of membranes or premature labor. Researchers who advocate for amniocentesis state that early diagnosis and management are possible and may result in better perinatal outcome. However, spontaneous labor within 24 hours after membrane rupture is very common and thus there are no data to support amniocentesis as a clinical standard in cases of PROM.

\section{Biochemistry}

Amniotic fluid collection and estimation of enzyme level were used in order to diagnose metabolic diseases and other conditions such as cystic fibrosis or congenital adrenal hyperplasia before DNA techniques were applied. This practice has been replaced by the most accurate detection of gene mutation responsible for the disease in fetal cells collected by amniocentesis or CVS.

Another past indication of amniocentesis is the evaluation of fetal hemolytic anemia in cases of Rhesus alloimmunization. Amniocentesis and amniotic fluid spectrophotometry are performed less since middle cerebral artery Doppler velocimetry has been proposed as a routine test for the evaluation of hemolysis and fetal anemia.

\section{Obstetric Cholestasis}

Amniocentesis is used in obstetric cholestasis in order to diagnose meconium-stained amniotic fluid, which is a common complication of this pregnancy-related disorder and related to intrauterine fetal demise. However, improvements in understanding both the pathophysiology of the disease and the management of these cases have led to decreased need for amniocentesis in this context.

\section{Fetal Therapy}

Amniocentesis is used for uterine decompression in case of hydramnios and twin-to-twin transfusion syndrome in 
Table 1 Indications for Amniocentesis

Chromosome analysis
Advanced maternal age
Abnormal biochemical screen
Ultrasound findings
Family or personal history of trisomy
Abnormal parental karyotype
DNA analysis
Genetic testing
Endocrine disorders
Suspected fetal anemia
Rhesus sensitization
Fetal hydrops
Fetal infection
Lung maturity
Chorioamnionitis
Biochemistry
Obstetric cholestasis
Fetal therapy

monochorionic (MC) twins. Amnioinfusion has been used in cases of severe oligohydramnios, but research is needed in order to define both the indications and the outcome of such an intervention. Finally, thyroxin has been injected into the amniotic sac to treat goitrous hypothyroidism of the fetus.

\section{TECHNIQUE}

Amniocentesis is mainly performed during the second trimester but can be performed also during the third trimester when certain indications stand. Amniocentesis for prenatal diagnosis of genetic diseases of the fetus is traditionally carried out between 15 and 18 weeks of gestation when the fundus of the uterus is easily accessible. Additionally in this gestational age, the volume of amniotic fluid is 150 to $200 \mathrm{~mL}$, which permits the removal of 15 to $20 \mathrm{~mL}$ of amniotic fluid, which is adequate for this test without creating problems. Moreover, at this gestational stage, the relationship between the viable and nonviable cells of the amniotic fluid is the optimal in order to have results. For the above reasons, amniocentesis should be carried out at this time (6). Early amniocentesis (between 11 and 14 weeks) has been associated with increased risk of fetal malformations, mainly clubfeet, and has been abandoned.

In any case, before amniocentesis is performed, pregnant women should receive appropriate genetic counseling concerning the risks, the limitations, and the benefits of the procedure and the tests. Informed consent should be obtained before the procedure. A detailed ultrasound examination must be done in order to determine the number of fetuses, gestation age, cardiac function, possible abnormal formation, amniotic fluid quantity, position of the placenta, existence of fibromas in the uterus, existence of adnexal masses, and finally possible cervical inadequacy. When this ultrasound examination has been completed, the entry site of the needle into the amniotic cavity is determined $(7,8)$. Preferred pockets are located away from the fetal face. Sites that must also be determined and avoided as much as possible during amniocentesis is the umbilical cord — depending on its position — and the placenta. However, none of these is absolutely necessary to obtain amniotic fluid safely. The skin around the entry site of the needle is carefully cleaned with an antiseptic solution.

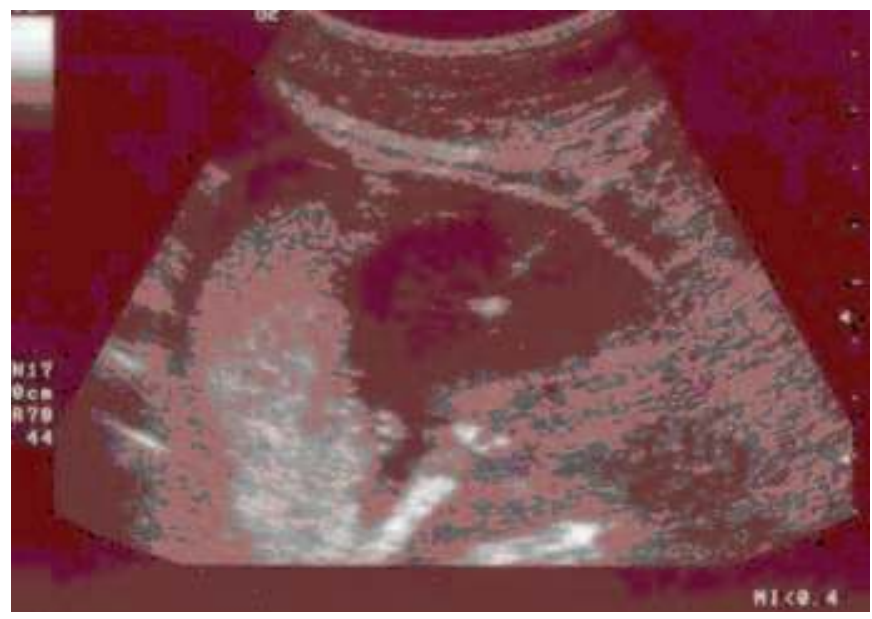

Figure 1 Amniocentesis in singleton. Note that the whole length and the tip of the needle can be identified when the procedure is done under ultrasound guidance.

A disposable 22-G spinal needle (Fig. 1) is carefully guided into the amniotic cavity under continuous ultrasound monitoring. When the needle enters the corresponding area, the inner needle is removed and a syringe is attached to the needle hub. If freely flowing fluid is not obtained, the needle is then repositioned. Failure to aspirate amniotic fluid is most commonly due to membrane tenting. When this occurs, the needle appears inside the amniotic cavity, but the needle tip has not actually penetrated the amniotic sac. This usually occurs when an early amniocentesis is carried out, when the amnion and the chorion have not yet fused. In this case, it is recommended that the inner needle is replaced and the needle's entry point to the amniotic cavity is carefully changed so as to avoid injuring the fetus or the umbilical cord. Another reason for failing to take a sample of amniotic fluid is when the fetal membranes plug up the opening of the needle, due to the negative pressure that is exerted here. If this occurs, we then slightly change the needle site without exerting negative pressure. The first 1 to $2 \mathrm{cc}$ of amniotic fluid that is aspirated is discarded because in theory it may contain maternal cells and thus gives us inaccurate results. An amount of approximately 20-cc amniotic fluid is considered to be enough for the results we require when amniocentesis is carried out between the 15 th and 20th weeks of gestation. An ultrasound examination is performed after the amniocentesis is completed to document fetal cardiac activity and to look for evidence of bleeding into the amniotic cavity. The pregnant woman is then advised to rest and avoid intercourse for 15 days and informed about possible complications, such as persistent uterine cramping, fever, bleeding, or leakage of the amniotic fluid, which she must report timely.

\section{AMNIOCENTESIS COMPLICATIONS}

Amniocentesis is an interventional procedure, and since the feto-maternal unit is injured, a number of complications can ensue. Maternal complications are rare and include perforation of the intra-abdominal viscera with subsequent intra-abdominal infection, sepsis, bleeding, blood group sensitization, and uterine contractions (9). On the other hand, fetal complications are more of a concern and include fetal 
Table 2 Fetal Loss Rate in Review by Seeds (15)

$\begin{array}{lll}\text { Ultrasound use } & \text { Preamniocentesis scan } & \text { Concurrent ultrasound guidance } \\ \text { All studies } & & \\ \text { Losses (n/N) } & 714 / 33,975(2.1 \%) & 478 / 34,144(1.40 \%) \\ \text { Amniocentesis } & 88 / 6457(1.4 \%) & 130 / 12,097(1.08 \%) \\ \text { Control subjects } & 0.74 & 0.33 \\ \text { Difference (\%) } & <0.0001 & <0.0007 \\ p \text { Value } & 0.37,1.1 & 0.09,0.56 \\ 95 \% \text { CI for difference } & & \\ \text { Only controlled studies } & 170 / 8607(2.0 \%) & 191 / 11,372(1.68 \%) \\ \text { Losses (n/N) } & 88 / 6457(1.4 \%) & 130 / 12,097(1.08 \%) \\ \text { Amniocentesis } & 0.0042 & <0.0001 \\ \text { Control subjects } & 1.46 & 1.57 \\ p \text { Value } & 0.6 & 0.6 \\ \text { Odds ratio } & 0.19,1.03 & 0.31,0.9 \\ \text { Difference (\%) } & & \end{array}$

loss, needle puncture injuries, placenta abruption, preterm labor, injury due to withdrawal of amniotic fluid (e.g., amniotic bands), and rupture of the membranes. Amniocentesis may also cause intra-amniotic infections through the introduction of microorganisms into the amniotic cavity via the needle. This may eventually lead to the abortion or death of the fetus. In order to eliminate the possibility of an infectious complication, rules of aseptic procedures, which apply in all surgical procedures, must be applied here, such as the aseptic cleaning of the skin and using septic gloves and needles. However, endometrial infections are not always related to amniocentesis, but may exist before the prenatal intervention $(10,11)$.

\section{Fetal Loss}

Fetal loss is the major risk of second-trimester genetic amniocentesis. When amniocentesis was first introduced in the clinical practice, the risk of miscarriage related to the procedure was not clear mainly because there was lack of ultrasound guidance and lack of determination of fetal viability before the procedure. Since fetal miscarriage does not occur only in association with amniocentesis, it is essential to take into consideration the background loss rate, which is associated with the gestation age, parity, and any other underlying risk factors such as pathologic conditions of the mother (e.g., uncontrolled diabetes mellitus, severe hypertension induced by lupus, etc.) and the fetus (e.g., structural anomalies, chromosomal abnormalities, etc.) as well. Gestational age at the procedure is an important determinant of the observed fetal loss rate, since the earlier the pregnancy, the greater is the pre-procedural risk of miscarriage.

The safety of amniocentesis has been evaluated by several series from single centers and a number of multicentric studies. The difficulties in evaluating the procedure-related loss rate have been shown by the controversial results of these studies.

In 1976, the National Institute of Child Health and Human Development conducted a case-control/cohort study to evaluate the safety and accuracy of second-trimester amniocentesis for prenatal diagnosis and reported that the study group that underwent amniocentesis had a 3.5\% total loss rate while the controls had $3.2 \%$ (9). The authors also concluded that the loss risk was unrelated to previous miscarriage, volume of aspirated fluid, or number of attempts. In the same year, the Canadian Collaborative Study reported a loss rate of $3.2 \%$, but there were no controls (12). In this study, an increased risk of loss was reported in cases of failed attempts and large bore needles $(>19)$. The third collaborative casecontrol study was conducted by the British Working Party on amniocentesis in 1978 (13) and the writers reported a loss rate of $1.5 \%$ related to amniocentesis. They reported a $2.4 \%$ fetal loss rate $<28$ weeks in the study group and $1.2 \%$ in controls. This study however was strongly criticized mainly on the selection of control individuals who were recruited in the study later in gestation and on the replacement of some of the matched controls who had aborted.

The only randomized trial for the estimation of miscarriage risk related to amniocentesis was done in Denmark in 1986 (14). This study randomized 4606 low-risk women aged 24 to 35 years to have or not to have an amniocentesis, which was carried out using a 20-gauge needle under real-time ultrasound guidance. Most procedures were performed between 16 and 18 weeks of gestation. The amniocentesis group had a loss rate, which exceeded the control group by $1 \%(1.7 \%$ and $0.7 \%$ respectively), a figure which has often been quoted in counseling. More than half of the procedures were performed by one operator and the rest by four less experienced operators. The placenta was avoided whenever possible but was perforated in $15 \%$ of cases and the study indicated that perforation of the placental was associated with a relative risk of 2.6 for miscarriage.

Since then, a lot of studies reported amniocentesis-related fetal loss rate between $0.2 \%$ and $0.9 \%$.

In 2001, Seeds performed a systematic review of 29 controlled and uncontrolled studies with a total of 68,119 amniocenteses (15) and he concluded that amniocentesis with concurrent ultrasound guidance in controlled studies appears to be associated with a procedure-related rate of excess pregnancy loss of $0.6 \%$ (95\% CI, $0.31,0.90$ ) (Table 2). The use of concurrent ultrasound guidance appears to reduce the number of punctures and the incidence of blood-stained fluid and eliminates the direct fetal needle trauma. Finally, the review did not substantiate an increased rate of pregnancy loss if placental puncture is required. 
In 2006, another trial was published in order to quantify the contemporary procedure-related loss rate after midtrimester amniocentesis using a database generated from patients who were recruited to the First- and Second-Trimester Evaluation of Risk for Aneuploidy trial (16). A total of 35,003 unselected patients from the general population with viable singleton pregnancies were enrolled in this trial and 3096 of them underwent midtrimester amniocentesis. The total spontaneous fetal loss rate less than 24 weeks of gestation in the study group was $1.0 \%$ and was not statistically different from the background $0.94 \%$ rate seen in the control group $(p=0.74$, $95 \%$ CI, $0.26 \%, 0.49 \%)$. The researchers stated that the odds of pregnancy loss were actually lower in patients who underwent amniocentesis because of advanced maternal age or screen positive results compared with those who did not. They concluded that this is likely due to the fact that spontaneous pregnancy loss is so strongly associated with aneuploidy, and patients who have an amniocentesis would presumably terminate aneuploid fetuses in most cases before a spontaneous loss could occur. They stated that it is not surprising that patients in these categories who elected not to undergo amniocentesis had a higher rate of spontaneous loss because they were carrying a higher proportion of aneuploid fetuses.

Recently, Mujezinovic and Alfirevic compiled a systematic review of complications related to genetic amniocentesis and CVS to provide benchmark data for counseling and performance assessment of individual operators (17). Twenty-nine articles of amniocentesis were included and pooled pregnancy loss within 14 days was $0.6 \%$ (95\% CI $0.5-0.7$ ), rising to $0.9 \%$ (95\% CI 0.6-1.3) for pregnancy loss before 24 weeks and 1.9\% (95\% CI 1.4-2.5) for total pregnancy loss (Table 3). However, the authors noted that since most of the reviewed studies do not take into account the background risk and therefore do not address the issue of added procedure-related risk, these figures may be useful only for counseling purposes in a broad sense. The authors identified five studies that have included control groups in order to quantify the background risk. Overall, these studies reported higher pregnancy losses compared with uncontrolled case series and the pooled results showed a relative increase of $25 \%$ for total pregnancy loss after amniocentesis and $46 \%$ for pregnancy losses before 24 or 28 weeks of gestation. However, the results for the latter were quite heterogeneous, resulting in very wide, statistically no significant confidence intervals. The absolute risk difference between cases and controls was quite similar, showing that second-trimester pregnancy loss and total fetal loss after an amniocentesis increase by approximately $0.6 \%$. The reviewers noted that although the risk of pregnancy loss is relatively low, lack of adequate controls tends to underestimate the true added risk of prenatal invasive procedures.

Midtrimester amniocentesis does not appear to further increase the already increased risk for second-trimester spontaneous abortion in women with uterine myomas (18).

\section{Preterm Delivery}

The association of second-trimester amniocentesis and delivery before 37 weeks was evaluated by a case-control study in 2003 by the EUROPOP Group (19). During 1994 to 1997, 3091 preterm births and 5298 controls randomly selected from singleton births born at term were analyzed. An increased risk of preterm delivery was found in women having secondtrimester genetic amniocentesis after taking into account other risk factors and confounding variables (OR 1:59, 95\% CI 1.31-1.92). The association was statistically significant and similar for spontaneous preterm delivery and induced preterm delivery. Since the data are limited, further research is needed on the causative role of amniocentesis.

\section{Fetal Trauma}

The risk of direct fetal needle injury often is presumed to be reduced with the use of simultaneous ultrasound guidance, but several case reports document or attribute cases of fetal injury to the amniocentesis needle, despite the use of simultaneous guidance for the procedure (15). Although several case reports document or attribute cases of fetal injury to the amniocentesis needle (20-22), the attribution has been generally by association only and not on the basis of direct evidence $(21,23-31)$.

\section{Alloimmunization}

Feto-maternal hemorrhage occurs in approximately $50 \%$ of all women (32) and during amniocentesis on one out of six (33). The attributed risk is $1 \%$ greater than the background risk of $1.5 \%$ (34). As for preventing the Rhesus sensitization of the mother and when the husband is Rhesus positive, every

Table 3 Pregnancy Loss After Amniocentesis

\begin{tabular}{|c|c|c|c|}
\hline Type of pregnancy loss & Combined total $(\mathbf{n} / \mathrm{N})$ & Combined proportion $\%$ & $95 \% \mathrm{CI}$ \\
\hline Less than 20 weeks of pregnancy & $22 / 2133$ & 1.1 & $0.7-1.5$ \\
\hline More than 20 weeks of pregnancy & $17 / 1775$ & 0.9 & $0.5-1.4$ \\
\hline Less than 24 weeks of pregnancy & $122 / 14,057$ & 0.9 & $0.6-1.3$ \\
\hline Less than 28 weeks of pregnancy & $283 / 14,915$ & 1.7 & $1.3-2.2$ \\
\hline More than 28 weeks of pregnancy & $134 / 14,596$ & 0.9 & $0.7 .7-1.2$ \\
\hline Less than 14 days after AC & $102 / 17,047$ & 0.6 & $0.5-0.7$ \\
\hline Less than 60 days after $\mathrm{AC}$ & $66 / 9406$ & 0.7 & $0.5-0.9$ \\
\hline Total pregnancy loss & $627 / 49,413$ & 1.9 & $1.4-2.5$ \\
\hline Multiple insertions & $226 / 12,142$ & 2.0 & $0.9-3.6$ \\
\hline Bloody or discolored tap & $468 / 10,908$ & 4.1 & $1.1-8.7$ \\
\hline
\end{tabular}


Rhesus-negative mother must be given $300-\mu \mathrm{g}$ anti-D immunoglobulin after amniocentesis, provided of course the indirect Coombs is negative $(35,36)$.

Other means to minimize the risk is the use of small-gauge needles and to avoid transplacental approach (36).

\section{Respiratory Distress}

A significant although small increased risk of neonatal respiratory distress was noted in some studies but not in others $(9,37)$. This complication has been attributed to chronic oligohydramnios, which might follow amniocentesis.

\section{Orthopedic Abnormalities}

The British collaboration study (13) implied that amniocentesis is associated with talipes equinovarus, congenital dislocation, and subluxation of the hip. This trend was not confirmed by other studies. This complication has been attributed to chronic oligohydramnios, which might follow amniocentesis.

\section{Transmission of Viral Diseases}

Before antiretroviral therapy (ART) use, maternal HIV infection was considered a relative contraindication for amniocentesis and invasive prenatal procedures. Since maternal ART has been used, amniotic fluid viral load is usually undetectable even if the virus was detectable in maternal blood samples. This finding raises the possibility to perform amniocentesis, when it is indicated, to provide the mother with an adequate antiretroviral treatment (38).

\section{Leakage of Amniotic Fluid and Vaginal Bleeding}

Uterine contractions and vaginal spotting are quite common (2-3\%) after amniocentesis; however, they are almost always transient and self-limited. Leakage of amniotic fluid occurs in $1 \%$ of women who underwent second-trimester amniocentesis. It is usually small amount and the outcome is normal in $90 \%$ of the patients who experienced fluid leakage (39).

\section{SPECIAL CIRCUMSTANCES}

\section{Discolored Amniotic Fluid}

The presence of blood in the amniotic fluid occurs in a very small number of cases. It is usually of maternal origin and under no circumstances does it affect the culture or multiplication of the cells. However, the presence of blood in the amniotic fluid is related to the increased possibility that the outcome of the pregnancy will not be good. Thus, the percentage of fetal losses in these cases reaches $33 \%$ of all losses (40-42). If at the same time the blood-stained or discolored amniotic fluid is accompanied by increased levels of alphafetoprotein (AFP), then in almost all instances the prenatal results will not be good.

\section{Amniocentesis in the Presence of Bleeding}

The fetal loss following amniocentesis in women who had bleeding before the procedure is probably increased. Antsaklis et al. found an excess loss rate of $0.6 \%$ in women who had experienced bleeding before amniocentesis (43).

History of bleeding is not a contraindication for the procedure but patients should be counseled about the risks.

\section{AMNIOCENTESIS IN MULTIPLE GESTATIONS}

Amniocentesis has been recognized as a relatively safe procedure for prenatal diagnosis in singletons. It has been a common practice to extrapolate data derived from singletons to multiples. However, implementation of invasive prenatal diagnostic modalities in twins or higher order pregnancies seems to be more complex. Amniocentesis can be safely performed on twins or higher multiple gestations as long as the sacs have been defined through ultrasound examination. The management and the prenatal diagnosis of a multiple pregnancy are challenging, not only related to the number of fetuses, but also strongly influenced by chorionicity. Chorionicity refers to the placentation whereas zygosity implies the genetic profile of the pregnancy and therefore determines the degree of risk and whether or not the fetuses may be concordant or discordant for chromosomal abnormalities.

The amniocentesis technique in multiple gestations is different from that used in a single gestation and therefore women should be referred to experienced centers. The number of fetuses, their positions, the number of gestation sacs and placentas, the gender, the age and the degree of development of each fetus, and finally the fetal anatomy are all-important and necessary elements of the ultrasound examination that precedes amniocentesis. An important element before amniocentesis is determining the position of each fetus, which is achieved by mapping the site of the placenta that is feeding the specific fetus, because the position of the placenta changes very little over time while the position of the fetus never remains stable. Consequently, in the case where after amniocentesis the problem of selective feticide arises due to an abnormal result, we must know which is the specific fetus in question and its reference point so that we can pinpoint it. This is nothing more than determining the position of the placenta, as determined by the mapping that was carried out before amniocentesis.

\section{The Risk of Aneuploidy in Multiple Gestations}

As far as zygosity represents the genetic makeup of the developing entity, accurate determination of this parameter is considered a prerequisite in multiple pregnancy prenatal screening for aneuploidies. In the clinical setting, zygosity is usually inferred from the ultrasound diagnosis of chorionicity (44), the latter best achieved in the first trimester. In diamniotic (DA) pregnancies with fused placentas, measurements of the thickness of the dividing membrane using a cutoff value of $2 \mathrm{~mm}$ can differentiate MC from dichorionic (DC) twinning, though a high inter- and intra-observer variability has been reported. Sonographic detection of the "lambda" or "twin peak" sign is reported as a more reliable indicator of DC placentation with an accuracy of $100 \%$ in 10 to 14 weeks of gestation (45). Delayed sonographic evaluation in the second trimester is associated with a $10 \%$ to $12 \%$ chorionicity misinterpretation rate $(46,47)$, while after 20 weeks of gestation, the determination may turn out impossible. Therefore, in the absence of the "lambda" or "twin peak" sign in a DA twin pregnancy, single placentation and monozygosity is concluded, when the rare cases of MC-DZ (dizygotic) gestations following ART reported are not taken into consideration. However, when a single amniotic sac is 
detected, monochorionicity is indisputable. Given that the great proportion $(80-90 \%)$ of DC twins are DZ $(48,49)$, chorionicity may roughly correspond to zygosity (50).

Monozygotic (MZ) twins are always of the same sex and genetically identical and therefore the risk for chromosomal abnormalities does not differ from that in singletons. Very infrequently, mutations can cause genetic discordance between MZ siblings, involving mosaicism, skewed Xinactivation, differential gene imprinting, and small-scale mutation (51). Heterokaryotypia is used to define the rare karyotypic discordance, most commonly expressed by one fetus affected by Turner syndrome whereas the other presents either a normal male or normal female karyotype (52-54). MZ discordance for trisomy 21, Klinefelter syndrome, Patau syndrome, trisomy 21, and 22q11 deletion syndrome has also been described (54-58).

However, these unusual discrepancies are not taken into consideration when calculating aneuploid risk, though should always be assumed when invasive prenatal diagnosis is performed dictating sampling from both sacs.

In DZ twins, each embryo has an independent risk for aneuploidy and therefore the risk that at least one fetus being affected will be almost twice the maternal age risk for a singleton. The probability of both fetuses being involved is minimal (59). In cases with uncertain chorionicity and thus zygosity, aneuploidy risk assessment requires an estimation of the most likely zygosity, which may vary according to maternal age and race. In general, given that one-third of all twin pairs are $M Z$, the risk for one twin being aneuploid in case of unknown zygosity is calculated to five-thirds that of the singleton risk $(46,50)$.

Based on these estimations, a 33-year-old woman bearing twins has a risk for at least one aneuploid offspring, comparable with the risk of a 35-year-old woman bearing a singleton. On this assumption, such women should be offered prenatal testing (60). However, despite these aspects, reported series show a lower risk for fetal chromosomal abnormalities in live-born twins and this fact is probably due to the higher incidence of spontaneous loss of fetuses with aneuploidy.

\section{Technique}

Three methods of tapping multiple sacs have been described so far. The first and most common one, initially described by Elias et al. in 1980, involves two or more needle insertions, one for each sac, also called the technique of double amniocentesis (61-63) (Fig. 2). In a twin or higher order multiple pregnancy, two or more 22-gauge 3.5-inch spinal needles are separately and sequentially inserted transabdominally under ultrasound visualization into each sac and about $20 \mathrm{~mL}$ of amniotic fluid is readily aspirated and sent for cytogenetic evaluation or fetal karyotyping. A problem not infrequently faced with this technique is erroneously sampling the same amniotic sac twice. In order to eliminate this possibility, the sampled sac is marked with a blue dye ensuring that the sac is tapped only once. For this purpose, indigo carmine has been successfully used without any adverse effects (64), though a mild vasoconstrictive effect following intravenous injection has been described. However, the instillation of a foreign substance into the amniotic cavity is of concern. A technical disadvantage with the instillation of indigo carmine is that the dye tends to concentrate at the bottom of the sac taking some time before the stained fluid surrounds the fetus. Methylene blue used as a marker dye in the past has been linked to certain toxic manifestations such as fetal hemolysis, fetal small bowel atresias, and fetal death (65-70). Nevertheless, the highresolution ultrasound equipment currently available, in expert hands, may ensure accurate sampling from each sac $(71,72)$, reserving the installation of dye for cases of amniotic volume discordance where detection of the septum is uncertain or high-order pregnancies where documentation and "labeling" of sacs turn out insecure (73).

An alternative approach first described by Jeanty et al. in 1990 is the single-needle insertion technique (74). The needle entry is made into the proximal sac near the insertion of the dividing membrane and $20 \mathrm{~mL}$ of amniotic fluid is retrieved. After the stylet is replaced, the needle is advanced through the second sac under direct ultrasound guidance. In order to avoid contamination, the first few milliliters of amniotic fluid are discarded and aspiration of $20 \mathrm{~mL}$ from the second sac integrates the procedure. Many advantages linked to this technique have been reported: requiring only one needle insertion and being swifter and shorter reduces woman's discomfort as well as the risk of post-procedural complications. Moreover, advancing the needle through the septum between the two sacs under ultrasound guidance provides positive proof of tapping both of them, diminishing the need for dye insertion. However, potential disadvantages render this approach less popular. Possible contamination of the second sample with amniotic fluid and fetal cells from the first one may lead to an incorrect diagnosis of mosaicism in the second fetus. This complication can be avoided by strictly adhering to the technique, by replacing the stylet prior to intertwin membrane penetration and by discarding the first few milliliters from the second sac. Besides, the possibility of converting DA to pseudo-MA twin pregnancy with the correspondence risks for cord entanglement and the formation of the amniotic band syndrome cannot be precluded (75). In addition, a technical difficulty in penetrating a "tenting" dividing membrane has been reported.

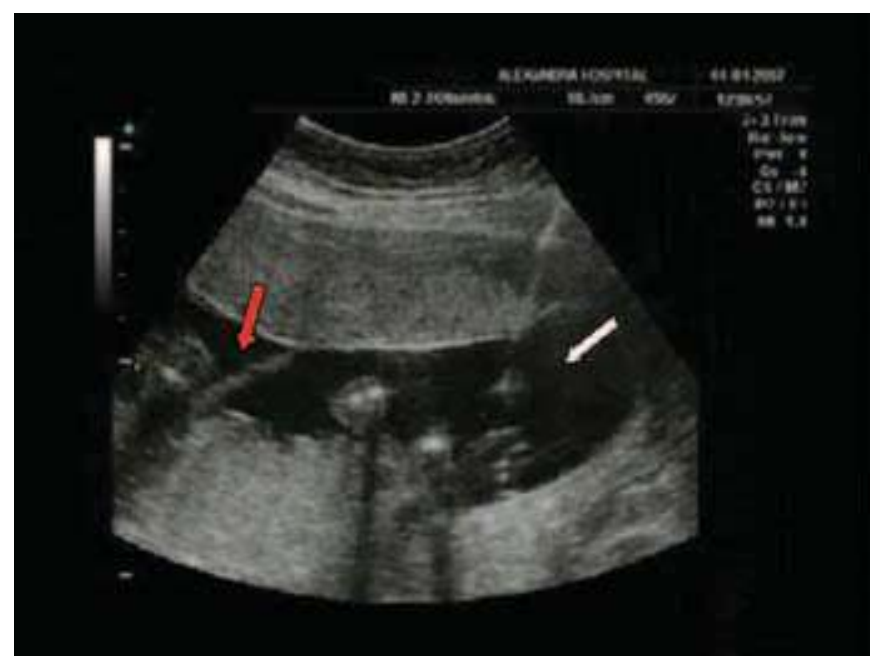

Figure 2 Amniocentesis in twin gestation, which is performed on each gestation sac separately and sequentially (white arrow pointing tip of the needle in one sac and red arrow pointing septum). 
Another approach called double simultaneous amniocentesis was introduced by Bahado-Singh et al. in 1992 (76). This technique involves two needles inserted separately into the amniotic sacs under ultrasound visualization like in the technique of double amniocentesis. The difference is that after aspiration of the amniotic fluid from the first sac, the needle is left in place indicating the sampled cavity and the second insertion is made into the other sac. The main advantage seems to be the documentation of correct sampling from each sac. However, it is not widely used mainly because it is more time consuming and thereby the experience with this approach is limited.

Amniocentesis, like all prenatal diagnostic invasive procedures, must be preceded by a detailed ultrasound evaluation of the multiple pregnancy involving chorionicity and amnionicity determination and documentation of the location of the placenta(s). Moreover, relative position, size, and anatomy of each fetus should be specified and "labeling" of the multiples using text and diagram should be performed to ensure correct sampling from each of them. Recently, the role of amniotic fluid alpha-fetoprotein values was evaluated in confirmation of both sacs in a dichorionic pregnancy being sampled (77).

Concern regarding potential post-amniocentesis increase in fetal loss rate in multiples has led to a plethora of studies evaluating this parameter.

The percentage of total fetal loss, as expressed by spontaneous abortions, stillbirths, and neonatal deaths, is approximately $10.8 \%$, a percentage that is almost the same as the statistical miscarriage percentage that is observed in every twin gestation after the 17th week of gestation $(42,78)$.

Early reports suggested a higher fetal loss rate in twin pregnancies than in those with singletons (79-81). However, these studies did not take into account the possibility that the increased fetal wastage might be attributed to the twin pregnancy itself rather than the invasive procedure. Later on, it was reported that the maternal history of twins per se carries a pregnancy loss rate up to 24 weeks of about $6.3 \%$ and severe prematurity (24-28 weeks) rate of about $8 \%$ (82). Most series of pregnancy outcome following secondtrimester amniocentesis report loss rates before 20 weeks of gestation of between $1 \%$ and $2.5 \%$ and a much higher loss rate before 28 weeks. In a multicenter European study, the pregnancy loss rate was estimated to be $2.3 \%$ and $3.7 \%$ before 20 and 28 weeks of gestation respectively (83). In a casecontrol study, a similar fetal loss rate was reported between sampled twins and unsampled matched twin controls (3.5\% vs. $3.2 \%)(84)$.

According to other studies, the percentage of fetal losses up to the 28th week of gestation in twin gestations after amniocentesis ranges from $3.57 \%$ to $3.78 \%$ versus $0.6 \%$ for single gestations. This means that the comparatively increased fetal losses observed in twin gestations versus singletons after amniocentesis are not due to amniocentesis, but rather due to the normally increased statistical percentage for abortions seen in twin gestations.

In a recent review by Weisz and Rodeck (85), the pooled data regarding the pregnancy loss rate after amniocentesis are comparable with these background loss rates for twin pregnancies (Table 4).

Finally, amniocentesis post multifetal pregnancy reduction (MFPR) does not seem to significantly increase the rate of fetal loss. However, one group has shown that amniocentesis post MFPR was significantly associated with increased risk of preterm delivery at 25 to 28 weeks (86).

\section{Early Amniocentesis}

Early amniocentesis was thought as an alternative option for early prenatal diagnosis in centers in which CVS was not offered. It was suggested to be performed between the 12th and 15th gestational weeks. The technique used for

Table 4 Fetal Loss Rate in Twin Pregnancies Following Amniocentesis

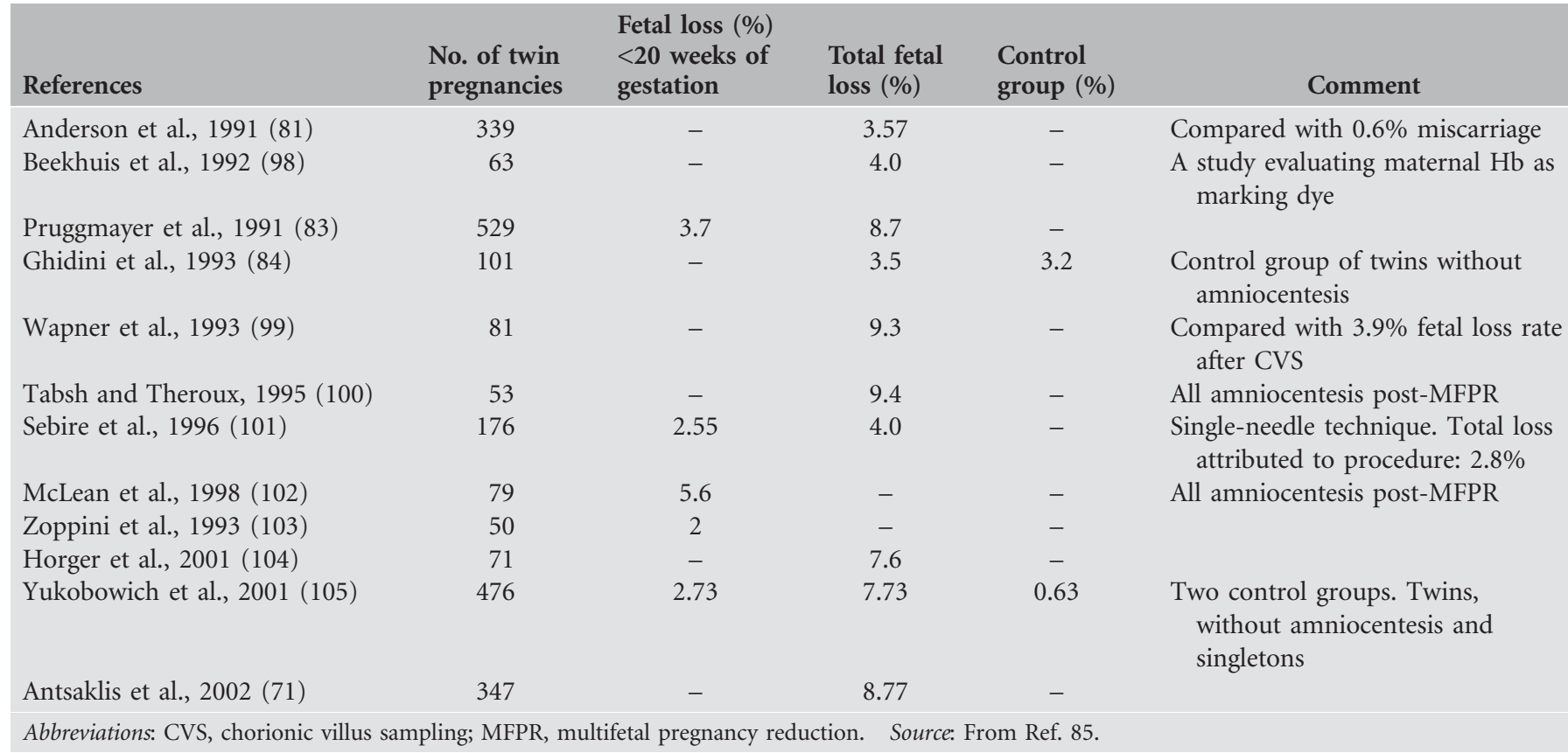


performing early amniocentesis is similar to that used in classical amniocentesis and the amount of amniotic fluid removed is equal in milliliters to the weeks of gestation (87). Failure or difficulty in taking out the sample was due to tenting of the membrane caused by the needle (88). Since amniocentesis was performed quite early, it was thought that it could give the opportunity to examine the karyotype of the fetus and determine the AFP and acetylcholinesterase for the early detection of those cases that show increased risk for nervous system abnormalities (89-91) with the same safety as midtrimester amniocentesis. These expectations were confirmed by observational studies although definite conclusions could not be drawn because of the small numbers (92).

The safety of early amniocentesis was not completely clear until the Canadian trial. In two randomized studies $(93,94)$, fetal loss rate was significantly higher for early amniocentesis when compared with transabdominal CVS (TA-CVS). In another randomized trial (95), which compared the firsttrimester and midtrimester amniocentesis, fetal losses were more frequently observed after first-trimester than after midtrimester amniocentesis. Additionally, early amniocentesis had a significantly higher rate of amniotic fluid leakage than TA-CVS and midtrimester amniocentesis.

Excessive risk for talipes equinovarus has been uniformly observed in all early amniocentesis studies, with an even greater likelihood when physician confirms amniotic fluid leakage $(17.7 \%$ vs. $0.5 \%)$ (93). Talipes can occur without apparent leakage of amniotic fluid, which likely results from a reduced amniotic fluid volume via transfer of amniotic fluid to the extra-embryonic coelomic space. TA-CVS did not have any effect on malformation rate. The Canadian study therefore indicated that early amniocentesis should be performed only if there is no alternative.

In 2005, Alfirevic et al. conducted a systematic review (96) for the Cochrane collaboration, and the pooled total pregnancy loss after early amniocentesis was $7.6 \%$ compared with $5.9 \%$ after midtrimester amniocentesis. Similarly, the number of talipes equinovarus was higher in cases of early amniocentesis $(1.3 \%$ vs. $0.09 \%)$. In the same review, combined total loss of pregnancy after early amniocentesis was $6.3 \%$ compared with $5 \%$ after CVS.

It is therefore justified to say that early amniocentesis should be performed only in extenuating circumstances, especially since TA-CVS has undoubtedly been proved a safe and more effective alternative (97).

\section{REFERENCES}

1. Von Schatz F. Gine besondere art von einseitiger polyhydramnie mit anderseitiger oligohydramnie bie eineiigen zwillingen. Arch Gynaecol 1882; 19: 329.

2. Beris DCA. Composition of liquor amnii in haemolytic disease of newborn. Lancet 1950; 2: 443.

3. Fuchs F, Riis P. Antenatal sex determination. Nature 1956; 177: 330.

4. Steele MW, Breg WR. Chromosome analysis of human amniotic fluid cells. Lancet 1966; 1: 383.

5. Dodson MG, Forrtunat SJ. Microorganisms and premature labor. J Reprod Med 1988; 33: 87-96.

6. Emery AEH. Antenatal diagnosis of genetic disease. Mod Trends Hum Genet 1970; 1: 267.

7. Jeanty P, Rodesch F, Romero R, et al. How to improve your amniocentesis technique. Am J Obstet Gynecol 1983; 146: 593.
8. Romero R, Jeanty P, Reece EA, et al. Sonographically monitored amniocentesis to decrease intraoperative complication. Obstet Gynecol 1985; 65: 426.

9. NICHD National Registry for amniocentesis study group. Mid trimester amniocentesis for prenatal diagnosis: safety and accuracy. J Am Med Assoc 1976; 236: 1471.

10. Cassell GH, Davis RO, Waiter KB, et al. Isolation of mycoplasma hominis and ureaplasma urealyticum from amniotic fluid at 16-20 weeks of gestation: potential effect on outcome of pregnancy. Sex Transm Dis 1983; 10: 294.

11. Gray DJ, Robinson H, Malone J, et al. Adverse outcome in pregnancy following amniotic fluid isolation of ureaplasma urealyticum. Prenat Diagn 1992; 12: 111-17.

12. Simpson NE, Dallaire L, Miller JR, et al. Prenatal diagnosis of genetic disease in Canada: report of a collaborative study. CMAJ 1976; 115: 739-48.

13. Medical Research Council. An assessment of the hazards of amniocentesis. Br J Obstet Gynecol 1978; 85(Suppl 2): 1-41.

14. Tabor A, Philip J, Madsen M, et al. Randomised controlled trial of genetic amniocentesis in 4606 low-risk women. Lancet 1986; 1: 1287-93.

15. Seeds JW. Diagnostic mid trimester amniocentesis: how safe? Am J Obstet Gynecol 2004; 191: 608-16.

16. FASTER Trial Research Consortium. Pregnancy loss rates after midtrimester amniocentesis. Obstet Gynecol 2006; 108: 1067-72.

17. Mujezinovic F, Alfirevic Z. Procedure-related complications of amniocentesis and chorionic villous sampling a systematic review. Obstet Gynecol 2007; 110: 687-94.

18. Salvador E, Bienstock J, Blakemore KJ, Pressman E. Leiomyomata uteri, genetic amniocentesis, and the risk of second-trimester spontaneous abortion. Am J Obstet Gynecol 2002; 186: 913-15.

19. Medda E, Donati S, Spinelli A, Di Renzo GC. EUROPOP Group Genetic amniocentesis: a risk factor for preterm delivery? Eu J Obstet Gynecol Reprod Biol 2003; 110: 153-8.

20. Williamson RA, Varner MW, Grant SS. Reduction in amniocentesis risks using a real-time needle guide procedure. Obstet Gynecol 1985; 65: 751-5.

21. Therkelsen AJ, Rehder H. Intestinal atresia caused by second trimester amniocentesis. BJOG 1981; 88: 559-62.

22. Admoni MM, BenEzra D. Ocular trauma following amniocentesis as the cause of leukocoria. J Pediatr Ophthalmol Strab 1988; 25: 196-7.

23. Youroukos S, Papadelis F, Matsaniotis N. Porencephalic cysts after amniocentesis. Arch Dis Child 1980; 55: 814-15.

24. Stock RJ. Fetal death secondary to needle laceration during 2 nd trimester amniocentesis: a case report. Prenat Diagn 1982; 2: 133-7.

25. Bruce S, Duffy JO, Wolf JE. Skin dimpling associated with midtrimester amniocentesis. Pediatr Dermatol 1984; 2: 140-2.

26. Raimer SS, Raimer BG. Needle puncture scars from midtrimester amniocentesis. Arch Dermatol 1984; 120: 1360-2.

27. Finegan JK, Quarrington BJ, Hughes HE, et al. Infant outcome following midtrimester amniocentesis: development and physical status at age six months. BJOG 1985; 92: 1015-23.

28. BenEzra D, Sela M, Peer J. Bilateral anophthalmia and unilateral microphthalmia in two siblings. Ophthalmologica 1989; 198: 140-4.

29. Chong SKF, Levitt GA, Lawson J, Lloyd U, Newman CGH. Subarachnoid cyst with hydrocephalus-a complication of midtrimester amniocentesis. Prenatal Diagn 1989; 9: 677-9.

30. Eller KM, Kuller JA. Porencephaly secondary to fetal trauma during amniocentesis. Obstet Gynecol 1995; 85: 865-7.

31. Raymond GV. Rare neurologic injury from amniocentesis. Birth Defects Res Clin Mol Teratol 2003; 67: 205-6.

32. Clayton EM, Feldhaus WD, Whitacre FE. Fetal erythrocytes in maternal circulation of pregnant women. Obstet Gynecol 1964; 23: 915-19.

33. Tabor A, Bang J, Norgaard-Pedersem B. Feto-maternal Haemorrhage associated with genetic amniocentesis: results of a randomized trial. $\mathrm{Br}$ J Obstet Gynecol 1987; 94: 528-35.

34. Murray JC, Karp LE, Williamson RA, Cheng EY, Luth DA. Rh isoimmunization related to amniocentesis. Am J Med Genet 1983; 16: 527-34.

35. American College of Obstetricians and Gynecologists: Prevention of D Isoimmunization. ACOG Tech. Bull No 147, 1990. 
36. WHO. Prevention of rhesus sensitization. Who Technical Report Series 1971; No 468.

37. Hunter AG. Neonatal lung function following mid-trimester amniocentesis. Prenat Diagn 1987; 7: 433-41.

38. Maiques V, Garcia-Tejedor A, Perales A, Cordoba J, Esteban RJ. HIV detection in amniotic fluid samples. Amniocentesis can be performed in HIV pregnant women? Eur J Obstet Gynecol Reprod Biol 2003; 108: $137-41$.

39. Borgida AF, Mills AA, Feldman DM, Rodis JF, Ergan JF. Outcome of pregnancies complicated with rupture of membranes after genetic amniocentesis. Am J Obstet Gynecol 2000; 183: 937-9.

40. Milunsky A. The of neural tube and other congenital defects. In: Milunsky A, ed. Genetic Disorders and the Fetus. New York: Plenum Press, 1986: 453.

41. Karpa E, Schiller HS. Meconium staining of amniotic fluid of mid trimester amniocentesis. Obstet Gynecol 1977; 50: 475.

42. Imaizumi Y, Asaka A, Inouye E. Analysis of multiple birth rates in Japan. Rates of spontaneous and induced termination of pregnancy in twins. Jpn J Hum Genet 1982; 27: 235.

43. Antsaklis A, Papantoniou N, Xygakis A, et al. Genetic amniocentesis in women $20 \pm 34$ years old: associated risks. Prenat Diagn 2000; 20: $247-50$.

44. Kohl SG, Casey G. Twin gestation. Mt Sinai J Med 1975; 42: 523-3.

45. Sepulveda W, Sebire NJ, Hughes K, Kalogeropoulos A, Nicolaides KH. Evolution of the lambda or twin-chorionic peak sign in dichorionic twin pregnancies. Obstet Gynecol 1997; 89: 439-41.

46. Jenkins TM, Wapner RJ. The challenge of prenatal diagnosis in twin pregnancies. Curr Opin Obstet Gynecol 2000; 12: 87-92.

47. Wood SL, St Onge R, Connors G, Elliot PD. Evaluation of the twin peak or lambda sign in determining chorionicity in multiple pregnancy. Obstet Gynecol 1996; 88: 6-9.

48. Rodis JF, Egan JF, Craffey A, et al. Calculated risk of chromosomal abnormalities in twin gestations. Obstet Gynecol 1990; 76: 1037-41.

49. Fisk NM, Bennett PR. Prenatal determination of chorionicity and zygosity. In: Ward RH, Whittle W, eds. Multiple Pregnancy. London: RCOG Press, 1995: 56-66.

50. Matias A, Montenegro N, Blickstein I. Down syndrome screening in multiple pregnancies. Obstet Gynecol Clin North Am 2005; 32 81-96, ix.

51. Machin GA. Some causes of genotypic and phenotypic discordance in monozygotic twin pairs. Am J Med Genet 1996; 61: 216-28.

52. Rogers JG, Voullaire L, Gold H. Monozygotic twins discordant for trisomy 21. Am J Med Genet 1982; 11: 143-6.

53. Dallapiccola B, Stomeo C, Ferranti G, Di Lecce A, Purpura M. Discordant sex in one of three monozygotic triplets. J Med Genet 1985; 22: 6-11.

54. Perlman EJ, Stetten G, Tuck-Müller CM, et al. Sexual discordance in monozygotic twins. Am J Med Genet 1990; 37: 551-7.

55. Schmid O, Trautmann U, Ashour $\mathrm{H}$, et al. Prenatal diagnosis of heterokaryotypic mosaic twins discordant for fetal sex. Prenat Diagn 2000; 20: 999-1003.

56. Wachtel SS, Somkuti SG, Schinfeld JS. Monozygotic twins of opposite sex. Cytogenet Cell Genet 2000; 91: 293-5.

57. Lespinasse J, Gicquel C, Robert M, Le Bouc Y. Phenotypic and genotypic variability in monozygotic triplets with Turner syndrome. Clin Genet 1998; 54: 56-9.

58. Nieuwint A, Van Zalen-Sprock R, Hummel P, et al. Identical' twins with discordant karyotypes. Prenat Diagn 1999; 19: 72-6.

59. Rodis JF, Egan JF, Craffey A, et al. Calculated risk of chromosomal abnormalities in twin gestations. Obstet Gynecol 1990; 76: 1037-41.

60. Weinblatt V, Wapner RJ. Chorionic villus sampling and amniocentesis in multiple pregnancy. In: Creasy RK, Resnik R, eds. Maternal-Fetal Medicine Principles and Practice, 4th edn. Philadelphia: WB Saunders, 1999: 201-11.

61. Elias S, Gerbie AB, Simpson JL, et al. Genetic amniocentesis in twin gestations. Am J Obstet Gynecol 1980; 138: 169

62. Bahado-Singh R, Schmitt R, Hobbins JC. New technique for genetic amniocentesis in twins. Obstet Gynecol 1992; 79: 304

63. Fribourg S. Safety of intra-amniotic injection of indigo carmine. Am J Obstet Gynecol 1981; 140: 350.

64. Cragan JD, Martin ML, Khoury MJ, Fernhoff PM. Dye use during amniocentesis and birth defects. Lancet 1993; 341: 1352.
65. Nicolini U, Monni G. Intestinal obstruction in babies exposed in utero to methylene blue. Lancet 1990; 336: 1258-9.

66. Kidd SA, Lancaster PA, Anderson JC, et al. Fetal death after exposure to methylene blue dye during mid-trimester amniocentesis in twin pregnancy. Prenat Diagn 1996; 16: 39-47.

67. McEnerney JK, McEnerney LN. Unfavorable neonatal outcome after intraamniotic injection of methylene blue. Obstet Gynecol 1983; 61 (3 Suppl): 35S-7S.

68. McFadyen I. The dangers of intra-amniotic methylene blue. Br J Obstet Gynaecol 1992; 99: 89-90.

69. van der Pol JG, Wolf $\mathrm{H}$, Boer $\mathrm{K}$, et al. Jejunal atresia related to the use of methylene blue in genetic amniocentesis in twins. Br J Obstet Gynaecol 1992; 99: 141-3.

70. Vincer MJ, Allen AC, Evans JR, Nwaesei C, Stinson DA. Methyleneblue-induced hemolytic anemia in a neonate. CMAJ 1987; 136: $503-435,40$

71. Antsaklis A, Souka AP, Daskalakis G, Kavalakis Y, Michalas S. Secondtrimester amniocentesis vs. chorionic villus sampling for prenatal diagnosis in multiple gestations. Ultrasound Obstet Gynecol 2002; 20: 476-81.

72. Taylor MJ, Fisk NM. Prenatal diagnosis in multiple pregnancy. Baillieres Best Pract Res Clin Obstet Gynaecol 2000; 14: 663-75.

73. Weisz $\mathrm{B}$, Rodeck $\mathrm{CH}$. Invasive diagnostic procedures in twin pregnancies. Prenat Diagn 2005; 25: 751-8.

74. Jeanty P, Shah D, Roussis P. Single-needle insertion in twin amniocentesis. J Ultrasound Med 1990; 9: 511-17.

75. Megory E, Weiner E, Shalev E, Ohel G. Pseudomonoamniotic twins with cord entanglement following genetic funipuncture. Obstet Gynecol 1991; 78(5 Pt 2): 915-17.

76. Bahado-Singh R, Schmitt R, Hobbins JC. New technique for genetic amniocentesis in twins. Obstet Gynecol 1992; 79: 304-7.

77. Delisle MF, Brosseuk L, Wilson RD. Amniocentesis for twin pregnancies: is alpha-fetoprotein useful in confirming that the two sacs were sampled? Fetal Diagn Ther 2007; 22: 221-5.

78. Ager RP, Oliver RWA. The risk of mid trimester amniocentesis. Lancashire, UK: University of Salford. Monograph, 1986

79. Palle C, Andersen JW, Tabor A, et al. Increased risk of abortion after genetic amniocentesis in twin pregnancies. Prenat Diagn 1983; 3: 83-9.

80. Pijpers L, Jahoda MG, Vosters RP, Niermeijer MF, Sachs ES. Genetic amniocentesis in twin pregnancies. Br J Obstet Gynaecol 1988; 95: 323-6.

81. Anderson RL, Goldberg JD, Golbus MS. Prenatal diagnosis in multiple gestation: 20 years experience with amniocentesis. Prenat Diagn 1991; 11: 263-70.

82. Yaron Y, Bryant-Greenwood PK, Dave N, et al. Multifetal pregnancy reductions of triplets to twins: comparison with nonreduced triplets and twins. Am J Obstet Gynecol 1999; 180: 1268-71.

83. Pruggmayer MR, Jahoda MG, Van der Pol JG, et al. Genetic amniocentesis in twin pregnancies: results of a multicenter study of 529 cases. Ultrasound Obstet Gynecol 1992; 2: 6-10.

84. Ghidini A, Lynch L, Hicks C, Alvarez M, Lockwood CJ. The risk of second-trimester amniocentesis in twin gestations: a case-control study. Am J Obstet Gynecol 1993; 169: 1013-16.

85. Weisz B, Rodeck $\mathrm{CH}$. Invasive diagnostic procedures in twin pregnancies (review). Prenat Diagn 2005; 25: 751-8.

86. Antsaklis A, Daskalakis G, Papantoniou N, Mesogitis S, Michalas S. Genetic amniocentesis in multifetal pregnancies reduced to twins compared with nonreduced twin gestations. Fertil Steril 2000; 74: $1051-2$.

87. Simpson JL, Elias S. Essentials of Prenatal Diagnosis Churchill Livingstone, 1993: 39 .

88. Henry GP, Miller WA. Early amniocentesis. J Reprod Med 1992; 37: 396.

89. Elejalde BR, de Elejlde MM, Acuna JM, et al. Prospective study of amniocentesis performed between weeks 9 and 16 of gestation: 1st feasibility, risk, complications and use in early genetic prenatal diagnosis. Am J Med Genet 1990; 35: 188-96

90. Penso CA, Sandstrom MM, Garber MF, et al. Early amniocentesis: report of 407 cases with neonatal follow-up. Obstet Gynecol 1990; 76: 1032.

91. Jorgensen FS, Bang I, Lind A, et al. Genetic amniocentesis at 7-14 weeks of gestation. Prenat Diagn 1992; 12: 277. 
92. Elias S, Simpson JL. Amniocentesis and fetal blood sampling. In: Milunsky A, ed. Genetic disorders and the fetus, 5th edn. Baltimore and London: The Johns Hopkins University Press, 2004: 66-99.

93. Philip J, Silver RK, Wilson RD, et al. Late first-trimester invasive prenatal diagnosis: results of an international randomized trial. Obstet Gynecol 2004; 103: 1164-73.

94. Nicolaides K, Brizot M de L, Patel F, Snijders R. Comparison of chorionic villus sampling and amniocentesis for fetal karyotyping at 10-13 weeks' gestation. Lancet 1994; 344: 435-9.

95. The Canadian Early and Mid-trimester Amniocentesis Trial (CEMAT) Group. Randomized trial to assess safety and fetal outcome of early and midtrimester amniocentesis. Lancet 1998; 351: $242-7$

96. Alfirevic Z, Sundberg K, Brigham S. Amniocentesis and chorionic villus sampling for prenatal diagnosis (review) the Cochrane Collaboration, 2005.

97. Brambati B, Tului L. Chorionic villus sampling and amniocentesis. Curr Opin Obstet Gynecol 2005; 17: 197-201.

98. Beekhuis JR, De Bruijn HW, van Lith JM, Mantigh A. Second trimester amniocentesis in twin pregnancies: maternal haemoglobin as a dye marker to differentiate diamniotic twins. Br J Obstet Gynaecol 1992; 99: 126-7.
99. Wapner RJ, Johnson A, Davis G, et al. Prenatal diagnosis in twin gestations: a comparison between second-trimester amniocentesis and first-trimester chorionic villus sampling. Obstet Gynecol 1993; 82: 49-56.

100. Tabsh KM, Theroux NL. Genetic amniocentesis following multifetal pregnancy reduction to twins: assessing the risk. Prenat Diagn 1995; 15: 221-3.

101. Sebire NJ, Noble PL, Odibo A, Malligiannis P, Nicolaides KH. Single uterine entry for genetic amniocentesis in twin pregnancies. Ultrasound Obstet Gynecol 1996; 7: 26-31.

102. McLean LK, Evans MI, Carpenter, RJ Jr, Johnson MP, Goldberg JD. Genetic amniocentesis following multifetal pregnancy reduction does not increase the risk of pregnancy loss. Prenat Diagn 1998; 18: 186-8.

103. Zoppini C, Ludomirsky A, Godmilow L, et al. Acute hemodynamic effects induced by chorionic villus sampling: a preliminary investigation. Am J Obstet Gynecol 1993; 169: 902-7.

104. Horger EO III, Finch H, Vincent VA. A single physician's experience with four thousand six hundred genetic amniocenteses. Am J Obstet Gynecol 2001; 185: 279-8.

105. Yukobowich E, Anteby EY, Cohen SM, Lavy Y, Granat M, Yagel S. Risk of fetal loss in twin pregnancies undergoing second trimester amniocentesis. Obstet Gynecol 2001; 98: 231-4. 


\section{Fetal echocardiography \\ Caroline K. Lee, Erik C. Michelfelder, and Gautam K. Singh}

\section{INTRODUCTION}

Two-dimensional (2D) ultrasound imaging of the fetal heart has been steadily evolving since its original descriptions in the early 1980s $(1,2)$. Using current ultrasound platforms, it is now possible to image the fetal heart with a high degree of spatial and temporal resolution, which allows clinicians to make precise anatomic and functional diagnoses in the fetus with acquired or congenital heart disease (CHD). Use of color flow and pulsed-wave Doppler techniques in fetal cardiac imaging has further augmented the diagnostic capabilities of fetal echocardiography, to the point where diagnostic accuracy in many regards rivals that of trans-thoracic echocardiography. Understanding the capabilities and limitations of fetal cardiac assessment is particularly important in the current era, where the possibility of fetal therapy is now possible. It is also important in the current era to understand the broadening indications for fetal echocardiography, particularly as it pertains to fetal therapy.

It is the purpose of this chapter to review the current status of fetal echocardiography with regard to (i) indications for and timing of fetal cardiac evaluation, (ii) standard imaging views and anatomic assessment, (iii) methods of assessing fetal cardiac function and rhythm, (iv) counseling the patient with fetal heart disease, and (v) highlight some of the newer advances and innovation in fetal echocardiography and fetal cardiology.

\section{INDICATIONS FOR FETAL ECHOCARDIOGRAPHY}

Cardiac defects in fetuses are one of the most frequently missed findings on prenatal ultrasound evaluation with significant complex consequences. This is partly related to the experience of the scanning examiner, capability of the scanning equipment used, natural history of evolution of $\mathrm{CHD}$, and the number and timing of the scan. Therefore, specific indications for a detailed fetal echocardiographic evaluation at centers with appropriate expertise and facilities to screen for cardiac anomalies in fetuses at high risk have evolved. Although most of the infants are born to mothers who have no known risk factors, the yields of detection of $\mathrm{CHD}$ in fetuses in high-risk groups have been significantly higher than those in low-risk groups (3). A detailed fetal echocardiographic evaluation has been indicated in high-risk conditions (Table 1). The yield for the detection of CHD is variable with different indications. The family history as indication has $2 \%$ to $10 \%$ yield for detecting fetal heart defects, depending upon the number of previous siblings affected, type of CHD in the index sibling, associated syndrome, extracardiac malformations with CHD in the siblings, or parental heart defects $(4,5)$. In contrast to this low-yielding indication, an abnormal obstetric sonogram suspecting anomalies of cardiac position or structures on screening examination has the highest yield of $50 \%$ to $60 \%$ as an indicator (6). Fetal arrhythmias and extracardiac anomalies of gastrointestinal system and genitourinary system have intermediate yield of $25 \%$ to $30 \%$ as indicators $(7,8)$. Nuchal translucency thickness of more than $5.5 \mathrm{~mm}$ (above 95th percentile) has a prevalence of major CHD $\sim 20 \%$ (9). Table 1 does not include the indications for fetal echocardiography in low-risk groups. Fetal echocardiographic screening in low-risk groups has been undertaken in some centers and countries with conflicting results. With advancement in screening program in all groups, early detection of CHDs in fetuses may prove beneficial in terms of outcomes of fetal cardiac intervention in the future.

Early fetal echocardiography with the use of high-resolution transvaginal ultrasound between 13 and 16 weeks of gestation has provided the capability to demonstrate all cardiac structures and the majority of major CHDs. However, the reported sensitivity for detection of CHDs by early fetal echocardiography is up to $\sim 65 \%$ (10). Limitation to the detection of some CHDs such as ventricular septal defect (VSD), double-outlet right ventricle (RV), as well as late-onset appearance of cardiac anomalies such as hypoplasia of one cardiac chamber/great artery in the presence of outflow tract obstruction and cardiac tumors suggest that early screening by transvaginal echocardiography should be followed by midgestational transabdominal echocardiography, which may yield an additional $\sim 20 \%$ detection of CHDs (10).

\section{STANDARD IMAGING VIEWS AND ANATOMIC ASSESSMENT OF THE FETAL HEART}

Fetal echocardiography is commonly performed between 18 and 22 weeks of gestational age (11). In later gestation, imaging may be more difficult due to increasing calcification of fetal ribs and a relative decrease in amniotic fluid volume. Highresolution ultrasound equipment with transducer frequencies from 5 to $7 \mathrm{MHz}$ should be used, with capabilities to perform M-mode, color Doppler, and spectral Doppler interrogation.

A comprehensive fetal echocardiogram includes 2D imaging of the cardiac anatomy, spectral and color Doppler assessment of blood flows, and evaluation of cardiac function and rhythm. Multiple tomographic planes used to image the fetal cardiovascular system have been described $(11,12)$ (Figs. 1,2).

\section{Fetal Position and Abdominal Situs}

The first step in the evaluation of the fetal heart is to establish fetal position within the maternal abdomen (vertex, breech, transverse, supine, prone) and to establish fetal left and right sides. From a transverse section of the fetal abdomen, visceral situs is assessed by demonstrating the relative position of the stomach, hepatic vessels, abdominal aorta, and inferior vena cava (IVC). Normal abdominal situs (situs solitus) is characterized by the stomach bubble on the fetal left side, 
Table 1 Indications for Fetal Echocardiography in Subpopulation at High Risk for Congenital Heart Disease (CHD)

Increased risk based on family history

Positive family history of CHD in previous child/children and parents

Positive family history of syndromes or malformations associated with CHD

CHARGE, DiGeorge, Holt-Oram, Marfan, Noonan, Williams-Beuren

Increased risk due to maternal conditions

Maternal diseases

Diabetes mellitus

Phenylketonuria

Collagen vascular disease

Exposure to teratogens in pregnancy

Teratogenic drugs: alcohol, amphetamine, anticonvulsants, lithium, retinoic acid, warfarin

Intrauterine infections

Rubella, myocarditis from cytomegalovirus, coxsackievirus, and parvovirus

High doses of ionizing radiation

Increased risk due to fetal conditions

Documented fetal anomaly

Abnormal obstetric sonogram

Suspected cardiac anomaly, abnormal cardiac position

Extracardiac malformations frequently associated with cardiac anomaly

Ectopia cordis, microcephaly, VACTERL, omphalocele, horseshoe kidney, single umbilical artery

Arrhythmia

Bradyarrhythmia

Tachyarrhythmia

Complete heart block

Nonimmune hydrops fetalis

Nuchal edema or hygromata, increased thickness of nuchal translucency

Amniotic fluid abnormality

In utero diagnosed chromosome anomaly

Twin gestation

Monozygous twin

Conjoint twin

Abbreviation: VACTERL, vertebral abnormalities, anal atresia, cardiac abnormalities, tracheoesophageal fistula and/or esophageal atresia, renal agenesis and dysplasia, and limb defects.

liver with hepatic vessels on the right side, abdominal aorta to the left of the fetal spine, and IVC to the right of the fetal spine and slightly anterior to the aorta (Fig. 3). In situs inversus, there is a mirror-image arrangement of these organs and vessels. Situs ambiguous denotes when neither situs solitus nor inversus is present; this is also referred to as heterotaxy and is commonly associated with complex congenital heart defects and splenic abnormalities (asplenia or polysplenia).

\section{Four-Chamber View}

Moving the transducer cephalad from the abdominal transverse view to a transverse view of the fetal chest should demonstrate the heart in the left hemithorax with the apex pointing to the left (levocardia). Also with this sweep, entrance of the IVC into the right atrium (RA) is demonstrated. Dextroposition of the heart is present when the fetal heart is found in the right hemithorax, such as when the heart is externally shifted to the right by a left-sided diaphragmatic hernia or cystic adenomatous malformation, or when hypoplasia of the right lung is present; however, the cardiac apex still points to the left. The term "dextrocardia" denotes the apex of the heart is pointed to the right. Dextrocardia in the presence of normal situs solitus is associated with CHD in $95 \%$ of cases (13). By contrast, in situs inversus totalis, where both stomach and heart are on the right side, heart defects are rare.

A transverse section of the fetal chest provides a fourchamber view of the heart, and this is usually easily obtained (Fig. 4). The heart should fill about one-third of the thoracic area or about half the thoracic circumference $(14,15)$. Cardiac axis refers to the angle between a line along the interventricular septum and the thoracic anteroposterior midline and is normally around $45^{\circ} \pm 20^{\circ}$ (16-18). Significant levorotation of the cardiac axis is often the first clue to the presence of a congenital heart defect $(17,18)$.

In the normal four-chamber view, the RA and left atrium (LA) are approximately equal in size, and the RV and left ventricle (LV) are also approximately equal in size and thickness, with the RV becoming slightly larger than the LV as gestation progresses (14). The LA is the most posterior cardiac chamber and lies anterior to the descending aorta. The flap of the foramen ovale (septum primum) moves from the RA into LA, billowing about half-way into the LA, consistent with the right-to-left direction of foramen ovale flow in fetal circulation. The RV is the most anterior cardiac chamber and sits just posterior to the sternum. The LV cavity comprises the cardiac apex. The RV can be identified by the moderator band that courses across the lower part of the RV, from the interventricular septum to the RV free wall. The RV is also identified by the tricuspid valve that inserts into it. The atrioventricular valves are slightly offset from each other, with the tricuspid valve attaching slightly more apically than the mitral valve. From the four-chamber view, a posterior sweep will show the coronary sinus, which should be a thin structure that courses within the LA (Fig. 5). A dilated coronary sinus is seen when a persistent left superior vena cava (SVC) or anomalous pulmonary veins are present.

Addition of color Doppler in the four-chamber view will demonstrate inflow from atria to ventricles. A small amount of tricuspid regurgitation may be seen in the normal fetus (19). Pulmonary venous flow is seen entering the LA; at least one pulmonary vein from both the left and the right should be demonstrated. Evaluation of atrial and ventricular septa by color Doppler to assess for septal defects is best undertaken in planes perpendicular to the septa. Foramen ovale flow is seen from RA to LA, and VSDs in the muscular and inlet parts of the ventricular septum may be seen in this view. Small muscular VSDs may be seen throughout gestation, and many spontaneously close prior to delivery.

In addition to VSDs, other heart defects may be identified in the four-chamber view (20). Ebstein's anomaly of the tricuspid valve is characterized by apical displacement of the septal leaflet of the tricuspid valve with associated tricuspid regurgitation and atrialization of the RV. Atrioventricular canal (endocardial cushion) defects result in a spectrum of defects ranging from a primum atrial septal defect (ASD) with cleft mitral valve, to a complete atrioventricular canal defect 


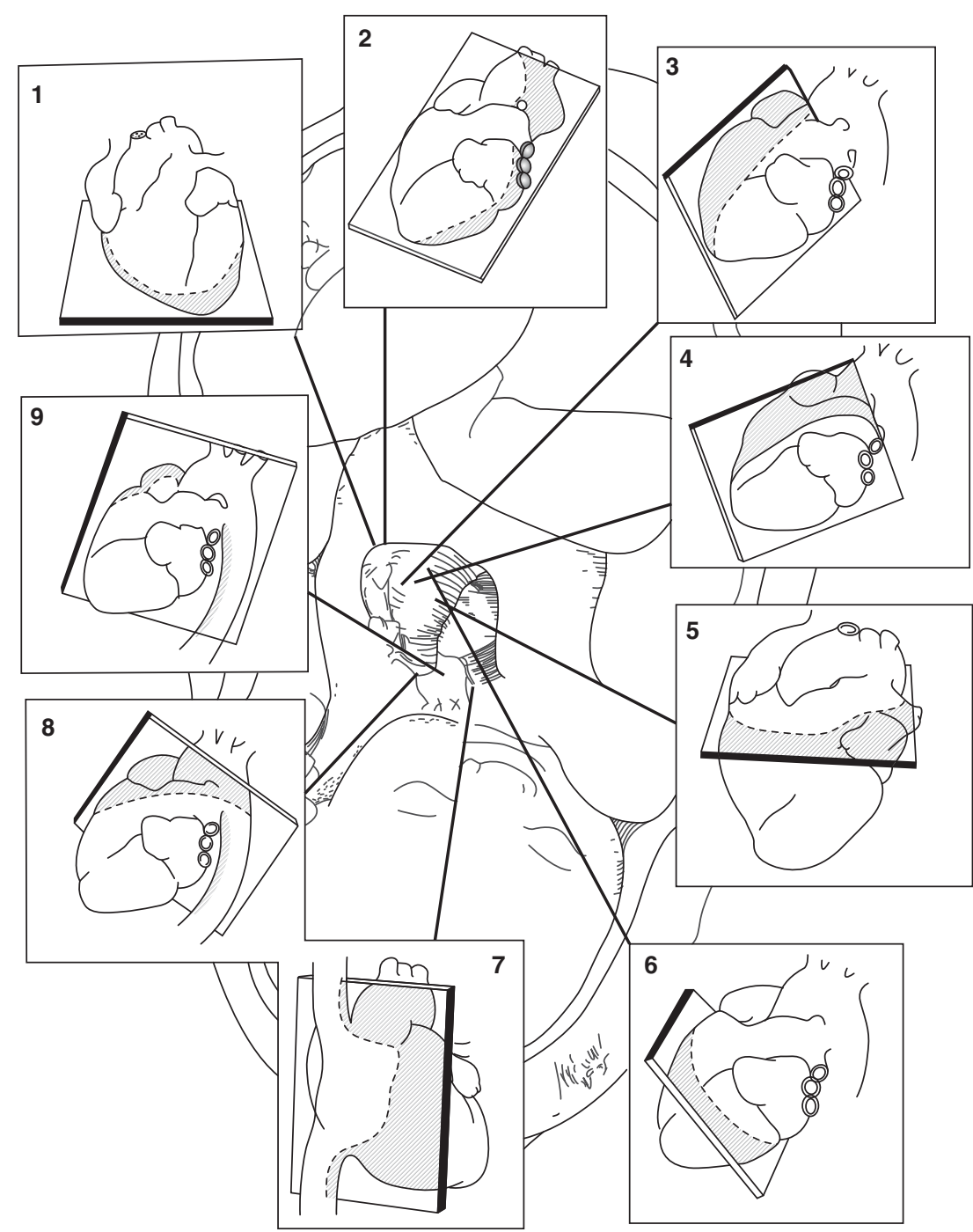

Figure 1 Illustration of the tomographic planes used to image the fetal cardiovascular system. 1, apical (four-chamber) view; 2, apical (four-chamber) view angled toward the aorta; 3 , long-axis view of the left ventricular outflow tract; 4 , long-axis view of the right ventricular outflow tract; 5 , short-axis view at the level of the great vessels; 6, short-axis view with caudad angling at level of ventricles; 7, caval long-axis view; 8, ductal arch view; 9, aortic arch view. Source: From American Society of Echocardiography.

with a single common atrioventricular (AV) valve, primum $\mathrm{ASD}$, and inlet VSD. A primum ASD is seen when the inferior aspect of the atrial septum is deficient. An inlet VSD is seen when the ventricular septum adjacent to the $\mathrm{AV}$ valves is deficient. An atrioventricular canal defect should be suspected when the AV valves appear at the same level in the fourchamber view, without the usual offset of tricuspid and mitral valves.

Ventricular hypoplasia or dilation is easily appreciated in the four-chamber view. A hypoplastic RV is seen in tricuspid atresia and in pulmonary atresia with intact ventricular septum. A small LV is seen in hypoplastic left heart syndrome; this is characterized by severe stenosis or atresia of the mitral and aortic valves and severe hypoplasia of the aortic arch. Other complex, functionally single ventricle lesions include double-inlet LV and unbalanced atrioventricular canal defects with hypoplasia of either ventricle. The latter are commonly associated with heterotaxy syndromes (situs ambiguous with asplenia or polysplenia) (13).

Ventricular dilation may signify the presence of regurgitation of the respective $\mathrm{AV}$ valve or obstruction of the respective proximal outflow tract, and the addition of color and spectral Doppler should be used to assess these areas. RV dilation with RA dilation and tricuspid regurgitation is seen when there is obstruction to the ductal arch (e.g., premature ductal closure) or aortic arch (e.g., coarctation of the aorta).

Importantly, the four-chamber view is inadequate for excluding transposition of the great arteries and conotruncal defects such as tetralogy of Fallot and truncus arteriosus.

\section{Long-Axis View}

While the four-chamber view is the only view of the heart required for standard second-and third-trimester fetal examinations, the addition of outflow tracts has been shown to yield higher detection of congenital heart anomalies than the fourchamber view alone (21-23). From the four-chamber view, sweeping cephalad will demonstrate the ventricular outflow tracts and the relationship of the great arteries. The aorta arises from the posterior LV and aims toward the fetal right shoulder (Fig. 6). The pulmonary artery (PA) arises from the anterior RV and courses leftward and posteriorly, crossing the aorta, extending to the ductus arteriosus, which then meets the 

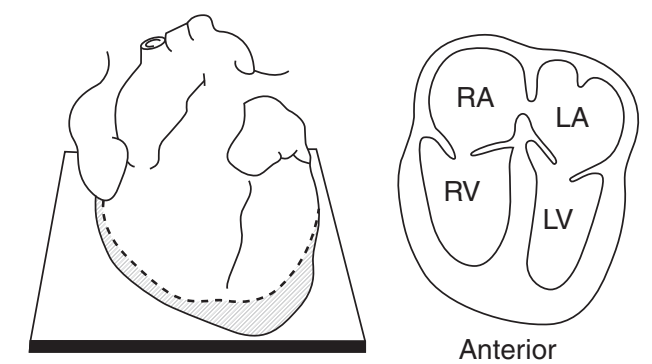

Anterior
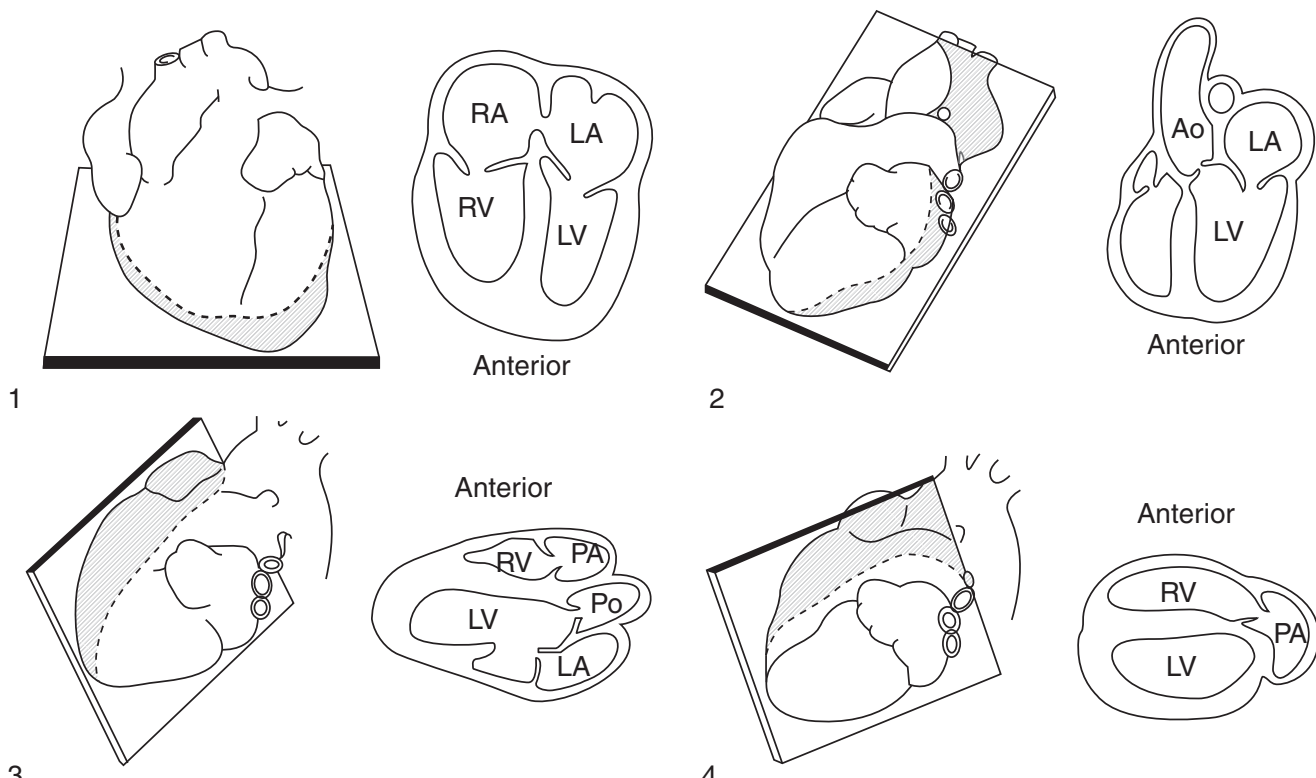

3
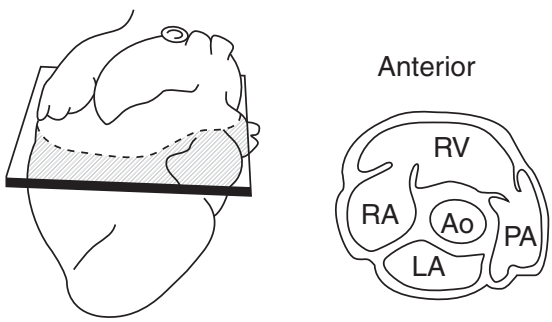

5
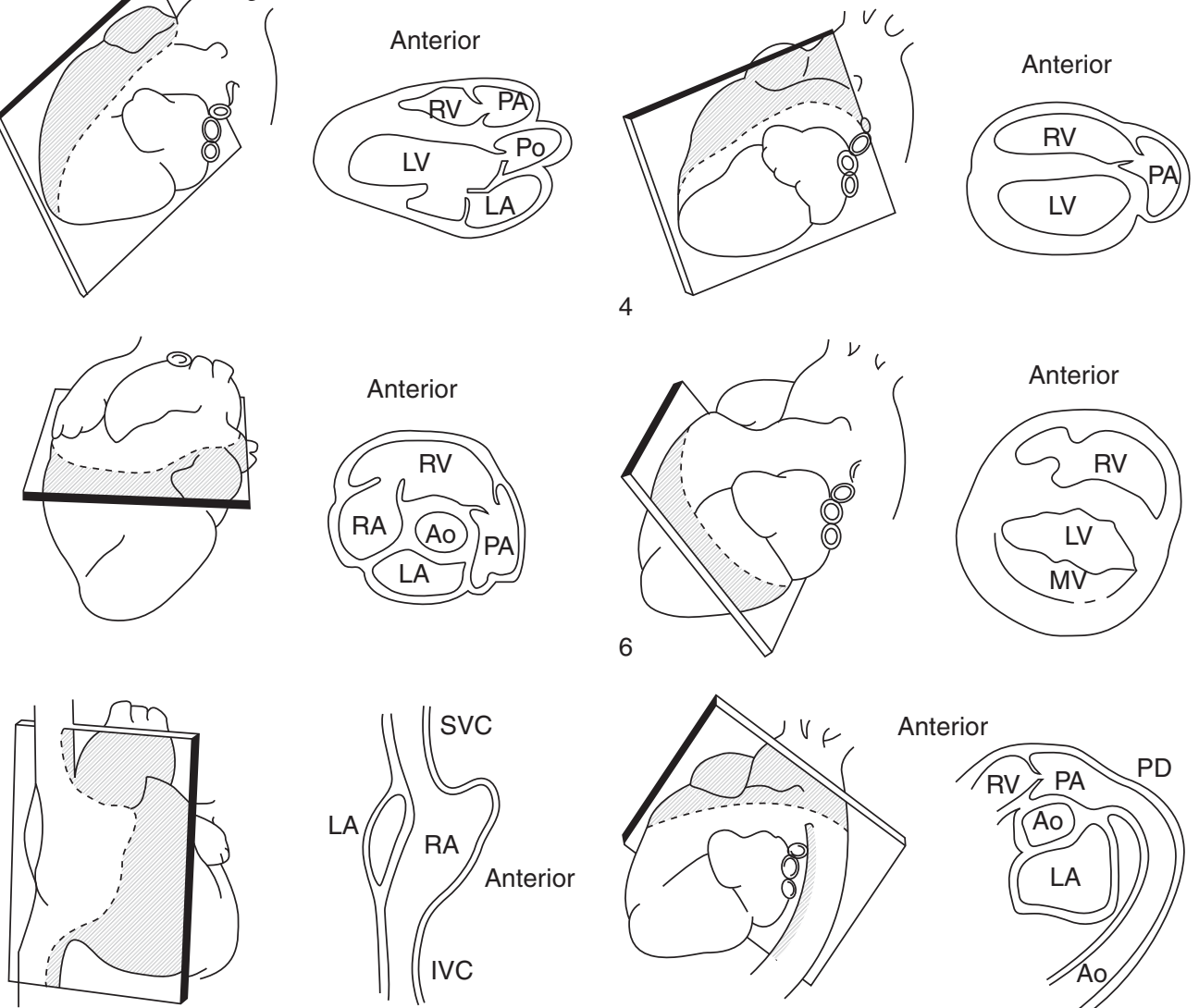

4
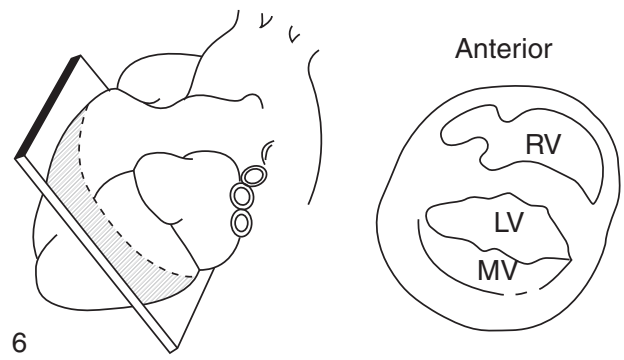

7

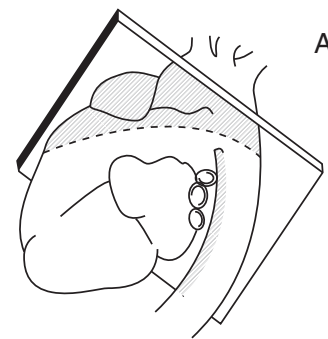

Anterior

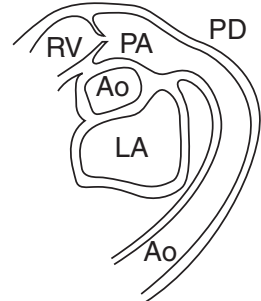

8

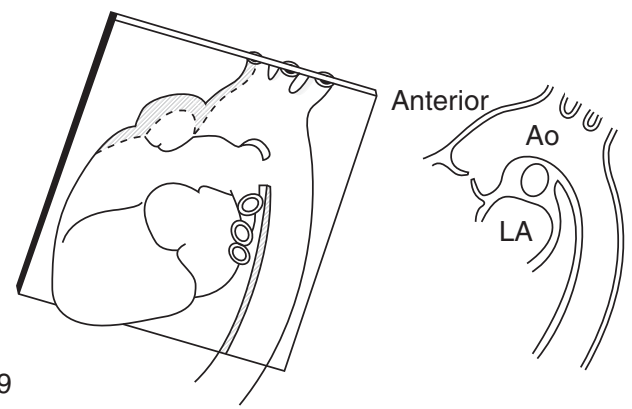

Figure 2 Illustrations of the anatomical correlates for each of the designated tomographic imaging planes used for imaging the fetal cardiovascular system. Each numbered view relates to the clockwise illustration of the fetal heart in Figure 1. Abbreviations: Ao, Aorta; IVC, inferior vena cava; LA, left atrium; LV, left ventricle; MV, mitral valve; PA, pulmonary artery; PD, patent ductus; RA, right atrium; RV, right ventricle; SVC, superior vena cava. Source: From American Society of Echocardiography.

descending aorta. Continuity between the mitral and aortic valves is demonstrated in this view, and the anterosuperior aspect of the ventricular septum can be interrogated to rule out a VSD. Discontinuity of the mitral and aortic valves is characteristic of double-outlet RV lesions.

An aorta that overrides a large malalignment VSD is seen in tetralogy of Fallot, where there is associated pulmonary outflow tract obstruction, and in truncus arteriosus, where there is a single semilunar (truncal) valve and the branch pulmonary arteries usually arise from the ascending aorta.

The subaortic area is also visualized in the long-axis view. Posterior malalignment of the ventricular septum produces a VSD and crowding of the left ventricular outflow tract producing varying degrees of subaortic obstruction. In these 


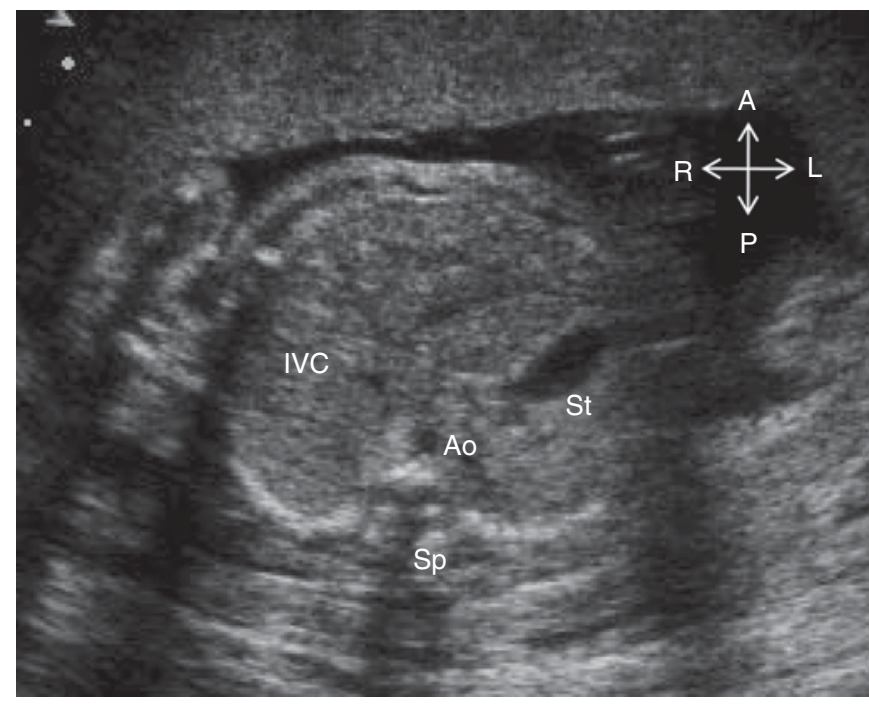

Figure 3 Abdominal situs. Transverse view of abdomen. Abbreviations: St, stomach; Ao, Aorta; IVC, inferior vena cava; Sp, spine.

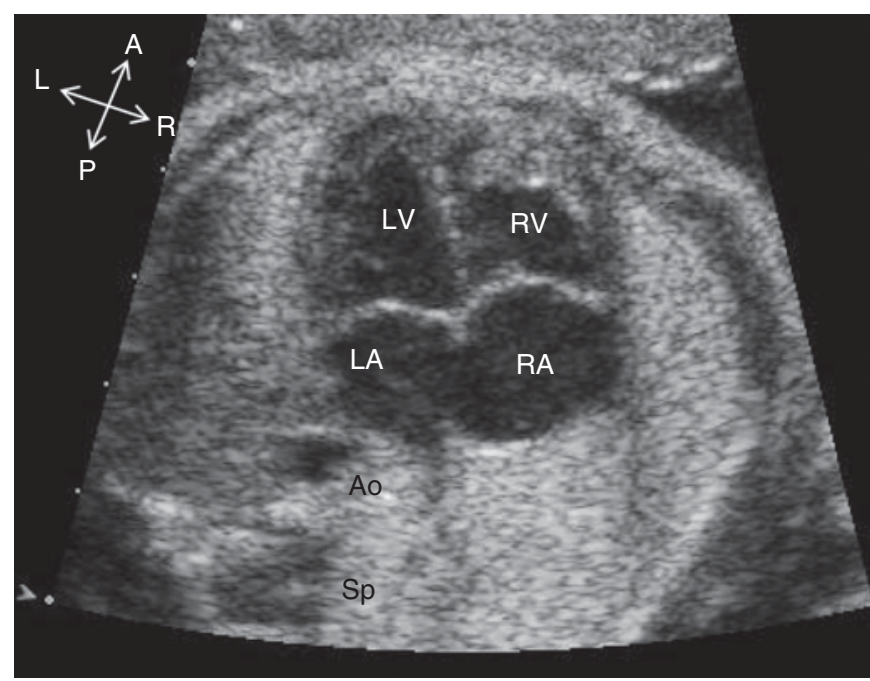

Figure 4 Four-chamber view. Abbreviations: RA, right atrium; RV, right ventricle; LA, left atrium; LV, left ventricle; Ao, aorta; Sp, spine. Note the moderator band traversing the inferior aspect of the RV.

instances, obstruction or interruption of the aortic arch may also be present.

If the anterior vessel is the aorta, then transposition or malposition of the great arteries is present. D-Transposition of the great arteries is recognized by the anterior RV giving rise to the aorta and the PA arising from the LV. The outflow tracts do not cross normally and instead travel in a parallel fashion. VSDs may also be seen with transposition of the great arteries.

In the normal fetal heart, the pulmonary valve and PA are slightly larger than the aortic valve and proximal aorta, and normal reference values based on gestational age have been reported (24). If the PA is hypoplastic, pulmonary stenosis or tetralogy of Fallot should be suspected, and the valve morphology and Doppler gradient be carefully evaluated. The aorta is often dilated in tetralogy of Fallot and truncus arteriosus. If the aorta is small, aortic stenosis and/or other left heart obstructive lesions such as coarctation of the aorta or interrupted aortic arch may be present.

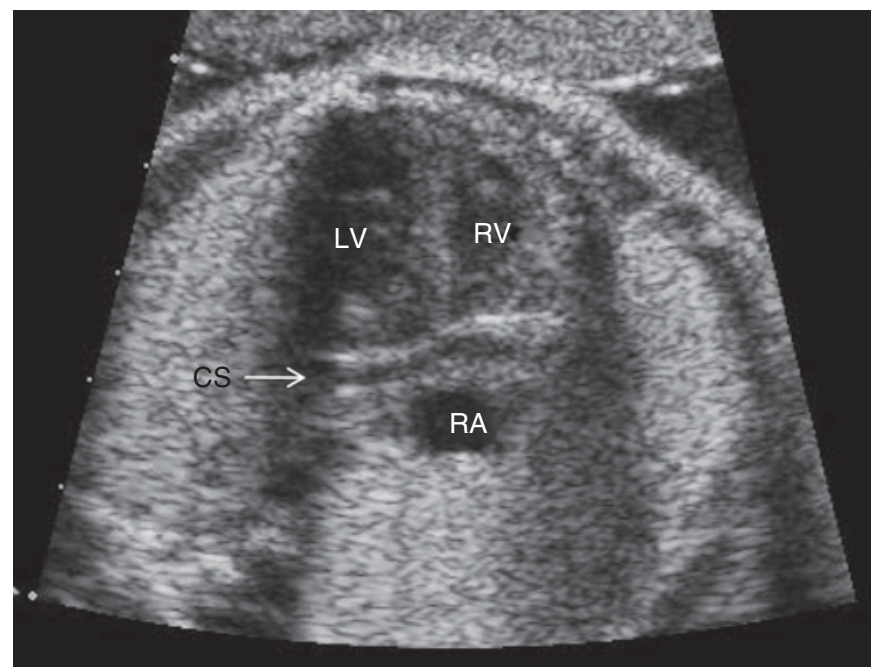

Figure 5 Coronary sinus. Abbreviations: RA, right atrium; RV, right ventricle; $\mathrm{LV}$, left ventricle; $\mathrm{CS}$, coronary sinus.

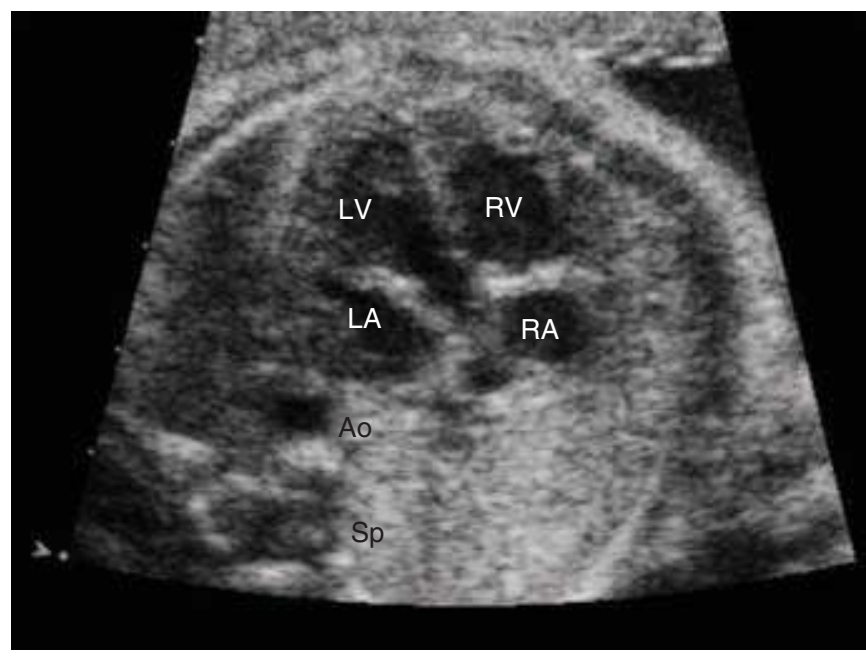

Figure 6 Slight cephalad angulation from the four-chamber view shows the left ventricular outflow tract. Abbreviations: RA, right atrium; RV, right ventricle; LA, left atrium; LV, left ventricle; Ao, aorta; Sp, spine.

\section{Three-Vessel View}

Sweeping further cephalad in a transverse plane will produce the three-vessel view. This is a cross-sectional view at the level of the superior mediastinum, displaying the normal relationship and size differences of the PA, aorta, and SVC (Fig. 7). The PA is seen in long axis in this view and is the larger, most anterior, and leftward vessel; the aorta is slightly smaller, to the right, and posterior to the PA; and the SVC is the smallest, most posterior, and rightward vessel. Discrepancies in the size of the PA and aorta may signify subtle pathology of the outflow tracts or arches, and a dilated SVC may be seen when an interrupted IVC with azygous continuation or vein of Galen aneurysm is present (25).

\section{Short-Axis View}

The short-axis view is obtained by rotating perpendicular to the long-axis view. This view is important in confirming the normal relationship of the great arteries. The aorta is seen in 


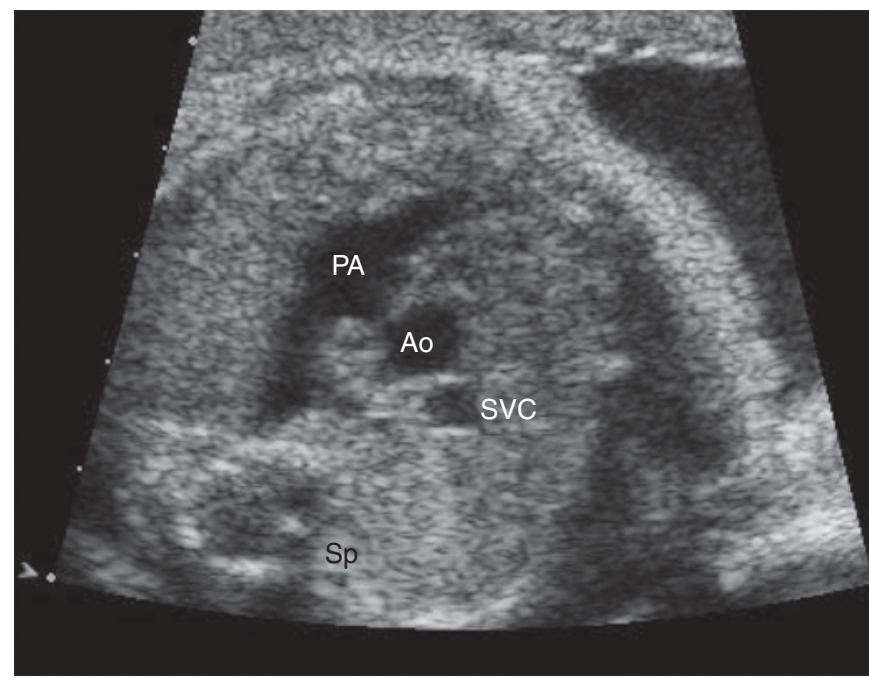

Figure 7 Three-vessel view. Abbreviations: PA, pulmonary artery; Ao, aorta; SVC, superior vena cava; $\mathrm{Sp}$, spine.

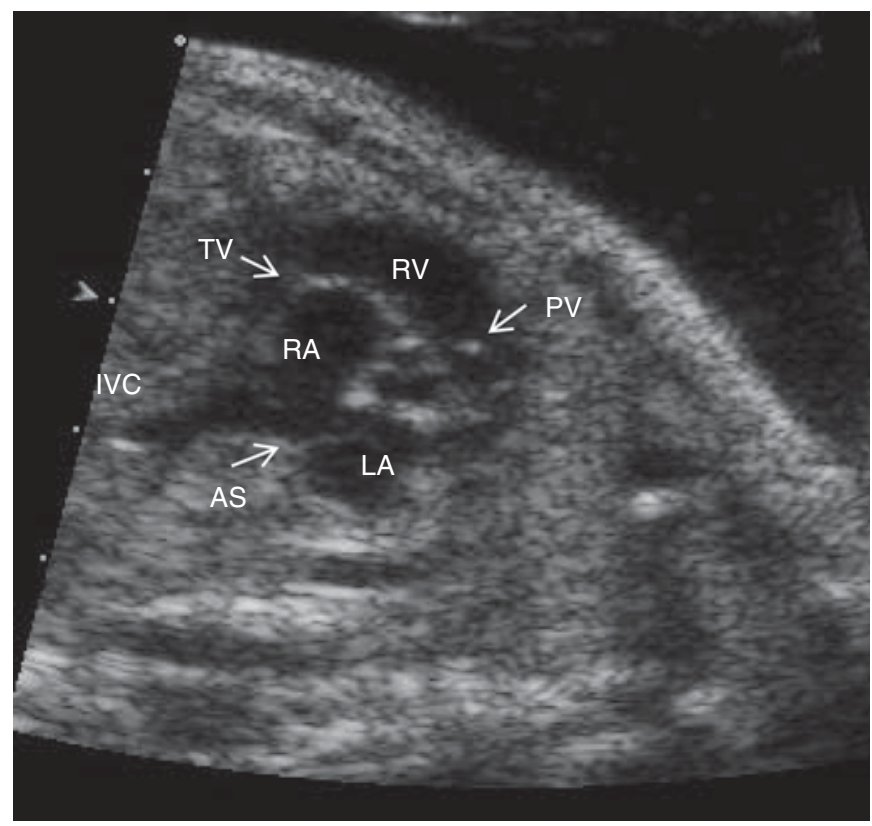

Figure 8 Short-axis view. Abbreviations: LA, left atrium; AS, atrial septum; IVC, inferior vena cava; RA, right atrium; TV, tricuspid valve; RV, right ventricle; PV, pulmonary valve. The aortic valve is seen in cross section in the middle of the short-axis view.

cross section as a circular structure, with the PA wrapping around the aorta as it arises from the anterior ventricle (Fig. 8). The main PA can be seen bifurcating into the ductus arteriosus and right PA and slight angulation to fetal left will show the left PA.

Slight inferior angulation from the short-axis view of the great arteries shows the ventricles in short axis. Quantitative assessment of RV and LV function can be made from this view, and $\mathrm{M}$-mode interrogation through the ventricles allows measurement of RV and LV systolic and diastolic dimensions (26). These dimensions can be used to quantitatively assess ventricular function by calculating a shortening fraction (end-diastolic dimension - end-systolic dimension/enddiastolic dimension). Normal shortening fraction for the RV and $\mathrm{LV}$ is $34 \pm 3 \%$ (27).

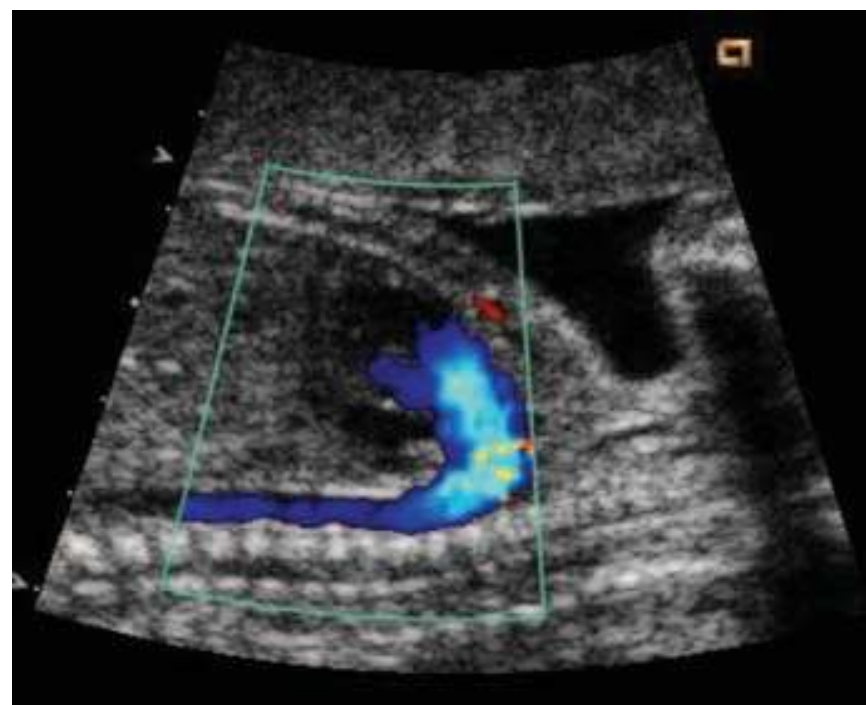

Figure 9 Ductal arch view. The ductus arteriosus arises from the anterior right ventricle and connects to the descending aorta, making a "hockey-stick" configuration.

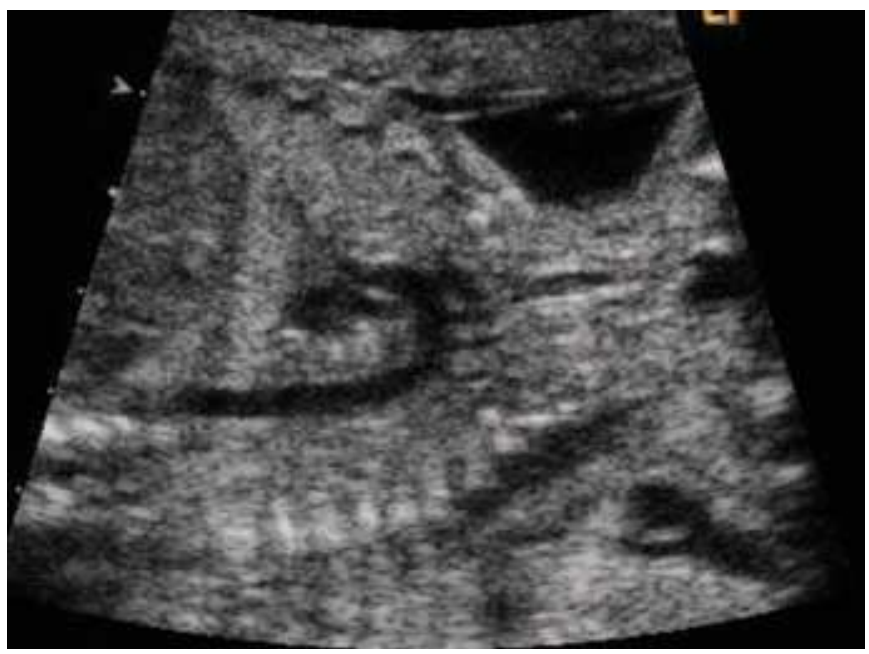

Figure 10 Aortic arch view. The aorta arises from the central thorax and normally gives rise to three brachiocephalic vessels. The aortic arch makes a "candy cane" configuration.

\section{Ductal View}

The ductal view is obtained in the fetal sagittal plane. The ductal arch has a flatter contour ("hockey-stick" shape) than the aortic arch as it arises anteriorly from the PA and connects to the descending aorta (Fig. 9). It lies more caudally than the aortic arch. Color Doppler interrogation demonstrates the normal right-to-left ductal flow (PA to descending aorta). Direction of ductal flow is reversed (aorta to PA) when there is obstruction to pulmonary blood flow, as in pulmonary atresia or severe pulmonary stenosis.

\section{Aortic Arch View}

The aortic arch is also best viewed in a sagittal plane and is obtained from the ductal view by slight angulation of the transducer. The aortic arch arises centrally from the heart in the mid-thorax and aims toward the fetal head. It has a "candy cane" contour and is identified by the three brachiocephalic vessels that arise from it (Fig. 10). Color Doppler interrogation confirms antegrade flow through the ascending aorta and 


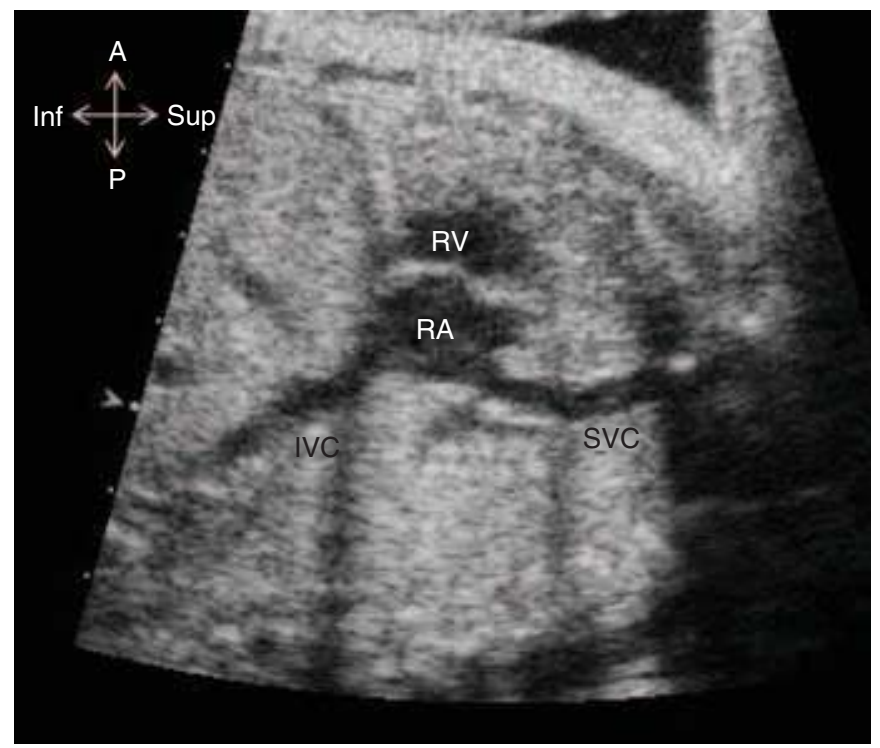

Figure 11 Bicaval view. Abbreviations: IVC, inferior vena cava; SVC, superior vena cava; RA, right atrium; RV, right ventricle.

aortic arch. Both spectral and color Doppler are used to assess for obstruction; however, even in the setting of a normalappearing arch, coarctation of the aorta cannot be ruled out owing to the presence of a large ductus arteriosus. Retrograde aortic arch flow from the ductus arteriosus is seen in critical left heart obstructive lesions such as hypoplastic left heart syndrome and critical aortic stenosis.

\section{Caval Long-Axis View}

This view is obtained from the aortic arch view by sliding from the left parasagittal chest to the right. Bicaval return to the RA can be seen (Fig. 11). The septum primum is seen extending into the LA and use of color Doppler demonstrates the normal right-to-left flow across the foramen ovale. Reversal of foramen ovale flow, from LA to RA, is seen in critical left heart hypoplasia/obstructive lesions. The ductus venosus, a fetal shunt connecting the umbilical vein (UV) with the IVC, can be seen entering the left hepatic vein-IVC junction just below the diaphragm and RA.

It is important to note than even with a comprehensive fetal echocardiogram, there are congenital heart defects that cannot be ruled out. As flow normally occurs across the atrial septum via the foramen ovale, the presence of an ASD cannot be excluded, particularly of the secundum type. Coarctation of the aorta may be difficult to exclude in the presence of a large ductus arteriosus; secondary findings of RV dilation and tricuspid regurgitation may be seen with a significant coarctation and are clues to its presence.

\section{FETAL CARDIAC FUNCTIONAL ASSESSMENT}

The 2D and Doppler echocardiography have become not only an integral component in the assessment of anatomic anomalies of the fetal heart, but also an important tool in the assessment of fetal hemodynamic status. Various techniques for assessing fetal cardiovascular function exist and can be utilized to assess fetuses with cardiovascular compromise due to a variety of cardiac and noncardiac causes (Table 2). As many of the conditions associated with fetal cardiac
Table 2 Examples of Conditions Associated with Fetal Cardiac Compromise

\begin{tabular}{|c|c|}
\hline & Condition \\
\hline \multirow[t]{11}{*}{ Cardiac disease } & Severe valve regurgitations \\
\hline & $\begin{array}{l}\text { Tetralogy of Fallot with absent pulmonary } \\
\text { valve }\end{array}$ \\
\hline & AV septal defect with AV valve regurgitation \\
\hline & Ebstein anomaly of the tricuspid valve \\
\hline & Elevated ventricular afterload \\
\hline & Bilateral semilunar valve stenosis \\
\hline & Constriction of the ductus arteriosus \\
\hline & Fetal arrhythmia \\
\hline & Fetal tachyarrhythmia \\
\hline & Complete AV block \\
\hline & Restrictive foramen ovale \\
\hline \multirow{10}{*}{$\begin{array}{l}\text { Noncardiac } \\
\text { disease }\end{array}$} & Volume overload/high cardiac output lesions \\
\hline & Fetal anemia \\
\hline & Arteriovenous fistulae \\
\hline & Sacrococcygeal or cervical teratoma \\
\hline & TRAP sequence \\
\hline & Placental chorioangioma \\
\hline & High cardiac afterload \\
\hline & Recipient twin in TTTS \\
\hline & Placental insufficiency \\
\hline & Congenital cystic adenomatoid malformation \\
\hline
\end{tabular}

compromise now have fetal therapeutic options, understanding and identifying fetal cardiac dysfunction in the current era assume even greater importance, because accurate description of cardiac natural history should become an important component in determining patient selection and timing of intervention.

This section reviews common 2D and Doppler methods of assessing fetal cardiovascular status, describes the changes in these indices that are characteristic of fetal cardiac compromise, and offers a clinical approach to the comprehensive assessment of fetal cardiovascular function. The components of the complete fetal cardiac assessment are outlined in Table 3.

\section{Assessment of Systolic Ventricular Function in the Fetus}

The fetal myocardium differs maturationally from the "adult" state, and these features must be considered when evaluating cardiac function in the setting of altered loading conditions $(28,29)$. The fetal myocardium responds to preload with higher resting and reduced active tension generation, due in part to higher concentrations of noncontractile proteins in the fetal versus the mature heart. The fetal response to afterload is also different, with a significant reduction in fiber shortening in the setting of increased afterload (29). These differences must be kept in mind when evaluating ventricular systolic function in settings where pathologic loading conditions likely exist.

\section{D Assessment of Fetal Systolic Function}

In the past, assessment of fetal ventricular systolic function has often consisted of qualitative assessments. Current practice is generally more quantitative. On initial 2D imaging of the fetus, it is important to note the presence of hydrops fetalis and/or 
its component findings. Overall, cardiac size is often a sign of altered hemodynamics in the fetus; in addition to qualitative assessment, the cardiothoracic ratio can be used to quantitatively express the degree of cardiac enlargement. In our lab, we utilize the ratio of the cardiac area (in the four-chamber view) to the thoracic area in the same view (Fig. 12), with normal values generally being $<0.35$ (30). It is also useful to examine why the heart is large, which may be due to ventricular enlargement, atrial dilation, ventricular hypertrophy, or a combination of these findings, as this may provide further clues as to the etiology of the cardiac abnormality. On 2D and/or M-mode imaging of the RV and LV, quantitative assessment of both RV and LV shortening fraction is also possible (27,31-33) (Fig. 12). In addition to subjective assessment of ventricular wall thickness, quantitation of RV and LV wall thickness is possible, and there are published normal values against which measurements can be compared (34).

\section{Doppler Assessment of Fetal Cardiac Function: General Comments}

The 2D and M-mode imaging can be limited on fetal echocardiography due to marginal acoustic windows or fetal position, sometimes limiting the utility of these methods. There are now a number of Doppler indices-sometimes more readily obtained in the setting of marginal $2 \mathrm{D}$ imaging windows - on fetal echocardiography that describe systolic and diastolic cardiac functions. Classically, Doppler characterization of systemic venous flow-in the IVC, ductus venosus, UV, and artery-has been utilized to identify changes associated with elevations of central venous pressure and changes in vascular flow distribution. More recently, the Doppler myocardial performance index has emerged as a promising new index for assessment of global—systolic and diastolic-ventricular function.

\section{Doppler Assessment of Fetal Systolic Function \\ Myocardial Performance Index}

The myocardial performance index (MPI) was originally described by Tei and colleagues in 1995 (35). The MPI is in actuality an index of global ventricular function, as calculation of the index incorporates both diastolic and systolic time intervals into assessment of ventricular function. The MPI is calculated as

$$
(\mathrm{IRT}+\mathrm{ICT}) / \mathrm{ET}
$$

Table 3 Elements of Comprehensive Cardiovascular Functional Exam in the Fetus

$\begin{array}{ll}\text { Echocardiographic Assessment } \\ \text { General } & \text { Presence of hydrops fetalis } \\ & \text { Cardiothoracic area ratio } \\ \text { Color flow Doppler evaluation of AV valve regurgitation } & \text { The 2D or M-mode assessment of RV and LV shortening fraction } \\ \text { Systolic function } & \text { Myocardial performance index (global function) } \\ & \text { Ventricular dP/dt (when AV valve regurgitation present) } \\ \text { Tricuspid and mitral AV valve inflow profile (E and A velocity, E/A wave } & \text { ratio, presence of monophasic inflow profile) } \\ \text { Diastolic function } & \text { Myocardial performance index (global function) } \\ & \text { IVC or hepatic venous flow (ratio of reverse flow VTI and forward flow VTI) } \\ & \text { Ductus venosus flow (presence or absence of absent or reverse end-diastolic } \\ \text { flow, increased pulsatility index) } & \text { Umbilical venous flow (presence or absence of pulsations) }\end{array}$

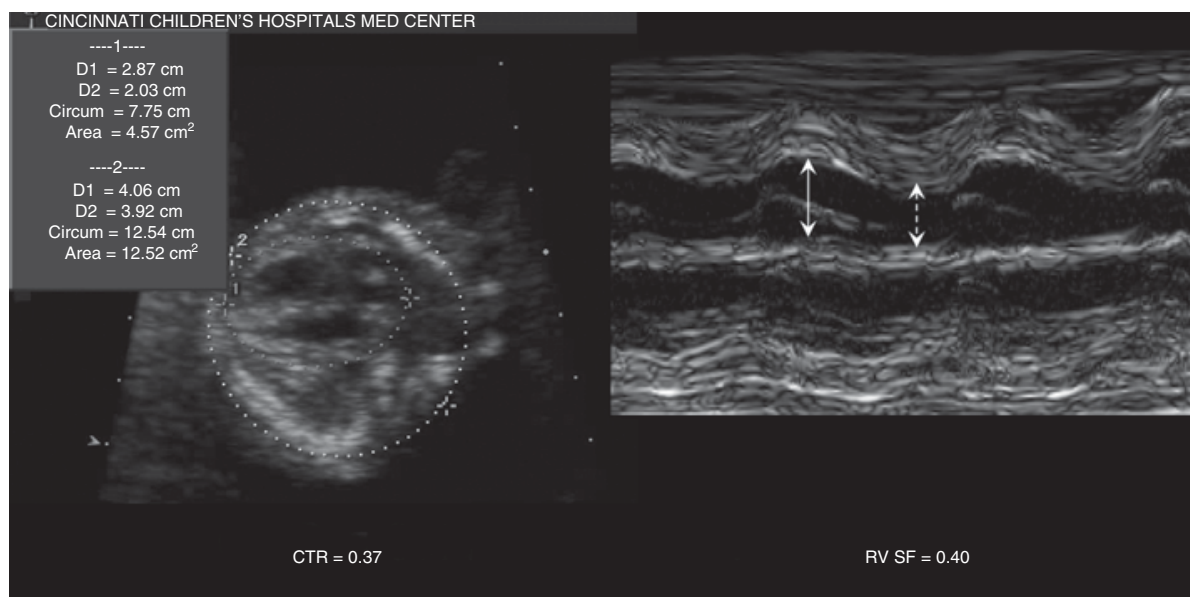

Figure 12 Left panel: Assessment of cardiothoracic ratio (CTR). The cardiac area in the four-chamber view is divided by the thoracic area. In this example, the CTR is mildly elevated at 0.37 (normal, $\leq 0.35$ ). Right panel: Quantitation of ventricular shortening fraction (SF). In this example, M-mode through the right ventricular (RV) short axis allows measurement of the RV end-diastolic dimension (solid arrow) and end-systolic dimension (dashed arrow). The RVSF is calculated as (RV end-diastolic dimension - RV end-systolic dimension)/(RV end-diastolic dimension). 
where IRT is the isovolumic relaxation time, ICT is the isovolumic contraction time, and ET is the ventricular ejection time. The sum of IRT and ICT can be derived from subtracting the ET from the time period " $\mathrm{A}$ " between cessation of atrioventricular valve inflow and onset of atrioventricular valve inflow in the next cardiac cycle (Fig. 13). With systolic dysfunction, ICT lengthens and ET shortens; diastolic dysfunction will manifest as prolonged IRT. Therefore, an elevation in the MPI indicates ventricular dysfunction, although the index does not allow specific identification of diastolic or systolic dysfunction. Normal values for both RV and LV MPI have been reported. In our laboratory, normal RV and LV MPI are $0.32 \pm 0.08$ and $0.33 \pm 0.05$, respectively. Both Eidem and Mori et al. reported comparable normal values for RV MPI to be approximately $0.35 \pm 0.06(36,37)$, although higher LV MPI in normal fetuses $(0.55 \pm 0.13)$ has been reported (38). The MPI in the fetus has shown to be significantly elevated in a number of disease states, including twin-twin transfusion syndrome $(39,40)$, fetuses of diabetic mothers (41), constriction of the ductus arteriosus (37), and cardiomyopathy (42).

\section{Color Flow Doppler Assessment}

In addition to the quantitative techniques described above, it is important to evaluate atrioventricular valve functionspecifically, for evidence of valve regurgitation-by color flow Doppler imaging. Tricuspid and mitral regurgitation-with normal valve anatomy-often develops in the setting of fetal cardiac compromise $(27,39,43-46)$ and should be evaluated during echocardiography in any fetus with suspected cardiac dysfunction.

\section{Assessment of Diastolic Ventricular Function in the Fetus} Diastolic ventricular function is also "immature" in the fetal heart $(28,47,48)$. Fetal ventricular relaxation is reduced in the fetus, although there are progressive changes in ventricular inflow Doppler patterns that demonstrate improving ventricular relaxation throughout gestation $(47,49)$.
Ventricular compliance characteristics demonstrate increased "stiffness" of the fetal heart (50). In fact, it is likely that diastolic dysfunction precedes overt ventricular systolic dysfunction or hydrops in a number of settings, making an understanding of the Doppler manifestations of fetal diastolic dysfunction important. For example, it has been reported that Doppler evidence of diastolic dysfunction will often be present prior to onset of ventricular systolic dysfunction in recipient twins in the setting of twin-twin transfusion syndrome $(39,40)$, as well as in fetuses with growth restriction (31).

Doppler assessment of diastolic function in the fetus consists of two primary components: (i) assessment of Doppler flow patterns in the fetal venous system and (ii) assessment of Doppler flow patterns across the atrioventricular valves.

Assessment of Doppler Flow Patterns in the Fetal Venous System There is a large body of literature describing changes in fetal venous Doppler flow patterns in a variety of disease states. In all of these conditions, changes in Doppler venous flow patterns are felt to be a reflection of elevated central venous pressure related to increased ventricular filling pressures. The magnitude of these changes has been correlated to severity of compromise in several studies (51-55).

The central finding in the compromised fetus is an increase in the magnitude of the blood flow away from the heart during atrial systole. This is manifested as changes in the Doppler flow profile in several important vessels:

1. IVC/hepatic veins

2. Ductus venosus

3. UV

It should be noted that as fetal cardiac compromise leads to elevation of central venous and ventricular filling pressure, venous flow reversals during atrial systole will manifest first proximally, in the IVC and hepatic veins. As compromise worsens, findings will then move distally—or "upstream"into the ductus venosus and UV (43).

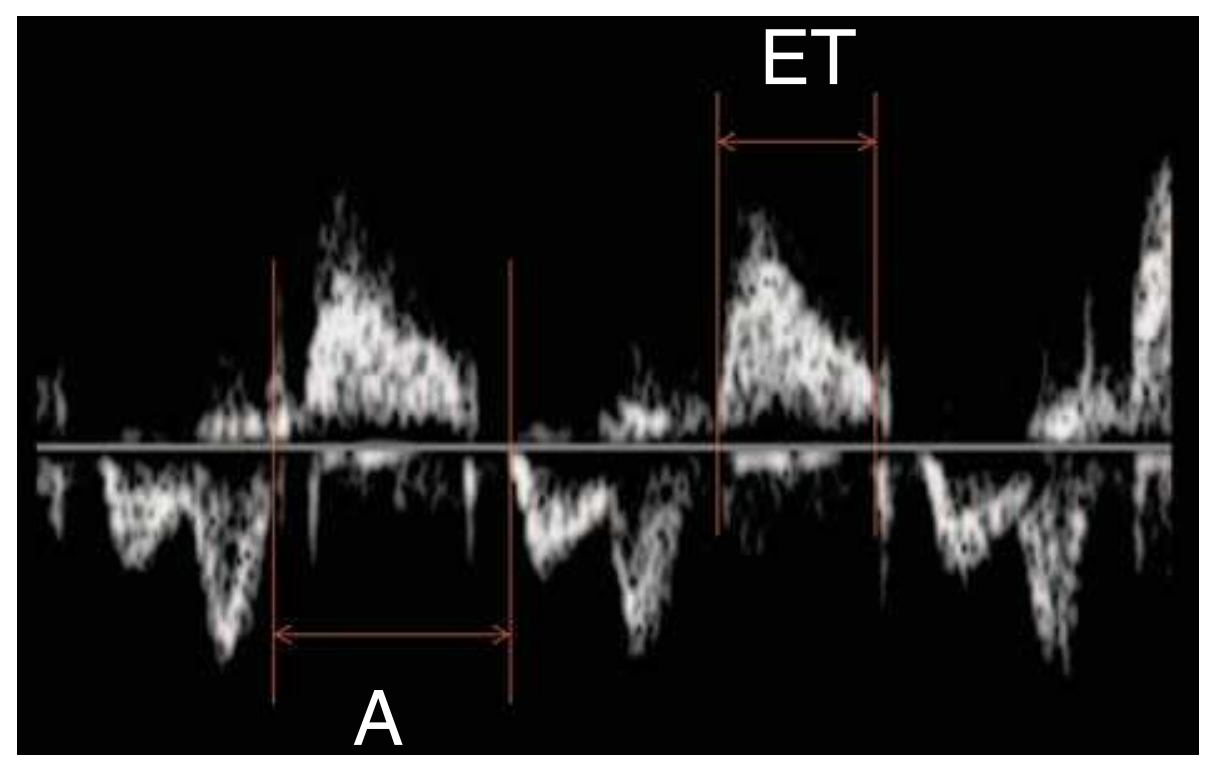

Figure 13 Simultaneous pulsed-wave Doppler of left ventricular inflow (below baseline) and outflow (above baseline). The mitral valve inflow is characterized by an early diastolic E-wave and a late diastolic A-wave (see text). The myocardial performance index is calculated as: (A - ET)/ET (see text). 


\section{IVC and Hepatic Veins}

In the IVC and hepatic veins, normal venous flow consists of a phasic low-velocity pattern consisting of an $\mathrm{S}$ wave during ventricular systole, a $\mathrm{D}$ wave during passive diastolic filling, and a small reversal in flow-the A wave-during atrial systole (Fig. 14). Normal values for IVC and hepatic venous S, D, and A waves throughout gestation have been reported (56); interestingly, the magnitude of the atrial systolic flow reversal diminishes in the course of normal development, suggesting improvements in ventricular compliance $(57,58)$. Another method of evaluating the magnitude of the atrial systolic flow reversal is as a ratio of reverse flow to forward flow. The timevelocity integral of both reverse and forward flow are measured and expressed as a percentage; it has been reported that ratios in excess of $7 \%$ are abnormal (43) (Fig. 14).

\section{Ductus Venosus}

Doppler assessment of the ductus venosus (DV) is important to incorporate into the comprehensive fetal echocardiogram, particularly if cardiac dysfunction is suspected, as changes in the DV and UV are reflective of the central venous and/or right atrial pressures. In some cases, this is not always a poor prognostic sign, as in the case of right heart obstructive lesions (59), such as tricuspid atresia or pulmonary atresia with intact ventricular septum, where the entire systemic and umbilical venous return is forced to cross the foramen ovale. In this setting, abnormalities of venous Doppler flow may be noted in a setting where fetal cardiovascular compromise is quite rare.

UV

The presence of umbilical venous pulsations is a finding associated with severe cardiovascular compromise and has been correlated to poor prognosis in the setting of nonimmune hydrops due to cardiomyopathy (44), twin-twin transfusion syndrome, arrhythmia, and structural heart disease $(55,60)$. It has been suggested, however, that, although specific, UV pulsations are not sensitive enough to be clinically useful in identifying fetal cardiovascular compromise at a time when poor outcome could be avoided (61). Further work should probably be focused on which venous Doppler changes - in IVC, DV, or UV-have the greatest clinical utility in early identification of the fetus in distress, so that management may be initiated before the fetus presents in a pre-morbid state.

\section{Doppler Assessment of AV Valve Inflow Doppler Flow Profile}

Pulsed-wave Doppler assessment of both trans-mitral and trans-tricuspid flow patterns should be performed during fetal cardiac functional evaluation. The Doppler E-wave velocity reflects early diastolic ventricular filling, where the A wave reflects ventricular filling that occurs during atrial contraction (Fig. 13). In the fetus, the AV valve Doppler Ewave velocity is lower than the A-wave velocity. The ratio of E wave to A-wave velocity increases progressively during gestation $(49,62)$, reflecting more rapid myocardial relaxation in diastole. In pathologic circumstances, a number of changes in AV valve inflow Doppler profiles have been described. In twin-twin transfusion syndrome and fetal cardiomyopathy, the AV inflow $\mathrm{E}$ - and A-wave velocity ratio increases (39). However, in other settings, the E- and A-wave ratio has been noted to decrease, such as in the setting of intrauterine growth restriction $(31,63)$ or fetuses of diabetic mothers (64). In the most severe cases of

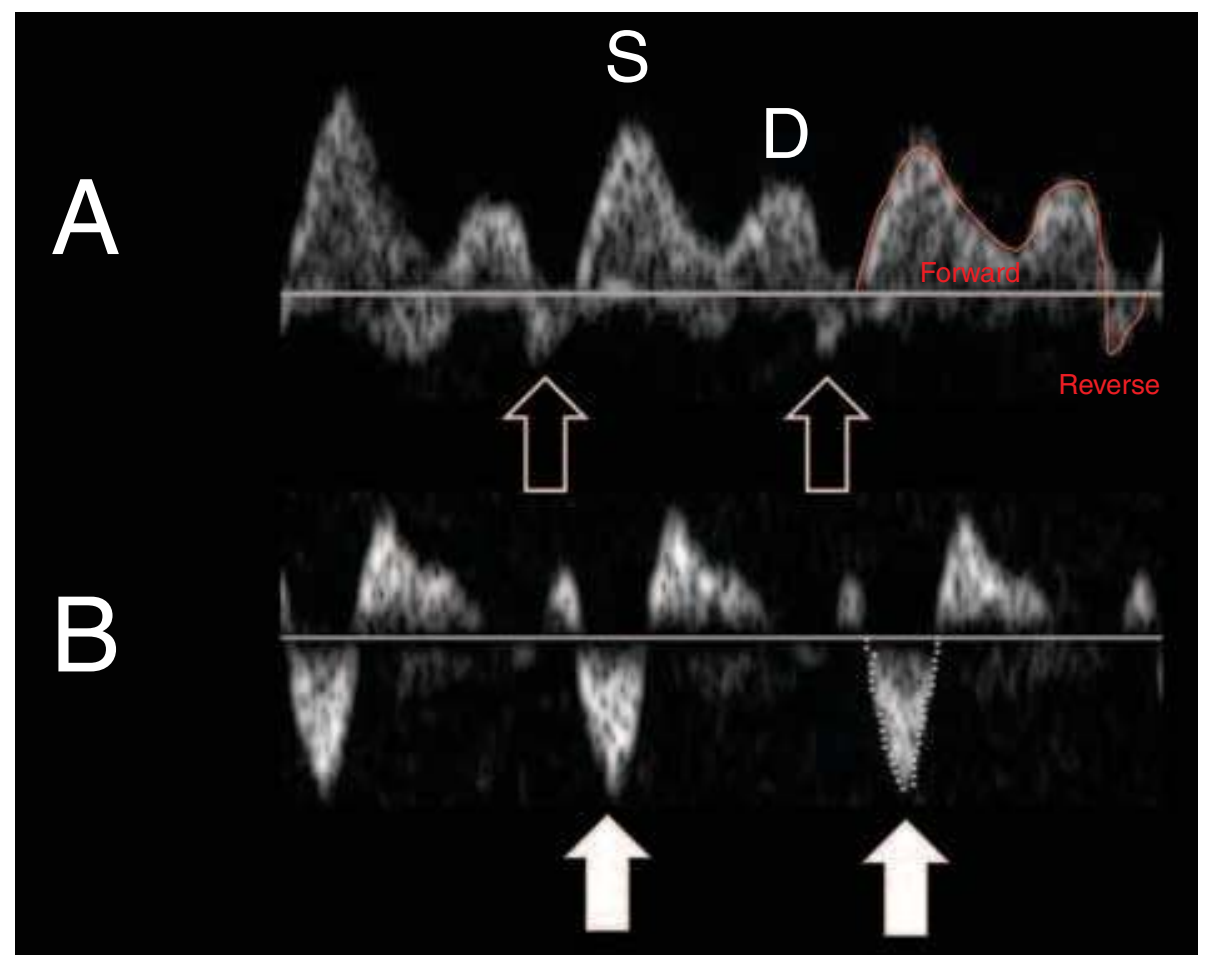

Figure 14 Hepatic venous Doppler evaluation. Normal hepatic venous flow (panel A) is characterized by a systolic S-wave, early diastolic D-wave, and an A-wave of reversed flow during atrial contraction (open arrows). The calculation of the forward and reverse (A-wave) velocity-time integral (VTI) is shown in red. With increasing central venous pressure, the magnitude of the hepatic venous A-wave will increase (solid white arrows, panel B). 
ventricular dysfunction, the AV inflow profile becomes monophasic (Fig. 15), which is a finding consistent with severe diastolic dysfunction.

\section{Doppler Assessment of "Critical" Outflow Tract Obstructive Lesions}

Another "functional" aspect of fetal echocardiographic assessment is identification of critical outflow tract obstruction, for example, in the setting of severe pulmonary stenosis (RV outflow tract obstruction) or severe aortic valve stenosis (LV outflow tract obstruction). In these settings, identification of "reversed" physiologic shunting in the fetus can allow clinicians to make the diagnosis of critical outflow tract obstruction and make the appropriate perinatal management decisions for each case.

In the setting of RV outflow tract-or more specifically, pulmonary outflow tract-obstruction, identification of reversed flow in the ductus arteriosus (from aorta to PA) heralds a fetus who will require maintenance of ductal patency to avoid critical hypoxemia related to pulmonary outflow tract obstruction after birth (65). In the setting of a left heart obstructive lesion such as aortic stenosis, left to right flow across the foramen ovale and reversal of flow in the aortic arch can be identified and used to confirm the diagnosis of critical left heart obstruction (65). In this setting, maintenance of ductal patency postnatally will prevent compromise of systemic perfusion and impending shock.
In all of these settings, the physiologic data obtained on fetal echocardiography can be used to determine severity of the anatomic lesion and make appropriate management decisions, for example, delivery at a level III perinatal center, institution of prostaglandin infusion to maintain ductal patency, and transfer to a pediatric cardiac center, in advance of birth.

\section{FETAL ARRHYTHMIAS}

The fetal arrhythmias encountered in fetal cardiology practice can be broadly categorized into tachyarrhythmias, bradyarrhythmias, and irregular cardiac rhythms with a normal heart rate.

\section{Fetal Tachyarrhythmias}

Fetal arrhythmia causing tachycardia is an important cause of fetal morbidity and mortality (66-69). Fetal tachycardia is defined as a fetal heart rate $\geq 200$ beats per minute (bpm) (69). Both sustained tachycardia (present $\geq 12$ consecutive hours) (70) as well as intermittent tachycardias are risk factors for fetal congestive heart failure $(67,71)$.

The majority of fetal tachycardias are due to supraventricular tachycardia (SVT). Characteristically, it shows 1:1 atrioventricular contraction sequence, abrupt onset and termination, and minimal (within $10 \mathrm{bpm}$ range) or no heart rate variability. The fetal heart rate can range from 220 to $300 \mathrm{bpm}$. Atrial flutter due to intra-atrial reentrant mechanism

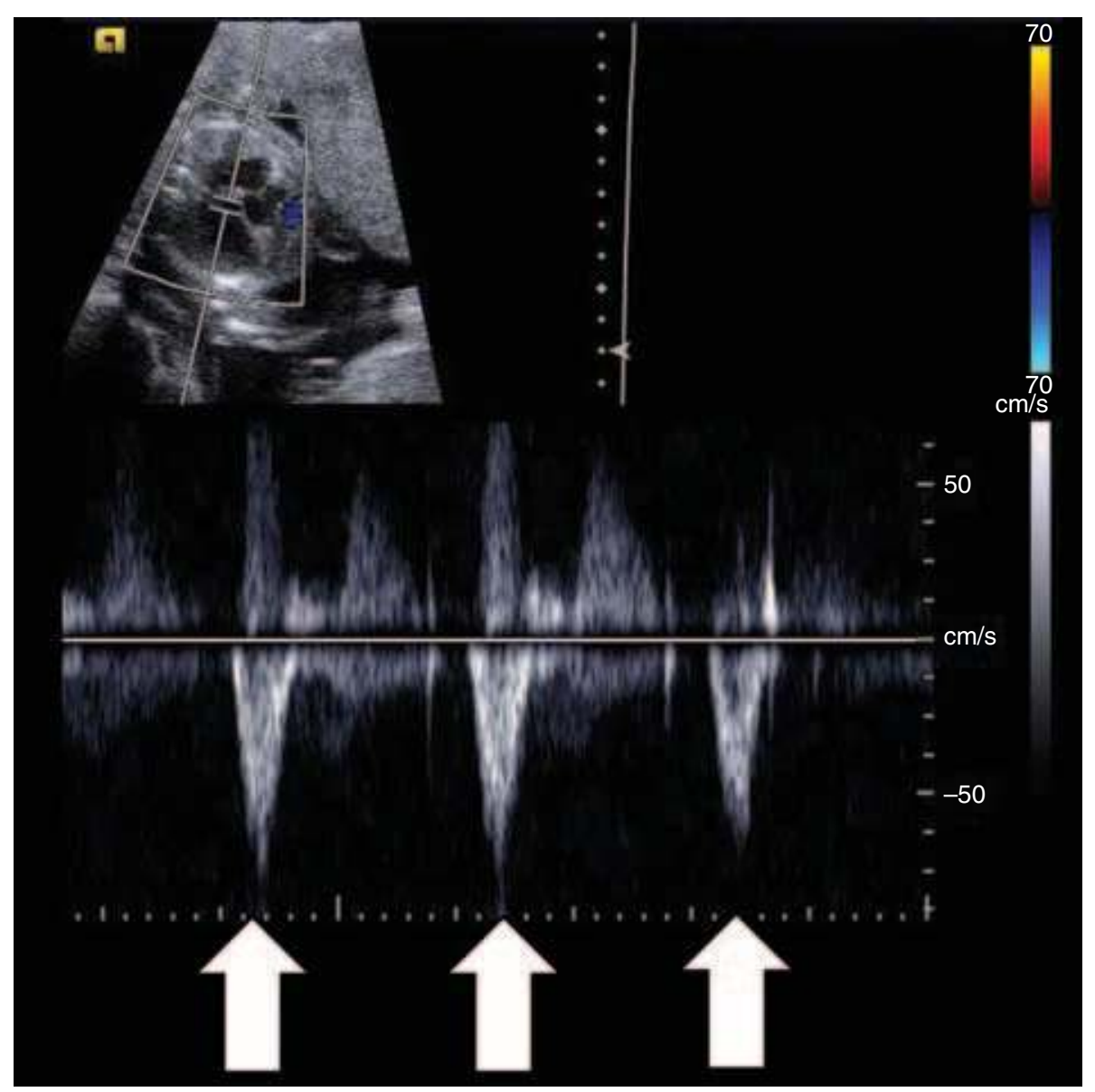

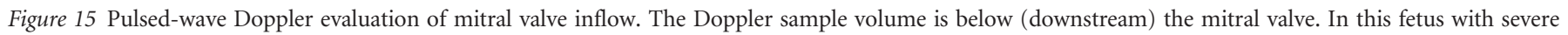

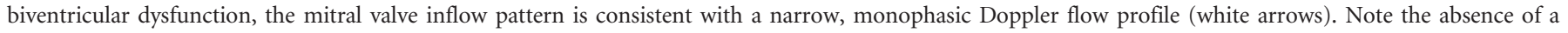
normal E-wave/A-wave complex (see also Fig. 12). 
is common next to atrioventricular reentrant SVT (68). In atrial flutter, the atrial rate is in excess of ventricular rate. It ranges from 300 to $500 \mathrm{bpm}$ with variable atrioventricular block leading to a ventricular rate ranging from normal to $\geq 300 \mathrm{bpm}$. The ventricular rate is often irregular in contrast to the minimal variability seen in SVT.

Mechanistically, almost $90 \%$ of SVT in fetuses are due to atrioventricular reentrant tachycardia via common type of accessory pathway (67). SVT can also be due to atrioventricular nodal, permanent junctional, or multiple reentrant pathways as well as due to automatic ectopic focus confined to the atrium. These types of SVTs are much less common during fetal age $(67,72,73)$.

Junctional ectopic tachycardia and ventricular tachycardia (VT) are rare in fetal life. In contrast to atrial flutter, they are characterized by ventricular rate in excess of the atrial rate and are often difficult to distinguish from each other. VT is often seen in association with fetal long QT syndrome, which arises due to gene mutations coding for different proteins in ion channels. Measurement of the QT interval by echocardiography is not possible, but this diagnosis can be suggested often by the presence of sinus bradycardia between the episodes of VT.

\section{Fetal Bradyarrhythmias}

Fetal arrhythmia causing bradycardia may be associated with significant fetal morbidity and mortality. Fetal bradycardia is defined as a persistent heart rate $<100$ beats/min. Most commonly, fetal bradycardia is due to sinus bradycardia. It shows 1:1 atrioventricular contraction sequence, slow onset, and beat-to-beat heart rate variability. It is often seen in fetuses suffering from significant hypoxic insult and raised intracardiac volume due to inhibitory vagal reflexes initiated by umbilical cord and other vessel compression. A sustained fetal sinus bradycardia with blunted or no heart rate variability is often a predictor of impending morbidity and mortality. Sinus bradycardia is often seen in fetuses with heterotaxia syndrome with atrial laterality abnormalities and AV canal defect. Fetuses with long QT syndrome may present with sinus bradycardia (74).

Fetuses with frequent or multiple premature atrial beats, which are not conducted to the ventricle, will have overall decreased ventricular rate and may have bradycardia due to it. This is a benign self-resolving condition and most of them resolve by the third trimester and certainly by the first weeks of postnatal life. Nonconducted premature atrial beats can occur after every two, three, or four normal beats setting up atrial bigeminy, atrial trigeminy, or atrial quadrigeminy patterns, respectively. Only occasionally, premature atrial beats can be a harbinger of SVT by initiating reentry mechanism in the presence of an accessory pathway.

Atrioventricular block due to AV node or ventricular conducting pathway abnormalities are the most common cause of persistent fetal bradycardia. The atrioventricular block can be of first degree, which demonstrates prolonged AV interval >150 msec (75); second degree, which has a fixed atrioventricular conduction block (2:1 or $3: 1$ block) or progressive prolongation of the AV interval until an atrial beat is nonconducted (Wenckebach); or third degree, which has permanent atrioventricular block (complete heart block) with complete dissociation between atrial and ventricular contractions.

Complete heart block can occur in fetuses either with malformed hearts or in otherwise structurally normal hearts. Fetuses with malformed hearts (corrected transposition of the great arteries, atrial isomerism, or atrioventricular canal defect) and complete heart block or with a ventricular rate $<55 \mathrm{bpm}$ carry a very poor prognosis $(76,77)$. Complete heart block, when found beyond the stage of embryogenesis in a structurally normal heart, has been associated with mass lesions of the conducting system, or more frequently as a consequence of initial insult by maternal antibodies to SS-A/Ro and SS-B/La antigens in fetal heart and subsequently leading to degeneration of the conduction system. Persistent bradycardia can occur in fetuses with long QT syndrome due to atrioventricular block secondary to prolonged refractory period of the ventricle (78).

\section{Diagnosis of Arrhythmia by Fetal Echocardiography}

In practice, fetal arrhythmias are diagnosed by M-mode, pulsed Doppler, or color flow encoded M-mode fetal echocardiography. The M-mode, with cursor oriented across atrial and ventricular cavities, reveals the relationship of atrial and ventricular contractions and their individual rate of contraction. Alternatively, pulse Doppler interrogation with a wide sample placed at mitral-aortic continuity can be employed, which demonstrates the rate of and relationship between inflow and outflow. In normal synchronized atrioventricular conduction, each atrial contraction is followed by a ventricular contraction, and both have the same and normal rate. In atrial flutter, ventricular contraction rate is a fraction of the atrial rate due to variable atrioventricular block (Fig. 16). In SVT, both atrial and ventricular contractions are synchronized and have the same rate, but their rates are between 220 and 300 beats/min (Fig. 17). Types of SVT can be differentiated according to the ventriculoatrial (VA) time interval (72) illustrated in Figure 18. The majority of SVT have short VA times, which are characteristic of atrioventricular reentry via common type of accessory pathway. The long VA time is typical of permanent junctional reentrant tachycardia or atrial ectopic tachycardia. Simultaneous pulse Doppler interrogation of the inflow and outflow can also reveal irregular heart rate due to blocked premature atrial beats (Fig. 19). Furthermore, a simultaneous Doppler interrogation of venous flow (superior vena) and an adjacent artery (aorta) can reveal AV and VA intervals (equivalent of PR interval and RP interval, respectively, on electrocardiogram) (Fig. 20).

In sinus bradycardia there is a 1:1 atrioventricular conduction with both atrial and ventricular rates the same but $<100 \mathrm{bpm}$ on M-mode and Doppler interrogation. Atrioventricular block of all degrees is assessed by using both M-mode and Doppler echocardiography to examine the relationship between contraction of the atrial and ventricular walls and their individual contraction rates. The latter provides the information about the (second or third) degree of heart block.

\section{Assessment of Fetal Cardiac Structure and Function}

In addition to discerning the diagnosis of the fetal arrhythmia, a thorough fetal echocardiographic evaluation should be undertaken to evaluate the cardiac structure, function, 


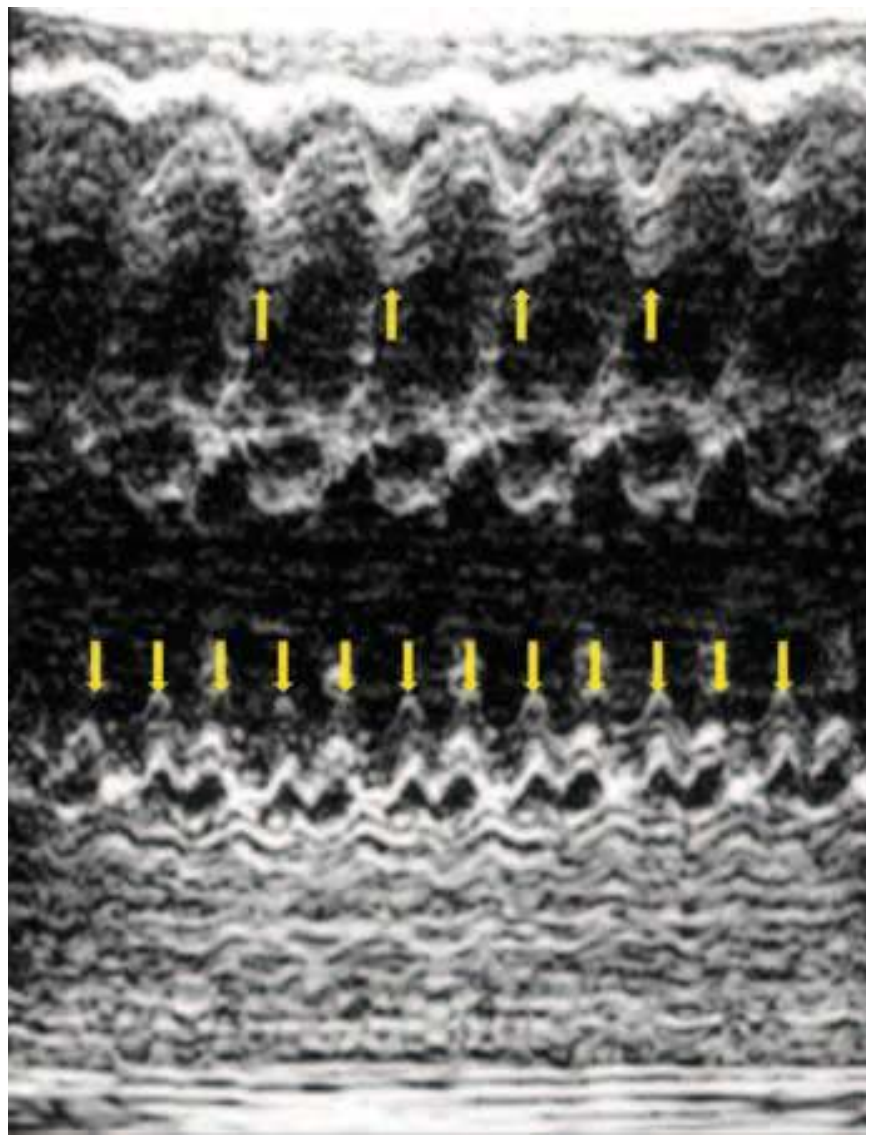

Figure $16 \mathrm{M}$-mode fetal echocardiogram shows both fast atrial and ventricular contractions in a 27-week-old fetus with atrial flutter. The atrial rate (inverted arrow) is $400 \mathrm{bpm}$, and ventricular rate (upright arrow) is $200 \mathrm{bpm}$, with 2:1 AV block.

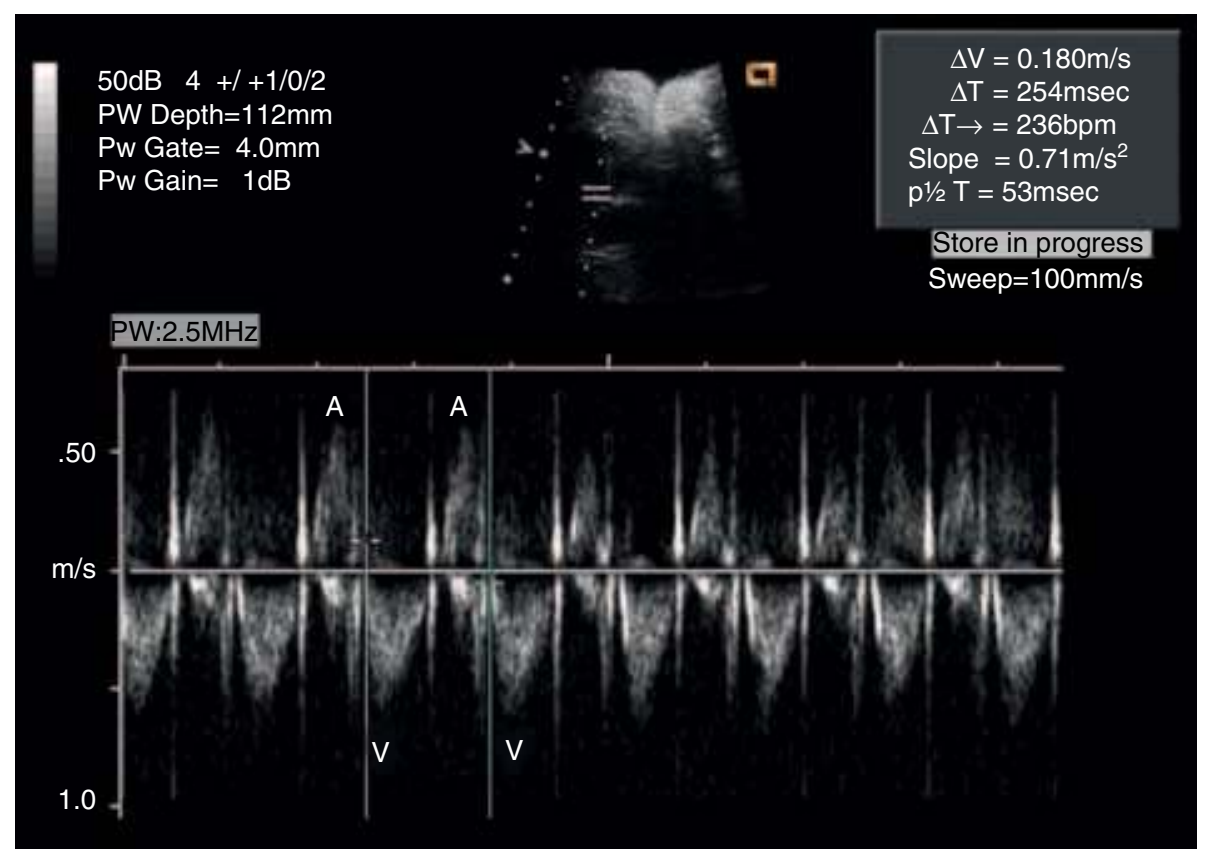

Figure 17 Pulsed-Doppler fetal echocardiogram shows both inflow waveforms due to atrial contractions (above the base line, labeled as A) and outflow waveforms due to ventricular contractions (below the base line, labeled as $\mathrm{V}$ ) at a rate of $236 \mathrm{bpm}$ with 1:1 atrioventricular conduction in a 30 -week-old fetus with supraventricular tachycardia.

associated cardiac defects, hemodynamics, and presence of fetal hydrops as outlined in the previous sections.

Structural malformations of the heart are seen in up to 5\% of the fetuses with tachyarrhythmia, which include Ebstein anomaly of the tricuspid valve, AV canal defect, hypoplastic left heart syndrome, and rhabdomyoma $(66,68,69)$. Ventricular dysfunction can occur. The severity of tachycardiainduced ventricular dysfunction can be assessed by fractional 


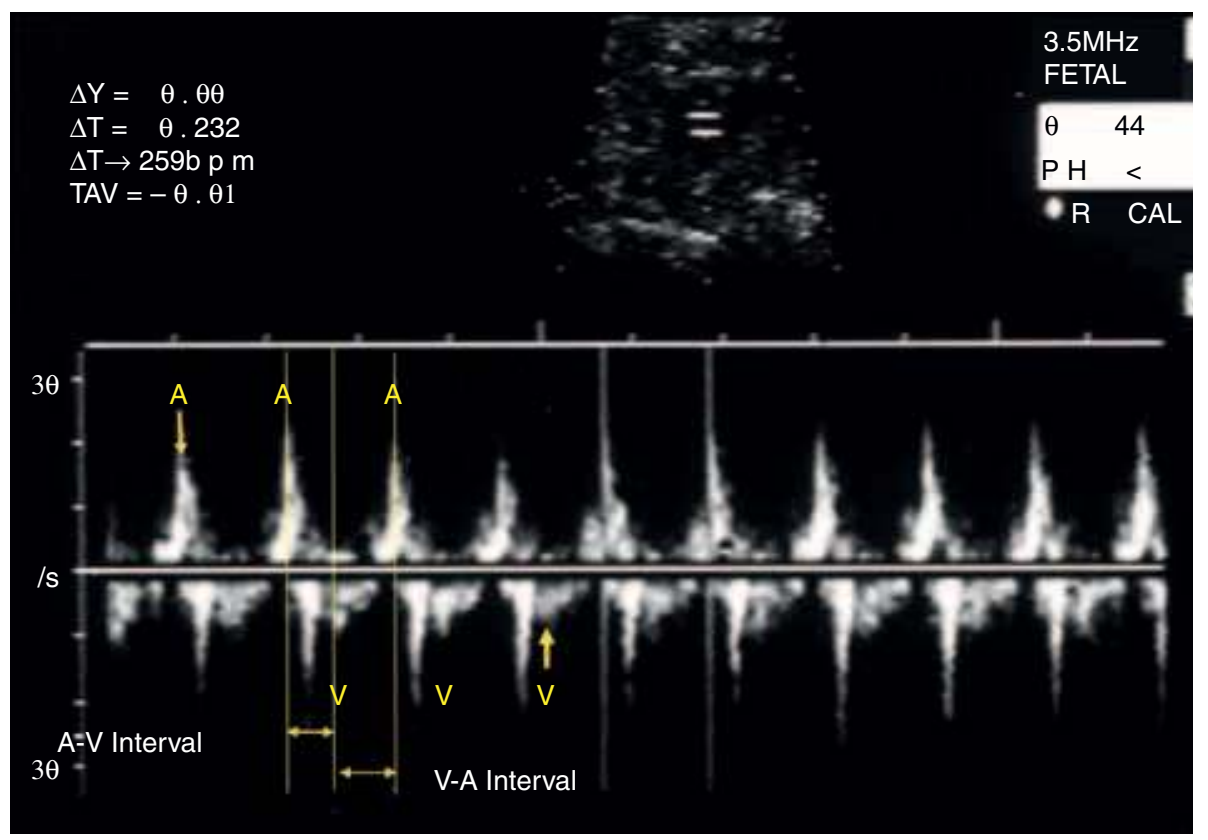

Figure 18 Pulsed-Doppler fetal echocardiogram shows the measurement s of atrioventricular intervals in a 27-week-old fetus with supraventricular tachycardia and a heart rate of $260 \mathrm{bpm}$. The inflow Doppler waveforms due to atrial contraction (above the base line, labeled as A) and outflow Doppler waveforms due to ventricular contractions ((below the base line, labeled as $\mathrm{V}$ ) with 1:1 atrioventricular conduction show that the A-V interval is shorter than the $\mathrm{V}$-A interval, which is characteristic of atrioventricular reentry via common type of accessory pathway.

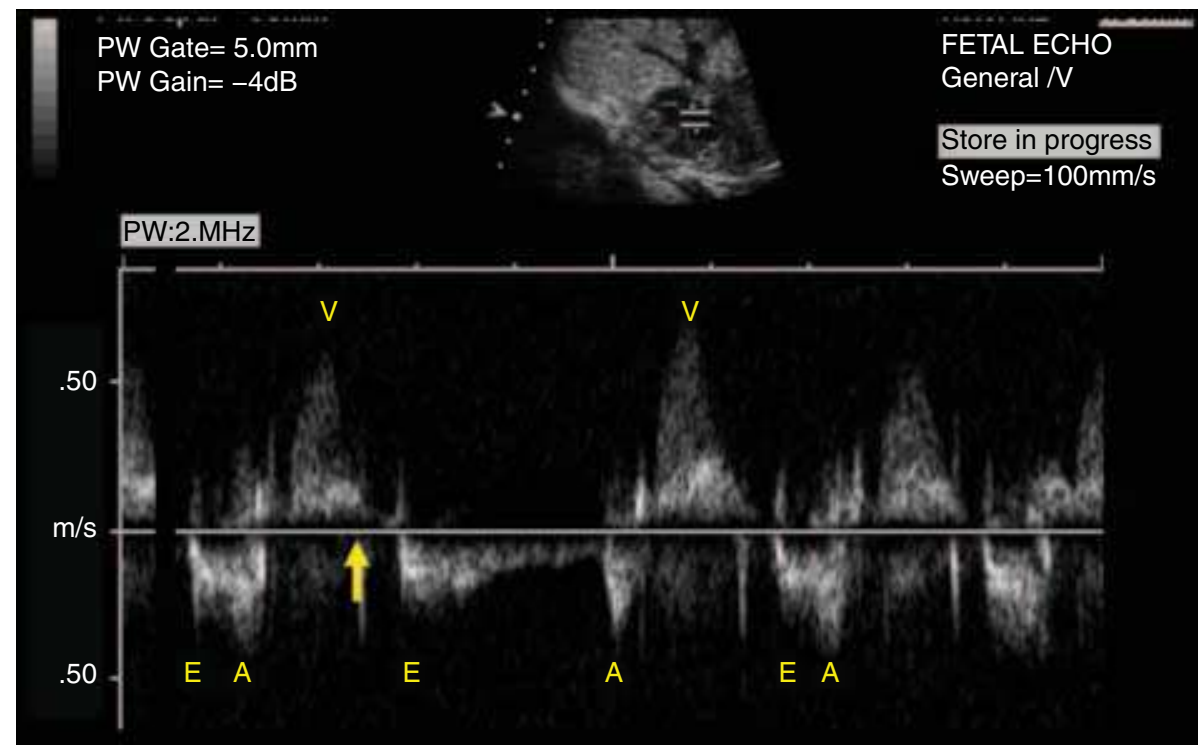

Figure 19 Pulsed-Doppler fetal echocardiogram shows inflow (below the baseline) and outflow (above the baseline) Doppler waveforms in a 30-week-old fetus with a nonconducted (blocked) premature atrial beat (buried in outflow waveform, marked by an arrow). Preceding and following inflow waveforms have normal early (E) and atrial (A) diastolic inflow waveforms. The premature atrial beat is blocked because of A-V node and ventricular refractory phase and is manifested by a late inflow valve opening (E-wave after the arrow), followed by a pause and normal atrial (first A-wave after the arrow) inflow waveform.

shortening, the degree and persistence of AV valve incompetence, and alternations of venous blood flow in IVC and ductus venosus $(79,80)$. Fetal hydrops is characterized by the presence of any two or all of the following signs: skin edema, ascites, pericardial effusion, and pleural effusion (Fig. 21) carry a much higher mortality than a nonhydropic fetus.

\section{Approach to the Treatment of Fetal Tachyarrhythmias}

In order to avoid significant morbidities associated with preterm birth, the approach to the management of fetal arrhythmias is to strive to deliver fetuses at or near term in normal sinus rhythm by intrauterine therapy, which mostly employs maternal administration of antiarrhythmic pharmaceutical agents. The mothers carrying fetuses with incessant tachycardia, tachycardia and major heart defects, or hydrops should be hospitalized to initiate the treatment with loading dose of antiarrhythmic agent, to monitor closely for adverse effects, and to modify the treatment. Continuous cardiac telemetry, serial ECG to assess the cardiac effects of the drug, serum chemistries, and repeated serum drug concentrations in the mother should be obtained. Both the mother and the fetus should be closely observed for any adverse effect of the 


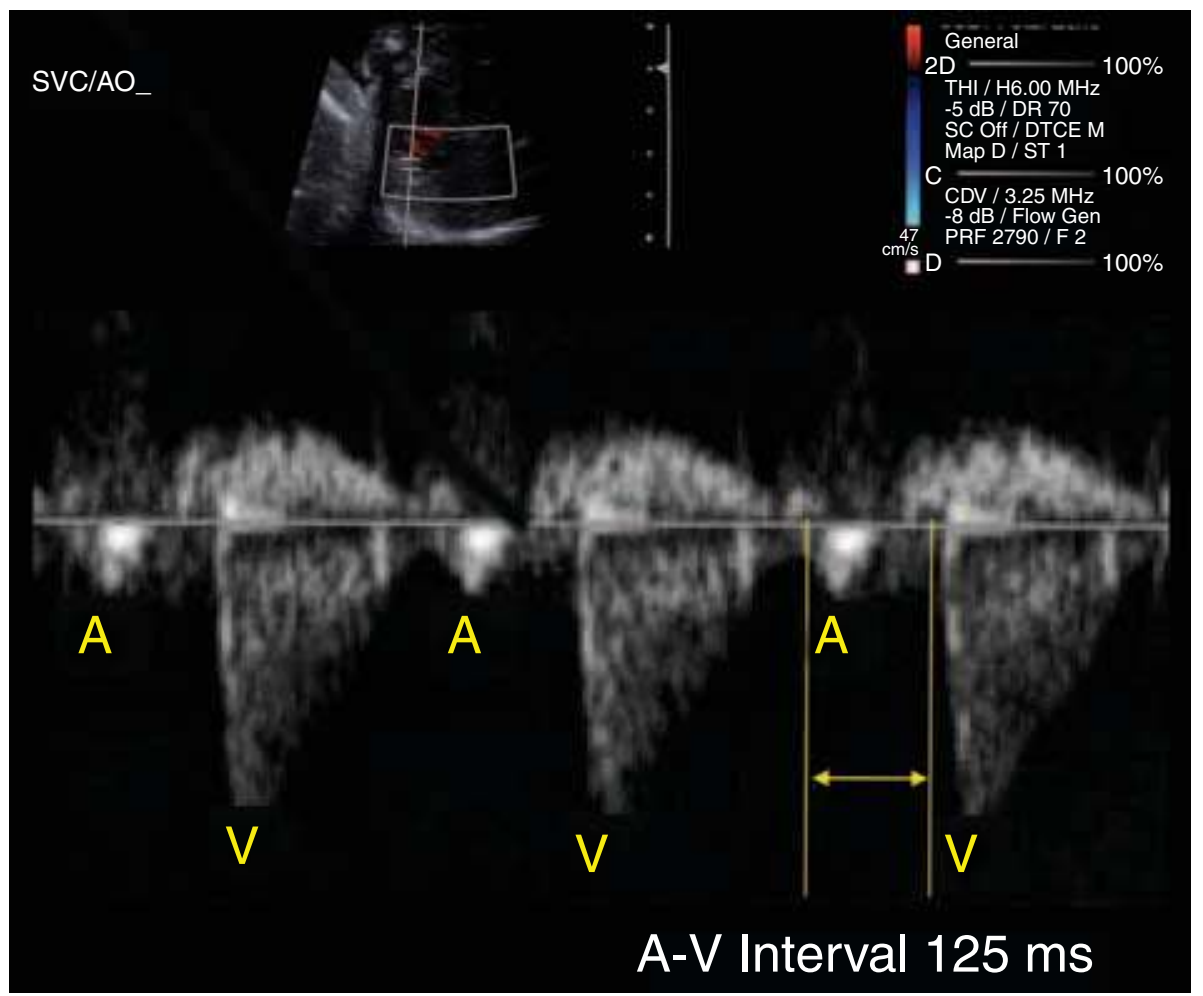

Figure 20 A simultaneous Doppler interrogation of superior vena cava and aortic flow shows reverse waveform due to atrial contraction (A) and outflow waveform in aorta with measured atrioventricular interval (A-V interval $125 \mathrm{~ms}$ ), which is equivalent to PR interval of electrocardiogram.

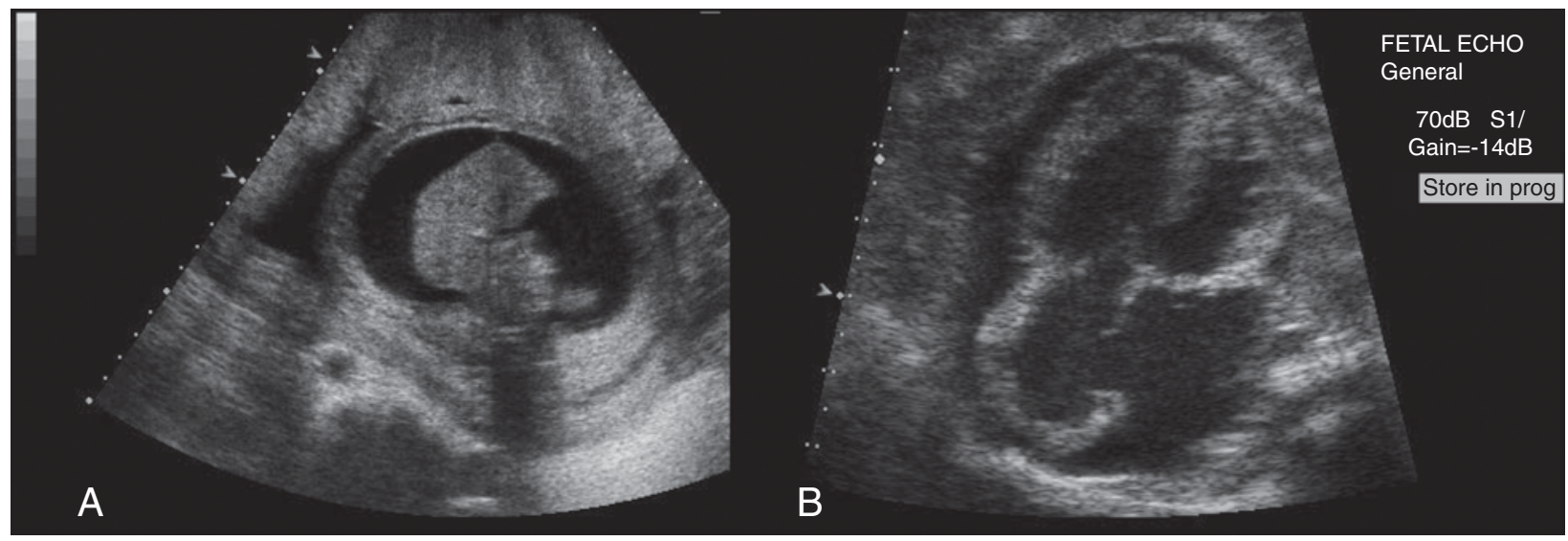

Figure 21 Two-dimensional fetal echocardiogram of a hydropic fetus shows pleural effusion in the chest cavity (A) and a rim of pericardial effusion around the heart (B).

treatment. Once the fetal cardiac rhythm or rate is controlled and maintenance therapy is instituted, the mother can be discharged for regular outpatient follow-up. A detailed discussion on antiarrhythmic drug treatment is out of the purview of this chapter. In general, digoxin has been the first choice followed by flecainide for the maternal administration of antiarrhythmic agent in nonhydropic fetuses and amiodarone in hydropic fetuses (81-83). Depending on the fetal gestation, cardiac function, success rate of triple antiarrhythmic drugs, and parental wishes, urgent delivery may have to be undertaken. A multidisciplinary team including perinatologist, pediatric and adult cardiologists, and neonatologist should be involved in the management of such fetuses and neonates.
PATIENT COUNSELING IN FETAL CARDIAC DISEASE Counseling of the mother who is carrying a fetus with significant CHD can be a complex and lengthy process. As accurate diagnosis is critical to reliable counseling, and as the medical and surgical management of cardiac anomalies is often complicated, collaboration with a fetal cardiac specialist is usually warranted, particularly in complex cases. Nonetheless, it is important for all fetal echocardiography providers to be prepared to provide basic counseling to patients in whom fetal cardiovascular anomalies are suspected.

In addition to accurate anatomic diagnosis of fetal cardiac anomalies, it is useful to categorize the various fetal cardiac lesions with regard to severity. One approach is to classify lesions based on need and complexity of 
Table 4 System for Classifying Severity of Fetal Congenital Heart Disease

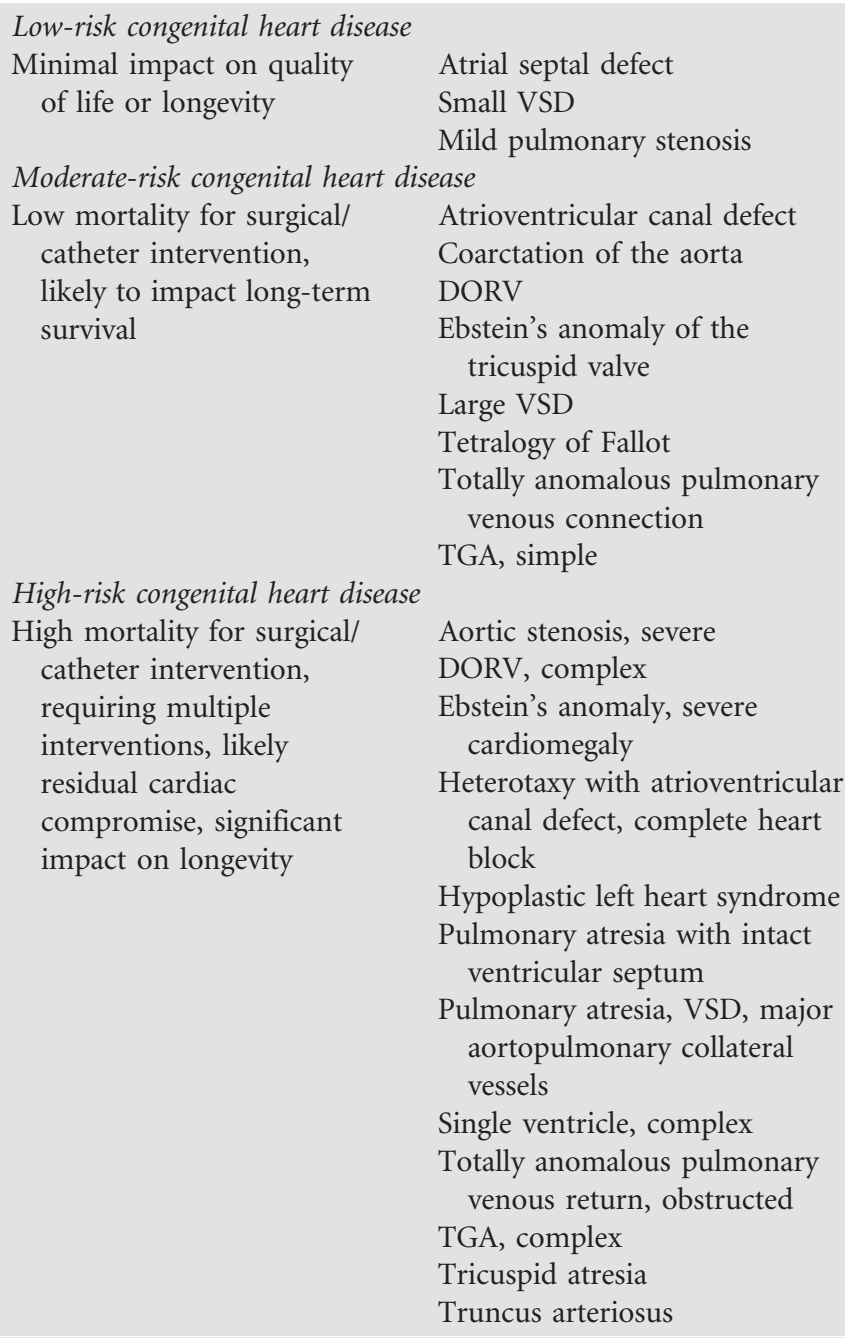

Abbreviations: VSD, ventricular septal defect; DORV, double-outlet right ventricle; TGA, transposition of the great arteries. Source: From Ref. 68.

surgical repair/palliation and the likelihood of residual cardiac disability. An approach for categorizing congenital heart defects in this fashion has been proposed by Allan and colleagues (84) and is presented in Table 4. This approach involves characterizing fetal cardiac anomalies as either low, moderate, or high risk based on expected impact on quality of life and longevity, as well as the complexity, mortality risk, and "completeness" of available surgical palliative procedures.

Making predictions regarding the need for perinatal medical and/or surgical management is also helpful. Identification of "reversed" physiologic shunting in the fetus with either right or left ventricular outflow tract obstruction can allow clinicians to make predictions about the need for prostaglandin infusion to maintain ductal patency and the need for transfer to a cardiac center for management of critical outflow tract obstruction. In our center, fetuses fitting this clinical description are routinely started on prostaglandin infusion following recommended delivery at a level III perinatal center and transferred to our cardiac intensive care unit for management. Examples of common lesions presenting in this

\section{Table 5 Risk of Chromosomal Anomalies in Common Congenital Heart Lesions}

High-risk congenital cardiac anomalies

Risk of chromosomal Absent pulmonary valve syndrome (20\%)

anomalies $>10 \% \quad$ Atrioventricular canal defect (35\%)

Coarctation of the aorta (29\%)

Double-outlet right ventricle (12\%)

Mitral valve atresia $(18 \%)$

Tetralogy of Fallot (27\%)

Totally anomalous pulmonary venous return $(30 \%)$

Truncus arteriosus (14\%)

Ventricular septal defect (48\%)

Low-risk congenital cardiac anomalies

Risk of chromosomal Hypoplastic left heart syndrome (4\%) anomalies $<10 \% \quad$ Pulmonary stenosis/atresia with intact ventricular septum (5\%)

Transposition of the great arteries $(0 \%)$

Tricuspid valve anomaly/Ebstein's (5\%)

Source: From Ref. 6.

manner include pulmonary stenosis/atresia, tetralogy of Fallot, tricuspid atresia, aortic stenosis, or variants of hypoplastic left heart syndrome. Other lesions that are routinely started on prostaglandin and transferred to the cardiac unit are severe coarctation and transposition of the great arteries. Generally, septal defects such as ASD, VSD, and AV canal defect do not require immediate intervention and can be evaluated more electively (after a confirmatory postnatal echocardiogram) at the birth hospital. In our center, all variants of single ventricle are delivered at a level III center and transferred to the cardiac unit for evaluation, given the complexity of cardiac issues involved in these cases. Given the complexity and diversity of congenital heart lesions, collaboration with a fetal cardiologist is important in arriving at a disposition for these complex fetuses.

Another major issue is genetic counseling for patients with the diagnosis of fetal CHD. Although we generally recommend genetic counseling for all mothers diagnosed with fetal CHD, it is known that certain cardiac lesions carry a particularly high risk of chromosomal abnormalities (6); these groups are outlined in Table 5. In addition to screening for aneuploidy, clinicians should also screen for microdeletions in chromosome 22, which is associated in high frequency with conotruncal defects and aortic arch anomalies. The presence of a significant chromosomal anomaly, as well as other extracardiac anomalies, has surgical implications, as most studies have identified these as risk factors for increased morbidity and mortality during surgical palliation, particularly in complex congenital heart defects.

\section{ADVANCES IN FETAL CARDIAC DIAGNOSIS AND THERAPY}

Evaluation of Fetal Arrhythmias by Magnetocardiography Magnetocardiography elucidates electrical signals in the fetal heart by detecting and analyzing the related magnetic fields. It processes the sequence of electrical events rather than mechanical events of the heart and can provide the capability of measuring different electrical time intervals such as QRS and QT intervals (85). Therefore, potentially it can reveal the 


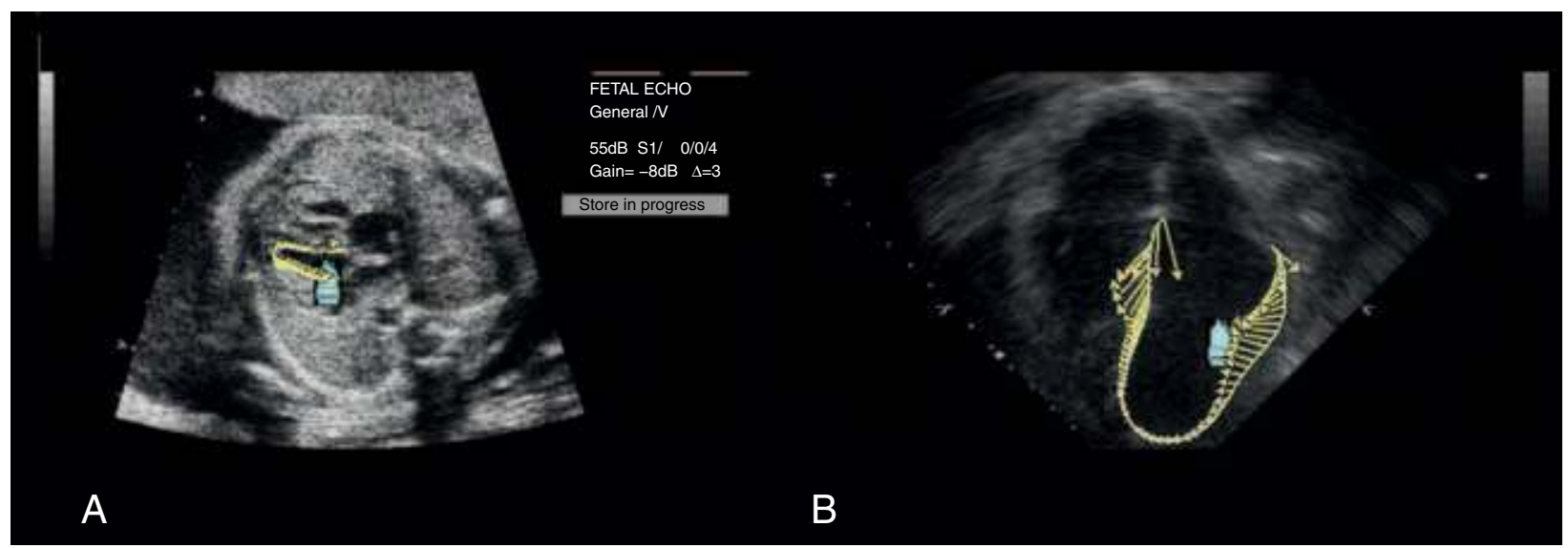

Figure 22 A vector velocity imaging (VVI) of 24-week gestation fetus of a diabetic mother shows the magnitude and direction of the longitudinal strain of the left ventricle (LV) indicated by the length and direction of arrows along the endocardial border of the LV (A) in transverse view. The VVI of the same infant at 1 week of age after birth shows the magnitude and direction of the longitudinal strain of the LV in four-chamber view (B).

mechanisms of fetal arrhythmias and aid in the differential diagnosis of fetal tachycardia such as in distinguishing VT with 1:1 conduction versus SVT as well as VT with AV dissociation versus junctional ectopic tachycardia (73). Discerning the mechanisms of fetal arrhythmias will help select the appropriate anti-tachyarrhythmic agents and unify the approach to the treatment of fetal arrhythmias. This modality is not in clinical use yet because of its need to be performed in a magnetically shielded room. Advancement has been made in the use of the technique at the bedside in an unshielded environment, which may bring it for wider use in the clinical practice (85).

\section{Evaluation of Fetal Cardiac Function by Strain Imaging} Cardiac function in fetuses has traditionally been measured by subjective assessment of contractility and observation for hydrops fetalis. Currently, myocardial functional assessment in the fetus has been limited by the reduced reproducibility due to the requirement for correct alignment to the structures being interrogated, lack of a concurrent electrocardiogram to time mechanical events accurately and unique properties of the fetal myocardium, which is made up of primarily noncontractile elements with fewer myocytes. Furthermore, most noninvasive methods of evaluation of cardiac function are load dependent (86). A novel method based on speckletracking techniques, including vector velocity imaging, has emerged that uses frame-by-frame tracking of myocardial speckles in 2D echocardiographic images to provide velocity and displacement data and permit quantification of the degree of myocardial deformation or strain (87) and strain rate, which is relatively load independent (88). The new method allows assessment of function independent of fetal lie, thus simplifying more sophisticated measures of function, such as strain and shear stress (89) (Fig. 22). This will help to improve the objectivity, reproducibility, and monitoring of fetal cardiac function.

\section{Fetal Cardiac Intervention}

Fetal cardiac intervention is a burgeoning field. Endeavors are being made in some centers to prevent fetal hydrops due to congenital heart defects, to recruit hypoplastic ventricles, to create a two-ventricle circulation after birth, and to remodel the fetal pulmonary vascular bed whose outlet is obstructed (90). A successful open heart surgery in the fetus has yet to be done, but catheter-based interventions for improved cardiac outcomes are now being taken up.

Fetal echocardiography can identify midgestation fetuses with aortic and pulmonary outflow obstruction, which are at high risk for developing hypoplastic left and right heart syndromes, respectively. With the development of novel techniques, risk reduction to the fetus and the mother, timely and successful valve dilation may prevent left or right heart growth arrest and may result in normal ventricular anatomy and function at birth $(91,92)$. There is an important learning curve for these procedures, and much remains to be learned about the selection of appropriate fetuses for prenatal intervention and further refinement in techniques of echocardiography, approach, and risk reduction in fetal intervention.

\section{REFERENCES}

1. Lange LW, et al. Qualitative real-time cross-sectional echocardiographic imaging of the human fetus during the second half of pregnancy. Circulation 1980; 62: 799-806.

2. Sahn DJ, et al. Quantitative real-time cross-sectional echocardiography in the developing normal human fetus and newborn. Circulation 1980; 62: 588-97.

3. Ott WJ. The accuracy of antenatal fetal echocardiography screening in high- and low-risk patients. Am J Obstet Gynecol 1995; 172: 1741-9.

4. Boughman JA, et al. Familial risks of congenital heart defect assessed in a population-based epidemiologic study. Am J Med Genet 1987; 26: 839-49.

5. Burn J, et al. Recurrence risk in offspring of adults with major heart defects: results from first cohort of British collaborative study. Lancet 1998; 351: 311-16.

6. Allan LD, et al. Prospective diagnosis of 1,006 consecutive cases of congenital heart disease in the fetus. J Am Coll Cardiol 1994; 23: 1452-8.

7. Copel JA, Pilu G, Kleinman CS. Congenital heart disease and extracardiac anomalies; associations and indications for fetal echocardiography. Am J Obstet Gynecol 1986; 154: 1121-32.

8. Respondek MI, et al. Extracardiac anomalies, aneuploidy, and growth retardation in 100 consecutive fetal congenital heart defects. Ultrasound Obstet Gynecol 1994; 4: 272-8.

9. Hyett JA, et al. Using fetal nuchal translucency to screen for major congenital heart defects at 10-14 weeks of gestation: population based cohort study. Br Med J 1999; 318: 81-5.

10. Yagel S, et al. Congenital heart defects: natural course and in utero development. Circulation 1997; 96: 550-5. 
11. AIUM Practice Guideline for the Performance of Fetal Echocardiography. J Ultrasound Med 2011; 30: 127-36.

12. Rychik J, et al. American Society of Echocardiography guidelines and standards for performance of the fetal echocardiogram. J Am Soc Echocardiogr 2004; 17: 803-10.

13. Winer-Muram HT, Tonkin ILD. The spectrum of heterotaxic syndromes. Radiol Clin North Am 1989; 27: 1147-70.

14. DeVore G. The prenatal diagnosis of congenital heart disease-a practical approach for the fetal sonographer. J Clin Ultrasound 1985; 13: 229-45.

15. Allan L. Fetal echocardiography. Clin Obstet Gynecol 1988; 31: 61-79.

16. Comstock C. Normal fetal heart axis and position. Obstet Gynecol 1987; 70: 255-9.

17. Shipp TD, et al. Levorotation of the fetal cardiac axis: a clue for the presence of congenital heart disease. Obstet Gynecol 1995; 85: 97-102.

18. Smith RS, et al. Ultrasonographic left cardiac axis deviation: a marker for fetal anomalies. Obstet Gynecol 1995; 85: 187-91.

19. Messing B, et al. Mild tricuspid regurgitation: a benign fetal finding at various stages of pregnancy. Ultrasound Obstet Gynecol 2005; 26: 606-10.

20. Copel JA, et al. Fetal echocardiographic screening for congenital heart disease: the importance of the four-chamber view. Am J Obstet Gynecol 1987; 157: 648-55.

21. AIUM Practice Guideline for the Performance of Obstetric Ultrasound Examinations. J Ultrasound Med 2010; 29: 157-66.

22. Carvalho JS, et al. Improving the effectiveness of routine prenatal screening for major congenital heart defects. Heart 2002; 88: 387-91.

23. Achiron R, et al. Extended fetal echocardiographic examination for detecting cardiac malformations in low risk pregnancies. Br Med J 1992; 304: 671-4.

24. Cartier MS, et al. The normal diameter of the fetal aorta and pulmonary artery: Echocardiographic evaluation in utero. AJR Am J Roentgenol 1987; 149: 1003-7.

25. Vinals F, Heredia F, Giuliano A. The role of the three vessels and trachea view (3VT) in the diagnosis of congenital heart defects. Ultrasound Obstet Gynecol 2003; 22: 358-67.

26. DeVore GR, Siassi B, Platt LD. M-mode assessment of ventricular size and contractility during the second and third trimesters of pregnancy in the normal fetus. Am J Obstet Gynecol 1984; 150: 981-8.

27. Schmidt KG, et al. Echocardiographic evaluation of dilated cardiomyopathy in the human fetus. Am J Cardiol 1989; 63: 599-605.

28. Friedman WF. The intrinsic physiologic properties of the developing heart. Prog Cardiovasc Dis 1972; 15: 87-111.

29. Nakanishi T, Jarmakani JM. Developmental changes in myocardial mechanical function and subcellular organelles. Am J Physiol 1984; 246 (4 Pt 2): H615-25.

30. Hofstaetter C, et al. A cardiovascular profile score in the surveillance of fetal hydrops. J Matern Fetal Neonatal Med 2006; 19: 407-13.

31. Figueras F, et al. Cardiac function monitoring of fetuses with growth restriction. Eur J Obstet Gynecol Reprod Biol 2003; 110: 159-63.

32. Goldinfeld $\mathrm{M}$, et al. Evaluation of fetal cardiac contractility by twodimensional ultrasonography. Prenat Diagn 2004; 24: 799-803.

33. Harada K, et al. Two-dimensional echocardiographic evaluation of ventricular systolic function in human fetuses with ductal constriction. Ultrasound Obstet Gynecol 1997; 10: 247-53.

34. Tan J, et al. Cardiac dimensions determined by cross-sectional echocardiography in the normal human fetus from 18 weeks to term. Am J Cardiol 1992; 70: 1459-67.

35. Tei $\mathrm{C}$, et al. New index of combined systolic and diastolic myocardial performance: a simple and reproducible measure of cardiac function-a study in normals and dilated cardiomyopathy. J Cardiol 1995; 26: 357-66.

36. Eidem BW, Edwards JM, Cetta F. Quantitative assessment of fetal ventricular function: establishing normal values of the myocardial performance index in the fetus. Echocardiography 2001; 18: 9-13.

37. Mori Y, et al. Evaluation of systolic and diastolic ventricular performance of the right ventricle in fetuses with ductal constriction using the Doppler Tei index. Am J Cardiol 2001; 88: 1173-8.

38. Friedman D, et al. Fetal cardiac function assessed by Doppler myocardial performance index (Tei Index). Ultrasound Obstet Gynecol 2003; 21: 33-6.

39. Barrea C, et al. Prenatal cardiovascular manifestations in the twin-to-twin transfusion syndrome recipients and the impact of therapeutic amnioreduction. Am J Obstet Gynecol 2005; 192: 892-902.
40. Michelfelder E, et al. Early manifestations and spectrum of recipient twin cardiomyopathy in twin-twin transfusion syndrome: relation to Quintero stage. Ultrasound Obstet Gynecol 2007; 30: 965-71.

41. Ichizuka $\mathrm{K}$, et al. The Tei index for evaluation of fetal myocardial performance in sick fetuses. Early Hum Dev 2005; 81: 273-9.

42. Aoki M, et al. Quantitative assessment of right ventricular function using Doppler tissue imaging in fetuses with and without heart failure. J Am Soc Echocardiogr 2004; 17: 28-35.

43. Huhta JC. Guidelines for the evaluation of heart failure in the fetus with or without hydrops. Pediatr Cardiol 2004; 25: 274-86.

44. Pedra SR, et al. Fetal cardiomyopathies: pathogenic mechanisms, hemodynamic findings, and clinical outcome. Circulation 2002; 106: 585-91.

45. Simpson LL, et al. Cardiac dysfunction in twin-twin transfusion syndrome: a prospective, longitudinal study. Obstet Gynecol 1998; 92 (4 Pt 1): 557-62.

46. Zosmer N, et al. Clinical and echographic features of in utero cardiac dysfunction in the recipient twin in twin-twin transfusion syndrome. $\mathrm{Br}$ Heart J 1994; 72: 74-9.

47. Carceller-Blanchard AM, Fouron JC. Determinants of the Doppler flow velocity profile through the mitral valve of the human fetus. Br Heart J 1993; 70: 457-60.

48. Kaufman TM, et al. Age-related changes in myocardial relaxation and sarcoplasmic reticulum function. Am J Physiol 1990; 259(2 Pt 2): H309-16.

49. Harada K, et al. Gestational age- and growth-related alterations in fetal right and left ventricular diastolic filling patterns. Am J Cardiol 1997; 79: 173-7.

50. Romero T, Covell J, Friedman WF. A comparison of pressure-volume relations of the fetal, newborn, and adult heart. Am J Physiol 1972; 222: 1285-90.

51. Hecher K, et al. Assessment of fetal compromise by Doppler ultrasound investigation of the fetal circulation. Arterial, intracardiac, and venous blood flow velocity studies. Circulation 1995; 91: 129-38.

52. Rizzo G, et al. Ductus venosus velocity waveforms in appropriate and small for gestational age fetuses. Early Hum Dev 1994; 39: 15-26.

53. Rizzo G, et al. The value of fetal arterial, cardiac and venous flows in predicting $\mathrm{pH}$ and blood gases measured in umbilical blood at cordocentesis in growth retarded fetuses. Br J Obstet Gynaecol 1995; 102: 963-9.

54. Rizzo G, et al. Ventricular ejection force in growth-retarded fetuses. Ultrasound Obstet Gynecol 1995; 5: 247-55.

55. Tulzer G, et al. Doppler in non-immune hydrops fetalis. Ultrasound Obstet Gynecol 1994; 4: 279-83.

56. Axt-Fliedner R, et al. Reference values of fetal ductus venosus, inferior vena cava and hepatic vein blood flow velocities and waveform indices during the second and third trimester of pregnancy. Arch Gynecol Obstet 2004; 270: 46-55.

57. Reed KL, et al. Doppler studies of vena cava flows in human fetuses. Insights into normal and abnormal cardiac physiology. Circulation 1990; 81: 498-505.

58. Reuss ML, Rudolph AM, Dae MW. Phasic blood flow patterns in the superior and inferior venae cavae and umbilical vein of fetal sheep. Am J Obstet Gynecol 1983; 145: 70-8.

59. Berg C, et al. Ductus venosus blood flow alterations in fetuses with obstructive lesions of the right heart. Ultrasound Obstet Gynecol 2006; 28: $137-42$.

60. Gudmundsson S, et al. Venous Doppler ultrasonography in the fetus with nonimmune hydrops. Am J Obstet Gynecol 1991; 164(1 Pt 1): 33-7.

61. Baschat AA, et al. Qualitative venous Doppler waveform analysis improves prediction of critical perinatal outcomes in premature growth-restricted fetuses. Ultrasound Obstet Gynecol 2003; 22: 240-5.

62. Tulzer G, et al. Diastolic function of the fetal heart during second and third trimester: a prospective longitudinal Doppler-echocardiographic study. Eur J Pediatr 1994; 153: 151-4.

63. Tsyvian $\mathrm{P}$, et al. Cardiac ventricular performance in the appropriate- forgestational age and small-for-gestational age fetus: relation to regional cardiac non-uniformity and peripheral resistance. Ultrasound Obstet Gynecol 2002; 20: 35-41. 
64. Wong SF, et al. Cardiac function in fetuses of poorly-controlled pregestational diabetic pregnancies-a pilot study. Gynecol Obstet Invest 2003; 56: 113-16.

65. Berning RA, et al. Reversed shunting across the ductus arteriosus or atrial septum in utero heralds severe congenital heart disease. J Am Coll Cardiol 1996; 27: 481-6.

66. Hansmann $M$, et al. Fetal tachyarrhythmias: transplacental and direct treatment of the fetus - a report of 60 cases. Ultrasound Obstet Gynecol 1991; 1: 62-70.

67. Naheed ZJ, et al. Fetal tachycardia: mechanism and predictors of hydrops fetalis. J Am Coll Cardiol 1996; 27: 1736-40.

68. vanEngelen A, et al. Management outcome and follow-up of fetal tachycardia. J Am Coll Cardiol 1994; 24: 1371-75.

69. Simpson JM, Sharland GK. Fetal tachycardias: management and outcome of 127 consecutive cases. Heart 1998; 79: 576-81.

70. Cuneo BF, Strasburger JF. Management strategy for fetal tachycardia. Obstet Gynecol 2000; 96: 575-81.

71. Simpson JM, et al. Outcome of intermittent tachyarrhythmias in the fetus. Pediatr Cardiol 1997; 18: 78-83.

72. Jaeggi E, et al. Ventriculoatrial time interval measured on M-mode echocardiography: a determining element in diagnosis, treatment, and prognosis of fetal supraventricular tachycardia. Heart 1998; 79: 582-87.

73. Wakai RT, et al. Magnetocardiographic rhythm patterns at initiation and termination of fetal supraventricular tachycardia. Circulation 2003; 107: 307.

74. Hofbeck M, et al. Prenatal findings in patients with prolonged QT interval in the neonatal period. Heart 1997; 77: 198-204.

75. Glickstein J, et al. The fetal Doppler mechanical PR interval: a validation study. Fetal Diagn Ther 2004; 19: 31-4.

76. Jaeggi E, et al. Prenatal diagnosis of complete atrioventricular block associated with structural heart disease: combined experience of two tertiary care centers and review of the literature. Ultrasound Obstet Gynecol 2005; 26: 16-21.

77. Schmidt KG, et al. Perinatal outcome of fetal complete atrioventricular block: a multicenter experience. J Am Coll Cardiol 1991; 17: 1360-6.
78. Lin MT, et al. Postnatal outcome of fetal bradycardia without significant cardiac abnormalities. Am Heart J 2004; 147: 540-4.

79. Gembruch $\mathrm{U}$, et al. Longitudinal study in 18 cases of fetal supraventricular tachycardia: Doppler echocardiographic findings and pathophysiologic implications. Am Heart J 1993; 125: 1290-301.

80. Gembruch U, et al. Venous Doppler in sonographic surveillance of fetuses with supraventricular tachycardia. Eur J Obstet Gynecol Reprod 1999; 84 187-92.

81. Allan LD, Chita SK, et al. Flecainide in the treatment of fetal tachycardias. Br Heart J 1991; 65: 46-8.

82. Strasburger JF, et al. Amiodarone therapy for drug-refractory fetal tachycardia. Circulation 2004; 109: 375-9.

83. Singh GK. Management of fetal tachyarrhythmias. Curr Treat Options Cardiovasc Med 2004; 6: 399-406.

84. Allan LD, Hornberger LK, Sharland G, eds. Textbook of Fetal Cardiology, 1st edn. Volume 1. London, UK: Greenwich Medical Media Ltd., 2000: 596.

85. Schneider U, et al. Prenatal diagnosis of long QT syndrome by fetal magnetocardiography in an unshielded bedside environment. Prenat Diagn 2005; 25: 704-8.

86. Suga H, Sagawa K, Shoukas AA. Load independence of the instantaneous pressure-volume ratio of the canine left ventricle and effects of epinephrine and heart rate on the ratio. Circ Res 1973; 32: 314-22.

87. Younoszai AK, et al. Evaluation of myocardial mechanics in the fetus by velocity vector imaging. J Am Soc Echocardiogr 2008; 21: 470-4.

88. Greenberg NL, et al. Doppler-derived myocardial systolic strain rate is a strong index of left ventricular contractility. Circulation 2002; 105: 99-105.

89. Matsui $\mathrm{H}$, et al. Temporal and spatial performance of vector velocity imaging in the human fetal heart. Ultrasound Obstet Gynecol 2011; 37: 150-7.

90. Huhta J, et al. Advances in fetal cardiac intervention. Curr Opin Pediatr 2004; 6: 487-93.

91. McElhinney DB, Tworetzky W, Lock JE. Current status of fetal cardiac intervention. Circulation 2010; 121: 1256-63.

92. Tworetzky W, et al. Balloon dilation of severe aortic stenosis in the fetus. Circulation 2004; 110: 2125-31. 


\section{Assessment of fetal behavior Asim Kurjak, Milan Stanojevic, Badreldeen Ahmed, Guillermo Azumendi, and Lara Spalldi-Barisic}

Three-dimensional (3D) ultrasound has been available for more than 15 years. During its continuous improvement and development, several different kinds of modes have been created. They include multiplanar imaging, volume rendering, surface rendering, 3D color Doppler, 3D volumetry, cine-loop animation, and post-processing and cutting. However, the 3D image freezes the object and therefore does not provide information on movements or any information about the dynamic changes of the object of interest. A technique was needed that would enable $3 \mathrm{D}$ imaging to be performed in a real-time mode. This technique can be called live $3 \mathrm{D}$ ultrasound (3D-US) or 4D ultrasound (4D-US), as coined by a manufacturer, because time becomes a parameter within the $3 \mathrm{D}$ imaging sequence. Human eyes are known to be able to differentiate between images up to a frequency of about 12 images per second; consequently, production of an appropriate frame rate with specially designed probes and a fast computer rendering device is required. At the moment, $4 \mathrm{D}-\mathrm{US}$ scanning is not real time and available machines can reach up to about 20 images per second, depending on volume size, resolution, and the mechanics of the probe. Nevertheless, even at these relatively slow frame rates, the ability to study fetal activity is strikingly good enabling the continuous monitoring of the fetal face and other surface features of the fetus such as fetal extremities. This was the beginning of investigation of the relatively unexplored area of fetal behavior as a possible measure of neurologic maturation.

Fetal behavior could be described as any fetal action or reaction observed by the mother or other more objective method, as actocardiotocography or ultrasonography. Although there are more than 100 years of curiosity in fetal behavior and more than three decades of sustained awareness and research, the study of fetal behavior has not achieved widespread acceptance in perinatal medicine. For centuries, maternal registration of fetal movements and obstetrician auscultation of fetal heartbeats were the only methods of the follow-up of fetal well-being in utero. It has been shown that fetal activity occurs as early as the late embryonic period, which is far earlier that a mother can sense it. A turning point in the assessment of fetal behavior was the introduction of real-time ultrasound. As it is not yet possible to assess functional development of the central nervous system (CNS) directly, investigators have started to analyze fetal behavior as a measure of neurologic maturation including properties of fetal hemodynamics and the muscular system, as well (1). This technique allowed the investigation of spontaneous fetal motor activity in utero. For the first time, studies of spontaneous prenatal movements and behavior were performed and published. Since fetal body movements give important information about the condition of the fetus, their quantitative as well as qualitative aspects were analyzed.

\section{NEW ADVANCES IN NEUROPHYSIOLOGY Development of the CNS and Prenatal Motor Development in the First Trimester}

Development of the human CNS begins in the early embryonic period and proceeds through a sequence of very complicated processes long after delivery. It is important to point out that the caudal region of the neural tube gives rise to the spinal cord, and the rostral region becomes the brain. The initial development is completed at 7 weeks of gestation (5 weeks after conception), when all five major subdivisions of the brain are clearly visible: the telencephalon, diencephalon, mesencephalon, metencephalon, and myelencephalon (Fig. 1) (2).

The earliest interneuronal connections, the synapses, can be detected in the spinal cord shortly before the onset of embryonic motility, at 6 to 7 weeks of gestation. Therefore, the neural activity leading to the first detectable movements is considered to originate from the spinal motor neurons. Another important prerequisite for the motility is the development and innervation of muscular fibers. It is well known that primitive muscle fibers (myotubes) are able to contract as soon as they are innervated by motor neurons. Between 6 and 8 weeks of gestation, muscle fibers can be formed by fusion of myoblasts, efferent and afferent neuromuscular connections can be developed, and spontaneous neural activity causing motility can begin.

The main events in the development of the CNS and motor development during the first 8 weeks of pregnancy are presented in Figure 1. The first spontaneous embryonic movements are gross body movements and they can be observed at the 7 to 7.5 th weeks of gestation. They consist of slow flexion and extension of the fetal trunk, accompanied by the passive displacement of arms and legs. These so-called vermicular movements appear in irregular sequences. Simultaneously with the onset of spontaneous movements, at the 7.5 th week of gestation, the earliest motor reflex activity can be observed, indicating the existence of the first afferent-efferent circuits in the spinal cord. The first reflex movements are massive and indicate a limited number of synapses in a reflex pathway. General movements (GMs) are the first complex, well-organized movement pattern, which involve head, trunk, and limb movements. This pattern has been interpreted as the first sign of a supraspinal control on motor activity and can be recognized from 8 to 9 weeks of gestation onward.

It is very important to note that even at this early stage of development, embryonic and fetal movements appear in recognizable temporal sequences, without any amorphous or 


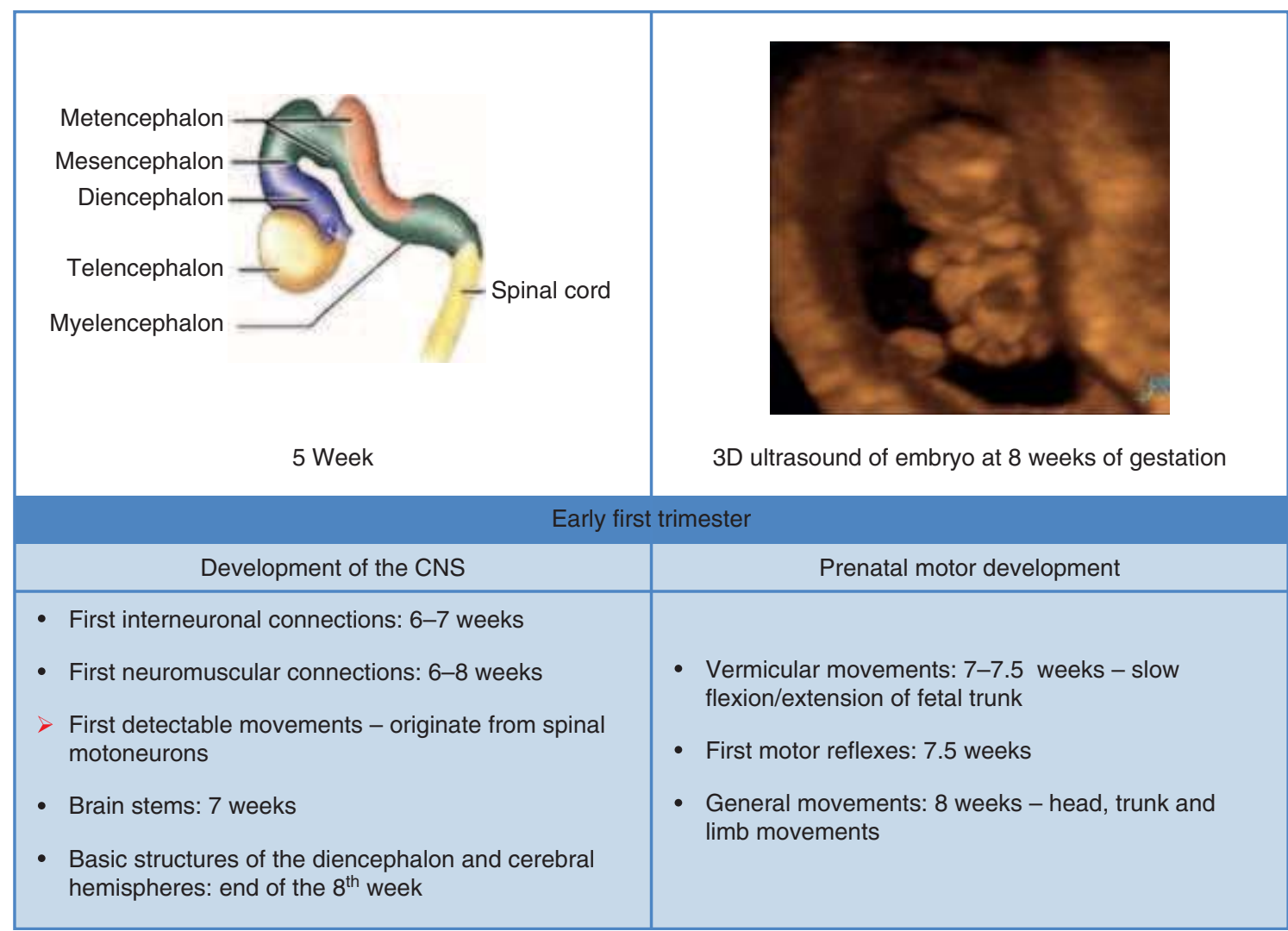

Figure 1 The main events in the development of the central nervous system (CNS) and motor development during the first 8 weeks of pregnancy.

random movement. The explanation for this fascinating phenomenon lies in the intrinsic properties of neurons. That means that neural cells begin to generate and propagate action potentials as soon as they interconnect. The interconnected neurons generate patterned activity because of endogenous properties of the neurons. Recent investigations have shown that neurons are able to communicate through non-synaptic mechanisms even before the onset of synaptogenesis.

The brain stem is fashioned around the 7 th week of gestation, and basic structures of the diencephalon and cerebral hemispheres are formed by the end of the 8th gestational week. The remarkable expansion of the cerebral hemispheres follows during the remainder of gestation. The development of synapses in the human cerebral cortex begins after the formation of the cortical plate, at the end of the 10th week of gestation.

The brain stem consists of the medulla oblongata, pons, and midbrain (Fig. 2). It forms and matures in a caudal to rostral direction. That means that the phylogenetically older structures, such as the medulla oblongata, will form and mature earlier in the gestation. The major structures of the medulla oblongata are fashioned by the 7 to 8 th week of gestation and are completely matured by the 7 months of gestation. In addition to its many subnuclei, the medulla gives rise to a variety of descending spinal motor tracts that reflexively trigger limb and body movements. It also hosts the five cranial nerves (VIII-XII), which exert tremendous influences on gross body movements, heart rate, respiration, and the head turning. As the medulla matures in advance of more rostral structures of the brain stem, reflexive movements of the head, body, extremities, as well as breathing movements (Fig. 2) and alterations in heart rate appear in advance of other functions.
The formation of pons begins almost simultaneously, but its maturation is more prolonged. The structures of the pons include the V to VIII cranial nerves (vestibular nuclei of the nerve VIII) and the medial longitudinal fasciculus, pontine tegmentum, raphe nucleus, and locus coeruleus, which exert widespread influences on arousal, including the sleep-wake cycles. Facial movements, which are also controlled by $\mathrm{V}$ and VII cranial nerves, appear around 10 to 11 weeks.

At 10 weeks of gestation, lateralized behavior may be observed, and the fetus begins to show the earliest signs of right- or left-handedness. Stimulation of the brain is known to influence brain organization and it is argued that fetal motor activity may eventually stimulate the brain to develop "handedness" and subsequent lateralization of the function. From 10 weeks onward, the number and frequency of fetal movements increase and the repertoire of movements begins to expand. Qualitative changes in GMs can be also observed. These movements, which are slow and of limited amplitude during 8 and 9 weeks, become more forceful at 10 to 12 weeks. After the 12th week, they become more variable in speed and amplitude. The isolated limb movements seen at the 9th week of gestation are followed by the appearance of the movements in the elbow joint at 10 weeks, changes in finger position at the 11th week, and easily recognizable clenching and unclenching of the fist at 12 to 13 weeks. Finally, at 13 to 14 weeks, isolated finger movements can be observed, as well increases in the activity and strength of the hand/finger movements. Using 4D sonography, Kurjak and collaborators have found that from 13 gestational weeks onward, a "goal orientation" of hand movements appears and a target point can be recognized for each hand movement (3). According to the spatial orientation, they classified the hand movements into several subtypes: 


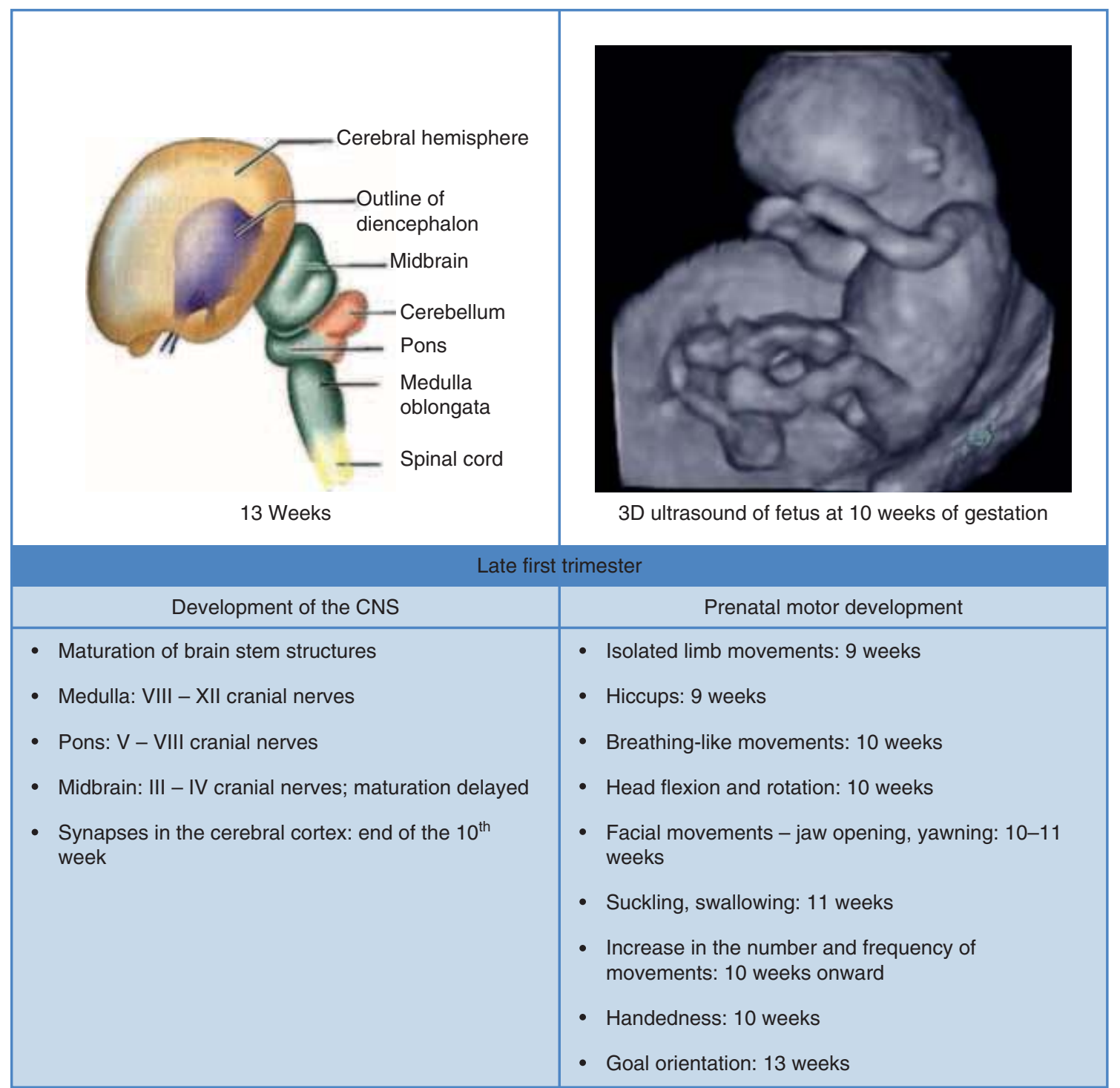

Figure 2 The main events in the development of the central nervous system (CNS) and motor development during the last 6 weeks of the first trimester.

hand to head, hand to mouth, hand near mouth, hand to face, hand near face, hand to eye, and hand to ear. Our recent longitudinal study, performed by $4 \mathrm{D}$ ultrasound in 100 fetuses from all trimesters of normal pregnancies, has shown increasing frequency of various movement patterns, such as GMs, isolated arm and leg movements, stretching, as well as head movements, during the first trimester (4). Only the startle movement pattern seemed to occur stagnantly in this period of gestation. Using 4D sonography, GMs were found to be the most frequent movement pattern between 9 and 14 weeks of gestation. Furthermore, the advanced ultrasonic techniques, 3D and 4D, significantly improve the assessment of structural and functional development of embryonic and fetal CNS. Figures 3 and 4 are showing the main events in the development of CNS and motor development during the second and third trimesters (2).

\section{Onset of Specific Fetal Behavioral Patterns}

\section{The First Trimester}

In the first trimester of pregnancy, 3D-US allows precise morphologic examinations, important for early detection of serious fetal malformations, such as anencephalic fetus and spina bifida (5). Using 4D-US, quantitative assessment of fetal motility can be performed almost equally precisely as by conventional 2D-US even at the very early period of gestation, at the onset of fetal motility. The qualitative assessment might be even more informative because 4D-US allows the simultaneous visualization of the whole body (Fig. 5).

However, our own study showed that we can use both 2D and 4D sonography to assess movements in the first trimester (6).

The early embryonic development is characterized by the immobility of an embryo. Most types of movement pattern emerge between 7 and 15 weeks of gestation. From the 15th week onward, distinct patterns can be seen (6). These movements remain present during the entire intrauterine development. Just discernible movements were found between 7 and 8 weeks of gestation by de Vries and coworkers (7). They reported not only how to describe a particular movement, but also how these movements were performed in terms of speed and amplitude $(7,8)$. Goldstein and colleagues as well as other investigators found embryonic body movements between 8 and 9 weeks of gestation by 2D transvaginal sonography (7,9-11). First spontaneous fetal movements were 


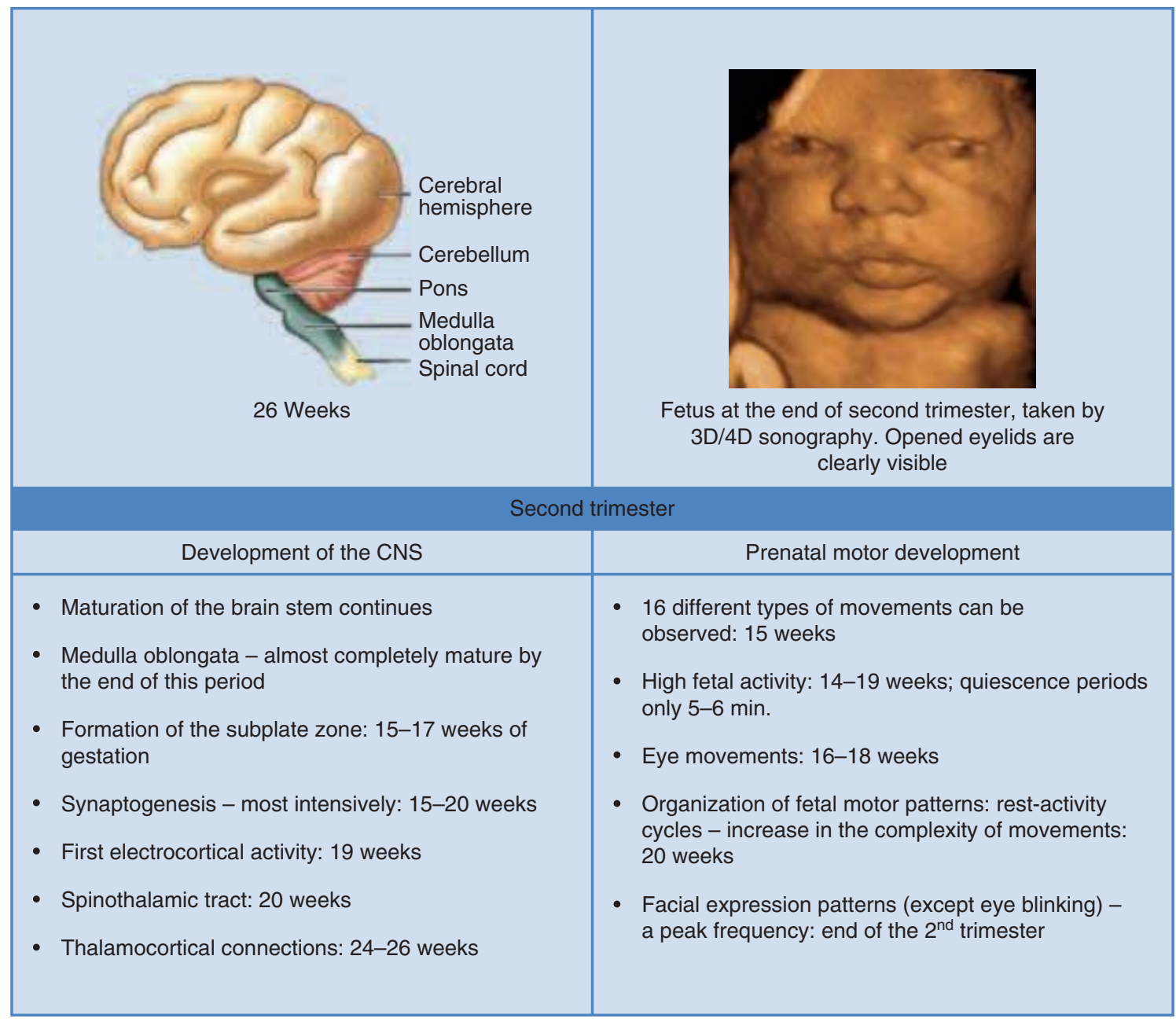

Figure 3 The main events in the development of the central nervous system (CNS) and motor development during second trimester.

described as consisted of slow flexion and extension of fetal trunk accompanied by the displacement of arms and legs and appearing in irregular sequences that were described as "vermicular" (12). The earliest signs of fetal motility coincide with the occurrence of first synapses, around the seventh postconceptional week and with the earliest electrical activity and transmission of information.

The aim of the first studies from Zagreb group on fetal behavior and $4 \mathrm{D}$ sonography was to classify first-trimester embryonic and fetal movements in normal pregnancies as seen by $4 \mathrm{D}$ sonography $(13,14)$. In order to achieve this goal, we examined a total of 98 pregnant women at gestational age (GA) from 6 to 12 weeks. Matters of interest were the GA at which the movements appeared and their visualization with $4 \mathrm{D}$-US. For each embryonic structure, there is a period between genesis and ultrasonic visualization. As technology advances, the period becomes shorter. Three types of movements can be visualized in the first trimester: gross body movements between 7 and 8 weeks, limb movements after 10 weeks, and complex movements after 11 weeks of gestation (Fig. 6).

An alteration from the given pattern of motoric development should be considered as an indication for further investigation (14).

By another study, the Zagreb group has evaluated the advantages of $4 \mathrm{D}$ over $2 \mathrm{D}$ real-time sonography in the assessment of early fetal behavior $(15,16)$. The aim of the study was to classify types of first-trimester embryonic and fetal movements in normal pregnancies as seen by $4 \mathrm{D}$ sonography. We found gross body movement at 7 weeks and limb movements at weeks 8 to 9 (Fig. 7).

Body and limb movements can be visualized by $4 \mathrm{D}$ sonography a week earlier than by $2 \mathrm{D}$. Since $4 \mathrm{D}$ ultrasound enables visualization of more details of the dynamics of small anatomical structures, it should thus enable the diagnosis of motoric development failure at the end of the first trimester.

With $4 \mathrm{D}$ transvaginal sonography, we found body movements at 7 weeks of pregnancy (15). The observed body movements consisted of the changing position of the head toward the body. However, embryonic movements are not frequent and consist mainly of the moving of the head toward the rest of the body. At 8 to 9 weeks, the head is less flexed and the changes of the position of the head toward the body are clearly visible (17). Vermicular movements are visible for less than 2 weeks. A startle is the next movement with participation of the fetal body. Startles were found between 8 and 9 weeks of gestation and lasted for about $1 \mathrm{sec}$ (18). Observations were performed using transabdominal sonography, and it is entirely possible that, with better resolution of the transvaginal transducer (either 2D or 4D), some of these movements may already be present at somewhat younger age.

In a little while, these first simple movements are replaced by different GMs that include head, trunk, and limb, such as "rippling" seen at the 8th week, "twitching" and "strong twitching" at 9th and 9.5th weeks respectively, and "floating," 


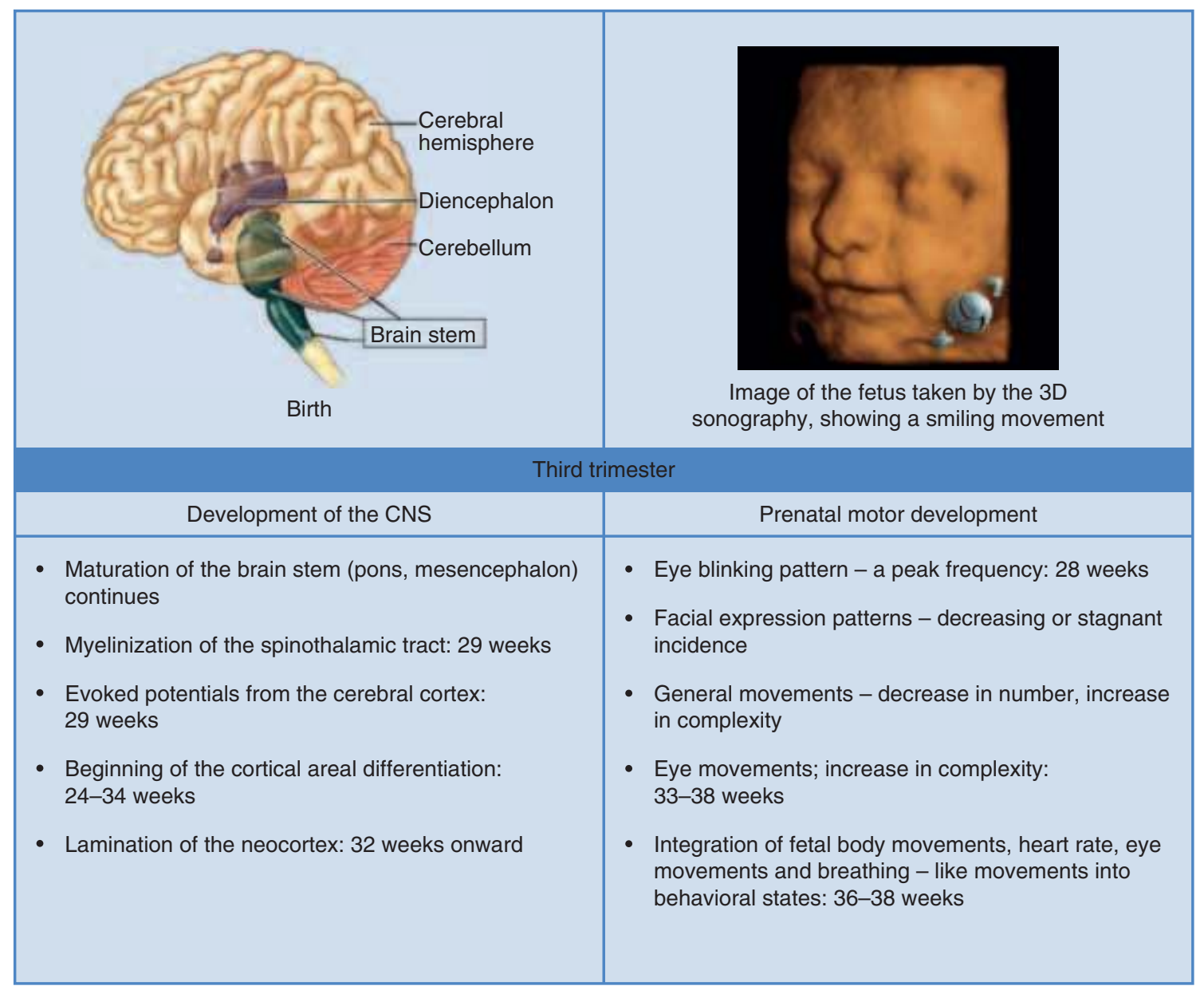

Figure 4 The main events in the development of the central nervous system (CNS) and motor development during third trimester.

"swimming," and "jumping" at 10th week (13). At first, GMs are slow and of limited amplitude. At 10 to 12 weeks, GMs become more forceful but are smooth in appearance and of large amplitude. We can suppose that the generating neural network responsible for GMs could be primarily located in the brain stem and spinal cord, higher structures of the brain playing more subtle role in modulating quality, and, perhaps, time patterning of the different movement patterns (19). Isolated limb movements emerge almost simultaneously with the generalized movements. In the literature, there is a range between the 8th and 12th weeks concerning the first appearance of limb movement. De Vries found isolated arm and leg movements at the 8th week of gestation (7). With 4DUS, limb movements at the 8th to 9th weeks were found. Organization of the appearance of the movement pattern occurs with the increasing frequency. It seems that fetal arms explore the surrounding environment and cross the midline, while the palmar surface is oriented toward the uterine wall. The fetal legs are extended to the uterine wall (Fig. 8).

More limb joints are active and move simultaneously, such as extension or flexion in arm and elbow or hip and knee. The elevation of the hand, extension of the elbow joint, with a slight change in direction and rotation, can be seen simultaneously. From the 10th week onward, the number and frequency of fetal movements increase and the repertoire of movements begins to expand. The isolated limb movements seen at the 9th week are followed by the appearance of the movements in the elbow joint at the 10th week, changes in finger position in the 11th week, and by easily recognizable clenching and unclenching of the fist at the 12th to 13th week.

After the 9th gestational week, the repertoire of movements expands rapidly. Hiccups appear, often in series, for up to several minutes, and isolated arm and leg movements can be observed. This is remarkable in two respects. First is that the young fetus is able to perform isolated movements of one limb at an age when one would expect a longer period of diffuse and generalized motor activity. The second is the unexpected finding of the simultaneous onset of arm and leg movements, unexpected because of the long-held principle of a cephalocaudal development in spinal motor functions. After 10 weeks, head movements of various types can be seen. They consist of lateral rotation of the head and overextension of the neck (20). At about the same age, hand-face contact is seen for the first time. Usually, this is an accidental contact of a hand with the face or the mouth. Between 10.5 and 12 weeks, the fetus starts to make breathing movements. At 11 weeks, three new patterns, namely, the opening of the jaw, bending forward of the head, and complex stretch movements, are added to the repertoire (Fig. 9).

Somewhat later than the irregular jaw movements, yawns occur. These have the same pattern as in children and adults and hence are easily recognizable (20).

To determine the accuracy of $4 \mathrm{D}$ sonography in the assessment of embryonic and early fetal motor activity in the first trimester of normal pregnancy, in comparison with the $2 \mathrm{D}$ sonography, the Zagreb group conducted a study with 


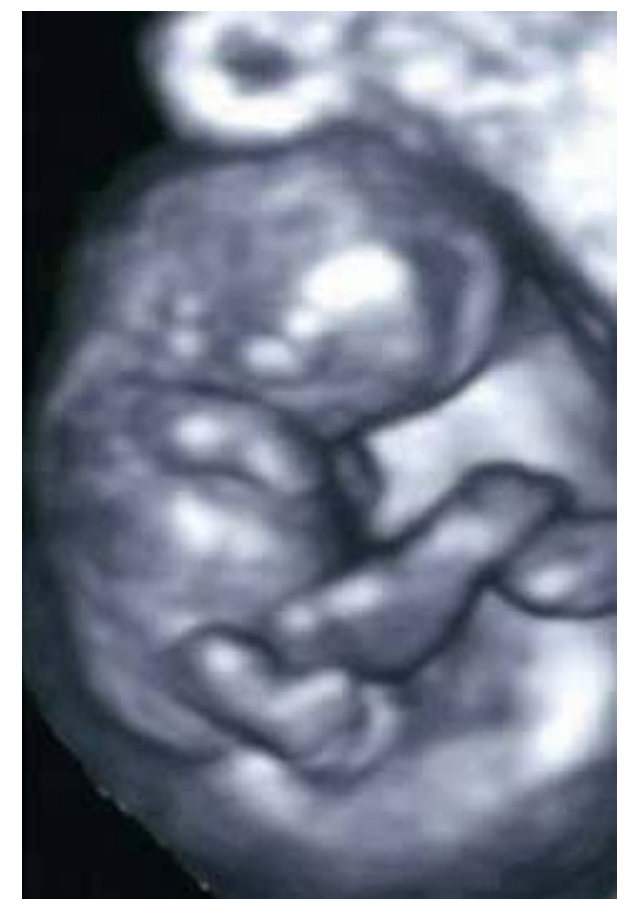

(A)

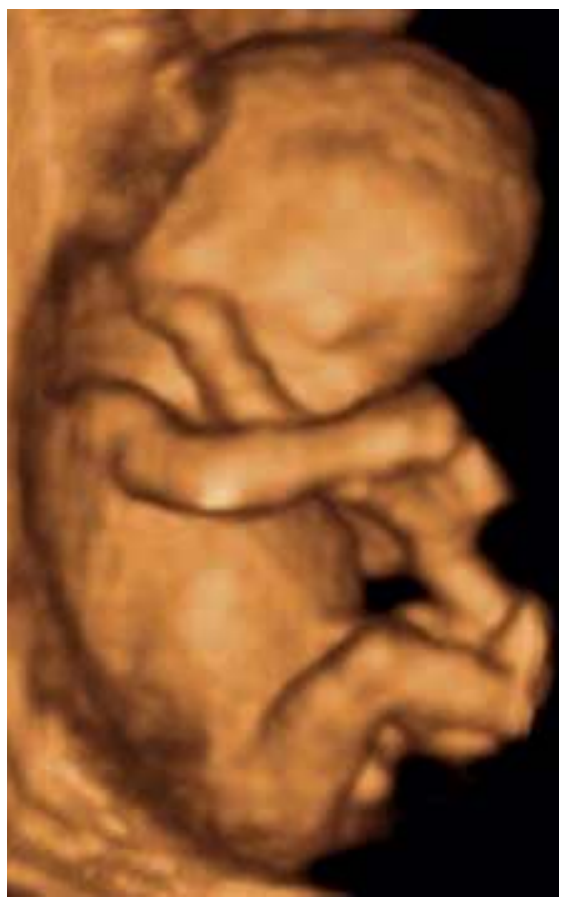

(B)

Figure 5 The whole body of the fetuses 9 (A) and 10 (B) weeks of gestation depicted by 3D ultrasound.

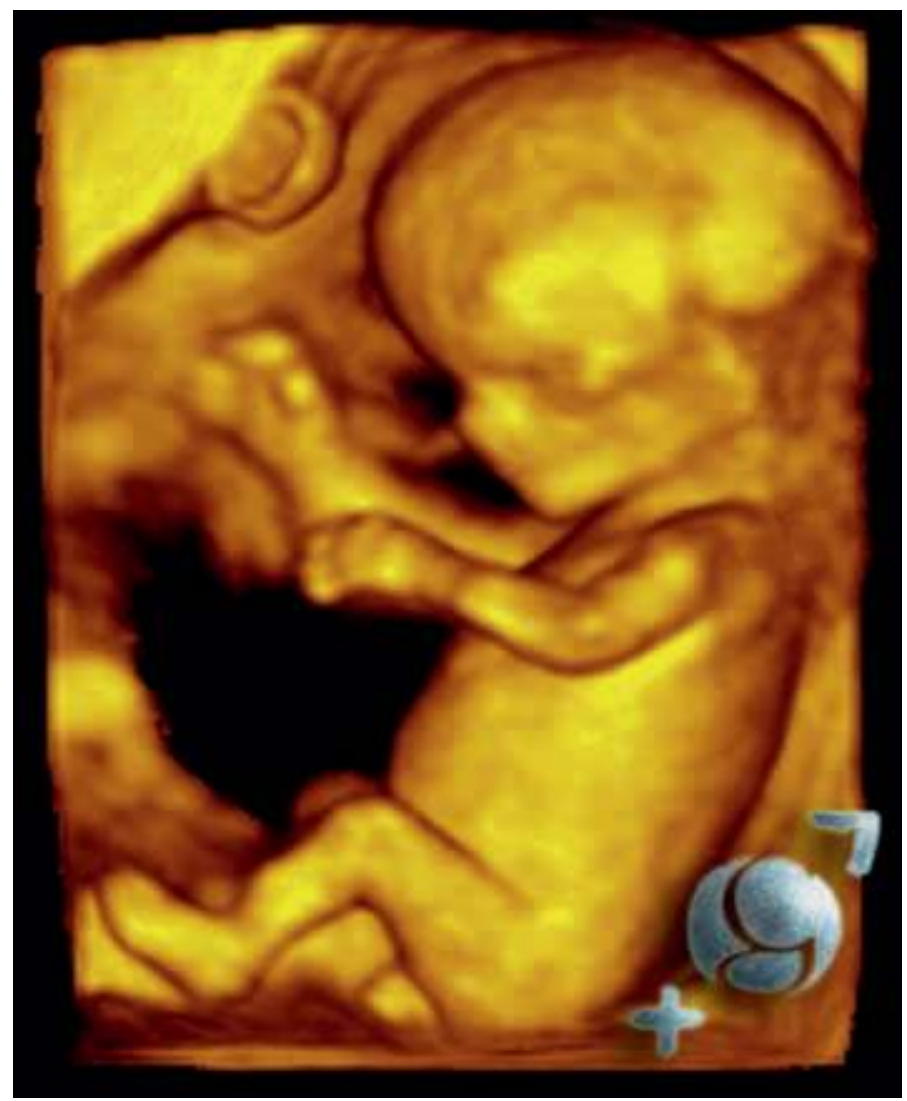

Figure 6 Complex movements of the trunk, limbs, and head in 12-week fetus depicted by 3D ultrasound.

50 pregnant women between 6 and 14 weeks of gestation. The $2 \mathrm{D}$ and $4 \mathrm{D}$ recordings were displayed on the screen and recorded on the videotape during the 15-minute observation period. The earliest embryonic movements were detected at the 7th week of gestation. The movements that involved the whole body, such as GMs and startles, occurred most frequently, although in 8-week-old embryos, isolated arm and leg movements could have also been observed. All the above described movement patterns were also recognizable by both $2 \mathrm{D}$ and $4 \mathrm{D}$ imaging methods in fetuses studied between 


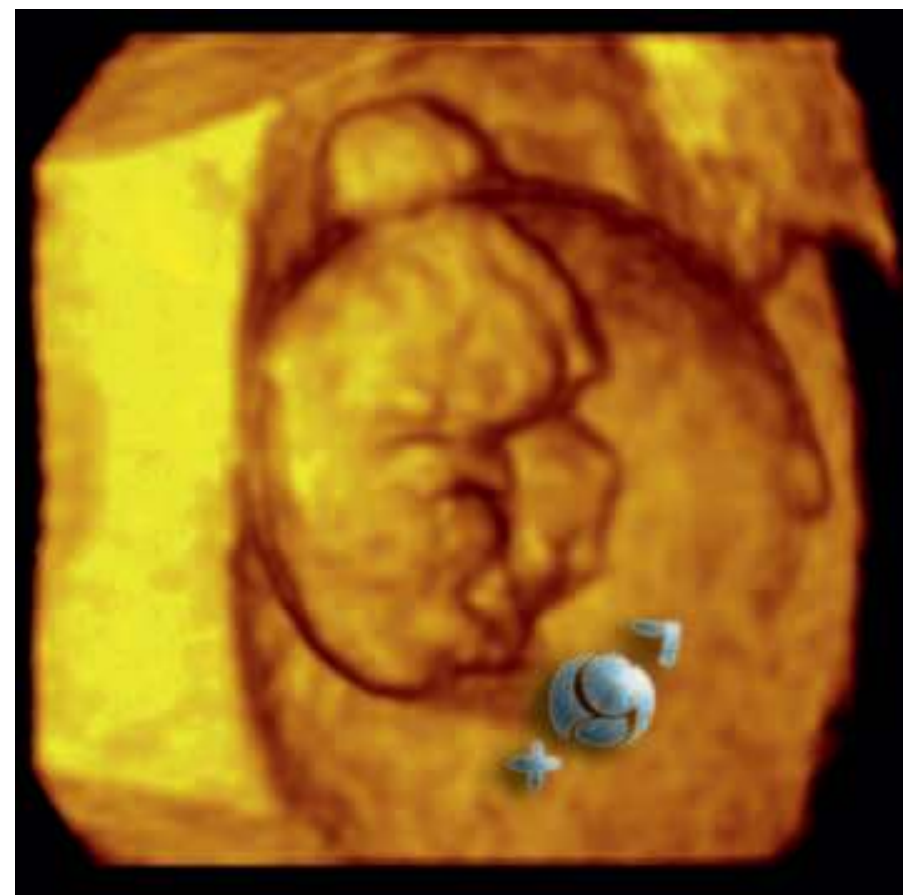

Figure 7 Gross body movements of the 8-week embryo depicted by 3D/4D ultrasound.

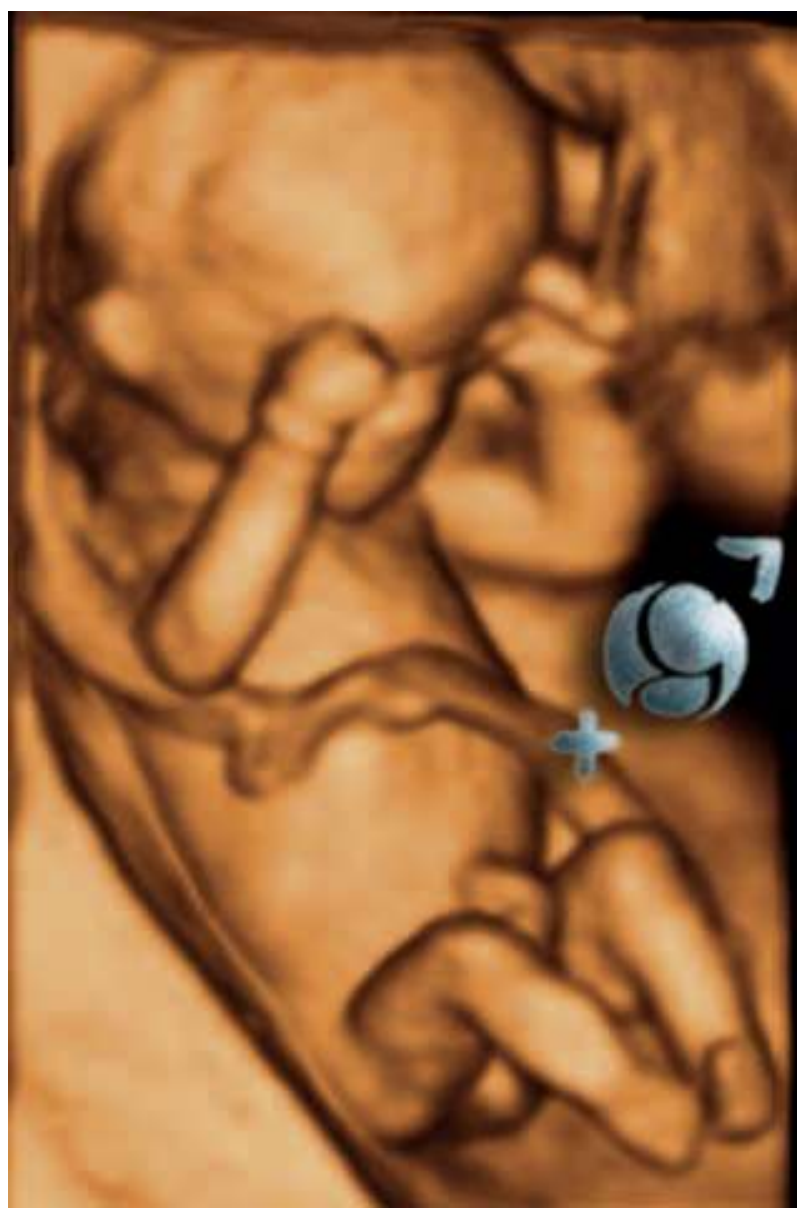

Figure 8 Fetal legs extended to the uterine wall (3D ultrasound of 10-week fetus).

the 9th and 14th weeks of gestation. Several movement patterns, such as sideway bending, hiccup, fetal breathing movements, and facial movements could not be observed by 4D-US imaging technique, although they were clearly visible by $2 \mathrm{D}$-US. The authors concluded that at the time, both $2 \mathrm{D}$ and $4 \mathrm{D}$ methods were required for the assessment of early fetal motor development and motor behavior. It was reasonable to expect that such technologic improvement may provide some new information about the intrauterine motor activity and facilitate the prenatal detection of some neurologic disorders (20).

To investigate the natural course of fetal hand and finger positioning, Pooh and Ogura examined 65 normal fetuses by 3D/4D ultrasound (21). Each appearance of fingers, thumb, and wrist was confirmed by viewing on three orthogonal planes. At 9 weeks and at the beginning of the 10th week, fetal hands were located in front of the chest and no movements of wrists and fingers were visualized. From the middle of 10th week of gestation, active arm movements were observed associated with active body and lower limb movement. Despite active movements of glenohumeral and elbow joints at this stage, wrist and finger movements were not visualized in most cases. At 11 weeks, a change in finger positioning was seen. At this stage, all fetuses still opened their palms, but five digits were no longer on the same layer. Mild adduction of the thumb and atonic fingers were observed and the palm appearance was clearly different from that at 9 and 10 weeks. At 12 weeks of gestation, fetuses started to mildly clench and unclench their fists (21). Confirming the feasibility of early dynamic observations in pregnancy, these studies also showed the capacity to obtain much better defined images as the whole fetus could be visualized.

In the first trimester, one could notice a tendency toward an increased frequency of fetal movement patterns with increasing GA. Only the startle movement pattern seemed to occur stagnantly during the first trimester (22).

\section{The Second Trimester}

Only a few studies are available on fetal movement patterns during the second trimester (3,7,23-27). De Vries and 


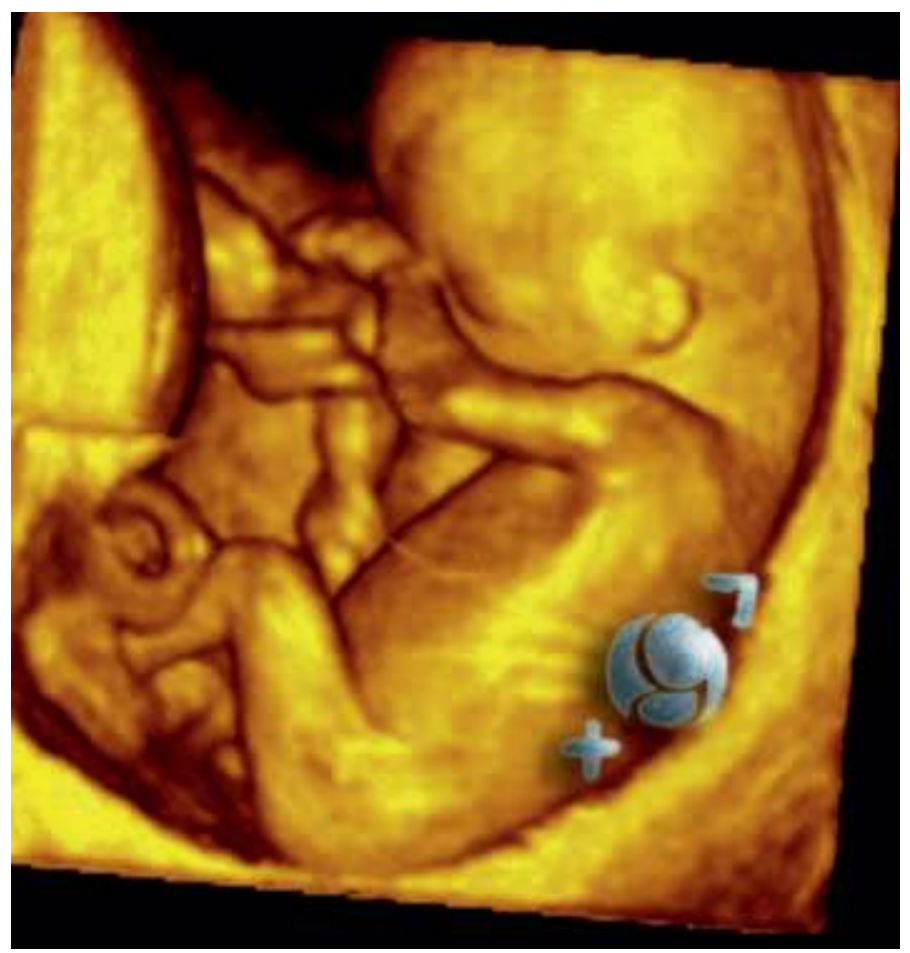

Figure 9 Fetus at 11 weeks depicted by 3D ultrasound showing addition of complex movements of the head, body, and limbs to the repertoire of movements.

colleagues studied fetal movements from 20 and 24 postmenstrual weeks onward (7). During the second trimester of pregnancy, the incidence of body movements increases considerably. The periods of quiescence become longer and eye movements (EMs) are clearly visible with significant trends in fetal EM organization (26). The earliest EMs appear at the developed by the end of the first trimester, indicating that some facial grimaces could appear at the 16th to 18th week of gestation, as do sporadic movements with a limited frequency. Isolated eye blinking patterns appear more frequently and begin to consolidate at the 24th to 26th week of gestation.

The incidence of hiccups startles and stretches decreased, whereas other movement patterns (jaw movement, hand-face contacts, and head movements) showed no clear developmental changes (24). At 28 postmenstrual weeks, Roodenburg and associates presented the following ranges and median values based on 1-hour observations: jaw movements, 60-460, median 300; hand-face contact, 30-190, median 95; head rotations, 20-125, median 37; head retroflexions, 4-29, median 12 (17). From a developmental point of view, one could say that in the second trimester the development continues, but there are no new movements appearing for the first time.

Spontaneous movements in fetuses from 12 to 35 weeks of gestation were described by Sparling and coworkers and they documented the typical hand movement (26). Many movements were shown to be straightforward, to a body part or uterine wall. The hands moved with different frequencies and force. Sparling also described repetition of head flexion movements, resulting in a "somersault" that makes possible for the fetus to change position within the uterine cavity throughout GAs. The hands of fetuses were extended and manipulated body parts and feature of the surroundings, for example, the umbilical cord. In a longitudinal study of fetal behavior from 14 weeks until neonatal period, Sparling and
Wilhelm concluded that fetal and neonatal movements appeared to be directed to specific targets to the head and face (27). They stated that the most useful predictors of neonatal movement were the observations performed at 32 weeks of gestation.

After 12 weeks, GMs become more variable in speed and amplitude. We already know that most of the qualitative features of GMs are already present during the 4 th to the 5 th months of gestation, with minor changes occurring in the last trimester of pregnancy.

Using $4 \mathrm{D}$ sonography, the Zagreb group has found that from the 13th gestational week onward, a "goal orientation" of hand movements appears and a target point can be recognized for each hand movement (3). According to the spatial orientation, the hand movements were classified into several subtypes: hand to head, hand to mouth, hand near mouth, hand to face, hand near face, hand to eye, and hand to ear and its incidence were determined. The investigation included 25 fetuses in uncomplicated pregnancies: 15 fetuses at 13 to 16 weeks and 10 fetuses at 30 to 33 weeks of gestation. After standard assessment in $2 \mathrm{D}$ real-time $\mathrm{B}$ mode, a $4 \mathrm{D}$ mode was switched on and further examination lasted a maximum of 15 minutes. All recordings were performed between 14 and 17.30hours, and no meals were taken within 2 hours of the beginning of the study. Patients with multiple or diabetic pregnancy at presentation were excluded from the study. In the first part of the study, GA was determined using biparietal diameter measurement, while in the second part of the study, GA was estimated from the first day of the last menstrual period and confirmed by the first-trimester sonographic examination.

Isolated hand movements with subtypes of hand movements and facial activities with different forms of expression were easily recognized by $4 \mathrm{D}-\mathrm{US}$. All subtypes of hand to head movement could be seen from 13 weeks of gestation, with 
fluctuating incidence. Among facial expressions, two types could be easily differentiated: smiling and scowling. One could recognize that the amount of isolated arm movements decreased gradually from 13 to 16 weeks. The incidence varied between 50 and 120 with a median value of 60 at 13 weeks, 17 and 27 with a median value of 23 at 14 weeks, 0 and 6 with a median value of 2 at 15 weeks, and 18 and 28 with a median value of 25 at 16 weeks. The highest range was registered at 13 weeks of gestation. The incidence of hand to head movement decreased, followed by a plateau at 14 weeks of gestation. The incidence varied from 4 to 29 at 13 weeks and from 0 to 7 at 16 weeks of gestation. The highest range of hand at mouth was found at 15 weeks of gestation; at 13 weeks, a plateau was observed and with mild fluctuations the plateau continued until 16 weeks. The incidence varied between 0 and 4 with a median value of 2 at 13 weeks, and between 0 and 2 with a median value of 2 at 16 weeks. In contrast to most other movement patterns, hand near mouth movements decreased gradually from 13 weeks onward with a single fluctuation in the 14th week. One can recognize that the incidence of hand near face movement is stable between 13 and 16 weeks of gestation with a slight increase at 14 and 15 weeks. At 13 weeks, the range was the widest. The incidence of hand to ear movement showed a rapid trend of decrease between 13 and 16 weeks while the incidence of the hand to eye movement pattern showed the same developmental trend as the hand to head and hand to face movement patterns (28). The incidence varied between 4 and 12 with a median value of 8 at 13 weeks, and 0 and 3 with a median value of 0 at 16 weeks. The incidence of the hand to eye movement pattern showed the same developmental trend as the hand to head and hand to face movement patterns. At 13 weeks, the incidence was between 4 and 12 occurrences per 15-minute observation time with a median value of 8 . At 16 weeks, the range was from 0 to 3 with a median value of 1 . The authors concluded that $4 \mathrm{D}$-US is superior over $2 \mathrm{D}$ real-time ultrasound for the qualitative, but inferior for quantitative analysis of hand movements. Thus, $4 \mathrm{D}$-US makes it possible to determine exactly the direction of the fetal hand, but the exact number of each type of hand movements still could not be determined. The advantage of $4 \mathrm{D}$ over real-time $2 \mathrm{D}$-US is that isolated hand movements can be determined with confidence. The $2 \mathrm{D}$ sonography easily recognizes hand movements associated with body movements, but there are problems in the recognition and differentiation of isolated hand movements and hand movements associated with leg movements. In this situation, 4D-US is the method of choice for the reliable recognition of the isolated hand movements. Another advantage of $4 \mathrm{D}$-US is the precise assessment of the direction of the hand movement and target of the fingers. Real-time 2D-US provides a tomographic image of the fetal head and visualizes hand movements in two dimensions. The 4D sonography provides surface-rendered images of the fetal head, and visualization of hand movements in three dimensions allows further differentiation of hand to head movements (3).

In another study, the same group of authors confirmed that in the second trimester, the number of head and hand movements decreased gradually compared with the first trimester. The highest incidence was registered for head retroflexion pattern, with a range of 15 to 42 and a median of 25 .
Among facial expressions, the highest incidence was found for sucking, with the range between 3 and 30 and a median of 9 (28).

Kuno and coworkers evaluated fetal behavioral patterns in the early second trimester in 11 healthy pregnant women at 14 to 18 weeks of gestation and they found that the most active fetal behavioral pattern was arm movement in each fetus, whereas the least active was mouth movement. Each fetal movement was synchronized and harmonized with other fetal movements during this period of pregnancy (24).

Kurjak et al. reported the first study with the 4D-US techniques used for obtaining longitudinal standard parameters of fetal neurologic development in all trimesters of a normal pregnancy (4). Valid reference ranges appropriate for GAs are essential for comparisons with former or future measurements of patients. For that purpose, a group of 100 healthy normal singleton pregnancies were recruited for longitudinal 4D-US examinations to evaluate fetal neurodevelopmental parameters between 7 and 40 weeks of gestation. The patients were assigned to the study if they met inclusion criteria, if the fetus and the mother were considered "normal," 2D ultrasound and clinical assessment were uneventful, and the neonates, eventually delivered at term, had normal 1- and 5-minute Apgar scores. Pregnancies that were subsequently found to be complicated by congenital abnormalities, gestational diabetes, and hypertensive disorders in pregnancy, preterm deliveries, and abnormal Apgar scores were excluded. Those fetuses from the second and third trimesters whose examined parts of the body were not able to be visualized in one region of interest were also excluded from the study. In the first trimester, eight fetal movement patterns were analyzed and 14 parameters of fetal movement and fetal facial expression patterns recorded thereafter for the construction of fetal neurologic charts. Standard parameters of fetal movements and facial expressions in all trimester of pregnancy are presented in Figures 10 and 11.

The statistical analysis of the incidence of fetal movements and facial expressions studied in the first trimester revealed statistically significant changes in GMs, stretching, isolated arm and leg movement, head retroflexion, head rotation, and head retroflexion. At the first trimester, a tendency toward increased frequency of fetal movement patterns with increasing GA has been noticed. Only in the startle movement pattern, it seemed to occur stagnantly during the first trimester (Fig. 10B). In this type of movement, there was no significant correlation with GA, as shown by the large dispersion of scatter points around the regression line ( $\mathrm{r} 0.673 ; p 0.506)$. At the first trimester, a tendency toward increased frequency of fetal movement patterns with increasing GA was noticed. During the second and third trimesters, multiple regression and polynomial regression revealed statistically significant changes in tongue expulsion, grimacing, swallowing, head movements, and all hand to body contact movements $(p<0.05)$. The authors found a tendency toward an increase in the frequency of fetal movement patterns at the beginning of the second trimester. They noticed fluctuation and dispersion of the incidence of all facial expressions as seen in the polynomial regression diagram (Figs. 11I-N). All types of facial expressions display a peak frequency at the end of the second trimester, except in isolated eye blinking, which increases at 
(A)

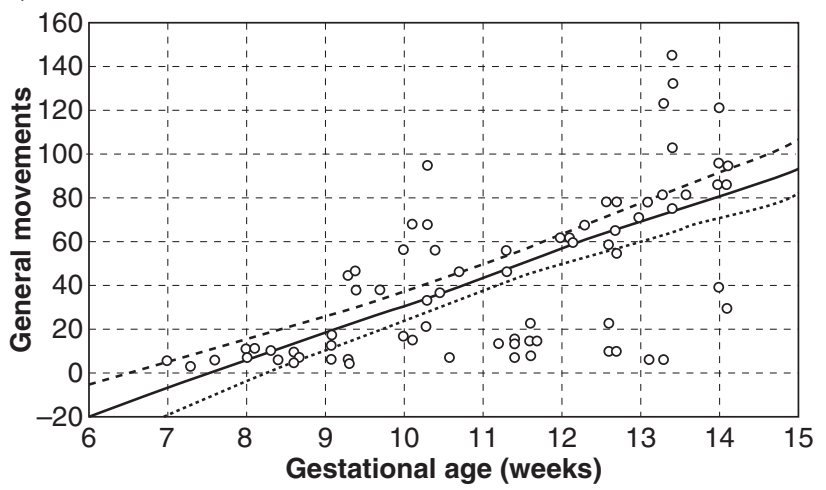

(C)

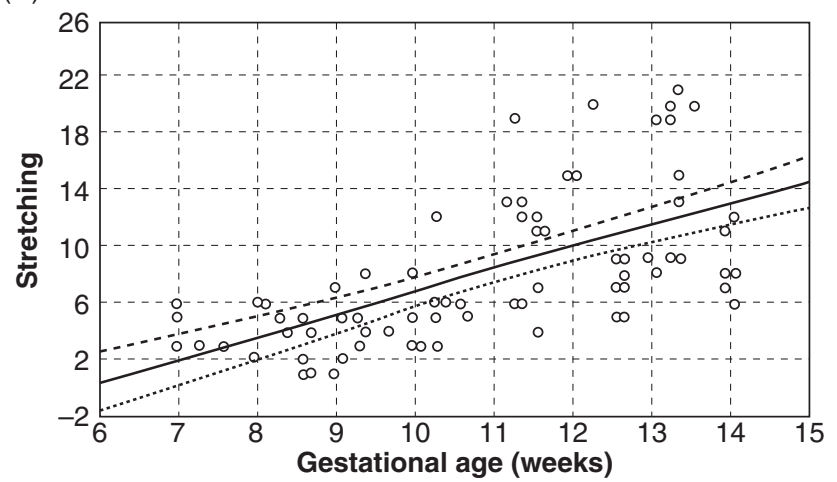

(E)

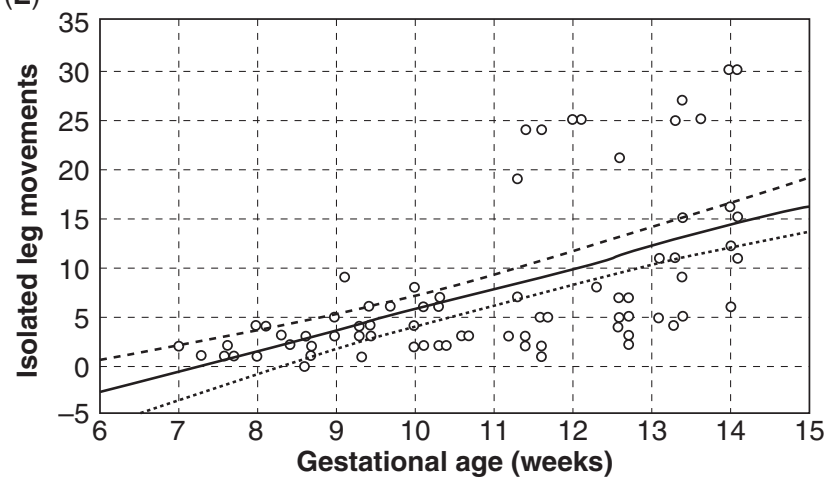

(G)

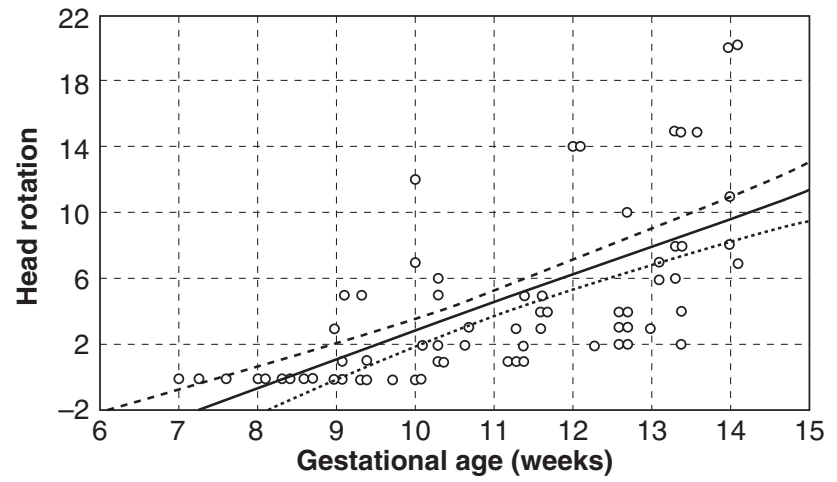

(B)

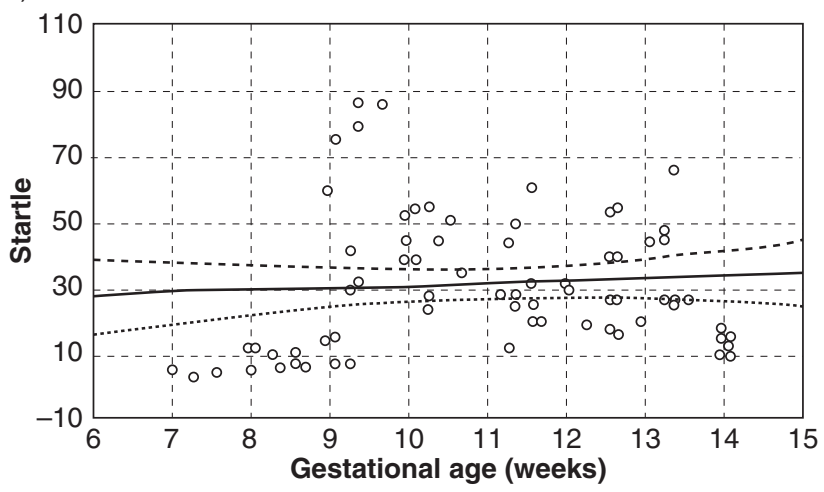

(D)

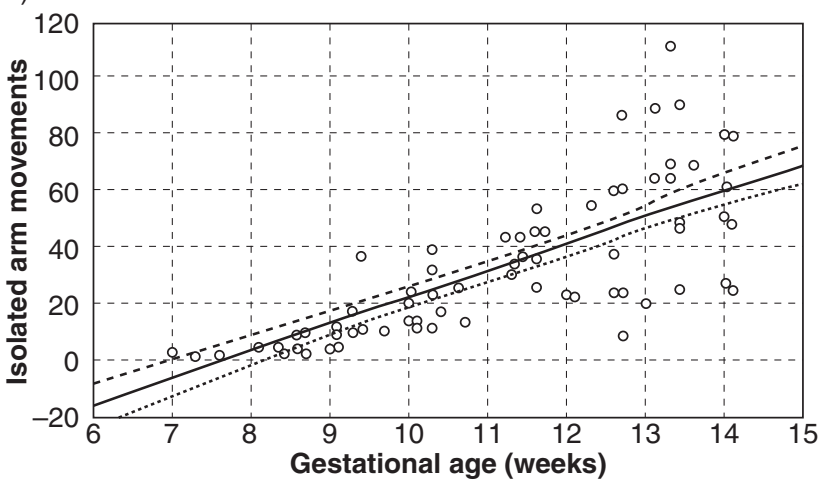

(F)

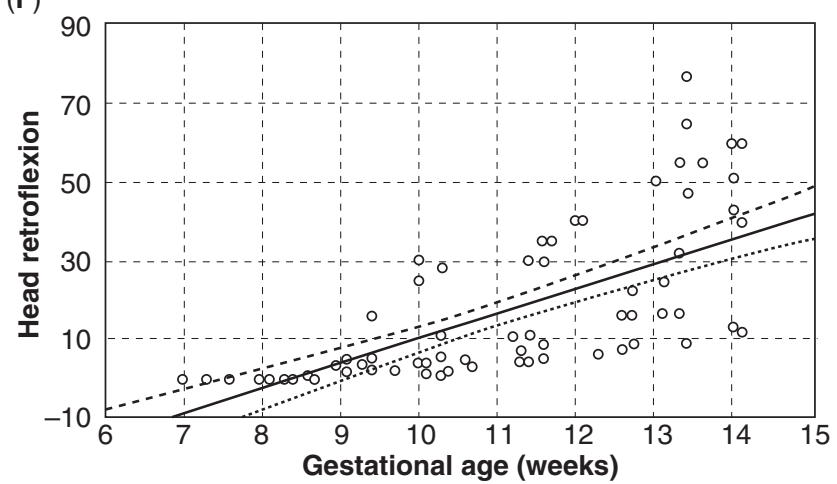

(H)

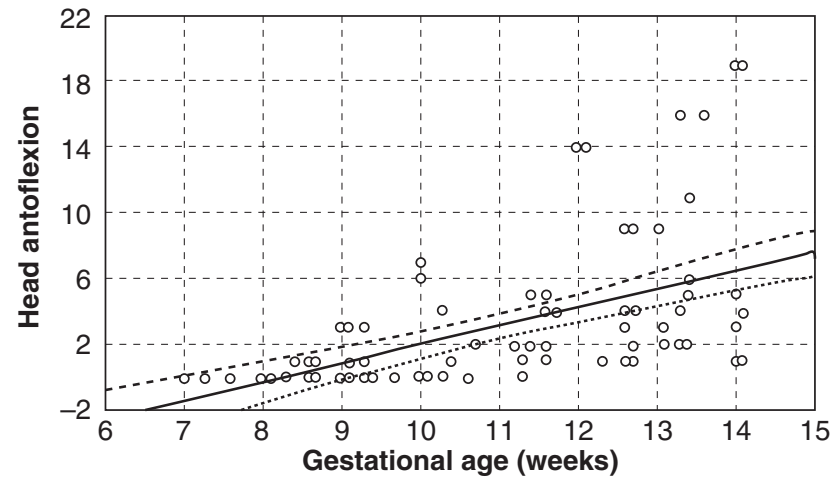

Figure 10 Scatter plot and multiple regression analysis of the first-trimester frequency of (A) general movements, (B) startle, (C) stretch, (D) isolated arm movement, (E) isolated leg movement, $(\mathbf{F})$ head retroflexion, $(\mathbf{G})$ head rotation, and (H) head anteflexion. 
(A)

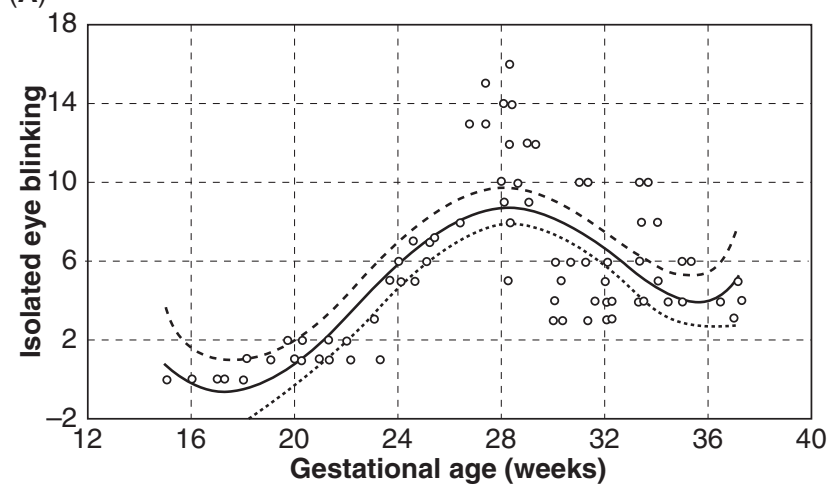

(C)

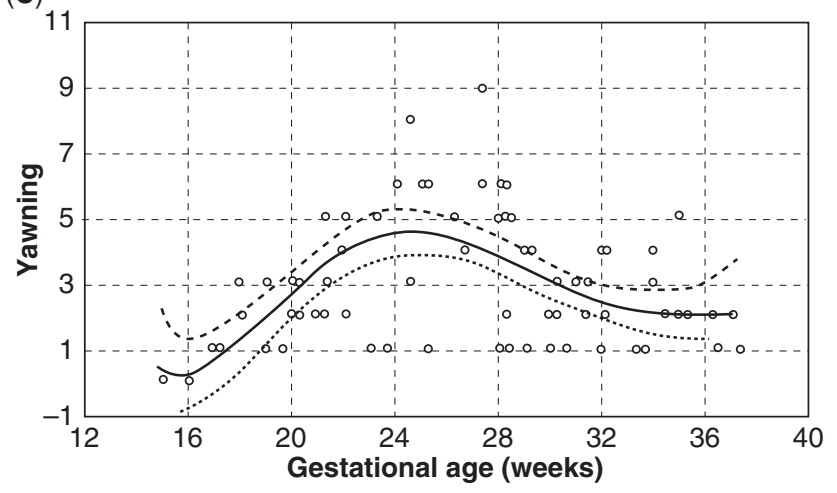

(E)

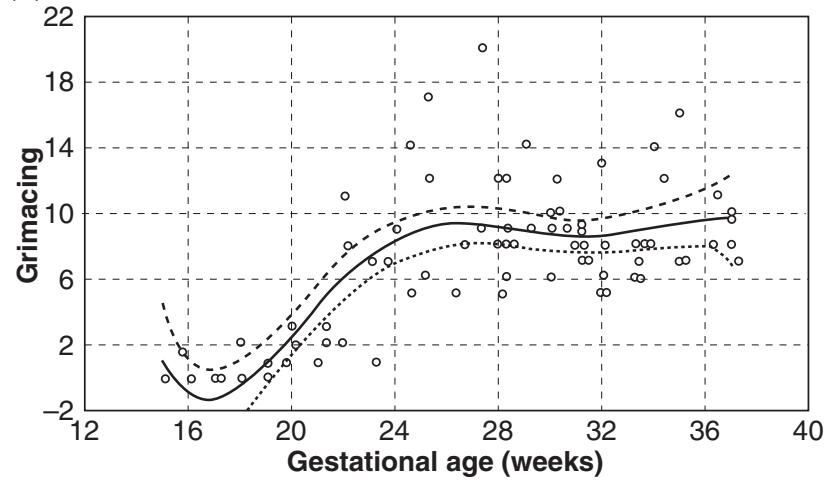

(G)

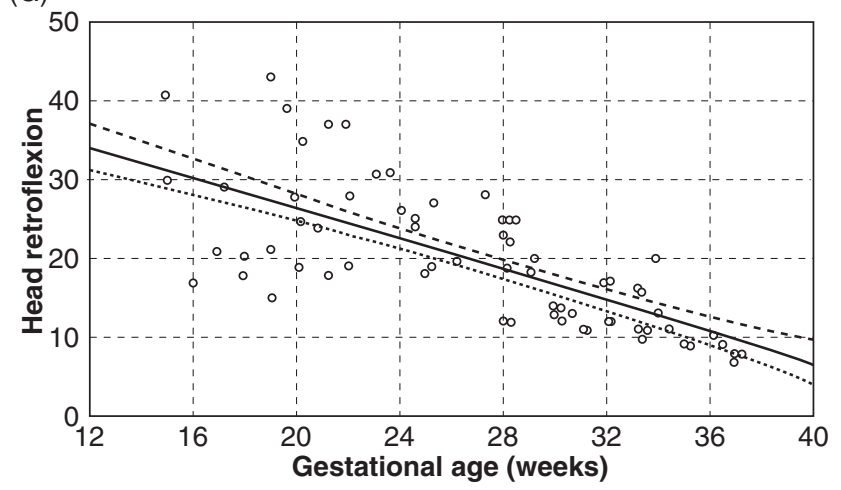

(B)

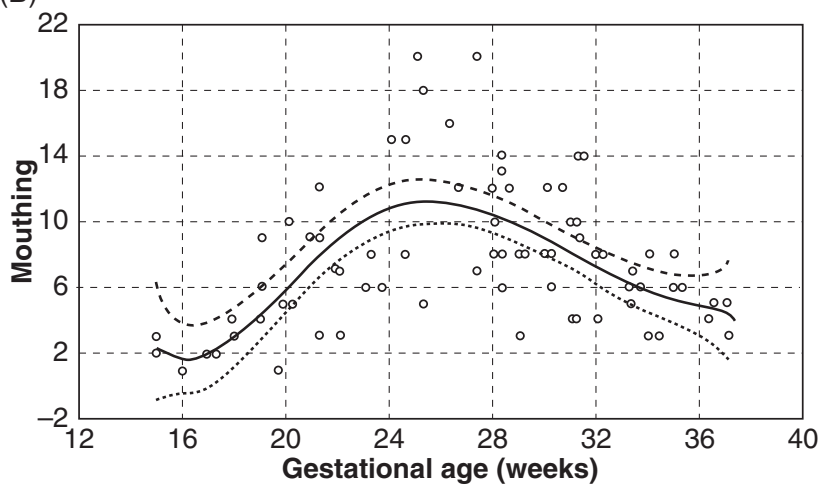

(D)

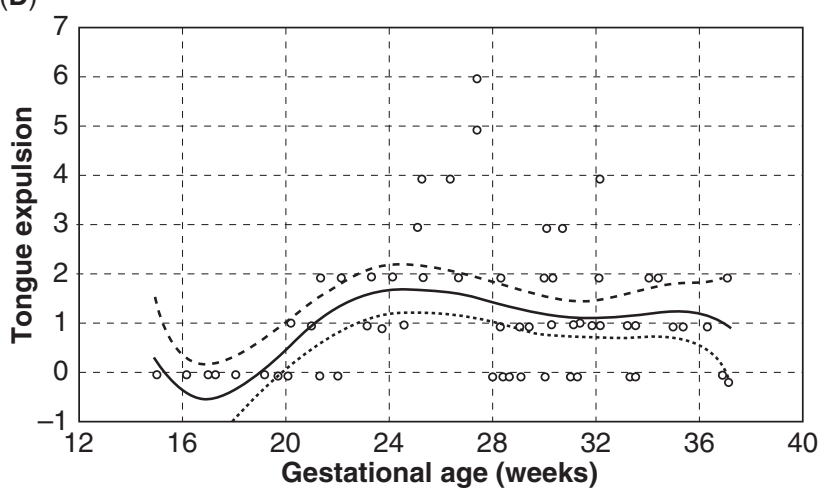

(F)

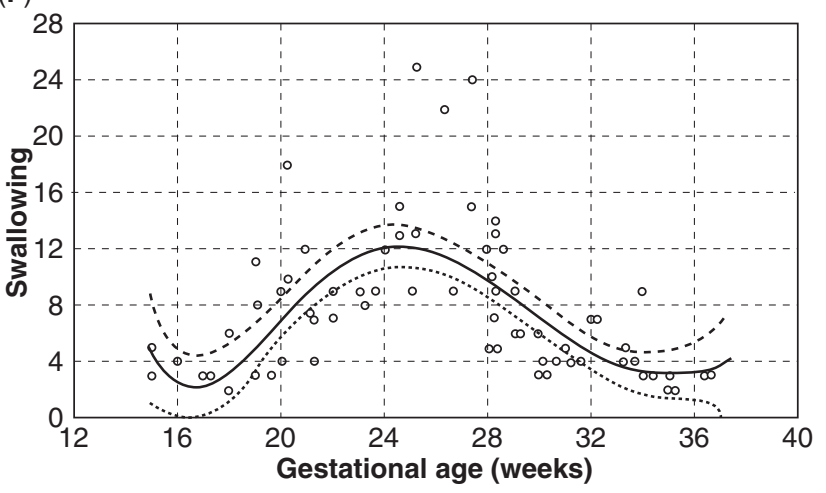

(H)

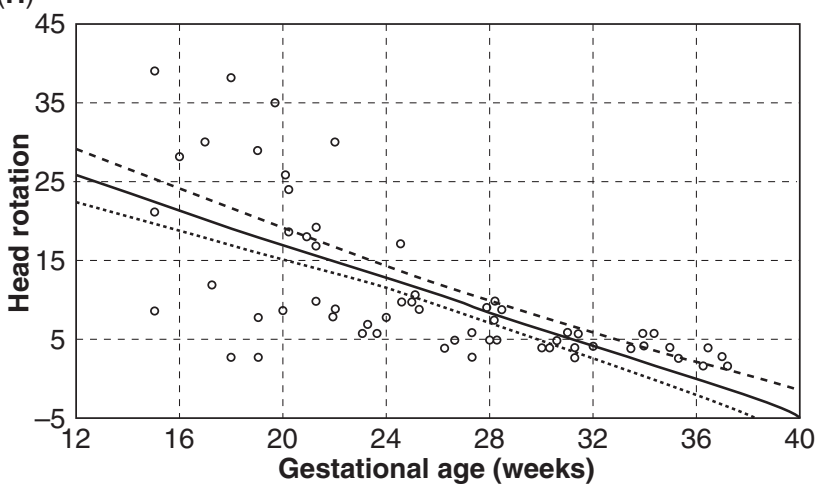

Figure 11 Scatter plot and multiple regression analysis of the second- to third-trimester frequency of (A) isolated eye blinking, (B) mouthing, (C) yawning, (D) tongue expulsion, (E) grimacing, (F) swallowing, (G) head retroflexion, (H) head rotation. (Continued) 


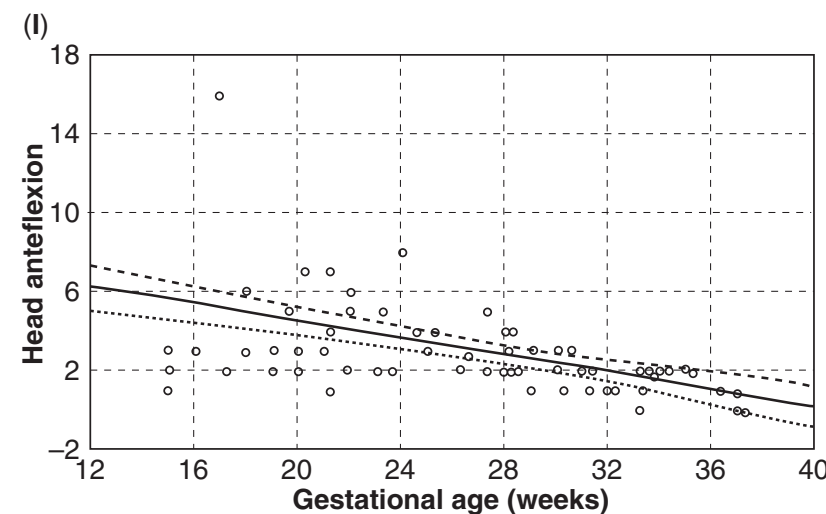

(K)
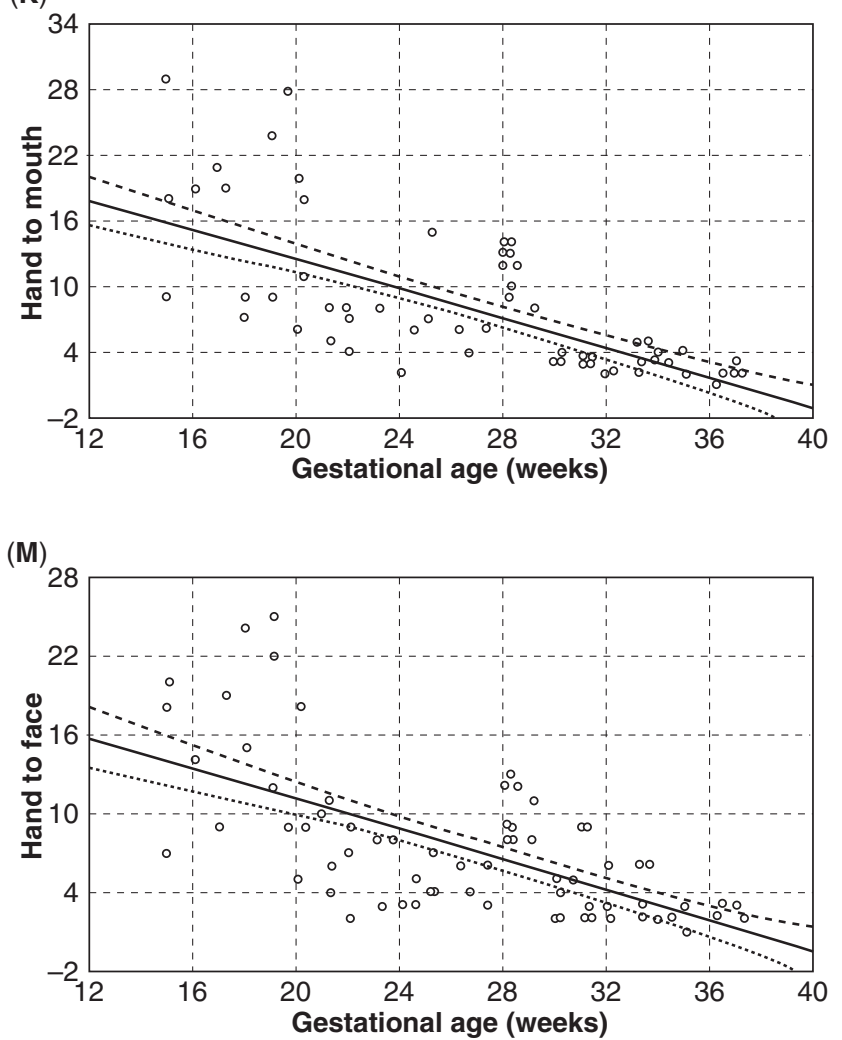

(J)

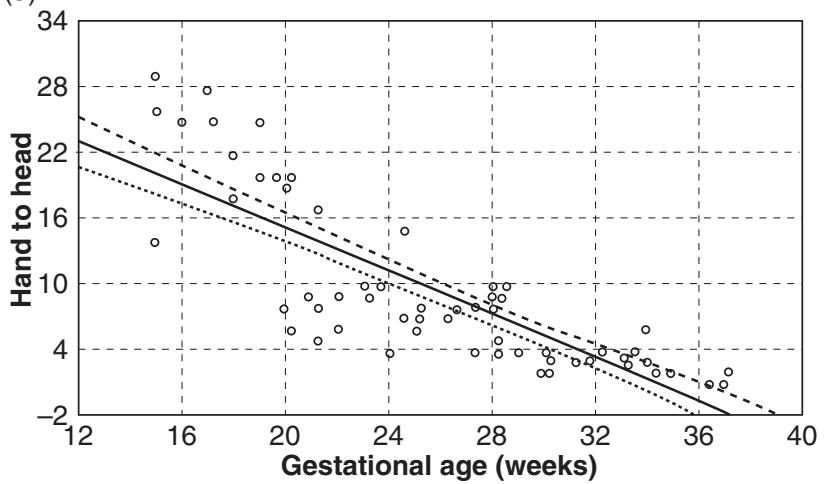

(L)

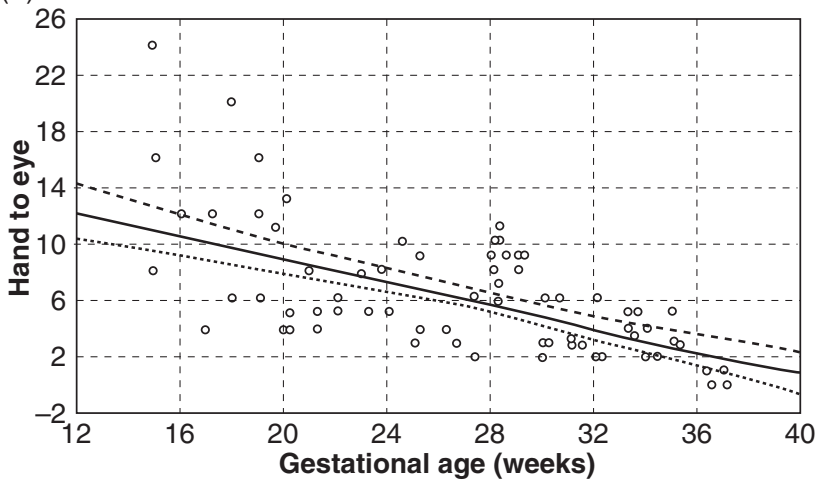

(N)

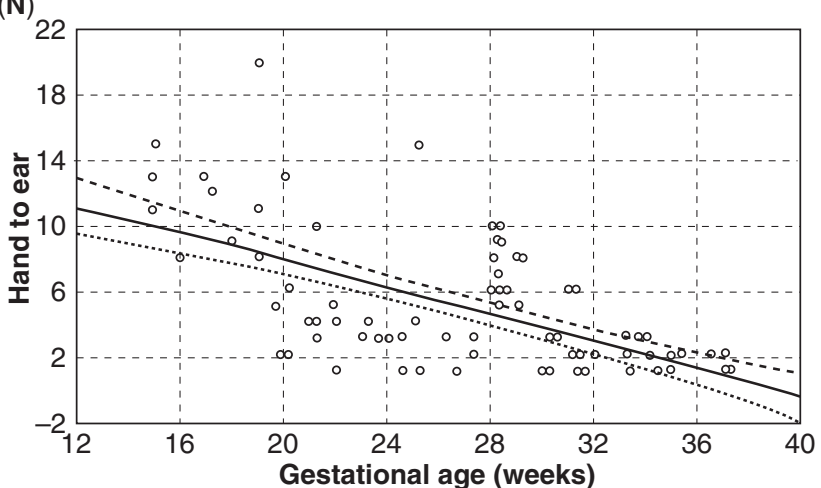

Figure 11 (Continued) (I) head anteflexion, (J) hand to head, (K) hand to mouth, (L) hand to eye, (M) hand to face, (N) hand to ear movements.

the beginning of 24th week. At the beginning of the third trimester, the fetuses display decreasing or stagnant incidence of fetal facial expression. However, all types of head movements and hand to body contact movements indicated a decrease in frequency from the beginning of the second trimester to the end of the third trimester. This longitudinal study establishes reference ranges with GA for suggestible used fetal neurobehavioral development parameters in respected number of normal singleton pregnancies. Standard of movement pattern and facial expression pattern curves are constructed through all the trimesters of pregnancy. Results from Yigiter et al. are similar to that study, as they found a significant correlation between all head movements and hand to body contact patterns during the second and third trimesters except for head anteflexion, which did not show a significant change during the second half of pregnancy (22). It has also been suggested that there is a tendency toward decreased frequency of observed facial expressions and movement patterns with increasing GA (4). All types of facial expressions display a peak frequency at the end of the second trimester, except in isolated eye blinking, which increases at the beginning of 24 th week (4).

\section{The Third Trimester}

During the second half of pregnancy, motor behavior becomes increasingly frequent and variable. Patrick and colleagues ultrasonically studied fetal stretching and rolling movements during the last 10 weeks of pregnancy and found that fetuses on average made 31 gross fetal body movements per hour that accounted for $10.2 \%$ of time (29). In a behavioral study of 20 healthy preterm infants (GA from 28 to 34 weeks), an obvious developmental improvement in orienting responses and in motor performance was assessed (29). Prechtl and colleagues showed that the development was intraindividually characteristic and consistent, but interindividually variable (30). In short, a rich 


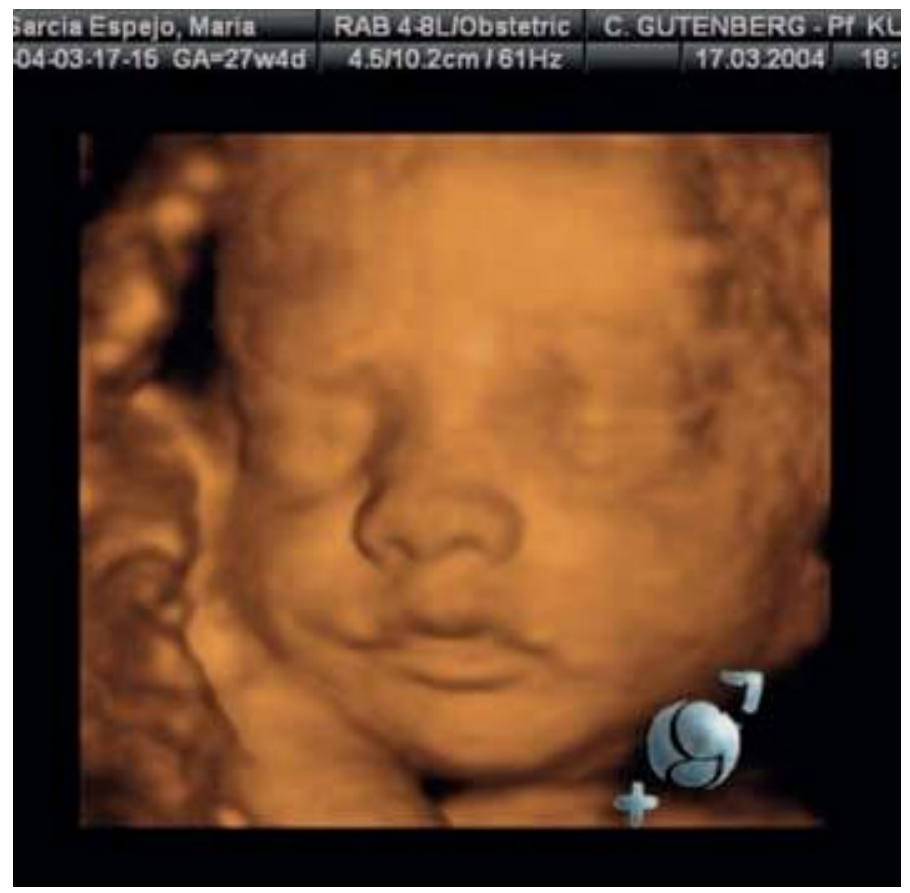

Figure 12 Fetal face depicted by 3D ultrasound with details and facial expression.

variety of fetal and premature movements has been described and it has been shown that the repertoire of fetal movements consists exclusively of motor patterns that can also be observed postnatally and that there is a high degree of continuity of behavior before and after birth. However, the newborn's behavioral repertoire rapidly expands with patterns never observed in the fetus, such as the Moro response. Significant developmental changes in specific movement patterns during the last trimester of pregnancy are in EMs: isolated EM was observed since the 16th week of gestation and rapid eye movements (REMs) since the 19th week (31).

The parameters that could be detected and analyzed during the third trimester are fetal heart rate (FHR) pattern, and eye and body movements (1). The association of these movements increases steadily, and in the last weeks of pregnancy, fetal behavior can almost completely be described in terms of behavioral states, which are stable over time and recur repeatedly, not only in the same infant, but also in similar forms in all infants (32-34). The concept of behavioral states has been used as a descriptive categorization of behavior, and also as an explanatory concept in which states are considered to reflect particular modes of nervous activity that modify the responsiveness of the infant (32).

By term, normal number of generalized movements per hour was found to be approximately 31 with the longest period between movements ranging from 50 to 75 minutes (7). This reduction is considered as a result of cerebral maturation processes, rather than a consequence of the decrease in the amniotic fluid volume (AFV). Simultaneously, with the decrease in the number of generalized movements, an increase in the facial movements, including opening or closing of the jaw, swallowing, and chewing can be observed. These movements can be seen mostly in the periods of absence of generalized movements and that pattern is considered to be the reflection of the normal neurologic development of the fetus (7). However, the changes not only in the quantity of movements, but also in their quality are shown to be the result of maturational processes.

The incorporation of 3D-US technology into clinical practice has resulted in remarkable progress in visualization and anatomic examination of the fetal face. 4D-US, in turn, provided for the first time an opportunity to evaluate subtle fetal facial expressions, which can be used to understand fetal behavior (35). The 4D-US has additional advantages in studying fetal activity in the surface-rendered mode and is particularly superior for fast fetal movements. Because of its curvature and small anatomic details, the fetal face can be visualized and analyzed only to a limited extent with 2D-US (Fig. 12) (5).

The entire face cannot be seen on a single 2D-US image, but 3D-US allows spatial reconstruction of the fetal face and simultaneous visualization of all facial structures such as the fetal nose, eyebrows, mouth, and eyelids. The standardized image display helps sonologists to understand fetal anatomy better and to communicate complex observations to both parents and less experienced observers. This technique does not replace conventional real-time 2D-US imaging, but rather supplements it. The 3D-US requires an investment of additional time in each case; therefore, it is predominately used, presently in conjunction with 2D-US, as a problem-solving tool.

Although facial movements, which are controlled by $\mathrm{V}$ and VII cranial nerves, appear around 10 and 11 weeks, the exact onset of facial expressions has not been determined and it is still unclear whether their appearance is GA related (35). The possibility of studying such subtle movements might open a new area of investigation.

Simultaneously, with the decrease in the number of GMs, an increase in facial movements, including opening or closing of the mouth and swallowing, can be observed. This pattern is considered to be a reflection of the normal neurologic development of the fetus. An important diagnostic aim of the observation of facial expression is prenatal diagnosis of facial paresis. The criterion for the diagnosis is asymmetric 
facial movement and detection of the movement is limited to only one side of the face. Unfortunately, during the relaxed phase, it is not possible to evaluate the status of the facial nerve. Therefore, during the active phase, the fetus should be scanned by $4 \mathrm{D}$-US. Because the origin of facial expression can be influenced by external forces, before the final diagnosis, examiners should be aware of this pitfall. For example, force of the fetal hand can alter the facial expression on one side of the face, causing asymmetry. This kind of asymmetry, however, should be differentiated from pathologic features such as unilateral facial paresis (35).

Zagreb group evaluated fetal behavioral patterns in the third trimester between 30 and 33 weeks of gestation in 10 gravidas (3). The incidence of eyelid movements ranged between 4 and 20 with a median value of 17 , mouthing movements ranged between 2 and 19 (Fig. 13) with a median value of 12, and mouth and eyelid movements ranged between 0 and 13 with a median value of 5 . The incidence of pure mouth movement such as mouth opening ranged between 4 and 13 with a median of 5, tongue expulsion (Fig. 14) ranged between 0 and 2 with a median of 2 , yawning ranged between 0 and 2 with a median of 1 , and pouting ranged between 0 and 9 with a median of 3 .

The incidence of facial expressions such as smiling ranged between 2 and 7 with a median of 2 , and scowling between 2 and 4 with a median of 2 . It is evident that eyelid and mouthing movements dominate at this GA (3). Pure mouth movements such as mouth opening, tongue expulsion, yawning, and pouting are present, but at a significantly lower incidence. Facial expressions such as smiling (Fig. 15) and scowling can be observed. Surface-rendered images that were used in this study depict the entire face and the relationship

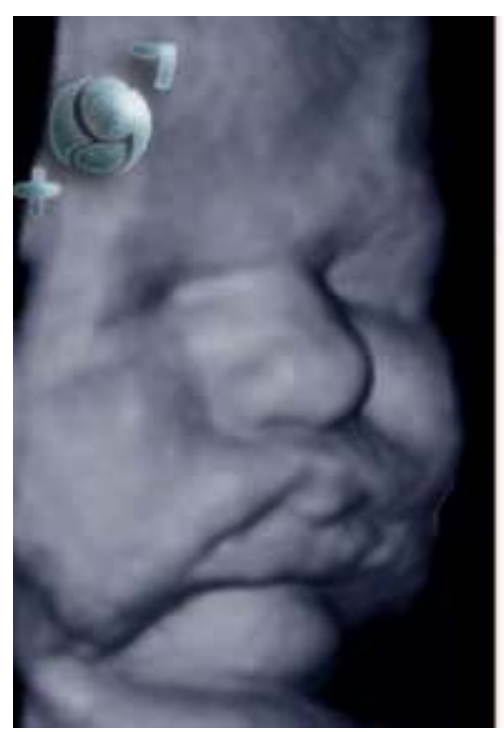

(A)

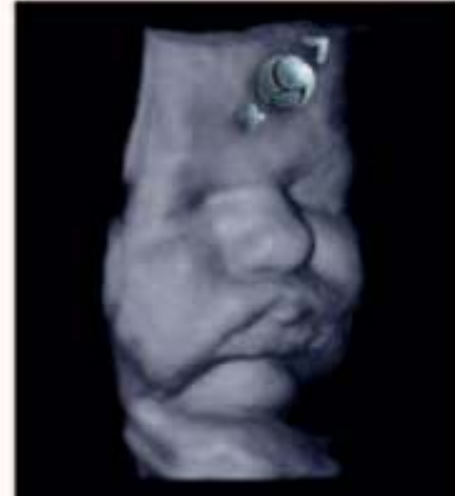

(B)

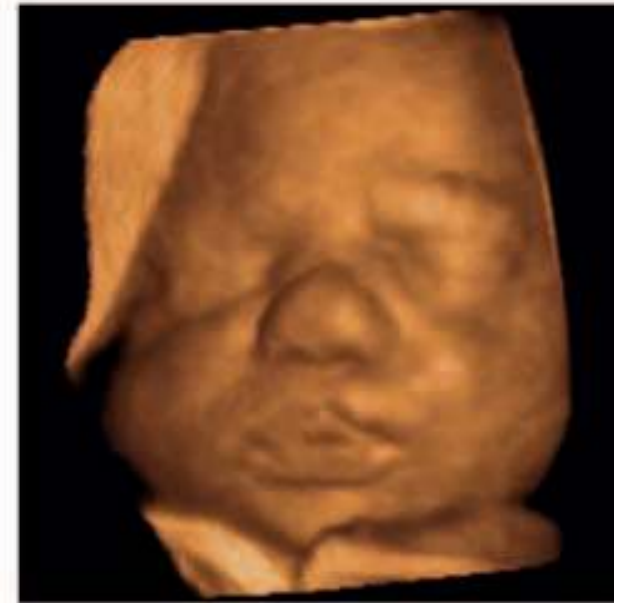

(C)

Figure 13 The 3D surface-rendering mode of the fetal mouthing in the third trimester. Mouthing movements dominate at this gestational age.

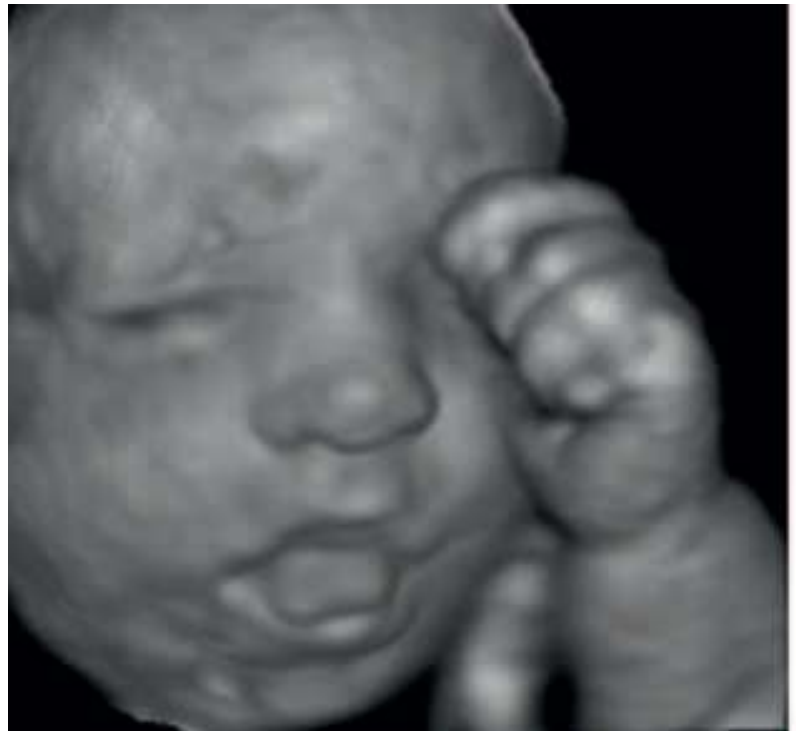

(A)

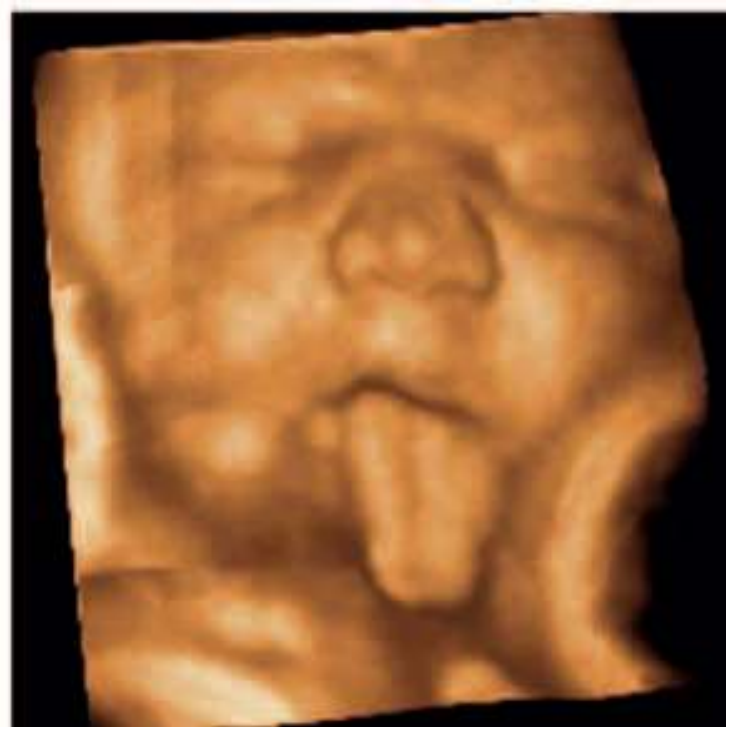

(B)

Figure 14 The 3D surface-rendering mode of the fetal tongue expulsion in the third trimester. 
between facial structures such as the nostrils, opened or closed eyelids, and mouth on a single image. Furthermore, depiction and observation of the orbital region and the status of fetal eyelids can be easily performed by this mode. However, EM cannot be registered because of the character of $4 \mathrm{D}$-US that allows visualization exclusively of superficial structures such as the eyelids. Simultaneous visualization of EM and mouthing can therefore not be achieved. The focus of interest in that study was the analysis of eyelid movements and mouthing both separately and together. Since there is a relationship between mouthing and non-REMs, the group of authors hypothesized that non-REM phases correlate with closed eyes and mouthing phenomenon, and that this pattern can be indirectly determined by $4 \mathrm{D}$-US. Using $3 \mathrm{D}$-US it was not possible to determine the beginning of mouth opening or smiling or the beginning of mouth opening, mouthing, or yawning. Therefore, 3D-US is not suitable for the observation of facial expression. Furthermore, as well as the duration of single facial movement or expression, complex facial movements such as the simultaneous movement of the two facial organs - mouth and eyelids - can be depicted. Mouthing, mouth opening, and yawning simultaneous with eyelid opening can thus be seen only using $4 \mathrm{D}$-US.
The next study by this team showed the ability of $4 \mathrm{D}$ sonography to depict different facial expressions and grimacing (Fig. 16), which might represent fetal awareness (36). This was based on the consideration that "the face predicts the brain" because of the same embryologic origin for many facial and encephalic structures (37). Very early in pregnancy fetuses are reactive to stimuli, but the reaction does not provide any evidence that the fetus actually experienced the stimulus. It has been shown that noxious stimuli can initiate physiologic, hormonal, and metabolic responses, but these neither imply nor preclude suffering, pain, or awareness. Is it the facial expressions of the fetus that can help to understand what the fetus would like to communicate? We can see on the fetal face whether it is satisfied or unhappy, smiling or worried, selfconfident or uncertain, but it is questionable whether the expression of the fetal face predicts its normal neurologic development. Whether the fetal face is an "organ that expresses awareness" with very complex functions is still questionable, because we need more scientific confirmation of what constitutes fetal awareness.

The study included 99 patients, 40 of whom were in the second and 59 in the third trimesters of pregnancy. Positive observation has been defined as a facial expression or

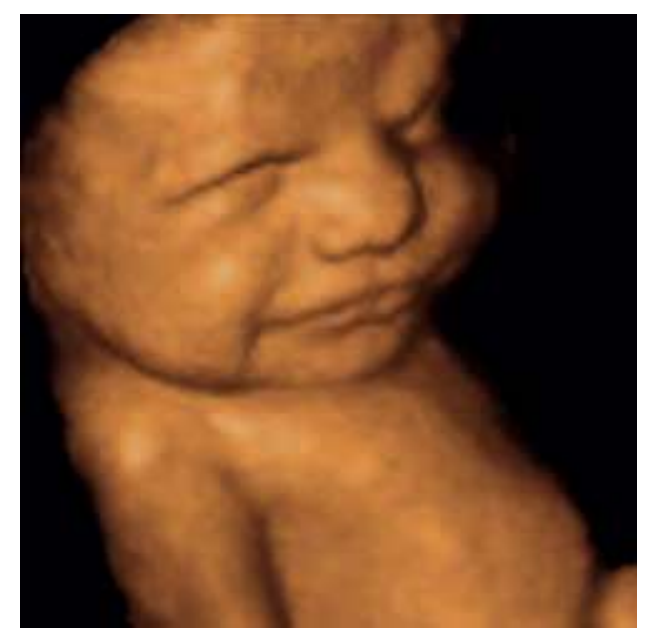

Figure 15 Smiling fetus (3D ultrasonography).

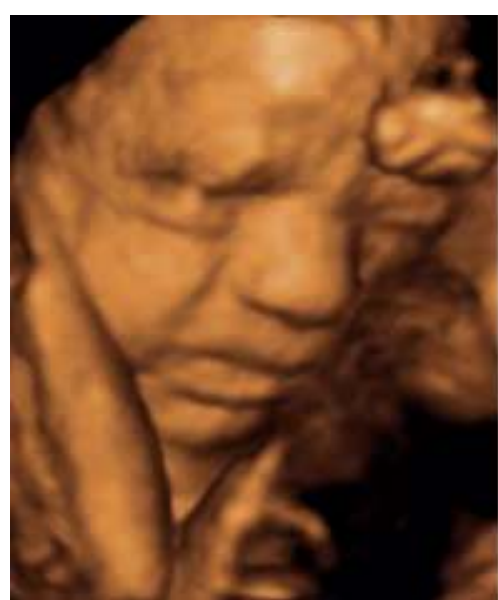

(A)

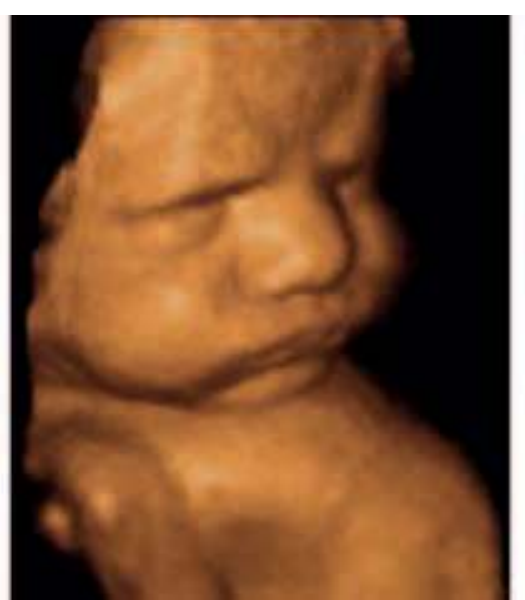

(B)

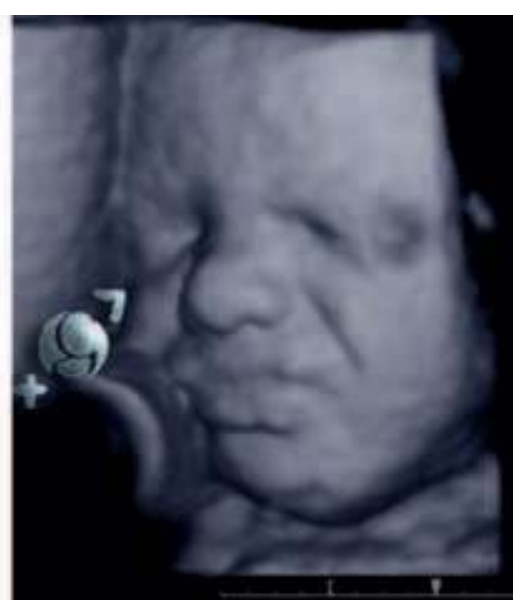

(C)

Figure 16 The 3D surface-rendering mode of the fetal grimacing in the third trimester. This movement is shown to have the continuity in its quantity and quality in the neonatal period. 
movement noted at least once during the observation of one examinee. A tendency toward increased frequency of observed facial expressions with increasing GA was noted, but the difference between the second- and third-trimester fetuses was not significant due to the low frequency of movements. As at that time the images were only near real time, they were able to study only the quantity and not the quality of facial movement patterns with the possibility that some very subtle facial movements may have been missed.

In the recent study by Yan and his group, 10 healthy fetuses aged from 28 to 34 weeks were recorded continuously for 15 minutes with a 4D ultrasonographic machine, and the occurrence rates of blinking, mouthing, yawning, tongue expulsion, smiling, scowling, and sucking were evaluated (38). As in previous reports, mouthing was found to be the most active facial expression during this gestational period $(3,36)$. However, the frequency of blinking was lower in this study. This could be due to the differences in the characteristics of the samples recruited and differences in interpreting the definition of each facial expression.

During the last trimester of pregnancy, significant developmental changes in specific movement patterns can be observed. Isolated EMs can be registered from 16 gestational weeks onward and REMs from 19 weeks (39). The EM patterns begin to consolidate at 24 to 26 weeks of gestation, and the periods of EMs begin to alternate with non-eye movement periods (NEM). During the last 10 weeks of gestation, both switching and maintaining mechanisms responsible for this ultradian rhythms mature, and constant mean values of duration of EM and NEM periods are achieved by 37 to 38 weeks. At that time, EM and NEM lasted 27 to 29 and 23 to 24 minutes respectively, which is similar to the values in the neonate (40). From 33 weeks onward, both REM pattern and slow rolling movement pattern can be registered, and the periods of REM alternate with periods of NEM. At 36 to 38 weeks of gestation, they become integrated with other parameters of fetal activity, such as heart rate and fetal movements, into organized and coherent behavioral states $(41,42)$. This indicates that the pontine and thalamocortical connection area neurons start functioning at that time. Regular mouthing concurred closely with the non-REM period from 35 weeks to term, whereas random mouthing movement was observed predominantly during the EM period and was unrelated to the advance of GA (3).

\section{Fetal Behavior as an Indicator of Fetal Brain Impairment}

The integrity of fetal nervous system could be assessed at least by two available methods. The first is assessment of previously described behavioral states, and the second is the qualitative assessment of GMs (43). Maturation of behavioral states was shown to be disturbed in risk pregnancies, like pregnancies with intrauterine growth restriction (IUGR), diabetes mellitus, or alcohol consumption. Even maternal emotions, like anxiety, have influence on the quantitative distribution of fetal behavioral states. The drawback is the unspecific nature of these changes in development limiting the clinical significance for predicting later impairment, which is in agreement with the findings on abnormal states in the neonate. Qualitative and quantitative changes of the state variables are unspecific sign of neural dysfunction and poor basis for prediction of later neurologic outcome. State assessment can be used as an indicator of an unspecific developmental delay. Qualitative assessment of GMs was underestimated for a long time due to the "subjectivity" of method that includes the observation of fetal movements. For many years, the interest of obstetricians was focused on the quantity of fetal movements, which was considered as an indicator of fetal well-being. Later studies on the subject have shown that movement counts are a poor indicator of brain damage, mainly because the great intra- and interindividual differences and the large overlap between normal and abnormal, which makes this method clinically useless. On the other hand, changes in the elegance and fluency, as well as the variability and fluctuation of intensity and speed of motor performances, were shown to be prominent in the sick preterm infants. It became evident that the qualitative changes in motor patterns of both the fetus and the neonate precede quantitative changes when the integrity of the nervous system is impaired. It must be admitted that it is more difficult to objectify complex qualitative changes than it is simply to count certain events when they occur, but the Gestalt perception is an excellent method for dealing with such phenomenon. Important step is video recording of movements of sufficient length to make a selection of several movements from one recording session. The observed GMs are diagnosed as normal if the movements are complex, including neck, trunk, and limb movements in a variable sequence, and are fluent and wax and wane in their intensity. GMs are selected for judgment only if they last for 20 seconds or longer. If the GMs are monotonous, have less complexity, and are repetitive in pattern, they are judged as abnormal and as being of "poor repertoire." Other abnormal patterns are the "cramped-synchronized" type, when the movements occur in block with almost simultaneous muscle contraction and relaxation. Besides that movements may occur in a jerky and exaggerated manner and in chaotic order. If the movements last very shortly and, hence, they are difficult to be judged, then we speak of "hypokinesis." Several studies employing this new assessment have been carried out and they showed that the early normal or abnormal findings of the GM quality are highly predictive for later outcome $(43,44)$.

Assessment of GMs is based on the concept of ontogenetic adaptation corresponding to the development of human organism, which is during each developmental stage adapted to the internal and external requirements. Prechtl stated that spontaneous motility, as the expression of spontaneous neural activity, is a marker of brain proper or disturbed function $(7,9)$. The observation of the unstimulated fetus or infant, which is the result of spontaneous behavior without sensory stimulation, is the best method to assess its CNS capacity. All endogenously generated movement patterns from an unstimulated CNS could be observed as early as from the 7 to 8 weeks of postmenstrual age, with a reach repertoire of movements developing within the next 2 or 3 weeks, continuing to be present for 5 to 6 months postnatally.

The identification of "CNS depression" during fetal life is based on pre-competences (opening of the eyes, variety of facial expressions), primary reflexes (rhythmical bursts in the sucking pattern), and quality of GMs (44-46). The addition of cranial signs (such as insufficient head growth and overlapping sutures) to neurologic signs could be a valuable complement 
$(44,45)$. Moreover, the identification of dynamic and static patterns of the symptoms may be as helpful to date the insult as it is postnatally: the more stable the signs, the more precise is the timing of the insult. In the presence of neurologic signs in fetuses, the next step is to proceed to the clinical synthesis. In order to do so, all examinees should be followed till the age of 2 years, when their categorization to disabling or nondisabling cerebral palsy (CP) can be possible, based on clinical neurologic findings and presence or absence of the ability to walk.

Obstetricians would have a great benefit if it were possible to assess the condition of the fetal nervous system especially due to the fact that in many cases obstetricians are held responsible for brain damage in neonates, regardless of a growing pool of evidence that most of such damages are consequences of prenatal complications. Even after the fetal brain anatomy can be visualized by ultrasound and the development of the fetal brain is well understood, not much is known about the functional development of the fetal CNS. In other words, the fetal CNS is not accessible. It is possible only to ascertain the output of the CNS, that is, "fetal behavior." Observation of fetal behavior provides a direct assessment of the most important human organ. It is possible to look closely at the functioning of the CNS and the brain. Prenatal motility is considered not only to reflect the developing nervous system but also to involve functional and maturational properties of fetal hemodynamic and the muscular systems (8). Prechtl and his coworkers have explored spontaneous motility during human development (43). They introduced the concept of ontogenic adaptation, meaning that during each developmental stage, the functional organization has to take into account internal and external requirements. Any fetal brain damage will interfere with endogenous motor activity. Therefore, spontaneous movements, as an expression of neural activity, could be used as a marker for fetal brain status. Consequently, the observation of the unstimulated fetus or infant should contribute significantly to the assessment of CNS function. Even after delivery, behavioral patterns frequently provide the most useful indicators of brain function in spite of having extending access to neurologic, physiologic, and pharmacologic measures (8). This remarkable continuity of endogenously generated activity from prenatal to postnatal life may allow identifying those fetuses and infants with emerging neurologic impairment.

During the 9 months of gestation, the repertoire of fetal activities constantly expands, correlating precisely with structural development of the CNS (19). Major developmental events, such as the establishment of neural connections in the different regions of the brain, are accompanied by the occurrence of new patterns of fetal activity or with the transformation of the existing patterns. The organization of behavioral states during the final weeks of pregnancy shows that the connection between cerebral cortex and periphery is established and that the cerebral cortex takes control over fetal activity. This also indicates the ability of the fetus to perceive and process external signals. Furthermore, the latest results indicate that even higher brain functions, such as learning, develop in utero during the last weeks of gestation (19).

The major problem with the study of fetal behavior is that it is very time consuming and not enough functional for routine clinical practice. The question of subjectivity should be overcome using recording of information. Nevertheless, there is no other possibility of assessing the function of the CNS in utero, and this is needed for the understanding of the hidden information in the neurodevelopmental pathways of the fetal CNS. Only if normal behavior is fairly understood, it is possible to identify and to perceive abnormal behavior before birth $(15,47)$.

First reports on fetal behavior obviously suggested that these studies should be standardized as much as possible. An objective analysis with strict application techniques and the use of valid reference ranges appropriate for the GA are essential (1). Without such standardization, comparisons with former or future measurements of patients and comparable studies cannot be made. In order to achieve this goal, the Zagreb group published the first study, which described the $4 \mathrm{D}$ sonographic techniques used for obtaining longitudinal standard parameters of fetal neurologic development in all trimesters of a normal pregnancy (4). Measurement of 7 parameters in the first trimester and 11 parameters in the second and third trimesters correlated with GA. Those parameters have been followed longitudinally through all trimesters and showed increasing frequency of fetal movements during the first trimester. A tendency toward decreased frequency of facial expressions and movement patterns with increasing GA from second to third trimesters has been confirmed (4).

Despite the long-standing conclusion that it is possible to make valid conclusion about brain function from observed, no generalized antenatal behavior screening has been developed to identify fetuses that may have CNS defects. Recent study from Morokuma tried to produce screening test that would be less time consuming and in that way cost effective as compared with their previous study (48). They devised a brief ultrasound examination to distinguish fetuses with compromised CNS function from the general population and evaluated it with their study (49). The study design compared findings on five behavioral patterns obtained by retrospectively reviewing the ultrasound examinations of 5 fetuses that had abnormal behavior with prospectively obtained findings of 29 normal fetuses. Median time for brief examination criteria was 50 minutes (range 30-60 minutes) with the only case undetectable by this brief ultrasound examination had an EM period significantly longer than the normal upper limit.

Improvements in technology and procedures that provide direct access to the fetus in utero are generating the impetus for prenatal developmental research to move beyond the simple documentation that behavioral abnormalities during pregnancy can produce effects that are evident in their offspring after birth. Rather, we see a growing need for developmental researchers to focus attention on how prenatal events affect the fetus, its behavior, and its relationship with environmental conditions in utero. Investigation of behavioral potentials in the fetus will promote understanding of the mechanisms of normal and abnormal development that lead to predictable behavioral outcomes after birth. In other words, behavioral study of the fetus will be necessary to understand the origins of motor and sensory capabilities of infants and the mechanisms of altered developmental outcomes (45).

Awareness of changing risk, and the potential for significant neurodevelopmental problem, is an underlying principle of 


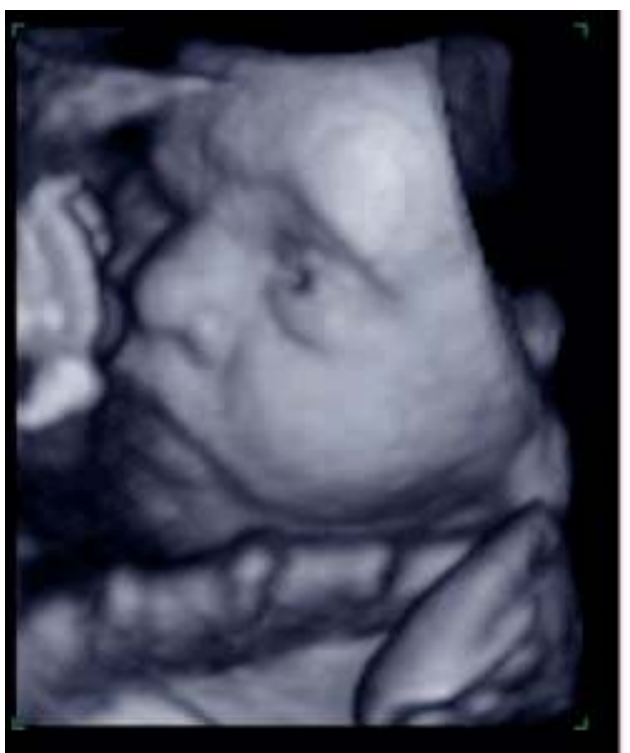

(A)

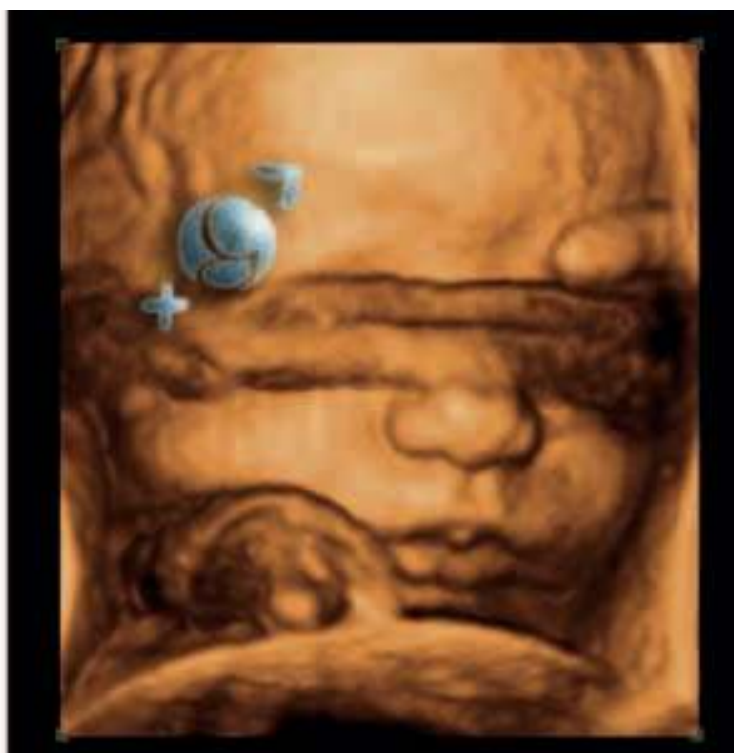

(B)

Figure 17 Nuchal cord around the (A) neck and (B) eyes.

perinatal medicine. Unfortunate neurologic outcomes often result from a delay in recognizing or responding to CNS developing risk. This factor may necessitate the timely referral of individuals to an appropriately staffed facility. The timing of referral can be critical, but there are obvious difficulties that may result in the decision to refer being considered unnecessary or, on the other hand, too late.

In the following chapter, we review the literature on the behavior in the high-risk pregnancies for CP that were defined according to our previous study $(50,51)$. Although we were aware that major prenatal pathologies associated with CP are prematurity, antepartum hemorrhage, complications of multiple pregnancy, genetic disorders, intrauterine infection, IUGR, multiple congenital anomalies, maternal diseases (hypothyroidism, diabetes, drug abuse, severe pre-eclampsia, viral illness), placental pathology, tight nuchal cord (Fig. 17), fetal hemorrhage, and fetal rhesus disease, we also included prenatal history of neuromuscular disease, which can adversely affect fetal movements and their inadequate interpretation (52). Main focus was on maternal pathologic conditions, while fetal behavior in multiple pregnancies is described in detail elsewhere in the book.

\section{Fetal Behavior in Pregnancies Complicated by Maternal Diabetes Mellitus Type I}

Among previously mentioned risk states for disturbed fetal behavior and motility, one of the most investigated is maternal diabetes mellitus, mostly type I. Those pregnancies were shown to be often complicated by early fetal growth delay and congenital malformations that frequently involve the nervous system. Both abnormal embryogenesis and early growth restriction are suggested to shear the common mechanism. Main findings on the subject are given in Table 1.

The new findings were made when the emergence of frequently occurring movement patterns was plotted against fetal crown-rump length, which is usually smaller in diabetic pregnancy, and there was still a general delay in comparison with the control group. It was concluded that delay in motor development therefore does not run completely parallel with the delay in growth, which indicates the possible existence of a specific diabetes-related influence on the functional development of the embryonic and fetal nervous system. Hyperglycemia was considered to be responsible, as the delay in the emergence of fetal GMs was most profound in the women whose periconceptional quality of glucose control was poor. When investigators looked into the developmental trends in the occurrence of movement patterns in diabetic pregnancies, they found the similarity to those in the control fetuses, with the exception of startles. It was found that before the 9th week of gestation, fetal movements occurred less frequently, which was related to the quality of maternal glucose control. After 12 weeks, the overall incidence was higher than that in the control group, due to an increase in the incidence of breathing movements that was generally slower than that in the control group (57).

For further investigation of the impact of early growth delay on subsequent growth (birth weight) and functional development near term (organizational level of fetal behavioral states), the same group investigated 21 and 10 fetuses of diabetic women, respectively (59). They found no relationship between the degree of early growth delay and birth weight, while the mean growth delay per fetus in early diabetic pregnancy was negatively correlated with the occurrence of no coincidence between behavioral state parameters at 36 weeks. Those results have shown that disorders occurring in early life may underlie abnormal functional development in later life, whereas (catch up) growth is mainly determined during the second half of pregnancy. These results corroborate their following findings in the fetuses at 32 to 38 weeks of gestation in type I diabetic pregnancy (60).

To assess the effect of type I diabetes on the quality of GMs, Kainer and coworkers analyzed GMs longitudinally in 12 human fetuses at 2-week intervals from 16 weeks until delivery (63). All fetuses showed normal GMs at 16 weeks, but from 20 weeks onward until delivery five fetuses developed abnormal GMs. The diabetes optimality score was significantly 
Table 1 Additional Findings of Fetal Behavior in High-Risk Diabetic Pregnancies in Published Reports

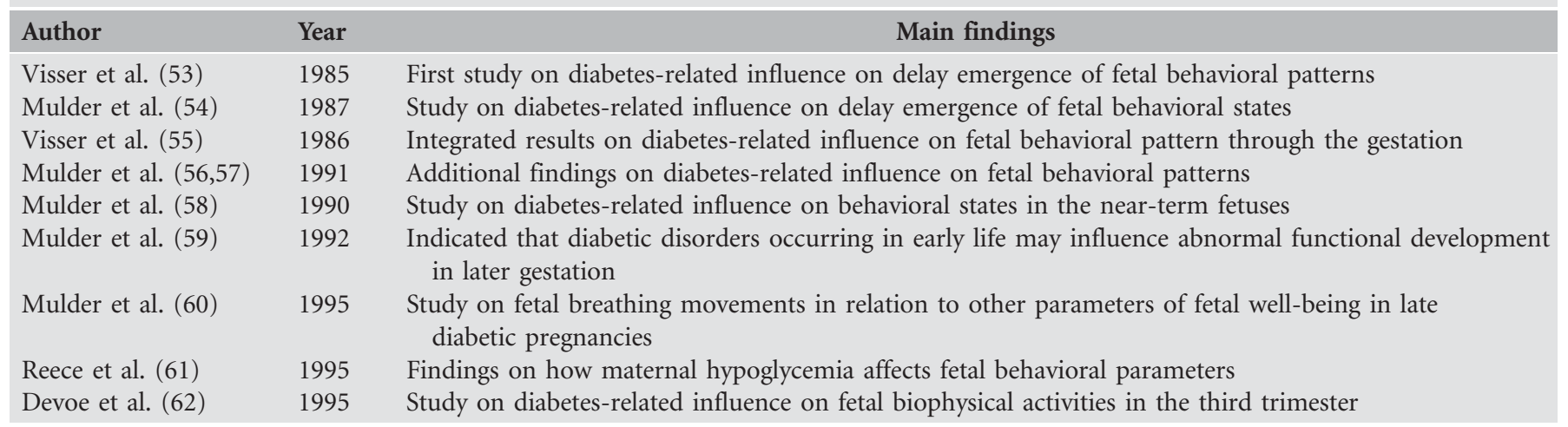

Table 2 Additional Findings of Fetal Behavior in High-Risk Intrauterine Growth Restriction (IUGR) Pregnancies in Published Reports

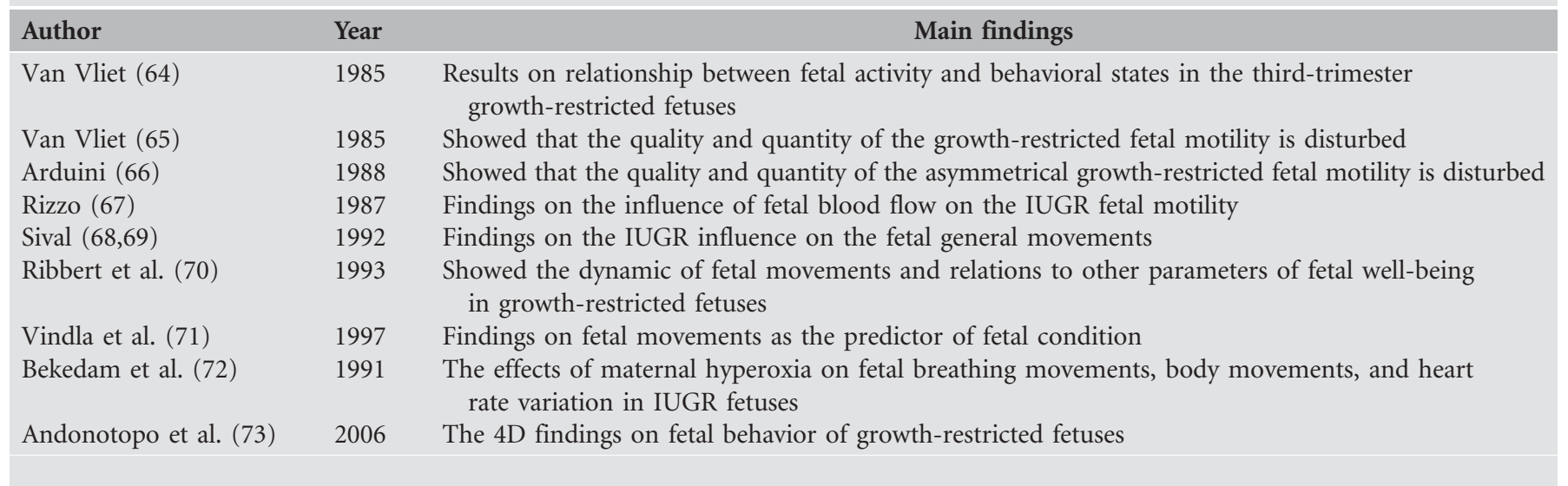

lower in the group with abnormal GMs whereas the pregnancy optimality score did not differ between fetuses with normal and abnormal GMs. These results indicate that type I diabetes can have a negative impact on prenatally observed GMs and that consistently normal GMs indicate normal neurodevelopmental outcome at 10 months. Although it is the very important observation on the effect of type I diabetes on the quality of GMs, relatively small number of observed fetuses cannot be denied.

To synthesize we can say that all mentioned published studied confirmed at least slight impact of maternal diabetes mellitus on fetal and even embryonic movements and behavior. The delay of mergence of spontaneous motility was shown to be independent on growth delay and changes of motility could not be explained by the glycemic control of the mother. Disturbed organization of behavioral states and GMs in fetuses in pregnancies complicated by the diabetes mellitus demand further investigation by the $3 \mathrm{D}$ and $4 \mathrm{D}$ technologies that will clarify the neurobehavioral changes in these fetuses.

\section{Fetal Behavior in Pregnancies Complicated by IUGR}

Studies dealing with fetal behavior in IUGR pregnancies are presented in Table 2.

One of the first studies on the impact of growth restriction on the fetal behavior focused on fetal breathing and on the course of behavioral state (67). To achieve that goal, Van Vliet and his group used real-time ultrasound scanners to detect fetal eye, body, and breathing movements, and the FHR of 12 growth-retarded fetuses between 36 and 40 weeks of gestation. The mean incidence of fetal breathing was greater during periods of fetal activity (body and eye movements present, greater heart rate variability) than during quiescence (body and eye movements absent, narrowed heart rate variability) at all GAs studied in both low-risk and growth-retarded fetuses. During periods when one of the state variables was in its active condition while the other two were quiet, or the reverse, the incidence of fetal breathing was intermediate between those found when all three state variables were in agreement. After behavioral states had developed, at 38 and 40 weeks, the mean incidence of fetal breathing in the low-risk fetuses was greater during active states than during the quiescence. There was no apparent increase in the degree of linkage between fetal breathing and other expressions of fetal activity after the emergence of behavioral states (67). In another study by the same group, behavioral state observations were carried out on 12 fetuses, which subsequently had birth weights below the 10th percentile (68). Their GAs at the time of study ranged from 32 to 40 weeks. Real-time ultrasound scanning was used to detect fetal body and eye movements, and the FHR was continuously recorded using a clinical fetal monitor. The appearance of states seemed to be delayed in the growthrestricted fetuses since states were present in only three of eight growth-restricted fetuses studied at 40 weeks. Also at 40 weeks, the proportion of discordant association of the state variables 
was increased in the growth-restricted fetuses as compared with the control. There were no consistent differences between the two groups in the occurrence of defined combinations of parameters of the state variables at earlier ages. The results from this study showed that the growth-restricted fetuses have impaired quality and quantity of somatic motility in comparison with low-risk fetuses of equivalent GA. These observations suggest that some aspects of CNS function are disturbed in growth-retarded fetuses, even in the absence of fetal distress (68).

The implementation of $4 \mathrm{D}$ sonography was necessary to find out whether the quantity of fetal facial expression and quality of body movements can be used as an additional diagnostic criterion for prenatal brain impairment in fetuses with growth restriction. For that purpose, a prospective study was conducted in 50 pregnant women with a growth-restricted fetus and in 50 uncomplicated healthy women in the third trimester of pregnancy (73). The 4D ultrasound observation was specially designed to assess whether functional brain impairment and fetal growth restriction had prenatally occurred by the utilization of several behavioral patterns. The results showed that the median value of all movement patterns in the normal fetuses differed from fetuses with IUGR. Statistical evaluation revealed significant differences in the distribution of the movements between these groups. A tendency that IUGR fetuses have less behavioral activity than normal fetuses was noted in all observed movement patterns. Correlation reached statistical significance between normal and IUGR fetuses in the third trimester in hand to head, hand to face, and head retroflexion. Statistically significant differences could be shown in the distribution of the median values of observation over the five qualitative categories of head and hand movements. These recent data on IUGR fetuses obtained by $4 \mathrm{D}$ sonography are stimulating and might result in a more effective strategy to assess development before birth and may encourage future use of 4D ultrasound for quantitative and qualitative assessment of fetal behavior as possible indicators of the neurologic condition in IUGR fetuses (73).

Fetal body movements and breathing movements as a variables of fetal biophysical profile (BPP) have been shown to be predictable of impending fetal infection in patients with premature rupture of the membranes (74) (Table 3).

Some viral and bacterial pathogens have been shown to influence fetal motility. For example, transplacental infection with coxsackie B3 confirmed by molecular techniques resulted in severe reduction of fetal movements at the 27th week detected by prenatal 2D ultrasound. Late onset of fetal akinesia deformation sequence with mild arthrogryposis was the finding at fetal autopsy following interruption of the pregnancy (75). Listeria monocytogenes has been increasingly recognized as a cause of intrauterine sepsis with associated perinatal wastage. The most common presentations in pregnancy include premature labor, an influenza-like illness, and reduced fetal movements (76).

Intraamniotic infections often result in preterm premature rupture of membranes (PROM). In a prospective study, fetal behavior was observed in 41 cases complicated with PROM (77). The length of time and the number of fetal breathing and gross body movements were correlated with the amniotic fluid culture results. An episode of fetal activity (body movements and breathing movements) of greater than or equal to 30 seconds during 30 minutes of observation was associated with the absence of intraamniotic infection in 100\% of the cases. On the other hand, the absence of fetal breathing movements and gross body movements of less than a 50 -second duration during 30 minutes of observation was associated with positive amniotic fluid cultures in all cases. If an episode of fetal breathing movements was present but lasted less than 30 seconds and/or the total time of gross body movements was greater than 50 seconds, $64 \%$ of patients had an intraamniotic infection. The conclusion was that the breathing movements could be used as a predictor of intraamniotic infection. On the contrary, study by Del Valle and his group, which enrolled 68 women with the diagnosis of PROM, showed that the BPP with breathing movements included and the nonstress test (NST) are poor predictors of perinatal infectious complications (78).

To investigate the relation between AFV and fetal movements at less than 32 weeks of gestation as assessed by routine BPP, the study included 352 consecutive nonhypertensive, nondiabetic patients (79). The results revealed a significant correlation between decreased AFV and decreased fetal movements. Fetal presentation and GA were not significantly different between patients based on the score of fetal movements. The authors concluded that decreased AFV is associated with decreased fetal movements irrespective of fetal presentation or GA (79).

Nicotine was shown to have impact on fetal motility and there are recently published studies on fetal movement in early pregnancy that were qualitatively and quantitatively observed by ultrasound monitoring in women smokers and in controls (Table 4) (80).

The study included three groups of 20 pregnant women each: nonsmokers (group 1), smokers of an average of 10 cigarettes daily (group 2), and smokers of an average of $>20$ cigarettes daily (chronic smokers; group 3). The 2D US study was performed once during gestational weeks 10 to 20 by the then standard method of fetal movement monitoring: (i) quantitative measurement of global fetal movements qualitatively verified as brisk or sluggish, (ii) quantitative measurement

Table 3 Additional Findings of Fetal Behavior in Pregnancies with Maternal Infection in Published Reports

\begin{tabular}{lll} 
Author & Year & \multicolumn{1}{c}{ Main findings } \\
Konstantinidou et al. (75) & 2007 & Case report on the effect of coxsackievirus B3 on fetal motility \\
Craig et al. (76) & 1996 & $\begin{array}{l}\text { Study on the effect of Listeria monocytogenes on fetal motility } \\
\text { Goldstein et al. (77) }\end{array}$ \\
$\begin{array}{l}\text { Del Valle et al. (78) } \\
\text { Sherer et al. (79) }\end{array}$ & 1988 & $\begin{array}{l}\text { Findings on fetal body and breathing movements as predictors of intraamniotic infections } \\
\text { Findings on fetal body and breathing movements as poor predictors of intraamniotic infections } \\
\text { Showed that decreased amniotic fluid volume before the third trimester is associated with } \\
\text { decreased fetal motility }\end{array}$
\end{tabular}


of isolated spontaneous head movements, (iii) quantitative measurement of isolated spontaneous arm movements, (iv) quantitative measurement of isolated spontaneous leg movements, and (v) M-mode measurement of FHR. The results showed that the ratio of brisk to sluggish fetal movements was higher in nonsmokers compared with smokers. The rate of isolated spontaneous head and arm movements and of the upper cerebral pattern (head and arm movements) was statistically significantly lower in group 3 as compared with groups 1 and 2, whereas no statistical significance was recorded in isolated spontaneous leg movements. The rate of fetal tachycardia was also significant in group 3, whereas tachyarrhythmia was recorded in seven children born to group 3 mothers. The described study of the effect of chronic tobacco hypoxia on the components of fetal behavior revealed a positive correlation between global and isolated fetal hypokinesia of the upper cerebral pattern, fetal tachycardia, and tachyarrhythmia in the group of mothers who were chronic smokers (80).

To determine the effects of maternal smoking on fetal spontaneous behavior and auditory processing, Cowperthwaite and coworkers examined 38 fetuses of smoking and nonsmoking mothers, stratified by GA and observed at least 1 hour following smoking (81). Observations included spontaneous FHR and body movements (20 minutes) followed by a 2-minute audiotape of the mother reading a story while FHR and body movements were recorded. They found that the fetuses less than 37 weeks of GA of mothers who smoke throughout pregnancy have a delayed onset of response to the maternal voice, a subtle difference that may have implications for later language development for prematurely born infants.

The relationships between maternal blood pressure (BP) and fetal behavior were examined in hypertensive compared with normotensive women at 33 and 36 weeks of GA (79). As expected, maternal BP was negatively related to GA at birth and birth weight (82). On average, fetuses of hypertensive women were born 2 weeks earlier (38 weeks of GA) and $340 \mathrm{~g}$ lighter. Maternal systolic BP was negatively related to the number of spontaneous body movements observed on ultrasound scan over 20 minutes and the magnitude of the FHR acceleration elicited by a vibroacoustic stimulus. At 36 weeks of GA, vibroacoustic stimulation elicited differential responding with fetuses in the hypertensive compared with the normotensive group having fewer body movements, a lower magnitude of FHR acceleration, and a lack of cardiac-body

Table 4 Additional Findings of Fetal Behavior in Pregnancies with Maternal Smoking in Published Reports

$\begin{array}{lll}\text { Author } & \text { Year } & \text { Main findings } \\ \begin{array}{l}\text { Habek D (80) } \\ \text { Cowperthwaite et al. (81) }\end{array} & 2007 & \begin{array}{l}\text { Revealed the effect of chronic tobacco hypoxia on the fetal behavior in early pregnancy } \\ \text { Showed that fetuses less than } 37 \text { weeks of GA of mothers who smoke throughout } \\ \text { pregnancy have a delayed onset of response to the maternal voice }\end{array}\end{array}$

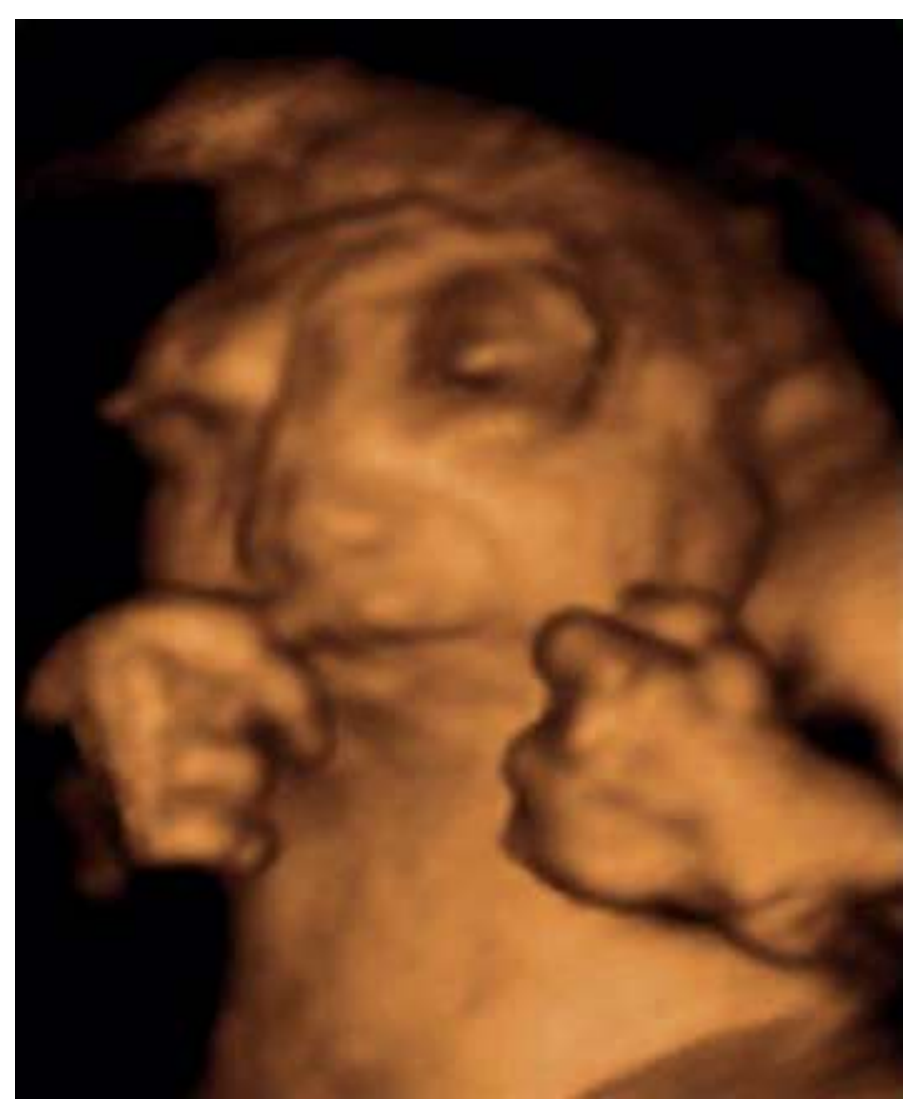

Figure 18 Anencephaly (3D image). 
movement coupled responses. These findings suggest a relationship between maternal $\mathrm{BP}$ and fetal behavior and differential functional development of sensory-motor response systems, which need to be characterized in the subgroups of hypertensive disorders observed during pregnancy.

Recent meta-analyses were conducted on archival data of human fetal behavior to identify differential behavior among high-risk fetuses in pregnancies complicated by threatened preterm delivery, maternal hypertension, or diabetes compared with low-risk fetuses in uneventful pregnancies, delivering as healthy, full-term infants (83). There were no differences in spontaneous behaviors when scored using clinical criteria for the NST and BPP; however, there were differences in the magnitude of the behaviors measured in the tests. Developmental differences were observed between those threatening to deliver early and the fetuses of hypertensive and diabetic mothers. The latter two groups differed little from one another but differed from low-risk fetuses in their response to auditory stimulation. It was concluded that differences in behavior among high-risk groups suggest that atypical fetal behaviors may represent adaptation to condition-specific insult rather than a generalized response to insult per se. The finding that high-risk fetuses showed atypical responses to auditory stimuli indicates a need to examine the relation between fetal auditory function and later language acquisition.

\section{Behavior in Fetuses with Congenital Disorders}

Although behavior of fetuses with congenital disorders has been investigated and published in many, mostly case report publications, systematic approach to the issue lacks mostly because of high diversity of disturbances. Although estimation of neurodevelopmental outcome of the fetuses based on the following studies could be possible, a direct precise prognosis is still not available during the prenatal period. The use of $4 \mathrm{D}$ US recording could provide more information about the quality of fetal movements leading to the better prediction of neuronal development.

De Vries conducted a literature search on motility in fetuses with congenital disorders. The review included 48 articles

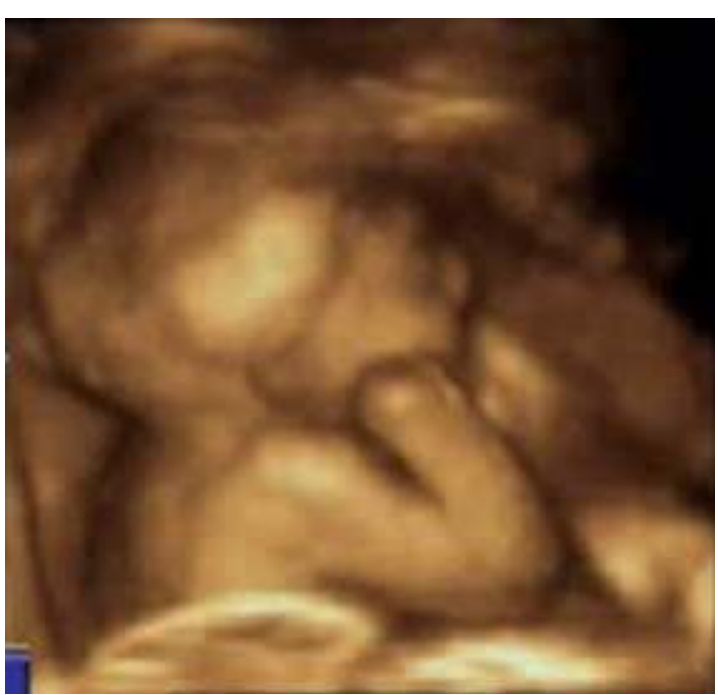

(A)

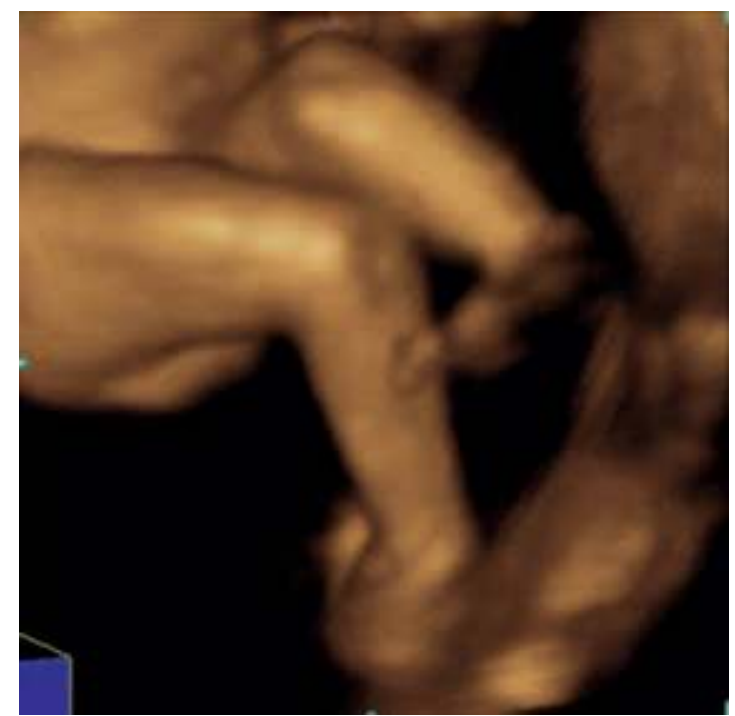

(B)

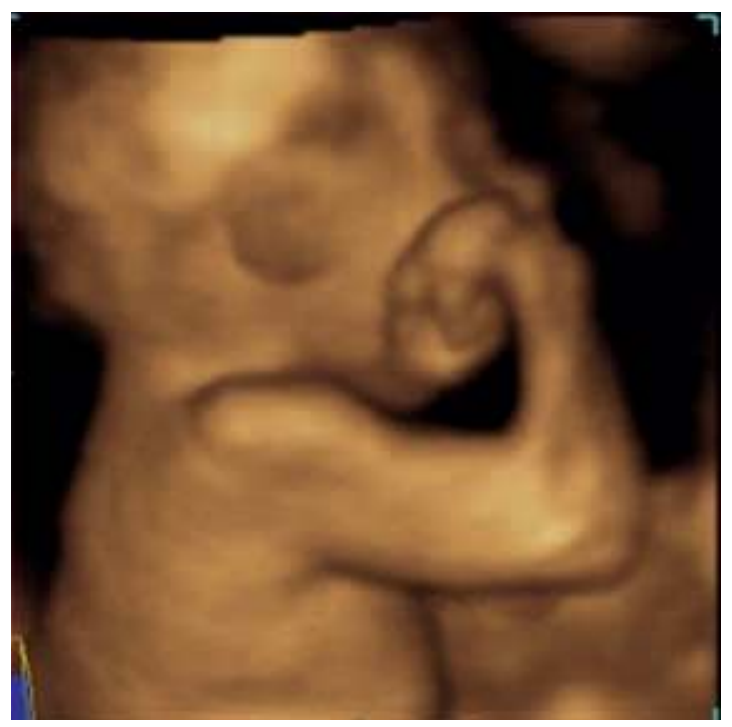

(C) 
describing motility of 104 fetuses (84). The author divided abnormal motility into two main subcategories: hypo- and hyperkinetic with posture affected in 40/60 hypokinetic and $4 / 44$ hyperkinetic moving fetuses. The majority of the disorders resulted in an adverse outcome. Fourteen percent survived with a handicap, depending on the underlying disorder. The 16 disorders with hypokinetic motility had mainly an autosomal recessive etiology with no possibility of invasive prenatal diagnosis or conclusive sonographic structural anomalies, in contrast to the 17 disorders with hyperkinetic motility. Within the limitations of the studies, a deeper understanding of affected milestones in motor development can be obtained. The author concluded that broadening motor assessment procedures from quantitative to qualitative aspects, including the assessment of behavioral states, and emphasizing onset and continuity of motility before and after birth will enhance the reliability and predictive value of motility as a parameter in the assessment of fetal condition (84).

Ahmed and his group established a behavioral state profile in eight high-risk fetuses by $4 \mathrm{D}$ US recording (85). In the study, the authors tried to observe all the behavioral parameters that are related to CNS function. In anencephalic case and cephalocele case, they noticed excessive hypertonic movements, while fetuses affected by homocystinuria and Meckel-Gruber syndrome demonstrated hypotonia. The sequence of occurrence of behavioral parameters was clearly abnormal in those fetuses affected by anencephaly (Fig. 18) and cephalocele.

These findings fully agree with their previous case report on the behavior of an anencephalic fetus (51).

Normal quantity and quality of behavioral parameters were noted in fetuses affected by prune belly, diaphragmatic hernia, achondrogenesis (Fig. 19), and hydrothorax. The quality and quantity of fetal movement in the fetus affected by achondrogenesis were normal, but it was difficult to observe the movement of extremities due to abnormal fetal posture.

In conclusion, one can say that fetal behavior serves as a good indicator of impaired CNS development. Since the frequency of different neurologic disturbances united in a term of $\mathrm{CP}$ has not declined in spite all improvements in prenatal care, fetal behavior assessment could be of great help in neurologic assessment particularly of fetuses in high-risk pregnancies.

\section{REFERENCES}

1. Nijhuis JG. Fetal Behaviour: Developmental and Perinatal Aspects. Oxford: Oxford University Press, 1992.

2. Salihagic Kadic A, Predojevic M, Kurjak A. Advances in fetal neurophysiology. In: Pooh RK, Kurjak A, eds. Fetal Neurology. New Delhi: Jaypee Brothers, 2009; In press.

3. Kurjak A, Azumendi G, Vecek N, et al. Fetal hand movements and facial expression in normal pregnancy studied by four dimensional sonography. J Perinat Med 2003; 31: 496-508.

4. Kurjak A, Andonotopo W, Hafner T, et al. Normal standards for fetal neurobehavioral developments-longitudinal quantification by four-dimensional sonography. J Perinat Med 2006; 34: 56-65.

5. Andonotopo W, Kurjak A, Ivancic Kosuta M. Behavior of an anencephalic fetus studied by 4D sonography. J Matern Fetal Neonatal Med 2005; 17: 165-8.

6. Andonotopo W, Stanojevic M, Kurjak A, et al. Assessment of fetal behavior and general movements by four-dimensional sonography. Ultrasound Rev Obstet Gynecol 2004; 4: 103-14.
7. de Vries JIP, Visser GH, Prechtl HF. The emergence of fetal behavior, I. Qualitative aspect. Early Hum Dev 1982; 7: 301-22.

8. Shawker TH, Schuette WH, Whitehouse W, et al. Early fetal movement: a real time ultrasound study. Obstet Gynecol 1980; 55: 194-8.

9. Back SA. Perinatal white matter injury: the changing spectrum of pathology and emerging insights into pathogenetic mechanisms. Ment Retard Dev Disabil Res Rev 2006; 12: 129-40.

10. Goldstein I, Zimmer EA, Tamir A, et al. Evaluation of normal gestational sac growth: appearance of embryonic heartbeat and embryo body movements using the transvaginal technique. Obstet Gynecol 1991; 77: 885-8.

11. de Vries JIP. The first trimester. In: Nijhuis JG, ed. Fetal Behaviour, Developmental and Perinatal Aspects. Oxford: Oxford University Press, 1992: 3-17.

12. Goto S, Kato TK. Early movements are useful for estimating the gestational weeks in the first trimester of pregnancy. In: Levski RA, Morley P, eds. Ultrasound '82. Oxford-New York: Pergamon Press, 1983: 577-82.

13. Kurjak A, Kupesic S, Banovic I, et al. The study of morphology and circulation of early embryo by three dimensional ultrasound and Power Doppler. J Perinat Med 1999; 27: 145-57.

14. Kurjak A, Pooh RK, Merce LT, et al. Structural and functional early human development assessed by three-dimensional and four-dimensional sonography. Fertil Steril 2005; 84: 1285-99.

15. Kurjak A, Vecek N, Hafner T, et al. Prenatal diagnosis: what does fourdimensional ultrasound add? J Perinat Med 2002; 30: 57-62.

16. Kurjak A, Carrera JM, Andonotopo W, et al. Behavioral Perinatology Assessed by Four-Dimensional Sonography. In: Kurjak A, ed. Textbook of Perinatal Medicine. New Delhi: Jaypee Brothers Medical Publishers.

17. Roodenburg PJ, Wladimiroff JW, van Es A, et al. Classification and quantitative aspects of fetal movements during the second half of normal pregnancy. Early Hum Dev 1991; 25: 19-35.

18. Salihagic-Kadic A, Medic M, Kurjak A, et al. Four-dimensional sonography in the assessment of fetal functional neurodevelopment and behavioral patterns. Ultrasound Rev Obstet Gynecol 2005; 5: 154-68.

19. Kurjak A, Carrera JM, Stanojevic M, et al. The role of $4 \mathrm{D}$ sonography in the neurological assessment of early human development. Ultrasound Rev Obstet Gynecol 2004; 4: 148-59.

20. Andonotopo W, Medic M, Salihagic-Kadic A, et al. The assessment of fetal behavior in early pregnancy: comparison between $2 \mathrm{D}$ and $4 \mathrm{D}$ sonographic scanning. J Perinat Med 2005; 33: 406-14.

21. Pooh RK, Ogura T. Normal and abnormal fetal hand position and movement in early pregnancy detected by three- and four-dimensional ultrasound. Ultrasound Rev Obstet Gynecol 2004; 4: 46-58.

22. Yigiter AB, Kavak ZN. Normal standards of fetal behavior assessed by four-dimensional sonography. J Matern Fetal Neonatal Med 2006; 19: $707-21$.

23. Visser GHA. The second trimester. In: Nijhuis JG, ed. Fetal Behaviour, Developmental and Perinatal Aspects. Oxford: Oxford University Press, 1992: 17-26.

24. Kuno A, Akiyama M, Yamashiro C, et al. Three dimensional sonographic assessment of fetal behavior in the early second trimester of pregnancy. J Ultrasound Med 2001; 20: 1271-5.

25. Bots RS, Nijhuis JG, Martin CB Jr, et al. Human fetal eye movements: detection in utero by ultrasonography. Early Hum Dev 1981; 5: 87-94.

26. Sparling JW, Van Tol J, Chescheir NC. Fetal and neonatal hand movement. Phys Ther 1999; 79: 24-39.

27. Sparling JW, Wilhelm IJ. Quantitative Measurement of Fetal Movement: Fetal-Post and Movement Assessment (F-PAM). Physical and Occupational Therapy in Pediatrics 1993; 12: 2-3.

28. Kurjak A, Stanojevic M, Andonotopo W, et al. Fetal behavior assessed in all three trimesters of normal pregnancy by four-dimensional ultrasonography. Croat Med J 2005; 46: 772-80.

29. Patrick J, Campbell K, Carmichael L, et al. Patterns of gross fetal body movements over 24-hours observation intervals during the last 10 weeks of pregnancy. Am J Obstet Gynecol 1982; 142: 363-71.

30. Prechtl HFR, Fargel JW, Weinmann HM, et al. Postures, motility and respiration of low risk preterm infants. Dev Med Child Neurol 1979; 21: 3-27.

31. Awoust J, Levi S. New aspects of fetal dynamics with a special emphasis on eye movements. Ultrasound Med Biol 1984; 10: 107-16. 
32. Nijhuis JG, Prechtl HF, Martin CB Jr, et al. Are there behavioural states in the human fetus? Early Hum Dev 1982; 6: 177-95.

33. Prechtl HFR, Weinmann HM, Akiyama Y. Organization of physiological parameters in normal and neurologically abnormal infants. Neuropaediatric 1969; 1: 101-29.

34. Nijhuis JG, Martin CB Jr, Gommers S, et al. The rhythmicity of fetal breathing varies with behavioural state in the human fetus. Early Hum Dev 1983; 9: 1-7.

35. Kurjak A, Azumendi G, Andonotopo W, et al. Three- and fourdimensional ultrasonography for the structural and functional evaluation of the fetal face. Am J Obstet Gynecol 2007; 196: 16-28.

36. Kurjak A, Stanojevic M, Azumendi G, et al. The potential of fourdimensional (4D) ultrasonography in the assessment of fetal awareness. J Perinat Med 2005; 33: 46-53.

37. De Meyer V, Zemen W, Palmer CG. The face predicts the brain: diagnostic significance of medial facial anomalies for holoprosencephaly (arhinencephaly). Pediatrics 1964; 34: 256-62.

38. Yan F, Dai SY, Akther N, et al. Four-dimensional sonographic assessment of fetal facial expression early in the third trimester. Int J Gynaecol Obstet 2006; 94: 108-13.

39. Awoust J, Levi S. Neurological maturation of the human fetus. Ultrasound Med Biol 1983; 9: 583-7.

40. Inoue M, Koyanagi T, Nakahara H. Functional development of human eye-movement in utero assessed quantitatively with real-time ultrasound. Am J Obstet Gynecol 1986; 155: 170-4.

41. Kurjak A, Carrera JM, Medic M, et al. The antenatal development of fetal behavioral patterns assessed by four-dimensional sonography. J Matern Fetal Neonatal Med 2005; 17: 401-16.

42. Parmelee AH, Stern E. Development of states in infants. In: Clemente CD, Purpura DP, Mayer FE, eds. Sleep and the maturing central nervous system. New York: Academic Press, 1972: 100-215.

43. Prechtl HFR. How can we assess the integrity of the fetal nervous system? In: Arbeille P, Manlik D, Laurini RN, editors. Fetal Hypoxia. New York, London: Parthenon Publishing Group, 1999: 109-15.

44. Einspieler C, Prechtl HFR, Bos AF, Ferrari F, Cioni G. Prechtl's method on the qualitative assessment of general movements in preterm, term and young infants. London: Mac Keith Press, 2004.

45. Amiel-Tison C. Clinical assessment of the infant nervous system. In: Levene MI, Chervenak FA, Whittle M, eds. Fetal and Neonatal Neurology and Neurosurgery, 3rd edn. London: Churchill Livingstone, 2001.

46. Sarnat HB. Functions of the corticospinal and corticobulbar tracts in the human newborn. J Pediatr Neurol 2003; 1: 3-10.

47. Azumendi G, Kurjak A. Three-dimensional and four dimensional sonography in the study of the fetal face. Ultrasound Rev Obstet Gynecol 2003; 3: 1-10.

48. Horimoto N, Koyanagi T, Maeda K, et al. Can brain impairment be detected by in utero behavioural patterns? Arch Dis Child 1993; 69: 3-8.

49. Morokuma S, Fukushima K, Yumoto Y, et al. Simplified ultrasound screening for fetal brain function based on behavioral pattern. Early Hum Dev 2007; 83: 177-81.

50. Ahmed B, Kurjak A, Andonotopo W, et al. Fetal behavioral and structural abnormalities in high-risk fetuses assessed by 4D sonography. Ultrasound Rev Obstet Gynecol 2005; 5: 275-87.

51. Kurjak A, Miskovic B, Stanojevic M, et al. New scoring system for fetal neurobehavior assessed by three- and four-dimensional sonography. J Perinat Med 2008; 36: 73-81.

52. Strijbis EMM, Oudman I, van Essen P, et al. Cerebral palsy and the application of criteria for acute intrapartum hypoxia. Obstet Gynecol 2006; 107: 1357-65.

53. Visser GH, Bekedam DJ, Mulder EJ, et al. Delayed emergence of fetal behaviour in type-1 diabetic women. Early Hum Dev 1985; 12: 167-72.

54. Mulder EJ, Visser GH, Bekedam DJ, et al. Emergence of behavioural states in fetuses of type-1-diabetic women. Early Hum Dev 1987; 15: 231-51.

55. Visser GH, Mulder EJ, Bekedam DJ, et al. Fetal behaviour in type-1 diabetic women. Eur J Obstet Gynecol Reprod Biol 1986; 21: 315-20.

56. Mulder EJ, Visser GH. Growth and motor development in fetuses of women with type-1 diabetes. II. Emergence of specific movement patterns. Early Hum Dev 1991; 25: 107-15.

57. Mulder EJ, Visser GH, Morssink LP, et al. Growth and motor development in fetuses of women with type-1 diabetes. III. First trimester quantity of fetal movement patterns. Early Hum Dev 1991; 25: 117-33.
58. Mulder EJ, O’Brien MJ, Lems YL, et al. Body and breathing movements in near-term fetuses and newborn infants of type-1 diabetic women. Early Hum Dev 1990; 24: 131-52.

59. Mulder EJ, Visser GH. Impact of early growth delay on subsequent fetal growth and functional development: a study on diabetic pregnancy. Early Hum Dev 1992; 31: 91-5.

60. Mulder EJ, Leiblum DM, Visser GH. Fetal breathing movements in late diabetic pregnancy: relationship to fetal heart rate patterns and Braxton Hicks' contractions. Early Hum Dev 1995; 43: 225-32.

61. Reece EA, Hagay Z, Roberts AB, et al. Fetal Doppler and behavioral responses during hypoglycemia induced with the insulin clamp technique in pregnant diabetic women. Am J Obstet Gynecol 1995; 172: 151-5.

62. Devoe LD, Youssef AA, Castillo RA, et al. Fetal biophysical activities in third-trimester pregnancies complicated by diabetes mellitus. Am J Obstet Gynecol 1994; 171: 298-303.

63. Kainer F, Prechtl HF, Engele H, et al. Assessment of the quality of general movements in fetuses and infants of women with type-I diabetes mellitus. Early Hum Dev 1997; 50: 13-25.

64. van Vliet MA, Martin CB Jr, Nijhuis JG, et al. The relationship between fetal activity and behavioral states and fetal breathing movements in normal and growth-retarded fetuses. Am J Obstet Gynecol 1985; 153: 582-8.

65. Van Vliet MA, Martin CB Jr, Nijhaus JG, et al. Behavioural states in growth-retarded human fetuses. Early Hum Dev 1985; 12: 183-97.

66. Arduini D, Rizzo G, Romanini C, et al. Computerized analysis of behavioural states in asymmetrical growth retarded fetuses. J Perinat Med 1988; 16: 357-63.

67. Rizzo G, Arduini D, Pennestri F, et al. Fetal behaviour in growth retardation: its relationship to fetal blood flow. Prenat Diagn 1987; 7: 229-38.

68. Sival DA, Visser GH, Prechtl HF. The effect of intrauterine growth retardation on the quality of general movements in the human fetus. Early Hum Dev 1992; 28: 119-32.

69. Sival DA, Visser GH, Prechtl HF. The relationship between the quantity and quality of prenatal movements in pregnancies complicated by intrauterine growth retardation and premature rupture of the membranes. Early Hum Dev 1992; 30: 193-209.

70. Ribbert LS, Visser GH, Mulder EJ, et al. Changes with time in fetal heart rate variation, movement incidences and haemodynamics in intrauterine growth retarded fetuses: a longitudinal approach to the assessment of fetal well being. Early Hum Dev 1993; 31: 195-208.

71. Vindla S, James DK, Sahota DS, et al. Computerised analysis of behaviour in normal and growth-retarded fetuses. Eur J Obstet Gynecol Reprod Biol 1997; 75: 169-75.

72. Bekedam DJ, Mulder EJ, Snijders RJ, et al. The effects of maternal hyperoxia on fetal breathing movements, body movements and heart rate variation in growth retarded fetuses. Early Hum Dev 1991; 27: 223-32.

73. Andonotopo W, Kurjak A. The assessment of fetal behavior of growth restricted fetuses by $4 \mathrm{D}$ sonography. J Perinat Med 2006; 34: 471-8.

74. Vintzileos AM, Campbell WA, Nochimson DJ, et al. The fetal biophysical profile in patients with premature rupture of the membranes-an early predictor of fetal infection. Am J Obstet Gynecol 1985; 152: 510-16.

75. Konstantinidou A, Anninos H, Spanakis N, et al. Transplacental infection of Coxsackievirus B3 pathological findings in the fetus. J Med Virol 2007 79: 754-7.

76. Craig S, Permezel M, Doyle L, et al. Perinatal infection with Listeria monocytogenes. Aust NZ J Obstet Gynaecol 1996; 36: 286-90.

77. Goldstein I, Romero R, Merrill S, et al. Fetal body and breathing movements as predictors of intraamniotic infection in preterm premature rupture of membranes. Am J Obstet Gynecol 1988; 159: 363-8.

78. Del Valle GO, Joffe GM, Izquierdo LA, et al. The biophysical profile and the nonstress test: poor predictors of chorioamnionitis and fetal infection in prolonged preterm premature rupture of membranes. Obstet Gynecol 1992; 80: 106-10.

79. Sherer DM, Spong CY, Minior VK, et al. Decreased amniotic fluid volume at $<32$ weeks of gestation is associated with decreased fetal movements. Am J Perinatol 1996; 13: 479-82. 
80. Habek D. Effects of smoking and fetal hypokinesia in early pregnancy. Arch Med Res 2007; 38: 864-7.

81. Cowperthwaite B, Hains SM, Kisilevsky BS. Fetal behavior in smoking compared to non-smoking pregnant women. Infant Behav Dev 2007; 30: $422-30$.

82. Warner J, Hains SM, Kisilevsky BS. An exploratory study of fetal behavior at 33 and 36 weeks gestational age in hypertensive women. Dev Psychobiol 2002; 41: 156-68.
83. Kisilevsky BS, Hains SM. Comparison of fetal behavior in low- and highrisk pregnancies. Fetal Pediatr Pathol 2005; 24: 1-20.

84. de Vries JI, Fong BF. Changes in fetal motility as a result of congenital disorders: an overview. Ultrasound Obstet Gynecol 2007; 29: 590-9.

85. Ahmed B, Kurjak A, Andonotopo W, et al. Fetal behavioral and structural abnormalities in high risk fetuses assessed by $4 \mathrm{~d}$ sonography. Ultrasound Rev Obstet Gynecol 2005; 5: 1-13. 


\section{Umbilical artery doppler sonography for fetal surveillance: Principles and practice Dev Maulik, David Mundy, and Timothy Bennett}

\section{INTRODUCTION}

Antenatal fetal monitoring is an essential component of modern obstetric care in pregnancies at high risk for adverse perinatal outcomes. Technical and clinical advances during the past few decades have resulted in the development of several methods of antepartum fetal surveillance that include fetal heart rate (FHR) monitoring, ultrasound assessment of fetal biophysical status, and Doppler velocimetry of fetal and uteroplacental circulation. This chapter presents a general background of Doppler ultrasound for fetal surveillance with emphasis on an evidence-based approach for its clinical application. The review is focused on the Doppler of the umbilical artery (UA), as it is the only fetal surveillance tool that has shown clinical effectiveness in improving perinatal outcome. Extensively revised since the last edition of the book, this chapter presents the following aspects of Doppler fetal surveillance:

- A brief history of diagnostic Doppler ultrasound

- Principles of Doppler velocimetry

- Doppler velocimetry of the UA

- Other fetal surveillance tests

- Clinical effectiveness of Doppler velocimetry for fetal surveillance

- Guideline for the clinical application of the Doppler fetal surveillance

For a more comprehensive review of the various clinical and basic aspects of Doppler sonography in obstetrics, a dedicated text or reference book is recommended (1).

\section{A BRIEF HISTORY OF DIAGNOSTIC DOPPLER ULTRASOUND}

Doppler sonography is based upon the physical phenomenon of Doppler shift first described by Austrian mathematician and physicist Christian Andreas Doppler in 1841. Doppler first reported this phenomenon at a meeting of the Natural Sciences Section of the Royal Bohemian Society in Prague, a meeting with only five members of the society in attendance and a transcriber. His paper, entitled "On the Colored Light of the Double Stars and Certain Other Stars of the Heavens," was published in 1843 in the society's proceedings (2). To Doppler's disappointment, however, the paper was not enthusiastically received by the scientific community of his time, although his principle has been extensively used in science and technology since the beginning of the twentieth century.

The history of diagnostic Doppler ultrasound has been reviewed in greater detail elsewhere (3). The premier medical use of Doppler sonography occurred during the late 1950s. The use of Doppler sonography for the investigation of human fetal circulation was first reported by FitzGerald and Drumm in 1977 (4). Maulik and coworkers further reported the feasibility of the Doppler ultrasound for fetal circulatory assessment (5). During the following years, many investigators used this technique to characterize most major fetal circulatory systems. In 1986, the National Institute of Child Health and Development organized a workshop that highlighted many issues pertaining to Doppler ultrasound measurement of maternal-fetal hemodynamics (6). These issues were also addressed by many investigators and discussed in several scientific societies around the world including the International Perinatal Doppler Society. For a decade and a half, the latter acted as a unique scientific forum dedicated to the obstetric applications of Doppler ultrasound and critically contributed to its introduction into the clinical practice through research and education. The diagnostic efficacy and clinical effectiveness of the Doppler technique has been extensively investigated. Of the various modalities of fetal surveillance, only the Doppler ultrasound of the UA has been shown to improve the perinatal outcome by randomized clinical trials (RCTs) and meta-analyses, and has now become a standard for fetal surveillance in most parts of the world.

\section{PRINCIPLES OF DOPPLER VELOCIMETRY} Doppler Frequency Shift

When relative motion occurs between a source of wave transmission and an observer, the observed changes in the frequency of energy wave transmission is defined as the Doppler effect. The change in the frequency is called the Doppler frequency shift or just the Doppler shift:

$$
f_{\mathrm{d}}=f_{\mathrm{t}}-f_{\mathrm{r}}
$$

Equation (1)

where $f_{\mathrm{d}}$ is the Doppler shift frequency, $f_{\mathrm{t}}$ is the transmitted frequency, and $f_{\mathrm{r}}$ is the received frequency. When the source and the observer move closer, the wavelength decreases and the frequency increases. Conversely, when the source and the observer move apart, the wavelength increases and the frequency decreases. The principle applies to all forms of energy wave propagation including sound. The utility of the Doppler effect originates from the fact that the shift in the frequency is proportional to the speed of movement between the source and the receiver and therefore can be used to assess this speed.

The phenomenon of the Doppler effect is observed when an ultrasound beam encounters a scatterer in motion. The scatterer acts first as a moving receiver and then as a moving source. This forms the basis for restating equation (1) as

$$
f_{\mathrm{d}}=2 f_{\mathrm{t}} \nu / c f_{r} \quad \text { Equation (2) }
$$

where $f_{\mathrm{d}}$ represents the Doppler frequency shift, $f_{\mathrm{t}}$ the frequency of the incident beam (transducer frequency), 
$v$ the velocity of the scatterer in a given direction, and $c$ the propagation speed of sound in the medium. Note that the transmitted ultrasound undergoes double Doppler shift before returning to the receiving transducer, the scatterer acting first as a receiver and then as a transmitter. This accounts for the factor of 2 in the above equation. In blood circulation, millions of red cells act as moving scatterers of an incident ultrasound, which consequently will undergo frequency shift proportional to the speed of red cell movement and therefore to blood flow velocity.

When the incident beam incurs an angle $(\theta)$ to the direction of blood flow, the $\mathrm{v}$ in the Doppler equation is replaced by the component of the velocity in the direction of the flow obtained by the cosine of the angle $(\cos \theta ;$ ) (Fig. 1):

$$
f_{\mathrm{d}}=2\left(f_{\mathrm{t}} \cos \theta \nu\right) / c \quad \text { Equation (3) }
$$

To determine the velocity of the scatterer, equation (3) can be rewritten as follows:

$$
\mathrm{v}=f_{\mathrm{d}} c / 2 f_{\mathrm{t}} \cos \theta \quad \text { Equation (4) }
$$

Thus, if the angle of beam incidence and the Doppler shift are known, the velocity of blood flow is also known assuming that the transducer frequency and the velocity of sound in tissue remain relatively constant. The above equation forms the basis for the clinical application of the Doppler principle.

\section{Circulatory Information from Doppler Shift}

Doppler sonography can yield a variety of clinically useful hemodynamic information. This includes quantification of flow and study of the flow velocity waveform (Table 1). Volumetric flow can be measured by integrating the average velocity across the vascular lumen with the vascular cross-sectional area. In the fetus, the Doppler technique has been used to measure umbilical venous flow (7), descending aortic flow (8), and fetal cardiac output (9). The clinical utility of Doppler flow quantification has remained limited because of its systemic unreliability related to errors in determining the vascular crosssectional area and the Doppler angle. Consequently, the main perinatal use for Doppler sonography has been the indirect assessment of downstream impedance by Doppler waveform analysis. However, several investigators have now improved the Doppler flow quantification method paving the way for its future potential clinical application (10). Current advances in Doppler and non-Doppler ultrasound methods for volumetric flow measurement have been recently reviewed (11).

\section{Doppler Waveform Analysis}

Doppler waveform analysis is usually based on the following characteristics of the maximum frequency shift envelope (Fig. 2): the peak systolic value (S), end-diastolic value (D), and the average value over the cardiac cycle (A). These three parameters have been used to develop indices describing the pulsatility of the Doppler waveform. A Doppler index is calculated as a ratio and is, therefore, virtually independent of the angle of insonation. Of the numerous indices, the pulsatility index (PI) (13), the resistance index (RI) (14), and the S/D ratio (15) are most commonly used in obstetric applications. Maulik and coinvestigators used the receiver

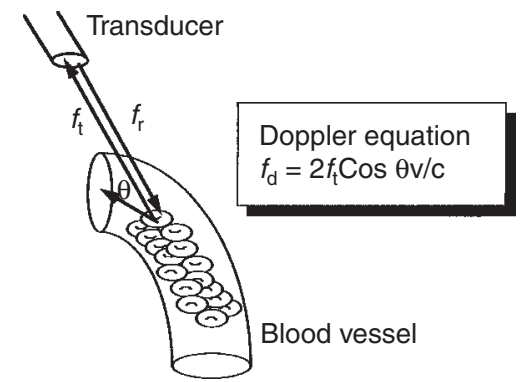

Figure 1 Graphic depiction of the principles of Doppler equation and the proportional relationship between the Doppler shift and the velocity of blood flow. Abbreviations: $f_{\mathrm{t}}$, Doppler frequency shift; $f_{\mathrm{r}}$, transmitted frequency; $f_{\mathrm{d}}$, Doppler frequency shift; $v$, velocity of blood flow; $\theta$, the angle of insonation between the ultrasound beam and the direction of flow; $c$, velocity of flow in tissue.

Table 1 Hemodynamic Information from Doppler Sonography

Presence of normal flow in expected location

Presence of flow in unexpected location

Absence of flow in expected location

Speed of blood flow

Direction of flow

Circulatory impedance in the arterial circulation

Volumetric flow

Disturbed hemodynamics, e.g., turbulent flow

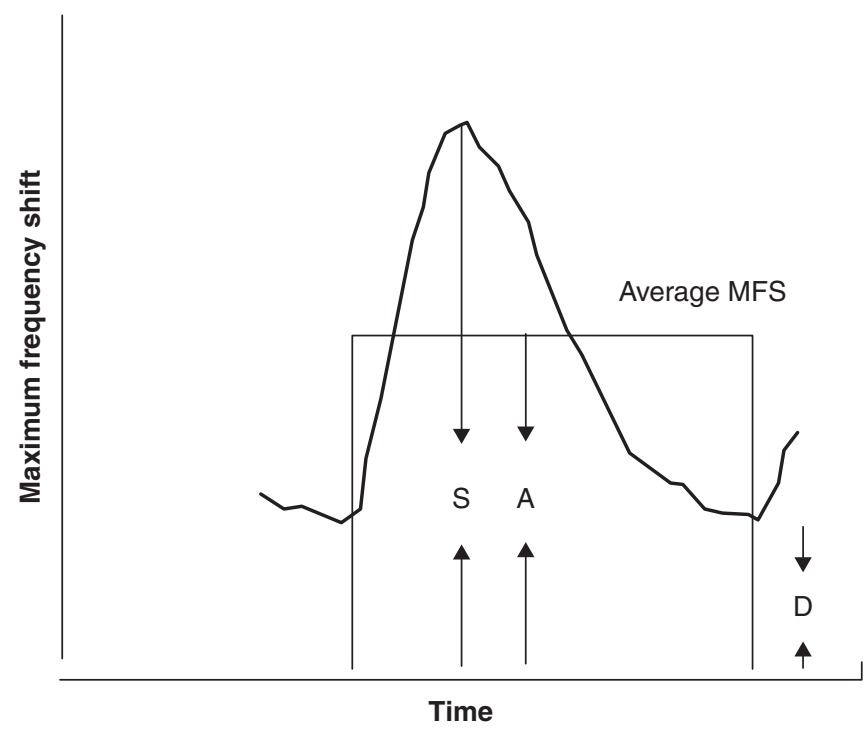

Figure 2 Doppler indices estimated from the maximum frequency shift (MFS) envelope. Abbreviations: S, peak systolic frequency shift value of the envelope; $\mathrm{D}$, end-diastolic frequency shift value of the envelope; $\mathrm{A}$, average value of the envelope over one cardiac cycle, $\mathrm{RI}=(\mathrm{S}-\mathrm{D}) / \mathrm{S} 6$; $\mathrm{PI}=(\mathrm{S}-\mathrm{D}) / \mathrm{A} 5$; S/D ratio 7; D/A ratio 9. Source: From Ref. 12.

operating characteristic (ROC) technique (Fig. 3) to investigate the comparative efficacy of the umbilical arterial Doppler indices for predicting adverse perinatal outcome and showed that the RI had the best discriminatory ability when compared with the S/D ratio $(p<0.05)$, the PI $(p<0.001)$, and the D/A ratio $(p<0.05)(16)$. Although more comprehensive approaches to Doppler waveform analysis have been described $(17,18)$, there is little evidence that these are superior to the traditional Doppler indices. The 
hemodynamic, angiomorphologic, and pathophysiologic justifications for Doppler waveform analysis are discussed below.

\section{Hemodynamic Rationale of Doppler Waveform Analysis}

Doppler waveform from an arterial source represents the arterial velocity waveform and is configured by both the upstream and downstream circulatory factors. Analysis of the waveform should, therefore, yield information on downstream impedance to flow. Obviously, this requires verification. Although the term "peripheral vascular resistance" has been traditionally used for describing opposition to flow in an arterial tree, it is applicable only to a steady non-pulsatile flow state. Opposition to flow in a pulsatile circulation is more accurately expressed by the concept of vascular impedance, which also includes the resistance. An integral component of this concept is the phenomenon of wave reflection in a vascular tree. Configuration of pressure and flow waves at a specific location results from the interaction of the forward propagating (orthograde) waves with the reflected backward propagating (retrograde) waves (19). The observed pressure waves, which are produced by the summation of orthograde and retrograde waves, show an "additive" effect; by contrast, the observed flow (or flow velocity) waves demonstrate a "subtractive" effect (Fig. 4). Wave reflections arise whenever there is a significant alteration or mismatching in vascular impedance in a circulation, and the arterial-arteriolar junctions serve as the main source of wave reflections in an arterial tree. Vasodilation leads to a fall and vasoconstriction to a rise in impedance and wave reflection.

The hemodynamic validation studies may be grouped as those relating to the peripheral resistance and those involving the impedance. However, as mentioned above, vascular impedance is the appropriate descriptor of opposition to flow in a pulsatile circulation. This was investigated by Downing and coworkers in a chronic lamb model and it was observed that a significant correlation exists between the PI and the impedance parameters, provided the reflex heart rate changes are suppressed. Evidently, the Doppler indices reflect changes in the downstream impedance (21). The greater the fetoplacental vascular impedance, the lower the end-diastolic flow in the UA and the higher the indices. A more comprehensive review of the hemodynamic interpretation of Doppler waveforms from an arterial system is available elsewhere (22).

\section{Angiomorphologic Validation of the Doppler Indices}

Several investigators have demonstrated that abnormal fetal placental angiogenesis is characterized by sparse, elongated, uncoiled, and less ramified terminal capillary loops as the major lesions in growth-restricted pregnancies with abnormal umbilical arterial Doppler $(23,24)$. These observations suggest that aberrant fetoplacental angiogenesis results in an increase in impedance, which is associated with enhanced pressure and flow velocity wave reflections. The reflected flow velocity waves propagate retrogradely and change the shape of arterial flow velocity waves, which show decreased end-diastolic flow and increased pulsatility. Doppler insonation of the appropriate arterial tree identifies this circulatory phenomenon by demonstrating abnormal Doppler waveforms and indices.

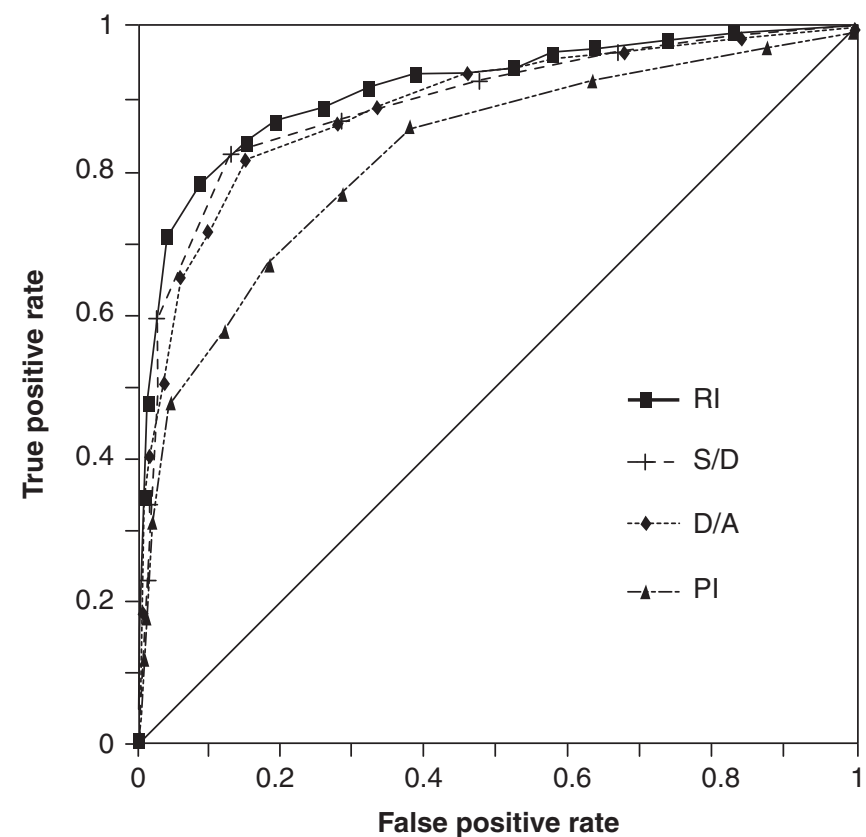

Figure 3 Receiver operating characteristic curves of umbilical arterial Doppler indices. Data points are the measured values of indices. Note that the resistance index shows the most and the pulsatility index shows the least diagnostic efficacy. RI;, S:D;, D:A;, PI. Source: From Ref. 12.

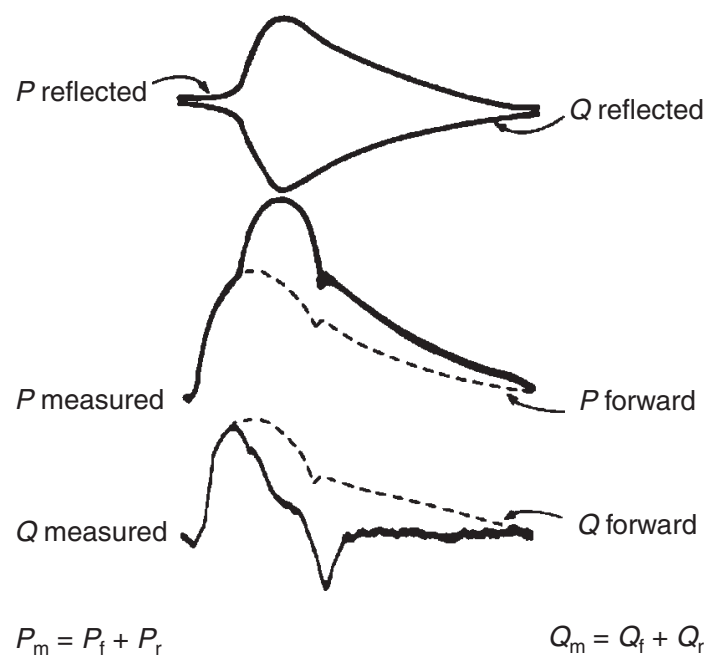

Figure 4 The influence of pulse wave reflections on ascending aortic pressure $(P)$ and flow $(Q)$ waveforms. Incident or forward (f) and backward or reflected $(\mathrm{r})$ pressure and flow waves are summed to yield measured $(\mathrm{m})$ pressure and flow waveforms. The forward pressure and flow waves are identical and so are the reflected waves except that the reflected wave form is inverted with impact to the reflected pressure wave. Source: From Ref. 20.

Pathophysiologic Rationale for Doppler Fetal Surveillance Antepartum challenge to fetal well-being may arise from chronic nutritive and respiratory deprivation. A spectrum of obstetric complications, including fetal growth restriction (FGR) and hypertension, may expose the fetus to such risks. Although an immense amount of information is available on acute and subacute fetal respiratory deficit, the pathophysiologic mechanism of chronic fetal stress has been less clear. However, significant advances have been made recently providing considerable insight into the mechanisms of fetal 


\section{Sequence of Fetal Response to Stress}

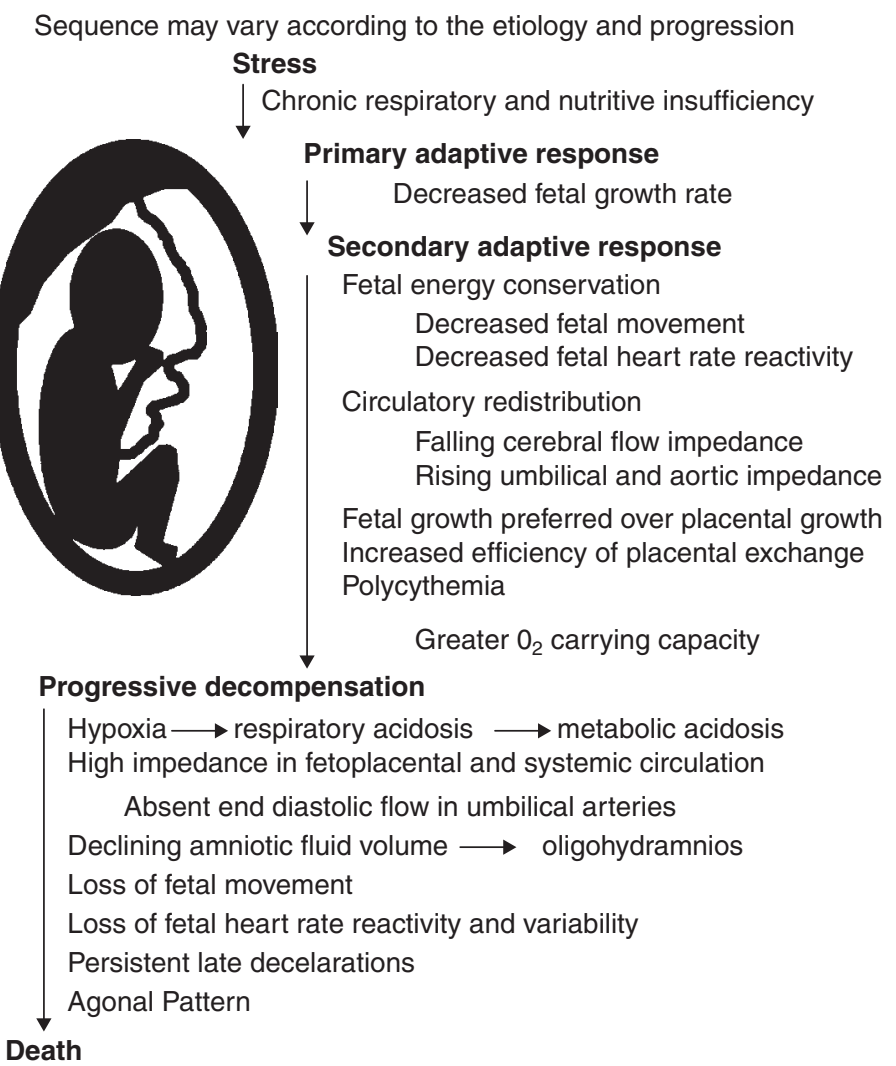

Figure 5 Summary of fetal sequential response to progressive stress. Note that the depicted sequence is an approximation and the actual course may vary depending on the characteristics of the chronic deprivation and the individual fetal ability to cope. Source: From Ref. 25.

compensation and decompensation. There is emerging evidence that, encountering sustained stress, the fetus appears to mobilize a spectrum of defensive responses that include preferential preservation of fetal growth over placental growth, changes in fetal movement pattern, and the eventual deceleration of the fetal growth rate (Fig. 5).

In the face of continuing deprivation, compensation gives way to decompensation. For example, growth-restricted human fetuses with abnormal UA waveforms have been shown to develop chronic hypoxia and acidosis. A critical component of fetal homeostatic response involves flow redistribution that favors perfusion of the vital organs (the brain, heart, and adrenals) at the expense of flow to muscle, viscera, skin, and other less critical tissues and organs (26). Underlying this phenomenon are the diverse changes in blood flow impedance in fetal regional circulations. Doppler velocimetry thus elucidates these circulatory changes associated with fetal compromise and allows perinatal prognostication. This constitutes the rationale for using Doppler ultrasound for fetal surveillance in complicated pregnancies.

\section{DOPPLER VELOCIMETRY OF THE UA}

\section{Normative Data}

The UA was the first fetal vessel to be evaluated by Doppler velocimetry and has since become the most widely investigated fetal circulation. Typically, Doppler interrogation of the umbilical arterial circulation is performed during fetal apnea (see below). In our experience, the inter- and intra-observer variances of the S:D ratio and the RI, as determined by continuous-wave Doppler, were $9.8 \%$ and $11.1 \%$, and $4 \%$ and $8 \%$, respectively (27). In normal pregnancy, the umbilical arterial Doppler indices are affected by gestational age, FHR, fetal breathing, and the site of measurement in the cord.

\section{Gestational Age}

As pregnancy advances, umbilical arterial Doppler waveforms show a progressive rise in the end-diastolic velocity, which results in a concomitant fall in the pulsatility (Fig. 6). This trend is consistent with a gradual decline in the fetoplacental flow impedance with the advancing gestation (29). The Doppler indices reflect these changes in the waveform (Fig. 7).

\section{FHR}

A statistically significant effect of the heart rate on Doppler indices has been reported by a number of investigators (31). It has been shown that changes in the heart rate occur primarily due to alterations in the duration of the diastolic component of the cardiac cycle (32) and that the effect of the FHR may contribute to $15 \%$ to $18 \%$ of the variance of the Doppler indices (27). However, it is not certain that correcting the Doppler indices for FHR changes would improve the diagnostic efficacy of the indices and may not be clinically significant so long as the heart rate remains within the normal range. 


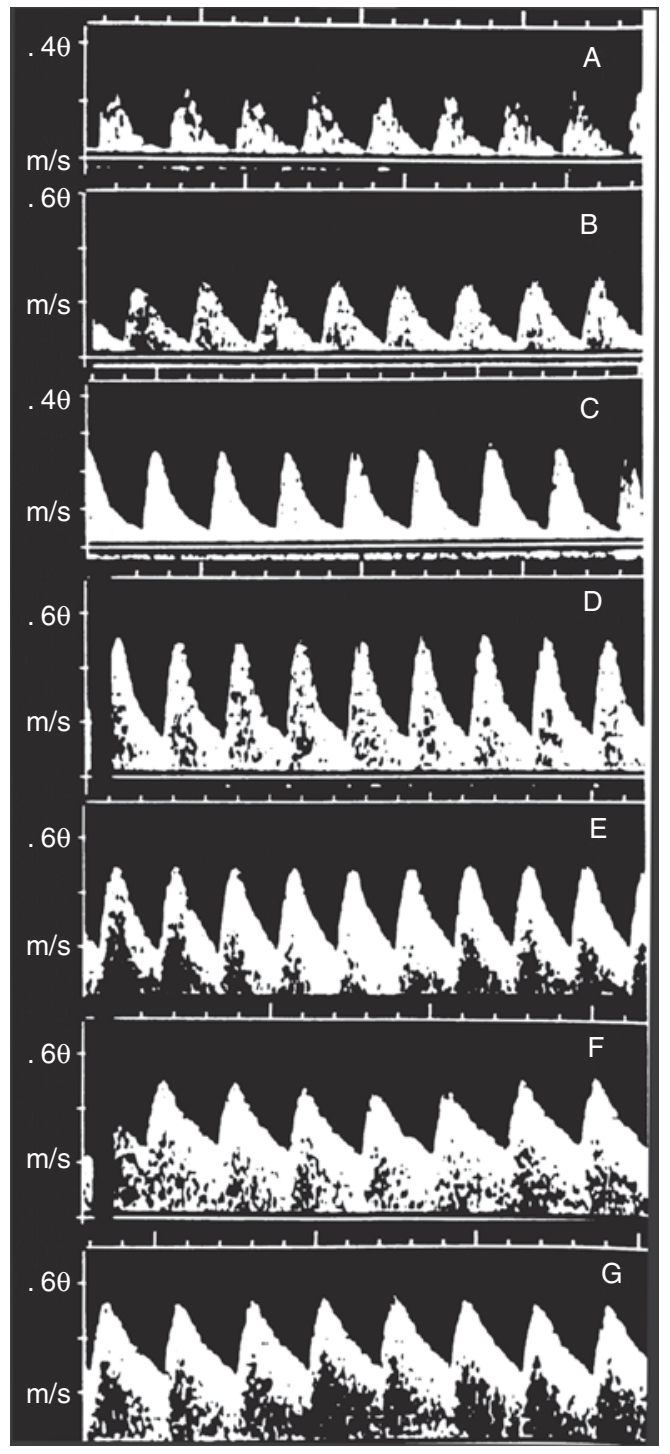

Figure 6 Gestational age effect on the umbilical arterial Doppler frequency shift waveforms. Panels are organized from the top to the bottom according to advancing gestation. Abbreviations: $\mathrm{A}$, waveforms at 16 weeks; $\mathrm{B}$, at 20 weeks; C, at 24 weeks; D, at 28 weeks; E, at 32 weeks; F, at 36 weeks; G, at 40 weeks. Note the progressive increase in the end-diastolic velocity and the concomitant fall in the pulsatility as gestation advances. Source: From Ref. 28.

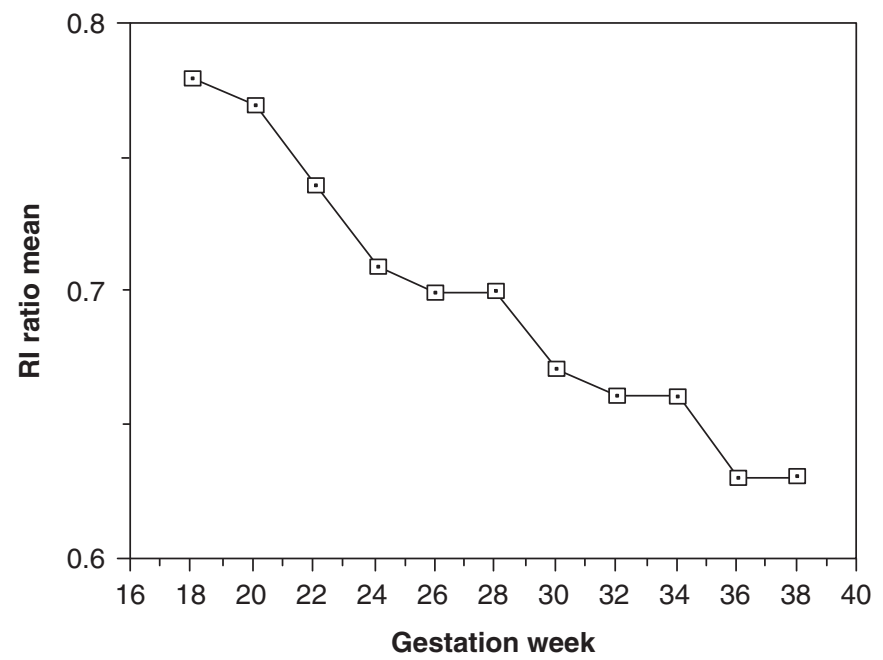

Figure 7 Variation in the umbilical arterial resistance index (RI) at different stages of gestation. Source: From Ref. 30.

\section{Fetal Breathing}

Substantial changes in the intrathoracic pressure and central hemodynamics occur during fetal breathing, which produce dynamic variability in the umbilical arterial Doppler waveform and, therefore, in the Doppler indices. This severely compromises the utility of the Doppler indices during fetal breathing and provides the rationale for measuring the indices only during fetal apnea. However, the indices do not appear to be affected by fetal behavioral states (33).

\section{Site of Doppler Interrogation}

The location of the Doppler sampling site in the umbilical cord affects the Doppler waveform and therefore the Doppler indices, which are higher at the fetal than at the placental end of the cord (27).

\section{Umbilical Arterial Doppler in FGR}

FGR is associated with defective development of placental vascular systems. FGR and pre-eclampsia are associated with aberrant angiogenesis of fetoplacental arterial system. The efficacy of UA Doppler indices for detecting suboptimal fetal growth has shown to be variable and has been reviewed (Table 2) (34). This may be partly explained by the considerable amount of ambiguity that surrounds the use of the terms "FGR" and "small for gestational age (SGA)." It is well recognized that neither all growth-restricted fetuses are SGA, nor are all SGA infants growth compromised. Furthermore, being SGA does not necessarily mean a compromised outcome. It is apparent that not all FGR or SGA fetuses may suffer from in utero compromise in terms of asphyxia, which correlates better with absent end-diastolic velocity (AEDV) in the UA than with subnormal fetal abdominal circumference ( $<5$ th percentile) as measured by ultrasound (35). This issue, however, is apparently controversial and is further discussed in the next section. With regard to the various sonographic modalities for diagnosing FGR, the majority of studies have demonstrated that conventional fetal ultrasound biometry is more sensitive than umbilical arterial Doppler velocimetry (36). This should not be surprising as fetal size is expected to be better expressed by sonographic measurement of fetal dimensions than by Doppler velocimetry, which assesses the hemodynamic state; unless the latter is compromised, the Doppler indices will not change.

\section{Umbilical Arterial Doppler Velocimetry and Antepartum Fetal Hypoxia-Asphyxia}

The purpose of fetal surveillance is to detect fetal compromise from intrauterine hypoxia and asphyxia. Fetal homeostatic response to hypoxic and asphyxial challenge has been well described in animals (37). A central manifestation of this response is the flow redistribution that favors perfusion of the vital organs (the brain, the heart, and the adrenals) at the expense of less critical organs and tissues. This compensatory phenomenon provided the justification for investigating the efficacy of the Doppler velocimetry in identifying fetal hypoxia and asphyxia. Both clinical and animal experimental studies have been reported to address this issue.

Further insight may be obtained from experimental studies conducted in animal models. Many of the relevant findings in this area have been reviewed (38). It has been noted that in the 
aorta of the fetal lamb, significant changes in the Doppler waveform occur only when the oxygen saturation declines to $10 \%$ to $15 \%$ or the fetal pH drops to $<7.15$ (39). Apparently, in an ovine fetus, hypoxia and acidosis may significantly affect umbilical and aortic velocity waveforms but only as a late phenomenon.

The association between the Doppler findings and fetal blood gases has been clinically investigated $(35,40-47)$. These have been reviewed and are summarized in Table 3. Some investigators performed umbilical cord blood sampling at the time of elective cesarean section. Obviously, these studies are limited because blood gases measured in this manner may not reflect fetal acid-base status in utero. Others determined fetal blood gases by cordocentesis. Most studies used Doppler assessment of the UA, whereas one used aortic mean velocity and another added aortic and carotid Doppler assessment. All studies measured $\mathrm{pH}$, most performed $\mathrm{PO}_{2}$, and some also measured $\mathrm{PCO}_{2}$ and lactate. Most investigators found a significant association between Doppler assessment of fetal circulation and fetal acid-base compromise. The response of fetal cerebral Doppler waveform presents another perspective on fetal hypoxia and acidosis. This is discussed later in the section dealing with fetal cerebral circulation.

\section{Umbilical Arterial Doppler Velocimetry and Adverse Perinatal Outcome}

The discriminatory efficacy of Doppler velocimetry for predicting perinatal outcome is briefly discussed in this section. A fetal surveillance test may be used either as a diagnostic test for identifying the fetal compromise in highrisk pregnancies or as a screening test in a general obstetric population at low risk for fetal compromise. Both are presented below. In evaluating this information, we need to recognize the imprecision inherent in the available criteria for defining adverse perinatal outcome.

\section{High-Risk Pregnancies}

Several preliminary studies indicated that the Doppler indices might be powerful predictors of adverse perinatal outcome in complicated pregnancies. The diagnostic efficacy of umbilical arterial A/B (systolic, diastolic) ratio was investigated by Trudinger and coworkers in 170 high-risk patients (48). The parameters of fetal compromise included birth weight below the 10th percentile or an Apgar score of $<7$ at 5 minutes. The FHR was assessed in terms of reactivity and a modified Fischer score. The Doppler results revealed a sensitivity of $60 \%$, a specificity of $85 \%$, and a positive predictive value of $64 \%$. For the FHR reactivity, the corresponding results were $17 \%, 97 \%$, and $69 \%$, respectively. The values for the Fischer score results were $36 \%, 88 \%$, and $58 \%$, respectively. In this study, the umbilical arterial S/D ratio appeared to be more sensitive but less specific than the electronic FHR monitoring techniques. Farmakides and others investigated the diagnostic efficacy of the nonstress test (NST) and of the umbilical arterial S/D ratio in 140 pregnancies (49). The measures of outcome included FGR, fetal distress, cesarean section for fetal distress, and

Table 2 Diagnostic Efficacy of Umbilical Arterial Doppler in Intrauterine Growth Retardation

\begin{tabular}{|c|c|c|c|c|c|}
\hline Investigators & Doppler index & Prevalence rate $(\%)$ & Sensitivity (\%) & Specificity (\%) & $\begin{array}{c}\text { Positive predictive } \\
\text { value }(\%)\end{array}$ \\
\hline Fleischer et al. (1985) & $\mathrm{S}: \mathrm{D}>3.0$ & 16.8 & 78 & 83 & 49 \\
\hline Arduini et al. (1987) & $\mathrm{PI}>1 \mathrm{SD}$ & 30.7 & 60.8 & 73 & 50 \\
\hline Divon et al. (1988) & $\mathrm{S}: \mathrm{D}>3.0$ & 35.4 & 49 & 94 & 81 \\
\hline Gaziano et al. (1988) & $\mathrm{S}: \mathrm{D}>4.0$ & 9.4 & 79 & 66 & 79 \\
\hline Lowery et al. (1990) & $\mathrm{S}: \mathrm{D}>4.0$ & 22.6 & 65 & 66 & 24 \\
\hline
\end{tabular}

Table 3 Association Between Fetal Doppler Results and Fetal Blood Gases

\begin{tabular}{|c|c|c|c|c|c|c|}
\hline $\begin{array}{l}\text { Author } \\
\text { (reference) }\end{array}$ & $\begin{array}{c}\text { Patient } \\
\text { population }\end{array}$ & $\begin{array}{l}\text { Patient risk } \\
\text { category }\end{array}$ & $\begin{array}{l}\text { Cord blood } \\
\text { sampling }\end{array}$ & Doppler assessment & $\begin{array}{l}\text { Acid-base } \\
\text { parameters }\end{array}$ & $\begin{array}{l}\text { Association } \\
\text { correlation }\end{array}$ \\
\hline Soothill (39) & 29 & SGA & Cordocentesis & Aortic MV & $\mathrm{pH}, \mathrm{PO}_{2}, \mathrm{PCO}_{2}$ & Present \\
\hline Nicolaides (40) & 59 & SGA & Cordocentesis & Umbilical arterial AEDV & $\mathrm{pH}, \mathrm{PO}_{2}$ & Present \\
\hline Tyrell (42) & 112 & Unselected & Cesarean section & Umbilical arterial AEDV & $\mathrm{pH}, \mathrm{PO}_{2}$ & Present \\
\hline Bilardo (43) & 51 & SGA, AGA & Cordocentesis & $\begin{array}{l}\text { Umbilical arterial, aortic, } \\
\text { carotid PI, RI, MV }\end{array}$ & $\mathrm{pH}, \mathrm{PO}_{2}$ & Present \\
\hline Yoon (45) & 105 & Unselected & Cesarean section & Umbilical arterial PI & $\mathrm{pH}, \mathrm{PO}_{2}, \mathrm{PCO}_{2}$ & Present \\
\hline Pardi (46) & 21 & SGA & Cordocentesis & Umbilical arterial PI lactate & $\mathrm{pH}, \mathrm{PO}_{2}, \mathrm{PCO}_{2}$ & Present \\
\hline Yoon (47) & 24 & High risk & Cordocentesis & Umbilical arterial PI & $\mathrm{pH}, \mathrm{PO}_{2}, \mathrm{PCO}_{2}$ & Present \\
\hline
\end{tabular}

Abbreviations: SGA, small for gestational age; AGA, appropriate for gestational age; AEDV, absent end-diastolic velocity; MV, mean velocity; PI, pulsatility index; RI, resistance index; S:D, systolic diastolic ratio. Source: From Ref. 1. 
admission to the neonatal intensive care unit. Fetuses with a normal NST but abnormal S/D ratio had an outcome worse than those with an abnormal NST and a normal S/D ratio; however, those with both tests abnormal experienced the worst outcome.

Maulik and coworkers studied the diagnostic efficacy of the umbilical arterial S/D ratio for predicting adverse perinatal outcome in 350 high-risk pregnant patients utilizing the ROC technique and other traditional parameters of testing a test (50). The latter included sensitivity, specificity, and the predictive values. The kappa index was used to investigate the degree of agreement between the test and the outcome. The abnormal outcome parameters included SGA $(<10$ th percentile), Apgar score at 5 minutes $>7$, fetal distress (late and severe variable decelerations, absent variability, fetal scalp $\mathrm{pH}$ $<7.20$ ), umbilical cord arterial $\mathrm{pH}<7.20$, presence of thick meconium, and admission to neonatal intensive care unit for more than 48 hours. The study indicated that the ratio predicted the general adverse perinatal outcomes well; however, the diagnostic efficacy was better when the SGA infants were excluded (Table 4).

\section{Low-Risk Pregnancies}

Although the above findings are very encouraging, there are a few recent reports that demonstrate the inadequate efficacy of Doppler velocimetry of the UA as a screening test in a general obstetric population at a low risk for adverse perinatal outcome. In a large prospective study involving 2097 singleton pregnancies, Beattie and Dornan evaluated the capability of umbilical arterial Doppler indices (PI, A/B ratio, RI) to detect FGR and perinatal compromise (52). It was noted that the indices did not adequately predict any of the parameters of adverse perinatal outcome. The suboptimal efficacy of umbilical Doppler insonation in a low-risk population has been corroborated by others (53). Beattie and associates (54) performed Bayesian analysis of the above four studies to determine the posttest probability of an abnormal outcome for an individual patient. The posttest probabilities of an adverse outcome was determined for an individual in terms of positive and negative likelihood ratios and are summarized in Table 5. It is evident that umbilical arterial Doppler velocimetry is of limited utility in a low-risk obstetric population. Clinical effectiveness of UA Doppler as a screening test in low-risk pregnancies is discussed later.

\section{Significance of AEDV in the UA}

Absence or reversal of end-diastolic flow velocity (Fig. 8) is associated with markedly adverse perinatal outcome including a high perinatal mortality rate with a higher prevalence of chromosomal abnormalities (especially trisomy 13, 18, and 21) and congenital anomalies. In the European multicenter study involving 245 cases with absent or reversed end-diastolic flow, the perinatal mortality was $28 \%$ and $96 \%$ to $98 \%$ of the infants required intensive care (55). In a review of 1126 cases of absent and reverse end-diastolic flow in the UA reported in the literature, it was observed that 170 per 1000 were stillborn and 280 per 1000 died during the neonatal period (Table 6) (56). Most deaths were due to obstetric complications such as growth restriction, prematurity, fetal anomalies, and aneuploidy. The perinatal mortality corrected for congenital malformations and chromosomal abnormalities was approximately 340 per 1000 births. This group demonstrates a high risk of chromosomal abnormalities with a predominance of trisomy 13,18 , and 21 . There is also a high association between absent end-diastolic flow and a variety of congenital anomalies (Table 7). The frequency of absent end-diastolic flow is approximately $2 \%$ in well-defined high-risk pregnancies and may be as low as $0.3 \%$ in a general obstetric population.

In a prospective study involving pregnancies with FGR, Baschat and associates (58) investigated the relationship between fetal Doppler (UA, middle cerebral artery, and ductus venosus), biophysical profile score (BPP), and neurodevelopmental outcome at 2 years of age. Of the various fetal tests, only the UA reverse end-diastolic flow independently predicted poor neurodevelopment.

Table 4 Diagnostic Efficacy of Systolic/Diastolic Ratio Cutoff Point of 3.0 to Predict the Various Abnormal Outcomes

$\begin{array}{lcccc}\text { Category } & \text { Sensitivity } & \text { Specificity } & \begin{array}{c}\text { Positive } \\ \text { predictive value }\end{array} & \begin{array}{c}\text { Kegative } \\ \text { predictive value }\end{array} \\ \text { index } \\ \text { General abnormal outcome } & 0.79 & 0.93 & 0.83 & 0.91 \\ \text { SGA only } & 0.75 & 0.77 & 0.32 & 0.95 \\ \text { Fetal distress, Apgar, pH, NICU } & 0.86 & 0.88 & 0.68 & 0.96 \\ \text { Fetal distress, Apgar, pH, NICU, } & 0.82 & 0.92 & 0.81 & 0.92 \\ \quad \text { and meconium } & & & 0.69 \\ \text { Abbreviations: NICU, neonatal intensive care unit admission; SGA, small for gestational age. Source: From Ref. 51. }\end{array}$

Table 5 Posttest Probability of an Adverse Outcome and Umbilical Arterial Doppler in Low-Risk Pregnancies: a Summary of Four Studies

\begin{tabular}{|c|c|c|}
\hline Outcome & $\begin{array}{l}\text { Positive } \\
\text { likelihood ratio }\end{array}$ & $\begin{array}{l}\text { Negative } \\
\text { likelihood ratio }\end{array}$ \\
\hline Small for gestational age (birth weight $<10$ th centile) & +1.1 to +4.2 & -0.8 to -1.0 \\
\hline $\begin{array}{l}\text { Adverse perinatal outcome (Apgar }<7 \text { at } 1 \mathrm{~min} \text {, umbilical arterial } \mathrm{pH}<7.2 \text {, } \\
\text { operative delivery for fetal distress, abnormal fetal heart rate) }\end{array}$ & +1.0 to +3.1 & -0.9 to -1.0 \\
\hline
\end{tabular}




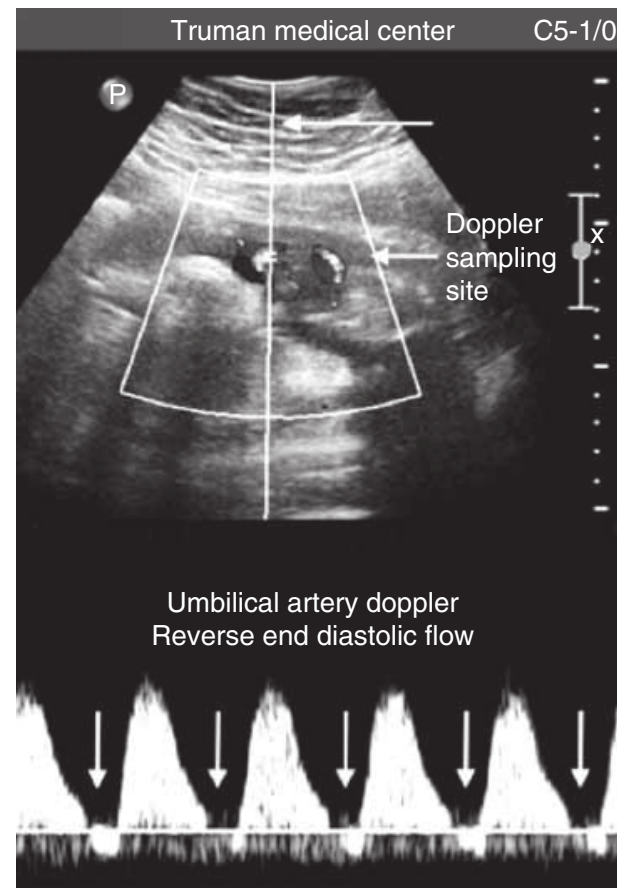

Figure 8 Doppler sonography depicting reverse end-diastolic flow in the umbilical artery (vertical arrows). The upper panel shows the twodimensional image of the umbilical artery and the directed placement of the Doppler sample volume. Color flow in the cord is shown in gray scale.

Table 6 Absent and Reverse End-Diastolic Frequency in the Umbilical Artery and Adverse Perinatal Outcome

\begin{tabular}{lll} 
Perinatal outcome & \multicolumn{1}{c}{ Mean } & \multicolumn{1}{c}{ Range } \\
Perinatal mortality & $45 \%$ & $17-100 \%$ \\
Gestational age & 31.6 weeks & $29-33$ weeks \\
Birth weight & $1056 \mathrm{~g}$ & $910-1481 \mathrm{~g}$ \\
Small for gestational age & $68 \%$ & $53-100 \%$ \\
Cesarean section for fetal distress & $73 \%$ & $24-100 \%$ \\
Apgar score at 5min $<7$ & $26 \%$ & $7-69 \%$ \\
Admission to neonatal intensive & $84 \%$ & $77-97 \%$ \\
$\quad$ care unit & & \\
Congenital anomalies & $10 \%$ & $0-24 \%$ \\
Aneuploidy & $6.4 \%$ & $0-18 \%$ \\
Source: From Ref. 34. & &
\end{tabular}

It has been observed that absent end-diastolic flow may improve, although often only transiently, and that weeks or more may elapse before the fetus may show additional evidence of compromise especially in preterm pregnancies. Thus, this finding is not necessarily an immediate indication for delivery in pregnancies before 34 weeks if fetal surveillance tests, such as the NST or biophysical profile, are reassuring (see "Guidelines for clinical practice" below).

\section{OTHER FETAL SURVEILLANCE TESTS}

Doppler sonography of ductus venosus, middle cerebral artery, and uterine artery has shown significant screening or diagnostic efficacy in high-risk pregnancies. However, their clinical effectiveness has not been adequately demonstrated by randomized trials. Ductus venosus Doppler does provide an additional tool for assessing serious fetal compromise (59).

Table 7 Absent End-Diastolic Velocity and Congenital
Malformations
Cardiovascular system
Ventricular septal defects
Hypoplastic left heart syndrome
Double-outlet right ventricle
Ebstein anomaly
Arrhythmia-congenital heart block
Central nervous system
Hydrocephaly
Holoprosencephaly
Agenesis of corpus callosum
Urogenital system
Renal agenesis
Hydronephrosis
Gastrointestinal system/abdominal wall
Esophageal atresia
Omphalocele
Gastroschisis
Skeletal system
Polydactyly
Dysplasia
Source: From Ref. 57.

The effectiveness of ductus venosus Doppler is under investigation at present in a multicenter randomized trial known as "Trial of Umbilical and Fetal Flow in Europe" (TRUFFLE), which is in the process of completion (60).

Current standard of practice for fetal monitoring includes antepartum FHR monitoring and fetal biophysical profile (BPP) or its modifications. The most common FHR monitoring technique is NST. The five-parameter BPP remains the main approach; however, modifications of this test have been proposed. Although diagnostic efficacy of NST and BPP has been demonstrated over the decades, there is insufficient evidence for their effectiveness in improving the outcome $(61,62)$. Their usefulness has been shown in clinical experience and they remain an essential part of the standard of practice in fetal surveillance.

It may be more prudent to use multiple tests of fetal wellbeing in high-risk pregnancies. Fetal response to chronic stress progresses from compensation to decompensation and involves multiple systems. However, there is no consensus in clinical practice at present regarding the optimal choice or sequence of fetal testing.

\section{CLINICAL EFFECTIVENESS OF DOPPLER VELOCIMETRY FOR FETAL SURVEILLANCE Randomized Trials}

Introduction of a new diagnostic test involves sequential and often parallel developmental processes that transform the potential of a new technique into an effective diagnostic tool making a measurable improvement in the outcome. The steps include the demonstration of feasibility, association with disease processes, and diagnostic efficacy of the technique, but in the end it must be shown without bias that the technique improves the clinical outcome. This level of effectiveness can be demonstrated only by RCTs.

It is encouraging that at the time of the writing of this revised chapter, 20 RCTs on fetal Doppler ultrasound have 
Table 8 Published Randomized Trials of Umbilical Artery Doppler Ultrasound in High-Risk, Low-Risk, and Unselected Populations

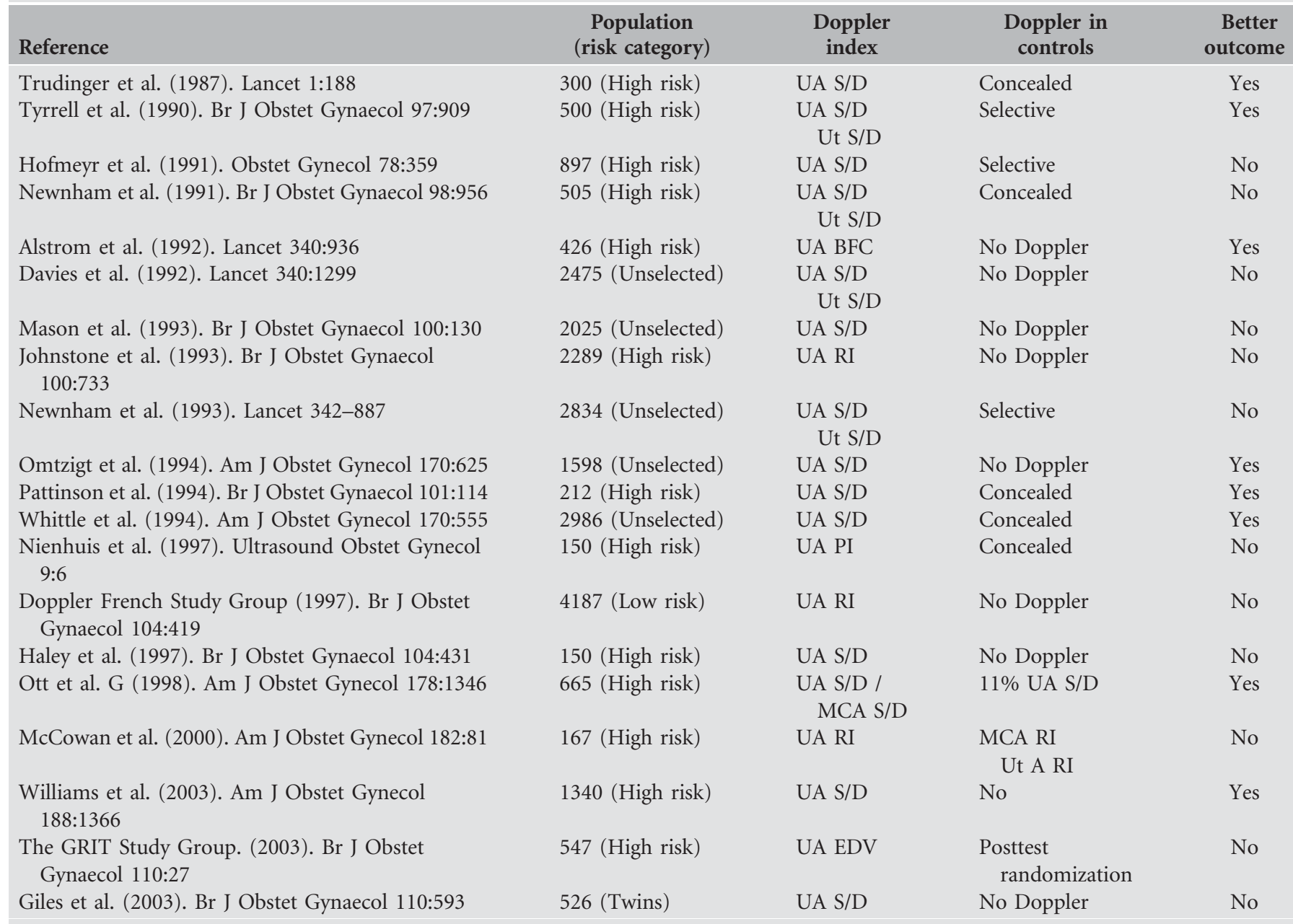

Abbreviations: BFC, blood flow classes; EDV, end-diastolic flow velocity; MCA, middle cerebral artery; PI, pulsatility index; RI, resistance index; S/D, systolic/diastolic ratio; UA, umbilical artery; Ut, uterine artery. Source: From Ref. 64.

been published involving a total population of almost 25,000 women. A detailed review of these studies is available elsewhere (63) and summarized in Table 8. Additional trials were reported only in the abstract form or as presentations. As noted before, the multicenter randomized trial TRUFFLE study has completed the enrollment and is awaiting further analysis and eventual publication.

It is noteworthy that most trials performed in high-risk populations showed improved outcome. As observed previously, fetal Doppler ultrasound is the only fetal surveillance modality that is supported by this level of evidence of effectiveness. All the RCT studies evaluated umbilical arterial velocimetry; a few also used uterine arterial velocimetry. While most were conducted in high-risk patients, many were used in low-risk mothers. Considerable heterogeneity exists among the trials regarding the selection criteria, the study design, and the measures of pregnancy outcome. Most studies did not have any protocol of management based on the test results. Finally, none of the studies had adequate sample size and therefore lacked sufficient power. Depending on the choice of the outcome parameter and the effect size, it will probably require thousands of patients involving multiple centers to conduct such a study. It would be a tremendous challenge to organize and obtain sufficient resources for a trial of this magnitude.

Several meta-analyses or systemic reviews of the Doppler trials have been conducted and are briefly summarized below.

\section{Meta-analysis of Doppler Trials}

Alfirevic and Neilson conducted a systematic review of 12 studies selected out of 20 identified studies and observed a 38\% decline in the odds of perinatal mortality, which was significant (95\% CI 15-55\%) (65). Additional benefits of the Doppler usage included significant reductions in antenatal admissions, inductions of labor, and cesarean sections for fetal distress. Maulik briefly reported a meta-analysis that looked only at trials involving high-risk pregnancies where there were guidelines for the use of Doppler data in the clinical management (66). Only three studies were identified with a total of 1575 patients. An average of $80 \%$ reduction in fetal deaths without malformations was noted (odds ratio $0.19,95 \%$ CI $0.06-0.63$ ).

Westergaard and associates reported a meta-analysis that included only well-defined high-risk pregnancies complicated with FGR or hypertension. Of the 13 trials of Doppler in highrisk pregnancies, only six had defined FGR and/pregnancy hypertension as indications for surveillance. The analysis 
showed that in the well-defined trials, there were significantly higher preventable perinatal deaths in the study than that in the control group (50\% and $20 \%$ respectively) (67).

The most recent Cochrane review (68) included 18 completed trials encompassing over 10,000 patients. The review demonstrated a $29 \%$ decrease in perinatal mortality, which was significant with a risk ratio (RR) of 0.71 and a $95 \%$ $\mathrm{CI}$ of 0.53 to 0.98 . This meant that 203 women needed to be treated for preventing one perinatal death. There were also less impressive but significant decreases in inductions of labor (RR $0.89,95 \%$ CI 0.80-0.99) and cesarean deliveries (RR 0.90, 95\% CI 0.84-0.97). No significant effects were noted in operative vaginal deliveries or Apgar scores $<7$ at 5 minutes.

In conclusion, most randomized trials of UA Doppler in high-risk pregnancies and their meta-analysis demonstrated a clinically and statistically significant improvement in the perinatal mortality. This constituted a level I evidence of effectiveness and that makes it unique among the other fetal surveillance tests.

\section{Low-Risk Pregnancies}

In contrast to high-risk pregnancies, trials of UA Doppler as a screening test in low-risk pregnancies did not show any improvement in pregnancy outcome. For example, a multicenter French randomized trial involving 4187 low-risk pregnant mothers failed to observe any improvements in the outcome with routine UA Doppler sonography between 28 and 34 weeks of gestation (69). Goffinet and coworkers conducted a systematic review of four trials selected out of seven studies and found no impact of UA Doppler on perinatal mortality in 11,451 low-risk pregnant mothers (odds ratio $0.51,95 \%$ CI $0.20-1.29$ ) (70). The Cochrane review of the topic in 2007 that included five trials with a total population of 14,338 women found no benefits of routine Doppler sonography in pregnancy (71). Moreover, the possibility of adverse outcome as suggested in two studies needs further investigation. In conclusion, routine Doppler ultrasound in low-risk pregnancies should be discouraged in clinical practice unless it is conducted under an approved experimental protocol.

\section{Fetal Doppler in Multiple Pregnancy}

The efficacy of Doppler velocimetry for identifying twin growth restriction has been variable with the sensitivity ranging from $29 \%$ to $82 \%$ (72). Fetal biometry remains the standard for the diagnosis of discordant twins. However, significant efficacy in predicting adverse perinatal outcome has been reported. Gaziano and associates (73) observed, in 94 twin pairs and 7 sets of triplets, that an abnormal pulsed Doppler velocimetry showed high correlation with adverse pregnancy events and that those with abnormal Doppler findings tended to be born 3 to 4 weeks earlier and to exhibit a greater number of stillbirths, malformations, and greater morbidity. Giles and coworkers (74) have suggested that the incorporation of the Doppler technique in the clinical management of twins may improve the perinatal outcome. A decline in corrected perinatal deaths from 42.1 in 1000 to 8.9 in 1000 was noted in their study. However, this was not a randomized trial.

A prospective randomized controlled multicenter trial of women with twin pregnancies was conducted that demonstrated no improvement in the perinatal mortality with the addition of UA Doppler to the standard management (75). The perinatal mortality was $9 / 1000$ live births in the Doppler group and 11/1000 live births in the control group. The study however was underpowered with a total of 526 women. The study did demonstrate that close monitoring in twin gestation is associated with a lower-than-expected fetal mortality in both the control and the Doppler groups.

Quintero and coworkers showed the prognostic value of Doppler of the UA, umbilical vein, and ductus venosus along with other ultrasound findings that constitute the staging of twin transfusion syndrome (76). The staging was proposed for individualizing the treatment options, especially the laser ablation procedure, and has become widely accepted in managing this complication.

In conclusion, fetal Doppler may be beneficial in managing multifetal gestations complicated with twin growth discordancy and those with twin transfusion syndrome. A comprehensive and relatively current review of the Doppler in multiple gestation is available elsewhere (72).

\section{GUIDELINE FOR THE CLINICAL APPLICATION OF THE DOPPLER FETAL SURVEILLANCE}

Based upon the above evidence of clinical efficacy of umbilical arterial Doppler indices, the following practical recommendations for its usage in managing high-risk pregnancy are suggested. These guidelines incorporate the existing modalities of fetal surveillance although some investigators advise the use of Doppler as the exclusive primary tool for fetal surveillance. Such a recommendation may not be pragmatic and pathophysiologic evidence suggests a more comprehensive approach for fetal surveillance.

\section{Indications for Doppler Fetal Surveillance}

Common indications for performing Doppler surveillance of the fetus at risk for chronic nutritive and respiratory stress are listed in Table 9 and are discussed below.

A variety of pregnancy complications such as FGR and preeclampsia can cause fetal stress. As discussed above, randomized trials have shown significant improvement in the rate of perinatal mortality in pregnancies complicated with clearly

Table 9 Indications for Umbilical Arterial Doppler Velocimetry

Primary indications

Fetal growth restriction by ultrasound fetal biometry (estimated fetal weight $<10$ th centile)

Pre-eclampsia

Primary

Superimposed

Multiple gestation

Discordancy

Fetal growth restriction

Twin transfusion syndrome

Extended indications

Autoimmune vascular disease: SLE, APL syndrome

Pregestational diabetes: $\geq$ class $\mathrm{C}$ or vasculopathy

Sickle cell disease

Abbreviations: SLE, systemic lupus erythematosus; APL, antiphospholipid. Source: From Ref. 77. 
defined FGR and hypertension. Although umbilical arterial Doppler has been recommended in the past for prenatal diagnosis of FGR, sonographic biometry is a better predictor of fetal size. Although the indices may be helpful in distinguishing a growth-restricted fetus from a constitutionally SGA fetus, there is a dearth of evidence guiding how this should be translated into a clinical management policy.

UA Doppler is helpful in recognizing fetal compromise in multiple pregnancies, particularly in those with discordant growth and twin transfusion syndrome. Current recommendations include the use of UA and ductus venosus Doppler for classifying the severity of fetal compromise in twin transfusion syndrome, known as the Quintero staging system, which assists in guiding the management options. Doppler velocimetry may also be useful in managing pregnancies affected by autoimmune disorders including systemic lupus erythematosus and antiphospholipid syndrome; however, its effectiveness is uncertain in the absence of fetal growth compromise. Finally, the utility of umbilical arterial Doppler in managing postdated pregnancies remains unproven.

\section{Technique of Umbilical Arterial Doppler Interrogation}

Before Doppler examination, the mother is appropriately counseled regarding the reason for the test, the nature of the information generated by the device, its reliability and safety, and other relevant issues. Similar to the procedure in FHR monitoring, the mother lies in a semirecumbent position with a slight lateral tilt to minimize the risk of significant caval compression. Examination should be conducted only during fetal apnea and in the absence of fetal hiccup or excessive movement. Doppler insonation can be performed by either a continuous-wave Doppler device or a pulsed Doppler duplex system; the latter is preferred for the UA.

With a pulsed-wave duplex Doppler system, an obstetric scan is initially performed to identify the loops of the cord (Fig. 9). Unlike the continuous-wave mode, the pulsed-wave Doppler insonation permits selection of the location in the umbilical cord for interrogation. Usually a free-floating loop of the cord is insonated. The cursor line representing the beam path is aligned to intersect the umbilical vessels at the selected location and the Doppler sample volume is placed in that location. The Doppler mode is then activated and the Doppler waveforms are recorded and analyzed.

\section{Management of High-Risk Pregnancy Using Doppler Sonography}

Principles of managing a high-risk pregnancy utilizing UA Doppler velocimetry in conjunction with other fetal surveillance test findings are described in this section. Management of high-risk pregnancies where fetal Doppler is indicated depends on several factors including the severity of fetal compromise as indicated by the Doppler and other fetal tests, and the underlying obstetric conditions such as pre-eclampsia, the gestational age, and the presence of fetal aneuploidy and malformations. These are further discussed below.

(i) Initial fetal assessment should rule out malformations. Such screening ultrasound is now in common practice and is typically performed at mid-pregnancy. If indicated, fetal karyotype should be determined especially in early-onset growth restriction with absent end-diastolic flow in the UA or with the presence of positive ultrasound or screening indicators. For those with FGR, appropriate follow-up growth

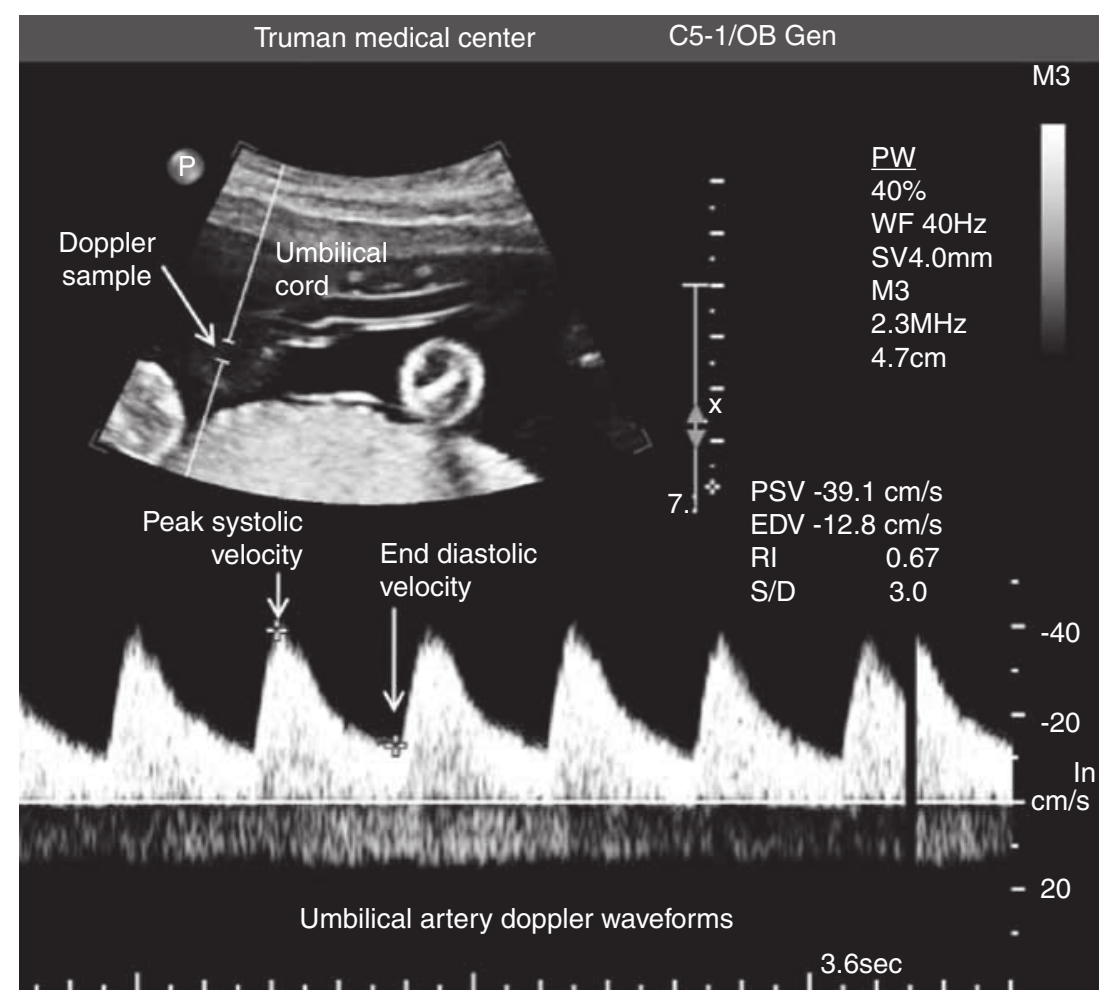

Figure 9 Duplex Doppler interrogation of the umbilical arterial flow. The upper panel shows gray scale and color (gray scale in the figure) depiction of the umbilical flow. The Doppler sample volume is placed in a free loop of the umbilical cord. The lower panel shows spectral Doppler waveforms from the umbilical artery. Cursors demonstrate the peak systolic and the end-diastolic velocity points in the wave. The inset shows the corresponding values and the Doppler indices. 
scan should be performed according to the prevailing standard of practice (usually 3-4 weeks interval).

(ii) Fetal surveillance should be multimodal including UA Doppler and full or modified biophysical profile. FHR pattern should also be used using the traditional NST or the computerized cardiotocography (cCTG). The frequency of these should be usually once a week. Daily fetal movement assessment by the mother may be added to this list. Many would also advise the incorporation of the ductus venosus Doppler.

(iii) As abnormal elevation of the umbilical arterial Doppler indices usually precedes ominous changes in other tests, a high or increasing UA Doppler index (S/D, RI, or PI) warrants more intense fetal surveillance, which consists of biweekly umbilical Doppler, and FHR monitoring (NST or cCTG) or the BPP. There is considerable variability in the choice, frequency, and sequence of these tests. As the fetal compromise involves multiple systems, it is more prudent to use a combination of tests rather than sequence of single tests.

If these tests are assuring, fetal surveillance should continue unless intervention is indicated because of other complications. On the other hand, if these tests are ominous, delivery should be planned.

(iv) The development of AEDV in the UA indicates more urgent action as it is associated with an unusually adverse perinatal outcome.

Preterm pregnancies: In preterm pregnancies greater than

28 weeks but less than 34 weeks of gestation, when the risk

of fetal lung immaturity is high, the management is conservative and further assurance of fetal well-being should be sought from daily surveillance by the UA Doppler and other fetal tests. Steroids should be administered for enhancing fetal lung maturity.

Delivery is recommended in the presence of ominous signs that include the reversal of UA end-diastolic flow by Doppler, non-reassuring FHR patterns (nonreactive NST, poor FHR baseline variability by NST or CCTG, persistent late decelerations), oligohydramnios, BPP score $<4$, and reversal or absence of the "a" wave of the ductus venosus Doppler. In many tertiary perinatal centers with the availability of advanced neonatal care, the threshold may be lowered to 32 weeks. A more conservative approach is advised in pregnancies less than 32 weeks.

There is controversy regarding conservative management versus immediate delivery in very preterm pregnancies. The growth restriction intervention trial project attempted to address this issue in a multicenter trial but failed to demonstrate any difference between immediate and delayed delivery groups (78). A follow-up study investigating the long-term outcome at 2 years showed a trend toward a higher rate of disability with immediate intervention but no differences in the median Griffiths test (79).

If the gestational age is less than 28 weeks, the management remains challenging. The validity of NST and BPP at this stage of gestation is not well established. Doppler of ductus may provide additional useful information, but there is no evidence of its effectiveness in this gestational group.

Term and near-term pregnancy: The development of AEDV should prompt consideration of delivery when fetal lung maturity is anticipated or proven. Although the prudence of emergency delivery for this complication has been questioned, RCTs and their meta-analyses have shown improved outcome from obstetric interventions based on UA Doppler. It is recommended therefore that the mother should be delivered when this complication develops in a pregnancy at or near term ( $>34$ weeks) when continuation of the pregnancy presents a greater threat to fetal safety and well-being.

Management of delivery: Standard guideline for intrapartum maternal and fetal monitoring in high-risk pregnancies should be followed. There is a higher risk of developing intrauterine asphyxia in fetuses with FGR or other associated complications arising from placental insufficiency. Close fetal surveillance during labor and delivery is therefore mandatory. The mode of delivery is determined by assessing relevant obstetric factors including cervical status, fetal presentation, and the severity of fetal compromise. If reversed end-diastolic velocity develops in the UA, the probability of impending fetal death is very high; therefore, it may be more prudent to deliver the infant promptly by cesarean section rather than to test its tolerance to the rigors of labor induction.

\section{CONCLUSION}

Development of Doppler ultrasound has allowed noninvasive assessment of the fetal circulatory state. Hemodynamic, angiomorphologic, and pathophysiologic evidence affirms the rationale for using the method for fetal surveillance. Numerous studies, as reviewed in this chapter, have established the efficacy of abnormal Doppler indices for identifying adverse perinatal outcome in various pregnancy disorders, especially FGR. More importantly, the majority of clinical trials have demonstrated the effectiveness of UA Doppler in improving the perinatal outcome in high-risk pregnancies. The most promising benefit is a significant reduction in preventable perinatal deaths. Current evidence clearly indicates that Doppler velocimetry should be an essential component of antepartum fetal surveillance in high-risk pregnancies especially those with FGR. Clearly, no single testing modality should be regarded as the exclusive method for fetal monitoring as the various fetal monitoring tests reveal different aspects of fetal compromise, often in a complementary manner. The recommendations in this chapter do not provide a solution for every contingency that may develop in the course of a high-risk pregnancy. The physician must individualize the care in light of the myriad of variations in the clinical situation. The information presented in this chapter should be used as a pragmatic guideline that integrates the new modality with the existing standards of fetal surveillance. As new evidence accumulates and experience grows, evidence-based integration of the various modalities of fetal monitoring in appropriate sequence and frequency will add further refinement to this plan of management.

\section{REFERENCES}

1. Maulik D. Doppler Ultrasound in Obstetrics and Gynecology, 2nd edn. Springer, 2005.

2. Doppler C. Uber das farbige Licht der Dopplesterne und einiger anderer Gestirne des Himmels. Abhandl Konigl Bohm Ges Ser 1843; 2: 465-82. 
3. Maulik D. Doppler sonography: a brief history. In: Maulik D, ed. Doppler Ultrasound in Obstetrics and Gynecology, 2nd edn. Springer, 2005: 1-7.

4. FitzGerald DE, Drumm JE. Noninvasive measurement of the fetal circulation using ultrasound: a new method. BMJ 1977; 2: 1450-1.

5. Maulik D, Saini VD, Nanda NC, Rosenzweig MS. Doppler evaluation of fetal hemodynamics. Ultrasound Med Biol 1982; 8: 705.

6. Maulik D, McNellis D, eds. Doppler Ultrasound Measurement of Maternal-Fetal Hemodynamics. Ithaca, NY: Perinatology Press, 1987.

7. Gill RW. Pulsed Doppler with B-mode imaging for quantitative blood flow measurements. Ultrasound Med Biol 1979; 5: 223-7.

8. Eik Nes SH, Brubakk AO, Ulstein MK. Measurement of human fetal blood flow. Br Med J 1980; 28: 283-4.

9. Maulik D, Nanda NC, Saini VD. Fetal Doppler echocardiography: methods and characterization of normal and abnormal hemodynamics. Am J Cardiol 1984; 53: 572-8.

10. Ferrazzi E, Rigano S. Doppler interrogation of umbilical venous flow. In: Maulik D, ed. Doppler Ultrasound in Obstetrics and Gynecology. Springer, 2005: 443-9.

11. Maulik D, Heitmann E, Maulik D. New horizons in Doppler ultrasound technology: relevance for obstetrical applications. Clin Obstet Gynecol 2010; In press.

12. Maulik D, Yarlagadda P, Youngblood JP, Ciston P. Comparative efficacy of umbilical arterial Doppler indices for predicting adverse perinatal outcome. Am J Obstet Gynecol 1991; 164: 1434.

13. Gosling RG, King DH. Ultrasound angiology. In: Marcus AW, Adamson J, eds. Arteries and Veins. Edinburgh: Churchill Livingstone, 1975: 61.

14. Pourcelot L. Applications cliniques de l'examen Dopple transcutane: velocimetric ultrasone Doppler. In: Pourcelot L, ed. Velocimetric Ultrasonore Doppler. Semin INSERM 1974; 34: 213-40.

15. Stuart B, Drumm J, FitzGerald DE, Duigan NM. Fetal blood velocity waveform in normal and complicated pregnancy. Br J Obstet Gynaecol 1980; 87: 780-5.

16. Maulik D, Yarlagadda P, Youngblood JP, Ciston P. Comparative efficacy of the umbilical arterial Doppler indices for predicting adverse perinatal outcome. Am J Obstet Gynecol 1991; 164: 1434-40.

17. Maulik D, Saini VD, Nanda NC, Rosenzweig MS. Doppler evaluation of fetal hemodynamics. Ultrasound Med Biol 1982; 8: 705-10.

18. Thompson RS, Trudinger JB, Cook CM. Doppler ultrasound waveforms in the fetal umbilical artery: quantitative analysis technique. Ultrasound Med Biol 1985; 11: 707-14.

19. Westerhof N, Sipkema P, Van den Bos GC, Elzinga G. Forward and backward waves in the arterial system. Cardiovasc Res 1972; 6: 648-56.

20. Nichols WW, O'Rourke MF, Avolio AP. Age related changes in left ventricular/arterial coupling. In Yin FCP, ed. Ventricular/Vascular Coupling. New York: Springer Verlag, 1987: 79.

21. Downing GJ, Yarlagadda AP, Maulik D. Comparison of the pulsatility index and input impedance parameters in a model of altered hemodynamics. J Ultrasound Med 1991; 10: 317-21.

22. Maulik D. Hemodynamic interpretation of the arterial Doppler waveform. Ultrasound Obstet Gynecol 1993; 3: 219.

23. Krebs C, Macara LM, Leiser R, et al. Intrauterine growth restriction with absent end-diastolic flow velocity in the umbilical artery is associated with maldevelopment of the placental terminal villous tree. Am J Obstet Gynecol 1996; 175: 1534.

24. Todros T, Sciarrone A, Piccoli E, et al. Umbilical Doppler waveforms and placental villous angiogenesis in pregnancies complicated by fetal growth restriction. Obstet Gynecol 1999; 93: 499-16, 17.

25. Maulik D. Doppler velocimetry for fetal surveillance: adverse perinatal outcome and fetal hypoxia. In: Maulik D, ed. Doppler Ultrasound in Obstetrics and Gynecology. New York: Springer Verlag, 1997: 349.

26. Peeters LLH, Sheldon RE, Jones MD, et al. Blood flow to fetal organs as a function of arterial oxygen content. Am J Obstet Gynecol 1979; 135: 637-46.

27. Maulik D, Yarlagdda AP, Youngblood JP, Willoughby L. Components of variability of umbilical arterial Doppler velocimetry - a prospective analysis. Am J Obstet Gynecol 1989; 160: 1406-12.

28. Maulik D. Umbilical arterial Doppler velocimetry: normative data and diagnostic efficacy. In: Maulik D, ed. Doppler Ultrasound in Obstetrics and Gynecology. New York: Springer Verlag, 1997: 129.

29. Dawes GS. Fetal and Neonatal Physiology. Chicago, IL: Yearbook Medical Publishers, 1968: 66
30. Maulik D. Basic principles of Doppler ultrasound as applied to obstetrics. Clin Obstet Gynecol 1989; 32: 641.

31. Yarlagadda AP, Willoughby L, Maulik D. Effect of fetal heart rate on umbilical arterial Doppler indices. J Ultrasound Med 1989; 8: 215-18.

32. Maulik D, Downing GJ, Yarlagadda AP. Umbilical arterial Doppler indices in acute uteroplacental flow occlusion. Echocardiography 1990; 7: 619-27.

33. van Eyck J, Wladimiroff JW, van den Wijngaard JA, et al. The blood flow velocity waveform in the fetal internal carotid and umbilical artery; its relationship to fetal behavioral states in normal pregnancy at 37-38 weeks of gestation. Br J Obstet Gynaecol 1987; 94: 736-41.

34. Maulik D. Doppler in ultrasound in Obstetrics. In: Cunningham FG, et al., eds. Williams' Obstetrics, 19th edn. Stamford CT: Appleton \& Lange, 1995.

35. Nicolaides KH, Bilardo CM, Soothill PW, et al. Absence of end diastolic frequencies in umbilical artery: a sign of fetal hypoxia and acidosis. Br Med J 1988; 297: 1026-7.

36. Chambers SE, Hoskins PR, Haddad NG, et al. A comparison of fetal abdominal circumference measurements and Doppler ultrasound in the prediction of small-for-dates babies and fetal compromise. Br J Obstet Gynaecol 1989; 96: 803-8.

37. Cohn H, Sachs E, Heymann M, Rudolph A. Cardiovascular responses to hypoxemia and acidemia in fetal lambs. Am J Obstet Gynecol 1974; 120: 817-24.

38. Hasaart THM, Maulik D, Morrow RJ. Validation of fetal flow velocimetry: a review of in vitro and in vivo modeling. J Matern Fetal Invest 1993; 3: 95-104.

39. Malcus P, Hokegard KH, Kjellmer I, et al. The relationship between arterial blood velocity waveforms and acid-base status in fetal lamb during acute experimental asphyxia. J Matern Fetal Invest 1991; 1: 29-34.

40. Soothill PW, Nicolaides KH, Bilardo CM, Campbell S. Relation of fetal hypoxia in growth retardation to mean blood velocity in the fetal aorta. Lancet 1986; 2: 1118-20.

41. Ferrazzi E, Pardi G, Bauscaglia M. The correlation of biochemical monitoring versus umbilical flow velocity measurements of the human fetus. Am J Obstet Gynecol 1988; 159: 1081-7.

42. Tyrrell S, Obaid AH, Lilford RJ. Umbilical artery Doppler velocimetry as a predictor of fetal hypoxia and acidosis at birth. Obstet Gynecol 1989; 74: 332-7.

43. Bilardo CM, Nicolaides KH, Campbell S. Doppler measurements of fetal and uteroplacental circulations: relationship with umbilical venous blood gases measured at cordocentesis. Am J Obstet Gynecol 1990; 162: 115-20.

44. Vintzileos AM, Campbell W, Rodis J, et al. The relationship between fetal biophysical assessment, umbilical artery velocimetry, and fetal acidosis. Obstet Gynecol 1991; 77: 622-6.

45. Yoon BH, Syn HC, Kim SW. The efficacy of Doppler umbilical velocimetry in identifying fetal acidosis. A comparison with fetal biophysical profile. J Ultrasound Med 1992; 11: 1-6.

46. Pardi G, Cetin I, Marconi AM, et al. Diagnostic value of blood sampling in fetuses with growth retardation. N Engl J Med 1993; 328: 692-6.

47. Yoon BH, Romero R, Roh CR, et al. Relationship between the fetal biophysical profile score, umbilical artery Doppler velocimetry, and fetal blood acid-base status determined by cordocentesis. Am J Obstet Gynecol 1993; 169: 1586

48. Trudinger BJ, Cook CM, Jones L, Giles WB. A comparison of fetal heart rate monitoring and umbilical artery waveforms in the recognition of fetal compromise. Br J Obstet Gynaecol 1986; 93: 171.

49. Farmakides G, Schulman H, Winter D, et al. Prenatal surveillance using non-stress testing and Doppler velocimetry. Obstet Gynecol 1988; 71: 184.

50. Maulik D, Yarlagadda AP, Youngblood JP, Ciston P. Efficacy of umbilical arterial Doppler velocimetry in predicting adverse perinatal outcome: a prospective study. Am J Obstet Gynecol 1990; 162: 1518

51. Maulik D, Yarlagadda P, Youngblood JP, Ciston P. The diagnostic efficacy of the umbilical arterial systolic/diastolic ratio as a screening tool: a prospective blinded study. Am J Obstet Gynecol 1990; 162: 1518.

52. Beattie RB, Dornan JC. Antenatal screening for intrauterine growth retardation with umbilical artery Doppler ultrasonography. Br Med J 1989; 298: 631. 
53. Hanretty KP, Primrose MH, Neilson JP, Whittle MJ. Pregnancy screening by Doppler uteroplacental and umbilical waveforms. Br J Obstet Gynaecol 1989; 96: 1163-7.

54. Beattie RB, Hannah ME, Dornan JC. Compound analysis of umbilical artery velocimetry in low-risk pregnancy. J Matern Fetal Invest 1992; 2: 269.

55. Karsdorp VH, van Vugt JM, van Geijn HP, et al. Clinical significance of absent or reversed end diastolic velocity waveforms in umbilical artery. Lancet 1994; 344: 1664-9.

56. Maulik D, Figueroa R. Absent end diastolic velocity in the umbilical artery and its clinical significance. In: Maulik D, Zalud I, eds. Doppler Ultrasound in Obstetrics and Gynecology. Springer, 2005: 375-86.

57. Maulik D. Doppler Ultrasound in Obstetrics and Gynecology. New York: Springer-Verlag, 1997.

58. Baschat AA, Viscardi RM, Hussey-Gardner B, Hashmi N, Harman C. Infant neurodevelopment following fetal growth restriction: relationship with antepartum surveillance parameters. Ultrasound Obstet Gynecol 2009; 33: 44-50.

59. Turan S, Turan OM, Berg C, et al. Computerized fetal heart rate analysis, Doppler ultrasound and biophysical profile score in the prediction of acid-base status of growth-restricted fetuses. Ultrasound Obstet Gynecol 2007; 30: 750-6.

60. Current Controlled Trials. ISRCTN56204499, Lancet protocol 02PRT/34, revised 2007: Trial of umbilical and foetal flow in Europe.

61. Grivell RM, Alfirevic Z, Gyte GM, Devane D. Antenatal cardiotocography for fetal assessment. Cochrane Database Syst Rev 2010: CD007863.

62. Lalor JG, Fawole B, Alfirevic Z, Devane D. Biophysical profile for fetal assessment in high risk pregnancies. Cochrane Database Syst Rev 2008: CD000038.

63. Maulik D, Figueroa R. Doppler velocimetry for fetal surveillance. In: Maulik D, ed. Doppler Ultrasound in Obstetrics and Gynecology. Springer, 2005: 161-76.

64. Maulik D, Figueroa R. Doppler velocimetry for fetal surveillance: randomized clinical trials and implications for practice. In: Maulik D, ed. Doppler Ultrasound in Obstetrics and Gynecology. Springer, 2005: 387-402.

65. Alfirevic Z, Nielson JP. Doppler ultrasonography in high risk pregnancies: systematic reviews with meta-analysis. Am J Obstet Gynecol 1995; 172: 1379-87.

66. Maulik D, Figueroa R, Sicuranza G, Garry D. Doppler fetal surveillance in clinical practice: evidence based guidelines. J Fertil Reprod 1998; SE 2: 5-6.
67. Westergaard HB, Langhoff-Roos J, Lingman G, Marsal K, Kreiner S. A critical appraisal of the use of umbilical artery Doppler ultrasound in high risk pregnancies: use of meta-analyses in evidence-based obstetrics. Ultrasound Obstet Gynecol 2001; 17: 466-76.

68. Alfirevic Z, Stampalija T, Gyte GM. Fetal and umbilical Doppler ultrasound in high risk pregnancies. Cochrane Database Syst Rev 2010: CD007529.

69. Doppler French Study Group. A randomised controlled trial of Doppler ultrasound velocimetry of the umbilical artery in low risk pregnancies. Br J Obstet Gynaecol 1997; 104: 419-22.

70. Goffinet F, Paris-Llado J, Nisand I, Breart G. Umbilical artery Doppler velocimetry in unselected and low risk pregnancies: a review of randomised controlled trials. Br J Obstet Gynaecol 1997; 104: 425-30.

71. Bricker L, Neilson JP. Routine Doppler ultrasound in pregnancy. Cochrane Database Syst Rev 2007: CD001450.

72. Gaziano E, Harkness EF. Doppler velocimetry and multiple gestation. In: Maulik D, ed. Doppler ultrasound in obstetrics and gynecology, 2nd edn. Springer, 2005.

73. Gaziano EP, Knox H, Ferrera B, et al. Is it time to reassess the risk for the growth-retarded fetus with normal Doppler velocimetry of the umbilical artery? Am J Obstet Gynecol 1994; 170: 1734-41.

74. Giles WB, Trudinger BJ, Cook CM, et al. Umbilical artery flow velocity waveforms and twin pregnancy outcome. Obstet Gynecol 1988; 72: 894-7.

75. Giles W, Bisits A, O'Callaghan S, Gill A, DAMP Study Group. The Doppler assessment in multiple pregnancy randomised controlled trial of ultrasound biometry versus umbilical artery Doppler ultrasound and biometry in twin pregnancy. BJOG 2003; 110: 593-7.

76. Quintero RA, Morales WJ, Allen $\mathrm{MH}$, et al. Staging of twin-twin transfusion syndrome. J Perinatol 1999; 19(8 Pt 1): 550-5.

77. Maulik D, Mundy D, Heitmann E, Maulik D. Evidence based approach to umbilical artery Doppler fetal surveillance in high risk pregnancies: an update. Clin Obstet Gynecol (In press).

78. GRIT Study Group. A randomized trial of timed delivery for the compromised preterm fetus: short term outcomes and Bayesian interpretation. BJOG 2003; 110: 27-32.

79. GRIT study group. Infant wellbeing at 2 years of age in the Growth Restriction Intervention Trial (GRIT): multicentred randomised controlled trial. Lancet 2004; 364: 513-20. 


\section{Assessment of fetal well-being: Fetal heart rate monitoring and the fetal biophysical profile Yinka Oyelese, Martin Chavez, and Anthony M. Vintzileos}

\section{INTRODUCTION}

The primary objective of antepartum fetal surveillance is the prevention of stillbirth. A second objective is the timely detection of fetal asphyxia that may result in long-term neurodevelopmental handicap. Antepartum fetal surveillance, for the most part, became possible only in the second half of the 20th century, mainly due to the advent of ultrasound and external continuous electronic fetal heart rate (FHR) monitoring (EFM). These technologies are now widely available and have become accepted as the mainstays of the evaluation of fetal well-being.

The use of any monitoring method to detect fetal compromise has, as a prerequisite, the clear understanding of the pathophysiology by which the particular condition results in adverse perinatal outcome. A test that is reliable for one condition may not be so valuable for predicting outcomes with another condition. Kontopoulos and Vintzileos (1) recently discussed how the efficacy of any antepartum screening test depends on the underlying pathophysiology of the condition and recommended condition-specific antenatal testing. Table 1A lists all the known pathophysiologic processes that can place the fetus at risk for in utero death or neurologic damage, whereas Table $1 \mathrm{~B}$ lists some of the more common indications for antepartum fetal surveillance. Importantly, no single list of indications could ever cover all the reasons for fetal surveillance. Essentially, any condition that puts the fetus at risk for antepartum death may be considered as an indication for fetal surveillance.

Until late in the 20th century, the main methods of antepartum fetal monitoring were the measurement of fundal height and maternal kick counts. The introduction of the FHR monitor by Hon around 1960 (2) ushered in a new era in fetal well-being assessment. EFM was initially designed for intrapartum assessment of fetal well-being, but it eventually became a tool for antepartum fetal monitoring too. However, it soon became obvious that monitoring of the FHR alone had limitations, especially a high false-positive rate. In 1980, Manning and colleagues introduced the fetal biophysical profile (BPP) (3), which utilizes real-time ultrasound examination of the fetus. While there are numerous other forms of fetal monitoring, such as Doppler for hemodynamic fetal evaluation, this chapter will discuss the role of antepartum FHR monitoring and the BPP in fetal assessment, including the limitations of each technique.

\section{EFM}

About 1960, Hon introduced the EFM originally for the purpose of intrapartum assessment of the fetus $(4,5)$. It was observed that certain FHR patterns were strongly predictive of a non-asphyxiated fetus, while other patterns suggested intrapartum fetal oxygen deprivation (5). Fetuses suffering from intrapartum hypoxia frequently exhibited a slowing of the FHR following a contraction, termed late deceleration. Following the routine introduction of the EFM during labor, there was a remarkable reduction in intrapartum fetal deaths. Subsequently, it was suggested that EFM might play a role in assessing fetal well-being way before the onset of labor. Since the presence of intrapartum late decelerations indicated fetal hypoxia, it was believed that stimulating uterine contractions in patients who were not in labor might help identify fetuses that were at risk of hypoxia in the antepartum period. This led to the "oxytocin challenge test" (OCT), the original antepartum continuous electronic FHR assessment of the fetus, introduced in the early 1970s by Ray and colleagues (6). Using oxytocin, uterine contractions were stimulated, and the presence of FHR late decelerations were considered a positive test and predictive of fetal compromise (7).

The OCT was considered positive if greater than $50 \%$ of contractions were followed by late decelerations, and negative if there were no late or significant variable decelerations. An OCT was described as "suspicious" if there were intermittent late or variable decelerations, while the test was deemed "unsatisfactory" if there were fewer than three contractions over a 10-minute period, or if the fetal heart tracing quality was uninterpretable. In cases where there were prolonged contractions that led to decelerations, the term "hyperstimulation" was used. Later, it became apparent that these decelerations could also result from spontaneous uterine contractions, and therefore the test was renamed the "contraction stress test" (CST). Others used nipple stimulation instead of oxytocin to provoke uterine contractions and found similar results (8-13). A positive CST (or OCT) was indicative of uteroplacental insufficiency. During contractions, blood flow to the intervillous space is impeded. A bradycardic response develops due to a chemoreceptor response that is provoked by fetal hypoxia. These late decelerations in the antepartum period were shown to predict subsequent adverse outcomes before, during, and after labor. Several studies demonstrated that though the OCT had a high false-positive rate, the false-negative rate was low, about $1 \%$ or less (14). In other words, the risk of fetal death within 1 week after a negative test is negligible. However, the false-positive rate of the OCT is at least $20 \%$ to $45 \%(15,16)$. Importantly, conditions associated with fetal demise after a negative CST are often umbilical cord accidents and placental abruption, conditions that occur suddenly, and may not necessarily be expected to be predicted by any antepartum surveillance modality (14). 
Table 1A Antepartum Maternal/Fetal Pathophysiologic

Processes That Can Place the Fetus at Risk for In Utero Death or Neurologic Damage

\begin{tabular}{|c|c|}
\hline $\begin{array}{l}\text { Pathophysiologic } \\
\text { process }\end{array}$ & Maternal/fetal condition \\
\hline $\begin{array}{l}\text { Decreased } \\
\text { uteroplacental } \\
\text { blood flow }\end{array}$ & $\begin{array}{l}\text { Chronic hypertension } \\
\text { Pre-eclampsia } \\
\text { Collagen/renal/vascular disease } \\
\text { Most cases of fetal growth restriction } \\
\quad \text { (i.e., }<32-34 \mathrm{wk} \text { ) }\end{array}$ \\
\hline $\begin{array}{l}\text { Decreased gas } \\
\text { exchange }\end{array}$ & $\begin{array}{l}\text { Postdate pregnancy, some fetal } \\
\text { growth-restricted cases } \\
\text { (i.e., }>32-34 \text { wk) }\end{array}$ \\
\hline $\begin{array}{l}\text { Metabolic } \\
\text { aberrations }\end{array}$ & $\begin{array}{l}\text { Fetal hyperglycemia } \\
\text { Fetal hyperinsulinemia }\end{array}$ \\
\hline Fetal sepsis & $\begin{array}{l}\text { PROM } \\
\text { Intra-amniotic infection } \\
\text { Maternal fever, primary subclinical } \\
\text { Intra-amniotic infection }\end{array}$ \\
\hline Fetal anemia & $\begin{array}{l}\text { Fetomaternal hemorrhage } \\
\text { Erythroblastosis fetalis } \\
\text { Parvovirus B19 infection }\end{array}$ \\
\hline Fetal heart failure & $\begin{array}{l}\text { Cardiac arrhythmia } \\
\text { Nonimmune hydrops } \\
\text { Placental chorioangioma } \\
\text { Aneurysm of the vein of Galen }\end{array}$ \\
\hline $\begin{array}{l}\text { Umbilical cord } \\
\text { accident }\end{array}$ & $\begin{array}{l}\text { Umbilical cord entanglement } \\
\text { (monoamniotic twins) } \\
\text { Velamentous cord insertion/Funic } \\
\text { presentation } \\
\text { Noncoiled umbilical cord } \\
\text { Oligohydramnios }\end{array}$ \\
\hline
\end{tabular}

Table $1 B$ Some Common Indications for Antepartum Fetal Surveillance

Intrauterine growth restriction
Prior stillbirth
Diabetes
Hypertension
Pre-eclampsia
Postdate pregnancies
Preterm premature rupture of the membranes
Oligohydramnios
Decreased fetal movements
Prior stillbirth
Maternal lupus
Multifetal pregnancies, particularly those with growth
disturbances or discordance
Cholestasis of pregnancy

Needless to say, the OCT was cumbersome and difficult to perform. In the process of performing OCTs, it was often observed that the presence of FHR accelerations had good predictive value for a non-asphyxiated fetus (17). Consequently, the concept of the nonstress test (NST) was proposed. Since monitoring of fetal movements (including maternal kick counts) had previously been used to assess fetal well-being, the NST was considered an objective method of fetal monitoring. A "reactive" NST was initially defined as one with two to five or more accelerations in 20 minutes. An acceleration is defined as a transient increase in FHR over the baseline of at least 15 beats/minute (bpm), lasting at least 15 seconds. For pregnancies less than 32 weeks of gestation, an acceleration is a transient increase over the baseline of at least $10 \mathrm{bpm}$, lasting at least 10 seconds.

There are other variables in the heart rate pattern that may suggest fetal well-being or compromise. The normal fetal baseline heart rate is 110 to 160 . The baseline heart rate decreases with gestational age, mainly as a consequence of vagal maturation. Fetal tachycardia (defined as a baseline FHR $>160 \mathrm{bpm}$ ) may occur in association with maternal fever, maternal ingestion of stimulants including beta-adrenergic agents. Uncomplicated fetal tachycardias (with accelerations and good variability) are generally associated with a good outcome. However, these require close and thorough evaluation. When there is a persistent complicated tachycardia (including a heart rate greater than $200 \mathrm{bpm}$, where there is minimal or no variability, or where there are decelerations), delivery should be considered, especially at term or near term. Obviously, one must take the entire clinical picture into consideration. For instance, treating maternal fever or stopping stimulant medications may be enough to resolve the tachycardia.

Bradycardia is defined as a baseline FHR $<110$ bpm. Again, this is generally benign if there are accelerations and good variability. However, FHRs of $<100 \mathrm{bpm}$ may be associated with fetal heart block and require further evaluation. The absence of accelerations or reduced variability may be due to periodicity. However, if this persists for $>45$ minutes, further evaluation should be undertaken. A sinusoidal FHR pattern (Fig. 1) is considered an ominous pattern (18) and is often associated with fetal anemia or hypovolemia, including that resulting from such causes as isoimmunization, parvovirus infection, placental abruption, twin-twin transfusion syndrome (19-21), and ruptured vasa previa (22). Generally, prompt delivery is indicated, unless the cause of the sinusoidal FHR (such as fetal anemia) can be treated in utero $(18,22)$.

The NST has several advantages over the CST - it is easy to perform and interpret, does not require oxytocin administration, and can be performed in shorter time than the CST. Furthermore, the CST has several absolute and relative contraindications, such as in patients with premature rupture of the membranes, prior classical cesarean delivery, multiple gestations, incompetent cervix, preterm labor, or vaginal bleeding. These important characteristics as well as the predictive reliability of the test made the NST the primary modality of fetal evaluation. Several studies have validated the role of the NST as a predictor of perinatal outcome $(23,24)$. The presence of accelerations is associated with a low risk of fetal hypoxia, while the absence of accelerations carries a significantly higher rate of fetal hypoxia and acidosis. The FHR reactivity is controlled by the autonomic nervous system and the percentage of reactive NSTs increases with fetal maturation. By term, over $90 \%$ of fetuses demonstrate reactive NSTs. FHR accelerations are associated with fetal movements. An FHR tracing may be nonreactive due to fetal immaturity, fetal sleep state, maternally administered sedatives, fetal neurologic and cardiac anomalies, fetal hypoxia/acidosis, uteroplacental insufficiency, or fetal infection. Unfortunately, the NST also has a high false-positive rate of about $50 \%$, while the 

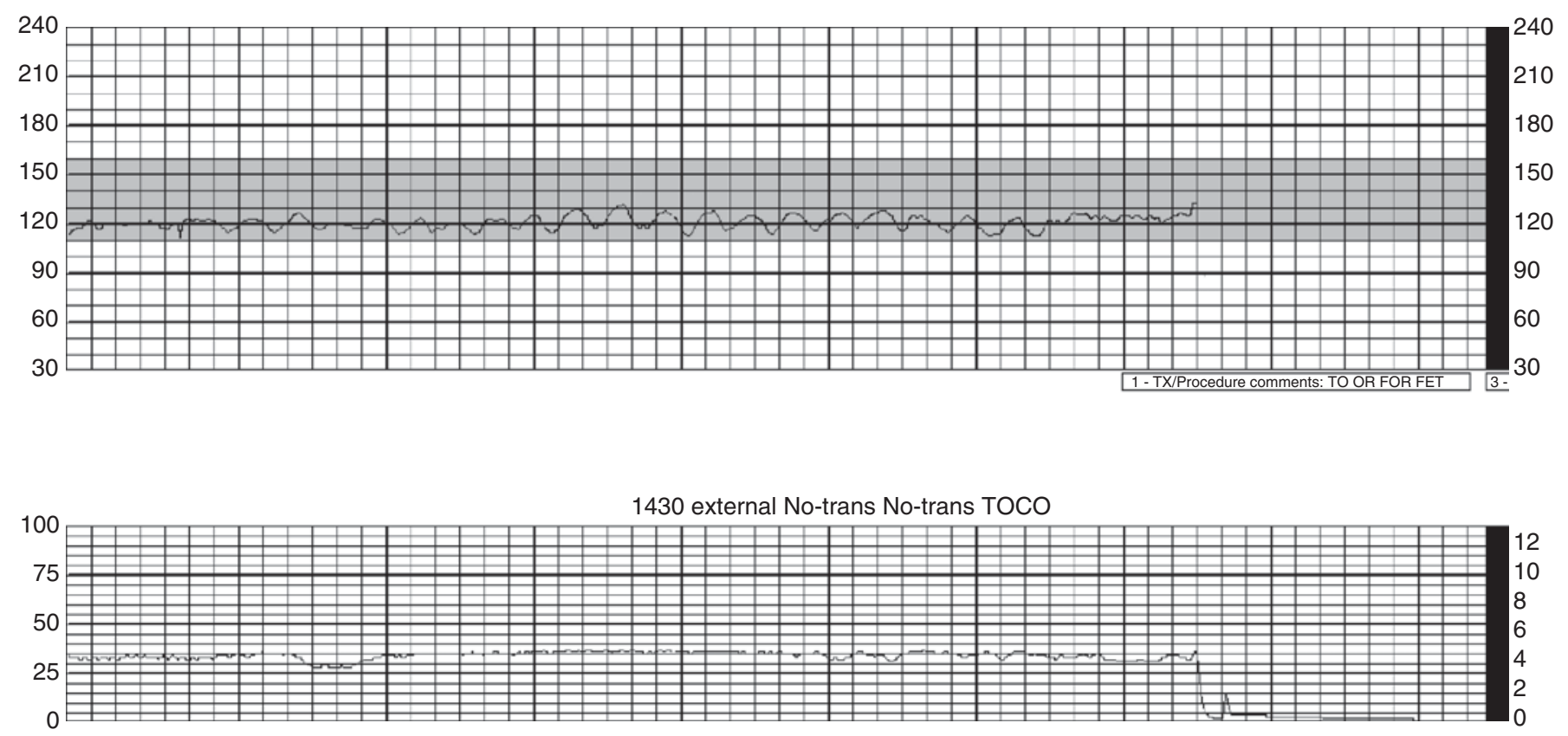

Figure 1 Fetal heart rate tracing demonstrating a sinusoidal fetal heart rate pattern.

false-negative rate is only about $0.3 \%$. Translated differently, a reactive NST carries a very low risk of fetal hypoxia, while a nonreactive NST is only associated with adverse outcomes in less than one-half of cases. Phelan and colleagues reviewed 2226 patients who had undergone 4000 NSTs. Of these, 1564 women with a reactive NST were delivered within 1 week of the test. There were four fetal deaths giving a false-negative rate of $0.026 \%$ - these resulted from placental abruption and cord accidents, events that may not be necessarily expected to be predicted by an NST (25).

In the late 1980s, it was observed that fetal auditory stimulation would often lead to FHR accelerations in a fetus that had previously lacked accelerations (26-30). This reduced testing time and also reduced the nonreactive NST rate. An artificial larynx was developed that produced a vibroacoustic signal (VAS). A rapidly occurring acceleration following the stimulation is considered a positive response. A positive VAS was shown to have similar accuracy in predicting good perinatal outcomes as a spontaneously reactive NST, with the advantage of a reduced testing time.

The OCT/CST and NST are performed using an external FHR monitor and a tocodynamometer strapped to the maternal abdomen. The FHR monitor derives its signals ultrasonically or via phonocardiography. Ultrasound is the most widely used technique and provides an accurate tracing in the majority of cases. Perhaps obesity is the major factor that may be associated with an inability to obtain a satisfactory fetal heart tracing. Uterine contractions are monitored via the tocodynamometer. The tocodynamometer monitors the frequency and duration of the contractions, but cannot assess the strength of the contractions. The tocodynamometer may not adequately detect contractions in obese patients.

Antepartum FHR testing has undergone considerable evaluation in several studies. It has been shown to have good prediction of fetal well-being when accelerations are present

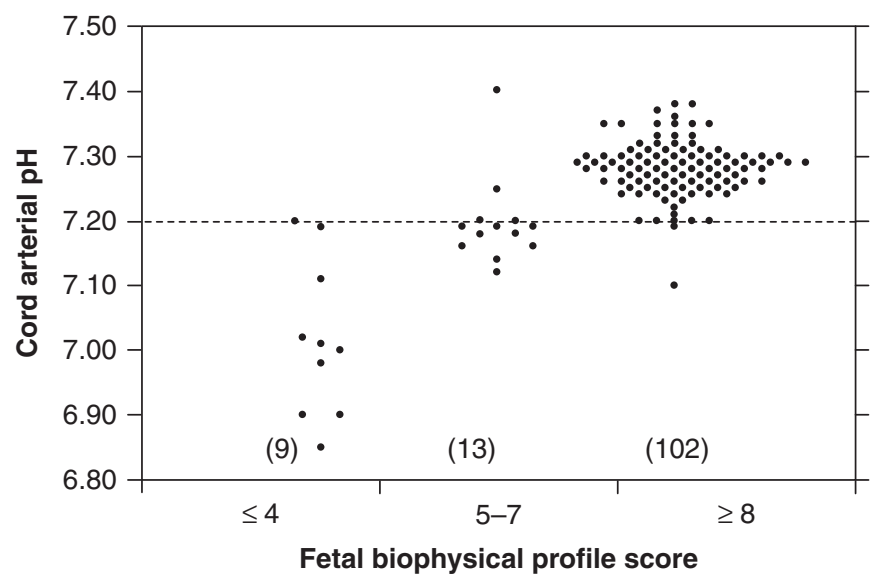

Figure 2 Relationship between the fetal biophysical score and umbilical cord arterial pH. Source: From Ref. 33. The American College of Obstetricians and Gynecologists.

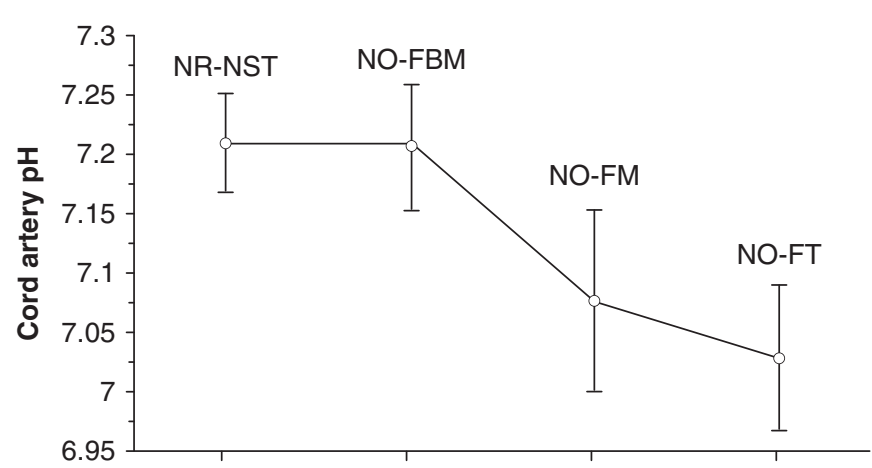

Figure 3 Relationship between cord artery $\mathrm{pH}$ and absent fetal biophysical activities. The $\mathrm{pH}$ tends to be lower in the absence of movements and/or tone compared with nonreactive nonstress test or absent fetal breathing. Results are expressed in means (95\% error bars). Abbreviations: No FM, absent fetal movements; No FT, absent fetal tone; No FBM, no fetal breathing movements; NR-NST, nonreactive nonstress test. Source: From Ref. 31. 


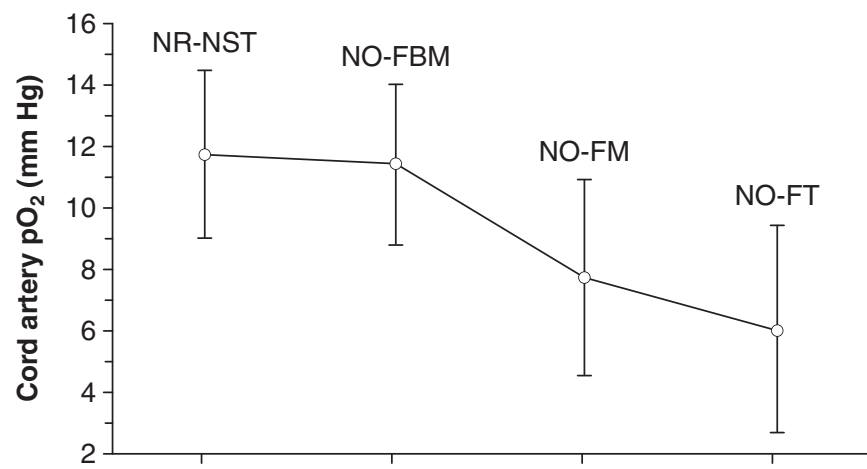

Figure 4 Relationship between cord artery oxygen partial pressure $\left(\mathrm{pO}_{2}\right)$ and absent fetal biophysical activities. The $\mathrm{pO}_{2}$ tends to be lower in the absence of movements and/or tone compared with nonreactive nonstress test or absent fetal breathing. Results are means expressed in millimeters of mercury (95\% error bars). Abbreviations: No FM, absent fetal movements; No FT, absent fetal tone; No FBM, no fetal breathing movements; NR-NST, nonreactive nonstress test. Source: From Ref. 31.

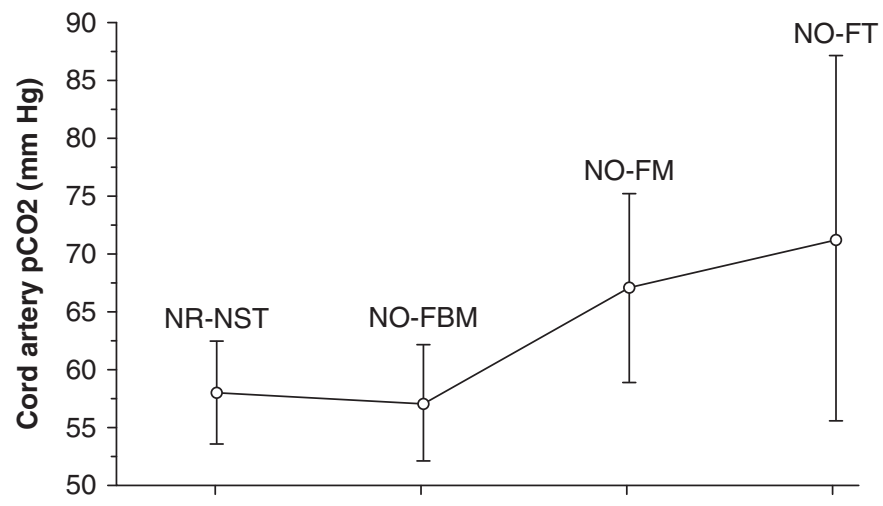

Figure 5 Relationship between cord artery carbon dioxide partial pressure $\left(\mathrm{pCO}_{2}\right)$ and absent fetal biophysical activities. The $\mathrm{pCO}_{2}$ tends to be higher in the absence of movements or tone compared with nonreactive nonstress test or absent fetal breathing. Results are means expressed in millimeters of mercury (95\% error bars). Abbreviations: No FM, absent fetal movements; No FT, absent fetal tone; No FBM, no fetal breathing movements; NR-NST, nonreactive nonstress test. Source: From Ref. 31. and decelerations are absent. However, the converse is not true; the absence of accelerations is not necessarily indicative of a compromised fetus. Thus, EFM was found to be associated with an exceedingly high false-positive rate, resulting in excessive unnecessary interventions such as inductions and cesarean deliveries. It became obvious that there was a need for some other form of surveillance that could reduce this falsepositive rate.

\section{THE FETAL BPP}

In 1980, Manning and colleagues introduced the BPP (Table 2) (3). This test was based on a more thorough evaluation of the fetus; the main advantage of the BPP over EFM is that the BPP evaluates several different biophysical markers. The FHR allowed the assessment of only a single biophysical marker. Just as a heart rate alone in an adult cannot distinguish between sleep and coma, the FHR, when used alone, has significant limitations, especially in the absence of accelerations. The BPP is analogous to an intrauterine Apgar score with its dynamic variables (acute markers) reflecting fetal CNS function. In addition to FHR reactivity, other acute fetal markers such as fetal movements, fetal breathing movements and tone, as well as assessment of the intrauterine environment, that is, estimation of amniotic fluid volume and placental grading could be objectively evaluated. The fetal BPP was developed to decrease the false-positive results associated with FHR monitoring. The most important element of this testing method is the combination of acute and chronic markers of the fetal condition. The acute markers include FHR reactivity, fetal breathing, fetal movements, and fetal tone, while fetal amniotic fluid volume is a chronic marker of fetal well-being. The BPP avoids the high false-positive rates of the NST and the CST and therefore helps reduce unnecessary interventions.

\section{The Physiologic Basis for the BPP}

\section{Acute Markers}

The acute fetal biophysical markers-FHR reactivity, fetal movements, fetal breathing movements, and fetal tone-are not random events, but are initiated and regulated by the fetal central nervous system (CNS) (Table 3). The presence of any one of these markers implies that, at the time of testing, the CNS center that controls the particular activity is

Table 2 Fetal Biophysical Profile Scoring (Manning and Coworkers)

\begin{tabular}{|c|c|c|}
\hline & Score 2 & Score 0 \\
\hline Movements & $\begin{array}{l}\text { Three or more gross body movements in a } 30 \text {-min } \\
\text { period. Simultaneous trunk and limb movements } \\
\text { count as a single movement }\end{array}$ & $\begin{array}{l}\text { Less than three gross body movements in a } 30 \text {-min } \\
\text { period }\end{array}$ \\
\hline Tone & $\begin{array}{l}\text { At least one movement of a limb from a position of } \\
\text { flexion to one of extension, with a rapid return to } \\
\text { flexion }\end{array}$ & $\begin{array}{l}\text { Fetal limb in extension with no return to flexion with } \\
\text { movement }\end{array}$ \\
\hline Amniotic fluid & $\begin{array}{l}\text { At least a single amniotic fluid pocket measuring } 2 \mathrm{~cm} \times \\
2 \mathrm{~cm} \text { in two perpendicular planes }\end{array}$ & $\begin{array}{l}\text { No amniotic fluid pocket that measures at least } \\
2 \mathrm{~cm} \times 2 \mathrm{~cm} \text { in two perpendicular planes }\end{array}$ \\
\hline Fetal heart rate & $\begin{array}{l}\text { At least two accelerations (of }>15 \text { beats per minute, } \\
\text { lasting at least } 15 \mathrm{sec} \text { ) in a } 40-\mathrm{min} \text { period }\end{array}$ & Less than two accelerations in $40 \mathrm{~min}$ \\
\hline
\end{tabular}


Table 3 Fetal Central Nervous System Centers Governing Biophysical Activities and Therefore Tested by the Biophysical Profile

\begin{tabular}{ll} 
Test & \multicolumn{1}{c}{ Center } \\
Fetal tone & Cortex-subcortical area \\
Fetal movements & Cortex nuclei \\
$\begin{array}{c}\text { Fetal breathing } \\
\text { movements }\end{array}$ & Ventral surface of the fourth ventricle \\
Nonstress test & Posterior hypothalamus, medulla
\end{tabular}

intact, functional, and therefore non-hypoxic. The converse, however, is not necessarily true; the absence of a single fetal biophysical activity is not always due to hypoxia and acidosis. Fetal biophysical activities such fetal breathing movements, fetal body movements, and FHR variability all undergo periodic changes. Thus, the absence of biophysical activities is more frequently the result of periodicity rather than a pathologic condition and needs to be interpreted with caution. The periodicity of these biophysical activities may be short term (20-40 minutes) or long term, similar to those diurnal rhythms of extrauterine life. Maternal administration of CNS depressants and sedatives, such as opiates, barbiturates, and benzodiazepines, may inhibit fetal biophysical activities, while factors that cause stimulation of the maternal CNS, for example, hyperglycemia or catecholamines, may result in increased biophysical activities. The effect of fetal hypoxia and acidosis, or fetal infection, on the fetal BPP depends on the severity, duration, chronicity, and frequency of the hypoxic insult(s). Fetal hypoxia may be transient without acidosis, or prolonged with associated metabolic or mixed acidosis, affecting multiple organs. Acute fetal hypoxia (or fetal infection) produces a definite decrease in FHR reactivity (nonreactive NST) and fetal breathing movements, and when severe hypoxia (or infection) abolishes fetal movements and tone (31-33).

The biophysical activities appear progressively in normal fetuses as gestation advances. The first biophysical activity to appear is tone; the fetal tone center begins functioning at $71 / 2-$ $8^{1 / 2}$ weeks of gestation. This is followed shortly thereafter by the fetal movement center, which begins functioning at approximately 9 weeks of gestation. The fetal breathing movement center starts operating at about 20 to 21 weeks of gestation, and finally the FHR reactivity center becomes functional by the end of the second trimester or early third trimester. These centers vary in their sensitivity to hypoxia and acidosis (31-33). It was first observed by Vintzileos and colleagues that the biophysical activities that become active first in fetal neurodevelopment are the last to become compromised in fetuses exposed to intrauterine asphyxia or intra-amniotic infection (31-33). Thus, the FHR reactivity and breathing centers, controlling the biophysical activities that appear latest in gestation, are the most sensitive and the first to become affected when the fetus is exposed to hypoxia or intraamniotic infection (31). Data from our prior studies indicate that FHR reactivity and fetal breathing are abolished when the fetal $\mathrm{pH}$ is $<7.20$, whereas fetal movement and tone are abolished at $\mathrm{pH}$ values $<7.10$. Thus, according to the "gradual hypoxia concept," the earliest biophysical manifestations of fetal compromise (fetal hypoxia or infection) are a loss of variability in the NST and absence of fetal breathing. As fetal condition deteriorates, fetal movements and then tone become abolished. In our experience, poor fetal tone is associated with the highest perinatal death rate $(42.8 \%)$ (32). An understanding of this progressive sensitivity of the biophysical activities to fetal hypoxia or infection of the fetal CNS centers is a prerequisite for the correct interpretation of the information derived from the biophysical assessment and allows for accurate assessment of the degree of fetal compromise in high-risk patients with intact as well as ruptured membranes.

\section{Chronic Markers}

The most important chronic marker of the BPP is the amniotic fluid volume, although placental grading was also included in the original studies by Vintzileos et al. (3,32). Chronic uteroplacental insufficiency that results in fetal hypoxia is frequently associated with redistribution of cardiac output away from non-vital to vital fetal organs. Thus, there is typically a reduction in renal perfusion, with consequent decreased urine production and, therefore, oligohydramnios. Oligohydramnios places the fetus at high risk for cord compression and in utero death. Several studies have demonstrated a strong correlation between oligohydramnios and increased incidence of adverse perinatal outcome. Chamberlain and colleagues demonstrated, in a study of 7582 referred high-risk patients, that in structurally normal fetuses, the perinatal mortality rates were 1.97 in 1000 in the presence of normal qualitative amniotic fluid volume (largest pocket $>2 \mathrm{~cm}$ and $<8 \mathrm{~cm}$ ), whereas rates in fetuses with marginal (largest pocket $1-2 \mathrm{~cm}$ ) and decreased amniotic fluid (largest pocket $<1 \mathrm{~cm}$ ) were 37.7 in 1000 and 109.4 in 1000, respectively (34). Another study of 147 pregnancies complicated by oligohydramnios in pregnancies at gestational ages $>34$ weeks found an increased risk of labor induction, nonreassuring FHR patterns, cesarean delivery, neonatal intensive care unit admission, meconium aspiration, stillbirth, and neonatal death (35). Amniotic fluid volume is not influenced by acute changes in fetal CNS function. Most investigators consider the finding of oligohydramnios, even when it occurs on its own, as an abnormal biophysical assessment even if when all the remaining biophysical parameters are normal and the presence of oligohydramnios is generally regarded as an indication for intensive surveillance (in preterm gestations) or delivery (term or near-term gestations) $(3,33,36,37)$. When spontaneous severe FHR decelerations and oligohydramnios coexist, delivery may be indicated, regardless of gestational age. Vintzileos and colleagues had initially included placental grading in the fetal BPP (32). These investigators observed that patients with grade 3 placentas had an increased incidence of abnormal intrapartum FHR patterns (44.4\%) and placental abruption (14.8\%) (32). Thus, inclusion of grade 3 placentas in the BPP alerts the obstetrician to the significant risk of intrapartum complications.

Since the early 1980s, attempts have been made to give a score to each biophysical variable to produce a "fetal BPP score." There are currently two types of scoring systems: one proposed by Manning and coworkers (Table 2) (3) and the other by Vintzileos and coworkers (Table 4) (32). In both scoring systems, a score of $>8$ is considered reassuring of fetal 
Table 4 Criteria for Scoring Biophysical Variables According to Vintzileos and Coworkers

\section{Nonstress test}

Score 2 (NST 2): five or more FHR accelerations of at least $15 \mathrm{bpm}$ in amplitude and at least 15 -sec duration associated with fetal movements in a 20 -min period

Score 1 (NST 1): two to four accelerations of at least $15 \mathrm{bpm}$ in amplitude and at least 15 -sec duration associated with fetal movements in a 20 -min period

Score 0 (NST 0): one or fewer accelerations in a 20 -min period Fetal movements

Score 2 (FM 2): at least three gross (trunk and limbs) episodes of fetal movements within $30 \mathrm{~min}$. Simultaneous limb and trunk movements were counted as a single movement Score 1 (FM 1): one or two fetal movements within $30 \mathrm{~min}$ Score 0 (FM 0): absence of fetal movements within $30 \mathrm{~min}$ Fetal breathing movements

Score 2 (FBM 2): at least one episode of fetal breathing of at least 60 -sec duration within a 30 -min observation period

Score 1 (FBM 1): at least one episode of fetal breathing lasting 30-60 sec within $30 \mathrm{~min}$

Score 0 (FBM 0 ): absence of fetal breathing or breathing lasting $<30 \mathrm{sec}$ within $30 \mathrm{~min}$

Fetal tone

Score 2 (FT 2): at least one episode of extension of extremities with return to position of flexion, and also one episode of extension of spine with return to position of flexion

Score 1 (FT 1): at least one episode of extension of extremities with return to position of flexion or one episode of extension of spine with return to flexion

Score 0 (FT 0): extremities in extension. Fetal movements not followed by return to flexion. Open hand

Amniotic fluid volume

Score 2 (AF 2): fluid evident throughout the uterine cavity. A pocket that measures $>2 \mathrm{~cm}$ in vertical diameter

Score 1 (AF 1 ): a pocket that measures $<2 \mathrm{~cm}$ but more than vertical diameter

Score 0 (AF 0): crowding of fetal small parts. Largest pocket $<1 \mathrm{~cm}$ in vertical diameter

Placental grading

Score 2 (PL 2): placental grading 0 , 1, or 2

Score 1 (PL 1): placenta posterior difficult to evaluate

Score 0 (PL 0): placental grading 3

Abbreviations: NST, nonstress test; FHR, fetal heart rate; bpm, beats per minute; FM, fetal movements; FBM, fetal breathing movements; FT, fetal tone; AF, amniotic fluid; PL, placental grading. Maximal score 12; minimal score 0. Source: From Ref. 32.

well-being. A score of $<8$ is non-reassuring and repeat testing or delivery is suggested. A limitation of the original BPP scoring system of Manning et al. is that biophysical activities are given an "all or none" score (3). For instance, if there are movements detected that are insufficient to meet to score of 2, the fetus is assigned a " 0 " for that biophysical activity. However, most would agree that a fetus that demonstrates two movements over 30 minutes may be different from one that exhibits no movements at all. Thus, the initial assignment of intermediate scores, as proposed by Vintzileos et al. (32), had as its main purpose to identify the minimum requirements for each biophysical component to meet normalcy criteria.

\section{The Fetal BPP and Fetal Acid-Base}

The BPP aims at assessing fetal acid-base status at the time of testing. However, in order to assess the accuracy of the fetal BPP in assessing antepartum fetal acid-base status, it is crucial that the acid-base status be assessed shortly after the BPP is performed. Furthermore, labor has a profound effect on acid-base status measured at birth. A major disadvantage of several studies has been the long interval between performing the biophysical assessment and the birth of the infant, as well as the effects of labor and drugs on the fetus. In order to overcome these limitations, Vintzileos and coworkers studied 124 consecutive patients with singleton pregnancies at gestational ages between 26 and 43 weeks, limiting their study to patients undergoing cesarean section prior to the onset of labor $(33,38)$. All patients had a BPP performed within 6 hours of the cesarean section. This BPP was correlated with fetal acid-base status as determined by umbilical cord blood $\mathrm{pH}$ measurements. Figure 2 illustrates the relationship between the fetal biophysical score and cord arterial $\mathrm{pH}$. Importantly, all nine fetuses with scores of $<4$ were acidemic with umbilical cord arterial $\mathrm{pH}$ values of $6.99 \pm 0.10$ (mean \pm SD). Thirteen fetuses with scores of 5 to 7 had a cord artery pH of $7.19 \pm 0.06$ (mean \pm SD); 9/13 of fetuses $(69 \%)$ were acidemic. One hundred and two fetuses had a score of $>8$; in this group of fetuses, the cord artery $\mathrm{pH}$ was $7.28 \pm 0.04$ (mean $\pm \mathrm{SD}$ ); only two of these $(2 \%)$ were acidemic. The three groups had statistically different cord arterial $\mathrm{pH}$ values. The relationship between the biophysical score and cord venous $\mathrm{pH}$ was similar.

As expected, based on the gradual hypoxia hypothesis, there was a relationship between the individual biophysical activities and the umbilical cord blood artery pH values (Fig. 3). When combined biophysical activities were considered, of the 96 fetuses with reactive NSTs and/or fetal breathing present (lasting $>30$ s), none was acidemic. All the remaining fetuses had nonreactive NSTs and absent fetal breathing. However, 17 fetuses had normal (three or more) body movements and tone; the mean $\mathrm{pH}$ of this group was 7.20 and 10 (59\%) of these fetuses were acidemic. Four fetuses had compromised movements (one to two body movements) or tone; the mean cord blood artery $\mathrm{pH}$ was 7.16 , and three fetuses (75\%) were acidemic. In the seven fetuses with all biophysical activities absent, the mean cord blood artery $\mathrm{pH}$ was 6.95 , with $100 \%$ of them having cord blood artery $\mathrm{pH}$ values $<7.20$. Careful observation of the results suggests that there are different levels of sensitivity to hypoxia and acidosis at which the fetal biophysical activities become compromised. The FHR reactivity and fetal breathing seem to be compromised when the $\mathrm{pH}$ is lower than 7.20. At $\mathrm{pH}$ values of 7.10 to 7.20 , fetal movements and tone are still present but not normal and at $\mathrm{pH}$ values $<7.10$, movements and tone are totally absent. The ability of the individual biophysical components alone and in combination to predict fetal acidemia is shown in Table 5. Of the individual biophysical components, FHR reactivity and fetal breathing had $100 \%$ sensitivities and negative predictive values, an observation that suggests that either test is a reasonable choice for primary fetal surveillance. The combination of nonreactive NST and absent fetal breathing movements (the "abnormal test") improved the specificity and positive predictive values to $92 \%$ 
Table 5 Efficacy of the Fetal Biophysical Variables to Predict Fetal acidemia

\begin{tabular}{|c|c|c|c|c|c|}
\hline Biophysical variable(s) & $\begin{array}{l}\text { Definition of the } \\
\text { abnormal test }\end{array}$ & $\begin{array}{l}\text { Sensitivity } \\
(\%)(n)\end{array}$ & $\begin{array}{l}\text { Specificity } \\
\qquad \%)(n)\end{array}$ & $\begin{array}{c}\text { Positive } \\
\text { predictive } \\
\text { value }(\%)(n)\end{array}$ & $\begin{array}{c}\text { Negative } \\
\text { predictive } \\
\text { value }(\%)(n)\end{array}$ \\
\hline Biophysical score & $<7$ & $90 \%(18 / 20)$ & $96 \%(100 / 104)$ & $82 \%(18 / 22)$ & $98 \%(100 / 102)$ \\
\hline Nonstress test & $\begin{array}{l}\text { Less than one } \\
\text { acceleration in } \\
20 \mathrm{~min}\end{array}$ & $100 \%(20 / 20)$ & $76 \%(79 / 104)$ & $44 \%(20 / 45)$ & $100 \%(79 / 79)$ \\
\hline $\begin{array}{c}\text { Fetal breathing } \\
\text { movements }\end{array}$ & $<30 \mathrm{sec}$ & $100 \%(20 / 20)$ & $64 \%(67 / 104)$ & $35 \%(20 / 57)$ & $100 \%(67 / 67)$ \\
\hline Fetal movements & Less than three & $50 \%(10 / 20)$ & $96 \%(100 / 104)$ & $71 \%(10 / 14)$ & $91 \%(100 / 110)$ \\
\hline Fetal tone & $\begin{array}{c}\text { Compromised } \\
\text { or absent }\end{array}$ & $45 \%(9 / 20)$ & $100 \%(104 / 104)$ & $100 \%(9 / 9)$ & $90 \%(104 / 115)$ \\
\hline
\end{tabular}

and $71 \%$, respectively. Fetal tone has the highest positive predictive value (100\%) for fetal acidemia, but low sensitivity (45\%), an observation that suggests that fetal tone alone should not be used for primary fetal surveillance.

Cordocentesis is a technique that allows accurate assessment of relationship between the fetal BPP and fetal umbilical cord blood gas values. Ribbert and colleagues studied 14 severely growth-retarded fetuses, performing a BPP assessment prior to fetal blood sampling by cordocentesis (39). The fetal BPP was correlated with fetal blood $\mathrm{pO}_{2}, \mathrm{pH}$, oxygen saturation, and oxygen content. It was found that the fetal BPP was a good indicator of the degree of fetal acidemia. Of the individual fetal biophysical activities, FHR reactivity was compromised for $\Delta \mathrm{pH}$ (observed $\mathrm{pH}$ subtracted from the appropriate mean for gestation) below -2 standard deviations (SD)s; fetal breathing movements were compromised for $\Delta \mathrm{pH}$ values below $-3 \mathrm{SDs}$; fetal body movements and fetal tone were compromised for $\Delta \mathrm{pH}$ values below -4 SDs. These preliminary data using fetal blood gas values obtained at cordocentesis confirmed the observations of Vintzileos and coworkers in that the fetal BPP can predict the degree of fetal acidemia and that the FHR reactivity and fetal breathing movements are the first, whereas fetal gross body movements and tone are the last, biophysical activities to be compromised during acidemia. In another study of 62 patients undergoing cesarean section prior to the onset of labor, the relationship between fetal biophysical assessment performed within 3 hours of delivery and cord blood gases was investigated (31). It was observed that nonreactive NSTs and absent fetal breathing movements were both associated with lower $\mathrm{pH}, \mathrm{pO}_{2}$, bicarbonate, base excess and higher $\mathrm{pCO}_{2}$ than that when the NST was reactive or fetal breathing was present. The absence of fetal movements and/or tone was associated with lower $\mathrm{pH}, \mathrm{pO}_{2}$, bicarbonate and base excess, and a higher $\mathrm{pCO}_{2}$ than when fetal body movements and tone were present (Figs. 3-7). These blood gas differences were noted in both cord arterial and venous blood. Thus, the first manifestations of fetal hypoxemia and acidemia are

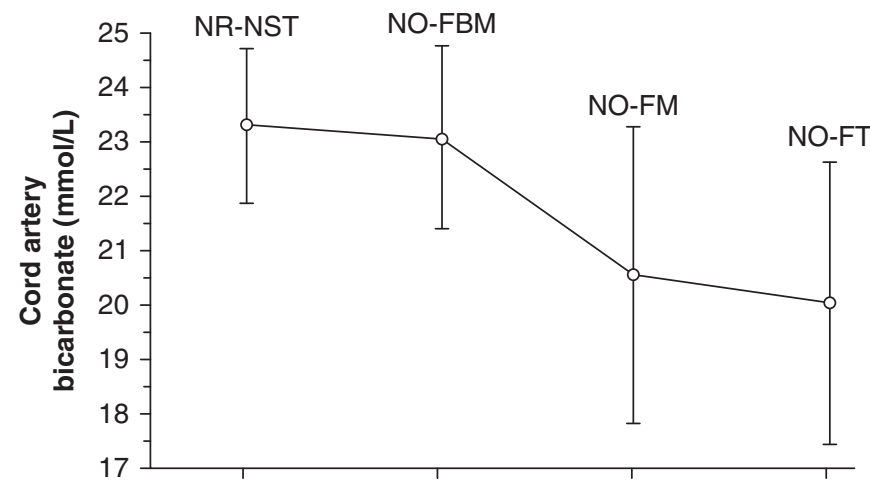

Figure 6 Relationship between cord artery bicarbonate level and absent fetal biophysical activities. The bicarbonate level tends to be lower in the absence of movements or tone compared with nonreactive nonstress test and absent fetal breathing. Results are means expressed in millimoles per liter (95\% error bars). Abbreviations: No FM, absent fetal movements; No FT, absent fetal tone; No FBM, no fetal breathing movements; NR-NST, nonreactive nonstress test; Source: From Ref. 31.

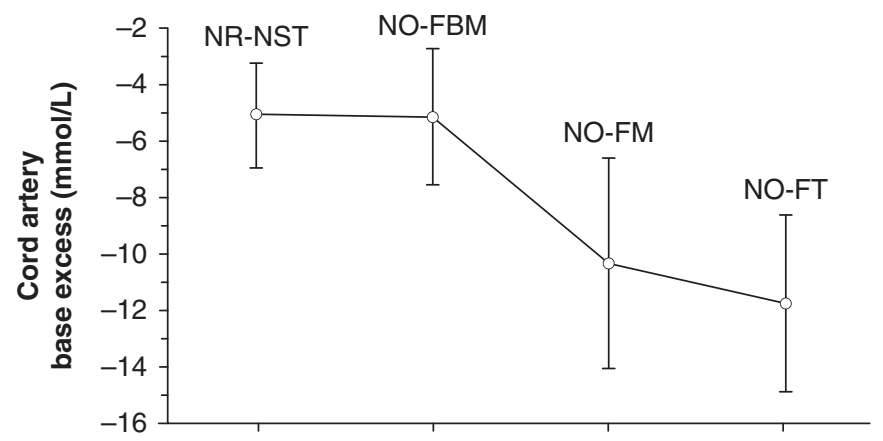

Figure 7 Relationship between cord artery base excess and absent fetal biophysical activities. The base excess tends to be lower in the absence of movements or tone compared with nonreactive nonstress test and absent fetal breathing. Results are means expressed in millimoles per liter (95\% error bars). Abbreviations: No FM, absent fetal movements; No FT, absent fetal tone; No FBM, no fetal breathing movements; NR-NST, nonreactive nonstress test. Source: From Ref. 31. 
nonreactive NST and the absence of fetal breathing. As hypoxemia becomes more advanced, and hypercapnia and acidemia develop, fetal movements and tone become absent. A similar sequence of events in the deteriorating fetus also occurs in the presence of fetal infection (40).

\section{The BPP: Impact on Perinatal Outcomes}

In their initial study of the use of the BPP in clinical practice, Manning et al. (3) measured five biophysical activities (fetal movement, fetal tone, fetal breathing, amniotic fluid volume, and fetal NST) in 216 patients, all of whom delivered within a week of the last BPP. The patients were managed based on their NSTs, not based on the results of the BPPs. These investigators found that each of the five individual biophysical markers had a low false-negative rate, but that all five had a high false-positive rate. However, this false-positive rate varied widely among the different markers. The authors found that combining the markers led to a significant improvement in the false-positive and false-negative rates. There was a significant correlation between abnormal BPP scores and low 5-minute Apgar scores, fetal distress in labor, and perinatal death rate. These authors found a perinatal mortality rate of $0 / 1000$ when all biophysical markers were normal (score 10), but that this rose to $600 / 1000$ when all biophysical activities were abnormal (score 0 ). The fetal death rate with a score of 0 was 400/1000.

Based on the results of this initial study, Manning and colleagues suggested a management protocol based on BPP scoring that is outlined in Table 6. These investigators used this protocol for patient management in a subsequent prospective study involving 1184 consecutive referred high-risk patients who had 2238 fetal biophysical scores performed (40). The purpose of the study was to assess the impact of using the biophysical score on perinatal death rate in high-risk patients. There were six perinatal deaths, in the study group, giving a perinatal mortality of 5.06 per 1000 . This was significantly lower than the predicted rate for a similar high-risk population (65 per 1000). Importantly, this perinatal death rate was also less than half of that in the general population in Manitoba (14.3 per 1000). Only one fetus died after a reassuring biophysical score, thus giving a true false-negative rate of 0.8 per 1000. As an added potential benefit, 13 of 19 (68.4\%) fetuses with previously undiagnosed major congenital anomalies,

Table 6 Management Scheme Based on Biophysical Profile Scoring

\begin{tabular}{ll}
\hline Score & \multicolumn{1}{c}{ Recommended management } \\
\hline $8-10$ & $\begin{array}{c}\text { Repeat in } 1 \text { week. In diabetic (insulin-dependent) } \\
\text { and postdate patients, repeat twice weekly. No } \\
\text { indication for active intervention }\end{array}$ \\
If fetal pulmonary maturity assured and cervix \\
favorable, delivery, otherwise repeat in 24 hours. \\
If there is persistent score of $4-6$, deliver if fetal \\
pulmonary maturity certain. Otherwise treat with \\
steroids and deliver in 48 hours \\
Evaluate for immediate delivery. In cases of certain \\
pulmonary immaturity, give steroids and deliver \\
in 48 hours
\end{tabular}

including 8 with lethal anomalies, were prenatally diagnosed as a result of real-time ultrasound. In two cases, the antepartum detection of the anomaly helped neonatal survival.

In 1983, Vintzileos and coworkers reported their initial experience using a BPP consisting of six variables in 150 patients with high-risk pregnancies during a 30-minute observation period (32). A total of 342 BPPs were performed. The patients were not managed based on the BPP results, but rather based on their nonstress or CSTs. There was a good correlation between the biophysical scores and adverse perinatal outcomes, including intrapartum fetal heart-rate pattern abnormalities, meconium during labor, fetal distress, and the perinatal mortality rate. Fetal biophysical scores $(>8)$ were associated with good pregnancy outcome in all cases. Very abnormal scores $(\leq 4)$ were almost always associated with fetal distress. The absence of fetal movements was the best predictor of abnormal intrapartum heart-rate patterns $(80 \%)$, while a nonreactive NST was the best predictor of meconiumstained fluid (33.3\%). Oligohydramnios was the best predictor of fetal distress (37.5\%) and absent fetal tone was the best predictor of fetal death $(42.8 \%)$. The 11 fetuses that were acidemic at birth (cord blood artery $\mathrm{pH}<7.20$ ) all had nonreactive NSTs and absent fetal breathing. There were two fetuses with normal fetal movement and both survived. Of five fetuses with movement present but compromised (one or two body movements in 30 minutes), only one died. Three out of the four fetuses with absent fetal movement died; all three also had absent fetal tone. These observations suggested that the biophysical components do not contribute to the predictive accuracy in an equally proportionate manner.

Baskett et al., using the Manning scoring criteria, reported on 5618 BPP scores performed on 2400 high-risk pregnancies (41). The perinatal mortality in the study population was only 9.2 per 1000 , compared with the overall perinatal mortality in the investigators' hospital over the study period of 14.5 per 1000 . Structurally normal fetuses with a normal biophysical score had a perinatal mortality of only 1 per 1000 . Fetuses with scores of 4 or less had a perinatal mortality rate of 292 per 1000. In this study, the positive predictive value of an abnormal biophysical score for abnormal perinatal outcome (defined as intrapartum fetal distress, 5-minute Apgar score $<7$, intrauterine growth restriction, or perinatal death) was $79.2 \%$. This predictive power was significantly better than that of the NST, fetal breathing movements, and fetal tone; however, it did not reach statistical significance when compared with fetal movement and amniotic fluid volume.

In the first prospective randomized blinded study of the BPP, Manning et al. randomized 735 women with high-risk pregnancies to BPPs or NSTs for fetal assessment (42). The results of the tests were used for management, but the physicians were not informed of which test had been used. The BPP was shown to have an improved predictive value over the NST for low Apgar scores. The negative predictive values were similar for both forms of fetal assessment. Sensitivity, specificity, and accuracy were not statistically different between the two methods of fetal assessment. In 1985, Platt and colleagues reported their results from a randomized prospective trial comparing the BPP with NST for the assessment of high-risk pregnancies, examining the ability of the tests to predict adverse perinatal outcomes (43). 
A number of 1628 tests were performed on 652 women. The authors found that the diagnostic values for all outcome parameters (apart from the negative predictive value for low 5-minute Apgar scores) were consistently higher with the BPP than the NST. However, only the positive prediction of overall abnormal outcome and negative prediction of smallfor-gestational-age infants achieved statistical significance.

Johnson and colleagues reviewed 307 consecutive post-term pregnancies assessed using twice-weekly BPPs and found that the BPP accurately differentiated fetuses with a normal outcome from those at risk for perinatal asphyxia (44). They proposed that conservative management of post-term pregnancies was made possible using the BPP for surveillance and that this had the potential to lead to a reduction in unnecessary inductions and cesarean deliveries. Meanwhile, Benacerraf and colleagues reported their observation on performing BPPs on 1000 women with high-risk pregnancies and found that four of five fetuses with oligohydramnios and absent or poor fetal tone and movements had evidence of fetal compromise at birth (45). Their finding reinforces the fact that oligohydramnios should be considered an ominous sign, regardless of the other variables, and generally should lead to intensive surveillance or delivery.

Vintzileos and coinvestigators described their experience using the BPP in 73 women with healthy pregnancies who had presented with preterm premature rupture of the membranes (PPROM) and were not in labor (46). They observed that PPROM did not affect the BPP. However, a low BPP $(<7)$ predicted impending fetal infection. Subsequently, these investigators performed a retrospective study of 1151 pregnancies complicated by PPROM (47). They found that PPROM was associated with a higher rate of reactive NSTs, absent fetal breathing, and reduced amniotic fluid compared with those pregnancies without PPROM. However, they observed that the overall BPP score was not affected by PPROM in otherwise healthy pregnancies. In a further study, Vintzileos and colleagues compared the BPP with amniocentesis for the diagnosis of chorioamnionitis in patients with PPROM (48). They found that daily BPP assessment was superior to amniocentesis in predicting infection outcome in these women. The role of the BPP in the evaluation of patients with PPROM will be discussed further later in this chapter.

In another study, Manning and coworkers reported on 12,620 high-risk patients who had 26,257 BPP scores (37). There were 93 perinatal deaths of which 24 occurred in structurally normal, non-isoimmunized fetuses (corrected perinatal mortality rate of 1.9 per 1000). Eight of these structurally normal fetuses died within one week of a normal BPP (corrected false-negative rate of 0.634 per 1000). The uncorrected stillbirth rate was 3.64 per 1000 and the uncorrected neonatal death rate was 3.72 per 1000 . When the perinatal mortality was correlated with the biophysical score results, it was found that the overall mortality ranged from 0.652 per 1000 tests in pregnancies with a BPP score $>8$ to 187 per 1000 tests for pregnancies with a BPP score of 0 . The overwhelming majority of the BPPs $(97.5 \%)$ were normal and only $0.76 \%$ had a score of $<4$. The same authors subsequently reported their experience with $44,828 \mathrm{BPPs}$ (49) performed in 19,221 high-risk pregnancies. The frequency of intrauterine death among structurally normal fetuses after a reassuring BPP was 0.726 per 1000 ( 14 deaths). Four of those deaths resulted from cord prolapse, an outcome not likely to be predicted by a normal BPP score. Thus, the false-negative rate of a normal test, excluding cases that the BPP would not have been expected to predict, could have been as low as 0.518 per 1000 (10 deaths). Almost half of the antepartum fetal deaths occurred 5 to 7 days after a normal BPP. Therefore, it is possible that increasing the frequency of testing to twice a week may have prevented some of these deaths. The usefulness of the fetal BPP in predicting perinatal death has also been investigated by Baskett and coworkers (50) in 4184 fetuses. The overall perinatal mortality in the study population was 7.6 per 1000. Fetuses with a normal biophysical score (8-10) had a perinatal mortality rate of 1 per 1000, while fetuses with an equivocal score of 6 had a perinatal mortality rate of 31.3/1000. Finally, fetuses with a BPP score of 0 to 4 had a perinatal mortality of 200 per 1000 . The false-negative rate of 0.7 per 1000 was very similar to the false-negative rate previously reported by Manning and coworkers.

Platt and coworkers (51) studied 283 patients who had a total of 1112 BPPs. Patients were managed on the basis of their NST results and not on their BPPs. The perinatal mortality rate for all patients delivered in their institution during the study period was 22.6 per 1000 as opposed to 14 per 1000 (corrected 7 per 1000) for the study population and 7.4 per 1000 for fetuses with a biophysical score of $>8$. The authors questioned the predictive accuracy of the biophysical score as compared with the NST alone.

\section{Outcomes with Very Low BPP Scores}

Manning and coworkers (52) reported on outcomes of pregnancies with BPP scores of zero. Twenty-nine of 28,655 fetuses $(0.092 \%)$ had a last BPP score of 0 prior to delivery. The perinatal death rate in this group of 29 fetuses was $48.3 \%$ (14 of 29 ) and the majority (11 of 14) were stillborn. Death occurred as early as 30 minutes and as long as 11 days after the last test. Three asphyxiarelated neonatal deaths occurred despite immediate and aggressive intervention. The positive predictive value of a BPP score of 0 for perinatal mortality or morbid perinatal outcome (defined as fetal distress in labor, 5-minute Apgar score $<7$, umbilical vein $\mathrm{pH}<7.20$, or admission to a neonatal intensive care unit for over 24 hours for reasons unrelated to prematurity) was $100 \%$. Thus, these authors argued that the very abnormal fetal BPP $($ score $=0)$ should be considered a perinatal emergency. Importantly, some fetuses will survive despite this extremely low biophysical score; thus, procrastination, when the BPP score is 0 , based upon the belief that the outcome will be dismal, should be discouraged.

In another study of 26,780 fetuses, Manning and coworkers (53) studied the relationship between the last BPP score and various perinatal outcomes including mortality and morbidity. A highly significant inverse linear correlation was observed between the BPP score and fetal distress, admission to the neonatal intensive care unit, intrauterine growth restriction, low 5-minute Apgar score $(<7)$, and umbilical cord $\mathrm{pH}<7.20$. No correlation was found between the fetal BPP score and the incidence of meconium or major anomaly. 
A highly significant inverse exponential relationship was observed between biophysical score and perinatal mortality. The authors interpreted the data as suggesting that the BPP scoring provides insight into the extent and degree of fetal compromise.

Several other investigators have subsequently found that the BPP, when used appropriately, leads to a reduction in perinatal mortality and adverse pregnancy outcomes in high-risk pregnancies. Furthermore, it has been useful in predicting those pregnancies at risk for an adverse outcome. The BPP was found to be an effective method of antepartum fetal surveillance in patients with diabetes mellitus, hypertension, multiple pregnancies, and other high-risk conditions. However, few years after its introduction into clinical practice, it became apparent that it was often used with inappropriate expectations or interpretation.

\section{Factors Affecting the BPP}

While the BPP is now widely used for fetal assessment, questions are often asked about how the BPP may be affected by several factors commonly encountered in obstetrics.

\section{Gestational Age}

Because the biophysical activities are gestational age dependent, it has been suggested that the BPP score would vary across gestation. Hence, Vintzileos and coworkers (47) performed 951 serial examinations from 25 to 44 weeks of gestation in 210 patients with intact membranes and normal pregnancy outcome. These investigators observed a significant increase in reactive NSTs after 32 weeks while both fetal breathing movements and amniotic fluid volume were decreased after 40 weeks. Fetal movements and fetal tone remained stable across these gestational ages. The incidence of grade 3 placentas increased significantly after 32 weeks. These variations in the frequencies of the fetal biophysical components from 25 to 44 weeks of gestation agree with the findings of several other investigators (54-57). It should be noted, however, that the frequency of reassuring biophysical scores $(>8)$ did not change significantly from 25 to 44 weeks of gestation. Using the scoring criteria of Manning and coworkers, Baskett (58) had similar findings regarding the changes of the biophysical components throughout gestation. Their study involved pregnancies with normal perinatal outcome (5582 fetuses/11,012 BPPs). There were an increased number of reactive NSTs and fetal breathing movements at 34 to 41 weeks as compared with earlier gestations. The NST, fetal breathing movements, fetal tone, and amniotic fluid volume were more likely to be abnormal in prolonged gestations (42-44 weeks) as compared with term gestations (37-41 weeks). Although changes in the individual biophysical components were noted, the frequency of normal biophysical scores $(>8) \mathrm{did}$ not change significantly throughout gestation. The understanding of the gestational age-dependent changes of the biophysical components is mandatory for the proper clinical application of the BPP, especially when one manages very preterm gestations.

Magnesium sulfate is probably the most commonly used tocolytic in the United States. It is also used for seizure prophylaxis in pre-eclampsia. Peaceman and colleagues performed BPPs on 16 women who were treated with magnesium sulfate tocolysis for preterm labor at gestational ages of 26 to 34 weeks of gestation (59). They found that magnesium sulfate did not affect fetal tone, gross body movements, or amniotic fluid volume. However, they found a reduction in fetal breathing movements and a higher proportion of nonreactive NSTs in fetuses exposed to magnesium sulfate. In another study, Carlan and colleagues (60) found that administration of magnesium sulfate for tocolysis was associated with a lowered score due to reduced fetal breathing movements. These investigators did not find that magnesium sulfate affected any other components of the BPP. Gray and colleagues evaluated the effect of magnesium sulfate on the BPP and failed to demonstrate any effect of magnesium sulfate on the BPP (61).

The corticosteroids betamethasone and dexamethasone are widely used to induce fetal lung maturation in fetuses at risk of preterm delivery. Rotmensch and colleagues found a transient but reversible suppression of fetal breathing movements, body movements, and FHR accelerations in pregnancies exposed to steroids for lung maturation (62). Jackson and colleagues similarly found a reduction in fetal breathing movements and gross body movements in fetuses in pregnancies where betamethasone was administered $(63,64)$. These authors also observed a reduction in amniotic fluid volume. Deren and coworkers observed that fetuses exposed to betamethasone had reduced fetal movements, breathing movements, and a higher number of nonreactive NSTs (64). In a study of 84 pregnancies in whom betamethasone was administered, Kelly and colleagues found a reduction in BPP scores, mainly caused by a reduction in fetal movements, fetal breathing movements, and a greater proportion of nonreactive NSTs (65). In summary, most investigators have found that steroids affect the BPP mainly through a reduction in reactive NSTs, fetal body movements, and fetal breathing movements. However, this reduction in BPP scores is transient (48-72 hours) and has not been associated with worse perinatal outcomes. This transient reduction in FHR reactivity and fetal breathing movements should be taken into consideration in evaluating preterm fetuses, when the mother has been administered corticosteroids, so iatrogenic prematurity can be avoided.

\section{Inappropriate Use of the BPP}

Vintzileos and colleagues pointed out several of the reasons that the BPP may be used inappropriately (36). First, these investigators emphasized that the most frequent error among clinicians is to base the management of the patient on the biophysical score alone. They argued that it is crucial that the clinician should utilize the individual components of the score rather than the total score in making management decisions. For instance, a fetus with a reactive NST and a normal amount of amniotic fluid, but demonstrating no tone or movements during the examination is assigned a score of 4 . However, both the acute marker, the most sensitive one (reactive NST), and the chronic marker (amniotic fluid volume) have indicated that this fetus is not hypoxemic. Unfortunately, such patients are often said to have failed their BPP and end up being delivered inappropriately and often prematurely. More than likely, the absence of fetal movements, tone, and/or breathing in such a case may be the result of periodicity. Conversely, a fetus with a BPP score of 8 , but with severe 
oligohydramnios, should be delivered if the gestational age is term or near term, despite the "favorable" BPP score of 8 because this fetus is a candidate for a cord accident and death in utero despite normal biophysical activities at the time of testing.

A second error that is often encountered, when using BPP to guide management, is failing to consider the overall clinical picture of the individual case. For instance, a fetus at term with severe growth restriction should be delivered, despite the presence of a reassuring BPP score.

Third, one should not use the same interval of testing (i.e., weekly) for all cases. Generally, a BPP score is considered reliable for 1 week. However, certain high-risk conditions, such as insulin-dependent diabetes, growth restriction, or postdate pregnancies may require more frequent testing. Manning and colleagues in evaluating 12,620 pregnancies with BPPs found that half of the fetal deaths after reassuring BPP results occur between 5 and 7 days after testing, suggesting that twice-weekly testing may reduce mortality. The interval between testing, therefore, should be individualized based on the circumstances of each particular case. When a test is nonreassuring, rather than repeat the test in 24 hours, as is widely advocated, it is appropriate to extend the period of testing beyond 30 minutes. Extending the testing duration will help differentiate between a compromised fetus and one where biophysical activities are absent due to periodicity, whereas just retesting in 24 hours, as is the usual advice, may result in fetal demise.

Next, some investigators advocate the use of BPP without performing an NST. However, the NST may detect FHR variable decelerations that may detect the fetuses at risk for cord accident. These fetuses may have a normal BPP score, and false reassurance may be provided if an NST is not performed.

Physicians may often delay delivery even with nonreassuring $\mathrm{BPP}$ testing due to such concerns as an unfavorable cervix or preterm gestation. This attitude may carry a significant risk of an adverse perinatal outcome. Clinicians should act based on the information derived from the biophysical assessment.

Finally, operator inexperience and faulty technique may lead to incorrect assessment of biophysical activities and inappropriate management decisions. Amniotic fluid measurements should occur in umbilical cord-free pockets. In addition, fetal breathing movements should be considered present only if they are continuous, lasting for more than 30 seconds. Fetal body movements should be considered present only if there are three or more rolling trunk movements observed in a 30-minute period.

\section{Selective Use of the NST}

The selective use of the NST was reported by Manning and coworkers (66), who omitted the NST from the initial biophysical evaluation and included only the other four biophysical components (fetal breathing movements, fetal movements, fetal tone, and amniotic fluid volume). The authors studied 2712 patients who had a total number of 7851 tests and showed that the test accuracy was not compromised. In addition, the need for NST was reduced to only $2.7 \%$ of the cases. The selective use of the NST improved the efficiency of the authors' testing unit, shortened the duration of the test, and increased the number of patients who could be tested daily. Another study by Eden and coworkers, however (67), questioned the wisdom of omitting the NST from the initial biophysical evaluation, as fetuses with all normal biophysical components but variable decelerations during NST testing had an increased frequency of abnormal perinatal outcome. Mills and coworkers (68) proposed a two-tier approach to biophysical assessment of the fetus. They performed a retrospective review of 2038 biophysical assessments in 500 high-risk pregnancies. The authors compared the efficacy of the NST and BPP in predicting fetal asphyxia in fetuses with normal and abnormal growth. The sensitivity, specificity, positive predictive value, and negative predictive value of the NST to predict lethal fetal asphyxia were $100 \%, 77.5 \%, 0.4 \%$, and $100 \%$, whereas that of the fetal BPP were $100 \%, 99.7 \%$, $33.3 \%$, and $100 \%$, respectively. The authors suggested that the appropriate biophysical evaluation of the fetus could be determined based on the clinical problem and the fetal growth. According to the authors, when fetal growth is normal, by ultrasound evaluations at every 4 weeks, no biophysical assessment is necessary. If the growth is abnormal, biweekly NSTs and fetal growth checks by ultrasound at every 2 weeks are indicated. The authors concluded that the fetal BPP should be reserved for those cases where the NST is non-reassuring or equivocal. By using this testing method, the authors speculated that they could theoretically reduce their workload of NSTs by $>60 \%$ and BPPs by $>75 \%$.

\section{The BPP in Diabetes and Postdate Pregnancies}

Johnson and coworkers (44) managed 307 consecutive postterm pregnancies with twice-weekly BPPs and suggested that if the biophysical score was reassuring, waiting for spontaneous onset of labor was advisable, resulting in good perinatal outcomes with a much lower cesarean section rate $(15 \%$ vs. $42 \%$ for induction patients). The same authors also reported on the use of the fetal BPP in managing 238 diabetic pregnancies who had a total number of 1028 profiles performed (69). In patients with an abnormal score, there was a high rate of intensive care nursery admissions and cesarean section rate $(50 \%)$. Of the fetuses with normal BPP scores, $57.4 \%$ entered spontaneous labor, $31.3 \%$ were induced, and the remaining $11.3 \%$ were delivered by elective repeat or primary cesarean section. Stillbirths were prevented and $87 \%$ of the patients delivered at or near term with minimal maternal or neonatal morbidity. Abnormal BPP scoring was associated with a significantly higher rate of operative intervention and neonatal morbidity. According to the authors, the fetal BPP allows for a safe expectant management in the diabetic pregnancy.

The usefulness of the fetal BPP in twin gestations has been studied by Lodeiro and coworkers (70). Forty-nine twin gestations with additional high-risk factors such as suspected growth retardation of one or both twins, maternal chronic hypertension, or pregnancy-induced hypertension were followed with weekly BPPs. The sensitivity, specificity, positive predictive value, and negative predictive value of the biophysical assessment in predicting fetal distress were $83.3 \%, 100 \%, 100 \%$, and $97.7 \%$, respectively. The falsenegative rate was $2.2 \%$. However, the only false-negative death resulted from extreme prematurity in a set of twins delivered at 
26 weeks of gestation. Four twin gestations had fetal distress in only one of the twins and all eight fetuses (four pairs) had nonreactive NSTs. The BPP accurately predicted the distressed twin of each of the four pairs, and it also correctly identified well-being for its counterpart twin. The authors concluded that the BPP is a safe and effective tool in the antepartum fetal surveillance of twin gestations.

The wisdom of arbitrary assignment of equal score weights for the various biophysical components was questioned by Manning and coworkers (71). In a study of 525 fetuses, they studied the relationship between the last abnormal BPP score, in total and by variable composition, and a variety of abnormal perinatal outcomes such as fetal distress in labor, low 5-minute Apgar score $(<7)$, intrauterine growth restriction, and admission to the neonatal intensive care unit for $>24$ hours. It was found that all variable combinations for the same score subsets (scores 6, 4, and 2) are not equal in predictive accuracy. For a biophysical score of 6 , the probability of fetal distress or death was significantly higher with either nonreactive NST/ fetal tone absent or nonreactive NST/absent fetal breathing movements, whereas the probability of fetal distress was significantly lower when fetal breathing movements were absent in the presence of oligohydramnios. Significant variations in positive predictive accuracy also occurred within subsets of variable combinations yielding a BPP score of 4; these variations, however, disappeared with a BPP score of 2 . Overall, the NST, amniotic fluid volume, and fetal breathing movements emerged as the most powerful variables for all perinatal outcome measures. Fetal tone seemed to play a lesser role, and fetal movement was found to be important only for predicting fetal distress.

\section{A Rational Approach to Antepartum Fetal Biophysical Assessment}

Considering the "gradual hypoxia concept," and also the $100 \%$ sensitivity of the combination of the nonreactive NST and absence of fetal breathing movements for predicting the acidemic fetus, Vintzileos and coworkers recommended a modified fetal evaluation scheme for patients with intact membranes (Fig. 8). This scheme is based on the individual biophysical components rather than the score $(32,36)$. It should be noted that this scheme is also supported by the data of Manning and coworkers (71), which suggest that the NST, amniotic fluid volume, and breathing movements are the most powerful predictors of fetal compromise.

This protocol includes assessment of an acute (NST or fetal breathing) and a chronic marker (amniotic fluid volume). A reactive NST or fetal breathing lasting $>30 \mathrm{sec}$ (even in the presence of a nonreactive NST) makes fetal acidemia at the time of testing highly unlikely. However, there is a caveat in patients with uncontrolled diabetes. Hyperglycemia may cause fetuses in diabetic pregnancies to exhibit fetal breathing even in the presence of (lactic) acidemia. Thus, the blood glucose should be checked in diabetic women at the time of the BPP assessment.

When the NST is nonreactive, it becomes crucial to make the differentiation between fetal sleep and fetal acidemia. If fetal breathing lasting greater than 30 seconds is detected, the examination is terminated and further management is based on the amniotic fluid volume assessment. If all biophysical activities (nonreactive NST, fetal breathing, fetal movements, and fetal tone) are absent after 30 minutes of continuous observation, the fetus should be delivered promptly. If the

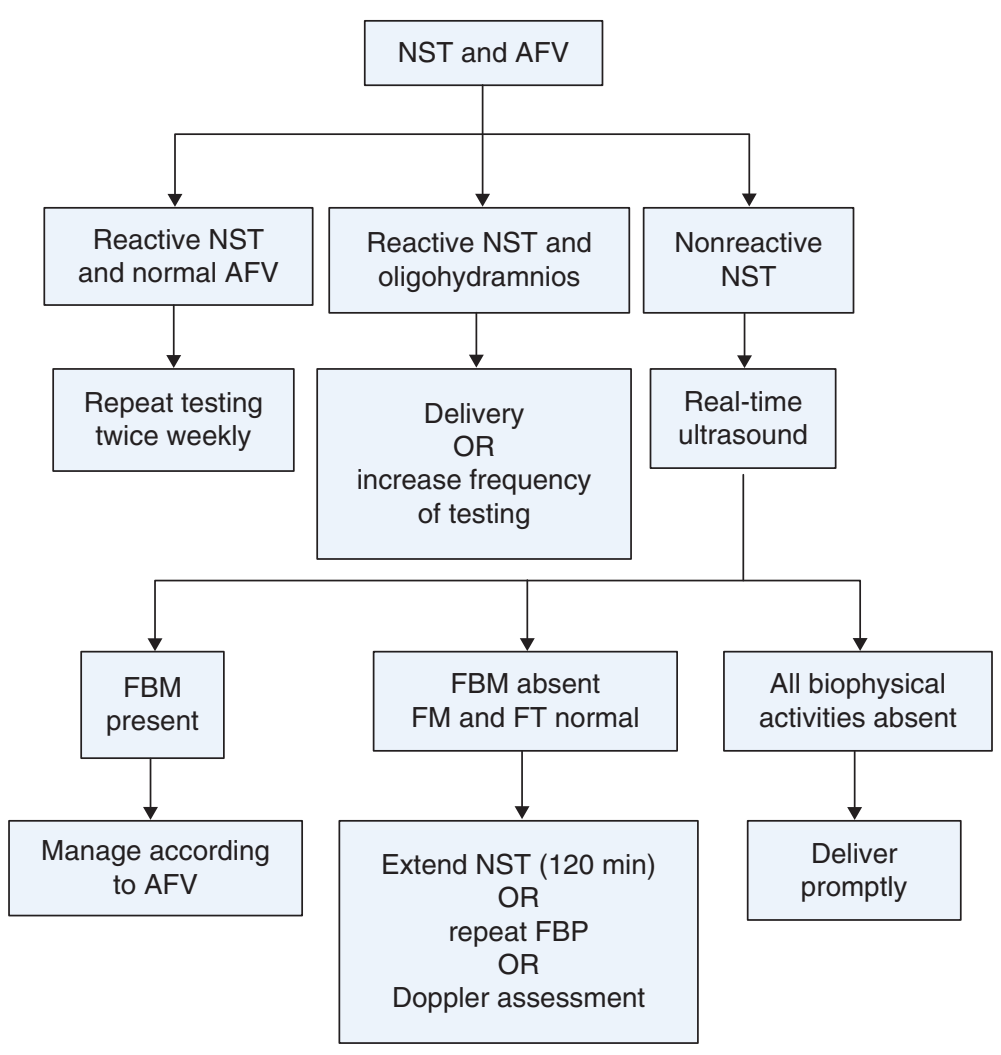

Figure 8 An algorithm for the use of the modified fetal biophysical profile in antepartum fetal surveillance. Abbreviations: AFV, amniotic fluid volume; FBM, fetal breathing movements; FM, fetal movements; FBP, fetal biophysical profile; FT, fetal tone; FHR, fetal heart rate; NST, nonstress test. $\quad$ Source: From Ref. 74. 
fetus has a nonreactive NST and breathing is absent, while movements and tone are normal, testing duration should be extended to differentiate fetal sleep from fetal compromise. The NST is continued until a reactive pattern is observed or until 120 minutes have elapsed. If the NST is still nonreactive, the real-time ultrasound examination should be repeated. If no fetal breathing is observed, and the gestational age is $>32$ weeks, additional evaluation (i.e., Doppler velocimetry) or delivery may be considered. In very preterm gestations ( $<32$ weeks) in the absence of FHR cyclicity, if there is a nonreactive NST and absent fetal breathing, additional evaluation or delivery should also be considered $(72,73)$. If FHR cyclicity and body movements are both present and normal, then expectant management is indicated. The presence of oligohydramnios and/or variable decelerations during NST suggests that that the fetus is at risk for umbilical cord accident, and the fetus should be delivered if the gestation is at or near term. The presence of a normal amount of amniotic fluid and the absence of variable decelerations is reassuring, and expectant management may be reasonable. In the presence of oligohydramnios in a term or a near-term gestation with intact membranes, delivery is indicated regardless of how reassuring the other biophysical components are. In our view, a largest amniotic fluid pocket less than $2 \mathrm{~cm}$ (or amniotic fluid index $<5 \mathrm{~cm}$ ) defines oligohydramnios. The combination of oligohydramnios and variable decelerations during the NST may indicate the need for delivery in viable, structurally normal fetuses with intact membranes, regardless of gestational age. In very preterm gestations ( $<32$ weeks) in the presence of oligohydramnios and no identifiable fetal anomalies, pregnancies should be managed on a case-by-case basis taking into consideration the results of Doppler velocimetry (if the underlying pathophysiology is reduced uteroplacental blood flow). However, it is crucial to recognize that these fetuses are at increased risk of stillbirth and that while it may be necessary to attempt to prolong gestation to improve maturity at birth, these fetuses may die while being managed conservatively. If delivery is not undertaken, frequent testing (every 24 hours) is an alternative logical plan of management. In this group of fetuses, Doppler studies may assist in deciding management.

\section{Computer-Assisted BPP}

The biophysical methods currently used in clinical practice are arbitrary since they do not provide exact quantification of the biophysical activities over a long period, nor do they account for the occasionally long intervals of fetal inactivity, which are frequently observed in normal fetuses. To circumvent these drawbacks, an extended period of concurrent observation of the biophysical activities has been attempted by using computer-assisted systems or phonographic transducers $(74,75)$. Devoe and coworkers (76) have reported their experience by using a computerized system that allowed analysis of the simultaneously acquired biophysical activities in 200 term high-risk fetuses. The biophysical activities obtained for each high-risk fetus were compared with previously established nomograms. The results correlated with the presence or absence of perinatal mortality, fetal distress, a low 5-minute Apgar score $(<7)$, and intrauterine growth retardation. A test was considered abnormal if two or more biophysical activities quantitatively fell $>2$ SD below the population mean. A decreased frequency of fetal body movements, decreased amniotic fluid volume, and decreased frequency of FHR accelerations were the most common abnormalities. When fetuses with normal or abnormal perinatal outcomes were classified by the last computerized biophysical test, the sensitivity was $86 \%$, specificity $89 \%$, positive predictive value $75 \%$, negative predictive value $93 \%$, and overall diagnostic accuracy $86.2 \%$. This diagnostic accuracy was significantly better as compared with the arbitrary scoring system of Manning and coworkers. However, this comparison was not based on a randomized trial; in addition, it was not clear whether or not the computer-assisted BPP results influenced patient management.

\section{THE FETAL BPP IN PATIENTS WITH PROM}

The effect of PROM on the BPP has been studied extensively. Vintzileos and colleagues performed a retrospective analysis of 1151 fetal BPPs performed between 25 and 44 weeks of gestation in pregnancies with good outcomes (defined as those with no congenital anomalies, infection, fetal distress, or perinatal mortality/morbidity) (47). These investigators compared the fetal BPPs and scores of patients with intact membranes with those who had PPROM, but had good pregnancy outcomes. The frequency of reactive NSTs, fetal breathing, fetal movements, and fetal tone did not change across gestation from 25 to 44 weeks in patients with PROM. However, when compared with patients with intact membranes, those pregnancies with PROM had a higher frequency of reactive NSTs, absent fetal breathing, and reduced amniotic fluid volume. The frequency of nonreactive NST at less than 32 weeks of gestation was only $13.5 \%$ in the presence of ruptured membranes. The overall biophysical scoring was not altered by the presence of PROM. The changes in the fetal BPP caused by PROM are summarized in Table 7.

In a prospective study, Vintzileos et al. (46) performed frequent fetal biophysical assessments on 73 patients with PROM with no clinical signs of labor or infection and demonstrated that an abnormal BPP was an early indicator of intra-amniotic infection. A fetal BPP was performed on admission and was repeated every 24 to 48 hours. The last biophysical assessment before delivery was correlated with the development of clinical amnionitis, possible neonatal sepsis, and neonatal sepsis. The diagnosis of possible neonatal sepsis was made when there was strong clinical and laboratory evidence of neonatal infection but the cultures were negative. The diagnosis of neonatal sepsis was made in the presence of positive cultures of blood, urine, or cerebrospinal fluid. There

Table 7 Summary of Changes in the Fetal Biophysical Profile Associated with Premature Rupture of the Membranes

Increase in fetal heart rate reactivity Decrease in fetal breathing movements

No change in fetal movements

No change in fetal tone

No change in the overall biophysical score 
was no correlation between the fetal biophysical assessment and intra-amniotic infection in the 20 of the 73 patients who delivered at greater than 24 hours following the last biophysical assessment. Fifty-three patients delivered within 24 hours of the last biophysical examination; in these patients, a biophysical score of $>8$ was associated with an infection rate of $2.7 \%$, while an abnormal biophysical score $(<8)$ was associated with an overall infection rate of $93.7 \%(46)$. The first manifestations of fetal intra-amniotic infection were nonreactive NST and absent fetal breathing. Loss of fetal movement and absent fetal tone occurred only in advanced infections. The best predictor of infection was the overall biophysical score. The fetal biophysical score range was $<8$ in all the 14 cases with possible neonatal sepsis or neonatal sepsis. No difference in the mean umbilical cord blood $\mathrm{pH}$ between the infected and non-infected cases was found, an observation that suggests that the low scores of the infected group were not mediated by hypoxia, but rather by infection. In patients with PROM, the degree of oligohydramnios, one of the components of the BPP, is strongly correlated with pregnancy prolongation, abnormal antepartum and intrapartum FHR patterns, cesarean section rates, fetal distress, infection, and perinatal mortality. Vintzileos and colleagues correlated the degree of oligohydramnios with pregnancy outcome in 90 patients with PROM and no signs of infection or labor (77). Patients with severe oligohydramnios (largest amniotic fluid pocket $<\mathrm{l} \mathrm{cm}$ ) had the highest frequency of amnionitis $(47.3 \%)$, possible neonatal sepsis $(26.3 \%)$, and neonatal sepsis (31.5\%), compared with $9.2 \%, 3.7 \%$, and $1.8 \%$, respectively, in patients with a largest amniotic fluid pocket $>2 \mathrm{~cm}$. Based on this finding, these investigators concluded that patients with PROM and severe oligohydramnios should have daily fetal BPPs performed. A retrospective analysis of 127 consecutive patients with PROM who had NSTs performed every 24 to 48 hours as part of the fetal BPP demonstrated that those who initially had a reactive NST and subsequently converted to a nonreactive NST developed clinical intraamniotic infection in almost $90 \%$ of the cases (78). The last NST prior to delivery had sensitivity, specificity, positive, and negative predictive values to predict infection outcome of $78.1 \%, 86.3 \%, 67.7 \%$, and $92.1 \%$, respectively. Fetal breathing movements (lasting $>30$ s) have also been shown to predict intra-amniotic infection. In a study of 130 patients with PROM who were followed with BPP assessments every 24-48 hours, the sensitivity, specificity, positive, and negative predictive values of fetal breathing to predict intra-amniotic infection were $91.6 \%, 64.8 \%, 50 \%$, and $95.3 \%$, respectively (79). Of the individual biophysical components, the presence of fetal breathing has been the most reliable in ruling out fetal infection, if observed within 24 hours prior to delivery. Vintzileos and coworkers compared daily fetal BPPs with amniocentesis (for Gram stain and culture) in 58 patients who presented with preterm PROM and no apparent infection or labor (48). In addition to the usual indications for delivery, a positive Gram stain or culture of the amniotic fluid, obtained by amniocentesis, or a persistently abnormal biophysical score $(<8)$ on two examinations, 2 hours apart, in the presence of a nonreactive NST and absent fetal breathing were also considered as the indications for delivery. The last BPP prior to delivery and amniocentesis results on admission were correlated with infection outcome. All but one of the 13 cases with neonatal infection were associated with an abnormal biophysical assessment. There were two cases with amnionitis without neonatal infection; both cases had normal BPPs. The sensitivity, specificity, positive predictive value, and negative predictive value of the BPP for infection were $80 \%, 97.6 \%, 92.3 \%$, and $93.3 \%$, respectively. The sensitivity, specificity, positive predictive value, and negative predictive value of the Gram stain were $60 \%, 81.3 \%, 52.9 \%$, and $85.3 \%$, respectively, whereas those of the amniotic fluid cultures were $60 \%, 86 \%, 60 \%$, and $86 \%$, respectively. The authors concluded that in preterm PROM daily BPPs are more efficacious than amniocentesis on admission in selecting those patients who either have or will develop intra-amniotic infection and therefore require prompt delivery. Importantly, the fetal BPP is simple, noninvasive, can be repeated daily, or even more frequently and is applicable to all patients. Vintzileos and coworkers (80) managed 73 consecutive patients with PROM with daily BPPs in an attempt to determine whether daily BPPs improved pregnancy outcomes. The pregnancy outcome of this group was compared with the outcomes of two other historic groups: 73 consecutive patients with PROM in the first control group were managed conservatively while the 73 consecutive patients with PROM in the second control group were managed with amniocentesis on admission. Maternal and neonatal infection rates, as well as low 5-minute Apgar scores were significantly less in the study group than among controls. The percentage of mother-infant pairs who developed infection in the study group (10.9\%) was significantly less than that in the control group (30.1\%). Clinical amnionitis was reduced from $20.5 \%$ to $5.4 \%$. The frequencies of possible neonatal sepsis and neonatal sepsis were also lower in the study group compared with the control group (5.4\% vs. $13.6 \%$ and $1.3 \%$ vs. $9.5 \%$, respectively). The study group also had significantly less neonatal sepsis $(1.3 \%$ vs. $12.3 \%)$. The authors concluded that the use of amniocentesis may decrease the incidence of clinical amnionitis, but it does not improve the neonatal infection outcome. The neonatal infection outcome was, however, reduced by using daily biophysical assessment.

In order to investigate the role of fetal acidemia in causing diminished biophysical activities in the setting of subclinical infection and PROM, Vintzileos and coworkers (81) performed a prospective evaluation of 53 consecutive patients with preterm PROM who were delivered solely on the basis of an abnormal fetal biophysical assessment. Fetal acidemia at birth was defined as cord artery $\mathrm{pH}<7.20$. Of the 53 fetuses, 19 developed no maternal or neonatal infection and none was acidemic at birth. Seventeen fetuses developed possible neonatal sepsis and none was acidemic at birth. Fifteen fetuses developed neonatal sepsis and only five were acidemic at birth. Two fetuses developed clinical amnionitis and only one was acidemic at birth. While fetal acidemia could explain the diminished fetal biophysical activities in 5 of the 15 fetuses who developed neonatal sepsis and in 1 with clinical amnionitis, this mechanism could not explain the compromised biophysical activities in the remaining 10 fetuses with neonatal sepsis and in all 17 fetuses who developed possible neonatal sepsis. A possible explanation for the diminished biophysical activities of the infected non-acidemic fetus may be an increased production of prostaglandins, particularly interleukin-1. Interleukin-1, the main fever 
mediator, induces changes in the electroencephalogram that may be responsible for the decreased motor activity during fever in both animals and humans (82). Since interleukin-1 is capable of stimulating prostaglandin production from macrophages and other cell types, it has been suggested that this may be responsible for the decrease in fetal breathing observed not only prior to labor but also in the setting of subclinical infection. This increased prostaglandin secretion may directly inhibit the fetal CNS centers or may indirectly lead to increased peripheral vascular resistance through vasoconstriction of the umbilical and chorionic vessels $(83,84)$. Since there are experimental data to suggest that prostaglandins increase placental vascular resistance through vasoconstriction of umbilical and chorionic vessels, the relationships among umbilical artery velocimetry, the fetal $\mathrm{BPP}$, and placental inflammation in patients with PROM have also been investigated (85). The study population involved 28 consecutive patients with preterm PROM who were followed with daily fetal BPPs and umbilical artery velocimetry. After delivery, placental pathologic examination for evidence of inflammation was performed in all cases. When only the last examination, within 24 hours of delivery, was considered, patients with placental inflammation (choriodeciduitis, umbilical and/or chorionic vasculitis) had higher mean umbilical artery Doppler systolic/diastolic (S:D) ratios (2.92 \pm 0.62 vs. $2.29 \pm 0.25)$ and lower biophysical scores ( $6.2 \pm 2.3$ vs. $9.1 \pm 2.1)$ compared with those without evidence of placental inflammation. It should be noted that the mean S:D ratio value 24 hours prior to delivery in cases with placental inflammation was 2.92, which is not necessarily higher than the normal range. Fetuses with placental infection increased their S:D ratio during the last 24 hours prior to delivery approximately 30\% (which was statistically significant) over the mean value of the preceding days. These data suggest that larger studies are needed to more accurately determine the value of the longitudinal trend in Doppler velocimetry indices in regard to being additional noninvasive means of identifying subclinical intra-amniotic infection. The increase in umbilical vascular resistance supports a role for vasoactive substances, such as prostaglandins, as mediators of infection causing vasoconstriction of umbilical and chorionic vessels. This increase in vascular resistance may compromise uteroplacental circulation and diminish biophysical activities prior to the development of fetal acidemia.

\section{CONCLUSIONS}

Antepartum fetal surveillance has led to a reduction in perinatal mortality. The techniques described in this chapter are now widely accepted and almost universally used. A clear understanding of the physiologic basis for these tests and of the pathophysiology of the conditions that can cause fetal death or intrauterine hypoxia is crucial to appropriate application of these surveillance methods. Unfortunately, there will still be some causes of death that cannot be predicted or prevented using these methods.

\section{REFERENCES}

1. Kontopoulos EV, Vintzileos AM. Condition-specific antepartum fetal testing. Am J Obstet Gynecol 2004; 191: 1546-51.

2. Hon EH. The foetal heart rate. Mod Trends Hum Reprod Physiol 1963; 15: 245-56.
3. Manning FA, Platt LD, Sipos L. Antepartum fetal evaluation: development of a fetal biophysical profile. Am J Obstet Gynecol 1980; 136: 787-95.

4. Hon EH, Reid BL, Hehre FW. The electronic evaluation of fetal heart rate. II. Changes with maternal hypotension. Am J Obstet Gynecol 1960; 79: 209-15.

5. Kubli FW, Hon EH, Khazin AF, Takemura H. Observations on heart rate and $\mathrm{pH}$ in the human fetus during labor. Am J Obstet Gynecol 1969; 104: 1190-206.

6. Ray M, Freeman R, Pine S, Hesselgesser R. Clinical experience with the oxytocin challenge test. Am J Obstet Gynecol 1972; 114: 1-9.

7. Freeman RK. The use of the oxytocin challenge test for antepartum clinical evaluation of uteroplacental respiratory function. Am J Obstet Gynecol 1975; 121: 481-9.

8. Oki EY, Keegan KA, Freeman RK, Dorchester WL. The breast-stimulated contraction stress test. J Reprod Med 1987; 32: 919-23.

9. Erkkola R, Rintala H, Gronroos M. Breast stimulation test in fetal surveillance. Acta Obstet Gynecol Scand 1984; 63: 719-22.

10. Capeless EL, Mann LI. Use of breast stimulation for antepartum stress testing. Obstet Gynecol 1984; 64: 641-5.

11. Copel JA, Otis CS, Stewart E, Rosetti C, Weiner S. Contraction stress testing with nipple stimulation. J Reprod Med 1985; 30: 465-71.

12. Chayen B, Scott E, Cheng CC, Perera C, Schiffer MA. Contraction stress test by breast stimulation as part of antepartum monitoring. Acta Obstet Gynecol Scand 1985; 64: 3-6.

13. Palmer SM, Martin JN, Moreland ML, et al. Contraction stress test by nipple stimulation: efficacy and safety. South Med J 1986; 79: 1102-5.

14. Evertson LR, Gauthier RJ, Collea JV. Fetal demise following negative contraction stress tests. Obstet Gynecol 1978; 51: 671-3.

15. Lagrew DC Jr. The contraction stress test. Clin Obstet Gynecol 1995; 38: $11-25$.

16. Gauthier RJ, Evertson LR, Paul RH. Antepartum fetal heart rate testing. II. Intrapartum fetal heart rate observation and newborn outcome following a positive contraction stress test. Am J Obstet Gynecol 1979; 133: 34-9.

17. Braly P, Freeman RK. The significance of fetal heart rate reactivity with a positive oxytocin challenge test. Obstet Gynecol 1977; 50: 689-93.

18. Modanlou HD, Murata Y. Sinusoidal heart rate pattern: reappraisal of its definition and clinical significance. J Obstet Gynaecol Res 2004; 30: 169-80.

19. Sadovsky G, Visser GH, Nicolaides KH. Heart rate patterns in fetal anemia. Fetal Ther 1988; 3: 216-23.

20. Birkenfeld A, Yaffe H, Sadovsky E. Sinusoidal fetal heart rate pattern with severe fetal anaemia. Case report. Br J Obstet Gynaecol 1980; 87: 916-19.

21. Antoine C, Young BK, Silverman F, Greco MA, Alvarez SP. Sinusoidal fetal heart rate pattern with vasa previa in twin pregnancy. J Reprod Med 1982; 27: 295-300.

22. Sherer DM, Ezra Y, Beyth Y, Sadovsky E. Sinusoidal fetal heart rate pattern associated with the twin to twin transfusion syndrome. Int J Gynaecol Obstet 1990; 31: 71-4.

23. Evertson LR, Gauthier RJ, Schifrin BS, Paul RH. Antepartum fetal heart rate testing. I. Evolution of the nonstress test. Am J Obstet Gynecol 1979; 133: 29-33.

24. Phelan JP. The nonstress test: a review of 3,000 tests. Am J Obstet Gynecol 1981; 139: 7-10.

25. Phelan JP, Cromartie AD, Smith CV. The nonstress test: the false negative test. Am J Obstet Gynecol 1982; 142: 293-6.

26. Davey DA, Dommisse J, Macnab M, Dacre D. The value of an auditory stimulatory test in antenatal fetal cardiotocography. Eur J Obstet Gynecol Reprod Biol 1984; 18: 273-7.

27. Nyman M, Westgren M. Maternal perception of sound-provoked fetal movements in low-risk pregnancies during the third trimester. Br J Obstet Gynaecol 1989; 96: 566-7.

28. Gagnon R. Acoustic stimulation: effect on heart rate and other biophysical variables. Clin Perinatol 1989; 16: 643-60.

29. Druzin ML, Edersheim TG, Hutson JM, Bond AL. The effect of vibroacoustic stimulation on the nonstress test at gestational ages of thirty-two weeks or less. Am J Obstet Gynecol 1989; 161(6 Pt 1): 1476-8.

30. Yao QW, Jakobsson J, Nyman M, et al. Fetal responses to different intensity levels of vibroacoustic stimulation. Obstet Gynecol 1990; 75: 206-9. 
31. Vintzileos AM, Fleming AD, Scorza WE, et al. Relationship between fetal biophysical activities and umbilical cord blood gas values. Am J Obstet Gynecol 1991; 165: 707-13.

32. Vintzileos AM, Campbell WA, Ingardia CJ, Nochimson DJ. The fetal biophysical profile and its predictive value. Obstet Gynecol 1983; 62: 271-8.

33. Vintzileos AM, Gaffney SE, Salinger LM, Campbell WA, Nochimson DJ. The relationship between fetal biophysical profile and cord $\mathrm{pH}$ in patients undergoing cesarean section before the onset of labor. Obstet Gynecol 1987; 70: 196-201.

34. Chamberlain PF, Manning FA, Morrison I, Harman CR, Lange IR. Ultrasound evaluation of amniotic fluid volume. I. The relationship of marginal and decreased amniotic fluid volumes to perinatal outcome. Am J Obstet Gynecol 1984; 150: 245-9.

35. Casey BM, McIntire DD, Bloom SL, et al. Pregnancy outcomes after antepartum diagnosis of oligohydramnios at or beyond 34 weeks' gestation. Am J Obstet Gynecol 2000; 182: 909-12.

36. Vintzileos AM, Campbell WA, Nochimson DJ, Weinbaum PJ. The use and misuse of the fetal biophysical profile. Am J Obstet Gynecol 1987; 156: 527-33.

37. Manning FA, Morrison I, Lange IR, Harman CR, Chamberlain PF. Fetal assessment based on fetal biophysical profile scoring: experience in 12,620 referred high-risk pregnancies. I. Perinatal mortality by frequency and etiology. Am J Obstet Gynecol 1985; 151: 343-50.

38. Vintzileos AM, Gaffney SE, Salinger LM, et al. The relationships among the fetal biophysical profile, umbilical cord $\mathrm{pH}$, and Apgar scores. Am J Obstet Gynecol 1987; 157: 627-31.

39. Ribbert LS, Snijders RJ, Nicolaides KH, Visser GH. Relationship of fetal biophysical profile and blood gas values at cordocentesis in severely growth-retarded fetuses. Am J Obstet Gynecol 1990; 163: 569-71.

40. Manning FA, Baskett TF, Morrison I, Lange I. Fetal biophysical profile scoring: a prospective study in 1,184 high-risk patients. Am J Obstet Gynecol 1981; 140: 289-94.

41. Baskett TF, Gray JH, Prewett SJ, Young LM, Allen AC. Antepartum fetal assessment using a fetal biophysical profile score. Am J Obstet Gynecol 1984; 148: 630-3.

42. Manning FA, Lange IR, Morrison I, Harman CR. Fetal biophysical profile score and the nonstress test: a comparative trial. Obstet Gynecol 1984; 64: 326-31.

43. Platt LD, Walla CA, Paul RH, et al. A prospective trial of the fetal biophysical profile versus the nonstress test in the management of highrisk pregnancies. Am J Obstet Gynecol 1985; 153: 624-33.

44. Johnson JM, Harman CR, Lange IR, Manning FA. Biophysical profile scoring in the management of the postterm pregnancy: an analysis of 307 patients. Am J Obstet Gynecol 1986; 154: 269-73.

45. Benacerraf BR, Frigoletto FD Jr. Fetal respiratory movements: only part of the biophysical profile. Obstet Gynecol 1986; 67: 556-7.

46. Vintzileos AM, Campbell WA, Nochimson DJ, et al. The fetal biophysical profile in patients with premature rupture of the membranes-an early predictor of fetal infection. Am J Obstet Gynecol 1985; 152: 510-16.

47. Vintzileos AM, Feinstein SJ, Lodeiro JG, et al. Fetal biophysical profile and the effect of premature rupture of the membranes. Obstet Gynecol 1986; 67: 818-23.

48. Vintzileos AM, Campbell WA, Nochimson DJ, et al. Fetal biophysical profile versus amniocentesis in predicting infection in preterm premature rupture of the membranes. Obstet Gynecol 1986; 68: 488-94.

49. Manning FA, Morrison I, Harman CR, Lange IR, Menticoglou S. Fetal assessment based on fetal biophysical profile scoring: experience in 19,221 referred high-risk pregnancies. II. An analysis of false-negative fetal deaths. Am J Obstet Gynecol 1987; 157(4 Pt 1): 880-4

50. Baskett TF, Allen AC, Gray JH, Young DC, Young LM. Fetal biophysical profile and perinatal death. Obstet Gynecol 1987; 70(3 Pt 1): 357-60.

51. Platt LD, Eglinton GS, Sipos L, Broussard PM, Paul RH. Further experience with the fetal biophysical profile. Obstet Gynecol 1983; 61: 480-5.

52. Manning FA, Harman CR, Morrison I, Menticoglou S. Fetal assessment based on fetal biophysical profile scoring. III. Positive predictive accuracy of the very abnormal test (biophysical profile score $=0$ ). Am J Obstet Gynecol 1990; 162: 398-402.
53. Manning FA, Harman CR, Morrison I, et al. Fetal assessment based on fetal biophysical profile scoring. IV. An analysis of perinatal morbidity and mortality. Am J Obstet Gynecol 1990; 162: 703-9.

54. Hopper KD, Komppa GH, Bice P, et al. A reevaluation of placental grading and its clinical significance. J Ultrasound Med 1984; 3: 261-6.

55. Lavin JP Jr, Miodovnik M, Barden TP. Relationship of nonstress test reactivity and gestational age. Obstet Gynecol 1984; 63: 338-44.

56. Queenan JT, Thompson W, Whitfield CR, Shah SI. Amniotic fluid volumes in normal pregnancies. Am J Obstet Gynecol 1972; 114: 34-8.

57. Sadovsky E, Laufer N, Allen JW. The incidence of different types of fetal movements during pregnancy. Br J Obstet Gynaecol 1979; 86: 10-14.

58. Baskett TF. Gestational age and fetal biophysical assessment. Am J Obstet Gynecol 1988; 158: 332-4.

59. Peaceman AM, Meyer BA, Thorp JA, Parisi VM, Creasy RK. The effect of magnesium sulfate tocolysis on the fetal biophysical profile. Am J Obstet Gynecol 1989; 161: 771-4.

60. Carlan SJ, O'Brien WF. The effect of magnesium sulfate on the biophysical profile of normal term fetuses. Obstet Gynecol 1991; 77: 681-4.

61. Gray SE, Rodis JF, Lettieri L, Egan JF, Vintzileos A. Effect of intravenous magnesium sulfate on the biophysical profile of the healthy preterm fetus. Am J Obstet Gynecol 1994; 170: 1131-5.

62. Rotmensch S, Liberati M, Celentano C, et al. The effect of betamethasone on fetal biophysical activities and Doppler velocimetry of umbilical and middle cerebral arteries. Acta Obstet Gynecol Scand 1999; 78: 768-73.

63. Jackson JR, Kleeman S, Doerzbacher M, Lambers DS. The effect of glucocorticosteroid administration on fetal movements and biophysical profile scores in normal pregnancies. J Matern Fetal Neonatal Med 2003; 13: $50-3$.

64. Deren O, Karaer C, Onderoglu L, et al. The effect of steroids on the biophysical profile and Doppler indices of umbilical and middle cerebral arteries in healthy preterm fetuses. Eur J Obstet Gynecol Reprod Biol 2001; 99: 72-6.

65. Kelly MK, Schneider EP, Petrikovsky BM, Lesser ML. Effect of antenatal steroid administration on the fetal biophysical profile. J Clin Ultrasound 2000; 28: 224-6.

66. Manning FA, Morrison I, Lange IR, Harman CR, Chamberlain PF. Fetal biophysical profile scoring: selective use of the nonstress test. Am J Obstet Gynecol 1987; 156: 709-12.

67. Eden RD, Seifert LS, Kodack LD, et al. A modified biophysical profile for antenatal fetal surveillance. Obstet Gynecol 1988; 71(3 Pt 1): 365-9.

68. Mills MS, James DK, Slade S. Two-tier approach to biophysical assessment of the fetus. Am J Obstet Gynecol 1990; 163(1 Pt 1): 12-17.

69. Johnson JM, Lange IR, Harman CR, Torchia MG, Manning FA. Biophysical profile scoring in the management of the diabetic pregnancy. Obstet Gynecol 1988; 72: 841-6.

70. Lodeiro JG, Vintzileos AM, Feinstein SJ, Campbell WA, Nochimson DJ. Fetal biophysical profile in twin gestations. Obstet Gynecol 1986; 67: 824-7.

71. Manning FA, Morrison I, Harman CR, Menticoglou SM. The abnormal fetal biophysical profile score. V. Predictive accuracy according to score composition. Am J Obstet Gynecol 1990; 162: 918-24; discussion 24-7.

72. Hanley ML, Vintzileos AM: Antepartum and intrapartum surveillance of fetal well being. Medicine of the Fetus and Mother, 2nd edn. Philadelphia: JB Lippincott, 1988: 793.

73. Vintzileos AM, Campbell WA, Bors-Koefoed R, et al. Relationship between cyclic variation of fetal heart rate patterns and cord $\mathrm{pH}$ in preterm gestations. Am J Perinatol 1989; 6: 310-13.

74. Colley N, Talbert DG, Southall DP. Biophysical profile in the fetus from a phonographic sensor. Eur J Obstet Gynecol Reprod Biol 1986; 23: 261-6.

75. Devoe LD, Searle N, Searle J, et al. Computer-assisted assessment of the fetal biophysical profile. Am J Obstet Gynecol 1985; 153: 317-21.

76. Devoe LD, Castillo RA, Searle N, Searle JS. Prognostic components of computerized fetal biophysical testing. Am J Obstet Gynecol 1988; 158: 1144-8.

77. Vintzileos AM, Campbell WA, Nochimson DJ, Weinbaum PJ. Degree of oligohydramnios and pregnancy outcome in patients with premature rupture of the membranes. Obstet Gynecol 1985; 66: 162-7. 
78. Vintzileos AM, Campbell WA, Nochimson DJ, Weinbaum PJ. The use of the nonstress test in patients with premature rupture of the membranes. Am J Obstet Gynecol 1986; 155: 149-53.

79. Vintzileos AM, Campbell WA, Nochimson DJ, Weinbaum PJ. Fetal breathing as a predictor of infection in premature rupture of the membranes. Obstet Gynecol 1986; 67: 813-17.

80. Vintzileos AM, Bors-Koefoed R, Pelegano JF, et al. The use of fetal biophysical profile improves pregnancy outcome in premature rupture of the membranes. Am J Obstet Gynecol 1987; 157: 236-40.

81. Vintzileos AM, Petrikovsky BM, Campbell WA, et al. Cord blood gases and abnormal fetal biophysical assessment in preterm premature rupture of the membranes. Am J Perinatol 1991; 8: 155-60.

82. Baracos V, Rodemann HP, Dinarello CA, Goldberg AL. Stimulation of muscle protein degradation and prostaglandin E2 release by leukocytic pyrogen (interleukin-1). A mechanism for the increased degradation of muscle proteins during fever. N Engl J Med 1983; 308: 553-8.

83. Howard RB, Hosokawa T, Maguire MH. Pressor and depressor actions of prostanoids in the intact human fetoplacental vascular bed. Prostaglandins Leukot Med 1986; 21: 323-30.

84. Mak KK, Gude NM, Walters WA, Boura AL. Effects of vasoactive autacoids on the human umbilical-fetal placental vasculature. Br J Obstet Gynaecol 1984; 91: 99-106.

85. Fleming AD, Salafia CM, Vintzileos AM, et al. The relationships among umbilical artery velocimetry, fetal biophysical profile, and placental inflammation in preterm premature rupture of the membranes. Am J Obstet Gynecol 1991; 164(1 Pt 1): 38-41. 


\section{Amnioinfusion \\ James Kerns, Erol Amon, and Hung N. Winn}

\section{INTRODUCTION}

Amnioinfusion is a technique in which a crystalloid solution, usually normal saline, is instilled into the amniotic cavity to replace amniotic fluid, which may be absent or low. This technique has been employed with increasing frequency and interest during the past three decades. It is used most often during the intrapartum period to prevent, decrease, or eliminate variable decelerations (1-5). During the antepartum period, amnioinfusion is used less frequently. It is mainly used in this setting to facilitate evaluation of fetal anatomy, less commonly in attempts to reduce fetal complications from prolonged oligohydramnios, and rarely to prevent infectious complications from preterm premature rupture of the membranes (PROM) (6-10).

\section{PATHOGENESIS AND CLINICAL ASPECTS OF OLIGOHYDRAMNIOS}

Near the end of the first trimester, the amnion and chorion fuse to create the amniotic cavity, which contains approximately $50 \mathrm{~mL}$ of amniotic fluid. In early pregnancy, the amniotic fluid is primarily the product of maternal transudate through the amniotic membranes. By the 16th week of gestation, the amniotic fluid volume is mainly regulated by the processes of fetal urination and fetal swallowing. As the pregnancy progresses, the normal amniotic fluid volume rapidly increases to a maximum (range 0.5-2.0liters, 95\% confidence interval) at about 34 weeks of gestation. The volume then normally decreases as a natural progression (11).

Oligohydramnios can result from

1. any condition that prevents the formation of urine or prevents the entry of urine into the amniotic sac, including bilateral renal agenesis, bilateral multicystic dysplastic kidneys, infantile polycystic kidney disease, and complete lower tract obstructive uropathies;

2. uteroplacental insufficiency, particularly in the setting of fetal growth restriction (FGR);

3. post-term pregnancies;

4. rupture of membranes (ROM);

5. prolonged maternal use of indomethacin or other nonsteroidal anti-inflammatory drugs;

6. abdominal pregnancy; and

7. reasons unknown, that is, idiopathic.

The amniotic fluid environment is necessary for normal fetal growth and development, as evidenced by fetal lung hypoplasia and deformations in the case of Potter's syndrome. Pulmonary hypoplasia may well develop if prolonged oligohydramnios ( $>2$ weeks) secondary to preterm PROM occurs prior to 24 weeks of gestation. The earlier the gestational age when the premature ROM occurs, the greater the incidence of lung hypoplasia (12). Oligohydramnios predisposes to umbilical cord compression, which can lead to fetal hypoxia if resultant interference with blood flow is severe, repetitive, and prolonged.

Clinical management involves obtaining a patient's history to determine:

1. leakage of amniotic fluid;

2. risk factors for FGR such as smoking, drug abuse, severe diabetes, or cardiovascular disorders;

3. medication history; and

4. family history of congenital anomalies, especially urinary tract abnormalities.

Physical examination may reveal the fundal height to be less than expected, leakage of amniotic fluid from the cervical canal, and/or positive tests for ROM. Fetal heart rate monitoring may reveal variable or prolonged decelerations.

Ultrasound evaluation is essential in assessing the amniotic fluid volume and fetal anatomy. Occasionally, amniocentesis with injection of indigo carmine dye may be necessary to diagnose ROM, especially when there is a history of leakage of fluid in the absence of confirmatory findings.

Ultrasound evaluation of the amniotic fluid includes the one largest vertical pocket and the amniotic fluid index (AFI). The latter is a summation of the highest vertical dimensions of the four quadrants of the uterine cavity. Oligohydramnios exists if the AFI is less than $5 \mathrm{~cm}$. If the vertical dimension of the largest pocket of fluid is $<1 \mathrm{~cm}$, severe oligohydramnios is present.

The initial step in the clinical management of oligohydramnios is to determine the etiology. Management is then based on gestational age, maternal health, fetal diagnosis and prognosis, and the patient and family's concerns.

\section{INDICATIONS FOR AMNIOINFUSION General Overview}

Amnioinfusion is generally classified according to the pregnancy status as antepartum or intrapartum. The procedure is performed either transcervically or transabdominally. Its purpose can be diagnostic, therapeutic, or prophylactic.

During the antepartum period, amnioinfusion may be used transabdominally to improve sonographic imaging, to obtain fluid for culture, to obtain fetal cells for karyotyping, and to restore adequate levels of fluid to reduce the likelihood of lung hypoplasia and arthrogryposis.

During the intrapartum period, amnioinfusion may be diagnostic (instilling and aspirating fluid to evaluate the microbiology of in utero infections), therapeutic (to alleviate cord compression), or prophylactic (selected cases to decrease the incidence of meconium aspiration syndrome). 


\section{Antepartum Indications: Diagnostic}

Oligohydramnios poses a diagnostic challenge for the physician, as the differential diagnosis is broad. Etiologies may include urethral outflow obstruction, absent or diminished renal function, PROM, FGR, congenital malformations, or chromosome anomalies. Conventional sonographic imaging may prove inadequate to view the fetal anatomy, owing to the absence of a satisfactory acoustic window. Fetal positioning, including crowding of small parts, may also impair visualization. Therefore, diagnostic imaging is an important step prior to selecting a course of therapy.

In 1988, Grembruch and Hansamnn described transabdominal amnioinfusion to improve ultrasound imaging (13). Quetel et al. used amnioinfusion in patients with oligohydramnios and were able to provide an adequate acoustic window and perform a comprehensive ultrasound evaluation (10). They confirmed the diagnosis of ruptured membranes by transabdominal instillation of indigo carmine and observed the passage of blue dye into the vagina. Fluid could be aspirated during the amnioinfusion procedure for evaluation and the cells cultured for karyotyping.

\section{Antepartum Indications: Therapeutic}

Fisk and colleagues (8) performed serial amnioinfusion in a few selected cases and found that they could avert lung hypoplasia. Serial infusions were initiated as soon as feasible after the diagnosis of oligohydramnios was made and terminated after the canalicular phase of lung development was complete. In the setting of PROM, some investigators demonstrated that successful amnioinfusion resulted in prolonged gestation, improved neonatal outcome, reduced incidence for pulmonary hypoplasia, and fewer abnormal neurologic sequelae compared with those patients with persistent oligohydramnios $(14,15)$. However, the Cochrane Collaboration review concluded that there was too little evidence to show the beneficial effects of amnioinfusion to the fetus following PROM (16). Since pulmonary hypoplasia remains the most common cause of neonatal death following vesico-amniotic shunting for obstructive uropathy, serial amnioinfusion may improve the neonatal outcomes for this condition.

Antepartum amnioinfusion has been proposed to improve the success rate of external cephalic version. Benifla et al. instilled about $800 \mathrm{~mL}$ of solution transabdominally into the amniotic cavity of the patients who had failed standard attempts at external version. In each case, $(n=6)$, a successful external version resulted after the amnioinfusion (17). However, others found that transabdominal amnioinfusion of $1000 \mathrm{~mL}$ of solution into the amniotic cavity did not improve the successful external version $(n=7)$ (18). The Cochrane Collaboration review concluded that there was too little evidence to show the benefit of transabdominal amnioinfusion in external cephalic version (19).

\section{Intrapartum Indications: Diagnostic}

An indwelling, intrauterine, fluid-filled pressure catheter was initially introduced to monitor uterine tone and uterine activity. The diagnostic use of this medical device has been expanded. In the clinical setting of chorioamnionitis, fluid may be aspirated from a catheter port for Gram-stain evaluation with or without culture and sensitivity, as clinically indicated. A paucity of amniotic fluid may be detected at the time of amniotomy. This could signify thick meconium or oligohydramnios.

\section{Intrapartum Indications: Therapeutic}

Using pregnant monkeys, Gabbe and coworkers demonstrated that removal of amniotic fluid produced variable decelerations, which were subsequently eliminated when the fluid volume was restored (20). Many have reported that a similar mechanism of using fluid as an umbilical cord cushion, via transcervical amnioinfusion in the setting of oligohydramnios, results in a significant improvement in variable and prolonged decelerations, which do not otherwise respond to conventional methods (intravenous fluids, repositioning, $\mathrm{O}_{2}$ administration, and manipulation of the presenting fetal part).

Accurate measurement of the amount of fluid retained is difficult, because unknown quantities of fluid may leak out during transcervical therapy. Serial ultrasound evaluation of the AFI may be useful in assessing the change as a guide for further infusion therapy including avoiding polyhydramnios $(2,3,10,21)$. It is important to define the end point of the amnioinfusion such as AFI of $8 \mathrm{~cm}$, total volume of solution infused, total rate infused, or the resting tone of the uterus.

\section{Intrapartum Indication: Prophylactic}

Oligohydramnios in the setting of structurally normal fetuses occurring in the intrapartum period may be caused by membrane rupture, FGR, or postdates. With the loss of the amniotic fluid cushion, there is an increased risk of cord compression, which may lead to variable or prolonged decelerations. These decelerations may be associated with hypoxia and acidosis. Using amnioinfusion, variable decelerations have been eliminated or reduced in frequency or severity during the first and second stages of labor, thereby reducing the associated hypoxia and acidosis and leading to an improved fetal acid-base status at delivery $(2,4,5,20)$.

With prophylactic restoration of amniotic fluid volume at term, Strong and colleagues $(3,21)$ reported a decreased incidence of meconium aspiration syndrome, severe variable decelerations, end-stage bradycardia, operative delivery for fetal distress, and low umbilical artery $\mathrm{pH}$ values. Other investigators (21-24) concluded that amnioinfusion prophylaxis at 36 weeks or greater in patients with membrane rupture and oligohydramnios (AFI $<5 \mathrm{~cm}$ ) resulted in reduced intrapartum morbidity, decreased rates of cesarean delivery, and decreased episodes of fetal intolerance to labor. They also concluded that there were improved umbilical cord gas values, neonatal outcomes, and improved Apgar scores.

The presence of meconium may be the natural response of the gastrointestinal system to maturation. It may also be the response of the fetus to a stressful condition such as hypoxia, acidosis, or vagal stimulation. Generally, these etiologies are distinguished by the normality of the fetal heart rate pattern, with completely normal patterns being indicative of normal maturation.

Meconium has been associated with increased perinatal morbidity and mortality, especially due to aspiration of meconium-stained amniotic fluid. Meconium aspiration syndrome, defined as respiratory distress requiring mechanical 
ventilation in a neonate with meconium aspiration, carries a mortality rate of approximately $25 \%$ and accounts for about $2 \%$ of all perinatal deaths. The passage of meconium in utero occurs in $8 \%$ to $16 \%$ of all deliveries; however, meconium aspiration syndrome occurs in only $1 \%$ to $3 \%$ of all cases of meconium-stained fluid (25). Meconium aspiration may occur before or during labor, or during the process of delivery. When meconium is aspirated into the lower respiratory tract, mechanical obstruction and chemical inflammation may occur.

Prior to the use of amnioinfusion, combined obstetric and pediatric suctioning of the neonate's airway during and after delivery was the mainstay of preventing meconium aspiration syndrome. This technique, as described by Carson and colleagues (26), was reported to decrease the frequency of meconium aspiration syndrome, assuming the aspiration occurred during the delivery process.

Some investigators reported amnioinfusion's beneficial effects in laboring patients who had meconium and oligohydramnios. Amnioinfusion resulted in a decreased incidence of:

1. 1- and 5-minute Apgar scores $<7$;

2. meconium visualized below the cords and in the oropharynx;

3. the presence of thick meconium in selected clinical settings;

4. umbilical arterial cord $\mathrm{pH}$ values $<7.20$;

5. intrapartum fetal distress;

6. operative delivery for fetal distress;

7. cesarean delivery rates; and

8. need for positive pressure ventilation of the newborn (27-29).

It was noted that the rates of cesarean delivery for arrest disorders were similar in the amnioinfusion and the control groups.

The Cochrane Collaboration recent review concluded that amnioinfusion during labor for meconium-stained amniotic fluid showed (i) no improvement in the perinatal outcomes if the labor and delivery unit has adequate monitoring of fetal condition and (ii) a significant improvement in the perinatal outcomes if the labor and delivery unit has limited capacity in monitoring fetal conditions during labor (27).

In summary, amnioinfusion provides an additional therapeutic option in labor to treat patients experiencing variable and prolonged decelerations that are unresponsive to conventional methods. Amnioinfusion also allows for prevention of fetal heart rate-related complications when used prophylactically in the intrapartum setting of oligohydramnios (30).

\section{CONTRAINDICATIONS}

In general, absolute contraindications to vaginal delivery are also absolute contraindications to intrapartum transcervical amnioinfusion. These include placenta previa and prior ruptured uterus. Other contraindications include conditions in which there would be no fetal benefit, such as lethal fetal anomalies and fetal death. The relative contraindications are listed in Table 1.

Clear evidence of impending fetal hypoxia or acidosis generally requires expedient delivery rather than temporization with amnioinfusion. Maternal exclusions include maternal
Table 1 Relative Contraindications to Amnioinfusion

Uterine anomalies

Chorioamnionitis

Maternal heart disease class II, III, IV

Fetal growth restriction

Fetal malformations

Malpresentation

Classical or unknown cesarean section scar

Abruptio placentae

Multi-fetal gestation

heart disease (New York Heart Association functional classification II or greater), as noted by Sadovsky and colleagues due to potential fluid overload from absorption (29). Abnormal uterus such as septate uterus may be less distensible and less pliable to amnioinfusion, thus less timely change in the pressure-volume curve. Whether chorioamnionitis is a contraindication is controversial, since some consider this condition to be an indication.

\section{PROTOCOLS}

A variety of protocols for amnioinfusion have emerged, all of which have common elements and guidelines. The common elements can be divided into pre-infusion preparation and evaluation, infusion equipment and monitoring, and postinfusion evaluation. The common guidelines are patient education and nursing in-service, evaluation of equipment, defining eligibility for infusion, and delineating the limits of the infusion. The ideal protocol defining the recommended infusion technique and rate has not been established. Various protocols are available, with variations defined by different authors for their own purposes. No controlled study has demonstrated the superiority of any given protocol.

In general, an eligible patient, during labor, has one or more of the following indications:

1. Variable deceleration with or without oligohydramnios;

2. Meconium amniotic fluid at term with limited monitoring capability;

3. Oligohydramnios either at term or preterm as a result of ROM; and/or

4. Oligohydramnios with intact membranes.

Variable decelerations, as previously discussed, are attributed to cord compression, with multiple studies showing that relieving the cord compression and improving the oxygen delivery to the fetus improve the intrauterine environment and the neonatal outcome.

The recommended protocol may include the following:

1. Informed consent concerning the clinical setting, risks and benefits, and alternatives. Oral consent may be legally sufficient, but it is best followed by written consent for amnioinfusion and cesarean delivery, if indicated.

2. A pelvic examination is performed to verify presentation, absence of cord prolapse, cervical dilatation and effacement, and fetal station.

3. Membrane rupture should be confirmed prior to transcervical amnioinfusion. An internal fetal heart 
rate electrode and an intrauterine pressure catheter, the latter ready for amnioinfusion, should be placed appropriately.

4. Some authors perform ultrasound for amniotic fluid volume index at the start as well as serially, but this may be unnecessary. Strong and coworkers (21) showed that $250 \mathrm{~mL}$ of infusion into a relatively closed system increases the amniotic fluid volume index by 4 to $4.3 \mathrm{~cm}$ (range $0-7.7 \mathrm{~cm}$ ).

5. The intrauterine catheter system chosen should be able to monitor intrauterine pressure and instill amniotic fluid.

6. The fluid chosen for infusion is either normal saline or lactated Ringer's.

7. Consider warming the infusion to $37^{\circ} \mathrm{C}$ for the preterm fetus or rapid infusion.

8. Infuse normal saline at a rate of 10 to $14 \mathrm{~mL} / \mathrm{min}$. If a faster rate is carried out (e.g., $15-25 \mathrm{~mL} / \mathrm{min}$ by gravity), then warming the fluid to $37^{\circ} \mathrm{C}$ is recommended. The initial infusion volume is generally 500 to $600 \mathrm{~mL}$. Some recommend stopping the infusion after a bolus, while others recommend continuous infusion at a lower rate (2-3 $\mathrm{mL} / \mathrm{min})$.

9. End points for the infusion are individualized, based on experience and the purpose to be achieved. These include:

(a) a predetermined volume of 600 to $1000 \mathrm{~mL}$;

(b) resolution of variable decelerations;

(c) an AFI of 8 to $10 \mathrm{~cm}$; and

(d) a slow continuous irrigation and lavage at 2 to $3 \mathrm{~mL} / \mathrm{min}$, as long as fluid is continually draining out of the uterus.

10. If sonographic monitoring is available, the following guidelines are recommended:

(a) If AFI $>10 \mathrm{~cm}$, no additional bolus is recommended.

(b) If AFI is 5 to $10 \mathrm{~cm}$, a second bolus of $250 \mathrm{~mL}$ is added and reassessment by sonogram is conducted.

(c) If AFI $<5 \mathrm{~cm}$, a second bolus of $500 \mathrm{~mL}$ is added and reassessment by sonogram is conducted.

11. Periodic re-bolus with 500 to $600 \mathrm{~mL}$ of fluid every 6 hours or a constant low infusion rate of 2 to $3 \mathrm{~mL} / \mathrm{min}$ may be used, depending on uterine tone and AFI.

12. Periodic assessment of the fetal heart rate pattern, uterine tone and activity, volume infused, vaginal leaking, and progress of labor is necessary.

13. Evaluation for complications.

Meconium-staining amniotic fluid presents potential meconium aspiration and the meconium aspiration syndrome. Amnioinfusion is effective in diluting meconium but may not reduce the incidence of the neonatal sequelae.

Preterm PROM presents two major concerns, namely, potential cord compression with its sequelae and intrauterine infection. There should be a low threshold to initiate therapeutic amnioinfusion and systemic chemoprophylaxis in the appropriate clinical setting.
Oligohydramnios at term, with membrane rupture occurring during labor, subjects the fetus to the potential of cord compression and its sequelae. The protocol recommended is the same as that for variable decelerations.

\section{COMPLICATIONS}

The adverse effects and potential complications of amnioinfusion are summarized in Table 2.

In a survey, $26 \%$ of centers reported at least one associated complication. The most frequent complication was uterine hypertonus (14\%), followed by fetal heart rate abnormalities (9\%) (30).

Although Posner and colleagues (31) found no change in uterine tone with controlled volumes of amnioinfusion and monitoring, they did report polyhydramnios with overinfusion. Two cases of polyhydramnios with uterine tenderness, one initially thought to be placental abruption (32) and the other with elevated uterine tone and fetal bradycardia from the amnioinfusion (33), were reported. In both cases, when the excess fluid was removed, the elevated uterine tone and uterine tenderness were relieved. In the case reported by Tabor and Maier, the bradycardia resolved after the excess fluid was removed (33). Over-distension and elevated uterine tone from amnioinfusion could disrupt a previous cesarean section scar. One case of a disrupted uterine scar was noted during a repeat cesarean delivery in which there was no resolution of the variable decelerations with amnioinfusion (1). Strong and colleagues (34) reported no change in the incidence of uterine scar disruption.

One case of cord prolapse was reported, but it was the same case in which the delivery was assisted by mid-forceps. No difference in the incidence of nuchal cords was demonstrated (31).

Miyazaki and Taylor (1) noted one case of fetal bradycardia, which occurred during rapid infusion of room temperature fluid ( $400 \mathrm{~mL}$ over 8 minutes). The proposed mechanism was sudden chilling, leading to vasoconstriction, hypertension, and reflex bradycardia in the fetus. They noted no episodes of bradycardia when the infusate was warmed to $37^{\circ} \mathrm{C}$, regardless of the infusion rate. Thus, warming of the fluid appears unnecessary if the infusion rates are limited to 10 to $15 \mathrm{~mL} / \mathrm{min}$ or less.

The rate of amnionitis as a result of amnioinfusion is variable (10,21). Patients with a higher rate of amnionitis also had more vaginal examinations. When this element was corrected, the rate was not significantly different from that of the control (non-infused) group (29). The majority of investigations reported no significant change in the rates of amnionitis associated with amnioinfusion.

Table 2 Complications of Amnioinfusion

Potential neonatal electrolyte imbalance

Potential neonatal hypothermia

Umbilical cord prolapse

Acute polyhydramnios

Fetal bradycardia

Elevated uterine tone

Previous cesarean section scar disruption

Amnionitis/endometritis

Amniotic fluid embolus 
Nageotte and coworkers (4) noted that there were no significant differences in the basic electrolytes $(\mathrm{Na}, \mathrm{K}, \mathrm{Cl}$, and $\mathrm{CO}_{2}$ ) in newborns delivered following amnioinfusion as compared with those not subjected to amnioinfusion. The infusate used in this study was normal saline.

No conclusive evidence of the effect of amnioinfusion on the length of labor is available. The evidence presented by Strong and colleagues (3) suggested that there was a significant increase in the length of labor from $10.1 \pm 6.5$ to $16.8 \pm 12.1$ hours in their group, whereas Schrimmer and coworkers (5) reported a shorter duration of labor in their group of patients. Macri and colleagues (35) noted no significant change in the lengths of labor between the control group and the amnioinfusion group.

Dibble and Elliott (36) reported possible amniotic fluid embolus in two cases following amnioinfusion. No direct association was made to this rare pregnancy complication and amnioinfusion. They proposed that these two cases were mild because of the dilutional effect of the infusate on the particulate matter in the amniotic fluid. Other authors have also reported maternal respiratory distress in association with amnioinfusion $(37,38)$.

\section{CONCLUSIONS}

Amnioinfusion is useful during the intrapartum period for relief of variable and prolonged decelerations, and obtaining fluid for evaluation of meconium staining and microbiology.

Amnioinfusion in the antepartum period may be useful in improving the ultrasound image, obtaining fluid for karyotype or culture and restoring fluid volume to decrease the likelihood of lung hypoplasia, arthrogryposis, or other fetal compression disorders in the setting of oligohydramnios.

Amnioinfusion has been an established part of the clinical management of complicated obstetric patients.

\section{REFERENCES}

1. Miyazaki FS, Taylor NA. Saline amnioinfusion for relief of variable or prolonged decelerations. Am J Obstet Gynecol 1983; 146: 670-8.

2. Nageotte MP, Freeman RK, Garitz TJ, Dorchester W. Prophylactic intrapartum amnioinfusion in patients with preterm premature rupture of membranes. Am J Obstet Gynecol 1985; 153: 557-62.

3. Strong TH, Hetzler G, Sarna AP, Paul RH. Prophylactic intrapartum amnioinfusion: a randomized clinical trial. Am J Obstet Gynecol 1990; 162: $1370-5$.

4. Nageotte MP, Bertucci L, Towers CV, et al. Prophylactic amnioinfusion in pregnancies complicated by oligohydramnios: a prospective study. Obstet Gynecol 1991; 77: 667-80.

5. Schrimmer DB, Macri CJ, Paul RH. Prophylactic amnioinfusion as a treatment for oligohydramnios in laboring patients: a prospective, randomized trial. Am J Obstet Gynecol 1991; 165: 972-5.

6. Ogita S, Imanaka M, Matsumoto M, et al. Transcervical amnioinfusion of antibiotics: a basic study for managing premature rupture of membranes. Am J Obstet Gynecol 1988; 158: 23-7.

7. Imanaka M, Ogita S, Sugawa T. Saline solution amnioinfusion for oligohydramnios after premature rupture of the membranes. Am J Obstet Gynecol 1989; 161: 102-6.

8. Fisk NM, Ronderos-Dumit D, Soliani A, et al. Diagnostic and therapeutic transabdominal amnioinfusion in oligohydramnios. Obset Gynecol 1991; 78: 270-8.

9. Sherer DM, McAndrew JA, Liberto L, Woods JR. Recurring bilateral renal agenesis diagnosed by ultrasound with the aid of amnioinfusion at 18 weeks gestation. Am J Perinatol 1992; 9: 49-51.

10. Quetel TA, Mejides AA, Salman FA, Torres-Rodriguez MM. Amnioinfusion: an aid in the ultrasonographic evaluation of severe oligohydramnios in pregnancy. AM J Obstet Gynecol 1992; 167: 333-6.
11. Brace RA, Wolf EJ. Normal amniotic fluid volume changes throughout pregnancy. Am J Obstet Gynecol 1989; 161: 382-8.

12. Thibeault DW, Beatty EC, Hall RT, et al. Neonatal pulmonary hypoplasia with premature rupture of fetal membranes and oligohydramnios. J Pediatr 1985; 107: 273-7.

13. Grembruch U, Hansamnn M. Artificial instillation of amniotic fluid as a new technique for the diagnostic evaluation of cases of oligohydramnios. Prenat Diagn 1988; 8: 33-45.

14. Locatelli A, Vergani P, Di Pirro G, et al. Role of amnioinfusion in the management of premature rupture of the membranes at $<26$ weeks gestation. Am J Obstet Gynecol 2000; 183: 878-82.

15. Tranquilli AL, Giannubilo SR, Bezzeccheri V, et al. Transabdominal amnioinfusion in preterm premature rupture of membranes: a randomized controlled trial. Br J Obstet Gynecol 2005; 112: 759-63.

16. Novikova N, Hofmeyr GJ, Essilfie-Appiah G. Prophylactic versus therapeutic amnioinfusion for oligohydramnios in labour. Cochrane Database Syst Rev 1996: CD000176.

17. Benifla JL, Goffinet F, Darai E, et al. Antepartum transabdominal amnioinfusion to facilitate external version after initial failure. Obstet Gynecol 1994; 84: 1041-2.

18. Adama van Scheltema PN, Feitsma AH, Middeldorp JM, et al. Amnioinfusion to facilitate external cephalic version after initial failure. Obstet Gynecol 2006; 108: 591-2.

19. Hofmeyr GJ, Gyte GML. Interventions to help external cephalic version for breech presentation at term. Cochrane Database Syst Rev 2004: CD000184.

20. Gabbe SG, Ettinger BB, Freeman RK, Martic CB. Umbilical cord compression associated with amniotomy: laboratory observations. Am J Obstet Gynecol 1976; 126: 353-8.

21. Strong TH, Hetzler G, Paul RH. Amniotic fluid volume increase after amnioinfusion of a fixed volume. Am J Obstet Gynecol 1990; 162: 746-8.

22. Ogundipe OA, Spong CY, Ross MG. Prophylactic amnioinfusion for oligohydramnios: a reevaluation. Obstet Gynecol 1994; 84: 544-8.

23. Hofmeyr GJ. Amnioinfusion for potential or suspected umbilical cord compression in labour. Cochrane Database Syst Rev 1998: CD000013.

24. Pitt C, Sanchez-Ramon L, Kaunitz AM, et al. Prophylactic amnioinfusion for intrapartum oligohydramnios: a meta-analysis of randomized controlled trials. Obstet Gynecol 2000; 96: 861-6.

25. Macri CJ, Schrimmer DB, Leung A, et al. Prophylactic amnioinfusion improves outcome of pregnancy complicated by thick meconium and oligohydramnios. Am J Obstet Gynecol 1992; 167: 117-21.

26. Carson BS, Losey R, Bowes WA, Simmons MA. Combined obstetrics and pediatric approach to prevent meconium aspiration syndrome. Am J Obstet Gynecol 1976; 126: 712-15.

27. Hofmeyr GJ, Xu H. Amnioinfusion for meconium-stained liquor in labour. Cochrane Database Syst Rev 2010: CD000014.

28. Wenstrom KD, Parsons MT. The prevention of meconium aspiration in labor using amnioinfusion. Obstet Gynecol 1989; 73: 642-51.

29. Sadovsky Y, Amon E, Bade ME, Petrie RH. Prophylactic amnioinfusion during labor complicated by meconium: a preliminary report. Am J Obstet Gynecol 1989; 161: 613-17.

30. Wenstrom K, Andrews WW, Maher JE. Amnioinfusion survey: prevalence, protocols, and complications. Obstet Gynecol 1995; 86: 572-6.

31. Posner MD, Ballagh SA, Paul RH. The effect of amnioinfusion on uterine pressure and activity: a preliminary report. Am J Obstet Gynecol 1990; 163: 813-18.

32. Sorensen T, Subeck J, Benedotte T. Intrauterine pressure in acute iatrogenic hydramnios. Obstet Gynecol 1991; 78: 917-19.

33. Tabor BL, Maier JA. Polyhydramnios and elevated intrauterine pressure during amnioinfusion. Am J Obstet Gynecol 1987; 156: 130-1.

34. Strong TH, Vage JS, O'Shaughnessy MJ, et al. Amnioinfusion among women attempting vaginal birth after Cesarean delivery. Obstet Gynecol 1992; 79: 673-4.

35. Macri CJ, Schrimmer DB, Greenspoon JS, et al. Amnioinfusion does not affect the length of labor. Am J Obstet Gynecol 1992; 167: 1134-6.

36. Dibble LA, Elliott JP. Possible amniotic fluid embolism associated with amnioinfusion. J Maternal Fetal Med 1992; 1: 263-6.

37. Dragich DA, Ross AF, Chestnut DH, et al. Respiratory failure associated with amnioinfusion during labor. Anesth Analg 1991; 72: 549-51.

38. Maher JE, Wenstrom KD, Hauth JC, et al. Amniotic fluid embolism after saline amnioinfusion: two cases and review of the literature. Obstet Gynecol 1994; 83: 851-9. 


\section{The maternal immune system during pregnancy Breton F. Barrier}

\section{INTRODUCTION}

The effects of pregnancy on the immune system are intriguing and clinically recognizable. A viral pharyngitis in pregnancy may persist for weeks on end while fulminate vulvar condyloma may suddenly regress in the postpartum period. At first glance, these changes seem to defy logic. Why does multiple sclerosis improve in pregnancy (1), while an active lupus flare is fueled? (2). Why does ankylosing spondylitis worsen (3), yet rheumatoid arthritis improves (4)?

Deepening the mystery is the remarkable coexistence of the fetus - a semi-allograph recognized by the maternal immune system-living freely within the maternal host without rejection. This phenomenon first caught the attention of Nobel laureate Sir Peter Medawar over 50 years ago, prompting him to advance his now-famous four nonexclusive postulates about maternal-fetal tolerance: (i) the fetus may not be immunogenic, (ii) the uterus may be immunoprivileged, (iii) the placenta may be an immunologic barrier, and (iv) the mother may be immunosuppressed (5). From the time that Medawar first enunciated these postulates, rapid advances in maternal-fetal immunology have evolved, giving great hope for an approaching era of practical clinical application.

The immune system is an exceptionally complex and elegant network of cells, cytokines, chemokines, antibodies, and regulatory proteins. Because of this complexity, it is useful to artificially partition the immune system to facilitate study and dialog. The broadest categories have been classically described as the immune system's innate and adaptive arms. The cellular constituents of these arms are summarized in Table 1.

\section{THE INNATE ARM OF THE IMMUNE SYSTEM}

The innate arm of the immune system is the first line of defense and includes (i) epithelial cells, (ii) macrophages, (iii) neutrophils, (iv) natural killer (NK) cells, and (v) complement. Innate sentinel cells include epithelial cells and macrophages that sample the local environment and rapidly respond when pathogens are detected. Neutrophils and NK cells are the primary effector cells of the innate immune system. Eosinophils and basophils play an important role in allergic and parasitic infections and will not be discussed in detail in this chapter. Serum complement does just that"complement" immune processes by weakening or marking pathogens for destruction.

\section{Epithelia}

The epithelial barrier to the environment provides the first line of defense against the intrusion of pathogens. Some epithelia, such as endometrium, possess the ability to synthesize and secrete cytokines, and to process and present antigens to adaptive immune cells, driving proliferation of autologous lymphocytes (6). Endometrial epithelial cells possess special pattern recognition receptors (PRRs) called Toll-like receptors (TLRs) that recognize "pathogen-associated molecular patterns” (PAMPs) (7). Different TLRs recognize various PAMPs, and their expression is highly tissue specific. TLRs are found in epithelial urogenital tissues, professional antigen-presenting cells (such as macrophages and dendritic cells), and trophoblast-derived tissues such as placenta and amnion (8).

\section{Complement}

Complement is a collection of serum proteins that bind pathogens, leading to immediate destruction, opsonization, or chemotaxis. Once activated, factors remain bound to the pathogen (i.e., factor $\mathrm{C} 3 \mathrm{~b}$ ) or released locally for neutrophil chemotaxis (i.e., factor C3a). Complement is activated through multiple mechanisms resulting in the formation of a "C3 convertase" (Fig. 1): (i) it is activated by antibodies present on pathogens through factor C1q (the "classical" pathway); (ii) it binds directly to the pathogen through factor $\mathrm{C} 3 \mathrm{~b}$ (the "alternative" pathway), or (iii) it is activated by the terminal mannose of bacterial $\mathrm{N}$-acetyl glucosamine through mannose-binding protein (the "lectin" pathway). The presence of $\mathrm{C} 3 \mathrm{~b}$ then opsonizes organisms for phagocytosis by neutrophils and macrophages. In some cases, such as with Neisseria sp., complement also forms a multicomponent membrane attack complex (MAC) that leads to direct perforation and lysis of the organism $(9,10)$.

Maternal complement levels in pregnancy are similar or greater than those of nonpregnant controls (11). Because of the potential of activation of complement by maternal antibodies toward paternal antigens, the trophoblast must have a mechanism in place to inhibit this process. At least three important complement regulatory proteins are expressed by the trophoblast as early as 6 weeks of gestation: decayaccelerating factor (DAF, CD55) (12), membrane cofactor protein (MCP, CD46), and CD59.

DAF inhibits formation and accelerates decay of $\mathrm{C} 3 / \mathrm{C} 5$ convertase, which inhibits the alternative pathway. MCP is an intrinsic-acting protein that binds efficiently to $\mathrm{C} 3 \mathrm{~b}$ attached to other molecules on the cell surface (13). MCP then cleaves the $\mathrm{C} 3 \mathrm{~b}$ or $\mathrm{C} 4 \mathrm{~b}$ components of complement to their inactive derivatives, which inhibits the classical complement pathway. Finally, CD59 inhibits the final common component of both complement pathways-the MAC. CD59 is expressed in greater amounts than either DAF or MCP, although the absence of CD59 is compatible with successful pregnancy, indicating that MCP and DAF are able to compensate for a lack of CD59.

Expression of all three proteins occurs on the terminally differentiated syncytiotrophoblast that is in direct contact with maternal blood. Villous cytotrophoblast cells express CD59 
Table 1 Commonly Encountered Cytokines

\begin{tabular}{|c|c|c|}
\hline Cytokine & Origin & Function \\
\hline IL-1 $\beta$ & $\begin{array}{l}\text { Epithelium, macrophages, } \\
\text { neutrophils }\end{array}$ & $\begin{array}{l}\text { Stimulates T- and B-lymphocytes; makes B-cells responsive to IL-5 and T-cells } \\
\text { responsive to IL-2 }\end{array}$ \\
\hline IL-2 & T-cells & Autocrine stimulation of T-cells \\
\hline IL-5 & T-cells $\left(\mathrm{Th}_{2}\right)$ & Stimulates B-cell growth and increases immunoglobulin secretion \\
\hline IL-6 & $\begin{array}{l}\text { Epithelium, macrophages, } \\
\text { T-cells }\end{array}$ & $\begin{array}{l}\text { Stimulates acute phase response; differentiation of B-cells (IgG1 antibody) and } \\
\text { T-cells (cytotoxic and } \mathrm{Th}_{17} \text { types) }\end{array}$ \\
\hline IL-12 & $\begin{array}{l}\text { Macrophages and dendritic } \\
\text { cells }\end{array}$ & $\begin{array}{l}\text { Induces synthesis of IFN } \gamma \text {, IL-2, and TNF by } \mathrm{Th}_{1} \text { cells; reduces synthesis of IL- } 4 \text {, } \\
\text { IL- } 5 \text {, and IL- } 10 \text { by } \mathrm{Th}_{2} \text { cells }\end{array}$ \\
\hline IL-13 & T-cells $\left(\mathrm{Th}_{2}\right)$ & Induces IgG4 and IgE isotype switching in human B-cells \\
\hline IL-17A & T-cells $\left(\mathrm{Th}_{17}\right)$ & Induces epithelia and macrophages to produce IL-6, IL-8, TNF, and TGF $\beta$ \\
\hline TGF $\beta$ & T-cells (Treg) & $\begin{array}{l}\text { Inhibits activation of lymphocytes and monocytes, promotes differentiation of } \\
\text { Treg cells and } \mathrm{Th}_{1} \text { cells, causes IgA isotype switching }\end{array}$ \\
\hline \multicolumn{3}{|r|}{ (1) } \\
\hline IL-8 (CXCL8) & $\begin{array}{l}\text { Epithelium, endothelia, } \\
\text { macrophages }\end{array}$ & Attracts neutrophils \\
\hline RANTES (CCL5) & Epithelium, T-lymphocytes & Attracts T-cells, eosinophils, basophils \\
\hline MCP-1 (CCL2) & $\begin{array}{l}\text { Macrophages, monocytes. } \\
\text { endothelium }\end{array}$ & Attracts monocytes/macrophages \\
\hline
\end{tabular}

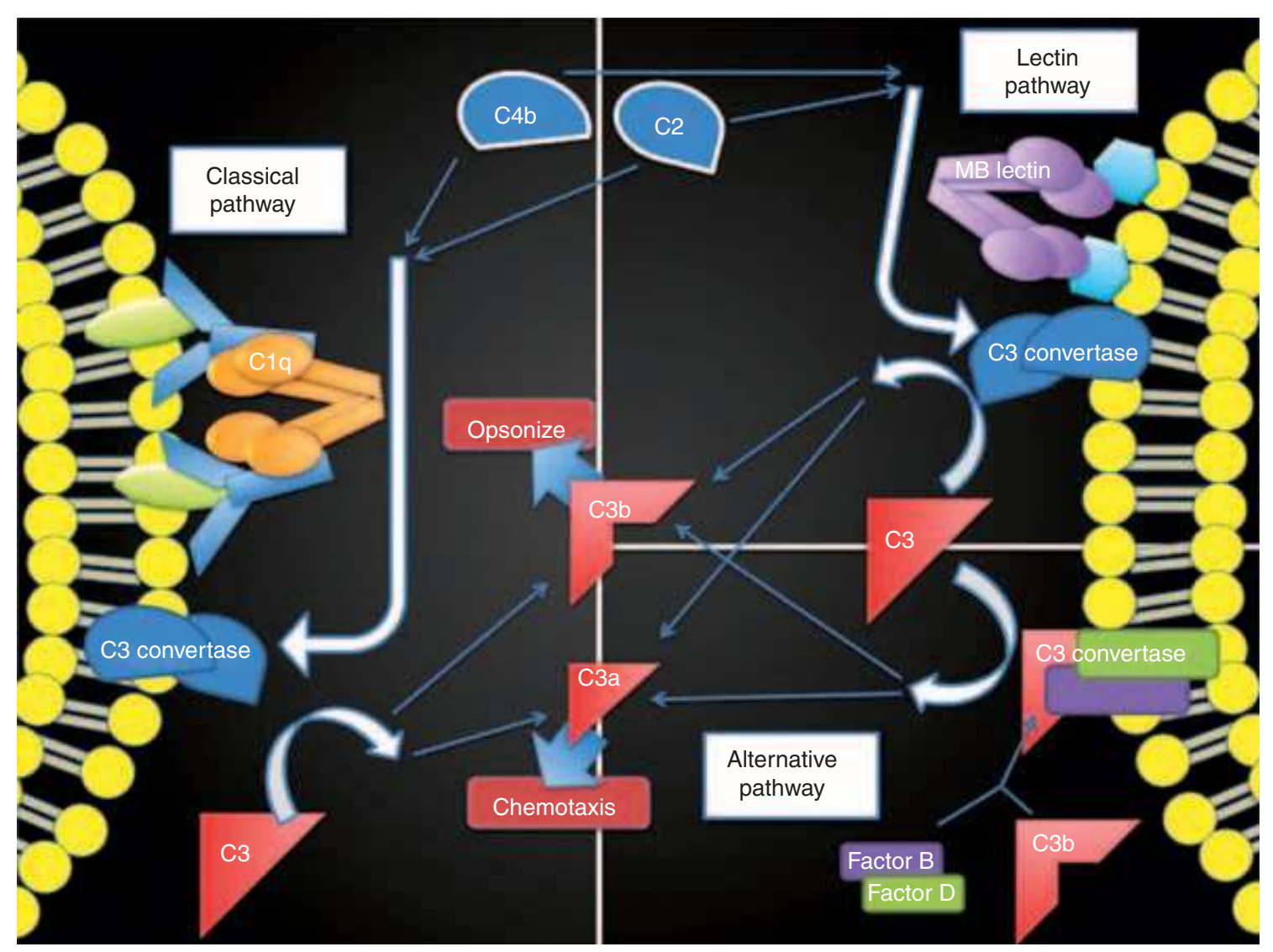

Figure 1 Complement pathways. All three pathways result in the formation of C3 convertase, which catalyzes C3 to split into C3b (binds pathogens) and C3a (chemotactic for neutrophils). Through the classical pathway, component $\mathrm{Clq}$ binds to antibodies on the bacterial cell surface, catalyzing $\mathrm{C} 4 \mathrm{~b}$ and $\mathrm{C} 2$ to combine into C3 convertase. Through the lectin pathway, mannose-binding lectin (MB lectin) binds mannose on the bacterial surface, catalyzing C4b and C2 to combine into C3 convertase. Through the alternative pathway, free factor C3b combines with factor B and factor D to form a C3 convertase. 
Table 2 Cells of the Innate and Adaptive Arms of the Immune System

Innate immune system

Epithelium

Macrophages

Neutrophils

NK cells

B-1 cells

$\gamma \delta$ T-cells

Adaptive immune system

Cytotoxic T-cell

T-helper (Th) cells

$\mathrm{Th}_{1}$

$\mathrm{Th}_{2}$

Treg

$\mathrm{Th}_{17}$

B-cell

Dendritic cell

Abbreviations: PRR, pattern recognition receptors; NK, natural killer.
Initial barrier to pathogens; present antigen; contain PRRs Immune sentinel; kill pathogens; present antigen; contain PRRs Immune foot soldiers; kill opsonized pathogens

Cytotoxic; kills infected cells in similar manner to cytotoxic T-cell Hybrid innate/adaptive B-cells; produce poly-reactive antibodies Hybrid innate/adaptive T-cells; T-cell receptors act like PRRs

Cytotoxic lymphocyte; directly kills infected cells

Cytokine-secreting lymphocytes; direct immune processes

Promotes inflammation; produces IFN $\gamma$ and IL-2

Promotes antibody responses; produces IL-4, IL-5, and IL-13

Suppresses inflammation; produces IL-10 and TGF $\beta$

Promotes inflammation; produces IL-17; recruits neutrophils

Antibody-secreting lymphocyte; directed by $\mathrm{Th}_{2}$ cells

Professional antigen-presenting cell; programs T-lymphocytes but are largely devoid of MCP and DAF after 8 weeks of gestation. Proliferation of this population to generate invasive cytotrophoblast cell columns is associated with continued expression of CD59 and an increased expression of DAF and MCP $(14,15)$. MCP is also expressed on the preimplantation embryo (16).

\section{Cytokines}

Cytokines are intracellular messengers of the immune response, triggering receptors on immune cells and local tissues that lead to downstream events such as cell differentiation, activation, and cytokine secretion. Chemokines—-derived from the words chemotactic cytokines-are cytokines that play a primary role in chemotaxis. They summon specific immune cell populations into a tissue to engage a pathogenic threat. Cytokines form the communication network for the immune system and are produced and utilized by cells in both arms. A list of commonly encountered cytokines, their cell of origin, and their common function is listed in Table 2.

\section{Macrophages}

Macrophages are innate sentinel cells that function as effector cells against certain pathogens. As an innate sentinel, the macrophage detects pathogens using surface receptors such as the mannose receptor, scavenger receptors (which bind many charged ligands), TLRs, and CD14, a TLR-4 co-receptor that recognizes bacterial lipopolysaccharide (LPS). Once activated, macrophages generate large cytoplasmic granules containing oxygen free radicals, antimicrobial peptides, and lysozyme that destroy phagocytized pathogens. They also secrete many important index cytokines and chemokines, such as interleukin (IL)-6, TNF, and IL-8.

The recognition of molecular patterns by sentinel cells should not be confused with antigen-initiated adaptive immune processes. The activation of the innate immune system does not directly lead to the development of immunologic memory. Activated sentinel cells release cytokines and chemokines. These cytokines and chemokines primarily recruit and activate innate effector cells, but they may also influence the process of antigen presentation to initiate adaptive processes (17).
Macrophages increase rapidly in number during early pregnancy, then remain relatively stable throughout gestation, representing $20 \%$ to $25 \%$ of CD $45+$ leukocytes and $10 \%$ of total decidual cells at the implantation site $(18,19)$. Villous macrophages have been demonstrated to secrete substances that promote the development of multinucleated syncytia from cytotrophoblast cells, which subsequently secrete human placental lactogen and human chorionic gonadotropin (hCG) (20).

Uterine macrophages are capable of presenting alloantigen and have a suppressive effect on mixed lymphocyte reactions and may contribute to protection of the fetus against immunologic rejection $(21,22)$. This may be at least in part due to expression of indoleamine 2,3-dioxygenase (IDO), which is suggested to have an immunosuppressive role in pregnancy. They exhibit lower IL-1 expression when compared with their peripheral counterparts, are capable of inducing interferon (IFN)- $\gamma$ expression by NK cells, and secrete IL-10 $(23,24)$.

Uterine macrophages may also regulate the population of uterine NK (uNK) cells, influencing differentiation and activation status through the production of IL-15, which is secreted by uterine macrophages isolated from both early and term gestations $(24,25)$.

\section{Neutrophils}

Innate effector cells include neutrophils, which are of myelocytic lineage. Neutrophils are hailed by activated macrophages using chemokines such as IL- 8 or indirectly by a population of "helper" T-cells through IL-17 secretion (26). Once neutrophils arrive, they rely on antibody binding and/or complement activation on the pathogen surface to direct chemotaxis and bind and phagocytose pathogens. Antibodies produced by B-lymphocytes tag (opsonize) pathogens for destruction. Neutrophils then use CD16, the Fc antibody receptor, to adhere to the opsonized pathogen.

The number of leukocytes in the peripheral blood increases during pregnancy, primarily due to an absolute increase in neutrophils (27). Peripheral neutrophils exhibit decreased lobularity index and myeloperoxidase activity at midgestation of normal pregnancy, suggesting a relative immaturity of the population as numbers increase (28). 
Both neutrophils $(29,30)$ and monocytes (31) display reduced chemotaxis, adherence, reactive oxygen metabolite release, phagocytosis, and microbial killing in pregnant compared with nonpregnant women. In seeming contradiction to this, neutrophils in pregnancy circulate in a chronically active state. Although pregnancy neutrophils cannot be fully activated for oxidant production (29), their basal rate of unstimulated oxidant production is the same as that of LPS-treated nonpregnancy neutrophils (30). Thus, while the maximum potential for activation of resting pregnancy neutrophils is suppressed, they circulate in a baseline-activated state.

Neutrophils have also been implicated in the process of cervical ripening and parturition $(32,33)$. Chemotaxis to the cervix and lower uterine segment are actively promoted by myometrial and cervical factors, chiefly by liberation of IL-8 and expression of cell adhesion molecules $(34,35)$. The resulting infiltration of neutrophils leads to a release of local inflammatory cytokines $(36,37)$ and proteolytic enzymes (38) that ripen the cervix and initiate parturition.

\section{NK Cells}

Innate effector cells include NK cells, which are of lymphocytic lineage. In contrast to other lymphocytes, NK cells do not possess the $\mathrm{CD} 3$ complex or clonally expand in response to antigen stimulation. NK cells do possess many activating and inhibitory receptors of two major structural classes, those of the immunoglobulin superfamily [killer immunoglobulin-like receptors (KIRs) and LIRs] and of the C-type lectin-like family
(Ly49, NKG2D, and CD94/NKG2) (39). These receptors recognize cell surface molecules, such as major histocompatibility complex (MHC)-1 glycoproteins and their structural relatives. The balance between activating and inhibiting signals determines the behavior of the NK cell. If the balance favors activation, the NK cell kills the target cell in much the same way as a cytotoxic T-lymphocyte, by forming an immunologic synapse, perforating the target cell membrane, and infusing granzyme into the cell, which initiates apoptosis of the target cell.

NK cell populations undergo marked changes in pregnancy and can be divided into two distinct subsets (Fig. 2). The majority (90\%) have low-density expression of CD56 (CD56 dim) and express high levels of CD16, the immunoglobulin receptor that is responsible for antibody-dependent cellular cytotoxicity. These are considered "peripheral" NK cells. The rest of NK cells (10\%), so-called uterine NK cells or large granular lymphocytes, have high-density expression of CD56 (CD56 bright) with low levels of CD16 (CD16 (-)) (40).

Peripheral NK cells are notably decreased in number in the decidua during normal pregnancy (41). Classic circulating CD57+/CD16+ NK cells have a $25 \%$ to $30 \%$ decrease in cytotoxicity during normal pregnancy (42-48). The decrease can be measured not only by decreases in cytotoxicity assays but also by a decrease in the expression of activation markers CD69, CD25, CD71, and HLA-DR (49). Though baseline cytotoxicity is lower, these peripheral NK cells are able to respond to infection with similar efficacy to nonpregnant controls (50).

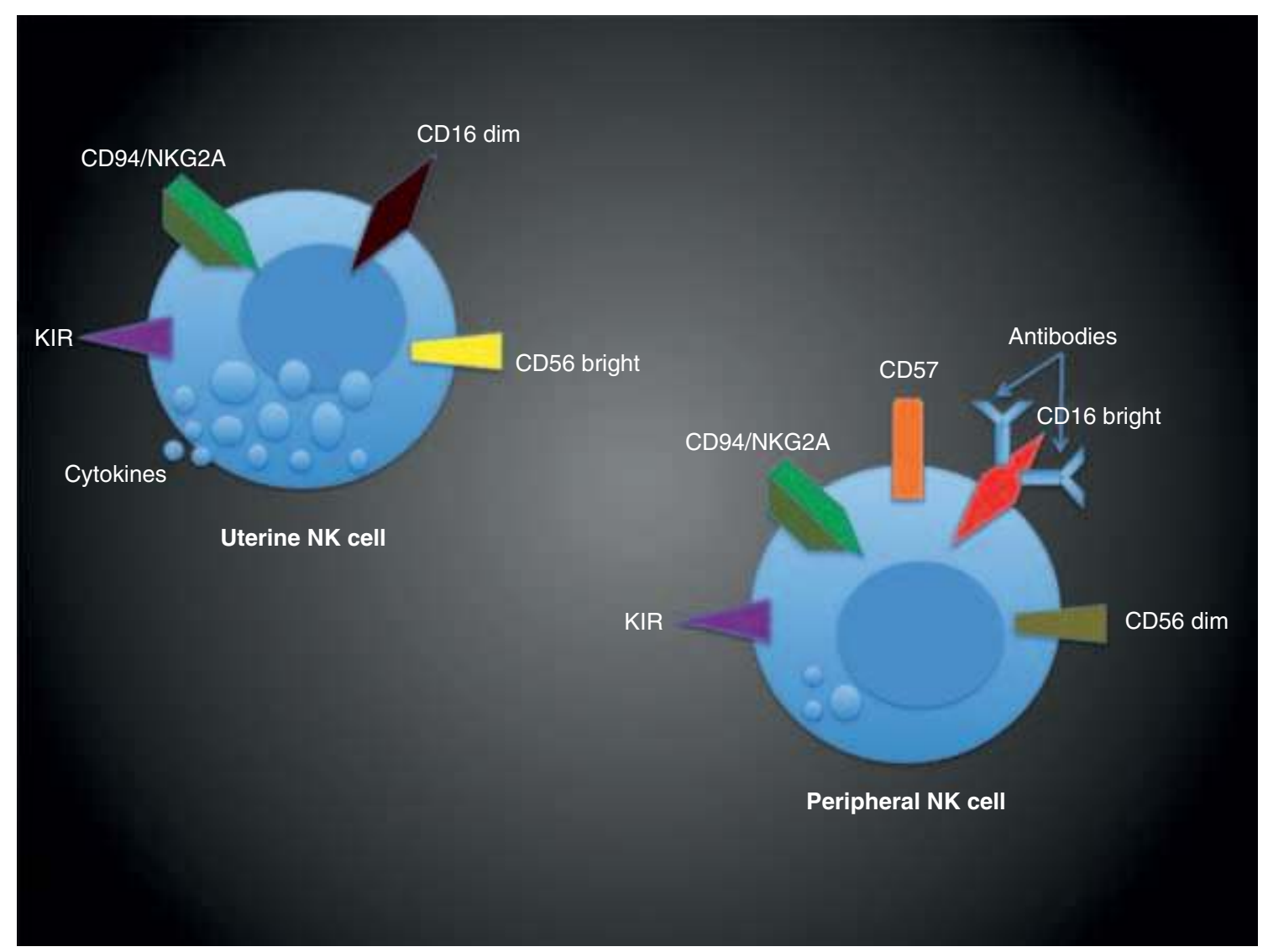

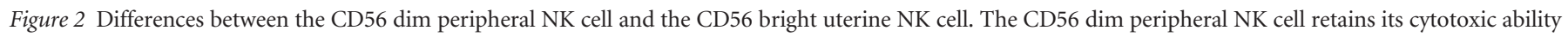

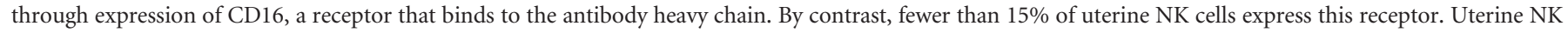
cells are also highly granulated and produce more cytokines than their peripheral counterparts, suggesting a regulatory function. 
Interestingly, peripheral NK cells maintain a nonpregnant phenotype during anembryonic gestations, ectopic pregnancies, and recurrent spontaneous abortions, all of which have been associated with increased NK activity $(42,51,52)$. Thus, classic NK appear to have a differing phenotype in normal versus abnormal pregnancies and could serve as potential effector cells during the elimination of abnormal pregnancy.

uNK cells with the phenotype CD56 bright/CD57-/CD16are actively recruited to the endometrium and decidua during the late secretory phase of menstruation and early normal pregnancy (53-56) and remain in the decidua if implantation is successful (57). These cells comprise $70 \%$ to $80 \%$ of decidual leukocytes, but less than $1 \%$ of peripheral blood leukocytes, existing side by side at the trophoblast margin with macrophages (20\% of leukocytes) and CD3+ T-cells (10\% of leukocytes) $(58,59)$. uNK cells possess the receptor for IL-15, which serves to activate them and promote their survival by increasing their expression of $b c l-2$ (60-63). IL-15 is expressed predominantly by the perivascular stromal cells surrounding spiral arteries during the mid to late secretory phase of the menstrual cycle and in early pregnancy (64).

uNK cells are considered to be poorly cytotoxic, yet potent cytokine producers, in contrast to classic NK cells that display higher levels of cytotoxicity but are poor cytokine producers. A small fraction of uNK cells (10-15\%) contain surface CD16 detectable by flow cytometry (65). CD16 is an antibody receptor, and only CD16+ cells exhibit cytotoxicity, and thus most uNK cells lack cytotoxic capability (66). All uNK cells remain at the implantation site in high numbers during early gestation, decrease after 20 weeks of gestation, and are practically absent in term decidua (67-69).

uNK cells are found in association with extravillous trophoblast in the placental bed (70). It has been suspected by some investigators that interactions between uNK cells and invading endovascular or perivascular trophoblast might promote spiral artery remodeling during human pregnancy. This suspicion is based on the close proximity of these uNK cells with spiral arteries and bolstered by placentation data from murine studies (71). However, in the human, trophoblast invasion along spiral arteries extends deeply into the inner myometrium, where uNK cells do not reside (72), and murine evidence that $\mathrm{uNK}$ cell production of IFN $\gamma$ promotes vascular remodeling remains to be established in the human (73). Thus, the role of uNK cells in the establishment of human hemochorial placentation is unclear.

\section{ADAPTIVE ARM OF THE IMMUNE SYSTEM}

The adaptive arm is the immune system's "intelligent" response to specific pathogens. This response is antigen dependent, occurring over weeks rather than minutes as in the innate response. The advantage of this more slowly evolving process is that it has time to "learn" through experience, developing a highly specialized, targeted response to specific antigens that results in the development of immunologic memory. The adaptive immune system consists of antigen-presenting cells, antibody-producing B-lymphocytes, "cytotoxic" CD8+ T-lymphocytes, and a variety of "helper" CD4+ T-lymphocytes. The primary factors determining the developmental fate of naive $\mathrm{T}$ - and B-lymphocytes include the type of antigen-presenting cell and the assortment of cytokines present in the local microenvironment. Cytokines that direct the specific adaptive response are primarily (but not exclusively) produced by T-helper (Th) lymphocytes and referred to as "Th" cytokines.

A core assortment of T-helper cells have been identified based on their cytokine secretion profiles, each "Th" type serving a different immunologic function. The four most commonly described types of T-helper cells are the $\mathrm{Th}_{1}, \mathrm{Th}_{2}$, Treg, and $\mathrm{Th}_{17}$ lymphocytes (Table 1 ).

$\mathrm{Th}_{1}$ lymphocytes promote cytotoxic cellular and antibodymediated responses through secretion of the $\mathrm{Th}_{1}$ cytokine IFN $\gamma$. IFN $\gamma$ activates effector cells such as "cytotoxic" T-lymphocytes, NK cells, and macrophages. It also promotes the production of dendritic cell IL-12, which in turn stimulates $\mathrm{Th}_{1}$ cells to auto-produce IFN $\gamma$, reinforcing the phenotype. IFN $\gamma$ inhibits the $\mathrm{Th}_{2}$ phenotype by downregulating IL-4 production. IFN $\gamma$ upregulates MHC class I antigen on target cell surfaces and activates CD8+ cytotoxic T-cells and NK cells, the sum of which promotes the recognition and clearance of intracellular pathogens.

$\mathrm{Th}_{2}$ lymphocytes promote humoral responses by B-cells through secretion of $\mathrm{Th}_{2}$ cytokines such as IL-4 and IL-5. These humoral responses result in the development of antibodies that target humoral factors for removal rather than cells for destruction. The presence of other local cytokines promotes antibody isotype switching. For example, IL-13 promotes switching to the IgE antibody class, TGF $\beta$ promotes switching to the IgA class, and the $\mathrm{Th}_{1}$ cytokine IFN $\gamma$ promotes switching to the $\operatorname{IgG}$ class.

Regulatory T-lymphocytes (Tregs) have been one of the more recently characterized helper-T-cell subsets. These cells are identified by the presence of a nuclear transcription factor called Forkhead box P3 (FoxP3) and characterized by their suppressive effect on effector T-cells, in part due to secretion of cytokines IL-10 and TGF $\beta$. IL-10 antagonizes $\mathrm{Th}_{1}$ cells and macrophages, while TGF $\beta$ is a potent suppressor of all other Th responses $\left(\mathrm{Th}_{1} / \mathrm{Th}_{2} / \mathrm{Th}_{17}\right)$ in the human. Tregs secrete TGF $\beta$ and/or IL-10 in response to specific antigen and are believed to be an important resident of the maternal-fetal interface necessary for suppressing any adaptive immune responses against the fetus.

$\mathrm{Th}_{17}$ lymphocytes are the most recently characterized class of T-helper cells. $\mathrm{Th}_{17}$ cells secrete cytokines including IL-17A, important for recruiting neutrophils to sites of inflammation, which makes them appear to function similarly to $\mathrm{Th}_{1}$ cells. Both IFN $\gamma$ and IL-4 have been shown to negatively regulate $\mathrm{Th}_{17}$ differentiation, while the presence of TGF $\beta$ and IL-6 together appear to promote this differentiation. $\mathrm{Th}_{17}$ cells appear to play an important role in autoimmune diseases, but if they play a role in inflammatory states during pregnancy, such as preterm labor and pre-eclampsia, this has not yet been defined.

\section{T-Helper (Th) Shift in Pregnancy}

Pregnancy has classically been described as having a " $\mathrm{Th}_{2}$ shift" $(74,75)$. In general terms, the systemic maternal immune response is noted to shift from a cytotoxic $\left(\mathrm{Th}_{1}\right)$ to a humoral $\left(\mathrm{Th}_{2}\right) \mathrm{T}$-helper paradigm of function. In light of evolving knowledge of T-helper-cell subsets, the $\mathrm{Th}_{1} / \mathrm{Th}_{2}$ balance paradigm has been recognized as an oversimplification $(76,77)$. 
It has become clear that additional regulatory mechanisms play an important role, including those mediated by the regulatory T-cell (Treg). As potent suppressors of inflammation, Tregs appear to be important for the maintenance of normal pregnancy (78).

If the shifting of immune cell subsets to a regulatory phenotype contributes to fetal protection, this may aid in our understanding of the observed maternal tolerance acquired against paternal antigens $(79,80)$. Innate processes also appear to promote local blunting of the adaptive immune system at the maternal fetal interface, including such factors as depletion of tryptophan at the maternal-fetal interface by IDO (81), and the expression of nonclassical placental MHC antigens such as HLA-G. It has been suggested that HLA-G might be an important determinant in the development of Tregs $(82,83,84)$.

\section{Peripheral T-Cells}

Pregnancy is accompanied by several unique changes in peripheral and decidual T-cells. Peripheral T-cells demonstrate decreased proliferative responses in serum from pregnant women, detected as a decreased T-cell response to mitogens (85). This may be due to a general state of suppression during pregnancy, as evidenced by a decrease in memory CD4+ T-cells and an increase in naive CD4+ T-cells, decreased expression of activation markers HLA-DR and $\mathrm{CD} 25$, and a decrease in precytotoxic $\mathrm{CD} 8+/ \mathrm{S} 6 \mathrm{~F} 1+$ cells with a concomitant increase in regulatory CD8+/S6F1-cells (86).

Peripheral blood CD4+/CD25+ lymphocytes, the putative regulatory $\mathrm{T}$-cell subset, are increased during the first and second trimesters (87).

\section{Uterine T-Cells}

Uterine T-cell changes are unique. T-cells comprise only about $10 \%$ of decidual lymphocytes $(88,89)$ and decrease in decidua by $>60 \%$ during pregnancy. This is due primarily to a decrease in CD8+ T-cells thereby increasing the CD4+/CD8+ ratio (90). Of the CD8+ T-cells in the decidua only a few express perforin, a suppressive phenotype among CD8+ T-cells (91).

Uterine decidual $\mathrm{T}$-cells have been reported to express multiple KIRs including both inhibitory and activating types. When both are expressed in a single T-cell, the inhibitory receptor has been suggested to be preferentially active and may contribute a level of immunosuppression (92).

Uterine decidual T-cells have been characterized as expressing CD25, a chief phenotypic characteristic shared by activated lymphocytes and Treg cells $(93,94)$. Up to $14 \%$ of uterine T-cells in the decidua possess a classic Treg phenotype (87). There is evidence for local expansion of decidual Treg cells and a low occurrence of $\mathrm{Th}_{17}$ cells in normal first-trimester decidua $(95,96)$. Furthermore, local moderate $\mathrm{Th}_{1}$ activity is reported, evidenced by a population of IFN $\gamma$-secreting T-cells. Local IFN $\gamma$ could serve the important purpose of upregulating MHC class II proteins on decidual stromal cells. Decidual stromal cells express ligands (B7-H1 and B7-DC) for the programmed death (PD)-1 receptor, which may play a crucial role by decreasing decidual T-helper cell production of IFN $\gamma$, TNF, and IL-2 (97).

During normal and anembryonic pregnancy, but not ectopic pregnancy, CD71 and CD25 increase, signifying activation of T-cells in response to successful implantation. The response may be augmented by the trophoblast production of IL-2, which, because of the lack of change in ectopic pregnancy, is probably decidua dependent $(98,99)$. Further evidence that T-cells are specifically involved with implantation and placental proliferation is derived from experiments with monoclonal antibodies against CD4 and CD8, which stimulates or inhibits placental proliferation, respectively (100).

\section{$\gamma \delta \mathrm{T}-$ Cells}

$\gamma \delta \mathrm{T}$-cells are an interesting intermediate cell type that appear to have both innate and adaptive function. They rearrange $\mathrm{T}$ cell receptor (TCR) genes to produce diversity and can develop a memory phenotype. However, various subsets contain a restricted TCR that appears be used as a PRR (101). These cells recognize not only nonclassical MHC class I molecules (102) but also heat shock proteins, soluble antigen, and carbohydrates (103).

$\gamma \delta \mathrm{T}$-cells appear in the first-trimester decidua $(104,105)$ and increase in the peripheral blood during pregnancy (106). They are also present in second-trimester placental villi (107). $\gamma \delta$ T-cells increase 2.4-fold in the decidua compared with peripheral blood, which is indicative of selective migration (105), and are twice as common as all other T-cells there (108), although the specific mechanism for their selection is unknown.

The specific function of $\gamma \delta \mathrm{T}$-cells is not clearly known. Because of their population kinetics and location, several roles have been proposed. They may protect the mother and fetus from infection, trophoblast from rejection, or limit passage of fetal leukocytes to the maternal circulation (105).

\section{B-Cells}

Maternal serum has been found to inhibit priming of B-cells in vitro via a molecule in the IgG fraction of serum, which reduces IL-2 receptor density. This inhibitory action is even more pronounced with retroplacental serum suggesting that it may be concentrated at the maternofetal interface (109).

Peripheral B-cells in pregnancy exhibit decreased synthesis of IgM with increases in IgA and IgE. As IgA and IgE do not cross the placenta, these changes may be a protective mechanism for the fetus. The increase of IgE and IgA (with concomitant decrease in IgM) may be explained, at least in part, by an increase in $\mathrm{CD} 23$ (low-affinity $\mathrm{IgE}$ receptor) expression on B-cells, which serves as an autocrine growth factor for pre-activated B-cells and is enhanced in the presence of maternal IgG (109).

The importance of these changes is illustrated by the fact that B-cell antibody synthesis can be implicated in pregnancy complications and spontaneous abortions. Antitrophoblastic IgG and IgM, which were better able to fix complement and lyse target cells than in controls, have been demonstrated in significantly higher numbers in patients with recurrent spontaneous abortions (110). Antibodies against fetal endothelial cells have also been demonstrated in patients with recurrent spontaneous abortion (111).

Modulation of B-cells also occurs with regard to B-cell subsets. B-1 (CD5+) cells are downregulated during pregnancy while numbers of conventional B-cells (CD5-) remain constant. B-1 cells are the main producers of polyreactive 
autoantibodies (112) and this antibody production can be enhanced in the presence of estrogen (in mice) (113). This downregulation may thus represent a protective mechanism for the fetus and the mother. During pregnancy, B-cells are generally either absent from or inactive in the uterus and decidua $(114,115)$.

\section{EFFECT OF PREGNANCY ON MATERNAL CELL- MEDIATED IMMUNITY}

Cell-mediated immunity is promoted by IFN $\gamma$, is carried out by CD8+ T-lymphocytes and NK cells, and results in the programmed cell death (apoptosis) of the foreign cell or infected host cell. Decreased cell-mediated immune function in pregnancy is manifested by a tendency toward reactivation of chronic viral infections, such as cytomegalovirus (116), human papillomavirus (117), and herpes simplex virus (HSV) (118), and by an increased frequency in acquiring new viral infections (119). Circulating NK cells maintain their population size (120), and though they express a general decrease in cytotoxic activity (121-127), they can still respond appropriately to pathogens (128).

Pregnant women are more prone to contract HSV (129), have a longer course of infection (130), and suffer increased morbidity $(131,132)$. Prior to polio vaccine, the risk of paralytic polio was increased in pregnancy (133). Malaria has been shown to have a prolonged course as well as increased morbidity and mortality during pregnancy (134). Varicella pneumonia has also been noted to occur almost exclusively in pregnant females or those otherwise immunosuppressed (135).

Impairment in cell-mediated immune function in pregnancy is associated with improvement in autoimmune diseases such as multiple sclerosis $(136,137)$, rheumatoid arthritis (138), and psoriasis (139).

\section{EFFECT OF PREGNANCY ON MATERNAL HUMORAL IMMUNITY}

In contrast to cell-mediated immunity, humoral immunity, promoted by $\mathrm{Th}_{2}$ cytokines such as IL- 4 and IL-5, is preserved in pregnancy. Circulating B-cell numbers are unchanged $(140,141)$. T-cell-independent B-cell functions remain intact as manifested by normal IgG stimulation with Staphylococcus aureus (142). There is an appropriate antibody response to vaccines (143). Serum complement concentrations remain normal or slightly elevated (11). T- helper cell activity is intact, evidenced by the maintenance of PPD positivity during normal pregnancy (144).

The shift favoring antibody production may help explain the persistence or worsening of immune-complex-mediated autoimmune disease in pregnancy. While the development of a de novo lupus flare during pregnancy may not frequently occur, exacerbation of existing lupus nephritis can be problematic. Grave's disease, seasonal allergies, myasthenia gravis, idiopathic thrombocytopenic purpura, and IgA nephropathy-antibody-dependent diseases-worsen.

The key difference between the pregnant and nonpregnant states is in the development and function of the maternalplacental-fetal unit. Trophoblast-immune cell interactions result in the differentiation of specialized subpopulations of lymphocytes. Moreover, peptide and steroid hormones generated by the placenta have demonstrable effects on immune cells.

\section{INFLUENCE OF PLACENTAL HORMONES ON IMMUNITY}

Placental hormones appear to play a role in immune function during pregnancy. The hCG can inhibit mitogen-induced Tcell and T-cell-dependent B-cell responses (145-149). These actions appear to be accomplished directly through T-cell hCG receptors (150), but further delineation of this mechanism is still the subject of ongoing investigation.

Pregnancy induces thymic cortical involution, possibly contributing a mechanistic explanation for altered T-cell function $(151,152)$. The view that such involution represents a quiescence of the gland is in conflict with the data that the medullary portion of the gland actually appears to become more active (153). Murine thymic epithelial cells express the progesterone receptor, and this receptor is necessary for thymic involution to occur in pregnancy in mice (154).

Progesterone appears to be a very important immunoregulatory hormone of pregnancy, but the pathways by which it mediates its effect are still unclear. It is unlikely that specific progesterone receptors on lymphocytes and NK cells exist, as they have not yet been identified $(155,156)$. However, decidual stromal cells have progesterone receptors, and a mechanistic link between these cells and various leukocytes has been suggested (157).

Despite lack of evidence of classic progesterone receptors in these cells, T-lymphocytes and decidual NK cells are reported to produce a peptide called progesterone-induced blocking factor (PIBF) in response to this hormone (158-160). PIBF has been reported to decrease activated lymphocyte production of IL-12 and TNF-alpha and cause a concomitant increase in IL-10 (161). PIBF is also reported to inhibit perforin liberation from the granules of stimulated NK cells (162), thereby limiting their cytotoxicity. The PIBF receptor has been characterized as joining with the IL-4 receptor alpha chain to form a novel type of IL-4 receptor (163).

Decreased levels of PIBF have been associated with recurrent spontaneous abortion (164), and monoclonal antibodies against PIBF have been demonstrated to cause decreased IL-10 levels with an associated immune-mediated resorption of pregnancies in mice (165).

Administration of the progesterone receptor antagonist RU486 has been reported to cause a decrease in the production of PIBF (166) and to be associated with at least a threefold increase in NK cytotoxicity $(167,168)$.

TROPHOBLAST DEFENSE: ALTERED MHC EXPRESSION An interesting mechanism of protection of the fetal allograft from maternal defenses appears to be the altered expression of MHC class I molecules by both syncytiotrophoblast and cytotrophoblast. All trophoblast cells are devoid of "classical" MHC class I molecules, except for the transient expression of HLA-C during early pregnancy. This inhibition of expression of HLA-A and HLA-B is apparently not affected by genomic imprinting, since both maternal and paternal alleles are inducible by IFN $\gamma$ treatment in vitro (169).

In addition, there is an absence of expression of MHC class II antigens, which appears to be caused by hypermethylation of the IFN $\gamma$-responsive promoter that regulates expression of the class II transactivator (CIITA) gene. In vitro introduction of exogenous CIITA can induce cell surface expression of HLA- 
$\mathrm{DR},-\mathrm{DP}$, and -DQ, leading to an acquired capacity to present antigen to antigen-specific T-helper cells (170).

Once formation of the cytotrophoblastic shell is complete, the syncytiotrophoblasts and villous cytotrophoblasts remain free of classical MHC class I molecules and downregulate the expression of the nonclassical MHC class I molecules. The invasive cytotrophoblasts, on the other hand, provide the primary contact point for maternal blood and continue to express an assortment of nonclassical MHC class I antigens, which may be helpful in deterring a maternal immune response toward paternal antigens.

Nonclassical MHC molecules that are present throughout pregnancy include HLA-E and HLA-G. Like classical MHC antigens, these molecules appear to be involved in the abrogation of NK-cell-mediated lysis of trophoblast via interactions with inhibitory NK cell receptors (171-173).

Many studies have focused on HLA-G, first discovered in 1987 (174). It is an inhibitory nonclassical MHC class I molecule that is found on both invasive cytotrophoblastic and amniotic epithelial cells (175). Like the classical MHC class I molecules, it comprises an alpha chain associated with $\beta 2$ microglobulin. Unlike the classical MHC class I molecules, HLA-G contains limited polymorphism and a truncated cytoplasmic tail as a result of a stop codon in exon 5/6. It can appear in 6 isoforms: two soluble and four membrane bound.

The membrane-bound isoforms have been shown to inhibit NK cell migration and NK-mediated cell lysis through interactions with various lectin family and immunoglobulinlike receptors on NK cells and macrophages (176-178). HLA$\mathrm{G}$ binds the CD94/NKG2A and p49 receptors, one or both of which are found on all $\mathrm{NK}$ cells isolated from maternal decidua during the first trimester (179). It may also bind the ILT2 and ILT4 (immunoglobulin-like transcript) inhibitory receptor on macrophages (180).

Soluble isoforms of HLA-G may serve an immunoregulatory role during pregnancy. Soluble HLA-G appears to induce apoptosis of CD8+ T-cells through the Fas/FasL pathways (181), and a low concentration is detectable in the serum of pregnant women throughout gestation. Although soluble HLA-G is present only in small amounts, it is more than twice as abundant in the serum of pregnant women as in nonpregnant and is likely in even greater concentration at the maternal-fetal interface (182).

HLA-E is not found on the cell surface unless HLA-G is also present. It is co-expressed with HLA-G due to a specific nonapeptide transcribed from the leader sequence of HLA-G, which serves as a ligand for HLA-E to induce its surface expression $(183,184)$. HLA-E appears to have similar characteristics to HLA-G, for example, binding CD94/NKG2A $(185,186)$.

HLA-G and HLA-E have both been demonstrated to influence cytokine production by decidual NK cells. Decidual NK cells cocultured with K-562 leukemia cells lacking classical and nonclassical MHC molecules expressed TNF-alpha, IL-10, and GM-CSF. When the K-562 cells were transfected with HLA-G, these concentrations of these three cytokines were significantly decreased in culture supernatants. When this same cell line was transfected with HLA-E, only IL-10 was significantly decreased (187).
HLA-C has been shown to exist only transiently on trophoblasts in the first trimester. It is recognized by KIRs (killer immunoglobulin-like or inhibitory receptors) on NK cells. Decidual NK cells have a higher expression of KIRs specific for HLA-C than do peripheral NKs. HLA-C is also upregulated in the presence of IFN $\gamma$, which may serve as a protective mechanism for trophoblastic cells (188). Expression of HLA-C apart from the pregnant state has been shown to be deleterious in some circumstances, as expression of HLA-Cw4 has been associated with poor prognosis in HIV infection and rapid development of AIDS, suggesting potential immunosuppressive functions (189).

Because of these functional differences from other "classical" MHC class I molecules, some investigators have suggested that, from a conceptual standpoint, HLA-C may be better grouped with the "nonclassical" MHC class I molecules (190).

\section{TROPHOBLAST DEFENSE: OTHER MECHANISMS}

In addition to the nonclassical MHC molecules, the trophoblast expresses other antigens that may counter maternal immunosurveillance. The Fas ligand (FasL) binds to Fas expressed on target cells and results in the initiation of the target cell apoptosis pathway. Trophoblast constitutively expresses the FasL, while decidual NK cells express Fas. IFN $\gamma$ and TNF promote Fas expression and sensitivity, whereas IL-6 and IL-10 increase the resistance of trophoblast cells to Fasmediated apoptosis (191).

RCAS1 (receptor-binding cancer antigen expressed on SiSo cells), a membrane-associated homodimer, has been recognized to play a role in immune evasion of the tumor cells in a manner similar to the FasL (192). Both RCAS1 and the FasL appear to be expressed concomitantly in uterine endometrial glands and extravillous cytotrophoblasts (193).

IDO is an enzyme that catabolizes tryptophan and is expressed by syncytiotrophoblast in mice (194). In the murine model, it is released by macrophages in response to IFN $\gamma$, inhibits T-cell proliferation, and is able to prevent T-cellmediated rejection of normal pregnancies (194,195). IDO has also been demonstrated in human placental trophoblast cells and decidual macrophages, where its expression is stimulated by IFN $\gamma(196,197)$. The expression of IDO by syncytiotrophoblast is a likely contributor to decreased serum levels of tryptophan found during pregnancy (198).

\section{THE PLACENTAL SINK}

The apical surface of the syncytiotrophoblast contains specialized $F c(\gamma)$ receptors $(F c \gamma R)(199,200)$. Significant transport of the four classes of maternal IgG begins around 20 weeks of gestation. The placenta may also serve as an immunosorbent tissue to protect the fetus from antibodies directed toward paternal antigens (201).

\section{CONCLUSION}

In conclusion, research over the past three decades has identified a host of changes in the maternal immune system and fetal tissues that promote the initiation and maintenance of normal pregnancy. It is daunting to think that what is now known is probably the tip of the iceberg. However, through further study, the immunology of the maternal-fetal interface may hold the secrets of successful and healthy pregnancy. 


\section{REFERENCES}

1. Gilmore W, Arias M, Stroud N, et al. Preliminary studies of cytokine secretion patterns associated with pregnancy in MS patients. J Neurol Sci 2004; 224: 69-76.

2. Ruiz-Irastorza G, Lima F, Alves J, et al. Increased rate of lupus flare during pregnancy and the puerperium: a prospective study of 78 pregnancies. Br J Rheumatol 1996; 35: 133-8.

3. Ostensen M, Fuhrer L, Mathieu R, Seitz M, Villiger PM. A prospective study of pregnant patients with rheumatoid arthritis and ankylosing spondylitis using validated clinical instruments. Ann Rheum Dis 2004; 63: $1212-17$.

4. Ostensen M, Villiger PM. Immunology of pregnancy-pregnancy as a remission inducing agent in rheumatoid arthritis. Transpl Immunol 2002; 9: 155-60.

5. Medawar PB. Society for Experimental Biology, Evolution Symposium. Some immunological and endocrinological problems raised by the evolution of viviparity in vertebrates. Volume 7. New York, NY, USA: Academic Press, 1953: 320-8.

6. Wallace PK, Yeaman GR, Johnson K, et al. MHC class II expression and antigen presentation by human endometrial cells. J Steroid Biochem Mol Biol 2001; 76: 203-11.

7. Jorgenson RL, Young SL, Lesmeister MJ, Lyddon TD, Misfeldt ML. Human endometrial epithelial cells cyclically express Toll-like receptor 3 (TLR3) and exhibit TLR3-dependent responses to dsRNA. Hum Immunol 2005; 66: 469-82.

8. Koga K, Mor G. Expression and function of toll-like receptors at the maternal-fetal interface. Reprod Sci 2008; 15: 231-42.

9. Walport MJ. Complement. First of two parts. N Engl J Med 2001; 344 1058-66.

10. Walport MJ. Complement. Second of two parts. N Engl J Med 2001; 344: 1140.

11. Johnson U, Gustavii B. Complement components in normal pregnancy. Acta Pathol Microbiol Immunol Scand 1987; 95: 97-9.

12. Cunningham DS, Tichenor JR Jr. Decay-accelerating factor protects human trophoblast from complement-mediated attack. Clin Immunol Immunopathol 1995; 74: 156-61.

13. Atkinson JP, Farries TF. Separation of self from non-self in the complement system. Immunol Today 1987; 8: 212-15.

14. Holmes $\mathrm{CH}$, Simpson $\mathrm{KL}$, Okada $\mathrm{H}$, et al. Complement regulatory proteins at the feto-maternal interface during human placental development: distribution of CD59 by comparison with membrane cofactor protein (CD46) and decay accelerating factor (CD55). Eur J Immunol 1992; 22: 1579-85.

15. Nishikori K, Noma J, Hirakawa S, Amano T, Kudo T. The change of membrane complement regulatory protein in chorion of early pregnancy. Clin Immunol Immunopathol 1993; 69: 167-74.

16. Roberts JM, Taylor CT, Melling GC, Kingsland CR, Johnson PM. Expression of the CD46 antigen, and absence of class I MHC antigen, on the human oocyte and preimplantation blastocyst. Immunology 1992; 75: 202-5.

17. Liu G, Zhang L, Zhao Y. Modulation of immune responses through direct activation of Toll-like receptors to $\mathrm{T}$ cells. Clin Exp Immunol 2010; 160: 168-75.

18. Kabawat SE, Mostoufi-Zadeh M, Driscoll SG, Bhan AK. Implantation site in normal pregnancy. A study with monoclonal antibodies. Am J Pathol 1985; 118: 76-84.

19. Vince GS, Starkey PM, Jackson MC, Sargent IL, Redman CW. Flow cytometric characterisation of cell populations in human pregnancy decidua and isolation of decidual macrophages. J Immunol Methods 1990; 132: 181-9.

20. Khan S, Katabuchi H, Araki M, Nishimura R, Okamura H. Human villous macrophage-conditioned media enhance human trophoblast growth and differentiation in vitro. Bio Reprod 2000; 62: 1075-83.

21. Heikkinen J, Möttönen M, Komi J, Alanen A, Lassila O. Phenotypic characterization of human decidual macrophages. Clin Exp Immunol 2003; 131: 498-505.

22. Nagamatsu T, Schust DJ. The immunomodulatory roles of macrophages at the maternal-fetal interface. Reprod Sci 2010; 17: 209-18.

23. Marzusch K, Buchholz F, Ruck P, et al. Interleukin-12- and interleukin2-stimulated release of interferon-gamma by uterine CD56++ large granular lymphocytes is amplified by decidual macrophages. Hum Reprod 1997; 12: 921-4.
24. McIntire RH, Ganacias KG, Hunt JS. Programming of human monocytes by the uteroplacental environment. Reprod Sci 2008; 15: 437-47.

25. Kitaya K, Yasuda J, Yagi I, et al. IL-15 expression at human endometrium and decidua. Biol Reprod 2000; 63: 683-7.

26. Fouser LA, Wright JF, Dunussi-Joannopoulos K, Collins M. Th17 cytokines and their emerging roles in inflammation and autoimmunity. Immunol Rev 2008; 226: 87-102.

27. Pitkin RM, Witte DL. Platelet and leukocyte counts in pregnancy. JAMA 1979; 242: 2696-8.

28. Tsakonas DP, Tsakona CP, Worman CP, Goldstone AH, Nicolaides KH. Myeloperoxidase activity and nuclear segmentation of maternal neutrophils during normal pregnancy. Clin Lab Haematol 1994; 16: 337-42.

29. Crouch SP, Crocker IP, Fletcher J. The effect of pregnancy on polymorphonuclear leukocyte function. J Immunol 1995; 155: 5436-43.

30. Kindzelskii AL, Huang JB, Chaiworapongsa T, et al. Pregnancy alters glucose-6-phosphate dehydrogenase trafficking, cell metabolism, and oxidant release of maternal neutrophils. J Clin Invest 2002; 110: 1801-11.

31. Luft BJ, Remington JS. The adverse effect of pregnancy on macrophage activation. Cell Immunol 1984; 85: 94-9.

32. Winkler M, Fischer DC, Ruck P, et al. Parturition at term: parallel increases in interleukin- 8 and proteinase concentrations and neutrophil count in the lower uterine segment. Hum Reprod 1999; 14: 1096-100.

33. Osman I, Young A, Ledingham M, et al. Leukocyte density and proinflammatory cytokine expression in human fetal membranes, decidua, cervix and myometrium before and during labour at term. Mol Hum Reprod 2003; 9: 41-5.

34. Barclay CG, Brennand JE, Kelly RW, Calder AA. Interleukin-8 production by the human cervix. Am J Obstet Gynecol 1993; 169: 625-32.

35. Ledingham M, Thomson A, Jordan F, et al. Cell adhesion molecule expression in cervix and myometrium during pregnancy and parturition. Obstet Gynecol 2001; 97: 235-42.

36. Athayde N, Romero R, Maymon E, et al. A role for the novel cytokine RANTES in pregnancy and parturition. Am J Obstet Gynecol 1999; 181: 989-94.

37. Young A, Thomson A, Ledingham M, et al. Immunolocalization of proinflammatory cytokines in myometrium, cervix and fetal membranes during human parturition at term. Biol Reprod 2002; 66: 445-9.

38. Rath W, Winkler M, Kemp B. The importance of extracellular matrix in the induction of preterm delivery. J Perinat Med 1998; 26: 437-41.

39. Natarajan K, Dimasi N, Wang J, Mariuzza RA, Margulies DH. Structure and function of natural killer cell receptors: multiple molecular solutions to self, nonself discrimination. Annu Rev Immunol 2002; 20: 853-85.

40. Cooper MA, Fehniger TA, Caligiuri MA. The biology of human natural killer-cell subsets. Trends Immunol 2001; 22: 633.

41. Ho HN, Chao KH, Chen CK, Yang YS, Huang SC. Activation status of $\mathrm{T}$ and NK cells in the endometrium throughout menstrual cycle and normal and abnormal early pregnancy. Hum Immunol 1996; 49: 130-6.

42. Chao KH, Yang YS, Ho HN, et al. Decidual natural killer cytotoxicity decreased in normal pregnancy but not in anembryonic pregnancy and recurrent spontaneous abortion. Am J Reprod Immunol 1995; 34: 274-80.

43. Gonik B, Loo LS, West S, Kohl S. Natural killer cell cytotoxicity and antibody-dependent cellular cytotoxicity to herpes simplex virusinfected cells in human pregnancy. Am J Reprod Immunol Microbiol 1987; 13: 23-6.

44. Toder V, Blank M, Gleicher N, et al. Activity of natural killer cells in normal pregnancy and edema-proteinuria-hypertension gestosis. Am J Obstet Gynecol 1983; 145: 7-10.

45. Vaquer S, de la Hera A, Jorda J, et al. Diminished natural killer activity in pregnancy: modulation by interleukin 2 and interferon gamma. Scand J Immunol 1987; 26: 691-8.

46. Baley JE, Schacter BZ. Mechanisms of diminished natural killer cell activity in pregnant women and neonates. J Immunol 1985; 134: 3042-8.

47. Okamura K, Furukawa K, Nakakuki M, Yamada K, Suzuki M. Natural killer cell activity during pregnancy. Am J Obstet Gynecol 1984; 149: 396-9.

48. Eriksen NL, Gonik B, Loo LS. Natural killer cell cytotoxicity to herpes simplex virus-1-infected cells is not altered by pregnancy. Am J Obstet Gynecol 1991; 165: 965-8. 
49. Tsakonas DP, Tsakona CP, Worman CP, Goldstone AH, Nicolaides KH. Myeloperoxidase activity and nuclear segmentation of maternal neutrophils during normal pregnancy. Clin Lab Haematol 1994; 16: 337-42.

50. Eriksen NL, Gonik B, Loo LS. Natural killer cell cytotoxicity to herpes simplex virus-1-infected cells is not altered by pregnancy. Am J Obstet Gynecol 1991; 165: 965-8.

51. Vassiliadou N, Bulmer JN. Immunohistochemical evidence for increased numbers of 'classic' CD57+ natural killer cells in the endometrium of women suffering spontaneous early pregnancy loss. Hum Reprod 1996; 11: 1569-74.

52. Aoki K, Kajiura S, Matsumoto Y, et al. Preconceptional natural-killercell activity as a predictor of miscarriage. Lancet 1995; 345: 1340-2.

53. Starkey PM, Sargent IL, Redman CW. Cell populations in human early pregnancy decidua: characterization and isolation of large granular lymphocytes by flow cytometry. Immunology 1988; 65: 129-34.

54. Ritson A, Bulmer JN. Isolation and functional studies of granulated lymphocytes in first trimester human decidua. Clin Exp Immunol 1989; 77: 263-8.

55. King A, Wellings V, Gardner L, Loke YW. Immunocytochemical characterization of the unusual large granular lymphocytes in human endometrium throughout the menstrual cycle. Hum Immunol 1989; 24: 195-205.

56. King A, Balendran N, Wooding P, Carter NP, Loke YW. CD3leukocytes present in the human uterus during early placentation: phenotypic and morphologic characterization of the CD56++ population. Dev Immunol 1991; 1: 169-90.

57. Ho HN, Chao KH, Yang YS, Huang SC. Activation status of T and NK cells in the endometrium throughout the menstrual cycle and normal and abnormal early pregnancy. Hum Immunol 1996; 49: 130-6.

58. King A, Wellings V, Cardner L, Loke YW. Immunocytochemical characterization of the unusual large granular lymphocytes in human endometrium throughout the menstrual cycle. Hum Immunol 1989; 24: 195-205.

59. Bulmer JN. Decidual cellular responses. Curr Opin Immunol 1989; 1: 1141-7.

60. Verma S, Hiby SE, Loke YW, King A. Human decidual natural killer cells express the receptor for and respond to the cytokine interleukin 15. Biol Reprod 2000; 62: 959-68.

61. Carson WE, Haldar S, Baiocchi RA, Croce CM, Caligiuri MA. The c-kit ligand suppresses apoptosis of human natural killer cells through the upregulation of bcl-2. Proc Natl Acad Sci USA 1994; 91: 7553-7.

62. Bulfone-Paus S, Ungureanu D, Pohl T, et al. Interleukin-15 protects from lethal apoptosis in vivo. Nat Med 1997; 3: 1124-8.

63. King A, Gardner L, Loke YW. Co-stimulation of human decidual natural killer cells by interleukin-2 and stromal cells. Hum Reprod 1999; 14: 656-63.

64. Kitaya K, Yasuda J, Yagi I, et al. IL-15 expression at human endometrium and deciduas. Biol Reprod 2000; 63: 683-7.

65. King A, Balendran N, Wooding P, Carter NP, Loke YW. Phenotypic and morphologic characterization of novel CD3-, 56 bright + lymphocytes in the human pregnant uterus. Dev Immunol 1991; 1: 169-90.

66. Christmas SE, Bulmer JN, Meager A, Johnson PM. Phenotypic and functional analysis of human CD3- decidual leukocyte clones. Immunology 1990; 71: 182-9.

67. Bulmer JN, Sunderland CA. Immunohistological characterization of lymphoid cell populations in the early human placental bed. Immunology 1984; 52: 349.

68. Bulmer JN, Morrison L, Longfellow M, Ritson A, Pace D. Granulated lymphocytes in human endometrium: histochemical and immunohistochemical studies. Hum Reprod 1991; 6: 791.

69. Trundley A, Moffett A. Human uterine leukocytes and pregnancy. Tissue Antigens 2004; 63: 1.

70. Loke YW, King A. Human implantation. Cambridge University Press, 1995: 299.

71. Ashkar AA, Di Santo JP, Croy BA. Interferon gamma contributes to initiation of uterine vascular modification, decidual integrity, and uterine natural killer cell maturation during normal murine pregnancy. J Exp Med 2000; 192: 259-70.

72. Pijnenborg R, Bland JM, Robertson WB, Dixon G, Brosens I. The pattern of interstitial trophoblastic invasion of the myometrium in early human pregnancy. Placenta 1981; 2: 303e16.
73. Bulmer JN, Lash GE. Human uterine natural killer cells: a reappraisal. Mol Immunol 2005; 42: 511e21.

74. Wegmann TG, Lin H, Guilbert L, Mosmann TR. Bidirectional cytokine interactions in the maternal-fetal relationship: is successful pregnancy a TH2 phenomenon? Immunol Today 1993; 14: 353-6.

75. Lin H, Mosmann TR, Guilbert L, Tuntipopipat S, Wegmann TG. Synthesis of $\mathrm{T}$ helper 2-type cytokines at the maternal-fetal interface. J Immunol 1993; 151: 4562-73.

76. Chaouat G, Ledee-Bataille N, Dubanchet S, et al. TH1/TH2 paradigm in pregnancy: paradigm lost? Cytokines in pregnancy/early abortion: reexamining the $\mathrm{TH} 1 / \mathrm{TH} 2$ paradigm. Int Arch Allergy Immunol 2004; 134: 93-119.

77. Chaouat G. The Th1/Th2 paradigm: still important in pregnancy? Semin Immunopathol 2007; 29: 95-113.

78. Guerin LR, Prins JR, Robertson SA. Regulatory T-cells and immune tolerance in pregnancy: a new target for infertility treatment? Hum Reprod Update 2009; 15: 517-35.

79. Robertson SA, Sharkey DJ. The role of semen in induction of maternal immune tolerance to pregnancy. Semin Immunol 2001; 13: 243-54.

80. Johansson M, Bromfield JJ, Jasper MJ, Robertson SA. Semen activates the female immune response during early pregnancy in mice. Immunology 2004; 112: 290-300.

81. Munn DH, Zhou M, Attwood JT, et al. Prevention of allogeneic fetal rejection by tryptophan catabolism. Science 1998; 281: 1191-3.

82. Le Rond S, Azema C, Krawice-Radanne I, et al. Evidence to support the role of HLA-G5 in allograft acceptance through induction of immunosuppressive/regulatory T cells. J Immunol 2006; 176: 3266-76, 5018-26.

83. Ristich V, Liang S, Zhang W, Wu J, Horuzsko A. Tolerization of dendritic cells by HLA-G. Eur J Immunol 2005; 35: 1133-42.

84. LeMaoult J, Krawice-Radanne I, Dausset J, Carosella ED. HLA-G1expressing antigen-presenting cells induce immunosuppressive CD4+ T cells. Proc Natl Acad Sci USA 2004; 101: 7064-9.

85. Gehrz RC, Christianson WR, Linner KM, et al. A longitudinal analysis of lymphocyte proliferative responses to mitogens and antigens during human pregnancy. Am J Obstet Gynecol 1981; 140: 665-70.

86. Matthiesen L, Berg G, Ernerudh J, Hakansson L. Lymphocyte subsets and mitogen stimulation of blood lymphocytes in normal pregnancy. Am J Reprod Immunol 1996; 35: 70-9.

87. Heikkinen J, Mottonen M, Alanen A, Lassila O. Phenotypic characterization of regulatory $\mathrm{T}$ cells in the human decidua. Clin Exp Immunol 2004; 136: 373-8.

88. King A, Jokhi PP, Burrows TD, et al. Functions of human decidual NK cells. Am J Reprod Immunol 1996; 35: 258-60.

89. Pace D, Longfellow M, Bulmer JN. Characterization of intraepithelial lymphocytes in human endometrium. J Reprod Fertil 1991; 91: 165-74.

90. Tsakonas DP, Tsakona CP, Worman CP, Goldstone AH, Nicolaides KH. Myeloperoxidase activity and nuclear segmentation of maternal neutrophils during normal pregnancy. Clin Lab Haematol 1994; 16: 337-42.

91. Rukavina D, Rubesa G, Gudelj L, Haller H, Podack ER. Characteristics of perforin expressing lymphocytes within the first trimester decidua of human pregnancy. Am J Reprod Immunol 1995; 33: 394-404.

92. Mandelboim O, Davis DM, Reyburn HT, et al. Enhancement of class IIrestricted $\mathrm{T}$ cell responses by costimulatory $\mathrm{NK}$ receptors for class I MHC proteins. Science 1996; 274: 2097-100.

93. Saito S, Nishikawa K, Morii T, et al. Expression of activation antigens CD69, HLA-DR, interleukin-2 receptor-alpha (IL-2R alpha) and IL-2R $\beta$ on $\mathrm{T}$ cells of human decidua at an early stage of pregnancy. Immunology 1992; 75: 710-12.

94. Ho HN, Chao KH, Chen CK, Yang YS, Huang SC. Activation status of $\mathrm{T}$ and NK cells in the endometrium throughout menstrual cycle and normal and abnormal early pregnancy. Hum Immunol 1996; 49: 130-6.

95. Guerin LR, Prins JR, Robertson SA. Regulatory T-cells and immune tolerance in pregnancy: a new target for infertility treatment? Hum Reprod Update 2009; 15: 517-35.

96. Mjösberg J, Berg G, Jenmalm MC, Ernerudh J. FOXP3+ regulatory $\mathrm{T}$ cells and $\mathrm{T}$ helper $1, \mathrm{~T}$ helper 2 , and $\mathrm{T}$ helper 17 cells in human early pregnancy decidua. Biol Reprod 2010; 82: 698-705.

97. Nagamatsu T, Schust DJ, Sugimoto J, Barrier BF. Human decidual stromal cells suppress cytokine secretion by allogeneic CD4+ T cells via PD-1 ligand interactions. Hum Reprod 2009; 24: 3160-71. 
98. Soubiran P, Zapitelli JP, Schaffar L. IL2-like material is present in human placenta and amnion. J Reprod Immunol 1987; 12: 225-34.

99. Boehm KD, Kelley MF, Ilan J, Ilan J. The interleukin 2 gene is expressed in the syncytiotrophoblast of the human placenta. Proc Natl Acad Sci USA 1989; 86: 656-60.

100. Athanassakis I, Chaouat G, Wegmann TG. The effects of anti-CD4 and anti-CD8 antibody treatment on placental growth and function in allogeneic and syngeneic murine pregnancy. Cell Immunol 1990; 129: $13-21$.

101. Morita CT, Mariuzza RA, Brenner MB. Antigen recognition by human gamma delta $\mathrm{T}$ cells: pattern recognition by the adaptive immune system. Springer Semin Immunopathol 2000; 22: 191-217.

102. Davis MM, Chien Y. Issues concerning the nature of antigen recognition by alpha $\beta$ and gamma delta T-cell receptors. Immunol Today 1995; 16: 316-18.

103. Chien YH, Jores R, Crowley MP. Recognition by gamma/delta T cells. Annu Rev Immunol 1996; 14: 511-32.

104. Christmas SE. T-cell receptor gene expression by human gamma delta $\mathrm{T}$-cell clones from peripheral blood and reproductive tissues in relation to non-MHC-restricted cytotoxic function. Scand J Immunol 1991; 33: $627-37$.

105. Ditzian-Kadanoff R, Garon J, Verp MS, Zilberstein M. Gamma delta T cells in human decidua. Am J Obstet Gynecol 1993; 168: 831-6.

106. Polgar B, Barakonyi A, Xynos I, Szekeres-Bartho J. The role of gamma/ delta $\mathrm{T}$ cell receptor positive cells in pregnancy. Am J Reprod Immunol 1999; 41: 239-44.

107. Bonney EA, Pudney J, Anderson DJ, Hill JA. Gamma-delta T cells in midgestation human placental villi. Gynecol Obstet Invest 2000; 50: $153-7$.

108. Heyborne KD, Cranfill RL, Carding SR, Born WK, O’Brien RL. Characterization of gamma delta $\mathrm{T}$ lymphocytes at the maternal-fetal interface. J Immunol 1992; 149: 2872-8.

109. Vanderbeeken YE, Duchateau J, Gregoire M, et al. Modulation of B cell stimulation by maternal serum. Immunol Invest 1991; 20: 287-304.

110. Tedesco F, Pausa M, Nardon E, et al. Prevalence and biological effects of anti-trophoblast and anti-endothelial cell antibodies in patients with recurrent spontaneous abortions. Am J Reprod Immunol 1997; 38: $205-11$.

111. Roussev RG, Stern JJ, Kaider BD. Anti-endothelial cell antibodies: another cause for pregnancy loss? Am J Reprod Immunol 1998; 39: 89-95.

112. Dighiero G, Lymberi P, Guilbert B, Ternynck T, Avrameas S. Natural autoantibodies constitute a substantial part of normal circulating immunoglobulins. Ann NY Acad Sci 1986; 475: 135-45.

113. Ahmed SA, Aufdemorte TB, Chen JR, et al. Estrogen induces the development of autoantibodies and promotes salivary gland lymphoid infiltrates in normal mice. J Autoimmun 1989; 2: 543-52.

114. Haller H, Radillo O, Rukavina D, et al. An immunohistochemical study of leucocytes in human endometrium, first and third trimester basal decidua. J Reprod Immunol 1993; 23: 41-9.

115. Ho HN, Chao KH, Chen CK, Yang YS, Huang SC. Activation status of $\mathrm{T}$ and NK cells in the endometrium throughout menstrual cycle and normal and abnormal early pregnancy. Hum Immunol 1996; 49: $130-6$.

116. Gehrz RC, Christianson WR, Linner KM, et al. Cytomegalovirus specific humoral and cellular immune response in human pregnancy. J Infect Dis 1981; 143: 391-5.

117. Biedermann K, Flepp M, Fierz W, Joller-Jemelka H, Kleihues P. Pregnancy, immunosuppression and reactivation of latent toxoplasmosis. J Perinat Med 1995; 23: 191-203.

118. Frenkel LM, Garratty EM, Shen JP, et al. Clinical reactivation of herpes simplex virus type 2 infection in seropositive pregnant women with no history of genital herpes. Ann Int Med 1993; 118: 414-18.

119. Verhoef J. Transient immunodepression. J Antimicrob Chemother 1990; 26(Suppl C): 23-9.

120. Gregory CD, Shah LP, Lee H, Scott IV, Golding PR. Cytotoxic reactivity of human natural killer (NK) cells during normal pregnancy: a longitudinal study. J Clin Lab Immunol 1985; 18: 175-81.

121. Vinatier D. Monnier JC. The fetal-maternal interface. Description of its elements from an immunologic perspective [French]. J Gynecol Obstet Biol Reprod 1990; 19: 691-700.
122. Luft BJ, Remington JS. Effect of pregnancy on augmentation of natural killer cell activity by Corynebacterium parvum and Toxoplasma gondii. J Immunol 1984; 132: 2375-9.

123. Toder V, Nebel L, Gleicher N. Studies of natural killer cells in pregnancy. I. Analysis at the single cell level. Clin Lab Immunol 1984; 14: $123-7$.

124. Okamura K, Furukawa K, Nakakuki M, Yamada K, Suzuki M. Natural killer cell activity during pregnancy. Am J Obstet Gynecol 1984; 149: 396-9.

125. Baley JE, Schacter BZ. Mechanisms of diminished natural killer cell activity in pregnant women and neonates. J Immunol 1985; 134: $3042-8$.

126. Vaquer S, De la Hera A, Jorda J, et al. Diminished natural killer activity in pregnancy: modulation by interleukin 2 and interferon gamma. Scand J Immunol 1987; 26: 691-8.

127. Toder V, Blank M, Gleicher N, et al. Activity of natural killer cells in normal pregnancy and edema-proteinuria-hypertension gestosis. Am J Obstet Gynecol 1983; 145: 7-10.

128. Eriksen NL, Gonik B, Loo LS. Natural killer cell cytotoxicity to herpes simplex virus-1-infected cells is not altered by pregnancy. Am J Obstet Gynecol 1991; 165: 965-8.

129. Whitley RJ, Nahmias AJ, Visintine AM, Fleming CL, Alford CA. The natural history of herpes simplex virus infection of mother and newborn. Pediatrics 1980; 66: 489-94.

130. Brown ZA, Vontver LA, Benedetti J, et al. Genital herpes in pregnancy: risk factors associated with recurrences and asymptomatic viral shedding. Am J Obstet Gynecol 1985; 153: 24-30.

131. Kobbermann T, CLark L, Griffin WT. Maternal death secondary to disseminated herpesvirus hominis. Am J Obstet Gynecol 1980; 137: 742-3.

132. Young EJ, Killam AP, Green JF. Disseminated herpes virus infection: association with primary genital herpes in pregnancy. JAMA 1976; 235: 2731-3.

133. Priddle HD, Lenz WR, Young DC, Stevenson CS. Poliomyelitis in pregnancy and the puerperium. Am J Obstet Gynecol 1952; 63: 408.

134. Diro M, Beydoun SN. Malaria in pregnancy. South Med J 1982; 75: 959-62.

135. Paryani SG, Arvin AM. Intrauterine infection with varicella-zoster virus after maternal varicella. N Eng J Med 1986; 314: 1542-6.

136. Birk K, Rudick R. Pregnancy and multiple sclerosis. Arch Neurol 1986; 43: 719-26.

137. Thompson DS, Nelson LM, Burns A, Burks JS, Franklin GM. The effects of pregnancy in multiple sclerosis: a retrospective study. Neurology 1986; 36: 1097-9.

138. Nelson JL, Ostensen M. Pregnancy and rheumatoid arthritis. Rheum Dis Clin North Am 1997; 23: 195-212.

139. Boyd AS, Morris LF, Phillips CM, Menter MA. Psoriasis and pregnancy: hormone and immune system interaction. Int J Dermatol 1996; 35: 169-72.

140. Dodson MG, Kerman RH, Lange CF, Stefani SS, O'Leary JA. T and B cells in pregnancy. Obstet Gynecol 1977; 49: 299-302.

141. Sridama V, Pacini F, Yang SL, et al. Decreased levels of T helper cells: a possible cause of immunodeficiency in pregnancy. N Eng J Med 1982; 307: 352-6.

142. Bisset LR, Fiddes TM, Gillett WR, Wilson PD, Griffin JF. Altered humoral immunoregulation during human pregnancy. Am J Reprod Immunol 1990; 23: 4-9.

143. Brabin BJ. Epidemiology of infection in pregnancy. Rev Infect Dis 1985 7: 579-603.

144. Present PA, Comstock GW. Tuberculin sensitivity in pregnancy. Am Rev Respir Dis 1975; 112: 413-16.

145. Reinisch N, Sitte BA, Kahler CM, Wiedermann CJ. Human chorionic gonadotrophin: a chemoattractant for human blood monocytes, neutrophils and lymphocytes. J Endocrinol 1994; 142: 167-70.

146. Kaye MD, Jones WR. Effect of human chorionic gonadotropin on in vitro lymphocyte transformation. Am J Obstet Gynecol 1971; 109: 1029-31.

147. Jenkins DM, Acres MG, Peters J, Riley J. Human chorionic gonadotropin and the fetal allograft. Am J Obstet Gynecol 1972; 114: 13-15.

148. Fuchs T, Hammarstrom L, Smith CI, Brundin J. In vitro induction of human suppressor $\mathrm{T}$ cells by a chorionic gonadotropin preparation. J Reprod Immunol 1981; 3: 75-84. 
149. Fuchs T, Hammarstrom L, Smith CI, Brundin J. Sex-dependent induction of human suppressor $\mathrm{T}$ cells by chorionic gonadotropin. J Reprod Immunol 1982; 4: 185-90.

150. Lin J, Lojun S, Lei ZM, et al. Lymphocytes from pregnant women express human chorionic gonadotropin/luteinizing hormone receptor gene. Mol Cell Endocrinol 1995; 111: R13-17.

151. Phuc LH, Papiernik M, Berrih S, Duval D. Thymic involution in pregnant mice. I. Characterization of the remaining thymocyte subpopulations. Clin Exp Immunol 1981; 44: 247-52.

152. Clarke AG, Kendall MD. Histological changes in the thymus during mouse pregnancy. Thymus 1989; 14: 65-78.

153. Kendall MD, Clarke AG. The thymus in the mouse changes its activity during pregnancy: a study of the microenvironment. J Anat 2000; 197(Pt 3): 393-411.

154. Tibbetts TA, DeMayo F, Rich S, Conneely OM, O’Malley BW. Progesterone receptors in the thymus are required for thymic involution during pregnancy and for normal fertility. Proc Natl Acad Sci USA 1999; 96: 12021-6.

155. Neifeld JP, Lippman ME, Tormey DC. Steroid hormone receptors in normal human lymphocytes. Induction of glucocorticoid receptor activity by phytohemagglutinin stimulation. J Biol Chem 1977; 252: 2972-7.

156. Schust DJ, Anderson DJ, Hill JA. Progesterone-induced immunosuppression is not mediated through the progesterone receptor. Hum Reprod 1996; 11: 980-5.

157. King A, Gardner L, Loke YW. Evaluation of oestrogen and progesterone receptor expression in uterine mucosal lymphocytes. Hum Reprod 1996; 11: 1079-82.

158. Faust Z, Laskarin G, Rukavina D, Szekeres-Bartho J. Progesteroneinduced blocking factor inhibits degranulation of natural killer cells. Am J Reprod Immunol 1999; 42: 71-5.

159. Szekeres-Bartho J, Szekeres G, Debre P, Autran B, Chaouat G. Reactivity of lymphocytes to a progesterone receptor-specific monoclonal antibody. Cell Immunol 1990; 125: 273-83.

160. Roussev RG, Higgins NG, McIntyre JA. Phenotypic characterization of normal human placental mononuclear cells. Am J Reprod Immunol 1993; 25: 15-30.

161. Szekeres-Bartho J, Faust Z, Varga P, Szereday L, Kelemen K. The immunological pregnancy protective effect of progesterone is manifested via controlling cytokine production. Am J Reprod Immunol 1996; 35: 348-51.

162. Faust Z, Laskarin G, Rukavina D, Szekeres-Bartho J. Progesteroneinduced blocking factor inhibits degranulation of natural killer cells. Am J Reprod Immunol 1999; 42: 71-5.

163. Kozma N, Halasz M, Polgar B, et al. Progesterone-induced blocking factor activates STAT6 via binding to a novel IL-4 receptor. J Immunol 2006; 176: 819-26.

164. Szekeres-Bartho J, Faust Z, Varga P. The expression of a progesteroneinduced immunomodulatory protein in pregnancy lymphocytes. Am J Reprod Immunol 1995; 34: 342-8.

165. Szekeres-Bartho J, Faust Z, Varga P, Szereday L, Kelemen K. The immunological pregnancy protective effect of progesterone is manifested via controlling cytokine production. Am J Reprod Immunol 1996; 35: 348-51.

166. Szekeres-Bartho J, Autran B, Debre P, et al. Immunoregulatory effects of a suppressor factor from healthy pregnant women's lymphocytes after progesterone induction. Cell Immunol 1989; 122: 281-94.

167. Szekeres-Bartho J, Philibert D, Chaouat G. Progesterone suppression of pregnancy lymphocytes is not mediated by glucocorticoid effect. Am J Reprod Immunol 1990; 23: 42-3.

168. Hansen KA, Opsahl MS, Nieman LK, Baker JR, Klein TA. Natural killer cell activity from pregnant subjects is modulated by RU-486. Am J Obstet Gynecol 1992; 166: 87-90.

169. Lenfant F, Fort M, Rodriguez AM, et al. Absence of imprinting of HLA class Ia genes leads to co-expression of biparental alleles on term human trophoblast cells upon IFN-gamma induction. Immunogenetics 1998; 47: 297-304.

170. van den Elsen PJ, van der Stoep N, Vietor HE, et al. Lack of CIITA expression is central to the absence $\mathrm{f}$ antigen presentation functions of trophoblast cells and is caused by methylation of the IFN-gamma inducible promoter (PIV) of CIITA. Hum Immunol 2000; 61: 850-62.
171. Lanier LL. Follow the leader: NK cell receptors for classical and nonclassical MHC class I. Cell 1998; 92: 705-7.

172. Yokoyama WM. Natural killer cell receptors. Curr Opin Immunol 1998; 10: 298-305.

173. Pazmany L, Mandelboim O, Vales-Gomez M, et al. Protection from natural killer cell-mediated lysis by HLA-G expression on target cells. Science 1996; 274: 792-5.

174. Geraghty DE, Koller BH, Orr HT. A human major histocompatibility complex class I gene that encodes a protein with a shortened cytoplasmic segment. Proc Natl Acad Sci USA 1987; 84: 9145-9.

175. Carosella ED, Paul P, Moreau P, Rouas-Freiss N. HLA-G and HLA-E: fundamental and pathophysiological aspects. Immunol Today 2000; 21: 532-4.

176. King A, Allan DS, Bowen M, et al. HLA-E is expressed on trophoblast and interacts with CD94/NKG2 receptors on decidual NK cells. Eur J Immunol 2000; 30: 1623-31.

177. Pende D, Sivori S, Accame L, et al. HLA-G recognition by human natural killer cells. Involvement of CD94 both as inhibitory and as activating receptor complex. Eur J Immunol 1997; 27: 1875-80.

178. Rajagopalan S, Long EO. A human histocompatibility leukocyte antigen (HLA)-G-specific receptor expressed on all natural killer cells. J Exp Med 1999; 189: 1093-100.

179. Ponte M, Cantoni C, Biassoni R, et al. Inhibitory receptors sensing HLAG1 molecules in pregnancy: decidua-associated natural killer cells express LIR-1 and CD94/NKG2A and acquire p49, an HLA-G1-specific receptor. Proc Natl Acad Sci USA 1999; 11: 5674-9.

180. Petroff MG, Sedlmayr P, Azzola D, Hunt JS. Decidual macrophages are potentially susceptible to inhibition by class Ia and class Ib HLA molecules. J Reprod Immunol 2002; 56: 3-17.

181. Fournel S, Aguerre-Girr M, Huc X, et al. Cutting edge: soluble HLA-G1 triggers CD95/CD95 ligand-mediated apoptosis in activated CD8+ cells by interacting with CD8. J Immunol 2000; 164: 6100-4.

182. Hunt JS, Jadhav L, Chu W, Geraghty DE, Ober C. Soluble HLA-G circulates in maternal blood during pregnancy. Am J Obstet Gynecol 2000; 183: 682-8.

183. Lee N, Goodlett DR, Ishitani A, Marquardt H, Geraghty DE. HLA-E surface expression depends on binding of TAP-dependent peptides derived from certain HLA class I signal sequences. J Immunol 1998; 160: 4951-60.

184. Braud V, Jones EY, McMichael A. The human major histocompatibility complex class Ib molecule HLA-E binds signal sequence-derived peptides with primary anchor residues at positions 2 and 9. Eur J Immunol 1997; 27: 1164-9.

185. Lee N, Llano M, Carretero M, et al. HLA-E is a major ligand for the natural killer inhibitory receptor CD94/NKG2A. Proc Natl Acad Sci USA 1998; 95: 5199-204.

186. Navarro F, Llano M, Bellon T, et al. The ILT2 (LIR1) and CD94/NKG2A NK cell receptors respectively recognize HLA-G1 and HLA-E molecules co-expressed on target cells. Eur J Immunol 1999; 29: 277-83.

187. Rieger L, Hofmeister V, Probe C, et al. Th1- and Th2-like cytokine production by first trimester decidual large granular lymphocytes is influenced by HLA-G and HLA-E. Mol Hum Reprod 2002; 8: 255-61.

188. King A, Burrows TD, Hiby SE, et al. Surface expression of HLA-C antigen by human extravillous trophoblast. Placenta 2000; 21: 376-87.

189. Carrington M, Nelson GW, Martin MP, et al. HLA and HIV-1: heterozygote advantage and $\mathrm{B}^{\star} 35-\mathrm{Cw}^{\star} 04$ disadvantage. Science 1999; 283: $1748-52$.

190. Furman MH, Ploegh HL, Schust DJ. Can viruses help us to understand and classify the MHC class I molecules at the maternal-fetal interface? Hum Immunol 2000; 61: 1169-76.

191. Aschkenazi S, Straszewski S, Verwer KM, et al. Differential regulation and function of the Fas/Fas ligand system in human trophoblast cells. Biol Reprod 2002; 66: 1853-61.

192. Nakashima M, Sonoda K, Watanabe T. Inhibition of cell growth and induction of apoptotic cell death by the human tumor-associated antigen RCAS1. Nat Med 1999; 5: 938-42.

193. Ohshima K, Nakashima M, Sonoda K, Kikuchi M, Watanabe T. Expression of RCAS1 and FasL in human trophoblasts and uterine glands during pregnancy: the possible role in immune privilege. Clin Exp Immunol 2001; 123: 481-6. 
194. Munn DH, Zhou M, Attwood JT, et al. Prevention of allogeneic fetal rejection by tryptophan catabolism. Science 1998; 281: 1191-3.

195. Munn DH, Shafizadeh E, Attwood JT, et al. Inhibition of T cell proliferation by macrophage tryptophan catabolism. J Exp Med 1999; 189: 1363-72.

196. Sedlmayr P, Blaschitz A, Wintersteiger R, et al. Localization of indoleamine 2,3-dioxygenase in human female reproductive organs and the placenta. Mol Hum Reprod 2002; 8: 385-91.

197. Kudo Y, Boyd CA, Spyropoulou I, et al. Indoleamine 2,3-dioxygenase: distribution and function in the developing human placenta. J Reprod Immunol 2004; 61: 87-98.
198. Schrocksnadel H, Baier-Bitterlich G, Dapunt O, Wachter H, Fuchs D. Decreased plasma tryptophan in pregnancy. Obstet Gynecol 1996; 88: 47-50.

199. Johnson PM, Brown PJ. Fc(gamma) receptors in the human placenta. Placenta 1981; 2: 355-70.

200. Leach JL, Sedmak DD, Osborne JM, et al. Isolation from human placenta of the IgG transporter, FcRn, and localization to the syncytiotrophoblast: implications for maternal-fetal antibody transport. J Immunol 1996; 157: 3317-22.

201. Wegmann TG, Mosmann TR, Carlson GA, Olijnyk O, Singh B. The ability of the murine placenta to absorb monoclonal anti-fetal $\mathrm{H}-2 \mathrm{~K}$ antibody from the maternal circulation. J Immunol 1979; 123: 1020-3. 


\section{Placental transport and metabolism Jeffrey M. Dicke}

As a fetal organ, the placenta not only is obligated to the fetus but also answers to the mother. More than merely a conduit for the provision of oxygen and nutrients to the fetus, the placenta actively regulates the passage of substrates necessary for fetal growth and development. The importance of placental function and dysfunction is manifest in a variety of adult chronic illnesses that are now felt to be a consequence of in utero insults. This phenomenon is known as fetal origins of adult disease and includes cardiovascular disease and features of the metabolic syndrome, including dyslipidemia, type 2 diabetes, insulin resistance, and obesity. The finding that the placenta is capable of influencing an individual's general health years following its relatively brief service is a testament to its critical effect on intra- as well as extrauterine survival. Preventative care and overall health begin in utero and require a healthy placenta. This realization has implications not only for an individual's quality of life but also for societal approaches to public health.

Placental nutrient transport potential involves multiple variables including placental size, architecture, blood flow, nutrient production and consumption, and transporters for glucose, lipids, and amino acids.

The placenta grows continuously throughout gestation. This benefits the fetus in a number of ways and is one determinate of fetal growth. The larger the placenta, the larger the surface area available for nutrient exchange. There is also a decrease in trophoblast thickness, decreasing the diffusion distance between maternal and fetal circulations. These changes support the observations that placental growth in the midtrimester is associated with fetal weight in the third trimester and that overall there is a positive correlation of placental size with fetal size (1-3).

Environmental insults, which reduce placental weight, such as nutritional conditions and glucocorticoid administration, can also have an effect on placental morphology. Changes in barrier thickness, surface area available for exchange, vascularity, and rates of proliferation and demise of certain cell types are ways in which placental efficiency may be diminished or enhanced $(4,5)$. For example, hypoxia has been associated with a reduction in barrier thickness between maternal and fetal capillaries in the human placenta, presumably encouraging diffusional change (6). Maternal food restriction in guinea pigs, whose hemomonochorional placenta is more like the human placenta than other non-primates, alters placental size and structure. Undernutrition is associated with reduced placental weight, decreased surface area available for exchange, and increase in membrane thickness. The placenta under these circumstances not only is small but also has diminished substrate transport capacity (4).

Placental growth and function also depend upon adequate blood flow. This requires normal trophoblast invasion and remodeling of the spiral arteries, achieved by vasculogenesis, new blood vessel formation from endothelial precursor cells, and angiogenesis, new vessel formation from those already present. Deficient conversion of the spiral arteries leads to impaired villous perfusion and hypoxia, which itself can have a pejorative effect on vascular remodeling by limiting proliferation and growth of fetal vessels $(7,8)$. This results in increased resistance to blood flow in the umbilical arteries, which is reflected in abnormal flow velocity waveforms using Doppler ultrasound. Growth of the placenta, its blood vessels, and its blood supply are intertwined such that, under normal circumstances, fetoplacental growth correlates with the ability to provide oxygen and nutrients to the placenta for subsequent transfer to the fetus.

The consumption and production of nutrients by placental tissues impact the availability of nutrients for transport to the fetus. In humans, $40 \%$ to $70 \%$ of the glucose and oxygen taken up by the uterus is utilized by uteroplacental tissues, which also produce lactate and metabolize amino acids for fetal use $(9,10)$. Just as placental growth, morphology, and blood supply are subject to exogenous factors, uteroplacental management of carbohydrates and amino acids is influenced by a host of variables including placental size, nutrient availability, and concentrations of hormones such as glucocorticoids, growth hormone, and insulin growth factors in maternal and fetal blood (11-13). In sheep, hypoglycemia is associated with decreased placental glucose consumption (14) while preterm exposure to cortisol has the opposite effect (15). Similarly, placental disposition of essential amino acids is influenced by undernutrition, heat stress, and treatment with IGF-1 $(14,15)$, which enhances fetal and placental amino acid uptake (16). These are ways in which placental utilization of nutrients is influenced by environmental factors with subsequent effects on nutrient availability to the fetus.

Hormones are produced by the placenta and released to the mother and fetus. These include peptides, steroids, glycoproteins, and cytokines. Progesterone and placental lactogen have effects on the mother, which promote glucose delivery to the fetus (2). The placental variant of growth hormone (GHv) and IGF-1 and IGF-2 may influence nutrient provision by endocrine effects on maternal metabolism and/or paracrine effects on placental potential for simple and facilitated diffusion $(16,17)$. Binding of these hormones to placental receptors can change placental clearance of analogs of glucose and amino acids in vivo and in vitro (18). Maternal levels and placental expression of GHv are decreased in pregnancies with growth-restricted fetuses, implicating changes in placental endocrine function as a cause rather than a result of abnormal fetal growth (19).

Placental prostaglandins (PG) $\mathrm{PGE}_{2}$ and $\mathrm{PGF}_{2} \alpha$ affect fetal nutrient and oxygen provision indirectly by their effects on 
regional blood flow, uterine contractility, and fetal endocrine function $(20,21)$. In vitro studies using murine placenta subjected to oxidative stress through a process of hypoxia/ reoxygenation have demonstrated significant increases in the concentrations of cyclooxygenase, enzymes that stimulates PG production (22). In nonhuman primates, undernutrition increases activity of placental PGH synthase and levels of $\mathrm{PGE}_{2}$ and $\mathrm{PGF}_{2} \alpha$. These increases correlate with reduced uteroplacental glucose consumption and are reversible by restoration of normoglycemia (20). PG dehydrogenase (PGDH), an enzyme that converts active prostaglandins into their inactive metabolites, has also been demonstrated in placental tissue (23). Changes in PG synthesis and metabolism have been associated with cortisol concentrations as glucocorticoids enhance PGH synthase activity and reduce PGDH activity, which increases intrauterine production of $\mathrm{PGE}_{2}$ and $\mathrm{PGF}_{2} \alpha$ (21,23). These changes increase with advancing gestation, a time of rising fetal cortisol concentrations and myometrial contractility.

11- $\beta$-hydroxysteroid dehydrogenase type-2 (11- $\beta$-HSD-2) is a placental enzyme that inactivates glucocorticoids, compounds produced by the trophoblast that regulate organ growth and maturation. Fetal exposure to glucocorticoids is associated with growth restriction in both animals and humans. By converting cortisol to cortisone, 11- $\beta$-HSD-2 forms a placental barrier by limiting transfer of free cortisol from mother to fetus. Decreased 11- $\beta$-HSD-2 activity and increased levels of cortisol in the fetus have been associated with fetal growth restriction (24). It is presumed that the placenta mitigates the effects of steroids on fetal growth as experimental studies have shown that repeated doses of corticosteroids given to pregnant ewes resulted in reduced birth weight while direct administration to the fetus did not interfere with fetal growth $(25,26)$. In humans, placental activity of $11-\beta-\mathrm{HSD}-2$ correlates with birth weight (27). Additionally, gene expression of $11-\beta-\mathrm{HSD}-2$ is sensitive to environmental and nutritional stressors. Hypoxia (in vitro) and infection decrease activity, which may lead to increased cortisol levels and suboptimal growth (28). While in vitro data derived using a variety of cell models are inconclusive, evidence to date suggests that glucocorticoids may decrease glucose transfer and increase amino acid transport (29). Glucocorticoids also influence fetal growth via their effects on placental production and activity of other hormones, including prostaglandins, progesterone, and estrogens (28). This is another way in which the placenta adapts to conditions in utero in an attempt to maintain optimal fetal growth.

Insulin-like growth factors, especially IGF-1, are presumptive regulators of placental transport based on the observations that IGF receptors are expressed in the syncytiotrophoblast, which in vitro studies have demonstrated increased glucose uptake and system A activity following treatment with IGF-1 (30) and that reduced fetal levels of IGF-1 (31) and expression of the IGF receptor were associated with intrauterine growth restriction (IUGR) (32). Based on the above, it has been proposed that reduced levels of IGF-1 in the maternal circulation could result in diminished transplacental transport of glucose and amino acids while increased levels of IGF-1, as occurs in diabetes, would have the opposite effect.
A relatively recent addition to the armamentarium regulating placental transport activity (maternal nutritional status, placental blood flow, maternal/placental hormone production, hypoxia) is mammalian target of rapamycin (mTOR). A serine/threonine kinase, mTOR protein is expressed in the cytosol of the syncytiotrophoblast and is thought to regulate cell growth by its influence on translation and transcription through a mechanism of "nutrient sensing" (33). This refers to a proposed process whereby mTOR regulates production of proteins related to cell growth in response to the placental environment, including nutrient availability, oxygenation, and growth factors. These proteins regulate nutrient transporters and thereby affect fetal growth. In vitro data supporting the proposed role of mTOR in fetal growth include the finding that the activity of the mTOR signaling pathway is downregulated in IUGR and that inhibition of mTOR resulted in nearly complete inhibition of leucine uptake by the system $\mathrm{L}$ amino acid transporter (34).

In addition to the aforementioned influences on placental transport and fetal growth, the organization of the placental plasma membrane interface between the maternal and fetal circulations is considered to be a critical component of placental function. This interface is the syncytiotrophoblast that consists of a microvillous membrane (MVM), exposed to maternal venous blood and a basal membrane $(\mathrm{BM})$, which is in contact with the fetal circulation. Available and accumulating evidence indicate that the placenta employs multiple nutrient transporters to provide the sustenance necessary for fetal growth. The MVM and BM contain specific receptors and proteins that regulate the supply of glucose, lipids, and amino acids available to the fetus. Following is a review of the cellular mechanisms responsible for fetal nutrition.

\section{GLUCOSE}

Glucose transport is facilitated by a family of isoforms with the prefix GLUT. In human placenta, six GLUT isoforms have been identified at the mRNA level $(35,36)$. The major placental GLUTs are GLUT1 and GLUT3 (37-39), whose abundance and activity differ in response to a variety of environmental factors, including diabetes, maternal nutritional status, hypoxia, and reduced uterine blood flow (28). These GLUTs have different kinetic activity and are differentially expressed within the placental membranes. GLUT3 occurs in the MVM at the maternal-fetal interface and is presumed to be responsible for the rate of glucose delivery to the fetus. GLUT1 tends to be located in the plasma BM and may modulate placental glucose consumption as well as being the rate-limiting step in transplacental glucose transport $(39,40)$.

\section{FATTY ACIDS}

Long-chain polyunsaturated fatty acids (LC-PUFA) are important not only for maternal health but also for fetal growth and central nervous system development. The LC-PUFA arachidonic acid (AA) and docosahexaenoic acid (DHA) are present in high concentrations in the fetal brain and regulate the structure and function of neural membranes (41). Diminished DHA in brain lipids is associated with learning impairment in various species. Eicosanoids, which are derived from AA, are integral to cell division and other important physiologic processes (41). 
The observation that LC-PUFA are higher in plasma lipids of the cord than in maternal plasma lipids at birth supports the notion of active maternal-fetal transfer, which is a complex, incompletely understood process. Human placental receptors to very-low-density lipoprotein, low-density lipoprotein, and high-density lipoprotein facilitate uptake of maternal plasma lipoproteins (42).

In addition, placental lipoprotein lipase, which is localized in the MVM, hydrolyzes lipoproteins (43). The free fatty acids (FFA) released diffuse across the plasma membrane. Fatty acid transport proteins, fatty acid translocase, and plasma membrane fatty acid-binding proteins (FABPpm) facilitate unesterified fatty acid uptake into the placental cells (44). An isoform of FABPpm unique to the placenta occurs only in the MVM of the syncytiotrophoblast, suggesting that it may be important in placental FFA uptake (45). While they are thought to encourage transmembrane passage of FFA, the manner in which this is accomplished by FABP is uncertain. Once transported into the syncytial cytoplasm, FFA bind to FABP and are presented to the BM through which they are presumed to be transported to the fetus via either diffusion or facilitation by FABPs (46).

\section{AMINO ACIDS}

While glucose is the primary fuel for the fetus, amino acids are critical as they are precursors for protein synthesis as well as nonprotein compounds, such as nitric oxide, neurotransmitters, and polyamines. The types and balance of essential and nonessential amino acids supplied to the fetus are important determinates of fetal growth. Amino acid concentrations in the fetal plasma exceed those in the mother (47). The uptake and transport of amino acids across the trophoblast membrane is an active process regulated by at least 15 different transport proteins with different structures, overlapping substrate specificity, expression, and activity. Sodium-dependent transport systems and their substrates include systems A (alanine, serine, proline, glycine), ASC (alanine, serine, cystine), $\mathrm{N}$ (histidine, asparagine, glutamine), $\beta$ (taurine), $\mathrm{X}_{\mathrm{AG}}^{-}$ (glutamate and aspartate), and GLY (glycine, sarcosine). Cationic amino acids are transported via system $y^{+}$. Small neutral amino acids are handled by system ASC. System L transports neutral and branched-chain amino acids while aromatic amino acids are the substrate for system $\mathrm{T}$ (48).

There are two main types of amino acid transporters, both of which are present in the placenta: accumulative transporters and amino acid exchangers (49). The former regulate influx of amino acids and lead to increased concentrations within the cell. Amino acid exchangers swap amino acids from outside the cell with those inside the cell. This allows the composition of amino acids within the cell to be altered to meet fetal metabolic requirements, with no change in total concentration. Exchangers are one potential means by which nonessential amino acids in the fetal circulation can be exchanged for less readily available essential amino acids within the placenta. Such a process is one way in which the placenta meets the unique growth and energy demands of the fetus.

Net amino acid transfer across the placenta is necessary to sustain fetal growth. There is active transport of amino acids across the MVM into the syncytiotrophoblast via accumulative transporters (47). These include subtypes of system A, a sodium-dependent transporter, which transports small neutral amino acids (SNAT1, SNAT2, and SNAT4), such as alanine, serine, glycine, and proline and system $\mathrm{X}_{\mathrm{AG}}^{-}$, which regulate uptake of glutamate and aspartate. System A transporters are notable among MVM accumulative transporters in that their substrate amino acids are also subject to exchange transport (50).

Although the mechanisms of amino acid uptake by the maternal-facing MVM have been delineated, the means by which amino acids traverse the BM to the fetus are less well understood. In studies of isolated perfused placenta, exchange and nonexchange transport systems have been demonstrated. The activity of the exchange systems was suggestive of system ASC and isoforms of system L (LAT1, LAT2). Possible nonexchange transporters include system $\mathrm{N}$, system $\mathrm{T}$ (TAT1), and the system L isoform LAT4 (51).

In summary, the appropriate quantity and balance of amino acids are essential for fetal and placental health. Provision of amino acids from the maternal circulation into and through the placenta to the fetus requires the coordinated activity of multiple transport proteins, which function differentially in the MVM and BM to provide and maintain the appropriate levels of specific amino acids required for protein synthesis and energy metabolism.

\section{CHANGES ASSOCIATED WITH ABNORMAL FETAL GROWTH}

Fetal growth restriction connotes changes in the demonstrated and proposed mechanisms of nutrient transport sufficient to preclude normal fetal growth.

Using vesicles prepared from the MVM and BM of the syncytiotrophoblast from placentas of IUGR pregnancies, changes in amino acid transport systems have been demonstrated including both decreased volume and transport capacity for amino acids. Downregulation of systems A, L, and TAU in the MVM results in reduced provision of taurine, lysine, and leucine $(52,53)$. Additionally, reduced activity of lipoprotein lipase, necessary for placental transport of FFA, has been reported in MVM from IUGR pregnancies (54). Diminished transport of leucine and lysine has been detected in BM vesicles (55). The expression and activity of glucose transport proteins is unaltered in IUGR (56).

In vitro studies demonstrating alterations in amino acid transport have served as a prelude to a variety of in vivo investigations attempting to extend in vitro findings. While the numbers of patients in these studies is small, the results to date support the notion of the placenta as an arbiter of amino acid availability.

Fetal blood sampling of 4 AGA and 14 IUGR human pregnancies coincident with a constant infusion of a leucine isotope revealed that the change in steady-state fetal/maternal leucine enrichment was significantly lower for IUGR pregnancies than appropriate-size-for-gestational-age (AGA) pregnancies. Additionally, the magnitude of the decrease in IUGR pregnancies correlated with the clinical severity of IUGR. The conclusion from this work was that the decrease in the fetal/ maternal leucine ratio may be due to reduced placental leucine flux and/or increased protein catabolism in the fetal and placental tissues (57). 
In a different study of seven AGA and seven IUGR human pregnancies, fetal blood sampling was performed shortly following maternal intravenous bolus administration of a solution containing isotopes of leucine, phenylalanine, glycine, and proline. Leucine and phenylalanine use exchange transporters and are transferred transplacentally more rapidly than amino acids that do not use exchange transporters, such as glycine and proline. Following maternal administration, the fetal/maternal enrichment ratio for the essential amino acids leucine and phenylalanine was significantly reduced in IUGR versus AGA pregnancies, consistent with inhibited transplacental flux. The fetal/maternal enrichment ratio of the nonessential amino acids glycine and proline was not significantly different (58).

Intravenous infusion of a solution of amino acids in 10 patients with uncomplicated pregnancies prior to cesarean section revealed a significant increase in the maternal concentration of all amino acids versus 16 control patients. Umbilical venoarterial differences were measured as an index of placental amino acid provision to the fetus. For most amino acids, the fetal concentration and the venoarterial concentration difference per micromole of oxygen increased, indicating amino acids cross the placenta rapidly. However, the umbilical uptakes of three essential amino acids, threonine, histidine, and lysine, did not change significantly despite increases in the maternal concentration. Thus, increasing maternal concentrations led to an increase in the delivery of most, but not all, amino acids to the fetus (59).

A similar protocol was used to study whether maternal infusion of amino acids could increase umbilical uptake and fetal amino acid concentrations in 18 pregnancies complicated by IUGR. Eight patients received a pre-cesarean section intravenous infusion of a solution of multiple amino acids. The remaining 10 patients were controls. Similar to what was described in uncomplicated pregnancies, the maternal concentration of all amino acids was significantly increased in the infusion group versus the controls. The umbilical uptake in the infusion group was significantly higher for leucine, isoleucine, methionine, arginine, serine, proline, and glycine. By contrast, there was no increase in the uptake of three essential amino acids, lysine, histidine, and threonine, in these IUGR pregnancies (60).

In contradistinction to the attention afforded placental changes in IUGR pregnancies, alterations associated with accelerated fetal growth have been less extensively studied. Evidence to date suggests that surplus substrate alone incompletely explains the pathology of large-for-gestationalage (LGA) fetal growth patterns. Strict glucose control does not necessarily prevent LGA newborns in diabetic pregnancies (61), implicating altered placental transport capacity as another mechanism of abnormal growth. Increased expression and activity of glucose transporters in the BM of pregnancies complicated by type 1 diabetes have been demonstrated (62). Given the fact that glucose delivery to the fetus is mediated by the $\mathrm{BM}$, this is one potential means by which excess glucose is made available to the fetus in spite of maternal normoglycemia. Similar changes have not been reported in LGA pregnancies with gestational diabetes mellitus (GDM) (63). This implicates insulin as a factor in increased glucose transport. BM amino acid transport systems are reportedly unaltered in LGA diabetic pregnancies (64). There are conflicting reports on system A and system L activity in the MVM of these pregnancies (65). However, these studies involved different populations in one of which the placental weight was increased in proportion to the fetal weight (64), which was not the case in the other study (65). In vivo studies have revealed increased umbilical levels of amino acids in GDM pregnancies with good control (66) and increased umbilical vein and artery glucose levels in fetuses of GDM mothers with normal glucose levels (67).

In summary, as the purveyor of nutrient provision to the fetus, the placenta is a vital organ, not only in the sense of its necessity for fetal survival but also as an entity capable of regulating its own response to maternal and fetal conditions. In considering the maternal-placental-fetal trinity, evidence continues to accumulate delineating the active role of the placenta in adjusting nutrient provision based on a host of ever-changing influences that are normally balanced to sustain equilibrium between maternal supply and fetal demand. Under normal circumstances, the placenta provides the appropriate balance and quantity of glucose, amino acids, and lipids to support optimum or at least adequate fetal growth. Disturbances on either side of the placenta are known to stimulate changes within the MVM and BM of the syncytiotrophoblast intended to compensate so as to protect its charge, the fetus. The response of the placenta to an unfriendly environment is typically to autoregulate as much as possible to best promote fetal survival. This is accomplished via multiple transporters, hormones, and growth factors that coordinate fetal nutrient demands with maternal supply. In so doing, changes may be initiated which, while necessary to maintain fetal viability, result in signaling and long-term changes in nutrient metabolism sufficient to increase the postnatal risks for cardiovascular and metabolic disorders.

\section{REFERENCES}

1. Kloosterman GJ. On intrauterine growth, the significance of prenatal care. Int J Gynecol Obstet 1970; 8: 985-9.

2. Fowden AL, Forhead AJ. Endocrine mechanisms of intrauterine programming. Reproduction 2004; 127: 515-26.

3. Thame M, Osmond C, Bennett P, Wilks R, Forrester T. Fetal growth is directly related to maternal anthropometry and placental volume. Eur J Clin Nutr 2004; 58: 894-900.

4. Roberts CT, Sohlstrom A, Kind KL, et al. Maternal food restriction reduces the exchange surface area and increases the barrier thickness of placenta in the guinea-pig. Placenta 2001; 22: 177-185.

5. Gardner DS, Ward JW, Giussani DA, Fowden AL. The effects of a reversible period of adverse intrauterine conditions during late gestation on fetal and placental weight and placentome distribution in sheep. Placenta 2002; 23: 459-66.

6. Rishetnikova OS, Burton GJ, Miloranov AP. Effects of hypobaric hypoxia on the feto-placental unit; the morphometric diffusing capacity of the villous membrane at high altitude. Am J Obstet Gynecol 1994; 171: 1560-5.

7. Hung TH, Skipper TN, Burton GJ. In vitro ischemia-reperfusion injury in term human placenta as a model for oxidative stress in pathological pregnancies. Am J Pathol 2001; 159: 1031-43.

8. Kingdom JCP, Kaufmann P. Oxygen and placental villous development: origins of fetal hypoxia. Placenta 1997; 18: 613-21.

9. Carter AM. Placental oxygen consumption. Part I: in vivo studies - a review. Placenta 2000; 21(Suppl A): S31-7.

10. Hay WW. Regulation of placental metabolism by glucose supply. Reprod Fertil Dev 1995; 7: 362-75.

11. Owens JA, Falconer J, Robinson JS. Restriction of placental size in sheep enhances efficiency of placental transfer of antipyrine, 3-O-methyl-Dglucose but not of urea. J Dev Physiol 1987; 9: 457-64. 
12. Wallace JM, Bourke DA, Aitken RP, Milne S, Hay WW. Placental glucose transport in growth restricted pregnancies induced by overnourishing adolescent sheep. J Physiol 2002; 278: E839-45.

13. Harding JE, Evans PC, Gluckman PD. Maternal growth hormone treatment increases placental diffusion capacity but not fetal or placental growth in sheep. Endocrinology 1997; 138: 5352-8.

14. Hay WW. Regulation of placental metabolism by glucose supply. Reprod Fertil Dev 1995; 7: 365-75.

15. Ward JW, Wooding FBP, Fowden AL. The effect of cortisol on the binucleate cell population in the ovine placenta during late gestation. Placenta 2002; 23: 451-8.

16. Harding JE, Liu LMP, Evans PC, Gluckman PD. Insulin-like growth factor 1 alters feto-placental protein and carbohydrate metabolism in fetal sheep. Endocrinology 1994; 134: 1509-14.

17. Sferruzi-Perri AN, Owens JA, Pringle KG, Robinson JS, Roberts CT. Maternal insulin-like growth factors-I and -II act via different pathways to promote fetal growth. Endocrinology 2006; 147: 3344-55.

18. Bauer MK, Harding JE, Bassett NS, et al. Fetal growth and placental function. Mol Cell Endocrinol 1998; 140: 115-20.

19. Chowden JA, Evian-Brion D, Pozo J, et al. Decreased expression of placental growth hormone in intrauterine growth retardation. Pediatr Res 1996; 39: 736-9.

20. Binienda A, Marsmann A, Mitchell MD, et al. Effect of food withdrawal on arterial blood glucose and plasma 13,14-dihydro-15-keto-prostaglandin F2 alpha concentrations and nocturnal myometrial electromyographic activity in the pregnant rhesus monkey in the last third of gestation: a model for preterm labor? Am J Obstet Gynecol 1989; 160: 746-50.

21. Fowden AL, Ralph M, Silver M. Nutritional regulation of uteroplacental prostaglandin production and metabolism in pregnant ewes and mares during late gestation. Exp Clin Endocrinol 1994; 102: 212-21.

22. Burdon C, Mann C, Cindrova-Davies T, Ferguson-Smith AC, Burton JG. Oxidative stress and the induction of cyclooxygenase enzymes and apoptosis in murine placenta. Placenta 2007; 28: 724-33.

23. Whittle WL, Patel A, Alfaidy N, et al. Glucocorticoid regulation of human and ovine parturition: the relationship between fetal hypothalamicpituitary-adrenal axis activation and intrauterine prostaglandin production. Biol Reprod 2001; 64: 1019-32.

24. Seckl JR, Cleasby M, Nyirenda MJ. Glucocorticoids, 11-beta-hydroxysteroid dehydrogenase, and fetal programming. Kidney Int 2000; 57: 1412-17.

25. Newnham JP, Evans SF, Godfrey M, et al. Maternal, but not fetal administration of corticosteroids restricts fetal growth. J Matern Fetal Med 1999; 8: 81-7.

26. Moss TJM, Sloboda DM, Gurrin LC, et al. Programming effects in sheep of prenatal growth restriction and glucocorticoid exposure. Am J Physiol Regul Integr Comp Physiol 2001; 28: 960-970.

27. McTernan CL, Draper N, Nicholson H, et al. Reduced placental $11 \beta$ hydroxysteroid dehydrogenase Type 2 MRNA levels in human pregnancies complicated by intrauterine growth restriction: an analysis of possible mechanisms. J Clin Endocrinol Metab 2001; 86: 4979-83.

28. Fowden AL, Forhead AJ, Coan PM, Burton GJ. The placenta and intrauterine programming. J Neuroendocrinol 2008; 20: 439-50.

29. Jones HN, Powell TL, Jansson T. Regulation of placental nutrient transport - A review. Placenta 2007; 28: 763-74.

30. Kniss DA, Shubert PJ, Zimmerman PD, Landon MB, Gabbe SG. Insulinlike growth factors: their regulation of glucose and amino acid transport in placental trophoblasts isolated from first-trimester chorionic villi. J Reprod Med Obstet Gynecol 1994; 39: 249-56.

31. Chard T. Insulin-like growth factors and their binding proteins in normal and abnormal fetal growth. Growth Regul 1994; 4: 91-100.

32. Laviola LMP, Perrini S, Belsanti G, et al. Intrauterine growth restriction in humans is associated with abnormalities in placental insulin-like growth factor signaling. Endocrinology 2005; 146: 1498-505.

33. Jansson T, Powell TL. Role of the placenta in fetal programming: underlying mechanisms and potential interventional approaches. Clinical Sci 2007; 113: 1-13.

34. Roos S, Palmberg I, Saljo K, Powell TL, Jansson T. Expression of placental mammalian target of rapamycin (mTOR) is altered in relation to fetal growth and mTOR regulates leucine transport. Placenta 2005; 26 : A9.

35. Illsley NP. Current topic: glucose transporters in the human placenta. Placenta 2000; 21: 14-22.
36. Baumann MU, Deburde S, Illsley NP. Placental glucose transfer and fetal growth. Endocrine 2002; 19: 13-22.

37. Jansson T, Wennergren M, Powell TL. Placental glucose transport and GLUT 1 expression in insulin-dependent diabetes. Am J Obstet Gynecol 1999; 180: 163-8.

38. Bell AW, Hay WW, Ehrhardt RA. Placental transport of nutrients and its implications for fetal growth. J Reprod Fertil Suppl 1999; 54: 401-10.

39. Wooding BP, Fowden AL, Bell AW, et al. Localisation of glucose transport in the ruminant placenta: implications for sequential use of transporter isoforms. Placenta 2005; 26: 626-40.

40. Langdown ML, Sugden MC. Enhanced placental GLUT1 and GLUT3 expression in dexamethasone-induced fetal growth retardation. Mol Cell Endocrinol 2001; 185: 109-17.

41. Larque E, Demmelmair H, Koletzko B. Perinatal supply and metabolism of long-chain polyunsaturated fatty acids : importance for early development of the nervous system. In: Klimes I, Siboköva E, Howard BV, Ravussin E, . eds. Lipids and insulin resistance. The role of fatty acid metabolism and fuel partitioning. Ann NY Acad Sci 2002; 967: 299.

42. Koletzko B, Larque E, Demmelmair H. Placental transfer of long-chain polyunsaturated fatty acids (LC-PUFA). J Perinatal Med 2007; 35(Suppl 1): S5-11.

43. Lindegaard MLS, Olivecrona G, Christoffersen C, et al. Endothelial and lipoprotein lipases in human and se placenta. J Lipid Res 2005; 46: 2339-46.

44. Dutta-Roy AK. Transport mechanisms for long-chain polyunsaturated fatty acids in the human placenta. Am J Clin Nutr 2000; 71: 315S-22S.

45. Campbell FM, Bush PG, Veerkamp JH, Dutta-Roy AK. Detection and cellular localization of plasma membrane-associated and cytoplasmic fatty acid binding proteins in human placenta. Placenta 1998; 19: 409-15.

46. Haggarty P. Effect of placental function in fatty acid requirements during pregnancy. Eur J Clin Nutr 2004; 58: 1559-70.

47. Jansson T. Amino acid transporters in the human placenta. Pediatr Res 2001; 49: 141-7.

48. Grillo MA, Lanza A, Colombatto S. Transport of amino acids through the placenta and their role. Amino Acids 2008; 34: 517-23.

49. Broer S. Adaptation of plasma membrane amino acid transport mechanisms to physiological demands. Pflugers Arch 2002; 444: 457-66.

50. Cleal JK, Lewis RM. The mechanisms and regulation of placental amino acid transport to the human fetus. J Neuroendocrinol 2008; 20: 419-26.

51. Cleal JK, Brownbill P, Godfrey KM, et al. Modification of fetal plasma amino acid composition by placental amino acid exchangers in vitro. J Physiol 2007; 582: 871-82.

52. Glazier JD, Cetin I, Perugino G, et al. Association between the activity of the system A amino acid transporter in the microvillous plasma membrane of the human placenta and severity of fetal compromise in intrauterine growth restriction. Pediatr Res 1997; 42: 514-19.

53. Norberg S, Powell TL, Jansson T. Intrauterine growth restriction is associated with reduced activity of placental taurine transporters. Pediatr Res 1998; 44: 233-8.

54. Magnusson AL, Waterman IJ, Wennergren M, Jansson T, Powell TL. Triglyceride hydrolase activities and expression of fatty acid binding proteins in human placenta in pregnancies complicated by IUGR and diabetes. J Clin Endocrinol Metab 2004; 89: 4607-14.

55. Jansson T, Scholtbach V, Powell TL. Placental transport of leucine and lysine is reduced in intrauterine growth restriction. Pediatr Res 1998; 44: $532-7$.

56. Jansson T, Wennergren M, Illsley NP. Glucose transporter protein expression in human placenta throughout gestation and in intrauterine growth retardation. J Clin Endocrinol Metab 1993; 77: 1554-62.

57. Marconi AM, Paolini CL, Stramare LMP, et al. Steady state maternal-fetal leucine enrichments in normal and intrauterine growth-restricted pregnancies. Pediatr Res 1999; 46: 114-19.

58. Paoline CL, Marconi AM, Ronzoni S, et al. Placental transport of leucine, phenylalanine, glycine and proline in intrauterine growth-restricted pregnancies. J Clin Endocrinol Metab 2001; 86: 5427-32.

59. Ronzoni S, Marconi AM, Cetin I, et al. Umbilical amino acid uptake at increasing maternal amino acid concentrations: effect of a maternal amino acid infusate. Am J Obstet Gynecol 1999; 181: 477-83.

60. Ronzoni S, Marconi AM, Paolini CL, et al. The effect of a maternal infusion of amino acids on umbilical uptake in pregnancies complicated by intrauterine growth restriction. Am J Obstet Gynecol 2002; 187: 741-6. 
61. Jansson T, Powell TL. Placental nutrient transfer and fetal growth. Nutrition 2000; 16: 500-2.

62. Jansson T, Wennergren M, Powell TL. Placental glucose transport and GLUT 1 expression in insulin dependent diabetes. Am J Obstet Gynecol 1999; 180: 163-8.

63. Jansson T, Ekstrand Y, Wennergren M, Powell TL. Placental glucose transport in gestational diabetes. Am J Obstet Gynecol 2001; 184: 111-16.

64. Jansson T, Ekstrand Y, Bjorn C, Wennergren M, Powell TL. Alterations in the activity of placental amino acid transporters in pregnancies complicated by diabetes. Diabetes 2002; 51: 2214-19.
65. Kuruvilla AG, D'Souza SW, Glazier JD, et al. Altered activity of the system A amino acid transporter in microvillous membrane vesicles from placentas of macrosomic babies born to diabetic women. J Clin Invest 1994; 94: 689-95.

66. Cetin I, Nobile de Santis MS, Taricco E, et al. Maternal and fetal amino acid concentrations in normal pregnancies and in pregnancies with gestational diabetes mellitus. Am J Obstet Gynecol 2005; 192: 610-17.

67. Radaelli T, Taricco E, Rossi G, et al. Oxygenation, acid-base balance and glucose levels in fetuses from gestational diabetic pregnancies (abstract). J Soc Gynecol Invest 2005; 12: 425. 


\section{Fertilization and normal embryonic and early fetal development Asim Kurjak, Ritsuko K. Pooh, Aida Salihagic-Kadic, Iva Lausin, and Lara Spalldi-Barisic}

The anatomy and physiology of placental and embryonic development is a field where medicine exerts its greatest impact on early pregnancy at present time, and it opens fascinating aspects of embryonic differentiation. Clinical assessment of those stages of growth relies heavily on threedimensional (3D) and four-dimensional (4D) sonography, one of the most promising forms of noninvasive diagnostics today. The embryologic phenomenon, once matters for textbooks, is now routinely recorded with outstanding clarity.

It is clear that with the use of $3 \mathrm{D}$ and $4 \mathrm{D}$ ultrasound, both structural and functional developments in the first 12 weeks of gestation can be assessed more objectively and reliably. New technology moved embryology from postmortem studies to the in vivo environment. It is the purpose of this chapter to illustrate the potential of $3 \mathrm{D} / 4 \mathrm{D}$ sonography in the study of structural and functional early human development (1-11).

\section{FROM FERTILIZATION TO IMPLANTATION- THE FIRST 4 WEEKS}

Introduction of the color Doppler made it possible to visualize blood vessels that enabled detailed examination of small vessels such as arteries supplying preovulatory follicle, corpus luteum, or endometrium (Figs. 1-5). Perifollicular vascularization can help in the identification of follicles that are containing high-quality oocytes with a high probability of recuperating, fertilizing, cleaving, and implanting. The 3D ultrasound makes it also possible to visualize cumulus oophorus (Fig. 6).

Fertilization is the end of the complicated process of conjunction of gametes by entering one spermatozoon into the oocyte. The gamets, ovum and sperm, contain half of the number of the chromosomes (haploid) compared with the number present in somatic cells (diploid). They obtain haploid number of chromosomes through the process of meiosis during the gametogenesis (4). The oocyte is approximately $120 \mu \mathrm{m}$ in diameter and has a thick membrane known as zona pellucida. The spermatozoon moves using the flagellum or tail, and the total length of the spermatozoon including the tail is $60 \mu \mathrm{m}$. Both oocyte and spermatozoon are highly specialized sex cells. Oocytes are produced in the ovary and expelled from it during the ovulation after which fimbriae of the fallopian tube sweep the oocyte into the ampulla where it can be fertilized. Sperms are produced in seminiferous tubules of the testes and stored in epididymis and ductus deferens. Ejaculation of semen results in the deposit of million sperms in the vagina around the external uterine os. They pass through the uterus and tubes and in the ampulla they surround the secondary oocyte if it is present. When the sperm enters the oocyte, the nucleus of the mature oocyte consists of the female pronucleus and the head of the sperm separates from the tail and enlarges to become male pronucleus. Fertilization is complete when the pronuclei unite and the maternal and paternal chromosomes intermingle during metaphase of the first mitotic division of the zygote. That cell is the primordium of the human being. The inification of the pronuclei restores a haploid number of chromosomes and completes the fertilization. Therefore, fertilization is a distinct moment when the diploid cell is formed and the development of an individual human begins (3).

Transfer of the oocyte is facilitated by the changing endocrine milieu of the early luteal phase with its rising ratio of progesterone to estrogen, which affects the oviductal and uterine musculature and relaxes the isthmic sphincter. It is probable, however, that the cilia, rather than the musculature, are the primary active transporters of the conceptus. Thus, pharmacologic inhibition of oviductal muscles does not prevent transfer of the conceptus. Furthermore, if a segment of oviduct is excised, turned around, and replaced such that cilia beat away from the uterus, the conceptus moves to that part of the oviduct and then it stops (Figs. 7-9) (4).

Already few minutes after the ovulation, oocyte is located in the ampullar part of the fallopian tube. It is surrounded by the zona pellucida. On the surface of the zona pellucida, there are few rows of the granulosa cells that make corona radiata. At the periphery of the corona radiata are left cells of cumulus oophorus. Often, these three units are called oocyte-coronacumulus complex. Capability for the fertilization is limited: oocyte can be fertilized only 6 to 12 hours after the ovulation. Sperms are capable for fertilization 48 to 72 hours, until they are movable. Fertilization occurs in the ampullar part of the fallopian tube. The process of fertilization begins with conditioning of the spermatozoon in the male and female reproductive tracts. Thereafter, fertilization involves not only the egg itself but also the various investments that surround the egg at the time it is released from the ovary follicle. Fertilization, therefore, is not an event; it is a complex biochemical process requiring a minimum of 24 hours to complete syngamy (formation of a diploid set of chromosomes). During that process, there is no commingling of maternal and paternal chromosomes within a single nuclear membrane (pre-zygote); after this process, the paternal chromosome material is commingled (zygote). The most important activity of this new cell is the recognition of the new genome that presents the principal information center for the development of the human being and for all its further activities. 


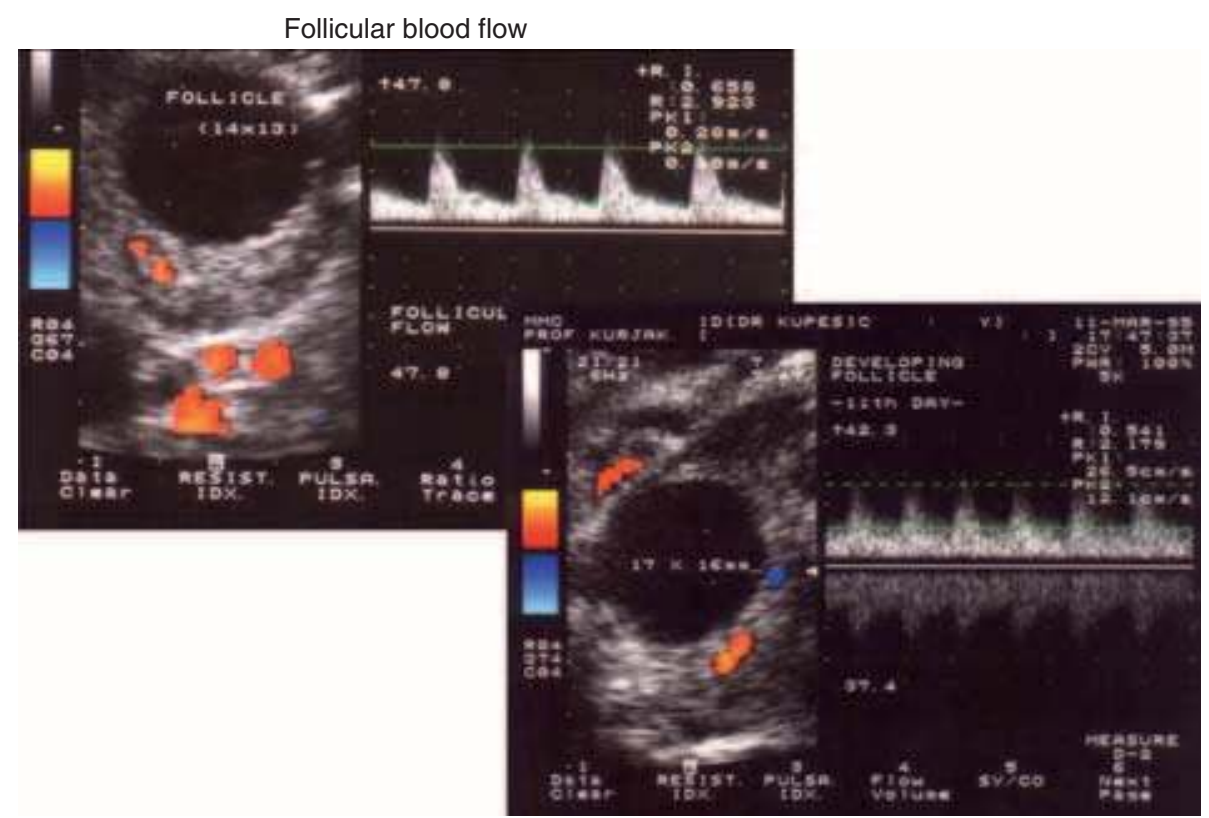

Figure 1 Visualization of morphology and vascularity of preovulatory ovarian follicle on the 10th and 11th day of menstrual cycle.

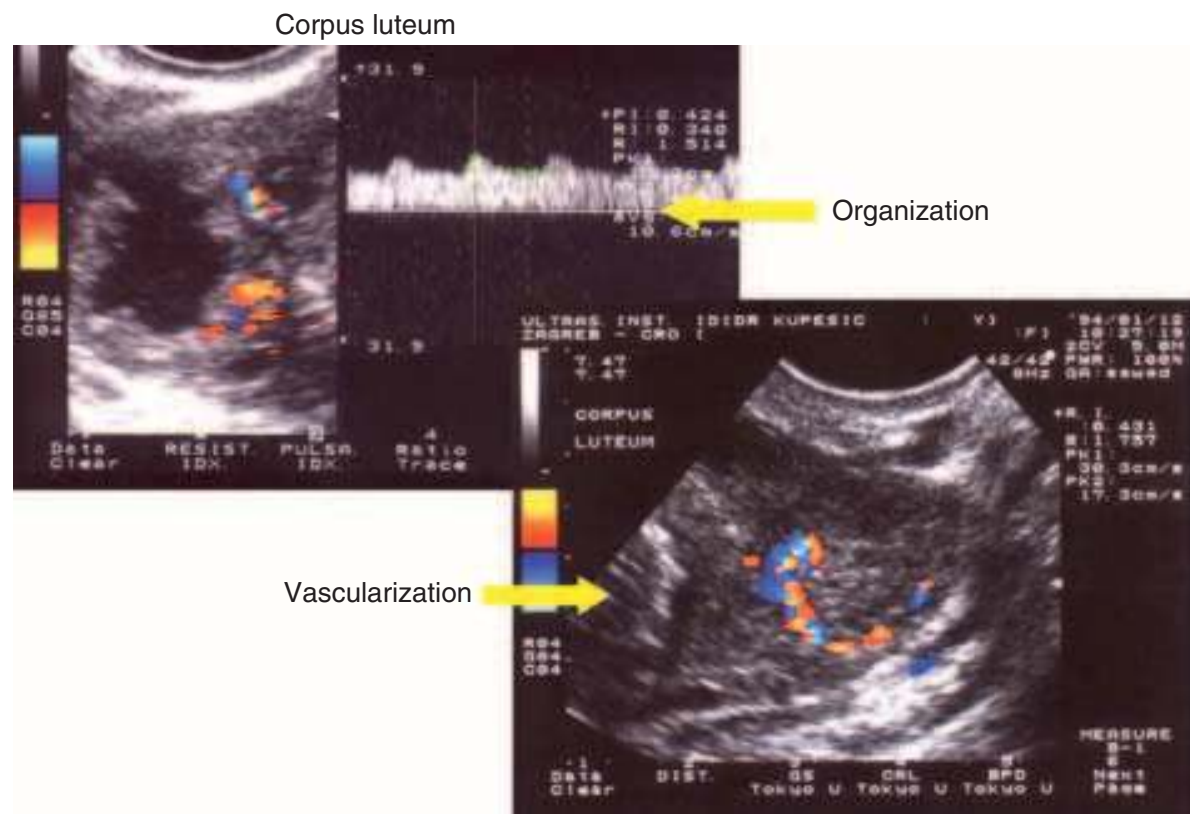

Figure 2 Developmental stages of fresh corpus luteum displayed by transvaginal color Doppler.

\section{Early Development of the Embryo}

After syngamy, the zygote undergoes mitotic cell division as it moves down the fallopian tube toward the uterus. Shortly after gene activation, conceptus shows marked quantitative increase in its biosynthetic capacity. Net synthesis of mRNA and protein increases, transport of amino acids and nucleotides into the cells rises, and changes occur in the synthetic patterns of phospholipids and cholesterol. The growth and metabolic activity of the preimplantation conceptus in vitro has been shown to be stimulated by a number of growth factors that vary between species and include insulin-like growth factors (IGF-1 and 2), transforming growth factors alpha (TGF-alpha) and beta (TGF beta 1 and 2), epidermal growth factor (EGF), and platelet-derived factor A. Receptors of those factors have been identified on human conceptus. Synthesis of many of those factors has been detected in early conceptus itself or in maternal tissues; therefore, it is reasonable to conclude that these factors act as autocrine or paracrine agents that promote the early development (3-5).

A series of mitotic divisions lead to the development of the pre-embryo. The newly divided cells are called blastomeres. 24 hours after fertilization from single-cell zygote two-celled embryo is developed. 2 days after the fertilization embryo consists of 6 to 8 blastomeres. Blastomeres form cellular aggregates of distinct, totipotent, undifferentiated cells that retain the capacity to develop independently into normal pre-embryos. After every division, blastomeres become half of their previous size keeping the total size the same as in the beginning when it was just one cell. All up to eight-cell phase, blastomeres are cluster of cells inside the zona pellucida. 


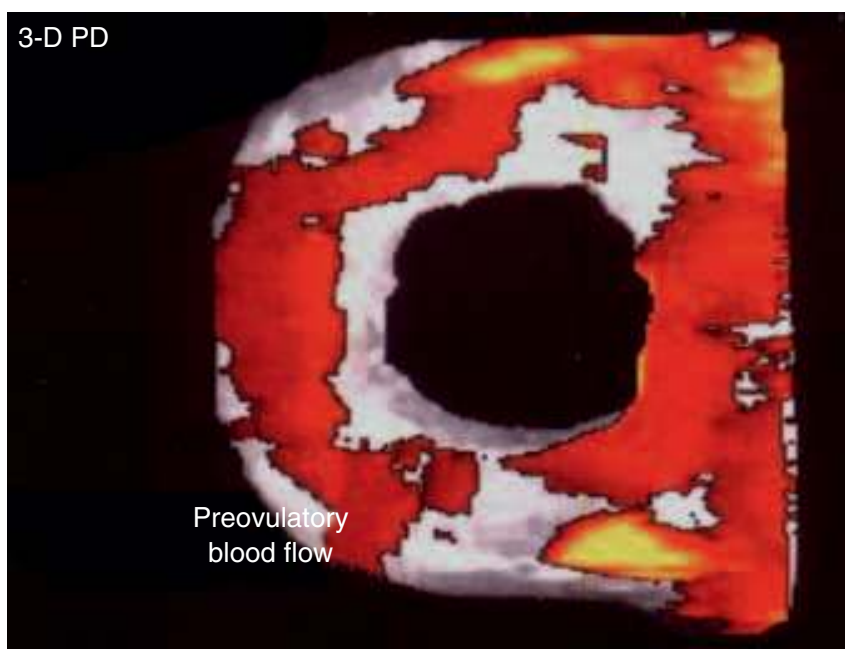

Figure 3 Improved visualization of perifollicular blood flow by threedimensional power Doppler.

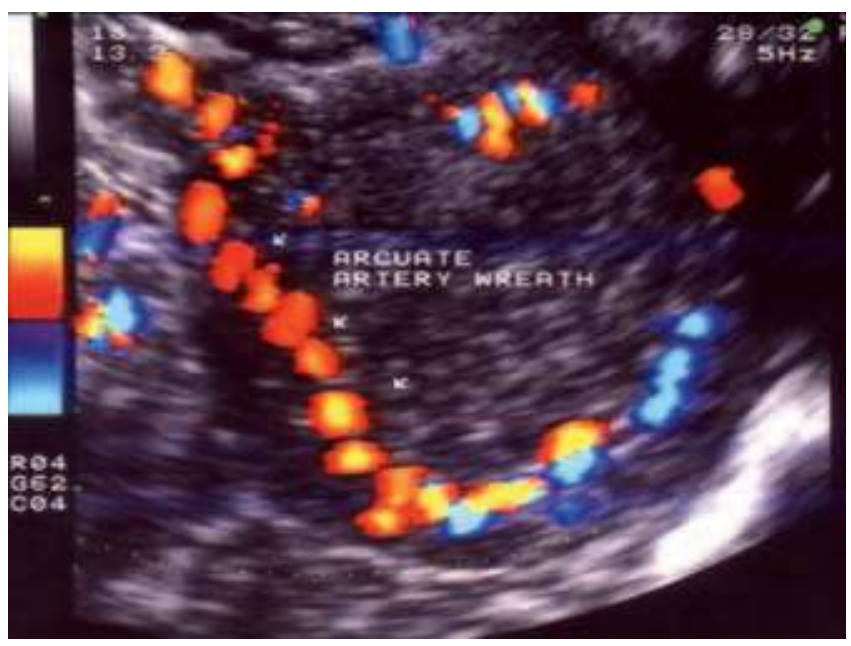

Figure 4 Vascularization of nonpregnant uterus.

But after the third division, they lose their round shape and they impact one to the other making compact round cluster of cells that are tightly connected by tight junctions. With that change, which is called compaction, cells from the outside start to differentiate from those inside. Third day after the fertilization, cells of the compact embryo divide again and morula arises. It contains 16 cells, outer part from which trophoblast will appear and inner cells from which embryo will appear.

Blastocyst enters the uterine cavity. In most species, it appears at the 32- to 64-cell stage. Inner part of cells is then called embryoblast and it is located on one side of the blastocyst. Outer part of cells is trophoblast and it makes epithelial surface of the blastocyst. The expansion of the blastocele is stimulated by growth factors EGF and TGF-alpha (4). Throughout the development, from fertilization to the blastocyst, conceptus remains enclosed in zona pellucida. Zona pellucida has two functions. First, it prevents blastomeres of the conceptus from falling apart during early cleavage period prior to compaction. If conceptus does divide into two distinct groups of cells at this time, monozygotic twins result. Second, it prevents two genetically distinct conceptuses from sticking together to make a single chimeric conceptus (4). Free-living

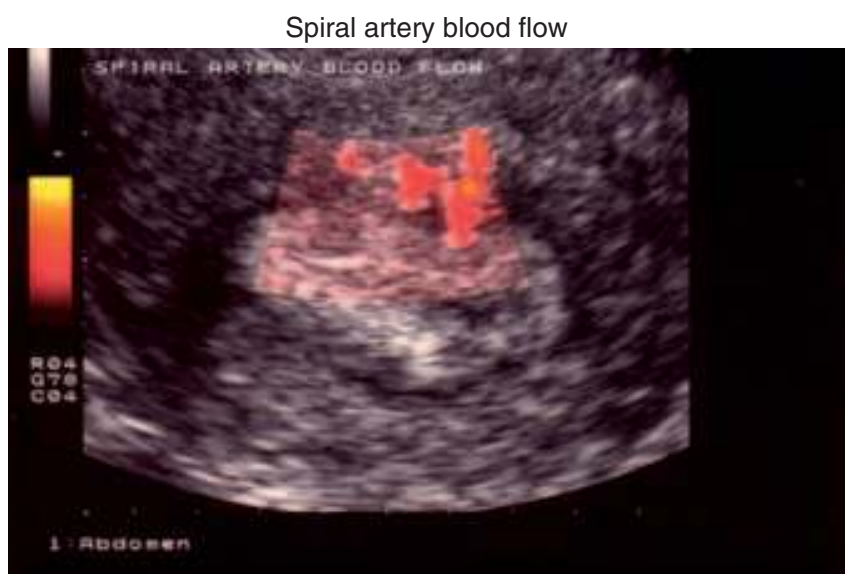

Figure 5 Richly vascularized thickened endometrium at the day of embryo transfer.

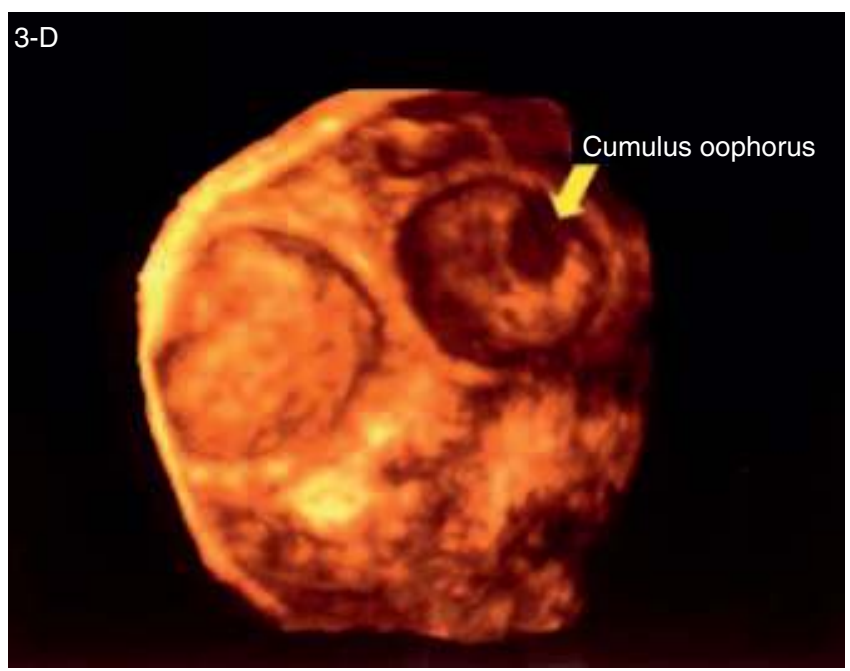

Figure 6 Surface of ovary of a young patient. Follicular cyst and follicle with intrafollicular structure visualized by three-dimensional sonography.

blastocyst is bathed in uterine secretions from which it draws the oxygen and metabolic substrates important for growth and survival. There is a limit of the size that free-living conceptus can attain before such exchanges become inadequate. Before that critical stage is reached, conceptus grows its own blood vascular system. It also grows highly vascularized zones at its extraembryonic surface through which the interchange of materials with maternal tissues is particularly facilitated. During the pre-, peri-, and postimplantation stages of pregnancy, conceptus utilizes maternal juices almost exclusively. That sort of nutrition is called histiotropic. However, this source becomes progressively inadequate and so with the successful conclusion of implantation, in maternal endometrial tissue, develops a corresponding specialized and vascularized region. That region will become a placenta, and this sort of nutrition is called hemotropic.

When zona pellucida disappears, it makes it possible for blastocyst to implant into the endometrium (Fig. 10).

Once entering the uterus, the conceptus engages in an elaborate interaction with the mother in which several messages are transmitted in both directions. First, conceptus establishes physical and nutritional contact with the maternal endometrium at the implantation. Failure to do so would 


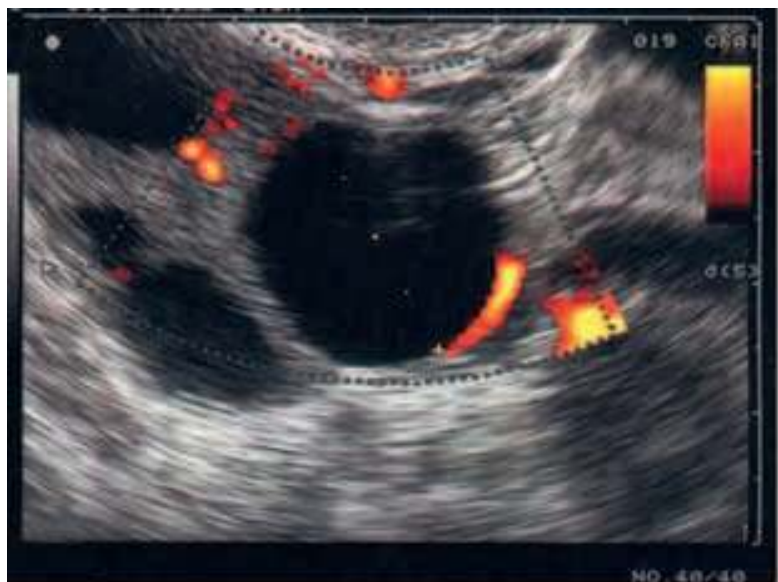

(A)

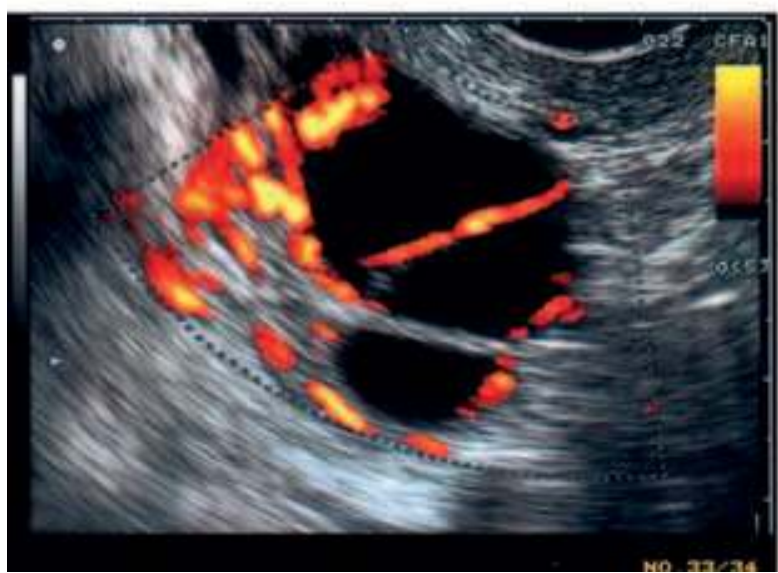

(C)

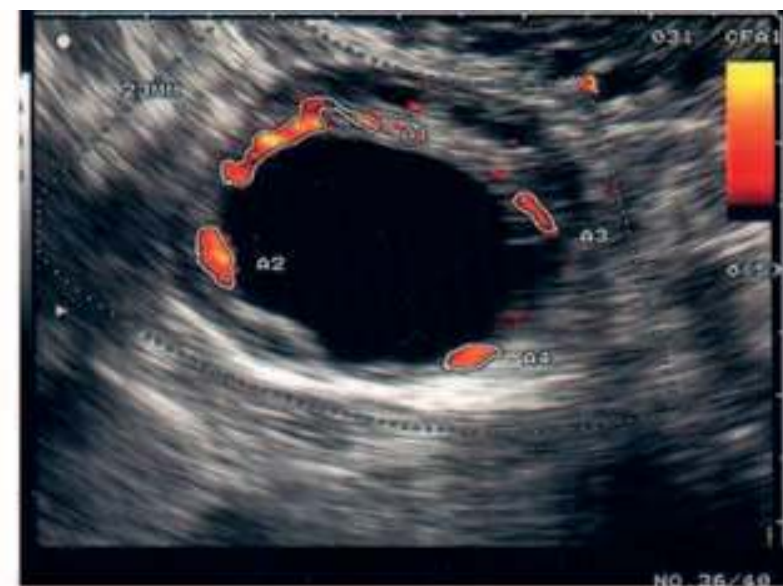

(B)

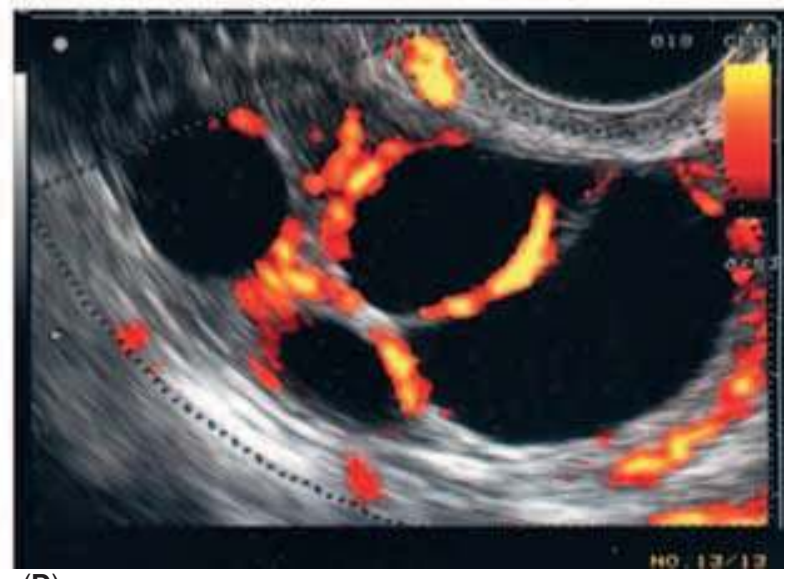

(D)

Classification of perifollicular vascularity according to the percentage of color by power doppler:

A) Grade I, when color mapping takes up less than $25 \%$ of the follicular circumference;

B) Grade II, when color mapping covers between 25 and 50\%;

C) Grade III, if color signal appears between 50 and $75 \%$; and

D) Grade IV, color mapping between 75 and $100 \%$ of the follicular perimeter.

Figure 7 Power Doppler display of vascularization of periovulatory follicle.

deprive the conceptus of essential nutritional substrates and arrest in growth. Second, conceptus signals its presence to maternal pituitary-ovarian axis; somehow it must convert the whole female reproductive system from cyclic pattern to a noncyclic in which progesterone dominates.

At the time when blastocyst is prepared for implantation, in the middle of the luteal phase of cycle, endometrium is about 10 to $14 \mathrm{~mm}$ thick. It is prepared for the implantation by various factors: EGF, IGF-I, and local paracrine and autocrine factors. From day 16 to 22 , the receptivity of the endometrium is the highest, which is called "implantation window." At that time, a large number of pinopodes (outgrowth of cytoplasm of endometrial cells) occur, and help blastocyst to implant. When zona pellucida disappears, it makes it possible for conceptus to make contact with decidual endometrium. Implantation consists of three phases: apposition, adhesion, and invasion. Apposition and adhesion are assisted with adhesive molecules of endometrium like laminin, fibronectin, and receptors like integrins. Conceptus produces signals that make change in mother's blood flow. It produces early pregnancy factor, which has immunosuppressive effect. By the beginning of the implantation, proteases and matrix metalloproteinases are activated. Conceptus that is stuck to the surface of the endometrium begins its invasion.
Enzymes of the trophoblast invade mother's circulation and build hemochorial placentation.

In some species, implantation is invasive (humans) in difference to some where it is noninvasive. Invasive conceptus tends to be smaller at attachment and only a few trophoblast cells are involved in making contact with the maternal endometrium. Within few hours, increased vascular permeability is observed. This is associated with edema and a progressive sprouting and ingrowth of capillaries. The stromal reaction is particularly marked in primates where it is called primary decidualization reaction. The uterine glandular and decidual tissue adjacent to the invading trophoblast of the conceptus is destroyed. It releases primary metabolic substrates: lipids, carbohydrates, nucleic acids, and proteins, which are taken by the growing conceptus. Decidual tissue thus functions like a large yolk reservoir.

The invasiveness of the conceptus is influenced by the effectiveness of the decidual response. The depth of the invasion varies between species. Those species in which the conceptus invades the stroma so deeply that the surface epithelium becomes restored over it are called to be implanted interstitially.

Endometrium that occurs in the attachment process involves leukemia inhibitory factor (LIF). It is produced by 


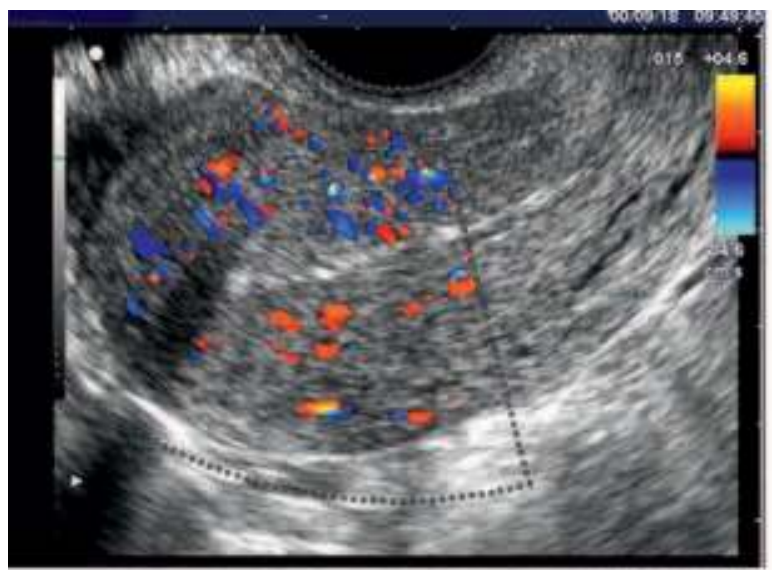

(A)

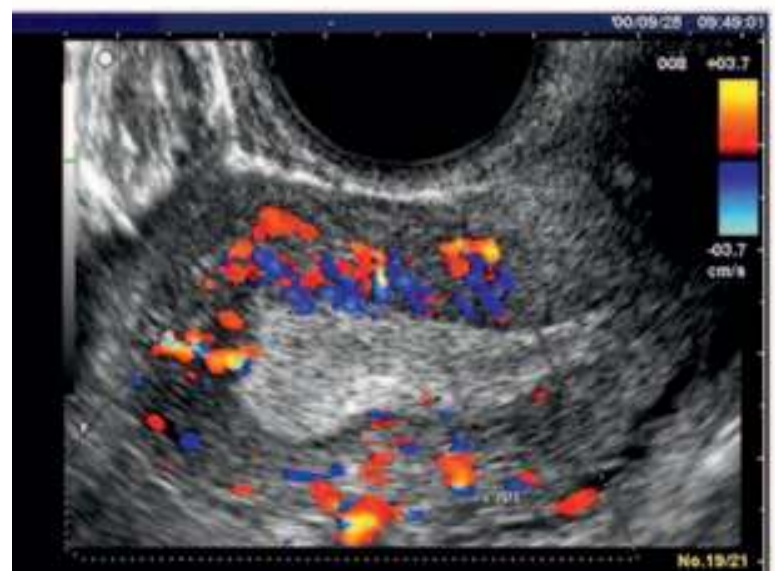

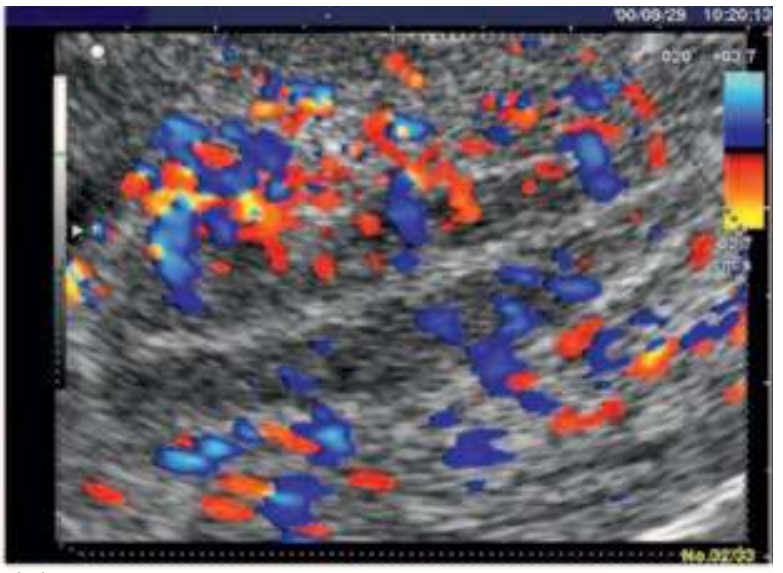

(B)

Patterns of endometrial echogenicity:
A) Non "triple-line" endometrium;
B) Proliferative "triple-line" endometrium
C) Secretory endometrium.

Figure 8 Endometrial thickness and rich vascularization indicating good uterine receptivity.

the cells of the endometrial glands under the influence of estrogen. Receptors for LIF also appear in both the epithelium and the stroma of the endometrium around the time of implantation. Seems like LIF promotes endometrial receptivity to attachment and/or subsequent decidualization.

In the presence of the estrogen, either the conceptus produces the signal or the local endometrium becomes sensitive to a signal being already sent. The nature of this putative signal is unknown, but it induces the HB-EGF production locally and the conceptus in turn responds in shedding its zona, attaching and invading (4).

The invasiveness of the trophoblast depends of the expression and regulation of the different proteolytic enzymes. TGF-beta promotes decidualization and controls trophoblastic invasion. Histamine and prostaglandins are involved in the stromal response: inhibition of either one of them reduces the decidualization.

An important feature of decidual response is increase in vascular permeability and growth of new capillaries (angiogenesis).

As implantation proceeds, trophoblast differentiates into two layers: inner cytotrophoblast and outer syncytiotrophoblast. Around day 9, syncytiotrophoblast cells begin to produce human chorionic gonadotropin (hCG). It maintains the thick endometrium and can be detected in maternal blood or urine, which is used for early pregnancy testing (3).

A small intradecidual gestation sac can be seen by transvaginal sonography between 32 and 34 days (Figs. 11 and 12). The secondary yolk sac is the earliest extraembryonic structure normally seen within the gestational sac at the beginning of the 5th gestational week (Fig. 13). Yolk sac volume increases from week 5 to 10 . When it reaches its maximum at week 10 , it would have already started to degenerate, which can be seen by the significant reduction in visualization rates of the yolk sac vascularity. The start of embryo-chorionic circulation changes the source of nourishment to all intraembryonic tissues. Development of the embryo becomes dependent on the circulation of embryonic blood. In the embryo, there are three distinct blood vascular systems: vitelline circulation (from yolk sac to embryo), intraembryonic circulation, and feto-placental circulation. It is possible to visualize and assess them virtually from conception.

At 5 weeks it is possible to obtain a 3D image of the developing intervillous circulation in the maternal site of the placenta (Fig.14). Doppler shows intense vascular activity surrounding the chorionic shell. At 7 weeks 3D power Doppler images depict aortic and umbilical blood flow.

\section{DEVELOPMENT OF EMBRYO THROUGH FIRST} 4 WEEKS

Along with the implantation process, changes occur in the embryoblast to produce a bilaminar embryonic disc, composed of the epiblast and the hypoblast. Early in the 2nd week, the amniotic cavity appears as a space lined with amnioblasts derived from the epiblast. By the end of the 2 nd week, the embryonic disc becomes oval in shape. Along the median line 


\section{Echographic characteristics of implantation and early placentation}
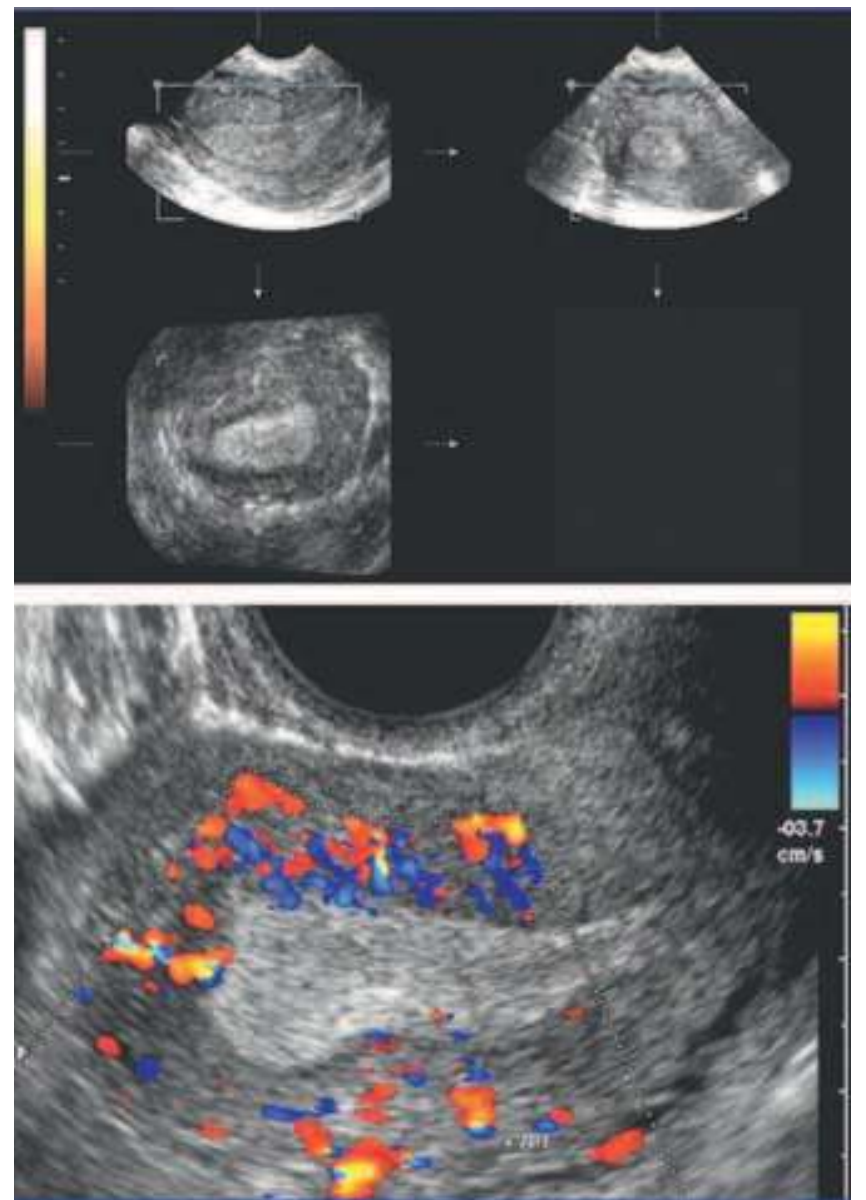

Endometrium - secretory phase (3-4 weeks)

- The "triple - line" disappears and is replaced by an echogenic pattern. At this stage the endometrium is at the maximum thickness as it reaches upto $14 \mathrm{~mm}$.

Figure 9 Vascularization is very obvious in the secretory phase of menstrual cycle.

in the posterior region of the embryonic disc, a thickening of the epiblast called the primitive streak appears, and it defines the longitudinal axis of the embryo. During the 3rd week, lateral epiblast cells migrate medially, enter the primitive streak, and then converge to form the primitive groove.

During the latter half of the 3rd week, drastic changes for morphogenesis (development of the body form) begin. The embryonic disc elongates craniocaudally to become pearshaped and then slipper like. Cranial to the primitive node, a thickening of the ectoderm appears bilaterally and is called the neural plate because it will eventually develop into the neural tissue. Between the bilateral neural plates, a longitudinal neural groove develops in the midline, flanked by the neural folds bilaterally. Around day 20, the bilateral neural folds begin to fuse with each other to form the neural tube, which is the primordium of the central nervous system (CNS; brain and spinal cord). The rostral (anterior) neuropore normally closes on days 24 to 26, and the caudal (posterior) neuropore closes by 28 days. When the neuropores close completely, three brain vesicles (forebrain or prosencephalon, mesencephalon or midbrain, and rhombencephalon) have already appeared. Rarely the neural tube fails to close completely, resulting in a variety of neural tube closure defects such as anencephaly, encephalocele, myeloschisis, and myelomeningocele.
By the end of the 3rd week, the paraxial mesoderm condenses segmentally along the midline to form somite pairs. First the somites are recognized as conspicuous surface elevations and gradually increase in number craniocaudally. The somites give rise to most of the axial skeleton, trunk and limb musculature, and dermis of the skin. The formation and specification of somite segments are controlled by a unique combination of Hox genes (Hox code). Concurrent with somite differentiation, the intraembryonic coelom arises as isolated spaces between the layers of the lateral plate mesoderm, which is the primordium of body cavities. The coelomic cavities subsequently coalesce to form a single, horseshoe-shaped cavity that eventually gives rise to the pericardial, pleural, and peritoneal cavities.

At the beginning of the 4th week, the embryo is almost straight. During the 4th and 5th weeks, the embryo body undergoes a drastic shape change as a result of embryonic folding. Embryonic folding occurs in both the longitudinal and the horizontal planes, resulting from the rapid growth of the embryo, especially the brain and spinal cord, as well as the expansion of the amniotic cavity. Folding of the embryo in the horizontal plane rolls the lateral parts of the embryonic disc ventrally and forms a cylindrical embryo. 


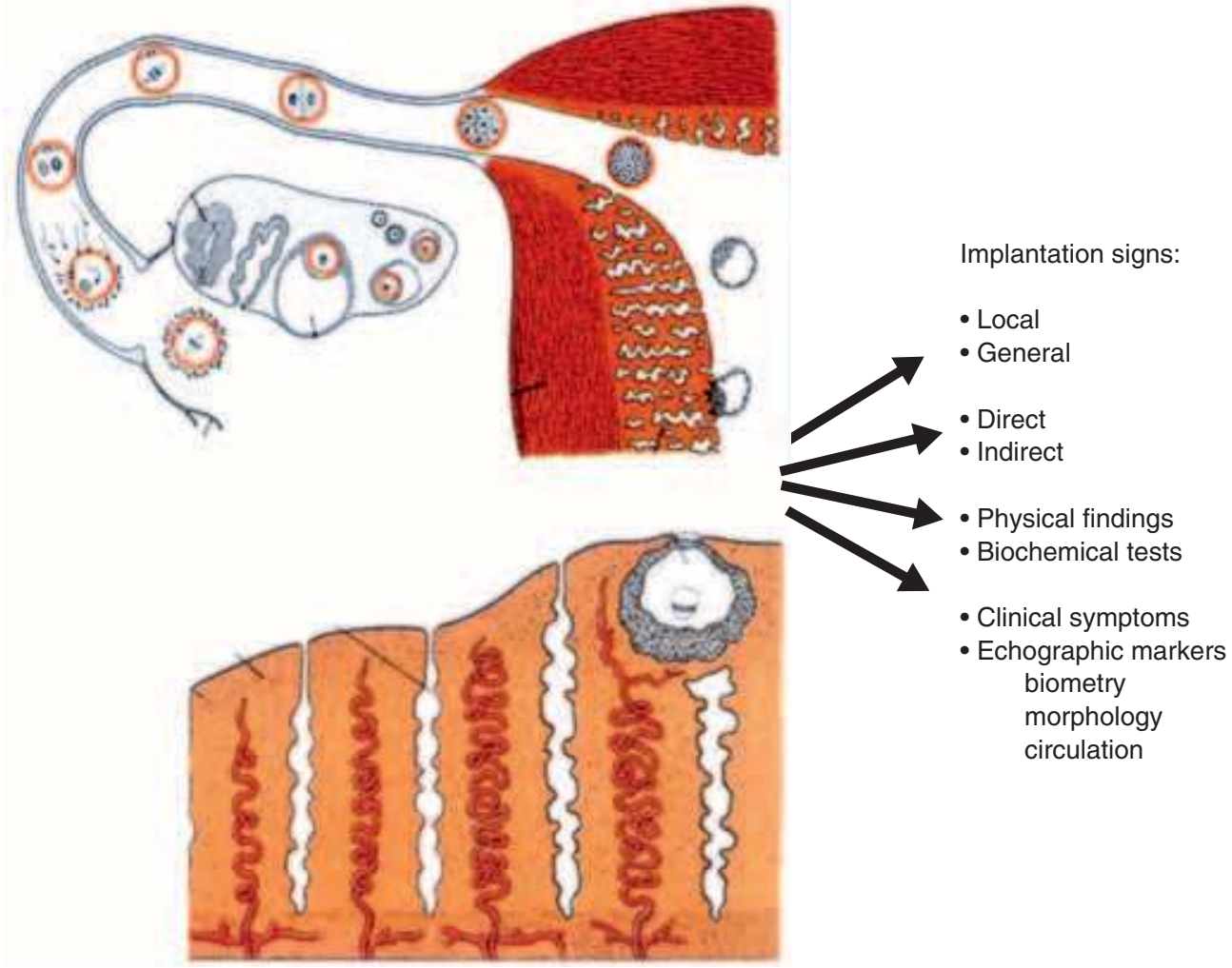

Figure 10 Schematic illustration of the process of implantation.

Echographic characteristics of implantation and early placentation

Gestrational sac (5th week)
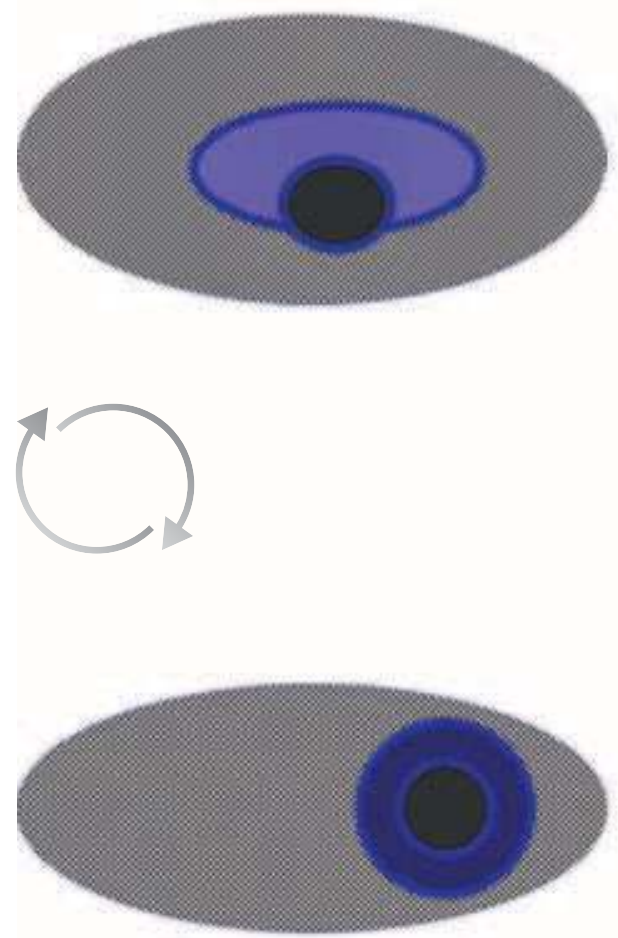
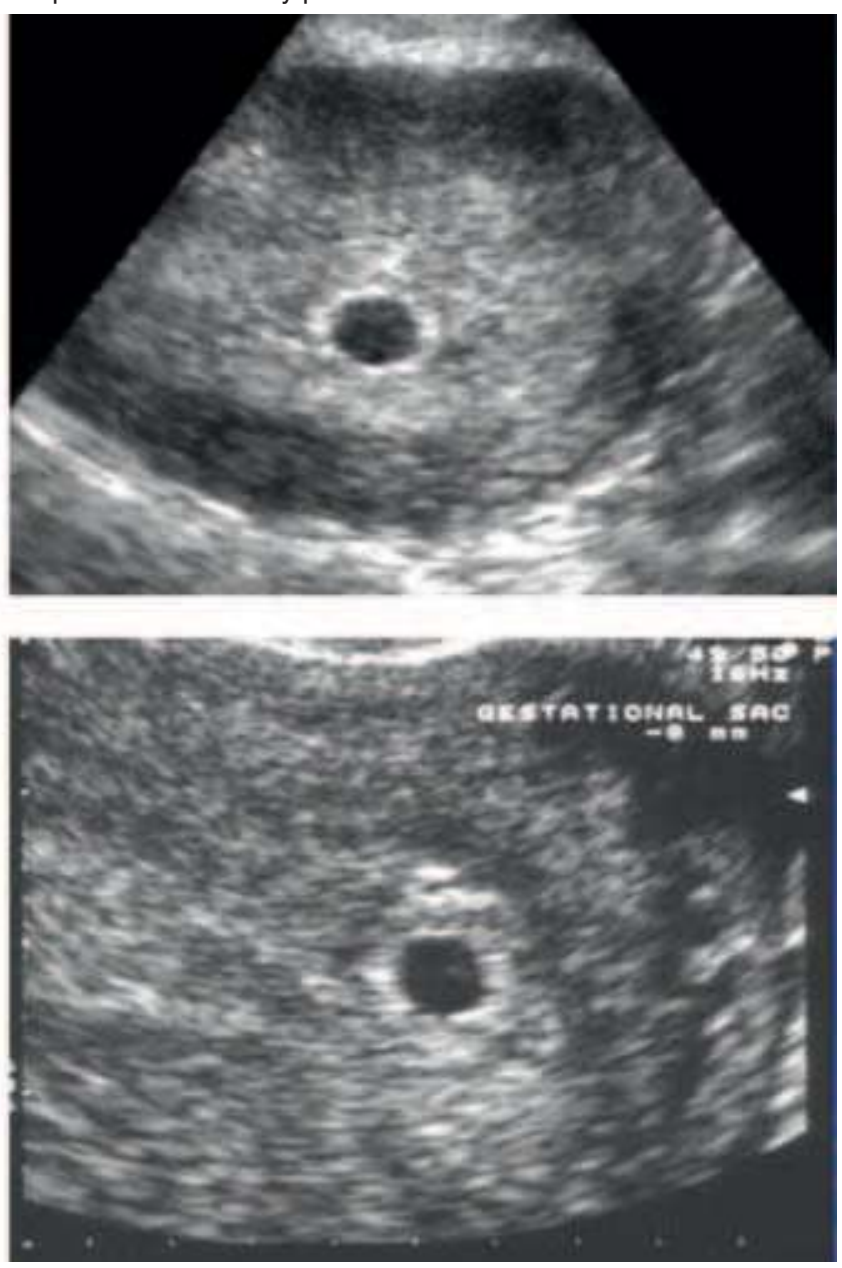

Figure 11 Gestational sac at 5 weeks visualized by two-dimensional sonography. 
Another important developmental event that occurs during the 4th week is the emergence of limb buds. Forelimb buds appear by 28 days and hindlimb buds about 2 days later. By the end of the 4th week, the embryo is in a C-shape.

During the 5th week, limb buds elongate and their distal ends become paddle shaped. The second pharyngeal arch overgrows the third and fourth arches and forms an ectodermal depression known as the cervical sinus. Ultrasonography reveals some primitive spontaneous movements of the embryo, such as twitching of the trunk, in the 7th week.
During the 6th week, the upper limbs undergo rapid morphologic differentiation, and digital rays, indicating that the future digits are recognizable. By the end of this week, the elbow and wrist regions become clearly identifiable. Retinal pigment is visible in the eyes. On the first and second pharyngeal arches, six swellings develop as the primordia of the auricle of the external ear. As a result of the rapid growth of the brain, the head is larger relative to the trunk and starts to lie over the cardiac prominence (1-6).
Echographic characteristics of implantation and early placentation

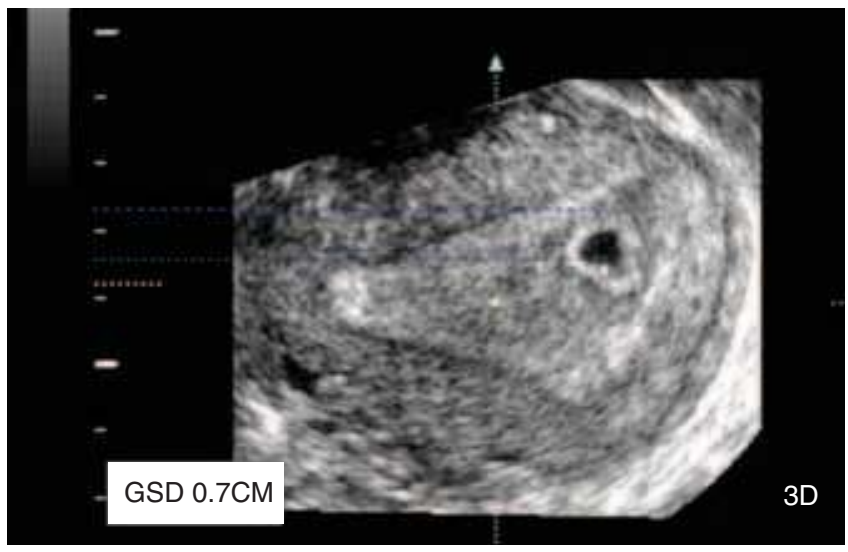

Figure 12 The same sac shown by three-dimensional sonography.
Echographic characteristics of implantation and early placentation

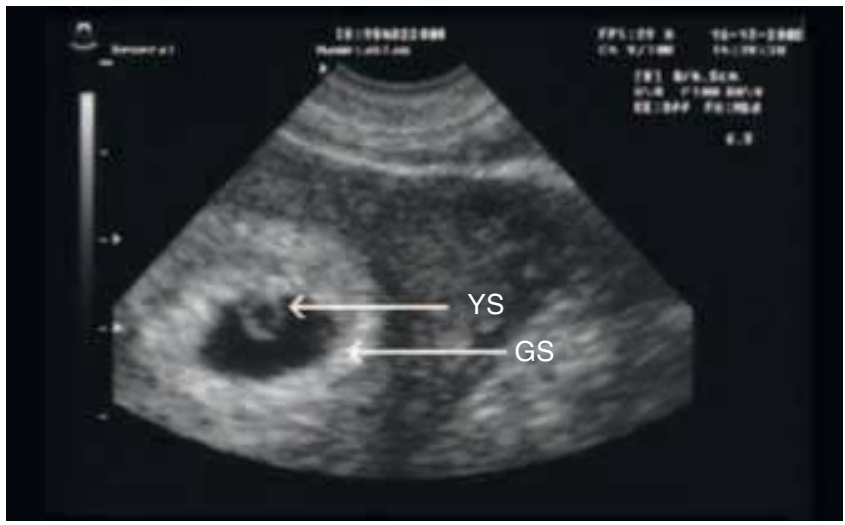

Figure 13 The first visible structure at 5 weeks is yolk sac (YS).

Establishment of intervillous circulation (IVS).

(A)
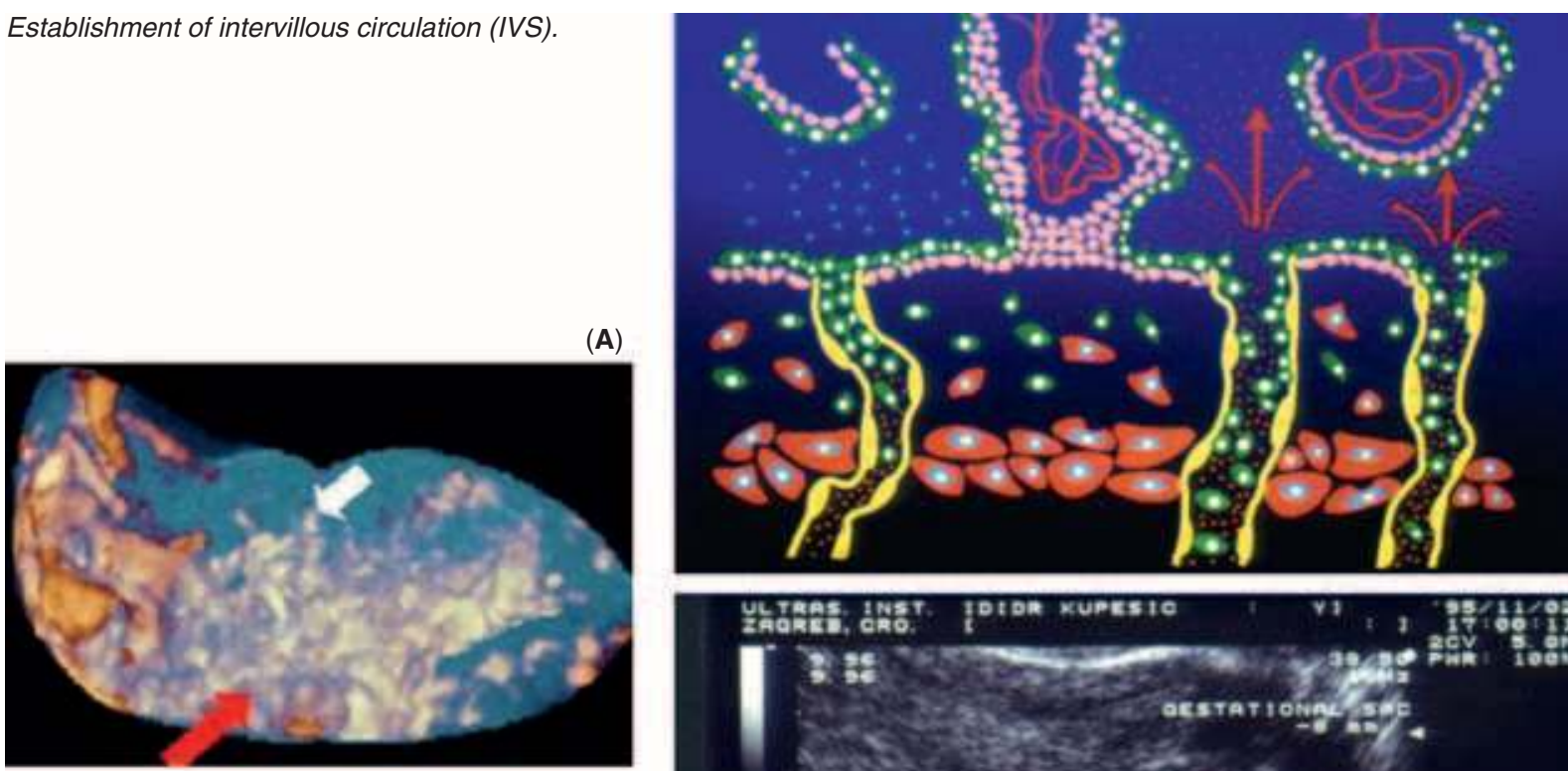

(B)
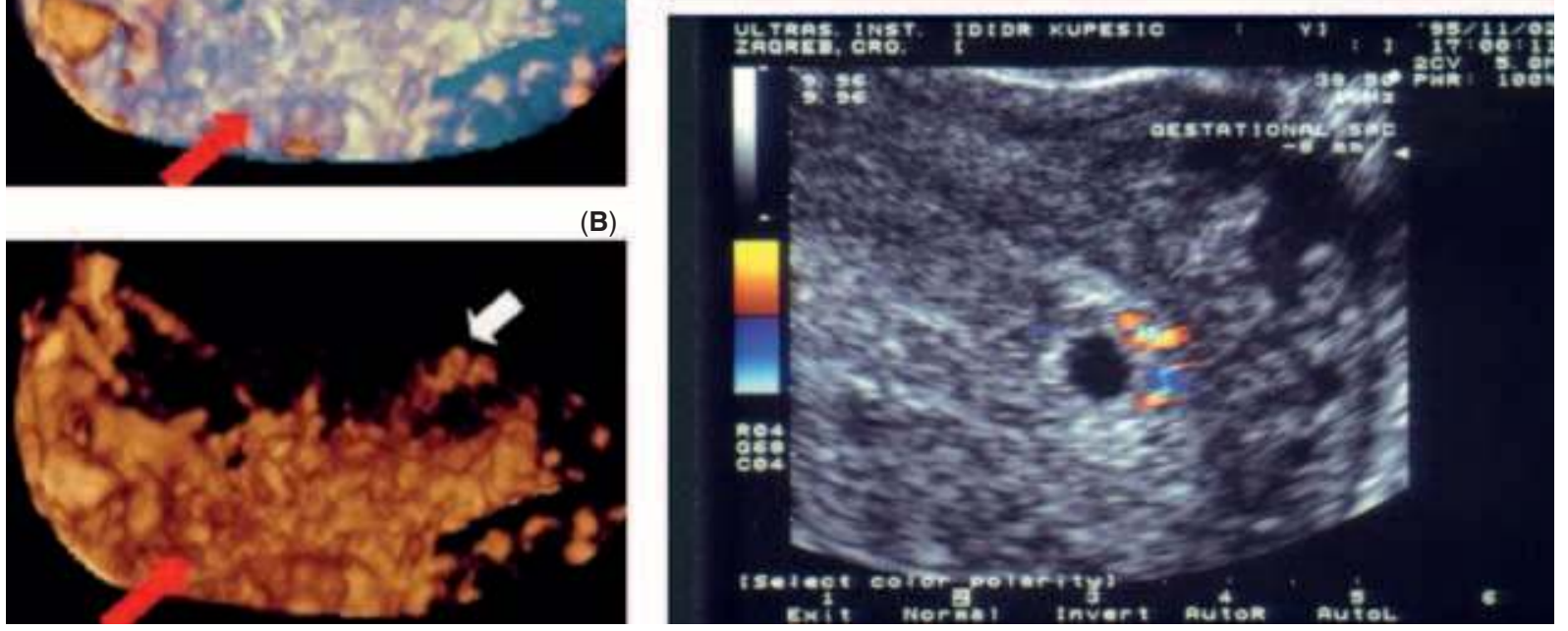

Figure 14 Development of intervillous circulation visualized by three-dimensional power Doppler (left) and conventional Doppler (right). 


\section{ULTRASONIC VISUALIZATION OF EARLY HUMAN DEVELOPMENT}

Gestational sac can be visualized from the middle of the 5th week of amenorrhea as a small spherical anechoic structure placed inside one of the endometrial leafs (7). Planar mode tomograms are helpful in distinguishing early intraendometrial gestational sac from collection of free fluid between the endometrial leafs (pseudogestational sac). The 3D sonography enables precise measurement of exponentially expanding gestational sac volume during the first trimester. At the beginning of the 5 th week, small secondary yolk sac is visible as the earliest sign of the developing embryo (Fig. 15). Adjacent to the yolk sac, embryo can be seen as a small straight line when it reaches 2 to $3 \mathrm{~mm}$ in length at the end of the 5 th week.

\section{Six Weeks}

Typical characteristics of the 6th week embryo include a rounded bulky head and a thinner body. The head is prominent due to the developing forebrain. Limb buds are rarely visible in this stage of pregnancy. However, umbilical cord and vitelline duct are always clearly visible. At this stage of pregnancy, the planar mode will allow for more accurate assessment of multiple pregnancies.

\section{Seven Weeks}

During the 7th week, fast development of rhombencephalon (hindbrain) takes place. This process gives even more prominence to the head. By the use of planar mode developing, vesicles of the brain can be depicted as anechoic structure inside the head. The biggest and usually the only visible part is rhombencephalon placed on the top of the head (vertex). Diencephalon and its cavity become visible few days later. The head is strongly flexed anteriorly being in the contact with the chest. Limb buds are often visible laterally to the body.

\section{Eight Weeks}

During the 8th week of pregnancy, there is expansion of the ventricular system of the brain (lateral, third, and midbrain ventricles). The shape of the face is becoming apparent, but the flexion of the cranial pole still makes it difficult to view the face entirely (Fig. 15). The vertex is now located over the position of the midbrain. Structures of the viscerocranium are not visible due to the small size. Arms and feet are clearly visible. Insertion of the umbilical cord is visible on the anterior abdominal wall. During the 8th and 9th weeks, developing intestine is being herniated into the proximal umbilical cord. The amnion can be clearly seen and the extraembryonic coelom is still larger in volume than the amniotic sac.
$5 w 2 d$
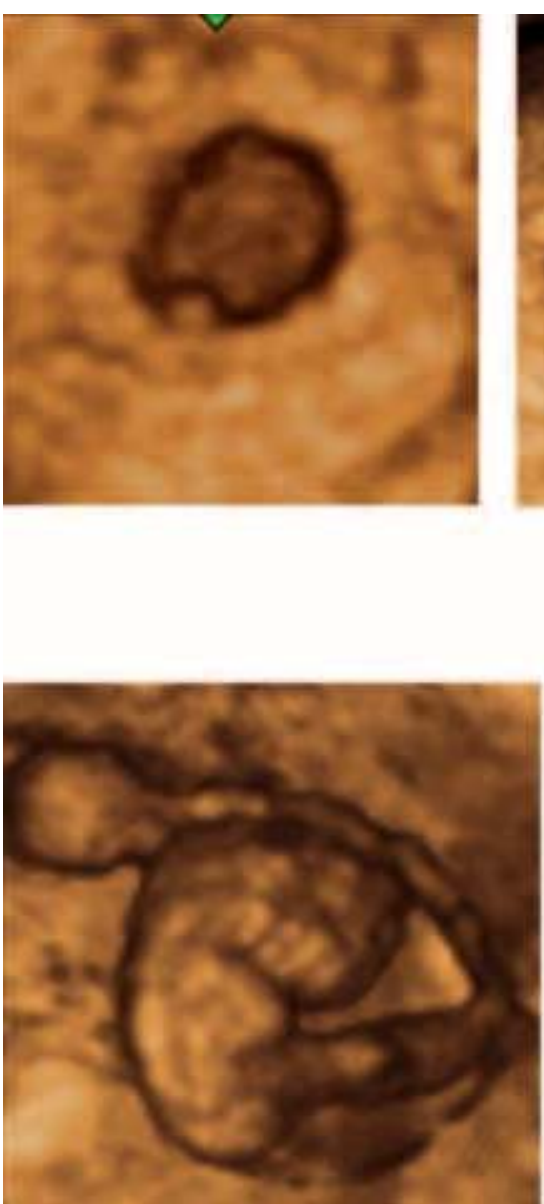

$8 w 3 d$
$6 w 3 d$
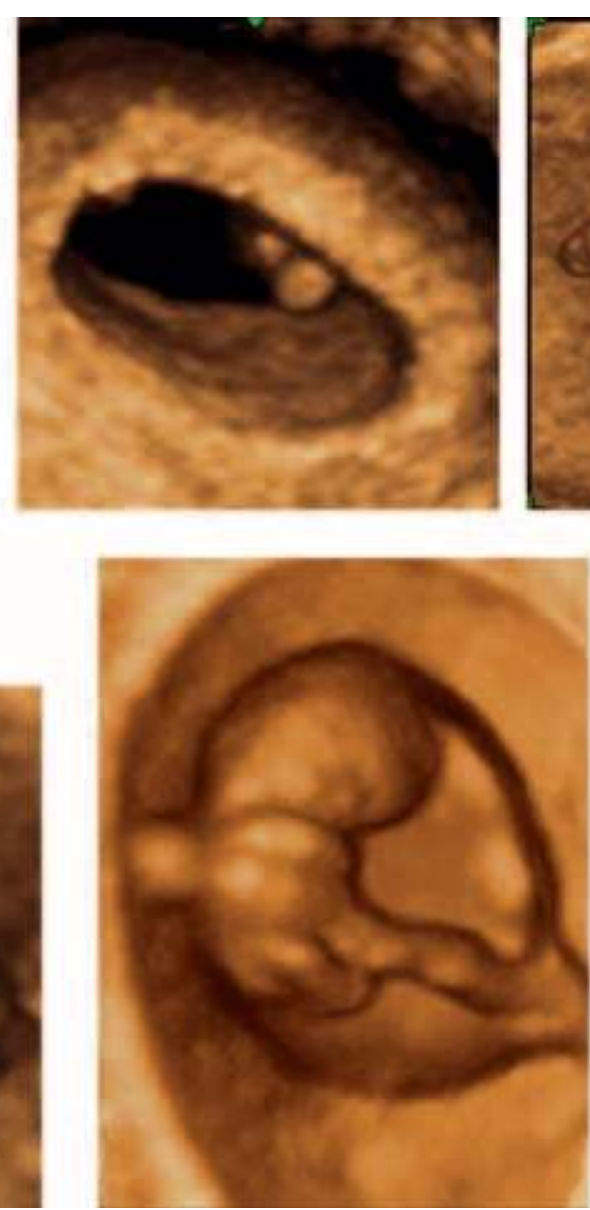

$9 w 0 d$
$7 w 3 d$

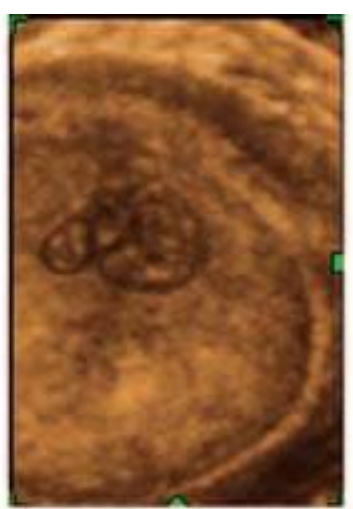

8w0d

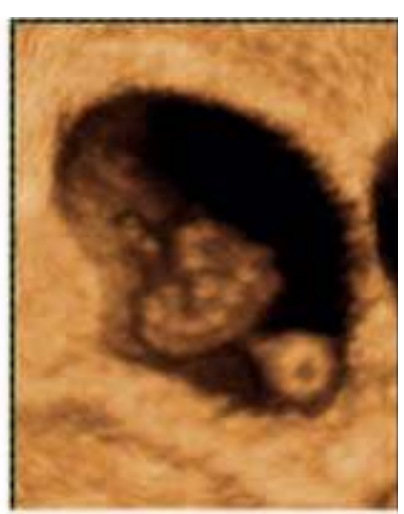

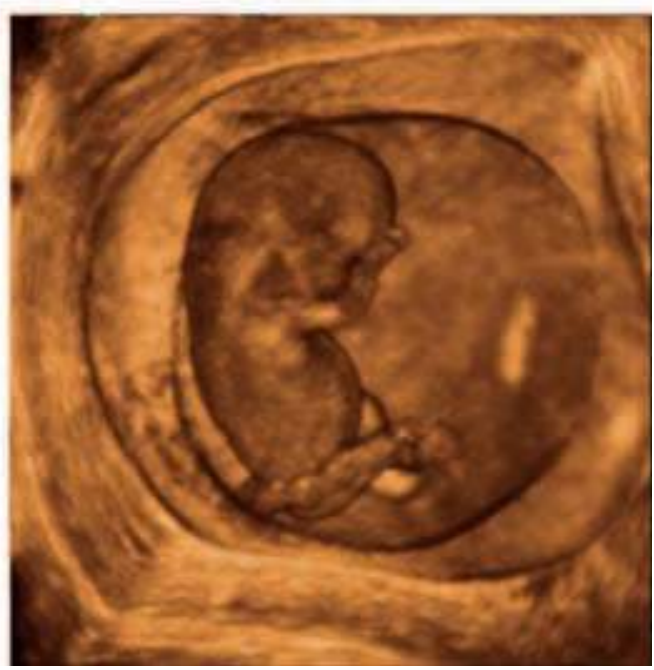

$10 w 3 d$

Figure 15 Normal morphologic development from 5 to 10 weeks of gestation. 
The extraembryonic space will appear more echogenic than the amniotic sac. The ventricular cavities are characteristically cystic, particularly the rhombencephalon. This should not be confused with an early diagnosis of Dandy Walker cyst.

\section{Nine to Ten Weeks}

Cerebral hemispheres continue with development during the 9th and 10th weeks of pregnancy. Visible are lateral ventricles containing hyperechoic choroid plexuses. The head is clearly divided from the body by the neck. External ear is sometimes depicted in the 3D surface image. Herniation of the midgut is present. Dorsal column, the early spine, can be examined in its whole length. The arms with elbow and legs with knee are now visible. Feet can be seen approaching the midline (Fig. 15).

\section{Eleven to Twelve Weeks}

During the last phases of the end of the first trimester, striking changes in the development of the head and neck continue. Details of the face such as the nose, orbits, maxilla, and mandible are visible. Herniated midgut returns to the abdominal cavity.

Planar mode enables detailed analysis of embryonic body with visualization of the stomach and urinary bladder. Kidneys are also often visible. Arms and legs continue with development. Long bones can be visualized as hyperechoic elongated structures inside upper and lower extremities. Fingers and toes are visible (Fig. 16). Detailed 3D analysis of fetal spine, chest, and limbs is obtainable by using the transparent X-ray-like mode.

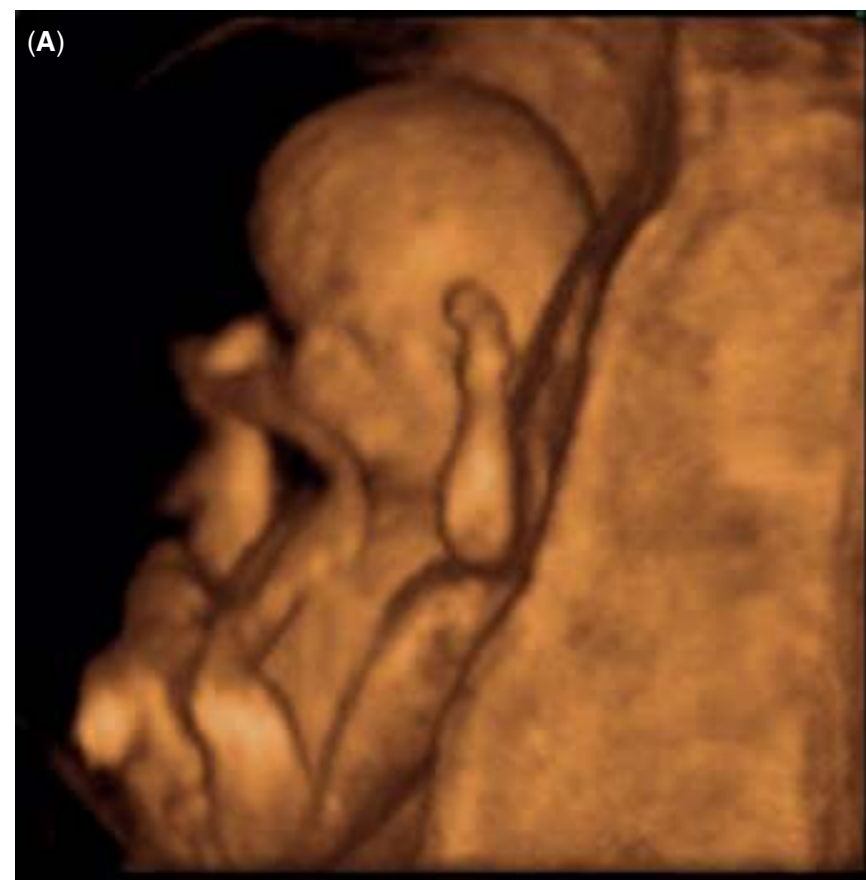

\section{(B)}

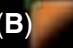

\section{(C)}

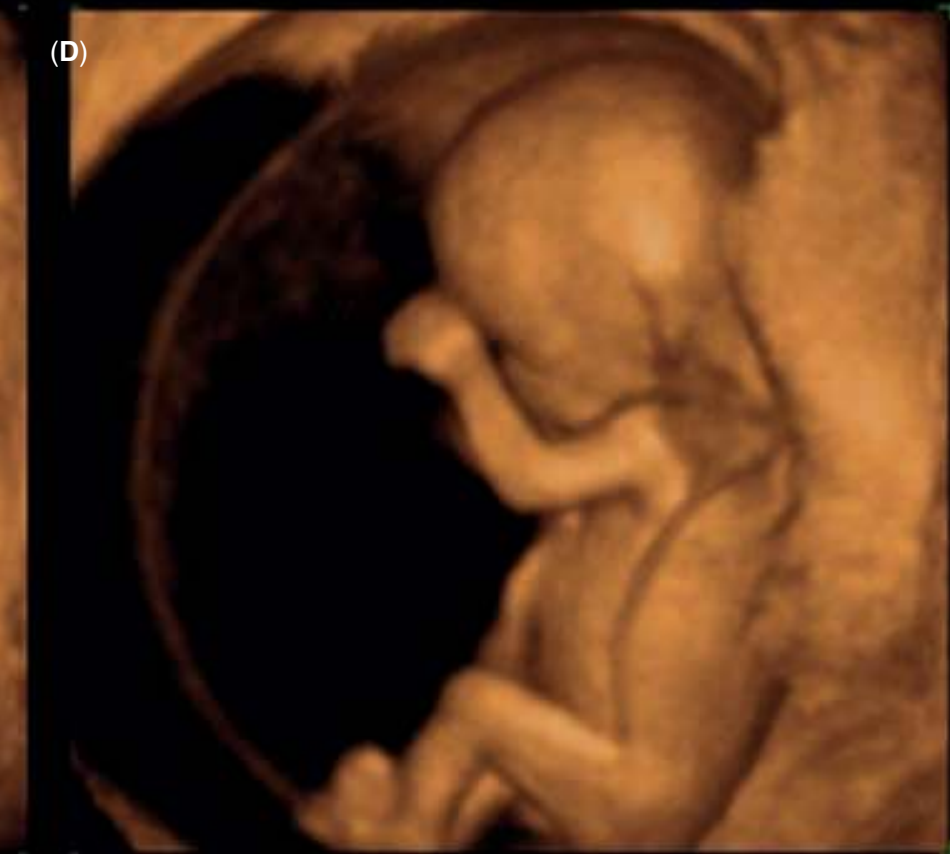

Figure 16 Fetus at 11 weeks showing movement of upper and lower extremities (A-D). 
Excellent visualization has been achieved by the $3 \mathrm{D}$ and $4 \mathrm{D}$ ultrasound combined with the transvaginal approach $(1,6,8,10,11)$. Demonstration of small embryo less than $10 \mathrm{~mm}$ is still difficult. Figure 17 shows $18.3 \mathrm{~mm}$-CRL (crown-rump length) embryo at 8 weeks of gestation and macrographic pictures of an aborted specimen at the same gestational age.

Maximum mode of 3D ultrasound can clearly demonstrate fetal bony structure. Rapid ossification of craniofacial bones dramatically occurs during the first trimester. Craniofacial bony structure can be demonstrated from 10 weeks of gestation by ultrasound. Figures 18 and 19 show premature structure of craniofacial bony structure visualized by using maximum mode of 3D ultrasound at 11 and 14 weeks of gestation respectively.

Difference of frontal bone appearance between 11 and 14 weeks indicates membranous ossification of cranium in this stage. Figure 20 demonstrates bony structure of the occipital region of head at 13 weeks of gestation.

During early embryonal period, the CNS structure rapidly changes its appearance. The $3 \mathrm{D}$ ultrasound produced high-resolutional images of embryos in early stage by tomographic ultrasound imaging and three-orthogonal imaging technologies (Figs. 21-26).

Indeed, advances described deserve the adjective "breathtaking," in particular 4D parallel study of the structural and functional early human development (12-23).

Those 3D neuron-sono-embryologic imaging in utero may change embryonal staging in vivo in future, from "gestational age based on menstrual period or measurement of CRL" into "morphologic staging system" such as Carnegie staging in embryology. Indeed, improvements in the visualization deserve the adjective "breathtaking" in the better inside into the early human development $(1,6,24-29)$.

\section{THE ASSESSMENT OF VASCULARITY BY 3D POWER} AND COLOR DOPPLER SONOGRAPHY

By adding colored blood flow signals to the gray scale image or extracting just the color-coded signals, it is possible to depict 3D image of vascular features of early pregnancy (Fig. 27).

This enables research of longitudinal changes in embryonic and early uteroplacental circulation. It has been found

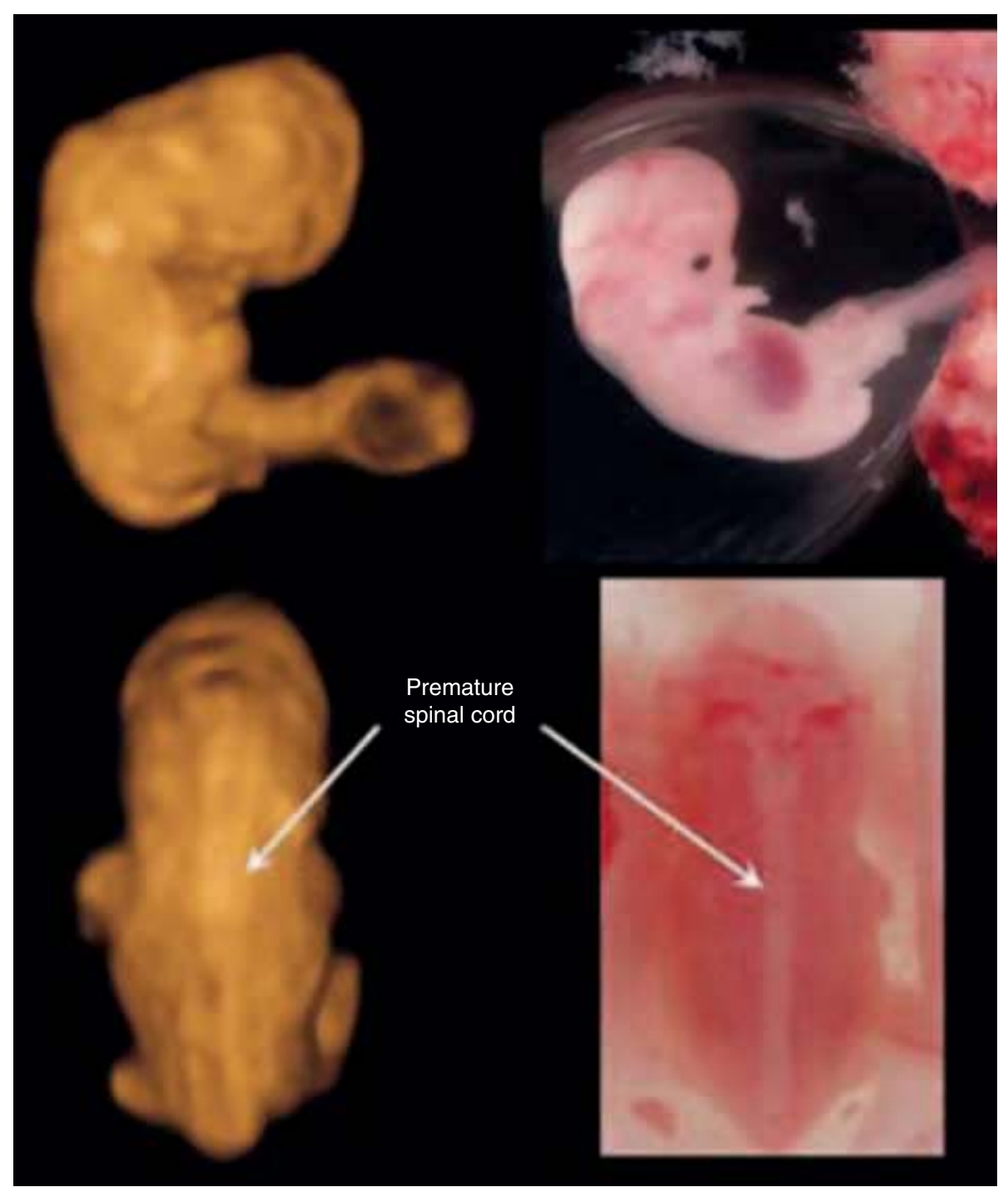

Figure 17 Three-dimensional ultrasound images of the embryo at 8 weeks of gestation (left) and macroscopic pictures of aborted fetus at the same gestation (right). Normal 8-week embryo. Lateral view (upper left) and occipital view (lower left). Occipital view shows the premature spinal cord that is seen in the right picture. Source: From Ref. 6. 


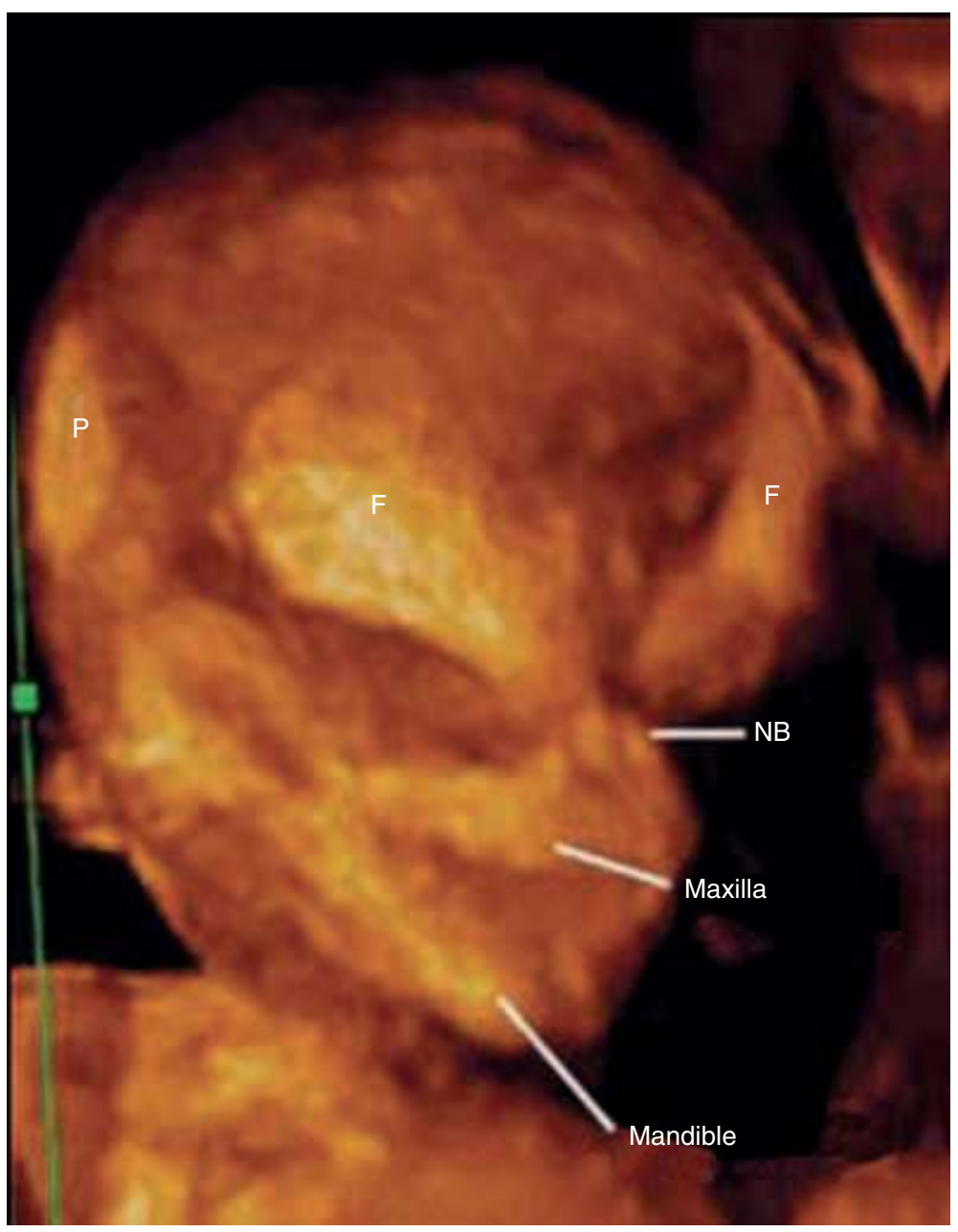

Figure 18 Three-dimensional maximum mode image of normal craniofacial structure at 11 weeks of gestation. Premature bony structure of frontal bone (F), parietal bone (P), nasal bone (NB), maxilla, and mandible are recognizable. Source: From Ref. 6.

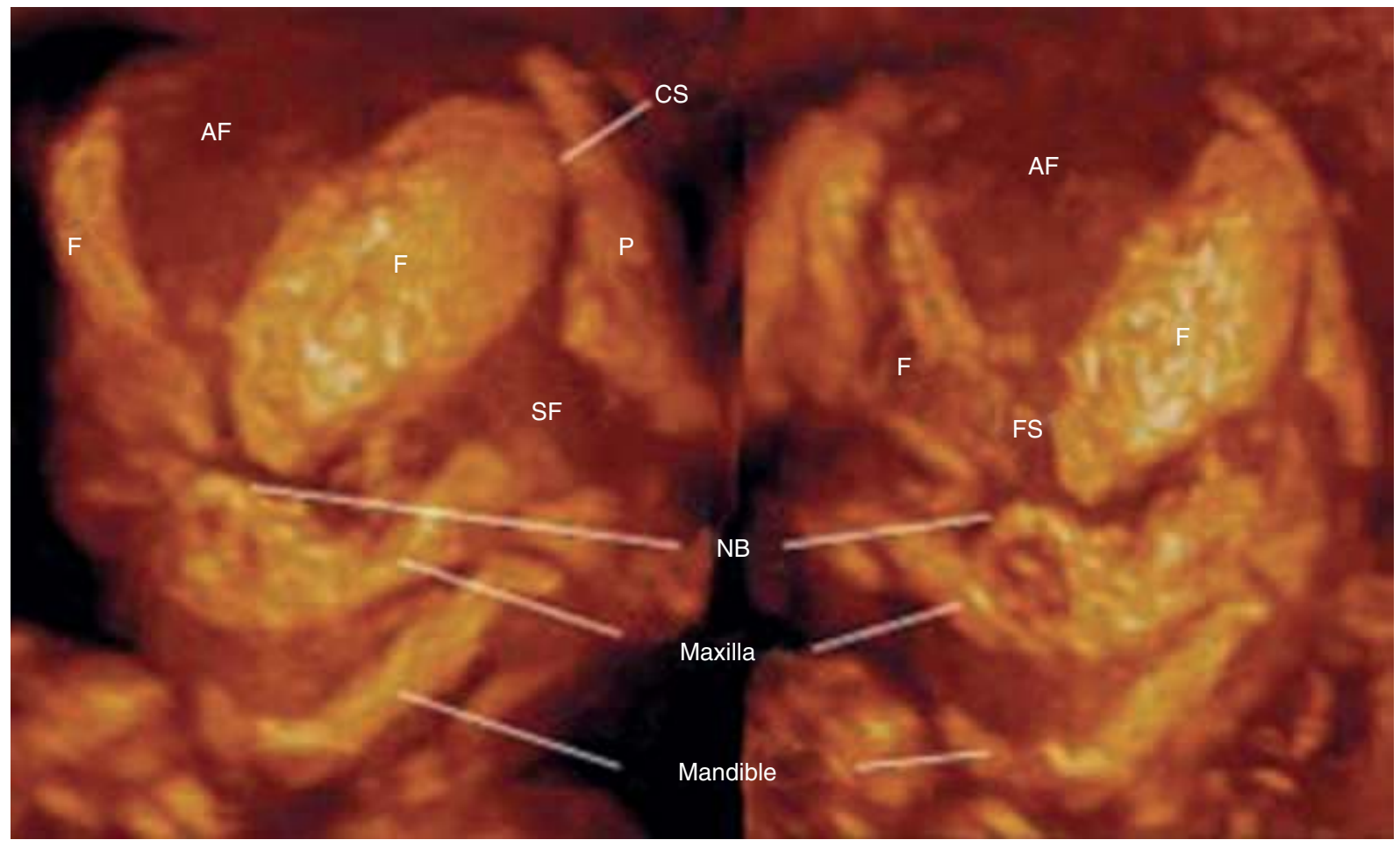

Figure 19 Three-dimensional maximum mode image of normal craniofacial structure at 14 weeks of gestation. (Left) oblique view. (Right) frontal view. Anterior fontanelle (AF), sphenoidal fontanelle (SF), frontal suture (FS), coronal suture (CS), nasal bone (NB), maxilla, and mandible are gradually formed according to cranial bony development. Source: From Ref. 6. 


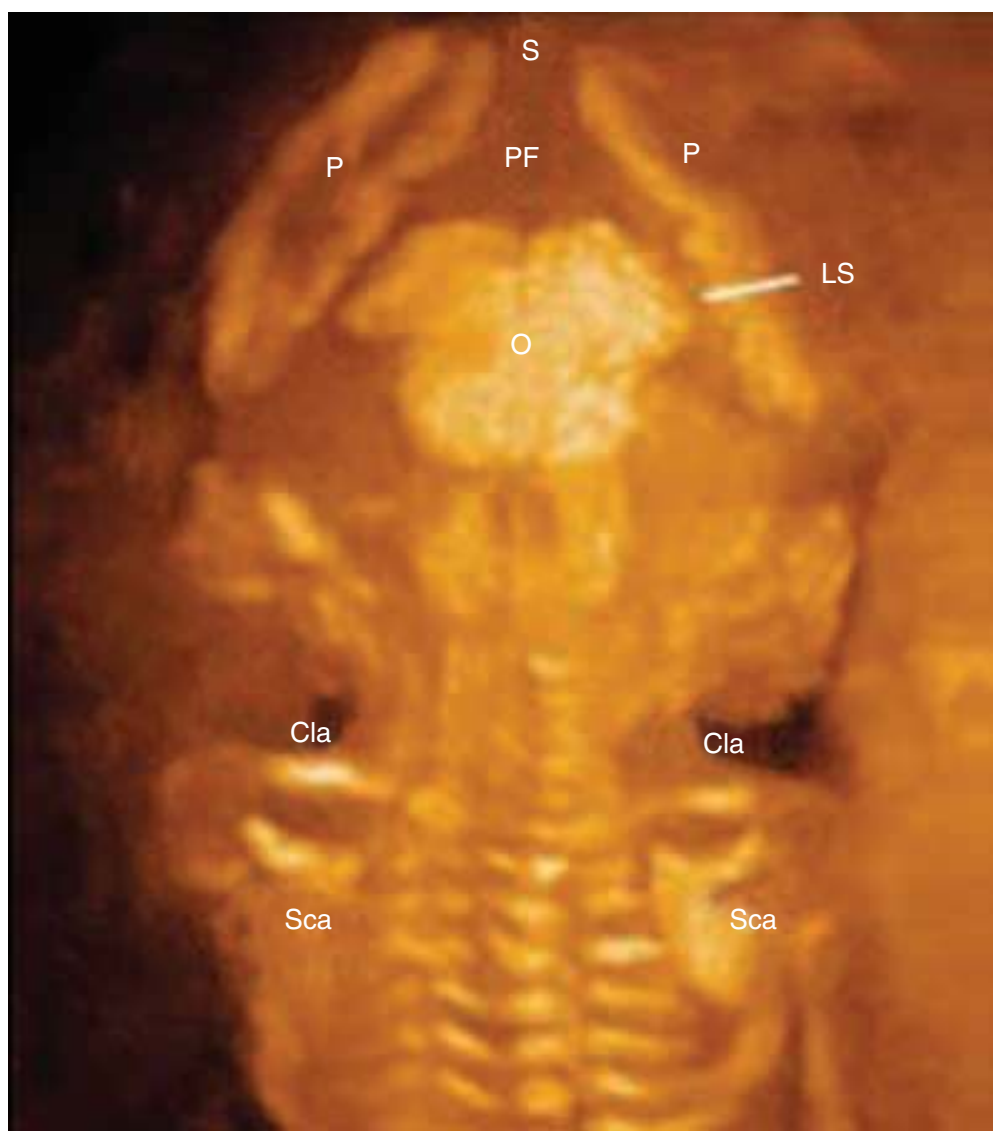

Figure 20 Three-dimensional maximum mode image of occipital view at 13 weeks of gestation. Note the premature occipital bone appearance. Midline crack is demonstrated. Abbreviations: S, Sagittal suture; P, parietal bone; PF, posterior fontanelle; O, occipital bone; Cla, clavicula; Sca, scapula; LS, lambdoid suture. Source: From Ref. 6.

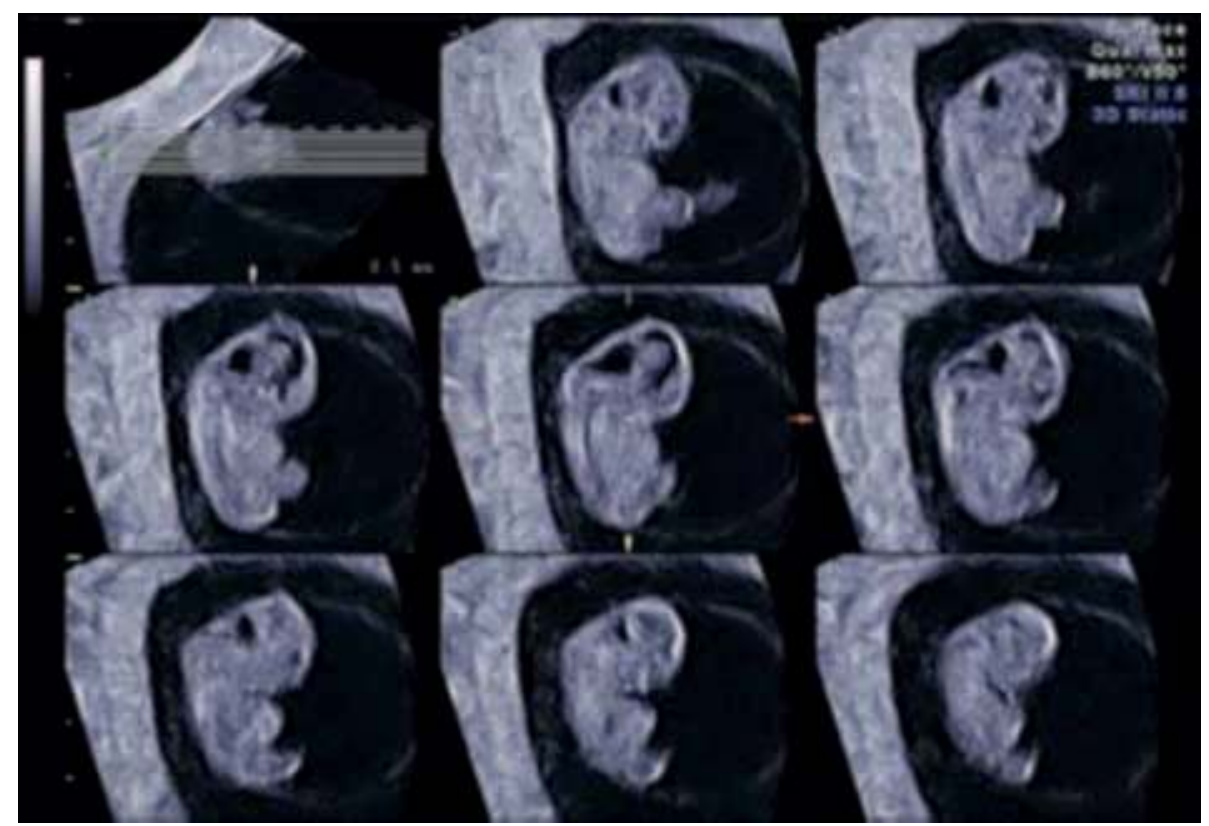

Figure 21 Tomographic sagittal imaging of normal fetus at the beginning of 8 weeks of gestation. Source: From Ref. 6.

that 3D power Doppler can clearly depict the full 3D continuation of early embryonic and umbilico-placental circulation. By adding the pulsed Doppler, pulsations from the embryonic aorta and umbilical artery can be identified as early as the 6th week of gestation. Umbilical artery is characterized by pulsations with absent end-diastolic velocities. Between the 11th and 14th weeks, diastolic velocities gradually emerge (24). Placental branches of umbilical artery are easily depicted by 3D power Doppler $(12,30-39)$.

Intracranial circulation becomes visible from the 8th week of gestation (Fig. 28). 


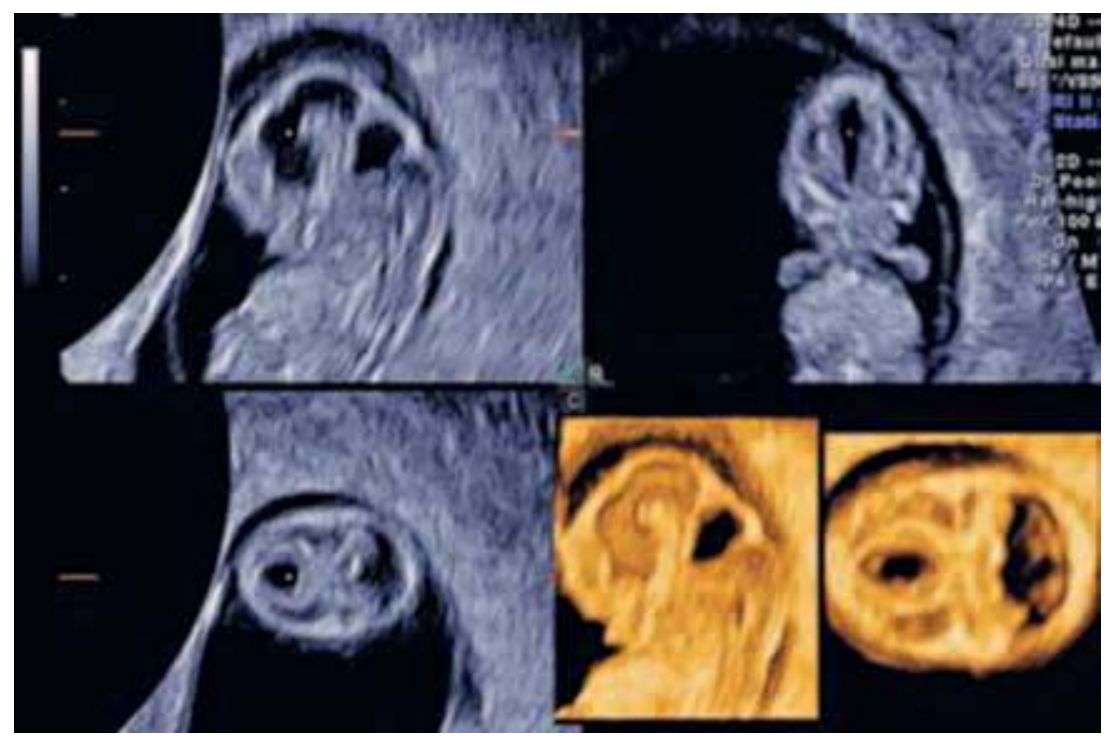

Figure 22 Three orthogonal image and thick slice of three-dimensional reconstructed image (right lower) of normal brain at the end of 8 weeks of gestation. The development of premature ventricular system is seen. Note the different appearance from the beginning of 8 weeks of gestation. Source: From Ref. 6.

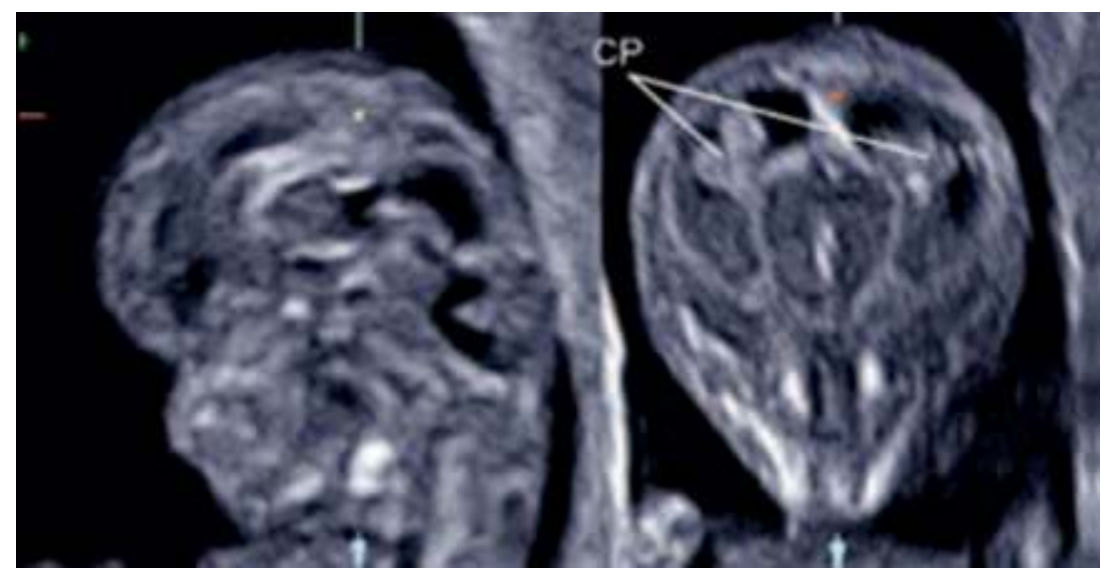

Figure 23 Sagittal section and coronal sections of 10-week fetus. CP, choroid plexus of the lateral ventricles. Source: From Ref. 6.

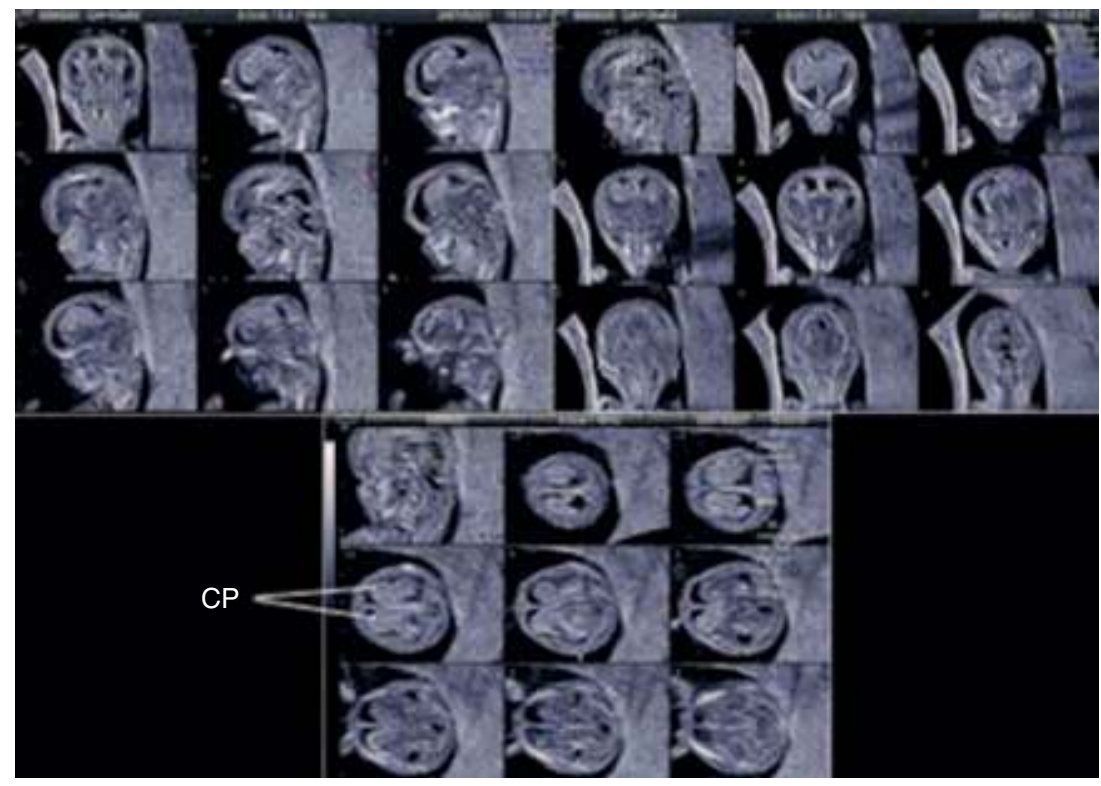

Figure 24 Normal brain at 10 weeks. Tomographic ultrasound imaging of sagittal (left upper), coronal (right upper), and axial (lower) sections. Choroid plexus (CP) occupies most of the lateral ventricles. Source: From Ref. 6. 


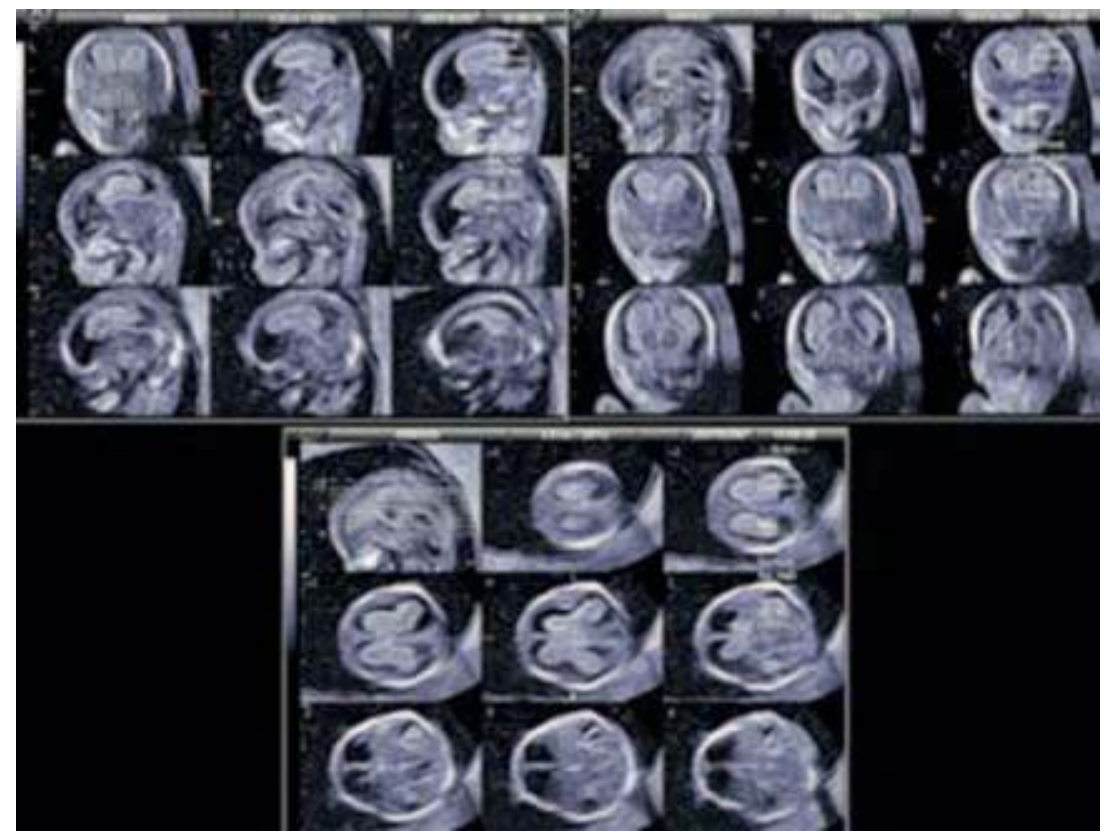

Figure 25 Normal brain at 12 weeks. Tomographic ultrasound imaging of sagittal (left upper), coronal (right upper), and axial (lower) sections. Choroid plexus (CP) still occupies most of the lateral ventricles. Source: From Ref. 6.

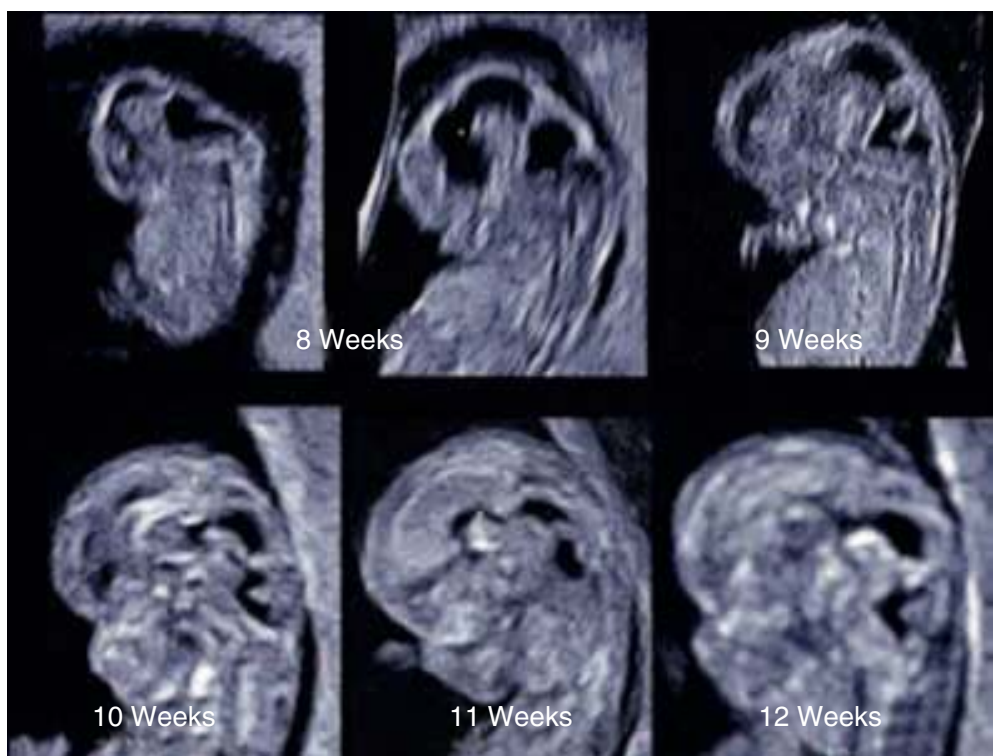

Figure 26 Normal brain development on the midsagittal section between 8 and 12 weeks of gestation. Source: From Ref. 6.

The 3D power Doppler image presents early vascular anatomy on the base of the skull with branches evolving laterally to the mesencephalon and cephalic flexure. Pulsed Doppler signals are initially characterized by the absent enddiastolic velocities. Continuous pan-diastolic flow emerges at the gestational age of 9 to 10 weeks. A significant decrease in impedance was observed in intracranial vessels with advancing gestational age, being more pronounced than that in the other parts of the fetal circulation $(12,22)$.

From the maternal side of placenta, it is possible to obtain the simultaneous 3D imaging of the developing intervillous circulation during the first trimester of pregnancy (Fig. 32A and 32B).

The 3D power Doppler reveals intensive vascular activity surrounding the chorionic shell starting from the first sonographic evidence of the developing pregnancy during the 5 th week of gestation.

Hyperechoic chorionic ring is interrupted by color-coded sprouts that penetrate its limits. These are areas of developing intervillous circulation. Pulsed Doppler reveals blood flow of two types in these areas: a pulsatile arterial like and a continuous venous like (27-29). All were in concordance with clear histomorphology evidence that spiral arteries began to open directly into the intervillous space during the second month of gestation (29).

\section{FUNCTIONAL DEVELOPMENT OF CNS}

The structural and functional development of the human CNS begins in the early embryonic period and proceeds through a sequence of very complicated processes long 

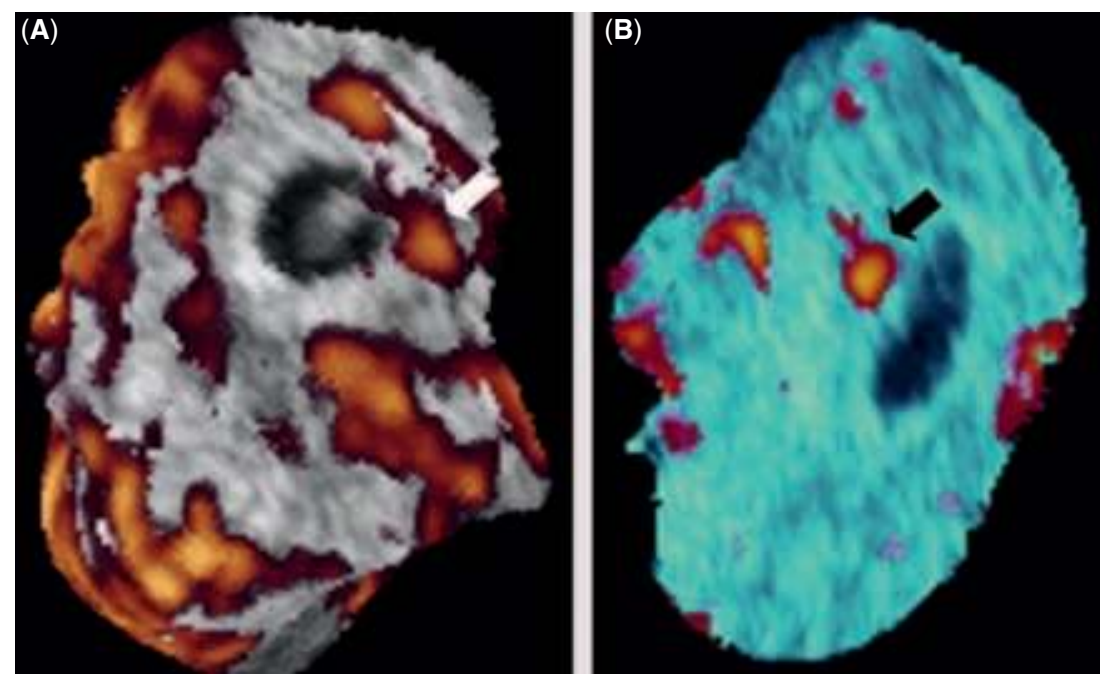

Figure 27 On 4th and 5th weeks of the normal pregnancy, endometrium more (A) or less (B) vascularized, but it is always possible to distinguish a threedimensional color signal penetrating to gestational sac. We named this finding the "comet sign" and it indicates the implantation site.

\section{Weeks}
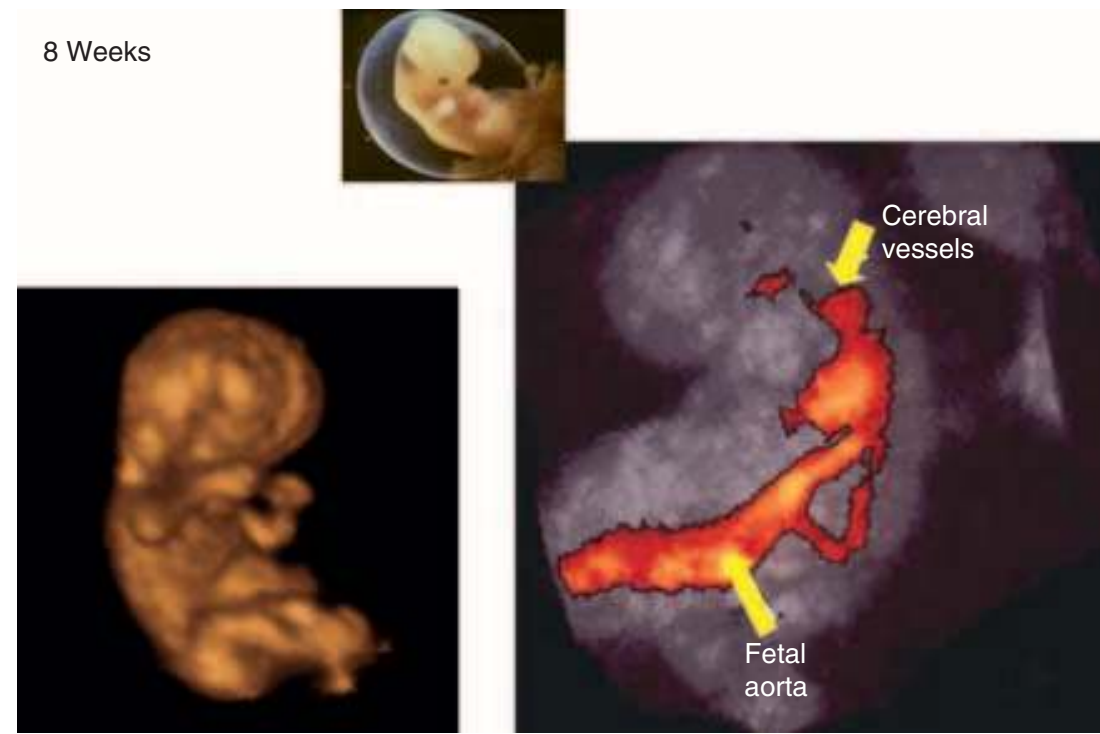

Figure 28 Embryo at 8 weeks. Left picture showing clear morphology with normal appearances of head, limbs, and body on the three-dimensional picture. Right part of picture is made by three-dimensional power Doppler technology visualizing fetal aorta and earliest appearance of middle cerebral artery.
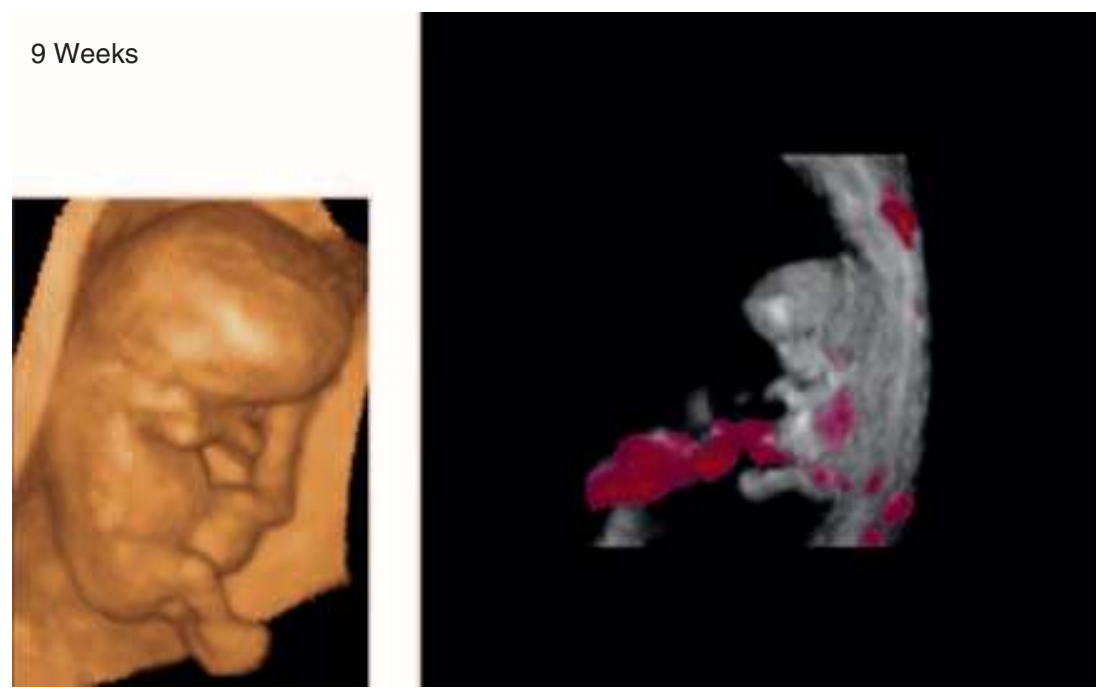

Figure 29 Three-dimensional (3D) static visualization of morphology of early fetus at 9 weeks (left) and 3D power Doppler visualization of umbilical cord vascularity, fetal aorta, and maternal part of circulation (right). 


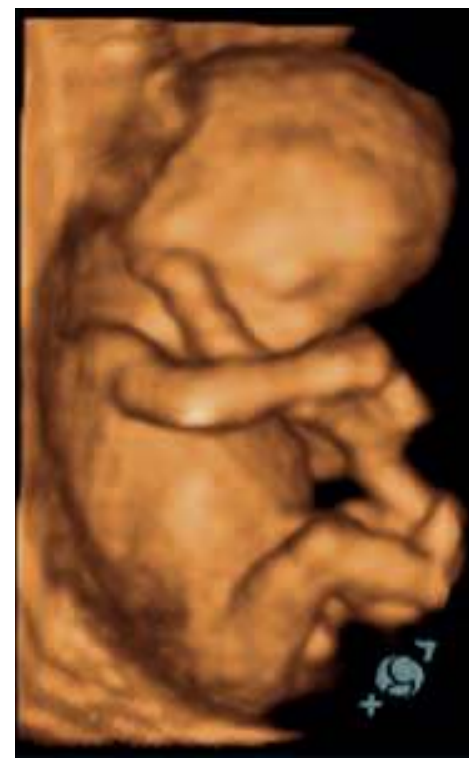

10 Weeks

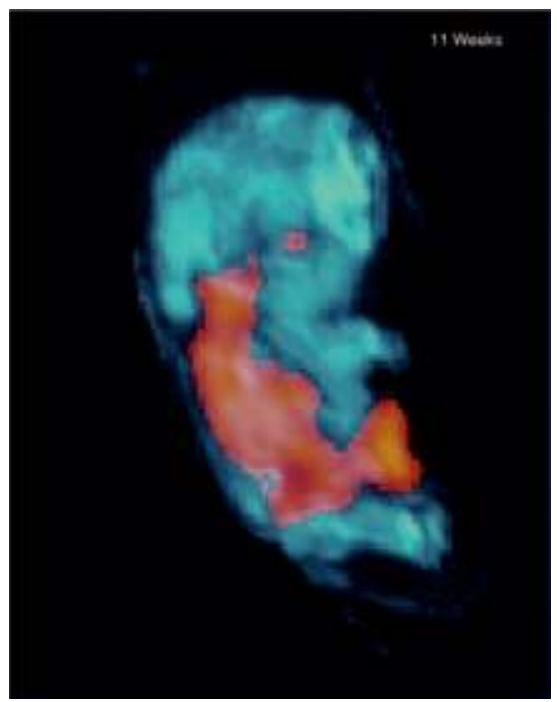

Figure 30 Morphology and vascularization of fetus at 10 weeks of gestation.
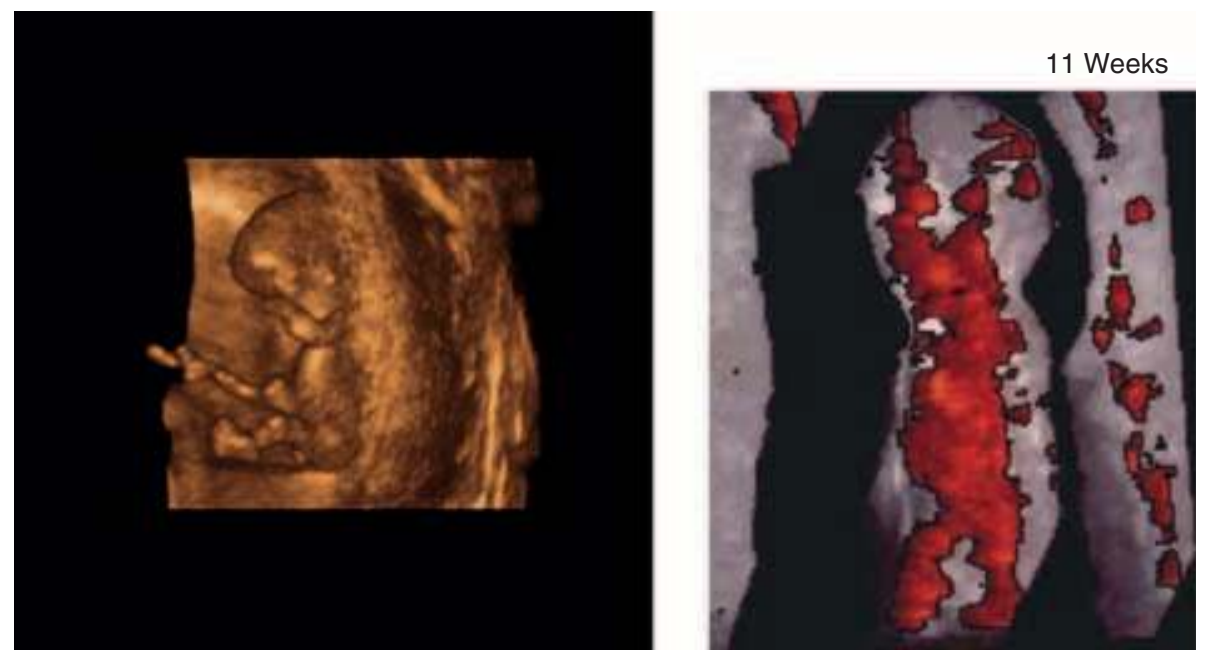

Figure 31 Illustrative examples of the anatomy of fetus and both parts of placental circulation: fetal (with bifurcation) and maternal uterine wall.
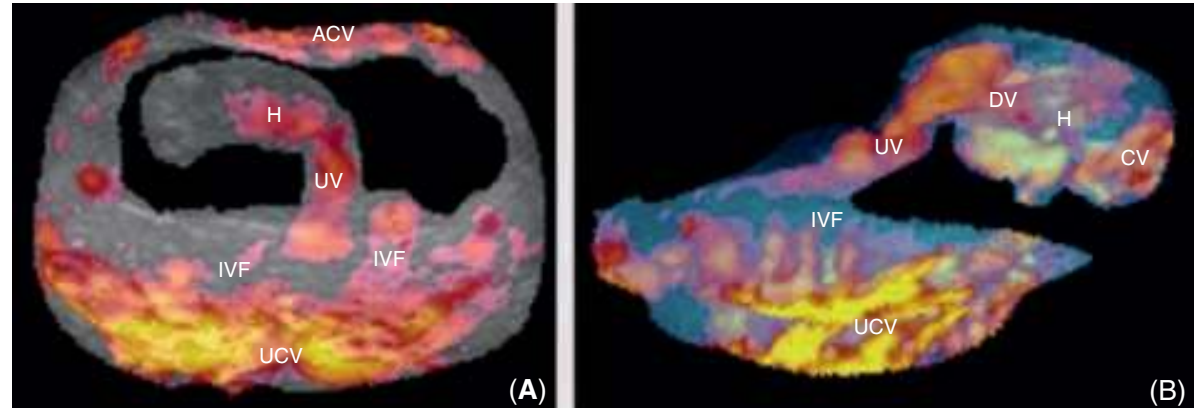

Figure 32 Uterochorionic, intervillous, and fetal circulation at 8th (A) and 10th (B) weeks of pregnancy. Abbreviations: UCV, uterochorionic (spiral and radial) vessels; IVF, intervillous blood flow; UV, umbilical vessels; $\mathrm{H}$, hear; DV, ductus venosus; CV, cerebral vessels; $\mathrm{ACV}$, antichorionic vessels.

after delivery (40). It is important to point out that the caudal region of the neural tube gives rise to the spinal cord and the rostral region becomes the brain. The initial development is completed at 7 weeks of gestation (5 weeks after conception), when all five major subdivisions of the brain are clearly visible: the telencephalon, diencephalon, mesencephalon, metencephalon, and myelencephalon (Fig. 33) (41).
The earliest interneuronal connections, the synapses, can be detected in the spinal cord shortly before the onset of embryonic motility, at 6 to 7 weeks of gestation (42). Therefore, the neural activity leading to the first detectable movements is considered to originate from the spinal motoneurons (43). Another important prerequisite for the motility is the development and innervation of muscular fibers. It is well known that primitive muscle fibers (myotubes) are able to 


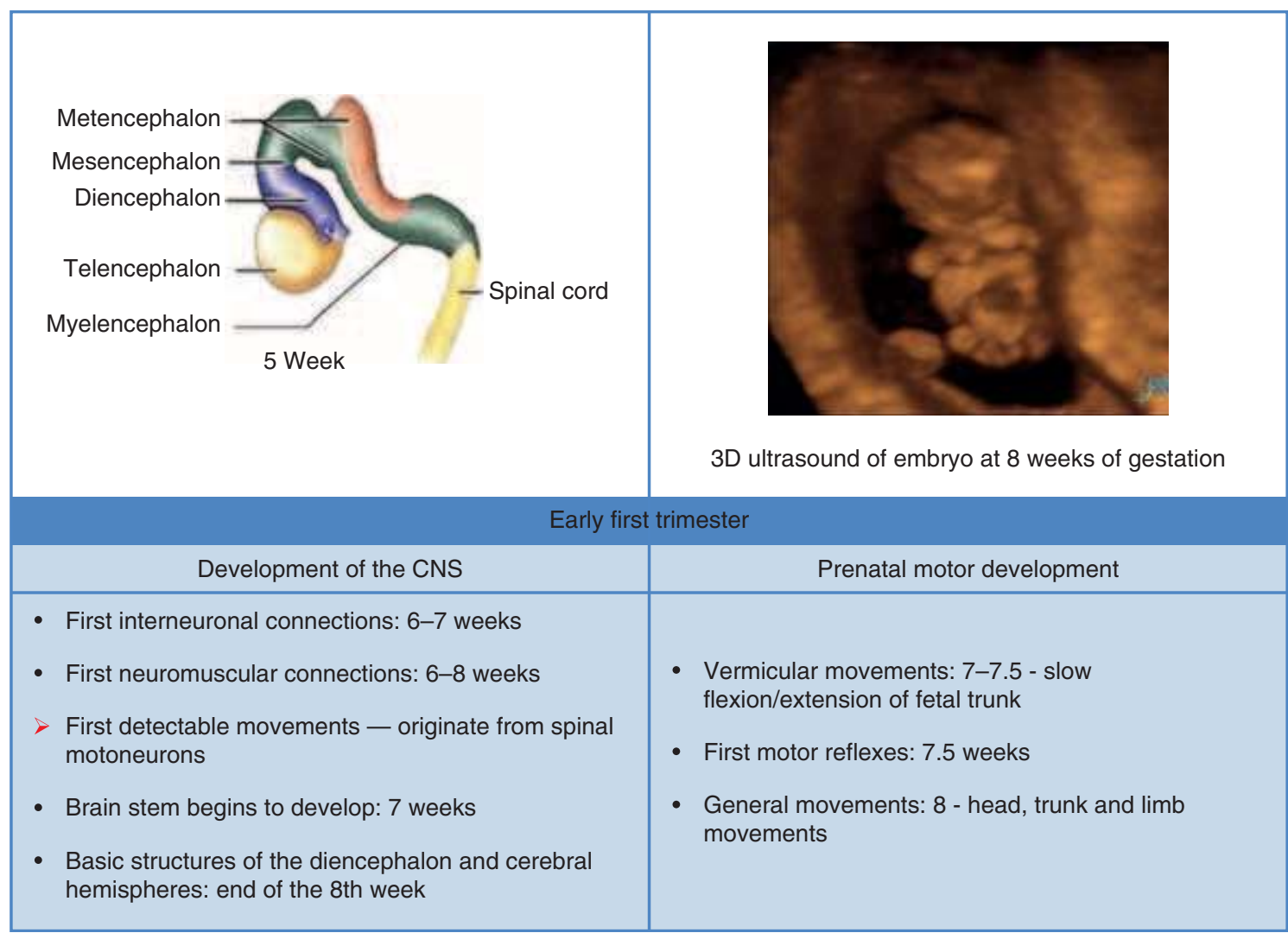

Figure 33 The main events in the development of the central nervous system (CNS) and motor development during the first 8 weeks of pregnancy. Source: From Ref. 40.

contract as soon as they are innervated by motoneurons (44). Between 6 and 8 weeks of gestation, muscle fibers have formed by fusion of myoblasts, efferent and afferent neuromuscular connections have developed, and spontaneous neural activity causing motility can begin.

The main events in the development of the CNS and motor development during the first 8 weeks of pregnancy are presented in Figure 33. The first spontaneous embryonic movements are gross body movements and they can be observed at the 7 to 7.5 th weeks of gestation. They consist of slow flexion and extension of the fetal trunk, accompanied by the passive displacement of arms and legs (45). These, so-called vermicular movements appear in irregular sequences (46). Simultaneous with the onset of spontaneous movements, at the 7.5th week of gestation, the earliest motor reflex activity can be observed, indicating the existence of the first afferent-efferent circuits in the spinal cord (47). The first reflex movements are massive and indicate a limited number of synapses in a reflex pathway. General movements are the first complex, wellorganized movement pattern, which involve head, trunk, and limb movements. This pattern has been interpreted as the first sign of a supraspinal control on motor activity $(48,49)$ and can be recognized from 8 to 9 weeks of gestation onward $(49,50)$.

It is very important to note that even at this early stage of development, embryonic and fetal movements appear in recognizable temporal sequences, without any amorphous or random movement. The explanation for this fascinating phenomenon lies in the intrinsic properties of neurons. That means that neural cells begin to generate and propagate action potentials as soon as they interconnect (51). The interconnected neurons generate patterned activity because of endogenous properties of the neurons (52). Recent investigations have shown that neurons are able to communicate through non-synaptic mechanisms even before the onset of synaptogenesis (53-55).

The brain stem is fashioned around the 7th week of gestation (48) and basic structures of the diencephalon and cerebral hemispheres are formed by the end of the 8th gestational week (56). The remarkable expansion of the cerebral hemispheres follows during the remainder of gestation. The development of synapses in the human cerebral cortex begins after the formation of the cortical plate, at the end of the 10th week of gestation $(53,57)$.

The brain stem consists of the medulla oblongata, pons, and midbrain (Fig. 34).

It forms and matures in a caudal to rostral direction. That means that the phylogenetically older structures, such as the medulla oblongata, will form and mature earlier in the gestation. The major structures of the medulla oblongata are fashioned by 7 th to 8 th week of gestation and are completely matured by 7 months of gestation (48). In addition to its many subnuclei, the medulla gives rise to a variety of descending spinal motor tracts that reflexively trigger limb and body movements. It also hosts the five cranial nerves (VIII-XII), which exert tremendous influences on gross body movements, heart rate, respiration, and the head turning. As the medulla matures in advance of more rostral structures of the brain stem, reflexive movements of the head, body, extremities, as well as breathing movements (Fig. 34) and alterations in heart rate, appear in advance of other functions. The formation of pons begins almost simultaneously, but its maturation is more prolonged. The 


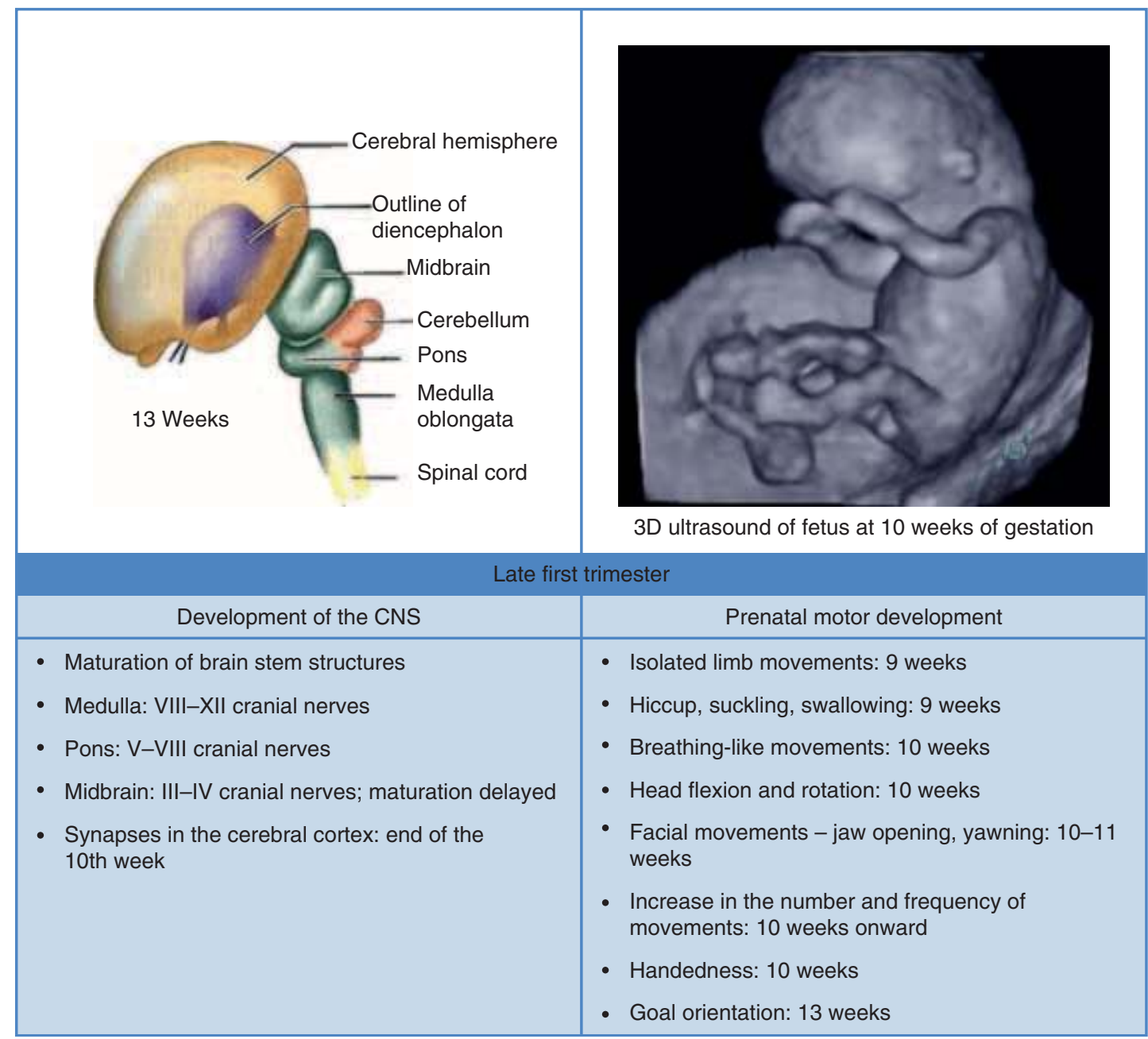

Figure 34 The main events in the development of the central nervous system (CNS) and motor development during the last 6 weeks of the first trimester. Source: From Ref. 40.

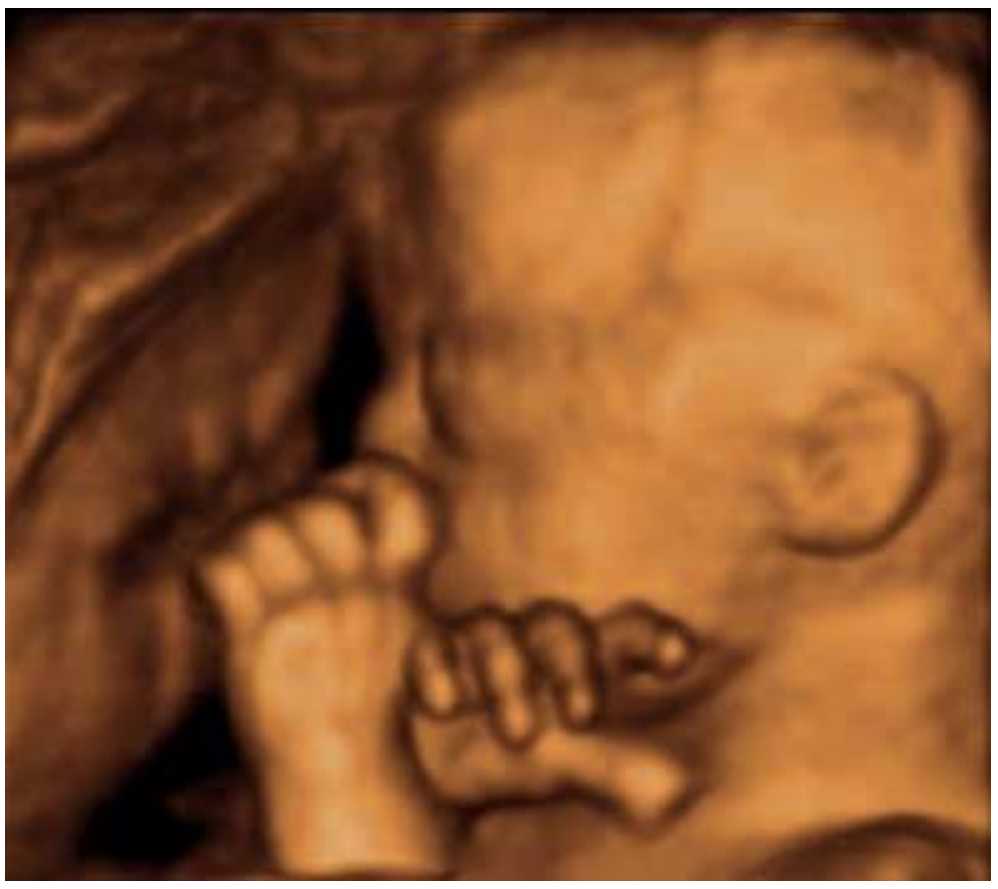

Figure 35 Image of the fetus recorded by three-dimensional/four-dimensional sonography, showing isolated finger movements. 

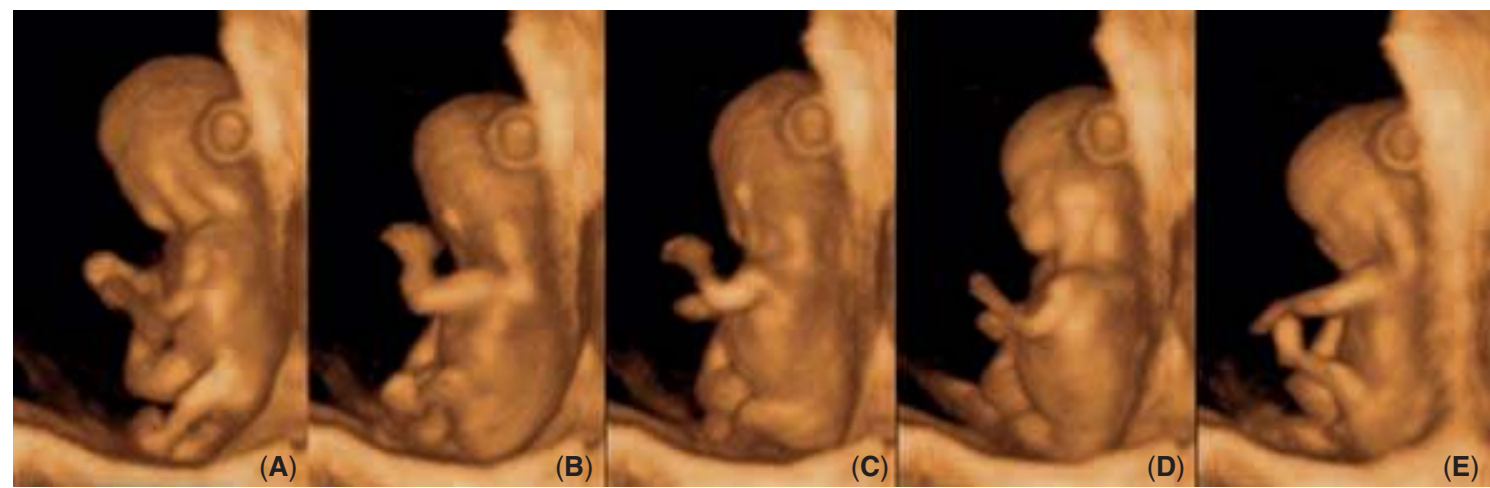

10 Weeks

Figure 36 Fetal movement at 10 weeks and 6 days. Active arm movements (A-E) are associated with active body and lower limb movements. Despite active movements of glenohumeral and elbow joints at this stage, wrist and finger movements were not visualized in most cases.

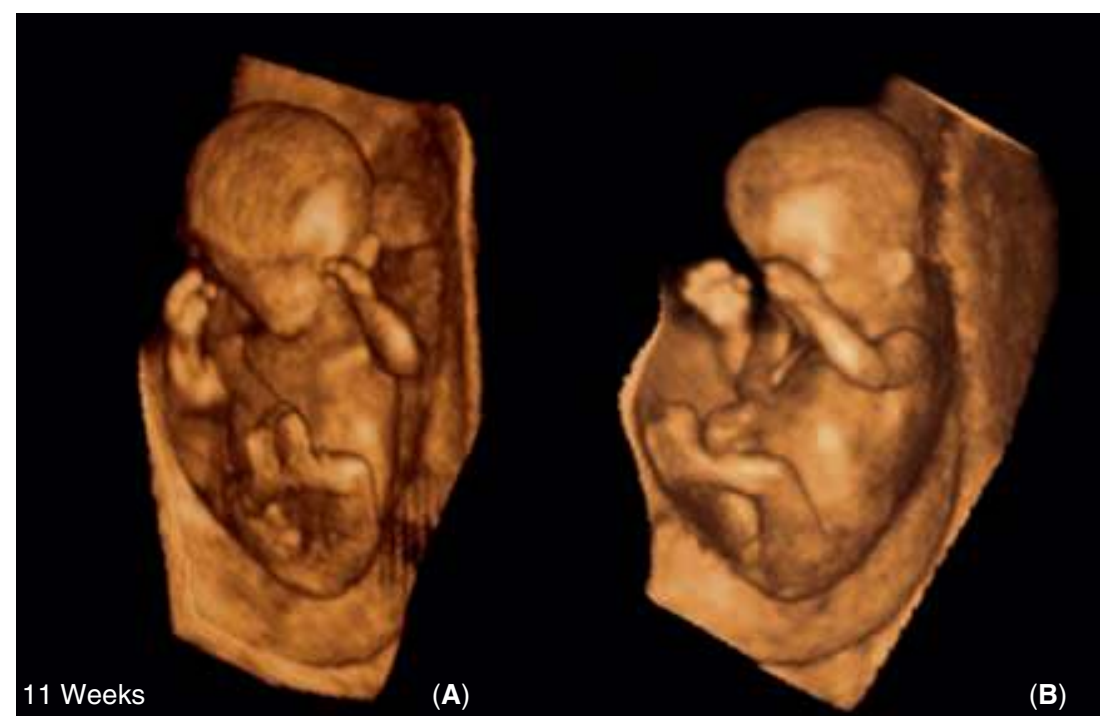

Figure 37 Fetus at 11 weeks still opens the palms but five digits are no longer on the same layer (A). Mild adduction of the thumb and atonic fingers were observed and the palm appearance was clearly different from that in 9 and 10 weeks (B).

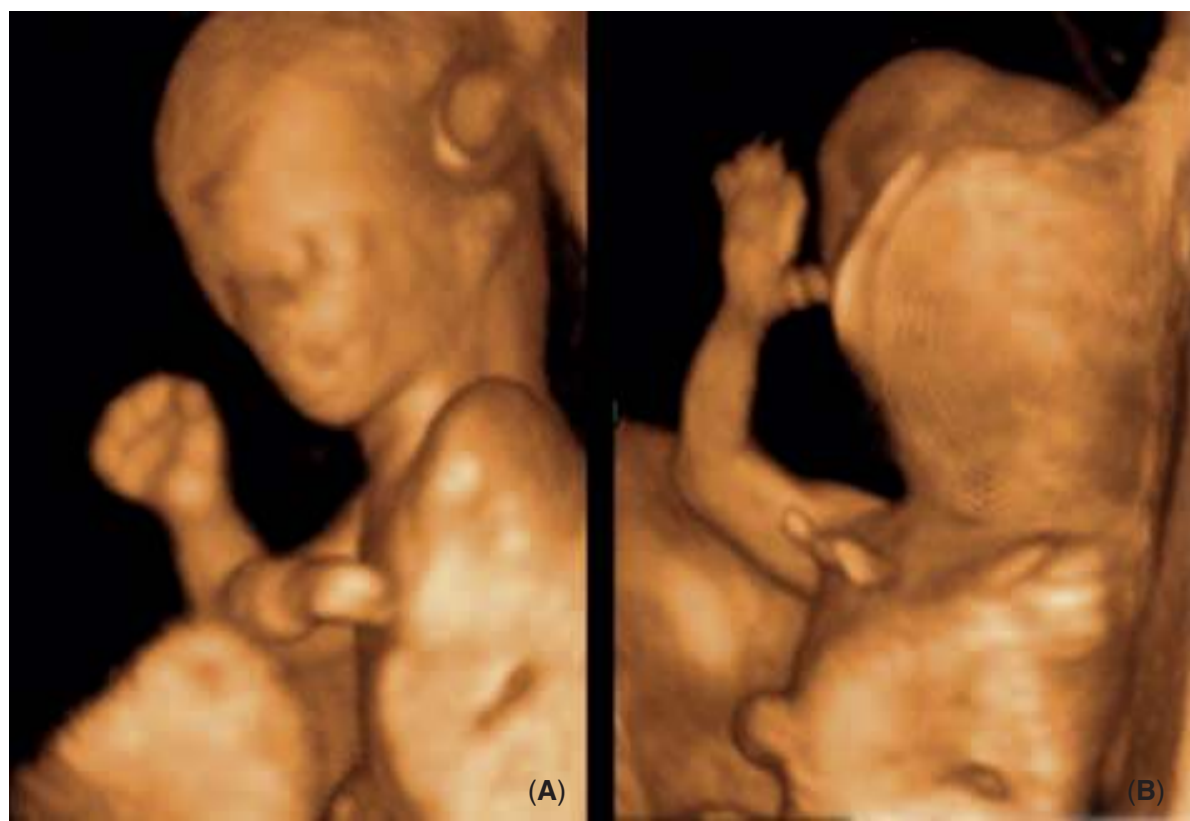

12 Weeks

Figure 38 Clench (A) and unclench (B) of the fist at 12 weeks. At this stage, fetuses start to clench and unclench their fists. 
structures of the pons include the V to VIII cranial nerves (vestibular nuclei of the nerve VIII) and the medial longitudinal fasciculus, pontine tegmentum, raphe nucleus, and locus coeruleus, which exert widespread influences on arousal, including the sleep-wake cycles. Facial movements, which are also controlled by V and VII cranial nerve, appear around 10 to 11 weeks.

At 10 weeks of gestation, lateralized behavior may be observed, and the fetus begins to show the earliest signs of right- or left-handedness. Stimulation of the brain is known to influence brain organization and it is argued that fetal motor activity may eventually stimulate the brain to develop "handedness" and subsequent lateralization of the function $(58,59)$. From 10 weeks onward, the number and frequency of fetal movements increase and the repertoire of movements begins to expand. Qualitative changes in general movements can also be observed. These movements, which are slow and of limited amplitude during 8 and 9 weeks, become more forceful at 10 to 12 weeks. After the 12th week, they become more variable in speed and amplitude (60). The isolated limb movements seen at the 9th week of gestation are followed by the appearance of the movements in the elbow joint at 10 weeks, changes in finger position at the 11th week, and easily recognizable clenching and unclenching of the fist at 12 to 13 weeks. Finally, at 13 to 14 weeks, isolated finger movements can be observed, as well as increases in the activity and strength of the hand/finger movements (61) (Fig. 35). Using 4D sonography, Kurjak and collaborators have found that from 13 gestational weeks onward, a "goal orientation" of hand movements appears and a target point can be recognized for each hand movement (62).

According to the spatial orientation, they classified the hand movements into several subtypes: hand to head, hand to mouth, hand near mouth, hand to face, hand near face, hand to eye, and hand to ear. Our recent longitudinal study, performed by $4 \mathrm{D}$ ultrasound in 100 fetuses from all trimesters of normal pregnancies, has shown increasing frequency of various movement patterns, such as general movements, isolated arm and leg movements, stretching, as well as head movements, during the first trimester. Only the startle movement pattern seemed to occur stagnantly in this period of gestation (63). Using $4 \mathrm{D}$ sonography, general movements were found to be the most frequent movement pattern between 9 and 14 weeks of gestation (64). Furthermore, the advanced ultrasonic techniques, $3 \mathrm{D}$ and $4 \mathrm{D}$ sonography, significantly improve the assessment of structural and functional development of embryonic and fetal CNS (65-67). Some illustrative examples are shown on Figures 36, 37, and 38.

The development of CNS begins rather early in the embryonic period and proceeds throughout the gestation and continues long after birth.

Although some histogenetic processes are not finished until puberty, the neurodevelopmental events are very active in the early pregnancy (56). The earliest signs of functional development of CNS, such as motility, can be registered as early as the late embryonic period $(62,68)$.

The sequential appearance of specific movement patterns, first described by de Vries et al., is presented in Table 1 (68).

Despite the rapid development and a fascinating variety of movements during this early period of gestation, dynamic
Table 1 Developmental Sequence of Fetal Behavioral Patterns in the First Trimester of Pregnancy (68)

\begin{tabular}{lccccc} 
& \multicolumn{5}{c}{ Postconceptional weeks } \\
\cline { 2 - 6 } Type of movements & $\mathbf{7}$ & $\mathbf{8}$ & $\mathbf{9}$ & $\mathbf{1 0}$ & $\mathbf{1 1}$ \\
Sideway bending & + & + & + & + & + \\
General movement & & + & + & + & + \\
Startle & & + & + & + & + \\
Isolated arm movement & & & + & + & + \\
Isolated leg movements & & & + & + & + \\
Hiccup & & + & + & + \\
Suckling & & + & + & + \\
Swallowing & & + & + & + \\
Hand to face contact & & & & + & + \\
Head retroflexion & & & & + & + \\
Head anteflexion & & & & + & + \\
Head rotation & & & + & + \\
Breathing movement & & & & + & + \\
Stretching & & & + & + \\
Jaw opening & & & & + & + \\
Yawning & & & & & +
\end{tabular}

pattern of neuronal production and migration, as well as the immature cerebral circuits, is considered too immature for the cerebral involvement in the motor behavior (69).

\section{REFERENCES}

1. Pooh RK, Shiota K, Kurjak A. Advances in ultrasonic visualization of early human development. Am J Obstet Gynecol 2009; 204: 77.e1-16.

2. Kurjak A. The beginning of human life and its modern scientific approach. Clin Perinatol 2003; 30: 27-44.

3. Shiota K. Early human development: an overview. In: Kurjak A, Chervenak FA, eds. Controversies on the beginning of the human life. New Delhi: Jaypee Brothers, 2008: 151-66.

4. Moore KL. The first two weeks of human development. In: Moore KL, Persaud TVN, Shiota K, eds. Color atlas of clinical embryology. Philadelphia: WB Saunders Company, 2000: 1-12.

5. Kurjak A, Chervenak FA, eds. Controversies on the Beginning of Human Life. New Delhi: Jaypee Brothers, 2008.

6. Pooh RK, Kurjak A, eds. Fetal Neurology. New Delhi: Jaypee Brothers, 2009 , in press.

7. Hertig AT, Rock J, Adams EC. A description of 34 human ova within the first 17 days of development. Am J Anat 1956; 98: 435-93.

8. Kurjak A, Pooh RK, Merce LT, et al. Structural and functional early human development assessed by three-dimensional and four-dimensional sonography. Fertil Steril 2005; 84: 1285-99.

9. de Vries JI, Visser GH, Prechtl HF. The emergence of fetal behaviour: I. Qualitative aspects. Early Hum Dev 1985; 12: 99-120.

10. Kurjak A, Azumendi G, Andonotopo W, Salihagic-Kadic A. Three- and four-dimensional ultrasonography for the structural and functional evaluation of the fetal face. Am J Obstet Gynecol 2007; 196: 16-28.

11. Kurjak A, Miskovic B, Stanojevic M, et al. New scoring system for fetal neurobehavior assessed by three- and four-dimensional sonography. J Perinat Med 2008; 36: 73-81.

12. Kupesic S, Kurjak A, Ivancic-Kosuta M. Volume and vascularity of the yolk sac studied by three-dimensional ultrasound and color Doppler. J Perinat Med 1999; 27: 91-6.

13. Lee A, Deutinger J, Bernaschek G. Three-dimensional ultrasound: abnormalities of the fetal face in surface and volume rendering mode. Br J Obstet Gynaecol 1995; 102: 40-3.

14. Bonilla-Musoles F, Raga F, Osbome N, et al. The use of three-dimensional (3D) ultrasound for study of normal pathologic morphology of the human embryo and fetus: preliminary report. J Ultrasound Med 1995; 14: 757-65.

15. Kurjak A, Kupesic S, Ivancic-Kosuta M. Three-dimensional transvaginal ultrasound improves measurement of nuchal translucency. J Perinat Med 1999; 27: 97-102. 
16. Budorick NE. The fetal musculoskeletal system. In: Callen PW, ed. Ultrasonography in Obstetric and Gynecology. A Harcourth Health Sciences Company: W.B Saunders, 2000: 369.

17. Takeuchi H. Sonoembryology in the central nervous system. In: Kurjak A, Chervenak FA, eds. The Fetus as a Patient. Carnforth UK: Parthenon Publishing, 1994: 141-50.

18. Bonilla-Musoles F. Three-dimensional visualization of the human embryo: a potential revolution in prenatal diagnosis. Editorial. Ultrasound Obstet Gynecol 1996; 7: 393-7.

19. Blaas H-G, Eik-Nes SH, Kiserud T, et al. Early development of the abdominal wall, stomach and heart from 7 to 12 weeks of gestation: a longitudinal study. Ultrasound Obstet Gynecol 1995; 6: 240-9.

20. Lindsay DJ, Lyons EA, Levi CS, et al. Endovaginal appearance of the yolk sac in early pregnancy: normal growth and usefulness as a predictor of abnormal pregnancy outcome. Radiology 1988; 166: 109-12.

21. Pretorius $\mathrm{DH}$, Nelson TR, Baergen $\mathrm{RN}$, et al. Imaging of placental vasculature using three-dimensional ultrasound and color power Doppler: a preliminary study. Ultrasound Obstet Gynecol 1998; 12: 45-9.

22. Kurjak A, Predanic M, Kupesic S. Transvaginal color Doppler study of middle cerebral artery blood flow in early normal and abnormal pregnancy. Ultrasound Obstet Gynecol 1992; 2: 424-8.

23. Kurjak A. Kupesic S. Doppler assessment of the intervillous blood flow in normal and abnormal early pregnancy. Obstet Gynecol 1997; 89: 252-6.

24. Pooh RK, Maeda K, Pooh KH, eds. An Atlas of Fetal Central Nervous System Disease. Diagnosis and Management. London-New York: Parthenon CRC Press, 2003.

25. Pooh RK, Nagao Y, Pooh KH. Fetal neuroimaging by transvaginal 3D ultrasound and MRI. Ultrasound Rev Obstet Gynecol 2006; 6: 123-34.

26. Pooh RK, Pooh KH. Fetal neuroimaging with new technology. Ultrasound Rev Obstet Gynecol 2002; 2: 178-81.

27. Pooh RK, Pooh KH. Transvaginal 3D and Doppler ultrasonography of the fetal brain. Semin Perinatol 2001; 25: 38-43.

28. Pooh RK, Pooh KH. The assessment of fetal brain morphology and circulation by transvaginal 3D sonography and power Doppler. J Perinat Med 2002; 30: 48-56.

29. Pooh RK, Aono T. Transvaginal power Doppler angiography of the fetal brain. Ultrasound Obstet Gynecol 1996; 8: 417-21.

30. Benoit B, Hafner T, Kurjak A, et al. Three-dimensional sonoembryology. J Perinat Med 2002; 30: 63-73.

31. Kurjak A, Pooh RK, Merce LT, et al. Structural and functional early human development assessed by three-dimensional and four-dimensional sonography. Fertil Steril 2005; 84: 1285-99.

32. Kurjak A, Zudenigo D, Predanic M, Kupesic S. Recent advances in the Doppler study of early fetomaternal circulation. J Perinat Med 1993; 21: 419-39.

33. Kurjak A, Schulman H, Predanic A, et al. Fetal choroid plexus vascularization assessed by color flow ultrasonography. J Ultrasound Med 1994; 13: 841-4.

34. Pooh RK, Aono T. Transvaginal power Doppler angiography of the fetal brain. Ultrasound Obstet Gynecol 1996; 8: 417-21.

35. Nicolaides KH. Nuchal translucency and other first-trimester sonographic markers of chromosomal abnormalities. Am J Obstet Gynecol 2004; 191 : 45-67.

36. Timor-Tritsch IE, Peisner DB, Raju S. Sonoembryology: an organoriented approach using a high-frequency vaginal probe. J Clin Ultrasound 1990; 18: 286-98.

37. Pooh RK, Maeda K, Pooh KH, Kurjak A. Sonographic assessment of the fetal brain morphology. Prenat Neonat Med 1999; 4: 18-38.

38. Pooh RK. Three-dimensional ultrasound of the fetal brain. In: Kurjak A, ed. Clinical application of 3D ultrasonography. Carnforth: Parthenon Publishing, 2000: 176-80.

39. Monteagudo A, Timor-Tritsch IE, Mayberry P. Three-dimensional transvaginal neurosonography of the fetal brain: 'navigating' in the volume scan. Ultrasound Obstet Gynecol 2000; 16: 307-13.

40. Salihagic Kadic A, Predojevic M, Kurjak A. Advances in fetal neurophysiology. In: Pooh RK, Kurjak A, eds. Fetal Neurology. New Delhi: Jaypee Brothers, 2009: 161-220.

41. O'Rahilly R, Muller F. Minireview: summary of the initial development of the human central nervous system. Teratology 1999; 60: 39-41.
42. Okado N, Kakimi S, Kojima T. Synaptogenesis in the cervical cord of the human embryo: sequence of synapse formation in a spinal reflex pathway. J Comp Neurol 1979; 184: 491-518.

43. Okado N, Kojima T. Ontogeny of the central nervous system: neurogenesis, fibre connection, synaptogenesis and myelination in the spinal cord. In: Prechtl HFR, ed. Continuity of neural function from prenatal to postnatal life. Oxford: Blackwell Science, 1984: 31-5.

44. Landmesser LT, Morris DG. The development of functional innervation in the hind limb of the chick embryo. J Physiol (Lond) 1975; 249: 301-26.

45. Prechtl HFR. Ultrasound studies of human fetal behaviour. Early Hum Dev 1985; 12: 91-8.

46. Ianniruberto A, Tajani E. Ultrasonographic study of fetal movements. Semin Perinatol 1981; 4: 175-81.

47. Okado N. Onset of synapse formation in the human spinal cord. J Comp Neurol 1981; 201: 211-19.

48. Joseph R. Fetal brain and cognitive development. Dev Rev 1999; 20: 81-98.

49. de Vries JIP, Visser GHA, Prechtl HFR. The emergence of fetal behavior I. Qualitative aspects. Early Human Dev 1982; 7: 301-22.

50. Einspieler C, Prechtl HF. Prechtl's assessment of general movements: a diagnostic tool for the functional assessment of the young nervous system. Ment Retard Dev Disabil Res Rev 2005; 11: 61-7.

51. Stafstrom CE, Johnston D, Wehner JM, Sheppard JR. Spontaneous neural activity in fetal brain reaggregate culture. Neuroscience 1980; 5: 1681-9.

52. Streit J. Regular oscillations of synaptic activity in spinal networks in vitro J Neurophysiol 1993; 70: 871-8.

53. Kostovic I, Judas M. Transient patterns of cortical lamination during prenatal life: do they have implications for treatment? Neurosci Biobehav Rev 2007; 31: 1157-68.

54. Voigt T, Opitz T, de Lima AD. Synchronous oscillatory activity in immature cortical network is driven by GABAergic preplate neurons. J Neurosci 2001; 21: 8895-905.

55. Albrieux M, Platel JC, Dupuis A, et al. Early expression of sodium channel transcripts and sodium current by cajal-retzius cells in the preplate of the embryonic mouse neocortex. J Neurosci 2004; 24: 1719-25.

56. Pomeroy SL, Volpe JJ. Development of the nervous system. In: Polin RA, Fox WW, eds. Fetal and neonatal physiology. Philadelphia-London-TorontoMontreal-Sydney-Tokyo: WB Saunders Company, 1992: 1491-509.

57. Molliver ME, Kostovic I, Van der Loos H. The development of synapses in cerebral cortex of the human fetus. Brain Res 1973; 50: 403-7.

58. McCartney G, Hepper P. Development of lateralized behavior in the human fetus from 12 to 27 weeks' gestation. Dev Med Child Neurol 1999; 41: 83-6.

59. Hepper PG, McCartney GR, Shannon EA. Lateralised behavior in first trimester human foetuses. Neuropsychologia 1998; 36: 531-4.

60. Lüchinger AB, Hadders-Algra M, Van Kan CM, de Vries JI. Fetal onset of general movements. Pediatr Res 2008; 63: 191-5.

61. Pooh RK, Ogura T. Normal and abnormal fetal hand positioning and movement in early pregnancy detected by three and four-dimensional ultrasound. Ultrasound Rev Obstet Gynecol 2004; 4: 46-51.

62. Kurjak A, Azumendi G, Vecek N, et al. Fetal hand movements and facial expression in normal pregnancy studied by four-dimensional sonography. J Perinat Med 2003; 31: 496-508.

63. Kurjak A, Andonotopo W, Hafner T, et al. Normal standards for fetal neurobehavioral developments-longitudinal quantification by fourdimensional sonography. J Perinat Med 2006; 34: 56-65.

64. Andonotopo W, Medic M, Salihagic-Kadic A, et al. The assessment of fetal behavior in early pregnancy: comparison between $2 \mathrm{D}$ and $4 \mathrm{D}$ sonographic scanning. J Perinat Med 2005; 33: 406-14.

65. Kurjak A, Pooh RK, Merce LT, et al. Structural and functional early human development assessed by three-dimensional and four-dimensional sonography. Fertil Steril 2005; 84: 1285-99.

66. Salihagic-Kadic A, Kurjak A, Medić M, et al. New data about embryonic and fetal neurodevelopment and behavior obtained by $3 \mathrm{D}$ and $4 \mathrm{D}$ sonography. J Perinat Med 2005; 33: 478-90.

67. Kurjak A, Miskovic B, Andonotopo W, et al. How useful is 3D and 4D ultrasound in perinatal medicine? J Perinat Med 2007; 35: 10-27.

68. de Vries JIP, Visser GHA, Prechtl HFR. Fetal motility in the first half of pregnancy. In: Prechtl HFR, ed. Continuity of Neural Functions from Prenatal to Postnatal Life. Philadelphia PA: Lippincott, 1984: 44-64.

69. Kostovic I, Judas M, Petanjek Z, et al. Ontogenesis of goal-directed behavior: anatomo-functional considerations. Int J Psychophysiol 1995; 19: 85-102. 


\section{Group B streptococcus infection Jessica Winn and Hung N. Winn}

Group B streptococcus (GBS) (Streptococcus agalactiae), a Gram-positive coccus, is one of the major causes of maternal or neonatal severe infection and sepsis. Maternal infection associated with GBS includes acute chorioamnionitis, endometritis, and urinary tract infection. Neonatal GBS infection is characterized as early onset if occurring within 7 days of age or late onset otherwise and involves bacteremia, pneumonia, or meningitis $(1,2)$.

\section{EPIDEMIOLOGY}

Of all pregnant women in the United States, 10\% to 30\% have recto-vaginal colonization with GBS (3-5). In patients with preterm labor or preterm premature rupture of membranes (PPROM), the incidence of genital colonization of GBS is $15 \%$ (6). Of urinary tract infections during pregnancy, $2 \%$ to $7 \%$ result from GBS colonization (7). The incidence of neonatal GBS infection (number of cases per 1000 live births) was gradually decreased between 1990 and 2008. It was 1.8, 0.5 , and 0.28 in 1990, 2000, and 2008, respectively (8-13). This decline can be attributed to screening of pregnant woman for GBS colonization and use of intrapartum antibiotic prophylaxis (14-17). Neonatal colonization of GBS may occur as a result of in utero ascending infection from the maternal genital tract or direct contact during delivery. Vertical transmission from the mother to the neonate by either method accounts for up to $50 \%$ (13) of cases of neonatal GBS colonization, and $1 \%$ to $2 \%$ of these infants will develop early-onset GBS infection $(14,18-22)$. The case fatality of early-onset GBS neonatal infection varies from $2 \%$ to $3 \%$ for term infants without meningitis to $20 \%$ for preterm infants $(13,23)$. Maternal risk factors that predispose a neonate to early-onset GBS infection include preterm delivery, prolonged rupture of membranes (>18 h), PPROM, chorioamnionitis, sustained intrapartum fetal tachycardia, GBS bacteriuria during the current pregnancy $\left(>10^{4} \mathrm{cfu} / \mathrm{mL}\right)$, intrapartum fever (temperature of at least $38^{\circ} \mathrm{C}$ or $100.4^{\circ} \mathrm{F}$ ), or prior infant with GBS infection $(15,16,24,25)$. The risk of having an infant with early-onset GBS is 6.5 times higher in a pregnant woman with maternal fever, prolonged rupture of membranes, or preterm birth when compared with women who displayed none of the above findings $(18,26,27)$. Late-onset GBS neonatal infection may occur from maternal-neonatal transmission, or nosocomial or community contacts $(28,29)$.

\section{DIAGNOSIS}

Group B streptococcal colonization can be detected by either culture or rapid diagnostic tests. Currently, culture remains the gold standard for diagnosing GBS colonization. The yield of positive GBS culture is increased by sampling the anorectum in addition to the vagina, since the gastrointestinal tract is a major reservoir of GBS $(5,30)$. This can be done in a single swab. Inhibition of competing organisms by using a selective medium such as Todd-Hewitt broth, which contains gentamicin, polymyxin B, and nalidixic acid or colistin (24), also increases the yield of the GBS culture. The main limitation of culture is time. Since culture results are not available for 24 to 48 hours, management may be problematic if delivery is imminent.

Rapid diagnostic tests of GBS directly detect the extracted specific polysaccharide antigen. The available tests use either latex particle agglutinization or enzyme immunoassay. These tests are relatively easy to perform, are generally less expensive than a culture, and produce results within a short period of time (usually within 1 hour). Although rapid detection tests are highly sensitive in patients who are heavily colonized with GBS, their overall rates of sensitivity and high false-negative results compared with those of cultures prevent their widespread clinical application (31-35). These drawbacks for the rapid diagnostic tests also exist in the setting of preterm labor or PPROM, demonstrating that the rapid latex agglutination test fails to identify GBS neonatal infection in (i) heavily, (ii) moderately, and (iii) lightly GBS-colonized mothers. More importantly, the overall sensitivity of the latex agglutination test in identifying maternal-neonatal pairs at risk for GBS neonatal infection is only $25 \%$ (6).

\section{TREATMENT}

Intrapartum chemoprophylaxis is effective in reducing the attack rate of early-onset neonatal GBS (26). In 2002, the Centers for Disease Control and Prevention (CDC) established new guidelines for GBS prevention. It recommended screening all pregnant women with culture of anogenital specimen at 35 to 37 weeks of gestation and providing intrapartum chemoprophylaxis for those with positive GBS culture. Specimen should be collected with swabbing the lower vagina and the lower rectum to obtain the best yield of GBS culture (36-39). Women with GBS bacteria during their current pregnancy and those with a prior history of having a neonate with invasive GBS infection do not need to be screened and should be given intrapartum chemoprophylaxis routinely (40). In 2010, the CDC maintained the same recommendations (24). Women with unknown GBS status at the time of delivery should be managed based on the presence of intrapartum risk factors [delivery at $<37$ weeks, rupture of membranes $>18$ hours, fever of $\left.\geq 100.4^{\circ} \mathrm{F}\left(38^{\circ} \mathrm{C}\right)\right]$ (24).

Currently, prenatal treatment of asymptomatically colonized pregnant patients with oral antibiotics is not recommended, since this approach is unlikely to eradicate maternal genital GBS colonization at the time of labor and delivery. Of pregnant patients who received antenatal ampicillin or aqueous penicillin for genital colonization of GBS, $30 \%$ to $70 \%$ remained colonized at delivery $(41,42)$. 


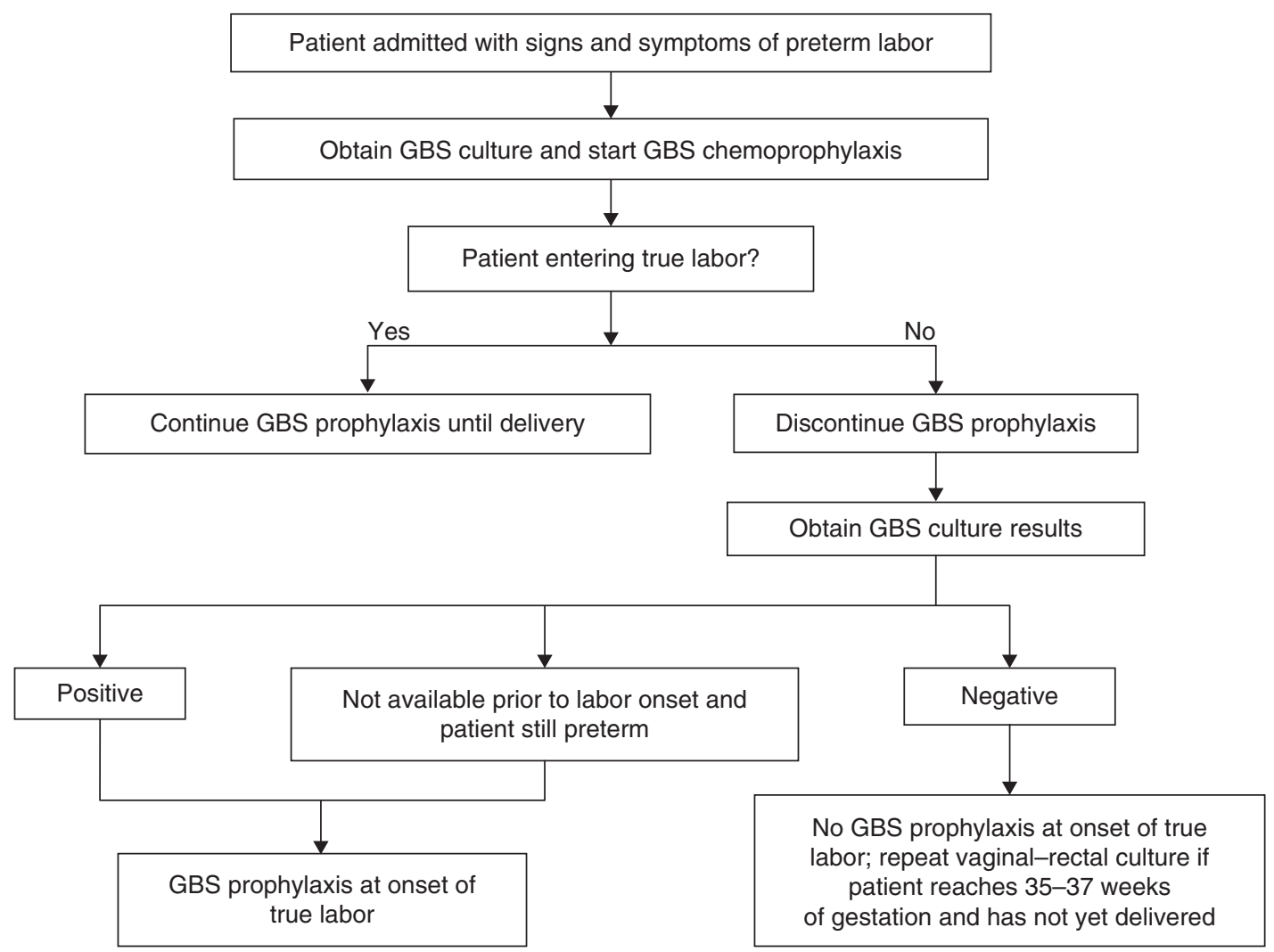

Figure 1 Algorithm for screening of group B streptococcal colonization and use of intrapartum prophylaxis in women with preterm labor. Abbreviation: GBS, group B streptococcus. Ref. 24.

In 2010, the CDC made recommendations regarding the prevention of early-onset neonatal GBS infection in the setting of preterm (less than 37 weeks) labor or PPROM (24). Patients who develop preterm labor without prior GBS screening during the preceding 5 weeks of admission should be screened for GBS colonization and provided GBS chemoprophylaxis. The latter should be stopped when the GBS screen is negative or preterm labor is resolved. GBS screening should be repeated at 35 to 37 weeks of gestation or when preterm labor recurs more than 5 weeks since the last GBS screening. Figure 1 summarizes the above process for management of patients with preterm labor.

Patients who develop PPROM should be screened for GBS colonization and provided antibiotics for prolonging latency period with adequate GBS coverage (ampicillin $2 \mathrm{~g}$ initially, then $1 \mathrm{~g}$ every 6 hours for 48 hours given intravenously) or GBS chemoprophylaxis otherwise. The GBS chemoprophylaxis can be discontinued when the GBS vaginal-rectal screening obtained on admission turns out to be negative. The patient with PPROM may not need GBS chemoprophylaxis on admission if her GBS screening was negative within 5 weeks of PPROM. Group B streptococcus (GBS) screening should be repeated at 35 to 37 weeks of gestation if she remains pregnant at that time. Figure 2 summarizes the above process. The patient should receive intrapartum chemoprophylaxis if she ever had genital GBS colonization during her pregnancy, regardless of the results of intervening GBS cultures and/or treatments in both situations of preterm labor and PPROM.

\section{INTRAPARTUM CHEMOPROPHYLAXIS}

Aqueous penicillin $\mathrm{G}$ is the drug of choice with the initial dose of 5 million units and maintenance dose of 2.5-3.0 million units every 4 hours. Ampicillin is the alternative with the initial dosage of $2 \mathrm{~g}$ and the maintenance dose of $1 \mathrm{~g}$ every 4 hours. Penicillin $\mathrm{G}$ is preferable to ampicillin because of its narrower spectrum of bacterial sensitivity, thus being less likely to cause the selective emergence of other pathogenic bacteria (15). Women who are penicillin allergic but do not have a high risk of anaphylaxis (defined as a history of anaphylaxis, angioedema, respiratory distress, or urticaria after administration of a penicillin or cephalosporin) should receive cefazolin $2 \mathrm{~g}$ IV initially then $1 \mathrm{~g}$ every 8 hours. Cefazolin crosses the placenta and attains effective amniotic concentrations. Women who are allergic to penicillin and at a high risk of anaphylaxis should receive clindamycin IV $900 \mathrm{mg}$ every 8 hours if (i) the GBS is susceptible to both clindamycin and erythromycin or (ii) GBS is susceptible to clindamycin, resistant to erythromycin but without inducible resistance to clindamycin. Women who are allergic to penicillin and at a high risk of anaphylaxis but do not meet the above conditions for clindamycin should receive vancomycin $1 \mathrm{~g}$ IV every 12 hours otherwise. Erythromycin is no longer used in women who are allergic to penicillin and are at high risk for anaphylaxis. Intrapartum chemoprophylaxis should be given intravenously until delivery. Figure 3 summarizes the GBS chemoprophylaxis (24).

\section{SUMMARY}

Although controversy exists regarding screening and treatments of GBS colonization in pregnant women, there is a consensus that chemoprophylaxis in the intrapartum period dramatically reduces the rate of infection in both the mother and the neonate. At present, the most cost-effective strategy of reducing early-onset GBS infection is universal screening of 


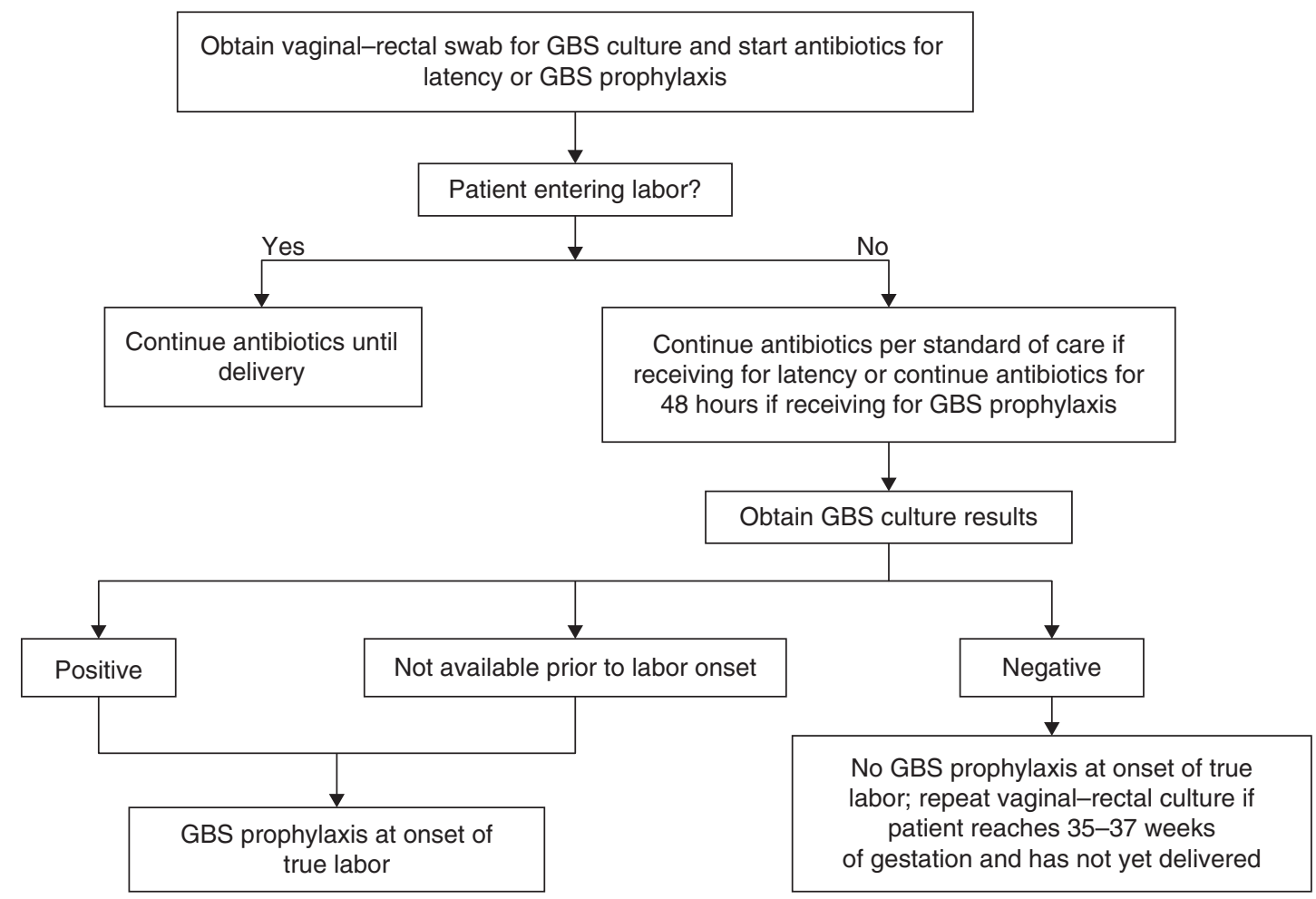

Figure 2 Algorithm for screening of group B streptococcal colonization and use of intrapartum prophylaxis in women with preterm premature rupture of membranes. Abbreviation: GBS, group B streptococcus. Ref. 24.

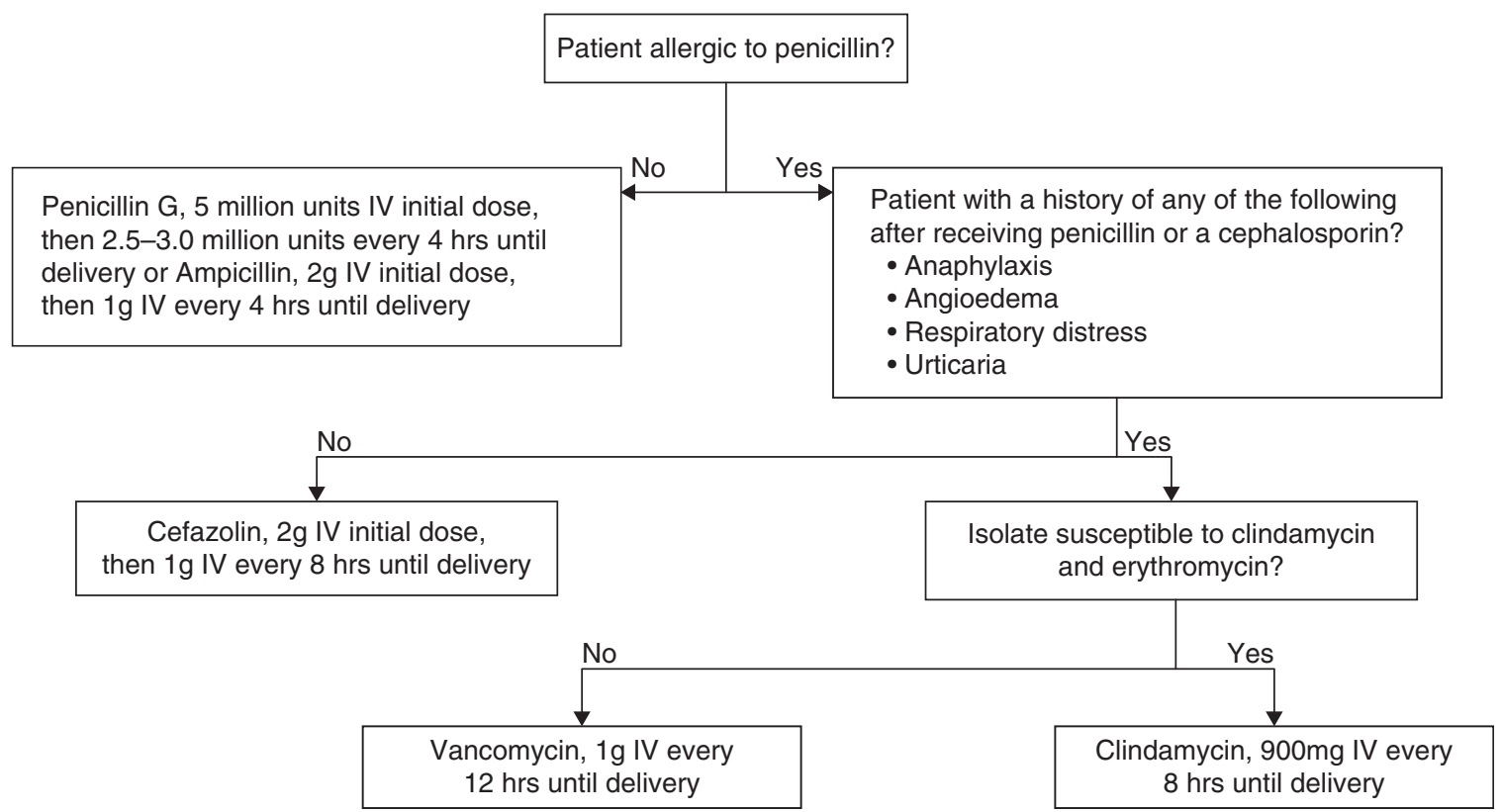

Figure 3 Recommended regimens for intrapartum antibiotic prophylaxis for prevention of early-onset group B streptococcal disease. Abbreviation: GBS, group B streptococcus. Ref. 24 .

maternal GBS vaginal-rectal colonization at 35-37 weeks of gestation. Intrapartum GBS chemoprophylaxis should be given to patients without antenatal screening who have one of the following risk factors: preterm delivery, prolonged rupture of membranes $(>18 \mathrm{~h})$, intrapartum fever, or prior infant with GBS infection. Penicillin $\mathrm{G}$ is the drug of choice for this purpose and ampicillin is the alternative. Cefazolin may be used in patients who are allergic to penicillin or cephalosporin without a high risk for anaphylaxis. Patients who have allergy to penicillin with a high risk of anaphylaxis should receive clindamycin if the bacterium is susceptible to both clindamycin and erythromycin or vancomycin otherwise. Maternal 
vaccination, being developed, appears to be the most costeffective measure in preventing early-onset GBS neonatal infection.

\section{REFERENCES}

1. Baker C. Summary of the workshop on perinatal infections due to group B streptococcus. J Infect Dis 1977; 136: 137-52.

2. Faro S. Group B beta-hemolytic streptococci and puerperal infections. Am J Obstet Gynecol 1981; 139: 686-9.

3. Anthony BF, Okada DM, Hobel CJ. Epidemiology of group B streptococcus: longitudinal observations during pregnancy. J Infect Dis 1978; 187: 524-30.

4. Regan J, Klebanoff M, Nugent R. The epidemiology of group B streptococcal colonization in pregnancy. Vaginal Infections and Prematurity Study Group. Obstet Gynecol 1991; 77: 604-10.

5. Dillon H, Gray E, Pass M, Gray B. Anorectal and vaginal carriage of group B streptococci during pregnancy. J Infect Dis 1982; 145: 794-9.

6. Winn H, McLennan M, Amon E. Clinical assessment of the rapid latex agglutination screening test for group B streptococcus. Int J Gynecol Obstet 1994; 47: 289-90.

7. Schwartz B, Schuchat A, Oxtoby M. Invasive group B streptococcal disease in adults. J Am Med Assoc 1991; 266: 1112-14.

8. Zangwill K, Schuchat A, Wenger J. Group B streptococcal disease in the United States, 1990: report from a multistate active surveillance system. MMWR CDC Surveill Summ 1992; 41: 25-32.

9. Centers for Disease Control and Prevention (CDC). Early-onset and lateonset neonatal group B streptococcal disease-United States, 1996-2004. Morb Mortal Wkly Rep 2005; 54: 1205-8.

10. Centers for Disease Control and Prevention (CDC). Perinatal group B streptococcal disease after universal screening recommendations-United States. Morb Mortal Wkly Rep 2007; 56: 701-5.

11. Phares C, Lynfield R, Farley M, et al. Epidemiology of invasive group B streptococcal disease in the United States, 1999-2005. J Am Med Assoc 2008; 299: 2056-65.

12. Centers for Disease Control and Prevention (CDC). Trends in perinatal group B streptococcal disease-United States, 2000-2006. Morb Mortal Wkly Rep 2009; 58: 109-12.

13. Centers for Disease Control and Prevention (CDC). Active Bacterial Core Surveillance Report, Emerging Infections Program Network, Group B Streptococcus, 2008. [Available from: http://www.cdc.gov/abcs/reportsfindings/survreports/gbs08.pdf], 2009.

14. Eichenwald E. Perinatally transmitted neonatal bacterial infections. Infect Dis Clin North Am 1997; 11: 223-39.

15. Schrag S, Gorwitz R, Fultz-Butts K, Schuchat A. Prevention of perinatal group B streptococcal disease. Revised Guidelines from CDC. Morb Mortal Wkly Rep 2002; 51: 1-22.

16. Centers for Disease Control and Prevention (CDC). Prevention of perinatal group B streptococcal disease: a public health perspective. Morb Mortal Wkly Rep 1996; 45(RR-7): 1-24.

17. Schrag S, Zywicki S, Farley M, et al. Group B streptococcal disease in the era of intrapartum antibiotic prophylaxis. N Engl J Med 2000; 342: 15-20.

18. Jones D, Kanarek K, Lim D. Group B streptococcal colonization patterns in mothers and their infants. J Clin Microbiol 1984; 20: 438-40.

19. Francois R, Knostman J, Zimmerman R. Group B streptococcal neonate and infant infections. J Pediatr 1973; 82: 707-18.

20. Hood M, Janney A, Dameron G. Beta hemolytic streptococcus group B associated with problems of the perinatal period. Am J Obstet Gynecol 1961; 82: 809-18.
21. Baker C, Barrett F. Transmission of group B streptococci among parturient women and their neonates. J Pediatr 1973; 83: 919-25.

22. Baker C, Edwards M. Group B streptococcus infections: perinatal impact and prevention methods. Ann N Y Acad Sci 1988; 549: 193-202.

23. Eberly M, Rajnik M. The effect of universal maternal screening on the incidence of neonatal early-onset group B streptococcal disease. Clin Pediatr (Phila) 2009; 48: 369.

24. Verani J, McGee L, Schrag S. Prevention of perinatal group B streptococcal disease. Revised guidelines from CDC, 2010. Morb Mortal Wkly Rep 2010; 59(RR-10): 1-36.

25. Baker C. Group B streptococcal infections. Clin Perinatol 1997; 24: 59-70.

26. Boyer K, Gotoff S. Prevention of early onset neonatal group B streptococcal disease with selective intrapartum chemoprophylaxis. N Engl J Med 1986; 314: 1665-9.

27. Boyer K, Gotoff S. Strategies for chemoprophylaxis of GBS early-onset infections. Antibiot Chemother 1985; 35: 267-80.

28. Yow M, Leeds L, Mason E, et al. The natural history of group B streptococcal colonization in the pregnant woman and her offspring. I. Colonization studies. Am J Obstet Gynecol 1980; 137: 34-8.

29. Anthony B, Okada D, Hobel C. Epidemiology of the group B streptococcus: maternal and nosocomial sources for the infant acquisitions. J Pediatr 1979; 135: 431-6.

30. Badri M, Zawaneh S, Cruz A, et al. Rectal colonization with group B streptococcus: relation to vaginal colonization of pregnant women. J Infect Dis 1977; 135: 308-12.

31. Kontrick C, Edberg S. Direct detection of group B streptococci from vaginal specimens compared with quantitative culture. J Clin Microbiol 1987; 25: 336-9.

32. Wald E, Dashefsky B, Green M, et al. Rapid detection of group B streptococci directly from vaginal swabs. J Clin Microbiol 1987; 25: 573-74.

33. Skoll M, Mercer B. Evaluation of two rapid group B streptococcal antigen tests in labor and delivery patients. Obstet Gynecol 1991; 77: 322-6.

34. Gentry Y, Hillier S. Evaluation of two rapid enzyme immunoassays for detection of group B streptococcus Obstet Gynecol 1991; 78: 397-401.

35. Granab P, Petosa M. Evaluation of a rapid screening test for detecting group B streptococci in pregnant women. J Clin Microbiol 1991; 29: 1536-8.

36. Philipson E, Palermino D, Robinson A. Enhanced antenatal detection of group B Streptococcus colonization. Obstet Gynecol 1995; 85: 437-9.

37. Platt M, McLaughlin J, Gilson G, Wellhone M, Nims L. Increased recovery of group B streptococcus by the inclusion of rectal culturing and enrichment Diagn Microbiol Infect Dis 1995; 21: 65-8.

38. Quinlan J, Hill D, Maxwell B, et al. The necessity of both anorectal and vaginal cultures for group B Streptococcus screening during pregnancy. J Fam Pract 2000; 49: 447-8.

39. Kovavisarach E, Sa-adying W, Kanjanahareutai S. Comparison of combined vaginal-anorectal, vaginal and anorectal culture in detecting of group B streptococci in pregnant women in labor. J Med Assoc Thai [Chotmaihet thangphaet] 2007; 90: 1710-14.

40. Center for Disease Control and Prevention (CDC). Prevention of perinatal group B streptococcal disease: revised guidelines from CDC. Morb Mortal Wkly Rep 2002; 51(RR-11): 1-22.

41. Hall R, Barnes W, Krishnan L, et al. Antibiotic treatment of parturient women colonized with group B streptococci. Am J Obstet Gynecol 1976; 124: $630-4$.

42. Gardner S, Yow M, Leeds L, et al. Failure of penicillin to eradicate group B streptococcal colonization in the pregnant woman: a couple study. Am J Obstet Gynecol 1979; 135: 1062-5. 


\section{Postpartum infections Dorothea Mostello}

Serious postpartum infections remain a significant cause of maternal morbidity and mortality. Some factors that may predispose the puerpera to infection include rapid colonization of the postpartum endometrial surface by vaginal flora (1), contamination of the perineal area by vaginal and fecal flora, introduction of bacteria through manipulations or instrumentation employed to effect delivery, as well as the physiologic adaptation of the breasts for lactation. Despite these risks, the overall incidence of clinical infection in the postpartum period is relatively low (less than 10\%). Because postpartum women are generally young and healthy, their general resilience may impede prompt diagnosis if the patient or physician underestimates the risks of infection. Identification and close surveillance of patients at risk allow for timely diagnosis and prompt treatment, which may minimize serious sequelae of infection.

The standard definition of "puerperal fever" as an oral temperature of $38.0^{\circ} \mathrm{C}$ or more in any 2 of the first 10 days postpartum, exclusive of the first 24 hours, is impractical and may not be a valid indicator of infection. While transient elevations in temperature occur commonly and resolve spontaneously, a temperature greater than $38.4^{\circ} \mathrm{C}$ or persistent low-grade fever during the first 24 hours postpartum is highly predictive of ensuing infection (2). Disregarding temperature elevations that do not fit into a standard definition or ignoring signs and symptoms of infection not accompanied by fever may delay proper evaluation and therapy.

Time of onset of symptoms may provide a clue to etiology. Infections occurring within 48 hours of delivery commonly involve the uterus and peritoneum following cesarean section, the urinary tract following catheterization, or the respiratory tract. The microorganisms responsible for early infection were frequently present or introduced at the time of delivery, and the rate of bacteremia is high. Abdominal and episiotomy wound infections, breast infections, complications of uterine infection, and uterine infection that follows vaginal delivery usually present later in the postpartum period. The rate of bacteremia is low and the organisms associated with late infection may be anaerobic, chlamydial, endogenous, or introduced after delivery (3).

\section{ENDOMETRITIS}

Endometritis, or infection arising from the uterine cavity, is the most common postpartum infection. The diagnosis, classically heralded by a tender uterus and foul lochia, is usually one of exclusion in the febrile postpartum woman without signs of infection from another source.

The pathophysiology of endometritis is similar to that of other pelvic infections-polymicrobial and ascending from the colonized lower genital tract. No risk factor is absolutely predictive; however, when more than one factor is present, risk is cumulative (4). Endometritis following vaginal delivery occurs in $1 \%$ to $3 \%$ of patients. Delivery by cesarean section increases the incidence 3 to 30 times when compared with the vaginal route $(5,6)$. Thus, cesarean delivery is the greatest risk factor for endometritis, with baseline rates of $9 \%$ following elective and $29 \%$ following nonelective procedures (7). Microorganisms from amniotic fluid or vagina directly inoculate the myometrium and peritoneal cavity at the time of surgery. The presence of suture material and surgically devitalized tissue, along with postoperative exudation and fluid collections, creates a favorable environment for subsequent bacterial growth (8). Placement of the uterine incision, however, has no effect on the incidence of postpartum infection (9).

Frank chorioamnionitis is an obvious antecedent of postpartum intrauterine infection, often despite intrapartum antibiotic therapy (10). Risk is compounded by the frequency of dysfunctional labor and failure to progress in women with chorioamnionitis, necessitating delivery by cesarean section (11). A positive amniotic fluid culture, even in the absence of overt signs of infection, is also highly predictive of subsequent endometritis, especially following cesarean section $(12,13)$. Bacterial contamination of amniotic fluid, rare when fetal membranes are intact, is almost always present after 6 hours of ruptured membranes (14). The quantity and virulence of the organisms that colonize the amniotic fluid following membrane rupture are primary determinants of subsequent infection $(6,15)$.

Major risk factors include prolonged duration of ruptured membranes (16), long labor with multiple vaginal exams, low socioeconomic status $(17)$, obesity $(17,18)$, and the presence of bacterial vaginosis (19). Untreated carriers of group B streptococci are also more likely to develop endometritis (20). Administration of glucocorticoids for acceleration of fetal lung maturation does not increase the risk of endometritis or other postpartum infections (21).

The diagnosis of endometritis is usually based upon clinical findings. Fever is the most useful sign and should prompt a physical examination even within the first 24 hours postpartum. In the absence of other signs of infection, continued observation may be appropriate, but further evaluation is indicated if fever persists. Endometritis after cesarean section typically presents within 48 hours of surgery. When endometritis follows vaginal delivery, the diagnosis is made within 7 days in $84 \%$ and within 14 days in 98\% (6).

The patient may complain of malaise, chills, abdominal pain, or foul-smelling discharge. On physical examination, tachycardia, decreased bowel sounds, lower abdominal tenderness, uterine and adnexal tenderness to bimanual palpation, and purulent or foul lochia may be found, although physical findings may be minimal. A pelvic mass on initial 
examination is unusual. Because the differential diagnosis for fever in the immediate postpartum period includes atelectasis, pneumonia, pyelonephritis, viral syndrome, mastitis, and appendicitis, the physical examination should be conducted to exclude these conditions.

If infection is suspected, laboratory evaluation should include complete blood count, urinalysis and urine culture from a catheterized specimen, and blood cultures in most cases. Routine endometrial cultures are rarely useful, as wellchosen antibiotic therapy results in a rapid recovery in most patients despite culture isolates. An endometrial culture may be helpful when hemolytic streptococci or clostridia are suspected or when endometritis develops in patients who are at risk for infection with unusual or resistant organisms (22). Prior to obtaining specimens transcervically, preparation by cervical cleansing is recommended to minimize contamination with lower genital tract flora (8).

With the aim of minimizing infectious morbidity, prevention of endometritis is paramount. Even though instrumental delivery is a risk factor for endometritis following vaginal delivery (23), data are too few and of insufficient quality to recommend routine use of prophylactic antibiotics for operative vaginal delivery (24). Antibiotic prophylaxis for cesarean section, however, reduces the rate of subsequent endometrial infection by more than half after both elective [relative risk (RR) 0.38, absolute risk reduction 4\%] and nonelective procedures (RR 0.39 , absolute risk reduction $18 \%$ ) $(25,26)$. The effect is evident even in nonlaboring patients with intact membranes (RR 0.05) (27) with significant cost savings per case (28).

Many antibiotics provide effective prophylaxis. The most recent systematic review (29) showed no benefit in efficacy of either single broad-spectrum agents over ampicillin or firstgeneration cephalosporins or multiple doses of antibiotics over a single dose. Clindamycin was recommended for penicillinallergic women. Recent concerns over the rising resistance of Gram-negative organisms have prompted a trend away from ampicillin toward first- and second-generation cephalosporins and ampicillin/sulbactam, although data supporting their superiority are lacking. Adding aztreonam to the clindamycin has been proposed in cases of penicillin allergy.

While delaying administration of the antibiotic until cord clamping was originally touted to avoid clouding the evaluation of the newborn, recent evidence shows significant maternal benefit to administration of the antibiotic 15 to 60 minutes preoperatively. Attaining therapeutic tissue levels of antibiotic before bacterial contamination decreases the risk of endometritis compared with dosing after cord clamping [adjusted odds ratio (aOR) 0.22] without increasing the neonatal length of stay or incidences of sepsis or septic workup in the newborn (30-32). The practice of giving intrapartum prophylaxis to women colonized with group B beta-hemolytic streptococci has also resulted in fewer cases of subsequent endometritis (33).

Other measures of prevention have been studied and warrant consideration. Delivery of the placenta at cesarean section by external uterine massage and gentle traction rather than manually has been shown to reduce the rate of endometritis in several randomized controlled trials (34-36). Administration of methylergonovine $0.2 \mathrm{mg} \mathrm{q} 6 \mathrm{~h}$ following cesarean section until discharge from the hospital (37) can decrease the rate of endometritis as well as minimize blood loss. A promising measure in the prevention of endometritis is extended spectrum antibiotic prophylaxis. The addition of an antibiotic (doxycycline or azithromycin) targeting Ureaplasma urealyticum, which has been shown to be a significant predictor of endometritis despite typical antibiotic prophylaxis and intact membranes, significantly reduced both endometritis and wound infection (38-40). Preoperative intravaginal treatments, such as intravaginal metronidazole gel (41) or a vaginal scrub with povidone-iodine (42), aimed at decreasing the inoculum of vaginal bacteria that may ascend, also show reduced rates of subsequent endometritis in controlled studies. Interventions with no effect on the rate of post-cesarean endometritis include in situ versus exteriorized repair of the uterine incision, glove change after delivery of the fetus before proceeding with the operation, and removal of uterine debris with tissue forceps versus uterine wiping with a laparotomy pad after placental delivery $(35,36,43)$.

Choice of antibiotic therapy for acute endometritis is directed by the bacteriology of the infection. Aerobic organisms are found in about $70 \%$ of cultures and anaerobic organisms in about $80 \%$ (44). Typically, more than one isolate per culture are identified (45). Gram-negative bacilli, particularly Escherichia coli, are the most common aerobes, followed by Gram-positive aerobes such as groups B and D streptococci. Group A streptococci and Staphylococcus aureus are recovered in less than $5 \%$ of cases. The most common anaerobic isolates are Bacteroides bivius and $B$. fragilis as well as the anaerobic streptococci peptococcus and peptostreptococcus. Genital mycoplasmas and chlamydia also may be recovered from patients with acute endometritis (3).

Empirical therapy with parenteral antibiotics should be initiated as soon as the diagnosis of endometritis has been made and cultures have been taken (Table 1). Most patients will show a clear response to treatment within 48 to 72 hours. Following vaginal delivery, 95\% respond to initial administration of a penicillin plus an aminoglycoside, despite incomplete anaerobic coverage. With additional antibiotics in those who fail initial therapy, less than 2\% develop major infectious complications such as abscess or septic pelvic thrombophlebitis (6). The most recent systematic review (46) did not specifically address endometritis following vaginal delivery due to insufficient data. Recommendations for treatment of endometritis following cesarean delivery are clear: regimens with poor activity against penicillin-resistant

\section{Table 1 First-Line Therapy for Endometritis}

After cesarean delivery

Clindamycin $900 \mathrm{mg}$ intravenously every $8 \mathrm{~h}$ plus Gentamicin $4 \mathrm{mg} / \mathrm{kg}$ intravenously once daily, (plus ampicillin $2 \mathrm{~g}$ intravenously every $6 \mathrm{~h}$ ) ${ }^{\mathrm{b}}$

Clindamycin (as above) plus aztreonam $2 \mathrm{~g}$

Intravenously every $6-8 \mathrm{~h}^{\mathrm{a}}$

Metronidazole $500 \mathrm{mg}$ intravenously every $6 \mathrm{~h}$

Plus gentamicin (as above), plus ampicillin (as above)

After vaginal delivery

Ampicillin (as above), plus gentamicin (as above)

${ }^{a}$ For penicillin-allergic patients. ${ }^{b}$ Depending on the agent used for prophylaxis. 
anaerobes (e.g., Bacteroides fragilis group) are more likely to fail. The combination of clindamycin and an aminoglycoside remains the gold standard. Although small studies comparing clindamycin/gentamicin with single broad-spectrum agents (such as second- or third-generation cephalosporins, ureidopenicillins, or penicillins combined with a beta-lactamase inhibitor) show them to be equally effective, meta-analysis shows that only 20 women would need to be treated with clindamycin and gentamicin to prevent one failure with other regimens (46). Metronidazole-penicillin-aminoglycoside and clindamycin-aztreonam may be appropriate choices for postcesarean infection as well (8).

Fewer failures occur with once-daily gentamicin dosing (46). Advantages of once-daily dosing include a superior bactericidal effect with higher peak serum concentrations, time for recovery of bacterial sensitivity between doses (post-antibiotic effect), lower trough levels resulting in less accumulation of the drug in the cochlear system, and cost savings $(47,48)$. Typical formulas for calculating the daily dose may be inadequate in the postpartum woman due to greater renal clearance, increased volume of distribution, and obesity. The pharmacokinetics has been investigated with the following recommendations: $4 \mathrm{mg} / \mathrm{kg}$ actual body weight, or $5 \mathrm{mg} / \mathrm{kg}$ adjusted body weight, or substituting a volume of distribution of 0.4 to $0.43 \mathrm{~L} / \mathrm{kg}$ in the formula pharmacies usually use to calculate the dose (49).

Changes in endometrial flora have been demonstrated following administration of prophylactic antibiotics and may affect treatment of subsequent infection. The use of cefazolin and other cephalosporins is associated with increases in infections with enterococci $(17,22,50,51)$, and the use of penicillin or ampicillin has resulted in an increased risk of infections caused by Gram-negative organisms $(50,52)$ and mycoplasmas (50). Unfortunately, no evidenced-based recommendations exist for treatment of women who develop endometritis after receiving antibiotic prophylaxis for cesarean section. In one study (53), only $54 \%$ of women, who had received cephalosporin prophylaxis and developed postcesarean endometritis, responded to clindamycin and gentamicin within 48 hours. An additional $40 \%$ responded to the addition of ampicillin or vancomycin. Thus, it seems prudent and pragmatic to consider initial treatment with clindamycin and once-daily gentamicin if a penicillin has been used for prophylaxis, but treatment with ampicillin, clindamycin, or metronidazole, and once-daily gentamicin if a cephalosporin has been used for prophylaxis.

Common sense guidelines and important caveats warrant mentioning. When the diagnosis of endometritis is suspected, it is unwise to extend the prophylactic agent for a longer time as the causative organism is likely resistant. In general, do not use an antibiotic for treatment to which the patient has been recently exposed (52). When high fever but no localizing signs are present, consider group A streptococcus infection (52) as two-thirds of patients present with a fever only (54). Similarly, group B streptococcal cultures are not $100 \%$ predictive; if a patient has an early-onset endometritis with high-spiking fever, group B streptococcus may be the causative organism even if her culture within 5 weeks was negative (33).

The appropriate duration of therapy has not been well established. Typically, parenteral antibiotics have been continued until the patient has been afebrile for 48 hours and the physical examination is benign. Discontinuation of treatment in a nonbacteremic patient when the patient's temperature has been $\leq 37.5^{\circ} \mathrm{C}$ for at least 24 hours has been shown to be safe and cost-effective in both private and indigent populations $(55,56)$. After treatment of uncomplicated endometritis, followup oral antibiotics, except in patients with known chlamydial infection, are generally unnecessary $(3,17,46,57)$.

Failure to respond within 48 to 72 hours warrants reevaluation of the patient. Common causes of failure include wound infection and resistant microorganisms. Culture results should be reviewed, if available, and cultures of blood, endometrium, and wound collected. Antibiotic therapy should be assessed. Increases in dose or frequency of administration may be required, as pregnancy-related changes in renal function, blood volume, and total body water affect elimination and volume of distribution of drugs, usually toward lower serum levels (58). In patients receiving clindamycin plus an aminoglycoside, aminoglycoside levels should be drawn and ampicillin added to provide coverage against enterococci (22). Anaerobic coverage should be added if lacking. Single-agent therapy may be changed to clindamycin plus an aminoglycoside plus a penicillin. For proven staphylococcal infection, specific therapy should be added (e.g., nafcillin $1-2 \mathrm{~g}$ intravenously every $4-6$ hours, cefazolin $2 \mathrm{~g}$ intravenously every 8 hours, or vancomycin $1 \mathrm{~g}$ intravenously every 12 hours). Wound infection, of course, warrants drainage.

If antibiotic therapy requires no modification, or if changes fail to produce response ( $80 \%$ respond) within another 48 to 72 hours, the case should be scrutinized for sources of continued fever. The differential diagnosis must be broadened to include wound infection, pelvic cellulitis, pelvic abscess, venous thromboembolism, drug fever, septic pelvic thrombophlebitis, enterocolitis, retained placental fragments, as well as viral or connective tissue disorders. Pelvic ultrasound may be helpful in visualizing retained placenta or a fluid collection. A computed tomographic (CT) scan may identify ovarian vein thrombosis, abscess, hematoma, or other masses.

The incidence of documented bacteremia following cesarean section is $3 \%$ to $4 \%$, compared with $0.1 \%$ to $0.4 \%$ in patients who have delivered vaginally. Endometritis accounts for the majority of cases, but wound, respiratory and urinary infections, as well as endocarditis may be responsible $(59,60)$. Bacteremia is more commonly found in post-cesarean endometritis (8-30\%) compared with endometritis following vaginal delivery $(5 \%)(5,6)$. Risk for positive blood cultures is correlated with length of labor, number of vaginal exams, duration of ruptured membranes, estimated blood loss, and intra-amniotic infection (61). Bacteremia is rare $(<1 \%)$ in the postpartum woman with a temperature peak $<38.8^{\circ} \mathrm{C}$, but occurs in more than $20 \%$ of those with higher fevers (61). Organisms isolated from the blood reflect organisms infecting the endometrium and are found in similar proportions (57). While blood culture results may guide antibiotic therapy for endometritis, failure to respond to initial therapy does not correlate with a resistant organism in blood culture (59). Identification of a pathogenic organism resistant to the antibiotics administered warrants broadening the antibiotic coverage despite apparent clinical response. Prolonged therapy may be indicated when S. aureus is isolated from the blood to 
avoid metastatic infection $(8,44)$. Mortality among obstetric bacteremic patients is extremely low $(<1 \%)$ compared with other medical or surgical populations (60).

Late postpartum endometritis, with onset from 7 to 42 days after delivery, is a clinically mild disease, which typically occurs in women who have delivered vaginally. Lower abdominal pain and an increasing vaginal discharge with odor are the presenting symptoms; systemic signs are rare. Chlamydia trachomatis, uncommon in acute endometritis (45), is isolated from the cervix or endometrium in $30 \%$ to $67 \%$ of cases $(62)$. In women with chlamydial infection detected in the antepartum period, late postpartum endometritis develops in $30 \%$ to $60 \%$ if untreated (62). When late-onset infection is suspected, a cervical or endometrial culture for chlamydia should be obtained. Azithromycin ( $1 \mathrm{~g}$ orally in a single dose), tetracycline (doxycycline $100 \mathrm{mg}$ orally twice a day for 7 days), or erythromycin (500 mg orally four times a day for 7 days) therapy is clinically effective. Treatment in the antepartum period may prevent late postpartum infection.

\section{WOUND INFECTIONS}

Abdominal wound infections occur in $1.6 \%$ to $10 \%$ of women delivered by cesarean section $(4,63,64)$. Antibiotic prophylaxis has been shown to reduce the rate of wound infection after both nonelective (RR 0.36) and elective (RR 0.73) cases (25). Extended spectrum prophylaxis with doxycycline and/or azithromycin (covering for Ureaplasma) reduces the risk even further $(38,40)$. Other basic measures, such as preoperative showering with chlorhexidine, clipping hair rather than shaving the area for incision, and preparation of the skin with an alcohol-based agent, are also advocated (65). Risk factors identified by prospective surveillance include skin closure with staples rather than subcuticular suture and maternal obesity (66), particularly in women with subcutaneous tissue thickness greater than $3 \mathrm{~cm}$ (67). One randomized trial showed a reduction in wound infections using the Joel-Cohen rather than Pfannenstiel incision (68). No effect on rate of infection has been demonstrated with prophylactic subcutaneous drainage (69).

The diagnosis of wound infection is based on the findings of local advancing erythema, induration, and/or drainage from the wound. Fever is not always present. Clinical signs typically manifest 4 to 8 days following delivery; $71 \%$ occur after discharge from the hospital (66). Many patients have had endometritis and present with recurrent fever after the initial response to treatment for the uterine infection. The majority of wound infections result from extension of polymicrobial contamination of the endometrium and amniotic fluid to the incision site (70), but the skin may also be the source of bacteria (65). Staphylococcal wound infections, accordingly, typically develop in women without obstetric risk factors. If wound infection presents with fever and spreading cellulitis within the first 48 hours after operation, a single bacterial pathogen, such as group A or B beta-hemolytic streptococci or Clostridium perfringens, may be responsible. A Gram stain of material aspirated from the active margin of infection may identify the causative organism.

Treatment for early-onset infection consists of antibiotic therapy such as penicillin 4 million units intravenously every 4 hours or cefazolin $2 \mathrm{~g}$ intravenously every 6 hours, and prompt debridement of necrotic tissue (71). Later infections usually respond to simple incision and drainage. Antibiotics are required only if there is extensive cellulitis, bacteremia, or failure to defervesce within 12 to 24 hours of opening the wound (71). Debridement under anesthesia is sometimes required (70). Fascial dehiscence of the wound occurs in $0.5 \%$ of post-cesarean patients and may be more common when wound infection is present $(64,70)$. Fascial repair under anesthesia may be necessary. Modern management of open wounds, including secondary closure within 1 to 4 days of disruption, negative pressure wound therapy (vacuumassisted closure), or healing by secondary intention with dressings that keep the wound moist and absorb drainage, has been shown to be superior to frequently changed, traditional "wet-to-dry" dressings and cleansers that disrupt wound healing (65).

Complications of wound infection such as necrotizing fasciitis, synergistic bacterial gangrene, and clostridial gas gangrene are extremely rare after cesarean section but should be suspected if the patient fails to respond following incision and drainage of the wound. Early recognition is essential to prevent mortality from these life-threatening conditions. Necrotizing fasciitis should be suspected if the patient appears critically ill or if the edema and induration surrounding the wound worsen rapidly despite treatment or are accompanied by local anesthesia or crepitus. At surgical exploration, there is undermining of the involved skin surrounding the wound and the superficial fascia lacks resistance to a blunt instrument. Synergistic bacterial gangrene is recognized by a central ulcer, a surrounding deep red zone, and peripheral erythema. The process is slowly progressive and is often marked by inordinate pain. Clostridial gangrene presents with sudden onset of severe pain, mild local edema, thin watery drainage from the wound, and systemic toxicity. In advanced stages, the skin appears bronzed with bullae, gangrene, and crepitation. Gas may be evident on X-ray (71). These destructive processes require extensive surgical debridement. Aerobic and anaerobic cultures and broad-spectrum parenteral antibiotics are appropriate in the management of these polymicrobial infections. Delays in treatment may lead to maternal mortality.

\section{SEPTIC PELVIC THROMBOPHLEBITIS}

Septic pelvic thrombophlebitis is a potentially life-threatening complication that occurs most commonly after obstetric procedures. The diagnosis is typically suspected in a woman with persistent fever after several days of adequate antibiotic therapy for presumed endometritis with no wound infection apparent (72). This persistent fever occurs in about 1 in 650 deliveries (72) or $0.5 \%$ to $2 \%$ of patients with postpartum endometritis (73).

The pathogenesis of pelvic thrombophlebitis rests on Virchow's triad of venous stasis, endothelial damage, and alterations in coagulation factors. Damage to the intima of pelvic veins may result from direct surgical trauma or from the inflammatory response to an infected uterus. The velocity of blood flow through the dilated ovarian veins declines sharply postpartum. The long veins have multiple valves, which are major sites of venous pooling and their thin walls make them subject to external compression. Pregnancy-related increases in levels of clotting factors and platelet adhesiveness, along 
with possible thromboplastin release from placental fragments and necrotic tissue, may generate a thrombotic process (73).

Pelvic thrombophlebitis encompasses two fairly distinct forms-ovarian vein thrombosis and thrombosis of multiple small pelvic vessels. In the acute ovarian vein syndrome, onset is usually within 2 to 4 days postoperatively, but may be weeks after delivery. The patient usually appears ill with a low-grade fever, tachycardia, flank or lower abdominal pain with guarding, and ileus with abdominal distention. Nausea and vomiting may be present. A tender, rope-like, abdominal mass originating near the uterine cornu and extending cephalad and laterally, palpable in one-half to two-thirds of patients, represents the thrombosed ovarian vein. Ovarian vein thrombosis commonly occurs in association with pelvic infection, but the patient's symptoms worsen, rather than improve, with continued antibiotic therapy. The syndrome may present in the absence of clinical infection, sometimes prompting laparotomy to exclude other disorders. The differential diagnosis includes acute appendicitis, broad ligament hematoma, degenerating fibroid, adnexal torsion, pyelonephritis, ureterolithiasis, and abscess.

Postpartum ovarian vein thrombosis is predominantly right sided $(80 \%)$, with bilateral and left-sided lesions unusual (14 and $6 \%$, respectively) $(74,75)$. The preponderance of rightsided involvement is explained by venous drainage, which, in the upright position, flows from the left ovarian vein and uterine venous plexus into the right ovarian vein, increasing venous stasis and the inoculum of pathogens on the right (3). Incidence is approximately 1 in 3000 deliveries, following 1 in 800 cesarean and 1 in 9000 vaginal deliveries (72). Although, when studied, $50 \%$ of women who had postpartum ovarian vein thrombosis were found to have inherited prothrombotic factors (76), no clinical risk profile could be established-prior thrombosis was rare, many had taken oral contraceptives without complication, and none had a family history of clots.

Septic pelvic thrombophlebitis with diffuse thrombosis of multiple small pelvic vessels usually presents with enigmatic fever and typically follows the diagnosis of endometritis or other pelvic infection. After several days of antibiotic therapy, recurrent high-spiking fevers occur despite resolution of other signs and symptoms of infection. The patient generally feels well when not febrile and may need to be convinced that she has a serious complication. Imaging techniques do not demonstrate the small uterine and vaginal veins, which have been shown at laparotomy to be involved (77). The diagnosis is usually presumptive, based on defervescence within 48 to 96 hours (average 37 hours) after addition of heparin to the antibiotic regimen (78). The incidence is estimated at 1 in 2000 deliveries, following 1 in 400 cesarean and 1 in 10,000 vaginal deliveries (79).

Diagnostic imaging may be helpful in evaluating patients with suspected septic pelvic thrombophlebitis as thrombus in ovarian and larger veins may be visualized by CT scan, ultrasound, or magnetic resonance imaging (MRI). Sonography has been suggested as a screening examination for postpartum ovarian vein thrombosis, but sensitivity is only $56 \%$, with imaging hampered by intervening bowel gas or obesity (80). CT and MRI are also superior to sonography at evaluating for other conditions in the differential diagnosis. CT remains the imaging procedure of choice (sensitivity
78-100\%), as MRI (sensitivity $92-100 \%$ ) offers no clinical advantages to balance its increased cost $(80,81)$. All imaging techniques are helpful only if positive and are inadequate in excluding the diagnosis.

Serious complications of pelvic thrombophlebitis are mainly due to extension of thrombus or embolization. About $45 \%$ of ovarian vein thromboses imaged are seen to extend to the inferior vena cava (76); extension to femoral, iliac, mesenteric veins, and even the right atrium is possible. Extension of clot from the ovarian to the renal vein may result in renal vein thrombosis with subsequent nephrotic syndrome and renal insufficiency. Clots in pelvic vessels may fragment, possibly owing to the presence of bacteria, and embolize. Showers of small infected emboli are characteristic with resulting septicemia and metastatic abscesses (82) (Fig. 1). Massive involvement of ovarian veins or the vena cava may precipitate large pulmonary emboli with symptoms of venous occlusion; in fact, respiratory distress from pulmonary embolism may be the presenting symptom (75). Mortality from pulmonary embolism or metastatic abscesses is estimated at 18 deaths per million (80). Ventilation-perfusion or CT scanning of the lung may be indicated to determine whether pulmonary embolization has occurred. Classic papers report evidence of emboli in one-third or more of patients tested $(73,82,83)$, even though symptomatic pulmonary embolism was rare (84). No modern reports contain a systematic assessment for emboli.

The diagnosis of pelvic thrombophlebitis, in either form, may not be confirmed by radiographic study. Clinical response to heparin may secure the diagnosis in both the acute and the enigmatic syndromes. Full anticoagulation should be instituted along with broad-spectrum antibiotics (Table 2) as microorganisms have been isolated from resected venous specimens and require treatment to promote resolution of the thrombus $(73,85)$. Resolution of the fever does not imply resolution of the clot, so a full course of anticoagulation is necessary. Substitution of low molecular weight for unfractionated heparin seems reasonable, but has not been systematically studied. Primary surgical ligation of ovarian veins and vena cava, employed 50 years ago, decreased mortality from $50 \%$ to $10 \%$ (86), but is fraught with risks of significant hemorrhage, embolization, and death (74). Today, a surgical approach should be reserved for patients who do not respond to anticoagulation plus antibiotics, who experience pulmonary

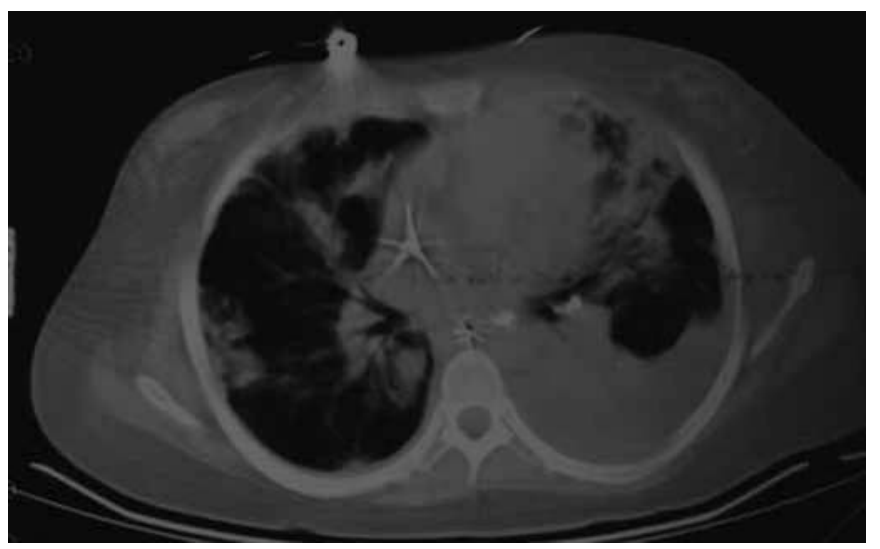

Figure 1 Septic emboli are seen as multiple peripheral and hilar infiltrates on the computed tomographic scan without contrast. 
Table 2 Treatment of Septic Pelvic Thrombophlebitis

Continuation of antibiotic therapy

Until the patient is afebrile for $48-72 \mathrm{~h}$

Full anticoagulation with heparin

$5000-10,000$ units loading dose, followed by $800-2000$

Units/h to maintain the PTT at $1.5-2.5 \times$ baseline value

Duration of anticoagulation therapy

Ovarian vein thrombosis

6 weeks to 6 months (no fewer than 3 weeks)

May change to oral anticoagulation after the first 5-7 days

Suspected thrombosis of smaller pelvic vessels (enigmatic fever) 7-10 days

Abbreviation: PTT, partial thromboplastin time.

embolism while on therapeutic anticoagulation, or in whom the diagnosis is uncertain. If pelvic vein thrombosis is found unexpectedly at postpartum laparotomy, closure of the abdomen without vein ligation and institution of medical therapy is probably the treatment of choice. Need for anticoagulation during or after subsequent pregnancy is probably not required.

The subject of anticoagulation has recently become controversial. The observation that some fevers eventually resolve without heparin has led some to argue that the superior efficacy of today's antibiotics may obviate the need for heparin. One trial in 14 women with CT-proven ovarian vein thromboses, randomized to receive either antibiotics and heparin or antibiotics alone, showed no difference in durations of fever or hospitalization and no evidence of symptomatic emboli (72). Unfortunately, the numbers were too small to preclude possible protection from embolization by heparin.

\section{EPISIOTOMY INFECTIONS}

Postpartum infection arising from an episiotomy site is a rare but potentially fatal condition. An episiotomy is a contaminated wound; yet clinical infection manifests in only $0.035 \%$ to $3 \%$ of patients $(63,87)$. In a randomized controlled trial, use of prophylactic antibiotics (second-generation cephalosporin) for repair of third- and fourth-degree tears was shown to decrease the rate of infectious complications (88). Life-threatening infection associated with episiotomy has occurred in 0.5 to 2 per 100,000 live births, accounting for $20 \%$ of maternal mortality. Perineal infection has been classified according to the depth of soft-tissue involvement (89). Successful management depends on accurate assessment of the infected tissues.

Infection limited to the skin and superficial fascia along the episiotomy incision may be managed conservatively. Episiotomy dehiscence or perineal pain may be the presenting complaint, with edema and erythema confined to the area immediately adjacent to the episiotomy (89). The previous repair should be taken down to allow careful inspection of the wound to exclude hematoma, abscess, or unsuspected rectovaginal communication. Following cleansing and debridement, the wound may be allowed to heal secondarily. With surrounding cellulitis, a course of broad-spectrum parenteral antibiotics (such as suggested in Table 1), in addition to local wound care, may be required. Early repair of episiotomy dehiscence, while contrary to traditional practice, has produced excellent anatomical and functional results (90).
Superficial fascial infection presents with erythema and edema, which extend beyond the area of episiotomy to the thigh, buttock, or abdominal wall. Appropriate therapy is dependent upon determination of associated necrotizing fasciitis. Diagnosis by inspection is unreliable, thus surgical exploration may be necessary to rule out fascial necrosis. In the absence of obvious signs of necrotic fascia, frozen-section biopsy has been employed to expedite diagnosis and avoid a possible lethal delay in treatment (91). In the absence of fascial necrosis, severe systemic involvement is uncommon and response to broad-spectrum antibiotics should occur within 24 to 48 hours. Lack of response or progression of the infection during antibiotic therapy may indicate a more severe infection.

When necrotizing fasciitis is present, aggressive management is necessary to avert mortality. Both Camper's and Colles' fascial layers become necrotic with this infection, which spreads along these fascial planes, undermining the skin, and eventually involves all tissues external to the deep fascia (92). The onset is acute with a rapidly progressive clinical course, systemic toxicity, and a fatality rate of $21 \%$ to $76 \%$ (89). Although the condition is classically described in patients with diabetes or atherosclerosis, affected obstetric patients are usually otherwise healthy (93). The patients appear severely ill, usually with fever and leukocytosis. Erythema with indistinct margins that gradually fade into normal areas may be present. As the disease progresses, skin color becomes blue or brown, with subsequent formation of bullae and gangrene. Progressive brawny edema and induration are evident; vulvar edema, especially unilateral, is characteristic (93). Marked local tenderness is common initially, but the lesions become hypesthetic or anesthetic as cutaneous nerves become ischemic (92). Crepitation may be detected. Exudate is serosanguinous "dirty dishwater" rather than purulent. Anemia due to hemolysis is common (92), but may be masked by massive fluid shifts into the extravascular compartment with resultant hemoconcentration (89). Hypocalcemia may develop due to saponification of liquified subcutaneous fat (92). At surgical exploration, the superficial fascia does not bleed and lacks resistance to blunt probing; the skin is easily separated from the underlying deep fascia.

Prompt surgical resection of the involved tissues is essential for effective management of necrotizing fasciitis. Mortality is $100 \%$ in patients treated with antibiotics alone or with incision and drainage only (94). Debridement should extend to where the subcutaneous tissues cannot be separated from the underlying deep fascia or from normal skin. Any tissue that is indurated, edematous, or crepitant, or that does not bleed readily when incised, should be removed (92). More than one surgical debridement may be necessary if subcutaneous necrosis is not arrested. Hyperbaric oxygen is an adjunctive measure and should not substitute for surgical debridement.

Similar to most pelvic infections, necrotizing fasciitis is a polymicrobial disorder. The pathophysiology involves bacterial synergism among various combinations of aerobic and anaerobic organisms $(92,94)$. Comprehensive antibiotic coverage with a penicillin, clindamycin, and an aminoglycoside is recommended as an adjunct to operative intervention until a healthy bed of granulation tissue is apparent. After extensive debridement, these patients resemble those with severe burns. 
Management in an intensive care setting with invasive monitoring, aggressive fluid management, and hemodynamic support may be required (92). The wound may be allowed to heal secondarily.

Infection beneath the deep fascia produces myonecrosis. A clostridial infection is the most common cause, although myonecrosis can occur from a neglected necrotizing fasciitis, which invades deep fascia. Clostridial infection may present early in the postpartum period with severe pain disproportionate to the physical findings. A wound or blood culture positive for clostridia should raise the index of suspicion for myonecrosis. Crepitation or clinical deterioration in the presence of an episiotomy infection warrants surgical exploration and radical excision of involved tissues, supplemented with antibiotic therapy. For clostridial infection, high-dose penicillin (4 million units intravenously every 4 hours) is indicated. Polyvalent gas gangrene antitoxin is thought to be ineffective (89). C. perfringens is the most notorious organism associated with myonecrosis. Clostridium sordellii has also been identified in serious episiotomy infections, but presents with massive malignant vulvar edema thought to be caused by toxin production and results in death from cardiovascular collapse (95).

\section{MASTITIS}

Lactation mastitis is a cellulitis of the interlobular connective tissue within the mammary gland that usually occurs within the first 6 weeks postpartum (96). Mastitis occurs in up to $9.5 \%$ of breastfeeding women $(96,97)$, but may rarely occur in women who are not nursing. Most cases are sporadic, presenting commonly in the second or third week following delivery $(98,99)$. The onset of symptoms is usually abrupt with chills, malaise, generalized achiness, and fever as high as 39 to $40^{\circ} \mathrm{C}$. The affected breast is tender, hot, swollen, and erythematous in a wedge-shaped segment (96) with its apex at the nipple and its base toward the periphery, demarcated by the divisions between the lobes of the breast. Decreased milk secretion may be noted (100), but expression of pus from the ducts is uncommon (99). Poor breastfeeding technique with attachment difficulties, injury to the nipple, nipple fissuring, and milk stasis (101) is considered to be the primary etiologic factor, along with lowered immune status from stress or sleep deprivation (96), but the cause of most cases is unclear (97). One study found fewer than 10 feedings/day to be protective (96), contrary to common belief. Mastitis is no more common among women who are nursing for the first time than among women who have previously nursed. Previous mastitis is a risk factor for recurrence in successive pregnancies (96), perhaps due to persistence of poor practices (102).

Breast infections should be distinguished from segmental breast inflammation due to milk stasis or focal engorgement. Milk contains pro-inflammatory cytokines, which may leak into the circulation when not drained and elicit a systemic response in the absence of infection (101). Fever and erythema are less marked in the absence of infection and will resolve with rest, frequent, complete emptying of the breast, and without antibiotics $(101,103)$. Local hot wet compresses or prone soaks in a hot tub may soften the indurated breast and allow drainage (99). Change in feeding position may permit drainage of blocked ducts. Antibiotics are indicated if symptoms do not resolve. Patients with obvious signs and symptoms of mastitis should be treated immediately with antibiotics, as delay in institution of antibiotic therapy is associated with abscess formation $(98,104)$. Diagnostic criteria based on leukocyte counts from the milk and quantitative bacterial cultures, helpful in differentiating mastitis in dairy cows, are not always valid in women, as bacterial counts have not been reliable (101).

Appropriate therapy for mastitis includes routine emptying of the breast and administration of antibiotics. Breastfeeding from both breasts should continue. If the infected breast is too sore for nursing, gentle pumping may be employed to reduce congestion. Weaning may increase the risk of abscess formation $(97,99,105)$. Choice of antibiotic may be empirical or based on culture of expressed breast milk. S. aureus is the organism isolated in $35 \%$ to $50 \%$ of breast infections, and $50 \%$ to $70 \%$ show penicillin resistance in vitro $(97,100,104,106)$. Other common pathogens include coagulase-negative staphylococci, streptococci, E. coli, Klebsiella pneumoniae, and Bacteroides fragilis (100). Cultures of breast milk may be prudent, since methicillinresistant S. aureus, primarily community-acquired, is emerging as a pathogen in mastitis (107), even in those without predisposing factors (108).

Therapy with a penicillinase-resistant penicillin, such as dicloxacillin (250-500 mg orally four times a day) or amoxicillin/clavulanic acid (500/125 $\mathrm{mg}$ three times a day), or a first-generation cephalosporin (cephalexin $500 \mathrm{mg}$ orally four times a day) should be started immediately after cultures are obtained. Erythromycin or azithromycin is the alternative in penicillin-allergic patients. In most cases, antibiotics may be given orally, and hospitalization is not required. Therapy should be continued for at least 10 days. Most patients become afebrile and asymptomatic within 36 to 48 hours of beginning treatment (97). If the patient is not improved within 48 hours, examination for abscess formation is warranted.

Breast abscesses develop in $4 \%$ to $11 \%$ of patients despite appropriate treatment $(96,97,106)$. An indurated, fluctuant area may be palpable, usually peripherally, and should be aspirated for culture if suspected. Axillary lymphadenitis is not typical, but the patient may be febrile and tachycardic, with leukocytosis. Bacteremia may occur (109). S. aureus is recovered from $38 \%$ of abscesses (104). Ultrasound is a useful adjunct when there is clinical suspicion of abscess or in cases of mastitis that do not promptly respond to appropriate therapy (110). Ultrasound imaging excludes abscess in one-third to one-half of suspected cases (104) and may allow guided aspiration of those that are confirmed (110). Serial aspiration has emerged as first-line therapy for abscesses, with a $91 \%$ cure rate. Aspiration and irrigation are performed with follow-up every 2 to 3 days for re-aspiration until the abscess is no longer visible by ultrasound (110). Incision and drainage have been the standard treatment, but risks ductal damage and a poor cosmetic result (110). If incision and drainage are required, radial or circumferential incision is made over the site of maximum tenderness and loculi are broken down, followed by insertion of a dependent drain or pack. Rapid resolution is invariable.

Antibiotic coverage is recommended preoperatively and susceptibility-directed antibiotic therapy is continued 
postoperatively (109). Milk stasis should be prevented by complete emptying of the breast with pumping to avoid secondary abscess formation. Continuation of breastfeeding from the affected breast is controversial. In the past, the presence of a staphylococcal breast abscess has been associated with infant deaths from lung abscesses, presumably due to aspiration of a large inoculum (3). Certainly, breastfeeding may continue from the unaffected breast and resume from the affected breast once the abscess has resolved.

Epidemic mastitis is a hospital-acquired infection caused by $S$. aureus. During the 1940s and 1950s, when lengthy postpartum hospitalization was practiced, up to $20 \%$ of women became infected (3). The infection involves the lactiferous glands and ducts of the breast, in contrast to the interlobular connective tissue in the sporadic form, and may affect multiple lobes. Abscess formation is common. Epidemic mastitis usually originated in hospital nurseries and was traceable to attendant carriers of the specific phage type that caused the infection $(3,98)$. Infants suffered significant mortality from staphylococcal pneumonia, meningitis, osteomyelitis, and pyoderma during these outbreaks (98). Fortunately, infection control measures and superior antibiotics have made epidemic mastitis an unlikely occurrence today.

\section{URINARY TRACT INFECTION}

Immediately postpartum, bacteriuria is found in up to $40 \%$ of women (111), but resolves spontaneously in most (112). By the third postpartum day, bacteriuria is found in $1.7 \%$ to $4 \%$ by suprapubic aspiration. Only one-fourth of women with bacteriuria complain of dysuria, but the symptom is more common following catheterization. Conditions and procedures that are associated with bladder catheterization-cesarean section, vaginal operative delivery, epidural anesthesia, tocolysis, pre-eclampsia, abruption, renal disease-significantly increase the risk of bacteriuria $(113,114)$. Antibiotic prophylaxis decreases the incidence of post-cesarean bacteriuria (115).

Treatment for urinary tract infection is similar to that for the nonpregnant woman. High rates of cure are achieved even with a short (3 days) course of therapy (113). The causative organism is E. coli in $80 \%$ to $90 \%$ of infections, but may be another coliform or staphylococcal or enterococcal species $(3,113)$. Culture and susceptibility testing may guide therapy, especially in patients who have recently received antibiotics. Suprapubic aspiration from a full bladder or urethral catheterization may be necessary to obtain samples of urine, as clean-catch midstream collections are frequently (46-69\%) contaminated (113).

Treatment of persistent asymptomatic bacteriuria, perhaps unwarranted in the nonpregnant patient, is recommended in the postpartum period (111). Decreased bladder tone, increased capacity, and incomplete emptying, along with a dilated collecting system and an enlarged uterus, capable of mechanical obstruction, may be present for weeks to months postpartum. These pregnancy-related changes predispose to vesicoureteric reflux and development of pyelonephritis. Infection of the urinary tract can be minimized by avoiding routine urethral catheterization of laboring or postpartum women. High-risk patients, who have received antibiotic suppression during the antepartum period to prevent pyelonephritis, should probably continue the regimen for at least 2 weeks postpartum.

\section{UNUSUAL INFECTIONS}

Infections resulting from spinal or epidural anesthesia occur following less than 1 in 10,000 blocks (116). The diagnosis should be considered in the febrile patient with backache and pain radiating from the spinal area. Evaluation may include neurologic examination, cerebrospinal fluid analysis, and myelography. Weakness, numbness, and finally paralysis will develop if the infection goes untreated (117).

Subgluteal or retropsoal infections have been reported following paracervical or pudendal block $(116,118)$. Patients presented with poorly localized severe hip pain and limited range of motion. Associated organisms were normal vaginal or bowel flora. Gas in the soft tissues was seen in half the cases, and abscess formation was common. Treatment may be delayed, owing to failure to suspect the diagnosis. In addition to antibiotic therapy, surgical drainage, debridement, and diverting colostomy may be necessary for appropriate treatment. Sequelae from these serious infections include persistent discomfort, impaired ambulation, and death.

Toxic shock syndrome has been reported in women with puerperal mastitis (119), postpartum endometritis (120), and abdominal wound infections (3) and may occur from several days to weeks after delivery. The organism responsible is typically $S$. aureus producing toxic shock syndrome toxin-1, but may alternatively be group A Streptococcus producing pyrogenic exotoxin A. Toxic shock syndrome presents with high fever, hypotension, rash, and multiorgan dysfunction. With proper antibiotic treatment and aggressive supportive care, most cases resolve in 48 to 72 hours, but mortality is $10 \%$ in cases of persistent hypotension, renal failure, or adult respiratory distress syndrome (ARDS) (120).

\section{SEPTIC SHOCK}

Rarely, septic shock may complicate a postpartum infection. Septic shock is characterized by hypotension and inadequate tissue perfusion, owing to overwhelming infection. The hypotension may be preceded by chills and fever and associated tachycardia, tachypnea, oliguria, or mental obtundation. Theories of pathogenesis and treatment of septic shock are reviewed elsewhere (121) (see further discussion in Chapter 11.

Microorganisms causing sepsis incite the formation or release of vasoactive and inflammatory mediators that lead to peripheral vasodilatation, regional microembolization, and endothelial cell injury. Severe multiorgan dysfunction involving the lungs, kidneys, liver, heart, and central nervous system may result from inadequate perfusion. Aggressive management strategies incorporate broad-spectrum antibiotic coverage, volume replacement, invasive hemodynamic monitoring, inotropic agents, and peripheral vasoconstrictors to maintain afterload. Rapid reversal of organ hypoperfusion, improvement in oxygen delivery, and correction of acidosis must be achieved for successful stabilization of the patient. A careful search for infected or necrotic foci that may be responsible for persistent bacteremia is warranted. Surgical intervention is sometimes required to remove the underlying cause of sepsis. 
Associated complications include ARDS, pulmonary edema, disseminated intravascular coagulation, thromboemboli, and cardiac arrest. The risk of maternal mortality increases as associated complications become superimposed on the septic hypotension. Fortunately, mortality due to septic shock is thought to be considerably lower than that in the nonobstetric population. Early identification of sepsis, prompt institution of antibiotic therapy, volume administration, and cardiopulmonary support when needed are essential to prevent complications and minimize mortality.

\section{REFERENCES}

1. Gibbs RS, O’Dell TN, MacGregor RR, et al. Puerperal endometritis: a prospective microbiologic study. Am J Obstet Gynecol 1975; 121: 919-25.

2. Filker R, Monif GRG. The significance of temperature during the first 24 hours postpartum. Obstet Gynecol 1979; 53: 358-61.

3. Eschenbach DA. Acute postpartum infections. Emerg Med Clin North Am 1985; 3: 87-115.

4. Nielsen TF, Hokegard K-H. Postoperative cesarean section morbidity: a prospective study. Am J Obstet Gynecol 1983; 146: 911-16.

5. Gibbs RS. Clinical risk factors for puerperal infection. Obstet Gynecol 1980; 55: 178S-83S.

6. Gibbs RS, Rodgers PJ, Castaneda YS, Ramzy I. Endometritis following vaginal delivery. Obstet Gynecol 1980; 56: 555-8.

7. Maharaj D. Puerperal pyrexia: a review. Part I. Obstet Gynecol Surv 2007; 62: 393-9.

8. Duff P. Pathophysiology and management of postcesarean endomyometritis. Obstet Gynecol 1986; 67: 269.

9. Blanco JD, Gibbs RS. Infections following classical cesarean section. Obstet Gynecol 1980; 55: 167-9.

10. Koh KS, Chan FH, Monfared AH, et al. The changing perinatal and maternal outcome in chorioamnionitis. Obstet Gynecol 1979; 53: 730-4.

11. Gilstrap LC, Cox SM. Acute chorioamnionitis. Obstet Gynecol Clin North Am 1989; 16: 373-9.

12. Blanco JD, Gibbs RS, Castaneda YS, St Clair PJ. Correlation of quantitative amniotic fluid cultures with endometritis after cesarean section. Am J Obstet Gynecol 1982; 143: 897-901.

13. Sherman D, Lurie S, Betzer M, et al. Uterine flora at cesarean and its relationship to postpartum endometritis. Obstet Gynecol 1999; 94: 787-91.

14. Gilstrap LC, Cunningham FG. The bacterial pathogenesis of infection following cesarean section. Obstet Gynecol 1979; 53: 545-9.

15. D’Angelo LJ, Sokol RJ. Determinants of postpartum morbidity in laboring monitored patients: a reassessment of the bacteriology of the amniotic fluid during labor. Am J Obstet Gynecol 1980; 136: 575-8.

16. Tran SH, Cheng YW, Kaimal AJ, Caughey AB. Length of rupture of membranes at term and infectious maternal morbidity. Am J Obstet Gynecol 2008; 198: 700.e1-5.

17. Casey BM, Cox SM. Chorioamnionitis and endometritis. Infect Dis Clin North Am 1997; 11: 203-22.

18. Carroll CS, Magann EF, Chauhan SP, et al. Vaginal birth after cesarean section versus elective repeat cesarean delivery: weight-based outcomes. Am J Obstet Gynecol 2003; 188: 1516-22.

19. Watts DH, Krohn MA, Hillier SL, Eschenbach DA. Bacterial vaginosis as a risk factor for post-cesarean endometritis. Obstet Gynecol 1990; 75: 52-8.

20. Christensen KK, Svenningsen N, Dahlander K, et al. Relation between neonatal pneumonia and maternal carriage of group $\mathrm{B}$ streptococci. Scand J Infect Dis 1982; 14: 261-6.

21. Curet LB, Morrison JC, Rao AV. Antenatal therapy with corticosteroids and postpartum complications. Am J Obstet Gynecol 1985; 152: 83-4.

22. Walmer D, Walmer KR, Gibbs RS. Enterococci in post-cesarean endometritis. Obstet Gynecol 1988; 71: 159-62.

23. Chaim W, Bashiri A, Bar-David J, et al. Prevalence and clinical significance of postpartum endometritis and wound infection. Infect Dis Obstet Gynecol 2000; 8: 77-82.
24. Liabsuetrakul T, Choobun T, Peeyananjarassri K, Islam M. Antibiotic prophylaxis for operative vaginal delivery. Cochrane Database Syst Rev 2004: CD004455.

25. Smaill F, Hofmeyr GJ. Antibiotic prophylaxis for cesarean section. Cochrane Database Syst Rev 2002: CD000933.

26. French L. Prevention and treatment of postpartum endometritis. Curr Womens Health Rep 2003; 3: 274-9.

27. Chelmow D, Ruehli MS, Huang E. Prophylactic use of antibiotics for nonlaboring patients undergoing cesarean delivery with intact membranes: a meta-analysis. Am J Obstet Gynecol 2001; 184: 656-61.

28. Chelmow D, Hennesy M, Evantash E. Prophylactic antibiotics for nonlaboring patients with intact membranes undergoing cesarean delivery: an economic analysis. Am J Obstet Gynecol 2004; 191: 1661-5.

29. Hopkins L, Smaill F. Antibiotic prophylaxis regimens and drugs for cesarean section. Cochrane Database Syst Rev 1999: CD001136.

30. Sullivan SA, Smith T, Chang E, et al. Administration of cefazolin prior to skin incision is superior to cefazolin at cord clamping in preventing postcesarean infectious morbidity: a randomized, controlled trial. Am J Obstet Gynecol 2007; 196: 455.e1-5.

31. Constantine MM, Rahman M, Ghulmiyah L, et al. Timing of perioperative antibiotics for cesarean delivery: a metaanalysis. Am J Obstet Gynecol 2008; 199: 301.e1-6.

32. Kaimal AJ, Zlatnik MG, Cheng YW, et al. Effect of a change in policy regarding the timing of prophylactic antibiotics on the rate of postcesarean delivery surgical-site infections. Am J Obstet Gynecol 2008; 199: 310.e1-5.

33. Muller AE, Oostvogel PM, Steegers E, Dorr PJ. Morbidity related to maternal group B streptococcal infections. Acta Obstet Gynecol Scand 2006; 85: 1027-37.

34. Dehbashi S, Honarvar M, Fardi FH. Manual removal or spontaneous placental delivery and postcesarean endometritis and bleeding. Int J Gynaecol Obstet 2004; 86: 12-15.

35. Baksu A, Kalan A, Ozkan A, et al. The effect of placental removal method and site of uterine repair on postcesarean endometritis and operative blood loss. Acta Obstet Gynecol Scand 2005; 84: 266-9.

36. Atkinson MW, Owen J, Wren A, Hauth JC. The effect of manual removal of the placenta on post-cesarean endometritis. Obstet Gynecol 1996; 87: 99-102.

37. Dweck MF, Lynch CM, Spellacy WN. Use of methergine for the prevention of postoperative endometritis in non-elective cesarean section patients. Infect Dis Obstet Gynecol 2000; 8: 151-4.

38. Tita AT, Owen J, Stamm AM, et al. Impact of extended-spectrum antibiotic prophylaxis on incidence of postcesarean surgical wound infection. Am J Obstet Gynecol 2008; 199: 303.e1-3.

39. Tita AT, Hauth JC, Grimes A, et al. Decreasing incidence of postcesarean endometritis with extended-spectrum antibiotic prophylaxis. Obstet Gynecol 2008; 111: 51-6.

40. Andrews WW, Hauth JC, Cliver SP, et al. Randomized clinical trial of extended spectrum antibiotic prophylaxis with coverage for Ureaplasma urealyticum to reduce post-cesarean delivery endometritis. Obstet Gynecol 2003; 101: 1183-9.

41. Pitt C, Sanchez-Ramos L, Kaunitz AM. Adjunctive intravaginal metronidazole for the prevention of postcesarean endometritis: a randomized controlled trial. Obstet Gynecol 2001; 98: 745-50.

42. Starr RV, Zurawski J, Ismail M. Preoperative vaginal preparation with povidone-iodine and the risk of postcesarean endometritis. Obstet Gynecol 2005; 105: 1024-9.

43. Magann EF, Chauhan SP, Martin JN, et al. Does uterine wiping influence the rate of post-cesarean endometritis? J Matern Fetal Med 2001; 10: 318-22.

44. Gibbs RS. Infection after cesarean section. Clin Obstet Gynecol 1985; 28: 697-10.

45. Watts DH, Eschenbach DA, Kenny GE. Early postpartum endometritis: the role of bacteria, genital mycoplasmas, and Chlamydia trachomatis. Obstet Gynecol 1989; 73: 52-60.

46. French LM, Smaill FM. Antibiotic regimens for endometritis after delivery. Cochrane Database Syst Rev 2004: CD001067.

47. Livingston JC, Llata E, Rinehart E, et al. Gentamicin and clindamycin therapy in postpartum endometritis: The efficacy of daily dosing versus dosing every 8 hours. Am J Obstet Gynecol 2003; 188: 149-52. 
48. Sunyecz JA, Wiesenfeld HC, Heine RP. The pharmacokinetics of oncedaily dosing with gentamicin in women with postpartum endometritis. Infect Dis Obstet Gynecol 1998; 6: 160-2.

49. Liu C, Abate B, Reyes M, Gonik B. Single daily dosing of gentamicin: pharmacokinetic comparison of two dosing methodologies for postpartum endometritis. Infect Dis Obstet Gynecol 1999; 7: 133-7.

50. Newton ER, Wallace PA. Effects of prophylactic antibiotics on endometrial flora in women with postcesarean endometritis. Obstet Gynecol 1998; 92: 262-8.

51. Faro S. Postpartum endometritis. Clin Perinatol 2005; 32: 803-14.

52. Ledger W. Post-partum endomyometritis diagnosis and treatment: a review. J Obstet Gynaecol Res 2003; 29: 364-73.

53. Brumfield CG, Hauth JC, Andrews WW. Puerperal infection after cesarean delivery: evaluation of a standardized protocol. Am J Obstet Gynecol 2000; 182: 1147-51.

54. Anteby EY, Yagel S, Hanoch J, et al. Puerperal and intrapartum group A streptococcal infection. Infect Dis Obstet Gynecol 1999; 7: 276-82.

55. Soper DE, Kemmer CT, Conover WB. Abbreviated antibiotic therapy for the treatment of postpartum endometritis. Obstet Gynecol 1987; 69: $127-30$.

56. Morales WJ, Collins EM, Angel JL, Knuppel RA. Short course of antibiotic therapy in treatment of postpartum endomyometritis. Am J Obstet Gynecol 1989; 161: 568-72.

57. Yonekura ML. Treatment of postcesarean endomyometritis. Clin Obstet Gynecol 1988; 31: 488-500.

58. Fortunato SJ, Dodson MG. Therapeutic considerations in postpartum endometritis. J Reprod Med 1988; 33: 101-6.

59. Blanco JD, Gibbs RS, Castaneda YS. Bacteremia in obstetrics: clinical course. Obstet Gynecol 1981; 58: 621-5.

60. Ledger WJ, Norman M, Gee C, Lewis W. Bacteremia on an obstetricgynecologic service. Am J Obstet Gynecol 1975; 121: 205-12.

61. Spandorfer SD, Graham E, Forouzan I. Postcesarean endometritis. J Reprod Med 1996; 41: 797-800.

62. Hoyme UB, Kiviat N, Eschenbach DA. Microbiology and treatment of late postpartum endometritis. Obstet Gynecol 1986; 68: 226-32.

63. Sweet RL, Ledger WJ. Puerperal infectious morbidity. Am J Obstet Gynecol 1973; 117: 1093-110.

64. Gibbs RS, Blanco JD, St Clair PJ. A case-control study of wound abscess after cesarean delivery. Obstet Gynecol 1983; 62: 498-501.

65. Sarsam SE, Elliott JP, Lam GK. Management of wound complications from cesarean delivery. Obstet Gynecol Surv 2005; 60: 462-73.

66. Johnson A, Young D, Reilly J. Caesarean section surgical site infection. J Hosp Infect 2006; 64: 30-5.

67. Vermillion ST, Lamoutte C, Soper DE, Verdeja A. Wound infection after cesarean: effect of subcutaneous tissue thickness. Obstet Gynecol 2000; 95: 923-6.

68. Franchi M, Ghezzi F, Balestreri D, et al. A randomized clinical trial of two surgical techniques for cesarean section. Am J Perinatol 1998; 15: 589-94.

69. Hellums EK, Lin MG, Ramsey PS. Prophylactic subcutaneous drainage for prevention of wound complications after cesarean delivery-a metaanalysis. Am J Obstet Gynecol 2007; 197: 229-35.

70. Emmons SL, Krohn M, Jackson M, Eschenbach DA. Development of wound infections among women undergoing cesarean section. Obstet Gynecol 1988; 72: 559-64

71. Gibbs RS. Severe infections in pregnancy. Med Clin North Am 1989; 73: 713-21.

72. Brown CE, Stettler RW, Twickler D. Puerperal septic pelvic thrombophlebitis: incidence and response to heparin therapy. Am J Obstet Gynecol 1999; 181: 143-8.

73. Duff P, Gibbs RS. Pelvic vein thrombophlebitis: diagnostic dilemma and therapeutic challenge. Obstet Gynecol Surv 1983; 38: 365-73.

74. Khurana BK, Rao J, Friedman SA, Cho KC. Computed tomographic features of puerperal ovarian vein thrombosis. Am J Obstet Gynecol 1988; 159: 905-8.

75. Munsick RA, Gillanders LA. A review of the syndrome of puerperal ovarian vein thrombophlebitis. Obstet Gynecol Surv 1981; 36: 57-66.

76. Salomon O, Apter S, Shaham D, et al. Risk factors associated with postpartum ovarian vein. Thromb Haemost 1999; 82: 1015-19.

77. Brown CEL, Lowe TW, Cunnigham FG, Weinreb JC. Puerperal pelvic thrombophlebitis: impact on diagnosis and treatment using $\mathrm{x}$-ray computed tomography and magnetic resonance imaging. Obstet Gynecol 1986; 68: 789-94.

78. Isler CM, Rinehart BK, Terrone DA, et al. Septic pelvic thrombophlebitis and preeclampsia are related disorders. Hypertens Pregnancy 2004; 23: 121-7.

79. Witlin AG, Mercer BM, Sibai BM. Septic pelvic thrombophlebitis or refractory postpartum fever of undetermined etiology. J Matern Fetal Med 1996; 5: 355-8.

80. Kubik-Huch RA, Hebisch G, Huch R, et al. Role of duplex color Doppler ultrasound, computed tomography, and MR angiography in the diagnosis of septic puerperal ovarian vein thrombosis. Abdom Imaging 1999; 24: 85-91.

81. Twickler DM, Setiawan AT, Evans RS, et al. Imaging of puerperal septic thrombophlebitis: prospective comparison of MR imaging, CT, and sonography. AJR Am J Roentgenol 1997; 169: 1039-43.

82. Josey WE, Staggers SR. Heparin therapy in septic pelvic thrombophlebitis: a study of 46 cases. Am J Obstet Gynecol 1974; 120: 228-32.

83. Collins CG, MacCallum EA, Nelson EW, et al. Suppurative pelvic thrombophlebitis I. Incidence, pathology, and etiology a study of 70 patients treated by ligation of the inferior vena cava and vessels. Surgery 1951; 30: 298-310.

84. Brown TK, Munsick RA. Puerperal ovarian vein thrombophlebitis: a syndrome. Am J Obstet Gynecol 1971; 109: 263-73.

85. Klima DA, Snyder TE. Postpartum ovarian vein thrombosis. Obstet Gynecol 2008; 111: 431-5.

86. Cohen MB, Pernoll ML, Gevirtz CM, Kerstein MD. Septic pelvic thrombophlebitis: an update. Obstet Gynecol 1983; 62: 83-9.

87. Thacker SB, Banta HD. Benefits and risks of episiotomy: an interpretative review of the English language literature, 1860-1980. Obstet Gynecol Surv 1983; 38: 322-38.

88. Duggal N, Mercado C, Daniels K, et al. Antibiotic prophylaxis for prevention of postpartum perineal wound complications. Obstet Gynecol 2008; 111: 1268-73.

89. Shy KK, Eschenbach DA. Fatal perineal cellulitis from an episiotomy site. Obstet Gynecol 1979; 54: 292-8.

90. Hankins GDV, Hauth JC, Gilstrap LC, et al. Early repair of episiotomy dehiscence. Obstet Gynecol 1990; 75: 48-51.

91. Stamenkovic I, Lew PD. Early recognition of potentially fatal necrotizing fasciitis. N Engl J Med 1984; 310: 1689-93.

92. Addison WA, Livengood CH, Hill GB, et al. Necrotizing fasciitis of vulvar origin in diabetic patients. Obstet Gynecol 1984; 63: 473-9.

93. Sutton GP, Smirz LR, Clark DH, Bennett JE. Group B streptococcal necrotizing fasciitis arising from an episiotomy. Obstet Gynecol 1985; 66: 733-6.

94. Stone HH, Martin JD. Synergistic necrotizing cellulitis. Ann Surg 1972; 175: 702-10.

95. McGregor JA, Soper DE, Lovell G, Todd JK. Maternal deaths associated with Clostridium sordellii infection. Am J Obstet Gynecol 1989; 161: 987-95.

96. Foxman B, D’Arcy H, Gillespie B, et al. Lactation mastitis: occurrence and medical management among 946 breastfeeding women in the United States. Am J Epidemiol 2002; 155: 103-14.

97. Marshall BR, Hepper JK, Zirbel CC. Sporadic puerperal mastitis. J Am Med Assoc 1975; 233: 1377-9.

98. Devereux WP. Acute puerperal mastitis. Am J Obstet Gynecol 1970; 108: 78-81.

99. Niebyl JR, Spence MR, Parmley TH. Sporadic (nonepidemic) puerperal mastitis. J Reprod Med 1978; 20: 97-100.

100. Thomsen AC, Hansen KB, Moller BR. Leukocyte counts and microbiologic cultivation in the diagnosis of puerperal mastitis. Am J Obstet Gynecol 1983; 146: 938-41.

101. Fetherston C. Mastitis in lactating women: physiology or pathology? Breastfeed Rev 2001; 9: 5-12.

102. Fetherston C. Risk factors for lactation mastitis. J Hum Lact 1998; 14: 101-9.

103. Osterman KL, Rahm VA. Lactation mastitis: bacterial cultivation of breast milk, symptoms, treatment, and outcome. J Hum Lact 2000; 16: 297-302.

104. Dener C, Inan A. Breast abscesses in lactating women. World J Surg 2003; 27: 130-3. 
105. Thomsen AC, Espersen T, Maigaard S. Course and treatment of milk stasis, noninfectious inflammation of the breast, and infectious mastitis in nursing women. Am J Obstet Gynecol 1984; 149: 492-5.

106. Matheson I, Aursnes I, Horgen M, et al. Bacteriological findings and clinical symptoms in relation to clinical outcome in puerperal mastitis. Acta Obstet Gynecol Scand 1988; 67: 723-6.

107. Reddy P, Qi C, Zembower T, et al. Postpartum mastitis and communityacquired methicillin-resistant Staphylococcus aureus. Emerg Infect Dis 2007; 13: 298-301.

108. Barbosa-Cesnik C, Schwartz K, Foxman B. Lactation mastitis. J Am Med Assoc 2003; 289: 1609-12.

109. Benson EA. Management of breast abscesses. World J Surg 1989; 13: 753-6.

110. Ozseker B, Ozean UA, Rasa K, Cizmeli OM. Treatment of breast abscesses with ultrasound-guided aspiration and irrigation in the emergency setting. Emerg Radiol 2008; 15: 105-8.

111. Orrett FA, Premanand N. Postpartum surveillance of bacteriuria in term vaginal deliveries. J Natl Med Assoc 1998; 90: 177-80.

112. Marraro RV, Harris RE. Incidence and spontaneous resolution of postpartum bacteriuria. Am J Obstet Gynecol 1977; 128: 722-3.

113. Stray-Pedersen B, Solberg VM, Torkildsen E, et al. Postpartum bacteriuria. A multicenter evaluation of different screening procedures and a controlled short-course treatment trial with amoxicillin. Eur J Obstet Gynecol Reprod Biol 1988; 31: 163-70.

114. Schwartz MA, Wang CC, Eckert LO, et al. Risk factors for urinary tract infection in the postpartum period. Am J Obstet Gynecol 1999; 181: 547-53.

115. Horowitz E, Yogev Y, Ben-Haroush A, et al. Urine culture at removal of indwelling catheter after cesarean section. Int J Gynaecol Obstet 2004; 85: 276-8.

116. Gibbs RS, Wienstein AJ. Puerperal infection in the antibiotic era. Am J Obstet Gynecol 1976; 124: 769-87.

117. Baker AS, Ojemann RG, Swartz MN, Richardson EP. Spinal epidural abscess. N Engl J Med 1975; 293: 463-8.

118. Hibbard LT, Synder EN, McVann RM. Subgluteal and retropsoal infection in obstetric practice. Obstet Gynecol 1972; 39: 137-50.

119. Demey HE, Hautekeete ML, Buytaert P, Bossaert LL. Mastitis and toxic shock syndrome. Acta Obstet Gynecol Scand 1989; 68: 87-8.

120. Davis D, Gash-Kim TL, Heffernan EJ. Toxic shock syndrome: case report of a postpartum female and a literature review. J Emerg Med 1998; 16: 607-14.

121. Lee W, Cotton DB, Hankins GDV, Faro S. Management of septic shock complicating pregnancy. Obstet Gynecol Clin North Am 1989; 16: 431-47. 


\section{Urinary tract infections in pregnancy Steven R. Allen}

Urinary tract infections (UTIs) commonly complicate pregnancy, present in approximately $10 \%$ of gestations. Asymptomatic bacteriuria (ASB) is encountered most frequently. Symptomatic infections, cystitis and pyelonephritis, complicate pregnancy less frequently. Of these three infections, the primary impact on pregnancy outcome derives from acute pyelonephritis, which is commonly preceded by ASB rather than acute cystitis (1). Clinical presentation, risk factors, common complications, and treatment for these three forms of UTI are summarized in Table 1.

\section{PATHOGENESIS}

Female anatomy and the physiologic changes of normal pregnancy impart risk factors for UTIs. Because of the short length (approximately $4 \mathrm{~cm}$ ) of female urethra and its termination beneath the labia and relatively near the anus, it is at increased risk of colonization by Gram-negative colonic bacteria. Urethral and periurethral bacteria may intermittently enter the bladder, typically after intercourse (2). Once within the urinary tract, attachment of bacterial pili or fimbriae to carbohydrate components of urothelial cell surface glycolipids and glycoproteins enhances virulence (3).

Half of women with UTIs during pregnancy have an enlarged bladder capacity, and 15\% have a significant postvoid residual volume $(50-150 \mathrm{~mL})(4)$. Normal physiologic changes of pregnancy include a mild hydronephrosis and hydroureter, potentially created by both the mechanical compression of the ureter by the enlarging uterus and pelvic blood vessels at the pelvic brim (right greater than left), and the relaxant effect of progesterone on ureteral smooth muscles. In combination, these factors account for the increased rate of both bladder and ascending infections during pregnancy.

Population-specific risk factors for UTIs in pregnancy include nonwhite race, younger maternal age, and antepartum genital tract infection (5).

The bacterial spectra responsible for all three types of UTIs in pregnancy are similar and also are similar to the spectra of bacteria causing UTIs in nonpregnant females. Escherichia coli is the most common isolate, present in $70 \%$ to $90 \%$ of positive urine cultures. Bacteria from other genuses of Enterobacteriaceae are the next most common: Klebsiella, Enterobacter, Citrobacter and Proteus. Gram-positive organisms cause $<10 \%$ of UTIs in pregnancy, with group B streptococcus the most common member of this group $(1,6-9)$.

Beyond the fetal risks discussed below in relation to antibiotic exposure and the potential complications of pyelonephritis, maternal UTIs may also increase the risk of fetal anomalies. Analyses of data from the U.S. National Birth Defects Prevention Study show associations between the firsttrimester maternal UTI and gastroschisis plus at least one other major anomaly (OR 3.1, 95\% CI 1.4-6.7) (10) and cardiovascular malformations (left ventricular outflow tract obstruction, OR 1.48, 95\% CI 1.02, 2.14; atrioventricular septal defect, OR 2.55, 95\% CI 1.19, 5.48) (11). For both of these noted associations, the risk appears to exist regardless of fever or antibiotic exposure.

\section{ANTIBACTERIAL CONSIDERATIONS}

Because of the similarity of bacterial spectra responsible for each of the three types of UTIs discussed within this chapter, similar antibacterial selection strategies can be applied for treating each. An understanding of the changing resistance pattern for $E$. coli is critical in selecting an appropriate antibiotic, since it is the predominant cause of UTIs. E. coli isolates from women with acute cystitis during an observation period ending in 1996 showed progressive resistance to ampicillin (34\%), cephalothin (28\%), trimethoprim (18\%), and trimethoprim-sulfamethoxazole (18\%). E. coli resistance to trimethoprim-sulfamethoxazole (TMP-SMX) had increased to over $20 \%$ in some regions of the United States (12). Results from a single institution in 2000 showed $E$. coli to be unacceptably resistant to ampicillin (75\% incidence of sensitivity) and marginally more likely to be sensitive to TMP-SMX. In that study population, nitrofurantoin had the highest likelihood of success for treating uropathogens (100\% susceptibility for E. coli and 89\% overall) (13).

Several antibiotics have proven to be effective for treating UTIs. When treating UTIs in pregnancy, the safety considerations must obviously include potential fetal effects. The following drugs may be considered during pregnancy.

\section{Penicillins and Cephalosporins}

High likelihood of resistance prevents these agents from being ideal empiric choices.

\section{Trimethoprim-Sulfamethoxazole}

Although TMP-SMX has been recommended as a primary treatment for uncomplicated UTIs (14), progressive bacterial resistance to it now potentially limits its effectiveness as an empiric therapy (13). The best predictor of resistance to TMP-SMX in urinary coliform bacteria is recent use of TMP-SMX (OR 5.9; 95\% CI 2.4, 14.3); other predictors include diabetes, recent hospitalization, and current use of antibiotics (15). In addition to declining efficacy, safety features should also be considered before using TMP-SMX to treat UTIs during pregnancy. Both trimethoprim and sulfonamide antibiotics inhibit nucleic acid synthesis by interfering with bacterial production of folic acid. A casecontrol study of birth defects in the United States and Canada reported an increased likelihood of first-trimester exposure to dihydrofolate reductase inhibitors (including TMP-SMX) among women giving birth to babies with cardiovascular 
Table 1 Summary of Urinary Tract Infections During Pregnancy

\begin{tabular}{|c|c|c|c|}
\hline & Acute cystitis & $\begin{array}{l}\text { Asymptomatic } \\
\text { bacteriuria }\end{array}$ & Pyelonephritis \\
\hline Symptoms & $\begin{array}{l}\text { Urgency } \\
\text { Frequency } \\
\text { Dysuria }\end{array}$ & No symptoms & $\begin{array}{l}\text { Back or flank pain } \\
\text { Fever } \\
\text { Chills }\end{array}$ \\
\hline Unique risk factors & $\begin{array}{l}\text { History of recurrent } \\
\text { cystitis }\end{array}$ & $\begin{array}{l}\text { Parity } \\
\text { Nonwhite race Lower } \\
\quad \text { socioeconomic status } \\
\text { Diabetes } \\
\text { Prior UTI }\end{array}$ & $\begin{array}{l}\text { Asymptomatic bacteriuria } \\
\text { Younger age } \\
\text { Nulliparity } \\
\text { Sickle cell trait or disease }\end{array}$ \\
\hline Complications & Recurrent acute cystitis & Pyelonephritis & $\begin{array}{l}\text { Bacteremia } \\
\text { Septic shock } \\
\text { Pulmonary edema }\end{array}$ \\
\hline Treatment & $\begin{array}{l}\text { Oral broad-spectrum } \\
\text { antibiotic, 3-7 days }\end{array}$ & $\begin{array}{l}\text { Oral broad-spectrum } \\
\text { antibiotic, 3-7 days }\end{array}$ & $\begin{array}{l}\text { Broad-spectrum antibiotic, parenteral } \\
\text { until clinically improved, then oral } \\
\text { to complete } 10 \text { - to } 14 \text {-day course, } \\
\text { followed by oral broad-spectrum } \\
\text { antibiotic prophylaxis until } 6 \text { weeks } \\
\text { postpartum }\end{array}$ \\
\hline
\end{tabular}

defects (OR 3.4; 95\% CI 1.8-6.4) and oral clefts (OR 2.6; 95\% CI 1.1-6.1) (16). The increased risk of cardiovascular defect associated with dihydrofolate reductase inhibitor consumption was offset in women who also took supplemental folic acid (400 $\mu \mathrm{g} /$ day). Although neonatal jaundice has occasionally been reported after fetal exposure to sulfonamides, a contemporary literature review shows no reported cases of kernicterus after such exposure (17). These findings support a recommendation to avoid TMP-SMX in both the first and late third trimesters, and to recommend a higher folic acid supplement dose $(4 \mathrm{mg} /$ day $)$ if TMP-SMX is prescribed in the first trimester (18).

\section{Nitrofurantoin}

This agent's primary benefit is its continued effectiveness against most strains of $E$. coli. It is also effective against many other gram-positive and gram-negative urinary pathogens, but has limited effectiveness against Klebsiella, Enterobacter, Pseudomonas, and Proteus. It reaches a high concentration in urine, but not in renal tissue, thus should not be used as primary treatment for pyelonephritis. Rare maternal complications include reversible sensory neuropathy (19) and reversible pneumonitis (occurring in fewer than $1 / 100,000$ exposures) (20). Nitrofurantoin is not teratogenic and does not need to be avoided in the first trimester (21). The extremely low risk for fetal or neonatal hemolytic anemia due to impaired glutathione reductase activity or glucose-6phosphate dehydrogenase deficiency (with an estimated risk of $0.001 \%$ ) (22) need not prohibit its use near term. However, these concerns still prompt some recommendations against its use near anticipated delivery (23).

\section{Gentamicin}

Aminoglycosides attain high renal tissue levels, thus may be particularly beneficial in the treatment of pyelonephritis. Although ototoxicity has been reported with other aminoglycosides, there are no clear human data supporting fetal ototoxicity $(24,25)$.

\section{Fluoroquinolones}

Although arthropathy may develop after fetal exposure in animals, limited human data have not shown arthropathy or other defects following fetal exposure (26). However, concerns about the potential development of drug resistance if this effective family of antimicrobials is overused logically keep fluoroquinolones from being first-line agents for the treatment of UTI in pregnancy.

\section{Fosfomycin}

Limited data for this agent in pregnancy preclude a specific recommendation for its use $(27,28)$.

\section{CYSTITIS}

Acute cystitis in pregnancy has a clinical presentation not unlike that of symptomatic bladder infection outside of pregnancy. The diagnosis should be suspected in a woman with urgency, frequency, dysuria, or hematuria, and without evidence of upper UTI or systemic illness. A culture of catheterized or midstream urine positive for a single organism confirms the diagnosis. Although the traditional diagnosis of UTI is based upon a quantitative culture containing $\geq 10^{5}$ bacteria/mL, a lower threshold may be appropriate in the presence of bladder symptoms. Comparing cultures of urine obtained directly from the bladder via suprapubic aspiration or urethral catheterization with those of midstream specimens in acutely dysuric (nonpregnant) women, midstream samples with $\geq 10^{2}$ bacteria/mL have a sensitivity of $95 \%$ and a specificity of $85 \%$ for the detection of coliform bacteria (29).

Acute cystitis occurs much less frequently in pregnancy than asymptomatic UTIs. Harris and Gilstrap (6) found the incidence of cystitis to be $1.3 \%$ among a population of military dependents. Two-thirds developed their symptomatic infection after the initial clinic visit, and of these, 95\% had negative urine cultures at their first prenatal visit. Although half of the patients with cystitis had a history of UTIs, after a 10 -day course of antibiotic only $17 \%$ had recurrence, and no patients developed pyelonephritis. 
The usual treatment for acute cystitis in pregnancy is with a short (3-7 days) course of broad-spectrum antibiotic. In nonpregnant women, without the cited physiologic genitourinary tract changes that predispose to UTI during pregnancy, multiple-dose regimens provide better treatment than a single dose of a broad-spectrum antibiotic (30). Logic predicts that multiple-dose regimens also will be more efficacious in pregnant women. Before the more common antimicrobial resistance patterns currently seen, a 10-day oral course of any one of the following-ampicillin $250 \mathrm{mg}$ four times a day, nitrofurantoin macrocrystals $100 \mathrm{mg}$ three times a day, sulfisoxazole $500 \mathrm{mg}$ four times a day, or cefazolin $250 \mathrm{mg}$ four times a day-demonstrated equal efficacy in pregnant women (6).

Taking into account the considerations listed in the prior section, any one of the typically effective drugs may be started empirically for the clinical diagnosis of cystitis, with appropriate changes in selection if directed by urine culture sensitivity results. Limited published data are available to specifically direct the best therapy for acute cystitis in pregnancy. Five days of twice-daily oral therapy with nitrofurantoin $100 \mathrm{mg}$ or ampicillin $500 \mathrm{mg}$ had similar cure rates $(87 \%$ and $90 \%)$ in 1989 (31), before recent increases in ampicillin resistance, and similar cure rates have also been shown in a small study comparing a single 3-g dose of fosfomycin trometamol and a 3-day course of 400-mg ceftibuten per day (95\% and 90\%) (28). Given the predominance of $E$. coli as a uropathogen and its ongoing susceptibility to nitrofurantoin, that agent is likely to be the best primary therapy in most cases (13).

A follow-up urine culture may be obtained after initial treatment to confirm appropriate therapy for cystitis, but because of the low rate of recurrence and its typical symptomatic presentation, serial screening urine cultures are not recommended.

Sexual intercourse is a risk factor for recurrent cystitis in some women. Pfau and Sacks (32) showed a 99\% reduction from the baseline (pre-pregnant) rate of recurrent cystitis among 33 pregnant women using post-coital cephalexin $250 \mathrm{mg}$ or nitrofurantoin $50 \mathrm{mg}$. As compared with daily prophylaxis, this form of therapy provides most at-risk women with the convenience and cost benefit of less frequent antibiotic administration.

\section{ASB}

$\mathrm{ASB}$, silent bacterial colonization of the urinary tract, is the most common UTI in pregnancy, with an incidence of $2 \%$ to $10 \%(7,33,34)$. Lower socioeconomic status, nonwhite race, increased parity (33), diabetes, and prior UTIs increase the risk (35).

For asymptomatic women, bacteriuria is diagnosed by quantitative urine culture: two consecutive voided specimens with isolation of the same bacterial strain in quantitative counts $\geq 10^{5}$ bacteria/mL or a single catheterized urine specimen containing $\geq 10^{2}$ bacteria/mL (36). Although only approximately $80 \%$ of women with a positive initial screening culture would have that confirmed on subsequent sampling, the detection of $\geq 10^{5}$ bacteria/mL in a single voided midstream urine is generally accepted as an adequate and more practical alternative to the requirement for serial positive samples (37).
The bacteria identified in ASB are generally similar to those of other UTIs, except that group B streptococcus is more common in this UTI, present in approximately one-quarter of positive screening cultures $(8,38)$.

The significance of ASB during pregnancy lies not in the primary infection but rather in the potential secondary complications. Although ASB is no more common in pregnant women, the physiologic changes of pregnancy that lead to urinary stasis increase the likelihood of ASB progressing into an ascending infection. Subsequent pyelonephritis occurs in approximately $40 \%$ of untreated women with ASB (39). Fortunately, the risk of pyelonephritis is reduced to approximately 3\% following the treatment for ASB (40). A metaanalysis evaluating the relationship between ASB and pregnancy outcomes showed low birth weight to be $3.4 \%$ more likely following ASB and preterm birth to be 3.8\% more likely after untreated ASB (41). The same analysis showed a reversal of ASB's impact upon the risk of low birth weight with antibiotic treatment (OR for low birth weight if given antibiotics $=0.52$, 95\% CI 0.38-0.7).

The risks of untreated ASB and the inability to identify it clinically prompt recommendations for routine screening in pregnancy $(36,42,43)$. As no rapid screening techniques have adequate sensitivity or predictive values, urine culture remains the recommended standard for detecting ASB $(42,44)$.

Current published guidelines for ASB screening recommend that a urine culture be obtained at least once in early pregnancy (36), such as at 12 to 16 weeks of gestation (or at the first prenatal visit, if later) (42). Screening on that schedule will not identify all cases of ASB. A prospective cohort study in which participants had serial urine cultures performed showed that only $43 \%$ of the overall positives occurred at $<20$ weeks of gestation (38). However, early studies showing a great reduction in risk for pyelonephritis following primary antimicrobial therapy for ASB and an average risk for subsequent pyelonephritis in initially nonbacteriuric women of only $1.4 \%$ (40) together suggest that serial screening to identify ASB later in pregnancy would have a relatively low yield and not be cost effective.

To accurately interpret the results of a quantitative urine culture, it must have been obtained under conditions that minimize contamination. Vulval cleansing with chlorhexidine may reduce the bacterial colony counts of a midstream urine sample, and cleansing with water provides more representative results. Additionally, the quantity of bacteriuria correlates with the time interval between episodes of voiding; although a first morning void is not necessary, the practice of having patients drink fluid to speed diuresis should be avoided (45).

Although early protocols used continuous treatment until delivery, by 1977 Whalley and Cunningham (46) had shown that short-term treatment (14 days) combined with surveillance for recurrent bacteriuria provided similar results. In 2000, a Cochrane systematic review concluded that published data are insufficient to determine the optimal duration of therapy for ASB in pregnancy (47). In 2005, the Infectious Diseases Society of America recommended 3 to 7 days of antimicrobial therapy for ASB, but did not identify an optimal drug for therapy (36).

A Cochrane systematic review of antibiotic treatments for ASB in pregnancy published in 2007, which included 
randomized controlled trials of a variety of antibiotics (sulfonamides, nitrofurantoin, penicillin, and ampicillin), concluded that antibiotic treatment is effective in reducing the risk of pyelonephritis, but no single antibiotic choice is superior (48). According to that review, the number of women needed to treat to prevent one case of pyelonephritis is seven.

Since the diagnosis of bacteriuria is made by urine culture and no optimal drug choice had been identified, antimicrobial selection for the treatment of ASB in pregnancy may be guided by susceptibility results and fetal safety considerations.

In response to the potential silent recurrence of ASB, the Infectious Diseases Society of America recommends periodic screening after initial therapy in pregnancy (36). Urine cultures obtained 1 to 2 weeks after initiation of therapy and additional screening per trimester would meet these guidelines.

Following a recurrent UTI, broad-spectrum antibiotic suppression for the remainder of the pregnancy may be helpful, such as with nitrofurantoin $100 \mathrm{mg}$ every day. For women with recurrent infections, urologic examination should be scheduled for 3 to 6 months postpartum, as up to half of these women will have a urinary tract structural abnormality (4). Although pyelographic abnormalities are more common in women with a history of ASB, it is unclear whether these are primarily or secondarily associated.

\section{PYELONEPHRITIS}

Affecting $1 \%$ to $2 \%$ of all pregnancies, pyelonephritis is one of the most common serious medical problems encountered during pregnancy. It will develop in $36 \%$ to $42 \%$ of untreated women with ASB; this risk falls to $\leq 3 \%$ with the treatment for bacteriuria $(39,49)$. Only approximately $1.4 \%$ of women without bacteriuria early in a pregnancy will subsequently develop pyelonephritis before delivery (40). Pyelonephritis occurs most commonly during the second or third trimester, although up to one-quarter of infections are diagnosed intrapartum or postpartum $(1,50,51)$. In addition to other risk factors for UTIs in general, younger age, nulliparity (51), and sickle cell trait (52) or disease (OR 1.3, 95\% CI 1.0-1.8) (53) make pyelonephritis more likely. It is usually an ascending infection, more likely to occur during pregnancy because of the physiologic changes predisposing to urinary stasis. Conditions unique to the renal medulla make the kidney particularly vulnerable to infection in the presence of stasis: high urine osmolarity, high urea and sodium concentrations, and low $\mathrm{pH}$ impair chemotaxis and inhibit polymorphonuclear leukocyte phagocytosis.

Unlike its usual precursor ASB, pyelonephritis is nearly always diagnosed clinically. Patients typically present with the symptoms of back pain and chills (82\%); approximately onequarter have nausea and vomiting. An elevated temperature is uniformly present, with a temperature of $\geq 40^{\circ} \mathrm{C}$ in $12 \%$ of patients. The majority of patients have costovertebral angle tenderness (predominantly right sided or bilateral) (1). Because none of these signs or symptoms is pathognomonic for pyelonephritis, the final diagnosis should depend on a urine culture positive for a known uropathogen.

To speed up diagnosis and treatment while awaiting the urine culture results, microscopic examination of uncentrifuged urine can be performed. One bacterium per high power field indicates a bacterial concentration of at least $10^{5} / \mathrm{mL}$. One or more white blood cells per high power field will also usually be seen in pyelonephritis. Although indicative of a UTI, none of these findings are specific for pyelonephritis. If the diagnosis of pyelonephritis is suspected, blood cultures may also be obtained before antibiotic treatment is begun. However, the additional cost of blood cultures may not be substantiated by clinical benefit, as the blood culture results of the patients with pyelonephritis who develop bacteremia usually parallel the urine culture results; MacMillan and Grimes changed antibiotic treatment based on blood cultures in only $2 \%$ of pregnant patients with pyelonephritis (54).

Traditional management recommendations for acute pyelonephritis in pregnancy include hospitalization and intravenous antibiotic administration until significant clinical improvement allows conversion to oral antibiotics, completing a 10- to 14-day course of therapy (55). Van Dorsten and associates showed the importance of appropriate initial antibiotic selection, demonstrating a $67 \%$ rate of successful treatment with an antibiotic proven effective by routine sensitivity methods, as compared with a $42 \%$ rate of successful treatment with an antibiotic to which the isolated bacteria were resistant (56). Although ampicillin has been used for primary therapy, the increasing resistance of $E$. coli to ampicillin compromises its status as the drug of choice. Gentamicin is nearly uniformly effective but because of associated costs for monitoring serum levels and the lack of an oral equivalent, it is not most commonly used for primary therapy. Gentamicin and ampicillin may be combined for efficacious empiric therapy of pyelonephritis in pregnancy; the half of women with ampicillin-resistant uropathogens have no higher rate of complications (anemia, renal dysfunction, respiratory insufficiency, or preterm birth) than the half with ampicillin-sensitive uropathogens (57). A variety of successful protocols may be used to treat pyelonephritis in pregnancy (Table 2).

Sanchez-Ramos and coworkers reported the single-daily intravenous administration of ceftriaxone $1 \mathrm{~g}$ to be equally effective and less costly than three-daily 2 -g doses of cefazolin intravenously (58). Wing and colleagues randomized gravidas with pyelonephritis to receive initial parenteral treatment with ampicillin (2g IV every 4 hours) plus gentamicin $(2 \mathrm{mg} / \mathrm{kg}$ IV loading then $1.75 \mathrm{mg}$ IV every 8 hours), cefazolin ( $2 \mathrm{~g}$ IV every 8 hours), or ceftriaxone ( $1 \mathrm{~g}$ IM every 24 hours) and identified no difference in the duration of fever, time to resolution of costovertebral angle tenderness, duration of hospitalization, or birth outcomes (59).

Table 2 Pyelonephritis Antibiotic Protocol Options

\begin{tabular}{ll} 
Antimicrobial agent & \multicolumn{1}{c}{ Suggested regimen } \\
$\begin{array}{l}\text { Cefazolin } \pm \text { gentamicin } \\
\text { (dose as below) }\end{array}$ & $1-2 \mathrm{~g} \mathrm{IV} \mathrm{q6-8h}$ \\
Cefuroxime & $0.75-1.5 \mathrm{~g} \mathrm{IV} \mathrm{q} 8 \mathrm{~h}$ \\
Ceftriaxone & $1-2 \mathrm{~g} \mathrm{IV}$ or IM q24h \\
$\begin{array}{l}\text { Ampicillin } \pm \text { gentamicin } \\
\text { (dose as below) }\end{array}$ & $2 \mathrm{~g} \mathrm{IV} \mathrm{q4h}$ \\
Gentamicin & $2 \mathrm{mg} / \mathrm{kg} \mathrm{IV} \mathrm{loading} \mathrm{dose,} \mathrm{then}$ \\
& $1.75 \mathrm{mg} / \mathrm{kg}$ q8h or per \\
& $\mathrm{pharmacokinetic} \mathrm{dosing}$ \\
Cephradine & $1 \mathrm{~g} \mathrm{IV} \mathrm{q6h}$
\end{tabular}


In addition to parenteral antibiotics, some gravidas with pyelonephritis also will initially require hydration to correct intravascular volume deficits and electrolyte imbalances, antipyretic treatment, and tocolysis.

Recognizing that outpatient oral treatment of acute pyelonephritis in nonpregnant patients has been proven safe and effective (60), successful trials of outpatient treatment for pregnant women with uncomplicated pyelonephritis also have been published (61-63). Angel and colleagues compared oral and intravenous cephalosporin treatments of pyelonephritis in pregnancy, reporting excellent results for both forms of administration (91\% and 93\% success rates, respectively) (61). Millar and colleagues demonstrated the efficacy of outpatient treatment for pyelonephritis in women with pregnancies of gestational age both less than 24 weeks and after 24 weeks $(62,63)$. Their protocol at less than 24 weeks compared initial parenteral ceftriaxone for outpatient treatment with cefazolin for inpatient treatment (both followed by oral cephalexin $500 \mathrm{mg}$ four times daily for 10 days), with similar culture-negative success rates (78-80\%) at follow-up 2 weeks after completion of therapy. However, inpatients receiving initial cefazolin were more likely to remain febrile for more than 3 days or require a change in antibiotic. Millar's subsequent study of women with pyelonephritis after 24 weeks of gestational age treated all participants with ceftriaxone $1 \mathrm{~g}$ IM twice (24 hours apart), then cephalexin $500 \mathrm{mg}$ orally four times daily for 10 days, and led to $91 \%$ culture-negative success at 2-week follow-up. The majority of the women randomized to outpatient treatment were discharged with follow-up within 2 to 3 days, but $28 \%$ remained hospitalized for complications including sepsis or preterm labor. The cited studies comparing inpatient and outpatient therapy for pyelonephritis in pregnancy share comparable success rates and lack of complications for both strategies. Furthermore, the published outpatient strategies, limiting the outpatient option to otherwise uncomplicated patients, share a conservative approach of initial hospital observation and prompt outpatient follow-up.

A meta-analysis of randomized control studies of treatments for symptomatic UTIs during pregnancy reviewed six pyelonephritis studies and concluded that data are insufficient to recommend any specific best strategy among the study protocols (64). Factors to consider in selecting an empiric regimen may then include local resistance patterns and costs.

The benefit of chronic antibiotic prophylaxis after primary treatment for pyelonephritis has been demonstrated by Harris and Gilstrap (9), who reduced the $60 \%$ risk of recurrent pyelonephritis in their population to $3 \%$ by continuing broadspectrum antibiotic prophylaxis after primary therapy throughout the duration of pregnancy. Nitrofurantoin macrocrystals $50 \mathrm{mg}$ every day is an excellent choice for prophylaxis. Note, however, that such prophylaxis is effective only if the primary antibiotic therapy is appropriate for the etiologic organism (56). Although most early studies on the potential benefit of prophylactic antibiotics following acute pyelonephritis in pregnancy ended prophylaxis at the time of delivery $(9,65)$, Sandberg extended the prophylaxis for a month postpartum (66), and many subsequent studies have extended prophylaxis until 6 weeks postpartum $(62,63)$.
Frequent follow-up urine cultures may suffice as an alternative to chronic antibiotic prophylaxis following pyelonephritis in pregnancy. In a study tracking urine cultures every 2 weeks and comparing the option of chronic nitrofurantoin following treated pyelonephritis, gravidas not receiving prophylaxis were no more likely to get recurrent symptomatic UTI ( $14 \%$ vs. $8 \%)$; however, they were nearly twice as likely to have a subsequent positive urine culture ( $59 \%$ vs. $33 \%, p<0.02)(66)$. The cost of multiple urine cultures will probably exceed the cost of prophylactic nitrofurantoin.

Following appropriate parenteral antimicrobial therapy, clinical improvement occurs quickly, with resolution of fever anticipated within 48 hours in $85 \%$ of patients (67). In the uncommon event of no improvement after 48 to 72 hours of appropriate antibiotic therapy, consideration should be given to complicating factors such as obstruction, cortical abscess, papillary necrosis, or perinephric abscess. A renal ultrasound scan will detect many of the treatable complications that could delay response to therapy. However, ultrasonography has only one-quarter of the sensitivity of non-enhanced helical computed tomography for the detection of renal calculi (68).

Transient renal dysfunction has been demonstrated during acute pyelonephritis, with a reduction of endogenous creatinine clearance to $\leq 80 \mathrm{~mL} / \mathrm{min}$ in $27 \%$ of patients, and an incidence of approximately $20 \%$ for elevated blood urea nitrogen $(>11 \mathrm{mg} / \mathrm{dL})$ and serum creatinine $(>1 \mathrm{mg} / \mathrm{dL})(1,69)$. The urinary tract is the most common site of origin for gram-negative sepsis, and bacteremia occurs in up to $17 \%$ of patients with pyelonephritis. Bacteremia in the presence of pyelonephritis is usually an incidental finding, but it may also present as septic shock. Cunningham and coworkers reported a $3 \%$ incidence of septic shock among gravidas with acute pyelonephritis (67). Septic shock occurs secondary to endotoxemia or exotoxemia, and may lead to diffuse organ failure. Acute renal failure secondary to acute tubular necrosis may further compromise the transient renal dysfunction commonly seen with pyelonephritis. Pulmonary insufficiency, including dyspnea, tachypnea, hypoxemia, and pulmonary infiltrates, complicates $7 \%$ of gravidas with pyelonephritis (51). Septic shock complicating pyelonephritis may also cause hemolysis, thrombocytopenia, hepatic dysfunction, and adult respiratory distress syndrome. A syndrome of noncardiogenic pulmonary edema, with or without renal, hepatic, or hematopoietic dysfunction, has been reported in $2 \%$ of pregnant patients with pyelonephritis (70). This syndrome typically appears within 30 hours of admission and manifests as dyspnea, tachypnea, hypoxemia, and pulmonary effusions and consolidation; $20 \%$ of patients require mechanical ventilation, and most recover within 2 to 3 days.

An association between pyelonephritis and both preterm delivery and increased perinatal mortality was recognized in the pre-antibiotic era; however, appropriate antibiotic therapy appears nearly to eliminate these risks. Gilstrap and coworkers reported a higher rate of low birth weight for women with pyelonephritis $(<2500 \mathrm{~g}, 15 \%$ incidence vs. $10 \%$ in controls) (71), but no increase in perinatal mortality rate, and Fan and associates found no increase in low birth weight, size small for gestational age, or preterm delivery (50). 


\section{REFERENCES}

1. Gilstrap LC III, Cunningham FG, Whalley PJ. Acute pyelonephritis in pregnancy: an anterospective study. Obstet Gynecol 1981; 57: 409-13.

2. Buckley RM Jr, McGuckin M, MacGregor RR. Urine bacterial counts after sexual intercourse. N Engl J Med 1978; 298: 321-4.

3. Lomberg H, Cedergren B, Leffler H, et al. Influence of blood group on the availability of receptors for attachment of uropathogenic Escherichia coli. Infect Immun 1986; 51: 919-26.

4. Diokno AC, Compton A, Seski J, Vinson R. Urologic evaluation of urinary tract infection in pregnancy. J Reprod Med 1986; 31: 23-6.

5. Schieve LA, Handler A, Hershow R, et al. Urinary tract infection during pregnancy: its association with maternal morbidity and perinatal outcome. Am J Public Health 1994; 84: 405-10.

6. Harris RE, Gilstrap LC III. Cystitis during pregnancy: a distinct clinical entity. Obstet Gynecol 1981; 57: 578-80.

7. Campbell-Brown M, McFadyen IR, Seal DV, Stephenson ML. Is screening for bacteriuria in pregnancy worthwhile? Br Med J 1987; 294: 1579-82.

8. Wood EG, Dillon HC Jr. A prospective study of group B streptococcal bacteriuria in pregnancy. Am J Obstet Gynecol 1981; 140: 515-20.

9. Harris RE, Gilstrap LC III. Prevention of recurrent pyelonephritis during pregnancy. Obstet Gynecol 1974; 44: 637-41.

10. Feldkamp ML, Reefjuis J, Kucik J, et al. Case-control study of self reported genitourinary infections and risk of gastroschisis: findings from the national birth defects prevention study, 1997-2003. BMJ 2008; 336: $1420-3$.

11. Cleves MA, Malik S, Yang S, et al. Maternal urinary tract infections and selected cardiovascular malformations. Birth Defects Res (Part A) 2008; 82: 464-73.

12. Karlowsky JA, Jones ME, Thornsberry C, et al. Prevalence of antimicrobial resistance among urinary tract pathogens isolated from female outpatients across the US in 1999. Int J Antimicrob Agents 2001; 18: $121-7$.

13. Jamie WE, Edward RK, Duff P. Antimicrobial susceptibility of Gramnegative uropathogens isolated form obstetric patients. Infect Dis Obstet Gynecol 2002; 10: 123-6.

14. Treatment of urinary tract infections in nonpregnant women. ACOG Practice Bulletin No. 91, American College of Obstetricians and Gynecologists. Obstet Gynecol 2008; 111: 785-94.

15. Wright SW, Wrenn KD, Haynes ML. Trimethoprim-sulfamethoxazole resistance among urinary coliform isolates. J Gen Intern Med 1999; 14: 606-9.

16. Hernandez-Diaz S, Werler MM, Walker AM, Mitchell AA. Folic acid antagonists during pregnancy and the risk of birth defects. N Engl J Med 2000; 343: 1608-14.

17. Forna F, McConnell M, Kitabire FN, et al. Systemic review of the safety of trimethoprim-sulfamethoxazole for prophylaxis in HIV-infected pregnant women: implications for resource-limited settings. AIDS Reviews 2006; 8: 24-36.

18. Sivojelezova A, Einarson A, Shuhaiber S, Koren G. Trimethoprimsulfonamide combination therapy in early pregnancy. Can Fam Physician 2003; 49: 1085-6.

19. Kammire LD, Donofrio PD. Nitrofurantoin neuropathy: a forgotten adverse effect. Obstet Gynecol 2007; 110: 510-12.

20. D’Arcy PF. Nitrofurantoin. Drug Intell Clin Pharm 1985; 19: 540-7.

21. Ben David S, Einarson T, Ben Daivd Y, et al. The safety of nitrofurantoin during the first trimester of pregnancy: meta-analysis. Fundam Clin Pharmacol 1995; 9: 503-7.

22. Gait JE. Hemolytic reactions to nitrofurantoin in patients with glucose-6phosphate dehydrogenase deficiency: theory and practice. Ann Pharmacother 1990; 24: 1210-13.

23. Cimolai N, Cimolai T. Nitrofurantoin and pregnancy. Cam Med Assoc J 2007; 176: 1860-1

24. Kirkwood A, Harris C, Timar N, Koren G. Is gentamycin ototoxic to the fetus? J Obstet Gynaecol Can 2007; 29: 140-5.

25. Czeizel AE, Rockenbauer N, Olsen J, Sorensen HT. A teratological study of aminoglycoside antibiotic treatment during pregnancy. Scand J Infect Dis 2000; 32: 309-13.

26. Larsen H, Nielsen GL, Schonheyder HC, et al. Birth outcome following maternal use of fluoroquinolones. Int J Antimicrob Agents 2001; 18: 259-62.
27. Bayrak O, Cimentepe E, Inegöl I, et al. Is single-dose fosfomycin trometamol a good alternative for asymptomatic bacteriuria in the second trimester of pregnancy? Int Urogynecol J Pelvic Floor Dysfunct 2007; 18: 525-9.

28. Krcmery S, Hromec J, Demesova D. Treatment of lower urinary tract infection in pregnancy. Intl J Antimicrob Agents 2001; 17: 279-82.

29. Stamm WE, Counts GW, Running KR, et al. Diagnosis of coliform infection in acutely dysuric women. N Engl J Med 1982; 307: 463-8.

30. Greenberg RN, Reilly PM, Luppen KL, et al. Randomized study of singledose, three-day, and seven-day treatment of cystitis in women. J Infect Dis 1986; 153: 277-82.

31. Jaimes EC, Garcia JLA, Salinas JO, et al. Cistouretritis aguda durante la gestacion. Ginec Obst Mex 1989; 57: 57-63.

32. Pfau A, Sacks TG. Effective prophylaxis for recurrent urinary tract infections during pregnancy. Clin Infect Dis 1992; 14: 810-14.

33. Turck M, Goffe BS, Petersdorf RG. Bacteriuria of pregnancy. Relation to socioeconomic factors. N Engl J Med 1962; 266: 857-60.

34. Van Dorsten JP, Bannister ER. Office diagnosis of asymptomatic bacteriuria in pregnant women. Am J Obstet Gynecol 1986; 1555: 777-80.

35. Golan A, Wexler S, Amit A, et al. Asymptomatic bacteriuria in normal and high-risk pregnancy. Eur J Obstet Gynecol Reprod Biol 1989; 33: 101-8.

36. Nicolle LE, Bradley S, Colgan R, et al. Infectious Diseases Society of America guidelines for the diagnosis and treatment of asymptomatic bacteriuria in adults. Clin Inf Dis 2005; 40: 643-54.

37. Smaill F. Asymptomatic bacteriuria in pregnancy. Best Pract Res Clin Obstet Gynaecol 2007; 21: 439-50.

38. McIsaac W, Carroll JC, Biringer, et al. Screening for asymptomatic bacteriuria in pregnancy. J Obstet Gynaecol Can 2005; 27: 20-4.

39. Kincaid-Smith P, Bullen M. Bacteriuria in pregnancy. Lancet 1965; 1: 395-9.

40. Sweet RL. Bacteriuria and pyelonephritis during pregnancy. Semin Perinatol 1977; 1: 25-40.

41. Romero R, Oyarzun E, Mazor M, et al. Meta-analysis of the relationship between asymptomatic bacteriuria and preterm delivery/low birth weight. Obstet Gynecol 1989; 73: 576-82.

42. US Preventive Services Task Force. Screening for asymptomatic bacteriuria in adults: US Preventive Services Task Force reaffirmation recommendation statement. Ann Intern Med 2008; 149: 43-7.

43. American Academy of Pediatrics, American College of Obstetricians and Gynecologists. Guidelines for Perinatal Care, 6th edn. Elk Grove Village, IL, and Washington, DC: American Academy of Pediatrics and American College of Obstetricians and Gynecologists 2007: 100-1.

44. Kacmaz B, Cakir O, Aksoy A, Biri A. Evaluation of rapid screening tests to detect asymptomatic bacteriuria in pregnancy. Jpn J Infect Dis 2006; 59: 261-3.

45. Roberts AP, Robinson RE, Beard RW. Some factors affecting bacterial colony counts in urinary infection. Br Med J 1967; 1: 400-3.

46. Whalley PJ, Cunningham FG. Short-term versus continuous antimicrobial therapy for asymptomatic bacteriuria in pregnancy. Obstet Gynecol 1977; 49: 262-5.

47. Villar J, Widmer M, Lydon-Rochelle MT, et al. Duration of treatment for asymptomatic bacteriuria during pregnancy. Cochrane Database Syst Rev 2000; (2): CD000491. DOI:10.1002/14651858.CD000491.

48. Smaill F, Vazquez JC. Antibiotics for asymptomatic bacteriuria in pregnancy. Cochrane Database Syst Rev 2007; (2): CD000490. DOI:10.1002/14651858.CD000490.pub2.

49. Kass EH. Bacteriuria and pyelonephritis of pregnancy. AMA Arch Int Med 1960; 105: 194-8.

50. Fan Y, Pastorek JG II, Miller JM Jr, et al. Acute pyelonephritis in pregnancy. Am J Perinatol 1987; 4: 324-6.

51. Hill JB, Sheffield JS, McIntire DD, Wendel GD. Acute pyelonephritis in pregnancy. Obstet Gynecol 2005; 105: 18-23.

52. Thurman AR, Steed LL, Hulsey T, Soper DE. Bacteriuria in pregnancy women with sickle cell trait. Am J Obstet Gynecol 2006; 194: 1366-70.

53. Villers MS, Jamison MG, DeCastro LM, James AH. Morbidity associated with sickle cell disease in pregnancy. Am J Obstet Gynecol 2008; 199: 125. e1-5.

54. MacMillan MC, Grimes DA. The limited usefulness of urine and blood cultures in treating pyelonephritis in pregnancy. Obstet Gynecol 1991; 78: 745-8.

55. Duff P. Pyelonephritis in pregnancy. Clin Obstet Gynecol 1984; 27: 17-31. 
56. Van Dorsten JP, Lenke RR, Schifrin BS. Pyelonephritis in pregnancy. The role of in-hospital management and nitrofurantoin suppression. J Reprod Med 1987; 32: 895-900.

57. Greer LG, Roberts SW, Sheffield JS, et al. Ampicillin resistance and outcome differences in acute antepartum pyelonephritis. Infect Dis Obstet Gynecol 2008; 2008: 891426. DOI:10.1155/2008/891426.

58. Sanchez-Ramos L, McAlpine JK, Adair CD, et al. Pyelonephritis in pregnancy: once-a-day ceftriaxone versus multiple doses of cefazolin. A randomized, double-blind trial. Am J Obstet Gynecol 1995; 172: 129-33.

59. Wing DA, Hendershott CA, Debuque L, Millar LK. A randomized trial of three antibiotic regimens for the treatment of pyelonephritis in pregnancy. Obstet Gynecol 1998; 92: 249-53.

60. Safrin S, Siegel D, Black D. Pyelonephritis in adult women: in-patient versus outpatient therapy. Am J Med 1988; 85: 793-8.

61. Angel JL, O’Brien WF, Finan MA, et al. Acute pyelonephritis in pregnancy: a prospective study of oral versus intravenous antibiotic therapy. Obstet Gynecol 1990; 76: 28-32.

62. Millar LK, Wing DA, Paul RH, Grimes DA. Outpatient treatment of pyelonephritis in pregnancy: a randomized controlled trial. Obstet Gynecol 1995; 86: 560-4.

63. Wing DA, Hendershott CA, Debuque L, Millar LK. Outpatient treatment of acute pyelonephritis in pregnancy after 24 weeks. Obstet Gynecol 1999; 94: 683-8.
64. Vazquez JC, Villar J. Treatments for symptomatic urinary tract infections during pregnancy. Cochrane Database Syst Rev 2003; (4): CD002256. DOI:10.1002/14651858.CD002256.

65. Lenke RR, VanDorsten JP, Schifrin BS. Pyelonephritis in pregnancy: a prospective randomized trial to prevent recurrent disease evaluating suppressive therapy with nitrofurantoin and close surveillance. Am J Obstet Gynecol 1983; 146: 953-7.

66. Sandberg T, Brorson J-E. Efficacy of long-term antimicrobial prophylaxis after acute pyelonephritis in pregnancy. Scand J Infect Dis 1991; 23: 221-3.

67. Cunningham FG, Morris GB, Mickal A. Acute pyelonephritis of pregnancy: a clinical review. Obstet Gynecol 1973; 42: 112-17.

68. Fowler KA, Locken JA, Duchesne JH, Williamson MR. US for detecting renal calculi with non-enhanced CT as a reference standard. Radiology 2002; 110: 109-13.

69. Whalley PJ, Cunningham FG, Martin FG. Transient renal dysfunction associated with acute pyelonephritis of pregnancy. Obstet Gynecol 1975; 46: 174-7.

70. Cunningham FG, Lucas MJ, Hankins GDV. Pulmonary injury complicating antepartum pyelonephritis. Am J Obstet Gynecol 1987; 156: 797-804.

71. Gilstrap LC, Leveno KJ, Cunningham FG, et al. Renal infection and pregnancy outcome. Am J Obstet Gynecol 1981; 141: 709-16. 


\section{Bacterial vaginosis in pregnancy: Evidence-based approaches James A. McGregor and Michael W. McCullough}

\section{INTRODUCTION}

Bacterial vaginosis is the most common cause of vaginal discharge and malodor among women of reproductive age $(1,2)$. This condition has also been termed Gardnerella vaginitis, nonspecific vaginitis, and anaerobic colpitis. Studies have shown an association with early and late pregnancy loss, preterm birth, preterm labor, premature rupture of membranes, chorioamnionitis, amniotic fluid infection, postpartum endometritis, and cesarean section infectious morbidity (3-18). These associated complications have resulted in the recommendation for screening all pregnancies as a way to prevent adverse events. Unfortunately, studies have often been conflicting as to the exact relationship between bacterial vaginosis and poor pregnancy outcomes. Recent large-scale analysis has shown screening in the asymptomatic general population to be ineffective. Many organizations including the U.S. Preventive Services Task Force (19), American College of Obstetrics and Gynecology (19), British Association for Sexual Health and HIV/Clinical Effectiveness Group (20), and the Society of Obstetricians and Gynaecologists of Canada do not recommend screening of bacterial vaginosis in low-risk pregnant populations (21). This chapter will review bacterial vaginosis in pregnancy, the pathophysiology involved in associated adverse pregnancy outcomes, and the screening recommendations based on the current literature.

\section{MICROBIOLOGY AND PATHOGENESIS OF BACTERIAL VAGINOSIS}

Bacterial vaginosis consists of microecologic alterations in which a characteristic set of bacterial species greatly expand their populations within the vagina. This causes dramatic shifts in the biochemical make-up of vaginal fluid (Table 1) $(22,23$ ).

In the healthy vagina, between 5 and 15 microbial species are usually recoverable. High concentrations $\left(10^{5}-10^{6} / \mathrm{g}\right.$ of fluid) of lactic acid-producing acidophilic facultative lactobacilli are normally the predominant microflora found throughout the menstrual cycle $(23,24)$. Lactobacillus crispatus, Lactobacillus jensenii, Lactobacillus fermentum, and Lactobacillus gasseri represent the predominant vaginal species (24). Many of these produce $\mathrm{H}_{2} \mathrm{O}_{2}$ along with lactic acid. Other bacteria account for the remaining $10 \%$ of bacteria recovered (23-25).

The acidic environment in the healthy vagina is maintained by microbial metabolism of glucose, produced by vaginal epithelial cells from glycogen, to lactic acid (25). In addition to maintaining an acidic vaginal environment, $\mathrm{H}_{2} \mathrm{O}_{2}$-producing lactobacilli contribute to the maintenance of a healthy, selfsustaining vaginal ecosystem $(26,27)$. In vitro experiments demonstrate that Lactobacillus species inhibit growth of bacteria by producing antimicrobial factors, including acidolin and lactacin $\mathrm{B}$, in addition to $\mathrm{H}_{2} \mathrm{O}_{2}$ (26-29). The microbicidal action of $\mathrm{H}_{2} \mathrm{O}_{2}$ is further enhanced in the presence of a halide ion, such as chloride, from cervical mucus, and peroxidase enzymes, such as myeloperoxidase, produced by neutrophils and monocytes (28).

Each of these factors is present in the vaginal fluid of healthy women in sufficient concentrations to produce in vitro bactericidal effects (28). In a longitudinal study, Hillier and colleagues (30) found that women without $\mathrm{H}_{2} \mathrm{O}_{2}$-producing lactobacilli more often developed bacterial vaginosis and more frequently relapsed after successful treatment. These findings support the hypothesis that an absence of $\mathrm{H}_{2} \mathrm{O}_{2}$-producing lactobacilli precedes the shift in the vaginal ecosystem toward development of bacterial vaginosis (31). This increase in $\mathrm{pH}$ tends to displace lactobacilli from receptor sites on vaginal epithelial cells and maximizes adherence of Gardnerella vaginalis and other bacterial vaginosis-associated microorganisms $(31,32)$.

The condition of bacterial vaginosis is characterized by this dramatic decrease in normal lactobacilli and subsequent high concentrations of aerobic and anaerobic bacteria, most notably Prevotella, Peptostreptococcus, Porphomonas, and Mobiluncus species, along with G. vaginalis, Mycoplasma hominis, and Ureaplama ureclyticum $(23,24)$.

Biophysical changes associated with bacterial vaginosis include elevated $\mathrm{pH}(>4.5)$, reduced redox potential, increased fluid concentrations of diamines, polyamines, and organic acids, as well as increased concentrations of enzymes, including mucinases, sialidases, IgA proteases, collagenases, nonspecific proteases, and phospholipases $\mathrm{A}_{2}$ and $\mathrm{C}(24,33-39)$. Endotoxin (lipopolysaccharide), cytokine interleukin-1a, and prostaglandins $E_{2}$ and $F_{2}$ a are also increased in the vaginal fluid of women with bacterial vaginosis $(40,41)$. Amines, primarily trimethylamine, putrescine, and cadaverine, are produced during amino acid metabolism by bacterial vaginosis-associated anaerobic bacteria $(37,42)$. These volatile amines are released as $\mathrm{pH}$ increases and are responsible for the "sharp" or "fishy" odor sometimes noticed in the presence of bacterial vaginosis $(37,43)$. Several short-chain fatty acids, including succinate, acetate, propionate, isobutyrate, butyrate, and isovalerate, are also increased in bacterial vaginosis (24). In vitro studies demonstrate that increased succinic acid dramatically impairs neutrophil phagocytic killing, response to chemotactic stimuli, and generation of respiratory bursts required for bacterial killing (44). Butyrate inhibits lymphocyte activation by release of an endotoxin $(45,46)$.

These virulence factors promote tissue attachment, overcome host defense mechanisms, and facilitate entrance of microorganisms and associated factors into the upper reproductive tract. Vaginal fluid protease and phospholipases probably play roles in bacterial vaginosis pathogenesis and ascent of microbes and into the uterus during pregnancy. 
Mucinases and sialidases lyse protective mucin and promote bacterial attachment, allowing invasion and spread to underlying epithelial cells. These enzymes may also play a role in disruption of cervical mucus, leading to upper genital tract spread of bacterial vaginosis-associated microflora (38). During pregnancy, bacterial phospholipases $\mathrm{A}_{2}$ and $\mathrm{C}$, as well as nonspecific proteases, may act on cervical and amniochorion tissues to promote cervical ripening and amniochorion weakening (40). In addition, phospholipases $\mathrm{A}_{2}$ and $\mathrm{C}$ may promote the release of arachidonic acid and prostaglandins, furthering the processes leading to preterm birth (40). Thus, these bacterial substances directly contribute to the initiation of a number of bacterial vaginosis-associated obstetric complications (38-42).

\section{EPIDEMIOLOGY AND NATURAL HISTORY OF \\ BACTERIAL VAGINOSIS}

Numerous studies have prospectively examined bacterial vaginosis among populations of pregnant women and demonstrated prevalence ranging from $6 \%$ to $32 \%$ (Table 2) $(5,7,9-$ $13,43,46,58)$. Studies from Canada, Sweden, and Denmark report bacterial vaginosis among $14 \%$ of pregnant women $(56,59,60)$, which is similar to the $16 \%$ prevalence reported in the United States (13). Epidemiologic factors showing increased prevalence in the United States are AfricanAmerican race, smoking, and multiple sexual partners and are the lowest among women with no history of heterosexual contact $(5,56,57,61,62)$. Among nonpregnant women of reproductive age, bacterial vaginosis is detected more often among those not using any method of contraception and among women using an intrauterine contraceptive device $(36,63)$.

Studies in pregnancy show that most women who test negative stay negative while women with bacterial vaginosis remain positive unless treated $(12,61,63,64)$. Among pregnant women initially tested negative for bacterial vaginosis in the first trimester, $2.9 \%$ of British women and no Swedish women developed bacterial vaginosis by the follow-up examination at 28 weeks of gestation, and $47 \%$ to $55 \%$ of initially positive women continued to have findings of bacterial vaginosis at the third-trimester follow-up visit. A further $2.4 \%$ of women without bacterial vaginosis in the first trimester had developed bacterial vaginosis by term $(61,65)$. In $31 \%$ of untreated women with bacterial vaginosis, the condition resolved by 28 weeks of gestation (63). Importantly, Joesoef and coworkers (12) determined that women with bacterial vaginosis at 16 to 20 weeks of gestation suffered

Table 1 Biochemical Factors in Bacterial Vaginosis and Their Potential Pathophysiologic Effects

\begin{tabular}{|c|c|c|}
\hline $\begin{array}{l}\text { Vaginal fluid } \\
\text { constituent }\end{array}$ & Known or potential actions & Potential effects in pregnancy \\
\hline $\mathrm{pH}$ & $\begin{array}{l}\text { Attachment of anaerobic bacteria and } \\
\text { Lactobacillus species attachment }\end{array}$ & Numbers of pathogenic anaerobic bacteria \\
\hline Succinic acid & $\begin{array}{l}\text { Impairs neutrophil phagocytic killing } \\
\text { and response to chemotactic stimuli }\end{array}$ & Vaginal fluid host defenses \\
\hline Butyrate & Toxic to fibroblasts in cell culture & $\begin{array}{l}\text { effects on cervical connective tissue and } \\
\text { amniochorion }\end{array}$ \\
\hline Sialidase/Mucinase & $\begin{array}{l}\text { Degrades protective surface mucin, } \\
\text { bacterial attachment to epithelial cells }\end{array}$ & Cervical mucus barrier \\
\hline IgA protease & Degrades IgA proteins & Cervical IgA \\
\hline $\begin{array}{l}\text { Collagenase } \\
\text { Nonspecific proteases }\end{array}$ & Degrades collagen types & Cervical ripening, amniochorion weakening \\
\hline Phospholipase $\mathrm{A}_{2}$ & $\begin{array}{l}\text { Induces matrix metalloproteinases, } \\
\text { induces prostaglandins }\end{array}$ & $\begin{array}{l}\text { Cervical ripening, amniochorion weakening, and } \\
\text { uterine contractions }\end{array}$ \\
\hline
\end{tabular}

Table 2 Prevalence of Bacterial Vaginosis in Pregnant Women Followed Prospectively

\begin{tabular}{|c|c|c|}
\hline \multirow{2}{*}{$\begin{array}{l}\text { Study population } \\
\text { USA }\end{array}$} & \multicolumn{2}{|c|}{ Diagnostic method prevalence } \\
\hline & & \\
\hline \multirow[t]{3}{*}{ Seattle, Washington $(10,47-49)$} & Gas-liquid chromatography & $14-28 \%$ \\
\hline & Clinical & $6-21 \%$ \\
\hline & Gram stain & $12-21 \%$ \\
\hline \multirow[t]{2}{*}{ Denver, Colorado $(13,38)$} & Gram stain & $18.7-23 \%$ \\
\hline & Clinical & $32 \%$ \\
\hline Vaginal Infections and Prematurity Study (50) & Gram stain and $\mathrm{pH}>4.5$ & $16 \%$ \\
\hline Maternal-Fetal Medicine Network Centers (51) & Gram stain and $\mathrm{pH}>4.5$ & $23.4 \%$ \\
\hline Halifax, Nova Scotia, Canada (52) & Clinical & $23 \%$ \\
\hline Finland (16) & Quantitative cultures & $21.4 \%$ \\
\hline Göteborg, Sweden (53) & Papanicolaou's smear and $\mathrm{pH}>4.5$ & $14.3 \%$ \\
\hline Harrow, UK $(54,55)$ & Gram stain & $12-14 \%$ \\
\hline Adelaide, Australia (15) & Gardnerella vaginalis culture (heavy growth) & $28 \%$ \\
\hline Jakarta, Indonesia (56) & Gram stain & $17 \%$ \\
\hline Canada (57) & Gram stain & $14 \%$ \\
\hline
\end{tabular}


increased risk for preterm birth, even if they no longer had findings of bacterial vaginosis at the later follow-up visit (28-32 weeks of gestation). Prospective treatment studies show that bacterial vaginosis recurs between $20 \%$ and $80 \%$ in pregnancy, regardless of initial successful treatment $(38,66)$. The individual factors that predispose to acquisition, persistence, resolution, and/or recurrence of bacterial vaginosis probably involve failure of $\mathrm{H}_{2} \mathrm{O}_{2}$-producing lactobacilli to recolonize and reestablish a normal, protective vaginal ecosystem following treatment $(26,31,67)$.

\section{DIAGNOSIS OF BACTERIAL VAGINOSIS Clinical Diagnosis}

The clinical "gold standard" established in 1983 by Amsel and colleagues (36) is based upon the presence of three of four clinical criteria:

1. homogeneous, thin vaginal fluid that adheres to the vaginal walls;

2. vaginal fluid $\mathrm{pH}>4.5$;

3. release of amine odor with alkalinization of vaginal fluid, the "whiff test"; and

4. presence of vaginal epithelial cells with borders obscured by adherent, small bacteria called "clue cells."

Of the four Amsel's criteria, the presence of clue cells on saline wet-mount examination is the most specific and sensitive indicator of bacterial vaginosis. Identification of clue cells accurately predicts $85 \%$ to $90 \%$ of women with clinical bacterial vaginosis (positive predictive value). Identification of clue cells may be hindered by adherence of normal bacteria, cellular debris, and availability and quality of the microscopy $(65,68)$.

Clue cells can be microscopically distinguished from normal vaginal epithelial cells by their characteristic stippled and ragged appearance (Fig. 1). The cell borders are obscured by adherent small coccobacilli-type bacteria, in contrast to normal vaginal epithelial cells where the cell borders are distinct and clearly seen (Fig. 2). In addition to the presence of clue cells, the characteristic long rods, of Lactobacillus morphotypes, are absent or rare. Background bacterial flora appears greatly increased and short rods and coccobacillary forms predominate. Mobiluncus species may be identified by their characteristic spiral or serpent-like motility (69).

The "whiff test" is similarly sensitive for the diagnosis of bacterial vaginosis, but may also be positive in trichomoniasis. Alkalinization of vaginal fluid releases volatile amines, putrescine, cadaverine, and trimethylamine, which give off a characteristically "fishy" or sharp odor. This odor may be noticed by a woman as amines are released spontaneously during activities that raise the vaginal $\mathrm{pH}$, such as sexual intercourse, douching, or menses $(37,43)$.

Normal vaginal $\mathrm{pH}$ from menarche to menopause ranges between 3.8 and 4.2. Amsel and colleagues (36) determined that a vaginal fluid $\mathrm{pH}$ over 4.5 discriminated between bacterial vaginosis and normal vaginal fluid. Identification of $\mathrm{pH}$ over 4.5 is highly sensitive for the diagnosis of bacterial vaginosis, but it is not specific $(2,36,70)$.

Other factors may raise vaginal $\mathrm{pH}$, including semen, cervical mucus, menses, trichomoniasis, and possibly recent douching. Samples for examination of vaginal fluid $\mathrm{pH}$ must be obtained from the lateral vaginal side wall or posterior fornices in order to reflect accurately vaginal, and not cervical, $\mathrm{pH}$. Similarly, the $\mathrm{pH}$ indicator paper must allow distinction between the normal vaginal $\mathrm{pH}(3.8-4.2)$ and a $\mathrm{pH}$ over 4.5. The characteristic thin, homogeneous, and adherent vaginal fluid is the most subjective indicator of bacterial vaginosis.

\section{Laboratory Diagnosis}

Gram stains of vaginal fluid are used in research settings to identify the shift from predominance of Lactobacillus morphotypes to predominance of coccobacillary morphotypes and gram-negative rods characteristic of bacterial vaginosis. The Gram-stain diagnosis involves quantitative estimations of

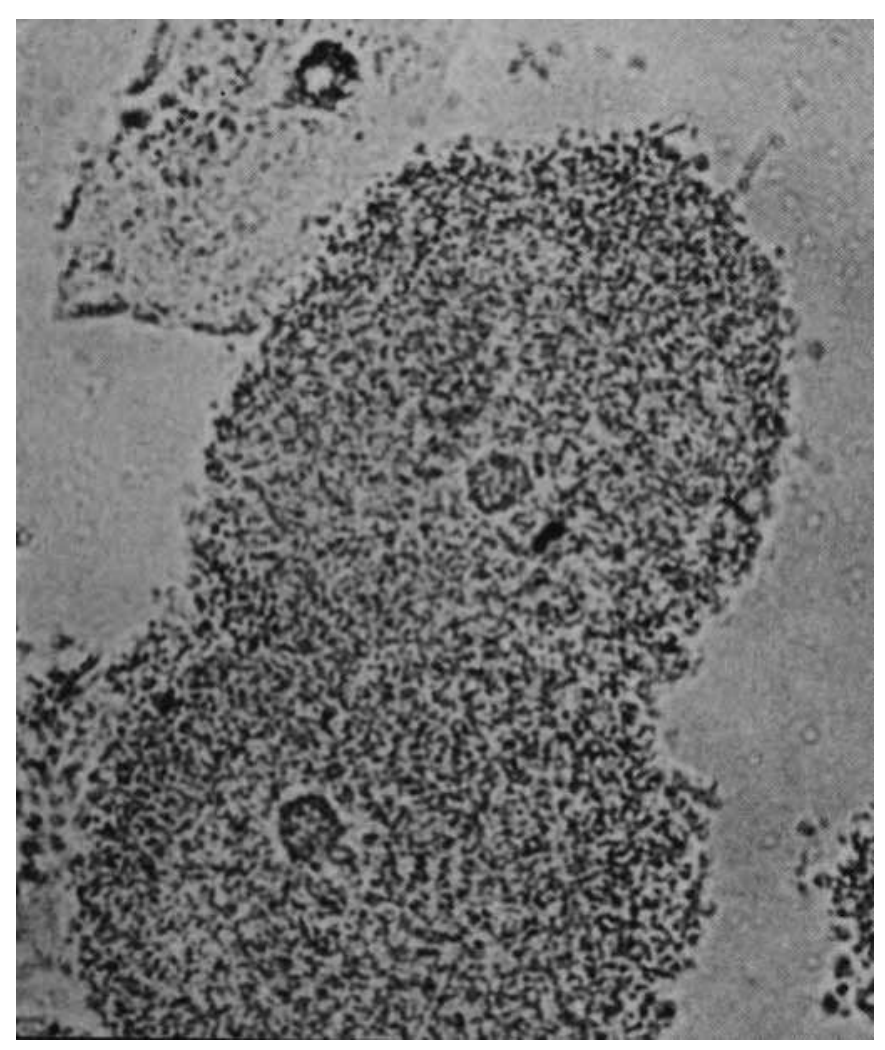

Figure 1 "Clue cells" identified on microscopic examination of saline preparation.

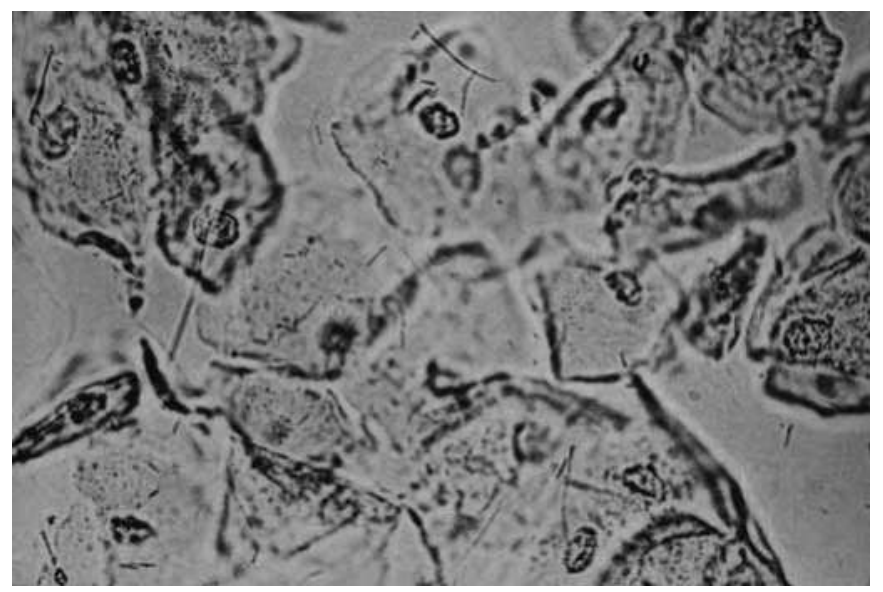

Figure 2 Normal vaginal epithelial cells identified on microscopic examination of saline preparation. 
these types of bacteria in the vaginal fluid (70-72). Normal vaginal fluid is described by a predominance of large grampositive rods, considered Lactobacillus morphotypes, with or without smaller gram-variable bacilli, considered Gardnerella morphotypes (Fig. 2) (73). A pattern of mixed vaginal flora, which includes Gardnerella morphotypes, gram-negative rods, fusiforms, curved rods, gram-positive cocci, and absent or reduced numbers of Lactobacillus morphotypes (less than five per high-power field), is consistent with bacterial vaginosis (Fig. 3) (73). The current laboratory "gold standard" incorporates Gram stain using a scoring system, the most prevalent being the "Nugent scoring" system with a sensitivity of $89 \%$ and a specificity of $83 \%$ when compared with Amsel's criteria (Fig. 4).

The technique of Nugent and coworkers (74) examines four bacterial morphotypes and assigns a summary score to the vaginal specimen based on the semiquantitative assessment for Lactobacillus morphotypes, G. vaginalis, Prevotella morphotypes, and Mobiluncus morphotypes. A score of 7 to 10 is considered to indicate bacterial vaginosis (74). Compared

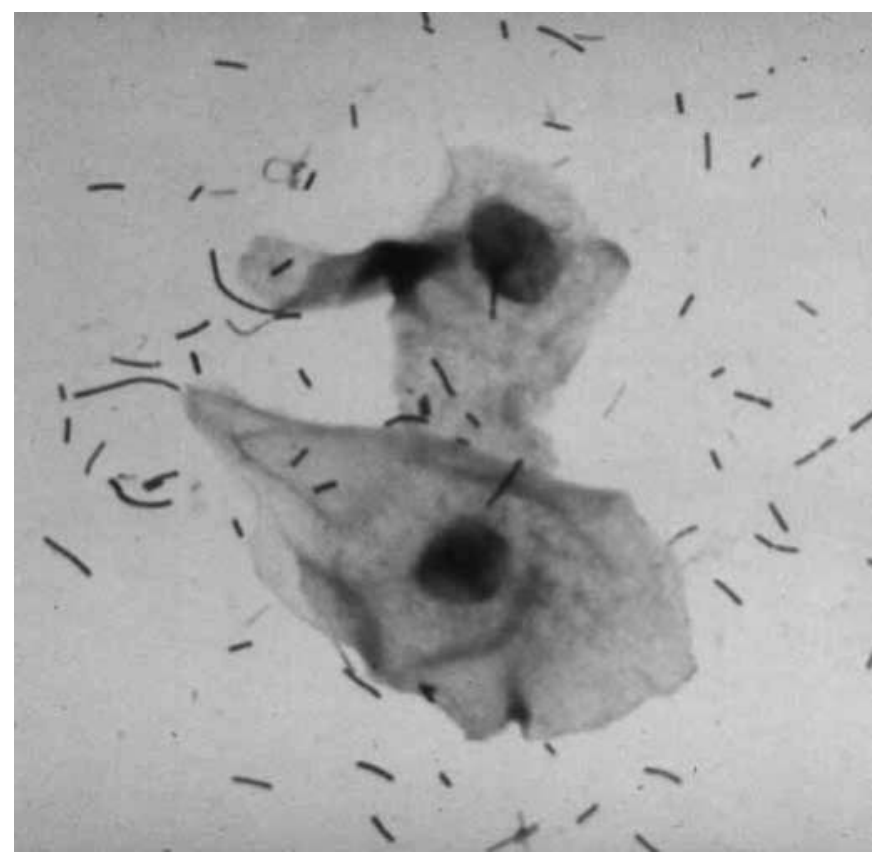

Figure 3 Normal vaginal fluid Gram stain.

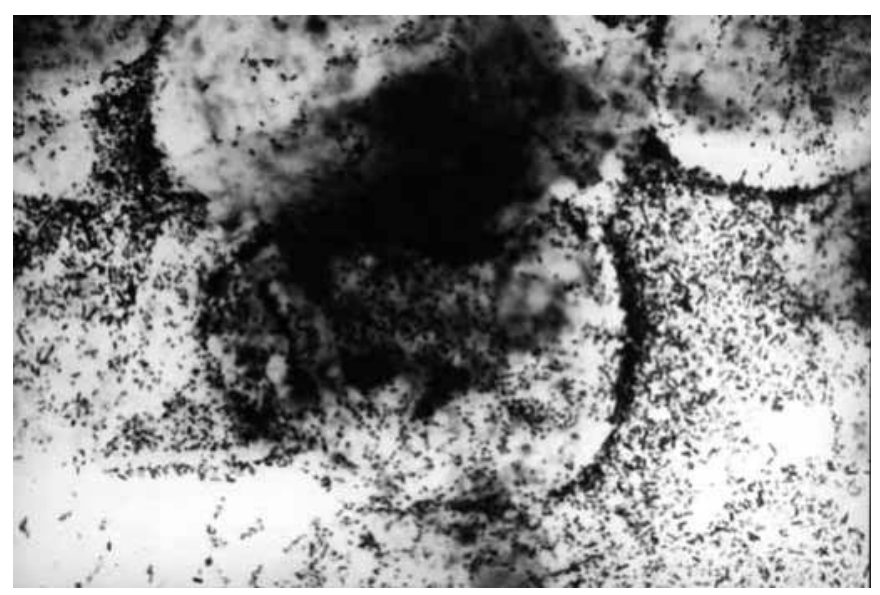

Figure 4 Gram stain of bacterial vaginosis. with other diagnostic methods, this standardized scheme has superior reproducibility (73).

This reproducibility has made the "Nugent score" the standard used in the majority of recent research. The required laboratory to perform the test and time delay prevent Gram-stain scoring systems from widespread clinical settings.

Nonquantitative microbial culture for G. vaginalis is both an inaccurate and expensive way to diagnose bacterial vaginosis. Clinical cultures should not be used to diagnose bacterial vaginosis. Since organisms such as $G$. vaginalis can be recovered from up to $60 \%$ of women with and without bacterial vaginosis, this leads to unacceptable false-positive rates $(2,59)$. Quantitative aerobic and anaerobic vaginal cultures have been used in clinical research studies to evaluate bacterial vaginosis, but this technique is costly and impractical for clinical use $(9,10)$.

Newer diagnostic techniques, including nucleic acid probes to detect high concentrations of G. vaginalis, have become available (71,75). Such tests as the Affirm VP III Microbial Identification Test (Becton Dickinson and Company, Sparks, MD) may provide a less subjective test for bacterial vaginosis than other current methods. Briselden, Hillier, and Sheiness reported accurate detection (sensitivity) of $95 \%$ to $97 \%$ of women with clinical criteria for bacterial vaginosis using the Affirm VP III test. Specificity for this test ranged from $71 \%$ to $98 \%(71,75)$. Identification of several different vaginal fluid biochemicals associated with bacterial vaginosis such as amines (FemExam) and sialidase activity (BV Blue) have recently become available commercially. $(5,6,24,37,76)$.

\section{OBSTETRIC COMPLICATIONS ASSOCIATED WITH BACTERIAL VAGINOSIS \\ Preterm Birth}

Associations between bacterial vaginosis and increased risk for adverse pregnancy outcomes have been investigated through case-control, cross-sectional, prospective cohort studies and randomized controlled treatment trials (Table 3) (4-13,38,50,54,56,60,74-76). Despite differences in study design, definitions of preterm birth, and the techniques used to identify bacterial vaginosis, research from around the world supports the association between bacterial vaginosis and increased risk of preterm labor, preterm birth, and preterm premature rupture of the membranes (Table 3 ) $(5-13,38,56,78)$. Crucially, bacterial vaginosis and associated microorganisms appear to increase the risk of preterm birth at the lowest viable gestational ages (8). Two published studies report an association between previable second-trimester pregnancy loss and bacterial vaginosis, suggesting that pathophysiologic processes associated with bacterial vaginosis cause a continuum of both previable loss and birth of severely premature newborns $(11,56)$.

Multiple prospective studies indicate that bacterial vaginosis may be detected months prior to the onset of preterm labor or preterm birth (5,7,9-13). Eschenbach and colleagues (4) and Gravett and coworkers $(5,6)$ were the first to implicate bacterial vaginosis as a risk factor for preterm labor and low birth weight. Bacterial vaginosis was identified using gasliquid chromatography among 19\% of 534 pregnant women studied (5). The presence of bacterial vaginosis in the midthird trimester (mean 32.6 weeks) was associated with 
increased risk for preterm labor [odds ratio (OR) 2.0, 95\% confidence interval (CI) 1.1-3.5] and preterm premature rupture of the membranes (OR 2.0, 95\% CI 1.1-3.7) (5). McDonald and colleagues (9) examined bacterial vaginosis (culture for heavy colonization with G. vaginalis) earlier in gestation (22-28 weeks) and demonstrated similar risks for preterm birth following labor (OR 1.8) and preterm premature rupture of the membranes (OR 2.7).

Studies that examine for bacterial vaginosis at the earliest gestational ages and carefully control for intervening antimicrobial treatment demonstrate the strongest association between bacterial vaginosis and preterm birth (Table 3) (10,11). The highest relative risks (sevenfold increase) between bacterial vaginosis and preterm birth and premature rupture of the membranes were noted in a Finnish study involving women who were initially examined in the first trimester (10). In an insightful analysis, Joesoef and colleagues (12) examined the risk of preterm birth for women found to have bacterial vaginosis between 16 and 20 weeks of gestation and/or between 28 and 32 weeks of gestation (12). Women with bacterial vaginosis in the early second trimester experienced a twofold increase in premature birth compared with women without bacterial vaginosis (12). Women with bacterial vaginosis at 28 to 32 weeks of gestation experienced somewhat less increased risk for premature birth (OR 1.5, $95 \%$ CI 0.7-3.0). Women who developed bacterial vaginosis between the screening intervals (i.e., those negative at 16-20 weeks of gestation and positive at 28-32 weeks of gestation) were not at increased risk for preterm birth (10.7\% preterm birth among women developing bacterial vaginosis vs. $11.8 \%$ among women never positive for bacterial vaginosis; calculated OR 0.9) (12). As discussed, women with findings of bacterial vaginosis at 16 to 20 weeks of gestation were at increased risk for preterm birth, even if they no longer had findings of bacterial vaginosis at the later followup visit $(20.5 \%$ preterm birth among women with bacterial

Table 3 Review of Prospective Cohort Studies Evaluating Bacterial Vaginosis and Adverse Pregnancy Outcomes

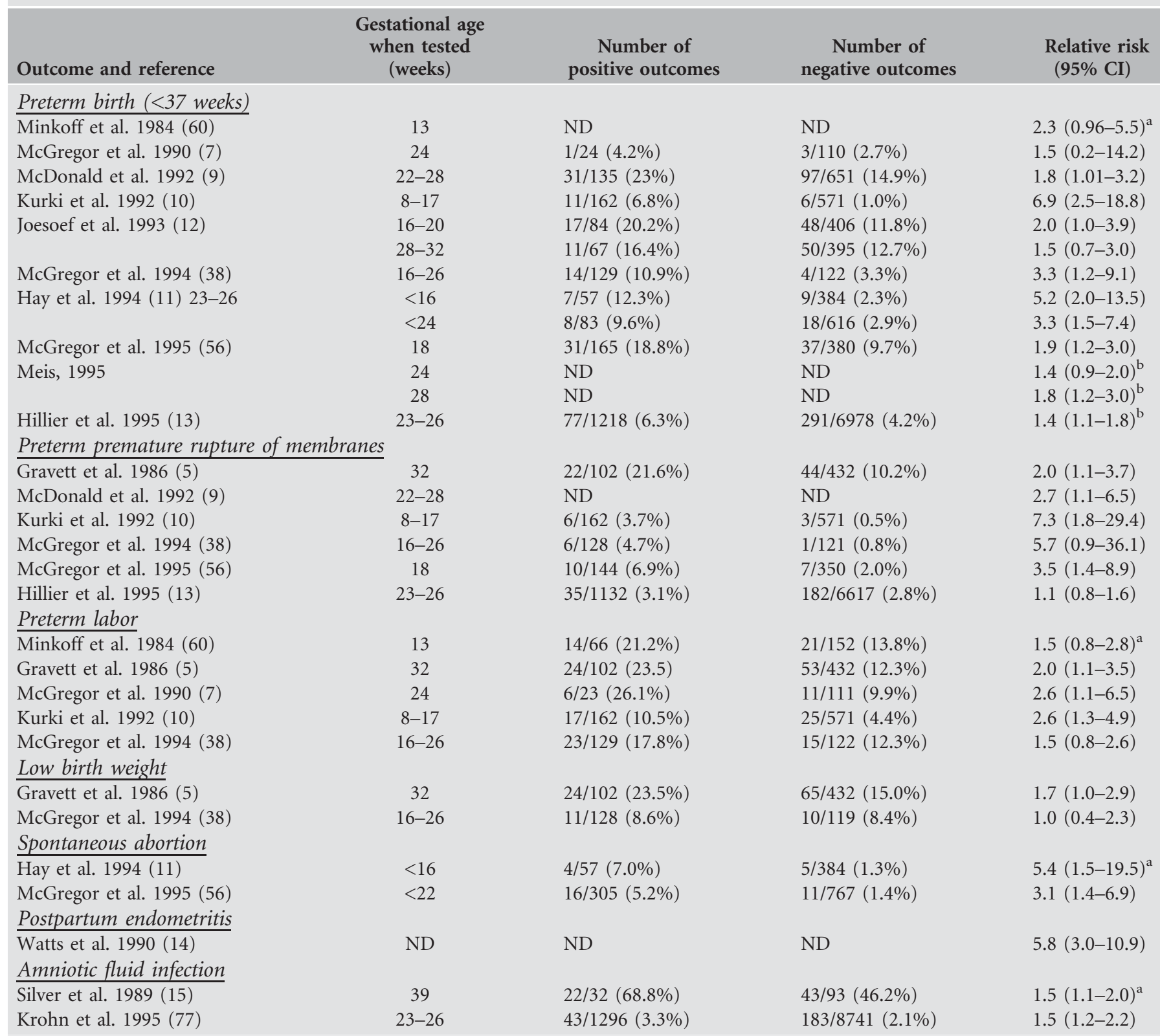

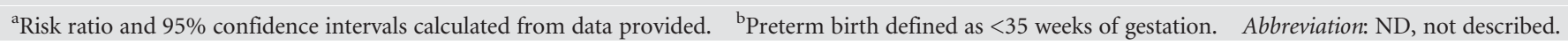


vaginosis at both visits and $20.5 \%$ preterm birth among women with bacterial vaginosis only at the first visit vs. $11.8 \%$ preterm birth among women without bacterial vaginosis) (12). These findings suggest that physiologic processes present or initiated at, or prior to, 16 to 20 weeks of gestation are important for the subsequent development of preterm birth. These authors concluded that "only bacterial vaginosis in early pregnancy plays a major role as a risk factor for preterm delivery" (12). Results of a large prospective cohort study from the National Institute of Child Health and Human Development-sponsored Vaginal Infections and Prematurity Study Group demonstrated a $40 \%$ increase in risk of delivering a preterm low-birth weight infant and a 10\% increase in risk of preterm premature rupture of the membranes among women with bacterial vaginosis detected between 23 and 26 weeks of gestation (13). These authors calculated a $9 \%$ attributable risk for prematurity in this study of over 10,000 subjects (13). Other populations could receive more or less benefits, depending on the prevalence of bacterial vaginosis.

\section{Intrapartum and Postpartum Infections}

Bacterial vaginosis has been linked to clinical chorioamnionitis, amniotic fluid infection, as well as postpartum endometritis and cesarean section infection. These associations were first suggested by observations that the microorganisms frequently recovered from women with these complications often include the common constituents of bacterial vaginosis, i.e., Prevotella bivius, G. vaginalis, M. hominis, U. urealyticum, and Peptostreptococcus species $(6,14-16)$. Clinical studies have examined bacterial vaginosis, identified by either Gram stain or gas-liquid chromatography, and shown that women with clinical amniotic fluid infection more commonly have findings of bacterial vaginosis compared with women without clinical infection. $(6,15,16)$ Among the $286(2.4 \%)$ women from the Vaginal Infections and Prematurity Study who developed amniotic fluid infection, antenatal carriage (23-26 weeks of gestation) of bacterial vaginosis (relative risk 1.5, 95\% CI 1.2-2.2) and bacterial vaginosis-associated microorganisms were significantly associated with amniotic fluid infection (78). This increase in risk for amniotic fluid infection associated with bacterial vaginosis was independent of duration of labor, duration of rupture of membranes, concurrent infection with Neisseria gonorrhoeae, Chlamydia trachomatis, Trichomonas vaginalis, group B streptococcus, and effective antimicrobial treatment (77). Postpartum endometritis occurs following $2 \%$ to $5 \%$ of vaginal births and $10 \%$ to $20 \%$ of cesarean deliveries (79). These infections are polymicrobial in nature for over $80 \%$ of affected women (80). In a series of 161 women with postpartum endometritis described by Watts and colleagues (81), the common constituents of bacterial vaginosis (i.e., G. vaginal is, P. bivius, Peptostreptococcus species, and Bacteroides species) were recovered from endometrial cultures in up to $60 \%$ of women (81). Wound infections developed among 18\% $(16 / 79)$ of women with endometrial bacterial vaginosisassociated flora and among 8\% (4/53) of women with other bacteria recovered from the endometrium (81). Subsequent work by Watts demonstrated a 5.8-fold increased risk for post-cesarean section endometritis among women with bacterial vaginosis (14).

\section{BACTERIAL VAGINOSIS TREATMENT IN PREGNANCY}

Oral (systemic) treatment with either metronidazole or clindamycin is preferred for the treatment of bacterial vaginosis during pregnancy. Only oral treatments provide adequate antimicrobial levels in both uterine and vaginal tissues and are associated with improved pregnancy outcome in published studies. Cure rates are similar to those obtained among nonpregnant women $(56,77)$. Initial cure rates within 1 to 2 weeks following completion of treatment are generally $25 \%$ to $35 \%$ higher than those among women examined 4 to 5 weeks posttreatment $(82,83)$.

Oral metronidazole, $500 \mathrm{mg}$ two times daily for 7 days, is the standard therapy recommended by the Centers for Disease Control in the United States for treatment of bacterial vaginosis (81). Resolution of bacterial vaginosis occurs among $85 \%$ to $100 \%$ of studied women examined approximately 1 week following completion of oral metronidazole treatment (84). The percentage of women without bacterial vaginosis 4 to 5 weeks following completion of treatment ranges from $60 \%$ to $95 \%$ and averages approximately $80 \%(82,84)$. Similarly, cure rates of $70 \%$ have been seen with the use of metronidazole and erythromycin (82). Different regimens of oral metronidazole have been examined in trials and observational reports; in general, cure rates at 4 weeks postdosing with a single 2 -g dose of metronidazole range from $45 \%$ to $69 \%$ (84).

Clindamycin is also an effective treatment for bacterial vaginitis, as well as its associated microflora, including some genital mycoplasmas. Greaves and coworkers (69) examined 49 women 7 to 10 days following completion of a 7-day course of oral clindamycin, $300 \mathrm{mg}$ twice daily, and demonstrated a cure rate of $94 \%$ for bacterial vaginosis. McGregor and associates (56) examined 194 pregnant women and found a $92 \%$ cure rate 2 to 4 weeks post-treatment (56). Oral treatment with clindamycin may be used throughout pregnancy, including the first trimester; it provides resolution of bacterial vaginosis equivalent to that of oral metronidazole.

Intravaginal treatments for bacterial vaginosis are similarly effective at eradication of bacterial vaginosis but have not been shown to improve pregnancy outcomes $(38,80,82,84)$. Metronidazole gel $0.75 \%$ one 5 -g applicator intravaginally once a day for 5 days or clindamycin $2 \%$ one 5 -g applicator for 7 days is recommended. Other treatments for bacterial vaginosis have been evaluated: ampicillin, augmentin, ofloxacin, erythromycin, triple sulfa cream, and vaginal acidification $(60,84)$. These treatments are less effective than both oral and vaginal preparations of metronidazole or clindamycin and are not recommended. Similarly, probiotic therapy with microbial products, such as lactobacillus powders and capsules, should be avoided; many of the nondairy products are contaminated with potentially harmful bacteria such as Clostridium sporogenes and Enterococcus species (85). Therapy with yogurt or acidophilus milk is also likely to be of little benefit, since the L. acidophilus strains are not easily established in the vagina (86).

Treatment of sexual partners of women with bacterial vaginosis is unnecessary: randomized controlled trials that examined co-treatment of male partners failed to demonstrate reduced recurrence rates of bacterial vaginosis for women $(87,88)$. 


\section{SCREENING IN PREGANCY}

In 2001, the U.S. Preventive Services Task Force published recommendations after review of seven randomized control trials. Screening in asymptomatic low-risk pregnancies was not recommended, and there was insufficient evidence to recommend screening for the high-risk population. In 2008, Nygren (52) and associates reviewed literature that was published following the 2001 task force findings. Seven randomized, controlled trials were included in their analysis and the results pooled with the 2001 report data. No evidence was found supporting screening in the asymptomatic low-to-moderate-risk patients. Screening in high-risk patients varied with three of five studies showing a benefit, one showing no benefit, and one showing harm with treatment (52).

Considering the current literature, there seems to be no benefit to screening the asymptomatic low-risk general population. Screening high-risk patients may provide benefit; however, there is no current consensus. The following are general guidelines that use current clinical evidence:

1. Test and treat all symptomatic patients.

2. Asymptomatic low-risk patients do not appear to benefit from universal screening.

3. Asymptomatic women at high risk for adverse pregnancy outcomes may benefit from screening and treatment. If screening is preformed, it should be done prior to 16 weeks of estimated gestational age.

4. Oral or vaginal preparations have similar organism eradication rates; however, vaginal treatments do not affect the preterm labor and delivery rates. Therefore, all patients at high risk for adverse outcomes should be treated with oral clindamycin $300 \mathrm{mg}$ twice a day for 7 days or metronidazole 500 mg two times a day for 7 days.

5. Test for cure should be done in 3 to 4 weeks after treatment.

\section{CONCLUSION}

Bacterial vaginosis is the most common cause of vaginal discharge in pregnant and nonpregnant women. This condition represents massive microbial and biochemical perturbations of the lower reproductive tract microecology. Diagnostic tests (Amsel's criteria) are easily performed. Treatments include oral or vaginal treatment with metronidazole or clindamycin. Descriptive and intervention studies carried out worldwide demonstrate increased risks of preterm birth and other adverse pregnancy outcomes associated with bacterial vaginosis; however, conclusive epidemiologic evidence to support screening in the general population has not been found. One explanation that has been proposed by Simhan et al. (89) involves molecular epidemiology looking at the individual patient's inflammatory response to a bacterial vaginosis infection based on genotypes. In the hypothesis by Simhan et al. (89), patients may be "hyperresponders" at risk for an overresponse of local immune factors resulting in pregnancy loss or preterm delivery, "normo-responders" with enough inflammatory mediators to promote clearing of the infection, or "hypo-responders" putting these patients at risk for ascending infection, preterm delivery, chorioamnionitis, and postsurgical infections $(89,90)$. These new ideas and technology may provide future researchers the tools in which to discover the contributing factors to the ongoing problem of being born "too soon."

\section{REFERENCES}

1. Eschenback DA, Hillier SL, Critchlow C, et al. Diagnosis and clinical manifestations of bacterial vaginosis. Am J Obstet Gynecol 1988; 158: 819.

2. Rein MF, Holmes KK. Nonspecific vaginitis, vulvovaginal candidiasis, and trichomoniasis: Clinical Topics in Infectious Diseases. 1983: 281-315.

3. Eschenback DA, Gravett MG, Chen KCS, et al. Bacterial vaginosis during pregnancy. An association with prematurity and post partum complications. In: Mårdh PA, Taylor-Robinson D, eds. Bacterial Vaginosis. Stockholm: Almqvist and Wiksill, 1984: 213-22.

4. Gravett MG, Nelson HP, DeRouen T, et al. Independent associations of bacterial vaginosis and Chlamydia trachomatis infection with adverse pregnancy outcome. J Am Med Assoc 1986; 256: 1899-903.

5. Gravett MG, Hummel D, Eschenback DA, Holmes KK. Preterm labor associated with subclinical amniotic fluid infection and with bacterial vaginosis. Obstet Gynecol 1986; 67: 229-37.

6. McGregor JA, French JI, Richter R, et al. Antenatal microbiologic and maternal risk factors associated with prematurity. Am J Obstet Gynecol 1990; 163: 1465-77.

7. Hillier SL, Martius J, Krohn MA, et al. A case-control study of chorioamnionic infection and histologic chorioamnionitis in prematurity. N Engl J Med 1988; 319: 972-8.

8. McDonald HM, O’Loughlin JA, Jolley P, et al. Prenatal microbiological risk factors associated with preterm birth. Br J Obstet Gynaecol 1992; 99: 190-6.

9. Kurki T, Sivonen A, Renkonen O-V, et al. Bacterial vaginosis in early pregnancy and pregnancy outcome. Obstet Gynecol 1992; 80: 173-7.

10. Hay PE, Lamont RF, Taylor-Robinson D, et al. Abnormal bacterial colonization of the genital tract and subsequent preterm delivery and late miscarriage. Br Med J 1994; 308: 295-8.

11. Joesoef MR, Hillier SL, Utomon B, et al. Bacterial vaginosis and prematurity in Indonesia: association in early and late pregnancy. Am J Obstet Gynecol 1993; 169: 175-8.

12. Hillier SL, Nugent RP, Eschenbach DA, et al.; for the Vaginal Infections and Prematurity Study Group. Association between bacterial vaginosis and preterm delivery of a low-birth-weight infant. N Engl J Med 1995; 333: $1737-42$.

13. Watts DH, Krohn MA, Hillier SL, Eschenbach DA. Bacterial vaginosis as a risk factor for post Cesarean endometritis. Obstet Gynecol 1990; 75: 52-8.

14. Silver HM, Sperling RS, St Clair P, Gibbs RS. Evidence relating bacterial vaginosis to intraamniotic infection. Am J Obstet Gynecol 1989; 161: 808-12.

15. Hillier SL, Krohn MA, Cassen R, et al. The role of bacterial vaginosis and vaginal bacteria in amniotic fluid infection in women in preterm labor with intact fetal membranes. Clin Infect Dis 1995; 20(Suppl 2): S276-8.

16. Leinich $\mathrm{H}$, Bodner-Adler $\mathrm{B}$, Brunbauer $\mathrm{M}$, et al. Bacterial vaginosis as a risk factor for preterm delivery: a meta-analysis. Am J Obstet Gynecol 2003; 189: 139-47.

17. Wiesenfield HC, Hillier SL, Krohn MA, et al. Lower genital tract infection and endometritis: insight into subclinical pelvic inflammatory disease. Obstet Gynecol 2002; 100: 456-63.

18. Nygren P, Bougatsos C, Freeman M, Helfand M. Screening and Treatment for Bacterial Vaginosis in Pregnancy: Systematic Review to Update the 2001 U.S. Preventive Service Task Force Recommendation Statement. Prepared for the Agency for Healthcare Research and Quality by the Oregon Evidence-based Practice Center under Contract No. 29002-0024. Evidence Synthesis No. 57. AHRQ Publication No. 08-05106. Rockville, MD: Agency for Healthcare Research and Quality, 2008.

19. American College of Obstetricians and Gynecologists, Assessment of risk factors for preterm birth. ACOG practice bulletin no 31. Washington, DC: American College of Obstetricians and Gynecologists, 2001.

20. Keane F, Ison C, Noble $\mathrm{H}$, et al. Sexually transmitted infections: UK national screening and testing guidelines. London (UK): British Association for Sexual Health and HIV (BASHH), 2006: 40-6.

21. Yudin M, Money D. Screening and management of bacterial vaginosis in pregnancy. SOGC CLINICAL Practice Guideline no 211. Ottawa, ON: Society of Obstetricians and Gynaecologists of Canada. 
22. Spiegel CA. Bacterial vaginosis. Clin Microbiol Rev 1991; 4: 485-502.

23. Spiegel CA, Amsel R, Eschenbach D, et al. Anaerobic bacteria in nonspecific vaginitis. N Engl J Med 1980; 303: 601-7.

24. Eschenbach DA, Davick PR, Williams BL, et al. Prevalence of hydrogen peroxide-producing Lactobacillus species in normal women and women with bacterial vaginosis. J Clin Microbiol 1989; 27: 251-6.

25. Redondo-Lopez V, Cook RL, Sobel JD. Emerging role of lactobacilli in the control and maintenance of vaginal bacterial microflora. Rev Infect Dis 1990; 12: 856-72.

26. Klebanoff SJ, Hillier SL, Eschenbach DA, Waltersdorph AM. Control of the microbial flora of the vagina by $\mathrm{H} 2 \mathrm{O} 2$-generating lactobacilli. J Infect Dis 1991; 164: 94-100.

27. Mårdh P-A, Soltesz LV. In vitro interactions between lactobacilli and other microorganisms occurring in the vaginal flora. Scand J Infect Dis 1983; 40(Suppl): 47-51.

28. Barefoot SF, Klaenhammer TR. Detection and activity of lactacin-b, a bacteriocin produced by Lactobacillus acidophilus. Appl Environ Microbiol 1983; 45: 1808-15.

29. Chan RCY, Reid G, Irvin RT, et al. Competitive exclusion of uropathogens from human uroepithelial cells by lactobacillus whole cells and cell wall fragments. Infect Immunol 1985; 47: 84-9.

30. Hillier SL, Krohn MA, Rabe LK, et al. The normal vaginal flora, H2O2producing lactobacilli, and bacterial vaginosis in pregnant women. Clin Infect Dis 1993; 16(Suppl 4): S273-81.

31. Peeters M, Piot P. Adhesion of Gardnerella vaginalis to vaginal epithelial cells: variables affecting adhesion and inhibition by metronidazole. Genitourin Med 1985; 61: 391-5.

32. Paavonen J. Physiology and ecology of the vagina. Scand J Infect Dis 1983; 40: 31-5.

33. Amsel R, Totten PA, Spiegel CA, et al. Nonspecific vaginitis. Diagnostic criteria and microbial and epidemiologic associations. Am J Med 1983; 74: 14

34. Chen KCS, Amsel R, Eschenbach DA, Holmes KK. Biochemical diagnosis of vaginitis: determination of diamines in vaginal fluid. J Infect Dis 1982; 145: 337-47.

35. McGregor JA, French JI, Jones W, et al. Bacterial vaginosis is associated with prematurity and vaginal fluid sialidase: results of a controlled trial of topical clindamycin cream. Am J Obstet Gynecol 1994; 170: 1048-60.

36. Glasson JH, Woods WH. Immunoglobulin proteases in bacteria associated with bacterial vaginosis. Aust J Med Lab Sci 1988; 9: 63-5.

37. McGregor JA, French JI, Jones W, et al. Association of cervico/vaginal infections with increased vaginal fluid phospholipase A2 activity. Am J Obstet Gynecol 1992; 167: 1588-94.

38. McGregor JA, Lawellin D, Franco-Buff A, Todd JK. Phospholipase C activity in microorganisms associated with reproductive tract infection. Am J Obstet Gynecol 1991; 164: 682-6.

39. McGregor JA, Lawellin D, Franco-Buff A, et al. Protease production by microorganisms associated with reproductive tract infection. Am J Obstet Gynecol 1986; 154: 109.

40. Platz-Christensen JJ, Brandberg A, Wiqvist N. Increased prostaglandin concentrations in the cervical mucus of pregnant women with bacterial vaginosis. Prostaglandins 1992; 43: 133-41.

41. Platz-Christensen JJ, Mattsby-Baltzer I, Thomsen P, Wiqvist N. Endotoxin and interleukin-1a in the cervical mucus and vaginal fluid of pregnant women with bacterial vaginosis. Am J Obstet Gynecol 1993; 169: 1161-6.

42. Brand JM, Galask RP. Trimethylamine: the substance mainly responsible for the fishy odor often associated with bacterial vaginosis. Obstet Gynecol 1986; 68: 682-5.

43. McGregor JA, French JI, Parker R, et al. Prevention of premature birth by screening and treatment for common genital tract infections: results of a prospective controlled evaluations. Am J Obstet Gynecol 1995; 173: 157-67.

44. Rotstein OD, Pruett TL, Fiegel VD, et al. Succinic acid, a metabolic byproduct of Bacteroides species, inhibits polymorphonuclear leukocyte function. Infect Immunol 1985; 48: 402-8.

45. Singer RE, Buckner BA. Butyrate and propionate: important components of toxic dental plaque extracts. Infect Immunol 1981; 32: 458-63.

46. Krohn MA, Hillier SL, Eschenbach DA. Comparison of methods for diagnosing bacterial vaginosis among pregnant women. J Clin Microbiol 1989; 27: 1266-71.

47. Krohn M, Hillier S, Eschenbach D. Comparison of methods for diagnosing bacterial vaginosis among pregnant women. J Clin Microbiol 1989; 27: 1266-71.
48. Hooton T, Fign S, Johnson C, Stamm W. Association between bacterial vaginosis and acute cystitis in women using diaphragms. Arch Intern Med 1989; 149: 1932-6.

49. Spiegel C, Eschenbach D, Amsel R, Holmes K. Curved anaerobic bacteria in bacterial vaginosis and their response to antimicrobial therapy. J Infect Dis 1983; 148: 817-22.

50. Joesoef MR, Hillier SL, Wiknjosastro G, et al. Intravaginal clindamycin treatment for bacterial vaginosis: effects on preterm delivery and low birth weight. Am J Obstet Gynecol 1995; 173: 1527-31.

51. Meis P, Goldenberg R, Mercer B, Moawad A. The preterm prediction study: Significance of vaginal infections. Am J Obstet Gynecol 1995; 173: 1231-5.

52. Nygren P, Rongwei F, Freeman M, et al. Evidence on the benefits and harms of screening and treating pregnant women who are asymptomatic for bacterial vaginosis: an update review for the U.S. Preventive Services Task Force. Ann Intern Med 2008; 148: 220-33.

53. Platz-Christensen J, Pernevi P, Hagmar B, et al. A longitudinal follow-up of bacterial vaginosis during pregnancy. Acta Obstet Gynecol Scand 1993; 72: 99-102.

54. Mc Donald HM, O’Loughlin JA, Jolley PT, et al. Changes in vaginal flora during pregnancy and association with preterm birth. J Infect Dis 1994; 170: 724-8.

55. Lamont R, Morgan D, Wilden S, Taylor-Robinson D. Prevalence of bacterial vaginosis in women attending one of three general practices for routine cervical cytology. Int J STD Aids 2000; 11: 495-8.

56. Wenman WM, Tatryn IV, Joffres MR, et al. Demographic, clinical and microbiological characteristics of maternity patients: a Canadian clinical cohort study. Can J Infect Dis 2002; 13: 311-18.

57. Hillier SL, Krohn MA, Nugent RP, Gibbs RS, for the Vaginal Infections and Prematurity Study Group. Characteristics of three vaginal flora patterns assessed by Gram stain among pregnant women. Am J Obstet Gynecol 1992; 166: 938-44.

58. Minkoff H, Grunebaum AN, Schwarz RH, et al. Risk factors for prematurity and premature rupture of membranes: a prospective study of the vaginal flora in pregnancy. Am J Obstet Gynecol 1984; 150: 965-72.

59. Platz-Christensen JJ, Pernevi P, Hagmar B, et al. A longitudinal follow-up of bacterial vaginosis during pregnancy. Acta Obstet Gynecol Scand 1993; 72: 99-102.

60. Thorsen P, Jensen IP, Molsted K, et al. An epidemiologic study of bacterial vaginosis in a population of 3600 pregnant women: first antenatal visit. Presented at 11th Annual meeting of the International Society for STD Research, New Orleans, Louisiana: 27-30 August 1995: abstract 204.

61. Tolosa JE, Chaithongwongwatthana S, Daly S, et al. The international Infections in Pregnancy (IIP) study: variations in the prevalence of bacterial vaginosis and distribution of morphotypes in vaginal smears among pregnant women. Am J Obstet Gynecol 2006; 195: 1198-204.

62. Klehanoff MA, Hillier SI, Nugent RP, et al.; National Institute of Child Health and Human Development Maternal-Fetal Medicine Units Network. Is bacterial vaginosis a stronger risk factor for preterm birth when it is diagnosed earlier in gestation? Am J Obstet Gynecol 2005; 192: 470-7.

63. Barbone F, Austin H, Louv WC, Alexander WJ. A follow-up study of methods of contraception, sexual activity, and rates of trichomoniasis, candidiasis and bacterial vaginosis. Am J Obstet Gynecol 1990; 163: 510-14.

64. Hay PE, Morgan DJ, Ison CA, et al. A longitudinal study of bacterial vaginosis during pregnancy. Br J Obstet Gynaecol 1994; 101: 1048-53.

65. Briselden AM, Hillier SL. Evaluation of affirm VP microbial identification test for Gardnerella vaginalis and Trichomonas vaginalis. J Clin Microbiol 1994; 32: 148-52.

66. Hillier SL, Holmes KK. Bacterial vaginosis. In: Holmes KK, Mårdh PA, Sparling PF, et al., eds. Sexually Transmitted Diseases, 2nd edn. New York: McGraw-Hill Information Services Company, 1990: 547-59.

67. Greaves WL, Chungafung J, Morris B, et al. Clindamycin versus metronidazole in the treatment of bacterial vaginosis. Obstet Gynecol 1988; 72: 799-802.

68. Thomason JL, Gelbart SM, Anderson RJ, et al. Statistical evaluation of diagnostic criteria for bacterial vaginosis. Am J Obstet Gynecol 1990; 162: 155-60. 
69. Thomason JL, Schreckenberger PA, Spellacy WN, et al. Clinical and microbiological characterization of patients with non-specific vaginosis having motile, curved anaerobic rods. J Infect Dis 1984; 149: 801-9.

70. Hay PE, Taylor-Robinson D, Lamont RF. Diagnosis of bacterial vaginosis in a gynaecology clinic. Br J Obstet Gynaecol 1992; 99: 63-6.

71. Spiegel CA, Amsel R, Holmes KK. Diagnosis of bacterial vaginosis by direct Gram stain of vaginal fluid. J Clin Microbiol 1983; 18: 170-7.

72. Nugent RP, Krohn MA, Hillier SL. Reliability of diagnosing bacterial vaginosis is improved by a standardized method of Gram stain interpretation. J Clin Microbiol 1991; 29: 297-301.

73. Joesoef MR, Hillier SL, Josodiwondo S, Linnan M. Reproducibility of a scoring system for Gram stain diagnosis of bacterial vaginosis. J Clin Microbiol 1991; 29: 1730-1.

74. Hauth JC, Goldenberg RL, Andrews WW, et al. Reduced incidence of preterm delivery with metronidazole and erythromycin in women with bacterial vaginosis. N Engl J Med 1995; 333: 1732-6.

75. McDonald HM, O’Loughlin JA, Jolley $\mathrm{P}$, et al. Vaginal infection and preterm labour. Br J Obstet Gynaecol 1991; 98: 427-35.

76. Morales WJ, Schorr S, Albritton J. Effects of metronidazole in patients with preterm birth in preceding pregnancy and bacterial vaginosis: a placebo-controlled, double-blind study. Am J Obstet Gynecol 1994; 171: 345-9.

77. McDonald HM, O’Loughlin JA, Vigneswaran R, et al. Bacterial vaginosis in pregnancy and efficacy of short-course oral metronidazole treatment: a randomized controlled trial. Obstet Gynecol 1994; 84: 343-8.

78. Krohn MA, Hillier SL, Nugent RP, et al.; for the Vaginal Infections and Prematurity Study Group. The genital flora of women with intraamniotic infection. J Infect Dis 1995; 171: 1475-80.

79. Gibbs RS. Infection after Cesarean section. Clin Obstet Gynecol 1985; 28: $697-710$.
80. Watts DH, Eschenbach DA, Kenny GE. Early postpartum endometritis: the role of bacteria, genital mycoplasmas, and Chlamydia trachomatis. Obstet Gynecol 1989; 73: 52-9.

81. Centers for Disease Control. 1993 Sexually transmitted diseases treatment guidelines. MMWR 1993; 42.

82. Livengood CH III, McGregor JA, Soper DE, et al. Bacterial vaginosis: efficacy and safety on intravaginal metronidazole treatment. Am J Obstet Gynecol 1994; 170: 759-64.

83. Larsson P-G. Treatment of bacterial vaginosis. Int J STD AIDS 1992; 3: 239-47.

84. Ferris DG, Litaker MS, Woodward L, et al. Treatment of bacterial vaginosis: a comparison of oral metronidazole, metronidazole vaginal gel, and clindamycin vaginal cream. J Fam Pract 1995; 41: 443-9.

85. Hughes VL, Hillier SL. Microbiologic characteristics of Lactobacillus products used for colonization of the vagina. Obstet Gynecol 1990; 75: 244-8.

86. Wood JR, Sweet RL, Catena A, et al. Invitro adherence of Lactobacillus species to vaginal epithelial cells. Am J Obstet Gynecol 1985; 153: 740-3.

87. Vejtorp M, Bollerup AC, Vejtorp L, et al. Bacterial vaginosis: a doubleblind randomized trial of the effect of treatment of the sexual partner. $\mathrm{Br}$ J Obstet Gynaecol 1988; 95: 920-6.

88. Moi H, Erkkola R, Jerve F, et al. Should male consorts of women with bacterial vaginosis be treated? Genitourin Med 1989; 65: 263-8.

89. Simhan HN, Caritis SN, Krohn MA, et al. Decreased cervical proinflammatory cytokines permit subsequent upper genital tract infection during pregnancy. Am J Obstet Gynecol 2003; 189: 560-7.

90. Romero R, Chaiworapongsa T, Kuivaniemi H, Tromp G. Bacterial vaginosis, the inflammatory response and the risk of preterm birth: a role for genetic epidemiology in the prevention of preterm birth. Am J Obstet Gynecol 2004; 190: 1509-19. 


\section{Viral hepatitis in pregnancy Dennis $\mathrm{Ng}$}

This chapter reviews two of the most common hepatitis affecting pregnancy, hepatitis B and C. It is catered toward a mixed audience of practicing obstetricians, obstetrics and gynecology residents, maternal-fetal medicine fellows, and health-care providers for pregnant patients. There has been an explosion of knowledge in disease pathophysiology and treatment options for hepatitis B and C in the last 10 years. Guidelines for treatment and management for hepatitis B and $\mathrm{C}$ are changing every 2 to 3 years. To the uninitiated, this can be a daunting subject.

My intention is to provide you with an easy-to-understand review of the subject, emphasizing on the main concepts and principles in the management of hepatitis $\mathrm{B}$ and $\mathrm{C}$, in the context of pregnancy and delivery. As such, no attempt has been made nor is it possible to be all-inclusive in the minute details of the subject. Instead, I attempt to provide you with an insight into the pertinent questions at hand related to pregnancy and delivery that may arise in your consultation. Particular emphasis will be placed on counseling and public health issues, a subject often forgotten by practitioners, but of utmost importance.

Interested readers may refer to the several excellent textbooks on hepatology, review articles, and guidelines published by American Association for the Study of Liver Diseases (AASLD). This chapter will focus only on hepatitis B and C. Brief mention of hepatitis $\mathrm{E}$ will be made. Other viral hepatitis like herpes simplex virus hepatitis will not be covered.

\section{PRESENTATION OF HEPATITIS B AND C AND GENERAL APPROACH}

The pregnant female may acquire hepatitis $\mathrm{A}, \mathrm{B}$, and $\mathrm{C}$ acutely. If symptomatic, they usually complain of flu-like symptoms, general aches and pains, fatigue, nausea, and vomiting. Physical exam may reveal icterus, jaundice, right upper quadrant tenderness, and change in the color of urine. Labs may reveal high levels of aspartate aminotransferase (AST) and alanine aminotransferase (ALT) in the thousand range. alkaline phosphatase is usually only slightly elevated. Bilirubin may be elevated. These pregnant patients are best admitted to the hospital, particularly if symptomatic with nausea, vomiting, dehydration, where an expedited workup can be performed to rule out other causes of liver diseases unique to pregnancy like acute fatty liver of pregnancy and hemolysis, elevated liver enzymes, and low platelet count (HELLP) syndrome.

More commonly, hepatitis B comes to the attention of the clinician from the adoption of the universal hepatitis $\mathrm{B}$ screening during prenatal visit in the United States. Hepatitis $C$ should be tested in high-risk patients. If found to be positive, they should be referred to the hepatologist/gastroenterologist to determine the status of hepatitis B or C, severity of liver disease, and to help with management of liver disease postdelivery. It is important to screen for these diseases because many times, for a young healthy female patient, the obstetrician may be the only doctor they will see. Once diagnosed, it allows the clinician the golden opportunity to talk about public health issues, mode of transmission of hepatitis, ways to prevent progression of liver disease (e.g., quit drugs and alcohol), referral to a hepatologist for possible treatment options, and cancer screening for the liver (if relevant).

\section{HEPATITIS B IN PREGNANCY Epidemiology}

Worldwide more than 350 million individuals are chronic hepatitis B carriers [1]. The prevalence of hepatitis B varies from region to region. Hepatitis $B$ prevalence can be divided into low-prevalence (e.g., North America, Western Europe), intermediate-prevalence, and high-prevalence areas (e.g., subSaharan Africa, Asia excluding Japan, the Pacific basin, parts of the Middle East, and the Amazon basin).

It is important to note that there is a recent increase in immigrants from highly prevalent countries over the last few decades into the United States. Between 1994 and 2003, the United States admitted about 40,000 individuals with chronic hepatitis B infection annually [2]. Hence, areas of high concentration of these immigrants will have a higher prevalence rate. For example, a recent study in 2009 examined the seroprevalence of HepBsAg among the Asian Chinese in an urban Chinatown internal medicine practice in Chicago [3]. The number was $11.1 \%$, suggesting that we should be even more vigilant about screening high-risk patients, because treatment where indicated has been shown to improve prognosis and decrease morbidity and mortality from hepatitis B in the long run.

Acute hepatitis B occurs in 1 to 2 out of 1000 pregnancies in the United States. Chronic carrier state occurs in 6 to 10 of 1000 pregnancies.

\section{Importance of Hepatitis B on a Global Scheme}

It is generally accepted that at least $50 \%$ of the hepatitis B carriers acquire infection either perinatally or during early childhood. Studies suggest that most of the infections occur around the time of delivery. This gives us the unique opportunity of intervening by providing active and passive hepatitis $B$ vaccination. The goal is to prevent infection in the newborn, hence decreasing the burden of hepatitis B infection worldwide.

When you acquire hepatitis B as an adult, you have a $90 \%$ to 95\% chance of clearing this virus (resolved hepatitis B infection). But when you acquire this as an infant, you have only a $5 \%$ to $10 \%$ chance of clearing this virus, that is, most of 
the infants will be chronic carriers of hepatitis B, with its disease-related morbidity and mortality. Therefore, it makes sense for us to intervene to decrease the rate of perinatal and childhood infection. Taiwan has implemented a mandatory hepatitis B vaccination program 20 years ago, with a significant impact on perinatal, childhood, and adolescent infection. Chronic liver disease and hepatocellular carcinoma attributed to hepatitis B have decreased since implementation [4].

In the United States, we have a mandatory screening for all pregnant females, and if positive for hepatitis B surface antigen, the infants are to get active and passive immunization.

Even in the best of hands, when vaccinations have been carried out appropriately, there is still about $10 \%$ of infants who will get hepatitis B infection, suggesting that perhaps they acquired this virus in utero $[5,6]$.

\section{Effect of Acute Hepatitis B Infection During Pregnancy}

Acute hepatitis B can occur during pregnancy; they present the same way as in the general population. Acute hepatitis B must be differentiated from hepatitis $\mathrm{A}$ and $\mathrm{C}$, and other causes of hepatitis unique to pregnancy (HELLP syndrome, acute fatty liver of pregnancy, etc.). It appears that acute hepatitis B does not increase mortality and has no teratogenic side effects. However, a higher incidence of low birth weight and prematurity has been reported [7].

\section{Effect of Pregnancy on Chronic Hepatitis B}

In general, patients do well during pregnancy. Patients have been known to have a flare of hepatitis B during or after delivery (one theory is that the high levels of adrenocorticosteroids in the body have now decreased significantly postpartum, just like abruptly removing steroids from the patient). Therefore, it is important to monitor patients for symptoms of hepatitis and also to test for liver function tests, during and also after delivery.

\section{Diagnosing Hepatitis B in the Mother}

Over the years, we have developed better tests for hepatitis B, including the ability to measure HBV DNA PCR quantitatively down to the most minute level, for example, $5 \mathrm{IU} / \mathrm{mL}$.

The most common mistake that I see clinicians make is to order an acute hepatitis panel. Usually this consists of HepBsAg, HepBcIgM, HepCAb, and HepAIgM. These are acute hepatitis markers, meaning good for diagnosing acute hepatitis infections, but tell you nothing about the chronic state of hepatitis B, which is what we are interested in, to determine treatment and management for the pregnant patient.

\section{Below Are Some Typical Hepatitis B Serology You Might Encounter in Clinical Practice \\ Case 1}

A 20-year-old Vietnamese pregnant college student, sent to you because tested positive for hepatitis B. Further workup reveals liver function tests (LFTs) normal, HepBsAg positive, $\mathrm{HepBsAb}$ negative, HepBcTotal positive, HepBcIgM negative, HepBeAg positive, HepBeAb negative, and HBV DNA $>2,000,000,000$. The ultrasound (US) of liver unremarkable.

Impression: She is in the immunotolerant phase of hepatitis B.
Case 2

A 30-year-old Malaysian female, pregnant, family history positive for hepatitis B in mother and father. Further workup reveals LFTs elevated, AST 60, ALT 70, HepBsAg positive, HepBsAb negative, HepBcTotal positive, HepBcIgM negative, HepBeAg positive, HepBeAb negative, HBV DNA 200,000. The US of liver with echogenic liver.

Impression: She is in immunoclearance phase of hepatitis B.

\section{Case 3}

A 38-year-old Chinese female, pregnant, family history positive of liver cancer in her dad. Further workup reveals LFTs normal, HepBsAg negative, HepBsAb positive, HepBcTotal positive, HepBcIgM negative, HepBeAg negative, $\mathrm{HepBeAb}$ positive, and HBV DNA undetectable. The US of liver unremarkable.

Impression: She has resolved hepatitis B infection.

\section{Case 4}

A 22-year-old white American female with the following labs: LFTs normal, HepBsAb positive, and every other marker for hepatitis B negative.

Impression: She has been vaccinated in the past and has shown immunity as evidenced by antibody formation.

\section{Vaccinations for Hepatitis B for the Mother and Baby}

The Centers for Disease Control and Prevention (CDC) recommends that all mothers be tested for hepatitis $\mathrm{B}$ during the initial prenatal visit [8]. This applies even if the patient has been vaccinated in the past or tested in the previous pregnancy.

If negative, the pregnant mother who is at risk for hepatitis $B$ infection (unsafe sex, multiple sexual partners, health-care workers, active intravenous drug user, etc.) should be vaccinated. The limited data we have indicate that there is no apparent risk for adverse events to developing fetuses. Current vaccines contain noninfectious HBsAg and should cause no risk to the fetus.

If HepBsAg is found to be negative, and the high-risk patient refuses vaccination, or is unable or unwilling to get vaccinated, then HepBsAg should be rechecked closer to the time of delivery because this will change management if found to be positive.

If HepBsAg positive, the patient is a carrier for hepatitis B. She should be referred to a gastroenterologist/hepatologist for further characterization of her liver disease and the need for treatment. Counseling should be provided regarding the risk of transmission to the newborn and sexual partners, and prevention of HBV transmission to newborns and sexual partners. They should be evaluated for other concomitant liver diseases including hepatitis A and hepatitis C. All sexual contacts and family members should be tested for hepatitis B. If the patient is not immune to hepatitis $A$ and is at high risk of acquiring it, then vaccines for hepatitis A should be given, because of the risk of severe hepatitis with hepatitis A on top of hepatitis B infection [9].

A patient who is not a hepatitis B treatment candidate during pregnancy may be a candidate for treatment at a later point in time. Therefore, it should be emphasized to the patient that she should continue to follow up with the 
gastroenterologist/hepatologist after delivery. Hepatitis B is a chronic disease that requires regular monitoring and liver cancer screening in at risk patients. The clinicians caring for these pregnant patients have the golden opportunity of providing education regarding hepatitis $\mathrm{B}$ and also to ensure that patients continue to follow up with specialists.

The pregnant patient with hepatitis B should be enrolled into a special program that ensures that active and passive vaccinations not only happen, but also happen in a timely manner. This includes making sure that there is ready access to the vaccines when needed, including at 3:00 in the morning or the weekend. We have heard of horror stories in smaller hospitals where the vaccines is available but locked up in the central pharmacy in the weekend. This program should also ensure that the hepatitis B vaccines are completed in a timely fashion postdelivery.

\section{Treatments Available for Hepatitis B in 2010}

The last 10 years have seen tremendous progress in the treatment of hepatitis B. We now have five FDA-approved nucleoside and nucleotide analogs and two interferon-based regimen:

Interferonalpha-2b recombinant (1992), lamivudine (1998), adefovir dipivoxil (2002), entecavir (2005), peginterferon alpha-2a (2005), telbivudine (2006), and tenofovir (2008).

It is beyond the scope of this chapter to discuss selection of hepatitis $\mathrm{B}$ drugs and the rationale behind this. For the interested readers, please refer to AASLD 2009 updated guidelines.

Tenofovir and telbivudine are pregnancy category class B, while the rests are class $\mathrm{C}$.

\section{Prevention of Hepatitis B Transmission to Newborns} Active and Passive Vaccination

CDC recommendation for hepatitis B in infants is stated below [8]:

\section{At Birth}

- Administer monovalent hep B to all newborns before hospital discharge.

- If mother is HBsAg positive, administer HepB and $0.5 \mathrm{~mL}$ of hepatitis B immunoglobulin (HBIG) within 12 hours of birth.

- If mother is HBsAg unknown, administer HepB within 12 hours of birth. Determine mother's HBsAg status as soon as possible and, if HBsAg positive, administer HBIG no later than age 1 week.

\section{After the Birth Dose}

- The HepB series should be completed with either monovalent $\mathrm{HepB}$ or a combination vaccine containing HepB. The second dose should be administered at age 1 or 2 months. Monovalent HepB vaccine should be used for doses administered before age 6 weeks. The final dose should be administered no earlier than age 24 weeks.

- Infants born to HBsAg-positive mothers should be tested for HBsAg and antibody to HBsAg 1 to
2 months after completion of at least three doses of the HepB series, at age 9 through 18 months.

- Administration of four doses of HepB to infants is permissible when a combination vaccine containing HepB is administered after the birth dose. The fourth dose should be administered no earlier than age 24 weeks.

\section{Breast-Feeding in Hepatitis B Patients}

Even though HepBsAg can be detected in breast milk in a large proportion of infected women, several studies fail to demonstrate increased risk of infection due to breast-feeding as opposed to infants who are formula-fed in hepatitis Binfected mother.

Breast-feeding is not considered a contraindication to breast-feeding by the CDC or American Academy of Pediatrics in infants who receive HBIG and active vaccination as indicated.

The nipples should be cared for properly, and mothers should not breast-feed if there are signs of cracked or bleeding nipple.

\section{Mode of Delivery}

Despite the attractive idea of decreasing the risk of perinatal transmission, studies have so far shown that the mode of delivery does not appear to have a significant effect on decreasing the risk of hepatitis B transmission [10]. The CDC or American College of Obstetricians and Gynecologists does not currently recommend caesarian section as the preferred mode of delivery purely for the purpose of reducing perinatal transmission.

\section{Role of Antiviral}

There is emerging evidence that patients who are put on antiviral therapy during the last trimester, to reduce hepatitis $\mathrm{B}$ viral load, could potentially reduce the risk of perinatal transmission. There are many factors to be taken into consideration, including compliance with therapy, severity of liver disease, history of prior treatment, resistance issues, the risk of flare of hepatitis B should the antiviral be stopped postpartum, mothers' preference, and so on. The major concern is that three of the medications we have for hepatitis B are in pregnancy category $\mathrm{C}$ and two in category $\mathrm{B}$. There is no category A medication for hepatitis at this point in time. These decisions are best made by the hepatologist and patient together.

\section{Summary for Hepatitis B}

Hepatitis B when diagnosed in mother prior to delivery affords us the opportunity to make a difference in reducing the risk of mother-to-infant transmission. Besides vaccination of the newborn, other important aspects of care of the young pregnant patients include counseling regarding their liver disease and mode of transmission and ways to reduce the risk of progression of liver disease. Patients who are not candidates for treatment now may become candidates in the future. Hepatitis B is not a disease to be diagnosed at a single point in time and be forgotten about. It requires lifelong follow-up with a clinician who is familiar with this disease. 


\section{HEPATITIS C}

\section{Background}

Hepatitis $\mathrm{C}$ is a major public health problem in the United States. It is estimated that 170 million people are infected worldwide [11]. This is the number one cause for liver transplantation in the United States today. The top three diagnoses in my liver clinic are hepatitis $\mathrm{C}$, nonalcoholic steatohepatitis, and alcoholic liver disease. The sad part is that the majority of the patients who are diagnosed with hepatitis $\mathrm{C}$ are unaware of their liver disease, because hepatitis $C$ mostly do not present with any specific symptoms. In fact, most patients will tell you that they do not feel any different from their normal healthy self. Therefore, it is even more important to screen high-risk patients for active disease.

\section{Why Do We Worry About Testing for Hepatitis C in High- Risk Patients?}

We worry because about $80 \%$ to $90 \%$ of patients who acquire acute hepatitis $\mathrm{C}$ infection fail to clear the virus. After 6 months from the time of exposure, if they still have virus in their system, they are considered to have chronic hepatitis $\mathrm{C}$ with active disease. Over time, this may lead to increase in fibrosis of the liver, progression of liver disease to cirrhosis, and finally, decompensated cirrhosis (ascites, edema, hepatic encephalopathy, etc.), and death. This is in contradistinction to hepatitis B, where if they get infected as an adult, about $90 \%$ to $95 \%$ of patients successfully clear the virus (resolved hepatitis B infection).

Practicing obstetricians and health-care providers during and after pregnancy have a unique opportunity to screen highrisk patients for hepatitis C. Many times, this may be the only time when young ladies touch base with the health-care system. They may not have a primary care provider. This is even more true in patients who are poor, have no insurance, and where drug and alcohol usage is a daily occurrence.

\section{Who Are High-Risk Patients?}

The AASLD 2009 guidelines suggest that the following people are high risk for having hepatitis $\mathrm{C}$ infection and should be screened for hepatitis C [12]:

1. persons who have injected illicit drugs in the recent and remote past, including those who injected only once and do not consider themselves to be drug users,

2. persons with conditions associated with a high prevalence of $\mathrm{HCV}$ infection including

o persons with HIV infection, persons with hemophilia who received clotting factor concentrates prior to 1987, persons who have ever been on hemodialysis, and persons with unexplained abnormal LFTs,

3. prior recipients of transfusions or organ transplants prior to July 1992 including

o persons who were notified that they had received blood from a donor who later tested positive for $\mathrm{HCV}$ infection, persons who received a transfusion of blood or blood products, and persons who received an organ transplant,

4. children born to HCV-infected mothers,
5. health-care, emergency medical, and public safety workers after a needlestick injury or mucosal exposure to $\mathrm{HCV}$-positive blood, and

6. current sexual partners of $\mathrm{HCV}$-infected persons

\section{How Do We Make the Diagnosis?}

Over the last 10 years, we have made a remarkable improvement in the diagnostic sensitivity of essays to detect hepatitis $\mathrm{C}$ RNA virus. We can now detect virus level as low as $5 \mathrm{IU} / \mathrm{mL}$.

For high-risk patients, screening for chronic hepatitis $\mathrm{C}$ should begin with anti-HCV ELISA test (using the latest generation). A positive result suggests exposure to the virus in the past. The next test that should be done is HCV RNA PCR and a genotype. If PCR is positive, this implies that the patient has active chronic hepatitis $\mathrm{C}$ and should be referred to the appropriate specialist for consideration of treatment and education.

\section{What Treatments Are Available Now?}

Hepatitis C treatment has come a long way since it was discovered. The perception in the past was that you cannot cure hepatitis C. Now we have effective treatments, but unfortunately, cure rate is not $100 \%$. On average, the quoted sustained virologic response (SVR; we think of this as cure) rate is about $50 \%$, but this is an oversimplistic view. Many factors affect the SVR, including gender, race, obesity, concurrent liver disease, severity of liver disease, coinfection with HIV, on-treatment viral kinetics, genotype, compliance with therapy, and so on. The latest STAT-C drugs that are projected to come out in 2011 look promising in increasing the SVR.

\section{What Does the Treatment Entail?}

It involves a subcutaneous shot (peginterferon) every week and ribavirin pills twice a day. Treatment duration depends on prior treatment response, genotype of virus, viral kinetics during response, and so on and can range anywhere from 6 months to 12 months to 18 months. This will be determined by the specialist based on prior treatment response, ontreatment response, and other factors. Treatment may also be terminated early because of development of intolerable side effects, or development of life-threatening side effects, or if the patient is deemed a nonresponder to interferon therapy.

\section{Who Are Good Candidates for Treatment?}

The AASLD guidelines suggest that every patient who has chronic hepatitis $\mathrm{C}$ should be considered treatment candidates, unless there is contraindication. This includes patients in prisons and those who are on methadone therapy. We know that treatment can lead to SVR (i.e., the HCV RNA remains undetectable at 6 months after stopping therapy, i.e., cured). This will prevent the progression of liver disease to cirrhosis, translating into a decrease in morbidity and mortality.

Unfortunately, this therapy is neither suitable nor possible for everyone because of the severe and sometimes lifethreatening side effects of interferon and ribavirin therapy. In addition, patients with certain preexisting medical conditions are not suitable candidates for treatment. Cost can be an issue. Also treatment requires the ability and willingness to take the medications faithfully, because SVR will be affected by 
lack of compliance. They need to be able to show up regularly for appointments for monitoring.

\section{Treatment with Regard to Pregnant Patients?}

Hepatitis $\mathrm{C}$ treatment is contraindicated during pregnancy. Pegylated interferon is a pregnancy category $\mathrm{C}$ drug, and ribavirin is a pregnancy category $\mathrm{X}$ drug. It is important that the patient be referred to see a specialist so that they can be followed up postpartum for possible treatment in the future.

In addition, she should be educated on the mode of transmission for hepatitis C and abstinence from alcohol, tested for other sexually transmitted diseases, and advised to get her partners tested [12].

During hepatitis $C$ treatment, patients are not allowed to get pregnant. It is recommended that they use two forms of contraception. I do not treat any patients who are unable or unwilling to comply with this. The package insert for pegylated interferon recommends that patients do not get pregnant until 6 months post therapy. Practically speaking, if patients are very worried about their hepatitis $\mathrm{C}$, and would like to get treated first before getting pregnant, this will usually mean a delay of 1 to 2 years depending on how they respond to therapy.

Another frequently asked question is whether they should get treated first before pregnancy? This will depend on the age of the patient, the severity of liver disease, patient's preference, and urgency of getting pregnant and is best discussed with the specialist.

It is important to note that treatment with interferon can cause or exacerbate preexisting depression. In addition, treatment is contraindicated when she develops postpartum depression.

Please bear in mind that patients who are not treatment candidates now may become suitable candidates later in time and, therefore, the importance of referring to specialists for follow-up.

\section{Transmission of Hepatitis C to Infants}

Hepatitis $\mathrm{C}$ is primarily transmitted by parenteral exposure to blood and blood products from HCV-infected persons. This used to be the most common cause of hepatitis $\mathrm{C}$ infection in infants and children, until we developed ways to screen for hepatitis C in blood. Now, vertical transmission is mainly responsible for infection in newborn infants. It is important to identify these infants because the majority of these patients will remain chronically infected with hepatitis $C$, hence the potential to develop chronic liver disease.

The rate of vertical transmission of hepatitis $\mathrm{C}$ is estimated to be $5 \%$. This number is reported to be higher in the $10 \%$ to $15 \%$ range in HIV-hepatitis C coinfected patients [13].

Numerous studies have suggested that the vertical risk of transmission for hepatitis $\mathrm{C}$ is increased in the following setting:

1. HIV-hepatitis C coinfection.

2. HCV viremia-mothers with positive virus load have increased risk of transmission, compared with mothers with undetectable viral load (suggesting resolved infection). The overwhelming majority of infants with HCV infection have mothers with positive hepatitis $\mathrm{C}$ virus in the blood.
3. Prolonged rupture of membrane for more than 6 hours has been associated with increased risk of perinatal transmission.

4. Obstetric procedures and intrapartum exposure to maternal blood infected by HCV-Steininger et al. have shown that invasive monitoring of fetus with scalp electrode increases the risk of transmission [14].

\section{What Can We Do to Decrease the Risk of Transmission to the Infant?}

Unlike hepatitis B, no effective intervention has been identified so far to decrease the risk of transmission of $\mathrm{HCV}$ to infants. There have been many studies looking to see if C-section will decrease the risk compared with vaginal delivery. Most of the studies suggest that the risk of perinatal transmission is not altered. The Cochrane Collaboration (Issue 2 2009) has published a review on this topic [15]. The authors suggest that there is no good evidence to support using C-section for reducing mother-to-baby transmission of hepatitis $\mathrm{C}$ during labor and birth.

To the mother-to-be who is absolutely horrified by the idea of hepatitis $C$ transmission to their newborn, a case could be made to treat their hepatitis $\mathrm{C}$ first prior to getting pregnant, because we know that the risk of hepatitis $\mathrm{C}$ transmission is much less in a patient who has no detectable virus in the blood versus active hepatitis $C$ infection with high viral load. They must be made to understand that this will delay pregnancy anywhere from 1 to 2 years (see above discussion).

\section{Testing for Hepatitis C in Infants}

The antibodies from the mother have been found to be in the infants even though the infants are not infected; therefore, finding positive $\mathrm{HCV}$ antibodies in the newly born infant means very little. Antibodies have been tested positive in infants as late as 15 months old [16].

The NIH consensus statement in 2002 suggested the following for diagnosis of perinatal infections [17]:

1. infants born to HCV-positive mothers should be tested for HCV infection by HCV RNA tests on two occasions between the ages of 2 and 6 months and/or

2. have tests for anti-HCV after 15 months of age.

\section{Breast-Feeding in Hepatitis C Patients}

Hepatitis $\mathrm{C}$ has been found in low levels in breast milk of HCV RNA-positive mothers. Multiple studies have not shown an increased risk of vertical transmission via breast-feeding.

Large cohorts of mothers infected with HCV suggest that in the absence of damaged, cracked, or bleeding nipples, breastfeeding can be performed safely without increasing the risk of transmission to the infant.

CDC currently (October 2009) suggests that it is safe for mother infected with HCV to breast-feed unless she has a cracked or bleeding nipple.

\section{Summary}

Hepatitis $\mathrm{C}$ is a common disease in high-risk patients. They should be screened for hepatitis $C$, even though there is no 
known intervention to decrease the risk of hepatitis $C$ from mother to infant. Pregnant patients are not candidates for treatment with interferon and ribavirin. They should be referred to specialist to determine whether they may be candidates for hepatitis $\mathrm{C}$ treatment in the future. Education should be provided regarding public health-related issues, including mode of transmission of hepatitis $\mathrm{C}$, avoidance of alcohol, vaccinations for hepatitis A and B if appropriate, and testing of sexual partners. It is fine to breast-feed the infant unless the nipples are cracked or bleeding.

\section{HEPATITIS E}

Clinically apparent acute hepatitis $\mathrm{E}$ is uncommon in the United States. A brief mention will be made here, because of its severity when acquired during pregnancy.

Hepatitis E causes acute hepatitis, just like hepatitis A. It is predominantly transmitted via the fecal-oral route, but less frequent mode of transmission has also been reported (ingestion of raw or undercooked shellfish, infrequently by blood products or blood transfusion).

Hepatitis E usually causes a self-limited acute hepatitis in men and nonpregnant females. However, HEV in pregnant females can result in fulminant hepatic failure, especially in the last trimester, resulting in death in as high as $25 \%$ of patients [18]. This is especially prominent in particular geographic areas in India.

This infection should be in the differential diagnosis of any female patient with a travel history to endemic areas, who presents with acute hepatitis. Vaccines for hepatitis E are not yet commercially available. Treatment remains supportive. It is best to educate pregnant patients who will travel to endemic areas (i.e., Asia, Africa, Middle East, and Central America) to avoid uncooked shellfish, avoid water that is not boiled, and avoid uncooked fruits and vegetables.

\section{ADDENDUM}

Since the writing of this chapter, 2 new drugs (telaprevir and boceprevir) have been approved by the FDA for the treatment of hepatitis $\mathrm{C}$ genotype 1 . They significantly improve the cure rate for hepatitis $\mathrm{C}$ treatment. However, these protease inhibitors must be used together with peg-interferon and ribavirin, which means that they cannot be used for pregnant patients. All the discussion for the treatment of hepatitis $\mathrm{C}$ in pregnant patients still applies. Those interested to read more may refer to the AASLD 2011 Hepatitis C Treatment Guidelines.

\section{ACKNOWLEDGMENTS}

I would like to acknowledge the help of Jessica Wynn, MD, Internal Medicine Resident PGY2, University of Missouri, Columbia, Missouri, in preparing the section on hepatitis $E$ in this chapter.

\section{REFERENCES}

1. Maynard JE. Hepatitis B: global importance and need for control. Vaccine 1990; 8(Suppl): S18-20.

2. Wasley A, Grytdal S, Gallagher K. Surveillance for acute viral hepatitisUnited States, 2006. MMWR Surveill Summ 2008; 57: 1-24.

3. Cotler SJ, Dhamija MK, Luc BJ, et al. The prevalence and clinical correlates of elevated ALT levels in an urban Chinatown community. J Viral Hepat 2010; 17: 148-52.

4. Chan CY, Lee SD, Lo KJ. Legend of hepatitis B vaccination: the Taiwan experience. J Gastroenterol Hepatol 2004; 19: 121-6.

5. Zhu Q, Lu Q, Gu X, Xu H, Duan S. A preliminary study on interruption of HBV transmission in uterus. Chin Med J (Engl) 1997; 110: 145-7.

6. Stevens CE, Toy PT, Tong MJ, et al. Perinatal hepatitis B virus transmission in the United States. Prevention by passive-active immunization. JAMA 1985; 253: 1740-5.

7. Jonas MM. Hepatitis B and pregnancy: an underestimated issue. Liver Int 2009; 29(Suppl 1): 133-9.

8. Mast EE, Margolis HS, Fiore AE, et al. A comprehensive immunization strategy to eliminate transmission of hepatitis B virus infection in the United States: recommendations of the Advisory Committee on Immunization Practices (ACIP) part 1: immunization of infants, children, and adolescents. MMWR Recomm Rep 2005; 54: 1-31. Erratum in: MMWR Morb Mortal Wkly Rep. 2006 Feb 17; 55(6): 158-9. MMWR Morb Mortal Wkly Rep. 2007 Dec 7; 56(48): 1267.

9. Lok AS, McMahon BJ. Chronic hepatitis B: update 2009. Hepatology 2009; 50: 661-2.

10. Yang J, Zeng XM, Men YL, Zhao LS. Elective caesarean section versus vaginal delivery for preventing mother to child transmission of hepatitis $B$ virus-a systematic review. Virol J 2008; 5: 100.

11. World Health Organization, "Hepatitis C. Epidemic and Pandemic Alert and Response," 2002. [Available from: http://www.who.int/csr/disease/ hepatitis/whocdscsrlyo2003/en/index.html.].

12. Ghany MG, Strader DB, Thomas DL, et al. Diagnosis, management, and treatment of hepatitis C: an update. Hepatology 2009; 49: 1335-74.

13. Su GL. itis C in pregnancy. Curr Gastroenterol Rep 2005; 7: 45-9.

14. Steininger C, Kundi M, Jatzko G, et al. Increased risk of mother-to-infant transmission of hepatitis $\mathrm{C}$ virus by intrapartum infantile exposure to maternal blood. J Infect Dis 2003; 187: 345-51.

15. McIntyre PG, Tosh K, McGuire W. Caesarean section versus vaginal delivery for preventing mother to infant hepatitis $\mathrm{C}$ virus transmission. Cochrane Database Syst Rev 2006: CD005546.

16. Indolfi G, Resti M. Fetal transmission of hepatitis $C$ virus infection. J Med Virol Fetal 2009; 81: 836-43.

17. Boyer JL, et al. NIH Consensus Statement on Management of Hepatitis C: 2002. NIH Consens State Sci Statements 2002; 19: 1-46.

18. Khuroo MS, Teli MR, Skidmore S, et al. Incidence and severity of viral hepatitis in pregnancy. Am J Med 1981; 70: 252-5. 


\section{INTRODUCTION}

Although toxoplasma encephalitis (1) has gained worldwide recognition as a major cause of death and morbidity in immunodeficient individuals, especially those with acquired immunodeficiency syndrome (AIDS), the fetus and child remain the major victims of toxoplasmic infection. Approximately 4000 pregnant women per year in the United States are infected with Toxoplasma gondii and $10 \%$ to $60 \%$ of their fetuses will be infected. Although the majority (>90\%) appear asymptomatic at birth, long-term studies suggest that a significant proportion (20-40\%) will demonstrate defects consistent with chronic toxoplasmosis. These defects include chorioretinitis (most common), hydrocephalus, deafness, and mental retardation. Unfortunately, problems in primary prevention, diagnosis, treatment, and follow-up limit the effectiveness of intervention programs.

\section{Biology}

T. gondii is an ubiquitous coccidian whose effects are felt in many animals in addition to humans (Table 1) (2). Toxoplasmosis is a member of the phylum Apicomplexa, similar to malaria and cryptosporia. Much of its life cycle is spent as an obligate intracellular parasite. There appear to be three distinct clonal genotypes with different human virulence; type II accounts for the majority of maternal to fetal infections (85-95\%); $5 \%$ to $15 \%$ are caused by clonal type I.

The organism has three distinct forms: sporozoites within an oocyst, and tachyzoites (also called endozoites or trophozoites) and brachyzoites (or cystozoites) within a tissue cyst. The life cycle of T. gondii is outlined in Figure 1. Members of the cat family, Felidae, are the only known definitive hosts. The sexual reproductive cycle occurs in the villi of the cat ileum. The time from primary inoculation to oocyst appearance in the feces varies by the mode of primary infection: ingestion of tissue cysts (chronic infection in prey animals), 3 to 5 days; ingestion of fecal oocysts, 20 to 24 days; or ingestion of tachyzoites, 7 to 10 days. Oocysts are shed for 1 to 3 weeks with peak production between 5 and 8 days $(4,5)$. Millions of oocysts are shed in a single day. Most cats are infected primarily between 6 and 12 weeks of age (6), corresponding to weaning and the development of hunting skills. Once a cat has been infected, its immune system severely curtails or eliminates future oocyst production. The previously infected cat may be a safer pet than curtailing the behavior of a seronegative cat.

After excretion with feces, the zygote within the oocysts $(13 \times$ $11 \mu \mathrm{m})$ undergoes sporulation. The zygote divides into two sporoblasts that form two sporocysts within the larger oocyst. Each sporoblast undergoes two divisions to produce four sporozoites $(8 \times 3 \mu \mathrm{m})$. Ultimately each oocyst contains eight infectious sporozoites. Sporulation takes 1 to 21 days (average, 3-4 days), depending upon temperature, moisture, and oxygenation. The oocysts can remain infectious for months $(4,5)$.

After elimination and within the 2 to 4 days prior to sporulation, the fecal oocysts are not infectious $(4,5,7)$. As a result, human infection from direct contact with a shedding cat is unusual. The major role of cats in human toxoplasmosis is to pollute transiently the environment with massive numbers of oocysts that may remain infectious for months or years. A small coastal community in California contained 7284 owned cats and 2046 feral cats. Forty-four percent of the owned cats defecated outside $>75 \%$ of the time. The annual fecal deposition of owned cats amounted to 76.4 tons (wet weight); the feral cats contributed about 29.5 tons of feces annually to the environment (8). In one estuarine in Northern California, degradation of the wetlands and runoff from nearby communities may have played a major role in the dieoff of sea otters from a toxoplasmosis epidemic (9). Global warming and environmental degradation may increase the incidence of human infections with toxoplasmosis in northern Europe $(10,11)$. In northern Poland, about $18 \%$ of sand pits or sand heaps in three cities contained toxoplasma DNA, which was identified by PCR (12).

The potential public health implications are concerning. The likelihood of human toxoplasmosis is related to environmental conditions that enhance or reduce oocyst survival, the prevalence of feline toxoplasmosis within that region, and culinary habits of the at-risk population. There have been several studies looking at sources of toxoplasma infection in pregnant women (13-15). All three studies demonstrated the same risk factors for toxoplasmosis infection: consumption of under-cooked meat or game, contact with soil (poor hand hygiene), consumption of poorly cleansed raw vegetables (poor kitchen hygiene), poor cleansing of meat preparation utensils or surfaces (poor kitchen hygiene), and changing the litter box of the pet cat. The odds ratio exceeded 3.0 with all risk factors. Consumption of undercooked meats (50\%), soil exposure (18\%), and work with animals (5\%) accounted for the major of maternal to fetal transmission of toxoplasma.

Higher rates of seropositivity to toxoplasmosis among pregnant women are associated with warm, moist climates: central Africa, 81\%; Lisbon Portugal, 64\%; Riyadh and Saudi Arabia, 30\%; western Scotland, 13\%; and Norway, 10\% (16). In the United States, the age-adjusted seroprevalence rate was northeast, 29\%; south, 23\%; Midwest, 21\%; and West, 18\% (3). These observations are supported by experimental data concerning oocyst survival related to humidity and temperature. Oocysts remain infectious for at least 17 months in water, at least 32 days in $100 \%$ relative humidity (at $22-26^{\circ} \mathrm{C}$ ), and 11 days at $37 \%$ relative humidity and lose their ability to infect at less than $19 \%$ relative humidity and when the moisture content of feces drops to $0 \%$ to $1 \%(17,18)$. Ambient 
temperatures affect both sporulation (infectivity) and survival of the oocysts. Unless combined with low relative humidity $(<20 \%)$, extremes in environmental temperature will not completely eliminate $T$. gondii from contaminated soil.

Survival and infectivity of sporulated oocysts after application of various cleaning agents have been examined experimentally by Frenkel and Dubey (5). High concentrations of ammonia (33\%) and iodine (7\%) killed all oocysts within 30 minutes, whereas ammonia (1.7\%) and iodine (20\%) were

Table 1 Seroprevalences of Toxoplasma gondii Among Various Animals in Developed Countries (2)

$\begin{array}{lr}\text { Animal } & \text { Seroprevalence } \\ \text { Cattle } & 15-50 \% \\ \text { Fattening pigs (intensive farming) } & 1-31 \% \\ \text { Pigs } & 1-21 \% \\ \text { Chickens } & 6-9 \% \\ \text { Sheep } & 16-40 \% \\ \text { Goats } & 19-47 \% \\ \text { Horses } & 1-17 \% \\ \text { Dogs } & 25-40 \% \\ \text { Rabbits } & 6-20 \% \\ \text { Pet cats } & 22-68 \% \\ \text { Feral or stray cats } & 33-68 \% \\ \text { Wild turkeys (United States) } & 17 \% \\ \text { Wild horses } & 16-37 \% \\ \text { Deer } & 30-70 \% \\ \text { Moose } & 15 \% \\ \text { Bears (United States) } & 30-80 \% \\ \text { Human (United States) (3) } & 18-29 \%\end{array}$

no more effective than drying alone. Household ammonia (4-6\%) requires more than 60 minutes of exposure to sterilize cat feces. Smaller particles of fecal matter (empty, uncleaned litter pan) exposed to water heated to boiling and poured into the pan $\left(75-80^{\circ} \mathrm{C}\right)$ are sterilized rapidly.

Despite strong experimental evidence, a direct relationship between oocyst shedding and human toxoplasmosis is not clear from epidemiologic studies. Although 14\% to 50\% of cats in the United States are seropositive, oocysts are found in the feces of less than $1 \%$ of cats examined, perhaps due to the limited age range and short duration (1-3 weeks) of oocyst shedding $(19,20)$. Likewise, the presence of oocysts in the soil correlates poorly with seropositivity in humans $(19,20)$. Additionally, serologic studies of veterinarians and cat owners do not support a strong relationship between a long, intensive exposure to cats and a greater risk of acute toxoplasmosis (21-23). Indeed, persons who report no contact with cats have seropositivity rates of $5 \%$ to $40 \%$ (21-23).

The latter discrepant information raises questions concerning the role of direct contact with cats and acute toxoplasmosis and our present methods of ascertaining the association. The correlation between seropositivity and house cats may be too crude to reveal a relationship. Studies limited to exposure to sick, young, or feral cats may identify a relationship.

On the other hand, oocysts may be so widely distributed in the environment and direct contact with a cat that is shedding oocysts is so rare that the risk of acute toxoplasmosis through oocysts is a function of age and nonspecific contact, such as outdoor gardening, culinary habits, food preparation, presence of insect vectors, and personal hygiene.

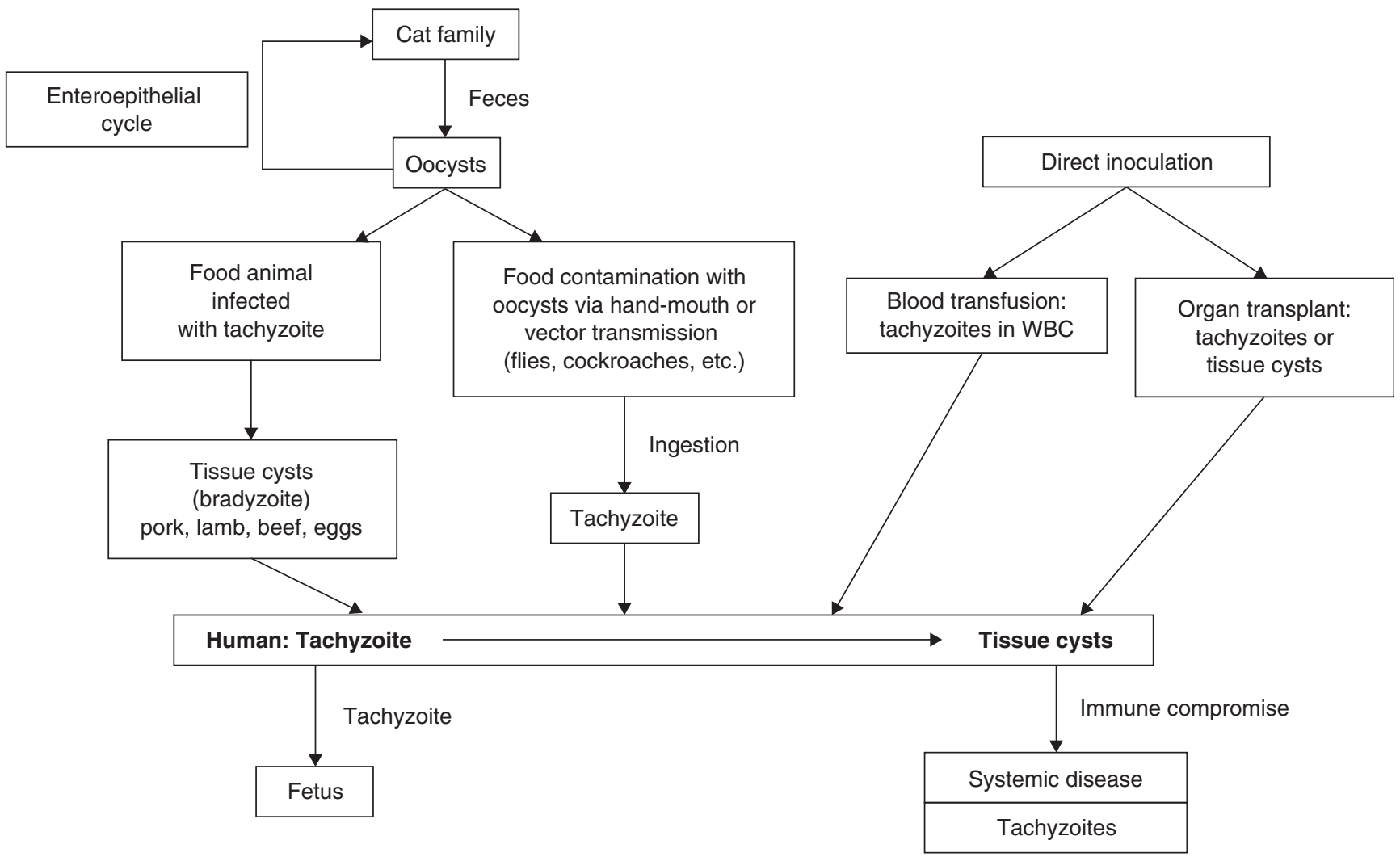

Figure 1 Life cycle of Toxoplasma gondii. 
The tachyzoite form of the parasite is seen in the acute stage of the infection. Tachyzoites result from ingestion of oocysts, that is, food contamination or tissue cysts (brachyzoites/ cystozoites), that is, infected food source. The liberated parasites can survive for 1 to 3 hours in pepsin-hydrochloric acid (stomach fluid) and 4 to 6 hours in trypsin (intestinal fluid) - enough time for transmural migration into the host. The tachyzoites are spread hematogenously and can be identified by Wright's or Giemsa's stains. Every cell type is susceptible to penetration.

The tachyzoite requires an intracellular habitat to survive. The intracellular, intravascular tachyzoite undergoes multiplication, endodyogeny, every 4 to 6 hours to form rosettes. These intracellular collections (tissue cysts) vary in size, but may contain as many as 3000 organisms and are $200 \mu \mathrm{m}$ in size. Rupture of the host cell results in continuing infection and tissue destruction. As the host develops an immune response to the parasite, parasitemia and systemic infection with tachyzoites decrease. In their "protected" intracellular cysts, the tachyzoites evolve into brachyzoites (cystozoites).

The latent or chronic stage is manifested by tissue cysts in most organs. The brain, skeletal muscle, cardiac muscle, and placenta are the most common sites for tissue cysts, which may be identified by periodic acid-Schiff stain. Recurrent parasitemia is rare in immunocompetent individuals. However, under immunodepressed states, especially AIDS, the cysts may reactivate and cause parasitemia and tissue destruction.

The ingestion of tissue cysts in undercooked or uncooked meat is an important source of infection in the United States. Serologic evidence of past infection and potential for tissue cyst inoculation $(2,24,25)$ is present in $15 \%$ of cattle, $15 \%$ of sheep, $30 \%$ of pigs, and $43 \%$ of goats. The reported frequency of tissue cysts in common cuts of meat is less frequent: fresh beef, less than $1 \%$; lamb, $5 \%$; mutton, $10 \%$; and pork, $30 \%$ $(2,24,25)$. However, these studies are based on older studies and may underestimate the risk. The selected samples used were too few and too small to estimate the prevalence accurately. It is also probable that modern farming techniques, intensive pen raising, and processed commercial feed reduce the risk. However, the recent movement back to local sources of meat, "reducing your carbon footprint," may lead to more beef, pork, lamb, or chicken that has been exposed to the barnyard cat and its feces.

There is clear evidence to support the relationship between infected meat ingestion and acute toxoplasmosis. Butchers and meat handlers have a higher seropositivity rate than does the general population, through oral ingestion or inoculation of open wounds. In 1965, Desmonts (26) reported a definitive study supporting the meat-to-human hypothesis. Seroconversion rates rose in response to adding undercooked mutton (as a part of therapy) to the diets of children in a French sanitarium. More recently, acute toxoplasmosis was associated with ingestion of undercooked hamburger (27).

Experimental data suggest that attention to food preparation will reduce transmission of tissue cysts. Cleansing food preparation surfaces and utensils with warm soapy water after meat preparation will limit contamination of other foodstuffs. Salting $(2 \% \mathrm{NaCl} /$ liter for 24 hours), drying (24 hours at $\left.18-20^{\circ} \mathrm{C}\right)$, freezing $\left(-15^{\circ} \mathrm{C}\right.$ for $12-24$ hours), or heating (to $60^{\circ} \mathrm{C}$ for $15-20$ minutes) sterilizes the meat (28).

\section{Clinical Presentations}

\section{Acute Infection}

Toxoplasmosis is asymptomatic or very mild (lymphadenopathy and/or fatigue) in $80 \%$ of immunocompetent individuals. Cervical, suboccipital, supraclavicular, axillary, or inquired lymph nodes are commonly enlarged. An enlarged single posterior cervical node is highly suspicious. In cases associated with a virulent strain of $T$. gondii or in cases associated with immunosuppressive therapy, AIDS or congenital immunodeficiency syndromes or an immature fetus, clinical signs, or symptoms are present in 30\% to $100 \%$ of infections.

The clinical syndrome in presumably immunocompetent individuals is best described by an epidemic related to oocyst exposure in a riding stable (29). Of 37 patients with serologically proven toxoplasmosis, $80 \%$ had fever, headache, and lymphadenopathy (flu-like syndrome). Myalgia, stiff neck, and anorexia were associated with $50 \%$ to $70 \%$ of patients. Additionally, arthralgia, rash, mental confusion, and hepatitis were more common than controls. Interestingly, only $3 / 25$ $(12 \%)$ of patients were correctly diagnosed by their physicians. The combination of lymphadenopathy, fatigue, malaise, atypical lymphocytosis, and/or occasional liver/spleen involvement is similar to mononucleosis or Epstein-Barr infections.

The clinical challenge is to differentiate the nonspecific symptoms of acute toxoplasmosis infection from the symptoms of pregnancy or common viral infections. An interesting study by the U.S. multicentered Toxoplasmosis Study Group (30) performed a detailed interview on 131 mothers of infants with proven congenital toxoplasmosis. Recognizing a serious recall bias, these women reported fever or night sweats $(27 \%)$, flu-like illness or myalgia $(26 \%)$, headache $(11 \%)$, or any "infectious illness" (48\%). Importantly, 52\% reported no maternal illnesses. Similarly, 35\% reported no cat or cat litter exposure and 50\% reported no exposure to undercooked meat or poor meat preparation hygiene. In a combined fashion, only $48 \%$ had exposure to cat litter, undercooked meat, or toxoplasmosis-like illness during pregnancy. These findings suggest that more than $50 \%$ of patients may not be amenable to serologic screening based on risk factors or symptoms.

In immunocompromised patients (AIDS, cancer therapy, transplant patients), reactivation of tissue cysts from prior infection leads to local tissue destruction and, ultimately, systemic parasitemia. As the central nervous system is a common site for tissue cysts, the clinical manifestations include central nervous system abscesses, encephalitis, cognitive impairment, headache, and chorioretinitis. Toxoplasma encephalitis occurs in $30 \%$ to $40 \%$ of AIDS patients (31).

Most pregnant women are immunocompetent, and clinical expression of the infection ("flu" or lymphadenopathy) is mild and occurs in a minority (5-15\%). Serologic evidence of maternal infection varies by maternal age and locale $(15,32)$. The age-related incidence is $20 \%$ at less than 25 years, $23 \%$ at 26 to 35 years, and $35 \%$ at greater than 36 years old. Warm, moist climates have high seropositivity rates $(40-60 \%)$ and cold, dry climates have much lower rates (10-20\%). These observations support the theory that toxoplasmosis is related to the ubiquitous distribution of infectious oocysts and/or food contamination with tissue cysts. 
Between $45 \%$ and $87 \%$ of women will be seronegative and at risk for primary infection during pregnancy. Between $0.5 \%$ and $2.3 \%$ of pregnant women will be described as being at high risk for fetal infection (probable maternal infection) as indicated by maternal seroconversion. Of these, $5 \%$ to $18 \%$ demonstrates fetal infection documented by neonatal signs or symptoms and/or positive neonatal T. gondii-specific IgM titers.

In a study by Zadik et al. (33), the rate of primary infection with toxoplasma was assessed by seroconversion. In that study, 1621 women had paired serum more than 500 days apart. Primary infection was determined by seroconversion alone. In 160 patients $(9.9 \%)$, initial titers were positive. Only one seroconversion was detected in 2966 women years. The projected rate for primary infections was 0.23 (95\% CI $0.0059-1.3)$ per 1000 pregnancies. This rate of maternal infection is much lower than predicted by combined serologic methods (i.e., high titer, rising titer, etc.). On the other hand, the neonatal infection rate may be higher as it is a primary infection and true infection is better defined.

While standard in vitro tests have shown little, if any, difference in humoral or cellular immunity between pregnant and nonpregnant women, later infection by certain organisms (i.e., varicella, human papilloma virus) may be reactivated during pregnancy. The combination of latent human immunodeficiency virus (or organ transplantation with immunosuppression), latent toxoplasmosis, and pregnancy often leads to maternal and fetal concerns of reactivation of the toxoplasmosis. In AIDS patients, reactivation usually occurs with $\mathrm{CD}_{4}$ counts $<100$ cells $/ \mathrm{mm}^{3}$. It appears that pregnant women have no greater risk than that defined by low $\mathrm{CD}_{4}$ counts. Among 48 pregnant women receiving immunosuppressive therapy after organ transplantation, no cerebral toxoplasmosis was recorded. Among 105 HIV-positive women, only one case of cerebral toxoplasmosis occurred with a $\mathrm{CD}_{4}$ count of 20 cells $/ \mathrm{mm}^{2}$ (30). Prophylactic treatment to prevent maternal reactivation and vertical transmission is not indicated for $\mathrm{CD}_{4}$ counts $>100$ cells $/ \mathrm{mm}^{3}$. At $\mathrm{CD}_{4}$ counts under 100 cells $/ \mathrm{mm}^{3}$, the risk is still small, but prophylaxis may be justified, although benefit is not proven. These observations raise a serious question of whether reactivation of latent toxoplasmosis actually occurs in immunocompetent pregnancies. Seroconversion, rather than high or rising titers, may be the major serologic marker for fetal infection.

Experimental infection of rhesus monkeys showed that transmission of T. gondii occurred in four of nine (44\%) in second-trimester maternal infections and three of nine $(33 \%)$ in the third trimester (34). Another four fetuses of mothers infected in the third trimester were probably infected, but the organism could not be isolated. The overall infection rate was $61 \%$. Likewise, pregnant cats were orally inoculated with T. gondii (17). Among 8 animals euthanized at 10 or 31 days post-inoculation, 7 of 33 (21\%) fetuses from all litters had T. gondii isolated. Eight additional pregnant cats were allowed to reach parturition and nurse their kittens. Of 40 kittens from all eight litters, 26 (65\%) had T. gondii isolated from tissues; 25 of 26 kittens were severely ill by 24 days of life. It is apparent that in susceptible species (cats, rhesus monkeys, and man), the rate of intrauterine fetal infection is $40 \%$ to $70 \%$.
Fetal infection is a combination of acute and persistent infection due to relative immune incompetence and reduced passive IgG transfer from the mother. Prior to 30 weeks of gestation, the fetal immune system is of little help; IgM, IgG, and cellular responses are limited and functionally immature (35). For example, in proven cases of in utero infection with toxoplasmosis in the first trimester, funicentesis at 20 to 24 weeks demonstrated four out of nine with toxoplasmaspecific IgM (36). Placental transfer of maternal IgG is limited by gestational age and target antigen. Immunoglobulin $G$ transport has been noted by 8 weeks, but remains less than $100 \mathrm{mg} / \mathrm{dL}$ until 20 weeks. Subsequently, the fetal levels of maternal IgG rise until they are greater than maternal levels.

Acute fetal toxoplasmosis results from initial placental involvement and subsequent systemic fetal parasitemia and infection. The most consistent histologic placental finding is chronic inflammation in the decidua capsularis and focal reaction in the villi. Tissue cysts as well as tachyzoites may be seen. Systemic involvement results in splenomegaly, hepatomegaly, jaundice, anemia, thrombocytopenia, chorioretinitis, and encephalitis. Persistent infection due to limited immune response leads to extramedullary hematopoiesis in the liver and decreased plasma protein production. When coupled with cardiac involvement, nonimmune hydrops may result. Widespread tissue destruction manifests as clinically apparent growth restriction. The most characteristic damage in the central nervous system is extensive necrosis of brain parenchyma secondary to vasculitis; microcephaly is a common sequela. Periventricular and periaqueductal vasculitis is common in toxoplasmosis. Subsequent calcification of these areas is apparent on roentgenographic or ultrasonographic examinations. Obstruction of the aqueduct and/or meningeal involvement leads to hydrocephalus. Chorioretinitis may be present at birth or may develop in the first two decades after birth through recrudescence or persistence of the disease.

Table 2 describes the incidence of organ system involvement in 210 of congenitally infected neonates who were followed prospectively for 1 year (37). Twenty-one (10\%) of the infants (including one death) had severe congenital toxoplasmosis

$\begin{array}{lr}\text { Table } 2 \text { Neonatal Toxoplasmosis }(n=210) & \\ \text { Prematurity }(\leq 2500 \mathrm{~g}) & 4 \% \\ \text { Intrauterine growth restriction } & 6 \% \\ \text { Jaundice } & 10 \% \\ \text { Hepatosplenomegaly } & 4 \% \\ \text { Thrombocytopenia } & 2 \% \\ \text { Anemia } & 4 \% \\ \text { Eosinophilia } & 2 \% \\ \text { Microcephaly } & 5 \% \\ \text { Hydrocephalus } & 4 \% \\ \text { Hypotonia } & 6 \% \\ \text { Seizures } & 4 \% \\ \text { Psychomotor retardation } & 5 \% \\ \text { Intracranial calcifications } & 11 \% \\ \text { Abnormal cerebrospinal fluid } & 35 \% \\ \text { Micro-ophthalmia } & 3 \% \\ \text { Bilateral chorioretinitis } & 6 \% \\ \text { Unilateral chorioretinitis } & 16 \% \\ \text { Normal } & 55 \% \\ \text { Source: From Ref. } 37 . & \end{array}$


with central nervous system involvement. Seventy-one (34\%) had a normal clinical exam except for peripheral retinal scarring or isolated asymptomatic intracranial calcifications. One hundred and sixteen (55\%) were normal. Caution should be exercised when evaluating the incidence of injury. Most series are populations of referred neonates and represent a population with more clinically apparent diseases. Unless all pregnant women and neonates are screened and all infected neonates are systematically examined, the risk is lower than that published. In addition, women countries, which use universal screening to identify maternal seroconversion and use amniocentesis and PCR to identify fetal infection, are more likely to initiate antibiotic therapy after a significant percentage of women (about 40\%) chose to abort (38). Antibiotic therapy may modify (lower) the incidence of severe disease at birth. In the latter study, 58 of $76(77 \%)$ had asymptomatic infection after $84 \%$ received antibiotics prior to amniocentesis.

The timing of acute fetal toxoplasmosis greatly affects the sequelae (39) (Table 3). When it occurs early in gestation, especially prior to 13 weeks, the infection is more widespread and destructive. At 11 to 13 weeks, the fetal immune system begins to identify antigens as "self" or "non-self." Toxoplasma antigens that are incorporated into fetal tissue may be misrecognized as "self" and harder to eliminate. In addition, the continuing immaturity of the fetal immune system and limited active transfer of toxoplasma-specific maternal IgG prior to 25 weeks poorly control the infection. Additionally, immune "protected" location, the central nervous system, and inner eye allow the organisms to avoid host responses.

\section{Chronic Infection}

Early reports of follow-up indicated that children with clinically apparent disease at birth had a poor prognosis: mental retardation, $93 \%$; seizures, $81 \%$; spasticity or palsies, $70 \%$; impaired vision, $61 \%$; hydrocephalus or microcephaly, $32 \%$; deafness, $15 \%$; and normal, 11\% (40). Fortunately, many infants with congenital toxoplasmosis are completely normal at birth (40-55\%). The prognosis in mildly affected or asymptomatic infection appears to be much better (Table 2).

A continued risk of damage is apparent in 20-year follow-up of congenitally infected children and in epidemiologic studies. Koppe et al. (41) followed 11 children for 20 years. One of five asymptomatic and treated infants and four of six (two with severe disabilities) symptomatic infants developed new chorioretinitis by 20 years. Wilson et al. (42) followed 23 children with subclinical infections at birth for 1.25 to 17.25 years. Of 23 children, $19(83 \%)$ had one or more episodes of chorioretinitis, $11(48 \%)$ had unilateral or bilateral blindness, and $12(53 \%)$ had a new diagnosis of central nervous system disorders. Sever et al. (32) performed a multivariate analysis of 22,845 patients in whom toxoplasmosis titers were correlated with poor outcomes during a standardized exam at 7 years of age. The results were controlled for race, maternal age, and socioeconomic index. Visual impairment, microcephaly, coordination problems, right and left identification, and soft neurologic signs correlated with toxoplasmosis titer. Bilateral deafness, extraocular movement, and IQ $<70$ correlated with the interaction variable, toxoplasmosis titer $\times$ maternal age.

A 20-year prospective study (1985-2005) of 676 pregnancies and 681 etuses gives more reassuring outcomes than prior data (43). Mothers who were IgG seronegative were followed with monthly serology. Antenatal therapy with spiramycin $9 \times 10^{6}$ SI units per day was initiated at recognition of maternal IgG seroconversion until an amniocentesis (4 weeks later) was performed to identify fetal infections by PCR. After definitive diagnosis of fetal infection, a regimen of sulfadoxine (1000 mg/wk), pyrimethamine $950 \mathrm{mg} /$ week, and folinic acid $50 \mathrm{mg} /$ week was initiated until delivery. The same regimen was used in pediatric doses until 2 years of age. They reported that 17\% (112 of 666) live borns had congenital toxoplasmosis after maternal seroconversion during pregnancy (monthly IgG serology). Of these, $74 \%$ were asymptomatic, $1.5 \%$ had severe manifestations at birth, and 26\% were diagnosed with chorioretinitis; 6 of 28 infants with chorioretinitis had macular disease (unilateral). Two children had additional neurologic issues, seizures, and psychomotor delay. The partial improvement in outcomes from earlier data suggests a benefit to early recognition for fetal infection and fetal therapy.

\section{Diagnosis \\ Behavioral Screening}

Ideally, all women who are at risk for pregnancy or are trying to get pregnant need assessment and education concerning the risk of toxoplasmosis during pregnancy, that is, preconceptual counseling. Each woman should be asked about her ongoing exposures to potentially contaminated soil or water, cat exposures, kitchen and personal hygiene, and predilection to eating and undercooked meats or game. In high-risk women (any positive response), a preconceptual measurement of toxoplasma-specific IgG will identify potential risk of fetal infection during a subsequent pregnancy. If the toxoplasmosis-specific IgG is positive, then the risk of fetal toxoplasmosis is very small in an immunocompetent individual. If patient is seronegative, a program of primary prevention education is critical (Table 5). During pregnancy, monthly serology will be

Table 3 Time of Maternal Infection and the Frequency of Fetal Infection

\begin{tabular}{lccr} 
& & Time of maternal infection & Third trimester $\boldsymbol{n = 1 2 8}$ \\
\cline { 2 - 4 } No infection & First trimester $\boldsymbol{n = 1 2 6}$ & Second trimester $\boldsymbol{n = 2 4 6}$ & $41 \%$ \\
Perinatal death & $86 \%$ & $71 \%$ & $0 \%$ \\
Fetal infection & $5 \%$ & $2 \%$ & $59 \%$ \\
Severe & $9 \%$ & $27 \%$ & $0 \%$ \\
Mild & $6 \%$ & $2 \%$ & $6 \%$ \\
Subclinical & $1 \%$ & $20 \%$ & $53 \%$ \\
Source: Adapted from Ref. 39 (Table 31.5). & $2 \%$ & &
\end{tabular}


helpful in the early identification of fetal infection and initiation of antibiotics.

\section{Serologic Screening}

Maternal antibody response is normal to a toxoplasmosis infection; IgM appears first and rises sharply for 1 to 2 weeks and is sustained for 4 to 6 months (IgM-IFA) or 6 to 12 months (or years) when measured by more sensitive methods [double-sandwiched IgM enzyme-linked immunosorbent assay (IgM-ELISA)]. After the start of the increase in IgM, IgG rises slowly for 1 to 3 weeks $(2-50 \mathrm{IU} / \mathrm{mL}$, 1:10-1:200). Between 3 and 6 weeks, IgG antibodies rise sharply to $>400 \mathrm{IU} / \mathrm{mL}$ or titers $>1: 1000$. In $2 \%$ to $4 \%$, the titers are $>1: 4000$ [indirect hemagglutination test (IHA)] and persist at high levels for years. Again, IgG tests vary in their sensitivity.

The majority of infected pregnant women are asymptomatic, and a combination of testing for immunoglobulin G ( $\operatorname{IgG}), \mathrm{M}$ (IgM), and IgA antibodies is used to diagnose toxoplasmosis. These tests include the Sabin-Feldman dye test (dye test), complement fixation, IHA, indirect fluorescence antibody test (IFA), agglutination test, double-sandwiched IgM-ELISA, and the IgM immunosorbent agglutination assay (IgM-ISAGA).

Although the Sabin-Feldman (dye test) is the time-honored gold standard, its technical complexity (mouse inoculation and observation of a biologic response) limits application outside of research settings. In the IHA test, red blood cells tagged with toxoplasma antigen agglutinate when exposed to serum containing IgG and IgM antibodies to toxoplasma. This response occurs several months after an infection. As a result, as many as $50 \%$ of infants with fetal infection will have negative IHA in the first 6 months of life. Complement fixing antibodies appear later than dye test antibodies and, depending on the antigen employed, the results vary. The agglutination test uses parasites fixed in formalin and is very sensitive to IgM antibodies. Nonspecific agglutination may occur due to "naturally" occurring IgM (in mothers negative for toxoplasma-specific IgG and IgM antibodies). Unless the laboratory corrects for these antibodies, false positives can occur. The IFA test uses slide preparations of fixed toxoplasma that are washed with the patient's serum at different dilutions and crossreacted with fluorescein-tagged antiserum prepared against IgG (anti-IgG). Although the IFA test is equal to the dye test in specificity, patients with connective tissue disorders, that is, positive rheumatoid factor, may test false positive. Positive tests should be confirmed with a dye or IHA test.

The IgM titer is measured in three ways: IgM-indirect fluorescent antibody (IgM-IFA), IgM-ELISA, and IgM-ISAGA. IgM-IFA relies on antiserum specific to IgM. A titer $>1: 160$ is diagnostic of an acute infection. If the antiserum cross-reacts with IgG, which is true of some testing kits, the results may be false positive. Additional problems include an inhibitory effect of high maternal IgG titers on fetal IgM titers and false positives created by autoimmune antibodies, that is, rheumatoid factor.

The conventional IgM-ELISA may also be false positive in patients with chronic infection or autoimmune disease. As a result, Naot et al. (44) developed a double-sandwiched IgMELISA. In a direct comparison with IgM-IFA in 55 serum samples from neonates with proven toxoplasmosis, the double-sandwiched IgM-ELISA was more likely to be positive ( $81 \%$ vs. $25 \%$ ) than was IgM-IFA.

The IgM-ISAGA combines the advantages of direct agglutination and double-sandwiched ELISA. The IgM-ISAGA does not use an enzyme conjugate and avoids cross-reactivity with rheumatic factor. It is more sensitive than the IgM-IFA or the IgM-ELISA and will be positive 1 to 2 weeks earlier than the latter tests.

Continual improvement in the specificity and sensitivity of toxoplasma-specific IgM testing has muted its usefulness. The more sensitive the test, the less likely it is to narrow the time of the maternal infection if preconceptual Toxoplasmosis IgG testing has not been performed. In the latter situation, the timing is critical. The earlier the infection, especially prior to 14 weeks, the less likely fetal infection has occurred, but it is more likely that severe disease will be manifest at birth. Diagnosis of fetal infection prior to 20 weeks allows the mother a more educated choice whether to carry or abort the pregnancy. In addition, earlier therapy may improve the efficacy of antibiotic therapy.

Commercial testing, the avenue for most clinicians in the United States, can create confusing and difficult decisions (45). The laboratory testing companies are most interested in producing a reliable result with the largest profit margin. Unless the laboratory pre-absorbs the naturally occurring antiIgG IgM (rheumatoid factor), false positive, low levels of IgM are identified and reported. This occurs more often when the IgG is very high. Overreading the results may create enough concern by the mother to terminate her pregnancy when no fetal infection is present; one study suggests this occurs in $17.5 \%$ of women misinformed about the risk of infection (46). Retesting the same serum using different IgM methodologies and identifying other elevated immunoglobulins (IgA, IgE), an abnormal differential agglutination test, or low IgG avidity can identify true positives and reduce the risk of abortion to $0.4 \%$ (47). Most commercial laboratories do not save the serum and are reluctant or are inexperienced in the performance of confirmatory testing. The American College of Obstetricians and Gynecologists and the Centers for Disease Control and Prevention recommend contacting a reference laboratory prior to an intervention as a result of the primary serologic screening test. Other useful contact information includes reference laboratory for serology, isolation, and PCR assay (650-853-4828), Congenital Toxoplasmosis Study Group (U.S.), and Educational information on the Internet www.toxoplasmosis.org.

Seroconversion of IgG in a prior seronegative provides unequivocal evidence of infection occurring in the period of time between the two tests. However, most women become pregnant without knowledge of their antibody status and diagnosis must rely on a skilled and experienced interpretation of positive IgG serology, a positive IgM serology, rising antibody titers, or, occasionally, persistent, high titers. Unfortunately, the numerous tests used to measure antibody response have considerable inter- and intra-test variability.

\section{Fetal Diagnosis}

The advent of sophisticated ultrasound diagnosis and invasive fetal testing (funicentesis/cordocentesis) has heralded new options in the diagnosis and management of toxoplasmosis. Although serologic testing may identify $0.5 \%$ to $2.5 \%$ of 
pregnant women at high risk of fetal toxoplasmosis, fetal infection is not an absolute diagnosis without measurement of fetal immunologic response or demonstration of the organism. By using advanced ultrasound diagnosis and invasive fetal testing, $5 \%$ to $20 \%$ of maternal infections in the first 20 weeks of pregnancy and $40 \%$ to $80 \%$ in the second 20 weeks of pregnancy result in fetal infection. The rapid identification of the fetal infections is the best management.

High-resolution obstetric ultrasound has a role in screening and diagnosis of fetal infection. The fetal findings include ventriculomegaly, microcephaly, intracranial calcifications, increased placental thickness, parenchymal liver calcifications, fetal hydrops, ascites, hyperechoic bowel, and fetal growth restriction. The presence of any one or a combination of these findings in the presence of positive serology has a sensitivity of $49 \%$, specificity of $99 \%$, positive predictive value of $98 \%$, and a negative predictive value of $89 \%$ (48). The unanticipated identification of these findings should initiate toxoplasma-specific IgG and IgM serology testing. If positive serology is positive, an amniocentesis for AF-PCR testing is warranted.

Access to fetal tissues (amniotic fluid, amniocytes, chorionic villi, or fetal blood) allows unequivocal fetal diagnosis. In utero fetal infection may be diagnosed through demonstration of toxoplasma antigens (parasites or DNA), fetal immune response (IgM), or physical or laboratory evidence of fetal infection. Inoculation of mice or tissue cultures with fetal specimens is $50 \%$ to $70 \%$ sensitive in documenting fetal infection. Polymerase chain reaction identification of toxoplasma DNA significantly reduces the time and laboratory requirements for antigen detection. However, adequate laboratory support is essential, but currently there is limited access and availability.

The demonstration of a fetal IgM through cordocentesis is limited by gestational age. Prior to 24 weeks, the upper age limit for abortion in the United States, less than half of fetuses, will mount an IgM response. Abnormal laboratory findings on fetal blood include elevated white cell count, eosinophil count, monocytes, gamma-glutamyl transpeptidase, and lactic dehydrogenase activity (Table 4) $(36,49)$.

Isolation of the parasite from the mother or infant provides unequivocal proof of infection. The parasite (bradyzoite or tachyzoite) can be isolated from blood, muscle, brain, amniotic fluid, or placental tissue through inoculation of mice or tissue culture. The frequency of isolation from the placenta varies by the trimester in which toxoplasmosis was acquired and untreated: first trimester, $25 \%$; second trimester, $54 \%$; third trimester, 65\%; and overall, 50\% (50). However, the placenta may be negative in the presence of a congenitally infected fetus. Parasitemia in infected newborns varies by clinical stage: acute/generalized, $71 \%$; neurologic or ocular, $17 \%$; and subclinical, $52 \%$ (51). When the diagnosis is attempted by funicentesis in the second trimester, the fetal blood is positive in $70 \%$ of cases (52). Overall (term and preterm), the amniotic fluid sediment will contain toxoplasma in $60 \%$ of samples from fetuses with congenital infection. Although isolation of the organism is a powerful investigational tool, it requires time and specialized laboratories not readily available to the practicing physician.

Molecular genetics has provided a powerful new tool in antigen identification, the polymerase chain reaction (PCR) (52,53). In 43 cases, PCR was compared with mouse inoculation (tissue/amniotic fluid cultures) and IgM-ISAGA (IgM immunosorbent agglutination) on fetal blood. PCR correctly diagnosed toxoplasmosis as positive in 8 out of 10 samples with no false-positive diagnosis. More recently, Hohlfeld et al. (52) compared a competitive PCR test of amniotic fluid with conventional diagnostic methods (ultrasonography, fetal blood sampling, and serologic diagnosis) in 339 women who had acquired toxoplasmosis during pregnancy (combined serologic diagnosis). Conventional methods identified 34 of 37 (sensitivity 89.7\%) neonates with serologically or pathologically confirmed toxoplasmosis at birth. The PCR test identified 36 of 37 infants (sensitivity 97.4\%). In a more recent analysis, real-time PCR was 92\% sensitive and had a $98 \%$ negative predictive value in a study 377 mothers with seroconversion during pregnancy (54). The PCR test on amniotic fluid is now the standard method for the diagnosis of fetal toxoplasmosis.

\section{Universal Screening}

The frequency of maternal $(0.8 \%)$ and fetal $(0.32 \%)$ infection, the frequency of long-term disability after fetal infection (50-90\%), and the cost of caring for those disabilities $(\$ 80,000$ $\$ 150,000$ per case) describe the potential benefits of a universal screening program. This potential benefit compares favorably

Table 4 Antenatal Diagnosis of Fetal Toxoplasma

$\begin{array}{lccc} & \text { Daffos et al. (36) } & \text { Foulon et al. (49) } & \text { Total } \\ \text { Number of high-risk mothers } & 746 & 50 & 796 \\ \text { Fetal infection } & 42 & 6 & 48(6 \%) \\ \text { Diagnosed in utero } & 39 / 42 & 5 / 6 & 44 / 48(92 \%) \\ \text { Positive specific IgM } & 9 / 42 & - & \\ \text { Elevated total IgM } & 22 / 37 & - & \\ \text { Elevated WBC } & 16 / 39 & 2 / 3 & \\ \text { Eosinophilia } & 8 / 33 & 3 / 3 & \\ \text { Elevated LDH } & 7 / 35 & 3 / 3 & 10 / 36(28 \%) \\ \text { Elevated GGT } & 24 / 34 & 0 / 3 & 10 / 38(26 \%) \\ \text { Abnormal ultrasound: ascites } & 2 / 42 & 1 / 3 & 27 / 36(75 \%) \\ \text { Ventricular dilation } & 17 / 42 & 1 / 3 & 2 / 45(4 \%) \\ \text { Intracranial calcification } & - & & 18 / 45(40 \%) \\ \text { Abbreviations: LDH, lactic dehydrogenase; GGT, gamma-glutamyl transpeptidase. } & \end{array}$


with other universal screening programs: syphilis, gonorrhea, phenylketonuria, or maternal serum alpha-fetoprotein. Commercial laboratories would easily and happily accommodate the demand for serologic testing in a universal screening program. Finally, respected researchers endorse the concept of universal screening (55).

On the other hand, the cost, unreliability in testing, and the inadequacy and cost of resources to interpret, diagnose, and treat fetal infection are major obstacles to implementation. The immunofluorescent antibody test, which is used in $80 \%$ of commercial laboratories (56), costs $\$ 80$ to $\$ 120$. Each patient would require two to three tests: two tests to document seroconversion and three tests (in $40 \%$ of patients) to document a rising titer (paired samples or IgM). Universal serologic screening would cost an estimated $\$ 320$ to $\$ 720$ million.

A cost benefit analysis based on data from a Finnish prospective study (57) and on Finnish cost data was performed to compare the no-screening and screening alternative for primary toxoplasma infection during pregnancy (58). Given a $20.3 \%$ seropositivity and an incidence of fetal infection to be $2.4 / 1000$ seronegative pregnancies, the total annual costs of congenital toxoplasmosis without screening were $\$ 128$ per pregnancy per year (U.S. dollars). With screening, the cost would be $\$ 95$. The authors concluded that universal screening, plus health education, is cost effective if the incidence of maternal primary infection exceeds $1.1 / 1000$ pregnancies and the effectiveness of treatment is greater than $22 \%$.

The diagnosis of fetal infection in high-risk mothers is necessary unless the practitioner and patient are willing to abort or treat 9 to 15 uninfected fetuses for each infected fetus. Amniocentesis and/or funicentesis would be needed in $296,000(0.8 \% \times 3.7$ million $)$ women per year, with each ultrasound-guided procedure costing a minimum of $\$ 1500$. The necessary expertise, laboratory support, and financial resources are not available for this demand.

In summary, while selected preconceptual screening for seronegativity and antenatal screening for seroconversion in behaviorally high-risk individuals may be warranted, firsttrimester universal screening for toxoplasmosis is not warranted at this time in the United States. However, serologic screening should be considered when the patient has a "flu"like syndrome, and/or has lymphadenopathy, or when the fetus, on ultrasonographic examination, has growth restriction, nonimmune hydrops, ascites, hydrocephalus, microcephaly, or intracranial calcification.

\section{Antibiotic Therapy}

Pyrimethamine, a substituted phenylpyrimidine antimalarial drug, cures toxoplasmosis in laboratory animals through folic acid antagonism. Pyrimethamine produces a reversible, doserelated bone marrow depression. Thrombocytopenia, with its associated bleeding sequelae, is the most common toxic affect, but anemia and leukopenia may occur as well. A semi-weekly complete blood cell and platelet count should be obtained while the patient is being treated.

Sulfadiazine (or sulfapyrazine, sulfamethazine, or sulfamerazine) acts synergistically with pyrimethamine with a combined activity against toxoplasmosis much greater than a merely additive affect. Like pyrimethamine, sulfa drugs disrupt folic acid metabolism in bacterial species. Renal toxicity (crystalluria and hematuria), toxic dermal reaction (StevensJohnson syndrome), and blood dyscrasias are reported with sulfa drug therapies. However, these agents are well tolerated in most individuals. In the neonate, sulfa drugs displace bilirubin from albumin, and kernicterus may occur. The risk of neonatal kernicterus from maternal intake of sulfa drugs is quite small.

The evaluation of antibiotic efficacy depends on the time between the onset of infection and initiation of therapy. In human studies, the timing of fetal toxoplasmosis is very difficult to determine because the infection is usually asymptomatic. Experimental inoculation of infra-human primates, rhesus monkeys, and treatment with anti-toxoplasmosis antibiotics provides powerful insight into the pharmacokinetics of pregnancy and the efficacy of the antibiotics.

Schoondermark-Van de Ven et al. (59) established the dosage regimen for pyrimethamine and sulfadiazine in pregnant rhesus monkeys. The distributions of both drugs followed a one-compartment model. The maximum concentrations in serum were $88.7 \mu \mathrm{g} / \mathrm{nL}, 0.22 \mu \mathrm{g} / \mathrm{nL}$ for sulfadiazine and pyrimethamine, respectively. The serum elimination halflives were much greater for pyrimethamine ( 44.4 hours) than sulfadiazine (5.2 hours).

Subsequent to the latter experiment, Back et al. used the dosage regimen to treat six rhesus monkeys that were infected with T. gondii at 90 days of gestation (second trimester) (60). Four similarly infected pregnant monkeys were used as controls. Infection was documented by isolation of T. gondii from amniotic fluid samples. The treated animals received pyrimethazine $1 \mathrm{mg} / \mathrm{kg}$ body weight and sulfadiazine $50 \mathrm{mg} / \mathrm{kg} /$ day for the remainder of the pregnancy. T. gondii was present at birth in three of four untreated neonates. In all animals treated with pyrimethazine and sulfadiazine, no parasite was identified in the amniotic fluid 10 to 13 days after initiation of therapy. No neonate demonstrated T. gondii at birth.

Spiramycin, a macrolide antibiotic, has an antibacterial spectrum similar to erythromycin and is active against toxoplasmosis in laboratory animals. With spiramycin $2 \mathrm{~g} /$ day, the concentrations were maternal serum, $1.19 \mu \mathrm{g} / \mathrm{nL}$; umbilical cord, $0.63 \mu \mathrm{g} / \mathrm{nL}$; and placenta, $2.75 \mu \mathrm{g} / \mathrm{nL}$. With spiramycin $3 \mathrm{~g} /$ day, the concentrations were maternal serum, $1.69 \mu \mathrm{g} / \mathrm{nL}$; umbilical cord serum, $0.78 \mu \mathrm{g} / \mathrm{nL}$; and placenta, $6.2 \mu \mathrm{g} / \mathrm{nL}$ (59,60). Persistent, high tissue levels account for its greater activity against toxoplasmosis than erythromycin. During pregnancy, spiramycin is concentrated in placental tissue two to four times the serum levels, an important advantage, as placental infection is sentinel to fetal infection. Spiramycin seems to be well tolerated. When used as an antineoplastic agent in doses greater than $35 \mathrm{mg} / \mathrm{kg}$, some patients experienced vertigo, nausea, vomiting, and anorexia. No impairment of the heart, blood, liver, or kidneys was noted (60).

Schoondermark-Van de Ven et al. (61) inoculated eight rhesus monkeys at 90 days of gestation (second trimester). The parasite was found in five of eight amniotic fluids at subsequent sampling. These monkeys with documented fetal infection were treated with intravenous spiramycin $20 \mathrm{mg} / \mathrm{kg} /$ day in two divided doses. At 14 days after initiation of spiramycin, T. gondii was still detectable by PCR analysis, 
but not by mouse inoculation in four of five amniotic fluids. In four monkeys that had received spiramycin for 7 weeks, no T. gondii was detected by PCR or mouse inoculation in the placenta, amniotic fluid, or neonatal organs. One fetus that delivered prematurely after 2 weeks of spiramycin had T. gondii associated with its placenta based on PCR results alone. Spiramycin accumulated in extra-central nervous system tissues at concentrations 5 to 28 times umbilical cord concentrations; but no spiramycin was found in fetal/neonatal brains. These data suggest that in primates early treatment with spiramycin may eliminate fetal infection with moderate success, but if there is fetal central nervous system involvement, spiramycin is likely to be ineffective.

Clinicians have come to expect that antibiotics will effectively treat infection. Unfortunately, effective antibiotic treatment of fetal toxoplasmosis has not been realized. In the last 10 years, three separate meta-analyses have concluded little benefit from antibiotic therapy. No trials with randomized assignment of women with confirmed fetal toxoplasmosis have been preformed; all are observational studies (62-64). The Systematic Review on Congenital Toxoplasmosis study group systematically reviewed 26 cohorts where spiramycin and/or pyrimethamine-sulfonamide were used to treat fetal toxoplasmosis (64). Overall, treatment did not change the frequency of any clinical manifestation: adjusted odds ratio $=$ $1.11(p=0.74)$. In a sub-analysis, there was a small reduction in maternal to fetal transmission when spiramycin was initiated less than 3 weeks after maternal seroconversion: adjusted odds ratio $(95$ th $\mathrm{CI})=0.48(0.28-0.80)$. Maternal to fetal transmission was strongly associated with gestational age at seroconversion. The odds of transmission increased by $12 \%$ per week of maternal gestation; the estimated rate of maternal to fetal transmission was $15 \%$ (95th CI $13-17 \%$ ) at 13 weeks, $44 \%$ $(40-47 \%)$ at 26 weeks, and $71 \%(66-76 \%)$ at 36 weeks.

The findings of early treatment after seroconversion and the results of the earlier quoted study of Berribi et al. (43), provide some guidance for therapy. In summary, a potential algorithm is preconceptual screening (or first prenatal visit) and monthly screening with Toxoplasma serology during pregnancy in seronegative women. If seroconversion occurs, then spiromycinis initiated until an amniocentesis can be performed for diagnosis of fetal infection. If fetal infection is diagnosed, then treatment with pyrimethamine/sulfonamide and folinic acid is initiated antenatally and neonatally.

\section{Prevention}

Primary prevention has the potential to greatly reduce the medical and economic costs of congenital toxoplasmosis. A combination of public education, that is, high school curriculum, preconceptual counseling, and early prenatal counseling, would be most effective in reducing congenital toxoplasmosis. In addition to material concerning the risks of toxoplasmosis, the role of cats and the general guidelines are listed in Table 5.

Foulon et al. (65) evaluated the reduction in the incidence of congenital toxoplasmosis using a primary prevention scheme (education) and a secondary prevention scheme (screening and treatment). Among the 11,286 consecutive pregnant women, primary prevention reduced the seroconversion rate by $63 \%(p=0.013)$. Of 76 women determined to be at risk for
Table 5 Primary Prevention of Toxoplasmosis During Pregnancy

\section{Oocyst exposure}

Avoid the purchase of a new cat just prior to or during pregnancy Avoid sick or feral cats

Use gloves for gardening

Have other family members clean the litter box

Clean the litter box daily

Sterilize the empty pan with boiling water for 20 minutes

Prevent flies and other vectors from contaminating food

Thoroughly wash fruits and vegetables

Tissue cyst exposure

Cook meat to $150^{\circ} \mathrm{F}\left(66^{\circ} \mathrm{C}\right)$

Cleanse the preparation surface immediately after meat preparation

Wash hands immediately after meat preparation

Avoid contact with mucous membranes of the mouth and eyes while handling raw meat

delivering a child with congenital toxoplasmosis, 8 (11\%) infected fetuses were detected by prenatal fetal diagnosis. Of these, secondary prevention (antibiotics) reduced the incidence of congenital toxoplasmosis by an additional $40 \%$. Unfortunately, a recent meta-analysis of randomized trials showed poor design quality and no benefit in reducing the incidence congenital toxoplasmosis. Regardless, given the benefit of the Foulon et al. study and the low cost of behavioral interventions, the author recommends their continued use (66).

\section{SUMMARY}

The medical costs of maternal toxoplasmosis warrant the development of effective programs in primary prevention, diagnosis, and therapy. Maternal toxoplasmosis occurs in $0.5 \%$ to $1 \%$ of pregnancies, and $10 \%$ to $60 \%$ of their fetuses will be infected. While the majority $(80-90 \%)$ of infected neonates will be asymptomatic at birth, a large proportion (20-90\%) will develop one or more disabilities. These include hydrocephalus, microcephaly, chorioretinitis, mental retardation, and deafness.

Primary prevention through education and monitoring of food sources has an unfulfilled potential. The effectiveness of primary prevention is measured by changes in behavior and seroconversion rates. A universal screening program, especially preconceptual screening, will improve diagnosis and allow abortion as an option. However, the lack of standardized tests and poor correlation between maternal infection and fetal infection may result in many unnecessary abortions.

Fetal diagnosis allows more focused therapy. It will significantly reduce unnecessary abortion and antibiotic therapy. Unfortunately, fetal diagnosis requires sophisticated clinical skills, equipment, and laboratory support that are currently available only at major medical centers.

The inability to pinpoint the onset or severity of fetal toxoplasmosis limits the effectiveness of antibiotic regimens used to treat fetal toxoplasmosis. Despite therapy, 10\% to 50\% of fetuses infected in the first half of pregnancy will manifest injury. This failure rate may be related to an ineffective antibiotic regimen or inadvertently delayed treatment. 
Comparative trials of antibiotics are critically important to the management of fetal toxoplasmosis in the future.

\section{REFERENCES}

1. Luft BJ, Remington JS. Toxoplasmic encephalitis. J Infect Dis 1988; 157: 1.

2. Tenter AM, Heckworth AR, Weiss LM. Toxoplasma gondii: from animal to human. Int J paraitol 2000; 30: 1217.

3. Jones JL, Krueszon-Moran D, Wilson M, et al. Toxoplasmosis gondii infection in the United States: seroprevalence and risk factors. Am J Epidemiol 2001; 154: 357-65.

4. Dubey JP, Miller NL, Frenkel JK. The Toxoplasma gondii oocyst from cat feces. J Exp Med 1970; 132: 636.

5. Frenkel JK, Dubey JP. Toxoplasmosis and its prevention in cats and man. J Infect Dis 1972; 126: 664.

6. Dubey JP, Hoover EA, Walls KW. Effect of age and sex on the acquisition of immunity to toxoplasmosis in cats. J Protozool 1977; 24: 184

7. Dubey JP, Frenkel JK. Experimental toxoplasma infection in mice with strains producing oocysts. J Parasitol 1973; 59: 505.

8. Dabritz HA, Atwill ER, Gardner IA, Miller MA, Conrad PA. Outdoor fecal deposition by the roaming cats and the attitudes of cat owners and nonowners toward stray pets, wildlife, and water pollution. J Am Vet Med Assoc 2006; 229: 74

9. Shapiro K, Conrad PA, Mazet JA, et al. Effect of estuarine wetland degradation on transport of Toxoplasma gondii surrogates from land to sea. Appl Environ Microbiol 2010; 76: 6821-8.

10. Meerburg BG, Kijlstra A. Changing climate-changing pathogens: Toxoplasma gondii in North-Western Europe. Parasitol Res 2009; 105: 17-24.

11. Lass A, Pietkiewicz H, Modzelewska E, et al. Detection of Toxoplasma gondii oocysts in environmental samples using molecular methods. Eur J Clin Microbiol Infect Dis 2009; 28: 599-605.

12. Kappareud G, Jenum PA, Stray-Peterson B, et al. Risk factors for Toxoplasma gondii infection in pregnancy. Am J Epidemiol 1996; 144: 405-12.

13. Cook AJC, Gilbert RE, Buffalano W, et al. Sources of toxoplasma infection in pregnant women: European multicentre case-control study. Br Med J 2000; 321: 142-7.

14. Boyer KM, Holfels E, Roizen N, et al. Risk factors for Toxoplasma gondii infection in mothers of infants with congenital toxoplasmosis: implications for prenatal management and screening. Am J Obstet Gynecol 2005; 192: 564-71.

15. Feldman HA. Epidemiology of toxoplasma infections. Epidemiol Rev 1982; 4: 204.

16. Remington JS, McLeod R, Thulliez P, Desmonts G. Toxoplasmosis. In: Remington JS, Klein JO, Wilson CB, Baker CT, eds. Infectious Disease of the Fetus and Newborn Infant, 6th edn. Chapter 31. Philadelphia, Pa: Elsevier 2006; Table 31-16: 968-969.

17. Dubey JP, Lappin MR, Thulliez P. Diagnosis of induced toxoplasmosis in neonatal cats. J Am Vet Med Assoc 1995; 207: 179-85.

18. Yilmaz SM, Hopkins SH. Effects of different conditions on duration of infectivity of Toxoplasma gondii oocysts. J Parasitol 1972; 58: 938.

19. Dubey JP. Toxoplasmosis in cats. Feline Pract 1986; 16: 12.

20. Dubey JP. Toxoplasmosis. J Am Vet Med Assoc 1986; 189: 166.

21. Behymer RD, Harlow DR, Behymer DE, Franti CE. Serologic diagnosis of toxoplasmosis and prevalence of Toxoplasma gondii antibodies in selected feline, canine, and human populations. J Am Vet Med Assoc 1973; 162: 959

22. Sengbusch HG, Sengbusch LA. Toxoplasma antibody prevalence in veterinary personnel and a selected population not exposed to cats. Am J Epidemiol 1976; 103: 595.

23. Ganley JP, Comstock GW. Association of cats and toxoplasmosis. Am J Epidemiol 1980; 111: 238.

24. Jacobs L, Remington JS, Melton ML. A survey of meat samples from swine, cattle, and sheep for the presence of encysted toxoplasma. J Parasitol 1960; 46: 23

25. Krogstad DJ, Juranek DD, Walls KW. Toxoplasmosis: with comments on risk of infection from cats. Ann Intern Med 1972; 77: 773

26. Desmonts G, Couvreur J, Alison F, et al. Itude JpidJmilogique sur la toxoplasmose: de l'influence de la cuisson des viandes de boucherie sur la frJquence de l'infection humaine. Rev Fr Itud Clin Biol 1965; 10: 952.
27. Kean BH, Kimball AC, Christianson WN. An epidemic of acute toxoplasmosis. JAMA 1969; 208: 1002.

28. Jacobs L, Remington JS, Melton ML. The resistance of the encysted form of Toxoplasma gondii. J Parasitol 1957; 46: 11.

29. Teutsch SM, Juranek DD, Sulzer A, Dubey JP, Sikes RK. Epidemic toxoplasmosis associated with infected cats. N Engl J Med 1979; 300: 695

30. Boyer KM, Holfels E, Roizen N, et al. Risk factors for Toxoplasmosis gondii infection of mothers of infant with congenital toxoplasmosis: Implications for prenatal management and screening. Am J Obstet Gynecol 2005; 192: 564-71.

31. Biedermann K, Flepp M, Fierz W, Joller-Jemelka H, Kleihues P. Pregnancy, immunosuppression and reactivation of latent toxoplasmosis. J Perinatal Med 1995; 23: 191-203.

32. Sever JL, Ellenbery JH, Ley AC, et al. Toxoplasmosis: maternal and pediatric findings in 23,000 pregnancies. Pediatrics 1988; 82: 181.

33. Zadik PM, Kudesia G, Siddons AD. Low incidence of primary infection with toxoplasma among women in Sheffield: a seroconversion study. $\mathrm{Br}$ J Obstet Gynecol 1995; 102: 608-10.

34. Schoondermark-Van de Ven E, Melchers W, Galama J, et al. Congenital toxoplasmosis: an experimental study in rhesus monkeys for transmission and prenatal diagnosis. Exp Parasitol 1993; 77: 200-11.

35. Wilson CB. Developmental immunology and the role of last defense in neonatal susceptibility. In: Remington JS, Klein JO, eds. Infectious Disease of the Fetus and Newborn Infant. 3rd edn. Philadelphia PA, W B Saunders, 1990: 17-67.

36. Daffos F, Forestier F, Capella-Pavlovsky M, et al. Prenatal management of 746 pregnancies at risk for congenital toxoplasmosis. N Engl J Med 1988; 318: 271.

37. Couvreur J, Desmonts G, Tournier G, et al. Itude d'une sJrie homogPne de 210 cases de toxoplasmose congJnitale chJz des nourrissons $>$ gJs de 0 a 11 mois et dJpistes de faHon prospective. Ann Pediatr 1984; 31: 815.

38. Romand S, Chosson M, Franck J, et al. Usefulness of quantitative polymerase reaction in amniotic fluid as an early marker for fetal infection with Toxoplasmosis gondii. Am J Obstet Gynecol 2004; 190: 797-802.

39. Remington JS, Desmonts G. Toxoplasmosis. In: Remington JS, Klein JO eds. Infectious Disease in the Fetus and Newborn Infant, 3rd edn. Philadelphia, PA: WB Saunders, 1990: 100.

40. Eichenwald H. A study of congenital toxoplasmosis. In: Siim JC, ed Human toxoplasmosis. Copenhagen: Munksgaard, 1960: 41-9.

41. Koppe JG, Loewer-Sieger DH, deRoever-Bonnet H. Results of 20-year follow-up of congenital toxoplasmosis. Lancet 1986; I: 254

42. Wilson CB, Remington JS, Stagno S, et al. Development of adverse sequelae in children born with subclinical congenital toxoplasma infection. Pediatrics 1980; 66: 767.

43. Berrebi A, Assouline C, Bessieres $\mathrm{MH}$, et al. Long-term outcome of children with congenital toxoplasmosis. Am J Obstetr Gynecol 2010; 203: 552.e1-6.

44. Naot Y, Desmonts G, Remington JS. IgM enzyme-linked immunosorbent assay test for the diagnosis of congenital toxoplasma infection. J Pediatr 1981; 98: 32 .

45. Newton ER. Diagnosis of perinatal TORCH infections. Clin Obstet Gynecol 1999; 42: 59-70.

46. Liesenfeld O, Montoya JG, Kinney S, Press C, Remington JC. Effect of testing for IgG avidity in the diagnosis of Toxoplasmosis gondii infection in pregnant women: experience in a US reference laboratory. J Inf Dis 2001; 183: 1248-53.

47. Liesenfeld O, Montoya JG, Tathineni NJ, et al. Confirmatory serologic testing for acute toxoplasmosis and the rate of induced abortions among women reported to have positive Toxoplasma immunoglobulin $\mathrm{M}$ antibody titers. Am J Obstet Gynecol 2001; 184: 140-5.

48. Crino JP. Ultrasound and fetal diagnosis of perinatal infection. Clin Obstet Gynecol 1999; 42: 71-80.

49. Foulon W, Naessens A, Mahler T, et al. Prenatal diagnosis of congenital toxoplasmosis. Obstet Gynecol 1990; 76: 769.

50. Couvreur J, Desmonts G, Thulliez PH. Prophylaxis of congenital toxoplasmosis: effects of spiramycin on placental infection. J Antimicrob Chemother 1988; 22: 193

51. Desmonts G, Couvreur J. Toxoplasmosis: epidemiologic and serologic aspects of perinatal infection. In: Krugman S, Gershon AA, eds. Infections 
of the fetus and the newborn infant. Progress in clinical and biological research. Volume 3. New York, NY: Alan R. Liss, Inc., 1975: 115-32.

52. Hohlfeld P, Daffos F, Costa JM, et al. Prenatal diagnosis of congenital toxoplasmosis with a polymerase-chain-reaction test on amniotic fluid. NEJM 1994; 331: 695-9.

53. Grover CM, Thulliez P, Remington JS, Boothroyd JC. Rapid prenatal diagnosis of congenital toxoplasma infection by using polymerase chain reaction and amniotic fluid. J Clin Microbiol 1990; 28: 2297.

54. Wallon M, Franck J, Thulliez P, et al. Accuracy of real-time polymerase chain reaction for Toxoplasma gondii in amniotic fluid. Obstet Gynecol 2010; 115: 727-33.

55. Wilson CB, Remington JS. What can be done to prevent congenital toxoplasmosis? Am J Obstet Gynecol 1980; 138: 357.

56. Sever JL. TORCH tests and what they mean. Am J Obstet Gynecol 1985; 152: 495.

57. Lappalainen $\mathrm{M}$, Koskela $\mathrm{P}$, Hedman $\mathrm{K}$, et al. Incidence of primary toxoplasma infections during pregnancy in southern Finland: a prospective cohort study. Scand J Infect Dis 1992; 24: 97-104.

58. Lappalainen M, Sintonen H, Koskiniemi M, et al. Cost-benefit analysis of screening for toxoplasmosis during pregnancy. Scand J Infect Dis 1995; 27: $265-72$.
59. Schoondermark-Van de Ven E, Melchers W, Camps W, et al. Effectiveness of spiramycin for treatment of congenital Toxoplasma gondii infection in rhesus monkeys. Antimicrob Agents Chemother 1994; 38: 1930-6.

60. Back N, Ambrus JL, Velasco H, et al. Clinical and experimental pharmacology of parenteral spiramycin. Clin Pharmacol Ther 1962; 3: 305.

61. Schoondermark-Van de Ven E, Galama J, Camps W, et al. Pharmacokinetics of spiramycin in the rhesus monkey: transplacental passage and distribution in tissue in the fetus. Antimicrob Agents Chemother 1994; 38: 1922-9.

62. Peyron F, Wallon M, Liou C, Garner P. Treatments for toxoplasmosis in pregnancy. Cochrane Database Syst Rev 2010: CD001684.

63. Peyron F, Wallon M, Liou C, Garner P. Treatments for toxoplasmosis in pregnancy. Cochrane Database Syst Rev 2001: CD001684.

64. Thiebaut R, Leproust S, Chene G, Gilbert R. Effectiveness of prenatal treatment for congenital toxoplasmosis: a meta-analysis of individual patients' data. SYROCOT (Systematic Review on Congenital Toxoplasmosis) study group. Lancet 2007; 369: 115-22.

65. Foulon W, Naessens A, Derde MP. Evaluation of the possibilities for preventing congenital toxoplasmosis. Am J Perinatol 1994; 11: 57-62.

66. Di Mario S, Basevi V, Gagliotti C, et al. Prenatal education for congenital toxoplasmosis (review). Cochrane Database Syst Rev 2009: CD006171. 


\section{Human immunodeficiency virus (HIV) Richard Basilan and William Salzer}

\section{EPIDEMIOLOGY}

The number of people living with human immunodeficiency virus (HIV) worldwide reached an estimated 33.4 million in 2008, with 2.7 million new infections and 2 million acquired immune deficiency syndrome (AIDS)-related deaths that same year. The total number of people living with the virus in 2008 was more than $20 \%$ higher than the number in 2000 , and the prevalence was roughly threefold higher than that in 1990 (1).

Meanwhile, an estimated 1.1 million adults and adolescents were living with diagnosed or undiagnosed HIV in the United States at the end of 2006, according to Centers for Disease Control and Prevention (CDC) estimates (2). During the same year, an estimated 54,230 new infections occurred in the United States (3). Although women make up only $25 \%$ of the HIV-infected population in the United States, certain subgroups, particularly African-Americans and Latinas, are disproportionately affected. African-American women, for example, have a prevalence rate nearly 18 times that of the rate of white women (2). In 2005, HIV disease was the third leading cause of death for African-American women aged 25 to 44 years and the fourth leading cause of death for African-American women aged 45 to 54 years (4).

Differences in the rates of HIV maternal to child transmission (MTCT) serve to highlight the disparities between resource-rich and resource-limited areas. While there were 390,000 children newly infected with HIV in sub-Saharan Africa in 2008, there were less than 500 new cases in North America, Western Europe, and central Europe combined (1). Analysis of national surveillance data shows that the number of pediatric AIDS cases that were reported to the CDC decreased from 858 in 1992 to only 41 in 2004, a decrease of about $95 \%$ (5). There has also been a marked decline in perinatal HIV transmission from an estimated 1650 in 1991 to between 144 and 236 in 2006 (6). The reduction in perinatal HIV transmission, now estimated to be less than $2 \%$ in the United States (7), is the result of a combination of universal maternal prenatal HIV counseling and testing, antiretroviral (ARV) prophylaxis, scheduled cesarean delivery when appropriate, and avoidance of breast-feeding. By contrast, perinatal transmission rates in resource-poor areas can be as high as $25 \%$ to $30 \%$ in the absence of any intervention (8).

\section{ETIOLOGY}

AIDS was first recognized in June 1981 when five previously healthy men who have sex with men (MSM) presented with Pneumocystis carinii pneumonia in Los Angeles (9). This was followed by a report of 26 MSM with Kaposi's sarcoma, with or without P. carinii pneumonia in New York and Los Angeles (10). The discovery of the HIV, initially named human T cell leukemia virus III, followed in 1983, and its causal relationship to AIDS was firmly established by the following year $(11,12)$. A reliable screening test (HIV ELISA) was developed in 1985, and since that time our understanding of HIV and its pathogenesis continues to increase and has led to successful treatment regimens not just for HIV but for other viral illnesses as well.

HIV belongs to the family of human retroviruses (Retroviridae) and the subfamily of lentiviruses. HIV-1 is the predominant cause of HIV disease in the world. HIV-2, which can also cause AIDS, is closely related to simian immunodeficiency virus and is largely confined to West Africa, although occasional cases occur worldwide. The HIV virion is an icosahedral structure containing numerous external spikes formed by the two major envelope proteins, the external gp120, and the transmembrane gp41. The virion attaches via gp120 to cells expressing the CD4 molecule, which acts as the cell receptor for the virus. The CD4 molecule is expressed mainly in a subset of $\mathrm{T}$ lymphocytes responsible for helper function in the immune system, but can also be found on the surface of monocytes/macrophages and dendritic/Langerhans cells (13). A conformational change then occurs in the gp120 molecule, allowing it to bind to one of two cellular coreceptors (CCR5 or CXCR4). Binding is followed by insertion of viral gp41 into the CD4 cell, resulting in membrane fusion followed by release of the viral core into the cell cytoplasm.

HIV, like other retroviruses, is an RNA virus. The virus transcribes its RNA using RNA-dependent DNA polymerase (reverse transcriptase) into DNA, which is then incorporated into the host DNA by a viral integrase. Following transcription, HIV mRNA is translated into proteins that are processed further by viral proteases, allowing for assembly of new virions that are then released to infect other cells. These steps in the viral replication cycle have been the target of highly active ARV therapy, which has revolutionized the management of HIV infection.

\section{PATHOPHYSIOLOGY}

The most common mode of HIV infection is sexual transmission through the genital mucosa (14), with initial infection of a small number of $\mathrm{T}$ lymphocytes, macrophages, and dendritic cells located in the lamina propria. Initial replication occurs in the regional lymph nodes, followed by migration of infected $\mathrm{T}$ lymphocytes or virions into the bloodstream, where secondary amplification in the gastrointestinal tract, spleen, and bone marrow results in massive infection of susceptible cells (15). The period of peak viremia, which occurs in the first 6 weeks, results in a symptomatic illness in $40 \%$ to $90 \%$ of patients, termed acute HIV infection. The most common symptoms include fever, fatigue, pharyngitis, lymphadenopathy, and myalgias. A maculopapular rash involving the trunk also occurs in $40 \%$ to $80 \%$ of patients (16). Oral or genital ulcers can also occur. Given the 
nonspecific mononucleosis like symptoms of acute HIV infection, the diagnosis is often missed. A high index of suspicion is required to make the diagnosis, because commonly used screening tests for HIV antibody are negative for the first 4 weeks following infection. Detection of the p24 antigen or HIV RNA by PCR in the absence of HIV antibodies would confirm the diagnosis. This has important public health implications as the patient is unaware of being infected with HIV and yet is highly infectious because of the very high viral load.

Despite the initial immune response during primary HIV infection, the virus continues to replicate at a high rate in untreated patients, with production of $10^{10}$ virions daily. Viral replication is error prone, producing multiple quasi-species. This helps the virus evade the immune system and facilitates development of resistance to ARV agents. The virus subsequently establishes a reservoir in resting memory CD4+ cells, as well as in immune-sequestered sites such as the central nervous system (CNS). The patient remains asymptomatic during this chronic phase, which on average lasts about 10 years. During this time, there is persistent viral replication and immune activation resulting in a gradual decrease in the number of circulating CD4+ cells. As the CD4+ count falls, patients become more susceptible to opportunistic infections, the hallmark of AIDS.

Transmission of HIV from mother to child can occur in utero, perinatally, or postpartum through breast-feeding (6). Multiple factors have been associated with an increased risk of MTCT, but the greatest risk is with higher maternal plasma HIV RNA levels. Although the risk of MTCT decreases with lower maternal plasma HIV RNA levels (8), particularly when it is $<1000$ copies $/ \mathrm{mL}$, it should be emphasized that MTCT can occur even at low or undetectable HIV RNA levels (17). Other factors that have been found to increase MTCT rates include vaginal delivery with plasma HIV RNA levels $>1000$ copies $/ \mathrm{mL}$, delivery at earlier gestation, prolonged interval between membrane rupture and delivery, chorioamnionitis, coinfection with other sexually transmitted diseases (STDs), illicit drug use, and cigarette smoking.

\section{DIAGNOSIS AND MANAGEMENT}

A critical component of caring for HIV-infected patients is knowledge of evidence-based treatment guidelines. Guidelines for management of HIV infection in pregnancy are published by the Department of Health and Human Services (DHHS) in the United States, the European AIDS Clinical Society (EACS) and the World Health Organization (WHO). Differences in CD4-based criteria for initiation of ARV therapy, preferred agents and regimens, and management of infant prophylaxis exist between these guidelines. The practitioner caring for HIV-infected patients should be familiar with the recommendations for their region of the world, as well as the availability of local resources, such as laboratory testing, ARV agents, prevalence of HIV drug resistance and coexisting infections (i. e., TB, malaria, Hepatitis B), demographic and sociocultural issues, and organizational support for the patients.

\section{LABORATORY TESTING}

Laboratory testing for HIV infection involves screening with a highly sensitive test, usually an enzyme-linked immunosorbent assay (ELISA), also known as enzyme immunoassay, for
HIV antibodies. The high sensitivity of the test makes it an effective screening tool, but requires a highly specific assay for confirmatory testing, such as western blot for antibodies to specific viral proteins. Virologic assays (PCR for HIV RNA) can be used for confirmatory testing when the western blot is indeterminate. Testing for HIV RNA by PCR is also used in neonates or when acute HIV infection is suspected. In recent years, multiple rapid tests using blood or saliva have been introduced for point-of-care testing, but it should be noted that all rapid tests require further confirmatory testing because of their high false-positive rate.

After a diagnosis of HIV infection is confirmed, further testing includes obtaining the patient's HIV RNA viral level (or viral load), CD4 count, as well as baseline complete blood count (CBC), comprehensive metabolic panel, and for African-Americans, urinalysis. Screening for coinfection with other STDs (gonorrhea, chlamydia, syphilis, hepatitis B and C), tuberculosis, and toxoplasma should also be performed.

The CDC recommends HIV screening for all patients aged 13 to 64 in health-care settings (18). Repeat HIV testing should be offered at least annually to women who use injection drugs, have had an STD in the past year, have had more than one sexual partner since their most recent HIV test, engage in sexual intercourse in exchange for drugs or money, or are partners of injection drug users or HIVinfected persons. Opt-out screening, in which the patient is notified that HIV testing be performed as a routine part of gynecologic and obstetric care unless she declines, should be performed (19). HIV-uninfected women with a known HIV (+) partner should be counseled regarding the risk for acquiring the infection and should have a second HIV test during the third trimester, before 36 weeks if possible. If such a patient presents in labor without a second HIV test, a rapid test should be done. Suspicion for acute seroconversion should prompt both rapid HIV testing and checking for plasma HIV RNA levels (20).

Laboratory testing for neonates must take into account that maternal antibodies cross the placenta and persist in the child's circulation, making tests for HIV antibodies unreliable. Exclusion of HIV infection therefore relies on virologic tests (i.e., p24 antigen testing or plasma HIV RNA levels) done on two separate samples drawn after 1 month of age, with at least one sample being drawn after 4 months of age. HIV antibody tests can be utilized after 6 months of age, but also require verification by repeat testing using a second, separately drawn sample. In addition to negative laboratory test, the child should also have no current or previous AIDS-defining condition before being declared as HIV uninfected (21).

Testing for ARV drug resistance should be done prior to starting ARV therapy (whether for therapy or prophylaxis), as well as when considering modification of a patient's drug regimen. Assays for HIV drug resistance require that the patient has a detectable viral load, which is usually the case in untreated individuals and patients failing therapy.

\section{ARV THERAPY}

The use of highly active ARV therapy has revolutionized the management of HIV infection, leading to better quality of life, decreased morbidity, prolonged survival, and decreased transmission, including MTCT. There are now five different 
classes of ARV drugs, with 24 individual ARV agents and 6 additional combination pills available for managing HIV infection in the United States. HIV therapy requires the use of drug combinations to decrease the development of drug resistance and requires a minimum of two, and ideally three, fully active agents at a time. As with most chronic infections requiring multidrug therapy, addition of a single agent to a failing regimen should be avoided as this fosters the development of resistance to the added agent.

Nucleoside reverse transcriptase inhibitors (NRTIs) were the first drugs active against HIV to be developed with the introduction of zidovudine or azidothymidine in 1987. The NRTIs are nucleoside analogs and require intracellular phosphorylation in order to become active. They are incorporated into the DNA chain and terminate DNA chain elongation by acting as nonactive substrates for the viral reverse transcriptase. Nonnucleoside reverse transcriptase inhibitors (NNRTIs), on the other hand, bind to the reverse transcriptase enzyme and induce conformational changes at the active site, thereby rendering the enzyme nonfunctional. Protease inhibitors (PIs) target the viral protease, an enzyme required for cleavage of precursor proteins (gag and gag-pol), thereby preventing the final assembly of the inner core of viral particles (22). Entry inhibitors block the penetration of HIV virions into their target cells by either preventing fusion (enfuvirtide) or by blocking the CCR5 coreceptor required for entry (Maraviroc). A newer class of ARVs act by inhibiting the enzyme required for integration of the transcribed viral genome into the host DNA (integrase), aptly named integrase inhibitors. Available ARV agents are summarized in Table 1.

The decision to initiate ARV therapy in adults is based on multiple factors, including the presence or absence of AIDSrelated conditions, other comorbidities (such as hepatitis $\mathrm{B}$ coinfection or HIV-associated nephropathy), CD4 count, as well as the patient's readiness to start lifelong therapy. There is a general consensus that ARV therapy should be started on all patients with symptomatic HIV infection as well as asymptomatic patients with a CD 4 count less than 500 cells $/ \mu \mathrm{L}$. Timing of initiation of ARV therapy in asymptomatic patients with CD4 counts $>500$ cells $/ \mu \mathrm{L}$ is a subject of continuing debate and is not recommended by the WHO or EACS in asymptomatic patients without significant coinfection or comorbidities $(23,24)$. However, in recent years, data on benefits of earlier initiation of ARV therapy have led to recommendations in the United States to start ARV therapy for all patients with $\mathrm{CD} 4$ counts $<500$ cells $/ \mu \mathrm{L}$ and to consider starting ARV therapy at CD 4 counts $>500$ cells $/ \mu \mathrm{L}$ (25). These newer recommendations are based on observational cohort studies showing a potential decrease in mortality as well as in the incidence of such conditions as HIV-associated nephropathy, liver disease progression from hepatitis B or hepatitis C, cardiovascular disease, malignancies (both AIDS defining and non-AIDS defining), neurocognitive decline, and blunted immunologic response in patients started on highly active ARV treatment therapy at CD4 counts $>500$ (20).

It is recommended that all HIV-infected pregnant women receive ARV therapy regardless of CD4 or viral load. Use of ARV agents in pregnant women should consider two separate, but related issues: treatment of maternal HIV infection and prevention of MTCT. As in all HIV-infected patients, evaluation of the patient's HIV viral load and CD4 count should be done, as well as initial testing for drug resistance if this has not been done previously. Patients with a CD4 count less than $200 \mathrm{cells} / \mu \mathrm{L}$ should be started on prophylaxis for Pneumocystis jiroveci (previously named Pneumocystis carinii) using trimethoprim sulfamethoxazole at a dose of $80 \mathrm{mg} / 400 \mathrm{mg}$ daily. Alternatives include dapsone, atovaquone, or aerosolized pentamidine. A patient with CD4 count less than 50 cells $/ \mu \mathrm{L}$ is at high risk for other opportunistic infections, including Mycobacterium avium complex, and prophylaxis using azithromycin $1200 \mathrm{mg}$ weekly is recommended. Like any medication taken during pregnancy, the potential benefits of therapy should be weighed against potential risk to both the mother and the fetus.

For ARV-naive, nonpregnant patients who meet criteria for initiation of ARV therapy, present guidelines recommend the use of an NNRTI-based, a PI-based, or an integrase inhibitorbased regimen. All regimens would also include two NRTIs. Purely NRTI-based regimens, such as Trizivir (a combination of zidovudine, lamivudine, and abacavir), are no longer recommended as they have been found to have higher rates of virologic failure. For an NNRTI-based regimen, the preferred agents include efavirenz with emtricitabine and tenofovir (available as combination pill Atripla ${ }^{\mathrm{TM}}$ ). Use of efavirenz should be avoided during the first trimester of pregnancy, as well as in women who are of reproductive age and are not taking sufficient steps to avoid pregnancy, given its known teratogenicity in animal and human studies. An alternative NNRTI-based regimen would include nevirapine with zidovudine and lamivudine (Combivir ${ }^{\mathrm{TM}}$ ). It should be noted that nevirapine should be avoided in patients with moderate-tosevere hepatic impairment (Child Pugh B or C), as well as females with a CD4 count $>250$ cells $/ \mu \mathrm{L}$ as both groups have an increased risk for potentially fatal hepatitis.

Protease-based regimens may also include emtricitabine and tenofovir, combined with either atazanavir or darunavir boosted with ritonavir. In the pregnant patient, lopinavir boosted with ritonavir (lopinavir/r or Kaletra ${ }^{\mathrm{TM}}$ ) is the preferred PI. The addition of ritonavir at a low dose (boosting) inhibits CYP450 3A4 resulting in an increase in the serum levels of most PIs. An integrase inhibitor-based regimen would use raltegravir in addition to two NRTIs.

A list of preferred initial ARV regimens is presented in Table 2.

The following management strategies have been adapted mainly from present DHHS guidelines (20) unless otherwise stated. Updated versions are available online at www.aidsinfo .nih.org.

\section{ANTEPARTUM CARE}

In reproductive age, nonpregnant HIV-infected women, preconception counseling should be done to minimize the risk of unintended pregnancies. ARV agents with known teratogenic potential, such as efavirenz, should be avoided if there is insufficient effort to avoid conception. Ideally, two methods of contraception should be used in such circumstances, noting that certain ARV agents decrease the efficacy of oral contraceptive pills.

If the patient desires to conceive, the risks of MTCT should be discussed with the patient. In couples with discordant HIV 
Table 1 Antiretroviral Agents and Common Adverse Effects

\begin{tabular}{|c|c|c|}
\hline Drug & $\begin{array}{l}\text { FDA } \\
\text { pregnancy } \\
\text { category }\end{array}$ & $\begin{array}{l}\text { Common side } \\
\text { effects }\end{array}$ \\
\hline NRTIs & & Lactic acidosis, hepatic steatosis, lipodystrophy \\
\hline Abacavir (ABC) & $\mathrm{C}$ & $\begin{array}{l}\text { Hypersensitivity reaction (can be fatal), rash, } \\
\text { possible risk of MI }\end{array}$ \\
\hline Didanosine (ddI) & $\mathrm{B}$ & $\begin{array}{l}\text { GI intolerance, peripheral neuropathy, } \\
\text { pancreatitis, lactic acidosis, noncirrhotic } \\
\text { portal hypertension }\end{array}$ \\
\hline Emtricitabine (FTC) & B & \\
\hline Lamivudine (3TC) & $\mathrm{C}$ & \\
\hline Stavudine $(\mathrm{d} 4 \mathrm{~T})$ & $\mathrm{C}$ & $\begin{array}{l}\text { Highest incidence of lactic acidosis, hepatic } \\
\text { steatosis, and lipodystrophy } \\
\text { Peripheral neuropathy, Pancreatitis }\end{array}$ \\
\hline Tenofovir (TDF) & B & $\begin{array}{l}\text { Renal impairment, decreased bone mineral } \\
\text { density, headache, GI intolerance }\end{array}$ \\
\hline $\begin{array}{l}\text { Zidovudine (AZT, } \\
\text { ZDV) }\end{array}$ & $\mathrm{C}$ & $\begin{array}{l}\text { Headache, lactic acidosis, GI intolerance, bone } \\
\text { marrow suppression }\end{array}$ \\
\hline NNRTIs & & $\begin{array}{l}\text { Rash, including Stevens-Johnson syndrome, } \\
\text { Drug-drug interactions }\end{array}$ \\
\hline Efavirenz (EFV) & $\mathrm{D}$ & $\begin{array}{l}\text { Neuropsychiatric, teratogenic in nonhuman } \\
\text { primates with reports of neural tube defects } \\
\text { in human infants following first-trimester } \\
\text { exposure }\end{array}$ \\
\hline
\end{tabular}

Etravirine (ETR)

B

Nevirapine (NVP)

Protease inhibitors

Atazanavir (ATV)

Darunavir (DRV)

Fosamprenavir

(f-APV)

Indinavir (IDV)

Lopinavir + ritonavir (LPV/r)

Nelfinavir (NFV)

Ritonavir (RTV)

Saquinavir (SQV)

Tipranavir (TPV)

Entry inhibitors

Enfuvirtide (T-20)

Maraviroc (MVC)

Integrase inhibitors

Raltegravir (RAL)
B

$\mathrm{B}$

B

Higher rate of rash, hepatotoxicity (may be severe and life threatening, especially in women w/CD4 count $>250$

Hyperlipidemia, insulin resistance and diabetes, lipodystrophy, increased LFTs, drug interactions, osteonecrosis

Hyperbilirubinemia, PR prolongation, nephrolithiasis

C Rash, liver toxicity

C GI intolerance, rash, possible increased risk of MI

C Nephrolithiasis, GI intolerance

C Possible increased risk of MI, PR, and QT prolongation, GI intolerance

Diarrhea

GI intolerance, hepatitis

GI intolerance

GI intolerance, rash, hyperlipidemia, liver toxicity, cases of intracranial hemorrhage

B Infection site reactions, hypersensitivity reactions, increased risk of bacterial pneumonia

Drug interactions, abdominal pain, hepatotoxicity, rash, URIs
Notes

Test for HLA-B ${ }^{\star} 5701$. Do not use if patient is HLA-B ${ }^{\star} 5701$ positive (high risk for hypersensitivity reaction)

Do not use with stavudine (additive toxicities such as lactic acidosis)

Preferred component of therapy during pregnancy

Do not use with didanosine or zidovudine

Preferred component of therapy during pregnancy.

Do not use with stavudine

(antagonistic effects on HIV activity)

A component of Atripla, a combination pill used commonly given preferred status. Should be used in pregnancy only if other agents not tolerated and benefits outweigh risk

Do not use with unboosted PIs, or ritonavir-boosted atazanavir, fosamprenavir, or tipranavir

Risk of hepatotoxicity higher in first 18 months of therapy, hepatitis B or $\mathrm{C}$ coinfection, women w/CD4 count $>250$, men w/CD4 count $>400$ cells $/ \mu \mathrm{L}$

Do not use with indinavir (additive hyperbilirubinemia)

Do not use with atazanavir

Preferred component of therapy during pregnancy

Abbreviations: LFTs, liver function tests; GI, gastrointestinal; MI, myocardial infarction; URIs, upper respiratory infections; CPK; creatine phosphokinase; PIs, protease inhibitors. Source: From Ref. 20. 


\begin{tabular}{|c|c|}
\hline NNRTI based & $\begin{array}{l}\text { Tenofovir/emtricitabine/efavirenz } \\
\text { daily }\end{array}$ \\
\hline \multirow[t]{3}{*}{ PI based } & $\begin{array}{l}\text { Atazanavir boosted with ritonavir }+ \\
\text { tenofovir/emtricitabine daily }\end{array}$ \\
\hline & Or \\
\hline & $\begin{array}{l}\text { Darunavir boosted with ritonavir }+ \\
\text { tenofovir/emtricitabine }\end{array}$ \\
\hline Integrase inhibitor based & Raltegravir + tenofovir/emtricitabine \\
\hline Pregnancy & $\begin{array}{l}\text { Lopinavir boosted with ritonavir }+ \\
\text { zidovudine/lamivudine }\end{array}$ \\
\hline
\end{tabular}

status, alternative methods for conception, such as in vitro fertilization, should be explored to decrease the risk to the HIV-uninfected partner. Ideally, the patient's HIV viral load should be suppressed to undetectable levels prior to conception. Modifiable factors that decrease MTCT, including avoiding alcohol and illicit drug use, smoking cessation, and STD prevention, should be addressed.

The present consensus for the management of HIV infection in pregnancy is that of recommending ARV therapy or prophylaxis to all pregnant HIV-infected women, regardless of plasma HIV RNA levels. The benefits and risks of therapy should be individualized and discussed with the patient, with particular emphasis on the importance of adherence to drug therapy. Every attempt should be made to coordinate comprehensive services for such patients before, during, and after delivery.

In pregnant HIV patients who have indications for initiation of ARV therapy, the goals of ARV therapy include control of HIV viremia to undetectable levels, immune reconstitution to avoid opportunistic infections, and decreased MTCT. As with other HIV patients, multiple factors should be considered when designing a drug regimen, including comorbidities, patient adherence potential, convenience, potential adverse effects, drug interactions, and results of genotypic resistance testing. In addition, pharmacokinetic (PK) changes in pregnancy (particularly with PIs) and potential teratogenic effects on the fetus and other adverse effects on the fetus or newborn would also need to be considered. ARV agents with teratogenic potential, such as efavirenz, should be avoided particularly during the first trimester unless the risk is outweighed by the anticipated benefits.

Patients who are already on effective therapy (i.e., with undetectable HIV viral load) for their HIV infection should continue the same regimen, with the exception of efavirenz in the first trimester. Patients with detectable viral load should have genotype testing for drug resistance in an effort to design an optimized drug regimen. Patients may require modifications of their ARV regimen, with particular attention to the factors previously noted. For patients who meet criteria for initiation of ARV therapy, the preferred initial regimen consists of zidovudine and lamivudine (available as a combination pill Combivir) and lopinavir boosted with ritonavir (available as a combination pill Kaletra) taken twice a day. All regimens should include zidovudine if possible, as this drug has the best evidence-based data to support efficacy in reducing MTCT and also has good safety data for use during pregnancy (8). Alternative regimens include zidovudine/ lamivudine with either (i) another PI, such as atazanavir/ ritonavir, indinavir/ritonavir, nelfinavir, or saquinavir/ritonavir or (ii) an NNRTI such as nevirapine. Efavirenz use after the first trimester can be considered if other alternatives are not tolerated. Nevirapine should not be started in pregnant women with CD4 counts $>250$ cells/ $\mu \mathrm{L}$ unless the anticipated benefits outweigh the potential risk of severe, sometime fatal hepatotoxicity. ARV therapy can be started during the first trimester if indicated for maternal health.

Patients who do not meet criteria for initiating ARV therapy (asymptomatic, CD4 counts $>500$ cells $/ \mu \mathrm{L}$, low-level viremia) should still be advised to take ARV therapy to decrease maternal viremia and provide prophylaxis for the fetus in an attempt to decrease MTCT. As most drug teratogenic effects occur early in the first trimester while majority (but not all) of maternal-to-child infections occur during late pregnancy and delivery, it would be worthwhile to wait until 10 to 12 weeks of gestation before starting antiviral therapy. However, this approach leaves the fetus exposed to persistent viremia for several weeks, and the risks and benefits of starting ARV therapy early versus waiting until the second trimester should be discussed with the patient.

Combination three-drug therapy with zidovudine/ lamivudine and lopinavir/ritonavir would still be recommended in the United States as this regimen appears to be safe and effective in decreasing MTCT. Alternative regimens (firstline regimens per WHO guidelines) include either zidovudine/ lamivudine or tenofovir/lamivudine plus either nevirapine or efavirenz, noting that efavirenz should be avoided during the first trimester of pregnancy). Concerns over fetal exposure to multiple drugs during pregnancy may prompt the use of monotherapy with zidovudine as an alternative if the patient's viral load is less than 1000 copies $/ \mathrm{mL}$. This approach, derived from the original Pediatric AIDS Clinical Trial Group (PACTG) study to reduce MTCT published in 1994, has been a mainstay in the WHO guidelines particularly because of its availability in resource-limited areas. In the United States, the use of zidovudine monotherapy as maternal prophylaxis for reduction of MTCT is now controversial and is generally not recommended particularly for patients with higher HIV viral RNA levels. ARV therapy given only to decrease MTCT can be discontinued following delivery if there is no indication for therapy for the mother and/or if there are no plans for breastfeeding.

There are conflicting data as to whether ARV drugs are associated with adverse outcomes such as preterm delivery, with some studies showing up to a twofold increase in risk while others fail to demonstrate any increased risk for preterm delivery. Patients on PIs were found in a recent meta-analysis to be at slightly higher risk for premature delivery (odds ratio 1.35) compared with those on regimens without a PI (26). There is also concern regarding mitochondrial dysfunction in neonates exposed to NRTIs, but this occurs rarely. These risks should be discussed with the patient, but are generally thought to be outweighed by the significant benefit of improved maternal health and decreased MTCT of HIV infection. Pregnant patients on ARV therapy should be enrolled in the ARV pregnancy registry available online at www.APRegistry.com.

As there remain limited data on the effects of ARV therapy on the fetus, a second ultrasound during the second trimester 
is generally recommended to assess fetal anatomy in addition to the staging ultrasound done in the first trimester. Although no perinatal transmission of HIV infection has been documented following amniocentesis in patients taking ARV therapy, a small risk of MTCT cannot be ruled out. Therefore, amniocentesis should be performed only after initiation of ARV therapy or prophylaxis and ideally with undetectable HIV viral RNA levels. Plasma RNA levels should be rechecked at 34 to 36 weeks to guide the decision regarding the preferred mode of delivery.

\section{INTRAPARTUM MANAGEMENT}

Patients already on ARV therapy should be continued on their regimen, with the oral route being preferred whenever possible. Intravenous zidovudine is recommended for all HIV-positive women during labor, even if drug resistance to this drug has been documented. If the patient was taking zidovudine as part of a combination regimen, the other components of her ARV therapy should also be continued. Zidovudine should be given at a dose of $2 \mathrm{mg} / \mathrm{kg}$ IV over 1 hour, followed by an infusion of $1 \mathrm{mg} / \mathrm{kg} /$ hour until the time of delivery, and should ideally be started 3 hours prior to surgery in the event of planned caesarian section. This dose is based on the original protocol used in the PACTG 076 (8) published in 1994, which demonstrated a marked reduction in MTCT from $25.5 \%$ in the placebo group to $8.3 \%$ infants in the zidovudine group. Stavudine $(\mathrm{d} 4 \mathrm{~T})$ should be discontinued when administering zidovudine because of antagonism between these two drugs.

Patients presenting in labor with unknown HIV status should have a rapid antibody test performed. A positive result should prompt administration of intravenous zidovudine to the mother and the infant, with continuation of infant prophylaxis for 6 weeks. Please see Table 3 for specific dosing. A confirmatory maternal HIV test should be done postpartum, with discontinuation of infant prophylaxis if the test is negative.

Patients should be counseled regarding risks and benefits of caesarian versus vaginal delivery. In general, HIV-infected patients undergoing caesarian delivery are at higher risk for surgical complications compared with HIV-infected women undergoing vaginal delivery, as well as compared with HIVuninfected women undergoing caesarian delivery. However, these risks do not outweigh the benefit of decreased transmission to those at high risk. Therefore, women whose HIV RNA levels are unknown or $>1000$ copies $/ \mathrm{mL}$ near the time of delivery should be scheduled for elective caesarian delivery around 38 weeks of gestation regardless of whether or not they are on ARV therapy. The benefits of caesarian delivery after rupture of membranes remain unclear, and the decision as to mode of delivery in such cases should be made after considering other clinical factors. Likewise, caesarian delivery for patients with HIV RNA levels $<1000$ copies/mL may not decrease the rate of perinatal transmission any further.

Procedures that risk fetal exposure to maternal blood have been suggested to increase MTCT by some authors. Older data have suggested up to a twofold increase in MTCT in these situations, but more recent observation studies have shown much less risk, particularly with the use of ARV therapy. Although limited data exist, invasive fetal monitoring (e.g., fetal scalp electrodes) should be avoided unless obstetrically indicated and the duration is expected to be short. Operative vaginal delivery using forceps or vacuum extractor as well as episiotomy would theoretically have an increased risk of transmission but should not be delayed if clear obstetric indications exist $(27,28)$.

The use of methergine, a drug metabolized through the hepatic CYP3A4 pathway, for postpartum hemorrhage should be avoided in women receiving PIs (potent CYP3A4 enzyme inhibitors) because of the risk of exaggerated vasoconstrictive response. If other alternatives (oxytocin, misoprostol, PGF2 alpha) are not available, a lower dose should be used and the duration of use should be minimized. Conversely, if methergine must be used in a patient taking a potent CYP3A4 inducer (such as efavirenz), additional uterotonic agents may be needed given the possibility of lower methergine levels.

Table 3 Intrapartum Maternal and Neonatal Zidovudine (ZDV) Dosing for Prevention of Mother-to-Child Transmission of HIV

\begin{tabular}{|c|c|c|}
\hline & Dosing & Duration \\
\hline Maternal intrapartum & $\begin{array}{l}2 \mathrm{mg} / \mathrm{kg} \text { IV over } 1 \text { hour, followed by continuous infusion of } \\
1 \mathrm{mg} / \mathrm{kg} / \mathrm{hr}\end{array}$ & Onset of labor until delivery of infant \\
\hline \multicolumn{3}{|c|}{ (1) } \\
\hline$<30$ weeks of gestation & $\begin{array}{l}2 \mathrm{mg} / \mathrm{kg} / \text { dose oral } \\
\text { Or } \\
1.5 \mathrm{mg} / \mathrm{kg} / \text { dose IV started within } 6-12 \text { hours of delivery, } \\
\text { then every } 12 \text { hours, advanced to every } 8 \text { hours at } 4 \text { weeks } \\
\text { of age }\end{array}$ & \\
\hline
\end{tabular}




\section{POSTPARTUM MANAGEMENT}

HIV-infected patients are commonly faced with multiple medical and social challenges, making the coordination of maternal postpartum follow-up between obstetric, pediatric, and HIV specialists even more important. The need for continued counseling and comprehensive medical care cannot be overemphasized in this period. Supportive services should ideally be assured prior to discharge, including obstetric/ gynecologic, pediatric, HIV specialty care and mental health services, substance abuse counseling, family planning services, and social work/case worker services.

The decision regarding continuation of maternal ARV therapy during this period would depend on the CD4 count nadir (lowest documented level), clinical symptoms, disease stage, presence of other indications for ARV therapy (e.g., chronic hepatitis B infection), and patient and provider preference. Patients who have had symptomatic HIV infection or are known to have had a CD4 count below the established criteria for starting treatment for asymptomatic patients should be encouraged to continue their ARV regimen. They should also be advised regarding potential challenges with regard to compliance in light of the physiologic and lifestyle changes associated with the postpartum period.

If the only indication for initiation of ARV therapy was to reduce MTCT, then consideration can be given to stopping ARV therapy after delivery. Because NNRTIs have much longer half-lives than drugs from other classes, patients on NNRTIs should be advised to take their NRTIs for at least 7 to 30 days after stopping their NNRTI (NRTI tail) to avoid development of NNRTI resistance. An alternative approach is to substitute a PI for the NNRTI, continuing the regimen for 7 to 30 days, and then stopping all ARV drugs at the same time. This becomes important when the patient presents with a need for ARV therapy, whether for maternal indications or for prophylaxis during subsequent pregnancies. Even singledose nevirapine, if given without an NRTI tail within the prior 12 months, would necessitate the empiric use of a nonNNRTI-based regimen (i.e., protease- or integrase-based regimen) (24).

Women with positive rapid HIV test result in labor should have their confirmatory tests done prior to discharge, as this would have important implications on infant prophylaxis and breast-feeding options. Further management of newly confirmed HIV infection at this stage would be similar to that of any other newly diagnosed HIV patient.

Ideally, the risk of HIV transmission through breast-feeding would have been discussed with the patient prior to delivery and would be reiterated at this phase. In resource-rich areas where safe alternatives are available and culturally acceptable, such as in the United States, breast-feeding is not recommended. MTCT through breast-feeding is a well-documented phenomenon even in patients on ARV therapy, and for patients on ARV therapy, limited data on pharmacokinetics of ARV drugs in breast milk can pose issues related to drug toxicity or drug resistance if drug levels in breast milk are too high or too low. It should be noted that in resource-limited areas where there is limited access to nutritional alternatives or clean water, the benefits of breast-feeding outweigh the risk of MTCT and should be encouraged. Withholding breast-feeding in such scenarios results in increased mortality not only from diarrheal illnesses, but also from other locally prevalent infectious diseases such as tuberculosis and malaria, as poor nutritional status increases susceptibility to these diseases.

Contraceptive counseling is critical to prevention of MTCT. Condom use is important for prevention of HIV and STD transmission, although the unintended pregnancy rate is high with condom use alone. It should be noted that drug interactions between oral contraceptives and many PIs and NNRTIs could potentially decrease the efficacy of oral contraceptive pills, so more than one method of contraception should be in place at any given time. For women who are certain they do not wish future childbearing, thorough counseling and discussion about permanent contraceptive methods should be done (20).

\section{NEONATAL POSTNATAL CARE}

Infants born to mothers with unknown HIV infection status should have rapid HIV antibody testing of the mother or infant performed. If the rapid test is found to be positive, ARV prophylaxis should be started immediately while waiting for the confirmatory tests. If the confirmatory test is negative, then ARV prophylaxis should be discontinued. A positive confirmatory test for HIV antibodies should be followed by a virologic test (HIV RNA by PCR) done on the neonate. A positive virologic test on the neonate should prompt immediate referral to a pediatric HIV specialist for confirmation and initiation of combination ARV therapy as well as pneumocystis pneumonia (PCP) prophylaxis.

All newborns with known ARV exposure in utero should have a CBC with differential performed as a baseline before starting zidovudine, with follow-up depending on the results as well as other clinical factors (gestational age, medications, maternal antepartum therapy, etc.). Liver function tests should also be checked in infants exposed to ARV therapy in utero. If the infant develops severe clinical symptoms of unknown etiology, particularly of the CNS, serum lactate levels should be checked, and ARV prophylaxis discontinued if levels are found to be elevated. Consultation with a pediatric HIV specialist is recommended if significant abnormalities are found with the above tests.

Diagnosis of HIV infection in infants $<18$ months of age involves the use of virologic tests (HIV RNA levels), which should be done at age 14 to 21 days, 1 to 2 months, and 4 to 6 months. Some experts would also test at birth. Oral PCP prophylaxis using trimethoprim sulfamethoxazole should be initiated at 4 to 6 weeks unless HIV infection has been ruled out. PCP prophylaxis is indicated in HIV-infected infants regardless of CD4 count and should be continued until they are 1 year of age. At 1 year of age, PCP prophylaxis should be continued if the child's CD4 count is $<500$ cells/ $\mu \mathrm{L}$ or $<15 \%$ (29). Physical examination of the newborn, as well as maternal risk factors and laboratory studies, should guide specific testing for coinfection with other agents (cytomegalovirus, herpes simplex virus, hepatitis B and C, syphilis, toxoplasmosis, or tuberculosis) as appropriate.

A 6-week course of zidovudine chemoprophylaxis is recommended for all HIV-exposed neonates in the United States, ideally initiated within 6 to 12 hours of delivery. It may be appropriate to shorten the course to 4 weeks in situations where concerns exist regarding adherence or toxicity, as the 
optimal duration for neonatal chemoprophylaxis is not known. In other countries, such as the United Kingdom and Ireland, a 4-week course is the recommended regimen, while WHO guidelines indicate a 4- to 6-week course of zidovudine to be appropriate. Please see Table 3 for specific dosing for zidovudine.

Consultation with a pediatric HIV specialist should be done when dealing with premature babies or when use of additional ARV agents is being considered because of increased transmission risk, such as those with higher maternal HIV RNA levels near delivery or had vaginal delivery.

Children with significant organ system abnormalities of unknown etiology (particularly of the CNS or heart) should be evaluated for mitochondrial dysfunction. As other possible early and late effects of in utero ARV drug exposure are not fully known, follow-up should include a yearly physical examination into adulthood, particularly given the theoretical risk of carcinogenicity of nucleoside analogs.

HIV MANAGEMENT IN RESOURCE-LIMITED SETTINGS HIV management in resource-limited settings, where the vast majority of perinatal transmission occurs, presents multiple challenges during all stages of pregnancy, and significant differences exist between U.S. (DHHS and UNAIDS) and WHO guidelines. These differences result from limited availability of maternal prenatal HIV testing and limited access to ARV drug therapy. The benefits of earlier initiation of ARV therapy in asymptomatic patients in much of the world is thwarted by limited availability and access to ARV drugs, systems for delivering medical care, monitoring therapy, management of drug toxicity, and assessing drug resistance and having alternative drug regimens for patients with known or suspected drug resistance.

An NNRTI-based regimen (nevirapine or efavirenz with either lamivudine/zidovudine or tenofovir/emtricitabine) is recommended in the WHO guidelines as first-line therapy for maternal prophylaxis to prevent MTCT (as opposed to the United States, where this regimen is considered as an alternative to a lopinavir/ritonavir-based regimen). In addition, monotherapy with zidovudine starting at 14 weeks remains an option, followed by single-dose intrapartum nevirapine and a 1-week NRTI tail consisting of lamivudine and zidovudine.

Despite the well-documented risk of MTCT of HIV infection through breast-feeding, the much greater risk of mortality from malnutrition and infections makes breastfeeding necessary in many areas. Multiple options have been studied in such a scenario where breast-feeding is deemed necessary. Breast-feeding mothers on triple ARV agents for their own health are advised to continue doing so, while the infant should continue to receive the standard twice-daily zidovudine for 4 to 6 weeks. Breast-feeding mothers who are on triple ARV agents for prophylaxis should continue doing so until 1 week after cessation of breast-feeding, while the infant continues 4 to 6 weeks of zidovudine. Breast-feeding mothers who were only on zidovudine for prophylaxis should receive a single dose of nevirapine at the start of labor, as well as lamivudine and zidovudine at the start of labor up to a week following delivery. In such cases, the infant should receive nevirapine from birth up 4 to 6 weeks, or up to a week after stopping breast-feeding, if cessation of breast-feeding is done earlier.

These recommendations were based on recent trials, including one done in Malawi comparing maternal with child HIV transmission during a 4-week breast-feeding period. This study showed a higher risk of transmission in the placebo group receiving only single-dose nevirapine and 7 days of zidovudine/lamivudine (5.7\%) compared with infants who received nevirapine for 28 days $(1.7 \%, p<0.001)$ and breast-feeding mothers on ARV therapy for 28 days (2.9\%, $p=0.009)$ (30). It has also been shown that three-drug maternal ARV therapy, whether NRTI, NNRTI, or PI based, showed similar rates of virologic suppression and decreased MTCT to $1.1 \%$ (31).

A simplified dosing regimen has been developed for use in resource-limited settings for infant prophylaxis. This regimen includes the use of zidovudine $10 \mathrm{mg}$ twice a day for infants weighing less than $2.5 \mathrm{~kg}$ at birth, and $15 \mathrm{mg}$ twice a day for infants weighing more than $2.5 \mathrm{~kg}$ at birth. Another alternative approach has been to give a daily dose of nevirapine for 4 to 6 weeks. Use of combination therapy has been used, but has not been proven to be additional benefit.

The field of HIV management continues to change rapidly. Updated guidelines are available online at www.aidsinfo.nih. gov as well as at the American College of Obstetricians and Gynecologists and WHO Web sites. In the United States, the National Perinatal HIV hotline (1-888-448-8765) also provides free clinical consultation on all aspects of perinatal HIV care.

\section{REFERENCES}

1. AIDS epidemic update: November 2009. From the UNAIDS website. [Available from: http://www.unaids.org/en/KnowledgeCentre/HIVData/ EpiUpdate/EpiUpdArchive/2009/default.asp].

2. Centers for Disease Control and Prevention. HIV Prevalence Estimates United States, 2006. MMWR 2008; 57: 1073-6.

3. Centers for Disease Control and Prevention. Subpopulation Estimates from the HIV Incidence Surveillance System - United States, 2006. MMWR 2008; 57: 985-9.

4. Centers for Disease Control and Prevention. Death Rates for Human Immunodeficiency Virus (HIV) Disease Among women, by Race and Age Group - United States, 1987-2005. MMWR 2009; 58: 286.

5. McKenna MT, Hu X. Recent trends in the incidence and morbidity that are associated with perinatal human immunodeficiency virus infection in the United States. Am J Obstet Gynecol 2007; 197: pS10.

6. Centers for Disease Control. Reduction in perinatal transmission of HIV infection - United States, 1985-2005. MMWR 2006; 55: 592-7.

7. Cooper ER, Charurat M, Mofenson L, et al. Combination antiretroviral strategies for the treatment of pregnant HIV-1 infected women and prevention of perinatal HIV-1 transmission. J Acquir Immune Defic Syndr Hum Retrovirol 2002; 29: 484-94.

8. Connor EM, Sperling RS, Gelber R, et al. Reduction of maternal infant transmission of human immunodeficiency virus type 1 with zidovudine treatment. Pediatric AIDS Clinical Trials Group Protocol 076 Study Group. N Engl J Med 1994; 331: 1173-80.

9. Centers for Disease Control. Pneumocystis Pneumonia - Los Angeles. MMWR 1981; 30: 250-2.

10. Durack DT. Opportunistic infections and Kaposi's sarcoma in homosexual men. N Engl J Med 1981; 305: 1465-7.

11. Barre-Sinoussi F, Chermann JC, Rey F, et al. Isolation of a T-lymphotropic retrovirus from a patient at risk for acquired immune deficiency syndrome (AIDS). Science 1983; 220: 868-71.

12. Gallo RC, Salahuddin SZ, Popovic M, et al. Frequent detection and isolation of cytopathic retroviruses (HTLV-III) from patients with AIDS and at risk for AIDS. Science 1984; 224: 500-3. 
13. Fauci Anthony S, Lane HC. Human immunodeficiency virus disease: AIDS and related disorders. Chapter 182. In: Fauci AS, Braunwald E, Kasper DL, Hauser SL, Longo DL, Jameson JL, Loscalzo J, eds. Harrison's Principles of Internal Medicine, New York, 2008: 17e.

14. Royce RA, Seña A, Cates W Jr, Cohen MS. Sexual transmission of HIV. N Engl J Med 1997; 336: 1072-8.

15. Simon V, Ho DH, Karim QA. HIV/AIDS epidemiology, pathogenesis, prevention, and treatment. Lancet 2006; 368: 489-504.

16. Cohen MS, Shaw GM, McMichael AJ, Haynes BF. Acute HIV-1 infection. N Engl J Med 2011; 364: 1943-54.

17. The European Collaborative Study. Maternal viral load and vertical transmission of HIV-1: an important factor but not the only one. AIDS 1999; 13: 1377-85.

18. Centers for Disease Control. Revised recommendations for HIV testing of adults, adolescents, and pregnant women in health care settings. MMWR 2006: 1-17.

19. Committee on Gynecologic Practice Routine Human Immunodeficiency Virus Screening ACOG Opinion Number 411. Routine Human Immunodeficiency Virus screening. Obstet Gynecol 2008; 112: 401-3.

20. Panel on Treatment of HIV-Infected Pregnant Women and Prevention of Perinatal Transmission. Recommendations for Use of Antiretroviral Drugs in Pregnant HIV-1-Infected Women for Maternal Health and Interventions to Reduce Perinatal HIV Transmission in the United States. Sep. 14, 2011; 1-207. [Available from: http://aidsinfo.nih.gov/ContentFiles/ PerinatalGL.pdf.]

21. Centers for Disease Control Revised Surveillance Case Definitions for HIV Infection Among Adults, Adolescents, and Children Aged $<18$ Months and for HIV Infection and AIDS Among Children Aged 18 Months to <13 Years - United States, 2008. MMWR 2008; 57: 1-12.

22. Clavel F, Hance AJ. HIV drug resistance. N Engl J Med 2004; 350: 10.
23. Clumeck N, Dedes N, Pozniak A, Raffi F, EACS Executive Committee. Clinical Management and Treatment of HIV Infected Adults in Europe. Published by the European Aids Clinical Society (EACS), 2008.

24. World Health Organization. Antiretroviral drugs for treating pregnant women and preventing HIV infection in infants: recommendations for a public health approach. 2010 version.

25. Panel on Antiretroviral Guidelines for Adults and Adolescents. Guidelines for the use of antiretroviral agents in HIV-1-infected adults and adolescents. Department of Health and Human Services. December 1, 2009: 1-161. [Available from: http://www.aidsinfo.nih.gov/ContentFiles/ AdultandAdolescentGL.pdf]

26. Kourtis AP, Schmid CH, Jamieson DJ, et al. Use of antiretroviral therapy in pregnant $\mathrm{HIV}$-infected women and the risk of premature delivery: a meta-analysis. AIDS 2007; 21: 607-15.

27. Shapiro DE, Sperling RS, Mandelbrot L, et al. Risk factors for perinatal human immunodeficiency virus transmission in patients receiving zidovudine prophylaxis. Pediatric AIDS Clinical Trials Group protocol 076 Study Group. Obstet Gynecol 1999; 94: 897-908.

28. Mandelbrot L, Mayaux MJ, Bongain A, et al. Obstetric factors and mother-to-child transmission of human immunodeficiency virus type 1: the French perinatal cohorts. SEROGEST French Pediatric HIV Infection Study Group. Am J Obstet Gynecol 1996; 175(3 Pt 1): 661-7.

29. Centers for Disease Control and Prevention (CDC). Guidelines for the Prevention and Treatment of Opportunistic Infections among HIVexposed and HIV-infected children. MMWR 2009; 58: 1-176.

30. Chasela CS, Hudgens MG, Jamieson DJ, et al. Maternal or infant antiretroviral drugs to reduce HIV-1 transmission. N Engl J Med 2010; 362: 2271-81.

31. Shapiro RL, Hughes MD, Ogwu A, et al. Antiretroviral regimens in pregnancy and breast-feeding in Botswana. N Engl J Med 2010; 362: 2282-94. 


\title{
85 Other viral infections
}

\author{
Stanley A. Gall
}

\section{HUMAN PAPILLOMA VIRUS}

Human papillomavirus (HPV) is an icosahedral, nonenveloped, double-stranded deoxyribonucleic acid (DNA) virus of approximately $55 \mathrm{~nm}$ in diameter (1). The genome of HPV s consists of approximately 8 kilobase paired molecules of circular, double-stranded DNA. All the proteins are encoded on one of the two DNA strands. All papilloma viruses share a similar genomic organization consisting of an early (E) gene region, a late (L) gene region, and a regulatory region. The five "early" proteins (E1, E2, E5, E6, E7) are required for viral replications and/or cellular transformation. Transformation of the "late" structural L1 and L2 transcripts and of the E1 to E4 spliced transcript is restricted to differentiating epithelium where viral assembly occurs $(2,3)$. The L1 protein comprises the majority of the virus shell. The L1 gene is the most highly conserved gene among individual types. The $\mathrm{L} 2$ protein of $77 \mathrm{kDa}$ is known as the minor capsid protein because it contributes a smaller percentage of the capsid mass than does the L1 protein.

More than 100 different types of HPV have been identified and fully sequenced and at least an additional 50 to 100 putative types have been partially characterized (4). HPV types are assigned numbers/designations when the DNA sequence has been established and a comparison with previously known types has found less than $90 \%$ sequence identity in the L1, E6, and E7 genes of the virus (5). An isolate with more than $90 \%$ homology to a known HPV type is classified as a subtype.

All HPV types are epitheliotropic and fully differentiated squamous epithelium is required for completion of the HPV life cycle. HPV infects keratinocytes, the predominate cells of epithelial surfaces. It is believed that only basal or undifferentiated keratinocytes are infected. The viral replication cycle is complicated as the keratinocytes undergoes the process of differentiation. Virus is assembled in the nuclei of most differentiated keratinocytes and can be detected in these cells as desquamation occurs from the epithelium (6).

HPV is transmitted by close contact with desquamated keratinocytes from an infected individual. The desquamated keratinocytes contain viral particles that are thought to infect a new host through microabrasions in the epithelial surface (7).

Approximately $40 \mathrm{HPV}$ types infect the genital tract. HPV infection of the human genitalia causes a range of clinical states including asymptomatic infections, genital warts, abnormal pap smears, preinvasive and invasive cervix, and vaginal and vulval cancers (8). The different HPV types can be subdivided into two categories: "high risk and "low risk" based on whether the HPV could be found in cancer specimens. The "low-risk" types such as HPV-6 and HPV-11 are associated with benign hyper-proliferative lesions commonly referred to as genital warts or condyloma acuminata and cause abnormal pap smears and low-grade dysplastic lesions (9). "High-risk" HPV types such as HPV-16 and HPV-18 cause low- and high-grade dysplastic lesions of the cervix and invasive cervical cancer. Other HPV-related genital malignancies include vulval cancer (10), vaginal cancer, and anal cancer (11). Approximately $45 \%$ of penile cancer is associated with HPV (12). HPV-16 causes more than $50 \%$ of the non-cervical genital tract cancers. The HPV types most commonly associated with anogenital lesions are HPV-6, -11, -16, -18, -31, -33, -35, -45, -52, and -58 (13).

\section{Epidemiology}

Genital HPV infection is primarily transmitted through sexual intercourse. In, virtually, all studies of HPV prevalence and incidence, the constant predicators of infection have been measures of sexual activity, most importantly the number of sex partners (lifetime and recent) $(14,15)$. Transmission of HPV through other types of genital contact in the absence of penetrative intercourse (i.e., oral genital, manual genital, genital-genital contact) has been described but is less common than that through sexual intercourse. Genital HPV transmission by nonsexual routes is uncommon. Nonsexual routes include transmission from a mother to a newborn baby (16).

The 2002 National Survey of Family Growth indicated that $24 \%$ of females in the United States were sexually active by age 15 years (17) increasing to $40 \%$ by age 16 years and $60 \%$ by age 18 years.

\section{Natural History of HPV Infection}

The majority of HPV infections are transient and asymptomatic and cause no clinical problems. The majority (70\%) of new HPV infections clear within 1 year and approximately $90 \%$ clear within 2 years $(15,18)$. The median duration of new infections is 8 months. Persistent infection with the same highrisk oncogenic HPV is a prerequisite for cervical cancer precursors and invasive cervical cancer (19).

\section{HPV Prevalence and Incidence in the United States}

It is estimated that 6.2 million new HPV infections occur every year among persons aged 14 to 44 years $(20,21)$.

This is an underestimate as HPV infections occur in persons younger than age 14 and over 44 years (22, 23). Modeling estimates suggest that $>80 \%$ of sexually active women will have acquired genital HPV by age 50 (24).

HPV infections are not reportable diseases. The HPV disease spectrum includes abnormal cytology, genital warts, low-grade squamous intraepithelial lesion (LGSIL), -highgrade squamous intraepithelial lesion (HGSIL), carcinoma in situ, adenocarcinoma, and invasive squamous cell carcinoma (Table 1). Evaluations from gynecologic exams from a variety of offices and clinics have documented the prevalence of HPV DNA ranging from $14 \%$ to $90 \%$ (25). Prevalence was the highest among sexually active females aged $<25$ years and 
Table 1 Incidence of Human Papillomavirus Spectrum Diseases Annually in the United States

$\begin{array}{lc}\text { Cervical cancer (21) } & 11,000 \\ \text { HGSIL (22) } & 330,000 \\ \text { LGSIL (22) } & 1,400,000 \\ \text { Genital warts (23) } & 1,000,000 \\ \text { Abnormal cytology } & 1.5-2.0 \text { million } \\ \text { Abbreviations: HGSIL, high-grade squamous intraepithelial lesion; LGSIL, } \\ \text { low-grade squamous intraepithelial lesion. }\end{array}$

decreasing with age (26). In a study of sexually active women aged 18 to 25 years, the prevalence of any HPV was $26.9 \%$. Prevalence of types 6 or 11 was $2.2 \%$ and prevalence of types 16 or 18 was $7.8 \%$ (27). Prevalence was the highest among women aged 20 to 24 years $(44.8 \%)$.

Studies of incident HPV infections that have evaluated HPV DNA detection over time demonstrate that acquisition occurs soon after sexual debut. In a prospective study of college women in the United States, the cumulative probability of incident infection was $38.9 \%$ by 24 months after first sexual intercourse. HPV-6 acquisition was highest (10.4\%); 5.6\% had acquired HPV-18 (14).

\section{Clinical Sequelae of HPV Infection}

In the United States, approximately 50 million women undergo annual pap cytologic testing; approximately 3.5 to 5.0 million of these tests will require follow-up including 2.0 to 30 million with atypical squamous cells of undetermined significance (ASC-US), 1.25 million LGSIL, 300,000 HGSIL, and 1.0 million with genital warts (28).

All anogenital warts (condylomata) are caused by HPV and approximately 90\% are associated with HPV types 6 and 11 . The average time for the development of new anogenital warts after infection with HPV types 6 and 11 is 2 to 3 months. Not all persons who acquire HPV types 6 and 11 develop warts. Approximately $30 \%$ of warts regress spontaneously except during pregnancy or in states of immunosuppression when the rate of regression is much lower.

Reoccurrence of anogenital warts tends to be more common following periods of immunosuppression, either exogenous or endogenous. Infection with HPV resulting in clinical genital warts will lead to activation of cell-mediated immunity, which will prevent infections with the same HPV type. However, since many HPV infections are not cleared but become latent, immunosuppression will be followed by either active genital warts or abnormal cytology. In a majority of women who develop genital warts during pregnancy, by 6 weeks postpartum, the genital warts are totally resolved or dramatically decreased in size.

Infection in the tracheobronchial tree with HPV types 6 ad 11 may result in recurrent respiratory papillomatosis (RRP), a disease characterized by recurrent warts or papillomas in the upper respiratory tract, particularly the larynx. RRP is divided into juvenile onset (JORRP) and adult onset based on diagnosis before or after 18. JORRP is believed to result from vertical transmission of HPV from mother to infant during delivery, although the median age of diagnosis is 4 years. A multicenter registry of JORRP during 1999 to 2003 demonstrated that although the clinical course of JORRP was variable, it is associated with extensive morbidity, requiring a median of
13 lifetime surgeries to remove warts and maintain an open airway. Estimates of the incidence of JORRP range from 0.12 to 2.1 cases per 100,000 children aged $<18$ years in two cities $(29,30)$.

\section{Prevention of HPV Infection}

Condom use may reduce the risk for HPV and HPV spectrum diseases (e.g., genital warts and cervical cancer). Pap testing can detect precancerous changes in the cervix by evaluation of cytology, and HPV vaccines can prevent HPV diseases due to HPV types in the HPV vaccines.

At the current time, two HPV vaccines are licensed by FDA. The vaccine licensed in June 2006 was the quadrivalent HPV vaccine (HPV types $6,11,16,18$ ) Gardasil ${ }^{\circledR}$ manufactured by Merck \& Co. The second HPV vaccine, a bivalent vaccine (HPV types 16,18) Cervarix ${ }^{\circledR}$ licensed by the FDA in October 2009, was manufactured by GlaxoSmith Kline. The quadrivalent vaccine is licensed for women for the prevention of HPV types 6, 11, 16, 18 related cervical, vaginal, and vulval cancer precursor and dysplastic lesions and genital warts. In October 2009, the quadrivalent vaccine was licensed by the FDA for the prevention of genital warts on males of age 9 to 26 years. The bivalent vaccine, Cervarix, has been licensed for the prevention of cervical cancer precursors and cervical cancer due to HPV types 16 and 18 (Table 2).

\section{Recommendations for Vaccine Use}

Advisory committee on immunization practices (ACIP) recommends routine vaccination of males and females aged 11 to 12 years with three doses of quadrivalent HPV vaccine. The vaccine series can start as early as age 9 years. Vaccinations are also recommended for males and females aged 13 to 26 who have not previously been vaccinated or who have not completed the full series. The recommendation for the bivalent vaccine is similar but does not have the male indication.

At this time, neither HPV vaccines are recommended for use in pregnancy. However, pregnancy testing is not needed before vaccination. Any exposure to the vaccine during pregnancy should be reported to the appropriate vaccine registry:

1-800-986-8999 (Merck + Co) for quadrivalent
vaccine
$\begin{aligned} & \text { 1-888-452-9622 (GlaxoSmith Kline) for bivalent } \\ & \text { vaccine }\end{aligned}$

\section{MOLLUSCUM CONTAGIOSUM}

Molluscum Contagiosum is a human disease with a worldwide distribution and is found in persons of all age and caused by members of the Poxvirus group (31). The disease is spread by sexual intercourse or fomites.

The disease is characterized by a long incubation period from 2 weeks to 2 months. The disease presents by the appearance of firm, shiny, flesh-colored lesions that become pearly white and umbilicated and discharge a caseous material. The lesions are characterized by proliferation, hyperplasia, thickening, and degeneration of the epidermis. The infected cells become enlarged and develop large intracytoplasmic eosinophilic hyaline inclusion bodies. The lesions are not pruritic and they measure 1 to $5 \mathrm{~mm}$ in diameter but may form larger clusters. The lesions typical of molluscum contagiosum 
Table 2 Differences in Human Papillomavirus Vaccines Currently Licensed by FDA

\begin{tabular}{|c|c|c|}
\hline & Quadrivalent, Gardasil ${ }^{\circledR a}$ & Bivalent, Cervarix $^{\bigotimes^{a}}$ \\
\hline Licensed by FDA for women & June 2006 & October 2009 \\
\hline Licensed by FDA for males & October 2009 & None \\
\hline HPV types in vaccine & $6,11,16,18$ & 16,18 \\
\hline Age of vaccine recipients & $9-26$ years & $9-26$ years \\
\hline Recommended by ACIP_women & June 2006 & October 2009 \\
\hline Vaccine administrators' schedule & $0,2,6$ months & $0,1,6$ months \\
\hline
\end{tabular}

are characterized by complete lack of immunocompetent cells in the epithelial component of the lesions (32).

The principal sites of involvement include the eyelids, face, trunk, and anogenital areas. Molluscum lesions evolve over 1 to 2 months and are frequently traumatized resulting in autoinoculation. Secondary bacterial infections may occur. The lesions may persist for as long as 1 year but have persisted longer. Spontaneous regression without scarring usually occurs and recurrences are rare. Patients who are immunosuppressed may develop extensive involvement (33).

The diagnosis of molluscum contagiosum is usually made by inspection of the characteristic umbilicated white papule. The differential diagnosis includes lichen planus, verrucous papules, basal cell epithelioma, and pyogenic granuloma. If the diagnosis is in doubt, the caseous material should be examined for intracytoplasmic eosinophilic inclusions. Treatment by sharp curettage or expression of porous material will facilitate healing without scarring.

\section{INFLUENZA}

See chapter on Maternal Immunization (Chapter 71).

\section{PARVOVIRUS}

\section{Epidemiology}

The human parvovirus B19 is a member of the DNA virus family Parvoviridae (34). This virus is one of the smallest known DNA viruses approximately 18 to $25 \mathrm{~nm}$ in size, about $5.5 \mathrm{~kb}$ in length, and with single-stranded DNA (34). The virus lacks an envelope, but does code for at least two major structural or capsid proteins and at least one nonstructural protein.

The human parvovirus B19 was first discovered by British investigators in 1975 while screening blood specimens for hepatitis B antigen. Subsequently, B19 was found to be associated with aplastic anemia crisis and erythema infectiosum or "fifth disease" $(34,35)$. This virus has also been associated with chronic bone marrow suppression, arthritis or arthropathy, fetal hydrops, and purpura.

Infection with parvovirus B19 is worldwide and is commonly seen in children aged 5 to 14 years. Outbreaks are most common in winter and spring, but there is a year-toyear variation in incidence. Estimates of positive seroprevalence rates in adults range from $28 \%$ to $72 \%(34,36)$.

Parvovirus is mildly contagious with an infection rate of $30 \%$ following primary exposure. The highest rates of secondary infection are seen in household exposure where the infection rate is as high as $50 \%$ (37).
Transmission of the virus is by respiratory droplets, and the incubation period is 4 to 14 days. Viremia develops 7 to 8 days after initial infection, lasting approximately 4 days (34). The rash associated with B19 appears following the viremic phase. The primary host is no longer infectious once the rash appears. Initial infection and seroconversion provide the host with lifelong immunity and reinfection is rare.

\section{Pathophysiology}

Parvovirus B19 viral replication and encapsulation take place in the host cells' nucleus. Host cell enzymes are used in viral replication. Rapidly dividing cells are preferentially infected, perhaps a product of $S$ phase of cell replication, a requirement for viral growth. Parvovirus B19 is particularly trophic and cytotoxic for erythroid progenitor cells $(34,35)$.

Following initial incubation and viremia, there is a transient inhibition of erythropoiesis, and reticulocytopenia occurs in the infected host. Similar changes are seen in other blood cell lines to a lesser degree. The bone marrow depression is first seen approximately 7 days after the initial infection and lasts for approximately 7 days. In the host with normal red cell survival or in a non-immunosuppressed state, this bone marrow depression is usually asymptomatic and self-limited. Aplastic crisis is seen particularly in patients with abnormally shortened red blood cell survival, in conditions such as sickle cell anemia, heredity spherocytosis, pyruvate kinase deficiency, thalassemia, and autoimmune hemolytic anemia. The immunocompromised host may develop bone marrow suppression of all cell lines secondary to an inability to clear the virus.

Transplacental fetal infection and subsequent fetal anemia may result in fetal hydrops and demise. The fetus is an ideal host for human parvovirus B19 because of an immature immune system and accelerated erythropoiesis with markedly shortened red cell life span $(34,35)$. It has also been proposed that the marked fetal anemia observed may lead to tissue anoxic and subsequent high output or hypoxic cardiac failure (37). While transplacental infection may lead to fetal anemia and hydrops, parvovirus B19 is not a common cause of congenital anomalies or neonatal disease. The period of peak fetal morbidity following maternal infection is approximately 4 to 6 weeks after maternal symptoms (37). The fetal loss rate following maternal infection has been estimated to be as low as $2.5 \%(38)$.

The rash associated with human parvovirus B19 infection occurs 17 to 21 days after infection and is probably secondary to antigen-antibody reaction (35). Arthritis or arthropathy, a 
probable postinfection phenomenon, may manifest with a purpuric rash-like illness, often associated with other signs of vasculitis. This rash may begin as a vesiculopapular eruption and is often distributed in the buttocks and lower extremities.

\section{Diagnosis and Management}

As with many viral infections, infection with human parvovirus B19 may be asymptomatic or carry symptoms of only a mild flu-like illness. The rash is more common in children than in adults but adults are more likely to develop arthritic complaints. The rash is characterized as a lacy rash especially on the face providing the "slapped cheek" appearance. A variable rash on the extremities may follow with a morbilliform, confluent, circinate, or annular appearance. This usually does not involve the trunk, palms, or soles and may be pruritic. The rash usually disappears within 1 to 2 weeks but may recur especially with stress, exercise, sunlight, or bathing $(34,35)$.

Cultured human parvovirus B19 requires bone marrow cells and therefore clinically difficult. Enzyme-linked immunoassay for B19 antigen and antibodies are available to aid in diagnosis. Diagnosis can be made through DNA hybridization techniques including DNA amplification via the polymerase chain reaction (37). Maternal IgM antibodies can be detected in 3 to 4 days after onset of clinical illness, whereas IgG antibodies are seen within 7 days. IgM antibodies persist for 3 to 4 months and IgG antibodies persist for years (36). Patients who demonstrate positive IgG with a negative IgM are considered immune and carry little risk from exposure to parvovirus B19. Those patients who have had an acute infection will have a positive IgM and are at significant risk for an adverse fetal outcome.

Fetal infection can be monitored by ultrasound for evidence of fetal hydrops, and percutaneous umbilical blood sampling can be used to diagnose fetal anemia. Demonstration of parvovirus B19 antigens or parvovirus-specific IgM antibody DNA will confirm the diagnosis.

Serial middle cerebral artery (MCA) blood flow analysis can detect early signs of fetal heart failure.

Infection is strongly suggested if eosinophilic inclusion and marginated chromatin are seen in infected fetal erythroid precursors (36).

If the initial ultrasound examination of the fetus is normal, including MCA measurements, the fetus should be followed with serial ultrasound examinations up to 12 weeks after maternal symptoms. Ultrasound-documented fetal hydrops resolves spontaneously in some cases of infection. While transplacental infection and hydrops fetalis have been successfully treated with percutaneous exchange transfusion, it has been observed that spontaneous resolution is frequent. Another observation that fetuses older than 20 weeks seem to have more spontaneous resolution may occur because of a more developed immune system (38).

Maternal serum alpha fetoprotein levels are elevated in association with intrauterine infection prior to the development of fetal hydrops as detected by ultrasound. The value of the test is limited by its nonspecific nature. Also the value of intravenous gammaglobulin for postexposure prevention of infection has not been fully evaluated. Vaccines or antiviral therapies specific for parvovirus B19 have not been developed.

\section{ENTEROVIRUSES \\ Etiology}

Human enteroviruses are members of the family of RNA Picornaviridae and are some of the most common human viruses (3). There are at least 72 serotypes of enteroviruses. The four recognized classes of enterovirus are poliovirus, coxsackie A virus, coxsackie B virus, and echovirus. To date, there are three polio subtypes, 23 coxsackie A serotypes, 6 coxsackie B serotypes, and more than 30 echovirus serotypes (39-41). Echovirus serotype 72 has been identified as hepatitis A virus. This chapter will focus on non-polio enteroviruses. Hepatitis A virus will be discussed in another chapter.

Viruses of the genus enterovirus are small $(27 \mathrm{~nm})$, singlestranded RNA with approximately 7500 bases. These viruses are icosahedral and lack an envelope. Enteroviruses have a nucleocapsid that is composed of 60 structural subunits $(39,40)$.

\section{Epidemiology}

The true incidences of disease due to enteroviruses are not known and those infections are not reportable. It is thought that between $50 \%$ and $80 \%$ of infections are asymptomatic. Infections occur throughout the year and are most prevalent from June to October in temperate climates $(39,40)$. Serotypes vary by region or year. The usual route of transmission is by either respiratory droplets or the gastrointestinal route.

Of all the infections, $80 \%$ to $90 \%$ are in children and adolescents 16 years old or younger (40). Adults account for approximately $8 \%$ of the non-polio enteroviral infections. Neonatal infections within the first month of life are common. In one study, $12.8 \%$ of newborns acquired enteroviral infection within 1 month of life and all infants recovered without difficulty (39). Women contracting enteroviral infection in late pregnancy are usually asymptomatic while those displaying symptoms are mild and nonspecific.

\section{Pathophysiology}

Diseases attributed to enteroviruses include aseptic meningitis, encephalitis, myocarditis, hepatitis, pneumonia, pleurodynia, and orchitis. Though often asymptomatic, infection may manifest with rashes, pharyngitis and conjunctivitis, parotitis, pericarditis, and pancreatitis. Rarely, the infection may be fulminant and fatal.

Following initial infection, viral replication occurs in the submucosal lymphoid tissue and within 1 to 3 days can be identified in regional lymph nodes (39) with subsequent viremia. The virus can be found in distant lymphoreticular tissue, that is, liver, spleen, and bone marrow (39). After replication in the lymphoreticular tissue, a more generalized infection with inflammation occurs, affecting multiple organs or tissues such as the central nervous system (CNS), striated muscle, and the heart. Animal studies have demonstrated a shorter incubation period and higher virus titers in pregnancy. Susceptibility to infection appears to increase with gestational age, but reverts rapidly to that of the nonpregnant state following delivery (39). Sites of primary infection in the gastrointestinal tract do not show evidence of this destructive process; therefore, diarrhea will not ensue in most patients. 
The host immune response to enteroviral infection includes the production of both humoral and mucosal antibodies as well as a cytotoxic T-lymphocyte cellular response (39).

Most perinatal infections occur secondary to transmission of the virus to the neonate during parturition or in the immediate postnatal period (41). Vertical transmission of enteroviruses is rare but has been documented $(39,41)$. As for infection of children and adults, the severity of disease in the neonate may range from mild to fatal, causing diseases of the CNS, liver, or heart (41). The neonate who has acquired an enteroviral infection by vertical transmission in the immediate peripartum period is thought to be at greater risk of serious disease when compared with the infant who has become infected following postnatal transmission. This serious infection is thought to result from a lack of maternal antibodies to the enteroviral infection.

Less is known about neonatal infection with coxsackie A virus. There are case reports of in utero fetal death with disseminated coxsackie A virus. It is rare for coxsackie A virus to be passed to the fetus (42). There appears to be no association between echovirus or coxsackie B virus infection and an increased spontaneous abortion rate. Stillbirth in late pregnancy increases with both maternal echovirus and coxsackie B viral infection. The association between maternal coxsackie B virus infection and a slightly greater risk of fetal urogenital defects and congenital heart defects is tenuous $(39,41)$. Coxsackie B virus, particularly serotypes B3 and B4, has been implicated in neonatal myocarditis (43).

\section{Diagnosis and Management}

The typical presenting symptoms of maternal enteroviral infection are those of an upper respiratory tract infection such as fever of a few days of duration with or without rash. The patient may have abdominal pain of such intensity that it may be mistaken for abruptio placentae or chorioamnionitis in the pregnant patient. Rarely, the patient presents with aseptic meningitis or myopericarditis. A presumptive diagnosis can be made by demonstrating virus-specific IgM antibodies or by documenting rising IgG titers in maternal serum. Definitive diagnosis requires culturing of the virus from either respiratory secretions or stool.

If maternal infection is established more than 5 to 7 days prior to delivery, the maternal immune response provides IgG antibody that crosses the placenta and protects the neonate against serious illness. Multiple studies have indicated that when the neonate has severe echovirus, or coxsackie B virus infection, approximately $60 \%$ of the pregnant patients will have been symptomatic during the perinatal period, usually within 1 week of delivery.

There is no specific treatment, effective vaccine or reliable immune globulin or specific vaccine for enteroviral infection. Vertical transmission at birth causes the most neonatal harm. Owing to the absence of passive immunization via maternal antibodies, it is suggested that delivery should be delayed for over 1 week after the onset of disease.

\section{MUMPS}

\section{Etiology}

The mumps virus is an RNA virus in the paramyxovirus family. The virus has a lipid membrane envelope with both the neuraminidase and the hemagglutinin. The envelope carries
"S" antigen; however, the antigen does not cross-react with other paramyxoviruses. The core antigen " $\mathrm{v}$ " is more specific to the mump virus (44).

\section{Epidemiology}

Mumps is found worldwide and the virus is spread by respiratory droplets or fomites (44). Epidemics may occur especially in urban settings or where susceptible individuals are in close quarters. The infected person is contagious from 6 days prior to and up to 9 days after the onset of disease (45). The virus may be isolated from the throat, saliva, and urine during the first 3 days of illness and may be found in the urine up to 2 weeks after it has disappeared from other sites. The virus may also be isolated from blood, breast milk, and the testis.

As in the pre-vaccine era, mumps is predominantly a disease of school age children of ages 5 to 14 years. A live virus vaccine was introduced in 1967 but uptake of the vaccine was scant until 1977 when less than 50\% of children had been immunized (46). The incidence of mumps in pregnancy is estimated to be between 0.8 and 10 cases per 10,000 pregnancies.

\section{Pathophysiology}

Mumps is usually a benign and self-limited disease. Following infection and incubation, viremia occurs. The disease is asymptomatic in $30 \%$ to $60 \%$ of patients. Most symptomatic patients experience moderate debilitation (46). Typical symptoms include fever, malaise, and swelling of one or both parotid glands. If the parotid glands are swollen, other salivary glands may also be involved. Viral replication occurs in multiple organs as the virus is disseminated. Pancreatitis, arthritis, and myocarditis are rare complications (45). Approximately $20 \%$ of postpubertal males will develop orchitis. Infection of the thyroid and breast may occur in the postpubertal females.

The virus may be cultured from cerebrospinal fluid in the asymptomatic patient and meningeal signs can be seen in 5\% to $25 \%$ of infections (46). The incidence of mumps encephalitis may be as high as 5 per 1000 reported cases. The cranial nerves may be involved and the most common residual CNS symptom is deafness.

Symptomatic reinfection has been known to occur (45). Although mumps infection may be fatal, the mortality rate is extremely low. Fatal cases of mumps are more common in adults with approximately one-half of mumps-associated deaths in patients 20 years.

The mumps virus can infect the placenta and infection can cause transplacental infection. One study reported a $27 \%$ spontaneous abortion rate when mumps occurred in the first trimester (46). Mumps have not been associated with prematurity.

\section{Diagnosis and Management}

The diagnosis of mumps may be made on clinical symptoms if present. The diagnosis can be confirmed by serologic studies demonstrating virus-specific IgM or using titer of virus-specific IgG. The virus can be easily cultured in African green monkey kidney cells, embryonated hen's eggs, and human cells (47).

In contrast to other viral infections such as influenza or varicella zoster, mumps does not demonstrate increased 
virulence or severity in the pregnant patient (44). Treatment is supportive.

Special precautions with delivery in isolation if possible has been suggested when the obstetric patient has overt mumps or if the patient has had proven or suspected onset of mumps within the 10 days preceding delivery (45). The same recommendations are suggested for the nonimmune patient exposed in the 3 weeks prior to labor. Postpartum care should be provided in isolation, though there is no need to separate the patient and her infant, and breast-feeding should be permitted. It is estimated that approximately one-half of newborns delivered to patients with overt mumps will acquire asymptomatic disease and be contagious up to 4 weeks of age. Nonimmune hospital personnel should not care for patients with mumps pointing out the need for all hospital personnel to be current with their measles-mumps-rubella vaccination.

The measles-mumps-rubella (MMR) vaccine is an effective live-attenuated viral vaccine that is part of the childhood vaccination schedule when the first dose of MMR is recommended at 12 to 15 months of age and a second dose at 4 to 6 years. Two doses of MMR vaccine are recommended for students attending colleges and one dose for health-care workers (47). In response to the resurgence of measles in 1989, this recommendation also had the effect of decreasing outbreak of mumps in high schools and college students. A study from the United Kingdom using MMR vaccine indicated vaccine effectiveness of $64 \%$ after one dose and $88 \%$ after two doses (48).

During the first 6 months of 2006, the United States reported a mumps outbreak of 2597 cases in 11 states (49). The outbreak highlighted the limitation of the 1998 recommendations and ACIP issued new recommendations (Table 3). The MMR vaccine is not indicated for use during pregnancy. Women should avoid pregnancy for 1 month following vaccination. Inadvertent immunization during pregnancy is not an indication for termination of pregnancy. Immune globulin has not been shown to be effective in the prevention of mumps and is not recommended $(45,46)$.

\section{MEASLES}

\section{Etiology}

Measles is an exanthematous disease caused by a member of the paramyxovirus family. This virus differs from other

Table 3 The 2006 Advisory Committee on Immunization Practices Recommendations on Mumps

Acceptable presumptive evidence of immunity

Documentation of adequate vaccination with two doses of live MMR vaccine for

Adults including health-care workers

Students at post-high school institution

Routine vaccination for health-care workers

Persons born after 1957: two doses of MMR vaccine

Persons born before 1957: one dose of MMR vaccine

For outbreak settings

Children aged 1-4 years and adults: consider a second dose of MMR vaccine

Health-care workers born before 1957: strongly consider two doses of MMR vaccine (50)

Abbreviation: MMR, measles-mumps-rubella. members of this family in that it does not demonstrate a neuraminidase.

Prior to the introduction of measles vaccination in 1963, epidemics of measles were seen every 2 to 3 years with 500,000 cases reported annually and approximately 400 to 500 deaths attributed to the complications of measles. In the 1980s, the reported incidence of measles fell to 1500 to 3000 cases per year (44). Measles is primarily a disease of children between ages 2 and 6 years. The incidence of measles in pregnancy is rare with estimates ranging from 6 to 40 cases per 100,000 pregnancies (51). Congenital measles are rare.

Prior to vaccination, measles were very contagious with an attack rate of approximately $90 \%$ in the nonimmune patient. Transmission is by respiratory droplets with the mucosa of the nose, oropharynx, or conjunctivas as the portal of entry. The usual incubation period is 10 to 14 days but may be as short as 7 days. The patient is infectious for 2 to 3 days prior to symptoms and 3 to 4 days after the appearance of the rash.

\section{Pathophysiology}

After the initial infection and incubation, viremia occurs and the patient demonstrates an influenza-like illness. There is usually a 3-day prodromal period with fever, inflammation of the mucosal membranes, cough, and coryza. Examination of the pharyngeal mucous membranes may reveal pathognomonic Koplik's spots. Subsequently, the patient develops a generalized maculopapular exanthema and malaise (52).

Complications of measles can be very serious. The most common complication is otitis media. Secondary bacterial pneumonia may follow measles viremia and is the most lethal complication. Encephalitis may seen at any age and occurs in 1 per 1000 cases. If encephalitis complicates measles, it usually appears within 3 to 7 days after the appearance of the rash and carries a mortality of approximately $10 \%$. The overall mortality rate of measles is estimated at less than $0.1 \%$ but is higher in children $<1$ year of age (53).

\section{Diagnosis and Management}

In an epidemic, the clinical signs and symptoms may allow a clinician to make a diagnosis. The differential diagnosis of measles includes a number of viral exanthematous diseases. The case definition includes fever $\left(>101^{\circ} \mathrm{F}\right)$ rash for more than 3 days and cough, coryza, or conjunctivitis. Hemagglutination inhibition (HI) serologic tests are the most widely used for diagnosis. A fourfold rise between acute serum (within 3 days of the rash) and convalescent serum up to 20 days later is diagnostic. The HI antibody persists for years and is useful to determine immune status. The presence of IgM HI-specific antibody will also diagnose a new case of measles. The IgM antibody will usually disappear 2 to 5 days after the appearance of the rash.

There is a higher complication and mortality rate in pregnant women. Pneumonia has been noted to be the most frequent fatal complication (44).

The virus infrequently crosses the placenta. Gestational measles has been linked to a higher incidence of premature labor. Maternal measles may lead to premature labor and delivery and therefore increase neonatal mortality. Pregnancies complicated by measles have not been firmly associated with congenital malformations or a higher rate of malformations (51). 
Newborns receive passive immunity from maternally derived IgG measles-specific antibody secondary to either maternal infection or immunization.

Nonimmune patients can be protected against measles following exposure if they are provided with passively acquired immunoglobulin within 3 days of exposure. The recommended dose of IgG is $0.2 \mathrm{ml} / \mathrm{kg}$ body weight intramuscularly $(51,52)$.

Measles can be prevented with administration of MMRattenuated live virus vaccine prior to pregnancy. In 1985, Gazala et al. reported the outcome of five pregnancies at 28 to 34 weeks of gestation complicated by a measles epidemic in Israel from December 1981 to May 1982. All five pregnancies were complicated by premature delivery with a mean duration between onset of illness and delivery of 3.5 days. The infants demonstrated low birth weight, and three of four live births had severe respiratory distress. The fifth infant was stillborn. Each live-born infant received IgG intramuscularly at a dose of $0.2 \mathrm{ml} / \mathrm{kg}$ on day 1 of life. None of the infants demonstrated any clinical signs or symptoms of measles.

\section{RUBELLA}

\section{Etiology}

The rubella virus is a member of the togavirus group and is a spherical, enveloped RNA virus, 50 to $100 \mathrm{~nm}$ in diameter (54).

\section{Epidemiology}

Rubella has worldwide distribution and prior to vaccine availability, pandemics occurred approximately every 20 years, with epidemics in 6- to 9-year cycles $(54,55)$. Rubella is endemic throughout the year but in temperature climates demonstrates a peak incidence from March to May. Prior to rubella vaccination, the primary disease occurred in children aged 5 to 9 years where as in the postvaccination era there is a major shift to persons older than age 15 (55).

Transmission of rubella is by aerosolized respiratory droplets. The usual incubation period is 16 to 18 days with a range of 14 to 21 days $(54,55)$. The infected individuals may shed virus for 1 week prior to and for approximately 1 week after the appearance of the typical rash. The virus may be isolated from respiratory secretions during this time, but the virus may be present in the urine and blood prior to the rash (55). A mild prodrome of malaise, low-grade fever, headache, and conjunctivitis may precede the rash by 1 to 5 days.

\section{Pathophysiology}

The initial infection occurs on the respiratory mucosa where the primary viral replication occurs (54). During incubation, for approximately 1 week prior to the onset of the rash, the virus can be isolated from leukocytes, conjunctiva, urine, and stool. The immune response of the host is both humoral and cell mediated. Viral-specific antibodies may be detected within 24 to 48 hours of the onset of the rash.

Infection with rubella is usually self-limited and mild. Postauricular, suboccipital, and posterior cervical lymphadenopathy are distinguishing features of the disease. The adenopathy will often herald the onset of the typical rash.

The most significant complication occurs secondary to transplacental transmission of the virus to the fetus during maternal viremia. In utero, infection may occur at any gestational age (31). The virus may infect the placenta and result in granulomatous changes and necrosis of chorionic villi. Fetal rubella infection may cause spontaneous abortion or stillbirth. Once established, fetal infection is chronic and persists well beyond birth (55). The rubella virus may be persistent in the most severely affected infants for up to 1 year. The exact pathologic mechanism of fetal infection and subsequent tissue damage is not well understood. Rubella is not cytolytic like measles and inflammation is not a prominent feature of infected cells. Infected cells do show reduced mitotic activity and there is a reduction in this total number of body cells.

The rate of congenital infection and the effect of transplacental infection appear to vary with gestational age at the time of infection. In a large prospective study of 1016 women with confirmed rubella infection, the rate of congenital infection was $81 \%$ in the first 12 weeks, $54 \%$ at 13 to 16 weeks, $36 \%$ at 17 to 22 weeks, $30 \%$ at 23 to 30 weeks, $60 \%$ at 31 to 36 weeks, and $100 \%$ from 37 weeks to delivery (55). No congenital anomalies were seen if infection occurred after 16 weeks. Another prospective study found no congenital anomalies if maternal infection occurred after 17 weeks of gestation $(56,57)$.

The congenital rubella syndrome (CRS) symptoms in decreasing frequency are hearing loss, mental retardation, cardiac malformations, and ocular defects $(56,57)$. The greatest risk to the fetus is when maternal infection occurs in the first trimester. Congenital anomalies of the heart, eye, and CNS have been seen only if maternal infection occurred between 3 and 12 weeks. Deafness is the most common anomaly occurring in 58\% of affected offspring and in $40 \%$ as a single defect (56). Patent ductus arteriosus was the most common cardiac defect and accounted for $79 \%$ of the cardiac anomalies seen $(56,57)$.

\section{Diagnosis and Management}

The symptomatic patient may experience a syndrome of malaise, low-grade fever, and tender and swollen lymphadenopathy of the suboccipital, postauricular, and posterior cervical lymph nodes prior to the onset of a rash. The rash of rubella initially appears over the face and posterior to the ears as a small, faint pink circular macular rash that eventually spreads to the neck, trunk, and extremities. The rash may also appear as a general blush in the face and may be described as petechial or purpuric. The rash generally disappears in 3 days.

Clinical history and examination are not reliable for making a diagnosis. Culture is slow and expensive and not recommended. The diagnosis of rubella is confirmed by serology. Rubella-specific IgM and IgG may be detected by a variety of techniques including HI, ELISA, compliment fixation, and radioimmunoassay. As with other diseases, the presence of IgM indicates acute illness as does a fourfold rise with IgG. IgM will disappear after 4 to 6 weeks.

All obstetric patients should be screened for rubella community on their first physician visit. If a patient suspects possible exposure and her immunity has not been established, serology should be obtained immediately and retested in 3 weeks.

The patient who shows a fourfold rise in titer of IgG antibody has a confirmed infection. If this occurs in the first 20 weeks of 
pregnancy, the patient should be counseled regarding implications for the fetus and should consider termination of pregnancy.

Vaccines for rubella were licensed in 1969 and are available with measles and mumps as MMR vaccine. ACIP recommends vaccination of all infants between 12 and 15 months and an additional dose for adolescents. All patients who are nonimmune on obstetric screening should receive MMR immediately postpartum. There is no risk to the postpartum patient or to the breast-feeding infant. Contraindication to MMR vaccine includes pregnancy, febrile illness, immunodeficient states, or receipt of immune serum globulin within preceding 3 months.

The risk to the fetus of inadvertent administration of MMR in pregnancy is not known. In a review, there were no defects compatible with CRS in 552 infants born to 635 women who received vaccine during pregnancy; women who receive MMR should not get pregnant within 1 month of vaccine administration.

\section{REFERENCES}

1. Rowson KE, Malik YBW. Human Papova (wart) virus. Bacterial RW 1967; 31: 110-31.

2. Zur Hausen H, deVilliers EM, Gissman L. Papillomavirus infections and human genital cancer. Gynecol Oncol 1981; 2(2 pt 2): 5124-8.

3. Bosch FX, Lorincz A, Munoz N, et al. The causal relation between human papillomaviruses and cervical cancer. J Clin Pathol 2002; 55: 244-65.

4. Trimble C, Hildesheim A, Brinton L, et al. Heterogeneous etiology of squamous cancer of the vulva. Obset Gynecol 1996; 87: 59-64.

5. deVilliers EM. Human Papillomaviruses-introduction. Semin Cancer Biol 1999; 9: 377.

6. Bryan JT, Brown DR. Transmission of human papillomavirus Type 11 infection by desquamated cornified cells. Virology 2001; 281: 35-42.

7. Evander M, Frazer IH, Payne E, et al. Identification of the alpha (6) integrin as a candidate receptor for papilloma viruses. J Virol 1997; 21: 2449-56.

8. Zur Hausen H. Papillomaviruses in human cancer. Proc AAP 1999; 111 : 581-7.

9. Brown DR, Schrader JM, Bryan JT, et al. Detection of multiple human papillomavirus types in condylomata acuminata lesions from otherwise healthy and immunosuppressed patients. J Clin Microbiol 1999; 37: 3316-22.

10. Hildesheim A, Han CL, Brinton LA, et al. Human papillomavirus type 16 and risk of pre-invasive and invasive vulvar cancer: results from a seroepidemiological case-control study. Obstet Gynecol 1997; 90: 748-54.

11. Bosch FX, Shiffman M, Solomon D, eds. Future directions in epidemiologic and preventive research on human papillomaviruses and cancer. J Nat'l Cancer Inst Mono. Vol. 31. Oxford, UK: Oxford University Press, 2003.

12. Dillner J, Von Krogh G, Horenblas S, et al. Etiology of squamous cell cancer of the penis. J Virol Nephrol Suppl 2000; 205: 189-93.

13. Smoltkins D. Virology of human papilloma virus. Clin Obstet Gynecol 1989; 32: 117-26.

14. Winer RL, Lee SK, Hughes JP, et al. Genital human papillomavirus infection: incidence and risk factors in a cohort of university students. Am J Epidemiol 2003; 157: 218-26.

15. Ho GY, Bierman R, Beardsley L, et al. Natural history of cervix vaginal papillomavirus infections in young women. N Engl J Med 1998; 338: 423-8.

16. Watts DH, Koutsky LA, Holmes KK, et al. Low risk of perinatal transmission of human papillomavirus: results from a prospective cohort study. Am J Obstet Gynecol 1998; 178: 365-73.

17. Mosher WD, Chandra A, Jones J. Sexual behavior and selected health measures: men and women 15-44 years of age. United States 2002. Adv Data 2006; 362: 1-55.

18. Franco EL, Villa LL, Sobrinho JP, et al. Epidemiology of acquisition and clearance of cervical human papillomavirus infection in women from a high-risk area for cervical cancer. J Infect Dis 1999; 180: 1415-23.

19. Molano M, Vanden BA, Plummer M, et al. Determinants of clearance of human papillomavirus infections in Colombian women with normal cytology: a population-based 5 year follow-up study. Am J Epidemiol 2003; 158: 486-94.

20. Weinstock H, Berman S, Cates W Jr. Sexually transmitted diseases among American youth: incidence and prevalence estimates 2000. Perspect Sex Reprod Health 2004; 36: 6-10.

21. American Cancer Society, Cancer Facts and Figures 2005. Atlanta, GA: ACS, 2005: 1-60.

22. Shiffman M, Soloman D. Findings to Date from the ASCUS-LSIL Triage study (ALTS). Arch Pathol LAB Med 2003; 127: 946-949.

23. Fleischer AB, Parrish CA, Glenn R, Feldman SR. Condylomata Acuminata (Genital Warts): Patient Demographics and Treating Physicians. Sex transmitted Dis 2001; 28: 643-647.

24. Myers ER, McCrory DC, Nanda K, et al. Mathematical model for the natural history of human papillomavirus infection and cervical carcinogenesis. Am J Epidemiol 2000; 157: 1158-71.

25. Revzina NV, Dielemento RJ. Prevalence and incidence of human papillomavirus infection in women in the USA: a systematic review. Int J STD AIDS 2005; 16: 528-37.

26. Burk RD, Kelly P, Feldman J, et al. Declining prevalence of cervicovaginal human papillomavirus infection with age is independent of other risk factors. Sex Trans Dis 1996; 23: 333-41.

27. Manhart LE, Holmes KK, Koutsky LA, et al. Human papillomavirus infection among sexually active young women in the United States: implications for developing a vaccine strategy. Sex Transm Dis 2006; 33: 502-8.

28. Wright TC Jr, Cox JT, Massad LS, et al. 2001 Consensus guidelines for the management of women with cervical cytological abnormalities. JAMA 2002; 287: 2120-9.

29. Reeves WC, Ruparelia SS, Swansen KI, et al. National registry for juvenile onset recurrent respiratory papillomatosis. Arch Otolaryngol Head Neck Surg 2003; 129: 976-82.

30. Armstrong LR, Preston EJ, Rechert M, et al. Incidence and prevalence of recurrent respiratory papillomatosis among children in Atlanta and Seattle. Clin Infect Dis 2000; 31: 107-9.

31. Davis BD, Dulbecco R, Eisen HN. Other Pox viruses that infect man. In: Hagerstown MD, ed. Microbiology. 2nd edn. Harper and Row, 1973: 1275.

32. Viac J, Churdonnet Y. Immuno competent cells and epithelial modifications in molluscum contagiosum. J Cutan Pathol 1990; 17: 202-5.

33. Cotton DWK, Cooper C, Barrett OF, et al. Severe atypical molluscan contagiosum infection in an immuno compromised host. Br J Dermatol 1987; 116: 871-6.

34. Thurn J. Human Parvovirus B19: historical and clinical review. Rev Infect Dis 1988; 10: 1005-11.

35. Botbart HA. Human parvovirus infections. Annu Rev Med 1990; 41: 25-34.

36. Rodis JF, Quinn DL, Gary WG, et al. Management and outcomes of pregnancies complicated by human B19 parvovirus infection: a prospective study. Am J Obstet Gynecol 1990; 163: 1168-71.

37. Torok TJ. Human Parvovirus B19 infections in pregnancy. Pediatr Infect Dis J 1990; 9: 770-6.

38. Humphrey W, Magoon M, O’Shaughnessey R. Severe nonimmune hydrops secondary to parvovirus B19 infection. Spontaneous reversal in utero and survival of a term infant. Obstet Gynecol 1991; 78: 900-2.

39. Modlin JF, Kinney JS. Perinatal enterovirus infections. Adv Pediatr Infect Dis 1987; 2: 57-78.

40. Cherry JD, Nelson DB. Enterovirus Infections: their epidemiology and pathogenesis. Clin Pediatr 1966; 5: 59-63.

41. Modlin JF. Perinatal echovirus and group B coxsackie virus infections. Clin Perinatal 1988; 15: 233-46.

42. Torfasion EG, Reimer CB, Keyserling HC. Subclass restriction of human enterovirus antibodies. J Clin Microbiol 1987; 8: 1376-9.

43. Rosenberg HS. Cardiovascular effects for congenital infections. Am J Cardiovasc Pathol 1987; 71: 147-56.

44. Korones SB. Uncommon virus infections of the mother, fetus and newborn: influenza, mumps and measles. Clin Perinatol 1988; 15: 259-72.

45. Sterner G, Grandien M. Mumps in pregnancy at term. Scand J Infet Dis 1990; 71: 36-8.

46. CDC. Mumps Prevention. Recommendations of the advisory committee on immunization practices (ACIP). MMWR 1991; 40: 1-91. 
47. CDC. Measles, mumps and rubella-vaccine use and strategies for elimination of measles, rubella and congenital rubella syndrome and control of mumps: recommendations of the advisory committee on immunization practices (ACIP). MMWR 1998; 47: RR-8.

48. Harling R, White JM, Ramsay ME, et al. The effectiveness of the mumps component of the MMR vaccine. A case control study. Vaccine 2005; 23: 4070-4.

49. CDC. Update: multistate outbreak of mumps-United States. January 1 May 2, 2006. MMWR 2006; 55: 1-5.

50. Notice to readers. Undated Recommendations of the advisory committee on immunization practices for the control and elimination of mumps. MMWR 2006; 55: 629-30.
51. Amstey MS. Measles in principles and practice of medical therapy in pregnancy. In: Gleicher NB, ed. 2nd edn. Stamford, CT: Appleton and Lange, 1998: 791-2.

52. Grandien M, Sterner G. Measles in pregnancy. Scand J Infect Dis 1990; 71: $45-8$.

53. Gazala E, Karplus M, Liberman JR, et al. The effect of maternal measles on the fetus. Pediatr Infect Dis 1985; 4: 203-4.

54. Horstmann DM. Rubella. Clin Obstet Gynecol 1982; 25: 585-98.

55. Freij BJ, South MA, Sever JL. Maternal rubella and the congenital rubella syndrome. Clin Perinatal 1988; 15: 247-57.

56. Munro ND, Sheppard S, Smithers RW, et al. Temporal relations between maternal rubella and congenital defects. Lancet 1987; 2: 201-4.

57. Gall SA. Rubella in pregnancy. Obstet Gynecol Rep 1990; 2: 161-9. 


\author{
Joyce A. Ibana and Danny J. Schust
}

Chlamydia trachomatis is an obligate intracellular pathogen, which is now recognized as the most prevalent sexually transmitted bacteria in the world (1). In 1999, the World Health Organization (WHO) estimated that of the 340 million cases of sexually transmitted infections, 92 million were due to C. trachomatis infection. This high prevalence rate makes it an important public health concern. In women, C. trachomatis infection has detrimental effects on reproductive health and carries considerable health-care costs. Genital infection in women is primarily localized to the endocervix, causing a clinical syndrome of mucopurulent cervicitis. The majority (70-90\%) of women infected with C. trachomatis are asymptomatic (2). In untreated women, this infection can ascend into the endometrium and fallopian tubes to establish a chronic infection that can result in pelvic inflammatory disease (PID) and salpingitis. Of women with a single episode of PID, 9\% develop ectopic pregnancy and $11 \%$ develop tubal factor infertility (3). In pregnant women, C. trachomatis infection is associated with premature rupture of membranes and an increased risk of preterm delivery (4). This infection can also be vertically transmitted, causing pneumonia and conjunctivitis in newborns.

In addition to its role in genital diseases, C. trachomatis infection of the eye (trachoma) is one of the world's leading causes of preventable blindness. In 2002, the WHO reported that this infection accounts for about 3.6\% (1.3 million) of total blindness worldwide. This disease is endemic in 56 countries including many in Africa, the Middle East and some parts of Asia, Latin America, and the Western Pacific. Trachoma is transmitted through contact with eye droplets from contaminated hands, clothing, and eye-seeking flies. The most affected populations are those living in poverty where resources are limited, hygiene is poor, and conditions permit facile transmission of the infection (5).

C. trachomatis can also cause a systemic sexually transmitted disease, Lymphogranuloma venereum (LGV), which is characterized by genital ulceration and inguinal lymphadenopathy (buboes). This disease can progress and result in hemorrhagic proctocolitis, rectogenital scarring, and lymphedema. LGV is endemic in some areas of Africa, Asia, South America, and the Caribbean but is uncommon in industrialized countries (6).

\section{TAXONOMY}

C. trachomatis is a member of the family chlamydiaceae. Three species under this family are commonly associated with human diseases: C. trachomatis, C. pneumoniae, and C. psittaci. Under a classification revised in 1999, chlamydiaceae is divided into two genera, chlamydia and Chlamydophila. C. trachomatis is under the genus chlamydia, while C. pneumoniae and C. psittaci are classified under the genus Chlamydophila (7). Some of the properties that differentiate the three chlamydiaceae species that represent important human pathogens are summarized in Table 1.

The two C. trachomatis biovars (trachoma and LGV) are further differentiated into 19 serovars based on antigenic differences in the major outer membrane proteins (MOMPs). The trachoma biovar includes serovars $\mathrm{A}, \mathrm{B}, \mathrm{Ba}$, and $\mathrm{C}$, which primarily infect the conjunctiva, and serovars $\mathrm{D}, \mathrm{Da}, \mathrm{E}, \mathrm{F}, \mathrm{G}$, Ga, H, I, Ia, J, and K, which primarily infect the urogenital tract. The LGV biovar consists of serovars L1, L2a, L2b, and L3 and primarily infects the inguinal lymph nodes.

In addition to these serovars, several other variants have been previously identified $(8,9)$. One new variant of C. trachomatis (nvCT) was discovered in Sweden in 2006 (10). It carries a deletion in the target sequences used for standard nucleic acid amplification-based diagnostic methods and escaped detection until recently. In 2007, nvCT spread throughout Sweden, accounting for $20 \%$ to $65 \%$ of chlamydia cases in that country (11). The majority of those cases that were detected in other countries had epidemiologic links to Swedish contacts (12).

\section{MICROBIOLOGY AND PATHOGENESIS}

The major disease symptoms associated with C. trachomatis infection are caused not by direct pathogen activity, but rather by the host immune response to infection. The conventional view holds that manifestations of chlamydial diseases result from classical delayed-type hypersensitivity (DTH) reactions. Clinical and animal studies suggest that DTH is stimulated by chlamydial heat shock protein 60 (13-16). However, other studies also suggest that the inflammatory response that is elicited by the infected host cells themselves is sufficient to cause chronic inflammation, tissue remodeling, and subsequent scarring. Following in vitro infection with C. trachomatis, genital epithelial cells produce and secrete large quantities of pro-inflammatory chemokines, including IL-8, GRO $\alpha$, granulocyte-macrophage colony-stimulating factor (GM-CSF), IL-1 $\alpha$, IL-11, and IL-6 $(17,18)$. IL-1 has been shown to initiate human fallopian tubal damage during C. trachomatis infection in an ex vivo model (19). Overall, the increase in epithelial secretion of pro-inflammatory chemokines results in the recruitment of immune cells to the site of infection. Persistence of the inflammatory response within the genital tract results in tissue scarring (20).

C. trachomatis infection can also directly destroy infected genital epithelial cells during replication. C. trachomatis is an obligate intracellular bacterium that has a unique developmental life cycle. It enters target host cells as a small (300-400 nm) elementary body (EB), metabolically inactive but infectious. chlamydial organisms never contact the host cell cytoplasm. Rather, the entry of chlamydial EB induces the formation of a 
Table 1 Human Pathogenic Chlamydiaceae

\begin{tabular}{|c|c|c|c|}
\hline Property & Chlamydia trachomatis & Chlamydia pneumonia & Chlamydia psittaci \\
\hline Host & Primarily human & Primarily human & $\begin{array}{l}\text { Primarily birds and lower } \\
\text { mammals; occasionally } \\
\text { humans }\end{array}$ \\
\hline Diseases & $\begin{array}{l}\text { Ocular trachoma, oculogenital } \\
\text { diseases, infant pneumonia and } \\
\text { lymphogranuloma venereum }\end{array}$ & $\begin{array}{l}\text { Upper and lower respiratory } \\
\text { tract infection }\end{array}$ & Psittacosis \\
\hline $\begin{array}{l}\text { Susceptibility to } \\
\text { sulfonamides }\end{array}$ & Yes & No & No \\
\hline
\end{tabular}

new intracellular compartment partially derived from the host cell's Golgi apparatus. This compartment, called an inclusion body, inhibits phagolysosomal fusion. chlamydial EBs within the inclusion body utilize host adenosine triphosphate for energy. Within 6 to 8 hours after entry, chlamydial EBs undergo primary differentiation and transform into larger $(800-1000 \mathrm{~nm})$ actively dividing reticulate bodies (RBs). RBs are metabolically active and noninfectious. RBs continuously divide over the next 18 hours by binary fission to produce 300 to 500 progeny. At 48 to 72 hours after infection, C. trachomatis RBs transform back into EBs through secondary differentiation. Upon completion of their developmental cycle within the host cell, EBs exit the epithelial cell to infect neighboring uninfected cells.

An alternate growth mode of chlamydia, commonly referred to as persistent growth, has also been observed in vitro. Persistent growth is induced by factors such as amino acid deprivation (21), penicillin treatment (22), and exposure to suboptimal levels of interferon gamma (23). This growth mode is characterized by enlarged and pleomorphic RBs that do not undergo binary fission and differentiation into EB. During persistent growth in the host cell, chlamydial organisms are viable but not cultivable. This condition, however, can be reversed upon removal of the persistenceinducing factor (24). Although direct evidence of persistent growth in vivo has not been clearly established, this phenomenon may explain chronic infection resulting from reactivation of these alternate persistent forms. Cycles of dormancy and reactivation may contribute to the pathologic changes observed in chronic disease (Fig. 1).

\section{EPIDEMIOLOGY}

In the United States, 1,108,374 chlamydial infections were reported to the Centers for Disease Control and Prevention (CDC) in 2007, corresponding to a rate of 370.2 cases per 100,000 people. This rate translates to a more than fourfold increase from 1988 when 87.1 cases were reported per 100,000 people (25). Independent studies in different cities in the United States and in different countries around the globe, however, have demonstrated variation in prevalence rates depending on several factors, including high-risk sexual behavior and age $(26,27)$. A comparative study conducted in the Philippines between high-risk female sex workers attending a social hygiene clinic and low-risk women attending an obstetrics and gynecology clinic showed that high-risk women have a fivefold higher chlamydial prevalence rate compared with low-risk women (28). In addition, multiple studies have shown that young age is the factor most closely associated with C. trachomatis infection $(29,30)$. Infection rates in sexually active adolescents are consistently at least two- to threefold higher than that in adult women (31).

In the same 2007 CDC report, the overall rate of chlamydial infection in women in the United States was 543.6 cases per 100,000 . The rate of chlamydial infection in men is lower than that in women with 190.0 reported cases per 100,000 people. This lower rate in men may reflect the higher number of women screened for C. trachomatis and may also indicate that sex partners of infected women may not be diagnosed or reported. This is a major deterrent to efforts at controlling the rate of infection in women because men provide a continuous, untreated reservoir for transmission of new and recurrent infections.

Approximately $5 \%$ to $15 \%$ of pregnant women in the United States are infected with C. trachomatis (32). Independent studies in other countries show that prevalence rates in pregnant women are widely divergent (Table 2). Assessment of $C$. trachomatis infection prevalence rates, however, is complicated by the use of several different diagnostic methods among different studies. These methods may vary markedly in their sensitivities (see section on Diagnosis and Screening) and specificities. Comparison among studies is also compounded by biases in the selection process and heterogeneity in inclusion criteria among investigations. An evaluation limited to those persons seeking medical care in clinics may not be representative of the entire population. Thus, there exist significant limitations in generalizations made based on these studies.

\section{Risk Factors of chlamydia Infection in Pregnancy}

The risk factors for chlamydial infection in pregnant women were summarized in a review article by Tiller et al. and include young age ( $<20$ years of age), coinfection with other sexually transmitted diseases, abacteriuric pyuria, unprotected sex within the past 3 months, a partner with nongonococcal urethritis, and more than one sexual partner in the last 3 months (41). Like studies in nonpregnant women, young age is consistently associated with chlamydial infection among pregnant women. 


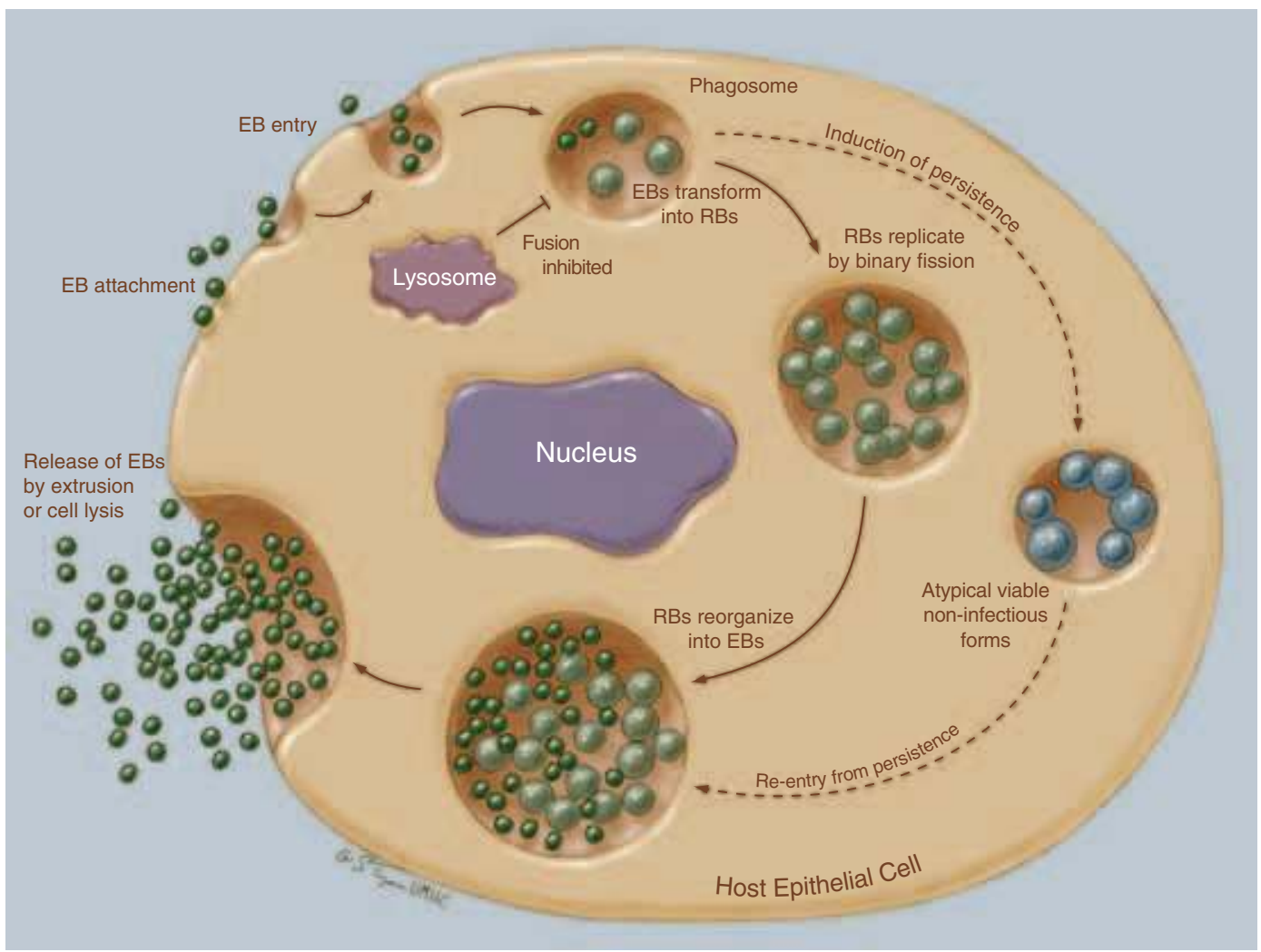

Figure 1 Chlamydial growth cycle. Chlamydia travels between susceptible cells as small, metabolically inert forms, called the elementary bodies (EBs). EBs attach to cells and enter into a phagosomal compartment partially derived from the host cell Golgi apparatus. Within the phagosomal compartment, now called an inclusion, EBs transform into larger, metabolically active forms called reticulate bodies (RBs). RBs reproduce by binary fission, filling the enlarging inclusion. Late in the chlamydial life cycle (36-48 hours), RBs reorganize into EBs and EBs are released into the extracellular space to infect surrounding susceptible cells. Under certain in vitro conditions, chlamydial RBs undergo differentiation into large atypical forms that may persist within the infected cell for prolonged periods of time. Clinical and epidemiologic characteristics of chlamydial infections also support persistence. [Reproduced with permission from The Curators of the University of Missouri (Copyright 2011, The Curators of the University of Missouri)].

\section{Table 2 Prevalence of Chlamydia trachomatis Infection in Pregnant Women}

\begin{tabular}{llr} 
Reference & \multicolumn{1}{c}{ Site } & Prevalence rate (\%) \\
Chen et al. $2009(33)$ & Melbourne, Australia & 3.2 \\
Lujan et al. $2008(34)$ & Tete, Mozambique & 4.1 \\
Lubbad et al. $2007(35)$ & Gaza, Palestine & 8.3 \\
Chen et al. 2006 (36) & Fuzhou, China & 10.1 \\
Kadzhaia et al. 2005 (37) & Tbilisi, Georgia & 33.0 \\
Apea-Kubi et al. 2004 (38) & Accra, Ghana & 3.0 \\
Shimano et al. 2004 (39) & Hokkaido, Japan & 4.2 \\
Chotnopparatpattara et al. 2003 (40) & Bangkok, Thailand & 10
\end{tabular}

C. trachomatis infection in pregnant women increases the risk of pre-term delivery, premature rupture of membranes, and perinatal mortality. Thus, prenatal screening of highrisk pregnant women and treatment of newly diagnosed infections can help to prevent adverse pregnancy outcomes and transmission to neonates. The CDC recommends screening at the onset of prenatal care, then again in the third trimester.

\section{Perinatal Risks of chlamydial Infection}

Based on a recent study of vertical transmission of C. trachomatis in China, the transmission rate of C. trachomatis from infected mother to infants born through vaginal passage is approximately $66.7 \%$. In comparison, the risk to those born by cesarean section is $8.3 \%$ (42)
Transmission of C. trachomatis infection from mother to infant may also occur via exposure to intrauterine infection during late pregnancy (43). Intrauterine infection may be responsible for chorioamnionitis, a frequent cause for prematurity and respiratory insufficiency at risk neonates (44). chlamydial infection may also result in neonatal conjunctivitis and in infant pneumonia that may progress to obstructive lung disease.

Early spontaneous pregnancy loss has been attributed to C. trachomatis infection-based seroepidemiologic evidence and on the detection of chlamydial antigens, in endometrial curettage samples $(45,46)$. This correlation, however, is not without controversy (47). An association between C. trachomatis infection and premature rupture of the membranes has also been suggested and intrauterine lung infection by 
C. trachomatis has been demonstrated has been demonstrated in premature infants (48).

\section{DIAGNOSIS AND SCREENING}

Traditional diagnostic testing for chlamydial infection involves cell culture techniques using urethral-, cervical-, or conjunctivalderived patient specimens. Specimens are inoculated on cycloheximide-treated McCoy cells, a susceptible tissue culture cell line, and examined after 24 and 72 hours of incubation. chlamydia inclusions are then stained using immunofluorescent chlamydia-specific antibodies and examination of chlamydial growth is performed using standard microscopy. In competent hands, this method can have a specificity as high as $100 \%$; therefore, culture-based detection of chlamydial infections has long been used as a reference standard for assessing the validity of new diagnostic tests and is the method of choice for medicolegal purposes. The cell culture method, however, is technically difficult, time consuming, and expensive. Furthermore, its sensitivity has been estimated to be only $60 \%$ to $85 \%(49,50)$. The performance of these techniques can vary between handlers and even within a laboratory. Thus, although some laboratories have maintained culture techniques for their high specificity, most others now use nonculture-based alternatives. These simpler techniques are more practical for routine work and offer higher sensitivity. They include detection of chlamydial antigens using directly labeled fluorescent antibodies (DFAs) and enzyme immunosorbent assays (EIAs) and detection of chlamydial genetic material (RNA/DNA) using nucleic acid amplification tests (NAATs).

DFA involves the use of fluorescein-conjugated antibodies to directly detect chlamydial EBs within patient specimens. This test is rapid and its sensitivities and specificities range from $70 \%$ to $100 \%$ and $87 \%$ to $99 \%$, respectively, for men and $68 \%$ to $100 \%$ and $82 \%$ to $100 \%$, respectively, for women $(51,52)$. The use of this technique is much faster than cell culture techniques but also requires highly skilled personnel. The EIA assay, on the other hand, does not require highly skilled personnel and has sensitivities and specificities that range from $62 \%$ to $97 \%$ and $92 \%$ to $100 \%$ for men and $64 \%$ to $100 \%$ and $89 \%$ to $100 \%$ for women (53). The sensitivity of the EIA method, however, has recently been questioned $(54,55)$.

Because chlamydial genetic material can be shed into the urine, NAAT can be performed on specimens collected noninvasively. The major target sequence used in nucleic acid amplification techniques for the diagnosis of chlamydial infection is the ompl gene, which codes for the MOMP present in all three human pathogenic chlamydial species (56). Because this method includes an amplification step that improves sensitivity, NAAT has a better sensitivity than EIA for detecting chlamydia in urine specimens. NAAT is now also considered an effective method for detecting asymptomatic chlamydial infections. Currently, there are several NAAT platforms available for chlamydial diagnostic and screening purposes. A recent evaluation using first void urine specimens and three automated NAAT systems [ProbeTec ET reagents on Viper platform (BD Diagnostics), Aptima Combo 2 reagents on Tigris platform (Gen-Probe) and Abbott RealTime CT/NG reagents on an m200 platform (Abbott Molecular Diagnostics)] revealed that these approaches are all suitable for
C. trachomatis detection and each offers high sensitivity and specificity (57).

The use of urine specimens for NAAT testing is also the method of choice for screening men for chlamydial infections. It is noninvasive and differences in sensitivity and specificity between urethral swabs and urine samples are not significant. Screening targeted to men is beneficial from a public health perspective, as men provide a reservoir for continuous transmission to women. The rates of C. trachomatis infection in men vary from $4 \%$ to $6 \%$ of which approximately $90 \%$ are asymptomatic (58-60). The availability of an effective noninvasive method for screening men may provide more widespread detection of asymptomatic C. trachomatis-infected individuals in the community. Furthermore, this methodology can be readily used in school-based health centers in highprevalence communities. Such noninvasive diagnostics could greatly increase the identification of infected individuals for treatment and help to prevent the spread of infection.

The use of vaginal swabs for NAAT testing offers an effective alternative for screening women for chlamydial infection. An evaluation of the use of vaginal swab specimens in the Aptima NAAT system revealed that NAAT provides excellent sensitivity and specificity. Using NAAT for detecting chlamydia on patient-collected vaginal swabs gives a sensitivity and specificity of $98.3 \%$ and $96.5 \%$ respectively, and for clinician-collected swabs, $97.2 \%$ and $95.2 \%$ respectively (61). Thus, the use of vaginal swab specimens for NAAT provides an excellent alternative to pelvic examination and endocervical specimen collection. To prevent the adverse effects of chlamydial infection on pregnancy outcomes, the screening of all pregnant women is recommended by the CDC. For these purposes, the noninvasive NAAT is the method of choice. In short, widespread, noninvasive screening for chlamydial infection in men and women should lead to early detection and treatment, thereby reducing reproductive tract morbidities and preventing vertical and horizontal transmission.

\section{TREATMENT AND MANAGEMENT}

Treatment of C. trachomatis-infected patients is essential not only for clearing infection and eliminating symptoms, but also for the prevention of transmission to sex partners. In pregnant women, treatment is also given to prevent vertical transmission to infants. Based on the latest CDC guidelines (62), the recommended firstline treatment for C. trachomatis is either a single 1-g oral dose of azithromycin or a twice daily, 100-mg oral dose of doxycycline for 7 total days. In populations in which adherence to the 7-day doxycycline regimen is typical, azithromycin and doxycycline are equally efficacious, with microbial cure rates of $97 \%$ and $98 \%$ respectively. However, in populations with poor compliance or unpredictable follow-up, cure rates for the azithromycin regimen exceeded those using doxycycline. Since the majority of gonococci in the United States are susceptible to azithromycin and doxycycline, either of these treatment regimens may also provide coverage for chlamydia patients coinfected with Neisseria gonorrhoeae. Recommended alternative regimens for C. trachomatis treatment include erythromycin, ofloxacin, and levofloxacin. Ofloxacin and levofloxacin, however, offer no dosage advantages and are more expensive than erythromycinbased treatments. 
For pregnant women, doxycycline, ofloxacin, and levofloxacin are contraindicated. Thus, the recommended firstline treatment for a chlamydia-infected pregnant woman is the single 1 -g oral dose of azithromycin or a 500-mg oral dose of amoxicillin, three times a day for 7 days. Alternative regimens include erythromycin base or erythromycin ethylsuccinate. Frequent occurrence of gastrointestinal side effects with use of erythromycins discourages compliance and consequently reduces treatment efficacy. Lower erythromycin doses for longer duration are alternative regimens that may improve gastrointestinal tolerance. Notably, erythromycin estolate is contraindicated during pregnancy because of drug-related hepatotoxicity. To ensure therapeutic cure, testing for chlamydial infection must be repeated 3 weeks after the completion of therapy in pregnant subjects. This reduces the risk of maternal and neonatal sequelae with persistent disease. It is also recommended that sexual partners of pregnant women be tested and treated if they have sexual contact within 60 days after treatment. If either partner is positive for chlamydial infection, he/she should be instructed to avoid sexual contact until both have been cured and are without symptoms.

For infants with ophthalmia neonatorum or pneumonia due to chlamydial infection, the recommended regimen is erythromycin base or erythromycin ethylsuccinate. Efficacy using

\section{Table 3 Treatment Recommendations for Chlamydial} Infections

\section{Recommended regimens}

Azithromycin $1 \mathrm{~g}$ orally, single dose, or

Doxycycline $100 \mathrm{mg}$ orally twice a day for 7 days

Alternative regimens

Erythromycin base $500 \mathrm{mg}$ orally four times a day for 7 days, or Erythromycin ethylsuccinate $800 \mathrm{mg}$ orally four times a day for 7 days, or

Ofloxacin $300 \mathrm{mg}$ orally twice a day for 7 days, or

Levofloxacin $500 \mathrm{mg}$ orally once daily for 7 days

For pregnant women

Recommended regimens

Azithromycin $1 \mathrm{~g}$ orally, single dose, or

Amoxicillin $500 \mathrm{mg}$ orally three times a day for 7 days

Alternative regimens

Erythromycin base $500 \mathrm{mg}$ orally four times a day for 7 days, or Erythromycin base $250 \mathrm{mg}$ orally four times a day for 14 days, or Erythromycin ethylsuccinate $800 \mathrm{mg}$ orally four times a day for 7 days, or

Erythromycin ethylsuccinate $400 \mathrm{mg}$ orally four times a day for 14 days

For newborns

Erythromycin base or ethylsuccinate $50 \mathrm{mg} / \mathrm{kg} /$ day orally divided into four doses daily for 14 days

For children who weigh $<45 \mathrm{~kg}$

Erythromycin base or ethylsuccinate $50 \mathrm{mg} / \mathrm{kg} /$ day orally divided into four doses daily for 14 days

For children who weigh $>45 \mathrm{~kg}$ but who are aged $<8$ years Azithromycin $1 \mathrm{~g}$ orally, single dose

For children aged $>8$ years

Azithromycin $1 \mathrm{~g}$ orally, single dose, or

Doxycycline $100 \mathrm{mg}$ orally twice a day for 7 days

Source: From Centers for Disease Control and Prevention 2010 treatment guidelines (62). erythromycin treatment is only $80 \%$, so follow-up testing on infants is recommended. If infection was not cleared, a second course of similar therapy is recommended (Table 3 ).

\section{PREVENTION AND FUTURE RESEARCH}

Implementation of a comprehensive $C$. trachomatis screening program can lead to a $60 \%$ reduction in the incidence of PID (63). With the availability of noninvasive, highly specific, and sensitive methods for the detection of $C$. trachomatis infection, opportunistic chlamydia screening has great potential for controlling the spread of infection through early detection and early treatment. Importantly, this includes the detection and treatment of subjects with asymptomatic infection. Combined, these screening efforts should help to prevent the development of adverse sequelae and further transmission of the infection.

Despite the efficacy of antibiotics in treating C. trachomatis infection, treatment is primarily given to those with symptomatic infections. Continued transmission by untreated individuals with asymptomatic infection hampers control efforts difficult. To achieve more effective control measures, the development of a safe and efficacious human vaccine is necessary, but has eluded researchers to date. Ongoing animal studies, however, do have promise. Novel antigens that induce protective immunity in $C$. trachomatis mouse models have been identified $(64,65)$ and provide vaccine candidates that may be suitable for human study. Also in murine models, the intranasal administration of a secreted chlamydial protein was found to enhance resolution of infection and provide protection against oviduct pathology (66). These advances in chlamydia vaccine development should ultimately lead to better chlamydial control measures and a reduction in the global prevalence rate of $C$. trachomatis infection.

\section{REFERENCES}

1. Manavi K. A review on infection with chlamydia trachomatis. Best Pract Res Clin Obstet Gynaecol 2006; 20: 941-51.

2. Peipert JF. Clinical practice. Genital chlamydial infections. N Engl J Med 2003; 349: 2424-30.

3. Cohen CR, Brunham RC. Pathogenesis of Chlamydia induced pelvic inflammatory disease. Sex Transm Infect 1999; 75: 21-4.

4. Blas MM, Canchihuaman FA, Alva IE, Hawes SE. Pregnancy outcomes in women infected with Chlamydia trachomatis: a population-based cohort study in Washington State. Sex Transm Infect 2007; 83: 314-18.

5. Wright HR, Turner A, Taylor HR. Trachoma and poverty: unnecessary blindness further disadvantages the poorest people in the poorest countries. Clin Exp Optom 2007; 90: 422-8.

6. Mabey D, Peeling RW. Lymphogranuloma venereum. Sex Transm Infect 2002; 78: 90-2.

7. Everett KD, Bush RM, Andersen AA. Emended description of the order Chlamydiales, proposal of Parachlamydiaceae fam. nov. and Simkaniaceae fam. nov., each containing one monotypic genus, revised taxonomy of the family Chlamydiaceae, including a new genus and five new species, and standards for the identification of organisms. Int J Syst Bacteriol 1999; 49(Pt 2): 415-40.

8. Dean D, Millman K. Molecular and mutation trends analyses of omp1 alleles for serovar $\mathrm{E}$ of Chlamydia trachomatis. Implications for the immunopathogenesis of disease. J Clin Invest 1997; 99: 475-83.

9. Lampe MF, Suchland RJ, Stamm WE. Nucleotide sequence of the variable domains within the major outer membrane protein gene from serovariants of Chlamydia trachomatis. Infect Immun 1993; 61: 213-19.

10. Ripa T, Nilsson P. A variant of Chlamydia trachomatis with deletion in cryptic plasmid: implications for use of PCR diagnostic tests. Euro Surveill 2006; 11: E061109. 2. 
11. Herrmann B. A new genetic variant of Chlamydia trachomatis. Sex Transm Infect 2007; 83: 253-4.

12. Savage EJ, Ison CA, van de Laar MJ. Results of a Europe-wide investigation to assess the presence of a new variant of Chlamydia trachomatis. Euro Surveill 2007; 12: E3-4.

13. Eckert LO, Hawes SE, Wolner-Hanssen P, et al. Prevalence and correlates of antibody to chlamydial heat shock protein in women attending sexually transmitted disease clinics and women with confirmed pelvic inflammatory disease. J Infect Dis 1997; 175: 1453-8.

14. Peeling RW, Kimani J, Plummer F, et al. Antibody to chlamydial hsp60 predicts an increased risk for chlamydial pelvic inflammatory disease. J Infect Dis 1997; 175: 1153-8.

15. Peeling RW, Patton DL, Cosgrove Sweeney YT, et al. Antibody response to the chlamydial heat-shock protein 60 in an experimental model of chronic pelvic inflammatory disease in monkeys (Macaca nemestrina). J Infect Dis 1999; 180: 774-9.

16. Lichtenwalner AB, Patton DL, Van Voorhis WC, Sweeney YT, Kuo CC. Heat shock protein 60 is the major antigen which stimulates delayed-type hypersensitivity reaction in the macaque model of Chlamydia trachomatis salpingitis. Infect Immun 2004; 72: 1159-61.

17. Rasmussen SJ, Eckmann L, Quayle AJ, et al. Secretion of proinflammatory cytokines by epithelial cells in response to Chlamydia infection suggests a central role for epithelial cells in chlamydial pathogenesis. J Clin Invest 1997; 99: 77-87.

18. Buchholz KR, Stephens RS. Activation of the host cell proinflammatory interleukin-8 response by Chlamydia trachomatis. Cell Microbiol 2006; 8: 1768-79.

19. Hvid M, Baczynska A, Deleuran B, et al. Interleukin-1 is the initiator of Fallopian tube destruction during Chlamydia trachomatis infection. Cell Microbiol 2007; 9: 2795-803.

20. Stephens RS. The cellular paradigm of chlamydial pathogenesis. Trends Microbiol 2003; 11: 44-51.

21. Coles AM, Reynolds DJ, Harper A, Devitt A, Pearce JH. Low-nutrient induction of abnormal chlamydial development: a novel component of chlamydial pathogenesis? FEMS Microbiol Lett 1993; 106: 193-200.

22. Matsumoto A, Manire GP. Electron microscopic observations on the effects of penicillin on the morphology of Chlamydia psittaci. J Bacteriol 1970; 101: 278-85.

23. Beatty WL, Byrne GI, Morrison RP. Morphologic and antigenic characterization of interferon gamma-mediated persistent Chlamydia trachomatis infection in vitro. Proc Natl Acad Sci USA 1993; 90: 3998-4002.

24. Hogan RJ, Mathews SA, Mukhopadhyay S, Summersgill JT, Timms P. Chlamydial persistence: beyond the biphasic paradigm. Infect Immun 2004; 72: 1843-55.

25. Centers for Disease Control and Prevention Sexually Transmitted Disease Surveillance 2007. Atlanta, GA: U.S. Department of Health and Human Services, 2008.

26. Miller WC, Ford CA, Morris M, et al. Prevalence of chlamydial and gonococcal infections among young adults in the United States. JAMA 2004; 291: 2229-36.

27. Ku L, St Louis M, Farshy C, et al. Risk behaviors, medical care, and chlamydial infection among young men in the United States. Am J Public Health 2002; 92: 1140-3.

28. Saison F, Mahilum-Tapay L, Michel CE, et al. Prevalence of Chlamydia trachomatis infection among low- and high-risk Filipino women and performance of Chlamydia rapid tests in resource-limited settings. J Clin Microbiol 2007; 45: 4011-17.

29. Macmillan S, McKenzie H, Flett G, Templeton A. Which women should be tested for Chlamydia trachomatis? BJOG 2000; 107: 1088-93.

30. Tarr ME, Gilliam ML. Sexually transmitted infections in adolescent women. Clin Obstet Gynecol 2008; 51: 306-18.

31. Shrier L. Bacterial sexually transmitted infections: gonorrhea, chlamydia, pelvic inflammatory disease, and syphilis. In: Emans SJLM, Goldstein DP, eds. Pediatric and Adolescent Gynecology, 5th edn. Philadelphia: Lippincott, Wiliams and Wilkins, 2005: 565-614.

32. Andrews WW, Klebanoff MA, Thom EA, et al. Midpregnancy genitourinary tract infection with Chlamydia trachomatis: association with subsequent preterm delivery in women with bacterial vaginosis and Trichomonas vaginalis. Am J Obstet Gynecol 2006; 194 : 493-500.
33. Chen MY, Fairley CK, De Guingand D, et al. Screening pregnant women for chlamydia: what are the predictors of infection? Sex Transm Infect 2009; 85: 31-5.

34. Lujan J, de Onate WA, Delva W, et al. Prevalence of sexually transmitted infections in women attending antenatal care in Tete province, Mozambique. S Afr Med J 2008; 98: 49-51.

35. Lubbad AM, Al-Hindi AI. Bacterial, viral and fungal genital tract infections in Palestinian pregnant women in Gaza, Palestine. West Afr J Med 2007; 26: 138-42.

36. Chen XS, Yin YP, Chen LP, et al. Sexually transmitted infections among pregnant women attending an antenatal clinic in Fuzhou, China. Sex Transm Dis 2006; 33: 296-301.

37. Kadzhaia D, Merabishvili N. Prevalence and risk factors for Chlamydia trachomatis infection in pregnant women. Georgian Med News 2005; 129: 33-6.

38. Apea-Kubi KA, Yamaguchi S, Sakyi B, et al. Neisseria gonorrhoea, Chlamydia trachomatis, and Treponema pallidum infection in antenatal and gynecological patients at Korle-Bu Teaching Hospital, Ghana. Jpn J Infect Dis 2004; 57: 253-6.

39. Shimano S, Nishikawa A, Sonoda T, Kudo R. Analysis of the prevalence of bacterial vaginosis and Chlamydia trachomatis infection in 6083 pregnant women at a hospital in Otaru, Japan. J Obstet Gynaecol Res 2004; 30: 230-6.

40. Chotnopparatpattara P, Limpongsanurak S, Wongprechasawas A. The prevalence of Chlamydia trachomatis infection in pregnant Thai women. J Med Assoc Thai 2003; 86(Suppl 2): S399-403.

41. Tiller CM. Chlamydia during pregnancy: implications and impact on perinatal and neonatal outcomes. J Obstet Gynecol Neonatal Nurs 2002; 31: 93-8.

42. Yu J, Wu S, Li F, Hu L. Vertical transmission of Chlamydia trachomatis in Chongqing China. Curr Microbiol 2009; 58: 315-20.

43. Numazaki K, Asanuma H, Niida Y. Chlamydia trachomatis infection in early neonatal period. BMC Infect Dis 2003; 3: 2.

44. Hillier SL, Martius J, Krohn M, et al. A case-control study of chorioamnionic infection and histologic chorioamnionitis in prematurity. N Engl J Med 1988; 319: 972-8.

45. Rastogi S, Salhan S, Mittal A. Detection of Chlamydia trachomatis antigen in spontaneous abortions. Is this organism a primary or secondary indicator of risk? Br J Biomed Sci 2000; 57: 126-9.

46. Witkin SS, Ledger WJ. Antibodies to Chlamydia trachomatis in sera of women with recurrent spontaneous abortions. Am J Obstet Gynecol 1992; 167: 135-9.

47. Rae R, Smith IW, Liston WA, Kilpatrick DC. Chlamydial serologic studies and recurrent spontaneous abortion. Am J Obstet Gynecol 1994; 170: 782-5.

48. Sweet RL, Landers DV, Walker C, Schachter J. Chlamydia trachomatis infection and pregnancy outcome. Am J Obstet Gynecol 1987; 156: 824-33.

49. Schachter J. Chlamydia trachomatis: the more you look, the more you find-how much is there? Sex Transm Dis 1998; 25: 229-31.

50. Johnson RE, Green TA, Schachter J, et al. Evaluation of nucleic acid amplification tests as reference tests for Chlamydia trachomatis infections in asymptomatic men. J Clin Microbiol 2000; 38: 4382-6.

51. Shafer MA, Vaughan E, Lipkin ES, Moscicki BA, Schachter J. Evaluation of fluorescein-conjugated monoclonal antibody test to detect Chlamydia trachomatis endocervical infections in adolescent girls. J Pediatr 1986; 108(5 Pt 1): 779-83.

52. Stamm WE, Harrison HR, Alexander ER, et al. Diagnosis of Chlamydia trachomatis infections by direct immunofluorescence staining of genital secretions. A multicenter trial. Ann Intern Med 1984; 101: 638-41.

53. Taylor-Robinson D, Thomas BJ. Laboratory techniques for the diagnosis of chlamydial infections. Genitourin Med 1991; 67: 256-66.

54. Van Der Pol B, Ferrero DV, Buck-Barrington L, et al. Multicenter evaluation of the BDProbeTec ET System for detection of Chlamydia trachomatis and Neisseria gonorrhoeae in urine specimens, female endocervical swabs, and male urethral swabs. J Clin Microbiol 2001; 39: 1008-16.

55. Watson EJ, Templeton A, Russell I, et al. The accuracy and efficacy of screening tests for Chlamydia trachomatis: a systematic review. J Med Microbiol 2002; 51: 1021-31. 
56. Fredlund H, Falk L, Jurstrand M, Unemo M. Molecular genetic methods for diagnosis and characterisation of Chlamydia trachomatis and Neisseria gonorrhoeae: impact on epidemiological surveillance and interventions. APMIS 2004; 112: 771-84.

57. Levett PN, Brandt K, Olenius K, et al. Evaluation of three automated nucleic acid amplification systems for detection of Chlamydia trachomatis and Neisseria gonorrhoeae in first-void urine specimens. J Clin Microbiol 2008; 46: 2109-11.

58. Cohen DA, Nsuami M, Etame RB, et al. A school-based Chlamydia control program using DNA amplification technology. Pediatrics 1998; 101: E1.

59. Cohen DA, Nsuami M, Martin DH, Farley TA. Repeated school-based screening for sexually transmitted diseases: a feasible strategy for reaching adolescents. Pediatrics 1999; 104: 1281-5.

60. Nsuami M, Cohen DA. Participation in a school-based sexually transmitted disease screening program. Sex Transm Dis 2000; 27: 473-9.

61. Schachter J, Chernesky MA, Willis DE, et al. Vaginal swabs are the specimens of choice when screening for Chlamydia trachomatis and
Neisseria gonorrhoeae: results from a multicenter evaluation of the APTIMA assays for both infections. Sex Transm Dis 2005; 32: 725-8.

62. Workowski KA, Berman S. Sexually transmitted disease treatment guidelines 2010. MMWR 2010; 59: 44-9.

63. Scholes D, Stergachis A, Heidrich FE, et al. Prevention of pelvic inflammatory disease by screening for cervical chlamydial infection. N Engl J Med 1996; 334: 1362-6.

64. Yu H, Jiang X, Shen C, Karunakaran KP, Brunham RC. Novel Chlamydia muridarum $\mathrm{T}$ cell antigens induce protective immunity against lung and genital tract infection in murine models. J Immunol 2009; 182: 1602-8.

65. Karunakaran KP, Rey-Ladino J, Stoynov N, et al. Immunoproteomic discovery of novel $\mathrm{T}$ cell antigens from the obligate intracellular pathogen Chlamydia. J Immunol 2008; 180: 2459-65.

66. Murthy AK, Chambers JP, Meier PA, Zhong G, Arulanandam BP. Intranasal vaccination with a secreted chlamydial protein enhances resolution of genital Chlamydia muridarum infection, protects against oviduct pathology, and is highly dependent upon endogenous gamma interferon production. Infect Immun 2007; 75: 666-76. 


\section{Candida and parasitic infection: Helminths, trichomoniasis, lice, scabies, and malaria Jeanna M. Piper}

\section{CANDIDIASIS IN PREGNANCY}

Yeast infection is an important issue in women's health, even though systemic fungal infection is exceedingly rare in women of childbearing age. The presence of Candida organisms is almost universal. Three-fourths of all women have symptomatic Candida vulvovaginitis during their lifetime and almost half of those with an initial attack have additional episodes (1). Candida organisms are also isolated by culture in $8 \%$ of asymptomatic females (2). The dysuria, itching, and inflammation that accompany symptomatic infections may be severe enough to interrupt daily activities and prompt the patient to seek medical attention or use of over-the-counter products to relieve symptoms.

More than 100 species of Candida have been identified, with many further classified to the strain level (3). Only seven species of Candida are identified frequently in human clinical cultures. Candida albicans and C. tropicalis form $80 \%$ of all clinical isolates $(4,5)$. C. glabrata and C. parapsilosis comprise an additional $10 \%$ to $15 \%$, leaving $5 \%$ to $10 \%$ distributed among other species $(5,6)$.

The species breakdown in vulvovaginal specimens differs in that C. albicans is identified in $80 \%$ to $85 \%$ of all positive vaginal cultures, C. glabrata in $10 \%$ to $15 \%$, and C. tropicalis in $5 \%$, with minimum identification of other species (7).

\section{Pathophysiology of Candida Infection}

Candida are dimorphic organisms with blastospore and mycelial forms. The blastospore form (budding yeast) is associated with transmission and colonization, and is the form found in the bloodstream in systemic infection. Germinated yeast with mycelia and pseudohyphae is the tissue-invasive form that causes symptomatic disease. The Candida life cycle is one of rapid budding, maturation, and degeneration. Budding occurs as a new cell outgrowth from the mother blastospore. Following mitosis, a septum partitions the two cells and budding resumes in each cell. Mycelium formation begins as a cylindrical outgrowth from the cell wall. Septa are laid down behind the apical tip as the hyphae shoot lengthens. Blastospores are then produced just behind the newly created septa. Pseudohyphae are a morphologic derivative between budding and hyphal growth that is found in all Candida species (8).

The attachment of Candida to vaginal cells has been reported to be mediated by mannose-containing receptors on the surface of the mucosal epithelial cells (3). The effect of estrogen in increasing vaginal epithelial avidity for candidal adherence may involve these surface receptors (1). Intracellular receptors for estradiol and corticosteroids have been identified in C. albicans and C. glabrata $(9,10)$. Although the mechanisms of action of these receptors have not yet been elucidated, high levels of estrogens and corticosteroids are associated with increased candidal virulence and tissue invasion.

The human host defense mechanism against candidal vaginitis is primarily based on the natural bacterial flora of the vagina, particularly lactobacilli. Lactobacillus is thought to decrease fungal growth via competition for nutrients, mechanical interference with candidal mucosal adherence, and chemical inhibition by lactobacillus secretions (1). Cellmediated immunity also plays a role in preventing infection. Monocytes and leukocytes prevent deep tissue invasion and systemic infection. IgG and IgM are elicited systemically and IgA is secreted in cervical mucus in response to acute candidal infection, but infection rates are not increased in patients with isolated immunoglobulin deficiencies (1).

Factors that predispose a patient to Candida vulvovaginitis can be categorized as systemic, localized, or exogenous. Systemic conditions associated with genital candidiasis include diabetes, nondiabetic glycosuria, endocrinopathies, debilitating disease, older age, and pregnancy. Local conditions that alter the barrier function of the perineal epithelium allow higher infection rates including tissue maceration (from moisture accumulation, urinary leakage, or abrasion from tight-fitting clothing) and thinning of the skin (from menopause or topical steroid use). Exogenous factors include commonly prescribed medications such as antibiotics (particularly tetracycline, ampicillin, and oral cephalosporins), immunosuppressive agents, and systemic steroids. Oral contraceptives were previously thought to increase infection rates, but recent studies with the low-dose combination pills have shown no effect (1).

\section{Diagnosis of Candidiasis}

Although the diagnosis of Candida infection is generally suspected on the basis of physical findings, confirmation should be obtained. The use of saline or potassium hydroxide $(\mathrm{KOH})$ wet mounts of the vaginal secretions is the most rapid and least expensive confirmatory test. If a saline preparation is used, dark-field or phase-contrast microscopy may aid in visualization of the fungal elements. Potassium hydroxide (10-20\% solution) lyses the epithelial cells, allowing the Candida to be seen more easily. Wet-mount analysis is highly specific, but sensitivity is poor (as low as 20\%) (11). Other microscopic methods of Candida detection include Gram stain of smears or touch preps and Papanicolaou smears. If microscopic analysis is negative despite persistent symptoms following therapy, culture should be performed prior to further treatment. Infected secretions are inoculated into tubes 
of candida culture media [Sabouraud's dextrose slants or Nickerson's medium have the highest sensitivity (90\%) and specificity (70\%) for Candida strains] (12) and incubated at $25^{\circ} \mathrm{C}$ to $37^{\circ} \mathrm{C}$ (3). Candida grows quickly, often within 24 hours. Isolates from sterile sources should be subcultured and identified to the species level, but vaginal specimens need only categorization to either albicans or non-albicans. Species differentiation is based on subculture characteristics. C. albicans exhibits both germ tube and chlamydospore growth (3). C. parapsilosis, tropicalis, and glabrata produce neither.

Culture of yeast from blood or tissue specimens is more challenging. Automated systems such as the Bactec radiometric system (Johnston Laboratories, Towson, MD) can detect Candida in 2 to 4 days. Use of biphasic media further improves sensitivity, but requires 5 to 9 days for adequate growth (3). Tissue biopsy may inadvertently reveal candidal infection (when performed for other indications, not recommended if candida suspected) with partial or total destruction of the surface epithelium and filaments of fungus extending into the underlying tissues, covered by a thick film composed of inflammatory cells, necrotic debris, yeast cells, and pseudohyphae (13).

\section{Clinical Presentations of Candidal Infections}

Vulvovaginal itching and burning are the most common symptoms of Candida vulvovaginitis (reported by $60 \%$ of women with positive yeast cultures) (11) along with external dysuria and a white, curdled vaginal discharge. One-third of females with positive vaginal Candida cultures are asymptomatic, while one-fourth of women with negative cultures have symptoms (11). The symptom spectrum of vulvovaginal candidiasis goes from acute/exudative (sudden onset, heavy discharge loaded with yeast) to progressive/inflammatory (minimal discharge, few organisms, severe itching). The pruritus may be a hypersensitivity reaction and may worsen with occlusive clothing and in the premenstrual phase of the cycle (14).

Classic physical findings for Candida vulvovaginitis are bright erythema and skin erosions with satellite pustules. The pustules are non-follicular and are scattered around the eroded areas. The erosions are superficial with a narrow white border of scale. In advanced cases, the entire perineum may appear scalded and oozing, with excoriations and fissures. Edema may be a finding in both early and advanced disease. Inguinal lymphadenopathy may accompany perineal involvement. Speculum examination reveals inflamed, erythematous, edematous mucosa with white thrush patches and pooling of the thick, white, curd-like discharge in the posterior fornix. The cervix may become red, inflamed, and quite friable.

Males with Candida balanitis (inflammation of the glans penis) have swelling, itching, and burning of the glans and shaft of the penis. Symptoms are more common in uncircumcised males (75\%); circumcised men with positive yeast cultures are usually asymptomatic (90\%) (2). Physical findings in the male with Candida balanitis include edema, erythema, and peeling of the glans penis and scrotum. Transient nonprogressive penile inflammation following intercourse may be due to a hypersensitivity reaction to Candida in the female partner's genital tract (2).

\section{Therapy of Vulvovaginal Candidiasis}

Current recommended therapy for vulvovaginal candidiasis is use of a topical imidazole. Imidazoles inhibit fungal cell wall synthesis and are absorbed in small amounts through mucous membranes (15). There are five topical azoles for the treatment of vulvovaginal candidiasis currently available in the United Sates (butoconazole, clotrimazole, miconazole, terconazole, and tioconazole) (16) many of which have multiple dosing forms as well as multiple brand names associated. Dosing information for current intravaginal azole treatments are listed in Table 1. There are no significant differences in efficacy or safety between the currently available topically applied azole products, with the caveat that longer duration of therapy typically results in slightly higher cure rates, so choice of product can be based on cost and convenience of dosing regimen.

Oral treatment of vulvovaginal candidiasis has become quite common with the approval of single dose, oral fluconazole $150 \mathrm{mg}$ (Diflucan ${ }^{\circledR}$ ). Fluconazole has been shown to be as effective as intravaginal clotrimazole ( $200 \mathrm{mg} /$ day for 3 days) (17), intravaginal miconazole (1200 $\mathrm{mg}$ single dose) (18), and oral ketoconazole $(400 \mathrm{mg} /$ day for 5 days) (19). Short-term response rates of $94 \%$ (symptomatic) and $85 \%$ (mycologic) have been reported (20). The older oral medication used for

Table 1 Topical Azoles for Treatment of Candida Vulvovaginitis

\begin{tabular}{|c|c|c|c|}
\hline Generic name & Formulation & Daily dose & Duration of dosing \\
\hline Butoconazole & $2 \%$ cream & $5 g$ & 3 days \\
\hline Butoconazole $^{\mathrm{a}}$ & $2 \%$ cream & $5 g$ & 1 day \\
\hline Clotrimazole & $1 \%$ cream & $5 \mathrm{~g}$ & $7-14$ days \\
\hline Miconazole & $2 \%$ cream & $5 g$ & 7 days \\
\hline Miconazole & $4 \%$ cream & $5 g$ & 3 days \\
\hline Miconazole & Suppository & $100 \mathrm{mg}$ & 7 days \\
\hline Miconazole & Suppository & $1200 \mathrm{mg}$ & 1 day \\
\hline Terconazole $^{a}$ & $0.4 \%$ cream & $5 g$ & 7 days \\
\hline Terconazole $^{\mathrm{a}}$ & $0.8 \%$ cream & $5 \mathrm{~g}$ & 3 days \\
\hline Terconazole $^{a}$ & Suppository & $80 \mathrm{mg}$ & 3 days \\
\hline Tioconazole & $6.5 \%$ ointment & $5 g$ & 1 day \\
\hline
\end{tabular}


vulvovaginal candidiasis, ketoconazole (Nizoral $\left.{ }^{\circledR}\right)$, may still be considered for use in recurrent or resistant cases, but its use is limited by gastrointestinal side effects and ability to cause idiosyncratic hepatitis. When taken daily $(400 \mathrm{mg}$ for 14 days, then $100 \mathrm{mg}$ for 6 months), ketoconazole has been shown to reduce recurrence rates in complex cases (21).

Currently recommended alternative treatments for use in resistant cases or women with azole allergies include nystatin, boric acid, and gentian violet. Nystatin (Mycostatin ${ }^{\circledR}$ ) is a polyene antifungal, which binds to sterols in the fungal membrane, causing membrane incompetence and allowing intracellular components to leak out (15). Polyenes are not well absorbed orally or via mucous membranes. Boric acid powder is placed in capsules, with a 30-day cure rate of $72 \%$ when given as $600 \mathrm{mg}$ daily for 14 days (22). Boric acid therapy can provide a very low-cost alternative for therapy of yeast vaginitis for resource-limited settings. Gentian violet ( $1 \%$ solution painted onto the affected areas two or three times a week) can provide rapid symptomatic relief, but is quite inconvenient due to permanent staining of skin and clothing. Gentian violet is fungicidal via interference with fungal enzymes (15).

\section{Vulvovaginal Candidiasis in Pregnancy}

Vaginal colonization with yeast occurs in $30 \%$ to $40 \%$ of all pregnancies (23). The attack rate is higher in the third trimester and symptomatic recurrence is more common than that in nonpregnant patients. The cause of increased prevalence in pregnancy is not known, but theories include fungal growth promotion via estrogen receptors on the vaginal mucosa and within the yeast cells, as well as improved fuel for growth due to higher mucosal glycogen content (23). Intraamniotic infection with yeast is exceedingly rare with intact membranes, but can be seen in the presence of a foreign body, such as a cerclage or an intrauterine device, and may cause preterm labor or spontaneous abortion by invading the umbilical cord and major fetal organs (15). Up to $80 \%$ of infants that pass through an infected birth canal at delivery are colonized with yeast typically resulting in oral colonization (thrush) or diaper dermatitis, both easily treated with local therapy (24). Congenital cutaneous candidiasis is a disseminated papulovesicular to pustular dermatitis involving mainly the head, neck, palms, and soles, which resolves rapidly with topical antifungal therapy and carries little risk of progression to systemic infection in normal neonates (24).

Treatment of Candida vulvovaginitis in pregnancy is typically limited to topical azoles and nystatin $(16,25)$. For many years, nystatin was the primary drug used for Candida vulvovaginitis in pregnancy because its extremely poor absorption was felt to improve its safety (pregnancy category B) (25). As studies have proven, the increased efficacy of the azole antifungal agents, miconazole, clotrimazole, butoconazole, and terconazole, has become the mainstays of therapy in pregnancy. They have been used extensively in human pregnancy with no adverse effects or increase in congenital malformations noted, but most remain pregnancy category $\mathrm{C}$ due to the absence of adequate controlled human studies. There is no clear leader from this group in terms of efficacy and patient satisfaction, so selection may be based on availability and cost. Seven-day treatment is usually needed in pregnancy due to the higher rates of treatment failure and recurrence. Cure rates are typically $5 \%$ to $10 \%$ lower than those in nonpregnant women for the same dosing regimen.

None of the alternative agents are recommended for use in pregnancy. Fluconazole is associated with fetal loss in animal studies with several small human studies that have shown no safety concerns, but are not adequate to recommend use in pregnancy (pregnancy category C) (25). Ketoconazole given orally has been shown to increase abortion and fetal loss in mice and rats, with no adequate human studies to document its effect on human pregnancy; therefore, its use for vaginitis in pregnancy is not recommended (pregnancy category C) (25). Boric acid is not recommended in pregnancy due to limited absorption of borate through the mucous membranes (less than $1 \mathrm{mg} / \mathrm{mL}$ serum levels). Gentian violet is not recommended for use in pregnancy because no adequate studies have been performed to judge its safety (pregnancy category C).

\section{Recurrent Vulvovaginal Candidiasis}

Almost one-half of women with an initial infection will have additional episodes. The subsequent attacks may be newly acquired cases or persistent infection due to incomplete eradication. Studies of culture-proven vaginal candidiasis with appropriate antifungal therapy show persistence of the same Candida strain in $20 \%$ to $25 \%$ of patients at 4 - to 6-week follow-up (1). Recurrent candidal vaginitis may be due to chronic recolonization of the vagina from the intestine. Studies have shown a high strain correlation between the vagina and the intestine in those with intestinal colonization (1), but several studies of intestinal treatment with nystatin and ketoconazole have shown reduction or complete eradication of intestinal yeast with no impact on vaginal recurrence rates $(21,26)$.

The role of male-partner penile colonization in promoting recurrent vaginitis is unresolved. Evidence in favor of a role for this includes a fourfold higher rate of asymptomatic penile colonization and the presence of Candida in the ejaculate of partners of women with recurrent vaginitis (1). Evidence against this includes the lack of documentation of sexual transmission previously outlined and the lack of improved recurrence rates following treatment of the male partner. Attention to hygiene should be recommended in all cases of Candida vaginitis. A trial of therapy in the male partner may be attempted if other therapy is unsuccessful in resolving recurrent infections.

All patients with recurrent episodes of vulvovaginal candidiasis without an obvious precipitating factor should be evaluated for underlying systemic disease (diabetes, HIV, or other debilitating disease) and use of over-the-counter or "home" remedies for the perineal and vaginal areas. Patients with continued pruritus without evidence of organisms may be experiencing an allergic reaction, not only to the Candida, but also to the antifungal medications in which case the inflammation should improve following cessation of therapy. Refractory vaginitis is a multifocal problem with no single solution. Inadequate therapy, resistant organisms, intestinal colonization, male-partner colonization, vaginal flora abnormalities, immunodeficiency, hypersensitivity reaction, and hampered barrier function may all be involved to a differing extent in individual patients with recurrent candidal 
infections. Adequate evaluation, with therapy aimed at individual components, is the best approach.

\section{Systemic Candidiasis}

Systemic candidiasis typically occurs in debilitated patients with diabetes, acquired immunodeficiency syndrome, intravenous drug use, malignancies, chemotherapy, prolonged antibiotic or steroid therapy, and intravenous hyperalimentation (27). Systemic infection can be subdivided into disseminated infection, fungemia, and single-organ infection (meningitis, endocarditis, pneumonia, arthritis, peritonitis, laryngitis, endophthalmitis, and urinary tract infections). Systemic candidal infection is usually associated with an indwelling foreign body (intravenous catheter, Foley catheter, endotracheal tube, etc.). Symptoms are variable; however, persistent fever, in spite of broad-spectrum antibiotic coverage, is the most frequent clue, but may be masked in patients on high-dose steroids. Hypotension and mental status changes (confusion to obtundation) are also common signs. The diagnosis of disseminated candidiasis may be difficult. Blood cultures may be negative in $40 \%$ to $60 \%$ of cases proven by autopsy. Tissue biopsy, culture of fluids from sterile spaces, and urine cultures may be used to enhance detection in those at risk.

Treatment of systemic candidiasis has been changed significantly with the recent approval of new medications including additional second-generation triazoles (voriconazole and posaconazole) and a novel class of antifungals, the echinocandins (caspofungin, micafungin, and anidulafungin) $(28,29)$. These new products have replaced amphotericin B and 5-fluocytosine for the treatment of candidemia with the echinocandins currently recommended as the drugs of choice due to higher efficacy and voriconazole/posaconazole as second-line treatment due to similar efficacy to amphotericin with a much improved side effect profile (28). Choice of echinocandins is usually based on local availability and cost. Unfortunately, survival rates for systemic candidiasis remain quite low despite improved therapy due to delays in diagnosis (resulting in delayed treatment) and the severity of underlying disease, particularly immunocompromise and diminished renal function (27). Since the new triazoles and echinocandins are quite expensive, amphotericin B and 5-fluocytosine continue to be commonly used in low-resource settings.

\section{Systemic Candidiasis in Pregnancy}

Systemic candidiasis in women of childbearing age is quite rare, but occasionally seen in cases with significant underlying disease (25). There are no adequate controlled studies during pregnancy for any of the medications used for systemic candidiasis; however, the life-threatening nature of this infection clearly outweighs any concerns over impact of the medications on pregnancy. Animal data available for both caspofungin and micafungin showed fetal loss and other issues (impairment of ossification for caspofungin and visceral abnormalities for micafungin) in both rats and rabbits with inadequate human data, resulting in both being labeled as pregnancy category $C$ (25). The most experience exists for amphotericin B (pregnancy category B) (25), but the increased efficacy of the echinocandins should still be considered in the selection of treatment. Consultation with infectious disease specialists and hospital infection control officers is recommended for these unusual life-threatening infections in pregnancy.

\section{HELMINTHIC INFECTION IN PREGNANCY Introduction}

Helminths are parasitic worms that spend at least a portion of their life cycle in an animal or human host. Helminths include cestodes (tapeworms), trematodes (flukes), and nematodes (roundworms). Helminthic infection has a worldwide distribution, but is more prevalent in underdeveloped countries. Although the prevalence rates have fallen in the developed world, helminthic infection has by no means disappeared. Life-threatening parasitic infection in pregnancy is uncommon in industrial nations of the world, but may be seen in individuals who have recently immigrated from less developed areas.

The effects of helminthic infection on pregnancy, although generally benign, should be recognized and treated. Anemia is a common feature of intestinal parasitic infection (owing to intestinal blood loss) and may compound anemia of pregnancy. Malnutrition may occur as a result of competition for nutrients. Chronic helminthic infection may lead to a debilitated state, with the possibility of superimposed bacterial infection. Since most helminthic infections in pregnancy are only mildly symptomatic, previously most were treated symptomatically until the postpartum period when definitive treatment was given. More recently, there have been increasing recommendations not to delay treatment, particularly in cases impacted by anemia. Each organism, however, has to be considered individually for therapeutic recommendations.

The diagnosis of helminthic infection must be individualized, but may be suspected on the basis of gastrointestinal symptoms (nausea, vomiting, and diarrhea) and a history compatible with exposure. With notable exceptions, helminthic infection is diagnosed by demonstration of eggs or larvae in feces. In heavy infection, eggs and larvae may be seen directly in saline wet mounts. To evaluate lighter infections, concentration must be performed via zinc sulfate flotation or formalin-ether sedimentation prior to saline wetmount examination. Traditionally, examination of stool specimens from 3 consecutive days has been recommended to increase sensitivity in detection of ova and parasites in lightly infected cases. In moderately to heavily infected cases, a single-specimen evaluation should be as effective as multiple testing (30).

A description of the classification system for helminths is followed by review of the more commonly identified helminths. The chapter is completed by a description of the commonly used anthelmintic agents and their status for use in pregnancy and lactation. Treatment recommendations for all of the helminths detailed in this chapter are based on (i) the current treatment guidelines in the $\mathrm{CDC}$ parasites $\mathrm{A}$ to $\mathrm{Z} \mathrm{Web}$ site (www.cdc.gov/parasites/az/index.html) section for each organism (obtained by selecting the organism from the index and then selecting "Resources for Health Care Professions") or (ii) The Medical Letter article entitled "Drugs for Parasitic Infections" available at The Medical Letter Web site (www.medicalletter.org). Additional information regarding laboratory diagnosis of helminthic infections can be obtained 
at the DPDx Web site (www.dpd.cdc.gov/dpdx/HTML/ DiagnosticProcedures.htm), which is a service of the CDC Division of Parasitic Diseases and Malaria. Links to the applicable sections of the Medical Letter "Drugs for Parasitic Infections" may also be accessed via the treatment tab for each organism on the DPDx Web site.

\section{Classification and Description of Common Helminthic Infections}

All helminths are classified as Platyhelminthes (flatworms) or Nematoda (roundworms). Flatworms are further divided into cestodes and trematodes (Table 2). Cestodes, or tapeworms, have segmented bodies consisting of proglottids attached via the neck zone to a head or scolex. The scolex provides attachment and locomotion via grooves, suckers, and hooks extending from its surface (Fig. 1). Tapeworms are hermaphroditic, with both male and female reproductive organs contained in each segment. There is no organized digestive tract; nutrients are absorbed via the integument. Excretory and nervous systems are present, but only in primitive form (31).

Trematodes, or flukes, have a flattened, leaf-like body with one or more ventral muscular suckers to provide attachment. Flukes are monoecious (hermaphroditic), with separate male and female reproductive organs that connect at the common genital atrium. Trematodes have a more advanced alimentary canal, nervous system, and excretory system (Fig. 2). All trematodes require a period of external development in a snail host (31). Nematodes are unsegmented worms with an external cuticle, developed internal organ structures, and somatic musculature (Fig. 3). Most nematodes are dioecious (heterosexual), with male and female sexual differentiation, and mating required for reproduction. The alimentary, excretory, and nervous systems of nematodes are more differentiated than those of other helminths. Most nematodes undergo four molts, with shedding or resorption of the old external cuticle, and formation of a new cuticle (31)

\section{Cestodes}

Taenia saginata and Taenia solium

Taenia saginata and T. solium have a worldwide distribution and cause taeniasis or tapeworm infection. Adult taenias are 3 to $8 \mathrm{~m}$ in length and comprise a small scolex $(1-2 \mathrm{~mm})$ and multiple proglottids, each about $0.5 \mathrm{~cm} \times 1-2 \mathrm{~mm}$ in size (32). Taenia eggs are yellow to brown and spherical, with thick shells (Fig. 4). The life cycle of the taenias involves a definitive host (humans) and an intermediate host (T. saginata, cattle; T. solium, pigs). Eggs are passed in feces and reach pastures where they are ingested by the intermediate host. The larvae hatch and migrate to the muscle tissue, where they remain until the raw beef or pork is ingested by the definitive host. The infective form (cysticerci) requires 3 to 5 months to mature to adulthood. Taenia adult worms may live in the human small intestine for 25 years. The diagnosis of taenia infection is based on identification of eggs or proglottid segments in the patient's stool specimen (32).

T. solium eggs can be directly infective if ingested by humans, causing cysticercosis. Extreme care must be taken in

Table 2 Classification of Helminths

\begin{tabular}{|c|c|c|}
\hline \multicolumn{3}{|c|}{ Platyhelminthes } \\
\hline Cestodes (tapeworms) & Trematodes (flukes) & Nematodes (roundworms) \\
\hline $\begin{array}{l}\text { Taenia saginata } \\
\text { Taenia solium } \\
\text { Diphyllobothrium latum } \\
\text { Hymenolepis nana } \\
\text { Hymenolepis diminuta } \\
\text { Echinococcus granulosus } \\
\text { Echinococcus multilocularis } \\
\text { Multiceps multiceps } \\
\text { Dipylidium caninum }\end{array}$ & $\begin{array}{l}\text { Schistosoma mansoni } \\
\text { Schistosoma japonicum } \\
\text { Schistosoma haematobium } \\
\text { Schistosoma intercalatum } \\
\text { Fasciola hepatica } \\
\text { Fasciola gigantica } \\
\text { Fasciolopsis buski } \\
\text { Clonorchis sinensis } \\
\text { Opisthorchis felineus } \\
\text { Opisthorchis viverrini } \\
\text { Gastrodiscoides hominis } \\
\text { Watsonius watsoni } \\
\text { Heterophyes heterophyes } \\
\text { Metagonimus yokogawai } \\
\text { Paragonimus westermani }\end{array}$ & $\begin{array}{l}\text { Enterobius vermicularis } \\
\text { Trichuris trichiura } \\
\text { Trichinella spiralis } \\
\text { Ascaris lumbricoides } \\
\text { Capillaria hepatica } \\
\text { Capillaria philippinensis } \\
\text { Strongyloides } \\
\text { Ancylostoma } \\
\text { Necator } \\
\text { Tricostrongylus } \\
\text { Toxocara } \\
\text { Ternidens } \\
\text { Angiostrongylus } \\
\text { Metastrongylus } \\
\text { Anisakis } \\
\text { Lagochilascaris } \\
\text { Gongylonema } \\
\text { Thelazia } \\
\text { Gnathostoma } \\
\text { Wuchereria } \\
\text { Brugia } \\
\text { Onchocerca } \\
\text { Loa loa } \\
\text { Dipetalonema } \\
\text { Mansonella } \\
\text { Dracunculus } \\
\text { Dirofilaria }\end{array}$ \\
\hline
\end{tabular}




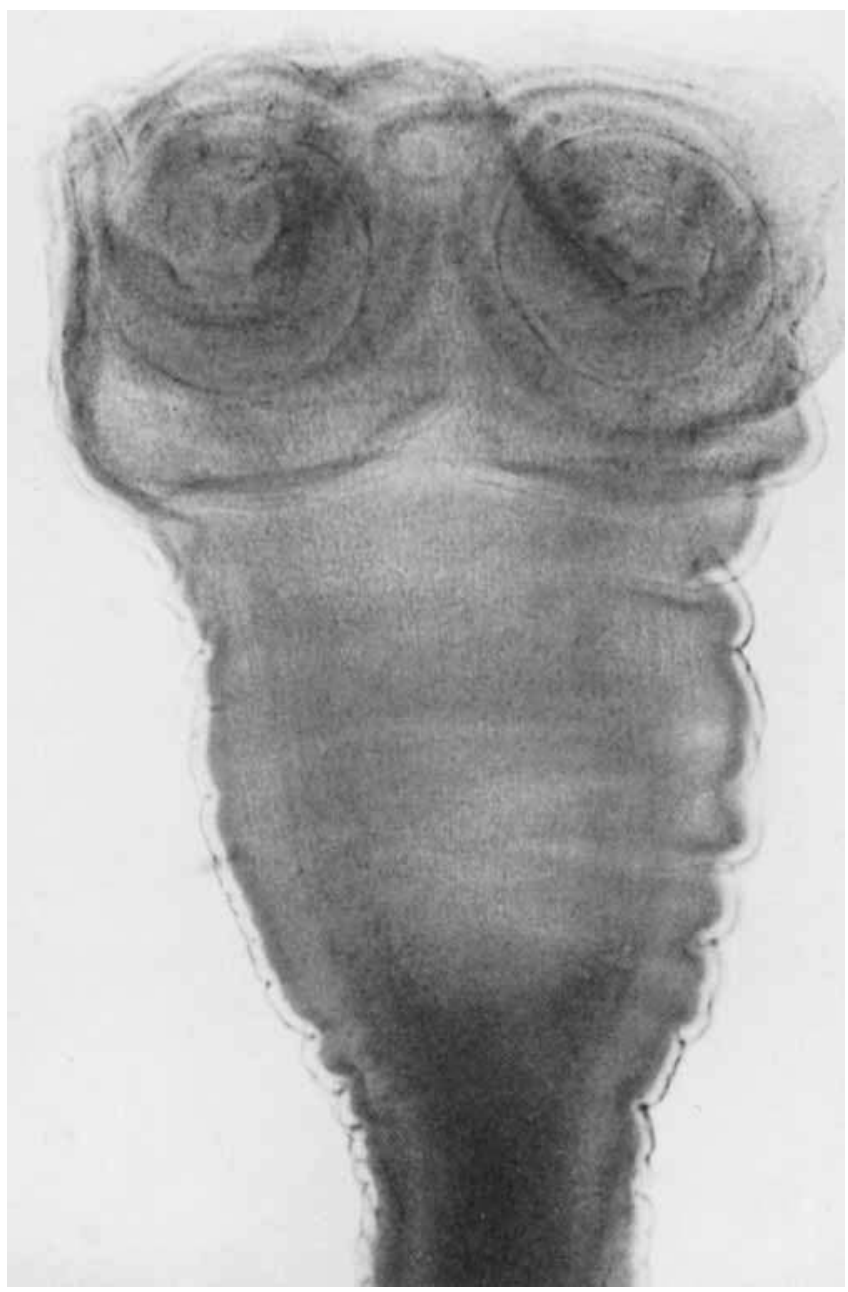

Figure 1 Taenia saginata adult scolex, showing suckers. Source: From Ref. 32.

handling all specimens with taenia eggs, as T. solium are not easily distinguished from T. saginata (32). Cysticercosis is the disease caused by larval migration throughout the body. Cysticercosis may be severe, even fatal, due to inflammation of vital organs, particularly cardiac muscle (33).

The clinical effects of taeniasis are usually minimal, but a minority of cases report abdominal discomfort, vomiting, and diarrhea (31). Rare cases of appendicitis and intestinal obstruction do occur. The treatment of choice is praziquantel (single $5-10 \mathrm{mg} / \mathrm{kg}$ oral dose), regardless of pregnancy status; niclosamide (single 2-g oral dose) is an alternative. Taenia infection generally has minimal effects on pregnancy and can typically be left untreated until the postpartum period, unless a symptomatic infection with T. solium is diagnosed (owing to the risk of cysticercosis).

\section{Diphyllobothrium latum}

Diphyllobothrium latum, or fish tapeworm, is found in northern Europe, North America, and Japan, specifically in temperate areas with cold, clear lakes. D. latum adult worms are 4 to $10 \mathrm{~m}$ long, with a small scolex $(3 \times 1 \mathrm{~mm})$ and wide, short proglottids (32). The eggs are yellow to brown and ovoid, with an operculum (a lid-like structure). The life cycle begins as eggs that are passed in feces into water, where they hatch 2 weeks later, with coracidium released. The coracidium are ingested by copepods, where they develop to the procercoid

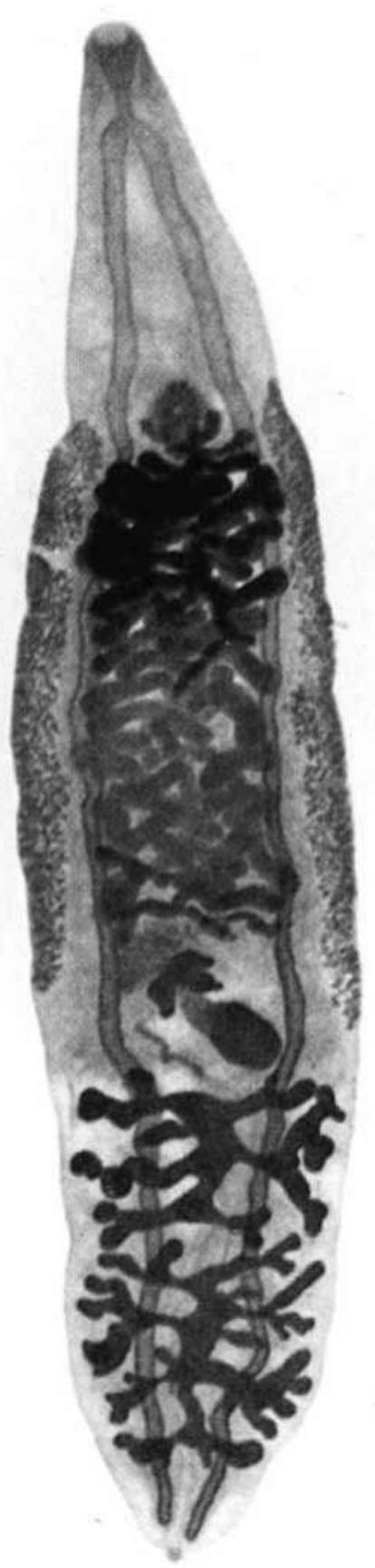

Figure 2 Clonorchis sinensis adult fluke (carmine stain). Source: From Ref. 32.

stage. Following ingestion of the copepod host by fish, the infective form (plerocercoid or sparganum) matures to await ingestion of raw fish by humans or other carnivores. D. latum migrates to the small intestine, where it attaches and matures to an adult. The adult worm may survive for up to 25 years, releasing its eggs in the feces (32). Diagnosis is based on finding eggs in feces (34).

Fish tapeworm infection may be asymptomatic or may have mild-to-severe gastrointestinal symptoms. Praziquantel (single $5-10 \mathrm{mg} / \mathrm{kg}$ oral dose) and niclosamide (single 2 -g oral dose) are the most commonly used therapies. Unless infection is severe, anthelmintic therapy is typically delayed until the postpartum period. Diphyllobothriasis may cause vitamin $B_{12}$ deficiency in pregnancy, owing to malabsorption; therefore, vitamin $B_{12}$ supplementation is recommended. 


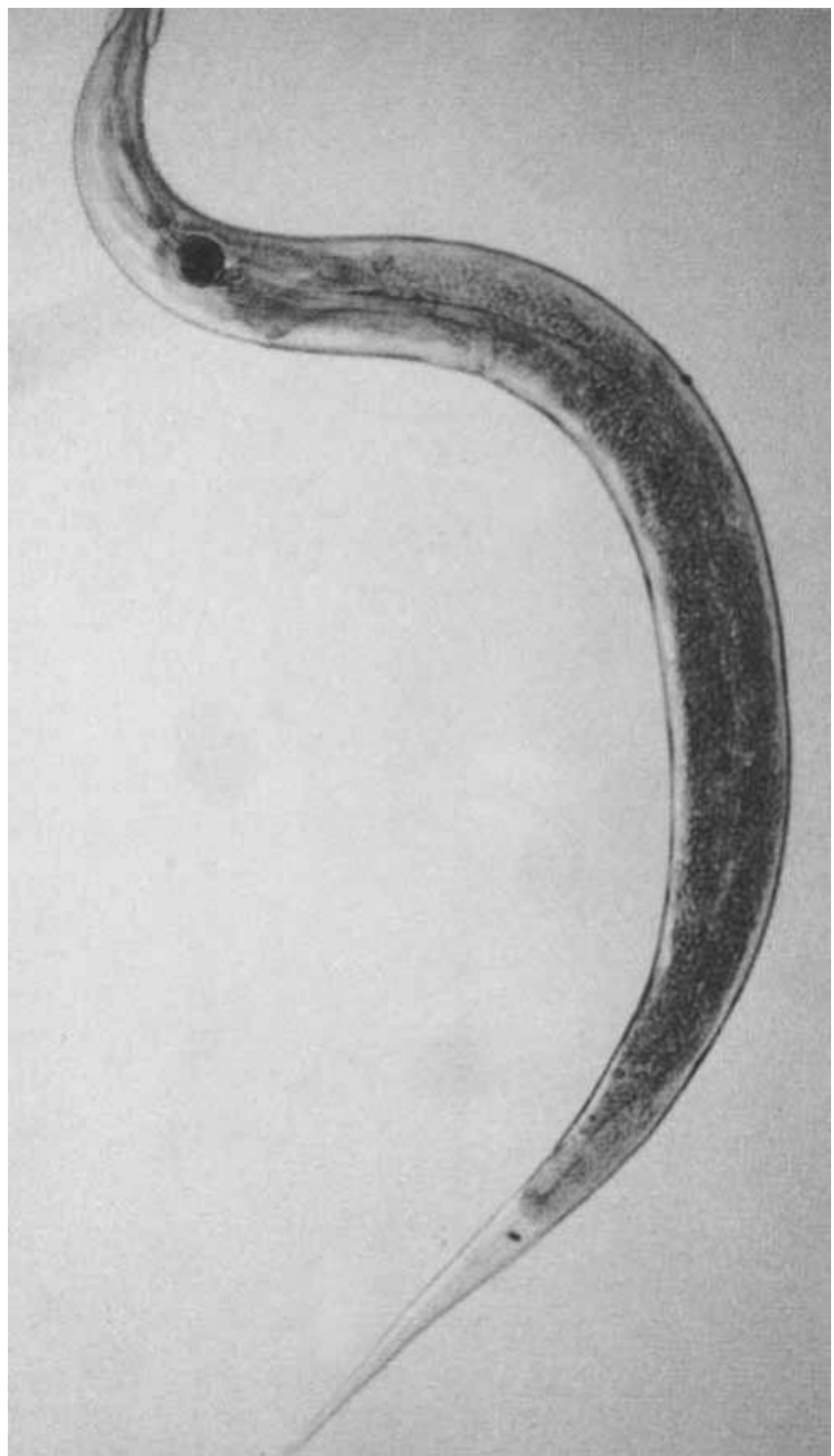

Figure 3 Enterobius vermicularis adult female. Source: From Ref. 32.

\section{Hymenolepis nana}

Hymenolepiasis or dwarf tapeworm infection is cosmopolitan in distribution. Hymenolepis nana adult worms are small tapeworms measuring $2 \times 4 \mathrm{~cm}$, with short wide proglottids (32). The eggs are spherical with a thin hyaline shell (Fig. 5). The definitive hosts of $H$. nana are mice and humans; beetles are intermediate hosts. The life cycle involves passage of eggs in feces with subsequent ingestion by beetles. The beetles are eaten by mice in the natural life cycle. Humans can be infected directly, by ingestion of the eggs. In humans, the eggs hatch, cysticercoids develop in the wall of the small intestine, and tapeworms emerge into the lumen of the intestine to mature to adulthood. Eggs are then passed in feces to restart the cycle. Hymenolepiasis is diagnosed by the finding of characteristic eggs in feces (32).

The clinical effect of dwarf tapeworm infection in humans is variable. There may be no symptoms or gastrointestinal complaints (diarrhea, nausea, anorexia, or vomiting) (31). The course is generally not severe. Praziquantel (single $25 \mathrm{mg} / \mathrm{kg}$ oral dose) is the drug of choice. Pregnancy is not threatened by hymenolepiasis, and treatment is typically deferred to the postpartum period.

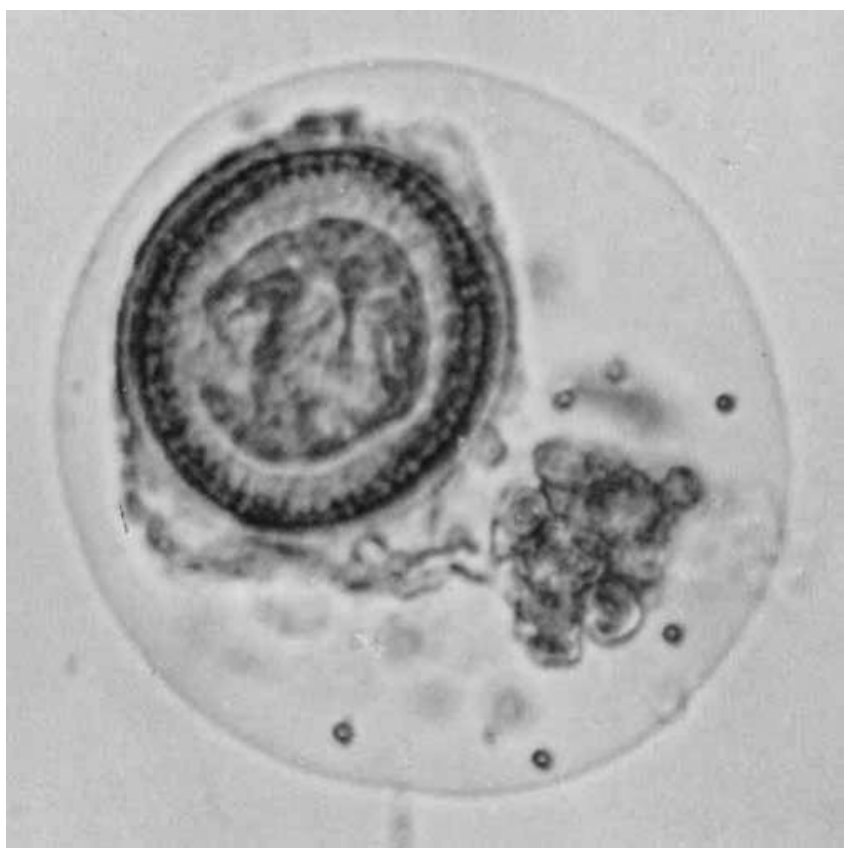

Figure 4 Taenia egg (T. solium and T. saginata eggs are indistinguishable). Source: From Ref. 32.

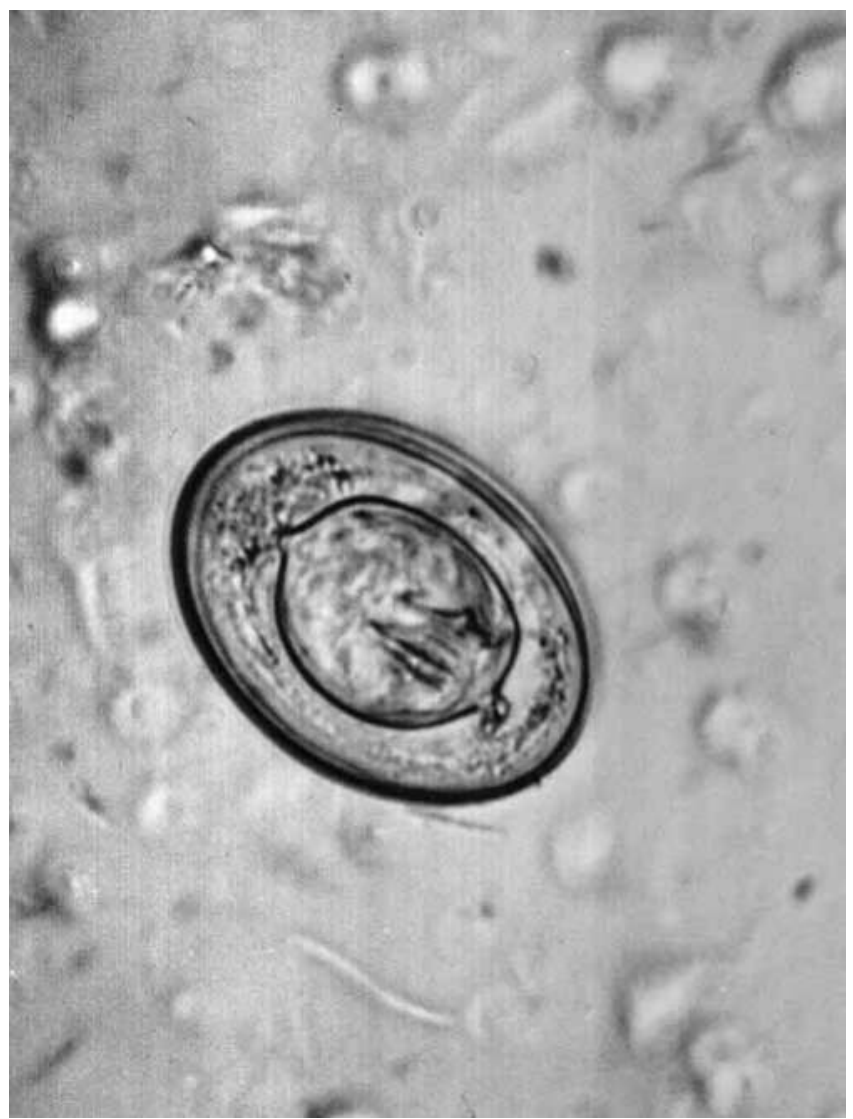

Figure 5 Hymenolepis nana egg. Source: From Ref. 32.

Echinococcus granulosus, Echinococcus multilocularis, and Multiceps multiceps

The definitive hosts of echinococcus and multiceps species are dogs and wild carnivores. On occasion, humans are an incidental host, infected by the ingestion of infective eggs. The embryos hatch in the intestine and migrate through the body to various organs, where cysts develop. Clinical diagnosis is 
based on biopsy of the cyst/granuloma area or on immunologic testing.

Symptoms occur as the cysts enlarge and create a spaceoccupying lesion (31). Multiceps multiceps cysts are commonly located in the subarachnoid space and may cause meningitis (31). Pregnancy can be threatened if the echinococcal cysts are located in the uterine muscle. Intrauterine growth retardation and even impairment of labor may result from uterine cysts. Migration and encystment in vital organs can be life-threatening. Treatment is immediate, regardless of pregnancy status. Surgery to resect the cyst is the best therapy. Albendazole ( $440 \mathrm{mg}$ orally BID $\times$ 1-6 months) can be used to reduce cyst size prior to surgery and may resolve smaller cysts without surgery. If surgery is not feasible and antepartum therapy is needed, praziquantel $[100 \mathrm{mg} / \mathrm{kg}$ orally (divided into three doses) $\times 1$ day, followed by $50 \mathrm{mg} / \mathrm{kg} /$ day orally (divided into three doses per day) $\times$ 28 days] is an alternative therapy that may be preferred for use in pregnancy (35), but it is not as effective in reducing cyst size.

\section{Trematodes}

Schistosoma mansoni, Schistosoma japonicum, and Schistosoma haematobium

Schistosomiasis or bilharziasis is mainly seen in the Middle East, Africa, and South America, but can be found in immigrant populations worldwide. Adult flukes are 0.5 to $2 \mathrm{~cm}$ in length, with females longer than males. Male flukes are wider with a posterior fold, the gynecophoric canal, in which the female rests. All schistosoma eggs are non-operculate with a transparent shell and either a lateral spine (S. mansoni) or terminal spine (S. japonicum) (32). Schistosoma adult worms live in the small blood vessels of the pelvic venous plexi. The female lies within the canal of the male for copulation and oviposition. The eggs migrate through the vessel wall and intervening tissues to reach the bladder or bowel, from which the eggs are excreted. If passed into water, the eggs hatch into a miracidial form that can penetrate and infect snails, the intermediate host. While in the snail, the miracidia mature to cercariae. The cercariae burst out of the snail host into water, where they directly infect humans through skin penetration. The schistosomule is transported via the venous system, through the heart and pulmonary vessels, to be propelled into the arterial system by the left ventricle. The schistosomules mature in the large vessels prior to migration to the terminal vascular beds, where the life cycle is completed (36). All therapies destroy only the flukes and have no effect on the migrating eggs (36). Diagnosis of schistosomal infection is based on the finding of eggs in urine, feces, or vaginal secretions, or the identification of adult worms or eggs in biopsy of the granulomas.

Initial infection (schistosome penetration) results in itching and papular rash. The migration of immature flukes causes fever, cough, allergic symptoms, and gastrointestinal complaints 2 to 4 weeks after initial exposure. Chronic schistosomiasis may cause anemia, cirrhosis, and urogenital disease. In females, this may include obstructive uropathy, abdominal pain, menorrhagia, salpingitis, oophoritis, sterility, and ectopic pregnancy (36). Praziquantel $(20 \mathrm{mg} / \mathrm{kg}$ orally BID $\times 1$ day for S. mansoni, S. haematobium or S. intercalatum, or $20 \mathrm{mg} / \mathrm{kg}$ orally TID $\times 1$ day for S. japonicum or $S$. mekongi) is the treatment of choice for schistosomiasis.

Schistosome infiltration of the placenta has been reported, but is quite rare (36). Infection of a fetus with schistosomiasis was last reported in 1920 (36). Schistosomiasis has been associated with a number of adverse pregnancy outcomes including spontaneous abortion, preterm labor, low birth weight, and maternal morbidity/mortality, but additional prospective data are needed to quantify the impact (37). Heavy maternal schistosomal infection has been associated with decreased hemoglobin levels and increased prevalence of anemia in pregnant women, additive upon the typical anemia of pregnancy. Maternal anemia is strongly associated with low birth weight and maternal mortality (particularly in cases with antepartum or postpartum hemorrhage). Chronic schistosomiasis has also been associated with increased release of pro-inflammatory cytokines, which have been implicated in both the decreased appetite seen with schistosomiasis (which may contribute to low birth weight through maternal malnutrition) and with inflammation of the placenta (which may cause abortion and preterm labor) (37).

In the past, treatment of schistosomiasis in pregnant women was typically postponed until after delivery, but with more information associating maternal infection with adverse pregnancy outcomes, World Health Organization (WHO) recommended in 2002 that pregnant women with schistosomiasis be treated with praziquantel (dose same as listed above for nonpregnant adults) upon diagnosis and that pregnant women not be excluded from mass drug administration (MDA) programs aimed at the elimination of schistosomiasis $(38,39)$. The "informal" WHO recommendation remains controversial since praziquantel is not specifically licensed for use in pregnancy, but efforts are underway to obtain the prospective human safety data needed to convince resistant incountry authorities to implement this recommendation (37).

\section{Nematodes}

Enterobius vermicularis

Enterobiasis or pinworm infection is universally distributed and is mainly seen in children. Adult male worms are small $(2-3 \mathrm{~mm})$ and females are larger $(8-13 \mathrm{~mm})$, both with long, pointed tails (40). Pinworm eggs are elongated with one flat side and have thick, colorless shells (Fig. 6). The definitive hosts for Enterobius vermicularis are humans. The female pinworms exit the rectum to lay eggs on the perianal skin overnight. The eggs embryonate in 4 to 6 hours to become infective. Eggs that are ingested hatch and develop in the lower intestinal tract (the cecum, appendix, and colon). Ingestion is facilitated by hand-to-mouth transmission or fomites. The adult worms may live for several months in the colon and rectum (32). Diagnosis is based on clinical suspicion and cellulose tape prep from perianal skin. Eggs are not found in feces. Cellulose tape prep is performed by taking clear cellulose tape, touching it to the perianal skin, and then adhering it to a microscope slide for examination (32). The tape prep is most accurate if done immediately upon arising, prior to bathing. The symptoms of pinworm infection are all related to the perianal egg deposition. Insomnia, restlessness, perianal itching/inflammation, and perianal bacterial infection 


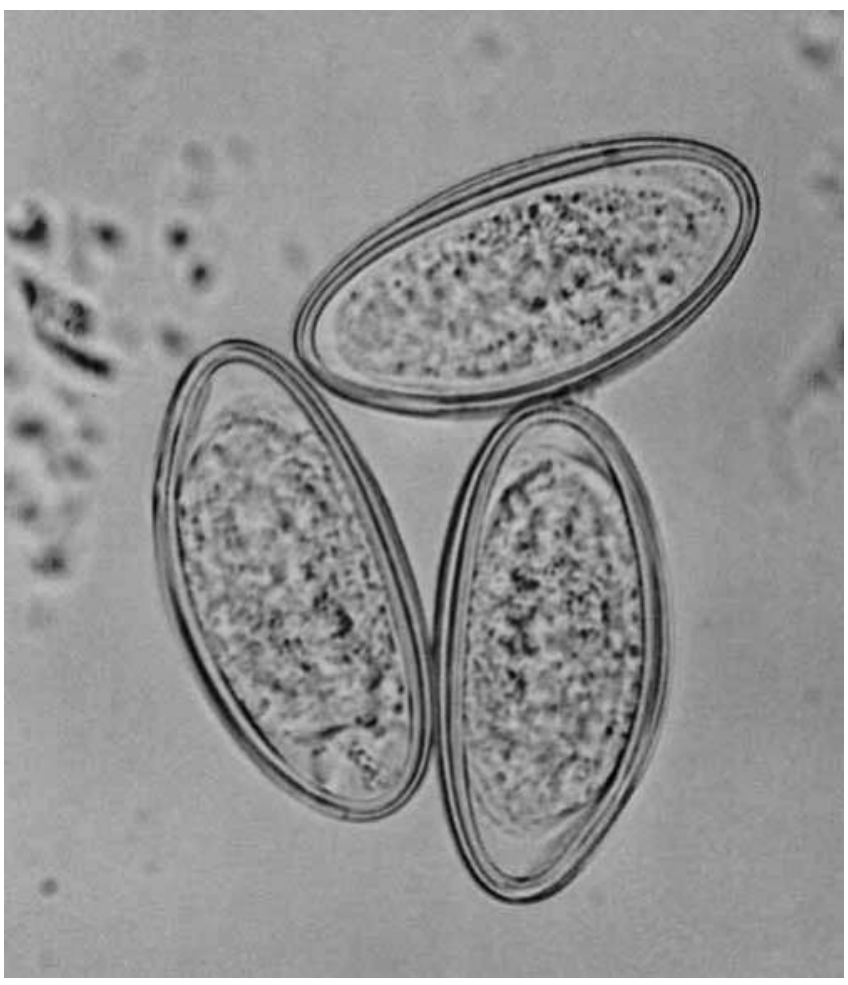

Figure 6 Enterobius vermicularis eggs. Source: From Ref. 32.

(secondary to excoriation) are the common clinical effects (31). Albendazole (single 400-mg oral dose followed by a second dose 14 days later), mebendazole (single 100-mg oral dose, followed by a second dose 14 days later), and pyrantel pamoate [single 11-mg base $/ \mathrm{kg}$ (maximum 1-g base) oral dose, followed by a second dose 14 days later] are the drugs most commonly used for treatment. The entire family should be treated simultaneously, and clothing and bedding should be washed with hot water or chlorine bleach.

Pinworms can migrate through the vagina, uterus, and fallopian tubes, but no detrimental effect on the fetus or the pregnancy has been reported (41). Therapy is typically delayed until after delivery, unless the infection is potentially compromising the pregnancy. Pyrantel pamoate can be used after the first trimester (41).

\section{Trichuris trichiura}

Trichuriasis, or whipworm infection, is cosmopolitan in warm, moist areas. Adult whipworms are 3 to $5 \mathrm{~mm}$ long, with a slender, whip-like anterior end used to thread its way into the colonic mucosa (32). Whipworm eggs are barrel shaped, with a thick shell and plugs at each end (Fig. 7). Humans are the definitive host for Trichuris trichiura. Whipworm eggs pass in feces into the soil for further development. Infective eggs are ingested and hatched. The larvae migrate to the large intestine, where they mature to adulthood. The adult worms may remain in the colon for more than 10 years. Whipworm infection is diagnosed by finding eggs in feces (32).

Symptoms vary from none, in light infection, to colitis with bloody, mucous diarrhea in heavier infestation (31). Chronic infection can result in rectal prolapse, due to inflammation. Whipworm may be treated with albendazole ( $400 \mathrm{mg}$ orally $\times$ 3 days), mebendazole ( $100 \mathrm{mg}$ orally BID $\times 3$ days), or

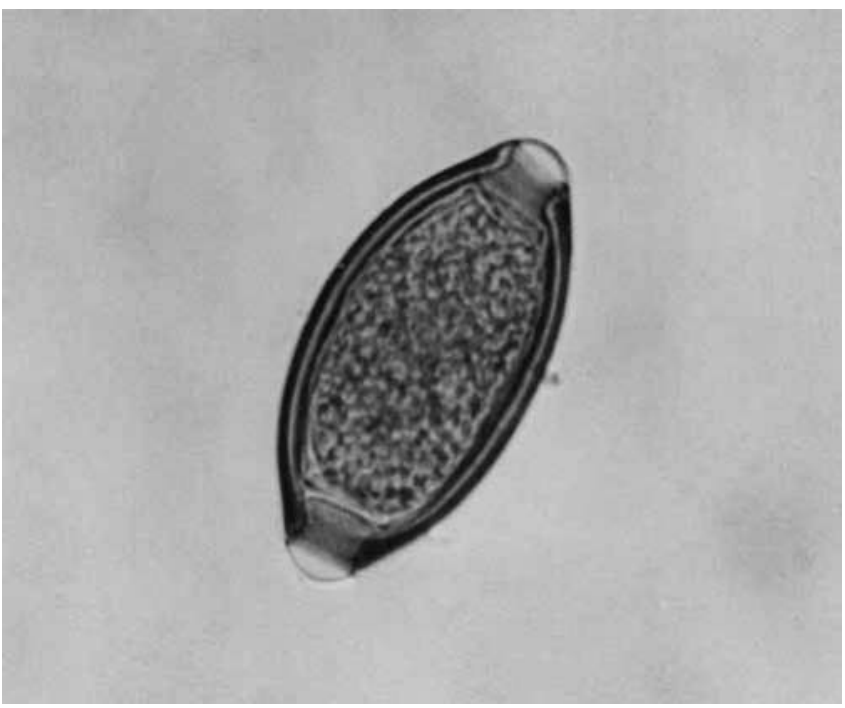

Figure 7 Trichuris trichiura egg. Source: From Ref. 32.

ivermectin $(200 \mu \mathrm{g} / \mathrm{kg} /$ day orally $\times 3$ days $)$. The impact of whipworm infection on pregnancy is mainly anemia due to increased gastrointestinal blood loss. Fetal infection has not been reported. Therapy for pregnant women is typically deferred until after delivery.

\section{Trichinella spiralis}

Trichinosis has a worldwide distribution, but is most prevalent in Europe and North America. The adult Trichinella spiralis are 1 to $4 \mathrm{~mm}$ in length (32). The female is viviparous, delivering larvae; thus, there is no external egg stage. All carnivorous species, including humans, are the definitive hosts. Most human infections result from ingestion of inadequately cooked meat. Bear, wild pig, boar, horse, and dog meat are the usual sources, but pork is the most common cause in the United States (42). Adult worms, living in the small intestine, release larvae into the mucosal epithelium. The larvae enter the bloodstream and migrate to muscle tissue, where they embed and mature to the infective stage. A cyst envelops the larva, and it remains infective until the cyst calcifies, killing the larva. When meat containing encysted larvae is ingested, the cysts are dissolved by gastric secretions, and the infective larvae are released. Within 36 hours, maturation occurs, and within a week larvae are produced, to become encysted in this new host. Diagnosis of trichinosis is based on the history of ingestion of undercooked meat and a compatible clinical picture (32). Definitive diagnosis requires either laboratory detection of specific antibodies or muscle biopsy finding encysted larvae (31).

The clinical effects of trichinosis in humans range from no symptoms to fatality. The initial ingestion of infected meat may cause diarrhea and gastroenteritis. The majority of symptomatology results from the larval migration and the resultant inflammatory response. Fever, edema, dyspnea, urticaria, weakness, and myositis are common manifestations (31). The ocular muscles are generally the most severely affected, but myocardial involvement is the most common cause of death (42). Therapy is a combination of anthelmintic agents, corticosteroids, anti-inflammatory agents, and supportive care (40). Mebendazole (200-400 mg orally TID $\times 3$ days followed by $400-500 \mathrm{mg}$ 
orally TID $\times 10$ days $)$ and albendazole $(400$ mg orally BID $\times 8-14$ days) are the recommended drugs. Anthelmintic destroy adult worms, not the larvae; however, once larval production ceases the disease is self-limited; symptoms resolve as soon as all larvae are encysted.

Trichinosis in pregnancy typically follows a moderate course, but may result in abortion, preterm labor, or intrauterine fetal death (42). Intrauterine infection has been reported twice, resulting in fetal/neonatal death (42). Trichinosis in pregnancy should be treated with mebendazole or albendazole as above when the maternal condition is such that the benefit to the mother outweighs the potential risk to the fetus.

\section{Ascaris lumbricoides}

Ascariasis or roundworm infection is most prevalent in warm, moist climates, but can be seen anywhere. Adult worms are quite large $(15-30 \mathrm{~cm}$ long), with males longer and thinner than females (32). Ascaris eggs have thick, bile-stained shells (Fig. 8). Humans are the definitive host. The life cycle begins as eggs are passed in feces into soil or water. The infective eggs are then ingested, with larvae released in the small intestine. The larvae migrate through the venous system to the lungs, where they cross into alveoli and climb the bronchial tree. The larvae are then swallowed, and return to the small intestine, to mature into adults (32). The life span of adult ascaris is less than 1 year (41). Ascariasis is diagnosed by finding eggs in feces or on barium enema, when elongated filling defects occur (31).

There are two types of clinical disease related to ascariasis. Loeffler's syndrome is heralded by inflammation and pulmonary symptoms due to the migration of the larvae. Migration of the adult worms may cause obstruction of the bile duct or the appendix (41). Migration of adult females into the hepatic duct allows eggs to be released into the liver,

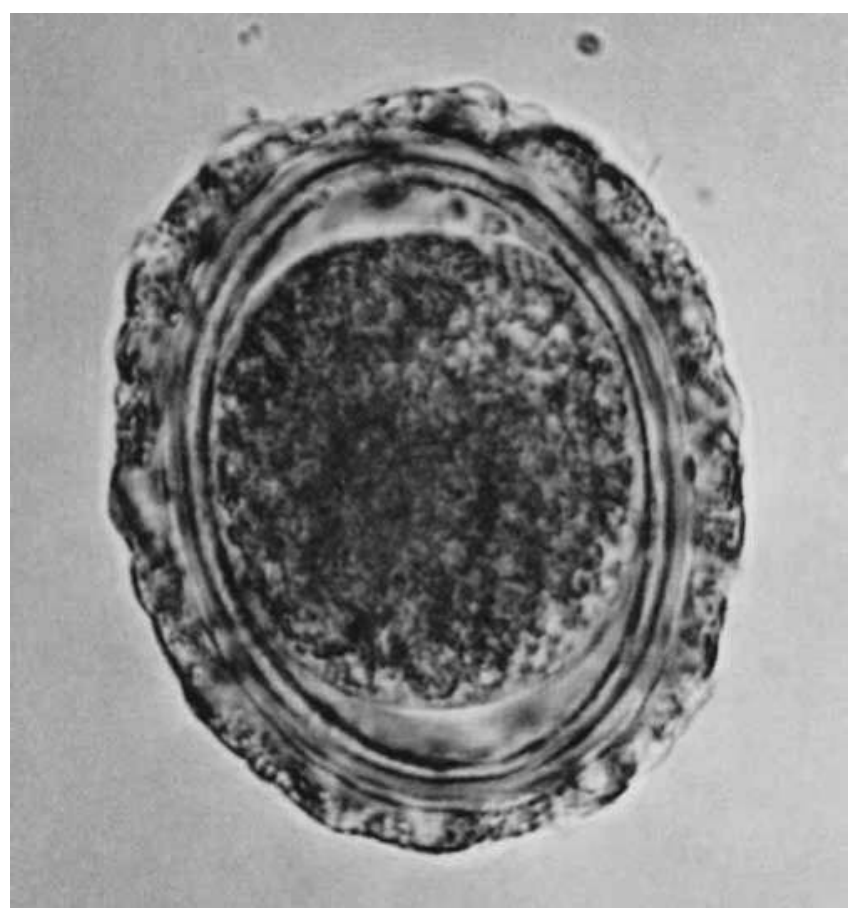

Figure 8 Ascaris lumbricoides egg. Source: From Ref. 32. resulting in multifocal abscess formation (31.) Most patients, however, have minimal or no symptoms. Albendazole (single 400 -mg oral dose), mebendazole ( $100 \mathrm{mg}$ orally BID $\times 3$ days or single 500-mg oral dose), and ivermectin (single 150$200 \mu \mathrm{g} / \mathrm{kg}$ oral dose) are recommended for treatment.

Ascariasis in pregnancy is generally asymptomatic. Transplacental infection has been reported, but is quite rare (41). Pregnant women should, however, be treated prior to labor or cesarean section, because of the risk of adult worm migration, stimulated by the stress of labor or general anesthetics.

\section{Capillaria hepatica and Capillaria philippinensis}

Hepatic capillariasis has a worldwide distribution, whereas intestinal capillariasis is seen mainly in the Philippines and Thailand. The adult worms are 2 to $4 \mathrm{~mm}$ long (32). Capillaria hepatica adults live in the liver parenchyma, whereas C. philippinensis adults remain in the small intestine. Capillaria eggs have striated shells and are unembryonated. Humans are incidental hosts; birds and rodents are the definitive hosts. The C. hepatica life cycle involves passage of eggs into feces, which then embryonate and are ingested by rodents (or humans). The larvae hatch and migrate to the liver to mature. The adult worms deposit eggs in the liver, where they remain until the rodent is consumed by a carnivore. In digestion, the eggs are released from the liver and pass in the carnivore's feces to restart the cycle (32). C. philippinensis eggs pass into water, where they are ingested by fish, and develop to the infective stage. Birds (or humans) are infected by eating raw fish. The adult worms live in the birds' (or human's) intestine and pass eggs in its feces (32).

Hepatic capillariasis is quite uncommon in humans and requires liver biopsy for diagnosis. Intestinal capillariasis is more common and can be diagnosed by finding eggs in feces (32). The clinical course resembles sprue, with diarrhea and severe gastrointestinal symptoms (31). Human autoinfection can occur, worsening the prognosis significantly.

Capillariasis in pregnancy may be left untreated until postpartum if symptoms are mild. Severe cases must be treated antepartum. Mebendazole ( $100 \mathrm{mg}$ twice a day for 3 days) is the drug of choice, regardless of pregnancy status (40).

\section{Strongyloides stercoralis}

Strongyloidiasis has a worldwide distribution, but is most common in warm climates with a high water table. Adult worms are all females. They measure 2 to $3 \mathrm{~mm}$ in length and live in the small intestine. Strongyloides eggs are thin shelled (32). Female worms deposit eggs in the intestinal mucosa, where they hatch, releasing larvae. The larvae migrate into the lumen and pass in the feces into soil. Following maturation in the soil, infective larvae infect humans through direct skin penetration. The larvae then migrate, via the venous system and lungs, to the intestine, where they mature to adulthood and lay eggs. The entire life cycle may occur outside the body (in soil) or inside the body (internal autoinfection) (32). Strongyloidiasis is difficult to diagnose. Fecal concentration techniques are required to find larvae in feces, owing to the low number present.

The clinical effects of Strongyloides stercoralis infection include rash or pruritus (penetration phase); pneumonitis and cough (migration phase); and abdominal pain, diarrhea, 
nausea, and vomiting (intestinal phase) (31). Immunosuppressed patients are at high risk from fulminant autoinfection. The drug of choice for treatment is ivermectin $(200 \mu \mathrm{g} / \mathrm{kg} /$ day orally $\times 2$ days $)$ with albendazole $(400 \mathrm{mg}$ orally BID $\times 7$ days $)$ listed as an alternative. Strongyloidiasis in pregnancy may be left untreated until postpartum if symptoms are mild. Severe cases should be treated antepartum.

\section{Necator americanus and Ancylostoma duodenale}

Hookworm infection is common throughout Africa, Asia, and the South Pacific. Adult worms are small (7-11 mm in length), with females being larger than males. Hookworm eggs are thin shelled and colorless (32). Adult hookworms may live in the human intestine for 5 to 15 years, with the eggs being passed in feces. The eggs enter soil, hatch, and mature to third-stage (infective) larvae (Fig. 9). Infective larvae directly penetrate human skin and migrate, via the lungs, to the small intestine, where they mature to adulthood (41). Hookworm infection is diagnosed by finding eggs in feces (32).

Light hookworm infection is asymptomatic. Heavy infection produces hypochromic macrocytic anemia, due to intestinal blood loss (31). Treatment is albendazole (single 400-mg oral dose), mebendazole $(100 \mathrm{mg}$ orally $\mathrm{BID} \times 3$ days or single $500-\mathrm{mg}$ oral dose), or pyrantel pamoate $[11 \mathrm{mg}$ base $/ \mathrm{kg} /$ day (maximum 1-g base) orally $\times 3$ days].

Prior to modern therapy, severe hookworm infection in pregnancy was associated with significant maternal (27\%)

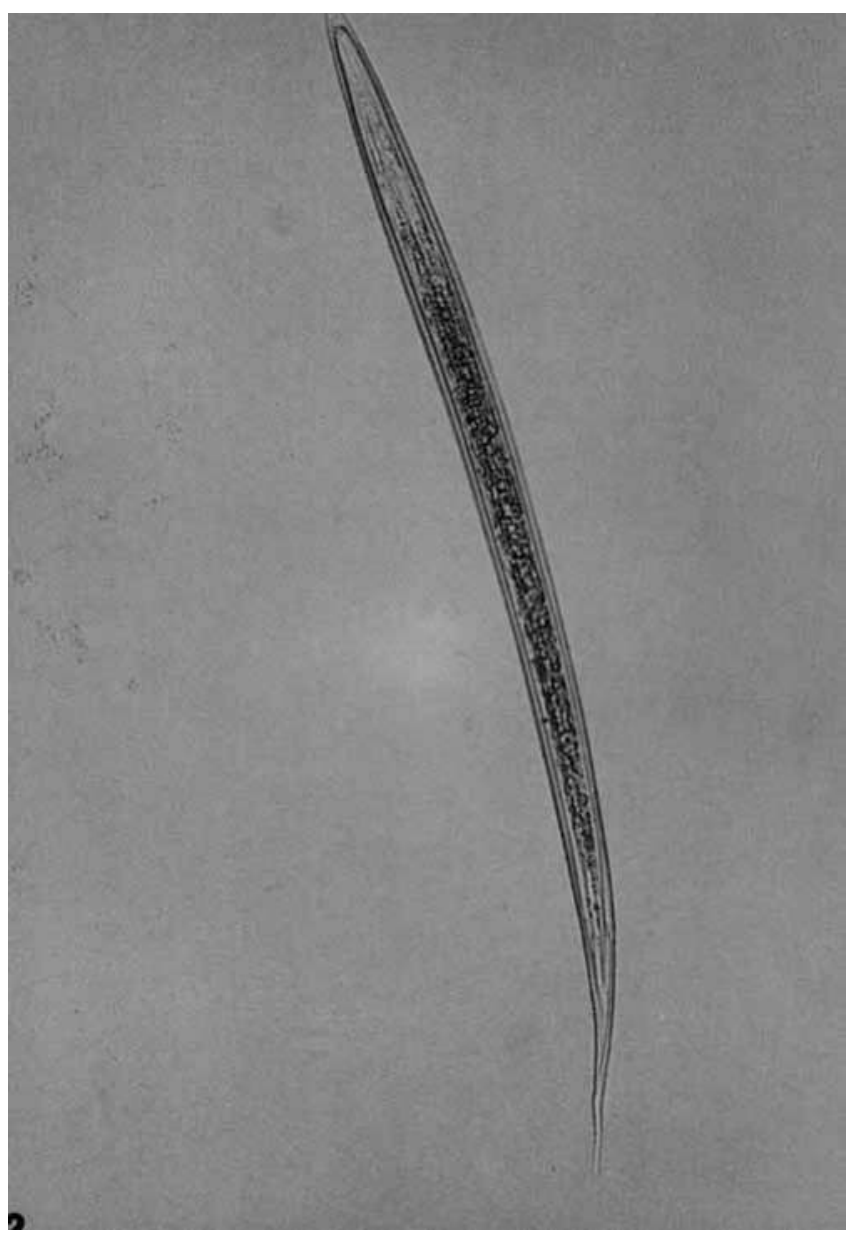

Figure 9 Necator/Ancylostoma (hookworm) infective third-stage larva. Source: From Ref. 32. and perinatal (23\%) mortality (41). Maternal anemia, if untreated, may result in debilitation and heart failure (43). This maternal morbidity may cause fetal loss through abortion, preterm delivery, or stillbirth. Congenital infection has been reported (41). In 2002, WHO published a new guidance document recommending immediate treatment of hookworm infections in pregnant women beyond the first trimester, based in part on 20 years of experience using mebendazole in antenatal clinics in Sri Lanka without excess adverse events $(38,39)$. Clinical trials of anthelmintic treatment for hookworm in pregnancy suggest that treatment is safe and may reduce maternal anemia, low birth weight, and infant mortality (44). Addition of iron therapy to anthelmintic treatment may further improve maternal anemia (44).

\section{Toxocara canis}

Toxocara canis (visceral larva migrans) is cosmopolitan, with dogs as the definitive host. The adult worms are 4 to $10 \mathrm{~cm}$ long and inhabit dog intestines. Toxocara eggs are thick shelled and spherical. Humans are incidental hosts (32). Toxocara eggs pass into soil, mature, and are ingested by rodents. Upon ingestion, the larvae are released and migrate throughout the body. Dogs ingest infected meat, with release of larvae in the stomach and maturation in the intestine. Human infection, called visceral larva migrans, occurs via ingestion of infective eggs with subsequent larval release and migration (32). Clinical diagnosis is based on history of contact with a pet and clinical examination. Laboratory diagnosis can be made by serologic testing for larval antigens, but there is some crossreactivity with Ascaris lumbricoides. Clinical diagnosis is considered sufficient for treatment.

Human infection generally occurs in children who have contact with outdoor pets. The clinical syndrome varies based on the number of migrating larvae and the degree of inflammation and immune response to those larvae. It may include fever, cough, wheezing, abdominal pain, and hepatomegaly. Eosinophilia is usually present. Treatment with steroids and anti-inflammatory medications is indicated to reduce the inflammation associated with migration of the larvae. Albendazole $(400 \mathrm{mg}$ orally BID $\times 5$ days $)$ and mebendazole $(100-200 \mathrm{mg}$ orally BID $\times 5$ days $)$ are the recommended anthelmintic treatments. Infections during pregnancy may be left untreated until postpartum if symptoms are mild. Severe cases must be treated antepartum.

Anthelmintic Agents and Their Status for Use in Pregnancy Anthelmintic agents are quite effective, with cure rates of $70 \%$ to $100 \%$ following a single course of therapy (34). Niclosamide, praziquantel, and pyrantel pamoate are the anthelmintic agents commonly used in pregnancy (Table 3). Mebendazole and albendazole are avoided, if possible, owing to the teratogenicity in rat and rabbit models but have quite a bit of reassuring human data despite the absence of large randomized trials to prove safety. Ivermectin is also avoided if possible due to teratogenicity in animals with very limited human experience thus far. Table 4 summarizes the classification of anthelmintic agents for use in pregnancy or lactation.

Pyrantel pamoate, a pyrimidine derivative, acts by paralyzing nematodes, allowing them to be expelled. Its action is similar to depolarizing neuromuscular blocking agents (41) 
Table 3 Summary of anthelmintic Therapy and Recommendations for Treatment in Pregnancy

\begin{tabular}{|c|c|c|}
\hline & First choice & Alternative \\
\hline \multicolumn{3}{|l|}{ Cestodes } \\
\hline Taenia $^{c}$ & Praziquantel & Niclosamide \\
\hline Diphyllobothrium $^{c}$ & Praziquantel + vitamin $B_{12}$ & Niclosamide+ vitamin $\mathrm{B}_{12}$ \\
\hline Echinococcus $^{\mathrm{a}}$ & Surgery & Albendazole/praziquantel \\
\hline \multicolumn{3}{|l|}{ Trematodes } \\
\hline Schistosoma ${ }^{\mathrm{b}}$ & Praziquantel & \\
\hline Enterobius (pinworm) $)^{\mathrm{c}}$ & Pyrantel pamoate & Albendazole/mebendazole \\
\hline Trichuris (whipworm) ${ }^{\mathrm{c}}$ & Mebendazole/albendazole & Ivermectin \\
\hline Trichinella $^{\mathrm{b}}$ & Steroids + mebendazole/albendazole & \\
\hline Ascaris $^{\mathrm{d}}$ & Albendazole/mebendazole & Ivermectin \\
\hline Capillaria $^{c}$ & Mebendazole & \\
\hline Strongyloides ${ }^{c}$ & Ivermectin & Albendazole \\
\hline
\end{tabular}

Table 4 Summary of Pregnancy/Lactation Recommendations

$\begin{array}{lcc}\text { Anthelmintic agent } & \text { Pregnancy use category } & \text { Recommendations for breastfeeding women } \\ \text { Albendazole } & \mathrm{C} & \text { NR (no data) } \\ \text { Mebendazole } & \mathrm{C} & \text { Compatible } \\ \text { Ivermectin } & \mathrm{C} & \text { NR (no data) } \\ \text { Pyrantel pamoate } & \mathrm{B}^{\mathrm{a}} & \text { Compatible } \\ \text { Niclosamide } & \mathrm{B} & \text { NR (no data) } \\ \text { Praziquantel } & \mathrm{B} & \text { Compatible } \\ \text { a'Based on available data, but never assigned by the FDA. Abbreviation: NR, Not recommended. } & \end{array}$

Pyrantel pamoate is very poorly absorbed, with half remaining in the intestinal tract unabsorbed. This poor absorption is the basis for its recommendation as first-line therapy in pregnancy. Blood levels of pyrantel pamoate are quite low, but it is not known whether it crosses the placenta. Animal teratology studies were negative, and no human malformations have been reported (compatible with pregnancy category B, but never labeled by the FDA). Pyrantel pamoate is considered safe for use while breastfeeding, due to its low absorption. Side effects (gastrointestinal complaints) are generally minimal and are not increased in pregnancy. Pyrantel doses can be calculated from the pamoate form or the free base ( $1 \mathrm{mg}$ base $=2.9 \mathrm{mg}$ pamoate form $)$. Anthelmintic doses are 5 to $10 \mathrm{mg} / \mathrm{kg}$ pyrantel base or 15 to $30 \mathrm{mg} / \mathrm{kg}$ pyrantel pamoate (maximum daily dose, 1 -g pyrantel base) (41).

Niclosamide, a salicylanilide, inhibits oxidative phosphorylation in the cestode mitochondria, killing the worm on contact. Niclosamide has been shown to have no teratogenic effect in rat and rabbit studies (45). No human malformations have been reported (pregnancy category B). The drug is poorly absorbed, but no information is available on niclosamide levels in breastmilk or on its ability to cross the placenta. There are inadequate data to assure the safety of uninterrupted breastfeeding following niclosamide therapy. Since single-dose therapy is used, temporary interruption (24 hours) of breastfeeding with disposal of milk can accommodate this therapy.
Praziquantel (an isoquinolone) is the drug of choice for most trematode infections in pregnancy (43). It is well tolerated with minimal side effects (36). Studies of teratogenicity have been negative in mice, rats, and rabbits $(46,47)$. There are no reports of human malformations (pregnancy category B). Praziquantel is secreted in breastmilk at $29 \%$ of the maternal serum level (34). Praziquantel use is considered compatible with breastfeeding. In order to reduce potential infant exposure with single-dose maternal therapy, the mother can pump and discard the milk for 1 to 2 days to allow clearance of the drug.

Ivermectin is an anthelmintic macrocyclic lactone similar to macrolide antibiotics but with no antibiotic activity. Its activity against helminths comes from affecting ion channels in the cell membrane to increase permeability causing cell death. Ivermectin is a pregnancy category $\mathrm{C}$ drug, due to animal data suggesting teratogenicity, without controlled data in humans. There are only a few case reports of ivermectin use in pregnancy thus far, but there were no issues noted in those cases. Use of ivermectin in pregnancy is thus recommended only when the benefit to the mother outweighs any potential risk to the fetus. Ivermectin is excreted into breastmilk at low levels so its use during breastfeeding is likewise recommended only if the benefit to the mother outweighs any risk to the infant.

Albendazole and mebendazole (benzimidazole derivatives) are the drugs of choice for treatment for most nematode infections. Both are pregnancy category $\mathrm{C}$ based on animal data suggesting teratogenicity without controlled data in 
humans. Mebendazole has been used extensively in the second and third trimesters in Sri Lanka, Nepal, and Madagascar, where it is routinely used for deworming in antenatal clinics without evidence of increased adverse outcomes (48). Both albendazole and mebendazole have been used in a number of second- and third-trimester clinical trials to study the benefit of deworming in pregnancy with more than 400 women having been exposed with no increase in adverse events reported (44). There was a case series from Sri Lanka reporting increased congenital anomalies with first-trimester mebendazole use so that is not recommended unless the maternal condition is severe enough to require immediate treatment without regard for fetal risks (49). Mebendazole has very limited excretion in breastmilk so is compatible with breastfeeding. There are inadequate data on albendazole to recommend use during breastfeeding.

\section{PROTOZOAN INFECTIONS IN PREGNANCY}

\section{Trichomoniasis}

Trichomonas vaginalis is an extracellular flagellated single-cell protozoon that colonizes the female lower urogenital tract and male urethra. Humans are the only host for T. vaginalis and the organisms cannot survive outside their human hosts for very long. $T$. vaginalis replicates by binary fusion of the trophozoite form. Trichomoniasis is transmitted from person to person, typically via genital contact at the time of sexual intercourse. Although transmission via fomites, such as towels or clothing, has been theorized, non-venereal transmission has not yet been documented.

Diagnosis of trichomoniasis has classically been performed by wet-mount microscopy, which is highly specific, but not very sensitive missing up to $40 \%$ of infections. There are two FDA-cleared rapid tests for detection of trichomoniasis in vaginal secretions, OSOM Trichomonas Rapid Test (Genzyme Diagnostics, Cambridge, Massachusetts), and the Affirm VIP III (Becton Dickenson, San Jose, California). Both were developed as point-of-care tests with results in 10 and 45 minutes respectively. Both have improved sensitivity over wet mount $(>83 \%)$, but even though the specificity is quite high (>97\%), false-positive test results can be a problem in lowprevalence settings (50). Trichomonas culture is also sensitive and highly specific. Several nucleic acid detection techniques are available for diagnosis of trichomoniasis and are particularly useful for diagnosis in men due to higher sensitivity than the previously used culture techniques.

Colonization with $T$. vaginalis is asymptomatic in most males and initially in many females, but it is estimated that $50 \%$ to $90 \%$ of women with vaginal colonization will ultimately become symptomatic if left untreated (23). Symptoms in women include a profuse, malodorous, frothy, yellow-green vaginal discharge and vulval itching or burning. Males have symptoms consistent with non-gonococcal urethritis, including urethral discharge, local itching/burning, and pain with urination. Treatment of trichomoniasis is typically limited to drugs from the nitroimidazole familymetronidazole (single 2 -g oral dose or $500 \mathrm{mg}$ orally BID $\times$ 7 days) and tinidazole (single 2-g oral dose) (50). Simultaneous treatment of all sexual partners prior to next sexual contact is recommended to prevent immediate recolonization. There has been some controversy over the legal status of providing trichomoniasis treatment to the patient to deliver to his/her partners in the absence of direct contact between the partners and the health-care system, but many U.S. states have now enacted legal protection for partner-delivered therapy for sexually transmitted diseases. Assessment of local standard of care and legal requirements is recommended prior to initiating a practice of partnerdelivered therapy.

Trichomoniasis in pregnancy has been associated with adverse pregnancy outcomes including premature rupture of membranes and preterm delivery. A large multicenter trial was conducted to see whether treatment of asymptomatic trichomoniasis at the end of the second trimester could reduce the rate of preterm birth (51). Unfortunately, this study reported a somewhat increased rate of preterm delivery in the treated women as compared with the placebo group. This increase has not been confirmed in other studies and there has been much discussion of potential limitations of this trial (including the timing and dosage of the treatment), but to date there has been no benefit identified for treatment of asymptomatic pregnant women (52). By contrast, treatment of symptomatic pregnant women with metronidazole (single 2-g oral dose) is recommended upon diagnosis (50). Metronidazole has no evidence of teratogenicity in animal studies and extensive use during human pregnancy (pregnancy category B). Some women may prefer to await completion of the first trimester prior to treatment to avoid exposure during organogenesis. Tinidazole has evidence of increased fetal mortality in animal studies with no controlled human studies (pregnancy category C). Since tinidazole has no advantage over metronidazole with potential for increased risks, tinidazole is not recommended for use during pregnancy. Metronidazole is excreted into breastmilk at levels similar to those found in maternal plasma after oral dosing, thus the American Academy of Pediatrics recommends that women pump and discard breastmilk for 12 to 24 hours following maternal single-dose oral metronidazole therapy to reduce infant exposure (50). It should be noted that the levels seen in the infant after maternal therapy during breastfeeding are lower than levels achieved in infants directly treated with metronidazole. Tinidazole is also excreted into breastmilk at levels similar to maternal plasma following oral dosing. Since tinidazole is still detectable in breastmilk up to 72 hours after the last dose, pumping and discarding milk for 3 days are recommended to reduce infant exposure.

\section{Lice}

\section{Head Lice}

Pediculosis capitis is caused by Pediculus humanus capitis, an ectoparasite that infests the human head. Humans are the only hosts. Adult lice require human blood to survive and die within 1 to 2 days if unable to feed. Adult lice live for about 30 days attached to the proximal hair shafts to provide close proximity to the scalp for feeding. Adult females may lay up to eight eggs (nits) per day adjacent to the scalp to allow the warmth from the head to incubate them. The nits are small $(<1 \mathrm{~mm})$ white-yellow oval eggs that hatch to form nymphs within a week, leaving the empty shell attached to the hair shaft. The nymphs are initially tiny, but grow rapidly to 
become adults in about 7 days when the lifecycle begins again. The diagnosis is based on seeing either adult lice or nits in the hair.

Pediculosis capitis is quite common with the estimated 6- to 12-million infestations per year in the United States alone, mostly in school-aged children. Head lice are usually transmitted via head to head contact with an infected individual, but may be transmitted via fomites such as hats, towels, combs, or bed linens recently used by an infected person. The high prevalence among school children is thought to be due to frequent contact through sports or play as well as the sharing of combs and hair brushes. Infestation with head lice is not related to the level of hygiene. Clinical manifestations of infection are typically itching of the scalp due to hypersensitivity to the louse bites. Itching may not occur until 4 to 6 weeks after the first infection due to the time required to develop an allergic reaction. Although adult lice move from person to person, head lice are not known to transmit other diseases (53).

The diagnosis of pediculosis is often made by the infected person or a family member seeing the adult lice or nits in the hair or on a comb or brush. A number of further diagnostic techniques have been employed including visual inspection (with or without magnification), use of combing (either dry with a special fine-toothed comb or wet following washing and application of a conditioner), shampooing with use of a strainer to collect the rinse water, and collection of hair clipping from hairdressers (54). In comparison, self-diagnosis is almost as sensitive ( $80 \%)$ as wet or dry combing, with visual inspection being by far the least sensitive technique, even with magnification. Since dry combing requires less equipment (only need the fine-toothed comb; no need to wash and condition the hair as required for wet combing) and can thus be performed in any setting, it is typically the diagnostic test of choice, particularly in school-based screening.

Treatment of head lice is recommended for all infected persons. It is important to assess household members of an infected individual for active infection (as described above) in order to treat all infected household members at the same time. Prophylactic treatment of anyone who shares the same bed with an infected person has been recommended, but mass treatment of the entire household is not. Treatment of head lice is performed using a pediculicide applied to the hair and scalp then washed off. Since the medications typically kill only adult and nymph lice (not the nits), retreatment is usually required in 7 to 9 days to kill any recently hatched lice. Commonly used products available over-the-counter in the United States are pyrethrins combined with piperonyl butoxide (Pronto ${ }^{\circledR}, \mathrm{RID}^{\circledR}$ ) and the synthetic pyrethrin, permethrin $1 \%$ lotion $\left(\mathrm{Nix}^{\circledR}\right)$. Pyrethrins are neurologic toxins to insects by alterations of the sodium channels resulting in neurologic paralysis and death to exposed insects. Worldwide resistance to pyrethrins appears to be increasing, so repeating treatment beyond a third dose is not recommended (55). Alternative products used primarily for resistant cases that do not respond to treatment with a pyrethrin include Malathion $0.5 \%$ lotion, benzyl alcohol 5\% lotion, and lindane $1 \%$ shampoo (all available only by prescription) (56). Supplemental measures, such as thorough combing to remove nits, washing of bed linens, bombs, and brushes in hot water, and sealing unwashable items in sealed plastic bags for 2 weeks, may assist in eradication of infection, but are not essential. Since the adult lice and nits die rapidly when removed from the human host, extensive household cleaning is not typically required. There is no role for household fumigation or other use of insecticides in the management of pediculosis.

Pediculosis capitis during pregnancy follows a similar course to nonpregnant adults so treatment prior to delivery is not required, but there is typically a desire to treat due to the itching and psychologic distress of carrying a parasite in a visible location. Permethrin is the drug of choice for treatment in pregnancy and breastfeeding due to the low systemic absorption ( $<2 \%$ of the applied dose) and rapid clearance (53). There was no teratogenicity noted in animal studies of permethrin and there are no controlled human trials so it is a pregnancy category B drug. Observational reviews of use in humans have not identified any increased risk of adverse pregnancy outcomes (53). Permethrin use is considered to be compatible with use during breastfeeding due to the very low levels found in breastmilk. Malathion is also a pregnancy category B drug, but is typically not used in pregnancy or breastfeeding. Lindane and benzyl alcohol have been associated with abnormal neurodevelopment in animal teratogenicity studies (pregnancy category C) so should not be used in pregnancy unless absolutely necessary.

\section{Body Lice}

Pediculosis humanus corporis is caused by the ectoparasite Pediculus humanus humanus. Similarly to head lice, humans are the only host for body lice. The life cycle is quite similar to head lice, with the entire lifecycle occurring on hair shafts in close proximity to the skin. Body lice infestations typically occur in conditions of poor hygiene and overcrowding, such as those seen among homeless individual or in refugee camps. The primary clinical manifestation is itching, with the potential for skin thickening in chronically infected areas.

A major difference between head lice and body lice is the ability of body lice to transmit other diseases. Typhus, trench fever, and endemic relapsing fever have all been transmitted by body lice (57). Pediculus humanus humanus has recently been shown to be capable of transmitting Yersinia pestis as well and may have contributed to the transmission of the Black Plague (58). Body lice are diagnosed by visualization on clothing or skin, which may require magnification. Body lice can typically be resolved by improved hygiene, including use of a clean change of clothing at least weekly with laundering of clothing and towels in hot water (59). Treatment with a pediculicide is not typically required, but if needed the same medications used for head lice are used for body lice. There are no specific issues related to pregnancy.

\section{Pubic Lice}

Pediculosis pubis is caused by the ectoparasite, Phthirus pubis. As was true for head and body lice, humans are the only host for pubic lice and the life cycle is quite similar. Similar to head lice, pubic lice have not been reported to transmit other diseases. A common lay term for pubic lice is "crabs" due to the more rounded crab-like appearance of pubic lice compared with the more elongated head and body lice. Pubic lice are found on the coarse body hair located in the pubic 
region, but may also be seen on other coarse body hairs including beards, mustaches, eyelashes, eyebrows, and axillary hair. Transmission of pubic lice is generally via body contact during sexual intercourse, thus pubic lice are most commonly found in adults. Finding of pubic lice on the eyelashes or head of a child raises the question of potential sexual abuse; however, fomites such as towels, clothing, and bed linens may be involved in transmission so sexual contact is not always involved.

Treatment of pubic lice is usually performed with permethrin $1 \%$ lotion or pyrethrin with piperonyl butoxide applied to the affected area and washed off 10 minutes later (60). Alternative therapies include Malathion 0.5\% lotion and ivermectin. Unlike head lice, a second treatment is required only after 9 to 10 days if live lice are noted at that time. A notable exception to the use of these medications is infection of eyebrows or eyelashes that are most commonly treated by physical removal of lice and nits, with application of ophthalmic-grade petrolatum ointment two to four times per day for 10 days. Supplemental measures including physical removal of nits, laundering of clothing and bed linens in hot water, and placement of unwashable items in sealed plastic bags for 2 weeks are more strongly recommended than that was the case for head lice. Also all sexual partners for the past month should be informed of their risk for infection and treated prior to resuming sexual contact. Since pubic lice is a sexually transmitted disease, it is prudent to evaluate for the presence of other sexually transmitted diseases (60). Pregnant women should be treated with the primary therapies of permethrin or pyrethrin; use of Malathion and ivermectin in pregnancy should be avoided if possible.

\section{Scabies}

Scabies is an ectoparasitic infestation caused by the human itch mite, Sarcoptes scabiei var hominis, for which humans are the only host in which replication can occur. Animal mites can infest human skin temporarily, but cannot replicate in humans and thus their infestation is self-limited. Adult female mites make characteristic serpentine burrows into the skin, usually between fingers and around the wrist in humans, where they lay 2 to 3 eggs per day as the burrow lengthens (61). Eggs hatch in 3 to 4 days to form larvae, which exit the serpentine burrow to dig a shallow molting pouch. Larvae develop into nymphs and then adults within 10 to 14 days inside the molting pouch. Adult male mites leave their molting pouch to burrow into the molting pouch of an adult female mite in order to fertilize the female who then continues to be fertile the remaining month of her life. Once impregnated, the adult female mite leaves the molting pouch to find a site to begin her own serpentine burrow and egg deposition, thus restarting the life cycle.

The diagnosis of scabies can be made clinically based on the presence of characteristic burrows or visible mites. Skin scraping can be performed to look for mite or eggs, but treatment should be provided for suspected cases without confirmation of the presence of mites since a typical infection may involve only 10 to 15 female mites (61). In addition to microscopic examination of skin scrapings with oil, skin scrapings can also be submitted for PCR to detect S. scabiei DNA. When using PCR to verify resolution of infection, it is important to wait at least 28 days after the last treatment to allow time for skin to shed with new epithelium developed, since the DNA from dead organisms will persist in the skin until it has been shed (62). Scabies is transmitted by close interpersonal contact, particularly in crowded living conditions. Outbreaks have been identified in nursing homes, hospitals, orphanages, and refugee camps (63). Transmission may occur with sharing of a bed and often occurs among adults via sexual contact. Children may be infected via sharing of beds or direct contact during play. Scabies is not considered to be an indication of potential sexual abuse due to its frequency in non-abused children. Fomites, such as clothing, towels, and sheets, may be involved with spread in some cases, but are not thought to be a major contributor.

The primary clinical manifestation of scabies is itching due to a hypersensitivity reaction to the organism, which may take several weeks to develop following first exposure, but may develop rapidly with subsequent exposures. A classic rash occurs with visible burrows on the hands, wrists, and ankles. Papules may occur in those locations as well as on the forearms, axilla, breasts, and penis. The visible burrows and papules are the sites from which mites could possibly be obtained via skin scraping. The treatment of choice for uncomplicated scabies is permethrin $5 \%$ cream (Elimite ${ }^{\circledR}$ ) applied to the entire body below the neck with specific attention to folds and creases including those between the fingers and toes as well as beneath the nails, which is washed off after 8 to 14 hours $(64,65)$. A second application a week later may be required to eliminate all mites. Note that this is a higher dose of permethrin than that used for lice and this product is not available over-the-counter in the United States. Alternative treatments include ivermectin $(200 \mu \mathrm{g} / \mathrm{kg} /$ day in 2 doses 14 days apart), crotamiton $10 \%$ lotion or cream, and lindane $1 \%$ lotion but all have a lower efficacy than topical permethrin for uncomplicated scabies $(64,65)$. Supplemental measures such as washing of clothes, bed linens, and towels in hot water may be helpful, but are not essential for uncomplicated scabies as there are few organisms involved.

Treatment of scabies in pregnancy is typically performed with permethrin (64) (pregnancy category B) for which there is no evidence of teratogenicity in animals and there are no controlled studies in humans. There is a retrospective study of women who received either permethrin $4 \%$ lotion $(n=196)$ or benzyl benzoate $25 \%$ lotion $(n=444)$ for treatment of scabies during pregnancy (primarily second and third trimesters, but $10 \%$ first trimester as well) (66). They found no difference in pregnancy outcomes between treated women and their matched controls. They did note a higher need for retreatment with benzyl benzoate lotion than with permethrin $(16.4 \%$ vs. 9.7\%), compatible with the higher efficacy for permethrin seen in nonpregnant adults.

In debilitated or immunocompromised individuals, an aggressive form of scabies called crusted or Norwegian scabies may develop. Crusted scabies is highly infectious with potentially more than a million organisms infecting a single individual. The crusting patches and gray flaky plaques may be difficult to distinguish from psoriasis or eczema and may be localized, extensively covering the hands, feet, trunk, face, and scalp. Optimal treatment for crusted scabies is not yet known, but current recommendations are combined treatment with a topical scabicide (daily dose as described above) and oral 
ivermectin $(200 \mu \mathrm{g} / \mathrm{kg} /$ day $)$ on days $1,2,8,9$, and 15 with additional treatments on days 22 and 29 if needed (64). Supplemental measures including washing of all bed linens, clothing towels, and other items of direct contact are essential to eliminate the infestation due to the high volume of organisms and the propensity for the flaky plaques to fall of bearing high numbers of mites. Close cropping of nails may also be of benefit. Increased rates of crusted scabies have not been described during pregnancy in the absence of underlying debilitation. Treatment of crusted scabies in pregnant women should be individualized with the use of ivermectin if clinically indicated for the health of the mother.

\section{Malaria}

Malaria is typically caused by infection with one of four protozoa, Plasmodium falciparum, Plasmodium vivax, Plasmodium ovale, or Plasmodium malariae. Humans are infected with malaria when bitten by an Anopheles mosquito carrying the organism. Plasmodium sporozoites are transferred from the mosquito to the human bloodstream, where they travel to the liver and infect liver cells. Inside the liver cells, the sporozoite matures to a schizont, which then ruptures releasing merozoites that invade erythrocytes and either multiple asexually to develop a ring stage schizont (which will again rupture releasing merozoites) or develop into a gametocyte. The gametocytes can then be taken up by the Anopheles mosquito to begin the mosquito portion of their life cycle, which includes fertilization of the female gametocyte in the mosquito stomach, maturation in the mosquito midgut wall, and migration of the mature sporozoites to the mosquito salivary gland for injection during the next mosquito blood meal to begin the human portion of the life cycle.

Clinical symptoms of malaria are similar to those of a flu-like illness, with fever, chills, headache, myalgia, malaise, arthralgia, weakness, nausea, vomiting, and diarrhea (67). The degree of symptoms and speed of disease progression varies depending on the infecting organism ( $P$. falciparum is typically most severe), the level of parasitemia, and the immune status of the individual. Symptoms can develop as quickly as 7 days or as long as 2 months following exposure. Severe infections with $P$. falciparum can progress rapidly to acute renal failure, central nervous system involvement, severe anemia, respiratory distress syndrome, and death. Malaria is estimated to cause about 1 million deaths per year, mostly due to $P$. falciparum infections in children in Africa, but there are deaths each year among developed world travelers who visit the developing world, particularly the African region. Physical finding associated with malaria include hepatomegaly, splenomegaly, fever, increased respiratory rate, weakness, and mild jaundice.

In the developed world, suspicion of malaria based on identification of potential exposure is the first key to diagnosis. Diagnosis is based on either identification of parasites in blood by microscopy or detection of malaria antigens or enzymes in blood. On microscopic examination of blood smears, the gametocyte form is typically the easiest to identify as other forms (such as ring-stage schizont) may be quite difficult to distinguish. More recently, a variety of rapid diagnostic tests (RDTs) have been developed to detect either antibodies or enzymes produced by the Plasmodium organisms, allowing expanded diagnosis in resource-poor settings (67). Assistance with diagnosis and treatment in the United States can be obtained from the U.S. Centers for Disease Control and Prevention (CDC, www.CDC.GOV), who maintain a malaria control hotline for emergency assistance. Outside the United States, The WHO (www.who.org) maintains guidelines for diagnosis and treatment of malaria and assists with drug procurement in the developing world.

Treatment for malaria is based on the infecting organism and the likelihood of resistance based on the location in which the infection was acquired. The WHO updated their malaria treatment guidelines (67) in 2010 to stress the need for diagnosis prior to treatment and to promote a move toward use of artemisinin-based combination therapy (ACT) as opposed to monotherapy to reduce the spread of artemisinin resistance already widely seen in Southeast Asia. In the United States, chloroquine-sensitive malaria is usually treated with chloroquine as the first-line therapy. Atovaquone-proguanil (Malarone $\left.{ }^{\circledR}\right)$, artemether-lumefantrine $\left(\right.$ Coartem $\left.^{\circledR}\right)$, or quinine plus doxycycline or tetracycline are currently the first-line regimens for $P$. falciparum infections likely to be chloroquine resistant. Since treatment guidelines have recently been undergoing frequent modification to keep up with changing patterns of resistance, it would be advised to check the WHO or CDC Web site or other frequently updated resources for current recommendations prior to initiating treatment.

The best treatment for malaria, however, is prevention. There are a number of recommended prophylactic regimens for travel into malaria-endemic areas. Atovaquone-proguanil (Malarone) and doxycycline can be used in all areas, with chloroquine and mefloquine (Lariam $\left.{ }^{\circledR}\right)$ limited to areas with no evidence of resistance and primaquine limited to areas with only $P$. vivax infections. Supplemental measures are also quite important to avoid contact with the Anopheles mosquito including using insecticide-treated bed nets, limiting exposed skin by wearing long pants and sleeves, using insecticide spray or lotion on exposed skin, and staying in a mosquito-resistant building in the evening when mosquitoes are most likely to bite.

Malaria in pregnancy is associated with high risk of maternal and perinatal morbidity and mortality, presumably due to impaired immune response to the infection. Increased adverse outcomes include spontaneous abortion, fetal death, preterm birth, low-birth weight infants, and maternal death (68). The increase in maternal mortality may be directly related to severe malarial infection or may be due to the impact of severe anemiacausing congestive heart failure or death due to postpartum hemorrhage. Malaria organisms can be sequestered in the placenta, where they may be resistant to treatment and may also increase fetal pathology.

Treatment of malaria in pregnancy is also impacted by type of organism and likelihood of chloroquine resistance. For chloroquine-sensitive malaria, chloroquine is the drug of choice, as it is for nonpregnant adults. For chloroquineresistant malaria, quinine plus clindamycin is currently the first-line therapy for the first trimester due to lack of experience with either of the other first-line combinations (atovaquone-proguanil or artemether-lumefantrine, both pregnancy category C) $(67,68)$, with the caveat that one of those regimens should be used if quinine/clindamycin is either unavailable or is not tolerated. The 2010 WHO guidelines recommend use of ACT known to be effective in the local area 
after the first trimester due to the enhanced efficacy (67), whereas the CDC recommendations are to continue the use of quinine plus clindamycin throughout pregnancy unless intolerance or treatment failure is seen. Quinine is pregnancy category $\mathrm{C}$ and is known to cross the placenta, but extensive experience has revealed no increase in adverse pregnancy outcomes with its use. Clindamycin is pregnancy category B with no teratogenicity seen in animal studies and extensive human experience with no increase in adverse outcomes seen.

Prophylaxis for pregnant travelers is very complicated since both primaquine and doxycycline are contraindicated during pregnancy and atovaquone-proguanil is also not recommended for pregnancy use. Chloroquine is considered safe, but has little benefit in areas with extensive resistance. Mefloquine is pregnancy category $\mathrm{C}$ and should not be used in pregnancy unless prophylaxis for travel to an area with chloroquine resistance is absolutely needed and only after the first trimester due to animal data showing embryotoxicity (69). Supplemental measures (as discussed above) are even more important for pregnant women due to the limitations of other prevention and treatment options.

Elimination of malaria will clearly require more than treatment of symptomatic individuals and prophylaxis for travelers. Two techniques recommended by $\mathrm{WHO}$ are mass screening and treating of asymptomatic individuals who are infected and MDA. Mass screening and treatment requires much higher levels of resources than MDA so it is the technique most commonly employed. To date, MDA campaigns have not been able to achieve complete eradication with the exception of one closed island community with limited contact with the outside world where P. falciparum was eradicated rapidly (5 weeks), but it took 5 years to eradicate $P$. vivax (67). Inclusion or exclusion of pregnant women from MDA has been quite controversial due to the frequent use of drugs not commonly used in pregnancy being contrasted with the severe consequences of malarial infection during pregnancy. Reviews of data to date indicate no increase in adverse pregnancy outcome with inclusion in MDA or the more specific use of intermittent preventive therapy (IPT) targeting pregnant women [usually performed as one dose at quickening (18-20 weeks) with a second dose a month or more later $(68,70)]$. One key issue raised has been the importance of using highly effective drugs if MDA or IPT are to be used in pregnancy as opposed to using older, less effective, or completely ineffective drugs simply because there is more experience with them in pregnancy. Exposure to an ineffective drug in pregnancy is all risk with no benefit.

\section{REFERENCES}

1. Sobel JD. Pathophysiology of vulvovaginitis candidiasis. J Reprod Med 1989; 34: 572-9.

2. McKay M. Cutaneous manifestations of candidiasis. Am J Obstet Gynecol 1988; 158: 991-3.

3. Hopfer RL. Mycology of Candida infections. In: Bodey GP, Fainstein V, eds. Candidiasis. New York: Raven Publishers, 1985.

4. Higashide A, Aman R, Yamamuro O. Clinical characteristics correlated with different fungi causing vulvovaginal mycosis. Mycoses 1988; 31: 213-25.

5. Oriel JD, Partridge BM, Denny MJ, Coleman JC. Genital yeast infections. Br J Med 1972; 4: 761-4.

6. Redondo-Lopez V, Lynch M, Schmitt C, et al. Torulopsis glabrata vaginitis: clinical aspects and susceptibility to antifungal agents. Obstet Gynecol 1990; 76: 651-5.
7. Das Neves J, Pinto E, Teixeira B, et al. Local treatment of vulvovaginal candidosis. Drugs 2008; 68: 1787-802.

8. Robertson WH. Mycology of vulvovaginitis. Am J Obstet Gynecol 1988; 158: 989-91.

9. Powell BL, Frey CL, Drutz DJ. Identification of a17b-estradiol binding protein in Candida albicans and Candida glabrata. Exp Mycol 1984; 8: 304-13.

10. Loose DS, Schurman DJ, Feldman D. A corticosteroid binding protein and endogenous ligand in C. albicans indicating a possible steroid receptor system. Nature 1981; 293: 477-9.

11. McCormack WM, Starko KM, Zinner SH. Symptoms associated with vaginal colonization with yeast. Am J Obstet Gynecol 1988; 158: 31-3.

12. Kaufman RH. Establishing a correct diagnosis of vulvovaginal infection. Am J Obstet Gynecol 1988; 158: 986-8.

13. Luna MA, Tortoledo ME. Histologic identification and pathologic patterns of disease due to Candida. In: Bodey GP, Fainstein V, eds. Candidiasis. New York: Raven Publishers, 1985.

14. Gentry LO, Price MF. Urinary and genital Candida infections. In: Bodey GP, Fainstein V, eds. Candidiasis. New York: Raven Publishers, 1985.

15. Hill LVH, Embil JA. Vaginitis: current microbiologic and clinical concepts. Can Med Assoc J 1986; 134: 321-31.

16. Centers for Disease Control and Prevention. Sexually Transmitted Diseases Treatment Guidelines, 2010. MMWR 2010; 59: 61-3.

17. Brammer KW. A comparison of single-dose oral fluconazole with 3-day intravaginal clotrimazole in the treatment of vaginal candidiasis. $\mathrm{Br}$ J Obstet Gynaecol 1989; 96: 226-32.

18. Van Heusden AM, Merkus HM, Corbeij RS, et al. Single-dose oral fluconazole versus single-dose topical miconazole for the treatment of acute vulvovaginal candidosis. Acta Obstet Gynecol Scand 1990; 69: 417-22.

19. Kutzer E, Oittner R, Leodolter S, Brammer KW. A comparison of fluconazole and ketoconazole in oral treatment of vulvovaginal candidiasis; report of a double-blind multicentre trial. Eur J Obstet Gynecol Reprod Biol 1988; 29: 305-13.

20. De Los Reyes C, Edelman DE, De Bruin MF. Clinical experience with single-dose fluconazole in vaginal candidiasis: a review of the worldwide database. Int J Gynecol Obstet 1992; 37(Suppl): 9-15.

21. Sobel JD. Recurrent vulvovaginal candidiasis: a prospective study of the efficacy of maintenance ketoconazole therapy. N Engl J Med 1986; 315: 1455-8.

22. Van Slyke KK, Michel VP, Rein MF. Treatment of vulvovaginal candidiasis with boric acid powder. Am J Obstet Gynecol 1981; 141: 145-8.

23. Hay P, Czeizel AE. Asymptomatic trichomonas and candida colonization and pregnancy outcome. Best Pract Res Clin Obstet Gynecol 2007; 21: 403-9.

24. Rosen T. Cutaneous candidiasis. In: Bodey GP, Fainstein V, eds. Candidiasis. New York: Raven Publishers, 1985.

25. Moudgal VV, Sobel JD. Antifungal drugs in pregnancy: a review. Expert Opin Drug Saf 2003; 2: 475-83.

26. Nystatin Multicenter Study Group. Therapy of candidal vaginitis: the effect of eliminating intestinal Candida. Am J Obstet Gynecol 1986; 155: 651-5.

27. Bodey GP, Fainstein V. Systemic candidiasis. In: Bodey GP, Fainstein V, eds. Candidiasis. New York: Raven Publishers, 1985.

28. Moudgal VV, Sobel JD. Antifungals to treat Candida albicans. Expert Opin Pharmacother 2010; 11: 2037-48.

29. Fera MT, La Camera E, De Sarro A. New triazoles and echinocandins: mode of action, in vitro activity and mechanisms of resistance. Expert Rev Anti Infect Ther 2009; 7: 981-98.

30. Gyorkos TW, MacLean JD, Lae CG. Absence of significant differences in intestinal parasite prevalence estimates after examination of either one or two stool specimens. Am J Epidemiol 1989; 130: 976-80.

31. Zaman V. Atlas of Medical Parasitology. Boston, MA: ADIS Health Science Press, 1978.

32. Ash LR, Orihel TC. Atlas of Human Parasitology, 3rd edn. Chicago: ASCP Press, 1990.

33. Powlowski ZS. Role of chemotherapy of taeniasis in prevention of neurocysticercosis. Parasitol Int 2006; 55(Suppl): s105-9.

34. MacLeod CL. Parasitic Infections of Pregnancy and the Newborn. New York: Oxford University Press, 1988.

35. Vuitton D. Alveolar echinococcosis of the liver: a parasitic disease in search of treatment. Hepatology 1990; 12: 617-18. 
36. McNeely DF, Magu MR. Schistosomiasis. In: MacLeod CL, ed. Parasitic Infections of Pregnancy and the Newborn. New York: Oxford University Press, 1988.

37. Friedman JF, Mital P, Kanzaria HK, Olds GR, Kurtis JD. Schistosomiasis and pregnancy. Trends Parasitol 2007; 23: 159-64.

38. WHO. Report of the WHO informal consultation on the use of praziquantel during pregnancy and albendazole/mebendazole in children under 24 months. Geneva: World Health Organization, WHO/CDS/CPE/ PVC/2002.4, 2002.

39. Allen HE. New policies for using anthelmintics in high risk groups. Trends Parasitol 2002; 18: 381-2.

40. Youssef FG, Mikhail EM, Mansour NS. Intestinal capillariasis in Egypt: a case report. Am J Trop Med Hyg 1989; 40: 195-6.

41. MacLeod CL. Intestinal nematode infections. In: MacLeod CL, ed. Parasitic Infections of Pregnancy and the Newborn. New York: Oxford University Press, 1988.

42. Kociecka W. Trichinosis. In: MacLeod CL, ed. Parasitic Infections of Pregnancy and the Newborn. New York: Oxford University Press, 1988.

43. Langer A, Hung CT. Hookworm disease in pregnancy with severe anemia. Obstet Gynecol 1973; 42: 564-7.

44. Brooker S, Hotez PJ, Bundy DAP. Hookworm-related anemia among pregnant women: a systematic review. PLoS Negl Trop Dis 2008; 2: e291.

45. Goldsmith R, Markell EK. Other trematode infections. In: MacLeod CL, ed Parasitic Infections of Pregnancy and the Newborn. New York: Oxford University Press, 1988.

46. Frohberg H, Schencking MS. Toxicological profile of praziquantel, a new drug against cestode and schistosome infections, as compared to some other schistosomicides. Arzneimittelforsch 1981; 31: 555-65.

47. Frohberg H. Results of toxicological studies on praziquantel. Arzneimittelforsch 1984; 34: 1137-44.

48. Gyorkos TW, Laroque R, Casapia M, Gotuzzo E. Lack of adverse birth outcomes after deworming in pregnant women. Pediatr Inf Dis J 2006; 25: 791-4.

49. Haider BA, Humayun Q, Bhutta ZA. Effect of administration of anthelmintics for soil transmitted helminths during pregnancy. Cochrane Database Syst Rev 2009: CD005547.

50. Centers for Disease Control and Prevention. Sexually Transmitted Diseases Treatment Guidelines, 2010. MMWR 2010; 59: 58-61.

51. Klebanoff MA, Carey JC, Hauth JC, et al. Failure of metronidazole to prevent preterm delivery among pregnant women with asymptomatic Trichomonas vaginalis infection. N Engl J Med 2001; 345: 487-93.
52. Gulmezoglu AM. Interventions for trichomoniasis in pregnancy. Cochrane Database Syst Rev 2002: CD000220.

53. Kennedy D, Hurst V, Konradsdottir E. Pregnancy outcome following exposure to permethrin and use of teratogen information. Am J Perinatol 2005; 22: 87-90.

54. Burgess IF. Current treatments for Pediculosis capitis. Curr Opin Infect Dis 2009; 22: 131-6.

55. Lebwohl M, Clark L, Levitt J. Therapy for head lice based on life cycle, resistance and safety considerations. Pediatrics 2007; 119: 965-74.

56. Frankowski BL, Bocchini JA. Head lice. Pediatrics 2010; 126: 392-403.

57. Bechah Y, Capo C, Mege L, Raoult D. Endemic typhus. Lancet Inf Dis 2008; 8: 417-26.

58. Ayyadurai S, Sebbane F, Raoult D, Drancourt M. Body lice, Yersinia pestis orientalis and black death. Emerg Infect Dis 2010; 16: 892-3.

59. Badiaga S, Raoult D, Brouqui P. Preventing and controlling emerging and reemerging transmissible diseases in the homeless. Emerg Infect Dis 2008; 14: 1353-9.

60. Centers for Disease Control and Prevention. Sexually Transmitted Diseases Treatment Guidelines, 2010. MMWR 2010; 59: 88-9.

61. Nordlund JJ. Cutaneous ectoparasites. Dermatol Ther 2009; 22: 503-17.

62. Hicks MI, Elston DM. Scabies. Dermatol Ther 2009; 22: 279-92.

63. Currie BJ, McCarthy JS. Permethrin and ivermectin for scabies. N Engl J Med 2010; 362: 717-25.

64. Centers for Disease Control and Prevention. Sexually Transmitted Diseases Treatment Guidelines, 2010. MMWR 2010; 59: 89-90.

65. Strong M, Johnstone P. Interventions for treating scabies. Cochrane Database Syst Rev 2007: CD000320.

66. Mytton OT, McGready R, Lee SJ, et al. Safety of benzyl benzoate lotion and permethrin in pregnancy: a retrospective matched cohort study. BJOG 2007; 114: 582-7.

67. WHO. Guidelines for the treatment of malaria, 2nd edn. Geneva: WHO, 2010.

68. McGready R, White NJ, Nosten F. Parasitological efficacy of antimalarials in the treatment and prevention of falciparum malaria in pregnancy 1998 to 2009. BJOG 2011; 118: 123-35.

69. Davis TME, Mueller I, Rogerson J. Prevention and treatment of malaria in pregnancy. Future Microbiol 2010; 5: 1599-613.

70. Garner P, Gulmezoglu AM. Drugs for preventing malaria in pregnant women. Cochrane Database Syst Rev 2003: CD000169. 


\section{Routine maternal immunizations for all pregnant women Stanley A. Gall}

The concept of adult immunization in general and maternal immunization specifically has been receiving an increased attention from clinicians and medical writers as new vaccines have come into the market. However, the general population's knowledge of the need for vaccination and which vaccines to obtain is very limited. Unfortunately, physicians who provide care to adults are also hampered by an incomplete knowledge of adult vaccines. The general public has an overall awareness of the significant reduction that has occurred in the incidence of measles, mumps, rubella, polio, diphtheria, and tetanus. However, the general public does not understand that failure to maintain immune status to diseases such as tetanus, diphtheria, hepatitis A, hepatitis B, herpes zoster, varicella influenza, pneumococcal disease, and human papillomavirus can result in serious diseases. Each of these diseases has an effective vaccine and need to be seriously considered for adult immunization.

The benefit of vaccination in pregnancy is to protect (i) the mother from disease and (ii) the neonate from disease with passive maternal antibodies. This is the best two for one deal in nature.

Active immunization is the administration of an antigen, which may be a live attenuated organism, inactivated viruses, recombinant viral particles, purified proteins, bacterial capsular polysaccharides, or exotoxin derivatives (toxoids) with the projected effect of preventing, abbreviating, or ameliorating natural infection.

Passive immunization is the transfer of performed antibodies from immune individuals by potential administration of pooled human or equine serum or by placental transfer of immunoglobulin G (IgG) from mother to fetus.

Current adult vaccines and their use in pregnancy are seen in Tables 1 and 2. Immunoglobulin G and antitoxins that are available in the United States are listed in Table 3.

Table 1 lists the vaccines that should be routine for every pregnancy. This assumes that there are universal recommendations for each of these vaccines. Table 2 lists other licensed vaccines that may be considered to be used during pregnancy in special circumstances.

\section{VACCINATION IN PREGNANCY}

It is seen from Tables 1 and 2 that a total of 6 vaccines should be used during each pregnancy and 12 vaccines that are licensed may be used if indicated. However, the vaccines that should be routinely administered to all pregnant women who have not been immunized previously are influenza, hepatitis A and hepatitis B, tetanus, diphtheria, acellular pertussis (TdaP), and pneumococcal and meningococcal vaccines. It is tragic for any pregnant women to develop an infectious disease that is vaccine preventable. This universal approach is a change in practice from ignoring the pregnant woman and assuming she is protected to a policy of active inquiry as to her vaccine status and a program of active immunization. The result is protection of the pregnant women from disease and protection of the neonate for a variable length of time from passive maternal antibodies. The inactive vaccines and toxoids are safe to administer during pregnancy, and practicing obstetricians should adopt this positive management advance (2).

An immunization history should be obtained at the first prenatal visit. In an ideal world, the patient should be immune to measles, mumps, rubella, tetanus, diphtheria, pertussis, poliomyelitis, varicella, and hepatitis A and B. However, in the real world, adults generally are not aware of their needs for vaccination and the release of new vaccines, and many physicians are not aware of vaccine requirements. As a result of this vast lack of knowledge and information, the majority of patients are either no longer protected from disease or they have never received the appropriate vaccines.

The Advisory Committee for Immunization Practices (ACIP) at the Centers for Disease Control and Prevention (CDC) is a 15 -member committee, which advises the CDC with recommendations for vaccine use. While the vaccine recommendations for pediatric vaccines are based on an agerelated schedule, the adult recommendations are risk based. The risk-based strategy causes confusion for the practitioner since as many as 26 risks are identified for hepatitis B and numerous risks for other vaccines. Few physicians will remember the many risks as indications for vaccine administration. A simplified approach is to select the six important vaccines to be routinely administered during pregnancy and routinely administer these vaccines to all pregnant women as universal vaccines. A simple vaccine questionnaire is seen in Table 4. This questionnaire assumes that the six vaccines listed should be administered to all patients who have not been previously immunized, asks about allergies to vaccines, and lists whether the vaccine information statement (VIS) has been given to the patient and whether the patient has been immunized or refused immunization on the visit day. The form then becomes a record of vaccine refusal (potential medical-legal benefit), VIS given (federal requirement), and vaccine administered.

\section{INFLUENZA EPIDEMIOLOGY}

Influenza is an acute febrile illness associated with headaches, migraines, upper airway respiratory complaints, and gastrointestinal symptoms. Primary influenza pneumonia, which is frequently fatal, may occur in patients who have not been vaccinated and/or have underlying cardiopulmonary disease. Most fatalities associated with influenza are due to secondary bacterial pneumonia, usually Streptococcus pneumoniae $(3,4)$. Influenza is caused by one of two viral isotypes: influenza A or influenza B. Influenza A and B are the two types that cause 
epidemic human disease. Influenza A is the most common and the most virulent strain. Influenza A viruses are further characterized into subtypes based on two surface antigens, hemagglutinin $(\mathrm{H})$ and neuraminidase $(\mathrm{N})$. Influenza $\mathrm{B}$ viruses are separated into two distinct genetic lineages (Yamagata and Victoria) but cannot categorize into subtypes.

Table 1 Licensed Vaccine Recommended to All Pregnant Women

\begin{tabular}{|c|c|c|c|}
\hline Vaccine & Type & Route & $\begin{array}{c}\text { Use in } \\
\text { pregnancy }\end{array}$ \\
\hline Hepatitis B & $\begin{array}{l}\text { Recombinant viral } \\
\text { particle }\end{array}$ & IM & Yes \\
\hline Hepatitis A & Killed virus & IM & Yes \\
\hline TdaP & $\begin{array}{l}\text { Toxoids, } \\
\text { inactivated } \\
\text { acellular pertussis } \\
\text { antigens }\end{array}$ & IM & Yes \\
\hline Pneumococcal & $\begin{array}{l}\text { Bacterial } \\
\text { polysaccharide }\end{array}$ & IM or SC & Yes \\
\hline Influenza & $\begin{array}{l}\text { Trivalent } \\
\text { inactivated viral }\end{array}$ & IM & Yes \\
\hline Meningococcal & Protein conjugate & IM & Yes \\
\hline
\end{tabular}

The hemagglutinin $(\mathrm{H})$ antigen on the influenza A viral surface enables entry of the viral genome into the host cell. The neuraminidase $(\mathrm{N})$ antigens lead to cell lysis after replication of the viral genome in a host cell. These viral antigens are critical components in the mechanisms by which influenza $\mathrm{A}$ viruses infect host cells and cause disease. However, the $\mathrm{H}$ and $\mathrm{N}$ antigens stimulate an immune response, acting as target antigens in developing prophylactic vaccines (5).

Influenza viruses are RNA viruses and undergo periodic genetic mutation. This alters the genetic composition of the virus allowing it to evade the natural host immunity acquired from previous exposure to vaccines or infection. The changes in the viral genome are usually minor and described as "antigenic drift." This minor change does not result in reclassification of the viral subtype. Antigenic drift results in a novel strain that can cause disease in a naive population despite previous exposure to a genetic variant outbreak. The continual process of antigenic drift necessitates the development of new influenza A vaccines on an annual basis. Influenza $\mathrm{B}$ viruses are more stable and undergo antigenic drift less frequently than influenza A viruses (6).

While antigenic drift describes minor genetic changes in the viral genome, "antigenic shift" refers to major genetic alteration. The major pandemic strain in 1917 to 1918 was caused by an H1N1 subtype influenza A virus and this

Table 2 Licensed Vaccines May Be Used During Pregnancy in Special Circumstances

\begin{tabular}{llll} 
Vaccine & \multicolumn{1}{c}{ Type } & Route & Circumstances for use in pregnancy \\
Anthrax & Inactivated bacteria & SC & For use in epidemics \\
BCG & Live bacteria & ID & None \\
Japanese encephalitis & Inactive virus & SC & Yes, if traveling to endemic area \\
Measles & Attenuated live virus & SC & No \\
Measles, mumps, rubella & Attenuated live virus & SC & No \\
Herpes zoster & Attenuated live virus & SC & No \\
Polio, inactive & Inactivated live virus (three types) & IM & Yes, if traveling to endemic area \\
Rabies & Inactivated virus & IM & Yes, use when indicated \\
Typhoid (parenteral) & Inactivated bacteria & SC & Yes, if epidemic \\
Typhoid (oral) & Live bacteria & Oral & No \\
Varicella & Attenuated live virus & SC & No, give postpartum if indicated \\
Yellow fever & Attenuated live virus & SC & No, use when indicated \\
Abbreviations: BCG, Bacillus Calmette-Guerin; ID, intradermal; IM, intramuscular; SC, subcutaneous. Source: From Ref. 1.
\end{tabular}

Table 3 Immunoglobulins and Antitoxins Available in the United States

\begin{tabular}{|c|c|c|}
\hline Immunobiologic & Type & Indication \\
\hline Botulinum antitoxin & Specific equine antibodies & Treatment of botulism \\
\hline $\begin{array}{l}\text { Cytomegalovirus } \\
\text { Immunoglobulin (IG) intravenous }\end{array}$ & Specific human antibodies & Prophylactic for bone marrow and kidney transplants \\
\hline IG intravenous & Pooled human antibodies & A \\
\hline Hepatitis B, IG & Specific human antibodies & $\mathrm{B}$ \\
\hline Hepatitis A, IG & Specific human antibodies & $\mathrm{C}$ \\
\hline Tetanus IG & Specific human antibodies & Tetanus exposure \\
\hline Varicella zoster IG & Specific human antibodies & $\mathrm{D}$ \\
\hline \multicolumn{3}{|c|}{$\begin{array}{l}\text { All preparations are given IM except when noted (A) Replacement therapy for antibody deficit, ITP, hypogammaglobulinemia CLL. (B) Hepatitis B } \\
\text { postexposure to infant of HBsAg-positive mother at birth. (C) Postexposure prophylaxis or pre-exposure prophylaxis. (D) Postexposure prophylaxis of } \\
\text { susceptible persons and certain susceptible pregnant women and their exposed newborns. Abbreviations: CLL, Chronic lymphocytic leukemia; ITP, idiopathic } \\
\text { thrombocytopenia purpura. Source: From Ref. 1. }\end{array}$} \\
\hline
\end{tabular}


presented until 1957 when the H2N2 subtype appeared. The H2N2 subtype underwent a major antigen shift in 1968 resulting in the $\mathrm{H} 3 \mathrm{~N} 2$, which has been circulating until the present time. An H1N1 subtype emerged in 1977 and has been co-circulating with the $\mathrm{H} 3 \mathrm{~N} 2$ subtype. Current influenza vaccine contains one $\mathrm{H} 3 \mathrm{~N} 2$ strain, one $\mathrm{H} 1 \mathrm{~N} 1$ strain, and one influenza B strain. In March 2009, a novel H1N1 subtype emerged in Mexico and is the result of influenza A strains from pigs, birds, and humans. Recombinant analysis showed triplereassessment swine $\mathrm{H} 1 \mathrm{~N} 1$ influenza virus coming from classic swine, North American lineage; avian, North American lineage; human (seasonal) $\mathrm{H} 3 \mathrm{~N} 2$ and human seasonal (H1N1) strain initiated a worldwide pandemic (7).

\section{INFLUENZA AND PREGNANCY}

Influenza increases the risk for morbidity and mortality in all pregnant women. While increased risk of influenza-associated morbidity and mortality is present throughout pregnancy, it increases dramatically in the third trimester $(3,8)$. Excess maternal deaths were reported during the influenza pandemic of 1917 to 1919 caused by an H1N1 subtype (9) and in 1957 to
1958 caused by an H2N2 subtype (10). Recently, it has been reported an increased risk for serious illness and hospitalization in pregnant women suffering from the respiratory complications of influenza during non-pandemic influenza seasons (11). The risk ratio for hospitalization was 5.1 among women without comorbidities and 7.9 among women with comorbidities. The relative risk for pregnant women without comorbidities rose from 1.7 in the first trimester to 5.1 in the third trimester for women with comorbidities. The relative risk for hospitalization for an influenza infection rose from 2.9 in the first trimester to 7.9 in the third trimester. ACIP and the American College of Obstetrics and Gynecologists (ACOG) have recommended that all pregnant women receive influenza vaccine during their pregnancy (4).

This pandemic H1N1 2009 influenza has shown an increased likelihood of adverse outcomes in pregnant women and has shown an increased risk of maternal mortality (3). A number of reasons for the increased morbidity and mortality of influenza A in pregnancy have been proposed. They include i) an increasing relative immunosuppression to viral antigens as the pregnancy progresses, ii) physiologic changes in pregnancy

Table 4 Office Vaccine Questionnaire

\section{Patient name or}

Facility Label

\section{Vaccine Questionnaire}

Date:

1. Do you have an allergy to: EGGS YEAST NEOMYCN

Vaccine Vaccine VIS

Today Refusal Given

2. Have you had your "FLU" shot this year? YES NO

3. Have you had your "pneumonia shot"? YES $\mathrm{NO}$

4. When was your last Tetanus shot?

Don't remember

More than 2yrs ago If yes give TdaP

5. Have you had the three shot Hepatitis B series?YES $\mathrm{NO}$

6. Have you had the three shot Hepatitis Aseries?YES $\mathrm{NO}$

7. For ages 11-19: 
with a $20 \%$ to $100 \%$ expansion of circulating blood volume having to traverse the lungs giving them a heavy quality, and iii) induction of a "cytokine storm" in patients without circulating influenza antibodies to the infectious strain (12).

It is important to remember that influenza vaccines are not teratogenic, and no convincing evidence has been published regarding fetal adverse outcomes (2). Some authors include "theoretic concerns" of fetal effects in their writing but when questioned about the theoretical risk, they are unable to locate supporting data.

ACIP and ACOG advise that all pregnant women receive trivalent inactivated influenza vaccine (TIV) at any time during the pregnancy. It is advisable to receive TIV earlier in the pregnancy so adequate time is available for the mother to develop an immune response and transfer passive antibodies to the fetus thereby protecting the fetus for the influenza season (13).

\section{TETANUS, DiPTHERIA, PERTUSSIS}

In the minds of many people, tetanus, diphtheria, and pertussis have been thought of as being eliminated or controlled. By the 1970s, widespread implementation of tetanus diphtheria $(\mathrm{Td})$ vaccine targeting pregnant women resulted in an almost universal disappearance of tetanus and diphtheria in the mother and the disappearance of neonatal tetanus when $\mathrm{Td}$ was used prior to or during pregnancy. Whole-cell pertussis vaccine was developed and brought into clinical practice in the late 1930s and 1940s, which led to a dramatic decrease in the incidence of pertussis. However, the number of cases of pertussis began to rise in the late 1970s primarily in adolescents and young adults. This was thought to be due to waning immunity to pertussis antigens. The vaccine available at that time was DPT and was indicated for persons up to 5 years of age. In 1992, a new formulation of DPT was developed with the introduction of " $T$ " (full strength), "d" (reduced strength), and acellular pertussis (TdaP) for children up to 5 years of age. The acellular pertussis vaccine was significantly less reactogenic. In 2005 , the TdaP vaccine was licensed for adults up to age 65 years.

Pertussis is an acute respiratory infection caused by Bordetella pertussis. The organism produces multiple toxins that damage the respiratory epithelium and can have symptomatic effects including lymphocytosis. B. pertussis infection in adolescents and adults covers a spectrum of severity from asymptomatic to a mild cough illness to classic pertussis (14).

The incubation period for pertussis is 7 to 10 days (range 5-21 days). Classic pertussis is characterized by three phases: Catarrhal, paroxysmal, and convalescent. The catarrhal phase lasts 1 to 2 weeks and consists of injection of the conjunctiva, frequent sneezing, a watery nasal discharge, and frequent cough. The cough suggests that tracheal irritation is short, hacking, isolated, and present equally day or night. Fever is uncommon during any phase.

The paroxysmal phase lasts 4 to 6 weeks. During this phase, the patient has intermittent periods of intense coughing (paroxysmal). The paroxysms are characterized by severe spasms of coughing, choking, post-tussive and inspiratory whoop. Adults and adolescents with pertussis make repeated visits for medical care and miss work and school $(15,16)$.
Table 5 Hospitalization and Complications Among 16,431 Infants Aged $<12$ Months with Pertussis (2000-2005)

\section{Complication}

Number (\% of total)

Hospitalization

Apnea

$8067(61.7)$

Pneumonia

$7377(56.3)$

Seizures

$1419(12.8)$

Deaths

$169(1.3)$

$130(0.8)$

Source: From Ref. 17.

Table 6 Relation and Age Reported Source of Pertussis Among Infants Aged $<12$ Months 1999-2002

\begin{tabular}{lcr} 
Relation & Number & $\%$ \\
\hline Unknown & 353 & 57 \\
Mother & 84 & 14 \\
Father & 39 & 6 \\
Grandparent & 22 & 4 \\
Sibling & 52 & 8 \\
Other & 67 & 11 \\
Total & 616 & 100 \\
Source: From Ref. 18. & &
\end{tabular}

Pertussis is transmitted from person to person via large respiratory droplets generated by coughing or sneezing. Persons with pertussis are highly infectious especially during the catarrhal and early paroxysmal phases. Patients can remain infected for up to 6 weeks or more.

Case reports suggest that morbidity from pertussis is not increased among pregnant women as compared with nonpregnant women. Reports of fetal morbidity among pregnant women with pertussis are rare and no causal relationship with abnormal fetal development, fetal morbidity, or adverse outcome of pregnancy has been confirmed.

Unfortunately, the brunt of burden of disease occurs in the infants less than 1 year of age (Table 5). It is to be noted that apnea is a prominent finding in children admitted to the hospital. Infant deaths are characterized by an increased rate of primary and secondary pneumonia and coinfection with bacterial or viral pathogens.

The source of pertussis for the neonatal patient is largely unknown (Table 6). Although the mother is the source in $14 \%$, the father in $6 \%$, and grandparents and sibling in an additional $12 \%$, immunization programs targeting mothers postpartum and their support groups are likely to be unsuccessful. The neonatal patient receives active pertussis vaccine at 2, 4, and 6 months of life and is not protected from disease until 6 months of life.

Therefore, immunization during pregnancy will protect the mother against pertussis disease and the passive antibodies that cross to the fetus will protect the neonate against disease. The newer recommendations for routine $\mathrm{TdaP}$ vaccinations are listed in Table 7. TdaP is recommended during the second and third trimesters and will likely need to be repeated in 10 years.

\section{HEPATITIS B}

Acute hepatitis B virus infection during pregnancy can result in severe disease for the mother and chronic infection in the 


\section{Table 7 Recommendations for Routine TdaP Vaccination}

Adolescents and adults should receive a single dose of tetanus, reduced diphtheria, and acellular pertussis (TdaP) to replace a single dose of $\mathrm{Td}$ for active booster vaccination if they have received their last dose of $\mathrm{Td}$ greater than 2 years ago

Although previous recommendations have indicated 10 -year intervals for $\mathrm{Td}$, the interval between $\mathrm{Td}$ and $\mathrm{TdaP}$ of 2 years is now recommended for a single dose of $\mathrm{TdaP}$

The American College of Obstetricians and Gynecologists recommend that TdaP may be administered during pregnancy

For those women who do not receive TdaP during pregnancy, they should be given $\mathrm{TdaP}$ in the postpartum period

Lactating women may receive $\mathrm{TdaP}$

Health-care workers who have direct patient contact should receive a single dose of $\mathrm{TdaP}$

Source: From Ref. 14.

Table 8 Risk Groups for Preexposure Prophylaxis for Hepatitis B

All persons age 0 to 19 years

All newborns

All heterosexual persons who are not in a long-term mutually monogamous relationship

Men who have sex with men (MSM)

Injecting drug users (IDU)

All persons screened for a sexually transmitted disease

All persons tested for a sexually transmitted disease

All persons receiving hemodialysis

All persons with end-stage renal disease not on dialysis

Persons with chronic liver disease of any type

HIV-infected persons

All health-care workers

All public safety workers at risk for blood exposure or containing bodily fluids

Staff of correctional facilities

Inmates in federal, state prisons, jails, or juvenile correctional facilities

Residents of institutions for developmentally disabled

Sexual and household contacts of HBsAg-positive persons

Family members of HBsAg-positive immigrants and international adoptees

International travelers to moderate or high endemic areas

Victims of sexual assault

Persons who are not previously vaccinated or immune to $\mathrm{HB}$ virus infectious

Persons who desire to become vaccinated

The ACIP has published recommendation for use of Hepatitis A virus based on risk (Table 10)

Source: From Ref. 20. Abbreviation: ACIP, Advisory Committee for Immunization Practices.

neonate if a live birth occurs. Pregnancy in a woman with chronic acute hepatitis B virus infection can result in vertical transmission to the fetus and chronic infection in the neonate if no postexposure therapy occurs. The acute infection is characterized by a prolonged viremic phase that persists weeks to months. Chronic hepatitis B infections occur in 1\% to 5\% of adult patients with acute hepatitis B infection. Maternal infection with $\mathrm{HBsAg}$ positive and $\mathrm{HBeAg}$ positive carries a fetal transmission rate of $90 \%$, with $50 \%$ of the neonates developing chronic hepatitis (19).
Table 9 Risk Groups for Past Exposure Prophylaxis for Hepatitis B

Occupational exposure to blood or bodily fluids that contain blood Sexual and household contacts of persons with acute hepatitis B infection

Sexual and household contacts of persons with chronic hepatitis B infection

Victims of sexual assault

Newborn infants whose mothers are HBsAg positive

Perinatal HB virus exposure

Source: From Ref. 20.

\section{Table 10 Current Risk-Based Recommendations for} Hepatitis A Vaccinations

International travelers

Men who have sex with men (MSM)

Household and sexual contacts of persons infected with HAV

Laboratory workers who handle live HAV

Laboratory workers who work with nonhuman primates

Illicit drug users (injecting and non-injecting)

Carries of chronic hepatitis B

Persons with chronic liver diseases

Persons with clotting-factor disorders

All persons who desire protection from HAV

All public food handlers

Source: From Ref. 22.

Pregnancy should be considered an indication for the initiation of the hepatitis B vaccine series. The ACIP has recommended hepatitis B vaccine for any person, pregnant or not, who fall into any of the 22 risk groups or 6 postexposure risk groups (Tables 8 and 9).

The ACIP recommendations are complex and almost impossible to remember all the risk groups (Table 10). It is important to remember that all pregnant women are screened for six sexually transmitted diseases and all pregnant women qualify for HBV vaccine. The American Academy of Pediatrics has stated that no adverse effect to the developing fetus has been observed when pregnant women are immunized. Because severe hepatitis B virus infection can cause chronic infection in the mother and the infant, all pregnant women should be considered candidates for the HBV vaccine series if they have not been previously immunized.

Ingardi and colleagues analyzed serum antihepatitis B levels in 37 maternal serum-umbilical cord pairs following maternal vaccination (21). At the time of delivery, 18 of $37(49 \%)$ had appropriate $\mathrm{HBsAb}$ levels of $10 \mathrm{MIU} / \mathrm{mL}$ or greater. The ratio of cord blood titer to maternal titer of HBgAb increased with the ratios for first, second, and third doses of vaccine were $0.55,0.67$, and 0.72 respectively. When maternal HBsAb levels exceeded $35 \mathrm{MIU} / \mathrm{mL}$, all cord blood liters were in the seroprotective range.

\section{HEPATITIS A VACCINE}

Hepatitis A virus is transmitted through the fecal-oral route, spreading primarily through close personal contact. The acute disease is characterized by nausea, malaise, abdominal pain, and jaundice. HA virus infection does not cause chronic 
Table 11 Acute and Chronic Disease Burden Estimates for Viral Hepatitis in the United States

\begin{tabular}{|c|c|c|c|}
\hline & $\begin{array}{c}\text { Hepatitis } \\
\text { A virus } \\
\text { (HAV) }\end{array}$ & $\begin{array}{c}\text { Hepatitis } \\
\text { B virus } \\
(\mathrm{HBV})\end{array}$ & $\begin{array}{c}\text { Hepatitis } \\
\mathrm{C} \text { virus } \\
(\mathrm{HCV})\end{array}$ \\
\hline Total infection & 32,000 & 46,000 & 19,000 \\
\hline Vaccine preventable & Yes & Yes & No \\
\hline $\begin{array}{l}\text { Chronic } \\
\text { infections }\end{array}$ & NA & $\begin{array}{l}1 \mathrm{million} / \\
1.25 \mathrm{mil}\end{array}$ & 3.2 million \\
\hline Chronic liver deaths & NA & 5000 & $8000-10,000$ \\
\hline Source: From Ref. 24. & & & \\
\hline
\end{tabular}

infection and vertical transmission has not been described. During the period 1987 to 1997 , an average of 28,000 cases of hepatitis A occurred annually in the United States. After induction of hepatitis A vaccine in 1995, a significant reduction in the annual number of cases reported has occurred.

The general public perceives HAV infection as either nonserious or very mild. One has to be reminded of a large outbreak of HAV infection in fast-food restaurant in Western Pennsylvania in 2004.

More than 600 persons became ill, with 194 admissions and four deaths. The source of HAV was not the employees but imported green onions that was used to make salsa $(23,24)$. This emphasized the vulnerability of the population to contaminated food and that universal protection is indicated. This is further indicated by the large burden of acute and chronic disease for viral hepatitis in the United States (Table 11) or that behavioral risk factors are not always predictable, thereby leaving individuals at risk until the time they come to medical attention. Importantly, the current recommendation have resulted in rates of $\mathrm{HBV}$ infection that are actually increasing among men aged $>20$ years and among women $>40$ years (25). Additionally, riskbased approaches have been abandoned for all pediatric vaccines and most of the adult vaccines. Unfortunately, the universal recommendations that pertain to infants, children, and adolescents have not yet been equally applied to adults (26).

Table 11 presents acute and chronic disease burdens estimated for viral hepatitis in the United States. The disease burden is great and the clinical presentation is similar for all the types of hepatitis. An active program of hepatitis A and B vaccination can be important in reducing the number of persons infected.

Problems are present with the current ACIP recommendations regarding hepatitis $\mathrm{A}$ and hepatitis $\mathrm{B}$ vaccinations. The major deficit in the current recommendations is that they are risk based and therefore likely to miss many persons at risk who would receive vaccination if the recommendation were universal. In addition, the risk categories are tedious and the majority of practitioners are unaware of multitude of risk categories. The ACIP recommendations ignore the prevailing epidemiology of these diseases. For example, $<50 \%$ of patients with HAV and HBV infections do not have an identifiable risk factor for infection, specifically, whether and when an individual becomes at risk, particularly, through a lifestyle change.
Table 12 Current Advisory Committee for Immunization Practices Recommendations for Pneumococcal (PS-23) Vaccine

persons aged 65 or more persons with surgical or functional splenectomy person with cardiac disease persons with pulmonary disease persons with asthma persons who are smokers persons with diabetes mellitus persons with thyroid disease persons with renal disease persons with metabolic syndrome persons with chronic liver disease persons with cerebrospinal fluid levels persons with cochlear implants persons who are alcoholics persons with immunocompromised status persons exposed to pneumococcal outbreaks persons living in a nursing home or other long-term care facilities persons with sickle cell disease

Source: From Ref. 28.

\section{PNEUMOCOCCAL VACCINE}

Streptococcus pneumoniae infection is a significant health problem in the United States (27). It is estimated that 3000 cases of meningitis, 500,000 cases of pneumonia, and 7 million cases of otitis media are due to the pneumococcus. This translates into 175,000 hospitalizations and an adult fatality rate of 40,000 persons per year. With aging populations, an increasing antibiotic oesistance to S. pneumonia, and more adults immunosuppressed, the annual number of fatalities will rise.

Pneumococcal pneumonia is the most serious clinical presentation of pneumococcal disease among adults. The incubation period is 1 to 3 days. Symptoms include an abrupt onset of fever and chills with other symptoms such as pleuritic chest pain, cough, productive of purulent, rusty sputum, dyspnea, tachypnea, hypoxia, tachycardia, malaise, and weakness.

Pneumococci account for up to $36 \%$ of adult communityacquired pneumonia and $50 \%$ of hospital-acquired pneumonia. Pneumonia is a common bacterial complication of influenza and measles. The case fatality rate is $5 \%$ to $7 \%$ and may be higher in elderly patients.

The ACIP recommendations for administration are largely risk based and therefore miss many persons (Table 12).

It is noted that there are 18 risk indications for the use of pneumococcal vaccine. The issue of risk-based recommendations is raised again as in hepatitis $A$ and hepatitis $B$ vaccine recommendations. The risk indication listed in Table 12 also applies to pregnant women. The VIS for pneumococcal vaccine states that there is no evidence that pneumococcal vaccine is harmful to either a pregnant women or to her fetus and women with the indications that put them at risk for pneumococcal disease should receive pneumococcal vaccine prior to pregnancy or during pregnancy (28).

\section{MENINGOCOCCAL VACCINE}

Neisseria meningitidis is the second most common cause of meningitis in the United States. Large epidemics are often the 
Table 13 Immunization During Pregnancy (Active Immunization)

\begin{tabular}{|c|c|c|c|c|c|c|c|}
\hline Vaccine & $\begin{array}{l}\text { Risk from disease } \\
\text { to pregnant } \\
\text { women }\end{array}$ & $\begin{array}{l}\text { Risk from disease } \\
\text { to fetus or neonate }\end{array}$ & $\begin{array}{c}\text { Type of } \\
\text { immunizing agent }\end{array}$ & $\begin{array}{c}\text { Risk of } \\
\text { immunization } \\
\text { to fetus }\end{array}$ & $\begin{array}{c}\text { Indication for } \\
\text { maternal } \\
\text { immunization }\end{array}$ & Dose schedule & Comments \\
\hline Influenza & $\begin{array}{l}\text { Increase in } \\
\text { morbidity and } \\
\text { mortality } \\
\text { especially during } \\
\text { epidemic with } \\
\text { new antigenic } \\
\text { strain }\end{array}$ & $\begin{array}{l}\text { Anatomical fetal } \\
\text { damage, } 50 \% \\
\text { mortality in } \\
\text { neonatal disease }\end{array}$ & $\begin{array}{l}\text { Trivalent } \\
\text { inactivated } \\
\text { vaccine (TIV) }\end{array}$ & $\begin{array}{l}\text { None. Fetus } \\
\text { receives passive } \\
\text { antibodies from } \\
\text { mother }\end{array}$ & $\begin{array}{l}\text { All women } \\
\text { pregnant during } \\
\text { influenza season, } \\
\text { any trimester }\end{array}$ & One dose annually & $\begin{array}{l}\text { Passive antibodies } \\
\text { to fetus protects } \\
\text { from influenza }\end{array}$ \\
\hline Hepatitis B & $\begin{array}{l}\text { Possibly increased } \\
\text { disease in third } \\
\text { trimester }\end{array}$ & $\begin{array}{l}\text { Increase rate, } \\
\text { spontaneous } \\
\text { abortion, and } \\
\text { preterm labor. } \\
\text { Neonatal } \\
\text { hepatitis. High } \\
\text { risk of chronic } \\
\text { carrier state }\end{array}$ & $\begin{array}{l}\text { Recombinant } \\
\text { vaccine }\end{array}$ & None & $\begin{array}{l}\text { All women should } \\
\text { receive vaccine }\end{array}$ & $\begin{array}{l}\text { Three dose series } \\
0,1,6-12 \text { months }\end{array}$ & $\begin{array}{l}\text { Birth dose strongly } \\
\text { advised. Also } \\
\text { HBIG for } \\
\text { infants born of } \\
\text { HBsAg-positive } \\
\text { mothers }\end{array}$ \\
\hline $\begin{array}{l}\text { Tetanus diphtheria } \\
\text { acellular } \\
\text { pertussis (TdaP) }\end{array}$ & $\begin{array}{l}\text { Severe morbidity } \\
\text { tetanus } \\
\text { morbidity } 30 \% \\
\text { diphtheria } \\
\text { mortality } 10 \% \\
\text { pertussis mild } \\
\text { morbidity }\end{array}$ & $\begin{array}{l}\text { Most mortality in } \\
\text { infants }<6 \\
\text { months } \\
\text { hospitalization } \\
70 \% \\
\text { Apnea } 60 \%\end{array}$ & $\begin{array}{l}\text { Tetanus }=\text { toxoid } \\
\text { Diphtheria }= \\
\text { toxoid } \\
\text { Pertussis }-3-5 \\
\text { antigens }\end{array}$ & None & $\begin{array}{l}\text { All pregnant } \\
\text { women who } \\
\text { received last } \mathrm{Td} \\
>2 \text { years ago }\end{array}$ & $\begin{array}{l}\text { Booster single IM } \\
\text { injection } \\
\text { Primary } 3 \text { doses } \\
0,1,6-12 \\
\text { months }\end{array}$ & $\begin{array}{l}\text { Fetus protected } \\
\text { with passive } \\
\text { antibodies; } \\
\text { immigrants } \\
\text { usually not } \\
\text { immunized }\end{array}$ \\
\hline Pneumococcal & $\begin{array}{l}\text { Severe morbidity } \\
\text { and mortality. } \\
\text { Increased risk of } \\
\text { pulmonary } \\
\text { complications }\end{array}$ & $\begin{array}{l}\text { Determined by } \\
\text { mothers' } \\
\text { morbidity, } \\
\text { vaccine reduces } \\
\text { otitis media in } \\
\text { neonate }\end{array}$ & $\begin{array}{l}\text { Polyvalent } \\
\text { polysaccharide } \\
\text { vaccine }\end{array}$ & None & $\begin{array}{l}\text { All pregnant } \\
\text { women in } 2-3 \\
\text { trimesters }\end{array}$ & $\begin{array}{l}1 \text { dose SC or IM, } \\
\text { repeat in } 6 \text { years } \\
\text { for medical high } \\
\text { risk }\end{array}$ & $\begin{array}{l}\text { Reduction in otitis } \\
\text { media first } 4 \\
\text { months of life }\end{array}$ \\
\hline $\begin{array}{l}\text { Meningococcal } \\
\text { vaccine }\end{array}$ & $\begin{array}{l}\text { Severe morbidity } \\
\text { and mortality }\end{array}$ & $\begin{array}{l}\text { Determined by } \\
\text { mothers' } \\
\text { morbidity }\end{array}$ & $\begin{array}{l}\text { Protein conjugate } \\
\text { vaccine }\end{array}$ & None & $\begin{array}{l}\text { Adolescent women } \\
\text { International } \\
\text { travel }\end{array}$ & One dose IM & \\
\hline
\end{tabular}


Table 14 Immunoglobulin Use in Pregnancy

\begin{tabular}{|c|c|c|c|c|c|c|c|}
\hline $\begin{array}{l}\text { Immunobiologic } \\
\text { agents }\end{array}$ & $\begin{array}{l}\text { Risk form disease } \\
\text { in pregnant } \\
\text { women }\end{array}$ & $\begin{array}{l}\text { Risk of disease to } \\
\text { fetus or neonate }\end{array}$ & $\begin{array}{c}\text { Type of } \\
\text { immunization } \\
\text { agent }\end{array}$ & $\begin{array}{c}\text { Risk of agent } \\
\text { to fetus }\end{array}$ & $\begin{array}{l}\text { Indication during } \\
\text { pregnancy }\end{array}$ & Dose schedule & Comments \\
\hline Measles & $\begin{array}{l}\text { Significant } \\
\text { morbidity }\end{array}$ & $\begin{array}{l}\text { Increase in } \\
\text { spontaneous } \\
\text { abortion, may } \\
\text { cause } \\
\text { malformation }\end{array}$ & $\begin{array}{l}\text { Standard immune } \\
\text { globulin }\end{array}$ & None & $\begin{array}{l}\text { Postexposure } \\
\text { prophylaxis }\end{array}$ & $\begin{array}{l}0.25 \mathrm{mg} / \mathrm{kg} \text { one } \\
\text { dose of IG up to } \\
15 \mathrm{~mL}\end{array}$ & $\begin{array}{l}\text { Must be given } \\
\text { within } 6 \text { days } \\
\text { of exposure }\end{array}$ \\
\hline Hepatitis B & $\begin{array}{l}\text { Increase severity in } \\
\text { third trimester }\end{array}$ & $\begin{array}{l}\text { Increase in } \\
\text { spontaneous } \\
\text { abortion, } \\
\text { preterm delivery. } \\
\text { Neonatal } \\
\text { hepatitis, high } \\
\text { chronic carrier } \\
\text { rate }\end{array}$ & $\begin{array}{l}\text { Hepatitis B } \\
\text { immune globulin }\end{array}$ & None & $\begin{array}{l}\text { Postexposure } \\
\text { prophylaxis }\end{array}$ & $0.06 \mathrm{~mL} / \mathrm{kg} \mathrm{IM}$ & $\begin{array}{l}\text { HBV vaccine is } \\
\text { given with } \\
\text { HBIG if } \\
\text { mother is } \\
\text { HBsAg positive }\end{array}$ \\
\hline Rabies & $\begin{array}{l}\text { Nearly } 100 \% \text { fatal } \\
\text { not altered by } \\
\text { pregnancy }\end{array}$ & $\begin{array}{l}\text { Determined by } \\
\text { maternal disease }\end{array}$ & Rabies IG & None & $\begin{array}{l}\text { Postexposure } \\
\text { prophylaxis }\end{array}$ & $\begin{array}{l}20 \mathrm{IU} / \mathrm{Kg} 1 / 2 \text { dose in } \\
\text { wound } 1 / 2 \text { dose in } \\
\text { Deltoid }\end{array}$ & $\begin{array}{l}\text { Use with rabies } \\
\text { viral vaccine }\end{array}$ \\
\hline Varicella & $\begin{array}{l}\text { Increase in } \\
\text { morbidity and } \\
\text { mortality }\end{array}$ & $\begin{array}{l}\text { Congenital varicella } \\
1-2 \% \text { first } \\
\text { trimester }\end{array}$ & $\begin{array}{l}\text { Varicella immune } \\
\text { globulin }\end{array}$ & None & $\begin{array}{l}\text { Postexposure } \\
\text { prophylaxis for } \\
\text { mother exposure }\end{array}$ & $\begin{array}{l}\text { One dose IM } 125 \mathrm{u} / \\
10 \mathrm{~kg} \\
\text { Max: } 625 \mathrm{U}\end{array}$ & $\begin{array}{l}\text { Dose within } 96 \\
\text { hours of } \\
\text { exposure. } \\
\text { Neonatal if } \\
\text { mother } \\
\text { developed } \\
\text { varicella within } \\
4 \text { days before } \\
\text { delivery or } \\
2 \text { days after } \\
\text { delivery }\end{array}$ \\
\hline Hepatitis A & $\begin{array}{l}\text { Severe morbidity } \\
\text { Low mortality }\end{array}$ & $\begin{array}{l}\text { Increase in } \\
\text { spontaneous } \\
\text { abortion }\end{array}$ & Standard IG & None & $\begin{array}{l}\text { Postexposure } \\
\text { prophylaxis }\end{array}$ & $\begin{array}{l}\text { One dose } 0.02 \mathrm{mg} / \\
\text { kg IM }\end{array}$ & $\begin{array}{l}\text { Give hepatitis A } \\
\text { vaccine with } \\
\text { immune } \\
\text { globulin }\end{array}$ \\
\hline Rh Immunoglobulin & $\begin{array}{l}\text { Death to fetus } \\
\text { None }\end{array}$ & $\begin{array}{l}\text { Rh } \\
\text { isoimmunization } \\
\text { with possible } \\
\text { hydrops and } \\
\text { deaths }\end{array}$ & $\begin{array}{l}\text { Rh immune } \\
\text { globulin }\end{array}$ & None & $\begin{array}{l}\text { Rh-negative } \\
\text { mother }\end{array}$ & $\begin{array}{l}\text { One dose IM } 300 \\
\text { mg }\end{array}$ & $\begin{array}{l}\text { Administrate } \\
28-30 \text { weeks }\end{array}$ \\
\hline
\end{tabular}


result of infection with N. Meningitidis serotype A and C. A protein conjugate meningococcal vaccine (MCV4) providing protection against serotypes $\mathrm{A}, \mathrm{C}, \mathrm{Y}$ and $\mathrm{W}-135$ is now available (29).

Approximately 2500 persons get meningococcal disease each year in the United States with fatality rate of $10 \%$ to $15 \%$ with another $1 \%$ to $19 \%$ losing arms or legs, becoming deaf, become mentally retarded, or suffer seizures or strokes.

$\mathrm{MCV} 4$ vaccine is recommended for the following persons:

- Adolescents aged 11 through 18 years

- College freshmen living in dormitories

- U.S. military recruits

- Anyone traveling to a part of the world where meningococcal disease is common

- Persons who have had a surgical splenectomy or have a functional splenectomy

- Persons with a terminal compliment disorder

- Persons exposed to meningitis during an outbreak

Meningitis vaccine should be administered to adolescent pregnant women. The major vaccines that should be administered to all pregnant not previously immunized are summarized on Table 13. The summary of immunoglobulin that can be used in pregnancy is seen on Table 14. This chapter strongly advocates for maternal immunization as a routine to protect the mother from disease and to protect the infant with passive maternal antibodies. The shortcomings and inadequacy of the CDC's recommendations on hepatitis A and B vaccines and pneumococcal as well as $\mathrm{TdaP}$ are emphasized. The goal should be to prevent all vaccine preventable diseases in the mother and thereby prevent the disease in the infant by timely maternal vaccinations.

\section{REFERENCES}

1. CDC General Recommendation on Immunization. Recommendations of the Advisory Committee or Immunization Practice (ACIP). MMWR 2011; 60: 1-64.

2. Tamma PO, Ault KA, del Rio C, et al. Safety of influenza vaccination during pregnancy. Am J Obstet Gynecol 2009; 201: 547-52.

3. Jamieson D, Honein MA, Rasmussen SA, et al. H1N1 2009 influenza virus infection during pregnancy. N Engl J Med 2009; 374: 451-8.

4. Fiore AE, Shay DK, Broder K, et al. Prevention and control of seasonal influenza with vaccines: recommendation of the Advisory Committee of Immunization Practice (ACIP), 2009. MMWR Recomm Rep 2009; 58: $1-52$.

5. MacDonald NE, Riley LE, Steinhuff MC. Influenza immunization in pregnancy. Obstet Gynecol 2009; 114: 365-8.

6. Rusmussen SA, Jamieson DJ, Breese JS. Pandemic influenza and pregnant women. Emerg Infect Dis 2008; 14: 95-100.

7. Shinde V, Bridges CB, Uyeki TM, et al. Triple-reassortant swine influenza A (H1) in humans in the United States, 2005-2009. N Engl J Med 2009; 361: $1-10$.
8. Neuzil KM, Reed GW, Michel EF Jr, et al. Impact of influenza on acute cardiopulmonary hospitalization in pregnant women. Am J Epidemiol 1998; 148: 1094-102.

9. Harris JW. Influenza occurring in pregnant women. JAMA 1919; 72: 978-80.

10. Freeman DW, Barno A. Deaths from Asian influenza associated with pregnancy. Am J Obstet Gynecol 1959; 78: 1172-5.

11. Dodds L, McNeil SA, Fell OB, et al. Impact of influenza exposure on rates of hospital admissions and physician visits because of respiratory illness among pregnant women. CMAJ 2007; 176: 463-8.

12. Chowell G, Bertozyi SN, Colchero NA, et al. Severe respiratory disease concurrent with circulation of H1N1 influenza. N Engl J Med 2009; 361: 674-9.

13. Zaman K, Roy E, Arifaen SE, et al. Effectiveness of maternal influenza immunization in mothers and infants. N Engl J Med 2008; 359: 1555-64.

14. CDC. Preventing Tetanus, diphtheria and Pertussis among adults: use of tetanus toxoid, reduced diphtheria toxoid and acellular Pertussis vaccine (TdaP). Recommendations of the Advisory Committee in Immunization Practice (ACIP). MMWR 2006; 55: 1-37.

15. Cherry JD, Gornbein J, Heininger U, et al. A search for serologic correlates of Immunity to Bordetella Pertussis cough illness. Vaccine 1998; 16: 1901-6.

16. Cherry JD. The epidemiology of pertussis: a comparison of the epidemiology of the disease pertussis with the epidemiology of Bordetella Pertussis infection. Pediatrics 2005; 115: 1422-7.

17. CDC. National Notifiable disease surveillance system and supplemental pertussis surveillance system 2000-2005. Atlanta, GA, 2005.

18. Bisgard KM, Pascual RB, Ehresmann KR, et al. Infant pertussis, who is the source? Pediatr infect Dis 2004; 23: 895-9.

19. Burk RD, Hwang LY, Ho GYF, et al. Outcome of perinatal hepatitis B exposure is dependent on maternal virus load. J Infect Dis 1994; 120: $1418-1423$.

20. CDC. A comprehensive immunization strategy to eliminate transmission of hepatitis B virus infections in the United States. Recommendations of the Advisory Committee on Immunization Practice (ACIP). MMWR 2006; 55: 1-25.

21. Ingardi CJ, Keeley L, Lerer T, et al. Correlation of maternal and fetal hepatitis B antibody titers following maternal vaccinations in pregnancy. Am J Perinatal 1999; 16: 129-32.

22. CDC. Prevention of hepatitis A through active or passive immunization: recommendation of the Advisory Committee in Immunization Practices (ACIP). MMWR 2006; 55: 1-23.

23. Wheeler C, Vogh TM, Armstrong GL, et al. An outbreak of hepatitis A associated with green onions. N Engl J Med 2005; 353: 890-7.

24. CDC. Epidemiology and Prevention of Vaccine Preventable Disease, 10th edn. 2008: 197-234.

25. CDC. Incidence of acute hepatitis B: United States 1990-2002. MMWR 2004; 52: 1252-4.

26. Poland GA. Evaluating existing recommendations for hepatitis A and B vaccination. Am J Med 2005; 118: 165-205.

27. CDC. Prevention of Pneumococcal disease. Recommendations of the Advisory Committee in Immunization Practice (ACIP). MMWR 1997; 46: $1-24$.

28. CDC. Pneumococcal vaccine Information Statement (VIS) dated 10/6/2009. [Available from: WWW.cdc.gov/VIS]

29. Lieberman JM, Chiu SS, Wong UK, et al. Safety and immunogenicity of a subgroup A/C Neisseria meningitidis oligosaccharide protein conjugate vaccine in young children. JAMA 1996; 225: 1499-503. 


\section{Neonatal diseases I John Pardalos}

\section{APNEA OF PREMATURITY}

Apnea of prematurity is a condition frequently seen in preterm infants. It is defined as a cessation in breathing for more than 20 seconds, or less than 20 seconds if it is associated with bradycardia and/or cyanosis (1). The incidence of this disorder increases with decreasing gestational age, but luckily most infants outgrow this disorder by the time they reach 35 to 36 weeks postconceptional age. Over the last 20 years, the prevalence of this disorder has increased as smaller, more preterm infants are now surviving. With advances in ventilator management and surfactant therapy, many preterm infants are currently being extubated within days of admission to the neonatal intensive care unit. The goal is to keep them off the ventilators to prevent potential trauma to the developing lungs. As a result, neonatologists are faced with a dilemma about when is the frequency of apnea high enough to require reintubation.

For decades, neonatologists have been looking for ways to treat these infants without resorting to mechanical ventilation. Many times these infants are placed on some type of positive pressure device post-extubation to help the infants maintain adequate respiratory dynamics while using minimal pressures to achieve this. Some of the various devices used to accomplish this include nasal prong continuous positive airway pressure [CPAP (NCPAP)], nasal prong CPAP with a rate (known as NIPPV), SiPAP, and high-flow nasal cannula. These devices provide positive pressure to the infant's airway that helps stent the airways open, allowing for easier flow of air into and out of the lungs, which helps prevent obstructive apnea. NIPPV generates increased pharyngeal pressure intermittently based on the peak inspiratory pressure and rate that may activate the dilator muscles of the pharynx and stimulates respiratory drives to abort the apnea spell (2). In studies comparing NIPPV with NCPAP, it was noted that those infants treated with NIPPV had a greater reduction in the number of apneic spells compared with those treated with NCPAP (2-5).

Since the 1970s, methylxanthines have been used to treat apnea of prematurity. Over the years, various methylxanthines have been used including theophylline, aminophylline, and caffeine. Currently, caffeine is most commonly used due to a wide therapeutic window with fewer risks of side effects. We do not have to draw levels with caffeine compared with theophylline and aminophylline. The mechanism of action of methylxanthines is unknown, but they have been shown to decrease apneic events, increase minute volumes, improve respiratory system compliance, reduce diaphragmatic fatigue, and act as a diuretic $(6,12,14)$. In fact those infants treated with caffeine required mechanical ventilation, noninvasive ventilation, and/or supplemental oxygen therapy for 1 week less on average compared with those infants treated with placebo. Those infants treated with caffeine, however, did show slower weight gain compared with the placebo group at least initially $(7,13,14)$.

Another concern with methylxanthines was that they may interfere with the neurodevelopmental outcome of infants. In animal models, adenosine was noted to protect the brain from energy failure or cell death during hypoxia and ischemia and methylxanthines inhibit adenosine receptors (8). The Caffeine for Apnea of Prematurity (CAP) trial was conducted to see what short- and long-term risks and benefits caffeine provided infants. The trial found that caffeine reduced the incidence of bronchopulmonary dysplasia and decreased the need for supplemental oxygen therapy and assisted ventilation of any type. The CAP trial followed these patients to 2 years of life and found that caffeine also improved the neurodevelopmental outcome at 18 to 21 months of age. Caffeine was noted to decrease the incidence of cerebral palsy and cognitive delay but no change in death, severe hearing loss, or bilateral blindness was found $(6,8,12,13)$. The study did find that those infants who were started on caffeine while they were still on some type of respiratory support had the greatest improvement in their neurodevelopmental outcomes (6).

Other medications used to attempt to treat apnea of prematurity include carnitine and doxapram. Carnitine deficiency can be a cause of apnea in infants and adults but no trial has shown that supplementation with carnitine made a difference in the frequency of apnea $(9,10)$. Doxapram is another respiratory stimulant that has been used to treat apnea of prematurity. Doxapram must be administered intravenously, however, and potentially has the following side effects: seizures, liver dysfunction, and gastrointestinal irritation, which has limited its use. In a recent Cochrane review that compared the use of doxapram versus methylxanthine in the treatment of apnea found no difference between the two medications (11).

\section{LATE PRETERM NEWBORNS}

Infants born at $340 / 7$ weeks to 36 6/7 weeks of gestation were called near-term until 2005 when the National Institute of Child Health and Human Development of the National Institutes of Health held a workshop entitled "Optimizing Care and Outcome of the Near-Term Pregnancy and the NearTerm Newborn Infant." At this workshop, a decision was made to call these infants late preterm infants to signify that even though these infants many times were of the physical size of term infants, physiologically and metabolically they were immature $(15,16)$. When compared with term infants, late preterm infants are more likely to have respiratory distress, temperature instability, hypoglycemia, hyperbilirubinemia, apnea, sepsis, and feeding problems. They were also noted to have higher mortality and rehospitalization rates during the newborn period (15-20). Therefore, it is important that late 
preterm infants not be treated like healthy term infants but monitored closely to make sure that if they develop any morbidities noted above, they are treated promptly.

Between 1990 and 2005, the overall preterm birth rate increased by $20 \%$ from $10.6 \%$ in 1990 to $12.7 \%$ in 2005 . During this same time period, the late preterm infant birth rate increased by $25 \%$ from $7.3 \%$ in 1990 to $9.1 \%$ in 2005 . In 2005, $71 \%$ of all preterm births ( $<370 / 7$ weeks of gestation) occurred in the late preterm gestation (34 0/7 to 36 6/7 weeks) group (21). Three factors that may have contributed to this increase in the overall preterm birth rate as well as the late preterm birth rate include increased percentage of women waiting until later in life to have children, increased use of assisted reproductive technology to have children, and increased rates of multiple births (twins, triplets, etc.). These three factors are interrelated, and it is difficult to ascertain the effect on the preterm birth rate by any one factor. Studies have shown that preterm delivery frequency is higher in women less than 16 years old and in those over 35 years old by $2 \%$ to $4 \%$ compared with the rate seen in women 21 to 24 years old. In addition, studies have shown that using assisted reproductive technology to become pregnant increases the risk of multiple births, which are more likely to deliver prematurely but this increased risk of preterm birth is also seen with singleton births conceived using assisted reproductive technology (22).

As stated previously, late preterm infants have a higher mortality rate compared with term infants $(16,17,21-25)$. From 1995 to 2002, the overall infant mortality rate (0-364 days) for late preterm infants decreased from 9.5 per 1000 to 7.9 per 1000 and for term infants it decreased from 3.0 per 1000 to 2.4 per 1000. Congenital malformations accounted for $42 \%$ of the deaths in the late preterm infants and $31 \%$ in the term infants (23). In a study conducted by Young et al., all infants born in Utah between 1999 and 2004 with a gestation age of 34 to 42 weeks were evaluated to see whether they died prior to their first birthday. They also found that the most common reason for the death of infants during their first year of life was due to birth defects. In addition, they determined the early neonatal ( $1-7$ days), neonatal (1-28 days), and infant (1-365 days) mortality rates by gestational age excluding infants with birth defects. In all three categories, they found that the 34-week-gestational-age infants had the highest mortality rate while the 39- to 41-week-gestational-age infants had the lowest rates. For example, infants without birth defects had an early neonatal mortality rate of 1.27 per 1000 at 34 weeks of gestation, 1.06 per 1000 at 35 weeks of gestation, 0.66 per 1000 at 36 weeks of gestation but a rate of 0.08 per 1000 at 40 weeks of gestation. This gave a risk ratio of 3.25 , 3.21 , and 2.69 at 34,35 , and 36 weeks of gestation respectively when compared with 40 weeks of gestation. Infants in this study had a neonatal mortality rate of 1.90 per 1000 at 34 weeks of gestation, 1.41 per 1000 at 35 weeks of gestation, 0.99 per 1000 at 36 weeks of gestation, but a rate of 0.22 per 1000 at 40 weeks of gestation. This gave a risk ratio of 3.24 , 3.20 , and 2.68 at 34,35 , and 36 weeks of gestation respectively when compared with 40 weeks of gestation. These infants had an infant mortality rate of 3.79 per 1000 at 34 weeks of gestation, 3.16 per 1000 at 35 weeks of gestation, 2.55 per 1000 at 36 weeks of gestation, but a rate of 0.80 per 1000 at 40 weeks of gestation. This gave a risk ratio of 4.62,3.91, and 3.14 at 34,
35 , and 36 weeks of gestation respectively when compared with 40 weeks of gestation (24). When birth weight was taken into account, the neonatal and infant mortality rates again decreased with increasing gestational age from 34 to 41 weeks of gestation. Those infants who were small for gestational age (SGA) at any gestational age had the highest mortality rate. Late preterm infants who were SGA were 44 times more likely to die during the first 28 days of life and 21 times more likely to die during the first year of life compared with term appropriate-for-gestational-age (AGA) infants. When infants with congenital anomalies were excluded, late preterm infants who were SGA continued to have a higher risk of dying compared with term AGA infants during both the neonatal and infant periods (25).

When late preterm infants are born, there are a variety of potential complications that they are at a higher risk of developing compared with their term counterparts for which they need to be monitored closely. In a study by Wang et al. in 2004, they looked at infants born between October 1997 and October 2000 at Massachusetts General Hospital and randomly selected 90 late preterm infants (35 0/7 to 36 6/7 weeks of gestational age) and 95 term infants (37 to 40 weeks of gestational age) who met the inclusion criteria. They found that the late preterm infants were more likely to have temperature instability ( $10 \%$ vs. $0 \%$; OR: infinite), hypoglycemia ( $15.6 \%$ vs. $5.3 \%$; OR: 3.30 ; $95 \%$ CI: $1.1-12.2$ ), require intravenous infusion $(26.7 \%$ vs. $5.3 \%$; OR: 6.48 ; $95 \%$ CI: 2.27-22.91), respiratory distress ( $28.9 \%$ vs. $4.2 \%$; OR: 9.14 ; $95 \%$ CI: $2.9-37.8$ ), and were clinically jaundiced (54.4\% vs. 37.9\%; OR 1.95; 95\% CI: 1.04-3.67) compared with the term infants. The late preterm infants were also more likely to require a sepsis evaluation compared with the term infants (36.7\% vs. $12.6 \%$; OR: 3.97 ; $95 \%$ CI: $1.82-9.21$ ). Even though more of the late preterm infants ended up receiving a full 7-day course of antibiotics, it was not statistically significant (20). Because of these morbidities, late preterm infants are more likely to have prolonged hospitalizations (required hospitalization after their mother was discharged). In a study by Pulver et al., they found that $75 \%$ of 34 -week-gestationalage infants, $50 \%$ of 35 -week-gestational age, and $25 \%$ of $36-$ week-gestational-age infants had a prolonged hospitalization. The average length of stay for these infants was 12.6, 6.1, and 3.8 days for 34-, 35-, and 36-week-gestational-age infants respectively (18).

In a study by Shapiro-Mendoza et al., they looked for risk factors that increased the morbidity rate in singleton late preterm infants who were considered healthy at birth. These infants were considered healthy if they were discharged before a fourth-night hospital stay, weighed at least $2000 \mathrm{~g}$ at birth, and did not have any significant health problems or procedures. They found that having an Asian/Pacific Islander mother, being firstborn, being breastfed at discharge, having a mother with complications at labor and delivery, and having a public payer source at the time of delivery all increased the risk of a neonatal morbidity. They also found that these healthy late preterm infants had a $4.8 \%$ risk of being readmitted to the hospital and $1.3 \%$ had an observation stay within 28 days of life. They found that those infants who were breastfed were more likely to require readmission to the hospital or have an observation stay due to inadequate weight gain, jaundice, or 
feeding problems (19). Therefore, it is important to make sure that healthy late preterm infants are truly feeding well prior to discharge from the hospital and have close follow-up after discharge to prevent the need for readmission to the hospital.

At 34 weeks of gestation, the brain weighs about $65 \%$ that of a term infant's brain and very few sulci and gyri are present, so studies have been conducted to look at the longterm neurologic outcome of late preterm infants. These studies have found that late preterm infants have worse neurologic outcomes compared with term infants. For example, in a study by Woythaler et al., they looked at 6300 term and 1200 late preterm infants at 24 months of age. They looked at the mental developmental index (MDI) and psychomotor developmental index (PDI) on the Bayley Scales of Infant Development Short Form-Research Edition. These indices have a mean score of 100 with a standard deviation of 15 , so a score less than 70 indicates significant delay and a score of 70 to 84 indicates mild delay. In this study, they found that the late preterm infants had a mean score of 85 and the term infants had a mean score of 89 on the MDI while on the PDI, the mean scores were 88 and 92 for the late preterm and term infants respectively. About $21.2 \%$ of the late preterm infants and $16.4 \%$ of the term infants had a score $<70$ on the MDI while $6.1 \%$ of the late preterm infants and $6.5 \%$ of the term infants had a score $<70$ on the PDI. When they controlled for gestational age, plurality, maternal race, education, marital status, depression, prenatal care, primary language, infant gender, poverty level, delivery type, fetal growth, and any breastmilk feedings, the adjusted odds $(95 \% \mathrm{CI})$ of a late preterm infant having more severe mental developmental delay (MDI score <70) was $1.51(95 \%$ CI: 1.26-1.82) and milder mental developmental delay (MDI score 70-84) was 1.43 (95\% CI: 1.22-1.67) compared with a term infant. In addition, the adjust odds ratio that a late preterm infant having more severe psychomotor developmental delay (PDI score <70) was 1.56 (95\% CI: 1.29-1.88) and milder psychomotor developmental delay (PDI score 70-84) was 1.58 (95\% CI: 1.37-1.83) compared with a term infant (26). In another study by Morse et al., they looked at healthy late preterm infants (spent $<72$ hours in the hospital at birth) to see whether they were ready for kindergarten. They looked at 7152 healthy late preterm infants and 152,661 term infants and found that the late preterm infants had a $36 \%$ higher risk of developmental delay or disability, a $19 \%$ higher risk of suspension in kindergarten, and a $10 \%$ to $13 \%$ higher risk of having disability in pre-kindergarten, requiring exceptional student education or requiring retention in kindergarten (27). These studies show that even though these late preterm infants appear healthy at birth, they should be monitored closely and placed in early educational interventions if they start to show delays to help improve their outcomes.

As you can see, late preterm infants should not be treated like term infants. They have a higher risk of developing neonatal morbidities, require rehospitalization, and have poor neurologic outcomes. These infants need to be monitored closely throughout their childhood, and if they start to develop any issues, they need to be treated quickly to allow them to have the best possible outcome.

\section{THERAPEUTIC HYPOTHERMIA}

Hypoxic-ischemic encephalopathy (HIE) occurs in infants when cerebral perfusion is impaired resulting in hypoxic injury to the brain. It occurs in 3 to 5 per 1000 live births with moderate or severe HIE occurring in 0.5 to 1 per 1000 live births $(28,29)$. HIE accounts for $23 \%$ of the 4 million neonatal deaths globally each year $(28,30)$.

Studies have shown that neuronal death following a hypoxic-ischemic injury occurs in two phases. Initially, the lack of oxygen causes primary energy failure that results in neuronal death via necrosis or apoptosis. Necrosis is characterized by swelling of the plasma membrane until the cell eventually ruptures releasing intracellular toxins while apoptosis is a slower form of neuronal death due to a less severe or prolonged hypoxic event often described as cell suicide. Once reperfusion of the brain occurs, the energy levels in the neurons "normalize." This period is characterized by normal blood pressure and intracellular $\mathrm{pH}$, transient improvement in cellular edema, and an absence of seizures. Then a second energy failure occurs during which delayed neuronal death occurs. This is caused by hyperemia, cytotoxic edema, mitochondrial failure, accumulation of excitotoxins, apoptosis, nitric oxide synthesis, free radical damage, and cytotoxic actions of activated microglia. It is this secondary energy failure that leads to the highest proportion of neuronal death and leads to encephalopathy and seizures. Therefore, the time between the primary and secondary energy failures provide a window of opportunity to provide some intervention that may prevent the more devastating secondary event from occurring $(28,31)$.

Scientists have been looking for interventions that could be initiated during this therapeutic window to try to improve the outcome of infants who suffer from HIE, but to date, nothing has shown promise except for hypothermia. Hypothermia has been shown to modify cells programmed for apoptosis leading to their survival. It may also protect neurons by reducing their metabolic rate, attenuating the release of excitatory amino acids (glutamate and dopamine), ameliorating the ischemiaimpaired uptake of glutamate and decreasing the production of toxic nitric oxide and free radicals $(28,29)$. Studies in animal models have shown that a $2^{\circ} \mathrm{C}$ to $3^{\circ} \mathrm{C}$ drop in the temperature of the brain after a hypoxic-ischemic event decreased energy expenditure, improved subsequent performance testing, and/ or decreased histologic neuronal loss (29). Other studies found that the earlier the hypothermia was initiated, the better the outcome. This neuroprotective effect of hypothermia was seen up to 6 hours post insult. Still other studies found that moderate hypothermia of the brain in the $32^{\circ} \mathrm{C}$ to $34^{\circ} \mathrm{C}$ range that was continued for 24 to 72 hours again had the best outcomes in animal models. Temperatures lower than this range revealed an increase in potential systemic adverse effects such as mortality, hypoglycemia, decreased cardiac contractility and cardiac output, sinus bradycardia, hypotension, ventilation-perfusion mismatch, increased blood viscosity, coagulopathy, acid/base imbalance, electrolyte imbalance, and increased risk of infections (31).

Currently there are two ways to provide hypothermia to neonates, selective brain cooling or total body cooling. Recently there were four large multicenter randomized control trials that were conducted to evaluate the effect of 
hypothermia in neonates with moderate or severe HIE using one of these two methods of hypothermia. The CoolCap trial included 234 infants who were $\geq 36$ weeks of gestational age with moderate to severe HIE and abnormal amplitudeintegrated electroencephalography (aEEG). These infants were randomized to either receive selective head cooling for 72 hours with a goal rectal temperature of $34^{\circ} \mathrm{C}$ to $35^{\circ} \mathrm{C}$ or standard care. Therapy with hypothermia was to be initiated within 6 hours of birth. The inclusion criteria were an Apgar score of $\leq 5$ at 10 minutes after birth, continued need for resuscitation including endotracheal or mask ventilation at 10 minutes after birth, or severe acidosis defined as a $\mathrm{pH}<7.00$ or a base deficit of $\geq 16 \mathrm{mmol} / \mathrm{L}$ on either a cord gas or an arterial or venous sample drawn within 60 minutes of birth. Eligible infants were then assessed for clinical evidence of moderate or severe HIE using the Sarnat criteria (lethargy, stupor, or coma with one or more of the following symptoms: hypotonia, abnormal reflexes (including oculomotor or pupillary), an absent or weak suck, or clinical evidence of seizures. Next an aEEG was used to determine whether the infant had a moderate or severely abnormal aEEG tracing for 20 minutes prior to initiation of hypothermia. Those infants who were randomized to hypothermia were kept cool for 72 hours and then gradually rewarmed by no more than $0.5^{\circ} \mathrm{C} / \mathrm{hr}$ until the infant's temperature was normal $\left(36.5-37.5^{\circ} \mathrm{C}\right)$. They ended up with 116 infants who were treated with hypothermia and 118 who received standard therapy. These infants were followed for 18 months and they found no difference in the primary outcomes of death or severe disability, but when they looked at the subset of infants with moderately abnormal aEEG, those who were cooled had an improved outcome ( $n=172$, odds ratio 0.42 : 95\% CI 0.22-0.80: $p=0.009)(32)$.

The NICHD Neonatal Research trial included 208 infants who were $\geq 36$ weeks of gestational age with moderate-tosevere HIE. These infants were randomized to either receive whole-body cooling for 72 hours with a goal esophageal temperature of $33.5^{\circ} \mathrm{C}$ or standard care. Therapy with hypothermia was to be initiated within 6 hours of birth. The inclusion criteria were infants with severe acidosis defined as a $\mathrm{pH}<7.00$ or a base deficit of $\geq 16 \mathrm{mmol} / \mathrm{L}$ on either a cord gas or any blood sample drawn within 60 minutes of birth or those infants with a $\mathrm{pH}$ of 7.01 to 7.15 , a base deficit of 10 to $15.9 \mathrm{mmol} / \mathrm{L}$, or no blood gas available with additional criteria of an acute perinatal event (e.g., late or variable decelerations, cord prolapse, cord rupture, uterine rupture, maternal trauma, hemorrhage, or cardiorespiratory arrest), and either an Apgar score of $\leq 5$ at 10 minutes after birth, or continued need for resuscitation including endotracheal or mask ventilation at 10 minutes after birth. Eligible infants were then assessed for clinical evidence of moderate or severe HIE or seizures. HIE was defined as having one or more signs in at least three of the following six categories: level of consciousness, spontaneous activity, posture, tone, primitive reflexes (suck or Moro), and autonomic nervous system (pupils, heart rate, or respirations). Those neonates that were randomized to hypothermia were kept cool for 72 hours and then gradually rewarmed by no more than $0.5^{\circ} \mathrm{C} / \mathrm{hr}$ until the infant's temperature was normal $\left(36.5-37.0^{\circ} \mathrm{C}\right)$. They ended up with 102 infants who were treated with hypothermia and 106 who received standard therapy. These infants were followed for 18 to 22 months and they found death or moderate/severe disability occurred in $44 \%$ of the hypothermia group and $62 \%$ of the control group (risk ratio 0.72: 95\% CI 0.54-0.95: $p=0.01$ ). Death occurred in $24 \%$ of the hypothermia group and $37 \%$ of the control group and cerebral palsy occurred in 19\% of the hypothermia group and $30 \%$ of the control group (33).

The TOBY trial included 325 infants who were $\geq 36$ weeks of gestational age with moderate to severe HIE. These infants were randomized to either receive whole-body cooling for 72 hours with a goal rectal temperature of $33.5^{\circ} \mathrm{C}$ or standard care. Therapy with hypothermia was to be initiated within 6 hours of birth. The inclusion criteria were infants with severe acidosis defined as a $\mathrm{pH}<7.00$ or a base deficit of $\geq 16 \mathrm{mmol} / \mathrm{L}$ on either a cord gas or any blood sample drawn within 60 minutes of birth or an Apgar score of $\leq 5$ at 10 minutes after birth or continued need for resuscitation including endotracheal or mask ventilation at 10 minutes after birth. Eligible infants were then assessed for clinical evidence of moderate or severe HIE (indicated by lethargy, stupor, or coma) and either hypotonia, abnormal reflexes (including oculomotor or pupillary), an absent or weak suck, or clinical seizures. Finally, they had to have an abnormal aEEG pattern on a 30-minute tracing. Those infants who were randomized to hypothermia were kept cool for 72 hours and then gradually rewarmed by no more than $0.5^{\circ} \mathrm{C} / \mathrm{hr}$ until the infant's temperature was normal $\left(37.0^{\circ} \mathrm{C}\right)$. They ended up with 163 infants who were treated with hypothermia and 162 who received standard therapy. These infants were followed for 18 months and they found death or moderate/severe disability occurred in $45 \%$ of the hypothermia group and $53 \%$ of the control group (risk ratio 0.86: 95\% CI 0.68-1.07: $p=0.17$ ). Survival without neurologic abnormalities was improved in the hypothermia group (risk ratio 1.57: 95\% CI 1.16-2.12: $p=0.003$ ). In addition, cerebral palsy was decreased in the hypothermia group (risk ratio 0.67: 95\% CI 0.47-0.96: $p=0.03$ ) and improved outcomes were noted on the MDI and the PDI of the Bayley Scales of Infant Development II ( $p=0.03$ for each) (34).

As you can see, when you look at these three randomized control trials, hypothermia appears to decrease the mortality rate or major disability at 18 months of age especially in those infants with only moderate HIE (28). This was confirmed in the neo.nEURO.network trial, which included 111 infants who were $\geq 36$ weeks of gestational age with moderate to severe HIE. These infants were randomized to either receive wholebody cooling for 72 hours with a goal rectal temperature of $33.5^{\circ} \mathrm{C}$ or standard care. Therapy with hypothermia was to be initiated within 6 hours of birth. The inclusion criteria were infants with at least one of the following criteria: severe acidosis defined as a $\mathrm{pH}<7.00$ or a base deficit of $\geq 16 \mathrm{mmol} / \mathrm{L}$ on either a cord gas or arterial blood gas drawn within 60 minutes of birth, or an Apgar score of less than 5 at 10 minutes after birth, or continued need for resuscitation including endotracheal or mask ventilation at 10 minutes after birth. Eligible infants were then assessed for clinical evidence of moderate or severe HIE (indicated by lethargy, stupor, or coma) and at least one of the following criteria: hypotonia, abnormal reflexes (including oculomotor or pupillary), an absent or weak suck, or clinical seizures. Finally, they had to have an abnormal standard EEG or aEEG pattern on a 
30-minute tracing. Those infants who were randomized to hypothermia were kept cool for 72 hours and then gradually rewarmed by no more than $0.5^{\circ} \mathrm{C} / \mathrm{hr}$ until the infant's temperature was normal $\left(37.0^{\circ} \mathrm{C}\right)$. They ended up with 53 infants who were treated with hypothermia and 58 who received standard therapy. Both groups were treated with either morphine or fentanyl to reduce the discomfort due to the HIE or the hypothermia. These infants were followed for 18 to 21 months, and they found death or severe disability occurred in $51 \%$ of the hypothermia group and $83 \%$ of the control group (odds ratio 0.21 : $95 \%$ CI 0.09-0.54: $p=0.001$ ). This was even true in the severe HIE group (odds ratio 0.17: 95\% CI 0.05-0.57: $p=0.005$ ) (35). As you can see from these studies, hypothermia appears to help improve the outcomes of infants with HIE more so with those who are moderately affected, but hypothermia is not a cure-all and more studies combining hypothermia with other neuroprotective interventions need to be conducted.

\section{REFERENCES}

1. American Academy of Pediatrics Committee on Fetus and Newborn Apnea, sudden infant death syndrome, and home monitoring. Pediatrics 2003; 111: 914-17.

2. Lin C, Wang S, Lin Y, Yeh T. Efficacy of nasal intermittent positive pressure ventilation in treating apnea of prematurity. Pediatr Pulmonol 1998; 26: 349-53.

3. Barrington KJ, Bull D, Finer NN. Randomized trial of nasal synchronized intermittent mandatory ventilation compared with continuous positive airway pressure after extubation of very low birth weight infants. Pediatrics 2001; 107: 638-41.

4. Lemyre B, Davis PG, DePaoli AG. Nasal intermittent positive pressure ventilation (NIPPV) versus nasal continuous positive airway pressure (NCPAP) for apnea of prematurity. Cochrane Database Syst Rev 2008.

5. Friedlich P, Lecart C, Posen R, et al. A randomized trial of nasopharyngealsynchronized intermittent mandatory ventilation versus nasopharyngeal continuous positive airway pressure in very low birth weight infants after extubation. J Perinatol 1999; 19: 413-18.

6. Davis PG, Schmidt B, Roberts RS, et al.; for the Caffeine for Apnea of Prematurity Trial Group. Caffeine for apnea of prematurity trial: benefits may vary in subgroups. J Pediatr 2010; 156: 382-7.

7. Schmidt B, Roberts RS, Davis P, et al.; for the Caffeine for Apnea of Prematurity Trial Group. Caffeine therapy for apnea of prematurity. N Engl J Med 2006; 354: 2112-21.

8. Schmidt B, Roberts RS, Davis P, et al.; for the Caffeine for Apnea of Prematurity Trial Group. Long-term effects of caffeine therapy for apnea of prematurity. N Engl J Med 2007; 357: 1893-902.

9. Kumar M, Kabra NS, Paes B. Role of carnitine supplementation in apnea of prematurity: a systematic review. J Perinatol 2004; 24: 158-63.

10. Kumar M, Jubra NS, Paes B. Carnitine supplementation for preterm infants with recurrent apnea. Cochrane Database Syst Rev 2008.

11. Henderson-Smart DJ, Steer PA. Doxapram versus methylxanthine for apnea in preterm infants. Cochrane Database Syst Rev 2011.

12. Henderson-Smart DJ, DePaoli AG. Methylxanthine treatment for apnoea in preterm infants. Cochrane Database Syst Rev 2011.

13. Henderson-Smart DJ, Davis PG. Prophylactic methylxanthines for endotracheal extubation in preterm infants. Cochrane Database Syst Rev 2011.

14. Henderson-Smart DJ, DePaoli AG. Prophylactic methylxanthine for prevention of apnoea in preterm infants. Cochrane Database Syst Rev 2011.
15. Raju TNK, Higgins RD, Stark AR, Leveno KJ. Optimizing care and outcomes for late-preterm (near-term) infants: a summary of the workshop sponsored by the national institute of child health and human development. Pediatrics 2006; 118: 1207-14.

16. Engle WA, Tomashek KM, Wallman C, the Committee on Fetus and Newborn. "Late-preterm" infants: a population at risk. Pediatrics 2007; 120: 1390-401.

17. Khashu M, Narayanan M, Bhargava S, Osiovich H. Perinatal outcomes associated with preterm birth at 33 to 36 weeks' gestation: a populationbased cohort study. Pediatrics 2009; 123: 109-13.

18. Pulver LS, Denney JM, Silver RM, Young PC. Morbidity and discharge timing of late preterm newborns. Clin Pediatr 2010; 49: 1061-7.

19. Shapiro-Mendoza CK, Tomashek KM, Kotelchuck M, et al. Risk factors for neonatal morbidity and mortality among "healthy," late preterm newborns. Semin Perinatol 2006; 30: 54-60.

20. Wang ML, Dorer DJ, Fleming MP, Catlin EA. Clinical outcomes of nearterm infants. Pediatrics 2004; 114: 372-6.

21. Reddy UM, Ko C, Raju TNK, Willinger M. Delivery indications at latepreterm gestations and infant mortality rates in the United States. Pediatrics 2009; 124: 234-40.

22. Raju TNK. Epidemiology of late preterm (near-term) births. Clin Perinatol 2006; 33: 751-63.

23. Tomashek KM, Shapiro-Mendoza CK, Davidoff MJ, Petrini JR. Differences in mortality between late-preterm and term singleton infants in the United States, 1995-2002. J Pediatr 2007; 151: 450-6.

24. Young PC, Glasgow TS, Li X, Guest-Warnick G, Stoddard G. Mortality of late-preterm (near-term) newborns in Utah. Pediatrics 2007; 119: e659-65.

25. Pulver LS, Guest-Warnick G, Stoddard GJ, Byington CL, Young PC. Weight for gestational age affects the mortality of late preterm infants. Pediatrics 2009; 123: e1072-7.

26. Woythaler MA, McCormick MC, Smith VC. Late preterm infants have worse 24-month neurodevelopmental outcomes than term infants. Pediatrics 2011; 127: e622-9.

27. Morse SB, Zheng H, Tang Y, Roth J. Early school-age outcomes of late preterm infants. Pediatrics 2009; 123: e622-9.

28. Jacobs SE, Tarnow-Mordi WO. Therapeutic hypothermia for newborn infants with hypoxic-ischeamic encephalopathy. J Paediatr Child Health 2010; 46: 568-76.

29. Jacobs SE, Hunt R, Tarnow-Mordi WO, Inder TE, Davis PG. Cooling for newborns with hypoxic ischaemic encephalopathy. Cochrane Database Syst Rev 2008.

30. Shah PS, Ohlsson A, Perlman M. Hypothermia to treat neonatal hypoxic ischemic encephalopathy: systematic review. Arch Pediatr Adolesc Med 2007; 161: 951-8.

31. Sahni R, Sanocka UM. Hypothermia for hypoxic-ischemic encephalopathy. Clin Perinatol 2008; 35: 717-34.

32. Gluckman PD, Wyatt JS, Azzopardi D, et al.; on behalf of the CoolCap Study Group. Selective head cooling with mild systemic hypothermia after neonatal encephalopathy: multicentre randomized trial. Lancet 2005; 365: 663-70.

33. Shankaran S, Laptook AR, Ehrenkranz RA, et al.; for the NICHD Neonatal Research Network. Whole-body hypothermia for neonates with hypoxic-ischemic encephalopathy. N Engl J Med 2005; 353: 1574-84.

34. Azzopardi DV, Strohm B, Edwards AD, et al.; for the TOBY Study Group. Moderate hypothermia to treat perinatal asphyxial encephalopathy. N Engl J Med 2009; 361: 1349-58.

35. Simbruner G, Mittal RA, Rohlmann F, Muche R, for the neo. nEURO.network trial. Systemic hypothermia after neonatal encephalopathy: outcomes of neo.nEURO.network rct. Pediatrics 2010; 126: e771-8. 


\title{
90 Neonatal diseases II
}

\author{
Brian P. Hackett, Jeffrey Dawson, Akshaya Vachharajani, Barbara Warner, \\ and F. Sessions Cole
}

\section{RESPIRATORY PROBLEMS IN TERM INFANTS}

Unlike the typical respiratory problems of prematurely delivered newborns that result from inadequate surfactant production, the mechanisms of respiratory problems in term infants ( $\geq 37$ weeks of gestation) are associated with inadequate pulmonary perfusion (e.g., persistent pulmonary hypertension of the newborn) as well as surfactant deficiency due to both inadequate production (e.g., in the late preterm infant) and inactivation [e.g., meconium aspiration syndrome (MAS), congenital pneumonia]. Finally, both spontaneous airleaks and congenital abnormalities of airway, pulmonary parenchyma, or pulmonary vascular development (e.g., congenital diaphragmatic hernia, alveolar-capillary dysplasia) present as respiratory distress in the term newborn. Although term infants are less likely to suffer from respiratory problems than preterm infants, their risk for respiratory distress has been estimated at $0.6 \%$ in one large prospective study (1) and their risk for severe respiratory distress that requires mechanical ventilation at $\sim 0.2 \%$ (2).

\section{MAS}

Although most infants delivered after exposure to meconiumstained amniotic fluid will exhibit no symptoms, a subgroup may develop severe respiratory failure. Understanding the pathophysiology of this subgroup has been enhanced by understanding fetal lung physiology. Net fluid flow in the fetal lung results from an egress of fluid from pulmonary epithelial cells into future airspaces and airways. The flow of fetal lung fluid across the glottis into the amniotic cavity keeps contaminants in the amniotic fluid (e.g., meconium, squamous cells, and blood) downstream from pulmonary epithelial cells in utero. Aspiration of these agents may occur if the fetus is "gasping," a fetal behavior associated with severe hypoxia or ischemia. Exposure of the pulmonary epithelium to meconium leads to evidence of significant injury with elaboration of cytokines associated with inflammation, altered surfactant metabolism, and histologic evidence of airspace overdistention. These pathophysiologic mechanisms help explain the ventilatory problems associated with MAS and increased risk of airleaks (3). The associated hypoxia and acidosis, particularly if preceded by prolonged intrauterine distress with increase in the thickness of the muscularis layer in the smaller pulmonary branch vessels (4), contribute to failure to achieve normal pulmonary vasorelaxation and to worsening hypoxia and hypercarbia in a cyclic fashion. Thus, both severe pulmonary parenchymal injury and pulmonary hypertension contribute to the presentation and must be treated.

MAS occurs in $0.6 \%$ to $0.7 \%$ of all newborn infants with any respiratory symptoms and in $0.04 \%$ of newborn infants who require mechanical ventilation $(2,5,6)$. Although risk for other common respiratory problems [e.g., transient tachypnea of the newborn (TTN) or respiratory distress syndrome (RDS)] decreases with advancing gestational age, risk for MAS increases with advancing gestational age. Approximately onethird of symptomatic infants will require mechanical ventilation, and despite surfactant replacement and availability of high-frequency oscillatory ventilation (HFOV) and nitric oxide (NO) treatment, mortality is reported in $2.5 \%$ of infants who require ventilatory support (down from 12\% before widespread adoption of HFOV and NO).

Over the last decade, approaches to intrapartum and immediate postpartum prevention of MAS have moved away from attempts to evacuate the upper airway of meconium by obstetricians at delivery of the head and by pediatricians after the delivery is complete. These interventions were found to be helpful in small historical control studies in the 1970s. However, larger randomized controlled trials reported in the 1990s failed to demonstrate any improvement in outcomes and raised concerns about associated iatrogenic injury to the upper airway in some infants. The official 2005 recommendation of the American Heart Association and the American Academy of Pediatrics makes clear that in the setting of infants born with thick meconium staining, the priority is to reserve intubation only for the infants who are most depressed at birth and not routinely to aspirate the mouth and nose (7). Similarly, although initial randomized studies of amnioinfusions for meconium-stained amniotic fluid found a beneficial effect on neonatal respiratory outcomes without increased maternal morbidity, a large multicenter randomized study (8) reported no routine benefit from amnioinfusion. The American College of Obstetrics and Gynecology revised its practice guideline to indicate that although amnioinfusion remains an appropriate intervention for treatment of fetal distress (with or without meconium-stained amniotic fluid) evaluated by heart rate monitoring, it is not effective for reduction of risk of MAS $(9,10)$.

\section{TTN}

First described by Mary Ellen Avery in 1966 (11), this selflimited (hours to a few days) condition is characterized by presentation within the first few hours of life with tachypnea, a radiographic picture of increased perihilar pulmonary vascular markings, fluid in the pulmonary lobar fissure, and increased lung volumes. Both the severity and duration of symptoms vary but are generally less intense than those seen in respiratory disorders like MAS or congenital pneumonia. As noted earlier, net fetal lung liquid production leads to egress of fluid into the amniotic cavity. Just prior to birth, this 
chloride-rich fluid production by the lung epithelium switches to fluid resorption driven by amiloride-sensitive sodium channels. The reversal of chloride and water transport leads to a decrease in total lung water before delivery. This process is dependent on long-term, developmentally regulated changes in the expression of sodium channels and their adrenoceptor sensitivity, more acute changes in ambient catecholamine levels associated with labor and delivery, and transition to the relatively oxygen-rich postnatal environment (12). Failure of this process of adaptation to the extrauterine environment in the absence of infection, surfactant deficiency or inactivation, or relevant birth defects is believed to cause the symptom complex known as TTN.

In keeping with this proposed mechanism, the risk factors associated with higher risk of TTN are those that interfere with normal adaptation from placenta-dependent gas exchange in utero to lung-dependent gas exchange in the extrauterine environment. Shorter gestation may lead to lower levels of expression of critical components of the sodium channel assembly, its catecholamine regulators, and surfactant (the risk of developing TTN increases with decreasing gestational age). Additionally, absent or shortened labor (e.g., in the settings of elective cesarean section without labor or of rapid second phase of labor) provides less time for alveolar fluid resorption and is associated with the development of TTN. Finally, disease risk may be increased by genetic factors, for example, polymorphisms in $B$ adrenoceptor gene (13). Recent studies have reinforced the association of TTN with both earlier gestational age and cesarean delivery mode (14-16).

\section{RESPIRATORY DISTRESS IN PRETERM INFANTS}

Despite advances in the care of infants born prematurely, prematurity (delivery at $<37$ weeks of gestation) remains one of the leading causes of neonatal death (17). The incidence of RDS, a major complication of prematurity, is directly related to lower gestational age at birth. The incidence of RDS in infants born at 25 weeks of gestation approaches $100 \%$. At 27 weeks of gestation, the incidence decreases to approximately $85 \%$, at 30 weeks of gestation to $55 \%$, at 32 weeks of gestation to $30 \%$, and by 35 weeks of gestation to $10 \%$ (18). In addition to prematurity, other factors that predispose to RDS include male gender, European ancestry, maternal diabetes, perinatal hypoxemia, hypothermia, multifetal pregnancy, and caesarean delivery (19). An increase in the rate of preterm deliveries over the last several years, attributable primarily to infants delivered between 34 and 37 weeks of gestation (late preterm infants) (See section below entitled "Late Preterm Neonates."), has contributed significantly to the burden of newborn respiratory distress (20).

In 1959, Mary Ellen Avery and Jere Mead described the relationship between preterm birth, lung surface tension, and RDS (21). Since that time, understanding the biology of pulmonary surfactant and its role in preterm respiratory distress has been one of the triumphs of newborn medicine. This understanding has led to the use of exogenous surfactant replacement as a primary treatment for preterm RDS. Available surfactant preparations include bovine- or porcinederived products and synthetic surfactant preparations that are administered to preterm infants via endotracheal tube. Multiple studies have demonstrated the efficacy of exogenous surfactant in reducing newborn mortality and airleaks, especially when given as multiple doses early after birth in a prophylactic, as opposed to rescue, manner $(22,23)$. Although a consistent reduction in the development of chronic lung disease (bronchopulmonary dysplasia) in preterm infants treated with surfactant has not been demonstrated, there is evidence that the severity of chronic lung disease has decreased in the era of surfactant therapy (24). Combined management strategies, for example, surfactant treatment and early extubation to continuous positive airway pressure (CPAP), show promise in decreasing not only the severity but the incidence of chronic lung disease in preterm infants with RDS as well $(25,26)$.

In the late 1960s, Graham Liggins made the seminal observation that lambs exposed in utero to corticosteroids and delivered prematurely had less respiratory distress, more mature appearing lungs, and decreased mortality (27). By 1972, the first human trial demonstrating the efficacy of antenatal corticosteroids in reducing RDS in preterm human infants had been completed (28). Since the 1970s, multiple clinical trials have demonstrated the effectiveness of antenatal glucocorticoid treatment of women in preterm labor in reducing the incidence and severity of RDS (29). Although significant concern has been raised regarding the association of postnatal steroid treatment of preterm infants with poorer neurodevelopmental outcomes, there has been no consistent evidence that administration of antenatal corticosteroids results in neurodevelopmental delay $(30,31)$.

The identification of genetic mutations that result in lethal respiratory failure in newborn infants has led to the discovery of genetic polymorphisms associated with an increased risk of respiratory distress in the premature newborn (32). Polymorphisms associated with an increased risk of RDS in preterm infants have been identified in the surfactant protein$A$, surfactant protein- $B$, surfactant protein- $C$, and the ATPbinding cassette transporter A3 (ABCA3) genes (33-35). Additionally, polymorphisms associated with preterm RDS have also been identified in genes not directly related to surfactant synthesis and secretion including mannose-binding lectin, interleukin-10, and the G protein-coupled receptor for asthma susceptibility (36-38). Elucidating the interactions between and among genetic variants, environment, and developmental factors may lead to novel management strategies in preterm infants with RDS.

\section{LATE PRETERM NEONATES}

\section{Terminology and Epidemiology}

An expert panel convened by the National Institute of Child Health and Human Development in July, 2005, recommended that infants born between 34 0/7 weeks (day 239) and 36 $6 / 7$ weeks (day 259) of gestation be referred to as late preterm $(39,40)$. Late preterm infants account for 360,000 of more than 4 million births in the United States (40). Davidoff and Dias et al. found that two-thirds of the last decade's increase in the rate of all preterm births was associated with an increase in the rate of late preterm births. Also noteworthy was that $74.1 \%$ of all singleton preterm births in 2002 occurred at 34, 35, and 36 weeks of gestation (41). Late preterm births also accounted for $61 \%$ of preterm multiple births (41). Since late preterm infants constitute such a large proportion of preterm infants, 
Table 1 Neonatal Infections by Timing of Onset

\begin{tabular}{lccc} 
Onset & Transmission & Risk Factors & Predominant Organisms \\
\hline $\begin{array}{l}\text { Early } \\
\text { Birth to 2-7 days }\end{array}$ & Vertical & Maternal colonization/infection & Group B Streptococcus \\
& & Prolonged premature rupture & Escherichia coli \\
Late & Prematurity & Coagulase-negative \\
$>2-7$ days & Verizontal & Invasive hardware/procedures & staphylococci \\
to 1 month & common & (vascular catheters, & Staphylococcus aureus \\
& & endotracheal tubes, surgery) & Escherichia coli \\
& & Environmental contact & Pseudomonas species \\
& & Prematurity & Candida
\end{tabular}

even a modest increase in their birth rate can have a significant impact on infant outcomes and health-care costs. Maternal and fetal indications commonly associated with late preterm delivery include pre-eclampsia, multiple gestation, and abnormal presentations (42). A portion of late preterm delivery may be preventable, especially those births that result from "maternal preference" $(43,44)$.

\section{Morbidity}

Although historically called "near term," multiple morbidities associated with delivery between 34 and 37 weeks of gestation including respiratory distress, temperature instability, hypoglycemia, hyperbilirubinemia, apnea, seizures, and feeding problems, as well as higher rates of rehospitalization suggest that infants at these gestational ages are not prepared for successful fetal-neonatal transition $(39,45)$. For example, temperature instability, hypoglycemia, and jaundice were observed more frequently in late preterm infants $(10 \%$, $15.6 \%$, and $54.5 \%$, respectively) than term babies $(0 \%, 5.3 \%$, and $37.9 \%$, respectively) (45). Similarly, poor feeding was observed more frequently in late preterm than term infants $(77.8 \%$ vs. $45.3 \%)$. Apnea and bradycardia are seen in late preterm infants (45) with prevalence rates as high as 10\% (46). Late preterm infants are more likely to be evaluated with a complete blood count and blood culture and treated with antibiotics for 48 hours or 7 days compared with term babies (45) due to the difficulty of excluding sepsis as an underlying cause for multiple transitional symptoms including respiratory distress, hypothermia, and poor feeding. Delay in discharge among late preterm infants is more common as a result of these problems, with poor feeding as the most common reason for the delay (45). Respiratory complications are also prevalent among late preterm infants. The most common respiratory diagnoses among late preterm infants whorequire assisted ventilation include RDS, MAS, and pneumonia/sepsis (20). When compared with infants born at 37 to 42 weeks of gestation, the frequency of RDS is greater in late preterm infants ( $62 \%$ vs. $43 \%$ ), while the frequency of MAS is less $(1.3 \%$ vs. $9.7 \%)$ (20). Earlier studies also demonstrate increased need for mechanical ventilation as gestational age decreases among late preterm infants (1). Need for extracorporeal membrane oxygenator (ECMO) therapy for respiratory failure among late preterm infants has also been increasing (47). Among 15,590 infants in the Extracorporeal Life Support Organization National Registry treated with ECMO between 1989 and 2006, 2258 (14.5\%) were late preterm infants (47).

Death is more common in late preterm infants than term infants: risk of early neonatal death (birth to 28 days of age) is 5.2-fold greater, late neonatal death (28 days to 1 year of life) is 2.9-fold greater, and total infant mortality (all deaths from birth to 1 year of age) is 2.5 -fold greater $(39,48)$. Risk for increased morbidity also persists as evidenced by increased emergency department visits and rehospitalizations (49-51). Studies by Kinney et al. suggest that although the brain is more mature in late preterm than very preterm infants, it is still immature and can be disrupted or damaged by late preterm delivery (52). Substantial brain growth occurs between 34 and 41 weeks of gestation in utero: brain size increases by $33 \%$ in the final 6 to 8 weeks of gestation accompanied by a fivefold increase in brain volume. Late preterm infants can also develop periventricular leukomalacia, a white matter injury marker for subsequent cerebral palsy. The exact prevalence of brain pathology is unknown as systematic brain imaging is not currently recommended at this gestational age (52). Longer term follow-up studies suggest that late preterm infants are at increased risk for lower reading and math scores from kindergarten through elementary school and for participation in special educational programs (53).

\section{Conclusion}

Late preterm infants are at significantly greater risk for shortterm and long-term morbidity and mortality than was previously recognized.

\section{NEONATAL BACTERIAL SEPSIS}

Despite improvements in perinatal and newborn care, sepsis continues to be a leading cause of neonatal mortality, ranked sixth among causes of neonatal deaths in the United States (54). Neonatal sepsis refers to systemic infections that occur in infants $<1$ month of age. While infection with bacterial, viral, or fungal organisms may present identically in the newborn infant, this section will focus on bacterial infections. Among developed countries, the reported incidence of systemic bacterial infection ranges from 1 to 8 per 1000 live births $(55,56)$, with increasing incidence as birth weight decreases $(55,57)$. Neonatal sepsis is typically classified by timing of onset: early-onset sepsis (EOS) occurs from birth to a week of life, and late-onset sepsis (LOS) after the first week through the first month. The two categories differ not only by age of onset, but also by mode of transmission, risk factors, and causative organism (Table 1). For EOS, despite recent advances in preventing perinatal transmission with selective intrapartum chemoprophylaxis (see Chapter 74), group B Streptococcus (GBS) continues to be the most common causative organism. As the incidence of GBS has decreased, 
the contribution from Escherichia coli, particularly among the very low-birth weight population (VLBW, <1500 g), has increased. These two organisms account for $60 \%$ to $70 \%$ of all EOS $(55,58,59)$. Other less common pathogens include Enterococcus species, Streptococcus viridans, Staphylococcus aureus, a variety of gram-negative bacilli, and Listeria monocytogenes. For EOS in VLBW infants, the profile of organisms has recently changed. Gram-negative organisms are now the predominant causative organisms with ampicillinresistant E. coli accounting for approximately $40 \%$ of cases (60,61). Gram-positive organisms including GBS and coagulase-negative staphylococcus (CONS), account for another $45 \%$ of EOS cases among VLBW infants.

In LOS, gram-positive organisms predominate in all weight and gestational-age categories. For infants discharged from the hospital, the incidence of LOS caused by GBS has remained unchanged despite the use of intrapartum prophylaxis (62). In addition, LOS also occurs commonly in infants who require prolonged hospitalization in the neonatal intensive care unit (NICU). Gram-positive bacteria are the primary causative organisms with CONS accounting for approximately $40 \%$ of cases $(55,60)$. Other organisms include $S$. aureus with increasing frequency of methicillin resistance (MRSA), E. coli, and other gram negatives.

The clinical presentation of sepsis in the newborn is distinct from older children and adults. Signs and symptoms may often be subtle, nonspecific, and similar to a variety of neonatal illnesses. Common manifestations include temperature instability, tachypnea, lethargy, apnea, poor feeding, and abdominal distention $(63,64)$. Temperature instability is more commonly manifested as hypothermia, although temperatures above $37.8^{\circ} \mathrm{C}$ can be significant in a newborn infant when not associated with iatrogenic causes. The overlap of symptoms with respiratory, gastrointestinal, central nervous system, and metabolic disorders means that septicemia should be part of the differential diagnosis for infants with any of these findings.

The definitive diagnosis of sepsis is made by isolation of microorganisms from blood. Diagnosis by blood culture, however, is limited by test sensitivity, blood volume drawn, and can be contaminated by skin flora that may also be pathogenic in this population $(65,66)$. As a result, diagnosis and treatment are often based on a combination of clinical symptomatology and other diagnostic markers. Evaluating the white blood cell count and peripheral differential is an important first step in assessment but has variable sensitivity and poor specificity (67). Additional hematologic parameters include the absolute neutrophil count and ratio of immature to total cells within the neutrophil series (I:T ratio) (67). Evaluation of biochemical markers associated with the host inflammatory response, such as C-reactive protein (CRP) (67,68), may also used. CRP requires 8 to 10 hours for synthesis making it much more sensitive in the later phase of infection. Serial CRPs (two levels obtained 24 hours apart, 8-48 hours after presentation) have a reported sensitivity of $80 \%$ to $90 \%$ and negative predictive value of $99 \%$ (69). The low sensitivity of CRP early in sepsis progression makes withholding initiation of antibiotics based on a low, early CRP value inadvisable, but serial CRPs may be useful in determining the duration of antibiotic course.

Approximately one-fourth to one-third of infants with sepsis also has meningitis (70). Among symptomatic infants, consideration of performance of lumbar puncture for examination and culture of cerebrospinal fluid is important as meningitis (pleocytosis, increased protein, hypoglycorrhachia, and positive culture) will determine duration and type of antibiotic treatment. Because viral and bacterial infections may present similarly, testing for viruses, particularly herpes simplex virus, in blood and spinal fluid should also be considered (see Chapter 74). Beyond the first few days of life, evaluation and culture of urine by catheterization or suprapubic bladder aspiration are also indicated to identify potential urinary tract foci of infection. Additional testing should be based on clinical findings and may include radiologic evaluation of the chest and abdomen.

Rapidity of progression of sepsis in the newborn infant requires prompt initiation of antibiotics once cultures have been obtained. The choice of empiric antibiotic therapy should be based on the timing and setting of infection (EOS vs. LOS, community vs. NICU) as well as the profile of microorganisms and their susceptibility patterns among specific populations. For EOS, a combination of ampicillin and gentamicin is frequently used. If meningitis is suspected, consideration should be given to addition of cefotaxime for its improved cerebrospinal fluid penetration. For LOS, therapy should be tailored to the individual patient, her/his risk factors, and past microbial flora. The frequencies of CONS, MRSA, and gramnegative organisms have resulted in the common use of vancomycin and an aminoglycoside. A worsening clinical course should result in consideration of broadening of coverage to include Pseudomonas species, drug-resistant gram negatives, and common fungi (e.g., Candida). Isolated bacteremia without meningitis or other focus is treated with 7 to 10 days of antibiotics. Treatment is otherwise largely supportive, with close monitoring and intervention for respiratory failure, hypotension, and central nervous system disturbances. Given the compromised immune response of the newborn infant, several immunologic therapies have also been investigated (71-73) of which only intravenous immune globulin has demonstrated a statistically significant reduction in mortality in cases of subsequently proven infection (71).

\section{NEWBORN SCREENING FOR METABOLIC DISORDERS}

Newborn screening for metabolic disorders is the most widely performed genetic testing in the United States. Nearly all of the more than 4 million infants born each year undergo newborn metabolic screening. The specific battery of screening tests is determined by individual state statute. As of 2005, the number of different screening tests performed varied from 4 to 46 among the states (74). Funding for the screening programs and follow-up services also varies from state to state. Ninety percent of the state screening programs have a fee paid by the family or third party payer, $61 \%$ receive funds from the Maternal and Child Health Services Title V block grant, 33\% rely on at least partial funding from state general revenues, and 24\% receive direct Medicaid reimbursement (75). In addition to the screening programs in the United States, most developed and many developing countries have newborn screening programs (76-79).

Newborn metabolic screening was introduced in the 1960s with the development by Robert Guthrie of a bacterial assay for phenylalanine used for the diagnosis of phenylketonuria. 
Table 2 ACMG Recommendations for Newborn Screening

Core panel

Secondary targets

Organic acid metabolic defects

Isovaleric acidemia

Glutaric acidemia type I

3-Hydroxy-3-methylglutaric aciduria

Multiple carboxylase deficiency

Methylmalonic academia (mutase)

3-Methylcrotonyl-CoA-carboxylase deficiency

Methylmalonic acidemia (Cbl A, B)

Propionic acidemia

$\beta$-Ketothiolase deficiency

Fatty acid oxidation disorders

Medium-chain acyl-CoA dehydrogenase deficiency

Very long-chain acyl-CoA dehydrogenase deficiency

Long chain L-3-hydroxyacyl-CoA dehydrogenase deficiency

Trifunctional protein deficiency

Carnitine uptake deficiency

Amino acid metabolism disorders

Phenylketonuria

Maple syrup disease

Homocystinuria

Citrullinemia

Argininosuccinic academia

Tyrosinemia type I

\section{Hemoglobinopathies}

Sickle cell anemia (Hb SS)

Hemoglobin $S / \beta$-thalassemia

Hemoglobin S/C disease

Other

Congenital hypothyroidism

Biotinidase deficiency

Congenital adrenal hyperplasia (21-hydroxylase deficiency)

Classic galactosemia

Hearing loss

Cystic fibrosis

Guthrie's other major contribution to newborn screening was a method for using a dried infant blood spot on filter paper (the eponymous "Guthrie card") that allows for easy collection and processing of a large number of samples (80). As additional assays were developed that could be performed on blood spots, the scope of newborn metabolic screening was expanded. Criteria for inclusion of a test in the screening panel include benefit of early detection due to availability of an effective treatment, availability of a test with appropriate sensitivity and specificity, and opportunity for early detection of a potentially treatable condition during the neonatal period when it might otherwise not be clinically apparent (81). Screening programs should also provide mechanisms for appropriate confirmatory testing as well as referral for counseling and management.

With the recent development of tandem mass spectroscopic (MS/MS) methods to detect metabolites on Guthrie cards, there has been a major expansion in the metabolic diseases for which screening is now available. In 2005, the American College of Medical Genetics recommended that all states begin
Methylmalonic acidemia (Cbl C,D)

Malonic acidemia

Isobutyryl-CoA dehydrogenase deficiency

2-Methylbutyrl-CoA dehydrogenase deficiency

2-Methyl-3-hydroxybutyric aciduria

3-Methylglutaconic aciduria

Short-chain acyl-CoA dehydrogenase deficiency

Glutaric acidemia type II

Medium/short-chain L-3-hydroxyacyl-CoA dehydrogenase deficiency

Medium-chain ketoacyl-CoA thiolase deficiency

Carnitine palmitoyltransferase II deficiency

Carnitine/acylcarnitine translocase deficiency

Carnitine palmitoyltransferase I deficiency

Dienoyl-CoA reductase deficiency

Benign hyperphenylalaninemia

Tyrosinemia type II

Biopterin cofactor metabolism defects

Argininemia

Tyrosinemia type III

Biopterin cofactor regeneration defects

Hypermethioninemia

Citrullinemia type II

Variant hemoglobinopathies

Galactokinase deficiency

Galactose epimerase deficiency screening for 29 core conditions plus an additional 25 conditions (secondary targets) that can be detected by the screening methods but may not have an effective therapy or their natural history may not be well understood (82). Table 2 lists the recommended screening tests. This list includes some assays that utilize methods other than MS/MS. Although this recommendation has been controversial, it has been endorsed by the American Academy of Pediatrics $(81,83,84)$. The American College of Obstetrics and Gynecology has also issued a supportive opinion (85). This building consensus has resulted in an increase in the number of tests included on the screening panels in most states (74). In the future, as DNA microarray technology advances and becomes less costly, newborn metabolic screening may also incorporate this technology (86).

\section{REFERENCES}

1. Rubaltelli FF, Dani C, Reali MF, et al. Acute neonatal respiratory distress in Italy: a one-year prospective study. Italian Group of Neonatal Pneumology. Acta Paediatr 1998; 87: 1261-8. 
2. Gouyon JB, Ribakovsky C, Ferdynus C, et al. Severe respiratory disorders in term neonates. Paediatr Perinat Epidemiol 2008; 22: 22-30.

3. Cayabyab RG, Kwong K, Jones C, Minoo P, Durand M. Lung inflammation and pulmonary function in infants with meconium aspiration syndrome. Pediatr Pulmonol 2007; 42: 898-905.

4. Thureen PJ, Hall DM, Hoffenberg A, Tyson RW. Fatal meconium aspiration in spite of appropriate perinatal airway management: pulmonary and placental evidence of prenatal disease. Am J Obstet Gynecol 1997; 176: 967-75.

5. Wiswell TE, Tuggle JM, Turner BS. Meconium aspiration syndrome: have we made a difference? Pediatrics 1990; 85: 715-21.

6. Dargaville PA, Copnell B. The epidemiology of meconium aspiration syndrome: incidence, risk factors, therapies, and outcome. Pediatrics 2006; 117: 1712-21.

7. International Consensus on Cardiopulmonary Resuscitation and Emergency Cardiovascular Care Science with Treatment Recommendations. Part 7: neonatal resuscitation. Resuscitation 2005; 67: 293-303.

8. Vain NE, Szyld EG, Prudent LM, et al. Oropharyngeal and nasopharyngeal suctioning of meconium-stained neonates before delivery of their shoulders: multicentre, randomised controlled trial. Lancet 2004; 364: 597-602.

9. ACOG Committee Opinion Number 346. Amnioninfusion does not prevent meconium aspiration syndrome. Obstet Gynecol 2006; 108: 1053

10. ACOG Committee Opinion No. 379. Management of delivery of a newborn with meconium-stained amniotic fluid. Obstet Gynecol 2007; 110: 739 .

11. Avery ME, Gatewood OB, Brumley G. Transient tachypnea of newborn. Possible delayed resorption of fluid at birth. Am J Dis Child 1966; 111: 380-5.

12. Barker PM, Olver RE. Invited review: clearance of lung liquid during the perinatal period. J Appl Physiol 2002; 93: 1542-8.

13. Aslan E, Tutdibi E, Martens S, et al. Transient tachypnea of the newborn (TTN): a role for polymorphisms in the beta-adrenergic receptor (ADRB) encoding genes? Acta Paediatr 2008; 97: 1346-50.

14. Villar J, Carroli G, Zavaleta N, et al. Maternal and neonatal individual risks and benefits associated with caesarean delivery: multicentre prospective study. BMJ 2007; 335: 1025.

15. Balchin I, Whittaker JC, Lamont RF, Steer PJ. Timing of planned cesarean delivery by racial group. Obstet Gynecol 2008; 111: 659-66.

16. Hansen AK, Wisborg K, Uldbjerg N, Henriksen TB. Risk of respiratory morbidity in term infants delivered by elective caesarean section: cohort study. BMJ 2008; 336: 85-7.

17. Martin JA, Kung HC, Mathews TJ, et al. Annual summary of vital statistics: 2006. Pediatrics 2008; 121: 788-801.

18. Stevens TP, Sinkin RA. Surfactant replacement therapy. Chest 2007; 131: 1577-82.

19. Fraser J, Walls M, McGuire W. Respiratory complications of preterm birth. BMJ 2004; 329: 962-5.

20. Clark RH. The epidemiology of respiratory failure in neonates born at an estimated gestational age of 34 weeks or more. J Perinatol 2005; 25: 251-7.

21. Avery ME, Mead J. Surface properties in relation to atelectasis and hyaline membrane disease. AMA J Dis Child 1959; 97(5 Pt 1): 517-23.

22. Soll RF, Morley CJ. Prophylactic versus selective use of surfactant in preventing morbidity and mortality in preterm infants. Cochrane Database Syst Rev 2001: CD000510.

23. Yost CC, Soll RF. Early versus delayed selective surfactant treatment for neonatal respiratory distress syndrome. Cochrane Database Syst Rev 2000: CD001456.

24. Cerny L, Torday JS, Rehan VK. Prevention and treatment of bronchopulmonary dysplasia: contemporary status and future outlook. Lung 2008; 186: 75-89.

25. Geary C, Caskey M, Fonseca R, Malloy M. Decreased incidence of bronchopulmonary dysplasia after early management changes, including surfactant and nasal continuous positive airway pressure treatment at delivery, lowered oxygen saturation goals, and early amino acid administration: a historical cohort study. Pediatrics 2008; 121: 89-96.

26. Stevens TP, Harrington EW, Blennow M, Soll RF. Early surfactant administration with brief ventilation vs. selective surfactant and continued mechanical ventilation for preterm infants with or at risk for respiratory distress syndrome. Cochrane Database Syst Rev 2007: CD003063.

27. Liggins GC. Premature delivery of foetal lambs infused with glucocorticoids. J Endocrinol 1969; 45: 515-23.

28. Liggins GC, Howie RN. A controlled trial of antepartum glucocorticoid treatment for prevention of the respiratory distress syndrome in premature infants. Pediatrics 1972; 50: 515-25.

29. Roberts D, Dalziel S. Antenatal corticosteroids for accelerating fetal lung maturation for women at risk of preterm birth. Cochrane Database Syst Rev 2006; 3: CD004454.

30. O'Shea TM, Washburn LK, Nixon PA, Goldstein DJ. Follow-up of a randomized, placebo-controlled trial of dexamethasone to decrease the duration of ventilator dependency in very low birth weight infants: neurodevelopmental outcomes at 4 to 11 years of age. Pediatrics 2007; 120: 594-602.

31. Wilson-Costello D, Friedman H, Minich N, et al. Improved neurodevelopmental outcomes for extremely low birth weight infants in 2000-2002. Pediatrics 2007; 119: 37-45.

32. Hamvas A, Cole FS, Nogee LM. Genetic disorders of surfactant proteins. Neonatology 2007; 91: 311-17.

33. Karjalainen MK, Haataja R, Hallman M. Haplotype analysis of ABCA3: association with respiratory distress in very premature infants. Ann Med 2008; 40: 56-65.

34. Lahti M, Marttila R, Hallman M. Surfactant protein C gene variation in the Finnish population - association with perinatal respiratory disease. Eur J Hum Genet 2004; 12: 312-20.

35. Marttila R, Haataja R, Guttentag S, Hallman M. Surfactant protein A and $\mathrm{B}$ genetic variants in respiratory distress syndrome in singletons and twins. Am J Respir Crit Care Med 2003; 168: 1216-22.

36. Capasso M, Avvisati RA, Piscopo C, et al. Cytokine gene polymorphisms in Italian preterm infants: association between interleukin-10 -1082 G/A polymorphism and respiratory distress syndrome. Pediatr Res 2007; 61: 313-17.

37. Hilgendorff A, Heidinger K, Pfeiffer A, et al. Association of polymorphisms in the mannose-binding lectin gene and pulmonary morbidity in preterm infants. Genes Immun 2007; 8: 671-7.

38. Pulkkinen V, Haataja R, Hannelius U, et al. G protein-coupled receptor for asthma susceptibility associates with respiratory distress syndrome. Ann Med 2006; 38: 357-66.

39. Raju TN, Higgins RD, Stark AR, Leveno KJ. Optimizing care and outcome for late-preterm (near-term) infants: a summary of the workshop sponsored by the National Institute of Child Health and Human Development. Pediatrics 2006; 118: 1207-14.

40. Raju TN. Epidemiology of late preterm (near-term) births. Clin Perinatol 2006; 33: 751-63; abstract vii.

41. Davidoff MJ, Dias T, Damus K, et al. Changes in the gestational age distribution among U.S. singleton births: impact on rates of late preterm birth, 1992 to 2002. Semin Perinatol 2006; 30: 8-15.

42. Sibai BM. Preeclampsia as a cause of preterm and late preterm (nearterm) births. Semin Perinatol 2006; 30: 16-19.

43. Florica M, Stephansson O, Nordstrom L. Indications associated with increased cesarean section rates in a Swedish hospital. Int J Gynaecol Obstet 2006; 92: 181-5.

44. Quinlivan JA, Petersen RW, Nichols CN. Patient preference the leading indication for elective Caesarean section in public patients-results of a 2-year prospective audit in a teaching hospital. Aust NZ J Obstet Gynaecol 1999; 39: 207-14.

45. Wang ML, Dorer DJ, Fleming MP, Catlin EA. Clinical outcomes of nearterm infants. Pediatrics 2004; 114: 372-6.

46. Hunt CE. Ontogeny of autonomic regulation in late preterm infants born at 34-37 weeks postmenstrual age. Semin Perinatol 2006; 30: 73-6.

47. Dudell GG, Jain L. Hypoxic respiratory failure in the late preterm infant. Clin Perinatol 2006; 33: 803-30; abstract viii-ix.

48. Kramer MS, Demissie K, Yang H, et al. The contribution of mild and moderate preterm birth to infant mortality. Fetal and Infant Health Study Group of the Canadian Perinatal Surveillance System. JAMA 2000; 284: 843-9.

49. Jain S, Cheng J. Emergency department visits and rehospitalizations in late preterm infants. Clin Perinatol 2006; 33: 935-45; abstract xi. 
50. Tomashek KM, Shapiro-Mendoza CK, Weiss J, et al. Early discharge among late preterm and term newborns and risk of neonatal morbidity. Semin Perinatol 2006; 30: 61-8.

51. Shapiro-Mendoza CK, Tomashek KM, Kotelchuck M, et al. Risk factors for neonatal morbidity and mortality among "healthy," late preterm newborns. Semin Perinatol 2006; 30: 54-60.

52. Kinney HC. The near-term (late preterm) human brain and risk for periventricular leukomalacia: a review. Semin Perinatol 2006; 30: 81-8.

53. Chyi LJ, Lee HC, Hintz SR, Gould JB, Sutcliffe TL. School outcomes of late preterm infants: special needs and challenges for infants born at 32 to 36 weeks gestation. J Pediatr 2008; 153: 25-31.

54. Heron M. Deaths: leading causes for 2004. Natl Vital Stat Rep 2007; 56: 1-95.

55. Bizzarro MJ, Raskind C, Baltimore RS, Gallagher PG. Seventy-five years of neonatal sepsis at Yale: 1928-2003. Pediatrics 2005; 116: 595-602.

56. Stoll BJ, Holman RC, Schuchat A. Decline in sepsis-associated neonatal and infant deaths in the United States, 1979 through 1994. Pediatrics 1998; 102: e18.

57. Stoll BJ, Hansen N. Infections in VLBW infants: studies from the NICHD Neonatal Research Network. Semin Perinatol 2003; 27: 293-301.

58. Baltimore RS, Huie SM, Meek JI, Schuchat A, O’Brien KL. Early-onset neonatal sepsis in the era of group B streptococcal prevention. Pediatrics 2001; 108: 1094-8.

59. Hyde TB, Hilger TM, Reingold A, et al. Trends in incidence and antimicrobial resistance of early-onset sepsis: population-based surveillance in San Francisco and Atlanta. Pediatrics 2002; 110: 690-5.

60. Stoll BJ, Hansen N, Fanaroff AA, et al. Changes in pathogens causing early-onset sepsis in very-low-birth-weight infants. N Engl J Med 2002; 347: 240-7.

61. Stoll BJ, Hansen NI, Higgins RD, et al. Very low birth weight preterm infants with early onset neonatal sepsis: the predominance of gramnegative infections continues in the National Institute of Child Health and Human Development Neonatal Research Network, 2002-2003. Pediatr Infect Dis J 2005; 24: 635-9.

62. Phares CR, Lynfield R, Farley MM, et al. Epidemiology of invasive group B streptococcal disease in the United States, 1999-2005. JAMA 2008; 299: 2056-65.

63. Voora S, Srinivasan G, Lilien LD, Yeh TF, Pildes RS. Fever in full-term newborns in the first four days of life. Pediatrics 1982; 69: 40-4.

64. Fanaroff AA, Korones SB, Wright LL, et al. Incidence, presenting features, risk factors and significance of late onset septicemia in very low birth weight infants. The National Institute of Child Health and Human Development Neonatal Research Network. Pediatr Infect Dis J 1998; 17: 593-8.

65. Sarkar S, Bhagat I, DeCristofaro JD, Wiswell TE, Spitzer AR. A study of the role of multiple site blood cultures in the evaluation of neonatal sepsis. J Perinatol 2006; 26: 18-22.

66. Wiswell TE, Hachey WE. Multiple site blood cultures in the initial evaluation for neonatal sepsis during the first week of life. Pediatr Infect Dis J 1991; 10: 365-9.
67. Ng PC. Diagnostic markers of infection in neonates. Arch Dis Child Fetal Neonatal Ed 2004; 89: F229-35.

68. Lam HS, Ng PC. Biochemical markers of neonatal sepsis. Pathology 2008; 40: $141-8$.

69. Benitz WE, Han MY, Madan A, Ramachandra P. Serial serum C-reactive protein levels in the diagnosis of neonatal infection. Pediatrics 1998; 102: E41.

70. Baltimore R. Perinatal bacterial and fungal infections. In: Jenson HB, Baltimore RS, eds. Pediatric Infectious Diseases: Principles and Practice, 2nd edn. Baltimore: W.B. Saunders, 2002.

71. Ohlsson A, Lacy JB. Intravenous immunoglobulin for suspected or subsequently proven infection in neonates. Cochrane Database Syst Rev 2004: CD001239.

72. Mohan P, Brocklehurst P. Granulocyte transfusions for neonates with confirmed or suspected sepsis and neutropaenia. Cochrane Database Syst Rev 2003: CD003956.

73. Carr R, Modi N, Dore C. G-CSF and GM-CSF for treating or preventing neonatal infections. Cochrane Database Syst Rev 2003: CD003066.

74. Tarini BA, Christakis DA, Welch HG. State newborn screening in the tandem mass spectrometry era: more tests, more false-positive results. Pediatrics 2006; 118: 448-56.

75. Therrell BL, Williams D, Johnson K, et al. Financing newborn screening: sources, issues, and future considerations. J Public Health Manag Pract 2007; 13: 207-13.

76. Bodamer OA, Hoffmann GF, Lindner M. Expanded newborn screening in Europe 2007. J Inherit Metab Dis 2007; 30: 439-44.

77. Borrajo GJ. Newborn screening in Latin America at the beginning of the 21st century. J Inherit Metab Dis 2007; 30: 466-81.

78. Padilla CD, Therrell BL. Newborn screening in the Asia Pacific region. J Inherit Metab Dis 2007; 30: 490-506.

79. Saadallah AA, Rashed MS. Newborn screening: experiences in the Middle East and North Africa. J Inherit Metab Dis 2007; 30: 482-9.

80. Guthrie R, Susi A. A simple phenylalanine method for detecting phenylketonuria in large populations of newborn infants. Pediatrics 1963; 32: 338-43.

81. Watson M, Mann MY, Lloyd-Puryear MA, et al. Newborn screening: toward a uniform screening panel and system-executive summary. Pediatrics 2006; 117(5 Pt 2): S296-307.

82. Watson M, Lloyd-Puryear MA, Mann MY, et al. Newborn screening: toward a uniform screening panel and system. Genet Med 2006; 8: 12S-252S

83. Baily MA, Murray TH. Ethics, evidence, and cost in newborn screening. Hastings Cent Rep 2008; 38: 23-31.

84. Moyer VA, Calonge N, Teutsch SM, Botkin JR. Expanding newborn screening: process, policy, and priorities. Hastings Cent Rep 2008; 38: 32-9.

85. ACOG Committee Opinion No. 393. Newborn screening. Obstet Gynecol 2007; 110: 1497-500.

86. Green NS, Pass KA. Neonatal screening by DNA microarray: spots and chips. Nat Rev Genet 2005; 6: 147-51. 